Argonne

ANL/ESD/11-4

\title{
Light-Duty Vehicle Fuel Consumption Displacement Potential up to 2045
}

\author{
Energy Systems Division
}

\section{U.S. Department of Energy}


For more information, please contact:

Aymeric Rousseau, Manager

Vehicle Modeling and Simulation

(630) 252-7261

arousseau@anl.gov

About Argonne National Laboratory

Argonne is a U.S. Department of Energy laboratory managed by UChicago Argonne, LLC under contract DE-AC02-06CH11357. The Laboratory's main facility is outside Chicago, at 9700 South Cass Avenue, Argonne, Illinois 60439. For information about Argonne and its pioneering science and technology programs, see www.anl.gov.

\title{
Availability of This Report
}

This report is available, at no cost, at http://www.osti.gov/bridge. It is also available on paper to the U.S. Department of Energy and its contractors, for a processing fee, from:

\author{
U.S. Department of Energy \\ Office of Scientific and Technical Information \\ P.O. Box 62 \\ Oak Ridge, TN 37831-0062 \\ phone (865) 576-8401 \\ fax (865) 576-5728 \\ reports@adonis.osti.gov
}

\section{Disclaimer}

This report was prepared as an account of work sponsored by an agency of the United States Government. Neither the United States Government nor any agency thereof, nor UChicago Argonne, LLC, nor any of their employees or officers, makes any warranty, express or implied, or assumes any legal liability or responsibility for the accuracy, completeness, or usefulness of any information, apparatus, product, or process disclosed, or represents that its use would not infringe privately owned rights. Reference herein to any specific commercial product, process, or service by trade name, trademark, manufacturer, or otherwise, does not necessarily constitute or imply its endorsement, recommendation, or favoring by the United States Government or any agency thereof. The views and opinions of document authors expressed herein do not necessarily state or reflect those of the United States Government or any agency thereof, Argonne National Laboratory, or UChicago Argonne, LLC. 


\section{Light-Duty Vehicle Fuel Consumption Displacement Potential up to 2045}

by

Ayman Moawad, Phil Sharer, and Aymeric Rousseau

Energy Systems Division, Argonne National Laboratory

July 2011 
Argonne National Laboratory Report - Light-Duty Vehicle Fuel Consumption Displacement Potential Up to 2045

\section{TABLE OF CONTENTS}

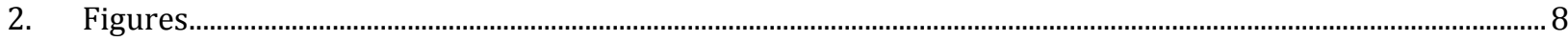

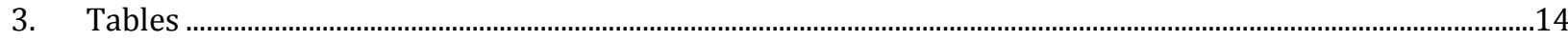

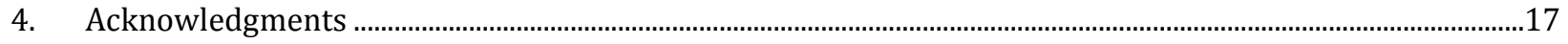

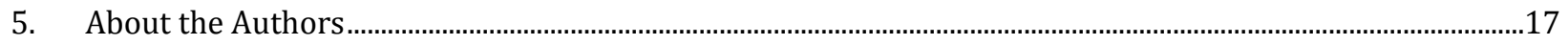

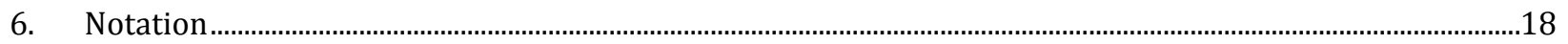

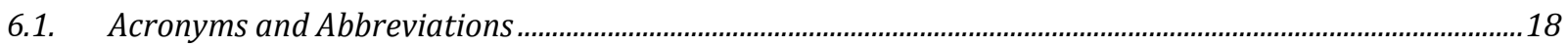

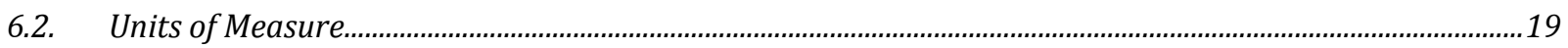

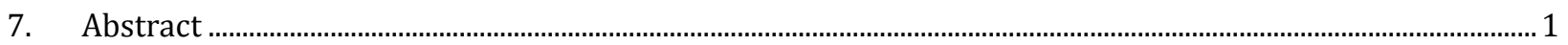

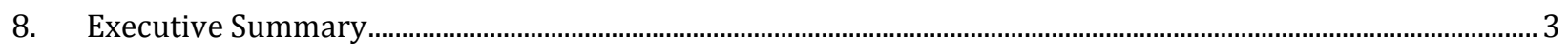

8.1. Vehicle Sizing Findings ..............................................................................................................................

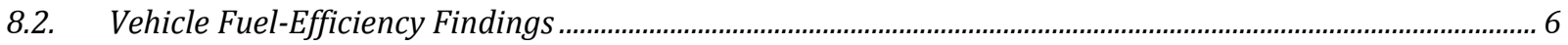

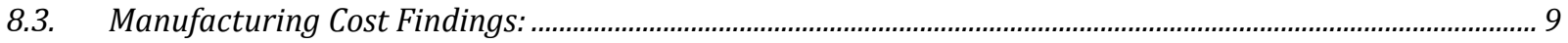

8.4. Conclusion

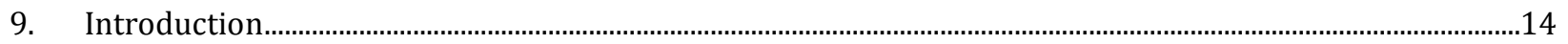

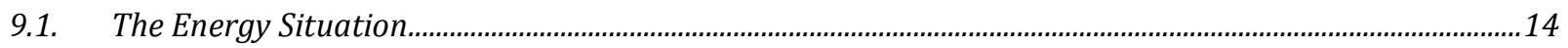

9.2. The American Automotive Market.............................................................................................................

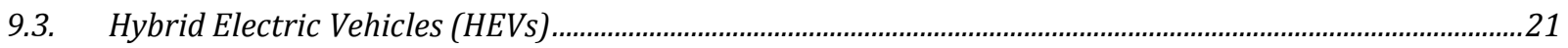

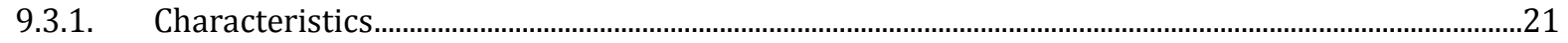

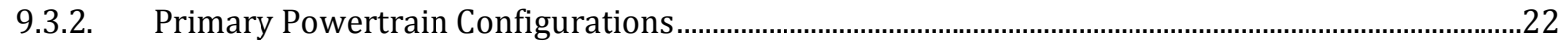

9.3.3. The HEV Market …………………………….......................................................................................24

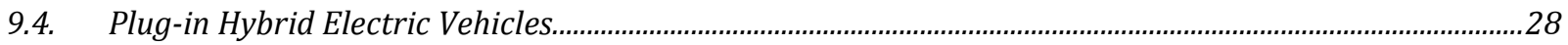

9.4.1. Definition and Characteristics................................................................................................................28

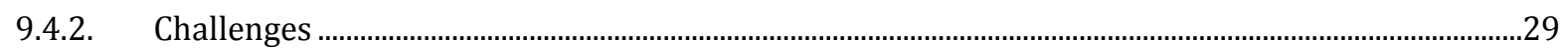

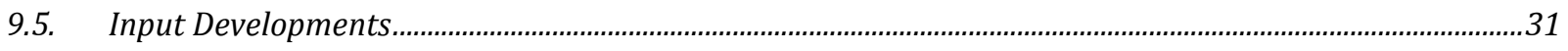

10. Methodology

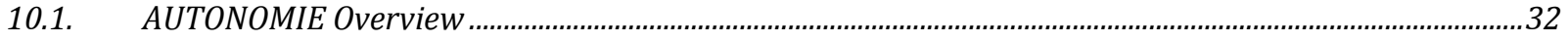

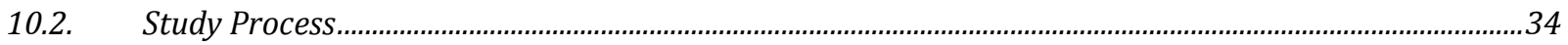

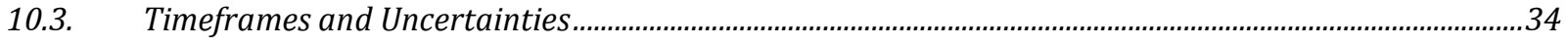

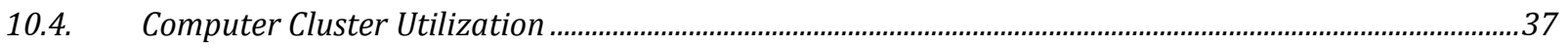

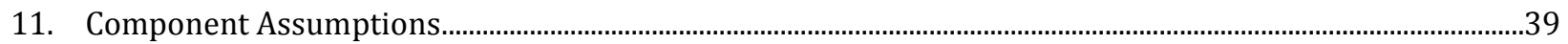


Argonne National Laboratory Report - Light-Duty Vehicle Fuel Consumption Displacement Potential Up to 2045

11.1. Engine

11.1.1. Reference Engine and Projections.............................................................................................

11.1.2. Determination OF Number of CylinderS........................................................................................ 44

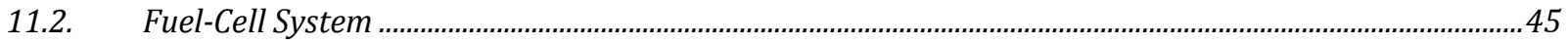

11.3. Hydrogen Storage

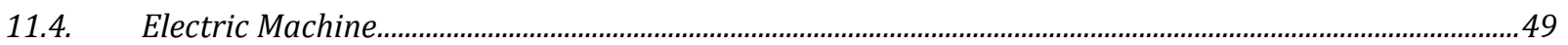

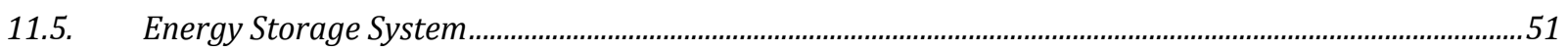

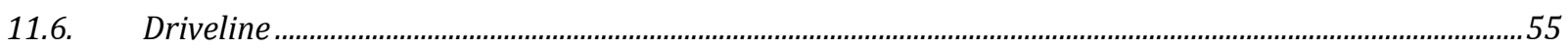

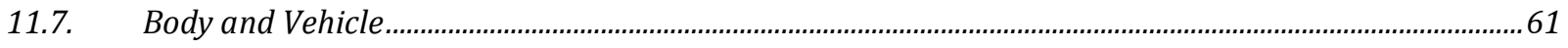

11.7.1. TechnologY Overview ............................................................................................................61

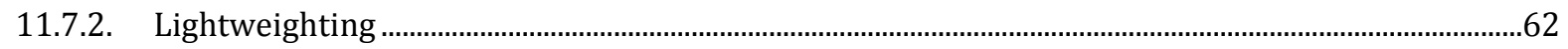

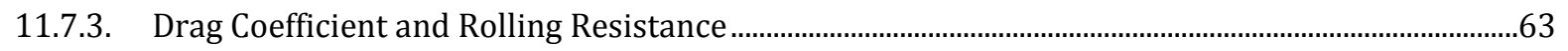

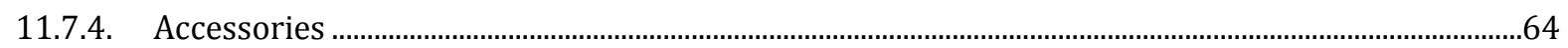

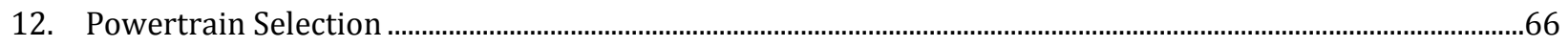

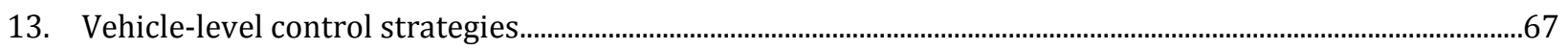

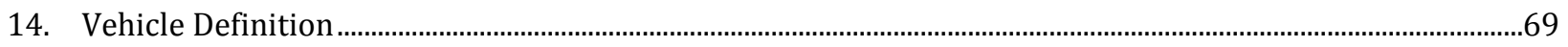

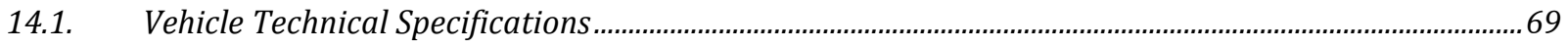

14.2. Sizing Algorithms....

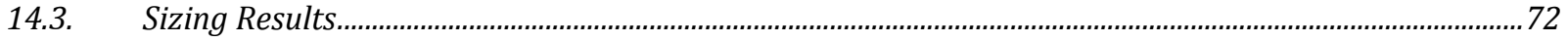

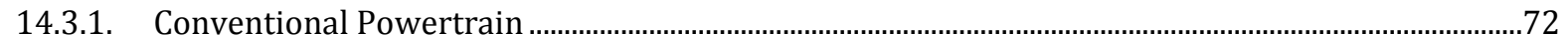

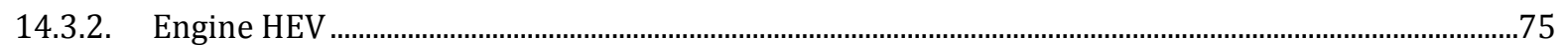

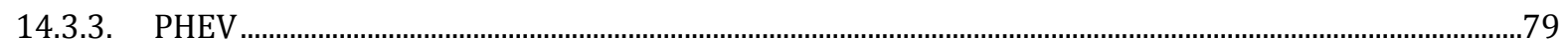

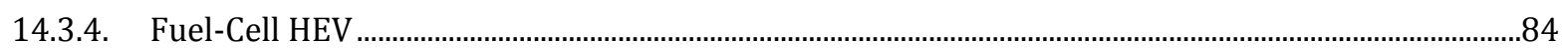

14.3.5. Fuel-Cell PHEV ......................................................................................................................................

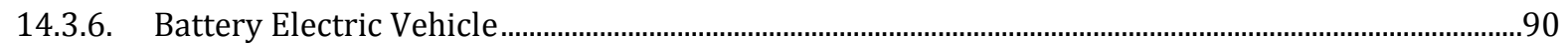

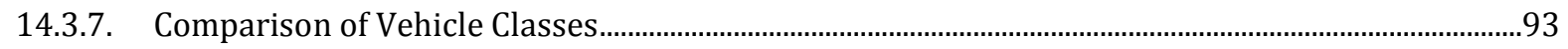

15. Test Procedure and Consumption Calculations.........................................................................................98

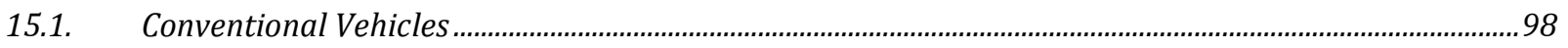

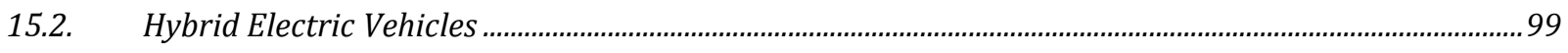

15.2.1. Two-Cycle Procedure Calculations for Conventional and Hybrid vehicles........................................101

15.3. Plug-in Hybrid Electric Vehicles............................................................................................................ 101

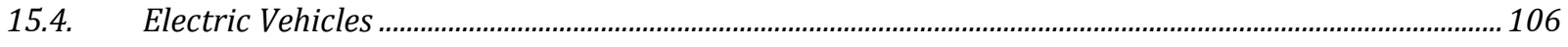

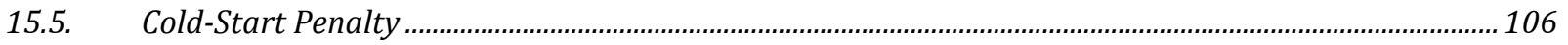

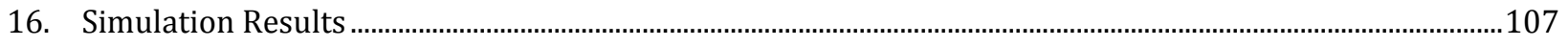

16.1. Evolution of Specific Powertrain Configurations............................................................................................. 107

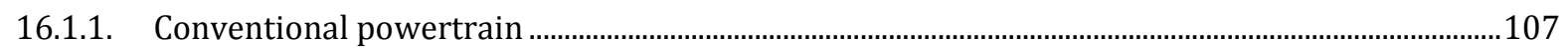

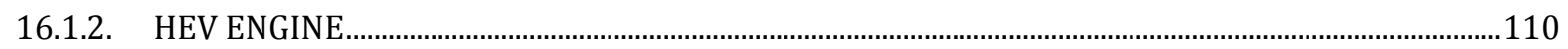


Argonne National Laboratory Report - Light-Duty Vehicle Fuel Consumption Displacement Potential Up to 2045

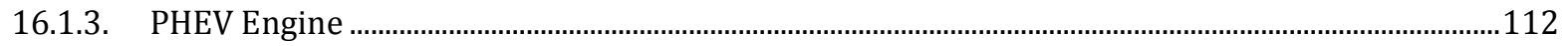

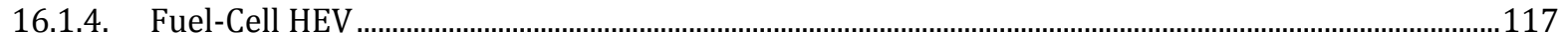

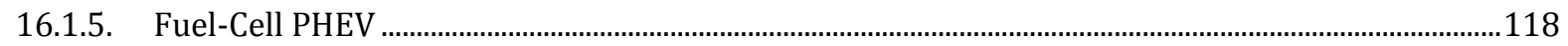

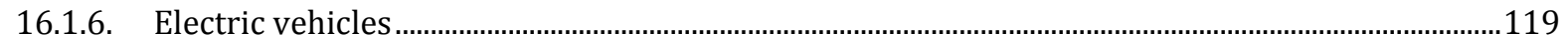

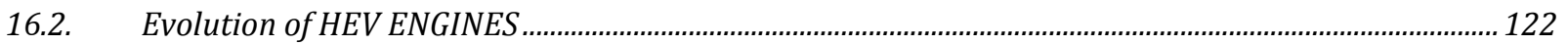

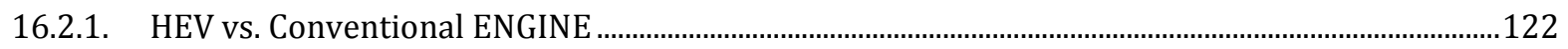

16.2.2. Engine HEV vs. Fuel-Cell HEV.........................................................................................................

16.3. Evolution of Hydrogen-Fueled Vehicles …….............................................................................................. 126

16.3.1. Fuel-Cell HEV vs. Gasoline Engine ................................................................................................126

16.3.2. Fuel-Cell HEV vs. Hydrogen Engine ……………...................................................................................129

17. Vehicle Fuel Consumption vs. Manufacturing Cost Results ............................................................................134

17.1. Evolution of Specific Powertrain Configurations........................................................................................... 134

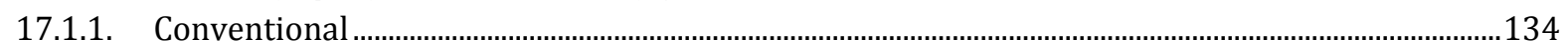

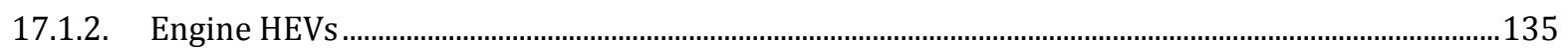

17.1.3. Engine PHEVs .................................................................................................................................137

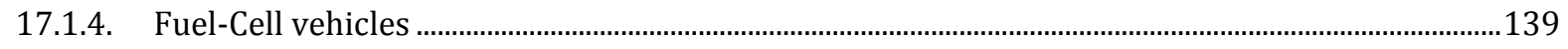

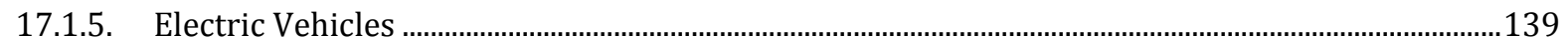

17.2. Powertrain Comparison …………………….......................................................................................... 140

18. Trade-off Between Vehicle Fuel Consumption and manufacturing Cost ............................................................143

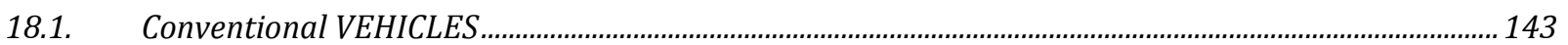

18.2. HEVS

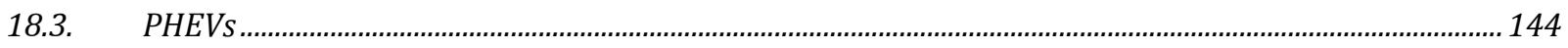

18.4. fuel-cell and other hydrogen-fueled Vehicles........................................................................................ 145

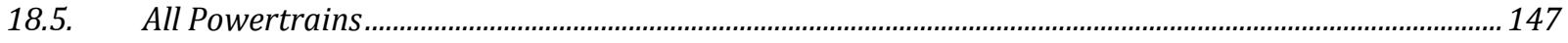

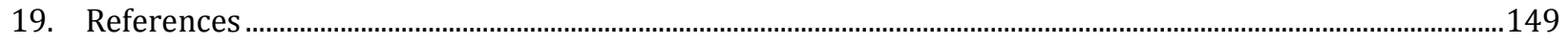

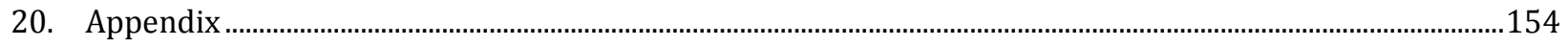




\section{FIGURES}

Figure 1 - Fraction of the world's oil consumption per country or continent .14

Figure 2 - Fraction of American imported oil per geographical region (International Energy Agency, 2010)...15

Figure 3 - U.S. consumption distribution (left) and role of transportation in global consumption (right) .........15

Figure 4 - Sources of energy used in major U.S. economic sectors in 1973 and 2005 (Mtoe = Million Tons of Oil Equivalent) (International Energy Agency, 2010) ......................................................................................................16

Figure 5 - Evolution of price of a barrel of oil from 1990 to 2010 [EIA]

Figure 6 - Light-duty automotive technology, fuel economy, and emission trends: 1975 through 2010 (Environmental Protection Agency, 2010).

Figure 7 - Light-duty automotive technology and market share trends: 1975 through 2009 (Environmental Protection Agency, 2010)

Figure 8 - Vehicle weight and performance evolution from 1975 to 2010

Figure 9 - Hybridization degree.

Figure 10 - Hybrid powertrain configurations.

Figure 11 - HEV sales in U.S. from 1999 to November 2010 (Source - Anant Vyas, personal communication).25

Figure 12 - U.S. HEV sales trends from 1999 to November 2010 (Source - Anant Vyas, personal communication).

Figure 13 - U.S. gasoline prices and HEV sales, 2005-2010 (Source - Anant Vyas, personal communication) ..27

Figure 14 - HEV car and light-truck sales as share of worldwide vehicle sales (Source - Anant Vyas, personal communication).

Figure 15 - Status of Li-Ion batteries vs. DOE goals (Source - Howell, 2008)............................................................30

Figure 16 - Process to evaluate fuel efficiency of advanced vehicle technologies ......................................................35

Figure 17 - Vehicle classes, timeframes, configurations, fuels, and risk levels considered .......................................36

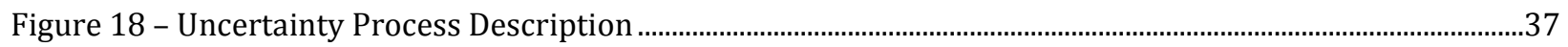

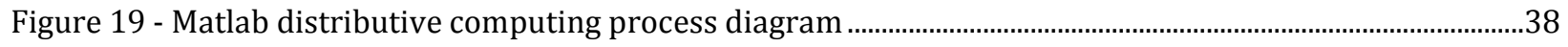

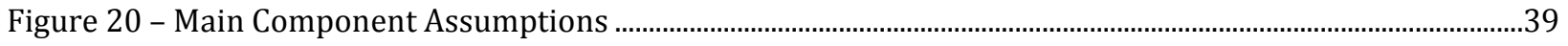

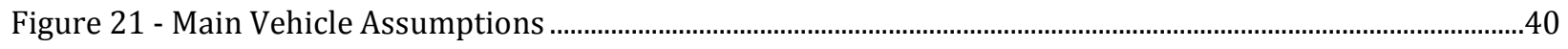

Figure 22 - ICE efficiency for diesel, hydrogen, and gasoline fuels .................................................................................42

Figure 23 - Emission control costs for gasoline, diesel and Flex-Fuel (ethanol) engines ........................................43 
Figure 24 - Number of cylinders vs. engine power for database gasoline engines. (Blue) values from the database; (red) thresholds chosen for the study

Figure 25 - Number of cylinders vs. engine power for database diesel engines. (Blue) values from the database; (red) thresholds chosen for the study ......................................................................................................4

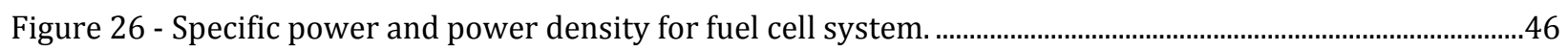

Figure 27 - Fuel-cell system efficiency and cost .......................................................................................................4

Figure 28 - Hydrogen storage energy: gravimetric and volumetric capacity............................................................47

Figure 29 - Hydrogen storage capacity in terms of hydrogen quantity ..................................................................48

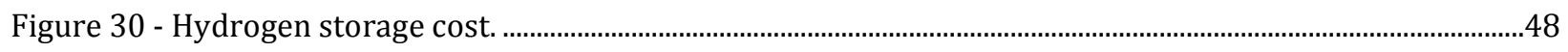

Figure 31 - Hydrogen storage vehicle range on UDDS cycle, based on unadjusted fuel consumption...................49

Figure 32 - Electric-machine and power-electronic specific power...............................................................................50

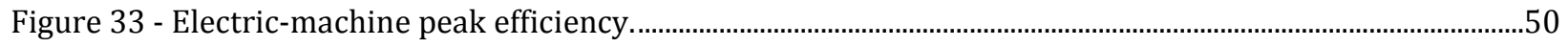

Figure 34 - Electric-machine and controller cost..................................................................................................... 51

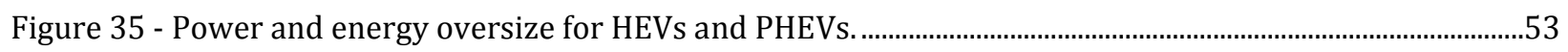

Figure 36 - Battery cost for (top left) HEVs, (top right) PHEVs, and (bottom) EV......................................................54

Figure 37 - Relation between power/energy ratio and battery energy cost.................................................................55

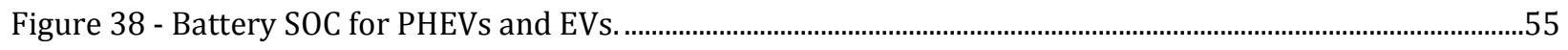

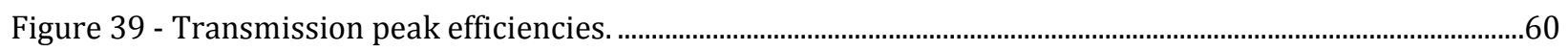

Figure 40 - Glider mass reduction for all vehicle classes. ......................................................................................62

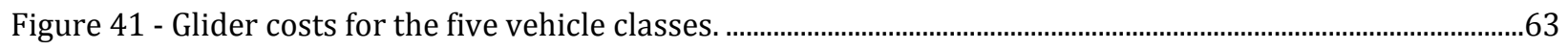

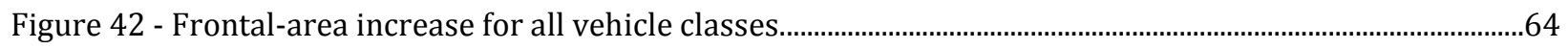

Figure 43 - (Left) drag coefficient and (right) rolling resistance values for the five vehicle classes.....................64

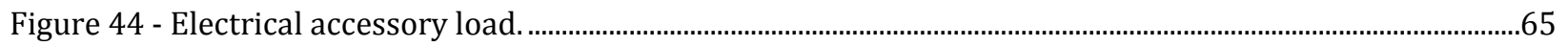

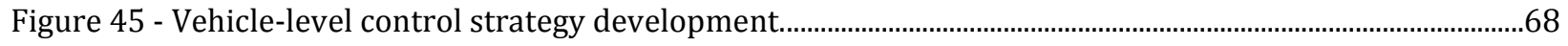

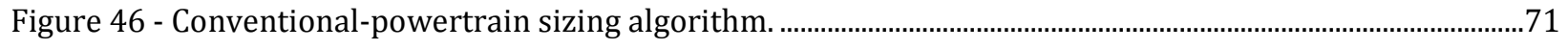

Figure 47 - Engine peak power with conventional powertrain for compact car...........................................................72

Figure 48 - Engine peak power compared to the same-year, same-case conventional gasoline engine for a

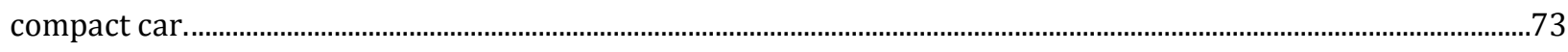

Figure 49 - Engine peak power as a function of vehicle mass for conventional gasoline engine............................73 
Figure 50 - Vehicle curb weights for compact cars with conventional powertrains. . .74

Figure 51 - Engine peak power for compact cars with conventional powertrains. . .75

Figure 52 - Engine peak power compared to the same-year, same-case gasoline split-HEV engine for a compact car. . .76

Figure 53 - Electric-machine power for compact split HEVs. . .76

Figure 54 - Electric-machine peak power compared to the gasoline HEV engine for a compact car. . .77

Figure 55 - Battery power for compact gasoline HEVs.

Figure 56 - Total battery energy for compact HEVs. . .78

Figure 57 - Vehicle curb weight for compact engine-HEV powertrains. . .78

Figure 58 - Engine peak power for compact PHEV powertrains. . .79

Figure 59 - ICE power for compact PHEV10s and PHEV20s relative to gasoline PHEV with matching AER. .....80

Figure 60 - Electric machine power for compact PHEVs . .80

Figure 61 - Battery power for compact gasoline HEV and PHEVs. . .81

Figure 62 - Usable battery energy for compact PHEV10, 20, 30, and 40. .82

Figure 63 - Total battery energy as a function of vehicle mass for gasoline PHEVs. .83

Figure 64 - Vehicle curb weights for compact PHEVs. . .84

Figure 65 - Fuel-cell system power for compact fuel-cell HEVs. . .85

Figure 66 - Electric-machine power for compact fuel-cell HEVs. .85

Figure 67 - Total battery energy for compact fuel-cell HEVs. . .86

Figure 68 - Vehicle curb weight for compact fuel-cell HEVs. . .86

Figure 69 - Fuel-cell system power for compact fuel-cell PHEVs. . .87

Figure 70 - Usable battery energy for compact fuel-cell PHEVs. . .87

Figure 71 - Electric-machine power for compact fuel-cell PHEVs. .88

Figure 72 - Vehicle curb weights for compact fuel-cell PHEVs. .89

Figure 73 - Battery energy as a function of vehicle mass for compact fuel-cell PHEVs. .89

Figure 74 - Electric-machine power for compact BEVs. . .91

Figure 75 - Battery power for compact BEVs. . .91

Figure 76 - Usable battery energy for compact BEVs. 
Figure 77 - Vehicle curb weights for compact BEVs.

Figure 78 - Engine peak power for compact vehicles with various gasoline powertrains.

Figure 79 - Battery power as a function of vehicle mass for compact vehicles with five types of powertrains. 94

Figure 80 - Vehicle curb weights for compact vehicles with gasoline engine powertrains . .95

Figure 81 - Impact of hybridization degree on weight for compact vehicles. . .95

Figure 82 - Electric-machine peak power for compact gasoline HEVs and PHEVs. . .96

Figure 83 - Fuel converter power as a function of vehicle mass for compact HEVs and PHEVs. . .97

Figure 84 - The urban cycle for a non-hybrid vehicle. . .98

Figure 85 - The highway cycle for a non-hybrid vehicle.

Figure 86 - The urban cycle for a hybrid vehicle. 100

Figure 87 - The highway cycle for a hybrid vehicle. Only the results from the second cycle are used... 100

Figure 88 - Fuel economy vs. fuel consumption.

Figure 89 - Fuel consumption (gasoline equivalent) for conventional compact cars. 108

Figure 90 - Gasoline-equivalent fuel consumption for conventional compact cars, compared to the reference conventional gasoline vehicle. 109

Figure 91 - Gasoline-equivalent fuel consumption for conventional compact cars, compared to the same-year conventional gasoline vehicle.

Figure 92 - Gasoline-equivalent fuel consumption for compact split HEVs.

Figure 93 - Gasoline-equivalent fuel consumption of compact split HEVs, compared to the reference gasoline split-HEV.

Figure 94 - Gasoline-equivalent fuel consumption for split-HEV compact cars, compared to the same-year, same-case gasoline HEV.

Figure 95 - Gasoline-equivalent fuel consumption for compact split PHEV10s and PHEV20s. All the fuelconsumption values are $\mathrm{CD}+\mathrm{CS}$.

Figure 96 - Gasoline-equivalent fuel consumption for compact split PHEV30s and PHEV40s. All the fuelconsumption values are $\mathrm{CD}+\mathrm{CS}$.

Figure 97 - Gasoline-equivalent fuel consumption for split PHEV10 and -20 compact cars, compared to the same-year, same-case gasoline PHEV with matching AER. All the fuel-consumption values are CD+CS...........115

Figure 98 - Gasoline-equivalent fuel consumption for split PHEV10 and -20 compact cars, compared to the reference PHEV gasoline vehicle. All the fuel consumption values are CD+CS. 
Figure 99 - Gasoline-equivalent fuel consumption for split PHEV30 and -40 compact cars, compared to the reference PHEV gasoline vehicle.. All the fuel consumption values are CD+CS.

Figure 100 - Gasoline-equivalent fuel consumption for split PHEV30 and -40 compact cars, compared to the same-year, same-case gasoline PHEV with matching AER. All the fuel consumption values are CD+CS. ..........116

Figure 101 - Electric consumption in CD+CS mode for gasoline-powered-split PHEVs. . .117

Figure 102 - Gasoline-equivalent fuel consumption for compact fuel-cell HEVs. 117

Figure 103 - Gasoline-equivalent fuel consumption compared to the compact fuel cell HEV reference case..118 Figure 104 - Gasoline-equivalent fuel consumption for compact fuel-cell PHEVs. The fuel consumption values are $\mathrm{CD}+\mathrm{CS}$.

Figure 105 - Gasoline-equivalent fuel consumption for compact fuel-cell PHEV10s compared to the reference case.

Figure 106 - Electric consumption in CD+CS mode for compact fuel-cell PHEVs.

Figure 107 - Electric consumption by compact EVs operating on UDDS and HWFET cycles. 120

Figure 108 - Electric consumption by EVs.

Figure 109 - Gasoline-equivalent fuel consumption for compact power-split HEVs, compared to the sameyear, same-case conventional gasoline vehicle.

Figure 110 - Gasoline-equivalent fuel consumption for compact power-split HEVs, compared to same-fuel, same-year conventional compact vehicle.

Figure 111 - Gasoline-equivalent fuel consumption for compact power-split HEVs compared to same-year, same-case compact fuel-cell HEV.

Figure 112 - Gasoline-equivalent fuel consumption for compact fuel-cell HEV compared to same-year, samecase compact gasoline conventional vehicle.

Figure 113 - Gasoline-equivalent fuel consumption for compact fuel-cell HEVs compared to same-year, samecase compact gasoline split HEV.

Figure 114 - Gasoline-equivalent fuel consumption for compact fuel-cell HEV compared to the compact gasoline split-HEV reference case...

Figure 115 - Gasoline-equivalent fuel consumption for compact fuel-cell HEV compared to the same-year, same-case compact gasoline split-HEV.

Figure 116 - Gasoline-equivalent fuel consumption for compact fuel-cell HEV compared to the same-year, same-case compact hydrogen conventional vehicle.

Figure 117 - Gasoline-equivalent fuel consumption for compact fuel-cell HEV compared to reference-case hydrogen power-split HEV.

Figure 118 - Gasoline-equivalent fuel consumption for compact fuel-cell HEVs compared to same-year, samecase compact hydrogen power split HEV. 
Figure 119 - WSFC as a function of vehicle mass for compact cars.

Figure 120 - WSFC as a function of vehicle mass for gasoline conventional vehicles.

Figure 121 - WSFC for fuel-cell compact cars.

Figure 122 - WSFC as a function of vehicle mass for gasoline conventional vehicles.

Figure 123 - Manufacturing Costs of diesel, hydrogen, and ethanol conventional compact cars compared to same-year gasoline conventional compact car. 135

Figure 124 - Incremental manufacturing Costs of compact HEVs.

Figure 125 - Manufacturing Costs of compact HEVs compared with same-year conventional gasoline-powered vehicles. . .136

Figure 126 - Manufacturing Costs of compact PHEVs. 137

Figure 127 - Manufacturing Costs of compact PHEVs compared with same-year gasoline-powered HEV.......138

Figure 128 - Manufacturing Costs of compact fuel-cell vehicles.

Figure 129 - Manufacturing Costs for compact EVs.

Figure 130 - Manufacturing Costs of compact gasoline-powered vehicles. 141

Figure 131 - Manufacturing costs of compact H2 ICE vehicles compared with same-year conventional gasoline vehicles.

Figure 132 - Manufacturing Cost of compact fuel-cell HEV compared with same-year conventional gasoline vehicle.

Figure 133 - Incremental manufacturing cost (in comparison to the reference conventional gasoline vehicle manufacturing cost) as a function of fuel consumption for compact conventional vehicles...

Figure 134 - Incremental manufacturing cost (in comparison to the reference conventional gasoline vehicle manufacturing cost) as a function of fuel consumption for compact HEVs.

Figure 135 - Incremental manufacturing cost (in comparison to the reference conventional gasoline vehicle manufacturing cost) as a function of fuel consumption for PHEV10 vehicles.

Figure 136 - Incremental manufacturing cost (in comparison to the reference conventional gasoline vehicle manufacturing cost) as a function of fuel consumption for PHEV40 vehicles.

Figure 137 - Incremental manufacturing cost (in comparison to the reference conventional gasoline vehicle manufacturing cost) as a function of fuel consumption for fuel-cell vehicles..

Figure 138 - Incremental manufacturing cost (in comparison to the reference conventional gasoline vehicle manufacturing cost) as a function of fuel consumption for hydrogen-fueled vehicles..

Figure 139 - Incremental manufacturing cost (in comparison to the gasoline conventional reference vehicle) as a function of fuel consumption for all powertrains.. 
Figure 140 - Incremental manufacturing cost (in comparison to the reference conventional gasoline vehicle manufacturing cost) as a function of fuel consumption for gasoline vehicles.

Figure 141 - Conventional vehicle. 488

Figure 142 - Electric vehicle. 488

Figure 143 - Hybrid vehicle. 488

\section{TABLES}

Table 1 - ES - Percentage Fuel-Consumption Reduction (mi/gal gasoline equivalent or MPGGE) of Each Powertrain by 2045, Compared to 2010 Gasoline Conventional Powertrain. (Electrical consumption is not taken into account for PHEVs)

Table 2 - ES - Percentage Fuel Consumption Reduction for Each Powertrain by 2045, Compared to the Respective Current Status (Values show uncertainty range).

Table 3 - ES - Additional manufacturing Cost (\$) of Each Powertrain by 2045 compared to Reference 2010 Gasoline Conventional Engine for Compact Cars

Table 4 - ES - Percentage manufacturing cost Reduction for Each Powertrain by 2045, Compared to the Respective Current manufacturing cost, for Compact Cars.

Table 5: Definition of the baseline engines used in the present study..

Table 6: Description of Reference Battery Characteristics..

Table 7: Battery Technology Selection for Each Timeframe..

Table 8: Transmission technologies modeled for different vehicle classes*

Table 9: Gear ratios, final drive and cost for all transmissions. . .58

Table 10: Power-split transmission characteristics for all vehicle classes . .59

Table 11: Fuel-cell and electric-vehicle transmission characteristics for all vehicle classes . .59

Table 12: Main characteristics of the different vehicle classes . .61

Table 13: Incremental cost and glider composition associated with glider mass reduction

Table 14: Summary of travel distances for all vehicles on the UDDS cycle based on unadjusted consumption 69

Table 15: Relationship between engine power and vehicle weight . .74

Table 16: Relationship between battery energy and vehicle weight for gasoline PHEVs . .83

Table 17: Relationship between battery energy and vehicle weight for fuel-cell PHEVs. . .90

Table 18: Relationship between battery power and vehicle weight for different gasoline-fueled powertrains 94

Table 19: Relationship between fuel converter and vehicle weight for different powertrains . .97 
Argonne National Laboratory Report - Light-Duty Vehicle Fuel Consumption Displacement Potential Up to 2045

Table 20: Cold-start $\left(20^{\circ} \mathrm{C}\right)$ penalties for the different powertrain configurations (\%) .........................................106

Table 21: Fuel consumption of conventional compact vehicles for reference and 2045 technologies...............109

Table 22: Fuel consumption of compact HEVs for reference and 2045 technologies .............................................111

Table 23: Fuel consumption of compact PHEV10s with 2010 and 2045 technologies ...........................................114

Table 24: Comparison of fuel consumption with reference and 2045 technologies for conventional vehicles and HEVs 
Argonne National Laboratory Report - Light-Duty Vehicle Fuel Consumption Displacement Potential Up to 2045 


\section{ACKNOWLEDGMENTS}

This study was supported by the United States Department of Energy's (DOE's) FreedomCAR and Vehicle Technology Office under the direction of David Anderson and Lee Slezak. The submitted report has been created by UChicago Argonne, LLC, Operator of Argonne National Laboratory (Argonne). Argonne, a DOE Office of Science laboratory, is operated under Contract No. DE-AC02-06CH11357. The U.S. Government retains for itself, and others acting on its behalf, a paid-up, nonexclusive, irrevocable worldwide license in said article to reproduce, prepare derivative works, distribute copies to the public, and perform publicly and display publicly, by or on behalf of the Government.

We would like also to express special thanks to Jake Ward, Ann Schlenker, Tom Stephens, David Anderson, Michael Duoba, Anant Vyas, and Dan Santini for their review, comments and contributions to this report.

\section{ABOUT THE AUTHORS}

The authors are members of the Vehicle Modeling and Simulation group at Argonne.

Aymeric Rousseau is the manager of the Vehicle Modeling and Simulation Section at Argonne. He received his engineering diploma at the Industrial System Engineering School in La Rochelle, France, in 1997. After working for PSA Peugeot Citroen in the Hybrid Electric Vehicle research department, he joined Argonne in 1999, where he is now responsible for the development of Autonomie. He received an R\&D 100 Award in 2004 and a Vehicle Technologies Program R\&D Award in 2010. He has authored more than 40 technical papers in the area of advanced vehicle technologies.

Ayman Moawad is a research engineer in the Vehicle Modeling and Simulation Section at Argonne National Laboratory. He graduated from the Ecole des Mines de Nantes, France, in 2009 with a Master's Degree in Science, majoring in Automatics, Control Systems, and Industrial Computer Science. He focuses his research on light-duty vehicle fuelconsumption analysis as well as powertrain costs to support the Government Performance and Results Act.
Phil Sharer is the Principal Investigator behind the Autonomie Process Architecture. He wrote the Automated Model Building Algorithm and authored the XML Argonne Model Descriptor Specification. He has been a Research Engineer at Argonne for 11 years. During this time, he also co-authored the Powertrain Systems Analysis Toolkit. He received a Master of Science degree in engineering from Purdue University Calumet. 


\section{NOTATION}

\subsection{ACRONYMS AND ABBREVIATIONS}

AEO

AER

APRF

BEV

$\mathrm{BOL}$

CAFE

$C D$

$\mathrm{Cl}$

CS

DOE

E85

EIA

EOL

EPA

E-REV

EVS

FC

FCV

GDI

GHG

GPRA

GREET

GUI

GVW

H2

HEV

HWFET

ICE

IEA

IVM
Annual Energy Outlook

all-electric range

Advanced Powertrain Research Facility

battery-powered electric vehicle

beginning-of-life

Corporate Average Fuel Economy

charge depleting

compression ignition

charge sustaining

U.S. Department of Energy

blend of $85 \%$ ethanol and $15 \%$ gasoline by weight

Energy Information Administration

end of life

U.S. Environmental Protection Agency

extended-range EV

International Electric Vehicle Symposium

fuel cell

fuel cell vehicle

gasoline direct injection

greenhouse gas

Government Performance and Results Act

Greenhouse gases, Regulated Emissions, and Energy use in Transportation

graphical user interface

gross vehicle weight

hydrogen

hybrid electric vehicle

Highway Federal Emissions Test

internal combustion engine

International Energy Agency

initial vehicle movement 
Argonne National Laboratory Report - Light-Duty Vehicle Fuel Consumption Displacement Potential Up to 2045

\begin{tabular}{|c|c|}
\hline LHV & lower heating value \\
\hline Li-ion & lithium ion \\
\hline MPGGE & miles per gallon gasoline equivalent \\
\hline MC2/GC & Motor2/Generator \\
\hline costMY & model year \\
\hline NEMS & National Energy Modeling System \\
\hline NHTS & National Household Travel Survey \\
\hline $\mathrm{NiMH}$ & nickel metal hydride \\
\hline OEM & original equipment manufacturer \\
\hline PHEV & plug-in hybrid electric vehicle \\
\hline PHEV 10 and 20 & PHEV with 10 or 20 miles of all-electric range \\
\hline PHEV 30 and 40 & PHEV with 30 or 40 miles of all-electric range \\
\hline PSAT & Powertrain System Analysis Toolkit \\
\hline $\mathrm{P} / \mathrm{W}$ & power to weight ratio \\
\hline SAE & Society of Automotive Engineers \\
\hline SI & spark ignition \\
\hline SOC & state of charge \\
\hline SUV & sport utility vehicle \\
\hline UDDS & Urban Dynamometer Driving Schedule \\
\hline UF & utility factor \\
\hline US06 & duty cycle with aggressive highway driving \\
\hline VCR & variable compression ratio \\
\hline VTP & Vehicle Technologies Program \\
\hline VVT & variable valve timing \\
\hline WSFC & weight-specific fuel consumption \\
\hline WTP & well to pump \\
\hline WTW & well-to-wheels \\
\hline
\end{tabular}

\subsection{UNITS OF MEASURE}

$\begin{array}{llll}\mathrm{A} & \text { ampere(s) } & \text { MPG } & \text { mile(s) per gallon } \\ \mathrm{bbl} & \text { barrel(s) } & \text { MPGGE } & \text { mile(s) per gallon of gasoline equivalent } \\ \mathrm{Gal} & \text { gallon(s) } & \mathrm{MW} & \text { megawatt(s) } \\ \mathrm{H} & \text { hour(s) } & \mathrm{V} & \text { volt(s) } \\ \mathrm{Mi} & \text { mile(s) } & \mathrm{Wh} / \mathrm{mi} & \text { Watt hour(s) per mile }\end{array}$


Argonne National Laboratory Report - Light-Duty Vehicle Fuel Consumption Displacement Potential Up to 2045 


\section{ABSTRACT}

The U.S. Department of Energy (DOE) Vehicle Technologies Program (VTP) is developing more energyefficient and environmentally friendly highway transportation technologies that will enable America to use less petroleum. The long-term aim is to develop "leapfrog" technologies that will provide Americans with greater freedom of mobility and energy security, while lowering costs and reducing impacts on the environment. The U.S. Department of Energy Vehicle Technologies Program examines pre-competitive, high-risk research needed to develop

- Component and infrastructure technologies necessary to enable a full range of affordable cars and light trucks.

- Fueling infrastructure to reduce the dependence of the nation's personal transportation system on imported oil and minimize harmful vehicle emissions, without sacrificing freedom of mobility and freedom of vehicle choice.

As part of this ambitious program, numerous technologies are addressed, including engines, energy storage systems, fuel-cell systems, hydrogen storage, electric machines, and materials, among others.

The 1993 Government Performance and Results Act (GPRA) holds federal agencies accountable for using resources wisely and achieving program results. GPRA requires agencies to develop plans for what they intend to accomplish, measure how well they are doing, make appropriate decisions on the basis of the information they have gathered, and communicate information about their performance to Congress and to the public. The present study evaluates the benefits of the light-duty vehicle research conducted at DOE from fuel-efficiency and cost perspectives, to support GPRA activities.

Owing to the large number of component and powertrain technologies considered, the benefits were simulated using Autonomie. Argonne designed Autonomie to serve as a single tool that can be used to meet the requirements of automotive engineering throughout the development process, from modeling to control. Autonomie, a forward-looking model developed using MathWorks tools, offers the ability to quickly compare powertrain configurations and component technologies from a performance and fuel-efficiency point of view.

This report reviews the results of the DOE VTP, assessing the fuel and light-duty vehicle technologies that are most likely to be established, developed and eventually commercialized during the next 35 years (up to 2045). Owing to the rapid evolution of component technologies, this study is performed on a yearly basis to continuously update the results based on the latest state-of-the-art technologies.

While it is not possible to simulate all the different combinations, more than 2000 vehicles were simulated in the study to take the following into account: 
- Major powertrain configurations (i.e., conventional, power-split, series and battery electric drive)

- Major vehicle classes (i.e., compact car, midsize car, small sport utility vehicle [SUV], large SUV and pickup)

- Major fuels (i.e., gasoline, diesel, hydrogen and ethanol)

These technologies were assessed for four different timeframes (2010, 2015, 2030 and 2045). Finally, uncertainties were included for both performance and cost aspects by considering three cases:

- Low case (10\% uncertainty) - aligned with original-equipment-manufacturer improvements based on regulations.

- Average case (50\% uncertainty).

- High case (90\% uncertainty) - aligned with aggressive technology advancement based on the U.S. DOE Vehicle Technologies program.

The objective of the report is to provide an assessment of the conventional-fuel displacement and cost-reduction potentials of advanced technologies up to the year 2045 including uncertainties.

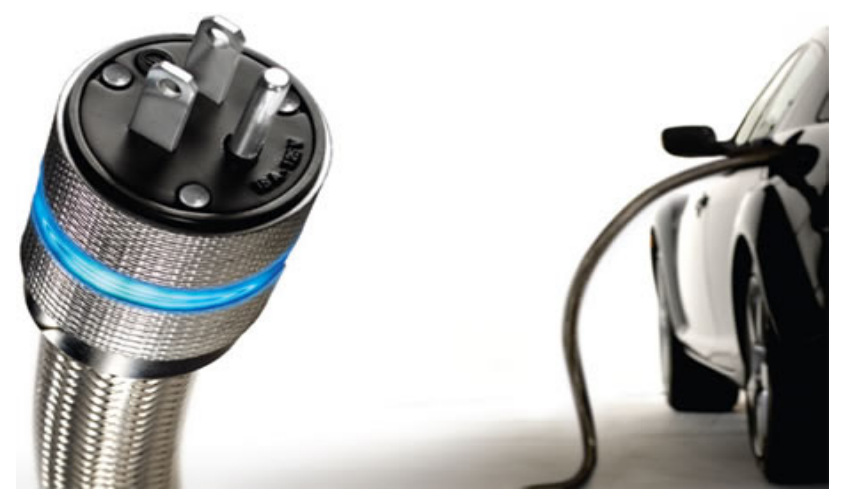




\section{EXECUTIVE SUMMARY}

The U. S. Department of Energy (DOE) Vehicle Technologies Program (VTP) supports new technologies to increase energy security in the transportation sector at a critical time for global petroleum supply, demand, and pricing. Consequences of our vehicles' dependence on oil as their source of energy were shown by the "first oil shock" brought on by the petroleum embargo of October 1973 and the "second oil shock" of 1979. However, this oil dependence continues to increase unabated to the present, and the oil price run-up of July 2008 (\$147 per barrel of crude) illustrated the rapidity with which these discontinuities can occur. As such, the lack of widely available and viable alternative non-petroleum-based fueling options for ground transport vehicles constitutes a high risk to stable economic activity. Some means of providing energy to move vehicles that greatly reduces or eliminates petroleum consumption must be developed. This challenge is greatly complicated by the fact that virtually all alternatives have some inherent fossil-fuel component. The U.S. transportation sector used about 13.5 million barrels of oil equivalent per day in 2009. It consumed more oil than the total U.S. domestic oil production. On-highway vehicles (passenger and commercial vehicles) used over 11 million barrels of oil equivalent per day, which accounts for over 79 percent of the total transportation oil use and over 59 percent of total U.S. oil use. The Vehicle Technologies Program focuses on ground transportation vehicles because of their dominant contribution to the nation's oil use.

VTP works in collaboration with industry to identify the priority areas of research needed to develop advanced vehicle technologies to reduce and eventually eliminate petroleum use, and reduce emissions of greenhouse gases, primarily carbon dioxide from carbon-based fuels. The VTP works on numerous technologies, including the following:

- Development of hybrid electric vehicles (HEVs) and plug-in HEVs (PHEVs) can provide significant improvement in fuel economy and petroleum displacement. This research supports President Obama's goal of 1 million plug-in hybrid vehicles (PHEVs) and electric vehicles (EVs) by 2015.

- $\quad$ Deployment of alternative fuels can rapidly reduce oil imports.

- Reducing vehicle weight directly improves vehicle efficiency and fuel economy, and can potentially reduce vehicle operating costs.

- Improved combustion technologies and optimized fuel systems can improve near- and mid-term fuel economy for passenger vehicles by 25-40\% for passenger vehicles by 2015 .

The objective of the present study is to evaluate the benefits of the DOE VTP for a wide range of vehicle applications, powertrain configurations and component technologies for different timeframes and quantify the potential future petroleum displacement up to 2045 as well as the cost evolution. More than 2000 light-duty vehicles were simulated with Autonomie, Argonne's vehicle simulation tool. 
Argonne National Laboratory Report - Light-Duty Vehicle Fuel Consumption Displacement Potential Up to 2045

Owing to the large number of powertrain and component technologies, only a limited number of combinations were taken into account (i.e., micro or "mild" HEVs were not included), leading to the consideration of more than 2000 vehicles. To address performance and cost uncertainties, three cases were considered: low $(10 \%)$, average $(50 \%)$, and high $(90 \%)$ uncertainty. When available, the high-case assumptions are based on the FreedomCAR and Fuel Partnership program goals. The other assumptions have been developed through discussions with experts from companies, universities, and the national laboratories. While the uncertainties are expected to provide a range, it should be noted that several ongoing research projects or lack of data for specific technologies could lead to significantly higher fuel-efficiency gains than considered in the study. For example, the engine gains could be considered less aggressive than for other technologies, and readers should take this parameter into account during the analysis. More than 400 assumptions are necessary to define each vehicle. Some of the main assumptions are highlighted below:

- The difference in peak efficiency between gasoline and diesel engines is expected to narrow in the future owing to the combination of advanced gasoline engine technologies and the impact of ever more stringent after-treatment for diesel.

- Coupling ultra-capacitors with batteries was not considered, owing to higher cost and expected increase in Li-ion battery life and cold start performance in the short term.

- Because of the drive quality requirements in North America, automated manual transmissions were not included in the study. Continuously variable transmissions (CVT) have also shown issues with reliability and fuel-efficiency gains and are not considered either.

- The peak efficiencies of fuel-cell systems remain constant in the future, as most research is expected to focus on reducing cost and increasing durability. The costs used are based on the assumption that 500,000 units are produced per year.

The main results related to vehicle sizing, fuel efficiency, and cost are highlighted in the following sections.

\subsection{VEHICLE SIZING FINDINGS}

Advances in material substitution will play a significant role in reducing overall vehicle weight and, consequently, component power and energy requirements.

- Owing to the impact of the component max-torque shapes, maintaining a constant power-to-weight $(\mathrm{P} / \mathrm{W})$ ratio between all configurations leads to an inconsistent comparison between technologies due to different performances. Each vehicle should be sized independently to meet specific Vehicle Technical Specifications (VTS).

- Reducing the vehicle weight ("lightweighting") has greater influence on conventional vehicles than on their electric-drive counterparts. 
Argonne National Laboratory Report - Light-Duty Vehicle Fuel Consumption Displacement Potential Up to 2045

- While performance (i.e., time for 0-60 mph) is the primary factor used to size components for current technologies, aggressive future lightweighting can make gradeability requirements the critical sizing criteria.

- Vehicle weight decreases in the range from 1 to $51 \%$ by 2045 across powertrain configurations. The weight reduction, however, varies with the configuration. For the configurations using an engine, the weight reduction for the gasoline conventional powertrain ranges from 1 to $30 \%$, power-split HEVs from 2 to 33\%, low-energy PHEVs (with all-electric ranges, or AERs, of 10 and 20 miles) from 3 to 34\%, and high energy PHEVs (30- and 40-mile AERs) from 6 to 37\%. Configurations with fuel-cell systems demonstrate a larger weight reduction, with fuel-cell HEV weight reductions ranging from 21 to 51\%, low-energy PHEV10s and 20s (i.e., 10- and 20-mile AERs) from 19 to 49\% and high-energy PHEV30s and 40s (30- and 40-mile AERs) from 18 to 47\%. Finally, battery electric vehicles achieve a weight reduction ranging from 7 to $44 \%$. Overall, significant weight reductions can be achieved compared to current technologies, especially for vehicles with large batteries and/or using hydrogen fuel

- Most of the component peak powers show a strong linear correlation with the vehicle weight. As a result, it is necessary to include secondary effects when analyzing the lightweighting benefits.

- Owing to lightweighting and component efficiency improvements, the peak power of engine and fuel-cell systems could be significantly reduced over time to meet current Vehicle Technical Specifications. Engine peak power could be reduced by 2045 over a 2-to-28\% range for conventional gasoline, 5 to $34 \%$ for gasoline power-split HEVs, 4 to $28 \%$ for low-energy PHEVs, and 8 to $36 \%$ for high-energy PHEVs. As seen for vehicle weight, hydrogen-fueled vehicles demonstrate a larger peak-power improvement than gasoline-fueled vehicles over time, with fuel-cell system power decreasing in the range from 22 to $53 \%$ for HEVs, 18 to $48 \%$ for low-energy PHEVs and 15 to $38 \%$ for high-energy PHEVs.

- Battery peak power is also expected to decrease over time to meet current vehicle performance. The battery power is expected to decrease up to $34 \%$ for gasoline-engine HEVs and PHEVs. For fuel-cell systems, the decrease could be as high as $48 \%$.

- Battery total energy will be decreasing significantly owing to other component improvements as well as a wider usable state-of-charge range. The reduction in energy required for PHEVs and battery-powered electric vehicles (BEVs) could range from 4 to $60 \%$.

- While the fuel selection influences the engine size for conventional vehicles (i.e., diesel has lower peak power than gasoline to higher maximum torque at low speed), the power required to meet the Vehicle Technical Specifications for electric-drive vehicles is comparable across all fuels.

The different PHEVs show a linear relationship between the usable battery energy and the vehicle mass, with the slope increasing with the All Electric Range. 
Argonne National Laboratory Report - Light-Duty Vehicle Fuel Consumption Displacement Potential Up to 2045

\subsection{VEHICLE FUEL-EFFICIENCY FINDINGS}

Overall, the combination of technology improvements leads to significant fuel-consumption reduction across vehicle applications. As a result, significant fuel can be displaced over the next few decades. There is a linear relationship between lightweighting and fuel and electrical consumption. However, as previously discussed, that relationship differs depending on the powertrain configuration.

\section{Evolution of Fuel Consumption Compared to Reference 2010 Gasoline Conventional Vehicle}

Table ES-1 shows the adjusted fuel-consumption reduction by 2045 on the combined driving cycle for each powertrain configuration and fuel, compared to the reference gasoline conventional vehicle.

The results demonstrate significant improvements over time across all powertrain configurations and fuels (Table ES-1). When considering the high-uncertainty case across all engines, conventional vehicles can achieve a 41-47\% fuel-consumption improvement, engine HEVs, 62 to $67 \%$, engine PHEV10s, 69 to 73\%, and engine PHEV40s, 80 to $82 \%$. Fuel-cell vehicles achieve an improvement of up to $70 \%$ for HEVs, $75 \%$ for PHEV10s, and $84 \%$ for PHEV40s.

Table 1 - ES - Percentage Fuel-Consumption Reduction (mi/gal gasoline equivalent or MPGGE) of Each Powertrain by 2045,

Compared to 2010 Gasoline Conventional Powertrain. (Electrical consumption is not taken into account for PHEVs)

\begin{tabular}{|c|c|c|c|c|}
\hline Fuel $\backslash$ Powertrain & Conventional & HEV & PHEV10 & PHEV40 \\
\hline Gasoline & $2-43$ & $37-64$ & $49-70$ & $64-81$ \\
\hline Diesel & $16-42$ & $42-62$ & $51-69$ & $65-80$ \\
\hline $\begin{array}{c}\text { Hydrogen Internal } \\
\text { Combustion Engine } \\
\text { (ICE) }\end{array}$ & $4-41$ & $50-67$ & $56-73$ & $69-82$ \\
\hline $\begin{array}{c}\text { Ethanol } \\
\text { Fuel Cell }\end{array}$ & $1-47$ & $32-62$ & $46-69$ & $62-80$ \\
\hline
\end{tabular}

\section{Evolution of Specific Powertrains}

Table ES-2 shows the 2045 adjusted fuel-consumption reduction, on the combined driving cycle, for each powertrain configuration and fuel, compared to each configuration's current status in 2010 (e.g., the diesel HEV in 2045 is compared to the reference diesel HEV in 2010). 
Argonne National Laboratory Report - Light-Duty Vehicle Fuel Consumption Displacement Potential Up to 2045

The results demonstrate that the maximum improvement expected for each powertrain technology compared to its current status ranges from 33 to $52 \%$. The range depends on fuels (i.e., diesel vehicles show less improvement than gasoline vehicles) and powertrain (i.e., conventional engines have a lower maximum improvement than PHEV40s). When considering the entire uncertainty range, fuel-cell vehicles show the greatest improvement over time.

Table 2 - ES - Percentage Fuel Consumption Reduction for Each Powertrain by 2045, Compared to the Respective Current Status (Values show uncertainty range)

\begin{tabular}{|c|c|c|c|c|}
\hline Fuel $\backslash$ Powertrain & Conventional & HEV & PHEV10 & PHEV40 \\
\hline Gasoline & $2-43$ & $9-45$ & $8-43$ & $11-49$ \\
\hline Diesel & $5-33$ & $10-39$ & $9-39$ & $12-43$ \\
\hline Hydrogen ICE & $19-49$ & $24-52$ & $23-50$ & $25-50$ \\
\hline Ethanol & $1-41$ & $10-46$ & $8-44$ & $10-50$ \\
\hline Fuel Cell & & $23-48$ & $20-45$ & $18-42$ \\
\hline Electricity (BEV) & $6-41$ & & & \\
\hline
\end{tabular}

\section{Powertrain Comparisons}

- Conventional Gasoline Vehicles vs. Engine HEVs

0 The comparison between these powertrains shows that the fuel-consumption reductions due to hybridization stay fairly constant over time for all power-split HEVs.

o For gasoline HEVs, fuel-consumption reductions range from 36 to $40 \%$ for compact cars, 34 to $40 \%$ for midsize cars, 60 to $36 \%$ for small sport utility vehicles (SUVs), 29 to $35 \%$ for large SUVs and 28 to $33 \%$ for pickup trucks.

- Conventional Gasoline Vehicles vs. Engine PHEVs

O As is the case for power-split HEVs, the fuel-consumption reduction observed for PHEVs relative to conventional gasoline vehicles remains fairly constant over time, ranging from 59 to $71 \%$.

o However, while the percentages decreased for higher vehicle weight classes, the benefits remain fairly constant across platforms, with 63 to $69 \%$ fuel consumption reduction for compacts, 65 to $71 \%$ for midsize cars, and 59 to $64 \%$ for small SUVs, large SUVs and pickup trucks.

- Conventional Gasoline Vehicles vs. Fuel-Cell HEVs 
Argonne National Laboratory Report - Light-Duty Vehicle Fuel Consumption Displacement Potential Up to 2045

o The current fuel-consumption reductions for fuel-cell HEVs compared to conventional gasoline vehicles are $45 \%$ for compacts, $43 \%$ for midsize cars, $39 \%$ for small and large SUVs, and $30 \%$ for pickups.

o Owing to the expected improvements in fuel-cell system and hydrogen-storage technologies, the fuel-consumption percentage improvements are expected to increase over time. By 2045, the benefits increase from 5 to $20 \%$, depending upon the vehicle class and uncertainties considered

- Engine HEVs vs. Fuel-Cell HEVs

O Fuel-cell system technology offers consistently lower fuel consumption than power-split HEV technology.

o The current fuel-consumption benefits of fuel-cell HEVs compared to gasoline power-split HEVs are fairly constant across all vehicle classes, ranging from 12 to $14 \%$

0 Owing to the engine and fuel-cell system operating conditions for HEVs, the fuel consumption improvement remains constant across all vehicle classes. However, owing to the expected improvement of hydrogen technologies, the percentage is expected to increase by 18 to $26 \%$ by 2045.

- Conventional Hydrogen Engines vs. Fuel-Cell HEVs

o The percentage of fuel-consumption reduction for fuel-cell vehicles compared to hydrogen conventional vehicles remains at around $50 \%$.

o The percentage of fuel-consumption reduction remains fairly constant over time for each vehicle class: 50 to $52 \%$ for compacts, 50 to $55 \%$ for midsize cars, 46 to $48 \%$ for small SUVs, 44 to $47 \%$ for large SUVs, and 42 to $46 \%$ for pickup trucks

- Hydrogen Engine HEVs vs. Fuel Cell HEVs

o The fuel-consumption reduction for fuel-cell HEVs compared to hydrogen engine HEVs remains fairly constant across vehicle classes for the reference case ranging from 13 to $17 \%$.

0 The percentage decreases over time owing to the faster improvement of hydrogen engine performance compared to fuel-cell system performance, reaching 8 to $15 \%$ by 2045 .

\section{Evolution of Fuel Comparisons}

- Gasoline vs. diesel

o The differences between gasoline and diesel-engine fuel consumption for conventional vehicles will tend to decrease in the future.

- For conventional vehicles, the fuel consumption advantage of diesel engines, when considering MPGGE, goes from 11-15\% in 2010 to $-1-15 \%$ by 2045. 
Argonne National Laboratory Report - Light-Duty Vehicle Fuel Consumption Displacement Potential Up to 2045

o For HEVs, the fuel consumption benefit of diesel is smaller than for conventional vehicles, ranging from 5 to $8 \%$ in 2010. However, the gap between these fuels is also expected to decrease over time, with gasoline achieving lower fuel consumption for high-uncertainty cases.

o For PHEVs, the benefits of diesel compared to gasoline are minimal, ranging from 1 to $2 \%$ in 2010.

o However, the diesel engine retains the best fuel consumption for the vast majority of uncertainties and timeframes.

- Ethanol

o In most cases, ethanol-engine vehicles have the highest fuel consumption among conventional vehicles.

o Ethanol-fuel conventional vehicles are expected to narrow their fuel consumption penalty over gasoline engines with time. In 2010, the penalty ranges from 7 to $8 \%$; it could be lowered to $5-7 \%$ by 2045 .

o The fuel-consumption penalty for ethanol deceases for increased hybridization degree and battery energy

- Hydrogen Engine

o The hydrogen-engine conventional vehicle shows the greatest improvements in fuel consumption over time, owing to the introduction of direct injection. The fuel penalty compared to its gasoline counterpart is $10-17 \%$ in 2010 ; by 2045 , hydrogen-fueled conventional vehicles could range from a fuel-consumption penalty of $3 \%$ up to an advantage of $12 \%$.

o By 2045, the hydrogen-fueled engine configurations consistently achieve the lowest fuel consumption of any configuration.

\subsection{MANUFACTURING COST FINDINGS:}

Overall, the combination of technology improvements leads to significant manufacturing cost reduction across vehicle applications. As a result, advanced technologies are expected to have significant market penetration over the next decades.

\section{Cost Evolution Compared to Reference 2010 Gasoline Conventional Vehicle}

Table ES-3 shows the additional manufacturing cost by 2045, compared to the reference gasoline conventional vehicle. The table shows a significant uncertainty range for the additional manufacturing cost across all technologies. This high uncertainty highlights the need to pursue aggressive research over the next decades to bring the cost of advanced technologies to a level that will favor high market penetrations. 
Argonne National Laboratory Report - Light-Duty Vehicle Fuel Consumption Displacement Potential Up to 2045

Table 3 - ES - Additional manufacturing Cost (\$) of Each Powertrain by 2045 compared to Reference 2010 Gasoline Conventional Engine for Compact Cars

\begin{tabular}{|c|c|c|c|c|}
\hline Fuel\Powertrain & Conventional & HEV & PHEV10 & PHEV40 \\
\hline Gasoline & $584-1240$ & $560-3091$ & $1029-4086$ & $2188-8402$ \\
\hline Diesel & $1782-2609$ & $2037-4831$ & $2290-5543$ & $3700-10159$ \\
\hline Hydrogen ICE & $452-951$ & $828-2706$ & $1099-3500$ & $2432-7935$ \\
\hline Ethanol & $511-1209$ & $583-3111$ & $802-3695$ & $2205-8426$ \\
\hline Fuel Cell & & $-264-1812$ & $-186-2661$ & $721-6035$ \\
\hline BEV & $1446-10649$ & & & \\
\hline
\end{tabular}

Evolution of Costs for Specific Powertrains

Table ES-4 compares the percentage change in the manufacturing cost between 2010 and 2045 for each configuration relative to its current value.

Vehicle manufacturing costs for gasoline, diesel, and ethanol conventional vehicles increase over time because of several factors, including lightweighting (vehicle body mass decreases up to $37 \%$ by 2045 and its cost increases owing to the use of aluminum or carbon fiber) and advanced component technologies such as direct injection. In contrast, the greatest reductions are noticed for the vehicles with high-energy batteries, fuel-cell systems and hydrogen storage.

Owing to the expected improvements in batteries, the higher the battery energy, the greater will be the manufacturing cost reduction. As a result, PHEV40 demonstrates a larger cost reduction than PHEV10 across all fuels. PHEV40s with gasoline engines show cost reductions ranging from 32 to $41 \%$ from 2010 to 2045, while PHEV10s only show a cost reduction ranging from 11 to $18 \%$.

The fuel-cell vehicle manufacturing costs decrease significantly over time. From 2010 to 2045, the manufacturing for the fuel-cell HEV decreases by 29 to $41 \%$, for the fuel-cell PHEV10 by 30 to $42 \%$, and for the fuel-cell PHEV40 by 35 to $45 \%$. 
Argonne National Laboratory Report - Light-Duty Vehicle Fuel Consumption Displacement Potential Up to 2045

Table 4 - ES - Percentage manufacturing cost Reduction for Each Powertrain by 2045, Compared to the Respective Current manufacturing cost, for Compact Cars

\begin{tabular}{|c|c|c|c|c|}
\hline Fuel\Powertrain & Conventional & HEV & PHEV10 & PHEV40 \\
\hline Gasoline & -9 to 4 & -1 to -2 & -18 to -11 & -41 to -32 \\
\hline Diesel & 1 to 4 & -12 to -5 & -20 to -13 & -40 to -33 \\
\hline Hydrogen ICE & -10 to 7 & -21 to -5 & -27 to -14 & -45 to -35 \\
\hline Ethanol & 4 to 9 & -10 to -2 & -18 to -11 & -40 to -33 \\
\hline Fuel Cell & -66 to -55 & -41 to -29 & -42 to -30 & -45 to -35 \\
\hline Electricity (BEV) & & & \\
\hline
\end{tabular}

\section{Powertrain Comparison}

- The manufacturing cost differences between different powertrain options tend to decrease over time. In 2010, for midsize cars, the gasoline power-split HEV is $26 \%$ more expensive than the conventional vehicle, the PHEV10 45\% more expensive and the PHEV40 150\% more expensive. By 2045, these percentages are 8-13\% for HEVs, $11-17 \%$ for PHEV10s, and $32-50 \%$ for PHEV40s.

- While vehicles with hydrogen engines will remain more expensive than fuel-cell vehicles, the technology will become more cost-competitive with the fuel-cell system over time. By 2045 , conventional hydrogenengine vehicles will be slightly cheaper to manufacture than their gasoline counterpart.

\section{Fuel-Comparison Evolution}

- Gasoline vs. diesel

o The conventional diesel vehicle manufacturing cost will remain between 10 and $13 \%$ more expensive than gasoline vehicles.

0 The diesel HEV is between 10 and $14 \%$ more expensive to manufacture than the gasoline vehicle, but this difference tends to decrease after 2010.

- Ethanol

o Gasoline and ethanol vehicles have similar manufacturing costs across timeframes. Their manufacturing cost increases between 4 and $8 \%$ between 2010 and 2045, whereas the dieselvehicle manufacturing cost increases by only 1 to $5 \%$ in that timeframe. 
Argonne National Laboratory Report - Light-Duty Vehicle Fuel Consumption Displacement Potential Up to 2045

o From 2010 forward, the relative cost of ethanol vehicles decreases, reaching almost the same level as gasoline HEVs in 2045.

- Hydrogen Engine

o Vehicle cost for the hydrogen engine decreases as the additional cost of reducing the vehicle weight ("lightweighting") is compensated by larger cost reductions in the hydrogen storage system.

\subsection{CONCLUSION}

The combination of the technology improvements leads to significant fuel consumption and cost reduction across light duty vehicle applications. Due to the uncertainty of the evolution of the technologies considered, research should continue to be conducted in the different area showing high fuel displacement potential.

Due to expected improvements, advanced technologies are expected to have significant market penetration over the next decades. In the short term, both engine HEVs and PHEVs allow for significant fuel displacement with acceptable additional cost. While electric vehicles do provide a promising solution, they are likely to remain expensive and range limited in the near future. In the medium term, hydrogen engine HEVs will offer significant fuel improvements and could potentially offer a bridging technology that would help establish the infrastructure required for fuel cell vehicles. For the long term, fuel cell vehicles demonstrate very high fuel displacement potential at a competitive cost

This research will be updated on a yearly bases to include the latest powertrain technologies (i.e., multi-mode HEV, E-REV...), component technologies, as well as additional timeframes (i.e., 2020) and vehicle applications. 
Argonne National Laboratory Report - Light-Duty Vehicle Fuel Consumption Displacement Potential Up to 2045 


\section{INTRODUCTION}

\subsection{THE ENERGY SITUATION}

The current international energy situation has brought about serious concerns in most of the developed countries about their use of fossil fuels and their need for developing renewable energy sources. This energy crisis takes place in the context of oil-stock reduction and a dramatic demand increase from developing countries. A study by M. Wang (2006) shows that Chinese on-road vehicles could consume up to 20.6 million bbl of oil per day by 2050. Moreover, China could face a tremendous increase in highway vehicles (including cars, trucks, and buses) in the next 40 years. Indeed, depending upon the case scenario developed in the study, there could be between 486 and 662 million highway vehicles in China in 2050, compared to roughly 27 million in 2004. Such a dramatic evolution could have severe impacts on climate change and on the oil market. The dilemma cannot be solved without creating new energy and/or transportation systems that either consume less oil or are not dependent on oil.

With a consumption of almost 20 million bbl/day, the United States is by far the world's highest-oil-consuming country (Central Intelligence Agency, 2008). As illustrated in Error! Reference source not found., the United States, with only $4.5 \%$ of the world's population, consumes almost a quarter of the world's oil.

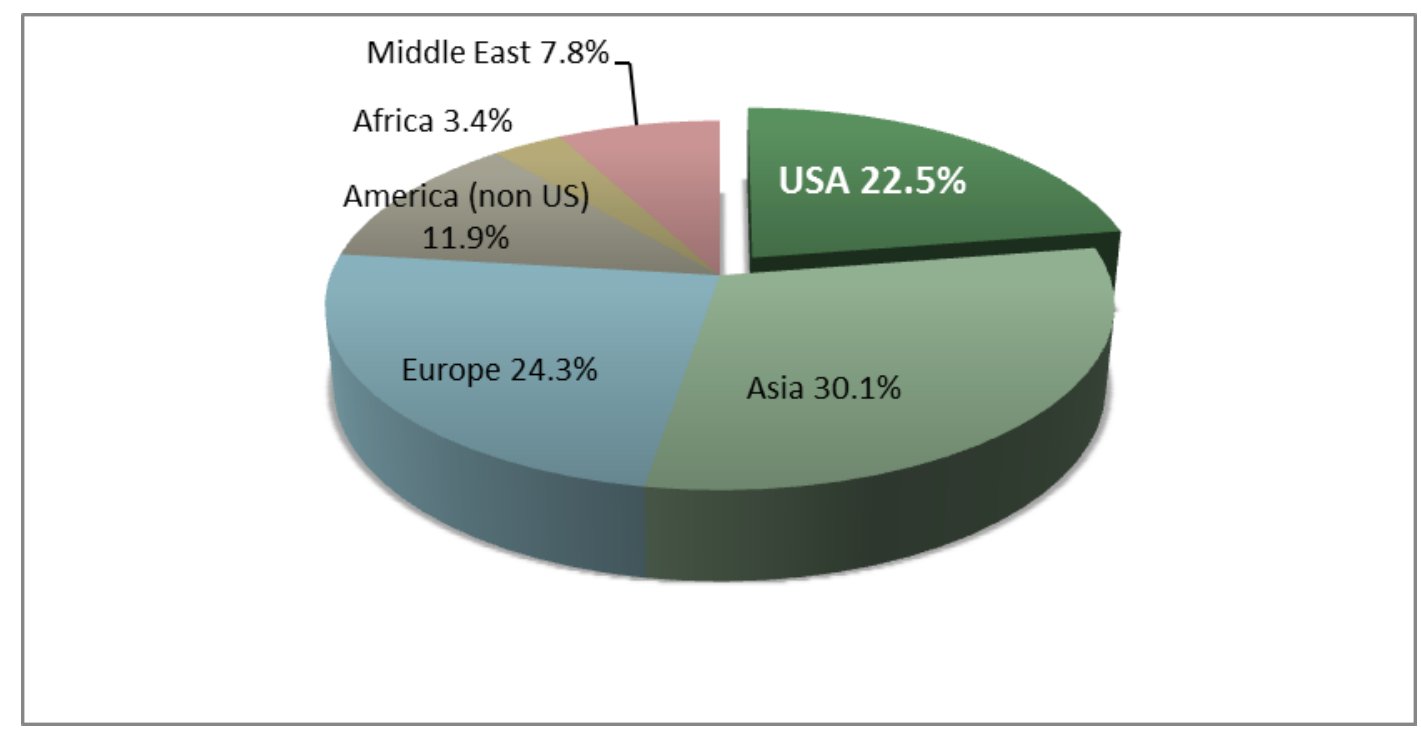

Figure 1 - Fraction of the world's oil consumption per country or continent

As shown in Figure 2, only $49 \%$ of the oil imported by the U.S. (8.1 million bbl/day) comes from the Western hemisphere, (World energy keys statistics from IEA), and the rest is imported from other regions of the world such as Africa and the Persian Gulf ( $20 \%$ and $16 \%$, respectively, of the imported oil). The unstable and unpredictable political situations in these regions have led the U.S. to focus on reducing its oil dependency through various programs in different sectors. 
Argonne National Laboratory Report - Light-Duty Vehicle Fuel Consumption Displacement Potential Up to 2045

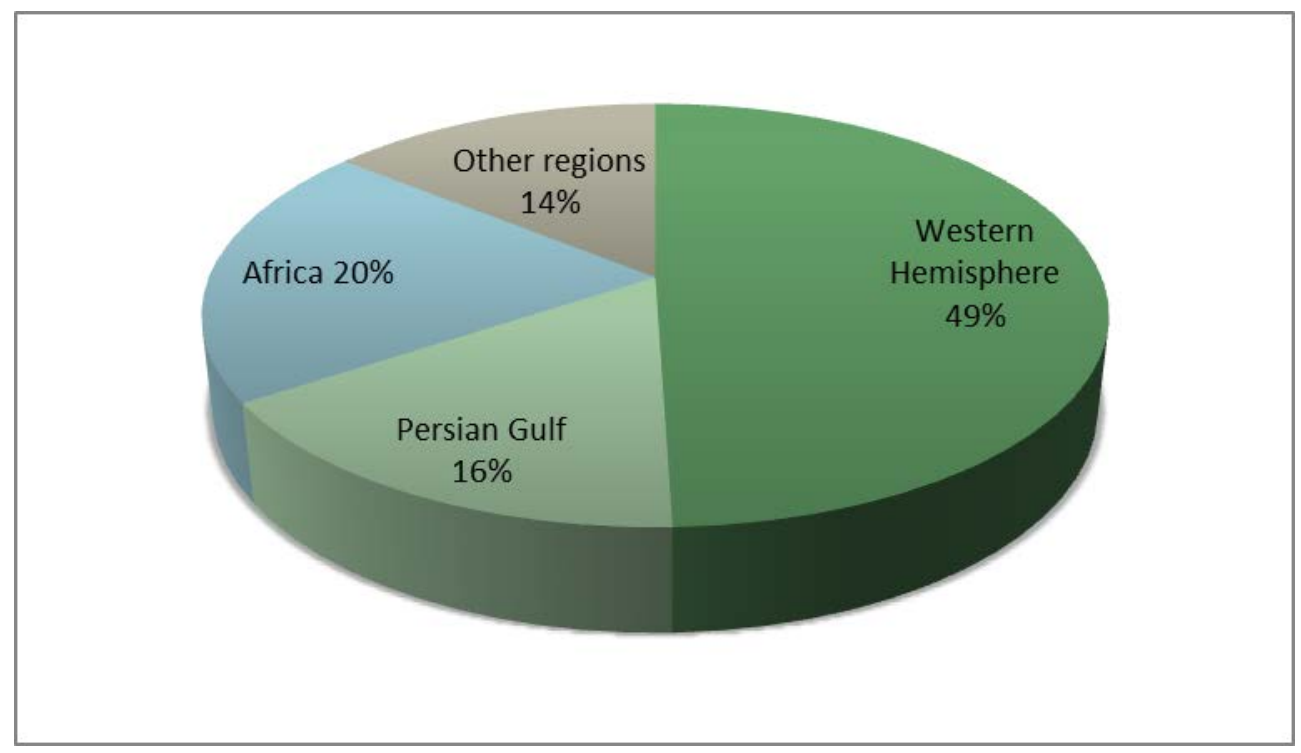

Figure 2 - Fraction of American imported oil per geographical region (International Energy Agency, 2010)

The data shown in Figures 3 and 4 separate the total energy consumption by the transportation, industry, and other sectors according to energy type (coal, natural gas, oil, nuclear, etc.). Although the development of nuclear, coal, and renewable energy could in the future help decrease the need for oil in the industrial sector, the primary focus should be the transportation sector, which is almost entirely dependent on oil as its primary energy source.
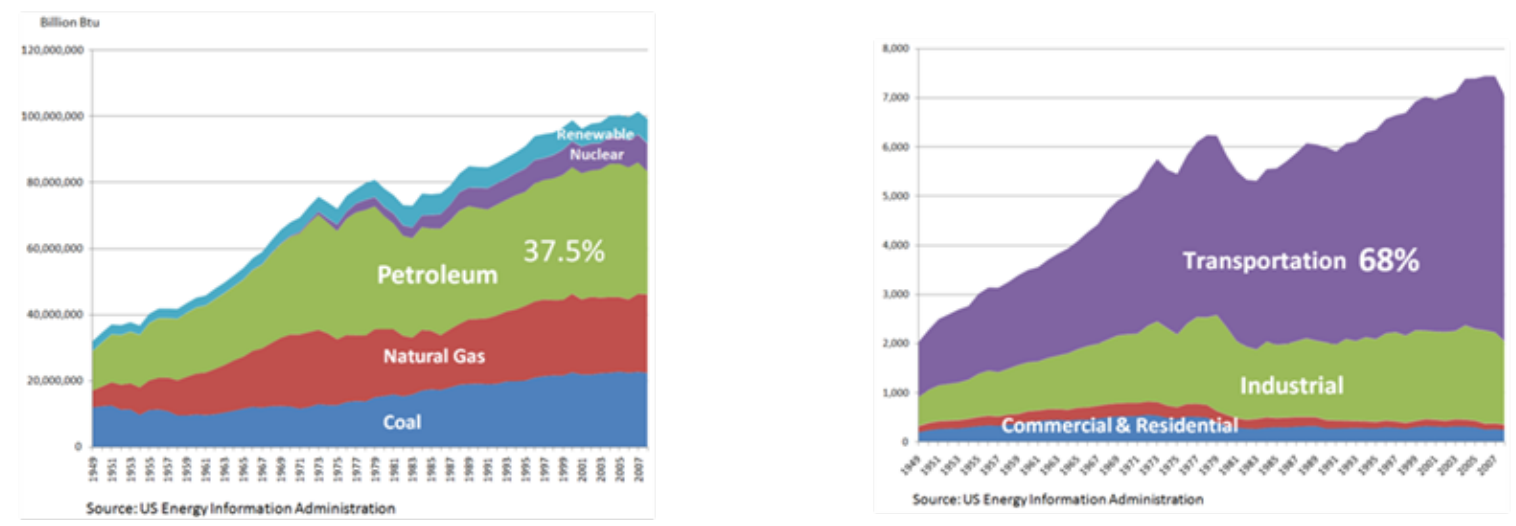

Figure 3 - U.S. consumption distribution (left) and role of transportation in global consumption (right) 
Breakdown of Sectorial Final Consumption by Source in 1973 and $2005^{\star}$

\section{United States}
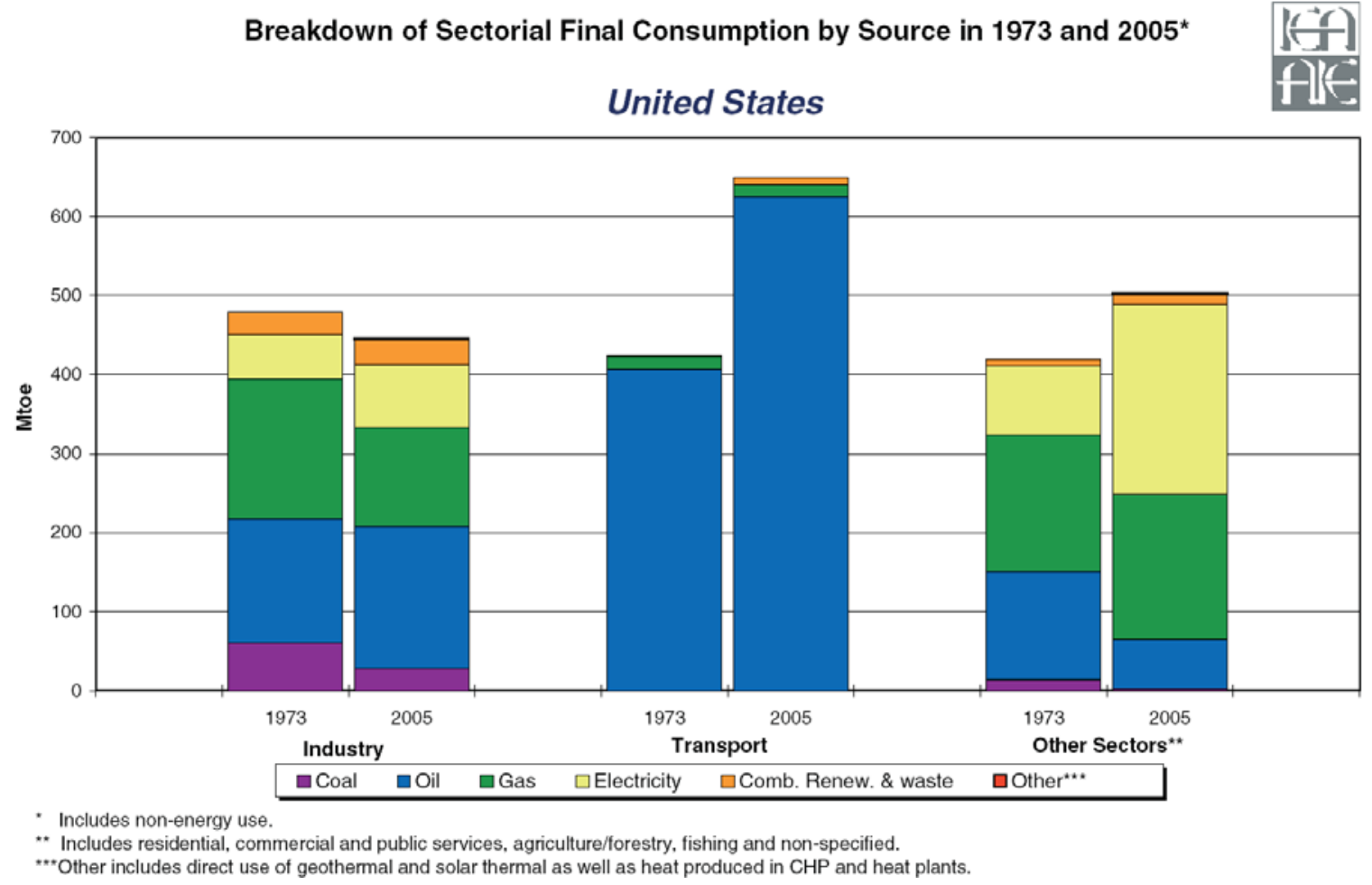

Figure 4 - Sources of energy used in major U.S. economic sectors in 1973 and 2005 (Mtoe = Million Tons of Oil Equivalent) (International Energy Agency, 2010)

Such a strong dependence on oil has important consequences for the current world energy situation. As shown in Figure 5, in July 2008, the price of oil barrel exceeded \$140/bbl, having doubled in less than a year. Consequently, the gasoline consumer price has also dramatically increased in past years, reaching the historic threshold of \$4/gallon in the United States in June 2008. Even with a huge decrease at the end of 2008, the oil barrel price remains high in 2010 compared to what it was before the crisis. 


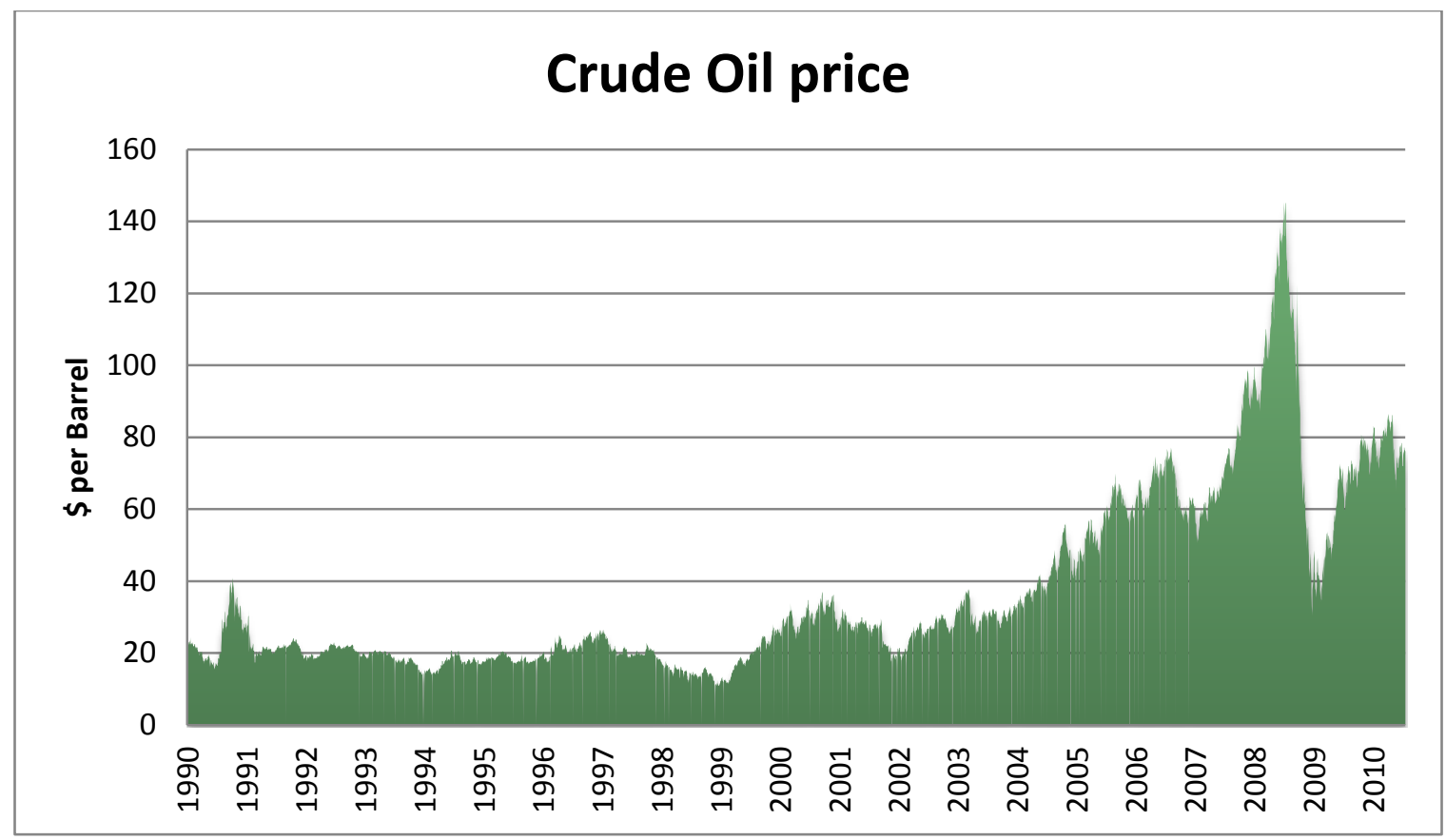

Figure 5 - Evolution of price of a barrel of oil from 1990 to 2010 [EIA]

To address the issue, the U.S. government and, in particular, the U.S. Department of Energy (DOE) have developed various projects to find alternative energy solutions for the transportation domain. Among the different possibilities that could be the key for the future, three main categories can be highlighted:

- The development of inexpensive and high-energy batteries to enable the commercialization of hybrid electric vehicles (HEVs) and plug-in hybrid electric vehicles (PHEVs). PHEVs would allow the driver to travel certain distances in electric-only mode and charge the vehicle overnight by plugging the car into the regular electric network.

- The development of biofuels such as ethanol. The current ethanol vehicles (also called flex-fuel or E85 vehicles) use a fuel made of $85 \%$ ethanol and $15 \%$ gasoline.

- The development of hydrogen/fuel-cell vehicles that would entirely eliminate oil dependency.

A DOE report (U.S. Department of Energy, 2010) states that the Obama Administration is investing in a broad portfolio of advanced vehicle technologies:

"As part of the Department of Energy's \$12 billion investment in advanced vehicle technologies, the Department is investing more than \$5 billion to electrify America's transportation sector. These investments under the American Recovery and Reinvestment Act and DOE's Advanced Technology Vehicle Manufacturing (ATVM) Loan Program are supporting the development, manufacturing, and deployment of the batteries, components, vehicles, and chargers necessary to put millions of electric vehicles on America's roads." 


\subsection{THE AMERICAN AUTOMOTIVE MARKET}

The automotive market in the United States has greatly evolved over the past 30 years. These modifications include not only the distribution of vehicle types sold but also changes in performance, weight, and thus fuel consumption for all light-duty vehicles. Prior to the oil embargo of 1973, domestic oil was inexpensive and abundant, and car companies produced large and heavy cars with powerful engines and poor fuel economy. A combination of events, including increasing public desire for better fuel economy, increasing concern about carbon emissions that resulted in state regulations on fuel economy and carbon emissions, key court decisions, and a stated desire by the federal executive branch to decrease gasoline consumption, led to increasing Corporate Average Fuel Economy (CAFE) standards, which automakers preferred to state-level regulations.

The fuel-economy evolution for both cars and trucks is shown in Figure 6. In 1975, a dramatic increase in the miles per gallon (mpg) began, and passenger-car fuel economy reached its peak in 1988, when cars averaged 24 mpg (a 71\% improvement compared to 1975). However, since 1988, fuel economy has remained constant at around $23 \mathrm{mpg}$ for cars, and if we consider all light-duty vehicles, it has even gradually declined from 1988 to 2004. Finally, since 2004, the light-duty vehicle adjusted fuel economy has increased from $20.2 \mathrm{mpg}$ in 2006 to $21.2 \mathrm{mpg}$ in 2009. Since tailpipe $\mathrm{CO}_{2}$ emissions have an inverse relationship to fuel economy, emissions showed a rapid decrease from 1975 through 1981; a slower decrease to a valley in 1987; a gradual increase until 2004; and a decrease for the six years beginning in 2005, with the largest decrease in 2009. It is interesting to see that model year (MY) 2009 had the lowest $\mathrm{CO}_{2}$ emission rate and highest fuel economy.

Adjusted $\mathrm{CO}_{2}$ Emissions by Model Year

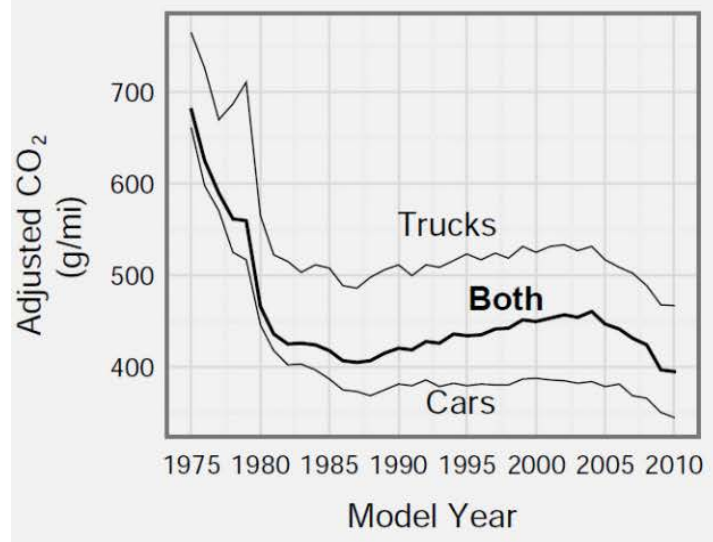

Adjusted Fuel Economy by Model Year

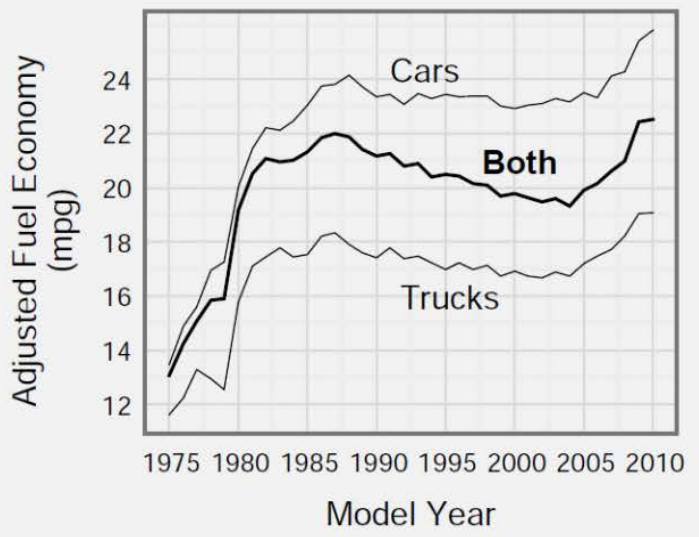

Figure 6 - Light-duty automotive technology, fuel economy, and emission trends: 1975 through 2010 (Environmental Protection Agency, 2010)

A study from the Civil Society Institute (Civil Society Institute [CSI], 2007) states that the average fuel economy of European light-duty vehicles is around $40 \mathrm{mpg}$, twice as high as in the United States. Also, CSI reports 
that, in 2008, only two cars in the U.S. (the Honda Civic Hybrid and the Toyota Prius) got 40+ mpg, whereas 113 cars in Europe could claim such an achievement. This means that more efficient vehicle technologies are available in the world, but because American customers prefer larger and more powerful cars such as pickup trucks and sport utility vehicles (SUVS), the more efficient vehicles do not penetrate the U.S. automotive market.

Figure 7 shows the U.S. sales fractions for four different classes of light-duty vehicles: cars, SUVs, vans, and pickups. If we group the last three vehicle types under the truck category, we notice that nearly half of the light-duty vehicles sold in 2007 were "trucks." In addition, the truck sales fraction has been increasing for the past 20 years. However, owing to higher gasoline prices in 2008, the pickup-truck sales fraction decreased from $13 \%$ in February 2008 to 9.1\% in May 2008 (DOE AutolnfoBank). Also, from June 2007 to June 2008, SUV sales dropped $54.7 \%$ and pickup-truck sales dropped $35.6 \%$, reflecting a deep change in consumers' behavior and expectations. Van and pickup-truck sales continued to decrease in the following years until 2009. Truck market share is now at the lowest level since model year (MY) 1995. The MY 2010 light-truck market share is projected to be $41 \%$, based on pre-MY production projections by automakers.

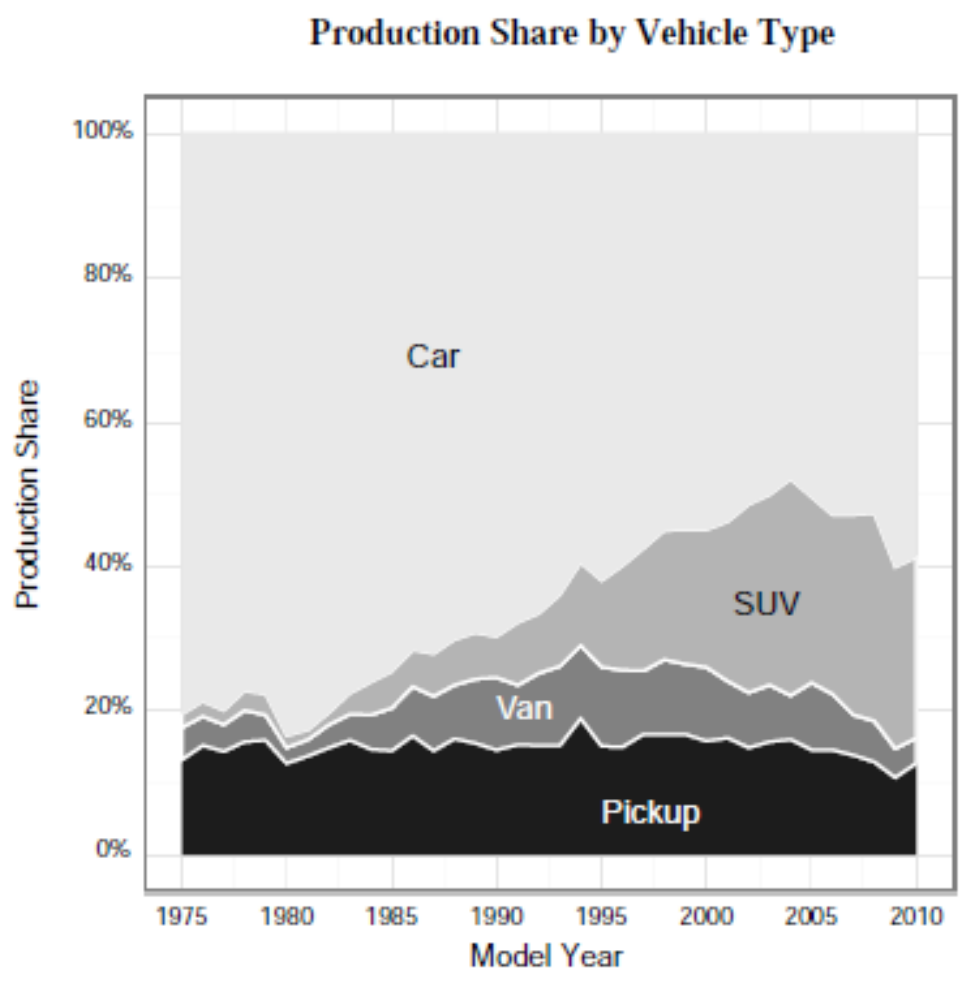

Figure 7 - Light-duty automotive technology and market share trends: 1975 through 2009 (Environmental Protection Agency, 2010) 
Argonne National Laboratory Report - Light-Duty Vehicle Fuel Consumption Displacement Potential Up to 2045

In May 2008, for the first time in 17 years, the Ford F-150 pickup truck was not the best-selling light-duty vehicle in the U.S. With 42,973 vehicles sold that month, it fell to fifth place after the Honda Accord $(43,728)$, the Toyota Camry $(51,291)$, the Toyota Corolla $(52,826)$, and the Honda Civic $(53,299)$ (Autoblog, 2008).

Vehicle weight and performance are two of the most important engineering parameters that help determine a vehicle's $\mathrm{CO}_{2}$ emissions and fuel consumption. Figure 8 shows that the MY 2009 light-vehicle weight averaged 3917 pounds, the lowest average weight since MY 2001. This weight reflects a decrease of 168 pounds (4 percent) from MY 2008, and the largest annual decrease since MY 1980. The average truck weight dropped by about 100 pounds, the average car weight dropped by about 60 pounds, and the remaining difference was due to lower truck market share. In MY 2009, the average vehicle power was 208 horsepower, the lowest value since MY 2003. Average horsepower dropped by 11 (5 percent), the largest annual decrease since MY 1980, with most of the decrease explained by cars having lower horsepower levels and trucks having a lower market share. The fourcylinder-engine market share grew from 38 percent in MY 2008 to 51 percent in MY 2009 (and to nearly 70 percent for the car market). Estimated MY 2009 0-to-60 acceleration time remained constant at 9.7 seconds.

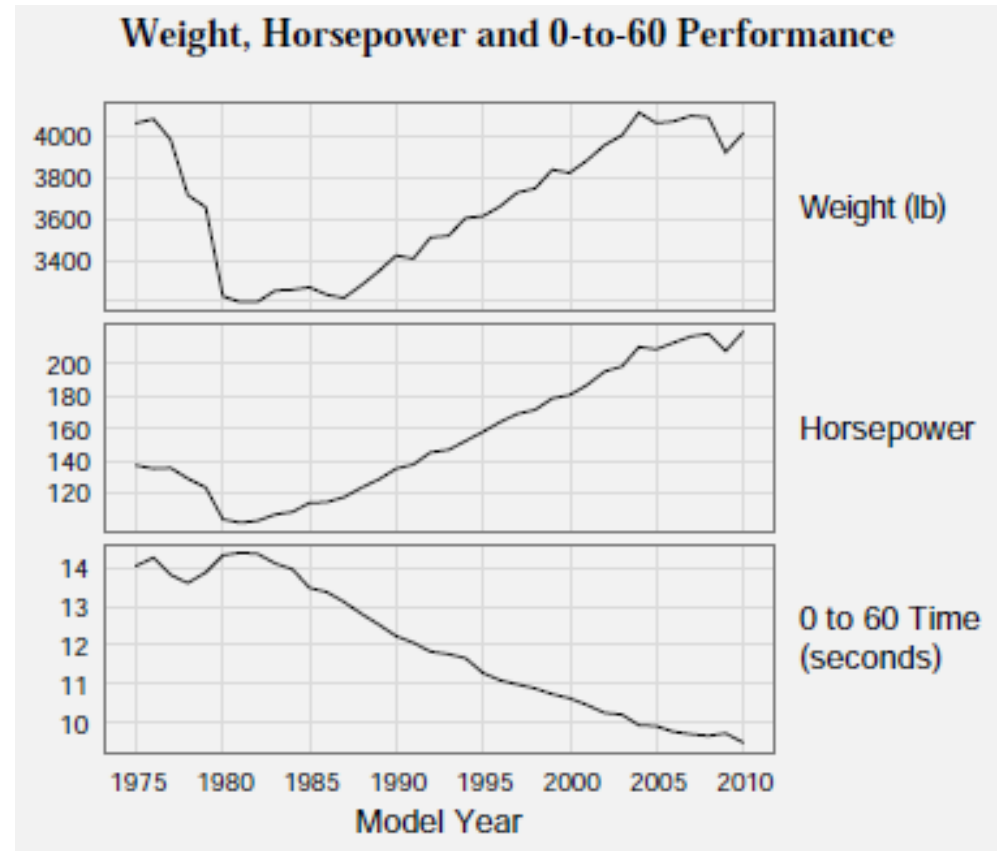

Figure 8 - Vehicle weight and performance evolution from 1975 to 2010

In summary, the American automotive market is steadily changing, with the automakers trying to adapt their light-duty vehicle offerings to consumers' needs. 
Argonne National Laboratory Report - Light-Duty Vehicle Fuel Consumption Displacement Potential Up to 2045

\subsection{HYBRID ELECTRIC VEHICLES (HEVS)}

\subsubsection{CHARACTERISTICS}

Hybrid electric vehicles are powered by at least two different sources of energy. In general, they combine an electrical storage system (i.e., battery, ultra-capacitor, etc.) and thermal source (i.e., engine, fuel-cell system, etc.).

The idea behind HEVs is to combine the advantages of conventional vehicles and battery electric vehicles (BEVs), so as to limit the drawbacks of each. EVs have excellent efficiency, owing to high electric-machine efficiency (usually above $80 \%$ average on a cycle) and low battery losses. Furthermore, they can recover part of the energy usually lost during deceleration. For electric vehicles (EVs), batteries are the critical component owing to their cost and life.

An HEV offers the following features:

- Idling stop: The engine is turned off at zero vehicle speed to avoid idling. The engine is started using the electric machine. Depending on the electrical power available, the engine is started as soon as the vehicle moves (low power) or at higher vehicle speeds (high power).

- Braking energy recovery: The energy usually wasted by friction during deceleration can be recovered as electrical energy by an electric machine. The process is often called regenerative braking, as it regenerates (part of) the energy that the vehicle had to provide to overcome the effect of inertia when accelerating.

- Low-speed electric machine propelling: When the electric machine has sufficient power, it can be used alone to propel the vehicle, to avoid operating the engine at low load and low efficiency.

- Electric machine assist: At high power demand (i.e., when accelerating), the electric machine can assist the engine, allowing downsizing as well as lower transients and emissions.

The features mentioned above are not all available for all HEVs and depend on the powertrain configurations considered. The following section provides an overview of the main families of HEVs. 


\subsubsection{PRIMARY POWERTRAIN CONFIGURATIONS}

The various HEV powertrain configurations can be classified on the basis of their hybridization degree, as shown in Figure 9. The hybridization degree is defined as the percentage of total power that can be delivered electrically. The higher the hybridization degree, the greater the ability to propel the vehicle using electrical energy.
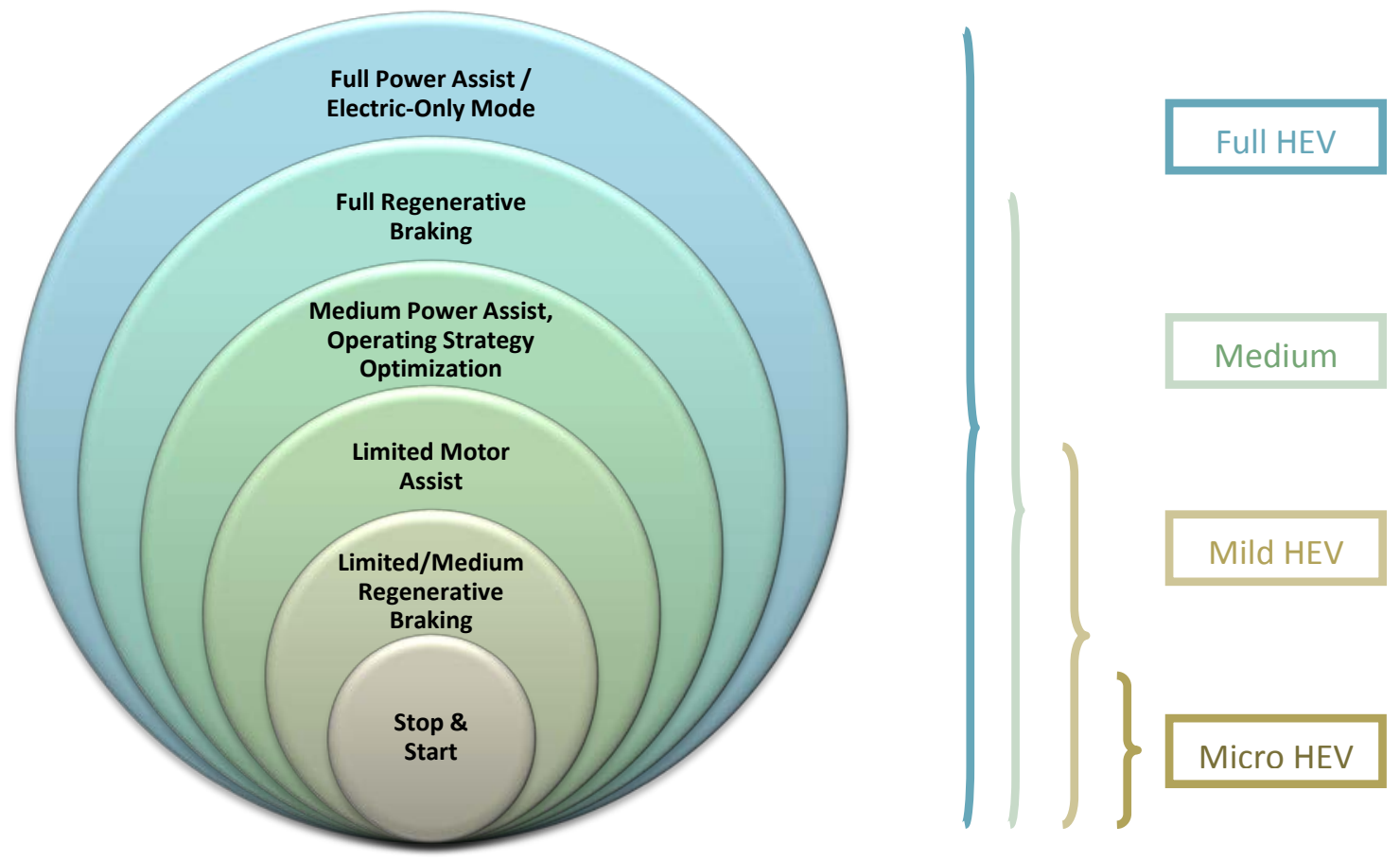

Figure 9 - Hybridization degree

There are numerous hybrid powertrain designs. The main families are described below and illustrated in Figure 10.

\section{Series Configuration}

The first hybrids were generally based on a series configuration. In this case, the vehicle is propelled solely by electrical energy. When an engine is used, it provides a generator with mechanical power, which then converts it into electricity. In the case of a fuel-cell system, the electrical energy is directly used by the electric machine. The main advantage is that the engine speed is decoupled from the vehicle speed, allowing an operating condition at or close to its most efficient operating point. The main drawback is that the main components have to be oversized to be able to maintain the same performance, leading to higher vehicle weight. Finally, the large number of components leads to a low powertrain efficiency.

\section{Parallel Configuration}


Argonne National Laboratory Report - Light-Duty Vehicle Fuel Consumption Displacement Potential Up to 2045

In a parallel configuration, the vehicle can be directly propelled by either electrical or mechanical power. Direct connection between the energy sources and the wheels leads to lower powertrain losses compared to the pure series configuration. However, since all of the components' speeds are linked to the vehicle's speed, the engine cannot constantly be operated close to its best efficiency curve.

Several subcategories exist within the parallel configuration:

- Start-stop: A small electric machine is used to turn the engine OFF when the vehicle is stopped. Examples include the Citroen $\mathrm{C3}$.

- Starter-alternator: This configuration is based on a small electric machine (usually 5 to $15 \mathrm{~kW}$ ) located between the engine and the transmission. Because of the low electric-machine power, this configuration is mostly focused on reducing consumption by eliminating idling. While some energy can be recuperated through regenerative braking, most of the negative electric-machine torque available is usually used to absorb the engine's negative torque. Examples include the Honda Civic, Honda Accord, and Citroen C3.

- Pre- and post-transmission: Both configurations allow the driver to propel the vehicle in electric-only mode as well as recover energy through regenerative braking. The electric-machine power usually ranges from 20 to $50 \mathrm{~kW}$. The main difference between these two options is the location of the electric machine (before or after the transmission). The post-transmission configuration has the advantage of maximizing the regenerative energy path by avoiding transmission losses. On the other hand, the pre-transmission configuration can take advantage of different gear ratios that allow the electric machine to operate at higher efficiency and provide high torque for a longer operating range.

\section{Power-Split Configuration}

The power-split configuration, composed of an engine and two electric machines, allows both parallel and series paths. The main feature is that all component speeds are decoupled, allowing a higher degree of control.

Each configuration is represented in Figure 10. 


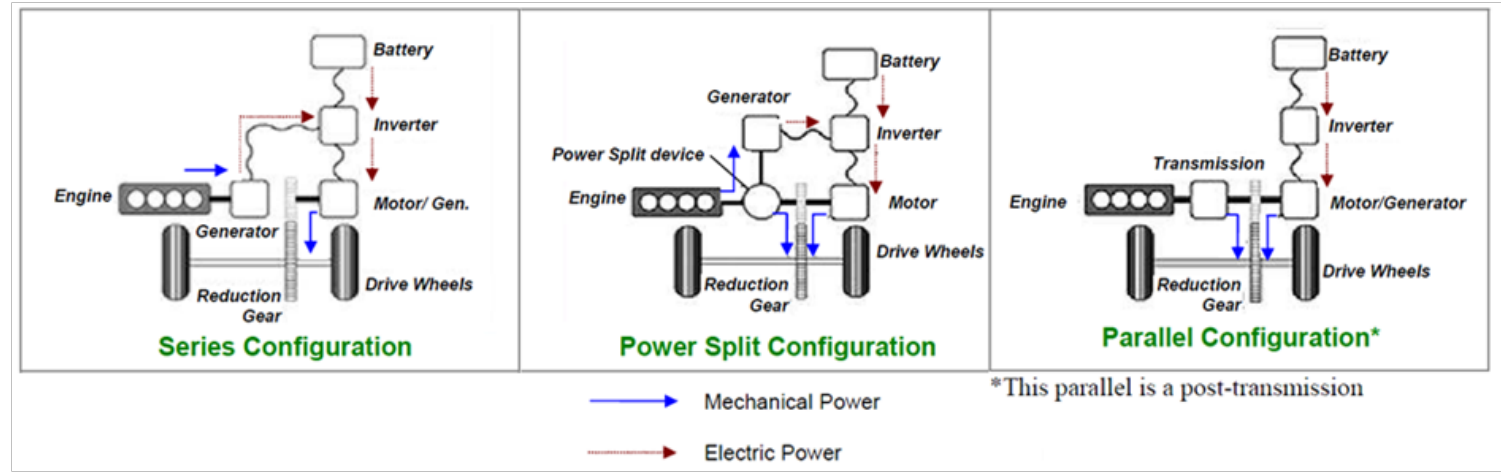

Figure 10 - Hybrid powertrain configurations

It is important to note that many different variations exist within each configuration (i.e., power-split configurations can be single-mode, two-mode, three-mode, etc.) and between configurations (i.e., several configurations are considered to be a mix of series, parallel and/or power-split). Overall, several hundred configurations are feasible for electric-drive vehicles.

\subsubsection{THE HEV MARKET}

Figure 11 shows the evolution of the hybrid-vehicle market from 1999 to November 2010. The first HEV introduced in the American market was the Honda Insight in 1999, a small two-seater with an aggressive design that has had limited success despite its excellent fuel economy (49 mpg city and $61 \mathrm{mpg}$ highway in MY 2000, according to the U.S. Environmental Protection Agency [EPA]).

Toyota released its first Prius in the U.S. in 2000, and Honda released its hybrid version of the popular Civic in 2002. After a redesigned, larger version of the Prius was released in 2004, Prius sales significantly increased and exceeded sales of the hybrid Civic. 


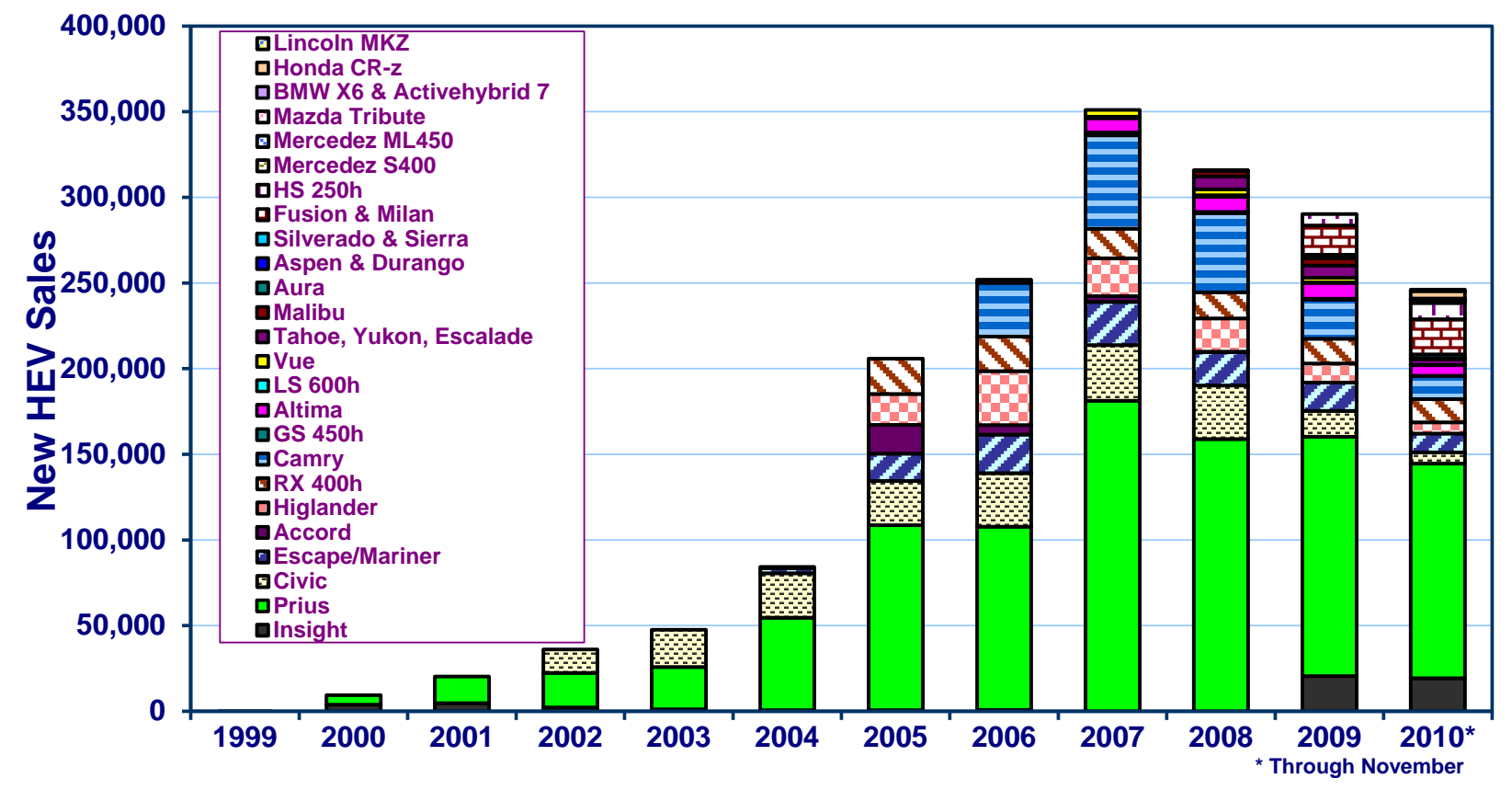

Figure 11 - HEV sales in U.S. from 1999 to November 2010 (Source - Anant Vyas, personal communication)

Since 2000, the hybrid vehicles offered have expanded across multiple carmakers and vehicle classes. Some SUVs (e.g., Toyota Highlander, Ford Escape, Tahoe HEV, etc.) are now hybrid vehicles. In 2007, hybrid-vehicle sales increased by $38 \%$ compared to 2006 and represented $2.2 \%$ of the total vehicle sales in the U.S. Several reasons explain the decrease of hybrid-vehicle sales since 2007:

1. Total vehicle sales decreased during that period.

2. Economic conditions made people cautious about investing in a more expensive technology.

3. The price of fuel dropped.

The results can be seen clearly in Figure 12 . 
Argonne National Laboratory Report - Light-Duty Vehicle Fuel Consumption Displacement Potential Up to 2045

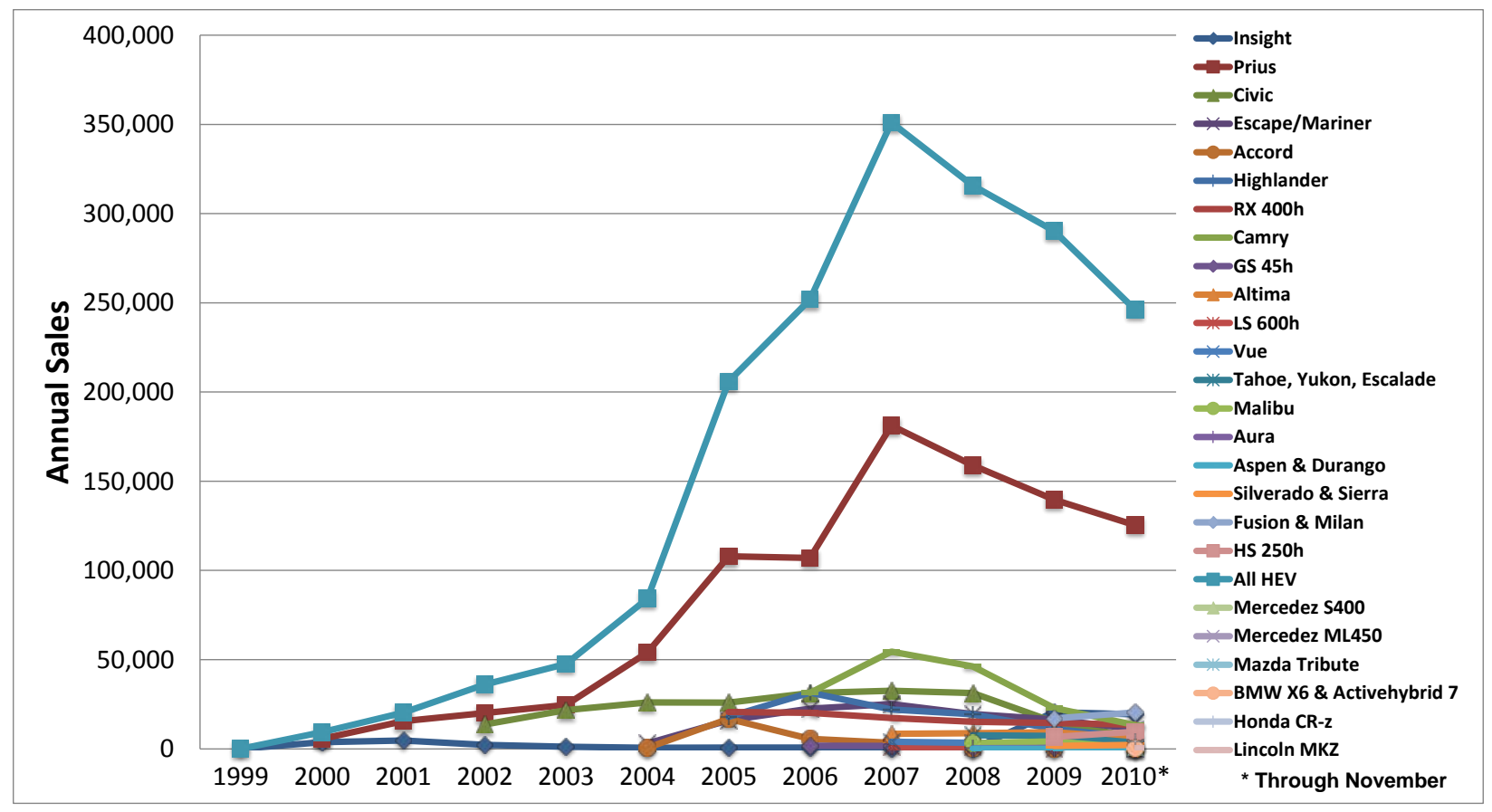

Figure 12 - U.S. HEV sales trends from 1999 to November 2010 (Source - Anant Vyas, personal communication)

As shown in Figure 13, hybrid vehicle sales also correlate with gasoline prices, since people are more likely to invest in an electric-drive vehicle if gasoline prices are high. For example, between April 2008 and October 2008, the U.S. average gasoline price went down from $\$ 4.10$ per gallon to $\$ 1.80$ per gallon. Simultaneously, hybridvehicle sales decreased by more than 50\%, with only 15,000 vehicles sold in January 2009.

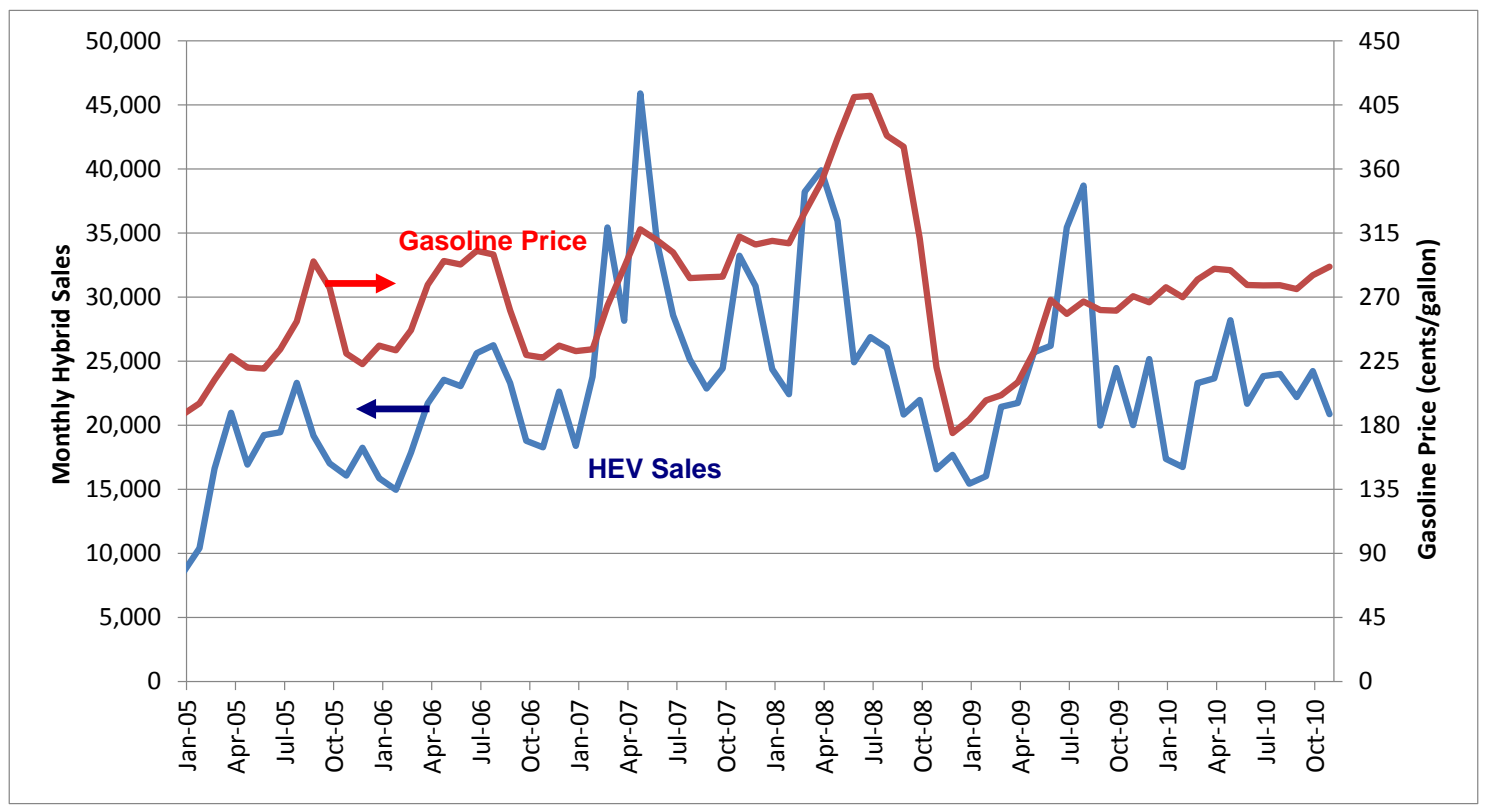


Argonne National Laboratory Report - Light-Duty Vehicle Fuel Consumption Displacement Potential Up to 2045

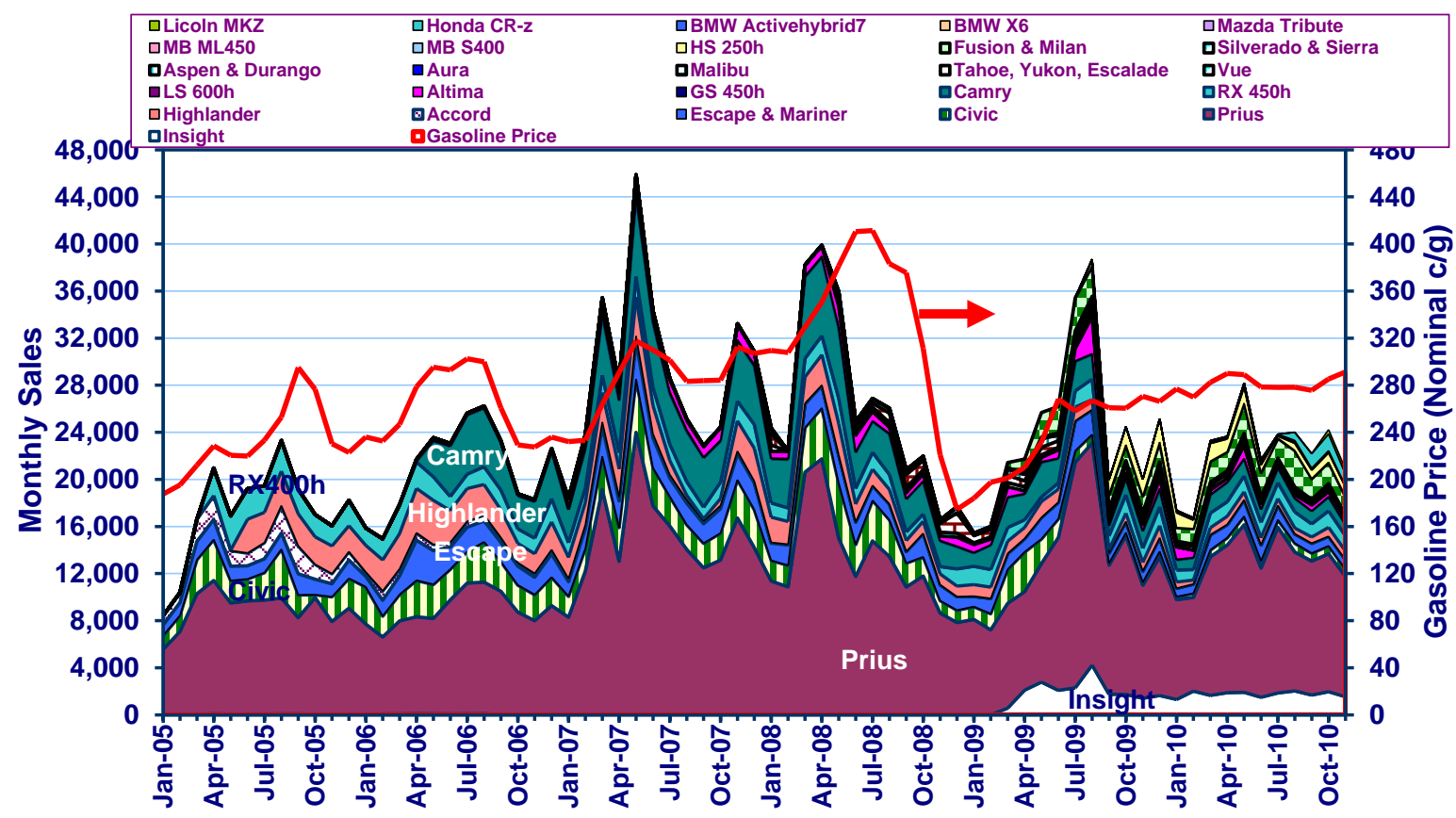

Figure 13 - U.S. gasoline prices and HEV sales, 2005-2010 (Source - Anant Vyas, personal communication)

Figure 14 shows worldwide sales of various types of hybrid vehicles as percentages of total sales from 1999 to 2010. In 2009, 4.4\% of cars (midsize and large) were HEVs, whereas roughly 1\% of light trucks (SUVs, trucks, vans) were HEVs. In total, 290,000 hybrid vehicles were sold worldwide in 2009 and cumulative sales data show that slightly more than 1,612,000 hybrid vehicles have been sold since HEVs have been on the market.

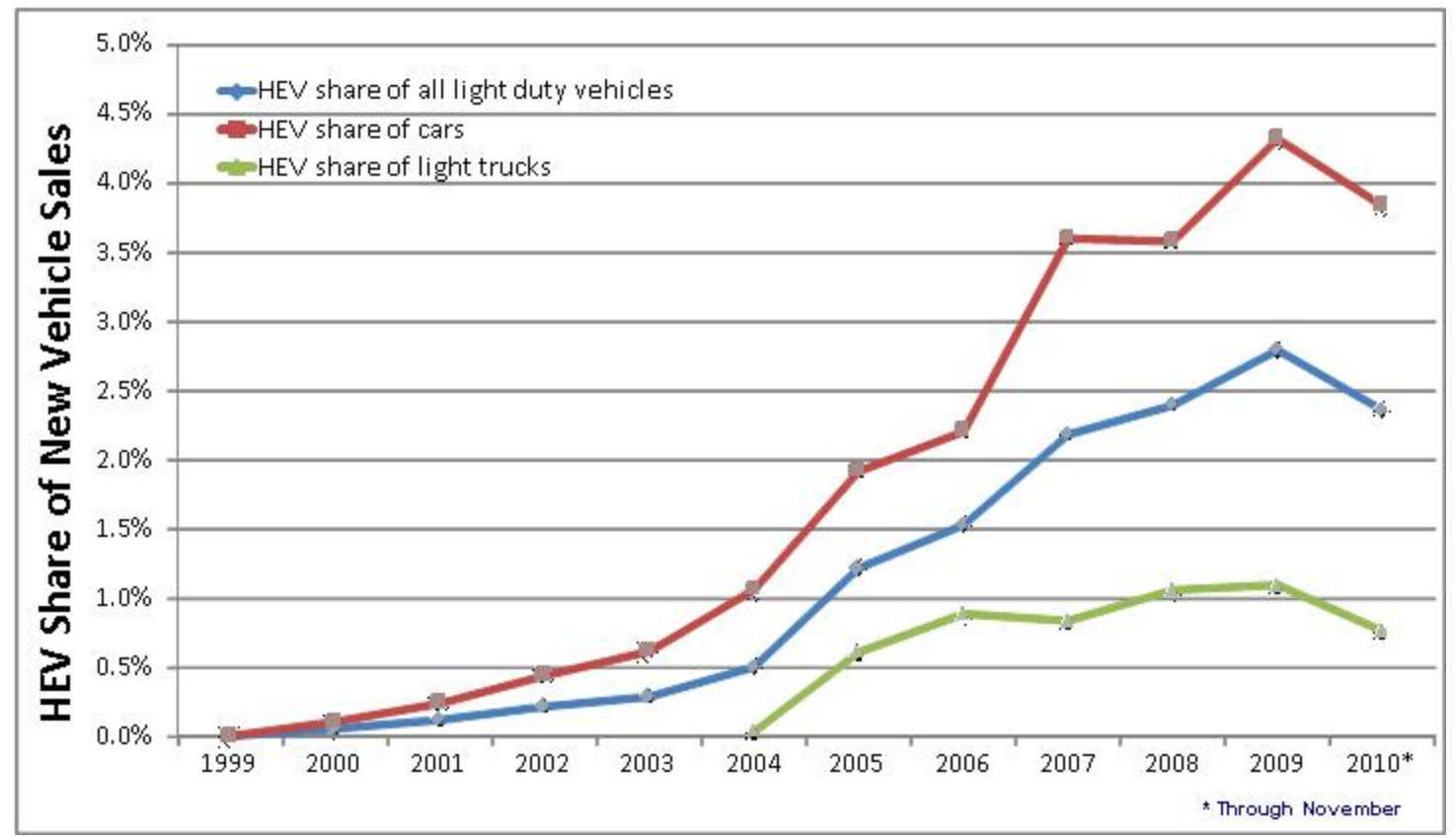

Figure 14 - HEV car and light-truck sales as share of worldwide vehicle sales (Source - Anant Vyas, personal communication) 


\subsection{PLUG-IN HYBRID ELECTRIC VEHICLES}

\subsubsection{DEFINITION AND CHARACTERISTICS}

A plug-in hybrid electric vehicle (PHEV), also called grid-connected HEV or extended-range EV (E-REV), is a HEV whose batteries can be charged from a wall-outlet. In other words, the energy storage system can be plugged into an external electric-power source. Owing to their ability to be recharged from an outlet, PHEV batteries have a lower power-to-energy ratio compared to their HEV counterparts. In addition, the increase in energy capacity for PHEV batteries vs. HEV batteries is more substantial than the increase in power requirements for PHEV batteries vs. HEV batteries. Their higher energy and power allow extended usage of the electric-only mode, leading to fewer engine ON/OFF cycles. While the engine is started at a power demand of $\sim 7-9 \mathrm{~kW}$ at the wheel for most HEVs, PHEVs offer the ability to start it later, at from 10 to $30 \mathrm{~kW}$, depending on the battery's available energy, its state of charge (SOC), and the trip distance.

Because of their ability to operate mostly in all-electric mode, PHEVs offer a very promising solution to fuel displacement. PHEVs share the same powertrain components as HEVs, with a higher hybridization degree. However, the vehicle's ability to operate in electric mode requires different energy storage system technology compared to current hybrids:

- Higher energy: the batteries have higher capacity and discharge range, as a function of all-electric-mode range $(A E R)$;

- Higher power: the electric system is in general more powerful, to be able to be the only source of power in a wider range of situations; and

- Increased control freedom: the higher degree of hybridization allows a greater number of possible electric-machine/engine-power combinations, leading to significant added complexity in determining the optimal control strategy compared to HEVs.

The DOE envisions a key future role for HEVs and PHEVs in reducing oil consumption and enabling a dramatic shift from petroleum-based transportation fuels to electricity, taking advantage of U.S. investments in renewable energy that will result in a flexible, clean and reliable power generation and distribution system in the future. A draft plan was released in June 2007 (U.S. Department of Energy, 2007); it was based on early benchmark testing of PHEV conversion vehicles (i.e., stock hybrids with added battery capacity and control modifications), vehicle modeling and simulation, and the status of batteries, power electronics, and electric motors in the DOE technology R\&D programs. 


\subsubsection{CHALLENGES}

\section{Battery Technology}

Batteries are the most critical technology for PHEVs. They are characterized by

- High capacity, for greater AER (charge depleting [CD]);

- High power, to meet the power demand in all-electric mode;

- High range of state-of-charge (SOC) use; while conventional hybrids use between 10 and $15 \%$ of the total battery energy, PHEVs use a larger percentage (e.g., 65\% for the GM Volt or higher for other prototypes);

- Longer continuous periods of discharge and recharging; and

- Thermal management issues (heating).

The last two points have a major impact on battery life and performance variation.

Lithium batteries are believed to be the best solution at the present time, as they possess twice the specific energy of nickel metal hydride $(\mathrm{NiMH})$ batteries. However, they require sophisticated batterymanagement systems and significant circuitry to prevent overcharging and over-discharge. Some safety problems remain to be solved. Further battery R\&D is still needed before long-life, compact, and inexpensive batteries are available. The future market penetration of PHEVs will depend greatly on the success of battery R\&D. Figure 15 shows the current status of lithium-ion (Li-ion) batteries compared to DOE goals. 


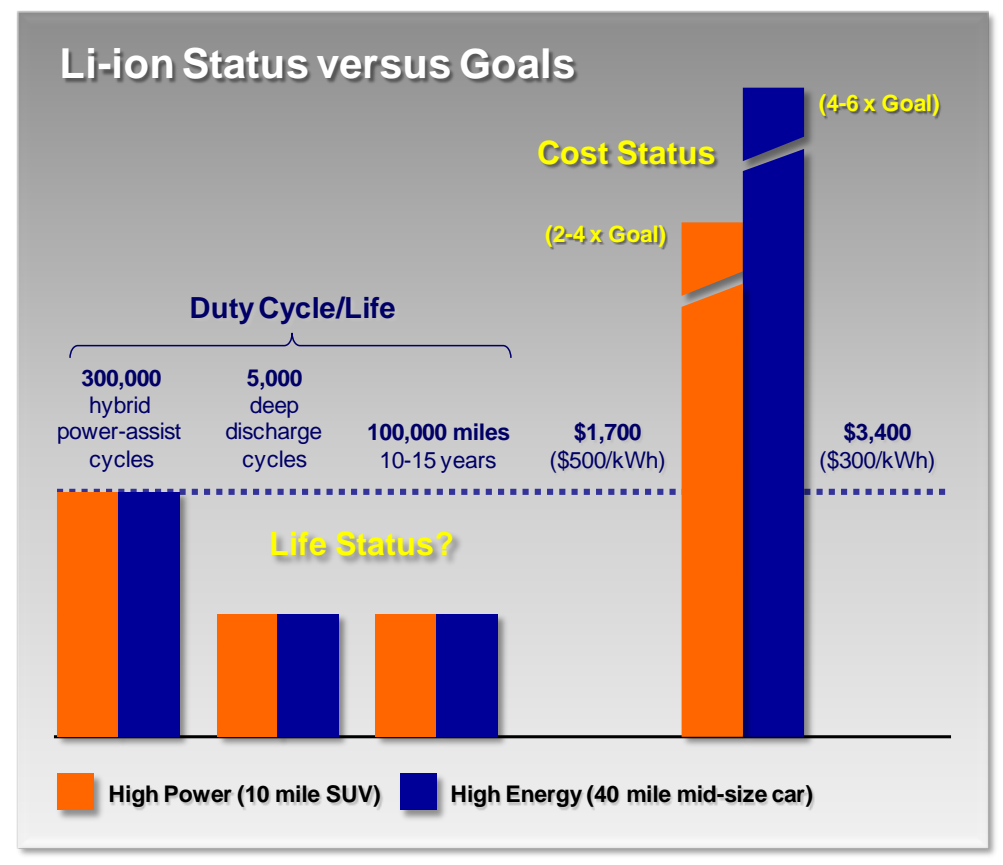

Figure 15 - Status of Li-lon batteries vs. DOE goals (Source - Howell, 2008)

\section{Electric Machine and Power Electronics}

The electric-drive system entails substantial technical and economic challenges as well. PHEVs with full performance (i.e., competitive acceleration and top speed) in electric mode require power electronics and electric motors with twice the power of today's hybrids-at lower cost (this requirement is, however, not considered a "showstopper"). And the need for "smart" onboard battery chargers (to ensure efficient and cost-effective recharging) adds more pressure for cost reduction.

\section{Vehicle System}

Special attention has to be paid to the design of the vehicle as a system. Because of the existence of two power sources, multiple design and control choices affect overall efficiency. For PHEVs, one of the key questions is the degree of hybridization, and more particularly, the "zero emission mode." Will the electric machine provide all the mechanical power or will it be assisted by the intermittent use of the engine? If so, what will be the level of this assistance? Each possible solution has to be considered, and environmental/economic benefits assessed. Several studies have been published to assess the impact of powertrain configuration and component sizing on fuel efficiency (Sharer, 2007; Fellah et al., 2009), but more work needs to be performed.

Advanced energy management control strategies will provide the opportunity to use the vehicle at its optimum efficiency (Karbowski et al., 2006; Rousseau and Moawad, 2010), but additional applied R\&D will be required. One of the current research approaches is to adapt the control strategy to the trip conditions and even to the driver's behavior through global optimization. 
Argonne National Laboratory Report - Light-Duty Vehicle Fuel Consumption Displacement Potential Up to 2045

The ultimate goal is to develop a vehicle that would be beneficial from a macroscopic as well as a customer point of view. Driving habits and patterns have to be reviewed to design one or several types of PHEVs that would be potentially adapted to a significant number of customers.

\section{Charging Infrastructure}

Charging at home will require appropriate circuits: $220 \mathrm{~V}$ AC is preferred for Level 2 charging of vehicles with longer electric ranges, compared to the standard U.S. household receptacle voltage of $110 \mathrm{~V}$. Innovative solutions will have to be found to provide convenient charging locations (e.g., in garages, parking lots and other structures) for those who do not have private garages.

In order to prepare for a significant market penetration of PHEVs, utilities have to develop their generation facilities as well as specific management of their generation, transmission, and distribution assets to balance the impact of PHEVs on the grid. Specific generation sites can also be developed, such as wind turbines for night charging, or "solar" parking for day-charging. The possibility of bidirectional energy flow between the grid and PHEVs will require communication systems to optimize the leveling effect. Numerous studies have been performed and are currently under way to examine these issues (Morrow et al., 2008).

\subsection{INPUT DEVELOPMENTS}

The inputs for the present study (i.e., component assumptions, control strategies, vehicle technical specifications, sizing algorithms, etc.) were developed over several years through numerous discussions with both components and systems experts.

To define an assumption, several experts were contacted independently to provide their inputs related to their area of expertise for each uncertainty and timeframe considered. At least three experts were contacted before defining an input. 


\section{METHODOLOGY}

\subsection{AUTONOMIE OVERVIEW}

Many of today's automotive control-system simulation tools are suitable for simulation, but they provide rather limited support for model building and management. Setting up a simulation model requires more than writing down state equations and running them on a computer. With the introduction of electric-drive vehicles, the number of components that can populate a vehicle has increased considerably, and more components translate into more possible drivetrain configurations. In addition, building hardware is expensive. Traditional design paradigms in the automotive industry often delay control-system design until late in the process-in some cases requiring several costly hardware iterations. To reduce costs and improve time to market, it is imperative that greater emphasis be placed on modeling and simulation. This only becomes truer as time goes on because of the increasing complexity of vehicles and the greater number of vehicle configurations.

Because of the large number of possible advanced vehicle architectures and time and cost constraints, it is impossible to manually build every powertrain configuration model. As a result, processes have to be automated.

Autonomie (Argonne National Laboratory, 2011a; Rousseau, n.d.) is a MATLAB ${ }^{\circledR}$-based software environment and framework for automotive control-system design, simulation, and analysis. The tool is designed for rapid and easy integration of models with varying levels of detail (low to high fidelity) and abstraction (from subsystems to systems and entire architectures), as well as processes (calibration, validation, etc.). Developed by Argonne in collaboration with General Motors, Autonomie was designed to serve as a single tool that can be used to meet the requirements of automotive engineering throughout the development process from modeling to control. Autonomie was built to accomplish the following:

- Support proper methods, from model-in-the-loop, software-in-the-loop, and hardware-in-theloop to rapid-control-prototyping;

- Integrate math-based engineering activities through all stages of development, from feasibility studies to production release;

- Promote re-use and exchange of models industry-wide through its modeling architecture and framework;

- Support users' customization of the entire software package, including system architecture, processes, and post-processing;

- Mix and match models of different levels of abstraction for execution efficiency with higherfidelity models where analysis and high-detail understanding is critical; 
Argonne National Laboratory Report - Light-Duty Vehicle Fuel Consumption Displacement Potential Up to 2045

- Link with commercial off-the-shelf software applications, including GT-Power ${ }^{\circ}$, AMESim $^{\circ}$, and CarSim $^{\odot}$, for detailed, physically-based models;

- Provide configuration and database management; and

- Protect proprietary models and processes.

By building models automatically, Autonomie allows the simulation of a very large number of component technologies and powertrain configurations. Autonomie can

- Simulate subsystems, systems, or entire vehicles;

- Predict and analyze fuel efficiency and performance;

- Perform analyses and tests for virtual calibration, verification, and validation of hardware models and algorithms;

- Support system hardware and software requirements;

- Link to optimization algorithms; and

- Supply libraries of models for propulsion architectures of conventional powertrains as well as electric-drive vehicles.

Autonomie will be used to assess the fuel consumption and cost of advanced powertrain technologies. Autonomie has been validated for several powertrain configurations and vehicle classes using Argonne's Advanced Powertrain Research Facility (APRF) vehicle test data (Kim et al., 2009; Rousseau et al., 2006; Cao, 2007; Rousseau et al.,2000; Pasquier, 2001).

With more than 400 different pre-defined powertrain configurations, Autonomie is an ideal tool to analyze the advantages and drawbacks of the different options within each family, including conventional, parallel, series, and power-split hybrid vehicles. Various approaches have been used in previous studies to compare options ranging from global optimization (Karbowski et al., 2009) to rule-based control (Freyermuth, 2008).

Autonomie also allows users to evaluate the impact of component sizing on fuel consumption for different powertrain technologies (Nelson et al., 2007; Karbowski et al., 2007) as well as to define the component requirements (power, energy, etc.) to maximize fuel displacement for a specific application (Fellah, 2009; Rousseau et al., 2004). To properly evaluate any powertrain-configuration or component-sizing impact, the vehicle-level control is critical, especially for electric drives. Argonne has extensive expertise in developing vehicle-level controls based on different approaches, from global optimization [7] to instantaneous optimization (Karbowski et al., 2010), rule-based optimization (Sharer et al., 2008) and heuristic optimization (Rousseau et al., 2008).

The ability to simulate a large number of powertrain configurations, component technologies, and vehiclelevel controls over numerous drive cycles has been used to support many DOE as well as manufacturers' studies, focusing on fuel efficiency (Delorme et al., 2008), cost-benefit analysis (Rousseau, 2005), or greenhouse gases 
Argonne National Laboratory Report - Light-Duty Vehicle Fuel Consumption Displacement Potential Up to 2045

(Elgowainy et al., 2009; Wu, 2006). All the development performed in simulation can then be implemented in hardware to take into account non-modeled parameters such as emissions and temperature (Vijayagopal et al., 2010).

Autonomie is the primary vehicle simulation tool selected by DOE to support its FreedomCAR program and VTP (U.S. Department of Energy, n.d.[b]; Vehicle Systems Analysis Technical Team [2006]). Autonomie has been used for numerous studies to provide the U.S. government with guidance for future research (www.autonomie.net). More than 150 companies and research entities, including major automotive companies and suppliers, are also using Autonomie to support advanced vehicle development programs.

\subsection{STUDY PROCESS}

The process to estimate the fuel consumption of various advanced powertrains can be divided into three steps:

\section{- Define the Architecture}

The vehicle architecture is built using the different components available in the main database. In this study, each component is associated with different uncertainties (low, average, and high), which will be presented later.

\section{- Size the Components}

Algorithms are used to size the vehicle components to compare vehicles with the same vehicle technical specifications. Once the sizing is complete, all the components' features are known, and thus it is possible to estimate the retail price of the vehicle. The sizing algorithms are specific for each configuration and will be discussed later in detail.

\section{- Run the Simulation}

The third step calculates the vehicle fuel consumption by simulating the different standard U.S. test procedures.

\subsection{TIMEFRAMES AND UNCERTAINTIES}

To evaluate the fuel-efficiency benefits of advanced vehicles, each vehicle is designed from the ground up based on each component's assumptions. The fuel efficiency is then simulated using the Urban Dynamometer Driving Schedule (UDDS) and Highway Federal Emissions Test (HWFET) cycles. The vehicle costs are calculated from the component characteristics (power, energy, weight, etc.). Both cost and fuel efficiency are then used to define the market penetration of each technology to finally estimate the amount of fuel saved. The process is highlighted in Figure 16. This report will focus on the first phase of the project: fuel efficiency and cost. 


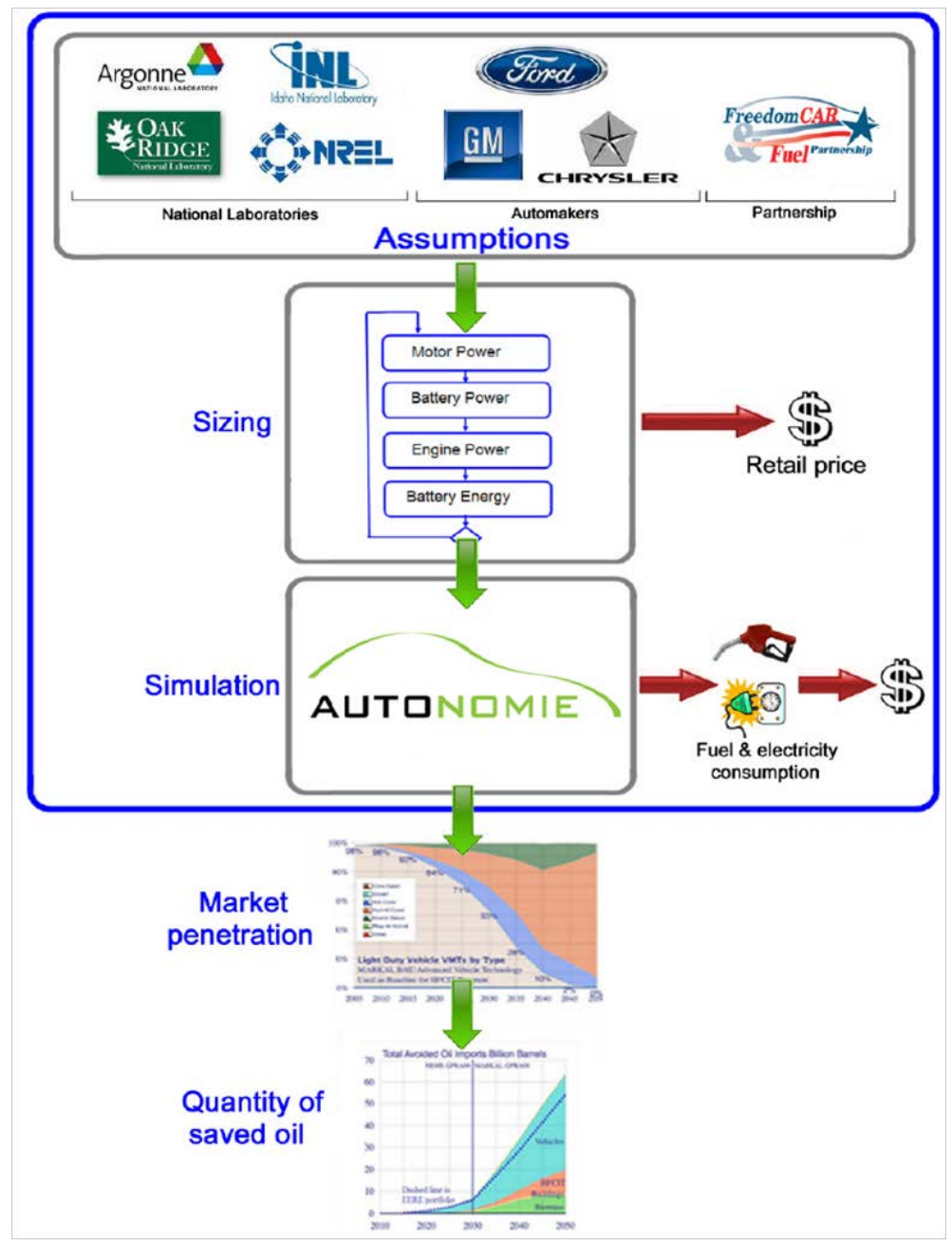

Figure 16 - Process to evaluate fuel efficiency of advanced vehicle technologies

To properly assess the benefits of future technologies, several options were considered, as shown in Figure 17:

- Five vehicle classes: compact, midsize car, small SUV, medium SUV, and pickup truck

- Five timeframes: reference (same as 2010 low), 2010, 2015, 2030, and 2045

- Five powertrain configurations: conventional, HEV, PHEV, fuel-cell HEV, and EV

- Four fuels: gasoline, diesel, ethanol, and hydrogen

- Three risk levels: low, average, and high cases. These correspond, respectively, to $10 \%$ uncertainty (aligned with original equipment-manufacturer [OEM] improvements based on regulations), 50\% uncertainty, and $90 \%$ uncertainty (aligned with aggressive technology advancement based on the DOE VTP). These levels are explained more fully below.

Overall, more than 2000 vehicles were defined and simulated in Autonomie. The study does not include micro or mild hybrids and does not focus on emissions. 


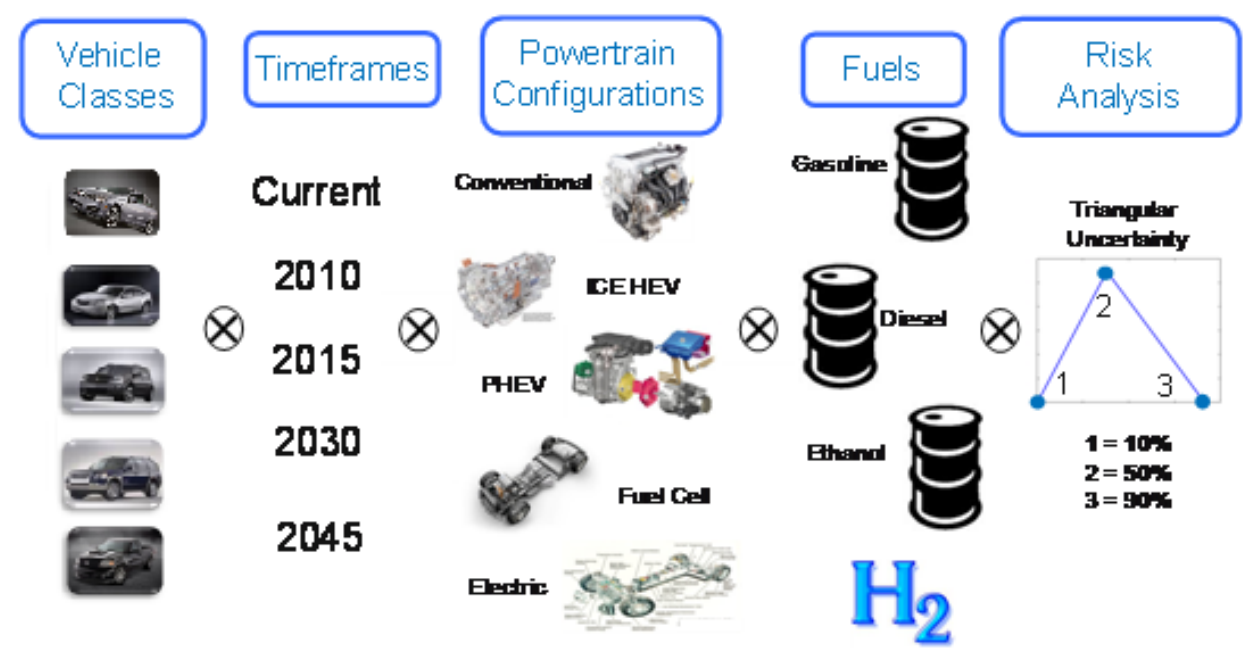

Figure 17 - Vehicle classes, timeframes, configurations, fuels, and risk levels considered

When dealing with uncertainties, numerous methodologies are available. In previous studies, Argonne has compared Monte Carlo simulation with a triangular distribution analysis (Faron et al., 2009). By allowing the introduction of uncertainty into our algorithm inputs, the Monte Carlo method increases the amount of useful information to describe a vehicle's possible behaviors. The major improvement concerns the introduction of the risk notion associated with each result. Instead of providing a single forecast value, Monte Carlo simulation provides the probability of occurrences associated with every possible output value. As a result, forecasts are more fully and accurately described and confidence intervals can be derived for each output.

The results from Monte Carlo simulations based on a midsize PHEV were defined, providing a mode for both fuel economy and cost within a certain confidence interval. The approach was then compared with the existing three-point option. Results demonstrated that, as expected, Monte Carlo simulation provided a narrower range. However, increasing the amount of information available in the results has a computational cost. The experiments carried out so far led us to a first evaluation of the number of points required to simulate. This number varies from 100 to 200 , depending on the number of uncertain inputs considered. While computational time varies for each configuration, the average time required to simulate a PHEV on all these points was 150 minutes.

Owing to the large number of vehicles considered in the study, the triangular distribution approach (low, average, and high) was employed, as shown in Figure 18. For each component, assumptions were made (i.e., efficiency, power density), and three separate values were defined to represent the 1) 90th percentile, 2) 50th percentile, and 3) 10th percentile. A $90 \%$ probability means that the technology has a $90 \%$ chance of being available at the time considered. For each vehicle considered, the cost assumptions also follow the triangular uncertainty. Each set of assumptions is, however, used for each vehicle, and the most efficient components are not 
automatically the cheapest ones. As a result, for each vehicle considered, we simulated three options for fuel efficiency. Each of these three options also has three values representing the cost uncertainties. A more detailed description of the uncertainty process is available (Henrion, 2008).

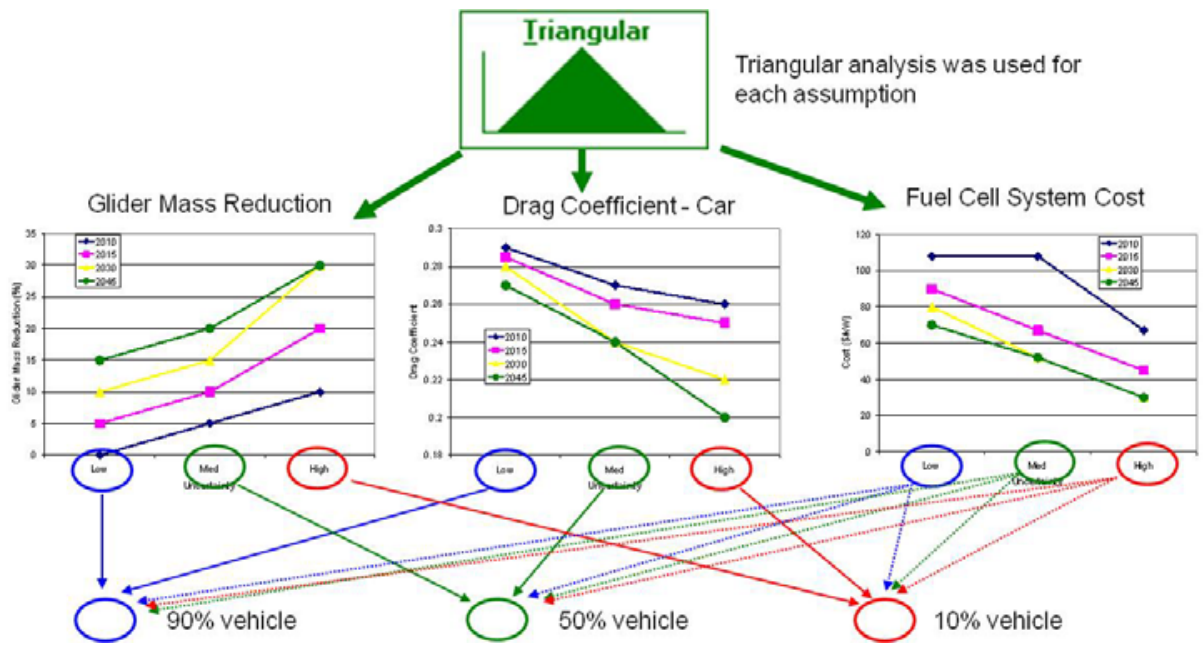

Figure 18 - Uncertainty Process Description

The "reference case" used in the study is considered to be the low-uncertainty 2010 case.

\subsection{COMPUTER CLUSTER UTILIZATION}

As shown previously, the number of combinations is very large, since a very large number of vehicles need to be sized and simulated. Taking into account that the sizing algorithms run as an iterative process, it becomes clear that a lot of simulating and calculating power is required from the computer hardware system. For such applications, a high-performance computing center is available at Argonne. It is composed of a stack of 32 calculating machines and a server computer.

An algorithm was developed for optimizing the distribution of jobs for vehicle simulations and parametric studies (Mathworks, n.d.; Pagerit, 2007). This system (Figure 19) was used to run the entire study. However, even with this powerful tool, the total simulation time is about 20 hours for sizing and 15 hours for simulation. This distributive computing operation tremendously decreased the sizing and simulation time, which otherwise would have taken many weeks. The distributed computing also greatly facilitated the reruns of simulations, which occurred numerous times during this study. 
Argonne National Laboratory Report - Light-Duty Vehicle Fuel Consumption Displacement Potential Up to 2045

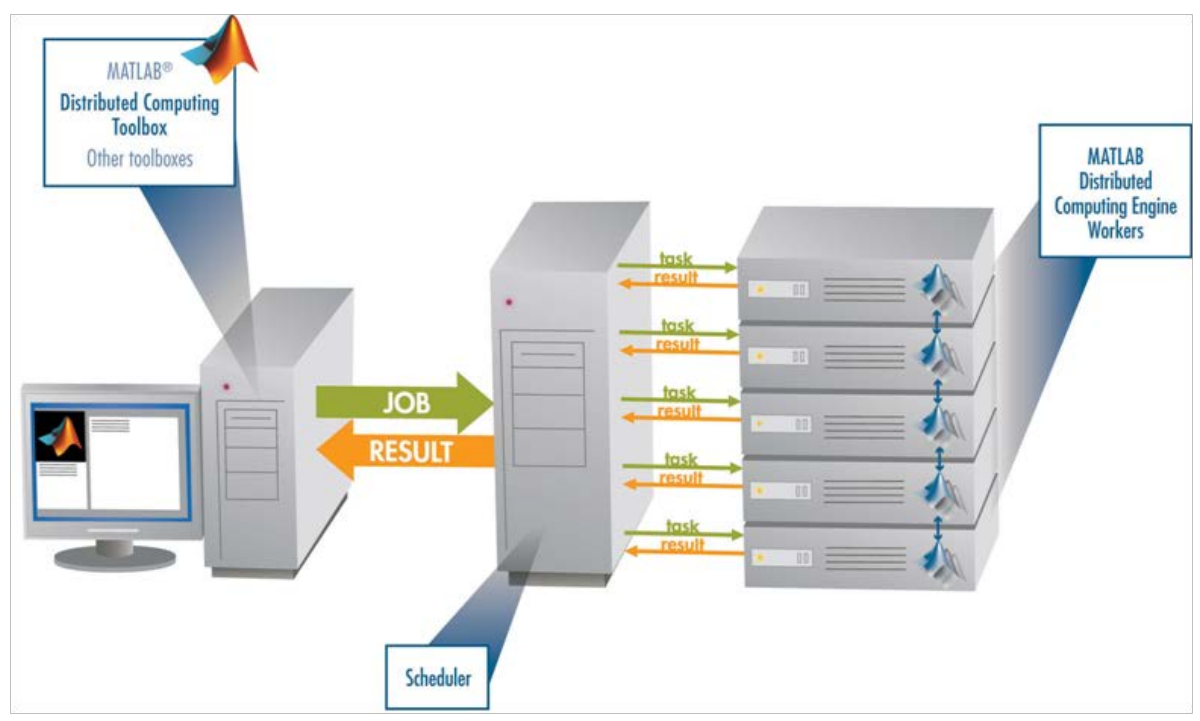

Figure 19 - Matlab distributive computing process diagram 


\section{COMPONENT ASSUMPTIONS}

The assumptions for each component were developed in collaboration with experts from the DOE, national laboratories, industry, and academia. The following paragraph represents a compromise reached by the authors of the study and should not be attributed to any specific company.

Several hundred assumptions are required to run a single vehicle simulation. Figures 20 and 21 show a short list of these assumptions for the components and vehicles, respectively. The following section only provides information regarding a very limited set of assumptions, since most of the assumptions have been provided by industry partners and are considered proprietary.

\section{Engine}

Technology

Fuel

Peak Torque

Specific Power

Efficiency

Time response

Cost...
Electric Machine

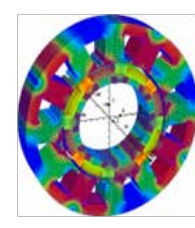

Technology

Peak Torque

Specific Power

Efficiency

Time response

Cost...

\section{Fuel Cell}

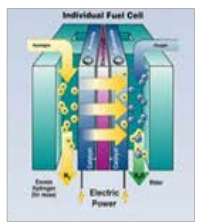

Technology

Specific Power

Efficiency

Time response

Cost...

\section{Transmission}

Technology

Gear Number

Mass

Efficiency

Cost...

\section{Energy Storage}

Technology Specific power

Power and energy oversize

Efficiency (Rint, Voc...)

SOC window

Cost...

\section{Hydrogen Storage}

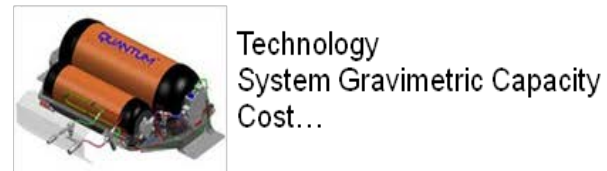

Figure 20 - Main Component Assumptions 


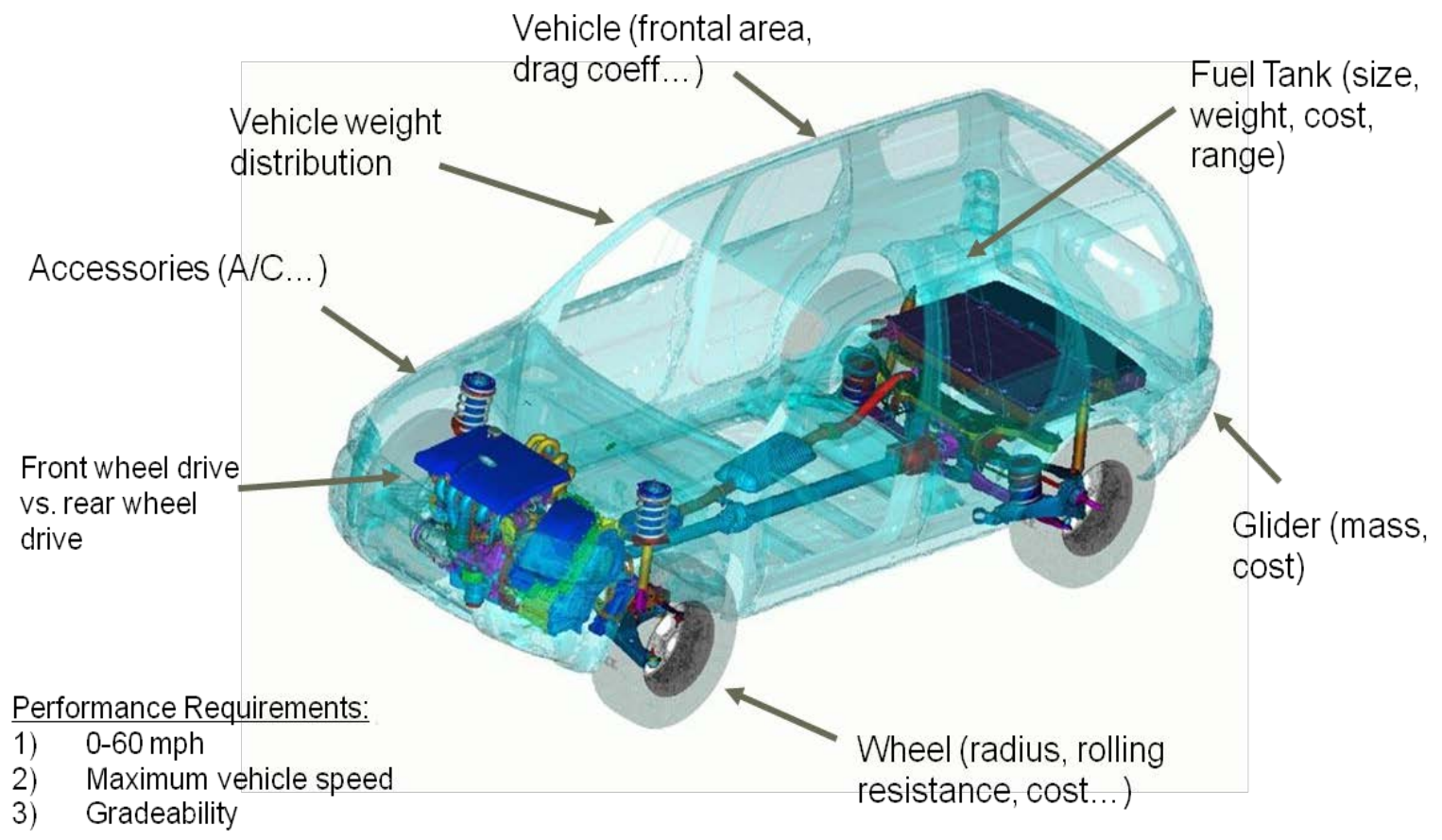

Figure 21 - Main Vehicle Assumptions

When available, the high-case assumptions are based on the FreedomCAR and Fuel Partnership program goals (Ward)The other assumptions have been developed through discussions with experts from companies, universities, and the national laboratories.

\subsection{ENGINE}

\subsubsection{REFERENCE ENGINE AND PROJECTIONS}

Several state-of-the-art internal combustion engines (ICEs) were selected as the baseline for the fuels considered: gasoline (spark ignition or $\mathrm{SI}$ ), diesel (compression ignition or $\mathrm{Cl}$ ), ethanol (E85), and hydrogen (H2). The gasoline, diesel, and ethanol engines used for reference conventional vehicles were provided by automotive car manufacturers, while the port-injected hydrogen engine data were generated at Argonne (Wallner and LohseBusch, 2007). The engines used for HEVs and PHEVs are based on Atkinson cycles, generated from test data collected at Argonne's dynamometer testing facility (Bohn, 2005). Table 1 shows the engines selected as a baseline for the study. 
Argonne National Laboratory Report - Light-Duty Vehicle Fuel Consumption Displacement Potential Up to 2045

Table 5: Definition of the baseline engines used in the present study

\begin{tabular}{|l|l|c|c|}
\hline Fuel & Source & Displacement (L) & Peak Power (kW) \\
\hline SI (Conv) & Car Manufacturer & 2.4 & 123 \\
\hline CI & Car Manufacturer & 1.9 & 110 \\
\hline H2 & Argonne & 2.2 & 84 \\
\hline E85 (Conv) & Car Manufacturer & 2.2 & 106 \\
\hline SI/E85 (HEV) & Argonne & 1.5 & 57 \\
\hline
\end{tabular}

Technologies available to increase the efficiency of engines include

- Low-friction lubricants

- Reduced engine friction losses

- Cylinder deactivation

- Variable valve timing (VVT) and variable valve lift

- Turbocharging and downsizing

- Variable compression ratio (VCR)

- Stoichiometric and lean-burn gasoline direct injection (GDI)

- Diesel engine

A literature review (Morrow et al., 2008) was performed to select the preceding technologies and define their impacts on both peak efficiency and engine map. Since all the technologies could not be represented owing to lack of data, a few technologies were selected, including low-friction lubricants, reduced friction losses, direct injection and VVT. Cylinder deactivation, turbocharging, and VCR were not included. It should be noted that several ongoing engine research projects could lead to technologies affording significantly higher fuel-efficiency gains than those considered in this study (Ciatti and Subramanian, 2010). As such, the engine gains should be considered less aggressive than gains for other technologies and this should be taken into account during the analysis.

The peak efficiencies of the different fuels and technologies are shown in Figure 22 - ICE efficiency for diesel, hydrogen, and gasoline fuels 
. Among the different ICEs, the hydrogen engine shows the most significant efficiency increase, from $38 \%$ in the reference case to $51 \%$ in the 2045 high case (a 34\% improvement), owing to the introduction of direct injection. The other engines show a lower increase in efficiency, since they are already well-developed technologies. The efficiencies of the other ICEs increase from $41 \%$ in the reference case to $45 \%$ in the 2045 high case (diesel), $38 \%$ to $45 \%$ (gasoline), and $36 \%$ to $43 \%$ (ethanol for HEV and PHEV applications). One also notes that the difference in peak efficiency between gasoline and diesel is expected to narrow in the future, owing to the combination of advanced gasoline engine technologies and the impact of ever more stringent after-treatments for diesel.

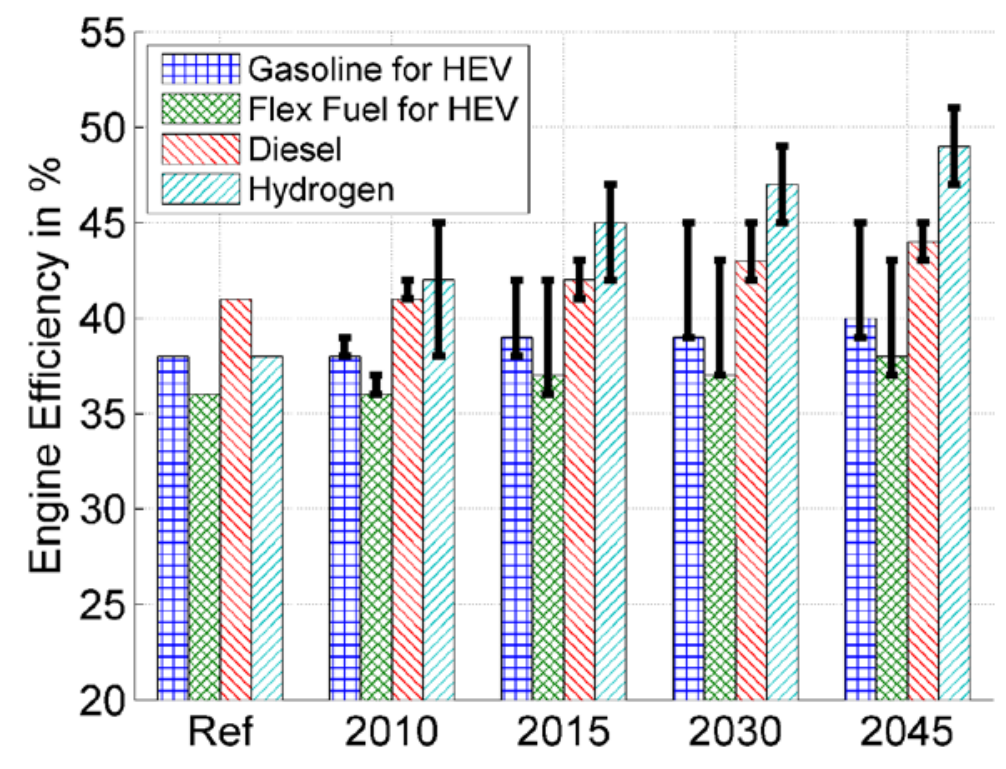

Figure 22 - ICE efficiency for diesel, hydrogen, and gasoline fuels

Figure 23 shows the evolution of the emission control systems for each fuel. Diesel emission-system costs are expected to increase in the short term, because of stricter regulations, and decrease later with improved technology. 


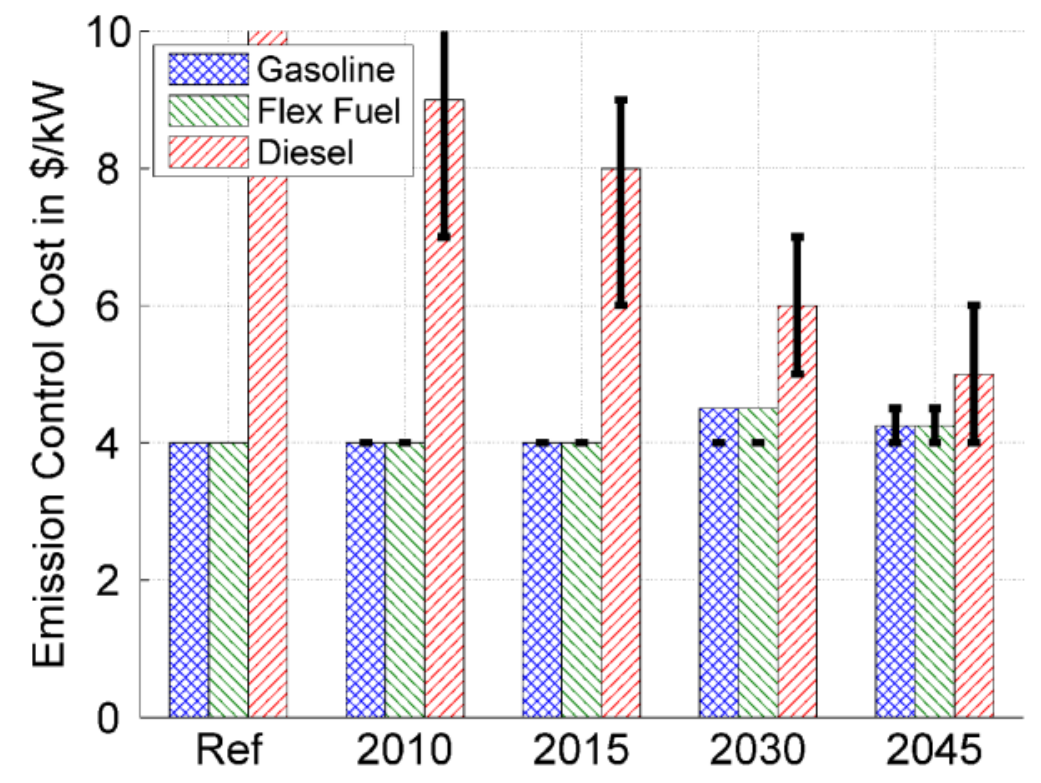

Figure 23 - Emission control costs for gasoline, diesel and Flex-Fuel (ethanol) engines 


\subsubsection{DETERMINATION OF NUMBER OF CYLINDERS}

To properly select the reference engine and calculate its cost, it is necessary to decide how many cylinders are needed for a given power.

Figure 24 shows the relationship between the number of cylinders in a gasoline engine and the peak power. This figure is based on data in the literature. On the basis of Figure 24, 4 cylinder engines were used up to a power of $140 \mathrm{~kW}, 6$ cylinder engines for a power between $140 \mathrm{~kW}$ and $220 \mathrm{~kW}$, and 8 cylinder engines for a power above $220 \mathrm{~kW}$.

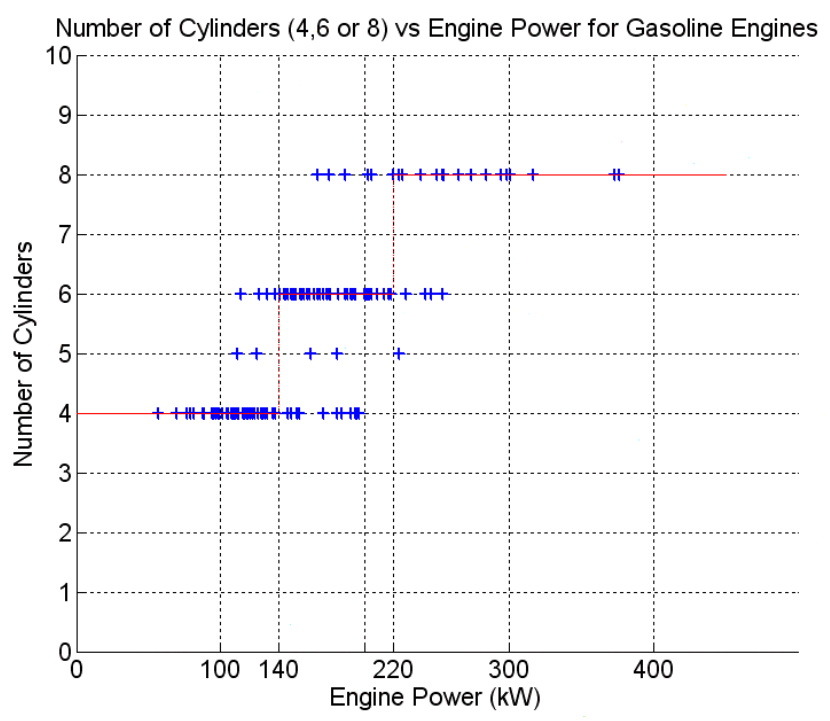

Figure 24 - Number of cylinders vs. engine power for database gasoline engines.

(Blue) values from the database; (red) thresholds chosen for the study

The same approach was taken for diesel engines, as shown in Figure 25. The small number of diesel engines in the U.S. database does not provide as clear a distribution as the gasoline case, but from the distribution shown in Figure 24, the same thresholds have been used. The hydrogen and ethanol engines will use the same cylinder/engine-power equation as the gasoline and the diesel engines. 


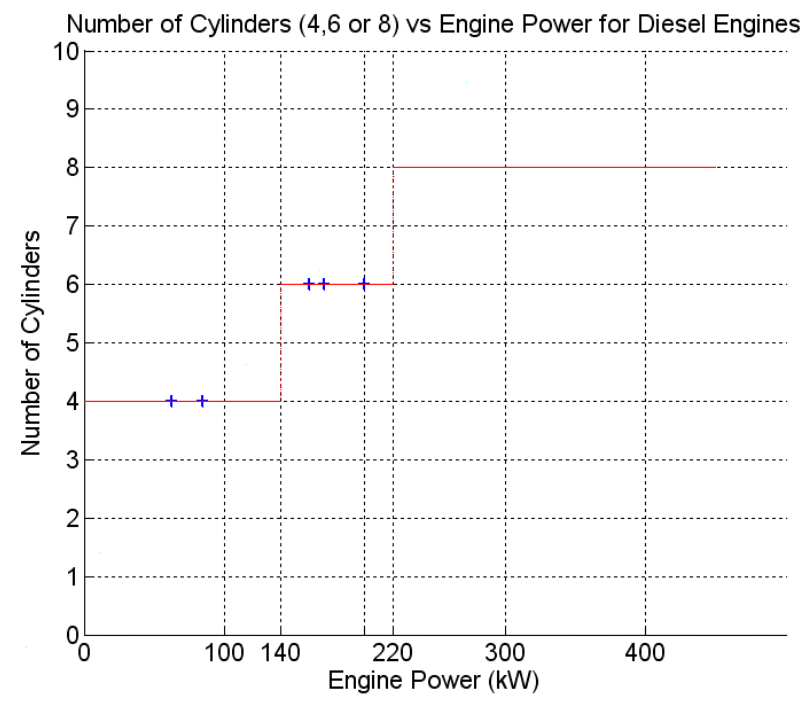

Figure 25 - Number of cylinders vs. engine power for database diesel engines. (Blue) values from the database; (red) thresholds chosen for the study.

\subsection{FUEL-CELL SYSTEM}

Fuel-cell vehicles are undergoing extensive R\&D because of their potential for high efficiency and low emissions (zero-emission). Because fuel-cell vehicles remain expensive and there is limited demand for hydrogen at present, very few fueling stations are being built. To try to accelerate the development of a hydrogen economy, some original equipment manufacturers (OEMs in the automotive industry have been working on a hydrogenfueled ICE as an intermediate step.

Figure 26 - Specific power and power density for fuel cell system.

shows the specific power and specific energy of the fuel-cell system. As shown, the specific power and power density continuously increase. Between the reference case and 2045, the specific power increases by $113 \%$ to $178 \%$ and the power density increases by $132 \%$ to $310 \%$. It should be noted that in the case of the fuel-cell systems, all the assumptions other than the efficiency curve were provided by DOE. 


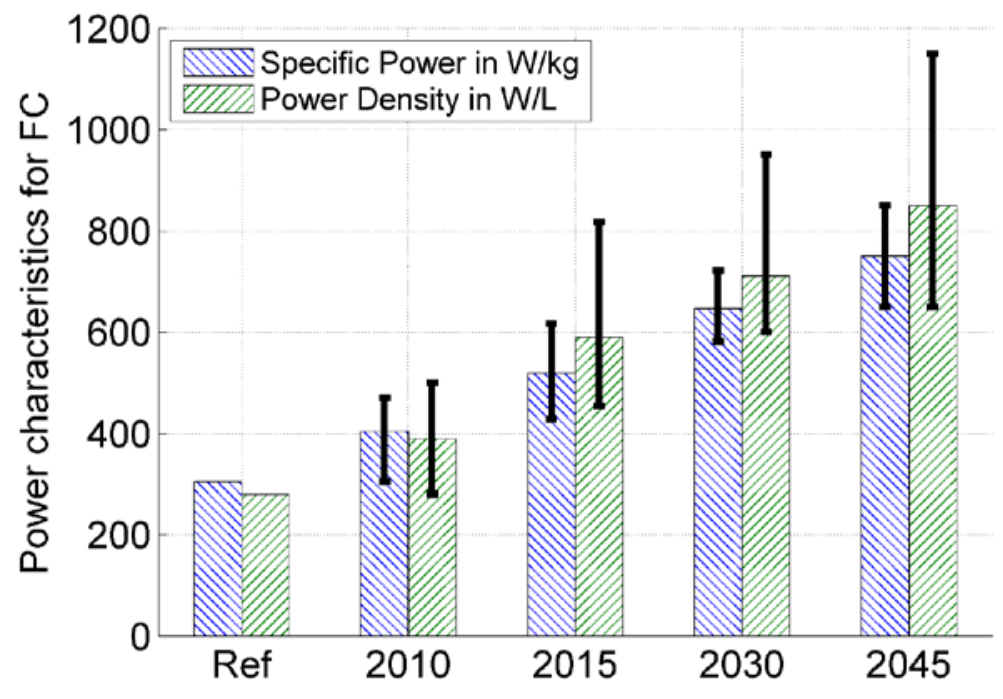

Figure 26 - Specific power and power density for fuel cell system.

The fuel-cell system model used for the study is based on a steady-state look-up table. The efficiency curve has been provided by a car manufacturer. As a result, the additional losses from the balance of plant due to transient operating conditions are not taken into account.

Figure 27 shows the evolution of the fuel-cell system peak efficiencies and cost. The peak fuel-cell efficiency is assumed to be at $55 \%$ currently, and it will increase to $60 \%$ by 2015 . A value of $60 \%$ has already been demonstrated in laboratories and therefore is expected to be implemented soon in vehicles. The peak efficiencies will remain constant in the future, as most research is expected to focus on reducing cost and increasing durability. The costs are projected to decrease from $\$ 80 / \mathrm{kW}$ currently (values based on high production volume) to an average of $\$ 25 / \mathrm{kW}$ in 2045 (uncertainty from $\$ 15$ to $\$ 30 / \mathrm{kW}$ ). These costs are based on an assumed production of 500,000 units per year.
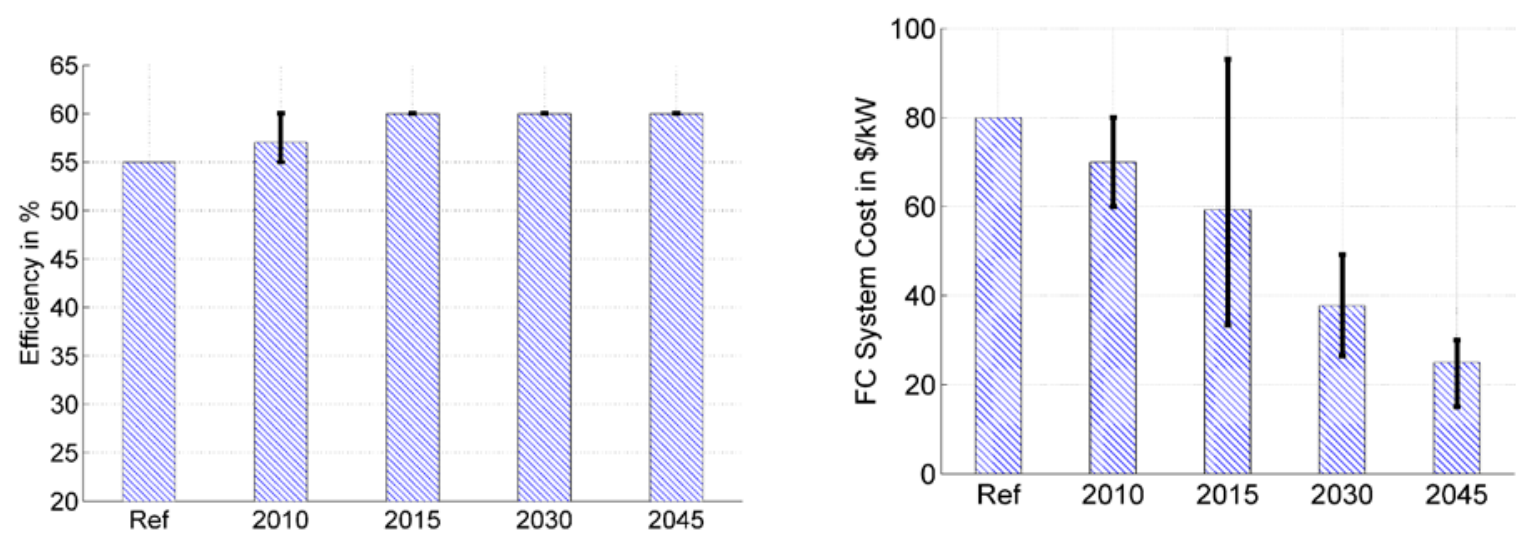

Figure 27 - Fuel-cell system efficiency and cost. 


\subsection{HYDROGEN STORAGE}

The evolution of hydrogen storage systems is vital to the introduction of hydrogen-powered vehicles. As in the case of the fuel-cell systems, all the assumptions used for hydrogen storage were based on values provided by DOE.

Figures 28 and 29 show the evolution of the hydrogen storage capacity. One notices a large uncertainty for both parameters. Consequently, there will be an important storage-system mass difference between the vehicles corresponding to these cases. Overall, the volumetric capacity dramatically increases by $177 \%$ to $288 \%$ between the reference case and 2045.

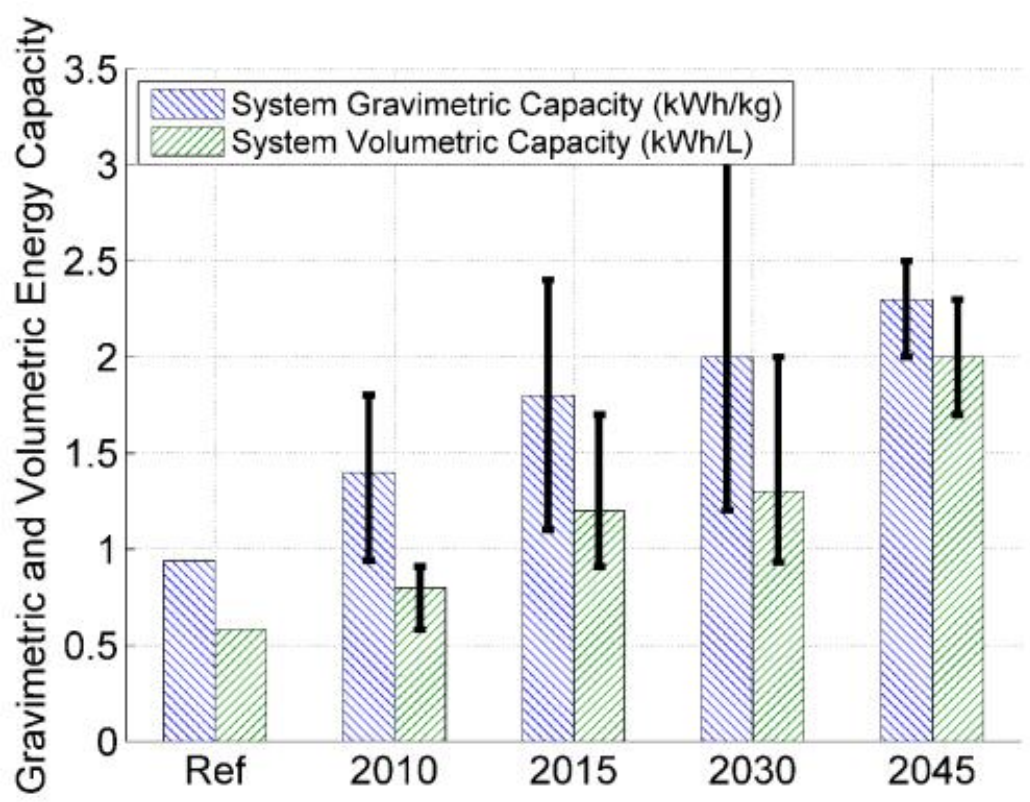

Figure 28 - Hydrogen storage energy: gravimetric and volumetric capacity. 


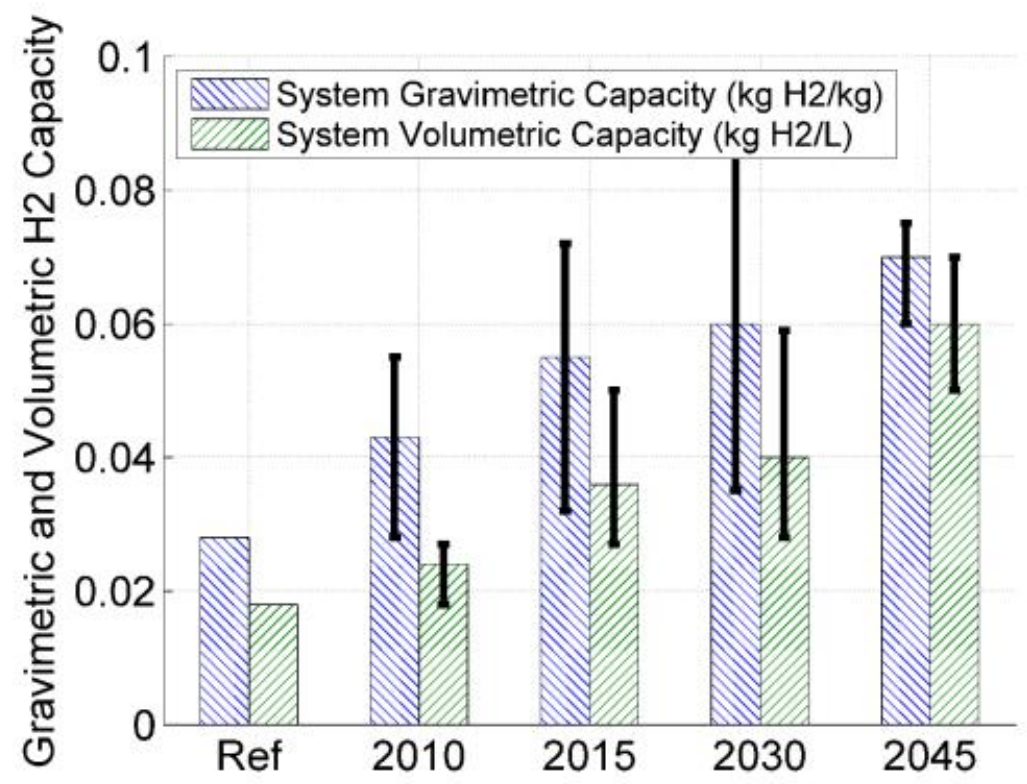

Figure 29 - Hydrogen storage capacity in terms of hydrogen quantity.

Figure 30 shows the evolution of the hydrogen storage cost from $\$ 1000 / \mathrm{kg} \mathrm{H} 2$ in the reference case to an average of $\$ 120 / \mathrm{kg} \mathrm{H} 2$ by 2045 . The values provided are based on production of 500,000 units per year.

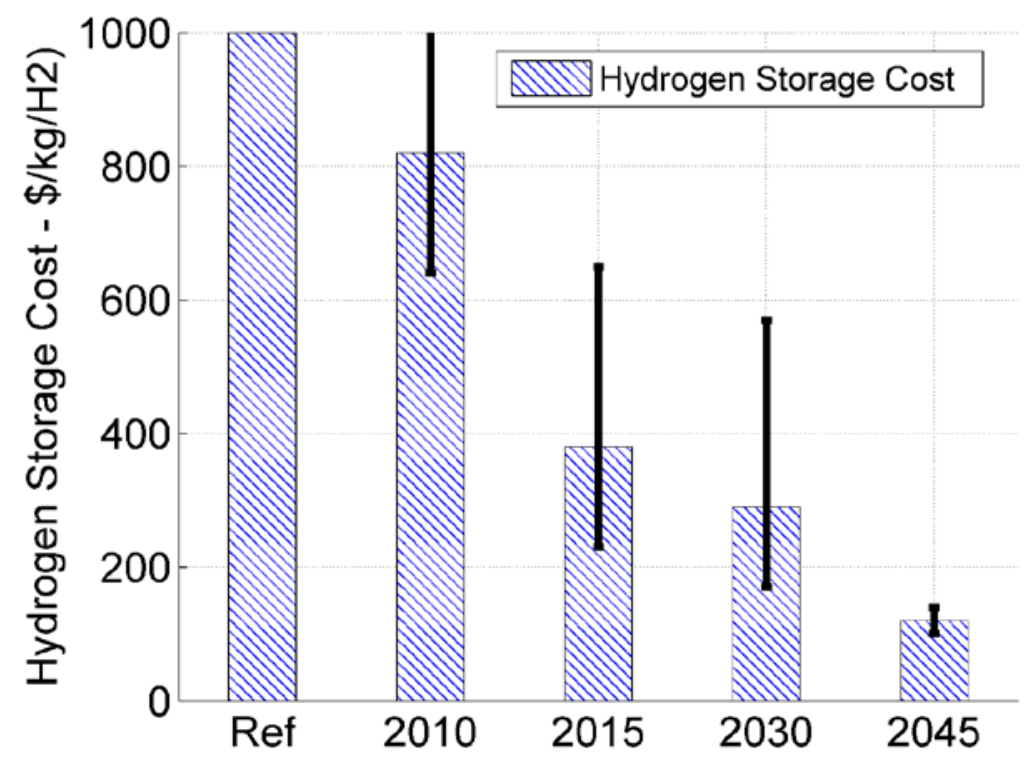

Figure 30 - Hydrogen storage cost.

To compare hydrogen-fueled vehicles with their ICE counterparts, driving ranges of 300 miles in 2010 and 2015 and 500 miles in 2030 and 2045 on the UDDS driving cycle have been selected. No fuel-consumption 
adjustments were performed. The amount of on-board hydrogen will be defined for each vehicle to meet these requirements. For the PHEVs, the total range of the vehicle is the value shown below plus the AER. Figure 31 summarizes the target range for each timeframe.

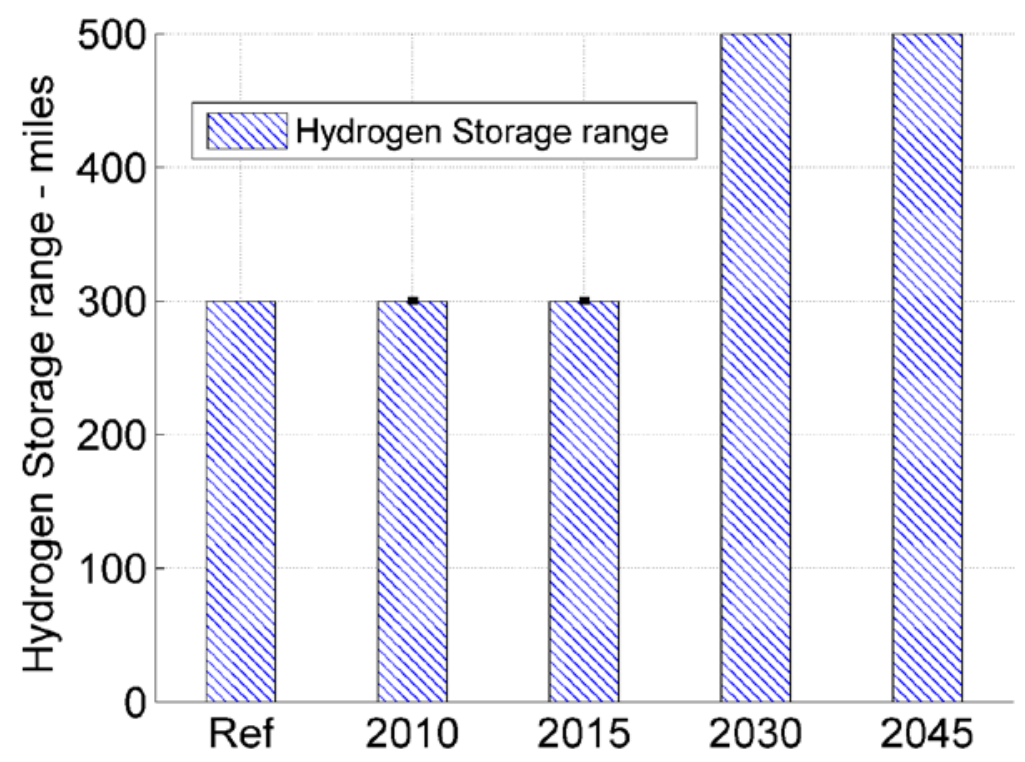

Figure 31 - Hydrogen storage vehicle range on UDDS cycle, based on unadjusted fuel consumption.

\subsection{ELECTRIC MACHINE}

Two different electric machines will be used as references in this study:

- Power-split vehicles operate with a permanent-magnet electric machine (similar to that used in the Toyota Camry) with a peak efficiency of $95 \%$.

- Series configurations (both engine and fuel cell) and EVs use an induction primary electric machine with a peak efficiency of $95 \%$.

The reference electric-machine data have been provided by car companies, suppliers, and Oak Ridge National Laboratory.

As shown in Figure 32, the power-electronic specific power will significantly increase between 2010 and 2015; the low case will be multiplied by 2. From the reference case to 2045, the power-electronic specific power will be multiplied by more than 2 to 3 . The electric-machine specific power does not show such an aggressive increase: from the reference case to 2045 ; it increases by $36 \%$. 


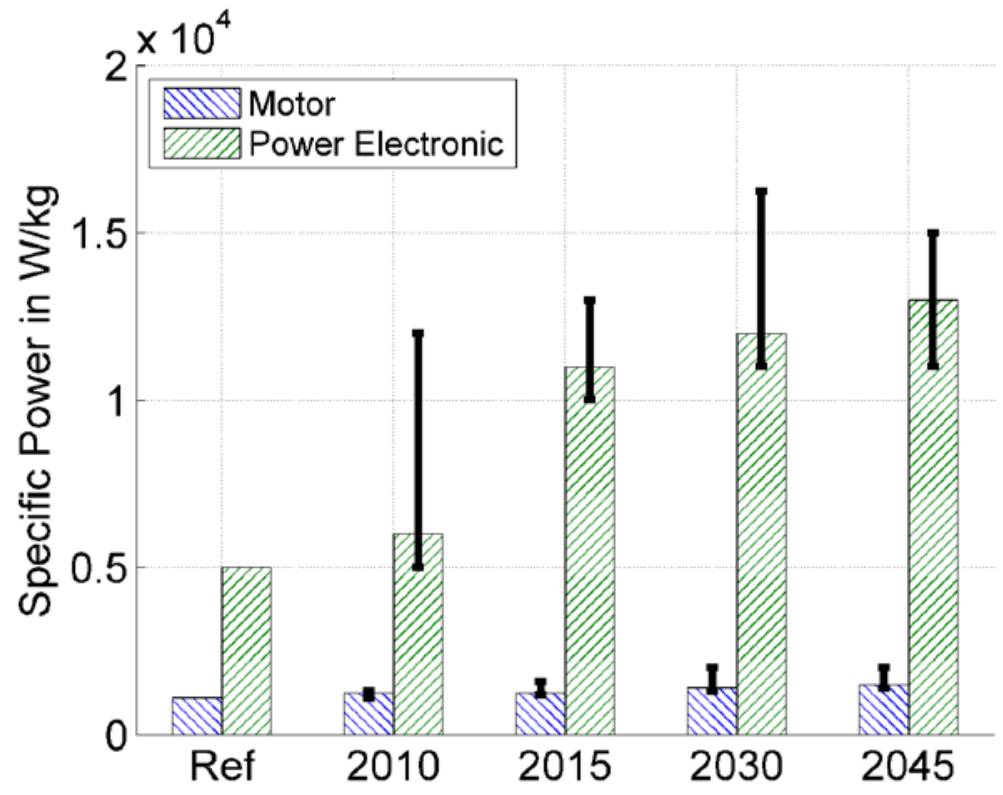

Figure 32 - Electric-machine and power-electronic specific power.

Both electric machines used in the study have a reference peak efficiency of $95 \%$. As shown in Figure 33 , it will increase from 95\% to 98\% between 2010 and 2030 and will remain at that value in 2045.

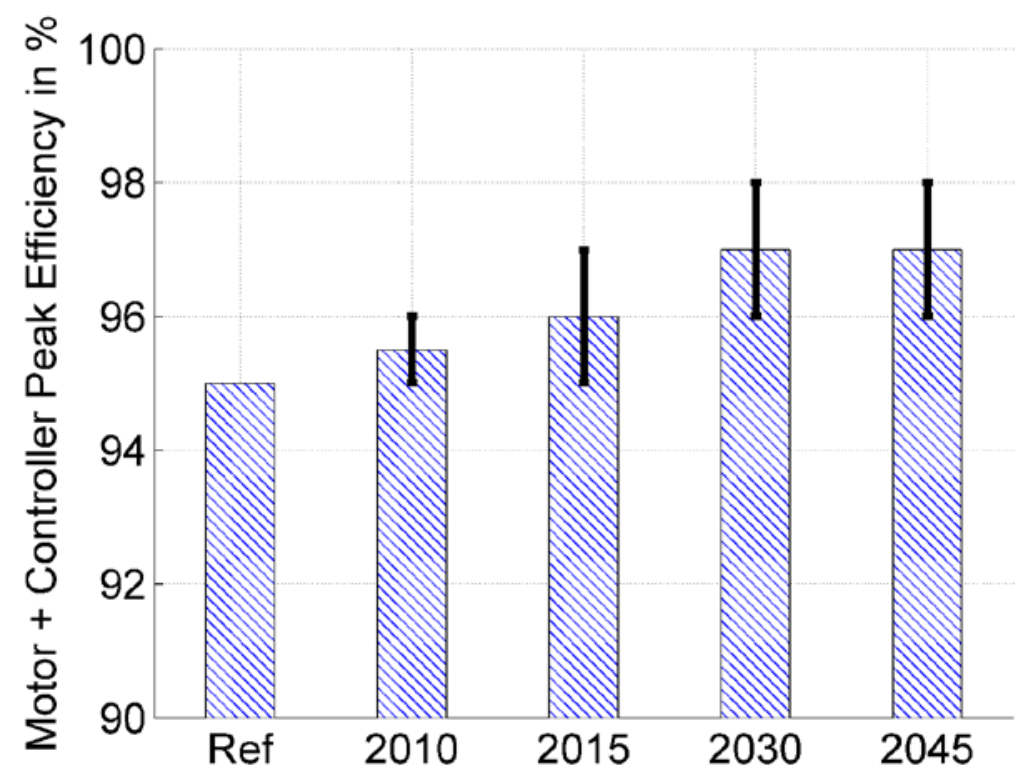

Figure 33 - Electric-machine peak efficiency.

As shown in Figure 34, the controller cost is decreased by 25\%-58\% between 2010 and 2015, whereas the electric-machine cost is only decreased by $15 \%-46 \%$. For both the electric machine and the controller, the cost decreases slightly from 2015 to 2030 and then stays constant through 2045. 


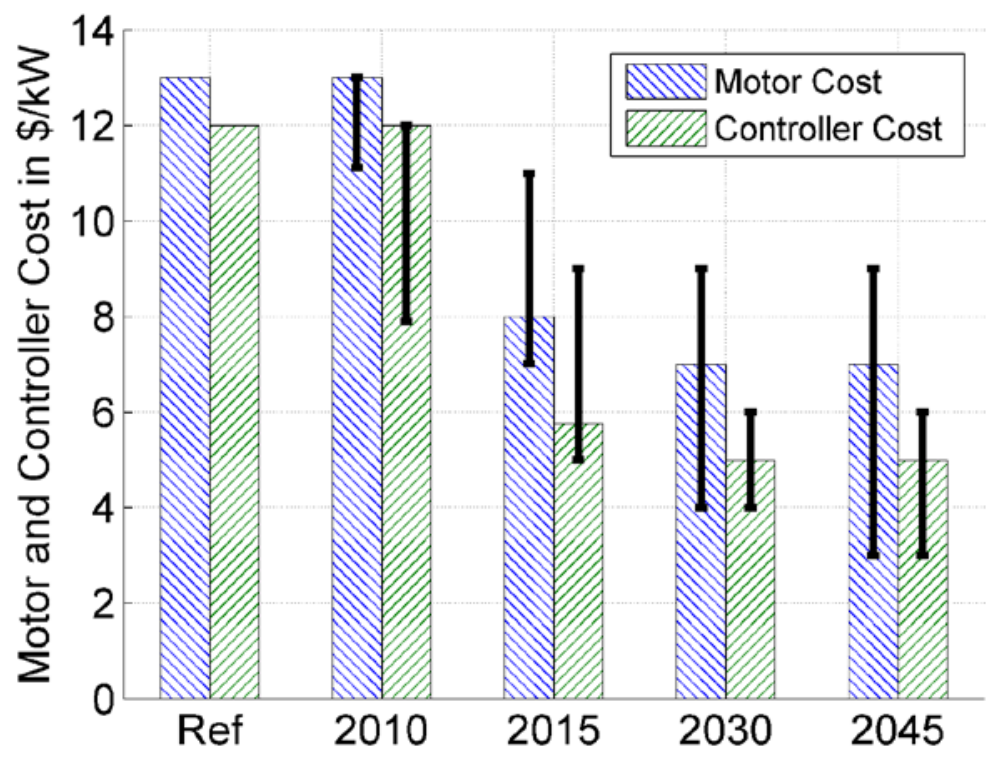

Figure 34 - Electric-machine and controller cost.

\subsection{ENERGY STORAGE SYSTEM}

Only batteries were used in the present study, on the assumption that ultra-capacitors alone could not provide sufficient available energy for full hybrid applications. We also considered that coupling ultra-capacitors with batteries would be cost-prohibitive and that Li-ion battery life would be significantly improved in the short term, making the combination ineffective.

The batteries used in the study as the reference have been provided by Argonne, Idaho National Laboratory, and major battery suppliers. A scaling algorithm developed by Argonne's battery experts is used for the highenergy cases (Nelson et al., 2007).

The battery used for the HEV reference case is a NiMH battery. It is assumed that this technology is the most likely to be used until 2015. This is why we simulated the HEVs with this battery for the reference case; 2010 low, average, and high cases; and the 2015 low case. The model used is similar to the one found in the Toyota Prius. For PHEV applications, all the vehicles are run with a Li-ion battery from Argonne. Tables 2 and 3 provide a summary of the battery characteristics and technologies. 
Argonne National Laboratory Report - Light-Duty Vehicle Fuel Consumption Displacement Potential Up to 2045

Table 6: Description of Reference Battery Characteristics

\begin{tabular}{|c|c|c|c|}
\hline & Source & Technology & Reference Cell Capacity [Ah] \\
\hline \multirow{2}{*}{ HEV } & Idaho National Laboratory & NiMH & 6.5 \\
& Battery manufacturer & Li-ion & 6 \\
\hline PHEV & Argonne National Laboratory & Li-ion & 41 \\
\hline
\end{tabular}

Table 7: Battery Technology Selection for Each Timeframe

\begin{tabular}{|l|c|c|c|c|c|c|c|c|c|c|c|c|}
\hline & \multicolumn{3}{|c|}{2010} & \multicolumn{2}{c|}{2015} & \multicolumn{2}{c|}{2030} & \multicolumn{2}{c|}{2045} \\
\hline & ref/low & avg & high & low & avg & high & low & avg & high & low & avg & high \\
\hline HEV & NiMH & NiMH & NiMH & NiMH & \multicolumn{2}{|c|}{ Li-ion } & \multicolumn{3}{|c|}{ Li-ion } & \multicolumn{2}{c|}{ Li-ion } \\
\hline
\end{tabular}

After a long period of time, batteries lose some of their power and energy capacity. To be able to maintain the same performance at the end of life (EOL) compared to the beginning of life (BOL), an oversize factor is applied while sizing the batteries for both power and energy. These factors are supposed to represent the percentage of power and energy that will not be provided by the battery at the EOL compared to the initial power and energy given by the manufacturer. The oversize factor is decreased over time to reflect an improvement in the ability of batteries to uniformly deliver the same performance throughout their life cycles. Figure 35 shows that the reference vehicles are sized with a $20 \%$ power oversize factor for all hybrid vehicles and energy oversize factors of $20 \%$ for HEVs and $30 \%$ for PHEVs. In 2045 , these values will be reduced to $10 \%-14 \%$ for power oversize, $10 \%-16 \%$ for HEV energy oversize, and $20 \%-24 \%$ for PHEV energy oversize. These oversizing factors influence the cost and weight; however, all the simulations are run at EOL (i.e., the additional weight is taken into account, but the power and energy used for the simulation are the ones from EOL). 


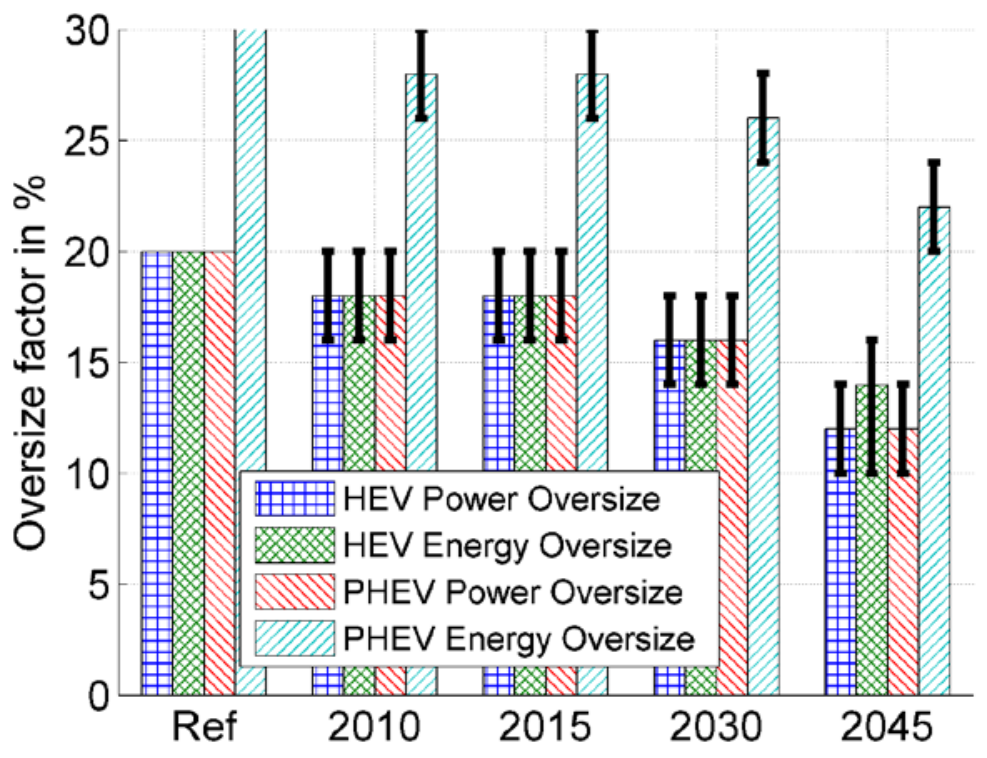

Figure 35 - Power and energy oversize for HEVs and PHEVs.

The battery cost for HEV applications will decrease over time for all cases, but the decrease is more aggressive for the high case between 2010 and 2015, with a 37.5\% cost reduction (Figure 36). The batteries are expected to be less than $1 / 3$ the cost in the 2045 high case $(\$ 18 / \mathrm{kW})$ in comparison to the reference case $(\$ 60 / \mathrm{kW})$. PHEV and EV battery energy costs are very close to each other. For both of them, the battery cost significantly decreases over time, starting at $\$ 600 / \mathrm{kWh}$ and ending up, in the 2045 high case, at $\$ 150 / \mathrm{kWh}$ for PHEVs and \$100/kWh for EVs; these correspond, respectively, to a $75 \%$ decrease and a $83 \%$ decrease. 

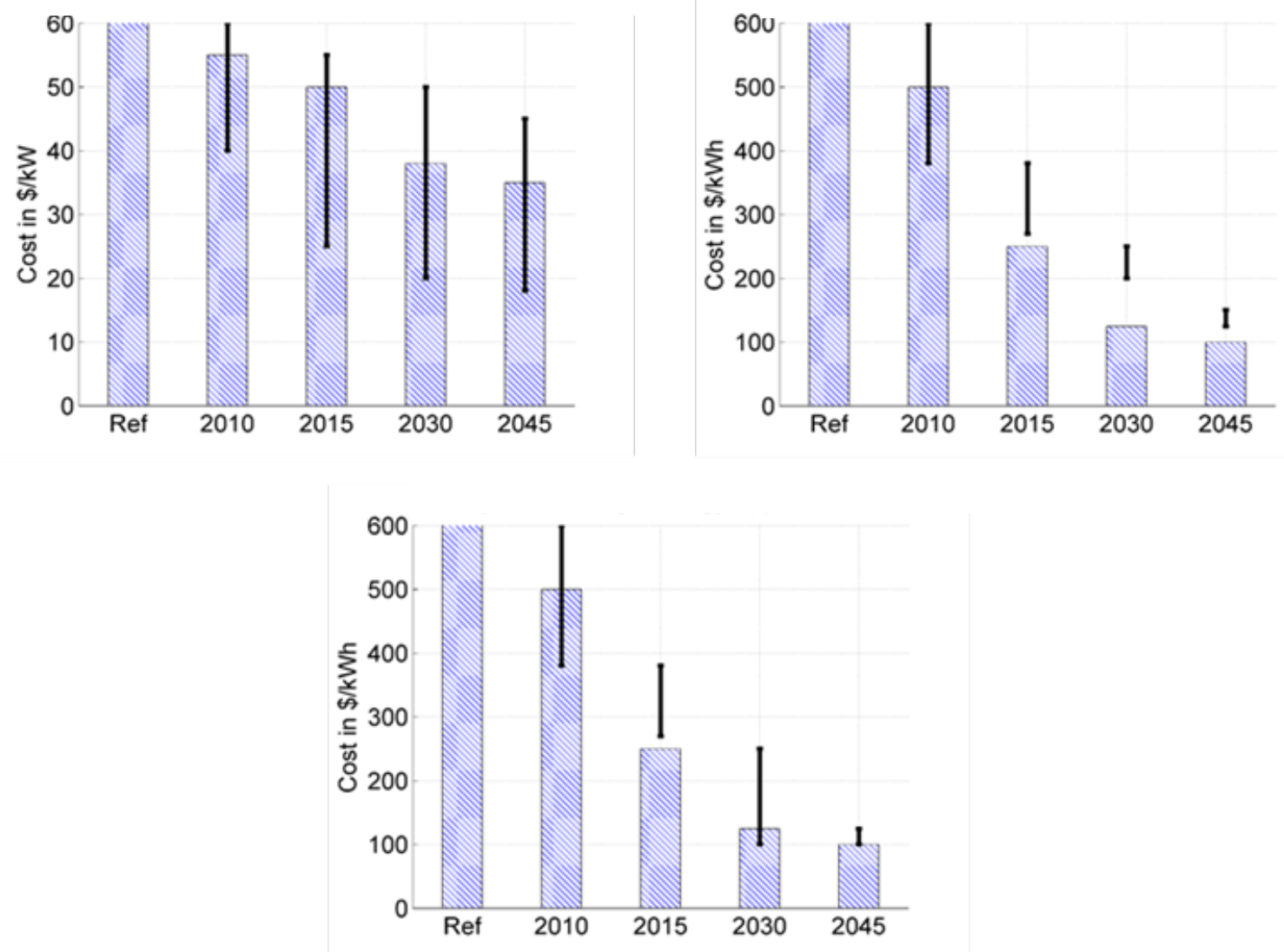

Figure 36 - Battery cost for (top left) HEVs, (top right) PHEVs, and (bottom) EV.

Figure 37 shows the relationship between the power/energy ratio and the battery energy cost.

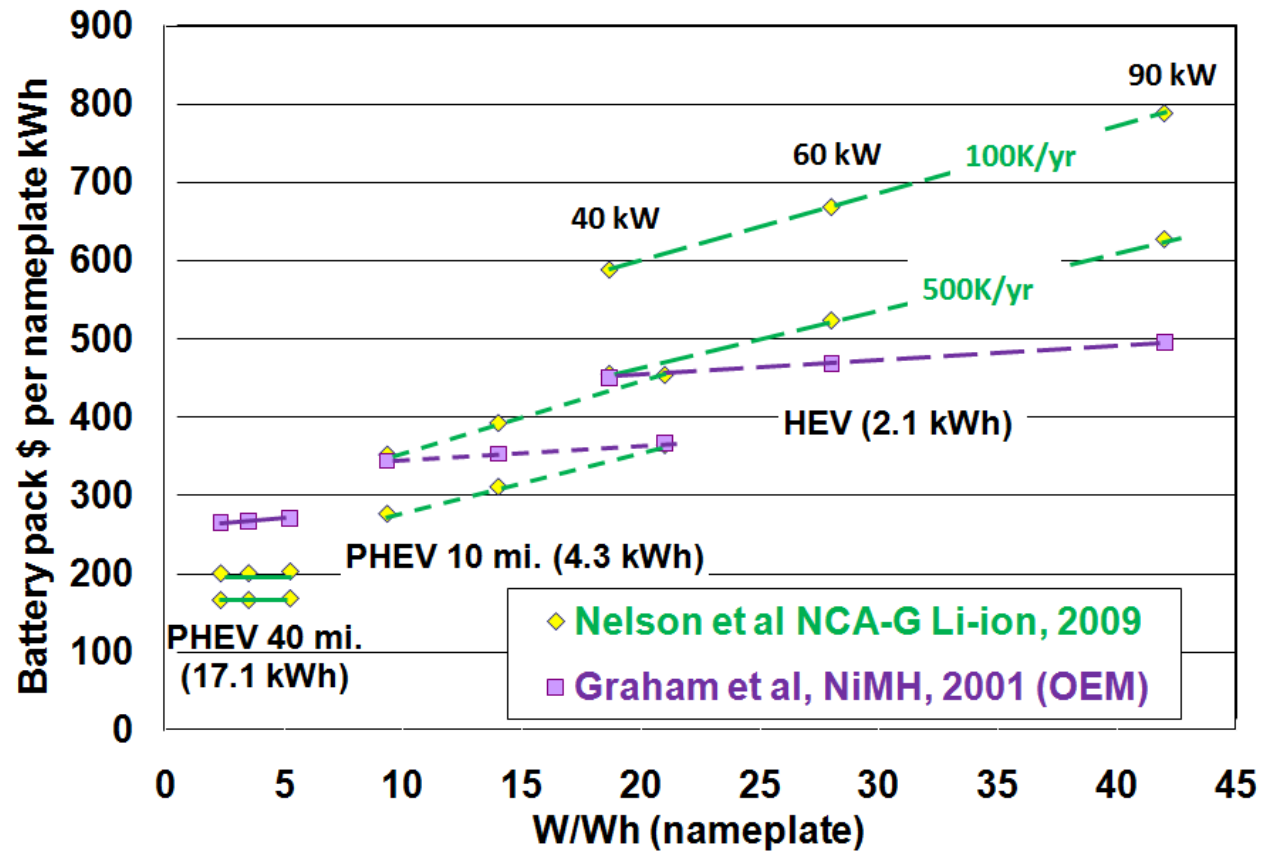


Argonne National Laboratory Report - Light-Duty Vehicle Fuel Consumption Displacement Potential Up to 2045

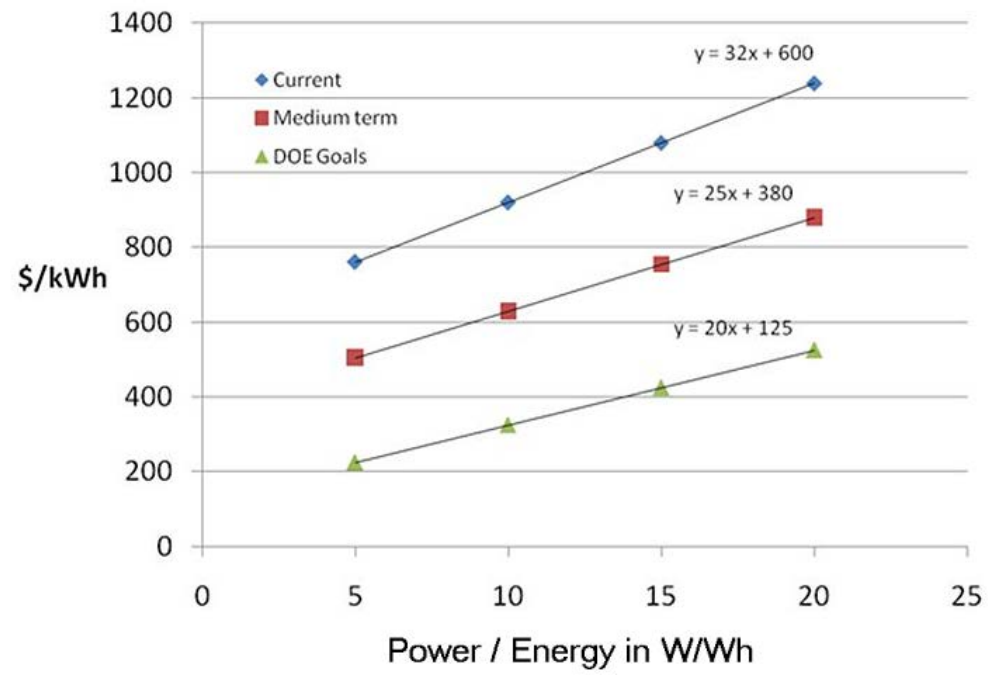

Figure 37 - Relation between power/energy ratio and battery energy cost.

Figure 38 shows that the SOC values used for PHEVs and EVs are $30 \%$ for the minimum and $90 \%$ for the maximum in the reference case and they change slightly over time to reach $20 \%$ for the minimum and $100 \%$ for the maximum in the 2045 high case.

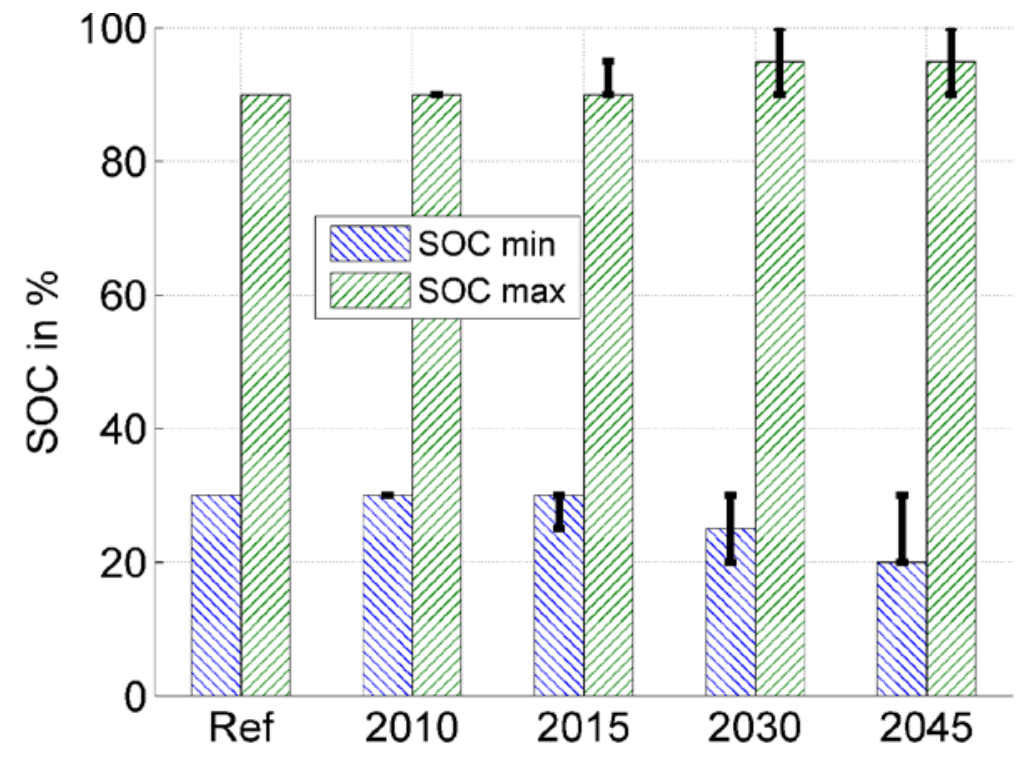

Figure 38 - Battery SOC for PHEVs and EVs.

\subsection{DRIVELINE}

Several types of transmission technologies are considered in this study:

- Increased number of gears for automatic transmissions. Additional gears allow the engine to be operated closer to its best efficiency line. While they are now limited to high-end vehicles, high- 
Argonne National Laboratory Report - Light-Duty Vehicle Fuel Consumption Displacement Potential Up to 2045

speed transmissions (i.e., up to eight gears) are expected to be used in a larger number of vehicles in the near future.

- Dual-clutch transmission (DCT). Every car manufacturer, if it does not already have some DCT models in production, is working on developing the technology. DCTs are likely to be the next dominant transmission technology in the future, since they combine the advantages of automatic transmissions (drive quality-no torque interruption) and manual transmissions (efficiency-no torque converter).

Owing to the drive-quality requirements in North America, automated manual transmissions have not been included in this study. Continuously variable transmissions have issues related to reliability and fuel-efficiency gains (the engine gains are often offset by higher transmission losses) and are not considered either.

Conventional vehicles are simulated with an automatic transmission, since that option best represents the American car market. However, a midsize car with a DCT is simulated for a few timeframes.

Power-split HEVs and PHEVs both have a planetary gearset with 78 ring teeth and 30 sun teeth. Finally, the fuel-cell vehicles and EVs use a two-speed manual transmission to increase the powertrain efficiency as well as allow them to achieve a maximum vehicle speed of at least $100 \mathrm{mph}$. Characteristics of all transmissions used in the study are shown Tables 4-7. 
Table 8: Transmission technologies modeled for different vehicle classes*

\begin{tabular}{|c|c|c|c|c|c|c|c|c|c|c|c|c|c|}
\hline \multirow{2}{*}{ Parameter } & \multirow{2}{*}{ Current } & \multicolumn{3}{|c|}{2010} & \multicolumn{3}{|c|}{2015} & \multicolumn{3}{|c|}{2030} & \multicolumn{3}{|c|}{2045} \\
\hline & & Low & Med & High & Low & Med & High & Low & Med & High & Low & Med & High \\
\hline \multicolumn{14}{|l|}{ Peak Efficiency (\%) } \\
\hline Automatic Trans & 95 & 95 & 96 & 97 & 96 & 97 & 97.5 & 97 & 97 & 97.5 & 97 & 97 & 97.5 \\
\hline DCT & 97.5 & 97.5 & 97.5 & 97.5 & 97.5 & 97.5 & 98 & 97.5 & 98 & 98 & 97.5 & 98 & 98 \\
\hline Planetary gearset & 97 & 97 & 97 & 98 & 97 & 97.5 & 98 & 97.5 & 98 & 98.5 & 97.5 & 98 & 98.5 \\
\hline Final Drive Peak Efficiency (\%) & 97.5 & 97.5 & 97.5 & 97.5 & 97.5 & 97.5 & 98 & 97.5 & 98 & 98 & 97.5 & 98 & 98 \\
\hline \multicolumn{14}{|l|}{ Conventional Vehicle (1) } \\
\hline gb Compact & MCOM4 & MCOM4 & MCOM4 & MCOM5 & MCOM4 & MCOM5 & MCOM5 & MCOM5 & MCOM5 & MCOM6 & MCOM6 & MCOM6 & MCOM6 \\
\hline gb Midsize & MCAR5 & MCAR5 & MCAR5 & MCAR6 & MCAR6 & MCAR6D & AA80E & MCAR6 & MCAR6D & AA80E & MCAR6D & AA80E & AA80E \\
\hline gb Small_SUV & SSUV4 & SSUV4 & 5-45RFE & 5-45RFE & SSUV4 & 5-45RFE & SSUV6 & 5-45RFE & SSUV6 & SSUV6 & SSUV6 & SSUV6 & SSUV6 \\
\hline gb Midsize_SUV & $4 \mathrm{~L} 60-\mathrm{E}$ & $4 \mathrm{~L} 60-\mathrm{E}$ & MSUV5 & MSUV5 & 4L60-E & MSUV5 & MSUV6 & MSUV5 & MSUV6 & MSUV6 & MSUV6 & MSUV6 & MSUV6 \\
\hline gb Pickup & 45RFE & 45RFE & 45RFE & 5-45RFE & 45RFE & 45RFE & 5-45RFE & 45RFE & 5-45RFE & 5-45RFE & 5-45RFE & 5-45RFE & 5-45RFE \\
\hline
\end{tabular}

*The code names of the transmissions are based on the vehicle class (i.e., COM for compact, MCAR for midsize car) and the number of gears. For example, SSUV4 means a small SUV with a 4 gear transmission. 
Argonne National Laboratory Report - Light-Duty Vehicle Fuel Consumption Displacement Potential Up to 2045

Table 9: Gear ratios, final drive and cost for all transmissions

\begin{tabular}{|c|c|c|c|c|c|c|c|c|c|c|c|c|c|}
\hline & Name & Type & Cost $\$$ & & & & & & & & & & \\
\hline \multirow{6}{*}{ Compact car } & MCOM4 & $\mathrm{AU}$ & 1100 & Gear Ratios & 3.64 & 2.01 & 1.3 & 0.89 & & & & & \\
\hline & & & & Final Drive & 2.96 & & & & & & & & \\
\hline & MCOM5 & $A U$ & 1150 & Gear Ratios & 2.66 & 1.533 & 1.02 & 0.72 & 0.52 & & & & \\
\hline & & & & Final Drive & 4.44 & & & & & & & & \\
\hline & MCOM6 & $A U$ & 1200 & Gear Ratios & 4.15 & 2.34 & 1.52 & 1.14 & 0.86 & 0.69 & & & \\
\hline & & & & Final Drive & 2.63 & & & & & & & & \\
\hline \multirow{8}{*}{ Midsize CAR } & MCAR5 & $A U$ & 1200 & Gear Ratios & 2.563 & 1.552 & 1.022 & 0.727 & 0.52 & & & & \\
\hline & & & & Final Drive & 4.43 & & & & & & & & \\
\hline & MCAR6 & $A U$ & 1236 & Gear Ratios & 4.15 & 2.37 & 1.56 & 1.16 & 0.86 & 0.69 & & & \\
\hline & & & & Final Drive & 2.74 & & & & & & & & \\
\hline & MCAR6D & DCT & 1273 & Gear Ratios & 3.454 & 2.045 & 1.452 & 1.114 & 1.078 & 0.921 & & & \\
\hline & & & & Final Drive & 3.29 & & & & & & & & \\
\hline & AA80E & $A U$ & 1311 & Gear Ratios & 4.596 & 2.72 & 1.863 & 1.464 & 1.23 & 1 & 0.824 & 0.82 & 0.685 \\
\hline & & & & Final Drive & 2.47 & & & & & & & & \\
\hline \multirow{8}{*}{ Small SUV } & SSUV4 & $A U$ & 1220 & Gear Ratios & 2.89 & 1.57 & 1 & 0.7 & & & & & \\
\hline & & & & Final Drive & 3.78 & & & & & & & & \\
\hline & SSUV5 & $A U$ & 1257 & Gear Ratios & 3.5 & 1.94 & 1.26 & 0.95 & 0.81 & & & & \\
\hline & & & & Final Drive & 3.12 & & & & & & & & \\
\hline & SSUV6 & $A U$ & 1294 & Gear Ratios & 4.15 & 2.34 & 1.52 & 1.14 & 0.86 & 0.69 & & & \\
\hline & & & & Final Drive & 2.63 & & & & & & & & \\
\hline & 5-45RFE & $A U$ & 1368 & Gear Ratios & 3 & 1.67 & 1 & 0.75 & 0.67 & & & & \\
\hline & & & & Final Drive & 3.64 & & & & & & & & \\
\hline \multirow{6}{*}{ Midsize SUV } & $4 \mathrm{~L} 60-\mathrm{E}$ & $A U$ & 1320 & Gear Ratios & 3.06 & 1.63 & 1 & 0.7 & & & & & \\
\hline & & & & Final Drive & 3.42 & & & & & & & & \\
\hline & MSUV5 & $A U$ & 1360 & Gear Ratios & 3.22 & 2.32 & 1.55 & 1 & 0.71 & & & & \\
\hline & & & & Final Drive & 3.25 & & & & & & & & \\
\hline & MSUV6 & $\mathrm{AU}$ & 1400 & Gear Ratios & 4.15 & 2.34 & 1.52 & 1.14 & 0.86 & 0.69 & & & \\
\hline & & & & Final Drive & 2.52 & & & & & & & & \\
\hline \multirow{4}{*}{ Pickup Truck } & 45RFE & $A U$ & 1409 & Gear Ratios & 3 & 1.67 & 1 & 0.75 & & & & & \\
\hline & & & & Final Drive & 3.55 & & & & & & & & \\
\hline & 5-45RFE & $A U$ & 1368 & Gear Ratios & 3 & 1.67 & 1 & 0.75 & 0.67 & & & & \\
\hline & & & & Final Drive & 3.55 & & & & & & & & \\
\hline
\end{tabular}


Argonne National Laboratory Report - Light-Duty Vehicle Fuel Consumption Displacement Potential Up to 2045

Table 10: Power-split transmission characteristics for all vehicle classes

\begin{tabular}{|c|c|}
\hline Cost & 840 \\
\hline Gear Ratios & Sun number of teeth $=30$. Ring number of teeth $=78$ \\
\hline Final Drive & 4.059 \\
\hline
\end{tabular}

Table 11: Fuel-cell and electric-vehicle transmission characteristics for all vehicle classes

\begin{tabular}{|c|c|}
\hline Cost & 840 \\
\hline Gear Ratios & $1.86 / 1$ \\
\hline Final Drive & 4.44 \\
\hline
\end{tabular}


The efficiencies of the transmission types other than automatic, and of the final drive, are already very high and will increase only slightly over time, as shown in Figure 39. The planetary gearset will have the best efficiency in 2030 and 2045 and will constitute the high case, with $98.5 \%$ efficiency.

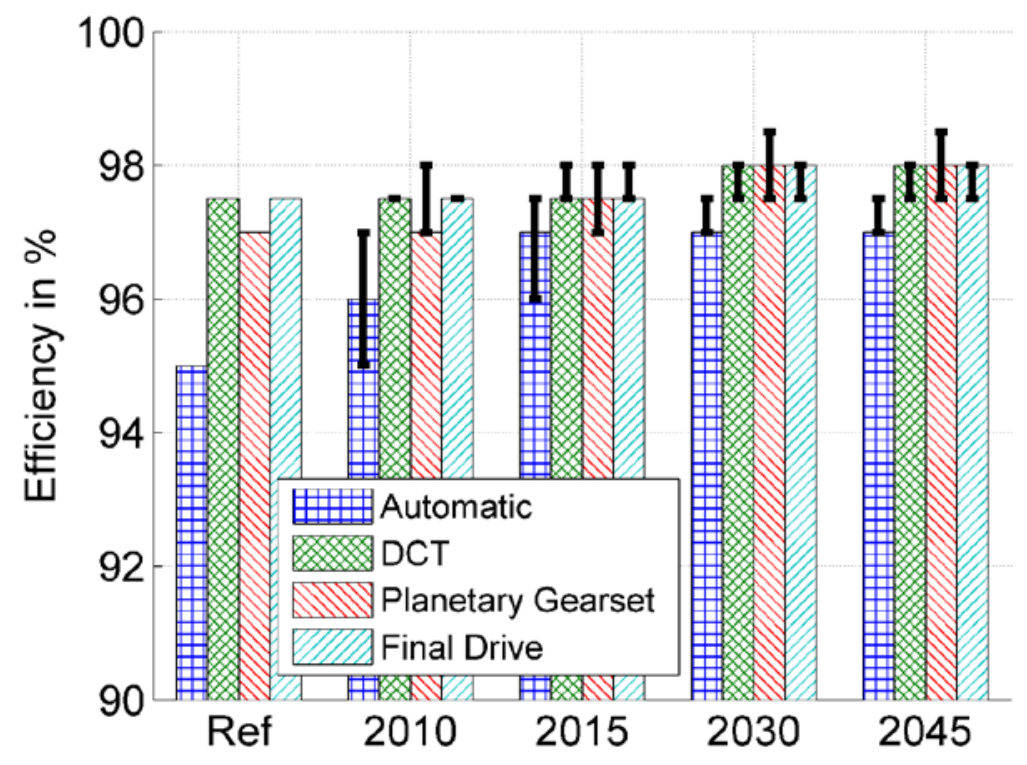

Figure 39 - Transmission peak efficiencies.

The shifting algorithm that is used automatically defines the shifting strategy based on the powertrain ratios and the component efficiencies to minimize fuel consumption while maintaining acceptable drive quality (i.e., torque reserve). The algorithm has been validated using APRF test data with several conventional vehicles over the past 10 years. 
Argonne National Laboratory Report - Light-Duty Vehicle Fuel Consumption Displacement Potential Up to 2045

\subsection{BODY AND VEHICLE}

\subsubsection{TECHNOLOGY OVERVIEW}

One of the main factors affecting fuel consumption is vehicle weight. Lowering the weight ("lightweighting") reduces the forces required to follow the vehicle speed trace. As a result, the components can be downsized, resulting in smaller components and decreased fuel consumption. However, the impact of lightweighting is not the same for all the powertrain configurations, with studies showing that the technology has greater influence in conventional vehicles than their electric-drive counterparts (Pagerit et al., 2006).

The principal methodologies include material substitution (i.e., high-strength low-alloy steel, aluminum, magnesium, etc.), improved packaging (i.e., ratio of interior volume to exterior size and weight), and unit body construction (elimination of conventional chassis/body structure). Several studies have shown the potential to decrease the weight by as much as $20 \%$ without cost penalties, which highlights the great potential of the technology (USCAR, 2010).

Reductions in rolling resistance, frontal area, and drag coefficient also have the potential to significantly improve fuel consumption as they also lead to a reduction in the force required at the wheel. In this study, the assumption is that frontal area will be increased rather than decreased because American consumers have demanded vehicles with greater passenger and cargo volume, i.e., more room inside the vehicle.

The main characteristics used as a reference are shown in Table 8.

Table 12: Main characteristics of the different vehicle classes

\begin{tabular}{|l|l|l|l|l|}
\hline & $\begin{array}{l}\text { Glider Mass (Ref) } \\
\text { in } \mathbf{~ k g}\end{array}$ & $\begin{array}{l}\text { Frontal Area } \\
\text { (Ref) in } \mathbf{~ m}^{2}\end{array}$ & Tire & $\begin{array}{l}\text { Wheel Radius in } \\
\mathbf{m}\end{array}$ \\
\hline Compact & 805 & 2.19 & P195/65/R15 & 0.317 \\
\hline Midsize & 996 & 2.24 & P195/65/R15 & 0.317 \\
\hline Small SUV & 1006 & 2.57 & P225/75/R15 & 0.359 \\
\hline Midsize SUV & 1268 & 2.93 & P235/70/R16 & 0.367 \\
\hline Pickup & 1509 & 3.27 & P255/65/R17 & 0.381 \\
\hline
\end{tabular}




\subsubsection{LIGHTWEIGHTING}

The same glider mass reduction factor will be applied to all vehicle classes (Figure 40 - Glider mass reduction for all vehicle classes.

The glider mass will be linearly reduced by up to $37 \%$ in the 2045 high case relative to the reference case. The reduction is due to the use of different materials and technologies like aluminum. Note that the mass reduction of the glider has been separated into three sections: body mass, chassis mass, and rest of weight. First, the glider weight in this study is considered as the vehicle weight minus the powertrain components: engine, aftertreatment, electric machines, fuel cell, fuel storage, energy storage system, transmission, final drive, wheels, and accessories. A fixed percentage of the glider mass was used for each section. It is assumed that $44 \%$ of the glider is due to its body weight, $26 \%$ to its chassis weight, and $30 \%$ to other components (e.g., seats).

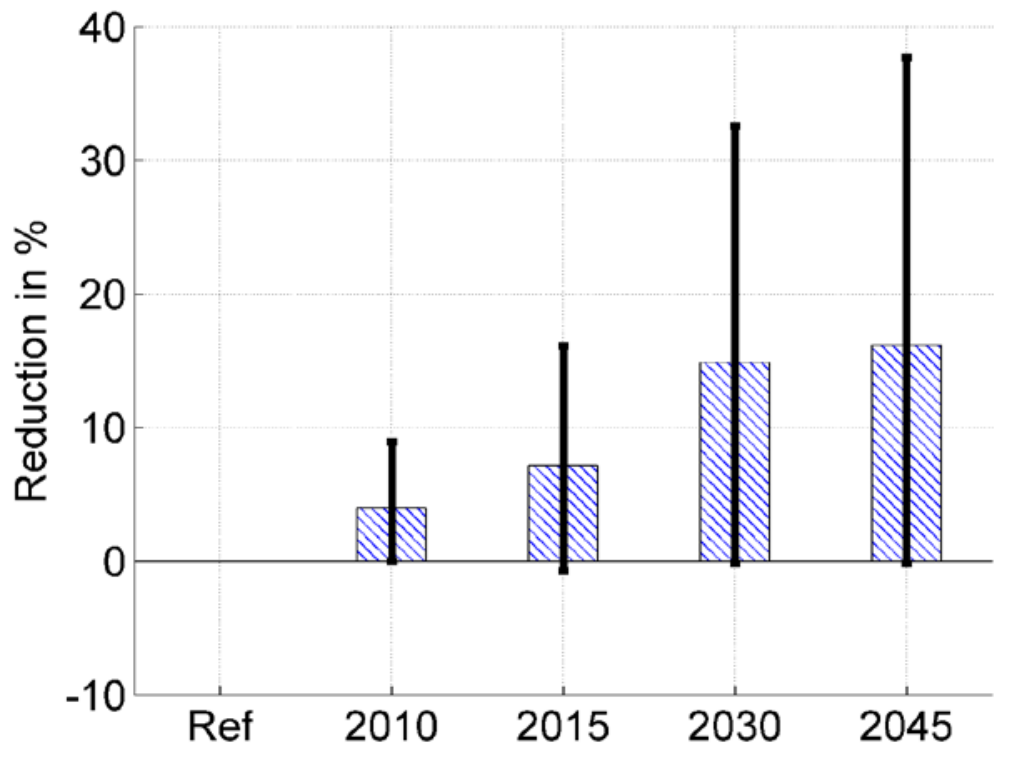

Figure 40 - Glider mass reduction for all vehicle classes.

The glider cost changes are due to the technologies used to achieve the glider mass reduction (Figure 41). For a midsize car, the reference-case glider costs $\$ 9955$ and is steel unibody. The other mass-reduction characteristics are shown in Table 9. 


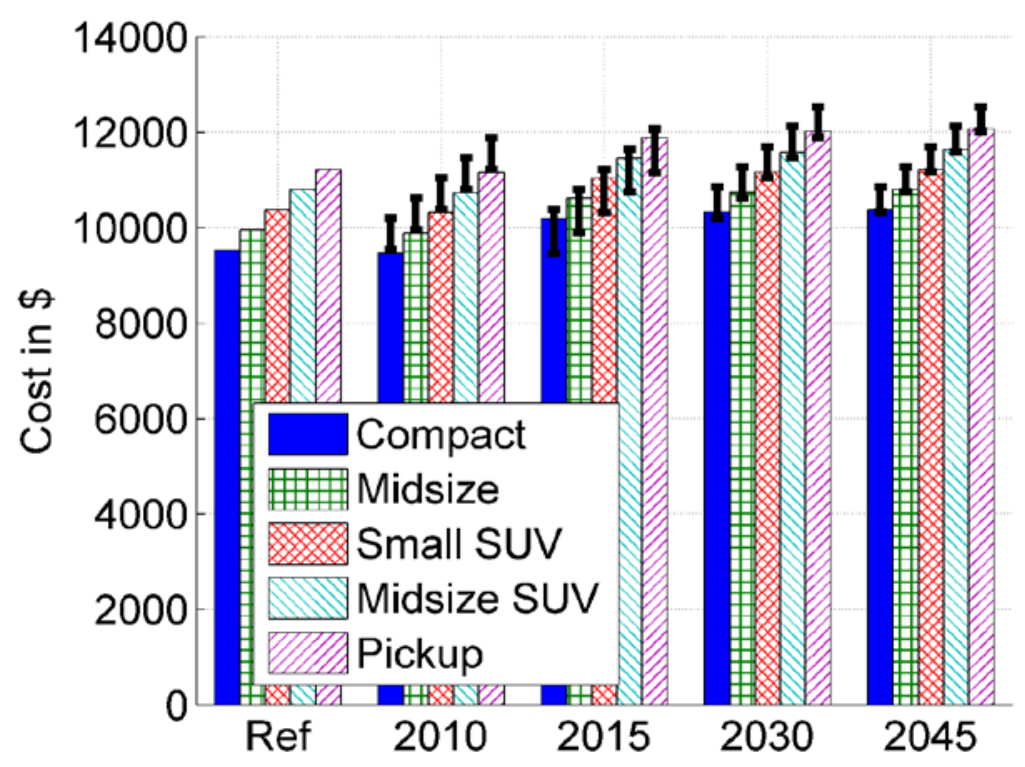

Figure 41 - Glider costs for the five vehicle classes.

Table 13: Incremental cost and glider composition associated with glider mass reduction

\begin{tabular}{|l|l|l|}
\hline & Incremental cost & Glider composition \\
\hline $5 \%$ reduction & $-\$ 65$ & Ultra-light steel auto body \\
\hline $10 \%$ reduction & $+\$ 660$ & Combination of ultra-light steel and aluminum components \\
\hline $15 \%$ reduction & $+\$ 790$ & All-aluminum unibody structure \\
\hline $20 \%$ reduction & $+\$ 844$ & Aluminum unibody structure, panels, bumper beam, etc. \\
\hline $30 \%$ reduction & $+\$ 1311$ & Carbon fiber polymer composites \\
\hline
\end{tabular}

\subsubsection{DRAG COEFFICIENT AND ROLLING RESISTANCE}

The same frontal-area increase factor will be applied to all vehicle classes (see Figure 42). The frontal area is expected to be increased up to $6 \%$ in the 2045 low case relative to the reference case. The increase pattern is not the same between cases. Whereas the average and low cases will continuously increase, the high-case increase will be maintained at $0 \%$ until 2015 and will be at $2 \%$ for the rest of the timeframes. 


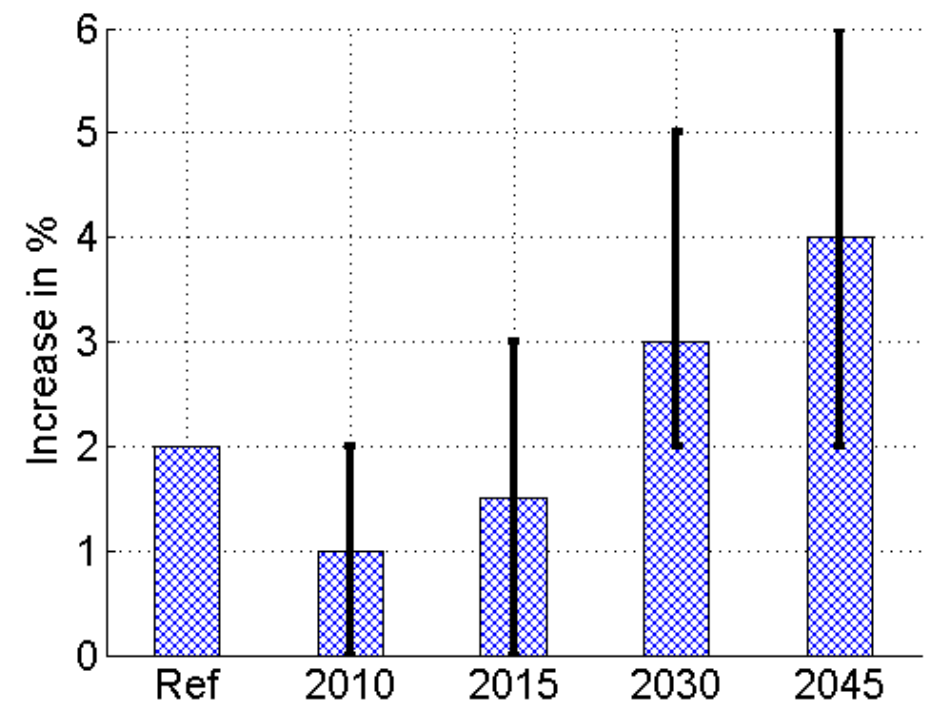

Figure 42 - Frontal-area increase for all vehicle classes.

As shown in Figure 43, the drag coefficient and rolling resistance show similar evolutions across vehicle classes.
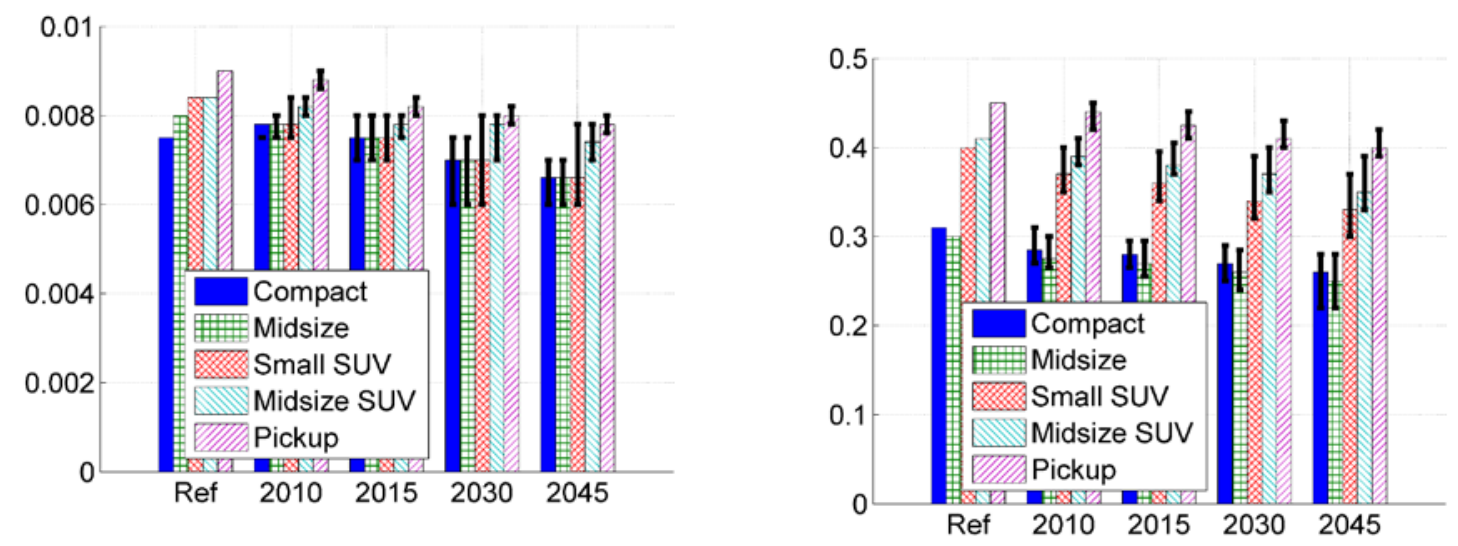

Figure 43 - (Left) drag coefficient and (right) rolling resistance values for the five vehicle classes.

\subsubsection{ACCESSORIES}

As shown in Figure 44, the accessory load is expected to increase over time as the power needed to supply electrical and electronic components increases in accordance with customers' expectations (i.e., GPS, etc.) and powertrain complexity (i.e., added controllers, etc.). However, in all timeframes, the non-conventional powertrains generally consume less power, except in the high cases. The values shown in Figure 44 are representative of an average consumption during the standard cycle testing (i.e., dynamometer test). Real-world accessory consumption would be higher. 
Argonne National Laboratory Report - Light-Duty Vehicle Fuel Consumption Displacement Potential Up to 2045

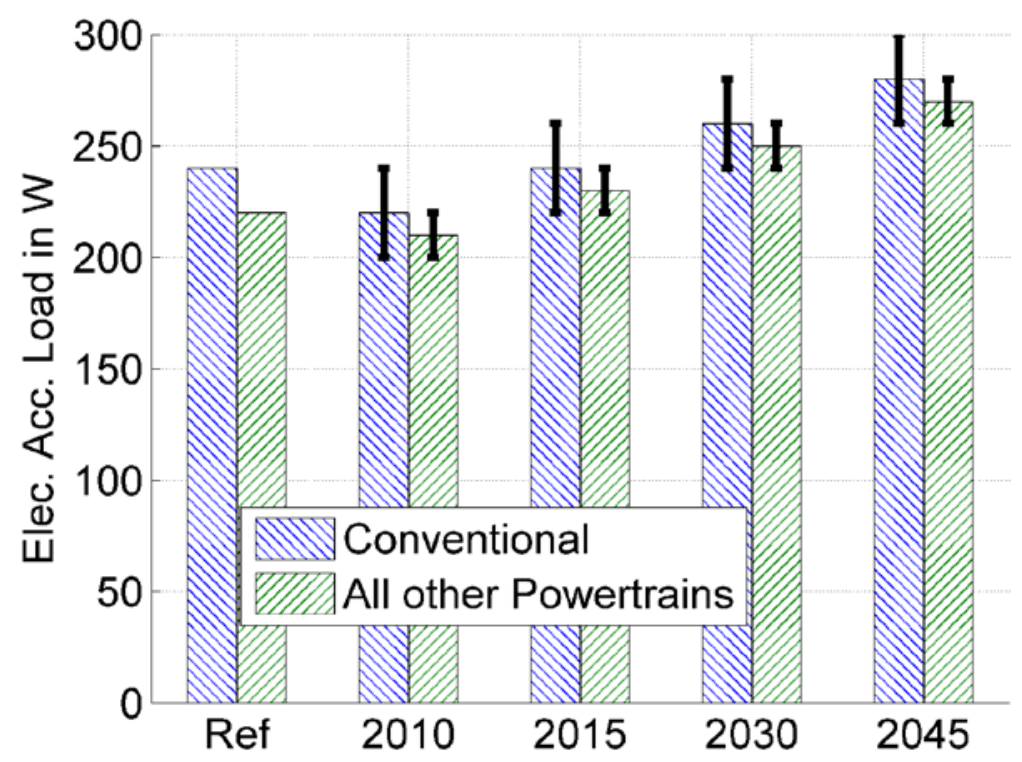

Figure 44 - Electrical accessory load. 


\section{POWERTRAIN SELECTION}

As discussed previously, hundreds of powertrain options are possible. The following powertrains were selected for the electric-drive vehicles:

- $\quad$ Single mode power-split hybrid with fixed gear ratio (HEV, PHEV10, PHEV20)

- Series engine with two-speed gearbox (PHEV30, PHEV40)

- Series fuel cell with two-speed gearbox (HEV, PHEVs)

- $\quad$ Electric drive with two-speed gearbox for BEV

The selection of the single-mode power-split hybrid was based on the current sales volume of both Toyota and Ford hybrid vehicles, which makes that configuration the dominant one on the market. However, multi-mode configurations, such as the one implemented in the GM Tahoe (Grewe et al., 2007), are interesting options, especially for SUV applications (Kim et al., 2010), and will be added in future studies.

The series engine configuration selected is the simplest one and has been used by many companies. For this option, the E-REV powertrain used in the GM Volt (Tate and Savagian, 2009) offers significant advantages, especially during high-vehicle-speed operations. Since the Volt configuration was not yet public at the beginning of this project, it has not been used but will also be added in future updates.

All the vehicles driven solely by electrical power use a two-speed gearbox. This choice was made to reach the vehicle maximum-speed requirement of at least $100 \mathrm{mph}$. This transmission also allows an increase in the powertrain efficiency. Another option to improve the electrical consumption is to use two electric machines (as is the case in the GM Volt), but the multi-speed option was preferred in order to meet the vehicle maximum-speed requirements.

This study will be regularly updated, and the authors are planning to use both the multi-mode transmission and E-REV configurations in future work. A significant amount of work has already been done with the two-mode hybrid as the powertrain, and vehicle-level controls have been developed and validated using vehicle test data from the APRF at Argonne (Kim et al., 2009; Karbowski et al., 2010). Models of the E-REV as well as its preliminary vehicle-level energy management have also been developed and will be validated in the upcoming months. 


\section{VEHICLE-LEVEL CONTROL STRATEGIES}

It is easy to create a bad HEV, fuel-cell vehicle, etc., if all one is doing is assembling the various parts, but it takes significant knowledge to design one that meets all customers' expectations, from performance to fuel consumption and drive quality. The vehicle-level control strategies used for the powertrain described previously have been developed over the past 12 years (Pasquier, 2001; Pagerit et al., 2005; Sharer, 2008; Cao, 2007; Karbowski et al., 2006). Generic processes were developed over the years to not only create but also validate the vehicle-level control strategies.

Figure 45 shows the generic process developed by Argonne for energy management. The process is defined in three steps:

- Global Optimization (Karbowski et al., 2006): The objective of this step is to define the main rules. For example, the engine turns ON based on the battery SOC, vehicle speed, and wheel torque demand.

- Rule-Based Control: In this step, the rules previously defined are implemented into an algorithm (generally Simulink and StateFlow) and exercised to make sure they operate properly.

- Heuristic Optimization: The objective of the last step is to define the values of the parameters of the main control strategy. For example, at which wheel torque does the engine turn ON for a specific SOC? The algorithm Argonne generally uses to automatically define the parameters is DIRECT (Divided RECTangles).

Other approaches, such as instantaneous optimizations (Karbowski et al., 2010) have also been developed and implemented into Autonomie, but were not used in the present study. 
Global Optimization (Dynamic Programming)

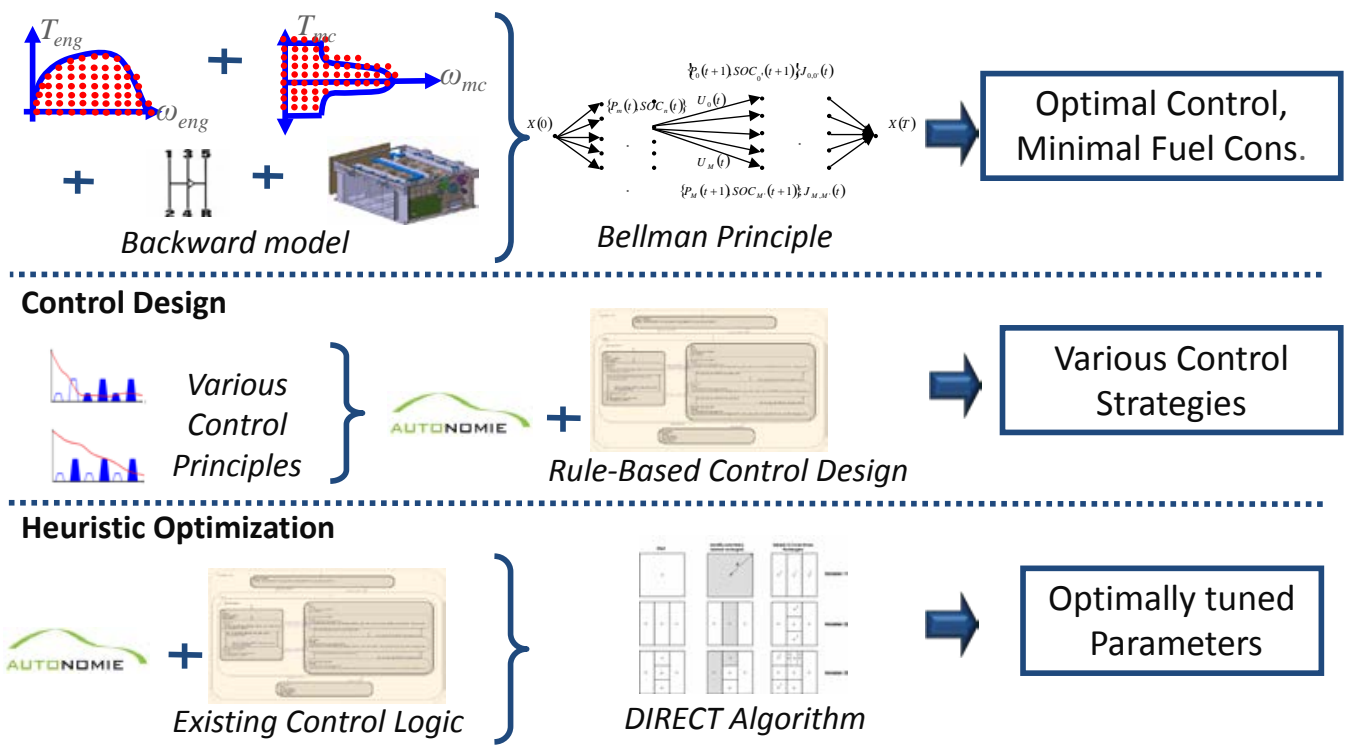

Figure 45 - Vehicle-level control strategy development.

Argonne has several state-of-the-art vehicle dynamometers and has been involved in testing and developing test procedures for advanced vehicles for many years. Over the past 12 years, numerous vehicle configurations from different classes have been tested at the APRF. More than 20 of them were modeled and validated into the Powertrain System Analysis Toolkit (PSAT) and now Autonomie, including conventional starter-alternators, full hybrids (input power-split, dual-mode power-split), plug-in hybrids (both after-market and manufacturer prototypes), and EVs. The vehicles were validated within $1 \%$ for the conventional vehicles, $2 \%$ for starteralternators, and $5 \%$ for hybrids, which in all cases is within the test-to-test repeatability. 


\section{VEHICLE DEFINITION}

\subsection{VEHICLE TECHNICAL SPECIFICATIONS}

All the vehicles have been sized to meet the same requirements:

- Initial vehicle movement (IVM) to $60 \mathrm{mi} / \mathrm{h}$ in $9 \mathrm{sec}+/-0.1 \mathrm{sec}$

- Maximum grade of $6 \%$ at $65 \mathrm{mi} / \mathrm{h}$ at gross vehicle weight (GVW)

- Maximum vehicle speed $>100 \mathrm{mi} / \mathrm{h}$

These requirements are a good representation of the current American automotive market as well as American drivers' expectations. A relationship between curb weight and gross vehicle weight (GVW was developed based on current technologies.

Table 10 summarizes the travel distances with a full tank of fuel for the different powertrains. The vehicles using gasoline, diesel or ethanol fuel have been sized for a distance of 500 miles on the combined driving cycle based on unadjusted fuel consumption. All vehicles have a range of at least 320 miles except the EV (150 miles) and the hydrogen vehicles.

Table 14: Summary of travel distances for all vehicles on the UDDS cycle based on unadjusted consumption

\begin{tabular}{|c|c|c|c|c|c|}
\hline & Timeframe & & & & \\
\hline Vehicle Type & Ref & 2010 & 2015 & 2030 & 2045 \\
\hline Conv H2 & 300 & 300 & 300 & 500 & 500 \\
\hline HEV H2, FC & 300 & 300 & 300 & 500 & 500 \\
\hline PHEV H2, FC & $300+$ AER & $300+\mathrm{AER}$ & $300+\mathrm{AER}$ & $500+\mathrm{AER}$ & $500+\mathrm{AER}$ \\
\hline EV & 150 & 150 & 150 & 150 & 150 \\
\hline
\end{tabular}

\subsection{SIZING ALGORITHMS}

Owing to the large number of vehicles (several thousand) and the diversity of powertrain options, it is not feasible to manually size each vehicle's components to match the performance targets. Some studies (Kromer and Heywood, 2008) defined their vehicles by maintaining a constant power-to-weight (P/W) ratio between all powertrain configurations. Owing to the impact of the component max torque shapes, maintaining a constant 
Argonne National Laboratory Report - Light-Duty Vehicle Fuel Consumption Displacement Potential Up to 2045

power to weight $(\mathrm{P} / \mathrm{W})$ ratio between all configurations leads to an inconsistent comparison between technologies because of different performances. Each vehicle should be sized independently to meet specific vehicle technical specifications.

Not properly sizing the components will lead to differences in both fuel consumption and cost and will influence the results. For example, the $\mathrm{P} / \mathrm{W}$ ratio for a 2010 midsize vehicle with IVM $=60 \mathrm{mi} / \mathrm{h}$ in $9 \mathrm{sec}$ varies from 85 to $75 \mathrm{~W} / \mathrm{kg}$, depending on the powertrain configuration. This difference will increase in the future because of a decrease in weight penalty for the electrified powertrains.

On this basis, we developed several automated sizing algorithms to provide a fair comparison between technologies. Different algorithms have been defined depending on the powertrain (i.e., conventional, powersplit, series, electric) and the application (i.e., HEV, PHEV).

All algorithms are based on the same concept: the vehicle is built from the bottom up, meaning each component assumption (i.e., specific power, efficiency, etc.) is taken into account to define the entire set of vehicle attributes (i.e., weight, etc.). This process is always iterative in the sense that the main component characteristics (i.e., maximum power, vehicle weight, etc.) are changed until all the vehicle technical specifications are met. On average, the algorithm takes between 5 and 10 iterations to converge. Figure 46 shows an example of the iterative process for a conventional vehicle. 


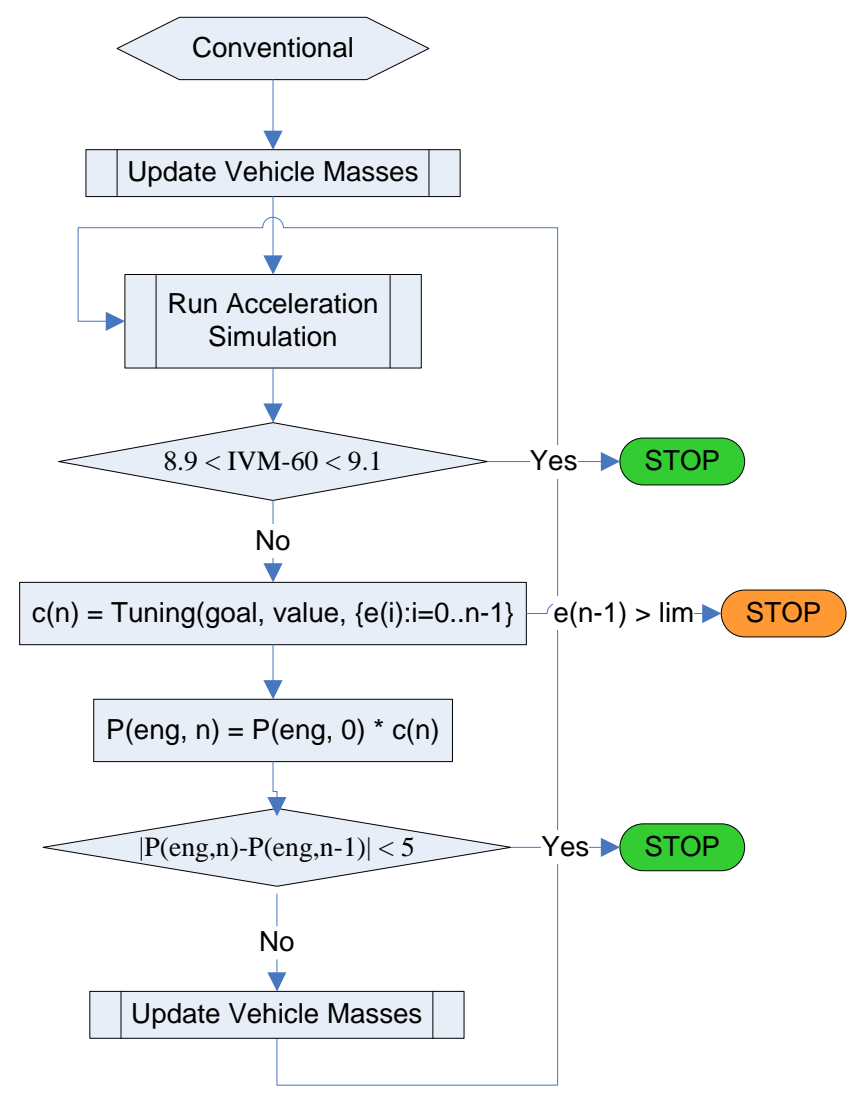

Figure 46 - Conventional-powertrain sizing algorithm.

Since each powertrain and application is different, the rules are specific. For example:

- For HEVs, the electric-machine and battery powers are determined to capture all the regenerative energy from a UDDS cycle. The engine and the generator are then sized to meet the grade ability and performance (IVM-60 $\mathrm{mi} / \mathrm{h}$ ) requirements.

- For PHEV10s and PHEV20s, the electric machine and battery powers are sized to be able to follow the UDDS cycle in electric-only mode (this control is only used for the sizing; a blended approach is used to evaluate consumptions). The battery usable energy is defined to follow the UDDS drive cycle for 10 or 20 miles, depending on the requirements. The engine is then sized to meet both performance and grade ability requirements (usually, grade ability is the determining factor for PHEVs).

- For PHEV30s and PHEV40s, the main electric-machine and battery powers are sized to be able to follow the aggressive US06 drive cycle (duty cycle with aggressive highway driving) in electriconly mode. The battery usable energy is defined to follow the UDDS drive cycle for 30 or 40 miles, depending on the requirements. The genset (engine + generator) or the fuel-cell systems are sized to meet the grade ability requirements. 
It is important to note that the sizing algorithms provide the optimum component sizes when OEMs would have to select among the available choices.

\subsection{SIZING RESULTS}

This section describes the maximum power, energy, and weight of the different vehicles after sizing. The details for all the vehicles simulated are located in Appendix 1.

\subsubsection{CONVENTIONAL POWERTRAIN}

The component characteristics of each vehicle class evolve similarly. In the following section, to avoid having too many figures and plots, only the compact class is presented.

Figure 47 shows the gasoline-engine peak power. One notices a small decrease in peak power due to lightweighting.

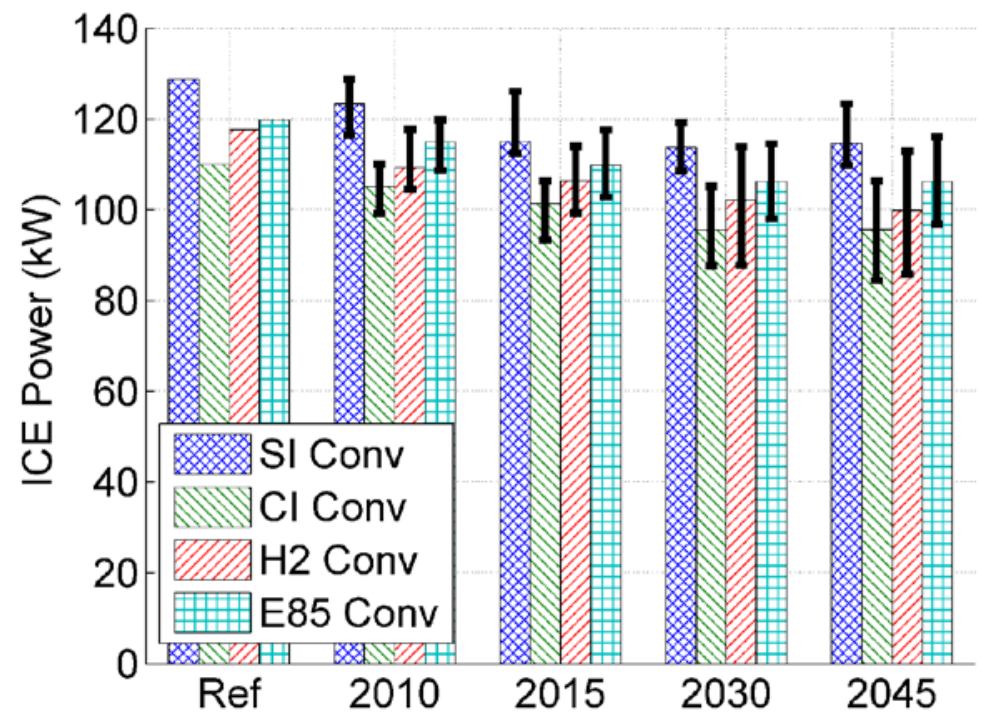

Figure 47 - Engine peak power with conventional powertrain for compact car.

Figure 48 shows the peak power of the diesel, hydrogen, and ethanol engines compared to the gasoline engine. One notices that the diesel, hydrogen and ethanol ratios stay roughly constant over time at around 0.9, indicating that technology improvements (i.e., weight reduction, aerodynamics, etc.) influence all engine technologies similarly with respect to engine peak efficiency. 


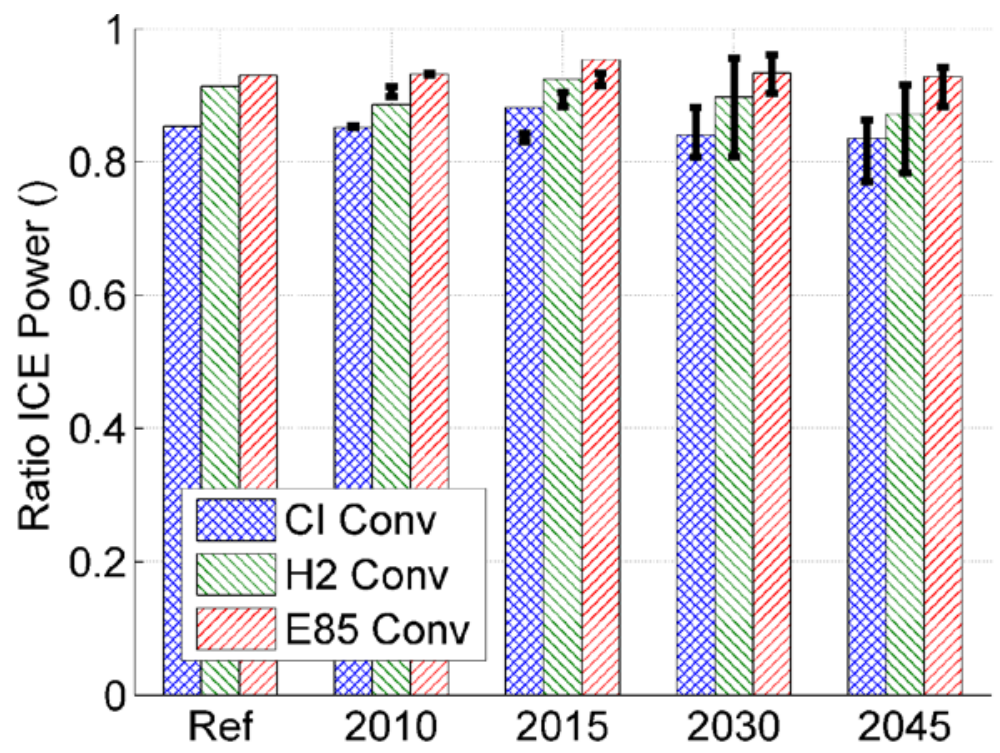

Figure 48 - Engine peak power compared to the same-year, same-case conventional gasoline engine for a compact car.

Figure 49 shows that engine power changes linearly with vehicle weight. The fuel order tracks the power ratios previously described. All engine technologies cover the same mass range but do not require the same power; higher torque is present at lower engine speed in the diesel case.

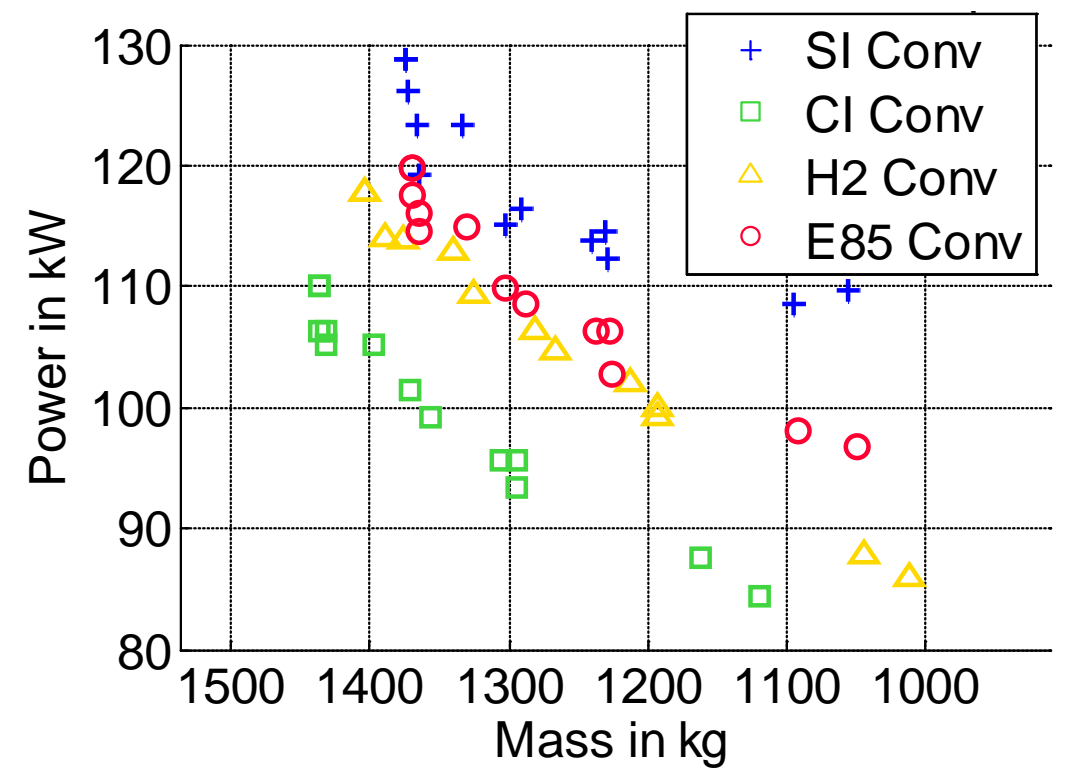

Figure 49 - Engine peak power as a function of vehicle mass for conventional gasoline engine.

The linear interpolation follows the equation

ICE Power $(k W)=a \times \operatorname{Mass}(k g)+b$ 
Argonne National Laboratory Report - Light-Duty Vehicle Fuel Consumption Displacement Potential Up to 2045

Table 11 gives the values of $a$ and $b$ for the different vehicle classes and fuels for conventional engines.

Table 15: Relationship between engine power and vehicle weight

\begin{tabular}{|l|l|l|l|l|l|l|l|l|}
\hline & \multicolumn{2}{l}{ SI } & \multicolumn{2}{l}{ CI } & \multicolumn{2}{l|}{ H2 } & \multicolumn{2}{l|}{ E85 } \\
\hline & a & b & a & b & a & b & a & b \\
\hline Compact & 0.0575 & 44.9358 & 0.0756 & -1.6804 & 0.0797 & 4.7481 & 0.0700 & 20.7229 \\
\hline Midsize & 0.0791 & 10.6411 & 0.0777 & -10.5299 & 0.0994 & -24.7923 & 0.0853 & -5.0726 \\
\hline Small SUV & 0.1009 & -9.6079 & 0.0725 & 0.6402 & 0.0934 & -12.4518 & 0.0927 & -4.6159 \\
\hline Midsize SUV & 0.0949 & -3.7142 & 0.0792 & -15.4696 & 0.0954 & -16.3857 & 0.0947 & -9.9181 \\
\hline Pickup & 0.1030 & -17.5006 & 0.0746 & -10.2896 & 0.0935 & -13.9417 & 0.1004 & -20.5003 \\
\hline
\end{tabular}

Figure 50 shows the vehicle curb weights.

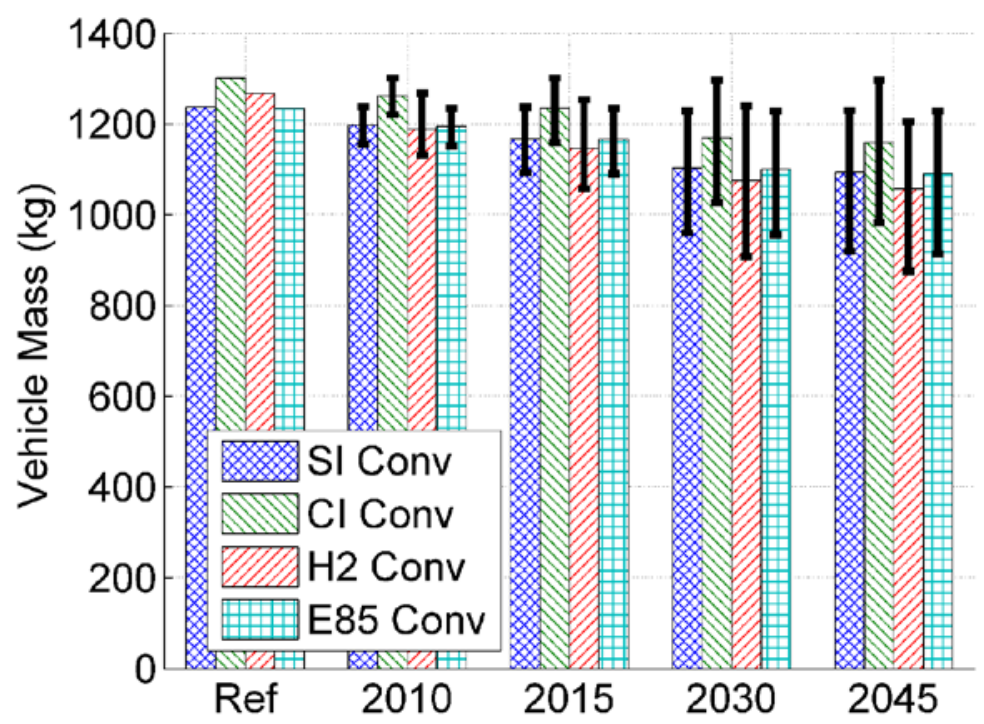

Figure 50 - Vehicle curb weights for compact cars with conventional powertrains. 


\subsubsection{ENGINE HEV}

\section{ENGINE}

Figure 51 shows the peak power for compact HEVs with gasoline engines. The engine power for HEVs is determined by both the performance and grade requirements. While performance is the primary factor for current technologies, future lightweighting makes grade ability requirements critical for some cases.

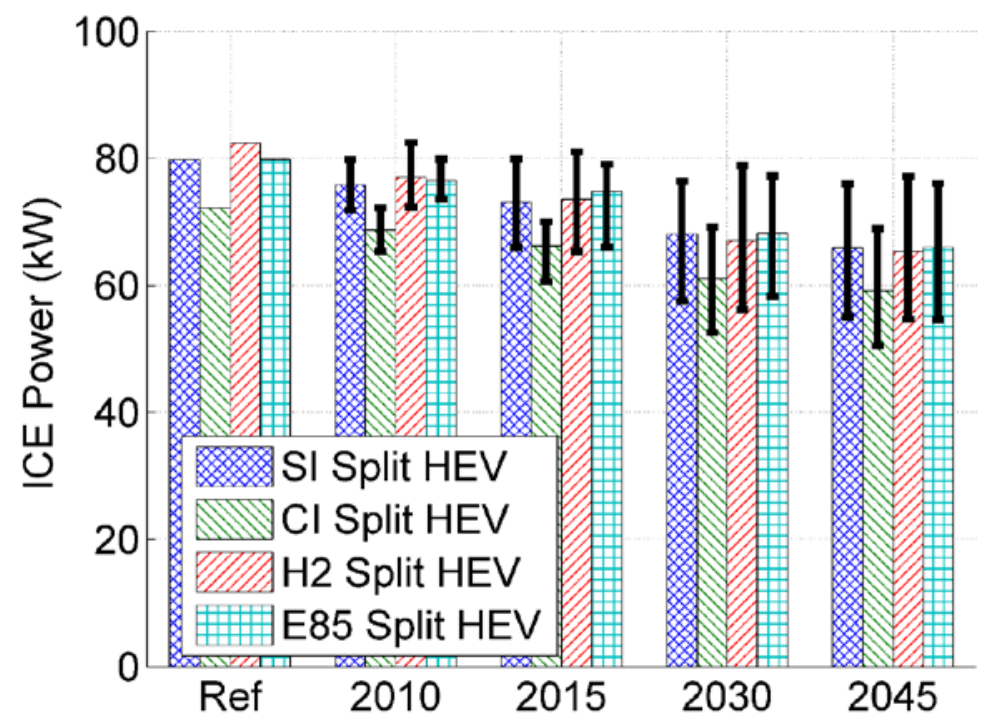

Figure 51 - Engine peak power for compact cars with conventional powertrains.

The ICE peak-power ratios also stay roughly constant over time for the power-split HEVs (Figure 52). Hydrogen and ethanol have approximately the same ratio over time, around 1, whereas diesel stays at 0.9. Engine sizes are comparable across fuels for HEVs over time, unlike for conventional engines (cf. Fig 49), where using diesel, hydrogen or ethanol fuels lead to engine downsizing. 
Argonne National Laboratory Report - Light-Duty Vehicle Fuel Consumption Displacement Potential Up to 2045

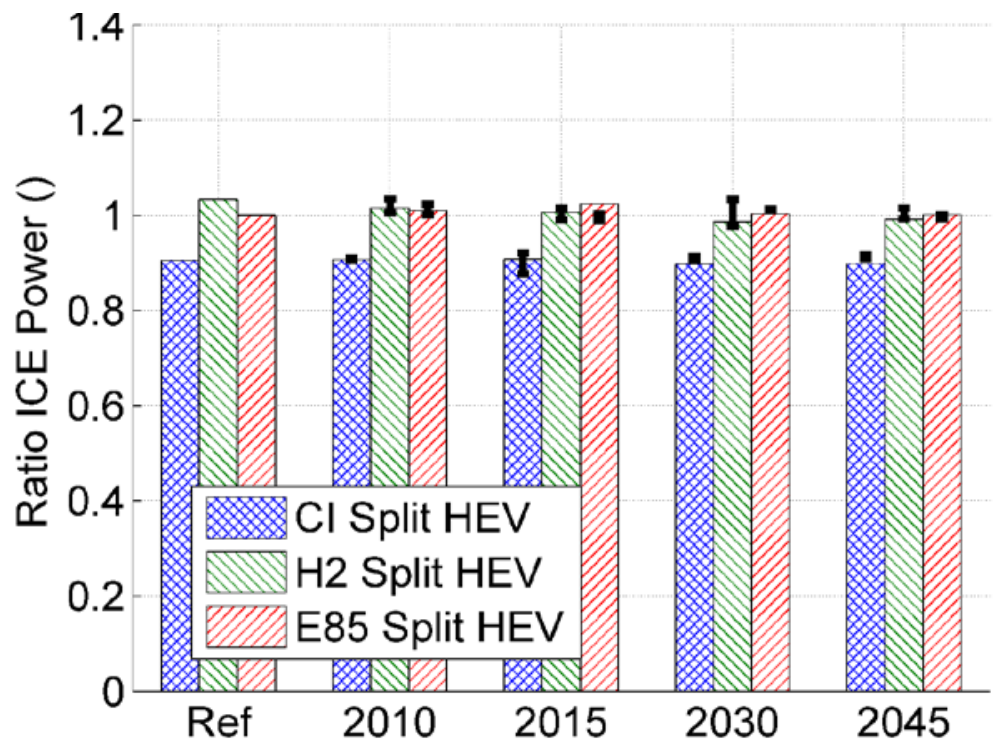

Figure 52 - Engine peak power compared to the same-year, same-case gasoline split-HEV engine for a compact car.

\section{ELECTRIC MACHINE}

Figure 53 shows the electric-machine power for HEVs with different fuels.

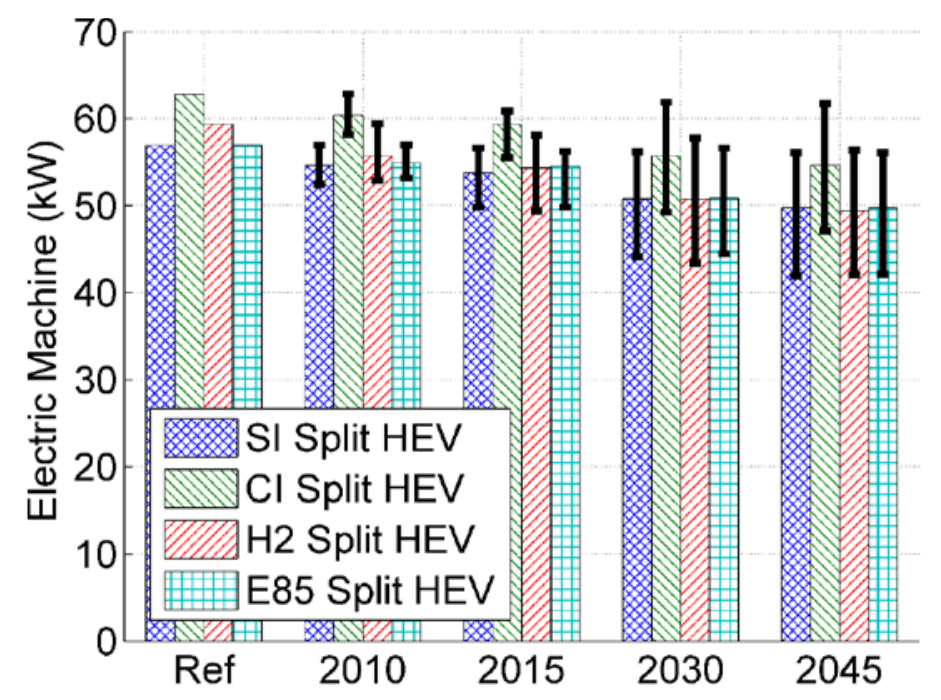

Figure 53 - Electric-machine power for compact split HEVs.

As shown in Figure 54, the peak-power ratios stay roughly constant over time. The diesel split HEV has a more powerful electric machine than the gasoline split HEV, but on the other hand, the hydrogen split HEV electric machine is less powerful than the gasoline vehicle or equal to it, in some cases. For the ethanol vehicle, the electric-machine ratios are in the same range as the ICE power ratios. We also noticed that the hydrogen vehicle 
has a peak power ratio of 1 in 2030 and 2045; high cases are explained by the bigger hydrogen storage system needed starting in 2030, which requires more electric machine power during the sizing.

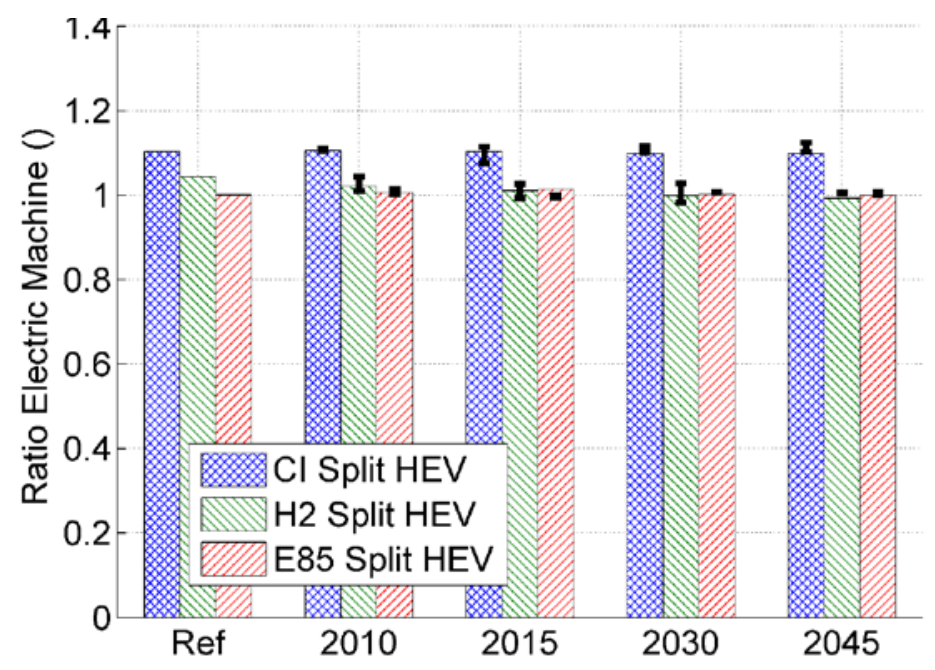

Figure 54 - Electric-machine peak power compared to the gasoline HEV engine for a compact car.

\section{BATTERY}

Figure 55 shows the HEV battery power. The powers are determined to capture the entire energy during deceleration on the UDDS drive cycle. Lightweighting and increased component efficiencies contribute to lower battery peak power.

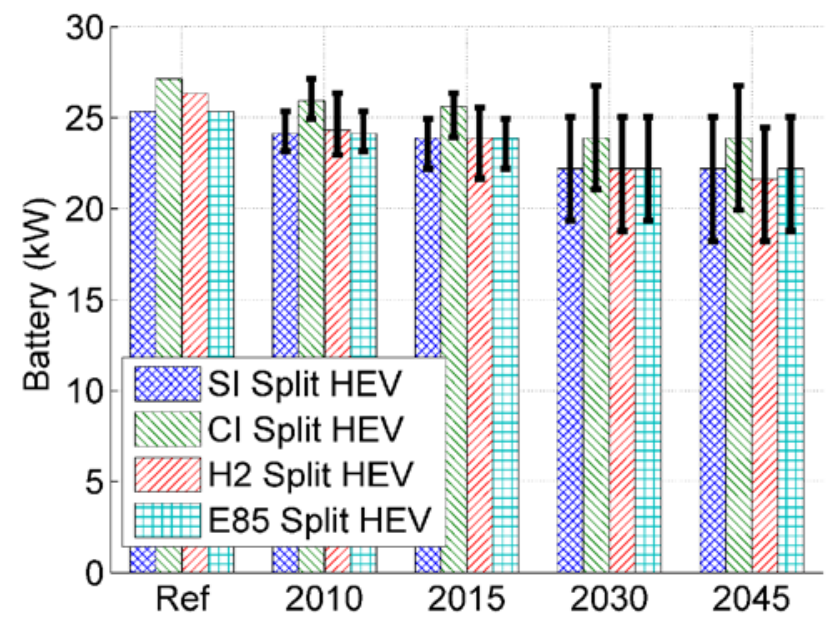

Figure 55 - Battery power for compact gasoline HEVs.

Since the sizing algorithm for HEVs does not modify the battery capacity, the trend of the total energy follows the total power. 


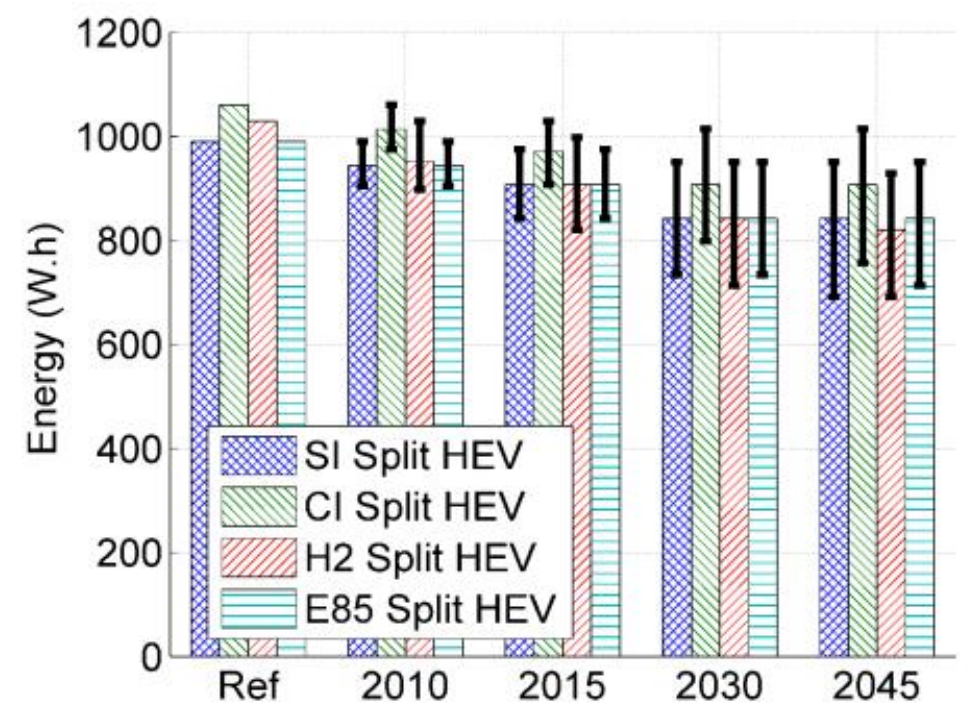

Figure 56 - Total battery energy for compact HEVs.

\section{VEHICLE WEIGHT}

Figure 57 shows the compact vehicles' curb weights. Vehicle weight reductions could range from $2 \%$ to $30 \%$ for gasoline, from $3 \%$ to $28 \%$ for diesel, from $7 \%$ to $34 \%$ for hydrogen and from $2 \%$ to $30 \%$ for ethanol.

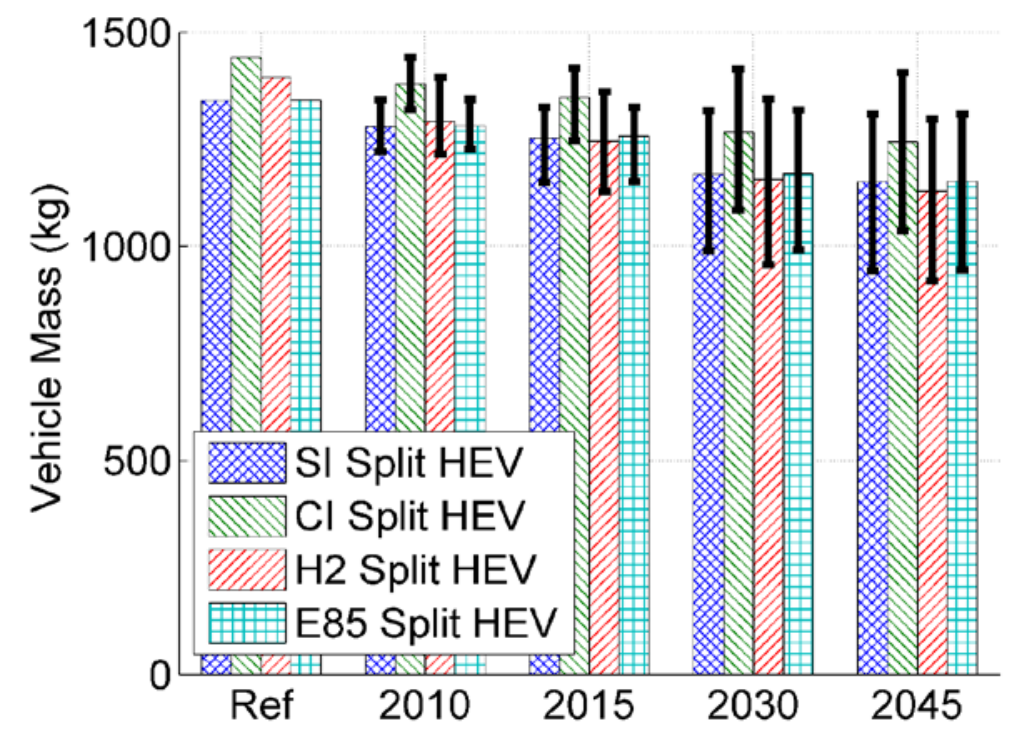

Figure 57 - Vehicle curb weight for compact engine-HEV powertrains. 
14.3.3. PHEV

ENGINE

Figure 58 shows the gasoline-engine peak power for the various PHEV powertrains and timeframes. In this case, owing to the large electric machine, the engines are all sized to provide gradeability.
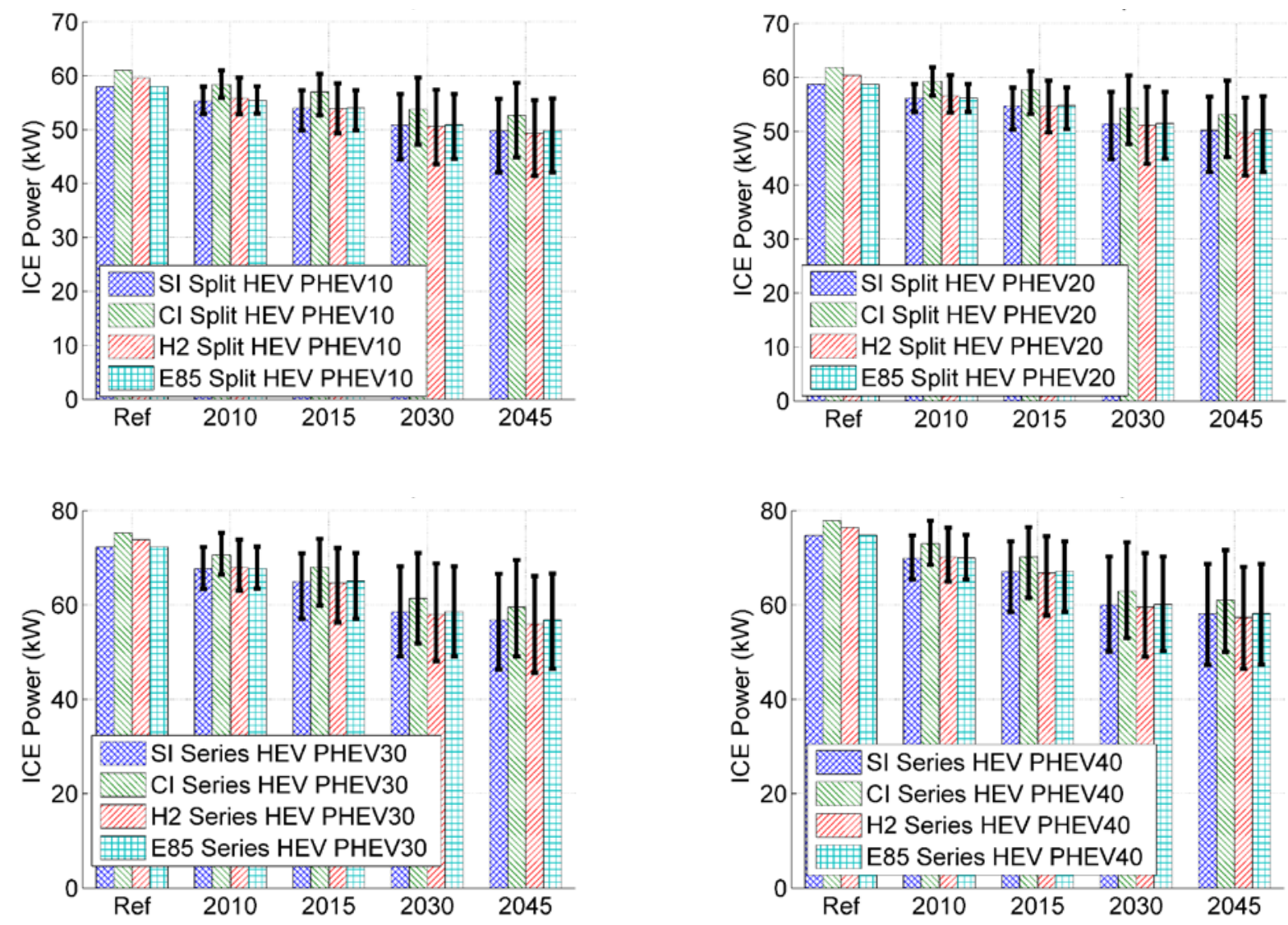

Figure 58 - Engine peak power for compact PHEV powertrains.

Across all the AERs for power-split PHEVs, the hydrogen engines consistently have a lower peak power than the gasoline engines except for the reference case, owing to the introduction of direct injection. Flex-Fuel engines have similar power to their gasoline counterpart while diesel are the most powerful. The power ratios between the various engines and the gasoline engine (Figure 59) are stable over time and from one AER to another. The trends are similar for the PHEV30 and PHEV40. 


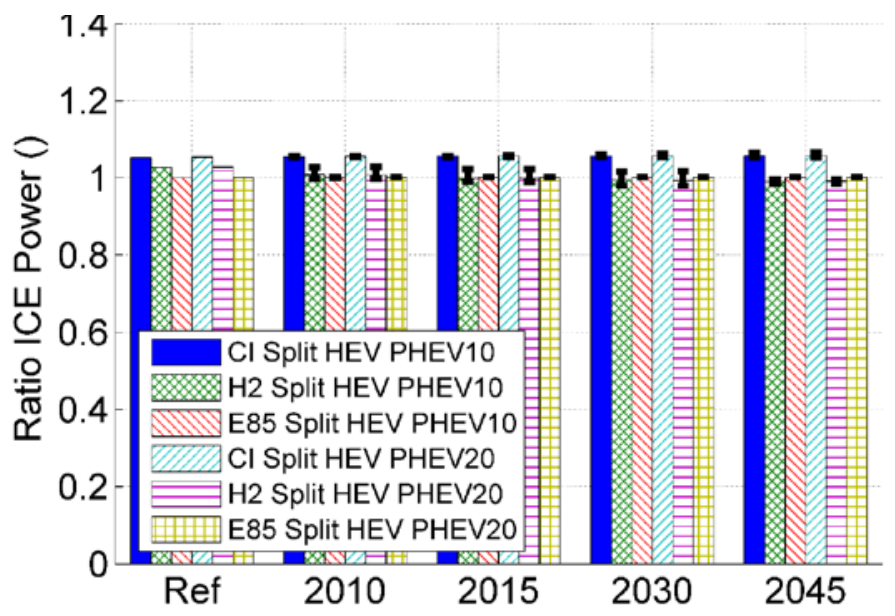

Figure 59 - ICE power for compact PHEV10s and PHEV20s relative to gasoline PHEV with matching AER.

\section{ELECTRIC MACHINE}

Figure 60 shows the peak power of the different electric machines for the PHEVs. The electric machines for the PHEV10 and PHEV20 cases are sized to have the capability to follow the UDDS drive cycle in EV mode, while the ones for the PHEV30 and PHEV40 cases allow the vehicles to follow the US06 drive cycle. Technology evolution leads to power reductions ranging from $3 \%$ to $28 \%$ by 2045 for PHEV10s, from $3 \%$ to $28 \%$ for PHEV20s, from $5 \%$ to $28 \%$ for PHEV30s, and from $5 \%$ to $29 \%$ for PHEV40s (gasoline).
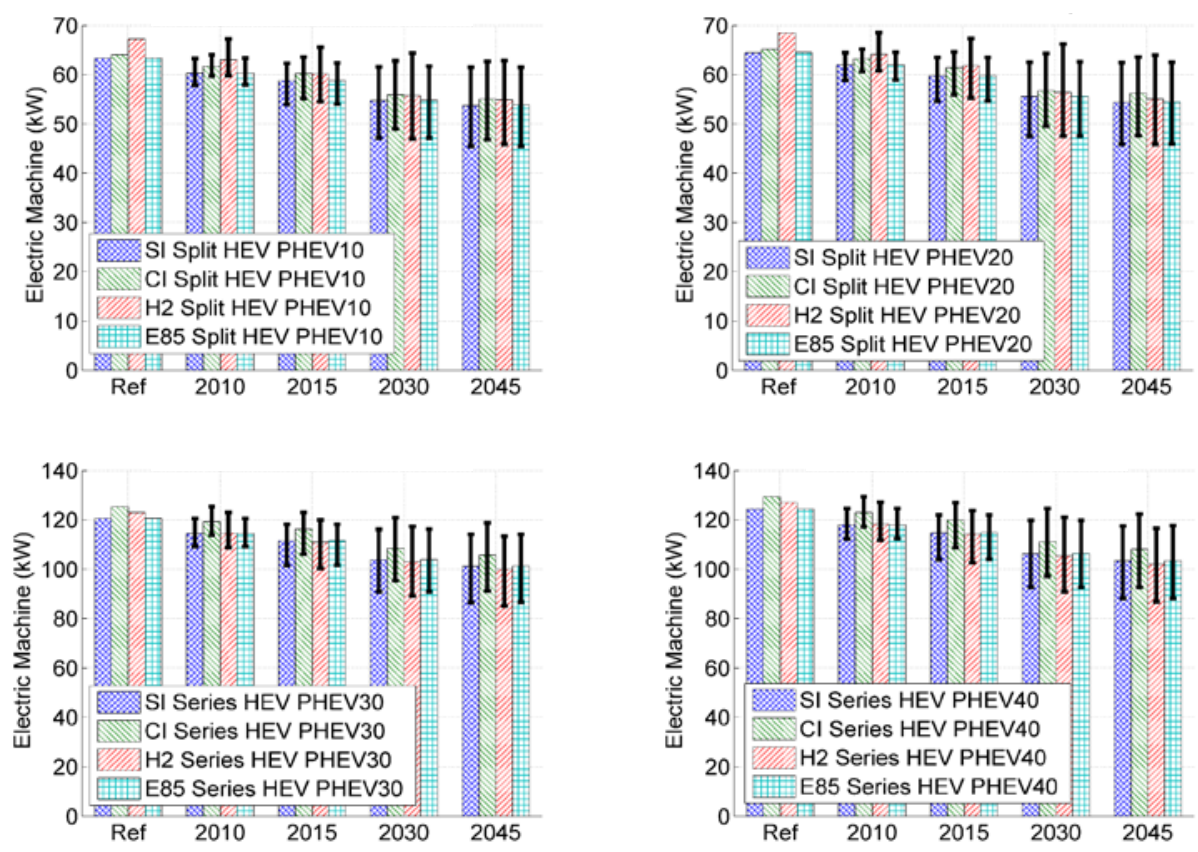

Figure 60 - Electric machine power for compact PHEVs. 


\section{BATTERY}

Figure 61 shows that the battery power for the PHEV10 and PHEV20 decreases by $16 \%$ over time. The battery for the PHEV30 and PHV40 has nearly 3 times more power than for the PHEV10 and PHEV20, owing to the need to follow the US06 cycle in electric-only mode. From one AER to the next, the battery power increases by an average of $1.8 \%$ for power-split and by an average of $2.6 \%$ for series powertrains.

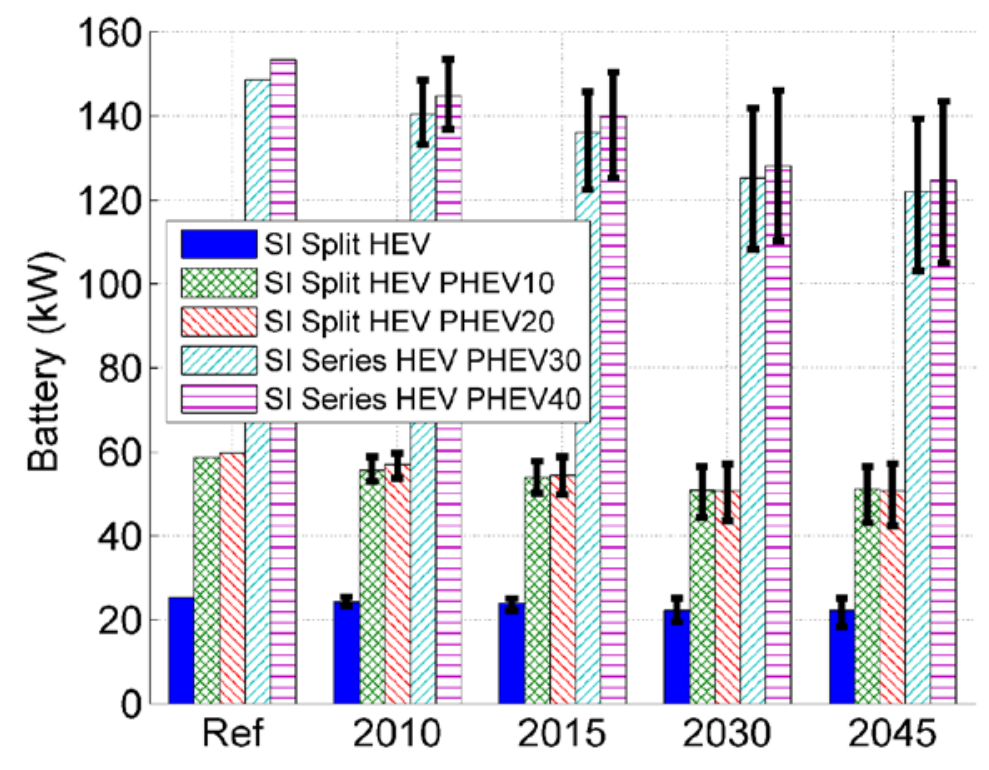

Figure 61 - Battery power for compact gasoline HEV and PHEVs.

Figure 62 shows that the usable battery energy is proportional to the AER for the various PHEVs. If the AER is multiplied by 2 , the usable battery energy will also be multiplied by 2 . For all of the AERs, the usable-energy decrease ranges from $4 \%$ to $32 \%$ by 2045 . 

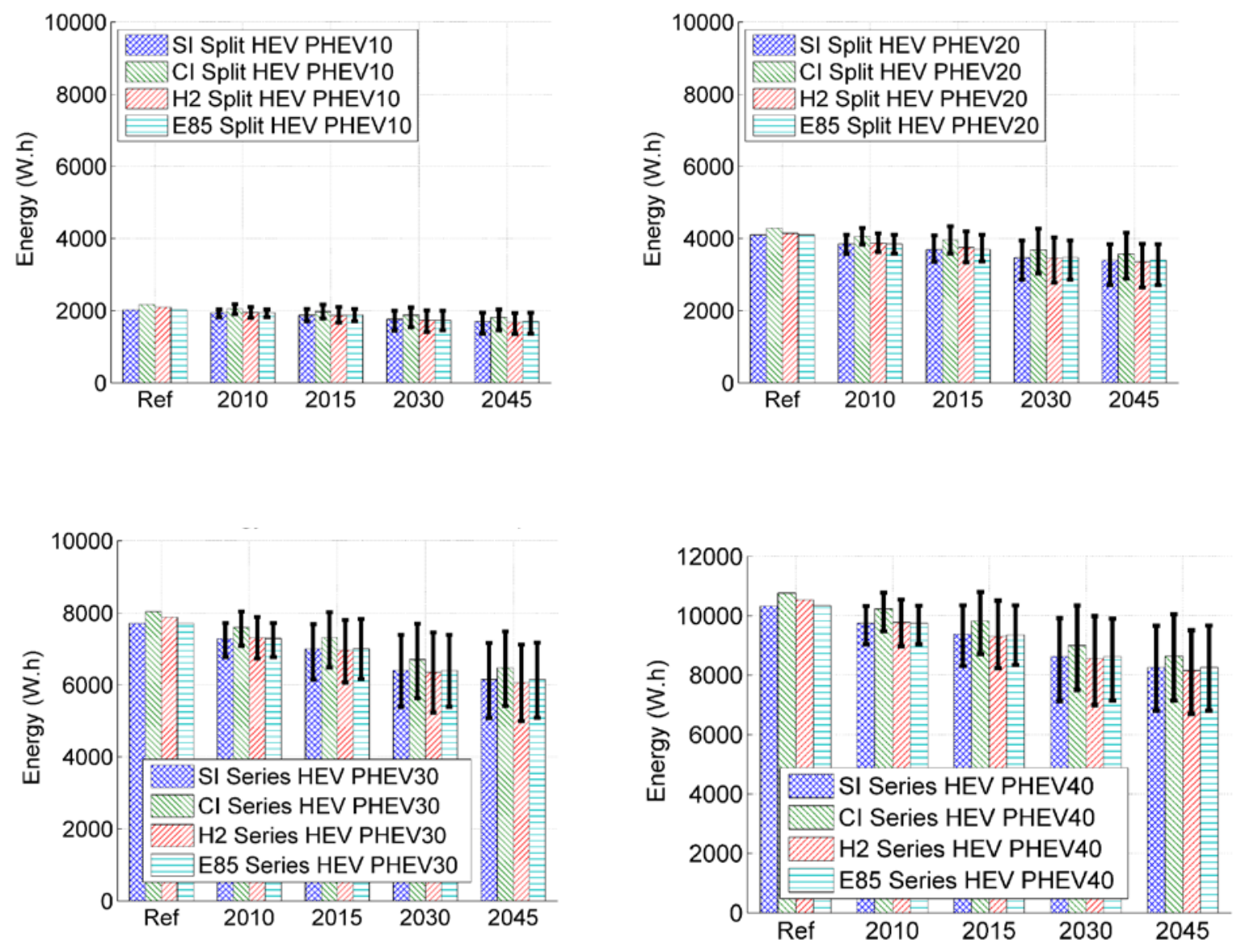

Figure 62 - Usable battery energy for compact PHEV10, 20, 30, and 40.

The PHEVs all show a linear relationship between the usable battery energy and the vehicle mass (Figure 63). However, it appears that the higher the AER, the greater the slope. 
Argonne National Laboratory Report - Light-Duty Vehicle Fuel Consumption Displacement Potential Up to 2045

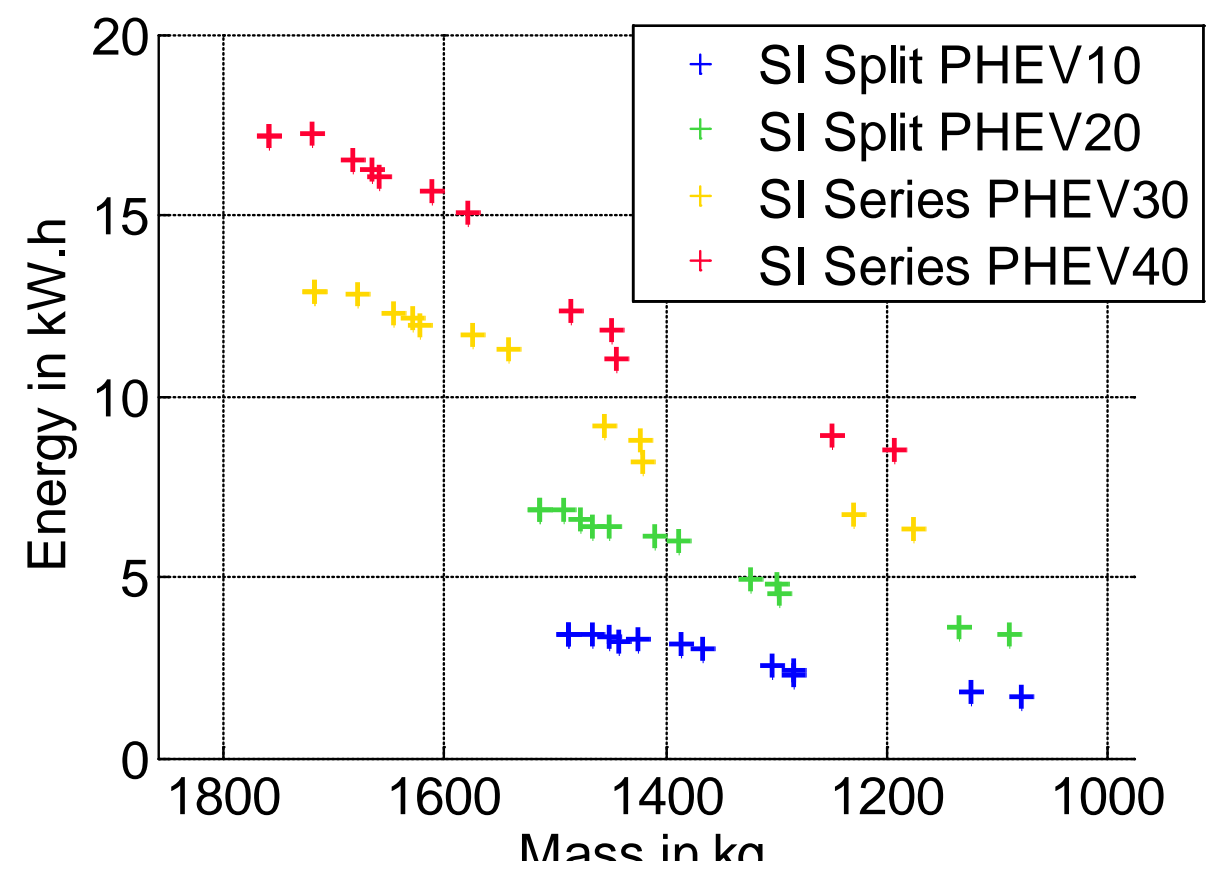

Figure 63 - Total battery energy as a function of vehicle mass for gasoline PHEVs.

The linear interpolation follows the equation

ESS Power $(k W)=a \times \operatorname{Mass}(k g)+b$

Table 12 gives the values of $a$ and $b$ for the different vehicle classes and powertrains for gasoline fuel.

Table 16: Relationship between battery energy and vehicle weight for gasoline PHEVs

\begin{tabular}{|l|l|l|l|l|l|l|l|l|}
\hline & \multicolumn{2}{l}{ Split PHEV10 } & \multicolumn{2}{l}{ Split PHEV20 } & \multicolumn{2}{l}{ Split PHEV30 } & \multicolumn{2}{l|}{ Split PHEV40 } \\
\hline & $\mathbf{a}$ & $\mathbf{b}$ & $\mathbf{a}$ & $\mathbf{b}$ & $\mathbf{a}$ & $\mathbf{b}$ & $\mathbf{a}$ & $\mathbf{b}$ \\
\hline Compact & 0.0045 & -3.2846 & 0.0088 & -6.4548 & 0.0133 & -9.7926 & 0.0171 & -12.5408 \\
\hline Midsize & 0.0046 & -3.9992 & 0.0089 & -7.8101 & 0.0136 & -12.1493 & 0.0177 & -15.7462 \\
\hline Small SUV & 0.0049 & -4.0780 & 0.0101 & -9.0512 & 0.0142 & -12.1689 & 0.0184 & -16.1091 \\
\hline Midsize SUV & 0.0037 & -2.7155 & 0.0094 & -9.0786 & 0.0135 & -12.5776 & 0.0172 & -16.0397 \\
\hline Pickup & 0.0040 & -3.6998 & 0.0104 & -12.0598 & 0.0153 & -18.1235 & 0.0181 & -19.9827 \\
\hline
\end{tabular}


VEHICLE WEIGHT

Figure 64 shows the vehicles' curb weights.
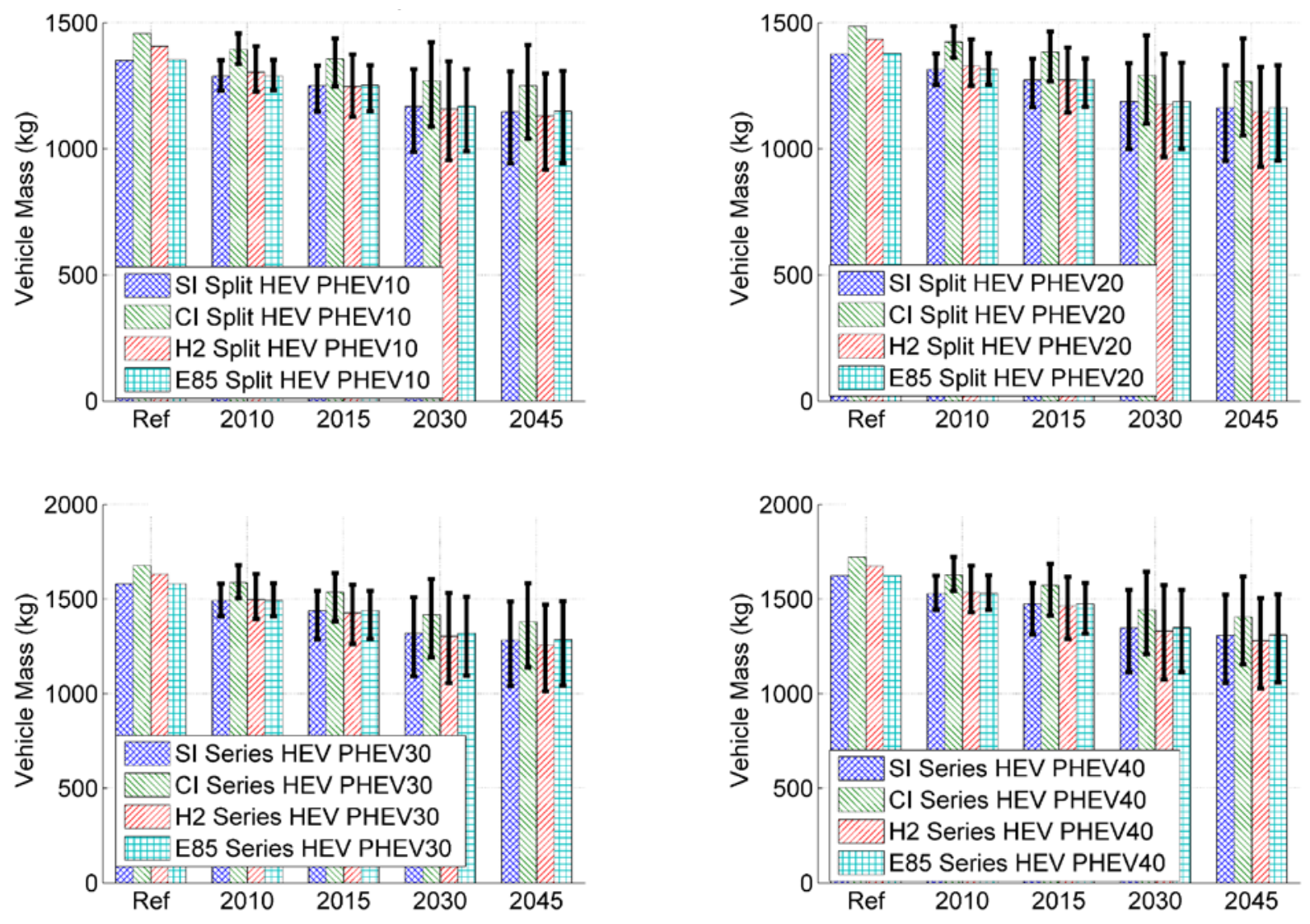

Figure 64 - Vehicle curb weights for compact PHEVs.

\subsubsection{FUEL-CELL HEV}

Fuel-cell systems, like other components, show a decrease in peak power over time (Figure 65), which is mostly due to vehicle lightweighting. The total decrease from the reference case to the 2045 case ranges from $26 \%$ to $52 \%$. 


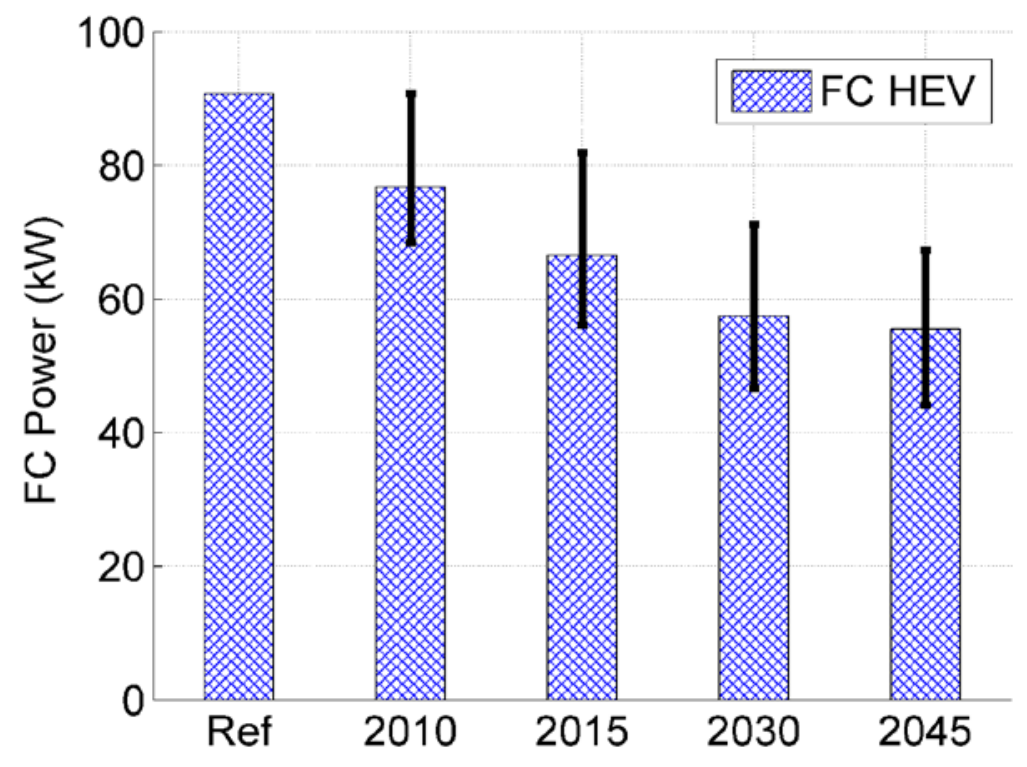

Figure 65 - Fuel-cell system power for compact fuel-cell HEVs.

Figure 66 shows that the electric-machine peak power shows a decrease ranging from $20 \%$ to $43 \%$ by 2045.

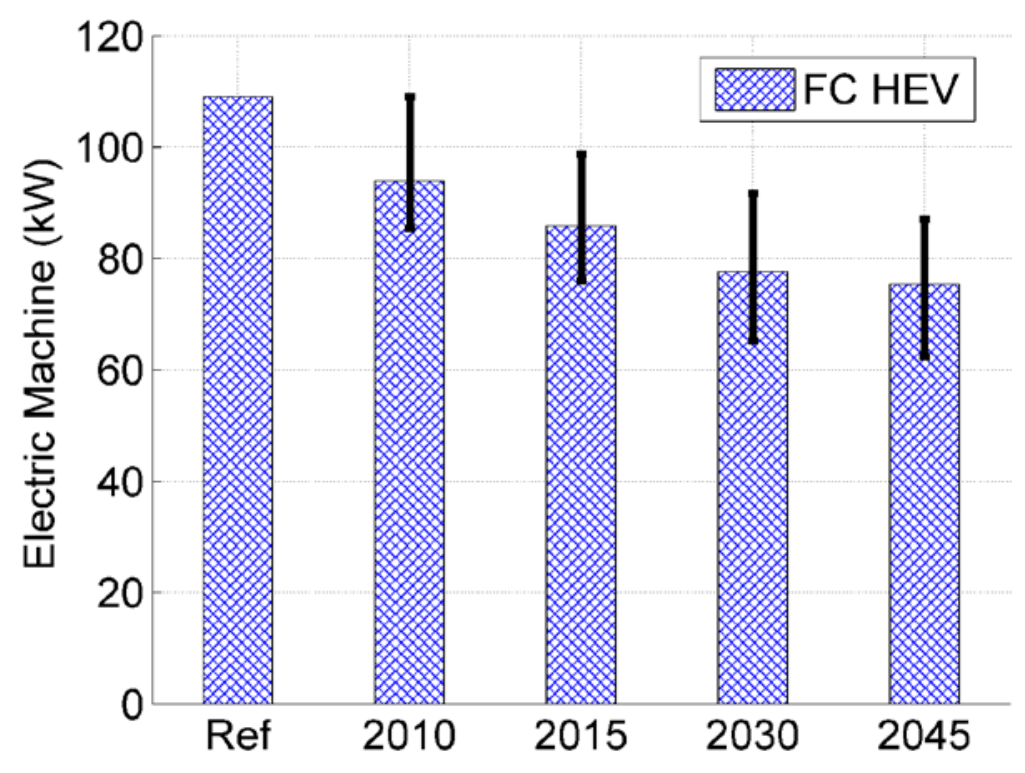

Figure 66 - Electric-machine power for compact fuel-cell HEVs.

The total battery energy does not show a continuous decrease over time. The pattern shown in 2010 and 2015 (Figure 67) can be explained by the battery technology used. HEV applications use a NiMH battery in the 2010 low and average cases and in the 2015 low case. For the rest of the timeframes, a Li-ion battery is used for 
HEVs. The cell voltage is much higher for the Li-ion battery than for the NiMH battery and thus the battery energy is a little higher

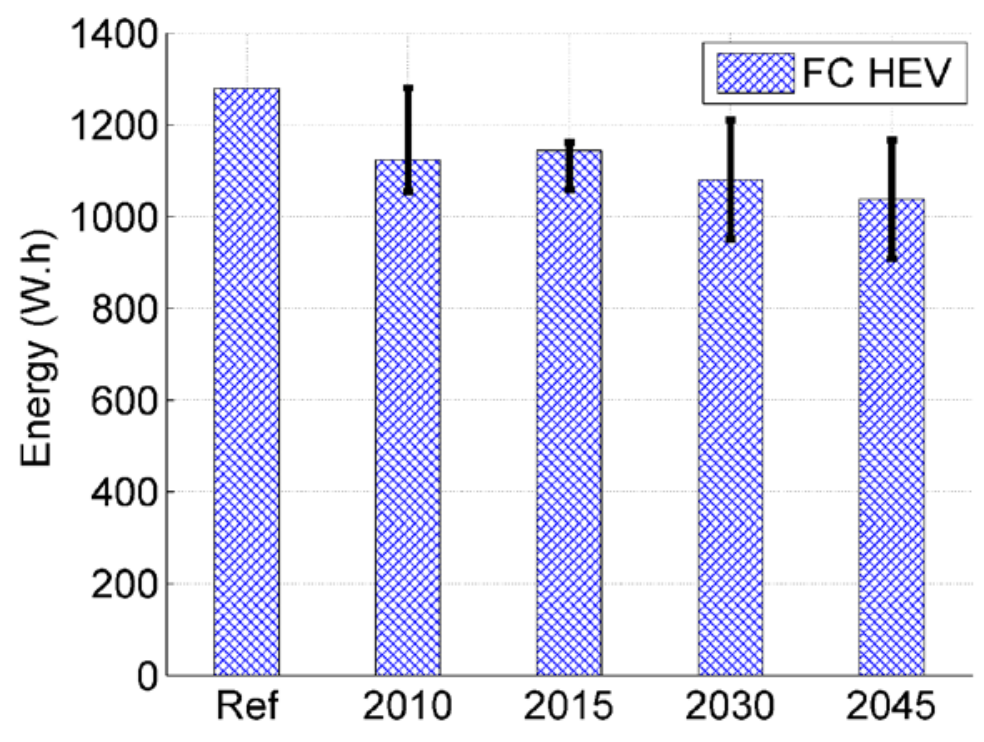

Figure 67 - Total battery energy for compact fuel-cell HEVs.

Figure 68 shows the vehicle curb weights. The fuel-cell vehicles show one of the most aggressive weight decreases compared to other powertrain configurations, owing to the hydrogen storage and fuel-cell system specific-power predictions. The vehicle-weight decrease by 2045 ranges from $20 \%$ to $43 \%$.

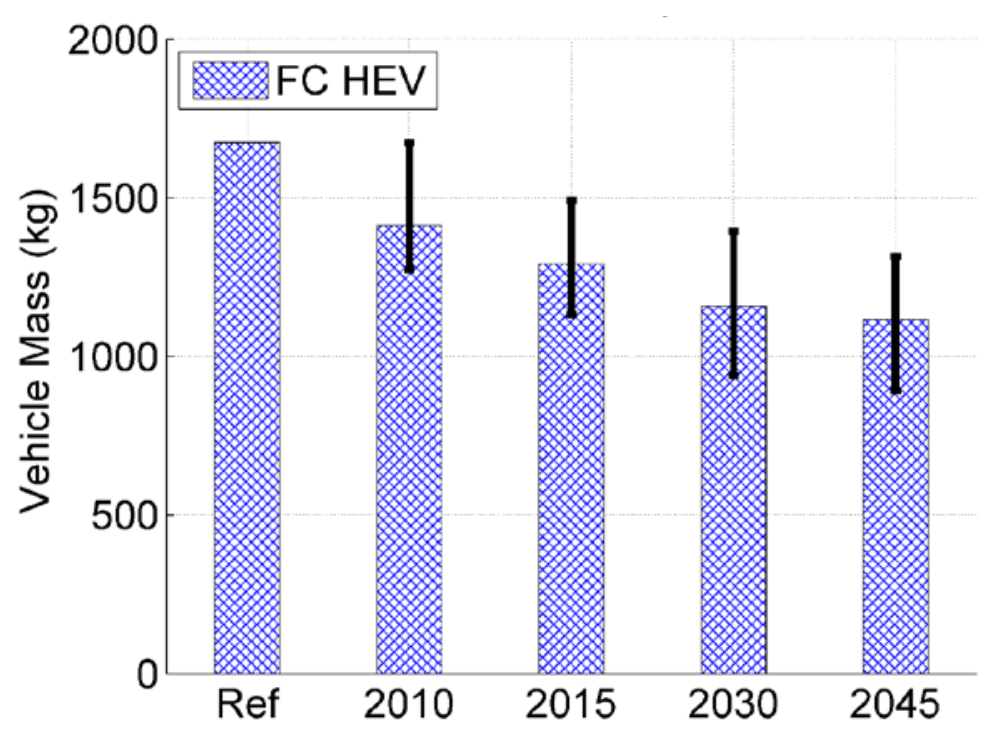

Figure 68 - Vehicle curb weight for compact fuel-cell HEVs. 

2045

\subsubsection{FUEL-CELL PHEV}

The fuel-cell system power decreases over time for all the AERs, with a reduction that ranges from $18 \%$ to 41\% (Figure 69). From one AER to another, the changes in fuel-cell power are very small.

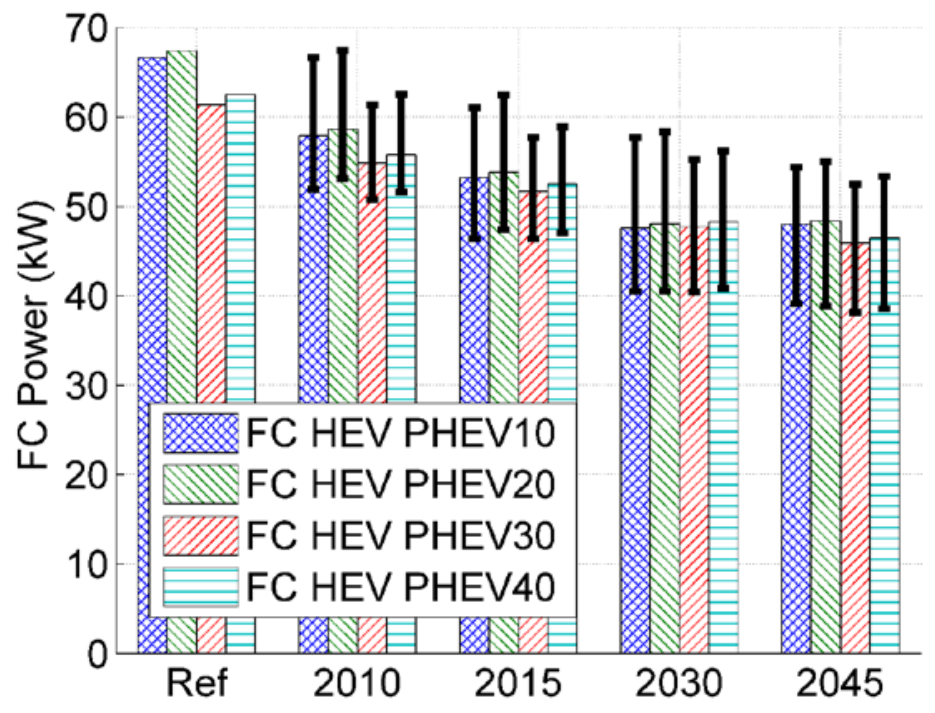

Figure 69 - Fuel-cell system power for compact fuel-cell PHEVs.

As shown in Figure 70, in terms of usable battery energy, the same pattern described for power-split PHEVs can be observed for fuel-cell PHEVs. The energy is proportional to the AER and the energy decreases continuously over time. For all of the AERs, the usable battery energy is from $14 \%$ to $42 \%$ lower by 2045 compared to the reference case.

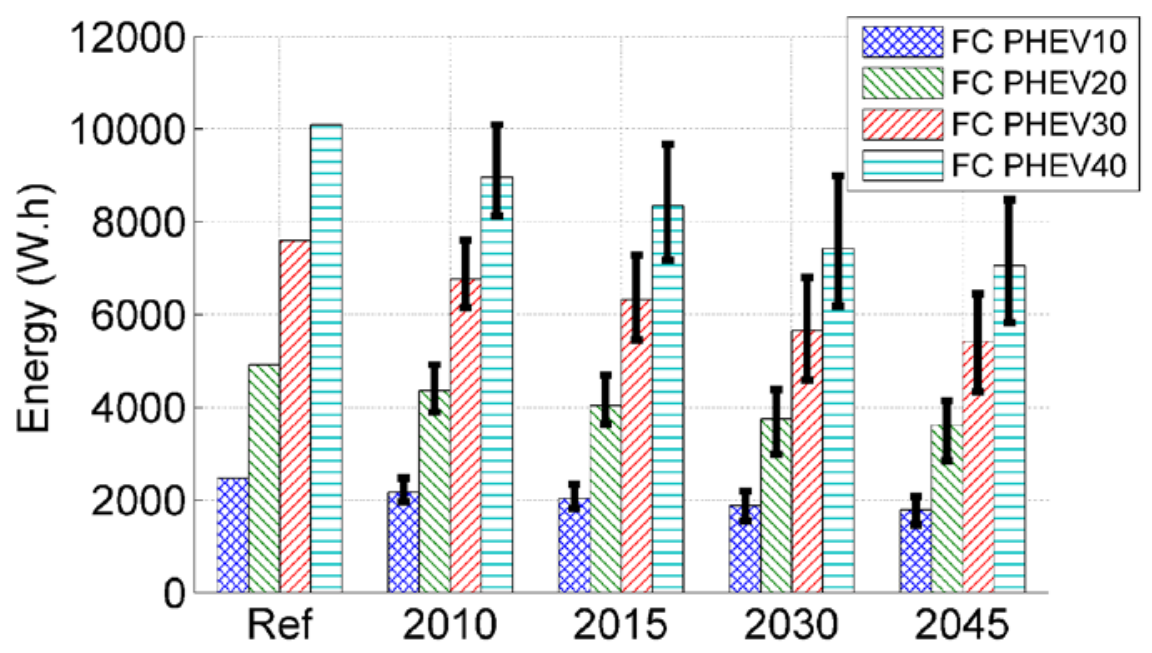

Figure 70 - Usable battery energy for compact fuel-cell PHEVs. 
The electric-machine power continuously decreases by between $11 \%$ and $41 \%$ over time from the reference case to 2045 (Figure 71). The higher the AER, the higher the electric-machine power within the same year and case.

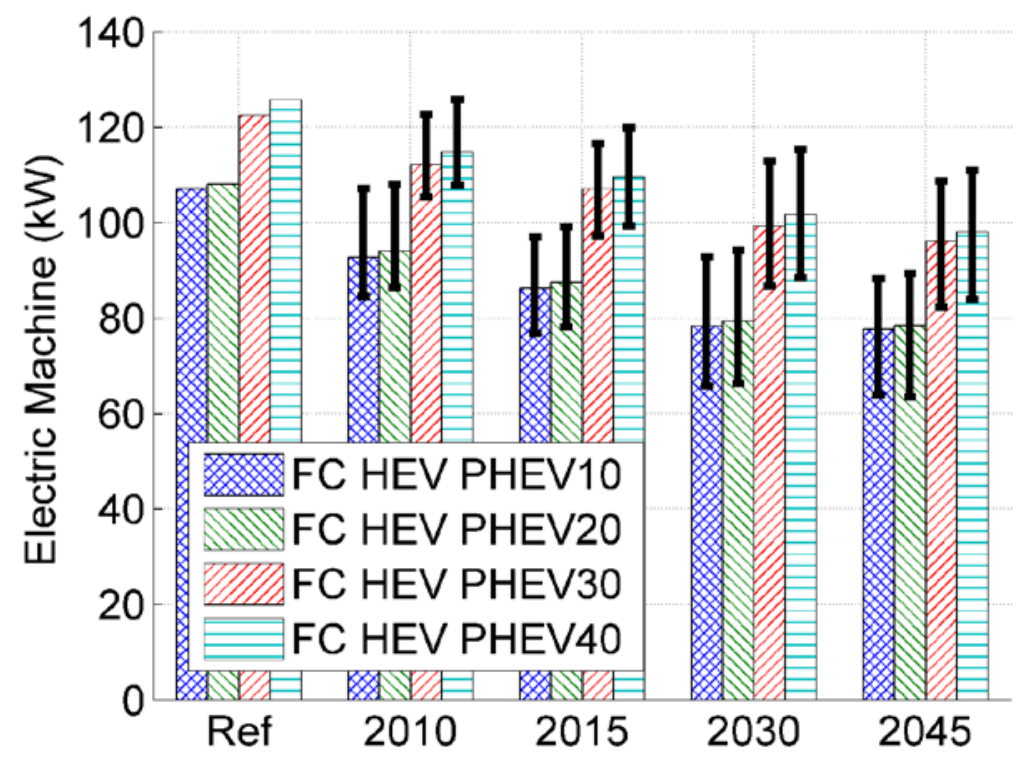

Figure 71 - Electric-machine power for compact fuel-cell PHEVs.

Figure 72 shows the vehicle curb weights. 

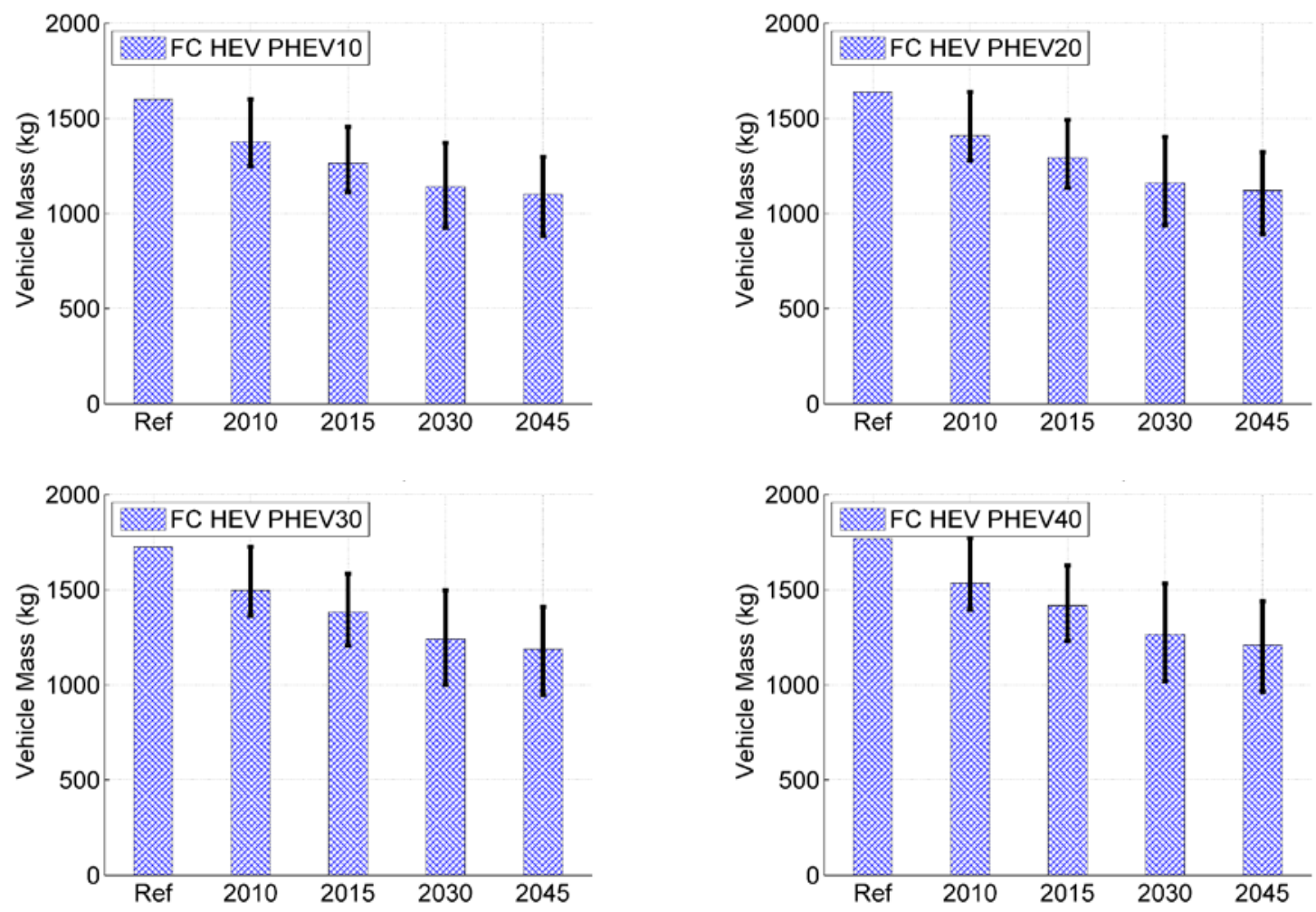

Figure 72 - Vehicle curb weights for compact fuel-cell PHEVs.

As shown in Figure 73, the battery energy has a linear relationship to vehicle mass, with a slope that increases with the AER.

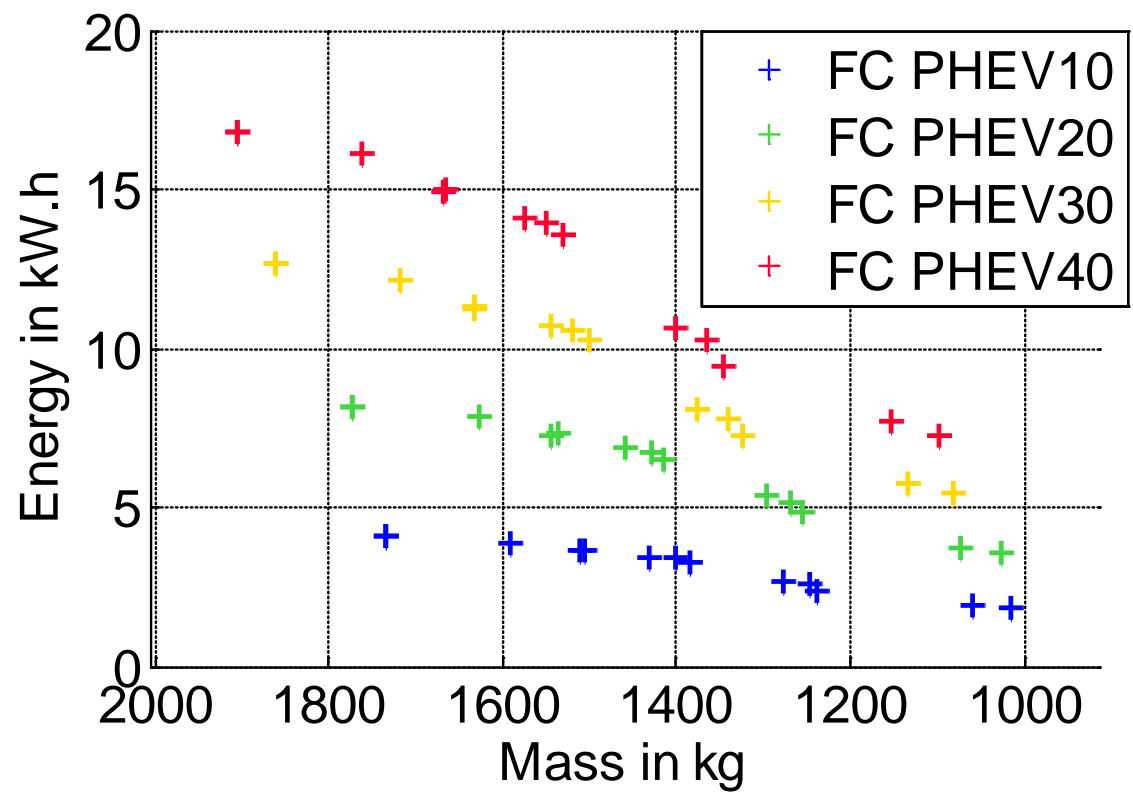

Figure 73 - Battery energy as a function of vehicle mass for compact fuel-cell PHEVs. 
Argonne National Laboratory Report - Light-Duty Vehicle Fuel Consumption Displacement Potential Up to 2045

The relationship follows the equation

ESS Power $(k W)=a \times \operatorname{Mass}(k g)+b$

Table 13 gives the values of $a$ and $b$ for the various fuel-cell vehicle classes and powertrains.

Table 17: Relationship between battery energy and vehicle weight for fuel-cell PHEVs

\begin{tabular}{|l|l|l|l|l|l|l|l|l|}
\hline & \multicolumn{2}{l}{ FC PHEV10 } & \multicolumn{2}{l}{ FC PHEV20 } & \multicolumn{2}{l}{ FC PHEV30 } & \multicolumn{2}{l|}{ FC PHEV40 } \\
\hline & a & $\mathbf{b}$ & $\mathbf{a}$ & $\mathbf{b}$ & $\mathbf{a}$ & $\mathbf{b}$ & $\mathbf{a}$ & b \\
\hline Compact & 0.0034 & -1.6171 & 0.0067 & -3.1784 & 0.0101 & -5.5256 & 0.0129 & -6.9619 \\
\hline Midsize & 0.0040 & -2.9492 & 0.0066 & -3.8277 & 0.0101 & -6.5327 & 0.0130 & -8.5352 \\
\hline Small SUV & 0.0036 & -1.8772 & 0.0069 & -3.5100 & 0.0115 & -7.6782 & 0.0138 & -8.6667 \\
\hline Midsize SUV & 0.0041 & -3.0393 & 0.0065 & -3.3493 & 0.0110 & -7.8634 & 0.0131 & -8.6881 \\
\hline Pickup & 0.0040 & -2.7255 & 0.0065 & -3.1824 & 0.0113 & -8.9508 & 0.0134 & -9.7746 \\
\hline
\end{tabular}

14.3.6. BATTERY ELECTRIC VEHICLE

Figure 74 shows the impact of lightweighting and improved aerodynamics and tires on the electricmachine peak power for BEVs. The electric machine is from $7 \%$ to $36 \%$ less powerful by 2045 compared to the reference case. The battery-power decrease between these timeframes ranges from $8 \%$ to $38 \%$, as shown in Figure 75. 
Argonne National Laboratory Report - Light-Duty Vehicle Fuel Consumption Displacement Potential Up to 2045

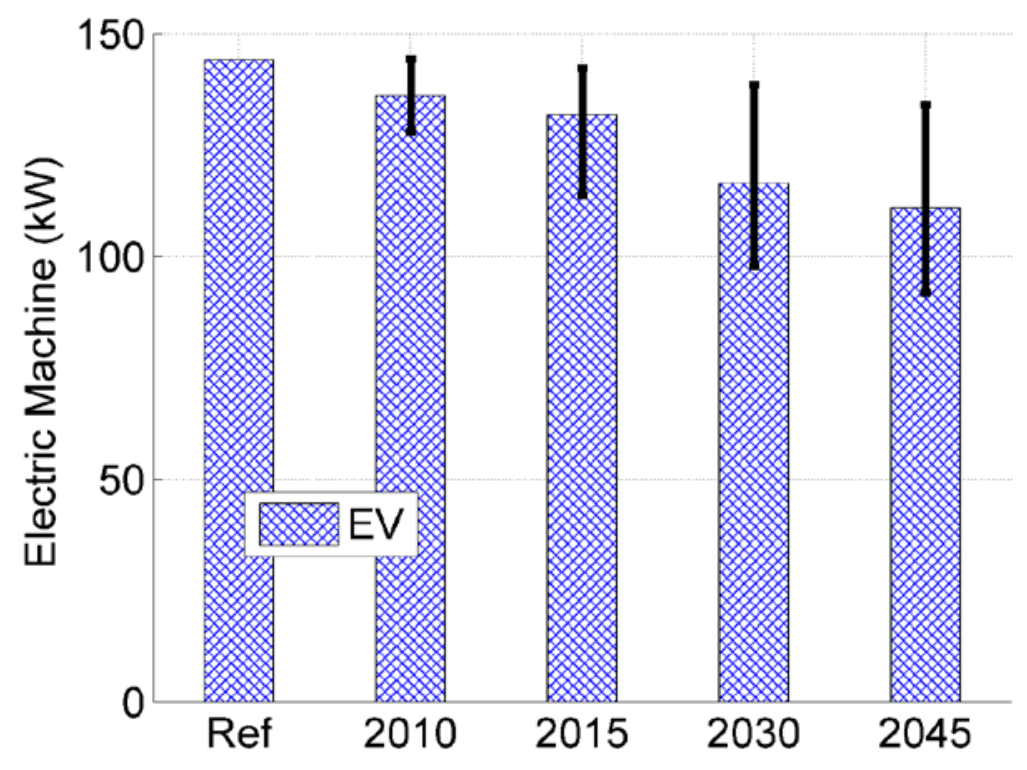

Figure 74 - Electric-machine power for compact BEVs.

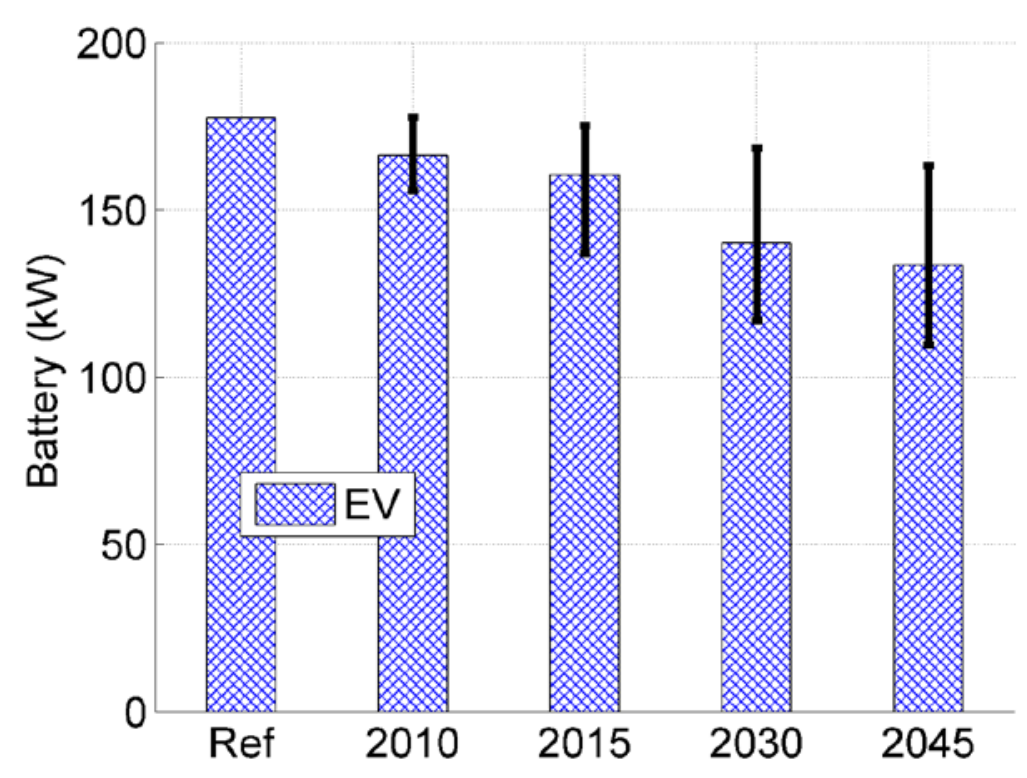

Figure 75 - Battery power for compact BEVs.

The decrease in usable energy for BEVs between the reference case and 2045 ranges from $8 \%$ to $42 \%$ (Figure 76). 


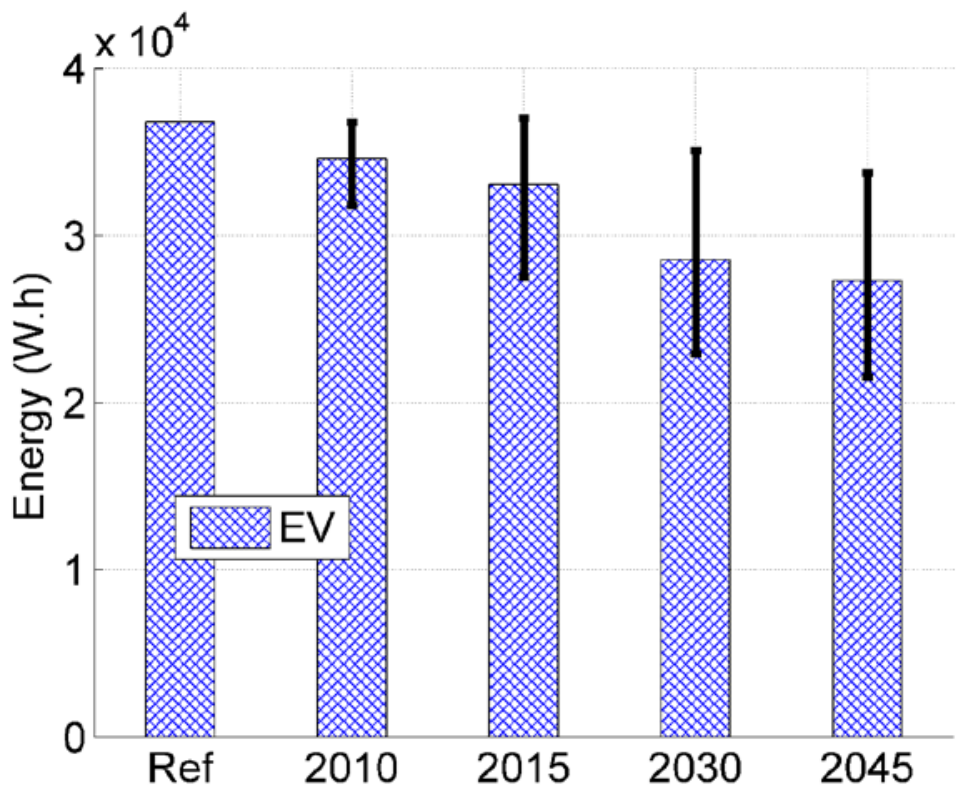

Figure 76 - Usable battery energy for compact BEVs.

Figure 77 shows the vehicles' curb weight.

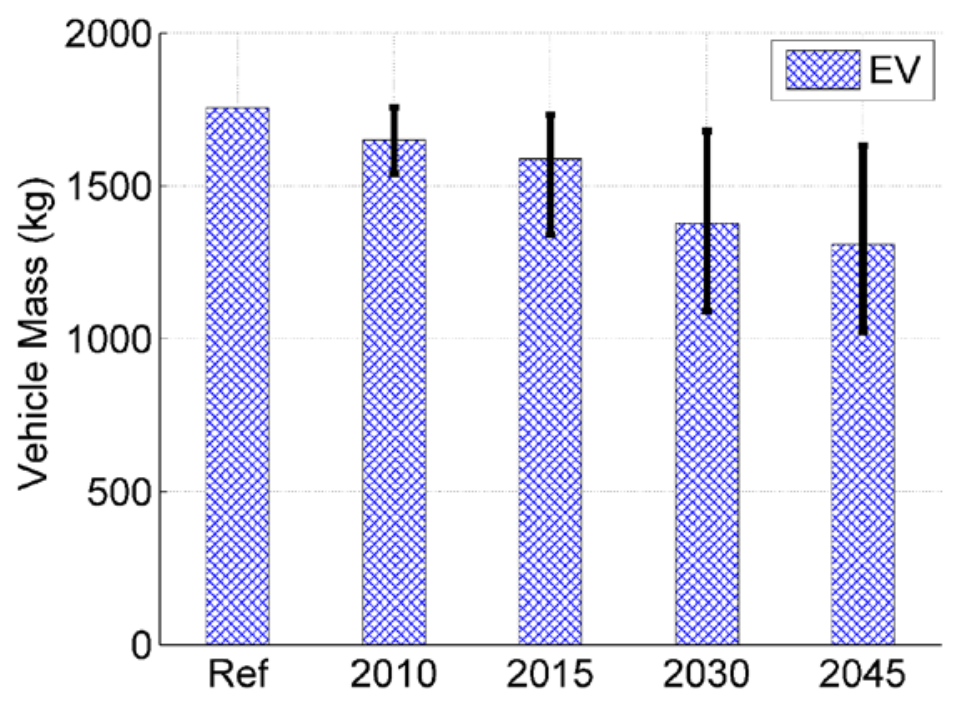

Figure 77 - Vehicle curb weights for compact BEVs. 


\subsubsection{COMPARISON OF VEHICLE CLASSES}

\section{IMPACT ON ENGINE POWER}

Since all the engine technologies and fuels show similar trends, only the compact-vehicle results will be shown. Figure 78 shows the gasoline-engine peak power for different powertrains and timeframes. One notices that the power-split HEV has the highest engine-power reduction. In addition, the engine power for PHEVs is higher than that for HEVs, owing to added vehicle weight and powertrain losses for the series configuration

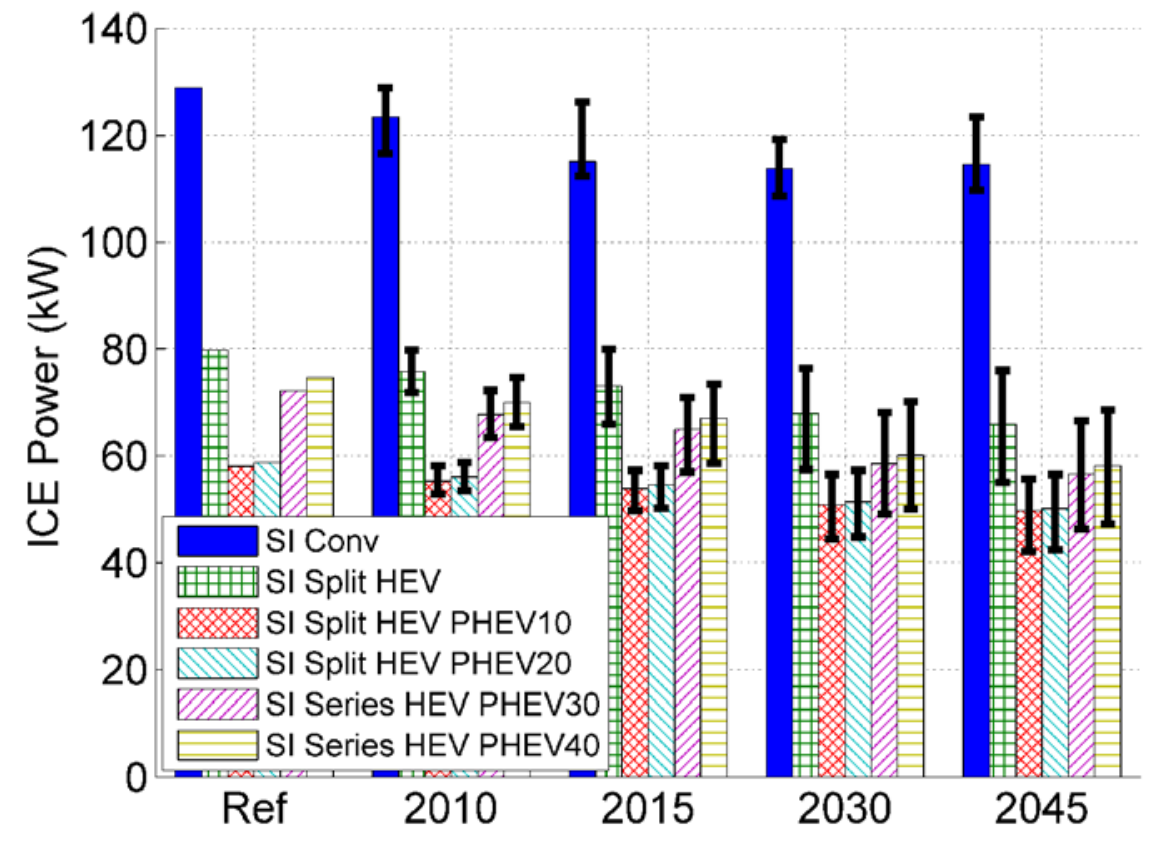

Figure 78 - Engine peak power for compact vehicles with various gasoline powertrains.

\section{IMPACT ON BATTERY POWER}

Each powertrain shows a linear relationship between the battery power and the vehicle mass (Figure 79). The figure shows similar peak power for each specific configuration type (i.e., engine and fuel-cell HEVs). Powersplit PHEVs show a slightly higher power compared to the HEVs, owing to the required ability to follow the UDDS cycle in $\mathrm{EV}$ mode. All configurations with high-energy batteries are close in value, owing to the required ability to follow the USO6 drive cycle. The remaining difference between the configurations is due to the powertrain efficiencies. 
Argonne National Laboratory Report - Light-Duty Vehicle Fuel Consumption Displacement Potential Up to 2045

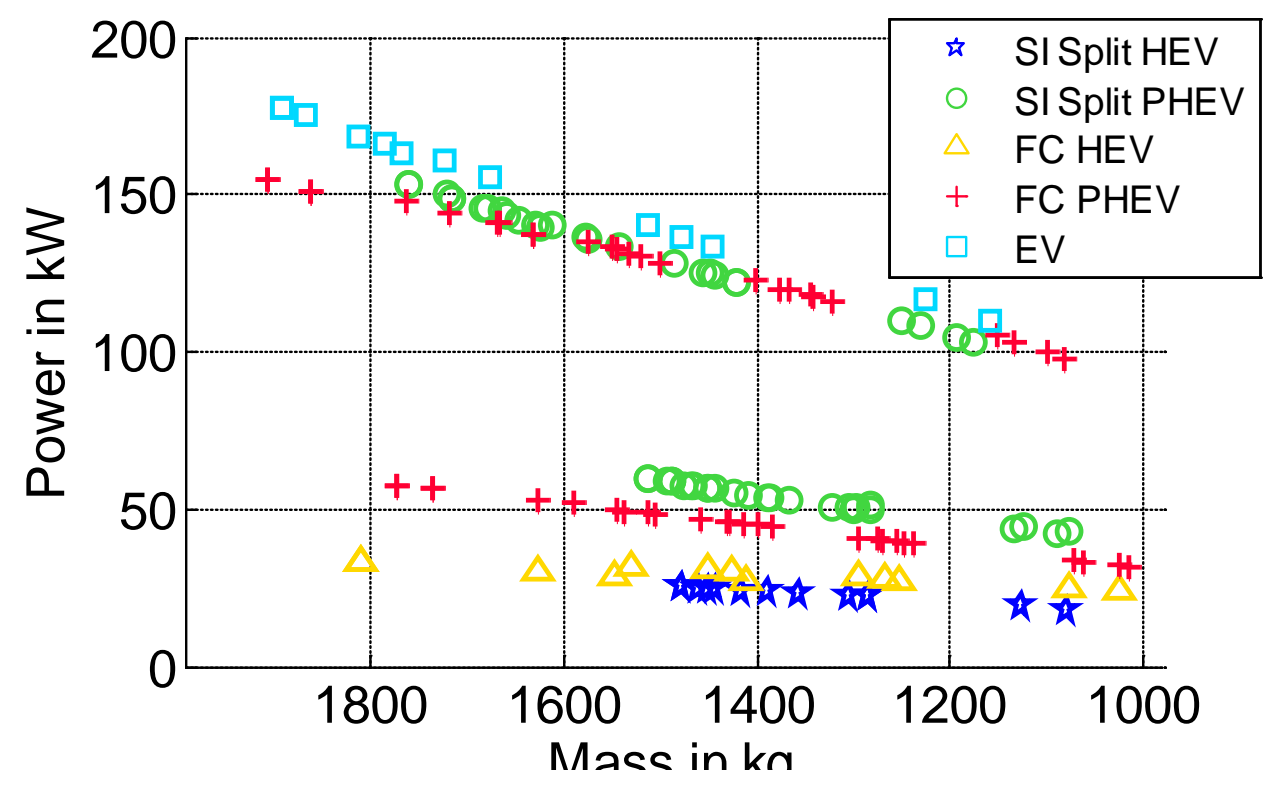

Figure 79 - Battery power as a function of vehicle mass for compact vehicles with five types of powertrains.

The linear interpolation follows the equation

ESS Power $(k W)=a \times \operatorname{Mass}(k g)+b$

Table 14 gives the values of $a$ and $b$ for the different vehicle classes and powertrains for gasoline fuel.

Table 18: Relationship between battery power and vehicle weight for different gasoline-fueled powertrains

\begin{tabular}{|l|l|l|l|l|l|l|l|l|l|l|}
\hline & \multicolumn{2}{l}{ SI Split HEV } & \multicolumn{2}{l}{ SI Split PHEV } & \multicolumn{2}{l}{ FC HEV } & \multicolumn{2}{l}{ FC PHEV } & \multicolumn{2}{l|}{ EV } \\
\hline & a & b & a & b & a & b & a & b & a & b \\
\hline Compact & 0.0176 & -0.5508 & 0.1647 & -145.7609 & 0.0102 & 14.4036 & 0.0907 & -45.2499 & 0.0923 & 1.7059 \\
\hline Midsize & 0.0174 & -0.3014 & 0.1768 & -193.1543 & 0.0096 & 16.9290 & 0.0893 & -54.7386 & 0.0899 & -1.4800 \\
\hline Small SUV & 0.0167 & 0.1445 & 0.1827 & -203.0809 & 0.0096 & 15.8316 & 0.0941 & -62.2804 & 0.0972 & -2.4403 \\
\hline Midsize SUV & 0.0173 & -1.1403 & 0.1632 & -199.2899 & 0.0100 & 16.5342 & 0.0829 & -52.7671 & 0.0920 & 6.4817 \\
\hline Pickup & 0.0168 & -0.3424 & 0.2194 & -368.3199 & 0.0098 & 18.2793 & 0.0943 & -93.6248 & 0.0969 & -5.9693 \\
\hline
\end{tabular}


IMPACT ON VEHICLE WEIGHT

Figure 80 shows the vehicle curb weights across powertrain configurations.

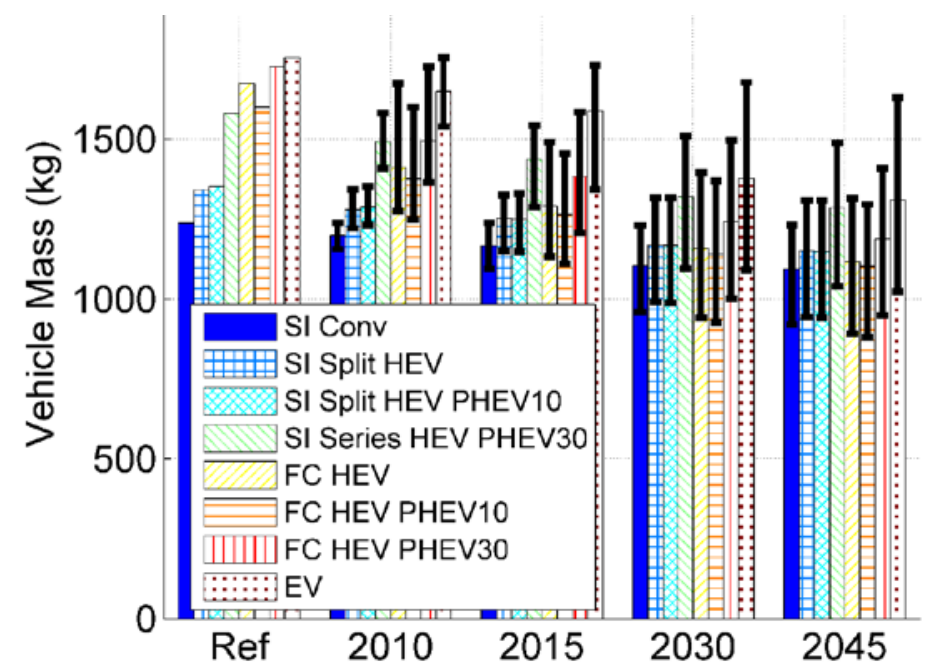

Figure 80 - Vehicle curb weights for compact vehicles with gasoline engine powertrains.

Figure 81 shows incremental weight compared to the reference conventional gasoline vehicle. One notices that significant weight savings can be achieved as early as 2015 when considering the high case for numerous configurations, including HEVs and PHEVs.

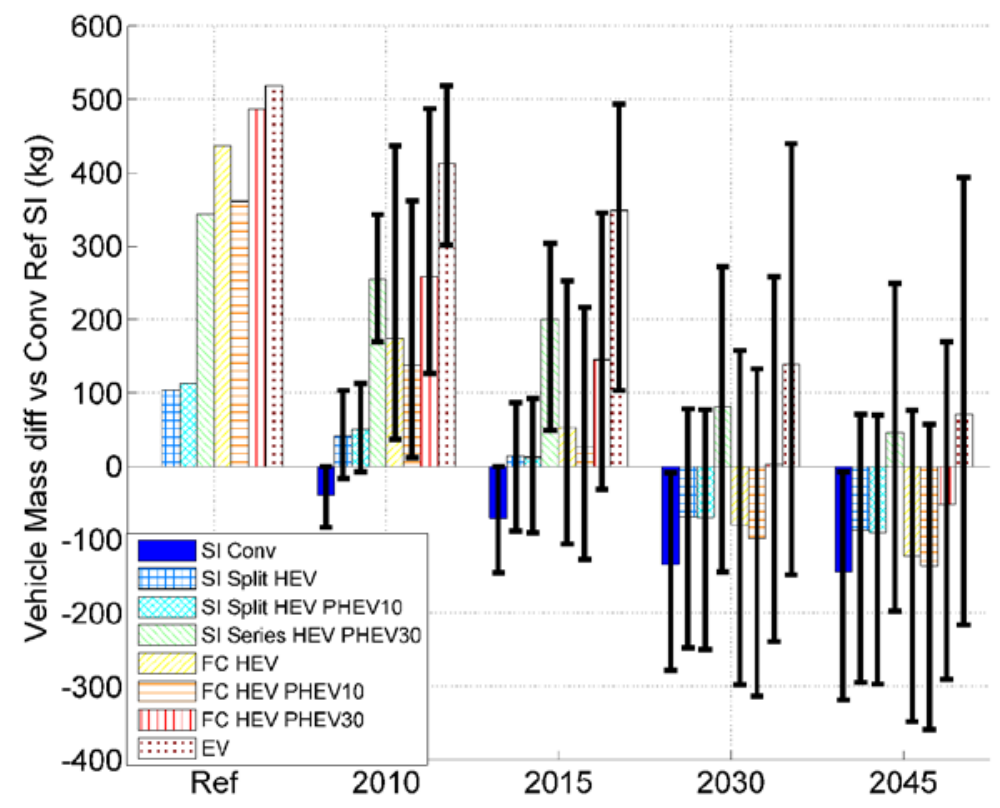

Figure 81 - Impact of hybridization degree on weight for compact vehicles. 


\section{IMPACT ON ELECTRIC MACHINE}

Figure 82 shows that like the engine power, the electric-machine power continuously and linearly decreases over time for all the powertrains. The PHEVs' electric machines are sized on the UDDS (10- and 20-mile AERs) or the USO6 (30- and 40-mile AERs) cycle and must provide enough power to complete the drive cycles. Since increased AER leads to higher vehicle weight, the electric-machine power needs to be higher for a PHEV30 and for a PHEV40.

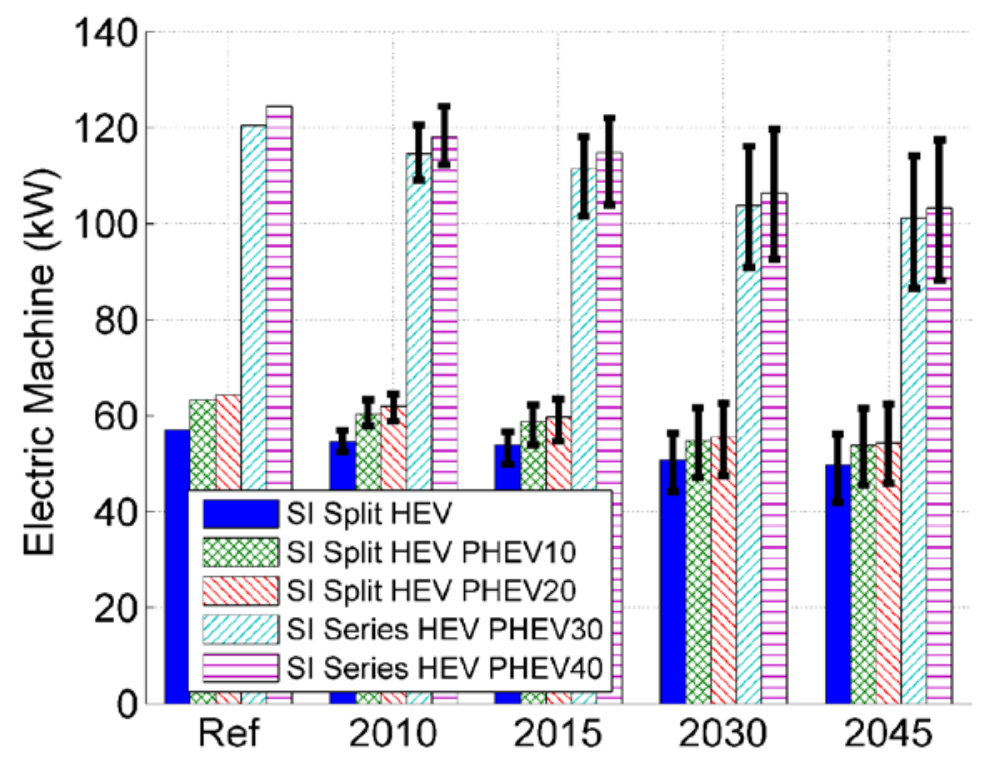

Figure 82 - Electric-machine peak power for compact gasoline HEVs and PHEVs.

\section{IMPACT OF VEHICLE WEIGHT ON FUEL CONVERTER POWER}

Figure 83 shows the engine and fuel-cell system power as a function of vehicle mass for HEVs and PHEVs. Since the electric machines used for PHEVs have higher power than the ones for HEVs, the engine is only sized for the grade ability requirement for PHEVs, while it is also sized by performance for both power-split and fuel-cell HEVs. As a result, both engine and fuel-cell powers are smaller for PHEVs than for HEVs. The different AER ranges for PHEVs have been plotted using the same colors (green for gasoline split PHEVs and red for fuel-cell PHEVs). 
Argonne National Laboratory Report - Light-Duty Vehicle Fuel Consumption Displacement Potential Up to 2045

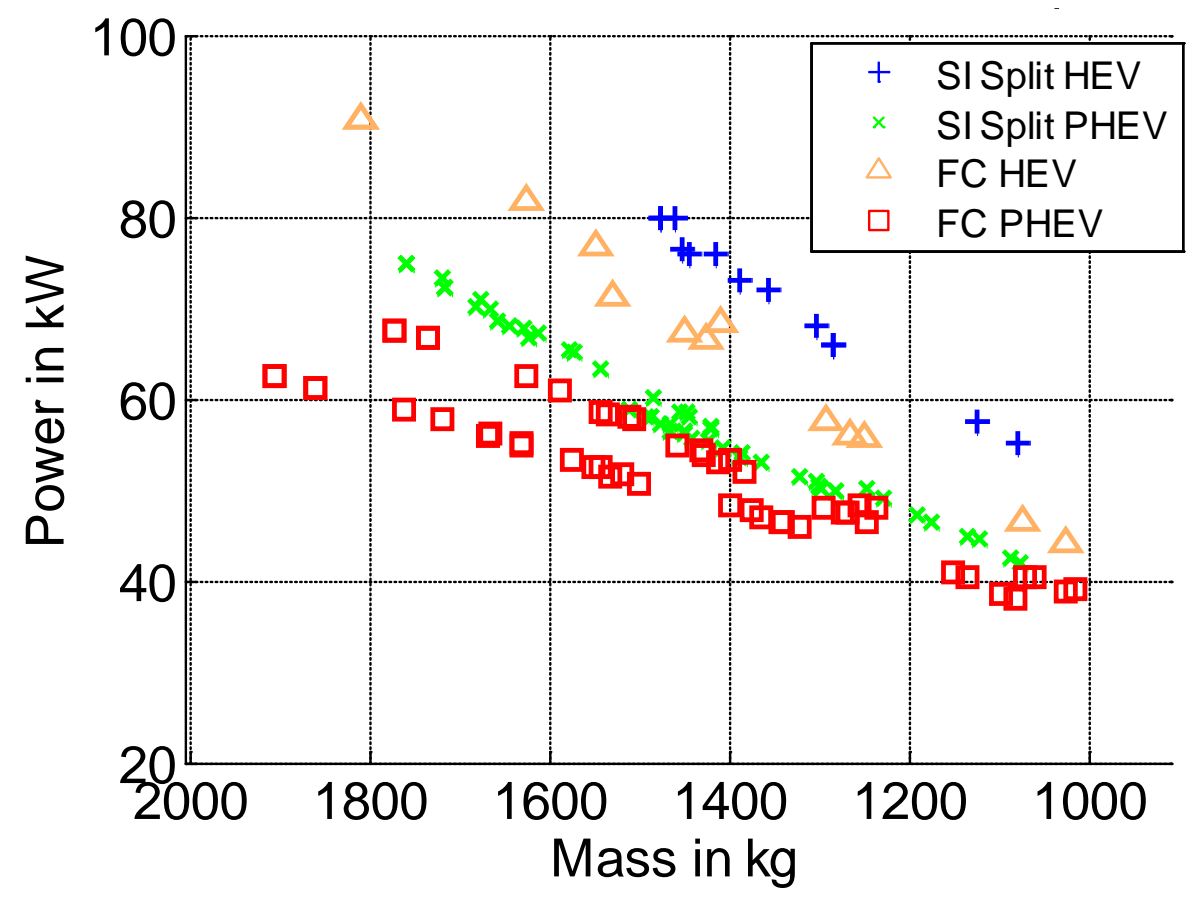

Figure 83 - Fuel converter power as a function of vehicle mass for compact HEVs and PHEVs.

The relationship follows the equation

ICE Power $(k W)=a \times$ Mass $(k g)+b$

Table 15 gives the values of $a$ and $b$ for the different vehicle classes and powertrains.

Table 19: Relationship between fuel converter and vehicle weight for different powertrains

\begin{tabular}{|l|l|l|l|l|l|l|l|l|}
\hline & \multicolumn{2}{l}{ SI Split HEV } & \multicolumn{2}{l}{ SI Split PHEV } & \multicolumn{2}{l}{ FC HEV } & \multicolumn{2}{l|}{ FC PHEV } \\
\hline & a & b & a & b & a & b & a & b \\
\hline Compact & 0.0628 & -13.6199 & 0.0483 & -11.8809 & 0.0616 & -20.6343 & 0.0309 & 7.2258 \\
\hline Midsize & 0.0623 & -15.8684 & 0.0491 & -17.3049 & 0.0618 & -24.5270 & 0.0302 & 8.4857 \\
\hline Small SUV & 0.0727 & -21.4843 & 0.0527 & -18.5476 & 0.0628 & -19.8390 & 0.0322 & 10.6523 \\
\hline Midsize SUV & 0.0692 & -16.3279 & 0.0495 & -15.3066 & 0.0642 & -24.0012 & 0.0320 & 13.3286 \\
\hline Pickup & 0.0763 & -29.4236 & 0.0559 & -31.8264 & 0.0661 & -28.1510 & 0.0307 & 21.1423 \\
\hline
\end{tabular}




\section{TEST PROCEDURE AND CONSUMPTION CALCULATIONS}

All the simulations were performed under hot conditions. The cold-start penalties were assessed after the simulations, on the basis of test data collected at Argonne's APRF and literature search. A two-cycle test procedure, based on the UDDS and HWFET drive cycles, was used.

\subsection{CONVENTIONAL VEHICLES}

The conventional-vehicle test procedure follows the current EPA two-cycle test procedure (U.S. Environmental Protection Agency, n.d.).

The urban cycle for a non-hybrid vehicle (Figure 84) is composed of four parts:

- Bag 1: cold start

- Bag 2: stop and go

- Idling

- Bag 3: hot start

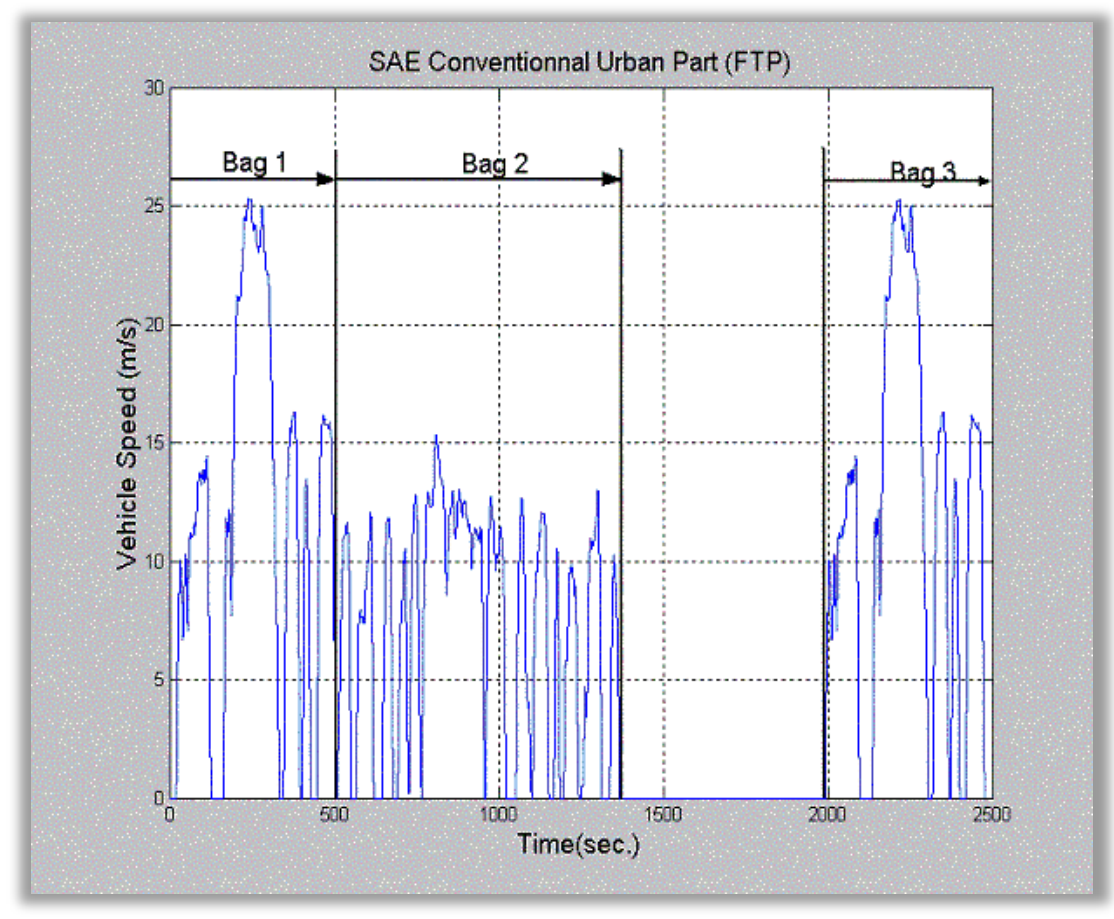

Figure 84 - The urban cycle for a non-hybrid vehicle.

The highway cycle for a non-hybrid vehicle is composed of only one part, the HWFET (Figure 85). 
Argonne National Laboratory Report - Light-Duty Vehicle Fuel Consumption Displacement Potential Up to 2045

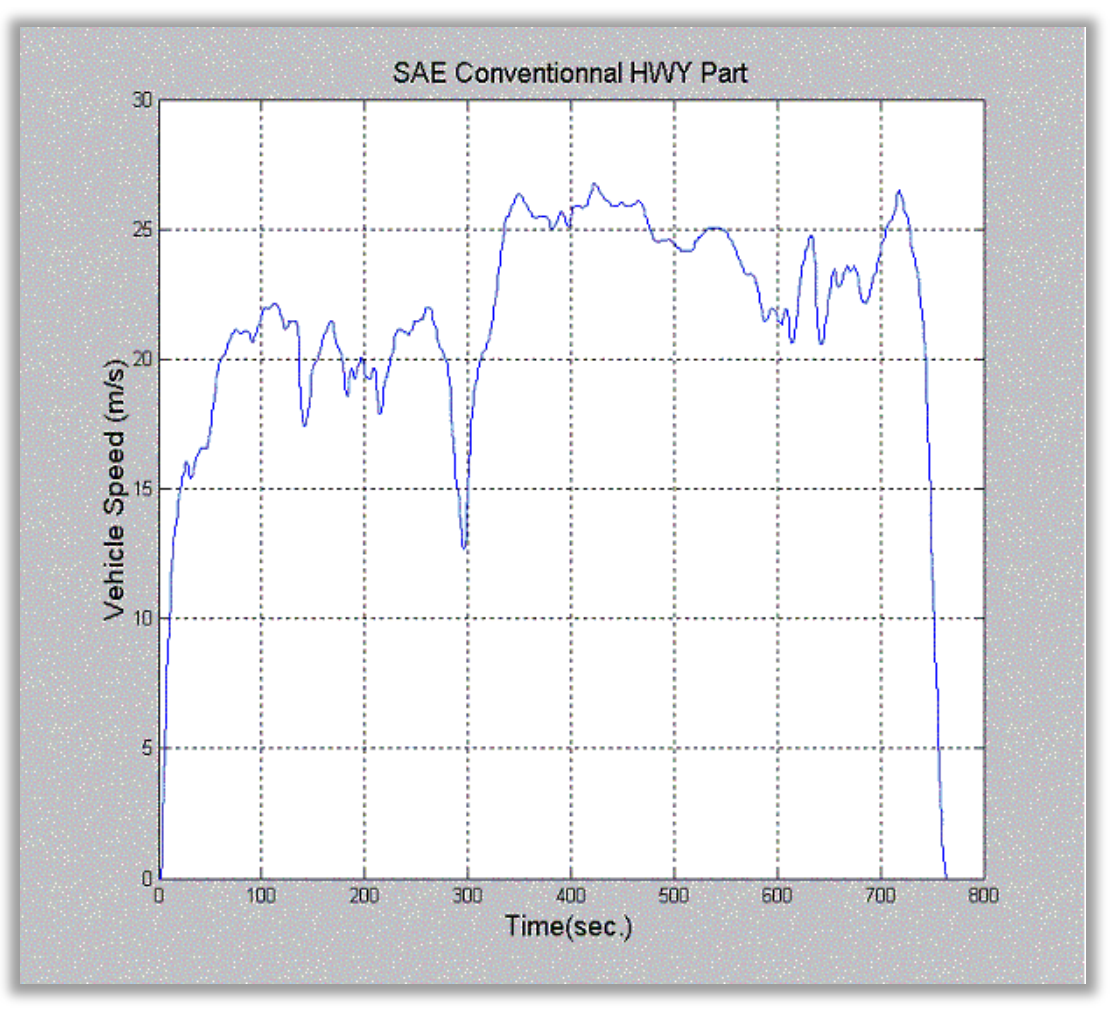

Figure 85 - The highway cycle for a non-hybrid vehicle.

\subsection{HYBRID ELECTRIC VEHICLES}

The HEV procedure is similar to the conventional-vehicle procedure except that the drive cycles are repeated until the initial and final battery SOCs are within a tolerance of $0.5 \%$. (See Figures 86 and 87. .) 


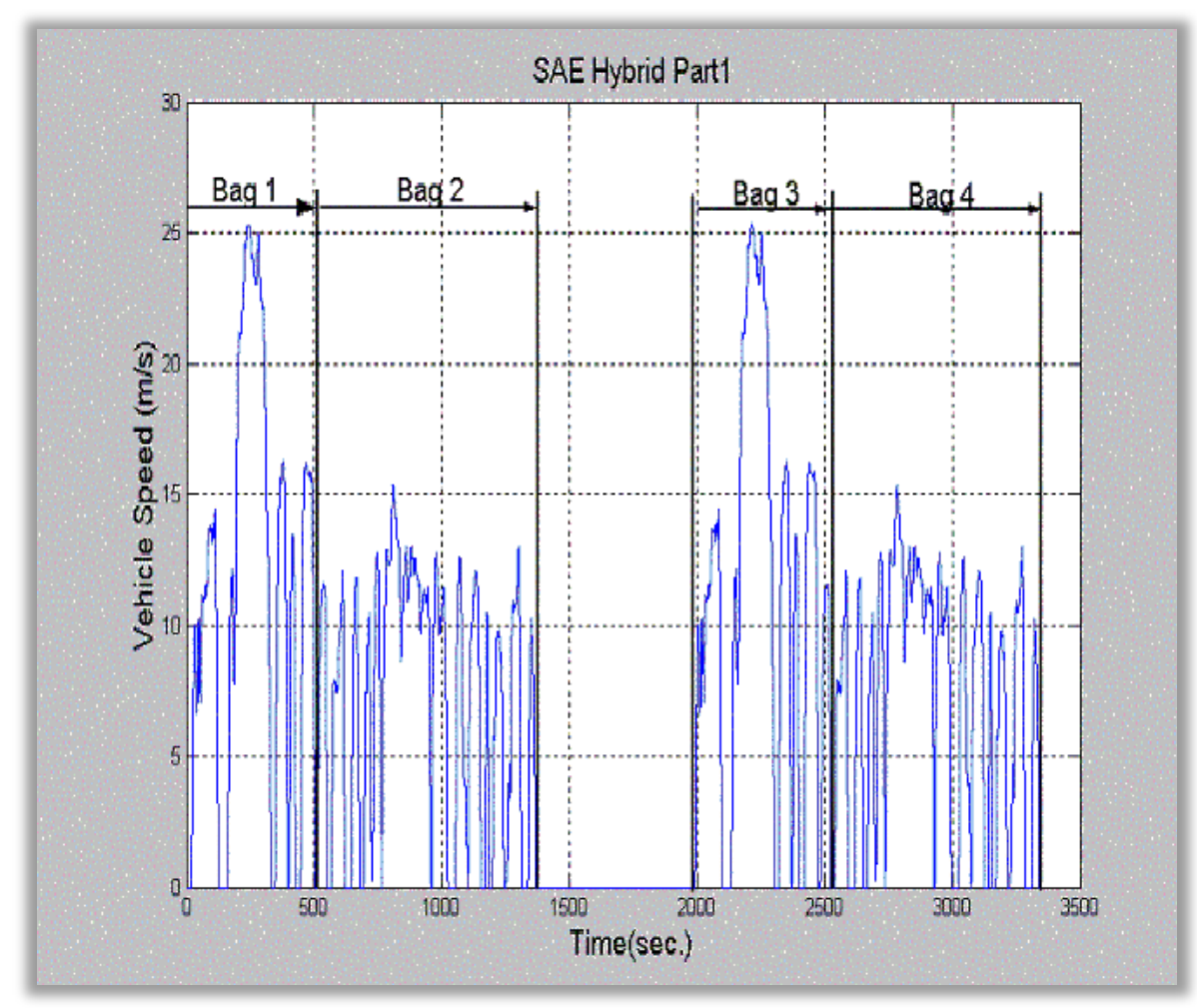

Figure 86 - The urban cycle for a hybrid vehicle.

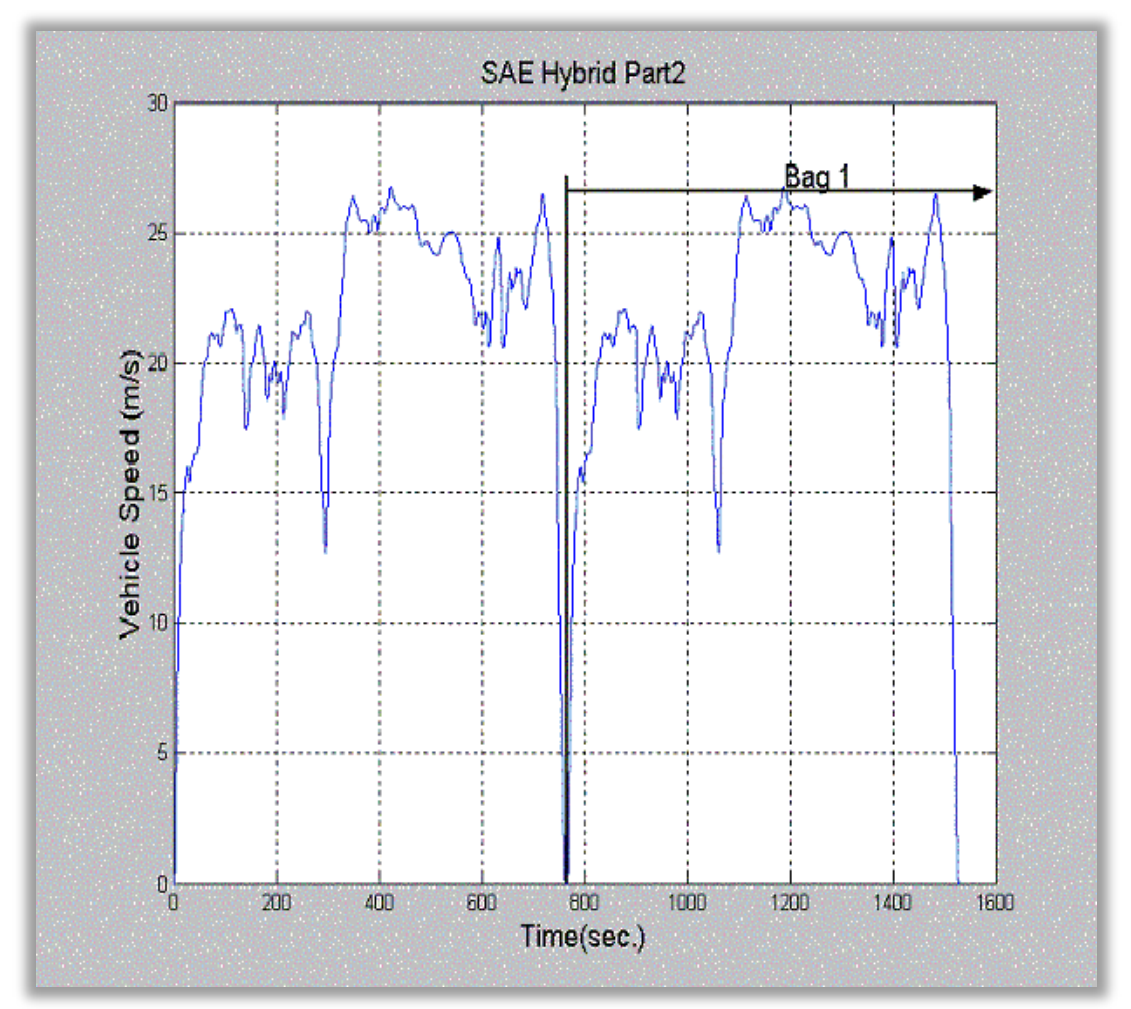

Figure 87 - The highway cycle for a hybrid vehicle. Only the results from the second cycle are used. 


\subsubsection{TWO-CYCLE PROCEDURE CALCULATIONS FOR CONVENTIONAL} AND HYBRID VEHICLES

\section{Fuel Consumption}

For the urban procedure, the fuel consumption is computed via Equation (1):

(1) $\quad F C=0.43 \frac{V_{\text {Fuel }}^{1}+V_{\text {Fuel }}^{2}}{\text { Dist }_{1}+\text { Dist }_{2}}+0.57 \frac{V_{\text {Fuel }}^{3}+V_{\text {Fuel }}^{Z}}{\text { Dist }_{3}+\text { Dist }_{Z}}$

Where

- $V_{\text {Fuel }}^{y}=$ Volume of fuel from Bag $y$

- Dist $_{y}=$ Distance driven by the vehicle for the Bag $y$ part of the cycle

- $Z$ = Bag 2 for a non-hybrid vehicle and Bag 4 for a hybrid

The same equation is used to compute the gas-equivalent fuel consumption as well as the SOC-adjusted fuel consumption by replacing $V_{F u e l}$ with the corresponding physical quantity.

The highway procedure results are the same as for a simple cycle, except for the hybrid case, where only the results from Bag 1 are used to compute the values:

(2) $\quad F C=\frac{V_{\text {Fuel }}^{2}}{\text { Dist }}$

\section{Combined Fuel Consumption}

The combined fuel consumption is a weighted value lying between the urban and highway cycles:

(3) $F C^{\text {combined }}=0.55 \times F C^{\text {urban }}+0.45 \times F C^{\text {highway }}$

\subsection{PLUG-IN HYBRID ELECTRIC VEHICLES}

This section describes the methodology currently implemented in Autonomie to support the GPRA. The implementation is based on the J1711 procedure (Duoba, 2010). The procedure is divided into several phases, as described below.

\section{Charge-Sustaining on the UDDS Cycle}

1. Set battery SOC to charge-sustaining (CS) value.

2. Run UDDS.

3. 10 minute soak with the key off.

4. Run UDDS.

5. Assume the cycle charge is balanced. Display warning if it does not meet $1 \%$. 
Weightings and cold factor correction:

The following equations demonstrate the cold compensation:

(4) $\quad M_{0-505}^{*}=\frac{M_{0-505}}{1-C F_{75 F}}$

Where

$M_{0-505}=$ fuel mass consumed during the time window between 0 and 505 seconds.

$C F_{75 F}=$ cold-factor correction at $75^{\circ} \mathrm{F}$.

$M_{0-505}^{*}=$ cold-corrected mass of fuel.

(5) $\quad$ Vol $_{0-505}^{*}=\frac{M_{0-505}^{*}}{\delta_{\text {gasoline }}}$

Where

$V o l_{0-505}^{*}=$ volume of fuel consumed during the time window between 0 and 505 seconds.

$\delta_{\text {gasoline }}=$ density of gasoline.

One can then calculate $\mathrm{FC}^{\mathrm{UDDS}}$, the fuel consumed on the UDDS cycle:

(6) $\quad F C^{U D D S}=0.43 \times\left(\frac{V o l_{0-505}^{*}+V o l_{506-1372}}{D_{0-505}+D_{506-1372}}\right)+0.57 \times\left(\frac{V o l_{1972-2477}+V o l_{2478-3340}}{D_{1972-2477}+D_{2478-3340}}\right)$

\section{Charge-Sustaining on the HWFET Cycle}

1. Set battery SOC to CS value.

2. RUn HWFET.

3. Wait4 sec.

4. Run HWFET.

5. Assume the cycle is charge balanced.

6. Perform calculations on the second HWFET cycle.

(7) $\quad F C^{H W F E T}=\frac{V^{H o l_{765-1529}}}{D_{765-1529}}$

Where

$\operatorname{Vol}_{765-1529}=$ volume of fuel consumed during the time window between 765 and 1529 seconds.

$D_{765-1529}=$ distance traveled during the time window between 765 and 1529 seconds.

$F C^{H W F E T}=$ highway fuel consumption 


\section{Charge-Depleting on the UDDS and HWFET Cycles}

1. The calculations are identical for the UDDS and HWFET cycles.

2. Set battery SOC to full charge test initial SOC.

3. Run UDDS (HWFET).

4. 10-minute soak with the key off (15-sec pause with key on).

5. Run UDDS (HWFET).

6. 10-minute soak with the key off (15-sec pause with key on).

7. Repeat until SOC reaches the $\mathrm{CD} / \mathrm{CS}$ crossover point and the last cycle is completed.

8. Round down the number of cycles unless the $C D$ range is less than one cycle. In that case, round up the number of cycles. At least $1 \mathrm{CD}$ cycle is required to run the analysis.

Cold weighting calculation:

The user specifies the number of cycles over which to apply the cold correction factor.

(8) $\quad N_{\text {cold }}=\min \left(\mathrm{N}_{\text {cold }}^{\mathrm{user}}, \mathrm{N}_{\mathrm{cd}}\right)$

(9) $\quad N_{\text {hot }}=N_{c d}-N_{\text {cold }}$

Wher:

$N_{\text {cold }}=$ number of cold cycles.

$N_{\text {hot }}=$ number of hot cycles.

$\mathrm{N}_{\text {cold }}^{\text {user }}=$ number of user-specified cold cycles.

$\mathrm{N}_{\mathrm{cd}}=$ total number of $\mathrm{CD}$ cycles.

$$
M_{c d}=\left[\frac{\alpha_{c o l d} M_{c d-c o l d}^{1}}{1-C F_{75 F}}, \cdots, \frac{\alpha_{c o l d} M_{c d-c o l d}^{N_{c o l d}}}{1-C F_{75 F}}, \alpha_{h o t} M_{c d-h o t}^{1}, \cdots, \alpha_{h o t} \mathrm{M}_{c d-h o t}^{N_{h o t}}\right]^{T}
$$

Where

$M_{c d-c o l d}^{1}=$ mass of fuel consumed during the first cold CD cycle.

$M_{c d-c o l d}^{N_{\text {cold }}}=$ mass of fuel consumed during the last cold CD cycle.

$C F_{75 F}=$ cold-start fuel economy penalty at $75^{\circ} \mathrm{F}$.

$M_{c d-h o t}^{1}=$ mass of fuel consumed during the first hot CD cycle.

$\mathrm{M}_{c d-h o t}^{N_{h o t}}=$ mass of fuel consumed during the last hot $\mathrm{CD}$ cycle.

$\alpha_{\text {cold }}=$ user-specified cold weighting factor (default value $=0.43$ ) .

$\alpha_{\text {hot }}=$ user-specified hot weighting factor (default value $=0.57$ ) .

$M_{c d}=$ column vector of cold-corrected fuel mass. 
(11) $\quad$ Vol $_{c d}=\frac{M_{c d}}{\delta_{\text {gasoline }}}$

Where

$\operatorname{Vol}_{c d}=$ column vector of cold-corrected fuel volumes.

Note that each element in the $V o l_{c d}$ vector is divided by its respective distance.

(12) $\quad F C_{c d}=\frac{V o l_{c d}}{D_{u d d s}}$

Where

$F C_{c d}=$ column vector of cold-corrected fuel consumptions.

The net battery energy used is calculated for each cycle using the open-circuit voltage and the current.

(13) for $i=1, \cdots, N_{c d} ; E_{c d}^{i}=\int_{(i-1) T_{u d d s}}^{(i) T_{u d d s}+t} V_{o c}(\tau) * I(\tau) d \tau$

Where

$E_{c d}^{i}=$ net battery energy used during the $i^{\text {th }} \mathrm{CD}$ cycle.

$T_{u d d s}=$ duration of the UDDS cycle + soak time or (HWFET + $15 \mathrm{sec}$ ).

$i=$ index of the CD cycle.

$N_{c d}=$ total number of CD cycles.

$V_{o c}=$ open-circuit voltage as a function of time during the cycle.

$I=$ battery current as a function of time during the cycle.

$$
E_{c d}=\left[E_{c d}^{1}, \cdots, E_{c d}^{N_{c d}}\right]^{T}
$$

Where

$E_{c d}=$ column vector of net battery energy used on each cycle.

Note that each element in the $E_{c d}$ vector is divided by its respective distance.

(15) $E C_{c d}=\frac{E_{c d}}{D_{\text {udds }} * \eta_{c h g}^{e s s} * \eta_{\text {charger }}}$

Where

$E C_{c d}=$ column vector of electrical-energy consumption in AC-Joules (wall outlet).

$D_{u d d s}=$ distance traveled on a UDDS (or HWFET $-D_{H W F E T}$ ) cycle. 
Argonne National Laboratory Report - Light-Duty Vehicle Fuel Consumption Displacement Potential Up to 2045

$\eta_{c h g}^{e S S}=$ user-definable efficiency of the battery during charging (default value $=0.99$ ).

$\eta_{\text {charger }}=$ user-definable efficiency of the charger (wall or in-vehicle) (default value $\left.=0.94\right)$.

$$
\text { for } i=1, \cdots, N_{c d} ; \mu_{i}=\mu\left(i * D_{u d d s}^{i}\right)-\mu(i-1) * D_{u d d s}^{i}
$$

$\mu_{c d}=\left[\mu_{1}, \cdots, \mu_{N_{c d}}\right]$

Where

$\mu_{c d}=$ row vector of utility factors.

$\mu_{1}=$ utility factor on the first CD cycle.

$\mu_{i}=$ utility factor on the $i^{\text {th }} \mathrm{CD}$ cycle.

$\mu_{N_{c d}}=$ utility factor on the last CD cycle.

$\mu=$ fleet Mileage Fraction Utility Factor as a function of distance.

(17) $\quad F C=\mu_{c d} F C_{c d}+\left(1-\sum_{i}^{N_{c d}} \mu_{i}\right) F C_{c s}$

Where

$F C=$ fuel consumed on the city or highway portion of the PHEV procedure.

$$
E C=\mu_{c d} E C_{c d}
$$

Where

$E C=$ electrical energy consumed during the city or highway portion of the PHEV procedure.

\section{Consumption adjustment factors:}

Although ONLY unadjusted values are used to support NEMS, MARKAL, and SEDS, this section describes the adjusted fuel-consumption values provided:

$$
\begin{aligned}
& F E_{a d j}^{u d d s}=0.003259+1.1805 * F E^{u d d s} \\
& F E_{a d j}^{h w f e t}=0.001376+1.3466 * F E^{h w f e t} \\
& F C_{a d j}^{\text {combined }}=0.55 * F C_{a d j}^{u d d s}+0.45 * F C_{a d j}^{h w f e t}
\end{aligned}
$$

Electrical consumption (corrected) $=0.7 *$ electrical consumption, per communication with U.S. EPA. 


\subsection{ELECTRIC VEHICLES}

Start the battery at full SOC and run until minimum SOC is reached.

$$
C=\frac{\int V_{o c} * I_{\text {ess }}}{\eta_{\text {ess }} \eta_{\text {charger }}}
$$

Where

$\eta_{\text {ess }}=$ efficiency of the battery while charging.

$\eta_{\text {charger }}=$ average efficiency of the charger while charging.

$V_{o c}=$ open-circuit voltage as a function of time over the cycle.

$I_{e s s}=$ current as a function of time over the cycle.

\subsection{COLD-START PENALTY}

A cold-start penalty of $20 \%$ is applied over 4 UDDS cycles for the CD results.

Table 20 summarizes the cold-start penalties applied to the UDDS CS results for the different powertrains.

Table 20: Cold-start $\left(20^{\circ} \mathrm{C}\right)$ penalties for the different powertrain configurations (\%)

\begin{tabular}{|c|c|c|c|c|c|c|c|c|c|c|c|c|c|}
\hline \multirow[t]{2}{*}{ Powertrain } & \multirow[t]{2}{*}{ Ref } & \multicolumn{3}{|c|}{2010} & \multicolumn{3}{|l|}{2015} & \multicolumn{3}{|c|}{2030} & \multicolumn{3}{|c|}{2045} \\
\hline & & Low & Med & High & Low & Med & High & Low & Med & High & Low & Med & High \\
\hline Conventional & 15 & 15 & 15 & 15 & 15 & 15 & 15 & 15 & 15 & 15 & 15 & 15 & 15 \\
\hline $\begin{array}{l}\text { Power-Split } \\
\text { HEV }\end{array}$ & 18 & 18 & 18 & 18 & 18 & 18 & 18 & 18 & 18 & 18 & 18 & 18 & 18 \\
\hline $\begin{array}{l}\text { Power-Split } \\
\text { PHEV }\end{array}$ & 14 & 14 & 14 & 14 & 14 & 14 & 14 & 14 & 14 & 14 & 14 & 14 & 14 \\
\hline Fuel Cell HEV & 25 & 25 & 25 & 25 & 25 & 25 & 25 & 25 & 25 & 25 & 25 & 25 & 25 \\
\hline Fuel Cell PHEV & 15 & 15 & 15 & 15 & 15 & 15 & 15 & 15 & 15 & 15 & 15 & 15 & 15 \\
\hline Electric & 10 & 10 & 10 & 10 & 10 & 10 & 10 & 10 & 10 & 10 & 10 & 10 & 10 \\
\hline
\end{tabular}




\section{SIMULATION RESULTS}

All the fuel-consumption results shown in this report are expressed in liters per $100 \mathrm{~km}$. The reasons behind this decision came from the analysis of the data shown in Figure 88, which shows the relationship between fuel economy (expressed in miles per gallon) and fuel consumption (expressed in gallons per 1000 miles). There is no linear relationship between fuel consumption and fuel economy. For example, if you improve your fuel economy from 15 to $30 \mathrm{mpg}$, you will save approximately 37 gallons of fuel per 1000 miles, whereas if you improve your fuel economy from 50 to $100 \mathrm{mpg}$, you will save only 10 gallons per 1000 miles. By comparing two different values of fuel consumption, one immediately knows the amount of fuel saved and thus the amounts of money and emissions saved, since they are linearly linked to the fuel consumption.

Moreover, whereas different vehicles can drive miles at a different pace, they all have roughly the same lifetime mileage.

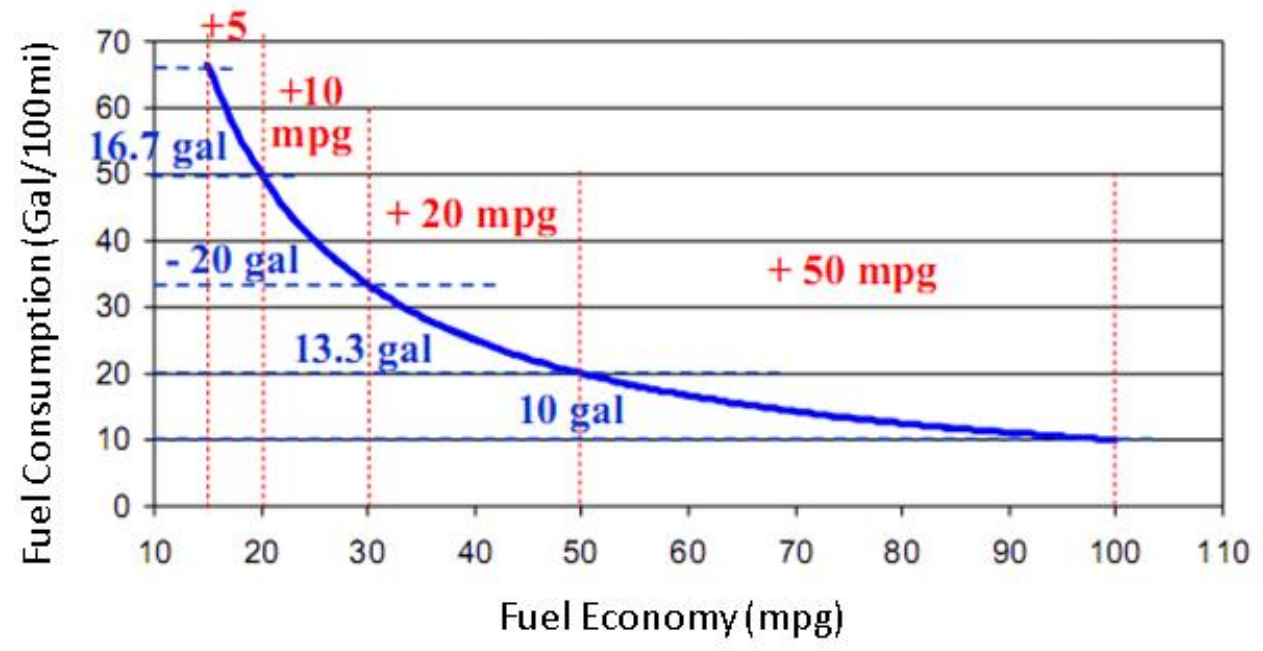

Figure 88 - Fuel economy vs. fuel consumption.

Unless otherwise specified, all the fuel-consumption results are provided for the combined drive cycle using unadjusted values based on gasoline equivalent. The details for all the vehicles are located in Appendix 2.

\subsection{EVOLUTION OF SPECIFIC POWERTRAIN CONFIGURATIONS}

\subsubsection{CONVENTIONAL POWERTRAIN}

Figure 89 shows that fuel consumption decreases over time across fuels. Gasoline conventional compact vehicles consume from $10 \%$ to $42 \%$ less fuel by 2045 compared to the reference case; the change is different for diesel vehicles, with a reduction in fuel consumption ranging from $10 \%$ to $33 \%$. Hydrogen vehicles will achieve the 
highest improvements in fuel consumption between the reference case and 2045, with a decrease ranging from $26 \%$ to $48 \%$, whereas ethanol shows the widest range of improvement, with a decrease ranging from $4 \%$ to $41 \%$.

The ethanol-engine vehicle has the highest fuel consumption among conventional vehicles for all timeframes (except for the reference case). The hydrogen ICE vehicle starts with very high fuel consumption but decreases quickly to end up at $4.9 \mathrm{~L} / 100 \mathrm{~km}$, owing to the introduction of direct injection. Note that its fuel consumption is roughly $15 \%$ higher than for the gasoline vehicle in the reference case; the difference between these two fuels becomes smaller with time. As expected, diesel ICE shows the lowest fuel consumption across timeframes.

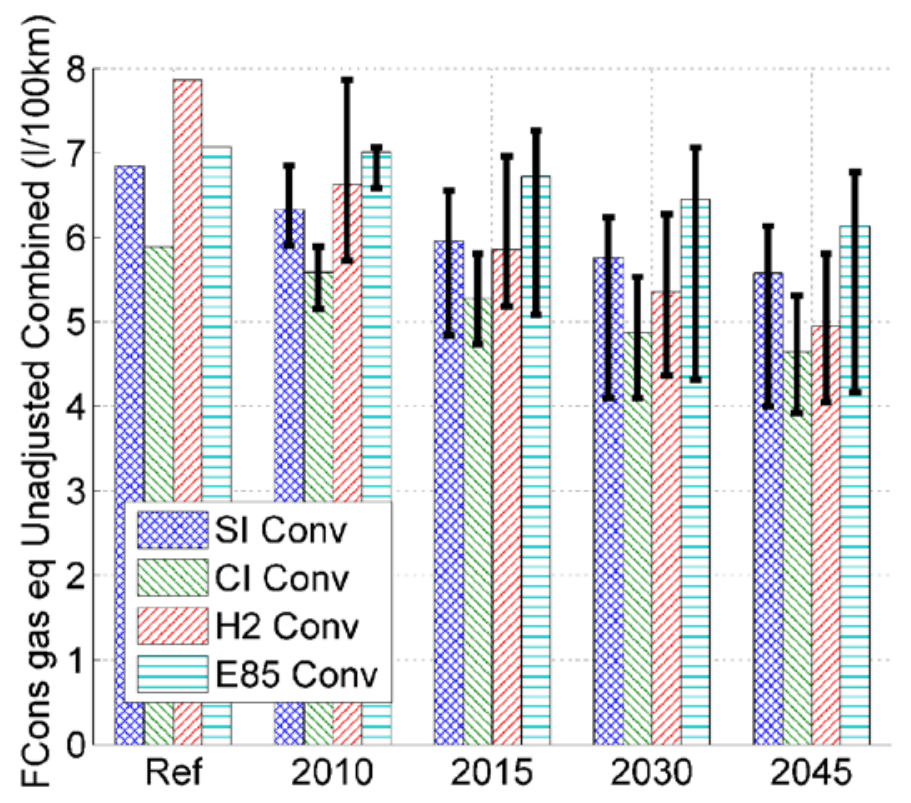

Figure 89 - Fuel consumption (gasoline equivalent) for conventional compact cars.

Table 21 shows the evolution of fuel consumption for the different fuels from the reference to the 2045 case. 
Argonne National Laboratory Report - Light-Duty Vehicle Fuel Consumption Displacement Potential Up to 2045

Table 21: Fuel consumption of conventional compact vehicles for reference and 2045 technologies

\begin{tabular}{|l|l|l|l|}
\hline & \multicolumn{2}{|l|}{ Fuel Cons. (1/100 $\mathbf{~}$ ) } & \multirow{2}{*}{ \% Fuel Cons. improvement } \\
\cline { 2 - 4 } & Ref & $\mathbf{2 0 4 5}$ low/avg/high & \\
\hline SI & 6.85 & $6.13 / 5.58 / 4$ & $10 \% / 18.5 \% / 42 \%$ \\
\hline CI & 5.89 & $5.3 / 4.65 / 3.9$ & $10 \% / 21 \% / 33 \%$ \\
\hline H2 & 7.86 & $5.8 / 4.95 / 4$ & $26 \% / 37 \% / 48 \%$ \\
\hline E85 & 7.07 & $6.8 / 6.14 / 4.2$ & $4 \% / 13.1 \% / 41 \%$ \\
\hline
\end{tabular}

Figure 90 shows the fuel consumption relative to the reference gasoline conventional vehicle. In 2045, compared to the gasoline reference case, the ranges of improvement are as follows: gasoline engine, $10 \%$ to $42 \%$; dieselengine, $23 \%$ to $43 \%$; hydrogen engine, $15 \%$ to $40 \%$; and ethanol engine, $1 \%$ to $39 \%$.

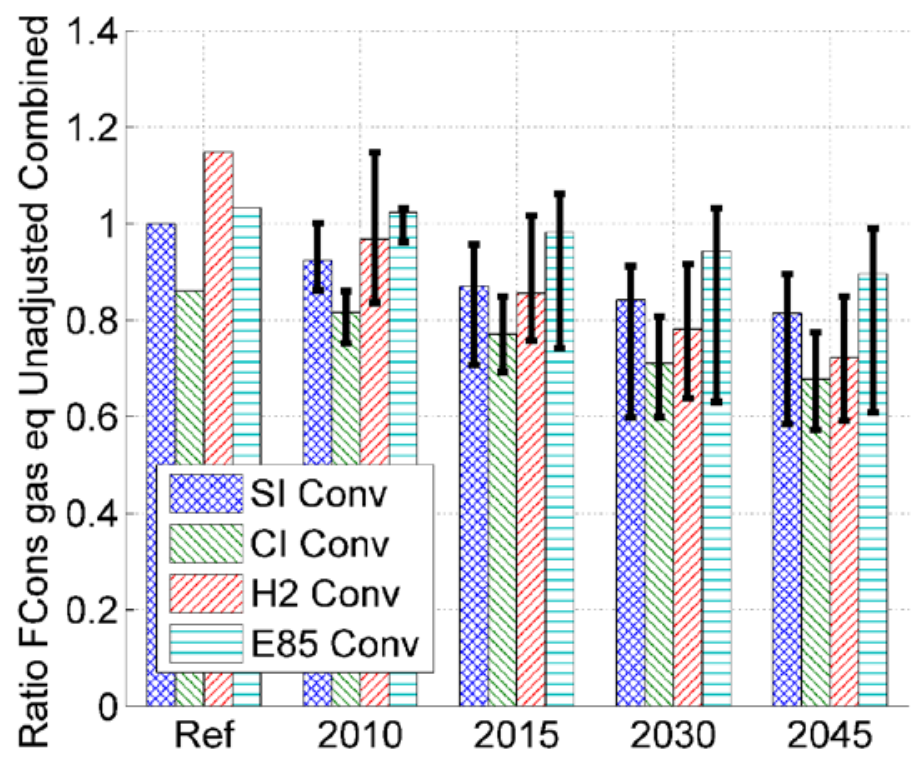

Figure 90 - Gasoline-equivalent fuel consumption for conventional compact cars, compared to the reference conventional gasoline vehicle.

Figure 91 shows the fuel consumption relative to that of the conventional gasoline vehicle of the same year. Notice that the differences between gasoline and diesel will tend to decrease in the future. In some cases (i.e., 2045 high 
case), both fuels achieve similar gasoline-equivalent fuel consumption. In addition, improvements in the hydrogen engine lead it to become more efficient than the gasoline engines. Overall, even if the fuel consumption of the ethanol engine improves in the future (Figure 90), ethanol will still remain less efficient than any other fuel on a volumetric basis.

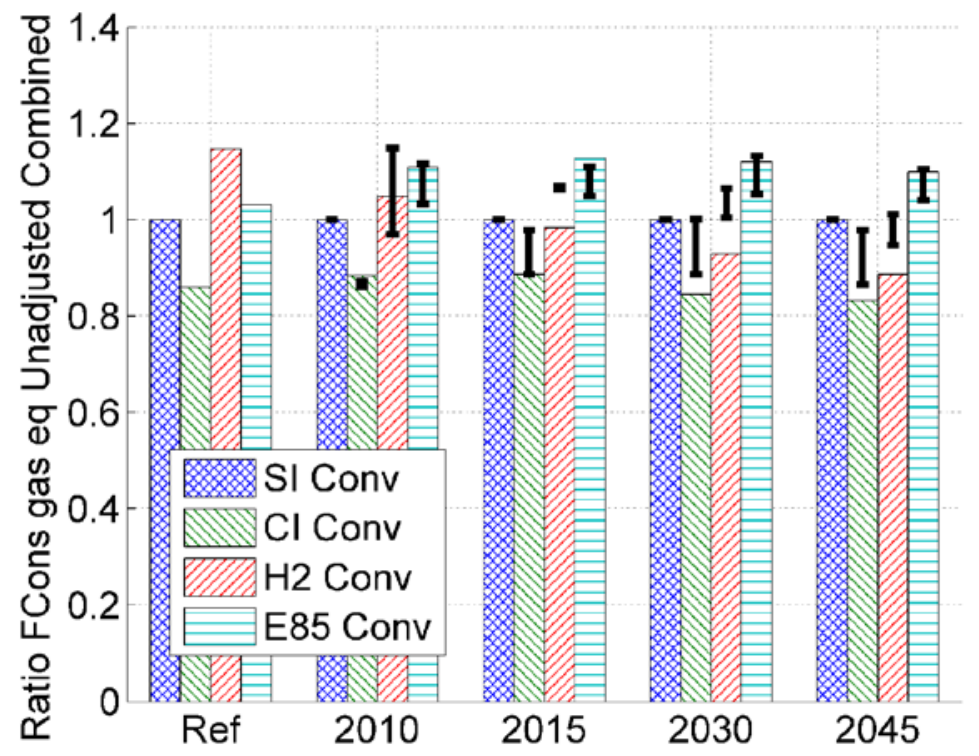

Figure 91 - Gasoline-equivalent fuel consumption for conventional compact cars, compared to the same-year conventional gasoline vehicle.

\subsubsection{HEV ENGINE}

Figure 92 shows that fuel consumption for HEVs is expected to decrease significantly over time. For HEVs, the fuelconsumption reduction with diesel fuel, ranging from $5 \%$ to $8 \%$ by 2010 , is smaller than with gasoline. However, the gap between the two fuels is also expected to decrease over time. 


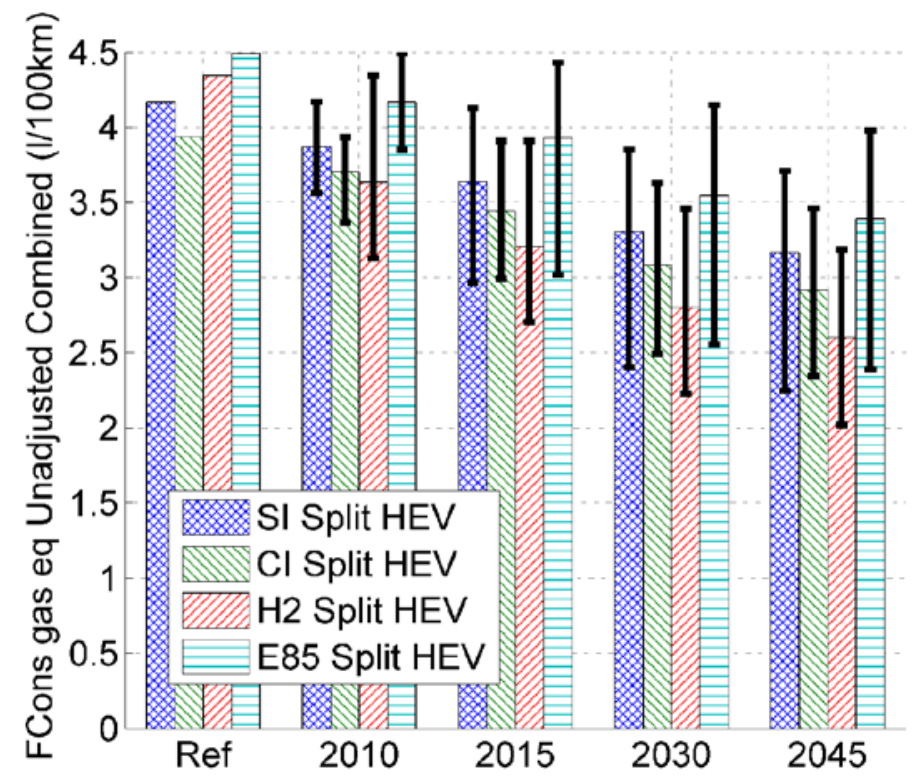

Figure 92 - Gasoline-equivalent fuel consumption for compact split HEVs.

Table 22 shows the fuel consumption of HEVs for reference and 2045 technologies. The results show consistent improvements for all fuels.

Table 22: Fuel consumption of compact HEVs for reference and 2045 technologies

\begin{tabular}{|l|l|l|l|}
\hline & \multicolumn{2}{|l|}{ Power-Split HEV } & \multicolumn{2}{l}{ Improvement } \\
\hline & \multicolumn{2}{|l|}{ Fuel Cons. (L/100 km) } & \multicolumn{1}{l|}{} \\
\hline & $\mathbf{2 0 1 0}$ & $\mathbf{2 0 4 5}$ low/avg/high & \\
\hline SI & 4.17 & $3.7 / 3.16 / 2.2$ & $11 \% / 24.2 \% / 46 \%$ \\
\hline CI & 3.93 & $3.5 / 2.92 / 2.3$ & $12 \% / 25.7 \% / 40 \%$ \\
\hline H2 & 4.34 & $3.2 / 2.6 / 2$ & $27 \% / 40 \% / 54 \%$ \\
\hline E85 & 4.49 & $4 / 3.39 / 2.4$ & $11 \% / 24.5 \% / 47 \%$ \\
\hline
\end{tabular}

Figure 93 shows the fuel consumption compared to the HEV reference gasoline vehicle. The ratio between hydrogen and gasoline reaches 0.624 in the 2045 high-uncertainty case, which shows the dramatic improvements that can be expected from hydrogen ICE power-split vehicles. 


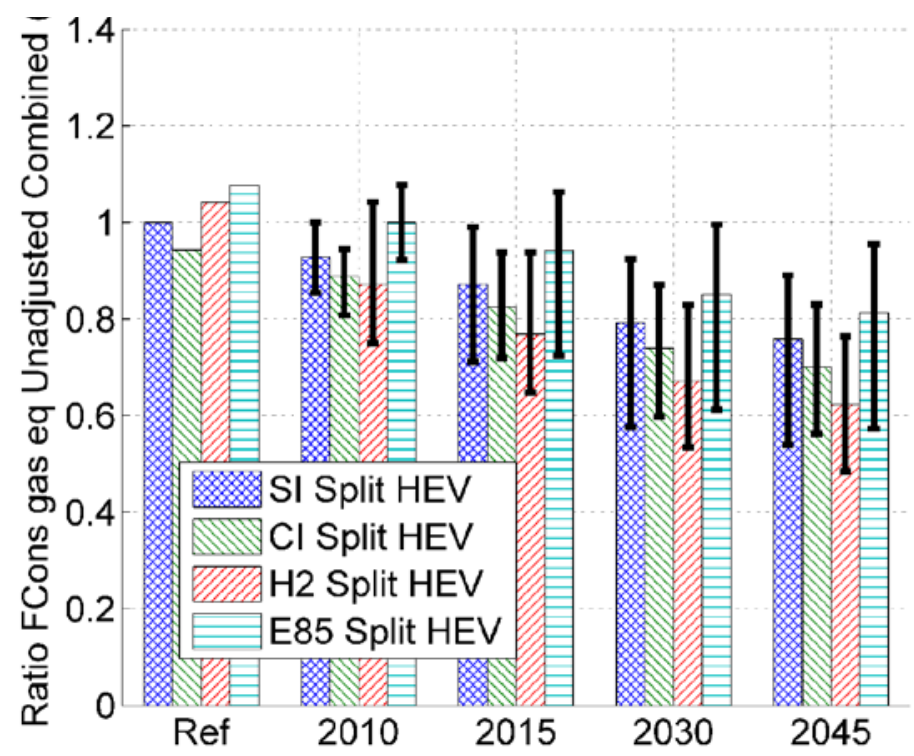

Figure 93 - Gasoline-equivalent fuel consumption of compact split HEVs, compared to the reference gasoline split-HEV.

Figure 94 shows the fuel-consumption ratios for HEVs with various fuels compared to the HEV gasoline vehicle of the same year. The results show that the ethanol fuel will maintain a fuel consumption between $6.4 \%$ and $8 \%$ higher than the gasoline case and it will have the highest fuel consumption over all the timeframes. Finally, the diesel power-split vehicles will have about $7 \%$ lower fuel consumption than the gasoline vehicles across all timeframes.

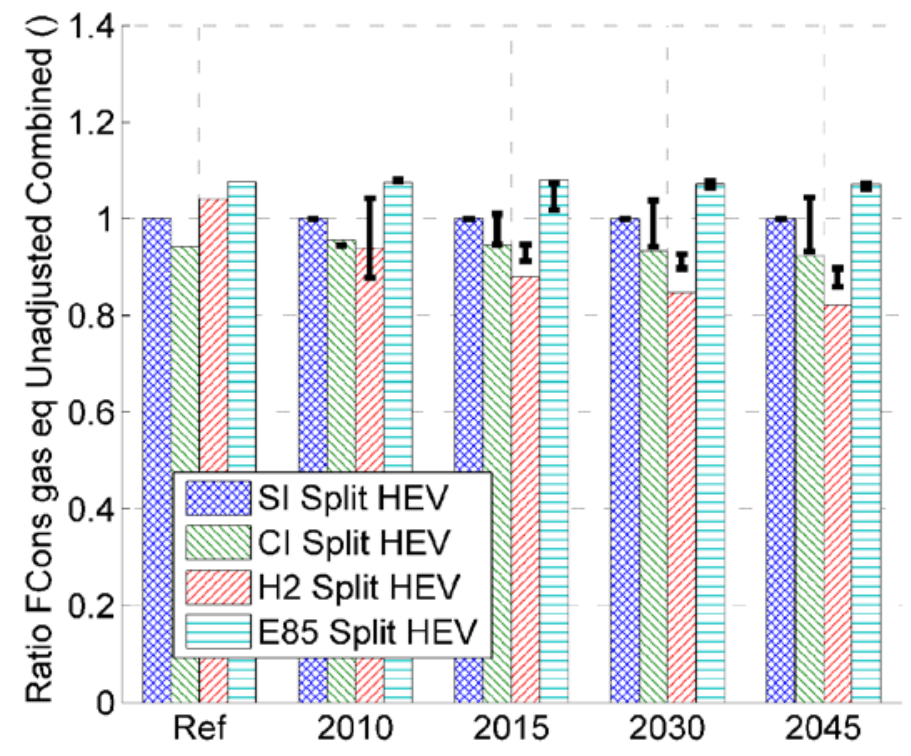

Figure 94 - Gasoline-equivalent fuel consumption for split-HEV compact cars, compared to the same-year, same-case gasoline HEV.

16.1.3.

PHEV ENGINE 
The fuel-consumption evolution for power-split PHEVs is similar to that for power-split HEVs. The ethanol vehicles always have the highest fuel consumption ( $\sim \%$ more than the gasoline vehicles), whereas the diesel vehicles' fuel consumption is between 6 and $9 \%$ lower than for gasoline vehicles. As is the case for HEVs, the hydrogen PHEVs have higher fuel consumption than gasoline PHEVs in 2010, but the ratio between these two fuels then goes below 1 and reaches 0.77 in 2045; i.e., hydrogen PHEVs have 23\% lower fuel consumption than gasoline PHEVs in 2045.

For the same fuel, the fuel consumption decreases slowly with the AER. The bigger the battery, the less fuel is consumed. However, there is no clear relationship between battery size and specific fuel-consumption improvement. For instance, between the reference case and 2045, the fuel-consumption improvement of gasoline engines is about $21.6 \%$ for PHEV10, 16.8\% for PHEV20, 26.2\% for PHEV30, and 34.2\% for PHEV40. These variations do not show a trend related to battery size and improvement over the years. Data for PHEV10s and PHEV20s are shown in Figure 95, and for PHEV30s and 40s in Figure 96.

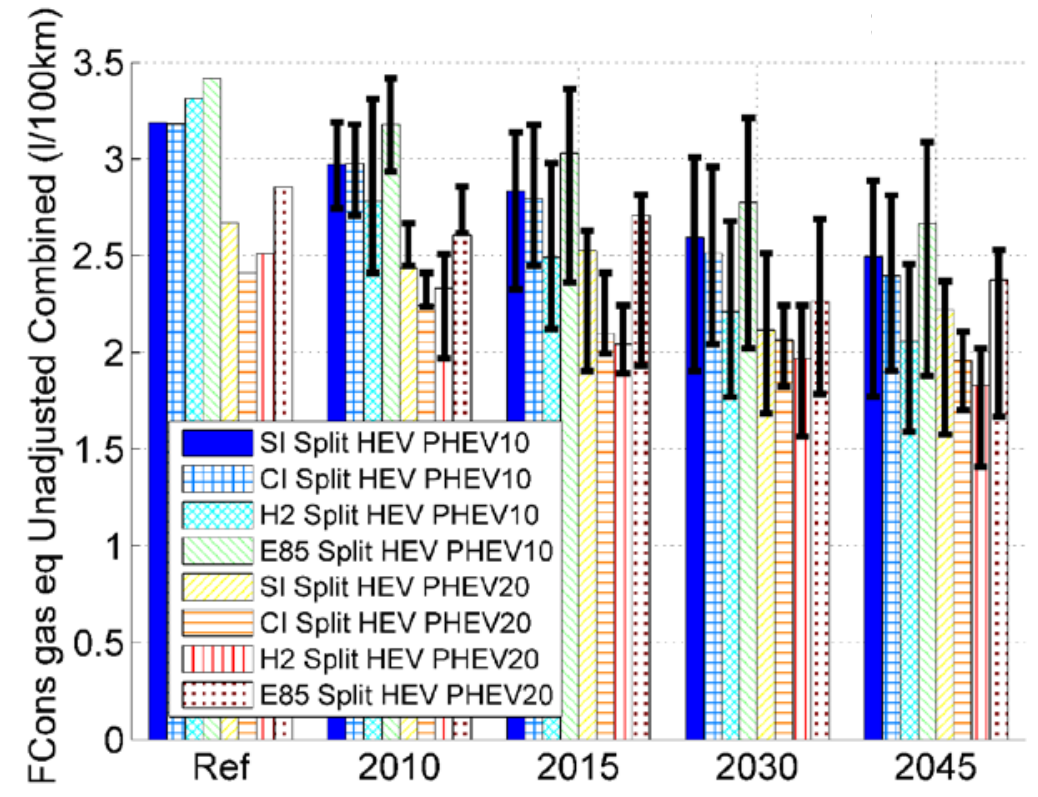

Figure 95 - Gasoline-equivalent fuel consumption for compact split PHEV10s and PHEV20s. All the fuel-consumption values are CD+CS. 


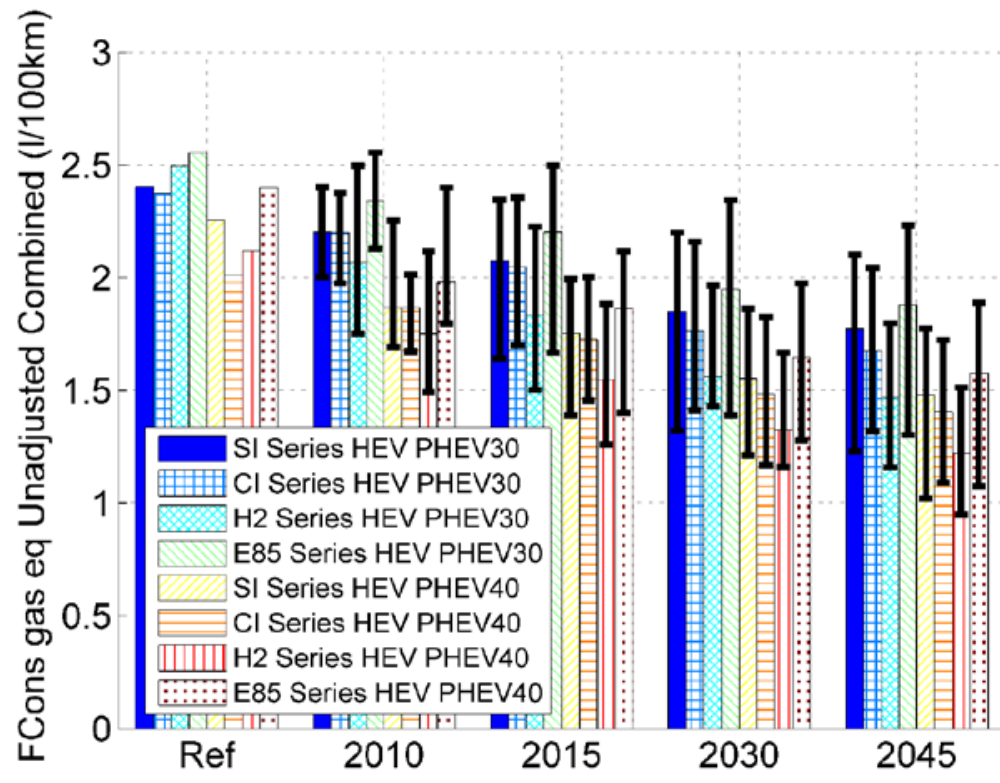

Figure 96 - Gasoline-equivalent fuel consumption for compact split PHEV30s and PHEV40s. All the fuel-consumption values are CD+CS.

Table 23 shows the PHEV10 fuel consumption for the reference and 2045 technologies; as in the HEV case, H2 shows the most improvement of any fuel between 2010 and 2045. The results for HEVs and PHEVs are very close to each other, since the engines are used under similar conditions.

Table 23: Fuel consumption of compact PHEV10s with 2010 and 2045 technologies

\begin{tabular}{|l|l|l|l|}
\hline & \multicolumn{2}{|l|}{ Power-Split PHEV10 } & \multicolumn{2}{l|}{ Improvement } \\
\hline & \multicolumn{2}{|l|}{ Fuel Cons. (L/100 km) } & \multicolumn{1}{l|}{} \\
\hline & Ref & $\mathbf{2 0 4 5}$ low/med/high & \\
\hline SI & 3.19 & $2.9 / 2.5 / 1.8$ & $9 \% / 21.6 \% / 44 \%$ \\
\hline CI & 3.18 & $2.8 / 2.4 / 1.9$ & $12 \% / 24.5 \% / 40 \%$ \\
\hline H2 & 3.31 & $2.5 / 2.06 / 1.6$ & $24 \% / 37.7 \% / 54 \%$ \\
\hline E85 & 3.42 & $3.1 / 2.67 / 1.9$ & $11 \% / 21.9 \% / 47 \%$ \\
\hline
\end{tabular}

Figure 97 shows the fuel consumption for the PHEV10 and PHEV20 compared to the same-year PHEV gasoline vehicle. 


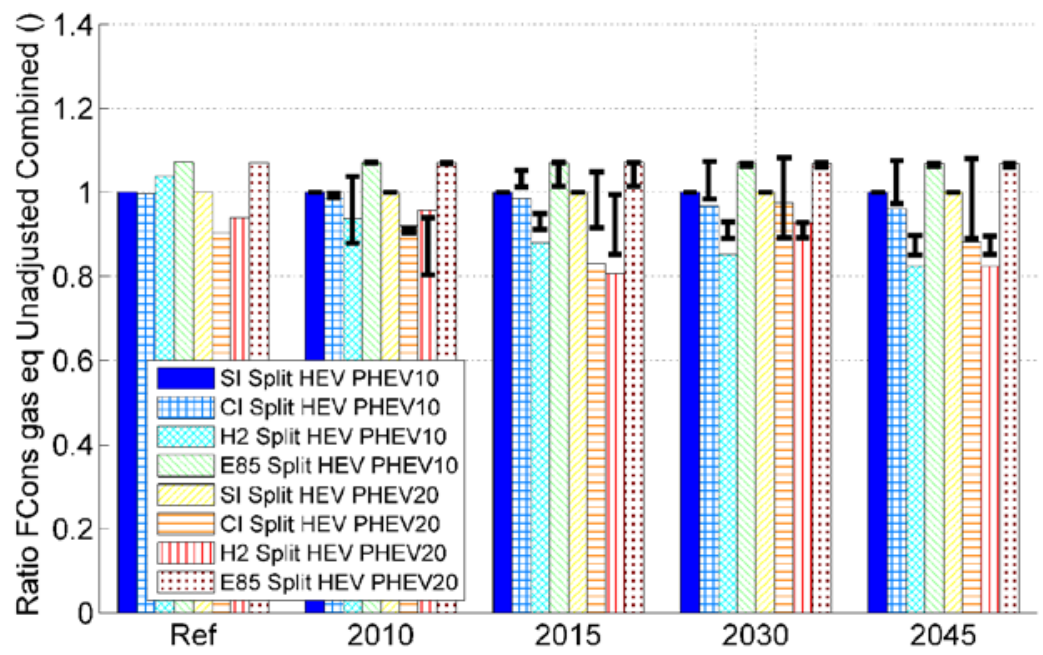

Figure 97 - Gasoline-equivalent fuel consumption for split PHEV10 and -20 compact cars, compared to the same-year, same-case gasoline PHEV with matching AER. All the fuel-consumption values are CD+CS.

Figure 98 shows the fuel-consumption ratios for the PHEV10 and PHEV20 compared to the reference PHEV gasoline vehicle. Overall, as in the HEV case, the H2 vehicle remains the most efficient vehicle in 2045, with improvements of $35 \%$ for PHEV10, 31\% for PHEV20, 38\% for PHEV30, and $45 \%$ for PHEV40 compared to the reference PHEV gasoline vehicle.

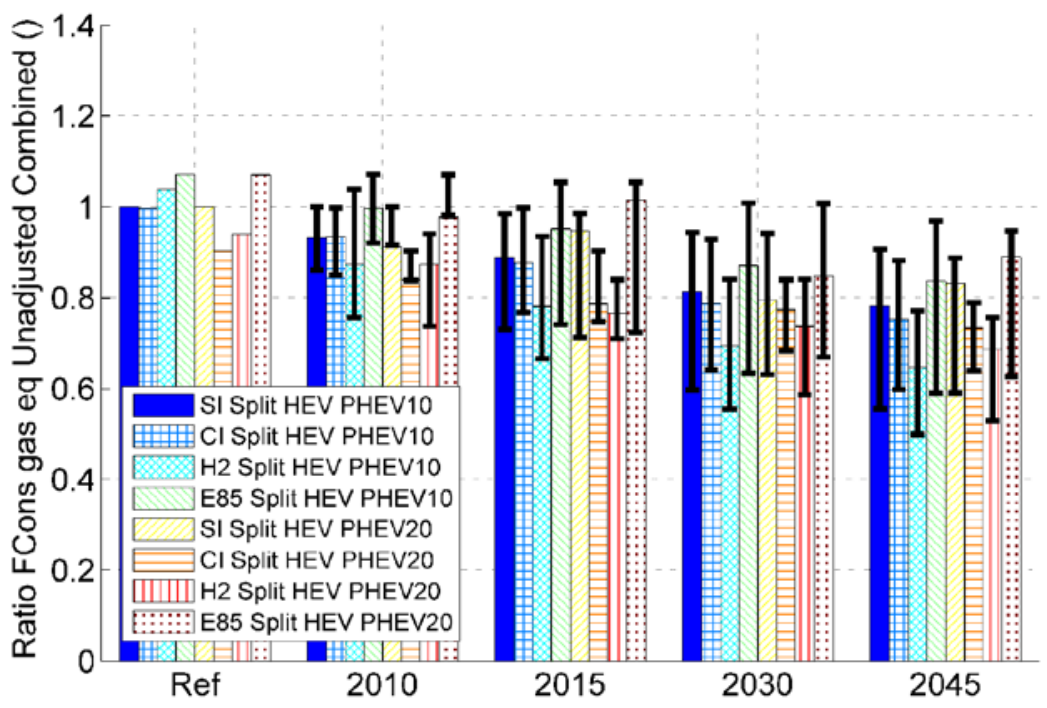

Figure 98 - Gasoline-equivalent fuel consumption for split PHEV10 and -20 compact cars, compared to the reference PHEV gasoline vehicle. All the fuel consumption values are CD+CS.

Figure 99 shows the fuel consumption for the PHEV30 and PHEV40 compared to the same-year PHEV gasoline vehicle. It is interesting to see that there is not really a linear trend for fuel-consumption ratios vs. the same-year gasoline PHEV. 


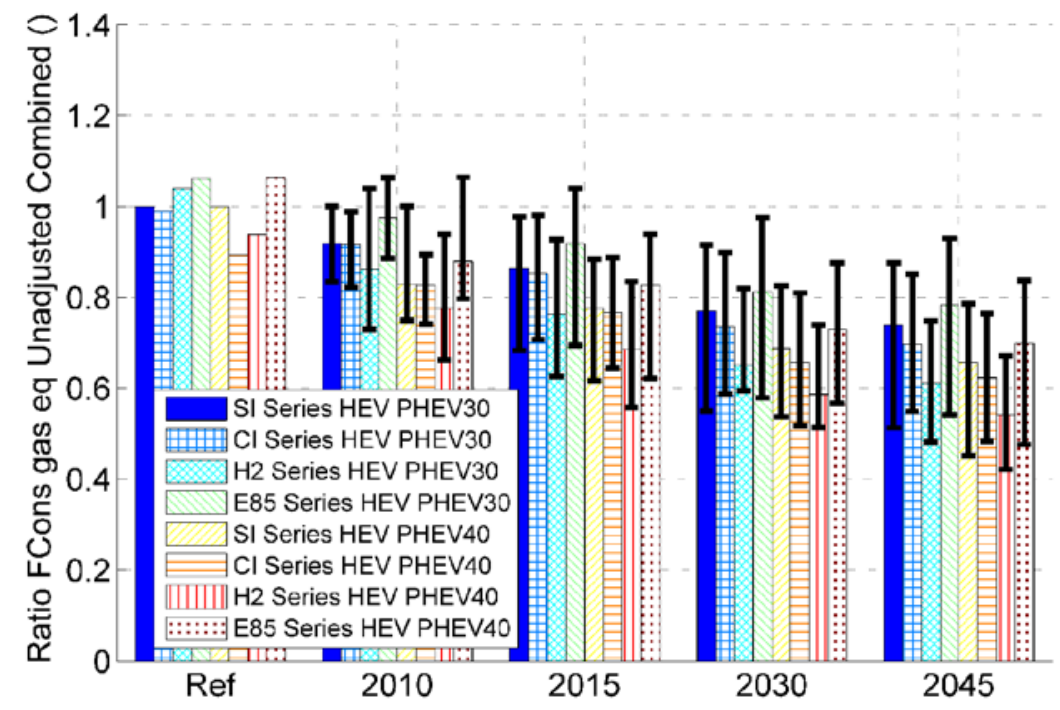

Figure 99 - Gasoline-equivalent fuel consumption for split PHEV 30 and -40 compact cars, compared to the reference PHEV gasoline vehicle.. All the fuel consumption values are CD+CS.

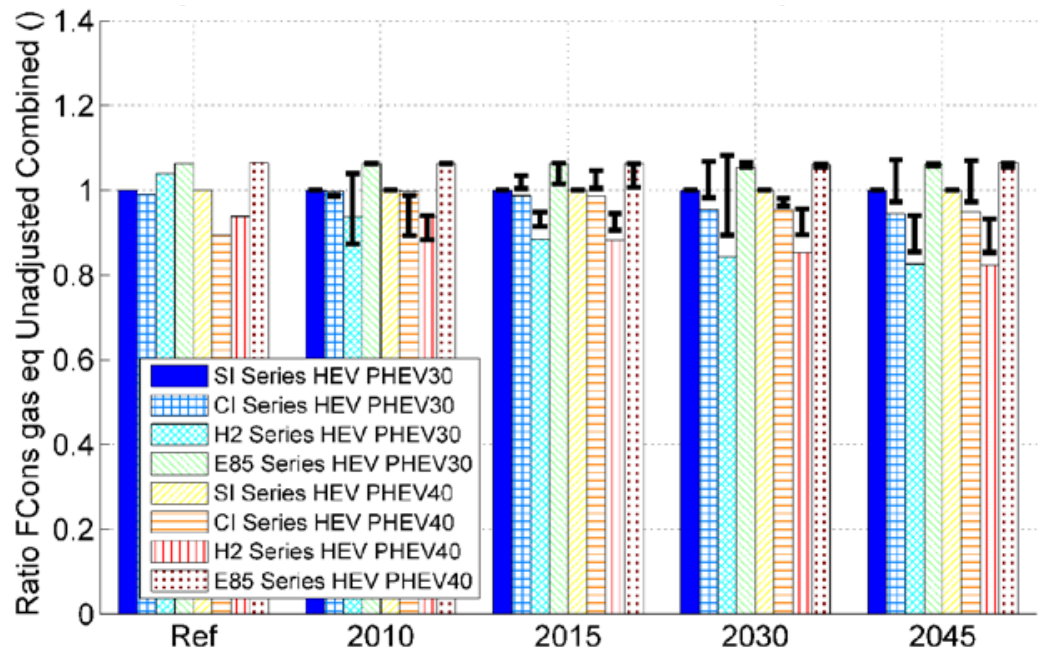

Figure 100 - Gasoline-equivalent fuel consumption for split PHEV30 and -40 compact cars, compared to the same-year, same-case gasoline PHEV with matching AER. All the fuel consumption values are CD+CS.

Figure 101 shows that there is a linear relationship between vehicle mass and electric consumption. The bigger the vehicle, the higher the electrical consumption. This observation is consistent with the fuel-consumption increase with vehicle mass. 
Argonne National Laboratory Report - Light-Duty Vehicle Fuel Consumption Displacement Potential Up to 2045

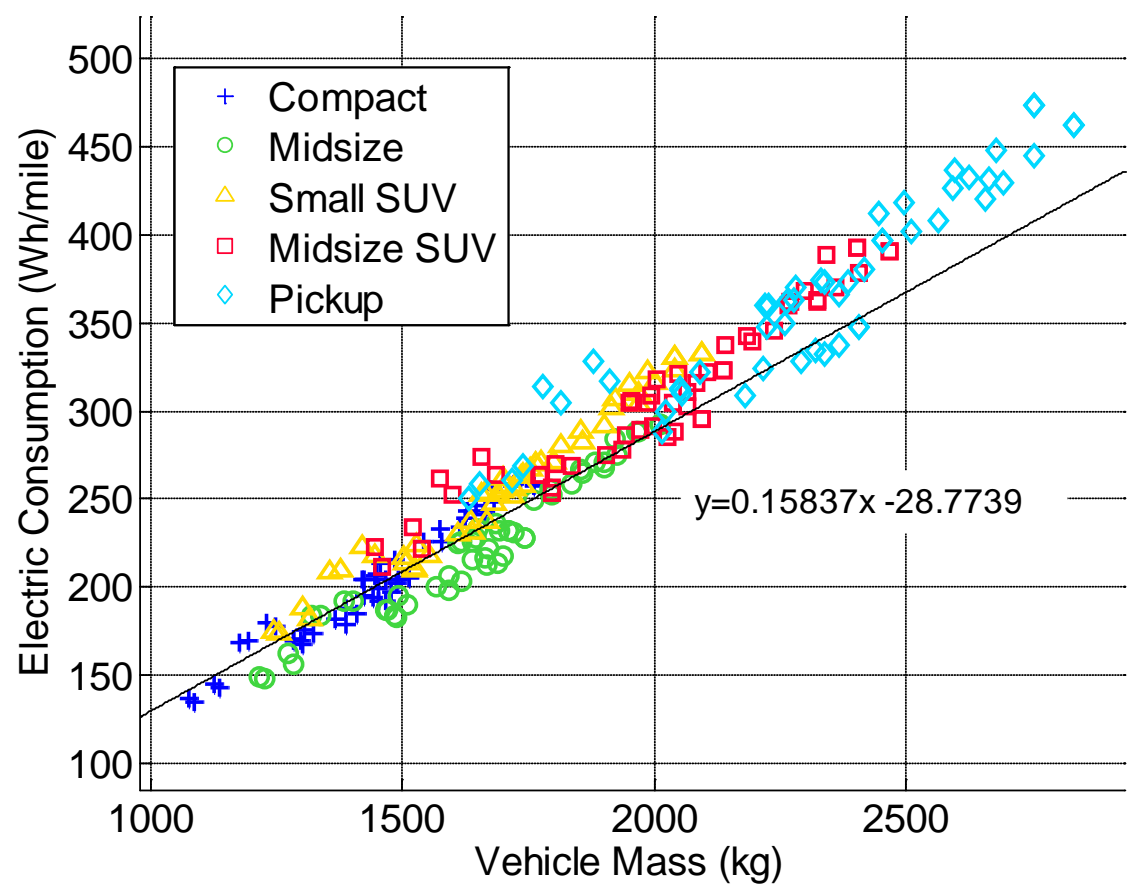

Figure 101 - Electric consumption in CD+CS mode for gasoline-powered-split PHEVs.

\subsubsection{FUEL-CELL HEV}

The fuel-cell HEV's fuel consumption (Figures 102 and 103) decreases at a higher rate from 2010 to 2030 than from 2030 to 2045, when the average and high cases hardly change. In 2045, the fuel consumption is from $27 \%$ to $50 \%$ lower than in the reference case, which is in the same range as for power-split HEVs H2.

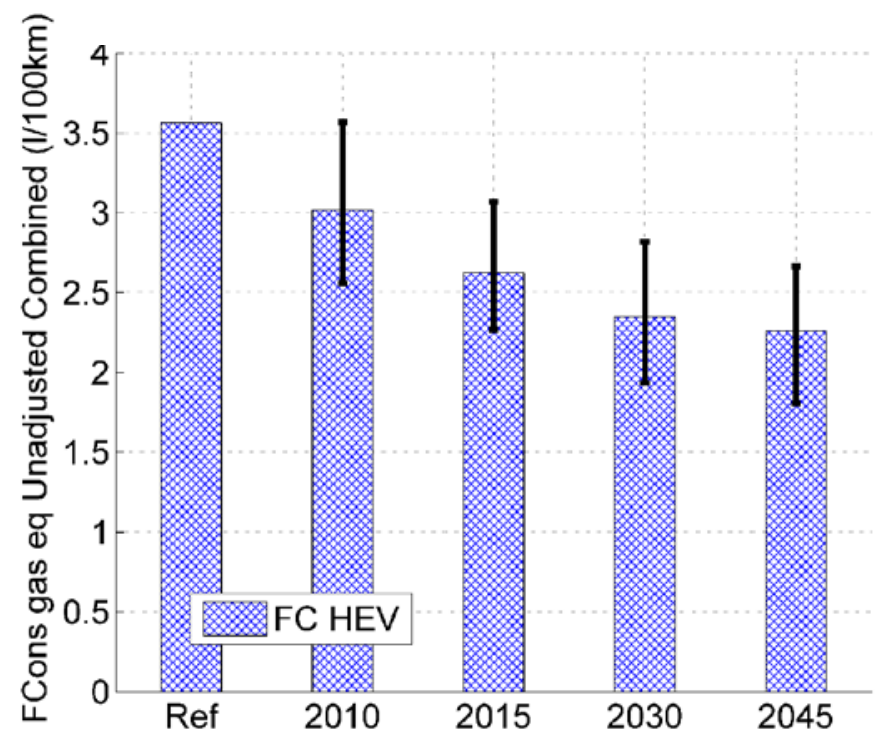

Figure 102 - Gasoline-equivalent fuel consumption for compact fuel-cell HEVs. 
Argonne National Laboratory Report - Light-Duty Vehicle Fuel Consumption Displacement Potential Up to 2045

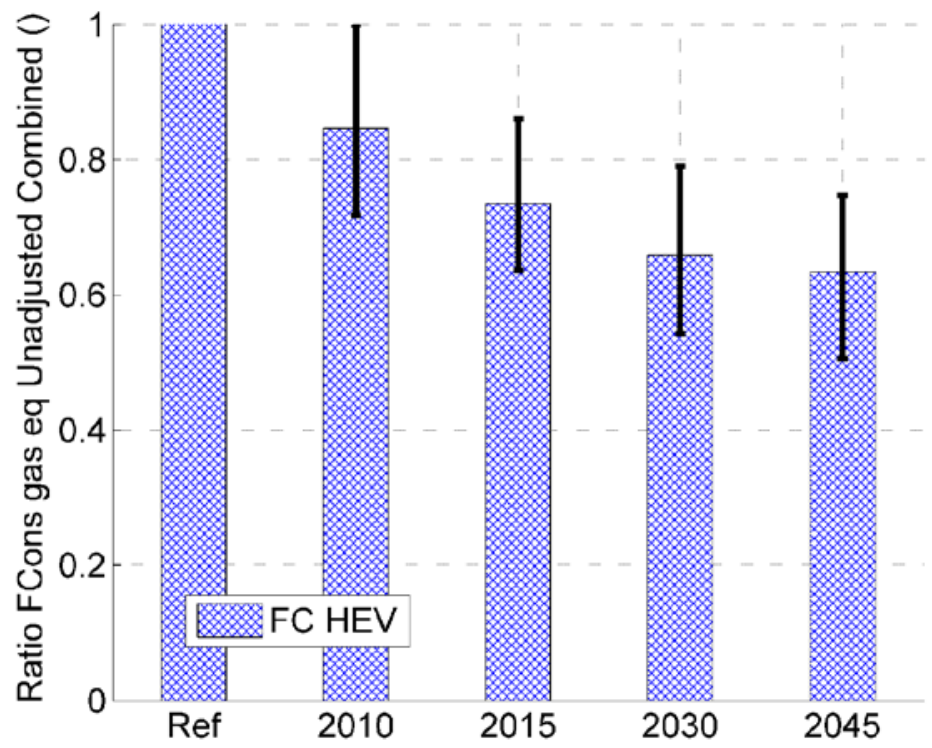

Figure 103 - Gasoline-equivalent fuel consumption compared to the compact fuel cell HEV reference case.

\subsubsection{FUEL-CELL PHEV}

For fuel-cell PHEVs, the fuel consumption decreases slowly (Figures 104 and 105) as the AER goes from one range to the next higher range, for the same reasons discussed for power-split PHEVs. From the reference case to 2045 , the consumption decreases by $22 \%-47 \%$ for all the AERs.

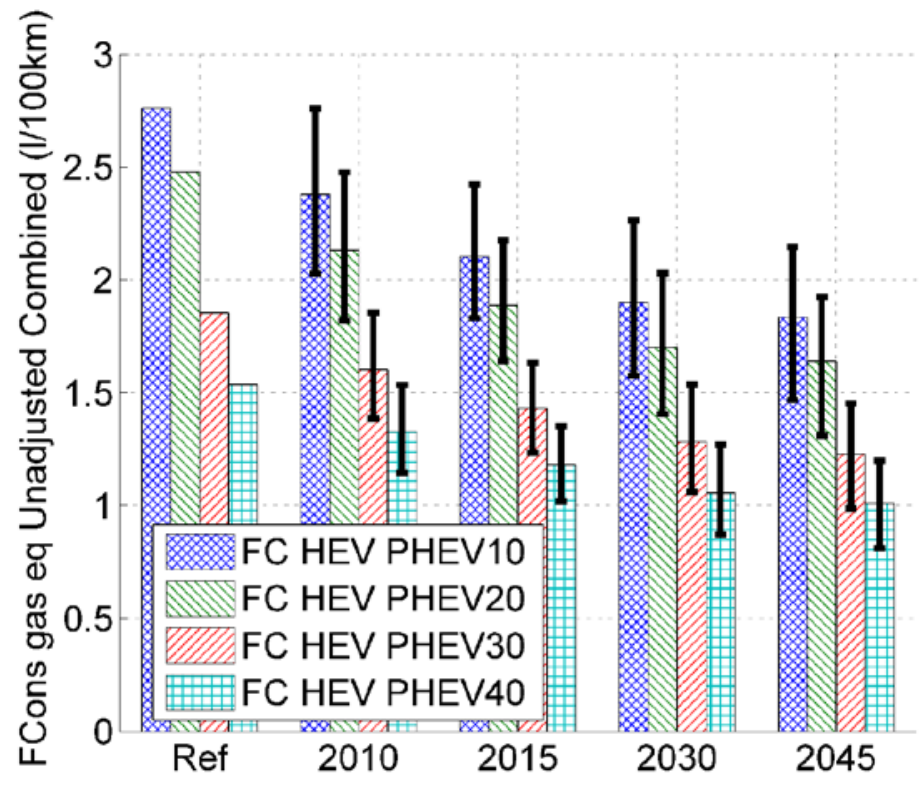

Figure 104 - Gasoline-equivalent fuel consumption for compact fuel-cell PHEVs. The fuel consumption values are CD+CS. 
Argonne National Laboratory Report - Light-Duty Vehicle Fuel Consumption Displacement Potential Up to 2045

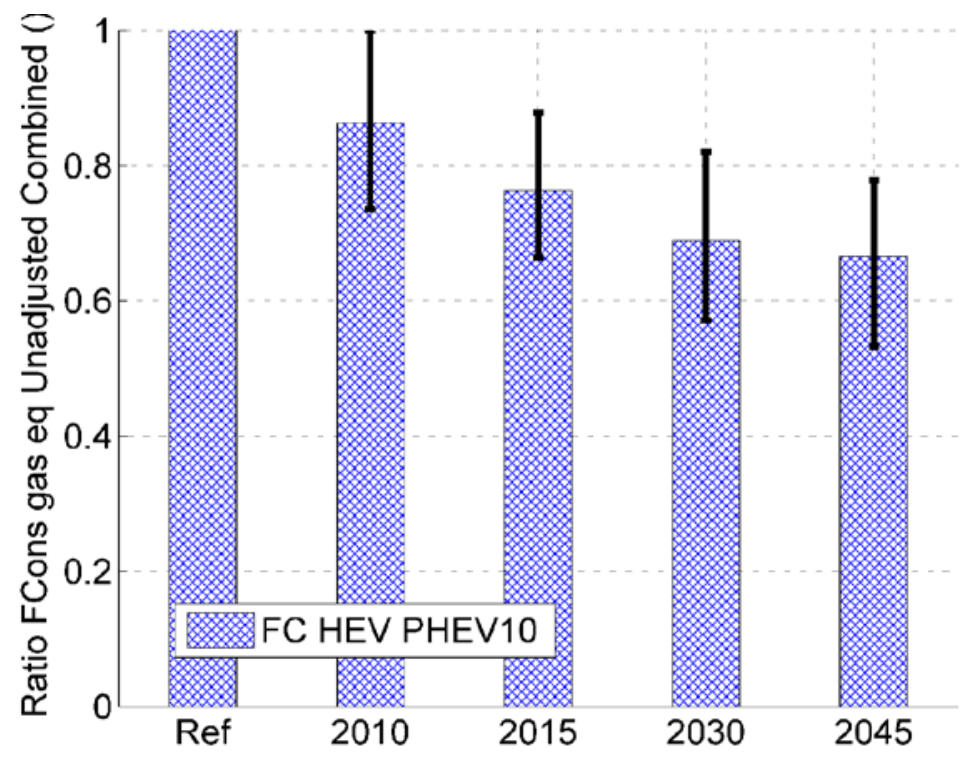

Figure 105 - Gasoline-equivalent fuel consumption for compact fuel-cell PHEV10s compared to the reference case.

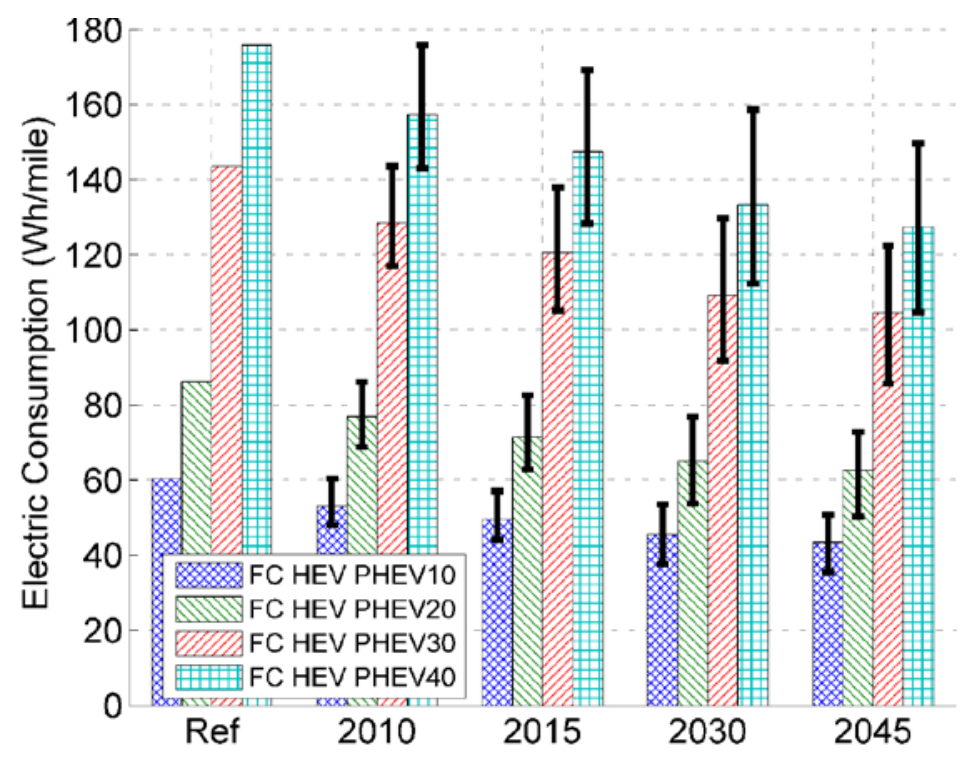

Figure 106 - Electric consumption in CD+CS mode for compact fuel-cell PHEVs.

\subsection{6. $\quad$ ELECTRIC VEHICLES}

For electric vehicles, the results are given in terms of electric-energy consumption for the two drive cycles used in the simulations: UDDS and HWFET. The combination of lightweighting and component improvements leads to a significant decrease in electrical consumption over time: from $8 \%$ to $41 \%$ on UDDS and from $9 \%$ to $39 \%$ on HWFET. 
The values expressed in $\mathrm{Wh} / \mathrm{mile}$ represent the average energy provided by the battery to drive the vehicle for 1 mile. As shown in Figure 107, the HWFET electric consumption is consistently higher than for a UDDS cycle. This can be explained by looking at the two drive-cycle shapes and the energy recoverable by regenerative braking. The UDDS cycle has many strong and steep braking periods, which offer ample opportunities to recover some energy through braking. On the other hand, the HWFET cycle features more stable speeds and only limited braking times. Consequently, the battery recuperates more energy through regenerative braking during a UDDS cycle than during a HWFET cycle.

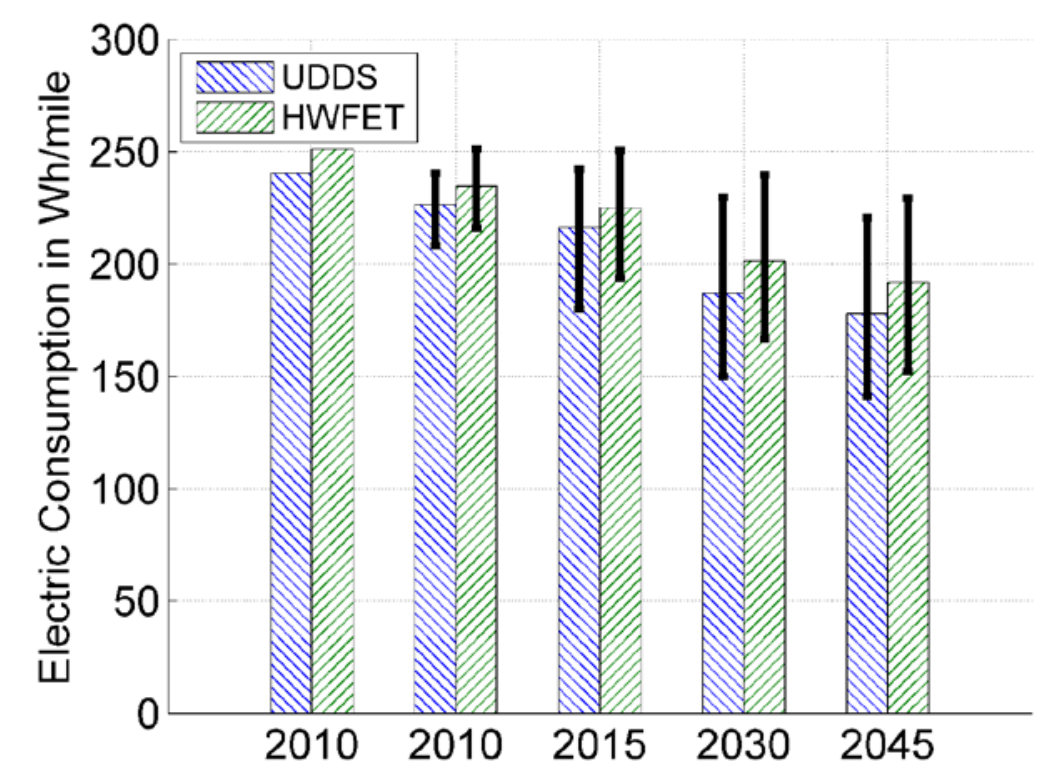

Figure 107 - Electric consumption by compact EVs operating on UDDS and HWFET cycles.

Figure 108 shows the strong relationship between vehicle lightweighting and electrical consumption. 
Argonne National Laboratory Report - Light-Duty Vehicle Fuel Consumption Displacement Potential Up to 2045

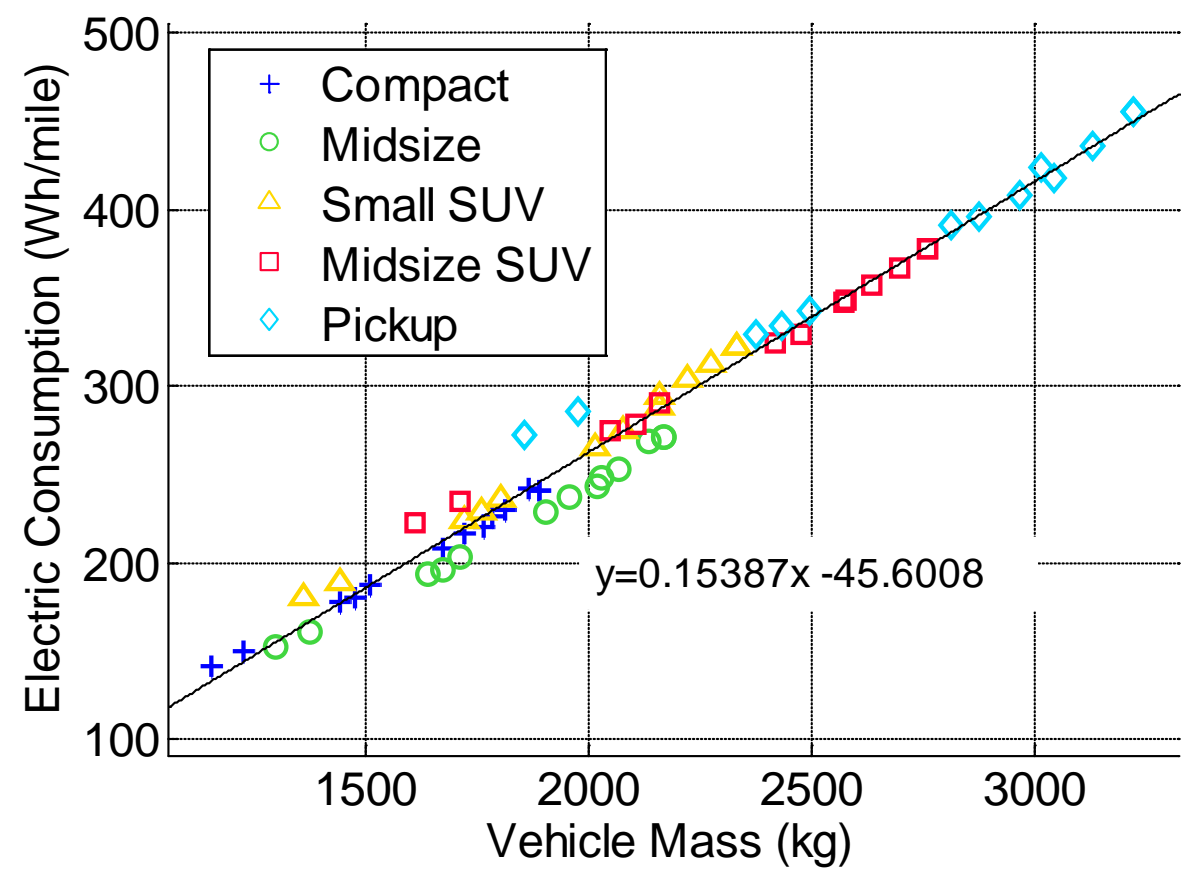

Figure 108 - Electric consumption by EVs. 


\subsection{EVOLUTION OF HEV ENGINES}

\subsubsection{HEV VS. CONVENTIONAL ENGINE}

The comparison between power-split HEVs and conventional gasoline vehicles (same year, same case) in Figure 109 shows that the ratios stay fairly constant for diesel, gasoline, and ethanol. Indeed, the gasoline powersplit compact vehicle consumes between 40 and $44 \%$ less fuel than the conventional gasoline vehicle, whereas the diesel consumes between 43 and $48 \%$ less. The ethanol power-split decrease is between 35 and $40 \%$. However, the hydrogen case shows more significant variations. In the reference case, the hydrogen power-split vehicle consumes roughly $37 \%$ less fuel than the conventional gasoline vehicle, but in 2045 , this advantage increases by $48 \%-54 \%$. This observation confirms that hydrogen vehicles will benefit more from hybridization in the future. It would be interesting to study the same kinds of ratios, but comparing fuel to fuel. This would again demonstrate which fuel would be most advantageous, in terms of reduced consumption, in the transition from a conventional to a power-split HEV powertrain.

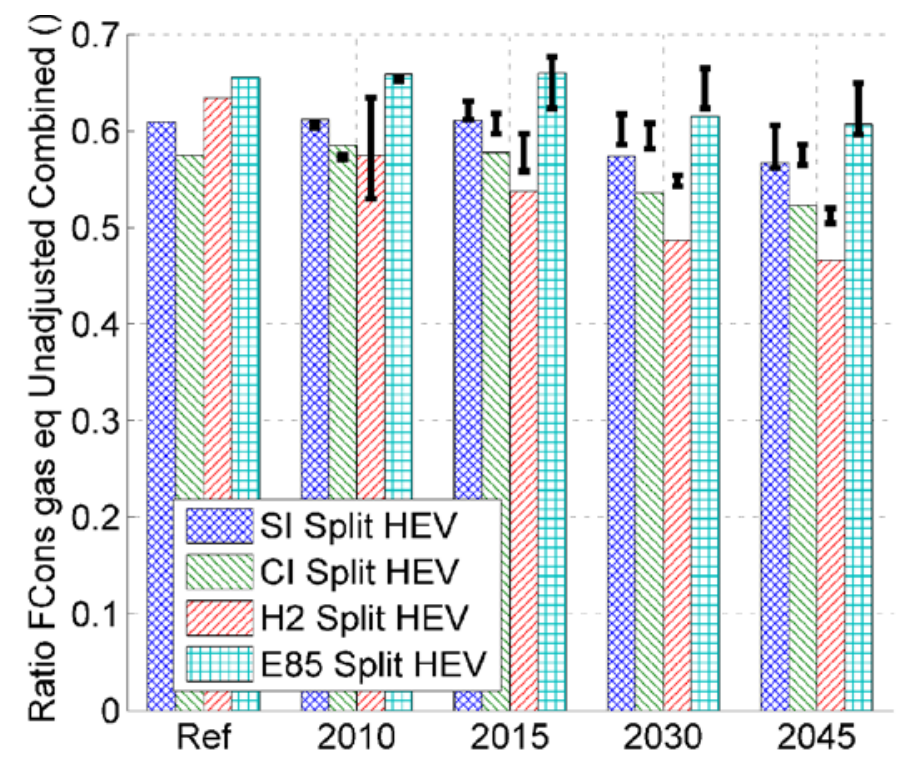

Figure 109 - Gasoline-equivalent fuel consumption for compact power-split HEVs, compared to the same-year, same-case conventional gasoline vehicle.

The power-split HEV fuel consumption changes differently over time from its conventional counterpart, as shown in Table 24. For gasoline, the fuel consumption is improved by $11 \%-47 \%$ in 2045 compared to 2010 . For the other fuels, the fuel-consumption changes are slightly different than for the conventional case. For example, the diesel and hydrogen power-split HEVs show larger improvements than conventional powertrains, with an 11\%$41 \%$ fuel-consumption improvement for diesel and $26 \%-54 \%$ for hydrogen (using the same two timeframes). On 
Argonne National Laboratory Report - Light-Duty Vehicle Fuel Consumption Displacement Potential Up to 2045

the other hand, the ethanol-vehicle fuel consumption decreases by $11 \%-47 \%$ between 2010 and 2045 , which is a faster decrease than for the ethanol conventional vehicle (4\%-41\%). Overall, improvements are greater for HEVs than for conventional vehicles.

Table 24: Comparison of fuel consumption with reference and 2045 technologies for conventional vehicles and HEVs

\begin{tabular}{|c|c|c|c|c|c|c|}
\hline & \multicolumn{3}{|c|}{ Conventional } & \multicolumn{3}{|c|}{ Power-Split HEV } \\
\hline & \multicolumn{2}{|c|}{ Fuel Cons. (L/100 km) } & \multirow{2}{*}{ Improvement } & \multicolumn{2}{|c|}{ Fuel Cons. (L/100 km) } & \multirow{2}{*}{ Improvement } \\
\hline & Ref & $\begin{array}{c}2045 \\
\text { low/avg/high }\end{array}$ & & Ref & $\begin{array}{c}2045 \\
\text { low/avg/high }\end{array}$ & \\
\hline SI & 6.85 & $6.13 / 5.58 / 4$ & $10 \% / 18.5 \% / 42 \%$ & 4.17 & $3.7 / 3.16 / 2.2$ & $\begin{array}{c}11 \% / 24.2 \% / \\
46 \%\end{array}$ \\
\hline $\mathrm{CI}$ & 5.89 & $5.3 / 4.65 / 3.9$ & $10 \% / 21 \% / 33 \%$ & 3.93 & $3.5 / 2.92 / 2.3$ & $\begin{array}{c}12 \% / 25.7 \% / \\
40 \%\end{array}$ \\
\hline $\mathrm{H} 2$ & 7.86 & $5.8 / 4.95 / 4$ & $26 \% / 37 \% / 48 \%$ & 4.34 & $3.2 / 2.6 / 2$ & $27 \% / 40 \% / 54 \%$ \\
\hline E85 & 7.07 & $6.8 / 6.14 / 4.2$ & $4 \% / 13.1 \% / 41 \%$ & 4.49 & $4 / 3.39 / 2.4$ & $\begin{array}{c}11 \% / 24.5 \% / \\
47 \%\end{array}$ \\
\hline
\end{tabular}

In Figure 110, the reference is the same-year, same-fuel conventional vehicle (the reference was always the conventional gasoline vehicle in Figure 109). Figure 110 shows how much the transition of hydrogen vehicles from a conventional to a power-split powertrain affects the fuel consumption. On the other hand, it also clearly appears that ethanol power-split vehicles do not have such a significant fuel-consumption improvement compared to the corresponding conventional-powertrain vehicles. The ratio between the two vehicles even increases over time, whereas it remains stable or decreases for the other fuels. Finally, the fuel-consumption ratio for power-split versus conventional vehicles will be lower for diesel than for gasoline starting in 2015. 
Argonne National Laboratory Report - Light-Duty Vehicle Fuel Consumption Displacement Potential Up to 2045

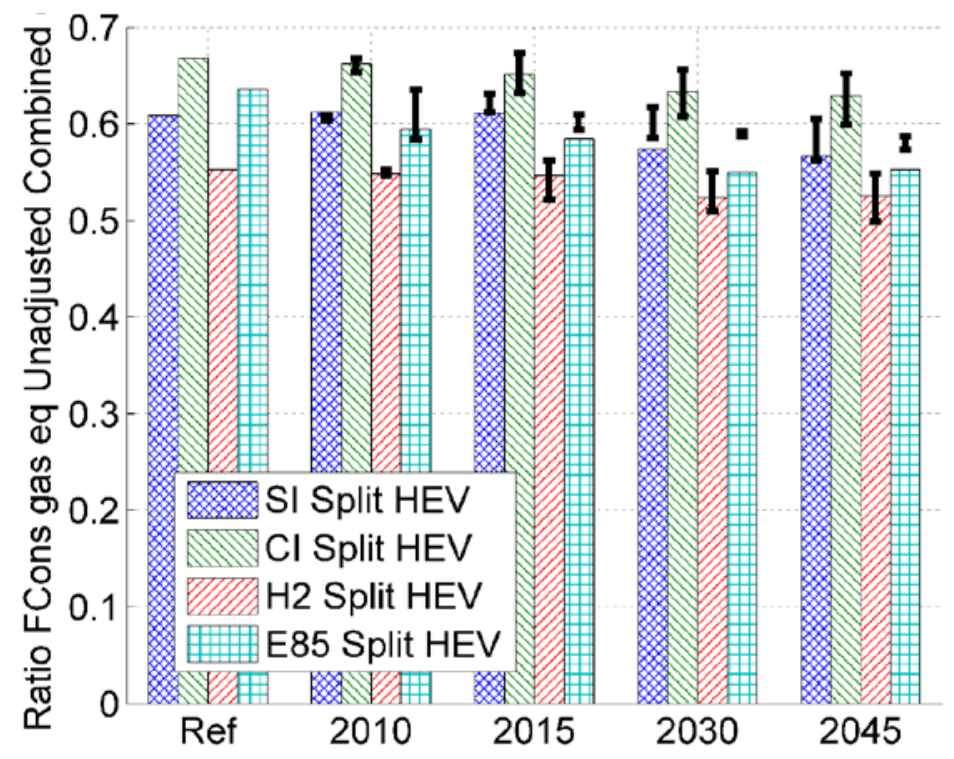

Figure 110 - Gasoline-equivalent fuel consumption for compact power-split HEVs, compared to same-fuel, same-year conventional compact vehicle. 


\subsubsection{ENGINE HEV VS. FUEL-CELL HEV}

The fuel-consumption ratios for all types of power-split HEVs vs. fuel-cell HEVs (Figure 111) are higher than 1, showing that fuel-cell technology offers consistently lower fuel consumption than power-split HEV technology. However, the ratios vary over time and it is pertinent to study the evolution for each fuel. As previously discussed, hydrogen power-split technology shows the best fuel-consumption improvement compared to fuel-cell technology. In the reference case, this vehicle consumes nearly $22 \%$ more fuel than a fuel-cell HEV, but in 2045 , this difference is reduced to the $12 \%-19 \%$ range. If we consider only the UDDS fuel consumption instead of the combined-cycle values, we find that the hydrogen power-split vehicle consumes only $2.5 \%$ more fuel than a fuel-cell HEV in the 2045 high case.

Ethanol, diesel, and gasoline power-split vehicles show similar trends. The ratios for these fuels increase from the reference case to 2030 before decreasing in 2045. For example, the gasoline-HEV fuel consumption is $26 \%$ higher than for the fuel-cell HEV in the reference case, and from 32\% to 50\% higher by 2045.

In 2045, one could expect power-split hydrogen vehicles with fuel consumption close to that of fuel-cell HEVs on an urban cycle and only around $10 \%$ higher on a combined cycle.

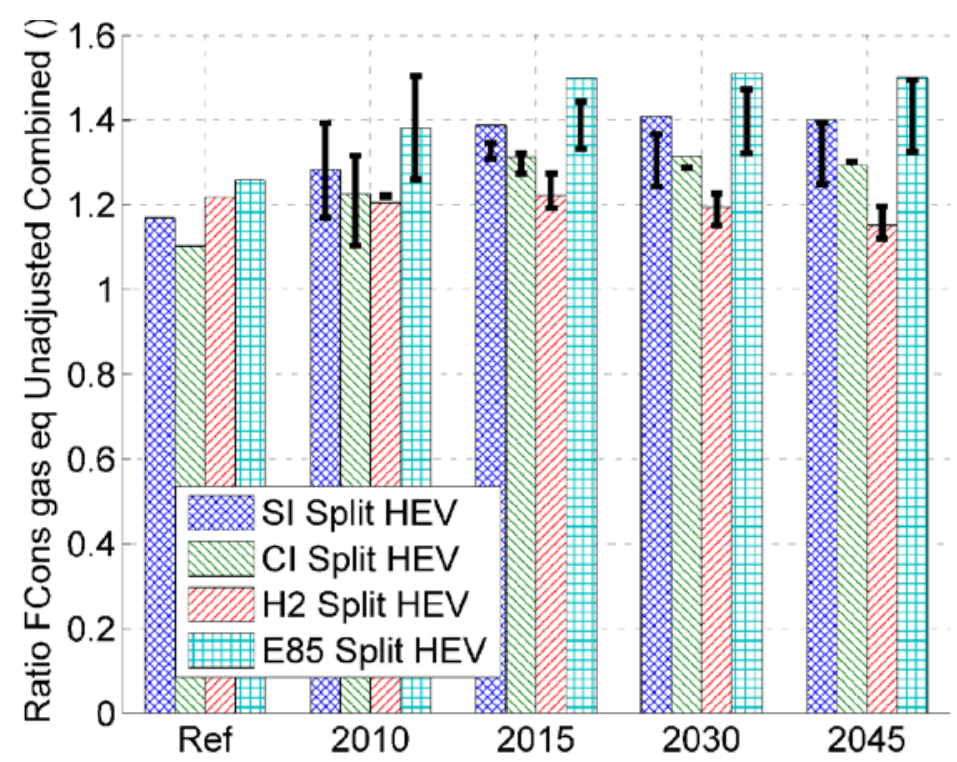

Figure 111 - Gasoline-equivalent fuel consumption for compact power-split HEVs compared to same-year, same-case compact fuel-cell HEV. 


\subsection{EVOLUTION OF HYDROGEN-FUELED VEHICLES}

\subsubsection{FUEL-CELL HEV VS. GASOLINE ENGINE}

In the reference case, the fuel-cell HEVs consume about $48 \%$ less fuel than conventional gasoline vehicles, and this difference in fuel consumption increases to the 55\%-60\% range in 2045 (Figure 112), indicating that the gasoline conventional vehicle will not improve its fuel consumption as fast as the fuel-cell HEV.

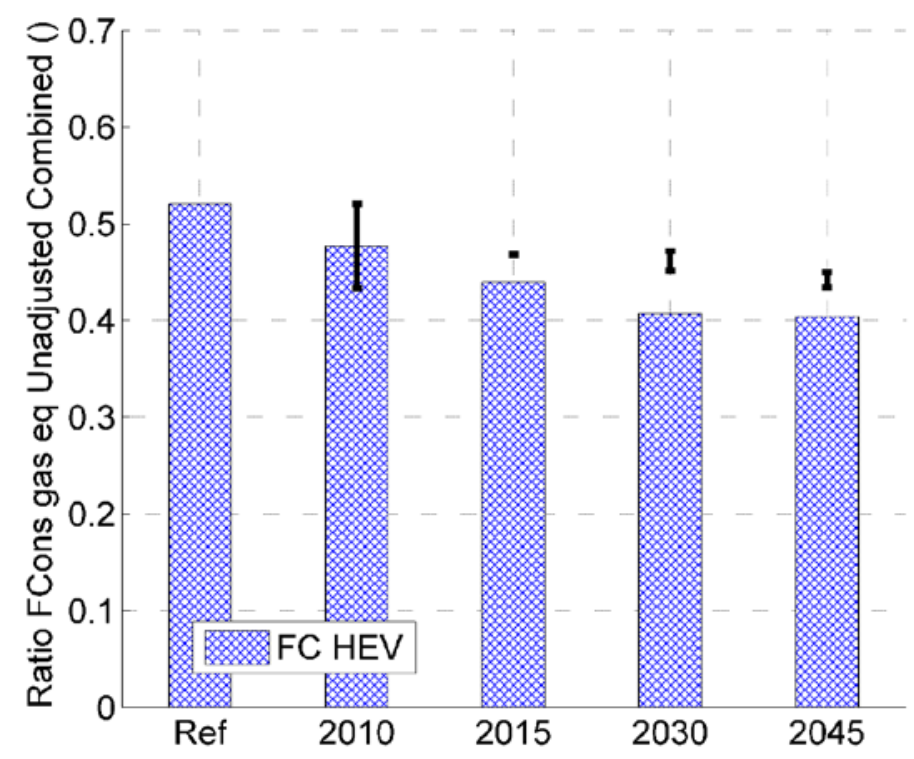

Figure 112 - Gasoline-equivalent fuel consumption for compact fuel-cell HEV compared to same-year, same-case compact gasoline conventional vehicle.

Whereas the fuel-consumption ratios between fuel-cell HEVs and hydrogen power-split HEVs increase over time, indicating some real improvements in the hydrogen power-split powertrain, the same conclusion cannot be drawn from the comparison with the gasoline power-split powertrain. Indeed, the evolution pattern will be very similar to the one shown in Figure 111 for gasoline conventional vehicles, with increasing ratio values. The fuel-cell HEV will go from 25\% lower fuel consumption in the reference case to $20 \%-29 \%$ lower in 2045 (Figure 113). From 2030 to 2045, the ratio will increase slightly. 
Argonne National Laboratory Report - Light-Duty Vehicle Fuel Consumption Displacement Potential Up to 2045

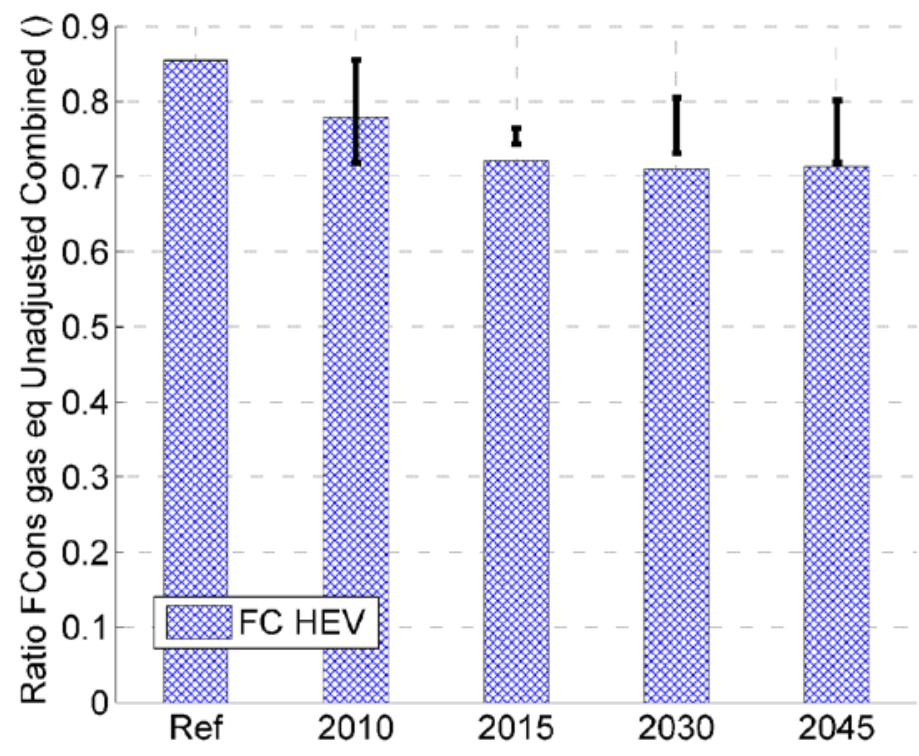

Figure 113 - Gasoline-equivalent fuel consumption for compact fuel-cell HEVs compared to same-year, same-case compact gasoline split HEV.

On the other hand, Figure 114 shows that in the reference case, fuel-cell HEVs consume about $15 \%$ less fuel than gasoline HEVs. This difference in fuel consumption increases in the next two timeframes to reach $33 \%$ to $54 \%$ in 2030 . In 2045 , the trend remains almost constant. In 2045 , the fuel-cell vehicle consumes from $45 \%$ to $57 \%$ less fuel than the gasoline HEV vehicle. This value is still higher than for the reference year, which means that the gasoline HEV vehicle will not improve its fuel consumption as fast as the fuel-cell HEV.

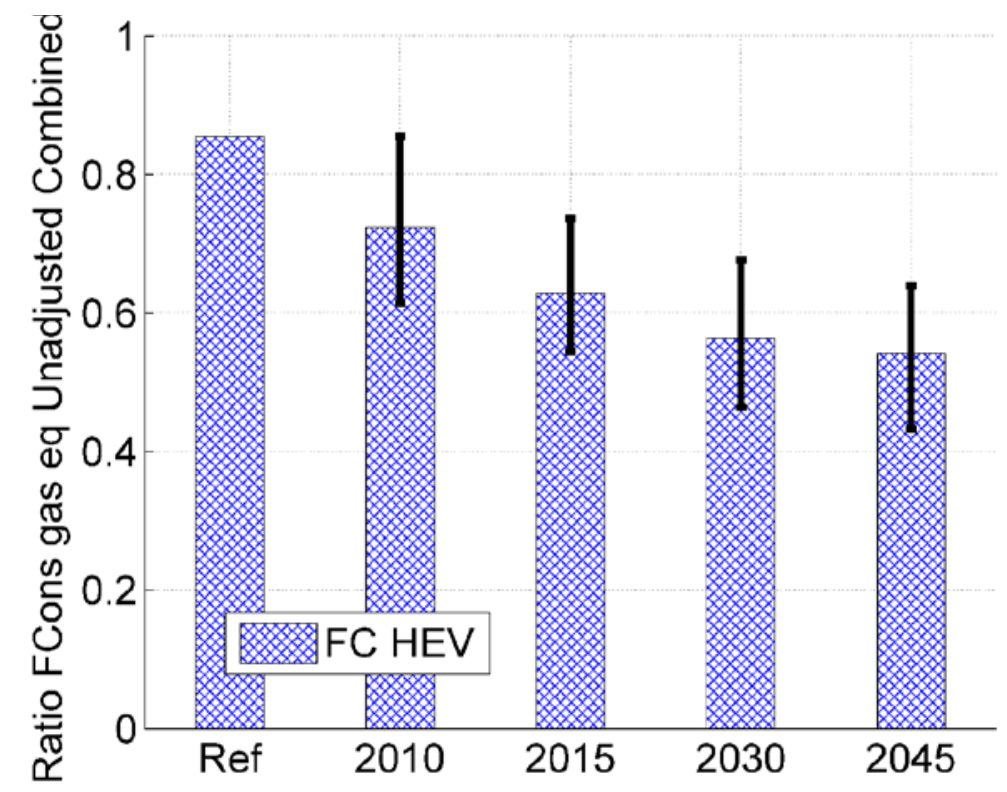

Figure 114 - Gasoline-equivalent fuel consumption for compact fuel-cell HEV compared to the compact gasoline split-HEV reference case. 
Argonne National Laboratory Report - Light-Duty Vehicle Fuel Consumption Displacement Potential Up to 2045

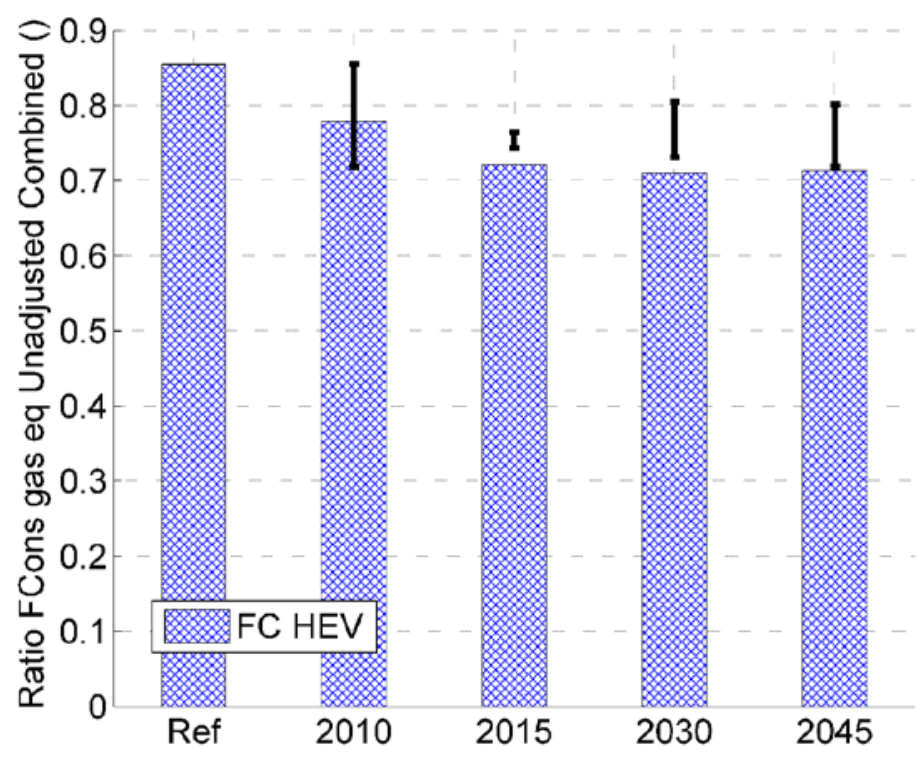

Figure 115 - Gasoline-equivalent fuel consumption for compact fuel-cell HEV compared to the same-year, same-case compact gasoline splitHEV. 


\subsubsection{FUEL-CELL HEV VS. HYDROGEN ENGINE}

The fuel consumption ratios between fuel-cell vehicles and hydrogen conventional vehicles (Figure 116) remain below 0.5 through 2045, meaning that the fuel-cell vehicle always has less than half the fuel consumption of the hydrogen conventional vehicle. From the reference case to 2045, the fuel-cell vehicle uses approximately $55 \%$ as much fuel as the hydrogen vehicle.

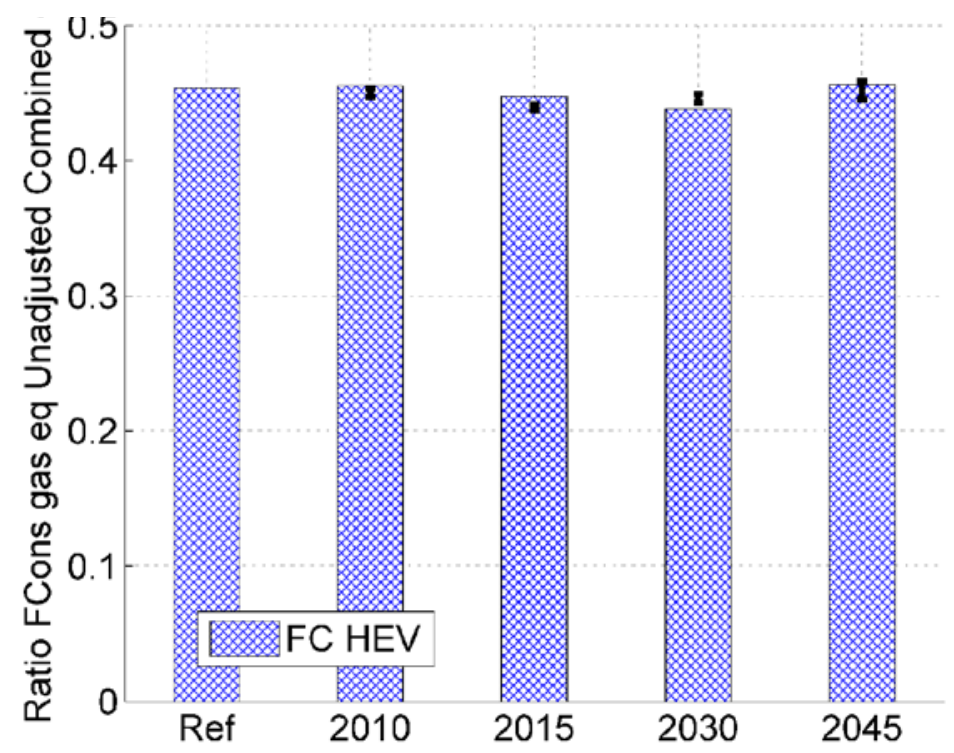

Figure 116 - Gasoline-equivalent fuel consumption for compact fuel-cell HEV compared to the same-year, same-case compact hydrogen conventional vehicle.

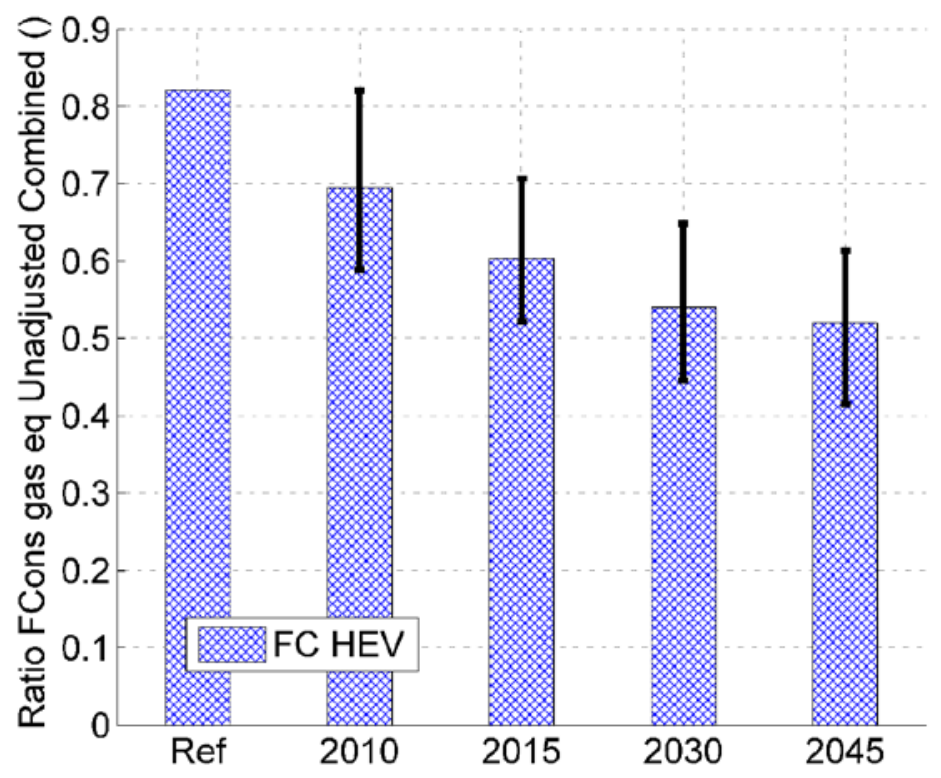

Figure 117 - Gasoline-equivalent fuel consumption for compact fuel-cell HEV compared to reference-case hydrogen power-split HEV. 
The evolution of fuel-consumption ratios for fuel-cell HEVs in comparison to hydrogen power-split HEVs (Figure 118) is different than with the hydrogen conventional vehicle. The ratios increase over time, reflecting the faster improvement made by hydrogen power-split HEVs compared to fuel-cell HEVs. In the reference case, the fuel-cell vehicles consume about $18 \%$ less fuel than the hydrogen power-split HEVs. but this advantage decreases to the $11 \%-17 \%$ range in 2045 , which confirms the trends described in the previous section.

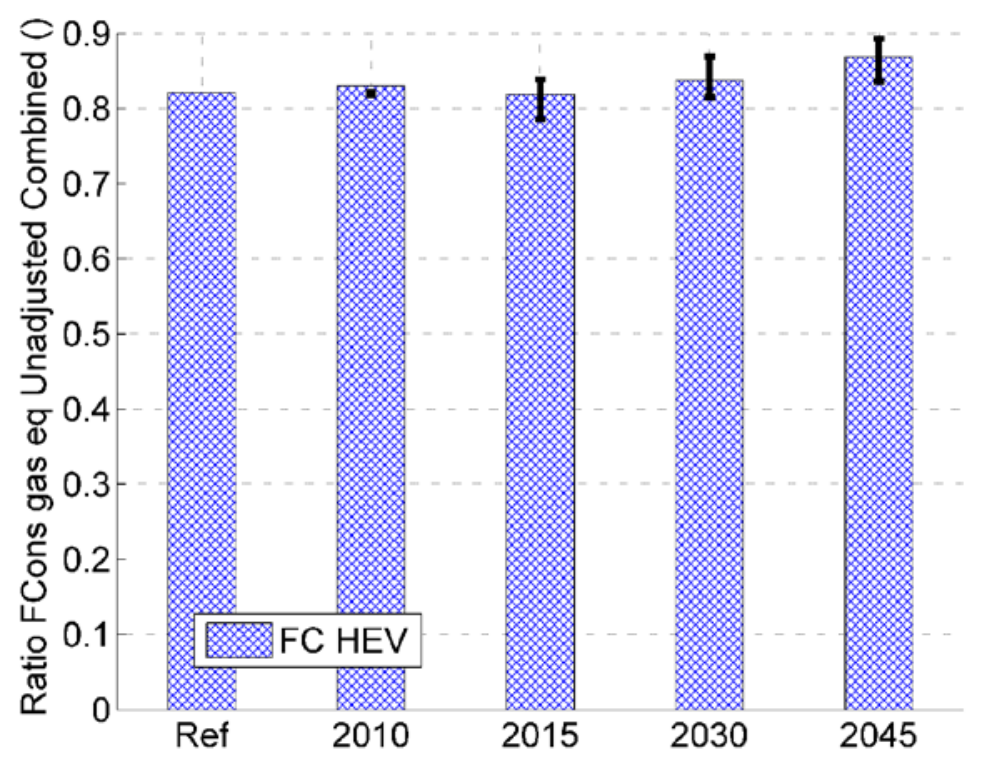

Figure 118 - Gasoline-equivalent fuel consumption for compact fuel-cell HEVs compared to same-year, same-case compact hydrogen power split HEV.

\subsection{All Powertrain Configurations}

Weight-specific fuel consumption (WSFC) is used to compare vehicle efficiencies independently of their weight. Figure 119 shows the WSFC of a compact car as a function of vehicle mass. 
Argonne National Laboratory Report - Light-Duty Vehicle Fuel Consumption Displacement Potential Up to 2045

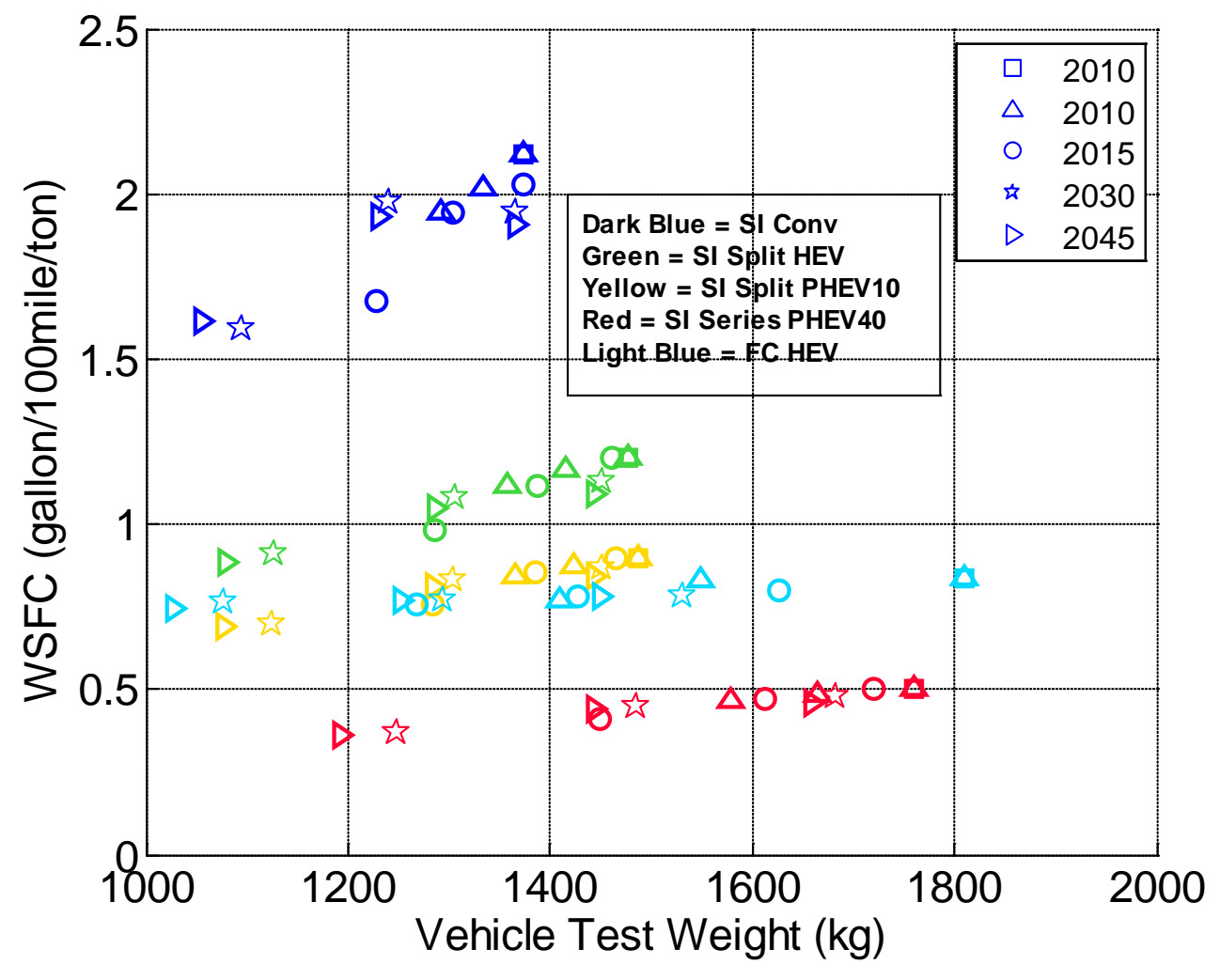

Figure 119 - WSFC as a function of vehicle mass for compact cars.

Figure 120 shows the WSFC for different vehicle classes with a conventional gasoline configuration. Midsize vehicles in 2030 and 2045 achieve the lowest WSFC, followed by compacts and small SUVs from the same timeframe. 
Argonne National Laboratory Report - Light-Duty Vehicle Fuel Consumption Displacement Potential Up to 2045

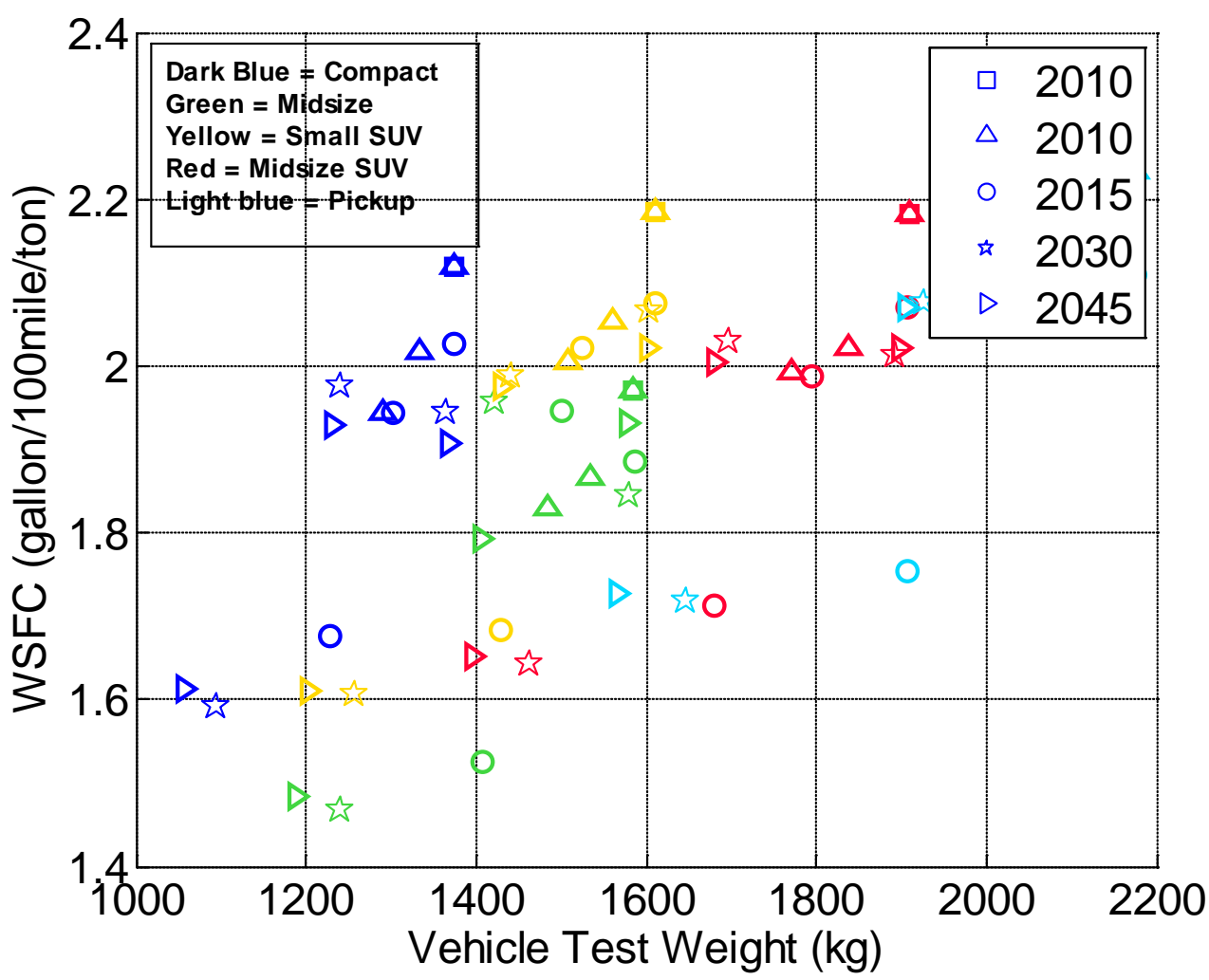

Figure 120 - WSFC as a function of vehicle mass for gasoline conventional vehicles.

Figure 121 shows the WSFC of various fuel-cell vehicles. The introduction of higher-energy batteries leads to a significant improvement. However, going from PHEV30 to PHEV40 provides a much smaller benefit than going from PHEV10 to PHEV20. This observation is consistent with previous studies that have shown a diminishing return on investment for PHEVs with a longer electric range. 


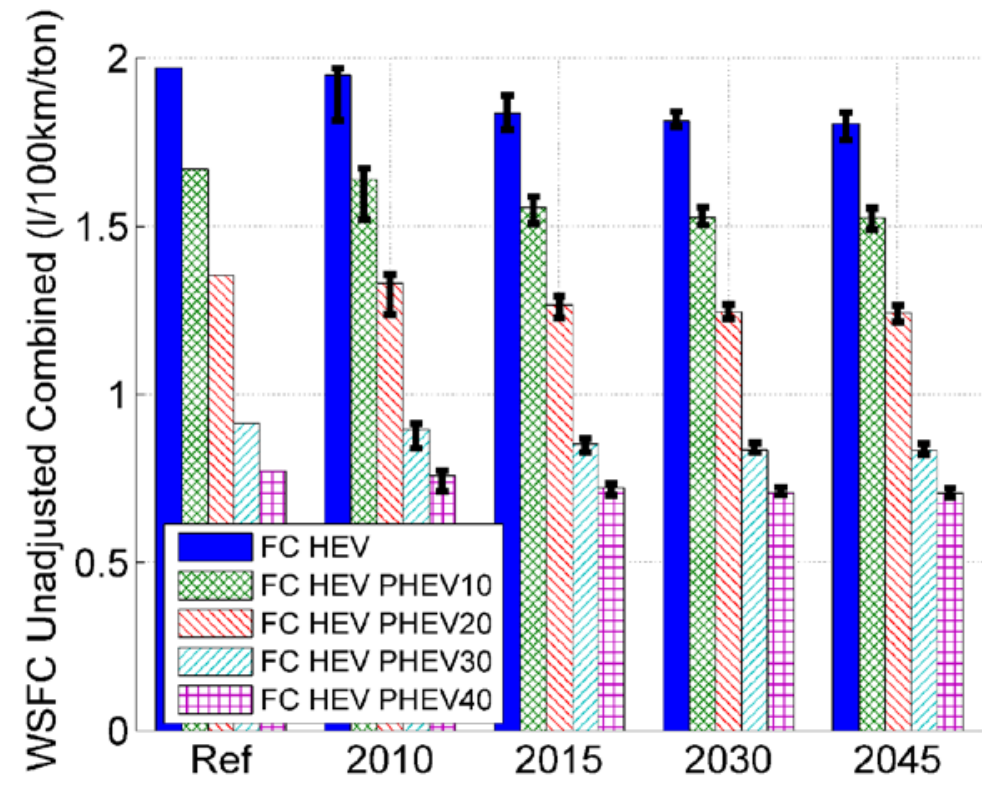

Figure 121 - WSFC for fuel-cell compact cars. 


\section{VEHICLE FUEL CONSUMPTION VS. MANUFACTURING COST RESULTS}

All costs are manufacturing costs. The details for all the vehicles are located in Appendix 3.

\subsection{EVOLUTION OF SPECIFIC POWERTRAIN CONFIGURATIONS}

\subsubsection{CONVENTIONAL}

Figure 122 shows incremental manufacturing cost for conventional compact vehicles. While the prices of gasoline, diesel, and ethanol vehicles increase from 2010 to 2045, the hydrogen vehicle becomes cheaper over time. The increase for the first three types is due to several factors, including lightweighting (the decrease in vehicle body mass by up to $37 \%$ by 2045 entails cost increases, owing to the use of aluminum or carbon fiber) and advanced component technologies such as direct injection. For the hydrogen engine, the increase due to lightweighting is compensated by larger cost reductions due to the hydrogen storage system. The cost of such a system for the reference vehicle is very significant. For example, the cost of a 4-kg hydrogen storage system is currently about $\$ 3600$. However, the technology improvements that are expected for hydrogen storage systems will significantly decrease their costs. Overall, even if the ICE cost as well as the body cost increases over time, the hydrogen-vehicle manufacturing cost will decrease because the hydrogen storage cost decrease will be more aggressive.

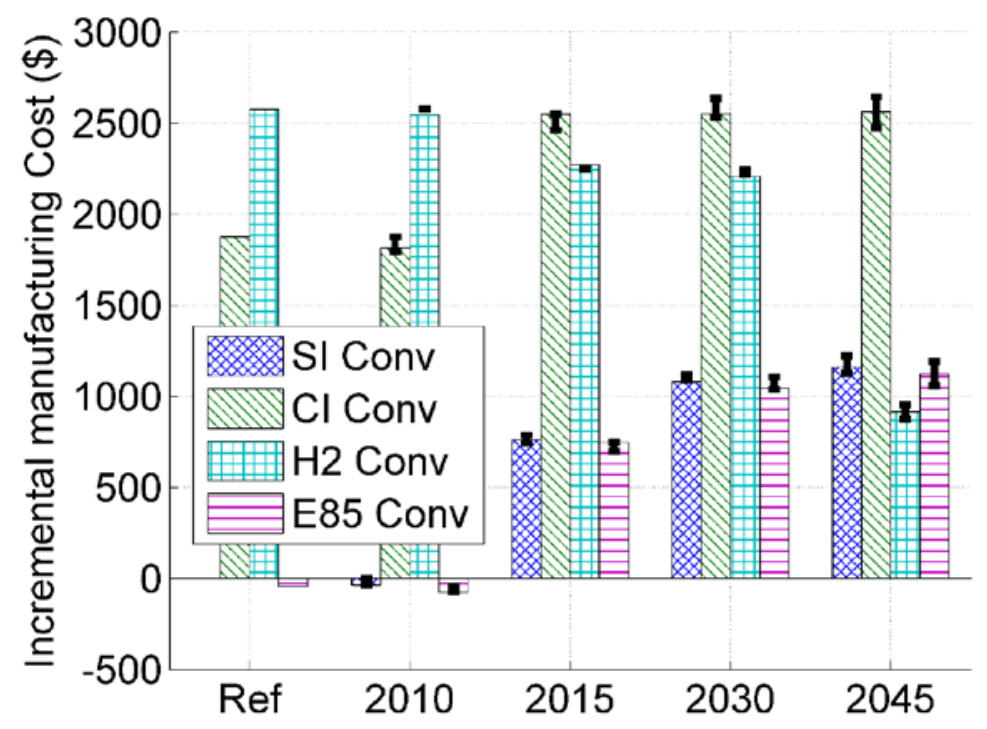

Figure 122 - WSFC as a function of vehicle mass for gasoline conventional vehicles.

Figure 123 shows the manufacturing cost of conventional compact diesel, hydrogen and ethanol vehicles compared to same-year conventional compact gasoline vehicles. The gasoline and ethanol vehicles have similar manufacturing costs for all the timeframes. Their manufacturing costs increase by $8 \%$ between the 2010 and 2045 
timeframes, whereas the diesel-vehicle manufacturing cost increases by only $4 \%$. However, the diesel vehicle remains between 10 and $13 \%$ more expensive than the ethanol and gasoline vehicles.

Finally, the hydrogen-vehicle manufacturing cost decreases by $10 \%$ from the reference case to 2045 . In the reference case, this vehicle is about $18 \%-19 \%$ more expensive than the gasoline vehicle, but this difference is reduced with time until the trend is reversed, with the hydrogen vehicle 2\% cheaper in 2045.

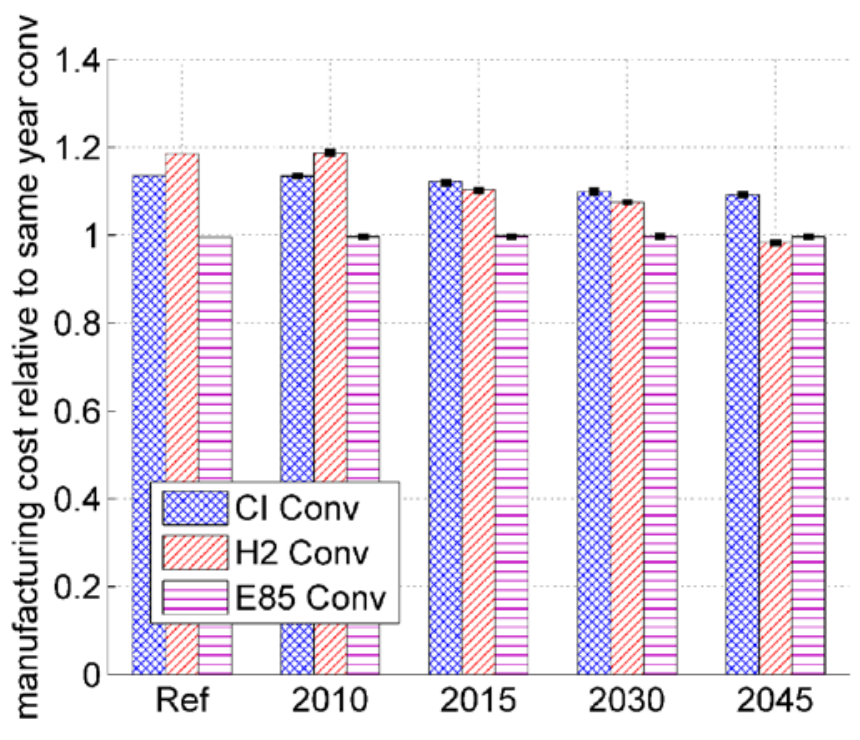

Figure 123 - Manufacturing Costs of diesel, hydrogen, and ethanol conventional compact cars compared to same-year gasoline conventional compact car.

\subsubsection{ENGINE HEVS}

Figure 124 shows the vehicle incremental manufacturing costs for the power-split HEVs. The gasoline power-split HEV is always the cheapest vehicle among all the HEVs except in the 2045 high case, where the hydrogen vehicle is cheaper. Figure 125 shows that overall, the diesel HEV is between 10 and $14 \%$ more expensive than the gasoline HEV, but this difference tends to decrease after 2010. From 2010 on, the ethanol-vehicle ratio decreases, reaching almost 1 in 2045. The same observation can be made for hydrogen vehicles, for which the average and high cases continuously decrease over time. 


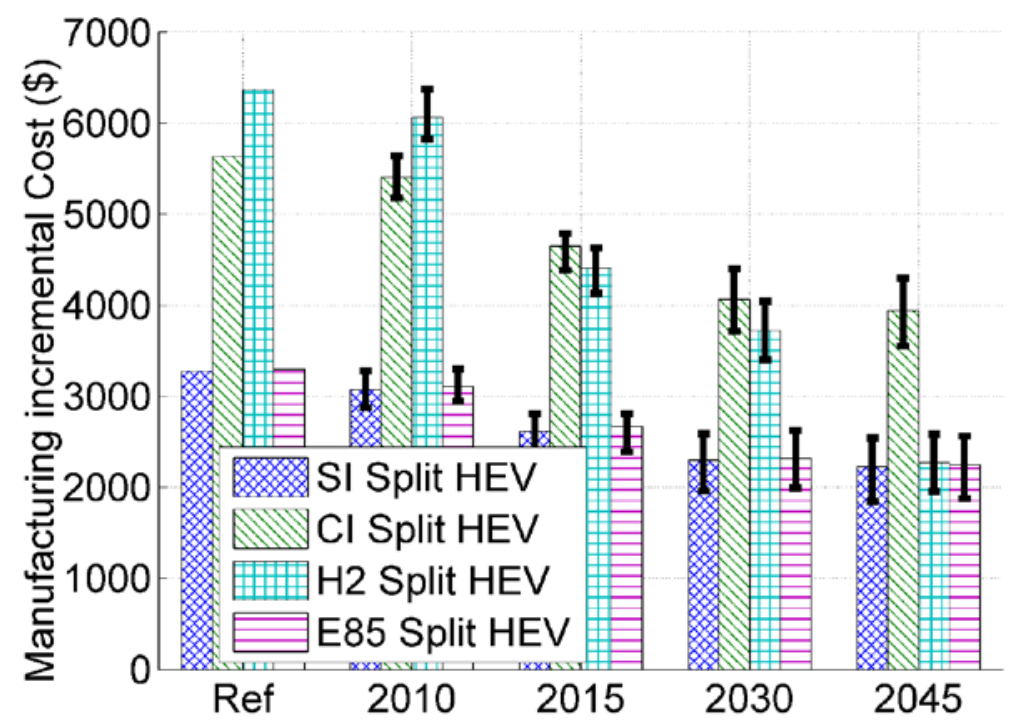

Figure 124 - Incremental manufacturing Costs of compact HEVs.

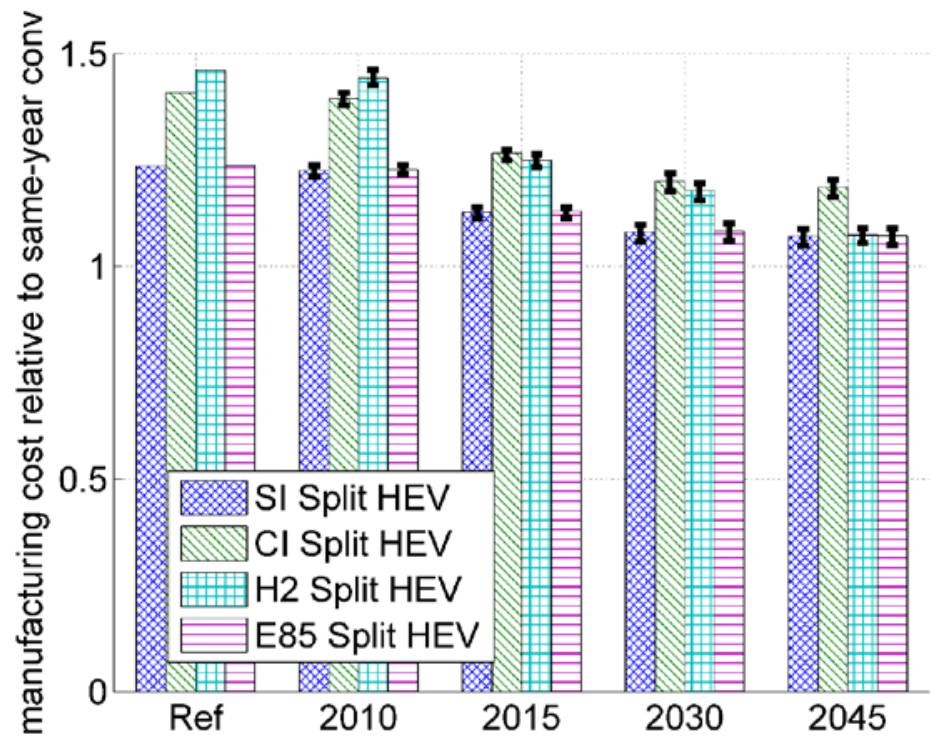

Figure 125 - Manufacturing Costs of compact HEVs compared with same-year conventional gasoline-powered vehicles. 


\subsubsection{ENGINE PHEVS}

Figure 126 - Manufacturing Costs of compact PHEVs.

shows the incremental manufacturing cost evolution of PHEVs with different fuels. The overall trend is the same for all fuels; only the actual costs vary. PHEV40 costs show a sharp decrease over time, whereas PHEV10s show a very slight decrease over time. This observation can be explained by improvements in batteries over time.
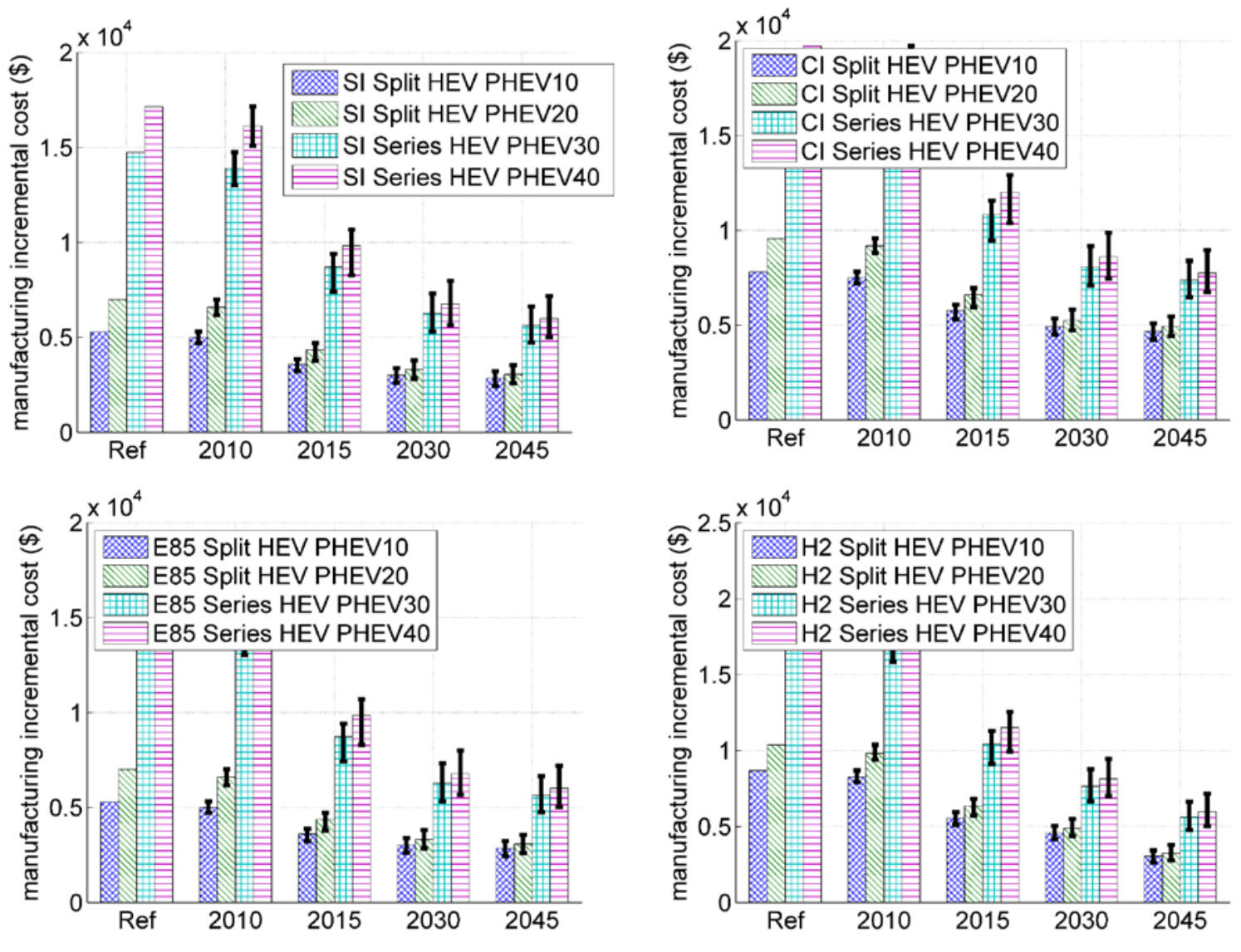

Figure 126 - Manufacturing Costs of compact PHEVs.

Figure 127 shows the manufacturing cost of PHEVs compared to gasoline HEVs. Again, the PHEVs become more cost-competitive over time owing to improvements in batteries. 

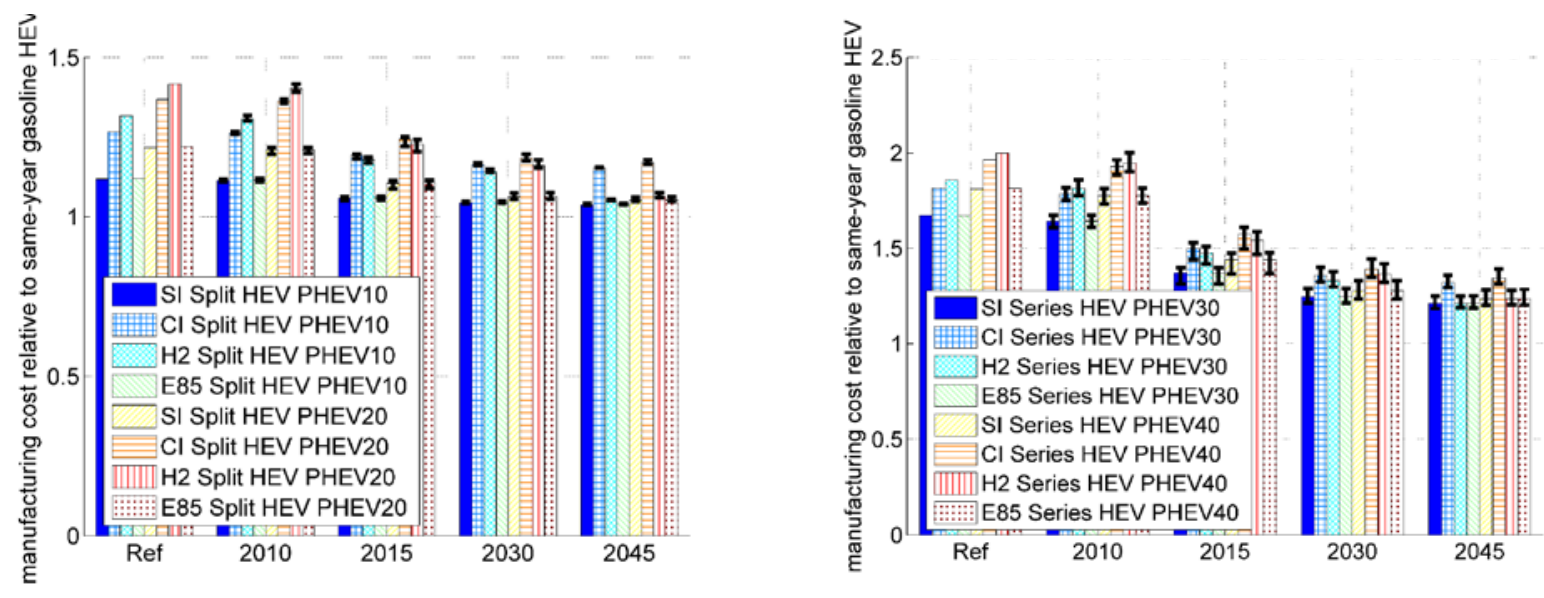

Figure 127 - Manufacturing Costs of compact PHEVs compared with same-year gasoline-powered HEV. 


\subsubsection{FUEL-CELL VEHICLES}

Fuel-cell vehicle incremental manufacturing costs (Figure 128) show a pattern similar to that of previously described gasoline-vehicle incremental manufacturing cost. Indeed, as time goes on, the different vehicles' manufacturing costs become closer and closer to each other. In 2010, a fuel-cell PHEV40 is approximately 39\% more expensive than a fuel-cell HEV, whereas it is only $20 \%$ more expensive in 2045. From 2010 to 2045, manufacturing cost reductions for fuel-cell vehicles are as follows: HEV, 40\%-44\%; PHEV10, 39\%-44\%; PHEV20, 42\%-47\%; PHEV30, 44\%-50\%; and PHEV40, 47\%-53\%. This finding confirms the pattern observed for power-split PHEVs, where the manufacturing cost reduction between the reference case and 2045 increases as the AER increases. Finally, it is interesting to note that in 2045, the fuel-cell vehicle manufacturing costs are under $\$ 20,000$ for all the average and high cases.

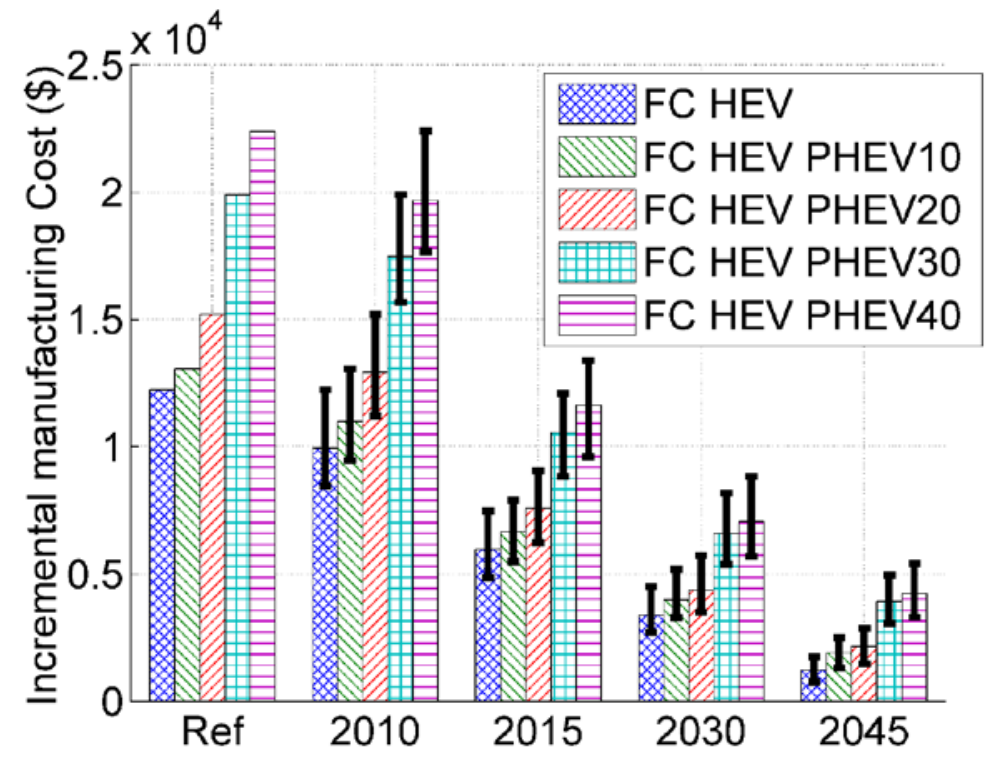

Figure 128 - Manufacturing Costs of compact fuel-cell vehicles.

\subsubsection{ELECTRIC VEHICLES}

As shown in Figure 129, improvements in battery costs and lightweighting affect EV costs, which are expected to decrease by a factor greater than 2 . 


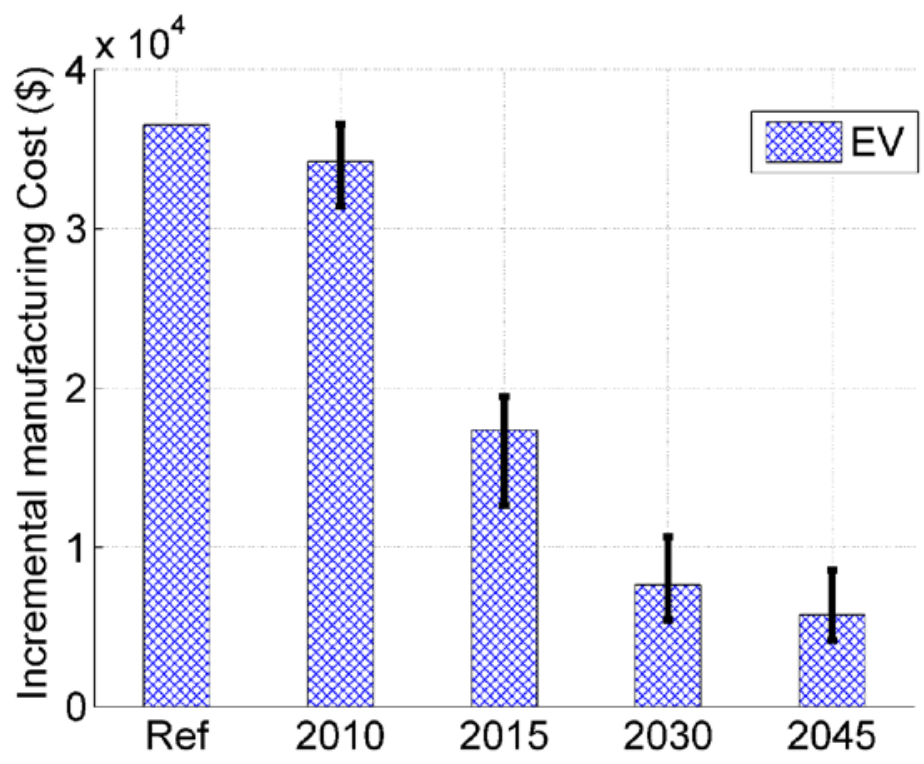

Figure 129 - Manufacturing Costs for compact EVs.

\subsection{POWERTRAIN COMPARISON}

Figure 130 shows the manufacturing costs for all the gasoline-powertrain vehicles (conventional, powersplit HEV and power-split PHEVs). The manufacturing costs tend to get closer to each other as time goes on. For example, in 2010, the power-split HEV is $23 \%$ more expensive than the conventional vehicle and the PHEV40 is $124 \%$ more expensive than the conventional vehicle. However, in the 2045 , the situation has dramatically changed; only a $5 \%$ to $7 \%$ difference in manufacturing cost is observed for the power-split HEV, and $26 \%$ to $40 \%$ for the power-split PHEV40.

Whereas the conventional-vehicle manufacturing cost increases slightly over time, the opposite pattern is observed for power-split vehicles. The higher the AER, the greater the manufacturing cost reduction over time: the difference between the reference case and 2045 is $11 \%-15 \%$ for the PHEV10, $16 \%-21 \%$ for the PHEV $20,28 \%-35 \%$ for the PHEV30, and 32\%-39\% for the PHEV40. 


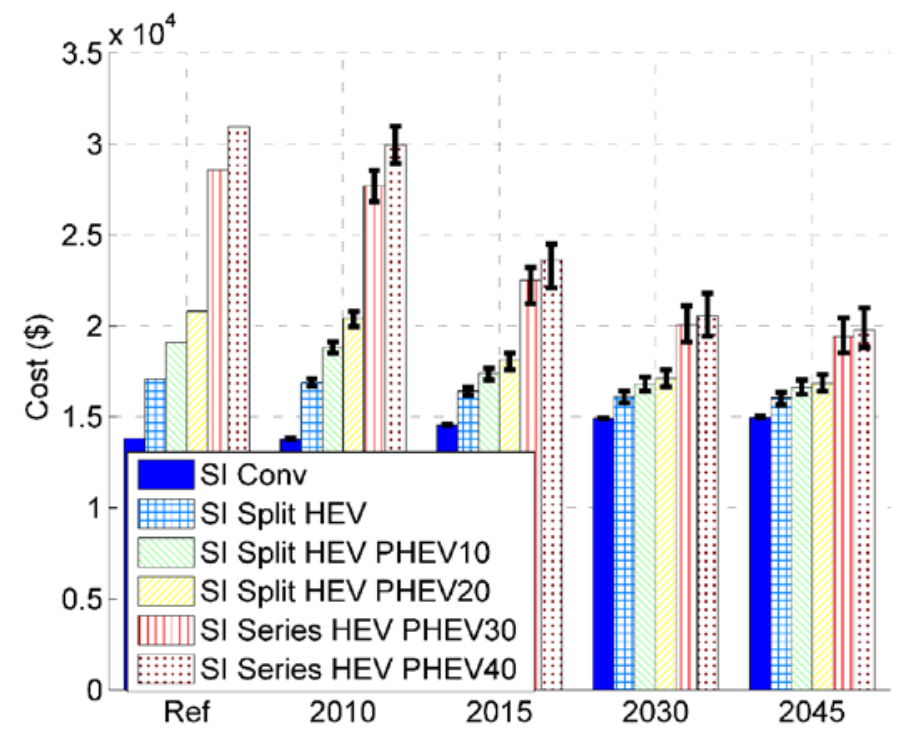

Figure 130 - Manufacturing Costs of compact gasoline-powered vehicles.

Figure 131 shows the relative manufacturing costs of hydrogen-engine vehicles and conventional gasoline vehicles. While hydrogen engines will remain more expensive, the technology will become more cost-competitive over time. The main reason is the development of a cheaper fuel-cell system and less costly hydrogen storage. Note that in 2045, a conventional hydrogen vehicle will be slightly cheaper than a conventional gasoline vehicle.

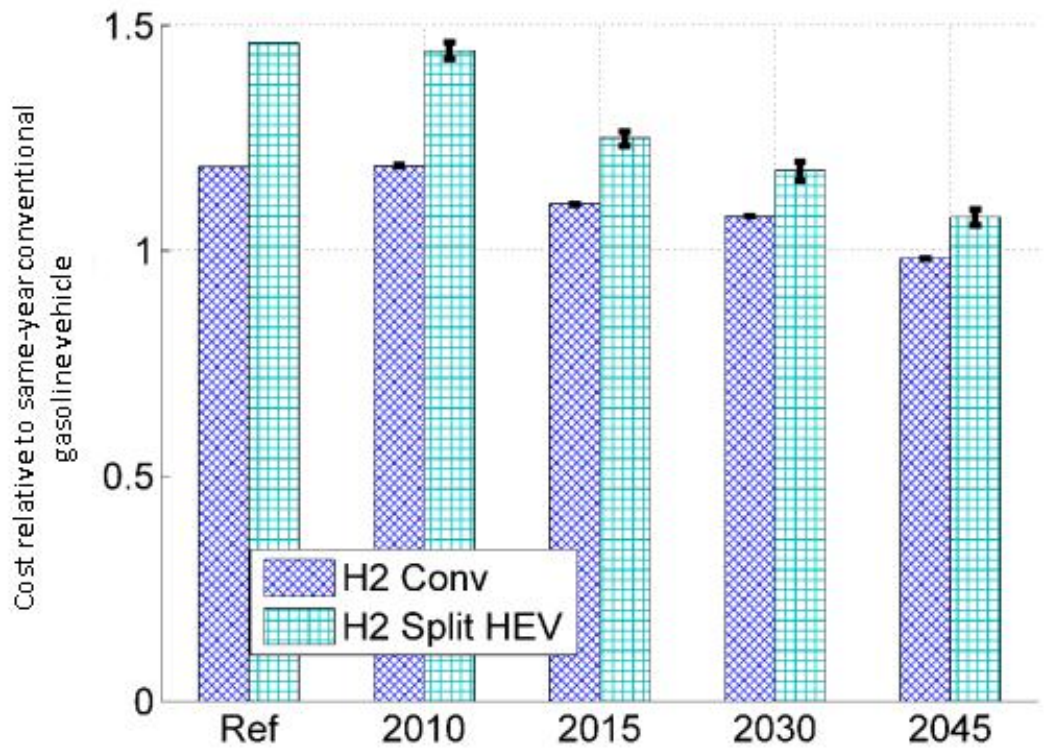

Figure 131 - Manufacturing costs of compact H2 ICE vehicles compared with same-year conventional gasoline vehicles. 
Figure 132 shows the manufacturing cost ratio between fuel-cell and conventional gasoline vehicles. The trend is similar to that for the hydrogen engine in the sense that the manufacturing cost ratio will decrease in the future. However, this decrease is more pronounced for the fuel-cell HEV because in addition to a significant manufacturing cost reduction for the hydrogen tanks, the vehicle also benefits from manufacturing cost reductions related to the fuel-cell system.

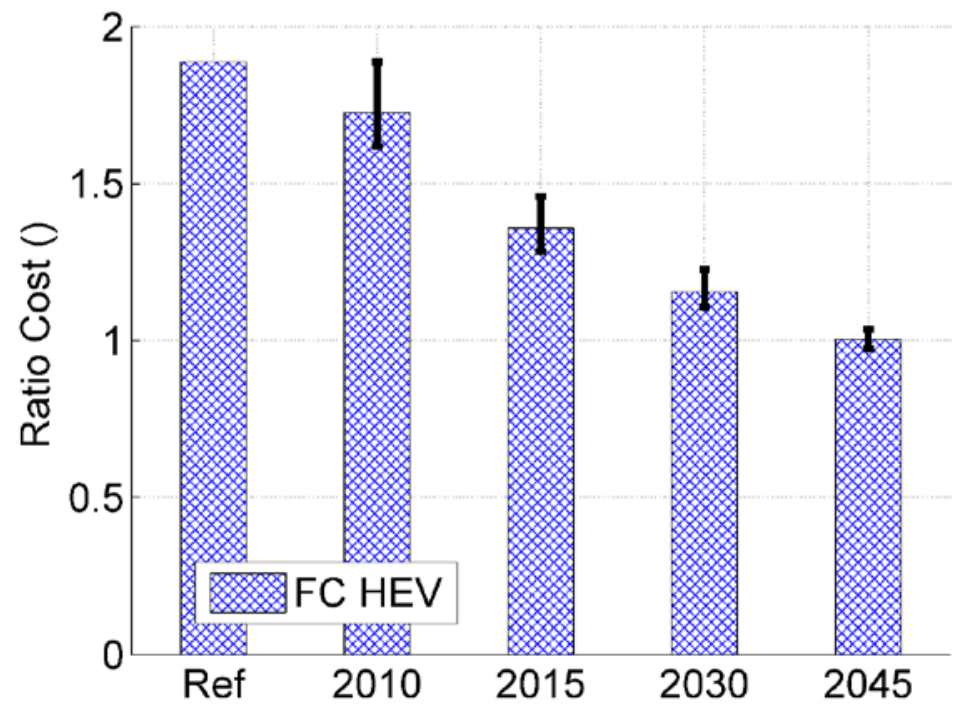

Figure 132 - Manufacturing Cost of compact fuel-cell HEV compared with same-year conventional gasoline vehicle. 


\section{TRADE-OFF BETWEEN VEHICLE FUEL CONSUMPTION AND MANUFACTURING}

\section{COST}

All costs are manufacturing costs.

\subsection{CONVENTIONAL VEHICLES}

It can be seen from Figure 133 that diesel vehicles remain more expensive relative to other conventional vehicles over time, with no advantage in fuel consumption, whereas hydrogen vehicles improve significantly, becoming similar to the conventional gasoline vehicle in cost and fuel consumption at some point.

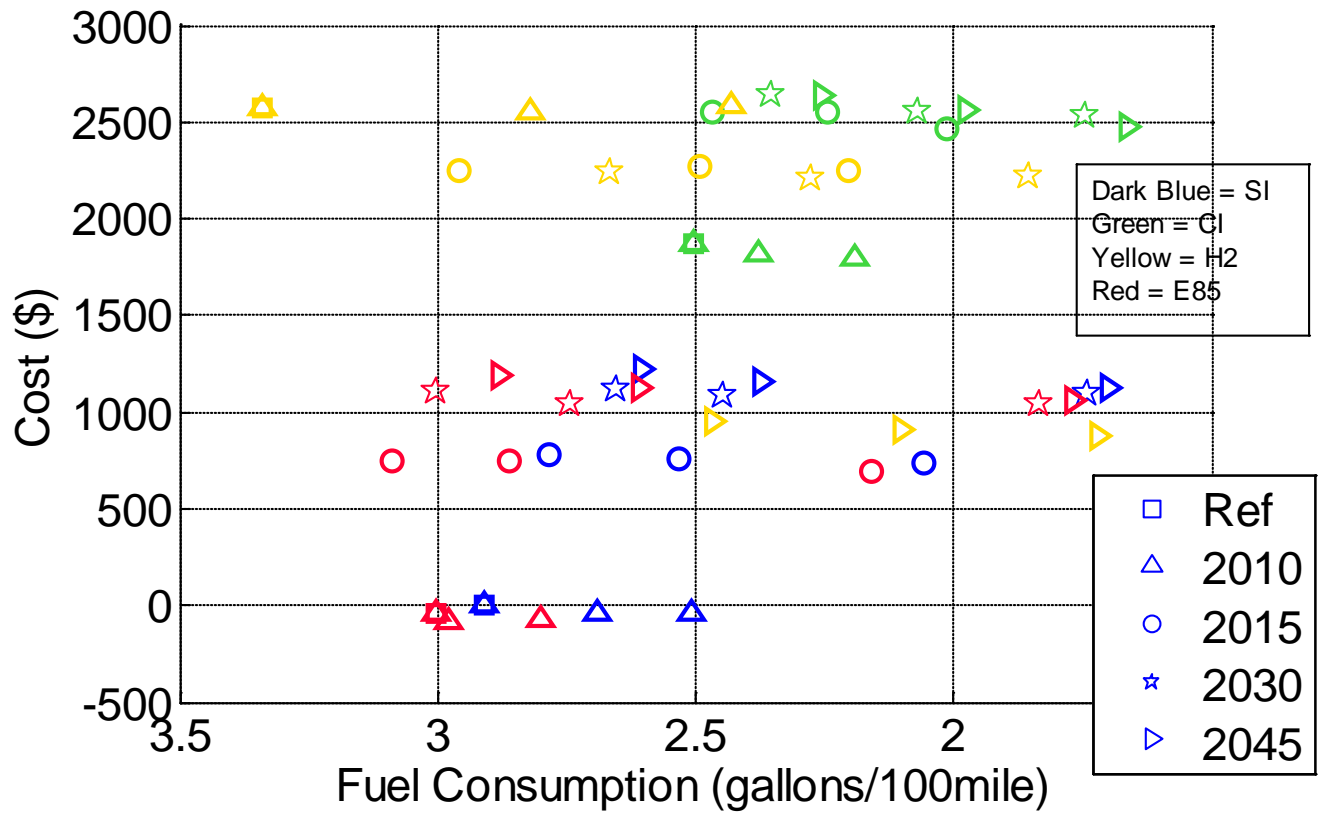

Figure 133 - Incremental manufacturing cost (in comparison to the reference conventional gasoline vehicle manufacturing cost) as a function of fuel consumption for compact conventional vehicles.

\subsection{HEVS}

Figure 134 shows similar trends for HEVs independently of ICE technology. The overall trend is decreasing, which means lower fuel consumption and lower cost. Gasoline and ethanol HEVs offer the best trade-offs over time, with the hydrogen HEV becoming competitive in the 2045 timeframe. 
Argonne National Laboratory Report - Light-Duty Vehicle Fuel Consumption Displacement Potential Up to 2045

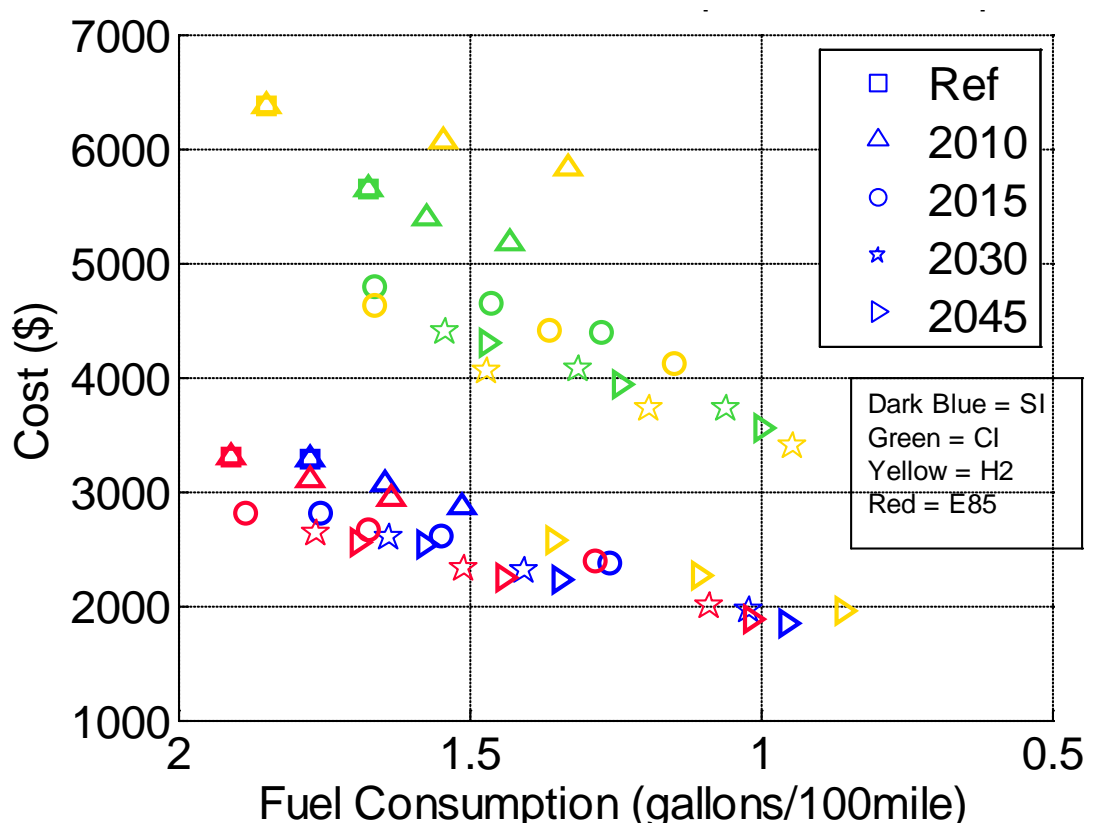

Figure 134 - Incremental manufacturing cost (in comparison to the reference conventional gasoline vehicle manufacturing cost) as a function of fuel consumption for compact HEVs.

\subsection{PHEVS}

Figures 135 and 136 show, respectively, that PHEV10 and PHEV40 vehicles offer less future benefit than HEVs and conventional vehicles, although the overall trend is promising.

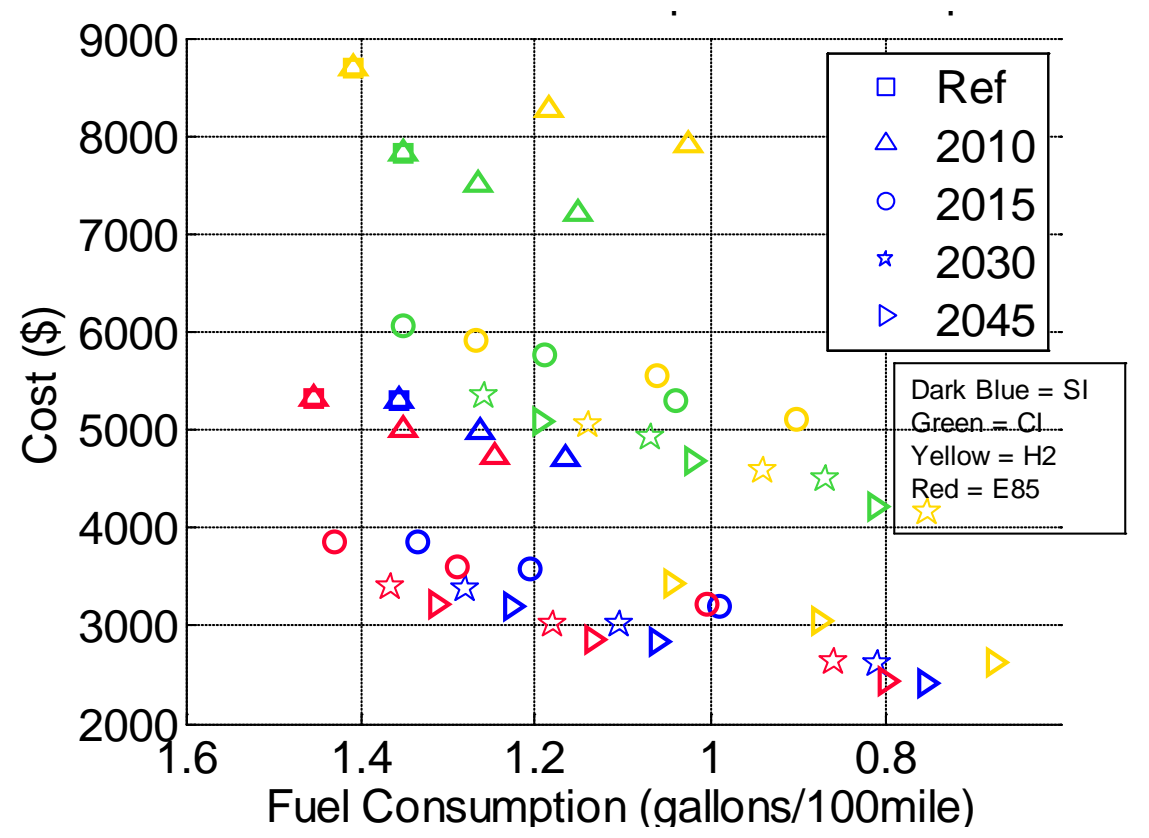

Figure 135 - Incremental manufacturing cost (in comparison to the reference conventional gasoline vehicle manufacturing cost) as a function of fuel consumption for PHEV10 vehicles. 


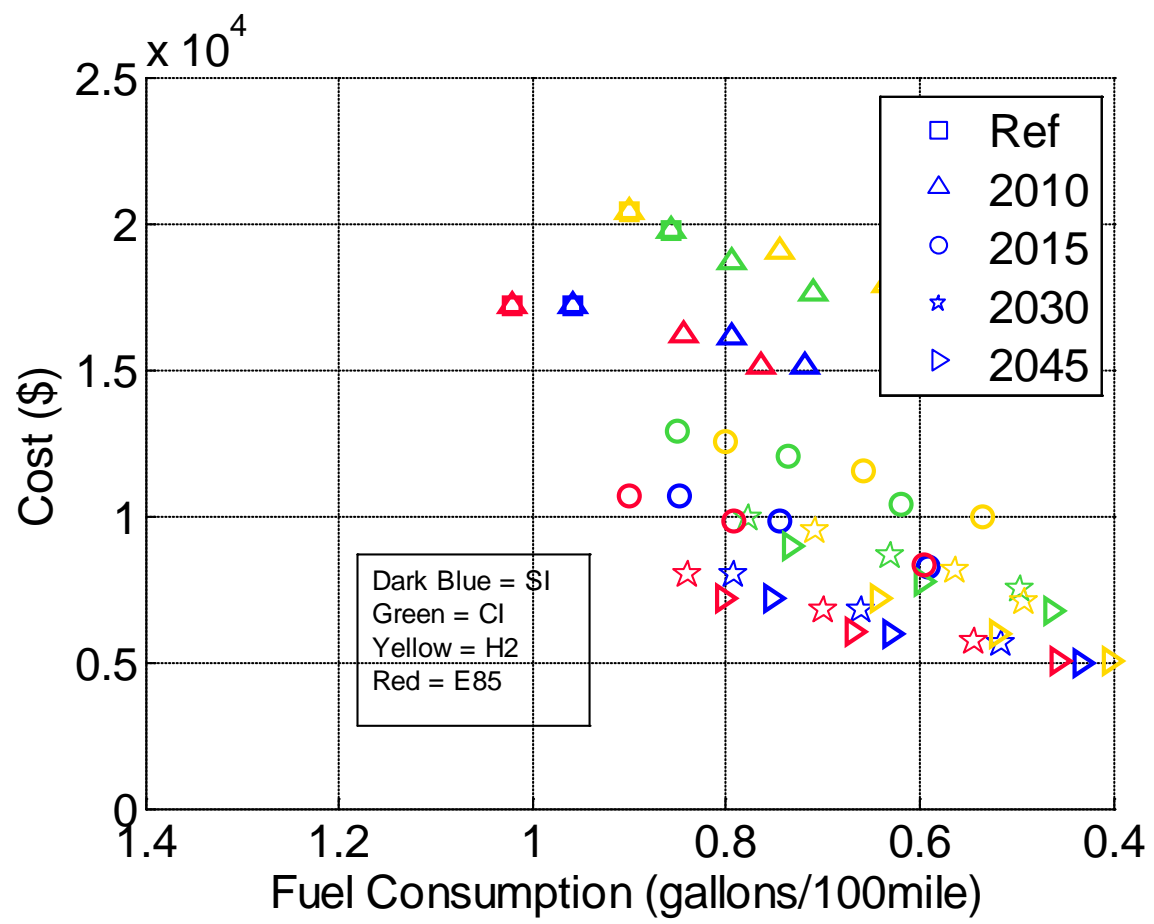

Figure 136 - Incremental manufacturing cost (in comparison to the reference conventional gasoline vehicle manufacturing cost) as a function of fuel consumption for PHEV40 vehicles.

\subsection{FUEL-CELL AND OTHER HYDROGEN-FUELED VEHICLES}

Figure 137 shows the tradeoffs of incremental manufacturing cost vs. fuel consumption for fuel-cell HEVs and PHEVs compared to the reference conventional gasoline vehicles. For the HEV in 2045, at $0.76 \mathrm{gal} / 100$ miles, the additional manufacturing cost is higher (ranging from $\$ 12,230$ to $\$ 740$ ) with the lowest fuel efficiency. For the PHEVs, we find a diminishing return on investment, since little fuel-efficiency gain is achieved for the higher AER despite a higher manufacturing cost. Overall, all configurations are trending towards good fuel efficiency at a low manufacturing cost. 


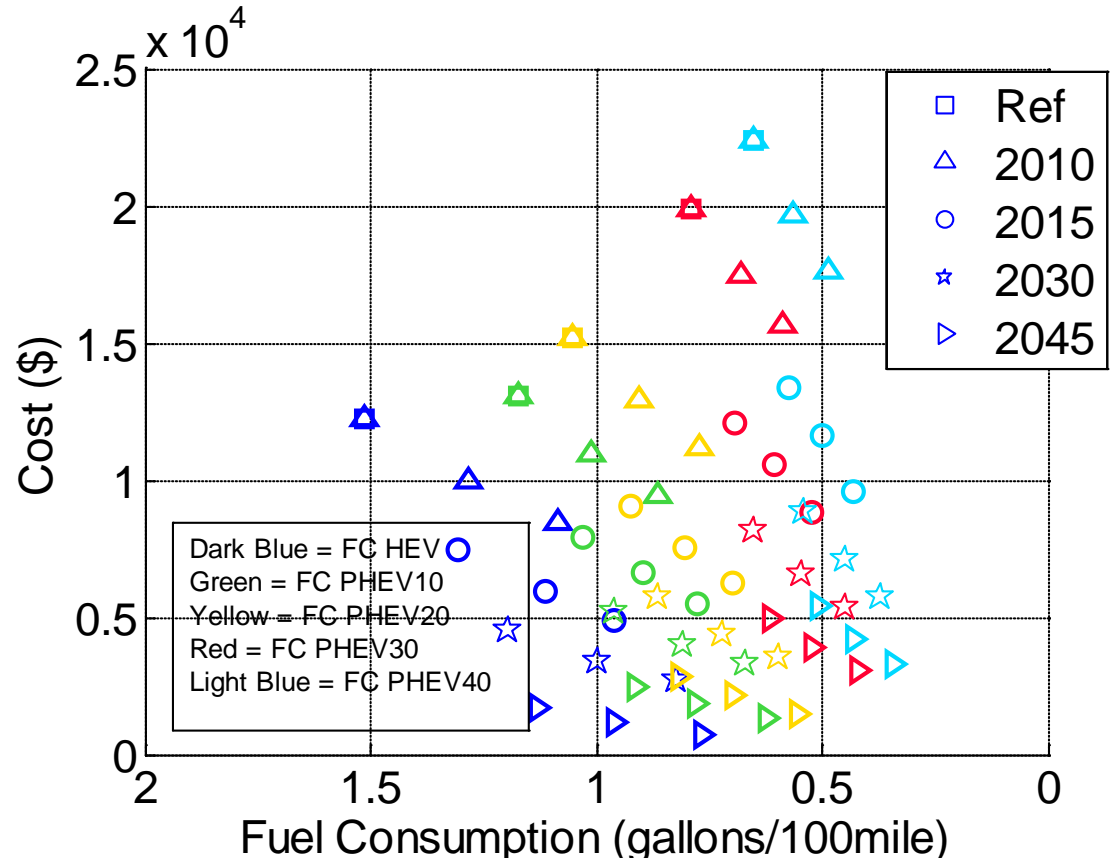

Figure 137 - Incremental manufacturing cost (in comparison to the reference conventional gasoline vehicle manufacturing cost) as a function of fuel consumption for fuel-cell vehicles.

Figure 138 shows the trade-off between fuel efficiency and manufacturing cost for all the hydrogen-fueled vehicles, including hydrogen engines. The figure shows how the same fuel efficiency can be achieved with different technologies at different manufacturing costs.

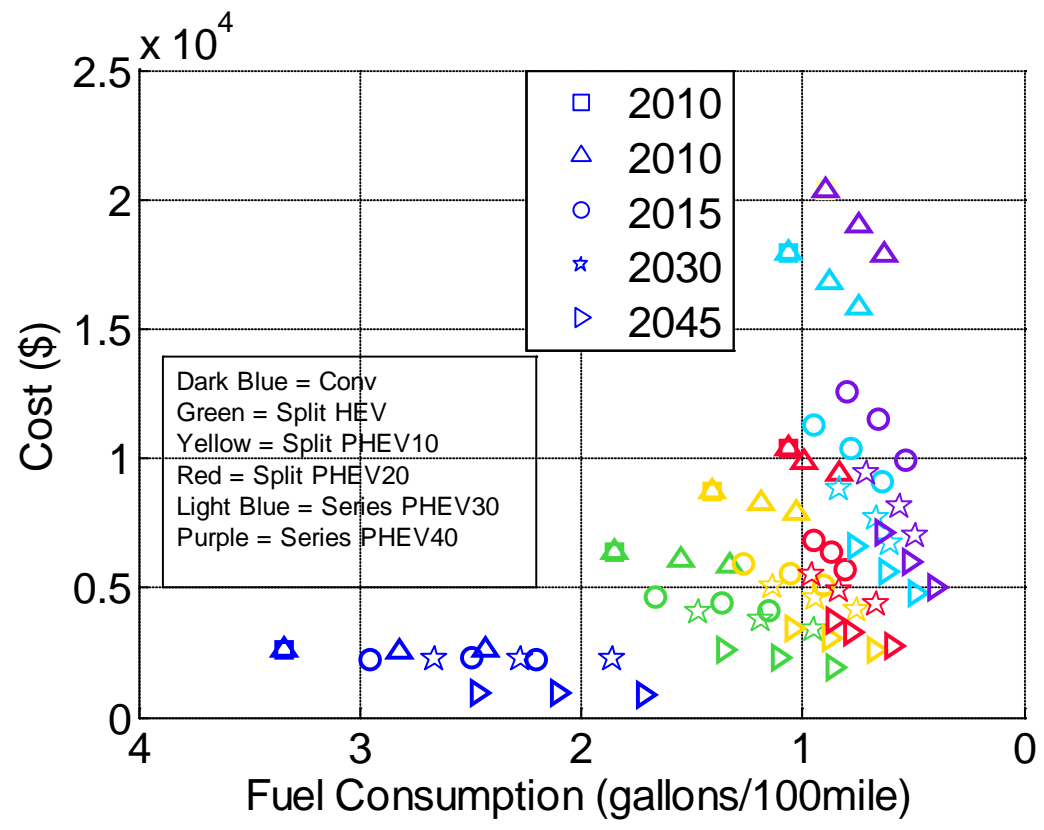

Figure 138 - Incremental manufacturing cost (in comparison to the reference conventional gasoline vehicle manufacturing cost) as a function of fuel consumption for hydrogen-fueled vehicles. 


\subsection{ALL POWERTRAINS}

Figure 139 shows the trade-offs between fuel consumption and increased manufacturing costs for all powertrains and fuels compared to the conventional gasoline reference. Overall, the vehicles on the bottom right would provide the best fuel consumption for the least additional cost. All years, all cases, and all fuels are presented.

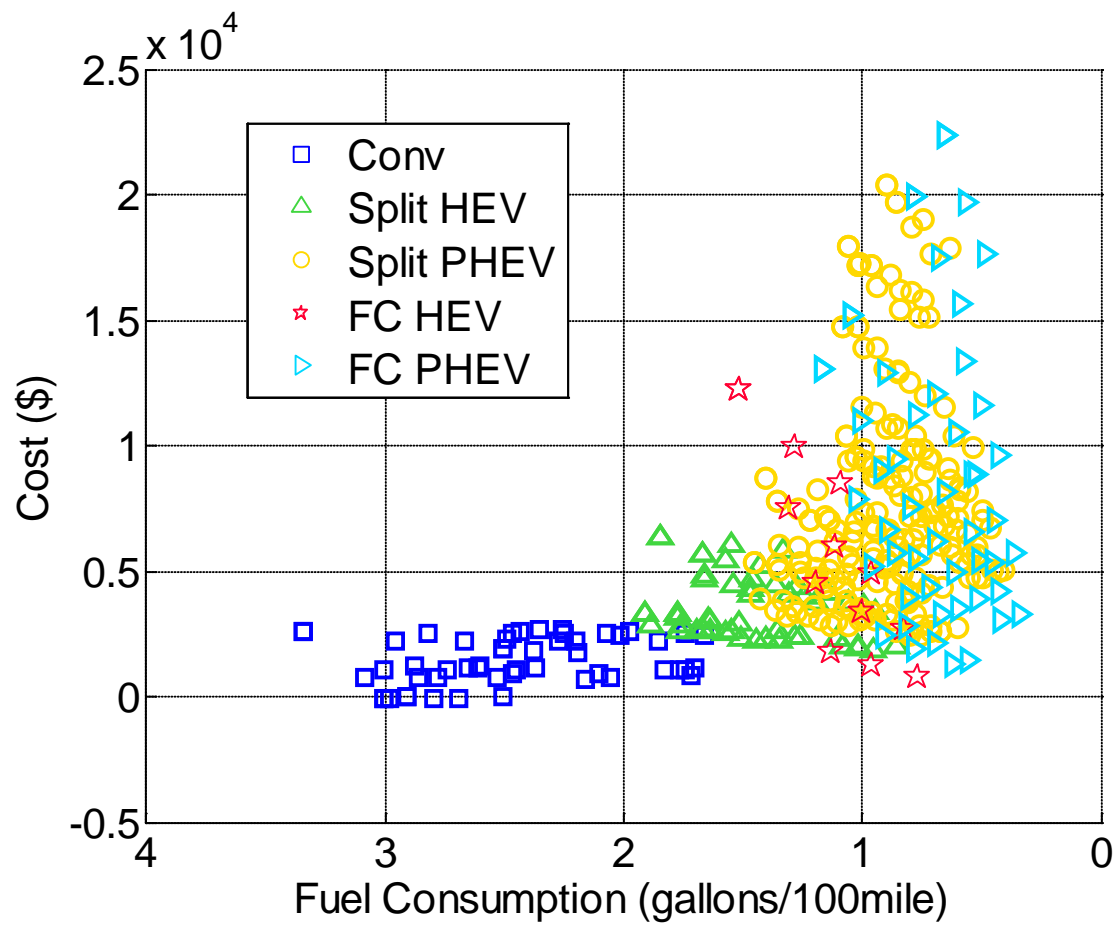

Figure 139 - Incremental manufacturing cost (in comparison to the gasoline conventional reference vehicle) as a function of fuel consumption for all powertrains. 
Argonne National Laboratory Report - Light-Duty Vehicle Fuel Consumption Displacement Potential Up to 2045

Figure 140 shows a comparison of all the powertrains, considering gasoline fuel only. The main conclusion from Figure 140 is that conventional vehicles are more likely to improve in fuel efficiency than in cost, whereas the higher the electrification level, the more the improvement focuses on cost. For example, the incremental manufacturing cost for the PHEV40 decreases from $\$ 17,150$ to $\$ 4991$ between 2010 and 2045, whereas the incremental manufacturing cost for the conventional gasoline vehicle increases from $\$ 0$ to $\$ 2000$ over the same period.

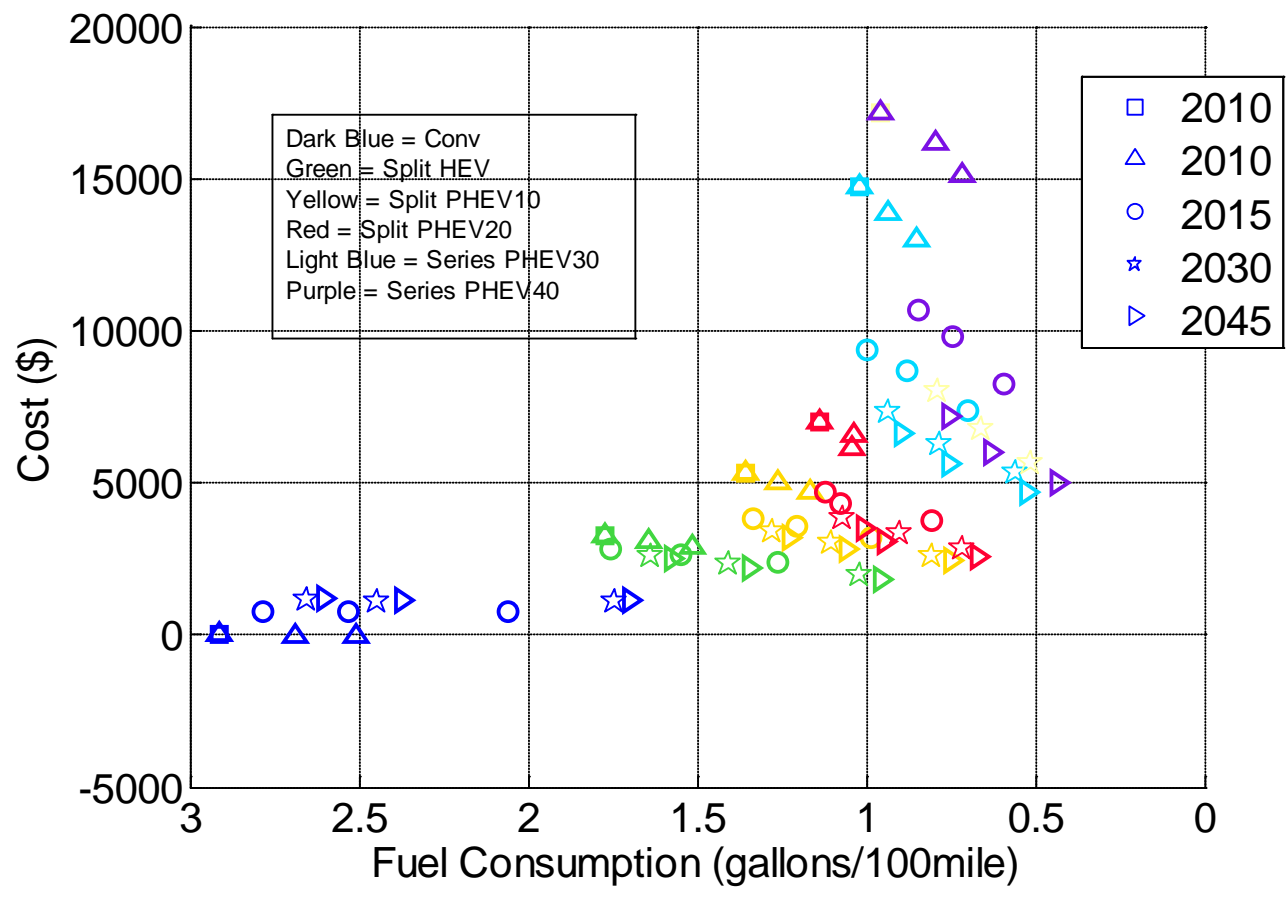

Figure 140 - Incremental manufacturing cost (in comparison to the reference conventional gasoline vehicle manufacturing cost) as a function of fuel consumption for gasoline vehicles. 
Argonne National Laboratory Report - Light-Duty Vehicle Fuel Consumption Displacement Potential Up to 2045

\section{REFERENCES}

Argonne National Laboratory (2011a). Autonomie. http://www.autonomie.net/

Argonne National Laboratory (2011b). Autonomie/Papers.

http://www.autonomie.net/overview/papers_software.html

Autoblog (2008). By the Numbers May 2008: F-150 Falls Edition.

http://www.autoblog.com/2008/06/03/by-the-numbers-may-2008-f-150-falls-edition.

Bandel, W. (2006). The Turbocharged GDI Engine: Boosted Synergies for High Fuel Economy plus Ultra-Low Emissions. SAE paper 2006-01-1266, presented at the SAE World Congress \& Exhibition, Detroit, MI, April 2006.

Bohn, T.A. (2005). Implementation of a Non-Intrusive In-Vehicle Engine Torque Sensor for Benchmarking the Toyota Prius HEV. SAE paper 2005-01-1046, presented at the SAE World Congress \& Exhibition, Detroit, MI, April 2005.

Cao, Q.P. (2007). PHEV Hymotion Prius Model Validation and Control Improvements. Presented at the the 23rd International Electric Vehicle Symposium (EVS23), Anaheim, CA, December 2007.

Central Intelligence Agency (2008). The World Factbook 2008. Washington, DC: Central Intelligence Agency.

Ciatti, S., and Subramanian, S. (2010). An Experimental Investigation of Low Octane Gasoline in Diesel Engines. http://www1.eere.energy.gov/vehiclesandfuels/pdfs/deer_2010/wednesday/presentations/deer10_ciatti.pdf

Civil Society Institute (2007). CSI/40mpg.org: "Fuel-Efficient Car Gap" Growing as U.S. Falls to Two 40mpg+ Vehicles, While Number of Gas-Sipping Cars Unavailable in U.S. Rises to Well Over 100.

http://www.civilsocietyinstitute.org/media/pdfs/021407_CSI_40mpg_fuel_efficiency_news_release.pdf

Delorme, A., Rousseau, A., and Pagerit, R. (2008 ). Fuel Economy Potential of Advanced Configurations from 2010 to 2045. Presented at the IFP HEV Conference, Paris, November 2008.

Duoba, M. (2010). SAE_J1711-HEV and PHEV Test Procedures-15Mar10-BallotDraft.pdf (unpublished; for additional information, contact M. Duoba at mduoba@anl.gov).

Elgowainy, A., Burnham, A., Wang, M., Molburg, J., and Rousseau, A. (2009). Well-To-Wheels Energy Use and Greenhouse Gas Emissions of Plug-in Hybrid Electric Vehicles. SAE paper 2009-01-1309, presented at the SAE World Congress \& Exhibition, Detroit, MI, April 2009.

Environmental Protection Agency (2010). Light Duty Automotive Technology, Carbon Dioxide Emissions and Fuel Economy Trends: 1975 through 2010. EPA-420-R10-023, November 2010.

Faron, G., Pagerit, S., and Rousseau, A. (2009). Evaluation of PHEVs Fuel Efficiency and Cost Using MonteCarlo Analysis. Presented at the 24th International Electric Vehicle Symposium (EVS24), Stavanger, Norway, May 2009. http://www.transportation.anl.gov/pdfs/HV/565.pdf

Fellah, M., Singh, G., Rousseau, A., Pagerit, S., Nam, E., and Hoffman, G. (2009). Impact of Real-World Drive Cycles on PHEV Battery Requirements. SAE paper 2009-01-1383, presented at the SAE World Congress \& Exhibition, Detroit, MI, April 2009. http://www.autonomie.net/docs/6\%20\%20Papers/HEVs\%20\&\%20PHEVs/Component\%20Requirements/impact_of_real_world_drive_cycles.pdf 
Argonne National Laboratory Report - Light-Duty Vehicle Fuel Consumption Displacement Potential Up to 2045

Freyermuth, V., Fallas, E., and Rousseau, A. (2008). Comparison of Powertrain Configuration for Plug-in HEVs from a Fuel Economy Perspective. SAE paper 2008-01-0461, presented at the SAE World Congress \& Exhibition, Detroit, MI, April 2008. (Selected for SAE International Journal of Engines.) http://papers.sae.org/2008-01-0461/

Grewe, T.M., Conlon, B.M., and Holmes, A.G. (2007). Defining the General Motors 2-Mode Hybrid Transmission. SAE paper 2007-01-0273, presented at the SAE World Congress \& Exhibition, Detroit, MI, April 2007.

Henrion, M. (2008). Guide to Estimating Unbiased Probability Distributions for Energy R\&D Results. DOE Risk Analysis Group.

International Energy Agency (2010). 2010 Key World Energy Statistics.

http://www.iea.org/textbase/nppdf/free/2010/key_stats_2010.pdf

Karbowski, D., Haliburton, C., and Rousseau, A. (2007). Impact of Component Size on Plug-In Hybrid Vehicle Energy Consumption Using Global Optimization. Presented at the 23rd International Electric Vehicle Symposium (EVS23), Anaheim, CA, December 2007. http://www.transportation.anl.gov/pdfs/HV/460.pdf

Karbowski, D., Kwon, J., Kim, N., and Rousseau, A. (2010). Instantaneously Optimized Controller for a Multimode Hybrid Electric Vehicle. SAE paper 2010-01-0816, presented at the SAE World Congress \& Exhibition, Detroit, MI, April 2010. http://papers.sae.org/2010-01-0816/

Karbowski, D., Pagerit, S., Kwon, J., Rousseau, A., and Freiherr von Pechmann, K-F. (2009). Fair Comparison of Powertrain Configurations for Plug-In Hybrid Operation using Global Optimization. SAE paper 2009-01-1334, presented at the SAE World Congress \& Exhibition, Detroit, MI, April 2009. http://papers.sae.org/2009-01-1334/

Karbowski, D., Rousseau, A., Pagerit, S., and Sharer, P. (2006). Plug-in Vehicle Control Strategy: From Global Optimization to Real-Time Application. Presented at the 22nd International Electric Vehicle Symposium (EVS22), Yokohama, Japan, October 2006. http://www.transportation.anl.gov/pdfs/HV/435.pdf

Karnopp, D.M. (1990). System Dynamics: A Unified Approach, 2nd edition. New York: John Wiley \& Sons, Inc.

Kim, N., Carlson, R., Jehlik, F., and Rousseau, A. (2009). Tahoe HEV Model Development in PSAT. Detroit: SAE paper 2009-01-1307, presented at the SAE World Congress \& Exhibition, Detroit, MI, April 2009.

http://papers.sae.org/2009-01-1307

Kim, N., Kwon, J., and Rousseau, A. (2010). Trade-off Between Multi-mode Powertrain Complexity and Fuel Consumption. Presented at the 25th International Electric Vehicle Symposium (EVS25), Shenzhen, China, November 5-9, 2010. http://www.autonomie.net/docs/6\%20-

\%20Papers/HEVs\%20\&\%20PHEVs/Powertrain\%20Configurations/trade-off_between_multimode.pdf

Kromer, M.A., and Heywood, J.B. (2008). A Comparative Assessment of Electric Propulsion Systems in the 2030 US Light-Duty Vehicle Fleet. SAE paper 2008-01-0459, presented at the SAE World Congress \& Exhibition, Detroit, MI, April 2008.

Mathworks (n.d.). Argonne National Laboratory Develops Powertrain System Analysis Toolkit with MathWorks' Tools. http://www.mathworks.com/company/user_stories/userstory45700.html

Mathworks (2007). Matlab Release 4.7, User's Guide. 
Argonne National Laboratory Report - Light-Duty Vehicle Fuel Consumption Displacement Potential Up to 2045

Morrow, K., Karner, D., and Francfort, J. (2008). Plug-in Hybrid Electric Vehicle Charging Infrastructure Review. Report No. INL/EXT-08-15058. http://avt.inl.gov/pdf/phev/phevInfrastructureReport08.pdf.

National Research Council (2011). Assessment of Fuel Economy Technologies for Light-Duty Vehicles. Washington, D.C.: The National Academies Press (in press).

Nelson, P., Amine, K., Rousseau, A., and Yomoto, H. (2007). Advanced Lithium-lon Batteries for Plug-in HybridElectric Vehicles. Presented at the 23rd International Electric Vehicle Symposium (EVS23), Anaheim, CA, December 2007. http://www.transportation.anl.gov/pdfs/HV/461.pdf

Pagerit, S. (2007). Using Matlab Distributed Computing for Vehicle Simulations.

Pagerit, S., Rousseau, A., and Sharer, P. (2005). Global Optimization to Real Time Control of HEV Power Flow: Example of a Fuel Cell Hybrid Vehicle. Presented at the 21st International Electric Vehicle Symposium (EVS21), Monaco, April 2005. http://www.transportation.anl.gov/pdfs/HV/441.pdf

Pagerit, S., Sharer, P., and Rousseau, A. (2006). Fuel Economy Sensitivity to Vehicle Mass for Advanced Vehicle Powertrains. SAE paper 2006-01-0665, presented at the SAE World Congress \& Exhibition, Detroit, MI, April 2006.

Pasquier, M., Duoba, M., and Rousseau, A. (2001). Validating Simulation Tools for Vehicle System Studies Using Advanced Control and Testing Procedure. Presented at the 18th International Electric Vehicle Symposium (EVS18), Berlin, Germany, October 20-24, 2001. http://www.autonomie.net/docs/6\%20-

\%20Papers/Validation/validating_simulation_tools.pdf

Rousseau, A. (n.d.). What Can Autonomie Do for You? http://www.autonomie.net/docs/5\%20$\% 20$ Presentations/Software/what_can_autonomie.pdf

Rousseau, A., (2000). Simulation and Validation of Hybrid Electric Vehicles Using AUTONOMIE. Presented at the 3rd Global Powertrain Congress, Detroit, MI, June 6-8, 2000.

Rousseau, A., Kwon, J., Sharer, P., Pagerit, S., and Duoba, M. (2006). Integrating Data, Performing Quality Assurance, and Validating the Vehicle Model for the 2004 Prius Using AUTONOMIE. SAE paper 2006-01-0667, presented at the SAE World Congress \& Exhibition, Detroit, MI, April 2006. http://papers.sae.org/2006-01-0667

Rousseau, A., and Moawad, A. (2010). Impact of Control Strategies on Fuel Efficiency of Different PHEVs using Real World Driving Conditions. Presented at IAMF 2010, Geneva, Switzerland, March 9-10, 2010.

http://www.autonomie.net/docs/6\%20-

\%20Papers/HEVs\%20\&\%20PHEVs/Control\%20Strategy/impact_of_control_strategies.pdf

Rousseau, A., Pagerit, S., and Gao, D.W. (2008). Plug-in Hybrid Electric Vehicle Control Strategy Parameter Optimization. Journal of Asian Electric Vehicles, 6(2), pp. 1125-1133.

Rousseau, A., Sharer, P., and Ahluwalia, R. (2004). Energy Storage Requirements for Fuel Cell Vehicles. SAE paper 2004-01-1302, presented at the SAE World Congress \& Exhibition, Detroit, MI, March 2004.

http://www.autonomie.net/docs/6\%20-\%20Papers/Hydrogen_fueled/energy_storage_requirements.pdf

Rousseau, A., Sharer, P., and Pagerit, S. (2005). Trade-off Between Fuel Economy and Cost for Advanced Vehicle Configurations. Presented at the 20th International Electric Vehicle Symposium (EVS20), Monaco, April 2005. http://www.transportation.anl.gov/pdfs/HV/340.pdf 
Argonne National Laboratory Report - Light-Duty Vehicle Fuel Consumption Displacement Potential Up to 2045

Sharer, P., Rousseau, A., Karbowski, D., and Pagerit, S. (2008). Plug-in Hybrid Electric Vehicle Control Strategy: Comparison between EV and Charge-Depleting Options. SAE paper 2008-01-0460, presented at the SAE World Congress \& Exhibition, Detroit, MI, April 2008.

Sharer, P., Rousseau, A., Pagerit, S., and Nelson, P. (2007). Midsize and SUV Vehicle Simulation Results for Plug-in HEV Component Requirements. SAE paper 2007-01-0295, presented at the SAE World Congress \& Exhibition, Detroit, MI, April 2007. http://www.autonomie.net/docs/6\%20\%20Papers/HEVs\%20\&\%20PHEVs/Component\%20Requirements/midsize_and_suv_vehicle_simulation.pdf

Slezak, L. (2008). The U.S. Hybrid and Plug-in Hybrid Vehicle Research Program, presented the International Conference on Advances in Hybrid Powertrains, Ruell-Malmaison, France, November 25-26, 2008.

Tate, E.D., and Savagian, P.J. (2009). The CO2 Benefits of Electrification E-REVs, PHEVs and Charging Scenarios. SAE paper 2009-01-1311, presented at the SAE World Congress \& Exhibition, April 2009, Detroit, MI.

USCAR (2010). Press Release: USAMP-A/SP-VALIDATION-PROJECT-YIELDS-PASSENGER-COMPARTMENT-MASSSAVINGS-OF-15-20-PERCENT. http://www.uscar.org/guest/news/465/Press-Release-USAMP-A-SP-VALIDATIONPROJECT-YIELDS-PASSENGER-COMPARTMENT-MASS-SAVINGS-OF-15-20-PERCENT.

U.S. Department of Energy (n.d.[a]). Energy Efficiency and Renewable Energy/Planning, Budget, \& Analysis/Data \& Analysis. http://www1.eere.energy.gov/ba/pba/program_benefits.html

U.S. Department of Energy (n.d.[b]). Vehicle Technologies Program.

http://www1.eere.energy.gov/vehiclesandfuels/resources/fcvt_software.html.

U.S. Department of Energy (2007). Plug-In Hybrid Electric Vehicle R\&D Plan, Working Draft, June 2007.

http://www1.eere.energy.gov/vehiclesandfuels/pdfs/program/phev_rd_plan_june_2007.pdf

U.S. Department of Energy (2010). The Recovery Act: Transforming America's Transportation Sector-Batteries and Electric Vehicles.

U.S. Environmental Protection Agency (n.d.). Fuel Economy.

http://www.epa.gov/fueleconomy/regulations.htmVehicle Systems Analysis Technical Team (2006). FreedomCAR and Fuel Partnership.

http://www1.eere.energy.gov/vehiclesandfuels/pdfs/program/vehicle_sys_analysis_team_tech_roadmap.pdf Vijayagopal, R., Shidore, N., Halbach, S., Michaels, L., and Rousseau, A. (2010). Automated Model Based Design Process to Evaluate Advanced Component Technologies. SAE paper 2010-01-0936, presented at the SAE World Congress \& Exhibition, Detroit, MI, April 2010. http://www.autonomie.net/docs/6\%20-

$\% 20$ Papers/CIL/automated_model_based_design.pdf

Wallner, T., and Lohse-Busch, H. (2007). Performance, Efficiency, and Emissions Evaluation of a Supercharged, Hydrogen-Powered, 4-Cylinder Engine. SAE paper 2007-01-0016, presented at the SAE Fuels and Emissions Conference, Capetown, South Africa, January 2007. http://papers.sae.org/2007-01-0016

Wang, M. (2006) Projection of Chinese Electric Machine Vehicle Growth, Oil Demand and CO2 Emissions Through 2050. http://www.transportation.anl.gov/pdfs/TA/398.pdf

Ward, J. (2010) VTP FY12 Goals to Autonomie Crosswalk 20100414.xlsx. Personal Communication. 
Argonne National Laboratory Report - Light-Duty Vehicle Fuel Consumption Displacement Potential Up to 2045

Wu, Y., Wang, M.Q., Dharer, P.B., and Rousseau, A. (2006). Well-to-Wheels Results of Energy Use, Greenhouse Gas Emissions and Criteria Air Pollutant Emissions of Selected Vehicle/Fuel Systems. SAE 2006 Transactions (Journal of Engines), Paper No. 2006-01-0377. http://www.transportation.anl.gov/pdfs/TA/382.pdf 
Argonne National Laboratory Report - Light-Duty Vehicle Fuel Consumption Displacement Potential Up to 2045 


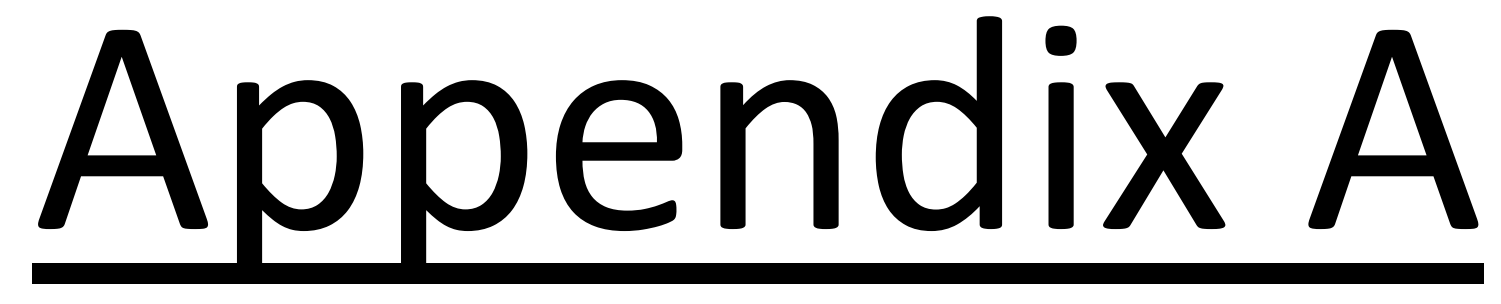

\section{Vehicle Sizing Tables}


Compact Class 


\section{Power}

\begin{tabular}{|c|c|c|c|c|c|c|c|c|c|c|c|c|c|c|}
\hline & & \multicolumn{13}{|c|}{ Compact } \\
\hline & & 2010 & \multicolumn{3}{|c|}{2010} & \multicolumn{3}{|c|}{2015} & \multicolumn{3}{|c|}{2030} & \multicolumn{3}{|c|}{2045} \\
\hline & & Ref & low & avg & high & low & avg & high & low & avg & high & low & avg & high \\
\hline \multirow{5}{*}{ SI Conv } & ICE Power & 128822 & 128822 & 123340 & 116488 & 126081 & 115117 & 112376 & 119229 & 113747 & 108539 & 123340 & 114569 & 109636 \\
\hline & Electric Machine & & & & & & & & & & & & & \\
\hline & $\mathrm{MC} 2 / \mathrm{GC}$ & & & & & & & & & & & & & \\
\hline & Battery & & & & & & & & & & & & & \\
\hline & Specific Power & 94 & 94 & 92 & 90 & 92 & 88 & 91 & 87 & 92 & 99 & 90 & 93 & 104 \\
\hline \multirow{5}{*}{$\mathrm{Cl}$ Conv } & ICE Power & 110000 & 110000 & 105111 & 99190 & 106333 & 101444 & 93337 & 105111 & 95605 & 87593 & 106333 & 95700 & 84415 \\
\hline & Electric Machine & & & & & & & & & & & & & \\
\hline & $\mathrm{MC} 2 / \mathrm{GC}$ & & & & & & & & & & & & & \\
\hline & Battery & & & & & & & & & & & & & \\
\hline & Specific Power & 77 & 77 & 75 & 73 & 74 & 74 & 72 & 73 & 73 & 75 & 74 & 74 & 75 \\
\hline \multirow{5}{*}{ H2 Conv } & ICE Power & 117693 & 117693 & 109320 & 104533 & 113970 & 106400 & 99224 & 113867 & 102086 & 87733 & 112933 & 99867 & 85867 \\
\hline & Electric Machine & & & & & & & & & & & & & \\
\hline & MC2/GC & & & & & & & & & & & & & \\
\hline & Battery & & & & & & & & & & & & & \\
\hline & Specific Power & 84 & 84 & 83 & 83 & 82 & 83 & 83 & 83 & 84 & 84 & 84 & 84 & 85 \\
\hline \multirow{5}{*}{ E85 Conv } & ICE Power & 119861 & 119861 & 114943 & 108611 & 117583 & 109792 & 102708 & 114514 & 106250 & 97986 & 116088 & 106250 & 96806 \\
\hline & Electric Machine & & & & & & & & & & & & & \\
\hline & MC2/GC & & & & & & & & & & & & & \\
\hline & Battery & & & & & & & & & & & & & \\
\hline & Specific Power & 87 & 87 & 86 & 84 & 86 & 84 & 84 & 84 & 86 & 90 & 85 & 87 & 92 \\
\hline \multirow{5}{*}{ SI Split HEV } & ICE Power & 79802 & 79802 & 75794 & 71856 & 79900 & 73098 & 65899 & 76360 & 68050 & 57516 & 75994 & 65931 & 55016 \\
\hline & Electric Machine & 56951 & 56951 & 54615 & 52409 & 56626 & 53772 & 49826 & 56222 & 50784 & 44119 & 56041 & 49790 & 41898 \\
\hline & MC2/GC & 46182 & 46182 & 43862 & 41583 & 46238 & 42302 & 38136 & 44190 & 39381 & 33285 & 43978 & 38155 & 31838 \\
\hline & Battery & 25337 & 25337 & 24140 & 23142 & 24938 & 23902 & 22195 & 25040 & 22195 & 19349 & 25040 & 22195 & 18211 \\
\hline & Specific Power & 71 & 71 & 71 & 70 & 72 & 70 & 69 & 70 & 69 & 68 & 70 & 69 & 68 \\
\hline \multirow{5}{*}{ SI Split HEV PHEV10 } & ICE Power & 57997 & 57997 & 55370 & 52945 & 57277 & 53972 & 49813 & 56552 & 50875 & 44494 & 55726 & 49780 & 42090 \\
\hline & Electric Machine & 63292 & 63292 & 60305 & 57875 & 62273 & 58776 & 53887 & 61589 & 54753 & 47108 & 61439 & 53705 & 45403 \\
\hline & MC2/GC & 34809 & 34809 & 33232 & 31743 & 34376 & 32393 & 29897 & 33941 & 30534 & 26704 & 33446 & 29877 & 25262 \\
\hline & Battery & 58701 & 58701 & 55512 & 52893 & 57727 & 53768 & 50015 & 56363 & 50796 & 44335 & 56380 & 51045 & 42948 \\
\hline & Specific Power & 78 & 78 & 78 & 77 & 78 & 78 & 78 & 78 & 78 & 79 & 78 & 79 & 79 \\
\hline
\end{tabular}


Argonne National Laboratory Report - Light-Duty Vehicle Fuel Consumption Displacement Potential Up to 2045

\begin{tabular}{|c|c|c|c|c|c|c|c|c|c|c|c|c|c|c|}
\hline & & \multicolumn{13}{|c|}{ Compact } \\
\hline & & \multirow{2}{*}{$\frac{2010}{\text { Ref }}$} & \multicolumn{3}{|c|}{2010} & \multicolumn{3}{|c|}{2015} & \multicolumn{3}{|c|}{2030} & \multicolumn{3}{|c|}{2045} \\
\hline & & & low & avg & high & low & avg & high & low & avg & high & low & avg & high \\
\hline \multirow{5}{*}{ SI Split HEV PHEV2O } & ICE Power & 58733 & 58733 & 56167 & 53569 & 58075 & 54708 & 50317 & 57288 & 51435 & 44825 & 56457 & 50197 & 42393 \\
\hline & Electric Machine & 64445 & 64445 & 61979 & 58809 & 63419 & 59741 & 54557 & 62567 & 55551 & 47480 & 62419 & 54381 & 45870 \\
\hline & $\mathrm{MC} 2 / \mathrm{GC}$ & 35250 & 35250 & 33693 & 32134 & 34839 & 32784 & 30182 & 34366 & 30853 & 26886 & 33851 & 30127 & 25443 \\
\hline & Battery & 59622 & 59622 & 56972 & 53592 & 58634 & 54431 & 49782 & 57088 & 50742 & 43563 & 57104 & 50709 & 42325 \\
\hline & Specific Power & 78 & 78 & 78 & 77 & 78 & 77 & 77 & 77 & 77 & 78 & 77 & 78 & 78 \\
\hline \multirow{5}{*}{ SI Series HEV PHEV30 } & ICE Power & 72284 & 72284 & 67720 & 63385 & 70932 & 65001 & 57014 & 68099 & 58534 & 49118 & 66605 & 56747 & 46372 \\
\hline & Electric Machine & 120498 & 120498 & 114516 & 109163 & 118131 & 111488 & 101504 & 116153 & 103842 & 90913 & 114126 & 101144 & 86514 \\
\hline & $\mathrm{MC} 2 / \mathrm{GC}$ & 70115 & 70115 & 65688 & 61483 & 68804 & 63051 & 55304 & 66056 & 56778 & 47644 & 64607 & 55045 & 44981 \\
\hline & Battery & 148515 & 148515 & 140406 & 133119 & 145629 & 135998 & 122320 & 141722 & 125178 & 108161 & 139274 & 121967 & 102942 \\
\hline & Specific Power & 70 & 70 & 70 & 71 & 70 & 71 & 71 & 71 & 71 & 74 & 70 & 71 & 74 \\
\hline \multirow{5}{*}{ SI Series HEV PHEV 40} & ICE Power & 74773 & 74773 & 69982 & 65406 & 73408 & 67111 & 58502 & 70238 & 60079 & 50168 & 68670 & 58141 & 47312 \\
\hline & Electric Machine & 124494 & 124494 & 118047 & 112252 & 122011 & 114801 & 103930 & 119694 & 106393 & 92616 & 117528 & 103426 & 88160 \\
\hline & $\mathrm{MC} 2 / \mathrm{GC}$ & 72530 & 72530 & 67883 & 63444 & 71206 & 65098 & 56747 & 68131 & 58277 & 48663 & 66610 & 56397 & 45893 \\
\hline & Battery & 153385 & 153385 & 144658 & 136829 & 150357 & 139981 & 125146 & 145983 & 128153 & 110100 & 143360 & 124619 & 104814 \\
\hline & Specific Power & 71 & 71 & 71 & 71 & 71 & 71 & 72 & 71 & 72 & 74 & 71 & 72 & 74 \\
\hline \multirow{5}{*}{ Cl Split HEV } & ICE Power & 72137 & 72137 & 68684 & 65339 & 69961 & 66296 & 60588 & 69177 & 61059 & 52534 & 68940 & 59193 & 50429 \\
\hline & Electric Machine & 62795 & 62795 & 60389 & 58151 & 60869 & 59298 & 55518 & 61887 & 55778 & 49213 & 61712 & 54688 & 47086 \\
\hline & MC2/GC & 60371 & 60371 & 57481 & 54682 & 58550 & 55483 & 50706 & 57894 & 51100 & 43965 & 57695 & 49538 & 42204 \\
\hline & Battery & 27132 & 27132 & 25935 & 24938 & 26334 & 25610 & 23902 & 26748 & 23902 & 21057 & 26748 & 23902 & 19919 \\
\hline & Specific Power & 63 & 63 & 62 & 62 & 62 & 62 & 61 & 62 & 61 & 60 & 62 & 60 & 60 \\
\hline \multirow{5}{*}{ CI Split HEV PHEV10 } & ICE Power & 61028 & 61028 & 58385 & 55891 & 60329 & 57002 & 52639 & 59582 & 53729 & 47225 & 58651 & 52646 & 44793 \\
\hline & Electric Machine & 64027 & 64027 & 61693 & 59699 & 63509 & 60219 & 55128 & 62800 & 55957 & 48924 & 62657 & 55026 & 46802 \\
\hline & MC2/GC & 51074 & 51074 & 48862 & 46775 & 50489 & 47705 & 44053 & 49864 & 44965 & 39522 & 49085 & 44059 & 37487 \\
\hline & Battery & 66875 & 66875 & 64095 & 61706 & 66366 & 62231 & 57496 & 64907 & 58341 & 51671 & 64838 & 58676 & 49470 \\
\hline & Specific Power & 80 & 80 & 80 & 80 & 81 & 80 & 80 & 80 & 80 & 81 & 80 & 80 & 80 \\
\hline \multirow{5}{*}{ CI Split HEV PHEV2O } & ICE Power & 61878 & 61878 & 59296 & 56599 & 61185 & 57796 & 53199 & 60376 & 54317 & 47611 & 59382 & 53119 & 45179 \\
\hline & Electric Machine & 65162 & 65162 & 63189 & 60604 & 64608 & 61431 & 55890 & 64227 & 56720 & 49488 & 63523 & 56229 & 47626 \\
\hline & MC2/GC & 51785 & 51785 & 49624 & 47367 & 51205 & 48369 & 44522 & 50528 & 45457 & 39845 & 49696 & 44455 & 37810 \\
\hline & Battery & 67881 & 67881 & 65485 & 62465 & 67333 & 63313 & 57654 & 66219 & 58521 & 51389 & 65553 & 59071 & 49540 \\
\hline & Specific Power & 80 & 80 & 80 & 80 & 80 & 80 & 79 & 80 & 79 & 80 & 79 & 80 & 80 \\
\hline
\end{tabular}


Argonne National Laboratory Report - Light-Duty Vehicle Fuel Consumption Displacement Potential Up to 2045

\begin{tabular}{|c|c|c|c|c|c|c|c|c|c|c|c|c|c|c|}
\hline & & \multicolumn{13}{|c|}{ Compact } \\
\hline & & \multirow{2}{*}{$\begin{array}{r}2010 \\
\text { Ref }\end{array}$} & \multicolumn{3}{|c|}{2010} & \multicolumn{3}{|c|}{2015} & \multicolumn{3}{|c|}{2030} & \multicolumn{3}{|c|}{2045} \\
\hline & & & low & avg & high & low & avg & high & low & avg & high & low & avg & high \\
\hline \multirow{5}{*}{ CI Series HEV PHEV 30} & ICE Power & 75288 & 75288 & 70675 & 66387 & 73958 & 68033 & 59847 & 71011 & 61395 & 51853 & 69526 & 59592 & 49108 \\
\hline & Electric Machine & 125310 & 125310 & 119258 & 113835 & 122930 & 116309 & 106146 & 120902 & 108549 & 95385 & 118831 & 105874 & 91164 \\
\hline & $\mathrm{MC} 2 / \mathrm{GC}$ & 73029 & 73029 & 68555 & 64395 & 71739 & 65992 & 58052 & 68881 & 59553 & 50297 & 67440 & 57804 & 47635 \\
\hline & Battery & 154426 & 154426 & 146185 & 138808 & 151523 & 141853 & 127898 & 147494 & 130831 & 113461 & 144979 & 127648 & 108456 \\
\hline & Specific Power & 69 & 69 & 69 & 69 & 69 & 70 & 70 & 69 & 70 & 72 & 69 & 70 & 72 \\
\hline \multirow{5}{*}{$\mathrm{Cl}$ Series HEV PHEV40 } & ICE Power & 77838 & 77838 & 72998 & 68467 & 76495 & 70202 & 61420 & 73270 & 62911 & 52958 & 71592 & 60986 & 50020 \\
\hline & Electric Machine & 129327 & 129327 & 122975 & 117217 & 126925 & 119842 & 108719 & 124604 & 111016 & 97135 & 122241 & 108070 & 92621 \\
\hline & $\mathrm{MC} 2 / \mathrm{GC}$ & 75503 & 75503 & 70808 & 66413 & 74200 & 68096 & 59577 & 71072 & 61024 & 51369 & 69444 & 59156 & 48519 \\
\hline & Battery & 159320 & 159320 & 150683 & 142861 & 156384 & 146103 & 130894 & 151954 & 133703 & 115450 & 149078 & 130192 & 110099 \\
\hline & Specific Power & 70 & 70 & 70 & 70 & 70 & 70 & 70 & 70 & 70 & 72 & 70 & 70 & 72 \\
\hline \multirow{5}{*}{ H2 Split HEV } & ICE Power & 82423 & 82423 & 76998 & 72255 & 81013 & 73517 & 65314 & 78853 & 67086 & 56140 & 77140 & 65347 & 54606 \\
\hline & Electric Machine & 59410 & 59410 & 55747 & 52848 & 58060 & 54332 & 49439 & 57723 & 50754 & 43345 & 56382 & 49404 & 42050 \\
\hline & $\mathrm{MC} 2 / \mathrm{GC}$ & 51953 & 51953 & 48534 & 45544 & 51064 & 46340 & 41169 & 49703 & 42286 & 35386 & 48623 & 41190 & 34420 \\
\hline & Battery & 26334 & 26334 & 24339 & 22943 & 25536 & 23902 & 21626 & 25040 & 22195 & 18780 & 24471 & 21626 & 18211 \\
\hline & Specific Power & 71 & 71 & 71 & 70 & 71 & 71 & 69 & 70 & 69 & 69 & 71 & 69 & 69 \\
\hline \multirow{5}{*}{ H2 Split HEV PHEV10 } & ICE Power & 59583 & 59583 & 55825 & 52804 & 58560 & 53887 & 49310 & 57430 & 50623 & 43584 & 55473 & 49335 & 41455 \\
\hline & Electric Machine & 67293 & 67293 & 63023 & 59750 & 65542 & 60065 & 54452 & 64332 & 55708 & 47014 & 62816 & 54907 & 45815 \\
\hline & MC2/GC & 41122 & 41122 & 38528 & 36443 & 40396 & 37191 & 33993 & 39636 & 34919 & 30080 & 38285 & 34049 & 28611 \\
\hline & Battery & 65808 & 65808 & 61157 & 57593 & 63933 & 57641 & 52143 & 61845 & 53297 & 45225 & 60612 & 54108 & 44589 \\
\hline & Specific Power & 81 & 81 & 81 & 81 & 81 & 81 & 80 & 80 & 80 & 81 & 81 & 82 & 82 \\
\hline \multirow{5}{*}{ H2 Split HEV PHEV2O } & ICE Power & 60405 & 60405 & 56565 & 53455 & 59359 & 54623 & 49757 & 58251 & 51099 & 43915 & 56232 & 49752 & 41731 \\
\hline & Electric Machine & 68479 & 68479 & 64124 & 60720 & 67360 & 61772 & 55187 & 66174 & 56446 & 47570 & 63911 & 55091 & 45835 \\
\hline & MC2/GC & 41669 & 41669 & 39020 & 36873 & 40947 & 37699 & 34321 & 40203 & 35267 & 30308 & 38809 & 34337 & 28801 \\
\hline & Battery & 66829 & 66829 & 62096 & 58389 & 65773 & 59369 & 52184 & 63687 & 53330 & 44932 & 61521 & 53137 & 43510 \\
\hline & Specific Power & 81 & 81 & 81 & 81 & 81 & 81 & 80 & 81 & 80 & 81 & 81 & 80 & 80 \\
\hline \multirow{5}{*}{ H2 Series HEV PHEV30 } & ICE Power & 73832 & 73832 & 67931 & 63058 & 72033 & 64674 & 56270 & 68753 & 58048 & 48068 & 66103 & 56036 & 45599 \\
\hline & Electric Machine & 123005 & 123005 & 114750 & 108599 & 119832 & 110974 & 100256 & 117271 & 103034 & 89095 & 113337 & 99946 & 85227 \\
\hline & MC2/GC & 71617 & 71617 & 65893 & 61166 & 69872 & 62734 & 54582 & 66690 & 56307 & 46626 & 64120 & 54355 & 44231 \\
\hline & Battery & 151593 & 151593 & 140681 & 132438 & 147715 & 135371 & 120822 & 143081 & 124211 & 106007 & 138311 & 120528 & 101420 \\
\hline & Specific Power & 70 & 70 & 70 & 71 & 70 & 71 & 72 & 70 & 72 & 75 & 71 & 72 & 74 \\
\hline
\end{tabular}


Argonne National Laboratory Report - Light-Duty Vehicle Fuel Consumption Displacement Potential Up to 2045

\begin{tabular}{|c|c|c|c|c|c|c|c|c|c|c|c|c|c|c|}
\hline & & \multicolumn{13}{|c|}{ Compact } \\
\hline & & \multirow{2}{*}{$\begin{array}{r}2010 \\
\text { Ref }\end{array}$} & \multicolumn{3}{|c|}{2010} & \multicolumn{3}{|c|}{2015} & \multicolumn{3}{|c|}{2030} & \multicolumn{3}{|c|}{2045} \\
\hline & & & low & avg & high & low & avg & high & low & avg & high & low & avg & high \\
\hline \multirow{5}{*}{ H2 Series HEV PHEV40 } & ICE Power & 76411 & 76411 & 70163 & 64960 & 74508 & 66755 & 57701 & 70981 & 59564 & 49035 & 68080 & 57344 & 46483 \\
\hline & Electric Machine & 127035 & 127035 & 118386 & 111645 & 123702 & 114241 & 102649 & 120949 & 105545 & 90820 & 116561 & 102145 & 86798 \\
\hline & $\mathrm{MC} 2 / \mathrm{GC}$ & 74119 & 74119 & 68058 & 63011 & 72273 & 64752 & 55970 & 68852 & 57777 & 47564 & 66038 & 55624 & 45089 \\
\hline & Battery & 156496 & 156496 & 145080 & 136096 & 152421 & 139302 & 123610 & 147510 & 127139 & 107974 & 142174 & 123079 & 103205 \\
\hline & Specific Power & 70 & 70 & 71 & 71 & 71 & 71 & 72 & 71 & 72 & 75 & 71 & 72 & 75 \\
\hline \multirow{5}{*}{ E85 Split HEV } & ICE Power & 79843 & 79843 & 76482 & 73492 & 79025 & 74801 & 65973 & 77203 & 68228 & 58217 & 76033 & 66004 & 54552 \\
\hline & Electric Machine & 56975 & 56975 & 54918 & 53131 & 56250 & 54517 & 49830 & 56593 & 50854 & 44429 & 56037 & 49795 & 42161 \\
\hline & $\mathrm{MC} 2 / \mathrm{GC}$ & 46205 & 46205 & 44260 & 42530 & 45732 & 43288 & 38179 & 44678 & 39484 & 33690 & 44001 & 38197 & 31569 \\
\hline & Battery & 25337 & 25337 & 24140 & 23142 & 24938 & 23902 & 22195 & 25040 & 22195 & 19349 & 25040 & 22195 & 18780 \\
\hline & Specific Power & 71 & 71 & 71 & 71 & 71 & 71 & 69 & 70 & 69 & 69 & 70 & 68 & 68 \\
\hline \multirow{5}{*}{ E85 Split HEV PHEV10 } & ICE Power & 58025 & 58025 & 55427 & 52974 & 57305 & 54028 & 49869 & 56580 & 50903 & 44522 & 55754 & 49836 & 42117 \\
\hline & Electric Machine & 63361 & 63361 & 60322 & 57943 & 62342 & 58834 & 53991 & 61698 & 54818 & 47168 & 61507 & 53811 & 45463 \\
\hline & MC2/GC & 34825 & 34825 & 33249 & 31760 & 34393 & 32426 & 29930 & 33958 & 30551 & 26721 & 33462 & 29910 & 25278 \\
\hline & Battery & 58738 & 58738 & 55427 & 52930 & 57763 & 53731 & 50092 & 56443 & 50813 & 44394 & 56416 & 51146 & 43007 \\
\hline & Specific Power & 78 & 78 & 78 & 77 & 78 & 78 & 78 & 78 & 78 & 79 & 78 & 79 & 79 \\
\hline \multirow{5}{*}{ E85 Split HEV PHEV20 } & ICE Power & 58762 & 58762 & 56195 & 53597 & 58104 & 54765 & 50373 & 57316 & 51463 & 44853 & 56485 & 50253 & 42421 \\
\hline & Electric Machine & 64515 & 64515 & 62049 & 58876 & 63488 & 59849 & 54662 & 62583 & 55616 & 47541 & 62530 & 54484 & 45930 \\
\hline & MC2/GC & 35268 & 35268 & 33710 & 32151 & 34855 & 32818 & 30216 & 34383 & 30870 & 26903 & 33867 & 30161 & 25460 \\
\hline & Battery & 59657 & 59657 & 57009 & 53627 & 58669 & 54506 & 49855 & 57024 & 50785 & 43610 & 57187 & 50792 & 42363 \\
\hline & Specific Power & 78 & 78 & 78 & 77 & 78 & 77 & 77 & 77 & 77 & 78 & 77 & 78 & 78 \\
\hline \multirow{5}{*}{ E85 Series HEV PHEV30 } & ICE Power & 72314 & 72314 & 67750 & 63445 & 70963 & 65061 & 57071 & 68128 & 58591 & 49145 & 66634 & 56804 & 46400 \\
\hline & Electric Machine & 120546 & 120546 & 114563 & 109210 & 118180 & 111582 & 101599 & 116202 & 103937 & 90959 & 114173 & 101239 & 86608 \\
\hline & MC2/GC & 70145 & 70145 & 65718 & 61542 & 68834 & 63109 & 55359 & 66084 & 56833 & 47671 & 64635 & 55100 & 45008 \\
\hline & Battery & 148574 & 148574 & 140463 & 133176 & 145689 & 136111 & 122435 & 141781 & 125293 & 108216 & 139330 & 122082 & 103058 \\
\hline & Specific Power & 70 & 70 & 70 & 71 & 70 & 71 & 71 & 71 & 71 & 74 & 70 & 71 & 74 \\
\hline \multirow{5}{*}{ E85 Series HEV PHEV40 } & ICE Power & 74833 & 74833 & 70012 & 65436 & 73439 & 67171 & 58559 & 70268 & 60107 & 50195 & 68700 & 58198 & 47340 \\
\hline & Electric Machine & 124542 & 124542 & 118095 & 112299 & 122060 & 114947 & 104072 & 119742 & 106440 & 92662 & 117576 & 103521 & 88207 \\
\hline & MC2/GC & 72588 & 72588 & 67912 & 63473 & 71236 & 65156 & 56802 & 68160 & 58304 & 48689 & 66639 & 56452 & 45920 \\
\hline & Battery & 153439 & 153439 & 144718 & 136885 & 150416 & 140156 & 125320 & 146042 & 128209 & 110154 & 143419 & 124732 & 104870 \\
\hline & Specific Power & 71 & 71 & 71 & 71 & 71 & 71 & 72 & 71 & 72 & 74 & 71 & 72 & 74 \\
\hline
\end{tabular}


Argonne National Laboratory Report - Light-Duty Vehicle Fuel Consumption Displacement Potential Up to 2045

\begin{tabular}{|c|c|c|c|c|c|c|c|c|c|c|c|c|c|c|}
\hline & & \multicolumn{13}{|c|}{ Compact } \\
\hline & & \multirow{2}{*}{$\frac{2010}{\operatorname{Ref}}$} & \multicolumn{3}{|c|}{2010} & \multicolumn{3}{|c|}{2015} & \multicolumn{3}{|c|}{2030} & \multicolumn{3}{|c|}{2045} \\
\hline & & & low & avg & high & low & avg & high & low & avg & high & low & avg & high \\
\hline \multirow{5}{*}{ FC HEV } & FC Power & 90737 & 90737 & 76778 & 68351 & 81791 & 66505 & 56049 & 71241 & 57491 & 46520 & 67215 & 55527 & 44024 \\
\hline & Electric Machine & 108983 & 108983 & 93920 & 85434 & 98726 & 85857 & 75846 & 91712 & 77652 & 65238 & 87051 & 75327 & 62409 \\
\hline & $\mathrm{MC} 2 / \mathrm{GC}$ & & & & & & & & & & & & & \\
\hline & Battery & 32718 & 32718 & 28728 & 26933 & 29726 & 30162 & 27886 & 31870 & 28455 & 25040 & 30731 & 27317 & 23902 \\
\hline & Specific Power & 60 & 60 & 61 & 61 & 61 & 60 & 60 & 60 & 60 & 61 & 60 & 60 & 61 \\
\hline \multirow{5}{*}{ FC HEV PHEV10 } & FC Power & 66597 & 66597 & 57939 & 51900 & 60984 & 53186 & 46387 & 57673 & 47543 & 40501 & 54349 & 48015 & 39076 \\
\hline & Electric Machine & 107116 & 107116 & 92713 & 84509 & 97033 & 86212 & 76684 & 92786 & 78389 & 65873 & 88194 & 77712 & 63947 \\
\hline & $\mathrm{MC} 2 / \mathrm{GC}$ & & & & & & & & & & & & & \\
\hline & Battery & 56383 & 56383 & 48985 & 44275 & 51772 & 45089 & 39507 & 48399 & 40413 & 33267 & 45990 & 39293 & 31845 \\
\hline & Specific Power & 62 & 62 & 61 & 61 & 61 & 62 & 61 & 62 & 61 & 62 & 62 & 63 & 63 \\
\hline \multirow{5}{*}{ FC HEV PHEV 20} & FC Power & 67394 & 67394 & 58629 & 53071 & 62370 & 53794 & 47413 & 58302 & 48057 & 40466 & 54935 & 48404 & 38803 \\
\hline & Electric Machine & 108076 & 108076 & 94103 & 86258 & 99135 & 87518 & 78083 & 94108 & 79379 & 66257 & 89398 & 78490 & 63385 \\
\hline & $\mathrm{MC} 2 / \mathrm{GC}$ & & & & & & & & & & & & & \\
\hline & Battery & 57521 & 57521 & 49987 & 45153 & 52950 & 45983 & 40135 & 49383 & 41027 & 33674 & 46833 & 39815 & 32175 \\
\hline & Specific Power & 61 & 61 & 61 & 61 & 61 & 61 & 61 & 61 & 61 & 62 & 61 & 62 & 62 \\
\hline \multirow{5}{*}{ FC HEV PHEV 30} & FC Power & 61314 & 61314 & 54816 & 50702 & 57681 & 51674 & 46354 & 55218 & 47689 & 40400 & 52465 & 45895 & 38143 \\
\hline & Electric Machine & 122524 & 122524 & 112180 & 105338 & 116668 & 107067 & 97135 & 112769 & 99334 & 86675 & 108667 & 96129 & 82211 \\
\hline & $\mathrm{MC} 2 / \mathrm{GC}$ & & & & & & & & & & & & & \\
\hline & Battery & 150942 & 150942 & 137482 & 128412 & 143769 & 130564 & 117121 & 137561 & 119809 & 103138 & 132572 & 115998 & 97841 \\
\hline & Specific Power & 66 & 66 & 69 & 70 & 68 & 70 & 72 & 69 & 72 & 76 & 70 & 73 & 76 \\
\hline \multirow{5}{*}{ FC HEV PHEV40 } & FC Power & 62526 & 62526 & 55805 & 51561 & 58851 & 52532 & 46956 & 56152 & 48290 & 40821 & 53292 & 46444 & 38539 \\
\hline & Electric Machine & 125742 & 125742 & 114842 & 107799 & 119833 & 109566 & 99292 & 115380 & 101696 & 88436 & 110962 & 98025 & 83828 \\
\hline & $\mathrm{MC} 2 / \mathrm{GC}$ & & & & & & & & & & & & & \\
\hline & Battery & 154838 & 154838 & 140700 & 131357 & 147609 & 133567 & 119610 & 140694 & 122544 & 105150 & 135321 & 118172 & 99685 \\
\hline & Specific Power & 66 & 66 & 69 & 70 & 68 & 71 & 73 & 69 & 73 & 77 & 70 & 73 & 76 \\
\hline \multirow{5}{*}{ BEV } & ICE Power & & & & & & & & & & & & & \\
\hline & Electric Machine & 144236 & 144236 & 136072 & 127921 & 142191 & 131804 & 113585 & 138418 & 116359 & 97847 & 134086 & 110853 & 91756 \\
\hline & $\mathrm{MC} 2 / \mathrm{GC}$ & 0 & 0 & 0 & 0 & 0 & 0 & 0 & 0 & 0 & 0 & 0 & 0 & 0 \\
\hline & Battery & 177718 & 177718 & 166472 & 155682 & 175117 & 160594 & 136861 & 168528 & 140228 & 116760 & 163264 & 133632 & 109512 \\
\hline & Specific Power & 94 & 94 & 93 & 93 & 94 & 93 & 93 & 93 & 93 & 95 & 92 & 92 & 95 \\
\hline
\end{tabular}




\section{Percentage of power reduction based on the respective 2010 reference case}

\begin{tabular}{|c|c|c|c|c|c|c|c|c|c|c|c|c|c|c|}
\hline & & \multicolumn{13}{|c|}{ compact } \\
\hline & & \multirow{2}{*}{$\frac{2010}{\operatorname{Ref}}$} & \multicolumn{3}{|c|}{2010} & \multicolumn{3}{|c|}{2015} & \multicolumn{3}{|c|}{2030} & \multicolumn{3}{|c|}{2045} \\
\hline & & & low & avg & high & low & avg & high & low & avg & high & low & avg & high \\
\hline \multirow{5}{*}{ SI Conv } & ICE Power & $0 \%$ & $0 \%$ & $-4 \%$ & $-10 \%$ & $-2 \%$ & $-11 \%$ & $-13 \%$ & $-7 \%$ & $-12 \%$ & $-16 \%$ & $-4 \%$ & $-11 \%$ & $-15 \%$ \\
\hline & Electric Machine & & & & & & & & & & & & & \\
\hline & $\mathrm{MC} 2 / \mathrm{GC}$ & & & & & & & & & & & & & \\
\hline & Battery & & & & & & & & & & & & & \\
\hline & Specific Power & $0 \%$ & $0 \%$ & $-1 \%$ & $-4 \%$ & $-2 \%$ & $-6 \%$ & $-2 \%$ & $-7 \%$ & $-2 \%$ & $6 \%$ & $-4 \%$ & $-1 \%$ & $11 \%$ \\
\hline \multirow{5}{*}{$\mathrm{Cl}$ Conv } & ICE Power & $0 \%$ & $0 \%$ & $-4 \%$ & $-10 \%$ & $-3 \%$ & $-8 \%$ & $-15 \%$ & $-4 \%$ & $-13 \%$ & $-20 \%$ & $-3 \%$ & $-13 \%$ & $-23 \%$ \\
\hline & Electric Machine & & & & & & & & & & & & & \\
\hline & $\mathrm{MC} 2 / \mathrm{GC}$ & & & & & & & & & & & & & \\
\hline & Battery & & & & & & & & & & & & & \\
\hline & Specific Power & $0 \%$ & $0 \%$ & $-2 \%$ & $-5 \%$ & $-3 \%$ & $-3 \%$ & $-6 \%$ & $-4 \%$ & $-4 \%$ & $-2 \%$ & $-3 \%$ & $-3 \%$ & $-1 \%$ \\
\hline \multirow{5}{*}{ H2 Conv } & ICE Power & $0 \%$ & $0 \%$ & $-7 \%$ & $-11 \%$ & $-3 \%$ & $-10 \%$ & $-16 \%$ & $-3 \%$ & $-13 \%$ & $-25 \%$ & $-4 \%$ & $-15 \%$ & $-27 \%$ \\
\hline & Electric Machine & & & & & & & & & & & & & \\
\hline & $\mathrm{MC} 2 / \mathrm{GC}$ & & & & & & & & & & & & & \\
\hline & Battery & & & & & & & & & & & & & \\
\hline & Specific Power & $0 \%$ & $0 \%$ & $-2 \%$ & $-2 \%$ & $-2 \%$ & $-1 \%$ & $-1 \%$ & $-1 \%$ & $0 \%$ & $0 \%$ & $1 \%$ & $0 \%$ & $1 \%$ \\
\hline \multirow{5}{*}{ E85 Conv } & ICE Power & $0 \%$ & $0 \%$ & $-4 \%$ & $-9 \%$ & $-2 \%$ & $-8 \%$ & $-14 \%$ & $-4 \%$ & $-11 \%$ & $-18 \%$ & $-3 \%$ & $-11 \%$ & $-19 \%$ \\
\hline & Electric Machine & & & & & & & & & & & & & \\
\hline & $\mathrm{MC} 2 / \mathrm{GC}$ & & & & & & & & & & & & & \\
\hline & Battery & & & & & & & & & & & & & \\
\hline & Specific Power & $0 \%$ & $0 \%$ & $-1 \%$ & $-4 \%$ & $-2 \%$ & $-4 \%$ & $-4 \%$ & $-4 \%$ & $-2 \%$ & $3 \%$ & $-3 \%$ & $-1 \%$ & $5 \%$ \\
\hline \multirow{5}{*}{ SI Split HEV } & ICE Power & $0 \%$ & $0 \%$ & $-5 \%$ & $-10 \%$ & $0 \%$ & $-8 \%$ & $-17 \%$ & $-4 \%$ & $-15 \%$ & $-28 \%$ & $-5 \%$ & $-17 \%$ & $-31 \%$ \\
\hline & Electric Machine & $0 \%$ & $0 \%$ & $-4 \%$ & $-8 \%$ & $-1 \%$ & $-6 \%$ & $-13 \%$ & $-1 \%$ & $-11 \%$ & $-23 \%$ & $-2 \%$ & $-13 \%$ & $-26 \%$ \\
\hline & $\mathrm{MC} 2 / \mathrm{GC}$ & $0 \%$ & $0 \%$ & $-5 \%$ & $-10 \%$ & $0 \%$ & $-8 \%$ & $-17 \%$ & $-4 \%$ & $-15 \%$ & $-28 \%$ & $-5 \%$ & $-17 \%$ & $-31 \%$ \\
\hline & Battery & $0 \%$ & $0 \%$ & $-5 \%$ & $-9 \%$ & $-2 \%$ & $-6 \%$ & $-12 \%$ & $-1 \%$ & $-12 \%$ & $-24 \%$ & $-1 \%$ & $-12 \%$ & $-28 \%$ \\
\hline & Specific Power & $0 \%$ & $0 \%$ & $-1 \%$ & $-2 \%$ & $1 \%$ & $-2 \%$ & $-4 \%$ & $-2 \%$ & $-3 \%$ & $-4 \%$ & $-2 \%$ & $-4 \%$ & $-5 \%$ \\
\hline \multirow{5}{*}{ SI Split HEV PHEV10 } & ICE Power & $0 \%$ & $0 \%$ & $-5 \%$ & $-9 \%$ & $-1 \%$ & $-7 \%$ & $-14 \%$ & $-2 \%$ & $-12 \%$ & $-23 \%$ & $-4 \%$ & $-14 \%$ & $-27 \%$ \\
\hline & Electric Machine & $0 \%$ & $0 \%$ & $-5 \%$ & $-9 \%$ & $-2 \%$ & $-7 \%$ & $-15 \%$ & $-3 \%$ & $-13 \%$ & $-26 \%$ & $-3 \%$ & $-15 \%$ & $-28 \%$ \\
\hline & $\mathrm{MC} 2 / \mathrm{GC}$ & $0 \%$ & $0 \%$ & $-5 \%$ & $-9 \%$ & $-1 \%$ & $-7 \%$ & $-14 \%$ & $-2 \%$ & $-12 \%$ & $-23 \%$ & $-4 \%$ & $-14 \%$ & $-27 \%$ \\
\hline & Battery & $0 \%$ & $0 \%$ & $-5 \%$ & $-10 \%$ & $-2 \%$ & $-8 \%$ & $-15 \%$ & $-4 \%$ & $-13 \%$ & $-24 \%$ & $-4 \%$ & $-13 \%$ & $-27 \%$ \\
\hline & Specific Power & $0 \%$ & $0 \%$ & $-1 \%$ & $-1 \%$ & $0 \%$ & $-1 \%$ & $-1 \%$ & $-1 \%$ & $-1 \%$ & $1 \%$ & $-1 \%$ & $0 \%$ & $1 \%$ \\
\hline
\end{tabular}


Argonne National Laboratory Report - Light-Duty Vehicle Fuel Consumption Displacement Potential Up to 2045

\begin{tabular}{|c|c|c|c|c|c|c|c|c|c|c|c|c|c|c|}
\hline & & \multicolumn{13}{|c|}{ compact } \\
\hline & & \multirow{2}{*}{$\begin{array}{r}2010 \\
\text { Ref }\end{array}$} & \multicolumn{3}{|c|}{2010} & \multicolumn{3}{|c|}{2015} & \multicolumn{3}{|c|}{2030} & \multicolumn{3}{|c|}{2045} \\
\hline & & & low & avg & high & low & avg & high & low & avg & high & low & avg & high \\
\hline \multirow{5}{*}{ SI Split HEV PHEV2O } & ICE Power & $0 \%$ & $0 \%$ & $-4 \%$ & $-9 \%$ & $-1 \%$ & $-7 \%$ & $-14 \%$ & $-2 \%$ & $-12 \%$ & $-24 \%$ & $-4 \%$ & $-15 \%$ & $-28 \%$ \\
\hline & Electric Machine & $0 \%$ & $0 \%$ & $-4 \%$ & $-9 \%$ & $-2 \%$ & $-7 \%$ & $-15 \%$ & $-3 \%$ & $-14 \%$ & $-26 \%$ & $-3 \%$ & $-16 \%$ & $-29 \%$ \\
\hline & $\mathrm{MC} 2 / \mathrm{GC}$ & $0 \%$ & $0 \%$ & $-4 \%$ & $-9 \%$ & $-1 \%$ & $-7 \%$ & $-14 \%$ & $-3 \%$ & $-12 \%$ & $-24 \%$ & $-4 \%$ & $-15 \%$ & $-28 \%$ \\
\hline & Battery & $0 \%$ & $0 \%$ & $-4 \%$ & $-10 \%$ & $-2 \%$ & $-9 \%$ & $-17 \%$ & $-4 \%$ & $-15 \%$ & $-27 \%$ & $-4 \%$ & $-15 \%$ & $-29 \%$ \\
\hline & Specific Power & $0 \%$ & $0 \%$ & $0 \%$ & $-1 \%$ & $0 \%$ & $-1 \%$ & $-2 \%$ & $-1 \%$ & $-1 \%$ & $0 \%$ & $-1 \%$ & $-1 \%$ & $0 \%$ \\
\hline \multirow{5}{*}{ SI Series HEV PHEV 30} & ICE Power & $0 \%$ & $0 \%$ & $-6 \%$ & $-12 \%$ & $-2 \%$ & $-10 \%$ & $-21 \%$ & $-6 \%$ & $-19 \%$ & $-32 \%$ & $-8 \%$ & $-21 \%$ & $-36 \%$ \\
\hline & Electric Machine & $0 \%$ & $0 \%$ & $-5 \%$ & $-9 \%$ & $-2 \%$ & $-7 \%$ & $-16 \%$ & $-4 \%$ & $-14 \%$ & $-25 \%$ & $-5 \%$ & $-16 \%$ & $-28 \%$ \\
\hline & $\mathrm{MC} 2 / \mathrm{GC}$ & $0 \%$ & $0 \%$ & $-6 \%$ & $-12 \%$ & $-2 \%$ & $-10 \%$ & $-21 \%$ & $-6 \%$ & $-19 \%$ & $-32 \%$ & $-8 \%$ & $-21 \%$ & $-36 \%$ \\
\hline & Battery & $0 \%$ & $0 \%$ & $-5 \%$ & $-10 \%$ & $-2 \%$ & $-8 \%$ & $-18 \%$ & $-5 \%$ & $-16 \%$ & $-27 \%$ & $-6 \%$ & $-18 \%$ & $-31 \%$ \\
\hline & Specific Power & $0 \%$ & $0 \%$ & $0 \%$ & $1 \%$ & $0 \%$ & $1 \%$ & $2 \%$ & $1 \%$ & $2 \%$ & $5 \%$ & $0 \%$ & $1 \%$ & $5 \%$ \\
\hline \multirow{5}{*}{ SI Series HEV PHEV40 } & ICE Power & $0 \%$ & $0 \%$ & $-6 \%$ & $-13 \%$ & $-2 \%$ & $-10 \%$ & $-22 \%$ & $-6 \%$ & $-20 \%$ & $-33 \%$ & $-8 \%$ & $-22 \%$ & $-37 \%$ \\
\hline & Electric Machine & $0 \%$ & $0 \%$ & $-5 \%$ & $-10 \%$ & $-2 \%$ & $-8 \%$ & $-17 \%$ & $-4 \%$ & $-15 \%$ & $-26 \%$ & $-6 \%$ & $-17 \%$ & $-29 \%$ \\
\hline & $\mathrm{MC} 2 / \mathrm{GC}$ & $0 \%$ & $0 \%$ & $-6 \%$ & $-13 \%$ & $-2 \%$ & $-10 \%$ & $-22 \%$ & $-6 \%$ & $-20 \%$ & $-33 \%$ & $-8 \%$ & $-22 \%$ & $-37 \%$ \\
\hline & Battery & $0 \%$ & $0 \%$ & $-6 \%$ & $-11 \%$ & $-2 \%$ & $-9 \%$ & $-18 \%$ & $-5 \%$ & $-16 \%$ & $-28 \%$ & $-7 \%$ & $-19 \%$ & $-32 \%$ \\
\hline & Specific Power & $0 \%$ & $0 \%$ & $0 \%$ & $1 \%$ & $0 \%$ & $1 \%$ & $1 \%$ & $1 \%$ & $1 \%$ & $5 \%$ & $0 \%$ & $1 \%$ & $4 \%$ \\
\hline \multirow{5}{*}{ CI Split HEV } & ICE Power & $0 \%$ & $0 \%$ & $-5 \%$ & $-9 \%$ & $-3 \%$ & $-8 \%$ & $-16 \%$ & $-4 \%$ & $-15 \%$ & $-27 \%$ & $-4 \%$ & $-18 \%$ & $-30 \%$ \\
\hline & Electric Machine & $0 \%$ & $0 \%$ & $-4 \%$ & $-7 \%$ & $-3 \%$ & $-6 \%$ & $-12 \%$ & $-1 \%$ & $-11 \%$ & $-22 \%$ & $-2 \%$ & $-13 \%$ & $-25 \%$ \\
\hline & $\mathrm{MC} 2 / \mathrm{GC}$ & $0 \%$ & $0 \%$ & $-5 \%$ & $-9 \%$ & $-3 \%$ & $-8 \%$ & $-16 \%$ & $-4 \%$ & $-15 \%$ & $-27 \%$ & $-4 \%$ & $-18 \%$ & $-30 \%$ \\
\hline & Battery & $0 \%$ & $0 \%$ & $-4 \%$ & $-8 \%$ & $-3 \%$ & $-6 \%$ & $-12 \%$ & $-1 \%$ & $-12 \%$ & $-22 \%$ & $-1 \%$ & $-12 \%$ & $-27 \%$ \\
\hline & Specific Power & $0 \%$ & $0 \%$ & $-1 \%$ & $-1 \%$ & $-1 \%$ & $-2 \%$ & $-3 \%$ & $-2 \%$ & $-4 \%$ & $-4 \%$ & $-1 \%$ & $-4 \%$ & $-5 \%$ \\
\hline \multirow{5}{*}{ CI Split HEV PHEV10 } & ICE Power & $0 \%$ & $0 \%$ & $-4 \%$ & $-8 \%$ & $-1 \%$ & $-7 \%$ & $-14 \%$ & $-2 \%$ & $-12 \%$ & $-23 \%$ & $-4 \%$ & $-14 \%$ & $-27 \%$ \\
\hline & Electric Machine & $0 \%$ & $0 \%$ & $-4 \%$ & $-7 \%$ & $-1 \%$ & $-6 \%$ & $-14 \%$ & $-2 \%$ & $-13 \%$ & $-24 \%$ & $-2 \%$ & $-14 \%$ & $-27 \%$ \\
\hline & $\mathrm{MC} 2 / \mathrm{GC}$ & $0 \%$ & $0 \%$ & $-4 \%$ & $-8 \%$ & $-1 \%$ & $-7 \%$ & $-14 \%$ & $-2 \%$ & $-12 \%$ & $-23 \%$ & $-4 \%$ & $-14 \%$ & $-27 \%$ \\
\hline & Battery & $0 \%$ & $0 \%$ & $-4 \%$ & $-8 \%$ & $-1 \%$ & $-7 \%$ & $-14 \%$ & $-3 \%$ & $-13 \%$ & $-23 \%$ & $-3 \%$ & $-12 \%$ & $-26 \%$ \\
\hline & Specific Power & $0 \%$ & $0 \%$ & $0 \%$ & $-1 \%$ & $0 \%$ & $-1 \%$ & $-1 \%$ & $0 \%$ & $-1 \%$ & $1 \%$ & $-1 \%$ & $0 \%$ & $0 \%$ \\
\hline \multirow{5}{*}{ CI Split HEV PHEV2O } & ICE Power & $0 \%$ & $0 \%$ & $-4 \%$ & $-9 \%$ & $-1 \%$ & $-7 \%$ & $-14 \%$ & $-2 \%$ & $-12 \%$ & $-23 \%$ & $-4 \%$ & $-14 \%$ & $-27 \%$ \\
\hline & Electric Machine & $0 \%$ & $0 \%$ & $-3 \%$ & $-7 \%$ & $-1 \%$ & $-6 \%$ & $-14 \%$ & $-1 \%$ & $-13 \%$ & $-24 \%$ & $-3 \%$ & $-14 \%$ & $-27 \%$ \\
\hline & $\mathrm{MC} 2 / \mathrm{GC}$ & $0 \%$ & $0 \%$ & $-4 \%$ & $-9 \%$ & $-1 \%$ & $-7 \%$ & $-14 \%$ & $-2 \%$ & $-12 \%$ & $-23 \%$ & $-4 \%$ & $-14 \%$ & $-27 \%$ \\
\hline & Battery & $0 \%$ & $0 \%$ & $-4 \%$ & $-8 \%$ & $-1 \%$ & $-7 \%$ & $-15 \%$ & $-2 \%$ & $-14 \%$ & $-24 \%$ & $-3 \%$ & $-13 \%$ & $-27 \%$ \\
\hline & Specific Power & $0 \%$ & $0 \%$ & $0 \%$ & $-1 \%$ & $0 \%$ & $0 \%$ & $-1 \%$ & $0 \%$ & $-1 \%$ & $0 \%$ & $-1 \%$ & $0 \%$ & $0 \%$ \\
\hline
\end{tabular}


Argonne National Laboratory Report - Light-Duty Vehicle Fuel Consumption Displacement Potential Up to 2045

\begin{tabular}{|c|c|c|c|c|c|c|c|c|c|c|c|c|c|c|}
\hline & & \multicolumn{13}{|c|}{ compact } \\
\hline & & \multirow{2}{*}{$\begin{array}{r}2010 \\
\text { Ref }\end{array}$} & \multicolumn{3}{|c|}{2010} & \multicolumn{3}{|c|}{2015} & \multicolumn{3}{|c|}{2030} & \multicolumn{3}{|c|}{2045} \\
\hline & & & low & avg & high & low & avg & high & low & avg & high & low & avg & high \\
\hline \multirow{5}{*}{ Cl Series HEV PHEV 30} & ICE Power & $0 \%$ & $0 \%$ & $-6 \%$ & $-12 \%$ & $-2 \%$ & $-10 \%$ & $-21 \%$ & $-6 \%$ & $-18 \%$ & $-31 \%$ & $-8 \%$ & $-21 \%$ & $-35 \%$ \\
\hline & Electric Machine & $0 \%$ & $0 \%$ & $-5 \%$ & $-9 \%$ & $-2 \%$ & $-7 \%$ & $-15 \%$ & $-4 \%$ & $-13 \%$ & $-24 \%$ & $-5 \%$ & $-16 \%$ & $-27 \%$ \\
\hline & $\mathrm{MC} 2 / \mathrm{GC}$ & $0 \%$ & $0 \%$ & $-6 \%$ & $-12 \%$ & $-2 \%$ & $-10 \%$ & $-21 \%$ & $-6 \%$ & $-18 \%$ & $-31 \%$ & $-8 \%$ & $-21 \%$ & $-35 \%$ \\
\hline & Battery & $0 \%$ & $0 \%$ & $-5 \%$ & $-10 \%$ & $-2 \%$ & $-8 \%$ & $-17 \%$ & $-4 \%$ & $-15 \%$ & $-27 \%$ & $-6 \%$ & $-17 \%$ & $-30 \%$ \\
\hline & Specific Power & $0 \%$ & $0 \%$ & $0 \%$ & $0 \%$ & $0 \%$ & $1 \%$ & $1 \%$ & $0 \%$ & $1 \%$ & $4 \%$ & $0 \%$ & $1 \%$ & $4 \%$ \\
\hline \multirow{5}{*}{$\mathrm{Cl}$ Series HEV PHEV40 } & ICE Power & $0 \%$ & $0 \%$ & $-6 \%$ & $-12 \%$ & $-2 \%$ & $-10 \%$ & $-21 \%$ & $-6 \%$ & $-19 \%$ & $-32 \%$ & $-8 \%$ & $-22 \%$ & $-36 \%$ \\
\hline & Electric Machine & $0 \%$ & $0 \%$ & $-5 \%$ & $-9 \%$ & $-2 \%$ & $-7 \%$ & $-16 \%$ & $-4 \%$ & $-14 \%$ & $-25 \%$ & $-5 \%$ & $-16 \%$ & $-28 \%$ \\
\hline & $\mathrm{MC} 2 / \mathrm{GC}$ & $0 \%$ & $0 \%$ & $-6 \%$ & $-12 \%$ & $-2 \%$ & $-10 \%$ & $-21 \%$ & $-6 \%$ & $-19 \%$ & $-32 \%$ & $-8 \%$ & $-22 \%$ & $-36 \%$ \\
\hline & Battery & $0 \%$ & $0 \%$ & $-5 \%$ & $-10 \%$ & $-2 \%$ & $-8 \%$ & $-18 \%$ & $-5 \%$ & $-16 \%$ & $-28 \%$ & $-6 \%$ & $-18 \%$ & $-31 \%$ \\
\hline & Specific Power & $0 \%$ & $0 \%$ & $0 \%$ & $0 \%$ & $0 \%$ & $1 \%$ & $1 \%$ & $1 \%$ & $1 \%$ & $4 \%$ & $0 \%$ & $1 \%$ & $3 \%$ \\
\hline \multirow{5}{*}{ H2 Split HEV } & ICE Power & $0 \%$ & $0 \%$ & $-7 \%$ & $-12 \%$ & $-2 \%$ & $-11 \%$ & $-21 \%$ & $-4 \%$ & $-19 \%$ & $-32 \%$ & $-6 \%$ & $-21 \%$ & $-34 \%$ \\
\hline & Electric Machine & $0 \%$ & $0 \%$ & $-6 \%$ & $-11 \%$ & $-2 \%$ & $-9 \%$ & $-17 \%$ & $-3 \%$ & $-15 \%$ & $-27 \%$ & $-5 \%$ & $-17 \%$ & $-29 \%$ \\
\hline & $\mathrm{MC2} / \mathrm{GC}$ & $0 \%$ & $0 \%$ & $-7 \%$ & $-12 \%$ & $-2 \%$ & $-11 \%$ & $-21 \%$ & $-4 \%$ & $-19 \%$ & $-32 \%$ & $-6 \%$ & $-21 \%$ & $-34 \%$ \\
\hline & Battery & $0 \%$ & $0 \%$ & $-8 \%$ & $-13 \%$ & $-3 \%$ & $-9 \%$ & $-18 \%$ & $-5 \%$ & $-16 \%$ & $-29 \%$ & $-7 \%$ & $-18 \%$ & $-31 \%$ \\
\hline & Specific Power & $0 \%$ & $0 \%$ & $0 \%$ & $-1 \%$ & $0 \%$ & $-1 \%$ & $-3 \%$ & $-1 \%$ & $-3 \%$ & $-3 \%$ & $0 \%$ & $-3 \%$ & $-3 \%$ \\
\hline \multirow{5}{*}{ H2 Split HEV PHEV10 } & ICE Power & $0 \%$ & $0 \%$ & $-6 \%$ & $-11 \%$ & $-2 \%$ & $-10 \%$ & $-17 \%$ & $-4 \%$ & $-15 \%$ & $-27 \%$ & $-7 \%$ & $-17 \%$ & $-30 \%$ \\
\hline & Electric Machine & $0 \%$ & $0 \%$ & $-6 \%$ & $-11 \%$ & $-3 \%$ & $-11 \%$ & $-19 \%$ & $-4 \%$ & $-17 \%$ & $-30 \%$ & $-7 \%$ & $-18 \%$ & $-32 \%$ \\
\hline & $\mathrm{MC} 2 / \mathrm{GC}$ & $0 \%$ & $0 \%$ & $-6 \%$ & $-11 \%$ & $-2 \%$ & $-10 \%$ & $-17 \%$ & $-4 \%$ & $-15 \%$ & $-27 \%$ & $-7 \%$ & $-17 \%$ & $-30 \%$ \\
\hline & Battery & $0 \%$ & $0 \%$ & $-7 \%$ & $-12 \%$ & $-3 \%$ & $-12 \%$ & $-21 \%$ & $-6 \%$ & $-19 \%$ & $-31 \%$ & $-8 \%$ & $-18 \%$ & $-32 \%$ \\
\hline & Specific Power & $0 \%$ & $0 \%$ & $0 \%$ & $0 \%$ & $0 \%$ & $-1 \%$ & $-1 \%$ & $-1 \%$ & $-1 \%$ & $0 \%$ & $0 \%$ & $0 \%$ & $0 \%$ \\
\hline \multirow{5}{*}{ H2 Split HEV PHEV2O } & ICE Power & $0 \%$ & $0 \%$ & $-6 \%$ & $-12 \%$ & $-2 \%$ & $-10 \%$ & $-18 \%$ & $-4 \%$ & $-15 \%$ & $-27 \%$ & $-7 \%$ & $-18 \%$ & $-31 \%$ \\
\hline & Electric Machine & $0 \%$ & $0 \%$ & $-6 \%$ & $-11 \%$ & $-2 \%$ & $-10 \%$ & $-19 \%$ & $-3 \%$ & $-18 \%$ & $-31 \%$ & $-7 \%$ & $-20 \%$ & $-33 \%$ \\
\hline & $\mathrm{MC} 2 / \mathrm{GC}$ & $0 \%$ & $0 \%$ & $-6 \%$ & $-12 \%$ & $-2 \%$ & $-10 \%$ & $-18 \%$ & $-4 \%$ & $-15 \%$ & $-27 \%$ & $-7 \%$ & $-18 \%$ & $-31 \%$ \\
\hline & Battery & $0 \%$ & $0 \%$ & $-7 \%$ & $-13 \%$ & $-2 \%$ & $-11 \%$ & $-22 \%$ & $-5 \%$ & $-20 \%$ & $-33 \%$ & $-8 \%$ & $-20 \%$ & $-35 \%$ \\
\hline & Specific Power & $0 \%$ & $0 \%$ & $0 \%$ & $0 \%$ & $0 \%$ & $0 \%$ & $-2 \%$ & $0 \%$ & $-2 \%$ & $-1 \%$ & $0 \%$ & $-1 \%$ & $-1 \%$ \\
\hline \multirow{5}{*}{ H2 Series HEV PHEV 30} & ICE Power & $0 \%$ & $0 \%$ & $-8 \%$ & $-15 \%$ & $-2 \%$ & $-12 \%$ & $-24 \%$ & $-7 \%$ & $-21 \%$ & $-35 \%$ & $-10 \%$ & $-24 \%$ & $-38 \%$ \\
\hline & Electric Machine & $0 \%$ & $0 \%$ & $-7 \%$ & $-12 \%$ & $-3 \%$ & $-10 \%$ & $-18 \%$ & $-5 \%$ & $-16 \%$ & $-28 \%$ & $-8 \%$ & $-19 \%$ & $-31 \%$ \\
\hline & $\mathrm{MC} 2 / \mathrm{GC}$ & $0 \%$ & $0 \%$ & $-8 \%$ & $-15 \%$ & $-2 \%$ & $-12 \%$ & $-24 \%$ & $-7 \%$ & $-21 \%$ & $-35 \%$ & $-10 \%$ & $-24 \%$ & $-38 \%$ \\
\hline & Battery & $0 \%$ & $0 \%$ & $-7 \%$ & $-13 \%$ & $-3 \%$ & $-11 \%$ & $-20 \%$ & $-6 \%$ & $-18 \%$ & $-30 \%$ & $-9 \%$ & $-20 \%$ & $-33 \%$ \\
\hline & Specific Power & $0 \%$ & $0 \%$ & $1 \%$ & $2 \%$ & $1 \%$ & $2 \%$ & $3 \%$ & $1 \%$ & $3 \%$ & $7 \%$ & $1 \%$ & $3 \%$ & $7 \%$ \\
\hline
\end{tabular}


Argonne National Laboratory Report - Light-Duty Vehicle Fuel Consumption Displacement Potential Up to 2045

\begin{tabular}{|c|c|c|c|c|c|c|c|c|c|c|c|c|c|c|}
\hline & & \multicolumn{13}{|c|}{ compact } \\
\hline & & \multirow{2}{*}{$\begin{array}{r}2010 \\
\text { Ref }\end{array}$} & \multicolumn{3}{|c|}{2010} & \multicolumn{3}{|c|}{2015} & \multicolumn{3}{|c|}{2030} & \multicolumn{3}{|c|}{2045} \\
\hline & & & low & avg & high & low & avg & high & low & avg & high & low & avg & high \\
\hline \multirow{5}{*}{ H2 Series HEV PHEV40 } & ICE Power & $0 \%$ & $0 \%$ & $-8 \%$ & $-15 \%$ & $-2 \%$ & $-13 \%$ & $-24 \%$ & $-7 \%$ & $-22 \%$ & $-36 \%$ & $-11 \%$ & $-25 \%$ & $-39 \%$ \\
\hline & Electric Machine & $0 \%$ & $0 \%$ & $-7 \%$ & $-12 \%$ & $-3 \%$ & $-10 \%$ & $-19 \%$ & $-5 \%$ & $-17 \%$ & $-29 \%$ & $-8 \%$ & $-20 \%$ & $-32 \%$ \\
\hline & $\mathrm{MC} 2 / \mathrm{GC}$ & $0 \%$ & $0 \%$ & $-8 \%$ & $-15 \%$ & $-2 \%$ & $-13 \%$ & $-24 \%$ & $-7 \%$ & $-22 \%$ & $-36 \%$ & $-11 \%$ & $-25 \%$ & $-39 \%$ \\
\hline & Battery & $0 \%$ & $0 \%$ & $-7 \%$ & $-13 \%$ & $-3 \%$ & $-11 \%$ & $-21 \%$ & $-6 \%$ & $-19 \%$ & $-31 \%$ & $-9 \%$ & $-21 \%$ & $-34 \%$ \\
\hline & Specific Power & $0 \%$ & $0 \%$ & $1 \%$ & $2 \%$ & $1 \%$ & $2 \%$ & $3 \%$ & $1 \%$ & $3 \%$ & $7 \%$ & $1 \%$ & $3 \%$ & $6 \%$ \\
\hline \multirow{5}{*}{ E85 Split HEV } & ICE Power & $0 \%$ & $0 \%$ & $-4 \%$ & $-8 \%$ & $-1 \%$ & $-6 \%$ & $-17 \%$ & $-3 \%$ & $-15 \%$ & $-27 \%$ & $-5 \%$ & $-17 \%$ & $-32 \%$ \\
\hline & Electric Machine & $0 \%$ & $0 \%$ & $-4 \%$ & $-7 \%$ & $-1 \%$ & $-4 \%$ & $-13 \%$ & $-1 \%$ & $-11 \%$ & $-22 \%$ & $-2 \%$ & $-13 \%$ & $-26 \%$ \\
\hline & $\mathrm{MC} 2 / \mathrm{GC}$ & $0 \%$ & $0 \%$ & $-4 \%$ & $-8 \%$ & $-1 \%$ & $-6 \%$ & $-17 \%$ & $-3 \%$ & $-15 \%$ & $-27 \%$ & $-5 \%$ & $-17 \%$ & $-32 \%$ \\
\hline & Battery & $0 \%$ & $0 \%$ & $-5 \%$ & $-9 \%$ & $-2 \%$ & $-6 \%$ & $-12 \%$ & $-1 \%$ & $-12 \%$ & $-24 \%$ & $-1 \%$ & $-12 \%$ & $-26 \%$ \\
\hline & Specific Power & $0 \%$ & $0 \%$ & $0 \%$ & $0 \%$ & $0 \%$ & $0 \%$ & $-4 \%$ & $-1 \%$ & $-3 \%$ & $-3 \%$ & $-2 \%$ & $-4 \%$ & $-5 \%$ \\
\hline \multirow{5}{*}{ E85 Split HEV PHEV10 } & ICE Power & $0 \%$ & $0 \%$ & $-4 \%$ & $-9 \%$ & $-1 \%$ & $-7 \%$ & $-14 \%$ & $-2 \%$ & $-12 \%$ & $-23 \%$ & $-4 \%$ & $-14 \%$ & $-27 \%$ \\
\hline & Electric Machine & $0 \%$ & $0 \%$ & $-5 \%$ & $-9 \%$ & $-2 \%$ & $-7 \%$ & $-15 \%$ & $-3 \%$ & $-13 \%$ & $-26 \%$ & $-3 \%$ & $-15 \%$ & $-28 \%$ \\
\hline & $\mathrm{MC} 2 / \mathrm{GC}$ & $0 \%$ & $0 \%$ & $-5 \%$ & $-9 \%$ & $-1 \%$ & $-7 \%$ & $-14 \%$ & $-2 \%$ & $-12 \%$ & $-23 \%$ & $-4 \%$ & $-14 \%$ & $-27 \%$ \\
\hline & Battery & $0 \%$ & $0 \%$ & $-6 \%$ & $-10 \%$ & $-2 \%$ & $-9 \%$ & $-15 \%$ & $-4 \%$ & $-13 \%$ & $-24 \%$ & $-4 \%$ & $-13 \%$ & $-27 \%$ \\
\hline & Specific Power & $0 \%$ & $0 \%$ & $-1 \%$ & $-1 \%$ & $0 \%$ & $-1 \%$ & $-1 \%$ & $-1 \%$ & $-1 \%$ & $1 \%$ & $-1 \%$ & $0 \%$ & $1 \%$ \\
\hline \multirow{5}{*}{ E85 Split HEV PHEV20 } & ICE Power & $0 \%$ & $0 \%$ & $-4 \%$ & $-9 \%$ & $-1 \%$ & $-7 \%$ & $-14 \%$ & $-2 \%$ & $-12 \%$ & $-24 \%$ & $-4 \%$ & $-14 \%$ & $-28 \%$ \\
\hline & Electric Machine & $0 \%$ & $0 \%$ & $-4 \%$ & $-9 \%$ & $-2 \%$ & $-7 \%$ & $-15 \%$ & $-3 \%$ & $-14 \%$ & $-26 \%$ & $-3 \%$ & $-16 \%$ & $-29 \%$ \\
\hline & $\mathrm{MC} 2 / \mathrm{GC}$ & $0 \%$ & $0 \%$ & $-4 \%$ & $-9 \%$ & $-1 \%$ & $-7 \%$ & $-14 \%$ & $-3 \%$ & $-12 \%$ & $-24 \%$ & $-4 \%$ & $-14 \%$ & $-28 \%$ \\
\hline & Battery & $0 \%$ & $0 \%$ & $-4 \%$ & $-10 \%$ & $-2 \%$ & $-9 \%$ & $-16 \%$ & $-4 \%$ & $-15 \%$ & $-27 \%$ & $-4 \%$ & $-15 \%$ & $-29 \%$ \\
\hline & Specific Power & $0 \%$ & $0 \%$ & $0 \%$ & $-1 \%$ & $0 \%$ & $-1 \%$ & $-2 \%$ & $-1 \%$ & $-1 \%$ & $0 \%$ & $-1 \%$ & $-1 \%$ & $0 \%$ \\
\hline \multirow{5}{*}{ E85 Series HEV PHEV30 } & ICE Power & $0 \%$ & $0 \%$ & $-6 \%$ & $-12 \%$ & $-2 \%$ & $-10 \%$ & $-21 \%$ & $-6 \%$ & $-19 \%$ & $-32 \%$ & $-8 \%$ & $-21 \%$ & $-36 \%$ \\
\hline & Electric Machine & $0 \%$ & $0 \%$ & $-5 \%$ & $-9 \%$ & $-2 \%$ & $-7 \%$ & $-16 \%$ & $-4 \%$ & $-14 \%$ & $-25 \%$ & $-5 \%$ & $-16 \%$ & $-28 \%$ \\
\hline & $\mathrm{MC} 2 / \mathrm{GC}$ & $0 \%$ & $0 \%$ & $-6 \%$ & $-12 \%$ & $-2 \%$ & $-10 \%$ & $-21 \%$ & $-6 \%$ & $-19 \%$ & $-32 \%$ & $-8 \%$ & $-21 \%$ & $-36 \%$ \\
\hline & Battery & $0 \%$ & $0 \%$ & $-5 \%$ & $-10 \%$ & $-2 \%$ & $-8 \%$ & $-18 \%$ & $-5 \%$ & $-16 \%$ & $-27 \%$ & $-6 \%$ & $-18 \%$ & $-31 \%$ \\
\hline & Specific Power & $0 \%$ & $0 \%$ & $0 \%$ & $1 \%$ & $0 \%$ & $1 \%$ & $2 \%$ & $1 \%$ & $2 \%$ & $5 \%$ & $0 \%$ & $1 \%$ & $5 \%$ \\
\hline \multirow{5}{*}{ E85 Series HEV PHEV40 } & ICE Power & $0 \%$ & $0 \%$ & $-6 \%$ & $-13 \%$ & $-2 \%$ & $-10 \%$ & $-22 \%$ & $-6 \%$ & $-20 \%$ & $-33 \%$ & $-8 \%$ & $-22 \%$ & $-37 \%$ \\
\hline & Electric Machine & $0 \%$ & $0 \%$ & $-5 \%$ & $-10 \%$ & $-2 \%$ & $-8 \%$ & $-16 \%$ & $-4 \%$ & $-15 \%$ & $-26 \%$ & $-6 \%$ & $-17 \%$ & $-29 \%$ \\
\hline & $\mathrm{MC} 2 / \mathrm{GC}$ & $0 \%$ & $0 \%$ & $-6 \%$ & $-13 \%$ & $-2 \%$ & $-10 \%$ & $-22 \%$ & $-6 \%$ & $-20 \%$ & $-33 \%$ & $-8 \%$ & $-22 \%$ & $-37 \%$ \\
\hline & Battery & $0 \%$ & $0 \%$ & $-6 \%$ & $-11 \%$ & $-2 \%$ & $-9 \%$ & $-18 \%$ & $-5 \%$ & $-16 \%$ & $-28 \%$ & $-7 \%$ & $-19 \%$ & $-32 \%$ \\
\hline & Specific Power & $0 \%$ & $0 \%$ & $0 \%$ & $1 \%$ & $0 \%$ & $1 \%$ & $1 \%$ & $1 \%$ & $1 \%$ & $5 \%$ & $0 \%$ & $1 \%$ & $5 \%$ \\
\hline
\end{tabular}


Argonne National Laboratory Report - Light-Duty Vehicle Fuel Consumption Displacement Potential Up to 2045

\begin{tabular}{|c|c|c|c|c|c|c|c|c|c|c|c|c|c|c|}
\hline & & \multicolumn{13}{|c|}{ compact } \\
\hline & & \multirow{2}{*}{$\frac{2010}{\text { Ref }}$} & \multicolumn{3}{|c|}{2010} & \multicolumn{3}{|c|}{2015} & \multicolumn{3}{|c|}{2030} & \multicolumn{3}{|c|}{2045} \\
\hline & & & low & avg & high & low & avg & high & low & avg & high & low & avg & high \\
\hline \multirow{5}{*}{ FC HEV } & FC Power & $0 \%$ & $0 \%$ & $-15 \%$ & $-25 \%$ & $-10 \%$ & $-27 \%$ & $-38 \%$ & $-21 \%$ & $-37 \%$ & $-49 \%$ & $-26 \%$ & $-39 \%$ & $-51 \%$ \\
\hline & Electric Machine & $0 \%$ & $0 \%$ & $-14 \%$ & $-22 \%$ & $-9 \%$ & $-21 \%$ & $-30 \%$ & $-16 \%$ & $-29 \%$ & $-40 \%$ & $-20 \%$ & $-31 \%$ & $-43 \%$ \\
\hline & $\mathrm{MC} 2 / \mathrm{GC}$ & & & & & & & & & & & & & \\
\hline & Battery & $0 \%$ & $0 \%$ & $-12 \%$ & $-18 \%$ & $-9 \%$ & $-8 \%$ & $-15 \%$ & $-3 \%$ & $-13 \%$ & $-23 \%$ & $-6 \%$ & $-17 \%$ & $-27 \%$ \\
\hline & Specific Power & $0 \%$ & $0 \%$ & $1 \%$ & $1 \%$ & $1 \%$ & $0 \%$ & $-1 \%$ & $-1 \%$ & $0 \%$ & $1 \%$ & $0 \%$ & $0 \%$ & $1 \%$ \\
\hline \multirow{5}{*}{ FC HEV PHEV 10} & FC Power & $0 \%$ & $0 \%$ & $-13 \%$ & $-22 \%$ & $-8 \%$ & $-20 \%$ & $-30 \%$ & $-13 \%$ & $-29 \%$ & $-39 \%$ & $-18 \%$ & $-28 \%$ & $-41 \%$ \\
\hline & Electric Machine & $0 \%$ & $0 \%$ & $-13 \%$ & $-21 \%$ & $-9 \%$ & $-20 \%$ & $-28 \%$ & $-13 \%$ & $-27 \%$ & $-39 \%$ & $-18 \%$ & $-27 \%$ & $-40 \%$ \\
\hline & $\mathrm{MC} 2 / \mathrm{GC}$ & & & & & & & & & & & & & \\
\hline & Battery & $0 \%$ & $0 \%$ & $-13 \%$ & $-21 \%$ & $-8 \%$ & $-20 \%$ & $-30 \%$ & $-14 \%$ & $-28 \%$ & $-41 \%$ & $-18 \%$ & $-30 \%$ & $-44 \%$ \\
\hline & Specific Power & $0 \%$ & $0 \%$ & $-1 \%$ & $-1 \%$ & $-1 \%$ & $0 \%$ & $0 \%$ & $0 \%$ & $0 \%$ & $1 \%$ & $0 \%$ & $2 \%$ & $2 \%$ \\
\hline \multirow{5}{*}{ FC HEV PHEV 20} & FC Power & $0 \%$ & $0 \%$ & $-13 \%$ & $-21 \%$ & $-7 \%$ & $-20 \%$ & $-30 \%$ & $-13 \%$ & $-29 \%$ & $-40 \%$ & $-18 \%$ & $-28 \%$ & $-42 \%$ \\
\hline & Electric Machine & $0 \%$ & $0 \%$ & $-13 \%$ & $-20 \%$ & $-8 \%$ & $-19 \%$ & $-28 \%$ & $-13 \%$ & $-27 \%$ & $-39 \%$ & $-17 \%$ & $-27 \%$ & $-41 \%$ \\
\hline & $\mathrm{MC} 2 / \mathrm{GC}$ & & & & & & & & & & & & & \\
\hline & Battery & $0 \%$ & $0 \%$ & $-13 \%$ & $-22 \%$ & $-8 \%$ & $-20 \%$ & $-30 \%$ & $-14 \%$ & $-29 \%$ & $-41 \%$ & $-19 \%$ & $-31 \%$ & $-44 \%$ \\
\hline & Specific Power & $0 \%$ & $0 \%$ & $0 \%$ & $0 \%$ & $0 \%$ & $0 \%$ & $1 \%$ & $0 \%$ & $0 \%$ & $1 \%$ & $1 \%$ & $3 \%$ & $1 \%$ \\
\hline \multirow{5}{*}{ FC HEV PHEV 30} & FC Power & $0 \%$ & $0 \%$ & $-11 \%$ & $-17 \%$ & $-6 \%$ & $-16 \%$ & $-24 \%$ & $-10 \%$ & $-22 \%$ & $-34 \%$ & $-14 \%$ & $-25 \%$ & $-38 \%$ \\
\hline & Electric Machine & $0 \%$ & $0 \%$ & $-8 \%$ & $-14 \%$ & $-5 \%$ & $-13 \%$ & $-21 \%$ & $-8 \%$ & $-19 \%$ & $-29 \%$ & $-11 \%$ & $-22 \%$ & $-33 \%$ \\
\hline & $\mathrm{MC} 2 / \mathrm{GC}$ & & & & & & & & & & & & & \\
\hline & Battery & $0 \%$ & $0 \%$ & $-9 \%$ & $-15 \%$ & $-5 \%$ & $-14 \%$ & $-22 \%$ & $-9 \%$ & $-21 \%$ & $-32 \%$ & $-12 \%$ & $-23 \%$ & $-35 \%$ \\
\hline & Specific Power & $0 \%$ & $0 \%$ & $4 \%$ & $7 \%$ & $3 \%$ & $7 \%$ & $10 \%$ & $5 \%$ & $10 \%$ & $16 \%$ & $7 \%$ & $10 \%$ & $15 \%$ \\
\hline \multirow{5}{*}{ FC HEV PHEV40 } & FC Power & $0 \%$ & $0 \%$ & $-11 \%$ & $-18 \%$ & $-6 \%$ & $-16 \%$ & $-25 \%$ & $-10 \%$ & $-23 \%$ & $-35 \%$ & $-15 \%$ & $-26 \%$ & $-38 \%$ \\
\hline & Electric Machine & $0 \%$ & $0 \%$ & $-9 \%$ & $-14 \%$ & $-5 \%$ & $-13 \%$ & $-21 \%$ & $-8 \%$ & $-19 \%$ & $-30 \%$ & $-12 \%$ & $-22 \%$ & $-33 \%$ \\
\hline & $\mathrm{MC} 2 / \mathrm{GC}$ & & & & & & & & & & & & & \\
\hline & Battery & $0 \%$ & $0 \%$ & $-9 \%$ & $-15 \%$ & $-5 \%$ & $-14 \%$ & $-23 \%$ & $-9 \%$ & $-21 \%$ & $-32 \%$ & $-13 \%$ & $-24 \%$ & $-36 \%$ \\
\hline & Specific Power & $0 \%$ & $0 \%$ & $4 \%$ & $7 \%$ & $3 \%$ & $7 \%$ & $10 \%$ & $5 \%$ & $10 \%$ & $16 \%$ & $7 \%$ & $10 \%$ & $16 \%$ \\
\hline \multirow{5}{*}{ EV } & ICE Power & & & & & & & & & & & & & \\
\hline & Electric Machine & $0 \%$ & $0 \%$ & $-6 \%$ & $-11 \%$ & $-1 \%$ & $-9 \%$ & $-21 \%$ & $-4 \%$ & $-19 \%$ & $-32 \%$ & $-7 \%$ & $-23 \%$ & $-36 \%$ \\
\hline & $\mathrm{MC2} / \mathrm{GC}$ & & & & & & & & & & & & & \\
\hline & Battery & $0 \%$ & $0 \%$ & $-6 \%$ & $-12 \%$ & $-1 \%$ & $-10 \%$ & $-23 \%$ & $-5 \%$ & $-21 \%$ & $-34 \%$ & $-8 \%$ & $-25 \%$ & $-38 \%$ \\
\hline & Specific Power & $0 \%$ & $0 \%$ & $-1 \%$ & $-1 \%$ & $0 \%$ & $-1 \%$ & $-1 \%$ & $-1 \%$ & $-1 \%$ & $1 \%$ & $-2 \%$ & $-2 \%$ & $1 \%$ \\
\hline
\end{tabular}




\section{Energy}

\begin{tabular}{|c|c|c|c|c|c|c|c|c|c|c|c|c|c|c|}
\hline & & \multicolumn{13}{|c|}{ Compact } \\
\hline & & \multirow{2}{*}{$\frac{2010}{\operatorname{Ref}}$} & \multicolumn{3}{|c|}{2010} & \multicolumn{3}{|c|}{2015} & \multicolumn{3}{|c|}{2030} & \multicolumn{3}{|c|}{2045} \\
\hline & & & low & avg & high & low & avg & high & low & avg & high & low & avg & high \\
\hline \multirow{2}{*}{ SI Split HEV } & Total Energy & 991 & 991 & 944 & 905 & 975 & 907 & 842 & 950 & 842 & 734 & 950 & 842 & 691 \\
\hline & Usable Energy & & & & & & & & & & & & & \\
\hline \multirow{2}{*}{ SI Split HEV PHEV10 } & Total Energy & 3379 & 3379 & 3250 & 3032 & 3411 & 3132 & 2433 & 3326 & 2518 & 1810 & 3240 & 2268 & 1709 \\
\hline & Usable Energy & 2028 & 2028 & 1950 & 1819 & 2047 & 1879 & 1703 & 1996 & 1762 & 1448 & 1944 & 1701 & 1367 \\
\hline \multirow{2}{*}{ SI Split HEV PHEV2O } & Total Energy & 6839 & 6839 & 6422 & 5961 & 6822 & 6157 & 4798 & 6568 & 4962 & 3577 & 6401 & 4528 & 3384 \\
\hline & Usable Energy & 4104 & 4104 & 3853 & 3577 & 4093 & 3694 & 3359 & 3941 & 3474 & 2861 & 3841 & 3396 & 2707 \\
\hline \multirow{2}{*}{ SI Series HEV PHEV30 } & Total Energy & 12856 & 12856 & 12151 & 11285 & 12799 & 11675 & 8777 & 12306 & 9148 & 6733 & 11950 & 8194 & 6344 \\
\hline & Usable Energy & 7713 & 7713 & 7291 & 6771 & 7680 & 7005 & 6144 & 7384 & 6404 & 5386 & 7170 & 6146 & 5076 \\
\hline \multirow{2}{*}{ SI Series HEV PHEV40 } & Total Energy & 17195 & 17195 & 16249 & 15052 & 17252 & 15640 & 11854 & 16531 & 12328 & 8915 & 16087 & 11009 & 8489 \\
\hline & Usable Energy & 10317 & 10317 & 9749 & 9031 & 10351 & 9384 & 8298 & 9918 & 8630 & 7132 & 9652 & 8257 & 6792 \\
\hline \multirow{2}{*}{ CI Split HEV } & Total Energy & 1061 & 1061 & 1014 & 975 & 1030 & 972 & 907 & 1015 & 907 & 799 & 1015 & 907 & 756 \\
\hline & Usable Energy & & & & & & & & & & & & & \\
\hline \multirow{2}{*}{ CI Split HEV PHEV10 } & Total Energy & 3633 & 3633 & 3419 & 3188 & 3621 & 3295 & 2542 & 3482 & 2678 & 1917 & 3392 & 2417 & 1822 \\
\hline & Usable Energy & 2180 & 2180 & 2052 & 1913 & 2173 & 1977 & 1780 & 2089 & 1874 & 1534 & 2035 & 1813 & 1458 \\
\hline \multirow{2}{*}{ CI Split HEV PHEV2O } & Total Energy & 7148 & 7148 & 6776 & 6386 & 7229 & 6602 & 5118 & 7116 & 5263 & 3777 & 6931 & 4766 & 3610 \\
\hline & Usable Energy & 4289 & 4289 & 4066 & 3832 & 4337 & 3961 & 3582 & 4270 & 3684 & 3021 & 4158 & 3575 & 2888 \\
\hline \multirow{2}{*}{ CI Series HEV PHEV30 } & Total Energy & 13398 & 13398 & 12662 & 11812 & 13350 & 12185 & 9264 & 12830 & 9579 & 7028 & 12461 & 8643 & 6762 \\
\hline & Usable Energy & 8039 & 8039 & 7597 & 7087 & 8010 & 7311 & 6485 & 7698 & 6705 & 5623 & 7476 & 6482 & 5409 \\
\hline \multirow{2}{*}{ CI Series HEV PHEV40 } & Total Energy & 17949 & 17949 & 17055 & 15788 & 17995 & 16372 & 12432 & 17234 & 12845 & 9372 & 16742 & 11518 & 8923 \\
\hline & Usable Energy & 10769 & 10769 & 10233 & 9473 & 10797 & 9823 & 8702 & 10341 & 8991 & 7498 & 10045 & 8639 & 7138 \\
\hline \multirow{2}{*}{ H2 Split HEV } & Total Energy & 1030 & 1030 & 952 & 897 & 998 & 907 & 821 & 950 & 842 & 713 & 929 & 821 & 691 \\
\hline & Usable Energy & & & & & & & & & & & & & \\
\hline \multirow{2}{*}{ H2 Split HEV PHEV10 } & Total Energy & 3501 & 3501 & 3262 & 3014 & 3511 & 3115 & 2389 & 3358 & 2484 & 1765 & 3218 & 2240 & 1684 \\
\hline & Usable Energy & 2101 & 2101 & 1957 & 1808 & 2106 & 1869 & 1672 & 2015 & 1739 & 1412 & 1931 & 1680 & 1347 \\
\hline
\end{tabular}


Argonne National Laboratory Report - Light-Duty Vehicle Fuel Consumption Displacement Potential Up to 2045

\begin{tabular}{|c|c|c|c|c|c|c|c|c|c|c|c|c|c|c|}
\hline & & \multicolumn{13}{|c|}{ Compact } \\
\hline & & \multirow{2}{*}{$\begin{array}{c}2010 \\
\text { Ref }\end{array}$} & \multicolumn{3}{|c|}{2010} & \multicolumn{3}{|c|}{2015} & \multicolumn{3}{|c|}{2030} & \multicolumn{3}{|c|}{2045} \\
\hline & & & low & avg & high & low & avg & high & low & avg & high & low & avg & high \\
\hline \multirow{2}{*}{ H2 Split HEV PHEV2O } & Total Energy & 6912 & 6912 & 6452 & 6041 & 7002 & 6248 & 4762 & 6712 & 4951 & 3477 & 6420 & 4478 & 3307 \\
\hline & Usable Energy & 4147 & 4147 & 3871 & 3625 & 4201 & 3749 & 3333 & 4027 & 3465 & 2782 & 3852 & 3358 & 2646 \\
\hline \multirow{2}{*}{ H2 Series HEV PHEV 30} & Total Energy & 13145 & 13145 & 12183 & 11229 & 13003 & 11619 & 8664 & 12434 & 9070 & 6531 & 11860 & 8092 & 6243 \\
\hline & Usable Energy & 7887 & 7887 & 7310 & 6738 & 7802 & 6972 & 6065 & 7460 & 6349 & 5225 & 7116 & 6069 & 4994 \\
\hline \multirow{2}{*}{ H2 Series HEV PHEV40 } & Total Energy & 17569 & 17569 & 16283 & 14949 & 17502 & 15504 & 11749 & 16635 & 12221 & 8737 & 15847 & 10875 & 8357 \\
\hline & Usable Energy & 10541 & 10541 & 9770 & 8970 & 10501 & 9303 & 8224 & 9981 & 8555 & 6989 & 9508 & 8156 & 6685 \\
\hline \multirow{2}{*}{ E85 Split HEV } & Total Energy & 991 & 991 & 944 & 905 & 975 & 907 & 842 & 950 & 842 & 734 & 950 & 842 & 713 \\
\hline & Usable Energy & & & & & & & & & & & & & \\
\hline \multirow{2}{*}{ E85 Split HEV PHEV10 } & Total Energy & 3383 & 3383 & 3252 & 3034 & 3415 & 3135 & 2438 & 3328 & 2487 & 1811 & 3245 & 2270 & 1710 \\
\hline & Usable Energy & 2030 & 2030 & 1951 & 1820 & 2049 & 1881 & 1706 & 1997 & 1741 & 1449 & 1947 & 1702 & 1368 \\
\hline \multirow{2}{*}{ E85 Split HEV PHEV20 } & Total Energy & 6842 & 6842 & 6425 & 5964 & 6824 & 6165 & 4803 & 6571 & 4966 & 3571 & 6403 & 4533 & 3386 \\
\hline & Usable Energy & 4105 & 4105 & 3855 & 3578 & 4094 & 3699 & 3362 & 3943 & 3476 & 2856 & 3842 & 3400 & 2709 \\
\hline \multirow{2}{*}{ E85 Series HEV PHEV30 } & Total Energy & 12861 & 12861 & 12153 & 11291 & 13045 & 11686 & 8786 & 12312 & 9150 & 6737 & 11955 & 8202 & 6350 \\
\hline & Usable Energy & 7716 & 7716 & 7292 & 6774 & 7827 & 7011 & 6150 & 7387 & 6405 & 5389 & 7173 & 6152 & 5080 \\
\hline \multirow{2}{*}{ E85 Series HEV PHEV40 } & Total Energy & 17231 & 17231 & 16252 & 15060 & 17255 & 15597 & 11918 & 16489 & 12334 & 8920 & 16098 & 11017 & 8492 \\
\hline & Usable Energy & 10338 & 10338 & 9751 & 9036 & 10353 & 9358 & 8343 & 9893 & 8634 & 7136 & 9659 & 8263 & 6794 \\
\hline \multirow{2}{*}{ FC HEV } & Total Energy & 1279 & 1279 & 1123 & 1053 & 1162 & 1145 & 1058 & 1210 & 1080 & 950 & 1166 & 1037 & 907 \\
\hline & Usable Energy & & & & & & & & & & & & & \\
\hline \multirow{2}{*}{ FC HEV PHEV 10} & Total Energy & 4119 & 4119 & 3623 & 3266 & 3885 & 3386 & 2597 & 3648 & 2686 & 1932 & 3456 & 2373 & 1821 \\
\hline & Usable Energy & 2472 & 2472 & 2174 & 1960 & 2331 & 2031 & 1818 & 2189 & 1880 & 1546 & 2074 & 1780 & 1457 \\
\hline \multirow{2}{*}{ FC HEV PHEV 20} & Total Energy & 8191 & 8191 & 7274 & 6482 & 7825 & 6715 & 5187 & 7300 & 5372 & 3722 & 6902 & 4834 & 3561 \\
\hline & Usable Energy & 4914 & 4914 & 4364 & 3889 & 4695 & 4029 & 3631 & 4380 & 3760 & 2977 & 4141 & 3625 & 2849 \\
\hline \multirow{2}{*}{ FC HEV PHEV 30} & Total Energy & 12672 & 12672 & 11275 & 10248 & 12116 & 10554 & 7792 & 11334 & 8092 & 5731 & 10725 & 7233 & 5416 \\
\hline & Usable Energy & 7603 & 7603 & 6765 & 6149 & 7270 & 6333 & 5454 & 6800 & 5665 & 4585 & 6435 & 5424 & 4333 \\
\hline \multirow{2}{*}{ FC HEV PHEV40 } & Total Energy & 16825 & 16825 & 14947 & 13544 & 16123 & 13927 & 10248 & 14988 & 10607 & 7729 & 14127 & 9408 & 7275 \\
\hline & Usable Energy & 10095 & 10095 & 8968 & 8126 & 9674 & 8356 & 7174 & 8993 & 7425 & 6183 & 8476 & 7056 & 5820 \\
\hline \multirow{2}{*}{ BEV } & Total Energy & 61349 & 61349 & 57720 & 53052 & 61719 & 55181 & 39332 & 58470 & 40807 & 28635 & 56289 & 36461 & 26910 \\
\hline & Usable Energy & 36810 & 36810 & 34632 & 31831 & 37031 & 33108 & 27533 & 35082 & 28565 & 22908 & 33773 & 27346 & 21528 \\
\hline
\end{tabular}




\section{Percentage of Energy reduction based on the respective 2010 reference case}

\begin{tabular}{|c|c|c|c|c|c|c|c|c|c|c|c|c|c|c|}
\hline & & \multicolumn{13}{|c|}{ Compact } \\
\hline & & \multirow{2}{*}{$\begin{array}{c}2010 \\
\text { Ref }\end{array}$} & \multicolumn{3}{|c|}{2010} & \multicolumn{3}{|c|}{2015} & \multicolumn{3}{|c|}{2030} & \multicolumn{3}{|c|}{2045} \\
\hline & & & low & avg & high & low & avg & high & low & avg & high & low & avg & high \\
\hline \multirow{2}{*}{ SI Split HEV } & Total Energy & $0.0 \%$ & $0.0 \%$ & $-4.7 \%$ & $-8.7 \%$ & $-1.6 \%$ & $-8.4 \%$ & $-15.0 \%$ & $-4.1 \%$ & $-15.0 \%$ & $-25.9 \%$ & $-4.1 \%$ & $-15.0 \%$ & $-30.2 \%$ \\
\hline & Usable Energy & & & & & & & & & & & & & \\
\hline \multirow{2}{*}{ SI Split HEV PHEV10 } & Total Energy & $0.0 \%$ & $0.0 \%$ & $-3.8 \%$ & $-10.3 \%$ & $0.9 \%$ & $-7.3 \%$ & $-28.0 \%$ & $-1.6 \%$ & $-25.5 \%$ & $-46.4 \%$ & $-4.1 \%$ & $-32.9 \%$ & $-49.4 \%$ \\
\hline & Usable Energy & $0.0 \%$ & $0.0 \%$ & $-3.8 \%$ & $-10.3 \%$ & $0.9 \%$ & $-7.3 \%$ & $-16.0 \%$ & $-1.6 \%$ & $-13.1 \%$ & $-28.6 \%$ & $-4.1 \%$ & $-16.1 \%$ & $-32.6 \%$ \\
\hline \multirow{2}{*}{ SI Split HEV PHEV2O } & Total Energy & $0.0 \%$ & $0.0 \%$ & $-6.1 \%$ & $-12.8 \%$ & $-0.3 \%$ & $-10.0 \%$ & $-29.8 \%$ & $-4.0 \%$ & $-27.4 \%$ & $-47.7 \%$ & $-6.4 \%$ & $-33.8 \%$ & $-50.5 \%$ \\
\hline & Usable Energy & $0.0 \%$ & $0.0 \%$ & $-6.1 \%$ & $-12.8 \%$ & $-0.3 \%$ & $-10.0 \%$ & $-18.2 \%$ & $-4.0 \%$ & $-15.4 \%$ & $-30.3 \%$ & $-6.4 \%$ & $-17.2 \%$ & $-34.0 \%$ \\
\hline \multirow{2}{*}{ SI Series HEV PHEV30 } & Total Energy & $0.0 \%$ & $0.0 \%$ & $-5.5 \%$ & $-12.2 \%$ & $-0.4 \%$ & $-9.2 \%$ & $-31.7 \%$ & $-4.3 \%$ & $-28.8 \%$ & $-47.6 \%$ & $-7.0 \%$ & $-36.3 \%$ & $-50.6 \%$ \\
\hline & Usable Energy & $0.0 \%$ & $0.0 \%$ & $-5.5 \%$ & $-12.2 \%$ & $-0.4 \%$ & $-9.2 \%$ & $-20.3 \%$ & $-4.3 \%$ & $-17.0 \%$ & $-30.2 \%$ & $-7.0 \%$ & $-20.3 \%$ & $-34.2 \%$ \\
\hline \multirow{2}{*}{ SI Series HEV PHEV40 } & Total Energy & $0.0 \%$ & $0.0 \%$ & $-5.5 \%$ & $-12.5 \%$ & $0.3 \%$ & $-9.0 \%$ & $-31.1 \%$ & $-3.9 \%$ & $-28.3 \%$ & $-48.2 \%$ & $-6.4 \%$ & $-36.0 \%$ & $-50.6 \%$ \\
\hline & Usable Energy & $0.0 \%$ & $0.0 \%$ & $-5.5 \%$ & $-12.5 \%$ & $0.3 \%$ & $-9.0 \%$ & $-19.6 \%$ & $-3.9 \%$ & $-16.4 \%$ & $-30.9 \%$ & $-6.4 \%$ & $-20.0 \%$ & $-34.2 \%$ \\
\hline \multirow{2}{*}{ CI Split HEV } & Total Energy & $0.0 \%$ & $0.0 \%$ & $-4.4 \%$ & $-8.1 \%$ & $-2.9 \%$ & $-8.4 \%$ & $-14.5 \%$ & $-4.3 \%$ & $-14.5 \%$ & $-24.7 \%$ & $-4.3 \%$ & $-14.5 \%$ & $-28.7 \%$ \\
\hline & Usable Energy & & & & & & & & & & & & & \\
\hline \multirow{2}{*}{ CI Split HEV PHEV10 } & Total Energy & $0.0 \%$ & $0.0 \%$ & $-5.9 \%$ & $-12.3 \%$ & $-0.3 \%$ & $-9.3 \%$ & $-30.0 \%$ & $-4.2 \%$ & $-26.3 \%$ & $-47.2 \%$ & $-6.6 \%$ & $-33.5 \%$ & $-49.8 \%$ \\
\hline & Usable Energy & $0.0 \%$ & $0.0 \%$ & $-5.9 \%$ & $-12.3 \%$ & $-0.3 \%$ & $-9.3 \%$ & $-18.4 \%$ & $-4.2 \%$ & $-14.0 \%$ & $-29.6 \%$ & $-6.6 \%$ & $-16.9 \%$ & $-33.1 \%$ \\
\hline \multirow{2}{*}{ CI Split HEV PHEV2O } & Total Energy & $0.0 \%$ & $0.0 \%$ & $-5.2 \%$ & $-10.7 \%$ & $1.1 \%$ & $-7.6 \%$ & $-28.4 \%$ & $-0.4 \%$ & $-26.4 \%$ & $-47.2 \%$ & $-3.0 \%$ & $-33.3 \%$ & $-49.5 \%$ \\
\hline & Usable Energy & $0.0 \%$ & $0.0 \%$ & $-5.2 \%$ & $-10.7 \%$ & $1.1 \%$ & $-7.6 \%$ & $-16.5 \%$ & $-0.4 \%$ & $-14.1 \%$ & $-29.6 \%$ & $-3.0 \%$ & $-16.7 \%$ & $-32.7 \%$ \\
\hline \multirow{2}{*}{ CI Series HEV PHEV 30} & Total Energy & $0.0 \%$ & $0.0 \%$ & $-5.5 \%$ & $-11.8 \%$ & $-0.4 \%$ & $-9.1 \%$ & $-30.9 \%$ & $-4.2 \%$ & $-28.5 \%$ & $-47.5 \%$ & $-7.0 \%$ & $-35.5 \%$ & $-49.5 \%$ \\
\hline & Usable Energy & $0.0 \%$ & $0.0 \%$ & $-5.5 \%$ & $-11.8 \%$ & $-0.4 \%$ & $-9.1 \%$ & $-19.3 \%$ & $-4.2 \%$ & $-16.6 \%$ & $-30.1 \%$ & $-7.0 \%$ & $-19.4 \%$ & $-32.7 \%$ \\
\hline \multirow{2}{*}{$\mathrm{Cl}$ Series HEV PHEV40 } & Total Energy & $0.0 \%$ & $0.0 \%$ & $-5.0 \%$ & $-12.0 \%$ & $0.3 \%$ & $-8.8 \%$ & $-30.7 \%$ & $-4.0 \%$ & $-28.4 \%$ & $-47.8 \%$ & $-6.7 \%$ & $-35.8 \%$ & $-50.3 \%$ \\
\hline & Usable Energy & $0.0 \%$ & $0.0 \%$ & $-5.0 \%$ & $-12.0 \%$ & $0.3 \%$ & $-8.8 \%$ & $-19.2 \%$ & $-4.0 \%$ & $-16.5 \%$ & $-30.4 \%$ & $-6.7 \%$ & $-19.8 \%$ & $-33.7 \%$ \\
\hline \multirow{2}{*}{ H2 Split HEV } & Total Energy & $0.0 \%$ & $0.0 \%$ & $-7.6 \%$ & $-12.9 \%$ & $-3.0 \%$ & $-11.9 \%$ & $-20.3 \%$ & $-7.7 \%$ & $-18.2 \%$ & $-30.8 \%$ & $-9.8 \%$ & $-20.3 \%$ & $-32.9 \%$ \\
\hline & Usable Energy & & & & & & & & & & & & & \\
\hline \multirow{2}{*}{ H2 Split HEV PHEV10 } & Total Energy & $0.0 \%$ & $0.0 \%$ & $-6.8 \%$ & $-13.9 \%$ & $0.3 \%$ & $-11.0 \%$ & $-31.8 \%$ & $-4.1 \%$ & $-29.1 \%$ & $-49.6 \%$ & $-8.1 \%$ & $-36.0 \%$ & $-51.9 \%$ \\
\hline & Usable Energy & $0.0 \%$ & $0.0 \%$ & $-6.8 \%$ & $-13.9 \%$ & $0.3 \%$ & $-11.0 \%$ & $-20.4 \%$ & $-4.1 \%$ & $-17.2 \%$ & $-32.8 \%$ & $-8.1 \%$ & $-20.0 \%$ & $-35.9 \%$ \\
\hline \multirow{2}{*}{ H2 Split HEV PHEV 20} & Total Energy & $0.0 \%$ & $0.0 \%$ & $-6.6 \%$ & $-12.6 \%$ & $1.3 \%$ & $-9.6 \%$ & $-31.1 \%$ & $-2.9 \%$ & $-28.4 \%$ & $-49.7 \%$ & $-7.1 \%$ & $-35.2 \%$ & $-52.2 \%$ \\
\hline & Usable Energy & $0.0 \%$ & $0.0 \%$ & $-6.6 \%$ & $-12.6 \%$ & $1.3 \%$ & $-9.6 \%$ & $-19.6 \%$ & $-2.9 \%$ & $-16.4 \%$ & $-32.9 \%$ & $-7.1 \%$ & $-19.0 \%$ & $-36.2 \%$ \\
\hline
\end{tabular}


Argonne National Laboratory Report - Light-Duty Vehicle Fuel Consumption Displacement Potential Up to 2045

\begin{tabular}{|c|c|c|c|c|c|c|c|c|c|c|c|c|c|c|}
\hline & & \multicolumn{13}{|c|}{ Compact } \\
\hline & & \multirow{2}{*}{$\frac{2010}{\text { Ref }}$} & \multicolumn{3}{|c|}{2010} & \multicolumn{3}{|c|}{2015} & \multicolumn{3}{|c|}{2030} & \multicolumn{3}{|c|}{2045} \\
\hline & & & low & avg & high & low & avg & high & low & avg & high & low & avg & high \\
\hline \multirow{2}{*}{ H2 Series HEV PHEV30 } & Total Energy & $0.0 \%$ & $0.0 \%$ & $-7.3 \%$ & $-14.6 \%$ & $-1.1 \%$ & $-11.6 \%$ & $-34.1 \%$ & $-5.4 \%$ & $-31.0 \%$ & $-50.3 \%$ & $-9.8 \%$ & $-38.4 \%$ & $-52.5 \%$ \\
\hline & Usable Energy & $0.0 \%$ & $0.0 \%$ & $-7.3 \%$ & $-14.6 \%$ & $-1.1 \%$ & $-11.6 \%$ & $-23.1 \%$ & $-5.4 \%$ & $-19.5 \%$ & $-33.8 \%$ & $-9.8 \%$ & $-23.1 \%$ & $-36.7 \%$ \\
\hline \multirow{2}{*}{ H2 Series HEV PHEV 40} & Total Energy & $0.0 \%$ & $0.0 \%$ & $-7.3 \%$ & $-14.9 \%$ & $-0.4 \%$ & $-11.8 \%$ & $-33.1 \%$ & $-5.3 \%$ & $-30.4 \%$ & $-50.3 \%$ & $-9.8 \%$ & $-38.1 \%$ & $-52.4 \%$ \\
\hline & Usable Energy & $0.0 \%$ & $0.0 \%$ & $-7.3 \%$ & $-14.9 \%$ & $-0.4 \%$ & $-11.8 \%$ & $-22.0 \%$ & $-5.3 \%$ & $-18.8 \%$ & $-33.7 \%$ & $-9.8 \%$ & $-22.6 \%$ & $-36.6 \%$ \\
\hline \multirow{2}{*}{ E85 Split HEV } & Total Energy & $0.0 \%$ & $0.0 \%$ & $-4.7 \%$ & $-8.7 \%$ & $-1.6 \%$ & $-8.4 \%$ & $-15.0 \%$ & $-4.1 \%$ & $-15.0 \%$ & $-25.9 \%$ & $-4.1 \%$ & $-15.0 \%$ & $-28.0 \%$ \\
\hline & Usable Energy & & & & & & & & & & & & & \\
\hline \multirow{2}{*}{ E85 Split HEV PHEV10 } & Total Energy & $0.0 \%$ & $0.0 \%$ & $-3.9 \%$ & $-10.3 \%$ & $1.0 \%$ & $-7.3 \%$ & $-27.9 \%$ & $-1.6 \%$ & $-26.5 \%$ & $-46.5 \%$ & $-4.1 \%$ & $-32.9 \%$ & $-49.5 \%$ \\
\hline & Usable Energy & $0.0 \%$ & $0.0 \%$ & $-3.9 \%$ & $-10.3 \%$ & $1.0 \%$ & $-7.3 \%$ & $-15.9 \%$ & $-1.6 \%$ & $-14.2 \%$ & $-28.6 \%$ & $-4.1 \%$ & $-16.1 \%$ & $-32.6 \%$ \\
\hline \multirow{2}{*}{ E85 Split HEV PHEV20 } & Total Energy & $0.0 \%$ & $0.0 \%$ & $-6.1 \%$ & $-12.8 \%$ & $-0.3 \%$ & $-9.9 \%$ & $-29.8 \%$ & $-4.0 \%$ & $-27.4 \%$ & $-47.8 \%$ & $-6.4 \%$ & $-33.7 \%$ & $-50.5 \%$ \\
\hline & Usable Energy & $0.0 \%$ & $0.0 \%$ & $-6.1 \%$ & $-12.8 \%$ & $-0.3 \%$ & $-9.9 \%$ & $-18.1 \%$ & $-4.0 \%$ & $-15.3 \%$ & $-30.4 \%$ & $-6.4 \%$ & $-17.2 \%$ & $-34.0 \%$ \\
\hline \multirow{2}{*}{ E85 Series HEV PHEV30 } & Total Energy & $0.0 \%$ & $0.0 \%$ & $-5.5 \%$ & $-12.2 \%$ & $1.4 \%$ & $-9.1 \%$ & $-31.7 \%$ & $-4.3 \%$ & $-28.9 \%$ & $-47.6 \%$ & $-7.0 \%$ & $-36.2 \%$ & $-50.6 \%$ \\
\hline & Usable Energy & $0.0 \%$ & $0.0 \%$ & $-5.5 \%$ & $-12.2 \%$ & $1.4 \%$ & $-9.1 \%$ & $-20.3 \%$ & $-4.3 \%$ & $-17.0 \%$ & $-30.2 \%$ & $-7.0 \%$ & $-20.3 \%$ & $-34.2 \%$ \\
\hline \multirow{2}{*}{ E85 Series HEV PHEV40 } & Total Energy & $0.0 \%$ & $0.0 \%$ & $-5.7 \%$ & $-12.6 \%$ & $0.1 \%$ & $-9.5 \%$ & $-30.8 \%$ & $-4.3 \%$ & $-28.4 \%$ & $-48.2 \%$ & $-6.6 \%$ & $-36.1 \%$ & $-50.7 \%$ \\
\hline & Usable Energy & $0.0 \%$ & $0.0 \%$ & $-5.7 \%$ & $-12.6 \%$ & $0.1 \%$ & $-9.5 \%$ & $-19.3 \%$ & $-4.3 \%$ & $-16.5 \%$ & $-31.0 \%$ & $-6.6 \%$ & $-20.1 \%$ & $-34.3 \%$ \\
\hline \multirow{2}{*}{ FC HEV } & Total Energy & $0.0 \%$ & $0.0 \%$ & $-12.2 \%$ & $-17.7 \%$ & $-9.1 \%$ & $-10.5 \%$ & $-17.3 \%$ & $-5.4 \%$ & $-15.6 \%$ & $-25.7 \%$ & $-8.8 \%$ & $-18.9 \%$ & $-29.1 \%$ \\
\hline & Usable Energy & & & & & & & & & & & & & \\
\hline \multirow{2}{*}{ FC HEV PHEV10 } & Total Energy & $0.0 \%$ & $0.0 \%$ & $-12.0 \%$ & $-20.7 \%$ & $-5.7 \%$ & $-17.8 \%$ & $-37.0 \%$ & $-11.4 \%$ & $-34.8 \%$ & $-53.1 \%$ & $-16.1 \%$ & $-42.4 \%$ & $-55.8 \%$ \\
\hline & Usable Energy & $0.0 \%$ & $0.0 \%$ & $-12.0 \%$ & $-20.7 \%$ & $-5.7 \%$ & $-17.8 \%$ & $-26.5 \%$ & $-11.4 \%$ & $-23.9 \%$ & $-37.5 \%$ & $-16.1 \%$ & $-28.0 \%$ & $-41.1 \%$ \\
\hline \multirow{2}{*}{ FC HEV PHEV20 } & Total Energy & $0.0 \%$ & $0.0 \%$ & $-11.2 \%$ & $-20.9 \%$ & $-4.5 \%$ & $-18.0 \%$ & $-36.7 \%$ & $-10.9 \%$ & $-34.4 \%$ & $-54.6 \%$ & $-15.7 \%$ & $-41.0 \%$ & $-56.5 \%$ \\
\hline & Usable Energy & $0.0 \%$ & $0.0 \%$ & $-11.2 \%$ & $-20.9 \%$ & $-4.5 \%$ & $-18.0 \%$ & $-26.1 \%$ & $-10.9 \%$ & $-23.5 \%$ & $-39.4 \%$ & $-15.7 \%$ & $-26.2 \%$ & $-42.0 \%$ \\
\hline \multirow{2}{*}{ FC HEV PHEV 30} & Total Energy & $0.0 \%$ & $0.0 \%$ & $-11.0 \%$ & $-19.1 \%$ & $-4.4 \%$ & $-16.7 \%$ & $-38.5 \%$ & $-10.6 \%$ & $-36.1 \%$ & $-54.8 \%$ & $-15.4 \%$ & $-42.9 \%$ & $-57.3 \%$ \\
\hline & Usable Energy & $0.0 \%$ & $0.0 \%$ & $-11.0 \%$ & $-19.1 \%$ & $-4.4 \%$ & $-16.7 \%$ & $-28.3 \%$ & $-10.6 \%$ & $-25.5 \%$ & $-39.7 \%$ & $-15.4 \%$ & $-28.7 \%$ & $-43.0 \%$ \\
\hline \multirow{2}{*}{ FC HEV PHEV 40} & Total Energy & $0.0 \%$ & $0.0 \%$ & $-11.2 \%$ & $-19.5 \%$ & $-4.2 \%$ & $-17.2 \%$ & $-39.1 \%$ & $-10.9 \%$ & $-37.0 \%$ & $-54.1 \%$ & $-16.0 \%$ & $-44.1 \%$ & $-56.8 \%$ \\
\hline & Usable Energy & $0.0 \%$ & $0.0 \%$ & $-11.2 \%$ & $-19.5 \%$ & $-4.2 \%$ & $-17.2 \%$ & $-28.9 \%$ & $-10.9 \%$ & $-26.4 \%$ & $-38.7 \%$ & $-16.0 \%$ & $-30.1 \%$ & $-42.3 \%$ \\
\hline \multirow{2}{*}{ EV } & Total Energy & $0.0 \%$ & $0.0 \%$ & $-5.9 \%$ & $-13.5 \%$ & $0.6 \%$ & $-10.1 \%$ & $-35.9 \%$ & $-4.7 \%$ & $-33.5 \%$ & $-53.3 \%$ & $-8.2 \%$ & $-40.6 \%$ & $-56.1 \%$ \\
\hline & Usable Energy & $0.0 \%$ & $0.0 \%$ & $-5.9 \%$ & $-13.5 \%$ & $0.6 \%$ & $-10.1 \%$ & $-25.2 \%$ & $-4.7 \%$ & $-22.4 \%$ & $-37.8 \%$ & $-8.2 \%$ & $-25.7 \%$ & $-41.5 \%$ \\
\hline
\end{tabular}


Curb weight

\begin{tabular}{|c|c|c|c|c|c|c|c|c|c|c|c|c|c|c|}
\hline & & \multicolumn{13}{|c|}{ Compact } \\
\hline & & 2010 & \multicolumn{3}{|c|}{2010} & \multicolumn{3}{|c|}{2015} & \multicolumn{3}{|c|}{2030} & \multicolumn{3}{|c|}{2045} \\
\hline & & Ref & low & avg & high & low & avg & high & low & avg & high & low & avg & high \\
\hline \multirow{3}{*}{ SI Conv } & Vehicle Mass & 1238 & 1238 & 1198 & 1155 & 1237 & 1167 & 1093 & 1229 & 1104 & 959 & 1230 & 1094 & 919 \\
\hline & $\%$ of Glider mass & 65.0 & 65.0 & 64.1 & 63.1 & 65.1 & 63.6 & 61.4 & 65.2 & 61.7 & 56.3 & 65.2 & 61.3 & 54.3 \\
\hline & $\%$ of Powertrain mass & 35.0 & 35.0 & 35.9 & 36.9 & 34.9 & 36.4 & 38.6 & 34.8 & 38.3 & 43.7 & 34.8 & 38.7 & 45.7 \\
\hline \multirow{3}{*}{$\mathrm{Cl}$ Conv } & Vehicle Mass & 1301 & 1301 & 1262 & 1221 & 1301 & 1235 & 1159 & 1296 & 1170 & 1026 & 1296 & 1159 & 983 \\
\hline & $\%$ of Glider mass & 61.9 & 61.9 & 60.8 & 59.7 & 61.9 & 60.1 & 57.9 & 61.9 & 58.2 & 52.6 & 61.9 & 57.8 & 50.7 \\
\hline & $\%$ of Powertrain mass & 38.1 & 38.1 & 39.2 & 40.3 & 38.1 & 39.9 & 42.1 & 38.1 & 41.8 & 47.4 & 38.1 & 42.2 & 49.3 \\
\hline \multirow{3}{*}{ H2 Conv } & Vehicle Mass & 1268 & 1268 & 1189 & 1131 & 1253 & 1146 & 1057 & 1240 & 1076 & 908 & 1204 & 1057 & 875 \\
\hline & $\%$ of Glider mass & 63.5 & 63.5 & 64.6 & 64.4 & 64.3 & 64.8 & 63.5 & 64.6 & 63.3 & 59.4 & 66.6 & 63.4 & 57.0 \\
\hline & $\%$ of Powertrain mass & 36.5 & 36.5 & 35.4 & 35.6 & 35.7 & 35.2 & 36.5 & 35.4 & 36.7 & 40.6 & 33.4 & 36.6 & 43.0 \\
\hline \multirow{3}{*}{ E85 Conv } & Vehicle Mass & 1234 & 1234 & 1194 & 1151 & 1234 & 1166 & 1090 & 1228 & 1101 & 955 & 1228 & 1091 & 913 \\
\hline & $\%$ of Glider mass & 65.2 & 65.2 & 64.3 & 63.3 & 65.3 & 63.7 & 61.6 & 65.3 & 61.8 & 56.5 & 65.3 & 61.4 & 54.6 \\
\hline & $\%$ of Powertrain mass & 34.8 & 34.8 & 35.7 & 36.7 & 34.7 & 36.3 & 38.4 & 34.7 & 38.2 & 43.5 & 34.7 & 38.6 & 45.4 \\
\hline \multirow{3}{*}{ SI Split HEV } & Vehicle Mass & 1341 & 1341 & 1279 & 1221 & 1325 & 1252 & 1149 & 1316 & 1169 & 990 & 1308 & 1150 & 943 \\
\hline & $\%$ of Glider mass & 60.0 & 60.0 & 60.0 & 59.7 & 60.8 & 59.3 & 58.4 & 60.9 & 58.2 & 54.5 & 61.3 & 58.3 & 52.9 \\
\hline & $\%$ of Powertrain mass & 40.0 & 40.0 & 40.0 & 40.3 & 39.2 & 40.7 & 41.6 & 39.1 & 41.8 & 45.5 & 38.7 & 41.7 & 47.1 \\
\hline \multirow{3}{*}{ SI Split HEV PHEV10 } & Vehicle Mass & 1351 & 1351 & 1288 & 1230 & 1330 & 1250 & 1147 & 1315 & 1168 & 988 & 1307 & 1147 & 941 \\
\hline & $\%$ of Glider mass & 59.6 & 59.6 & 59.6 & 59.3 & 60.6 & 59.4 & 58.5 & 61.0 & 58.3 & 54.6 & 61.3 & 58.4 & 53.0 \\
\hline & $\%$ of Powertrain mass & 40.4 & 40.4 & 40.4 & 40.7 & 39.4 & 40.6 & 41.5 & 39.0 & 41.7 & 45.4 & 38.7 & 41.6 & 47.0 \\
\hline \multirow{3}{*}{ SI Split HEV PHEV20 } & Vehicle Mass & 1377 & 1377 & 1315 & 1253 & 1357 & 1273 & 1164 & 1340 & 1187 & 999 & 1331 & 1162 & 952 \\
\hline & $\%$ of Glider mass & 58.4 & 58.4 & 58.4 & 58.2 & 59.4 & 58.3 & 57.7 & 59.8 & 57.4 & 54.0 & 60.2 & 57.7 & 52.4 \\
\hline & $\%$ of Powertrain mass & 41.6 & 41.6 & 41.6 & 41.8 & 40.6 & 41.7 & 42.3 & 40.2 & 42.6 & 46.0 & 39.8 & 42.3 & 47.6 \\
\hline \multirow{3}{*}{ SI Series HEV PHEV30 } & Vehicle Mass & 1581 & 1581 & 1493 & 1407 & 1542 & 1438 & 1286 & 1510 & 1320 & 1094 & 1487 & 1284 & 1040 \\
\hline & $\%$ of Glider mass & 50.9 & 50.9 & 51.4 & 51.8 & 52.3 & 51.6 & 52.2 & 53.1 & 51.6 & 49.3 & 53.9 & 52.2 & 47.9 \\
\hline & $\%$ of Powertrain mass & 49.1 & 49.1 & 48.6 & 48.2 & 47.7 & 48.4 & 47.8 & 46.9 & 48.4 & 50.7 & 46.1 & 47.8 & 52.1 \\
\hline \multirow{3}{*}{ SI Series HEV PHEV40 } & Vehicle Mass & 1624 & 1624 & 1529 & 1442 & 1584 & 1476 & 1313 & 1547 & 1349 & 1113 & 1523 & 1308 & 1057 \\
\hline & $\%$ of Glider mass & 49.6 & 49.6 & 50.2 & 50.5 & 50.9 & 50.3 & 51.1 & 51.8 & 50.5 & 48.5 & 52.6 & 51.3 & 47.2 \\
\hline & $\%$ of Powertrain mass & 50.4 & 50.4 & 49.8 & 49.5 & 49.1 & 49.7 & 48.9 & 48.2 & 49.5 & 51.5 & 47.4 & 48.7 & 52.8 \\
\hline \multirow{3}{*}{ CI Split HEV } & Vehicle Mass & 1442 & 1442 & 1378 & 1319 & 1416 & 1348 & 1246 & 1414 & 1266 & 1083 & 1405 & 1244 & 1036 \\
\hline & $\%$ of Glider mass & 55.8 & 55.8 & 55.7 & 55.3 & 56.9 & 55.1 & 53.9 & 56.7 & 53.8 & 49.8 & 57.1 & 53.9 & 48.1 \\
\hline & $\%$ of Powertrain mass & 44.2 & 44.2 & 44.3 & 44.7 & 43.1 & 44.9 & 46.1 & 43.3 & 46.2 & 50.2 & 42.9 & 46.1 & 51.9 \\
\hline
\end{tabular}




\begin{tabular}{|c|c|c|c|c|c|c|c|c|c|c|c|c|c|c|}
\hline & & \multicolumn{13}{|c|}{ Compact } \\
\hline & & \multirow{2}{*}{$\begin{array}{c}2010 \\
\operatorname{Ref} \\
\end{array}$} & \multicolumn{3}{|c|}{2010} & \multicolumn{3}{|c|}{2015} & \multicolumn{3}{|c|}{2030} & \multicolumn{3}{|c|}{2045} \\
\hline & & & low & avg & high & low & avg & high & low & avg & high & low & avg & high \\
\hline \multirow{3}{*}{ CI Split HEV PHEV10 } & Vehicle Mass & 1457 & 1457 & 1394 & 1336 & 1437 & 1357 & 1248 & 1422 & 1270 & 1087 & 1411 & 1250 & 1039 \\
\hline & $\%$ of Glider mass & 55.2 & 55.2 & 55.1 & 54.6 & 56.1 & 54.7 & 53.8 & 56.4 & 53.6 & 49.6 & 56.8 & 53.6 & 48.0 \\
\hline & $\%$ of Powertrain mass & 44.8 & 44.8 & 44.9 & 45.4 & 43.9 & 45.3 & 46.2 & 43.6 & 46.4 & 50.4 & 43.2 & 46.4 & 52.0 \\
\hline \multirow{3}{*}{ CI Split HEV PHEV 20} & Vehicle Mass & 1486 & 1486 & 1424 & 1360 & 1465 & 1384 & 1268 & 1449 & 1291 & 1100 & 1437 & 1267 & 1053 \\
\hline & $\%$ of Glider mass & 54.2 & 54.2 & 53.9 & 53.6 & 55.0 & 53.6 & 52.9 & 55.3 & 52.7 & 49.1 & 55.8 & 52.9 & 47.3 \\
\hline & $\%$ of Powertrain mass & 45.8 & 45.8 & 46.1 & 46.4 & 45.0 & 46.4 & 47.1 & 44.7 & 47.3 & 50.9 & 44.2 & 47.1 & 52.7 \\
\hline \multirow{3}{*}{ CI Series HEV PHEV30 } & Vehicle Mass & 1677 & 1677 & 1587 & 1505 & 1638 & 1537 & 1382 & 1605 & 1417 & 1190 & 1582 & 1381 & 1137 \\
\hline & $\%$ of Glider mass & 48.0 & 48.0 & 48.4 & 48.4 & 49.2 & 48.3 & 48.6 & 49.9 & 48.0 & 45.4 & 50.7 & 48.5 & 43.8 \\
\hline & $\%$ of Powertrain mass & 52.0 & 52.0 & 51.6 & 51.6 & 50.8 & 51.7 & 51.4 & 50.1 & 52.0 & 54.6 & 49.3 & 51.5 & 56.2 \\
\hline \multirow{3}{*}{ CI Series HEV PHEV40 } & Vehicle Mass & 1723 & 1723 & 1628 & 1542 & 1684 & 1574 & 1412 & 1645 & 1444 & 1210 & 1620 & 1405 & 1153 \\
\hline & $\%$ of Glider mass & 46.7 & 46.7 & 47.2 & 47.3 & 47.8 & 47.2 & 47.5 & 48.7 & 47.1 & 44.6 & 49.5 & 47.7 & 43.2 \\
\hline & $\%$ of Powertrain mass & 53.3 & 53.3 & 52.8 & 52.7 & 52.2 & 52.8 & 52.5 & 51.3 & 52.9 & 55.4 & 50.5 & 52.3 & 56.8 \\
\hline \multirow{3}{*}{ H2 Split HEV } & Vehicle Mass & 1394 & 1394 & 1291 & 1215 & 1360 & 1245 & 1127 & 1343 & 1156 & 956 & 1297 & 1129 & 919 \\
\hline & $\%$ of Glider mass & 57.7 & 57.7 & 59.5 & 60.0 & 59.2 & 59.6 & 59.6 & 59.7 & 58.9 & 56.5 & 61.8 & 59.4 & 54.3 \\
\hline & $\%$ of Powertrain mass & 42.3 & 42.3 & 40.5 & 40.0 & 40.8 & 40.4 & 40.4 & 40.3 & 41.1 & 43.5 & 38.2 & 40.6 & 45.7 \\
\hline \multirow{3}{*}{ H2 Split HEV PHEV10 } & Vehicle Mass & 1407 & 1407 & 1304 & 1227 & 1374 & 1247 & 1127 & 1346 & 1158 & 955 & 1298 & 1131 & 918 \\
\hline & $\%$ of Glider mass & 57.2 & 57.2 & 58.9 & 59.4 & 58.6 & 59.5 & 59.6 & 59.6 & 58.8 & 56.5 & 61.8 & 59.3 & 54.3 \\
\hline & $\%$ of Powertrain mass & 42.8 & 42.8 & 41.1 & 40.6 & 41.4 & 40.5 & 40.4 & 40.4 & 41.2 & 43.5 & 38.2 & 40.7 & 45.7 \\
\hline \multirow{3}{*}{ H2 Split HEV PHEV2O } & Vehicle Mass & 1435 & 1435 & 1329 & 1249 & 1402 & 1273 & 1144 & 1375 & 1176 & 967 & 1325 & 1146 & 928 \\
\hline & $\%$ of Glider mass & 56.1 & 56.1 & 57.8 & 58.4 & 57.5 & 58.3 & 58.7 & 58.3 & 57.9 & 55.8 & 60.5 & 58.5 & 53.7 \\
\hline & $\%$ of Powertrain mass & 43.9 & 43.9 & 42.2 & 41.6 & 42.5 & 41.7 & 41.3 & 41.7 & 42.1 & 44.2 & 39.5 & 41.5 & 46.3 \\
\hline \multirow{3}{*}{ H2 Series HEV PHEV3O } & Vehicle Mass & 1632 & 1632 & 1498 & 1396 & 1576 & 1427 & 1261 & 1532 & 1303 & 1056 & 1470 & 1258 & 1012 \\
\hline & $\%$ of Glider mass & 49.3 & 49.3 & 51.3 & 52.2 & 51.1 & 52.0 & 53.2 & 52.3 & 52.2 & 51.1 & 54.5 & 53.3 & 49.3 \\
\hline & $\%$ of Powertrain mass & 50.7 & 50.7 & 48.7 & 47.8 & 48.9 & 48.0 & 46.8 & 47.7 & 47.8 & 48.9 & 45.5 & 46.7 & 50.7 \\
\hline \multirow{3}{*}{ H2 Series HEV PHEV 40} & Vehicle Mass & 1675 & 1675 & 1536 & 1429 & 1618 & 1463 & 1288 & 1573 & 1331 & 1074 & 1505 & 1281 & 1029 \\
\hline & $\%$ of Glider mass & 48.0 & 48.0 & 50.0 & 51.0 & 49.8 & 50.7 & 52.1 & 51.0 & 51.1 & 50.2 & 53.3 & 52.3 & 48.5 \\
\hline & $\%$ of Powertrain mass & 52.0 & 52.0 & 50.0 & 49.0 & 50.2 & 49.3 & 47.9 & 49.0 & 48.9 & 49.8 & 46.7 & 47.7 & 51.5 \\
\hline \multirow{3}{*}{ E85 Split HEV } & Vehicle Mass & 1342 & 1342 & 1281 & 1226 & 1324 & 1257 & 1151 & 1318 & 1170 & 991 & 1309 & 1152 & 945 \\
\hline & $\%$ of Glider mass & 60.0 & 60.0 & 59.9 & 59.4 & 60.9 & 59.1 & 58.3 & 60.8 & 58.2 & 54.5 & 61.2 & 58.2 & 52.8 \\
\hline & $\%$ of Powertrain mass & 40.0 & 40.0 & 40.1 & 40.6 & 39.1 & 40.9 & 41.7 & 39.2 & 41.8 & 45.5 & 38.8 & 41.8 & 47.2 \\
\hline
\end{tabular}




\begin{tabular}{|c|c|c|c|c|c|c|c|c|c|c|c|c|c|c|}
\hline & & \multicolumn{13}{|c|}{ Compact } \\
\hline & & \multirow{2}{*}{$\frac{2010}{\operatorname{Ref}}$} & \multicolumn{3}{|c|}{2010} & \multicolumn{3}{|c|}{2015} & \multicolumn{3}{|c|}{2030} & \multicolumn{3}{|c|}{2045} \\
\hline & & & low & avg & high & low & avg & high & low & avg & high & low & avg & high \\
\hline \multirow{3}{*}{ E85 Split HEV PHEV10 } & Vehicle Mass & 1352 & 1352 & 1289 & 1231 & 1331 & 1252 & 1149 & 1316 & 1169 & 989 & 1308 & 1149 & 942 \\
\hline & $\%$ of Glider mass & 59.5 & 59.5 & 59.6 & 59.2 & 60.5 & 59.3 & 58.4 & 60.9 & 58.2 & 54.6 & 61.3 & 58.3 & 52.9 \\
\hline & $\%$ of Powertrain mass & 40.5 & 40.5 & 40.4 & 40.8 & 39.5 & 40.7 & 41.6 & 39.1 & 41.8 & 45.4 & 38.7 & 41.7 & 47.1 \\
\hline \multirow{3}{*}{ E85 Split HEV PHEV20 } & Vehicle Mass & 1378 & 1378 & 1316 & 1254 & 1358 & 1275 & 1166 & 1341 & 1188 & 1000 & 1332 & 1164 & 953 \\
\hline & $\%$ of Glider mass & 58.4 & 58.4 & 58.3 & 58.1 & 59.3 & 58.2 & 57.6 & 59.8 & 57.3 & 54.0 & 60.2 & 57.6 & 52.3 \\
\hline & $\%$ of Powertrain mass & 41.6 & 41.6 & 41.7 & 41.9 & 40.7 & 41.8 & 42.4 & 40.2 & 42.7 & 46.0 & 39.8 & 42.4 & 47.7 \\
\hline \multirow{3}{*}{ E85 Series HEV PHEV30 } & Vehicle Mass & 1582 & 1582 & 1494 & 1408 & 1543 & 1440 & 1288 & 1511 & 1321 & 1095 & 1488 & 1286 & 1042 \\
\hline & $\%$ of Glider mass & 50.9 & 50.9 & 51.4 & 51.8 & 52.2 & 51.6 & 52.1 & 53.1 & 51.5 & 49.3 & 53.9 & 52.1 & 47.8 \\
\hline & $\%$ of Powertrain mass & 49.1 & 49.1 & 48.6 & 48.2 & 47.8 & 48.4 & 47.9 & 46.9 & 48.5 & 50.7 & 46.1 & 47.9 & 52.2 \\
\hline \multirow{3}{*}{ E85 Series HEV PHEV40 } & Vehicle Mass & 1626 & 1626 & 1530 & 1443 & 1585 & 1476 & 1316 & 1548 & 1350 & 1114 & 1525 & 1310 & 1058 \\
\hline & $\%$ of Glider mass & 49.5 & 49.5 & 50.2 & 50.5 & 50.8 & 50.3 & 51.0 & 51.8 & 50.4 & 48.4 & 52.6 & 51.2 & 47.1 \\
\hline & $\%$ of Powertrain mass & 50.5 & 50.5 & 49.8 & 49.5 & 49.2 & 49.7 & 49.0 & 48.2 & 49.6 & 51.6 & 47.4 & 48.8 & 52.9 \\
\hline \multirow{3}{*}{ FC HEV } & Vehicle Mass & 1674 & 1674 & 1412 & 1274 & 1490 & 1291 & 1132 & 1395 & 1158 & 940 & 1314 & 1116 & 890 \\
\hline & $\%$ of Glider mass & 48.1 & 48.1 & 54.4 & 57.2 & 54.1 & 57.5 & 59.3 & 57.5 & 58.8 & 57.4 & 61.0 & 60.1 & 56.0 \\
\hline & $\%$ of Powertrain mass & 51.9 & 51.9 & 45.6 & 42.8 & 45.9 & 42.5 & 40.7 & 42.5 & 41.2 & 42.6 & 39.0 & 39.9 & 44.0 \\
\hline \multirow{3}{*}{ FC HEV PHEV10 } & Vehicle Mass & 1599 & 1599 & 1376 & 1249 & 1454 & 1264 & 1111 & 1370 & 1140 & 925 & 1295 & 1102 & 879 \\
\hline & $\%$ of Glider mass & 50.3 & 50.3 & 55.8 & 58.4 & 55.4 & 58.7 & 60.4 & 58.5 & 59.7 & 58.3 & 61.9 & 60.8 & 56.7 \\
\hline & $\%$ of Powertrain mass & 49.7 & 49.7 & 44.2 & 41.6 & 44.6 & 41.3 & 39.6 & 41.5 & 40.3 & 41.7 & 38.1 & 39.2 & 43.3 \\
\hline \multirow{3}{*}{ FC HEV PHEV 20} & Vehicle Mass & 1637 & 1637 & 1409 & 1278 & 1491 & 1293 & 1134 & 1401 & 1160 & 937 & 1322 & 1120 & 890 \\
\hline & $\%$ of Glider mass & 49.2 & 49.2 & 54.5 & 57.0 & 54.0 & 57.4 & 59.2 & 57.2 & 58.7 & 57.6 & 60.6 & 59.9 & 56.0 \\
\hline & $\%$ of Powertrain mass & 50.8 & 50.8 & 45.5 & 43.0 & 46.0 & 42.6 & 40.8 & 42.8 & 41.3 & 42.4 & 39.4 & 40.1 & 44.0 \\
\hline \multirow{3}{*}{ FC HEV PHEV 30} & Vehicle Mass & 1725 & 1725 & 1496 & 1364 & 1583 & 1383 & 1206 & 1496 & 1241 & 999 & 1408 & 1187 & 947 \\
\hline & $\%$ of Glider mass & 46.7 & 46.7 & 51.3 & 53.4 & 50.9 & 53.7 & 55.7 & 53.6 & 54.9 & 54.0 & 56.9 & 56.5 & 52.6 \\
\hline & $\%$ of Powertrain mass & 53.3 & 53.3 & 48.7 & 46.6 & 49.1 & 46.3 & 44.3 & 46.4 & 45.1 & 46.0 & 43.1 & 43.5 & 47.4 \\
\hline \multirow{3}{*}{ FC HEV PHEV40 } & Vehicle Mass & 1770 & 1770 & 1533 & 1396 & 1627 & 1415 & 1230 & 1531 & 1265 & 1016 & 1439 & 1209 & 963 \\
\hline & $\%$ of Glider mass & 45.5 & 45.5 & 50.1 & 52.2 & 49.5 & 52.5 & 54.6 & 52.4 & 53.8 & 53.1 & 55.7 & 55.5 & 51.8 \\
\hline & $\%$ of Powertrain mass & 54.5 & 54.5 & 49.9 & 47.8 & 50.5 & 47.5 & 45.4 & 47.6 & 46.2 & 46.9 & 44.3 & 44.5 & 48.2 \\
\hline \multirow{3}{*}{ BEV } & Vehicle Mass & 1756 & 1756 & 1650 & 1539 & 1731 & 1587 & 1341 & 1677 & 1377 & 1090 & 1631 & 1309 & 1022 \\
\hline & $\%$ of Glider mass & 45.8 & 45.8 & 46.5 & 47.4 & 46.5 & 46.8 & 50.1 & 47.8 & 49.4 & 49.5 & 49.1 & 51.2 & 48.8 \\
\hline & $\%$ of Powertrain mass & 54.2 & 54.2 & 53.5 & 52.6 & 53.5 & 53.2 & 49.9 & 52.2 & 50.6 & 50.5 & 50.9 & 48.8 & 51.2 \\
\hline
\end{tabular}


Percentage of vehicle curbs weight reduction based on the respective 2010 reference case

\begin{tabular}{|c|c|c|c|c|c|c|c|c|c|c|c|c|c|c|}
\hline & & \multicolumn{13}{|c|}{ Compact } \\
\hline & & 2010 & & 2010 & & & 2015 & & & 2030 & & & 2045 & \\
\hline & & Ref & low & avg & high & low & avg & high & low & avg & high & low & avg & high \\
\hline SI Conv & Vehicle Mass & $0.00 \%$ & $0.00 \%$ & $-3.23 \%$ & $-6.70 \%$ & $-0.08 \%$ & $-5.74 \%$ & $-11.71 \%$ & $-0.73 \%$ & $-10.82 \%$ & $-22.54 \%$ & $-0.65 \%$ & $-11.63 \%$ & $-25.77 \%$ \\
\hline $\mathrm{Cl}$ Conv & Vehicle Mass & $0.00 \%$ & $0.00 \%$ & $-3.00 \%$ & $-6.15 \%$ & $0.00 \%$ & $-5.07 \%$ & $-10.91 \%$ & $-0.38 \%$ & $-10.07 \%$ & $-21.14 \%$ & $-0.38 \%$ & $-10.91 \%$ & $-24.44 \%$ \\
\hline H2 Conv & Vehicle Mass & $0.00 \%$ & $0.00 \%$ & $-6.23 \%$ & $-10.80 \%$ & $-1.18 \%$ & $-9.62 \%$ & $-16.64 \%$ & $-2.21 \%$ & $-15.14 \%$ & $-28.39 \%$ & $-5.05 \%$ & $-16.64 \%$ & $-30.99 \%$ \\
\hline E85 Conv & Vehicle Mass & $0.00 \%$ & $0.00 \%$ & $-3.24 \%$ & $-6.73 \%$ & $0.00 \%$ & $-5.51 \%$ & $-11.67 \%$ & $-0.49 \%$ & $-10.78 \%$ & $-22.61 \%$ & $-0.49 \%$ & $-11.59 \%$ & $-26.01 \%$ \\
\hline SI Split HEV & Vehicle Mass & $0.00 \%$ & $0.00 \%$ & $-4.62 \%$ & $-8.95 \%$ & $-1.19 \%$ & $-6.64 \%$ & $-14.32 \%$ & $-1.86 \%$ & $-12.83 \%$ & $-26.17 \%$ & $-2.46 \%$ & $-14.24 \%$ & $-29.68 \%$ \\
\hline SI Split HEV PHEV10 & Vehicle Mass & $0.00 \%$ & $0.00 \%$ & $-4.66 \%$ & $-8.96 \%$ & $-1.55 \%$ & $-7.48 \%$ & $-15.10 \%$ & $-2.66 \%$ & $-13.55 \%$ & $-26.87 \%$ & $-3.26 \%$ & $-15.10 \%$ & $-30.35 \%$ \\
\hline SI Split HEV PHEV20 & Vehicle Mass & $0.00 \%$ & $0.00 \%$ & $-4.50 \%$ & $-9.01 \%$ & $-1.45 \%$ & $-7.55 \%$ & $-15.47 \%$ & $-2.69 \%$ & $-13.80 \%$ & $-27.45 \%$ & $-3.34 \%$ & $-15.61 \%$ & $-30.86 \%$ \\
\hline SI Series HEV PHEV30 & Vehicle Mass & $0.00 \%$ & $0.00 \%$ & $-5.57 \%$ & $-11.01 \%$ & $-2.47 \%$ & $-9.04 \%$ & $-18.66 \%$ & $-4.49 \%$ & $-16.51 \%$ & $-30.80 \%$ & $-5.95 \%$ & $-18.79 \%$ & $-34.22 \%$ \\
\hline SI Series HEV PHEV40 & Vehicle Mass & $0.00 \%$ & $0.00 \%$ & $-5.85 \%$ & $-11.21 \%$ & $-2.46 \%$ & $-9.11 \%$ & $-19.15 \%$ & $-4.74 \%$ & $-16.93 \%$ & $-31.47 \%$ & $-6.22 \%$ & $-19.46 \%$ & $-34.91 \%$ \\
\hline $\mathrm{Cl}$ Split HEV & Vehicle Mass & $0.00 \%$ & $0.00 \%$ & $-4.44 \%$ & $-8.53 \%$ & $-1.80 \%$ & $-6.52 \%$ & $-13.59 \%$ & $-1.94 \%$ & $-12.21 \%$ & $-24.90 \%$ & $-2.57 \%$ & $-13.73 \%$ & $-28.16 \%$ \\
\hline $\mathrm{Cl}$ Split HEV PH & Vehicle Mass & $00 \%$ & $00 \%$ & $-4.32 \%$ & $-8.30 \%$ & $-1.37 \%$ & $-6.86 \%$ & $-14.34 \%$ & $-2.40 \%$ & $-12.83 \%$ & $-25.39 \%$ & $-3.16 \%$ & $-14.21 \%$ & $-28.69 \%$ \\
\hline CI Split HEV PHEV2O & Vehicle Mass & $0.00 \%$ & $0.00 \%$ & $-4.17 \%$ & $-8.48 \%$ & $-1.41 \%$ & $-6.86 \%$ & $-14.67 \%$ & $-2.49 \%$ & $-13.12 \%$ & $-25.98 \%$ & $-3.30 \%$ & $-14.74 \%$ & $-29.14 \%$ \\
\hline CI Series HEV PHEV 30 & Vehicle Mass & $0.00 \%$ & $0.00 \%$ & $-5.37 \%$ & $-10.26 \%$ & $-2.33 \%$ & $-8.35 \%$ & $-17.59 \%$ & $-4.29 \%$ & $-15.50 \%$ & $-29.04 \%$ & $-5.66 \%$ & $-17.65 \%$ & $-32.20 \%$ \\
\hline CI Series HEV PHEV40 & Vehicle Mass & $0.00 \%$ & $0.00 \%$ & $-5.51 \%$ & $-10.50 \%$ & $-2.26 \%$ & $-8.65 \%$ & $-18.05 \%$ & $-4.53 \%$ & $-16.19 \%$ & $-29.77 \%$ & $-5.98 \%$ & $-18.46 \%$ & $-33.08 \%$ \\
\hline H2 Split HEV & Vehicle Mass & $0.00 \%$ & $0.00 \%$ & $-7.39 \%$ & $-12.84 \%$ & $-2.44 \%$ & $-10.69 \%$ & $-19.15 \%$ & $-3.66 \%$ & $-17.07 \%$ & $-31.42 \%$ & $-6.96 \%$ & $-19.01 \%$ & $-34.07 \%$ \\
\hline H2 Split HEV PHEV10 & Vehicle Mass & $0.00 \%$ & $0.00 \%$ & $-7.32 \%$ & $-12.79 \%$ & $-2.35 \%$ & $-11.37 \%$ & $-19.90 \%$ & $-4.34 \%$ & $-17.70 \%$ & $-32.13 \%$ & $-7.75 \%$ & $-19.62 \%$ & $-34.75 \%$ \\
\hline H2 Split HEV PHEV2O & Vehicle Mass & $0.00 \%$ & $0.00 \%$ & $-7.39 \%$ & $-12.96 \%$ & $-2.30 \%$ & $-11.29 \%$ & $-20.28 \%$ & $-4.18 \%$ & $-18.05 \%$ & $-32.61 \%$ & $-7.67 \%$ & $-20.14 \%$ & $-35.33 \%$ \\
\hline H2 Series HEV PHEV 30 & Vehicle Mass & $0.00 \%$ & $0.00 \%$ & $-8.21 \%$ & $-14.46 \%$ & $-3.43 \%$ & $-12.56 \%$ & $-22.73 \%$ & $-6.13 \%$ & $-20.16 \%$ & $-35.29 \%$ & $-9.93 \%$ & $-22.92 \%$ & $-37.99 \%$ \\
\hline H2 Series HEV PHEV40 & Vehicle Mass & $0.00 \%$ & $0.00 \%$ & $-8.30 \%$ & $-14.69 \%$ & $-3.40 \%$ & $-12.66 \%$ & $-23.10 \%$ & $-6.09 \%$ & $-20.54 \%$ & $-35.88 \%$ & $-10.15 \%$ & $-23.52 \%$ & $-38.57 \%$ \\
\hline E85 Split HEV & Vehicle Mass & $0.00 \%$ & $0.00 \%$ & $-4.55 \%$ & $-8.64 \%$ & $-1.34 \%$ & $-6.33 \%$ & $-14.23 \%$ & $-1.79 \%$ & $-12.82 \%$ & $-26.15 \%$ & $-2.46 \%$ & $-14.16 \%$ & $-29.58 \%$ \\
\hline E85 Split HEV PHEV10 & Vehicle Mass & $0.00 \%$ & $0.00 \%$ & $-4.66 \%$ & $-8.95 \%$ & $-1.55 \%$ & $-7.40 \%$ & $-15.01 \%$ & $-2.66 \%$ & $-13.54 \%$ & $-26.85 \%$ & $-3.25 \%$ & $-15.01 \%$ & $-30.33 \%$ \\
\hline E85 Split HEV PHEV20 & Vehicle Mass & $0.00 \%$ & $0.00 \%$ & $-4.50 \%$ & $-9.00 \%$ & $-1.45 \%$ & $-7.47 \%$ & $-15.38 \%$ & $-2.69 \%$ & $-13.79 \%$ & $-27.43 \%$ & $-3.34 \%$ & $-15.53 \%$ & $-30.84 \%$ \\
\hline E85 Series HEV PHEV30 & Vehicle Mass & $0.00 \%$ & $0.00 \%$ & $-5.56 \%$ & $-11.00 \%$ & $-2.47 \%$ & $-8.98 \%$ & $-18.58 \%$ & $-4.49 \%$ & $-16.50 \%$ & $-30.78 \%$ & $-5.94 \%$ & $-18.71 \%$ & $-34.13 \%$ \\
\hline E85 Series HEV PHEV40 & Vehicle Mass & $0.00 \%$ & $0.00 \%$ & $-5.90 \%$ & $-11.25 \%$ & $-2.52 \%$ & $-9.23 \%$ & $-19.07 \%$ & $-4.80 \%$ & $-16.97 \%$ & $-31.49 \%$ & $-6.21 \%$ & $-19.43 \%$ & $-34.93 \%$ \\
\hline FC HEV & Vehicle Mass & $0.00 \%$ & $0.00 \%$ & $-15.65 \%$ & $-23.89 \%$ & $-10.99 \%$ & $-22.88 \%$ & $-32.38 \%$ & $-16.67 \%$ & $-30.82 \%$ & $-43.85 \%$ & $-21.51 \%$ & $-33.33 \%$ & $-46.83 \%$ \\
\hline FC HEV PHEV 10 & Vehicle Mass & $0.00 \%$ & $0.00 \%$ & $-13.95 \%$ & $-21.89 \%$ & $-9.07 \%$ & $-20.95 \%$ & $-30.52 \%$ & $-14.32 \%$ & $-28.71 \%$ & $-42.15 \%$ & $-19.01 \%$ & $-31.08 \%$ & $-45.03 \%$ \\
\hline FC HEV PHEV 20 & Vehicle Mass & $0.00 \%$ & $0.00 \%$ & $-13.93 \%$ & $-21.93 \%$ & $-8.92 \%$ & $-21.01 \%$ & $-30.73 \%$ & $-14.42 \%$ & $-29.14 \%$ & $-42.76 \%$ & $-19.24 \%$ & $-31.58 \%$ & $-45.63 \%$ \\
\hline FC HEV PHEV 30 & Vehicle Mass & $0.00 \%$ & $0.00 \%$ & $-13.28 \%$ & $-20.93 \%$ & $-8.23 \%$ & $-19.83 \%$ & $-30.09 \%$ & $-13.28 \%$ & $-28.06 \%$ & $-42.09 \%$ & $-18.38 \%$ & $-31.19 \%$ & $-45.10 \%$ \\
\hline FC HEV PHEV 40 & Vehicle Mass & $0.00 \%$ & $0.00 \%$ & $-13.39 \%$ & $-21.13 \%$ & $-8.08 \%$ & $-20.06 \%$ & $-30.51 \%$ & $-13.50 \%$ & $-28.53 \%$ & $-42.60 \%$ & $-18.70 \%$ & $-31.69 \%$ & $-45.59 \%$ \\
\hline EV & Vehicle Mass & $0.00 \%$ & $0.00 \%$ & $-6.04 \%$ & $-12.36 \%$ & $-1.42 \%$ & $-9.62 \%$ & $-23.63 \%$ & $-4.50 \%$ & $-21.50 \%$ & & $-7.12 \%$ & $-25.46 \%$ & \\
\hline
\end{tabular}


Argonne National Laboratory Report - Light-Duty Vehicle Fuel Consumption Displacement Potential Up to 2045 


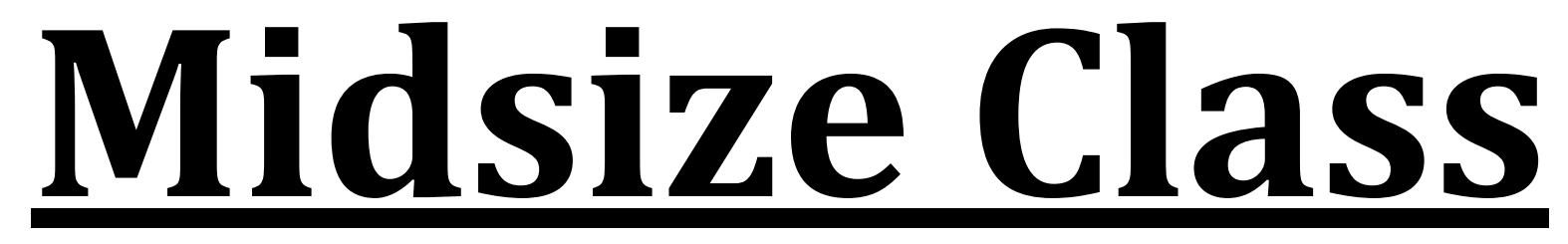




\section{Power}

\begin{tabular}{|c|c|c|c|c|c|c|c|c|c|c|c|c|c|c|}
\hline & & \multicolumn{13}{|c|}{ Midsize } \\
\hline & & \multirow{2}{*}{$\begin{array}{c}2010 \\
\operatorname{Ref}\end{array}$} & \multicolumn{3}{|c|}{2010} & \multicolumn{3}{|c|}{2015} & \multicolumn{3}{|c|}{2030} & \multicolumn{3}{|c|}{2045} \\
\hline & & & low & avg & high & low & avg & high & low & avg & high & low & avg & high \\
\hline \multirow{5}{*}{ SI Conv } & ICE Power & 137815 & 137815 & 132111 & 128234 & 137815 & 128822 & 119229 & 134760 & 123340 & 111006 & 133431 & 120599 & 109636 \\
\hline & Electric Machine & & & & & & & & & & & & & \\
\hline & $\mathrm{MC} 2 / \mathrm{GC}$ & & & & & & & & & & & & & \\
\hline & Battery & & & & & & & & & & & & & \\
\hline & Specific Power & 87 & 87 & 86 & 86 & 87 & 86 & 85 & 85 & 87 & 90 & 85 & 86 & 92 \\
\hline \multirow{5}{*}{ Cl Conv } & ICE Power & 119778 & 119778 & 113667 & 110000 & 118556 & 110000 & 102667 & 116111 & 105111 & 92156 & 113667 & 103889 & 88760 \\
\hline & Electric Machine & & & & & & & & & & & & & \\
\hline & $\mathrm{MC2} / \mathrm{GC}$ & & & & & & & & & & & & & \\
\hline & Battery & & & & & & & & & & & & & \\
\hline & Specific Power & 73 & 73 & 71 & 71 & 72 & 70 & 70 & 71 & 71 & 70 & 69 & 70 & 71 \\
\hline \multirow{5}{*}{ H2 Conv } & ICE Power & 138133 & 138133 & 126933 & 118533 & 134400 & 118533 & 108267 & 129733 & 112000 & 95200 & 124133 & 109200 & 91467 \\
\hline & Electric Machine & & & & & & & & & & & & & \\
\hline & $\mathrm{MC} 2 / \mathrm{GC}$ & & & & & & & & & & & & & \\
\hline & Battery & & & & & & & & & & & & & \\
\hline & Specific Power & 86 & 86 & 84 & 82 & 84 & 81 & 80 & 82 & 81 & 81 & 81 & 80 & 81 \\
\hline \multirow{5}{*}{ E85 Conv } & ICE Power & 131693 & 131693 & 125926 & 121007 & 131693 & 122156 & 112677 & 129106 & 114943 & 102708 & 126781 & 113806 & 100347 \\
\hline & Electric Machine & & & & & & & & & & & & & \\
\hline & $\mathrm{MC} 2 / \mathrm{GC}$ & & & & & & & & & & & & & \\
\hline & Battery & & & & & & & & & & & & & \\
\hline & Specific Power & 83 & 83 & 82 & 82 & 83 & 81 & 80 & 82 & 81 & 83 & 80 & 81 & 85 \\
\hline \multirow{5}{*}{ SI Split HEV } & ICE Power & 91008 & 91008 & 85882 & 81563 & 89218 & 82736 & 76020 & 88952 & 76768 & 64896 & 87946 & 74271 & 61174 \\
\hline & Electric Machine & 65240 & 65240 & 62440 & 59956 & 64223 & 61150 & 57530 & 65434 & 57858 & 49646 & 64944 & 56684 & 47355 \\
\hline & $\mathrm{MC} 2 / \mathrm{GC}$ & 52667 & 52667 & 49700 & 47201 & 51631 & 47880 & 43993 & 51477 & 44426 & 37556 & 50895 & 42981 & 35402 \\
\hline & Battery & 29127 & 29127 & 27930 & 26733 & 28928 & 27317 & 25610 & 29024 & 25610 & 21626 & 29024 & 25610 & 21057 \\
\hline & Specific Power & 70 & 70 & 70 & 69 & 70 & 69 & 69 & 70 & 68 & 68 & 70 & 68 & 68 \\
\hline \multirow{5}{*}{ SI Split HEV PHEV10 } & ICE Power & 64631 & 64631 & 61382 & 58653 & 63980 & 59698 & 54843 & 62874 & 55988 & 48413 & 62136 & 54798 & 46058 \\
\hline & Electric Machine & 72314 & 72314 & 69920 & 66860 & 71860 & 68044 & 61964 & 70824 & 62427 & 53403 & 70807 & 61846 & 51002 \\
\hline & $\mathrm{MC} 2 / \mathrm{GC}$ & 38773 & 38773 & 36823 & 35202 & 38399 & 35829 & 32916 & 37718 & 33603 & 29056 & 37293 & 32889 & 27643 \\
\hline & Battery & 67223 & 67223 & 64809 & 61564 & 66933 & 62677 & 57988 & 65072 & 58218 & 50851 & 65292 & 59605 & 48593 \\
\hline & Specific Power & 77 & 77 & 77 & 77 & 77 & 77 & 77 & 77 & 77 & 78 & 77 & 78 & 78 \\
\hline
\end{tabular}


Argonne National Laboratory Report - Light-Duty Vehicle Fuel Consumption Displacement Potential Up to 2045

\begin{tabular}{|c|c|c|c|c|c|c|c|c|c|c|c|c|c|c|}
\hline & & \multicolumn{13}{|c|}{ Midsize } \\
\hline & & \multirow{2}{*}{$\begin{array}{c}2010 \\
\text { Ref }\end{array}$} & \multicolumn{3}{|c|}{2010} & \multicolumn{3}{|c|}{2015} & \multicolumn{3}{|c|}{2030} & \multicolumn{3}{|c|}{2045} \\
\hline & & & low & avg & high & low & avg & high & low & avg & high & low & avg & high \\
\hline \multirow{5}{*}{ SI Split HEV PHEV20 } & ICE Power & 65572 & 65572 & 62178 & 59389 & 64836 & 60434 & 55374 & 63723 & 56520 & 48744 & 62952 & 55271 & 46389 \\
\hline & Electric Machine & 74399 & 74399 & 71012 & 67898 & 73105 & 68972 & 62819 & 71980 & 63785 & 54081 & 71925 & 62576 & 51639 \\
\hline & $\mathrm{MC} 2 / \mathrm{GC}$ & 39304 & 39304 & 37301 & 35610 & 38896 & 36254 & 33234 & 38194 & 33922 & 29255 & 37749 & 33172 & 27842 \\
\hline & Battery & 69134 & 69134 & 65612 & 62322 & 67895 & 63328 & 57775 & 65929 & 58654 & 50091 & 66108 & 58870 & 47893 \\
\hline & Specific Power & 77 & 77 & 77 & 76 & 77 & 76 & 76 & 76 & 76 & 77 & 76 & 77 & 77 \\
\hline \multirow{5}{*}{ SI Series HEV PHEV30 } & ICE Power & 80287 & 80287 & 74697 & 69998 & 78910 & 71574 & 62585 & 75297 & 64077 & 53237 & 73980 & 62105 & 50623 \\
\hline & Electric Machine & 132818 & 132818 & 125621 & 119742 & 130552 & 122101 & 110604 & 127852 & 112939 & 97759 & 126041 & 110076 & 93564 \\
\hline & $\mathrm{MC} 2 / \mathrm{GC}$ & 77878 & 77878 & 72456 & 67898 & 76543 & 69427 & 60707 & 73038 & 62155 & 51640 & 71761 & 60242 & 49104 \\
\hline & Battery & 163663 & 163663 & 153954 & 145968 & 160896 & 148893 & 133253 & 155944 & 136102 & 116266 & 153752 & 132689 & 111301 \\
\hline & Specific Power & 68 & 68 & 68 & 68 & 68 & 68 & 68 & 68 & 68 & 71 & 68 & 68 & 71 \\
\hline \multirow{5}{*}{ SI Series HEV PHEV40 } & ICE Power & 83068 & 83068 & 77079 & 72256 & 81507 & 73832 & 64159 & 77733 & 65793 & 54370 & 76282 & 63641 & 51673 \\
\hline & Electric Machine & 137016 & 137016 & 129449 & 123298 & 134708 & 125744 & 113195 & 131763 & 115716 & 99672 & 129752 & 112524 & 95228 \\
\hline & $\mathrm{MC} 2 / \mathrm{GC}$ & 80576 & 80576 & 74767 & 70088 & 79062 & 71617 & 62234 & 75401 & 63819 & 52739 & 73994 & 61732 & 50123 \\
\hline & Battery & 168763 & 168763 & 158595 & 150251 & 165949 & 153274 & 136266 & 160652 & 139337 & 118451 & 158219 & 135534 & 113194 \\
\hline & Specific Power & 68 & 68 & 68 & 69 & 68 & 69 & 69 & 68 & 69 & 71 & 68 & 69 & 71 \\
\hline \multirow{5}{*}{ CI Split HEV } & ICE Power & 81462 & 81462 & 77040 & 71786 & 80127 & 74261 & 67570 & 77947 & 68086 & 57933 & 77146 & 67424 & 55937 \\
\hline & Electric Machine & 71396 & 71396 & 68359 & 64608 & 70301 & 66815 & 62055 & 70314 & 62840 & 54383 & 69846 & 62406 & 52687 \\
\hline & $\mathrm{MC} 2 / \mathrm{GC}$ & 68175 & 68175 & 64474 & 60077 & 67058 & 62148 & 56549 & 65233 & 56981 & 48484 & 64563 & 56427 & 46813 \\
\hline & Battery & 31122 & 31122 & 29726 & 28130 & 30723 & 29024 & 26748 & 30731 & 27317 & 23333 & 30731 & 27317 & 22764 \\
\hline & Specific Power & 62 & 62 & 62 & 60 & 62 & 61 & 60 & 61 & 60 & 59 & 61 & 60 & 60 \\
\hline \multirow{5}{*}{ CI Split HEV PHEV10 } & ICE Power & 67826 & 67826 & 64482 & 61712 & 67090 & 62785 & 57753 & 65904 & 58926 & 51116 & 65174 & 57691 & 48844 \\
\hline & Electric Machine & 73559 & 73559 & 70948 & 67961 & 72828 & 69109 & 63099 & 71667 & 64065 & 54646 & 71808 & 62975 & 52302 \\
\hline & $\mathrm{MC} 2 / \mathrm{GC}$ & 56763 & 56763 & 53964 & 51646 & 56147 & 52544 & 48333 & 55155 & 49315 & 42779 & 54544 & 48281 & 40877 \\
\hline & Battery & 76661 & 76661 & 73874 & 70364 & 76227 & 71578 & 66076 & 74200 & 67050 & 57860 & 74425 & 67422 & 55333 \\
\hline & Specific Power & 79 & 79 & 79 & 79 & 80 & 79 & 79 & 79 & 79 & 80 & 79 & 80 & 79 \\
\hline \multirow{5}{*}{ CI Split HEV PHEV2O } & ICE Power & 68738 & 68738 & 65336 & 62448 & 67974 & 110000 & 58284 & 66839 & 59514 & 51613 & 65961 & 58192 & 49257 \\
\hline & Electric Machine & 74751 & 74751 & 72067 & 69034 & 74020 & 50000 & 63849 & 72904 & 64892 & 55620 & 72960 & 63684 & 53524 \\
\hline & $\mathrm{MC} 2 / \mathrm{GC}$ & 57526 & 57526 & 54679 & 52262 & 56887 & 30000 & 48777 & 55937 & 49807 & 43195 & 55202 & 48700 & 41223 \\
\hline & Battery & 77671 & 77671 & 74820 & 71273 & 77256 & 62677 & 66027 & 75202 & 67113 & 57932 & 75417 & 67072 & 55804 \\
\hline & Specific Power & 79 & 79 & 79 & 79 & 79 & 101 & 78 & 78 & 78 & 79 & 79 & 79 & 79 \\
\hline
\end{tabular}


Argonne National Laboratory Report - Light-Duty Vehicle Fuel Consumption Displacement Potential Up to 2045

\begin{tabular}{|c|c|c|c|c|c|c|c|c|c|c|c|c|c|c|}
\hline & & \multicolumn{13}{|c|}{ Midsize } \\
\hline & & \multirow{2}{*}{$\begin{array}{c}2010 \\
\text { Ref } \\
\end{array}$} & \multicolumn{3}{|c|}{2010} & \multicolumn{3}{|c|}{2015} & \multicolumn{3}{|c|}{2030} & \multicolumn{3}{|c|}{2045} \\
\hline & & & low & avg & high & low & avg & high & low & avg & high & low & avg & high \\
\hline \multirow{5}{*}{ CI Series HEV PHEV 30} & ICE Power & 83373 & 83373 & 77592 & 72940 & 81874 & 74635 & 65389 & 78239 & 66995 & 55945 & 76842 & 64978 & 53331 \\
\hline & Electric Machine & 137587 & 137587 & 130309 & 124496 & 135280 & 126987 & 115184 & 132572 & 117688 & 102417 & 130702 & 114686 & 98125 \\
\hline & $\mathrm{MC} 2 / \mathrm{GC}$ & 80872 & 80872 & 75264 & 70752 & 79418 & 72396 & 63427 & 75892 & 64985 & 54267 & 74537 & 63029 & 51731 \\
\hline & Battery & 169522 & 169522 & 159684 & 151750 & 166714 & 154826 & 138744 & 161685 & 141803 & 121787 & 159416 & 138230 & 116708 \\
\hline & Specific Power & 67 & 67 & 67 & 67 & 67 & 67 & 67 & 67 & 67 & 69 & 67 & 67 & 69 \\
\hline \multirow{5}{*}{ CI Series HEV PHEV40 } & ICE Power & 86155 & 86155 & 80065 & 75258 & 84625 & 76953 & 67049 & 80735 & 68711 & 57188 & 79233 & 66457 & 54436 \\
\hline & Electric Machine & 141916 & 141916 & 134217 & 128174 & 139472 & 130753 & 117970 & 136516 & 120560 & 104434 & 134493 & 117165 & 99979 \\
\hline & $\mathrm{MC} 2 / \mathrm{GC}$ & 83570 & 83570 & 77663 & 73000 & 82086 & 74644 & 65038 & 78313 & 66650 & 55472 & 76856 & 64463 & 52803 \\
\hline & Battery & 174781 & 174781 & 164412 & 156181 & 171803 & 159360 & 141989 & 166428 & 145140 & 124085 & 163979 & 141095 & 118816 \\
\hline & Specific Power & 67 & 67 & 67 & 68 & 68 & 68 & 68 & 68 & 68 & 70 & 67 & 67 & 70 \\
\hline \multirow{5}{*}{ H2 Split HEV } & ICE Power & 93359 & 93359 & 86527 & 80954 & 91180 & 82784 & 74302 & 88529 & 75144 & 63143 & 85987 & 73398 & 60485 \\
\hline & Electric Machine & 67580 & 67580 & 63188 & 59817 & 65893 & 61585 & 56655 & 65673 & 57041 & 48686 & 63998 & 56233 & 46897 \\
\hline & $\mathrm{MC} 2 / \mathrm{GC}$ & 58846 & 58846 & 54540 & 51027 & 57473 & 52181 & 46834 & 55802 & 47365 & 39801 & 54200 & 46265 & 38125 \\
\hline & Battery & 30125 & 30125 & 27930 & 26334 & 29327 & 27317 & 25040 & 29024 & 25040 & 21057 & 28455 & 25040 & 20488 \\
\hline & Specific Power & 71 & 71 & 70 & 69 & 70 & 70 & 69 & 69 & 68 & 68 & 69 & 68 & 68 \\
\hline \multirow{5}{*}{ H2 Split HEV PHEV10 } & ICE Power & 66086 & 66086 & 61553 & 58313 & 64922 & 59415 & 54031 & 63638 & 55401 & 47255 & 61743 & 54046 & 45175 \\
\hline & Electric Machine & 76918 & 76918 & 72011 & 68209 & 74920 & 69273 & 61848 & 74419 & 63205 & 53165 & 72107 & 62443 & 50989 \\
\hline & $\mathrm{MC} 2 / \mathrm{GC}$ & 45610 & 45610 & 42481 & 40245 & 44767 & 41006 & 37290 & 43920 & 38236 & 32614 & 42613 & 37300 & 31178 \\
\hline & Battery & 75851 & 75851 & 70559 & 66348 & 73669 & 67337 & 59937 & 72445 & 61197 & 52099 & 70184 & 62392 & 49934 \\
\hline & Specific Power & 81 & 81 & 80 & 80 & 80 & 80 & 79 & 80 & 79 & 81 & 80 & 81 & 80 \\
\hline \multirow{5}{*}{ H2 Split HEV PHEV2O } & ICE Power & 67027 & 67027 & 62349 & 59021 & 65863 & 60123 & 54563 & 64432 & 56044 & 47613 & 62474 & 54547 & 45506 \\
\hline & Electric Machine & 78379 & 78379 & 73213 & 69193 & 77027 & 70307 & 62718 & 75577 & 64774 & 53818 & 73259 & 62908 & 51640 \\
\hline & $\mathrm{MC} 2 / \mathrm{GC}$ & 46220 & 46220 & 43031 & 40734 & 45436 & 41495 & 37657 & 44468 & 38660 & 32861 & 43117 & 37608 & 31406 \\
\hline & Battery & 77153 & 77153 & 71580 & 67116 & 75832 & 68159 & 59904 & 73380 & 62043 & 51588 & 71131 & 61407 & 49486 \\
\hline & Specific Power & 80 & 80 & 80 & 80 & 81 & 80 & 79 & 80 & 79 & 80 & 80 & 80 & 80 \\
\hline \multirow{5}{*}{ H2 Series HEV PHEV30 } & ICE Power & 81601 & 81601 & 74456 & 69344 & 79612 & 70831 & 61470 & 75802 & 63333 & 51938 & 73124 & 61137 & 49546 \\
\hline & Electric Machine & 134822 & 134822 & 125332 & 118680 & 131747 & 120947 & 108837 & 128664 & 111690 & 95562 & 124705 & 108498 & 91839 \\
\hline & $\mathrm{MC} 2 / \mathrm{GC}$ & 79153 & 79153 & 72222 & 67264 & 77224 & 68706 & 59626 & 73528 & 61433 & 50380 & 70930 & 59303 & 48060 \\
\hline & Battery & 166124 & 166124 & 153601 & 144681 & 162369 & 147481 & 131129 & 156937 & 134604 & 113668 & 152129 & 130800 & 109257 \\
\hline & Specific Power & 67 & 67 & 68 & 68 & 68 & 68 & 69 & 68 & 69 & 72 & 68 & 69 & 72 \\
\hline
\end{tabular}


Argonne National Laboratory Report - Light-Duty Vehicle Fuel Consumption Displacement Potential Up to 2045

\begin{tabular}{|c|c|c|c|c|c|c|c|c|c|c|c|c|c|c|}
\hline & & \multicolumn{13}{|c|}{ Midsize } \\
\hline & & \multirow{2}{*}{$\frac{2010}{\text { Ref }}$} & \multicolumn{3}{|c|}{2010} & \multicolumn{3}{|c|}{2015} & \multicolumn{3}{|c|}{2030} & \multicolumn{3}{|c|}{2045} \\
\hline & & & low & avg & high & low & avg & high & low & avg & high & low & avg & high \\
\hline \multirow{5}{*}{ H2 Series HEV PHEV40 } & ICE Power & 84321 & 84321 & 76929 & 71484 & 82302 & 73119 & 63072 & 78239 & 65021 & 53044 & 75397 & 62617 & 50596 \\
\hline & Electric Machine & 139062 & 139062 & 129210 & 122099 & 135948 & 124643 & 111438 & 132524 & 114417 & 97374 & 128374 & 110816 & 93473 \\
\hline & $\mathrm{MC} 2 / \mathrm{GC}$ & 81791 & 81791 & 74621 & 69339 & 79833 & 70925 & 61180 & 75892 & 63070 & 51453 & 73135 & 60738 & 49078 \\
\hline & Battery & 171278 & 171278 & 158301 & 148789 & 167480 & 151935 & 134158 & 161577 & 137782 & 115724 & 156544 & 133484 & 111119 \\
\hline & Specific Power & 68 & 68 & 68 & 69 & 68 & 69 & 69 & 68 & 69 & 72 & 69 & 69 & 72 \\
\hline \multirow{5}{*}{ E85 Split HEV } & ICE Power & 91090 & 91090 & 85963 & 81644 & 90239 & 84655 & 74444 & 87137 & 76979 & 65105 & 88066 & 75998 & 62086 \\
\hline & Electric Machine & 65436 & 65436 & 62487 & 59983 & 64700 & 62019 & 56790 & 64623 & 57933 & 50232 & 64986 & 57458 & 47767 \\
\hline & $\mathrm{MC} 2 / \mathrm{GC}$ & 52714 & 52714 & 49747 & 47248 & 52222 & 48990 & 43081 & 50427 & 44548 & 37677 & 50964 & 43980 & 35929 \\
\hline & Battery & 29327 & 29327 & 27930 & 26733 & 28928 & 27317 & 25610 & 29024 & 25610 & 22195 & 29024 & 25610 & 21057 \\
\hline & Specific Power & 71 & 71 & 70 & 69 & 71 & 70 & 68 & 69 & 68 & 68 & 70 & 69 & 68 \\
\hline \multirow{5}{*}{ E85 Split HEV PHEV10 } & ICE Power & 64688 & 64688 & 61439 & 58738 & 64037 & 59783 & 54899 & 62987 & 56044 & 48468 & 62221 & 54853 & 46140 \\
\hline & Electric Machine & 72428 & 72428 & 70035 & 66972 & 71976 & 68157 & 62073 & 71020 & 62535 & 53508 & 70961 & 61955 & 51144 \\
\hline & $\mathrm{MC} 2 / \mathrm{GC}$ & 38807 & 38807 & 36874 & 35253 & 38434 & 35880 & 32949 & 37787 & 33636 & 29089 & 37344 & 32922 & 27692 \\
\hline & Battery & 67297 & 67297 & 64885 & 61632 & 67010 & 62745 & 58080 & 65224 & 58304 & 50933 & 65404 & 59725 & 48737 \\
\hline & Specific Power & 77 & 77 & 77 & 77 & 77 & 77 & 77 & 77 & 77 & 78 & 77 & 78 & 78 \\
\hline \multirow{5}{*}{ E85 Split HEV PHEV20 } & ICE Power & 65629 & 65629 & 62235 & 59446 & 64893 & 60491 & 55430 & 63780 & 56632 & 48799 & 63036 & 55326 & 46472 \\
\hline & Electric Machine & 74437 & 74437 & 71127 & 68011 & 73219 & 69085 & 62928 & 72713 & 63896 & 54224 & 72082 & 62567 & 51736 \\
\hline & $\mathrm{MC} 2 / \mathrm{GC}$ & 39338 & 39338 & 37335 & 35644 & 38931 & 36305 & 33268 & 38263 & 33956 & 29288 & 37799 & 33205 & 27891 \\
\hline & Battery & 69124 & 69124 & 65688 & 62396 & 67968 & 63399 & 57848 & 66735 & 58713 & 50213 & 66223 & 58815 & 47895 \\
\hline & Specific Power & 77 & 77 & 77 & 76 & 77 & 76 & 76 & 77 & 76 & 77 & 76 & 77 & 77 \\
\hline \multirow{5}{*}{ E85 Series HEV PHEV30 } & ICE Power & 80409 & 80409 & 74757 & 70117 & 78910 & 71633 & 62671 & 75505 & 64220 & 53320 & 74069 & 62162 & 50706 \\
\hline & Electric Machine & 132914 & 132914 & 125717 & 119838 & 130648 & 122197 & 110743 & 128044 & 113079 & 97855 & 126184 & 110168 & 93699 \\
\hline & $\mathrm{MC} 2 / \mathrm{GC}$ & 77997 & 77997 & 72514 & 68013 & 76543 & 69484 & 60791 & 73240 & 62293 & 51720 & 71847 & 60297 & 49185 \\
\hline & Battery & 163771 & 163771 & 154070 & 146084 & 161012 & 149004 & 133415 & 156178 & 136272 & 116380 & 153925 & 132800 & 111462 \\
\hline & Specific Power & 68 & 68 & 68 & 68 & 68 & 68 & 68 & 68 & 68 & 71 & 68 & 68 & 71 \\
\hline \multirow{5}{*}{ E85 Series HEV PHEV40 } & ICE Power & 83129 & 83129 & 77140 & 72316 & 81568 & 73892 & 64216 & 77942 & 65879 & 54425 & 76370 & 63726 & 51756 \\
\hline & Electric Machine & 137206 & 137206 & 129545 & 123394 & 134804 & 125839 & 113333 & 131954 & 115861 & 99815 & 129942 & 112616 & 95363 \\
\hline & $\mathrm{MC} 2 / \mathrm{GC}$ & 80635 & 80635 & 74826 & 70147 & 79121 & 71675 & 62290 & 75604 & 63903 & 52792 & 74079 & 61814 & 50203 \\
\hline & Battery & 169000 & 169000 & 158707 & 150367 & 166066 & 153389 & 136435 & 160881 & 139506 & 118619 & 158452 & 135644 & 113353 \\
\hline & Specific Power & 68 & 68 & 68 & 69 & 68 & 69 & 69 & 68 & 69 & 71 & 68 & 69 & 71 \\
\hline
\end{tabular}


Argonne National Laboratory Report - Light-Duty Vehicle Fuel Consumption Displacement Potential Up to 2045

\begin{tabular}{|c|c|c|c|c|c|c|c|c|c|c|c|c|c|c|}
\hline & & \multicolumn{13}{|c|}{ Midsize } \\
\hline & & \multirow{2}{*}{$\begin{array}{c}2010 \\
\text { Ref }\end{array}$} & \multicolumn{3}{|c|}{2010} & \multicolumn{3}{|c|}{2015} & \multicolumn{3}{|c|}{2030} & \multicolumn{3}{|c|}{2045} \\
\hline & & & low & avg & high & low & avg & high & low & avg & high & low & avg & high \\
\hline \multirow{5}{*}{ FC HEV } & FC Power & 104050 & 104050 & 88019 & 78035 & 96505 & 76723 & 64474 & 82622 & 66935 & 52362 & 77746 & 63650 & 49443 \\
\hline & Electric Machine & 124590 & 124590 & 107383 & 97235 & 113102 & 99769 & 86620 & 107109 & 89845 & 73258 & 100110 & 85292 & 69515 \\
\hline & $\mathrm{MC} 2 / \mathrm{GC}$ & & & & & & & & & & & & & \\
\hline & Battery & 36908 & 36908 & 32519 & 30324 & 33516 & 34146 & 31301 & 36422 & 31870 & 27317 & 34715 & 31301 & 26179 \\
\hline & Specific Power & 59 & 59 & 60 & 60 & 60 & 61 & 59 & 60 & 60 & 60 & 60 & 59 & 60 \\
\hline \multirow{5}{*}{ FC HEV PHEV10 } & FC Power & 76391 & 76391 & 66089 & 59488 & 70247 & 60702 & 52736 & 65493 & 53965 & 45936 & 62805 & 54463 & 44162 \\
\hline & Electric Machine & 121688 & 121688 & 105550 & 96445 & 111157 & 98186 & 86942 & 105476 & 88796 & 75059 & 101348 & 88020 & 70127 \\
\hline & $\mathrm{MC} 2 / \mathrm{GC}$ & & & & & & & & & & & & & \\
\hline & Battery & 64879 & 64879 & 56091 & 50670 & 59493 & 51573 & 44891 & 55463 & 45917 & 37373 & 52881 & 44657 & 35527 \\
\hline & Specific Power & 61 & 61 & 60 & 60 & 60 & 61 & 61 & 61 & 61 & 62 & 61 & 62 & 61 \\
\hline \multirow{5}{*}{ FC HEV PHEV 20} & FC Power & 78252 & 78252 & 68330 & 60873 & 72745 & 61369 & 53926 & 66968 & 54586 & 45636 & 63478 & 54954 & 43201 \\
\hline & Electric Machine & 124396 & 124396 & 107035 & 98435 & 112943 & 99533 & 88561 & 107580 & 89911 & 74403 & 102686 & 88963 & 70613 \\
\hline & $\mathrm{MC} 2 / \mathrm{GC}$ & & & & & & & & & & & & & \\
\hline & Battery & 66428 & 66428 & 57445 & 51676 & 61043 & 52478 & 45621 & 56622 & 46601 & 37797 & 53833 & 45176 & 35985 \\
\hline & Specific Power & 61 & 61 & 60 & 60 & 60 & 60 & 61 & 61 & 60 & 61 & 61 & 62 & 61 \\
\hline \multirow{5}{*}{ FC HEV PHEV 30} & FC Power & 68439 & 68439 & 60582 & 56144 & 64345 & 56956 & 50962 & 61284 & 52296 & 43864 & 58431 & 50392 & 41576 \\
\hline & Electric Machine & 134537 & 134537 & 122497 & 115240 & 128589 & 116695 & 105703 & 123686 & 108013 & 93347 & 119624 & 104476 & 88983 \\
\hline & $\mathrm{MC} 2 / \mathrm{GC}$ & & & & & & & & & & & & & \\
\hline & Battery & 165695 & 165695 & 150070 & 140433 & 158409 & 142250 & 127410 & 150804 & 130239 & 111041 & 145890 & 126022 & 105872 \\
\hline & Specific Power & 63 & 63 & 66 & 67 & 65 & 67 & 69 & 66 & 69 & 73 & 67 & 69 & 73 \\
\hline \multirow{5}{*}{ FC HEV PHEV 40} & FC Power & 69736 & 69736 & 61673 & 57054 & 65566 & 57941 & 51564 & 62345 & 53023 & 44309 & 59309 & 50965 & 41971 \\
\hline & Electric Machine & 137968 & 137968 & 125477 & 117907 & 131796 & 119501 & 107997 & 126658 & 110436 & 95022 & 122265 & 106588 & 90648 \\
\hline & $\mathrm{MC} 2 / \mathrm{GC}$ & & & & & & & & & & & & & \\
\hline & Battery & 169858 & 169858 & 153652 & 143629 & 162297 & 145615 & 130067 & 154374 & 133045 & 112950 & 149046 & 128442 & 107765 \\
\hline & Specific Power & 63 & 63 & 66 & 67 & 65 & 68 & 69 & 66 & 69 & 73 & 68 & 70 & 74 \\
\hline \multirow{5}{*}{ BEV } & ICE Power & & & & & & & & & & & & & \\
\hline & Electric Machine & 160089 & 160089 & 148705 & 139383 & 157749 & 143474 & 122979 & 151888 & 125841 & 104899 & 147273 & 119932 & 98934 \\
\hline & $\mathrm{MC} 2 / \mathrm{GC}$ & 0 & 0 & 0 & 0 & 0 & 0 & 0 & 0 & 0 & 0 & 0 & 0 & 0 \\
\hline & Battery & 194624 & 194624 & 180668 & 169594 & 192167 & 174251 & 148106 & 183881 & 151620 & 125107 & 178954 & 144527 & 118453 \\
\hline & Specific Power & 90 & 90 & 89 & 89 & 90 & 89 & 88 & 89 & 89 & 91 & 89 & 88 & 91 \\
\hline
\end{tabular}




\section{Percentage of power reduction based on the respective 2010 reference case}

\begin{tabular}{|c|c|c|c|c|c|c|c|c|c|c|c|c|c|c|}
\hline & & \multicolumn{13}{|c|}{ Midsize } \\
\hline & & \multirow{2}{*}{$\frac{2010}{\operatorname{Ref}}$} & \multicolumn{3}{|c|}{2010} & \multicolumn{3}{|c|}{2015} & \multicolumn{3}{|c|}{2030} & \multicolumn{3}{|c|}{2045} \\
\hline & & & low & avg & high & low & avg & high & low & avg & high & low & avg & high \\
\hline \multirow{5}{*}{ SI Conv } & ICE Power & $0 \%$ & $0 \%$ & $-4 \%$ & $-7 \%$ & $0 \%$ & $-7 \%$ & $-13 \%$ & $-2 \%$ & $-11 \%$ & $-19 \%$ & $-3 \%$ & $-12 \%$ & $-20 \%$ \\
\hline & Electric Machine & & & & & & & & & & & & & \\
\hline & $\mathrm{MC} 2 / \mathrm{GC}$ & & & & & & & & & & & & & \\
\hline & Battery & & & & & & & & & & & & & \\
\hline & Specific Power & $0 \%$ & $0 \%$ & $-1 \%$ & $-1 \%$ & $0 \%$ & $-1 \%$ & $-3 \%$ & $-2 \%$ & $0 \%$ & $3 \%$ & $-3 \%$ & $-1 \%$ & $6 \%$ \\
\hline \multirow{5}{*}{$\mathrm{Cl}$ Conv } & ICE Power & $0 \%$ & $0 \%$ & $-5 \%$ & $-8 \%$ & $-1 \%$ & $-8 \%$ & $-14 \%$ & $-3 \%$ & $-12 \%$ & $-23 \%$ & $-5 \%$ & $-13 \%$ & $-26 \%$ \\
\hline & Electric Machine & & & & & & & & & & & & & \\
\hline & $\mathrm{MC} 2 / \mathrm{GC}$ & & & & & & & & & & & & & \\
\hline & Battery & & & & & & & & & & & & & \\
\hline & Specific Power & $0 \%$ & $0 \%$ & $-2 \%$ & $-2 \%$ & $-1 \%$ & $-3 \%$ & $-4 \%$ & $-3 \%$ & $-3 \%$ & $-3 \%$ & $-5 \%$ & $-3 \%$ & $-3 \%$ \\
\hline \multirow{5}{*}{ H2 Conv } & ICE Power & $0 \%$ & $0 \%$ & $-8 \%$ & $-14 \%$ & $-3 \%$ & $-14 \%$ & $-22 \%$ & $-6 \%$ & $-19 \%$ & $-31 \%$ & $-10 \%$ & $-21 \%$ & $-34 \%$ \\
\hline & Electric Machine & & & & & & & & & & & & & \\
\hline & $\mathrm{MC} 2 / \mathrm{GC}$ & & & & & & & & & & & & & \\
\hline & Battery & & & & & & & & & & & & & \\
\hline & Specific Power & $0 \%$ & $0 \%$ & $-3 \%$ & $-5 \%$ & $-2 \%$ & $-6 \%$ & $-7 \%$ & $-4 \%$ & $-6 \%$ & $-6 \%$ & $-6 \%$ & $-6 \%$ & $-6 \%$ \\
\hline \multirow{5}{*}{ E85 Conv } & ICE Power & $0 \%$ & $0 \%$ & $-4 \%$ & $-8 \%$ & $0 \%$ & $-7 \%$ & $-14 \%$ & $-2 \%$ & $-13 \%$ & $-22 \%$ & $-4 \%$ & $-14 \%$ & $-24 \%$ \\
\hline & Electric Machine & & & & & & & & & & & & & \\
\hline & $\mathrm{MC} 2 / \mathrm{GC}$ & & & & & & & & & & & & & \\
\hline & Battery & & & & & & & & & & & & & \\
\hline & Specific Power & $0 \%$ & $0 \%$ & $-1 \%$ & $-2 \%$ & $0 \%$ & $-2 \%$ & $-4 \%$ & $-2 \%$ & $-3 \%$ & $0 \%$ & $-3 \%$ & $-3 \%$ & $2 \%$ \\
\hline \multirow{5}{*}{ SI Split HEV } & ICE Power & $0 \%$ & $0 \%$ & $-6 \%$ & $-10 \%$ & $-2 \%$ & $-9 \%$ & $-16 \%$ & $-2 \%$ & $-16 \%$ & $-29 \%$ & $-3 \%$ & $-18 \%$ & $-33 \%$ \\
\hline & Electric Machine & $0 \%$ & $0 \%$ & $-4 \%$ & $-8 \%$ & $-2 \%$ & $-6 \%$ & $-12 \%$ & $0 \%$ & $-11 \%$ & $-24 \%$ & $0 \%$ & $-13 \%$ & $-27 \%$ \\
\hline & $\mathrm{MC} 2 / \mathrm{GC}$ & $0 \%$ & $0 \%$ & $-6 \%$ & $-10 \%$ & $-2 \%$ & $-9 \%$ & $-16 \%$ & $-2 \%$ & $-16 \%$ & $-29 \%$ & $-3 \%$ & $-18 \%$ & $-33 \%$ \\
\hline & Battery & $0 \%$ & $0 \%$ & $-4 \%$ & $-8 \%$ & $-1 \%$ & $-6 \%$ & $-12 \%$ & $0 \%$ & $-12 \%$ & $-26 \%$ & $0 \%$ & $-12 \%$ & $-28 \%$ \\
\hline & Specific Power & $0 \%$ & $0 \%$ & $-1 \%$ & $-1 \%$ & $0 \%$ & $-2 \%$ & $-2 \%$ & $0 \%$ & $-3 \%$ & $-4 \%$ & $0 \%$ & $-4 \%$ & $-4 \%$ \\
\hline \multirow{5}{*}{ SI Split HEV PHEV10 } & ICE Power & $0 \%$ & $0 \%$ & $-5 \%$ & $-9 \%$ & $-1 \%$ & $-8 \%$ & $-15 \%$ & $-3 \%$ & $-13 \%$ & $-25 \%$ & $-4 \%$ & $-15 \%$ & $-29 \%$ \\
\hline & Electric Machine & $0 \%$ & $0 \%$ & $-3 \%$ & $-8 \%$ & $-1 \%$ & $-6 \%$ & $-14 \%$ & $-2 \%$ & $-14 \%$ & $-26 \%$ & $-2 \%$ & $-14 \%$ & $-29 \%$ \\
\hline & $\mathrm{MC} 2 / \mathrm{GC}$ & $0 \%$ & $0 \%$ & $-5 \%$ & $-9 \%$ & $-1 \%$ & $-8 \%$ & $-15 \%$ & $-3 \%$ & $-13 \%$ & $-25 \%$ & $-4 \%$ & $-15 \%$ & $-29 \%$ \\
\hline & Battery & $0 \%$ & $0 \%$ & $-4 \%$ & $-8 \%$ & $0 \%$ & $-7 \%$ & $-14 \%$ & $-3 \%$ & $-13 \%$ & $-24 \%$ & $-3 \%$ & $-11 \%$ & $-28 \%$ \\
\hline & Specific Power & $0 \%$ & $0 \%$ & $0 \%$ & $-1 \%$ & $0 \%$ & $0 \%$ & $0 \%$ & $-1 \%$ & $-1 \%$ & $1 \%$ & $-1 \%$ & $1 \%$ & $1 \%$ \\
\hline
\end{tabular}




\begin{tabular}{|c|c|c|c|c|c|c|c|c|c|c|c|c|c|c|}
\hline & & \multicolumn{13}{|c|}{ Midsize } \\
\hline & & \multirow{2}{*}{$\frac{2010}{\operatorname{Ref}}$} & \multicolumn{3}{|c|}{2010} & \multicolumn{3}{|c|}{2015} & \multicolumn{3}{|c|}{2030} & \multicolumn{3}{|c|}{2045} \\
\hline & & & low & avg & high & low & avg & high & low & avg & high & low & avg & high \\
\hline \multirow{5}{*}{ SI Split HEV PHEV2O } & ICE Power & $0 \%$ & $0 \%$ & $-5 \%$ & $-9 \%$ & $-1 \%$ & $-8 \%$ & $-16 \%$ & $-3 \%$ & $-14 \%$ & $-26 \%$ & $-4 \%$ & $-16 \%$ & $-29 \%$ \\
\hline & Electric Machine & $0 \%$ & $0 \%$ & $-5 \%$ & $-9 \%$ & $-2 \%$ & $-7 \%$ & $-16 \%$ & $-3 \%$ & $-14 \%$ & $-27 \%$ & $-3 \%$ & $-16 \%$ & $-31 \%$ \\
\hline & $\mathrm{MC} 2 / \mathrm{GC}$ & $0 \%$ & $0 \%$ & $-5 \%$ & $-9 \%$ & $-1 \%$ & $-8 \%$ & $-15 \%$ & $-3 \%$ & $-14 \%$ & $-26 \%$ & $-4 \%$ & $-16 \%$ & $-29 \%$ \\
\hline & Battery & $0 \%$ & $0 \%$ & $-5 \%$ & $-10 \%$ & $-2 \%$ & $-8 \%$ & $-16 \%$ & $-5 \%$ & $-15 \%$ & $-28 \%$ & $-4 \%$ & $-15 \%$ & $-31 \%$ \\
\hline & Specific Power & $0 \%$ & $0 \%$ & $-1 \%$ & $-1 \%$ & $0 \%$ & $-1 \%$ & $-2 \%$ & $-1 \%$ & $-1 \%$ & $0 \%$ & $-1 \%$ & $-1 \%$ & $-1 \%$ \\
\hline \multirow{5}{*}{ SI Series HEV PHEV30 } & ICE Power & $0 \%$ & $0 \%$ & $-7 \%$ & $-13 \%$ & $-2 \%$ & $-11 \%$ & $-22 \%$ & $-6 \%$ & $-20 \%$ & $-34 \%$ & $-8 \%$ & $-23 \%$ & $-37 \%$ \\
\hline & Electric Machine & $0 \%$ & $0 \%$ & $-5 \%$ & $-10 \%$ & $-2 \%$ & $-8 \%$ & $-17 \%$ & $-4 \%$ & $-15 \%$ & $-26 \%$ & $-5 \%$ & $-17 \%$ & $-30 \%$ \\
\hline & $\mathrm{MC} 2 / \mathrm{GC}$ & $0 \%$ & $0 \%$ & $-7 \%$ & $-13 \%$ & $-2 \%$ & $-11 \%$ & $-22 \%$ & $-6 \%$ & $-20 \%$ & $-34 \%$ & $-8 \%$ & $-23 \%$ & $-37 \%$ \\
\hline & Battery & $0 \%$ & $0 \%$ & $-6 \%$ & $-11 \%$ & $-2 \%$ & $-9 \%$ & $-19 \%$ & $-5 \%$ & $-17 \%$ & $-29 \%$ & $-6 \%$ & $-19 \%$ & $-32 \%$ \\
\hline & Specific Power & $0 \%$ & $0 \%$ & $0 \%$ & $1 \%$ & $1 \%$ & $1 \%$ & $1 \%$ & $1 \%$ & $1 \%$ & $5 \%$ & $0 \%$ & $1 \%$ & $5 \%$ \\
\hline \multirow{5}{*}{ SI Series HEV PHEV40 } & ICE Power & $0 \%$ & $0 \%$ & $-7 \%$ & $-13 \%$ & $-2 \%$ & $-11 \%$ & $-23 \%$ & $-6 \%$ & $-21 \%$ & $-35 \%$ & $-8 \%$ & $-23 \%$ & $-38 \%$ \\
\hline & Electric Machine & $0 \%$ & $0 \%$ & $-6 \%$ & $-10 \%$ & $-2 \%$ & $-8 \%$ & $-17 \%$ & $-4 \%$ & $-16 \%$ & $-27 \%$ & $-5 \%$ & $-18 \%$ & $-30 \%$ \\
\hline & $\mathrm{MC} 2 / \mathrm{GC}$ & $0 \%$ & $0 \%$ & $-7 \%$ & $-13 \%$ & $-2 \%$ & $-11 \%$ & $-23 \%$ & $-6 \%$ & $-21 \%$ & $-35 \%$ & $-8 \%$ & $-23 \%$ & $-38 \%$ \\
\hline & Battery & $0 \%$ & $0 \%$ & $-6 \%$ & $-11 \%$ & $-2 \%$ & $-9 \%$ & $-19 \%$ & $-5 \%$ & $-17 \%$ & $-30 \%$ & $-6 \%$ & $-20 \%$ & $-33 \%$ \\
\hline & Specific Power & $0 \%$ & $0 \%$ & $0 \%$ & $1 \%$ & $1 \%$ & $1 \%$ & $1 \%$ & $1 \%$ & $1 \%$ & $4 \%$ & $0 \%$ & $1 \%$ & $5 \%$ \\
\hline \multirow{5}{*}{ CI Split HEV } & ICE Power & $0 \%$ & $0 \%$ & $-5 \%$ & $-12 \%$ & $-2 \%$ & $-9 \%$ & $-17 \%$ & $-4 \%$ & $-16 \%$ & $-29 \%$ & $-5 \%$ & $-17 \%$ & $-31 \%$ \\
\hline & Electric Machine & $0 \%$ & $0 \%$ & $-4 \%$ & $-10 \%$ & $-2 \%$ & $-6 \%$ & $-13 \%$ & $-2 \%$ & $-12 \%$ & $-24 \%$ & $-2 \%$ & $-13 \%$ & $-26 \%$ \\
\hline & $\mathrm{MC} 2 / \mathrm{GC}$ & $0 \%$ & $0 \%$ & $-5 \%$ & $-12 \%$ & $-2 \%$ & $-9 \%$ & $-17 \%$ & $-4 \%$ & $-16 \%$ & $-29 \%$ & $-5 \%$ & $-17 \%$ & $-31 \%$ \\
\hline & Battery & $0 \%$ & $0 \%$ & $-4 \%$ & $-10 \%$ & $-1 \%$ & $-7 \%$ & $-14 \%$ & $-1 \%$ & $-12 \%$ & $-25 \%$ & $-1 \%$ & $-12 \%$ & $-27 \%$ \\
\hline & Specific Power & $0 \%$ & $0 \%$ & $-1 \%$ & $-3 \%$ & $0 \%$ & $-2 \%$ & $-3 \%$ & $-2 \%$ & $-4 \%$ & $-5 \%$ & $-2 \%$ & $-3 \%$ & $-4 \%$ \\
\hline \multirow{5}{*}{ CI Split HEV PHEV10 } & ICE Power & $0 \%$ & $0 \%$ & $-5 \%$ & $-9 \%$ & $-1 \%$ & $-7 \%$ & $-15 \%$ & $-3 \%$ & $-13 \%$ & $-25 \%$ & $-4 \%$ & $-15 \%$ & $-28 \%$ \\
\hline & Electric Machine & $0 \%$ & $0 \%$ & $-4 \%$ & $-8 \%$ & $-1 \%$ & $-6 \%$ & $-14 \%$ & $-3 \%$ & $-13 \%$ & $-26 \%$ & $-2 \%$ & $-14 \%$ & $-29 \%$ \\
\hline & $\mathrm{MC} 2 / \mathrm{GC}$ & $0 \%$ & $0 \%$ & $-5 \%$ & $-9 \%$ & $-1 \%$ & $-7 \%$ & $-15 \%$ & $-3 \%$ & $-13 \%$ & $-25 \%$ & $-4 \%$ & $-15 \%$ & $-28 \%$ \\
\hline & Battery & $0 \%$ & $0 \%$ & $-4 \%$ & $-8 \%$ & $-1 \%$ & $-7 \%$ & $-14 \%$ & $-3 \%$ & $-13 \%$ & $-25 \%$ & $-3 \%$ & $-12 \%$ & $-28 \%$ \\
\hline & Specific Power & $0 \%$ & $0 \%$ & $0 \%$ & $-1 \%$ & $0 \%$ & $0 \%$ & $-1 \%$ & $-1 \%$ & $0 \%$ & $0 \%$ & $0 \%$ & $0 \%$ & $0 \%$ \\
\hline \multirow{5}{*}{ CI Split HEV PHEV20 } & ICE Power & $0 \%$ & $0 \%$ & $-5 \%$ & $-9 \%$ & $-1 \%$ & $60 \%$ & $-15 \%$ & $-3 \%$ & $-13 \%$ & $-25 \%$ & $-4 \%$ & $-15 \%$ & $-28 \%$ \\
\hline & Electric Machine & $0 \%$ & $0 \%$ & $-4 \%$ & $-8 \%$ & $-1 \%$ & $-33 \%$ & $-15 \%$ & $-2 \%$ & $-13 \%$ & $-26 \%$ & $-2 \%$ & $-15 \%$ & $-28 \%$ \\
\hline & $\mathrm{MC} 2 / \mathrm{GC}$ & $0 \%$ & $0 \%$ & $-5 \%$ & $-9 \%$ & $-1 \%$ & $-48 \%$ & $-15 \%$ & $-3 \%$ & $-13 \%$ & $-25 \%$ & $-4 \%$ & $-15 \%$ & $-28 \%$ \\
\hline & Battery & $0 \%$ & $0 \%$ & $-4 \%$ & $-8 \%$ & $-1 \%$ & $-19 \%$ & $-15 \%$ & $-3 \%$ & $-14 \%$ & $-25 \%$ & $-3 \%$ & $-14 \%$ & $-28 \%$ \\
\hline & Specific Power & $0 \%$ & $0 \%$ & $0 \%$ & $0 \%$ & $0 \%$ & $28 \%$ & $-1 \%$ & $-1 \%$ & $-1 \%$ & $0 \%$ & $0 \%$ & $0 \%$ & $0 \%$ \\
\hline
\end{tabular}




\begin{tabular}{|c|c|c|c|c|c|c|c|c|c|c|c|c|c|c|}
\hline & & \multicolumn{13}{|c|}{ Midsize } \\
\hline & & \multirow{2}{*}{$\frac{2010}{\operatorname{Ref}}$} & \multicolumn{3}{|c|}{2010} & \multicolumn{3}{|c|}{2015} & \multicolumn{3}{|c|}{2030} & \multicolumn{3}{|c|}{2045} \\
\hline & & & low & avg & high & low & avg & high & low & avg & high & low & avg & high \\
\hline \multirow{5}{*}{ CI Series HEV PHEV30 } & ICE Power & $0 \%$ & $0 \%$ & $-7 \%$ & $-13 \%$ & $-2 \%$ & $-10 \%$ & $-22 \%$ & $-6 \%$ & $-20 \%$ & $-33 \%$ & $-8 \%$ & $-22 \%$ & $-36 \%$ \\
\hline & Electric Machine & $0 \%$ & $0 \%$ & $-5 \%$ & $-10 \%$ & $-2 \%$ & $-8 \%$ & $-16 \%$ & $-4 \%$ & $-14 \%$ & $-26 \%$ & $-5 \%$ & $-17 \%$ & $-29 \%$ \\
\hline & $\mathrm{MC} 2 / \mathrm{GC}$ & $0 \%$ & $0 \%$ & $-7 \%$ & $-13 \%$ & $-2 \%$ & $-10 \%$ & $-22 \%$ & $-6 \%$ & $-20 \%$ & $-33 \%$ & $-8 \%$ & $-22 \%$ & $-36 \%$ \\
\hline & Battery & $0 \%$ & $0 \%$ & $-6 \%$ & $-10 \%$ & $-2 \%$ & $-9 \%$ & $-18 \%$ & $-5 \%$ & $-16 \%$ & $-28 \%$ & $-6 \%$ & $-18 \%$ & $-31 \%$ \\
\hline & Specific Power & $0 \%$ & $0 \%$ & $0 \%$ & $1 \%$ & $1 \%$ & $0 \%$ & $1 \%$ & $0 \%$ & $1 \%$ & $4 \%$ & $0 \%$ & $1 \%$ & $4 \%$ \\
\hline \multirow{5}{*}{ CI Series HEV PHEV40 } & ICE Power & $0 \%$ & $0 \%$ & $-7 \%$ & $-13 \%$ & $-2 \%$ & $-11 \%$ & $-22 \%$ & $-6 \%$ & $-20 \%$ & $-34 \%$ & $-8 \%$ & $-23 \%$ & $-37 \%$ \\
\hline & Electric Machine & $0 \%$ & $0 \%$ & $-5 \%$ & $-10 \%$ & $-2 \%$ & $-8 \%$ & $-17 \%$ & $-4 \%$ & $-15 \%$ & $-26 \%$ & $-5 \%$ & $-17 \%$ & $-30 \%$ \\
\hline & $\mathrm{MC} 2 / \mathrm{GC}$ & $0 \%$ & $0 \%$ & $-7 \%$ & $-13 \%$ & $-2 \%$ & $-11 \%$ & $-22 \%$ & $-6 \%$ & $-20 \%$ & $-34 \%$ & $-8 \%$ & $-23 \%$ & $-37 \%$ \\
\hline & Battery & $0 \%$ & $0 \%$ & $-6 \%$ & $-11 \%$ & $-2 \%$ & $-9 \%$ & $-19 \%$ & $-5 \%$ & $-17 \%$ & $-29 \%$ & $-6 \%$ & $-19 \%$ & $-32 \%$ \\
\hline & Specific Power & $0 \%$ & $0 \%$ & $0 \%$ & $1 \%$ & $1 \%$ & $0 \%$ & $1 \%$ & $0 \%$ & $1 \%$ & $3 \%$ & $0 \%$ & $0 \%$ & $4 \%$ \\
\hline \multirow{5}{*}{ H2 Split HEV } & ICE Power & $0 \%$ & $0 \%$ & $-7 \%$ & $-13 \%$ & $-2 \%$ & $-11 \%$ & $-20 \%$ & $-5 \%$ & $-20 \%$ & $-32 \%$ & $-8 \%$ & $-21 \%$ & $-35 \%$ \\
\hline & Electric Machine & $0 \%$ & $0 \%$ & $-6 \%$ & $-11 \%$ & $-2 \%$ & $-9 \%$ & $-16 \%$ & $-3 \%$ & $-16 \%$ & $-28 \%$ & $-5 \%$ & $-17 \%$ & $-31 \%$ \\
\hline & $\mathrm{MC} 2 / \mathrm{GC}$ & $0 \%$ & $0 \%$ & $-7 \%$ & $-13 \%$ & $-2 \%$ & $-11 \%$ & $-20 \%$ & $-5 \%$ & $-20 \%$ & $-32 \%$ & $-8 \%$ & $-21 \%$ & $-35 \%$ \\
\hline & Battery & $0 \%$ & $0 \%$ & $-7 \%$ & $-13 \%$ & $-3 \%$ & $-9 \%$ & $-17 \%$ & $-4 \%$ & $-17 \%$ & $-30 \%$ & $-6 \%$ & $-17 \%$ & $-32 \%$ \\
\hline & Specific Power & $0 \%$ & $0 \%$ & $-1 \%$ & $-2 \%$ & $0 \%$ & $-1 \%$ & $-2 \%$ & $-2 \%$ & $-4 \%$ & $-3 \%$ & $-2 \%$ & $-3 \%$ & $-3 \%$ \\
\hline \multirow{5}{*}{ H2 Split HEV PHEV10 } & ICE Power & $0 \%$ & $0 \%$ & $-7 \%$ & $-12 \%$ & $-2 \%$ & $-10 \%$ & $-18 \%$ & $-4 \%$ & $-16 \%$ & $-28 \%$ & $-7 \%$ & $-18 \%$ & $-32 \%$ \\
\hline & Electric Machine & $0 \%$ & $0 \%$ & $-6 \%$ & $-11 \%$ & $-3 \%$ & $-10 \%$ & $-20 \%$ & $-3 \%$ & $-18 \%$ & $-31 \%$ & $-6 \%$ & $-19 \%$ & $-34 \%$ \\
\hline & $\mathrm{MC} 2 / \mathrm{GC}$ & $0 \%$ & $0 \%$ & $-7 \%$ & $-12 \%$ & $-2 \%$ & $-10 \%$ & $-18 \%$ & $-4 \%$ & $-16 \%$ & $-28 \%$ & $-7 \%$ & $-18 \%$ & $-32 \%$ \\
\hline & Battery & $0 \%$ & $0 \%$ & $-7 \%$ & $-13 \%$ & $-3 \%$ & $-11 \%$ & $-21 \%$ & $-4 \%$ & $-19 \%$ & $-31 \%$ & $-7 \%$ & $-18 \%$ & $-34 \%$ \\
\hline & Specific Power & $0 \%$ & $0 \%$ & $0 \%$ & $0 \%$ & $0 \%$ & $-1 \%$ & $-2 \%$ & $-1 \%$ & $-2 \%$ & $0 \%$ & $-1 \%$ & $0 \%$ & $0 \%$ \\
\hline \multirow{5}{*}{ H2 Split HEV PHEV 20} & ICE Power & $0 \%$ & $0 \%$ & $-7 \%$ & $-12 \%$ & $-2 \%$ & $-10 \%$ & $-19 \%$ & $-4 \%$ & $-16 \%$ & $-29 \%$ & $-7 \%$ & $-19 \%$ & $-32 \%$ \\
\hline & Electric Machine & $0 \%$ & $0 \%$ & $-7 \%$ & $-12 \%$ & $-2 \%$ & $-10 \%$ & $-20 \%$ & $-4 \%$ & $-17 \%$ & $-31 \%$ & $-7 \%$ & $-20 \%$ & $-34 \%$ \\
\hline & $\mathrm{MC} 2 / \mathrm{GC}$ & $0 \%$ & $0 \%$ & $-7 \%$ & $-12 \%$ & $-2 \%$ & $-10 \%$ & $-19 \%$ & $-4 \%$ & $-16 \%$ & $-29 \%$ & $-7 \%$ & $-19 \%$ & $-32 \%$ \\
\hline & Battery & $0 \%$ & $0 \%$ & $-7 \%$ & $-13 \%$ & $-2 \%$ & $-12 \%$ & $-22 \%$ & $-5 \%$ & $-20 \%$ & $-33 \%$ & $-8 \%$ & $-20 \%$ & $-36 \%$ \\
\hline & Specific Power & $0 \%$ & $0 \%$ & $0 \%$ & $-1 \%$ & $0 \%$ & $-1 \%$ & $-2 \%$ & $-1 \%$ & $-2 \%$ & $-1 \%$ & $-1 \%$ & $-1 \%$ & $-1 \%$ \\
\hline \multirow{5}{*}{ H2 Series HEV PHEV 30} & ICE Power & $0 \%$ & $0 \%$ & $-9 \%$ & $-15 \%$ & $-2 \%$ & $-13 \%$ & $-25 \%$ & $-7 \%$ & $-22 \%$ & $-36 \%$ & $-10 \%$ & $-25 \%$ & $-39 \%$ \\
\hline & Electric Machine & $0 \%$ & $0 \%$ & $-7 \%$ & $-12 \%$ & $-2 \%$ & $-10 \%$ & $-19 \%$ & $-5 \%$ & $-17 \%$ & $-29 \%$ & $-8 \%$ & $-20 \%$ & $-32 \%$ \\
\hline & $\mathrm{MC} 2 / \mathrm{GC}$ & $0 \%$ & $0 \%$ & $-9 \%$ & $-15 \%$ & $-2 \%$ & $-13 \%$ & $-25 \%$ & $-7 \%$ & $-22 \%$ & $-36 \%$ & $-10 \%$ & $-25 \%$ & $-39 \%$ \\
\hline & Battery & $0 \%$ & $0 \%$ & $-8 \%$ & $-13 \%$ & $-2 \%$ & $-11 \%$ & $-21 \%$ & $-6 \%$ & $-19 \%$ & $-32 \%$ & $-8 \%$ & $-21 \%$ & $-34 \%$ \\
\hline & Specific Power & $0 \%$ & $0 \%$ & $1 \%$ & $2 \%$ & $1 \%$ & $2 \%$ & $3 \%$ & $1 \%$ & $2 \%$ & $6 \%$ & $1 \%$ & $2 \%$ & $7 \%$ \\
\hline
\end{tabular}




\begin{tabular}{|c|c|c|c|c|c|c|c|c|c|c|c|c|c|c|}
\hline & & \multicolumn{13}{|c|}{ Midsize } \\
\hline & & \multirow{2}{*}{$\frac{2010}{\operatorname{Ref}}$} & \multicolumn{3}{|c|}{2010} & \multicolumn{3}{|c|}{2015} & \multicolumn{3}{|c|}{2030} & \multicolumn{3}{|c|}{2045} \\
\hline & & & low & avg & high & low & avg & high & low & avg & high & low & avg & high \\
\hline \multirow{5}{*}{ H2 Series HEV PHEV40 } & ICE Power & $0 \%$ & $0 \%$ & $-9 \%$ & $-15 \%$ & $-2 \%$ & $-13 \%$ & $-25 \%$ & $-7 \%$ & $-23 \%$ & $-37 \%$ & $-11 \%$ & $-26 \%$ & $-40 \%$ \\
\hline & Electric Machine & $0 \%$ & $0 \%$ & $-7 \%$ & $-12 \%$ & $-2 \%$ & $-10 \%$ & $-20 \%$ & $-5 \%$ & $-18 \%$ & $-30 \%$ & $-8 \%$ & $-20 \%$ & $-33 \%$ \\
\hline & $\mathrm{MC} 2 / \mathrm{GC}$ & $0 \%$ & $0 \%$ & $-9 \%$ & $-15 \%$ & $-2 \%$ & $-13 \%$ & $-25 \%$ & $-7 \%$ & $-23 \%$ & $-37 \%$ & $-11 \%$ & $-26 \%$ & $-40 \%$ \\
\hline & Battery & $0 \%$ & $0 \%$ & $-8 \%$ & $-13 \%$ & $-2 \%$ & $-11 \%$ & $-22 \%$ & $-6 \%$ & $-20 \%$ & $-32 \%$ & $-9 \%$ & $-22 \%$ & $-35 \%$ \\
\hline & Specific Power & $0 \%$ & $0 \%$ & $1 \%$ & $2 \%$ & $1 \%$ & $2 \%$ & $2 \%$ & $1 \%$ & $2 \%$ & $6 \%$ & $1 \%$ & $2 \%$ & $6 \%$ \\
\hline \multirow{5}{*}{ E85 Split HEV } & ICE Power & $0 \%$ & $0 \%$ & $-6 \%$ & $-10 \%$ & $-1 \%$ & $-7 \%$ & $-18 \%$ & $-4 \%$ & $-15 \%$ & $-29 \%$ & $-3 \%$ & $-17 \%$ & $-32 \%$ \\
\hline & Electric Machine & $0 \%$ & $0 \%$ & $-5 \%$ & $-8 \%$ & $-1 \%$ & $-5 \%$ & $-13 \%$ & $-1 \%$ & $-11 \%$ & $-23 \%$ & $-1 \%$ & $-12 \%$ & $-27 \%$ \\
\hline & $\mathrm{MC} 2 / \mathrm{GC}$ & $0 \%$ & $0 \%$ & $-6 \%$ & $-10 \%$ & $-1 \%$ & $-7 \%$ & $-18 \%$ & $-4 \%$ & $-15 \%$ & $-29 \%$ & $-3 \%$ & $-17 \%$ & $-32 \%$ \\
\hline & Battery & $0 \%$ & $0 \%$ & $-5 \%$ & $-9 \%$ & $-1 \%$ & $-7 \%$ & $-13 \%$ & $-1 \%$ & $-13 \%$ & $-24 \%$ & $-1 \%$ & $-13 \%$ & $-28 \%$ \\
\hline & Specific Power & $0 \%$ & $0 \%$ & $-1 \%$ & $-2 \%$ & $0 \%$ & $-1 \%$ & $-4 \%$ & $-2 \%$ & $-3 \%$ & $-3 \%$ & $-1 \%$ & $-2 \%$ & $-3 \%$ \\
\hline \multirow{5}{*}{ E85 Split HEV PHEV10 } & ICE Power & $0 \%$ & $0 \%$ & $-5 \%$ & $-9 \%$ & $-1 \%$ & $-8 \%$ & $-15 \%$ & $-3 \%$ & $-13 \%$ & $-25 \%$ & $-4 \%$ & $-15 \%$ & $-29 \%$ \\
\hline & Electric Machine & $0 \%$ & $0 \%$ & $-3 \%$ & $-8 \%$ & $-1 \%$ & $-6 \%$ & $-14 \%$ & $-2 \%$ & $-14 \%$ & $-26 \%$ & $-2 \%$ & $-14 \%$ & $-29 \%$ \\
\hline & $\mathrm{MC} 2 / \mathrm{GC}$ & $0 \%$ & $0 \%$ & $-5 \%$ & $-9 \%$ & $-1 \%$ & $-8 \%$ & $-15 \%$ & $-3 \%$ & $-13 \%$ & $-25 \%$ & $-4 \%$ & $-15 \%$ & $-29 \%$ \\
\hline & Battery & $0 \%$ & $0 \%$ & $-4 \%$ & $-8 \%$ & $0 \%$ & $-7 \%$ & $-14 \%$ & $-3 \%$ & $-13 \%$ & $-24 \%$ & $-3 \%$ & $-11 \%$ & $-28 \%$ \\
\hline & Specific Power & $0 \%$ & $0 \%$ & $0 \%$ & $-1 \%$ & $0 \%$ & $0 \%$ & $0 \%$ & $-1 \%$ & $-1 \%$ & $1 \%$ & $-1 \%$ & $1 \%$ & $1 \%$ \\
\hline \multirow{5}{*}{ E85 Split HEV PHEV 20} & ICE Power & $0 \%$ & $0 \%$ & $-5 \%$ & $-9 \%$ & $-1 \%$ & $-8 \%$ & $-16 \%$ & $-3 \%$ & $-14 \%$ & $-26 \%$ & $-4 \%$ & $-16 \%$ & $-29 \%$ \\
\hline & Electric Machine & $0 \%$ & $0 \%$ & $-4 \%$ & $-9 \%$ & $-2 \%$ & $-7 \%$ & $-15 \%$ & $-2 \%$ & $-14 \%$ & $-27 \%$ & $-3 \%$ & $-16 \%$ & $-30 \%$ \\
\hline & $\mathrm{MC} 2 / \mathrm{GC}$ & $0 \%$ & $0 \%$ & $-5 \%$ & $-9 \%$ & $-1 \%$ & $-8 \%$ & $-15 \%$ & $-3 \%$ & $-14 \%$ & $-26 \%$ & $-4 \%$ & $-16 \%$ & $-29 \%$ \\
\hline & Battery & $0 \%$ & $0 \%$ & $-5 \%$ & $-10 \%$ & $-2 \%$ & $-8 \%$ & $-16 \%$ & $-3 \%$ & $-15 \%$ & $-27 \%$ & $-4 \%$ & $-15 \%$ & $-31 \%$ \\
\hline & Specific Power & $0 \%$ & $0 \%$ & $-1 \%$ & $-1 \%$ & $0 \%$ & $-1 \%$ & $-2 \%$ & $-1 \%$ & $-1 \%$ & $0 \%$ & $-1 \%$ & $-1 \%$ & $-1 \%$ \\
\hline \multirow{5}{*}{ E85 Series HEV PHEV30 } & ICE Power & $0 \%$ & $0 \%$ & $-7 \%$ & $-13 \%$ & $-2 \%$ & $-11 \%$ & $-22 \%$ & $-6 \%$ & $-20 \%$ & $-34 \%$ & $-8 \%$ & $-23 \%$ & $-37 \%$ \\
\hline & Electric Machine & $0 \%$ & $0 \%$ & $-5 \%$ & $-10 \%$ & $-2 \%$ & $-8 \%$ & $-17 \%$ & $-4 \%$ & $-15 \%$ & $-26 \%$ & $-5 \%$ & $-17 \%$ & $-30 \%$ \\
\hline & $\mathrm{MC} 2 / \mathrm{GC}$ & $0 \%$ & $0 \%$ & $-7 \%$ & $-13 \%$ & $-2 \%$ & $-11 \%$ & $-22 \%$ & $-6 \%$ & $-20 \%$ & $-34 \%$ & $-8 \%$ & $-23 \%$ & $-37 \%$ \\
\hline & Battery & $0 \%$ & $0 \%$ & $-6 \%$ & $-11 \%$ & $-2 \%$ & $-9 \%$ & $-19 \%$ & $-5 \%$ & $-17 \%$ & $-29 \%$ & $-6 \%$ & $-19 \%$ & $-32 \%$ \\
\hline & Specific Power & $0 \%$ & $0 \%$ & $0 \%$ & $1 \%$ & $1 \%$ & $1 \%$ & $1 \%$ & $1 \%$ & $1 \%$ & $5 \%$ & $0 \%$ & $1 \%$ & $5 \%$ \\
\hline \multirow{5}{*}{ E85 Series HEV PHEV40 } & ICE Power & $0 \%$ & $0 \%$ & $-7 \%$ & $-13 \%$ & $-2 \%$ & $-11 \%$ & $-23 \%$ & $-6 \%$ & $-21 \%$ & $-35 \%$ & $-8 \%$ & $-23 \%$ & $-38 \%$ \\
\hline & Electric Machine & $0 \%$ & $0 \%$ & $-6 \%$ & $-10 \%$ & $-2 \%$ & $-8 \%$ & $-17 \%$ & $-4 \%$ & $-16 \%$ & $-27 \%$ & $-5 \%$ & $-18 \%$ & $-30 \%$ \\
\hline & $\mathrm{MC} 2 / \mathrm{GC}$ & $0 \%$ & $0 \%$ & $-7 \%$ & $-13 \%$ & $-2 \%$ & $-11 \%$ & $-23 \%$ & $-6 \%$ & $-21 \%$ & $-35 \%$ & $-8 \%$ & $-23 \%$ & $-38 \%$ \\
\hline & Battery & $0 \%$ & $0 \%$ & $-6 \%$ & $-11 \%$ & $-2 \%$ & $-9 \%$ & $-19 \%$ & $-5 \%$ & $-17 \%$ & $-30 \%$ & $-6 \%$ & $-20 \%$ & $-33 \%$ \\
\hline & Specific Power & $0 \%$ & $0 \%$ & $0 \%$ & $1 \%$ & $0 \%$ & $1 \%$ & $1 \%$ & $0 \%$ & $1 \%$ & $4 \%$ & $0 \%$ & $1 \%$ & $4 \%$ \\
\hline
\end{tabular}




\begin{tabular}{|c|c|c|c|c|c|c|c|c|c|c|c|c|c|c|}
\hline & & \multicolumn{13}{|c|}{ Midsize } \\
\hline & & \multirow{2}{*}{$\frac{2010}{\text { Ref }}$} & \multicolumn{3}{|c|}{2010} & \multicolumn{3}{|c|}{2015} & \multicolumn{3}{|c|}{2030} & \multicolumn{3}{|c|}{2045} \\
\hline & & & low & avg & high & low & avg & high & low & avg & high & low & avg & high \\
\hline \multirow{5}{*}{ FC HEV } & FC Power & $0 \%$ & $0 \%$ & $-15 \%$ & $-25 \%$ & $-7 \%$ & $-26 \%$ & $-38 \%$ & $-21 \%$ & $-36 \%$ & $-50 \%$ & $-25 \%$ & $-39 \%$ & $-52 \%$ \\
\hline & Electric Machine & $0 \%$ & $0 \%$ & $-14 \%$ & $-22 \%$ & $-9 \%$ & $-20 \%$ & $-30 \%$ & $-14 \%$ & $-28 \%$ & $-41 \%$ & $-20 \%$ & $-32 \%$ & $-44 \%$ \\
\hline & $\mathrm{MC} 2 / \mathrm{GC}$ & & & & & & & & & & & & & \\
\hline & Battery & $0 \%$ & $0 \%$ & $-12 \%$ & $-18 \%$ & $-9 \%$ & $-7 \%$ & $-15 \%$ & $-1 \%$ & $-14 \%$ & $-26 \%$ & $-6 \%$ & $-15 \%$ & $-29 \%$ \\
\hline & Specific Power & $0 \%$ & $0 \%$ & $1 \%$ & $1 \%$ & $1 \%$ & $2 \%$ & $0 \%$ & $1 \%$ & $1 \%$ & $1 \%$ & $0 \%$ & $-1 \%$ & $1 \%$ \\
\hline \multirow{5}{*}{ FC HEV PHEV 10} & FC Power & $0 \%$ & $0 \%$ & $-13 \%$ & $-22 \%$ & $-8 \%$ & $-21 \%$ & $-31 \%$ & $-14 \%$ & $-29 \%$ & $-40 \%$ & $-18 \%$ & $-29 \%$ & $-42 \%$ \\
\hline & Electric Machine & $0 \%$ & $0 \%$ & $-13 \%$ & $-21 \%$ & $-9 \%$ & $-19 \%$ & $-29 \%$ & $-13 \%$ & $-27 \%$ & $-38 \%$ & $-17 \%$ & $-28 \%$ & $-42 \%$ \\
\hline & $\mathrm{MC} 2 / \mathrm{GC}$ & & & & & & & & & & & & & \\
\hline & Battery & $0 \%$ & $0 \%$ & $-14 \%$ & $-22 \%$ & $-8 \%$ & $-21 \%$ & $-31 \%$ & $-15 \%$ & $-29 \%$ & $-42 \%$ & $-18 \%$ & $-31 \%$ & $-45 \%$ \\
\hline & Specific Power & $0 \%$ & $0 \%$ & $0 \%$ & $-1 \%$ & $0 \%$ & $0 \%$ & $0 \%$ & $0 \%$ & $0 \%$ & $3 \%$ & $1 \%$ & $2 \%$ & $1 \%$ \\
\hline \multirow{5}{*}{ FC HEV PHEV 20} & FC Power & $0 \%$ & $0 \%$ & $-13 \%$ & $-22 \%$ & $-7 \%$ & $-22 \%$ & $-31 \%$ & $-14 \%$ & $-30 \%$ & $-42 \%$ & $-19 \%$ & $-30 \%$ & $-45 \%$ \\
\hline & Electric Machine & $0 \%$ & $0 \%$ & $-14 \%$ & $-21 \%$ & $-9 \%$ & $-20 \%$ & $-29 \%$ & $-14 \%$ & $-28 \%$ & $-40 \%$ & $-17 \%$ & $-28 \%$ & $-43 \%$ \\
\hline & $\mathrm{MC} 2 / \mathrm{GC}$ & & & & & & & & & & & & & \\
\hline & Battery & $0 \%$ & $0 \%$ & $-14 \%$ & $-22 \%$ & $-8 \%$ & $-21 \%$ & $-31 \%$ & $-15 \%$ & $-30 \%$ & $-43 \%$ & $-19 \%$ & $-32 \%$ & $-46 \%$ \\
\hline & Specific Power & $0 \%$ & $0 \%$ & $-1 \%$ & $0 \%$ & $-1 \%$ & $0 \%$ & $0 \%$ & $0 \%$ & $0 \%$ & $1 \%$ & $1 \%$ & $2 \%$ & $1 \%$ \\
\hline \multirow{5}{*}{ FC HEV PHEV 30} & FC Power & $0 \%$ & $0 \%$ & $-11 \%$ & $-18 \%$ & $-6 \%$ & $-17 \%$ & $-26 \%$ & $-10 \%$ & $-24 \%$ & $-36 \%$ & $-15 \%$ & $-26 \%$ & $-39 \%$ \\
\hline & Electric Machine & $0 \%$ & $0 \%$ & $-9 \%$ & $-14 \%$ & $-4 \%$ & $-13 \%$ & $-21 \%$ & $-8 \%$ & $-20 \%$ & $-31 \%$ & $-11 \%$ & $-22 \%$ & $-34 \%$ \\
\hline & $\mathrm{MC} 2 / \mathrm{GC}$ & & & & & & & & & & & & & \\
\hline & Battery & $0 \%$ & $0 \%$ & $-9 \%$ & $-15 \%$ & $-4 \%$ & $-14 \%$ & $-23 \%$ & $-9 \%$ & $-21 \%$ & $-33 \%$ & $-12 \%$ & $-24 \%$ & $-36 \%$ \\
\hline & Specific Power & $0 \%$ & $0 \%$ & $4 \%$ & $7 \%$ & $3 \%$ & $7 \%$ & $9 \%$ & $5 \%$ & $9 \%$ & $15 \%$ & $7 \%$ & $10 \%$ & $16 \%$ \\
\hline \multirow{5}{*}{ FC HEV PHEV40 } & FC Power & $0 \%$ & $0 \%$ & $-12 \%$ & $-18 \%$ & $-6 \%$ & $-17 \%$ & $-26 \%$ & $-11 \%$ & $-24 \%$ & $-36 \%$ & $-15 \%$ & $-27 \%$ & $-40 \%$ \\
\hline & Electric Machine & $0 \%$ & $0 \%$ & $-9 \%$ & $-15 \%$ & $-4 \%$ & $-13 \%$ & $-22 \%$ & $-8 \%$ & $-20 \%$ & $-31 \%$ & $-11 \%$ & $-23 \%$ & $-34 \%$ \\
\hline & $\mathrm{MC} 2 / \mathrm{GC}$ & & & & & & & & & & & & & \\
\hline & Battery & $0 \%$ & $0 \%$ & $-10 \%$ & $-15 \%$ & $-4 \%$ & $-14 \%$ & $-23 \%$ & $-9 \%$ & $-22 \%$ & $-34 \%$ & $-12 \%$ & $-24 \%$ & $-37 \%$ \\
\hline & Specific Power & $0 \%$ & $0 \%$ & $4 \%$ & $7 \%$ & $3 \%$ & $7 \%$ & $10 \%$ & $5 \%$ & $10 \%$ & $16 \%$ & $7 \%$ & $10 \%$ & $17 \%$ \\
\hline \multirow{5}{*}{ EV } & ICE Power & & & & & & & & & & & & & \\
\hline & Electric Machine & $0 \%$ & $0 \%$ & $-7 \%$ & $-13 \%$ & $-1 \%$ & $-10 \%$ & $-23 \%$ & $-5 \%$ & $-21 \%$ & $-34 \%$ & $-8 \%$ & $-25 \%$ & $-38 \%$ \\
\hline & $\mathrm{MC} 2 / \mathrm{GC}$ & & & & & & & & & & & & & \\
\hline & Battery & $0 \%$ & $0 \%$ & $-7 \%$ & $-13 \%$ & $-1 \%$ & $-10 \%$ & $-24 \%$ & $-6 \%$ & $-22 \%$ & $-36 \%$ & $-8 \%$ & $-26 \%$ & $-39 \%$ \\
\hline & Specific Power & $0 \%$ & $0 \%$ & $-1 \%$ & $-1 \%$ & $0 \%$ & $-1 \%$ & $-1 \%$ & $-1 \%$ & $-1 \%$ & $1 \%$ & $-1 \%$ & $-2 \%$ & $2 \%$ \\
\hline
\end{tabular}




\section{Energy}

\begin{tabular}{|c|c|c|c|c|c|c|c|c|c|c|c|c|c|c|}
\hline & & \multicolumn{13}{|c|}{ Midsize } \\
\hline & & \multirow{2}{*}{$\begin{array}{l}2010 \\
\text { Ref }\end{array}$} & \multicolumn{3}{|c|}{2010} & \multicolumn{3}{|c|}{2015} & \multicolumn{3}{|c|}{2030} & \multicolumn{3}{|c|}{2045} \\
\hline & & & low & avg & high & low & avg & high & low & avg & high & low & avg & high \\
\hline \multirow{2}{*}{ SI Split HEV } & Total Energy & 1139 & 1139 & 1092 & 1045 & 1131 & 1037 & 972 & 1102 & 972 & 821 & 1102 & 972 & 799 \\
\hline & Usable Energy & & & & & & & & & & & & & \\
\hline \multirow{2}{*}{ SI Split HEV PHEV10 } & Total Energy & 3872 & 3872 & 3600 & 3334 & 3863 & 3447 & 2671 & 3694 & 2784 & 2033 & 3615 & 2489 & 1869 \\
\hline & Usable Energy & 2323 & 2323 & 2160 & 2000 & 2318 & 2068 & 1870 & 2216 & 1949 & 1627 & 2169 & 1867 & 1495 \\
\hline \multirow{2}{*}{ SI Split HEV PHEV2O } & Total Energy & 7593 & 7593 & 7097 & 6610 & 7716 & 6795 & 5238 & 7263 & 5425 & 3901 & 7106 & 4907 & 3700 \\
\hline & Usable Energy & 4556 & 4556 & 4258 & 3966 & 4630 & 4077 & 3667 & 4358 & 3797 & 3121 & 4264 & 3680 & 2960 \\
\hline \multirow{2}{*}{ SI Series HEV PHEV30 } & Total Energy & 14431 & 14431 & 13337 & 12456 & 14230 & 12799 & 9677 & 13561 & 9987 & 7214 & 13234 & 8980 & 6895 \\
\hline & Usable Energy & 8659 & 8659 & 8002 & 7474 & 8538 & 7680 & 6774 & 8137 & 6991 & 5771 & 7940 & 6735 & 5516 \\
\hline \multirow{2}{*}{ SI Series HEV PHEV40 } & Total Energy & 19472 & 19472 & 18044 & 16829 & 19196 & 17267 & 13041 & 18323 & 13476 & 9627 & 17834 & 12056 & 9223 \\
\hline & Usable Energy & 11683 & 11683 & 10827 & 10097 & 11518 & 10360 & 9129 & 10994 & 9433 & 7702 & 10700 & 9042 & 7378 \\
\hline \multirow{2}{*}{ Cl Split HEV } & Total Energy & 1217 & 1217 & 1162 & 1100 & 1201 & 1102 & 1015 & 1166 & 1037 & 886 & 1166 & 1037 & 864 \\
\hline & Usable Energy & & & & & & & & & & & & & \\
\hline \multirow{2}{*}{ CI Split HEV PHEV10 } & Total Energy & 4136 & 4136 & 3857 & 3548 & 4095 & 3689 & 2831 & 3928 & 2920 & 2178 & 3862 & 2645 & 2087 \\
\hline & Usable Energy & 2482 & 2482 & 2314 & 2129 & 2457 & 2213 & 1982 & 2357 & 2044 & 1742 & 2317 & 1983 & 1670 \\
\hline \multirow{2}{*}{ CI Split HEV PHEV20 } & Total Energy & 8151 & 8151 & 7461 & 6984 & 7955 & 7287 & 5629 & 7609 & 5810 & 4098 & 7439 & 5165 & 3927 \\
\hline & Usable Energy & 4891 & 4891 & 4477 & 4191 & 4773 & 4372 & 3940 & 4566 & 4067 & 3278 & 4463 & 3873 & 3141 \\
\hline \multirow{2}{*}{ CI Series HEV PHEV30 } & Total Energy & 14994 & 14994 & 13887 & 12973 & 14770 & 13342 & 10114 & 14077 & 10433 & 7616 & 13757 & 9400 & 7307 \\
\hline & Usable Energy & 8997 & 8997 & 8332 & 7784 & 8862 & 8005 & 7080 & 8446 & 7303 & 6093 & 8254 & 7050 & 5846 \\
\hline \multirow{2}{*}{ CI Series HEV PHEV40 } & Total Energy & 20292 & 20292 & 18797 & 17493 & 19896 & 18026 & 13638 & 19075 & 14068 & 10134 & 18603 & 12598 & 9680 \\
\hline & Usable Energy & 12175 & 12175 & 11278 & 10496 & 11937 & 10815 & 9547 & 11445 & 9847 & 8107 & 11162 & 9449 & 7744 \\
\hline \multirow{2}{*}{ H2 Split HEV } & Total Energy & 1178 & 1178 & 1092 & 1030 & 1147 & 1037 & 950 & 1102 & 950 & 799 & 1080 & 950 & 778 \\
\hline & Usable Energy & & & & & & & & & & & & & \\
\hline \multirow{2}{*}{ H2 Split HEV PHEV10 } & Total Energy & 4013 & 4013 & 3604 & 3340 & 3969 & 3436 & 2636 & 3755 & 2759 & 1906 & 3579 & 2459 & 1829 \\
\hline & Usable Energy & 2408 & 2408 & 2162 & 2004 & 2381 & 2061 & 1845 & 2253 & 1932 & 1525 & 2147 & 1844 & 1464 \\
\hline \multirow{2}{*}{ H2 Split HEV PHEV2O } & Total Energy & 7779 & 7779 & 7082 & 6738 & 7601 & 6891 & 5156 & 7358 & 5345 & 3804 & 7019 & 4830 & 3608 \\
\hline & Usable Energy & 4668 & 4668 & 4249 & 4043 & 4561 & 4134 & 3609 & 4415 & 3741 & 3043 & 4211 & 3622 & 2886 \\
\hline
\end{tabular}


Argonne National Laboratory Report - Light-Duty Vehicle Fuel Consumption Displacement Potential Up to 2045

\begin{tabular}{|c|c|c|c|c|c|c|c|c|c|c|c|c|c|c|}
\hline & & \multicolumn{13}{|c|}{ Midsize } \\
\hline & & \multirow{2}{*}{$\begin{array}{c}2010 \\
\text { Ref }\end{array}$} & \multicolumn{3}{|c|}{2010} & \multicolumn{3}{|c|}{2015} & \multicolumn{3}{|c|}{2030} & \multicolumn{3}{|c|}{2045} \\
\hline & & & low & avg & high & low & avg & high & low & avg & high & low & avg & high \\
\hline \multirow{2}{*}{ H2 Series HEV PHEV3O } & Total Energy & 14672 & 14672 & 13323 & 12342 & 14367 & 12683 & 9510 & 13653 & 9866 & 7039 & 13095 & 8852 & 6826 \\
\hline & Usable Energy & 8803 & 8803 & 7994 & 7405 & 8620 & 7610 & 6657 & 8192 & 6906 & 5631 & 7857 & 6639 & 5461 \\
\hline \multirow{2}{*}{ H2 Series HEV PHEV40 } & Total Energy & 19878 & 19878 & 17945 & 16610 & 19462 & 17109 & 12814 & 18481 & 13313 & 9390 & 17620 & 11860 & 9024 \\
\hline & Usable Energy & 11927 & 11927 & 10767 & 9966 & 11677 & 10265 & 8970 & 11088 & 9319 & 7512 & 10572 & 8895 & 7219 \\
\hline \multirow{2}{*}{ E85 Split HEV } & Total Energy & 1147 & 1147 & 1092 & 1045 & 1131 & 1037 & 972 & 1102 & 972 & 842 & 1102 & 972 & 799 \\
\hline & Usable Energy & & & & & & & & & & & & & \\
\hline \multirow{2}{*}{ E85 Split HEV PHEV10 } & Total Energy & 3928 & 3928 & 3606 & 3337 & 3864 & 3452 & 2673 & 3697 & 2801 & 2036 & 3620 & 2491 & 1872 \\
\hline & Usable Energy & 2357 & 2357 & 2164 & 2002 & 2319 & 2071 & 1871 & 2218 & 1960 & 1628 & 2172 & 1868 & 1497 \\
\hline \multirow{2}{*}{ E85 Split HEV PHEV20 } & Total Energy & 7593 & 7593 & 7097 & 6615 & 7719 & 6939 & 5243 & 7225 & 5430 & 3909 & 7108 & 4913 & 3707 \\
\hline & Usable Energy & 4556 & 4556 & 4258 & 3969 & 4632 & 4163 & 3670 & 4335 & 3801 & 3127 & 4265 & 3684 & 2965 \\
\hline \multirow{2}{*}{ E85 Series HEV PHEV30 } & Total Energy & 14443 & 14443 & 13362 & 12467 & 14237 & 12810 & 9687 & 13578 & 9997 & 7222 & 13245 & 8989 & 6906 \\
\hline & Usable Energy & 8666 & 8666 & 8017 & 7480 & 8542 & 7686 & 6781 & 8147 & 6998 & 5777 & 7947 & 6741 & 5525 \\
\hline \multirow{2}{*}{ E85 Series HEV PHEV40 } & Total Energy & 19456 & 19456 & 18104 & 16828 & 19211 & 17282 & 13062 & 18348 & 13494 & 9632 & 17851 & 12067 & 9222 \\
\hline & Usable Energy & 11674 & 11674 & 10862 & 10097 & 11527 & 10369 & 9143 & 11009 & 9445 & 7706 & 10711 & 9050 & 7378 \\
\hline \multirow{2}{*}{ FC HEV } & Total Energy & 1443 & 1443 & 1271 & 1186 & 1310 & 1296 & 1188 & 1382 & 1210 & 1037 & 1318 & 1188 & 994 \\
\hline & Usable Energy & & & & & & & & & & & & & \\
\hline \multirow{2}{*}{ FC HEV PHEV 10} & Total Energy & 5072 & 5072 & 4016 & 3585 & 4756 & 3696 & 2755 & 4000 & 2856 & 2068 & 3800 & 2566 & 1979 \\
\hline & Usable Energy & 3043 & 3043 & 2410 & 2151 & 2853 & 2218 & 1928 & 2400 & 1999 & 1654 & 2280 & 1925 & 1583 \\
\hline \multirow{2}{*}{ FC HEV PHEV 20} & Total Energy & 9405 & 9405 & 8065 & 7195 & 8738 & 7405 & 5679 & 8029 & 5880 & 4049 & 7613 & 5295 & 3865 \\
\hline & Usable Energy & 5643 & 5643 & 4839 & 4317 & 5243 & 4443 & 3975 & 4818 & 4116 & 3239 & 4568 & 3971 & 3092 \\
\hline \multirow{2}{*}{ FC HEV PHEV 30} & Total Energy & 14317 & 14317 & 12478 & 11318 & 13518 & 11590 & 8508 & 12539 & 8860 & 6313 & 11822 & 7875 & 6022 \\
\hline & Usable Energy & 8590 & 8590 & 7487 & 6791 & 8111 & 6954 & 5956 & 7524 & 6202 & 5050 & 7093 & 5906 & 4817 \\
\hline \multirow{2}{*}{ FC HEV PHEV 40} & Total Energy & 19052 & 19052 & 16494 & 14947 & 17925 & 15292 & 11216 & 16603 & 11635 & 8271 & 15770 & 10365 & 7858 \\
\hline & Usable Energy & 11431 & 11431 & 9897 & 8968 & 10755 & 9175 & 7851 & 9962 & 8145 & 6617 & 9462 & 7773 & 6286 \\
\hline \multirow{2}{*}{ BEV } & Total Energy & 69036 & 69036 & 63218 & 58559 & 68344 & 60421 & 42981 & 64591 & 44481 & 30736 & 62186 & 39717 & 29242 \\
\hline & Usable Energy & 41422 & 41422 & 37931 & 35136 & 41006 & 36253 & 30087 & 38755 & 31137 & 24589 & 37312 & 29788 & 23394 \\
\hline
\end{tabular}




\section{Percentage of Energy reduction based on the respective 2010 reference case}

\begin{tabular}{|c|c|c|c|c|c|c|c|c|c|c|c|c|c|c|}
\hline & & \multicolumn{13}{|c|}{ Midsize } \\
\hline & & \multirow{2}{*}{$\frac{2010}{\text { Ref }}$} & \multicolumn{3}{|c|}{2010} & \multicolumn{3}{|c|}{2015} & \multicolumn{3}{|c|}{2030} & \multicolumn{3}{|c|}{2045} \\
\hline & & & low & avg & high & low & avg & high & low & avg & high & low & avg & high \\
\hline \multirow{2}{*}{ SI Split HEV } & Total Energy & $0.0 \%$ & $0.0 \%$ & $-4.1 \%$ & $-8.2 \%$ & $-0.7 \%$ & $-9.0 \%$ & $-14.6 \%$ & $-3.3 \%$ & $-14.6 \%$ & $-27.9 \%$ & $-3.3 \%$ & $-14.6 \%$ & $-29.8 \%$ \\
\hline & Usable Energy & & & & & & & & & & & & & \\
\hline \multirow{2}{*}{ SI Split HEV PHEV10 } & Total Energy & $0.0 \%$ & $0.0 \%$ & $-7.0 \%$ & $-13.9 \%$ & $-0.3 \%$ & $-11.0 \%$ & $-31.0 \%$ & $-4.6 \%$ & $-28.1 \%$ & $-47.5 \%$ & $-6.6 \%$ & $-35.7 \%$ & $-51.7 \%$ \\
\hline & Usable Energy & $0.0 \%$ & $0.0 \%$ & $-7.0 \%$ & $-13.9 \%$ & $-0.3 \%$ & $-11.0 \%$ & $-19.5 \%$ & $-4.6 \%$ & $-16.1 \%$ & $-30.0 \%$ & $-6.6 \%$ & $-19.7 \%$ & $-35.7 \%$ \\
\hline \multirow{2}{*}{ SI Split HEV PHEV2O } & Total Energy & $0.0 \%$ & $0.0 \%$ & $-6.5 \%$ & $-12.9 \%$ & $1.6 \%$ & $-10.5 \%$ & $-31.0 \%$ & $-4.4 \%$ & $-28.6 \%$ & $-48.6 \%$ & $-6.4 \%$ & $-35.4 \%$ & $-51.3 \%$ \\
\hline & Usable Energy & $0.0 \%$ & $0.0 \%$ & $-6.5 \%$ & $-12.9 \%$ & $1.6 \%$ & $-10.5 \%$ & $-19.5 \%$ & $-4.4 \%$ & $-16.6 \%$ & $-31.5 \%$ & $-6.4 \%$ & $-19.2 \%$ & $-35.0 \%$ \\
\hline \multirow{2}{*}{ SI Series HEV PHEV30 } & Total Energy & $0.0 \%$ & $0.0 \%$ & $-7.6 \%$ & $-13.7 \%$ & $-1.4 \%$ & $-11.3 \%$ & $-32.9 \%$ & $-6.0 \%$ & $-30.8 \%$ & $-50.0 \%$ & $-8.3 \%$ & $-37.8 \%$ & $-52.2 \%$ \\
\hline & Usable Energy & $0.0 \%$ & $0.0 \%$ & $-7.6 \%$ & $-13.7 \%$ & $-1.4 \%$ & $-11.3 \%$ & $-21.8 \%$ & $-6.0 \%$ & $-19.3 \%$ & $-33.3 \%$ & $-8.3 \%$ & $-22.2 \%$ & $-36.3 \%$ \\
\hline \multirow{2}{*}{ SI Series HEV PHEV40 } & Total Energy & $0.0 \%$ & $0.0 \%$ & $-7.3 \%$ & $-13.6 \%$ & $-1.4 \%$ & $-11.3 \%$ & $-33.0 \%$ & $-5.9 \%$ & $-30.8 \%$ & $-50.6 \%$ & $-8.4 \%$ & $-38.1 \%$ & $-52.6 \%$ \\
\hline & Usable Energy & $0.0 \%$ & $0.0 \%$ & $-7.3 \%$ & $-13.6 \%$ & $-1.4 \%$ & $-11.3 \%$ & $-21.9 \%$ & $-5.9 \%$ & $-19.3 \%$ & $-34.1 \%$ & $-8.4 \%$ & $-22.6 \%$ & $-36.8 \%$ \\
\hline \multirow{2}{*}{ CI Split HEV } & Total Energy & $0.0 \%$ & $0.0 \%$ & $-4.5 \%$ & $-9.6 \%$ & $-1.3 \%$ & $-9.5 \%$ & $-16.6 \%$ & $-4.1 \%$ & $-14.8 \%$ & $-27.2 \%$ & $-4.1 \%$ & $-14.8 \%$ & $-29.0 \%$ \\
\hline & Usable Energy & & & & & & & & & & & & & \\
\hline \multirow{2}{*}{ CI Split HEV PHEV10 } & Total Energy & $0.0 \%$ & $0.0 \%$ & $-6.7 \%$ & $-14.2 \%$ & $-1.0 \%$ & $-10.8 \%$ & $-31.6 \%$ & $-5.0 \%$ & $-29.4 \%$ & $-47.3 \%$ & $-6.6 \%$ & $-36.1 \%$ & $-49.5 \%$ \\
\hline & Usable Energy & $0.0 \%$ & $0.0 \%$ & $-6.7 \%$ & $-14.2 \%$ & $-1.0 \%$ & $-10.8 \%$ & $-20.1 \%$ & $-5.0 \%$ & $-17.6 \%$ & $-29.8 \%$ & $-6.6 \%$ & $-20.1 \%$ & $-32.7 \%$ \\
\hline \multirow{2}{*}{ CI Split HEV PHEV2O } & Total Energy & $0.0 \%$ & $0.0 \%$ & $-8.5 \%$ & $-14.3 \%$ & $-2.4 \%$ & $-10.6 \%$ & $-30.9 \%$ & $-6.7 \%$ & $-28.7 \%$ & $-49.7 \%$ & $-8.7 \%$ & $-36.6 \%$ & $-51.8 \%$ \\
\hline & Usable Energy & $0.0 \%$ & $0.0 \%$ & $-8.5 \%$ & $-14.3 \%$ & $-2.4 \%$ & $-10.6 \%$ & $-19.4 \%$ & $-6.7 \%$ & $-16.8 \%$ & $-33.0 \%$ & $-8.7 \%$ & $-20.8 \%$ & $-35.8 \%$ \\
\hline \multirow{2}{*}{ CI Series HEV PHEV 30} & Total Energy & $0.0 \%$ & $0.0 \%$ & $-7.4 \%$ & $-13.5 \%$ & $-1.5 \%$ & $-11.0 \%$ & $-32.5 \%$ & $-6.1 \%$ & $-30.4 \%$ & $-49.2 \%$ & $-8.3 \%$ & $-37.3 \%$ & $-51.3 \%$ \\
\hline & Usable Energy & $0.0 \%$ & $0.0 \%$ & $-7.4 \%$ & $-13.5 \%$ & $-1.5 \%$ & $-11.0 \%$ & $-21.3 \%$ & $-6.1 \%$ & $-18.8 \%$ & $-32.3 \%$ & $-8.3 \%$ & $-21.6 \%$ & $-35.0 \%$ \\
\hline \multirow{2}{*}{$\mathrm{Cl}$ Series HEV PHEV40 } & Total Energy & $0.0 \%$ & $0.0 \%$ & $-7.4 \%$ & $-13.8 \%$ & $-2.0 \%$ & $-11.2 \%$ & $-32.8 \%$ & $-6.0 \%$ & $-30.7 \%$ & $-50.1 \%$ & $-8.3 \%$ & $-37.9 \%$ & $-52.3 \%$ \\
\hline & Usable Energy & $0.0 \%$ & $0.0 \%$ & $-7.4 \%$ & $-13.8 \%$ & $-2.0 \%$ & $-11.2 \%$ & $-21.6 \%$ & $-6.0 \%$ & $-19.1 \%$ & $-33.4 \%$ & $-8.3 \%$ & $-22.4 \%$ & $-36.4 \%$ \\
\hline \multirow{2}{*}{ H2 Split HEV } & Total Energy & $0.0 \%$ & $0.0 \%$ & $-7.3 \%$ & $-12.6 \%$ & $-2.6 \%$ & $-12.0 \%$ & $-19.3 \%$ & $-6.5 \%$ & $-19.3 \%$ & $-32.1 \%$ & $-8.3 \%$ & $-19.3 \%$ & $-34.0 \%$ \\
\hline & Usable Energy & & & & & & & & & & & & & \\
\hline \multirow{2}{*}{ H2 Split HEV PHEV10 } & Total Energy & $0.0 \%$ & $0.0 \%$ & $-10.2 \%$ & $-16.8 \%$ & $-1.1 \%$ & $-14.4 \%$ & $-34.3 \%$ & $-6.4 \%$ & $-31.2 \%$ & $-52.5 \%$ & $-10.8 \%$ & $-38.7 \%$ & $-54.4 \%$ \\
\hline & Usable Energy & $0.0 \%$ & $0.0 \%$ & $-10.2 \%$ & $-16.8 \%$ & $-1.1 \%$ & $-14.4 \%$ & $-23.4 \%$ & $-6.4 \%$ & $-19.8 \%$ & $-36.7 \%$ & $-10.8 \%$ & $-23.4 \%$ & $-39.2 \%$ \\
\hline \multirow{2}{*}{ H2 Split HEV PHEV 20} & Total Energy & $0.0 \%$ & $0.0 \%$ & $-9.0 \%$ & $-13.4 \%$ & $-2.3 \%$ & $-11.4 \%$ & $-33.7 \%$ & $-5.4 \%$ & $-31.3 \%$ & $-51.1 \%$ & $-9.8 \%$ & $-37.9 \%$ & $-53.6 \%$ \\
\hline & Usable Energy & $0.0 \%$ & $0.0 \%$ & $-9.0 \%$ & $-13.4 \%$ & $-2.3 \%$ & $-11.4 \%$ & $-22.7 \%$ & $-5.4 \%$ & $-19.8 \%$ & $-34.8 \%$ & $-9.8 \%$ & $-22.4 \%$ & $-38.2 \%$ \\
\hline
\end{tabular}




\begin{tabular}{|c|c|c|c|c|c|c|c|c|c|c|c|c|c|c|}
\hline & & \multicolumn{13}{|c|}{ Midsize } \\
\hline & & \multirow{2}{*}{$\frac{2010}{\operatorname{Ref}}$} & \multicolumn{3}{|c|}{2010} & \multicolumn{3}{|c|}{2015} & \multicolumn{3}{|c|}{2030} & \multicolumn{3}{|c|}{2045} \\
\hline & & & low & avg & high & low & avg & high & low & avg & high & low & avg & high \\
\hline \multirow{2}{*}{ H2 Series HEV PHEV30 } & Total Energy & $0.0 \%$ & $0.0 \%$ & $-9.2 \%$ & $-15.9 \%$ & $-2.1 \%$ & $-13.6 \%$ & $-35.2 \%$ & $-6.9 \%$ & $-32.8 \%$ & $-52.0 \%$ & $-10.7 \%$ & $-39.7 \%$ & $-53.5 \%$ \\
\hline & Usable Energy & $0.0 \%$ & $0.0 \%$ & $-9.2 \%$ & $-15.9 \%$ & $-2.1 \%$ & $-13.6 \%$ & $-24.4 \%$ & $-6.9 \%$ & $-21.5 \%$ & $-36.0 \%$ & $-10.7 \%$ & $-24.6 \%$ & $-38.0 \%$ \\
\hline \multirow{2}{*}{ H2 Series HEV PHEV40 } & Total Energy & $0.0 \%$ & $0.0 \%$ & $-9.7 \%$ & $-16.4 \%$ & $-2.1 \%$ & $-13.9 \%$ & $-35.5 \%$ & $-7.0 \%$ & $-33.0 \%$ & $-52.8 \%$ & $-11.4 \%$ & $-40.3 \%$ & $-54.6 \%$ \\
\hline & Usable Energy & $0.0 \%$ & $0.0 \%$ & $-9.7 \%$ & $-16.4 \%$ & $-2.1 \%$ & $-13.9 \%$ & $-24.8 \%$ & $-7.0 \%$ & $-21.9 \%$ & $-37.0 \%$ & $-11.4 \%$ & $-25.4 \%$ & $-39.5 \%$ \\
\hline \multirow{2}{*}{ E85 Split HEV } & Total Energy & $0.0 \%$ & $0.0 \%$ & $-4.8 \%$ & $-8.8 \%$ & $-1.4 \%$ & $-9.6 \%$ & $-15.2 \%$ & $-3.9 \%$ & $-15.2 \%$ & $-26.5 \%$ & $-3.9 \%$ & $-15.2 \%$ & $-30.3 \%$ \\
\hline & Usable Energy & & & & & & & & & & & & & \\
\hline \multirow{2}{*}{ E85 Split HEV PHEV10 } & Total Energy & $0.0 \%$ & $0.0 \%$ & $-8.2 \%$ & $-15.0 \%$ & $-1.6 \%$ & $-12.1 \%$ & $-31.9 \%$ & $-5.9 \%$ & $-28.7 \%$ & $-48.2 \%$ & $-7.8 \%$ & $-36.6 \%$ & $-52.3 \%$ \\
\hline & Usable Energy & $0.0 \%$ & $0.0 \%$ & $-8.2 \%$ & $-15.0 \%$ & $-1.6 \%$ & $-12.1 \%$ & $-20.6 \%$ & $-5.9 \%$ & $-16.8 \%$ & $-30.9 \%$ & $-7.8 \%$ & $-20.7 \%$ & $-36.5 \%$ \\
\hline \multirow{2}{*}{ E85 Split HEV PHEV20 } & Total Energy & $0.0 \%$ & $0.0 \%$ & $-6.5 \%$ & $-12.9 \%$ & $1.7 \%$ & $-8.6 \%$ & $-31.0 \%$ & $-4.9 \%$ & $-28.5 \%$ & $-48.5 \%$ & $-6.4 \%$ & $-35.3 \%$ & $-51.2 \%$ \\
\hline & Usable Energy & $0.0 \%$ & $0.0 \%$ & $-6.5 \%$ & $-12.9 \%$ & $1.7 \%$ & $-8.6 \%$ & $-19.4 \%$ & $-4.9 \%$ & $-16.6 \%$ & $-31.4 \%$ & $-6.4 \%$ & $-19.1 \%$ & $-34.9 \%$ \\
\hline \multirow{2}{*}{ E85 Series HEV PHEV30 } & Total Energy & $0.0 \%$ & $0.0 \%$ & $-7.5 \%$ & $-13.7 \%$ & $-1.4 \%$ & $-11.3 \%$ & $-32.9 \%$ & $-6.0 \%$ & $-30.8 \%$ & $-50.0 \%$ & $-8.3 \%$ & $-37.8 \%$ & $-52.2 \%$ \\
\hline & Usable Energy & $0.0 \%$ & $0.0 \%$ & $-7.5 \%$ & $-13.7 \%$ & $-1.4 \%$ & $-11.3 \%$ & $-21.8 \%$ & $-6.0 \%$ & $-19.2 \%$ & $-33.3 \%$ & $-8.3 \%$ & $-22.2 \%$ & $-36.2 \%$ \\
\hline \multirow{2}{*}{ E85 Series HEV PHEV40 } & Total Energy & $0.0 \%$ & $0.0 \%$ & $-7.0 \%$ & $-13.5 \%$ & $-1.3 \%$ & $-11.2 \%$ & $-32.9 \%$ & $-5.7 \%$ & $-30.6 \%$ & $-50.5 \%$ & $-8.2 \%$ & $-38.0 \%$ & $-52.6 \%$ \\
\hline & Usable Energy & $0.0 \%$ & $0.0 \%$ & $-7.0 \%$ & $-13.5 \%$ & $-1.3 \%$ & $-11.2 \%$ & $-21.7 \%$ & $-5.7 \%$ & $-19.1 \%$ & $-34.0 \%$ & $-8.2 \%$ & $-22.5 \%$ & $-36.8 \%$ \\
\hline \multirow{2}{*}{ FC HEV } & Total Energy & $0.0 \%$ & $0.0 \%$ & $-11.9 \%$ & $-17.8 \%$ & $-9.2 \%$ & $-10.2 \%$ & $-17.7 \%$ & $-4.2 \%$ & $-16.2 \%$ & $-28.1 \%$ & $-8.7 \%$ & $-17.7 \%$ & $-31.1 \%$ \\
\hline & Usable Energy & & & & & & & & & & & & & \\
\hline \multirow{2}{*}{ FC HEV PHEV10 } & Total Energy & $0.0 \%$ & $0.0 \%$ & $-20.8 \%$ & $-29.3 \%$ & $-6.2 \%$ & $-27.1 \%$ & $-45.7 \%$ & $-21.1 \%$ & $-43.7 \%$ & $-59.2 \%$ & $-25.1 \%$ & $-49.4 \%$ & $-61.0 \%$ \\
\hline & Usable Energy & $0.0 \%$ & $0.0 \%$ & $-20.8 \%$ & $-29.3 \%$ & $-6.2 \%$ & $-27.1 \%$ & $-36.6 \%$ & $-21.1 \%$ & $-34.3 \%$ & $-45.6 \%$ & $-25.1 \%$ & $-36.8 \%$ & $-48.0 \%$ \\
\hline \multirow{2}{*}{ FC HEV PHEV20 } & Total Energy & $0.0 \%$ & $0.0 \%$ & $-14.2 \%$ & $-23.5 \%$ & $-7.1 \%$ & $-21.3 \%$ & $-39.6 \%$ & $-14.6 \%$ & $-37.5 \%$ & $-57.0 \%$ & $-19.0 \%$ & $-43.7 \%$ & $-58.9 \%$ \\
\hline & Usable Energy & $0.0 \%$ & $0.0 \%$ & $-14.2 \%$ & $-23.5 \%$ & $-7.1 \%$ & $-21.3 \%$ & $-29.6 \%$ & $-14.6 \%$ & $-27.1 \%$ & $-42.6 \%$ & $-19.0 \%$ & $-29.6 \%$ & $-45.2 \%$ \\
\hline \multirow{2}{*}{ FC HEV PHEV 30} & Total Energy & $0.0 \%$ & $0.0 \%$ & $-12.8 \%$ & $-20.9 \%$ & $-5.6 \%$ & $-19.0 \%$ & $-40.6 \%$ & $-12.4 \%$ & $-38.1 \%$ & $-55.9 \%$ & $-17.4 \%$ & $-45.0 \%$ & $-57.9 \%$ \\
\hline & Usable Energy & $0.0 \%$ & $0.0 \%$ & $-12.8 \%$ & $-20.9 \%$ & $-5.6 \%$ & $-19.0 \%$ & $-30.7 \%$ & $-12.4 \%$ & $-27.8 \%$ & $-41.2 \%$ & $-17.4 \%$ & $-31.2 \%$ & $-43.9 \%$ \\
\hline \multirow{2}{*}{ FC HEV PHEV 40} & Total Energy & $0.0 \%$ & $0.0 \%$ & $-13.4 \%$ & $-21.5 \%$ & $-5.9 \%$ & $-19.7 \%$ & $-41.1 \%$ & $-12.9 \%$ & $-38.9 \%$ & $-56.6 \%$ & $-17.2 \%$ & $-45.6 \%$ & $-58.8 \%$ \\
\hline & Usable Energy & $0.0 \%$ & $0.0 \%$ & $-13.4 \%$ & $-21.5 \%$ & $-5.9 \%$ & $-19.7 \%$ & $-31.3 \%$ & $-12.9 \%$ & $-28.7 \%$ & $-42.1 \%$ & $-17.2 \%$ & $-32.0 \%$ & $-45.0 \%$ \\
\hline \multirow{2}{*}{ EV } & Total Energy & $0.0 \%$ & $0.0 \%$ & $-8.4 \%$ & $-15.2 \%$ & $-1.0 \%$ & $-12.5 \%$ & $-37.7 \%$ & $-6.4 \%$ & $-35.6 \%$ & $-55.5 \%$ & $-9.9 \%$ & $-42.5 \%$ & $-57.6 \%$ \\
\hline & Usable Energy & $0.0 \%$ & $0.0 \%$ & $-8.4 \%$ & $-15.2 \%$ & $-1.0 \%$ & $-12.5 \%$ & $-27.4 \%$ & $-6.4 \%$ & $-24.8 \%$ & $-40.6 \%$ & $-9.9 \%$ & $-28.1 \%$ & $-43.5 \%$ \\
\hline
\end{tabular}


Curb Weight

\begin{tabular}{|c|c|c|c|c|c|c|c|c|c|c|c|c|c|c|}
\hline & & \multicolumn{13}{|c|}{ Compact } \\
\hline & & 2010 & \multicolumn{3}{|c|}{2010} & \multicolumn{3}{|c|}{2015} & \multicolumn{3}{|c|}{2030} & \multicolumn{3}{|c|}{2045} \\
\hline & & Ref & low & avg & high & low & avg & high & low & avg & high & low & avg & high \\
\hline \multirow{3}{*}{ SI Conv } & Vehicle Mass & 1238 & 1238 & 1198 & 1155 & 1237 & 1167 & 1093 & 1229 & 1104 & 959 & 1230 & 1094 & 919 \\
\hline & $\%$ of Glider mass & 65.0 & 65.0 & 64.1 & 63.1 & 65.1 & 63.6 & 61.4 & 65.2 & 61.7 & 56.3 & 65.2 & 61.3 & 54.3 \\
\hline & $\%$ of Powertrain mass & 35.0 & 35.0 & 35.9 & 36.9 & 34.9 & 36.4 & 38.6 & 34.8 & 38.3 & 43.7 & 34.8 & 38.7 & 45.7 \\
\hline \multirow{3}{*}{$\mathrm{Cl}$ Conv } & Vehicle Mass & 1301 & 1301 & 1262 & 1221 & 1301 & 1235 & 1159 & 1296 & 1170 & 1026 & 1296 & 1159 & 983 \\
\hline & $\%$ of Glider mass & 61.9 & 61.9 & 60.8 & 59.7 & 61.9 & 60.1 & 57.9 & 61.9 & 58.2 & 52.6 & 61.9 & 57.8 & 50.7 \\
\hline & $\%$ of Powertrain mass & 38.1 & 38.1 & 39.2 & 40.3 & 38.1 & 39.9 & 42.1 & 38.1 & 41.8 & 47.4 & 38.1 & 42.2 & 49.3 \\
\hline \multirow{3}{*}{ H2 Conv } & Vehicle Mass & 1268 & 1268 & 1189 & 1131 & 1253 & 1146 & 1057 & 1240 & 1076 & 908 & 1204 & 1057 & 875 \\
\hline & $\%$ of Glider mass & 63.5 & 63.5 & 64.6 & 64.4 & 64.3 & 64.8 & 63.5 & 64.6 & 63.3 & 59.4 & 66.6 & 63.4 & 57.0 \\
\hline & $\%$ of Powertrain mass & 36.5 & 36.5 & 35.4 & 35.6 & 35.7 & 35.2 & 36.5 & 35.4 & 36.7 & 40.6 & 33.4 & 36.6 & 43.0 \\
\hline \multirow{3}{*}{ E85 Conv } & Vehicle Mass & 1234 & 1234 & 1194 & 1151 & 1234 & 1166 & 1090 & 1228 & 1101 & 955 & 1228 & 1091 & 913 \\
\hline & $\%$ of Glider mass & 65.2 & 65.2 & 64.3 & 63.3 & 65.3 & 63.7 & 61.6 & 65.3 & 61.8 & 56.5 & 65.3 & 61.4 & 54.6 \\
\hline & $\%$ of Powertrain mass & 34.8 & 34.8 & 35.7 & 36.7 & 34.7 & 36.3 & 38.4 & 34.7 & 38.2 & 43.5 & 34.7 & 38.6 & 45.4 \\
\hline \multirow{3}{*}{ SI Split HEV } & Vehicle Mass & 1341 & 1341 & 1279 & 1221 & 1325 & 1252 & 1149 & 1316 & 1169 & 990 & 1308 & 1150 & 943 \\
\hline & $\%$ of Glider mass & 60.0 & 60.0 & 60.0 & 59.7 & 60.8 & 59.3 & 58.4 & 60.9 & 58.2 & 54.5 & 61.3 & 58.3 & 52.9 \\
\hline & $\%$ of Powertrain mass & 40.0 & 40.0 & 40.0 & 40.3 & 39.2 & 40.7 & 41.6 & 39.1 & 41.8 & 45.5 & 38.7 & 41.7 & 47.1 \\
\hline \multirow{3}{*}{ SI Split HEV PHEV10 } & Vehicle Mass & 1351 & 1351 & 1288 & 1230 & 1330 & 1250 & 1147 & 1315 & 1168 & 988 & 1307 & 1147 & 941 \\
\hline & $\%$ of Glider mass & 59.6 & 59.6 & 59.6 & 59.3 & 60.6 & 59.4 & 58.5 & 61.0 & 58.3 & 54.6 & 61.3 & 58.4 & 53.0 \\
\hline & $\%$ of Powertrain mass & 40.4 & 40.4 & 40.4 & 40.7 & 39.4 & 40.6 & 41.5 & 39.0 & 41.7 & 45.4 & 38.7 & 41.6 & 47.0 \\
\hline \multirow{3}{*}{ SI Split HEV PHEV20 } & Vehicle Mass & 1377 & 1377 & 1315 & 1253 & 1357 & 1273 & 1164 & 1340 & 1187 & 999 & 1331 & 1162 & 952 \\
\hline & $\%$ of Glider mass & 58.4 & 58.4 & 58.4 & 58.2 & 59.4 & 58.3 & 57.7 & 59.8 & 57.4 & 54.0 & 60.2 & 57.7 & 52.4 \\
\hline & $\%$ of Powertrain mass & 41.6 & 41.6 & 41.6 & 41.8 & 40.6 & 41.7 & 42.3 & 40.2 & 42.6 & 46.0 & 39.8 & 42.3 & 47.6 \\
\hline \multirow{3}{*}{ SI Series HEV PHEV30 } & Vehicle Mass & 1581 & 1581 & 1493 & 1407 & 1542 & 1438 & 1286 & 1510 & 1320 & 1094 & 1487 & 1284 & 1040 \\
\hline & $\%$ of Glider mass & 50.9 & 50.9 & 51.4 & 51.8 & 52.3 & 51.6 & 52.2 & 53.1 & 51.6 & 49.3 & 53.9 & 52.2 & 47.9 \\
\hline & $\%$ of Powertrain mass & 49.1 & 49.1 & 48.6 & 48.2 & 47.7 & 48.4 & 47.8 & 46.9 & 48.4 & 50.7 & 46.1 & 47.8 & 52.1 \\
\hline \multirow{3}{*}{ SI Series HEV PHEV40 } & Vehicle Mass & 1624 & 1624 & 1529 & 1442 & 1584 & 1476 & 1313 & 1547 & 1349 & 1113 & 1523 & 1308 & 1057 \\
\hline & $\%$ of Glider mass & 49.6 & 49.6 & 50.2 & 50.5 & 50.9 & 50.3 & 51.1 & 51.8 & 50.5 & 48.5 & 52.6 & 51.3 & 47.2 \\
\hline & $\%$ of Powertrain mass & 50.4 & 50.4 & 49.8 & 49.5 & 49.1 & 49.7 & 48.9 & 48.2 & 49.5 & 51.5 & 47.4 & 48.7 & 52.8 \\
\hline \multirow{3}{*}{ CI Split HEV } & Vehicle Mass & 1442 & 1442 & 1378 & 1319 & 1416 & 1348 & 1246 & 1414 & 1266 & 1083 & 1405 & 1244 & 1036 \\
\hline & $\%$ of Glider mass & 55.8 & 55.8 & 55.7 & 55.3 & 56.9 & 55.1 & 53.9 & 56.7 & 53.8 & 49.8 & 57.1 & 53.9 & 48.1 \\
\hline & $\%$ of Powertrain mass & 44.2 & 44.2 & 44.3 & 44.7 & 43.1 & 44.9 & 46.1 & 43.3 & 46.2 & 50.2 & 42.9 & 46.1 & 51.9 \\
\hline
\end{tabular}




\begin{tabular}{|c|c|c|c|c|c|c|c|c|c|c|c|c|c|c|}
\hline & & \multicolumn{13}{|c|}{ Compact } \\
\hline & & \multirow{2}{*}{$\begin{array}{c}2010 \\
\operatorname{Ref} \\
\end{array}$} & \multicolumn{3}{|c|}{2010} & \multicolumn{3}{|c|}{2015} & \multicolumn{3}{|c|}{2030} & \multicolumn{3}{|c|}{2045} \\
\hline & & & low & avg & high & low & avg & high & low & avg & high & low & avg & high \\
\hline \multirow{3}{*}{ CI Split HEV PHEV10 } & Vehicle Mass & 1457 & 1457 & 1394 & 1336 & 1437 & 1357 & 1248 & 1422 & 1270 & 1087 & 1411 & 1250 & 1039 \\
\hline & $\%$ of Glider mass & 55.2 & 55.2 & 55.1 & 54.6 & 56.1 & 54.7 & 53.8 & 56.4 & 53.6 & 49.6 & 56.8 & 53.6 & 48.0 \\
\hline & $\%$ of Powertrain mass & 44.8 & 44.8 & 44.9 & 45.4 & 43.9 & 45.3 & 46.2 & 43.6 & 46.4 & 50.4 & 43.2 & 46.4 & 52.0 \\
\hline \multirow{3}{*}{ CI Split HEV PHEV 20} & Vehicle Mass & 1486 & 1486 & 1424 & 1360 & 1465 & 1384 & 1268 & 1449 & 1291 & 1100 & 1437 & 1267 & 1053 \\
\hline & $\%$ of Glider mass & 54.2 & 54.2 & 53.9 & 53.6 & 55.0 & 53.6 & 52.9 & 55.3 & 52.7 & 49.1 & 55.8 & 52.9 & 47.3 \\
\hline & $\%$ of Powertrain mass & 45.8 & 45.8 & 46.1 & 46.4 & 45.0 & 46.4 & 47.1 & 44.7 & 47.3 & 50.9 & 44.2 & 47.1 & 52.7 \\
\hline \multirow{3}{*}{ CI Series HEV PHEV30 } & Vehicle Mass & 1677 & 1677 & 1587 & 1505 & 1638 & 1537 & 1382 & 1605 & 1417 & 1190 & 1582 & 1381 & 1137 \\
\hline & $\%$ of Glider mass & 48.0 & 48.0 & 48.4 & 48.4 & 49.2 & 48.3 & 48.6 & 49.9 & 48.0 & 45.4 & 50.7 & 48.5 & 43.8 \\
\hline & $\%$ of Powertrain mass & 52.0 & 52.0 & 51.6 & 51.6 & 50.8 & 51.7 & 51.4 & 50.1 & 52.0 & 54.6 & 49.3 & 51.5 & 56.2 \\
\hline \multirow{3}{*}{ CI Series HEV PHEV40 } & Vehicle Mass & 1723 & 1723 & 1628 & 1542 & 1684 & 1574 & 1412 & 1645 & 1444 & 1210 & 1620 & 1405 & 1153 \\
\hline & $\%$ of Glider mass & 46.7 & 46.7 & 47.2 & 47.3 & 47.8 & 47.2 & 47.5 & 48.7 & 47.1 & 44.6 & 49.5 & 47.7 & 43.2 \\
\hline & $\%$ of Powertrain mass & 53.3 & 53.3 & 52.8 & 52.7 & 52.2 & 52.8 & 52.5 & 51.3 & 52.9 & 55.4 & 50.5 & 52.3 & 56.8 \\
\hline \multirow{3}{*}{ H2 Split HEV } & Vehicle Mass & 1394 & 1394 & 1291 & 1215 & 1360 & 1245 & 1127 & 1343 & 1156 & 956 & 1297 & 1129 & 919 \\
\hline & $\%$ of Glider mass & 57.7 & 57.7 & 59.5 & 60.0 & 59.2 & 59.6 & 59.6 & 59.7 & 58.9 & 56.5 & 61.8 & 59.4 & 54.3 \\
\hline & $\%$ of Powertrain mass & 42.3 & 42.3 & 40.5 & 40.0 & 40.8 & 40.4 & 40.4 & 40.3 & 41.1 & 43.5 & 38.2 & 40.6 & 45.7 \\
\hline \multirow{3}{*}{ H2 Split HEV PHEV10 } & Vehicle Mass & 1407 & 1407 & 1304 & 1227 & 1374 & 1247 & 1127 & 1346 & 1158 & 955 & 1298 & 1131 & 918 \\
\hline & $\%$ of Glider mass & 57.2 & 57.2 & 58.9 & 59.4 & 58.6 & 59.5 & 59.6 & 59.6 & 58.8 & 56.5 & 61.8 & 59.3 & 54.3 \\
\hline & $\%$ of Powertrain mass & 42.8 & 42.8 & 41.1 & 40.6 & 41.4 & 40.5 & 40.4 & 40.4 & 41.2 & 43.5 & 38.2 & 40.7 & 45.7 \\
\hline \multirow{3}{*}{ H2 Split HEV PHEV2O } & Vehicle Mass & 1435 & 1435 & 1329 & 1249 & 1402 & 1273 & 1144 & 1375 & 1176 & 967 & 1325 & 1146 & 928 \\
\hline & $\%$ of Glider mass & 56.1 & 56.1 & 57.8 & 58.4 & 57.5 & 58.3 & 58.7 & 58.3 & 57.9 & 55.8 & 60.5 & 58.5 & 53.7 \\
\hline & $\%$ of Powertrain mass & 43.9 & 43.9 & 42.2 & 41.6 & 42.5 & 41.7 & 41.3 & 41.7 & 42.1 & 44.2 & 39.5 & 41.5 & 46.3 \\
\hline \multirow{3}{*}{ H2 Series HEV PHEV3O } & Vehicle Mass & 1632 & 1632 & 1498 & 1396 & 1576 & 1427 & 1261 & 1532 & 1303 & 1056 & 1470 & 1258 & 1012 \\
\hline & $\%$ of Glider mass & 49.3 & 49.3 & 51.3 & 52.2 & 51.1 & 52.0 & 53.2 & 52.3 & 52.2 & 51.1 & 54.5 & 53.3 & 49.3 \\
\hline & $\%$ of Powertrain mass & 50.7 & 50.7 & 48.7 & 47.8 & 48.9 & 48.0 & 46.8 & 47.7 & 47.8 & 48.9 & 45.5 & 46.7 & 50.7 \\
\hline \multirow{3}{*}{ H2 Series HEV PHEV 40} & Vehicle Mass & 1675 & 1675 & 1536 & 1429 & 1618 & 1463 & 1288 & 1573 & 1331 & 1074 & 1505 & 1281 & 1029 \\
\hline & $\%$ of Glider mass & 48.0 & 48.0 & 50.0 & 51.0 & 49.8 & 50.7 & 52.1 & 51.0 & 51.1 & 50.2 & 53.3 & 52.3 & 48.5 \\
\hline & $\%$ of Powertrain mass & 52.0 & 52.0 & 50.0 & 49.0 & 50.2 & 49.3 & 47.9 & 49.0 & 48.9 & 49.8 & 46.7 & 47.7 & 51.5 \\
\hline \multirow{3}{*}{ E85 Split HEV } & Vehicle Mass & 1342 & 1342 & 1281 & 1226 & 1324 & 1257 & 1151 & 1318 & 1170 & 991 & 1309 & 1152 & 945 \\
\hline & $\%$ of Glider mass & 60.0 & 60.0 & 59.9 & 59.4 & 60.9 & 59.1 & 58.3 & 60.8 & 58.2 & 54.5 & 61.2 & 58.2 & 52.8 \\
\hline & $\%$ of Powertrain mass & 40.0 & 40.0 & 40.1 & 40.6 & 39.1 & 40.9 & 41.7 & 39.2 & 41.8 & 45.5 & 38.8 & 41.8 & 47.2 \\
\hline
\end{tabular}




\begin{tabular}{|c|c|c|c|c|c|c|c|c|c|c|c|c|c|c|}
\hline & & \multicolumn{13}{|c|}{ Compact } \\
\hline & & \multirow{2}{*}{$\frac{2010}{\operatorname{Ref}}$} & \multicolumn{3}{|c|}{2010} & \multicolumn{3}{|c|}{2015} & \multicolumn{3}{|c|}{2030} & \multicolumn{3}{|c|}{2045} \\
\hline & & & low & avg & high & low & avg & high & low & avg & high & low & avg & high \\
\hline \multirow{3}{*}{ E85 Split HEV PHEV10 } & Vehicle Mass & 1352 & 1352 & 1289 & 1231 & 1331 & 1252 & 1149 & 1316 & 1169 & 989 & 1308 & 1149 & 942 \\
\hline & $\%$ of Glider mass & 59.5 & 59.5 & 59.6 & 59.2 & 60.5 & 59.3 & 58.4 & 60.9 & 58.2 & 54.6 & 61.3 & 58.3 & 52.9 \\
\hline & $\%$ of Powertrain mass & 40.5 & 40.5 & 40.4 & 40.8 & 39.5 & 40.7 & 41.6 & 39.1 & 41.8 & 45.4 & 38.7 & 41.7 & 47.1 \\
\hline \multirow{3}{*}{ E85 Split HEV PHEV20 } & Vehicle Mass & 1378 & 1378 & 1316 & 1254 & 1358 & 1275 & 1166 & 1341 & 1188 & 1000 & 1332 & 1164 & 953 \\
\hline & $\%$ of Glider mass & 58.4 & 58.4 & 58.3 & 58.1 & 59.3 & 58.2 & 57.6 & 59.8 & 57.3 & 54.0 & 60.2 & 57.6 & 52.3 \\
\hline & $\%$ of Powertrain mass & 41.6 & 41.6 & 41.7 & 41.9 & 40.7 & 41.8 & 42.4 & 40.2 & 42.7 & 46.0 & 39.8 & 42.4 & 47.7 \\
\hline \multirow{3}{*}{ E85 Series HEV PHEV30 } & Vehicle Mass & 1582 & 1582 & 1494 & 1408 & 1543 & 1440 & 1288 & 1511 & 1321 & 1095 & 1488 & 1286 & 1042 \\
\hline & $\%$ of Glider mass & 50.9 & 50.9 & 51.4 & 51.8 & 52.2 & 51.6 & 52.1 & 53.1 & 51.5 & 49.3 & 53.9 & 52.1 & 47.8 \\
\hline & $\%$ of Powertrain mass & 49.1 & 49.1 & 48.6 & 48.2 & 47.8 & 48.4 & 47.9 & 46.9 & 48.5 & 50.7 & 46.1 & 47.9 & 52.2 \\
\hline \multirow{3}{*}{ E85 Series HEV PHEV40 } & Vehicle Mass & 1626 & 1626 & 1530 & 1443 & 1585 & 1476 & 1316 & 1548 & 1350 & 1114 & 1525 & 1310 & 1058 \\
\hline & $\%$ of Glider mass & 49.5 & 49.5 & 50.2 & 50.5 & 50.8 & 50.3 & 51.0 & 51.8 & 50.4 & 48.4 & 52.6 & 51.2 & 47.1 \\
\hline & $\%$ of Powertrain mass & 50.5 & 50.5 & 49.8 & 49.5 & 49.2 & 49.7 & 49.0 & 48.2 & 49.6 & 51.6 & 47.4 & 48.8 & 52.9 \\
\hline \multirow{3}{*}{ FC HEV } & Vehicle Mass & 1674 & 1674 & 1412 & 1274 & 1490 & 1291 & 1132 & 1395 & 1158 & 940 & 1314 & 1116 & 890 \\
\hline & $\%$ of Glider mass & 48.1 & 48.1 & 54.4 & 57.2 & 54.1 & 57.5 & 59.3 & 57.5 & 58.8 & 57.4 & 61.0 & 60.1 & 56.0 \\
\hline & $\%$ of Powertrain mass & 51.9 & 51.9 & 45.6 & 42.8 & 45.9 & 42.5 & 40.7 & 42.5 & 41.2 & 42.6 & 39.0 & 39.9 & 44.0 \\
\hline \multirow{3}{*}{ FC HEV PHEV10 } & Vehicle Mass & 1599 & 1599 & 1376 & 1249 & 1454 & 1264 & 1111 & 1370 & 1140 & 925 & 1295 & 1102 & 879 \\
\hline & $\%$ of Glider mass & 50.3 & 50.3 & 55.8 & 58.4 & 55.4 & 58.7 & 60.4 & 58.5 & 59.7 & 58.3 & 61.9 & 60.8 & 56.7 \\
\hline & $\%$ of Powertrain mass & 49.7 & 49.7 & 44.2 & 41.6 & 44.6 & 41.3 & 39.6 & 41.5 & 40.3 & 41.7 & 38.1 & 39.2 & 43.3 \\
\hline \multirow{3}{*}{ FC HEV PHEV 20} & Vehicle Mass & 1637 & 1637 & 1409 & 1278 & 1491 & 1293 & 1134 & 1401 & 1160 & 937 & 1322 & 1120 & 890 \\
\hline & $\%$ of Glider mass & 49.2 & 49.2 & 54.5 & 57.0 & 54.0 & 57.4 & 59.2 & 57.2 & 58.7 & 57.6 & 60.6 & 59.9 & 56.0 \\
\hline & $\%$ of Powertrain mass & 50.8 & 50.8 & 45.5 & 43.0 & 46.0 & 42.6 & 40.8 & 42.8 & 41.3 & 42.4 & 39.4 & 40.1 & 44.0 \\
\hline \multirow{3}{*}{ FC HEV PHEV 30} & Vehicle Mass & 1725 & 1725 & 1496 & 1364 & 1583 & 1383 & 1206 & 1496 & 1241 & 999 & 1408 & 1187 & 947 \\
\hline & $\%$ of Glider mass & 46.7 & 46.7 & 51.3 & 53.4 & 50.9 & 53.7 & 55.7 & 53.6 & 54.9 & 54.0 & 56.9 & 56.5 & 52.6 \\
\hline & $\%$ of Powertrain mass & 53.3 & 53.3 & 48.7 & 46.6 & 49.1 & 46.3 & 44.3 & 46.4 & 45.1 & 46.0 & 43.1 & 43.5 & 47.4 \\
\hline \multirow{3}{*}{ FC HEV PHEV40 } & Vehicle Mass & 1770 & 1770 & 1533 & 1396 & 1627 & 1415 & 1230 & 1531 & 1265 & 1016 & 1439 & 1209 & 963 \\
\hline & $\%$ of Glider mass & 45.5 & 45.5 & 50.1 & 52.2 & 49.5 & 52.5 & 54.6 & 52.4 & 53.8 & 53.1 & 55.7 & 55.5 & 51.8 \\
\hline & $\%$ of Powertrain mass & 54.5 & 54.5 & 49.9 & 47.8 & 50.5 & 47.5 & 45.4 & 47.6 & 46.2 & 46.9 & 44.3 & 44.5 & 48.2 \\
\hline \multirow{3}{*}{ BEV } & Vehicle Mass & 1756 & 1756 & 1650 & 1539 & 1731 & 1587 & 1341 & 1677 & 1377 & 1090 & 1631 & 1309 & 1022 \\
\hline & $\%$ of Glider mass & 45.8 & 45.8 & 46.5 & 47.4 & 46.5 & 46.8 & 50.1 & 47.8 & 49.4 & 49.5 & 49.1 & 51.2 & 48.8 \\
\hline & $\%$ of Powertrain mass & 54.2 & 54.2 & 53.5 & 52.6 & 53.5 & 53.2 & 49.9 & 52.2 & 50.6 & 50.5 & 50.9 & 48.8 & 51.2 \\
\hline
\end{tabular}


Percentage of vehicle curbs weight reduction based on the respective 2010 reference case

\begin{tabular}{|c|c|c|c|c|c|c|c|c|c|c|c|c|c|c|}
\hline & & \multicolumn{13}{|c|}{ Midsize } \\
\hline & & 2010 & & 2010 & & & 2015 & & & 2030 & & & 2045 & \\
\hline & & Ref & low & avg & high & low & avg & high & low & avg & high & low & avg & high \\
\hline SI Conv & Vehicle Mass & $0.00 \%$ & $0.00 \%$ & $-3.45 \%$ & $-6.90 \%$ & $0.07 \%$ & $-5.80 \%$ & $-12.22 \%$ & $-0.41 \%$ & $-11.32 \%$ & $-23.81 \%$ & $-0.55 \%$ & $-12.35 \%$ & $-27.40 \%$ \\
\hline $\mathrm{Cl}$ Conv & Vehicle Mass & $0.00 \%$ & $0.00 \%$ & $-3.24 \%$ & $-6.48 \%$ & $0.00 \%$ & $-5.42 \%$ & $-11.44 \%$ & $-0.40 \%$ & $-10.65 \%$ & $-22.49 \%$ & $-0.53 \%$ & $-11.51 \%$ & $-25.93 \%$ \\
\hline H2 Conv & Vehicle Mass & $0.00 \%$ & $0.00 \%$ & $-6.12 \%$ & $-10.81 \%$ & $-0.95 \%$ & $-9.65 \%$ & $-17.00 \%$ & $-2.04 \%$ & $-15.50 \%$ & $-29.30 \%$ & $-4.83 \%$ & $-16.93 \%$ & $-32.29 \%$ \\
\hline E85 Conv & Vehicle Mass & $0.00 \%$ & $0.00 \%$ & $-3.32 \%$ & $-6.91 \%$ & $0.14 \%$ & $-5.74 \%$ & $-12.23 \%$ & $-0.41 \%$ & $-11.33 \%$ & $-23.91 \%$ & $-0.55 \%$ & $-12.30 \%$ & $-27.57 \%$ \\
\hline SI Split HEV & Vehicle Mass & $0.00 \%$ & $0.00 \%$ & $-4.72 \%$ & $-9.24 \%$ & $-1.40 \%$ & $-7.01 \%$ & $-14.79 \%$ & $-1.66 \%$ & $-13.26 \%$ & $-27.41 \%$ & $-2.36 \%$ & $-14.72 \%$ & $-31.10 \%$ \\
\hline SI Split HEV PHEV10 & Vehicle Mass & $0.00 \%$ & $0.00 \%$ & $-4.57 \%$ & $-9.08 \%$ & $-1.21 \%$ & $-7.49 \%$ & $-15.43 \%$ & $-2.54 \%$ & $-13.90 \%$ & $-27.87 \%$ & $-3.11 \%$ & $-15.37 \%$ & $-31.56 \%$ \\
\hline SI Split HEV PHEV20 & Vehicle Mass & $0.00 \%$ & $0.00 \%$ & $-4.67 \%$ & $-9.34 \%$ & $-1.31 \%$ & $-7.72 \%$ & $-15.88 \%$ & $-2.68 \%$ & $-14.38 \%$ & $-28.52 \%$ & $-3.30 \%$ & $-15.94 \%$ & $-32.13 \%$ \\
\hline SI Series HEV PHEV30 & Vehicle Mass & $0.00 \%$ & $0.00 \%$ & $-5.96 \%$ & $-11.21 \%$ & $-2.41 \%$ & $-9.30 \%$ & $-18.93 \%$ & $-4.54 \%$ & $-17.12 \%$ & $-31.78 \%$ & $-5.91 \%$ & $-19.31 \%$ & $-35.34 \%$ \\
\hline SI Series HEV PHEV40 & Vehicle Mass & $0.00 \%$ & $0.00 \%$ & $-5.97 \%$ & $-11.41 \%$ & $-2.40 \%$ & $-9.43 \%$ & $-19.51 \%$ & $-4.64 \%$ & $-17.48 \%$ & $-32.52 \%$ & $-6.02 \%$ & $-19.88 \%$ & $-36.03 \%$ \\
\hline $\mathrm{Cl}$ Split HEV & Vehicle Mass & $0.00 \%$ & $0.00 \%$ & $-4.55 \%$ & $-9.10 \%$ & $-1.62 \%$ & $-6.71 \%$ & $-14.37 \%$ & $-2.10 \%$ & $-12.81 \%$ & $-26.35 \%$ & $-2.63 \%$ & $-14.07 \%$ & $-29.58 \%$ \\
\hline CI Split HEV PHEV10 & Vehicle Mass & $00 \%$ & $.00 \%$ & $-4.44 \%$ & $-8.77 \%$ & $-1.36 \%$ & $-7.23 \%$ & $-14.93 \%$ & $-2.67 \%$ & $-13.45 \%$ & $-26.90 \%$ & $-3.20 \%$ & $-14.87 \%$ & $-30.15 \%$ \\
\hline CI Split HEV PHEV2O & Vehicle Mass & $0.00 \%$ & $0.00 \%$ & $-4.59 \%$ & $-8.95 \%$ & $-1.40 \%$ & $-7.38 \%$ & $-15.41 \%$ & $-2.67 \%$ & $-13.84 \%$ & $-27.27 \%$ & $-3.43 \%$ & $-15.41 \%$ & $-30.58 \%$ \\
\hline CI Series HEV PHEV 30 & Vehicle Mass & $0.00 \%$ & $0.00 \%$ & $-5.71 \%$ & $-10.75 \%$ & $-2.34 \%$ & $-8.72 \%$ & $-18.07 \%$ & $-4.41 \%$ & $-16.20 \%$ & $-30.32 \%$ & $-5.61 \%$ & $-18.33 \%$ & $-33.54 \%$ \\
\hline CI Series HEV PHEV40 & Vehicle Mass & $0.00 \%$ & $0.00 \%$ & $-5.82 \%$ & $-10.84 \%$ & $-2.43 \%$ & $-8.86 \%$ & $-18.58 \%$ & $-4.56 \%$ & $-16.66 \%$ & $-30.89 \%$ & $-5.87 \%$ & $-18.94 \%$ & $-34.18 \%$ \\
\hline H2 Split HEV & Vehicle Mass & $0.00 \%$ & $0.00 \%$ & $-7.19 \%$ & $-12.76 \%$ & $-2.35 \%$ & $-10.47 \%$ & $-19.14 \%$ & $-3.35 \%$ & $-17.16 \%$ & $-32.16 \%$ & $-6.38 \%$ & $-19.02 \%$ & $-35.07 \%$ \\
\hline H2 Split HEV PHEV10 & Vehicle Mass & $0.00 \%$ & $0.00 \%$ & $-7.19 \%$ & $-12.72 \%$ & $-2.46 \%$ & $-11.06 \%$ & $-19.91 \%$ & $-3.93 \%$ & $-17.95 \%$ & $-32.76 \%$ & $-7.07 \%$ & $-19.73 \%$ & $-35.71 \%$ \\
\hline H2 Split HEV PHEV2O & Vehicle Mass & $0.00 \%$ & $0.00 \%$ & $-7.24 \%$ & $-12.85 \%$ & $-2.23 \%$ & $-11.22 \%$ & $-20.27 \%$ & $-4.04 \%$ & $-18.15 \%$ & $-33.23 \%$ & $-7.24 \%$ & $-20.27 \%$ & $-36.19 \%$ \\
\hline H2 Series HEV PHEV 30 & Vehicle Mass & $0.00 \%$ & $0.00 \%$ & $-8.24 \%$ & $-14.34 \%$ & $-3.21 \%$ & $-12.52 \%$ & $-22.79 \%$ & $-5.72 \%$ & $-20.39 \%$ & $-35.85 \%$ & $-9.36 \%$ & $-22.90 \%$ & $-38.68 \%$ \\
\hline H2 Series HEV PHEV40 & Vehicle Mass & $0.00 \%$ & $0.00 \%$ & $-8.34 \%$ & $-14.60 \%$ & $-3.18 \%$ & $-12.57 \%$ & $-23.20 \%$ & $-5.94 \%$ & $-20.75 \%$ & $-36.39 \%$ & $-9.54 \%$ & $-23.46 \%$ & $-39.31 \%$ \\
\hline E85 Split HEV & Vehicle Mass & $0.00 \%$ & $0.00 \%$ & $-4.71 \%$ & $-9.23 \%$ & $-1.34 \%$ & $-6.81 \%$ & $-14.89 \%$ & $-1.72 \%$ & $-13.24 \%$ & $-27.31 \%$ & $-2.29 \%$ & $-14.64 \%$ & $-31.00 \%$ \\
\hline E85 Split HEV PHEV10 & Vehicle Mass & $0.00 \%$ & $0.00 \%$ & $-4.50 \%$ & $-9.00 \%$ & $-1.20 \%$ & $-7.42 \%$ & $-15.41 \%$ & $-2.41 \%$ & $-13.89 \%$ & $-27.84 \%$ & $-3.04 \%$ & $-15.35 \%$ & $-31.45 \%$ \\
\hline E85 Split HEV PHEV20 & Vehicle Mass & $0.00 \%$ & $0.00 \%$ & $-4.66 \%$ & $-9.33 \%$ & $-1.31 \%$ & $-7.65 \%$ & $-15.86 \%$ & $-2.55 \%$ & $-14.37 \%$ & $-28.48 \%$ & $-3.23 \%$ & $-15.92 \%$ & $-32.03 \%$ \\
\hline E85 Series HEV PHEV30 & Vehicle Mass & $0.00 \%$ & $0.00 \%$ & $-5.85 \%$ & $-11.20 \%$ & $-2.40 \%$ & $-9.29 \%$ & $-18.91 \%$ & $-4.48 \%$ & $-17.05 \%$ & $-31.75 \%$ & $-5.85 \%$ & $-19.29 \%$ & $-35.25 \%$ \\
\hline E85 Series HEV PHEV40 & Vehicle Mass & $0.00 \%$ & $0.00 \%$ & $-5.91 \%$ & $-11.29 \%$ & $-2.34 \%$ & $-9.38 \%$ & $-19.34 \%$ & $-4.48 \%$ & $-17.37 \%$ & $-32.34 \%$ & $-5.91 \%$ & $-19.82 \%$ & $-35.80 \%$ \\
\hline FC HEV & Vehicle Mass & $0.00 \%$ & $0.00 \%$ & $-15.68 \%$ & $-23.90 \%$ & $-10.52 \%$ & $-22.73 \%$ & $-32.48 \%$ & $-16.29 \%$ & $-30.80 \%$ & $-44.59 \%$ & $-21.04 \%$ & $-33.35 \%$ & $-47.75 \%$ \\
\hline FC HEV PHEV 10 & Vehicle Mass & $0.00 \%$ & $0.00 \%$ & $-13.90 \%$ & $-21.82 \%$ & $-8.88 \%$ & $-20.80 \%$ & $-30.64 \%$ & $-14.17 \%$ & $-28.93 \%$ & $-42.78 \%$ & $-18.66 \%$ & $-31.18 \%$ & $-45.99 \%$ \\
\hline FC HEV PHEV 20 & Vehicle Mass & $0.00 \%$ & $0.00 \%$ & $-14.11 \%$ & $-22.14 \%$ & $-9.11 \%$ & $-21.30 \%$ & $-31.20 \%$ & $-14.53 \%$ & $-29.53 \%$ & $-43.65 \%$ & $-19.17 \%$ & $-31.98 \%$ & $-46.77 \%$ \\
\hline FC HEV PHEV 30 & Vehicle Mass & $0.00 \%$ & $0.00 \%$ & $-13.33 \%$ & $-20.95 \%$ & $-8.02 \%$ & $-19.95 \%$ & $-30.08 \%$ & $-13.13 \%$ & $-28.22 \%$ & $-42.61 \%$ & $-17.94 \%$ & $-31.18 \%$ & $-45.91 \%$ \\
\hline FC HEV PHEV 40 & Vehicle Mass & $0.00 \%$ & $0.00 \%$ & $-13.58 \%$ & $-21.25 \%$ & $-8.11 \%$ & $-20.18 \%$ & $-30.68 \%$ & $-13.34 \%$ & $-28.72 \%$ & $-43.23 \%$ & $-18.32 \%$ & $-31.80 \%$ & $-46.51 \%$ \\
\hline EV & Vehicle Mass & $0.00 \%$ & $0.00 \%$ & $-6.93 \%$ & $-12.98 \%$ & $-1.82 \%$ & $-10.4 / \%$ & $-24.29 \%$ & $-4.97 \%$ & $-22.41 \%$ & $-38.99 \%$ & $-7.42 \%$ & $-26.06 \%$ & \\
\hline
\end{tabular}




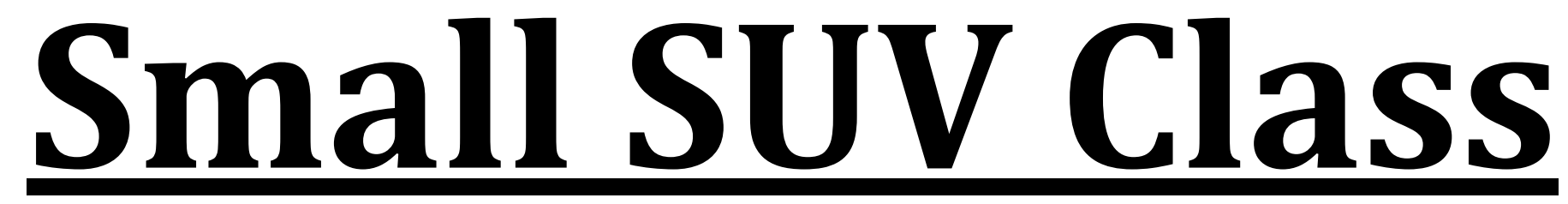




\section{Power}

\begin{tabular}{|c|c|c|c|c|c|c|c|c|c|c|c|c|c|c|}
\hline & & \multicolumn{13}{|c|}{ Small_SUV } \\
\hline & & 2010 & & 2010 & & & 2015 & & & 2030 & & & 2045 & \\
\hline & & Ref & low & avg & high & low & avg & high & low & avg & high & low & avg & high \\
\hline \multirow{5}{*}{ SI Conv } & ICE Power & 155898 & 155898 & 148851 & 141805 & 152875 & 143143 & 132111 & 150191 & 134760 & 119229 & 149872 & 133431 & 115117 \\
\hline & Electric Machine & & & & & & & & & & & & & \\
\hline & $\mathrm{MC} 2 / \mathrm{GC}$ & & & & & & & & & & & & & \\
\hline & Battery & & & & & & & & & & & & & \\
\hline & Specific Power & 97 & 97 & 95 & 94 & 95 & 94 & 93 & 94 & 93 & 95 & 94 & 93 & 96 \\
\hline \multirow{5}{*}{$\mathrm{Cl}$ Conv } & ICE Power & 122222 & 122222 & 119778 & 113667 & 119778 & 114889 & 107556 & 121000 & 110000 & 96800 & 119778 & 110000 & 94410 \\
\hline & Electric Machine & & & & & & & & & & & & & \\
\hline & $\mathrm{MC} 2 / \mathrm{GC}$ & & & & & & & & & & & & & \\
\hline & Battery & & & & & & & & & & & & & \\
\hline & Specific Power & 73 & 73 & 74 & 73 & 72 & 73 & 72 & 73 & 73 & 73 & 72 & 74 & 74 \\
\hline \multirow{5}{*}{ H2 Conv } & ICE Power & 141547 & 141547 & 132026 & 124426 & 137726 & 126282 & 114088 & 134950 & 116872 & 100970 & 130328 & 115832 & 97274 \\
\hline & Electric Machine & & & & & & & & & & & & & \\
\hline & $\mathrm{MC} 2 / \mathrm{GC}$ & & & & & & & & & & & & & \\
\hline & Battery & & & & & & & & & & & & & \\
\hline & Specific Power & 87 & 87 & 86 & 85 & 86 & 85 & 83 & 85 & 84 & 85 & 84 & 84 & 85 \\
\hline \multirow{5}{*}{ E85 Conv } & ICE Power & 145972 & 145972 & 141243 & 134012 & 143648 & 136336 & 125375 & 143332 & 127943 & 113333 & 142164 & 126781 & 109792 \\
\hline & Electric Machine & & & & & & & & & & & & & \\
\hline & $\mathrm{MC} 2 / \mathrm{GC}$ & & & & & & & & & & & & & \\
\hline & Battery & & & & & & & & & & & & & \\
\hline & Specific Power & 91 & 91 & 91 & 89 & 89 & 90 & 88 & 90 & 89 & 90 & 89 & 89 & 91 \\
\hline \multirow{5}{*}{ SI Split HEV } & ICE Power & 105888 & 105888 & 100215 & 94848 & 104471 & 97892 & 86370 & 101980 & 89644 & 73487 & 101364 & 86014 & 70349 \\
\hline & Electric Machine & 71956 & 71956 & 68839 & 65946 & 70952 & 68461 & 62301 & 70830 & 63743 & 53577 & 70559 & 62077 & 51539 \\
\hline & $\mathrm{MC} 2 / \mathrm{GC}$ & 61278 & 61278 & 57995 & 54889 & 60458 & 56650 & 49983 & 59016 & 51877 & 42527 & 58660 & 49777 & 40711 \\
\hline & Battery & 29327 & 29327 & 27930 & 26733 & 28928 & 27886 & 25610 & 28455 & 25610 & 21626 & 28455 & 25610 & 21057 \\
\hline & Specific Power & 77 & 77 & 77 & 76 & 78 & 77 & 75 & 76 & 75 & 73 & 76 & 74 & 74 \\
\hline
\end{tabular}


Argonne National Laboratory Report - Light-Duty Vehicle Fuel Consumption Displacement Potential Up to 2045

\begin{tabular}{|c|c|c|c|c|c|c|c|c|c|c|c|c|c|c|}
\hline & & \multicolumn{13}{|c|}{ Small_SUV } \\
\hline & & 2010 & \multicolumn{3}{|c|}{2010} & \multicolumn{3}{|c|}{2015} & \multicolumn{3}{|c|}{2030} & \multicolumn{3}{|c|}{2045} \\
\hline & & Ref & low & avg & high & low & avg & high & low & avg & high & low & avg & high \\
\hline \multirow{5}{*}{ SI Split HEV PHEV10 } & ICE Power & 72399 & 72399 & 68201 & 64874 & 71330 & 66295 & 60774 & 70958 & 61962 & 53994 & 69701 & 60758 & 51529 \\
\hline & Electric Machine & 80378 & 80378 & 77168 & 73745 & 79449 & 74975 & 67484 & 78900 & 68670 & 58805 & 78130 & 67387 & 56199 \\
\hline & $\mathrm{MC} 2 / \mathrm{GC}$ & 51069 & 51069 & 48107 & 45761 & 50315 & 46763 & 42849 & 50052 & 43687 & 38086 & 49165 & 42838 & 36347 \\
\hline & Battery & 76633 & 76633 & 73302 & 69646 & 75853 & 70776 & 64018 & 74322 & 65167 & 60019 & 73658 & 68578 & 57305 \\
\hline & Specific Power & 85 & 85 & 84 & 84 & 85 & 84 & 83 & 84 & 83 & 88 & 84 & 86 & 88 \\
\hline \multirow{5}{*}{ SI Split HEV PHEV2O } & ICE Power & 73460 & 73460 & 69168 & 65724 & 72357 & 67202 & 61418 & 71928 & 62634 & 54435 & 70640 & 61286 & 51887 \\
\hline & Electric Machine & 82157 & 82157 & 78640 & 74991 & 81003 & 76398 & 69310 & 80410 & 70380 & 59149 & 80086 & 69035 & 56924 \\
\hline & $\mathrm{MC} 2 / \mathrm{GC}$ & 51797 & 51797 & 48770 & 46360 & 51039 & 47383 & 43303 & 50717 & 44181 & 38378 & 49807 & 43211 & 36581 \\
\hline & Battery & 77565 & 77565 & 73906 & 70029 & 76536 & 71353 & 65266 & 74982 & 66264 & 56220 & 74829 & 66679 & 54282 \\
\hline & Specific Power & 84 & 84 & 83 & 83 & 84 & 83 & 83 & 84 & 83 & 84 & 84 & 84 & 85 \\
\hline \multirow{5}{*}{ SI Series HEV PHEV30 } & ICE Power & 90268 & 90268 & 83103 & 77565 & 88282 & 79685 & 69451 & 85369 & 71100 & 59518 & 83297 & 68938 & 56684 \\
\hline & Electric Machine & 148822 & 148822 & 139609 & 132531 & 145821 & 135677 & 122457 & 144100 & 124974 & 108450 & 141020 & 121733 & 103596 \\
\hline & $\mathrm{MC} 2 / \mathrm{GC}$ & 87560 & 87560 & 80610 & 75238 & 85634 & 77294 & 67367 & 82808 & 68967 & 57732 & 80798 & 66870 & 54983 \\
\hline & Battery & 180052 & 180052 & 167985 & 158611 & 176444 & 162427 & 144816 & 172585 & 147825 & 126594 & 168904 & 144035 & 120948 \\
\hline & Specific Power & 73 & 73 & 73 & 73 & 73 & 73 & 74 & 74 & 74 & 76 & 73 & 74 & 77 \\
\hline \multirow{5}{*}{ SI Series HEV PHEV40 } & ICE Power & 93557 & 93557 & 86028 & 80121 & 91430 & 82360 & 71368 & 88302 & 73132 & 60872 & 86013 & 70674 & 57927 \\
\hline & Electric Machine & 154014 & 154014 & 144347 & 136683 & 150853 & 140069 & 125630 & 148842 & 128384 & 110783 & 145514 & 124611 & 105801 \\
\hline & $\mathrm{MC} 2 / \mathrm{GC}$ & 90750 & 90750 & 83447 & 77717 & 88687 & 79889 & 69227 & 85653 & 70938 & 59046 & 83433 & 68554 & 56189 \\
\hline & Battery & 186251 & 186251 & 173614 & 163514 & 182448 & 167609 & 148445 & 178185 & 151732 & 129205 & 174214 & 147316 & 123417 \\
\hline & Specific Power & 74 & 74 & 73 & 74 & 74 & 74 & 74 & 74 & 74 & 77 & 74 & 74 & 77 \\
\hline \multirow{5}{*}{ Cl Split HEV } & ICE Power & 91855 & 91855 & 87213 & 82607 & 90792 & 83927 & 75527 & 88407 & 76688 & 65174 & 87876 & 75326 & 63091 \\
\hline & Electric Machine & 77305 & 77305 & 74361 & 71203 & 76356 & 72594 & 66997 & 76080 & 68143 & 58948 & 75756 & 67331 & 56679 \\
\hline & $\mathrm{MC} 2 / \mathrm{GC}$ & 76873 & 76873 & 72988 & 69133 & 75983 & 70238 & 63208 & 73987 & 64180 & 54544 & 73543 & 63040 & 52800 \\
\hline & Battery & 30923 & 30923 & 29726 & 28329 & 30524 & 29024 & 26748 & 30162 & 27317 & 23333 & 30162 & 27317 & 22195 \\
\hline & Specific Power & 67 & 67 & 66 & 66 & 67 & 66 & 64 & 66 & 64 & 64 & 66 & 64 & 64 \\
\hline \multirow{5}{*}{ CI Split HEV PHEV10 } & ICE Power & 75382 & 75382 & 71159 & 67763 & 74240 & 69156 & 63544 & 73782 & 64788 & 56753 & 72489 & 63457 & 54232 \\
\hline & Electric Machine & 81180 & 81180 & 78193 & 74812 & 80070 & 76079 & 69379 & 78896 & 70446 & 60099 & 78758 & 69042 & 57518 \\
\hline & $\mathrm{MC} 2 / \mathrm{GC}$ & 63087 & 63087 & 59552 & 56710 & 62131 & 57876 & 53179 & 61748 & 54221 & 47496 & 60665 & 53107 & 45386 \\
\hline & Battery & 85638 & 85638 & 82155 & 78198 & 84525 & 79533 & 73863 & 82325 & 75027 & 67489 & 82264 & 77532 & 64486 \\
\hline & Specific Power & 86 & 86 & 86 & 85 & 86 & 86 & 86 & 86 & 86 & 89 & 86 & 88 & 89 \\
\hline
\end{tabular}


Argonne National Laboratory Report - Light-Duty Vehicle Fuel Consumption Displacement Potential Up to 2045

\begin{tabular}{|c|c|c|c|c|c|c|c|c|c|c|c|c|c|c|}
\hline & & \multicolumn{13}{|c|}{ Small_SUV } \\
\hline & & 2010 & \multicolumn{3}{|c|}{2010} & \multicolumn{3}{|c|}{2015} & \multicolumn{3}{|c|}{2030} & \multicolumn{3}{|c|}{2045} \\
\hline & & Ref & low & avg & high & low & avg & high & low & avg & high & low & avg & high \\
\hline \multirow{5}{*}{ CI Split HEV PHEV20 } & ICE Power & 76501 & 76501 & 72126 & 68556 & 75267 & 70063 & 64216 & 74809 & 65460 & 57139 & 73484 & 64041 & 54618 \\
\hline & Electric Machine & 82757 & 82757 & 79606 & 75840 & 81599 & 77105 & 70152 & 80947 & 71537 & 60846 & 80159 & 69918 & 58296 \\
\hline & $\mathrm{MC} 2 / \mathrm{GC}$ & 64023 & 64023 & 60362 & 57374 & 62990 & 58635 & 53742 & 62607 & 54783 & 47819 & 61498 & 53595 & 45709 \\
\hline & Battery & 86821 & 86821 & 83126 & 78763 & 85658 & 80089 & 73312 & 84048 & 74777 & 64529 & 83270 & 74891 & 61816 \\
\hline & Specific Power & 86 & 86 & 85 & 85 & 86 & 85 & 85 & 86 & 85 & 86 & 85 & 86 & 86 \\
\hline \multirow{5}{*}{$\mathrm{Cl}$ Series HEV PHEV 30} & ICE Power & 93249 & 93249 & 86119 & 80448 & 91094 & 82508 & 72226 & 88183 & 73904 & 62143 & 86102 & 71726 & 59336 \\
\hline & Electric Machine & 153479 & 153479 & 144445 & 137213 & 150369 & 140311 & 127024 & 148696 & 129726 & 113052 & 145611 & 126384 & 108333 \\
\hline & $\mathrm{MC} 2 / \mathrm{GC}$ & 90452 & 90452 & 83535 & 78035 & 88361 & 80033 & 70059 & 85538 & 71687 & 60279 & 83519 & 69574 & 57556 \\
\hline & Battery & 185663 & 185663 & 173780 & 164200 & 181923 & 167947 & 150188 & 178068 & 153428 & 131939 & 174377 & 149521 & 126454 \\
\hline & Specific Power & 72 & 72 & 72 & 72 & 72 & 72 & 72 & 73 & 72 & 75 & 72 & 72 & 75 \\
\hline \multirow{5}{*}{$\mathrm{Cl}$ Series HEV PHEV40 } & ICE Power & 96722 & 96722 & 89044 & 83093 & 94364 & 85272 & 74172 & 91235 & 75907 & 63607 & 88937 & 73433 & 60607 \\
\hline & Electric Machine & 158913 & 158913 & 149180 & 141509 & 155592 & 144845 & 130333 & 153584 & 133034 & 115510 & 150201 & 129207 & 110466 \\
\hline & $\mathrm{MC} 2 / \mathrm{GC}$ & 93820 & 93820 & 86373 & 80600 & 91533 & 82714 & 71947 & 88498 & 73630 & 61699 & 86269 & 71230 & 58789 \\
\hline & Battery & 192155 & 192155 & 179399 & 169264 & 188159 & 173297 & 153979 & 183837 & 157208 & 134691 & 179795 & 152726 & 128837 \\
\hline & Specific Power & 73 & 73 & 72 & 72 & 73 & 73 & 73 & 73 & 73 & 75 & 73 & 73 & 75 \\
\hline \multirow{5}{*}{ H2 Split HEV } & ICE Power & 108272 & 108272 & 100207 & 93400 & 106540 & 95075 & 84301 & 103806 & 86225 & 71657 & 99642 & 84210 & 69466 \\
\hline & Electric Machine & 74164 & 74164 & 69484 & 65620 & 72862 & 67165 & 61337 & 72582 & 62709 & 52750 & 70182 & 61247 & 51163 \\
\hline & $\mathrm{MC} 2 / \mathrm{GC}$ & 68246 & 68246 & 63163 & 58872 & 67155 & 59928 & 53137 & 65431 & 54350 & 45167 & 62807 & 53080 & 43786 \\
\hline & Battery & 30125 & 30125 & 28130 & 26534 & 29526 & 27317 & 25040 & 29024 & 25610 & 21057 & 28455 & 25040 & 20488 \\
\hline & Specific Power & 77 & 77 & 77 & 76 & 78 & 76 & 74 & 77 & 74 & 74 & 76 & 74 & 75 \\
\hline \multirow{5}{*}{ H2 Split HEV PHEV10 } & ICE Power & 73862 & 73862 & 68400 & 64506 & 72272 & 65956 & 59935 & 71557 & 61403 & 52891 & 69189 & 60007 & 50646 \\
\hline & Electric Machine & 85158 & 85158 & 78854 & 74498 & 82474 & 75891 & 67868 & 81370 & 69757 & 58312 & 78865 & 68214 & 55956 \\
\hline & $\mathrm{MC} 2 / \mathrm{GC}$ & 57970 & 57970 & 53683 & 50627 & 56699 & 51765 & 47039 & 56161 & 48191 & 41511 & 54280 & 47008 & 39727 \\
\hline & Battery & 82723 & 82723 & 75953 & 71253 & 79806 & 72506 & 63897 & 77436 & 65756 & 58142 & 75133 & 68571 & 55617 \\
\hline & Specific Power & 86 & 86 & 85 & 85 & 86 & 85 & 84 & 86 & 84 & 88 & 85 & 87 & 88 \\
\hline \multirow{5}{*}{ H2 Split HEV PHEV2O } & ICE Power & 74952 & 74952 & 69310 & 65299 & 73327 & 66862 & 60606 & 72584 & 62102 & 53305 & 70128 & 60507 & 51032 \\
\hline & Electric Machine & 86814 & 86814 & 80269 & 75770 & 84562 & 77254 & 69478 & 82824 & 70875 & 59141 & 80851 & 69147 & 56502 \\
\hline & $\mathrm{MC} 2 / \mathrm{GC}$ & 58802 & 58802 & 54375 & 51226 & 57528 & 52454 & 47544 & 56967 & 48696 & 41792 & 54994 & 47466 & 40031 \\
\hline & Battery & 83927 & 83927 & 76914 & 72089 & 81581 & 73423 & 65627 & 78482 & 66793 & 55903 & 76924 & 66743 & 53320 \\
\hline & Specific Power & 86 & 86 & 85 & 85 & 86 & 85 & 84 & 85 & 84 & 86 & 85 & 85 & 85 \\
\hline
\end{tabular}


Argonne National Laboratory Report - Light-Duty Vehicle Fuel Consumption Displacement Potential Up to 2045

\begin{tabular}{|c|c|c|c|c|c|c|c|c|c|c|c|c|c|c|}
\hline & & \multicolumn{13}{|c|}{ Small_SUV } \\
\hline & & 2010 & \multicolumn{3}{|c|}{2010} & \multicolumn{3}{|c|}{2015} & \multicolumn{3}{|c|}{2030} & \multicolumn{3}{|c|}{2045} \\
\hline & & Ref & low & avg & high & low & avg & high & low & avg & high & low & avg & high \\
\hline \multirow{5}{*}{ H2 Series HEV PHEV3O } & ICE Power & 91559 & 91559 & 83012 & 76882 & 88924 & 78942 & 68335 & 85728 & 70242 & 58164 & 82401 & 67943 & 55606 \\
\hline & Electric Machine & 150812 & 150812 & 139464 & 131370 & 146981 & 134470 & 120530 & 144681 & 123578 & 106010 & 139617 & 120051 & 101672 \\
\hline & $\mathrm{MC} 2 / \mathrm{GC}$ & 88812 & 88812 & 80522 & 74576 & 86256 & 76574 & 66285 & 83156 & 68135 & 56419 & 79929 & 65905 & 53938 \\
\hline & Battery & 182447 & 182447 & 167812 & 157227 & 177843 & 160981 & 142544 & 173274 & 146186 & 123754 & 167229 & 142053 & 118712 \\
\hline & Specific Power & 73 & 73 & 73 & 73 & 73 & 73 & 74 & 74 & 74 & 77 & 74 & 74 & 77 \\
\hline \multirow{5}{*}{ H2 Series HEV PHEV40 } & ICE Power & 94878 & 94878 & 85938 & 79437 & 92133 & 81617 & 70281 & 88781 & 72245 & 59490 & 85147 & 69621 & 56822 \\
\hline & Electric Machine & 156099 & 156099 & 144202 & 135572 & 152014 & 138911 & 123757 & 149617 & 126943 & 108401 & 144016 & 122885 & 103842 \\
\hline & $\mathrm{MC} 2 / \mathrm{GC}$ & 92032 & 92032 & 83360 & 77054 & 89369 & 79168 & 68173 & 86118 & 70078 & 57705 & 82593 & 67532 & 55117 \\
\hline & Battery & 188761 & 188761 & 173439 & 162191 & 183848 & 166230 & 146237 & 179109 & 150041 & 126444 & 172426 & 145284 & 121141 \\
\hline & Specific Power & 73 & 73 & 73 & 74 & 74 & 74 & 75 & 74 & 74 & 78 & 74 & 74 & 78 \\
\hline \multirow{5}{*}{ E85 Split HEV } & ICE Power & 107091 & 107091 & 100343 & 94933 & 104556 & 98768 & 87270 & 104332 & 88541 & 73562 & 101449 & 88006 & 70425 \\
\hline & Electric Machine & 72594 & 72594 & 69148 & 66060 & 71052 & 68896 & 62668 & 72396 & 63328 & 53635 & 70656 & 62973 & 51601 \\
\hline & $\mathrm{MC} 2 / \mathrm{GC}$ & 61974 & 61974 & 58069 & 54938 & 60507 & 57157 & 50503 & 60377 & 51239 & 42571 & 58709 & 50929 & 40755 \\
\hline & Battery & 29327 & 29327 & 28130 & 26733 & 28928 & 27886 & 25610 & 29024 & 25610 & 21626 & 28455 & 25610 & 21057 \\
\hline & Specific Power & 78 & 78 & 77 & 76 & 77 & 77 & 75 & 78 & 75 & 73 & 76 & 75 & 74 \\
\hline \multirow{5}{*}{ E85 Split HEV PHEV10 } & ICE Power & 72456 & 72456 & 68343 & 64931 & 71387 & 66352 & 60830 & 71044 & 62018 & 54049 & 69758 & 60813 & 51584 \\
\hline & Electric Machine & 80496 & 80496 & 77285 & 73858 & 79565 & 75090 & 67638 & 79062 & 68782 & 58913 & 78246 & 68231 & 56305 \\
\hline & $\mathrm{MC} 2 / \mathrm{GC}$ & 51109 & 51109 & 48208 & 45801 & 50355 & 46803 & 42888 & 50113 & 43726 & 38125 & 49206 & 42877 & 36386 \\
\hline & Battery & 76712 & 76712 & 73406 & 69723 & 75946 & 70877 & 64131 & 74446 & 65247 & 60145 & 73759 & 69802 & 57449 \\
\hline & Specific Power & 85 & 85 & 84 & 84 & 85 & 84 & 83 & 84 & 83 & 88 & 84 & 87 & 88 \\
\hline \multirow{5}{*}{ E85 Split HEV PHEV20 } & ICE Power & 73518 & 73518 & 69225 & 65780 & 72414 & 67258 & 61474 & 71985 & 62690 & 54491 & 70697 & 61342 & 51970 \\
\hline & Electric Machine & 82321 & 82321 & 78757 & 75106 & 81165 & 76515 & 69422 & 80528 & 70492 & 59900 & 80249 & 69146 & 57030 \\
\hline & $\mathrm{MC} 2 / \mathrm{GC}$ & 51837 & 51837 & 48810 & 46400 & 51079 & 47422 & 43343 & 50757 & 44220 & 38417 & 49848 & 43249 & 36658 \\
\hline & Battery & 77711 & 77711 & 73993 & 70114 & 76681 & 71447 & 65361 & 75070 & 66360 & 57133 & 74965 & 66780 & 54393 \\
\hline & Specific Power & 84 & 84 & 83 & 83 & 84 & 83 & 83 & 84 & 83 & 85 & 84 & 84 & 84 \\
\hline \multirow{5}{*}{ E85 Series HEV PHEV30 } & ICE Power & 90299 & 90299 & 83163 & 77625 & 88251 & 79685 & 69508 & 85429 & 71158 & 59601 & 83356 & 68995 & 56767 \\
\hline & Electric Machine & 148918 & 148918 & 139754 & 132626 & 145917 & 135773 & 122553 & 144197 & 125118 & 108548 & 141117 & 121829 & 103743 \\
\hline & $\mathrm{MC} 2 / \mathrm{GC}$ & 87590 & 87590 & 80668 & 75296 & 85603 & 77294 & 67423 & 82866 & 69023 & 57813 & 80855 & 66925 & 55064 \\
\hline & Battery & 180173 & 180173 & 168159 & 158722 & 176556 & 162534 & 144929 & 172700 & 147997 & 126704 & 169019 & 144149 & 121119 \\
\hline & Specific Power & 73 & 73 & 73 & 73 & 73 & 73 & 74 & 74 & 74 & 76 & 73 & 73 & 76 \\
\hline
\end{tabular}




\begin{tabular}{|c|c|c|c|c|c|c|c|c|c|c|c|c|c|c|}
\hline & & \multirow{2}{*}{\multicolumn{13}{|c|}{ Midsize }} \\
\hline & & & & & & & & & & & & & & \\
\hline & & \multirow{2}{*}{$\begin{array}{c}2010 \\
\text { Ref }\end{array}$} & \multicolumn{3}{|c|}{2010} & \multicolumn{3}{|c|}{2015} & \multicolumn{3}{|c|}{2030} & \multicolumn{3}{|c|}{2045} \\
\hline & & & low & avg & high & low & avg & high & low & avg & high & low & avg & high \\
\hline \multirow{4}{*}{ E85 Split HEV PHEV20 } & Electric Machine & 74437 & 74437 & 71127 & 68011 & 73219 & 69085 & 62928 & 72713 & 63896 & 54224 & 72082 & 62567 & 51736 \\
\hline & $\mathrm{MC2} / \mathrm{GC}$ & 39338 & 39338 & 37335 & 35644 & 38931 & 36305 & 33268 & 38263 & 33956 & 29288 & 37799 & 33205 & 27891 \\
\hline & Battery & 69124 & 69124 & 65688 & 62396 & 67968 & 63399 & 57848 & 66735 & 58713 & 50213 & 66223 & 58815 & 47895 \\
\hline & Specific Power & 77 & 77 & 77 & 76 & 77 & 76 & 76 & 77 & 76 & 77 & 76 & 77 & 77 \\
\hline \multirow{5}{*}{ E85 Series HEV PHEV30 } & ICE Power & 80409 & 80409 & 74757 & 70117 & 78910 & 71633 & 62671 & 75505 & 64220 & 53320 & 74069 & 62162 & 50706 \\
\hline & Electric Machine & 132914 & 132914 & 125717 & 119838 & 130648 & 122197 & 110743 & 128044 & 113079 & 97855 & 126184 & 110168 & 93699 \\
\hline & $\mathrm{MC} 2 / \mathrm{GC}$ & 77997 & 77997 & 72514 & 68013 & 76543 & 69484 & 60791 & 73240 & 62293 & 51720 & 71847 & 60297 & 49185 \\
\hline & Battery & 163771 & 163771 & 154070 & 146084 & 161012 & 149004 & 133415 & 156178 & 136272 & 116380 & 153925 & 132800 & 111462 \\
\hline & Specific Power & 68 & 68 & 68 & 68 & 68 & 68 & 68 & 68 & 68 & 71 & 68 & 68 & 71 \\
\hline \multirow{5}{*}{ E85 Series HEV PHEV40 } & ICE Power & 83129 & 83129 & 77140 & 72316 & 81568 & 73892 & 64216 & 77942 & 65879 & 54425 & 76370 & 63726 & 51756 \\
\hline & Electric Machine & 137206 & 137206 & 129545 & 123394 & 134804 & 125839 & 113333 & 131954 & 115861 & 99815 & 129942 & 112616 & 95363 \\
\hline & $\mathrm{MC} 2 / \mathrm{GC}$ & 80635 & 80635 & 74826 & 70147 & 79121 & 71675 & 62290 & 75604 & 63903 & 52792 & 74079 & 61814 & 50203 \\
\hline & Battery & 169000 & 169000 & 158707 & 150367 & 166066 & 153389 & 136435 & 160881 & 139506 & 118619 & 158452 & 135644 & 113353 \\
\hline & Specific Power & 68 & 68 & 68 & 69 & 68 & 69 & 69 & 68 & 69 & 71 & 68 & 69 & 71 \\
\hline \multirow{5}{*}{ FC HEV } & FC Power & 104050 & 104050 & 88019 & 78035 & 96505 & 76723 & 64474 & 82622 & 66935 & 52362 & 77746 & 63650 & 49443 \\
\hline & Electric Machine & 124590 & 124590 & 107383 & 97235 & 113102 & 99769 & 86620 & 107109 & 89845 & 73258 & 100110 & 85292 & 69515 \\
\hline & $\mathrm{MC} 2 / \mathrm{GC}$ & & & & & & & & & & & & & \\
\hline & Battery & 36908 & 36908 & 32519 & 30324 & 33516 & 34146 & 31301 & 36422 & 31870 & 27317 & 34715 & 31301 & 26179 \\
\hline & Specific Power & 59 & 59 & 60 & 60 & 60 & 61 & 59 & 60 & 60 & 60 & 60 & 59 & 60 \\
\hline \multirow{5}{*}{ FC HEV PHEV 10} & FC Power & 76391 & 76391 & 66089 & 59488 & 70247 & 60702 & 52736 & 65493 & 53965 & 45936 & 62805 & 54463 & 44162 \\
\hline & Electric Machine & 121688 & 121688 & 105550 & 96445 & 111157 & 98186 & 86942 & 105476 & 88796 & 75059 & 101348 & 88020 & 70127 \\
\hline & $\mathrm{MC} 2 / \mathrm{GC}$ & & & & & & & & & & & & & \\
\hline & Battery & 64879 & 64879 & 56091 & 50670 & 59493 & 51573 & 44891 & 55463 & 45917 & 37373 & 52881 & 44657 & 35527 \\
\hline & Specific Power & 61 & 61 & 60 & 60 & 60 & 61 & 61 & 61 & 61 & 62 & 61 & 62 & 61 \\
\hline \multirow{5}{*}{ FC HEV PHEV 20} & FC Power & 78252 & 78252 & 68330 & 60873 & 72745 & 61369 & 53926 & 66968 & 54586 & 45636 & 63478 & 54954 & 43201 \\
\hline & Electric Machine & 124396 & 124396 & 107035 & 98435 & 112943 & 99533 & 88561 & 107580 & 89911 & 74403 & 102686 & 88963 & 70613 \\
\hline & $\mathrm{MC} 2 / \mathrm{GC}$ & & & & & & & & & & & & & \\
\hline & Battery & 66428 & 66428 & 57445 & 51676 & 61043 & 52478 & 45621 & 56622 & 46601 & 37797 & 53833 & 45176 & 35985 \\
\hline & Specific Power & 61 & 61 & 60 & 60 & 60 & 60 & 61 & 61 & 60 & 61 & 61 & 62 & 61 \\
\hline \multirow{5}{*}{ FC HEV PHEV 30} & FC Power & 68439 & 68439 & 60582 & 56144 & 64345 & 56956 & 50962 & 61284 & 52296 & 43864 & 58431 & 50392 & 41576 \\
\hline & Electric Machine & 134537 & 134537 & 122497 & 115240 & 128589 & 116695 & 105703 & 123686 & 108013 & 93347 & 119624 & 104476 & 88983 \\
\hline & $\mathrm{MC} 2 / \mathrm{GC}$ & & & & & & & & & & & & & \\
\hline & Battery & 165695 & 165695 & 150070 & 140433 & 158409 & 142250 & 127410 & 150804 & 130239 & 111041 & 145890 & 126022 & 105872 \\
\hline & Specific Power & 63 & 63 & 66 & 67 & 65 & 67 & 69 & 66 & 69 & 73 & 67 & 69 & 73 \\
\hline \multirow{5}{*}{ FC HEV PHEV 40} & FC Power & 69736 & 69736 & 61673 & 57054 & 65566 & 57941 & 51564 & 62345 & 53023 & 44309 & 59309 & 50965 & 41971 \\
\hline & Electric Machine & 137968 & 137968 & 125477 & 117907 & 131796 & 119501 & 107997 & 126658 & 110436 & 95022 & 122265 & 106588 & 90648 \\
\hline & $\mathrm{MC} 2 / \mathrm{GC}$ & & & & & & & & & & & & & \\
\hline & Battery & 169858 & 169858 & 153652 & 143629 & 162297 & 145615 & 130067 & 154374 & 133045 & 112950 & 149046 & 128442 & 107765 \\
\hline & Specific Power & 63 & 63 & 66 & 67 & 65 & 68 & 69 & 66 & 69 & 73 & 68 & 70 & 74 \\
\hline \multirow{5}{*}{ BEV } & ICE Power & & & & & & & & & & & & & \\
\hline & Electric Machine & 160089 & 160089 & 148705 & 139383 & 157749 & 143474 & 122979 & 151888 & 125841 & 104899 & 147273 & $11993 \not$ & ge99340 \\
\hline & $\mathrm{MC} 2 / \mathrm{GC}$ & 0 & 0 & 0 & 0 & 0 & 0 & 0 & 0 & 0 & 0 & 0 & 0 & 0 \\
\hline & Battery & 194624 & 194624 & 180668 & 169594 & 192167 & 174251 & 148106 & 183881 & 151620 & 125107 & 178954 & 144527 & 118453 \\
\hline & Specific Power & 90 & 90 & 89 & 89 & 90 & 89 & 88 & 89 & 89 & 91 & 89 & 88 & 91 \\
\hline
\end{tabular}




\section{Percentage of power reduction based on the respective 2010 reference case}

\begin{tabular}{|c|c|c|c|c|c|c|c|c|c|c|c|c|c|c|}
\hline & & \multicolumn{13}{|c|}{ Small_SUV } \\
\hline & & \multirow{2}{*}{$\frac{2010}{\text { Ref }}$} & \multicolumn{3}{|c|}{2010} & \multicolumn{3}{|c|}{2015} & \multicolumn{3}{|c|}{2030} & \multicolumn{3}{|c|}{2045} \\
\hline & & & low & avg & high & low & avg & high & low & avg & high & low & avg & high \\
\hline \multirow{5}{*}{ SI Conv } & ICE Power & $0 \%$ & $0 \%$ & $-5 \%$ & $-9 \%$ & $-2 \%$ & $-8 \%$ & $-15 \%$ & $-4 \%$ & $-14 \%$ & $-24 \%$ & $-4 \%$ & $-14 \%$ & $-26 \%$ \\
\hline & Electric Machine & & & & & & & & & & & & & \\
\hline & $\mathrm{MC} 2 / \mathrm{GC}$ & & & & & & & & & & & & & \\
\hline & Battery & & & & & & & & & & & & & \\
\hline & Specific Power & $0 \%$ & $0 \%$ & $-1 \%$ & $-3 \%$ & $-2 \%$ & $-3 \%$ & $-4 \%$ & $-3 \%$ & $-3 \%$ & $-2 \%$ & $-3 \%$ & $-3 \%$ & $-1 \%$ \\
\hline \multirow{5}{*}{ Cl Conv } & ICE Power & $0 \%$ & $0 \%$ & $-2 \%$ & $-7 \%$ & $-2 \%$ & $-6 \%$ & $-12 \%$ & $-1 \%$ & $-10 \%$ & $-21 \%$ & $-2 \%$ & $-10 \%$ & $-23 \%$ \\
\hline & Electric Machine & & & & & & & & & & & & & \\
\hline & $\mathrm{MC} 2 / \mathrm{GC}$ & & & & & & & & & & & & & \\
\hline & Battery & & & & & & & & & & & & & \\
\hline & Specific Power & $0 \%$ & $0 \%$ & $1 \%$ & $-1 \%$ & $-2 \%$ & $-1 \%$ & $-2 \%$ & $-1 \%$ & $0 \%$ & $0 \%$ & $-2 \%$ & $1 \%$ & $1 \%$ \\
\hline \multirow{5}{*}{ H2 Conv } & ICE Power & $0 \%$ & $0 \%$ & $-7 \%$ & $-12 \%$ & $-3 \%$ & $-11 \%$ & $-19 \%$ & $-5 \%$ & $-17 \%$ & $-29 \%$ & $-8 \%$ & $-18 \%$ & $-31 \%$ \\
\hline & Electric Machine & & & & & & & & & & & & & \\
\hline & $\mathrm{MC} 2 / \mathrm{GC}$ & & & & & & & & & & & & & \\
\hline & Battery & & & & & & & & & & & & & \\
\hline & Specific Power & $0 \%$ & $0 \%$ & $-1 \%$ & $-3 \%$ & $-2 \%$ & $-2 \%$ & $-5 \%$ & $-3 \%$ & $-4 \%$ & $-3 \%$ & $-4 \%$ & $-3 \%$ & $-3 \%$ \\
\hline \multirow{5}{*}{ E85 Conv } & ICE Power & $0 \%$ & $0 \%$ & $-3 \%$ & $-8 \%$ & $-2 \%$ & $-7 \%$ & $-14 \%$ & $-2 \%$ & $-12 \%$ & $-22 \%$ & $-3 \%$ & $-13 \%$ & $-25 \%$ \\
\hline & Electric Machine & & & & & & & & & & & & & \\
\hline & $\mathrm{MC} 2 / \mathrm{GC}$ & & & & & & & & & & & & & \\
\hline & Battery & & & & & & & & & & & & & \\
\hline & Specific Power & $0 \%$ & $0 \%$ & $0 \%$ & $-2 \%$ & $-2 \%$ & $-1 \%$ & $-3 \%$ & $-1 \%$ & $-2 \%$ & $-1 \%$ & $-2 \%$ & $-2 \%$ & $1 \%$ \\
\hline \multirow{5}{*}{ SI Split HEV } & ICE Power & $0 \%$ & $0 \%$ & $-5 \%$ & $-10 \%$ & $-1 \%$ & $-8 \%$ & $-18 \%$ & $-4 \%$ & $-15 \%$ & $-31 \%$ & $-4 \%$ & $-19 \%$ & $-34 \%$ \\
\hline & Electric Machine & $0 \%$ & $0 \%$ & $-4 \%$ & $-8 \%$ & $-1 \%$ & $-5 \%$ & $-13 \%$ & $-2 \%$ & $-11 \%$ & $-26 \%$ & $-2 \%$ & $-14 \%$ & $-28 \%$ \\
\hline & $\mathrm{MC} 2 / \mathrm{GC}$ & $0 \%$ & $0 \%$ & $-5 \%$ & $-10 \%$ & $-1 \%$ & $-8 \%$ & $-18 \%$ & $-4 \%$ & $-15 \%$ & $-31 \%$ & $-4 \%$ & $-19 \%$ & $-34 \%$ \\
\hline & Battery & $0 \%$ & $0 \%$ & $-5 \%$ & $-9 \%$ & $-1 \%$ & $-5 \%$ & $-13 \%$ & $-3 \%$ & $-13 \%$ & $-26 \%$ & $-3 \%$ & $-13 \%$ & $-28 \%$ \\
\hline & Specific Power & $0 \%$ & $0 \%$ & $-1 \%$ & $-2 \%$ & $0 \%$ & $-1 \%$ & $-4 \%$ & $-2 \%$ & $-3 \%$ & $-5 \%$ & $-2 \%$ & $-4 \%$ & $-5 \%$ \\
\hline \multirow{5}{*}{ SI Split HEV PHEV10 } & ICE Power & $0 \%$ & $0 \%$ & $-6 \%$ & $-10 \%$ & $-1 \%$ & $-8 \%$ & $-16 \%$ & $-2 \%$ & $-14 \%$ & $-25 \%$ & $-4 \%$ & $-16 \%$ & $-29 \%$ \\
\hline & Electric Machine & $0 \%$ & $0 \%$ & $-4 \%$ & $-8 \%$ & $-1 \%$ & $-7 \%$ & $-16 \%$ & $-2 \%$ & $-15 \%$ & $-27 \%$ & $-3 \%$ & $-16 \%$ & $-30 \%$ \\
\hline & $\mathrm{MC} 2 / \mathrm{GC}$ & $0 \%$ & $0 \%$ & $-6 \%$ & $-10 \%$ & $-1 \%$ & $-8 \%$ & $-16 \%$ & $-2 \%$ & $-14 \%$ & $-25 \%$ & $-4 \%$ & $-16 \%$ & $-29 \%$ \\
\hline & Battery & $0 \%$ & $0 \%$ & $-4 \%$ & $-9 \%$ & $-1 \%$ & $-8 \%$ & $-16 \%$ & $-3 \%$ & $-15 \%$ & $-22 \%$ & $-4 \%$ & $-11 \%$ & $-25 \%$ \\
\hline & Specific Power & $0 \%$ & $0 \%$ & $-1 \%$ & $-1 \%$ & $0 \%$ & $-1 \%$ & $-2 \%$ & $0 \%$ & $-2 \%$ & $4 \%$ & $-1 \%$ & $2 \%$ & $4 \%$ \\
\hline
\end{tabular}




\begin{tabular}{|c|c|c|c|c|c|c|c|c|c|c|c|c|c|c|}
\hline & & \multicolumn{13}{|c|}{ Small_SUV } \\
\hline & & \multirow{2}{*}{$\frac{2010}{\text { Ref }}$} & \multicolumn{3}{|c|}{2010} & \multicolumn{3}{|c|}{2015} & \multicolumn{3}{|c|}{2030} & \multicolumn{3}{|c|}{2045} \\
\hline & & & low & avg & high & low & avg & high & low & avg & high & low & avg & high \\
\hline \multirow{5}{*}{ SI Split HEV PHEV2O } & ICE Power & $0 \%$ & $0 \%$ & $-6 \%$ & $-11 \%$ & $-2 \%$ & $-9 \%$ & $-16 \%$ & $-2 \%$ & $-15 \%$ & $-26 \%$ & $-4 \%$ & $-17 \%$ & $-29 \%$ \\
\hline & Electric Machine & $0 \%$ & $0 \%$ & $-4 \%$ & $-9 \%$ & $-1 \%$ & $-7 \%$ & $-16 \%$ & $-2 \%$ & $-14 \%$ & $-28 \%$ & $-3 \%$ & $-16 \%$ & $-31 \%$ \\
\hline & $\mathrm{MC} 2 / \mathrm{GC}$ & $0 \%$ & $0 \%$ & $-6 \%$ & $-10 \%$ & $-1 \%$ & $-9 \%$ & $-16 \%$ & $-2 \%$ & $-15 \%$ & $-26 \%$ & $-4 \%$ & $-17 \%$ & $-29 \%$ \\
\hline & Battery & $0 \%$ & $0 \%$ & $-5 \%$ & $-10 \%$ & $-1 \%$ & $-8 \%$ & $-16 \%$ & $-3 \%$ & $-15 \%$ & $-28 \%$ & $-4 \%$ & $-14 \%$ & $-30 \%$ \\
\hline & Specific Power & $0 \%$ & $0 \%$ & $-1 \%$ & $-1 \%$ & $0 \%$ & $-1 \%$ & $-1 \%$ & $0 \%$ & $-1 \%$ & $0 \%$ & $0 \%$ & $0 \%$ & $1 \%$ \\
\hline \multirow{5}{*}{ SI Series HEV PHEV30 } & ICE Power & $0 \%$ & $0 \%$ & $-8 \%$ & $-14 \%$ & $-2 \%$ & $-12 \%$ & $-23 \%$ & $-5 \%$ & $-21 \%$ & $-34 \%$ & $-8 \%$ & $-24 \%$ & $-37 \%$ \\
\hline & Electric Machine & $0 \%$ & $0 \%$ & $-6 \%$ & $-11 \%$ & $-2 \%$ & $-9 \%$ & $-18 \%$ & $-3 \%$ & $-16 \%$ & $-27 \%$ & $-5 \%$ & $-18 \%$ & $-30 \%$ \\
\hline & $\mathrm{MC} 2 / \mathrm{GC}$ & $0 \%$ & $0 \%$ & $-8 \%$ & $-14 \%$ & $-2 \%$ & $-12 \%$ & $-23 \%$ & $-5 \%$ & $-21 \%$ & $-34 \%$ & $-8 \%$ & $-24 \%$ & $-37 \%$ \\
\hline & Battery & $0 \%$ & $0 \%$ & $-7 \%$ & $-12 \%$ & $-2 \%$ & $-10 \%$ & $-20 \%$ & $-4 \%$ & $-18 \%$ & $-30 \%$ & $-6 \%$ & $-20 \%$ & $-33 \%$ \\
\hline & Specific Power & $0 \%$ & $0 \%$ & $0 \%$ & $0 \%$ & $0 \%$ & $0 \%$ & $1 \%$ & $1 \%$ & $1 \%$ & $5 \%$ & $1 \%$ & $1 \%$ & $5 \%$ \\
\hline \multirow{5}{*}{ SI Series HEV PHEV40 } & ICE Power & $0 \%$ & $0 \%$ & $-8 \%$ & $-14 \%$ & $-2 \%$ & $-12 \%$ & $-24 \%$ & $-6 \%$ & $-22 \%$ & $-35 \%$ & $-8 \%$ & $-24 \%$ & $-38 \%$ \\
\hline & Electric Machine & $0 \%$ & $0 \%$ & $-6 \%$ & $-11 \%$ & $-2 \%$ & $-9 \%$ & $-18 \%$ & $-3 \%$ & $-17 \%$ & $-28 \%$ & $-6 \%$ & $-19 \%$ & $-31 \%$ \\
\hline & $\mathrm{MC} 2 / \mathrm{GC}$ & $0 \%$ & $0 \%$ & $-8 \%$ & $-14 \%$ & $-2 \%$ & $-12 \%$ & $-24 \%$ & $-6 \%$ & $-22 \%$ & $-35 \%$ & $-8 \%$ & $-24 \%$ & $-38 \%$ \\
\hline & Battery & $0 \%$ & $0 \%$ & $-7 \%$ & $-12 \%$ & $-2 \%$ & $-10 \%$ & $-20 \%$ & $-4 \%$ & $-19 \%$ & $-31 \%$ & $-6 \%$ & $-21 \%$ & $-34 \%$ \\
\hline & Specific Power & $0 \%$ & $0 \%$ & $0 \%$ & $0 \%$ & $1 \%$ & $0 \%$ & $1 \%$ & $1 \%$ & $1 \%$ & $4 \%$ & $0 \%$ & $0 \%$ & $5 \%$ \\
\hline \multirow{5}{*}{ CI Split HEV } & ICE Power & $0 \%$ & $0 \%$ & $-5 \%$ & $-10 \%$ & $-1 \%$ & $-9 \%$ & $-18 \%$ & $-4 \%$ & $-17 \%$ & $-29 \%$ & $-4 \%$ & $-18 \%$ & $-31 \%$ \\
\hline & Electric Machine & $0 \%$ & $0 \%$ & $-4 \%$ & $-8 \%$ & $-1 \%$ & $-6 \%$ & $-13 \%$ & $-2 \%$ & $-12 \%$ & $-24 \%$ & $-2 \%$ & $-13 \%$ & $-27 \%$ \\
\hline & $\mathrm{MC} 2 / \mathrm{GC}$ & $0 \%$ & $0 \%$ & $-5 \%$ & $-10 \%$ & $-1 \%$ & $-9 \%$ & $-18 \%$ & $-4 \%$ & $-17 \%$ & $-29 \%$ & $-4 \%$ & $-18 \%$ & $-31 \%$ \\
\hline & Battery & $0 \%$ & $0 \%$ & $-4 \%$ & $-8 \%$ & $-1 \%$ & $-6 \%$ & $-14 \%$ & $-2 \%$ & $-12 \%$ & $-25 \%$ & $-2 \%$ & $-12 \%$ & $-28 \%$ \\
\hline & Specific Power & $0 \%$ & $0 \%$ & $0 \%$ & $-1 \%$ & $0 \%$ & $-2 \%$ & $-4 \%$ & $-1 \%$ & $-4 \%$ & $-4 \%$ & $-1 \%$ & $-4 \%$ & $-4 \%$ \\
\hline \multirow{5}{*}{ CI Split HEV PHEV10 } & ICE Power & $0 \%$ & $0 \%$ & $-6 \%$ & $-10 \%$ & $-2 \%$ & $-8 \%$ & $-16 \%$ & $-2 \%$ & $-14 \%$ & $-25 \%$ & $-4 \%$ & $-16 \%$ & $-28 \%$ \\
\hline & Electric Machine & $0 \%$ & $0 \%$ & $-4 \%$ & $-8 \%$ & $-1 \%$ & $-6 \%$ & $-15 \%$ & $-3 \%$ & $-13 \%$ & $-26 \%$ & $-3 \%$ & $-15 \%$ & $-29 \%$ \\
\hline & $\mathrm{MC} 2 / \mathrm{GC}$ & $0 \%$ & $0 \%$ & $-6 \%$ & $-10 \%$ & $-2 \%$ & $-8 \%$ & $-16 \%$ & $-2 \%$ & $-14 \%$ & $-25 \%$ & $-4 \%$ & $-16 \%$ & $-28 \%$ \\
\hline & Battery & $0 \%$ & $0 \%$ & $-4 \%$ & $-9 \%$ & $-1 \%$ & $-7 \%$ & $-14 \%$ & $-4 \%$ & $-12 \%$ & $-21 \%$ & $-4 \%$ & $-9 \%$ & $-25 \%$ \\
\hline & Specific Power & $0 \%$ & $0 \%$ & $-1 \%$ & $-1 \%$ & $0 \%$ & $-1 \%$ & $0 \%$ & $-1 \%$ & $-1 \%$ & $3 \%$ & $-1 \%$ & $2 \%$ & $3 \%$ \\
\hline \multirow{5}{*}{ CI Split HEV PHEV20 } & ICE Power & $0 \%$ & $0 \%$ & $-6 \%$ & $-10 \%$ & $-2 \%$ & $-8 \%$ & $-16 \%$ & $-2 \%$ & $-14 \%$ & $-25 \%$ & $-4 \%$ & $-16 \%$ & $-29 \%$ \\
\hline & Electric Machine & $0 \%$ & $0 \%$ & $-4 \%$ & $-8 \%$ & $-1 \%$ & $-7 \%$ & $-15 \%$ & $-2 \%$ & $-14 \%$ & $-26 \%$ & $-3 \%$ & $-16 \%$ & $-30 \%$ \\
\hline & $\mathrm{MC} 2 / \mathrm{GC}$ & $0 \%$ & $0 \%$ & $-6 \%$ & $-10 \%$ & $-2 \%$ & $-8 \%$ & $-16 \%$ & $-2 \%$ & $-14 \%$ & $-25 \%$ & $-4 \%$ & $-16 \%$ & $-29 \%$ \\
\hline & Battery & $0 \%$ & $0 \%$ & $-4 \%$ & $-9 \%$ & $-1 \%$ & $-8 \%$ & $-16 \%$ & $-3 \%$ & $-14 \%$ & $-26 \%$ & $-4 \%$ & $-14 \%$ & $-29 \%$ \\
\hline & Specific Power & $0 \%$ & $0 \%$ & $-1 \%$ & $-1 \%$ & $0 \%$ & $-1 \%$ & $-1 \%$ & $0 \%$ & $-1 \%$ & $0 \%$ & $-1 \%$ & $0 \%$ & $0 \%$ \\
\hline
\end{tabular}




\begin{tabular}{|c|c|c|c|c|c|c|c|c|c|c|c|c|c|c|}
\hline & & \multicolumn{13}{|c|}{ Small_SUV } \\
\hline & & \multirow{2}{*}{$\frac{2010}{\operatorname{Ref}}$} & \multicolumn{3}{|c|}{2010} & \multicolumn{3}{|c|}{2015} & \multicolumn{3}{|c|}{2030} & \multicolumn{3}{|c|}{2045} \\
\hline & & & low & avg & high & low & avg & high & low & avg & high & low & avg & high \\
\hline \multirow{5}{*}{ CI Series HEV PHEV30 } & ICE Power & $0 \%$ & $0 \%$ & $-8 \%$ & $-14 \%$ & $-2 \%$ & $-12 \%$ & $-23 \%$ & $-5 \%$ & $-21 \%$ & $-33 \%$ & $-8 \%$ & $-23 \%$ & $-36 \%$ \\
\hline & Electric Machine & $0 \%$ & $0 \%$ & $-6 \%$ & $-11 \%$ & $-2 \%$ & $-9 \%$ & $-17 \%$ & $-3 \%$ & $-15 \%$ & $-26 \%$ & $-5 \%$ & $-18 \%$ & $-29 \%$ \\
\hline & $\mathrm{MC} 2 / \mathrm{GC}$ & $0 \%$ & $0 \%$ & $-8 \%$ & $-14 \%$ & $-2 \%$ & $-12 \%$ & $-23 \%$ & $-5 \%$ & $-21 \%$ & $-33 \%$ & $-8 \%$ & $-23 \%$ & $-36 \%$ \\
\hline & Battery & $0 \%$ & $0 \%$ & $-6 \%$ & $-12 \%$ & $-2 \%$ & $-10 \%$ & $-19 \%$ & $-4 \%$ & $-17 \%$ & $-29 \%$ & $-6 \%$ & $-19 \%$ & $-32 \%$ \\
\hline & Specific Power & $0 \%$ & $0 \%$ & $0 \%$ & $0 \%$ & $1 \%$ & $0 \%$ & $1 \%$ & $1 \%$ & $0 \%$ & $4 \%$ & $1 \%$ & $0 \%$ & $4 \%$ \\
\hline \multirow{5}{*}{ CI Series HEV PHEV40 } & ICE Power & $0 \%$ & $0 \%$ & $-8 \%$ & $-14 \%$ & $-2 \%$ & $-12 \%$ & $-23 \%$ & $-6 \%$ & $-22 \%$ & $-34 \%$ & $-8 \%$ & $-24 \%$ & $-37 \%$ \\
\hline & Electric Machine & $0 \%$ & $0 \%$ & $-6 \%$ & $-11 \%$ & $-2 \%$ & $-9 \%$ & $-18 \%$ & $-3 \%$ & $-16 \%$ & $-27 \%$ & $-5 \%$ & $-19 \%$ & $-30 \%$ \\
\hline & $\mathrm{MC} 2 / \mathrm{GC}$ & $0 \%$ & $0 \%$ & $-8 \%$ & $-14 \%$ & $-2 \%$ & $-12 \%$ & $-23 \%$ & $-6 \%$ & $-22 \%$ & $-34 \%$ & $-8 \%$ & $-24 \%$ & $-37 \%$ \\
\hline & Battery & $0 \%$ & $0 \%$ & $-7 \%$ & $-12 \%$ & $-2 \%$ & $-10 \%$ & $-20 \%$ & $-4 \%$ & $-18 \%$ & $-30 \%$ & $-6 \%$ & $-21 \%$ & $-33 \%$ \\
\hline & Specific Power & $0 \%$ & $0 \%$ & $0 \%$ & $0 \%$ & $1 \%$ & $0 \%$ & $0 \%$ & $1 \%$ & $0 \%$ & $3 \%$ & $0 \%$ & $0 \%$ & $4 \%$ \\
\hline \multirow{5}{*}{ H2 Split HEV } & ICE Power & $0 \%$ & $0 \%$ & $-7 \%$ & $-14 \%$ & $-2 \%$ & $-12 \%$ & $-22 \%$ & $-4 \%$ & $-20 \%$ & $-34 \%$ & $-8 \%$ & $-22 \%$ & $-36 \%$ \\
\hline & Electric Machine & $0 \%$ & $0 \%$ & $-6 \%$ & $-12 \%$ & $-2 \%$ & $-9 \%$ & $-17 \%$ & $-2 \%$ & $-15 \%$ & $-29 \%$ & $-5 \%$ & $-17 \%$ & $-31 \%$ \\
\hline & $\mathrm{MC} 2 / \mathrm{GC}$ & $0 \%$ & $0 \%$ & $-7 \%$ & $-14 \%$ & $-2 \%$ & $-12 \%$ & $-22 \%$ & $-4 \%$ & $-20 \%$ & $-34 \%$ & $-8 \%$ & $-22 \%$ & $-36 \%$ \\
\hline & Battery & $0 \%$ & $0 \%$ & $-7 \%$ & $-12 \%$ & $-2 \%$ & $-9 \%$ & $-17 \%$ & $-4 \%$ & $-15 \%$ & $-30 \%$ & $-6 \%$ & $-17 \%$ & $-32 \%$ \\
\hline & Specific Power & $0 \%$ & $0 \%$ & $-1 \%$ & $-2 \%$ & $1 \%$ & $-2 \%$ & $-4 \%$ & $-1 \%$ & $-4 \%$ & $-4 \%$ & $-1 \%$ & $-4 \%$ & $-4 \%$ \\
\hline \multirow{5}{*}{ H2 Split HEV PHEV10 } & ICE Power & $0 \%$ & $0 \%$ & $-7 \%$ & $-13 \%$ & $-2 \%$ & $-11 \%$ & $-19 \%$ & $-3 \%$ & $-17 \%$ & $-28 \%$ & $-6 \%$ & $-19 \%$ & $-31 \%$ \\
\hline & Electric Machine & $0 \%$ & $0 \%$ & $-7 \%$ & $-13 \%$ & $-3 \%$ & $-11 \%$ & $-20 \%$ & $-4 \%$ & $-18 \%$ & $-32 \%$ & $-7 \%$ & $-20 \%$ & $-34 \%$ \\
\hline & $\mathrm{MC} 2 / \mathrm{GC}$ & $0 \%$ & $0 \%$ & $-7 \%$ & $-13 \%$ & $-2 \%$ & $-11 \%$ & $-19 \%$ & $-3 \%$ & $-17 \%$ & $-28 \%$ & $-6 \%$ & $-19 \%$ & $-31 \%$ \\
\hline & Battery & $0 \%$ & $0 \%$ & $-8 \%$ & $-14 \%$ & $-4 \%$ & $-12 \%$ & $-23 \%$ & $-6 \%$ & $-21 \%$ & $-30 \%$ & $-9 \%$ & $-17 \%$ & $-33 \%$ \\
\hline & Specific Power & $0 \%$ & $0 \%$ & $-1 \%$ & $-1 \%$ & $0 \%$ & $-1 \%$ & $-3 \%$ & $-1 \%$ & $-2 \%$ & $2 \%$ & $-1 \%$ & $1 \%$ & $2 \%$ \\
\hline \multirow{5}{*}{ H2 Split HEV PHEV 20} & ICE Power & $0 \%$ & $0 \%$ & $-8 \%$ & $-13 \%$ & $-2 \%$ & $-11 \%$ & $-19 \%$ & $-3 \%$ & $-17 \%$ & $-29 \%$ & $-6 \%$ & $-19 \%$ & $-32 \%$ \\
\hline & Electric Machine & $0 \%$ & $0 \%$ & $-8 \%$ & $-13 \%$ & $-3 \%$ & $-11 \%$ & $-20 \%$ & $-5 \%$ & $-18 \%$ & $-32 \%$ & $-7 \%$ & $-20 \%$ & $-35 \%$ \\
\hline & $\mathrm{MC} 2 / \mathrm{GC}$ & $0 \%$ & $0 \%$ & $-8 \%$ & $-13 \%$ & $-2 \%$ & $-11 \%$ & $-19 \%$ & $-3 \%$ & $-17 \%$ & $-29 \%$ & $-6 \%$ & $-19 \%$ & $-32 \%$ \\
\hline & Battery & $0 \%$ & $0 \%$ & $-8 \%$ & $-14 \%$ & $-3 \%$ & $-13 \%$ & $-22 \%$ & $-6 \%$ & $-20 \%$ & $-33 \%$ & $-8 \%$ & $-20 \%$ & $-36 \%$ \\
\hline & Specific Power & $0 \%$ & $0 \%$ & $-1 \%$ & $-1 \%$ & $0 \%$ & $-1 \%$ & $-2 \%$ & $-1 \%$ & $-2 \%$ & $0 \%$ & $0 \%$ & $-1 \%$ & $-1 \%$ \\
\hline \multirow{5}{*}{ H2 Series HEV PHEV 30} & ICE Power & $0 \%$ & $0 \%$ & $-9 \%$ & $-16 \%$ & $-3 \%$ & $-14 \%$ & $-25 \%$ & $-6 \%$ & $-23 \%$ & $-36 \%$ & $-10 \%$ & $-26 \%$ & $-39 \%$ \\
\hline & Electric Machine & $0 \%$ & $0 \%$ & $-8 \%$ & $-13 \%$ & $-3 \%$ & $-11 \%$ & $-20 \%$ & $-4 \%$ & $-18 \%$ & $-30 \%$ & $-7 \%$ & $-20 \%$ & $-33 \%$ \\
\hline & $\mathrm{MC} 2 / \mathrm{GC}$ & $0 \%$ & $0 \%$ & $-9 \%$ & $-16 \%$ & $-3 \%$ & $-14 \%$ & $-25 \%$ & $-6 \%$ & $-23 \%$ & $-36 \%$ & $-10 \%$ & $-26 \%$ & $-39 \%$ \\
\hline & Battery & $0 \%$ & $0 \%$ & $-8 \%$ & $-14 \%$ & $-3 \%$ & $-12 \%$ & $-22 \%$ & $-5 \%$ & $-20 \%$ & $-32 \%$ & $-8 \%$ & $-22 \%$ & $-35 \%$ \\
\hline & Specific Power & $0 \%$ & $0 \%$ & $0 \%$ & $1 \%$ & $1 \%$ & $1 \%$ & $2 \%$ & $2 \%$ & $2 \%$ & $7 \%$ & $2 \%$ & $2 \%$ & $6 \%$ \\
\hline
\end{tabular}




\begin{tabular}{|c|c|c|c|c|c|c|c|c|c|c|c|c|c|c|}
\hline & & \multicolumn{13}{|c|}{ Small_SUV } \\
\hline & & \multirow{2}{*}{$\frac{2010}{\operatorname{Ref}}$} & \multicolumn{3}{|c|}{2010} & \multicolumn{3}{|c|}{2015} & \multicolumn{3}{|c|}{2030} & \multicolumn{3}{|c|}{2045} \\
\hline & & & low & avg & high & low & avg & high & low & avg & high & low & avg & high \\
\hline \multirow{5}{*}{ H2 Series HEV PHEV40 } & ICE Power & $0 \%$ & $0 \%$ & $-9 \%$ & $-16 \%$ & $-3 \%$ & $-14 \%$ & $-26 \%$ & $-6 \%$ & $-24 \%$ & $-37 \%$ & $-10 \%$ & $-27 \%$ & $-40 \%$ \\
\hline & Electric Machine & $0 \%$ & $0 \%$ & $-8 \%$ & $-13 \%$ & $-3 \%$ & $-11 \%$ & $-21 \%$ & $-4 \%$ & $-19 \%$ & $-31 \%$ & $-8 \%$ & $-21 \%$ & $-33 \%$ \\
\hline & $\mathrm{MC} 2 / \mathrm{GC}$ & $0 \%$ & $0 \%$ & $-9 \%$ & $-16 \%$ & $-3 \%$ & $-14 \%$ & $-26 \%$ & $-6 \%$ & $-24 \%$ & $-37 \%$ & $-10 \%$ & $-27 \%$ & $-40 \%$ \\
\hline & Battery & $0 \%$ & $0 \%$ & $-8 \%$ & $-14 \%$ & $-3 \%$ & $-12 \%$ & $-23 \%$ & $-5 \%$ & $-21 \%$ & $-33 \%$ & $-9 \%$ & $-23 \%$ & $-36 \%$ \\
\hline & Specific Power & $0 \%$ & $0 \%$ & $1 \%$ & $1 \%$ & $1 \%$ & $1 \%$ & $2 \%$ & $2 \%$ & $2 \%$ & $6 \%$ & $2 \%$ & $2 \%$ & $6 \%$ \\
\hline \multirow{5}{*}{ E85 Split HEV } & ICE Power & $0 \%$ & $0 \%$ & $-6 \%$ & $-11 \%$ & $-2 \%$ & $-8 \%$ & $-19 \%$ & $-3 \%$ & $-17 \%$ & $-31 \%$ & $-5 \%$ & $-18 \%$ & $-34 \%$ \\
\hline & Electric Machine & $0 \%$ & $0 \%$ & $-5 \%$ & $-9 \%$ & $-2 \%$ & $-5 \%$ & $-14 \%$ & $0 \%$ & $-13 \%$ & $-26 \%$ & $-3 \%$ & $-13 \%$ & $-29 \%$ \\
\hline & $\mathrm{MC} 2 / \mathrm{GC}$ & $0 \%$ & $0 \%$ & $-6 \%$ & $-11 \%$ & $-2 \%$ & $-8 \%$ & $-19 \%$ & $-3 \%$ & $-17 \%$ & $-31 \%$ & $-5 \%$ & $-18 \%$ & $-34 \%$ \\
\hline & Battery & $0 \%$ & $0 \%$ & $-4 \%$ & $-9 \%$ & $-1 \%$ & $-5 \%$ & $-13 \%$ & $-1 \%$ & $-13 \%$ & $-26 \%$ & $-3 \%$ & $-13 \%$ & $-28 \%$ \\
\hline & Specific Power & $0 \%$ & $0 \%$ & $-1 \%$ & $-2 \%$ & $-1 \%$ & $-1 \%$ & $-4 \%$ & $-1 \%$ & $-4 \%$ & $-6 \%$ & $-2 \%$ & $-3 \%$ & $-5 \%$ \\
\hline \multirow{5}{*}{ E85 Split HEV PHEV10 } & ICE Power & $0 \%$ & $0 \%$ & $-6 \%$ & $-10 \%$ & $-1 \%$ & $-8 \%$ & $-16 \%$ & $-2 \%$ & $-14 \%$ & $-25 \%$ & $-4 \%$ & $-16 \%$ & $-29 \%$ \\
\hline & Electric Machine & $0 \%$ & $0 \%$ & $-4 \%$ & $-8 \%$ & $-1 \%$ & $-7 \%$ & $-16 \%$ & $-2 \%$ & $-15 \%$ & $-27 \%$ & $-3 \%$ & $-15 \%$ & $-30 \%$ \\
\hline & $\mathrm{MC} 2 / \mathrm{GC}$ & $0 \%$ & $0 \%$ & $-6 \%$ & $-10 \%$ & $-1 \%$ & $-8 \%$ & $-16 \%$ & $-2 \%$ & $-14 \%$ & $-25 \%$ & $-4 \%$ & $-16 \%$ & $-29 \%$ \\
\hline & Battery & $0 \%$ & $0 \%$ & $-4 \%$ & $-9 \%$ & $-1 \%$ & $-8 \%$ & $-16 \%$ & $-3 \%$ & $-15 \%$ & $-22 \%$ & $-4 \%$ & $-9 \%$ & $-25 \%$ \\
\hline & Specific Power & $0 \%$ & $0 \%$ & $-1 \%$ & $-1 \%$ & $0 \%$ & $-1 \%$ & $-2 \%$ & $0 \%$ & $-2 \%$ & $4 \%$ & $-1 \%$ & $3 \%$ & $4 \%$ \\
\hline \multirow{5}{*}{ E85 Split HEV PHEV 20} & ICE Power & $0 \%$ & $0 \%$ & $-6 \%$ & $-11 \%$ & $-2 \%$ & $-9 \%$ & $-16 \%$ & $-2 \%$ & $-15 \%$ & $-26 \%$ & $-4 \%$ & $-17 \%$ & $-29 \%$ \\
\hline & Electric Machine & $0 \%$ & $0 \%$ & $-4 \%$ & $-9 \%$ & $-1 \%$ & $-7 \%$ & $-16 \%$ & $-2 \%$ & $-14 \%$ & $-27 \%$ & $-3 \%$ & $-16 \%$ & $-31 \%$ \\
\hline & $\mathrm{MC} 2 / \mathrm{GC}$ & $0 \%$ & $0 \%$ & $-6 \%$ & $-10 \%$ & $-1 \%$ & $-9 \%$ & $-16 \%$ & $-2 \%$ & $-15 \%$ & $-26 \%$ & $-4 \%$ & $-17 \%$ & $-29 \%$ \\
\hline & Battery & $0 \%$ & $0 \%$ & $-5 \%$ & $-10 \%$ & $-1 \%$ & $-8 \%$ & $-16 \%$ & $-3 \%$ & $-15 \%$ & $-26 \%$ & $-4 \%$ & $-14 \%$ & $-30 \%$ \\
\hline & Specific Power & $0 \%$ & $0 \%$ & $-1 \%$ & $-1 \%$ & $0 \%$ & $-1 \%$ & $-1 \%$ & $0 \%$ & $-1 \%$ & $1 \%$ & $0 \%$ & $0 \%$ & $1 \%$ \\
\hline \multirow{5}{*}{ E85 Series HEV PHEV30 } & ICE Power & $0 \%$ & $0 \%$ & $-8 \%$ & $-14 \%$ & $-2 \%$ & $-12 \%$ & $-23 \%$ & $-5 \%$ & $-21 \%$ & $-34 \%$ & $-8 \%$ & $-24 \%$ & $-37 \%$ \\
\hline & Electric Machine & $0 \%$ & $0 \%$ & $-6 \%$ & $-11 \%$ & $-2 \%$ & $-9 \%$ & $-18 \%$ & $-3 \%$ & $-16 \%$ & $-27 \%$ & $-5 \%$ & $-18 \%$ & $-30 \%$ \\
\hline & $\mathrm{MC} 2 / \mathrm{GC}$ & $0 \%$ & $0 \%$ & $-8 \%$ & $-14 \%$ & $-2 \%$ & $-12 \%$ & $-23 \%$ & $-5 \%$ & $-21 \%$ & $-34 \%$ & $-8 \%$ & $-24 \%$ & $-37 \%$ \\
\hline & Battery & $0 \%$ & $0 \%$ & $-7 \%$ & $-12 \%$ & $-2 \%$ & $-10 \%$ & $-20 \%$ & $-4 \%$ & $-18 \%$ & $-30 \%$ & $-6 \%$ & $-20 \%$ & $-33 \%$ \\
\hline & Specific Power & $0 \%$ & $0 \%$ & $0 \%$ & $0 \%$ & $0 \%$ & $0 \%$ & $1 \%$ & $1 \%$ & $1 \%$ & $5 \%$ & $1 \%$ & $1 \%$ & $5 \%$ \\
\hline \multirow{5}{*}{ E85 Series HEV PHEV40 } & ICE Power & $0 \%$ & $0 \%$ & $-8 \%$ & $-14 \%$ & $-2 \%$ & $-12 \%$ & $-24 \%$ & $-6 \%$ & $-22 \%$ & $-35 \%$ & $-8 \%$ & $-24 \%$ & $-38 \%$ \\
\hline & Electric Machine & $0 \%$ & $0 \%$ & $-6 \%$ & $-11 \%$ & $-2 \%$ & $-9 \%$ & $-18 \%$ & $-3 \%$ & $-17 \%$ & $-28 \%$ & $-6 \%$ & $-19 \%$ & $-31 \%$ \\
\hline & $\mathrm{MC} 2 / \mathrm{GC}$ & $0 \%$ & $0 \%$ & $-8 \%$ & $-14 \%$ & $-2 \%$ & $-12 \%$ & $-24 \%$ & $-6 \%$ & $-22 \%$ & $-35 \%$ & $-8 \%$ & $-24 \%$ & $-38 \%$ \\
\hline & Battery & $0 \%$ & $0 \%$ & $-7 \%$ & $-12 \%$ & $-2 \%$ & $-10 \%$ & $-20 \%$ & $-4 \%$ & $-19 \%$ & $-31 \%$ & $-6 \%$ & $-21 \%$ & $-34 \%$ \\
\hline & Specific Power & $0 \%$ & $0 \%$ & $0 \%$ & $0 \%$ & $1 \%$ & $0 \%$ & $1 \%$ & $1 \%$ & $0 \%$ & $4 \%$ & $0 \%$ & $0 \%$ & $4 \%$ \\
\hline
\end{tabular}




\begin{tabular}{|c|c|c|c|c|c|c|c|c|c|c|c|c|c|c|}
\hline & & \multicolumn{13}{|c|}{ Small_SUV } \\
\hline & & \multirow{2}{*}{$\frac{2010}{\operatorname{Ref}}$} & \multicolumn{3}{|c|}{2010} & \multicolumn{3}{|c|}{2015} & \multicolumn{3}{|c|}{2030} & \multicolumn{3}{|c|}{2045} \\
\hline & & & low & avg & high & low & avg & high & low & avg & high & low & avg & high \\
\hline \multirow{5}{*}{ FC HEV } & FC Power & $0 \%$ & $0 \%$ & $-15 \%$ & $-26 \%$ & $-11 \%$ & $-27 \%$ & $-38 \%$ & $-20 \%$ & $-36 \%$ & $-49 \%$ & $-25 \%$ & $-39 \%$ & $-52 \%$ \\
\hline & Electric Machine & $0 \%$ & $0 \%$ & $-15 \%$ & $-23 \%$ & $-10 \%$ & $-22 \%$ & $-32 \%$ & $-16 \%$ & $-30 \%$ & $-42 \%$ & $-20 \%$ & $-33 \%$ & $-45 \%$ \\
\hline & $\mathrm{MC} 2 / \mathrm{GC}$ & & & & & & & & & & & & & \\
\hline & Battery & $0 \%$ & $0 \%$ & $-14 \%$ & $-19 \%$ & $-10 \%$ & $-9 \%$ & $-17 \%$ & $-4 \%$ & $-15 \%$ & $-28 \%$ & $-9 \%$ & $-18 \%$ & $-31 \%$ \\
\hline & Specific Power & $0 \%$ & $0 \%$ & $1 \%$ & $1 \%$ & $1 \%$ & $2 \%$ & $0 \%$ & $0 \%$ & $0 \%$ & $2 \%$ & $1 \%$ & $1 \%$ & $2 \%$ \\
\hline \multirow{5}{*}{ FC HEV PHEV 10} & FC Power & $0 \%$ & $0 \%$ & $-15 \%$ & $-24 \%$ & $-8 \%$ & $-22 \%$ & $-32 \%$ & $-16 \%$ & $-30 \%$ & $-44 \%$ & $-20 \%$ & $-33 \%$ & $-47 \%$ \\
\hline & Electric Machine & $0 \%$ & $0 \%$ & $-12 \%$ & $-20 \%$ & $-7 \%$ & $-19 \%$ & $-28 \%$ & $-12 \%$ & $-27 \%$ & $-40 \%$ & $-17 \%$ & $-29 \%$ & $-43 \%$ \\
\hline & $\mathrm{MC} 2 / \mathrm{GC}$ & & & & & & & & & & & & & \\
\hline & Battery & $0 \%$ & $0 \%$ & $-14 \%$ & $-23 \%$ & $-9 \%$ & $-22 \%$ & $-32 \%$ & $-15 \%$ & $-30 \%$ & $-44 \%$ & $-19 \%$ & $-33 \%$ & $-47 \%$ \\
\hline & Specific Power & $0 \%$ & $0 \%$ & $2 \%$ & $2 \%$ & $2 \%$ & $2 \%$ & $3 \%$ & $2 \%$ & $3 \%$ & $4 \%$ & $3 \%$ & $3 \%$ & $4 \%$ \\
\hline \multirow{5}{*}{ FC HEV PHEV2O } & FC Power & $0 \%$ & $0 \%$ & $-15 \%$ & $-25 \%$ & $-8 \%$ & $-23 \%$ & $-33 \%$ & $-16 \%$ & $-31 \%$ & $-44 \%$ & $-20 \%$ & $-34 \%$ & $-47 \%$ \\
\hline & Electric Machine & $0 \%$ & $0 \%$ & $-13 \%$ & $-21 \%$ & $-7 \%$ & $-19 \%$ & $-29 \%$ & $-13 \%$ & $-27 \%$ & $-40 \%$ & $-16 \%$ & $-30 \%$ & $-43 \%$ \\
\hline & $\mathrm{MC} 2 / \mathrm{GC}$ & & & & & & & & & & & & & \\
\hline & Battery & $0 \%$ & $0 \%$ & $-14 \%$ & $-23 \%$ & $-9 \%$ & $-22 \%$ & $-32 \%$ & $-15 \%$ & $-31 \%$ & $-44 \%$ & $-19 \%$ & $-33 \%$ & $-47 \%$ \\
\hline & Specific Power & $0 \%$ & $0 \%$ & $2 \%$ & $2 \%$ & $3 \%$ & $3 \%$ & $3 \%$ & $2 \%$ & $3 \%$ & $4 \%$ & $4 \%$ & $3 \%$ & $4 \%$ \\
\hline \multirow{5}{*}{ FC HEV PHEV 30} & FC Power & $0 \%$ & $0 \%$ & $-12 \%$ & $-19 \%$ & $-7 \%$ & $-18 \%$ & $-27 \%$ & $-10 \%$ & $-25 \%$ & $-36 \%$ & $-15 \%$ & $-27 \%$ & $-39 \%$ \\
\hline & Electric Machine & $0 \%$ & $0 \%$ & $-9 \%$ & $-15 \%$ & $-5 \%$ & $-14 \%$ & $-22 \%$ & $-8 \%$ & $-21 \%$ & $-32 \%$ & $-11 \%$ & $-23 \%$ & $-35 \%$ \\
\hline & $\mathrm{MC} 2 / \mathrm{GC}$ & & & & & & & & & & & & & \\
\hline & Battery & $0 \%$ & $0 \%$ & $-10 \%$ & $-16 \%$ & $-5 \%$ & $-15 \%$ & $-24 \%$ & $-8 \%$ & $-22 \%$ & $-34 \%$ & $-12 \%$ & $-25 \%$ & $-37 \%$ \\
\hline & Specific Power & $0 \%$ & $0 \%$ & $5 \%$ & $7 \%$ & $4 \%$ & $8 \%$ & $11 \%$ & $6 \%$ & $10 \%$ & $17 \%$ & $8 \%$ & $11 \%$ & $17 \%$ \\
\hline \multirow{5}{*}{ FC HEV PHEV 40} & FC Power & $0 \%$ & $0 \%$ & $-12 \%$ & $-20 \%$ & $-7 \%$ & $-18 \%$ & $-27 \%$ & $-10 \%$ & $-25 \%$ & $-37 \%$ & $-15 \%$ & $-28 \%$ & $-40 \%$ \\
\hline & Electric Machine & $0 \%$ & $0 \%$ & $-10 \%$ & $-16 \%$ & $-5 \%$ & $-14 \%$ & $-23 \%$ & $-8 \%$ & $-21 \%$ & $-32 \%$ & $-11 \%$ & $-24 \%$ & $-35 \%$ \\
\hline & $\mathrm{MC} 2 / \mathrm{GC}$ & & & & & & & & & & & & & \\
\hline & Battery & $0 \%$ & $0 \%$ & $-10 \%$ & $-16 \%$ & $-5 \%$ & $-15 \%$ & $-24 \%$ & $-9 \%$ & $-23 \%$ & $-34 \%$ & $-12 \%$ & $-26 \%$ & $-38 \%$ \\
\hline & Specific Power & $0 \%$ & $0 \%$ & $5 \%$ & $8 \%$ & $4 \%$ & $8 \%$ & $11 \%$ & $6 \%$ & $11 \%$ & $17 \%$ & $9 \%$ & $11 \%$ & $18 \%$ \\
\hline \multirow{5}{*}{ EV } & ICE Power & & & & & & & & & & & & & \\
\hline & Electric Machine & $0 \%$ & $0 \%$ & $-8 \%$ & $-14 \%$ & $-2 \%$ & $-11 \%$ & $-24 \%$ & $-4 \%$ & $-23 \%$ & $-35 \%$ & $-7 \%$ & $-26 \%$ & $-39 \%$ \\
\hline & $\mathrm{MC} 2 / \mathrm{GC}$ & & & & & & & & & & & & & \\
\hline & Battery & $0 \%$ & $0 \%$ & $-8 \%$ & $-15 \%$ & $-2 \%$ & $-12 \%$ & $-26 \%$ & $-5 \%$ & $-24 \%$ & $-37 \%$ & $-8 \%$ & $-28 \%$ & $-41 \%$ \\
\hline & Specific Power & $0 \%$ & $0 \%$ & $-1 \%$ & $-1 \%$ & $0 \%$ & $-1 \%$ & $-2 \%$ & $0 \%$ & $-2 \%$ & $1 \%$ & $-1 \%$ & $-2 \%$ & $1 \%$ \\
\hline
\end{tabular}




\section{Energy}

\begin{tabular}{|c|c|c|c|c|c|c|c|c|c|c|c|c|c|c|}
\hline & & \multicolumn{13}{|c|}{ Small_SUV } \\
\hline & & \multirow{2}{*}{$\begin{array}{l}2010 \\
\text { Ref }\end{array}$} & \multicolumn{3}{|c|}{2010} & \multicolumn{3}{|c|}{2015} & \multicolumn{3}{|c|}{2030} & \multicolumn{3}{|c|}{2045} \\
\hline & & & low & avg & high & low & avg & high & low & avg & high & low & avg & high \\
\hline \multirow{2}{*}{ SI Split HEV } & Total Energy & 1147 & 1147 & 1092 & 1045 & 1131 & 1058 & 972 & 1080 & 972 & 821 & 1080 & 972 & 799 \\
\hline & Usable Energy & & & & & & & & & & & & & \\
\hline \multirow{2}{*}{ SI Split HEV PHEV10 } & Total Energy & 4501 & 4501 & 4125 & 3837 & 4398 & 3955 & 3099 & 4252 & 3204 & 2349 & 4270 & 2851 & 2187 \\
\hline & Usable Energy & 2700 & 2700 & 2475 & 2302 & 2639 & 2373 & 2169 & 2551 & 2243 & 1879 & 2562 & 2138 & 1749 \\
\hline \multirow{2}{*}{ SI Split HEV PHEV2O } & Total Energy & 9013 & 9013 & 8388 & 7687 & 9035 & 7922 & 6006 & 8890 & 6221 & 4548 & 8588 & 5581 & 4334 \\
\hline & Usable Energy & 5408 & 5408 & 5033 & 4612 & 5421 & 4753 & 4204 & 5334 & 4355 & 3639 & 5153 & 4186 & 3468 \\
\hline \multirow{2}{*}{ SI Series HEV PHEV30 } & Total Energy & 16533 & 16533 & 15059 & 14000 & 16085 & 14439 & 10881 & 15730 & 11206 & 8374 & 15360 & 10102 & 7830 \\
\hline & Usable Energy & 9920 & 9920 & 9035 & 8400 & 9651 & 8663 & 7617 & 9438 & 7844 & 6699 & 9216 & 7577 & 6264 \\
\hline \multirow{2}{*}{ SI Series HEV PHEV40 } & Total Energy & 22157 & 22157 & 20273 & 18808 & 21533 & 19415 & 14561 & 21034 & 15031 & 10865 & 20643 & 13473 & 10457 \\
\hline & Usable Energy & 13294 & 13294 & 12164 & 11285 & 12920 & 11649 & 10193 & 12620 & 10522 & 8692 & 12386 & 10105 & 8366 \\
\hline \multirow{2}{*}{ CI Split HEV } & Total Energy & 1209 & 1209 & 1162 & 1108 & 1193 & 1102 & 1015 & 1145 & 1037 & 886 & 1145 & 1037 & 842 \\
\hline & Usable Energy & & & & & & & & & & & & & \\
\hline \multirow{2}{*}{ CI Split HEV PHEV10 } & Total Energy & 4570 & 4570 & 4380 & 4021 & 4586 & 4207 & 3267 & 4506 & 3358 & 2465 & 4507 & 2978 & 2365 \\
\hline & Usable Energy & 2742 & 2742 & 2628 & 2413 & 2751 & 2524 & 2287 & 2704 & 2350 & 1972 & 2704 & 2233 & 1892 \\
\hline \multirow{2}{*}{ CI Split HEV PHEV2O } & Total Energy & 9378 & 9378 & 8779 & 8114 & 9418 & 8373 & 6286 & 9257 & 6459 & 4741 & 9044 & 5842 & 4546 \\
\hline & Usable Energy & 5627 & 5627 & 5267 & 4868 & 5651 & 5024 & 4400 & 5554 & 4521 & 3793 & 5427 & 4381 & 3636 \\
\hline \multirow{2}{*}{ CI Series HEV PHEV30 } & Total Energy & 17346 & 17346 & 15610 & 14533 & 17137 & 14961 & 11309 & 16495 & 11644 & 8584 & 15883 & 10499 & 8387 \\
\hline & Usable Energy & 10408 & 10408 & 9366 & 8720 & 10282 & 8977 & 7917 & 9897 & 8151 & 6867 & 9530 & 7874 & 6709 \\
\hline \multirow{2}{*}{ CI Series HEV PHEV40 } & Total Energy & 22995 & 22995 & 20997 & 19510 & 22331 & 20126 & 15182 & 21749 & 15665 & 11397 & 21351 & 13966 & 10918 \\
\hline & Usable Energy & 13797 & 13797 & 12598 & 11706 & 13399 & 12076 & 10627 & 13049 & 10965 & 9117 & 12811 & 10474 & 8735 \\
\hline \multirow{2}{*}{ H2 Split HEV } & Total Energy & 1178 & 1178 & 1100 & 1037 & 1154 & 1037 & 950 & 1102 & 972 & 799 & 1080 & 950 & 778 \\
\hline & Usable Energy & & & & & & & & & & & & & \\
\hline \multirow{2}{*}{ H2 Split HEV PHEV10 } & Total Energy & 4577 & 4577 & 4131 & 3816 & 4510 & 3947 & 3060 & 4352 & 3171 & 2303 & 4233 & 2796 & 2125 \\
\hline & Usable Energy & 2746 & 2746 & 2478 & 2289 & 2706 & 2368 & 2142 & 2611 & 2219 & 1842 & 2540 & 2097 & 1700 \\
\hline \multirow{2}{*}{ H2 Split HEV PHEV2O } & Total Energy & 9122 & 9122 & 8256 & 7576 & 9134 & 7822 & 5958 & 8954 & 6165 & 4459 & 8572 & 5557 & 4190 \\
\hline & Usable Energy & 5473 & 5473 & 4954 & 4545 & 5480 & 4693 & 4171 & 5372 & 4315 & 3567 & 5143 & 4168 & 3352 \\
\hline
\end{tabular}


Argonne National Laboratory Report - Light-Duty Vehicle Fuel Consumption Displacement Potential Up to 2045

\begin{tabular}{|c|c|c|c|c|c|c|c|c|c|c|c|c|c|c|}
\hline & & \multicolumn{13}{|c|}{ Small_SUV } \\
\hline & & \multirow{2}{*}{$\begin{array}{c}2010 \\
\text { Ref }\end{array}$} & \multicolumn{3}{|c|}{2010} & \multicolumn{3}{|c|}{2015} & \multicolumn{3}{|c|}{2030} & \multicolumn{3}{|c|}{2045} \\
\hline & & & low & avg & high & low & avg & high & low & avg & high & low & avg & high \\
\hline \multirow{2}{*}{ H2 Series HEV PHEV3O } & Total Energy & 16771 & 16771 & 15042 & 13868 & 16210 & 14326 & 10704 & 15809 & 11085 & 8186 & 15203 & 9962 & 7683 \\
\hline & Usable Energy & 10062 & 10062 & 9025 & 8321 & 9726 & 8595 & 7493 & 9486 & 7759 & 6548 & 9122 & 7472 & 6147 \\
\hline \multirow{2}{*}{ H2 Series HEV PHEV40 } & Total Energy & 22499 & 22499 & 20149 & 18641 & 21720 & 19248 & 14367 & 21150 & 14857 & 10631 & 20433 & 13396 & 10261 \\
\hline & Usable Energy & 13500 & 13500 & 12089 & 11184 & 13032 & 11549 & 10057 & 12690 & 10400 & 8505 & 12260 & 10047 & 8209 \\
\hline \multirow{2}{*}{ E85 Split HEV } & Total Energy & 1147 & 1147 & 1100 & 1045 & 1131 & 1058 & 972 & 1102 & 972 & 821 & 1080 & 972 & 799 \\
\hline & Usable Energy & & & & & & & & & & & & & \\
\hline \multirow{2}{*}{ E85 Split HEV PHEV10 } & Total Energy & 4506 & 4506 & 4134 & 3840 & 4537 & 3959 & 3127 & 4416 & 3209 & 2353 & 4273 & 2858 & 2224 \\
\hline & Usable Energy & 2704 & 2704 & 2481 & 2304 & 2722 & 2375 & 2189 & 2650 & 2246 & 1882 & 2564 & 2143 & 1779 \\
\hline \multirow{2}{*}{ E85 Split HEV PHEV20 } & Total Energy & 8978 & 8978 & 8267 & 7636 & 9047 & 7928 & 6011 & 8897 & 6222 & 4546 & 8592 & 5587 & 4341 \\
\hline & Usable Energy & 5387 & 5387 & 4960 & 4582 & 5428 & 4757 & 4208 & 5338 & 4355 & 3637 & 5155 & 4190 & 3473 \\
\hline \multirow{2}{*}{ E85 Series HEV PHEV30 } & Total Energy & 16544 & 16544 & 15080 & 14011 & 16096 & 14445 & 10890 & 15737 & 11212 & 8382 & 15371 & 10107 & 7838 \\
\hline & Usable Energy & 9927 & 9927 & 9048 & 8406 & 9658 & 8667 & 7623 & 9442 & 7848 & 6705 & 9222 & 7580 & 6271 \\
\hline \multirow{2}{*}{ E85 Series HEV PHEV40 } & Total Energy & 22151 & 22151 & 20300 & 18827 & 21543 & 19430 & 14580 & 21048 & 15036 & 10875 & 20662 & 13484 & 10464 \\
\hline & Usable Energy & 13291 & 13291 & 12180 & 11296 & 12926 & 11658 & 10206 & 12629 & 10525 & 8700 & 12397 & 10113 & 8372 \\
\hline \multirow{2}{*}{ FC HEV } & Total Energy & 1443 & 1443 & 1248 & 1162 & 1303 & 1274 & 1166 & 1339 & 1188 & 1015 & 1274 & 1145 & 972 \\
\hline & Usable Energy & & & & & & & & & & & & & \\
\hline \multirow{2}{*}{ FC HEV PHEV 10} & Total Energy & 5474 & 5474 & 4670 & 4161 & 5063 & 4298 & 3230 & 4786 & 3331 & 2388 & 4549 & 2995 & 2284 \\
\hline & Usable Energy & 3285 & 3285 & 2802 & 2496 & 3038 & 2579 & 2261 & 2872 & 2332 & 1910 & 2729 & 2246 & 1827 \\
\hline \multirow{2}{*}{ FC HEV PHEV 20} & Total Energy & 10997 & 10997 & 9366 & 8306 & 10170 & 8596 & 6539 & 9555 & 6750 & 4737 & 9111 & 6061 & 4520 \\
\hline & Usable Energy & 6598 & 6598 & 5619 & 4984 & 6102 & 5158 & 4577 & 5733 & 4725 & 3790 & 5466 & 4545 & 3616 \\
\hline \multirow{2}{*}{ FC HEV PHEV 30} & Total Energy & 17800 & 17800 & 15186 & 13724 & 15810 & 14098 & 9974 & 15125 & 10300 & 7166 & 14307 & 9215 & 6848 \\
\hline & Usable Energy & 10680 & 10680 & 9112 & 8235 & 9486 & 8459 & 6982 & 9075 & 7210 & 5732 & 8584 & 6911 & 5478 \\
\hline \multirow{2}{*}{ FC HEV PHEV 40} & Total Energy & 22698 & 22698 & 18993 & 17152 & 20663 & 17618 & 12850 & 19719 & 13305 & 9608 & 18645 & 11892 & 9161 \\
\hline & Usable Energy & 13619 & 13619 & 11396 & 10291 & 12398 & 10571 & 8995 & 11831 & 9314 & 7687 & 11187 & 8919 & 7329 \\
\hline \multirow{2}{*}{ BEV } & Total Energy & 81994 & 81994 & 73591 & 67488 & 79539 & 70008 & 49862 & 77268 & 51340 & 35971 & 74726 & 45950 & 34246 \\
\hline & Usable Energy & 49196 & 49196 & 44155 & 40493 & 47723 & 42005 & 34904 & 46361 & 35938 & 28777 & 44835 & 34462 & 27397 \\
\hline
\end{tabular}




\section{Percentage of Energy reduction based on the respective 2010 reference case}

\begin{tabular}{|c|c|c|c|c|c|c|c|c|c|c|c|c|c|c|}
\hline & & \multicolumn{13}{|c|}{ Small_SUV } \\
\hline & & \multirow{2}{*}{$\frac{2010}{\operatorname{Ref}}$} & \multicolumn{3}{|c|}{2010} & \multicolumn{3}{|c|}{2015} & \multicolumn{3}{|c|}{2030} & \multicolumn{3}{|c|}{2045} \\
\hline & & & low & avg & high & low & avg & high & low & avg & high & low & avg & high \\
\hline \multirow{2}{*}{ SI Split HEV } & Total Energy & $0.0 \%$ & $0.0 \%$ & $-4.8 \%$ & $-8.8 \%$ & $-1.4 \%$ & $-7.7 \%$ & $-15.2 \%$ & $-5.8 \%$ & $-15.2 \%$ & $-28.4 \%$ & $-5.8 \%$ & $-15.2 \%$ & $-30.3 \%$ \\
\hline & Usable Energy & & & & & & & & & & & & & \\
\hline \multirow{2}{*}{ SI Split HEV PHEV10 } & Total Energy & $0.0 \%$ & $0.0 \%$ & $-8.3 \%$ & $-14.8 \%$ & $-2.3 \%$ & $-12.1 \%$ & $-31.1 \%$ & $-5.5 \%$ & $-28.8 \%$ & $-47.8 \%$ & $-5.1 \%$ & $-36.6 \%$ & $-51.4 \%$ \\
\hline & Usable Energy & $0.0 \%$ & $0.0 \%$ & $-8.3 \%$ & $-14.8 \%$ & $-2.3 \%$ & $-12.1 \%$ & $-19.7 \%$ & $-5.5 \%$ & $-16.9 \%$ & $-30.4 \%$ & $-5.1 \%$ & $-20.8 \%$ & $-35.2 \%$ \\
\hline \multirow{2}{*}{ SI Split HEV PHEV2O } & Total Energy & $0.0 \%$ & $0.0 \%$ & $-6.9 \%$ & $-14.7 \%$ & $0.2 \%$ & $-12.1 \%$ & $-33.4 \%$ & $-1.4 \%$ & $-31.0 \%$ & $-49.5 \%$ & $-4.7 \%$ & $-38.1 \%$ & $-51.9 \%$ \\
\hline & Usable Energy & $0.0 \%$ & $0.0 \%$ & $-6.9 \%$ & $-14.7 \%$ & $0.2 \%$ & $-12.1 \%$ & $-22.3 \%$ & $-1.4 \%$ & $-19.5 \%$ & $-32.7 \%$ & $-4.7 \%$ & $-22.6 \%$ & $-35.9 \%$ \\
\hline \multirow{2}{*}{ SI Series HEV PHEV 30} & Total Energy & $0.0 \%$ & $0.0 \%$ & $-8.9 \%$ & $-15.3 \%$ & $-2.7 \%$ & $-12.7 \%$ & $-34.2 \%$ & $-4.9 \%$ & $-32.2 \%$ & $-49.3 \%$ & $-7.1 \%$ & $-38.9 \%$ & $-52.6 \%$ \\
\hline & Usable Energy & $0.0 \%$ & $0.0 \%$ & $-8.9 \%$ & $-15.3 \%$ & $-2.7 \%$ & $-12.7 \%$ & $-23.2 \%$ & $-4.9 \%$ & $-20.9 \%$ & $-32.5 \%$ & $-7.1 \%$ & $-23.6 \%$ & $-36.9 \%$ \\
\hline \multirow{2}{*}{ SI Series HEV PHEV40 } & Total Energy & $0.0 \%$ & $0.0 \%$ & $-8.5 \%$ & $-15.1 \%$ & $-2.8 \%$ & $-12.4 \%$ & $-34.3 \%$ & $-5.1 \%$ & $-32.2 \%$ & $-51.0 \%$ & $-6.8 \%$ & $-39.2 \%$ & $-52.8 \%$ \\
\hline & Usable Energy & $0.0 \%$ & $0.0 \%$ & $-8.5 \%$ & $-15.1 \%$ & $-2.8 \%$ & $-12.4 \%$ & $-23.3 \%$ & $-5.1 \%$ & $-20.9 \%$ & $-34.6 \%$ & $-6.8 \%$ & $-24.0 \%$ & $-37.1 \%$ \\
\hline \multirow{2}{*}{ CI Split HEV } & Total Energy & $0.0 \%$ & $0.0 \%$ & $-3.9 \%$ & $-8.4 \%$ & $-1.3 \%$ & $-8.9 \%$ & $-16.0 \%$ & $-5.3 \%$ & $-14.2 \%$ & $-26.7 \%$ & $-5.3 \%$ & $-14.2 \%$ & $-30.3 \%$ \\
\hline & Usable Energy & & & & & & & & & & & & & \\
\hline \multirow{2}{*}{ CI Split HEV PHEV10 } & Total Energy & $0.0 \%$ & $0.0 \%$ & $-4.2 \%$ & $-12.0 \%$ & $0.4 \%$ & $-7.9 \%$ & $-28.5 \%$ & $-1.4 \%$ & $-26.5 \%$ & $-46.1 \%$ & $-1.4 \%$ & $-34.8 \%$ & $-48.2 \%$ \\
\hline & Usable Energy & $0.0 \%$ & $0.0 \%$ & $-4.2 \%$ & $-12.0 \%$ & $0.4 \%$ & $-7.9 \%$ & $-16.6 \%$ & $-1.4 \%$ & $-14.3 \%$ & $-28.1 \%$ & $-1.4 \%$ & $-18.5 \%$ & $-31.0 \%$ \\
\hline \multirow{2}{*}{ CI Split HEV PHEV 20} & Total Energy & $0.0 \%$ & $0.0 \%$ & $-6.4 \%$ & $-13.5 \%$ & $0.4 \%$ & $-10.7 \%$ & $-33.0 \%$ & $-1.3 \%$ & $-31.1 \%$ & $-49.4 \%$ & $-3.6 \%$ & $-37.7 \%$ & $-51.5 \%$ \\
\hline & Usable Energy & $0.0 \%$ & $0.0 \%$ & $-6.4 \%$ & $-13.5 \%$ & $0.4 \%$ & $-10.7 \%$ & $-21.8 \%$ & $-1.3 \%$ & $-19.7 \%$ & $-32.6 \%$ & $-3.6 \%$ & $-22.1 \%$ & $-35.4 \%$ \\
\hline \multirow{2}{*}{ CI Series HEV PHEV30 } & Total Energy & $0.0 \%$ & $0.0 \%$ & $-10.0 \%$ & $-16.2 \%$ & $-1.2 \%$ & $-13.7 \%$ & $-34.8 \%$ & $-4.9 \%$ & $-32.9 \%$ & $-50.5 \%$ & $-8.4 \%$ & $-39.5 \%$ & $-51.7 \%$ \\
\hline & Usable Energy & $0.0 \%$ & $0.0 \%$ & $-10.0 \%$ & $-16.2 \%$ & $-1.2 \%$ & $-13.7 \%$ & $-23.9 \%$ & $-4.9 \%$ & $-21.7 \%$ & $-34.0 \%$ & $-8.4 \%$ & $-24.3 \%$ & $-35.5 \%$ \\
\hline \multirow{2}{*}{$\mathrm{Cl}$ Series HEV PHEV40 } & Total Energy & $0.0 \%$ & $0.0 \%$ & $-8.7 \%$ & $-15.2 \%$ & $-2.9 \%$ & $-12.5 \%$ & $-34.0 \%$ & $-5.4 \%$ & $-31.9 \%$ & $-50.4 \%$ & $-7.1 \%$ & $-39.3 \%$ & $-52.5 \%$ \\
\hline & Usable Energy & $0.0 \%$ & $0.0 \%$ & $-8.7 \%$ & $-15.2 \%$ & $-2.9 \%$ & $-12.5 \%$ & $-23.0 \%$ & $-5.4 \%$ & $-20.5 \%$ & $-33.9 \%$ & $-7.1 \%$ & $-24.1 \%$ & $-36.7 \%$ \\
\hline \multirow{2}{*}{ H2 Split HEV } & Total Energy & $0.0 \%$ & $0.0 \%$ & $-6.6 \%$ & $-11.9 \%$ & $-2.0 \%$ & $-12.0 \%$ & $-19.3 \%$ & $-6.5 \%$ & $-17.5 \%$ & $-32.1 \%$ & $-8.3 \%$ & $-19.3 \%$ & $-34.0 \%$ \\
\hline & Usable Energy & & & & & & & & & & & & & \\
\hline \multirow{2}{*}{ H2 Split HEV PHEV10 } & Total Energy & $0.0 \%$ & $0.0 \%$ & $-9.8 \%$ & $-16.6 \%$ & $-1.5 \%$ & $-13.8 \%$ & $-33.1 \%$ & $-4.9 \%$ & $-30.7 \%$ & $-49.7 \%$ & $-7.5 \%$ & $-38.9 \%$ & $-53.6 \%$ \\
\hline & Usable Energy & $0.0 \%$ & $0.0 \%$ & $-9.8 \%$ & $-16.6 \%$ & $-1.5 \%$ & $-13.8 \%$ & $-22.0 \%$ & $-4.9 \%$ & $-19.2 \%$ & $-32.9 \%$ & $-7.5 \%$ & $-23.6 \%$ & $-38.1 \%$ \\
\hline \multirow{2}{*}{ H2 Split HEV PHEV2O } & Total Energy & $0.0 \%$ & $0.0 \%$ & $-9.5 \%$ & $-17.0 \%$ & $0.1 \%$ & $-14.2 \%$ & $-34.7 \%$ & $-1.8 \%$ & $-32.4 \%$ & $-51.1 \%$ & $-6.0 \%$ & $-39.1 \%$ & $-54.1 \%$ \\
\hline & Usable Energy & $0.0 \%$ & $0.0 \%$ & $-9.5 \%$ & $-17.0 \%$ & $0.1 \%$ & $-14.2 \%$ & $-23.8 \%$ & $-1.8 \%$ & $-21.2 \%$ & $-34.8 \%$ & $-6.0 \%$ & $-23.9 \%$ & $-38.8 \%$ \\
\hline
\end{tabular}


Argonne National Laboratory Report - Light-Duty Vehicle Fuel Consumption Displacement Potential Up to 2045

\begin{tabular}{|c|c|c|c|c|c|c|c|c|c|c|c|c|c|c|}
\hline & & \multicolumn{13}{|c|}{ Small_SUV } \\
\hline & & \multirow{2}{*}{$\frac{2010}{\text { Ref }}$} & \multicolumn{3}{|c|}{2010} & \multicolumn{3}{|c|}{2015} & \multicolumn{3}{|c|}{2030} & \multicolumn{3}{|c|}{2045} \\
\hline & & & low & avg & high & low & avg & high & low & avg & high & low & avg & high \\
\hline \multirow{2}{*}{ H2 Series HEV PHEV30 } & Total Energy & $0.0 \%$ & $0.0 \%$ & $-10.3 \%$ & $-17.3 \%$ & $-3.3 \%$ & $-14.6 \%$ & $-36.2 \%$ & $-5.7 \%$ & $-33.9 \%$ & $-51.2 \%$ & $-9.3 \%$ & $-40.6 \%$ & $-54.2 \%$ \\
\hline & Usable Energy & $0.0 \%$ & $0.0 \%$ & $-10.3 \%$ & $-17.3 \%$ & $-3.3 \%$ & $-14.6 \%$ & $-25.5 \%$ & $-5.7 \%$ & $-22.9 \%$ & $-34.9 \%$ & $-9.3 \%$ & $-25.7 \%$ & $-38.9 \%$ \\
\hline \multirow{2}{*}{ H2 Series HEV PHEV40 } & Total Energy & $0.0 \%$ & $0.0 \%$ & $-10.4 \%$ & $-17.2 \%$ & $-3.5 \%$ & $-14.5 \%$ & $-36.1 \%$ & $-6.0 \%$ & $-34.0 \%$ & $-52.8 \%$ & $-9.2 \%$ & $-40.5 \%$ & $-54.4 \%$ \\
\hline & Usable Energy & $0.0 \%$ & $0.0 \%$ & $-10.4 \%$ & $-17.2 \%$ & $-3.5 \%$ & $-14.5 \%$ & $-25.5 \%$ & $-6.0 \%$ & $-23.0 \%$ & $-37.0 \%$ & $-9.2 \%$ & $-25.6 \%$ & $-39.2 \%$ \\
\hline \multirow{2}{*}{ E85 Split HEV } & Total Energy & $0.0 \%$ & $0.0 \%$ & $-4.1 \%$ & $-8.8 \%$ & $-1.4 \%$ & $-7.7 \%$ & $-15.2 \%$ & $-3.9 \%$ & $-15.2 \%$ & $-28.4 \%$ & $-5.8 \%$ & $-15.2 \%$ & $-30.3 \%$ \\
\hline & Usable Energy & & & & & & & & & & & & & \\
\hline \multirow{2}{*}{ E85 Split HEV PHEV10 } & Total Energy & $0.0 \%$ & $0.0 \%$ & $-8.2 \%$ & $-14.8 \%$ & $0.7 \%$ & $-12.1 \%$ & $-30.6 \%$ & $-2.0 \%$ & $-28.8 \%$ & $-47.8 \%$ & $-5.2 \%$ & $-36.6 \%$ & $-50.6 \%$ \\
\hline & Usable Energy & $0.0 \%$ & $0.0 \%$ & $-8.2 \%$ & $-14.8 \%$ & $0.7 \%$ & $-12.1 \%$ & $-19.0 \%$ & $-2.0 \%$ & $-16.9 \%$ & $-30.4 \%$ & $-5.2 \%$ & $-20.7 \%$ & $-34.2 \%$ \\
\hline \multirow{2}{*}{ E85 Split HEV PHEV20 } & Total Energy & $0.0 \%$ & $0.0 \%$ & $-7.9 \%$ & $-14.9 \%$ & $0.8 \%$ & $-11.7 \%$ & $-33.1 \%$ & $-0.9 \%$ & $-30.7 \%$ & $-49.4 \%$ & $-4.3 \%$ & $-37.8 \%$ & $-51.6 \%$ \\
\hline & Usable Energy & $0.0 \%$ & $0.0 \%$ & $-7.9 \%$ & $-14.9 \%$ & $0.8 \%$ & $-11.7 \%$ & $-21.9 \%$ & $-0.9 \%$ & $-19.2 \%$ & $-32.5 \%$ & $-4.3 \%$ & $-22.2 \%$ & $-35.5 \%$ \\
\hline \multirow{2}{*}{ E85 Series HEV PHEV30 } & Total Energy & $0.0 \%$ & $0.0 \%$ & $-8.8 \%$ & $-15.3 \%$ & $-2.7 \%$ & $-12.7 \%$ & $-34.2 \%$ & $-4.9 \%$ & $-32.2 \%$ & $-49.3 \%$ & $-7.1 \%$ & $-38.9 \%$ & $-52.6 \%$ \\
\hline & Usable Energy & $0.0 \%$ & $0.0 \%$ & $-8.8 \%$ & $-15.3 \%$ & $-2.7 \%$ & $-12.7 \%$ & $-23.2 \%$ & $-4.9 \%$ & $-20.9 \%$ & $-32.5 \%$ & $-7.1 \%$ & $-23.6 \%$ & $-36.8 \%$ \\
\hline \multirow{2}{*}{ E85 Series HEV PHEV40 } & Total Energy & $0.0 \%$ & $0.0 \%$ & $-8.4 \%$ & $-15.0 \%$ & $-2.7 \%$ & $-12.3 \%$ & $-34.2 \%$ & $-5.0 \%$ & $-32.1 \%$ & $-50.9 \%$ & $-6.7 \%$ & $-39.1 \%$ & $-52.8 \%$ \\
\hline & Usable Energy & $0.0 \%$ & $0.0 \%$ & $-8.4 \%$ & $-15.0 \%$ & $-2.7 \%$ & $-12.3 \%$ & $-23.2 \%$ & $-5.0 \%$ & $-20.8 \%$ & $-34.5 \%$ & $-6.7 \%$ & $-23.9 \%$ & $-37.0 \%$ \\
\hline \multirow{2}{*}{ FC HEV } & Total Energy & $0.0 \%$ & $0.0 \%$ & $-13.5 \%$ & $-19.5 \%$ & $-9.7 \%$ & $-11.7 \%$ & $-19.2 \%$ & $-7.2 \%$ & $-17.7 \%$ & $-29.6 \%$ & $-11.7 \%$ & $-20.7 \%$ & $-32.6 \%$ \\
\hline & Usable Energy & & & & & & & & & & & & & \\
\hline \multirow{2}{*}{ FC HEV PHEV10 } & Total Energy & $0.0 \%$ & $0.0 \%$ & $-14.7 \%$ & $-24.0 \%$ & $-7.5 \%$ & $-21.5 \%$ & $-41.0 \%$ & $-12.6 \%$ & $-39.2 \%$ & $-56.4 \%$ & $-16.9 \%$ & $-45.3 \%$ & $-58.3 \%$ \\
\hline & Usable Energy & $0.0 \%$ & $0.0 \%$ & $-14.7 \%$ & $-24.0 \%$ & $-7.5 \%$ & $-21.5 \%$ & $-31.2 \%$ & $-12.6 \%$ & $-29.0 \%$ & $-41.8 \%$ & $-16.9 \%$ & $-31.6 \%$ & $-44.4 \%$ \\
\hline \multirow{2}{*}{ FC HEV PHEV20 } & Total Energy & $0.0 \%$ & $0.0 \%$ & $-14.8 \%$ & $-24.5 \%$ & $-7.5 \%$ & $-21.8 \%$ & $-40.5 \%$ & $-13.1 \%$ & $-38.6 \%$ & $-56.9 \%$ & $-17.2 \%$ & $-44.9 \%$ & $-58.9 \%$ \\
\hline & Usable Energy & $0.0 \%$ & $0.0 \%$ & $-14.8 \%$ & $-24.5 \%$ & $-7.5 \%$ & $-21.8 \%$ & $-30.6 \%$ & $-13.1 \%$ & $-28.4 \%$ & $-42.6 \%$ & $-17.2 \%$ & $-31.1 \%$ & $-45.2 \%$ \\
\hline \multirow{2}{*}{ FC HEV PHEV 30} & Total Energy & $0.0 \%$ & $0.0 \%$ & $-14.7 \%$ & $-22.9 \%$ & $-11.2 \%$ & $-20.8 \%$ & $-44.0 \%$ & $-15.0 \%$ & $-42.1 \%$ & $-59.7 \%$ & $-19.6 \%$ & $-48.2 \%$ & $-61.5 \%$ \\
\hline & Usable Energy & $0.0 \%$ & $0.0 \%$ & $-14.7 \%$ & $-22.9 \%$ & $-11.2 \%$ & $-20.8 \%$ & $-34.6 \%$ & $-15.0 \%$ & $-32.5 \%$ & $-46.3 \%$ & $-19.6 \%$ & $-35.3 \%$ & $-48.7 \%$ \\
\hline \multirow{2}{*}{ FC HEV PHEV 40} & Total Energy & $0.0 \%$ & $0.0 \%$ & $-16.3 \%$ & $-24.4 \%$ & $-9.0 \%$ & $-22.4 \%$ & $-43.4 \%$ & $-13.1 \%$ & $-41.4 \%$ & $-57.7 \%$ & $-17.9 \%$ & $-47.6 \%$ & $-59.6 \%$ \\
\hline & Usable Energy & $0.0 \%$ & $0.0 \%$ & $-16.3 \%$ & $-24.4 \%$ & $-9.0 \%$ & $-22.4 \%$ & $-34.0 \%$ & $-13.1 \%$ & $-31.6 \%$ & $-43.6 \%$ & $-17.9 \%$ & $-34.5 \%$ & $-46.2 \%$ \\
\hline \multirow{2}{*}{ EV } & Total Energy & $0.0 \%$ & $0.0 \%$ & $-10.2 \%$ & $-17.7 \%$ & $-3.0 \%$ & $-14.6 \%$ & $-39.2 \%$ & $-5.8 \%$ & $-37.4 \%$ & $-56.1 \%$ & $-8.9 \%$ & $-44.0 \%$ & $-58.2 \%$ \\
\hline & Usable Energy & $0.0 \%$ & $0.0 \%$ & $-10.2 \%$ & $-17.7 \%$ & $-3.0 \%$ & $-14.6 \%$ & $-29.1 \%$ & $-5.8 \%$ & $-26.9 \%$ & $-41.5 \%$ & $-8.9 \%$ & $-29.9 \%$ & $-44.3 \%$ \\
\hline
\end{tabular}


curb weight

\begin{tabular}{|c|c|c|c|c|c|c|c|c|c|c|c|c|c|c|}
\hline & & \multicolumn{13}{|c|}{ Small_SUV } \\
\hline & & \multirow{2}{*}{$\frac{2010}{\operatorname{Ref}}$} & \multicolumn{3}{|c|}{2010} & \multicolumn{3}{|c|}{2015} & \multicolumn{3}{|c|}{2030} & \multicolumn{3}{|c|}{2045} \\
\hline & & & low & avg & high & low & avg & high & low & avg & high & low & avg & high \\
\hline \multirow{3}{*}{ SI Conv } & Vehicle Mass & 1475 & 1475 & 1424 & 1371 & 1474 & 1389 & 1292 & 1467 & 1306 & 1120 & 1466 & 1292 & 1066 \\
\hline & $\%$ of Glider mass & 68.2 & 68.2 & 67.4 & 66.4 & 68.3 & 66.8 & 65.0 & 68.3 & 65.2 & 60.2 & 68.3 & 64.9 & 58.5 \\
\hline & $\%$ of Powertrain mass & 31.8 & 31.8 & 32.6 & 33.6 & 31.7 & 33.2 & 35.0 & 31.7 & 34.8 & 39.8 & 31.7 & 35.1 & 41.5 \\
\hline \multirow{3}{*}{$\mathrm{Cl}$ Conv } & Vehicle Mass & 1528 & 1528 & 1481 & 1429 & 1528 & 1447 & 1354 & 1523 & 1367 & 1185 & 1522 & 1354 & 1133 \\
\hline & $\%$ of Glider mass & 65.8 & 65.8 & 64.8 & 63.8 & 65.9 & 64.1 & 62.0 & 65.8 & 62.3 & 56.9 & 65.8 & 61.9 & 55.0 \\
\hline & $\%$ of Powertrain mass & 34.2 & 34.2 & 35.2 & 36.2 & 34.1 & 35.9 & 38.0 & 34.2 & 37.7 & 43.1 & 34.2 & 38.1 & 45.0 \\
\hline \multirow{3}{*}{ H2 Conv } & Vehicle Mass & 1488 & 1488 & 1399 & 1331 & 1474 & 1349 & 1238 & 1459 & 1260 & 1056 & 1419 & 1239 & 1010 \\
\hline & $\%$ of Glider mass & 67.6 & 67.6 & 68.6 & 68.4 & 68.3 & 68.8 & 67.8 & 68.7 & 67.5 & 63.9 & 70.6 & 67.6 & 61.7 \\
\hline & $\%$ of Powertrain mass & 32.4 & 32.4 & 31.4 & 31.6 & 31.7 & 31.2 & 32.2 & 31.3 & 32.5 & 36.1 & 29.4 & 32.4 & 38.3 \\
\hline \multirow{3}{*}{ E85 Conv } & Vehicle Mass & 1471 & 1471 & 1422 & 1368 & 1471 & 1387 & 1291 & 1465 & 1304 & 1119 & 1463 & 1290 & 1066 \\
\hline & $\%$ of Glider mass & 68.4 & 68.4 & 67.5 & 66.6 & 68.5 & 66.9 & 65.0 & 68.4 & 65.3 & 60.3 & 68.5 & 65.0 & 58.5 \\
\hline & $\%$ of Powertrain mass & 31.6 & 31.6 & 32.5 & 33.4 & 31.5 & 33.1 & 35.0 & 31.6 & 34.7 & 39.7 & 31.5 & 35.0 & 41.5 \\
\hline \multirow{3}{*}{ SI Split HEV } & Vehicle Mass & 1610 & 1610 & 1531 & 1459 & 1585 & 1498 & 1366 & 1574 & 1393 & 1161 & 1566 & 1368 & 1103 \\
\hline & $\%$ of Glider mass & 62.5 & 62.5 & 62.7 & 62.4 & 63.5 & 61.9 & 61.4 & 63.7 & 61.1 & 58.1 & 64.0 & 61.3 & 56.5 \\
\hline & $\%$ of Powertrain mass & 37.5 & 37.5 & 37.3 & 37.6 & 36.5 & 38.1 & 38.6 & 36.3 & 38.9 & 41.9 & 36.0 & 38.7 & 43.5 \\
\hline \multirow{3}{*}{ SI Split HEV PHEV10 } & Vehicle Mass & 1627 & 1627 & 1547 & 1473 & 1602 & 1500 & 1365 & 1586 & 1392 & 1165 & 1572 & 1369 & 1107 \\
\hline & $\%$ of Glider mass & 61.8 & 61.8 & 62.0 & 61.8 & 62.9 & 61.9 & 61.5 & 63.2 & 61.1 & 57.9 & 63.7 & 61.2 & 56.3 \\
\hline & $\%$ of Powertrain mass & 38.2 & 38.2 & 38.0 & 38.2 & 37.1 & 38.1 & 38.5 & 36.8 & 38.9 & 42.1 & 36.3 & 38.8 & 43.7 \\
\hline \multirow{3}{*}{ SI Split HEV PHEV2O } & Vehicle Mass & 1663 & 1663 & 1580 & 1503 & 1638 & 1531 & 1388 & 1618 & 1417 & 1180 & 1604 & 1388 & 1119 \\
\hline & $\%$ of Glider mass & 60.5 & 60.5 & 60.7 & 60.6 & 61.5 & 60.6 & 60.5 & 61.9 & 60.1 & 57.2 & 62.5 & 60.4 & 55.7 \\
\hline & $\%$ of Powertrain mass & 39.5 & 39.5 & 39.3 & 39.4 & 38.5 & 39.4 & 39.5 & 38.1 & 39.9 & 42.8 & 37.5 & 39.6 & 44.3 \\
\hline \multirow{3}{*}{ SI Series HEV PHEV30 } & Vehicle Mass & 1903 & 1903 & 1780 & 1678 & 1852 & 1717 & 1527 & 1815 & 1564 & 1285 & 1786 & 1520 & 1218 \\
\hline & $\%$ of Glider mass & 52.9 & 52.9 & 53.9 & 54.3 & 54.4 & 54.0 & 55.0 & 55.2 & 54.4 & 52.5 & 56.1 & 55.1 & 51.2 \\
\hline & $\%$ of Powertrain mass & 47.1 & 47.1 & 46.1 & 45.7 & 45.6 & 46.0 & 45.0 & 44.8 & 45.6 & 47.5 & 43.9 & 44.9 & 48.8 \\
\hline \multirow{3}{*}{ SI Series HEV PHEV40 } & Vehicle Mass & 1959 & 1959 & 1831 & 1721 & 1905 & 1763 & 1560 & 1865 & 1601 & 1308 & 1834 & 1552 & 1241 \\
\hline & $\%$ of Glider mass & 51.4 & 51.4 & 52.4 & 52.9 & 52.9 & 52.6 & 53.8 & 53.7 & 53.2 & 51.6 & 54.6 & 54.0 & 50.2 \\
\hline & $\%$ of Powertrain mass & 48.6 & 48.6 & 47.6 & 47.1 & 47.1 & 47.4 & 46.2 & 46.3 & 46.8 & 48.4 & 45.4 & 46.0 & 49.8 \\
\hline \multirow{3}{*}{ CI Split HEV } & \begin{tabular}{|l|} 
Vehicle Mass \\
\end{tabular} & 1704 & 1704 & 1625 & 1550 & 1676 & 1588 & 1455 & 1667 & 1483 & 1251 & 1657 & 1459 & 1195 \\
\hline & $\%$ of Glider mass & 59.0 & 59.0 & 59.1 & 58.8 & 60.1 & 58.4 & 57.7 & 60.1 & 57.4 & 53.9 & 60.5 & 57.4 & 52.2 \\
\hline & \% of Powertrain mass & 41.0 & 41.0 & 40.9 & 41.2 & 39.9 & 41.6 & 42.3 & 39.9 & 42.6 & 46.1 & 39.5 & 42.6 & 47.8 \\
\hline
\end{tabular}




\begin{tabular}{|c|c|c|c|c|c|c|c|c|c|c|c|c|c|c|}
\hline & & \multicolumn{13}{|c|}{ Small_SUV } \\
\hline & & \multirow{2}{*}{$\frac{2010}{\text { Ref }}$} & \multicolumn{3}{|c|}{2010} & \multicolumn{3}{|c|}{2015} & \multicolumn{3}{|c|}{2030} & \multicolumn{3}{|c|}{2045} \\
\hline & & & low & avg & high & low & avg & high & low & avg & high & low & avg & high \\
\hline \multirow{3}{*}{ CI Split HEV PHEV10 } & Vehicle Mass & 1731 & 1731 & 1651 & 1575 & 1704 & 1601 & 1465 & 1684 & 1494 & 1265 & 1670 & 1467 & 1205 \\
\hline & $\%$ of Glider mass & 58.1 & 58.1 & 58.1 & 57.8 & 59.1 & 58.0 & 57.3 & 59.5 & 57.0 & 53.3 & 60.0 & 57.1 & 51.7 \\
\hline & $\%$ of Powertrain mass & 41.9 & 41.9 & 41.9 & 42.2 & 40.9 & 42.0 & 42.7 & 40.5 & 43.0 & 46.7 & 40.0 & 42.9 & 48.3 \\
\hline \multirow{3}{*}{ CI Split HEV PHEV20 } & Vehicle Mass & 1769 & 1769 & 1684 & 1602 & 1738 & 1633 & 1489 & 1719 & 1517 & 1279 & 1704 & 1488 & 1219 \\
\hline & $\%$ of Glider mass & 56.9 & 56.9 & 57.0 & 56.9 & 58.0 & 56.8 & 56.4 & 58.3 & 56.1 & 52.7 & 58.8 & 56.3 & 51.1 \\
\hline & $\%$ of Powertrain mass & 43.1 & 43.1 & 43.0 & 43.1 & 42.0 & 43.2 & 43.6 & 41.7 & 43.9 & 47.3 & 41.2 & 43.7 & 48.9 \\
\hline \multirow{3}{*}{ CI Series HEV PHEV30 } & Vehicle Mass & 1998 & 1998 & 1877 & 1773 & 1943 & 1811 & 1621 & 1909 & 1661 & 1380 & 1878 & 1616 & 1313 \\
\hline & $\%$ of Glider mass & 50.4 & 50.4 & 51.1 & 51.4 & 51.8 & 51.2 & 51.8 & 52.5 & 51.2 & 48.9 & 53.4 & 51.9 & 47.5 \\
\hline & $\%$ of Powertrain mass & 49.6 & 49.6 & 48.9 & 48.6 & 48.2 & 48.8 & 48.2 & 47.5 & 48.8 & 51.1 & 46.6 & 48.1 & 52.5 \\
\hline \multirow{3}{*}{$\mathrm{CI}$ Series HEV PHEV40 } & Vehicle Mass & 2055 & 2055 & 1927 & 1818 & 1998 & 1860 & 1656 & 1958 & 1696 & 1405 & 1928 & 1645 & 1334 \\
\hline & $\%$ of Glider mass & 49.0 & 49.0 & 49.8 & 50.1 & 50.4 & 49.9 & 50.7 & 51.2 & 50.2 & 48.0 & 52.0 & 50.9 & 46.7 \\
\hline & $\%$ of Powertrain mass & 51.0 & 51.0 & 50.2 & 49.9 & 49.6 & 50.1 & 49.3 & 48.8 & 49.8 & 52.0 & 48.0 & 49.1 & 53.3 \\
\hline \multirow{3}{*}{ H2 Split HEV } & Vehicle Mass & 1656 & 1656 & 1536 & 1441 & 1615 & 1478 & 1333 & 1598 & 1369 & 1118 & 1545 & 1337 & 1071 \\
\hline & $\%$ of Glider mass & 60.7 & 60.7 & 62.5 & 63.2 & 62.4 & 62.8 & 63.0 & 62.7 & 62.2 & 60.3 & 64.9 & 62.7 & 58.2 \\
\hline & $\%$ of Powertrain mass & 39.3 & 39.3 & 37.5 & 36.8 & 37.6 & 37.2 & 37.0 & 37.3 & 37.8 & 39.7 & 35.1 & 37.3 & 41.8 \\
\hline \multirow{3}{*}{ H2 Split HEV PHEV10 } & Vehicle Mass & 1678 & 1678 & 1554 & 1460 & 1634 & 1488 & 1336 & 1606 & 1373 & 1125 & 1553 & 1339 & 1074 \\
\hline & $\%$ of Glider mass & 60.0 & 60.0 & 61.8 & 62.4 & 61.6 & 62.4 & 62.8 & 62.4 & 62.0 & 60.0 & 64.5 & 62.6 & 58.0 \\
\hline & $\%$ of Powertrain mass & 40.0 & 40.0 & 38.2 & 37.6 & 38.4 & 37.6 & 37.2 & 37.6 & 38.0 & 40.0 & 35.5 & 37.4 & 42.0 \\
\hline \multirow{3}{*}{ H2 Split HEV PHEV2O } & Vehicle Mass & 1715 & 1715 & 1586 & 1487 & 1671 & 1519 & 1359 & 1642 & 1396 & 1138 & 1585 & 1360 & 1088 \\
\hline & $\%$ of Glider mass & 58.7 & 58.7 & 60.5 & 61.3 & 60.3 & 61.1 & 61.8 & 61.0 & 61.0 & 59.3 & 63.2 & 61.6 & 57.3 \\
\hline & $\%$ of Powertrain mass & 41.3 & 41.3 & 39.5 & 38.7 & 39.7 & 38.9 & 38.2 & 39.0 & 39.0 & 40.7 & 36.8 & 38.4 & 42.7 \\
\hline \multirow{3}{*}{ H2 Series HEV PHEV30 } & Vehicle Mass & 1943 & 1943 & 1777 & 1654 & 1874 & 1694 & 1488 & 1829 & 1537 & 1236 & 1757 & 1486 & 1181 \\
\hline & $\%$ of Glider mass & 51.8 & 51.8 & 54.0 & 55.1 & 53.7 & 54.8 & 56.4 & 54.8 & 55.4 & 54.6 & 57.0 & 56.4 & 52.8 \\
\hline & $\%$ of Powertrain mass & 48.2 & 48.2 & 46.0 & 44.9 & 46.3 & 45.2 & 43.6 & 45.2 & 44.6 & 45.4 & 43.0 & 43.6 & 47.2 \\
\hline \multirow{3}{*}{ H2 Series HEV PHEV40 } & Vehicle Mass & 2000 & 2000 & 1827 & 1698 & 1927 & 1740 & 1523 & 1880 & 1572 & 1261 & 1805 & 1515 & 1203 \\
\hline & $\%$ of Glider mass & 50.3 & 50.3 & 52.5 & 53.7 & 52.3 & 53.3 & 55.1 & 53.3 & 54.1 & 53.5 & 55.5 & 55.3 & 51.8 \\
\hline & $\%$ of Powertrain mass & 49.7 & 49.7 & 47.5 & 46.3 & 47.7 & 46.7 & 44.9 & 46.7 & 45.9 & 46.5 & 44.5 & 44.7 & 48.2 \\
\hline \multirow{3}{*}{ E85 Split HEV } & Vehicle Mass & 1614 & 1614 & 1534 & 1462 & 1587 & 1501 & 1369 & 1584 & 1393 & 1163 & 1568 & 1373 & 1105 \\
\hline & $\%$ of Glider mass & 62.3 & 62.3 & 62.6 & 62.3 & 63.5 & 61.8 & 61.3 & 63.3 & 61.1 & 58.0 & 63.9 & 61.0 & 56.4 \\
\hline & $\%$ of Powertrain mass & 37.7 & 37.7 & 37.4 & 37.7 & 36.5 & 38.2 & 38.7 & 36.7 & 38.9 & 42.0 & 36.1 & 39.0 & 43.6 \\
\hline
\end{tabular}




\begin{tabular}{|c|c|c|c|c|c|c|c|c|c|c|c|c|c|c|}
\hline & & \multicolumn{13}{|c|}{ Small_SUV } \\
\hline & & \multirow{2}{*}{$\frac{2010}{\text { Ref }}$} & \multicolumn{3}{|c|}{2010} & \multicolumn{3}{|c|}{2015} & \multicolumn{3}{|c|}{2030} & \multicolumn{3}{|c|}{2045} \\
\hline & & & low & avg & high & low & avg & high & low & avg & high & low & avg & high \\
\hline \multirow{3}{*}{ E85 Split HEV PHEV10 } & Vehicle Mass & 1629 & 1629 & 1552 & 1475 & 1604 & 1502 & 1367 & 1588 & 1394 & 1167 & 1574 & 1371 & 1109 \\
\hline & $\%$ of Glider mass & 61.8 & 61.8 & 61.8 & 61.8 & 62.8 & 61.8 & 61.4 & 63.1 & 61.0 & 57.8 & 63.7 & 61.1 & 56.2 \\
\hline & $\%$ of Powertrain mass & 38.2 & 38.2 & 38.2 & 38.2 & 37.2 & 38.2 & 38.6 & 36.9 & 39.0 & 42.2 & 36.3 & 38.9 & 43.8 \\
\hline \multirow{3}{*}{ E85 Split HEV PHEV20 } & Vehicle Mass & 1665 & 1665 & 1582 & 1505 & 1640 & 1533 & 1390 & 1620 & 1419 & 1182 & 1606 & 1390 & 1123 \\
\hline & $\%$ of Glider mass & 60.4 & 60.4 & 60.7 & 60.5 & 61.4 & 60.5 & 60.4 & 61.9 & 60.0 & 57.1 & 62.4 & 60.3 & 55.5 \\
\hline & $\%$ of Powertrain mass & 39.6 & 39.6 & 39.3 & 39.5 & 38.6 & 39.5 & 39.6 & 38.1 & 40.0 & 42.9 & 37.6 & 39.7 & 44.5 \\
\hline \multirow{3}{*}{ E85 Series HEV PHEV30 } & Vehicle Mass & 1905 & 1905 & 1783 & 1680 & 1854 & 1719 & 1529 & 1817 & 1566 & 1287 & 1788 & 1522 & 1221 \\
\hline & $\%$ of Glider mass & 52.8 & 52.8 & 53.8 & 54.2 & 54.3 & 54.0 & 54.9 & 55.1 & 54.3 & 52.4 & 56.0 & 55.1 & 51.0 \\
\hline & $\%$ of Powertrain mass & 47.2 & 47.2 & 46.2 & 45.8 & 45.7 & 46.0 & 45.1 & 44.9 & 45.7 & 47.6 & 44.0 & 44.9 & 49.0 \\
\hline \multirow{3}{*}{ E85 Series HEV PHEV40 } & Vehicle Mass & 1961 & 1961 & 1834 & 1723 & 1907 & 1765 & 1562 & 1869 & 1603 & 1310 & 1836 & 1554 & 1243 \\
\hline & $\%$ of Glider mass & 51.3 & 51.3 & 52.3 & 52.9 & 52.8 & 52.6 & 53.7 & 53.6 & 53.1 & 51.5 & 54.6 & 53.9 & 50.1 \\
\hline & $\%$ of Powertrain mass & 48.7 & 48.7 & 47.7 & 47.1 & 47.2 & 47.4 & 46.3 & 46.4 & 46.9 & 48.5 & 45.4 & 46.1 & 49.9 \\
\hline \multirow{3}{*}{ FC HEV } & Vehicle Mass & 2065 & 2065 & 1720 & 1539 & 1817 & 1562 & 1360 & 1702 & 1395 & 1111 & 1594 & 1338 & 1045 \\
\hline & $\%$ of Glider mass & 48.7 & 48.7 & 55.8 & 59.2 & 55.4 & 59.4 & 61.7 & 58.9 & 61.0 & 60.7 & 62.9 & 62.6 & 59.6 \\
\hline & $\%$ of Powertrain mass & 51.3 & 51.3 & 44.2 & 40.8 & 44.6 & 40.6 & 38.3 & 41.1 & 39.0 & 39.3 & 37.1 & 37.4 & 40.4 \\
\hline \multirow{3}{*}{ FC HEV PHEV 10} & Vehicle Mass & 1976 & 1976 & 1679 & 1518 & 1783 & 1533 & 1339 & 1674 & 1373 & 1093 & 1575 & 1322 & 1031 \\
\hline & $\%$ of Glider mass & 50.9 & 50.9 & 57.2 & 60.0 & 56.5 & 60.5 & 62.7 & 59.9 & 62.0 & 61.7 & 63.6 & 63.4 & 60.4 \\
\hline & $\%$ of Powertrain mass & 49.1 & 49.1 & 42.8 & 40.0 & 43.5 & 39.5 & 37.3 & 40.1 & 38.0 & 38.3 & 36.4 & 36.6 & 39.6 \\
\hline \multirow{3}{*}{ FC HEV PHEV2O } & Vehicle Mass & 2034 & 2034 & 1722 & 1550 & 1832 & 1570 & 1366 & 1717 & 1401 & 1112 & 1614 & 1344 & 1047 \\
\hline & $\%$ of Glider mass & 49.5 & 49.5 & 55.7 & 58.8 & 55.0 & 59.1 & 61.4 & 58.4 & 60.7 & 60.7 & 62.1 & 62.4 & 59.5 \\
\hline & $\%$ of Powertrain mass & 50.5 & 50.5 & 44.3 & 41.2 & 45.0 & 40.9 & 38.6 & 41.6 & 39.3 & 39.3 & 37.9 & 37.6 & 40.5 \\
\hline \multirow{3}{*}{ FC HEV PHEV 30} & Vehicle Mass & 2120 & 2120 & 1814 & 1647 & 1930 & 1671 & 1448 & 1829 & 1489 & 1185 & 1714 & 1425 & 1119 \\
\hline & $\%$ of Glider mass & 47.5 & 47.5 & 52.9 & 55.3 & 52.2 & 55.5 & 58.0 & 54.8 & 57.2 & 56.9 & 58.5 & 58.8 & 55.7 \\
\hline & $\%$ of Powertrain mass & 52.5 & 52.5 & 47.1 & 44.7 & 47.8 & 44.5 & 42.0 & 45.2 & 42.8 & 43.1 & 41.5 & 41.2 & 44.3 \\
\hline \multirow{3}{*}{ FC HEV PHEV40 } & Vehicle Mass & 2186 & 2186 & 1863 & 1688 & 1985 & 1713 & 1478 & 1878 & 1520 & 1208 & 1758 & 1450 & 1138 \\
\hline & $\%$ of Glider mass & 46.0 & 46.0 & 51.5 & 54.0 & 50.7 & 54.2 & 56.8 & 53.4 & 56.0 & 55.8 & 57.0 & 57.8 & 54.8 \\
\hline & $\%$ of Powertrain mass & 54.0 & 54.0 & 48.5 & 46.0 & 49.3 & 45.8 & 43.2 & 46.6 & 44.0 & 44.2 & 43.0 & 42.2 & 45.2 \\
\hline \multirow{3}{*}{ BEV } & Vehicle Mass & 2194 & 2194 & 2023 & 1881 & 2137 & 1941 & 1627 & 2084 & 1669 & 1310 & 2025 & 1585 & 1228 \\
\hline & $\%$ of Glider mass & 45.9 & 45.9 & 47.4 & 48.4 & 47.1 & 47.8 & 51.6 & 48.1 & 51.0 & 51.5 & 49.5 & 52.9 & 50.7 \\
\hline & $\%$ of Powertrain mass & 54.1 & 54.1 & 52.6 & 51.6 & 52.9 & 52.2 & 48.4 & 51.9 & 49.0 & 48.5 & 50.5 & 47.1 & 49.3 \\
\hline
\end{tabular}


Percentage of vehicle curbs weight reduction based on the respective 2010 reference case

\begin{tabular}{|c|c|c|c|c|c|c|c|c|c|c|c|c|c|c|}
\hline & & \multicolumn{13}{|c|}{ Small_SUV } \\
\hline & & \multirow{2}{*}{$\begin{array}{c}2010 \\
\text { Ref }\end{array}$} & \multicolumn{3}{|c|}{2010} & \multicolumn{3}{|c|}{2015} & \multicolumn{3}{|c|}{2030} & \multicolumn{3}{|c|}{2045} \\
\hline & & & low & avg & high & low & avg & high & low & avg & high & low & avg & high \\
\hline SI Conv & Vehicle Mass & $0.00 \%$ & $0.00 \%$ & $-3.46 \%$ & $-7.05 \%$ & $-0.07 \%$ & $-5.83 \%$ & $-12.41 \%$ & $-0.54 \%$ & $-11.46 \%$ & $-24.07 \%$ & $-0.61 \%$ & $-12.41 \%$ & $-27.73 \%$ \\
\hline $\mathrm{Cl}$ Conv & Vehicle Mass & $0.00 \%$ & $0.00 \%$ & $-3.08 \%$ & $-6.48 \%$ & $0.00 \%$ & $-5.30 \%$ & $-11.39 \%$ & $-0.33 \%$ & $-10.54 \%$ & $-22.45 \%$ & $-0.39 \%$ & $-11.39 \%$ & $-25.85 \%$ \\
\hline H2 Conv & Vehicle Mass & $0.00 \%$ & $0.00 \%$ & $-5.98 \%$ & $-10.55 \%$ & $-0.94 \%$ & $-9.34 \%$ & $-16.80 \%$ & $-1.95 \%$ & $-15.32 \%$ & $-29.03 \%$ & $-4.64 \%$ & $-16.73 \%$ & $-32.12 \%$ \\
\hline E85 Conv & Vehicle Mass & $0.00 \%$ & $0.00 \%$ & $-3.33 \%$ & & $0.00 \%$ & $-5.71 \%$ & $-12.24 \%$ & $-0.41 \%$ & $-11.35 \%$ & $-23.93 \%$ & $-0.54 \%$ & $-12.30 \%$ & $-27.53 \%$ \\
\hline SI Split HEV & Vehicle Mass & $0.00 \%$ & $0.00 \%$ & $-4.91 \%$ & $-9.38 \%$ & $-1.55 \%$ & $-6.96 \%$ & $-15.16 \%$ & $-2.24 \%$ & $-13.48 \%$ & $-27.89 \%$ & $-2.73 \%$ & $-15.03 \%$ & $-31.49 \%$ \\
\hline SI Split HEV PHEV10 & Vehicle Mass & $0.00 \%$ & $0.00 \%$ & $-4.92 \%$ & $-9.47 \%$ & $-1.54 \%$ & $-7.81 \%$ & $-16.10 \%$ & $-2.52 \%$ & $-14.44 \%$ & $-28.40 \%$ & $-3.38 \%$ & $-15.86 \%$ & $-31.96 \%$ \\
\hline SI Split HEV PHEV20 & & $0.00 \%$ & $0.00 \%$ & $-4.99 \%$ & & $-1.50 \%$ & $-7.94 \%$ & $-16.54 \%$ & $-2.71 \%$ & $-14.79 \%$ & $-29.04 \%$ & $-3.55 \%$ & $-16.54 \%$ & $-32.71 \%$ \\
\hline SI Series HEV PHEV30 & Vehicle Mass & $0.00 \%$ & $0.00 \%$ & $-6.46 \%$ & $-11.82 \%$ & $-2.68 \%$ & $-9.77 \%$ & $-19.76 \%$ & $-4.62 \%$ & $-17.81 \%$ & $-32.48 \%$ & $-6.15 \%$ & $-20.13 \%$ & $-36.00 \%$ \\
\hline SI Series HEV PHEV40 & Mass & $0.00 \%$ & $0.00 \%$ & $-6.53 \%$ & $-12.15 \%$ & $-2.76 \%$ & $-10.01 \%$ & $-20.37 \%$ & $-4.80 \%$ & $-18.27 \%$ & $-33.23 \%$ & $-6.38 \%$ & $-20.78 \%$ & $-36.65 \%$ \\
\hline CI Split HEV & le Mass & $0.00 \%$ & $0.00 \%$ & $-4.64 \%$ & $-9.04 \%$ & $-1.64 \%$ & $-6.81 \%$ & $-14.61 \%$ & $-2.17 \%$ & $-12.97 \%$ & $-26.58 \%$ & $-2.76 \%$ & $-14.38 \%$ & $-29.87 \%$ \\
\hline CI Split HEV PHEV10 & & & $0.00 \%$ & & & $-1.56 \%$ & $-7.51 \%$ & $-15.37 \%$ & $-2.72 \%$ & $-13.69 \%$ & $-26.92 \%$ & $-3.52 \%$ & $-15.25 \%$ & $-30.39 \%$ \\
\hline CI Split HEV PHEV20 & Vehicle Mass & $0.00 \%$ & $0.00 \%$ & $-4.80 \%$ & $-9.44 \%$ & $-1.75 \%$ & $-7.69 \%$ & $-15.83 \%$ & $-2.83 \%$ & $-14.25 \%$ & $-27.70 \%$ & $-3.67 \%$ & $-15.88 \%$ & $-31.09 \%$ \\
\hline $\mathrm{CI}$ Series HEV PHEV30 & Vehicle Mass & $0.00 \%$ & $0.00 \%$ & $-6.06 \%$ & $-11.26 \%$ & $-2.75 \%$ & $-9.36 \%$ & $-18.87 \%$ & $-4.45 \%$ & $-16.87 \%$ & $-30.93 \%$ & $-6.01 \%$ & $-19.12 \%$ & $-34.28 \%$ \\
\hline CI Series HEV PHEV40 & Vehicle Mass & & $0.00 \%$ & $-6.23 \%$ & & $-2.77 \%$ & $-9.49 \%$ & $-19.42 \%$ & $-4.72 \%$ & $-17.47 \%$ & $-31.63 \%$ & $-6.18 \%$ & $-19.95 \%$ & $-35.09 \%$ \\
\hline H2 Split HEV & & & $0.00 \%$ & $-7.25 \%$ & $-12.98 \%$ & $-2.48 \%$ & $-10.75 \%$ & $-19.50 \%$ & $-3.50 \%$ & $-17.33 \%$ & $-32.49 \%$ & $-6.70 \%$ & $-19.26 \%$ & $-35.33 \%$ \\
\hline H2 Split HEV PHEV10 & Vehicle Mass & $0.00 \%$ & $0.00 \%$ & $-7.39 \%$ & $-12.99 \%$ & $-2.62 \%$ & $-11.32 \%$ & $-20.38 \%$ & $-4.29 \%$ & $-18.18 \%$ & $-32.96 \%$ & $-7.45 \%$ & $-20.20 \%$ & $-36.00 \%$ \\
\hline H2 Split HEV PHEV2O & Vehicle Mass & $0.00 \%$ & $0.00 \%$ & $-7.52 \%$ & $-13.29 \%$ & $-2.57 \%$ & $-11.43 \%$ & $-20.76 \%$ & $-4.26 \%$ & $-18.60 \%$ & $-33.64 \%$ & $-7.58 \%$ & $-20.70 \%$ & $-36.56 \%$ \\
\hline H2 Series HEV PHEV30 & Vehicle Mass & $0.00 \%$ & $0.00 \%$ & $-8.54 \%$ & & $-3.55 \%$ & $-12.82 \%$ & $-23.42 \%$ & $-5.87 \%$ & $-20.90 \%$ & $-36.39 \%$ & $-9.57 \%$ & $-23.52 \%$ & $-39.22 \%$ \\
\hline H2 Series HEV PHEV40 & Vehicle Mass & $0.00 \%$ & $0.00 \%$ & $-8.65 \%$ & $-15.10 \%$ & $-3.65 \%$ & $-13.00 \%$ & $-23.85 \%$ & $-6.00 \%$ & $-21.40 \%$ & $-36.95 \%$ & $-9.75 \%$ & $-24.25 \%$ & $-39.85 \%$ \\
\hline E85 Split HEV & Vehicle Mass & $0.00 \%$ & $0.00 \%$ & $-4.96 \%$ & $-9.42 \%$ & $-1.67 \%$ & $-7.00 \%$ & $-15.18 \%$ & $-1.86 \%$ & $-13.69 \%$ & $-27.94 \%$ & $-2.85 \%$ & $-14.93 \%$ & $-31.54 \%$ \\
\hline E85 Split HEV PHEV10 & Vehicle Mass & $0.00 \%$ & $0.00 \%$ & $-4.73 \%$ & $-9.45 \%$ & $-1.53 \%$ & $-7.80 \%$ & $-16.08 \%$ & $-2.52 \%$ & $-14.43 \%$ & $-28.36 \%$ & $-3.38 \%$ & $-15.84 \%$ & $-31.92 \%$ \\
\hline E85 Split HEV PHEV20 & Vehicle Mass & $0.00 \%$ & $0.00 \%$ & $-4.98 \%$ & $-9.61 \%$ & $-1.50 \%$ & $-7.93 \%$ & $-16.52 \%$ & $-2.70 \%$ & $-14.77 \%$ & $-29.01 \%$ & $-3.54 \%$ & $-16.52 \%$ & $-32.55 \%$ \\
\hline E85 Series HEV PHEV30 & Vehicle Mass & $0.00 \%$ & $0.00 \%$ & $-6.40 \%$ & $-11.81 \%$ & $-2.68 \%$ & $-9.76 \%$ & $-19.74 \%$ & $-4.62 \%$ & $-17.80 \%$ & $-32.44 \%$ & $-6.14 \%$ & $-20.10 \%$ & $-35.91 \%$ \\
\hline E85 Series HEV PHEV40 & Vehicle Mass & $0.00 \%$ & $0.00 \%$ & $-6.48 \%$ & $-12.14 \%$ & $-2.75 \%$ & $-9.99 \%$ & $-20.35 \%$ & $-4.69 \%$ & $-18.26 \%$ & $-33.20 \%$ & $-6.37 \%$ & $-20.75 \%$ & $-36.61 \%$ \\
\hline FC HEV & Vehicle Mass & $0.00 \%$ & $0.00 \%$ & $-16.71 \%$ & $-25.47 \%$ & $-12.01 \%$ & $-24.36 \%$ & $-34.14 \%$ & $-17.58 \%$ & $-32.45 \%$ & $-46.20 \%$ & $-22.81 \%$ & $-35.21 \%$ & $-49.39 \%$ \\
\hline FC HEV PHEV 10 & Vehicle Mass & $0.00 \%$ & $0.00 \%$ & $-15.03 \%$ & $-23.18 \%$ & $-9.77 \%$ & $-22.42 \%$ & $-32.24 \%$ & $-15.28 \%$ & $-30.52 \%$ & $-44.69 \%$ & $-20.29 \%$ & $-33.10 \%$ & $-47.82 \%$ \\
\hline FC HEV PHEV20 & Vehicle Mass & $0.00 \%$ & $0.00 \%$ & $-15.34 \%$ & $-23.80 \%$ & $-9.93 \%$ & $-22.81 \%$ & $-32.84 \%$ & $-15.59 \%$ & $-31.12 \%$ & $-45.33 \%$ & $-20.65 \%$ & $-33.92 \%$ & $-48.53 \%$ \\
\hline FC HEV PHEV 30 & Vehicle Mass & $0.00 \%$ & $0.00 \%$ & $-14.43 \%$ & $-22.31 \%$ & $-8.96 \%$ & $-21.18 \%$ & $-31.70 \%$ & $-13.73 \%$ & $-29.76 \%$ & $-44.10 \%$ & $-19.15 \%$ & $-32.78 \%$ & $-47.22 \%$ \\
\hline FC HEV PHEV 40 & Vehicle Mass & $0.00 \%$ & $0.00 \%$ & $-14.78 \%$ & $-22.78 \%$ & $-9.19 \%$ & $-21.64 \%$ & $-32.39 \%$ & $-14.09 \%$ & $-30.47 \%$ & $-44.74 \%$ & $-19.58 \%$ & $-33.67 \%$ & $-47.94 \%$ \\
\hline $\mathrm{EV}$ & Vehicle Mass & $0.00 \%$ & $0.00 \%$ & $-7.79 \%$ & $-14.27 \%$ & $-2.60 \%$ & $-11.53 \%$ & $-25.84 \%$ & $-5.01 \%$ & $-23.93 \%$ & $-40.29 \%$ & $-7.70 \%$ & $-27.76 \%$ & $-44.03 \%$ \\
\hline
\end{tabular}




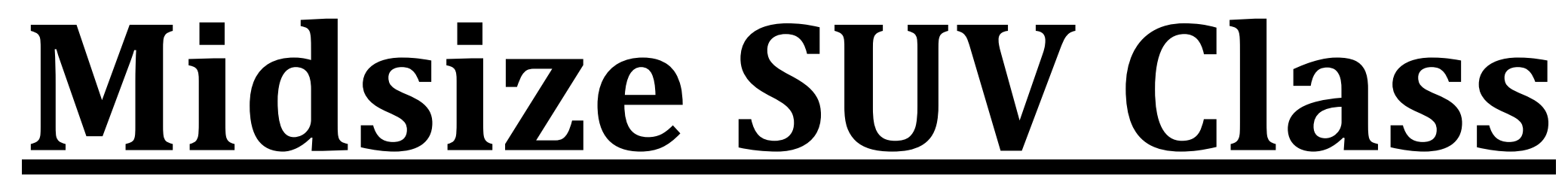




\section{Power}

\begin{tabular}{|c|c|c|c|c|c|c|c|c|c|c|c|c|c|c|}
\hline & & \multicolumn{13}{|c|}{ Midsize_SUV } \\
\hline & & 2010 & \multicolumn{3}{|c|}{2010} & \multicolumn{3}{|c|}{2015} & \multicolumn{3}{|c|}{2030} & \multicolumn{3}{|c|}{2045} \\
\hline & & Ref & low & avg & high & low & avg & high & low & avg & high & low & avg & high \\
\hline \multirow{5}{*}{ SI Conv } & ICE Power & 186156 & 186156 & 165030 & 156916 & 181884 & 158266 & 156916 & 167519 & 159616 & 138780 & 177566 & 158266 & 134304 \\
\hline & Electric Machine & & & & & & & & & & & & & \\
\hline & $\mathrm{MC} 2 / \mathrm{GC}$ & & & & & & & & & & & & & \\
\hline & Battery & & & & & & & & & & & & & \\
\hline & Specific Power & 98 & 98 & 90 & 89 & 95 & 88 & 93 & 88 & 94 & 95 & 94 & 94 & 96 \\
\hline \multirow{5}{*}{ Cl Conv } & ICE Power & 142122 & 142122 & 134868 & 127383 & 138742 & 129800 & 121000 & 136074 & 123444 & 106333 & 136074 & 122222 & 103889 \\
\hline & Electric Machine & & & & & & & & & & & & & \\
\hline & $\mathrm{MC} 2 / \mathrm{GC}$ & & & & & & & & & & & & & \\
\hline & Battery & & & & & & & & & & & & & \\
\hline & Specific Power & 73 & 73 & 71 & 70 & 71 & 70 & 70 & 70 & 71 & 70 & 70 & 71 & 71 \\
\hline \multirow{5}{*}{ H2 Conv } & ICE Power & 170788 & 170788 & 153145 & 144323 & 166667 & 146322 & 136800 & 156078 & 139856 & 118865 & 157921 & 137848 & 115015 \\
\hline & Electric Machine & & & & & & & & & & & & & \\
\hline & $\mathrm{MC} 2 / \mathrm{GC}$ & & & & & & & & & & & & & \\
\hline & Battery & & & & & & & & & & & & & \\
\hline & Specific Power & 90 & 90 & 85 & 84 & 88 & 84 & 85 & 83 & 85 & 86 & 86 & 86 & 87 \\
\hline \multirow{5}{*}{ E85 Conv } & ICE Power & 178069 & 178069 & 158803 & 152528 & 175573 & 153866 & 149179 & 161143 & 152694 & 131693 & 169525 & 150350 & 126544 \\
\hline & Electric Machine & & & & & & & & & & & & & \\
\hline & $\mathrm{MC} 2 / \mathrm{GC}$ & & & & & & & & & & & & & \\
\hline & Battery & & & & & & & & & & & & & \\
\hline & Specific Power & 93 & 93 & 86 & 86 & 92 & 86 & 89 & 85 & 90 & 90 & 89 & 90 & 91 \\
\hline \multirow{5}{*}{ SI Split HEV } & ICE Power & 128655 & 128655 & 120914 & 115075 & 126047 & 115949 & 104367 & 121873 & 106694 & 90382 & 121977 & 103953 & 86177 \\
\hline & Electric Machine & 86749 & 86749 & 82659 & 79285 & 85214 & 80730 & 74170 & 84844 & 75720 & 64615 & 84910 & 73929 & 61570 \\
\hline & $\mathrm{MC} 2 / \mathrm{GC}$ & 74453 & 74453 & 69973 & 66594 & 72944 & 67100 & 60398 & 70528 & 61744 & 52304 & 70589 & 60158 & 49871 \\
\hline & Battery & 34713 & 34713 & 33117 & 31521 & 34314 & 32439 & 29593 & 34146 & 30162 & 25040 & 34146 & 29593 & 23902 \\
\hline & Specific Power & 79 & 79 & 78 & 78 & 78 & 77 & 76 & 77 & 76 & 76 & 77 & 75 & 76 \\
\hline \multirow{5}{*}{ SI Split HEV PHEV10 } & ICE Power & 84876 & 84876 & 80766 & 77264 & 83638 & 78195 & 72199 & 83064 & 73901 & 64191 & 82169 & 72029 & 61131 \\
\hline & Electric Machine & 96980 & 96980 & 92698 & 88778 & 96035 & 89978 & 80938 & 94184 & 82599 & 69427 & 94375 & 80770 & 66052 \\
\hline & $\mathrm{MC} 2 / \mathrm{GC}$ & 61419 & 61419 & 58404 & 55911 & 60524 & 56585 & 52246 & 60108 & 53436 & 46451 & 59461 & 52061 & 44216 \\
\hline & Battery & 93584 & 93584 & 88917 & 84670 & 92846 & 85862 & 76855 & 89589 & 78449 & 70921 & 90084 & 83931 & 67570 \\
\hline & Specific Power & 85 & 85 & 85 & 85 & 85 & 85 & 84 & 85 & 85 & 89 & 85 & 88 & 89 \\
\hline
\end{tabular}




\begin{tabular}{|c|c|c|c|c|c|c|c|c|c|c|c|c|c|c|}
\hline & & \multicolumn{13}{|c|}{ Midsize_SUV } \\
\hline & & \multirow{2}{*}{$\begin{array}{l}2010 \\
\operatorname{Ref}\end{array}$} & \multicolumn{3}{|c|}{2010} & \multicolumn{3}{|c|}{2015} & \multicolumn{3}{|c|}{2030} & \multicolumn{3}{|c|}{2045} \\
\hline & & & low & avg & high & low & avg & high & low & avg & high & low & avg & high \\
\hline \multirow{5}{*}{ SI Split HEV PHEV2O } & ICE Power & 86081 & 86081 & 81910 & 78348 & 84837 & 79219 & 73017 & 84262 & 74778 & 64694 & 83307 & 72620 & 61607 \\
\hline & Electric Machine & 98986 & 98986 & 94650 & 90457 & 97852 & 91611 & 82265 & 96980 & 84707 & 70132 & 96276 & 82007 & 66963 \\
\hline & $\mathrm{MC} 2 / \mathrm{GC}$ & 62291 & 62291 & 59253 & 56696 & 61370 & 57305 & 52797 & 60975 & 54112 & 46774 & 60263 & 52509 & 44520 \\
\hline & Battery & 94086 & 94086 & 89409 & 84904 & 93088 & 86036 & 77776 & 91063 & 80252 & 67154 & 90470 & 79539 & 64122 \\
\hline & Specific Power & 84 & 84 & 84 & 84 & 84 & 84 & 84 & 84 & 84 & 86 & 84 & 85 & 86 \\
\hline \multirow{5}{*}{ SI Series HEV PHEV3O } & ICE Power & 105296 & 105296 & 98183 & 92276 & 102967 & 93674 & 82393 & 99567 & 84752 & 70731 & 97990 & 81630 & 67240 \\
\hline & Electric Machine & 171089 & 171089 & 162058 & 154515 & 167798 & 156745 & 142282 & 165688 & 145793 & 125627 & 163303 & 141013 & 119898 \\
\hline & MC2/GC & 102137 & 102137 & 95238 & 89508 & 99878 & 90864 & 79921 & 96580 & 82209 & 68609 & 95050 & 79181 & 65223 \\
\hline & Battery & 207175 & 207175 & 195197 & 185134 & 203221 & 187834 & 168438 & 198614 & 172640 & 146805 & 195771 & 167025 & 140136 \\
\hline & Specific Power & 71 & 71 & 72 & 72 & 72 & 72 & 73 & 72 & 73 & 76 & 72 & 72 & 76 \\
\hline \multirow{5}{*}{ SI Series HEV PHEV40 } & ICE Power & 109138 & 109138 & 101670 & 95419 & 106604 & 96987 & 84698 & 103039 & 87096 & 72413 & 101213 & 83644 & 68726 \\
\hline & Electric Machine & 177182 & 177182 & 167568 & 159583 & 173584 & 162095 & 146067 & 171330 & 149682 & 128504 & 168554 & 144365 & 122492 \\
\hline & $\mathrm{MC} 2 / \mathrm{GC}$ & 105864 & 105864 & 98620 & 92556 & 103406 & 94077 & 82157 & 99948 & 84483 & 70241 & 98177 & 81135 & 66664 \\
\hline & Battery & 214459 & 214459 & 201748 & 191124 & 210132 & 194157 & 172779 & 205280 & 177096 & 150036 & 201975 & 170844 & 143042 \\
\hline & Specific Power & 72 & 72 & 72 & 73 & 72 & 72 & 73 & 73 & 73 & 76 & 73 & 73 & 77 \\
\hline \multirow{5}{*}{ CI Split HEV } & ICE Power & 109565 & 109565 & 104080 & 99159 & 107420 & 100670 & 89618 & 105735 & 91361 & 78096 & 104782 & 90079 & 73840 \\
\hline & Electric Machine & 91932 & 91932 & 88131 & 84744 & 90222 & 86543 & 79177 & 90847 & 80656 & 69606 & 89799 & 79387 & 66084 \\
\hline & $\mathrm{MC} 2 / \mathrm{GC}$ & 91694 & 91694 & 87104 & 82985 & 89899 & 84250 & 75001 & 88489 & 76459 & 65358 & 87691 & 75386 & 61796 \\
\hline & Battery & 36509 & 36509 & 34713 & 33117 & 35910 & 34146 & 31301 & 35853 & 31870 & 26748 & 35284 & 31301 & 25610 \\
\hline & Specific Power & 67 & 67 & 67 & 67 & 67 & 67 & 65 & 67 & 65 & 65 & 66 & 65 & 65 \\
\hline \multirow{5}{*}{ CI Split HEV PHEV10 } & ICE Power & 87859 & 87859 & 83684 & 80173 & 86491 & 81011 & 74961 & 85917 & 76731 & 66905 & 84985 & 74758 & 63873 \\
\hline & Electric Machine & 97586 & 97586 & 93299 & 89317 & 96280 & 90247 & 81781 & 94593 & 83260 & 70532 & 93878 & 82382 & 67329 \\
\hline & $\mathrm{MC} 2 / \mathrm{GC}$ & 73529 & 73529 & 70034 & 67096 & 72384 & 67797 & 62734 & 71903 & 64216 & 55992 & 71123 & 62564 & 53455 \\
\hline & $\begin{array}{l}\text { Battery } \\
\end{array}$ & 103706 & 103706 & 98617 & 93910 & 102431 & 94930 & 86854 & 99463 & 88519 & 80353 & 98757 & 94261 & 76689 \\
\hline & Specific Power & 87 & 87 & 87 & 87 & 87 & 86 & 87 & 87 & 87 & 91 & 86 & 90 & 91 \\
\hline \multirow{5}{*}{ CI Split HEV PHEV2O } & ICE Power & 89122 & 89122 & 84828 & 81229 & 87689 & 82120 & 75806 & 87115 & 77580 & 67437 & 86151 & 75406 & 64405 \\
\hline & Electric Machine & 99133 & 99133 & 94726 & 90624 & 97784 & 91895 & 83014 & 96320 & 84579 & 71536 & 96142 & 83347 & 68332 \\
\hline & $\mathrm{MC2} / \mathrm{GC}$ & 74586 & 74586 & 70992 & 67980 & 73386 & 68726 & 63441 & 72906 & 64926 & 56437 & 72099 & 63107 & 53900 \\
\hline & Battery & 104287 & 104287 & 99103 & 94284 & 102928 & 95647 & 87050 & 100239 & 88670 & 76249 & 100118 & 89740 & 72828 \\
\hline & Specific Power & 86 & 86 & 86 & 86 & 86 & 86 & 86 & 86 & 86 & 88 & 86 & 87 & 88 \\
\hline
\end{tabular}


Argonne National Laboratory Report - Light-Duty Vehicle Fuel Consumption Displacement Potential Up to 2045

\begin{tabular}{|c|c|c|c|c|c|c|c|c|c|c|c|c|c|c|}
\hline & & \multicolumn{13}{|c|}{ Midsize_SUV } \\
\hline & & \multirow{2}{*}{$\begin{array}{l}2010 \\
\operatorname{Ref}\end{array}$} & \multicolumn{3}{|c|}{2010} & \multicolumn{3}{|c|}{2015} & \multicolumn{3}{|c|}{2030} & \multicolumn{3}{|c|}{2045} \\
\hline & & & low & avg & high & low & avg & high & low & avg & high & low & avg & high \\
\hline \multirow{5}{*}{ CI Series HEV PHEV3O } & ICE Power & 108247 & 108247 & 101064 & 95180 & 105809 & 96539 & 85188 & 102351 & 87616 & 73394 & 100706 & 84335 & 69931 \\
\hline & Electric Machine & 175732 & 175732 & 166602 & 159150 & 172331 & 161514 & 146882 & 170221 & 150451 & 130275 & 167734 & 145562 & 124553 \\
\hline & $\mathrm{MC} 2 / \mathrm{GC}$ & 105000 & 105000 & 98032 & 92325 & 102635 & 93643 & 82632 & 99280 & 84988 & 71192 & 97685 & 81805 & 67833 \\
\hline & Battery & 212779 & 212779 & 200649 & 190666 & 208678 & 193528 & 173868 & 204022 & 178139 & 152218 & 201057 & 172393 & 145548 \\
\hline & Specific Power & 70 & 70 & 71 & 71 & 71 & 71 & 72 & 71 & 72 & 74 & 71 & 71 & 75 \\
\hline \multirow{5}{*}{ CI Series HEV PHEV40 } & ICE Power & 112119 & 112119 & 104612 & 98382 & 109690 & 99822 & 87551 & 105912 & 89931 & 75132 & 104078 & 86464 & 71585 \\
\hline & Electric Machine & 181919 & 181919 & 172302 & 164266 & 178358 & 166720 & 150809 & 175911 & 154385 & 133147 & 173176 & 149057 & 127332 \\
\hline & $\mathrm{MC2} / \mathrm{GC}$ & 108755 & 108755 & 101474 & 95431 & 106399 & 96827 & 84924 & 102735 & 87233 & 72878 & 100956 & 83870 & 69437 \\
\hline & Battery & 220172 & 220172 & 207421 & 196711 & 215884 & 199675 & 178368 & 210750 & 182638 & 155433 & 207489 & 176375 & 148672 \\
\hline & Specific Power & 71 & 71 & 71 & 72 & 71 & 71 & 72 & 72 & 72 & 75 & 72 & 72 & 75 \\
\hline \multirow{5}{*}{ H2 Split HEV } & ICE Power & 129757 & 129757 & 120179 & 112884 & 126099 & 114690 & 100983 & 123213 & 103629 & 85855 & 120403 & 101397 & 82162 \\
\hline & Electric Machine & 88222 & 88222 & 82635 & 78315 & 86057 & 80087 & 72638 & 85928 & 74335 & 62051 & 84133 & 72774 & 59781 \\
\hline & $\mathrm{MC} 2 / \mathrm{GC}$ & 81789 & 81789 & 75752 & 71154 & 79483 & 72292 & 63652 & 77664 & 65320 & 54117 & 75893 & 63913 & 51789 \\
\hline & Battery & 35312 & 35312 & 32918 & 30923 & 34713 & 31870 & 29024 & 34146 & 29593 & 23902 & 33577 & 29024 & 23333 \\
\hline & Specific Power & 78 & 78 & 78 & 77 & 78 & 77 & 75 & 77 & 75 & 75 & 77 & 75 & 76 \\
\hline \multirow{5}{*}{ H2 Split HEV PHEV10 } & ICE Power & 86024 & 86024 & 80566 & 76579 & 84209 & 77512 & 71016 & 83407 & 72995 & 62652 & 81401 & 70847 & 59872 \\
\hline & Electric Machine & 100781 & 100781 & 94233 & 89222 & 98707 & 90587 & 80758 & 97446 & 83066 & 68720 & 94930 & 81446 & 65709 \\
\hline & $\mathrm{MC} 2 / \mathrm{GC}$ & 68929 & 68929 & 64555 & 61315 & 67474 & 62108 & 56903 & 66809 & 58489 & 50201 & 65224 & 56745 & 47951 \\
\hline & Battery & 98345 & 98345 & 91195 & 85568 & 96348 & 86997 & 74679 & 93563 & 77056 & 69420 & 91127 & 83578 & 66258 \\
\hline & Specific Power & 86 & 86 & 86 & 86 & 87 & 86 & 84 & 86 & 85 & 90 & 86 & 89 & 90 \\
\hline \multirow{5}{*}{ H2 Split HEV PHEV2O } & ICE Power & 87314 & 87314 & 81710 & 77578 & 85436 & 78565 & 71833 & 84576 & 73844 & 63127 & 82510 & 71466 & 60292 \\
\hline & Electric Machine & 103625 & 103625 & 96042 & 90797 & 100559 & 92251 & 82913 & 99299 & 84122 & 69937 & 96733 & 82631 & 66735 \\
\hline & $\mathrm{MC} 2 / \mathrm{GC}$ & 69940 & 69940 & 65450 & 62161 & 68434 & 62929 & 57535 & 67746 & 59124 & 50582 & 66067 & 57241 & 48288 \\
\hline & Battery & 100618 & 100618 & 92187 & 86351 & 97332 & 87871 & 78388 & 94590 & 79141 & 66088 & 92116 & 79996 & 62921 \\
\hline & Specific Power & 86 & 86 & 86 & 86 & 86 & 85 & 85 & 86 & 85 & 87 & 86 & 86 & 87 \\
\hline \multirow{5}{*}{ H2 Series HEV PHEV3O } & ICE Power & 106187 & 106187 & 97637 & 91139 & 103242 & 92600 & 80924 & 99627 & 83537 & 68966 & 96736 & 80192 & 65783 \\
\hline & Electric Machine & 172587 & 172587 & 161190 & 152680 & 168233 & 155057 & 139838 & 165783 & 143777 & 122701 & 161328 & 138618 & 117393 \\
\hline & $\mathrm{MC} 2 / \mathrm{GC}$ & 103001 & 103001 & 94708 & 88405 & 100145 & 89822 & 78496 & 96638 & 81031 & 66897 & 93834 & 77786 & 63810 \\
\hline & Battery & 208990 & 208990 & 194156 & 182944 & 203739 & 185822 & 165553 & 198731 & 170261 & 143397 & 193414 & 164201 & 137224 \\
\hline & Specific Power & 71 & 71 & 72 & 73 & 72 & 72 & 73 & 72 & 73 & 77 & 72 & 73 & 77 \\
\hline
\end{tabular}


Argonne National Laboratory Report - Light-Duty Vehicle Fuel Consumption Displacement Potential Up to 2045

\begin{tabular}{|c|c|c|c|c|c|c|c|c|c|c|c|c|c|c|}
\hline & & \multicolumn{13}{|c|}{ Midsize_SUV } \\
\hline & & \multirow{2}{*}{$\frac{2010}{\operatorname{Ref}}$} & \multicolumn{3}{|c|}{2010} & \multicolumn{3}{|c|}{2015} & \multicolumn{3}{|c|}{2030} & \multicolumn{3}{|c|}{2045} \\
\hline & & & low & avg & high & low & avg & high & low & avg & high & low & avg & high \\
\hline \multirow{5}{*}{ H2 Series HEV PHEV40 } & ICE Power & 110091 & 110091 & 101003 & 94252 & 106909 & 95763 & 83200 & 103069 & 86025 & 70647 & 99930 & 82206 & 67268 \\
\hline & Electric Machine & 178681 & 178681 & 166601 & 157748 & 174067 & 160166 & 143623 & 171378 & 147810 & 125532 & 166530 & 142019 & 119993 \\
\hline & $\mathrm{MC} 2 / \mathrm{GC}$ & 106788 & 106788 & 97973 & 91424 & 103702 & 92890 & 80704 & 99977 & 83444 & 68528 & 96932 & 79740 & 65250 \\
\hline & Battery & 216268 & 216268 & 200589 & 188940 & 210709 & 191856 & 169897 & 205337 & 174896 & 146579 & 199560 & 168075 & 140133 \\
\hline & Specific Power & 72 & 72 & 72 & 73 & 72 & 73 & 74 & 73 & 74 & 77 & 73 & 73 & 77 \\
\hline \multirow{5}{*}{ E85 Split HEV } & ICE Power & 128835 & 128835 & 121978 & 115162 & 127099 & 116986 & 104491 & 123285 & 106558 & 91223 & 122108 & 106348 & 86106 \\
\hline & Electric Machine & 86932 & 86932 & 83225 & 79422 & 85779 & 81300 & 74312 & 85605 & 75709 & 65071 & 85052 & 74999 & 61587 \\
\hline & $\mathrm{MC} 2 / \mathrm{GC}$ & 74557 & 74557 & 70589 & 66645 & 73553 & 67700 & 60469 & 71345 & 61666 & 52791 & 70664 & 61544 & 49830 \\
\hline & Battery & 34713 & 34713 & 33117 & 31521 & 34314 & 32439 & 29593 & 34146 & 30162 & 25040 & 34146 & 29593 & 23902 \\
\hline & Specific Power & 79 & 79 & 78 & 78 & 79 & 77 & 76 & 77 & 76 & 77 & 77 & 77 & 76 \\
\hline \multirow{5}{*}{ E85 Split HEV PHEV10 } & ICE Power & 84962 & 84962 & 80852 & 77321 & 83696 & 78280 & 72284 & 83207 & 73986 & 64247 & 82311 & 72113 & 61215 \\
\hline & Electric Machine & 97192 & 97192 & 92864 & 88898 & 96157 & 90144 & 81099 & 95375 & 82760 & 69539 & 94543 & 81764 & 66204 \\
\hline & $\mathrm{MC} 2 / \mathrm{GC}$ & 61482 & 61482 & 58487 & 55952 & 60566 & 56646 & 52307 & 60191 & 53539 & 46492 & 59563 & 52123 & 44277 \\
\hline & Battery & 93818 & 93818 & 89083 & 84766 & 92961 & 86029 & 76928 & 90977 & 78598 & 71110 & 90231 & 85249 & 67676 \\
\hline & Specific Power & 85 & 85 & 85 & 85 & 85 & 85 & 84 & 85 & 84 & 89 & 85 & 89 & 89 \\
\hline \multirow{5}{*}{ E85 Split HEV PHEV20 } & ICE Power & 86224 & 86224 & 81996 & 78433 & 84894 & 79333 & 73129 & 84348 & 74920 & 64778 & 83392 & 72676 & 61719 \\
\hline & Electric Machine & 99156 & 99156 & 94817 & 90577 & 97975 & 91778 & 82467 & 97237 & 84869 & 71007 & 96443 & 83014 & 67117 \\
\hline & $\mathrm{MC} 2 / \mathrm{GC}$ & 62354 & 62354 & 59315 & 56737 & 61412 & 57408 & 52878 & 61037 & 54174 & 46835 & 60325 & 52571 & 44601 \\
\hline & Battery & 94225 & 94225 & 89558 & 84994 & 93202 & 86179 & 77967 & 91317 & 80392 & 68244 & 90621 & 80793 & 64291 \\
\hline & Specific Power & 84 & 84 & 84 & 84 & 84 & 84 & 84 & 84 & 85 & 86 & 84 & 85 & 86 \\
\hline \multirow{5}{*}{ E85 Series HEV PHEV30 } & ICE Power & 105388 & 105388 & 98273 & 92336 & 103028 & 93793 & 82537 & 99687 & 84839 & 70788 & 98079 & 81688 & 67296 \\
\hline & Electric Machine & 171235 & 171235 & 162204 & 154611 & 167894 & 156985 & 142475 & 165880 & 145938 & 125723 & 163448 & 141109 & 120042 \\
\hline & $\mathrm{MC} 2 / \mathrm{GC}$ & 102226 & 102226 & 95325 & 89566 & 99937 & 90979 & 80061 & 96696 & 82294 & 68664 & 95137 & 79237 & 65277 \\
\hline & Battery & 207348 & 207348 & 195367 & 185249 & 203336 & 188124 & 168670 & 198846 & 172809 & 146916 & 195943 & 167138 & 140306 \\
\hline & Specific Power & 71 & 71 & 72 & 72 & 72 & 72 & 73 & 72 & 73 & 76 & 72 & 72 & 76 \\
\hline \multirow{5}{*}{ E85 Series HEV PHEV40 } & ICE Power & 109261 & 109261 & 101761 & 95569 & 106695 & 97076 & 84785 & 103219 & 87269 & 72469 & 101333 & 83702 & 68810 \\
\hline & Electric Machine & 177376 & 177376 & 167713 & 159730 & 173730 & 162239 & 146211 & 171572 & 149922 & 128648 & 168746 & 144461 & 122636 \\
\hline & $\mathrm{MC} 2 / \mathrm{GC}$ & 105983 & 105983 & 98708 & 92702 & 103494 & 94164 & 82241 & 100122 & 84651 & 70295 & 98293 & 81191 & 66746 \\
\hline & Battery & 214692 & 214692 & 201919 & 191299 & 210302 & 194329 & 172947 & 205572 & 177383 & 150203 & 202205 & 170956 & 143208 \\
\hline & Specific Power & 72 & 72 & 72 & 73 & 72 & 72 & 73 & 73 & 73 & 76 & 72 & 73 & 77 \\
\hline
\end{tabular}


Argonne National Laboratory Report - Light-Duty Vehicle Fuel Consumption Displacement Potential Up to 2045

\begin{tabular}{|c|c|c|c|c|c|c|c|c|c|c|c|c|c|c|}
\hline & & \multicolumn{13}{|c|}{ Midsize_SUV } \\
\hline & & \multirow{2}{*}{$\begin{array}{c}2010 \\
\text { Ref }\end{array}$} & \multicolumn{3}{|c|}{2010} & \multicolumn{3}{|c|}{2015} & \multicolumn{3}{|c|}{2030} & \multicolumn{3}{|c|}{2045} \\
\hline & & & low & avg & high & low & avg & high & low & avg & high & low & avg & high \\
\hline \multirow{5}{*}{ FC HEV } & FC Power & 145073 & 145073 & 122437 & 106103 & 129971 & 102984 & 89099 & 113351 & 91353 & 71759 & 107556 & 88105 & 68484 \\
\hline & Electric Machine & 166468 & 166468 & 139482 & 126241 & 146286 & 125613 & 111731 & 136409 & 114268 & 93264 & 130155 & 110240 & 88631 \\
\hline & $\mathrm{MC} 2 / \mathrm{GC}$ & & & & & & & & & & & & & \\
\hline & Battery & 43292 & 43292 & 36908 & 34314 & 38304 & 38699 & 35284 & 40975 & 35853 & 30162 & 39268 & 34715 & 28455 \\
\hline & Specific Power & 63 & 63 & 63 & 63 & 62 & 62 & 63 & 62 & 63 & 64 & 63 & 63 & 64 \\
\hline \multirow{5}{*}{ FC HEV PHEV10 } & FC Power & 107021 & 107021 & 91069 & 81808 & 97539 & 81701 & 72340 & 89764 & 74052 & 59614 & 84751 & 71268 & 56621 \\
\hline & Electric Machine & 158328 & 158328 & 138890 & 126821 & 146705 & 127352 & 113916 & 138355 & 116420 & 95167 & 132091 & 112298 & 90281 \\
\hline & $\mathrm{MC} 2 / \mathrm{GC}$ & & & & & & & & & & & & & \\
\hline & Battery & 81500 & 81500 & 70267 & 63328 & 74459 & 63977 & 55667 & 69484 & 57142 & 45559 & 65994 & 54971 & 43165 \\
\hline & Specific Power & 63 & 63 & 64 & 64 & 64 & 64 & 65 & 64 & 65 & 66 & 65 & 65 & 66 \\
\hline \multirow{5}{*}{ FC HEV PHEV 20} & FC Power & 109488 & 109488 & 93197 & 83584 & 99829 & 83496 & 72256 & 91807 & 75570 & 60180 & 85768 & 72693 & 57188 \\
\hline & Electric Machine & 161877 & 161877 & 141986 & 129442 & 150012 & 130023 & 114625 & 141359 & 118608 & 96439 & 134097 & 114265 & 91423 \\
\hline & $\mathrm{MC} 2 / \mathrm{GC}$ & & & & & & & & & & & & & \\
\hline & Battery & 83572 & 83572 & 71945 & 64663 & 76205 & 65403 & 56511 & 71081 & 58187 & 46346 & 67343 & 55869 & 43894 \\
\hline & Specific Power & 63 & 63 & 64 & 64 & 64 & 64 & 64 & 64 & 65 & 66 & 64 & 65 & 66 \\
\hline \multirow{5}{*}{ FC HEV PHEV 30} & FC Power & 90438 & 90438 & 80397 & 74647 & 84594 & 75214 & 67715 & 81713 & 69766 & 58634 & 77947 & 66628 & 55687 \\
\hline & Electric Machine & 173556 & 173556 & 158821 & 149590 & 165483 & 150815 & 136817 & 160671 & 139886 & 120394 & 155785 & 134304 & 114394 \\
\hline & $\mathrm{MC} 2 / \mathrm{GC}$ & & & & & & & & & & & & & \\
\hline & Battery & 210067 & 210067 & 191233 & 179184 & 200341 & 180680 & 162054 & 192552 & 165731 & 140712 & 186733 & 159166 & 133727 \\
\hline & Specific Power & 65 & 65 & 68 & 70 & 68 & 70 & 73 & 69 & 72 & 77 & 71 & 73 & 78 \\
\hline \multirow{5}{*}{ FC HEV PHEV 40} & FC Power & 92382 & 92382 & 81927 & 75918 & 86197 & 76456 & 68625 & 83162 & 70704 & 59386 & 79266 & 67435 & 56289 \\
\hline & Electric Machine & 178923 & 178923 & 163413 & 153744 & 170260 & 154962 & 140221 & 165158 & 143441 & 123277 & 159979 & 137420 & 117006 \\
\hline & $\mathrm{MC} 2 / \mathrm{GC}$ & & & & & & & & & & & & & \\
\hline & Battery & 216467 & 216467 & 196679 & 184083 & 206035 & 185574 & 165921 & 197838 & 169776 & 143962 & 191665 & 162690 & 136665 \\
\hline & Specific Power & 65 & 65 & 69 & 71 & 68 & 71 & 73 & 69 & 73 & 78 & 71 & 73 & 78 \\
\hline \multirow{5}{*}{ BEV } & ICE Power & & & & & & & & & & & & & \\
\hline & Electric Machine & 216254 & 216254 & 202345 & 191209 & 211884 & 194626 & 167179 & 207959 & 171797 & 142700 & 202213 & 161398 & 134555 \\
\hline & $\mathrm{MC} 2 / \mathrm{GC}$ & 0 & 0 & 0 & 0 & 0 & 0 & 0 & 0 & 0 & 0 & 0 & 0 & 0 \\
\hline & Battery & 261257 & 261257 & 243172 & 228580 & 256006 & 232699 & 197866 & 248683 & 203372 & 167262 & 241846 & 191093 & 157761 \\
\hline & Specific Power & 95 & 95 & 94 & 95 & 95 & 94 & 94 & 94 & 94 & 98 & 94 & 93 & 98 \\
\hline
\end{tabular}




\section{Percentage of power reduction based on the respective 2010 reference case}

\begin{tabular}{|c|c|c|c|c|c|c|c|c|c|c|c|c|c|c|}
\hline & & \multicolumn{13}{|c|}{ Midsize_SUV } \\
\hline & & \multirow{2}{*}{$\frac{2010}{\operatorname{Ref}}$} & \multicolumn{3}{|c|}{2010} & \multicolumn{3}{|c|}{2015} & \multicolumn{3}{|c|}{2030} & \multicolumn{3}{|c|}{2045} \\
\hline & & & low & avg & high & low & avg & high & low & avg & high & low & avg & high \\
\hline \multirow{5}{*}{ SI Conv } & ICE Power & $0 \%$ & $0 \%$ & $-11 \%$ & $-16 \%$ & $-2 \%$ & $-15 \%$ & $-16 \%$ & $-10 \%$ & $-14 \%$ & $-25 \%$ & $-5 \%$ & $-15 \%$ & $-28 \%$ \\
\hline & Electric Machine & & & & & & & & & & & & & \\
\hline & $\mathrm{MC} 2 / \mathrm{GC}$ & & & & & & & & & & & & & \\
\hline & Battery & & & & & & & & & & & & & \\
\hline & Specific Power & $0 \%$ & $0 \%$ & $-8 \%$ & $-9 \%$ & $-2 \%$ & $-10 \%$ & $-4 \%$ & $-9 \%$ & $-4 \%$ & $-3 \%$ & $-4 \%$ & $-3 \%$ & $-1 \%$ \\
\hline \multirow{5}{*}{ Cl Conv } & ICE Power & $0 \%$ & $0 \%$ & $-5 \%$ & $-10 \%$ & $-2 \%$ & $-9 \%$ & $-15 \%$ & $-4 \%$ & $-13 \%$ & $-25 \%$ & $-4 \%$ & $-14 \%$ & $-27 \%$ \\
\hline & Electric Machine & & & & & & & & & & & & & \\
\hline & $\mathrm{MC} 2 / \mathrm{GC}$ & & & & & & & & & & & & & \\
\hline & Battery & & & & & & & & & & & & & \\
\hline & Specific Power & $0 \%$ & $0 \%$ & $-2 \%$ & $-4 \%$ & $-2 \%$ & $-4 \%$ & $-4 \%$ & $-4 \%$ & $-3 \%$ & $-4 \%$ & $-4 \%$ & $-3 \%$ & $-2 \%$ \\
\hline \multirow{5}{*}{ H2 Conv } & ICE Power & $0 \%$ & $0 \%$ & $-10 \%$ & $-15 \%$ & $-2 \%$ & $-14 \%$ & $-20 \%$ & $-9 \%$ & $-18 \%$ & $-30 \%$ & $-8 \%$ & $-19 \%$ & $-33 \%$ \\
\hline & Electric Machine & & & & & & & & & & & & & \\
\hline & $\mathrm{MC} 2 / \mathrm{GC}$ & & & & & & & & & & & & & \\
\hline & Battery & & & & & & & & & & & & & \\
\hline & Specific Power & $0 \%$ & $0 \%$ & $-5 \%$ & $-6 \%$ & $-2 \%$ & $-6 \%$ & $-5 \%$ & $-7 \%$ & $-4 \%$ & $-4 \%$ & $-4 \%$ & $-4 \%$ & $-3 \%$ \\
\hline \multirow{5}{*}{ E85 Conv } & ICE Power & $0 \%$ & $0 \%$ & $-11 \%$ & $-14 \%$ & $-1 \%$ & $-14 \%$ & $-16 \%$ & $-10 \%$ & $-14 \%$ & $-26 \%$ & $-5 \%$ & $-16 \%$ & $-29 \%$ \\
\hline & Electric Machine & & & & & & & & & & & & & \\
\hline & $\mathrm{MC} 2 / \mathrm{GC}$ & & & & & & & & & & & & & \\
\hline & Battery & & & & & & & & & & & & & \\
\hline & Specific Power & $0 \%$ & $0 \%$ & $-7 \%$ & $-8 \%$ & $-1 \%$ & $-8 \%$ & $-5 \%$ & $-9 \%$ & $-4 \%$ & $-3 \%$ & $-4 \%$ & $-4 \%$ & $-3 \%$ \\
\hline \multirow{5}{*}{ SI Split HEV } & ICE Power & $0 \%$ & $0 \%$ & $-6 \%$ & $-11 \%$ & $-2 \%$ & $-10 \%$ & $-19 \%$ & $-5 \%$ & $-17 \%$ & $-30 \%$ & $-5 \%$ & $-19 \%$ & $-33 \%$ \\
\hline & Electric Machine & $0 \%$ & $0 \%$ & $-5 \%$ & $-9 \%$ & $-2 \%$ & $-7 \%$ & $-15 \%$ & $-2 \%$ & $-13 \%$ & $-26 \%$ & $-2 \%$ & $-15 \%$ & $-29 \%$ \\
\hline & $\mathrm{MC} 2 / \mathrm{GC}$ & $0 \%$ & $0 \%$ & $-6 \%$ & $-11 \%$ & $-2 \%$ & $-10 \%$ & $-19 \%$ & $-5 \%$ & $-17 \%$ & $-30 \%$ & $-5 \%$ & $-19 \%$ & $-33 \%$ \\
\hline & Battery & $0 \%$ & $0 \%$ & $-5 \%$ & $-9 \%$ & $-1 \%$ & $-7 \%$ & $-15 \%$ & $-2 \%$ & $-13 \%$ & $-28 \%$ & $-2 \%$ & $-15 \%$ & $-31 \%$ \\
\hline & Specific Power & $0 \%$ & $0 \%$ & $-1 \%$ & $-1 \%$ & $0 \%$ & $-2 \%$ & $-4 \%$ & $-2 \%$ & $-4 \%$ & $-3 \%$ & $-2 \%$ & $-4 \%$ & $-3 \%$ \\
\hline \multirow{5}{*}{ SI Split HEV PHEV10 } & ICE Power & $0 \%$ & $0 \%$ & $-5 \%$ & $-9 \%$ & $-1 \%$ & $-8 \%$ & $-15 \%$ & $-2 \%$ & $-13 \%$ & $-24 \%$ & $-3 \%$ & $-15 \%$ & $-28 \%$ \\
\hline & Electric Machine & $0 \%$ & $0 \%$ & $-4 \%$ & $-8 \%$ & $-1 \%$ & $-7 \%$ & $-17 \%$ & $-3 \%$ & $-15 \%$ & $-28 \%$ & $-3 \%$ & $-17 \%$ & $-32 \%$ \\
\hline & $\mathrm{MC} 2 / \mathrm{GC}$ & $0 \%$ & $0 \%$ & $-5 \%$ & $-9 \%$ & $-1 \%$ & $-8 \%$ & $-15 \%$ & $-2 \%$ & $-13 \%$ & $-24 \%$ & $-3 \%$ & $-15 \%$ & $-28 \%$ \\
\hline & Battery & $0 \%$ & $0 \%$ & $-5 \%$ & $-10 \%$ & $-1 \%$ & $-8 \%$ & $-18 \%$ & $-4 \%$ & $-16 \%$ & $-24 \%$ & $-4 \%$ & $-10 \%$ & $-28 \%$ \\
\hline & Specific Power & $0 \%$ & $0 \%$ & $0 \%$ & $0 \%$ & $0 \%$ & $-1 \%$ & $-1 \%$ & $-1 \%$ & $-1 \%$ & $4 \%$ & $0 \%$ & $3 \%$ & $4 \%$ \\
\hline
\end{tabular}




\begin{tabular}{|c|c|c|c|c|c|c|c|c|c|c|c|c|c|c|}
\hline & & \multicolumn{13}{|c|}{ Midsize_SUV } \\
\hline & & \multirow{2}{*}{$\frac{2010}{\operatorname{Ref}}$} & \multicolumn{3}{|c|}{2010} & \multicolumn{3}{|c|}{2015} & \multicolumn{3}{|c|}{2030} & \multicolumn{3}{|c|}{2045} \\
\hline & & & low & avg & high & low & avg & high & low & avg & high & low & avg & high \\
\hline \multirow{5}{*}{ SI Split HEV PHEV2O } & ICE Power & $0 \%$ & $0 \%$ & $-5 \%$ & $-9 \%$ & $-1 \%$ & $-8 \%$ & $-15 \%$ & $-2 \%$ & $-13 \%$ & $-25 \%$ & $-3 \%$ & $-16 \%$ & $-28 \%$ \\
\hline & Electric Machine & $0 \%$ & $0 \%$ & $-4 \%$ & $-9 \%$ & $-1 \%$ & $-7 \%$ & $-17 \%$ & $-2 \%$ & $-14 \%$ & $-29 \%$ & $-3 \%$ & $-17 \%$ & $-32 \%$ \\
\hline & $\mathrm{MC} 2 / \mathrm{GC}$ & $0 \%$ & $0 \%$ & $-5 \%$ & $-9 \%$ & $-1 \%$ & $-8 \%$ & $-15 \%$ & $-2 \%$ & $-13 \%$ & $-25 \%$ & $-3 \%$ & $-16 \%$ & $-29 \%$ \\
\hline & Battery & $0 \%$ & $0 \%$ & $-5 \%$ & $-10 \%$ & $-1 \%$ & $-9 \%$ & $-17 \%$ & $-3 \%$ & $-15 \%$ & $-29 \%$ & $-4 \%$ & $-15 \%$ & $-32 \%$ \\
\hline & Specific Power & $0 \%$ & $0 \%$ & $0 \%$ & $0 \%$ & $0 \%$ & $-1 \%$ & $-1 \%$ & $0 \%$ & $0 \%$ & $2 \%$ & $0 \%$ & $0 \%$ & $2 \%$ \\
\hline \multirow{5}{*}{ SI Series HEV PHEV30 } & ICE Power & $0 \%$ & $0 \%$ & $-7 \%$ & $-12 \%$ & $-2 \%$ & $-11 \%$ & $-22 \%$ & $-5 \%$ & $-20 \%$ & $-33 \%$ & $-7 \%$ & $-22 \%$ & $-36 \%$ \\
\hline & Electric Machine & $0 \%$ & $0 \%$ & $-5 \%$ & $-10 \%$ & $-2 \%$ & $-8 \%$ & $-17 \%$ & $-3 \%$ & $-15 \%$ & $-27 \%$ & $-5 \%$ & $-18 \%$ & $-30 \%$ \\
\hline & $\mathrm{MC} 2 / \mathrm{GC}$ & $0 \%$ & $0 \%$ & $-7 \%$ & $-12 \%$ & $-2 \%$ & $-11 \%$ & $-22 \%$ & $-5 \%$ & $-20 \%$ & $-33 \%$ & $-7 \%$ & $-22 \%$ & $-36 \%$ \\
\hline & Battery & $0 \%$ & $0 \%$ & $-6 \%$ & $-11 \%$ & $-2 \%$ & $-9 \%$ & $-19 \%$ & $-4 \%$ & $-17 \%$ & $-29 \%$ & $-6 \%$ & $-19 \%$ & $-32 \%$ \\
\hline & Specific Power & $0 \%$ & $0 \%$ & $0 \%$ & $1 \%$ & $1 \%$ & $1 \%$ & $2 \%$ & $1 \%$ & $2 \%$ & $6 \%$ & $1 \%$ & $1 \%$ & $7 \%$ \\
\hline \multirow{5}{*}{ SI Series HEV PHEV40 } & ICE Power & $0 \%$ & $0 \%$ & $-7 \%$ & $-13 \%$ & $-2 \%$ & $-11 \%$ & $-22 \%$ & $-6 \%$ & $-20 \%$ & $-34 \%$ & $-7 \%$ & $-23 \%$ & $-37 \%$ \\
\hline & Electric Machine & $0 \%$ & $0 \%$ & $-5 \%$ & $-10 \%$ & $-2 \%$ & $-9 \%$ & $-18 \%$ & $-3 \%$ & $-16 \%$ & $-27 \%$ & $-5 \%$ & $-19 \%$ & $-31 \%$ \\
\hline & $\mathrm{MC} 2 / \mathrm{GC}$ & $0 \%$ & $0 \%$ & $-7 \%$ & $-13 \%$ & $-2 \%$ & $-11 \%$ & $-22 \%$ & $-6 \%$ & $-20 \%$ & $-34 \%$ & $-7 \%$ & $-23 \%$ & $-37 \%$ \\
\hline & Battery & $0 \%$ & $0 \%$ & $-6 \%$ & $-11 \%$ & $-2 \%$ & $-9 \%$ & $-19 \%$ & $-4 \%$ & $-17 \%$ & $-30 \%$ & $-6 \%$ & $-20 \%$ & $-33 \%$ \\
\hline & Specific Power & $0 \%$ & $0 \%$ & $0 \%$ & $1 \%$ & $0 \%$ & $1 \%$ & $2 \%$ & $1 \%$ & $2 \%$ & $6 \%$ & $1 \%$ & $1 \%$ & $7 \%$ \\
\hline \multirow{5}{*}{ CI Split HEV } & ICE Power & $0 \%$ & $0 \%$ & $-5 \%$ & $-9 \%$ & $-2 \%$ & $-8 \%$ & $-18 \%$ & $-3 \%$ & $-17 \%$ & $-29 \%$ & $-4 \%$ & $-18 \%$ & $-33 \%$ \\
\hline & Electric Machine & $0 \%$ & $0 \%$ & $-4 \%$ & $-8 \%$ & $-2 \%$ & $-6 \%$ & $-14 \%$ & $-1 \%$ & $-12 \%$ & $-24 \%$ & $-2 \%$ & $-14 \%$ & $-28 \%$ \\
\hline & $\mathrm{MC} 2 / \mathrm{GC}$ & $0 \%$ & $0 \%$ & $-5 \%$ & $-9 \%$ & $-2 \%$ & $-8 \%$ & $-18 \%$ & $-3 \%$ & $-17 \%$ & $-29 \%$ & $-4 \%$ & $-18 \%$ & $-33 \%$ \\
\hline & Battery & $0 \%$ & $0 \%$ & $-5 \%$ & $-9 \%$ & $-2 \%$ & $-6 \%$ & $-14 \%$ & $-2 \%$ & $-13 \%$ & $-27 \%$ & $-3 \%$ & $-14 \%$ & $-30 \%$ \\
\hline & Specific Power & $0 \%$ & $0 \%$ & $-1 \%$ & $-1 \%$ & $0 \%$ & $-1 \%$ & $-3 \%$ & $-1 \%$ & $-3 \%$ & $-3 \%$ & $-1 \%$ & $-3 \%$ & $-4 \%$ \\
\hline \multirow{5}{*}{ CI Split HEV PHEV10 } & ICE Power & $0 \%$ & $0 \%$ & $-5 \%$ & $-9 \%$ & $-2 \%$ & $-8 \%$ & $-15 \%$ & $-2 \%$ & $-13 \%$ & $-24 \%$ & $-3 \%$ & $-15 \%$ & $-27 \%$ \\
\hline & Electric Machine & $0 \%$ & $0 \%$ & $-4 \%$ & $-8 \%$ & $-1 \%$ & $-8 \%$ & $-16 \%$ & $-3 \%$ & $-15 \%$ & $-28 \%$ & $-4 \%$ & $-16 \%$ & $-31 \%$ \\
\hline & $\mathrm{MC} 2 / \mathrm{GC}$ & $0 \%$ & $0 \%$ & $-5 \%$ & $-9 \%$ & $-2 \%$ & $-8 \%$ & $-15 \%$ & $-2 \%$ & $-13 \%$ & $-24 \%$ & $-3 \%$ & $-15 \%$ & $-27 \%$ \\
\hline & Battery & $0 \%$ & $0 \%$ & $-5 \%$ & $-9 \%$ & $-1 \%$ & $-8 \%$ & $-16 \%$ & $-4 \%$ & $-15 \%$ & $-23 \%$ & $-5 \%$ & $-9 \%$ & $-26 \%$ \\
\hline & Specific Power & $0 \%$ & $0 \%$ & $0 \%$ & $0 \%$ & $0 \%$ & $-1 \%$ & $-1 \%$ & $-1 \%$ & $0 \%$ & $4 \%$ & $-1 \%$ & $3 \%$ & $4 \%$ \\
\hline \multirow{5}{*}{ Cl Split HEV PHEV20 } & ICE Power & $0 \%$ & $0 \%$ & $-5 \%$ & $-9 \%$ & $-2 \%$ & $-8 \%$ & $-15 \%$ & $-2 \%$ & $-13 \%$ & $-24 \%$ & $-3 \%$ & $-15 \%$ & $-28 \%$ \\
\hline & Electric Machine & $0 \%$ & $0 \%$ & $-4 \%$ & $-9 \%$ & $-1 \%$ & $-7 \%$ & $-16 \%$ & $-3 \%$ & $-15 \%$ & $-28 \%$ & $-3 \%$ & $-16 \%$ & $-31 \%$ \\
\hline & $\mathrm{MC} 2 / \mathrm{GC}$ & $0 \%$ & $0 \%$ & $-5 \%$ & $-9 \%$ & $-2 \%$ & $-8 \%$ & $-15 \%$ & $-2 \%$ & $-13 \%$ & $-24 \%$ & $-3 \%$ & $-15 \%$ & $-28 \%$ \\
\hline & Battery & $0 \%$ & $0 \%$ & $-5 \%$ & $-10 \%$ & $-1 \%$ & $-8 \%$ & $-17 \%$ & $-4 \%$ & $-15 \%$ & $-27 \%$ & $-4 \%$ & $-14 \%$ & $-30 \%$ \\
\hline & Specific Power & $0 \%$ & $0 \%$ & $0 \%$ & $0 \%$ & $0 \%$ & $-1 \%$ & $-1 \%$ & $-1 \%$ & $0 \%$ & $2 \%$ & $0 \%$ & $1 \%$ & $2 \%$ \\
\hline
\end{tabular}




\begin{tabular}{|c|c|c|c|c|c|c|c|c|c|c|c|c|c|c|}
\hline & & \multicolumn{13}{|c|}{ Midsize_SUV } \\
\hline & & \multirow{2}{*}{$\frac{2010}{\operatorname{Ref}}$} & \multicolumn{3}{|c|}{2010} & \multicolumn{3}{|c|}{2015} & \multicolumn{3}{|c|}{2030} & \multicolumn{3}{|c|}{2045} \\
\hline & & & low & avg & high & low & avg & high & low & avg & high & low & avg & high \\
\hline \multirow{5}{*}{ CI Series HEV PHEV30 } & ICE Power & $0 \%$ & $0 \%$ & $-7 \%$ & $-12 \%$ & $-2 \%$ & $-11 \%$ & $-21 \%$ & $-5 \%$ & $-19 \%$ & $-32 \%$ & $-7 \%$ & $-22 \%$ & $-35 \%$ \\
\hline & Electric Machine & $0 \%$ & $0 \%$ & $-5 \%$ & $-9 \%$ & $-2 \%$ & $-8 \%$ & $-16 \%$ & $-3 \%$ & $-14 \%$ & $-26 \%$ & $-5 \%$ & $-17 \%$ & $-29 \%$ \\
\hline & $\mathrm{MC} 2 / \mathrm{GC}$ & $0 \%$ & $0 \%$ & $-7 \%$ & $-12 \%$ & $-2 \%$ & $-11 \%$ & $-21 \%$ & $-5 \%$ & $-19 \%$ & $-32 \%$ & $-7 \%$ & $-22 \%$ & $-35 \%$ \\
\hline & Battery & $0 \%$ & $0 \%$ & $-6 \%$ & $-10 \%$ & $-2 \%$ & $-9 \%$ & $-18 \%$ & $-4 \%$ & $-16 \%$ & $-28 \%$ & $-6 \%$ & $-19 \%$ & $-32 \%$ \\
\hline & Specific Power & $0 \%$ & $0 \%$ & $0 \%$ & $1 \%$ & $1 \%$ & $1 \%$ & $2 \%$ & $1 \%$ & $2 \%$ & $6 \%$ & $1 \%$ & $1 \%$ & $6 \%$ \\
\hline \multirow{5}{*}{ CI Series HEV PHEV40 } & ICE Power & $0 \%$ & $0 \%$ & $-7 \%$ & $-12 \%$ & $-2 \%$ & $-11 \%$ & $-22 \%$ & $-6 \%$ & $-20 \%$ & $-33 \%$ & $-7 \%$ & $-23 \%$ & $-36 \%$ \\
\hline & Electric Machine & $0 \%$ & $0 \%$ & $-5 \%$ & $-10 \%$ & $-2 \%$ & $-8 \%$ & $-17 \%$ & $-3 \%$ & $-15 \%$ & $-27 \%$ & $-5 \%$ & $-18 \%$ & $-30 \%$ \\
\hline & $\mathrm{MC} 2 / \mathrm{GC}$ & $0 \%$ & $0 \%$ & $-7 \%$ & $-12 \%$ & $-2 \%$ & $-11 \%$ & $-22 \%$ & $-6 \%$ & $-20 \%$ & $-33 \%$ & $-7 \%$ & $-23 \%$ & $-36 \%$ \\
\hline & Battery & $0 \%$ & $0 \%$ & $-6 \%$ & $-11 \%$ & $-2 \%$ & $-9 \%$ & $-19 \%$ & $-4 \%$ & $-17 \%$ & $-29 \%$ & $-6 \%$ & $-20 \%$ & $-32 \%$ \\
\hline & Specific Power & $0 \%$ & $0 \%$ & $0 \%$ & $1 \%$ & $0 \%$ & $1 \%$ & $2 \%$ & $1 \%$ & $2 \%$ & $5 \%$ & $1 \%$ & $1 \%$ & $6 \%$ \\
\hline \multirow{5}{*}{ H2 Split HEV } & ICE Power & $0 \%$ & $0 \%$ & $-7 \%$ & $-13 \%$ & $-3 \%$ & $-12 \%$ & $-22 \%$ & $-5 \%$ & $-20 \%$ & $-34 \%$ & $-7 \%$ & $-22 \%$ & $-37 \%$ \\
\hline & Electric Machine & $0 \%$ & $0 \%$ & $-6 \%$ & $-11 \%$ & $-2 \%$ & $-9 \%$ & $-18 \%$ & $-3 \%$ & $-16 \%$ & $-30 \%$ & $-5 \%$ & $-18 \%$ & $-32 \%$ \\
\hline & $\mathrm{MC} 2 / \mathrm{GC}$ & $0 \%$ & $0 \%$ & $-7 \%$ & $-13 \%$ & $-3 \%$ & $-12 \%$ & $-22 \%$ & $-5 \%$ & $-20 \%$ & $-34 \%$ & $-7 \%$ & $-22 \%$ & $-37 \%$ \\
\hline & Battery & $0 \%$ & $0 \%$ & $-7 \%$ & $-12 \%$ & $-2 \%$ & $-10 \%$ & $-18 \%$ & $-3 \%$ & $-16 \%$ & $-32 \%$ & $-5 \%$ & $-18 \%$ & $-34 \%$ \\
\hline & Specific Power & $0 \%$ & $0 \%$ & $-1 \%$ & $-1 \%$ & $0 \%$ & $-2 \%$ & $-4 \%$ & $-1 \%$ & $-4 \%$ & $-4 \%$ & $-1 \%$ & $-4 \%$ & $-3 \%$ \\
\hline \multirow{5}{*}{ H2 Split HEV PHEV10 } & ICE Power & $0 \%$ & $0 \%$ & $-6 \%$ & $-11 \%$ & $-2 \%$ & $-10 \%$ & $-17 \%$ & $-3 \%$ & $-15 \%$ & $-27 \%$ & $-5 \%$ & $-18 \%$ & $-30 \%$ \\
\hline & Electric Machine & $0 \%$ & $0 \%$ & $-6 \%$ & $-11 \%$ & $-2 \%$ & $-10 \%$ & $-20 \%$ & $-3 \%$ & $-18 \%$ & $-32 \%$ & $-6 \%$ & $-19 \%$ & $-35 \%$ \\
\hline & $\mathrm{MC} 2 / \mathrm{GC}$ & $0 \%$ & $0 \%$ & $-6 \%$ & $-11 \%$ & $-2 \%$ & $-10 \%$ & $-17 \%$ & $-3 \%$ & $-15 \%$ & $-27 \%$ & $-5 \%$ & $-18 \%$ & $-30 \%$ \\
\hline & Battery & $0 \%$ & $0 \%$ & $-7 \%$ & $-13 \%$ & $-2 \%$ & $-12 \%$ & $-24 \%$ & $-5 \%$ & $-22 \%$ & $-29 \%$ & $-7 \%$ & $-15 \%$ & $-33 \%$ \\
\hline & Specific Power & $0 \%$ & $0 \%$ & $0 \%$ & $0 \%$ & $0 \%$ & $0 \%$ & $-2 \%$ & $0 \%$ & $-2 \%$ & $4 \%$ & $0 \%$ & $3 \%$ & $4 \%$ \\
\hline \multirow{5}{*}{ H2 Split HEV PHEV 20} & ICE Power & $0 \%$ & $0 \%$ & $-6 \%$ & $-11 \%$ & $-2 \%$ & $-10 \%$ & $-18 \%$ & $-3 \%$ & $-15 \%$ & $-28 \%$ & $-6 \%$ & $-18 \%$ & $-31 \%$ \\
\hline & Electric Machine & $0 \%$ & $0 \%$ & $-7 \%$ & $-12 \%$ & $-3 \%$ & $-11 \%$ & $-20 \%$ & $-4 \%$ & $-19 \%$ & $-33 \%$ & $-7 \%$ & $-20 \%$ & $-36 \%$ \\
\hline & $\mathrm{MC} 2 / \mathrm{GC}$ & $0 \%$ & $0 \%$ & $-6 \%$ & $-11 \%$ & $-2 \%$ & $-10 \%$ & $-18 \%$ & $-3 \%$ & $-15 \%$ & $-28 \%$ & $-6 \%$ & $-18 \%$ & $-31 \%$ \\
\hline & Battery & $0 \%$ & $0 \%$ & $-8 \%$ & $-14 \%$ & $-3 \%$ & $-13 \%$ & $-22 \%$ & $-6 \%$ & $-21 \%$ & $-34 \%$ & $-8 \%$ & $-20 \%$ & $-37 \%$ \\
\hline & Specific Power & $0 \%$ & $0 \%$ & $-1 \%$ & $-1 \%$ & $0 \%$ & $-1 \%$ & $-1 \%$ & $-1 \%$ & $-2 \%$ & $1 \%$ & $-1 \%$ & $0 \%$ & $1 \%$ \\
\hline \multirow{5}{*}{ H2 Series HEV PHEV 30} & ICE Power & $0 \%$ & $0 \%$ & $-8 \%$ & $-14 \%$ & $-3 \%$ & $-13 \%$ & $-24 \%$ & $-6 \%$ & $-21 \%$ & $-35 \%$ & $-9 \%$ & $-24 \%$ & $-38 \%$ \\
\hline & Electric Machine & $0 \%$ & $0 \%$ & $-7 \%$ & $-12 \%$ & $-3 \%$ & $-10 \%$ & $-19 \%$ & $-4 \%$ & $-17 \%$ & $-29 \%$ & $-7 \%$ & $-20 \%$ & $-32 \%$ \\
\hline & $\mathrm{MC} 2 / \mathrm{GC}$ & $0 \%$ & $0 \%$ & $-8 \%$ & $-14 \%$ & $-3 \%$ & $-13 \%$ & $-24 \%$ & $-6 \%$ & $-21 \%$ & $-35 \%$ & $-9 \%$ & $-24 \%$ & $-38 \%$ \\
\hline & Battery & $0 \%$ & $0 \%$ & $-7 \%$ & $-12 \%$ & $-3 \%$ & $-11 \%$ & $-21 \%$ & $-5 \%$ & $-19 \%$ & $-31 \%$ & $-7 \%$ & $-21 \%$ & $-34 \%$ \\
\hline & Specific Power & $0 \%$ & $0 \%$ & $1 \%$ & $2 \%$ & $1 \%$ & $2 \%$ & $3 \%$ & $2 \%$ & $3 \%$ & $8 \%$ & $2 \%$ & $3 \%$ & $9 \%$ \\
\hline
\end{tabular}




\begin{tabular}{|c|c|c|c|c|c|c|c|c|c|c|c|c|c|c|}
\hline & & \multicolumn{13}{|c|}{ Midsize_SUV } \\
\hline & & \multirow{2}{*}{$\frac{2010}{\operatorname{Ref}}$} & \multicolumn{3}{|c|}{2010} & \multicolumn{3}{|c|}{2015} & \multicolumn{3}{|c|}{2030} & \multicolumn{3}{|c|}{2045} \\
\hline & & & low & avg & high & low & avg & high & low & avg & high & low & avg & high \\
\hline \multirow{5}{*}{ H2 Series HEV PHEV40 } & ICE Power & $0 \%$ & $0 \%$ & $-8 \%$ & $-14 \%$ & $-3 \%$ & $-13 \%$ & $-24 \%$ & $-6 \%$ & $-22 \%$ & $-36 \%$ & $-9 \%$ & $-25 \%$ & $-39 \%$ \\
\hline & Electric Machine & $0 \%$ & $0 \%$ & $-7 \%$ & $-12 \%$ & $-3 \%$ & $-10 \%$ & $-20 \%$ & $-4 \%$ & $-17 \%$ & $-30 \%$ & $-7 \%$ & $-21 \%$ & $-33 \%$ \\
\hline & $\mathrm{MC} 2 / \mathrm{GC}$ & $0 \%$ & $0 \%$ & $-8 \%$ & $-14 \%$ & $-3 \%$ & $-13 \%$ & $-24 \%$ & $-6 \%$ & $-22 \%$ & $-36 \%$ & $-9 \%$ & $-25 \%$ & $-39 \%$ \\
\hline & Battery & $0 \%$ & $0 \%$ & $-7 \%$ & $-13 \%$ & $-3 \%$ & $-11 \%$ & $-21 \%$ & $-5 \%$ & $-19 \%$ & $-32 \%$ & $-8 \%$ & $-22 \%$ & $-35 \%$ \\
\hline & Specific Power & $0 \%$ & $0 \%$ & $1 \%$ & $2 \%$ & $1 \%$ & $2 \%$ & $3 \%$ & $1 \%$ & $3 \%$ & $8 \%$ & $2 \%$ & $2 \%$ & $8 \%$ \\
\hline \multirow{5}{*}{ E85 Split HEV } & ICE Power & $0 \%$ & $0 \%$ & $-5 \%$ & $-11 \%$ & $-1 \%$ & $-9 \%$ & $-19 \%$ & $-4 \%$ & $-17 \%$ & $-29 \%$ & $-5 \%$ & $-17 \%$ & $-33 \%$ \\
\hline & Electric Machine & $0 \%$ & $0 \%$ & $-4 \%$ & $-9 \%$ & $-1 \%$ & $-6 \%$ & $-15 \%$ & $-2 \%$ & $-13 \%$ & $-25 \%$ & $-2 \%$ & $-14 \%$ & $-29 \%$ \\
\hline & $\mathrm{MC} 2 / \mathrm{GC}$ & $0 \%$ & $0 \%$ & $-5 \%$ & $-11 \%$ & $-1 \%$ & $-9 \%$ & $-19 \%$ & $-4 \%$ & $-17 \%$ & $-29 \%$ & $-5 \%$ & $-17 \%$ & $-33 \%$ \\
\hline & Battery & $0 \%$ & $0 \%$ & $-5 \%$ & $-9 \%$ & $-1 \%$ & $-7 \%$ & $-15 \%$ & $-2 \%$ & $-13 \%$ & $-28 \%$ & $-2 \%$ & $-15 \%$ & $-31 \%$ \\
\hline & Specific Power & $0 \%$ & $0 \%$ & $-1 \%$ & $-1 \%$ & $0 \%$ & $-2 \%$ & $-4 \%$ & $-2 \%$ & $-4 \%$ & $-3 \%$ & $-2 \%$ & $-3 \%$ & $-3 \%$ \\
\hline \multirow{5}{*}{ E85 Split HEV PHEV10 } & ICE Power & $0 \%$ & $0 \%$ & $-5 \%$ & $-9 \%$ & $-1 \%$ & $-8 \%$ & $-15 \%$ & $-2 \%$ & $-13 \%$ & $-24 \%$ & $-3 \%$ & $-15 \%$ & $-28 \%$ \\
\hline & Electric Machine & $0 \%$ & $0 \%$ & $-4 \%$ & $-9 \%$ & $-1 \%$ & $-7 \%$ & $-17 \%$ & $-2 \%$ & $-15 \%$ & $-28 \%$ & $-3 \%$ & $-16 \%$ & $-32 \%$ \\
\hline & $\mathrm{MC} 2 / \mathrm{GC}$ & $0 \%$ & $0 \%$ & $-5 \%$ & $-9 \%$ & $-1 \%$ & $-8 \%$ & $-15 \%$ & $-2 \%$ & $-13 \%$ & $-24 \%$ & $-3 \%$ & $-15 \%$ & $-28 \%$ \\
\hline & Battery & $0 \%$ & $0 \%$ & $-5 \%$ & $-10 \%$ & $-1 \%$ & $-8 \%$ & $-18 \%$ & $-3 \%$ & $-16 \%$ & $-24 \%$ & $-4 \%$ & $-9 \%$ & $-28 \%$ \\
\hline & Specific Power & $0 \%$ & $0 \%$ & $0 \%$ & $0 \%$ & $0 \%$ & $-1 \%$ & $-1 \%$ & $0 \%$ & $-1 \%$ & $4 \%$ & $0 \%$ & $4 \%$ & $4 \%$ \\
\hline \multirow{5}{*}{ E85 Split HEV PHEV 20} & ICE Power & $0 \%$ & $0 \%$ & $-5 \%$ & $-9 \%$ & $-2 \%$ & $-8 \%$ & $-15 \%$ & $-2 \%$ & $-13 \%$ & $-25 \%$ & $-3 \%$ & $-16 \%$ & $-28 \%$ \\
\hline & Electric Machine & $0 \%$ & $0 \%$ & $-4 \%$ & $-9 \%$ & $-1 \%$ & $-7 \%$ & $-17 \%$ & $-2 \%$ & $-14 \%$ & $-28 \%$ & $-3 \%$ & $-16 \%$ & $-32 \%$ \\
\hline & $\mathrm{MC} 2 / \mathrm{GC}$ & $0 \%$ & $0 \%$ & $-5 \%$ & $-9 \%$ & $-2 \%$ & $-8 \%$ & $-15 \%$ & $-2 \%$ & $-13 \%$ & $-25 \%$ & $-3 \%$ & $-16 \%$ & $-28 \%$ \\
\hline & Battery & $0 \%$ & $0 \%$ & $-5 \%$ & $-10 \%$ & $-1 \%$ & $-9 \%$ & $-17 \%$ & $-3 \%$ & $-15 \%$ & $-28 \%$ & $-4 \%$ & $-14 \%$ & $-32 \%$ \\
\hline & Specific Power & $0 \%$ & $0 \%$ & $0 \%$ & $0 \%$ & $0 \%$ & $-1 \%$ & $-1 \%$ & $0 \%$ & $0 \%$ & $2 \%$ & $0 \%$ & $1 \%$ & $2 \%$ \\
\hline \multirow{5}{*}{ E85 Series HEV PHEV30 } & ICE Power & $0 \%$ & $0 \%$ & $-7 \%$ & $-12 \%$ & $-2 \%$ & $-11 \%$ & $-22 \%$ & $-5 \%$ & $-19 \%$ & $-33 \%$ & $-7 \%$ & $-22 \%$ & $-36 \%$ \\
\hline & Electric Machine & $0 \%$ & $0 \%$ & $-5 \%$ & $-10 \%$ & $-2 \%$ & $-8 \%$ & $-17 \%$ & $-3 \%$ & $-15 \%$ & $-27 \%$ & $-5 \%$ & $-18 \%$ & $-30 \%$ \\
\hline & $\mathrm{MC} 2 / \mathrm{GC}$ & $0 \%$ & $0 \%$ & $-7 \%$ & $-12 \%$ & $-2 \%$ & $-11 \%$ & $-22 \%$ & $-5 \%$ & $-19 \%$ & $-33 \%$ & $-7 \%$ & $-22 \%$ & $-36 \%$ \\
\hline & Battery & $0 \%$ & $0 \%$ & $-6 \%$ & $-11 \%$ & $-2 \%$ & $-9 \%$ & $-19 \%$ & $-4 \%$ & $-17 \%$ & $-29 \%$ & $-6 \%$ & $-19 \%$ & $-32 \%$ \\
\hline & Specific Power & $0 \%$ & $0 \%$ & $0 \%$ & $1 \%$ & $1 \%$ & $1 \%$ & $2 \%$ & $1 \%$ & $2 \%$ & $6 \%$ & $1 \%$ & $1 \%$ & $7 \%$ \\
\hline \multirow{5}{*}{ E85 Series HEV PHEV40 } & ICE Power & $0 \%$ & $0 \%$ & $-7 \%$ & $-13 \%$ & $-2 \%$ & $-11 \%$ & $-22 \%$ & $-6 \%$ & $-20 \%$ & $-34 \%$ & $-7 \%$ & $-23 \%$ & $-37 \%$ \\
\hline & Electric Machine & $0 \%$ & $0 \%$ & $-5 \%$ & $-10 \%$ & $-2 \%$ & $-9 \%$ & $-18 \%$ & $-3 \%$ & $-15 \%$ & $-27 \%$ & $-5 \%$ & $-19 \%$ & $-31 \%$ \\
\hline & $\mathrm{MC} 2 / \mathrm{GC}$ & $0 \%$ & $0 \%$ & $-7 \%$ & $-13 \%$ & $-2 \%$ & $-11 \%$ & $-22 \%$ & $-6 \%$ & $-20 \%$ & $-34 \%$ & $-7 \%$ & $-23 \%$ & $-37 \%$ \\
\hline & Battery & $0 \%$ & $0 \%$ & $-6 \%$ & $-11 \%$ & $-2 \%$ & $-9 \%$ & $-19 \%$ & $-4 \%$ & $-17 \%$ & $-30 \%$ & $-6 \%$ & $-20 \%$ & $-33 \%$ \\
\hline & Specific Power & $0 \%$ & $0 \%$ & $0 \%$ & $1 \%$ & $1 \%$ & $1 \%$ & $2 \%$ & $1 \%$ & $2 \%$ & $6 \%$ & $1 \%$ & $1 \%$ & $7 \%$ \\
\hline
\end{tabular}




\begin{tabular}{|c|c|c|c|c|c|c|c|c|c|c|c|c|c|c|}
\hline & & \multicolumn{13}{|c|}{ Midsize_SUV } \\
\hline & & \multirow{2}{*}{$\frac{2010}{\operatorname{Ref}}$} & \multicolumn{3}{|c|}{2010} & \multicolumn{3}{|c|}{2015} & \multicolumn{3}{|c|}{2030} & \multicolumn{3}{|c|}{2045} \\
\hline & & & low & avg & high & low & avg & high & low & avg & high & low & avg & high \\
\hline \multirow{5}{*}{ FC HEV } & FC Power & $0 \%$ & $0 \%$ & $-16 \%$ & $-27 \%$ & $-10 \%$ & $-29 \%$ & $-39 \%$ & $-22 \%$ & $-37 \%$ & $-51 \%$ & $-26 \%$ & $-39 \%$ & $-53 \%$ \\
\hline & Electric Machine & $0 \%$ & $0 \%$ & $-16 \%$ & $-24 \%$ & $-12 \%$ & $-25 \%$ & $-33 \%$ & $-18 \%$ & $-31 \%$ & $-44 \%$ & $-22 \%$ & $-34 \%$ & $-47 \%$ \\
\hline & $\mathrm{MC} 2 / \mathrm{GC}$ & & & & & & & & & & & & & \\
\hline & Battery & $0 \%$ & $0 \%$ & $-15 \%$ & $-21 \%$ & $-12 \%$ & $-11 \%$ & $-18 \%$ & $-5 \%$ & $-17 \%$ & $-30 \%$ & $-9 \%$ & $-20 \%$ & $-34 \%$ \\
\hline & Specific Power & $0 \%$ & $0 \%$ & $0 \%$ & $0 \%$ & $-1 \%$ & $-1 \%$ & $0 \%$ & $-1 \%$ & $0 \%$ & $1 \%$ & $0 \%$ & $0 \%$ & $2 \%$ \\
\hline \multirow{5}{*}{ FC HEV PHEV 10} & FC Power & $0 \%$ & $0 \%$ & $-15 \%$ & $-24 \%$ & $-9 \%$ & $-24 \%$ & $-32 \%$ & $-16 \%$ & $-31 \%$ & $-44 \%$ & $-21 \%$ & $-33 \%$ & $-47 \%$ \\
\hline & Electric Machine & $0 \%$ & $0 \%$ & $-12 \%$ & $-20 \%$ & $-7 \%$ & $-20 \%$ & $-28 \%$ & $-13 \%$ & $-26 \%$ & $-40 \%$ & $-17 \%$ & $-29 \%$ & $-43 \%$ \\
\hline & $\mathrm{MC} 2 / \mathrm{GC}$ & & & & & & & & & & & & & \\
\hline & Battery & $0 \%$ & $0 \%$ & $-14 \%$ & $-22 \%$ & $-9 \%$ & $-22 \%$ & $-32 \%$ & $-15 \%$ & $-30 \%$ & $-44 \%$ & $-19 \%$ & $-33 \%$ & $-47 \%$ \\
\hline & Specific Power & $0 \%$ & $0 \%$ & $2 \%$ & $2 \%$ & $2 \%$ & $2 \%$ & $4 \%$ & $2 \%$ & $3 \%$ & $5 \%$ & $3 \%$ & $3 \%$ & $5 \%$ \\
\hline \multirow{5}{*}{ FC HEV PHEV 20} & FC Power & $0 \%$ & $0 \%$ & $-15 \%$ & $-24 \%$ & $-9 \%$ & $-24 \%$ & $-34 \%$ & $-16 \%$ & $-31 \%$ & $-45 \%$ & $-22 \%$ & $-34 \%$ & $-48 \%$ \\
\hline & Electric Machine & $0 \%$ & $0 \%$ & $-12 \%$ & $-20 \%$ & $-7 \%$ & $-20 \%$ & $-29 \%$ & $-13 \%$ & $-27 \%$ & $-40 \%$ & $-17 \%$ & $-29 \%$ & $-44 \%$ \\
\hline & $\mathrm{MC} 2 / \mathrm{GC}$ & & & & & & & & & & & & & \\
\hline & Battery & $0 \%$ & $0 \%$ & $-14 \%$ & $-23 \%$ & $-9 \%$ & $-22 \%$ & $-32 \%$ & $-15 \%$ & $-30 \%$ & $-45 \%$ & $-19 \%$ & $-33 \%$ & $-47 \%$ \\
\hline & Specific Power & $0 \%$ & $0 \%$ & $2 \%$ & $3 \%$ & $2 \%$ & $2 \%$ & $3 \%$ & $2 \%$ & $3 \%$ & $5 \%$ & $3 \%$ & $4 \%$ & $5 \%$ \\
\hline \multirow{5}{*}{ FC HEV PHEV 30} & FC Power & $0 \%$ & $0 \%$ & $-11 \%$ & $-17 \%$ & $-6 \%$ & $-17 \%$ & $-25 \%$ & $-10 \%$ & $-23 \%$ & $-35 \%$ & $-14 \%$ & $-26 \%$ & $-38 \%$ \\
\hline & Electric Machine & $0 \%$ & $0 \%$ & $-8 \%$ & $-14 \%$ & $-5 \%$ & $-13 \%$ & $-21 \%$ & $-7 \%$ & $-19 \%$ & $-31 \%$ & $-10 \%$ & $-23 \%$ & $-34 \%$ \\
\hline & $\mathrm{MC} 2 / \mathrm{GC}$ & & & & & & & & & & & & & \\
\hline & Battery & $0 \%$ & $0 \%$ & $-9 \%$ & $-15 \%$ & $-5 \%$ & $-14 \%$ & $-23 \%$ & $-8 \%$ & $-21 \%$ & $-33 \%$ & $-11 \%$ & $-24 \%$ & $-36 \%$ \\
\hline & Specific Power & $0 \%$ & $0 \%$ & $5 \%$ & $8 \%$ & $4 \%$ & $8 \%$ & $12 \%$ & $6 \%$ & $11 \%$ & $19 \%$ & $9 \%$ & $12 \%$ & $20 \%$ \\
\hline \multirow{5}{*}{ FC HEV PHEV40 } & FC Power & $0 \%$ & $0 \%$ & $-11 \%$ & $-18 \%$ & $-7 \%$ & $-17 \%$ & $-26 \%$ & $-10 \%$ & $-23 \%$ & $-36 \%$ & $-14 \%$ & $-27 \%$ & $-39 \%$ \\
\hline & Electric Machine & $0 \%$ & $0 \%$ & $-9 \%$ & $-14 \%$ & $-5 \%$ & $-13 \%$ & $-22 \%$ & $-8 \%$ & $-20 \%$ & $-31 \%$ & $-11 \%$ & $-23 \%$ & $-35 \%$ \\
\hline & $\mathrm{MC} 2 / \mathrm{GC}$ & & & & & & & & & & & & & \\
\hline & Battery & $0 \%$ & $0 \%$ & $-9 \%$ & $-15 \%$ & $-5 \%$ & $-14 \%$ & $-23 \%$ & $-9 \%$ & $-22 \%$ & $-33 \%$ & $-11 \%$ & $-25 \%$ & $-37 \%$ \\
\hline & Specific Power & $0 \%$ & $0 \%$ & $5 \%$ & $9 \%$ & $4 \%$ & $8 \%$ & $12 \%$ & $6 \%$ & $12 \%$ & $19 \%$ & $9 \%$ & $12 \%$ & $20 \%$ \\
\hline \multirow{5}{*}{ EV } & ICE Power & & & & & & & & & & & & & \\
\hline & Electric Machine & $0 \%$ & $0 \%$ & $-6 \%$ & $-12 \%$ & $-2 \%$ & $-10 \%$ & $-23 \%$ & $-4 \%$ & $-21 \%$ & $-34 \%$ & $-6 \%$ & $-25 \%$ & $-38 \%$ \\
\hline & $\mathrm{MC} 2 / \mathrm{GC}$ & & & & & & & & & & & & & \\
\hline & Battery & $0 \%$ & $0 \%$ & $-7 \%$ & $-13 \%$ & $-2 \%$ & $-11 \%$ & $-24 \%$ & $-5 \%$ & $-22 \%$ & $-36 \%$ & $-7 \%$ & $-27 \%$ & $-40 \%$ \\
\hline & Specific Power & $0 \%$ & $0 \%$ & $0 \%$ & $0 \%$ & $0 \%$ & $-1 \%$ & $-1 \%$ & $0 \%$ & $-1 \%$ & $3 \%$ & $-1 \%$ & $-2 \%$ & $3 \%$ \\
\hline
\end{tabular}




\section{Energy}

\begin{tabular}{|c|c|c|c|c|c|c|c|c|c|c|c|c|c|c|}
\hline & & \multicolumn{13}{|c|}{ Midsize_SUV } \\
\hline & & \multirow{2}{*}{$\frac{2010}{\operatorname{Ref}}$} & \multicolumn{3}{|c|}{2010} & \multicolumn{3}{|c|}{2015} & \multicolumn{3}{|c|}{2030} & \multicolumn{3}{|c|}{2045} \\
\hline & & & low & avg & high & low & avg & high & low & avg & high & low & avg & high \\
\hline \multirow{2}{*}{ SI Split HEV } & Total Energy & 1357 & 1357 & 1295 & 1232 & 1342 & 1231 & 1123 & 1296 & 1145 & 950 & 1296 & 1123 & 907 \\
\hline & Usable Energy & & & & & & & & & & & & & \\
\hline \multirow{2}{*}{ SI Split HEV PHEV10 } & Total Energy & 4919 & 4919 & 4861 & 4578 & 5046 & 4637 & 3761 & 4809 & 3853 & 2928 & 4747 & 3517 & 2791 \\
\hline & Usable Energy & 2951 & 2951 & 2917 & 2747 & 3027 & 2782 & 2633 & 2886 & 2697 & 2342 & 2848 & 2638 & 2232 \\
\hline \multirow{2}{*}{ SI Split HEV PHEV 20} & Total Energy & 10770 & 10770 & 10145 & 9532 & 10720 & 9645 & 7338 & 10515 & 7693 & 5539 & 10366 & 6765 & 5294 \\
\hline & Usable Energy & 6462 & 6462 & 6087 & 5719 & 6432 & 5787 & 5137 & 6309 & 5385 & 4431 & 6220 & 5074 & 4235 \\
\hline \multirow{2}{*}{ SI Series HEV PHEV30 } & Total Energy & 19633 & 19633 & 18014 & 16882 & 19408 & 17129 & 13100 & 18384 & 13636 & 10275 & 18091 & 12173 & 9821 \\
\hline & Usable Energy & 11780 & 11780 & 10808 & 10129 & 11645 & 10277 & 9170 & 11030 & 9545 & 8220 & 10855 & 9129 & 7857 \\
\hline \multirow{2}{*}{ SI Series HEV PHEV40 } & Total Energy & 26016 & 26016 & 24118 & 22615 & 25190 & 23038 & 17620 & 24697 & 18332 & 13200 & 24191 & 16235 & 12601 \\
\hline & Usable Energy & 15610 & 15610 & 14471 & 13569 & 15114 & 13823 & 12334 & 14818 & 12832 & 10560 & 14515 & 12176 & 10081 \\
\hline \multirow{2}{*}{ Cl Split HEV } & Total Energy & 1427 & 1427 & 1357 & 1295 & 1404 & 1296 & 1188 & 1361 & 1210 & 1015 & 1339 & 1188 & 972 \\
\hline & Usable Energy & & & & & & & & & & & & & \\
\hline \multirow{2}{*}{ CI Split HEV PHEV10 } & Total Energy & 5098 & 5098 & 4906 & 4476 & 4982 & 4766 & 3861 & 4898 & 3799 & 2945 & 4836 & 3592 & 2915 \\
\hline & Usable Energy & 3059 & 3059 & 2943 & 2686 & 2989 & 2860 & 2702 & 2939 & 2659 & 2356 & 2901 & 2694 & 2332 \\
\hline \multirow{2}{*}{ CI Split HEV PHEV20 } & Total Energy & 11131 & 11131 & 10700 & 9891 & 10992 & 10022 & 7757 & 10846 & 7933 & 5874 & 10629 & 7071 & 5547 \\
\hline & Usable Energy & 6678 & 6678 & 6420 & 5935 & 6595 & 6013 & 5430 & 6507 & 5553 & 4700 & 6378 & 5303 & 4437 \\
\hline \multirow{2}{*}{ CI Series HEV PHEV30 } & Total Energy & 20203 & 20203 & 18819 & 17678 & 19642 & 17930 & 13551 & 19190 & 14075 & 10367 & 18877 & 12585 & 9930 \\
\hline & Usable Energy & 12122 & 12122 & 11291 & 10607 & 11785 & 10758 & 9486 & 11514 & 9852 & 8294 & 11326 & 9438 & 7944 \\
\hline \multirow{2}{*}{$\mathrm{Cl}$ Series HEV PHEV 40} & Total Energy & 26763 & 26763 & 24947 & 23392 & 26011 & 23731 & 18125 & 25395 & 18932 & 13759 & 24994 & 16781 & 13176 \\
\hline & Usable Energy & 16058 & 16058 & 14968 & 14035 & 15607 & 14239 & 12687 & 15237 & 13252 & 11008 & 14996 & 12585 & 10541 \\
\hline \multirow{2}{*}{ H2 Split HEV } & Total Energy & 1381 & 1381 & 1287 & 1209 & 1357 & 1210 & 1102 & 1296 & 1123 & 907 & 1274 & 1102 & 886 \\
\hline & Usable Energy & & & & & & & & & & & & & \\
\hline \multirow{2}{*}{ H2 Split HEV PHEV10 } & Total Energy & 5181 & 5181 & 4850 & 4522 & 4925 & 4596 & 3676 & 4882 & 3794 & 2825 & 4785 & 3482 & 2712 \\
\hline & Usable Energy & 3108 & 3108 & 2910 & 2713 & 2955 & 2758 & 2573 & 2929 & 2656 & 2260 & 2871 & 2612 & 2169 \\
\hline \multirow{2}{*}{ H2 Split HEV PHEV2O } & Total Energy & 10982 & 10982 & 10122 & 9480 & 10811 & 9546 & 7231 & 10323 & 7594 & 5449 & 10249 & 6669 & 5231 \\
\hline & Usable Energy & 6589 & 6589 & 6073 & 5688 & 6487 & 5727 & 5061 & 6194 & 5316 & 4359 & 6149 & 5002 & 4185 \\
\hline
\end{tabular}




\begin{tabular}{|c|c|c|c|c|c|c|c|c|c|c|c|c|c|c|}
\hline & & \multicolumn{13}{|c|}{ Midsize_SUV } \\
\hline & & 2010 & \multicolumn{3}{|c|}{2010} & \multicolumn{3}{|c|}{2015} & \multicolumn{3}{|c|}{2030} & \multicolumn{3}{|c|}{2045} \\
\hline & & Ref & low & avg & high & low & avg & high & low & avg & high & low & avg & high \\
\hline \multirow{2}{*}{ H2 Series HEV PHEV3O } & Total Energy & 19830 & 19830 & 18478 & 16685 & 19462 & 16945 & 12876 & 18978 & 13447 & 9743 & 17868 & 11969 & 9667 \\
\hline & Usable Energy & 11898 & 11898 & 11087 & 10011 & 11677 & 10167 & 9013 & 11387 & 9413 & 7794 & 10721 & 8977 & 7734 \\
\hline \multirow{2}{*}{ H2 Series HEV PHEV40 } & Total Energy & 26246 & 26246 & 24070 & 22333 & 25366 & 22668 & 17249 & 24718 & 17997 & 12879 & 23890 & 15968 & 12343 \\
\hline & Usable Energy & 15748 & 15748 & 14442 & 13400 & 15220 & 13601 & 12074 & 14831 & 12598 & 10303 & 14334 & 11976 & 9874 \\
\hline \multirow{2}{*}{ E85 Split HEV } & Total Energy & 1357 & 1357 & 1295 & 1232 & 1342 & 1231 & 1123 & 1296 & 1145 & 950 & 1296 & 1123 & 907 \\
\hline & Usable Energy & & & & & & & & & & & & & \\
\hline \multirow{2}{*}{ E85 Split HEV PHEV10 } & Total Energy & 5166 & 5166 & 4870 & 4663 & 5068 & 4737 & 3851 & 4814 & 3855 & 2930 & 4757 & 3518 & 2795 \\
\hline & Usable Energy & 3100 & 3100 & 2922 & 2798 & 3041 & 2842 & 2696 & 2889 & 2699 & 2344 & 2854 & 2639 & 2236 \\
\hline \multirow{2}{*}{ E85 Split HEV PHEV2O } & Total Energy & 10719 & 10719 & 10170 & 9528 & 10724 & 9648 & 7316 & 10548 & 7682 & 5536 & 10315 & 6751 & 5301 \\
\hline & Usable Energy & 6431 & 6431 & 6102 & 5717 & 6435 & 5789 & 5121 & 6329 & 5378 & 4429 & 6189 & 5063 & 4241 \\
\hline \multirow{2}{*}{ E85 Series HEV PHEV30 } & Total Energy & 19820 & 19820 & 18031 & 16888 & 19250 & 17146 & 13119 & 18412 & 13645 & 10267 & 18518 & 12181 & 9820 \\
\hline & Usable Energy & 11892 & 11892 & 10819 & 10133 & 11550 & 10288 & 9183 & 11047 & 9552 & 8214 & 11111 & 9136 & 7856 \\
\hline \multirow{2}{*}{ E85 Series HEV PHEV40 } & Total Energy & 26047 & 26047 & 24135 & 22596 & 25186 & 22953 & 17638 & 24642 & 18270 & 13210 & 24232 & 16254 & 12617 \\
\hline & Usable Energy & 15628 & 15628 & 14481 & 13557 & 15112 & 13772 & 12347 & 14785 & 12789 & 10568 & 14539 & 12190 & 10093 \\
\hline \multirow{2}{*}{ FC HEV } & Total Energy & 1693 & 1693 & 1443 & 1342 & 1498 & 1469 & 1339 & 1555 & 1361 & 1145 & 1490 & 1318 & 1080 \\
\hline & Usable Energy & & & & & & & & & & & & & \\
\hline \multirow{2}{*}{ FC HEV PHEV10 } & Total Energy & 7188 & 7188 & 6147 & 5079 & 6016 & 5141 & 3929 & 6154 & 4088 & 2930 & 5398 & 3651 & 2798 \\
\hline & Usable Energy & 4313 & 4313 & 3688 & 3047 & 3610 & 3085 & 2750 & 3692 & 2862 & 2344 & 3239 & 2738 & 2238 \\
\hline \multirow{2}{*}{ FC HEV PHEV 20} & Total Energy & 13011 & 13011 & 11321 & 10168 & 12002 & 10300 & 7956 & 11575 & 8302 & 5835 & 10797 & 7387 & 5567 \\
\hline & Usable Energy & 7807 & 7807 & 6793 & 6101 & 7201 & 6180 & 5569 & 6945 & 5811 & 4668 & 6478 & 5540 & 4454 \\
\hline \multirow{2}{*}{ FC HEV PHEV 30} & Total Energy & 20979 & 20979 & 17522 & 16053 & 19485 & 16907 & 12122 & 17761 & 12662 & 9157 & 16931 & 11207 & 8732 \\
\hline & Usable Energy & 12588 & 12588 & 10513 & 9632 & 11691 & 10144 & 8485 & 10656 & 8863 & 7326 & 10158 & 8405 & 6986 \\
\hline \multirow{2}{*}{ FC HEV PHEV40 } & Total Energy & 26665 & 26665 & 23018 & 20967 & 24410 & 21075 & 15684 & 23284 & 16387 & 11838 & 22104 & 14486 & 11244 \\
\hline & Usable Energy & 15999 & 15999 & 13811 & 12580 & 14646 & 12645 & 10979 & 13970 & 11471 & 9471 & 13263 & 10865 & 8995 \\
\hline \multirow{2}{*}{ BEV } & Total Energy & 96339 & 96339 & 88868 & 82677 & 93491 & 83942 & 61018 & 90828 & 63572 & 44521 & 88658 & 56146 & 42243 \\
\hline & Usable Energy & 57803 & 57803 & 53321 & 49606 & 56095 & 50365 & 42713 & 54497 & 44501 & 35617 & 53195 & 42110 & 33794 \\
\hline
\end{tabular}




\section{Percentage of Energy reduction based on the respective 2010 reference case}

\begin{tabular}{|c|c|c|c|c|c|c|c|c|c|c|c|c|c|c|}
\hline & & \multicolumn{13}{|c|}{ Midsize_SUV } \\
\hline & & \multirow{2}{*}{$\frac{2010}{\text { Ref }}$} & \multicolumn{3}{|c|}{2010} & \multicolumn{3}{|c|}{2015} & \multicolumn{3}{|c|}{2030} & \multicolumn{3}{|c|}{2045} \\
\hline & & & low & avg & high & low & avg & high & low & avg & high & low & avg & high \\
\hline \multirow{2}{*}{ SI Split HEV } & Total Energy & $0.0 \%$ & $0.0 \%$ & $-4.6 \%$ & $-9.2 \%$ & $-1.1 \%$ & $-9.3 \%$ & $-17.2 \%$ & $-4.5 \%$ & $-15.6 \%$ & $-30.0 \%$ & $-4.5 \%$ & $-17.2 \%$ & $-33.2 \%$ \\
\hline & Usable Energy & & & & & & & & & & & & & \\
\hline \multirow{2}{*}{ SI Split HEV PHEV10 } & Total Energy & $0.0 \%$ & $0.0 \%$ & $-1.2 \%$ & $-6.9 \%$ & $2.6 \%$ & $-5.7 \%$ & $-23.5 \%$ & $-2.2 \%$ & $-21.7 \%$ & $-40.5 \%$ & $-3.5 \%$ & $-28.5 \%$ & $-43.3 \%$ \\
\hline & Usable Energy & $0.0 \%$ & $0.0 \%$ & $-1.2 \%$ & $-6.9 \%$ & $2.6 \%$ & $-5.7 \%$ & $-10.8 \%$ & $-2.2 \%$ & $-8.6 \%$ & $-20.6 \%$ & $-3.5 \%$ & $-10.6 \%$ & $-24.4 \%$ \\
\hline \multirow{2}{*}{ SI Split HEV PHEV2O } & Total Energy & $0.0 \%$ & $0.0 \%$ & $-5.8 \%$ & $-11.5 \%$ & $-0.5 \%$ & $-10.4 \%$ & $-31.9 \%$ & $-2.4 \%$ & $-28.6 \%$ & $-48.6 \%$ & $-3.7 \%$ & $-37.2 \%$ & $-50.8 \%$ \\
\hline & Usable Energy & $0.0 \%$ & $0.0 \%$ & $-5.8 \%$ & $-11.5 \%$ & $-0.5 \%$ & $-10.4 \%$ & $-20.5 \%$ & $-2.4 \%$ & $-16.7 \%$ & $-31.4 \%$ & $-3.7 \%$ & $-21.5 \%$ & $-34.5 \%$ \\
\hline \multirow{2}{*}{ SI Series HEV PHEV30 } & Total Energy & $0.0 \%$ & $0.0 \%$ & $-8.2 \%$ & $-14.0 \%$ & $-1.1 \%$ & $-12.8 \%$ & $-33.3 \%$ & $-6.4 \%$ & $-30.5 \%$ & $-47.7 \%$ & $-7.9 \%$ & $-38.0 \%$ & $-50.0 \%$ \\
\hline & Usable Energy & $0.0 \%$ & $0.0 \%$ & $-8.2 \%$ & $-14.0 \%$ & $-1.1 \%$ & $-12.8 \%$ & $-22.2 \%$ & $-6.4 \%$ & $-19.0 \%$ & $-30.2 \%$ & $-7.9 \%$ & $-22.5 \%$ & $-33.3 \%$ \\
\hline \multirow{2}{*}{ SI Series HEV PHEV40 } & Total Energy & $0.0 \%$ & $0.0 \%$ & $-7.3 \%$ & $-13.1 \%$ & $-3.2 \%$ & $-11.4 \%$ & $-32.3 \%$ & $-5.1 \%$ & $-29.5 \%$ & $-49.3 \%$ & $-7.0 \%$ & $-37.6 \%$ & $-51.6 \%$ \\
\hline & Usable Energy & $0.0 \%$ & $0.0 \%$ & $-7.3 \%$ & $-13.1 \%$ & $-3.2 \%$ & $-11.4 \%$ & $-21.0 \%$ & $-5.1 \%$ & $-17.8 \%$ & $-32.4 \%$ & $-7.0 \%$ & $-22.0 \%$ & $-35.4 \%$ \\
\hline \multirow{2}{*}{ CI Split HEV } & Total Energy & $0.0 \%$ & $0.0 \%$ & $-4.9 \%$ & $-9.3 \%$ & $-1.6 \%$ & $-9.2 \%$ & $-16.8 \%$ & $-4.7 \%$ & $-15.3 \%$ & $-28.9 \%$ & $-6.2 \%$ & $-16.8 \%$ & $-31.9 \%$ \\
\hline & Usable Energy & & & & & & & & & & & & & \\
\hline \multirow{2}{*}{ CI Split HEV PHEV10 } & Total Energy & $0.0 \%$ & $0.0 \%$ & $-3.8 \%$ & $-12.2 \%$ & $-2.3 \%$ & $-6.5 \%$ & $-24.3 \%$ & $-3.9 \%$ & $-25.5 \%$ & $-42.2 \%$ & $-5.2 \%$ & $-29.6 \%$ & $-42.8 \%$ \\
\hline & Usable Energy & $0.0 \%$ & $0.0 \%$ & $-3.8 \%$ & $-12.2 \%$ & $-2.3 \%$ & $-6.5 \%$ & $-11.7 \%$ & $-3.9 \%$ & $-13.1 \%$ & $-23.0 \%$ & $-5.2 \%$ & $-11.9 \%$ & $-23.8 \%$ \\
\hline \multirow{2}{*}{ CI Split HEV PHEV 20} & Total Energy & $0.0 \%$ & $0.0 \%$ & $-3.9 \%$ & $-11.1 \%$ & $-1.2 \%$ & $-10.0 \%$ & $-30.3 \%$ & $-2.6 \%$ & $-28.7 \%$ & $-47.2 \%$ & $-4.5 \%$ & $-36.5 \%$ & $-50.2 \%$ \\
\hline & Usable Energy & $0.0 \%$ & $0.0 \%$ & $-3.9 \%$ & $-11.1 \%$ & $-1.2 \%$ & $-10.0 \%$ & $-18.7 \%$ & $-2.6 \%$ & $-16.8 \%$ & $-29.6 \%$ & $-4.5 \%$ & $-20.6 \%$ & $-33.6 \%$ \\
\hline \multirow{2}{*}{ Cl Series HEV PHEV30 } & Total Energy & $0.0 \%$ & $0.0 \%$ & $-6.9 \%$ & $-12.5 \%$ & $-2.8 \%$ & $-11.3 \%$ & $-32.9 \%$ & $-5.0 \%$ & $-30.3 \%$ & $-48.7 \%$ & $-6.6 \%$ & $-37.7 \%$ & $-50.8 \%$ \\
\hline & Usable Energy & $0.0 \%$ & $0.0 \%$ & $-6.9 \%$ & $-12.5 \%$ & $-2.8 \%$ & $-11.3 \%$ & $-21.7 \%$ & $-5.0 \%$ & $-18.7 \%$ & $-31.6 \%$ & $-6.6 \%$ & $-22.1 \%$ & $-34.5 \%$ \\
\hline \multirow{2}{*}{ Cl Series HEV PHEV40 } & Total Energy & $0.0 \%$ & $0.0 \%$ & $-6.8 \%$ & $-12.6 \%$ & $-2.8 \%$ & $-11.3 \%$ & $-32.3 \%$ & $-5.1 \%$ & $-29.3 \%$ & $-48.6 \%$ & $-6.6 \%$ & $-37.3 \%$ & $-50.8 \%$ \\
\hline & Usable Energy & $0.0 \%$ & $0.0 \%$ & $-6.8 \%$ & $-12.6 \%$ & $-2.8 \%$ & $-11.3 \%$ & $-21.0 \%$ & $-5.1 \%$ & $-17.5 \%$ & $-31.4 \%$ & $-6.6 \%$ & $-21.6 \%$ & $-34.4 \%$ \\
\hline \multirow{2}{*}{ H2 Split HEV } & Total Energy & $0.0 \%$ & $0.0 \%$ & $-6.8 \%$ & $-12.4 \%$ & $-1.7 \%$ & $-12.4 \%$ & $-20.2 \%$ & $-6.1 \%$ & $-18.6 \%$ & $-34.3 \%$ & $-7.7 \%$ & $-20.2 \%$ & $-35.9 \%$ \\
\hline & Usable Energy & & & & & & & & & & & & & \\
\hline \multirow{2}{*}{ H2 Split HEV PHEV10 } & Total Energy & $0.0 \%$ & $0.0 \%$ & $-6.4 \%$ & $-12.7 \%$ & $-4.9 \%$ & $-11.3 \%$ & $-29.0 \%$ & $-5.8 \%$ & $-26.8 \%$ & $-45.5 \%$ & $-7.6 \%$ & $-32.8 \%$ & $-47.7 \%$ \\
\hline & Usable Energy & $0.0 \%$ & $0.0 \%$ & $-6.4 \%$ & $-12.7 \%$ & $-4.9 \%$ & $-11.3 \%$ & $-17.2 \%$ & $-5.8 \%$ & $-14.6 \%$ & $-27.3 \%$ & $-7.6 \%$ & $-16.0 \%$ & $-30.2 \%$ \\
\hline \multirow{2}{*}{ H2 Split HEV PHEV2O } & Total Energy & $0.0 \%$ & $0.0 \%$ & $-7.8 \%$ & $-13.7 \%$ & $-1.6 \%$ & $-13.1 \%$ & $-34.2 \%$ & $-6.0 \%$ & $-30.8 \%$ & $-50.4 \%$ & $-6.7 \%$ & $-39.3 \%$ & $-52.4 \%$ \\
\hline & Usable Energy & $0.0 \%$ & $0.0 \%$ & $-7.8 \%$ & $-13.7 \%$ & $-1.6 \%$ & $-13.1 \%$ & $-23.2 \%$ & $-6.0 \%$ & $-19.3 \%$ & $-33.8 \%$ & $-6.7 \%$ & $-24.1 \%$ & $-36.5 \%$ \\
\hline
\end{tabular}


Argonne National Laboratory Report - Light-Duty Vehicle Fuel Consumption Displacement Potential Up to 2045

\begin{tabular}{|c|c|c|c|c|c|c|c|c|c|c|c|c|c|c|}
\hline & & \multicolumn{13}{|c|}{ Midsize_SUV } \\
\hline & & \multirow{2}{*}{$\frac{2010}{\text { Ref }}$} & \multicolumn{3}{|c|}{2010} & \multicolumn{3}{|c|}{2015} & \multicolumn{3}{|c|}{2030} & \multicolumn{3}{|c|}{2045} \\
\hline & & & low & avg & high & low & avg & high & low & avg & high & low & avg & high \\
\hline \multirow{2}{*}{ H2 Series HEV PHEV30 } & Total Energy & $0.0 \%$ & $0.0 \%$ & $-6.8 \%$ & $-15.9 \%$ & $-1.9 \%$ & $-14.5 \%$ & $-35.1 \%$ & $-4.3 \%$ & $-32.2 \%$ & $-50.9 \%$ & $-9.9 \%$ & $-39.6 \%$ & $-51.2 \%$ \\
\hline & Usable Energy & $0.0 \%$ & $0.0 \%$ & $-6.8 \%$ & $-15.9 \%$ & $-1.9 \%$ & $-14.5 \%$ & $-24.2 \%$ & $-4.3 \%$ & $-20.9 \%$ & $-34.5 \%$ & $-9.9 \%$ & $-24.5 \%$ & $-35.0 \%$ \\
\hline \multirow{2}{*}{ H2 Series HEV PHEV40 } & Total Energy & $0.0 \%$ & $0.0 \%$ & $-8.3 \%$ & $-14.9 \%$ & $-3.4 \%$ & $-13.6 \%$ & $-34.3 \%$ & $-5.8 \%$ & $-31.4 \%$ & $-50.9 \%$ & $-9.0 \%$ & $-39.2 \%$ & $-53.0 \%$ \\
\hline & Usable Energy & $0.0 \%$ & $0.0 \%$ & $-8.3 \%$ & $-14.9 \%$ & $-3.4 \%$ & $-13.6 \%$ & $-23.3 \%$ & $-5.8 \%$ & $-20.0 \%$ & $-34.6 \%$ & $-9.0 \%$ & $-23.9 \%$ & $-37.3 \%$ \\
\hline \multirow{2}{*}{ E85 Split HEV } & Total Energy & $0.0 \%$ & $0.0 \%$ & $-4.6 \%$ & $-9.2 \%$ & $-1.1 \%$ & $-9.3 \%$ & $-17.2 \%$ & $-4.5 \%$ & $-15.6 \%$ & $-30.0 \%$ & $-4.5 \%$ & $-17.2 \%$ & $-33.2 \%$ \\
\hline & Usable Energy & & & & & & & & & & & & & \\
\hline \multirow{2}{*}{ E85 Split HEV PHEV10 } & Total Energy & $0.0 \%$ & $0.0 \%$ & $-5.7 \%$ & $-9.7 \%$ & $-1.9 \%$ & $-8.3 \%$ & $-25.5 \%$ & $-6.8 \%$ & $-25.4 \%$ & $-43.3 \%$ & $-7.9 \%$ & $-31.9 \%$ & $-45.9 \%$ \\
\hline & Usable Energy & $0.0 \%$ & $0.0 \%$ & $-5.7 \%$ & $-9.7 \%$ & $-1.9 \%$ & $-8.3 \%$ & $-13.0 \%$ & $-6.8 \%$ & $-12.9 \%$ & $-24.4 \%$ & $-7.9 \%$ & $-14.9 \%$ & $-27.9 \%$ \\
\hline \multirow{2}{*}{ E85 Split HEV PHEV20 } & Total Energy & $0.0 \%$ & $0.0 \%$ & $-5.1 \%$ & $-11.1 \%$ & $0.1 \%$ & $-10.0 \%$ & $-31.8 \%$ & $-1.6 \%$ & $-28.3 \%$ & $-48.4 \%$ & $-3.8 \%$ & $-37.0 \%$ & $-50.5 \%$ \\
\hline & Usable Energy & $0.0 \%$ & $0.0 \%$ & $-5.1 \%$ & $-11.1 \%$ & $0.1 \%$ & $-10.0 \%$ & $-20.4 \%$ & $-1.6 \%$ & $-16.4 \%$ & $-31.1 \%$ & $-3.8 \%$ & $-21.3 \%$ & $-34.1 \%$ \\
\hline \multirow{2}{*}{ E85 Series HEV PHEV30 } & Total Energy & $0.0 \%$ & $0.0 \%$ & $-9.0 \%$ & $-14.8 \%$ & $-2.9 \%$ & $-13.5 \%$ & $-33.8 \%$ & $-7.1 \%$ & $-31.2 \%$ & $-48.2 \%$ & $-6.6 \%$ & $-38.5 \%$ & $-50.5 \%$ \\
\hline & Usable Energy & $0.0 \%$ & $0.0 \%$ & $-9.0 \%$ & $-14.8 \%$ & $-2.9 \%$ & $-13.5 \%$ & $-22.8 \%$ & $-7.1 \%$ & $-19.7 \%$ & $-30.9 \%$ & $-6.6 \%$ & $-23.2 \%$ & $-33.9 \%$ \\
\hline \multirow{2}{*}{ E85 Series HEV PHEV40 } & Total Energy & $0.0 \%$ & $0.0 \%$ & $-7.3 \%$ & $-13.2 \%$ & $-3.3 \%$ & $-11.9 \%$ & $-32.3 \%$ & $-5.4 \%$ & $-29.9 \%$ & $-49.3 \%$ & $-7.0 \%$ & $-37.6 \%$ & $-51.6 \%$ \\
\hline & Usable Energy & $0.0 \%$ & $0.0 \%$ & $-7.3 \%$ & $-13.2 \%$ & $-3.3 \%$ & $-11.9 \%$ & $-21.0 \%$ & $-5.4 \%$ & $-18.2 \%$ & $-32.4 \%$ & $-7.0 \%$ & $-22.0 \%$ & $-35.4 \%$ \\
\hline \multirow{2}{*}{ FC HEV } & Total Energy & $0.0 \%$ & $0.0 \%$ & $-14.7 \%$ & $-20.7 \%$ & $-11.5 \%$ & $-13.2 \%$ & $-20.9 \%$ & $-8.1 \%$ & $-19.6 \%$ & $-32.4 \%$ & $-11.9 \%$ & $-22.2 \%$ & $-36.2 \%$ \\
\hline & Usable Energy & & & & & & & & & & & & & \\
\hline \multirow{2}{*}{ FC HEV PHEV10 } & Total Energy & $0.0 \%$ & $0.0 \%$ & $-14.5 \%$ & $-29.3 \%$ & $-16.3 \%$ & $-28.5 \%$ & $-45.3 \%$ & $-14.4 \%$ & $-43.1 \%$ & $-59.2 \%$ & $-24.9 \%$ & $-49.2 \%$ & $-61.1 \%$ \\
\hline & Usable Energy & $0.0 \%$ & $0.0 \%$ & $-14.5 \%$ & $-29.3 \%$ & $-16.3 \%$ & $-28.5 \%$ & $-36.2 \%$ & $-14.4 \%$ & $-33.7 \%$ & $-45.7 \%$ & $-24.9 \%$ & $-36.5 \%$ & $-48.1 \%$ \\
\hline \multirow{2}{*}{ FC HEV PHEV20 } & Total Energy & $0.0 \%$ & $0.0 \%$ & $-13.0 \%$ & $-21.9 \%$ & $-7.8 \%$ & $-20.8 \%$ & $-38.9 \%$ & $-11.0 \%$ & $-36.2 \%$ & $-55.2 \%$ & $-17.0 \%$ & $-43.2 \%$ & $-57.2 \%$ \\
\hline & Usable Energy & $0.0 \%$ & $0.0 \%$ & $-13.0 \%$ & $-21.9 \%$ & $-7.8 \%$ & $-20.8 \%$ & $-28.7 \%$ & $-11.0 \%$ & $-25.6 \%$ & $-40.2 \%$ & $-17.0 \%$ & $-29.0 \%$ & $-42.9 \%$ \\
\hline \multirow{2}{*}{ FC HEV PHEV 30} & Total Energy & $0.0 \%$ & $0.0 \%$ & $-16.5 \%$ & $-23.5 \%$ & $-7.1 \%$ & $-19.4 \%$ & $-42.2 \%$ & $-15.3 \%$ & $-39.6 \%$ & $-56.4 \%$ & $-19.3 \%$ & $-46.6 \%$ & $-58.4 \%$ \\
\hline & Usable Energy & $0.0 \%$ & $0.0 \%$ & $-16.5 \%$ & $-23.5 \%$ & $-7.1 \%$ & $-19.4 \%$ & $-32.6 \%$ & $-15.3 \%$ & $-29.6 \%$ & $-41.8 \%$ & $-19.3 \%$ & $-33.2 \%$ & $-44.5 \%$ \\
\hline \multirow{2}{*}{ FC HEV PHEV 40} & Total Energy & $0.0 \%$ & $0.0 \%$ & $-13.7 \%$ & $-21.4 \%$ & $-8.5 \%$ & $-21.0 \%$ & $-41.2 \%$ & $-12.7 \%$ & $-38.5 \%$ & $-55.6 \%$ & $-17.1 \%$ & $-45.7 \%$ & $-57.8 \%$ \\
\hline & Usable Energy & $0.0 \%$ & $0.0 \%$ & $-13.7 \%$ & $-21.4 \%$ & $-8.5 \%$ & $-21.0 \%$ & $-31.4 \%$ & $-12.7 \%$ & $-28.3 \%$ & $-40.8 \%$ & $-17.1 \%$ & $-32.1 \%$ & $-43.8 \%$ \\
\hline \multirow{2}{*}{ EV } & Total Energy & $0.0 \%$ & $0.0 \%$ & $-7.8 \%$ & $-14.2 \%$ & $-3.0 \%$ & $-12.9 \%$ & $-36.7 \%$ & $-5.7 \%$ & $-34.0 \%$ & $-53.8 \%$ & $-8.0 \%$ & $-41.7 \%$ & $-56.2 \%$ \\
\hline & Usable Energy & $0.0 \%$ & $0.0 \%$ & $-7.8 \%$ & $-14.2 \%$ & $-3.0 \%$ & $-12.9 \%$ & $-26.1 \%$ & $-5.7 \%$ & $-23.0 \%$ & $-38.4 \%$ & $-8.0 \%$ & $-27.1 \%$ & $-41.5 \%$ \\
\hline
\end{tabular}




\section{Curb weight}

\begin{tabular}{|c|c|c|c|c|c|c|c|c|c|c|c|c|c|c|}
\hline & & \multicolumn{13}{|c|}{ Midsize_SUV } \\
\hline & & \multirow{2}{*}{$\frac{2010}{\text { Ref }}$} & \multicolumn{3}{|c|}{2010} & \multicolumn{3}{|c|}{2015} & \multicolumn{3}{|c|}{2030} & \multicolumn{3}{|c|}{2045} \\
\hline & & & low & avg & high & low & avg & high & low & avg & high & low & avg & high \\
\hline \multirow{3}{*}{ SI Conv } & Vehicle Mass & 1773 & 1773 & 1702 & 1636 & 1772 & 1658 & 1544 & 1757 & 1561 & 1326 & 1762 & 1544 & 1259 \\
\hline & $\%$ of Glider mass & 71.5 & 71.5 & 71.1 & 70.2 & 71.6 & 70.5 & 68.5 & 71.9 & 68.7 & 64.1 & 71.7 & 68.4 & 62.4 \\
\hline & $\%$ of Powertrain mass & 28.5 & 28.5 & 28.9 & 29.8 & 28.4 & 29.5 & 31.5 & 28.1 & 31.3 & 35.9 & 28.3 & 31.6 & 37.6 \\
\hline \multirow{3}{*}{ Cl Conv } & Vehicle Mass & 1818 & 1818 & 1757 & 1692 & 1818 & 1715 & 1598 & 1810 & 1614 & 1386 & 1809 & 1597 & 1320 \\
\hline & $\%$ of Glider mass & 69.7 & 69.7 & 68.8 & 67.8 & 69.8 & 68.2 & 66.2 & 69.8 & 66.4 & 61.3 & 69.8 & 66.1 & 59.5 \\
\hline & $\%$ of Powertrain mass & 30.3 & 30.3 & 31.2 & 32.2 & 30.2 & 31.8 & 33.8 & 30.2 & 33.6 & 38.7 & 30.2 & 33.9 & 40.5 \\
\hline \multirow{3}{*}{ H2 Conv } & Vehicle Mass & 1772 & 1772 & 1666 & 1584 & 1758 & 1607 & 1475 & 1737 & 1500 & 1246 & 1702 & 1476 & 1188 \\
\hline & $\%$ of Glider mass & 71.5 & 71.5 & 72.6 & 72.5 & 72.2 & 72.8 & 71.7 & 72.7 & 71.5 & 68.2 & 74.2 & 71.5 & 66.1 \\
\hline & $\%$ of Powertrain mass & 28.5 & 28.5 & 27.4 & 27.5 & 27.8 & 27.2 & 28.3 & 27.3 & 28.5 & 31.8 & 25.8 & 28.5 & 33.9 \\
\hline \multirow{3}{*}{ E85 Conv } & Vehicle Mass & 1772 & 1772 & 1702 & 1637 & 1771 & 1659 & 1544 & 1757 & 1560 & 1325 & 1761 & 1542 & 1257 \\
\hline & $\%$ of Glider mass & 71.5 & 71.5 & 71.1 & 70.1 & 71.7 & 70.5 & 68.5 & 71.9 & 68.7 & 64.2 & 71.7 & 68.5 & 62.5 \\
\hline & $\%$ of Powertrain mass & 28.5 & 28.5 & 28.9 & 29.9 & 28.3 & 29.5 & 31.5 & 28.1 & 31.3 & 35.8 & 28.3 & 31.5 & 37.5 \\
\hline \multirow{3}{*}{ SI Split HEV } & Vehicle Mass & 1938 & 1938 & 1842 & 1752 & 1908 & 1795 & 1634 & 1894 & 1665 & 1379 & 1885 & 1634 & 1307 \\
\hline & $\%$ of Glider mass & 65.4 & 65.4 & 65.7 & 65.5 & 66.5 & 65.1 & 64.7 & 66.7 & 64.4 & 61.6 & 67.0 & 64.6 & 60.1 \\
\hline & $\%$ of Powertrain mass & 34.6 & 34.6 & 34.3 & 34.5 & 33.5 & 34.9 & 35.3 & 33.3 & 35.6 & 38.4 & 33.0 & 35.4 & 39.9 \\
\hline \multirow{3}{*}{ SI Split HEV PHEV10 } & Vehicle Mass & 1958 & 1958 & 1860 & 1769 & 1929 & 1801 & 1635 & 1904 & 1666 & 1385 & 1890 & 1639 & 1310 \\
\hline & $\%$ of Glider mass & 64.7 & 64.7 & 65.0 & 64.9 & 65.8 & 64.9 & 64.7 & 66.3 & 64.4 & 61.4 & 66.8 & 64.4 & 59.9 \\
\hline & $\%$ of Powertrain mass & 35.3 & 35.3 & 35.0 & 35.1 & 34.2 & 35.1 & 35.3 & 33.7 & 35.6 & 38.6 & 33.2 & 35.6 & 40.1 \\
\hline \multirow{3}{*}{ SI Split HEV PHEV20 } & Vehicle Mass & 2000 & 2000 & 1901 & 1807 & 1970 & 1836 & 1662 & 1946 & 1699 & 1401 & 1929 & 1661 & 1325 \\
\hline & $\%$ of Glider mass & 63.4 & 63.4 & 63.6 & 63.5 & 64.4 & 63.7 & 63.6 & 64.9 & 63.1 & 60.7 & 65.4 & 63.6 & 59.3 \\
\hline & $\%$ of Powertrain mass & 36.6 & 36.6 & 36.4 & 36.5 & 35.6 & 36.3 & 36.4 & 35.1 & 36.9 & 39.3 & 34.6 & 36.4 & 40.7 \\
\hline \multirow{3}{*}{ SI Series HEV PHEV30 } & Vehicle Mass & 2265 & 2265 & 2129 & 2005 & 2207 & 2046 & 1819 & 2163 & 1869 & 1521 & 2135 & 1814 & 1439 \\
\hline & $\%$ of Glider mass & 56.0 & 56.0 & 56.8 & 57.2 & 57.5 & 57.1 & 58.1 & 58.4 & 57.4 & 55.9 & 59.1 & 58.2 & 54.6 \\
\hline & $\%$ of Powertrain mass & 44.0 & 44.0 & 43.2 & 42.8 & 42.5 & 42.9 & 41.9 & 41.6 & 42.6 & 44.1 & 40.9 & 41.8 & 45.4 \\
\hline \multirow{3}{*}{ SI Series HEV PHEV40 } & Vehicle Mass & 2330 & 2330 & 2187 & 2059 & 2268 & 2103 & 1859 & 2223 & 1910 & 1550 & 2188 & 1849 & 1464 \\
\hline & $\%$ of Glider mass & 54.4 & 54.4 & 55.3 & 55.7 & 56.0 & 55.6 & 56.9 & 56.8 & 56.1 & 54.8 & 57.7 & 57.1 & 53.6 \\
\hline & $\%$ of Powertrain mass & 45.6 & 45.6 & 44.7 & 44.3 & 44.0 & 44.4 & 43.1 & 43.2 & 43.9 & 45.2 & 42.3 & 42.9 & 46.4 \\
\hline \multirow{3}{*}{ CI Split HEV } & Vehicle Mass & 2029 & 2029 & 1935 & 1841 & 1995 & 1885 & 1720 & 1986 & 1755 & 1469 & 1971 & 1725 & 1395 \\
\hline & $\%$ of Glider mass & 62.5 & 62.5 & 62.5 & 62.3 & 63.6 & 62.0 & 61.5 & 63.6 & 61.1 & 57.9 & 64.1 & 61.2 & 56.3 \\
\hline & $\%$ of Powertrain mass & 37.5 & 37.5 & 37.5 & 37.7 & 36.4 & 38.0 & 38.5 & 36.4 & 38.9 & 42.1 & 35.9 & 38.8 & 43.7 \\
\hline
\end{tabular}




\begin{tabular}{|c|c|c|c|c|c|c|c|c|c|c|c|c|c|c|}
\hline & & \multicolumn{13}{|c|}{ Midsize_SUV } \\
\hline & & \multirow{2}{*}{$\begin{array}{l}2010 \\
\text { Ref } \\
\end{array}$} & \multicolumn{3}{|c|}{2010} & \multicolumn{3}{|c|}{2015} & \multicolumn{3}{|c|}{2030} & \multicolumn{3}{|c|}{2045} \\
\hline & & & low & avg & high & low & avg & high & low & avg & high & low & avg & high \\
\hline \multirow{3}{*}{ CI Split HEV PHEV10 } & Vehicle Mass & 2062 & 2062 & 1964 & 1871 & 2029 & 1900 & 1733 & 2004 & 1768 & 1481 & 1989 & 1739 & 1409 \\
\hline & $\%$ of Glider mass & 61.5 & 61.5 & 61.6 & 61.4 & 62.5 & 61.5 & 61.0 & 63.0 & 60.6 & 57.4 & 63.5 & 60.7 & 55.7 \\
\hline & $\%$ of Powertrain mass & 38.5 & 38.5 & 38.4 & 38.6 & 37.5 & 38.5 & 39.0 & 37.0 & 39.4 & 42.6 & 36.5 & 39.3 & 44.3 \\
\hline \multirow{3}{*}{ CI Split HEV PHEV2O } & Vehicle Mass & 2104 & 2104 & 2003 & 1906 & 2070 & 1939 & 1762 & 2045 & 1796 & 1500 & 2029 & 1762 & 1426 \\
\hline & $\%$ of Glider mass & 60.2 & 60.2 & 60.4 & 60.2 & 61.3 & 60.3 & 60.0 & 61.7 & 59.7 & 56.7 & 62.2 & 59.9 & 55.1 \\
\hline & $\%$ of Powertrain mass & 39.8 & 39.8 & 39.6 & 39.8 & 38.7 & 39.7 & 40.0 & 38.3 & 40.3 & 43.3 & 37.8 & 40.1 & 44.9 \\
\hline \multirow{3}{*}{ CI Series HEV PHEV30 } & Vehicle Mass & 2359 & 2359 & 2221 & 2099 & 2298 & 2143 & 1914 & 2254 & 1963 & 1616 & 2225 & 1907 & 1535 \\
\hline & $\%$ of Glider mass & 53.7 & 53.7 & 54.5 & 54.7 & 55.2 & 54.6 & 55.2 & 56.0 & 54.6 & 52.6 & 56.7 & 55.4 & 51.2 \\
\hline & $\%$ of Powertrain mass & 46.3 & 46.3 & 45.5 & 45.3 & 44.8 & 45.4 & 44.8 & 44.0 & 45.4 & 47.4 & 43.3 & 44.6 & 48.8 \\
\hline \multirow{3}{*}{ CI Series HEV PHEV40 } & Vehicle Mass & 2426 & 2426 & 2283 & 2152 & 2364 & 2196 & 1955 & 2315 & 2005 & 1645 & 2283 & 1943 & 1563 \\
\hline & $\%$ of Glider mass & 52.2 & 52.2 & 53.0 & 53.3 & 53.7 & 53.2 & 54.1 & 54.5 & 53.5 & 51.7 & 55.3 & 54.3 & 50.2 \\
\hline & $\%$ of Powertrain mass & 47.8 & 47.8 & 47.0 & 46.7 & 46.3 & 46.8 & 45.9 & 45.5 & 46.5 & 48.3 & 44.7 & 45.7 & 49.8 \\
\hline \multirow{3}{*}{ H2 Split HEV } & Vehicle Mass & 1972 & 1972 & 1832 & 1723 & 1924 & 1766 & 1588 & 1904 & 1629 & 1322 & 1853 & 1594 & 1258 \\
\hline & $\%$ of Glider mass & 64.3 & 64.3 & 66.0 & 66.6 & 66.0 & 66.2 & 66.6 & 66.3 & 65.8 & 64.3 & 68.1 & 66.2 & 62.4 \\
\hline & $\%$ of Powertrain mass & 35.7 & 35.7 & 34.0 & 33.4 & 34.0 & 33.8 & 33.4 & 33.7 & 34.2 & 35.7 & 31.9 & 33.8 & 37.6 \\
\hline \multirow{3}{*}{ H2 Split HEV PHEV10 } & Vehicle Mass & 1998 & 1998 & 1855 & 1743 & 1949 & 1777 & 1593 & 1915 & 1636 & 1330 & 1863 & 1599 & 1265 \\
\hline & $\%$ of Glider mass & 63.4 & 63.4 & 65.2 & 65.9 & 65.1 & 65.8 & 66.4 & 65.9 & 65.5 & 63.9 & 67.8 & 66.0 & 62.1 \\
\hline & $\%$ of Powertrain mass & 36.6 & 36.6 & 34.8 & 34.1 & 34.9 & 34.2 & 33.6 & 34.1 & 34.5 & 36.1 & 32.2 & 34.0 & 37.9 \\
\hline \multirow{3}{*}{ H2 Split HEV PHEV2O } & Vehicle Mass & 2042 & 2042 & 1894 & 1780 & 1991 & 1813 & 1621 & 1956 & 1664 & 1347 & 1900 & 1621 & 1280 \\
\hline & $\%$ of Glider mass & 62.1 & 62.1 & 63.9 & 64.5 & 63.7 & 64.5 & 65.2 & 64.5 & 64.4 & 63.1 & 66.4 & 65.1 & 61.3 \\
\hline & $\%$ of Powertrain mass & 37.9 & 37.9 & 36.1 & 35.5 & 36.3 & 35.5 & 34.8 & 35.5 & 35.6 & 36.9 & 33.6 & 34.9 & 38.7 \\
\hline \multirow{3}{*}{ H2 Series HEV PHEV 30} & Vehicle Mass & 2296 & 2296 & 2111 & 1968 & 2216 & 2012 & 1770 & 2165 & 1828 & 1460 & 2094 & 1764 & 1388 \\
\hline & $\%$ of Glider mass & 55.2 & 55.2 & 57.3 & 58.3 & 57.3 & 58.1 & 59.7 & 58.3 & 58.7 & 58.2 & 60.3 & 59.9 & 56.6 \\
\hline & $\%$ of Powertrain mass & 44.8 & 44.8 & 42.7 & 41.7 & 42.7 & 41.9 & 40.3 & 41.7 & 41.3 & 41.8 & 39.7 & 40.1 & 43.4 \\
\hline \multirow{3}{*}{ H2 Series HEV PHEV 40} & Vehicle Mass & 2359 & 2359 & 2169 & 2021 & 2279 & 2065 & 1810 & 2225 & 1870 & 1488 & 2147 & 1800 & 1414 \\
\hline & $\%$ of Glider mass & 53.7 & 53.7 & 55.8 & 56.8 & 55.7 & 56.6 & 58.4 & 56.7 & 57.3 & 57.1 & 58.8 & 58.7 & 55.5 \\
\hline & $\%$ of Powertrain mass & 46.3 & 46.3 & 44.2 & 43.2 & 44.3 & 43.4 & 41.6 & 43.3 & 42.7 & 42.9 & 41.2 & 41.3 & 44.5 \\
\hline \multirow{3}{*}{ E85 Split HEV } & Vehicle Mass & 1941 & 1941 & 1846 & 1754 & 1910 & 1798 & 1637 & 1900 & 1668 & 1383 & 1888 & 1640 & 1310 \\
\hline & $\%$ of Glider mass & 65.3 & 65.3 & 65.5 & 65.4 & 66.4 & 65.0 & 64.6 & 66.4 & 64.3 & 61.5 & 66.9 & 64.4 & 59.9 \\
\hline & $\%$ of Powertrain mass & 34.7 & 34.7 & 34.5 & 34.6 & 33.6 & 35.0 & 35.4 & 33.6 & 35.7 & 38.5 & 33.1 & 35.6 & 40.1 \\
\hline
\end{tabular}




\begin{tabular}{|c|c|c|c|c|c|c|c|c|c|c|c|c|c|c|}
\hline & & \multicolumn{13}{|c|}{ Midsize_SUV } \\
\hline & & \multirow{2}{*}{$\frac{2010}{\text { Ref }}$} & \multicolumn{3}{|c|}{2010} & \multicolumn{3}{|c|}{2015} & \multicolumn{3}{|c|}{2030} & \multicolumn{3}{|c|}{2045} \\
\hline & & & low & avg & high & low & avg & high & low & avg & high & low & avg & high \\
\hline \multirow{3}{*}{ E85 Split HEV PHEV10 } & Vehicle Mass & 1961 & 1961 & 1864 & 1771 & 1931 & 1804 & 1638 & 1908 & 1671 & 1387 & 1895 & 1642 & 1313 \\
\hline & $\%$ of Glider mass & 64.6 & 64.6 & 64.9 & 64.8 & 65.7 & 64.8 & 64.6 & 66.2 & 64.2 & 61.3 & 66.6 & 64.3 & 59.8 \\
\hline & $\%$ of Powertrain mass & 35.4 & 35.4 & 35.1 & 35.2 & 34.3 & 35.2 & 35.4 & 33.8 & 35.8 & 38.7 & 33.4 & 35.7 & 40.2 \\
\hline \multirow{3}{*}{ E85 Split HEV PHEV20 } & Vehicle Mass & 2003 & 2003 & 1904 & 1809 & 1972 & 1841 & 1666 & 1949 & 1702 & 1404 & 1932 & 1664 & 1329 \\
\hline & $\%$ of Glider mass & 63.3 & 63.3 & 63.5 & 63.5 & 64.4 & 63.5 & 63.5 & 64.8 & 63.0 & 60.5 & 65.3 & 63.5 & 59.1 \\
\hline & $\%$ of Powertrain mass & 36.7 & 36.7 & 36.5 & 36.5 & 35.6 & 36.5 & 36.5 & 35.2 & 37.0 & 39.5 & 34.7 & 36.5 & 40.9 \\
\hline \multirow{3}{*}{ E85 Series HEV PHEV30 } & Vehicle Mass & 2268 & 2268 & 2132 & 2007 & 2209 & 2050 & 1823 & 2168 & 1872 & 1523 & 2138 & 1816 & 1442 \\
\hline & $\%$ of Glider mass & 55.9 & 55.9 & 56.7 & 57.2 & 57.5 & 57.0 & 58.0 & 58.2 & 57.3 & 55.8 & 59.1 & 58.1 & 54.5 \\
\hline & $\%$ of Powertrain mass & 44.1 & 44.1 & 43.3 & 42.8 & 42.5 & 43.0 & 42.0 & 41.8 & 42.7 & 44.2 & 40.9 & 41.9 & 45.5 \\
\hline \multirow{3}{*}{ E85 Series HEV PHEV40 } & Vehicle Mass & 2334 & 2334 & 2190 & 2061 & 2270 & 2106 & 1862 & 2227 & 1913 & 1552 & 2192 & 1851 & 1467 \\
\hline & $\%$ of Glider mass & 54.3 & 54.3 & 55.2 & 55.7 & 55.9 & 55.5 & 56.8 & 56.7 & 56.1 & 54.8 & 57.6 & 57.0 & 53.5 \\
\hline & $\%$ of Powertrain mass & 45.7 & 45.7 & 44.8 & 44.3 & 44.1 & 44.5 & 43.2 & 43.3 & 43.9 & 45.2 & 42.4 & 43.0 & 46.5 \\
\hline \multirow{3}{*}{ FC HEV } & Vehicle Mass & 2511 & 2511 & 2087 & 1866 & 2207 & 1882 & 1643 & 2055 & 1687 & 1332 & 1931 & 1616 & 1250 \\
\hline & $\%$ of Glider mass & 50.5 & 50.5 & 57.9 & 61.5 & 57.5 & 62.1 & 64.4 & 61.4 & 63.6 & 63.8 & 65.4 & 65.3 & 62.8 \\
\hline & $\%$ of Powertrain mass & 49.5 & 49.5 & 42.1 & 38.5 & 42.5 & 37.9 & 35.6 & 38.6 & 36.4 & 36.2 & 34.6 & 34.7 & 37.2 \\
\hline \multirow{3}{*}{ FC HEV PHEV10 } & Vehicle Mass & 2389 & 2389 & 2033 & 1839 & 2155 & 1852 & 1619 & 2024 & 1662 & 1312 & 1907 & 1596 & 1235 \\
\hline & $\%$ of Glider mass & 53.1 & 53.1 & 59.5 & 62.4 & 58.9 & 63.1 & 65.3 & 62.4 & 64.5 & 64.8 & 66.2 & 66.2 & 63.6 \\
\hline & $\%$ of Powertrain mass & 46.9 & 46.9 & 40.5 & 37.6 & 41.1 & 36.9 & 34.7 & 37.6 & 35.5 & 35.2 & 33.8 & 33.8 & 36.4 \\
\hline \multirow{3}{*}{ FC HEV PHEV20 } & Vehicle Mass & 2454 & 2454 & 2086 & 1883 & 2211 & 1896 & 1649 & 2074 & 1698 & 1335 & 1950 & 1625 & 1254 \\
\hline & $\%$ of Glider mass & 51.7 & 51.7 & 58.0 & 61.0 & 57.4 & 61.7 & 64.1 & 60.9 & 63.1 & 63.7 & 64.7 & 65.0 & 62.6 \\
\hline & $\%$ of Powertrain mass & 48.3 & 48.3 & 42.0 & 39.0 & 42.6 & 38.3 & 35.9 & 39.1 & 36.9 & 36.3 & 35.3 & 35.0 & 37.4 \\
\hline \multirow{3}{*}{ FC HEV PHEV 30} & Vehicle Mass & 2533 & 2533 & 2183 & 1987 & 2313 & 2008 & 1745 & 2194 & 1797 & 1419 & 2063 & 1713 & 1336 \\
\hline & $\%$ of Glider mass & 50.0 & 50.0 & 55.4 & 57.8 & 54.9 & 58.2 & 60.6 & 57.5 & 59.7 & 59.9 & 61.2 & 61.6 & 58.8 \\
\hline & $\%$ of Powertrain mass & 50.0 & 50.0 & 44.6 & 42.2 & 45.1 & 41.8 & 39.4 & 42.5 & 40.3 & 40.1 & 38.8 & 38.4 & 41.2 \\
\hline \multirow{3}{*}{ FC HEV PHEV 40} & Vehicle Mass & 2610 & 2610 & 2242 & 2038 & 2376 & 2057 & 1780 & 2251 & 1833 & 1448 & 2115 & 1745 & 1360 \\
\hline & $\%$ of Glider mass & 48.6 & 48.6 & 53.9 & 56.3 & 53.4 & 56.8 & 59.4 & 56.1 & 58.5 & 58.7 & 59.7 & 60.5 & 57.7 \\
\hline & $\%$ of Powertrain mass & 51.4 & 51.4 & 46.1 & 43.7 & 46.6 & 43.2 & 40.6 & 43.9 & 41.5 & 41.3 & 40.3 & 39.5 & 42.3 \\
\hline \multirow{3}{*}{ BEV } & Vehicle Mass & 2623 & 2623 & 2441 & 2281 & 2558 & 2337 & 1968 & 2496 & 2024 & 1579 & 2434 & 1914 & 1475 \\
\hline & $\%$ of Glider mass & 48.3 & 48.3 & 49.5 & 50.3 & 49.6 & 50.0 & 53.7 & 50.6 & 53.0 & 53.8 & 51.9 & 55.2 & 53.2 \\
\hline & $\%$ of Powertrain mass & 51.7 & 51.7 & 50.5 & 49.7 & 50.4 & 50.0 & 46.3 & 49.4 & 47.0 & 46.2 & 48.1 & 44.8 & 46.8 \\
\hline
\end{tabular}


Percentage of vehicle curbs weight reduction based on the respective 2010 reference case

\begin{tabular}{|c|c|c|c|c|c|c|c|c|c|c|c|c|c|c|}
\hline & & \multicolumn{13}{|c|}{ Midsize_SUV } \\
\hline & & \multirow{2}{*}{$\begin{array}{c}2010 \\
\text { Ref }\end{array}$} & \multicolumn{3}{|c|}{2010} & \multicolumn{3}{|c|}{2015} & \multicolumn{3}{|c|}{2030} & \multicolumn{3}{|c|}{2045} \\
\hline & & & low & avg & high & low & avg & high & low & avg & high & low & avg & high \\
\hline SI Conv & Vehicle Mass & $0.00 \%$ & $0.00 \%$ & $-4.00 \%$ & $-7.73 \%$ & $-0.06 \%$ & $-6.49 \%$ & $-12.92 \%$ & $-0.90 \%$ & $-11.96 \%$ & $-25.21 \%$ & $-0.62 \%$ & $-12.92 \%$ & $-28.99 \%$ \\
\hline $\mathrm{Cl}$ Conv & Vehicle Mass & $0.00 \%$ & $0.00 \%$ & $-3.36 \%$ & $-6.93 \%$ & $0.00 \%$ & $-5.67 \%$ & $-12.10 \%$ & $-0.44 \%$ & $-11.22 \%$ & $-23.76 \%$ & $-0.50 \%$ & $-12.16 \%$ & $-27.39 \%$ \\
\hline H2 Conv & Vehicle Mass & $0.00 \%$ & $0.00 \%$ & $-5.98 \%$ & $-10.61 \%$ & $-0.79 \%$ & $-9.31 \%$ & $-16.76 \%$ & $-1.98 \%$ & $-15.35 \%$ & $-29.68 \%$ & $-3.95 \%$ & $-16.70 \%$ & $-32.96 \%$ \\
\hline E85 Conv & Vehicle Mass & $0.00 \%$ & $0.00 \%$ & $-3.95 \%$ & & $-0.06 \%$ & $-6.38 \%$ & $-12.87 \%$ & $-0.85 \%$ & $-11.96 \%$ & $-25.23 \%$ & $-0.62 \%$ & $-12.98 \%$ & $-29.06 \%$ \\
\hline SI Split HEV & Vehicle Mass & $0.00 \%$ & $0.00 \%$ & $-4.95 \%$ & $-9.60 \%$ & $-1.55 \%$ & $-7.38 \%$ & $-15.69 \%$ & $-2.27 \%$ & $-14.09 \%$ & $-28.84 \%$ & $-2.73 \%$ & $-15.69 \%$ & $-32.56 \%$ \\
\hline SI Split HEV PHEV10 & Vehicle Mass & $0.00 \%$ & $0.00 \%$ & $-5.01 \%$ & $-9.65 \%$ & $-1.48 \%$ & $-8.02 \%$ & $-16.50 \%$ & $-2.76 \%$ & $-14.91 \%$ & $-29.26 \%$ & $-3.47 \%$ & $-16.29 \%$ & $-33.09 \%$ \\
\hline SI Split HEV PHEV20 & & $0.00 \%$ & $0.00 \%$ & $-4.95 \%$ & & $-1.50 \%$ & $-8.20 \%$ & $-16.90 \%$ & $-2.70 \%$ & $-15.05 \%$ & $-29.95 \%$ & $-3.55 \%$ & $-16.95 \%$ & $-33.75 \%$ \\
\hline SI Series HEV PHEV30 & Vehicle Mass & $0.00 \%$ & $0.00 \%$ & $-6.00 \%$ & $-11.48 \%$ & $-2.56 \%$ & $-9.67 \%$ & $-19.69 \%$ & $-4.50 \%$ & $-17.48 \%$ & $-32.85 \%$ & $-5.74 \%$ & $-19.91 \%$ & $-36.47 \%$ \\
\hline SI Series HEV PHEV40 & Vehic & $0.00 \%$ & $0.00 \%$ & $-6.14 \%$ & $-11.63 \%$ & $-2.66 \%$ & $-9.74 \%$ & $-20.21 \%$ & $-4.59 \%$ & $-18.03 \%$ & $-33.48 \%$ & $-6.09 \%$ & $-20.64 \%$ & $-37.17 \%$ \\
\hline CI Split HEV & Vehicle Mass & $0.00 \%$ & $0.00 \%$ & $-4.63 \%$ & $-9.27 \%$ & $-1.68 \%$ & $-7.10 \%$ & $-15.23 \%$ & $-2.12 \%$ & $-13.50 \%$ & $-27.60 \%$ & $-2.86 \%$ & $-14.98 \%$ & $-31.25 \%$ \\
\hline CI Split HEV PHEV10 & & & $0.00 \%$ & $-4.75 \%$ & & $-1.60 \%$ & $-7.86 \%$ & $-15.96 \%$ & $-2.81 \%$ & $-14.26 \%$ & $-28.18 \%$ & $-3.54 \%$ & $-15.66 \%$ & \\
\hline CI Split HEV PHEV20 & Vehicle Mass & $0.00 \%$ & $0.00 \%$ & $-4.80 \%$ & $-9.41 \%$ & $-1.62 \%$ & $-7.84 \%$ & $-16.25 \%$ & $-2.80 \%$ & $-14.64 \%$ & $-28.71 \%$ & $-3.56 \%$ & $-16.25 \%$ & $-32.22 \%$ \\
\hline $\mathrm{CI}$ Series HEV PHEV30 & Vehicle Mass & $0.00 \%$ & $0.00 \%$ & $-5.85 \%$ & $-11.02 \%$ & $-2.59 \%$ & $-9.16 \%$ & $-18.86 \%$ & $-4.45 \%$ & $-16.79 \%$ & $-31.50 \%$ & $-5.68 \%$ & $-19.16 \%$ & $-34.93 \%$ \\
\hline CI Series HEV PHEV40 & Vehicle Mass & & $0.00 \%$ & $-5.89 \%$ & & $-2.56 \%$ & $-9.48 \%$ & $-19.41 \%$ & $-4.58 \%$ & $-17.35 \%$ & $-32.19 \%$ & $-5.89 \%$ & $-19.91 \%$ & $-35.57 \%$ \\
\hline H2 Split HEV & & & $0.00 \%$ & $-7.10 \%$ & & $-2.43 \%$ & $-10.45 \%$ & $-19.47 \%$ & $-3.45 \%$ & $-17.39 \%$ & $-32.96 \%$ & $-6.03 \%$ & $-19.17 \%$ & $-36.21 \%$ \\
\hline H2 Split HEV PHEV10 & Vehicle Mass & $0.00 \%$ & $0.00 \%$ & $-7.16 \%$ & $-12.76 \%$ & $-2.45 \%$ & $-11.06 \%$ & $-20.27 \%$ & $-4.15 \%$ & $-18.12 \%$ & $-33.43 \%$ & $-6.76 \%$ & $-19.97 \%$ & $-36.69 \%$ \\
\hline H2 Split HEV PHEV2O & Vehicle Mass & $0.00 \%$ & $0.00 \%$ & $-7.25 \%$ & $-12.83 \%$ & $-2.50 \%$ & $-11.21 \%$ & $-20.62 \%$ & $-4.21 \%$ & $-18.51 \%$ & $-34.04 \%$ & $-6.95 \%$ & $-20.62 \%$ & $-37.32 \%$ \\
\hline H2 Series HEV PHEV30 & Vehicle Mass & $0.00 \%$ & $0.00 \%$ & $-8.06 \%$ & & $-3.48 \%$ & $-12.37 \%$ & $-22.91 \%$ & $-5.71 \%$ & $-20.38 \%$ & $-36.41 \%$ & $-8.80 \%$ & $-23.17 \%$ & $-39.55 \%$ \\
\hline H2 Series HEV PHEV40 & Vehicle Mass & $0.00 \%$ & $0.00 \%$ & $-8.05 \%$ & $-14.33 \%$ & $-3.39 \%$ & $-12.46 \%$ & $-23.27 \%$ & $-5.68 \%$ & $-20.73 \%$ & $-36.92 \%$ & $-8.99 \%$ & $-23.70 \%$ & $-40.06 \%$ \\
\hline E85 Split HEV & Vehicle Mass & $0.00 \%$ & $0.00 \%$ & $-4.89 \%$ & $-9.63 \%$ & $-1.60 \%$ & $-7.37 \%$ & $-15.66 \%$ & $-2.11 \%$ & $-14.06 \%$ & $-28.75 \%$ & $-2.73 \%$ & $-15.51 \%$ & $-32.51 \%$ \\
\hline E85 Split HEV PHEV10 & Vehicle Mass & $0.00 \%$ & $0.00 \%$ & $-4.95 \%$ & $-9.69 \%$ & $-1.53 \%$ & $-8.01 \%$ & $-16.47 \%$ & $-2.70 \%$ & $-14.79 \%$ & $-29.27 \%$ & $-3.37 \%$ & $-16.27 \%$ & $-33.04 \%$ \\
\hline E85 Split HEV PHEV20 & Vehicle Mass & $0.00 \%$ & $0.00 \%$ & $-4.94 \%$ & $-9.69 \%$ & $-1.55 \%$ & $-8.09 \%$ & $-16.82 \%$ & $-2.70 \%$ & $-15.03 \%$ & $-29.91 \%$ & $-3.54 \%$ & $-16.92 \%$ & $-33.65 \%$ \\
\hline E85 Series HEV PHEV30 & Vehicle Mass & $0.00 \%$ & $0.00 \%$ & $-6.00 \%$ & $-11.51 \%$ & $-2.60 \%$ & $-9.61 \%$ & $-19.62 \%$ & $-4.41 \%$ & $-17.46 \%$ & $-32.85 \%$ & $-5.73 \%$ & $-19.93 \%$ & $-36.42 \%$ \\
\hline E85 Series HEV PHEV40 & Vehicle Mass & $0.00 \%$ & $0.00 \%$ & $-6.17 \%$ & $-11.70 \%$ & $-2.74 \%$ & $-9.77 \%$ & $-20.22 \%$ & $-4.58 \%$ & $-18.04 \%$ & $-33.50 \%$ & $-6.08 \%$ & $-20.69 \%$ & $-37.15 \%$ \\
\hline FC HEV & Vehicle Mass & $0.00 \%$ & $0.00 \%$ & $-16.89 \%$ & $-25.69 \%$ & $-12.11 \%$ & $-25.05 \%$ & $-34.57 \%$ & $-18.16 \%$ & $-32.82 \%$ & $-46.95 \%$ & $-23.10 \%$ & $-35.64 \%$ & $-50.22 \%$ \\
\hline FC HEV PHEV 10 & Vehicle Mass & $0.00 \%$ & $0.00 \%$ & $-14.90 \%$ & $-23.02 \%$ & $-9.79 \%$ & $-22.48 \%$ & $-32.23 \%$ & $-15.28 \%$ & $-30.43 \%$ & $-45.08 \%$ & $-20.18 \%$ & $-33.19 \%$ & $-48.30 \%$ \\
\hline FC HEV PHEV20 & Vehicle Mass & $0.00 \%$ & $0.00 \%$ & $-15.00 \%$ & $-23.27 \%$ & $-9.90 \%$ & $-22.74 \%$ & $-32.80 \%$ & $-15.48 \%$ & $-30.81 \%$ & $-45.60 \%$ & $-20.54 \%$ & $-33.78 \%$ & $-48.90 \%$ \\
\hline FC HEV PHEV 30 & Vehicle Mass & $0.00 \%$ & $0.00 \%$ & $-13.82 \%$ & $-21.56 \%$ & $-8.69 \%$ & $-20.73 \%$ & $-31.11 \%$ & $-13.38 \%$ & $-29.06 \%$ & $-43.98 \%$ & $-18.56 \%$ & $-32.37 \%$ & $-47.26 \%$ \\
\hline FC HEV PHEV 40 & Vehicle Mass & $0.00 \%$ & $0.00 \%$ & $-14.10 \%$ & $-21.92 \%$ & $-8.97 \%$ & $-21.19 \%$ & $-31.80 \%$ & $-13.75 \%$ & $-29.77 \%$ & $-44.52 \%$ & $-18.97 \%$ & $-33.14 \%$ & $-47.89 \%$ \\
\hline $\mathrm{EV}$ & Vehicle Mass & $0.00 \%$ & $0.00 \%$ & $-6.94 \%$ & $-13.04 \%$ & $-2.48 \%$ & $-10.90 \%$ & $-24.97 \%$ & $-4.84 \%$ & $-22.84 \%$ & $-39.80 \%$ & $-7.21 \%$ & $-27.03 \%$ & $-43.77 \%$ \\
\hline
\end{tabular}




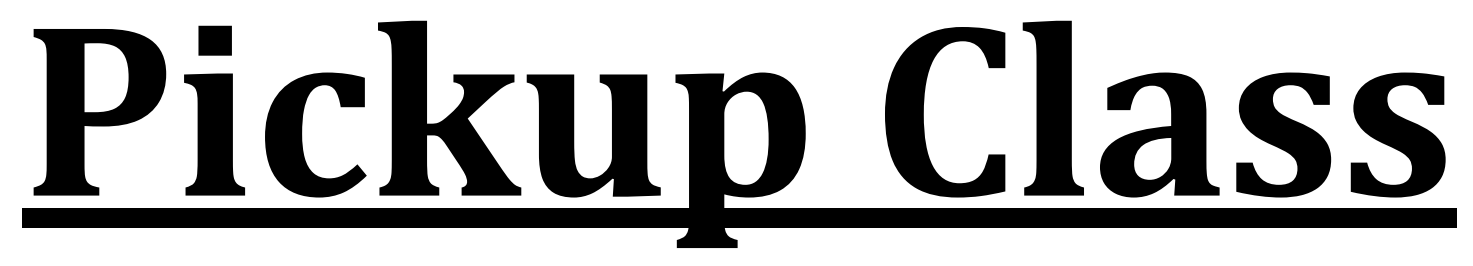




\section{Power}

\begin{tabular}{|c|c|c|c|c|c|c|c|c|c|c|c|c|c|c|}
\hline & & \multicolumn{13}{|c|}{ Pickup } \\
\hline & & 2010 & \multicolumn{3}{|c|}{2010} & \multicolumn{3}{|c|}{2015} & \multicolumn{3}{|c|}{2030} & \multicolumn{3}{|c|}{2045} \\
\hline & & Ref & low & avg & high & low & avg & high & low & avg & high & low & avg & high \\
\hline \multirow{5}{*}{ SI Conv } & ICE Power & 209224 & 209224 & 200211 & 192973 & 204889 & 190482 & 177831 & 201561 & 182107 & 158266 & 205614 & 180533 & 150191 \\
\hline & Electric Machine & & & & & & & & & & & & & \\
\hline & $\mathrm{MC} 2 / \mathrm{GC}$ & & & & & & & & & & & & & \\
\hline & Battery & & & & & & & & & & & & & \\
\hline & Specific Power & 96 & 96 & 95 & 95 & 94 & 93 & 93 & 93 & 95 & 96 & 95 & 95 & 96 \\
\hline \multirow{5}{*}{ Cl Conv } & ICE Power & 157606 & 157606 & 148992 & 145026 & 154011 & 144765 & 135143 & 150189 & 137541 & 121000 & 152875 & 136341 & 116111 \\
\hline & Electric Machine & & & & & & & & & & & & & \\
\hline & $\mathrm{MC} 2 / \mathrm{GC}$ & & & & & & & & & & & & & \\
\hline & Battery & & & & & & & & & & & & & \\
\hline & Specific Power & 71 & 71 & 70 & 70 & 70 & 69 & 69 & 68 & 70 & 71 & 69 & 70 & 72 \\
\hline \multirow{5}{*}{ H2 Conv } & ICE Power & 191010 & 191010 & 177101 & 169870 & 185928 & 168035 & 156396 & 180846 & 161194 & 136168 & 180048 & 158395 & 129727 \\
\hline & Electric Machine & & & & & & & & & & & & & \\
\hline & $\mathrm{MC} 2 / \mathrm{GC}$ & & & & & & & & & & & & & \\
\hline & Battery & & & & & & & & & & & & & \\
\hline & Specific Power & 88 & 88 & 87 & 87 & 87 & 85 & 86 & 85 & 87 & 88 & 86 & 87 & 87 \\
\hline \multirow{5}{*}{ E85 Conv } & ICE Power & 201330 & 201330 & 190432 & 183032 & 196480 & 181867 & 170856 & 192577 & 174568 & 149464 & 196254 & 173236 & 144500 \\
\hline & Electric Machine & & & & & & & & & & & & & \\
\hline & $\mathrm{MC} 2 / \mathrm{GC}$ & & & & & & & & & & & & & \\
\hline & Battery & & & & & & & & & & & & & \\
\hline & Specific Power & 93 & 93 & 91 & 91 & 90 & 89 & 90 & 89 & 91 & 91 & 91 & 91 & 92 \\
\hline \multirow{5}{*}{ SI Split HEV } & ICE Power & 152084 & 152084 & 143808 & 136377 & 149923 & 138707 & 123260 & 145833 & 127497 & 105588 & 144462 & 122573 & 100410 \\
\hline & Electric Machine & 101251 & 101251 & 96548 & 92496 & 99963 & 94584 & 86381 & 99753 & 88706 & 74372 & 99130 & 86000 & 70377 \\
\hline & $\mathrm{MC} 2 / \mathrm{GC}$ & 88012 & 88012 & 83222 & 78922 & 86761 & 80270 & 71331 & 84394 & 73783 & 61104 & 83601 & 70933 & 58108 \\
\hline & Battery & 39501 & 39501 & 37307 & 35511 & 39102 & 36422 & 33577 & 38699 & 34146 & 27886 & 38699 & 33577 & 26179 \\
\hline & Specific Power & 81 & 81 & 80 & 80 & 81 & 79 & 78 & 79 & 79 & 78 & 79 & 78 & 78 \\
\hline \multirow{5}{*}{ SI Split HEV PHEV10 } & ICE Power & 98963 & 98963 & 94677 & 89992 & 96915 & 91151 & 83696 & 95820 & 85496 & 75067 & 94851 & 84131 & 71911 \\
\hline & Electric Machine & 114142 & 114142 & 109059 & 103444 & 111939 & 104876 & 94535 & 110669 & 96149 & 80359 & 109843 & 94451 & 76431 \\
\hline & $\mathrm{MC} 2 / \mathrm{GC}$ & 74400 & 74400 & 71177 & 67632 & 72860 & 68525 & 62941 & 72037 & 64294 & 56430 & 71265 & 63225 & 54057 \\
\hline & Battery & 112418 & 112418 & 106850 & 100431 & 110239 & 101967 & 86860 & 107641 & 88322 & 82024 & 106865 & 98785 & 78028 \\
\hline & Specific Power & 88 & 88 & 88 & 87 & 88 & 87 & 85 & 87 & 85 & 91 & 87 & 90 & 92 \\
\hline
\end{tabular}




\begin{tabular}{|c|c|c|c|c|c|c|c|c|c|c|c|c|c|c|}
\hline & & \multicolumn{13}{|c|}{ Pickup } \\
\hline & & \multirow{2}{*}{\begin{tabular}{|c|}
2010 \\
Ref \\
\end{tabular}} & \multicolumn{3}{|c|}{2010} & \multicolumn{3}{|c|}{2015} & \multicolumn{3}{|c|}{2030} & \multicolumn{3}{|c|}{2045} \\
\hline & & & low & avg & high & low & avg & high & low & avg & high & low & avg & high \\
\hline \multirow{5}{*}{ SI Split HEV PHEV2O } & ICE Power & 100438 & 100438 & 96090 & 91257 & 98349 & 92381 & 84802 & 97222 & 86602 & 75746 & 96078 & 84980 & 72532 \\
\hline & Electric Machine & 117186 & 117186 & 111924 & 106537 & 115355 & 108073 & 96163 & 114019 & 98801 & 81651 & 112010 & 95941 & 77588 \\
\hline & $\mathrm{MC} 2 / \mathrm{GC}$ & 75487 & 75487 & 72218 & 68562 & 73939 & 69450 & 63730 & 73027 & 65062 & 56898 & 72230 & 63821 & 54524 \\
\hline & Battery & 112330 & 112330 & 106615 & 100830 & 110712 & 102376 & 92199 & 108037 & 95021 & 78970 & 105975 & 94456 & 74930 \\
\hline & Specific Power & 87 & 87 & 87 & 86 & 87 & 86 & 86 & 86 & 87 & 89 & 85 & 88 & 89 \\
\hline \multirow{5}{*}{ SI Series HEV PHEV 30} & ICE Power & 122583 & 122583 & 115052 & 107353 & 119170 & 109132 & 95570 & 114516 & 97996 & 82830 & 112735 & 95378 & 79162 \\
\hline & Electric Machine & 195797 & 195797 & 186307 & 176389 & 190995 & 179400 & 161888 & 187765 & 165690 & 143788 & 185092 & 161525 & 137806 \\
\hline & $\mathrm{MC} 2 / \mathrm{GC}$ & 118906 & 118906 & 111600 & 104132 & 115595 & 105858 & 92703 & 111081 & 95056 & 80345 & 109353 & 92517 & 76787 \\
\hline & Battery & 237429 & 237429 & 224734 & 211640 & 231610 & 215282 & 191910 & 225354 & 196457 & 168262 & 222166 & 191584 & 161306 \\
\hline & Specific Power & 71 & 71 & 72 & 72 & 71 & 72 & 73 & 72 & 73 & 77 & 71 & 73 & 77 \\
\hline \multirow{5}{*}{ SI Series HEV PHEV40 } & ICE Power & 127293 & 127293 & 119273 & 111095 & 123535 & 112974 & 98385 & 118598 & 100868 & 84928 & 116596 & 97866 & 81112 \\
\hline & Electric Machine & 203148 & 203148 & 192979 & 182429 & 197929 & 185659 & 166539 & 194309 & 170436 & 147288 & 191244 & 165643 & 141113 \\
\hline & $\mathrm{MC} 2 / \mathrm{GC}$ & 123474 & 123474 & 115695 & 107762 & 119829 & 109585 & 95433 & 115040 & 97842 & 82380 & 113098 & 94930 & 78679 \\
\hline & Battery & 246223 & 246223 & 232668 & 218796 & 239913 & 222696 & 197256 & 233103 & 201909 & 172207 & 229443 & 196291 & 165019 \\
\hline & Specific Power & 72 & 72 & 72 & 73 & 72 & 72 & 73 & 72 & 73 & 77 & 72 & 73 & 78 \\
\hline \multirow{5}{*}{ CI Split HEV } & ICE Power & 130224 & 130224 & 123091 & 115206 & 126744 & 118370 & 106058 & 124281 & 106865 & 90409 & 123172 & 105137 & 85943 \\
\hline & Electric Machine & 107291 & 107291 & 102554 & 97310 & 105037 & 100028 & 91685 & 105299 & 92637 & 79472 & 104164 & 91130 & 75315 \\
\hline & $\mathrm{MC} 2 / \mathrm{GC}$ & 108983 & 108983 & 103014 & 96415 & 106071 & 99063 & 88759 & 104010 & 89434 & 75663 & 103082 & 87988 & 71925 \\
\hline & Battery & 41097 & 41097 & 39102 & 37107 & 40698 & 38130 & 34715 & 40406 & 35284 & 29593 & 39837 & 34715 & 27886 \\
\hline & Specific Power & 69 & 69 & 69 & 68 & 69 & 68 & 67 & 68 & 67 & 67 & 68 & 67 & 67 \\
\hline \multirow{5}{*}{ CI Split HEV PHEV10 } & ICE Power & 101797 & 101797 & 97475 & 92753 & 99698 & 93925 & 86534 & 98509 & 88277 & 77840 & 97532 & 86876 & 74649 \\
\hline & Electric Machine & 113511 & 113511 & 109146 & 104138 & 111801 & 105100 & 94887 & 110619 & 96443 & 81158 & 109774 & 94887 & 77294 \\
\hline & $\mathrm{MC} 2 / \mathrm{GC}$ & 85193 & 85193 & 81576 & 77624 & 83436 & 78605 & 72420 & 82441 & 73878 & 65144 & 81624 & 72706 & 62473 \\
\hline & Battery & 122226 & 122226 & 117090 & 111162 & 120545 & 112099 & 99071 & 117994 & 101167 & 92098 & 117152 & 110247 & 88193 \\
\hline & Specific Power & 90 & 90 & 90 & 90 & 89 & 89 & 88 & 89 & 88 & 94 & 89 & 93 & 94 \\
\hline \multirow{5}{*}{ CI Split HEV PHEV2O } & ICE Power & 103243 & 103243 & 98830 & 94019 & 101103 & 95241 & 87555 & 99940 & 89327 & 78491 & 98845 & 87640 & 75270 \\
\hline & Electric Machine & 115911 & 115911 & 110818 & 105560 & 114064 & 107050 & 96452 & 112700 & 98048 & 82574 & 111767 & 96279 & 78622 \\
\hline & MC2/GC & 86403 & 86403 & 82710 & 78684 & 84612 & 79706 & 73274 & 83639 & 74757 & 65689 & 82723 & 73345 & 62993 \\
\hline & Battery & 122378 & 122378 & 116352 & 110203 & 120502 & 111909 & 102030 & 117762 & 103750 & 89373 & 116817 & 104818 & 85211 \\
\hline & Specific Power & 88 & 88 & 88 & 88 & 88 & 88 & 88 & 88 & 88 & 91 & 88 & 90 & 92 \\
\hline
\end{tabular}


Argonne National Laboratory Report - Light-Duty Vehicle Fuel Consumption Displacement Potential Up to 2045

\begin{tabular}{|c|c|c|c|c|c|c|c|c|c|c|c|c|c|c|}
\hline & & \multicolumn{13}{|c|}{ Pickup } \\
\hline & & \multirow{2}{*}{$\begin{array}{c}2010 \\
\text { Ref } \\
\end{array}$} & \multicolumn{3}{|c|}{2010} & \multicolumn{3}{|c|}{2015} & \multicolumn{3}{|c|}{2030} & \multicolumn{3}{|c|}{2045} \\
\hline & & & low & avg & high & low & avg & high & low & avg & high & low & avg & high \\
\hline \multirow{5}{*}{ CI Series HEV PHEV3O } & ICE Power & 125496 & 125496 & 117927 & 110190 & 121998 & 111953 & 98385 & 117307 & 100723 & 85494 & 115429 & 98040 & 81904 \\
\hline & Electric Machine & 200297 & 200297 & 190852 & 180884 & 195521 & 183927 & 166445 & 192241 & 170196 & 148246 & 189418 & 165930 & 142406 \\
\hline & $\mathrm{MC} 2 / \mathrm{GC}$ & 121731 & 121731 & 114389 & 106884 & 118338 & 108594 & 95433 & 113788 & 97701 & 82929 & 111966 & 95099 & 79447 \\
\hline & Battery & 242857 & 242857 & 230194 & 217013 & 237074 & 220690 & 197295 & 230703 & 201775 & 173460 & 227329 & 196784 & 166662 \\
\hline & Specific Power & 71 & 71 & 71 & 71 & 71 & 71 & 72 & 71 & 72 & 75 & 71 & 72 & 76 \\
\hline \multirow{5}{*}{ CI Series HEV PHEV40 } & ICE Power & 130268 & 130268 & 122239 & 114083 & 126485 & 115795 & 101141 & 121539 & 103625 & 87592 & 119379 & 100528 & 83882 \\
\hline & Electric Machine & 207691 & 207691 & 197617 & 187115 & 202455 & 190137 & 171094 & 198974 & 174988 & 151791 & 195806 & 170140 & 145805 \\
\hline & $\mathrm{MC} 2 / \mathrm{GC}$ & 126360 & 126360 & 118572 & 110661 & 122690 & 112321 & 98107 & 117893 & 100516 & 84964 & 115798 & 97512 & 81366 \\
\hline & Battery & 251705 & 251705 & 238243 & 224391 & 245371 & 228039 & 202628 & 238673 & 207281 & 177447 & 234889 & 201598 & 170484 \\
\hline & Specific Power & 71 & 71 & 72 & 72 & 71 & 72 & 72 & 71 & 72 & 76 & 71 & 72 & 76 \\
\hline \multirow{5}{*}{ H2 Split HEV } & ICE Power & 152647 & 152647 & 142587 & 133126 & 149386 & 135434 & 120354 & 145452 & 123161 & 101435 & 142350 & 120719 & 96803 \\
\hline & Electric Machine & 102337 & 102337 & 96315 & 90974 & 100354 & 92981 & 84639 & 100015 & 86406 & 71984 & 97579 & 84751 & 68748 \\
\hline & $\mathrm{MC} 2 / \mathrm{GC}$ & 96217 & 96217 & 89876 & 83913 & 94162 & 85367 & 75862 & 91682 & 77631 & 63937 & 89727 & 76092 & 61017 \\
\hline & Battery & 39900 & 39900 & 37107 & 34913 & 39302 & 35853 & 32439 & 38699 & 33008 & 26748 & 37561 & 32439 & 25610 \\
\hline & Specific Power & 80 & 80 & 80 & 79 & 80 & 79 & 78 & 79 & 78 & 78 & 79 & 78 & 78 \\
\hline \multirow{5}{*}{ H2 Split HEV PHEV10 } & ICE Power & 99859 & 99859 & 94186 & 88956 & 97374 & 90178 & 82277 & 95935 & 84360 & 73284 & 93710 & 82716 & 70387 \\
\hline & Electric Machine & 118214 & 118214 & 110307 & 104147 & 115823 & 105355 & 94056 & 114038 & 96257 & 79222 & 110229 & 94201 & 75584 \\
\hline & $\mathrm{MC} 2 / \mathrm{GC}$ & 82541 & 82541 & 77850 & 73503 & 80463 & 74560 & 68028 & 79296 & 69750 & 60545 & 77458 & 68391 & 58151 \\
\hline & Battery & 116044 & 116044 & 107089 & 100285 & 114079 & 101496 & 84596 & 110618 & 86313 & 77220 & 106471 & 96361 & 73485 \\
\hline & Specific Power & 89 & 89 & 89 & 88 & 89 & 88 & 85 & 88 & 85 & 91 & 88 & 91 & 91 \\
\hline \multirow{5}{*}{ H2 Split HEV PHEV2O } & ICE Power & 101392 & 101392 & 95629 & 90193 & 98780 & 91494 & 83327 & 97337 & 85496 & 73935 & 95051 & 83565 & 71008 \\
\hline & Electric Machine & 120656 & 120656 & 113236 & 105689 & 117929 & 108590 & 95631 & 116141 & 98822 & 80727 & 113470 & 96465 & 76902 \\
\hline & $\mathrm{MC} 2 / \mathrm{GC}$ & 83760 & 83760 & 78971 & 74478 & 81578 & 75601 & 68826 & 80384 & 70595 & 61083 & 78495 & 68998 & 58641 \\
\hline & Battery & 116728 & 116728 & 108595 & 100163 & 114170 & 103401 & 90109 & 110715 & 93314 & 76113 & 108288 & 93463 & 72484 \\
\hline & Specific Power & 88 & 88 & 88 & 87 & 88 & 88 & 87 & 87 & 87 & 89 & 87 & 89 & 90 \\
\hline \multirow{5}{*}{ H2 Series HEV PHEV30 } & ICE Power & 123110 & 123110 & 114196 & 105904 & 119201 & 107662 & 93800 & 114276 & 96545 & 80789 & 111269 & 93642 & 77466 \\
\hline & Electric Machine & 196668 & 196668 & 184954 & 174116 & 191090 & 177133 & 158964 & 187380 & 163292 & 140336 & 182641 & 158699 & 134883 \\
\hline & $\mathrm{MC} 2 / \mathrm{GC}$ & 119417 & 119417 & 110770 & 102727 & 115625 & 104432 & 90986 & 110848 & 93649 & 78365 & 107931 & 90833 & 75142 \\
\hline & Battery & 238472 & 238472 & 223110 & 208932 & 231728 & 212577 & 188457 & 224898 & 193631 & 164245 & 219236 & 188251 & 157897 \\
\hline & Specific Power & 71 & 71 & 72 & 73 & 71 & 72 & 73 & 72 & 73 & 78 & 72 & 73 & 78 \\
\hline
\end{tabular}




\begin{tabular}{|c|c|c|c|c|c|c|c|c|c|c|c|c|c|c|}
\hline & & \multicolumn{13}{|c|}{ Pickup } \\
\hline & & \multirow{2}{*}{\begin{tabular}{|c|}
2010 \\
Ref \\
\end{tabular}} & \multicolumn{3}{|c|}{2010} & \multicolumn{3}{|c|}{2015} & \multicolumn{3}{|c|}{2030} & \multicolumn{3}{|c|}{2045} \\
\hline & & & low & avg & high & low & avg & high & low & avg & high & low & avg & high \\
\hline \multirow{5}{*}{ H2 Series HEV PHEV40 } & ICE Power & 127913 & 127913 & 118355 & 109767 & 123565 & 111653 & 96586 & 118388 & 99417 & 82858 & 114950 & 96159 & 79388 \\
\hline & Electric Machine & 204115 & 204115 & 191528 & 180160 & 197977 & 183446 & 163567 & 193924 & 167944 & 143837 & 188744 & 162864 & 138190 \\
\hline & $\mathrm{MC} 2 / \mathrm{GC}$ & 124076 & 124076 & 114804 & 106474 & 119858 & 108303 & 93688 & 114836 & 96434 & 80372 & 111502 & 93274 & 77006 \\
\hline & Battery & 247390 & 247390 & 230935 & 216081 & 239971 & 220047 & 193756 & 232641 & 198970 & 168189 & 226464 & 193016 & 161623 \\
\hline & Specific Power & 72 & 72 & 73 & 73 & 72 & 73 & 74 & 72 & 74 & 78 & 72 & 74 & 79 \\
\hline \multirow{5}{*}{ E85 Split HEV } & ICE Power & 153966 & 153966 & 145458 & 137592 & 150062 & 138841 & 123389 & 147073 & 125835 & 105709 & 145692 & 123666 & 101361 \\
\hline & Electric Machine & 102547 & 102547 & 97925 & 93283 & 100424 & 95354 & 86606 & 100494 & 88212 & 74501 & 99828 & 86677 & 71408 \\
\hline & $\mathrm{MC2} / \mathrm{GC}$ & 89101 & 89101 & 84177 & 79625 & 86841 & 80348 & 71406 & 85112 & 72821 & 61174 & 84313 & 71566 & 58658 \\
\hline & Battery & 39501 & 39501 & 37506 & 35511 & 39102 & 36992 & 33577 & 38699 & 34146 & 27886 & 38699 & 33577 & 26748 \\
\hline & Specific Power & 81 & 81 & 81 & 80 & 81 & 80 & 78 & 80 & 78 & 78 & 80 & 78 & 79 \\
\hline \multirow{5}{*}{ E85 Split HEV PHEV10 } & ICE Power & 99107 & 99107 & 94763 & 90078 & 97059 & 91294 & 83781 & 95906 & 85581 & 75152 & 94965 & 84215 & 71996 \\
\hline & Electric Machine & 115059 & 115059 & 108758 & 103614 & 113330 & 106188 & 94700 & 110839 & 96315 & 80563 & 110058 & 94661 & 76591 \\
\hline & $\mathrm{MC} 2 / \mathrm{GC}$ & 74530 & 74530 & 71241 & 67696 & 72968 & 68633 & 63005 & 72123 & 64358 & 56494 & 71351 & 63310 & 54142 \\
\hline & Battery & 113375 & 113375 & 106259 & 100619 & 111910 & 103573 & 86874 & 107842 & 88473 & 82339 & 107102 & 98926 & 78235 \\
\hline & Specific Power & 88 & 88 & 88 & 87 & 88 & 88 & 85 & 87 & 85 & 92 & 87 & 90 & 92 \\
\hline \multirow{5}{*}{ E85 Split HEV PHEV20 } & ICE Power & 100525 & 100525 & 96177 & 91344 & 98435 & 92552 & 84888 & 97308 & 86688 & 75831 & 96249 & 85064 & 72617 \\
\hline & Electric Machine & 117454 & 117454 & 112097 & 106709 & 115529 & 108245 & 96329 & 114240 & 98969 & 81810 & 113402 & 96106 & 77746 \\
\hline & MC2/GC & 75596 & 75596 & 72283 & 68627 & 74004 & 69558 & 63794 & 73134 & 65148 & 56983 & 72338 & 63884 & 54588 \\
\hline & Battery & 112595 & 112595 & 106773 & 100992 & 110880 & 102526 & 92369 & 108254 & 95190 & 79166 & 107526 & 94665 & 75122 \\
\hline & Specific Power & 87 & 87 & 87 & 86 & 87 & 86 & 86 & 86 & 87 & 89 & 86 & 88 & 89 \\
\hline \multirow{5}{*}{ E85 Series HEV PHEV30 } & ICE Power & 122676 & 122676 & 115144 & 107534 & 119263 & 109222 & 95657 & 114636 & 98112 & 82915 & 112825 & 95465 & 79247 \\
\hline & Electric Machine & 195943 & 195943 & 186452 & 176583 & 191187 & 179593 & 162032 & 188005 & 165834 & 143932 & 185237 & 161668 & 137998 \\
\hline & $\mathrm{MC} 2 / \mathrm{GC}$ & 118996 & 118996 & 111690 & 104308 & 115685 & 105945 & 92787 & 111197 & 95169 & 80428 & 109440 & 92601 & 76870 \\
\hline & Battery & 237601 & 237601 & 224904 & 211879 & 231844 & 215511 & 192080 & 225645 & 196624 & 168429 & 222336 & 191753 & 161528 \\
\hline & Specific Power & 71 & 71 & 72 & 72 & 71 & 72 & 73 & 71 & 73 & 77 & 71 & 73 & 77 \\
\hline \multirow{5}{*}{ E85 Series HEV PHEV40 } & ICE Power & 127386 & 127386 & 119487 & 111336 & 123627 & 113124 & 98472 & 118748 & 101042 & 85013 & 116686 & 97982 & 81197 \\
\hline & Electric Machine & 203293 & 203293 & 193269 & 182624 & 198074 & 185852 & 166683 & 194549 & 170676 & 147431 & 191435 & 165834 & 141256 \\
\hline & MC2/GC & 123564 & 123564 & 115902 & 107996 & 119918 & 109730 & 95518 & 115186 & 98011 & 82463 & 113185 & 95043 & 78761 \\
\hline & Battery & 246393 & 246393 & 233023 & 219025 & 240086 & 222925 & 197426 & 233384 & 202195 & 172374 & 229676 & 196517 & 165186 \\
\hline & Specific Power & 72 & 72 & 72 & 73 & 72 & 72 & 73 & 72 & 73 & 77 & 72 & 73 & 78 \\
\hline
\end{tabular}


Argonne National Laboratory Report - Light-Duty Vehicle Fuel Consumption Displacement Potential Up to 2045

\begin{tabular}{|c|c|c|c|c|c|c|c|c|c|c|c|c|c|c|}
\hline & & \multicolumn{13}{|c|}{ Pickup } \\
\hline & & \multirow{2}{*}{$\begin{array}{c}2010 \\
\text { Ref } \\
\end{array}$} & \multicolumn{3}{|c|}{2010} & \multicolumn{3}{|c|}{2015} & \multicolumn{3}{|c|}{2030} & \multicolumn{3}{|c|}{2045} \\
\hline & & & low & avg & high & low & avg & high & low & avg & high & low & avg & high \\
\hline \multirow{5}{*}{ FC HEV } & FC Power & 174206 & 174206 & 146897 & 126630 & 155644 & 124430 & 105596 & 135664 & 108754 & 86392 & 127799 & 105363 & 82056 \\
\hline & Electric Machine & 196411 & 196411 & 164087 & 145284 & 172000 & 150359 & 129629 & 159824 & 132977 & 108607 & 151709 & 128855 & 103001 \\
\hline & $\mathrm{MC} 2 / \mathrm{GC}$ & & & & & & & & & & & & & \\
\hline & Battery & 49077 & 49077 & 41297 & 37905 & 43092 & 43252 & 38699 & 46097 & 39268 & 33008 & 43821 & 38130 & 31301 \\
\hline & Specific Power & 64 & 64 & 64 & 63 & 63 & 64 & 64 & 63 & 64 & 65 & 64 & 64 & 65 \\
\hline \multirow{5}{*}{ FC HEV PHEV10 } & FC Power & 127808 & 127808 & 107943 & 96009 & 114747 & 97217 & 84055 & 106993 & 86629 & 69550 & 100403 & 85628 & 66176 \\
\hline & Electric Machine & 185982 & 185982 & 162597 & 147471 & 170592 & 149203 & 131494 & 161936 & 134818 & 110159 & 154100 & 132292 & 104503 \\
\hline & $\mathrm{MC} 2 / \mathrm{GC}$ & & & & & & & & & & & & & \\
\hline & Battery & 94917 & 94917 & 81650 & 73138 & 86062 & 74029 & 64062 & 80217 & 65693 & 52369 & 76089 & 63560 & 49585 \\
\hline & Specific Power & 63 & 63 & 65 & 65 & 64 & 65 & 65 & 65 & 65 & 67 & 65 & 66 & 67 \\
\hline \multirow{5}{*}{ FC HEV PHEV2O } & FCPower & 130827 & 130827 & 110482 & 98185 & 117459 & 99439 & 85818 & 109439 & 88478 & 70327 & 102665 & 86382 & 66867 \\
\hline & Electric Machine & 190268 & 190268 & 166260 & 150626 & 174474 & 152513 & 133956 & 165461 & 137408 & 111693 & 157387 & 133794 & 105893 \\
\hline & $\mathrm{MC} 2 / \mathrm{GC}$ & & & & & & & & & & & & & \\
\hline & Battery & 97376 & 97376 & 83656 & 74854 & 88132 & 75717 & 65254 & 81993 & 66882 & 53328 & 77718 & 64527 & 50405 \\
\hline & Specific Power & 63 & 63 & 65 & 65 & 64 & 65 & 65 & 65 & 65 & 67 & 65 & 66 & 67 \\
\hline \multirow{5}{*}{ FC HEV PHEV 30} & FC Power & 105904 & 105904 & 94669 & 87196 & 98226 & 87790 & 78801 & 94331 & 80955 & 69025 & 89980 & 78219 & 65910 \\
\hline & Electric Machine & 198508 & 198508 & 182826 & 171120 & 188344 & 172799 & 155892 & 182085 & 159266 & 138175 & 176822 & 154198 & 131955 \\
\hline & $\mathrm{MC} 2 / \mathrm{GC}$ & & & & & & & & & & & & & \\
\hline & Battery & 240604 & 240604 & 220466 & 205277 & 228320 & 207315 & 184892 & 218487 & 188932 & 161725 & 212202 & 182989 & 154488 \\
\hline & Specific Power & 64 & 64 & 68 & 70 & 67 & 70 & 72 & 68 & 72 & 78 & 70 & 73 & 79 \\
\hline \multirow{5}{*}{ FC HEV PHEV40 } & FC Power & 108251 & 108251 & 96546 & 88760 & 100247 & 89422 & 79946 & 96015 & 82099 & 69836 & 91608 & 79157 & 66669 \\
\hline & Electric Machine & 204889 & 204889 & 188435 & 176196 & 194175 & 177858 & 160019 & 187381 & 163533 & 141536 & 181775 & 157885 & 135074 \\
\hline & $\mathrm{MC} 2 / \mathrm{GC}$ & & & & & & & & & & & & & \\
\hline & Battery & 248228 & 248228 & 227132 & 211271 & 235289 & 213291 & 189602 & 224738 & 193806 & 165510 & 218050 & 187173 & 157993 \\
\hline & Specific Power & 64 & 64 & 68 & 70 & 67 & 70 & 73 & 68 & 72 & 78 & 70 & 73 & 79 \\
\hline \multirow{5}{*}{ BEV } & ICE Power & & & & & & & & & & & & & \\
\hline & Electric Machine & 252830 & 252830 & 238393 & 223095 & 245706 & 227574 & 194034 & 239034 & 198489 & 166874 & 232485 & 188747 & 158480 \\
\hline & $\mathrm{MC} 2 / \mathrm{GC}$ & 0 & 0 & 0 & 0 & 0 & 0 & 0 & 0 & 0 & 0 & 0 & 0 & 0 \\
\hline & Battery & 305838 & 305838 & 286879 & 267061 & 297236 & 272427 & 229959 & 286567 & 235281 & 195890 & 278376 & 223770 & 186105 \\
\hline & Specific Power & 95 & 95 & 95 & 95 & 95 & 95 & 94 & 94 & 94 & 99 & 94 & 94 & 100 \\
\hline
\end{tabular}




\section{Percentage of power reduction based on the respective 2010 reference case}

\begin{tabular}{|c|c|c|c|c|c|c|c|c|c|c|c|c|c|c|}
\hline & & \multicolumn{13}{|c|}{ Pickup } \\
\hline & & \multirow{2}{*}{$\begin{array}{c}2010 \\
\text { Ref }\end{array}$} & \multicolumn{3}{|c|}{2010} & \multicolumn{3}{|c|}{2015} & \multicolumn{3}{|c|}{2030} & \multicolumn{3}{|c|}{2045} \\
\hline & & & low & avg & high & low & avg & high & low & avg & high & low & avg & high \\
\hline \multirow{5}{*}{ SI Conv } & ICE Power & $0 \%$ & $0 \%$ & $-4 \%$ & $-8 \%$ & $-2 \%$ & $-9 \%$ & $-15 \%$ & $-4 \%$ & $-13 \%$ & $-24 \%$ & $-2 \%$ & $-14 \%$ & $-28 \%$ \\
\hline & Electric Machine & & & & & & & & & & & & & \\
\hline & $\mathrm{MC} 2 / \mathrm{GC}$ & & & & & & & & & & & & & \\
\hline & Battery & & & & & & & & & & & & & \\
\hline & Specific Power & $0 \%$ & $0 \%$ & $-1 \%$ & $-1 \%$ & $-2 \%$ & $-3 \%$ & $-3 \%$ & $-3 \%$ & $-2 \%$ & $0 \%$ & $-1 \%$ & $-1 \%$ & $0 \%$ \\
\hline \multirow{5}{*}{ Cl Conv } & ICE Power & $0 \%$ & $0 \%$ & $-5 \%$ & $-8 \%$ & $-2 \%$ & $-8 \%$ & $-14 \%$ & $-5 \%$ & $-13 \%$ & $-23 \%$ & $-3 \%$ & $-13 \%$ & $-26 \%$ \\
\hline & Electric Machine & & & & & & & & & & & & & \\
\hline & $\mathrm{MC} 2 / \mathrm{GC}$ & & & & & & & & & & & & & \\
\hline & Battery & & & & & & & & & & & & & \\
\hline & Specific Power & $0 \%$ & $0 \%$ & $-2 \%$ & $-1 \%$ & $-2 \%$ & $-3 \%$ & $-3 \%$ & $-4 \%$ & $-2 \%$ & $0 \%$ & $-3 \%$ & $-2 \%$ & $1 \%$ \\
\hline \multirow{5}{*}{$\mathrm{H} 2$ Conv } & ICE Power & $0 \%$ & $0 \%$ & $-7 \%$ & $-11 \%$ & $-3 \%$ & $-12 \%$ & $-18 \%$ & $-5 \%$ & $-16 \%$ & $-29 \%$ & $-6 \%$ & $-17 \%$ & $-32 \%$ \\
\hline & Electric Machine & & & & & & & & & & & & & \\
\hline & $\mathrm{MC} 2 / \mathrm{GC}$ & & & & & & & & & & & & & \\
\hline & Battery & & & & & & & & & & & & & \\
\hline & Specific Power & $0 \%$ & $0 \%$ & $-2 \%$ & $-2 \%$ & $-2 \%$ & $-4 \%$ & $-3 \%$ & $-4 \%$ & $-2 \%$ & $-1 \%$ & $-3 \%$ & $-2 \%$ & $-1 \%$ \\
\hline \multirow{5}{*}{ E85 Conv } & ICE Power & $0 \%$ & $0 \%$ & $-5 \%$ & $-9 \%$ & $-2 \%$ & $-10 \%$ & $-15 \%$ & $-4 \%$ & $-13 \%$ & $-26 \%$ & $-3 \%$ & $-14 \%$ & $-28 \%$ \\
\hline & Electric Machine & & & & & & & & & & & & & \\
\hline & $\mathrm{MC} 2 / \mathrm{GC}$ & & & & & & & & & & & & & \\
\hline & Battery & & & & & & & & & & & & & \\
\hline & Specific Power & $0 \%$ & $0 \%$ & $-2 \%$ & $-2 \%$ & $-2 \%$ & $-4 \%$ & $-3 \%$ & $-4 \%$ & $-2 \%$ & $-2 \%$ & $-2 \%$ & $-2 \%$ & $0 \%$ \\
\hline \multirow{5}{*}{ SI Split HEV } & ICE Power & $0 \%$ & $0 \%$ & $-5 \%$ & $-10 \%$ & $-1 \%$ & $-9 \%$ & $-19 \%$ & $-4 \%$ & $-16 \%$ & $-31 \%$ & $-5 \%$ & $-19 \%$ & $-34 \%$ \\
\hline & Electric Machine & $0 \%$ & $0 \%$ & $-5 \%$ & $-9 \%$ & $-1 \%$ & $-7 \%$ & $-15 \%$ & $-1 \%$ & $-12 \%$ & $-27 \%$ & $-2 \%$ & $-15 \%$ & $-30 \%$ \\
\hline & $\mathrm{MC} 2 / \mathrm{GC}$ & $0 \%$ & $0 \%$ & $-5 \%$ & $-10 \%$ & $-1 \%$ & $-9 \%$ & $-19 \%$ & $-4 \%$ & $-16 \%$ & $-31 \%$ & $-5 \%$ & $-19 \%$ & $-34 \%$ \\
\hline & Battery & $0 \%$ & $0 \%$ & $-6 \%$ & $-10 \%$ & $-1 \%$ & $-8 \%$ & $-15 \%$ & $-2 \%$ & $-14 \%$ & $-29 \%$ & $-2 \%$ & $-15 \%$ & $-34 \%$ \\
\hline & Specific Power & $0 \%$ & $0 \%$ & $-1 \%$ & $-1 \%$ & $0 \%$ & $-2 \%$ & $-3 \%$ & $-2 \%$ & $-2 \%$ & $-3 \%$ & $-2 \%$ & $-4 \%$ & $-3 \%$ \\
\hline \multirow{5}{*}{ SI Split HEV PHEV10 } & ICE Power & $0 \%$ & $0 \%$ & $-4 \%$ & $-9 \%$ & $-2 \%$ & $-8 \%$ & $-15 \%$ & $-3 \%$ & $-14 \%$ & $-24 \%$ & $-4 \%$ & $-15 \%$ & $-27 \%$ \\
\hline & Electric Machine & $0 \%$ & $0 \%$ & $-4 \%$ & $-9 \%$ & $-2 \%$ & $-8 \%$ & $-17 \%$ & $-3 \%$ & $-16 \%$ & $-30 \%$ & $-4 \%$ & $-17 \%$ & $-33 \%$ \\
\hline & $\mathrm{MC} 2 / \mathrm{GC}$ & $0 \%$ & $0 \%$ & $-4 \%$ & $-9 \%$ & $-2 \%$ & $-8 \%$ & $-15 \%$ & $-3 \%$ & $-14 \%$ & $-24 \%$ & $-4 \%$ & $-15 \%$ & $-27 \%$ \\
\hline & Battery & $0 \%$ & $0 \%$ & $-5 \%$ & $-11 \%$ & $-2 \%$ & $-9 \%$ & $-23 \%$ & $-4 \%$ & $-21 \%$ & $-27 \%$ & $-5 \%$ & $-12 \%$ & $-31 \%$ \\
\hline & Specific Power & $0 \%$ & $0 \%$ & $0 \%$ & $-1 \%$ & $0 \%$ & $-1 \%$ & $-4 \%$ & $-1 \%$ & $-4 \%$ & $4 \%$ & $-1 \%$ & $3 \%$ & $5 \%$ \\
\hline
\end{tabular}




\begin{tabular}{|c|c|c|c|c|c|c|c|c|c|c|c|c|c|c|}
\hline & & \multicolumn{13}{|c|}{ Pickup } \\
\hline & & \multirow{2}{*}{$\frac{2010}{\operatorname{Ref}}$} & \multicolumn{3}{|c|}{2010} & \multicolumn{3}{|c|}{2015} & \multicolumn{3}{|c|}{2030} & \multicolumn{3}{|c|}{2045} \\
\hline & & & low & avg & high & low & avg & high & low & avg & high & low & avg & high \\
\hline \multirow{5}{*}{ SI Split HEV PHEV2O } & ICE Power & $0 \%$ & $0 \%$ & $-4 \%$ & $-9 \%$ & $-2 \%$ & $-8 \%$ & $-16 \%$ & $-3 \%$ & $-14 \%$ & $-25 \%$ & $-4 \%$ & $-15 \%$ & $-28 \%$ \\
\hline & Electric Machine & $0 \%$ & $0 \%$ & $-4 \%$ & $-9 \%$ & $-2 \%$ & $-8 \%$ & $-18 \%$ & $-3 \%$ & $-16 \%$ & $-30 \%$ & $-4 \%$ & $-18 \%$ & $-34 \%$ \\
\hline & $\mathrm{MC} 2 / \mathrm{GC}$ & $0 \%$ & $0 \%$ & $-4 \%$ & $-9 \%$ & $-2 \%$ & $-8 \%$ & $-16 \%$ & $-3 \%$ & $-14 \%$ & $-25 \%$ & $-4 \%$ & $-15 \%$ & $-28 \%$ \\
\hline & Battery & $0 \%$ & $0 \%$ & $-5 \%$ & $-10 \%$ & $-1 \%$ & $-9 \%$ & $-18 \%$ & $-4 \%$ & $-15 \%$ & $-30 \%$ & $-6 \%$ & $-16 \%$ & $-33 \%$ \\
\hline & Specific Power & $0 \%$ & $0 \%$ & $0 \%$ & $0 \%$ & $0 \%$ & $-1 \%$ & $0 \%$ & $-1 \%$ & $0 \%$ & $3 \%$ & $-1 \%$ & $1 \%$ & $3 \%$ \\
\hline \multirow{5}{*}{ SI Series HEV PHEV30 } & ICE Power & $0 \%$ & $0 \%$ & $-6 \%$ & $-12 \%$ & $-3 \%$ & $-11 \%$ & $-22 \%$ & $-7 \%$ & $-20 \%$ & $-32 \%$ & $-8 \%$ & $-22 \%$ & $-35 \%$ \\
\hline & Electric Machine & $0 \%$ & $0 \%$ & $-5 \%$ & $-10 \%$ & $-2 \%$ & $-8 \%$ & $-17 \%$ & $-4 \%$ & $-15 \%$ & $-27 \%$ & $-5 \%$ & $-18 \%$ & $-30 \%$ \\
\hline & $\mathrm{MC} 2 / \mathrm{GC}$ & $0 \%$ & $0 \%$ & $-6 \%$ & $-12 \%$ & $-3 \%$ & $-11 \%$ & $-22 \%$ & $-7 \%$ & $-20 \%$ & $-32 \%$ & $-8 \%$ & $-22 \%$ & $-35 \%$ \\
\hline & Battery & $0 \%$ & $0 \%$ & $-5 \%$ & $-11 \%$ & $-2 \%$ & $-9 \%$ & $-19 \%$ & $-5 \%$ & $-17 \%$ & $-29 \%$ & $-6 \%$ & $-19 \%$ & $-32 \%$ \\
\hline & Specific Power & $0 \%$ & $0 \%$ & $1 \%$ & $1 \%$ & $0 \%$ & $1 \%$ & $2 \%$ & $1 \%$ & $2 \%$ & $8 \%$ & $0 \%$ & $2 \%$ & $9 \%$ \\
\hline \multirow{5}{*}{ SI Series HEV PHEV40 } & ICE Power & $0 \%$ & $0 \%$ & $-6 \%$ & $-13 \%$ & $-3 \%$ & $-11 \%$ & $-23 \%$ & $-7 \%$ & $-21 \%$ & $-33 \%$ & $-8 \%$ & $-23 \%$ & $-36 \%$ \\
\hline & Electric Machine & $0 \%$ & $0 \%$ & $-5 \%$ & $-10 \%$ & $-3 \%$ & $-9 \%$ & $-18 \%$ & $-4 \%$ & $-16 \%$ & $-27 \%$ & $-6 \%$ & $-18 \%$ & $-31 \%$ \\
\hline & $\mathrm{MC} 2 / \mathrm{GC}$ & $0 \%$ & $0 \%$ & $-6 \%$ & $-13 \%$ & $-3 \%$ & $-11 \%$ & $-23 \%$ & $-7 \%$ & $-21 \%$ & $-33 \%$ & $-8 \%$ & $-23 \%$ & $-36 \%$ \\
\hline & Battery & $0 \%$ & $0 \%$ & $-6 \%$ & $-11 \%$ & $-3 \%$ & $-10 \%$ & $-20 \%$ & $-5 \%$ & $-18 \%$ & $-30 \%$ & $-7 \%$ & $-20 \%$ & $-33 \%$ \\
\hline & Specific Power & $0 \%$ & $0 \%$ & $1 \%$ & $1 \%$ & $0 \%$ & $1 \%$ & $2 \%$ & $1 \%$ & $2 \%$ & $7 \%$ & $0 \%$ & $2 \%$ & $8 \%$ \\
\hline \multirow{5}{*}{ CI Split HEV } & ICE Power & $0 \%$ & $0 \%$ & $-5 \%$ & $-12 \%$ & $-3 \%$ & $-9 \%$ & $-19 \%$ & $-5 \%$ & $-18 \%$ & $-31 \%$ & $-5 \%$ & $-19 \%$ & $-34 \%$ \\
\hline & Electric Machine & $0 \%$ & $0 \%$ & $-4 \%$ & $-9 \%$ & $-2 \%$ & $-7 \%$ & $-15 \%$ & $-2 \%$ & $-14 \%$ & $-26 \%$ & $-3 \%$ & $-15 \%$ & $-30 \%$ \\
\hline & $\mathrm{MC} 2 / \mathrm{GC}$ & $0 \%$ & $0 \%$ & $-5 \%$ & $-12 \%$ & $-3 \%$ & $-9 \%$ & $-19 \%$ & $-5 \%$ & $-18 \%$ & $-31 \%$ & $-5 \%$ & $-19 \%$ & $-34 \%$ \\
\hline & Battery & $0 \%$ & $0 \%$ & $-5 \%$ & $-10 \%$ & $-1 \%$ & $-7 \%$ & $-16 \%$ & $-2 \%$ & $-14 \%$ & $-28 \%$ & $-3 \%$ & $-16 \%$ & $-32 \%$ \\
\hline & Specific Power & $0 \%$ & $0 \%$ & $-1 \%$ & $-2 \%$ & $-1 \%$ & $-2 \%$ & $-3 \%$ & $-2 \%$ & $-4 \%$ & $-4 \%$ & $-2 \%$ & $-4 \%$ & $-4 \%$ \\
\hline \multirow{5}{*}{ CI Split HEV PHEV10 } & ICE Power & $0 \%$ & $0 \%$ & $-4 \%$ & $-9 \%$ & $-2 \%$ & $-8 \%$ & $-15 \%$ & $-3 \%$ & $-13 \%$ & $-24 \%$ & $-4 \%$ & $-15 \%$ & $-27 \%$ \\
\hline & Electric Machine & $0 \%$ & $0 \%$ & $-4 \%$ & $-8 \%$ & $-2 \%$ & $-7 \%$ & $-16 \%$ & $-3 \%$ & $-15 \%$ & $-29 \%$ & $-3 \%$ & $-16 \%$ & $-32 \%$ \\
\hline & $\mathrm{MC} 2 / \mathrm{GC}$ & $0 \%$ & $0 \%$ & $-4 \%$ & $-9 \%$ & $-2 \%$ & $-8 \%$ & $-15 \%$ & $-3 \%$ & $-13 \%$ & $-24 \%$ & $-4 \%$ & $-15 \%$ & $-27 \%$ \\
\hline & Battery & $0 \%$ & $0 \%$ & $-4 \%$ & $-9 \%$ & $-1 \%$ & $-8 \%$ & $-19 \%$ & $-3 \%$ & $-17 \%$ & $-25 \%$ & $-4 \%$ & $-10 \%$ & $-28 \%$ \\
\hline & Specific Power & $0 \%$ & $0 \%$ & $0 \%$ & $0 \%$ & $0 \%$ & $-1 \%$ & $-2 \%$ & $-1 \%$ & $-2 \%$ & $5 \%$ & $-1 \%$ & $4 \%$ & $5 \%$ \\
\hline \multirow{5}{*}{ CI Split HEV PHEV2O } & ICE Power & $0 \%$ & $0 \%$ & $-4 \%$ & $-9 \%$ & $-2 \%$ & $-8 \%$ & $-15 \%$ & $-3 \%$ & $-13 \%$ & $-24 \%$ & $-4 \%$ & $-15 \%$ & $-27 \%$ \\
\hline & Electric Machine & $0 \%$ & $0 \%$ & $-4 \%$ & $-9 \%$ & $-2 \%$ & $-8 \%$ & $-17 \%$ & $-3 \%$ & $-15 \%$ & $-29 \%$ & $-4 \%$ & $-17 \%$ & $-32 \%$ \\
\hline & $\mathrm{MC} 2 / \mathrm{GC}$ & $0 \%$ & $0 \%$ & $-4 \%$ & $-9 \%$ & $-2 \%$ & $-8 \%$ & $-15 \%$ & $-3 \%$ & $-13 \%$ & $-24 \%$ & $-4 \%$ & $-15 \%$ & $-27 \%$ \\
\hline & Battery & $0 \%$ & $0 \%$ & $-5 \%$ & $-10 \%$ & $-2 \%$ & $-9 \%$ & $-17 \%$ & $-4 \%$ & $-15 \%$ & $-27 \%$ & $-5 \%$ & $-14 \%$ & $-30 \%$ \\
\hline & Specific Power & $0 \%$ & $0 \%$ & $0 \%$ & $0 \%$ & $0 \%$ & $-1 \%$ & $0 \%$ & $-1 \%$ & $0 \%$ & $3 \%$ & $-1 \%$ & $1 \%$ & $4 \%$ \\
\hline
\end{tabular}




\begin{tabular}{|c|c|c|c|c|c|c|c|c|c|c|c|c|c|c|}
\hline & & \multicolumn{13}{|c|}{ Pickup } \\
\hline & & \multirow{2}{*}{$\frac{2010}{\operatorname{Ref}}$} & \multicolumn{3}{|c|}{2010} & \multicolumn{3}{|c|}{2015} & \multicolumn{3}{|c|}{2030} & \multicolumn{3}{|c|}{2045} \\
\hline & & & low & avg & high & low & avg & high & low & avg & high & low & avg & high \\
\hline \multirow{5}{*}{ CI Series HEV PHEV30 } & ICE Power & $0 \%$ & $0 \%$ & $-6 \%$ & $-12 \%$ & $-3 \%$ & $-11 \%$ & $-22 \%$ & $-7 \%$ & $-20 \%$ & $-32 \%$ & $-8 \%$ & $-22 \%$ & $-35 \%$ \\
\hline & Electric Machine & $0 \%$ & $0 \%$ & $-5 \%$ & $-10 \%$ & $-2 \%$ & $-8 \%$ & $-17 \%$ & $-4 \%$ & $-15 \%$ & $-26 \%$ & $-5 \%$ & $-17 \%$ & $-29 \%$ \\
\hline & $\mathrm{MC} 2 / \mathrm{GC}$ & $0 \%$ & $0 \%$ & $-6 \%$ & $-12 \%$ & $-3 \%$ & $-11 \%$ & $-22 \%$ & $-7 \%$ & $-20 \%$ & $-32 \%$ & $-8 \%$ & $-22 \%$ & $-35 \%$ \\
\hline & Battery & $0 \%$ & $0 \%$ & $-5 \%$ & $-11 \%$ & $-2 \%$ & $-9 \%$ & $-19 \%$ & $-5 \%$ & $-17 \%$ & $-29 \%$ & $-6 \%$ & $-19 \%$ & $-31 \%$ \\
\hline & Specific Power & $0 \%$ & $0 \%$ & $1 \%$ & $1 \%$ & $0 \%$ & $1 \%$ & $2 \%$ & $0 \%$ & $2 \%$ & $7 \%$ & $0 \%$ & $2 \%$ & $8 \%$ \\
\hline \multirow{5}{*}{$\mathrm{CI}$ Series HEV PHEV40 } & ICE Power & $0 \%$ & $0 \%$ & $-6 \%$ & $-12 \%$ & $-3 \%$ & $-11 \%$ & $-22 \%$ & $-7 \%$ & $-20 \%$ & $-33 \%$ & $-8 \%$ & $-23 \%$ & $-36 \%$ \\
\hline & Electric Machine & $0 \%$ & $0 \%$ & $-5 \%$ & $-10 \%$ & $-3 \%$ & $-8 \%$ & $-18 \%$ & $-4 \%$ & $-16 \%$ & $-27 \%$ & $-6 \%$ & $-18 \%$ & $-30 \%$ \\
\hline & $\mathrm{MC} 2 / \mathrm{GC}$ & $0 \%$ & $0 \%$ & $-6 \%$ & $-12 \%$ & $-3 \%$ & $-11 \%$ & $-22 \%$ & $-7 \%$ & $-20 \%$ & $-33 \%$ & $-8 \%$ & $-23 \%$ & $-36 \%$ \\
\hline & Battery & $0 \%$ & $0 \%$ & $-5 \%$ & $-11 \%$ & $-3 \%$ & $-9 \%$ & $-19 \%$ & $-5 \%$ & $-18 \%$ & $-30 \%$ & $-7 \%$ & $-20 \%$ & $-32 \%$ \\
\hline & Specific Power & $0 \%$ & $0 \%$ & $1 \%$ & $1 \%$ & $0 \%$ & $1 \%$ & $2 \%$ & $1 \%$ & $2 \%$ & $7 \%$ & $0 \%$ & $2 \%$ & $8 \%$ \\
\hline \multirow{5}{*}{ H2 Split HEV } & ICE Power & $0 \%$ & $0 \%$ & $-7 \%$ & $-13 \%$ & $-2 \%$ & $-11 \%$ & $-21 \%$ & $-5 \%$ & $-19 \%$ & $-34 \%$ & $-7 \%$ & $-21 \%$ & $-37 \%$ \\
\hline & Electric Machine & $0 \%$ & $0 \%$ & $-6 \%$ & $-11 \%$ & $-2 \%$ & $-9 \%$ & $-17 \%$ & $-2 \%$ & $-16 \%$ & $-30 \%$ & $-5 \%$ & $-17 \%$ & $-33 \%$ \\
\hline & $\mathrm{MC} 2 / \mathrm{GC}$ & $0 \%$ & $0 \%$ & $-7 \%$ & $-13 \%$ & $-2 \%$ & $-11 \%$ & $-21 \%$ & $-5 \%$ & $-19 \%$ & $-34 \%$ & $-7 \%$ & $-21 \%$ & $-37 \%$ \\
\hline & Battery & $0 \%$ & $0 \%$ & $-7 \%$ & $-13 \%$ & $-2 \%$ & $-10 \%$ & $-19 \%$ & $-3 \%$ & $-17 \%$ & $-33 \%$ & $-6 \%$ & $-19 \%$ & $-36 \%$ \\
\hline & Specific Power & $0 \%$ & $0 \%$ & $0 \%$ & $-1 \%$ & $0 \%$ & $-1 \%$ & $-3 \%$ & $-1 \%$ & $-3 \%$ & $-3 \%$ & $-1 \%$ & $-3 \%$ & $-3 \%$ \\
\hline \multirow{5}{*}{ H2 Split HEV PHEV10 } & ICE Power & $0 \%$ & $0 \%$ & $-6 \%$ & $-11 \%$ & $-2 \%$ & $-10 \%$ & $-18 \%$ & $-4 \%$ & $-16 \%$ & $-27 \%$ & $-6 \%$ & $-17 \%$ & $-30 \%$ \\
\hline & Electric Machine & $0 \%$ & $0 \%$ & $-7 \%$ & $-12 \%$ & $-2 \%$ & $-11 \%$ & $-20 \%$ & $-4 \%$ & $-19 \%$ & $-33 \%$ & $-7 \%$ & $-20 \%$ & $-36 \%$ \\
\hline & $\mathrm{MC} 2 / \mathrm{GC}$ & $0 \%$ & $0 \%$ & $-6 \%$ & $-11 \%$ & $-3 \%$ & $-10 \%$ & $-18 \%$ & $-4 \%$ & $-15 \%$ & $-27 \%$ & $-6 \%$ & $-17 \%$ & $-30 \%$ \\
\hline & Battery & $0 \%$ & $0 \%$ & $-8 \%$ & $-14 \%$ & $-2 \%$ & $-13 \%$ & $-27 \%$ & $-5 \%$ & $-26 \%$ & $-33 \%$ & $-8 \%$ & $-17 \%$ & $-37 \%$ \\
\hline & Specific Power & $0 \%$ & $0 \%$ & $0 \%$ & $0 \%$ & $0 \%$ & $-1 \%$ & $-4 \%$ & $0 \%$ & $-4 \%$ & $3 \%$ & $-1 \%$ & $2 \%$ & $3 \%$ \\
\hline \multirow{5}{*}{ H2 Split HEV PHEV20 } & ICE Power & $0 \%$ & $0 \%$ & $-6 \%$ & $-11 \%$ & $-3 \%$ & $-10 \%$ & $-18 \%$ & $-4 \%$ & $-16 \%$ & $-27 \%$ & $-6 \%$ & $-18 \%$ & $-30 \%$ \\
\hline & Electric Machine & $0 \%$ & $0 \%$ & $-6 \%$ & $-12 \%$ & $-2 \%$ & $-10 \%$ & $-21 \%$ & $-4 \%$ & $-18 \%$ & $-33 \%$ & $-6 \%$ & $-20 \%$ & $-36 \%$ \\
\hline & $\mathrm{MC} 2 / \mathrm{GC}$ & $0 \%$ & $0 \%$ & $-6 \%$ & $-11 \%$ & $-3 \%$ & $-10 \%$ & $-18 \%$ & $-4 \%$ & $-16 \%$ & $-27 \%$ & $-6 \%$ & $-18 \%$ & $-30 \%$ \\
\hline & Battery & $0 \%$ & $0 \%$ & $-7 \%$ & $-14 \%$ & $-2 \%$ & $-11 \%$ & $-23 \%$ & $-5 \%$ & $-20 \%$ & $-35 \%$ & $-7 \%$ & $-20 \%$ & $-38 \%$ \\
\hline & Specific Power & $0 \%$ & $0 \%$ & $0 \%$ & $-1 \%$ & $0 \%$ & $0 \%$ & $-1 \%$ & $-1 \%$ & $-1 \%$ & $2 \%$ & $0 \%$ & $1 \%$ & $2 \%$ \\
\hline \multirow{5}{*}{ H2 Series HEV PHEV30 } & ICE Power & $0 \%$ & $0 \%$ & $-7 \%$ & $-14 \%$ & $-3 \%$ & $-13 \%$ & $-24 \%$ & $-7 \%$ & $-22 \%$ & $-34 \%$ & $-10 \%$ & $-24 \%$ & $-37 \%$ \\
\hline & Electric Machine & $0 \%$ & $0 \%$ & $-6 \%$ & $-11 \%$ & $-3 \%$ & $-10 \%$ & $-19 \%$ & $-5 \%$ & $-17 \%$ & $-29 \%$ & $-7 \%$ & $-19 \%$ & $-31 \%$ \\
\hline & $\mathrm{MC} 2 / \mathrm{GC}$ & $0 \%$ & $0 \%$ & $-7 \%$ & $-14 \%$ & $-3 \%$ & $-13 \%$ & $-24 \%$ & $-7 \%$ & $-22 \%$ & $-34 \%$ & $-10 \%$ & $-24 \%$ & $-37 \%$ \\
\hline & Battery & $0 \%$ & $0 \%$ & $-6 \%$ & $-12 \%$ & $-3 \%$ & $-11 \%$ & $-21 \%$ & $-6 \%$ & $-19 \%$ & $-31 \%$ & $-8 \%$ & $-21 \%$ & $-34 \%$ \\
\hline & Specific Power & $0 \%$ & $0 \%$ & $1 \%$ & $2 \%$ & $0 \%$ & $2 \%$ & $3 \%$ & $1 \%$ & $3 \%$ & $9 \%$ & $1 \%$ & $3 \%$ & $10 \%$ \\
\hline
\end{tabular}




\begin{tabular}{|c|c|c|c|c|c|c|c|c|c|c|c|c|c|c|}
\hline & & \multicolumn{13}{|c|}{ Pickup } \\
\hline & & \multirow{2}{*}{$\frac{2010}{\operatorname{Ref}}$} & \multicolumn{3}{|c|}{2010} & \multicolumn{3}{|c|}{2015} & \multicolumn{3}{|c|}{2030} & \multicolumn{3}{|c|}{2045} \\
\hline & & & low & avg & high & low & avg & high & low & avg & high & low & avg & high \\
\hline \multirow{5}{*}{ H2 Series HEV PHEV40 } & ICE Power & $0 \%$ & $0 \%$ & $-7 \%$ & $-14 \%$ & $-3 \%$ & $-13 \%$ & $-24 \%$ & $-7 \%$ & $-22 \%$ & $-35 \%$ & $-10 \%$ & $-25 \%$ & $-38 \%$ \\
\hline & Electric Machine & $0 \%$ & $0 \%$ & $-6 \%$ & $-12 \%$ & $-3 \%$ & $-10 \%$ & $-20 \%$ & $-5 \%$ & $-18 \%$ & $-30 \%$ & $-8 \%$ & $-20 \%$ & $-32 \%$ \\
\hline & $\mathrm{MC} 2 / \mathrm{GC}$ & $0 \%$ & $0 \%$ & $-7 \%$ & $-14 \%$ & $-3 \%$ & $-13 \%$ & $-24 \%$ & $-7 \%$ & $-22 \%$ & $-35 \%$ & $-10 \%$ & $-25 \%$ & $-38 \%$ \\
\hline & Battery & $0 \%$ & $0 \%$ & $-7 \%$ & $-13 \%$ & $-3 \%$ & $-11 \%$ & $-22 \%$ & $-6 \%$ & $-20 \%$ & $-32 \%$ & $-8 \%$ & $-22 \%$ & $-35 \%$ \\
\hline & Specific Power & $0 \%$ & $0 \%$ & $1 \%$ & $2 \%$ & $0 \%$ & $2 \%$ & $3 \%$ & $1 \%$ & $3 \%$ & $9 \%$ & $1 \%$ & $3 \%$ & $10 \%$ \\
\hline \multirow{5}{*}{ E85 Split HEV } & ICE Power & $0 \%$ & $0 \%$ & $-6 \%$ & $-11 \%$ & $-3 \%$ & $-10 \%$ & $-20 \%$ & $-4 \%$ & $-18 \%$ & $-31 \%$ & $-5 \%$ & $-20 \%$ & $-34 \%$ \\
\hline & Electric Machine & $0 \%$ & $0 \%$ & $-5 \%$ & $-9 \%$ & $-2 \%$ & $-7 \%$ & $-16 \%$ & $-2 \%$ & $-14 \%$ & $-27 \%$ & $-3 \%$ & $-15 \%$ & $-30 \%$ \\
\hline & $\mathrm{MC} 2 / \mathrm{GC}$ & $0 \%$ & $0 \%$ & $-6 \%$ & $-11 \%$ & $-3 \%$ & $-10 \%$ & $-20 \%$ & $-4 \%$ & $-18 \%$ & $-31 \%$ & $-5 \%$ & $-20 \%$ & $-34 \%$ \\
\hline & Battery & $0 \%$ & $0 \%$ & $-5 \%$ & $-10 \%$ & $-1 \%$ & $-6 \%$ & $-15 \%$ & $-2 \%$ & $-14 \%$ & $-29 \%$ & $-2 \%$ & $-15 \%$ & $-32 \%$ \\
\hline & Specific Power & $0 \%$ & $0 \%$ & $-1 \%$ & $-1 \%$ & $-1 \%$ & $-2 \%$ & $-4 \%$ & $-2 \%$ & $-4 \%$ & $-4 \%$ & $-2 \%$ & $-4 \%$ & $-3 \%$ \\
\hline \multirow{5}{*}{ E85 Split HEV PHEV10 } & ICE Power & $0 \%$ & $0 \%$ & $-4 \%$ & $-9 \%$ & $-2 \%$ & $-8 \%$ & $-15 \%$ & $-3 \%$ & $-14 \%$ & $-24 \%$ & $-4 \%$ & $-15 \%$ & $-27 \%$ \\
\hline & Electric Machine & $0 \%$ & $0 \%$ & $-5 \%$ & $-10 \%$ & $-2 \%$ & $-8 \%$ & $-18 \%$ & $-4 \%$ & $-16 \%$ & $-30 \%$ & $-4 \%$ & $-18 \%$ & $-33 \%$ \\
\hline & $\mathrm{MC} 2 / \mathrm{GC}$ & $0 \%$ & $0 \%$ & $-4 \%$ & $-9 \%$ & $-2 \%$ & $-8 \%$ & $-15 \%$ & $-3 \%$ & $-14 \%$ & $-24 \%$ & $-4 \%$ & $-15 \%$ & $-27 \%$ \\
\hline & Battery & $0 \%$ & $0 \%$ & $-6 \%$ & $-11 \%$ & $-1 \%$ & $-9 \%$ & $-23 \%$ & $-5 \%$ & $-22 \%$ & $-27 \%$ & $-6 \%$ & $-13 \%$ & $-31 \%$ \\
\hline & Specific Power & $0 \%$ & $0 \%$ & $-1 \%$ & $-1 \%$ & $0 \%$ & $0 \%$ & $-4 \%$ & $-1 \%$ & $-4 \%$ & $4 \%$ & $-1 \%$ & $3 \%$ & $4 \%$ \\
\hline \multirow{5}{*}{ E85 Split HEV PHEV20 } & ICE Power & $0 \%$ & $0 \%$ & $-4 \%$ & $-9 \%$ & $-2 \%$ & $-8 \%$ & $-16 \%$ & $-3 \%$ & $-14 \%$ & $-25 \%$ & $-4 \%$ & $-15 \%$ & $-28 \%$ \\
\hline & Electric Machine & $0 \%$ & $0 \%$ & $-5 \%$ & $-9 \%$ & $-2 \%$ & $-8 \%$ & $-18 \%$ & $-3 \%$ & $-16 \%$ & $-30 \%$ & $-3 \%$ & $-18 \%$ & $-34 \%$ \\
\hline & $\mathrm{MC} 2 / \mathrm{GC}$ & $0 \%$ & $0 \%$ & $-4 \%$ & $-9 \%$ & $-2 \%$ & $-8 \%$ & $-16 \%$ & $-3 \%$ & $-14 \%$ & $-25 \%$ & $-4 \%$ & $-15 \%$ & $-28 \%$ \\
\hline & Battery & $0 \%$ & $0 \%$ & $-5 \%$ & $-10 \%$ & $-2 \%$ & $-9 \%$ & $-18 \%$ & $-4 \%$ & $-15 \%$ & $-30 \%$ & $-5 \%$ & $-16 \%$ & $-33 \%$ \\
\hline & Specific Power & $0 \%$ & $0 \%$ & $0 \%$ & $0 \%$ & $0 \%$ & $-1 \%$ & $0 \%$ & $-1 \%$ & $0 \%$ & $3 \%$ & $-1 \%$ & $1 \%$ & $3 \%$ \\
\hline \multirow{5}{*}{ E85 Series HEV PHEV30 } & ICE Power & $0 \%$ & $0 \%$ & $-6 \%$ & $-12 \%$ & $-3 \%$ & $-11 \%$ & $-22 \%$ & $-7 \%$ & $-20 \%$ & $-32 \%$ & $-8 \%$ & $-22 \%$ & $-35 \%$ \\
\hline & Electric Machine & $0 \%$ & $0 \%$ & $-5 \%$ & $-10 \%$ & $-2 \%$ & $-8 \%$ & $-17 \%$ & $-4 \%$ & $-15 \%$ & $-27 \%$ & $-5 \%$ & $-17 \%$ & $-30 \%$ \\
\hline & $\mathrm{MC} 2 / \mathrm{GC}$ & $0 \%$ & $0 \%$ & $-6 \%$ & $-12 \%$ & $-3 \%$ & $-11 \%$ & $-22 \%$ & $-7 \%$ & $-20 \%$ & $-32 \%$ & $-8 \%$ & $-22 \%$ & $-35 \%$ \\
\hline & Battery & $0 \%$ & $0 \%$ & $-5 \%$ & $-11 \%$ & $-2 \%$ & $-9 \%$ & $-19 \%$ & $-5 \%$ & $-17 \%$ & $-29 \%$ & $-6 \%$ & $-19 \%$ & $-32 \%$ \\
\hline & Specific Power & $0 \%$ & $0 \%$ & $1 \%$ & $1 \%$ & $0 \%$ & $1 \%$ & $2 \%$ & $0 \%$ & $2 \%$ & $8 \%$ & $0 \%$ & $2 \%$ & $9 \%$ \\
\hline \multirow{5}{*}{ E85 Series HEV PHEV40 } & ICE Power & $0 \%$ & $0 \%$ & $-6 \%$ & $-13 \%$ & $-3 \%$ & $-11 \%$ & $-23 \%$ & $-7 \%$ & $-21 \%$ & $-33 \%$ & $-8 \%$ & $-23 \%$ & $-36 \%$ \\
\hline & Electric Machine & $0 \%$ & $0 \%$ & $-5 \%$ & $-10 \%$ & $-3 \%$ & $-9 \%$ & $-18 \%$ & $-4 \%$ & $-16 \%$ & $-27 \%$ & $-6 \%$ & $-18 \%$ & $-31 \%$ \\
\hline & $\mathrm{MC} 2 / \mathrm{GC}$ & $0 \%$ & $0 \%$ & $-6 \%$ & $-13 \%$ & $-3 \%$ & $-11 \%$ & $-23 \%$ & $-7 \%$ & $-21 \%$ & $-33 \%$ & $-8 \%$ & $-23 \%$ & $-36 \%$ \\
\hline & Battery & $0 \%$ & $0 \%$ & $-5 \%$ & $-11 \%$ & $-3 \%$ & $-10 \%$ & $-20 \%$ & $-5 \%$ & $-18 \%$ & $-30 \%$ & $-7 \%$ & $-20 \%$ & $-33 \%$ \\
\hline & Specific Power & $0 \%$ & $0 \%$ & $1 \%$ & $1 \%$ & $0 \%$ & $1 \%$ & $2 \%$ & $1 \%$ & $2 \%$ & $7 \%$ & $0 \%$ & $2 \%$ & $8 \%$ \\
\hline
\end{tabular}




\begin{tabular}{|c|c|c|c|c|c|c|c|c|c|c|c|c|c|c|}
\hline & & \multicolumn{13}{|c|}{ Pickup } \\
\hline & & \multirow{2}{*}{$\frac{2010}{\operatorname{Ref}}$} & \multicolumn{3}{|c|}{2010} & \multicolumn{3}{|c|}{2015} & \multicolumn{3}{|c|}{2030} & \multicolumn{3}{|c|}{2045} \\
\hline & & & low & avg & high & low & avg & high & low & avg & high & low & avg & high \\
\hline \multirow{5}{*}{ FC HEV } & FC Power & $0 \%$ & $0 \%$ & $-16 \%$ & $-27 \%$ & $-11 \%$ & $-29 \%$ & $-39 \%$ & $-22 \%$ & $-38 \%$ & $-50 \%$ & $-27 \%$ & $-40 \%$ & $-53 \%$ \\
\hline & Electric Machine & $0 \%$ & $0 \%$ & $-16 \%$ & $-26 \%$ & $-12 \%$ & $-23 \%$ & $-34 \%$ & $-19 \%$ & $-32 \%$ & $-45 \%$ & $-23 \%$ & $-34 \%$ & $-48 \%$ \\
\hline & $\mathrm{MC} 2 / \mathrm{GC}$ & & & & & & & & & & & & & \\
\hline & Battery & $0 \%$ & $0 \%$ & $-16 \%$ & $-23 \%$ & $-12 \%$ & $-12 \%$ & $-21 \%$ & $-6 \%$ & $-20 \%$ & $-33 \%$ & $-11 \%$ & $-22 \%$ & $-36 \%$ \\
\hline & Specific Power & $0 \%$ & $0 \%$ & $0 \%$ & $-1 \%$ & $0 \%$ & $1 \%$ & $0 \%$ & $-1 \%$ & $0 \%$ & $2 \%$ & $0 \%$ & $1 \%$ & $3 \%$ \\
\hline \multirow{5}{*}{ FC HEV PHEV 10} & FC Power & $0 \%$ & $0 \%$ & $-16 \%$ & $-25 \%$ & $-10 \%$ & $-24 \%$ & $-34 \%$ & $-16 \%$ & $-32 \%$ & $-46 \%$ & $-21 \%$ & $-33 \%$ & $-48 \%$ \\
\hline & Electric Machine & $0 \%$ & $0 \%$ & $-13 \%$ & $-21 \%$ & $-8 \%$ & $-20 \%$ & $-29 \%$ & $-13 \%$ & $-28 \%$ & $-41 \%$ & $-17 \%$ & $-29 \%$ & $-44 \%$ \\
\hline & $\mathrm{MC} 2 / \mathrm{GC}$ & & & & & & & & & & & & & \\
\hline & Battery & $0 \%$ & $0 \%$ & $-14 \%$ & $-23 \%$ & $-9 \%$ & $-22 \%$ & $-33 \%$ & $-15 \%$ & $-31 \%$ & $-45 \%$ & $-20 \%$ & $-33 \%$ & $-48 \%$ \\
\hline & Specific Power & $0 \%$ & $0 \%$ & $2 \%$ & $3 \%$ & $2 \%$ & $3 \%$ & $3 \%$ & $3 \%$ & $3 \%$ & $5 \%$ & $3 \%$ & $5 \%$ & $6 \%$ \\
\hline \multirow{5}{*}{ FC HEV PHEV2O } & FC Power & $0 \%$ & $0 \%$ & $-16 \%$ & $-25 \%$ & $-10 \%$ & $-24 \%$ & $-34 \%$ & $-16 \%$ & $-32 \%$ & $-46 \%$ & $-22 \%$ & $-34 \%$ & $-49 \%$ \\
\hline & Electric Machine & $0 \%$ & $0 \%$ & $-13 \%$ & $-21 \%$ & $-8 \%$ & $-20 \%$ & $-30 \%$ & $-13 \%$ & $-28 \%$ & $-41 \%$ & $-17 \%$ & $-30 \%$ & $-44 \%$ \\
\hline & $\mathrm{MC} 2 / \mathrm{GC}$ & & & & & & & & & & & & & \\
\hline & Battery & $0 \%$ & $0 \%$ & $-14 \%$ & $-23 \%$ & $-9 \%$ & $-22 \%$ & $-33 \%$ & $-16 \%$ & $-31 \%$ & $-45 \%$ & $-20 \%$ & $-34 \%$ & $-48 \%$ \\
\hline & Specific Power & $0 \%$ & $0 \%$ & $2 \%$ & $3 \%$ & $2 \%$ & $3 \%$ & $4 \%$ & $3 \%$ & $4 \%$ & $5 \%$ & $4 \%$ & $5 \%$ & $6 \%$ \\
\hline \multirow{5}{*}{ FC HEV PHEV 30} & FC Power & $0 \%$ & $0 \%$ & $-11 \%$ & $-18 \%$ & $-7 \%$ & $-17 \%$ & $-26 \%$ & $-11 \%$ & $-24 \%$ & $-35 \%$ & $-15 \%$ & $-26 \%$ & $-38 \%$ \\
\hline & Electric Machine & $0 \%$ & $0 \%$ & $-8 \%$ & $-14 \%$ & $-5 \%$ & $-13 \%$ & $-21 \%$ & $-8 \%$ & $-20 \%$ & $-30 \%$ & $-11 \%$ & $-22 \%$ & $-34 \%$ \\
\hline & $\mathrm{MC} 2 / \mathrm{GC}$ & & & & & & & & & & & & & \\
\hline & Battery & $0 \%$ & $0 \%$ & $-8 \%$ & $-15 \%$ & $-5 \%$ & $-14 \%$ & $-23 \%$ & $-9 \%$ & $-21 \%$ & $-33 \%$ & $-12 \%$ & $-24 \%$ & $-36 \%$ \\
\hline & Specific Power & $0 \%$ & $0 \%$ & $6 \%$ & $9 \%$ & $4 \%$ & $9 \%$ & $13 \%$ & $6 \%$ & $12 \%$ & $21 \%$ & $9 \%$ & $13 \%$ & $22 \%$ \\
\hline \multirow{5}{*}{ FC HEV PHEV40 } & FC Power & $0 \%$ & $0 \%$ & $-11 \%$ & $-18 \%$ & $-7 \%$ & $-17 \%$ & $-26 \%$ & $-11 \%$ & $-24 \%$ & $-35 \%$ & $-15 \%$ & $-27 \%$ & $-38 \%$ \\
\hline & Electric Machine & $0 \%$ & $0 \%$ & $-8 \%$ & $-14 \%$ & $-5 \%$ & $-13 \%$ & $-22 \%$ & $-9 \%$ & $-20 \%$ & $-31 \%$ & $-11 \%$ & $-23 \%$ & $-34 \%$ \\
\hline & $\mathrm{MC} 2 / \mathrm{GC}$ & & & & & & & & & & & & & \\
\hline & Battery & $0 \%$ & $0 \%$ & $-8 \%$ & $-15 \%$ & $-5 \%$ & $-14 \%$ & $-24 \%$ & $-9 \%$ & $-22 \%$ & $-33 \%$ & $-12 \%$ & $-25 \%$ & $-36 \%$ \\
\hline & Specific Power & $0 \%$ & $0 \%$ & $6 \%$ & $9 \%$ & $4 \%$ & $9 \%$ & $13 \%$ & $6 \%$ & $12 \%$ & $21 \%$ & $9 \%$ & $13 \%$ & $23 \%$ \\
\hline \multirow{5}{*}{ EV } & ICE Power & & & & & & & & & & & & & \\
\hline & Electric Machine & $0 \%$ & $0 \%$ & $-6 \%$ & $-12 \%$ & $-3 \%$ & $-10 \%$ & $-23 \%$ & $-5 \%$ & $-21 \%$ & $-34 \%$ & $-8 \%$ & $-25 \%$ & $-37 \%$ \\
\hline & $\mathrm{MC} 2 / \mathrm{GC}$ & & & & & & & & & & & & & \\
\hline & Battery & $0 \%$ & $0 \%$ & $-6 \%$ & $-13 \%$ & $-3 \%$ & $-11 \%$ & $-25 \%$ & $-6 \%$ & $-23 \%$ & $-36 \%$ & $-9 \%$ & $-27 \%$ & $-39 \%$ \\
\hline & Specific Power & $0 \%$ & $0 \%$ & $0 \%$ & $0 \%$ & $0 \%$ & $0 \%$ & $-1 \%$ & $-1 \%$ & $-1 \%$ & $4 \%$ & $-1 \%$ & $-1 \%$ & $6 \%$ \\
\hline
\end{tabular}




\section{Energy}

\begin{tabular}{|c|c|c|c|c|c|c|c|c|c|c|c|c|c|c|}
\hline & & \multicolumn{13}{|c|}{ Pickup } \\
\hline & & \multirow{2}{*}{$\begin{array}{l}2010 \\
\text { Ref }\end{array}$} & \multicolumn{3}{|c|}{2010} & \multicolumn{3}{|c|}{2015} & \multicolumn{3}{|c|}{2030} & \multicolumn{3}{|c|}{2045} \\
\hline & & & low & avg & high & low & avg & high & low & avg & high & low & avg & high \\
\hline \multirow{2}{*}{ SI Split HEV } & Total Energy & 1544 & 1544 & 1459 & 1388 & 1529 & 1382 & 1274 & 1469 & 1296 & 1058 & 1469 & 1274 & 994 \\
\hline & Usable Energy & & & & & & & & & & & & & \\
\hline \multirow{2}{*}{ SI Split HEV PHEV10 } & Total Energy & 5791 & 5791 & 5474 & 5139 & 5627 & 5395 & 4113 & 5537 & 4426 & 3264 & 5570 & 4000 & 3131 \\
\hline & Usable Energy & 3474 & 3474 & 3285 & 3083 & 3376 & 3237 & 2879 & 3322 & 3098 & 2612 & 3342 & 3000 & 2505 \\
\hline \multirow{2}{*}{ SI Split HEV PHEV2O } & Total Energy & 13206 & 13206 & 12450 & 11569 & 12671 & 11669 & 8947 & 12452 & 9188 & 6710 & 12205 & 8317 & 6471 \\
\hline & Usable Energy & 7924 & 7924 & 7470 & 6942 & 7602 & 7002 & 6263 & 7471 & 6432 & 5368 & 7323 & 6237 & 5177 \\
\hline \multirow{2}{*}{ SI Series HEV PHEV30 } & Total Energy & 23645 & 23645 & 21802 & 20624 & 22381 & 20886 & 15421 & 21645 & 15880 & 12298 & 21306 & 14404 & 11762 \\
\hline & Usable Energy & 14187 & 14187 & 13081 & 12375 & 13429 & 12531 & 10795 & 12987 & 11116 & 9838 & 12784 & 10803 & 9410 \\
\hline \multirow{2}{*}{ SI Series HEV PHEV40 } & Total Energy & 30815 & 30815 & 28759 & 26752 & 29615 & 27174 & 20754 & 28618 & 21368 & 15846 & 28039 & 19293 & 15255 \\
\hline & Usable Energy & 18489 & 18489 & 17256 & 16051 & 17769 & 16304 & 14528 & 17171 & 14958 & 12677 & 16824 & 14470 & 12204 \\
\hline \multirow{2}{*}{ Cl Split HEV } & Total Energy & 1607 & 1607 & 1529 & 1451 & 1591 & 1447 & 1318 & 1534 & 1339 & 1123 & 1512 & 1318 & 1058 \\
\hline & Usable Energy & & & & & & & & & & & & & \\
\hline \multirow{2}{*}{ CI Split HEV PHEV10 } & Total Energy & 5985 & 5985 & 5654 & 5320 & 5807 & 5486 & 4295 & 5678 & 4396 & 3390 & 5727 & 3922 & 3249 \\
\hline & Usable Energy & 3591 & 3591 & 3393 & 3192 & 3484 & 3292 & 3007 & 3407 & 3077 & 2712 & 3436 & 2941 & 2599 \\
\hline \multirow{2}{*}{ CI Split HEV PHEV2O } & Total Energy & 13712 & 13712 & 12923 & 12076 & 13248 & 12160 & 9305 & 12868 & 9571 & 6853 & 12710 & 8644 & 6653 \\
\hline & Usable Energy & 8227 & 8227 & 7754 & 7246 & 7949 & 7296 & 6514 & 7721 & 6700 & 5482 & 7626 & 6483 & 5323 \\
\hline \multirow{2}{*}{ Cl Series HEV PHEV3O } & Total Energy & 24190 & 24190 & 22690 & 20839 & 23286 & 21066 & 16394 & 22518 & 16863 & 12324 & 22145 & 14803 & 12233 \\
\hline & Usable Energy & 14514 & 14514 & 13614 & 12504 & 13971 & 12640 & 11476 & 13511 & 11804 & 9859 & 13287 & 11102 & 9786 \\
\hline \multirow{2}{*}{ CI Series HEV PHEV40 } & Total Energy & 31544 & 31544 & 29570 & 27563 & 30326 & 27852 & 21236 & 29326 & 21864 & 16339 & 28838 & 19891 & 15666 \\
\hline & Usable Energy & 18927 & 18927 & 17742 & 16538 & 18195 & 16711 & 14865 & 17595 & 15305 & 13071 & 17303 & 14918 & 12533 \\
\hline \multirow{2}{*}{ H2 Split HEV } & Total Energy & 1560 & 1560 & 1451 & 1365 & 1537 & 1361 & 1231 & 1469 & 1253 & 1015 & 1426 & 1231 & 972 \\
\hline & Usable Energy & & & & & & & & & & & & & \\
\hline \multirow{2}{*}{ H2 Split HEV PHEV10 } & Total Energy & 5842 & 5842 & 5481 & 5074 & 5649 & 5133 & 4134 & 5619 & 4232 & 3193 & 5377 & 3865 & 3114 \\
\hline & Usable Energy & 3505 & 3505 & 3289 & 3044 & 3389 & 3080 & 2894 & 3371 & 2963 & 2555 & 3226 & 2899 & 2491 \\
\hline \multirow{2}{*}{ H2 Split HEV PHEV2O } & Total Energy & 13371 & 13371 & 12417 & 11507 & 12800 & 11613 & 8869 & 12452 & 9151 & 6611 & 12099 & 8082 & 6335 \\
\hline & Usable Energy & 8023 & 8023 & 7450 & 6904 & 7680 & 6968 & 6208 & 7471 & 6406 & 5289 & 7259 & 6062 & 5068 \\
\hline
\end{tabular}


Argonne National Laboratory Report - Light-Duty Vehicle Fuel Consumption Displacement Potential Up to 2045

\begin{tabular}{|c|c|c|c|c|c|c|c|c|c|c|c|c|c|c|}
\hline & & \multicolumn{13}{|c|}{ Pickup } \\
\hline & & 2010 & \multicolumn{3}{|c|}{2010} & \multicolumn{3}{|c|}{2015} & \multicolumn{3}{|c|}{2030} & \multicolumn{3}{|c|}{2045} \\
\hline & & Ref & low & avg & high & low & avg & high & low & avg & high & low & avg & high \\
\hline \multirow{2}{*}{ H2 Series HEV PHEV3O } & Total Energy & 23580 & 23580 & 21828 & 20375 & 22722 & 20626 & 15556 & 21969 & 16077 & 12061 & 21020 & 14145 & 11583 \\
\hline & Usable Energy & 14148 & 14148 & 13097 & 12225 & 13633 & 12376 & 10889 & 13181 & 11254 & 9648 & 12612 & 10609 & 9266 \\
\hline \multirow{2}{*}{ H2 Series HEV PHEV40 } & Total Energy & 30966 & 30966 & 28619 & 26511 & 29630 & 26741 & 20367 & 28571 & 21050 & 15467 & 27762 & 18961 & 14789 \\
\hline & Usable Energy & 18579 & 18579 & 17171 & 15907 & 17778 & 16045 & 14257 & 17143 & 14735 & 12373 & 16657 & 14221 & 11831 \\
\hline \multirow{2}{*}{ E85 Split HEV } & Total Energy & 1544 & 1544 & 1466 & 1388 & 1529 & 1404 & 1274 & 1469 & 1296 & 1058 & 1469 & 1274 & 1015 \\
\hline & Usable Energy & & & & & & & & & & & & & \\
\hline \multirow{2}{*}{ E85 Split HEV PHEV10 } & Total Energy & 5793 & 5793 & 5481 & 5157 & 5635 & 5395 & 4120 & 5547 & 4394 & 3270 & 5572 & 4007 & 3137 \\
\hline & Usable Energy & 3476 & 3476 & 3288 & 3094 & 3381 & 3237 & 2884 & 3328 & 3075 & 2616 & 3343 & 3005 & 2510 \\
\hline \multirow{2}{*}{ E85 Split HEV PHEV20 } & Total Energy & 13205 & 13205 & 12475 & 11586 & 12767 & 11695 & 8945 & 12439 & 9215 & 6769 & 12246 & 8323 & 6481 \\
\hline & Usable Energy & 7923 & 7923 & 7485 & 6951 & 7660 & 7017 & 6262 & 7463 & 6450 & 5415 & 7347 & 6242 & 5185 \\
\hline \multirow{2}{*}{ E85 Series HEV PHEV30 } & Total Energy & 23634 & 23634 & 22181 & 20526 & 22392 & 20882 & 15435 & 21686 & 15899 & 12280 & 21656 & 14412 & 11742 \\
\hline & Usable Energy & 14181 & 14181 & 13309 & 12316 & 13435 & 12529 & 10805 & 13011 & 11129 & 9824 & 12994 & 10809 & 9394 \\
\hline \multirow{2}{*}{ E85 Series HEV PHEV40 } & Total Energy & 30839 & 30839 & 28790 & 26768 & 29631 & 27197 & 20712 & 28571 & 21387 & 15859 & 28055 & 19316 & 15272 \\
\hline & Usable Energy & 18504 & 18504 & 17274 & 16061 & 17779 & 16318 & 14498 & 17143 & 14971 & 12687 & 16833 & 14487 & 12217 \\
\hline \multirow{2}{*}{ FC HEV } & Total Energy & 1919 & 1919 & 1615 & 1482 & 1685 & 1642 & 1469 & 1750 & 1490 & 1253 & 1663 & 1447 & 1188 \\
\hline & Usable Energy & & & & & & & & & & & & & \\
\hline \multirow{2}{*}{ FC HEV PHEV 10} & Total Energy & 8746 & 8746 & 7555 & 6117 & 7888 & 6672 & 5098 & 7349 & 5256 & 3581 & 6953 & 4367 & 3428 \\
\hline & Usable Energy & 5248 & 5248 & 4533 & 3670 & 4733 & 4003 & 3568 & 4409 & 3679 & 2865 & 4172 & 3275 & 2743 \\
\hline \multirow{2}{*}{ FC HEV PHEV 20} & Total Energy & 15961 & 15961 & 13781 & 12466 & 14336 & 12600 & 9476 & 13576 & 9779 & 7192 & 12976 & 8834 & 6878 \\
\hline & Usable Energy & 9577 & 9577 & 8268 & 7480 & 8602 & 7560 & 6634 & 8146 & 6845 & 5754 & 7785 & 6625 & 5503 \\
\hline \multirow{2}{*}{ FC HEV PHEV 30} & Total Energy & 25131 & 25131 & 22163 & 19210 & 23062 & 20202 & 14417 & 21734 & 14885 & 11096 & 20746 & 13413 & 10602 \\
\hline & Usable Energy & 15079 & 15079 & 13298 & 11526 & 13837 & 12121 & 10092 & 13041 & 10420 & 8876 & 12448 & 10060 & 8482 \\
\hline \multirow{2}{*}{ FC HEV PHEV 40} & Total Energy & 32250 & 32250 & 27795 & 25217 & 28902 & 25271 & 18670 & 27200 & 19269 & 14366 & 25945 & 17328 & 13680 \\
\hline & Usable Energy & 19350 & 19350 & 16677 & 15130 & 17341 & 15163 & 13069 & 16320 & 13488 & 11493 & 15567 & 12996 & 10944 \\
\hline \multirow{2}{*}{ BEV } & Total Energy & 116026 & 116026 & 107968 & 99700 & 111194 & 100831 & 73394 & 106600 & 75634 & 54374 & 103851 & 68012 & 51446 \\
\hline & Usable Energy & 69615 & 69615 & 64781 & 59820 & 66716 & 60499 & 51376 & 63960 & 52944 & 43499 & 62311 & 51009 & 41157 \\
\hline
\end{tabular}




\section{Percentage of Energy reduction based on the respective 2010 reference case}

\begin{tabular}{|c|c|c|c|c|c|c|c|c|c|c|c|c|c|c|}
\hline & & \multicolumn{13}{|c|}{ Pickup } \\
\hline & & \multirow{2}{*}{$\frac{2010}{\operatorname{Ref}}$} & \multicolumn{3}{|c|}{2010} & \multicolumn{3}{|c|}{2015} & \multicolumn{3}{|c|}{2030} & \multicolumn{3}{|c|}{2045} \\
\hline & & & low & avg & high & low & avg & high & low & avg & high & low & avg & high \\
\hline \multirow{2}{*}{ SI Split HEV } & Total Energy & $0.0 \%$ & $0.0 \%$ & $-5.6 \%$ & $-10.1 \%$ & $-1.0 \%$ & $-10.5 \%$ & $-17.5 \%$ & $-4.9 \%$ & $-16.1 \%$ & $-31.5 \%$ & $-4.9 \%$ & $-17.5 \%$ & $-35.7 \%$ \\
\hline & Usable Energy & & & & & & & & & & & & & \\
\hline \multirow{2}{*}{ SI Split HEV PHEV10 } & Total Energy & $0.0 \%$ & $0.0 \%$ & $-5.5 \%$ & $-11.3 \%$ & $-2.8 \%$ & $-6.8 \%$ & $-29.0 \%$ & $-4.4 \%$ & $-23.6 \%$ & $-43.6 \%$ & $-3.8 \%$ & $-30.9 \%$ & $-45.9 \%$ \\
\hline & Usable Energy & $0.0 \%$ & $0.0 \%$ & $-5.5 \%$ & $-11.3 \%$ & $-2.8 \%$ & $-6.8 \%$ & $-17.1 \%$ & $-4.4 \%$ & $-10.8 \%$ & $-24.8 \%$ & $-3.8 \%$ & $-13.6 \%$ & $-27.9 \%$ \\
\hline \multirow{2}{*}{ SI Split HEV PHEV2O } & Total Energy & $0.0 \%$ & $0.0 \%$ & $-5.7 \%$ & $-12.4 \%$ & $-4.1 \%$ & $-11.6 \%$ & $-32.3 \%$ & $-5.7 \%$ & $-30.4 \%$ & $-49.2 \%$ & $-7.6 \%$ & $-37.0 \%$ & $-51.0 \%$ \\
\hline & Usable Energy & $0.0 \%$ & $0.0 \%$ & $-5.7 \%$ & $-12.4 \%$ & $-4.1 \%$ & $-11.6 \%$ & $-21.0 \%$ & $-5.7 \%$ & $-18.8 \%$ & $-32.3 \%$ & $-7.6 \%$ & $-21.3 \%$ & $-34.7 \%$ \\
\hline \multirow{2}{*}{ SI Series HEV PHEV 30} & Total Energy & $0.0 \%$ & $0.0 \%$ & $-7.8 \%$ & $-12.8 \%$ & $-5.3 \%$ & $-11.7 \%$ & $-34.8 \%$ & $-8.5 \%$ & $-32.8 \%$ & $-48.0 \%$ & $-9.9 \%$ & $-39.1 \%$ & $-50.3 \%$ \\
\hline & Usable Energy & $0.0 \%$ & $0.0 \%$ & $-7.8 \%$ & $-12.8 \%$ & $-5.3 \%$ & $-11.7 \%$ & $-23.9 \%$ & $-8.5 \%$ & $-21.6 \%$ & $-30.7 \%$ & $-9.9 \%$ & $-23.9 \%$ & $-33.7 \%$ \\
\hline \multirow{2}{*}{ SI Series HEV PHEV40 } & Total Energy & $0.0 \%$ & $0.0 \%$ & $-6.7 \%$ & $-13.2 \%$ & $-3.9 \%$ & $-11.8 \%$ & $-32.7 \%$ & $-7.1 \%$ & $-30.7 \%$ & $-48.6 \%$ & $-9.0 \%$ & $-37.4 \%$ & $-50.5 \%$ \\
\hline & Usable Energy & $0.0 \%$ & $0.0 \%$ & $-6.7 \%$ & $-13.2 \%$ & $-3.9 \%$ & $-11.8 \%$ & $-21.4 \%$ & $-7.1 \%$ & $-19.1 \%$ & $-31.4 \%$ & $-9.0 \%$ & $-21.7 \%$ & $-34.0 \%$ \\
\hline \multirow{2}{*}{ CI Split HEV } & Total Energy & $0.0 \%$ & $0.0 \%$ & $-4.9 \%$ & $-9.7 \%$ & $-1.0 \%$ & $-9.9 \%$ & $-18.0 \%$ & $-4.6 \%$ & $-16.7 \%$ & $-30.1 \%$ & $-5.9 \%$ & $-18.0 \%$ & $-34.1 \%$ \\
\hline & Usable Energy & & & & & & & & & & & & & \\
\hline \multirow{2}{*}{ CI Split HEV PHEV10 } & Total Energy & $0.0 \%$ & $0.0 \%$ & $-5.5 \%$ & $-11.1 \%$ & $-3.0 \%$ & $-8.3 \%$ & $-28.2 \%$ & $-5.1 \%$ & $-26.6 \%$ & $-43.4 \%$ & $-4.3 \%$ & $-34.5 \%$ & $-45.7 \%$ \\
\hline & Usable Energy & $0.0 \%$ & $0.0 \%$ & $-5.5 \%$ & $-11.1 \%$ & $-3.0 \%$ & $-8.3 \%$ & $-16.3 \%$ & $-5.1 \%$ & $-14.3 \%$ & $-24.5 \%$ & $-4.3 \%$ & $-18.1 \%$ & $-27.6 \%$ \\
\hline \multirow{2}{*}{ CI Split HEV PHEV 20} & Total Energy & $0.0 \%$ & $0.0 \%$ & $-5.8 \%$ & $-11.9 \%$ & $-3.4 \%$ & $-11.3 \%$ & $-32.1 \%$ & $-6.2 \%$ & $-30.2 \%$ & $-50.0 \%$ & $-7.3 \%$ & $-37.0 \%$ & $-51.5 \%$ \\
\hline & Usable Energy & $0.0 \%$ & $0.0 \%$ & $-5.8 \%$ & $-11.9 \%$ & $-3.4 \%$ & $-11.3 \%$ & $-20.8 \%$ & $-6.2 \%$ & $-18.6 \%$ & $-33.4 \%$ & $-7.3 \%$ & $-21.2 \%$ & $-35.3 \%$ \\
\hline \multirow{2}{*}{ CI Series HEV PHEV30 } & Total Energy & $0.0 \%$ & $0.0 \%$ & $-6.2 \%$ & $-13.9 \%$ & $-3.7 \%$ & $-12.9 \%$ & $-32.2 \%$ & $-6.9 \%$ & $-30.3 \%$ & $-49.1 \%$ & $-8.5 \%$ & $-38.8 \%$ & $-49.4 \%$ \\
\hline & Usable Energy & $0.0 \%$ & $0.0 \%$ & $-6.2 \%$ & $-13.9 \%$ & $-3.7 \%$ & $-12.9 \%$ & $-20.9 \%$ & $-6.9 \%$ & $-18.7 \%$ & $-32.1 \%$ & $-8.5 \%$ & $-23.5 \%$ & $-32.6 \%$ \\
\hline \multirow{2}{*}{ CI Series HEV PHEV40 } & Total Energy & $0.0 \%$ & $0.0 \%$ & $-6.3 \%$ & $-12.6 \%$ & $-3.9 \%$ & $-11.7 \%$ & $-32.7 \%$ & $-7.0 \%$ & $-30.7 \%$ & $-48.2 \%$ & $-8.6 \%$ & $-36.9 \%$ & $-50.3 \%$ \\
\hline & Usable Energy & $0.0 \%$ & $0.0 \%$ & $-6.3 \%$ & $-12.6 \%$ & $-3.9 \%$ & $-11.7 \%$ & $-21.5 \%$ & $-7.0 \%$ & $-19.1 \%$ & $-30.9 \%$ & $-8.6 \%$ & $-21.2 \%$ & $-33.8 \%$ \\
\hline \multirow{2}{*}{ H2 Split HEV } & Total Energy & $0.0 \%$ & $0.0 \%$ & $-7.0 \%$ & $-12.5 \%$ & $-1.5 \%$ & $-12.8 \%$ & $-21.1 \%$ & $-5.8 \%$ & $-19.7 \%$ & $-34.9 \%$ & $-8.6 \%$ & $-21.1 \%$ & $-37.7 \%$ \\
\hline & Usable Energy & & & & & & & & & & & & & \\
\hline \multirow{2}{*}{ H2 Split HEV PHEV10 } & Total Energy & $0.0 \%$ & $0.0 \%$ & $-6.2 \%$ & $-13.1 \%$ & $-3.3 \%$ & $-12.1 \%$ & $-29.2 \%$ & $-3.8 \%$ & $-27.6 \%$ & $-45.3 \%$ & $-8.0 \%$ & $-33.8 \%$ & $-46.7 \%$ \\
\hline & Usable Energy & $0.0 \%$ & $0.0 \%$ & $-6.2 \%$ & $-13.1 \%$ & $-3.3 \%$ & $-12.1 \%$ & $-17.4 \%$ & $-3.8 \%$ & $-15.5 \%$ & $-27.1 \%$ & $-8.0 \%$ & $-17.3 \%$ & $-28.9 \%$ \\
\hline \multirow{2}{*}{ H2 Split HEV PHEV2O } & Total Energy & $0.0 \%$ & $0.0 \%$ & $-7.1 \%$ & $-13.9 \%$ & $-4.3 \%$ & $-13.1 \%$ & $-33.7 \%$ & $-6.9 \%$ & $-31.6 \%$ & $-50.6 \%$ & $-9.5 \%$ & $-39.6 \%$ & $-52.6 \%$ \\
\hline & Usable Energy & $0.0 \%$ & $0.0 \%$ & $-7.1 \%$ & $-13.9 \%$ & $-4.3 \%$ & $-13.1 \%$ & $-22.6 \%$ & $-6.9 \%$ & $-20.2 \%$ & $-34.1 \%$ & $-9.5 \%$ & $-24.4 \%$ & $-36.8 \%$ \\
\hline
\end{tabular}


Argonne National Laboratory Report - Light-Duty Vehicle Fuel Consumption Displacement Potential Up to 2045

\begin{tabular}{|c|c|c|c|c|c|c|c|c|c|c|c|c|c|c|}
\hline & & \multicolumn{13}{|c|}{ Pickup } \\
\hline & & \multirow{2}{*}{$\frac{2010}{\text { Ref }}$} & \multicolumn{3}{|c|}{2010} & \multicolumn{3}{|c|}{2015} & \multicolumn{3}{|c|}{2030} & \multicolumn{3}{|c|}{2045} \\
\hline & & & low & avg & high & low & avg & high & low & avg & high & low & avg & high \\
\hline \multirow{2}{*}{ H2 Series HEV PHEV30 } & Total Energy & $0.0 \%$ & $0.0 \%$ & $-7.4 \%$ & $-13.6 \%$ & $-3.6 \%$ & $-12.5 \%$ & $-34.0 \%$ & $-6.8 \%$ & $-31.8 \%$ & $-48.9 \%$ & $-10.9 \%$ & $-40.0 \%$ & $-50.9 \%$ \\
\hline & Usable Energy & $0.0 \%$ & $0.0 \%$ & $-7.4 \%$ & $-13.6 \%$ & $-3.6 \%$ & $-12.5 \%$ & $-23.0 \%$ & $-6.8 \%$ & $-20.5 \%$ & $-31.8 \%$ & $-10.9 \%$ & $-25.0 \%$ & $-34.5 \%$ \\
\hline \multirow{2}{*}{ H2 Series HEV PHEV40 } & Total Energy & $0.0 \%$ & $0.0 \%$ & $-7.6 \%$ & $-14.4 \%$ & $-4.3 \%$ & $-13.6 \%$ & $-34.2 \%$ & $-7.7 \%$ & $-32.0 \%$ & $-50.1 \%$ & $-10.3 \%$ & $-38.8 \%$ & $-52.2 \%$ \\
\hline & Usable Energy & $0.0 \%$ & $0.0 \%$ & $-7.6 \%$ & $-14.4 \%$ & $-4.3 \%$ & $-13.6 \%$ & $-23.3 \%$ & $-7.7 \%$ & $-20.7 \%$ & $-33.4 \%$ & $-10.3 \%$ & $-23.5 \%$ & $-36.3 \%$ \\
\hline \multirow{2}{*}{ E85 Split HEV } & Total Energy & $0.0 \%$ & $0.0 \%$ & $-5.1 \%$ & $-10.1 \%$ & $-1.0 \%$ & $-9.1 \%$ & $-17.5 \%$ & $-4.9 \%$ & $-16.1 \%$ & $-31.5 \%$ & $-4.9 \%$ & $-17.5 \%$ & $-34.3 \%$ \\
\hline & Usable Energy & & & & & & & & & & & & & \\
\hline \multirow{2}{*}{ E85 Split HEV PHEV10 } & Total Energy & $0.0 \%$ & $0.0 \%$ & $-5.4 \%$ & $-11.0 \%$ & $-2.7 \%$ & $-6.9 \%$ & $-28.9 \%$ & $-4.2 \%$ & $-24.2 \%$ & $-43.6 \%$ & $-3.8 \%$ & $-30.8 \%$ & $-45.8 \%$ \\
\hline & Usable Energy & $0.0 \%$ & $0.0 \%$ & $-5.4 \%$ & $-11.0 \%$ & $-2.7 \%$ & $-6.9 \%$ & $-17.0 \%$ & $-4.2 \%$ & $-11.5 \%$ & $-24.8 \%$ & $-3.8 \%$ & $-13.5 \%$ & $-27.8 \%$ \\
\hline \multirow{2}{*}{ E85 Split HEV PHEV20 } & Total Energy & $0.0 \%$ & $0.0 \%$ & $-5.5 \%$ & $-12.3 \%$ & $-3.3 \%$ & $-11.4 \%$ & $-32.3 \%$ & $-5.8 \%$ & $-30.2 \%$ & $-48.7 \%$ & $-7.3 \%$ & $-37.0 \%$ & $-50.9 \%$ \\
\hline & Usable Energy & $0.0 \%$ & $0.0 \%$ & $-5.5 \%$ & $-12.3 \%$ & $-3.3 \%$ & $-11.4 \%$ & $-21.0 \%$ & $-5.8 \%$ & $-18.6 \%$ & $-31.7 \%$ & $-7.3 \%$ & $-21.2 \%$ & $-34.6 \%$ \\
\hline \multirow{2}{*}{ E85 Series HEV PHEV30 } & Total Energy & $0.0 \%$ & $0.0 \%$ & $-6.1 \%$ & $-13.2 \%$ & $-5.3 \%$ & $-11.6 \%$ & $-34.7 \%$ & $-8.2 \%$ & $-32.7 \%$ & $-48.0 \%$ & $-8.4 \%$ & $-39.0 \%$ & $-50.3 \%$ \\
\hline & Usable Energy & $0.0 \%$ & $0.0 \%$ & $-6.1 \%$ & $-13.2 \%$ & $-5.3 \%$ & $-11.6 \%$ & $-23.8 \%$ & $-8.2 \%$ & $-21.5 \%$ & $-30.7 \%$ & $-8.4 \%$ & $-23.8 \%$ & $-33.8 \%$ \\
\hline \multirow{2}{*}{ E85 Series HEV PHEV40 } & Total Energy & $0.0 \%$ & $0.0 \%$ & $-6.6 \%$ & $-13.2 \%$ & $-3.9 \%$ & $-11.8 \%$ & $-32.8 \%$ & $-7.4 \%$ & $-30.6 \%$ & $-48.6 \%$ & $-9.0 \%$ & $-37.4 \%$ & $-50.5 \%$ \\
\hline & Usable Energy & $0.0 \%$ & $0.0 \%$ & $-6.6 \%$ & $-13.2 \%$ & $-3.9 \%$ & $-11.8 \%$ & $-21.6 \%$ & $-7.4 \%$ & $-19.1 \%$ & $-31.4 \%$ & $-9.0 \%$ & $-21.7 \%$ & $-34.0 \%$ \\
\hline \multirow{2}{*}{ FC HEV } & Total Energy & $0.0 \%$ & $0.0 \%$ & $-15.9 \%$ & $-22.8 \%$ & $-12.2 \%$ & $-14.4 \%$ & $-23.5 \%$ & $-8.8 \%$ & $-22.3 \%$ & $-34.7 \%$ & $-13.3 \%$ & $-24.6 \%$ & $-38.1 \%$ \\
\hline & Usable Energy & & & & & & & & & & & & & \\
\hline \multirow{2}{*}{ FC HEV PHEV10 } & Total Energy & $0.0 \%$ & $0.0 \%$ & $-13.6 \%$ & $-30.1 \%$ & $-9.8 \%$ & $-23.7 \%$ & $-41.7 \%$ & $-16.0 \%$ & $-39.9 \%$ & $-59.1 \%$ & $-20.5 \%$ & $-50.1 \%$ & $-60.8 \%$ \\
\hline & Usable Energy & $0.0 \%$ & $0.0 \%$ & $-13.6 \%$ & $-30.1 \%$ & $-9.8 \%$ & $-23.7 \%$ & $-32.0 \%$ & $-16.0 \%$ & $-29.9 \%$ & $-45.4 \%$ & $-20.5 \%$ & $-37.6 \%$ & $-47.7 \%$ \\
\hline \multirow{2}{*}{ FC HEV PHEV20 } & Total Energy & $0.0 \%$ & $0.0 \%$ & $-13.7 \%$ & $-21.9 \%$ & $-10.2 \%$ & $-21.1 \%$ & $-40.6 \%$ & $-14.9 \%$ & $-38.7 \%$ & $-54.9 \%$ & $-18.7 \%$ & $-44.7 \%$ & $-56.9 \%$ \\
\hline & Usable Energy & $0.0 \%$ & $0.0 \%$ & $-13.7 \%$ & $-21.9 \%$ & $-10.2 \%$ & $-21.1 \%$ & $-30.7 \%$ & $-14.9 \%$ & $-28.5 \%$ & $-39.9 \%$ & $-18.7 \%$ & $-30.8 \%$ & $-42.5 \%$ \\
\hline \multirow{2}{*}{ FC HEV PHEV 30} & Total Energy & $0.0 \%$ & $0.0 \%$ & $-11.8 \%$ & $-23.6 \%$ & $-8.2 \%$ & $-19.6 \%$ & $-42.6 \%$ & $-13.5 \%$ & $-40.8 \%$ & $-55.8 \%$ & $-17.4 \%$ & $-46.6 \%$ & $-57.8 \%$ \\
\hline & Usable Energy & $0.0 \%$ & $0.0 \%$ & $-11.8 \%$ & $-23.6 \%$ & $-8.2 \%$ & $-19.6 \%$ & $-33.1 \%$ & $-13.5 \%$ & $-30.9 \%$ & $-41.1 \%$ & $-17.4 \%$ & $-33.3 \%$ & $-43.8 \%$ \\
\hline \multirow{2}{*}{ FC HEV PHEV 40} & Total Energy & $0.0 \%$ & $0.0 \%$ & $-13.8 \%$ & $-21.8 \%$ & $-10.4 \%$ & $-21.6 \%$ & $-42.1 \%$ & $-15.7 \%$ & $-40.3 \%$ & $-55.5 \%$ & $-19.6 \%$ & $-46.3 \%$ & $-57.6 \%$ \\
\hline & Usable Energy & $0.0 \%$ & $0.0 \%$ & $-13.8 \%$ & $-21.8 \%$ & $-10.4 \%$ & $-21.6 \%$ & $-32.5 \%$ & $-15.7 \%$ & $-30.3 \%$ & $-40.6 \%$ & $-19.6 \%$ & $-32.8 \%$ & $-43.4 \%$ \\
\hline \multirow{2}{*}{ EV } & Total Energy & $0.0 \%$ & $0.0 \%$ & $-6.9 \%$ & $-14.1 \%$ & $-4.2 \%$ & $-13.1 \%$ & $-36.7 \%$ & $-8.1 \%$ & $-34.8 \%$ & $-53.1 \%$ & $-10.5 \%$ & $-41.4 \%$ & $-55.7 \%$ \\
\hline & Usable Energy & $0.0 \%$ & $0.0 \%$ & $-6.9 \%$ & $-14.1 \%$ & $-4.2 \%$ & $-13.1 \%$ & $-26.2 \%$ & $-8.1 \%$ & $-23.9 \%$ & $-37.5 \%$ & $-10.5 \%$ & $-26.7 \%$ & $-40.9 \%$ \\
\hline
\end{tabular}


Curb weight

\begin{tabular}{|c|c|c|c|c|c|c|c|c|c|c|c|c|c|c|}
\hline & & \multicolumn{13}{|c|}{ Pickup } \\
\hline & & \multirow{2}{*}{2010} & \multicolumn{3}{|c|}{2010} & \multicolumn{3}{|c|}{2015} & \multicolumn{3}{|c|}{2030} & \multicolumn{3}{|c|}{2045} \\
\hline & & & low & avg & high & low & avg & high & low & avg & high & low & avg & high \\
\hline \multirow{3}{*}{ SI Conv } & Vehicle Mass & 2040 & 2040 & 1965 & 1888 & 2039 & 1911 & 1770 & 2029 & 1790 & 1511 & 2030 & 1769 & 1429 \\
\hline & $\%$ of Glider mass & 74.0 & 74.0 & 73.3 & 72.4 & 74.1 & 72.8 & 71.1 & 74.1 & 71.3 & 67.0 & 74.0 & 71.1 & 65.4 \\
\hline & $\%$ of Powertrain mass & 26.0 & 26.0 & 26.7 & 27.6 & 25.9 & 27.2 & 28.9 & 25.9 & 28.7 & 33.0 & 26.0 & 28.9 & 34.6 \\
\hline \multirow{3}{*}{ Cl Conv } & Vehicle Mass & 2078 & 2078 & 2006 & 1931 & 2079 & 1956 & 1818 & 2069 & 1837 & 1565 & 2069 & 1817 & 1486 \\
\hline & $\%$ of Glider mass & 72.6 & 72.6 & 71.8 & 70.8 & 72.7 & 71.2 & 69.2 & 72.6 & 69.5 & 64.7 & 72.6 & 69.2 & 62.9 \\
\hline & $\%$ of Powertrain mass & 27.4 & 27.4 & 28.2 & 29.2 & 27.3 & 28.8 & 30.8 & 27.4 & 30.5 & 35.3 & 27.4 & 30.8 & 37.1 \\
\hline \multirow{3}{*}{ H2 Conv } & Vehicle Mass & 2026 & 2026 & 1910 & 1818 & 2012 & 1843 & 1688 & 1993 & 1718 & 1419 & 1955 & 1689 & 1347 \\
\hline & $\%$ of Glider mass & 74.5 & 74.5 & 75.4 & 75.2 & 75.1 & 75.5 & 74.6 & 75.4 & 74.3 & 71.3 & 76.9 & 74.4 & 69.4 \\
\hline & $\%$ of Powertrain mass & 25.5 & 25.5 & 24.6 & 24.8 & 24.9 & 24.5 & 25.4 & 24.6 & 25.7 & 28.7 & 23.1 & 25.6 & 30.6 \\
\hline \multirow{3}{*}{ E85 Conv } & Vehicle Mass & 2039 & 2039 & 1963 & 1886 & 2038 & 1910 & 1770 & 2028 & 1790 & 1510 & 2029 & 1769 & 1430 \\
\hline & $\%$ of Glider mass & 74.0 & 74.0 & 73.3 & 72.5 & 74.1 & 72.9 & 71.1 & 74.1 & 71.3 & 67.0 & 74.1 & 71.1 & 65.4 \\
\hline & $\%$ of Powertrain mass & 26.0 & 26.0 & 26.7 & 27.5 & 25.9 & 27.1 & 28.9 & 25.9 & 28.7 & 33.0 & 25.9 & 28.9 & 34.6 \\
\hline \multirow{3}{*}{ SI Split HEV } & Vehicle Mass & 2239 & 2239 & 2127 & 2019 & 2204 & 2069 & 1877 & 2191 & 1916 & 1575 & 2178 & 1877 & 1487 \\
\hline & $\%$ of Glider mass & 67.4 & 67.4 & 67.7 & 67.7 & 68.5 & 67.3 & 67.1 & 68.6 & 66.6 & 64.2 & 69.0 & 67.0 & 62.9 \\
\hline & $\%$ of Powertrain mass & 32.6 & 32.6 & 32.3 & 32.3 & 31.5 & 32.7 & 32.9 & 31.4 & 33.4 & 35.8 & 31.0 & 33.0 & 37.1 \\
\hline \multirow{3}{*}{ SI Split HEV PHEV10 } & Vehicle Mass & 2268 & 2268 & 2154 & 2043 & 2229 & 2080 & 1878 & 2201 & 1918 & 1582 & 2184 & 1886 & 1495 \\
\hline & $\%$ of Glider mass & 66.5 & 66.5 & 66.8 & 66.9 & 67.8 & 66.9 & 67.0 & 68.3 & 66.6 & 64.0 & 68.8 & 66.6 & 62.5 \\
\hline & $\%$ of Powertrain mass & 33.5 & 33.5 & 33.2 & 33.1 & 32.2 & 33.1 & 33.0 & 31.7 & 33.4 & 36.0 & 31.2 & 33.4 & 37.5 \\
\hline \multirow{3}{*}{ SI Split HEV PHEV20 } & Vehicle Mass & 2318 & 2318 & 2202 & 2086 & 2279 & 2123 & 1915 & 2247 & 1954 & 1604 & 2229 & 1914 & 1517 \\
\hline & $\%$ of Glider mass & 65.1 & 65.1 & 65.4 & 65.5 & 66.3 & 65.6 & 65.7 & 66.9 & 65.3 & 63.1 & 67.4 & 65.7 & 61.6 \\
\hline & $\%$ of Powertrain mass & 34.9 & 34.9 & 34.6 & 34.5 & 33.7 & 34.4 & 34.3 & 33.1 & 34.7 & 36.9 & 32.6 & 34.3 & 38.4 \\
\hline \multirow{3}{*}{ SI Series HEV PHEV30 } & Vehicle Mass & 2616 & 2616 & 2459 & 2310 & 2543 & 2359 & 2089 & 2488 & 2144 & 1742 & 2456 & 2085 & 1643 \\
\hline & $\%$ of Glider mass & 57.7 & 57.7 & 58.5 & 59.2 & 59.4 & 59.0 & 60.3 & 60.4 & 59.5 & 58.1 & 61.2 & 60.3 & 56.9 \\
\hline & $\%$ of Powertrain mass & 42.3 & 42.3 & 41.5 & 40.8 & 40.6 & 41.0 & 39.7 & 39.6 & 40.5 & 41.9 & 38.8 & 39.7 & 43.1 \\
\hline \multirow{3}{*}{ SI Series HEV PHEV40 } & Vehicle Mass & 2695 & 2695 & 2529 & 2373 & 2616 & 2426 & 2139 & 2557 & 2195 & 1776 & 2520 & 2127 & 1677 \\
\hline & $\%$ of Glider mass & 56.0 & 56.0 & 56.9 & 57.6 & 57.8 & 57.4 & 58.8 & 58.8 & 58.2 & 57.0 & 59.6 & 59.1 & 55.7 \\
\hline & $\%$ of Powertrain mass & 44.0 & 44.0 & 43.1 & 42.4 & 42.2 & 42.6 & 41.2 & 41.2 & 41.8 & 43.0 & 40.4 & 40.9 & 44.3 \\
\hline \multirow{3}{*}{ CI Split HEV } & Vehicle Mass & 2332 & 2332 & 2214 & 2102 & 2291 & 2157 & 1960 & 2276 & 1999 & 1660 & 2259 & 1965 & 1574 \\
\hline & $\%$ of Glider mass & 64.7 & 64.7 & 65.0 & 65.0 & 65.9 & 64.5 & 64.2 & 66.0 & 63.9 & 61.0 & 66.5 & 64.0 & 59.4 \\
\hline & $\%$ of Powertrain mass & 35.3 & 35.3 & 35.0 & 35.0 & 34.1 & 35.5 & 35.8 & 34.0 & 36.1 & 39.0 & 33.5 & 36.0 & 40.6 \\
\hline
\end{tabular}




\begin{tabular}{|c|c|c|c|c|c|c|c|c|c|c|c|c|c|c|}
\hline & & \multicolumn{13}{|c|}{ Pickup } \\
\hline & & \multirow{2}{*}{$\begin{array}{l}2010 \\
\text { Ref }\end{array}$} & \multicolumn{3}{|c|}{2010} & \multicolumn{3}{|c|}{2015} & \multicolumn{3}{|c|}{2030} & \multicolumn{3}{|c|}{2045} \\
\hline & & & low & avg & high & low & avg & high & low & avg & high & low & avg & high \\
\hline \multirow{3}{*}{ CI Split HEV PHEV10 } & Vehicle Mass & 2367 & 2367 & 2252 & 2141 & 2327 & 2178 & 1978 & 2296 & 2016 & 1680 & 2279 & 1983 & 1592 \\
\hline & $\%$ of Glider mass & 63.8 & 63.8 & 63.9 & 63.8 & 64.9 & 63.9 & 63.6 & 65.5 & 63.3 & 60.2 & 65.9 & 63.4 & 58.7 \\
\hline & $\%$ of Powertrain mass & 36.2 & 36.2 & 36.1 & 36.2 & 35.1 & 36.1 & 36.4 & 34.5 & 36.7 & 39.8 & 34.1 & 36.6 & 41.3 \\
\hline \multirow{3}{*}{ CI Split HEV PHEV2O } & Vehicle Mass & 2417 & 2417 & 2299 & 2184 & 2375 & 2221 & 2011 & 2344 & 2052 & 1702 & 2326 & 2011 & 1612 \\
\hline & $\%$ of Glider mass & 62.4 & 62.4 & 62.6 & 62.6 & 63.6 & 62.7 & 62.6 & 64.1 & 62.2 & 59.5 & 64.6 & 62.5 & 58.0 \\
\hline & $\%$ of Powertrain mass & 37.6 & 37.6 & 37.4 & 37.4 & 36.4 & 37.3 & 37.4 & 35.9 & 37.8 & 40.5 & 35.4 & 37.5 & 42.0 \\
\hline \multirow{3}{*}{$\mathrm{Cl}$ Series HEV PHEV30 } & Vehicle Mass & 2705 & 2705 & 2550 & 2400 & 2635 & 2451 & 2183 & 2580 & 2237 & 1833 & 2544 & 2176 & 1739 \\
\hline & $\%$ of Glider mass & 55.8 & 55.8 & 56.5 & 56.9 & 57.3 & 56.8 & 57.7 & 58.3 & 57.1 & 55.2 & 59.1 & 57.8 & 53.8 \\
\hline & $\%$ of Powertrain mass & 44.2 & 44.2 & 43.5 & 43.1 & 42.7 & 43.2 & 42.3 & 41.7 & 42.9 & 44.8 & 40.9 & 42.2 & 46.2 \\
\hline \multirow{3}{*}{$\mathrm{Cl}$ Series HEV PHEV40 } & Vehicle Mass & 2786 & 2786 & 2621 & 2465 & 2707 & 2515 & 2230 & 2649 & 2287 & 1868 & 2611 & 2219 & 1772 \\
\hline & $\%$ of Glider mass & 54.2 & 54.2 & 54.9 & 55.4 & 55.8 & 55.3 & 56.4 & 56.7 & 55.8 & 54.2 & 57.6 & 56.6 & 52.8 \\
\hline & $\%$ of Powertrain mass & 45.8 & 45.8 & 45.1 & 44.6 & 44.2 & 44.7 & 43.6 & 43.3 & 44.2 & 45.8 & 42.4 & 43.4 & 47.2 \\
\hline \multirow{3}{*}{ H2 Split HEV } & Vehicle Mass & 2264 & 2264 & 2107 & 1981 & 2212 & 2031 & 1822 & 2190 & 1870 & 1509 & 2134 & 1829 & 1432 \\
\hline & $\%$ of Glider mass & 66.7 & 66.7 & 68.3 & 69.0 & 68.3 & 68.5 & 69.1 & 68.6 & 68.3 & 67.1 & 70.4 & 68.7 & 65.3 \\
\hline & $\%$ of Powertrain mass & 33.3 & 33.3 & 31.7 & 31.0 & 31.7 & 31.5 & 30.9 & 31.4 & 31.7 & 32.9 & 29.6 & 31.3 & 34.7 \\
\hline \multirow{3}{*}{ H2 Split HEV PHEV10 } & \begin{tabular}{|l|} 
Vehicle Mass \\
\end{tabular} & 2299 & 2299 & 2137 & 2007 & 2244 & 2047 & 1828 & 2205 & 1878 & 1518 & 2146 & 1838 & 1440 \\
\hline & $\%$ of Glider mass & 65.6 & 65.6 & 67.4 & 68.1 & 67.3 & 68.0 & 68.9 & 68.2 & 68.0 & 66.7 & 70.0 & 68.4 & 64.9 \\
\hline & $\%$ of Powertrain mass & 34.4 & 34.4 & 32.6 & 31.9 & 32.7 & 32.0 & 31.1 & 31.8 & 32.0 & 33.3 & 30.0 & 31.6 & 35.1 \\
\hline \multirow{3}{*}{ H2 Split HEV PHEV2O } & \begin{tabular}{|l|} 
Vehicle Mass \\
\end{tabular} & 2350 & 2350 & 2184 & 2048 & 2291 & 2091 & 1862 & 2251 & 1914 & 1541 & 2190 & 1864 & 1461 \\
\hline & $\%$ of Glider mass & 64.2 & 64.2 & 65.9 & 66.7 & 65.9 & 66.6 & 67.6 & 66.8 & 66.7 & 65.7 & 68.6 & 67.4 & 64.0 \\
\hline & $\%$ of Powertrain mass & 35.8 & 35.8 & 34.1 & 33.3 & 34.1 & 33.4 & 32.4 & 33.2 & 33.3 & 34.3 & 31.4 & 32.6 & 36.0 \\
\hline \multirow{3}{*}{ H2 Series HEV PHEV 30} & Vehicle Mass & 2633 & 2633 & 2430 & 2264 & 2544 & 2314 & 2030 & 2481 & 2096 & 1670 & 2405 & 2027 & 1585 \\
\hline & $\%$ of Glider mass & 57.3 & 57.3 & 59.2 & 60.4 & 59.4 & 60.2 & 62.0 & 60.6 & 60.9 & 60.6 & 62.5 & 62.0 & 59.0 \\
\hline & $\%$ of Powertrain mass & 42.7 & 42.7 & 40.8 & 39.6 & 40.6 & 39.8 & 38.0 & 39.4 & 39.1 & 39.4 & 37.5 & 38.0 & 41.0 \\
\hline \multirow{3}{*}{ H2 Series HEV PHEV 40} & Vehicle Mass & 2713 & 2713 & 2500 & 2327 & 2617 & 2379 & 2078 & 2549 & 2144 & 1705 & 2468 & 2070 & 1617 \\
\hline & $\%$ of Glider mass & 55.6 & 55.6 & 57.6 & 58.7 & 57.7 & 58.5 & 60.6 & 59.0 & 59.5 & 59.3 & 60.9 & 60.7 & 57.8 \\
\hline & $\%$ of Powertrain mass & 44.4 & 44.4 & 42.4 & 41.3 & 42.3 & 41.5 & 39.4 & 41.0 & 40.5 & 40.7 & 39.1 & 39.3 & 42.2 \\
\hline \multirow{3}{*}{ E85 Split HEV } & Vehicle Mass & 2246 & 2246 & 2132 & 2024 & 2208 & 2073 & 1880 & 2195 & 1918 & 1578 & 2181 & 1883 & 1492 \\
\hline & $\%$ of Glider mass & 67.2 & 67.2 & 67.5 & 67.5 & 68.4 & 67.1 & 67.0 & 68.5 & 66.6 & 64.1 & 68.9 & 66.8 & 62.7 \\
\hline & $\%$ of Powertrain mass & 32.8 & 32.8 & 32.5 & 32.5 & 31.6 & 32.9 & 33.0 & 31.5 & 33.4 & 35.9 & 31.1 & 33.2 & 37.3 \\
\hline
\end{tabular}




\begin{tabular}{|c|c|c|c|c|c|c|c|c|c|c|c|c|c|c|}
\hline & & \multicolumn{13}{|c|}{ Pickup } \\
\hline & & \multirow{2}{*}{$\frac{2010}{\text { Ref }}$} & \multicolumn{3}{|c|}{2010} & \multicolumn{3}{|c|}{2015} & \multicolumn{3}{|c|}{2030} & \multicolumn{3}{|c|}{2045} \\
\hline & & & low & avg & high & low & avg & high & low & avg & high & low & avg & high \\
\hline \multirow{3}{*}{ E85 Split HEV PHEV10 } & Vehicle Mass & 2274 & 2274 & 2157 & 2046 & 2234 & 2085 & 1881 & 2205 & 1921 & 1585 & 2188 & 1890 & 1499 \\
\hline & $\%$ of Glider mass & 66.4 & 66.4 & 66.7 & 66.8 & 67.6 & 66.8 & 66.9 & 68.2 & 66.4 & 63.8 & 68.7 & 66.5 & 62.4 \\
\hline & $\%$ of Powertrain mass & 33.6 & 33.6 & 33.3 & 33.2 & 32.4 & 33.2 & 33.1 & 31.8 & 33.6 & 36.2 & 31.3 & 33.5 & 37.6 \\
\hline \multirow{3}{*}{ E85 Split HEV PHEV20 } & Vehicle Mass & 2323 & 2323 & 2205 & 2089 & 2282 & 2128 & 1918 & 2252 & 1958 & 1608 & 2234 & 1917 & 1520 \\
\hline & $\%$ of Glider mass & 65.0 & 65.0 & 65.3 & 65.4 & 66.2 & 65.4 & 65.6 & 66.7 & 65.2 & 62.9 & 67.3 & 65.6 & 61.5 \\
\hline & $\%$ of Powertrain mass & 35.0 & 35.0 & 34.7 & 34.6 & 33.8 & 34.6 & 34.4 & 33.3 & 34.8 & 37.1 & 32.7 & 34.4 & 38.5 \\
\hline \multirow{3}{*}{ E85 Series HEV PHEV30 } & \begin{tabular}{|l|} 
Vehicle Mass \\
\end{tabular} & 2619 & 2619 & 2462 & 2314 & 2546 & 2364 & 2092 & 2495 & 2149 & 1745 & 2459 & 2088 & 1647 \\
\hline & $\%$ of Glider mass & 57.6 & 57.6 & 58.5 & 59.1 & 59.3 & 58.9 & 60.2 & 60.2 & 59.4 & 58.0 & 61.1 & 60.2 & 56.8 \\
\hline & $\%$ of Powertrain mass & 42.4 & 42.4 & 41.5 & 40.9 & 40.7 & 41.1 & 39.8 & 39.8 & 40.6 & 42.0 & 38.9 & 39.8 & 43.2 \\
\hline \multirow{3}{*}{ E85 Series HEV PHEV40 } & Vehicle Mass & 2698 & 2698 & 2533 & 2376 & 2619 & 2429 & 2142 & 2562 & 2198 & 1779 & 2523 & 2131 & 1680 \\
\hline & $\%$ of Glider mass & 55.9 & 55.9 & 56.8 & 57.5 & 57.7 & 57.3 & 58.8 & 58.7 & 58.1 & 56.9 & 59.6 & 59.0 & 55.6 \\
\hline & $\%$ of Powertrain mass & 44.1 & 44.1 & 43.2 & 42.5 & 42.3 & 42.7 & 41.2 & 41.3 & 41.9 & 43.1 & 40.4 & 41.0 & 44.4 \\
\hline \multirow{3}{*}{ FC HEV } & Vehicle Mass & 2949 & 2949 & 2441 & 2168 & 2578 & 2198 & 1902 & 2397 & 1953 & 1534 & 2246 & 1875 & 1441 \\
\hline & $\%$ of Glider mass & 51.2 & 51.2 & 59.0 & 63.0 & 58.6 & 63.3 & 66.2 & 62.7 & 65.4 & 66.0 & 66.9 & 67.0 & 64.9 \\
\hline & $\%$ of Powertrain mass & 48.8 & 48.8 & 41.0 & 37.0 & 41.4 & 36.7 & 33.8 & 37.3 & 34.6 & 34.0 & 33.1 & 33.0 & 35.1 \\
\hline \multirow{3}{*}{ FC HEV PHEV10 } & Vehicle Mass & 2800 & 2800 & 2374 & 2135 & 2510 & 2156 & 1874 & 2357 & 1925 & 1513 & 2219 & 1854 & 1423 \\
\hline & $\%$ of Glider mass & 53.9 & 53.9 & 60.6 & 64.0 & 60.2 & 64.6 & 67.2 & 63.8 & 66.3 & 66.9 & 67.7 & 67.8 & 65.7 \\
\hline & $\%$ of Powertrain mass & 46.1 & 46.1 & 39.4 & 36.0 & 39.8 & 35.4 & 32.8 & 36.2 & 33.7 & 33.1 & 32.3 & 32.2 & 34.3 \\
\hline \multirow{3}{*}{ FC HEV PHEV2O } & Vehicle Mass & 2879 & 2879 & 2437 & 2190 & 2574 & 2211 & 1913 & 2414 & 1966 & 1542 & 2270 & 1886 & 1448 \\
\hline & $\%$ of Glider mass & 52.4 & 52.4 & 59.1 & 62.4 & 58.7 & 63.0 & 65.8 & 62.3 & 64.9 & 65.6 & 66.2 & 66.6 & 64.6 \\
\hline & $\%$ of Powertrain mass & 47.6 & 47.6 & 40.9 & 37.6 & 41.3 & 37.0 & 34.2 & 37.7 & 35.1 & 34.4 & 33.8 & 33.4 & 35.4 \\
\hline \multirow{3}{*}{ FC HEV PHEV 30} & Vehicle Mass & 2953 & 2953 & 2545 & 2309 & 2688 & 2332 & 2018 & 2540 & 2078 & 1642 & 2391 & 1988 & 1543 \\
\hline & $\%$ of Glider mass & 51.1 & 51.1 & 56.6 & 59.2 & 56.2 & 59.7 & 62.4 & 59.2 & 61.4 & 61.6 & 62.9 & 63.2 & 60.6 \\
\hline & $\%$ of Powertrain mass & 48.9 & 48.9 & 43.4 & 40.8 & 43.8 & 40.3 & 37.6 & 40.8 & 38.6 & 38.4 & 37.1 & 36.8 & 39.4 \\
\hline \multirow{3}{*}{ FC HEV PHEV40 } & Vehicle Mass & 3046 & 3046 & 2618 & 2369 & 2761 & 2391 & 2063 & 2606 & 2123 & 1674 & 2452 & 2025 & 1572 \\
\hline & $\%$ of Glider mass & 49.5 & 49.5 & 55.0 & 57.7 & 54.7 & 58.2 & 61.0 & 57.7 & 60.1 & 60.4 & 61.3 & 62.1 & 59.5 \\
\hline & $\%$ of Powertrain mass & 50.5 & 50.5 & 45.0 & 42.3 & 45.3 & 41.8 & 39.0 & 42.3 & 39.9 & 39.6 & 38.7 & 37.9 & 40.5 \\
\hline \multirow{3}{*}{ BEV } & Vehicle Mass & 3081 & 3081 & 2875 & 2674 & 2990 & 2738 & 2298 & 2903 & 2357 & 1840 & 2829 & 2237 & 1719 \\
\hline & $\%$ of Glider mass & 49.0 & 49.0 & 50.1 & 51.1 & 50.5 & 50.8 & 54.8 & 51.8 & 54.2 & 55.0 & 53.1 & 56.2 & 54.4 \\
\hline & $\%$ of Powertrain mass & 51.0 & 51.0 & 49.9 & 48.9 & 49.5 & 49.2 & 45.2 & 48.2 & 45.8 & 45.0 & 46.9 & 43.8 & 45.6 \\
\hline
\end{tabular}


Percentage of vehicle curbs weight reduction based on the respective 2010 reference case

\begin{tabular}{|c|c|c|c|c|c|c|c|c|c|c|c|c|c|c|}
\hline & & \multicolumn{13}{|c|}{ Pickup } \\
\hline & & 2010 & & 2010 & & & 2015 & & & 2030 & & & 2045 & \\
\hline & & Ref & low & avg & high & low & avg & high & low & avg & high & low & avg & high \\
\hline SI Conv & Vehicle Mass & $0.00 \%$ & $0.00 \%$ & $-3.68 \%$ & $-7.45 \%$ & $-0.05 \%$ & $-6.32 \%$ & $-13.24 \%$ & $-0.54 \%$ & $-12.25 \%$ & $-25.93 \%$ & $-0.49 \%$ & $-13.28 \%$ & $-29.95 \%$ \\
\hline $\mathrm{Cl}$ Conv & Vehicle Mass & $0.00 \%$ & $0.00 \%$ & $-3.46 \%$ & $-7.07 \%$ & $0.05 \%$ & $-5.87 \%$ & $-12.51 \%$ & $-0.43 \%$ & $-11.60 \%$ & $-24.69 \%$ & $-0.43 \%$ & $-12.56 \%$ & $-28.49 \%$ \\
\hline H2 Conv & Vehicle Mass & $0.00 \%$ & $0.00 \%$ & $-5.73 \%$ & $-10.27 \%$ & $-0.69 \%$ & $-9.03 \%$ & $-16.68 \%$ & $-1.63 \%$ & $-15.20 \%$ & $-29.96 \%$ & $-3.50 \%$ & $-16.63 \%$ & $-33.51 \%$ \\
\hline E85 Conv & Vehicle Mass & $0.00 \%$ & $0.00 \%$ & $-3.73 \%$ & $-7.50 \%$ & $-0.05 \%$ & $-6.33 \%$ & $-13.19 \%$ & $-0.54 \%$ & $-12.21 \%$ & $-25.94 \%$ & $-0.49 \%$ & $-13.24 \%$ & $-29.87 \%$ \\
\hline SI Split HEV & Vehicle Mass & $0.00 \%$ & $0.00 \%$ & $-5.00 \%$ & $-9.83 \%$ & $-1.56 \%$ & $-7.59 \%$ & $-16.17 \%$ & $-2.14 \%$ & $-14.43 \%$ & $-29.66 \%$ & $-2.72 \%$ & $-16.17 \%$ & $-33.59 \%$ \\
\hline SI Split HEV PHEV10 & Vehicle Mass & $0.00 \%$ & $0.00 \%$ & $-5.03 \%$ & $-9.92 \%$ & $-1.72 \%$ & $-8.29 \%$ & $-17.20 \%$ & $-2.95 \%$ & $-15.43 \%$ & $-30.25 \%$ & $-3.70 \%$ & $-16.84 \%$ & $-34.08 \%$ \\
\hline SI Split HEV PHEV20 & Vehicle Mass & $0.00 \%$ & $0.00 \%$ & $-5.00 \%$ & $-10.01 \%$ & $-1.68 \%$ & $-8.41 \%$ & $-17.39 \%$ & $-3.06 \%$ & $-15.70 \%$ & $-30.80 \%$ & $-3.84 \%$ & $-17.43 \%$ & $-34.56 \%$ \\
\hline SI Series HEV PHEV30 & Vehicle Mass & $0.00 \%$ & $0.00 \%$ & $-6.00 \%$ & $-11.70 \%$ & $-2.79 \%$ & $-9.82 \%$ & $-20.15 \%$ & $-4.89 \%$ & $-18.04 \%$ & $-33.41 \%$ & $-6.12 \%$ & $-20.30 \%$ & $-37.19 \%$ \\
\hline SI Series HEV PHEV40 & Vehicle Mass & $0.00 \%$ & $0.00 \%$ & $-6.16 \%$ & $-11.95 \%$ & $-2.93 \%$ & $-9.98 \%$ & $-20.63 \%$ & $-5.12 \%$ & $-18.55 \%$ & $-34.10 \%$ & $-6.49 \%$ & $-21.08 \%$ & $-37.77 \%$ \\
\hline $\mathrm{Cl}$ Split HEV & Vehicle Mass & $0.00 \%$ & $0.00 \%$ & $-5.06 \%$ & $-9.86 \%$ & $-1.76 \%$ & $-7.50 \%$ & $-15.95 \%$ & $-2.40 \%$ & $-14.28 \%$ & $-28.82 \%$ & $-3.13 \%$ & $-15.74 \%$ & $-32.50 \%$ \\
\hline $\mathrm{Cl}$ Split HEV PH & Vehicle Mass & $00 \%$ & $00 \%$ & $-4.86 \%$ & $-9.55 \%$ & $-1.69 \%$ & $-7.98 \%$ & $-16.43 \%$ & $-3.00 \%$ & $-14.83 \%$ & $-29.02 \%$ & $-3.72 \%$ & $-16.22 \%$ & $-32.74 \%$ \\
\hline CI Split HEV PHEV2O & Vehicle Mass & $0.00 \%$ & $0.00 \%$ & $-4.88 \%$ & $-9.64 \%$ & $-1.74 \%$ & $-8.11 \%$ & $-16.80 \%$ & $-3.02 \%$ & $-15.10 \%$ & $-29.58 \%$ & $-3.76 \%$ & $-16.80 \%$ & $-33.31 \%$ \\
\hline CI Series HEV PHEV 30 & Vehicle Mass & $0.00 \%$ & $0.00 \%$ & $-5.73 \%$ & $-11.28 \%$ & $-2.59 \%$ & $-9.39 \%$ & $-19.30 \%$ & $-4.62 \%$ & $-17.30 \%$ & $-32.24 \%$ & $-5.95 \%$ & $-19.56 \%$ & $-35.71 \%$ \\
\hline CI Series HEV PHEV40 & Vehicle Mass & $0.00 \%$ & $0.00 \%$ & $-5.92 \%$ & $-11.52 \%$ & $-2.84 \%$ & $-9.73 \%$ & $-19.96 \%$ & $-4.92 \%$ & $-17.91 \%$ & $-32.95 \%$ & $-6.28 \%$ & $-20.35 \%$ & $-36.40 \%$ \\
\hline H2 Split HEV & Vehicle Mass & $0.00 \%$ & $0.00 \%$ & $-6.93 \%$ & $-12.50 \%$ & $-2.30 \%$ & $-10.29 \%$ & $-19.52 \%$ & $-3.27 \%$ & $-17.40 \%$ & $-33.35 \%$ & $-5.74 \%$ & $-19.21 \%$ & $-36.75 \%$ \\
\hline H2 Split HEV PHEV10 & Vehicle Mass & $0.00 \%$ & $0.00 \%$ & $-7.05 \%$ & $-12.70 \%$ & $-2.39 \%$ & $-10.96 \%$ & $-20.49 \%$ & $-4.09 \%$ & $-18.31 \%$ & $-33.97 \%$ & $-6.66 \%$ & $-20.05 \%$ & $-37.36 \%$ \\
\hline H2 Split HEV PHEV2O & Vehicle Mass & $0.00 \%$ & $0.00 \%$ & $-7.06 \%$ & $-12.85 \%$ & $-2.51 \%$ & $-11.02 \%$ & $-20.77 \%$ & $-4.21 \%$ & $-18.55 \%$ & $-34.43 \%$ & $-6.81 \%$ & $-20.68 \%$ & $-37.83 \%$ \\
\hline H2 Series HEV PHEV 30 & Vehicle Mass & $0.00 \%$ & $0.00 \%$ & $-7.71 \%$ & $-14.01 \%$ & $-3.38 \%$ & $-12.12 \%$ & $-22.90 \%$ & $-5.77 \%$ & $-20.39 \%$ & $-36.57 \%$ & $-8.66 \%$ & $-23.02 \%$ & $-39.80 \%$ \\
\hline H2 Series HEV PHEV40 & Vehicle Mass & $0.00 \%$ & $0.00 \%$ & $-7.85 \%$ & $-14.23 \%$ & $-3.54 \%$ & $-12.31 \%$ & $-23.41 \%$ & $-6.04 \%$ & $-20.97 \%$ & $-37.15 \%$ & $-9.03 \%$ & $-23.70 \%$ & $-40.40 \%$ \\
\hline E85 Split HEV & Vehicle Mass & $0.00 \%$ & $0.00 \%$ & $-5.08 \%$ & $-9.88 \%$ & $-1.69 \%$ & $-7.70 \%$ & $-16.30 \%$ & $-2.27 \%$ & $-14.60 \%$ & $-29.74 \%$ & $-2.89 \%$ & $-16.16 \%$ & $-33.57 \%$ \\
\hline E85 Split HEV PHEV10 & Vehicle Mass & $0.00 \%$ & $0.00 \%$ & $-5.15 \%$ & $-10.03 \%$ & $-1.76 \%$ & $-8.31 \%$ & $-17.28 \%$ & $-3.03 \%$ & $-15.52 \%$ & $-30.30 \%$ & $-3.78 \%$ & $-16.89 \%$ & $-34.08 \%$ \\
\hline E85 Split HEV PHEV20 & Vehicle Mass & $0.00 \%$ & $0.00 \%$ & $-5.08 \%$ & $-10.07 \%$ & $-1.76 \%$ & $-8.39 \%$ & $-17.43 \%$ & $-3.06 \%$ & $-15.71 \%$ & $-30.78 \%$ & $-3.83 \%$ & $-17.48 \%$ & $-34.57 \%$ \\
\hline E85 Series HEV PHEV30 & Vehicle Mass & $0.00 \%$ & $0.00 \%$ & $-5.99 \%$ & $-11.65 \%$ & $-2.79 \%$ & $-9.74 \%$ & $-20.12 \%$ & $-4.73 \%$ & $-17.95 \%$ & $-33.37 \%$ & $-6.11 \%$ & $-20.27 \%$ & $-37.11 \%$ \\
\hline E85 Series HEV PHEV40 & Vehicle Mass & $0.00 \%$ & $0.00 \%$ & $-6.12 \%$ & $-11.93 \%$ & $-2.93 \%$ & $-9.97 \%$ & $-20.61 \%$ & $-5.04 \%$ & $-18.53 \%$ & $-34.06 \%$ & $-6.49 \%$ & $-21.02 \%$ & $-37.73 \%$ \\
\hline FC HEV & Vehicle Mass & $0.00 \%$ & $0.00 \%$ & $-17.23 \%$ & $-26.48 \%$ & $-12.58 \%$ & $-25.47 \%$ & $-35.50 \%$ & $-18.72 \%$ & $-33.77 \%$ & $-47.98 \%$ & $-23.84 \%$ & $-36.42 \%$ & $-51.14 \%$ \\
\hline FC HEV PHEV 10 & Vehicle Mass & $0.00 \%$ & $0.00 \%$ & $-15.21 \%$ & $-23.75 \%$ & $-10.36 \%$ & $-23.00 \%$ & $-33.07 \%$ & $-15.82 \%$ & $-31.25 \%$ & $-45.96 \%$ & $-20.75 \%$ & $-33.79 \%$ & $-49.18 \%$ \\
\hline FC HEV PHEV 20 & Vehicle Mass & $0.00 \%$ & $0.00 \%$ & $-15.35 \%$ & $-23.93 \%$ & $-10.59 \%$ & $-23.20 \%$ & $-33.55 \%$ & $-16.15 \%$ & $-31.71 \%$ & $-46.44 \%$ & $-21.15 \%$ & $-34.49 \%$ & $-49.70 \%$ \\
\hline FC HEV PHEV 30 & Vehicle Mass & $0.00 \%$ & $0.00 \%$ & $-13.82 \%$ & $-21.81 \%$ & $-8.97 \%$ & $-21.03 \%$ & $-31.66 \%$ & $-13.99 \%$ & $-29.63 \%$ & $-44.40 \%$ & $-19.03 \%$ & $-32.68 \%$ & $-47.75 \%$ \\
\hline FC HEV PHEV 40 & Vehicle Mass & $0.00 \%$ & $0.00 \%$ & $-14.05 \%$ & $-22.23 \%$ & $-9.36 \%$ & $-21.50 \%$ & $-32.27 \%$ & $-14.45 \%$ & $-30.30 \%$ & $-45.04 \%$ & $-19.50 \%$ & $-33.52 \%$ & $-48.39 \%$ \\
\hline EV & Vehicle N & $0.00 \%$ & $0.00 \%$ & $-6.69 \%$ & $-13.21 \%$ & $-2.95 \%$ & $-11.13 \%$ & $-25.41 \%$ & $-5.78 \%$ & $-25.00 \%$ & $-40.28 \%$ & $-8.18 \%$ & $-27.39 \%$ & \\
\hline
\end{tabular}




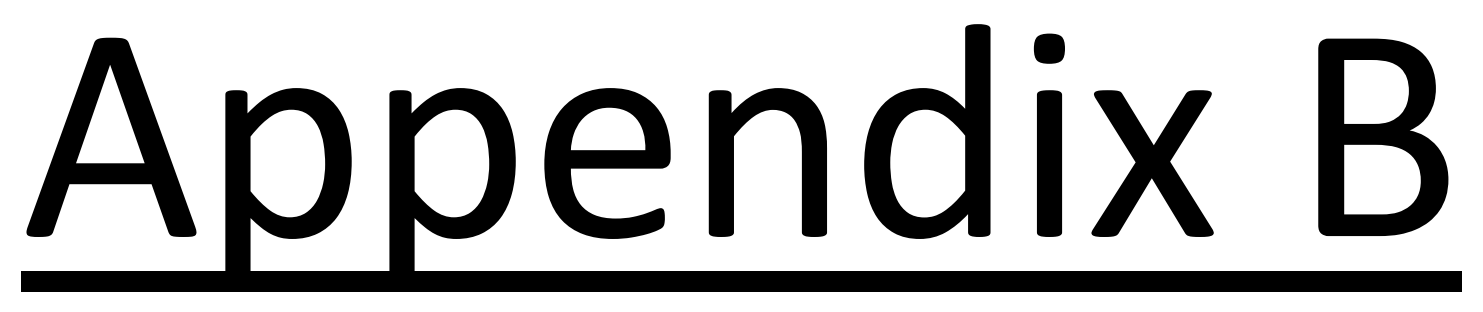

\section{Vehicle Energy Consumption Tables}


Compact Class 
Fuel economy

\begin{tabular}{|c|c|c|c|c|c|c|c|c|c|c|c|c|c|c|c|}
\hline & & & \multicolumn{13}{|c|}{ Compact } \\
\hline & & & 2010 & & 2010 & & & 2015 & & & 2030 & & & 2045 & \\
\hline & & & Ref & low & avg & high & low & avg & high & low & avg & high & low & avg & high \\
\hline \multirow{6}{*}{ SI Conv } & \multirow{3}{*}{ Unadjusted } & UDDS & 30.1 & 30.1 & 32.4 & 34.0 & 31.4 & 33.8 & 41.4 & 32.3 & 34.9 & 49.3 & 32.9 & 36.1 & 50.0 \\
\hline & & HWFET & 41.5 & 41.5 & 45.2 & 50.6 & 43.6 & 49.7 & 61.6 & 47.3 & 51.6 & 71.8 & 48.0 & 52.9 & 74.8 \\
\hline & & Combined & 34.3 & 34.3 & 37.2 & 39.9 & 35.9 & 39.5 & 48.6 & 37.7 & 40.8 & 57.4 & 38.4 & 42.1 & 58.8 \\
\hline & \multirow{3}{*}{ Adjusted } & \begin{tabular}{|l|} 
UDDS \\
\end{tabular} & 23.1 & 23.1 & 24.8 & 25.9 & 24.1 & 25.7 & 31.1 & 24.7 & 26.5 & 36.4 & 25.2 & 27.4 & 36.9 \\
\hline & & HWFET & 29.3 & 29.3 & 31.9 & 35.6 & 30.8 & 35.1 & 43.3 & 33.4 & 36.3 & 50.4 & 33.9 & 37.2 & 52.4 \\
\hline & & Combined & 25.5 & 25.5 & 27.6 & 29.5 & 26.7 & 29.2 & 35.6 & 28.0 & 30.2 & 41.6 & 28.4 & 31.1 & 42.6 \\
\hline \multirow{6}{*}{$\mathrm{Cl}$ Conv } & \multirow{3}{*}{ Unadjusted } & \begin{tabular}{|l|} 
UDDS \\
\end{tabular} & 35.2 & 35.2 & 36.9 & 39.8 & 35.6 & 38.9 & 43.3 & 37.2 & 42.2 & 50.1 & 38.8 & 44.2 & 52.1 \\
\hline & & HWFET & 47.9 & 47.9 & 50.8 & 55.7 & 48.7 & 54.1 & 60.6 & 51.5 & 58.7 & 69.5 & 53.7 & 61.5 & 74.0 \\
\hline & & Combined & 39.9 & 39.9 & 42.1 & 45.7 & 40.5 & 44.5 & 49.6 & 42.5 & 48.3 & 57.3 & 44.3 & 50.6 & 60.1 \\
\hline & \multirow{3}{*}{ Adjusted } & UDDS & 26.7 & 26.7 & 27.9 & 30.0 & 27.1 & 29.3 & 32.4 & 28.2 & 31.6 & 37.0 & 29.3 & 33.0 & 38.3 \\
\hline & & HWFET & 33.8 & 33.8 & 35.8 & 39.2 & 34.4 & 38.1 & 42.6 & 36.3 & 41.3 & 48.8 & 37.8 & 43.3 & 51.9 \\
\hline & & Combined & 29.5 & 29.5 & 31.0 & 33.5 & 29.9 & 32.7 & 36.3 & 31.3 & 35.4 & 41.5 & 32.6 & 37.0 & 43.4 \\
\hline \multirow{6}{*}{ H2 Conv } & \multirow{3}{*}{ Unadjusted } & UDDS & 25.6 & 25.6 & 30.3 & 34.2 & 28.9 & 33.5 & 37.8 & 31.3 & 36.6 & 45.2 & 33.8 & 39.7 & 48.3 \\
\hline & & HWFET & 37.7 & 37.7 & 44.8 & 54.6 & 42.6 & 53.1 & 60.2 & 49.6 & 58.2 & 70.5 & 53.3 & 62.6 & 77.2 \\
\hline & & Combined & 29.9 & 29.9 & 35.5 & 41.1 & 33.8 & 40.2 & 45.4 & 37.5 & 43.9 & 53.9 & 40.5 & 47.5 & 58.1 \\
\hline & \multirow{3}{*}{ Adjusted } & UDDS & 19.8 & 19.8 & 23.3 & 26.0 & 22.3 & 25.5 & 28.6 & 23.9 & 27.8 & 33.7 & 25.8 & 29.9 & 35.8 \\
\hline & & HWFET & 26.6 & 26.6 & 31.6 & 38.4 & 30.1 & 37.4 & 42.4 & 35.0 & 40.9 & 49.5 & 37.6 & 44.0 & 54.1 \\
\hline & & Combined & 22.4 & 22.4 & 26.4 & 30.5 & 25.2 & 29.8 & 33.5 & 27.9 & 32.5 & 39.3 & 30.0 & 35.0 & 42.2 \\
\hline \multirow{6}{*}{ E85 Conv } & \multirow{3}{*}{ Unadjusted } & \begin{tabular}{|l|} 
UDDS \\
\end{tabular} & 29.5 & 29.5 & 29.6 & 31.2 & 28.6 & 30.6 & 40.4 & 29.2 & 31.9 & 47.5 & 30.2 & 33.4 & 48.8 \\
\hline & & HWFET & 39.5 & 39.5 & 40.1 & 43.4 & 38.6 & 42.4 & 56.3 & 40.3 & 44.3 & 66.5 & 42.4 & 46.9 & 69.9 \\
\hline & & Combined & 33.3 & 33.3 & 33.5 & 35.7 & 32.4 & 35.0 & 46.3 & 33.3 & 36.5 & 54.5 & 34.7 & 38.3 & 56.5 \\
\hline & \multirow{3}{*}{ Adjusted } & UDDS & 22.7 & 22.7 & 22.8 & 23.9 & 22.0 & 23.5 & 30.4 & 22.4 & 24.4 & 35.3 & 23.2 & 25.4 & 36.2 \\
\hline & & HWFET & 27.9 & 27.9 & 28.3 & 30.7 & 27.3 & 29.9 & 39.7 & 28.5 & 31.2 & 46.7 & 29.9 & 33.1 & 49.1 \\
\hline & & Combined & 24.8 & 24.8 & 25.0 & 26.5 & 24.1 & 26.0 & 34.0 & 24.8 & 27.0 & 39.6 & 25.8 & 28.4 & 41.0 \\
\hline \multirow{6}{*}{ SI Split HEV } & \multirow{3}{*}{ Unadjusted } & UDDS & 60.0 & 60.0 & 64.2 & 69.7 & 60.3 & 68.9 & 84.8 & 65.1 & 76.1 & 105.9 & 67.4 & 79.3 & 111.5 \\
\hline & & HWFET & 52.6 & 52.6 & 57.0 & 62.1 & 53.4 & 60.2 & 73.6 & 56.8 & 65.9 & 89.7 & 59.1 & 69.0 & 97.2 \\
\hline & & Combined & 56.4 & 56.4 & 60.8 & 66.1 & 57.0 & 64.6 & 79.4 & 61.1 & 71.1 & 98.0 & 63.4 & 74.3 & 104.6 \\
\hline & \multirow{3}{*}{ Adjusted } & \begin{tabular}{|l|} 
UDDS \\
\end{tabular} & 43.5 & 43.5 & 46.2 & 49.7 & 43.7 & 49.1 & 58.8 & 46.8 & 53.6 & 70.8 & 48.2 & 55.6 & 73.9 \\
\hline & & HWFET & 37.0 & 37.0 & 40.1 & 43.7 & 37.6 & 42.3 & 51.6 & 40.0 & 46.3 & 62.7 & 41.6 & 48.4 & 67.8 \\
\hline & & Combined & 40.3 & 40.3 & 43.3 & 46.8 & 40.7 & 45.8 & 55.3 & 43.4 & 50.0 & 66.9 & 45.0 & 52.1 & 71.0 \\
\hline \multirow{6}{*}{ SI Split HEV PHEV10 } & \multirow{3}{*}{ Unadjusted } & \begin{tabular}{|l|} 
UDDS \\
\end{tabular} & 75.1 & 75.1 & 80.3 & 86.8 & 75.8 & 84.4 & 102.9 & 79.4 & 92.2 & 127.8 & 82.4 & 95.7 & 134.8 \\
\hline & & HWFET & 72.2 & 72.2 & 77.9 & 84.5 & 73.9 & 81.4 & 99.1 & 76.8 & 88.8 & 119.1 & 80.4 & 92.5 & 130.5 \\
\hline & & Combined & 73.8 & 73.8 & 79.2 & 85.7 & 74.9 & 83.0 & 101.2 & 78.2 & 90.7 & 123.7 & 81.5 & 94.3 & 132.8 \\
\hline & \multirow{3}{*}{ Adjusted } & UDDS & 53.0 & 53.0 & 56.1 & 60.0 & 53.4 & 58.6 & 69.2 & 55.6 & 63.1 & 82.3 & 57.4 & 65.1 & 85.9 \\
\hline & & HWFET & 50.7 & 50.7 & 54.6 & 59.1 & 51.9 & 57.0 & 69.1 & 53.8 & 62.1 & 82.7 & 56.3 & 64.6 & 90.4 \\
\hline & & Combined & 51.9 & 51.9 & 55.4 & 59.6 & 52.7 & 57.8 & 69.1 & 54.8 & 62.7 & 82.5 & 56.9 & 64.9 & 87.9 \\
\hline
\end{tabular}




\begin{tabular}{|c|c|c|c|c|c|c|c|c|c|c|c|c|c|c|c|}
\hline & & & \multicolumn{13}{|c|}{ Compact } \\
\hline & & & \multirow{2}{*}{$\begin{array}{l}2010 \\
\operatorname{Ref} \\
\end{array}$} & \multicolumn{3}{|c|}{2010} & \multicolumn{3}{|c|}{2015} & \multicolumn{3}{|c|}{2030} & \multicolumn{3}{|c|}{2045} \\
\hline & & & & low & avg & high & low & avg & high & low & avg & high & low & avg & high \\
\hline \multirow{6}{*}{ SI Split HEV PHEV2O } & \multirow{3}{*}{ Unadjusted } & UDDS & 108.2 & 108.2 & 115.5 & 104.8 & 108.9 & 101.6 & 149.1 & 114.5 & 134.1 & 156.0 & 119.0 & 115.7 & 164.2 \\
\hline & & HWFET & 71.8 & 71.8 & $\begin{array}{lll}80.4 \\
\end{array}$ & 87.3 & 73.4 & 84.3 & 102.4 & 76.6 & $\begin{array}{ll}91.8 \\
\end{array}$ & 123.7 & 82.7 & 96.0 & 134.2 \\
\hline & & Combined & 88.1 & 88.1 & 96.5 & 96.1 & 89.4 & 93.0 & 123.7 & 93.6 & 111.1 & 139.6 & 99.4 & 105.9 & 149.2 \\
\hline & \multirow{3}{*}{ Adjusted } & \begin{tabular}{|l|} 
UDDS \\
\end{tabular} & 72.0 & 72.0 & 76.0 & 70.2 & 72.4 & 68.4 & 92.9 & 75.4 & 85.5 & 96.1 & 77.8 & 76.1 & 99.9 \\
\hline & & HWFET & 50.4 & 50.4 & 56.3 & 61.0 & 51.5 & 59.0 & 71.4 & 53.7 & 64.1 & 85.9 & 57.9 & 67.0 & 92.9 \\
\hline & & Combined & 60.4 & 60.4 & 65.7 & 65.8 & 61.2 & 63.8 & 81.8 & 63.8 & 74.4 & 91.2 & 67.4 & 71.7 & 96.6 \\
\hline \multirow{6}{*}{ SI Series HEV PHEV30 } & \multirow{3}{*}{ Unadjusted } & \begin{tabular}{|l|} 
UDDS \\
\end{tabular} & 99.5 & 99.5 & 108.1 & 118.8 & 101.5 & 114.9 & 146.4 & 108.7 & 129.2 & 183.4 & 113.3 & 133.9 & 192.9 \\
\hline & & \begin{tabular}{|l|} 
HWFET \\
\end{tabular} & 96.2 & 96.2 & 105.1 & 115.9 & 98.8 & 111.6 & 139.5 & 104.9 & 124.8 & 172.0 & 110.3 & 131.1 & 189.3 \\
\hline & & \begin{tabular}{|l|} 
Combined \\
\end{tabular} & 98.0 & 98.0 & 106.7 & 117.4 & 100.3 & 113.4 & 143.2 & 106.9 & 127.2 & 178.1 & 111.9 & 132.6 & 191.3 \\
\hline & \multirow{3}{*}{ Adjusted } & \begin{tabular}{|l|} 
UDDS \\
\end{tabular} & 67.2 & 67.2 & 72.0 & 77.7 & 68.4 & 75.7 & 91.6 & 72.3 & 83.1 & 108.3 & 74.8 & 85.4 & 112.3 \\
\hline & & \begin{tabular}{|l|} 
HWFET \\
\end{tabular} & 67.1 & 67.1 & 73.2 & 80.5 & 68.9 & 77.7 & 96.5 & 73.1 & 86.6 & 118.2 & 76.8 & 90.8 & 129.6 \\
\hline & & \begin{tabular}{|l|} 
Combined \\
\end{tabular} & 67.2 & 67.2 & 72.5 & 78.9 & 68.6 & 76.6 & 93.7 & 72.7 & 84.6 & 112.5 & 75.7 & 87.8 & 119.5 \\
\hline \multirow{6}{*}{ SI Series HEV PHEV40 } & \multirow{3}{*}{ Unadjusted } & \begin{tabular}{|l|} 
UDDS \\
\end{tabular} & 113.7 & 113.7 & 123.8 & 136.8 & 115.9 & 132.1 & 167.5 & 124.5 & 150.1 & 217.9 & 130.5 & 156.8 & 230.0 \\
\hline & & HWFET & 94.8 & 94.8 & 128.8 & 142.1 & 120.7 & 136.9 & 171.7 & 128.8 & 153.5 & 171.3 & 135.4 & 161.6 & 231.9 \\
\hline & & Combined & 104.3 & 104.3 & 126.0 & 139.1 & 118.0 & 134.2 & 169.3 & 126.4 & 151.6 & 194.1 & 132.7 & 158.9 & 230.9 \\
\hline & \multirow{3}{*}{ Adjusted } & \begin{tabular}{|l|} 
UDDS \\
\end{tabular} & 75.0 & 75.0 & 80.3 & 86.9 & 76.2 & 84.6 & 101.3 & 80.7 & 93.3 & 122.3 & 83.7 & 96.5 & 126.9 \\
\hline & & HWFET & 66.2 & 66.2 & 89.3 & 98.2 & 83.8 & 94.7 & 117.9 & 89.3 & 105.8 & 117.6 & 93.7 & 111.2 & 157.3 \\
\hline & & Combined & 70.8 & 70.8 & 84.1 & 91.6 & 79.5 & 88.9 & 108.2 & 84.3 & 98.5 & 120.2 & 87.9 & 102.6 & 139.0 \\
\hline \multirow{6}{*}{ CI Split HEV } & \multirow{3}{*}{ Unadjusted } & \begin{tabular}{|l|} 
UDDS \\
\end{tabular} & 64.2 & 64.2 & 67.6 & 74.4 & 63.9 & 73.3 & 84.5 & 69.5 & 82.1 & 102.4 & 72.7 & 86.4 & 107.3 \\
\hline & & HWFET & 55.1 & 55.1 & 59.2 & 65.1 & 56.1 & 63.1 & 72.3 & 59.9 & 70.2 & 86.2 & 63.0 & 74.4 & 92.9 \\
\hline & & Combined & 59.8 & 59.8 & 63.6 & 69.9 & 60.2 & 68.4 & 78.6 & 64.8 & 76.2 & 94.4 & 68.0 & 80.5 & 100.3 \\
\hline & \multirow{3}{*}{ Adjusted } & UDDS & 46.2 & 46.2 & 48.3 & 52.6 & 46.0 & 51.9 & 58.7 & 49.6 & 57.2 & 68.8 & 51.5 & 59.7 & 71.6 \\
\hline & & HWFET & 38.8 & 38.8 & 41.7 & 45.8 & 39.5 & 44.4 & 50.8 & 42.1 & 49.2 & 60.3 & 44.3 & 52.2 & 64.9 \\
\hline & & Combined & 42.6 & 42.6 & 45.1 & 49.3 & 42.9 & 48.2 & 54.8 & 45.9 & 53.3 & 64.7 & 48.0 & 56.1 & 68.4 \\
\hline \multirow{6}{*}{ CI Split HEV PHEV10 } & \multirow{3}{*}{ Unadjusted } & UDDS & 75.7 & 75.7 & 80.1 & 87.9 & 75.4 & 85.2 & 97.8 & 80.8 & 95.2 & 118.6 & 84.8 & 99.3 & 124.8 \\
\hline & & HWFET & 72.0 & 72.0 & 77.7 & 85.6 & 72.4 & 82.8 & 94.4 & 78.0 & 91.8 & 111.2 & 82.3 & 96.6 & 121.9 \\
\hline & & \begin{tabular}{|l|} 
Combined \\
\end{tabular} & 74.0 & 74.0 & 79.0 & 86.8 & 74.0 & 84.1 & 96.2 & 79.5 & 93.6 & 115.2 & 83.7 & 98.1 & 123.5 \\
\hline & \multirow{3}{*}{ Adjusted } & \begin{tabular}{|l|} 
UDDS \\
\end{tabular} & 53.3 & 53.3 & 56.0 & 60.6 & 53.2 & 59.0 & 66.3 & 56.4 & 64.8 & 77.6 & 58.8 & 67.1 & 80.8 \\
\hline & & \begin{tabular}{|l|} 
HWFET \\
\end{tabular} & 50.5 & 50.5 & 54.5 & 59.9 & 50.8 & 58.0 & 65.9 & 54.7 & 64.1 & 77.4 & 57.6 & 67.4 & 84.6 \\
\hline & & Combined & 52.0 & 52.0 & 55.3 & 60.3 & 52.1 & 58.6 & 66.1 & 55.6 & 64.5 & 77.5 & 58.3 & 67.3 & 82.5 \\
\hline \multirow{6}{*}{ CI Split HEV PHEV2O } & & UDDS & 107.9 & 107.9 & 115.1 & 126.7 & 107.4 & 122.8 & 142.3 & 116.3 & 137.7 & 143.6 & 122.2 & 144.7 & 152.1 \\
\hline & Unadjusted & HWFET & 87.3 & 87.3 & 94.6 & 87.0 & 87.7 & 101.1 & 97.5 & 93.8 & 94.1 & 114.8 & 101.1 & 99.5 & 124.2 \\
\hline & & Combined & 97.5 & 97.5 & 104.9 & 105.1 & 97.6 & 112.0 & 117.9 & 104.9 & 113.9 & 129.0 & 111.7 & 120.1 & 138.1 \\
\hline & & \begin{tabular}{|l|} 
UDDS \\
\end{tabular} & 71.9 & 71.9 & 75.8 & 81.8 & 71.6 & 79.8 & 89.6 & 76.4 & 87.3 & 90.2 & 79.5 & 90.7 & 94.2 \\
\hline & Adjusted & HWFET & 61.0 & 61.0 & 66.1 & 60.9 & 61.3 & 70.5 & 68.1 & 65.5 & 65.7 & 79.8 & 70.5 & 69.4 & 86.2 \\
\hline & & Combined & 66.6 & 66.6 & 71.1 & 70.8 & 66.6 & 75.3 & 78.4 & 71.1 & 76.0 & 85.2 & 75.2 & 79.7 & 90.4 \\
\hline
\end{tabular}




\begin{tabular}{|c|c|c|c|c|c|c|c|c|c|c|c|c|c|c|c|}
\hline & & & \multicolumn{13}{|c|}{ Compact } \\
\hline & & & \multirow{2}{*}{$\begin{array}{l}2010 \\
\operatorname{Ref} \\
\end{array}$} & \multicolumn{3}{|c|}{2010} & \multicolumn{3}{|c|}{2015} & \multicolumn{3}{|c|}{2030} & \multicolumn{3}{|c|}{2045} \\
\hline & & & & low & avg & high & low & avg & high & low & avg & high & low & avg & high \\
\hline \multirow{6}{*}{$\mathrm{Cl}$ Series HEV PHEV3O } & \multirow{3}{*}{ Unadjusted } & UDDS & 100.3 & 100.3 & 108.0 & 120.0 & 100.8 & 116.2 & 142.4 & 110.4 & 136.4 & 171.3 & 116.2 & 143.0 & 180.3 \\
\hline & & HWFET & 97.5 & 97.5 & 105.5 & 118.1 & 98.8 & 113.3 & 134.2 & 107.2 & 129.4 & 161.5 & 113.8 & 137.4 & 176.2 \\
\hline & & Combined & 99.0 & 99.0 & 106.8 & 119.1 & 99.9 & 114.9 & 138.6 & 108.9 & 133.2 & 166.8 & 115.1 & 140.4 & 178.5 \\
\hline & \multirow{3}{*}{ Adjusted } & \begin{tabular}{|l|} 
UDDS \\
\end{tabular} & 67.7 & 67.7 & 71.9 & 78.3 & 68.0 & 76.4 & 89.6 & 73.2 & 86.7 & 103.0 & 76.4 & 89.9 & 107.0 \\
\hline & & HWFET & 68.1 & 68.1 & 73.5 & 82.1 & 68.9 & 78.8 & 92.9 & 74.6 & 89.7 & 111.2 & 79.1 & 95.0 & 120.9 \\
\hline & & Combined & 67.9 & 67.9 & 72.6 & 80.0 & 68.4 & 77.4 & 91.1 & 73.9 & 88.0 & 106.6 & 77.6 & 92.1 & 112.8 \\
\hline \multirow{6}{*}{$\mathrm{Cl}$ Series HEV PHEV40 } & \multirow{3}{*}{ Unadjusted } & \begin{tabular}{|l|} 
UDDS \\
\end{tabular} & 114.7 & 114.7 & 123.5 & 138.2 & 115.1 & 133.8 & 159.2 & 126.7 & 158.0 & 202.6 & 134.0 & 165.9 & 214.2 \\
\hline & & \begin{tabular}{|l|} 
HWFET \\
\end{tabular} & 119.5 & 119.5 & 129.3 & 144.3 & 120.5 & 139.0 & 165.1 & 131.5 & 159.2 & 200.3 & 139.6 & 169.2 & 217.8 \\
\hline & & Combined & 116.8 & 116.8 & 126.1 & 140.9 & 117.5 & 136.1 & 161.8 & 128.8 & 158.5 & 201.5 & 136.4 & 167.4 & 215.8 \\
\hline & \multirow{3}{*}{ Adjusted } & \begin{tabular}{|l|} 
UDDS \\
\end{tabular} & 75.5 & 75.5 & 80.2 & 87.6 & 75.8 & 85.4 & 97.6 & 81.8 & 97.0 & 116.3 & 85.5 & 100.6 & 120.9 \\
\hline & & \begin{tabular}{|l|} 
HWFET \\
\end{tabular} & 83.0 & 83.0 & 89.6 & 99.7 & 83.7 & 96.1 & 113.5 & 91.1 & 109.6 & 136.7 & 96.5 & 116.3 & 148.1 \\
\hline & & \begin{tabular}{|l|} 
Combined \\
\end{tabular} & 78.7 & 78.7 & 84.2 & 92.6 & 79.2 & 89.9 & 104.2 & 85.7 & 102.3 & 124.7 & 90.1 & 107.1 & 131.8 \\
\hline \multirow{6}{*}{ H2 Split HEV } & \multirow{3}{*}{ Unadjusted } & \begin{tabular}{|l|} 
UDDS \\
\end{tabular} & 57.8 & 57.8 & 68.8 & 80.1 & 63.9 & 78.8 & 93.7 & 72.9 & 90.4 & 115.3 & 78.9 & 97.4 & 125.2 \\
\hline & & HWFET & 50.3 & 50.3 & 60.3 & 70.1 & 56.2 & 67.7 & 80.1 & 62.9 & 77.2 & 96.0 & 68.5 & 83.2 & 107.5 \\
\hline & & Combined & 54.1 & 54.1 & 64.7 & 75.3 & 60.2 & 73.4 & 87.1 & 68.1 & 83.9 & 105.7 & 73.8 & 90.5 & 116.5 \\
\hline & \multirow{3}{*}{ Adjusted } & UDDS & 42.1 & 42.1 & 49.1 & 56.0 & 46.0 & 55.2 & 64.0 & 51.6 & 62.1 & 75.8 & 55.3 & 66.0 & 81.0 \\
\hline & & HWFET & 35.4 & 35.4 & 42.4 & 49.2 & 39.6 & 47.6 & 56.1 & 44.3 & 54.1 & 67.0 & 48.1 & 58.3 & 74.8 \\
\hline & & Combined & 38.8 & 38.8 & 45.8 & 52.7 & 42.9 & 51.5 & 60.2 & 48.0 & 58.2 & 71.6 & 51.8 & 62.3 & 78.1 \\
\hline \multirow{6}{*}{ H2 Split HEV PHEV10 } & \multirow{3}{*}{ Unadjusted } & UDDS & 72.1 & 72.1 & 85.6 & 99.1 & 79.6 & 96.0 & 113.4 & 88.9 & 108.7 & 138.2 & 97.2 & 116.2 & 151.2 \\
\hline & & HWFET & 69.8 & 69.8 & 83.1 & 95.8 & 78.1 & 92.5 & 107.9 & 86.5 & 103.8 & 127.3 & 94.1 & 111.9 & 144.5 \\
\hline & & Combined & 71.0 & 71.0 & 84.5 & 97.6 & 78.9 & 94.4 & 110.9 & 87.8 & 106.4 & 133.1 & 95.8 & 114.2 & 148.1 \\
\hline & \multirow{3}{*}{ Adjusted } & UDDS & 51.2 & 51.2 & 59.3 & 67.0 & 55.7 & 65.3 & 74.9 & 61.2 & 72.3 & 87.6 & 65.9 & 76.3 & 93.8 \\
\hline & & HWFET & 49.0 & 49.0 & 58.2 & 66.8 & 54.7 & 64.6 & 75.1 & 60.5 & 72.3 & 88.3 & 65.7 & 77.8 & 99.8 \\
\hline & & Combined & 50.2 & 50.2 & 58.8 & 66.9 & 55.3 & 65.0 & 75.0 & 60.9 & 72.3 & 87.9 & 65.8 & 77.0 & 96.4 \\
\hline \multirow{6}{*}{ H2 Split HEV PHEV2O } & \multirow{3}{*}{ Unadjusted } & UDDS & 103.6 & 103.6 & 123.3 & 143.8 & 114.6 & 138.7 & 136.7 & 128.2 & 131.6 & 168.8 & 139.7 & 140.8 & 184.2 \\
\hline & & HWFET & 83.9 & 83.9 & 82.6 & 99.1 & 95.0 & 95.3 & 111.9 & 85.8 & 107.7 & 132.6 & 96.9 & 116.1 & 149.3 \\
\hline & & \begin{tabular}{|l|} 
Combined \\
\end{tabular} & 93.7 & 93.7 & 100.9 & 119.5 & 104.9 & 115.1 & 124.3 & 104.9 & 119.6 & 150.4 & 116.5 & 128.5 & 166.7 \\
\hline & \multirow{3}{*}{ Adjusted } & \begin{tabular}{|l|} 
UDDS \\
\end{tabular} & 69.6 & 69.6 & 80.1 & 90.3 & 75.5 & 87.8 & 86.8 & 82.6 & 84.3 & 101.9 & 88.3 & 88.8 & 108.7 \\
\hline & & \begin{tabular}{|l|} 
HWFET \\
\end{tabular} & 58.7 & 58.7 & 57.8 & 69.1 & 66.4 & 66.6 & 77.8 & 60.0 & 75.0 & 91.9 & 67.6 & 80.7 & 103.1 \\
\hline & & Combined & 64.2 & 64.2 & 68.2 & 79.4 & 71.1 & 76.8 & 82.5 & 70.6 & 79.8 & 97.1 & 77.6 & 85.0 & 106.1 \\
\hline \multirow{6}{*}{ H2 Series HEV PHEV3O } & & UDDS & 94.5 & 94.5 & 114.1 & 136.1 & 105.8 & 129.0 & 159.3 & 120.2 & 153.6 & 187.8 & 131.3 & 161.3 & 202.0 \\
\hline & Unadjusted & HWFET & 94.0 & 94.0 & 113.3 & 132.3 & 105.5 & 127.6 & 153.4 & 118.9 & 147.2 & 143.0 & 130.3 & 159.4 & 205.6 \\
\hline & & Combined & 94.2 & 94.2 & 113.7 & 134.3 & 105.7 & 128.4 & 156.6 & 119.6 & 150.7 & 164.6 & 130.9 & 160.4 & 203.6 \\
\hline & & UDDS & 64.4 & 64.4 & 75.2 & 86.5 & 70.8 & 83.0 & 97.6 & 78.5 & 95.0 & 110.2 & 84.1 & 98.5 & 116.0 \\
\hline & Adjusted & HWFET & 65.6 & 65.6 & 78.8 & 91.6 & 73.5 & 88.5 & 105.7 & 82.6 & 101.7 & 98.8 & 90.3 & 109.7 & 140.2 \\
\hline & & Combined & 64.9 & 64.9 & 76.8 & 88.7 & 72.0 & 85.4 & 101.1 & 80.3 & 97.9 & 104.7 & 86.8 & 103.3 & 125.8 \\
\hline
\end{tabular}




\begin{tabular}{|c|c|c|c|c|c|c|c|c|c|c|c|c|c|c|c|}
\hline & & & \multicolumn{13}{|c|}{ Compact } \\
\hline & & & 2010 & & 2010 & & & 2015 & & & 2030 & & & 2045 & \\
\hline & & & Ref & low & avg & high & low & avg & high & low & avg & high & low & avg & high \\
\hline \multirow{6}{*}{ H2 Series HEV PHEV40 } & \multirow{3}{*}{ Unadjusted } & UDDS & 108.2 & 108.2 & 131.2 & 153.9 & 121.7 & 148.6 & 185.4 & 137.8 & 175.3 & 223.9 & 151.8 & 189.6 & 241.9 \\
\hline & & HWFET & 114.8 & 114.8 & 138.5 & 162.6 & 129.2 & 156.4 & 188.8 & 145.6 & 181.1 & 182.6 & 160.3 & 196.6 & 255.2 \\
\hline & & Combined & 111.1 & 111.1 & 134.3 & 157.7 & 124.9 & 152.0 & 186.9 & 141.2 & 177.8 & 203.2 & 155.5 & 192.7 & 247.7 \\
\hline & \multirow{3}{*}{ Adjusted } & UDDS & 72.1 & 72.1 & 84.1 & 95.1 & 79.2 & 92.6 & 109.1 & 87.3 & 104.8 & 124.6 & 94.1 & 110.9 & 131.3 \\
\hline & & \begin{tabular}{|l|} 
HWFET \\
\end{tabular} & 79.8 & 79.8 & 95.8 & 111.9 & 89.5 & 107.8 & 129.2 & 100.5 & 124.1 & 125.2 & 110.4 & 134.3 & 172.2 \\
\hline & & Combined & 75.4 & 75.4 & 89.0 & 102.0 & 83.5 & 98.9 & 117.3 & 92.8 & 112.7 & 124.9 & 100.8 & 120.4 & 147.0 \\
\hline \multirow{6}{*}{ E85 Split HEV } & \multirow{3}{*}{ Unadjusted } & UDDS & 55.9 & 55.9 & 59.8 & 64.7 & 56.3 & 63.8 & 83.4 & 60.6 & 71.0 & 99.6 & 63.0 & 74.2 & 105.3 \\
\hline & & HWFET & 48.6 & 48.6 & 52.8 & 57.3 & 49.7 & 55.6 & 72.2 & 52.6 & 61.4 & 84.3 & 54.9 & 64.3 & 91.4 \\
\hline & & Combined & 52.4 & 52.4 & 56.4 & 61.1 & 53.1 & 59.8 & 78.0 & 56.7 & 66.3 & 92.1 & 59.1 & 69.4 & 98.6 \\
\hline & \multirow{3}{*}{ Adjusted } & UDDS & 40.8 & 40.8 & 43.4 & 46.5 & 41.1 & 46.0 & 58.0 & 43.9 & 50.5 & 67.3 & 45.4 & 52.4 & 70.5 \\
\hline & & \begin{tabular}{|l|} 
HWFET \\
\end{tabular} & 34.3 & 34.3 & 37.2 & 40.4 & 35.0 & 39.1 & 50.6 & 37.1 & 43.2 & 59.0 & 38.7 & 45.2 & 63.9 \\
\hline & & Combined & 37.6 & 37.6 & 40.4 & 43.5 & 38.1 & 42.6 & 54.4 & 40.5 & 46.9 & 63.3 & 42.1 & 48.9 & 67.3 \\
\hline \multirow{6}{*}{ E85 Split HEV PHEV10 } & \multirow{3}{*}{ Unadjusted } & UDDS & 70.3 & 70.3 & 75.2 & 81.4 & 71.0 & 79.0 & 101.6 & 74.6 & 86.5 & 120.6 & 77.3 & 89.7 & 127.3 \\
\hline & & HWFET & 67.2 & 67.2 & 72.5 & 78.7 & 68.8 & 76.0 & 97.4 & 71.6 & 82.6 & 112.0 & 74.9 & 86.5 & 122.8 \\
\hline & & Combined & 68.9 & 68.9 & 74.0 & 80.2 & 70.0 & 77.6 & 99.7 & 73.2 & 84.7 & 116.5 & 76.2 & 88.2 & 125.2 \\
\hline & \multirow{3}{*}{ Adjusted } & UDDS & 50.0 & 50.0 & 53.1 & 56.8 & 50.4 & 55.3 & 68.4 & 52.7 & 59.8 & 78.6 & 54.3 & 61.6 & 82.1 \\
\hline & & HWFET & 47.2 & 47.2 & 50.9 & 55.1 & 48.3 & 53.3 & 68.0 & 50.2 & 57.8 & 77.9 & 52.5 & 60.5 & 85.2 \\
\hline & & Combined & 48.7 & 48.7 & 52.1 & 56.0 & 49.5 & 54.4 & 68.2 & 51.5 & 58.9 & 78.3 & 53.5 & 61.1 & 83.5 \\
\hline \multirow{6}{*}{ E85 Split HEV PHEV20 } & \multirow{3}{*}{ Unadjusted } & UDDS & 101.3 & 101.3 & 108.3 & 98.4 & 102.1 & 95.2 & 147.7 & 107.3 & 125.8 & 147.4 & 111.7 & 108.6 & 155.2 \\
\hline & & HWFET & 66.9 & 66.9 & 74.8 & 81.4 & 68.4 & 78.4 & 100.5 & 71.4 & 85.7 & 116.5 & 77.3 & 89.5 & 126.6 \\
\hline & & Combined & 82.3 & 82.3 & 90.1 & 89.9 & 83.5 & 86.8 & 121.9 & 87.5 & 103.9 & 131.7 & 93.0 & 99.1 & 140.9 \\
\hline & \multirow{3}{*}{ Adjusted } & UDDS & 68.3 & 68.3 & 72.1 & 66.6 & 68.7 & 64.8 & 92.2 & 71.6 & 81.4 & 92.0 & 73.9 & 72.3 & 95.7 \\
\hline & & HWFET & 47.0 & 47.0 & 52.4 & 57.0 & 48.0 & 54.9 & 70.1 & 50.1 & 59.9 & 81.0 & 54.2 & 62.5 & 87.8 \\
\hline & & Combined & 56.7 & 56.7 & 61.7 & 61.9 & 57.5 & 60.0 & 80.7 & 60.0 & 70.1 & 86.7 & 63.5 & 67.5 & 92.0 \\
\hline \multirow{6}{*}{ E85 Series HEV PHEV30 } & \multirow{3}{*}{ Unadjusted } & UDDS & 93.6 & 93.6 & 101.8 & 111.9 & 95.5 & 108.3 & 143.6 & 101.9 & 123.6 & 174.5 & 106.8 & 126.5 & 183.5 \\
\hline & & HWFET & 90.3 & 90.3 & 98.8 & 109.0 & 92.6 & 105.0 & 138.4 & 98.7 & 117.4 & 163.1 & 103.8 & 123.4 & 177.8 \\
\hline & & \begin{tabular}{|l|} 
Combined \\
\end{tabular} & 92.1 & 92.1 & 100.4 & 110.6 & 94.2 & 106.8 & 141.2 & 100.4 & 120.7 & 169.1 & 105.4 & 125.1 & 180.9 \\
\hline & \multirow{3}{*}{ Adjusted } & UDDS & 63.9 & 63.9 & 68.5 & 74.1 & 65.0 & 72.1 & 90.2 & 68.6 & 80.2 & 104.4 & 71.3 & 81.7 & 108.3 \\
\hline & & HWFET & 63.1 & 63.1 & 68.9 & 75.8 & 64.7 & 73.2 & 95.7 & 68.8 & 81.6 & 112.2 & 72.3 & 85.7 & 122.0 \\
\hline & & Combined & 63.6 & 63.6 & 68.7 & 74.9 & 64.8 & 72.6 & 92.6 & 68.7 & 80.8 & 107.8 & 71.8 & 83.4 & 114.1 \\
\hline \multirow{6}{*}{ E85 Series HEV PHEV40 } & \multirow{3}{*}{ Unadjusted } & UDDS & 106.9 & 106.9 & 116.8 & 128.9 & 109.1 & 124.5 & 166.4 & 117.5 & 141.8 & 207.0 & 122.4 & 147.1 & 218.5 \\
\hline & & HWFET & 89.0 & 89.0 & 121.0 & 133.7 & 113.7 & 128.4 & 170.2 & 121.0 & 144.4 & 162.4 & 127.4 & 152.1 & 220.0 \\
\hline & & Combined & 98.0 & 98.0 & 118.6 & 131.0 & 111.1 & 126.2 & 168.1 & 119.1 & 143.0 & 184.2 & 124.6 & 149.3 & 219.2 \\
\hline & \multirow{3}{*}{ Adjusted } & UDDS & 71.4 & 71.4 & 76.6 & 82.9 & 72.6 & 80.7 & 100.8 & 77.0 & 89.3 & 118.0 & 79.6 & 91.9 & 122.5 \\
\hline & & HWFET & 62.2 & 62.2 & 84.0 & 92.6 & 79.1 & 89.0 & 117.0 & 84.0 & 99.8 & 111.7 & 88.3 & 104.9 & 149.6 \\
\hline & & Combined & 66.9 & 66.9 & 79.8 & 87.0 & 75.3 & 84.2 & 107.5 & 80.0 & 93.7 & 115.1 & 83.3 & 97.3 & 133.4 \\
\hline
\end{tabular}




\begin{tabular}{|c|c|c|c|c|c|c|c|c|c|c|c|c|c|c|c|}
\hline & & & \multicolumn{13}{|c|}{ Compact } \\
\hline & & & \multirow{2}{*}{$\begin{array}{l}2010 \\
\operatorname{Ref} \\
\end{array}$} & \multicolumn{3}{|c|}{2010} & \multicolumn{3}{|c|}{2015} & \multicolumn{3}{|c|}{2030} & \multicolumn{3}{|c|}{2045} \\
\hline & & & & low & avg & high & low & avg & high & low & avg & high & low & avg & high \\
\hline \multirow{6}{*}{ FC HEV } & \multirow{3}{*}{ Unadjusted } & UDDS & 64.5 & 64.5 & 76.8 & 91.1 & 75.4 & 89.5 & 104.7 & 83.2 & 101.2 & 124.7 & 88.2 & 105.0 & 131.8 \\
\hline & & HWFET & 67.8 & 67.8 & 79.5 & 93.0 & 78.3 & 89.9 & 102.7 & 83.8 & 99.0 & 118.3 & 88.5 & 103.1 & 129.0 \\
\hline & & Combined & 66.0 & 66.0 & 78.0 & 92.0 & 76.7 & 89.7 & 103.8 & 83.5 & 100.2 & 121.7 & 88.3 & 104.1 & 130.5 \\
\hline & \multirow{3}{*}{ Adjusted } & \begin{tabular}{|l|} 
UDDS \\
\end{tabular} & 46.4 & 46.4 & 54.0 & 62.5 & 53.1 & 61.6 & 70.2 & 57.9 & 68.2 & 80.8 & 60.8 & 70.3 & 84.4 \\
\hline & & \begin{tabular}{|l|} 
HWFET \\
\end{tabular} & 47.6 & 47.6 & 55.7 & 65.0 & 54.9 & 62.9 & 71.6 & 58.7 & 69.1 & 82.2 & 61.9 & 71.8 & 89.4 \\
\hline & & \begin{tabular}{|l|} 
Combined \\
\end{tabular} & 46.9 & 46.9 & 54.7 & 63.6 & 53.9 & 62.1 & 70.8 & 58.2 & 68.6 & 81.4 & 61.3 & 71.0 & 86.6 \\
\hline \multirow{6}{*}{ FC PHEV10 } & \multirow{3}{*}{ Unadjusted } & UDDS & 81.2 & 81.2 & 94.8 & 111.8 & 93.2 & 108.0 & 125.1 & 100.3 & 120.7 & 147.6 & 105.8 & 124.6 & 155.6 \\
\hline & & HWFET & 90.8 & 90.8 & 104.1 & 121.6 & 102.5 & 116.7 & 133.1 & 108.7 & 127.7 & 151.6 & 114.4 & 132.6 & 166.6 \\
\hline & & Combined & 85.2 & 85.2 & 98.8 & 116.0 & 97.1 & 111.8 & 128.6 & 103.9 & 123.8 & 149.3 & 109.5 & 128.1 & 160.4 \\
\hline & \multirow{3}{*}{ Adjusted } & \begin{tabular}{|l|} 
UDDS \\
\end{tabular} & 56.7 & 56.7 & 64.6 & 74.0 & 63.7 & 72.0 & 81.0 & 67.7 & 78.7 & 92.1 & 70.8 & 80.7 & 95.9 \\
\hline & & HWFET & 63.5 & 63.5 & 72.5 & 84.4 & 71.5 & 81.1 & 92.2 & 75.7 & 88.6 & 104.5 & 79.6 & 91.9 & 114.6 \\
\hline & & Combined & 59.5 & 59.5 & 68.0 & 78.3 & 66.9 & 75.8 & 85.7 & 71.1 & 82.9 & 97.3 & 74.5 & 85.4 & 103.5 \\
\hline \multirow{6}{*}{ FC PHEV2O } & \multirow{3}{*}{ Unadjusted } & \begin{tabular}{|l|} 
UDDS \\
\end{tabular} & 97.9 & 97.9 & 114.4 & 134.6 & 111.8 & 130.0 & 150.9 & 120.8 & 145.9 & 179.0 & 127.6 & 150.7 & 188.9 \\
\hline & & HWFET & 91.6 & 91.6 & 105.7 & 123.4 & 104.0 & 118.5 & 135.1 & 110.4 & 130.0 & 155.4 & 116.4 & 135.5 & 169.7 \\
\hline & & \begin{tabular}{|l|} 
Combined \\
\end{tabular} & 95.0 & 95.0 & 110.3 & 129.3 & 108.1 & 124.6 & 143.3 & 115.8 & 138.3 & 167.5 & 122.3 & 143.4 & 179.7 \\
\hline & \multirow{3}{*}{ Adjusted } & \begin{tabular}{|l|} 
UDDS \\
\end{tabular} & 66.4 & 66.4 & 75.4 & 85.8 & 74.0 & 83.5 & 93.7 & 78.7 & 91.3 & 106.4 & 82.3 & 93.6 & 110.6 \\
\hline & & HWFET & 64.0 & 64.0 & 73.6 & 85.6 & 72.5 & 82.3 & 93.5 & 76.8 & 90.1 & 107.1 & 80.9 & 93.8 & 116.6 \\
\hline & & \begin{tabular}{|c|} 
Combined \\
\end{tabular} & 65.3 & 65.3 & 74.6 & 85.7 & 73.3 & 83.0 & 93.6 & 77.9 & 90.7 & 106.7 & 81.6 & 93.7 & 113.2 \\
\hline \multirow{6}{*}{ FC PHEV30 } & \multirow{3}{*}{ Unadjusted } & \begin{tabular}{|l|} 
UDDS \\
\end{tabular} & 138.4 & 138.4 & 160.9 & 186.6 & 157.8 & 181.6 & 212.3 & 168.8 & 204.6 & 250.7 & 178.8 & 213.2 & 264.9 \\
\hline & & HWFET & 115.1 & 115.1 & 132.5 & 152.9 & 130.3 & 147.6 & 169.4 & 137.5 & 162.7 & 194.3 & 145.4 & 170.1 & 212.1 \\
\hline & & Combined & 126.8 & 126.8 & 146.8 & 169.8 & 144.1 & 164.5 & 190.6 & 153.1 & 183.3 & 221.7 & 162.0 & 191.4 & 238.2 \\
\hline & \multirow{3}{*}{ Adjusted } & \begin{tabular}{|l|} 
UDDS \\
\end{tabular} & 87.7 & 87.7 & 98.4 & 109.7 & 96.9 & 107.5 & 120.1 & 101.9 & 117.1 & 134.4 & 106.3 & 120.5 & 139.3 \\
\hline & & HWFET & 80.0 & 80.0 & 91.8 & 105.4 & 90.3 & 101.9 & 116.4 & 95.1 & 112.0 & 132.8 & 100.4 & 116.9 & 144.5 \\
\hline & & Combined & 84.0 & 84.0 & 95.3 & 107.7 & 93.8 & 104.9 & 118.4 & 98.7 & 114.7 & 133.7 & 103.6 & 118.8 & 141.6 \\
\hline \multirow{6}{*}{ FC PHEV40 } & \multirow{3}{*}{ Unadjusted } & \begin{tabular}{|l|} 
UDDS \\
\end{tabular} & 159.7 & 159.7 & 185.9 & 215.9 & 181.9 & 210.1 & 246.4 & 195.3 & 237.5 & 291.7 & 207.1 & 247.8 & 308.2 \\
\hline & & HWFET & 145.8 & 145.8 & 168.1 & 194.0 & 165.0 & 187.5 & 215.4 & 174.5 & 207.0 & 247.7 & 184.7 & 216.6 & 270.4 \\
\hline & & \begin{tabular}{|l|} 
Combined \\
\end{tabular} & 153.1 & 153.1 & 177.4 & 205.5 & 173.9 & 199.3 & 231.4 & 185.4 & 222.7 & 270.1 & 196.4 & 232.7 & 289.9 \\
\hline & \multirow{3}{*}{ Adjusted } & \begin{tabular}{|l|} 
UDDS \\
\end{tabular} & 97.8 & 97.8 & 109.4 & 121.5 & 107.7 & 119.3 & 132.9 & 113.3 & 129.7 & 148.1 & 118.1 & 133.4 & 153.2 \\
\hline & & HWFET & 100.7 & 100.7 & 115.5 & 132.7 & 113.5 & 128.3 & 146.6 & 119.8 & 141.1 & 167.5 & 126.5 & 147.4 & 181.9 \\
\hline & & Combined & 99.1 & 99.1 & 112.1 & 126.3 & 110.2 & 123.2 & 138.8 & 116.1 & 134.6 & 156.2 & 121.7 & 139.3 & 164.9 \\
\hline
\end{tabular}




\section{$\underline{\text { PHEV details }}$}

\begin{tabular}{|c|c|c|c|c|c|c|c|c|c|c|c|c|c|c|c|c|}
\hline & & & & \multicolumn{13}{|c|}{ Compact } \\
\hline & & & & \multirow{2}{*}{$\begin{array}{c}2010 \\
\text { Ref }\end{array}$} & \multicolumn{3}{|c|}{2010} & \multicolumn{3}{|c|}{2015} & \multicolumn{3}{|c|}{2030} & \multicolumn{3}{|c|}{2045} \\
\hline & & & & & low & avg & high & low & avg & high & low & avg & high & low & avg & high \\
\hline \multirow{25}{*}{ SI Split HEV PHEV10 } & \multirow{9}{*}{ UDDS } & \multirow{4}{*}{$C D$} & Wh/mile & 178.0 & 178.0 & 172.0 & 160.3 & 179.4 & 165.7 & 149.2 & 172.5 & 154.3 & 142.0 & 171.3 & 150.0 & 134.4 \\
\hline & & & Distance & 11.4 & 11.4 & 11.3 & 11.4 & 11.4 & 11.3 & 11.4 & 11.6 & 11.4 & 10.2 & 11.4 & 11.3 & 10.2 \\
\hline & & & MPGGE Unadjusted & 1614.7 & 1614.7 & 2205.9 & 2373.8 & 1667.1 & 2321.3 & 2885.5 & 1730.2 & 2590.4 & N/A & 2247.8 & 2723.9 & $\mathrm{~N} / \mathrm{A}$ \\
\hline & & & MPGGE Adjusted & 302.3 & 302.3 & 322.1 & 326.2 & 304.5 & 325.0 & 336.3 & 307.0 & 330.9 & N/A & 323.2 & 333.4 & N/A \\
\hline & & \multirow{2}{*}{ CS } & MPGGE Unadjusted & 62.8 & 62.8 & 67.1 & 72.5 & 63.4 & 70.5 & 86.0 & 66.4 & 77.0 & 106.0 & 68.8 & 79.9 & 111.9 \\
\hline & & & MPGGE Adjusted & 45.3 & 45.3 & 48.0 & 51.4 & 45.7 & 50.1 & 59.5 & 47.6 & 54.1 & 70.9 & 49.1 & 55.9 & 74.1 \\
\hline & & \multirow{3}{*}{$\mathrm{CD}+\mathrm{CS}$} & Wh/mile & 37.1 & 37.1 & 35.8 & 33.4 & 37.4 & 34.5 & 31.1 & 35.9 & 32.2 & 29.6 & 35.7 & 31.2 & 28.0 \\
\hline & & & MPGGE Unadjusted & 75.1 & 75.1 & 80.3 & 86.8 & 75.8 & 84.4 & 102.9 & 79.4 & 92.2 & 127.8 & 82.4 & 95.7 & 134.8 \\
\hline & & & MPGGE Adjusted & 53.0 & 53.0 & 56.1 & 60.0 & 53.4 & 58.6 & 69.2 & 55.6 & 63.1 & 82.3 & 57.4 & 65.1 & 85.9 \\
\hline & \multirow{9}{*}{ HWFET } & \multirow{4}{*}{$C D$} & Wh/mile & 198.7 & 198.7 & 188.9 & 176.4 & 199.5 & 182.1 & 165.0 & 193.4 & 170.7 & 143.5 & 188.6 & 164.3 & 135.7 \\
\hline & & & Distance & 9.9 & 9.9 & 9.1 & 9.1 & 10.5 & 9.1 & 9.0 & 9.1 & 9.0 & 8.9 & 9.1 & 8.9 & 9.0 \\
\hline & & & MPGGE Unadjusted & 456.3 & 456.3 & 505.3 & 559.3 & 505.9 & 519.5 & 678.3 & 489.2 & 588.4 & 724.9 & 536.6 & 601.7 & 1004.2 \\
\hline & & & MPGGE Adjusted & 295.6 & 295.6 & 324.2 & 355.0 & 324.5 & 332.4 & 420.8 & 314.8 & 371.4 & 445.8 & 342.2 & 378.8 & 586.4 \\
\hline & & \multirow{2}{*}{ CS } & MPGGE Unadjusted & 57.1 & 57.1 & 61.5 & 66.6 & 58.2 & 64.3 & 78.0 & 60.6 & 70.1 & 94.2 & 63.4 & 73.0 & 102.4 \\
\hline & & & MPGGE Adjusted & 40.2 & 40.2 & 43.2 & 46.8 & 41.0 & 45.2 & 54.7 & 42.7 & 49.2 & 65.8 & 44.5 & 51.2 & 71.4 \\
\hline & & \multirow{3}{*}{$\mathrm{CD}+\mathrm{CS}$} & Wh/mile & 58.4 & 58.4 & 55.6 & 51.9 & 58.7 & 53.6 & 48.5 & 56.9 & 50.2 & 42.2 & 55.5 & 48.3 & 39.9 \\
\hline & & & MPGGE Unadjusted & 72.2 & 72.2 & 77.9 & 84.5 & 73.9 & 81.4 & 99.1 & 76.8 & 88.8 & 119.1 & 80.4 & 92.5 & 130.5 \\
\hline & & & MPGGE Adjusted & 50.7 & 50.7 & 54.6 & 59.1 & 51.9 & 57.0 & 69.1 & 53.8 & 62.1 & 82.7 & 56.3 & 64.6 & 90.4 \\
\hline & \multirow{7}{*}{ Combined } & \multirow{2}{*}{$C D$} & MPGGE Unadjusted & 753.7 & 753.7 & 877.2 & 964.9 & 820.0 & 906.5 & 1171.0 & 807.9 & 1023.4 & 1589.5 & 923.1 & 1052.8 & 2190.4 \\
\hline & & & MPGGE Adjusted & 299.2 & 299.2 & 323.0 & 338.6 & 313.2 & 328.3 & 369.7 & 310.5 & 348.0 & 412.9 & 331.4 & 352.4 & 458.8 \\
\hline & & \multirow{2}{*}{ CS } & MPGGE Unadjusted & 60.1 & 60.1 & 64.4 & 69.7 & 61.0 & 67.5 & 82.2 & 63.7 & 73.7 & 100.4 & 66.2 & 76.7 & 107.4 \\
\hline & & & MPGGE Adjusted & 42.8 & 42.8 & 45.7 & 49.2 & 43.4 & 47.8 & 57.2 & 45.2 & 51.8 & 68.5 & 46.9 & 53.7 & 72.8 \\
\hline & & \multirow{3}{*}{$\mathrm{CD}+\mathrm{CS}$} & Wh/mile & 46.7 & 46.7 & 44.7 & 41.7 & 47.0 & 43.1 & 38.9 & 45.4 & 40.3 & 35.3 & 44.6 & 38.9 & 33.4 \\
\hline & & & MPGGE Unadjusted & 73.8 & 73.8 & 79.2 & 85.7 & 74.9 & 83.0 & 101.2 & 78.2 & 90.7 & 123.7 & 81.5 & 94.3 & 132.8 \\
\hline & & & MPGGE Adjusted & 51.9 & 51.9 & 55.4 & 59.6 & 52.7 & 57.8 & 69.1 & 54.8 & 62.7 & 82.5 & 56.9 & 64.9 & 87.9 \\
\hline
\end{tabular}


Argonne National Laboratory Report - Light-Duty Vehicle Fuel Consumption Displacement Potential Up to 2045

\begin{tabular}{|c|c|c|c|c|c|c|c|c|c|c|c|c|c|c|c|c|}
\hline & & & & \multicolumn{13}{|c|}{ Compact } \\
\hline & & & & \multirow{2}{*}{$\begin{array}{c}2010 \\
\text { Ref }\end{array}$} & \multicolumn{3}{|c|}{2010} & \multicolumn{3}{|c|}{2015} & \multicolumn{3}{|c|}{2030} & \multicolumn{3}{|c|}{2045} \\
\hline \multirow{26}{*}{ SI Split HEV PHEV2O } & & \multirow{5}{*}{$C D$} & & & low & avg & high & low & avg & high & low & avg & high & low & avg & high \\
\hline & \multirow{9}{*}{ UDDS } & & Wh/mile & 180.2 & 180.2 & 170.7 & 162.1 & 181.3 & 167.6 & 150.3 & 174.7 & 155.4 & 139.5 & 170.2 & 152.6 & 132.1 \\
\hline & & & Distance & 22.8 & 22.8 & 22.6 & 22.1 & 22.6 & 22.0 & 22.4 & 22.6 & 22.4 & 20.5 & 22.6 & 22.3 & 20.5 \\
\hline & & & MPGGE Unadjusted & 1570.4 & 1570.4 & 1654.3 & 2329.6 & 1563.5 & 2277.2 & 2638.7 & 1684.4 & 2342.3 & N/A & 1740.3 & 2665.7 & $\mathrm{~N} / \mathrm{A}$ \\
\hline & & & MPGGE Adjusted & 300.3 & 300.3 & 303.9 & 325.2 & 300.0 & 323.9 & 331.8 & 305.2 & 325.5 & N/A & 307.4 & 332.3 & N/A \\
\hline & & \multirow{2}{*}{ CS } & MPGGE Unadjusted & 62.4 & 62.4 & 66.7 & 72.3 & 62.9 & 70.1 & 85.6 & 66.0 & 77.0 & 106.1 & 68.7 & 79.8 & 111.7 \\
\hline & & & MPGGE Adjusted & 45.1 & 45.1 & 47.8 & 51.3 & 45.3 & 49.9 & 59.3 & 47.4 & 54.1 & 70.9 & 49.0 & 55.8 & 73.9 \\
\hline & & \multirow{3}{*}{$\mathrm{CD}+\mathrm{CS}$} & Wh/mile & 95.0 & 95.0 & 89.9 & 61.6 & 95.5 & 63.7 & 79.5 & 92.0 & 82.2 & 53.1 & 89.6 & 58.1 & 50.3 \\
\hline & & & MPGGE Unadjusted & 108.2 & 108.2 & 115.5 & 104.8 & 108.9 & 101.6 & 149.1 & 114.5 & 134.1 & 156.0 & 119.0 & 115.7 & 164.2 \\
\hline & & & MPGGE Adjusted & 72.0 & 72.0 & 76.0 & 70.2 & 72.4 & $\begin{array}{ll}68.4 \\
\end{array}$ & 92.9 & 75.4 & 85.5 & 96.1 & 77.8 & 76.1 & 99.9 \\
\hline & \multirow{9}{*}{ HWFET } & \multirow{4}{*}{$C D$} & Wh/mile & 203.0 & 203.0 & 217.2 & 202.2 & 201.9 & 209.9 & 187.8 & 195.5 & 195.1 & 167.6 & 215.6 & 187.9 & 153.6 \\
\hline & & & Distance & 20.2 & 20.2 & 17.4 & 17.4 & 20.2 & 17.4 & 17.5 & 20.1 & 17.5 & 16.9 & 17.5 & 17.5 & 17.4 \\
\hline & & & MPGGE Unadjusted & 505.6 & 505.6 & N/A & N/A & 519.9 & N/A & N/A & 538.8 & N/A & N/A & N/A & N/A & N/A \\
\hline & & & MPGGE Adjusted & 324.4 & 324.4 & N/A & $\mathrm{N} / \mathrm{A}$ & 332.6 & N/A & N/A & 343.4 & N/A & $\mathrm{N} / \mathrm{A}$ & N/A & $\mathrm{N} / \mathrm{A}$ & N/A \\
\hline & & \multirow{2}{*}{ CS } & MPGGE Unadjusted & 56.5 & 56.5 & 61.1 & 66.3 & 57.8 & 64.1 & 77.9 & 60.3 & 69.8 & 94.0 & 62.9 & 72.9 & 102.0 \\
\hline & & & MPGGE Adjusted & 39.8 & 39.8 & 43.0 & 46.6 & 40.6 & 45.0 & 54.6 & 42.4 & 49.0 & 65.7 & 44.2 & 51.2 & 71.1 \\
\hline & & \multirow{3}{*}{$\mathrm{CD}+\mathrm{CS}$} & Wh/mile & 59.7 & 59.7 & 63.9 & 59.5 & 59.4 & 61.7 & 55.2 & 57.5 & 57.4 & 49.3 & 63.4 & 55.3 & 45.2 \\
\hline & & & MPGGE Unadjusted & 71.8 & 71.8 & 80.4 & 87.3 & 73.4 & 84.3 & 102.4 & 76.6 & 91.8 & 123.7 & 82.7 & 96.0 & 134.2 \\
\hline & & & MPGGE Adjusted & 50.4 & 50.4 & 56.3 & 61.0 & 51.5 & 59.0 & 71.4 & 53.7 & 64.1 & 85.9 & 57.9 & 67.0 & 92.9 \\
\hline & \multirow{7}{*}{ Combined } & \multirow{2}{*}{$C D$} & MPGGE Unadjusted & 806.3 & 806.3 & 2947.0 & 4115.8 & 821.4 & 4025.9 & 4644.7 & 860.8 & 4137.7 & N/A & 3096.8 & 4690.6 & N/A \\
\hline & & & MPGGE Adjusted & 310.7 & 310.7 & 510.8 & 543.6 & 313.8 & 541.6 & 553.8 & 321.3 & 544.1 & 640.8 & 516.1 & 554.6 & 640.8 \\
\hline & & \multirow{2}{*}{ CS } & MPGGE Unadjusted & 59.6 & 59.6 & 64.0 & 69.5 & 60.4 & 67.2 & 81.9 & 63.3 & 73.5 & 100.3 & 65.9 & 76.5 & 107.1 \\
\hline & & & MPGGE Adjusted & 42.5 & 42.5 & 45.5 & 49.1 & 43.1 & 47.6 & 57.0 & 45.0 & 51.7 & 68.4 & 46.7 & 53.6 & 72.6 \\
\hline & & \multirow{3}{*}{$C D+C S$} & Wh/mile & 79.1 & 79.1 & 78.2 & 60.6 & 79.3 & 62.8 & 68.6 & 76.5 & 71.0 & 51.4 & 77.8 & 56.8 & 48.0 \\
\hline & & & MPGGE Unadjusted & 88.1 & 88.1 & 96.5 & 96.1 & 89.4 & 93.0 & 123.7 & 93.6 & 111.1 & 139.6 & 99.4 & 105.9 & 149.2 \\
\hline & & & MPGGE Adjusted & 60.4 & 60.4 & 65.7 & 65.8 & 61.2 & 63.8 & 81.8 & 63.8 & 74.4 & 91.2 & 67.4 & 71.7 & 96.6 \\
\hline
\end{tabular}


Argonne National Laboratory Report - Light-Duty Vehicle Fuel Consumption Displacement Potential Up to 2045

\begin{tabular}{|c|c|c|c|c|c|c|c|c|c|c|c|c|c|c|c|c|}
\hline & & & & \multicolumn{13}{|c|}{ Compact } \\
\hline & & & & \multirow{2}{*}{$\begin{array}{c}2010 \\
\text { Ref } \\
\end{array}$} & \multicolumn{3}{|c|}{2010} & \multicolumn{3}{|c|}{2015} & \multicolumn{3}{|c|}{2030} & \multicolumn{3}{|c|}{2045} \\
\hline & & & & & low & avg & high & low & avg & high & low & avg & high & low & avg & high \\
\hline \multirow{25}{*}{ SI Series HEV PHEV3O } & \multirow{9}{*}{ UDDS } & \multirow{4}{*}{$C D$} & Wh/mile & 249.4 & 249.4 & 235.6 & 218.6 & 248.5 & 226.3 & 200.0 & 238.7 & 207.0 & 174.4 & 231.6 & 200.3 & 165.5 \\
\hline & & & Distance & 30.9 & 30.9 & 30.9 & 31.0 & 30.9 & 31.0 & 30.7 & 30.9 & 30.9 & 30.9 & 31.0 & 30.7 & 30.7 \\
\hline & & & MPGGE Unadjusted & $\mathrm{N} / \mathrm{A}$ & N/A & N/A & N/A & N/A & N/A & $\mathrm{N} / \mathrm{A}$ & $\mathrm{N} / \mathrm{A}$ & N/A & $\mathrm{N} / \mathrm{A}$ & $\mathrm{N} / \mathrm{A}$ & N/A & N/A \\
\hline & & & MPGGE Adjusted & $\mathrm{N} / \mathrm{A}$ & $\mathrm{N} / \mathrm{A}$ & N/A & N/A & N/A & N/A & N/A & $\mathrm{N} / \mathrm{A}$ & N/A & N/A & $\mathrm{N} / \mathrm{A}$ & N/A & N/A \\
\hline & & \multirow{2}{*}{ CS } & MPGGE Unadjusted & 46.8 & 46.8 & 50.8 & 55.8 & 47.7 & 54.0 & 68.8 & 51.1 & 60.7 & 86.2 & 53.2 & 62.9 & 90.7 \\
\hline & & & MPGGE Adjusted & 34.8 & 34.8 & 37.5 & 40.8 & 35.4 & 39.6 & 49.1 & 37.7 & 44.0 & 59.6 & 39.1 & 45.4 & 62.2 \\
\hline & & \multirow{3}{*}{$C D+C S$} & Wh/mile & 160.7 & 160.7 & 151.8 & 140.8 & 160.0 & 145.9 & 129.4 & 153.8 & 133.9 & 113.2 & 149.1 & 129.5 & 107.4 \\
\hline & & & MPGGE Unadjusted & 99.5 & 99.5 & 108.1 & 118.8 & 101.5 & 114.9 & 146.4 & 108.7 & 129.2 & 183.4 & 113.3 & 133.9 & 192.9 \\
\hline & & & MPGGE Adjusted & 67.2 & 67.2 & 72.0 & 77.7 & 68.4 & 75.7 & 91.6 & 72.3 & 83.1 & 108.3 & 74.8 & 85.4 & 112.3 \\
\hline & \multirow{9}{*}{ HWFET } & \multirow{4}{*}{$C D$} & Wh/mile & 249.4 & 249.4 & 233.3 & 216.2 & 247.0 & 224.6 & 198.1 & 238.1 & 205.9 & 173.8 & 229.5 & 198.2 & 159.7 \\
\hline & & & \begin{tabular}{|l} 
Distance \\
\end{tabular} & 30.9 & 30.9 & 31.2 & 31.3 & 31.1 & 31.2 & 31.2 & 31.0 & 31.1 & 31.2 & 31.2 & 31.2 & 31.8 \\
\hline & & & MPGGE Unadjusted & $\mathrm{N} / \mathrm{A}$ & N/A & N/A & N/A & N/A & N/A & N/A & N/A & $\mathrm{N} / \mathrm{A}$ & N/A & N/A & $\mathrm{N} / \mathrm{A}$ & N/A \\
\hline & & & MPGGE Adjusted & N/A & N/A & N/A & N/A & N/A & N/A & N/A & N/A & N/A & N/A & N/A & N/A & N/A \\
\hline & & \multirow{2}{*}{ CS } & MPGGE Unadjusted & 44.3 & 44.3 & 48.4 & 53.3 & 45.5 & 51.4 & 64.2 & 48.3 & 57.4 & 79.1 & 50.8 & 60.3 & 87.1 \\
\hline & & & MPGGE Adjusted & 31.2 & 31.2 & 34.1 & 37.5 & 32.1 & 36.2 & 45.1 & 34.0 & 40.4 & 55.4 & 35.8 & 42.4 & 60.9 \\
\hline & & \multirow{3}{*}{$C D+C S$} & Wh/mile & 168.5 & 168.5 & 157.6 & 146.1 & 166.8 & 151.7 & 133.8 & 160.8 & 139.1 & 117.4 & 155.1 & 133.9 & 107.9 \\
\hline & & & MPGGE Unadjusted & 96.2 & 96.2 & 105.1 & 115.9 & 98.8 & 111.6 & 139.5 & 104.9 & 124.8 & 172.0 & 110.3 & 131.1 & 189.3 \\
\hline & & & MPGGE Adjusted & 67.1 & 67.1 & 73.2 & 80.5 & 68.9 & 77.7 & 96.5 & 73.1 & 86.6 & 118.2 & 76.8 & 90.8 & 129.6 \\
\hline & \multirow{7}{*}{ Combined } & \multirow{2}{*}{$C D$} & MPGGE Unadjusted & $\mathrm{N} / \mathrm{A}$ & $\mathrm{N} / \mathrm{A}$ & $\mathrm{N} / \mathrm{A}$ & $\mathrm{N} / \mathrm{A}$ & $\mathrm{N} / \mathrm{A}$ & $\mathrm{N} / \mathrm{A}$ & $\mathrm{N} / \mathrm{A}$ & N/A & $\mathrm{N} / \mathrm{A}$ & $\mathrm{N} / \mathrm{A}$ & $\mathrm{N} / \mathrm{A}$ & $\mathrm{N} / \mathrm{A}$ & $\mathrm{N} / \mathrm{A}$ \\
\hline & & & MPGGE Adjusted & N/A & N/A & N/A & $\mathrm{N} / \mathrm{A}$ & N/A & N/A & $\mathrm{N} / \mathrm{A}$ & N/A & N/A & N/A & $\mathrm{N} / \mathrm{A}$ & N/A & N/A \\
\hline & & \multirow{2}{*}{ CS } & MPGGE Unadjusted & 45.6 & 45.6 & 49.7 & 54.7 & 46.7 & 52.8 & 66.7 & 49.8 & 59.2 & 82.9 & 52.1 & 61.7 & 89.0 \\
\hline & & & MPGGE Adjusted & 33.1 & 33.1 & 35.9 & 39.3 & 33.8 & 38.0 & 47.2 & 35.9 & 42.3 & 57.7 & 37.5 & 44.0 & 61.6 \\
\hline & & \multirow{3}{*}{$C D+C S$} & Wh/mile & 164.2 & 164.2 & 154.4 & 143.2 & 163.1 & 148.5 & 131.4 & 157.0 & 136.2 & 115.1 & 151.8 & 131.5 & 107.6 \\
\hline & & & MPGGE Unadjusted & 98.0 & 98.0 & 106.7 & 117.4 & 100.3 & 113.4 & 143.2 & 106.9 & 127.2 & 178.1 & 111.9 & 132.6 & 191.3 \\
\hline & & & MPGGE Adjusted & 67.2 & 67.2 & 72.5 & 78.9 & 68.6 & 76.6 & 93.7 & 72.7 & 84.6 & 112.5 & 75.7 & 87.8 & 119.5 \\
\hline
\end{tabular}


Argonne National Laboratory Report - Light-Duty Vehicle Fuel Consumption Displacement Potential Up to 2045

\begin{tabular}{|c|c|c|c|c|c|c|c|c|c|c|c|c|c|c|c|c|}
\hline & & & & \multicolumn{13}{|c|}{ Compact } \\
\hline & & & & \multirow{2}{*}{$\begin{array}{r}2010 \\
\text { Ref } \\
\end{array}$} & \multicolumn{3}{|c|}{2010} & \multicolumn{3}{|c|}{2015} & \multicolumn{3}{|c|}{2030} & \multicolumn{3}{|c|}{2045} \\
\hline \multirow{26}{*}{ SI Series HEV PHEV40 } & & \multirow{5}{*}{$C D$} & & & low & avg & high & low & avg & high & low & avg & high & low & avg & high \\
\hline & \multirow{9}{*}{ UDDS } & & Wh/mile & 254.6 & 254.6 & 240.1 & 223.1 & 253.7 & 230.9 & 204.0 & 243.2 & 211.5 & 176.1 & 236.6 & 203.7 & 167.3 \\
\hline & & & Distance & 40.5 & 40.5 & 40.6 & 40.5 & 40.8 & 40.6 & 40.7 & 40.8 & 40.8 & 40.5 & 40.8 & 40.5 & 40.6 \\
\hline & & & \begin{tabular}{|l} 
MPGGE Unadjusted \\
\end{tabular} & $\mathrm{N} / \mathrm{A}$ & $\mathrm{N} / \mathrm{A}$ & $\mathrm{N} / \mathrm{A}$ & $\mathrm{N} / \mathrm{A}$ & $\mathrm{N} / \mathrm{A}$ & $\mathrm{N} / \mathrm{A}$ & $\mathrm{N} / \mathrm{A}$ & $\mathrm{N} / \mathrm{A}$ & $\mathrm{N} / \mathrm{A}$ & $\mathrm{N} / \mathrm{A}$ & $\mathrm{N} / \mathrm{A}$ & $\mathrm{N} / \mathrm{A}$ & N/A \\
\hline & & & MPGGE Adjusted & $\mathrm{N} / \mathrm{A}$ & $\mathrm{N} / \mathrm{A}$ & $\mathrm{N} / \mathrm{A}$ & $\mathrm{N} / \mathrm{A}$ & $\mathrm{N} / \mathrm{A}$ & $\mathrm{N} / \mathrm{A}$ & $\mathrm{N} / \mathrm{A}$ & $\mathrm{N} / \mathrm{A}$ & $\mathrm{N} / \mathrm{A}$ & $\mathrm{N} / \mathrm{A}$ & $\mathrm{N} / \mathrm{A}$ & $\mathrm{N} / \mathrm{A}$ & N/A \\
\hline & & \multirow{2}{*}{ CS } & MPGGE Unadjusted & 45.5 & 45.5 & 49.5 & 54.7 & 46.4 & 52.9 & 67.0 & 49.8 & 60.0 & 87.2 & 52.2 & 62.7 & 92.0 \\
\hline & & & MPGGE Adjusted & 33.9 & 33.9 & 36.6 & 40.1 & 34.5 & 38.8 & 48.0 & 36.8 & 43.5 & 60.2 & 38.4 & 45.3 & 63.0 \\
\hline & & \multirow{3}{*}{$C D+C S$} & Wh/mile & 187.2 & 187.2 & 176.6 & 164.0 & 186.4 & 169.9 & 151.1 & 178.8 & 156.6 & 130.6 & 174.1 & 150.6 & 124.1 \\
\hline & & & MPGGE Unadjusted & 113.7 & 113.7 & 123.8 & 136.8 & 115.9 & 132.1 & 167.5 & 124.5 & 150.1 & 217.9 & 130.5 & 156.8 & 230.0 \\
\hline & & & MPGGE Adjusted & 75.0 & 75.0 & 80.3 & 86.9 & 76.2 & 84.6 & 101.3 & 80.7 & 93.3 & 122.3 & 83.7 & 96.5 & 126.9 \\
\hline & \multirow{9}{*}{ HWFET } & \multirow{4}{*}{$C D$} & Wh/mile & 252.2 & 252.2 & 235.4 & 218.2 & 249.5 & 226.7 & 200.0 & 240.1 & 208.0 & 177.2 & 231.4 & 199.4 & 162.1 \\
\hline & & & Distance & 40.5 & 40.5 & 41.3 & 41.5 & 41.3 & 41.3 & 41.5 & 41.1 & 41.4 & 40.3 & 41.5 & 41.5 & 41.8 \\
\hline & & & MPGGE Unadjusted & $\mathrm{N} / \mathrm{A}$ & N/A & $\mathrm{N} / \mathrm{A}$ & N/A & N/A & N/A & $\mathrm{N} / \mathrm{A}$ & $\mathrm{N} / \mathrm{A}$ & N/A & $\mathrm{N} / \mathrm{A}$ & $\mathrm{N} / \mathrm{A}$ & N/A & N/A \\
\hline & & & MPGGE Adjusted & $\mathrm{N} / \mathrm{A}$ & N/A & N/A & $\mathrm{N} / \mathrm{A}$ & $\mathrm{N} / \mathrm{A}$ & N/A & $\mathrm{N} / \mathrm{A}$ & $\mathrm{N} / \mathrm{A}$ & N/A & $\mathrm{N} / \mathrm{A}$ & $\mathrm{N} / \mathrm{A}$ & $\mathrm{N} / \mathrm{A}$ & $\mathrm{N} / \mathrm{A}$ \\
\hline & & \multirow{2}{*}{ CS } & MPGGE Unadjusted & 43.6 & 43.6 & 47.7 & 52.6 & 44.7 & 50.7 & 63.5 & 47.7 & 56.8 & 78.8 & 50.1 & 59.8 & 85.8 \\
\hline & & & MPGGE Adjusted & 30.8 & 30.8 & 33.6 & 37.1 & 31.5 & 35.7 & 44.7 & 33.6 & 40.0 & 55.2 & 35.3 & 42.1 & 60.0 \\
\hline & & \multirow{3}{*}{$C D+C S$} & Wh/mile & 170.4 & 170.4 & 185.1 & 171.6 & 196.2 & 178.3 & 157.6 & 188.8 & 163.8 & 120.3 & 182.0 & 156.9 & 128.2 \\
\hline & & & MPGGE Unadjusted & 94.8 & 94.8 & 128.8 & 142.1 & 120.7 & 136.9 & 171.7 & 128.8 & 153.5 & 171.3 & 135.4 & 161.6 & 231.9 \\
\hline & & & MPGGE Adjusted & 66.2 & 66.2 & 89.3 & 98.2 & 83.8 & 94.7 & 117.9 & 89.3 & 105.8 & 117.6 & 93.7 & 111.2 & 157.3 \\
\hline & \multirow{7}{*}{ Combined } & \multirow{2}{*}{$C D$} & MPGGE Unadjusted & $\mathrm{N} / \mathrm{A}$ & $\mathrm{N} / \mathrm{A}$ & N/A & N/A & $\mathrm{N} / \mathrm{A}$ & N/A & N/A & $\mathrm{N} / \mathrm{A}$ & $\mathrm{N} / \mathrm{A}$ & $\mathrm{N} / \mathrm{A}$ & $\mathrm{N} / \mathrm{A}$ & $\mathrm{N} / \mathrm{A}$ & $\mathrm{N} / \mathrm{A}$ \\
\hline & & & MPGGE Adjusted & $\mathrm{N} / \mathrm{A}$ & $\mathrm{N} / \mathrm{A}$ & $\mathrm{N} / \mathrm{A}$ & $\mathrm{N} / \mathrm{A}$ & $\mathrm{N} / \mathrm{A}$ & $\mathrm{N} / \mathrm{A}$ & $\mathrm{N} / \mathrm{A}$ & $\mathrm{N} / \mathrm{A}$ & $\mathrm{N} / \mathrm{A}$ & $\mathrm{N} / \mathrm{A}$ & $\mathrm{N} / \mathrm{A}$ & $\mathrm{N} / \mathrm{A}$ & $\mathrm{N} / \mathrm{A}$ \\
\hline & & \multirow{2}{*}{ CS } & MPGGE Unadjusted & 44.6 & 44.6 & 48.7 & 53.7 & 45.6 & 51.8 & 65.4 & 48.8 & 58.5 & 83.2 & 51.2 & 61.4 & 89.1 \\
\hline & & & MPGGE Adjusted & 32.4 & 32.4 & 35.2 & 38.7 & 33.1 & 37.4 & 46.4 & 35.3 & 41.9 & 57.8 & 37.0 & 43.8 & 61.6 \\
\hline & & \multirow{3}{*}{$C D+C S$} & Wh/mile & 179.7 & 179.7 & 180.4 & 167.5 & 190.8 & 173.7 & 154.0 & 183.3 & 159.8 & 125.9 & 177.6 & 153.4 & 126.0 \\
\hline & & & MPGGE Unadjusted & 104.3 & 104.3 & 126.0 & 139.1 & 118.0 & 134.2 & 169.3 & 126.4 & 151.6 & 194.1 & 132.7 & 158.9 & 230.9 \\
\hline & & & MPGGE Adjusted & 70.8 & 70.8 & 84.1 & 91.6 & 79.5 & 88.9 & 108.2 & 84.3 & 98.5 & 120.2 & 87.9 & 102.6 & 139.0 \\
\hline
\end{tabular}


Argonne National Laboratory Report - Light-Duty Vehicle Fuel Consumption Displacement Potential Up to 2045

\begin{tabular}{|c|c|c|c|c|c|c|c|c|c|c|c|c|c|c|c|c|}
\hline & & & & \multicolumn{13}{|c|}{ Compact } \\
\hline & & & & \multirow{2}{*}{$\begin{array}{c}2010 \\
\text { Ref }\end{array}$} & \multicolumn{3}{|c|}{2010} & \multicolumn{3}{|c|}{2015} & \multicolumn{3}{|c|}{2030} & \multicolumn{3}{|c|}{2045} \\
\hline & & & & & low & avg & high & low & avg & high & low & avg & high & low & avg & high \\
\hline \multirow{25}{*}{ CI Split HEV PHEV10 } & \multirow{9}{*}{ UDDS } & \multirow{4}{*}{$C D$} & Wh/mile & 179.3 & 179.3 & 177.0 & 165.3 & 187.5 & 170.9 & 156.7 & 180.4 & 162.0 & 150.4 & 175.6 & 157.5 & 142.9 \\
\hline & & & Distance & 12.2 & 12.2 & 11.6 & 11.6 & 11.6 & 11.6 & 11.4 & 11.6 & 11.6 & 10.2 & 11.6 & 11.5 & 10.2 \\
\hline & & & MPGGE Unadjusted & 1111.5 & 1111.5 & 1675.1 & 1849.9 & 1560.6 & 1801.4 & 2642.8 & 1670.8 & 2578.1 & 65207.3 & 1754.1 & 2707.5 & 65207.3 \\
\hline & & & MPGGE Adjusted & 273.8 & 273.8 & 304.8 & 311.4 & 299.9 & 309.6 & 331.9 & 304.6 & 330.6 & 389.4 & 307.9 & 333.1 & 389.4 \\
\hline & & \multirow{2}{*}{ CS } & MPGGE Unadjusted & 63.6 & 63.6 & 67.1 & 73.5 & 63.1 & 71.3 & 81.6 & 67.6 & 79.5 & 98.4 & 71.0 & 82.9 & 103.6 \\
\hline & & & MPGGE Adjusted & 45.8 & 45.8 & 48.0 & 52.0 & 45.5 & 50.7 & 56.9 & 48.4 & 55.7 & 66.6 & 50.5 & 57.7 & 69.5 \\
\hline & & \multirow{3}{*}{$C D+C S$} & Wh/mile & 37.4 & 37.4 & 36.9 & 34.4 & 39.1 & 35.6 & 32.6 & 37.6 & 33.8 & 31.3 & 36.6 & 32.8 & 29.8 \\
\hline & & & MPGGE Unadjusted & 75.7 & 75.7 & 80.1 & 87.9 & 75.4 & 85.2 & 97.8 & 80.8 & 95.2 & 118.6 & 84.8 & 99.3 & 124.8 \\
\hline & & & MPGGE Adjusted & 53.3 & 53.3 & 56.0 & 60.6 & 53.2 & 59.0 & 66.3 & 56.4 & 64.8 & 77.6 & 58.8 & 67.1 & 80.8 \\
\hline & \multirow{9}{*}{ HWFET } & \multirow{4}{*}{$C D$} & Wh/mile & 198.0 & 198.0 & 195.2 & 185.9 & 197.2 & 192.3 & 171.3 & 198.8 & 180.0 & 151.9 & 193.5 & 173.5 & 144.1 \\
\hline & & & \begin{tabular}{|l} 
Distance \\
\end{tabular} & 11.0 & 11.0 & 10.6 & 9.6 & 11.1 & 9.6 & 9.1 & 10.6 & 9.1 & 9.1 & 10.7 & 9.1 & 9.4 \\
\hline & & & MPGGE Unadjusted & 393.7 & 393.7 & 570.2 & 712.4 & 399.5 & 663.5 & 690.5 & 537.7 & 752.7 & 829.7 & 602.3 & 776.5 & 1277.5 \\
\hline & & & MPGGE Adjusted & 258.3 & 258.3 & 361.2 & 439.1 & 261.8 & 412.8 & 427.4 & 342.8 & 460.4 & 500.3 & 379.1 & 472.9 & 711.1 \\
\hline & & \multirow{2}{*}{ CS } & MPGGE Unadjusted & 57.2 & 57.2 & 61.1 & 67.0 & 57.5 & 64.9 & 74.1 & 61.4 & 71.8 & 87.4 & 64.7 & 75.7 & 94.8 \\
\hline & & & MPGGE Adjusted & 40.3 & 40.3 & 43.0 & 47.1 & 40.5 & 45.6 & 52.0 & 43.2 & 50.4 & 61.1 & 45.5 & 53.0 & 66.2 \\
\hline & & \multirow{3}{*}{$C D+C S$} & Wh/mile & 58.2 & 58.2 & 57.4 & 54.7 & 58.0 & 56.6 & 50.4 & 58.5 & 53.0 & 44.7 & 56.9 & 51.0 & 42.4 \\
\hline & & & MPGGE Unadjusted & 72.0 & 72.0 & 77.7 & 85.6 & 72.4 & 82.8 & 94.4 & 78.0 & 91.8 & 111.2 & 82.3 & 96.6 & 121.9 \\
\hline & & & MPGGE Adjusted & 50.5 & 50.5 & 54.5 & 59.9 & 50.8 & 58.0 & 65.9 & 54.7 & 64.1 & 77.4 & 57.6 & 67.4 & 84.6 \\
\hline & \multirow{7}{*}{ Combined } & \multirow{2}{*}{$C D$} & MPGGE Unadjusted & 610.6 & 610.6 & 894.8 & 1076.5 & 676.2 & 1016.8 & 1163.0 & 857.6 & 1232.8 & 1815.5 & 942.7 & 1277.7 & 2772.5 \\
\hline & & & MPGGE Adjusted & 266.6 & 266.6 & 327.8 & 358.3 & 281.5 & 348.9 & 369.0 & 320.7 & 378.7 & 432.6 & 336.3 & 384.2 & 489.0 \\
\hline & & \multirow{2}{*}{ CS } & MPGGE Unadjusted & 60.5 & 60.5 & 64.2 & 70.4 & 60.4 & 68.3 & 78.1 & 64.7 & 75.9 & 93.1 & 68.0 & 79.5 & 99.4 \\
\hline & & & MPGGE Adjusted & 43.1 & 43.1 & 45.6 & 49.7 & 43.1 & 48.2 & 54.6 & 45.9 & 53.2 & 64.0 & 48.1 & 55.5 & 68.0 \\
\hline & & \multirow{3}{*}{$\mathrm{CD}+\mathrm{CS}$} & Wh/mile & 46.7 & 46.7 & 46.1 & 43.6 & 47.6 & 45.0 & 40.6 & 47.0 & 42.4 & 37.3 & 45.7 & 41.0 & 35.4 \\
\hline & & & MPGGE Unadjusted & 74.0 & 74.0 & 79.0 & 86.8 & 74.0 & 84.1 & 96.2 & 79.5 & 93.6 & 115.2 & 83.7 & 98.1 & 123.5 \\
\hline & & & MPGGE Adjusted & 52.0 & 52.0 & 55.3 & 60.3 & 52.1 & 58.6 & 66.1 & 55.6 & 64.5 & 77.5 & 58.3 & 67.3 & 82.5 \\
\hline
\end{tabular}


Argonne National Laboratory Report - Light-Duty Vehicle Fuel Consumption Displacement Potential Up to 2045

\begin{tabular}{|c|c|c|c|c|c|c|c|c|c|c|c|c|c|c|c|c|}
\hline & & & & \multicolumn{13}{|c|}{ Compact } \\
\hline & & & & 2010 & & 2010 & & & 2015 & & & 2030 & & & 2045 & \\
\hline \multirow{26}{*}{ CI Split HEV PHEV2O } & & \multirow{5}{*}{$C D$} & & Ref & low & avg & high & low & avg & high & low & avg & high & low & avg & high \\
\hline & \multirow{9}{*}{ UDDS } & & Wh/mile & 181.2 & 181.2 & 179.4 & 167.4 & 183.2 & 173.1 & 158.7 & 182.2 & 164.2 & 143.5 & 177.6 & 159.3 & 140.3 \\
\hline & & & Distance & 23.7 & 23.7 & 22.7 & 22.9 & 23.7 & 22.9 & 22.6 & 23.4 & 22.4 & 21.1 & 23.4 & 22.4 & 20.6 \\
\hline & & & \begin{tabular}{|l} 
MPGGE Unadjusted \\
\end{tabular} & 1071.5 & 1071.5 & 1619.2 & 1806.1 & 1097.8 & 1753.5 & 2599.3 & 1606.4 & 2530.9 & 7801.3 & 1695.8 & 2668.4 & 65207.3 \\
\hline & & & MPGGE Adjusted & 270.8 & 270.8 & 302.5 & 309.8 & 272.8 & 307.9 & 331.1 & 301.9 & 329.7 & 369.5 & 305.6 & 332.4 & 389.4 \\
\hline & & \multirow{2}{*}{ CS } & MPGGE Unadjusted & 63.2 & 63.2 & 66.5 & 73.2 & 62.8 & 70.9 & 81.6 & 67.2 & 79.0 & 98.1 & 70.6 & 82.9 & 103.4 \\
\hline & & & MPGGE Adjusted & 45.6 & 45.6 & 47.7 & 51.8 & 45.3 & 50.4 & 56.9 & 48.1 & 55.3 & 66.5 & 50.2 & 57.7 & 69.4 \\
\hline & & \multirow{3}{*}{$C D+C S$} & Wh/mile & 95.5 & 95.5 & 94.5 & 88.2 & 96.5 & 91.2 & 83.9 & 96.0 & 86.8 & 54.8 & 93.6 & 84.2 & 53.4 \\
\hline & & & MPGGE Unadjusted & 107.9 & 107.9 & 115.1 & 126.7 & 107.4 & 122.8 & 142.3 & 116.3 & 137.7 & 143.6 & 122.2 & 144.7 & 152.1 \\
\hline & & & MPGGE Adjusted & 71.9 & 71.9 & 75.8 & 81.8 & 71.6 & 79.8 & 89.6 & 76.4 & 87.3 & 90.2 & 79.5 & 90.7 & 94.2 \\
\hline & \multirow{9}{*}{ HWFET } & \multirow{4}{*}{$C D$} & Wh/mile & 199.8 & 199.8 & 194.0 & 201.3 & 199.0 & 189.1 & 193.2 & 194.0 & 200.4 & 172.5 & 194.2 & 193.1 & 158.6 \\
\hline & & & Distance & 21.3 & 21.3 & 21.1 & 19.3 & 21.4 & 21.1 & 17.9 & 21.4 & 17.8 & 17.4 & 21.1 & 18.0 & 17.8 \\
\hline & & & MPGGE Unadjusted & 386.6 & 386.6 & 498.6 & 2292.2 & 392.6 & 558.7 & 65207.3 & 435.7 & 65207.3 & 65207.3 & 570.9 & 65207.3 & 65207.3 \\
\hline & & & MPGGE Adjusted & 254.0 & 254.0 & 320.3 & 1086.9 & 257.6 & 354.7 & 3034.8 & 283.4 & 3034.8 & 3034.8 & 361.6 & 3034.8 & 3034.8 \\
\hline & & \multirow{2}{*}{ CS } & MPGGE Unadjusted & 56.9 & 56.9 & 60.6 & 66.7 & 57.1 & 64.5 & 74.1 & 60.8 & 71.5 & 87.3 & 64.4 & 75.6 & 94.4 \\
\hline & & & MPGGE Adjusted & 40.0 & 40.0 & 42.6 & 46.9 & 40.2 & 45.3 & 52.0 & 42.8 & 50.2 & 61.0 & 45.3 & 53.0 & 65.9 \\
\hline & & \multirow{3}{*}{$\mathrm{CD}+\mathrm{CS}$} & Wh/mile & 102.8 & 102.8 & 100.0 & 59.2 & 102.4 & 97.4 & 56.8 & 99.8 & 59.0 & 50.7 & 100.0 & 56.8 & 46.7 \\
\hline & & & MPGGE Unadjusted & 87.3 & 87.3 & 94.6 & 87.0 & 87.7 & 101.1 & 97.5 & 93.8 & 94.1 & 114.8 & 101.1 & 99.5 & 124.2 \\
\hline & & & MPGGE Adjusted & 61.0 & 61.0 & 66.1 & 60.9 & 61.3 & 70.5 & 68.1 & 65.5 & 65.7 & 79.8 & 70.5 & 69.4 & 86.2 \\
\hline & \multirow{7}{*}{ Combined } & \multirow{2}{*}{$C D$} & MPGGE Unadjusted & 596.3 & 596.3 & 805.0 & 1996.6 & 607.0 & 893.6 & 4576.7 & 727.1 & 4460.0 & 12919.5 & 898.8 & 4694.5 & 65207.3 \\
\hline & & & MPGGE Adjusted & 263.0 & 263.0 & 310.2 & 456.8 & 265.7 & 327.3 & 552.6 & 293.3 & 550.5 & 611.0 & 328.5 & 554.7 & 640.8 \\
\hline & & \multirow{2}{*}{ CS } & MPGGE Unadjusted & 60.2 & 60.2 & 63.7 & 70.1 & 60.1 & 67.9 & 78.1 & 64.2 & 75.4 & 92.9 & 67.7 & 79.5 & 99.1 \\
\hline & & & MPGGE Adjusted & 42.9 & 42.9 & 45.3 & 49.5 & 42.9 & 48.0 & 54.6 & 45.6 & 52.9 & 63.9 & 47.9 & 55.5 & 67.8 \\
\hline & & \multirow{3}{*}{$C D+C S$} & Wh/mile & 98.7 & 98.7 & 97.0 & 75.1 & 99.1 & 94.0 & 71.7 & 97.7 & 74.2 & 53.0 & 96.5 & 71.9 & 50.4 \\
\hline & & & MPGGE Unadjusted & 97.5 & 97.5 & 104.9 & 105.1 & 97.6 & 112.0 & 117.9 & 104.9 & 113.9 & 129.0 & 111.7 & 120.1 & 138.1 \\
\hline & & & MPGGE Adjusted & 66.6 & 66.6 & 71.1 & 70.8 & 66.6 & 75.3 & 78.4 & 71.1 & 76.0 & 85.2 & 75.2 & 79.7 & 90.4 \\
\hline
\end{tabular}


Argonne National Laboratory Report - Light-Duty Vehicle Fuel Consumption Displacement Potential Up to 2045

\begin{tabular}{|c|c|c|c|c|c|c|c|c|c|c|c|c|c|c|c|c|}
\hline & & & & \multicolumn{13}{|c|}{ Compact } \\
\hline & & & & \multirow{2}{*}{$\frac{2010}{\operatorname{Ref}}$} & \multicolumn{3}{|c|}{2010} & \multicolumn{3}{|c|}{2015} & \multicolumn{3}{|c|}{2030} & \multicolumn{3}{|c|}{2045} \\
\hline & & & & & low & avg & high & low & avg & high & low & avg & high & low & avg & high \\
\hline \multirow{25}{*}{ CI Series HEV PHEV30 } & \multirow{9}{*}{ UDDS } & \multirow{4}{*}{$C D$} & Wh/mile & 259.7 & 259.7 & 245.5 & 228.9 & 259.0 & 236.1 & 209.7 & 248.7 & 216.9 & 183.3 & 241.3 & 209.9 & 175.1 \\
\hline & & & Distance & 31.0 & 31.0 & 31.0 & 31.0 & 30.9 & 31.0 & 30.9 & 31.0 & 30.9 & 30.7 & 31.0 & 30.9 & 30.9 \\
\hline & & & MPGGE Unadjusted & $\mathrm{N} / \mathrm{A}$ & N/A & N/A & N/A & N/A & N/A & $\mathrm{N} / \mathrm{A}$ & $\mathrm{N} / \mathrm{A}$ & N/A & $\mathrm{N} / \mathrm{A}$ & $\mathrm{N} / \mathrm{A}$ & N/A & $\mathrm{N} / \mathrm{A}$ \\
\hline & & & MPGGE Adjusted & $\mathrm{N} / \mathrm{A}$ & $\mathrm{N} / \mathrm{A}$ & N/A & N/A & N/A & N/A & N/A & $\mathrm{N} / \mathrm{A}$ & N/A & N/A & $\mathrm{N} / \mathrm{A}$ & N/A & N/A \\
\hline & & \multirow{2}{*}{ CS } & MPGGE Unadjusted & 47.1 & 47.1 & 50.7 & 56.4 & 47.4 & 54.6 & 66.9 & 51.9 & 64.1 & 80.5 & 54.6 & 67.2 & 84.8 \\
\hline & & & MPGGE Adjusted & 35.0 & 35.0 & 37.4 & 41.2 & 35.2 & 40.0 & 47.9 & 38.2 & 46.1 & 56.3 & 40.0 & 48.1 & 58.8 \\
\hline & & \multirow{3}{*}{$C D+C S$} & Wh/mile & 167.3 & 167.3 & 158.1 & 147.5 & 166.8 & 152.1 & 135.7 & 160.3 & 140.3 & 119.1 & 155.5 & 135.7 & 113.8 \\
\hline & & & MPGGE Unadjusted & 100.3 & 100.3 & 108.0 & 120.0 & 100.8 & 116.2 & 142.4 & 110.4 & 136.4 & 171.3 & 116.2 & 143.0 & 180.3 \\
\hline & & & MPGGE Adjusted & 67.7 & 67.7 & 71.9 & 78.3 & 68.0 & 76.4 & 89.6 & 73.2 & 86.7 & 103.0 & 76.4 & 89.9 & 107.0 \\
\hline & \multirow{9}{*}{ HWFET } & \multirow{4}{*}{$C D$} & Wh/mile & 256.2 & 256.2 & 240.0 & 223.0 & 254.0 & 231.4 & 204.3 & 244.7 & 212.2 & 179.4 & 235.9 & 204.3 & 165.3 \\
\hline & & & \begin{tabular}{|l} 
Distance \\
\end{tabular} & 31.3 & 31.3 & 31.5 & 31.7 & 31.3 & 31.5 & 31.6 & 31.3 & 31.4 & 31.4 & 31.6 & 31.5 & 33.0 \\
\hline & & & MPGGE Unadjusted & $\mathrm{N} / \mathrm{A}$ & N/A & N/A & N/A & N/A & N/A & N/A & N/A & $\mathrm{N} / \mathrm{A}$ & N/A & N/A & N/A & $\mathrm{N} / \mathrm{A}$ \\
\hline & & & MPGGE Adjusted & N/A & N/A & N/A & N/A & N/A & N/A & N/A & N/A & N/A & N/A & N/A & N/A & N/A \\
\hline & & \multirow{2}{*}{ CS } & MPGGE Unadjusted & 44.9 & 44.9 & 48.5 & 54.3 & 45.5 & 52.1 & 61.7 & 49.3 & 59.5 & 74.3 & 52.3 & 63.2 & 81.1 \\
\hline & & & MPGGE Adjusted & 31.7 & 31.7 & 34.2 & 38.3 & 32.1 & 36.7 & 43.4 & 34.8 & 41.9 & 52.1 & 36.9 & 44.4 & 56.8 \\
\hline & & \multirow{3}{*}{$C D+C S$} & Wh/mile & 173.1 & 173.1 & 162.2 & 150.7 & 171.6 & 156.4 & 138.1 & 165.3 & 143.4 & 121.2 & 159.4 & 138.0 & 111.8 \\
\hline & & & MPGGE Unadjusted & 97.5 & 97.5 & 105.5 & 118.1 & 98.8 & 113.3 & 134.2 & 107.2 & 129.4 & 161.5 & 113.8 & 137.4 & 176.2 \\
\hline & & & MPGGE Adjusted & 68.1 & 68.1 & 73.5 & 82.1 & 68.9 & 78.8 & 92.9 & 74.6 & 89.7 & 111.2 & 79.1 & 95.0 & 120.9 \\
\hline & \multirow{7}{*}{ Combined } & \multirow{2}{*}{$C D$} & MPGGE Unadjusted & $\mathrm{N} / \mathrm{A}$ & $\mathrm{N} / \mathrm{A}$ & $\mathrm{N} / \mathrm{A}$ & $\mathrm{N} / \mathrm{A}$ & $\mathrm{N} / \mathrm{A}$ & $\mathrm{N} / \mathrm{A}$ & $\mathrm{N} / \mathrm{A}$ & N/A & $\mathrm{N} / \mathrm{A}$ & $\mathrm{N} / \mathrm{A}$ & $\mathrm{N} / \mathrm{A}$ & $\mathrm{N} / \mathrm{A}$ & $\mathrm{N} / \mathrm{A}$ \\
\hline & & & MPGGE Adjusted & $\mathrm{N} / \mathrm{A}$ & N/A & $\mathrm{N} / \mathrm{A}$ & $\mathrm{N} / \mathrm{A}$ & N/A & N/A & $\mathrm{N} / \mathrm{A}$ & N/A & N/A & N/A & $\mathrm{N} / \mathrm{A}$ & N/A & N/A \\
\hline & & \multirow{2}{*}{ CS } & MPGGE Unadjusted & 46.1 & 46.1 & 49.7 & 55.4 & 46.5 & 53.5 & 64.5 & 50.7 & 62.0 & 77.6 & 53.6 & 65.3 & 83.1 \\
\hline & & & MPGGE Adjusted & 33.4 & 33.4 & 35.9 & 39.8 & 33.7 & 38.5 & 45.8 & 36.6 & 44.1 & 54.3 & 38.5 & 46.4 & 57.9 \\
\hline & & \multirow{3}{*}{$C D+C S$} & Wh/mile & 169.9 & 169.9 & 160.0 & 148.9 & 169.0 & 154.0 & 136.8 & 162.5 & 141.7 & 120.0 & 157.2 & 136.8 & 112.9 \\
\hline & & & MPGGE Unadjusted & 99.0 & 99.0 & 106.8 & 119.1 & 99.9 & 114.9 & 138.6 & 108.9 & 133.2 & 166.8 & 115.1 & 140.4 & 178.5 \\
\hline & & & MPGGE Adjusted & 67.9 & 67.9 & 72.6 & 80.0 & 68.4 & 77.4 & 91.1 & 73.9 & 88.0 & 106.6 & 77.6 & 92.1 & 112.8 \\
\hline
\end{tabular}


Argonne National Laboratory Report - Light-Duty Vehicle Fuel Consumption Displacement Potential Up to 2045

\begin{tabular}{|c|c|c|c|c|c|c|c|c|c|c|c|c|c|c|c|c|}
\hline & & & & \multicolumn{13}{|c|}{ Compact } \\
\hline & & & & \multirow{2}{*}{$\begin{array}{c}2010 \\
\text { Ref }\end{array}$} & \multicolumn{3}{|c|}{2010} & \multicolumn{3}{|c|}{2015} & \multicolumn{3}{|c|}{2030} & \multicolumn{3}{|c|}{2045} \\
\hline \multirow{26}{*}{$\mathrm{Cl}$ Series HEV PHEV40 } & & \multirow{5}{*}{$C D$} & & & low & avg & high & low & avg & high & low & avg & high & low & avg & high \\
\hline & \multirow{9}{*}{ UDDS } & & Wh/mile & 265.3 & 265.3 & 250.8 & 233.7 & 264.6 & 240.8 & 214.1 & 253.5 & 221.0 & 185.5 & 246.1 & 213.4 & 176.6 \\
\hline & & & Distance & 40.6 & 40.6 & 40.8 & 40.5 & 40.8 & 40.8 & 40.6 & 40.8 & 40.7 & 40.4 & 40.8 & 40.5 & 40.4 \\
\hline & & & \begin{tabular}{|l|} 
MPGGE Unadjusted \\
\end{tabular} & $\mathrm{N} / \mathrm{A}$ & N/A & N/A & $\mathrm{N} / \mathrm{A}$ & $\mathrm{N} / \mathrm{A}$ & N/A & N/A & $\mathrm{N} / \mathrm{A}$ & $\mathrm{N} / \mathrm{A}$ & $\mathrm{N} / \mathrm{A}$ & $\mathrm{N} / \mathrm{A}$ & N/A & N/A \\
\hline & & & MPGGE Adjusted & N/A & N/A & N/A & N/A & N/A & N/A & N/A & N/A & N/A & N/A & N/A & N/A & N/A \\
\hline & & \multirow{2}{*}{ CS } & MPGGE Unadjusted & 45.9 & 45.9 & 49.4 & 55.3 & 46.1 & 53.5 & 63.7 & 50.7 & 63.2 & 81.0 & 53.6 & 66.4 & 85.7 \\
\hline & & & MPGGE Adjusted & 34.2 & 34.2 & 36.6 & 40.4 & 34.3 & 39.3 & 45.9 & 37.4 & 45.6 & 56.6 & 39.3 & 47.6 & 59.3 \\
\hline & & \multirow{3}{*}{$\mathrm{CD}+\mathrm{CS}$} & Wh/mile & 195.1 & 195.1 & 184.5 & 171.9 & 194.6 & 177.1 & 158.7 & 186.5 & 163.7 & 137.6 & 181.0 & 157.8 & 131.1 \\
\hline & & & MPGGE Unadjusted & 114.7 & 114.7 & 123.5 & 138.2 & 115.1 & 133.8 & 159.2 & 126.7 & 158.0 & 202.6 & 134.0 & 165.9 & 214.2 \\
\hline & & & MPGGE Adjusted & 75.5 & 75.5 & 80.2 & 87.6 & 75.8 & 85.4 & 97.6 & 81.8 & 97.0 & 116.3 & 85.5 & 100.6 & 120.9 \\
\hline & \multirow{9}{*}{ HWFET } & \multirow{4}{*}{$C D$} & Wh/mile & 258.8 & 258.8 & 242.4 & 225.1 & 256.8 & 233.4 & 206.4 & 246.9 & 214.2 & 182.3 & 237.8 & 205.4 & 167.9 \\
\hline & & & Distance & 41.4 & 41.4 & 41.8 & 42.0 & 41.9 & 41.7 & 42.0 & 41.5 & 41.8 & 41.3 & 41.9 & 42.0 & 42.7 \\
\hline & & & MPGGE Unadjusted & N/A & N/A & N/A & N/A & N/A & N/A & N/A & N/A & N/A & N/A & N/A & N/A & N/A \\
\hline & & & MPGGE Adjusted & N/A & N/A & N/A & N/A & N/A & N/A & N/A & N/A & N/A & N/A & N/A & N/A & N/A \\
\hline & & \multirow{2}{*}{ CS } & MPGGE Unadjusted & 44.2 & 44.2 & 47.8 & 53.4 & 44.6 & 51.4 & 61.1 & 48.7 & 58.9 & 74.1 & 51.6 & 62.6 & 80.6 \\
\hline & & & MPGGE Adjusted & 31.2 & 31.2 & 33.7 & 37.6 & 31.5 & 36.2 & 42.9 & 34.3 & 41.4 & 52.0 & 36.4 & 44.0 & 56.4 \\
\hline & & \multirow{3}{*}{$\mathrm{CD}+\mathrm{CS}$} & Wh/mile & 203.6 & 203.6 & 190.7 & 177.1 & 201.9 & 183.7 & 162.7 & 194.2 & 168.7 & 144.2 & 187.1 & 161.7 & 132.9 \\
\hline & & & MPGGE Unadjusted & 119.5 & 119.5 & 129.3 & 144.3 & 120.5 & 139.0 & 165.1 & 131.5 & 159.2 & 200.3 & 139.6 & 169.2 & 217.8 \\
\hline & & & MPGGE Adjusted & 83.0 & 83.0 & 89.6 & 99.7 & 83.7 & 96.1 & 113.5 & 91.1 & 109.6 & 136.7 & 96.5 & 116.3 & 148.1 \\
\hline & \multirow{7}{*}{ Combined } & \multirow{2}{*}{$C D$} & MPGGE Unadjusted & N/A & $\mathrm{N} / \mathrm{A}$ & $\mathrm{N} / \mathrm{A}$ & $\mathrm{N} / \mathrm{A}$ & $\mathrm{N} / \mathrm{A}$ & $\mathrm{N} / \mathrm{A}$ & $\mathrm{N} / \mathrm{A}$ & $\mathrm{N} / \mathrm{A}$ & $\mathrm{N} / \mathrm{A}$ & $\mathrm{N} / \mathrm{A}$ & $\mathrm{N} / \mathrm{A}$ & $\mathrm{N} / \mathrm{A}$ & $\mathrm{N} / \mathrm{A}$ \\
\hline & & & MPGGE Adjusted & N/A & N/A & N/A & N/A & N/A & N/A & N/A & N/A & N/A & N/A & N/A & N/A & N/A \\
\hline & & \multirow{2}{*}{ CS } & MPGGE Unadjusted & 45.1 & 45.1 & 48.7 & 54.4 & 45.4 & 52.6 & 62.5 & 49.7 & 61.2 & 77.8 & 52.7 & 64.6 & 83.3 \\
\hline & & & MPGGE Adjusted & 32.8 & 32.8 & 35.2 & 39.1 & 33.0 & 37.9 & 44.5 & 35.9 & 43.6 & 54.4 & 38.0 & 45.9 & 58.0 \\
\hline & & \multirow{3}{*}{$\mathrm{CD}+\mathrm{CS}$} & Wh/mile & 198.9 & 198.9 & 187.3 & 174.2 & 197.9 & 180.0 & 160.5 & 190.0 & 165.9 & 140.6 & 183.8 & 159.6 & 131.9 \\
\hline & & & MPGGE Unadjusted & 116.8 & 116.8 & 126.1 & 140.9 & 117.5 & 136.1 & 161.8 & 128.8 & 158.5 & 201.5 & 136.4 & 167.4 & 215.8 \\
\hline & & & MPGGE Adjusted & 78.7 & 78.7 & 84.2 & 92.6 & 79.2 & 89.9 & 104.2 & 85.7 & 102.3 & 124.7 & 90.1 & 107.1 & 131.8 \\
\hline
\end{tabular}


Argonne National Laboratory Report - Light-Duty Vehicle Fuel Consumption Displacement Potential Up to 2045

\begin{tabular}{|c|c|c|c|c|c|c|c|c|c|c|c|c|c|c|c|c|}
\hline & & & & \multicolumn{13}{|c|}{ Compact } \\
\hline & & & & \multirow{2}{*}{$\begin{array}{c}2010 \\
\text { Ref } \\
\end{array}$} & \multicolumn{3}{|c|}{2010} & \multicolumn{3}{|c|}{2015} & \multicolumn{3}{|c|}{2030} & \multicolumn{3}{|c|}{2045} \\
\hline & & & & & low & avg & high & low & avg & high & low & avg & high & low & avg & high \\
\hline \multirow{25}{*}{ H2 Split HEV PHEV10 } & \multirow{9}{*}{ UDDS } & \multirow{4}{*}{$C D$} & Wh/mile & 181.4 & 181.4 & 169.2 & 159.0 & 182.1 & 164.7 & 147.1 & 174.0 & 152.9 & 138.8 & 169.7 & 148.0 & 132.1 \\
\hline & & & Distance & 11.6 & 11.6 & 11.6 & 11.4 & 11.6 & 11.4 & 11.4 & 11.6 & 11.4 & 10.2 & 11.4 & 11.4 & 10.2 \\
\hline & & & MPGGE Unadjusted & 1514.3 & 1514.3 & 1874.4 & 2716.3 & 1722.7 & 2655.7 & 3253.9 & 1915.6 & 3087.9 & N/A & 2662.4 & 3381.3 & $\mathrm{~N} / \mathrm{A}$ \\
\hline & & & MPGGE Adjusted & 297.7 & 297.7 & 312.2 & 333.3 & 306.7 & 332.2 & 341.8 & 313.6 & 339.4 & N/A & 332.3 & 343.5 & $\mathrm{~N} / \mathrm{A}$ \\
\hline & & \multirow{2}{*}{ CS } & MPGGE Unadjusted & 60.3 & 60.3 & 71.6 & 82.8 & 66.6 & 80.2 & 94.7 & 74.4 & 90.7 & 114.7 & 81.2 & 97.0 & 125.5 \\
\hline & & & MPGGE Adjusted & 43.7 & 43.7 & 50.9 & 57.6 & 47.7 & 56.1 & 64.5 & 52.6 & 62.3 & 75.5 & 56.7 & 65.9 & 81.2 \\
\hline & & \multirow{3}{*}{$C D+C S$} & Wh/mile & 37.8 & 37.8 & 35.3 & 33.1 & 37.9 & 34.3 & 30.6 & 36.3 & 31.9 & 28.9 & 35.4 & 30.8 & 27.5 \\
\hline & & & MPGGE Unadjusted & 72.1 & 72.1 & 85.6 & 99.1 & 79.6 & 96.0 & 113.4 & 88.9 & 108.7 & 138.2 & 97.2 & 116.2 & 151.2 \\
\hline & & & MPGGE Adjusted & 51.2 & 51.2 & 59.3 & 67.0 & 55.7 & 65.3 & 74.9 & 61.2 & 72.3 & 87.6 & 65.9 & 76.3 & 93.8 \\
\hline & \multirow{9}{*}{ HWFET } & \multirow{4}{*}{$C D$} & Wh/mile & 202.8 & 202.8 & 189.9 & 175.4 & 202.1 & 181.6 & 162.0 & 194.5 & 168.5 & 140.8 & 187.7 & 162.7 & 133.7 \\
\hline & & & Distance & 10.5 & 10.5 & 9.1 & 9.0 & 10.5 & 9.0 & 8.9 & 10.5 & 8.9 & 8.9 & 9.1 & 8.9 & 9.0 \\
\hline & & & MPGGE Unadjusted & 471.2 & 471.2 & 555.2 & 630.1 & 548.0 & 593.2 & 691.3 & 592.4 & 657.7 & 749.6 & 635.4 & 720.0 & 1079.6 \\
\hline & & & MPGGE Adjusted & 304.4 & 304.4 & 352.7 & 394.5 & 348.7 & 374.1 & 427.8 & 373.6 & 409.6 & 458.8 & 397.4 & 443.1 & 622.0 \\
\hline & & \multirow{2}{*}{ CS } & MPGGE Unadjusted & 55.0 & 55.0 & 65.5 & 75.5 & 61.5 & 73.0 & 85.2 & 68.1 & 82.0 & 100.9 & 74.1 & 88.3 & 113.4 \\
\hline & & & MPGGE Adjusted & 38.7 & 38.7 & 46.0 & 52.9 & 43.2 & 51.2 & 59.6 & 47.8 & 57.4 & 70.4 & 52.0 & 61.8 & 78.9 \\
\hline & & \multirow{3}{*}{$C D+C S$} & Wh/mile & 59.7 & 59.7 & 55.9 & 51.6 & 59.4 & 53.4 & 47.6 & 57.2 & 49.6 & 41.4 & 55.2 & 47.8 & 39.3 \\
\hline & & & MPGGE Unadjusted & 69.8 & 69.8 & 83.1 & 95.8 & 78.1 & 92.5 & 107.9 & 86.5 & 103.8 & 127.3 & 94.1 & 111.9 & 144.5 \\
\hline & & & MPGGE Adjusted & 49.0 & 49.0 & 58.2 & 66.8 & 54.7 & 64.6 & 75.1 & 60.5 & 72.3 & 88.3 & 65.7 & 77.8 & 99.8 \\
\hline & \multirow{7}{*}{ Combined } & \multirow{2}{*}{$C D$} & MPGGE Unadjusted & 758.6 & 758.6 & 905.8 & 1090.9 & 876.9 & 1035.6 & 1219.6 & 955.3 & 1159.6 & 1642.9 & 1093.1 & 1269.5 & 2351.7 \\
\hline & & & MPGGE Adjusted & 300.7 & 300.7 & 329.2 & 358.3 & 324.3 & 349.8 & 375.8 & 338.0 & 367.8 & 417.9 & 358.7 & 382.1 & 468.2 \\
\hline & & \multirow{2}{*}{ CS } & MPGGE Unadjusted & 57.8 & 57.8 & 68.7 & 79.3 & 64.2 & 76.8 & 90.2 & 71.4 & 86.6 & 108.0 & 77.8 & 92.9 & 119.8 \\
\hline & & & MPGGE Adjusted & 41.3 & 41.3 & 48.6 & 55.4 & 45.6 & 53.8 & 62.2 & 50.3 & 60.0 & 73.1 & 54.5 & 63.9 & 80.1 \\
\hline & & \multirow{3}{*}{$C D+C S$} & Wh/mile & 47.6 & 47.6 & 44.5 & 41.4 & 47.6 & 42.9 & 38.3 & 45.7 & 39.8 & 34.5 & 44.3 & 38.5 & 32.8 \\
\hline & & & MPGGE Unadjusted & 71.0 & 71.0 & 84.5 & 97.6 & 78.9 & 94.4 & 110.9 & 87.8 & 106.4 & 133.1 & 95.8 & 114.2 & 148.1 \\
\hline & & & MPGGE Adjusted & 50.2 & 50.2 & 58.8 & 66.9 & 55.3 & 65.0 & 75.0 & 60.9 & 72.3 & 87.9 & 65.8 & 77.0 & 96.4 \\
\hline
\end{tabular}


Argonne National Laboratory Report - Light-Duty Vehicle Fuel Consumption Displacement Potential Up to 2045

\begin{tabular}{|c|c|c|c|c|c|c|c|c|c|c|c|c|c|c|c|c|}
\hline & & & & \multicolumn{13}{|c|}{ Compact } \\
\hline & & & & \multirow{2}{*}{$\begin{array}{c}2010 \\
\text { Ref }\end{array}$} & \multicolumn{3}{|c|}{2010} & \multicolumn{3}{|c|}{2015} & \multicolumn{3}{|c|}{2030} & \multicolumn{3}{|c|}{2045} \\
\hline \multirow{26}{*}{ H2 Split HEV PHEV2O } & & & & & low & avg & high & low & avg & high & low & avg & high & low & avg & high \\
\hline & \multirow{9}{*}{ UDDS } & \multirow{4}{*}{$C D$} & Wh/mile & 182.5 & 182.5 & 171.4 & 160.5 & 183.6 & 166.1 & 149.7 & 176.2 & 155.8 & 136.4 & 168.9 & 150.9 & 129.8 \\
\hline & & & Distance & 22.7 & 22.7 & 22.6 & 22.6 & 22.9 & 22.6 & 22.3 & 22.9 & 22.3 & 20.4 & 22.8 & 22.3 & 20.4 \\
\hline & & & MPGGE Unadjusted & 1370.5 & 1370.5 & 1820.8 & 2529.9 & 1608.1 & 2404.9 & 3170.3 & 1858.2 & 3022.2 & N/A & 2068.7 & 3310.7 & $\mathrm{~N} / \mathrm{A}$ \\
\hline & & & MPGGE Adjusted & 290.4 & 290.4 & 310.3 & 329.6 & 302.0 & 326.9 & 340.6 & 311.7 & 338.5 & N/A & 318.3 & 342.6 & N/A \\
\hline & & \multirow{2}{*}{ CS } & MPGGE Unadjusted & 60.0 & 60.0 & 71.1 & 82.6 & 66.2 & 79.6 & 94.3 & 74.0 & 90.7 & 114.8 & 80.6 & 97.0 & 125.3 \\
\hline & & & MPGGE Adjusted & 43.5 & 43.5 & 50.6 & 57.5 & 47.5 & 55.7 & 64.3 & 52.3 & 62.2 & 75.6 & 56.3 & 65.9 & 81.1 \\
\hline & & \multirow{3}{*}{$C D+C S$} & Wh/mile & 96.1 & 96.1 & 90.3 & 84.6 & 96.7 & 87.6 & 57.0 & 92.9 & 59.3 & 52.0 & 89.0 & 57.5 & 49.4 \\
\hline & & & MPGGE Unadjusted & 103.6 & 103.6 & 123.3 & 143.8 & 114.6 & 138.7 & 136.7 & 128.2 & 131.6 & 168.8 & 139.7 & 140.8 & 184.2 \\
\hline & & & MPGGE Adjusted & 69.6 & 69.6 & 80.1 & 90.3 & 75.5 & 87.8 & 86.8 & 82.6 & 84.3 & 101.9 & 88.3 & 88.8 & 108.7 \\
\hline & \multirow{9}{*}{ HWFET } & \multirow{4}{*}{$C D$} & Wh/mile & 198.3 & 198.3 & 190.6 & 201.0 & 201.2 & 208.9 & 186.0 & 196.5 & 193.9 & 165.4 & 214.4 & 186.4 & 151.9 \\
\hline & & & Distance & 21.1 & 21.1 & 20.2 & 17.6 & 21.1 & 17.6 & 17.5 & 20.3 & 17.5 & 16.7 & 17.7 & 17.5 & 17.3 \\
\hline & & & MPGGE Unadjusted & 382.7 & 382.7 & 586.5 & N/A & 492.8 & N/A & $\mathrm{N} / \mathrm{A}$ & 601.6 & N/A & N/A & N/A & $\mathrm{N} / \mathrm{A}$ & $\mathrm{N} / \mathrm{A}$ \\
\hline & & & MPGGE Adjusted & 251.6 & 251.6 & 370.3 & $\mathrm{~N} / \mathrm{A}$ & 317.0 & N/A & $\mathrm{N} / \mathrm{A}$ & 378.8 & $\mathrm{~N} / \mathrm{A}$ & N/A & N/A & $\mathrm{N} / \mathrm{A}$ & $\mathrm{N} / \mathrm{A}$ \\
\hline & & \multirow{2}{*}{ CS } & MPGGE Unadjusted & 54.5 & 54.5 & 65.0 & 75.3 & 60.9 & 72.5 & 85.0 & 67.5 & 81.9 & 100.8 & 73.6 & 88.2 & 113.5 \\
\hline & & & MPGGE Adjusted & 38.4 & 38.4 & 45.6 & 52.8 & 42.9 & 50.8 & 59.5 & 47.4 & 57.3 & 70.3 & 51.7 & 61.7 & 78.9 \\
\hline & & \multirow{3}{*}{$C D+C S$} & Wh/mile & 102.0 & 102.0 & 56.1 & 59.1 & 103.7 & 61.5 & 54.7 & 57.8 & 57.0 & 48.6 & 63.1 & 54.8 & 44.7 \\
\hline & & & MPGGE Unadjusted & 83.9 & 83.9 & 82.6 & 99.1 & 95.0 & 95.3 & 111.9 & 85.8 & 107.7 & 132.6 & 96.9 & 116.1 & 149.3 \\
\hline & & & MPGGE Adjusted & 58.7 & 58.7 & 57.8 & 69.1 & 66.4 & 66.6 & 77.8 & 60.0 & 75.0 & 91.9 & 67.6 & 80.7 & 103.1 \\
\hline & \multirow{7}{*}{ Combined } & \multirow{2}{*}{$C D$} & MPGGE Unadjusted & 634.1 & 634.1 & 935.1 & 4459.0 & 796.7 & 4245.1 & 5544.7 & 957.9 & 5295.1 & N/A & 3666.6 & 5780.6 & N/A \\
\hline & & & MPGGE Adjusted & 271.6 & 271.6 & 334.7 & 550.5 & 308.6 & 546.3 & 567.3 & 338.7 & 563.9 & 640.8 & 533.0 & 570.2 & 640.8 \\
\hline & & \multirow{2}{*}{ CS } & MPGGE Unadjusted & 57.4 & 57.4 & 68.2 & 79.1 & 63.7 & 76.2 & 89.9 & 70.9 & 86.5 & 108.1 & 77.3 & 92.9 & 119.7 \\
\hline & & & MPGGE Adjusted & 41.1 & 41.1 & 48.2 & 55.3 & 45.3 & 53.4 & 62.0 & 50.0 & 59.9 & 73.1 & 54.1 & 63.9 & 80.1 \\
\hline & & \multirow{3}{*}{$\mathrm{CD}+\mathrm{CS}$} & Wh/mile & 98.8 & 98.8 & 74.9 & 73.1 & 99.9 & 75.8 & 56.0 & 77.1 & 58.3 & 50.5 & 77.3 & 56.3 & 47.3 \\
\hline & & & MPGGE Unadjusted & 93.7 & 93.7 & 100.9 & 119.5 & 104.9 & 115.1 & 124.3 & 104.9 & 119.6 & 150.4 & 116.5 & 128.5 & 166.7 \\
\hline & & & MPGGE Adjusted & 64.2 & 64.2 & 68.2 & 79.4 & 71.1 & 76.8 & 82.5 & 70.6 & 79.8 & 97.1 & 77.6 & 85.0 & 106.1 \\
\hline
\end{tabular}


Argonne National Laboratory Report - Light-Duty Vehicle Fuel Consumption Displacement Potential Up to 2045

\begin{tabular}{|c|c|c|c|c|c|c|c|c|c|c|c|c|c|c|c|c|}
\hline & & & & \multicolumn{13}{|c|}{ Compact } \\
\hline & & & & \multirow{2}{*}{$\begin{array}{c}2010 \\
\text { Ref } \\
\end{array}$} & \multicolumn{3}{|c|}{2010} & \multicolumn{3}{|c|}{2015} & \multicolumn{3}{|c|}{2030} & \multicolumn{3}{|c|}{2045} \\
\hline & & & & & low & avg & high & low & avg & high & low & avg & high & low & avg & high \\
\hline \multirow{25}{*}{ H2 Series HEV PHEV3O } & \multirow{9}{*}{ UDDS } & \multirow{4}{*}{$C D$} & Wh/mile & 254.8 & 254.8 & 236.2 & 217.5 & 252.2 & 225.2 & 197.5 & 241.0 & 205.3 & 170.4 & 229.8 & 197.7 & 162.8 \\
\hline & & & Distance & 31.0 & 31.0 & 31.0 & 31.0 & 30.9 & 31.0 & 30.7 & 31.0 & 30.9 & 30.7 & 31.0 & 30.7 & 30.7 \\
\hline & & & MPGGE Unadjusted & $\mathrm{N} / \mathrm{A}$ & N/A & $\mathrm{N} / \mathrm{A}$ & N/A & $\mathrm{N} / \mathrm{A}$ & $\mathrm{N} / \mathrm{A}$ & N/A & $\mathrm{N} / \mathrm{A}$ & N/A & N/A & $\mathrm{N} / \mathrm{A}$ & N/A & N/A \\
\hline & & & MPGGE Adjusted & $\mathrm{N} / \mathrm{A}$ & N/A & $\mathrm{N} / \mathrm{A}$ & $\mathrm{N} / \mathrm{A}$ & $\mathrm{N} / \mathrm{A}$ & $\mathrm{N} / \mathrm{A}$ & $\mathrm{N} / \mathrm{A}$ & $\mathrm{N} / \mathrm{A}$ & $\mathrm{N} / \mathrm{A}$ & $\mathrm{N} / \mathrm{A}$ & $\mathrm{N} / \mathrm{A}$ & $\mathrm{N} / \mathrm{A}$ & $\mathrm{N} / \mathrm{A}$ \\
\hline & & \multirow{2}{*}{ CS } & MPGGE Unadjusted & 44.4 & 44.4 & 53.6 & 64.0 & 49.7 & 60.6 & 74.9 & 56.5 & 72.2 & 88.3 & 61.7 & 75.8 & 94.9 \\
\hline & & & MPGGE Adjusted & 33.2 & 33.2 & 39.3 & 46.0 & 36.8 & 43.9 & 52.9 & 41.2 & 51.2 & 60.8 & 44.6 & 53.4 & 64.7 \\
\hline & & \multirow{3}{*}{$C D+C S$} & Wh/mile & 164.2 & 164.2 & 152.2 & 140.1 & 162.4 & 145.1 & 127.7 & 155.3 & 132.8 & 110.6 & 148.0 & 127.8 & 105.7 \\
\hline & & & MPGGE Unadjusted & 94.5 & 94.5 & 114.1 & 136.1 & 105.8 & 129.0 & 159.3 & 120.2 & 153.6 & 187.8 & 131.3 & 161.3 & 202.0 \\
\hline & & & MPGGE Adjusted & 64.4 & 64.4 & 75.2 & 86.5 & 70.8 & 83.0 & 97.6 & 78.5 & 95.0 & 110.2 & 84.1 & 98.5 & 116.0 \\
\hline & \multirow{9}{*}{ HWFET } & \multirow{4}{*}{$C D$} & Wh/mile & 253.0 & 253.0 & 233.7 & 215.5 & 249.4 & 223.8 & 196.5 & 239.6 & 204.8 & 172.0 & 228.4 & 196.6 & 158.0 \\
\hline & & & Distance & 31.2 & 31.2 & 31.2 & 31.2 & 31.2 & 31.2 & 31.1 & 31.2 & 31.0 & 30.2 & 31.2 & 31.1 & 31.6 \\
\hline & & & MPGGE Unadjusted & $\mathrm{N} / \mathrm{A}$ & N/A & $\mathrm{N} / \mathrm{A}$ & $\mathrm{N} / \mathrm{A}$ & N/A & N/A & $\mathrm{N} / \mathrm{A}$ & N/A & $\mathrm{N} / \mathrm{A}$ & $\mathrm{N} / \mathrm{A}$ & $\mathrm{N} / \mathrm{A}$ & $\mathrm{N} / \mathrm{A}$ & N/A \\
\hline & & & MPGGE Adjusted & $\mathrm{N} / \mathrm{A}$ & $\mathrm{N} / \mathrm{A}$ & $\mathrm{N} / \mathrm{A}$ & $\mathrm{N} / \mathrm{A}$ & $\mathrm{N} / \mathrm{A}$ & $\mathrm{N} / \mathrm{A}$ & $\mathrm{N} / \mathrm{A}$ & N/A & $\mathrm{N} / \mathrm{A}$ & $\mathrm{N} / \mathrm{A}$ & N/A & $\mathrm{N} / \mathrm{A}$ & $\mathrm{N} / \mathrm{A}$ \\
\hline & & \multirow{2}{*}{ CS } & MPGGE Unadjusted & 43.2 & 43.2 & 52.1 & 60.9 & 48.5 & 58.7 & 70.5 & 54.7 & 67.7 & 84.3 & 60.0 & 73.3 & 94.6 \\
\hline & & & MPGGE Adjusted & 30.5 & 30.5 & 36.7 & 42.8 & 34.2 & 41.3 & 49.5 & 38.5 & 47.6 & 59.0 & 42.2 & 51.4 & 66.0 \\
\hline & & \multirow{3}{*}{$C D+C S$} & Wh/mile & 170.9 & 170.9 & 157.9 & 145.6 & 168.5 & 151.2 & 132.7 & 161.9 & 138.4 & 88.7 & 154.3 & 132.8 & 106.7 \\
\hline & & & MPGGE Unadjusted & 94.0 & 94.0 & 113.3 & 132.3 & 105.5 & 127.6 & 153.4 & 118.9 & 147.2 & 143.0 & 130.3 & 159.4 & 205.6 \\
\hline & & & MPGGE Adjusted & 65.6 & 65.6 & 78.8 & 91.6 & 73.5 & 88.5 & 105.7 & 82.6 & 101.7 & 98.8 & 90.3 & 109.7 & 140.2 \\
\hline & \multirow{7}{*}{ Combined } & \multirow{2}{*}{$C D$} & MPGGE Unadjusted & $\mathrm{N} / \mathrm{A}$ & $\mathrm{N} / \mathrm{A}$ & $\mathrm{N} / \mathrm{A}$ & $\mathrm{N} / \mathrm{A}$ & N/A & $\mathrm{N} / \mathrm{A}$ & $\mathrm{N} / \mathrm{A}$ & $\mathrm{N} / \mathrm{A}$ & $\mathrm{N} / \mathrm{A}$ & $\mathrm{N} / \mathrm{A}$ & N/A & $\mathrm{N} / \mathrm{A}$ & $\mathrm{N} / \mathrm{A}$ \\
\hline & & & MPGGE Adjusted & N/A & $\mathrm{N} / \mathrm{A}$ & $\mathrm{N} / \mathrm{A}$ & $\mathrm{N} / \mathrm{A}$ & $\mathrm{N} / \mathrm{A}$ & $\mathrm{N} / \mathrm{A}$ & $\mathrm{N} / \mathrm{A}$ & N/A & $\mathrm{N} / \mathrm{A}$ & $\mathrm{N} / \mathrm{A}$ & $\mathrm{N} / \mathrm{A}$ & $\mathrm{N} / \mathrm{A}$ & $\mathrm{N} / \mathrm{A}$ \\
\hline & & \multirow{2}{*}{ CS } & MPGGE Unadjusted & 43.9 & 43.9 & 52.9 & 62.5 & 49.2 & 59.7 & 72.9 & 55.7 & 70.1 & 86.5 & 60.9 & 74.7 & 94.8 \\
\hline & & & MPGGE Adjusted & 31.9 & 31.9 & 38.1 & 44.5 & 35.6 & 42.7 & 51.3 & 40.0 & 49.5 & 60.0 & 43.5 & 52.5 & 65.3 \\
\hline & & \multirow{3}{*}{$\mathrm{CD}+\mathrm{CS}$} & Wh/mile & 167.2 & 167.2 & 154.8 & 142.6 & 165.2 & 147.9 & 130.0 & 158.2 & 135.3 & 100.7 & 150.9 & 130.0 & 106.1 \\
\hline & & & MPGGE Unadjusted & 94.2 & 94.2 & 113.7 & 134.3 & 105.7 & 128.4 & 156.6 & 119.6 & 150.7 & 164.6 & 130.9 & 160.4 & 203.6 \\
\hline & & & MPGGE Adjusted & 64.9 & 64.9 & 76.8 & 88.7 & 72.0 & 85.4 & 101.1 & 80.3 & 97.9 & 104.7 & 86.8 & 103.3 & 125.8 \\
\hline
\end{tabular}


Argonne National Laboratory Report - Light-Duty Vehicle Fuel Consumption Displacement Potential Up to 2045

\begin{tabular}{|c|c|c|c|c|c|c|c|c|c|c|c|c|c|c|c|c|}
\hline & & & & \multicolumn{13}{|c|}{ Compact } \\
\hline & & & & \multirow{2}{*}{$\frac{2010}{\text { Ref }}$} & \multicolumn{3}{|c|}{2010} & \multicolumn{3}{|c|}{2015} & \multicolumn{3}{|c|}{2030} & \multicolumn{3}{|c|}{2045} \\
\hline \multirow{26}{*}{ H2 Series HEV PHEV4O } & & & & & low & avg & high & low & avg & high & low & avg & high & low & avg & high \\
\hline & \multirow{9}{*}{ UDDS } & \multirow{4}{*}{$C D$} & Wh/mile & 260.1 & 260.1 & 240.9 & 221.9 & 257.4 & 229.1 & 201.5 & 245.9 & 209.6 & 172.3 & 234.7 & 201.0 & 164.6 \\
\hline & & & Distance & 40.5 & 40.5 & 40.6 & 40.4 & 40.8 & 40.6 & 40.8 & 40.6 & 40.8 & 40.6 & 40.5 & 40.6 & 40.6 \\
\hline & & & MPGGE Unadjusted & N/A & N/A & $\mathrm{N} / \mathrm{A}$ & N/A & $\mathrm{N} / \mathrm{A}$ & $\mathrm{N} / \mathrm{A}$ & $\mathrm{N} / \mathrm{A}$ & $\mathrm{N} / \mathrm{A}$ & N/A & $\mathrm{N} / \mathrm{A}$ & $\mathrm{N} / \mathrm{A}$ & N/A & $\mathrm{N} / \mathrm{A}$ \\
\hline & & & MPGGE Adjusted & N/A & N/A & N/A & N/A & N/A & $\mathrm{N} / \mathrm{A}$ & N/A & N/A & N/A & N/A & N/A & N/A & N/A \\
\hline & & \multirow{2}{*}{ CS } & MPGGE Unadjusted & 43.3 & 43.3 & 52.5 & 61.5 & 48.7 & 59.4 & 74.1 & 55.1 & 70.1 & 89.6 & 60.7 & 75.9 & 96.8 \\
\hline & & & MPGGE Adjusted & 32.4 & 32.4 & 38.6 & 44.5 & 36.0 & 43.1 & 52.4 & 40.3 & 49.9 & 61.6 & 44.0 & 53.4 & 65.7 \\
\hline & & \multirow{3}{*}{$\mathrm{CD}+\mathrm{CS}$} & Wh/mile & 191.3 & 191.3 & 177.2 & 163.2 & 189.2 & 168.4 & 149.2 & 180.9 & 155.2 & 127.7 & 172.7 & 148.6 & 122.1 \\
\hline & & & MPGGE Unadjusted & 108.2 & 108.2 & 131.2 & 153.9 & 121.7 & 148.6 & 185.4 & 137.8 & 175.3 & 223.9 & 151.8 & 189.6 & 241.9 \\
\hline & & & MPGGE Adjusted & 72.1 & 72.1 & 84.1 & 95.1 & 79.2 & 92.6 & 109.1 & 87.3 & 104.8 & 124.6 & 94.1 & 110.9 & 131.3 \\
\hline & \multirow{9}{*}{ HWFET } & \multirow{4}{*}{$C D$} & Wh/mile & 255.5 & 255.5 & 235.9 & 217.3 & 252.0 & 225.8 & 198.4 & 241.9 & 206.8 & 173.1 & 230.2 & 197.7 & 160.4 \\
\hline & & & Distance & 41.1 & 41.1 & 41.3 & 41.4 & 41.4 & 41.1 & 41.4 & 41.1 & 41.3 & 40.3 & 41.4 & 41.4 & 41.6 \\
\hline & & & MPGGE Unadjusted & N/A & N/A & $\mathrm{N} / \mathrm{A}$ & N/A & $\mathrm{N} / \mathrm{A}$ & N/A & $\mathrm{N} / \mathrm{A}$ & N/A & N/A & $\mathrm{N} / \mathrm{A}$ & N/A & $\mathrm{N} / \mathrm{A}$ & N/A \\
\hline & & & MPGGE Adjusted & $\mathrm{N} / \mathrm{A}$ & $\mathrm{N} / \mathrm{A}$ & $\mathrm{N} / \mathrm{A}$ & N/A & N/A & N/A & $\mathrm{N} / \mathrm{A}$ & N/A & N/A & N/A & N/A & $\mathrm{N} / \mathrm{A}$ & N/A \\
\hline & & \multirow{2}{*}{ CS } & MPGGE Unadjusted & 42.5 & 42.5 & 51.2 & 60.2 & 47.8 & 57.9 & 69.9 & 53.9 & 67.0 & 84.0 & 59.3 & 72.7 & 94.4 \\
\hline & & & MPGGE Adjusted & 30.0 & 30.0 & 36.1 & 42.3 & 33.7 & 40.7 & 49.0 & 37.9 & 47.1 & 58.8 & 41.7 & 51.0 & 65.9 \\
\hline & & \multirow{3}{*}{$C D+C S$} & Wh/mile & 200.9 & 200.9 & 185.5 & 170.9 & 198.2 & 177.6 & 156.3 & 190.3 & 162.9 & 117.1 & 181.1 & 155.5 & 126.9 \\
\hline & & & MPGGE Unadjusted & 114.8 & 114.8 & 138.5 & 162.6 & 129.2 & 156.4 & 188.8 & 145.6 & 181.1 & 182.6 & 160.3 & 196.6 & 255.2 \\
\hline & & & MPGGE Adjusted & 79.8 & 79.8 & 95.8 & 111.9 & 89.5 & 107.8 & 129.2 & 100.5 & 124.1 & 125.2 & 110.4 & 134.3 & 172.2 \\
\hline & \multirow{7}{*}{ Combined } & \multirow{2}{*}{$C D$} & MPGGE Unadjusted & $\mathrm{N} / \mathrm{A}$ & $\mathrm{N} / \mathrm{A}$ & $\mathrm{N} / \mathrm{A}$ & $N / A$ & N/A & $\mathrm{N} / \mathrm{A}$ & $\mathrm{N} / \mathrm{A}$ & $\mathrm{N} / \mathrm{A}$ & $\mathrm{N} / \mathrm{A}$ & $\mathrm{N} / \mathrm{A}$ & $\mathrm{N} / \mathrm{A}$ & $\mathrm{N} / \mathrm{A}$ & $\mathrm{N} / \mathrm{A}$ \\
\hline & & & MPGGE Adjusted & $\mathrm{N} / \mathrm{A}$ & N/A & $\mathrm{N} / \mathrm{A}$ & $\mathrm{N} / \mathrm{A}$ & N/A & N/A & N/A & N/A & N/A & N/A & $\mathrm{N} / \mathrm{A}$ & N/A & $\mathrm{N} / \mathrm{A}$ \\
\hline & & \multirow{2}{*}{ CS } & MPGGE Unadjusted & 42.9 & 42.9 & 51.9 & 60.9 & 48.3 & 58.7 & 72.2 & 54.5 & 68.7 & 87.0 & 60.1 & 74.4 & 95.7 \\
\hline & & & MPGGE Adjusted & 31.3 & 31.3 & 37.4 & 43.5 & 35.0 & 42.0 & 50.8 & 39.2 & 48.6 & 60.3 & 42.9 & 52.3 & 65.8 \\
\hline & & \multirow{3}{*}{$C D+C S$} & Wh/mile & 195.6 & 195.6 & 180.9 & 166.7 & 193.2 & 172.5 & 152.4 & 185.1 & 158.7 & 123.0 & 176.5 & 151.7 & 124.2 \\
\hline & & & MPGGE Unadjusted & 111.1 & 111.1 & 134.3 & 157.7 & 124.9 & 152.0 & 186.9 & 141.2 & 177.8 & 203.2 & 155.5 & 192.7 & 247.7 \\
\hline & & & MPGGE Adjusted & 75.4 & 75.4 & 89.0 & 102.0 & 83.5 & 98.9 & 117.3 & 92.8 & 112.7 & 124.9 & 100.8 & 120.4 & 147.0 \\
\hline
\end{tabular}


Argonne National Laboratory Report - Light-Duty Vehicle Fuel Consumption Displacement Potential Up to 2045

\begin{tabular}{|c|c|c|c|c|c|c|c|c|c|c|c|c|c|c|c|c|}
\hline & & & & \multicolumn{13}{|c|}{ Compact } \\
\hline & & & & \multirow{2}{*}{$\begin{array}{c}2010 \\
\text { Ref } \\
\end{array}$} & \multicolumn{3}{|c|}{2010} & \multicolumn{3}{|c|}{2015} & \multicolumn{3}{|c|}{2030} & \multicolumn{3}{|c|}{2045} \\
\hline & & & & & low & avg & high & low & avg & high & low & avg & high & low & avg & high \\
\hline \multirow{25}{*}{ E85 Split HEV PHEV10 } & \multirow{9}{*}{ UDDS } & \multirow{4}{*}{$C D$} & Wh/mile & 178.1 & 178.1 & 172.1 & 160.4 & 179.4 & 165.9 & 149.3 & 172.6 & 154.4 & 142.0 & 168.3 & 150.1 & 134.5 \\
\hline & & & Distance & 11.4 & 11.4 & 11.3 & 11.4 & 11.4 & 11.3 & 11.4 & 11.6 & 11.3 & 10.2 & 11.6 & 11.3 & 10.2 \\
\hline & & & MPGGE Unadjusted & 1519.7 & 1519.7 & 2079.5 & 2239.9 & 1571.1 & 2188.5 & 2864.6 & 1633.1 & 2439.7 & N/A & 1688.3 & 2571.2 & $\mathrm{~N} / \mathrm{A}$ \\
\hline & & & MPGGE Adjusted & 298.0 & 298.0 & 318.6 & 323.0 & 300.4 & 321.6 & 335.9 & 303.1 & 327.7 & $\mathrm{~N} / \mathrm{A}$ & 305.3 & 330.5 & $\mathrm{~N} / \mathrm{A}$ \\
\hline & & \multirow{2}{*}{ CS } & MPGGE Unadjusted & 58.8 & 58.8 & 62.8 & 68.0 & 59.4 & 65.9 & 84.8 & 62.4 & 72.2 & 100.1 & 64.6 & 74.9 & 105.7 \\
\hline & & & MPGGE Adjusted & 42.7 & 42.7 & 45.3 & 48.6 & 43.1 & 47.3 & 58.8 & 45.0 & 51.2 & 67.6 & 46.5 & 52.8 & 70.7 \\
\hline & & \multirow{3}{*}{$C D+C S$} & Wh/mile & 37.1 & 37.1 & 35.9 & 33.4 & 37.4 & 34.6 & 31.1 & 36.0 & 32.2 & 29.6 & 35.1 & 31.3 & 28.0 \\
\hline & & & MPGGE Unadjusted & 70.3 & 70.3 & 75.2 & 81.4 & 71.0 & 79.0 & 101.6 & 74.6 & 86.5 & 120.6 & 77.3 & 89.7 & 127.3 \\
\hline & & & MPGGE Adjusted & 50.0 & 50.0 & 53.1 & 56.8 & 50.4 & 55.3 & 68.4 & 52.7 & 59.8 & 78.6 & 54.3 & 61.6 & 82.1 \\
\hline & \multirow{9}{*}{ HWFET } & \multirow{4}{*}{$C D$} & Wh/mile & 198.9 & 198.9 & 189.0 & 176.3 & 199.7 & 182.4 & 165.3 & 193.6 & 168.7 & 143.6 & 188.7 & 164.5 & 135.8 \\
\hline & & & Distance & 9.9 & 9.9 & 9.1 & 9.1 & 10.5 & 9.1 & 9.0 & 9.1 & 9.0 & 8.9 & 9.1 & 8.9 & 9.0 \\
\hline & & & MPGGE Unadjusted & 426.0 & 426.0 & 472.2 & 514.7 & 473.6 & 486.4 & 669.5 & 457.3 & 507.1 & 683.7 & 501.4 & 564.9 & 947.8 \\
\hline & & & MPGGE Adjusted & 277.6 & 277.6 & 304.9 & 329.6 & 305.7 & 313.2 & 416.0 & 296.2 & 325.2 & 423.7 & 321.9 & 358.2 & 559.2 \\
\hline & & \multirow{2}{*}{ CS } & MPGGE Unadjusted & 53.1 & 53.1 & 57.2 & 62.1 & 54.2 & 60.0 & 76.7 & 56.5 & 65.3 & 88.6 & 59.0 & 68.2 & 96.3 \\
\hline & & & MPGGE Adjusted & 37.4 & 37.4 & 40.3 & 43.7 & 38.2 & 42.2 & 53.8 & 39.8 & 45.9 & 61.9 & 41.5 & 47.9 & 67.2 \\
\hline & & \multirow{3}{*}{$C D+C S$} & Wh/mile & 58.5 & 58.5 & 55.6 & 51.9 & 58.7 & 53.6 & 48.6 & 56.9 & 49.6 & 42.2 & 55.5 & 48.4 & 39.9 \\
\hline & & & MPGGE Unadjusted & 67.2 & 67.2 & 72.5 & 78.7 & 68.8 & 76.0 & 97.4 & 71.6 & 82.6 & 112.0 & 74.9 & 86.5 & 122.8 \\
\hline & & & MPGGE Adjusted & 47.2 & 47.2 & 50.9 & 55.1 & 48.3 & 53.3 & 68.0 & 50.2 & 57.8 & 77.9 & 52.5 & 60.5 & 85.2 \\
\hline & \multirow{7}{*}{ Combined } & \multirow{2}{*}{$C D$} & MPGGE Unadjusted & 705.0 & 705.0 & 821.3 & 892.9 & 769.0 & 850.0 & 1157.2 & 757.1 & 898.5 & 1500.2 & 817.5 & 989.7 & 2069.7 \\
\hline & & & MPGGE Adjusted & 288.5 & 288.5 & 312.3 & 325.9 & 302.8 & 317.8 & 367.8 & 299.9 & 326.6 & 404.2 & 312.6 & 342.4 & 451.1 \\
\hline & & \multirow{2}{*}{ CS } & MPGGE Unadjusted & 56.1 & 56.1 & 60.2 & 65.2 & 56.9 & 63.1 & 81.0 & 59.6 & 68.9 & 94.5 & 62.0 & 71.7 & 101.3 \\
\hline & & & MPGGE Adjusted & 40.2 & 40.2 & 42.9 & 46.2 & 40.7 & 44.9 & 56.4 & 42.5 & 48.7 & 64.9 & 44.1 & 50.5 & 69.1 \\
\hline & & \multirow{3}{*}{$C D+C S$} & Wh/mile & 46.7 & 46.7 & 44.7 & 41.7 & 47.0 & 43.1 & 39.0 & 45.4 & 40.0 & 35.3 & 44.3 & 39.0 & 33.4 \\
\hline & & & MPGGE Unadjusted & 68.9 & 68.9 & 74.0 & 80.2 & 70.0 & 77.6 & 99.7 & 73.2 & 84.7 & 116.5 & 76.2 & 88.2 & 125.2 \\
\hline & & & MPGGE Adjusted & 48.7 & 48.7 & 52.1 & 56.0 & 49.5 & 54.4 & 68.2 & 51.5 & 58.9 & 78.3 & 53.5 & 61.1 & 83.5 \\
\hline
\end{tabular}


Argonne National Laboratory Report - Light-Duty Vehicle Fuel Consumption Displacement Potential Up to 2045

\begin{tabular}{|c|c|c|c|c|c|c|c|c|c|c|c|c|c|c|c|c|}
\hline & & & & \multicolumn{13}{|c|}{ Compact } \\
\hline & & & & \multirow{2}{*}{$\begin{array}{c}2010 \\
\operatorname{Ref}\end{array}$} & \multicolumn{3}{|c|}{2010} & \multicolumn{3}{|c|}{2015} & \multicolumn{3}{|c|}{2030} & \multicolumn{3}{|c|}{2045} \\
\hline \multirow{26}{*}{ E85 Split HEV PHEV20 } & & & & & low & avg & high & low & avg & high & low & avg & high & low & avg & high \\
\hline & \multirow{9}{*}{ UDDS } & \multirow{4}{*}{$C D$} & Wh/mile & 180.3 & 180.3 & 170.7 & 162.2 & 181.3 & 167.8 & 150.4 & 174.8 & 155.5 & 139.6 & 170.2 & 152.7 & 132.1 \\
\hline & & & Distance & 22.8 & 22.8 & 22.6 & 22.1 & 22.6 & 22.1 & 22.4 & 22.6 & 22.4 & 20.5 & 22.6 & 22.3 & 20.5 \\
\hline & & & MPGGE Unadjusted & 1480.6 & 1480.6 & 1558.7 & 2194.4 & 1471.8 & 2141.2 & 2621.0 & 1587.0 & 2209.2 & N/A & 1643.3 & 2519.5 & N/A \\
\hline & & & MPGGE Adjusted & 296.1 & 296.1 & 299.8 & 321.8 & 295.7 & 320.4 & 331.5 & 301.1 & 322.2 & N/A & 303.5 & 329.4 & $\mathrm{~N} / \mathrm{A}$ \\
\hline & & \multirow{2}{*}{ CS } & MPGGE Unadjusted & 58.4 & 58.4 & 62.5 & 67.8 & 58.9 & 65.6 & 84.7 & 61.9 & 72.2 & 100.2 & 64.4 & 74.8 & 105.5 \\
\hline & & & MPGGE Adjusted & 42.5 & 42.5 & 45.1 & 48.5 & 42.8 & 47.1 & 58.8 & 44.7 & 51.2 & 67.7 & 46.3 & 52.8 & 70.6 \\
\hline & & \multirow{3}{*}{$\mathrm{CD}+\mathrm{CS}$} & Wh/mile & 95.0 & 95.0 & 90.0 & 61.6 & 95.6 & 63.7 & 79.5 & 92.1 & 82.2 & 53.2 & 89.7 & 58.2 & 50.3 \\
\hline & & & MPGGE Unadjusted & 101.3 & 101.3 & 108.3 & 98.4 & 102.1 & 95.2 & 147.7 & 107.3 & 125.8 & 147.4 & 111.7 & 108.6 & 155.2 \\
\hline & & & MPGGE Adjusted & 68.3 & 68.3 & 72.1 & 66.6 & 68.7 & 64.8 & 92.2 & 71.6 & 81.4 & 92.0 & 73.9 & 72.3 & 95.7 \\
\hline & \multirow{9}{*}{ HWFET } & \multirow{4}{*}{$C D$} & Wh/mile & 203.3 & 203.3 & 217.2 & 202.3 & 202.2 & 210.0 & 188.0 & 195.8 & 195.2 & 167.7 & 215.7 & 188.0 & 153.6 \\
\hline & & & Distance & 20.2 & 20.2 & 17.4 & 17.4 & 20.2 & 17.3 & 17.5 & 20.0 & 17.5 & 16.9 & 17.5 & 17.5 & 17.4 \\
\hline & & & MPGGE Unadjusted & 474.7 & 474.7 & N/A & N/A & 488.2 & N/A & N/A & 506.5 & N/A & N/A & N/A & N/A & N/A \\
\hline & & & MPGGE Adjusted & 306.4 & 306.4 & N/A & N/A & 314.3 & N/A & N/A & 324.9 & N/A & N/A & N/A & N/A & N/A \\
\hline & & \multirow{2}{*}{ CS } & MPGGE Unadjusted & 52.7 & 52.7 & 56.8 & 61.8 & 53.8 & 59.6 & 76.4 & 56.2 & 65.1 & 88.6 & 58.8 & 68.0 & 96.2 \\
\hline & & & MPGGE Adjusted & 37.1 & 37.1 & 40.0 & 43.5 & 37.9 & 41.9 & 53.5 & 39.6 & 45.8 & 61.9 & 41.3 & 47.8 & 67.1 \\
\hline & & \multirow{3}{*}{$\mathrm{CD}+\mathrm{CS}$} & Wh/mile & 59.8 & 59.8 & 63.9 & 59.5 & 59.5 & 61.8 & 55.3 & 57.6 & 57.4 & 49.3 & 63.4 & 55.3 & 45.2 \\
\hline & & & MPGGE Unadjusted & 66.9 & 66.9 & 74.8 & 81.4 & 68.4 & 78.4 & 100.5 & 71.4 & 85.7 & 116.5 & 77.3 & 89.5 & 126.6 \\
\hline & & & MPGGE Adjusted & 47.0 & 47.0 & 52.4 & 57.0 & 48.0 & 54.9 & 70.1 & 50.1 & 59.9 & 81.0 & 54.2 & 62.5 & 87.8 \\
\hline & \multirow{7}{*}{ Combined } & \multirow{2}{*}{$C D$} & MPGGE Unadjusted & 757.9 & 757.9 & 2780.0 & 3883.4 & 772.0 & 3791.8 & 4614.5 & 809.7 & 3908.9 & $\mathrm{~N} / \mathrm{A}$ & 2927.7 & 4441.3 & N/A \\
\hline & & & MPGGE Adjusted & 300.7 & 300.7 & 504.4 & 538.4 & 303.8 & 536.2 & 553.3 & 311.3 & 539.0 & 640.8 & 510.1 & 550.1 & 640.8 \\
\hline & & \multirow{2}{*}{ CS } & MPGGE Unadjusted & 55.7 & 55.7 & 59.8 & 65.0 & 56.5 & 62.8 & 80.8 & 59.2 & 68.8 & 94.6 & 61.7 & 71.6 & 101.1 \\
\hline & & & MPGGE Adjusted & 39.9 & 39.9 & 42.7 & 46.1 & 40.4 & 44.6 & 56.3 & 42.2 & 48.6 & 65.0 & 43.9 & 50.4 & 69.0 \\
\hline & & \multirow{3}{*}{$\mathrm{CD}+\mathrm{CS}$} & Wh/mile & 79.1 & 79.1 & 78.2 & 60.7 & 79.3 & 62.8 & 68.6 & 76.6 & 71.1 & 51.4 & 77.9 & 56.9 & 48.0 \\
\hline & & & MPGGE Unadjusted & 82.3 & 82.3 & 90.1 & 89.9 & 83.5 & 86.8 & 121.9 & 87.5 & 103.9 & 131.7 & 93.0 & 99.1 & 140.9 \\
\hline & & & MPGGE Adjusted & 56.7 & 56.7 & 61.7 & 61.9 & 57.5 & 60.0 & 80.7 & 60.0 & 70.1 & 86.7 & 63.5 & 67.5 & 92.0 \\
\hline
\end{tabular}


Argonne National Laboratory Report - Light-Duty Vehicle Fuel Consumption Displacement Potential Up to 2045

\begin{tabular}{|c|c|c|c|c|c|c|c|c|c|c|c|c|c|c|c|c|}
\hline & & & & \multicolumn{13}{|c|}{ Compact } \\
\hline & & & & \multirow{2}{*}{$\begin{array}{c}2010 \\
\text { Ref } \\
\end{array}$} & \multicolumn{3}{|c|}{2010} & \multicolumn{3}{|c|}{2015} & \multicolumn{3}{|c|}{2030} & \multicolumn{3}{|c|}{2045} \\
\hline & & & & & low & avg & high & low & avg & high & low & avg & high & low & avg & high \\
\hline \multirow{25}{*}{ E85 Series HEV PHEV30 } & \multirow{9}{*}{ UDDS } & \multirow{4}{*}{$C D$} & Wh/mile & 249.5 & 249.5 & 235.8 & 218.7 & 248.6 & 226.5 & 200.2 & 238.8 & 207.1 & 174.5 & 231.7 & 200.5 & 165.7 \\
\hline & & & Distance & 30.9 & 30.9 & 30.9 & 31.0 & 31.5 & 31.0 & 30.7 & 30.9 & 30.9 & 30.9 & 31.0 & 30.7 & 30.7 \\
\hline & & & MPGGE Unadjusted & $\mathrm{N} / \mathrm{A}$ & N/A & $\mathrm{N} / \mathrm{A}$ & $\mathrm{N} / \mathrm{A}$ & $\mathrm{N} / \mathrm{A}$ & $\mathrm{N} / \mathrm{A}$ & $\mathrm{N} / \mathrm{A}$ & $\mathrm{N} / \mathrm{A}$ & N/A & $\mathrm{N} / \mathrm{A}$ & $\mathrm{N} / \mathrm{A}$ & N/A & $\mathrm{N} / \mathrm{A}$ \\
\hline & & & MPGGE Adjusted & $\mathrm{N} / \mathrm{A}$ & $\mathrm{N} / \mathrm{A}$ & $\mathrm{N} / \mathrm{A}$ & $\mathrm{N} / \mathrm{A}$ & $\mathrm{N} / \mathrm{A}$ & $\mathrm{N} / \mathrm{A}$ & $\mathrm{N} / \mathrm{A}$ & $\mathrm{N} / \mathrm{A}$ & $\mathrm{N} / \mathrm{A}$ & $\mathrm{N} / \mathrm{A}$ & $\mathrm{N} / \mathrm{A}$ & $\mathrm{N} / \mathrm{A}$ & $\mathrm{N} / \mathrm{A}$ \\
\hline & & \multirow{2}{*}{ CS } & MPGGE Unadjusted & 44.0 & 44.0 & 47.8 & 52.6 & 44.9 & 50.9 & 67.5 & 47.9 & 58.1 & 82.0 & 50.2 & 59.5 & 86.2 \\
\hline & & & MPGGE Adjusted & 32.9 & 32.9 & 35.5 & 38.7 & 33.5 & 37.5 & 48.3 & 35.5 & 42.3 & 57.1 & 37.1 & 43.2 & 59.6 \\
\hline & & \multirow{3}{*}{$C D+C S$} & Wh/mile & 160.7 & 160.7 & 151.9 & 140.9 & 160.1 & 146.0 & 129.5 & 153.9 & 133.9 & 113.3 & 149.2 & 129.6 & 107.6 \\
\hline & & & MPGGE Unadjusted & 93.6 & 93.6 & 101.8 & 111.9 & 95.5 & 108.3 & 143.6 & 101.9 & 123.6 & 174.5 & 106.8 & 126.5 & 183.5 \\
\hline & & & MPGGE Adjusted & 63.9 & 63.9 & 68.5 & 74.1 & 65.0 & 72.1 & 90.2 & 68.6 & 80.2 & 104.4 & 71.3 & 81.7 & 108.3 \\
\hline & \multirow{9}{*}{ HWFET } & \multirow{4}{*}{$C D$} & Wh/mile & 249.5 & 249.5 & 233.4 & 216.3 & 247.0 & 224.7 & 198.2 & 238.2 & 206.0 & 173.9 & 229.6 & 198.3 & 159.8 \\
\hline & & & Distance & 30.9 & 30.9 & 31.2 & 31.3 & 31.1 & 31.2 & 31.2 & 31.0 & 31.1 & 31.2 & 31.2 & 31.2 & 31.8 \\
\hline & & & MPGGE Unadjusted & $\mathrm{N} / \mathrm{A}$ & N/A & $\mathrm{N} / \mathrm{A}$ & $\mathrm{N} / \mathrm{A}$ & N/A & $\mathrm{N} / \mathrm{A}$ & $\mathrm{N} / \mathrm{A}$ & $\mathrm{N} / \mathrm{A}$ & $\mathrm{N} / \mathrm{A}$ & $\mathrm{N} / \mathrm{A}$ & $\mathrm{N} / \mathrm{A}$ & $\mathrm{N} / \mathrm{A}$ & $\mathrm{N} / \mathrm{A}$ \\
\hline & & & MPGGE Adjusted & $\mathrm{N} / \mathrm{A}$ & $\mathrm{N} / \mathrm{A}$ & $\mathrm{N} / \mathrm{A}$ & $\mathrm{N} / \mathrm{A}$ & $\mathrm{N} / \mathrm{A}$ & $\mathrm{N} / \mathrm{A}$ & N/A & $\mathrm{N} / \mathrm{A}$ & $\mathrm{N} / \mathrm{A}$ & $\mathrm{N} / \mathrm{A}$ & $\mathrm{N} / \mathrm{A}$ & $\mathrm{N} / \mathrm{A}$ & $\mathrm{N} / \mathrm{A}$ \\
\hline & & \multirow{2}{*}{ CS } & MPGGE Unadjusted & 41.6 & 41.6 & 45.5 & 50.1 & 42.6 & 48.3 & 63.7 & 45.4 & 54.0 & 75.0 & 47.8 & 56.8 & 81.8 \\
\hline & & & MPGGE Adjusted & 29.3 & 29.3 & 32.1 & 35.3 & 30.1 & 34.1 & 44.8 & 32.0 & 38.0 & 52.6 & 33.7 & 40.0 & 57.3 \\
\hline & & \multirow{3}{*}{$C D+C S$} & Wh/mile & 168.6 & 168.6 & 157.7 & 146.2 & 166.9 & 151.8 & 133.9 & 160.9 & 139.2 & 117.4 & 155.1 & 134.0 & 108.0 \\
\hline & & & MPGGE Unadjusted & 90.3 & 90.3 & 98.8 & 109.0 & 92.6 & 105.0 & 138.4 & 98.7 & 117.4 & 163.1 & 103.8 & 123.4 & 177.8 \\
\hline & & & MPGGE Adjusted & 63.1 & 63.1 & 68.9 & 75.8 & 64.7 & 73.2 & 95.7 & 68.8 & 81.6 & 112.2 & 72.3 & 85.7 & 122.0 \\
\hline & \multirow{7}{*}{ Combined } & \multirow{2}{*}{$C D$} & MPGGE Unadjusted & $\mathrm{N} / \mathrm{A}$ & $\mathrm{N} / \mathrm{A}$ & $\mathrm{N} / \mathrm{A}$ & $\mathrm{N} / \mathrm{A}$ & $\mathrm{N} / \mathrm{A}$ & $\mathrm{N} / \mathrm{A}$ & $\mathrm{N} / \mathrm{A}$ & $\mathrm{N} / \mathrm{A}$ & $\mathrm{N} / \mathrm{A}$ & $\mathrm{N} / \mathrm{A}$ & $\mathrm{N} / \mathrm{A}$ & $\mathrm{N} / \mathrm{A}$ & $\mathrm{N} / \mathrm{A}$ \\
\hline & & & MPGGE Adjusted & $\mathrm{N} / \mathrm{A}$ & $\mathrm{N} / \mathrm{A}$ & $\mathrm{N} / \mathrm{A}$ & $\mathrm{N} / \mathrm{A}$ & $\mathrm{N} / \mathrm{A}$ & $\mathrm{N} / \mathrm{A}$ & $\mathrm{N} / \mathrm{A}$ & $\mathrm{N} / \mathrm{A}$ & $\mathrm{N} / \mathrm{A}$ & $\mathrm{N} / \mathrm{A}$ & $\mathrm{N} / \mathrm{A}$ & $\mathrm{N} / \mathrm{A}$ & $\mathrm{N} / \mathrm{A}$ \\
\hline & & \multirow{2}{*}{ CS } & MPGGE Unadjusted & 42.9 & 42.9 & 46.7 & 51.5 & 43.8 & 49.7 & 65.7 & 46.7 & 56.2 & 78.7 & 49.1 & 58.2 & 84.2 \\
\hline & & & MPGGE Adjusted & 31.2 & 31.2 & 33.9 & 37.1 & 31.9 & 35.9 & 46.6 & 33.9 & 40.3 & 55.0 & 35.5 & 41.7 & 58.5 \\
\hline & & \multirow{3}{*}{$C D+C S$} & Wh/mile & 164.3 & 164.3 & 154.5 & 143.3 & 163.2 & 148.6 & 131.5 & 157.0 & 136.3 & 115.2 & 151.9 & 131.6 & 107.8 \\
\hline & & & MPGGE Unadjusted & 92.1 & 92.1 & 100.4 & 110.6 & 94.2 & 106.8 & 141.2 & 100.4 & 120.7 & 169.1 & 105.4 & 125.1 & 180.9 \\
\hline & & & MPGGE Adjusted & 63.6 & 63.6 & 68.7 & 74.9 & 64.8 & 72.6 & 92.6 & 68.7 & 80.8 & 107.8 & 71.8 & 83.4 & 114.1 \\
\hline
\end{tabular}


Argonne National Laboratory Report - Light-Duty Vehicle Fuel Consumption Displacement Potential Up to 2045

\begin{tabular}{|c|c|c|c|c|c|c|c|c|c|c|c|c|c|c|c|c|}
\hline & & & & \multicolumn{13}{|c|}{ Compact } \\
\hline & & & & \multirow{2}{*}{$\begin{array}{c}2010 \\
\text { Ref }\end{array}$} & \multicolumn{3}{|c|}{2010} & \multicolumn{3}{|c|}{2015} & \multicolumn{3}{|c|}{2030} & \multicolumn{3}{|c|}{2045} \\
\hline \multirow{26}{*}{ E85 Series HEV PHEV40 } & & & & & low & avg & high & low & avg & high & low & avg & high & low & avg & high \\
\hline & \multirow{9}{*}{ UDDS } & \multirow{4}{*}{$C D$} & Wh/mile & 254.8 & 254.8 & 240.2 & 223.2 & 253.8 & 230.5 & 204.3 & 243.3 & 211.6 & 176.2 & 236.8 & 203.9 & 167.4 \\
\hline & & & Distance & 40.6 & 40.6 & 40.6 & 40.5 & 40.8 & 40.6 & 40.8 & 40.7 & 40.8 & 40.5 & 40.8 & 40.5 & 40.6 \\
\hline & & & MPGGE Unadjusted & $\mathrm{N} / \mathrm{A}$ & N/A & N/A & $\mathrm{N} / \mathrm{A}$ & $\mathrm{N} / \mathrm{A}$ & N/A & N/A & $\mathrm{N} / \mathrm{A}$ & $\mathrm{N} / \mathrm{A}$ & N/A & $\mathrm{N} / \mathrm{A}$ & N/A & N/A \\
\hline & & & MPGGE Adjusted & N/A & N/A & N/A & N/A & N/A & N/A & N/A & N/A & N/A & N/A & N/A & N/A & N/A \\
\hline & & \multirow{2}{*}{ CS } & MPGGE Unadjusted & 42.8 & 42.8 & 46.7 & 51.6 & 43.7 & 49.8 & 66.6 & 47.0 & 56.7 & 82.8 & 48.9 & 58.8 & 87.4 \\
\hline & & & MPGGE Adjusted & 32.0 & 32.0 & 34.7 & 38.0 & 32.6 & 36.8 & 47.7 & 34.9 & 41.4 & 57.6 & 36.2 & 42.8 & 60.3 \\
\hline & & \multirow{3}{*}{$\mathrm{CD}+\mathrm{CS}$} & Wh/mile & 187.4 & 187.4 & 176.7 & 164.1 & 186.5 & 169.4 & 151.3 & 178.9 & 156.6 & 130.7 & 174.2 & 150.7 & 124.2 \\
\hline & & & MPGGE Unadjusted & 106.9 & 106.9 & 116.8 & 128.9 & 109.1 & 124.5 & 166.4 & 117.5 & 141.8 & 207.0 & 122.4 & 147.1 & 218.5 \\
\hline & & & MPGGE Adjusted & 71.4 & 71.4 & 76.6 & 82.9 & 72.6 & 80.7 & 100.8 & 77.0 & 89.3 & 118.0 & 79.6 & 91.9 & 122.5 \\
\hline & \multirow{9}{*}{ HWFET } & \multirow{4}{*}{$C D$} & Wh/mile & 252.4 & 252.4 & 235.4 & 218.2 & 249.6 & 226.7 & 200.2 & 240.2 & 208.0 & 177.3 & 231.5 & 199.5 & 162.2 \\
\hline & & & \begin{tabular}{|l} 
Distance \\
\end{tabular} & 40.5 & 40.5 & 41.3 & 41.5 & 41.3 & 41.2 & 41.5 & 41.1 & 41.4 & 40.3 & 41.5 & 41.5 & 41.8 \\
\hline & & & MPGGE Unadjusted & N/A & N/A & N/A & N/A & N/A & N/A & N/A & N/A & N/A & N/A & N/A & N/A & N/A \\
\hline & & & MPGGE Adjusted & N/A & N/A & N/A & N/A & N/A & N/A & N/A & N/A & N/A & N/A & N/A & N/A & N/A \\
\hline & & \multirow{2}{*}{ CS } & MPGGE Unadjusted & 40.9 & 40.9 & 44.8 & 49.5 & 42.1 & 47.5 & 63.0 & 44.8 & 53.4 & 74.7 & 47.1 & 56.3 & 81.4 \\
\hline & & & MPGGE Adjusted & 28.9 & 28.9 & 31.6 & 34.9 & 29.7 & 33.5 & 44.3 & 31.6 & 37.6 & 52.4 & 33.3 & 39.6 & 57.0 \\
\hline & & \multirow{3}{*}{$\mathrm{CD}+\mathrm{CS}$} & Wh/mile & 170.5 & 170.5 & 185.2 & 171.7 & 196.3 & 178.3 & 157.7 & 188.9 & 163.9 & 120.3 & 182.1 & 157.0 & 128.3 \\
\hline & & & MPGGE Unadjusted & 89.0 & 89.0 & 121.0 & 133.7 & 113.7 & 128.4 & 170.2 & 121.0 & 144.4 & 162.4 & 127.4 & 152.1 & 220.0 \\
\hline & & & MPGGE Adjusted & 62.2 & 62.2 & 84.0 & 92.6 & 79.1 & 89.0 & 117.0 & 84.0 & 99.8 & 111.7 & 88.3 & 104.9 & 149.6 \\
\hline & \multirow{7}{*}{ Combined } & \multirow{2}{*}{$C D$} & MPGGE Unadjusted & N/A & $\mathrm{N} / \mathrm{A}$ & $\mathrm{N} / \mathrm{A}$ & $\mathrm{N} / \mathrm{A}$ & $\mathrm{N} / \mathrm{A}$ & N/A & $\mathrm{N} / \mathrm{A}$ & $\mathrm{N} / \mathrm{A}$ & $\mathrm{N} / \mathrm{A}$ & $\mathrm{N} / \mathrm{A}$ & $\mathrm{N} / \mathrm{A}$ & $\mathrm{N} / \mathrm{A}$ & $\mathrm{N} / \mathrm{A}$ \\
\hline & & & MPGGE Adjusted & N/A & N/A & N/A & N/A & N/A & N/A & N/A & N/A & N/A & N/A & N/A & N/A & N/A \\
\hline & & \multirow{2}{*}{ CS } & MPGGE Unadjusted & 41.9 & 41.9 & 45.8 & 50.6 & 42.9 & 48.7 & 64.9 & 46.0 & 55.2 & 78.9 & 48.1 & 57.7 & 84.6 \\
\hline & & & MPGGE Adjusted & 30.6 & 30.6 & 33.2 & 36.5 & 31.3 & 35.3 & 46.1 & 33.4 & 39.6 & 55.1 & 34.8 & 41.3 & 58.8 \\
\hline & & \multirow{3}{*}{$\mathrm{CD}+\mathrm{CS}$} & Wh/mile & 179.8 & 179.8 & 180.5 & 167.5 & 190.9 & 173.4 & 154.2 & 183.4 & 159.9 & 126.0 & 177.8 & 153.5 & 126.0 \\
\hline & & & MPGGE Unadjusted & 98.0 & 98.0 & 118.6 & 131.0 & 111.1 & 126.2 & 168.1 & 119.1 & 143.0 & 184.2 & 124.6 & 149.3 & 219.2 \\
\hline & & & MPGGE Adjusted & 66.9 & 66.9 & 79.8 & 87.0 & 75.3 & 84.2 & 107.5 & 80.0 & 93.7 & 115.1 & 83.3 & 97.3 & 133.4 \\
\hline
\end{tabular}


Argonne National Laboratory Report - Light-Duty Vehicle Fuel Consumption Displacement Potential Up to 2045

\begin{tabular}{|c|c|c|c|c|c|c|c|c|c|c|c|c|c|c|c|c|}
\hline & & & & \multicolumn{13}{|c|}{ Compact } \\
\hline & & & & 2010 & \multicolumn{3}{|c|}{2010} & \multicolumn{3}{|c|}{2015} & \multicolumn{3}{|c|}{2030} & \multicolumn{3}{|c|}{2045} \\
\hline & & & & Ref & low & avg & high & low & avg & high & low & avg & high & low & avg & high \\
\hline \multirow{25}{*}{ FC HEV PHEV10 } & \multirow{9}{*}{ UDDS } & \multirow{4}{*}{$C D$} & Wh/mile & 241.8 & 241.8 & 214.8 & 191.6 & 230.6 & 198.6 & 176.3 & 214.0 & 182.9 & 150.5 & 202.7 & 173.8 & 142.8 \\
\hline & & & Distance & 10.2 & 10.2 & 10.1 & 10.2 & 10.1 & 10.2 & 10.3 & 10.2 & 10.3 & 10.3 & 10.2 & 10.2 & 10.2 \\
\hline & & & MPGGE Unadjusted & $\mathrm{N} / \mathrm{A}$ & N/A & $\mathrm{N} / \mathrm{A}$ & N/A & $\mathrm{N} / \mathrm{A}$ & N/A & N/A & $\mathrm{N} / \mathrm{A}$ & N/A & N/A & $\mathrm{N} / \mathrm{A}$ & N/A & $\mathrm{N} / \mathrm{A}$ \\
\hline & & & MPGGE Adjusted & $\mathrm{N} / \mathrm{A}$ & N/A & $\mathrm{N} / \mathrm{A}$ & $\mathrm{N} / \mathrm{A}$ & $\mathrm{N} / \mathrm{A}$ & $\mathrm{N} / \mathrm{A}$ & $\mathrm{N} / \mathrm{A}$ & $\mathrm{N} / \mathrm{A}$ & N/A & $\mathrm{N} / \mathrm{A}$ & $\mathrm{N} / \mathrm{A}$ & $\mathrm{N} / \mathrm{A}$ & $\mathrm{N} / \mathrm{A}$ \\
\hline & & \multirow{2}{*}{ CS } & MPGGE Unadjusted & 67.4 & 67.4 & 78.7 & 92.8 & 77.3 & 89.7 & 103.8 & 83.3 & 100.2 & 122.5 & 87.8 & 103.4 & 129.1 \\
\hline & & & MPGGE Adjusted & 48.2 & 48.2 & 55.2 & 63.5 & 54.3 & 61.7 & 69.7 & 57.9 & 67.6 & 79.6 & 60.6 & 69.4 & 83.0 \\
\hline & & \multirow{3}{*}{$C D+C S$} & Wh/mile & 50.4 & 50.4 & 44.8 & 39.9 & 48.0 & 41.4 & 36.7 & 44.6 & 38.1 & 31.4 & 42.2 & 36.2 & 29.8 \\
\hline & & & MPGGE Unadjusted & 81.2 & 81.2 & 94.8 & 111.8 & 93.2 & 108.0 & 125.1 & 100.3 & 120.7 & 147.6 & 105.8 & 124.6 & 155.6 \\
\hline & & & MPGGE Adjusted & 56.7 & 56.7 & 64.6 & 74.0 & 63.7 & 72.0 & 81.0 & 67.7 & 78.7 & 92.1 & 70.8 & 80.7 & 95.9 \\
\hline & \multirow{9}{*}{ HWFET } & \multirow{4}{*}{$C D$} & Wh/mile & 246.2 & 246.2 & 215.2 & 195.6 & 230.8 & 202.9 & 180.5 & 218.6 & 186.9 & 154.0 & 207.1 & 177.8 & 144.4 \\
\hline & & & Distance & 9.5 & 9.5 & 9.3 & 9.4 & 9.3 & 9.3 & 9.4 & 9.3 & 9.4 & 9.1 & 9.3 & 9.3 & 9.4 \\
\hline & & & MPGGE Unadjusted & 1229.2 & 1229.2 & 979.5 & 1240.2 & 989.9 & 1124.2 & 1459.0 & 1074.0 & 1304.1 & 1247.6 & 1109.5 & 1201.9 & 1814.8 \\
\hline & & & MPGGE Adjusted & 690.0 & 690.0 & 574.6 & 694.8 & 579.6 & 642.7 & 787.6 & 619.4 & 722.6 & 698.0 & 635.9 & 677.8 & 925.0 \\
\hline & & \multirow{2}{*}{ CS } & MPGGE Unadjusted & 70.3 & 70.3 & 81.2 & 94.6 & 79.9 & 90.9 & 103.4 & 84.7 & 99.4 & 118.6 & 89.2 & 103.6 & 129.5 \\
\hline & & & MPGGE Adjusted & 49.3 & 49.3 & 56.8 & 66.1 & 56.0 & 63.5 & 72.1 & 59.2 & 69.3 & 82.4 & 62.3 & 72.2 & 89.7 \\
\hline & & \multirow{3}{*}{$C D+C S$} & Wh/mile & 72.4 & 72.4 & 63.3 & 57.5 & 67.9 & 59.7 & 53.1 & 64.3 & 55.0 & 45.3 & 60.9 & 52.3 & 42.5 \\
\hline & & & MPGGE Unadjusted & 90.8 & 90.8 & 104.1 & 121.6 & 102.5 & 116.7 & 133.1 & 108.7 & 127.7 & 151.6 & 114.4 & 132.6 & 166.6 \\
\hline & & & MPGGE Adjusted & 63.5 & 63.5 & 72.5 & 84.4 & 71.5 & 81.1 & 92.2 & 75.7 & 88.6 & 104.5 & 79.6 & 91.9 & 114.6 \\
\hline & \multirow{7}{*}{ Combined } & \multirow{2}{*}{$C D$} & MPGGE Unadjusted & $\mathrm{N} / \mathrm{A}$ & $\mathrm{N} / \mathrm{A}$ & $\mathrm{N} / \mathrm{A}$ & $\mathrm{N} / \mathrm{A}$ & N/A & $\mathrm{N} / \mathrm{A}$ & N/A & N/A & $\mathrm{N} / \mathrm{A}$ & N/A & $\mathrm{N} / \mathrm{A}$ & N/A & N/A \\
\hline & & & MPGGE Adjusted & N/A & $\mathrm{N} / \mathrm{A}$ & $\mathrm{N} / \mathrm{A}$ & N/A & N/A & N/A & $\mathrm{N} / \mathrm{A}$ & $\mathrm{N} / \mathrm{A}$ & N/A & $\mathrm{N} / \mathrm{A}$ & N/A & N/A & N/A \\
\hline & & \multirow{2}{*}{ CS } & MPGGE Unadjusted & 68.6 & 68.6 & 79.8 & 93.6 & 78.5 & 90.2 & 103.6 & 83.9 & 99.8 & 120.7 & 88.4 & 103.5 & 129.3 \\
\hline & & & MPGGE Adjusted & 48.7 & 48.7 & 55.9 & 64.6 & 55.1 & 62.5 & 70.7 & 58.5 & 68.4 & 80.9 & 61.4 & 70.6 & 85.9 \\
\hline & & \multirow{3}{*}{$C D+C S$} & Wh/mile & 60.3 & 60.3 & 53.1 & 47.8 & 57.0 & 49.6 & 44.1 & 53.4 & 45.7 & 37.6 & 50.6 & 43.5 & 35.5 \\
\hline & & & MPGGE Unadjusted & 85.2 & 85.2 & 98.8 & 116.0 & 97.1 & 111.8 & 128.6 & 103.9 & 123.8 & 149.3 & 109.5 & 128.1 & 160.4 \\
\hline & & & MPGGE Adjusted & 59.5 & 59.5 & 68.0 & 78.3 & 66.9 & 75.8 & 85.7 & 71.1 & 82.9 & 97.3 & 74.5 & 85.4 & 103.5 \\
\hline
\end{tabular}


Argonne National Laboratory Report - Light-Duty Vehicle Fuel Consumption Displacement Potential Up to 2045

\begin{tabular}{|c|c|c|c|c|c|c|c|c|c|c|c|c|c|c|c|c|}
\hline & & & & \multicolumn{13}{|c|}{ Compact } \\
\hline & & & & \multirow{2}{*}{$\frac{2010}{\text { Ref }}$} & \multicolumn{3}{|c|}{2010} & \multicolumn{3}{|c|}{2015} & \multicolumn{3}{|c|}{2030} & \multicolumn{3}{|c|}{2045} \\
\hline \multirow{26}{*}{ FC HEV PHEV2O } & & \multirow{5}{*}{$C D$} & & & low & avg & high & low & avg & high & low & avg & high & low & avg & high \\
\hline & \multirow{9}{*}{ UDDS } & & Wh/mile & 245.5 & 245.5 & 218.0 & 194.3 & 234.3 & 201.3 & 176.5 & 216.8 & 182.7 & 148.5 & 205.1 & 176.3 & 140.8 \\
\hline & & & Distance & 20.0 & 20.0 & 20.0 & 20.0 & 20.0 & 20.0 & 20.6 & 20.2 & 20.6 & 20.1 & 20.2 & 20.6 & 20.2 \\
\hline & & & MPGGE Unadjusted & $\mathrm{N} / \mathrm{A}$ & N/A & N/A & N/A & $\mathrm{N} / \mathrm{A}$ & N/A & N/A & $\mathrm{N} / \mathrm{A}$ & N/A & N/A & N/A & N/A & N/A \\
\hline & & & MPGGE Adjusted & N/A & N/A & N/A & N/A & N/A & N/A & N/A & N/A & N/A & N/A & N/A & N/A & N/A \\
\hline & & \multirow{2}{*}{ CS } & MPGGE Unadjusted & 66.6 & 66.6 & 77.8 & 91.5 & 76.0 & 88.4 & 102.6 & 82.1 & 99.2 & 121.7 & 86.8 & 102.5 & 128.4 \\
\hline & & & MPGGE Adjusted & 47.7 & 47.7 & 54.6 & 62.7 & 53.5 & 60.9 & 69.0 & 57.2 & 67.1 & 79.2 & 60.0 & 68.9 & 82.7 \\
\hline & & \multirow{3}{*}{$C D+C S$} & Wh/mile & 93.3 & 93.3 & 82.8 & 73.8 & 89.0 & 76.5 & 67.2 & 82.4 & 69.5 & 56.5 & 77.9 & 67.1 & 53.6 \\
\hline & & & MPGGE Unadjusted & 97.9 & 97.9 & 114.4 & 134.6 & 111.8 & 130.0 & 150.9 & 120.8 & 145.9 & 179.0 & 127.6 & 150.7 & 188.9 \\
\hline & & & MPGGE Adjusted & 66.4 & 66.4 & 75.4 & 85.8 & 74.0 & 83.5 & 93.7 & 78.7 & 91.3 & 106.4 & 82.3 & 93.6 & 110.6 \\
\hline & \multirow{9}{*}{ HWFET } & \multirow{4}{*}{$C D$} & Wh/mile & 263.0 & 263.0 & 236.4 & 213.2 & 253.1 & 222.1 & 194.9 & 238.7 & 202.7 & 171.3 & 226.3 & 194.6 & 156.7 \\
\hline & & & Distance & 19.5 & 19.5 & 18.5 & 18.2 & 19.0 & 18.0 & 18.3 & 18.3 & 18.2 & 17.2 & 18.2 & 18.3 & 18.0 \\
\hline & & & MPGGE Unadjusted & N/A & N/A & N/A & N/A & N/A & N/A & N/A & N/A & N/A & N/A & N/A & N/A & N/A \\
\hline & & & MPGGE Adjusted & N/A & N/A & N/A & $\mathrm{N} / \mathrm{A}$ & N/A & $\mathrm{N} / \mathrm{A}$ & $\mathrm{N} / \mathrm{A}$ & N/A & $\mathrm{N} / \mathrm{A}$ & $\mathrm{N} / \mathrm{A}$ & N/A & $\mathrm{N} / \mathrm{A}$ & N/A \\
\hline & & \multirow{2}{*}{ CS } & MPGGE Unadjusted & 69.6 & 69.6 & 80.4 & 93.8 & 79.0 & 90.1 & 102.7 & 83.9 & 98.8 & 118.1 & 88.4 & 102.9 & 129.0 \\
\hline & & & MPGGE Adjusted & 48.9 & 48.9 & 56.3 & 65.5 & 55.4 & 63.0 & 71.6 & 58.7 & 68.9 & 82.0 & 61.8 & 71.7 & 89.4 \\
\hline & & \multirow{3}{*}{$C D+C S$} & Wh/mile & 77.4 & 77.4 & 69.5 & 62.7 & 74.4 & 65.3 & 57.3 & 70.2 & 59.6 & 50.4 & 66.6 & 57.2 & 46.1 \\
\hline & & & MPGGE Unadjusted & 91.6 & 91.6 & 105.7 & 123.4 & 104.0 & 118.5 & 135.1 & 110.4 & 130.0 & 155.4 & 116.4 & 135.5 & 169.7 \\
\hline & & & MPGGE Adjusted & 64.0 & 64.0 & 73.6 & 85.6 & 72.5 & 82.3 & 93.5 & 76.8 & 90.1 & 107.1 & 80.9 & 93.8 & 116.6 \\
\hline & \multirow{7}{*}{ Combined } & \multirow{2}{*}{$C D$} & MPGGE Unadjusted & N/A & $\mathrm{N} / \mathrm{A}$ & N/A & N/A & N/A & N/A & N/A & N/A & $\mathrm{N} / \mathrm{A}$ & N/A & N/A & $\mathrm{N} / \mathrm{A}$ & N/A \\
\hline & & & MPGGE Adjusted & N/A & N/A & N/A & N/A & N/A & N/A & N/A & N/A & N/A & N/A & $\mathrm{N} / \mathrm{A}$ & N/A & N/A \\
\hline & & \multirow{2}{*}{ CS } & MPGGE Unadjusted & 67.9 & 67.9 & 78.9 & 92.5 & 77.3 & 89.2 & 102.6 & 82.9 & 99.0 & 120.1 & 87.5 & 102.7 & 128.7 \\
\hline & & & MPGGE Adjusted & 48.2 & 48.2 & 55.4 & 63.9 & 54.3 & 61.8 & 70.1 & 57.9 & 67.9 & 80.5 & 60.8 & 70.2 & 85.6 \\
\hline & & \multirow{3}{*}{$C D+C S$} & Wh/mile & 86.1 & 86.1 & 76.8 & 68.8 & 82.4 & 71.4 & 62.7 & 76.9 & 65.1 & 53.8 & 72.8 & 62.7 & 50.2 \\
\hline & & & MPGGE Unadjusted & 95.0 & 95.0 & 110.3 & 129.3 & 108.1 & 124.6 & 143.3 & 115.8 & 138.3 & 167.5 & 122.3 & 143.4 & 179.7 \\
\hline & & & MPGGE Adjusted & 65.3 & 65.3 & 74.6 & $\begin{array}{ll}85.7 \\
\end{array}$ & 73.3 & 83.0 & 93.6 & 77.9 & 90.7 & 106.7 & 81.6 & 93.7 & 113.2 \\
\hline
\end{tabular}


Argonne National Laboratory Report - Light-Duty Vehicle Fuel Consumption Displacement Potential Up to 2045

\begin{tabular}{|c|c|c|c|c|c|c|c|c|c|c|c|c|c|c|c|c|}
\hline & & & & \multicolumn{13}{|c|}{ Compact } \\
\hline & & & & \multirow{2}{*}{$\begin{array}{c}2010 \\
\text { Ref } \\
\end{array}$} & \multicolumn{3}{|c|}{2010} & \multicolumn{3}{|c|}{2015} & \multicolumn{3}{|c|}{2030} & \multicolumn{3}{|c|}{2045} \\
\hline & & & & & low & avg & high & low & avg & high & low & avg & high & low & avg & high \\
\hline \multirow{25}{*}{ FC HEV PHEV30 } & \multirow{9}{*}{ UDDS } & \multirow{4}{*}{$C D$} & Wh/mile & 240.7 & 240.7 & 214.7 & 194.8 & 230.9 & 200.4 & 173.1 & 215.7 & 179.8 & 149.2 & 203.4 & 172.4 & 141.3 \\
\hline & & & Distance & 31.6 & 31.6 & 31.5 & 31.6 & 31.5 & 31.6 & 31.5 & 31.5 & 31.5 & 30.7 & 31.6 & 31.5 & 30.7 \\
\hline & & & MPGGE Unadjusted & $\mathrm{N} / \mathrm{A}$ & N/A & $\mathrm{N} / \mathrm{A}$ & N/A & $\mathrm{N} / \mathrm{A}$ & $\mathrm{N} / \mathrm{A}$ & N/A & $\mathrm{N} / \mathrm{A}$ & N/A & N/A & $\mathrm{N} / \mathrm{A}$ & N/A & $\mathrm{N} / \mathrm{A}$ \\
\hline & & & MPGGE Adjusted & $\mathrm{N} / \mathrm{A}$ & N/A & $\mathrm{N} / \mathrm{A}$ & $\mathrm{N} / \mathrm{A}$ & $\mathrm{N} / \mathrm{A}$ & $\mathrm{N} / \mathrm{A}$ & N/A & $\mathrm{N} / \mathrm{A}$ & $\mathrm{N} / \mathrm{A}$ & $\mathrm{N} / \mathrm{A}$ & $\mathrm{N} / \mathrm{A}$ & $\mathrm{N} / \mathrm{A}$ & $\mathrm{N} / \mathrm{A}$ \\
\hline & & \multirow{2}{*}{ CS } & MPGGE Unadjusted & 65.1 & 65.1 & 75.6 & 87.7 & 74.2 & 85.3 & 99.8 & 79.3 & 96.1 & 117.8 & 84.1 & 100.2 & 124.5 \\
\hline & & & MPGGE Adjusted & 46.7 & 46.7 & 53.3 & 60.5 & 52.4 & 59.1 & 67.4 & 55.5 & 65.4 & 77.2 & 58.4 & 67.6 & 80.7 \\
\hline & & \multirow{3}{*}{$C D+C S$} & Wh/mile & 153.4 & 153.4 & 136.8 & 124.1 & 147.1 & 127.7 & 110.7 & 137.4 & 115.0 & 95.9 & 129.6 & 110.2 & 90.9 \\
\hline & & & MPGGE Unadjusted & 138.4 & 138.4 & 160.9 & 186.6 & 157.8 & 181.6 & 212.3 & 168.8 & 204.6 & 250.7 & 178.8 & 213.2 & 264.9 \\
\hline & & & MPGGE Adjusted & 87.7 & 87.7 & 98.4 & 109.7 & 96.9 & 107.5 & 120.1 & 101.9 & 117.1 & 134.4 & 106.3 & 120.5 & 139.3 \\
\hline & \multirow{9}{*}{ HWFET } & \multirow{4}{*}{$C D$} & Wh/mile & 255.5 & 255.5 & 229.9 & 209.9 & 246.2 & 217.4 & 190.6 & 233.5 & 198.5 & 167.7 & 220.8 & 189.7 & 153.7 \\
\hline & & & Distance & 29.5 & 29.5 & 28.6 & 28.5 & 28.7 & 28.2 & 27.8 & 28.2 & 27.7 & 27.4 & 28.3 & 27.8 & 28.1 \\
\hline & & & MPGGE Unadjusted & $\mathrm{N} / \mathrm{A}$ & N/A & N/A & $\mathrm{N} / \mathrm{A}$ & N/A & N/A & $\mathrm{N} / \mathrm{A}$ & N/A & $\mathrm{N} / \mathrm{A}$ & N/A & $\mathrm{N} / \mathrm{A}$ & N/A & $\mathrm{N} / \mathrm{A}$ \\
\hline & & & MPGGE Adjusted & $\mathrm{N} / \mathrm{A}$ & $\mathrm{N} / \mathrm{A}$ & $\mathrm{N} / \mathrm{A}$ & $\mathrm{N} / \mathrm{A}$ & $\mathrm{N} / \mathrm{A}$ & $\mathrm{N} / \mathrm{A}$ & N/A & N/A & $\mathrm{N} / \mathrm{A}$ & $\mathrm{N} / \mathrm{A}$ & $\mathrm{N} / \mathrm{A}$ & $\mathrm{N} / \mathrm{A}$ & $\mathrm{N} / \mathrm{A}$ \\
\hline & & \multirow{2}{*}{ CS } & MPGGE Unadjusted & 67.9 & 67.9 & 78.2 & 90.2 & 76.9 & 87.1 & 99.9 & 81.1 & 96.0 & 114.6 & 85.8 & 100.4 & 125.2 \\
\hline & & & MPGGE Adjusted & 47.7 & 47.7 & 54.8 & 63.0 & 53.9 & 60.9 & 69.7 & 56.8 & 67.0 & 79.7 & 60.0 & 70.0 & 86.8 \\
\hline & & \multirow{3}{*}{$C D+C S$} & Wh/mile & 131.5 & 131.5 & 118.3 & 108.0 & 126.7 & 111.9 & 98.1 & 120.2 & 102.2 & 86.5 & 113.6 & 97.7 & 79.3 \\
\hline & & & MPGGE Unadjusted & 115.1 & 115.1 & 132.5 & 152.9 & 130.3 & 147.6 & 169.4 & 137.5 & 162.7 & 194.3 & 145.4 & 170.1 & 212.1 \\
\hline & & & MPGGE Adjusted & 80.0 & 80.0 & 91.8 & 105.4 & 90.3 & 101.9 & 116.4 & 95.1 & 112.0 & 132.8 & 100.4 & 116.9 & 144.5 \\
\hline & \multirow{7}{*}{ Combined } & \multirow{2}{*}{$C D$} & MPGGE Unadjusted & $\mathrm{N} / \mathrm{A}$ & $\mathrm{N} / \mathrm{A}$ & $\mathrm{N} / \mathrm{A}$ & N/A & $\mathrm{N} / \mathrm{A}$ & N/A & N/A & N/A & $\mathrm{N} / \mathrm{A}$ & N/A & $\mathrm{N} / \mathrm{A}$ & $\mathrm{N} / \mathrm{A}$ & N/A \\
\hline & & & MPGGE Adjusted & $\mathrm{N} / \mathrm{A}$ & $\mathrm{N} / \mathrm{A}$ & $\mathrm{N} / \mathrm{A}$ & $\mathrm{N} / \mathrm{A}$ & N/A & N/A & N/A & $\mathrm{N} / \mathrm{A}$ & N/A & $\mathrm{N} / \mathrm{A}$ & N/A & N/A & N/A \\
\hline & & \multirow{2}{*}{ CS } & MPGGE Unadjusted & 66.3 & 66.3 & 76.8 & 88.8 & 75.3 & 86.1 & 99.8 & 80.1 & 96.1 & 116.4 & 84.8 & 100.3 & 124.8 \\
\hline & & & MPGGE Adjusted & 47.2 & 47.2 & 54.0 & 61.6 & 53.1 & 59.9 & 68.4 & 56.1 & 66.1 & 78.3 & 59.1 & 68.7 & 83.3 \\
\hline & & \multirow{3}{*}{$C D+C S$} & Wh/mile & 143.5 & 143.5 & 128.5 & 116.9 & 137.9 & 120.6 & 105.0 & 129.7 & 109.2 & 91.7 & 122.4 & 104.6 & 85.6 \\
\hline & & & MPGGE Unadjusted & 126.8 & 126.8 & 146.8 & 169.8 & 144.1 & 164.5 & 190.6 & 153.1 & 183.3 & 221.7 & 162.0 & 191.4 & 238.2 \\
\hline & & & MPGGE Adjusted & 84.0 & 84.0 & 95.3 & 107.7 & 93.8 & 104.9 & 118.4 & 98.7 & 114.7 & 133.7 & 103.6 & 118.8 & 141.6 \\
\hline
\end{tabular}


Argonne National Laboratory Report - Light-Duty Vehicle Fuel Consumption Displacement Potential Up to 2045

\begin{tabular}{|c|c|c|c|c|c|c|c|c|c|c|c|c|c|c|c|c|}
\hline & & & & \multicolumn{13}{|c|}{ Compact } \\
\hline & & & & 2010 & & 2010 & & & 2015 & & & 2030 & & & 2045 & \\
\hline \multirow{26}{*}{ FC HEV PHEV40 } & & \multirow{5}{*}{$C D$} & & Ref & low & avg & high & low & avg & high & low & avg & high & low & avg & high \\
\hline & \multirow{9}{*}{ UDDS } & & Wh/mile & 245.0 & 245.0 & 218.2 & 197.7 & 235.1 & 203.4 & 174.8 & 218.9 & 181.5 & 151.4 & 206.2 & 173.8 & 143.4 \\
\hline & & & Distance & 41.2 & 41.2 & 41.1 & 41.1 & 41.1 & 41.1 & 41.0 & 41.1 & 40.9 & 40.8 & 41.1 & 40.6 & 40.6 \\
\hline & & & MPGGE Unadjusted & N/A & N/A & $\mathrm{N} / \mathrm{A}$ & N/A & N/A & N/A & N/A & N/A & N/A & N/A & N/A & N/A & $\mathrm{N} / \mathrm{A}$ \\
\hline & & & MPGGE Adjusted & $\mathrm{N} / \mathrm{A}$ & N/A & $\mathrm{N} / \mathrm{A}$ & $\mathrm{N} / \mathrm{A}$ & $\mathrm{N} / \mathrm{A}$ & $\mathrm{N} / \mathrm{A}$ & $\mathrm{N} / \mathrm{A}$ & $\mathrm{N} / \mathrm{A}$ & N/A & $\mathrm{N} / \mathrm{A}$ & $\mathrm{N} / \mathrm{A}$ & $\mathrm{N} / \mathrm{A}$ & $\mathrm{N} / \mathrm{A}$ \\
\hline & & \multirow{2}{*}{ CS } & MPGGE Unadjusted & 63.9 & 63.9 & 74.4 & 86.4 & 72.8 & 84.1 & 98.6 & 78.1 & 95.0 & 116.7 & 82.9 & 99.1 & 123.3 \\
\hline & & & MPGGE Adjusted & 46.0 & 46.0 & 52.5 & 59.7 & 51.6 & 58.4 & 66.7 & 54.8 & 64.7 & 76.6 & 57.7 & 67.0 & 80.0 \\
\hline & & \multirow{3}{*}{$C D+C S$} & Wh/mile & 177.1 & 177.1 & 157.7 & 142.9 & 170.0 & 147.0 & 126.9 & 158.2 & 131.8 & 111.2 & 149.1 & 126.2 & 105.3 \\
\hline & & & MPGGE Unadjusted & 159.7 & 159.7 & 185.9 & 215.9 & 181.9 & 210.1 & 246.4 & 195.3 & 237.5 & 291.7 & 207.1 & 247.8 & 308.2 \\
\hline & & & MPGGE Adjusted & 97.8 & 97.8 & 109.4 & 121.5 & 107.7 & 119.3 & 132.9 & 113.3 & 129.7 & 148.1 & 118.1 & 133.4 & 153.2 \\
\hline & \multirow{9}{*}{ HWFET } & \multirow{4}{*}{$C D$} & Wh/mile & 258.7 & 258.7 & 232.6 & 212.1 & 249.5 & 219.6 & 192.3 & 236.0 & 200.2 & 168.0 & 222.9 & 191.3 & 153.9 \\
\hline & & & Distance & 38.3 & 38.3 & 37.9 & 37.7 & 38.1 & 37.5 & 36.7 & 37.5 & 36.5 & 36.6 & 37.5 & 36.8 & 37.7 \\
\hline & & & MPGGE Unadjusted & N/A & N/A & N/A & N/A & N/A & N/A & N/A & N/A & N/A & N/A & N/A & N/A & N/A \\
\hline & & & MPGGE Adjusted & $\mathrm{N} / \mathrm{A}$ & $\mathrm{N} / \mathrm{A}$ & $\mathrm{N} / \mathrm{A}$ & $\mathrm{N} / \mathrm{A}$ & N/A & N/A & $\mathrm{N} / \mathrm{A}$ & N/A & $\mathrm{N} / \mathrm{A}$ & $\mathrm{N} / \mathrm{A}$ & N/A & N/A & N/A \\
\hline & & \multirow{2}{*}{ CS } & MPGGE Unadjusted & 67.1 & 67.1 & 77.3 & 89.3 & 75.9 & 86.2 & 99.1 & 80.3 & 95.2 & 114.0 & 85.0 & 99.6 & 124.4 \\
\hline & & & MPGGE Adjusted & 47.1 & 47.1 & 54.2 & 62.4 & 53.2 & 60.3 & 69.1 & 56.2 & 66.5 & 79.2 & 59.5 & 69.5 & 86.3 \\
\hline & & \multirow{3}{*}{$C D+C S$} & Wh/mile & 174.3 & 174.3 & 156.7 & 142.9 & 168.1 & 148.0 & 129.8 & 159.0 & 135.1 & 113.5 & 150.2 & 129.1 & 104.0 \\
\hline & & & MPGGE Unadjusted & 145.8 & 145.8 & 168.1 & 194.0 & 165.0 & 187.5 & 215.4 & 174.5 & 207.0 & 247.7 & 184.7 & 216.6 & 270.4 \\
\hline & & & MPGGE Adjusted & 100.7 & 100.7 & 115.5 & 132.7 & 113.5 & 128.3 & 146.6 & 119.8 & 141.1 & 167.5 & 126.5 & 147.4 & 181.9 \\
\hline & \multirow{7}{*}{ Combined } & \multirow{2}{*}{$C D$} & MPGGE Unadjusted & $\mathrm{N} / \mathrm{A}$ & N/A & $\mathrm{N} / \mathrm{A}$ & $\mathrm{N} / \mathrm{A}$ & $\mathrm{N} / \mathrm{A}$ & $\mathrm{N} / \mathrm{A}$ & N/A & $\mathrm{N} / \mathrm{A}$ & $\mathrm{N} / \mathrm{A}$ & N/A & $\mathrm{N} / \mathrm{A}$ & $\mathrm{N} / \mathrm{A}$ & $\mathrm{N} / \mathrm{A}$ \\
\hline & & & MPGGE Adjusted & N/A & N/A & $\mathrm{N} / \mathrm{A}$ & N/A & N/A & $\mathrm{N} / \mathrm{A}$ & $\mathrm{N} / \mathrm{A}$ & N/A & N/A & N/A & N/A & $\mathrm{N} / \mathrm{A}$ & N/A \\
\hline & & \multirow{2}{*}{ CS } & MPGGE Unadjusted & 65.3 & 65.3 & 75.7 & 87.6 & 74.1 & 85.0 & 98.8 & 79.1 & 95.1 & 115.4 & 83.8 & 99.3 & 123.8 \\
\hline & & & MPGGE Adjusted & 46.5 & 46.5 & 53.3 & 60.9 & 52.3 & 59.2 & 67.8 & 55.4 & 65.5 & 77.8 & 58.4 & 68.1 & 82.7 \\
\hline & & \multirow{3}{*}{$C D+C S$} & Wh/mile & 175.8 & 175.8 & 157.3 & 142.9 & 169.1 & 147.4 & 128.2 & 158.6 & 133.3 & 112.2 & 149.6 & 127.5 & 104.7 \\
\hline & & & MPGGE Unadjusted & 153.1 & 153.1 & 177.4 & 205.5 & 173.9 & 199.3 & 231.4 & 185.4 & 222.7 & 270.1 & 196.4 & 232.7 & 289.9 \\
\hline & & & MPGGE Adjusted & 99.1 & 99.1 & 112.1 & 126.3 & 110.2 & 123.2 & 138.8 & 116.1 & 134.6 & 156.2 & 121.7 & 139.3 & 164.9 \\
\hline \multirow{2}{*}{ EV } & UDDS & EV Only & Wh/mile & 240.4 & 240.4 & 226.3 & 208.1 & 242.0 & 216.3 & 180.2 & 229.4 & 186.9 & 149.9 & 220.5 & 178.1 & 140.9 \\
\hline & HWFET & EV Only & Wh/mile & 251.2 & 251.2 & 234.7 & 216.1 & 250.7 & 224.9 & 193.7 & 239.5 & 201.4 & 166.6 & 229.1 & 191.8 & 152.1 \\
\hline
\end{tabular}


Fuel consumption

\begin{tabular}{|c|c|c|c|c|c|c|c|c|c|c|c|c|c|c|c|}
\hline & & & \multicolumn{13}{|c|}{ Compact } \\
\hline & & & \multirow{2}{*}{$\frac{2010}{\text { Ref }}$} & \multicolumn{3}{|c|}{2010} & \multicolumn{3}{|c|}{2015} & \multicolumn{3}{|c|}{2030} & \multicolumn{3}{|c|}{2045} \\
\hline & & & & low & avg & high & low & avg & high & low & avg & high & low & avg & high \\
\hline \multirow{6}{*}{ SI Conv } & \multirow{3}{*}{ Unadjusted } & UDDS & 7.8 & 7.8 & 7.2 & 6.9 & 7.5 & 7.0 & 5.7 & 7.3 & 6.7 & 4.8 & 7.1 & 6.5 & 4.7 \\
\hline & & HWFET & 5.7 & 5.7 & 5.2 & 4.7 & 5.4 & 4.7 & 3.8 & 5.0 & 4.6 & 3.3 & 4.9 & 4.4 & 3.1 \\
\hline & & Combined & 6.8 & 6.8 & 6.3 & 5.9 & 6.5 & 6.0 & 4.8 & 6.2 & 5.8 & 4.1 & 6.1 & 5.6 & 4.0 \\
\hline & \multirow{3}{*}{ Adjusted } & UDDS & 10.2 & 10.2 & 9.5 & 9.1 & 9.8 & 9.1 & 7.6 & 9.5 & 8.9 & 6.5 & 9.4 & 8.6 & 6.4 \\
\hline & & HWFET & 8.0 & 8.0 & 7.4 & 6.6 & 7.6 & 6.7 & 5.4 & 7.0 & 6.5 & 4.7 & 6.9 & 6.3 & 4.5 \\
\hline & & Combined & 9.2 & 9.2 & 8.5 & 8.0 & 8.8 & 8.0 & 6.6 & 8.4 & 7.8 & 5.6 & 8.3 & 7.6 & 5.5 \\
\hline \multirow{6}{*}{$\mathrm{Cl}$ Conv } & \multirow{3}{*}{ Unadjusted } & UDDS & 6.7 & 6.7 & 6.4 & 5.9 & 6.6 & 6.0 & 5.4 & 6.3 & 5.6 & 4.7 & 6.1 & 5.3 & 4.5 \\
\hline & & HWFET & 4.9 & 4.9 & 4.6 & 4.2 & 4.8 & 4.3 & 3.9 & 4.6 & 4.0 & 3.4 & 4.4 & 3.8 & 3.2 \\
\hline & & Combined & 5.9 & 5.9 & 5.6 & 5.2 & 5.8 & 5.3 & 4.7 & 5.5 & 4.9 & 4.1 & 5.3 & 4.6 & 3.9 \\
\hline & \multirow{3}{*}{ Adjusted } & \begin{tabular}{|l|} 
UDDS \\
\end{tabular} & 8.8 & 8.8 & 8.4 & 7.8 & 8.7 & 8.0 & 7.3 & 8.4 & 7.4 & 6.4 & 8.0 & 7.1 & 6.1 \\
\hline & & HWFET & 7.0 & 7.0 & 6.6 & 6.0 & 6.8 & 6.2 & 5.5 & 6.5 & 5.7 & 4.8 & 6.2 & 5.4 & 4.5 \\
\hline & & Combined & 8.0 & 8.0 & 7.6 & 7.0 & 7.9 & 7.2 & 6.5 & 7.5 & 6.7 & 5.7 & 7.2 & 6.4 & 5.4 \\
\hline \multirow{6}{*}{ H2 Conv } & \multirow{3}{*}{ Unadjusted } & UDDS & 9.2 & 9.2 & 7.8 & 6.9 & 8.1 & 7.0 & 6.2 & 7.5 & 6.4 & 5.2 & 7.0 & 5.9 & 4.9 \\
\hline & & HWFET & 6.2 & 6.2 & 5.2 & 4.3 & 5.5 & 4.4 & 3.9 & 4.7 & 4.0 & 3.3 & 4.4 & 3.8 & 3.0 \\
\hline & & Combined & 7.9 & 7.9 & 6.6 & 5.7 & 7.0 & 5.9 & 5.2 & 6.3 & 5.4 & 4.4 & 5.8 & 4.9 & 4.0 \\
\hline & \multirow{3}{*}{ Adjusted } & \begin{tabular}{|l|} 
UDDS \\
\end{tabular} & 11.9 & 11.9 & 10.1 & 9.0 & 10.6 & 9.2 & 8.2 & 9.8 & 8.5 & 7.0 & 9.1 & 7.9 & 6.6 \\
\hline & & \begin{tabular}{|l|} 
HWFET \\
\end{tabular} & 8.8 & 8.8 & 7.4 & 6.1 & 7.8 & 6.3 & 5.6 & 6.7 & 5.7 & 4.8 & 6.3 & 5.3 & 4.3 \\
\hline & & Combined & 10.5 & 10.5 & 8.9 & 7.7 & 9.3 & 7.9 & 7.0 & 8.4 & 7.2 & 6.0 & 7.8 & 6.7 & 5.6 \\
\hline \multirow{6}{*}{ E85 Conv } & \multirow{3}{*}{ Unadjusted } & \begin{tabular}{|l|} 
UDDS \\
\end{tabular} & 8.0 & 8.0 & 7.9 & 7.5 & 8.2 & 7.7 & 5.8 & 8.1 & 7.4 & 5.0 & 7.8 & 7.1 & 4.8 \\
\hline & & \begin{tabular}{|c|} 
HWFET \\
\end{tabular} & 6.0 & 6.0 & 5.9 & 5.4 & 6.1 & 5.5 & 4.2 & 5.8 & 5.3 & 3.5 & 5.6 & 5.0 & 3.4 \\
\hline & & Combined & 7.1 & 7.1 & 7.0 & 6.6 & 7.3 & 6.7 & 5.1 & 7.1 & 6.5 & 4.3 & 6.8 & 6.1 & 4.2 \\
\hline & \multirow{3}{*}{ Adjusted } & \begin{tabular}{|l|} 
UDDS \\
\end{tabular} & 10.4 & 10.4 & 10.3 & 9.8 & 10.7 & 10.0 & 7.7 & 10.5 & 9.6 & 6.7 & 10.1 & 9.2 & 6.5 \\
\hline & & \begin{tabular}{|c|} 
HWFET \\
\end{tabular} & 8.4 & 8.4 & 8.3 & 7.7 & 8.6 & 7.9 & 5.9 & 8.3 & 7.5 & 5.0 & 7.9 & 7.1 & 4.8 \\
\hline & & Combined & 9.5 & 9.5 & 9.4 & 8.9 & 9.8 & 9.0 & 6.9 & 9.5 & 8.7 & 5.9 & 9.1 & 8.3 & 5.7 \\
\hline \multirow{6}{*}{ SI Split HEV } & \multirow{3}{*}{ Unadjusted } & \begin{tabular}{|l|} 
UDDS \\
\end{tabular} & 3.9 & 3.9 & 3.7 & 3.4 & 3.9 & 3.4 & 2.8 & 3.6 & 3.1 & 2.2 & 3.5 & 3.0 & 2.1 \\
\hline & & \begin{tabular}{|c|} 
HWFET \\
\end{tabular} & 4.5 & 4.5 & 4.1 & 3.8 & 4.4 & 3.9 & 3.2 & 4.1 & 3.6 & 2.6 & 4.0 & 3.4 & 2.4 \\
\hline & & Combined & 4.2 & 4.2 & 3.9 & 3.6 & 4.1 & 3.6 & 3.0 & 3.9 & 3.3 & 2.4 & 3.7 & 3.2 & 2.2 \\
\hline & \multirow{3}{*}{ Adjusted } & \begin{tabular}{|l|} 
UDDS \\
\end{tabular} & 5.4 & 5.4 & 5.1 & 4.7 & 5.4 & 4.8 & 4.0 & 5.0 & 4.4 & 3.3 & 4.9 & 4.2 & 3.2 \\
\hline & & \begin{tabular}{|c|} 
HWFET \\
\end{tabular} & 6.4 & 6.4 & 5.9 & 5.4 & 6.3 & 5.6 & 4.6 & 5.9 & 5.1 & 3.8 & 5.7 & 4.9 & 3.5 \\
\hline & & Combined & 5.8 & 5.8 & 5.4 & 5.0 & 5.8 & 5.1 & 4.3 & 5.4 & 4.7 & 3.5 & 5.2 & 4.5 & 3.3 \\
\hline \multirow{6}{*}{ SI Split HEV PHEV10 } & & \begin{tabular}{|l|} 
UDDS \\
\end{tabular} & 3.1 & 3.1 & 2.9 & 2.7 & 3.1 & 2.8 & 2.3 & 3.0 & 2.6 & 1.8 & 2.9 & 2.5 & 1.7 \\
\hline & Unadjusted & HWFET & 3.3 & 3.3 & 3.0 & 2.8 & 3.2 & 2.9 & 2.4 & 3.1 & 2.6 & 2.0 & 2.9 & 2.5 & 1.8 \\
\hline & & Combined & 3.2 & 3.2 & 3.0 & 2.7 & 3.1 & 2.8 & 2.3 & 3.0 & 2.6 & 1.9 & 2.9 & 2.5 & 1.8 \\
\hline & & \begin{tabular}{|l|} 
UDDS \\
\end{tabular} & 4.4 & 4.4 & 4.2 & 3.9 & 4.4 & 4.0 & 3.4 & 4.2 & 3.7 & 2.9 & 4.1 & 3.6 & 2.7 \\
\hline & Adjusted & \begin{tabular}{|l|} 
HWFET \\
\end{tabular} & 4.6 & 4.6 & 4.3 & 4.0 & 4.5 & 4.1 & 3.4 & 4.4 & 3.8 & 2.8 & 4.2 & 3.6 & 2.6 \\
\hline & & Combined & 4.5 & 4.5 & 4.2 & 3.9 & 4.5 & 4.1 & 3.4 & 4.3 & 3.8 & 2.9 & 4.1 & 3.6 & 2.7 \\
\hline
\end{tabular}




\begin{tabular}{|c|c|c|c|c|c|c|c|c|c|c|c|c|c|c|c|}
\hline & & & \multicolumn{13}{|c|}{ Compact } \\
\hline & & & \multirow{2}{*}{$\frac{2010}{\text { Ref }}$} & \multicolumn{3}{|c|}{2010} & \multicolumn{3}{|c|}{2015} & \multicolumn{3}{|c|}{2030} & \multicolumn{3}{|c|}{2045} \\
\hline & & & & low & avg & high & low & avg & high & low & avg & high & low & avg & high \\
\hline \multirow{6}{*}{ SI Split HEV PHEV20 } & \multirow{3}{*}{ Unadjusted } & UDDS & 2.2 & 2.2 & 2.0 & 2.2 & 2.2 & 2.3 & 1.6 & 2.1 & 1.8 & 1.5 & 2.0 & 2.0 & 1.4 \\
\hline & & HWFET & 3.3 & 3.3 & 2.9 & 2.7 & 3.2 & 2.8 & 2.3 & 3.1 & 2.6 & 1.9 & 2.8 & 2.5 & 1.8 \\
\hline & & Combined & 2.7 & 2.7 & 2.4 & 2.4 & 2.6 & 2.5 & 1.9 & 2.5 & 2.1 & 1.7 & 2.4 & 2.2 & 1.6 \\
\hline & \multirow{3}{*}{ Adjusted } & UDDS & 3.3 & 3.3 & 3.1 & 3.4 & 3.2 & 3.4 & 2.5 & 3.1 & 2.7 & 2.4 & 3.0 & 3.1 & 2.4 \\
\hline & & \begin{tabular}{|c|} 
HWFET \\
\end{tabular} & 4.7 & 4.7 & 4.2 & 3.9 & 4.6 & 4.0 & 3.3 & 4.4 & 3.7 & 2.7 & 4.1 & 3.5 & 2.5 \\
\hline & & Combined & 3.9 & 3.9 & 3.6 & 3.6 & 3.8 & 3.7 & 2.9 & 3.7 & 3.2 & 2.6 & 3.5 & 3.3 & 2.4 \\
\hline \multirow{6}{*}{ SI Series HEV PHEV30 } & \multirow{3}{*}{ Unadjusted } & UDDS & 2.4 & 2.4 & 2.2 & 2.0 & 2.3 & 2.0 & 1.6 & 2.2 & 1.8 & 1.3 & 2.1 & 1.8 & 1.2 \\
\hline & & \begin{tabular}{|l|} 
HWFET \\
\end{tabular} & 2.4 & 2.4 & 2.2 & 2.0 & 2.4 & 2.1 & 1.7 & 2.2 & 1.9 & 1.4 & 2.1 & 1.8 & 1.2 \\
\hline & & Combined & 2.4 & 2.4 & 2.2 & 2.0 & 2.3 & 2.1 & 1.6 & 2.2 & 1.8 & 1.3 & 2.1 & 1.8 & 1.2 \\
\hline & \multirow{3}{*}{ Adjusted } & UDDS & 3.5 & 3.5 & 3.3 & 3.0 & 3.4 & 3.1 & 2.6 & 3.3 & 2.8 & 2.2 & 3.1 & 2.8 & 2.1 \\
\hline & & \begin{tabular}{|l|} 
HWFET \\
\end{tabular} & 3.5 & 3.5 & 3.2 & 2.9 & 3.4 & 3.0 & 2.4 & 3.2 & 2.7 & 2.0 & 3.1 & 2.6 & 1.8 \\
\hline & & Combined & 3.5 & 3.5 & 3.2 & 3.0 & 3.4 & 3.1 & 2.5 & 3.2 & 2.8 & 2.1 & 3.1 & 2.7 & 2.0 \\
\hline \multirow{6}{*}{ SI Series HEV PHEV40 } & \multirow{3}{*}{ Unadjusted } & \begin{tabular}{|l|} 
UDDS \\
\end{tabular} & 2.1 & 2.1 & 1.9 & 1.7 & 2.0 & 1.8 & 1.4 & 1.9 & 1.6 & 1.1 & 1.8 & 1.5 & 1.0 \\
\hline & & \begin{tabular}{|l|} 
HWFET \\
\end{tabular} & 2.5 & 2.5 & 1.8 & 1.7 & 1.9 & 1.7 & 1.4 & 1.8 & 1.5 & 1.4 & 1.7 & 1.5 & 1.0 \\
\hline & & Combined & 2.3 & 2.3 & 1.9 & 1.7 & 2.0 & 1.8 & 1.4 & 1.9 & 1.6 & 1.2 & 1.8 & 1.5 & 1.0 \\
\hline & \multirow{3}{*}{ Adjusted } & \begin{tabular}{|l|} 
UDDS \\
\end{tabular} & 3.1 & 3.1 & 2.9 & 2.7 & 3.1 & 2.8 & 2.3 & 2.9 & 2.5 & 1.9 & 2.8 & 2.4 & 1.9 \\
\hline & & \begin{tabular}{|c|} 
HWFET \\
\end{tabular} & 3.6 & 3.6 & 2.6 & 2.4 & 2.8 & 2.5 & 2.0 & 2.6 & 2.2 & 2.0 & 2.5 & 2.1 & 1.5 \\
\hline & & Combined & 3.3 & 3.3 & 2.8 & 2.6 & 3.0 & 2.6 & 2.2 & 2.8 & 2.4 & 2.0 & 2.7 & 2.3 & 1.7 \\
\hline \multirow{6}{*}{ CI Split HEV } & \multirow{3}{*}{ Unadjusted } & \begin{tabular}{|l|} 
UDDS \\
\end{tabular} & 3.7 & 3.7 & 3.5 & 3.2 & 3.7 & 3.2 & 2.8 & 3.4 & 2.9 & 2.3 & 3.2 & 2.7 & 2.2 \\
\hline & & HWFET & 4.3 & 4.3 & 4.0 & 3.6 & 4.2 & 3.7 & 3.3 & 3.9 & 3.4 & 2.7 & 3.7 & 3.2 & 2.5 \\
\hline & & Combined & 3.9 & 3.9 & 3.7 & 3.4 & 3.9 & 3.4 & 3.0 & 3.6 & 3.1 & 2.5 & 3.5 & 2.9 & 2.3 \\
\hline & \multirow{3}{*}{ Adjusted } & \begin{tabular}{|l|} 
UDDS \\
\end{tabular} & 5.1 & 5.1 & 4.9 & 4.5 & 5.1 & 4.5 & 4.0 & 4.7 & 4.1 & 3.4 & 4.6 & 3.9 & 3.3 \\
\hline & & HWFET & 6.1 & 6.1 & 5.6 & 5.1 & 6.0 & 5.3 & 4.6 & 5.6 & 4.8 & 3.9 & 5.3 & 4.5 & 3.6 \\
\hline & & Combined & 5.5 & 5.5 & 5.2 & 4.8 & 5.5 & 4.9 & 4.3 & 5.1 & 4.4 & 3.6 & 4.9 & 4.2 & 3.4 \\
\hline \multirow{6}{*}{ CI Split HEV PHEV10 } & \multirow{3}{*}{ Unadjusted } & \begin{tabular}{|l|} 
UDDS \\
\end{tabular} & 3.1 & 3.1 & 2.9 & 2.7 & 3.1 & 2.8 & 2.4 & 2.9 & 2.5 & 2.0 & 2.8 & 2.4 & 1.9 \\
\hline & & \begin{tabular}{|l|} 
HWFET \\
\end{tabular} & 3.3 & 3.3 & 3.0 & 2.7 & 3.3 & 2.8 & 2.5 & 3.0 & 2.6 & 2.1 & 2.9 & 2.4 & 1.9 \\
\hline & & Combined & 3.2 & 3.2 & 3.0 & 2.7 & 3.2 & 2.8 & 2.4 & 3.0 & 2.5 & 2.0 & 2.8 & 2.4 & 1.9 \\
\hline & \multirow{3}{*}{ Adjusted } & \begin{tabular}{|l|} 
UDDS \\
\end{tabular} & 4.4 & 4.4 & 4.2 & 3.9 & 4.4 & 4.0 & 3.5 & 4.2 & 3.6 & 3.0 & 4.0 & 3.5 & 2.9 \\
\hline & & HWFET & 4.7 & 4.7 & 4.3 & 3.9 & 4.6 & 4.1 & 3.6 & 4.3 & 3.7 & 3.0 & 4.1 & 3.5 & 2.8 \\
\hline & & Combined & 4.5 & 4.5 & 4.3 & 3.9 & 4.5 & 4.0 & 3.6 & 4.2 & 3.6 & 3.0 & 4.0 & 3.5 & 2.9 \\
\hline \multirow{6}{*}{ CI Split HEV PHEV20 } & & UDDS & 2.2 & 2.2 & 2.0 & 1.9 & 2.2 & 1.9 & 1.7 & 2.0 & 1.7 & 1.6 & 1.9 & 1.6 & 1.5 \\
\hline & Unadjusted & \begin{tabular}{|c|} 
HWFET \\
\end{tabular} & 2.7 & 2.7 & 2.5 & 2.7 & 2.7 & 2.3 & 2.4 & 2.5 & 2.5 & 2.0 & 2.3 & 2.4 & 1.9 \\
\hline & & Combined & 2.4 & 2.4 & 2.2 & 2.2 & 2.4 & 2.1 & 2.0 & 2.2 & 2.1 & 1.8 & 2.1 & 2.0 & 1.7 \\
\hline & & \begin{tabular}{|l|} 
UDDS \\
\end{tabular} & 3.3 & 3.3 & 3.1 & 2.9 & 3.3 & 2.9 & 2.6 & 3.1 & 2.7 & 2.6 & 3.0 & 2.6 & 2.5 \\
\hline & Adjusted & \begin{tabular}{|c|} 
HWFET \\
\end{tabular} & 3.9 & 3.9 & 3.6 & 3.9 & 3.8 & 3.3 & 3.5 & 3.6 & 3.6 & 2.9 & 3.3 & 3.4 & 2.7 \\
\hline & & Combined & 3.5 & 3.5 & 3.3 & 3.3 & 3.5 & 3.1 & 3.0 & 3.3 & 3.1 & 2.8 & 3.1 & 3.0 & 2.6 \\
\hline
\end{tabular}




\begin{tabular}{|c|c|c|c|c|c|c|c|c|c|c|c|c|c|c|c|}
\hline & & & \multicolumn{13}{|c|}{ Compact } \\
\hline & & & \multirow{2}{*}{$\frac{2010}{\text { Ref }}$} & \multicolumn{3}{|c|}{2010} & \multicolumn{3}{|c|}{2015} & \multicolumn{3}{|c|}{2030} & \multicolumn{3}{|c|}{2045} \\
\hline & & & & low & avg & high & low & avg & high & low & avg & high & low & avg & high \\
\hline \multirow{6}{*}{ CI Series HEV PHEV30 } & \multirow{3}{*}{ Unadjusted } & UDDS & 2.3 & 2.3 & 2.2 & 2.0 & 2.3 & 2.0 & 1.7 & 2.1 & 1.7 & 1.4 & 2.0 & 1.6 & 1.3 \\
\hline & & HWFET & 2.4 & 2.4 & 2.2 & 2.0 & 2.4 & 2.1 & 1.8 & 2.2 & 1.8 & 1.5 & 2.1 & 1.7 & 1.3 \\
\hline & & Combined & 2.4 & 2.4 & 2.2 & 2.0 & 2.4 & 2.0 & 1.7 & 2.2 & 1.8 & 1.4 & 2.0 & 1.7 & 1.3 \\
\hline & \multirow{3}{*}{ Adjusted } & UDDS & 3.5 & 3.5 & 3.3 & 3.0 & 3.5 & 3.1 & 2.6 & 3.2 & 2.7 & 2.3 & 3.1 & 2.6 & 2.2 \\
\hline & & \begin{tabular}{|c|} 
HWFET \\
\end{tabular} & 3.5 & 3.5 & 3.2 & 2.9 & 3.4 & 3.0 & 2.5 & 3.2 & 2.6 & 2.1 & 3.0 & 2.5 & 1.9 \\
\hline & & Combined & 3.5 & 3.5 & 3.2 & 2.9 & 3.4 & 3.0 & 2.6 & 3.2 & 2.7 & 2.2 & 3.0 & 2.6 & 2.1 \\
\hline \multirow{6}{*}{ Cl Series HEV PHEV40 } & \multirow{3}{*}{ Unadjusted } & UDDS & 2.1 & 2.1 & 1.9 & 1.7 & 2.0 & 1.8 & 1.5 & 1.9 & 1.5 & 1.2 & 1.8 & 1.4 & 1.1 \\
\hline & & \begin{tabular}{|l|} 
HWFET \\
\end{tabular} & 2.0 & 2.0 & 1.8 & 1.6 & 2.0 & 1.7 & 1.4 & 1.8 & 1.5 & 1.2 & 1.7 & 1.4 & 1.1 \\
\hline & & Combined & 2.0 & 2.0 & 1.9 & 1.7 & 2.0 & 1.7 & 1.5 & 1.8 & 1.5 & 1.2 & 1.7 & 1.4 & 1.1 \\
\hline & \multirow{3}{*}{ Adjusted } & UDDS & 3.1 & 3.1 & 2.9 & 2.7 & 3.1 & 2.8 & 2.4 & 2.9 & 2.4 & 2.0 & 2.8 & 2.3 & 1.9 \\
\hline & & \begin{tabular}{|l|} 
HWFET \\
\end{tabular} & 2.8 & 2.8 & 2.6 & 2.4 & 2.8 & 2.4 & 2.1 & 2.6 & 2.1 & 1.7 & 2.4 & 2.0 & 1.6 \\
\hline & & Combined & 3.0 & 3.0 & 2.8 & 2.5 & 3.0 & 2.6 & 2.3 & 2.7 & 2.3 & 1.9 & 2.6 & 2.2 & 1.8 \\
\hline \multirow{6}{*}{ H2 Split HEV } & \multirow{3}{*}{ Unadjusted } & \begin{tabular}{|l|} 
UDDS \\
\end{tabular} & 4.1 & 4.1 & 3.4 & 2.9 & 3.7 & 3.0 & 2.5 & 3.2 & 2.6 & 2.0 & 3.0 & 2.4 & 1.9 \\
\hline & & \begin{tabular}{|l|} 
HWFET \\
\end{tabular} & 4.7 & 4.7 & 3.9 & 3.4 & 4.2 & 3.5 & 2.9 & 3.7 & 3.0 & 2.5 & 3.4 & 2.8 & 2.2 \\
\hline & & Combined & 4.3 & 4.3 & 3.6 & 3.1 & 3.9 & 3.2 & 2.7 & 3.5 & 2.8 & 2.2 & 3.2 & 2.6 & 2.0 \\
\hline & \multirow{3}{*}{ Adjusted } & UDDS & 5.6 & 5.6 & 4.8 & 4.2 & 5.1 & 4.3 & 3.7 & 4.6 & 3.8 & 3.1 & 4.3 & 3.6 & 2.9 \\
\hline & & \begin{tabular}{|c|} 
HWFET \\
\end{tabular} & 6.6 & 6.6 & 5.5 & 4.8 & 5.9 & 4.9 & 4.2 & 5.3 & 4.3 & 3.5 & 4.9 & 4.0 & 3.1 \\
\hline & & Combined & 6.1 & 6.1 & 5.1 & 4.5 & 5.5 & 4.6 & 3.9 & 4.9 & 4.0 & 3.3 & 4.5 & 3.8 & 3.0 \\
\hline \multirow{6}{*}{ H2 Split HEV PHEV10 } & \multirow{3}{*}{ Unadjusted } & \begin{tabular}{|l|} 
UDDS \\
\end{tabular} & 3.3 & 3.3 & 2.7 & 2.4 & 3.0 & 2.5 & 2.1 & 2.6 & 2.2 & 1.7 & 2.4 & 2.0 & 1.6 \\
\hline & & HWFET & 3.4 & 3.4 & 2.8 & 2.5 & 3.0 & 2.5 & 2.2 & 2.7 & 2.3 & 1.8 & 2.5 & 2.1 & 1.6 \\
\hline & & Combined & 3.3 & 3.3 & 2.8 & 2.4 & 3.0 & 2.5 & 2.1 & 2.7 & 2.2 & 1.8 & 2.5 & 2.1 & 1.6 \\
\hline & \multirow{3}{*}{ Adjusted } & UDDS & 4.6 & 4.6 & 4.0 & 3.5 & 4.2 & 3.6 & 3.1 & 3.8 & 3.3 & 2.7 & 3.6 & 3.1 & 2.5 \\
\hline & & HWFET & 4.8 & 4.8 & 4.0 & 3.5 & 4.3 & 3.6 & 3.1 & 3.9 & 3.3 & 2.7 & 3.6 & 3.0 & 2.4 \\
\hline & & Combined & 4.7 & 4.7 & 4.0 & 3.5 & 4.3 & 3.6 & 3.1 & 3.9 & 3.3 & 2.7 & 3.6 & 3.1 & 2.4 \\
\hline \multirow{6}{*}{ H2 Split HEV PHEV2O } & \multirow{3}{*}{ Unadjusted } & \begin{tabular}{|l|} 
UDDS \\
\end{tabular} & 2.3 & 2.3 & 1.9 & 1.6 & 2.1 & 1.7 & 1.7 & 1.8 & 1.8 & 1.4 & 1.7 & 1.7 & 1.3 \\
\hline & & \begin{tabular}{|l|} 
HWFET \\
\end{tabular} & 2.8 & 2.8 & 2.8 & 2.4 & 2.5 & 2.5 & 2.1 & 2.7 & 2.2 & 1.8 & 2.4 & 2.0 & 1.6 \\
\hline & & Combined & 2.5 & 2.5 & 2.3 & 2.0 & 2.2 & 2.0 & 1.9 & 2.2 & 2.0 & 1.6 & 2.0 & 1.8 & 1.4 \\
\hline & \multirow{3}{*}{ Adjusted } & \begin{tabular}{|l|} 
UDDS \\
\end{tabular} & 3.4 & 3.4 & 2.9 & 2.6 & 3.1 & 2.7 & 2.7 & 2.8 & 2.8 & 2.3 & 2.7 & 2.6 & 2.2 \\
\hline & & HWFET & 4.0 & 4.0 & 4.1 & 3.4 & 3.5 & 3.5 & 3.0 & 3.9 & 3.1 & 2.6 & 3.5 & 2.9 & 2.3 \\
\hline & & Combined & 3.7 & 3.7 & 3.4 & 3.0 & 3.3 & 3.1 & 2.8 & 3.3 & 2.9 & 2.4 & 3.0 & 2.8 & 2.2 \\
\hline \multirow{6}{*}{ H2 Series HEV PHEV 30} & & UDDS & 2.5 & 2.5 & 2.1 & 1.7 & 2.2 & 1.8 & 1.5 & 2.0 & 1.5 & 1.3 & 1.8 & 1.5 & 1.2 \\
\hline & Unadjusted & \begin{tabular}{|l|} 
HWFET \\
\end{tabular} & 2.5 & 2.5 & 2.1 & 1.8 & 2.2 & 1.8 & 1.5 & 2.0 & 1.6 & 1.6 & 1.8 & 1.5 & 1.1 \\
\hline & & Combined & 2.5 & 2.5 & 2.1 & 1.8 & 2.2 & 1.8 & 1.5 & 2.0 & 1.6 & 1.4 & 1.8 & 1.5 & 1.2 \\
\hline & & \begin{tabular}{|l|} 
UDDS \\
\end{tabular} & 3.7 & 3.7 & 3.1 & 2.7 & 3.3 & 2.8 & 2.4 & 3.0 & 2.5 & 2.1 & 2.8 & 2.4 & 2.0 \\
\hline & Adjusted & \begin{tabular}{|c|} 
HWFET \\
\end{tabular} & 3.6 & 3.6 & 3.0 & 2.6 & 3.2 & 2.7 & 2.2 & 2.8 & 2.3 & 2.4 & 2.6 & 2.1 & 1.7 \\
\hline & & Combined & 3.6 & 3.6 & 3.1 & 2.7 & 3.3 & 2.8 & 2.3 & 2.9 & 2.4 & 2.2 & 2.7 & 2.3 & 1.9 \\
\hline
\end{tabular}




\begin{tabular}{|c|c|c|c|c|c|c|c|c|c|c|c|c|c|c|c|}
\hline & & & \multicolumn{13}{|c|}{ Compact } \\
\hline & & & \multirow{2}{*}{$\frac{2010}{\text { Ref }}$} & \multicolumn{3}{|c|}{2010} & \multicolumn{3}{|c|}{2015} & \multicolumn{3}{|c|}{2030} & \multicolumn{3}{|c|}{2045} \\
\hline & & & & low & avg & high & low & avg & high & low & avg & high & low & avg & high \\
\hline \multirow{6}{*}{ H2 Series HEV PHEV40 } & \multirow{3}{*}{ Unadjusted } & UDDS & 2.2 & 2.2 & 1.8 & 1.5 & 1.9 & 1.6 & 1.3 & 1.7 & 1.3 & 1.1 & 1.6 & 1.2 & 1.0 \\
\hline & & HWFET & 2.0 & 2.0 & 1.7 & 1.4 & 1.8 & 1.5 & 1.2 & 1.6 & 1.3 & 1.3 & 1.5 & 1.2 & 0.9 \\
\hline & & Combined & 2.1 & 2.1 & 1.8 & 1.5 & 1.9 & 1.5 & 1.3 & 1.7 & 1.3 & 1.2 & 1.5 & 1.2 & 0.9 \\
\hline & \multirow{3}{*}{ Adjusted } & UDDS & 3.3 & 3.3 & 2.8 & 2.5 & 3.0 & 2.5 & 2.2 & 2.7 & 2.2 & 1.9 & 2.5 & 2.1 & 1.8 \\
\hline & & \begin{tabular}{|l|} 
HWFET \\
\end{tabular} & 2.9 & 2.9 & 2.5 & 2.1 & 2.6 & 2.2 & 1.8 & 2.3 & 1.9 & 1.9 & 2.1 & 1.8 & 1.4 \\
\hline & & Combined & 3.1 & 3.1 & 2.6 & 2.3 & 2.8 & 2.4 & 2.0 & 2.5 & 2.1 & 1.9 & 2.3 & 2.0 & 1.6 \\
\hline \multirow{6}{*}{ E85 Split HEV } & \multirow{3}{*}{ Unadjusted } & UDDS & 4.2 & 4.2 & 3.9 & 3.6 & 4.2 & 3.7 & 2.8 & 3.9 & 3.3 & 2.4 & 3.7 & 3.2 & 2.2 \\
\hline & & HWFET & 4.8 & 4.8 & 4.5 & 4.1 & 4.7 & 4.2 & 3.3 & 4.5 & 3.8 & 2.8 & 4.3 & 3.7 & 2.6 \\
\hline & & Combined & 4.5 & 4.5 & 4.2 & 3.8 & 4.4 & 3.9 & 3.0 & 4.1 & 3.5 & 2.6 & 4.0 & 3.4 & 2.4 \\
\hline & \multirow{3}{*}{ Adjusted } & UDDS & 5.8 & 5.8 & 5.4 & 5.1 & 5.7 & 5.1 & 4.1 & 5.4 & 4.7 & 3.5 & 5.2 & 4.5 & 3.3 \\
\hline & & \begin{tabular}{|l|} 
HWFET \\
\end{tabular} & 6.9 & 6.9 & 6.3 & 5.8 & 6.7 & 6.0 & 4.6 & 6.3 & 5.4 & 4.0 & 6.1 & 5.2 & 3.7 \\
\hline & & Combined & 6.3 & 6.3 & 5.8 & 5.4 & 6.2 & 5.5 & 4.3 & 5.8 & 5.0 & 3.7 & 5.6 & 4.8 & 3.5 \\
\hline \multirow{6}{*}{ E85 Split HEV PHEV10 } & \multirow{3}{*}{ Unadjusted } & UDDS & 3.3 & 3.3 & 3.1 & 2.9 & 3.3 & 3.0 & 2.3 & 3.2 & 2.7 & 2.0 & 3.0 & 2.6 & 1.8 \\
\hline & & \begin{tabular}{|l|} 
HWFET \\
\end{tabular} & 3.5 & 3.5 & 3.2 & 3.0 & 3.4 & 3.1 & 2.4 & 3.3 & 2.8 & 2.1 & 3.1 & 2.7 & 1.9 \\
\hline & & Combined & 3.4 & 3.4 & 3.2 & 2.9 & 3.4 & 3.0 & 2.4 & 3.2 & 2.8 & 2.0 & 3.1 & 2.7 & 1.9 \\
\hline & \multirow{3}{*}{ Adjusted } & UDDS & 4.7 & 4.7 & 4.4 & 4.1 & 4.7 & 4.3 & 3.4 & 4.5 & 3.9 & 3.0 & 4.3 & 3.8 & 2.9 \\
\hline & & \begin{tabular}{|l|} 
HWFET \\
\end{tabular} & 5.0 & 5.0 & 4.6 & 4.3 & 4.9 & 4.4 & 3.5 & 4.7 & 4.1 & 3.0 & 4.5 & 3.9 & 2.8 \\
\hline & & Combined & 4.8 & 4.8 & 4.5 & 4.2 & 4.8 & 4.3 & 3.4 & 4.6 & 4.0 & 3.0 & 4.4 & 3.8 & 2.8 \\
\hline \multirow{6}{*}{ E85 Split HEV PHEV20 } & \multirow{3}{*}{ Unadjusted } & \begin{tabular}{|l|} 
UDDS \\
\end{tabular} & 2.3 & 2.3 & 2.2 & 2.4 & 2.3 & 2.5 & 1.6 & 2.2 & 1.9 & 1.6 & 2.1 & 2.2 & 1.5 \\
\hline & & HWFET & 3.5 & 3.5 & 3.1 & 2.9 & 3.4 & 3.0 & 2.3 & 3.3 & 2.7 & 2.0 & 3.0 & 2.6 & 1.9 \\
\hline & & Combined & 2.9 & 2.9 & 2.6 & 2.6 & 2.8 & 2.7 & 1.9 & 2.7 & 2.3 & 1.8 & 2.5 & 2.4 & 1.7 \\
\hline & \multirow{3}{*}{ Adjusted } & UDDS & 3.4 & 3.4 & 3.3 & 3.5 & 3.4 & 3.6 & 2.6 & 3.3 & 2.9 & 2.6 & 3.2 & 3.3 & 2.5 \\
\hline & & HWFET & 5.0 & 5.0 & 4.5 & 4.1 & 4.9 & 4.3 & 3.4 & 4.7 & 3.9 & 2.9 & 4.3 & 3.8 & 2.7 \\
\hline & & Combined & 4.1 & 4.1 & 3.8 & 3.8 & 4.1 & 3.9 & 2.9 & 3.9 & 3.4 & 2.7 & 3.7 & 3.5 & 2.6 \\
\hline \multirow{6}{*}{ E85 Series HEV PHEV30 } & \multirow{3}{*}{ Unadjusted } & UDDS & 2.5 & 2.5 & 2.3 & 2.1 & 2.5 & 2.2 & 1.6 & 2.3 & 1.9 & 1.3 & 2.2 & 1.9 & 1.3 \\
\hline & & HWFET & 2.6 & 2.6 & 2.4 & 2.2 & 2.5 & 2.2 & 1.7 & 2.4 & 2.0 & 1.4 & 2.3 & 1.9 & 1.3 \\
\hline & & Combined & 2.6 & 2.6 & 2.3 & 2.1 & 2.5 & 2.2 & 1.7 & 2.3 & 1.9 & 1.4 & 2.2 & 1.9 & 1.3 \\
\hline & \multirow{3}{*}{ Adjusted } & UDDS & 3.7 & 3.7 & 3.4 & 3.2 & 3.6 & 3.3 & 2.6 & 3.4 & 2.9 & 2.3 & 3.3 & 2.9 & 2.2 \\
\hline & & \begin{tabular}{|c|} 
HWFET \\
\end{tabular} & 3.7 & 3.7 & 3.4 & 3.1 & 3.6 & 3.2 & 2.5 & 3.4 & 2.9 & 2.1 & 3.3 & 2.7 & 1.9 \\
\hline & & Combined & 3.7 & 3.7 & 3.4 & 3.1 & 3.6 & 3.2 & 2.5 & 3.4 & 2.9 & 2.2 & 3.3 & 2.8 & 2.1 \\
\hline \multirow{6}{*}{ E85 Series HEV PHEV40 } & & \begin{tabular}{|l|} 
UDDS \\
\end{tabular} & 2.2 & 2.2 & 2.0 & 1.8 & 2.2 & 1.9 & 1.4 & 2.0 & 1.7 & 1.1 & 1.9 & 1.6 & 1.1 \\
\hline & Unadjusted & \begin{tabular}{|l|} 
HWFET \\
\end{tabular} & 2.6 & 2.6 & 1.9 & 1.8 & 2.1 & 1.8 & 1.4 & 1.9 & 1.6 & 1.4 & 1.8 & 1.5 & 1.1 \\
\hline & & Combined & 2.4 & 2.4 & 2.0 & 1.8 & 2.1 & 1.9 & 1.4 & 2.0 & 1.6 & 1.3 & 1.9 & 1.6 & 1.1 \\
\hline & & UDDS & 3.3 & 3.3 & 3.1 & 2.8 & 3.2 & 2.9 & 2.3 & 3.1 & 2.6 & 2.0 & 3.0 & 2.6 & 1.9 \\
\hline & Adjusted & HWFET & 3.8 & 3.8 & 2.8 & 2.5 & 3.0 & 2.6 & 2.0 & 2.8 & 2.4 & 2.1 & 2.7 & 2.2 & 1.6 \\
\hline & & Combined & 3.5 & 3.5 & 2.9 & 2.7 & 3.1 & 2.8 & 2.2 & 2.9 & 2.5 & 2.0 & 2.8 & 2.4 & 1.8 \\
\hline
\end{tabular}


Argonne National Laboratory Report - Light-Duty Vehicle Fuel Consumption Displacement Potential Up to 2045

\begin{tabular}{|c|c|c|c|c|c|c|c|c|c|c|c|c|c|c|c|}
\hline & & & \multicolumn{13}{|c|}{ Compact } \\
\hline & & & \multirow{2}{*}{$\begin{array}{l}2010 \\
\text { Ref }\end{array}$} & \multicolumn{3}{|c|}{2010} & \multicolumn{3}{|c|}{2015} & \multicolumn{3}{|c|}{2030} & \multicolumn{3}{|c|}{2045} \\
\hline & & & & low & avg & high & low & avg & high & low & avg & high & low & avg & high \\
\hline \multirow{6}{*}{ FC HEV } & \multirow{3}{*}{ Unadjusted } & UDDS & 3.6 & 3.6 & 3.1 & 2.6 & 3.1 & 2.6 & 2.2 & 2.8 & 2.3 & 1.9 & 2.7 & 2.2 & 1.8 \\
\hline & & \begin{tabular}{|c|} 
HWFET \\
\end{tabular} & 3.5 & 3.5 & 3.0 & 2.5 & 3.0 & 2.6 & 2.3 & 2.8 & 2.4 & 2.0 & 2.7 & 2.3 & 1.8 \\
\hline & & Combined & 3.6 & 3.6 & 3.0 & 2.6 & 3.1 & 2.6 & 2.3 & 2.8 & 2.3 & 1.9 & 2.7 & 2.3 & 1.8 \\
\hline & \multirow{3}{*}{ Adjusted } & UDDS & 5.1 & 5.1 & 4.4 & 3.8 & 4.4 & 3.8 & 3.4 & 4.1 & 3.4 & 2.9 & 3.9 & 3.3 & 2.8 \\
\hline & & \begin{tabular}{|c|} 
HWFET \\
\end{tabular} & 4.9 & 4.9 & 4.2 & 3.6 & 4.3 & 3.7 & 3.3 & 4.0 & 3.4 & 2.9 & 3.8 & 3.3 & 2.6 \\
\hline & & Combined & 5.0 & 5.0 & 4.3 & 3.7 & 4.4 & 3.8 & 3.3 & 4.0 & 3.4 & 2.9 & 3.8 & 3.3 & 2.7 \\
\hline \multirow{6}{*}{ FC PHEV10 } & \multirow{3}{*}{ Unadjusted } & UDDS & 2.9 & 2.9 & 2.5 & 2.1 & 2.5 & 2.2 & 1.9 & 2.3 & 1.9 & 1.6 & 2.2 & 1.9 & 1.5 \\
\hline & & HWFET & 2.6 & 2.6 & 2.3 & 1.9 & 2.3 & 2.0 & 1.8 & 2.2 & 1.8 & 1.6 & 2.1 & 1.8 & 1.4 \\
\hline & & Combined & 2.8 & 2.8 & 2.4 & 2.0 & 2.4 & 2.1 & 1.8 & 2.3 & 1.9 & 1.6 & 2.1 & 1.8 & 1.5 \\
\hline & \multirow{3}{*}{ Adjusted } & UDDS & 4.2 & 4.2 & 3.6 & 3.2 & 3.7 & 3.3 & 2.9 & 3.5 & 3.0 & 2.6 & 3.3 & 2.9 & 2.5 \\
\hline & & \begin{tabular}{|l|} 
HWFET \\
\end{tabular} & 3.7 & 3.7 & 3.2 & 2.8 & 3.3 & 2.9 & 2.6 & 3.1 & 2.7 & 2.2 & 3.0 & 2.6 & 2.1 \\
\hline & & Combined & 4.0 & 4.0 & 3.5 & 3.0 & 3.5 & 3.1 & 2.7 & 3.3 & 2.8 & 2.4 & 3.2 & 2.8 & 2.3 \\
\hline \multirow{6}{*}{ FC PHEV2O } & \multirow{3}{*}{ Unadjusted } & UDDS & 2.4 & 2.4 & 2.1 & 1.7 & 2.1 & 1.8 & 1.6 & 1.9 & 1.6 & 1.3 & 1.8 & 1.6 & 1.2 \\
\hline & & HWFET & 2.6 & 2.6 & 2.2 & 1.9 & 2.3 & 2.0 & 1.7 & 2.1 & 1.8 & 1.5 & 2.0 & 1.7 & 1.4 \\
\hline & & Combined & 2.5 & 2.5 & 2.1 & 1.8 & 2.2 & 1.9 & 1.6 & 2.0 & 1.7 & 1.4 & 1.9 & 1.6 & 1.3 \\
\hline & \multirow{3}{*}{ Adjusted } & UDDS & 3.5 & 3.5 & 3.1 & 2.7 & 3.2 & 2.8 & 2.5 & 3.0 & 2.6 & 2.2 & 2.9 & 2.5 & 2.1 \\
\hline & & \begin{tabular}{|c|} 
HWFET \\
\end{tabular} & 3.7 & 3.7 & 3.2 & 2.7 & 3.2 & 2.9 & 2.5 & 3.1 & 2.6 & 2.2 & 2.9 & 2.5 & 2.0 \\
\hline & & Combined & 3.6 & 3.6 & 3.2 & 2.7 & 3.2 & 2.8 & 2.5 & 3.0 & 2.6 & 2.2 & 2.9 & 2.5 & 2.1 \\
\hline \multirow{6}{*}{ FC PHEV 30} & \multirow{3}{*}{ Unadjusted } & \begin{tabular}{|l|} 
UDDS \\
\end{tabular} & 1.7 & 1.7 & 1.5 & 1.3 & 1.5 & 1.3 & 1.1 & 1.4 & 1.1 & 0.9 & 1.3 & 1.1 & 0.9 \\
\hline & & HWFET & 2.0 & 2.0 & 1.8 & 1.5 & 1.8 & 1.6 & 1.4 & 1.7 & 1.4 & 1.2 & 1.6 & 1.4 & 1.1 \\
\hline & & Combined & 1.9 & 1.9 & 1.6 & 1.4 & 1.6 & 1.4 & 1.2 & 1.5 & 1.3 & 1.1 & 1.5 & 1.2 & 1.0 \\
\hline & \multirow{3}{*}{ Adjusted } & UDDS & 2.7 & 2.7 & 2.4 & 2.1 & 2.4 & 2.2 & 2.0 & 2.3 & 2.0 & 1.7 & 2.2 & 2.0 & 1.7 \\
\hline & & HWFET & 2.9 & 2.9 & 2.6 & 2.2 & 2.6 & 2.3 & 2.0 & 2.5 & 2.1 & 1.8 & 2.3 & 2.0 & 1.6 \\
\hline & & Combined & 2.8 & 2.8 & 2.5 & 2.2 & 2.5 & 2.2 & 2.0 & 2.4 & 2.1 & 1.8 & 2.3 & 2.0 & 1.7 \\
\hline \multirow{6}{*}{ FC PHEV 40} & \multirow{3}{*}{ Unadjusted } & \begin{tabular}{|l|} 
UDDS \\
\end{tabular} & 1.5 & 1.5 & 1.3 & 1.1 & 1.3 & 1.1 & 1.0 & 1.2 & 1.0 & 0.8 & 1.1 & 0.9 & 0.8 \\
\hline & & \begin{tabular}{|c|} 
HWFET \\
\end{tabular} & 1.6 & 1.6 & 1.4 & 1.2 & 1.4 & 1.3 & 1.1 & 1.3 & 1.1 & 0.9 & 1.3 & 1.1 & 0.9 \\
\hline & & Combined & 1.5 & 1.5 & 1.3 & 1.1 & 1.4 & 1.2 & 1.0 & 1.3 & 1.1 & 0.9 & 1.2 & 1.0 & 0.8 \\
\hline & \multirow{3}{*}{ Adjusted } & UDDS & 2.4 & 2.4 & 2.2 & 1.9 & 2.2 & 2.0 & 1.8 & 2.1 & 1.8 & 1.6 & 2.0 & 1.8 & 1.5 \\
\hline & & HWFET & 2.3 & 2.3 & 2.0 & 1.8 & 2.1 & 1.8 & 1.6 & 2.0 & 1.7 & 1.4 & 1.9 & 1.6 & 1.3 \\
\hline & & Combined & 2.4 & 2.4 & 2.1 & 1.9 & 2.1 & 1.9 & 1.7 & 2.0 & 1.7 & 1.5 & 1.9 & 1.7 & 1.4 \\
\hline
\end{tabular}


Fuel Consumption Improvement versus the Conventional 2010 Reference

\begin{tabular}{|c|c|c|c|c|c|c|c|c|c|c|c|c|c|c|c|}
\hline & & & \multicolumn{13}{|c|}{ Compact } \\
\hline & & & 2010 & & 2010 & & & 2015 & & & 2030 & & & 2045 & \\
\hline & & & Ref & low & avg & high & low & avg & high & low & avg & high & low & avg & high \\
\hline \multirow{6}{*}{ SI Conv } & \multirow{3}{*}{ Unadjusted } & UDDS & $0.0 \%$ & $0.0 \%$ & $-7.3 \%$ & $-11.4 \%$ & $-4.2 \%$ & $-10.9 \%$ & $-27.3 \%$ & $-6.8 \%$ & $-13.8 \%$ & $-38.9 \%$ & $-8.7 \%$ & $-16.7 \%$ & $-39.8 \%$ \\
\hline & & HWFET & $0.0 \%$ & $0.0 \%$ & $-8.2 \%$ & $-17.8 \%$ & $-4.6 \%$ & $-16.5 \%$ & $-32.6 \%$ & $-12.3 \%$ & $-19.5 \%$ & $-42.2 \%$ & $-13.5 \%$ & $-21.4 \%$ & $-44.5 \%$ \\
\hline & & Combined & $0.0 \%$ & $0.0 \%$ & $-7.6 \%$ & $-13.8 \%$ & $-4.4 \%$ & $-13.0 \%$ & $-29.3 \%$ & $-8.9 \%$ & $-15.9 \%$ & $-40.1 \%$ & $-10.5 \%$ & $-18.5 \%$ & $-41.5 \%$ \\
\hline & \multirow{3}{*}{ Adjusted } & UDDS & $0.0 \%$ & $0.0 \%$ & $-6.8 \%$ & $-10.8 \%$ & $-4.0 \%$ & $-10.3 \%$ & $-25.7 \%$ & $-6.4 \%$ & $-12.9 \%$ & $-36.6 \%$ & $-8.2 \%$ & $-15.8 \%$ & $-37.5 \%$ \\
\hline & & HWFET & $0.0 \%$ & $0.0 \%$ & $-8.1 \%$ & $-17.7 \%$ & $-4.6 \%$ & $-16.3 \%$ & $-32.3 \%$ & $-12.1 \%$ & $-19.3 \%$ & $-41.8 \%$ & $-13.4 \%$ & $-21.2 \%$ & $-44.1 \%$ \\
\hline & & Combined & $0.0 \%$ & $0.0 \%$ & $-7.4 \%$ & $-13.5 \%$ & $-4.2 \%$ & $-12.7 \%$ & $-28.3 \%$ & $-8.7 \%$ & $-15.4 \%$ & $-38.7 \%$ & $-10.2 \%$ & $-17.9 \%$ & $-40.1 \%$ \\
\hline \multirow{6}{*}{$\mathrm{Cl}$ Conv } & \multirow{3}{*}{ Unadjusted } & UDDS & $-14.4 \%$ & $-14.4 \%$ & $-18.4 \%$ & $-24.4 \%$ & $-15.5 \%$ & $-22.6 \%$ & $-30.4 \%$ & $-19.1 \%$ & $-28.7 \%$ & $-40.0 \%$ & $-22.4 \%$ & $-32.0 \%$ & $-42.2 \%$ \\
\hline & & HWFET & $-13.3 \%$ & $-13.3 \%$ & $-18.3 \%$ & $-25.5 \%$ & $-14.7 \%$ & $-23.3 \%$ & $-31.4 \%$ & $-19.3 \%$ & $-29.2 \%$ & $-40.2 \%$ & $-22.7 \%$ & $-32.5 \%$ & $-43.8 \%$ \\
\hline & & Combined & $-14.0 \%$ & $-14.0 \%$ & $-18.4 \%$ & $-24.8 \%$ & $-15.2 \%$ & $-22.9 \%$ & $-30.8 \%$ & $-19.2 \%$ & $-28.9 \%$ & $-40.1 \%$ & $-22.5 \%$ & $-32.2 \%$ & $-42.8 \%$ \\
\hline & \multirow{3}{*}{ Adjusted } & \begin{tabular}{|l|} 
UDDS \\
\end{tabular} & $-13.6 \%$ & $-13.6 \%$ & $-17.3 \%$ & $-22.9 \%$ & $-14.6 \%$ & $-21.3 \%$ & $-28.6 \%$ & $-18.0 \%$ & $-27.0 \%$ & $-37.6 \%$ & $-21.1 \%$ & $-30.1 \%$ & $-39.7 \%$ \\
\hline & & HWFET & $-13.1 \%$ & $-13.1 \%$ & $-18.1 \%$ & $-25.3 \%$ & $-14.6 \%$ & $-23.1 \%$ & $-31.2 \%$ & $-19.2 \%$ & $-29.0 \%$ & $-39.9 \%$ & $-22.5 \%$ & $-32.2 \%$ & $-43.4 \%$ \\
\hline & & Combined & $-13.4 \%$ & $-13.4 \%$ & $-17.6 \%$ & $-23.8 \%$ & $-14.6 \%$ & $-22.0 \%$ & $-29.6 \%$ & $-18.5 \%$ & $-27.7 \%$ & $-38.5 \%$ & $-21.7 \%$ & $-30.9 \%$ & $-41.2 \%$ \\
\hline \multirow{6}{*}{ H2 Conv } & \multirow{3}{*}{ Unadjusted } & \begin{tabular}{|l|} 
UDDS \\
\end{tabular} & $17.5 \%$ & $17.5 \%$ & $-0.7 \%$ & $-12.0 \%$ & $4.0 \%$ & $-10.1 \%$ & $-20.3 \%$ & $-3.8 \%$ & $-17.8 \%$ & $-33.4 \%$ & $-11.1 \%$ & $-24.2 \%$ & $-37.7 \%$ \\
\hline & & \begin{tabular}{|l|} 
HWFET \\
\end{tabular} & $10.2 \%$ & $10.2 \%$ & $-7.3 \%$ & $-23.9 \%$ & $-2.6 \%$ & $-21.8 \%$ & $-31.0 \%$ & $-16.3 \%$ & $-28.6 \%$ & $-41.1 \%$ & $-22.1 \%$ & $-33.6 \%$ & $-46.2 \%$ \\
\hline & & Combined & $14.8 \%$ & $14.8 \%$ & $-3.2 \%$ & $-16.4 \%$ & $1.6 \%$ & $-14.5 \%$ & $-24.3 \%$ & $-8.4 \%$ & $-21.8 \%$ & $-36.3 \%$ & $-15.2 \%$ & $-27.7 \%$ & $-40.9 \%$ \\
\hline & \multirow{3}{*}{ Adjusted } & \begin{tabular}{|l|} 
UDDS \\
\end{tabular} & $16.4 \%$ & $16.4 \%$ & $-0.7 \%$ & $-11.3 \%$ & $3.8 \%$ & $-9.5 \%$ & $-19.1 \%$ & $-3.5 \%$ & $-16.8 \%$ & $-31.4 \%$ & $-10.4 \%$ & $-22.8 \%$ & $-35.5 \%$ \\
\hline & & HWFET & $10.2 \%$ & $10.2 \%$ & $-7.2 \%$ & $-23.7 \%$ & $-2.6 \%$ & $-21.6 \%$ & $-30.8 \%$ & $-16.1 \%$ & $-28.3 \%$ & $-40.7 \%$ & $-21.9 \%$ & $-33.3 \%$ & $-45.8 \%$ \\
\hline & & Combined & $14.0 \%$ & $14.0 \%$ & $-3.3 \%$ & $-16.2 \%$ & $1.3 \%$ & $-14.2 \%$ & $-23.7 \%$ & $-8.5 \%$ & $-21.3 \%$ & $-35.1 \%$ & $-14.9 \%$ & $-26.9 \%$ & $-39.5 \%$ \\
\hline \multirow{6}{*}{ E85 Conv } & \multirow{3}{*}{ Unadjusted } & \begin{tabular}{|l|} 
UDDS \\
\end{tabular} & $2.1 \%$ & $2.1 \%$ & $1.6 \%$ & $-3.5 \%$ & $5.1 \%$ & $-1.7 \%$ & $-25.6 \%$ & $3.2 \%$ & $-5.6 \%$ & $-36.7 \%$ & $-0.5 \%$ & $-9.8 \%$ & $-38.4 \%$ \\
\hline & & \begin{tabular}{|c|} 
HWFET \\
\end{tabular} & $5.2 \%$ & $5.2 \%$ & $3.7 \%$ & $-4.4 \%$ & $7.7 \%$ & $-2.0 \%$ & $-26.3 \%$ & $3.1 \%$ & $-6.1 \%$ & $-37.5 \%$ & $-2.0 \%$ & $-11.4 \%$ & $-40.6 \%$ \\
\hline & & Combined & $3.2 \%$ & $3.2 \%$ & $2.4 \%$ & $-3.8 \%$ & $6.1 \%$ & $-1.8 \%$ & $-25.8 \%$ & $3.1 \%$ & $-5.8 \%$ & $-37.0 \%$ & $-1.0 \%$ & $-10.4 \%$ & $-39.2 \%$ \\
\hline & \multirow{3}{*}{ Adjusted } & UDDS & $1.9 \%$ & $1.9 \%$ & $1.5 \%$ & $-3.3 \%$ & $4.8 \%$ & $-1.6 \%$ & $-24.1 \%$ & $3.0 \%$ & $-5.2 \%$ & $-34.5 \%$ & $-0.5 \%$ & $-9.2 \%$ & $-36.1 \%$ \\
\hline & & \begin{tabular}{|c|} 
HWFET \\
\end{tabular} & $5.1 \%$ & $5.1 \%$ & $3.6 \%$ & $-4.3 \%$ & $7.6 \%$ & $-2.0 \%$ & $-26.0 \%$ & $3.1 \%$ & $-6.1 \%$ & $-37.2 \%$ & $-1.9 \%$ & $-11.3 \%$ & $-40.2 \%$ \\
\hline & & Combined & $3.2 \%$ & $3.2 \%$ & $2.3 \%$ & $-3.7 \%$ & $5.9 \%$ & $-1.7 \%$ & $-24.8 \%$ & $3.0 \%$ & $-5.6 \%$ & $-35.6 \%$ & $-1.0 \%$ & $-10.0 \%$ & $-37.7 \%$ \\
\hline \multirow{6}{*}{ SI Split HEV } & \multirow{3}{*}{ Unadjusted } & \begin{tabular}{|l|} 
UDDS \\
\end{tabular} & $-49.9 \%$ & $-49.9 \%$ & $-53.1 \%$ & $-56.9 \%$ & $-50.1 \%$ & $-56.3 \%$ & $-64.5 \%$ & $-53.8 \%$ & $-60.5 \%$ & $-71.6 \%$ & $-55.4 \%$ & $-62.1 \%$ & $-73.0 \%$ \\
\hline & & HWFET & $-21.0 \%$ & $-21.0 \%$ & $-27.1 \%$ & $-33.1 \%$ & $-22.2 \%$ & $-31.0 \%$ & $-43.6 \%$ & $-26.9 \%$ & $-37.0 \%$ & $-53.7 \%$ & $-29.7 \%$ & $-39.8 \%$ & $-57.3 \%$ \\
\hline & & Combined & $-39.1 \%$ & $-39.1 \%$ & $-43.5 \%$ & $-48.0 \%$ & $-39.7 \%$ & $-46.9 \%$ & $-56.7 \%$ & $-43.8 \%$ & $-51.7 \%$ & $-64.9 \%$ & $-45.8 \%$ & $-53.8 \%$ & $-67.2 \%$ \\
\hline & \multirow{3}{*}{ Adjusted } & \begin{tabular}{|l|} 
UDDS \\
\end{tabular} & $-46.9 \%$ & $-46.9 \%$ & $-50.0 \%$ & $-53.5 \%$ & $-47.2 \%$ & $-53.0 \%$ & $-60.7 \%$ & $-50.6 \%$ & $-56.9 \%$ & $-67.4 \%$ & $-52.1 \%$ & $-58.4 \%$ & $-68.7 \%$ \\
\hline & & HWFET & $-20.8 \%$ & $-20.8 \%$ & $-26.9 \%$ & $-32.8 \%$ & $-22.0 \%$ & $-30.7 \%$ & $-43.2 \%$ & $-26.6 \%$ & $-36.6 \%$ & $-53.2 \%$ & $-29.4 \%$ & $-39.4 \%$ & $-56.8 \%$ \\
\hline & & Combined & $-36.7 \%$ & $-36.7 \%$ & $-41.0 \%$ & $-45.4 \%$ & $-37.3 \%$ & $-44.2 \%$ & $-53.8 \%$ & $-41.2 \%$ & $-49.0 \%$ & $-61.8 \%$ & $-43.2 \%$ & $-51.0 \%$ & $-64.0 \%$ \\
\hline \multirow{6}{*}{ SI Split HEV PHEV10 } & \multirow{3}{*}{ Unadjusted } & UDDS & $-59.9 \%$ & $-59.9 \%$ & $-62.5 \%$ & $-65.3 \%$ & $-60.3 \%$ & $-64.3 \%$ & $-70.8 \%$ & $-62.1 \%$ & $-67.4 \%$ & $-76.4 \%$ & $-63.5 \%$ & $-68.6 \%$ & $-77.7 \%$ \\
\hline & & HWFET & $-42.5 \%$ & $-42.5 \%$ & $-46.7 \%$ & $-50.8 \%$ & $-43.8 \%$ & $-49.0 \%$ & $-58.1 \%$ & $-45.9 \%$ & $-53.2 \%$ & $-65.1 \%$ & $-48.3 \%$ & $-55.1 \%$ & $-68.2 \%$ \\
\hline & & Combined & $-53.4 \%$ & $-53.4 \%$ & $-56.6 \%$ & $-59.9 \%$ & $-54.2 \%$ & $-58.6 \%$ & $-66.0 \%$ & $-56.1 \%$ & $-62.1 \%$ & $-72.2 \%$ & $-57.8 \%$ & $-63.6 \%$ & $-74.1 \%$ \\
\hline & \multirow{3}{*}{ Adjusted } & \begin{tabular}{|l|} 
UDDS \\
\end{tabular} & $-56.4 \%$ & $-56.4 \%$ & $-58.8 \%$ & $-61.5 \%$ & $-56.8 \%$ & $-60.6 \%$ & $-66.6 \%$ & $-58.4 \%$ & $-63.4 \%$ & $-71.9 \%$ & $-59.7 \%$ & $-64.5 \%$ & $-73.1 \%$ \\
\hline & & HWFET & $-42.1 \%$ & $-42.1 \%$ & $-46.3 \%$ & $-50.4 \%$ & $-43.4 \%$ & $-48.5 \%$ & $-57.6 \%$ & $-45.5 \%$ & $-52.8 \%$ & $-64.5 \%$ & $-47.9 \%$ & $-54.6 \%$ & $-67.6 \%$ \\
\hline & & Combined & $-50.8 \%$ & $-50.8 \%$ & $-53.9 \%$ & $-57.1 \%$ & $-51.5 \%$ & $-55.8 \%$ & $-63.1 \%$ & $-53.4 \%$ & $-59.2 \%$ & $-69.0 \%$ & $-55.1 \%$ & $-60.6 \%$ & $-70.9 \%$ \\
\hline
\end{tabular}


Argonne National Laboratory Report - Light-Duty Vehicle Fuel Consumption Displacement Potential Up to 2045

\begin{tabular}{|c|c|c|c|c|c|c|c|c|c|c|c|c|c|c|c|}
\hline & & & \multicolumn{13}{|c|}{ Compact } \\
\hline & & & \multirow{2}{*}{$\begin{array}{c}2010 \\
\text { Ref }\end{array}$} & \multicolumn{3}{|c|}{2010} & \multicolumn{3}{|c|}{2015} & \multicolumn{3}{|c|}{2030} & \multicolumn{3}{|c|}{2045} \\
\hline & & & & low & avg & high & low & avg & high & low & avg & high & low & avg & high \\
\hline \multirow{6}{*}{ SI Split HEV PHEV2O } & \multirow{3}{*}{ Unadjusted } & UDDS & $-72.2 \%$ & $-72.2 \%$ & $-74.0 \%$ & $-71.3 \%$ & $-72.4 \%$ & $-70.4 \%$ & $-79.8 \%$ & $-73.7 \%$ & $-77.6 \%$ & $-80.7 \%$ & $-74.7 \%$ & $-74.0 \%$ & $-81.7 \%$ \\
\hline & & HWFET & $-42.1 \%$ & $-42.1 \%$ & $-48.3 \%$ & $-52.4 \%$ & $-43.4 \%$ & $-50.7 \%$ & $-59.5 \%$ & $-45.8 \%$ & $-54.8 \%$ & $-66.4 \%$ & $-49.8 \%$ & $-56.7 \%$ & $-69.0 \%$ \\
\hline & & Combined & $-61.0 \%$ & $-61.0 \%$ & $-64.4 \%$ & $-64.3 \%$ & $-61.6 \%$ & $-63.1 \%$ & $-72.2 \%$ & $-63.3 \%$ & $-69.1 \%$ & $-75.4 \%$ & $-65.4 \%$ & $-67.6 \%$ & $-77.0 \%$ \\
\hline & \multirow{3}{*}{ Adjusted } & \begin{tabular}{|l|} 
UDDS \\
\end{tabular} & $-67.9 \%$ & $-67.9 \%$ & $-69.6 \%$ & $-67.1 \%$ & $-68.1 \%$ & $-66.2 \%$ & $-75.1 \%$ & $-69.4 \%$ & $-73.0 \%$ & $-76.0 \%$ & $-70.3 \%$ & $-69.6 \%$ & $-76.9 \%$ \\
\hline & & HWFET & $-41.8 \%$ & $-41.8 \%$ & $-47.9 \%$ & $-51.9 \%$ & $-43.0 \%$ & $-50.3 \%$ & $-58.9 \%$ & $-45.3 \%$ & $-54.3 \%$ & $-65.8 \%$ & $-49.3 \%$ & $-56.2 \%$ & $-68.4 \%$ \\
\hline & & Combined & $-57.7 \%$ & $-57.7 \%$ & $-61.1 \%$ & $-61.2 \%$ & $-58.3 \%$ & $-60.0 \%$ & $-68.8 \%$ & $-60.0 \%$ & $-65.7 \%$ & $-72.0 \%$ & $-62.1 \%$ & $-64.4 \%$ & $-73.6 \%$ \\
\hline \multirow{6}{*}{ SI Series HEV PHEV 30} & \multirow{3}{*}{ Unadjusted } & \begin{tabular}{|l|} 
UDDS \\
\end{tabular} & $-69.7 \%$ & $-69.7 \%$ & $-72.2 \%$ & $-74.7 \%$ & $-70.4 \%$ & $-73.8 \%$ & $-79.5 \%$ & $-72.3 \%$ & $-76.7 \%$ & $-83.6 \%$ & $-73.4 \%$ & $-77.5 \%$ & $-84.4 \%$ \\
\hline & & HWFET & $-56.8 \%$ & $-56.8 \%$ & $-60.5 \%$ & $-64.1 \%$ & $-58.0 \%$ & $-62.8 \%$ & $-70.2 \%$ & $-60.4 \%$ & $-66.7 \%$ & $-75.9 \%$ & $-62.4 \%$ & $-68.3 \%$ & $-78.1 \%$ \\
\hline & & Combined & $-64.9 \%$ & $-64.9 \%$ & $-67.8 \%$ & $-70.8 \%$ & $-65.7 \%$ & $-69.7 \%$ & $-76.0 \%$ & $-67.9 \%$ & $-73.0 \%$ & $-80.7 \%$ & $-69.3 \%$ & $-74.1 \%$ & $-82.0 \%$ \\
\hline & \multirow{3}{*}{ Adjusted } & \begin{tabular}{|l|} 
UDDS \\
\end{tabular} & $-65.6 \%$ & $-65.6 \%$ & $-67.9 \%$ & $-70.3 \%$ & $-66.2 \%$ & $-69.5 \%$ & $-74.8 \%$ & $-68.1 \%$ & $-72.2 \%$ & $-78.7 \%$ & $-69.1 \%$ & $-73.0 \%$ & $-79.4 \%$ \\
\hline & & HWFET & $-56.3 \%$ & $-56.3 \%$ & $-59.9 \%$ & $-63.6 \%$ & $-57.4 \%$ & $-62.2 \%$ & $-69.6 \%$ & $-59.9 \%$ & $-66.1 \%$ & $-75.2 \%$ & $-61.8 \%$ & $-67.7 \%$ & $-77.4 \%$ \\
\hline & & Combined & $-62.0 \%$ & $-62.0 \%$ & $-64.8 \%$ & $-67.6 \%$ & $-62.8 \%$ & $-66.6 \%$ & $-72.7 \%$ & $-64.8 \%$ & $-69.8 \%$ & $-77.3 \%$ & $-66.2 \%$ & $-70.9 \%$ & $-78.6 \%$ \\
\hline \multirow{6}{*}{ SI Series HEV PHEV40 } & \multirow{3}{*}{ Unadjusted } & \begin{tabular}{|l|} 
UDDS \\
\end{tabular} & $-73.5 \%$ & $-73.5 \%$ & $-75.7 \%$ & $-78.0 \%$ & $-74.0 \%$ & $-77.2 \%$ & $-82.0 \%$ & $-75.8 \%$ & $-79.9 \%$ & $-86.2 \%$ & $-76.9 \%$ & $-80.8 \%$ & $-86.9 \%$ \\
\hline & & HWFET & $-56.2 \%$ & $-56.2 \%$ & $-67.8 \%$ & $-70.8 \%$ & $-65.6 \%$ & $-69.7 \%$ & $-75.8 \%$ & $-67.7 \%$ & $-72.9 \%$ & $-75.7 \%$ & $-69.3 \%$ & $-74.3 \%$ & $-82.1 \%$ \\
\hline & & Combined & $-67.1 \%$ & $-67.1 \%$ & $-72.7 \%$ & $-75.3 \%$ & $-70.9 \%$ & $-74.4 \%$ & $-79.7 \%$ & $-72.8 \%$ & $-77.3 \%$ & $-82.3 \%$ & $-74.1 \%$ & $-78.4 \%$ & $-85.1 \%$ \\
\hline & \multirow{3}{*}{ Adjusted } & \begin{tabular}{|l|} 
UDDS \\
\end{tabular} & $-69.2 \%$ & $-69.2 \%$ & $-71.2 \%$ & $-73.4 \%$ & $-69.7 \%$ & $-72.7 \%$ & $-77.2 \%$ & $-71.4 \%$ & $-75.2 \%$ & $-81.1 \%$ & $-72.4 \%$ & $-76.1 \%$ & $-81.8 \%$ \\
\hline & & HWFET & $-55.7 \%$ & $-55.7 \%$ & $-67.2 \%$ & $-70.1 \%$ & $-65.0 \%$ & $-69.0 \%$ & $-75.1 \%$ & $-67.1 \%$ & $-72.3 \%$ & $-75.1 \%$ & $-68.7 \%$ & $-73.6 \%$ & $-81.3 \%$ \\
\hline & & Combined & $-63.9 \%$ & $-63.9 \%$ & $-69.6 \%$ & $-72.1 \%$ & $-67.9 \%$ & $-71.3 \%$ & $-76.4 \%$ & $-69.7 \%$ & $-74.1 \%$ & $-78.7 \%$ & $-71.0 \%$ & $-75.1 \%$ & $-81.6 \%$ \\
\hline \multirow{6}{*}{ CI Split HEV } & \multirow{3}{*}{ Unadjusted } & \begin{tabular}{|l|} 
UDDS \\
\end{tabular} & $-53.2 \%$ & $-53.2 \%$ & $-55.5 \%$ & $-59.6 \%$ & $-52.9 \%$ & $-59.0 \%$ & $-64.4 \%$ & $-56.7 \%$ & $-63.3 \%$ & $-70.6 \%$ & $-58.6 \%$ & $-65.2 \%$ & $-72.0 \%$ \\
\hline & & HWFET & $-24.7 \%$ & $-24.7 \%$ & $-29.9 \%$ & $-36.2 \%$ & $-26.0 \%$ & $-34.2 \%$ & $-42.6 \%$ & $-30.6 \%$ & $-40.8 \%$ & $-51.8 \%$ & $-34.0 \%$ & $-44.2 \%$ & $-55.3 \%$ \\
\hline & & Combined & $-42.6 \%$ & $-42.6 \%$ & $-46.0 \%$ & $-50.9 \%$ & $-42.9 \%$ & $-49.8 \%$ & $-56.3 \%$ & $-47.0 \%$ & $-54.9 \%$ & $-63.6 \%$ & $-49.5 \%$ & $-57.4 \%$ & $-65.8 \%$ \\
\hline & \multirow{3}{*}{ Adjusted } & \begin{tabular}{|l|} 
UDDS \\
\end{tabular} & $-50.0 \%$ & $-50.0 \%$ & $-52.2 \%$ & $-56.0 \%$ & $-49.8 \%$ & $-55.5 \%$ & $-60.6 \%$ & $-53.4 \%$ & $-59.6 \%$ & $-66.4 \%$ & $-55.2 \%$ & $-61.3 \%$ & $-67.7 \%$ \\
\hline & & HWFET & $-24.5 \%$ & $-24.5 \%$ & $-29.6 \%$ & $-35.9 \%$ & $-25.8 \%$ & $-33.9 \%$ & $-42.2 \%$ & $-30.4 \%$ & $-40.4 \%$ & $-51.3 \%$ & $-33.7 \%$ & $-43.8 \%$ & $-54.8 \%$ \\
\hline & & Combined & $-40.0 \%$ & $-40.0 \%$ & $-43.4 \%$ & $-48.1 \%$ & $-40.4 \%$ & $-47.0 \%$ & $-53.4 \%$ & $-44.4 \%$ & $-52.1 \%$ & $-60.5 \%$ & $-46.8 \%$ & $-54.5 \%$ & $-62.7 \%$ \\
\hline \multirow{6}{*}{ CI Split HEV PHEV10 } & \multirow{3}{*}{ Unadjusted } & \begin{tabular}{|l|} 
UDDS \\
\end{tabular} & $-60.2 \%$ & $-60.2 \%$ & $-62.5 \%$ & $-65.8 \%$ & $-60.1 \%$ & $-64.7 \%$ & $-69.2 \%$ & $-62.8 \%$ & $-68.4 \%$ & $-74.6 \%$ & $-64.5 \%$ & $-69.7 \%$ & $-75.9 \%$ \\
\hline & & HWFET & $-42.3 \%$ & $-42.3 \%$ & $-46.6 \%$ & $-51.5 \%$ & $-42.6 \%$ & $-49.8 \%$ & $-56.0 \%$ & $-46.7 \%$ & $-54.7 \%$ & $-62.7 \%$ & $-49.6 \%$ & $-57.0 \%$ & $-65.9 \%$ \\
\hline & & Combined & $-53.6 \%$ & $-53.6 \%$ & $-56.5 \%$ & $-60.5 \%$ & $-53.6 \%$ & $-59.2 \%$ & $-64.3 \%$ & $-56.8 \%$ & $-63.3 \%$ & $-70.2 \%$ & $-59.0 \%$ & $-65.0 \%$ & $-72.2 \%$ \\
\hline & \multirow{3}{*}{ Adjusted } & \begin{tabular}{|l|} 
UDDS \\
\end{tabular} & $-56.7 \%$ & $-56.7 \%$ & $-58.8 \%$ & $-61.9 \%$ & $-56.6 \%$ & $-60.9 \%$ & $-65.1 \%$ & $-59.1 \%$ & $-64.4 \%$ & $-70.2 \%$ & $-60.7 \%$ & $-65.6 \%$ & $-71.4 \%$ \\
\hline & & \begin{tabular}{|l|} 
HWFET \\
\end{tabular} & $-41.9 \%$ & $-41.9 \%$ & $-46.1 \%$ & $-51.0 \%$ & $-42.2 \%$ & $-49.4 \%$ & $-55.5 \%$ & $-46.3 \%$ & $-54.2 \%$ & $-62.1 \%$ & $-49.1 \%$ & $-56.5 \%$ & $-65.3 \%$ \\
\hline & & Combined & $-50.9 \%$ & $-50.9 \%$ & $-53.8 \%$ & $-57.6 \%$ & $-50.9 \%$ & $-56.4 \%$ & $-61.4 \%$ & $-54.1 \%$ & $-60.4 \%$ & $-67.0 \%$ & $-56.2 \%$ & $-62.0 \%$ & $-69.0 \%$ \\
\hline \multirow{6}{*}{ CI Split HEV PHEV2O } & & UDDS & $-72.1 \%$ & $-72.1 \%$ & $-73.9 \%$ & $-76.3 \%$ & $-72.0 \%$ & $-75.5 \%$ & $-78.9 \%$ & $-74.1 \%$ & $-78.1 \%$ & $-79.0 \%$ & $-75.4 \%$ & $-79.2 \%$ & $-80.2 \%$ \\
\hline & Unadjusted & HWFET & $-52.4 \%$ & $-52.4 \%$ & $-56.1 \%$ & $-52.3 \%$ & $-52.7 \%$ & $-58.9 \%$ & $-57.4 \%$ & $-55.7 \%$ & $-55.8 \%$ & $-63.8 \%$ & $-58.9 \%$ & $-58.2 \%$ & $-66.6 \%$ \\
\hline & & Combined & $-64.8 \%$ & $-64.8 \%$ & $-67.3 \%$ & $-67.3 \%$ & $-64.8 \%$ & $-69.3 \%$ & $-70.9 \%$ & $-67.3 \%$ & $-69.8 \%$ & $-73.4 \%$ & $-69.3 \%$ & $-71.4 \%$ & $-75.1 \%$ \\
\hline & & \begin{tabular}{|l|} 
UDDS \\
\end{tabular} & $-67.9 \%$ & $-67.9 \%$ & $-69.5 \%$ & $-71.8 \%$ & $-67.8 \%$ & $-71.1 \%$ & $-74.2 \%$ & $-69.8 \%$ & $-73.5 \%$ & $-74.4 \%$ & $-70.9 \%$ & $-74.5 \%$ & $-75.5 \%$ \\
\hline & Adjusted & HWFET & $-51.9 \%$ & $-51.9 \%$ & $-55.6 \%$ & $-51.8 \%$ & $-52.2 \%$ & $-58.4 \%$ & $-56.9 \%$ & $-55.2 \%$ & $-55.3 \%$ & $-63.2 \%$ & $-58.4 \%$ & $-57.7 \%$ & $-66.0 \%$ \\
\hline & & Combined & $-61.6 \%$ & $-61.6 \%$ & $-64.1 \%$ & $-63.9 \%$ & $-61.7 \%$ & $-66.1 \%$ & $-67.4 \%$ & $-64.1 \%$ & $-66.4 \%$ & $-70.0 \%$ & $-66.0 \%$ & $-67.9 \%$ & $-71.8 \%$ \\
\hline
\end{tabular}




\begin{tabular}{|c|c|c|c|c|c|c|c|c|c|c|c|c|c|c|c|}
\hline & & & \multicolumn{13}{|c|}{ Compact } \\
\hline & & & \multirow{2}{*}{$\begin{array}{c}2010 \\
\operatorname{Ref}\end{array}$} & \multicolumn{3}{|c|}{2010} & \multicolumn{3}{|c|}{2015} & \multicolumn{3}{|c|}{2030} & \multicolumn{3}{|c|}{2045} \\
\hline & & & & low & avg & high & low & avg & high & low & avg & high & low & avg & high \\
\hline \multirow{6}{*}{$\mathrm{Cl}$ Series HEV PHEV 30} & \multirow{3}{*}{ Unadjusted } & UDDS & $-70.0 \%$ & $-70.0 \%$ & $-72.1 \%$ & $-74.9 \%$ & $-70.1 \%$ & $-74.1 \%$ & $-78.9 \%$ & $-72.7 \%$ & $-77.9 \%$ & $-82.4 \%$ & $-74.1 \%$ & $-79.0 \%$ & $-83.3 \%$ \\
\hline & & HWFET & $-57.4 \%$ & $-57.4 \%$ & $-60.6 \%$ & $-64.8 \%$ & $-58.0 \%$ & $-63.3 \%$ & $-69.1 \%$ & $-61.3 \%$ & $-67.9 \%$ & $-74.3 \%$ & $-63.5 \%$ & $-69.8 \%$ & $-76.4 \%$ \\
\hline & & Combined & $-65.3 \%$ & $-65.3 \%$ & $-67.8 \%$ & $-71.2 \%$ & $-65.6 \%$ & $-70.1 \%$ & $-75.2 \%$ & $-68.5 \%$ & $-74.2 \%$ & $-79.4 \%$ & $-70.2 \%$ & $-75.5 \%$ & $-80.8 \%$ \\
\hline & \multirow{3}{*}{ Adjusted } & UDDS & $-65.9 \%$ & $-65.9 \%$ & $-67.9 \%$ & $-70.5 \%$ & $-66.0 \%$ & $-69.8 \%$ & $-74.2 \%$ & $-68.5 \%$ & $-73.4 \%$ & $-77.6 \%$ & $-69.8 \%$ & $-74.3 \%$ & $-78.4 \%$ \\
\hline & & HWFET & $-56.9 \%$ & $-56.9 \%$ & $-60.1 \%$ & $-64.2 \%$ & $-57.4 \%$ & $-62.8 \%$ & $-68.4 \%$ & $-60.7 \%$ & $-67.3 \%$ & $-73.6 \%$ & $-62.9 \%$ & $-69.1 \%$ & $-75.7 \%$ \\
\hline & & Combined & $-62.4 \%$ & $-62.4 \%$ & $-64.8 \%$ & $-68.1 \%$ & $-62.7 \%$ & $-67.0 \%$ & $-72.0 \%$ & $-65.4 \%$ & $-71.0 \%$ & $-76.0 \%$ & $-67.1 \%$ & $-72.3 \%$ & $-77.4 \%$ \\
\hline \multirow{6}{*}{$\mathrm{CI}$ Series HEV PHEV 40} & \multirow{3}{*}{ Unadjusted } & UDDS & $-73.8 \%$ & $-73.8 \%$ & $-75.6 \%$ & $-78.2 \%$ & $-73.9 \%$ & $-77.5 \%$ & $-81.1 \%$ & $-76.2 \%$ & $-81.0 \%$ & $-85.2 \%$ & $-77.5 \%$ & $-81.9 \%$ & $-86.0 \%$ \\
\hline & & HWFET & $-65.2 \%$ & $-65.2 \%$ & $-67.9 \%$ & $-71.2 \%$ & $-65.5 \%$ & $-70.1 \%$ & $-74.8 \%$ & $-68.4 \%$ & $-73.9 \%$ & $-79.3 \%$ & $-70.2 \%$ & $-75.5 \%$ & $-80.9 \%$ \\
\hline & & Combined & $-70.6 \%$ & $-70.6 \%$ & $-72.8 \%$ & $-75.6 \%$ & $-70.8 \%$ & $-74.8 \%$ & $-78.8 \%$ & $-73.3 \%$ & $-78.3 \%$ & $-83.0 \%$ & $-74.8 \%$ & $-79.5 \%$ & $-84.1 \%$ \\
\hline & \multirow{3}{*}{ Adjusted } & UDDS & $-69.4 \%$ & $-69.4 \%$ & $-71.2 \%$ & $-73.6 \%$ & $-69.5 \%$ & $-73.0 \%$ & $-76.3 \%$ & $-71.8 \%$ & $-76.2 \%$ & $-80.1 \%$ & $-73.0 \%$ & $-77.0 \%$ & $-80.9 \%$ \\
\hline & & \begin{tabular}{|l|} 
HWFET \\
\end{tabular} & $-64.7 \%$ & $-64.7 \%$ & $-67.3 \%$ & $-70.6 \%$ & $-65.0 \%$ & $-69.5 \%$ & $-74.2 \%$ & $-67.8 \%$ & $-73.2 \%$ & $-78.5 \%$ & $-69.6 \%$ & $-74.8 \%$ & $-80.2 \%$ \\
\hline & & \begin{tabular}{|l|} 
Combined \\
\end{tabular} & $-67.6 \%$ & $-67.6 \%$ & $-69.7 \%$ & $-72.4 \%$ & $-67.7 \%$ & $-71.6 \%$ & $-75.5 \%$ & $-70.2 \%$ & $-75.0 \%$ & $-79.5 \%$ & $-71.7 \%$ & $-76.2 \%$ & $-80.6 \%$ \\
\hline \multirow{6}{*}{ H2 Split HEV } & \multirow{3}{*}{ Unadjusted } & UDDS & $-48.0 \%$ & $-48.0 \%$ & $-56.3 \%$ & $-62.4 \%$ & $-52.9 \%$ & $-61.8 \%$ & $-67.9 \%$ & $-58.7 \%$ & $-66.7 \%$ & $-73.9 \%$ & $-61.9 \%$ & $-69.1 \%$ & $-76.0 \%$ \\
\hline & & HWFET & $-17.4 \%$ & $-17.4 \%$ & $-31.1 \%$ & $-40.7 \%$ & $-26.1 \%$ & $-38.7 \%$ & $-48.2 \%$ & $-34.0 \%$ & $-46.2 \%$ & $-56.7 \%$ & $-39.3 \%$ & $-50.1 \%$ & $-61.4 \%$ \\
\hline & & \begin{tabular}{|l|} 
Combined \\
\end{tabular} & $-36.6 \%$ & $-36.6 \%$ & $-46.9 \%$ & $-54.4 \%$ & $-42.9 \%$ & $-53.2 \%$ & $-60.5 \%$ & $-49.5 \%$ & $-59.1 \%$ & $-67.5 \%$ & $-53.5 \%$ & $-62.0 \%$ & $-70.5 \%$ \\
\hline & \multirow{3}{*}{ Adjusted } & \begin{tabular}{|l|} 
UDDS \\
\end{tabular} & $-45.1 \%$ & $-45.1 \%$ & $-52.9 \%$ & $-58.8 \%$ & $-49.8 \%$ & $-58.2 \%$ & $-63.9 \%$ & $-55.3 \%$ & $-62.8 \%$ & $-69.5 \%$ & $-58.2 \%$ & $-65.0 \%$ & $-71.5 \%$ \\
\hline & & HWFET & $-17.2 \%$ & $-17.2 \%$ & $-30.8 \%$ & $-40.4 \%$ & $-25.8 \%$ & $-38.3 \%$ & $-47.7 \%$ & $-33.7 \%$ & $-45.8 \%$ & $-56.2 \%$ & $-39.0 \%$ & $-49.7 \%$ & $-60.8 \%$ \\
\hline & & Combined & $-34.2 \%$ & $-34.2 \%$ & $-44.3 \%$ & $-51.5 \%$ & $-40.4 \%$ & $-50.4 \%$ & $-57.6 \%$ & $-46.8 \%$ & $-56.1 \%$ & $-64.3 \%$ & $-50.7 \%$ & $-59.0 \%$ & $-67.3 \%$ \\
\hline \multirow{6}{*}{ H2 Split HEV PHEV 10} & \multirow{3}{*}{ Unadjusted } & UDDS & $-58.3 \%$ & $-58.3 \%$ & $-64.9 \%$ & $-69.6 \%$ & $-62.2 \%$ & $-68.7 \%$ & $-73.5 \%$ & $-66.2 \%$ & $-72.3 \%$ & $-78.2 \%$ & $-69.0 \%$ & $-74.1 \%$ & $-80.1 \%$ \\
\hline & & HWFET & $-40.5 \%$ & $-40.5 \%$ & $-50.0 \%$ & $-56.6 \%$ & $-46.8 \%$ & $-55.1 \%$ & $-61.5 \%$ & $-52.0 \%$ & $-60.0 \%$ & $-67.4 \%$ & $-55.8 \%$ & $-62.9 \%$ & $-71.2 \%$ \\
\hline & & \begin{tabular}{|l|} 
Combined \\
\end{tabular} & $-51.6 \%$ & $-51.6 \%$ & $-59.3 \%$ & $-64.8 \%$ & $-56.5 \%$ & $-63.6 \%$ & $-69.0 \%$ & $-60.9 \%$ & $-67.7 \%$ & $-74.2 \%$ & $-64.1 \%$ & $-69.9 \%$ & $-76.8 \%$ \\
\hline & \multirow{3}{*}{ Adjusted } & UDDS & $-54.8 \%$ & $-54.8 \%$ & $-61.0 \%$ & $-65.5 \%$ & $-58.6 \%$ & $-64.6 \%$ & $-69.1 \%$ & $-62.3 \%$ & $-68.1 \%$ & $-73.6 \%$ & $-65.0 \%$ & $-69.7 \%$ & $-75.4 \%$ \\
\hline & & HWFET & $-40.1 \%$ & $-40.1 \%$ & $-49.6 \%$ & $-56.1 \%$ & $-46.4 \%$ & $-54.6 \%$ & $-60.9 \%$ & $-51.5 \%$ & $-59.4 \%$ & $-66.8 \%$ & $-55.3 \%$ & $-62.3 \%$ & $-70.6 \%$ \\
\hline & & \begin{tabular}{|l|} 
Combined \\
\end{tabular} & $-49.1 \%$ & $-49.1 \%$ & $-56.5 \%$ & $-61.8 \%$ & $-53.8 \%$ & $-60.7 \%$ & $-65.9 \%$ & $-58.1 \%$ & $-64.7 \%$ & $-70.9 \%$ & $-61.2 \%$ & $-66.8 \%$ & $-73.5 \%$ \\
\hline \multirow{6}{*}{ H2 Split HEV PHEV2O } & \multirow{3}{*}{ Unadjusted } & UDDS & $-71.0 \%$ & $-71.0 \%$ & $-75.6 \%$ & $-79.1 \%$ & $-73.8 \%$ & $-78.3 \%$ & $-78.0 \%$ & $-76.5 \%$ & $-77.1 \%$ & $-82.2 \%$ & $-78.5 \%$ & $-78.6 \%$ & $-83.7 \%$ \\
\hline & & HWFET & $-50.5 \%$ & $-50.5 \%$ & $-49.7 \%$ & $-58.1 \%$ & $-56.3 \%$ & $-56.4 \%$ & $-62.9 \%$ & $-51.6 \%$ & $-61.4 \%$ & $-68.7 \%$ & $-57.1 \%$ & $-64.2 \%$ & $-72.2 \%$ \\
\hline & & Combined & $-63.4 \%$ & $-63.4 \%$ & $-66.0 \%$ & $-71.3 \%$ & $-67.3 \%$ & $-70.2 \%$ & $-72.4 \%$ & $-67.2 \%$ & $-71.3 \%$ & $-77.2 \%$ & $-70.5 \%$ & $-73.3 \%$ & $-79.4 \%$ \\
\hline & \multirow{3}{*}{ Adjusted } & UDDS & $-66.8 \%$ & $-66.8 \%$ & $-71.2 \%$ & $-74.4 \%$ & $-69.4 \%$ & $-73.7 \%$ & $-73.4 \%$ & $-72.0 \%$ & $-72.6 \%$ & $-77.3 \%$ & $-73.8 \%$ & $-74.0 \%$ & $-78.7 \%$ \\
\hline & & HWFET & $-50.1 \%$ & $-50.1 \%$ & $-49.2 \%$ & $-57.5 \%$ & $-55.8 \%$ & $-55.9 \%$ & $-62.3 \%$ & $-51.1 \%$ & $-60.9 \%$ & $-68.1 \%$ & $-56.6 \%$ & $-63.6 \%$ & $-71.5 \%$ \\
\hline & & Combined & $-60.2 \%$ & $-60.2 \%$ & $-62.6 \%$ & $-67.8 \%$ & $-64.1 \%$ & $-66.7 \%$ & $-69.1 \%$ & $-63.8 \%$ & $-68.0 \%$ & $-73.7 \%$ & $-67.1 \%$ & $-69.9 \%$ & $-75.9 \%$ \\
\hline \multirow{6}{*}{ H2 Series HEV PHEV3O } & & UDDS & $-68.1 \%$ & $-68.1 \%$ & $-73.6 \%$ & $-77.9 \%$ & $-71.6 \%$ & $-76.7 \%$ & $-81.1 \%$ & $-75.0 \%$ & $-80.4 \%$ & $-84.0 \%$ & $-77.1 \%$ & $-81.4 \%$ & $-85.1 \%$ \\
\hline & Unadjusted & HWFET & $-55.8 \%$ & $-55.8 \%$ & $-63.3 \%$ & $-68.6 \%$ & $-60.6 \%$ & $-67.5 \%$ & $-72.9 \%$ & $-65.1 \%$ & $-71.8 \%$ & $-70.9 \%$ & $-68.1 \%$ & $-73.9 \%$ & $-79.8 \%$ \\
\hline & & Combined & $-63.5 \%$ & $-63.5 \%$ & $-69.8 \%$ & $-74.4 \%$ & $-67.5 \%$ & $-73.2 \%$ & $-78.1 \%$ & $-71.3 \%$ & $-77.2 \%$ & $-79.1 \%$ & $-73.8 \%$ & $-78.6 \%$ & $-83.1 \%$ \\
\hline & & UDDS & $-64.1 \%$ & $-64.1 \%$ & $-69.3 \%$ & $-73.3 \%$ & $-67.4 \%$ & $-72.2 \%$ & $-76.3 \%$ & $-70.6 \%$ & $-75.7 \%$ & $-79.0 \%$ & $-72.5 \%$ & $-76.6 \%$ & $-80.1 \%$ \\
\hline & Adjusted & HWFET & $-55.3 \%$ & $-55.3 \%$ & $-62.8 \%$ & $-68.0 \%$ & $-60.1 \%$ & $-66.8 \%$ & $-72.3 \%$ & $-64.5 \%$ & $-71.1 \%$ & $-70.3 \%$ & $-67.5 \%$ & $-73.3 \%$ & $-79.1 \%$ \\
\hline & & \begin{tabular}{|l|} 
Combined \\
\end{tabular} & $-60.7 \%$ & $-60.7 \%$ & $-66.7 \%$ & $-71.2 \%$ & $-64.5 \%$ & $-70.1 \%$ & $-74.7 \%$ & $-68.2 \%$ & $-73.9 \%$ & $-75.6 \%$ & $-70.6 \%$ & $-75.3 \%$ & $-79.7 \%$ \\
\hline
\end{tabular}




\begin{tabular}{|c|c|c|c|c|c|c|c|c|c|c|c|c|c|c|c|}
\hline & & & \multicolumn{13}{|c|}{ Compact } \\
\hline & & & 2010 & & 2010 & & & 2015 & & & 2030 & & & 2045 & \\
\hline & & & Ref & low & avg & high & low & avg & high & low & avg & high & low & avg & high \\
\hline \multirow{6}{*}{ H2 Series HEV PHEV40 } & \multirow{3}{*}{ Unadjusted } & UDDS & $-72.2 \%$ & $-72.2 \%$ & $-77.1 \%$ & $-80.4 \%$ & $-75.3 \%$ & $-79.7 \%$ & $-83.8 \%$ & $-78.2 \%$ & $-82.8 \%$ & $-86.6 \%$ & $-80.2 \%$ & $-84.1 \%$ & $-87.6 \%$ \\
\hline & & HWFET & $-63.8 \%$ & $-63.8 \%$ & $-70.0 \%$ & $-74.5 \%$ & $-67.8 \%$ & $-73.4 \%$ & $-78.0 \%$ & $-71.5 \%$ & $-77.1 \%$ & $-77.3 \%$ & $-74.1 \%$ & $-78.9 \%$ & $-83.7 \%$ \\
\hline & & \begin{tabular}{|c|} 
Combined \\
\end{tabular} & $-69.1 \%$ & $-69.1 \%$ & $-74.4 \%$ & $-78.2 \%$ & $-72.5 \%$ & $-77.4 \%$ & $-81.6 \%$ & $-75.7 \%$ & $-80.7 \%$ & $-83.1 \%$ & $-77.9 \%$ & $-82.2 \%$ & $-86.1 \%$ \\
\hline & \multirow{3}{*}{ Adjusted } & UDDS & $-67.9 \%$ & $-67.9 \%$ & $-72.5 \%$ & $-75.7 \%$ & $-70.8 \%$ & $-75.1 \%$ & $-78.8 \%$ & $-73.6 \%$ & $-78.0 \%$ & $-81.5 \%$ & $-75.5 \%$ & $-79.2 \%$ & $-82.4 \%$ \\
\hline & & HWFET & $-63.3 \%$ & $-63.3 \%$ & $-69.4 \%$ & $-73.8 \%$ & $-67.2 \%$ & $-72.8 \%$ & $-77.3 \%$ & $-70.8 \%$ & $-76.4 \%$ & $-76.6 \%$ & $-73.4 \%$ & $-78.2 \%$ & $-83.0 \%$ \\
\hline & & \begin{tabular}{|l|} 
Combined \\
\end{tabular} & $-66.1 \%$ & $-66.1 \%$ & $-71.3 \%$ & $-75.0 \%$ & $-69.4 \%$ & $-74.2 \%$ & $-78.2 \%$ & $-72.5 \%$ & $-77.3 \%$ & $-79.5 \%$ & $-74.7 \%$ & $-78.8 \%$ & $-82.6 \%$ \\
\hline \multirow{6}{*}{ E85 Split HEV } & \multirow{3}{*}{ Unadjusted } & \begin{tabular}{|l|} 
UDDS \\
\end{tabular} & $-46.1 \%$ & $-46.1 \%$ & $-49.7 \%$ & $-53.5 \%$ & $-46.5 \%$ & $-52.9 \%$ & $-63.9 \%$ & $-50.3 \%$ & $-57.6 \%$ & $-69.8 \%$ & $-52.2 \%$ & $-59.4 \%$ & $-71.4 \%$ \\
\hline & & HWFET & $-14.6 \%$ & $-14.6 \%$ & $-21.4 \%$ & $-27.6 \%$ & $-16.4 \%$ & $-25.2 \%$ & $-42.5 \%$ & $-21.0 \%$ & $-32.4 \%$ & $-50.7 \%$ & $-24.4 \%$ & $-35.4 \%$ & $-54.6 \%$ \\
\hline & & Combined & $-34.4 \%$ & $-34.4 \%$ & $-39.1 \%$ & $-43.8 \%$ & $-35.3 \%$ & $-42.6 \%$ & $-55.9 \%$ & $-39.4 \%$ & $-48.2 \%$ & $-62.7 \%$ & $-41.9 \%$ & $-50.5 \%$ & $-65.1 \%$ \\
\hline & \multirow{3}{*}{ Adjusted } & UDDS & $-43.4 \%$ & $-43.4 \%$ & $-46.7 \%$ & $-50.3 \%$ & $-43.8 \%$ & $-49.8 \%$ & $-60.2 \%$ & $-47.4 \%$ & $-54.2 \%$ & $-65.7 \%$ & $-49.2 \%$ & $-55.9 \%$ & $-67.2 \%$ \\
\hline & & \begin{tabular}{|l|} 
HWFET \\
\end{tabular} & $-14.5 \%$ & $-14.5 \%$ & $-21.2 \%$ & $-27.3 \%$ & $-16.2 \%$ & $-25.0 \%$ & $-42.1 \%$ & $-20.8 \%$ & $-32.1 \%$ & $-50.2 \%$ & $-24.2 \%$ & $-35.1 \%$ & $-54.1 \%$ \\
\hline & & \begin{tabular}{|l|} 
Combined \\
\end{tabular} & $-32.1 \%$ & $-32.1 \%$ & $-36.7 \%$ & $-41.3 \%$ & $-33.0 \%$ & $-40.1 \%$ & $-53.1 \%$ & $-37.0 \%$ & $-45.6 \%$ & $-59.6 \%$ & $-39.4 \%$ & $-47.8 \%$ & $-62.1 \%$ \\
\hline \multirow{6}{*}{ E85 Split HEV PHEV10 } & \multirow{3}{*}{ Unadjusted } & UDDS & $-57.2 \%$ & $-57.2 \%$ & $-60.0 \%$ & $-63.1 \%$ & $-57.6 \%$ & $-61.9 \%$ & $-70.4 \%$ & $-59.7 \%$ & $-65.2 \%$ & $-75.0 \%$ & $-61.1 \%$ & $-66.4 \%$ & $-76.4 \%$ \\
\hline & & HWFET & $-38.2 \%$ & $-38.2 \%$ & $-42.7 \%$ & $-47.2 \%$ & $-39.6 \%$ & $-45.3 \%$ & $-57.4 \%$ & $-42.0 \%$ & $-49.7 \%$ & $-62.9 \%$ & $-44.5 \%$ & $-52.0 \%$ & $-66.2 \%$ \\
\hline & & \begin{tabular}{|l|} 
Combined \\
\end{tabular} & $-50.1 \%$ & $-50.1 \%$ & $-53.6 \%$ & $-57.2 \%$ & $-50.9 \%$ & $-55.7 \%$ & $-65.5 \%$ & $-53.1 \%$ & $-59.4 \%$ & $-70.5 \%$ & $-54.9 \%$ & $-61.1 \%$ & $-72.6 \%$ \\
\hline & \multirow{3}{*}{ Adjusted } & UDDS & $-53.8 \%$ & $-53.8 \%$ & $-56.5 \%$ & $-59.3 \%$ & $-54.2 \%$ & $-58.2 \%$ & $-66.2 \%$ & $-56.1 \%$ & $-61.4 \%$ & $-70.6 \%$ & $-57.5 \%$ & $-62.5 \%$ & $-71.9 \%$ \\
\hline & & HWFET & $-37.8 \%$ & $-37.8 \%$ & $-42.4 \%$ & $-46.8 \%$ & $-39.3 \%$ & $-44.9 \%$ & $-56.9 \%$ & $-41.6 \%$ & $-49.3 \%$ & $-62.3 \%$ & $-44.1 \%$ & $-51.5 \%$ & $-65.6 \%$ \\
\hline & & Combined & $-47.6 \%$ & $-47.6 \%$ & $-50.9 \%$ & $-54.4 \%$ & $-48.4 \%$ & $-53.0 \%$ & $-62.6 \%$ & $-50.4 \%$ & $-56.6 \%$ & $-67.4 \%$ & $-52.2 \%$ & $-58.2 \%$ & $-69.4 \%$ \\
\hline \multirow{6}{*}{ E85 Split HEV PHEV20 } & \multirow{3}{*}{ Unadjusted } & UDDS & $-70.3 \%$ & $-70.3 \%$ & $-72.2 \%$ & $-69.4 \%$ & $-70.5 \%$ & $-68.4 \%$ & $-79.6 \%$ & $-72.0 \%$ & $-76.1 \%$ & $-79.6 \%$ & $-73.1 \%$ & $-72.3 \%$ & $-80.6 \%$ \\
\hline & & HWFET & $-37.9 \%$ & $-37.9 \%$ & $-44.5 \%$ & $-48.9 \%$ & $-39.3 \%$ & $-47.0 \%$ & $-58.7 \%$ & $-41.8 \%$ & $-51.5 \%$ & $-64.4 \%$ & $-46.3 \%$ & $-53.6 \%$ & $-67.2 \%$ \\
\hline & & \begin{tabular}{|l|} 
Combined \\
\end{tabular} & $-58.3 \%$ & $-58.3 \%$ & $-61.9 \%$ & $-61.8 \%$ & $-58.9 \%$ & $-60.4 \%$ & $-71.8 \%$ & $-60.7 \%$ & $-66.9 \%$ & $-73.9 \%$ & $-63.1 \%$ & $-65.3 \%$ & $-75.6 \%$ \\
\hline & \multirow{3}{*}{ Adjusted } & UDDS & $-66.2 \%$ & $-66.2 \%$ & $-68.0 \%$ & $-65.3 \%$ & $-66.4 \%$ & $-64.4 \%$ & $-74.9 \%$ & $-67.7 \%$ & $-71.6 \%$ & $-74.9 \%$ & $-68.8 \%$ & $-68.0 \%$ & $-75.9 \%$ \\
\hline & & HWFET & $-37.6 \%$ & $-37.6 \%$ & $-44.1 \%$ & $-48.5 \%$ & $-38.9 \%$ & $-46.6 \%$ & $-58.2 \%$ & $-41.5 \%$ & $-51.1 \%$ & $-63.8 \%$ & $-45.9 \%$ & $-53.1 \%$ & $-66.6 \%$ \\
\hline & & \begin{tabular}{|l|} 
Combined \\
\end{tabular} & $-55.0 \%$ & $-55.0 \%$ & $-58.6 \%$ & $-58.7 \%$ & $-55.6 \%$ & $-57.4 \%$ & $-68.4 \%$ & $-57.4 \%$ & $-63.6 \%$ & $-70.5 \%$ & $-59.8 \%$ & $-62.2 \%$ & $-72.2 \%$ \\
\hline \multirow{6}{*}{ E85 Series HEV PHEV30 } & \multirow{3}{*}{ Unadjusted } & UDDS & $-67.8 \%$ & $-67.8 \%$ & $-70.4 \%$ & $-73.1 \%$ & $-68.5 \%$ & $-72.2 \%$ & $-79.0 \%$ & $-70.5 \%$ & $-75.6 \%$ & $-82.8 \%$ & $-71.8 \%$ & $-76.2 \%$ & $-83.6 \%$ \\
\hline & & HWFET & $-54.0 \%$ & $-54.0 \%$ & $-58.0 \%$ & $-61.9 \%$ & $-55.1 \%$ & $-60.4 \%$ & $-70.0 \%$ & $-57.9 \%$ & $-64.6 \%$ & $-74.5 \%$ & $-60.0 \%$ & $-66.4 \%$ & $-76.6 \%$ \\
\hline & & Combined & $-62.7 \%$ & $-62.7 \%$ & $-65.8 \%$ & $-68.9 \%$ & $-63.5 \%$ & $-67.8 \%$ & $-75.7 \%$ & $-65.8 \%$ & $-71.5 \%$ & $-79.7 \%$ & $-67.4 \%$ & $-72.5 \%$ & $-81.0 \%$ \\
\hline & \multirow{3}{*}{ Adjusted } & UDDS & $-63.9 \%$ & $-63.9 \%$ & $-66.3 \%$ & $-68.8 \%$ & $-64.5 \%$ & $-68.0 \%$ & $-74.4 \%$ & $-66.3 \%$ & $-71.2 \%$ & $-77.9 \%$ & $-67.6 \%$ & $-71.7 \%$ & $-78.7 \%$ \\
\hline & & HWFET & $-53.5 \%$ & $-53.5 \%$ & $-57.4 \%$ & $-61.3 \%$ & $-54.6 \%$ & $-59.9 \%$ & $-69.4 \%$ & $-57.4 \%$ & $-64.0 \%$ & $-73.9 \%$ & $-59.4 \%$ & $-65.8 \%$ & $-75.9 \%$ \\
\hline & & Combined & $-59.8 \%$ & $-59.8 \%$ & $-62.8 \%$ & $-65.9 \%$ & $-60.6 \%$ & $-64.8 \%$ & $-72.4 \%$ & $-62.8 \%$ & $-68.4 \%$ & $-76.3 \%$ & $-64.4 \%$ & $-69.4 \%$ & $-77.6 \%$ \\
\hline \multirow{6}{*}{ E85 Series HEV PHEV40 } & \multirow{3}{*}{ Unadjusted } & UDDS & $-71.9 \%$ & $-71.9 \%$ & $-74.2 \%$ & $-76.7 \%$ & $-72.4 \%$ & $-75.8 \%$ & $-81.9 \%$ & $-74.4 \%$ & $-78.8 \%$ & $-85.5 \%$ & $-75.4 \%$ & $-79.5 \%$ & $-86.2 \%$ \\
\hline & & HWFET & $-53.3 \%$ & $-53.3 \%$ & $-65.7 \%$ & $-68.9 \%$ & $-63.5 \%$ & $-67.6 \%$ & $-75.6 \%$ & $-65.7 \%$ & $-71.2 \%$ & $-74.4 \%$ & $-67.4 \%$ & $-72.7 \%$ & $-81.1 \%$ \\
\hline & & Combined & $-65.0 \%$ & $-65.0 \%$ & $-71.0 \%$ & $-73.8 \%$ & $-69.1 \%$ & $-72.8 \%$ & $-79.6 \%$ & $-71.2 \%$ & $-76.0 \%$ & $-81.4 \%$ & $-72.4 \%$ & $-77.0 \%$ & $-84.3 \%$ \\
\hline & \multirow{3}{*}{ Adjusted } & UDDS & $-67.6 \%$ & $-67.6 \%$ & $-69.9 \%$ & $-72.1 \%$ & $-68.2 \%$ & $-71.4 \%$ & $-77.1 \%$ & $-70.0 \%$ & $-74.1 \%$ & $-80.4 \%$ & $-71.0 \%$ & $-74.9 \%$ & $-81.2 \%$ \\
\hline & & HWFET & $-52.8 \%$ & $-52.8 \%$ & $-65.1 \%$ & $-68.3 \%$ & $-62.9 \%$ & $-67.0 \%$ & $-74.9 \%$ & $-65.1 \%$ & $-70.6 \%$ & $-73.7 \%$ & $-66.8 \%$ & $-72.0 \%$ & $-80.4 \%$ \\
\hline & & Combined & $-61.8 \%$ & $-61.8 \%$ & $-68.0 \%$ & $-70.6 \%$ & $-66.1 \%$ & $-69.7 \%$ & $-76.2 \%$ & $-68.1 \%$ & $-72.8 \%$ & $-77.8 \%$ & $-69.3 \%$ & $-73.8 \%$ & $-80.9 \%$ \\
\hline
\end{tabular}


Argonne National Laboratory Report - Light-Duty Vehicle Fuel Consumption Displacement Potential Up to 2045

\begin{tabular}{|c|c|c|c|c|c|c|c|c|c|c|c|c|c|c|c|}
\hline & & & \multicolumn{13}{|c|}{ Compact } \\
\hline & & & \multirow{2}{*}{$\frac{2010}{\operatorname{Ref}}$} & \multicolumn{3}{|c|}{2010} & \multicolumn{3}{|c|}{2015} & \multicolumn{3}{|c|}{2030} & \multicolumn{3}{|c|}{2045} \\
\hline & & & & low & avg & high & low & avg & high & low & avg & high & low & avg & high \\
\hline \multirow{6}{*}{ FC HEV } & \multirow{3}{*}{ Unadjusted } & UDDS & $-53.4 \%$ & $-53.4 \%$ & $-60.8 \%$ & $-67.0 \%$ & $-60.1 \%$ & $-66.4 \%$ & $-71.3 \%$ & $-63.9 \%$ & $-70.3 \%$ & $-75.9 \%$ & $-65.9 \%$ & $-71.3 \%$ & $-77.2 \%$ \\
\hline & & HWFET & $-38.8 \%$ & $-38.8 \%$ & $-47.7 \%$ & $-55.4 \%$ & $-46.9 \%$ & $-53.8 \%$ & $-59.6 \%$ & $-50.4 \%$ & $-58.0 \%$ & $-64.9 \%$ & $-53.1 \%$ & $-59.7 \%$ & $-67.8 \%$ \\
\hline & & Combined & $-47.9 \%$ & $-47.9 \%$ & $-55.9 \%$ & $-62.7 \%$ & $-55.2 \%$ & $-61.7 \%$ & $-66.9 \%$ & $-58.9 \%$ & $-65.7 \%$ & $-71.8 \%$ & $-61.1 \%$ & $-67.0 \%$ & $-73.7 \%$ \\
\hline & \multirow{3}{*}{ Adjusted } & UDDS & $-50.2 \%$ & $-50.2 \%$ & $-57.2 \%$ & $-63.0 \%$ & $-56.5 \%$ & $-62.5 \%$ & $-67.1 \%$ & $-60.1 \%$ & $-66.1 \%$ & $-71.4 \%$ & $-62.0 \%$ & $-67.1 \%$ & $-72.6 \%$ \\
\hline & & HWFET & $-38.4 \%$ & $-38.4 \%$ & $-47.3 \%$ & $-54.9 \%$ & $-46.5 \%$ & $-53.3 \%$ & $-59.0 \%$ & $-50.0 \%$ & $-57.5 \%$ & $-64.3 \%$ & $-52.6 \%$ & $-59.2 \%$ & $-67.2 \%$ \\
\hline & & Combined & $-45.6 \%$ & $-45.6 \%$ & $-53.3 \%$ & $-59.8 \%$ & $-52.6 \%$ & $-58.9 \%$ & $-63.9 \%$ & $-56.1 \%$ & $-62.8 \%$ & $-68.6 \%$ & $-58.3 \%$ & $-64.0 \%$ & $-70.5 \%$ \\
\hline \multirow{6}{*}{ FC PHEV10 } & \multirow{3}{*}{ Unadjusted } & \begin{tabular}{|l|} 
UDDS \\
\end{tabular} & $-62.9 \%$ & $-62.9 \%$ & $-68.3 \%$ & $-73.1 \%$ & $-67.7 \%$ & $-72.2 \%$ & $-75.9 \%$ & $-70.0 \%$ & $-75.1 \%$ & $-79.6 \%$ & $-71.6 \%$ & $-75.8 \%$ & $-80.7 \%$ \\
\hline & & HWFET & $-54.3 \%$ & $-54.3 \%$ & $-60.1 \%$ & $-65.8 \%$ & $-59.5 \%$ & $-64.4 \%$ & $-68.8 \%$ & $-61.8 \%$ & $-67.5 \%$ & $-72.6 \%$ & $-63.7 \%$ & $-68.7 \%$ & $-75.1 \%$ \\
\hline & & Combined & $-59.7 \%$ & $-59.7 \%$ & $-65.2 \%$ & $-70.4 \%$ & $-64.6 \%$ & $-69.3 \%$ & $-73.3 \%$ & $-67.0 \%$ & $-72.2 \%$ & $-77.0 \%$ & $-68.6 \%$ & $-73.2 \%$ & $-78.6 \%$ \\
\hline & \multirow{3}{*}{ Adjusted } & UDDS & $-59.2 \%$ & $-59.2 \%$ & $-64.3 \%$ & $-68.8 \%$ & $-63.7 \%$ & $-67.9 \%$ & $-71.5 \%$ & $-65.9 \%$ & $-70.7 \%$ & $-74.9 \%$ & $-67.4 \%$ & $-71.4 \%$ & $-75.9 \%$ \\
\hline & & \begin{tabular}{|l|} 
HWFET \\
\end{tabular} & $-53.8 \%$ & $-53.8 \%$ & $-59.5 \%$ & $-65.2 \%$ & $-58.9 \%$ & $-63.8 \%$ & $-68.2 \%$ & $-61.2 \%$ & $-66.9 \%$ & $-71.9 \%$ & $-63.1 \%$ & $-68.1 \%$ & $-74.4 \%$ \\
\hline & & \begin{tabular}{|l|} 
Combined \\
\end{tabular} & $-57.1 \%$ & $-57.1 \%$ & $-62.4 \%$ & $-67.4 \%$ & $-61.8 \%$ & $-66.3 \%$ & $-70.2 \%$ & $-64.1 \%$ & $-69.2 \%$ & $-73.8 \%$ & $-65.7 \%$ & $-70.1 \%$ & $-75.3 \%$ \\
\hline \multirow{6}{*}{ FC PHEV2O } & \multirow{3}{*}{ Unadjusted } & UDDS & $-69.3 \%$ & $-69.3 \%$ & $-73.7 \%$ & $-77.6 \%$ & $-73.1 \%$ & $-76.9 \%$ & $-80.1 \%$ & $-75.1 \%$ & $-79.4 \%$ & $-83.2 \%$ & $-76.4 \%$ & $-80.0 \%$ & $-84.1 \%$ \\
\hline & & HWFET & $-54.7 \%$ & $-54.7 \%$ & $-60.7 \%$ & $-66.3 \%$ & $-60.1 \%$ & $-65.0 \%$ & $-69.2 \%$ & $-62.4 \%$ & $-68.0 \%$ & $-73.3 \%$ & $-64.3 \%$ & $-69.3 \%$ & $-75.5 \%$ \\
\hline & & \begin{tabular}{|l|} 
Combined \\
\end{tabular} & $-63.8 \%$ & $-63.8 \%$ & $-68.9 \%$ & $-73.4 \%$ & $-68.2 \%$ & $-72.4 \%$ & $-76.0 \%$ & $-70.3 \%$ & $-75.2 \%$ & $-79.5 \%$ & $-71.9 \%$ & $-76.1 \%$ & $-80.9 \%$ \\
\hline & \multirow{3}{*}{ Adjusted } & UDDS & $-65.2 \%$ & $-65.2 \%$ & $-69.4 \%$ & $-73.1 \%$ & $-68.8 \%$ & $-72.3 \%$ & $-75.3 \%$ & $-70.7 \%$ & $-74.7 \%$ & $-78.3 \%$ & $-71.9 \%$ & $-75.3 \%$ & $-79.1 \%$ \\
\hline & & HWFET & $-54.2 \%$ & $-54.2 \%$ & $-60.2 \%$ & $-65.7 \%$ & $-59.5 \%$ & $-64.4 \%$ & $-68.6 \%$ & $-61.8 \%$ & $-67.4 \%$ & $-72.6 \%$ & $-63.7 \%$ & $-68.7 \%$ & $-74.8 \%$ \\
\hline & & Combined & $-60.9 \%$ & $-60.9 \%$ & $-65.8 \%$ & $-70.2 \%$ & $-65.2 \%$ & $-69.2 \%$ & $-72.7 \%$ & $-67.2 \%$ & $-71.9 \%$ & $-76.1 \%$ & $-68.7 \%$ & $-72.7 \%$ & $-77.4 \%$ \\
\hline \multirow{6}{*}{ FC PHEV30 } & \multirow{3}{*}{ Unadjusted } & UDDS & $-78.3 \%$ & $-78.3 \%$ & $-81.3 \%$ & $-83.9 \%$ & $-80.9 \%$ & $-83.4 \%$ & $-85.8 \%$ & $-82.2 \%$ & $-85.3 \%$ & $-88.0 \%$ & $-83.2 \%$ & $-85.9 \%$ & $-88.6 \%$ \\
\hline & & HWFET & $-63.9 \%$ & $-63.9 \%$ & $-68.7 \%$ & $-72.8 \%$ & $-68.1 \%$ & $-71.9 \%$ & $-75.5 \%$ & $-69.8 \%$ & $-74.5 \%$ & $-78.6 \%$ & $-71.4 \%$ & $-75.6 \%$ & $-80.4 \%$ \\
\hline & & \begin{tabular}{|l|} 
Combined \\
\end{tabular} & $-72.9 \%$ & $-72.9 \%$ & $-76.6 \%$ & $-79.8 \%$ & $-76.2 \%$ & $-79.1 \%$ & $-82.0 \%$ & $-77.6 \%$ & $-81.3 \%$ & $-84.5 \%$ & $-78.8 \%$ & $-82.1 \%$ & $-85.6 \%$ \\
\hline & \multirow{3}{*}{ Adjusted } & UDDS & $-73.7 \%$ & $-73.7 \%$ & $-76.5 \%$ & $-78.9 \%$ & $-76.2 \%$ & $-78.5 \%$ & $-80.8 \%$ & $-77.3 \%$ & $-80.3 \%$ & $-82.8 \%$ & $-78.3 \%$ & $-80.8 \%$ & $-83.4 \%$ \\
\hline & & HWFET & $-63.3 \%$ & $-63.3 \%$ & $-68.0 \%$ & $-72.2 \%$ & $-67.5 \%$ & $-71.2 \%$ & $-74.8 \%$ & $-69.2 \%$ & $-73.8 \%$ & $-77.9 \%$ & $-70.8 \%$ & $-74.9 \%$ & $-79.7 \%$ \\
\hline & & Combined & $-69.6 \%$ & $-69.6 \%$ & $-73.2 \%$ & $-76.3 \%$ & $-72.8 \%$ & $-75.7 \%$ & $-78.4 \%$ & $-74.1 \%$ & $-77.7 \%$ & $-80.9 \%$ & $-75.3 \%$ & $-78.5 \%$ & $-82.0 \%$ \\
\hline \multirow{6}{*}{ FC PHEV40 } & \multirow{3}{*}{ Unadjusted } & UDDS & $-81.2 \%$ & $-81.2 \%$ & $-83.8 \%$ & $-86.1 \%$ & $-83.5 \%$ & $-85.7 \%$ & $-87.8 \%$ & $-84.6 \%$ & $-87.3 \%$ & $-89.7 \%$ & $-85.5 \%$ & $-87.9 \%$ & $-90.2 \%$ \\
\hline & & HWFET & $-71.5 \%$ & $-71.5 \%$ & $-75.3 \%$ & $-78.6 \%$ & $-74.8 \%$ & $-77.8 \%$ & $-80.7 \%$ & $-76.2 \%$ & $-79.9 \%$ & $-83.2 \%$ & $-77.5 \%$ & $-80.8 \%$ & $-84.6 \%$ \\
\hline & & \begin{tabular}{|l|} 
Combined \\
\end{tabular} & $-77.6 \%$ & $-77.6 \%$ & $-80.6 \%$ & $-83.3 \%$ & $-80.2 \%$ & $-82.8 \%$ & $-85.2 \%$ & $-81.5 \%$ & $-84.6 \%$ & $-87.3 \%$ & $-82.5 \%$ & $-85.2 \%$ & $-88.2 \%$ \\
\hline & \multirow{3}{*}{ Adjusted } & UDDS & $-76.4 \%$ & $-76.4 \%$ & $-78.9 \%$ & $-81.0 \%$ & $-78.5 \%$ & $-80.6 \%$ & $-82.6 \%$ & $-79.6 \%$ & $-82.2 \%$ & $-84.4 \%$ & $-80.4 \%$ & $-82.7 \%$ & $-84.9 \%$ \\
\hline & & HWFET & $-70.9 \%$ & $-70.9 \%$ & $-74.6 \%$ & $-77.9 \%$ & $-74.1 \%$ & $-77.1 \%$ & $-80.0 \%$ & $-75.5 \%$ & $-79.2 \%$ & $-82.5 \%$ & $-76.8 \%$ & $-80.1 \%$ & $-83.9 \%$ \\
\hline & & Combined & $-74.2 \%$ & $-74.2 \%$ & $-77.2 \%$ & $-79.8 \%$ & $-76.8 \%$ & $-79.3 \%$ & $-81.6 \%$ & $-78.0 \%$ & $-81.0 \%$ & $-83.7 \%$ & $-79.0 \%$ & $-81.7 \%$ & $-84.5 \%$ \\
\hline
\end{tabular}




\section{$\underline{\text { Midsize Class }}$}

Fuel economy 


\begin{tabular}{|c|c|c|c|c|c|c|c|c|c|c|c|c|c|c|c|}
\hline & & & \multicolumn{13}{|c|}{ Midsize } \\
\hline & & & 2010 & & 2010 & & & 2015 & & & 2030 & & & 2045 & \\
\hline & & & Ref & low & avg & high & low & avg & high & low & avg & high & low & avg & high \\
\hline \multirow{6}{*}{ SI Conv } & \multirow{3}{*}{ Unadjusted } & UDDS & 27.3 & 27.3 & 29.7 & 31.3 & 28.6 & 30.3 & 39.8 & 29.3 & 31.9 & 47.0 & 29.1 & 33.8 & 48.2 \\
\hline & & HWFET & 40.5 & 40.5 & 44.5 & 46.8 & 42.2 & 40.7 & 58.9 & 43.4 & 42.8 & 69.5 & 39.0 & 50.2 & 72.3 \\
\hline & & \begin{tabular}{|l|} 
Combined \\
\end{tabular} & 32.0 & 32.0 & 34.9 & 36.8 & 33.5 & 34.2 & 46.6 & 34.3 & 36.0 & 55.0 & 32.8 & 39.7 & 56.8 \\
\hline & \multirow{3}{*}{ Adjusted } & \begin{tabular}{|l|} 
UDDS \\
\end{tabular} & 21.1 & 21.1 & 22.8 & 24.0 & 22.0 & 23.2 & 30.0 & 22.5 & 24.4 & 34.9 & 22.3 & 25.8 & 35.8 \\
\hline & & HWFET & 28.6 & 28.6 & 31.4 & 33.0 & 29.8 & 28.8 & 41.4 & 30.7 & 30.2 & 48.8 & 27.6 & 35.4 & 50.8 \\
\hline & & \begin{tabular}{|c|} 
Combined \\
\end{tabular} & 23.9 & 23.9 & 26.0 & 27.3 & 25.0 & 25.4 & 34.2 & 25.6 & 26.7 & 40.0 & 24.4 & 29.4 & 41.2 \\
\hline \multirow{6}{*}{ Cl Conv } & \multirow{3}{*}{ Unadjusted } & \begin{tabular}{|l|} 
UDDS \\
\end{tabular} & 31.6 & 31.6 & 33.5 & 36.4 & 32.2 & 34.7 & 39.8 & 34.0 & 37.6 & 46.4 & 34.2 & 40.3 & 48.1 \\
\hline & & HWFET & 44.5 & 44.5 & 47.9 & 53.0 & 45.9 & 47.2 & 56.1 & 48.9 & 51.1 & 65.4 & 46.4 & 57.0 & 68.8 \\
\hline & & \begin{tabular}{|c|} 
Combined \\
\end{tabular} & 36.3 & 36.3 & 38.7 & 42.4 & 37.2 & 39.4 & 45.8 & 39.4 & 42.7 & 53.4 & 38.8 & 46.4 & 55.7 \\
\hline & \multirow{3}{*}{ Adjusted } & \begin{tabular}{|l|} 
UDDS \\
\end{tabular} & 24.2 & 24.2 & 25.5 & 27.6 & 24.6 & 26.4 & 30.0 & 25.9 & 28.4 & 34.5 & 26.0 & 30.3 & 35.7 \\
\hline & & HWFET & 31.4 & 31.4 & 33.8 & 37.3 & 32.4 & 33.3 & 39.5 & 34.5 & 36.0 & 46.0 & 32.7 & 40.1 & 48.3 \\
\hline & & \begin{tabular}{|l|} 
Combined \\
\end{tabular} & 27.0 & 27.0 & 28.7 & 31.3 & 27.6 & 29.1 & 33.6 & 29.2 & 31.4 & 38.9 & 28.7 & 34.1 & 40.4 \\
\hline \multirow{6}{*}{ H2 Conv } & \multirow{3}{*}{ Unadjusted } & \begin{tabular}{|l|} 
UDDS \\
\end{tabular} & 22.3 & 22.3 & 26.8 & 30.6 & 25.2 & 29.6 & 35.3 & 27.9 & 32.7 & 41.9 & 29.5 & 36.4 & 45.1 \\
\hline & & HWFET & 36.1 & 36.1 & 43.5 & 51.5 & 42.1 & 40.9 & 57.1 & 46.7 & 45.2 & 67.2 & 40.8 & 59.1 & 73.5 \\
\hline & & \begin{tabular}{|l|} 
Combined \\
\end{tabular} & 26.9 & 26.9 & 32.4 & 37.4 & 30.7 & 33.8 & 42.7 & 34.0 & 37.4 & 50.4 & 33.7 & 44.0 & 54.6 \\
\hline & \multirow{3}{*}{ Adjusted } & \begin{tabular}{|l|} 
UDDS \\
\end{tabular} & 17.4 & 17.4 & 20.7 & 23.5 & 19.5 & 22.7 & 26.8 & 21.5 & 25.0 & 31.4 & 22.6 & 27.6 & 33.7 \\
\hline & & HWFET & 25.5 & 25.5 & 30.7 & 36.3 & 29.7 & 28.9 & 40.2 & 33.0 & 31.9 & 47.2 & 28.8 & 41.6 & 51.5 \\
\hline & & \begin{tabular}{|l|} 
Combined \\
\end{tabular} & 20.3 & 20.3 & 24.2 & 27.9 & 23.1 & 25.1 & 31.6 & 25.5 & 27.7 & 37.0 & 25.1 & 32.5 & 39.9 \\
\hline \multirow{6}{*}{ E85 Conv } & \multirow{3}{*}{ Unadjusted } & \begin{tabular}{|l|} 
UDDS \\
\end{tabular} & 26.5 & 26.5 & 26.8 & 29.1 & 26.4 & 27.7 & 38.2 & 27.0 & 29.3 & 44.5 & 26.5 & 30.7 & 45.8 \\
\hline & & HWFET & 37.0 & 37.0 & 37.9 & 40.9 & 36.7 & 37.0 & 53.0 & 37.8 & 39.1 & 61.6 & 35.4 & 42.8 & 64.3 \\
\hline & & Combined & 30.4 & 30.4 & 30.9 & 33.4 & 30.2 & 31.2 & 43.7 & 31.0 & 33.0 & 50.8 & 29.9 & 35.2 & 52.6 \\
\hline & \multirow{3}{*}{ Adjusted } & \begin{tabular}{|l|} 
UDDS \\
\end{tabular} & 20.5 & 20.5 & 20.7 & 22.4 & 20.4 & 21.3 & 28.9 & 20.9 & 22.5 & 33.2 & 20.5 & 23.6 & 34.1 \\
\hline & & HWFET & 26.2 & 26.2 & 26.8 & 28.9 & 26.0 & 26.1 & 37.4 & 26.7 & 27.7 & 43.3 & 25.1 & 30.2 & 45.2 \\
\hline & & Combined & 22.7 & 22.7 & 23.1 & 24.9 & 22.6 & 23.3 & 32.2 & 23.1 & 24.6 & 37.1 & 22.3 & 26.2 & 38.4 \\
\hline \multirow{6}{*}{ SI Split HEV } & \multirow{3}{*}{ Unadjusted } & \begin{tabular}{|l|} 
UDDS \\
\end{tabular} & 53.2 & 53.2 & 58.1 & 63.1 & 54.2 & 62.6 & 77.4 & 58.4 & 69.6 & 97.6 & 60.5 & 72.7 & 102.4 \\
\hline & & \begin{tabular}{|l|} 
HWFET \\
\end{tabular} & 47.8 & 47.8 & 52.8 & 57.3 & 49.2 & 55.9 & 68.4 & 52.1 & 61.5 & 84.8 & 53.9 & 64.7 & 90.4 \\
\hline & & Combined & 50.6 & 50.6 & 55.6 & 60.3 & 51.8 & 59.4 & 73.1 & 55.4 & 65.7 & 91.4 & 57.3 & 68.9 & 96.6 \\
\hline & \multirow{3}{*}{ Adjusted } & \begin{tabular}{|l|} 
UDDS \\
\end{tabular} & 39.0 & 39.0 & 42.3 & 45.5 & 39.8 & 45.2 & 54.4 & 42.5 & 49.6 & 66.2 & 43.8 & 51.5 & 68.9 \\
\hline & & HWFET & 33.7 & 33.7 & 37.2 & 40.3 & 34.7 & 39.4 & 48.0 & 36.7 & 43.3 & 59.4 & 38.0 & 45.5 & 63.2 \\
\hline & & Combined & 36.5 & 36.5 & 39.8 & 43.0 & 37.3 & 42.4 & 51.3 & 39.7 & 46.5 & 62.9 & 41.0 & 48.6 & 66.2 \\
\hline \multirow{6}{*}{ SI Split HEV PHEV10 } & \multirow{3}{*}{ Unadjusted } & \begin{tabular}{|l|} 
UDDS \\
\end{tabular} & 66.5 & 66.5 & 72.2 & 78.5 & 67.7 & 76.3 & 93.8 & 71.4 & 84.1 & 117.7 & 73.8 & 87.2 & 124.0 \\
\hline & & HWFET & 65.9 & 65.9 & 72.1 & 78.7 & 67.2 & 76.6 & 94.0 & 70.8 & 84.5 & 114.8 & 73.3 & 88.3 & 123.0 \\
\hline & & Combined & 66.2 & 66.2 & 72.2 & 78.6 & 67.5 & 76.4 & 93.9 & 71.1 & 84.3 & 116.4 & 73.6 & 87.7 & 123.5 \\
\hline & \multirow{3}{*}{ Adjusted } & \begin{tabular}{|l|} 
UDDS \\
\end{tabular} & 47.6 & 47.6 & 51.2 & 55.0 & 48.4 & 53.7 & 64.0 & 50.7 & 58.4 & 77.1 & 52.2 & 60.2 & 80.4 \\
\hline & & HWFET & 46.3 & 46.3 & 50.6 & 55.1 & 47.2 & 53.7 & 65.6 & 49.7 & 59.1 & 79.8 & 51.4 & 61.7 & 85.3 \\
\hline & & Combined & 47.0 & 47.0 & 50.9 & 55.1 & 47.9 & 53.7 & 64.7 & 50.3 & 58.7 & 78.3 & 51.8 & 60.9 & 82.6 \\
\hline
\end{tabular}




\begin{tabular}{|c|c|c|c|c|c|c|c|c|c|c|c|c|c|c|c|}
\hline & & & \multicolumn{13}{|c|}{ Midsize } \\
\hline & & & 2010 & & 2010 & & & 2015 & & & 2030 & & & 2045 & \\
\hline & & & Ref & low & avg & high & low & avg & high & low & avg & high & low & avg & high \\
\hline \multirow{6}{*}{ SI Split HEV PHEV2O } & \multirow{3}{*}{ Unadjusted } & UDDS & 94.1 & 94.1 & 103.0 & 113.1 & 96.7 & 109.9 & 135.5 & 101.8 & 122.2 & 142.4 & 105.4 & 126.7 & 151.0 \\
\hline & & HWFET & 79.6 & 79.6 & 87.2 & 96.3 & 82.2 & 92.1 & 96.3 & 85.7 & 85.6 & 118.2 & 88.7 & 90.5 & 125.9 \\
\hline & & \begin{tabular}{|c|} 
Combined \\
\end{tabular} & 87.0 & 87.0 & 95.2 & 104.9 & 89.6 & 101.1 & 114.5 & 93.9 & 102.5 & 130.4 & 97.2 & 107.4 & 138.6 \\
\hline & \multirow{3}{*}{ Adjusted } & \begin{tabular}{|l|} 
UDDS \\
\end{tabular} & 64.2 & 64.2 & 69.2 & 74.7 & 65.7 & 73.0 & 86.2 & 68.6 & 79.5 & 89.6 & 70.5 & 81.8 & 93.7 \\
\hline & & \begin{tabular}{|l|} 
HWFET \\
\end{tabular} & 55.8 & 55.8 & 61.0 & 67.2 & 57.5 & 64.4 & 67.2 & 60.0 & 59.9 & 82.1 & 62.0 & 63.2 & 87.3 \\
\hline & & \begin{tabular}{|c|} 
Combined \\
\end{tabular} & 60.1 & 60.1 & 65.3 & 71.1 & 61.7 & 68.8 & 76.5 & 64.4 & 69.3 & 86.1 & 66.4 & 72.2 & 90.7 \\
\hline \multirow{6}{*}{ SI Series HEV PHEV3O } & \multirow{3}{*}{ Unadjusted } & \begin{tabular}{|l|} 
UDDS \\
\end{tabular} & 86.6 & 86.6 & 97.0 & 106.8 & 90.3 & 103.2 & 131.8 & 97.0 & 118.4 & 170.1 & 100.9 & 122.8 & 177.9 \\
\hline & & HWFET & 88.3 & 88.3 & 98.1 & 107.3 & 90.7 & 104.0 & 133.3 & 97.3 & 118.0 & 164.7 & 101.4 & 124.2 & 175.3 \\
\hline & & \begin{tabular}{|c|} 
Combined \\
\end{tabular} & 87.3 & 87.3 & 97.5 & 107.0 & 90.5 & 103.5 & 132.4 & 97.1 & 118.2 & 167.6 & 101.1 & 123.4 & 176.7 \\
\hline & \multirow{3}{*}{ Adjusted } & \begin{tabular}{|l|} 
UDDS \\
\end{tabular} & 59.9 & 59.9 & 65.8 & 71.3 & 62.0 & 69.3 & 84.4 & 65.8 & 77.5 & 102.5 & 68.0 & 79.8 & 105.9 \\
\hline & & HWFET & 61.7 & 61.7 & 68.5 & 74.7 & 63.4 & 72.5 & 92.3 & 67.9 & 82.0 & 113.3 & 70.7 & 86.1 & 120.3 \\
\hline & & \begin{tabular}{|l|} 
Combined \\
\end{tabular} & 60.7 & 60.7 & 67.0 & 72.8 & 62.6 & 70.7 & 87.8 & 66.8 & 79.4 & 107.1 & 69.2 & 82.5 & 112.0 \\
\hline \multirow{6}{*}{ SI Series HEV PHEV40 } & \multirow{3}{*}{ Unadjusted } & \begin{tabular}{|l|} 
UDDS \\
\end{tabular} & 100.4 & 100.4 & 111.3 & 122.8 & 102.5 & 118.8 & 152.5 & 111.6 & 136.8 & 201.1 & 116.2 & 142.3 & 210.8 \\
\hline & & HWFET & 108.1 & 108.1 & 120.1 & 132.6 & 111.1 & 127.5 & 164.1 & 119.3 & 145.6 & 203.2 & 124.4 & 152.9 & 217.0 \\
\hline & & \begin{tabular}{|l|} 
Combined \\
\end{tabular} & 103.7 & 103.7 & 115.1 & 127.0 & 106.2 & 122.5 & 157.5 & 114.9 & 140.7 & 202.1 & 119.7 & 146.9 & 213.5 \\
\hline & \multirow{3}{*}{ Adjusted } & \begin{tabular}{|l|} 
UDDS \\
\end{tabular} & 67.8 & 67.8 & 73.7 & 79.8 & 68.9 & 77.7 & 94.4 & 73.9 & 86.9 & 115.7 & 76.3 & 89.6 & 119.6 \\
\hline & & HWFET & 75.3 & 75.3 & 83.4 & 91.9 & 77.3 & 88.4 & 112.9 & 82.9 & 100.6 & 138.7 & 86.3 & 105.4 & 147.6 \\
\hline & & \begin{tabular}{|l|} 
Combined \\
\end{tabular} & 70.9 & 70.9 & 77.8 & 84.8 & 72.5 & 82.2 & 101.9 & 77.7 & 92.6 & 125.0 & 80.5 & 96.1 & 130.7 \\
\hline \multirow{6}{*}{ CI Split HEV } & \multirow{3}{*}{ Unadjusted } & \begin{tabular}{|l|} 
UDDS \\
\end{tabular} & 57.3 & 57.3 & 61.5 & 67.8 & 57.8 & 66.8 & 77.6 & 63.0 & 75.3 & 95.3 & 65.9 & 79.1 & 99.1 \\
\hline & & HWFET & 50.6 & 50.6 & 55.2 & 60.8 & 51.2 & 59.3 & 68.2 & 55.5 & 66.1 & 82.3 & 58.1 & 69.8 & 87.0 \\
\hline & & Combined & 54.1 & 54.1 & 58.5 & 64.4 & 54.6 & 63.2 & 73.1 & 59.4 & 70.9 & 89.0 & 62.1 & 74.6 & 93.3 \\
\hline & \multirow{3}{*}{ Adjusted } & \begin{tabular}{|l|} 
UDDS \\
\end{tabular} & 41.8 & 41.8 & 44.5 & 48.5 & 42.1 & 47.9 & 54.5 & 45.4 & 53.1 & 64.9 & 47.3 & 55.4 & 67.0 \\
\hline & & HWFET & 35.7 & 35.7 & 38.9 & 42.7 & 36.1 & 41.7 & 47.9 & 39.1 & 46.5 & 57.6 & 40.9 & 49.0 & 60.9 \\
\hline & & Combined & 38.8 & 38.8 & 41.8 & 45.7 & 39.2 & 44.9 & 51.3 & 42.3 & 49.9 & 61.4 & 44.2 & 52.3 & 64.1 \\
\hline \multirow{6}{*}{ CI Split HEV PHEV10 } & \multirow{3}{*}{ Unadjusted } & \begin{tabular}{|l|} 
UDDS \\
\end{tabular} & 67.4 & 67.4 & 72.5 & 79.7 & 67.8 & 77.6 & 89.9 & 73.1 & 87.1 & 109.6 & 76.4 & 91.2 & 114.4 \\
\hline & & HWFET & 66.0 & 66.0 & 71.9 & 78.7 & 66.6 & 76.6 & 90.1 & 71.7 & 87.1 & 107.6 & 75.1 & 92.2 & 114.7 \\
\hline & & \begin{tabular}{|l|} 
Combined \\
\end{tabular} & 66.7 & 66.7 & 72.3 & 79.3 & 67.2 & 77.2 & 90.0 & 72.4 & 87.1 & 108.7 & 75.8 & 91.7 & 114.5 \\
\hline & \multirow{3}{*}{ Adjusted } & \begin{tabular}{|l|} 
UDDS \\
\end{tabular} & 48.2 & 48.2 & 51.4 & 55.8 & 48.5 & 54.5 & 61.8 & 51.7 & 60.2 & 72.8 & 53.8 & 62.6 & 75.4 \\
\hline & & HWFET & 46.4 & 46.4 & 50.5 & 55.2 & 46.8 & 53.7 & 63.0 & 50.3 & 60.9 & 74.9 & 52.6 & 64.4 & 79.7 \\
\hline & & Combined & 47.4 & 47.4 & 51.0 & 55.5 & 47.7 & 54.2 & 62.3 & 51.1 & 60.5 & 73.8 & 53.3 & 63.4 & 77.3 \\
\hline \multirow{6}{*}{ CI Split HEV PHEV2O } & \multirow{3}{*}{ Unadjusted } & \begin{tabular}{|l|} 
UDDS \\
\end{tabular} & 96.0 & 96.0 & 103.2 & 113.6 & 96.2 & 111.3 & 129.9 & 103.7 & 125.8 & 159.2 & 108.9 & 132.0 & 166.2 \\
\hline & & HWFET & 81.0 & 81.0 & 87.1 & $\begin{array}{l}95.8 \\
\end{array}$ & 80.6 & $\begin{array}{l}93.8 \\
\end{array}$ & 91.0 & 87.0 & 106.1 & 109.8 & 91.3 & $\begin{array}{l}93.2 \\
\end{array}$ & 116.4 \\
\hline & & Combined & 88.6 & 88.6 & 95.3 & 104.8 & 88.5 & 102.7 & 109.0 & 95.5 & 116.1 & 132.4 & 100.2 & 111.1 & 139.4 \\
\hline & \multirow{3}{*}{ Adjusted } & \begin{tabular}{|l|} 
UDDS \\
\end{tabular} & 65.3 & 65.3 & 69.3 & 75.0 & 65.4 & 73.7 & 83.4 & 69.6 & 81.3 & 97.6 & $\begin{array}{l}72.4 \\
\end{array}$ & 84.5 & 100.7 \\
\hline & & HWFET & 56.7 & 56.7 & 60.9 & 66.8 & 56.4 & 65.5 & 63.6 & 60.8 & 73.9 & 76.4 & 63.8 & 65.1 & 80.9 \\
\hline & & Combined & 61.1 & 61.1 & 65.3 & 71.1 & 61.0 & 69.8 & 73.2 & 65.4 & 77.8 & 86.8 & 68.3 & 74.5 & 90.7 \\
\hline
\end{tabular}




\begin{tabular}{|c|c|c|c|c|c|c|c|c|c|c|c|c|c|c|c|}
\hline & & & \multicolumn{13}{|c|}{ Midsize } \\
\hline & & & \multirow{2}{*}{$\begin{array}{l}2010 \\
\text { Ref }\end{array}$} & \multicolumn{3}{|c|}{2010} & \multicolumn{3}{|c|}{2015} & \multicolumn{3}{|c|}{2030} & \multicolumn{3}{|c|}{2045} \\
\hline & & & & low & avg & high & low & avg & high & low & avg & high & low & avg & high \\
\hline \multirow{6}{*}{ CI Series HEV PHEV 30} & \multirow{3}{*}{ Unadjusted } & UDDS & 88.7 & 88.7 & 97.2 & 108.3 & 90.3 & 104.7 & 126.1 & 99.0 & 122.1 & 159.2 & 103.9 & 128.5 & 166.5 \\
\hline & & HWFET & 89.7 & 89.7 & 98.7 & 110.8 & 91.0 & 107.6 & 128.5 & 99.7 & 123.5 & 154.9 & 105.0 & 130.0 & 164.5 \\
\hline & & Combined & 89.2 & 89.2 & 97.9 & 109.4 & 90.6 & 106.0 & 127.2 & 99.3 & 122.7 & 157.2 & 104.4 & 129.2 & 165.6 \\
\hline & \multirow{3}{*}{ Adjusted } & \begin{tabular}{|l|} 
UDDS \\
\end{tabular} & 61.1 & 61.1 & 65.9 & 72.1 & 62.0 & 70.1 & 81.5 & 67.0 & 79.4 & 97.6 & 69.7 & 82.7 & 100.9 \\
\hline & & HWFET & 62.7 & 62.7 & 68.9 & 77.1 & 63.6 & 74.9 & 89.1 & 69.5 & 85.7 & 106.7 & 73.1 & 90.1 & 113.2 \\
\hline & & \begin{tabular}{|c|} 
Combined \\
\end{tabular} & 61.8 & 61.8 & 67.2 & 74.3 & 62.7 & 72.2 & 84.8 & 68.1 & 82.1 & 101.5 & 71.2 & 85.9 & 106.1 \\
\hline \multirow{6}{*}{ CI Series HEV PHEV40 } & \multirow{3}{*}{ Unadjusted } & \begin{tabular}{|l|} 
UDDS \\
\end{tabular} & 102.1 & 102.1 & 111.9 & 124.1 & 103.7 & 120.6 & 145.9 & 114.1 & 141.2 & 186.5 & 119.8 & 148.8 & 197.3 \\
\hline & & HWFET & 109.9 & 109.9 & 121.9 & 135.7 & 111.4 & 131.3 & 158.1 & 122.2 & 151.9 & 190.9 & 128.8 & 159.9 & 205.2 \\
\hline & & \begin{tabular}{|c|} 
Combined \\
\end{tabular} & 105.4 & 105.4 & 116.2 & 129.1 & 107.1 & 125.2 & 151.2 & 117.6 & 145.8 & 188.5 & 123.7 & 153.6 & 200.8 \\
\hline & \multirow{3}{*}{ Adjusted } & \begin{tabular}{|l|} 
UDDS \\
\end{tabular} & 68.7 & 68.7 & 74.0 & 80.5 & 69.6 & 78.7 & 91.3 & 75.2 & 89.0 & 109.6 & 78.2 & 92.7 & 114.1 \\
\hline & & HWFET & 76.5 & 76.5 & 84.6 & 93.9 & 77.5 & 91.0 & 108.9 & 84.8 & 104.8 & 130.6 & 89.3 & 110.1 & 140.0 \\
\hline & & \begin{tabular}{|l|} 
Combined \\
\end{tabular} & 72.0 & 72.0 & 78.4 & 86.0 & 73.0 & 83.8 & 98.5 & 79.3 & 95.5 & 118.2 & 82.9 & 99.8 & 124.5 \\
\hline \multirow{6}{*}{ H2 Split HEV } & \multirow{3}{*}{ Unadjusted } & \begin{tabular}{|l|} 
UDDS \\
\end{tabular} & 51.7 & 51.7 & 62.8 & 72.5 & 57.8 & 71.6 & 85.6 & 66.0 & 82.6 & 106.3 & 71.7 & 89.0 & 115.3 \\
\hline & & HWFET & 45.8 & 45.8 & 55.8 & 64.7 & 51.4 & 63.1 & 74.8 & 58.1 & 72.4 & 90.9 & 63.1 & 78.1 & 100.2 \\
\hline & & \begin{tabular}{|l|} 
Combined \\
\end{tabular} & 48.9 & 48.9 & 59.4 & 68.8 & 54.8 & 67.5 & 80.4 & 62.2 & 77.6 & 98.8 & 67.5 & 83.8 & 108.0 \\
\hline & \multirow{3}{*}{ Adjusted } & \begin{tabular}{|l|} 
UDDS \\
\end{tabular} & 38.1 & 38.1 & 45.3 & 51.4 & 42.1 & 50.8 & 59.3 & 47.4 & 57.5 & 71.0 & 50.9 & 61.3 & 75.9 \\
\hline & & HWFET & 32.3 & 32.3 & 39.3 & 45.5 & 36.2 & 44.4 & 52.4 & 40.9 & 50.8 & 63.5 & 44.3 & 54.7 & 69.9 \\
\hline & & \begin{tabular}{|l|} 
Combined \\
\end{tabular} & 35.2 & 35.2 & 42.4 & 48.6 & 39.3 & 47.7 & 56.0 & 44.2 & 54.3 & 67.5 & 47.7 & 58.1 & 73.1 \\
\hline \multirow{6}{*}{ H2 Split HEV PHEV10 } & \multirow{3}{*}{ Unadjusted } & \begin{tabular}{|l|} 
UDDS \\
\end{tabular} & 63.9 & 63.9 & 77.4 & 89.8 & 71.6 & 87.1 & 103.1 & 80.2 & 99.5 & 128.6 & 87.0 & 106.6 & 139.4 \\
\hline & & HWFET & 63.6 & 63.6 & 77.2 & 89.4 & 71.3 & 87.1 & 102.8 & 79.5 & 99.0 & 123.0 & 85.9 & 106.8 & 136.4 \\
\hline & & Combined & 63.8 & 63.8 & 77.3 & 89.6 & 71.5 & 87.1 & 102.9 & 79.9 & 99.3 & 126.0 & 86.5 & 106.7 & 138.0 \\
\hline & \multirow{3}{*}{ Adjusted } & \begin{tabular}{|l|} 
UDDS \\
\end{tabular} & 46.0 & 46.0 & 54.4 & 61.7 & 50.8 & 60.1 & 69.2 & 56.1 & 67.2 & 82.7 & 60.1 & 71.2 & 88.1 \\
\hline & & HWFET & 44.7 & 44.7 & 54.1 & 62.5 & 50.0 & 60.9 & 71.6 & 55.7 & 69.1 & 85.4 & 60.1 & 74.4 & 94.4 \\
\hline & & Combined & 45.4 & 45.4 & 54.2 & 62.1 & 50.5 & 60.5 & 70.3 & 55.9 & 68.0 & 83.9 & 60.1 & 72.6 & 90.8 \\
\hline \multirow{6}{*}{ H2 Split HEV PHEV2O } & \multirow{3}{*}{ Unadjusted } & \begin{tabular}{|l|} 
UDDS \\
\end{tabular} & 91.0 & 91.0 & 110.4 & 129.4 & 101.6 & 125.6 & 149.5 & 114.9 & 144.1 & 154.3 & 124.3 & 154.6 & 169.0 \\
\hline & & $\begin{array}{l}\text { HWFET } \\
\end{array}$ & 76.9 & 76.9 & 93.5 & 109.7 & 86.0 & 104.7 & 105.5 & 97.4 & 100.7 & 126.7 & 104.2 & 109.7 & 140.0 \\
\hline & & Combined & 84.0 & 84.0 & 102.1 & 119.7 & 93.9 & 115.2 & 125.9 & 106.3 & 120.7 & 140.5 & 114.4 & 130.5 & 154.6 \\
\hline & \multirow{3}{*}{ Adjusted } & \begin{tabular}{|l|} 
UDDS \\
\end{tabular} & 62.4 & 62.4 & 73.2 & 83.2 & 68.4 & 81.2 & 93.0 & 75.7 & 90.4 & 95.3 & 80.6 & 95.4 & 102.0 \\
\hline & & HWFET & 53.9 & 53.9 & 65.3 & 76.3 & 60.2 & 72.9 & 73.5 & 68.0 & 70.2 & 87.9 & 72.6 & 76.3 & 96.8 \\
\hline & & Combined & 58.3 & 58.3 & 69.4 & 79.9 & 64.5 & 77.3 & 83.1 & 72.0 & 80.1 & 91.8 & 76.8 & 85.8 & 99.6 \\
\hline \multirow{6}{*}{ H2 Series HEV PHEV30 } & & \begin{tabular}{|l|} 
UDDS \\
\end{tabular} & 84.0 & 84.0 & 102.9 & 120.9 & 95.0 & 117.0 & 143.6 & 108.1 & 137.7 & 176.3 & 118.3 & 146.9 & 190.7 \\
\hline & Unadjusted & HWFET & 86.3 & 86.3 & 106.0 & 123.2 & 97.2 & 119.7 & 146.2 & 110.5 & 139.6 & 177.1 & 120.6 & 151.4 & 194.9 \\
\hline & & Combined & 85.1 & 85.1 & 104.3 & 121.9 & 96.0 & 118.2 & 144.7 & 109.2 & 138.6 & 176.7 & 119.3 & 148.9 & 192.6 \\
\hline & & \begin{tabular}{|l|} 
UDDS \\
\end{tabular} & 58.4 & 58.4 & 69.1 & 78.8 & 64.7 & 76.8 & 90.2 & 72.0 & 87.3 & 105.2 & 77.4 & $\begin{array}{ll}91.8 \\
\end{array}$ & 111.4 \\
\hline & Adjusted & HWFET & 60.4 & 60.4 & 73.8 & 85.5 & 67.8 & 83.1 & 101.0 & 76.9 & 96.6 & 121.5 & 83.7 & 104.5 & 133.2 \\
\hline & & Combined & 59.3 & 59.3 & 71.2 & 81.7 & 66.1 & 79.5 & 94.7 & 74.1 & 91.3 & 112.0 & 80.2 & 97.1 & 120.3 \\
\hline
\end{tabular}




\begin{tabular}{|c|c|c|c|c|c|c|c|c|c|c|c|c|c|c|c|}
\hline & & & \multicolumn{13}{|c|}{ Midsize } \\
\hline & & & 2010 & & 2010 & & & 2015 & & & 2030 & & & 2045 & \\
\hline & & & Ref & low & avg & high & low & avg & high & low & avg & high & low & avg & high \\
\hline \multirow{6}{*}{ H2 Series HEV PHEV 40} & \multirow{3}{*}{ Unadjusted } & UDDS & 95.5 & 95.5 & 118.5 & 138.8 & 109.2 & 134.9 & 165.4 & 124.7 & 159.2 & 208.9 & 135.9 & 170.6 & 226.2 \\
\hline & & HWFET & 105.8 & 105.8 & 129.9 & 152.1 & 119.0 & 146.5 & 179.9 & 135.6 & 171.6 & 218.5 & 147.4 & 186.6 & 241.1 \\
\hline & & \begin{tabular}{|c|} 
Combined \\
\end{tabular} & 99.9 & 99.9 & 123.4 & 144.4 & 113.4 & 139.9 & 171.6 & 129.4 & 164.5 & 213.1 & 140.8 & 177.4 & 232.7 \\
\hline & \multirow{3}{*}{ Adjusted } & \begin{tabular}{|l|} 
UDDS \\
\end{tabular} & 65.0 & 65.0 & 77.6 & 87.8 & 72.6 & 86.0 & 100.4 & 80.8 & 97.5 & 118.8 & 86.4 & 102.7 & 125.5 \\
\hline & & HWFET & 73.7 & 73.7 & 90.0 & 104.9 & 82.7 & 101.2 & 123.4 & 93.9 & 117.9 & 148.6 & 101.8 & 127.7 & 163.2 \\
\hline & & \begin{tabular}{|c|} 
Combined \\
\end{tabular} & 68.6 & 68.6 & 82.7 & 94.8 & 76.8 & 92.2 & 109.6 & 86.2 & 105.8 & 130.6 & 92.7 & 112.7 & 140.1 \\
\hline \multirow{6}{*}{ E85 Split HEV } & \multirow{3}{*}{ Unadjusted } & \begin{tabular}{|l|} 
UDDS \\
\end{tabular} & 49.3 & 49.3 & 53.9 & 58.7 & 50.3 & 58.1 & 76.5 & 54.7 & 64.9 & 92.0 & 56.4 & 67.6 & 96.1 \\
\hline & & HWFET & 44.3 & 44.3 & 49.0 & 53.2 & 45.3 & 51.5 & 67.4 & 48.6 & 57.3 & 79.6 & 50.0 & 59.8 & 84.6 \\
\hline & & \begin{tabular}{|c|} 
Combined \\
\end{tabular} & 46.9 & 46.9 & 51.6 & 56.1 & 47.9 & 54.9 & 72.2 & 51.8 & 61.3 & 86.0 & 53.3 & 63.9 & 90.6 \\
\hline & \multirow{3}{*}{ Adjusted } & \begin{tabular}{|l|} 
UDDS \\
\end{tabular} & 36.5 & 36.5 & 39.6 & 42.7 & 37.1 & 42.3 & 53.9 & 40.1 & 46.7 & 63.0 & 41.1 & 48.3 & 65.3 \\
\hline & & HWFET & 31.3 & 31.3 & 34.6 & 37.5 & 31.9 & 36.3 & 47.4 & 34.3 & 40.3 & 55.7 & 35.3 & 42.1 & 59.2 \\
\hline & & \begin{tabular}{|l|} 
Combined \\
\end{tabular} & 33.9 & 33.9 & 37.1 & 40.2 & 34.6 & 39.4 & 50.7 & 37.2 & 43.6 & 59.5 & 38.3 & 45.3 & 62.4 \\
\hline \multirow{6}{*}{ E85 Split HEV PHEV10 } & \multirow{3}{*}{ Unadjusted } & \begin{tabular}{|l|} 
UDDS \\
\end{tabular} & 62.1 & 62.1 & 67.6 & 73.6 & 63.4 & 71.5 & 92.5 & 66.9 & 78.8 & 111.3 & 69.2 & 81.9 & 117.0 \\
\hline & & HWFET & 61.3 & 61.3 & 67.2 & 73.3 & 62.7 & 71.3 & 92.4 & 65.9 & 78.8 & 108.0 & 68.3 & 82.3 & 115.5 \\
\hline & & Combined & 61.7 & 61.7 & 67.4 & 73.5 & 63.0 & 71.4 & 92.4 & 66.4 & 78.8 & 109.8 & 68.8 & 82.1 & 116.3 \\
\hline & \multirow{3}{*}{ Adjusted } & \begin{tabular}{|l|} 
UDDS \\
\end{tabular} & 44.8 & 44.8 & 48.3 & 52.1 & 45.7 & 50.8 & 63.3 & 47.9 & 55.2 & 73.7 & 49.3 & 57.1 & 76.8 \\
\hline & & HWFET & 43.1 & 43.1 & 47.2 & 51.4 & 44.0 & 50.1 & 64.5 & 46.3 & 55.2 & 75.2 & 47.9 & 57.6 & 80.3 \\
\hline & & \begin{tabular}{|c|} 
Combined \\
\end{tabular} & 44.0 & 44.0 & 47.8 & 51.8 & 44.9 & 50.4 & 63.8 & 47.2 & 55.2 & 74.4 & 48.7 & 57.3 & 78.3 \\
\hline \multirow{6}{*}{ E85 Split HEV PHEV20 } & \multirow{3}{*}{ Unadjusted } & \begin{tabular}{|l|} 
UDDS \\
\end{tabular} & 88.2 & 88.2 & 96.6 & 106.0 & 90.6 & 103.0 & 134.0 & 95.4 & 114.6 & 134.6 & 98.9 & 119.0 & 142.4 \\
\hline & & HWFET & 73.9 & 73.9 & 81.5 & 89.9 & 76.5 & 85.8 & 94.7 & 79.9 & 79.9 & 111.3 & 82.7 & 84.4 & 118.3 \\
\hline & & Combined & 81.1 & 81.1 & 89.1 & 98.1 & 83.7 & 94.5 & 112.9 & 87.8 & 95.9 & 123.0 & 90.9 & 100.5 & 130.5 \\
\hline & \multirow{3}{*}{ Adjusted } & \begin{tabular}{|l|} 
UDDS \\
\end{tabular} & 60.8 & 60.8 & 65.6 & 70.9 & 62.2 & 69.2 & 85.5 & 64.9 & 75.5 & 85.8 & 66.9 & 777.8 & 89.6 \\
\hline & & HWFET & 51.8 & 51.8 & 57.1 & 62.8 & 53.7 & 60.0 & 66.1 & 56.0 & 56.0 & 77.4 & 57.9 & 59.1 & 82.2 \\
\hline & & Combined & 56.4 & 56.4 & 61.5 & 67.0 & 58.0 & 64.8 & 75.5 & 60.6 & 65.2 & 81.8 & 62.5 & 68.1 & 86.1 \\
\hline \multirow{6}{*}{ E85 Series HEV PHEV30 } & \multirow{3}{*}{ Unadjusted } & \begin{tabular}{|l|} 
UDDS \\
\end{tabular} & 82.2 & 82.2 & 91.1 & 100.4 & 83.9 & 97.1 & 130.8 & 91.2 & 111.5 & 161.5 & 95.0 & 115.7 & 168.9 \\
\hline & & HWFET & 82.9 & 82.9 & 92.1 & 100.8 & 85.2 & 97.8 & 132.3 & 91.5 & 111.0 & 156.1 & 95.4 & 117.0 & 166.0 \\
\hline & & Combined & 82.5 & 82.5 & 91.5 & 100.6 & 84.5 & 97.4 & 131.5 & 91.3 & 111.3 & 159.0 & 95.2 & 116.3 & 167.5 \\
\hline & \multirow{3}{*}{ Adjusted } & \begin{tabular}{|l|} 
UDDS \\
\end{tabular} & 57.2 & 57.2 & 62.4 & 67.8 & 58.3 & 65.9 & 83.9 & 62.5 & 73.8 & 98.6 & 64.7 & 76.1 & 101.9 \\
\hline & & HWFET & 58.0 & 58.0 & 64.3 & 70.3 & 59.6 & 68.2 & 91.6 & 63.9 & 77.2 & 107.6 & 66.6 & 81.3 & 114.1 \\
\hline & & Combined & 57.6 & 57.6 & 63.3 & 68.9 & 58.9 & 66.9 & 87.2 & 63.2 & 75.3 & 102.5 & 65.5 & 78.3 & 107.1 \\
\hline \multirow{6}{*}{ E85 Series HEV PHEV40 } & \multirow{3}{*}{ Unadjusted } & \begin{tabular}{|l|} 
UDDS \\
\end{tabular} & 93.3 & 93.3 & 104.7 & 115.0 & 97.3 & 111.8 & 151.3 & 105.0 & 128.6 & 190.5 & 109.4 & 133.9 & 201.2 \\
\hline & & HWFET & 101.5 & 101.5 & 112.8 & 123.2 & 104.4 & 119.8 & 162.7 & 112.1 & 136.2 & 192.5 & 117.0 & 143.9 & 204.8 \\
\hline & & Combined & 96.8 & 96.8 & 108.2 & 118.5 & 100.4 & 115.3 & 156.2 & 108.1 & 131.9 & 191.4 & 112.7 & 138.3 & 202.8 \\
\hline & \multirow{3}{*}{ Adjusted } & \begin{tabular}{|l|} 
UDDS \\
\end{tabular} & 63.8 & 63.8 & 70.2 & 75.7 & 66.0 & 74.0 & 93.9 & 70.3 & 82.8 & 1111.3 & 72.7 & 85.4 & 115.7 \\
\hline & & HWFET & 70.7 & 70.7 & 78.5 & 85.5 & 72.7 & 83.2 & 112.0 & 78.0 & 94.3 & 131.6 & 81.3 & 99.5 & 139.7 \\
\hline & & Combined & 66.7 & 66.7 & 73.7 & 79.8 & 68.9 & 77.9 & 101.2 & 73.5 & 87.6 & 119.6 & 76.3 & 91.2 & 125.4 \\
\hline
\end{tabular}


Argonne National Laboratory Report - Light-Duty Vehicle Fuel Consumption Displacement Potential Up to 2045

\begin{tabular}{|c|c|c|c|c|c|c|c|c|c|c|c|c|c|c|c|}
\hline & & & \multicolumn{13}{|c|}{ Midsize } \\
\hline & & & \multirow{2}{*}{$\begin{array}{c}2010 \\
\text { Ref }\end{array}$} & \multicolumn{3}{|c|}{2010} & \multicolumn{3}{|c|}{2015} & \multicolumn{3}{|c|}{2030} & \multicolumn{3}{|c|}{2045} \\
\hline & & & & low & avg & high & low & avg & high & low & avg & high & low & avg & high \\
\hline \multirow{6}{*}{ FC HEV } & \multirow{3}{*}{ Unadjusted } & UDDS & 56.7 & 56.7 & 68.9 & 81.8 & 66.7 & 80.7 & 95.0 & 74.6 & 91.6 & 115.1 & 78.9 & 95.6 & 120.9 \\
\hline & & HWFET & 61.5 & 61.5 & 73.8 & 86.1 & 71.3 & 83.9 & 96.6 & 77.4 & 93.0 & 113.1 & 81.3 & 97.2 & 120.7 \\
\hline & & Combined & 58.8 & 58.8 & 71.0 & 83.7 & 68.7 & 82.1 & 95.7 & 75.8 & 92.2 & 114.2 & 80.0 & 96.3 & 120.8 \\
\hline & \multirow{3}{*}{ Adjusted } & \begin{tabular}{|l|} 
UDDS \\
\end{tabular} & 41.4 & 41.4 & 49.1 & 57.0 & 47.8 & 56.4 & 64.7 & 52.7 & 62.8 & 75.8 & 55.3 & 65.1 & 78.8 \\
\hline & & HWFET & 43.3 & 43.3 & 51.8 & 60.2 & 50.0 & 58.7 & 67.4 & 54.2 & 65.0 & 78.6 & 56.9 & 67.9 & 83.8 \\
\hline & & Combined & 42.2 & 42.2 & 50.3 & 58.4 & 48.8 & 57.4 & 65.9 & 53.4 & 63.7 & 77.0 & 56.0 & 66.3 & 81.0 \\
\hline \multirow{6}{*}{ FC PHEV10 } & \multirow{3}{*}{ Unadjusted } & \begin{tabular}{|l|} 
UDDS \\
\end{tabular} & 71.8 & 71.8 & 85.5 & 100.7 & 83.3 & 97.6 & 113.8 & 90.3 & 110.1 & 136.4 & 95.0 & 113.8 & 143.1 \\
\hline & & \begin{tabular}{|l|} 
HWFET \\
\end{tabular} & 83.6 & 83.6 & 98.4 & 113.7 & 95.1 & 110.3 & 126.2 & 101.6 & 121.4 & 146.4 & 106.1 & 126.5 & 157.5 \\
\hline & & Combined & 76.6 & 76.6 & 90.9 & 106.1 & 88.2 & 103.0 & 119.1 & 95.1 & 114.9 & 140.7 & 99.7 & 119.2 & 149.2 \\
\hline & \multirow{3}{*}{ Adjusted } & \begin{tabular}{|l|} 
UDDS \\
\end{tabular} & 50.9 & 50.9 & 59.2 & 67.9 & 57.9 & 66.2 & 75.1 & 62.0 & 73.1 & 86.7 & 64.7 & 75.0 & 89.9 \\
\hline & & \begin{tabular}{|l|} 
HWFET \\
\end{tabular} & 58.5 & 58.5 & 68.7 & 79.1 & 66.4 & 76.8 & 87.5 & 70.8 & 84.3 & 101.1 & 73.9 & 87.7 & 108.5 \\
\hline & & Combined & 54.1 & 54.1 & 63.1 & 72.5 & 61.4 & 70.6 & 80.2 & 65.7 & 77.7 & 92.6 & 68.5 & 80.3 & 97.5 \\
\hline \multirow{6}{*}{ FC PHEV2O } & \multirow{3}{*}{ Unadjusted } & \begin{tabular}{|l|} 
UDDS \\
\end{tabular} & 86.0 & 86.0 & 102.8 & 121.1 & 100.1 & 117.6 & 137.4 & 108.7 & 132.9 & 165.6 & 114.5 & 137.7 & 173.6 \\
\hline & & HWFET & 83.4 & 83.4 & 98.8 & 114.8 & 95.7 & 111.4 & 127.9 & 102.6 & 123.2 & 149.3 & 107.5 & 128.6 & 159.3 \\
\hline & & Combined & 84.8 & 84.8 & 100.9 & 118.1 & 98.1 & 114.8 & 132.9 & 105.9 & 128.3 & 157.8 & 111.2 & 133.4 & 166.9 \\
\hline & \multirow{3}{*}{ Adjusted } & \begin{tabular}{|l|} 
UDDS \\
\end{tabular} & 59.5 & 59.5 & 69.1 & 78.9 & 67.6 & 77.1 & 87.2 & 72.3 & 84.9 & 100.5 & 75.4 & 87.3 & 104.1 \\
\hline & & HWFET & 58.4 & 58.4 & 68.9 & 79.8 & 66.8 & 77.5 & 88.7 & 71.5 & 85.5 & 103.0 & 74.9 & 89.1 & 109.7 \\
\hline & & Combined & 59.0 & 59.0 & 69.0 & 79.3 & 67.2 & 77.3 & 87.8 & 72.0 & 85.2 & 101.6 & 75.2 & 88.1 & 106.5 \\
\hline \multirow{6}{*}{ FC PHEV30 } & \multirow{3}{*}{ Unadjusted } & UDDS & 122.9 & 122.9 & 146.0 & 169.0 & 141.8 & 165.4 & 194.1 & 152.8 & 187.4 & 232.9 & 161.3 & 195.7 & 244.6 \\
\hline & & \begin{tabular}{|c|} 
HWFET \\
\end{tabular} & 132.4 & 132.4 & 124.4 & 142.7 & 120.2 & 139.3 & 160.5 & 128.3 & 154.4 & 186.8 & 134.7 & 161.8 & 199.6 \\
\hline & & \begin{tabular}{|l|} 
Combined \\
\end{tabular} & 127.0 & 127.0 & 135.4 & 156.0 & 131.2 & 152.5 & 177.4 & 140.7 & 170.9 & 209.6 & 148.1 & 178.8 & 222.1 \\
\hline & \multirow{3}{*}{ Adjusted } & \begin{tabular}{|l|} 
UDDS \\
\end{tabular} & 79.8 & 79.8 & 91.3 & 102.0 & 89.3 & 100.4 & 112.8 & 94.6 & 110.0 & 128.0 & 98.5 & 113.5 & 132.3 \\
\hline & & \begin{tabular}{|c|} 
HWFET \\
\end{tabular} & 91.7 & 91.7 & 86.3 & 98.6 & 83.4 & 96.3 & 110.5 & 88.9 & 106.5 & 127.9 & 93.2 & 111.3 & 136.3 \\
\hline & & Combined & 84.8 & 84.8 & 89.0 & 100.4 & 86.6 & 98.5 & 111.8 & 91.9 & 108.4 & 128.0 & 96.1 & 112.5 & 134.0 \\
\hline \multirow{6}{*}{ FC PHEV40 } & \multirow{3}{*}{ Unadjusted } & \begin{tabular}{|l|} 
UDDS \\
\end{tabular} & 141.6 & 141.6 & 168.7 & 195.6 & 163.7 & 191.3 & 225.5 & 176.5 & 217.4 & 271.2 & 186.9 & 227.5 & 284.8 \\
\hline & & HWFET & 133.4 & 133.4 & 157.8 & 181.1 & 152.2 & 176.6 & 204.3 & 162.7 & 196.4 & 238.2 & 171.0 & 206.0 & 254.5 \\
\hline & & Combined & 137.8 & 137.8 & 163.6 & 188.8 & 158.3 & 184.4 & 215.4 & 170.0 & 207.4 & 255.3 & 179.4 & 217.3 & 270.3 \\
\hline & \multirow{3}{*}{ Adjusted } & \begin{tabular}{|l|} 
UDDS \\
\end{tabular} & 89.2 & 89.2 & 101.9 & 113.4 & 99.6 & 111.6 & 125.2 & 105.3 & 122.1 & 141.5 & 109.8 & 126.0 & 145.9 \\
\hline & & HWFET & 92.4 & 92.4 & 108.7 & 124.1 & 105.0 & 121.2 & 139.3 & 112.0 & 134.2 & 161.4 & 117.5 & 140.5 & 171.8 \\
\hline & & Combined & 90.6 & 90.6 & 104.8 & 118.0 & 102.0 & 115.7 & 131.2 & 108.2 & 127.3 & 149.8 & 113.1 & 132.1 & 156.5 \\
\hline
\end{tabular}


Argonne National Laboratory Report - Light-Duty Vehicle Fuel Consumption Displacement Potential Up to 2045

$\underline{\text { Pev details }}$ 
Argonne National Laboratory Report - Light-Duty Vehicle Fuel Consumption Displacement Potential Up to 2045

\begin{tabular}{|c|c|c|c|c|c|c|c|c|c|c|c|c|c|c|c|c|}
\hline & & & & \multicolumn{13}{|c|}{ Midsize } \\
\hline & & & & \multirow{2}{*}{$\frac{2010}{\text { Ref }}$} & \multicolumn{3}{|c|}{2010} & \multicolumn{3}{|c|}{2015} & \multicolumn{3}{|c|}{2030} & \multicolumn{3}{|c|}{2045} \\
\hline & & & & & low & avg & high & low & avg & high & low & avg & high & low & avg & high \\
\hline \multirow{25}{*}{ SI Split HEV PHEV10 } & \multirow{9}{*}{ UDDS } & \multirow{4}{*}{$C D$} & Wh/mile & 189.2 & 189.2 & 176.3 & 172.6 & 188.6 & 176.2 & 159.3 & 180.0 & 164.8 & 143.3 & 176.2 & 159.5 & 146.6 \\
\hline & & & Distance & 12.3 & 12.3 & 12.3 & 11.6 & 12.3 & 11.7 & 11.7 & 12.3 & 11.8 & 11.4 & 12.3 & 11.7 & 10.2 \\
\hline & & & MPGGE Unadjusted & 874.1 & 874.1 & 974.6 & 1547.0 & 901.6 & 1400.1 & 1921.7 & 944.5 & 1731.6 & 3224.9 & 979.3 & 1812.0 & N/A \\
\hline & & & MPGGE Adjusted & 253.1 & 253.1 & 262.7 & 299.3 & 255.8 & 292.0 & 313.8 & 259.9 & 307.0 & 341.4 & 263.1 & 310.0 & N/A \\
\hline & & \multirow{2}{*}{ CS } & MPGGE Unadjusted & 55.9 & 55.9 & 60.7 & 65.7 & 56.9 & 64.0 & 78.5 & 60.0 & 70.4 & 98.3 & 62.0 & 73.0 & 102.9 \\
\hline & & & MPGGE Adjusted & 40.8 & 40.8 & 44.0 & 47.2 & 41.5 & 46.0 & 55.0 & 43.5 & 50.1 & 66.6 & 44.8 & 51.7 & 69.1 \\
\hline & & \multirow{3}{*}{$C D+C S$} & Wh/mile & 39.4 & 39.4 & 36.7 & 36.0 & 39.3 & 36.7 & 33.2 & 37.5 & 34.3 & 29.9 & 36.7 & 33.2 & 30.5 \\
\hline & & & MPGGE Unadjusted & 66.5 & 66.5 & 72.2 & 78.5 & 67.7 & 76.3 & 93.8 & 71.4 & 84.1 & 117.7 & 73.8 & 87.2 & 124.0 \\
\hline & & & MPGGE Adjusted & 47.6 & 47.6 & 51.2 & 55.0 & 48.4 & 53.7 & 64.0 & 50.7 & 58.4 & 77.1 & 52.2 & 60.2 & 80.4 \\
\hline & \multirow{9}{*}{ HWFET } & \multirow{4}{*}{$C D$} & Wh/mile & 207.6 & 207.6 & 190.6 & 188.1 & 206.2 & 193.4 & 178.5 & 197.3 & 185.0 & 154.7 & 192.3 & 178.5 & 147.6 \\
\hline & & & \begin{tabular}{|l|} 
Distance \\
\end{tabular} & 11.1 & 11.1 & 11.1 & 10.6 & 11.1 & 10.6 & 9.2 & 11.1 & 9.6 & 9.1 & 11.1 & 9.2 & 9.4 \\
\hline & & & MPGGE Unadjusted & 353.5 & 353.5 & 384.8 & 564.1 & 362.2 & 550.3 & 822.3 & 377.3 & 744.8 & 926.9 & 389.7 & 777.9 & 1200.4 \\
\hline & & & MPGGE Adjusted & 233.8 & 233.8 & 252.9 & 357.8 & 239.2 & 350.0 & 496.5 & 248.3 & 456.3 & 548.9 & 255.9 & 473.6 & 677.2 \\
\hline & & \multirow{2}{*}{ CS } & MPGGE Unadjusted & 52.4 & 52.4 & 57.4 & 61.8 & 53.5 & 60.2 & 73.4 & 56.4 & 66.0 & 89.9 & 58.4 & 69.0 & 95.8 \\
\hline & & & MPGGE Adjusted & 37.0 & 37.0 & 40.4 & 43.5 & 37.7 & 42.4 & 51.5 & 39.7 & 46.4 & 62.8 & 41.1 & 48.4 & 66.9 \\
\hline & & \multirow{3}{*}{$\mathrm{CD}+\mathrm{CS}$} & Wh/mile & 61.1 & 61.1 & 56.1 & 55.3 & 60.7 & 56.9 & 52.5 & 58.0 & 54.4 & 45.5 & 56.6 & 52.5 & 43.4 \\
\hline & & & MPGGE Unadjusted & 65.9 & 65.9 & 72.1 & 78.7 & 67.2 & 76.6 & 94.0 & 70.8 & 84.5 & 114.8 & 73.3 & 88.3 & 123.0 \\
\hline & & & \begin{tabular}{|l|} 
MPGEE Adjusted \\
\end{tabular} & 46.3 & 46.3 & 50.6 & 55.1 & 47.2 & 53.7 & 65.6 & 49.7 & 59.1 & 79.8 & 51.4 & 61.7 & 85.3 \\
\hline & \multirow{7}{*}{ Combined } & \multirow{2}{*}{$C D$} & MPGGE Unadjusted & 525.7 & 525.7 & 576.7 & 867.1 & 539.9 & 826.1 & 1199.8 & 563.3 & 1084.8 & 1524.3 & 582.6 & 1133.7 & 2609.2 \\
\hline & & & MPGGE Adjusted & 244.0 & 244.0 & 258.2 & 323.0 & 248.1 & 315.5 & 376.0 & 254.6 & 360.0 & 411.4 & 259.8 & 367.1 & 481.5 \\
\hline & & \multirow{2}{*}{ CS } & MPGGE Unadjusted & 54.3 & 54.3 & 59.2 & 63.9 & 55.3 & 62.2 & 76.1 & 58.3 & 68.3 & 94.3 & 60.3 & 71.1 & 99.6 \\
\hline & & & MPGGE Adjusted & 39.0 & 39.0 & 42.3 & 45.4 & 39.7 & 44.3 & 53.4 & 41.7 & 48.3 & 64.8 & 43.1 & 50.2 & 68.1 \\
\hline & & \multirow{3}{*}{$\mathrm{CD}+\mathrm{CS}$} & Wh/mile & 49.2 & 49.2 & 45.4 & 44.7 & 48.9 & 45.8 & 41.9 & 46.7 & 43.4 & 36.9 & 45.6 & 41.9 & 36.3 \\
\hline & & & \begin{tabular}{|l|l} 
MPGE Unadjusted \\
\end{tabular} & 66.2 & 66.2 & 72.2 & 78.6 & 67.5 & 76.4 & 93.9 & 71.1 & 84.3 & 116.4 & 73.6 & 87.7 & 123.5 \\
\hline & & & MPGGE Adjusted & 47.0 & 47.0 & 50.9 & 55.1 & 47.9 & 53.7 & 64.7 & 50.3 & 58.7 & 78.3 & 51.8 & 60.9 & 82.6 \\
\hline
\end{tabular}


Argonne National Laboratory Report - Light-Duty Vehicle Fuel Consumption Displacement Potential Up to 2045

\begin{tabular}{|c|c|c|c|c|c|c|c|c|c|c|c|c|c|c|c|c|}
\hline & & & & \multicolumn{13}{|c|}{ Midsize } \\
\hline & & & & \multirow{2}{*}{$\begin{array}{c}2010 \\
\text { Ref }\end{array}$} & \multicolumn{3}{|c|}{2010} & \multicolumn{3}{|c|}{2015} & \multicolumn{3}{|c|}{2030} & \multicolumn{3}{|c|}{2045} \\
\hline \multirow{26}{*}{ SI Split HEV PHEV2O } & & \multirow{5}{*}{$C D$} & & & low & avg & high & low & avg & high & low & avg & high & low & avg & high \\
\hline & \multirow{9}{*}{ UDDS } & & Wh/mile & 190.3 & 190.3 & 178.3 & 173.4 & 194.5 & 178.2 & 161.1 & 182.0 & 166.4 & 140.4 & 178.0 & 161.6 & 143.9 \\
\hline & & & Distance & 23.9 & 23.9 & 23.9 & 22.9 & 23.8 & 22.9 & 22.8 & 23.9 & 22.8 & 22.2 & 24.0 & 22.8 & 20.6 \\
\hline & & & MPGGE Unadjusted & 817.3 & 817.3 & 945.9 & 1408.5 & 1001.0 & 1361.4 & 1879.9 & 921.4 & 1693.7 & 3143.9 & 953.4 & 1777.8 & N/A \\
\hline & & & MPGGE Adjusted & 247.0 & 247.0 & 260.1 & 292.5 & 265.0 & 289.9 & 312.4 & 257.8 & 305.5 & 340.3 & 260.8 & 308.8 & $\mathrm{~N} / \mathrm{A}$ \\
\hline & & \multirow{2}{*}{ CS } & MPGGE Unadjusted & 55.5 & 55.5 & 60.5 & 65.6 & 56.5 & 63.7 & 78.3 & 59.9 & 70.6 & 98.2 & 62.0 & 73.2 & 102.7 \\
\hline & & & MPGGE Adjusted & 40.6 & 40.6 & 43.8 & 47.1 & 41.3 & 45.9 & 54.9 & 43.4 & 50.2 & 66.5 & 44.8 & 51.8 & 69.0 \\
\hline & & \multirow{3}{*}{$\mathrm{CD}+\mathrm{CS}$} & Wh/mile & 100.3 & 100.3 & 94.0 & 91.3 & 102.2 & 93.9 & 85.2 & 95.9 & 88.0 & 53.5 & 93.8 & 85.4 & 54.8 \\
\hline & & & MPGGE Unadjusted & 94.1 & 94.1 & 103.0 & 113.1 & 96.7 & 109.9 & 135.5 & 101.8 & 122.2 & 142.4 & 105.4 & 126.7 & 151.0 \\
\hline & & & MPGGE Adjusted & 64.2 & 64.2 & 69.2 & 74.7 & 65.7 & 73.0 & 86.2 & 68.6 & 79.5 & 89.6 & 70.5 & 81.8 & 93.7 \\
\hline & \multirow{9}{*}{ HWFET } & \multirow{4}{*}{$C D$} & Wh/mile & 209.4 & 209.4 & 192.5 & 188.5 & 213.3 & 187.0 & 197.6 & 199.2 & 198.3 & 174.3 & 194.1 & 197.2 & 163.5 \\
\hline & & & Distance & 21.6 & 21.6 & 21.8 & 20.8 & 21.5 & 21.4 & 18.3 & 21.7 & 19.3 & 17.5 & 21.8 & 18.3 & 17.6 \\
\hline & & & MPGGE Unadjusted & 346.1 & 346.1 & 379.3 & 527.6 & 405.4 & 418.6 & $\mathrm{~N} / \mathrm{A}$ & 372.0 & 2210.9 & $\mathrm{~N} / \mathrm{A}$ & 384.5 & $\mathrm{~N} / \mathrm{A}$ & N/A \\
\hline & & & MPGGE Adjusted & 229.3 & 229.3 & 249.6 & 337.0 & 265.3 & 273.2 & $\mathrm{~N} / \mathrm{A}$ & 245.1 & 1060.9 & $\mathrm{~N} / \mathrm{A}$ & 252.7 & $\mathrm{~N} / \mathrm{A}$ & N/A \\
\hline & & \multirow{2}{*}{ CS } & MPGGE Unadjusted & 52.0 & 52.0 & 57.0 & 61.5 & 53.1 & 59.9 & 73.2 & 56.0 & 65.7 & 89.8 & 58.0 & 68.7 & 95.7 \\
\hline & & & MPGGE Adjusted & 36.6 & 36.6 & 40.1 & 43.3 & 37.4 & 42.1 & 51.3 & 39.4 & 46.1 & 62.8 & 40.8 & 48.3 & 66.8 \\
\hline & & \multirow{3}{*}{$C D+C S$} & Wh/mile & 107.7 & 107.7 & 99.0 & 97.1 & 109.4 & 96.2 & 58.1 & 102.5 & 58.3 & 51.3 & 99.8 & 58.0 & 48.1 \\
\hline & & & MPGGE Unadjusted & 79.6 & 79.6 & 87.2 & 96.3 & 82.2 & 92.1 & 96.3 & 85.7 & 85.6 & 118.2 & 88.7 & 90.5 & 125.9 \\
\hline & & & MPGGE Adjusted & 55.8 & 55.8 & 61.0 & 67.2 & 57.5 & 64.4 & 67.2 & 60.0 & 59.9 & 82.1 & 62.0 & 63.2 & 87.3 \\
\hline & \multirow{7}{*}{ Combined } & \multirow{2}{*}{$C D$} & MPGGE Unadjusted & 506.8 & 506.8 & 565.7 & 804.3 & 602.6 & 676.1 & 3339.6 & 553.5 & 1892.9 & 5500.2 & 572.4 & 3162.1 & N/A \\
\hline & & & MPGGE Adjusted & 238.7 & 238.7 & 255.2 & 311.0 & 265.1 & 282.1 & 523.9 & 251.9 & 449.6 & 566.7 & 257.1 & 518.3 & 640.8 \\
\hline & & \multirow{2}{*}{ CS } & MPGGE Unadjusted & 53.8 & 53.8 & 58.9 & 63.7 & 54.9 & 62.0 & 75.9 & 58.1 & 68.3 & 94.3 & 60.1 & 71.1 & 99.4 \\
\hline & & & MPGGE Adjusted & 38.7 & 38.7 & 42.1 & 45.3 & 39.4 & 44.1 & 53.3 & 41.5 & 48.3 & 64.8 & 42.9 & 50.2 & 68.0 \\
\hline & & \multirow{3}{*}{$C D+C S$} & Wh/mile & 103.6 & 103.6 & 96.2 & 93.9 & 105.5 & 94.9 & 73.0 & 98.9 & 74.6 & 52.5 & 96.5 & 73.1 & 51.8 \\
\hline & & & MPGGE Unadjusted & 87.0 & 87.0 & 95.2 & 104.9 & 89.6 & 101.1 & 114.5 & 93.9 & 102.5 & 130.4 & 97.2 & 107.4 & 138.6 \\
\hline & & & MPGGE Adjusted & 60.1 & 60.1 & 65.3 & 71.1 & 61.7 & 68.8 & 76.5 & 64.4 & 69.3 & 86.1 & 66.4 & 72.2 & 90.7 \\
\hline
\end{tabular}


Argonne National Laboratory Report - Light-Duty Vehicle Fuel Consumption Displacement Potential Up to 2045

\begin{tabular}{|c|c|c|c|c|c|c|c|c|c|c|c|c|c|c|c|c|}
\hline & & & & \multicolumn{13}{|c|}{ Midsize } \\
\hline & & & & \multirow{2}{*}{$\frac{2010}{\text { Ref }}$} & \multicolumn{3}{|c|}{2010} & \multicolumn{3}{|c|}{2015} & \multicolumn{3}{|c|}{2030} & \multicolumn{3}{|c|}{2045} \\
\hline & & & & & low & avg & high & low & avg & high & low & avg & high & low & avg & high \\
\hline \multirow{25}{*}{ SI Series HEV PHEV30 } & \multirow{9}{*}{ UDDS } & \multirow{4}{*}{$C D$} & Wh/mile & 280.1 & 280.1 & 258.7 & 241.5 & 276.1 & 248.2 & 219.2 & 262.9 & 226.2 & 188.2 & 256.6 & 218.1 & 180.0 \\
\hline & & & Distance & 30.9 & 30.9 & 30.9 & 31.0 & 30.9 & 30.9 & 30.9 & 31.0 & 30.9 & 30.7 & 31.0 & 30.9 & 30.7 \\
\hline & & & MPGGE Unadjusted & N/A & N/A & N/A & N/A & N/A & N/A & N/A & N/A & N/A & N/A & N/A & N/A & N/A \\
\hline & & & MPGGE Adjusted & N/A & N/A & N/A & N/A & N/A & N/A & N/A & N/A & N/A & N/A & N/A & N/A & N/A \\
\hline & & \multirow{2}{*}{ CS } & MPGGE Unadjusted & 40.7 & 40.7 & 45.6 & 50.2 & 42.4 & 48.5 & 61.9 & 45.6 & 55.6 & 80.0 & 47.4 & 57.7 & 83.6 \\
\hline & & & MPGGE Adjusted & 30.6 & 30.6 & 34.0 & 37.1 & 31.8 & 35.9 & 44.8 & 34.0 & 40.7 & 55.9 & 35.2 & 42.0 & 58.1 \\
\hline & & \multirow{3}{*}{$\mathrm{CD}+\mathrm{CS}$} & Wh/mile & 180.5 & 180.5 & 166.7 & 155.6 & 177.9 & 159.9 & 141.9 & 169.4 & 146.4 & 122.3 & 165.3 & 141.0 & 116.9 \\
\hline & & & MPGGE Unadjusted & 86.6 & 86.6 & 97.0 & 106.8 & 90.3 & 103.2 & 131.8 & 97.0 & 118.4 & 170.1 & 100.9 & 122.8 & 177.9 \\
\hline & & & MPGGE Adjusted & 59.9 & 59.9 & 65.8 & 71.3 & 62.0 & 69.3 & 84.4 & 65.8 & 77.5 & 102.5 & 68.0 & 79.8 & 105.9 \\
\hline & \multirow{9}{*}{ HWFET } & \multirow{4}{*}{$C D$} & Wh/mile & 271.0 & 271.0 & 248.1 & 231.7 & 267.4 & 238.4 & 209.2 & 255.0 & 217.0 & 181.1 & 247.7 & 208.5 & 170.0 \\
\hline & & & \begin{tabular}{|l|} 
Distance \\
\end{tabular} & 31.8 & 31.8 & 32.1 & 32.2 & 31.8 & 32.1 & 32.2 & 31.8 & 32.0 & 31.9 & 31.9 & 32.2 & 32.5 \\
\hline & & & MPGGE Unadjusted & $N / A$ & N/A & N/A & $\mathrm{N} / \mathrm{A}$ & $\mathrm{N} / \mathrm{A}$ & N/A & N/A & N/A & N/A & N/A & N/A & $\mathrm{N} / \mathrm{A}$ & $\mathrm{N} / \mathrm{A}$ \\
\hline & & & MPGGE Adjusted & N/A & N/A & N/A & N/A & N/A & N/A & N/A & N/A & N/A & N/A & N/A & N/A & N/A \\
\hline & & \multirow{2}{*}{ CS } & MPGGE Unadjusted & 40.6 & 40.6 & 45.1 & 49.4 & 41.7 & 47.8 & 61.3 & 44.8 & 54.3 & 75.8 & 46.7 & 57.1 & 80.6 \\
\hline & & & MPGGE Adjusted & 28.7 & 28.7 & 31.9 & 34.8 & 29.5 & 33.7 & 43.1 & 31.6 & 38.2 & 53.1 & 32.9 & 40.2 & 56.5 \\
\hline & & \multirow{3}{*}{$\mathrm{CD}+\mathrm{CS}$} & Wh/mile & 183.1 & 183.1 & 167.7 & 156.6 & 180.7 & 161.1 & 141.4 & 172.3 & 146.7 & 122.4 & 167.4 & 140.9 & 114.9 \\
\hline & & & MPGGE Unadjusted & 88.3 & 88.3 & 98.1 & 107.3 & 90.7 & 104.0 & 133.3 & 97.3 & 118.0 & 164.7 & 101.4 & 124.2 & 175.3 \\
\hline & & & MPGGE Adjusted & 61.7 & 61.7 & 68.5 & 74.7 & 63.4 & 72.5 & 92.3 & 67.9 & 82.0 & 113.3 & 70.7 & 86.1 & 120.3 \\
\hline & \multirow{7}{*}{ Combined } & \multirow{2}{*}{$C D$} & MPGGE Unadjusted & $\mathrm{N} / \mathrm{A}$ & $\mathrm{N} / \mathrm{A}$ & $\mathrm{N} / \mathrm{A}$ & $\mathrm{N} / \mathrm{A}$ & $\mathrm{N} / \mathrm{A}$ & N/A & $\mathrm{N} / \mathrm{A}$ & $\mathrm{N} / \mathrm{A}$ & $\mathrm{N} / \mathrm{A}$ & $N / A$ & $\mathrm{~N} / \mathrm{A}$ & $\mathrm{N} / \mathrm{A}$ & $\mathrm{N} / \mathrm{A}$ \\
\hline & & & MPGGE Adjusted & N/A & N/A & N/A & N/A & N/A & N/A & N/A & N/A & N/A & $\mathrm{N} / \mathrm{A}$ & N/A & N/A & N/A \\
\hline & & \multirow{2}{*}{ CS } & MPGGE Unadjusted & 40.7 & 40.7 & 45.4 & 49.8 & 42.1 & 48.2 & 61.6 & 45.2 & 55.0 & 78.0 & 47.1 & 57.4 & 82.3 \\
\hline & & & MPGGE Adjusted & 29.7 & 29.7 & 33.0 & 36.0 & 30.7 & 34.9 & 44.0 & 32.9 & 39.5 & 54.6 & 34.1 & 41.2 & 57.4 \\
\hline & & \multirow{3}{*}{$\mathrm{CD}+\mathrm{CS}$} & Wh/mile & 181.7 & 181.7 & 167.1 & 156.0 & 179.1 & 160.4 & 141.7 & 170.7 & 146.5 & 122.3 & 166.3 & 141.0 & 116.0 \\
\hline & & & \begin{tabular}{|l|} 
MPGGE Unadjusted \\
\end{tabular} & 87.3 & 87.3 & 97.5 & 107.0 & 90.5 & 103.5 & 132.4 & 97.1 & 118.2 & 167.6 & 101.1 & 123.4 & 176.7 \\
\hline & & & MPGGE Adjusted & 60.7 & 60.7 & 67.0 & 72.8 & 62.6 & 70.7 & 87.8 & 66.8 & 79.4 & 107.1 & 69.2 & 82.5 & 112.0 \\
\hline
\end{tabular}


Argonne National Laboratory Report - Light-Duty Vehicle Fuel Consumption Displacement Potential Up to 2045

\begin{tabular}{|c|c|c|c|c|c|c|c|c|c|c|c|c|c|c|c|c|}
\hline & & & & \multicolumn{13}{|c|}{ Midsize } \\
\hline \multirow{27}{*}{ SI Series HEV PHEV40 } & & & & 2010 & & 2010 & & & 2015 & & & 2030 & & & 2045 & \\
\hline & & \multirow{5}{*}{$C D$} & & Ref & low & avg & high & low & avg & high & low & avg & high & low & avg & high \\
\hline & \multirow{9}{*}{ UDDS } & & Wh/mile & 286.1 & 286.1 & 265.0 & 246.5 & 281.9 & 253.9 & 223.5 & 269.0 & 231.0 & 190.2 & 261.9 & 222.4 & 181.9 \\
\hline & & & Distance & 40.8 & 40.8 & 40.9 & 41.0 & 40.9 & 40.8 & 40.8 & 40.9 & 40.8 & 40.5 & 40.9 & 40.7 & 40.6 \\
\hline & & & MPGGE Unadjusted & N/A & N/A & N/A & N/A & N/A & N/A & $\mathrm{N} / \mathrm{A}$ & $\mathrm{N} / \mathrm{A}$ & N/A & N/A & N/A & N/A & N/A \\
\hline & & & MPGGE Adjusted & N/A & $\mathrm{N} / \mathrm{A}$ & N/A & N/A & N/A & N/A & N/A & $\mathrm{N} / \mathrm{A}$ & N/A & N/A & N/A & N/A & N/A \\
\hline & & \multirow{2}{*}{ CS } & MPGGE Unadjusted & 40.2 & 40.2 & 44.5 & 49.1 & 41.0 & 47.5 & 61.0 & 44.6 & 54.7 & 80.4 & 46.5 & 56.9 & 84.3 \\
\hline & & & MPGGE Adjusted & 30.2 & 30.2 & 33.2 & 36.4 & 30.8 & 35.3 & 44.1 & 33.3 & 40.1 & 56.2 & 34.6 & 41.5 & 58.5 \\
\hline & & \multirow{3}{*}{$C D+C S$} & Wh/mile & 210.4 & 210.4 & 195.0 & 181.4 & 207.3 & 186.9 & 165.7 & 198.0 & 171.2 & 141.2 & 192.7 & 164.6 & 135.0 \\
\hline & & & MPGGE Unadjusted & 100.4 & 100.4 & 111.3 & 122.8 & 102.5 & 118.8 & 152.5 & 111.6 & 136.8 & 201.1 & 116.2 & 142.3 & 210.8 \\
\hline & & & MPGGE Adjusted & 67.8 & 67.8 & 73.7 & 79.8 & 68.9 & 77.7 & 99.4 & 73.9 & 86.9 & 115.7 & 76.3 & 89.6 & 119.6 \\
\hline & \multirow{9}{*}{ HWFET } & \multirow{4}{*}{$C D$} & Wh/mile & 273.9 & 273.9 & 250.7 & 233.8 & 270.2 & 240.6 & 211.3 & 257.4 & 219.4 & 184.0 & 250.0 & 209.9 & 172.7 \\
\hline & & & Distance & 42.4 & 42.4 & 42.9 & 43.0 & 42.3 & 42.9 & 43.1 & 42.4 & 42.8 & 41.8 & 42.5 & 43.1 & 42.7 \\
\hline & & & MPGGE Unadjusted & $\mathrm{N} / \mathrm{A}$ & $\mathrm{N} / \mathrm{A}$ & N/A & N/A & $\mathrm{N} / \mathrm{A}$ & N/A & N/A & N/A & $\mathrm{N} / \mathrm{A}$ & $\mathrm{N} / \mathrm{A}$ & $\mathrm{N} / \mathrm{A}$ & $\mathrm{N} / \mathrm{A}$ & $\mathrm{N} / \mathrm{A}$ \\
\hline & & & MPGGE Adjusted & $\mathrm{N} / \mathrm{A}$ & $\mathrm{N} / \mathrm{A}$ & $\mathrm{N} / \mathrm{A}$ & $\mathrm{N} / \mathrm{A}$ & N/A & N/A & N/A & N/A & $\mathrm{N} / \mathrm{A}$ & $\mathrm{N} / \mathrm{A}$ & N/A & $\mathrm{N} / \mathrm{A}$ & $\mathrm{N} / \mathrm{A}$ \\
\hline & & \multirow{2}{*}{ CS } & MPGGE Unadjusted & 40.0 & 40.0 & 44.5 & 49.1 & 41.1 & 47.2 & 60.7 & 44.2 & 53.9 & 75.2 & 46.0 & 56.6 & 80.3 \\
\hline & & & MPGGE Adjusted & 28.3 & 28.3 & 31.4 & 34.6 & 29.0 & 33.3 & 42.7 & 31.2 & 38.0 & 52.7 & 32.5 & 39.8 & 56.2 \\
\hline & & \multirow{3}{*}{$C D+C S$} & Wh/mile & 215.4 & 215.4 & 197.2 & 184.0 & 212.5 & 189.3 & 166.6 & 202.6 & 173.0 & 145.6 & 196.7 & 165.3 & 136.7 \\
\hline & & & MPGGE Unadjusted & 108.1 & 108.1 & 120.1 & 132.6 & 111.1 & 127.5 & 164.1 & 119.3 & 145.6 & 203.2 & 124.4 & 152.9 & 217.0 \\
\hline & & & MPGGE Adjusted & 75.3 & 75.3 & 83.4 & 91.9 & 77.3 & 88.4 & 112.9 & 82.9 & 100.6 & 138.7 & 86.3 & 105.4 & 147.6 \\
\hline & \multirow{7}{*}{ Combined } & \multirow{2}{*}{$C D$} & MPGGE Unadjusted & N/A & $\mathrm{N} / \mathrm{A}$ & $\mathrm{N} / \mathrm{A}$ & N/A & $\mathrm{N} / \mathrm{A}$ & $\mathrm{N} / \mathrm{A}$ & N/A & N/A & N/A & $\mathrm{N} / \mathrm{A}$ & $\mathrm{N} / \mathrm{A}$ & $\mathrm{N} / \mathrm{A}$ & $\mathrm{N} / \mathrm{A}$ \\
\hline & & & MPGGE Adjusted & N/A & N/A & N/A & N/A & N/A & N/A & N/A & N/A & N/A & N/A & N/A & N/A & N/A \\
\hline & & \multirow{2}{*}{ CS } & MPGGE Unadjusted & 40.1 & 40.1 & 44.5 & 49.1 & 41.0 & 47.4 & 60.9 & 44.4 & 54.3 & 78.0 & 46.3 & 56.8 & 82.5 \\
\hline & & & MPGGE Adjusted & 29.3 & 29.3 & 32.4 & 35.5 & 30.0 & 34.3 & 43.5 & 32.3 & 39.1 & 54.6 & 33.6 & 40.7 & 57.5 \\
\hline & & \multirow{3}{*}{$C D+C S$} & Wh/mile & 212.7 & 212.7 & 196.0 & 182.6 & 209.7 & 188.0 & 166.1 & 200.0 & 172.0 & 143.2 & 194.5 & 164.9 & 135.8 \\
\hline & & & MPGGE Unadjusted & 103.7 & 103.7 & 115.1 & 127.0 & 106.2 & 122.5 & 157.5 & 114.9 & 140.7 & 202.1 & 119.7 & 146.9 & 213.5 \\
\hline & & & MPGGE Adjusted & 70.9 & 70.9 & 77.8 & 84.8 & 72.5 & 82.2 & 101.9 & 77.7 & 92.6 & 125.0 & 80.5 & 96.1 & 130.7 \\
\hline
\end{tabular}


Argonne National Laboratory Report - Light-Duty Vehicle Fuel Consumption Displacement Potential Up to 2045

\begin{tabular}{|c|c|c|c|c|c|c|c|c|c|c|c|c|c|c|c|c|}
\hline & & & & \multicolumn{13}{|c|}{ Midsize } \\
\hline & & & & \multirow{2}{*}{$\frac{2010}{\text { Ref }}$} & \multicolumn{3}{|c|}{2010} & \multicolumn{3}{|c|}{2015} & \multicolumn{3}{|c|}{2030} & \multicolumn{3}{|c|}{2045} \\
\hline & & & & & low & avg & high & low & avg & high & low & avg & high & low & avg & high \\
\hline \multirow{25}{*}{ CI Split HEV PHEV10 } & \multirow{9}{*}{ UDDS } & \multirow{4}{*}{$C D$} & Wh/mile & 195.7 & 195.7 & 182.5 & 173.6 & 194.8 & 178.1 & 166.7 & 185.9 & 171.8 & 150.6 & 181.8 & 166.8 & 144.7 \\
\hline & & & Distance & 12.7 & 12.7 & 12.7 & 12.3 & 12.6 & 12.4 & 11.9 & 12.7 & 11.9 & 11.6 & 12.8 & 11.9 & 11.5 \\
\hline & & & MPGGE Unadjusted & 816.7 & 816.7 & 896.4 & 1096.0 & 832.5 & 1058.2 & 1759.0 & 890.6 & 1683.5 & 2854.6 & 929.6 & 1806.3 & 3051.2 \\
\hline & & & MPGGE Adjusted & 246.9 & 246.9 & 255.3 & 272.7 & 248.7 & 269.7 & 308.1 & 254.7 & 305.1 & 335.7 & 258.5 & 309.8 & 338.9 \\
\hline & & \multirow{2}{*}{ CS } & MPGGE Unadjusted & 56.7 & 56.7 & 61.0 & 67.0 & 57.0 & 65.3 & 75.3 & 61.5 & 72.9 & 91.6 & 64.3 & 76.4 & 95.6 \\
\hline & & & MPGGE Adjusted & 41.4 & 41.4 & 44.2 & 48.0 & 41.6 & 46.9 & 53.1 & 44.5 & 51.7 & 62.8 & 46.3 & 53.8 & 65.0 \\
\hline & & \multirow{3}{*}{$C D+C S$} & Wh/mile & 40.8 & 40.8 & 38.0 & 36.2 & 40.6 & 37.1 & 34.7 & 38.7 & 35.8 & 31.4 & 37.9 & 34.8 & 30.2 \\
\hline & & & MPGGE Unadjusted & 67.4 & 67.4 & 72.5 & 79.7 & 67.8 & 77.6 & 89.9 & 73.1 & 87.1 & 109.6 & 76.4 & 91.2 & 114.4 \\
\hline & & & MPGGE Adjusted & 48.2 & 48.2 & 51.4 & 55.8 & 48.5 & 54.5 & 61.8 & 51.7 & 60.2 & 72.8 & 53.8 & 62.6 & 75.4 \\
\hline & \multirow{9}{*}{ HWFET } & \multirow{4}{*}{$C D$} & Wh/mile & 211.3 & 211.3 & 196.4 & 184.6 & 210.2 & 189.8 & 187.0 & 200.8 & 193.7 & 163.3 & 195.8 & 187.7 & 156.2 \\
\hline & & & \begin{tabular}{|l|} 
Distance \\
\end{tabular} & 11.3 & 11.3 & 11.3 & 11.2 & 11.1 & 11.2 & 9.8 & 11.3 & 9.8 & 9.5 & 11.3 & 9.7 & 9.6 \\
\hline & & & MPGGE Unadjusted & 341.1 & 341.1 & 391.6 & 430.3 & 347.7 & 419.8 & 992.0 & 370.8 & 957.0 & 1168.0 & 388.5 & 1080.3 & 1566.2 \\
\hline & & & MPGGE Adjusted & 226.2 & 226.2 & 257.0 & 280.3 & 230.3 & 273.9 & 580.6 & 244.4 & 563.7 & 662.6 & 255.1 & 622.4 & 830.7 \\
\hline & & \multirow{2}{*}{ CS } & MPGGE Unadjusted & 52.6 & 52.6 & 57.2 & 62.6 & 53.0 & 60.9 & 70.0 & 57.1 & 67.6 & 83.6 & 59.8 & 71.6 & 88.7 \\
\hline & & & MPGGE Adjusted & 37.1 & 37.1 & 40.2 & 44.0 & 37.4 & 42.8 & 49.1 & 40.2 & 47.5 & 58.5 & 42.1 & 50.2 & 62.0 \\
\hline & & \multirow{3}{*}{$\mathrm{CD}+\mathrm{CS}$} & Wh/mile & 62.2 & 62.2 & 57.8 & 54.3 & 61.8 & 55.8 & 55.0 & 59.1 & 57.0 & 48.0 & 57.6 & 55.2 & 46.0 \\
\hline & & & MPGGE Unadjusted & 66.0 & 66.0 & 71.9 & 78.7 & 66.6 & 76.6 & 90.1 & 71.7 & 87.1 & 107.6 & 75.1 & 92.2 & 114.7 \\
\hline & & & MPGGE Adjusted & 46.4 & 46.4 & 50.5 & 55.2 & 46.8 & 53.7 & 63.0 & 50.3 & 60.9 & 74.9 & 52.6 & 64.4 & 79.7 \\
\hline & \multirow{7}{*}{ Combined } & \multirow{2}{*}{$C D$} & MPGGE Unadjusted & 501.9 & 501.9 & 567.3 & 646.2 & 511.5 & 628.2 & 1305.0 & 546.1 & 1254.8 & 1730.2 & 571.4 & 1386.9 & 2138.7 \\
\hline & & & MPGGE Adjusted & 237.1 & 237.1 & 256.1 & 276.0 & 240.0 & 271.6 & 390.6 & 250.0 & 384.5 & 431.5 & 257.0 & 400.3 & 462.0 \\
\hline & & \multirow{2}{*}{ CS } & MPGGE Unadjusted & 54.8 & 54.8 & 59.2 & 65.0 & 55.2 & 63.2 & 72.8 & 59.4 & 70.4 & 87.8 & 62.2 & 74.1 & 92.4 \\
\hline & & & MPGGE Adjusted & 39.3 & 39.3 & 42.3 & 46.1 & 39.6 & 45.0 & 51.2 & 42.4 & 49.7 & 60.8 & 44.3 & 52.1 & 63.6 \\
\hline & & \multirow{3}{*}{$C D+C S$} & Wh/mile & 50.4 & 50.4 & 46.9 & 44.3 & 50.1 & 45.5 & 43.8 & 47.9 & 45.3 & 38.9 & 46.7 & 44.0 & 37.3 \\
\hline & & & \begin{tabular}{|l|} 
MPGEE Unadjusted \\
\end{tabular} & 66.7 & 66.7 & 72.3 & 79.3 & 67.2 & 77.2 & 90.0 & 72.4 & 87.1 & 108.7 & 75.8 & 91.7 & 114.5 \\
\hline & & & MPGGE Adjusted & 47.4 & 47.4 & 51.0 & 55.5 & 47.7 & 54.2 & 62.3 & 51.1 & 60.5 & 73.8 & 53.3 & 63.4 & 77.3 \\
\hline
\end{tabular}


Argonne National Laboratory Report - Light-Duty Vehicle Fuel Consumption Displacement Potential Up to 2045

\begin{tabular}{|c|c|c|c|c|c|c|c|c|c|c|c|c|c|c|c|c|}
\hline & & & & \multicolumn{13}{|c|}{ Midsize } \\
\hline & & & & \multirow{2}{*}{$\begin{array}{c}2010 \\
\text { Ref }\end{array}$} & \multicolumn{3}{|c|}{2010} & \multicolumn{3}{|c|}{2015} & \multicolumn{3}{|c|}{2030} & \multicolumn{3}{|c|}{2045} \\
\hline \multirow{26}{*}{ CI Split HEV PHEV2O } & & \multirow{5}{*}{$C D$} & & & low & avg & high & low & avg & high & low & avg & high & low & avg & high \\
\hline & \multirow{9}{*}{ UDDS } & & Wh/mile & 204.6 & 204.6 & 187.2 & 175.4 & 199.0 & 184.5 & 168.4 & 189.4 & 173.7 & 146.5 & 186.6 & 168.9 & 140.6 \\
\hline & & & Distance & 23.9 & 23.9 & 23.9 & 23.9 & 24.0 & 23.7 & 23.4 & 24.1 & 23.4 & 22.4 & 23.9 & 22.9 & 22.4 \\
\hline & & & MPGGE Unadjusted & 974.9 & 974.9 & 952.5 & 1067.2 & 855.4 & 1228.4 & 1708.8 & 901.9 & 1640.2 & 2812.4 & 1002.9 & 1759.4 & 2998.3 \\
\hline & & & MPGGE Adjusted & 262.7 & 262.7 & 260.7 & 270.4 & 251.1 & 281.9 & 306.1 & 255.9 & 303.4 & 335.0 & 265.2 & 308.1 & 338.1 \\
\hline & & \multirow{2}{*}{ CS } & MPGGE Unadjusted & 56.2 & 56.2 & 60.7 & 66.7 & 56.6 & 64.9 & 75.2 & 61.1 & 72.8 & 91.4 & 64.0 & 76.4 & 95.3 \\
\hline & & & MPGGE Adjusted & 41.1 & 41.1 & 43.9 & 47.8 & 41.3 & 46.6 & 53.0 & 44.2 & 51.6 & 62.6 & 46.1 & 53.8 & 64.9 \\
\hline & & \multirow{3}{*}{$\mathrm{CD}+\mathrm{CS}$} & Wh/mile & 107.4 & 107.4 & 98.3 & 92.4 & 104.8 & 97.0 & 89.0 & 99.7 & 91.8 & 77.5 & 98.2 & 89.3 & 74.3 \\
\hline & & & MPGGE Unadjusted & 96.0 & 96.0 & 103.2 & 113.6 & 96.2 & 111.3 & 129.9 & 103.7 & 125.8 & 159.2 & 108.9 & 132.0 & 166.2 \\
\hline & & & MPGGE Adjusted & 65.3 & 65.3 & 69.3 & 75.0 & 65.4 & 73.7 & $\begin{array}{l}83.4 \\
\end{array}$ & 69.6 & 81.3 & 97.6 & 72.4 & 84.5 & 100.7 \\
\hline & \multirow{9}{*}{ HWFET } & \multirow{4}{*}{$C D$} & Wh/mile & 220.8 & 220.8 & 199.0 & 186.2 & 213.1 & 196.2 & 196.0 & 205.3 & 185.1 & 179.5 & 199.9 & 195.5 & 168.8 \\
\hline & & & Distance & 21.7 & 21.7 & 22.5 & 22.5 & 22.5 & 21.8 & 19.5 & 22.5 & 21.4 & 17.9 & 22.4 & 19.4 & 18.3 \\
\hline & & & MPGGE Unadjusted & 401.6 & 401.6 & 392.4 & 424.1 & 349.3 & 472.3 & 2198.0 & 389.4 & 600.7 & 65207.3 & 409.7 & 2271.8 & 65207.3 \\
\hline & & & MPGGE Adjusted & 263.1 & 263.1 & 257.5 & 276.5 & 231.2 & 305.0 & 1056.7 & 255.7 & 378.2 & 3034.8 & 267.9 & 1080.4 & 3034.8 \\
\hline & & \multirow{2}{*}{ CS } & MPGGE Unadjusted & 52.3 & 52.3 & 56.7 & 62.4 & 52.7 & 60.5 & 69.9 & 56.6 & 67.6 & 83.4 & 59.4 & 71.5 & 88.5 \\
\hline & & & MPGGE Adjusted & 36.8 & 36.8 & 39.9 & 43.9 & 37.1 & 42.5 & 49.0 & 39.9 & 47.5 & 58.4 & 41.8 & 50.2 & 61.8 \\
\hline & & \multirow{3}{*}{$C D+C S$} & Wh/mile & 113.2 & 113.2 & 102.4 & 95.8 & 109.6 & 100.6 & 57.6 & 105.6 & 95.2 & 52.8 & 102.9 & 57.5 & 49.6 \\
\hline & & & MPGGE Unadjusted & 81.0 & 81.0 & 87.1 & 95.8 & 80.6 & 93.8 & 91.0 & 87.0 & 106.1 & 109.8 & 91.3 & 93.2 & 116.4 \\
\hline & & & MPGGE Adjusted & 56.7 & 56.7 & 60.9 & 66.8 & 56.4 & 65.5 & 63.6 & 60.8 & 73.9 & 76.4 & 63.8 & 65.1 & 80.9 \\
\hline & \multirow{7}{*}{ Combined } & \multirow{2}{*}{$C D$} & MPGGE Unadjusted & 593.6 & 593.6 & 580.0 & 634.3 & 517.8 & 714.0 & 1899.0 & 566.5 & 922.1 & 4939.1 & 607.3 & 1958.1 & 5253.7 \\
\hline & & & MPGGE Adjusted & 262.9 & 262.9 & 259.2 & 273.1 & 241.8 & 291.9 & 450.0 & 255.8 & 333.0 & 558.7 & 266.4 & 454.2 & 563.3 \\
\hline & & \multirow{2}{*}{ CS } & MPGGE Unadjusted & 54.4 & 54.4 & 58.8 & 64.7 & 54.8 & 62.8 & 72.7 & 59.0 & 70.4 & 87.6 & 61.9 & 74.1 & 92.1 \\
\hline & & & MPGGE Adjusted & 39.1 & 39.1 & 42.0 & 45.9 & 39.3 & 44.7 & 51.2 & 42.2 & 49.7 & 60.7 & 44.1 & 52.1 & 63.5 \\
\hline & & \multirow{3}{*}{$\mathrm{CD}+\mathrm{CS}$} & Wh/mile & 110.0 & 110.0 & 100.1 & 94.0 & 106.9 & 98.6 & 74.9 & 102.4 & 93.4 & 66.4 & 100.3 & 75.0 & 63.2 \\
\hline & & & MPGGE Unadjusted & 88.6 & 88.6 & 95.3 & 104.8 & 88.5 & 102.7 & 109.0 & 95.5 & 116.1 & 132.4 & 100.2 & 111.1 & 139.4 \\
\hline & & & MPGGE Adjusted & 61.1 & 61.1 & 65.3 & 71.1 & 61.0 & 69.8 & 73.2 & 65.4 & 77.8 & 86.8 & 68.3 & 74.5 & 90.7 \\
\hline
\end{tabular}


Argonne National Laboratory Report - Light-Duty Vehicle Fuel Consumption Displacement Potential Up to 2045

\begin{tabular}{|c|c|c|c|c|c|c|c|c|c|c|c|c|c|c|c|c|}
\hline & & & & \multicolumn{13}{|c|}{ Midsize } \\
\hline & & & & \multirow{2}{*}{$\frac{2010}{\text { Ref }}$} & \multicolumn{3}{|c|}{2010} & \multicolumn{3}{|c|}{2015} & \multicolumn{3}{|c|}{2030} & \multicolumn{3}{|c|}{2045} \\
\hline & & & & & low & avg & high & low & avg & high & low & avg & high & low & avg & high \\
\hline \multirow{25}{*}{ CI Series HEV PHEV 30} & \multirow{9}{*}{ UDDS } & \multirow{4}{*}{$C D$} & Wh/mile & 290.9 & 290.9 & 269.1 & 251.6 & 286.7 & 258.7 & 229.0 & 273.0 & 236.2 & 197.4 & 266.6 & 228.3 & 189.4 \\
\hline & & & Distance & 30.9 & 30.9 & 31.0 & 30.9 & 30.9 & 30.9 & 30.9 & 30.9 & 30.9 & 30.9 & 31.0 & 30.9 & 30.9 \\
\hline & & & MPGGE Unadjusted & N/A & N/A & N/A & N/A & N/A & N/A & N/A & N/A & N/A & N/A & N/A & N/A & N/A \\
\hline & & & MPGGE Adjusted & N/A & N/A & N/A & N/A & N/A & N/A & N/A & N/A & N/A & N/A & N/A & N/A & N/A \\
\hline & & \multirow{2}{*}{ CS } & MPGGE Unadjusted & 41.7 & 41.7 & 45.7 & 50.9 & 42.4 & 49.2 & 59.3 & 46.5 & 57.4 & 74.8 & 48.8 & 60.4 & 78.2 \\
\hline & & & MPGGE Adjusted & 31.3 & 31.3 & 34.0 & 37.5 & 31.8 & 36.4 & 43.0 & 34.6 & 41.8 & 52.8 & 36.2 & 43.8 & 54.9 \\
\hline & & \multirow{3}{*}{$\mathrm{CD}+\mathrm{CS}$} & Wh/mile & 187.5 & 187.5 & 173.4 & 162.1 & 184.8 & 166.7 & 148.2 & 175.9 & 152.9 & 128.3 & 171.8 & 147.7 & 123.1 \\
\hline & & & MPGGE Unadjusted & $\begin{array}{ll}88.7 \\
\end{array}$ & 88.7 & 97.2 & 108.3 & 90.3 & 104.7 & 126.1 & 99.0 & 122.1 & 159.2 & 103.9 & 128.5 & 166.5 \\
\hline & & & MPGGE Adjusted & 61.1 & 61.1 & 65.9 & 72.1 & 62.0 & 70.1 & 81.5 & 67.0 & 79.4 & 97.6 & 69.7 & 82.7 & 100.9 \\
\hline & \multirow{9}{*}{ HWFET } & \multirow{4}{*}{$C D$} & Wh/mile & 278.2 & 278.2 & 255.0 & 238.4 & 274.5 & 245.3 & 215.5 & 261.7 & 223.4 & 186.7 & 254.3 & 214.7 & 175.9 \\
\hline & & & \begin{tabular}{|l|} 
Distance \\
\end{tabular} & 32.2 & 32.2 & 32.6 & 32.5 & 32.1 & 32.5 & 32.9 & 32.1 & 32.6 & 32.5 & 32.4 & 33.3 & 33.5 \\
\hline & & & MPGGE Unadjusted & $N / A$ & N/A & N/A & $\mathrm{N} / \mathrm{A}$ & $\mathrm{N} / \mathrm{A}$ & N/A & N/A & N/A & N/A & N/A & N/A & $\mathrm{N} / \mathrm{A}$ & $\mathrm{N} / \mathrm{A}$ \\
\hline & & & MPGGE Adjusted & N/A & N/A & N/A & N/A & N/A & N/A & N/A & N/A & N/A & N/A & N/A & N/A & N/A \\
\hline & & \multirow{2}{*}{ CS } & MPGGE Unadjusted & 41.3 & 41.3 & 45.4 & 51.0 & 41.8 & 49.5 & 59.1 & 45.9 & 56.8 & 71.2 & 48.3 & 59.8 & 75.7 \\
\hline & & & MPGGE Adjusted & 29.1 & 29.1 & 32.1 & 35.9 & 29.6 & 34.9 & 41.6 & 32.4 & 40.0 & 50.0 & 34.1 & 42.1 & 53.1 \\
\hline & & \multirow{3}{*}{$\mathrm{CD}+\mathrm{CS}$} & Wh/mile & 188.0 & 188.0 & 172.4 & 161.1 & 185.5 & 165.8 & 145.7 & 176.9 & 151.0 & 126.3 & 171.9 & 145.1 & 119.0 \\
\hline & & & MPGGE Unadjusted & 89.7 & 89.7 & 98.7 & 110.8 & 91.0 & 107.6 & 128.5 & 99.7 & 123.5 & 154.9 & 105.0 & 130.0 & 164.5 \\
\hline & & & MPGGE Adjusted & 62.7 & 62.7 & 68.9 & 77.1 & 63.6 & 74.9 & 89.1 & 69.5 & 85.7 & 106.7 & 73.1 & 90.1 & 113.2 \\
\hline & \multirow{7}{*}{ Combined } & \multirow{2}{*}{$C D$} & MPGGE Unadjusted & $\mathrm{N} / \mathrm{A}$ & $\mathrm{N} / \mathrm{A}$ & $\mathrm{N} / \mathrm{A}$ & $\mathrm{N} / \mathrm{A}$ & $\mathrm{N} / \mathrm{A}$ & N/A & $\mathrm{N} / \mathrm{A}$ & $\mathrm{N} / \mathrm{A}$ & $\mathrm{N} / \mathrm{A}$ & $N / A$ & $\mathrm{~N} / \mathrm{A}$ & $\mathrm{N} / \mathrm{A}$ & $\mathrm{N} / \mathrm{A}$ \\
\hline & & & MPGGE Adjusted & N/A & N/A & N/A & N/A & N/A & N/A & N/A & N/A & N/A & $\mathrm{N} / \mathrm{A}$ & N/A & N/A & N/A \\
\hline & & \multirow{2}{*}{ CS } & MPGGE Unadjusted & 41.5 & 41.5 & 45.6 & 50.9 & 42.2 & 49.3 & 59.2 & 46.2 & 57.1 & 73.2 & 48.6 & 60.1 & 77.1 \\
\hline & & & MPGGE Adjusted & 30.3 & 30.3 & 33.1 & 36.8 & 30.8 & 35.7 & 42.4 & 33.6 & 41.0 & 51.5 & 35.2 & 43.0 & 54.1 \\
\hline & & \multirow{3}{*}{$\mathrm{CD}+\mathrm{CS}$} & Wh/mile & 187.7 & 187.7 & 172.9 & 161.7 & 185.1 & 166.3 & 147.1 & 176.3 & 152.1 & 127.4 & 171.8 & 146.5 & 121.3 \\
\hline & & & \begin{tabular}{|l|} 
MPGGE Unadjusted \\
\end{tabular} & 89.2 & 89.2 & 97.9 & 109.4 & 90.6 & 106.0 & 127.2 & 99.3 & 122.7 & 157.2 & 104.4 & 129.2 & 165.6 \\
\hline & & & MPGGE Adjusted & 61.8 & 61.8 & 67.2 & 74.3 & 62.7 & 72.2 & 84.8 & 68.1 & 82.1 & 101.5 & 71.2 & 85.9 & 106.1 \\
\hline
\end{tabular}


Argonne National Laboratory Report - Light-Duty Vehicle Fuel Consumption Displacement Potential Up to 2045

\begin{tabular}{|c|c|c|c|c|c|c|c|c|c|c|c|c|c|c|c|c|}
\hline & & & & \multicolumn{13}{|c|}{ Midsize } \\
\hline & & & & \multirow{2}{*}{$\begin{array}{c}2010 \\
\text { Ref }\end{array}$} & \multicolumn{3}{|c|}{2010} & \multicolumn{3}{|c|}{2015} & \multicolumn{3}{|c|}{2030} & \multicolumn{3}{|c|}{2045} \\
\hline \multirow{26}{*}{$\mathrm{Cl}$ Series HEV PHEV40 } & & \multirow{5}{*}{$C D$} & & & low & avg & high & low & avg & high & low & avg & high & low & avg & high \\
\hline & \multirow{9}{*}{ UDDS } & & Wh/mile & 297.0 & 297.0 & 275.4 & 256.9 & 292.4 & 264.1 & 233.5 & 279.2 & 241.0 & 199.8 & 271.8 & 232.2 & 191.6 \\
\hline & & & Distance & 41.0 & 41.0 & 41.0 & 40.9 & 40.8 & 41.0 & 40.9 & 41.0 & 40.9 & 40.6 & 41.1 & 40.7 & 40.4 \\
\hline & & & MPGGE Unadjusted & $\mathrm{N} / \mathrm{A}$ & $\mathrm{N} / \mathrm{A}$ & $\mathrm{N} / \mathrm{A}$ & $\mathrm{N} / \mathrm{A}$ & $\mathrm{N} / \mathrm{A}$ & $\mathrm{N} / \mathrm{A}$ & $\mathrm{N} / \mathrm{A}$ & $\mathrm{N} / \mathrm{A}$ & N/A & N/A & N/A & $\mathrm{N} / \mathrm{A}$ & $\mathrm{N} / \mathrm{A}$ \\
\hline & & & MPGGE Adjusted & $\mathrm{N} / \mathrm{A}$ & $\mathrm{N} / \mathrm{A}$ & $\mathrm{N} / \mathrm{A}$ & $\mathrm{N} / \mathrm{A}$ & $\mathrm{N} / \mathrm{A}$ & $\mathrm{N} / \mathrm{A}$ & $\mathrm{N} / \mathrm{A}$ & $\mathrm{N} / \mathrm{A}$ & $\mathrm{N} / \mathrm{A}$ & $\mathrm{N} / \mathrm{A}$ & $\mathrm{N} / \mathrm{A}$ & $\mathrm{N} / \mathrm{A}$ & $\mathrm{N} / \mathrm{A}$ \\
\hline & & \multirow{2}{*}{ CS } & MPGGE Unadjusted & 40.8 & 40.8 & 44.7 & 49.6 & 41.5 & 48.3 & 58.4 & 45.6 & 56.5 & 74.6 & 47.9 & 59.5 & 78.9 \\
\hline & & & MPGGE Adjusted & 30.7 & 30.7 & 33.4 & 36.7 & 31.2 & 35.8 & 42.5 & 34.0 & 41.2 & 52.7 & 35.6 & 43.2 & 55.3 \\
\hline & & \multirow{3}{*}{$\mathrm{CD}+\mathrm{CS}$} & Wh/mile & 218.5 & 218.5 & 202.7 & 189.1 & 215.1 & 194.3 & 173.2 & 205.5 & 178.7 & 148.5 & 200.1 & 172.0 & 142.3 \\
\hline & & & MPGGE Unadjusted & 102.1 & 102.1 & 111.9 & 124.1 & 103.7 & 120.6 & 145.9 & 114.1 & 141.2 & 186.5 & 119.8 & 148.8 & 197.3 \\
\hline & & & MPGGE Adjusted & 68.7 & 68.7 & 74.0 & 80.5 & 69.6 & 78.7 & 91.3 & 75.2 & 89.0 & 109.6 & 78.2 & 92.7 & 114.1 \\
\hline & \multirow{9}{*}{ HWFET } & \multirow{4}{*}{$C D$} & Wh/mile & 281.0 & 281.0 & 257.5 & 240.6 & 277.2 & 247.6 & 217.7 & 264.1 & 225.8 & 189.9 & 256.3 & 216.0 & 178.7 \\
\hline & & & Distance & 43.0 & 43.0 & 43.6 & 43.6 & 42.8 & 43.6 & 43.7 & 43.1 & 43.6 & 42.7 & 43.4 & 43.7 & 43.5 \\
\hline & & & MPGGE Unadjusted & N/A & N/A & $\mathrm{N} / \mathrm{A}$ & N/A & $\mathrm{N} / \mathrm{A}$ & $\mathrm{N} / \mathrm{A}$ & $\mathrm{N} / \mathrm{A}$ & N/A & N/A & N/A & $\mathrm{N} / \mathrm{A}$ & $\mathrm{N} / \mathrm{A}$ & N/A \\
\hline & & & MPGGE Adjusted & N/A & N/A & N/A & N/A & N/A & $\mathrm{N} / \mathrm{A}$ & $\mathrm{N} / \mathrm{A}$ & N/A & N/A & N/A & $\mathrm{N} / \mathrm{A}$ & $\mathrm{N} / \mathrm{A}$ & N/A \\
\hline & & \multirow{2}{*}{ CS } & MPGGE Unadjusted & 40.7 & 40.7 & 45.1 & 50.2 & 41.2 & 48.6 & 58.5 & 45.2 & 56.2 & 70.6 & 47.7 & 59.2 & 75.9 \\
\hline & & & MPGGE Adjusted & 28.7 & 28.7 & 31.8 & 35.4 & 29.1 & 34.3 & 41.2 & 31.9 & 39.6 & 49.6 & 33.6 & 41.6 & 53.2 \\
\hline & & \multirow{3}{*}{$C D+C S$} & Wh/mile & 221.1 & 221.1 & 202.7 & 189.4 & 218.1 & 194.9 & 171.7 & 207.8 & 178.0 & 150.4 & 201.8 & 170.1 & 141.6 \\
\hline & & & MPGGE Unadjusted & 109.9 & 109.9 & 121.9 & 135.7 & 111.4 & 131.3 & 158.1 & 122.2 & 151.9 & 190.9 & 128.8 & 159.9 & 205.2 \\
\hline & & & MPGGE Adjusted & 76.5 & 76.5 & 84.6 & 93.9 & 77.5 & 91.0 & 108.9 & 84.8 & 104.8 & 130.6 & 89.3 & 110.1 & 140.0 \\
\hline & \multirow{7}{*}{ Combined } & \multirow{2}{*}{$C D$} & MPGGE Unadjusted & $\mathrm{N} / \mathrm{A}$ & $\mathrm{N} / \mathrm{A}$ & $\mathrm{N} / \mathrm{A}$ & N/A & $\mathrm{N} / \mathrm{A}$ & $\mathrm{N} / \mathrm{A}$ & $\mathrm{N} / \mathrm{A}$ & N/A & $\mathrm{N} / \mathrm{A}$ & $\mathrm{N} / \mathrm{A}$ & $\mathrm{N} / \mathrm{A}$ & $\mathrm{N} / \mathrm{A}$ & N/A \\
\hline & & & MPGGE Adjusted & $\mathrm{N} / \mathrm{A}$ & N/A & $\mathrm{N} / \mathrm{A}$ & N/A & N/A & N/A & N/A & N/A & $\mathrm{N} / \mathrm{A}$ & N/A & N/A & N/A & $\mathrm{N} / \mathrm{A}$ \\
\hline & & \multirow{2}{*}{ CS } & MPGGE Unadjusted & 40.7 & 40.7 & 44.9 & 49.9 & 41.4 & 48.4 & 58.4 & 45.4 & 56.4 & 72.8 & 47.8 & 59.4 & 77.5 \\
\hline & & & MPGGE Adjusted & 29.8 & 29.8 & 32.7 & 36.1 & 30.2 & 35.1 & 41.9 & 33.0 & 40.5 & 51.2 & 34.7 & 42.5 & 54.4 \\
\hline & & \multirow{3}{*}{$\mathrm{CD}+\mathrm{CS}$} & Wh/mile & 219.7 & 219.7 & 202.7 & 189.2 & 216.5 & 194.6 & 172.5 & 206.6 & 178.4 & 149.3 & 200.8 & 171.1 & 142.0 \\
\hline & & & MPGGE Unadjusted & 105.4 & 105.4 & 116.2 & 129.1 & 107.1 & 125.2 & 151.2 & 117.6 & 145.8 & 188.5 & 123.7 & 153.6 & 200.8 \\
\hline & & & MPGGE Adjusted & 72.0 & 72.0 & 78.4 & 86.0 & 73.0 & 83.8 & 98.5 & 79.3 & 95.5 & 118.2 & 82.9 & 99.8 & 124.5 \\
\hline
\end{tabular}


Argonne National Laboratory Report - Light-Duty Vehicle Fuel Consumption Displacement Potential Up to 2045

\begin{tabular}{|c|c|c|c|c|c|c|c|c|c|c|c|c|c|c|c|c|}
\hline & & & & \multicolumn{13}{|c|}{ Midsize } \\
\hline & & & & \multirow{2}{*}{$\frac{2010}{\text { Ref }}$} & \multicolumn{3}{|c|}{2010} & \multicolumn{3}{|c|}{2015} & \multicolumn{3}{|c|}{2030} & \multicolumn{3}{|c|}{2045} \\
\hline & & & & & low & avg & high & low & avg & high & low & avg & high & low & avg & high \\
\hline \multirow{25}{*}{ H2 Split HEV PHEV10 } & \multirow{9}{*}{ UDDS } & \multirow{4}{*}{$C D$} & Wh/mile & 190.8 & 190.8 & 176.1 & 170.8 & 186.6 & 175.7 & 159.5 & 181.0 & 162.5 & 149.5 & 174.7 & 159.8 & 143.5 \\
\hline & & & Distance & 12.6 & 12.6 & 12.3 & 11.7 & 12.8 & 11.7 & 11.6 & 12.5 & 11.9 & 10.2 & 12.3 & 11.5 & 10.2 \\
\hline & & & MPGGE Unadjusted & 805.6 & 805.6 & 1056.5 & 1796.6 & 857.2 & 1721.8 & 2704.5 & 1064.8 & 2078.4 & $\mathrm{~N} / \mathrm{A}$ & 1183.2 & 2821.7 & N/A \\
\hline & & & MPGGE Adjusted & 245.7 & 245.7 & 269.6 & 309.5 & 251.3 & 306.7 & 333.1 & 270.2 & 318.6 & N/A & 278.9 & 335.2 & N/A \\
\hline & & \multirow{2}{*}{ CS } & MPGGE Unadjusted & 53.8 & 53.8 & 65.0 & 75.1 & 60.3 & 72.9 & 86.1 & 67.4 & 83.2 & 106.7 & 73.1 & 89.1 & 115.7 \\
\hline & & & MPGGE Adjusted & 39.5 & 39.5 & 46.7 & 53.0 & 43.7 & 51.6 & 59.6 & 48.2 & 57.9 & 71.2 & 51.8 & 61.3 & 76.1 \\
\hline & & \multirow{3}{*}{$\mathrm{CD}+\mathrm{CS}$} & Wh/mile & 39.8 & 39.8 & 36.7 & 35.6 & 38.9 & 36.6 & 33.2 & 37.7 & 33.9 & 31.1 & 36.4 & 33.3 & 29.9 \\
\hline & & & MPGGE Unadjusted & 63.9 & 63.9 & 77.4 & 89.8 & 71.6 & 87.1 & 103.1 & 80.2 & 99.5 & 128.6 & 87.0 & 106.6 & 139.4 \\
\hline & & & MPGGE Adjusted & 46.0 & 46.0 & 54.4 & 61.7 & 50.8 & 60.1 & 69.2 & 56.1 & 67.2 & 82.7 & 60.1 & 71.2 & 88.1 \\
\hline & \multirow{9}{*}{ HWFET } & \multirow{4}{*}{$C D$} & Wh/mile & 208.8 & 208.8 & 191.7 & 186.8 & 207.2 & 192.3 & 175.7 & 198.0 & 183.2 & 151.2 & 191.1 & 175.9 & 144.8 \\
\hline & & & \begin{tabular}{|l|} 
Distance \\
\end{tabular} & 11.1 & 11.1 & 11.1 & 10.6 & 11.1 & 10.6 & 9.1 & 11.1 & 9.1 & 9.0 & 11.1 & 9.1 & 9.2 \\
\hline & & & MPGGE Unadjusted & 337.2 & 337.2 & 434.0 & 647.6 & 386.4 & 631.0 & 855.9 & 426.0 & 843.3 & 936.2 & 463.1 & 895.3 & 1257.5 \\
\hline & & & MPGGE Adjusted & 223.8 & 223.8 & 282.4 & 404.1 & 253.8 & 395.1 & 513.5 & 277.7 & 507.2 & 553.5 & 299.6 & 533.3 & 702.4 \\
\hline & & \multirow{2}{*}{ CS } & MPGGE Unadjusted & 50.6 & 50.6 & 61.3 & 70.3 & 56.7 & 68.4 & 80.4 & 63.2 & 77.4 & 96.5 & 68.3 & 83.5 & 106.4 \\
\hline & & & MPGGE Adjusted & 35.7 & 35.7 & 43.1 & 49.3 & 39.9 & 48.1 & 56.3 & 44.5 & 54.3 & 67.4 & 48.0 & 58.5 & 74.1 \\
\hline & & \multirow{3}{*}{$\mathrm{CD}+\mathrm{CS}$} & Wh/mile & 61.4 & 61.4 & 56.4 & 55.0 & 60.9 & 56.6 & 51.7 & 58.2 & 53.9 & 44.5 & 56.2 & 51.7 & 42.6 \\
\hline & & & MPGGE Unadjusted & 63.6 & 63.6 & 77.2 & 89.4 & 71.3 & 87.1 & 102.8 & 79.5 & 99.0 & 123.0 & 85.9 & 106.8 & 136.4 \\
\hline & & & MPGGE Adjusted & 44.7 & 44.7 & 54.1 & 62.5 & 50.0 & 60.9 & 71.6 & 55.7 & 69.1 & 85.4 & 60.1 & 74.4 & 94.4 \\
\hline & \multirow{7}{*}{ Combined } & \multirow{2}{*}{$C D$} & MPGGE Unadjusted & 495.7 & 495.7 & 642.1 & 999.0 & 553.6 & 968.5 & 1371.4 & 635.8 & 1252.8 & 2044.8 & 696.1 & 1433.6 & 2730.4 \\
\hline & & & MPGGE Adjusted & 235.3 & 235.3 & 275.2 & 345.9 & 252.4 & 341.0 & 395.6 & 273.5 & 382.6 & 449.4 & 287.9 & 402.5 & 487.1 \\
\hline & & \multirow{2}{*}{ CS } & MPGGE Unadjusted & 52.3 & 52.3 & 63.3 & 72.9 & 58.6 & 70.8 & 83.4 & 65.5 & 80.5 & 101.9 & 70.9 & 86.5 & 111.3 \\
\hline & & & MPGGE Adjusted & 37.7 & 37.7 & 45.0 & 51.3 & 41.9 & 50.0 & 58.1 & 46.5 & 56.2 & 69.4 & 50.0 & 60.0 & 75.2 \\
\hline & & \multirow{3}{*}{$\mathrm{CD}+\mathrm{CS}$} & Wh/mile & 49.5 & 49.5 & 45.5 & 44.3 & 48.8 & 45.6 & 41.5 & 46.9 & 42.9 & 37.1 & 45.3 & 41.6 & 35.6 \\
\hline & & & \begin{tabular}{|l|} 
MPGGE Unadjusted \\
\end{tabular} & 63.8 & 63.8 & 77.3 & 89.6 & 71.5 & 87.1 & 102.9 & 79.9 & 99.3 & 126.0 & 86.5 & 106.7 & 138.0 \\
\hline & & & MPGGE Adjusted & 45.4 & 45.4 & 54.2 & 62.1 & 50.5 & 60.5 & 70.3 & 55.9 & 68.0 & 83.9 & 60.1 & 72.6 & 90.8 \\
\hline
\end{tabular}


Argonne National Laboratory Report - Light-Duty Vehicle Fuel Consumption Displacement Potential Up to 2045

\begin{tabular}{|c|c|c|c|c|c|c|c|c|c|c|c|c|c|c|c|c|}
\hline & & & & \multicolumn{13}{|c|}{ Midsize } \\
\hline & & & & \multirow{2}{*}{$\begin{array}{c}2010 \\
\text { Ref }\end{array}$} & \multicolumn{3}{|c|}{2010} & \multicolumn{3}{|c|}{2015} & \multicolumn{3}{|c|}{2030} & \multicolumn{3}{|c|}{2045} \\
\hline \multirow{26}{*}{ H2 Split HEV PHEV2O } & & \multirow{5}{*}{$C D$} & & & low & avg & high & low & avg & high & low & avg & high & low & avg & high \\
\hline & \multirow{9}{*}{ UDDS } & & Wh/mile & 195.0 & 195.0 & 178.1 & 172.8 & 191.4 & 176.5 & 158.5 & 186.5 & 164.5 & 138.5 & 176.4 & 159.1 & 140.8 \\
\hline & & & Distance & 23.9 & 23.9 & 23.9 & 23.4 & 23.8 & 23.4 & 22.8 & 23.7 & 22.8 & 22.0 & 23.9 & 22.8 & 20.5 \\
\hline & & & MPGGE Unadjusted & 833.0 & 833.0 & 1026.9 & 1733.3 & 896.7 & 1569.9 & 2121.2 & 1177.8 & 2035.4 & 4174.0 & 1152.5 & 2222.2 & $\mathrm{~N} / \mathrm{A}$ \\
\hline & & & MPGGE Adjusted & 248.7 & 248.7 & 267.2 & 307.1 & 255.4 & 300.3 & 319.8 & 278.6 & 317.3 & 351.8 & 276.8 & 322.5 & $\mathrm{~N} / \mathrm{A}$ \\
\hline & & \multirow{2}{*}{ CS } & MPGGE Unadjusted & 53.5 & 53.5 & 64.8 & 74.8 & 59.8 & 72.8 & 86.3 & 67.2 & 83.2 & 106.1 & 73.0 & 89.3 & 114.9 \\
\hline & & & MPGGE Adjusted & 39.3 & 39.3 & 46.6 & 52.8 & 43.4 & 51.6 & 59.7 & 48.1 & 57.9 & 70.9 & 51.7 & 61.4 & 75.7 \\
\hline & & \multirow{3}{*}{$\mathrm{CD}+\mathrm{CS}$} & Wh/mile & 102.6 & 102.6 & 93.8 & 91.0 & 100.8 & 93.0 & 83.8 & 98.0 & 86.9 & 52.7 & 93.0 & 84.1 & 53.6 \\
\hline & & & MPGGE Unadjusted & 91.0 & 91.0 & 110.4 & 129.4 & 101.6 & 125.6 & 149.5 & 114.9 & 144.1 & 154.3 & 124.3 & 154.6 & 169.0 \\
\hline & & & MPGGE Adjusted & 62.4 & 62.4 & 73.2 & 83.2 & 68.4 & 81.2 & 93.0 & 75.7 & 90.4 & 95.3 & 80.6 & 95.4 & 102.0 \\
\hline & \multirow{9}{*}{ HWFET } & \multirow{4}{*}{$C D$} & Wh/mile & 211.8 & 211.8 & 193.5 & 188.1 & 208.8 & 186.1 & 195.3 & 205.3 & 196.8 & 171.5 & 192.8 & 195.0 & 161.2 \\
\hline & & & Distance & 21.8 & 21.8 & 21.8 & 20.8 & 21.6 & 21.4 & 18.0 & 21.4 & 19.3 & 17.3 & 21.7 & 18.3 & 17.6 \\
\hline & & & MPGGE Unadjusted & 338.8 & 338.8 & 427.6 & 625.6 & 375.3 & 481.9 & $\mathrm{~N} / \mathrm{A}$ & 488.5 & 2709.3 & $\mathrm{~N} / \mathrm{A}$ & 457.1 & $\mathrm{~N} / \mathrm{A}$ & N/A \\
\hline & & & MPGGE Adjusted & 224.8 & 224.8 & 278.6 & 392.1 & 247.1 & 310.6 & $\mathrm{~N} / \mathrm{A}$ & 314.5 & 1210.9 & N/A & 296.1 & $\mathrm{~N} / \mathrm{A}$ & N/A \\
\hline & & \multirow{2}{*}{ CS } & MPGGE Unadjusted & 50.1 & 50.1 & 60.8 & 69.8 & 56.2 & 68.0 & 80.2 & 62.8 & 77.2 & 96.3 & 68.0 & 83.4 & 106.4 \\
\hline & & & MPGGE Adjusted & 35.3 & 35.3 & 42.7 & 49.0 & 39.5 & 47.7 & 56.2 & 44.2 & 54.1 & 67.2 & 47.7 & 58.3 & 74.1 \\
\hline & & \multirow{3}{*}{$C D+C S$} & Wh/mile & 108.9 & 108.9 & 99.6 & 96.8 & 107.4 & 95.7 & 57.5 & 105.3 & 57.9 & 50.4 & 99.2 & 57.4 & 47.4 \\
\hline & & & MPGGE Unadjusted & 76.9 & 76.9 & 93.5 & 109.7 & 86.0 & 104.7 & 105.5 & 97.4 & 100.7 & 126.7 & 104.2 & 109.7 & 140.0 \\
\hline & & & MPGGE Adjusted & 53.9 & 53.9 & 65.3 & 76.3 & 60.2 & 72.9 & 73.5 & 68.0 & 70.2 & 87.9 & 72.6 & 76.3 & 96.8 \\
\hline & \multirow{7}{*}{ Combined } & \multirow{2}{*}{$C D$} & MPGGE Unadjusted & 502.9 & 502.9 & 629.7 & 964.7 & 551.7 & 778.8 & 3757.1 & 720.4 & 2292.0 & 7213.1 & 684.2 & 3931.2 & N/A \\
\hline & & & MPGGE Adjusted & 237.3 & 237.3 & 272.2 & 340.3 & 251.6 & 304.9 & 535.3 & 293.6 & 475.1 & 584.2 & 285.2 & 539.5 & 640.8 \\
\hline & & \multirow{2}{*}{ CS } & MPGGE Unadjusted & 51.9 & 51.9 & 62.9 & 72.5 & 58.1 & 70.6 & 83.5 & 65.2 & 80.4 & 101.5 & 70.6 & 86.5 & 110.9 \\
\hline & & & MPGGE Adjusted & 37.4 & 37.4 & 44.8 & 51.0 & 41.6 & 49.8 & 58.1 & 46.3 & 56.1 & 69.2 & 49.8 & 60.0 & 75.0 \\
\hline & & \multirow{3}{*}{$C D+C S$} & Wh/mile & 105.5 & 105.5 & 96.4 & 93.6 & 103.8 & 94.2 & 71.9 & 101.3 & 73.8 & 51.7 & 95.7 & 72.1 & 50.8 \\
\hline & & & MPGGE Unadjusted & 84.0 & 84.0 & 102.1 & 119.7 & 93.9 & 115.2 & 125.9 & 106.3 & 120.7 & 140.5 & 114.4 & 130.5 & 154.6 \\
\hline & & & MPGGE Adjusted & 58.3 & 58.3 & 69.4 & 79.9 & 64.5 & 77.3 & 83.1 & 72.0 & 80.1 & 91.8 & 76.8 & 85.8 & 99.6 \\
\hline
\end{tabular}


Argonne National Laboratory Report - Light-Duty Vehicle Fuel Consumption Displacement Potential Up to 2045

\begin{tabular}{|c|c|c|c|c|c|c|c|c|c|c|c|c|c|c|c|c|}
\hline & & & & \multicolumn{13}{|c|}{ Midsize } \\
\hline & & & & \multirow{2}{*}{$\frac{2010}{\text { Ref }}$} & \multicolumn{3}{|c|}{2010} & \multicolumn{3}{|c|}{2015} & \multicolumn{3}{|c|}{2030} & \multicolumn{3}{|c|}{2045} \\
\hline & & & & & low & avg & high & low & avg & high & low & avg & high & low & avg & high \\
\hline \multirow{25}{*}{ H2 Series HEV PHEV 30} & \multirow{9}{*}{ UDDS } & \multirow{4}{*}{$C D$} & Wh/mile & 284.6 & 284.6 & 258.3 & 239.2 & 278.8 & 245.8 & 215.3 & 264.7 & 223.4 & 183.5 & 253.9 & 215.0 & 176.8 \\
\hline & & & Distance & 30.9 & 30.9 & 31.0 & 31.0 & 30.9 & 31.0 & 30.9 & 31.0 & 30.9 & 30.7 & 31.0 & 30.9 & 30.9 \\
\hline & & & MPGGE Unadjusted & N/A & N/A & $\mathrm{N} / \mathrm{A}$ & N/A & $\mathrm{N} / \mathrm{A}$ & N/A & $\mathrm{N} / \mathrm{A}$ & N/A & N/A & N/A & $\mathrm{N} / \mathrm{A}$ & N/A & N/A \\
\hline & & & MPGGE Adjusted & N/A & N/A & N/A & N/A & N/A & N/A & N/A & N/A & N/A & N/A & N/A & N/A & N/A \\
\hline & & \multirow{2}{*}{ CS } & MPGGE Unadjusted & 39.5 & 39.5 & 48.4 & 56.8 & 44.6 & 55.0 & 67.5 & 50.8 & 64.7 & 82.9 & 55.6 & 69.1 & 89.6 \\
\hline & & & MPGGE Adjusted & 29.8 & 29.8 & 35.9 & 41.5 & 33.3 & 40.3 & 48.3 & 37.5 & 46.5 & 57.7 & 40.7 & 49.3 & 61.6 \\
\hline & & \multirow{3}{*}{$\mathrm{CD}+\mathrm{CS}$} & Wh/mile & 183.4 & 183.4 & 166.4 & 154.1 & 179.7 & 158.4 & 139.3 & 170.5 & 144.6 & 119.2 & 163.6 & 139.0 & 114.9 \\
\hline & & & MPGGE Unadjusted & 84.0 & 84.0 & 102.9 & 120.9 & 95.0 & 117.0 & 143.6 & 108.1 & 137.7 & 176.3 & 118.3 & 146.9 & 190.7 \\
\hline & & & MPGGE Adjusted & 58.4 & 58.4 & 69.1 & 78.8 & 64.7 & 76.8 & 90.2 & 72.0 & 87.3 & 105.2 & 77.4 & 91.8 & 111.4 \\
\hline & \multirow{9}{*}{ HWFET } & \multirow{4}{*}{$C D$} & Wh/mile & 274.0 & 274.0 & 247.8 & 230.2 & 269.2 & 236.8 & 206.7 & 256.2 & 215.3 & 178.3 & 246.0 & 206.4 & 167.8 \\
\hline & & & Distance & 31.9 & 31.9 & 32.2 & 32.1 & 31.8 & 32.0 & 32.0 & 31.8 & 31.9 & 31.7 & 31.8 & 31.9 & 32.9 \\
\hline & & & MPGGE Unadjusted & $N / A$ & N/A & N/A & N/A & N/A & N/A & N/A & N/A & N/A & N/A & N/A & N/A & $\mathrm{N} / \mathrm{A}$ \\
\hline & & & MPGGE Adjusted & N/A & N/A & N/A & N/A & N/A & N/A & N/A & N/A & N/A & N/A & N/A & N/A & N/A \\
\hline & & \multirow{2}{*}{ CS } & MPGGE Unadjusted & 39.7 & 39.7 & 48.8 & 56.7 & 44.7 & 55.1 & 67.3 & 50.8 & 64.2 & 81.5 & 55.5 & 69.7 & 89.7 \\
\hline & & & \begin{tabular}{|l|} 
MPGEE Adjusted \\
\end{tabular} & 28.1 & 28.1 & 34.4 & 39.9 & 31.6 & 38.8 & 47.2 & 35.8 & 45.2 & 57.0 & 39.1 & 48.9 & 62.7 \\
\hline & & \multirow{3}{*}{$C D+C S$} & Wh/mile & 185.2 & 185.2 & 167.5 & 155.6 & 181.9 & 160.0 & 139.7 & 173.1 & 145.5 & 120.5 & 166.3 & 139.5 & 113.4 \\
\hline & & & MPGGE Unadjusted & 86.3 & 86.3 & 106.0 & 123.2 & 97.2 & 119.7 & 146.2 & 110.5 & 139.6 & 177.1 & 120.6 & 151.4 & 194.9 \\
\hline & & & MPGGE Adjusted & 60.4 & 60.4 & 73.8 & 85.5 & 67.8 & 83.1 & 101.0 & 76.9 & 96.6 & 121.5 & 83.7 & 104.5 & 133.2 \\
\hline & \multirow{7}{*}{ Combined } & \multirow{2}{*}{$C D$} & MPGGE Unadjusted & N/A & $\mathrm{N} / \mathrm{A}$ & $\mathrm{N} / \mathrm{A}$ & N/A & $\mathrm{N} / \mathrm{A}$ & N/A & N/A & $\mathrm{N} / \mathrm{A}$ & $\mathrm{N} / \mathrm{A}$ & $\mathrm{N} / \mathrm{A}$ & N/A & N/A & N/A \\
\hline & & & MPGGE Adjusted & N/A & $\mathrm{N} / \mathrm{A}$ & N/A & N/A & N/A & N/A & N/A & $\mathrm{N} / \mathrm{A}$ & N/A & N/A & N/A & N/A & N/A \\
\hline & & \multirow{2}{*}{ CS } & MPGGE Unadjusted & 39.6 & 39.6 & 48.5 & 56.8 & 44.7 & 55.0 & 67.4 & 50.8 & 64.5 & 82.2 & 55.5 & 69.3 & 89.7 \\
\hline & & & MPGGE Adjusted & 29.0 & 29.0 & 35.2 & 40.7 & 32.5 & 39.6 & 47.8 & 36.7 & 45.9 & 57.4 & 39.9 & 49.1 & 62.1 \\
\hline & & \multirow{3}{*}{$C D+C S$} & Wh/mile & 184.2 & 184.2 & 166.9 & 154.8 & 180.7 & 159.1 & 139.5 & 171.7 & 145.0 & 119.8 & 164.8 & 139.2 & 114.2 \\
\hline & & & MPGGE Unadjusted & 85.1 & 85.1 & 104.3 & 121.9 & 96.0 & 118.2 & 144.7 & 109.2 & 138.6 & 176.7 & 119.3 & 148.9 & 192.6 \\
\hline & & & \begin{tabular}{|l|} 
MPGGE Adjusted \\
\end{tabular} & 59.3 & 59.3 & 71.2 & 81.7 & 66.1 & 79.5 & 94.7 & 74.1 & 91.3 & 112.0 & 80.2 & 97.1 & 120.3 \\
\hline
\end{tabular}


Argonne National Laboratory Report - Light-Duty Vehicle Fuel Consumption Displacement Potential Up to 2045

\begin{tabular}{|c|c|c|c|c|c|c|c|c|c|c|c|c|c|c|c|c|}
\hline & & & & \multicolumn{13}{|c|}{ Midsize } \\
\hline & & & & 2010 & & 2010 & & & 2015 & & & 2030 & & & 2045 & \\
\hline \multirow{26}{*}{ H2 Series HEV PHEV40 } & & \multirow{5}{*}{$C D$} & & Ref & low & avg & high & low & avg & high & low & avg & high & low & avg & high \\
\hline & \multirow{9}{*}{ UDDS } & & Wh/mile & 290.7 & 290.7 & 263.8 & 244.0 & 284.7 & 251.6 & 219.7 & 270.6 & 228.1 & 185.8 & 259.1 & 219.0 & 178.4 \\
\hline & & & Distance & 41.0 & 41.0 & 40.8 & 40.9 & 41.0 & 40.8 & 40.8 & 41.0 & 40.9 & 40.4 & 40.8 & 40.6 & 40.5 \\
\hline & & & MPGGE Unadjusted & N/A & N/A & N/A & N/A & N/A & N/A & $\mathrm{N} / \mathrm{A}$ & $\mathrm{N} / \mathrm{A}$ & N/A & N/A & N/A & N/A & N/A \\
\hline & & & MPGGE Adjusted & N/A & $\mathrm{N} / \mathrm{A}$ & N/A & N/A & N/A & N/A & $\mathrm{N} / \mathrm{A}$ & $\mathrm{N} / \mathrm{A}$ & N/A & N/A & N/A & N/A & N/A \\
\hline & & \multirow{2}{*}{ CS } & MPGGE Unadjusted & 38.2 & 38.2 & 47.4 & 55.5 & 43.7 & 54.0 & 66.2 & 49.9 & 63.7 & 83.6 & 54.4 & 68.3 & 90.5 \\
\hline & & & MPGGE Adjusted & 28.9 & 28.9 & 35.2 & 40.6 & 32.7 & 39.6 & 47.4 & 36.9 & 45.9 & 58.1 & 39.8 & 48.8 & 62.1 \\
\hline & & \multirow{3}{*}{$C D+C S$} & Wh/mile & 213.8 & 213.8 & 194.0 & 179.5 & 209.4 & 185.2 & 162.9 & 199.2 & 169.0 & 137.9 & 190.6 & 162.1 & 132.4 \\
\hline & & & MPGGE Unadjusted & 95.5 & 95.5 & 118.5 & 138.8 & 109.2 & 134.9 & 165.4 & 124.7 & 159.2 & 208.9 & 135.9 & 170.6 & 226.2 \\
\hline & & & MPGGE Adjusted & 65.0 & 65.0 & 77.6 & 87.8 & 72.6 & 86.0 & 100.4 & 80.8 & 97.5 & 118.8 & 86.4 & 102.7 & 125.5 \\
\hline & \multirow{9}{*}{ HWFET } & \multirow{4}{*}{$C D$} & Wh/mile & 276.9 & 276.9 & 250.2 & 232.1 & 272.1 & 239.1 & 208.9 & 258.5 & 217.6 & 181.2 & 248.1 & 207.7 & 170.5 \\
\hline & & & Distance & 42.8 & 42.8 & 42.7 & 42.7 & 42.7 & 42.8 & 42.8 & 42.7 & 42.7 & 41.5 & 42.2 & 42.8 & 42.3 \\
\hline & & & MPGGE Unadjusted & $\mathrm{N} / \mathrm{A}$ & $\mathrm{N} / \mathrm{A}$ & N/A & N/A & $\mathrm{N} / \mathrm{A}$ & N/A & N/A & N/A & N/A & N/A & $\mathrm{N} / \mathrm{A}$ & $\mathrm{N} / \mathrm{A}$ & $\mathrm{N} / \mathrm{A}$ \\
\hline & & & MPGGE Adjusted & $\mathrm{N} / \mathrm{A}$ & $\mathrm{N} / \mathrm{A}$ & $\mathrm{N} / \mathrm{A}$ & N/A & N/A & N/A & N/A & N/A & $\mathrm{N} / \mathrm{A}$ & N/A & N/A & $\mathrm{N} / \mathrm{A}$ & $\mathrm{N} / \mathrm{A}$ \\
\hline & & \multirow{2}{*}{ CS } & MPGGE Unadjusted & 39.1 & 39.1 & 48.1 & 56.3 & 44.0 & 54.2 & 66.6 & 50.2 & 63.5 & 80.9 & 54.5 & 69.0 & 89.2 \\
\hline & & & MPGGE Adjusted & 27.7 & 27.7 & 33.9 & 39.6 & 31.1 & 38.2 & 46.8 & 35.4 & 44.6 & 56.6 & 38.4 & 48.5 & 62.4 \\
\hline & & \multirow{3}{*}{$C D+C S$} & Wh/mile & 217.9 & 217.9 & 196.9 & 182.7 & 214.0 & 188.1 & 164.7 & 203.4 & 171.5 & 143.4 & 195.2 & 163.5 & 135.0 \\
\hline & & & MPGGE Unadjusted & 105.8 & 105.8 & 129.9 & 152.1 & 119.0 & 146.5 & 179.9 & 135.6 & 171.6 & 218.5 & 147.4 & 186.6 & 241.1 \\
\hline & & & MPGGE Adjusted & 73.7 & 73.7 & 90.0 & 104.9 & 82.7 & 101.2 & 123.4 & 93.9 & 117.9 & 148.6 & 101.8 & 127.7 & 163.2 \\
\hline & \multirow{7}{*}{ Combined } & \multirow{2}{*}{$C D$} & MPGGE Unadjusted & N/A & $\mathrm{N} / \mathrm{A}$ & $\mathrm{N} / \mathrm{A}$ & $\mathrm{N} / \mathrm{A}$ & $\mathrm{N} / \mathrm{A}$ & $\mathrm{N} / \mathrm{A}$ & N/A & N/A & N/A & $\mathrm{N} / \mathrm{A}$ & $\mathrm{N} / \mathrm{A}$ & $\mathrm{N} / \mathrm{A}$ & $\mathrm{N} / \mathrm{A}$ \\
\hline & & & MPGGE Adjusted & N/A & N/A & N/A & N/A & N/A & N/A & N/A & N/A & N/A & N/A & N/A & N/A & N/A \\
\hline & & \multirow{2}{*}{ CS } & MPGGE Unadjusted & 38.6 & 38.6 & 47.7 & 55.8 & 43.8 & 54.1 & 66.3 & 50.0 & 63.6 & 82.3 & 54.4 & 68.6 & 89.9 \\
\hline & & & MPGGE Adjusted & 28.3 & 28.3 & 34.6 & 40.1 & 31.9 & 38.9 & 47.1 & 36.2 & 45.3 & 57.4 & 39.2 & 48.6 & 62.2 \\
\hline & & \multirow{3}{*}{$C D+C S$} & Wh/mile & 215.6 & 215.6 & 195.3 & 180.9 & 211.5 & 186.5 & 163.7 & 201.1 & 170.1 & 140.4 & 192.7 & 162.7 & 133.6 \\
\hline & & & MPGGE Unadjusted & 99.9 & 99.9 & 123.4 & 144.4 & 113.4 & 139.9 & 171.6 & 129.4 & 164.5 & 213.1 & 140.8 & 177.4 & 232.7 \\
\hline & & & MPGGE Adjusted & 68.6 & 68.6 & 82.7 & 94.8 & 76.8 & 92.2 & 109.6 & 86.2 & 105.8 & 130.6 & 92.7 & 112.7 & 140.1 \\
\hline
\end{tabular}


Argonne National Laboratory Report - Light-Duty Vehicle Fuel Consumption Displacement Potential Up to 2045

\begin{tabular}{|c|c|c|c|c|c|c|c|c|c|c|c|c|c|c|c|c|}
\hline & & & & \multicolumn{13}{|c|}{ Midsize } \\
\hline & & & & \multirow{2}{*}{$\frac{2010}{\text { Ref }}$} & \multicolumn{3}{|c|}{2010} & \multicolumn{3}{|c|}{2015} & \multicolumn{3}{|c|}{2030} & \multicolumn{3}{|c|}{2045} \\
\hline & & & & & low & avg & high & low & avg & high & low & avg & high & low & avg & high \\
\hline \multirow{25}{*}{ E85 Split HEV PHEV10 } & \multirow{9}{*}{ UDDS } & \multirow{4}{*}{$C D$} & Wh/mile & 186.0 & 186.0 & 176.6 & 172.8 & 188.8 & 176.4 & 159.4 & 180.3 & 164.9 & 143.5 & 176.4 & 159.7 & 146.8 \\
\hline & & & Distance & 12.7 & 12.7 & 12.3 & 11.6 & 12.3 & 11.7 & 11.7 & 12.3 & 11.9 & 11.4 & 12.3 & 11.7 & 10.2 \\
\hline & & & MPGGE Unadjusted & 735.8 & 735.8 & 918.8 & 1452.8 & 850.2 & 1319.5 & 1909.2 & 889.5 & 1628.7 & 3063.1 & 922.3 & 1710.8 & N/A \\
\hline & & & MPGGE Adjusted & 237.2 & 237.2 & 257.5 & 294.7 & 250.6 & 287.5 & 313.4 & 254.6 & 302.9 & 339.1 & 257.9 & 306.2 & N/A \\
\hline & & \multirow{2}{*}{ CS } & MPGGE Unadjusted & 52.3 & 52.3 & 56.8 & 61.6 & 53.3 & 59.9 & 77.4 & 56.2 & 66.0 & 93.0 & 58.1 & 68.5 & 97.1 \\
\hline & & & MPGGE Adjusted & 38.5 & 38.5 & 41.5 & 44.6 & 39.1 & 43.4 & 54.4 & 41.1 & 47.3 & 63.6 & 42.3 & 48.9 & 65.9 \\
\hline & & \multirow{3}{*}{$\mathrm{CD}+\mathrm{CS}$} & Wh/mile & 38.8 & 38.8 & 36.8 & 36.0 & 39.3 & 36.8 & 33.2 & 37.6 & 34.4 & 29.9 & 36.8 & 33.3 & 30.6 \\
\hline & & & MPGGE Unadjusted & 62.1 & 62.1 & 67.6 & 73.6 & 63.4 & 71.5 & 92.5 & 66.9 & 78.8 & 111.3 & 69.2 & 81.9 & 117.0 \\
\hline & & & MPGGE Adjusted & 44.8 & 44.8 & 48.3 & 52.1 & 45.7 & 50.8 & 63.3 & 47.9 & 55.2 & 73.7 & 49.3 & 57.1 & 76.8 \\
\hline & \multirow{9}{*}{ HWFET } & \multirow{4}{*}{$C D$} & Wh/mile & 208.0 & 208.0 & 192.4 & 188.5 & 206.7 & 193.8 & 178.7 & 197.8 & 185.1 & 154.9 & 192.7 & 178.7 & 147.9 \\
\hline & & & Distance & 11.0 & 11.0 & 11.0 & 10.6 & 11.0 & 10.6 & 9.2 & 11.1 & 9.6 & 9.1 & 11.1 & 9.2 & 9.4 \\
\hline & & & MPGGE Unadjusted & 332.1 & 332.1 & 378.3 & 530.0 & 340.5 & 516.9 & 810.7 & 354.3 & 698.4 & 875.6 & 366.5 & 729.1 & 1141.4 \\
\hline & & & MPGGE Adjusted & 220.6 & 220.6 & 248.9 & 338.4 & 225.8 & 330.9 & 490.5 & 234.3 & 431.6 & 523.5 & 241.8 & 448.0 & 650.6 \\
\hline & & \multirow{2}{*}{ CS } & MPGGE Unadjusted & 48.7 & 48.7 & 53.3 & 57.6 & 49.8 & 56.1 & 72.2 & 52.5 & 61.5 & 84.6 & 54.3 & 64.3 & 90.0 \\
\hline & & & \begin{tabular}{|l|} 
MPGEE Adjusted \\
\end{tabular} & 34.4 & 34.4 & 37.6 & 40.5 & 35.1 & 39.5 & 50.6 & 37.0 & 43.3 & 59.2 & 38.3 & 45.2 & 62.9 \\
\hline & & \multirow{3}{*}{$C D+C S$} & Wh/mile & 61.2 & 61.2 & 56.6 & 55.4 & 60.8 & 57.0 & 52.6 & 58.2 & 54.5 & 45.5 & 56.7 & 52.6 & 43.5 \\
\hline & & & MPGGE Unadjusted & 61.3 & 61.3 & 67.2 & 73.3 & 62.7 & 71.3 & 92.4 & 65.9 & 78.8 & 108.0 & 68.3 & 82.3 & 115.5 \\
\hline & & & MPGGE Adjusted & 43.1 & 43.1 & 47.2 & 51.4 & 44.0 & 50.1 & 64.5 & 46.3 & 55.2 & 75.2 & 47.9 & 57.6 & 80.3 \\
\hline & \multirow{7}{*}{ Combined } & \multirow{2}{*}{$C D$} & MPGGE Unadjusted & 475.6 & 475.6 & 559.2 & 814.6 & 508.0 & 776.8 & 1186.0 & 529.6 & 1018.3 & 1441.9 & 548.2 & 1065.4 & 2483.6 \\
\hline & & & MPGGE Adjusted & 229.4 & 229.4 & 253.6 & 312.9 & 238.8 & 305.5 & 374.2 & 245.1 & 349.8 & 402.9 & 250.4 & 357.1 & 475.3 \\
\hline & & \multirow{2}{*}{ CS } & MPGGE Unadjusted & 50.6 & 50.6 & 55.2 & 59.8 & 51.7 & 58.1 & 75.0 & 54.5 & 63.9 & 89.0 & 56.4 & 66.5 & 93.8 \\
\hline & & & MPGGE Adjusted & 36.5 & 36.5 & 39.6 & 42.7 & 37.2 & 41.6 & 52.6 & 39.1 & 45.4 & 61.5 & 40.4 & 47.2 & 64.5 \\
\hline & & \multirow{3}{*}{$C D+C S$} & Wh/mile & 48.8 & 48.8 & 45.7 & 44.7 & 49.0 & 45.9 & 41.9 & 46.8 & 43.4 & 36.9 & 45.7 & 42.0 & 36.4 \\
\hline & & & MPGGE Unadjusted & 61.7 & 61.7 & 67.4 & 73.5 & 63.0 & 71.4 & 92.4 & 66.4 & 78.8 & 109.8 & 68.8 & 82.1 & 116.3 \\
\hline & & & MPGGE Adjusted & 44.0 & 44.0 & 47.8 & 51.8 & 44.9 & 50.4 & 63.8 & 47.2 & 55.2 & 74.4 & 48.7 & 57.3 & 78.3 \\
\hline
\end{tabular}


Argonne National Laboratory Report - Light-Duty Vehicle Fuel Consumption Displacement Potential Up to 2045

\begin{tabular}{|c|c|c|c|c|c|c|c|c|c|c|c|c|c|c|c|c|}
\hline & & & & \multicolumn{13}{|c|}{ Midsize } \\
\hline & & & & \multirow{2}{*}{$\begin{array}{r}2010 \\
\operatorname{Ref}\end{array}$} & \multicolumn{3}{|c|}{2010} & \multicolumn{3}{|c|}{2015} & \multicolumn{3}{|c|}{2030} & \multicolumn{3}{|c|}{2045} \\
\hline \multirow{26}{*}{ E85 Split HEV PHEV20 } & & \multirow{5}{*}{$C D$} & & & low & avg & high & low & avg & high & low & avg & high & low & avg & high \\
\hline & \multirow{9}{*}{ UDDS } & & Wh/mile & 190.5 & 190.5 & 178.5 & 173.5 & 194.7 & 178.4 & 161.2 & 182.2 & 166.6 & 140.6 & 178.3 & 161.8 & 144.2 \\
\hline & & & Distance & 23.9 & 23.9 & 23.9 & 22.9 & 23.8 & 23.3 & 22.8 & 23.8 & 22.8 & 22.3 & 23.9 & 22.8 & 20.6 \\
\hline & & & MPGGE Unadjusted & 770.5 & 770.5 & 892.0 & 1327.0 & 943.2 & 1280.8 & 1869.8 & 867.3 & 1595.8 & 2992.7 & 900.0 & 1679.6 & N/A \\
\hline & & & MPGGE Adjusted & 241.5 & 241.5 & 254.9 & 288.0 & 259.8 & 285.2 & 312.0 & 252.4 & 301.5 & 338.0 & 255.7 & 305.0 & N/A \\
\hline & & \multirow{2}{*}{ CS } & MPGGE Unadjusted & 51.9 & 51.9 & 56.7 & 61.5 & 53.0 & 59.8 & 77.4 & 56.1 & 66.2 & 92.9 & 58.2 & 68.7 & 96.8 \\
\hline & & & MPGGE Adjusted & 38.2 & 38.2 & 41.4 & 44.5 & 38.9 & 43.4 & 54.4 & 41.0 & 47.5 & 63.5 & 42.3 & 49.0 & 65.8 \\
\hline & & \multirow{3}{*}{$C D+C S$} & Wh/mile & 100.4 & 100.4 & 94.1 & 91.4 & 102.3 & 94.0 & 85.2 & 96.0 & 88.0 & 53.5 & 93.9 & 85.5 & 54.9 \\
\hline & & & MPGGE Unadjusted & 88.2 & 88.2 & 96.6 & 106.0 & 90.6 & 103.0 & 134.0 & 95.4 & 114.6 & 134.6 & 98.9 & 119.0 & 142.4 \\
\hline & & & MPGGE Adjusted & 60.8 & 60.8 & 65.6 & 70.9 & 62.2 & 69.2 & 85.5 & 64.9 & 75.5 & 85.8 & 66.9 & 77.8 & 89.6 \\
\hline & \multirow{9}{*}{ HWFET } & \multirow{4}{*}{$C D$} & Wh/mile & 209.9 & 209.9 & 194.2 & 188.8 & 213.7 & 187.4 & 197.8 & 199.6 & 198.4 & 174.4 & 194.5 & 197.3 & 163.7 \\
\hline & & & Distance & 21.5 & 21.5 & 21.8 & 20.8 & 21.5 & 21.7 & 18.3 & 21.4 & 19.3 & 17.4 & 21.8 & 18.3 & 17.7 \\
\hline & & & MPGGE Unadjusted & 324.6 & 324.6 & 372.4 & 494.9 & 380.8 & 393.3 & N/A & 349.6 & 2076.8 & $\mathrm{~N} / \mathrm{A}$ & 361.7 & N/A & N/A \\
\hline & & & MPGGE Adjusted & 216.0 & 216.0 & 245.4 & 318.2 & 250.4 & 258.0 & N/A & 231.4 & 1016.7 & $\mathrm{~N} / \mathrm{A}$ & 238.8 & N/A & N/A \\
\hline & & \multirow{2}{*}{ CS } & MPGGE Unadjusted & 48.2 & 48.2 & 53.0 & 57.4 & 49.4 & 55.7 & 71.9 & 52.2 & 61.3 & 84.6 & 54.0 & 64.2 & 89.9 \\
\hline & & & MPGGE Adjusted & 34.0 & 34.0 & 37.3 & 40.4 & 34.8 & 39.2 & 50.5 & 36.7 & 43.1 & 59.2 & 38.0 & 45.1 & 62.8 \\
\hline & & \multirow{3}{*}{$\mathrm{CD}+\mathrm{CS}$} & Wh/mile & 108.0 & 108.0 & 99.9 & 97.2 & 109.6 & 96.4 & 58.2 & 102.7 & 58.4 & 51.3 & 100.1 & 58.0 & 48.1 \\
\hline & & & MPGGE Unadjusted & 73.9 & 73.9 & 81.5 & 89.9 & 76.5 & 85.8 & 94.7 & 79.9 & 79.9 & 111.3 & 82.7 & 84.4 & 118.3 \\
\hline & & & MPGGE Adjusted & 51.8 & 51.8 & 57.1 & 62.8 & 53.7 & 60.0 & 66.1 & 56.0 & 56.0 & 77.4 & 57.9 & 59.1 & 82.2 \\
\hline & \multirow{7}{*}{ Combined } & \multirow{2}{*}{$C D$} & MPGGE Unadjusted & 476.1 & 476.1 & 548.0 & 755.4 & 566.6 & 635.5 & 3322.0 & 520.5 & 1781.4 & 5245.3 & 539.0 & 2991.1 & N/A \\
\hline & & & MPGGE Adjusted & 229.3 & 229.3 & 250.5 & 300.8 & 255.5 & 272.3 & 523.3 & 242.5 & 441.1 & 563.2 & 247.8 & 512.4 & 640.8 \\
\hline & & \multirow{2}{*}{ CS } & MPGGE Unadjusted & 50.2 & 50.2 & 55.0 & 59.6 & 51.3 & 57.9 & 74.8 & 54.2 & 63.9 & 88.9 & 56.2 & 66.6 & 93.6 \\
\hline & & & MPGGE Adjusted & 36.2 & 36.2 & 39.4 & 42.5 & 37.0 & 41.4 & 52.6 & 39.0 & 45.4 & 61.5 & 40.3 & 47.2 & 64.4 \\
\hline & & \multirow{3}{*}{$\mathrm{CD}+\mathrm{CS}$} & Wh/mile & 103.8 & 103.8 & 96.7 & 94.0 & 105.6 & 95.1 & 73.0 & 99.0 & 74.7 & 52.5 & 96.7 & 73.2 & 51.9 \\
\hline & & & MPGGE Unadjusted & 81.1 & 81.1 & 89.1 & 98.1 & 83.7 & 94.5 & 112.9 & 87.8 & 95.9 & 123.0 & 90.9 & 100.5 & 130.5 \\
\hline & & & MPGGE Adjusted & 56.4 & 56.4 & 61.5 & 67.0 & 58.0 & 64.8 & 75.5 & 60.6 & 65.2 & 81.8 & 62.5 & 68.1 & 86.1 \\
\hline
\end{tabular}


Argonne National Laboratory Report - Light-Duty Vehicle Fuel Consumption Displacement Potential Up to 2045

\begin{tabular}{|c|c|c|c|c|c|c|c|c|c|c|c|c|c|c|c|c|}
\hline & & & & \multicolumn{13}{|c|}{ Midsize } \\
\hline & & & & \multirow{2}{*}{$\frac{2010}{\text { Ref }}$} & \multicolumn{3}{|c|}{2010} & \multicolumn{3}{|c|}{2015} & \multicolumn{3}{|c|}{2030} & \multicolumn{3}{|c|}{2045} \\
\hline & & & & & low & avg & high & low & avg & high & low & avg & high & low & avg & high \\
\hline \multirow{25}{*}{ E85 Series HEV PHEV30 } & \multirow{9}{*}{ UDDS } & \multirow{4}{*}{$C D$} & Wh/mile & 280.4 & 280.4 & 259.1 & 241.7 & 276.3 & 248.4 & 219.4 & 263.2 & 226.5 & 188.4 & 256.9 & 218.3 & 180.3 \\
\hline & & & Distance & 30.9 & 30.9 & 30.9 & 31.0 & 30.9 & 30.9 & 30.9 & 31.0 & 30.9 & 30.7 & 30.9 & 30.9 & 30.7 \\
\hline & & & MPGGE Unadjusted & N/A & N/A & N/A & N/A & $\mathrm{N} / \mathrm{A}$ & N/A & N/A & N/A & N/A & N/A & N/A & $\mathrm{N} / \mathrm{A}$ & $\mathrm{N} / \mathrm{A}$ \\
\hline & & & MPGGE Adjusted & N/A & N/A & N/A & N/A & N/A & N/A & N/A & N/A & $\mathrm{N} / \mathrm{A}$ & $\mathrm{N} / \mathrm{A}$ & N/A & N/A & N/A \\
\hline & & \multirow{2}{*}{ CS } & MPGGE Unadjusted & 38.6 & 38.6 & 42.8 & 47.2 & 39.4 & 45.7 & 61.5 & 42.9 & 52.4 & 75.9 & 44.7 & 54.4 & 79.4 \\
\hline & & & MPGGE Adjusted & 29.2 & 29.2 & 32.1 & 35.1 & 29.7 & 34.0 & 44.5 & 32.1 & 38.6 & 53.5 & 33.3 & 39.9 & 55.6 \\
\hline & & \multirow{3}{*}{$C D+C S$} & Wh/mile & 180.7 & 180.7 & 166.9 & 155.7 & 178.0 & 160.1 & 142.0 & 169.6 & 146.6 & 122.4 & 165.5 & 141.1 & 117.1 \\
\hline & & & MPGGE Unadjusted & 82.2 & 82.2 & 91.1 & 100.4 & 83.9 & 97.1 & 130.8 & 91.2 & 111.5 & 161.5 & 95.0 & 115.7 & 168.9 \\
\hline & & & MPGGE Adjusted & 57.2 & 57.2 & 62.4 & 67.8 & 58.3 & 65.9 & 83.9 & 62.5 & 73.8 & 98.6 & 64.7 & 76.1 & 101.9 \\
\hline & \multirow{9}{*}{ HWFET } & \multirow{4}{*}{$C D$} & Wh/mile & 271.1 & 271.1 & 248.3 & 231.8 & 267.5 & 238.5 & 209.4 & 255.2 & 217.2 & 181.3 & 247.9 & 208.6 & 170.1 \\
\hline & & & Distance & 31.8 & 31.8 & 32.2 & 32.2 & 31.8 & 32.1 & 32.3 & 31.8 & 32.1 & 31.9 & 31.9 & 32.2 & 32.5 \\
\hline & & & MPGGE Unadjusted & N/A & N/A & N/A & N/A & N/A & N/A & $\mathrm{N} / \mathrm{A}$ & N/A & N/A & N/A & N/A & N/A & $\mathrm{N} / \mathrm{A}$ \\
\hline & & & MPGGE Adjusted & N/A & N/A & N/A & N/A & N/A & N/A & N/A & N/A & N/A & N/A & N/A & N/A & N/A \\
\hline & & \multirow{2}{*}{ CS } & MPGGE Unadjusted & 38.1 & 38.1 & 42.4 & 46.4 & 39.2 & 45.0 & 60.9 & 42.1 & 51.1 & 71.8 & 43.9 & 53.8 & 76.4 \\
\hline & & & MPGGE Adjusted & 26.9 & 26.9 & 29.9 & 32.7 & 27.7 & 31.7 & 42.8 & 29.7 & 36.0 & 50.4 & 31.0 & 37.9 & 53.5 \\
\hline & & \multirow{3}{*}{$C D+C S$} & Wh/mile & 183.2 & 183.2 & 167.8 & 156.7 & 180.8 & 161.2 & 141.5 & 172.5 & 146.8 & 122.5 & 167.6 & 141.0 & 115.0 \\
\hline & & & MPGGE Unadjusted & 82.9 & 82.9 & 92.1 & 100.8 & 85.2 & 97.8 & 132.3 & 91.5 & 111.0 & 156.1 & 95.4 & 117.0 & 166.0 \\
\hline & & & MPGGE Adjusted & 58.0 & 58.0 & 64.3 & 70.3 & 59.6 & 68.2 & 91.6 & 63.9 & 77.2 & 107.6 & 66.6 & 81.3 & 114.1 \\
\hline & \multirow{7}{*}{ Combined } & \multirow{2}{*}{$C D$} & MPGGE Unadjusted & N/A & N/A & $\mathrm{N} / \mathrm{A}$ & N/A & N/A & N/A & N/A & N/A & $\mathrm{N} / \mathrm{A}$ & $\mathrm{N} / \mathrm{A}$ & $\mathrm{N} / \mathrm{A}$ & $\mathrm{N} / \mathrm{A}$ & N/A \\
\hline & & & MPGGE Adjusted & N/A & N/A & N/A & N/A & N/A & N/A & N/A & $\mathrm{N} / \mathrm{A}$ & N/A & N/A & N/A & N/A & N/A \\
\hline & & \multirow{2}{*}{ CS } & MPGGE Unadjusted & 38.4 & 38.4 & 42.6 & 46.8 & 39.3 & 45.3 & 61.2 & 42.5 & 51.8 & 74.0 & 44.3 & 54.1 & 78.0 \\
\hline & & & MPGGE Adjusted & 28.1 & 28.1 & 31.1 & 34.0 & 28.8 & 33.0 & 43.7 & 31.0 & 37.4 & 52.0 & 32.2 & 39.0 & 54.6 \\
\hline & & \multirow{3}{*}{$C D+C S$} & Wh/mile & 181.8 & 181.8 & 167.3 & 156.2 & 179.3 & 160.6 & 141.8 & 170.9 & 146.7 & 122.4 & 166.4 & 141.1 & 116.2 \\
\hline & & & MPGGE Unadjusted & 82.5 & 82.5 & 91.5 & 100.6 & 84.5 & 97.4 & 131.5 & 91.3 & 111.3 & 159.0 & 95.2 & 116.3 & 167.5 \\
\hline & & & MPGGE Adjusted & 57.6 & 57.6 & 63.3 & 68.9 & 58.9 & 66.9 & 87.2 & 63.2 & 75.3 & 102.5 & 65.5 & 78.3 & 107.1 \\
\hline
\end{tabular}


Argonne National Laboratory Report - Light-Duty Vehicle Fuel Consumption Displacement Potential Up to 2045

\begin{tabular}{|c|c|c|c|c|c|c|c|c|c|c|c|c|c|c|c|c|}
\hline & & & & \multicolumn{13}{|c|}{ Midsize } \\
\hline & & & & \multirow{2}{*}{$\begin{array}{r}2010 \\
\operatorname{Ref}\end{array}$} & \multicolumn{3}{|c|}{2010} & \multicolumn{3}{|c|}{2015} & \multicolumn{3}{|c|}{2030} & \multicolumn{3}{|c|}{2045} \\
\hline \multirow{26}{*}{ E85 Series HEV PHEV40 } & & \multirow{5}{*}{$C D$} & & & low & avg & high & low & avg & high & low & avg & high & low & avg & high \\
\hline & \multirow{9}{*}{ UDDS } & & Wh/mile & 286.2 & 286.2 & 265.2 & 246.8 & 282.1 & 254.1 & 223.9 & 269.4 & 231.3 & 190.6 & 262.3 & 222.6 & 182.4 \\
\hline & & & Distance & 40.8 & 40.8 & 41.0 & 40.9 & 40.9 & 40.8 & 40.8 & 40.9 & 40.8 & 40.4 & 40.8 & 40.7 & 40.5 \\
\hline & & & MPGGE Unadjusted & $\mathrm{N} / \mathrm{A}$ & $\mathrm{N} / \mathrm{A}$ & $\mathrm{N} / \mathrm{A}$ & $\mathrm{N} / \mathrm{A}$ & $\mathrm{N} / \mathrm{A}$ & $\mathrm{N} / \mathrm{A}$ & $\mathrm{N} / \mathrm{A}$ & $\mathrm{N} / \mathrm{A}$ & $\mathrm{N} / \mathrm{A}$ & $\mathrm{N} / \mathrm{A}$ & $\mathrm{N} / \mathrm{A}$ & $\mathrm{N} / \mathrm{A}$ & $\mathrm{N} / \mathrm{A}$ \\
\hline & & & MPGGE Adjusted & N/A & N/A & $\mathrm{N} / \mathrm{A}$ & N/A & N/A & N/A & N/A & $\mathrm{N} / \mathrm{A}$ & $\mathrm{N} / \mathrm{A}$ & N/A & N/A & $\mathrm{N} / \mathrm{A}$ & N/A \\
\hline & & \multirow{2}{*}{ CS } & MPGGE Unadjusted & 37.3 & 37.3 & 41.9 & 46.0 & 38.9 & 44.7 & 60.5 & 42.0 & 51.4 & 76.2 & 43.8 & 53.6 & 80.5 \\
\hline & & & MPGGE Adjusted & 28.3 & 28.3 & 31.4 & 34.2 & 29.4 & 33.4 & 43.8 & 31.5 & 37.9 & 53.7 & 32.7 & 39.3 & 56.2 \\
\hline & & \multirow{3}{*}{$\mathrm{CD}+\mathrm{CS}$} & Wh/mile & 210.5 & 210.5 & 195.2 & 181.6 & 207.5 & 187.1 & 166.0 & 198.3 & 171.4 & 141.5 & 192.9 & 164.8 & 135.4 \\
\hline & & & MPGGE Unadjusted & 93.3 & 93.3 & 104.7 & 115.0 & 97.3 & 111.8 & 151.3 & 105.0 & 128.6 & 190.5 & 109.4 & 133.9 & 201.2 \\
\hline & & & MPGGE Adjusted & 63.8 & 63.8 & 70.2 & 75.7 & 66.0 & 74.0 & 93.9 & 70.3 & 82.8 & 111.3 & 72.7 & 85.4 & 115.7 \\
\hline & \multirow{9}{*}{ HWFET } & \multirow{4}{*}{$C D$} & Wh/mile & 273.9 & 273.9 & 250.8 & 234.0 & 270.3 & 240.7 & 211.5 & 257.7 & 219.6 & 184.2 & 250.2 & 210.0 & 173.0 \\
\hline & & & Distance & 42.2 & 42.2 & 43.1 & 43.0 & 42.3 & 42.9 & 43.2 & 42.4 & 42.8 & 41.8 & 42.6 & 43.1 & 42.7 \\
\hline & & & MPGGE Unadjusted & N/A & N/A & N/A & N/A & N/A & N/A & $\mathrm{N} / \mathrm{A}$ & N/A & $\mathrm{N} / \mathrm{A}$ & N/A & N/A & N/A & N/A \\
\hline & & & MPGGE Adjusted & N/A & N/A & N/A & $\mathrm{N} / \mathrm{A}$ & $\mathrm{N} / \mathrm{A}$ & N/A & $\mathrm{N} / \mathrm{A}$ & N/A & N/A & N/A & $\mathrm{N} / \mathrm{A}$ & N/A & N/A \\
\hline & & \multirow{2}{*}{ CS } & MPGGE Unadjusted & 37.5 & 37.5 & 41.8 & 45.6 & 38.6 & 44.3 & 60.2 & 41.5 & 50.4 & 71.2 & 43.3 & 53.3 & 75.8 \\
\hline & & & MPGGE Adjusted & 26.5 & 26.5 & 29.5 & 32.2 & 27.3 & 31.3 & 42.4 & 29.3 & 35.5 & 50.0 & 30.6 & 37.5 & 53.1 \\
\hline & & \multirow{3}{*}{$\mathrm{CD}+\mathrm{CS}$} & Wh/mile & 215.5 & 215.5 & 197.4 & 184.2 & 212.7 & 189.5 & 166.8 & 202.8 & 173.1 & 145.8 & 196.9 & 165.4 & 137.0 \\
\hline & & & MPGGE Unadjusted & 101.5 & 101.5 & 112.8 & 123.2 & 104.4 & 119.8 & 162.7 & 112.1 & 136.2 & 192.5 & 117.0 & 143.9 & 204.8 \\
\hline & & & MPGGE Adjusted & 70.7 & 70.7 & 78.5 & 85.5 & 72.7 & 83.2 & 112.0 & 78.0 & 94.3 & 131.6 & 81.3 & 99.5 & 139.7 \\
\hline & \multirow{7}{*}{ Combined } & \multirow{2}{*}{$C D$} & MPGGE Unadjusted & N/A & $\mathrm{N} / \mathrm{A}$ & N/A & $\mathrm{N} / \mathrm{A}$ & $\mathrm{N} / \mathrm{A}$ & N/A & $\mathrm{N} / \mathrm{A}$ & $\mathrm{N} / \mathrm{A}$ & $\mathrm{N} / \mathrm{A}$ & N/A & $\mathrm{N} / \mathrm{A}$ & $\mathrm{N} / \mathrm{A}$ & N/A \\
\hline & & & MPGGE Adjusted & N/A & N/A & N/A & N/A & N/A & N/A & N/A & N/A & $\mathrm{N} / \mathrm{A}$ & N/A & N/A & N/A & N/A \\
\hline & & \multirow{2}{*}{ CS } & MPGGE Unadjusted & 37.4 & 37.4 & 41.8 & 45.8 & 38.8 & 44.6 & 60.4 & 41.7 & 51.0 & 73.9 & 43.5 & 53.4 & 78.3 \\
\hline & & & MPGGE Adjusted & 27.5 & 27.5 & 30.5 & 33.3 & 28.4 & 32.4 & 43.2 & 30.5 & 36.8 & 51.9 & 31.7 & 38.5 & 54.8 \\
\hline & & \multirow{3}{*}{$\mathrm{CD}+\mathrm{CS}$} & Wh/mile & 212.8 & 212.8 & 196.2 & 182.7 & 209.8 & 188.2 & 166.4 & 200.3 & 172.2 & 143.5 & 194.7 & 165.0 & 136.1 \\
\hline & & & MPGGE Unadjusted & 96.8 & 96.8 & 108.2 & 118.5 & 100.4 & 115.3 & 156.2 & 108.1 & 131.9 & 191.4 & 112.7 & 138.3 & 202.8 \\
\hline & & & MPGGE Adjusted & 66.7 & 66.7 & 73.7 & 79.8 & 68.9 & 77.9 & 101.2 & 73.5 & 87.6 & 119.6 & 76.3 & 91.2 & 125.4 \\
\hline
\end{tabular}


Argonne National Laboratory Report - Light-Duty Vehicle Fuel Consumption Displacement Potential Up to 2045

\begin{tabular}{|c|c|c|c|c|c|c|c|c|c|c|c|c|c|c|c|c|}
\hline & & & & \multicolumn{13}{|c|}{ Midsize } \\
\hline & & & & \multirow{2}{*}{$\begin{array}{c}2010 \\
\text { Ref }\end{array}$} & \multicolumn{3}{|c|}{2010} & \multicolumn{3}{|c|}{2015} & \multicolumn{3}{|c|}{2030} & \multicolumn{3}{|c|}{2045} \\
\hline & & & & & low & avg & high & low & avg & high & low & avg & high & low & avg & high \\
\hline \multirow{25}{*}{ FC HEV PHEV10 } & \multirow{9}{*}{ UDDS } & \multirow{4}{*}{$C D$} & Wh/mile & 273.9 & 273.9 & 237.9 & 212.3 & 257.5 & 219.1 & 190.2 & 237.1 & 197.0 & 162.8 & 225.3 & 190.0 & 155.2 \\
\hline & & & Distance & 11.1 & 11.1 & 10.1 & 10.1 & 11.1 & 10.1 & 10.1 & 10.1 & 10.2 & 10.2 & 10.1 & 10.1 & 10.2 \\
\hline & & & MPGGE Unadjusted & N/A & N/A & N/A & N/A & N/A & N/A & $\mathrm{N} / \mathrm{A}$ & N/A & N/A & N/A & $\mathrm{N} / \mathrm{A}$ & N/A & N/A \\
\hline & & & MPGGE Adjusted & N/A & N/A & N/A & N/A & N/A & N/A & N/A & N/A & N/A & N/A & N/A & N/A & N/A \\
\hline & & \multirow{2}{*}{ CS } & MPGGE Unadjusted & 59.6 & 59.6 & 71.0 & 83.6 & 69.1 & 81.0 & 94.5 & 75.0 & 91.4 & 113.2 & 78.9 & 94.4 & 118.8 \\
\hline & & & MPGGE Adjusted & 43.2 & 43.2 & 50.4 & 58.1 & 49.3 & 56.6 & 64.4 & 52.9 & 62.6 & 74.7 & 55.3 & 64.4 & 77.7 \\
\hline & & \multirow{3}{*}{$\mathrm{CD}+\mathrm{CS}$} & Wh/mile & 57.1 & 57.1 & 49.6 & 44.2 & 53.7 & 45.7 & 39.6 & 49.4 & 41.0 & 33.9 & 46.9 & 39.6 & 32.3 \\
\hline & & & MPGGE Unadjusted & 71.8 & 71.8 & 85.5 & 100.7 & 83.3 & 97.6 & 113.8 & 90.3 & 110.1 & 136.4 & 95.0 & 113.8 & 143.1 \\
\hline & & & \begin{tabular}{|l|} 
MPGGE Adjusted \\
\end{tabular} & 50.9 & 50.9 & 59.2 & 67.9 & 57.9 & 66.2 & 75.1 & 62.0 & 73.1 & 86.7 & 64.7 & 75.0 & 89.9 \\
\hline & \multirow{9}{*}{ HWFET } & \multirow{4}{*}{$C D$} & Wh/mile & 274.9 & 274.9 & 237.8 & 212.4 & 257.8 & 219.4 & 190.7 & 237.2 & 197.9 & 163.4 & 225.1 & 190.1 & 156.4 \\
\hline & & & Distance & 9.6 & 9.6 & 9.5 & 9.4 & 9.5 & 9.4 & 9.4 & 9.4 & 9.4 & 9.4 & 9.4 & 9.4 & 9.5 \\
\hline & & & MPGGE Unadjusted & 1851.9 & 1851.9 & 1589.5 & 1405.7 & 1388.2 & 1381.1 & 1475.5 & 1203.2 & 1377.5 & 1553.5 & 1142.0 & 1441.5 & 2350.3 \\
\hline & & & MPGGE Adjusted & 938.5 & 938.5 & 839.9 & 765.6 & 758.3 & 755.4 & 794.4 & 678.4 & 753.9 & 825.7 & 650.8 & 780.5 & 1105.0 \\
\hline & & \multirow{2}{*}{ CS } & MPGGE Unadjusted & 64.2 & 64.2 & 76.0 & 88.1 & 73.5 & 85.5 & 97.9 & 78.8 & 94.3 & 113.9 & 82.5 & 98.2 & 121.7 \\
\hline & & & \begin{tabular}{|l|} 
MPGGE Adjusted \\
\end{tabular} & 45.1 & 45.1 & 53.2 & 61.6 & 51.5 & 59.8 & 68.3 & 55.2 & 65.8 & 79.2 & 57.7 & 68.5 & 84.5 \\
\hline & & \multirow{3}{*}{$C D+C S$} & Wh/mile & 80.9 & 80.9 & 69.9 & 62.5 & 75.8 & 64.5 & 56.1 & 69.8 & 58.2 & 48.1 & 66.2 & 55.9 & 46.0 \\
\hline & & & MPGGE Unadjusted & 83.6 & 83.6 & 98.4 & 113.7 & 95.1 & 110.3 & 126.2 & 101.6 & 121.4 & 146.4 & 106.1 & 126.5 & 157.5 \\
\hline & & & MPGGE Adjusted & 58.5 & 58.5 & 68.7 & 79.1 & 66.4 & 76.8 & 87.5 & 70.8 & 84.3 & 101.1 & 73.9 & 87.7 & 108.5 \\
\hline & \multirow{7}{*}{ Combined } & \multirow{2}{*}{$C D$} & MPGGE Unadjusted & N/A & $\mathrm{N} / \mathrm{A}$ & $\mathrm{N} / \mathrm{A}$ & N/A & $\mathrm{N} / \mathrm{A}$ & N/A & N/A & $\mathrm{N} / \mathrm{A}$ & $\mathrm{N} / \mathrm{A}$ & N/A & N/A & N/A & N/A \\
\hline & & & MPGGE Adjusted & N/A & $\mathrm{N} / \mathrm{A}$ & N/A & N/A & N/A & N/A & N/A & $\mathrm{N} / \mathrm{A}$ & N/A & N/A & N/A & N/A & N/A \\
\hline & & \multirow{2}{*}{ CS } & MPGGE Unadjusted & 61.6 & 61.6 & 73.1 & 85.5 & 71.0 & 83.0 & 96.0 & 76.7 & 92.6 & 113.5 & 80.4 & 96.1 & 120.0 \\
\hline & & & MPGGE Adjusted & 44.1 & 44.1 & 51.7 & 59.6 & 50.3 & 58.0 & 66.1 & 53.9 & 64.0 & 76.7 & 56.3 & 66.2 & 80.6 \\
\hline & & \multirow{3}{*}{$C D+C S$} & Wh/mile & 67.8 & 67.8 & 58.7 & 52.4 & 63.6 & 54.1 & 47.0 & 58.6 & 48.8 & 40.3 & 55.6 & 46.9 & 38.5 \\
\hline & & & MPGGE Unadjusted & 76.6 & 76.6 & 90.9 & 106.1 & 88.2 & 103.0 & 119.1 & 95.1 & 114.9 & 140.7 & 99.7 & 119.2 & 149.2 \\
\hline & & & MPGGE Adjusted & 54.1 & 54.1 & 63.1 & 72.5 & 61.4 & 70.6 & 80.2 & 65.7 & 77.7 & 92.6 & 68.5 & 80.3 & 97.5 \\
\hline
\end{tabular}


Argonne National Laboratory Report - Light-Duty Vehicle Fuel Consumption Displacement Potential Up to 2045

\begin{tabular}{|c|c|c|c|c|c|c|c|c|c|c|c|c|c|c|c|c|}
\hline & & & & \multicolumn{13}{|c|}{ Midsize } \\
\hline & & & & \multirow{2}{*}{$\begin{array}{c}2010 \\
\text { Ref }\end{array}$} & \multicolumn{3}{|c|}{2010} & \multicolumn{3}{|c|}{2015} & \multicolumn{3}{|c|}{2030} & \multicolumn{3}{|c|}{2045} \\
\hline \multirow{26}{*}{ FC HEV PHEV2O } & & \multirow{5}{*}{$C D$} & & & low & avg & high & low & avg & high & low & avg & high & low & avg & high \\
\hline & \multirow{9}{*}{ UDDS } & & Wh/mile & 278.9 & 278.9 & 241.6 & 215.4 & 261.5 & 221.9 & 193.4 & 240.5 & 200.2 & 160.3 & 228.1 & 192.8 & 153.0 \\
\hline & & & Distance & 20.2 & 20.2 & 20.0 & 20.0 & 20.1 & 20.0 & 20.6 & 20.0 & 20.6 & 20.2 & 20.0 & 20.6 & 20.2 \\
\hline & & & MPGGE Unadjusted & $\mathrm{N} / \mathrm{A}$ & $\mathrm{N} / \mathrm{A}$ & $\mathrm{N} / \mathrm{A}$ & $\mathrm{N} / \mathrm{A}$ & $\mathrm{N} / \mathrm{A}$ & $\mathrm{N} / \mathrm{A}$ & $\mathrm{N} / \mathrm{A}$ & $\mathrm{N} / \mathrm{A}$ & $\mathrm{N} / \mathrm{A}$ & $\mathrm{N} / \mathrm{A}$ & $\mathrm{N} / \mathrm{A}$ & $\mathrm{N} / \mathrm{A}$ & $\mathrm{N} / \mathrm{A}$ \\
\hline & & & MPGGE Adjusted & $\mathrm{N} / \mathrm{A}$ & $\mathrm{N} / \mathrm{A}$ & $\mathrm{N} / \mathrm{A}$ & $\mathrm{N} / \mathrm{A}$ & $\mathrm{N} / \mathrm{A}$ & $\mathrm{N} / \mathrm{A}$ & $\mathrm{N} / \mathrm{A}$ & $\mathrm{N} / \mathrm{A}$ & $\mathrm{N} / \mathrm{A}$ & $\mathrm{N} / \mathrm{A}$ & $\mathrm{N} / \mathrm{A}$ & $\mathrm{N} / \mathrm{A}$ & $\mathrm{N} / \mathrm{A}$ \\
\hline & & \multirow{2}{*}{ CS } & MPGGE Unadjusted & 58.5 & 58.5 & 69.9 & 82.3 & 68.1 & 80.0 & 93.4 & 73.9 & 90.4 & 112.6 & 77.9 & 93.6 & 118.1 \\
\hline & & & MPGGE Adjusted & 42.5 & 42.5 & 49.8 & 57.3 & 48.6 & 56.0 & 63.8 & 52.3 & 62.0 & 74.4 & 54.7 & 63.9 & 77.3 \\
\hline & & \multirow{3}{*}{$\mathrm{CD}+\mathrm{CS}$} & Wh/mile & 106.0 & 106.0 & 91.8 & 81.9 & 99.3 & 84.3 & 73.6 & 91.4 & 76.2 & 61.1 & 86.6 & 73.4 & 58.3 \\
\hline & & & MPGGE Unadjusted & 86.0 & 86.0 & 102.8 & 121.1 & 100.1 & 117.6 & 137.4 & 108.7 & 132.9 & 165.6 & 114.5 & 137.7 & 173.6 \\
\hline & & & MPGGE Adjusted & 59.5 & 59.5 & 69.1 & 78.9 & 67.6 & 77.1 & 87.2 & 72.3 & 84.9 & 100.5 & 75.4 & 87.3 & 104.1 \\
\hline & \multirow{9}{*}{ HWFET } & \multirow{4}{*}{$C D$} & Wh/mile & 287.5 & 287.5 & 251.7 & 228.3 & 274.2 & 235.2 & 205.0 & 255.6 & 213.1 & 177.8 & 244.0 & 204.1 & 166.4 \\
\hline & & & Distance & 19.4 & 19.4 & 19.2 & 19.0 & 19.3 & 19.1 & 19.0 & 19.2 & 18.8 & 18.0 & 19.0 & 19.0 & 18.5 \\
\hline & & & MPGGE Unadjusted & N/A & $\mathrm{N} / \mathrm{A}$ & N/A & $\mathrm{N} / \mathrm{A}$ & $\mathrm{N} / \mathrm{A}$ & $\mathrm{N} / \mathrm{A}$ & N/A & N/A & N/A & N/A & $\mathrm{N} / \mathrm{A}$ & $\mathrm{N} / \mathrm{A}$ & N/A \\
\hline & & & MPGGE Adjusted & $\mathrm{N} / \mathrm{A}$ & $\mathrm{N} / \mathrm{A}$ & $\mathrm{N} / \mathrm{A}$ & $\mathrm{N} / \mathrm{A}$ & $\mathrm{N} / \mathrm{A}$ & $\mathrm{N} / \mathrm{A}$ & $\mathrm{N} / \mathrm{A}$ & $\mathrm{N} / \mathrm{A}$ & N/A & N/A & N/A & N/A & N/A \\
\hline & & \multirow{2}{*}{ CS } & MPGGE Unadjusted & 63.4 & 63.4 & 75.1 & 87.2 & 72.7 & 84.7 & 97.2 & 78.0 & 93.6 & 113.4 & 81.7 & 97.7 & 121.1 \\
\hline & & & MPGGE Adjusted & 44.6 & 44.6 & 52.7 & 61.0 & 51.0 & 59.3 & 67.9 & 54.7 & 65.4 & 78.9 & 57.2 & 68.2 & 84.1 \\
\hline & & \multirow{3}{*}{$\mathrm{CD}+\mathrm{CS}$} & Wh/mile & 84.6 & 84.6 & 74.0 & 67.1 & 80.7 & 69.2 & 60.3 & 75.2 & 62.7 & 52.3 & 71.8 & 60.0 & 48.9 \\
\hline & & & MPGGE Unadjusted & 83.4 & 83.4 & 98.8 & 114.8 & 95.7 & 111.4 & 127.9 & 102.6 & 123.2 & 149.3 & 107.5 & 128.6 & 159.3 \\
\hline & & & MPGGE Adjusted & 58.4 & 58.4 & 68.9 & 79.8 & 66.8 & 77.5 & 88.7 & 71.5 & 85.5 & 103.0 & 74.9 & 89.1 & 109.7 \\
\hline & \multirow{7}{*}{ Combined } & \multirow{2}{*}{$C D$} & MPGGE Unadjusted & $\mathrm{N} / \mathrm{A}$ & $\mathrm{N} / \mathrm{A}$ & $\mathrm{N} / \mathrm{A}$ & N/A & $\mathrm{N} / \mathrm{A}$ & N/A & N/A & N/A & $\mathrm{N} / \mathrm{A}$ & N/A & $\mathrm{N} / \mathrm{A}$ & $\mathrm{N} / \mathrm{A}$ & N/A \\
\hline & & & MPGGE Adjusted & $\mathrm{N} / \mathrm{A}$ & $\mathrm{N} / \mathrm{A}$ & $\mathrm{N} / \mathrm{A}$ & N/A & $\mathrm{N} / \mathrm{A}$ & $\mathrm{N} / \mathrm{A}$ & N/A & N/A & $\mathrm{N} / \mathrm{A}$ & N/A & $\mathrm{N} / \mathrm{A}$ & $\mathrm{N} / \mathrm{A}$ & N/A \\
\hline & & \multirow{2}{*}{ CS } & MPGGE Unadjusted & 60.6 & 60.6 & 72.1 & 84.5 & 70.1 & 82.0 & 95.1 & 75.7 & 91.8 & 113.0 & 79.5 & 95.4 & 119.4 \\
\hline & & & MPGGE Adjusted & 43.4 & 43.4 & 51.0 & 58.9 & 49.7 & 57.4 & 65.6 & 53.3 & 63.5 & 76.4 & 55.8 & 65.8 & 80.2 \\
\hline & & \multirow{3}{*}{$C D+C S$} & Wh/mile & 96.3 & 96.3 & 83.8 & 75.2 & 90.9 & 77.5 & 67.6 & 84.1 & 70.1 & 57.1 & 79.9 & 67.4 & 54.1 \\
\hline & & & MPGGE Unadjusted & 84.8 & 84.8 & 100.9 & 118.1 & 98.1 & 114.8 & 132.9 & 105.9 & 128.3 & 157.8 & 111.2 & 133.4 & 166.9 \\
\hline & & & MPGGE Adjusted & 59.0 & 59.0 & 69.0 & 79.3 & 67.2 & 77.3 & 87.8 & 72.0 & 85.2 & 101.6 & 75.2 & 88.1 & 106.5 \\
\hline
\end{tabular}


Argonne National Laboratory Report - Light-Duty Vehicle Fuel Consumption Displacement Potential Up to 2045

\begin{tabular}{|c|c|c|c|c|c|c|c|c|c|c|c|c|c|c|c|c|}
\hline & & & & \multicolumn{13}{|c|}{ Midsize } \\
\hline & & & & \multirow{2}{*}{$\frac{2010}{\text { Ref }}$} & \multicolumn{3}{|c|}{2010} & \multicolumn{3}{|c|}{2015} & \multicolumn{3}{|c|}{2030} & \multicolumn{3}{|c|}{2045} \\
\hline & & & & & low & avg & high & low & avg & high & low & avg & high & low & avg & high \\
\hline \multirow{25}{*}{ FC HEV PHEV 30} & \multirow{9}{*}{ UDDS } & \multirow{4}{*}{$C D$} & Wh/mile & 271.3 & 271.3 & 236.3 & 214.7 & 256.5 & 219.7 & 189.1 & 237.9 & 196.0 & 160.7 & 225.2 & 187.7 & 153.1 \\
\hline & & & Distance & 31.7 & 31.7 & 31.7 & 31.6 & 31.6 & 31.7 & 31.5 & 31.6 & 31.7 & 31.4 & 31.5 & 31.5 & 31.5 \\
\hline & & & MPGGE Unadjusted & N/A & N/A & N/A & N/A & N/A & N/A & $\mathrm{N} / \mathrm{A}$ & N/A & N/A & N/A & $\mathrm{N} / \mathrm{A}$ & N/A & N/A \\
\hline & & & MPGGE Adjusted & N/A & N/A & N/A & N/A & N/A & N/A & N/A & N/A & N/A & N/A & N/A & N/A & N/A \\
\hline & & \multirow{2}{*}{ CS } & MPGGE Unadjusted & 57.7 & 57.7 & 68.6 & 79.4 & 66.7 & 77.8 & 91.3 & 71.8 & 88.1 & 109.5 & 75.8 & 92.0 & 115.0 \\
\hline & & & MPGGE Adjusted & 42.1 & 42.1 & 49.0 & 55.6 & 47.8 & 54.6 & 62.6 & 51.0 & 60.7 & 72.7 & 53.4 & 63.0 & 75.7 \\
\hline & & \multirow{3}{*}{$\mathrm{CD}+\mathrm{CS}$} & Wh/mile & 172.9 & 172.9 & 150.6 & 136.8 & 163.4 & 139.9 & 120.9 & 151.6 & 125.3 & 103.4 & 143.5 & 120.0 & 98.5 \\
\hline & & & MPGGE Unadjusted & 122.9 & 122.9 & 146.0 & 169.0 & 141.8 & 165.4 & 194.1 & 152.8 & 187.4 & 232.9 & 161.3 & 195.7 & 244.6 \\
\hline & & & MPGGE Adjusted & 79.8 & 79.8 & 91.3 & 102.0 & 89.3 & 100.4 & 112.8 & 94.6 & 110.0 & 128.0 & 98.5 & 113.5 & 132.3 \\
\hline & \multirow{9}{*}{ HWFET } & \multirow{4}{*}{$C D$} & Wh/mile & 272.0 & 272.0 & 244.6 & 224.6 & 266.8 & 230.2 & 200.9 & 250.0 & 208.9 & 174.6 & 238.2 & 199.4 & 163.4 \\
\hline & & & Distance & 31.1 & 31.1 & 29.7 & 29.5 & 29.6 & 29.5 & 29.2 & 29.5 & 29.0 & 28.3 & 29.4 & 29.2 & 28.8 \\
\hline & & & MPGGE Unadjusted & N/A & N/A & N/A & N/A & N/A & N/A & N/A & N/A & N/A & N/A & N/A & N/A & $\mathrm{N} / \mathrm{A}$ \\
\hline & & & MPGGE Adjusted & N/A & N/A & N/A & N/A & N/A & N/A & N/A & N/A & N/A & N/A & N/A & N/A & N/A \\
\hline & & \multirow{2}{*}{ CS } & MPGGE Unadjusted & 62.2 & 62.2 & 73.4 & 84.2 & 70.9 & 82.2 & 94.7 & 75.7 & 91.1 & 110.2 & 79.5 & 95.4 & 117.8 \\
\hline & & & \begin{tabular}{|l|} 
MPGEE Adjusted \\
\end{tabular} & 43.8 & 43.8 & 51.5 & 58.9 & 49.8 & 57.5 & 66.1 & 53.1 & 63.7 & 76.7 & 55.7 & 66.6 & 81.8 \\
\hline & & \multirow{3}{*}{$\mathrm{CD}+\mathrm{CS}$} & Wh/mile & 184.6 & 184.6 & 125.9 & 115.6 & 137.3 & 118.5 & 103.5 & 128.7 & 107.6 & 90.0 & 122.6 & 102.7 & 84.3 \\
\hline & & & MPGGE Unadjusted & 132.4 & 132.4 & 124.4 & 142.7 & 120.2 & 139.3 & 160.5 & 128.3 & 154.4 & 186.8 & 134.7 & 161.8 & 199.6 \\
\hline & & & MPGGE Adjusted & 91.7 & 91.7 & 86.3 & 98.6 & 83.4 & 96.3 & 110.5 & 88.9 & 106.5 & 127.9 & 93.2 & 111.3 & 136.3 \\
\hline & \multirow{7}{*}{ Combined } & \multirow{2}{*}{$C D$} & MPGGE Unadjusted & N/A & $\mathrm{N} / \mathrm{A}$ & $\mathrm{N} / \mathrm{A}$ & N/A & $\mathrm{N} / \mathrm{A}$ & N/A & $\mathrm{N} / \mathrm{A}$ & $\mathrm{N} / \mathrm{A}$ & N/A & $\mathrm{N} / \mathrm{A}$ & N/A & N/A & N/A \\
\hline & & & MPGGE Adjusted & N/A & $\mathrm{N} / \mathrm{A}$ & N/A & N/A & N/A & N/A & N/A & $\mathrm{N} / \mathrm{A}$ & N/A & N/A & N/A & N/A & N/A \\
\hline & & \multirow{2}{*}{ CS } & MPGGE Unadjusted & 59.7 & 59.7 & 70.7 & 81.5 & 68.5 & 79.7 & 92.8 & 73.5 & 89.4 & 109.8 & 77.4 & 93.5 & 116.2 \\
\hline & & & MPGGE Adjusted & 42.8 & 42.8 & 50.1 & 57.0 & 48.6 & 55.9 & 64.1 & 51.9 & 62.0 & 74.5 & 54.4 & 64.6 & 78.3 \\
\hline & & \multirow{3}{*}{$C D+C S$} & Wh/mile & 178.1 & 178.1 & 139.5 & 127.2 & 151.7 & 130.3 & 113.1 & 141.3 & 117.3 & 97.4 & 134.1 & 112.2 & 92.1 \\
\hline & & & MPGGE Unadjusted & 127.0 & 127.0 & 135.4 & 156.0 & 131.2 & 152.5 & 177.4 & 140.7 & 170.9 & 209.6 & 148.1 & 178.8 & 222.1 \\
\hline & & & \begin{tabular}{|l|} 
MPGGE Adjusted \\
\end{tabular} & 84.8 & 84.8 & 89.0 & 100.4 & 86.6 & 98.5 & 111.8 & 91.9 & 108.4 & 128.0 & 96.1 & 112.5 & 134.0 \\
\hline
\end{tabular}


Argonne National Laboratory Report - Light-Duty Vehicle Fuel Consumption Displacement Potential Up to 2045

\begin{tabular}{|c|c|c|c|c|c|c|c|c|c|c|c|c|c|c|c|c|}
\hline & & & & \multicolumn{13}{|c|}{ Midsize } \\
\hline & & & & 2010 & & 2010 & & & 2015 & & & 2030 & & & 2045 & \\
\hline \multirow{26}{*}{ FC HEV PHEV 40} & & \multirow{5}{*}{$C D$} & & Ref & low & avg & high & low & avg & high & low & avg & high & low & avg & high \\
\hline & \multirow{9}{*}{ UDDS } & & Wh/mile & 276.4 & 276.4 & 240.2 & 217.9 & 261.0 & 223.1 & 190.7 & 241.7 & 197.9 & 163.0 & 228.3 & 189.2 & 155.3 \\
\hline & & & Distance & 41.4 & 41.4 & 41.2 & 41.2 & 41.2 & 41.1 & 41.2 & 41.2 & 41.2 & 40.6 & 41.4 & 41.1 & 40.5 \\
\hline & & & MPGGE Unadjusted & N/A & $\mathrm{N} / \mathrm{A}$ & $\mathrm{N} / \mathrm{A}$ & $\mathrm{N} / \mathrm{A}$ & $\mathrm{N} / \mathrm{A}$ & $\mathrm{N} / \mathrm{A}$ & N/A & $\mathrm{N} / \mathrm{A}$ & $\mathrm{N} / \mathrm{A}$ & $\mathrm{N} / \mathrm{A}$ & $\mathrm{N} / \mathrm{A}$ & $\mathrm{N} / \mathrm{A}$ & $\mathrm{N} / \mathrm{A}$ \\
\hline & & & MPGGE Adjusted & N/A & $\mathrm{N} / \mathrm{A}$ & N/A & $\mathrm{N} / \mathrm{A}$ & $\mathrm{N} / \mathrm{A}$ & $\mathrm{N} / \mathrm{A}$ & $\mathrm{N} / \mathrm{A}$ & N/A & N/A & $\mathrm{N} / \mathrm{A}$ & N/A & $\mathrm{N} / \mathrm{A}$ & N/A \\
\hline & & \multirow{2}{*}{ CS } & MPGGE Unadjusted & 56.6 & 56.6 & 67.5 & 78.2 & 65.5 & 76.5 & 90.2 & 70.6 & 87.0 & 108.5 & 74.7 & 91.0 & 113.9 \\
\hline & & & MPGGE Adjusted & 41.3 & 41.3 & 48.3 & 54.9 & 47.0 & 53.8 & 62.0 & 50.2 & 60.1 & 72.2 & 52.8 & 62.4 & 75.1 \\
\hline & & \multirow{3}{*}{$C D+C S$} & Wh/mile & 199.8 & 199.8 & 173.6 & 157.6 & 188.7 & 161.3 & 138.5 & 174.7 & 143.7 & 119.8 & 165.1 & 137.3 & 114.2 \\
\hline & & & MPGGE Unadjusted & 141.6 & 141.6 & 168.7 & 195.6 & 163.7 & 191.3 & 225.5 & 176.5 & 217.4 & 271.2 & 186.9 & 227.5 & 284.8 \\
\hline & & & MPGGE Adjusted & 89.2 & 89.2 & 101.9 & 113.4 & 99.6 & 111.6 & 125.2 & 105.3 & 122.1 & 141.5 & 109.8 & 126.0 & 145.9 \\
\hline & \multirow{9}{*}{ HWFET } & \multirow{4}{*}{$C D$} & Wh/mile & 282.4 & 282.4 & 247.5 & 227.1 & 270.2 & 232.8 & 202.7 & 252.9 & 210.9 & 174.7 & 240.5 & 201.1 & 163.5 \\
\hline & & & Distance & 40.0 & 40.0 & 39.8 & 39.0 & 39.7 & 38.9 & 38.1 & 38.9 & 38.0 & 37.8 & 39.7 & 38.0 & 38.4 \\
\hline & & & MPGGE Unadjusted & N/A & $\mathrm{N} / \mathrm{A}$ & N/A & $\mathrm{N} / \mathrm{A}$ & $\mathrm{N} / \mathrm{A}$ & $\mathrm{N} / \mathrm{A}$ & $\mathrm{N} / \mathrm{A}$ & N/A & $\mathrm{N} / \mathrm{A}$ & $\mathrm{N} / \mathrm{A}$ & $\mathrm{N} / \mathrm{A}$ & $\mathrm{N} / \mathrm{A}$ & $\mathrm{N} / \mathrm{A}$ \\
\hline & & & MPGGE Adjusted & $\mathrm{N} / \mathrm{A}$ & $\mathrm{N} / \mathrm{A}$ & $\mathrm{N} / \mathrm{A}$ & $\mathrm{N} / \mathrm{A}$ & $\mathrm{N} / \mathrm{A}$ & $\mathrm{N} / \mathrm{A}$ & N/A & $\mathrm{N} / \mathrm{A}$ & $\mathrm{N} / \mathrm{A}$ & $\mathrm{N} / \mathrm{A}$ & N/A & $\mathrm{N} / \mathrm{A}$ & N/A \\
\hline & & \multirow{2}{*}{ CS } & MPGGE Unadjusted & 61.4 & 61.4 & 72.6 & 83.3 & 70.0 & 81.3 & 94.0 & 74.9 & 90.3 & 109.6 & 78.7 & 94.7 & 117.1 \\
\hline & & & MPGGE Adjusted & 43.2 & 43.2 & 50.9 & 58.3 & 49.1 & 56.9 & 65.6 & 52.5 & 63.1 & 76.3 & 55.1 & 66.2 & 81.4 \\
\hline & & \multirow{3}{*}{$\mathrm{CD}+\mathrm{CS}$} & Wh/mile & 190.3 & 190.3 & 166.8 & 153.0 & 182.1 & 156.9 & 136.8 & 170.4 & 142.4 & 118.1 & 162.1 & 135.7 & 110.5 \\
\hline & & & MPGGE Unadjusted & 133.4 & 133.4 & 157.8 & 181.1 & 152.2 & 176.6 & 204.3 & 162.7 & 196.4 & 238.2 & 171.0 & 206.0 & 254.5 \\
\hline & & & MPGGE Adjusted & 92.4 & 92.4 & 108.7 & 124.1 & 105.0 & 121.2 & 139.3 & 112.0 & 134.2 & 161.4 & 117.5 & 140.5 & 171.8 \\
\hline & \multirow{7}{*}{ Combined } & \multirow{2}{*}{$C D$} & MPGGE Unadjusted & $\mathrm{N} / \mathrm{A}$ & $\mathrm{N} / \mathrm{A}$ & $\mathrm{N} / \mathrm{A}$ & $\mathrm{N} / \mathrm{A}$ & $\mathrm{N} / \mathrm{A}$ & $\mathrm{N} / \mathrm{A}$ & $\mathrm{N} / \mathrm{A}$ & N/A & $\mathrm{N} / \mathrm{A}$ & N/A & N/A & $\mathrm{N} / \mathrm{A}$ & N/A \\
\hline & & & MPGGE Adjusted & $\mathrm{N} / \mathrm{A}$ & $\mathrm{N} / \mathrm{A}$ & $\mathrm{N} / \mathrm{A}$ & $\mathrm{N} / \mathrm{A}$ & $\mathrm{N} / \mathrm{A}$ & $\mathrm{N} / \mathrm{A}$ & $\mathrm{N} / \mathrm{A}$ & $\mathrm{N} / \mathrm{A}$ & $\mathrm{N} / \mathrm{A}$ & $\mathrm{N} / \mathrm{A}$ & $\mathrm{N} / \mathrm{A}$ & $\mathrm{N} / \mathrm{A}$ & $\mathrm{N} / \mathrm{A}$ \\
\hline & & \multirow{2}{*}{ CS } & MPGGE Unadjusted & 58.7 & 58.7 & 69.7 & 80.4 & 67.5 & 78.6 & 91.8 & 72.5 & 88.5 & 109.0 & 76.5 & 92.6 & 115.3 \\
\hline & & & MPGGE Adjusted & 42.1 & 42.1 & 49.4 & 56.4 & 48.0 & 55.2 & 63.5 & 51.2 & 61.4 & 74.0 & 53.8 & 64.0 & 77.8 \\
\hline & & \multirow{3}{*}{$\mathrm{CD}+\mathrm{CS}$} & Wh/mile & 195.5 & 195.5 & 170.5 & 155.5 & 185.7 & 159.3 & 137.7 & 172.8 & 143.1 & 119.0 & 163.7 & 136.6 & 112.5 \\
\hline & & & MPGGE Unadjusted & 137.8 & 137.8 & 163.6 & 188.8 & 158.3 & 184.4 & 215.4 & 170.0 & 207.4 & 255.3 & 179.4 & 217.3 & 270.3 \\
\hline & & & MPGGE Adjusted & 90.6 & 90.6 & 104.8 & 118.0 & 102.0 & 115.7 & 131.2 & 108.2 & 127.3 & 149.8 & 113.1 & 132.1 & 156.5 \\
\hline \multirow{2}{*}{ EV } & UDDS & EV Only & Wh/mile & 271.1 & 271.1 & 248.2 & 229.1 & 268.3 & 236.9 & 196.3 & 252.8 & 203.1 & 160.8 & 243.8 & 193.4 & 152.7 \\
\hline & HWFET & EV Only & Wh/mile & 275.0 & 275.0 & 250.1 & 232.3 & 272.1 & 239.9 & 204.0 & 257.5 & 211.4 & \begin{tabular}{|l|l}
173.4 \\
\end{tabular} & 248.4 & 201.1 & 161.8 \\
\hline
\end{tabular}


Argonne National Laboratory Report - Light-Duty Vehicle Fuel Consumption Displacement Potential Up to 2045

\begin{tabular}{|c|c|c|c|c|c|c|c|c|c|c|c|c|c|c|c|c|}
\hline & & & & \multicolumn{13}{|c|}{ Midsize } \\
\hline & & & & \multirow{2}{*}{$\frac{2010}{\text { Ref }}$} & \multicolumn{3}{|c|}{2010} & \multicolumn{3}{|c|}{2015} & \multicolumn{3}{|c|}{2030} & \multicolumn{3}{|c|}{2045} \\
\hline & & & & & low & avg & high & low & avg & high & low & avg & high & low & avg & high \\
\hline \multirow{25}{*}{ H2 Series HEV PHEV 30} & \multirow{9}{*}{ UDDS } & \multirow{4}{*}{$C D$} & Wh/mile & 284.6 & 284.6 & 258.3 & 239.2 & 278.8 & 245.8 & 215.3 & 264.7 & 223.4 & 183.5 & 253.9 & 215.0 & 176.8 \\
\hline & & & Distance & 30.9 & 30.9 & 31.0 & 31.0 & 30.9 & 31.0 & 30.9 & 31.0 & 30.9 & 30.7 & 31.0 & 30.9 & 30.9 \\
\hline & & & MPGGE Unadjusted & N/A & N/A & N/A & N/A & N/A & N/A & N/A & N/A & N/A & N/A & N/A & N/A & N/A \\
\hline & & & MPGGE Adjusted & N/A & N/A & N/A & N/A & N/A & N/A & N/A & N/A & N/A & N/A & N/A & N/A & N/A \\
\hline & & \multirow{2}{*}{ CS } & MPGGE Unadjusted & 39.5 & 39.5 & 48.4 & 56.8 & 44.6 & 55.0 & 67.5 & 50.8 & 64.7 & 82.9 & 55.6 & 69.1 & 89.6 \\
\hline & & & MPGGE Adjusted & 29.8 & 29.8 & 35.9 & 41.5 & 33.3 & 40.3 & 48.3 & 37.5 & 46.5 & 57.7 & 40.7 & 49.3 & 61.6 \\
\hline & & \multirow{3}{*}{$\mathrm{CD}+\mathrm{CS}$} & Wh/mile & 183.4 & 183.4 & 166.4 & 154.1 & 179.7 & 158.4 & 139.3 & 170.5 & 144.6 & 119.2 & 163.6 & 139.0 & 114.9 \\
\hline & & & MPGGE Unadjusted & 84.0 & 84.0 & 102.9 & 120.9 & 95.0 & 117.0 & 143.6 & 108.1 & 137.7 & 176.3 & 118.3 & 146.9 & 190.7 \\
\hline & & & MPGGE Adjusted & 58.4 & 58.4 & 69.1 & 78.8 & 64.7 & 76.8 & 90.2 & 72.0 & 87.3 & 105.2 & 77.4 & 91.8 & 111.4 \\
\hline & \multirow{9}{*}{ HWFET } & \multirow{4}{*}{$C D$} & Wh/mile & 274.0 & 274.0 & 247.8 & 230.2 & 269.2 & 236.8 & 206.7 & 256.2 & 215.3 & 178.3 & 246.0 & 206.4 & 167.8 \\
\hline & & & \begin{tabular}{|l|} 
Distance \\
\end{tabular} & 31.9 & 31.9 & 32.2 & 32.1 & 31.8 & 32.0 & 32.0 & 31.8 & 31.9 & 31.7 & 31.8 & 31.9 & 32.9 \\
\hline & & & MPGGE Unadjusted & $N / A$ & N/A & N/A & $\mathrm{N} / \mathrm{A}$ & $\mathrm{N} / \mathrm{A}$ & N/A & N/A & N/A & N/A & N/A & N/A & $\mathrm{N} / \mathrm{A}$ & $\mathrm{N} / \mathrm{A}$ \\
\hline & & & MPGGE Adjusted & N/A & N/A & N/A & N/A & N/A & N/A & N/A & N/A & N/A & N/A & N/A & N/A & N/A \\
\hline & & \multirow{2}{*}{ CS } & MPGGE Unadjusted & 39.7 & 39.7 & 48.8 & 56.7 & 44.7 & 55.1 & 67.3 & 50.8 & 64.2 & 81.5 & 55.5 & 69.7 & 89.7 \\
\hline & & & MPGGE Adjusted & 28.1 & 28.1 & 34.4 & 39.9 & 31.6 & 38.8 & 47.2 & 35.8 & 45.2 & 57.0 & 39.1 & 48.9 & 62.7 \\
\hline & & \multirow{3}{*}{$\mathrm{CD}+\mathrm{CS}$} & Wh/mile & 185.2 & 185.2 & 167.5 & 155.6 & 181.9 & 160.0 & 139.7 & 173.1 & 145.5 & 120.5 & 166.3 & 139.5 & 113.4 \\
\hline & & & MPGGE Unadjusted & 86.3 & 86.3 & 106.0 & 123.2 & 97.2 & 119.7 & 146.2 & 110.5 & 139.6 & 177.1 & 120.6 & 151.4 & 194.9 \\
\hline & & & MPGGE Adjusted & 60.4 & 60.4 & 73.8 & 85.5 & 67.8 & 83.1 & 101.0 & 76.9 & 96.6 & 121.5 & 83.7 & 104.5 & 133.2 \\
\hline & \multirow{7}{*}{ Combined } & \multirow{2}{*}{$C D$} & MPGGE Unadjusted & $\mathrm{N} / \mathrm{A}$ & $\mathrm{N} / \mathrm{A}$ & $\mathrm{N} / \mathrm{A}$ & $\mathrm{N} / \mathrm{A}$ & $\mathrm{N} / \mathrm{A}$ & N/A & $\mathrm{N} / \mathrm{A}$ & $\mathrm{N} / \mathrm{A}$ & $\mathrm{N} / \mathrm{A}$ & $N / A$ & $\mathrm{~N} / \mathrm{A}$ & $\mathrm{N} / \mathrm{A}$ & $\mathrm{N} / \mathrm{A}$ \\
\hline & & & MPGGE Adjusted & N/A & N/A & N/A & N/A & N/A & N/A & N/A & N/A & N/A & $\mathrm{N} / \mathrm{A}$ & N/A & N/A & N/A \\
\hline & & \multirow{2}{*}{ CS } & MPGGE Unadjusted & 39.6 & 39.6 & 48.5 & 56.8 & 44.7 & 55.0 & 67.4 & 50.8 & 64.5 & 82.2 & 55.5 & 69.3 & 89.7 \\
\hline & & & MPGGE Adjusted & 29.0 & 29.0 & 35.2 & 40.7 & 32.5 & 39.6 & 47.8 & 36.7 & 45.9 & 57.4 & 39.9 & 49.1 & 62.1 \\
\hline & & \multirow{3}{*}{$\mathrm{CD}+\mathrm{CS}$} & Wh/mile & 184.2 & 184.2 & 166.9 & 154.8 & 180.7 & 159.1 & 139.5 & 171.7 & 145.0 & 119.8 & 164.8 & 139.2 & 114.2 \\
\hline & & & \begin{tabular}{|l|} 
MPGGE Unadjusted \\
\end{tabular} & 85.1 & 85.1 & 104.3 & 121.9 & 96.0 & 118.2 & 144.7 & 109.2 & 138.6 & 176.7 & 119.3 & 148.9 & 192.6 \\
\hline & & & MPGGE Adjusted & 59.3 & 59.3 & 71.2 & 81.7 & 66.1 & 79.5 & 94.7 & 74.1 & 91.3 & 112.0 & 80.2 & 97.1 & 120.3 \\
\hline
\end{tabular}

\section{Fuel consumption}




\begin{tabular}{|c|c|c|c|c|c|c|c|c|c|c|c|c|c|c|c|}
\hline & & & \multicolumn{13}{|c|}{ Midsize } \\
\hline & & & \multirow{2}{*}{$\frac{2010}{\operatorname{Ref}}$} & \multicolumn{3}{|c|}{2010} & \multicolumn{3}{|c|}{2015} & \multicolumn{3}{|c|}{2030} & \multicolumn{3}{|c|}{2045} \\
\hline & & & & low & avg & high & low & avg & high & low & avg & high & low & avg & high \\
\hline \multirow{6}{*}{ SI Conv } & \multirow{3}{*}{ Unadjusted } & UDDS & 8.6 & 8.6 & 7.9 & 7.5 & 8.2 & 7.8 & 5.9 & 8.0 & 7.4 & 5.0 & 8.1 & 7.0 & 4.9 \\
\hline & & HWFET & 5.8 & 5.8 & 5.3 & 5.0 & 5.6 & 5.8 & 4.0 & 5.4 & 5.5 & 3.4 & 6.0 & 4.7 & 3.3 \\
\hline & & Combined & 7.3 & 7.3 & 6.7 & 6.4 & 7.0 & 6.9 & 5.0 & 6.9 & 6.5 & 4.3 & 7.2 & 5.9 & 4.1 \\
\hline & \multirow{3}{*}{ Adjusted } & \begin{tabular}{|l|} 
UDDS \\
\end{tabular} & 11.2 & 11.2 & 10.3 & 9.8 & 10.7 & 10.1 & 7.8 & 10.4 & 9.7 & 6.7 & 10.5 & 9.1 & 6.6 \\
\hline & & HWFET & 8.2 & 8.2 & 7.5 & 7.1 & 7.9 & 8.2 & 5.7 & 7.7 & 7.8 & 4.8 & 8.5 & 6.6 & 4.6 \\
\hline & & Combined & 9.8 & 9.8 & 9.0 & 8.6 & 9.4 & 9.2 & 6.9 & 9.2 & 8.8 & 5.9 & 9.6 & 8.0 & 5.7 \\
\hline \multirow{6}{*}{$\mathrm{Cl}$ Conv } & \multirow{3}{*}{ Unadjusted } & \begin{tabular}{|l|} 
UDDS \\
\end{tabular} & 7.4 & 7.4 & 7.0 & 6.5 & 7.3 & 6.8 & 5.9 & 6.9 & 6.3 & 5.1 & 6.9 & 5.8 & 4.9 \\
\hline & & \begin{tabular}{|l|} 
HWFET \\
\end{tabular} & 5.3 & 5.3 & 4.9 & 4.4 & 5.1 & 5.0 & 4.2 & 4.8 & 4.6 & 3.6 & 5.1 & 4.1 & 3.4 \\
\hline & & Combined & 6.5 & 6.5 & 6.1 & 5.6 & 6.3 & 6.0 & 5.1 & 6.0 & 5.5 & 4.4 & 6.1 & 5.1 & 4.2 \\
\hline & \multirow{3}{*}{ Adjusted } & \begin{tabular}{|l|} 
UDDS \\
\end{tabular} & 9.7 & 9.7 & 9.2 & 8.5 & 9.6 & 8.9 & 7.9 & 9.1 & 8.3 & 6.8 & 9.0 & 7.8 & 6.6 \\
\hline & & \begin{tabular}{|c|} 
HWFET \\
\end{tabular} & 7.5 & 7.5 & 7.0 & 6.3 & 7.3 & 7.1 & 6.0 & 6.8 & 6.5 & 5.1 & 7.2 & 5.9 & 4.9 \\
\hline & & Combined & 8.7 & 8.7 & 8.2 & 7.5 & 8.5 & 8.1 & 7.0 & 8.1 & 7.5 & 6.1 & 8.2 & 6.9 & 5.8 \\
\hline \multirow{6}{*}{ H2 Conv } & \multirow{3}{*}{ Unadjusted } & \begin{tabular}{|l|} 
UDDS \\
\end{tabular} & 10.5 & 10.5 & 8.8 & 7.7 & 9.3 & 8.0 & 6.7 & 8.4 & 7.2 & 5.6 & 8.0 & 6.5 & 5.2 \\
\hline & & \begin{tabular}{|l|} 
HWFET \\
\end{tabular} & 6.5 & 6.5 & 5.4 & 4.6 & 5.6 & 5.7 & 4.1 & 5.0 & 5.2 & 3.5 & 5.8 & 4.0 & 3.2 \\
\hline & & Combined & 8.7 & 8.7 & 7.3 & 6.3 & 7.7 & 7.0 & 5.5 & 6.9 & 6.3 & 4.7 & 7.0 & 5.3 & 4.3 \\
\hline & \multirow{3}{*}{ Adjusted } & \begin{tabular}{|l|} 
UDDS \\
\end{tabular} & 13.5 & 13.5 & 11.4 & 10.0 & 12.0 & 10.4 & 8.8 & 10.9 & 9.4 & 7.5 & 10.4 & 8.5 & 7.0 \\
\hline & & HWFET & 9.2 & 9.2 & 7.7 & 6.5 & 7.9 & 8.1 & 5.8 & 7.1 & 7.4 & 5.0 & 8.2 & 5.7 & 4.6 \\
\hline & & Combined & 11.6 & 11.6 & 9.7 & 8.4 & 10.2 & 9.4 & 7.5 & 9.2 & 8.5 & 6.4 & 9.4 & 7.2 & 5.9 \\
\hline \multirow{6}{*}{ E85 Conv } & \multirow{3}{*}{ Unadjusted } & \begin{tabular}{|l|} 
UDDS \\
\end{tabular} & 8.9 & 8.9 & 8.8 & 8.1 & 8.9 & 8.5 & 6.2 & 8.7 & 8.0 & 5.3 & 8.9 & 7.7 & 5.1 \\
\hline & & \begin{tabular}{|c|} 
HWFET \\
\end{tabular} & 6.4 & 6.4 & 6.2 & 5.7 & 6.4 & 6.4 & 4.4 & 6.2 & 6.0 & 3.8 & 6.6 & 5.5 & 3.7 \\
\hline & & Combined & 7.7 & 7.7 & 7.6 & 7.0 & 7.8 & 7.5 & 5.4 & 7.6 & 7.1 & 4.6 & 7.9 & 6.7 & 4.5 \\
\hline & \multirow{3}{*}{ Adjusted } & UDDS & 11.5 & 11.5 & 11.3 & 10.5 & 11.5 & 11.0 & 8.1 & 11.3 & 10.4 & 7.1 & 11.5 & 10.0 & 6.9 \\
\hline & & \begin{tabular}{|c|} 
HWFET \\
\end{tabular} & 9.0 & 9.0 & 8.8 & 8.1 & 9.1 & 9.0 & 6.3 & 8.8 & 8.5 & 5.4 & 9.4 & 7.8 & 5.2 \\
\hline & & Combined & 10.4 & 10.4 & 10.2 & 9.4 & 10.4 & 10.1 & 7.3 & 10.2 & 9.6 & 6.3 & 10.5 & 9.0 & 6.1 \\
\hline \multirow{6}{*}{ SI Split HEV } & \multirow{3}{*}{ Unadjusted } & \begin{tabular}{|l|} 
UDDS \\
\end{tabular} & 4.4 & 4.4 & 4.0 & 3.7 & 4.3 & 3.8 & 3.0 & 4.0 & 3.4 & 2.4 & 3.9 & 3.2 & 2.3 \\
\hline & & \begin{tabular}{|c|} 
HWFET \\
\end{tabular} & 4.9 & 4.9 & 4.5 & 4.1 & 4.8 & 4.2 & 3.4 & 4.5 & 3.8 & 2.8 & 4.4 & 3.6 & 2.6 \\
\hline & & Combined & 4.6 & 4.6 & 4.2 & 3.9 & 4.5 & 4.0 & 3.2 & 4.2 & 3.6 & 2.6 & 4.1 & 3.4 & 2.4 \\
\hline & \multirow{3}{*}{ Adjusted } & \begin{tabular}{|l|} 
UDDS \\
\end{tabular} & 6.0 & 6.0 & 5.6 & 5.2 & 5.9 & 5.2 & 4.3 & 5.5 & 4.7 & 3.6 & 5.4 & 4.6 & 3.4 \\
\hline & & HWFET & 7.0 & 7.0 & 6.3 & 5.8 & 6.8 & 6.0 & 4.9 & 6.4 & 5.4 & 4.0 & 6.2 & 5.2 & 3.7 \\
\hline & & Combined & 6.5 & 6.5 & 5.9 & 5.5 & 6.3 & 5.5 & 4.6 & 5.9 & 5.1 & 3.7 & 5.7 & 4.8 & 3.6 \\
\hline \multirow{6}{*}{ SI Split HEV PHEV10 } & & UDDS & 3.5 & 3.5 & 3.3 & 3.0 & 3.5 & 3.1 & 2.5 & 3.3 & 2.8 & 2.0 & 3.2 & 2.7 & 1.9 \\
\hline & Unadjusted & HWFET & 3.6 & 3.6 & 3.3 & 3.0 & 3.5 & 3.1 & 2.5 & 3.3 & 2.8 & 2.0 & 3.2 & 2.7 & 1.9 \\
\hline & & Combined & 3.6 & 3.6 & 3.3 & 3.0 & 3.5 & 3.1 & 2.5 & 3.3 & 2.8 & 2.0 & 3.2 & 2.7 & 1.9 \\
\hline & & \begin{tabular}{|l|} 
UDDS \\
\end{tabular} & 4.9 & 4.9 & 4.6 & 4.3 & 4.9 & 4.4 & 3.7 & 4.6 & 4.0 & 3.0 & 4.5 & 3.9 & 2.9 \\
\hline & Adjusted & HWFET & 5.1 & 5.1 & 4.7 & 4.3 & 5.0 & 4.4 & 3.6 & 4.7 & 4.0 & 2.9 & 4.6 & 3.8 & 2.8 \\
\hline & & Combined & 5.0 & 5.0 & 4.6 & 4.3 & 4.9 & 4.4 & 3.6 & 4.7 & 4.0 & 3.0 & 4.5 & 3.9 & 2.8 \\
\hline
\end{tabular}




\begin{tabular}{|c|c|c|c|c|c|c|c|c|c|c|c|c|c|c|c|}
\hline & & & \multicolumn{13}{|c|}{ Midsize } \\
\hline & & & \multirow{2}{*}{$\begin{array}{l}2010 \\
\text { Ref }\end{array}$} & \multicolumn{3}{|c|}{2010} & \multicolumn{3}{|c|}{2015} & \multicolumn{3}{|c|}{2030} & \multicolumn{3}{|c|}{2045} \\
\hline & & & & low & avg & high & low & avg & high & low & avg & high & low & avg & high \\
\hline \multirow{6}{*}{ SI Split HEV PHEV2O } & \multirow{3}{*}{ Unadjusted } & UDDS & 2.5 & 2.5 & 2.3 & 2.1 & 2.4 & 2.1 & 1.7 & 2.3 & 1.9 & 1.7 & 2.2 & 1.9 & 1.6 \\
\hline & & HWFET & 3.0 & 3.0 & 2.7 & 2.4 & 2.9 & 2.6 & 2.4 & 2.7 & 2.7 & 2.0 & 2.7 & 2.6 & 1.9 \\
\hline & & Combined & 2.7 & 2.7 & 2.5 & 2.2 & 2.6 & 2.3 & 2.1 & 2.5 & 2.3 & 1.8 & 2.4 & 2.2 & 1.7 \\
\hline & \multirow{3}{*}{ Adjusted } & \begin{tabular}{|l|} 
UDDS \\
\end{tabular} & 3.7 & 3.7 & 3.4 & 3.1 & 3.6 & 3.2 & 2.7 & 3.4 & 3.0 & 2.6 & 3.3 & 2.9 & 2.5 \\
\hline & & HWFET & 4.2 & 4.2 & 3.9 & 3.5 & 4.1 & 3.7 & 3.5 & 3.9 & 3.9 & 2.9 & 3.8 & 3.7 & 2.7 \\
\hline & & Combined & 3.9 & 3.9 & 3.6 & 3.3 & 3.8 & 3.4 & 3.1 & 3.7 & 3.4 & 2.7 & 3.5 & 3.3 & 2.6 \\
\hline \multirow{6}{*}{ SI Series HEV PHEV30 } & \multirow{3}{*}{ Unadjusted } & \begin{tabular}{|l|} 
UDDS \\
\end{tabular} & 2.7 & 2.7 & 2.4 & 2.2 & 2.6 & 2.3 & 1.8 & 2.4 & 2.0 & 1.4 & 2.3 & 1.9 & 1.3 \\
\hline & & HWFET & 2.7 & 2.7 & 2.4 & 2.2 & 2.6 & 2.3 & 1.8 & 2.4 & 2.0 & 1.4 & 2.3 & 1.9 & 1.3 \\
\hline & & Combined & 2.7 & 2.7 & 2.4 & 2.2 & 2.6 & 2.3 & 1.8 & 2.4 & 2.0 & 1.4 & 2.3 & 1.9 & 1.3 \\
\hline & \multirow{3}{*}{ Adjusted } & UDDS & 3.9 & 3.9 & 3.6 & 3.3 & 3.8 & 3.4 & 2.8 & 3.6 & 3.0 & 2.3 & 3.5 & 2.9 & 2.2 \\
\hline & & \begin{tabular}{|l|} 
HWFET \\
\end{tabular} & 3.8 & 3.8 & 3.4 & 3.1 & 3.7 & 3.2 & 2.5 & 3.5 & 2.9 & 2.1 & 3.3 & 2.7 & 2.0 \\
\hline & & Combined & 3.9 & 3.9 & 3.5 & 3.2 & 3.8 & 3.3 & 2.7 & 3.5 & 3.0 & 2.2 & 3.4 & 2.9 & 2.1 \\
\hline \multirow{6}{*}{ SI Series HEV PHEV40 } & \multirow{3}{*}{ Unadjusted } & \begin{tabular}{|l|} 
UDDS \\
\end{tabular} & 2.3 & 2.3 & 2.1 & 1.9 & 2.3 & 2.0 & 1.5 & 2.1 & 1.7 & 1.2 & 2.0 & 1.7 & 1.1 \\
\hline & & HWFET & 2.2 & 2.2 & 2.0 & 1.8 & 2.1 & 1.8 & 1.4 & 2.0 & 1.6 & 1.2 & 1.9 & 1.5 & 1.1 \\
\hline & & Combined & 2.3 & 2.3 & 2.0 & 1.9 & 2.2 & 1.9 & 1.5 & 2.0 & 1.7 & 1.2 & 2.0 & 1.6 & 1.1 \\
\hline & \multirow{3}{*}{ Adjusted } & \begin{tabular}{|l|} 
UDDS \\
\end{tabular} & 3.5 & 3.5 & 3.2 & 2.9 & 3.4 & 3.0 & 2.5 & 3.2 & 2.7 & 2.0 & 3.1 & 2.6 & 2.0 \\
\hline & & HWFET & 3.1 & 3.1 & 2.8 & 2.6 & 3.0 & 2.7 & 2.1 & 2.8 & 2.3 & 1.7 & 2.7 & 2.2 & 1.6 \\
\hline & & Combined & 3.3 & 3.3 & 3.0 & 2.8 & 3.2 & 2.9 & 2.3 & 3.0 & 2.5 & 1.9 & 2.9 & 2.4 & 1.8 \\
\hline \multirow{6}{*}{ CI Split HEV } & \multirow{3}{*}{ Unadjusted } & \begin{tabular}{|l|} 
UDDS \\
\end{tabular} & 4.1 & 4.1 & 3.8 & 3.5 & 4.1 & 3.5 & 3.0 & 3.7 & 3.1 & 2.5 & 3.6 & 3.0 & 2.4 \\
\hline & & \begin{tabular}{|c|} 
HWFET \\
\end{tabular} & 4.7 & 4.7 & 4.3 & 3.9 & 4.6 & 4.0 & 3.4 & 4.2 & 3.6 & 2.9 & 4.1 & 3.4 & 2.7 \\
\hline & & Combined & 4.3 & 4.3 & 4.0 & 3.6 & 4.3 & 3.7 & 3.2 & 4.0 & 3.3 & 2.6 & 3.8 & 3.2 & 2.5 \\
\hline & \multirow{3}{*}{ Adjusted } & \begin{tabular}{|l|} 
UDDS \\
\end{tabular} & 5.6 & 5.6 & 5.3 & 4.9 & 5.6 & 4.9 & 4.3 & 5.2 & 4.4 & 3.6 & 5.0 & 4.2 & 3.5 \\
\hline & & \begin{tabular}{|c|} 
HWFET \\
\end{tabular} & 6.6 & 6.6 & 6.0 & 5.5 & 6.5 & 5.6 & 4.9 & 6.0 & 5.1 & 4.1 & 5.8 & 4.8 & 3.9 \\
\hline & & Combined & 6.1 & 6.1 & 5.6 & 5.1 & 6.0 & 5.2 & 4.6 & 5.6 & 4.7 & 3.8 & 5.3 & 4.5 & 3.7 \\
\hline \multirow{6}{*}{ CI Split HEV PHEV10 } & \multirow{3}{*}{ Unadjusted } & UDDS & 3.5 & 3.5 & 3.2 & 2.9 & 3.5 & 3.0 & 2.6 & 3.2 & 2.7 & 2.1 & 3.1 & 2.6 & 2.1 \\
\hline & & \begin{tabular}{|l|} 
HWFET \\
\end{tabular} & 3.6 & 3.6 & 3.3 & 3.0 & 3.5 & 3.1 & 2.6 & 3.3 & 2.7 & 2.2 & 3.1 & 2.6 & 2.1 \\
\hline & & Combined & 3.5 & 3.5 & 3.3 & 3.0 & 3.5 & 3.0 & 2.6 & 3.2 & 2.7 & 2.2 & 3.1 & 2.6 & 2.1 \\
\hline & \multirow{3}{*}{ Adjusted } & UDDS & 4.9 & 4.9 & 4.6 & 4.2 & 4.9 & 4.3 & 3.8 & 4.5 & 3.9 & 3.2 & 4.4 & 3.8 & 3.1 \\
\hline & & HWFET & 5.1 & 5.1 & 4.7 & 4.3 & 5.0 & 4.4 & 3.7 & 4.7 & 3.9 & 3.1 & 4.5 & 3.7 & 3.0 \\
\hline & & Combined & 5.0 & 5.0 & 4.6 & 4.2 & 4.9 & 4.3 & 3.8 & 4.6 & 3.9 & 3.2 & 4.4 & 3.7 & 3.0 \\
\hline \multirow{6}{*}{ CI Split HEV PHEV2O } & & UDDS & 2.4 & 2.4 & 2.3 & 2.1 & 2.4 & 2.1 & 1.8 & 2.3 & 1.9 & 1.5 & 2.2 & 1.8 & 1.4 \\
\hline & Unadjusted & HWFET & 2.9 & 2.9 & 2.7 & 2.5 & 2.9 & 2.5 & 2.6 & 2.7 & 2.2 & 2.1 & 2.6 & 2.5 & 2.0 \\
\hline & & Combined & 2.7 & 2.7 & 2.5 & 2.2 & 2.7 & 2.3 & 2.2 & 2.5 & 2.0 & 1.8 & 2.3 & 2.1 & 1.7 \\
\hline & & \begin{tabular}{|l|} 
UDDS \\
\end{tabular} & 3.6 & 3.6 & 3.4 & 3.1 & 3.6 & 3.2 & 2.8 & 3.4 & 2.9 & 2.4 & 3.2 & 2.8 & 2.3 \\
\hline & Adjusted & HWFET & 4.1 & 4.1 & 3.9 & 3.5 & 4.2 & 3.6 & 3.7 & 3.9 & 3.2 & 3.1 & 3.7 & 3.6 & 2.9 \\
\hline & & Combined & 3.8 & 3.8 & 3.6 & 3.3 & 3.9 & 3.4 & 3.2 & 3.6 & 3.0 & 2.7 & 3.4 & 3.2 & 2.6 \\
\hline
\end{tabular}




\begin{tabular}{|c|c|c|c|c|c|c|c|c|c|c|c|c|c|c|c|}
\hline & & & \multicolumn{13}{|c|}{ Midsize } \\
\hline & & & \multirow{2}{*}{$\begin{array}{l}2010 \\
\text { Ref }\end{array}$} & \multicolumn{3}{|c|}{2010} & \multicolumn{3}{|c|}{2015} & \multicolumn{3}{|c|}{2030} & \multicolumn{3}{|c|}{2045} \\
\hline & & & & low & avg & high & low & avg & high & low & avg & high & low & avg & high \\
\hline \multirow{6}{*}{ CI Series HEV PHEV 30} & \multirow{3}{*}{ Unadjusted } & UDDS & 2.7 & 2.7 & 2.4 & 2.2 & 2.6 & 2.2 & 1.9 & 2.4 & 1.9 & 1.5 & 2.3 & 1.8 & 1.4 \\
\hline & & HWFET & 2.6 & 2.6 & 2.4 & 2.1 & 2.6 & 2.2 & 1.8 & 2.4 & 1.9 & 1.5 & 2.2 & 1.8 & 1.4 \\
\hline & & Combined & 2.6 & 2.6 & 2.4 & 2.1 & 2.6 & 2.2 & 1.8 & 2.4 & 1.9 & 1.5 & 2.3 & 1.8 & 1.4 \\
\hline & \multirow{3}{*}{ Adjusted } & \begin{tabular}{|l|} 
UDDS \\
\end{tabular} & 3.8 & 3.8 & 3.6 & 3.3 & 3.8 & 3.4 & 2.9 & 3.5 & 3.0 & 2.4 & 3.4 & 2.8 & 2.3 \\
\hline & & HWFET & 3.8 & 3.8 & 3.4 & 3.1 & 3.7 & 3.1 & 2.6 & 3.4 & 2.7 & 2.2 & 3.2 & 2.6 & 2.1 \\
\hline & & Combined & 3.8 & 3.8 & 3.5 & 3.2 & 3.8 & 3.3 & 2.8 & 3.5 & 2.9 & 2.3 & 3.3 & 2.7 & 2.2 \\
\hline \multirow{6}{*}{ CI Series HEV PHEV40 } & \multirow{3}{*}{ Unadjusted } & UDDS & 2.3 & 2.3 & 2.1 & 1.9 & 2.3 & 1.9 & 1.6 & 2.1 & 1.7 & 1.3 & 2.0 & 1.6 & 1.2 \\
\hline & & \begin{tabular}{|l|} 
HWFET \\
\end{tabular} & 2.1 & 2.1 & 1.9 & 1.7 & 2.1 & 1.8 & 1.5 & 1.9 & 1.5 & 1.2 & 1.8 & 1.5 & 1.1 \\
\hline & & Combined & 2.2 & 2.2 & 2.0 & 1.8 & 2.2 & 1.9 & 1.6 & 2.0 & 1.6 & 1.2 & 1.9 & 1.5 & 1.2 \\
\hline & \multirow{3}{*}{ Adjusted } & \begin{tabular}{|l|} 
UDDS \\
\end{tabular} & 3.4 & 3.4 & 3.2 & 2.9 & 3.4 & 3.0 & 2.6 & 3.1 & 2.6 & 2.1 & 3.0 & 2.5 & 2.1 \\
\hline & & HWFET & 3.1 & 3.1 & 2.8 & 2.5 & 3.0 & 2.6 & 2.2 & 2.8 & 2.2 & 1.8 & 2.6 & 2.1 & 1.7 \\
\hline & & Combined & 3.3 & 3.3 & 3.0 & 2.7 & 3.2 & 2.8 & 2.4 & 3.0 & 2.5 & 2.0 & 2.8 & 2.4 & 1.9 \\
\hline \multirow{6}{*}{ H2 Split HEV } & \multirow{3}{*}{ Unadjusted } & \begin{tabular}{|l|} 
UDDS \\
\end{tabular} & 4.5 & 4.5 & 3.7 & 3.2 & 4.1 & 3.3 & 2.7 & 3.6 & 2.8 & 2.2 & 3.3 & 2.6 & 2.0 \\
\hline & & HWFET & 5.1 & 5.1 & 4.2 & 3.6 & 4.6 & 3.7 & 3.1 & 4.0 & 3.3 & 2.6 & 3.7 & 3.0 & 2.3 \\
\hline & & Combined & 4.8 & 4.8 & 4.0 & 3.4 & 4.3 & 3.5 & 2.9 & 3.8 & 3.0 & 2.4 & 3.5 & 2.8 & 2.2 \\
\hline & \multirow{3}{*}{ Adjusted } & \begin{tabular}{|l|} 
UDDS \\
\end{tabular} & 6.2 & 6.2 & 5.2 & 4.6 & 5.6 & 4.6 & 4.0 & 5.0 & 4.1 & 3.3 & 4.6 & 3.8 & 3.1 \\
\hline & & HWFET & 7.3 & 7.3 & 6.0 & 5.2 & 6.5 & 5.3 & 4.5 & 5.7 & 4.6 & 3.7 & 5.3 & 4.3 & 3.4 \\
\hline & & Combined & 6.7 & 6.7 & 5.5 & 4.8 & 6.0 & 4.9 & 4.2 & 5.3 & 4.3 & 3.5 & 4.9 & 4.0 & 3.2 \\
\hline \multirow{6}{*}{ H2 Split HEV PHEV10 } & \multirow{3}{*}{ Unadjusted } & \begin{tabular}{|l|} 
UDDS \\
\end{tabular} & 3.7 & 3.7 & 3.0 & 2.6 & 3.3 & 2.7 & 2.3 & 2.9 & 2.4 & 1.8 & 2.7 & 2.2 & 1.7 \\
\hline & & \begin{tabular}{|l|} 
HWFET \\
\end{tabular} & 3.7 & 3.7 & 3.0 & 2.6 & 3.3 & 2.7 & 2.3 & 3.0 & 2.4 & 1.9 & 2.7 & 2.2 & 1.7 \\
\hline & & Combined & 3.7 & 3.7 & 3.0 & 2.6 & 3.3 & 2.7 & 2.3 & 2.9 & 2.4 & 1.9 & 2.7 & 2.2 & 1.7 \\
\hline & \multirow{3}{*}{ Adjusted } & \begin{tabular}{|l|} 
UDDS \\
\end{tabular} & 5.1 & 5.1 & 4.3 & 3.8 & 4.6 & 3.9 & 3.4 & 4.2 & 3.5 & 2.8 & 3.9 & 3.3 & 2.7 \\
\hline & & \begin{tabular}{|l|} 
HWFET \\
\end{tabular} & 5.3 & 5.3 & 4.3 & 3.8 & 4.7 & 3.9 & 3.3 & 4.2 & 3.4 & 2.8 & 3.9 & 3.2 & 2.5 \\
\hline & & Combined & 5.2 & 5.2 & 4.3 & 3.8 & 4.7 & 3.9 & 3.3 & 4.2 & 3.5 & 2.8 & 3.9 & 3.2 & 2.6 \\
\hline \multirow{6}{*}{ H2 Split HEV PHEV 20} & \multirow{3}{*}{ Unadjusted } & UDDS & 2.6 & 2.6 & 2.1 & 1.8 & 2.3 & 1.9 & 1.6 & 2.0 & 1.6 & 1.5 & 1.9 & 1.5 & 1.4 \\
\hline & & HWFET & 3.1 & 3.1 & 2.5 & 2.1 & 2.7 & 2.2 & 2.2 & 2.4 & 2.3 & 1.9 & 2.3 & 2.1 & 1.7 \\
\hline & & Combined & 2.8 & 2.8 & 2.3 & 2.0 & 2.5 & 2.0 & 1.9 & 2.2 & 1.9 & 1.7 & 2.1 & 1.8 & 1.5 \\
\hline & \multirow{3}{*}{ Adjusted } & UDDS & 3.8 & 3.8 & 3.2 & 2.8 & 3.4 & 2.9 & 2.5 & 3.1 & 2.6 & 2.5 & 2.9 & 2.5 & 2.3 \\
\hline & & HWFET & 4.4 & 4.4 & 3.6 & 3.1 & 3.9 & 3.2 & 3.2 & 3.5 & 3.3 & 2.7 & 3.2 & 3.1 & 2.4 \\
\hline & & Combined & 4.0 & 4.0 & 3.4 & 2.9 & 3.6 & 3.0 & 2.8 & 3.3 & 2.9 & 2.6 & 3.1 & 2.7 & 2.4 \\
\hline \multirow{6}{*}{ H2 Series HEV PHEV30 } & & UDDS & 2.8 & 2.8 & 2.3 & 1.9 & 2.5 & 2.0 & 1.6 & 2.2 & 1.7 & 1.3 & 2.0 & 1.6 & 1.2 \\
\hline & Unadjusted & HWFET & 2.7 & 2.7 & 2.2 & 1.9 & 2.4 & 2.0 & 1.6 & 2.1 & 1.7 & 1.3 & 2.0 & 1.6 & 1.2 \\
\hline & & Combined & 2.8 & 2.8 & 2.3 & 1.9 & 2.5 & 2.0 & 1.6 & 2.2 & 1.7 & 1.3 & 2.0 & 1.6 & 1.2 \\
\hline & & \begin{tabular}{|l|} 
UDDS \\
\end{tabular} & 4.0 & 4.0 & 3.4 & 3.0 & 3.6 & 3.1 & 2.6 & 3.3 & 2.7 & 2.2 & 3.0 & 2.6 & 2.1 \\
\hline & Adjusted & HWFET & 3.9 & 3.9 & 3.2 & 2.8 & 3.5 & 2.8 & 2.3 & 3.1 & 2.4 & 1.9 & 2.8 & 2.3 & 1.8 \\
\hline & & Combined & 4.0 & 4.0 & 3.3 & 2.9 & 3.6 & 3.0 & 2.5 & 3.2 & 2.6 & 2.1 & 2.9 & 2.4 & 2.0 \\
\hline
\end{tabular}




\begin{tabular}{|c|c|c|c|c|c|c|c|c|c|c|c|c|c|c|c|}
\hline & & & \multicolumn{13}{|c|}{ Midsize } \\
\hline & & & \multirow{2}{*}{$\begin{array}{l}2010 \\
\text { Ref }\end{array}$} & \multicolumn{3}{|c|}{2010} & \multicolumn{3}{|c|}{2015} & \multicolumn{3}{|c|}{2030} & \multicolumn{3}{|c|}{2045} \\
\hline & & & & low & avg & high & low & avg & high & low & avg & high & low & avg & high \\
\hline \multirow{6}{*}{ H2 Series HEV PHEV40 } & \multirow{3}{*}{ Unadjusted } & UDDS & 2.5 & 2.5 & 2.0 & 1.7 & 2.2 & 1.7 & 1.4 & 1.9 & 1.5 & 1.1 & 1.7 & 1.4 & 1.0 \\
\hline & & HWFET & 2.2 & 2.2 & 1.8 & 1.5 & 2.0 & 1.6 & 1.3 & 1.7 & 1.4 & 1.1 & 1.6 & 1.3 & 1.0 \\
\hline & & Combined & 2.4 & 2.4 & 1.9 & 1.6 & 2.1 & 1.7 & 1.4 & 1.8 & 1.4 & 1.1 & 1.7 & 1.3 & 1.0 \\
\hline & \multirow{3}{*}{ Adjusted } & UDDS & 3.6 & 3.6 & 3.0 & 2.7 & 3.2 & 2.7 & 2.3 & 2.9 & 2.4 & 2.0 & 2.7 & 2.3 & 1.9 \\
\hline & & HWFET & 3.2 & 3.2 & 2.6 & 2.2 & 2.8 & 2.3 & 1.9 & 2.5 & 2.0 & 1.6 & 2.3 & 1.8 & 1.4 \\
\hline & & Combined & 3.4 & 3.4 & 2.8 & 2.5 & 3.1 & 2.6 & 2.1 & 2.7 & 2.2 & 1.8 & 2.5 & 2.1 & 1.7 \\
\hline \multirow{6}{*}{ E85 Split HEV } & \multirow{3}{*}{ Unadjusted } & \begin{tabular}{|l|} 
UDDS \\
\end{tabular} & 4.8 & 4.8 & 4.4 & 4.0 & 4.7 & 4.0 & 3.1 & 4.3 & 3.6 & 2.6 & 4.2 & 3.5 & 2.4 \\
\hline & & HWFET & 5.3 & 5.3 & 4.8 & 4.4 & 5.2 & 4.6 & 3.5 & 4.8 & 4.1 & 3.0 & 4.7 & 3.9 & 2.8 \\
\hline & & Combined & 5.0 & 5.0 & 4.6 & 4.2 & 4.9 & 4.3 & 3.3 & 4.5 & 3.8 & 2.7 & 4.4 & 3.7 & 2.6 \\
\hline & \multirow{3}{*}{ Adjusted } & UDDS & 6.4 & 6.4 & 5.9 & 5.5 & 6.3 & 5.6 & 4.4 & 5.9 & 5.0 & 3.7 & 5.7 & 4.9 & 3.6 \\
\hline & & \begin{tabular}{|l|} 
HWFET \\
\end{tabular} & 7.5 & 7.5 & 6.8 & 6.3 & 7.4 & 6.5 & 5.0 & 6.9 & 5.8 & 4.2 & 6.7 & 5.6 & 4.0 \\
\hline & & Combined & 6.9 & 6.9 & 6.3 & 5.9 & 6.8 & 6.0 & 4.6 & 6.3 & 5.4 & 4.0 & 6.1 & 5.2 & 3.8 \\
\hline \multirow{6}{*}{ E85 Split HEV PHEV10 } & \multirow{3}{*}{ Unadjusted } & \begin{tabular}{|l|} 
UDDS \\
\end{tabular} & 3.8 & 3.8 & 3.5 & 3.2 & 3.7 & 3.3 & 2.5 & 3.5 & 3.0 & 2.1 & 3.4 & 2.9 & 2.0 \\
\hline & & HWFET & 3.8 & 3.8 & 3.5 & 3.2 & 3.8 & 3.3 & 2.5 & 3.6 & 3.0 & 2.2 & 3.4 & 2.9 & 2.0 \\
\hline & & Combined & 3.8 & 3.8 & 3.5 & 3.2 & 3.7 & 3.3 & 2.5 & 3.5 & 3.0 & 2.1 & 3.4 & 2.9 & 2.0 \\
\hline & \multirow{3}{*}{ Adjusted } & UDDS & 5.2 & 5.2 & 4.9 & 4.5 & 5.2 & 4.6 & 3.7 & 4.9 & 4.3 & 3.2 & 4.8 & 4.1 & 3.1 \\
\hline & & \begin{tabular}{|l|} 
HWFET \\
\end{tabular} & 5.5 & 5.5 & 5.0 & 4.6 & 5.3 & 4.7 & 3.6 & 5.1 & 4.3 & 3.1 & 4.9 & 4.1 & 2.9 \\
\hline & & Combined & 5.3 & 5.3 & 4.9 & 4.5 & 5.2 & 4.7 & 3.7 & 5.0 & 4.3 & 3.2 & 4.8 & 4.1 & 3.0 \\
\hline \multirow{6}{*}{ E85 Split HEV PHEV 20} & \multirow{3}{*}{ Unadjusted } & \begin{tabular}{|l|} 
UDDS \\
\end{tabular} & 2.7 & 2.7 & 2.4 & 2.2 & 2.6 & 2.3 & 1.8 & 2.5 & 2.1 & 1.7 & 2.4 & 2.0 & 1.7 \\
\hline & & HWFET & 3.2 & 3.2 & 2.9 & 2.6 & 3.1 & 2.7 & 2.5 & 2.9 & 2.9 & 2.1 & 2.8 & 2.8 & 2.0 \\
\hline & & Combined & 2.9 & 2.9 & 2.6 & 2.4 & 2.8 & 2.5 & 2.1 & 2.7 & 2.5 & 1.9 & 2.6 & 2.3 & 1.8 \\
\hline & \multirow{3}{*}{ Adjusted } & UDDS & 3.9 & 3.9 & 3.6 & 3.3 & 3.8 & 3.4 & 2.8 & 3.6 & 3.1 & 2.7 & 3.5 & 3.0 & 2.6 \\
\hline & & \begin{tabular}{|c|} 
HWFET \\
\end{tabular} & 4.5 & 4.5 & 4.1 & 3.7 & 4.4 & 3.9 & 3.6 & 4.2 & 4.2 & 3.0 & 4.1 & 4.0 & 2.9 \\
\hline & & \begin{tabular}{|l|} 
Combined \\
\end{tabular} & 4.2 & 4.2 & 3.8 & 3.5 & 4.1 & 3.6 & 3.1 & 3.9 & 3.6 & 2.9 & 3.8 & 3.5 & 2.7 \\
\hline \multirow{6}{*}{ E85 Series HEV PHEV 30} & \multirow{3}{*}{ Unadjusted } & \begin{tabular}{|l|} 
UDDS \\
\end{tabular} & 2.9 & 2.9 & 2.6 & 2.3 & 2.8 & 2.4 & 1.8 & 2.6 & 2.1 & 1.5 & 2.5 & 2.0 & 1.4 \\
\hline & & HWFET & 2.8 & 2.8 & 2.6 & 2.3 & 2.8 & 2.4 & 1.8 & 2.6 & 2.1 & 1.5 & 2.5 & 2.0 & 1.4 \\
\hline & & Combined & 2.9 & 2.9 & 2.6 & 2.3 & 2.8 & 2.4 & 1.8 & 2.6 & 2.1 & 1.5 & 2.5 & 2.0 & 1.4 \\
\hline & \multirow{3}{*}{ Adjusted } & UDDS & 4.1 & 4.1 & 3.8 & 3.5 & 4.0 & 3.6 & 2.8 & 3.8 & 3.2 & 2.4 & 3.6 & 3.1 & 2.3 \\
\hline & & HWFET & 4.1 & 4.1 & 3.7 & 3.3 & 3.9 & 3.4 & 2.6 & 3.7 & 3.0 & 2.2 & 3.5 & 2.9 & 2.1 \\
\hline & & Combined & 4.1 & 4.1 & 3.7 & 3.4 & 4.0 & 3.5 & 2.7 & 3.7 & 3.1 & 2.3 & 3.6 & 3.0 & 2.2 \\
\hline \multirow{6}{*}{ E85 Series HEV PHEV40 } & & UDDS & 2.5 & 2.5 & 2.2 & 2.0 & 2.4 & 2.1 & 1.6 & 2.2 & 1.8 & 1.2 & 2.2 & 1.8 & 1.2 \\
\hline & Unadjusted & HWFET & 2.3 & 2.3 & 2.1 & 1.9 & 2.3 & 2.0 & 1.4 & 2.1 & 1.7 & 1.2 & 2.0 & 1.6 & 1.1 \\
\hline & & Combined & 2.4 & 2.4 & 2.2 & 2.0 & 2.3 & 2.0 & 1.5 & 2.2 & 1.8 & 1.2 & 2.1 & 1.7 & 1.2 \\
\hline & & UDDS & 3.7 & 3.7 & 3.4 & 3.1 & 3.6 & 3.2 & 2.5 & 3.3 & 2.8 & 2.1 & 3.2 & 2.8 & 2.0 \\
\hline & Adjusted & HWFET & 3.3 & 3.3 & 3.0 & 2.8 & 3.2 & 2.8 & 2.1 & 3.0 & 2.5 & 1.8 & 2.9 & 2.4 & 1.7 \\
\hline & & Combined & 3.5 & 3.5 & 3.2 & 2.9 & 3.4 & 3.0 & 2.3 & 3.2 & 2.7 & 2.0 & 3.1 & 2.6 & 1.9 \\
\hline
\end{tabular}


Argonne National Laboratory Report - Light-Duty Vehicle Fuel Consumption Displacement Potential Up to 2045

\begin{tabular}{|c|c|c|c|c|c|c|c|c|c|c|c|c|c|c|c|}
\hline & & & \multicolumn{13}{|c|}{ Midsize } \\
\hline & & & \multirow{2}{*}{$\frac{2010}{\text { Ref }}$} & \multicolumn{3}{|c|}{2010} & \multicolumn{3}{|c|}{2015} & \multicolumn{3}{|c|}{2030} & \multicolumn{3}{|c|}{2045} \\
\hline & & & & low & avg & high & low & avg & high & low & avg & high & low & avg & high \\
\hline \multirow{6}{*}{ FC HEV } & \multirow{3}{*}{ Unadjusted } & UDDS & 4.1 & 4.1 & 3.4 & 2.9 & 3.5 & 2.9 & 2.5 & 3.2 & 2.6 & 2.0 & 3.0 & 2.5 & 1.9 \\
\hline & & HWFET & 3.8 & 3.8 & 3.2 & 2.7 & 3.3 & 2.8 & 2.4 & 3.0 & 2.5 & 2.1 & 2.9 & 2.4 & 1.9 \\
\hline & & \begin{tabular}{|l|} 
Combined \\
\end{tabular} & 4.0 & 4.0 & 3.3 & 2.8 & 3.4 & 2.9 & 2.5 & 3.1 & 2.6 & 2.1 & 2.9 & 2.4 & 1.9 \\
\hline & \multirow{3}{*}{ Adjusted } & \begin{tabular}{|l|} 
UDDS \\
\end{tabular} & 5.7 & 5.7 & 4.8 & 4.1 & 4.9 & 4.2 & 3.6 & 4.5 & 3.7 & 3.1 & 4.3 & 3.6 & 3.0 \\
\hline & & HWFET & 5.4 & 5.4 & 4.5 & 3.9 & 4.7 & 4.0 & 3.5 & 4.3 & 3.6 & 3.0 & 4.1 & 3.5 & 2.8 \\
\hline & & Combined & 5.6 & 5.6 & 4.7 & 4.0 & 4.8 & 4.1 & 3.6 & 4.4 & 3.7 & 3.1 & 4.2 & 3.5 & 2.9 \\
\hline \multirow{6}{*}{ FC PHEV10 } & \multirow{3}{*}{ Unadjusted } & \begin{tabular}{|l|} 
UDDS \\
\end{tabular} & 3.3 & 3.3 & 2.8 & 2.3 & 2.8 & 2.4 & 2.1 & 2.6 & 2.1 & 1.7 & 2.5 & 2.1 & 1.6 \\
\hline & & HWFET & 2.8 & 2.8 & 2.4 & 2.1 & 2.5 & 2.1 & 1.9 & 2.3 & 1.9 & 1.6 & 2.2 & 1.9 & 1.5 \\
\hline & & Combined & 3.1 & 3.1 & 2.6 & 2.2 & 2.7 & 2.3 & 2.0 & 2.5 & 2.0 & 1.7 & 2.4 & 2.0 & 1.6 \\
\hline & \multirow{3}{*}{ Adjusted } & \begin{tabular}{|l|} 
UDDS \\
\end{tabular} & 4.6 & 4.6 & 4.0 & 3.5 & 4.1 & 3.6 & 3.1 & 3.8 & 3.2 & 2.7 & 3.6 & 3.1 & 2.6 \\
\hline & & \begin{tabular}{|c|} 
HWFET \\
\end{tabular} & 4.0 & 4.0 & 3.4 & 3.0 & 3.5 & 3.1 & 2.7 & 3.3 & 2.8 & 2.3 & 3.2 & 2.7 & 2.2 \\
\hline & & Combined & 4.3 & 4.3 & 3.7 & 3.2 & 3.8 & 3.3 & 2.9 & 3.6 & 3.0 & 2.5 & 3.4 & 2.9 & 2.4 \\
\hline \multirow{6}{*}{ FC PHEV2O } & \multirow{3}{*}{ Unadjusted } & \begin{tabular}{|l|} 
UDDS \\
\end{tabular} & 2.7 & 2.7 & 2.3 & 1.9 & 2.3 & 2.0 & 1.7 & 2.2 & 1.8 & 1.4 & 2.1 & 1.7 & 1.4 \\
\hline & & HWFET & 2.8 & 2.8 & 2.4 & 2.0 & 2.5 & 2.1 & 1.8 & 2.3 & 1.9 & 1.6 & 2.2 & 1.8 & 1.5 \\
\hline & & Combined & 2.8 & 2.8 & 2.3 & 2.0 & 2.4 & 2.0 & 1.8 & 2.2 & 1.8 & 1.5 & 2.1 & 1.8 & 1.4 \\
\hline & \multirow{3}{*}{ Adjusted } & \begin{tabular}{|l|} 
UDDS \\
\end{tabular} & 4.0 & 4.0 & 3.4 & 3.0 & 3.5 & 3.1 & 2.7 & 3.3 & 2.8 & 2.3 & 3.1 & 2.7 & 2.3 \\
\hline & & HWFET & 4.0 & 4.0 & 3.4 & 2.9 & 3.5 & 3.0 & 2.7 & 3.3 & 2.8 & 2.3 & 3.1 & 2.6 & 2.1 \\
\hline & & Combined & 4.0 & 4.0 & 3.4 & 3.0 & 3.5 & 3.0 & 2.7 & 3.3 & 2.8 & 2.3 & 3.1 & 2.7 & 2.2 \\
\hline \multirow{6}{*}{ FC PHEV30 } & \multirow{3}{*}{ Unadjusted } & \begin{tabular}{|l|} 
UDDS \\
\end{tabular} & 1.9 & 1.9 & 1.6 & 1.4 & 1.7 & 1.4 & 1.2 & 1.5 & 1.3 & 1.0 & 1.5 & 1.2 & 1.0 \\
\hline & & HWFET & 1.8 & 1.8 & 1.9 & 1.6 & 2.0 & 1.7 & 1.5 & 1.8 & 1.5 & 1.3 & 1.7 & 1.5 & 1.2 \\
\hline & & Combined & 1.9 & 1.9 & 1.7 & 1.5 & 1.8 & 1.5 & 1.3 & 1.7 & 1.4 & 1.1 & 1.6 & 1.3 & 1.1 \\
\hline & \multirow{3}{*}{ Adjusted } & \begin{tabular}{|l|} 
UDDS \\
\end{tabular} & 2.9 & 2.9 & 2.6 & 2.3 & 2.6 & 2.3 & 2.1 & 2.5 & 2.1 & 1.8 & 2.4 & 2.1 & 1.8 \\
\hline & & \begin{tabular}{|c|} 
HWFET \\
\end{tabular} & 2.6 & 2.6 & 2.7 & 2.4 & 2.8 & 2.4 & 2.1 & 2.6 & 2.2 & 1.8 & 2.5 & 2.1 & 1.7 \\
\hline & & Combined & 2.8 & 2.8 & 2.6 & 2.3 & 2.7 & 2.4 & 2.1 & 2.6 & 2.2 & 1.8 & 2.4 & 2.1 & 1.8 \\
\hline \multirow{6}{*}{ FC PHEV40 } & \multirow{3}{*}{ Unadjusted } & UDDS & 1.7 & 1.7 & 1.4 & 1.2 & 1.4 & 1.2 & 1.0 & 1.3 & 1.1 & 0.9 & 1.3 & 1.0 & 0.8 \\
\hline & & \begin{tabular}{|c|} 
HWFET \\
\end{tabular} & 1.8 & 1.8 & 1.5 & 1.3 & 1.5 & 1.3 & 1.2 & 1.4 & 1.2 & 1.0 & 1.4 & 1.1 & 0.9 \\
\hline & & Combined & 1.7 & 1.7 & 1.4 & 1.2 & 1.5 & 1.3 & 1.1 & 1.4 & 1.1 & 0.9 & 1.3 & 1.1 & 0.9 \\
\hline & \multirow{3}{*}{ Adjusted } & \begin{tabular}{|l|} 
UDDS \\
\end{tabular} & 2.6 & 2.6 & 2.3 & 2.1 & 2.4 & 2.1 & 1.9 & 2.2 & 1.9 & 1.7 & 2.1 & 1.9 & 1.6 \\
\hline & & HWFET & 2.5 & 2.5 & 2.2 & 1.9 & 2.2 & 1.9 & 1.7 & 2.1 & 1.8 & 1.5 & 2.0 & 1.7 & 1.4 \\
\hline & & Combined & 2.6 & 2.6 & 2.2 & 2.0 & 2.3 & 2.0 & 1.8 & 2.2 & 1.8 & 1.6 & 2.1 & 1.8 & 1.5 \\
\hline
\end{tabular}


Fuel Consumption Improvement versus the Conventional 2010 Reference

\begin{tabular}{|c|c|c|c|c|c|c|c|c|c|c|c|c|c|c|c|}
\hline & & & \multicolumn{13}{|c|}{ Midsize } \\
\hline & & & 2010 & & 2010 & & & 2015 & & & 2030 & & & 2045 & \\
\hline & & & Ref & low & avg & high & low & avg & high & low & avg & high & low & avg & high \\
\hline \multirow{6}{*}{ SI Conv } & \multirow{3}{*}{ Unadjusted } & UDDS & $0.0 \%$ & $0.0 \%$ & $-7.9 \%$ & $-12.7 \%$ & $-4.5 \%$ & $-9.8 \%$ & $-31.4 \%$ & $-6.8 \%$ & $-14.3 \%$ & $-41.9 \%$ & $-6.0 \%$ & $-19.3 \%$ & $-43.4 \%$ \\
\hline & & HWFET & $0.0 \%$ & $0.0 \%$ & $-9.1 \%$ & $-13.5 \%$ & $-4.0 \%$ & $-0.5 \%$ & $-31.2 \%$ & $-6.7 \%$ & $-5.3 \%$ & $-41.7 \%$ & $3.8 \%$ & $-19.3 \%$ & $-44.0 \%$ \\
\hline & & Combined & $0.0 \%$ & $0.0 \%$ & $-8.3 \%$ & $-13.0 \%$ & $-4.3 \%$ & $-6.5 \%$ & $-31.3 \%$ & $-6.8 \%$ & $-11.1 \%$ & $-41.8 \%$ & $-2.5 \%$ & $-19.3 \%$ & $-43.6 \%$ \\
\hline & \multirow{3}{*}{ Adjusted } & \begin{tabular}{|l|} 
UDDS \\
\end{tabular} & $0.0 \%$ & $0.0 \%$ & $-7.5 \%$ & $-12.0 \%$ & $-4.3 \%$ & $-9.3 \%$ & $-29.7 \%$ & $-6.4 \%$ & $-13.5 \%$ & $-39.6 \%$ & $-5.7 \%$ & $-18.3 \%$ & $-41.1 \%$ \\
\hline & & HWFET & $0.0 \%$ & $0.0 \%$ & $-9.0 \%$ & $-13.4 \%$ & $-4.0 \%$ & $-0.5 \%$ & $-31.0 \%$ & $-6.7 \%$ & $-5.2 \%$ & $-41.3 \%$ & $3.8 \%$ & $-19.2 \%$ & $-43.6 \%$ \\
\hline & & Combined & $0.0 \%$ & $0.0 \%$ & $-8.1 \%$ & $-12.5 \%$ & $-4.2 \%$ & $-6.0 \%$ & $-30.2 \%$ & $-6.5 \%$ & $-10.4 \%$ & $-40.3 \%$ & $-2.1 \%$ & $-18.6 \%$ & $-42.0 \%$ \\
\hline \multirow{6}{*}{$\mathrm{Cl}$ Conv } & \multirow{3}{*}{ Unadjusted } & \begin{tabular}{|l|} 
UDDS \\
\end{tabular} & $-13.6 \%$ & $-13.6 \%$ & $-18.5 \%$ & $-25.0 \%$ & $-15.1 \%$ & $-21.4 \%$ & $-31.3 \%$ & $-19.7 \%$ & $-27.3 \%$ & $-41.1 \%$ & $-20.0 \%$ & $-32.2 \%$ & $-43.2 \%$ \\
\hline & & HWFET & $-8.9 \%$ & $-8.9 \%$ & $-15.4 \%$ & $-23.5 \%$ & $-11.8 \%$ & $-14.2 \%$ & $-27.8 \%$ & $-17.2 \%$ & $-20.7 \%$ & $-38.1 \%$ & $-12.7 \%$ & $-28.9 \%$ & $-41.1 \%$ \\
\hline & & Combined & $-11.9 \%$ & $-11.9 \%$ & $-17.4 \%$ & $-24.5 \%$ & $-13.9 \%$ & $-18.8 \%$ & $-30.1 \%$ & $-18.8 \%$ & $-25.0 \%$ & $-40.1 \%$ & $-17.4 \%$ & $-31.0 \%$ & $-42.5 \%$ \\
\hline & \multirow{3}{*}{ Adjusted } & \begin{tabular}{|l|} 
UDDS \\
\end{tabular} & $-12.9 \%$ & $-12.9 \%$ & $-17.5 \%$ & $-23.6 \%$ & $-14.3 \%$ & $-20.2 \%$ & $-29.6 \%$ & $-18.7 \%$ & $-25.9 \%$ & $-38.9 \%$ & $-19.0 \%$ & $-30.4 \%$ & $-40.9 \%$ \\
\hline & & \begin{tabular}{|c|} 
HWFET \\
\end{tabular} & $-8.8 \%$ & $-8.8 \%$ & $-15.2 \%$ & $-23.3 \%$ & $-11.7 \%$ & $-14.1 \%$ & $-27.6 \%$ & $-17.1 \%$ & $-20.6 \%$ & $-37.8 \%$ & $-12.6 \%$ & $-28.7 \%$ & $-40.8 \%$ \\
\hline & & Combined & $-11.3 \%$ & $-11.3 \%$ & $-16.6 \%$ & $-23.5 \%$ & $-13.3 \%$ & $-17.9 \%$ & $-28.9 \%$ & $-18.1 \%$ & $-23.9 \%$ & $-38.5 \%$ & $-16.6 \%$ & $-29.8 \%$ & $-40.9 \%$ \\
\hline \multirow{6}{*}{ H2 Conv } & \multirow{3}{*}{ Unadjusted } & UDDS & $22.4 \%$ & $22.4 \%$ & $2.1 \%$ & $-10.7 \%$ & $8.5 \%$ & $-7.6 \%$ & $-22.7 \%$ & $-2.0 \%$ & $-16.5 \%$ & $-34.8 \%$ & $-7.3 \%$ & $-25.0 \%$ & $-39.5 \%$ \\
\hline & & \begin{tabular}{|c|} 
HWFET \\
\end{tabular} & $12.3 \%$ & $12.3 \%$ & $-6.9 \%$ & $-21.4 \%$ & $-3.7 \%$ & $-1.0 \%$ & $-29.1 \%$ & $-13.3 \%$ & $-10.4 \%$ & $-39.7 \%$ & $-0.7 \%$ & $-31.4 \%$ & $-44.9 \%$ \\
\hline & & Combined & $18.8 \%$ & $18.8 \%$ & $-1.1 \%$ & $-14.5 \%$ & $4.1 \%$ & $-5.3 \%$ & $-25.0 \%$ & $-6.0 \%$ & $-14.3 \%$ & $-36.5 \%$ & $-4.9 \%$ & $-27.3 \%$ & $-41.4 \%$ \\
\hline & \multirow{3}{*}{ Adjusted } & \begin{tabular}{|l|} 
UDDS \\
\end{tabular} & $21.2 \%$ & $21.2 \%$ & $2.0 \%$ & $-10.1 \%$ & $8.0 \%$ & $-7.2 \%$ & $-21.5 \%$ & $-1.9 \%$ & $-15.6 \%$ & $-32.9 \%$ & $-6.9 \%$ & $-23.7 \%$ & $-37.4 \%$ \\
\hline & & \begin{tabular}{|c|} 
HWFET \\
\end{tabular} & $12.2 \%$ & $12.2 \%$ & $-6.8 \%$ & $-21.2 \%$ & $-3.7 \%$ & $-1.0 \%$ & $-28.9 \%$ & $-13.2 \%$ & $-10.3 \%$ & $-39.4 \%$ & $-0.7 \%$ & $-31.2 \%$ & $-44.5 \%$ \\
\hline & & Combined & $17.8 \%$ & $17.8 \%$ & $-1.3 \%$ & $-14.3 \%$ & $3.6 \%$ & $-4.9 \%$ & $-24.2 \%$ & $-6.1 \%$ & $-13.6 \%$ & $-35.3 \%$ & $-4.6 \%$ & $-26.5 \%$ & $-40.0 \%$ \\
\hline \multirow{6}{*}{ E85 Conv } & \multirow{3}{*}{ Unadjusted } & \begin{tabular}{|l|} 
UDDS \\
\end{tabular} & $3.0 \%$ & $3.0 \%$ & $1.7 \%$ & $-6.1 \%$ & $3.3 \%$ & $-1.2 \%$ & $-28.5 \%$ & $1.1 \%$ & $-6.8 \%$ & $-38.6 \%$ & $3.1 \%$ & $-11.1 \%$ & $-40.4 \%$ \\
\hline & & \begin{tabular}{|c|} 
HWFET \\
\end{tabular} & $9.4 \%$ & $9.4 \%$ & $6.8 \%$ & $-1.0 \%$ & $10.3 \%$ & $9.5 \%$ & $-23.6 \%$ & $7.3 \%$ & $3.5 \%$ & $-34.2 \%$ & $14.4 \%$ & $-5.3 \%$ & $-37.0 \%$ \\
\hline & & Combined & $5.3 \%$ & $5.3 \%$ & $3.5 \%$ & $-4.3 \%$ & $5.8 \%$ & $2.6 \%$ & $-26.8 \%$ & $3.3 \%$ & $-3.1 \%$ & $-37.0 \%$ & $7.1 \%$ & $-9.1 \%$ & $-39.2 \%$ \\
\hline & \multirow{3}{*}{ Adjusted } & \begin{tabular}{|l|} 
UDDS \\
\end{tabular} & $2.9 \%$ & $2.9 \%$ & $1.6 \%$ & $-5.7 \%$ & $3.1 \%$ & $-1.2 \%$ & $-27.0 \%$ & $1.0 \%$ & $-6.4 \%$ & $-36.5 \%$ & $2.9 \%$ & $-10.5 \%$ & $-38.2 \%$ \\
\hline & & HWFET & $9.3 \%$ & $9.3 \%$ & $6.8 \%$ & $-1.0 \%$ & $10.2 \%$ & $9.5 \%$ & $-23.4 \%$ & $7.2 \%$ & $3.5 \%$ & $-33.9 \%$ & $14.2 \%$ & $-5.3 \%$ & $-36.7 \%$ \\
\hline & & Combined & $5.3 \%$ & $5.3 \%$ & $3.6 \%$ & $-4.0 \%$ & $5.8 \%$ & $2.8 \%$ & $-25.6 \%$ & $3.3 \%$ & $-2.7 \%$ & $-35.5 \%$ & $7.2 \%$ & $-8.6 \%$ & $-37.7 \%$ \\
\hline \multirow{6}{*}{ SI Split HEV } & \multirow{3}{*}{ Unadjusted } & \begin{tabular}{|l|} 
UDDS \\
\end{tabular} & $-48.6 \%$ & $-48.6 \%$ & $-53.0 \%$ & $-56.7 \%$ & $-49.6 \%$ & $-56.4 \%$ & $-64.7 \%$ & $-53.2 \%$ & $-60.8 \%$ & $-72.0 \%$ & $-54.8 \%$ & $-62.4 \%$ & $-73.3 \%$ \\
\hline & & HWFET & $-15.3 \%$ & $-15.3 \%$ & $-23.3 \%$ & $-29.3 \%$ & $-17.7 \%$ & $-27.6 \%$ & $-40.8 \%$ & $-22.2 \%$ & $-34.2 \%$ & $-52.3 \%$ & $-24.8 \%$ & $-37.4 \%$ & $-55.2 \%$ \\
\hline & & Combined & $-36.8 \%$ & $-36.8 \%$ & $-42.4 \%$ & $-47.0 \%$ & $-38.3 \%$ & $-46.2 \%$ & $-56.2 \%$ & $-42.2 \%$ & $-51.3 \%$ & $-65.0 \%$ & $-44.2 \%$ & $-53.5 \%$ & $-66.9 \%$ \\
\hline & \multirow{3}{*}{ Adjusted } & \begin{tabular}{|l|} 
UDDS \\
\end{tabular} & $-46.0 \%$ & $-46.0 \%$ & $-50.1 \%$ & $-53.7 \%$ & $-47.0 \%$ & $-53.4 \%$ & $-61.3 \%$ & $-50.4 \%$ & $-57.5 \%$ & $-68.1 \%$ & $-51.9 \%$ & $-59.1 \%$ & $-69.4 \%$ \\
\hline & & \begin{tabular}{|c|} 
HWFET \\
\end{tabular} & $-15.1 \%$ & $-15.1 \%$ & $-23.1 \%$ & $-29.0 \%$ & $-17.5 \%$ & $-27.4 \%$ & $-40.4 \%$ & $-22.0 \%$ & $-33.9 \%$ & $-51.8 \%$ & $-24.6 \%$ & $-37.1 \%$ & $-54.7 \%$ \\
\hline & & Combined & $-34.4 \%$ & $-34.4 \%$ & $-40.0 \%$ & $-44.4 \%$ & $-35.9 \%$ & $-43.6 \%$ & $-53.4 \%$ & $-39.7 \%$ & $-48.6 \%$ & $-62.0 \%$ & $-41.6 \%$ & $-50.8 \%$ & $-63.9 \%$ \\
\hline \multirow{6}{*}{ SI Split HEV PHEV10 } & \multirow{3}{*}{ Unadjusted } & \begin{tabular}{|l|} 
UDDS \\
\end{tabular} & $-58.9 \%$ & $-58.9 \%$ & $-62.2 \%$ & $-65.2 \%$ & $-59.7 \%$ & $-64.2 \%$ & $-70.9 \%$ & $-61.8 \%$ & $-67.5 \%$ & $-76.8 \%$ & $-63.0 \%$ & $-68.7 \%$ & $-78.0 \%$ \\
\hline & & \begin{tabular}{|l|} 
HWFET \\
\end{tabular} & $-38.5 \%$ & $-38.5 \%$ & $-43.8 \%$ & $-48.5 \%$ & $-39.7 \%$ & $-47.1 \%$ & $-56.9 \%$ & $-42.8 \%$ & $-52.1 \%$ & $-64.7 \%$ & $-44.8 \%$ & $-54.1 \%$ & $-67.1 \%$ \\
\hline & & \begin{tabular}{|l|} 
Combined \\
\end{tabular} & $-51.7 \%$ & $-51.7 \%$ & $-55.7 \%$ & $-59.3 \%$ & $-52.6 \%$ & $-58.1 \%$ & $-65.9 \%$ & $-55.0 \%$ & $-62.0 \%$ & $-72.5 \%$ & $-56.5 \%$ & $-63.5 \%$ & $-74.1 \%$ \\
\hline & \multirow{3}{*}{ Adjusted } & \begin{tabular}{|l|} 
UDDS \\
\end{tabular} & $-55.7 \%$ & $-55.7 \%$ & $-58.8 \%$ & $-61.7 \%$ & $-56.4 \%$ & $-60.8 \%$ & $-67.1 \%$ & $-58.4 \%$ & $-63.9 \%$ & $-72.7 \%$ & $-59.6 \%$ & $-65.0 \%$ & $-73.8 \%$ \\
\hline & & \begin{tabular}{|l|} 
HWFET \\
\end{tabular} & $-38.2 \%$ & $-38.2 \%$ & $-43.4 \%$ & $-48.1 \%$ & $-39.4 \%$ & $-46.7 \%$ & $-56.4 \%$ & $-42.4 \%$ & $-51.6 \%$ & $-64.1 \%$ & $-44.4 \%$ & $-53.6 \%$ & $-66.5 \%$ \\
\hline & & Combined & $-49.1 \%$ & $-49.1 \%$ & $-53.0 \%$ & $-56.6 \%$ & $-50.0 \%$ & $-55.5 \%$ & $-63.1 \%$ & $-52.4 \%$ & $-59.3 \%$ & $-69.5 \%$ & $-53.9 \%$ & $-60.7 \%$ & $-71.0 \%$ \\
\hline
\end{tabular}




\begin{tabular}{|c|c|c|c|c|c|c|c|c|c|c|c|c|c|c|c|}
\hline & & & \multicolumn{13}{|c|}{ Midsize } \\
\hline & & & 2010 & & 2010 & & & 2015 & & & 2030 & & & 2045 & \\
\hline & & & Ref & low & avg & high & low & avg & high & low & avg & high & low & avg & high \\
\hline \multirow{6}{*}{ SI Split HEV PHEV2O } & \multirow{3}{*}{ Unadjusted } & UDDS & $-71.0 \%$ & $-71.0 \%$ & $-73.5 \%$ & $-75.8 \%$ & $-71.8 \%$ & $-75.1 \%$ & $-79.8 \%$ & $-73.2 \%$ & $-77.6 \%$ & $-80.8 \%$ & $-74.1 \%$ & $-78.4 \%$ & $-81.9 \%$ \\
\hline & & HWFET & $-49.1 \%$ & $-49.1 \%$ & $-53.6 \%$ & $-58.0 \%$ & $-50.7 \%$ & $-56.0 \%$ & $-57.9 \%$ & $-52.8 \%$ & $-52.7 \%$ & $-65.7 \%$ & $-54.3 \%$ & $-55.2 \%$ & $-67.8 \%$ \\
\hline & & Combined & $-63.2 \%$ & $-63.2 \%$ & $-66.4 \%$ & $-69.5 \%$ & $-64.3 \%$ & $-68.3 \%$ & $-72.1 \%$ & $-65.9 \%$ & $-68.8 \%$ & $-75.5 \%$ & $-67.1 \%$ & $-70.2 \%$ & $-76.9 \%$ \\
\hline & \multirow{3}{*}{ Adjusted } & \begin{tabular}{|l|} 
UDDS \\
\end{tabular} & $-67.2 \%$ & $-67.2 \%$ & $-69.5 \%$ & $-71.8 \%$ & $-67.9 \%$ & $-71.1 \%$ & $-75.6 \%$ & $-69.2 \%$ & $-73.5 \%$ & $-76.5 \%$ & $-70.1 \%$ & $-74.2 \%$ & $-77.5 \%$ \\
\hline & & HWFET & $-48.7 \%$ & $-48.7 \%$ & $-53.1 \%$ & $-57.4 \%$ & $-50.3 \%$ & $-55.5 \%$ & $-57.4 \%$ & $-52.3 \%$ & $-52.2 \%$ & $-65.2 \%$ & $-53.9 \%$ & $-54.7 \%$ & $-67.2 \%$ \\
\hline & & \begin{tabular}{|l|} 
Combined \\
\end{tabular} & $-60.2 \%$ & $-60.2 \%$ & $-63.3 \%$ & $-66.4 \%$ & $-61.3 \%$ & $-65.3 \%$ & $-68.7 \%$ & $-62.9 \%$ & $-65.5 \%$ & $-72.2 \%$ & $-64.0 \%$ & $-66.9 \%$ & $-73.6 \%$ \\
\hline \multirow{6}{*}{ SI Series HEV PHEV30 } & \multirow{3}{*}{ Unadjusted } & \begin{tabular}{|l|} 
UDDS \\
\end{tabular} & $-68.5 \%$ & $-68.5 \%$ & $-71.8 \%$ & $-74.4 \%$ & $-69.7 \%$ & $-73.5 \%$ & $-79.3 \%$ & $-71.8 \%$ & $-76.9 \%$ & $-83.9 \%$ & $-72.9 \%$ & $-77.8 \%$ & $-84.6 \%$ \\
\hline & & HWFET & $-54.1 \%$ & $-54.1 \%$ & $-58.7 \%$ & $-62.2 \%$ & $-55.4 \%$ & $-61.1 \%$ & $-69.6 \%$ & $-58.4 \%$ & $-65.7 \%$ & $-75.4 \%$ & $-60.1 \%$ & $-67.4 \%$ & $-76.9 \%$ \\
\hline & & Combined & $-63.4 \%$ & $-63.4 \%$ & $-67.2 \%$ & $-70.1 \%$ & $-64.6 \%$ & $-69.1 \%$ & $-75.8 \%$ & $-67.1 \%$ & $-72.9 \%$ & $-80.9 \%$ & $-68.4 \%$ & $-74.1 \%$ & $-81.9 \%$ \\
\hline & \multirow{3}{*}{ Adjusted } & \begin{tabular}{|l|} 
UDDS \\
\end{tabular} & $-64.8 \%$ & $-64.8 \%$ & $-68.0 \%$ & $-70.4 \%$ & $-66.0 \%$ & $-69.6 \%$ & $-75.0 \%$ & $-68.0 \%$ & $-72.8 \%$ & $-79.4 \%$ & $-69.0 \%$ & $-73.6 \%$ & $-80.1 \%$ \\
\hline & & HWFET & $-53.6 \%$ & $-53.6 \%$ & $-58.2 \%$ & $-61.7 \%$ & $-54.9 \%$ & $-60.5 \%$ & $-69.0 \%$ & $-57.9 \%$ & $-65.1 \%$ & $-74.7 \%$ & $-59.5 \%$ & $-66.8 \%$ & $-76.2 \%$ \\
\hline & & Combined & $-60.6 \%$ & $-60.6 \%$ & $-64.3 \%$ & $-67.1 \%$ & $-61.8 \%$ & $-66.2 \%$ & $-72.7 \%$ & $-64.2 \%$ & $-69.9 \%$ & $-77.7 \%$ & $-65.5 \%$ & $-71.0 \%$ & $-78.6 \%$ \\
\hline \multirow{6}{*}{ SI Series HEV PHEV40 } & \multirow{3}{*}{ Unadjusted } & \begin{tabular}{|l|} 
UDDS \\
\end{tabular} & $-72.8 \%$ & $-72.8 \%$ & $-75.5 \%$ & $-77.8 \%$ & $-73.3 \%$ & $-77.0 \%$ & $-82.1 \%$ & $-75.5 \%$ & $-80.0 \%$ & $-86.4 \%$ & $-76.5 \%$ & $-80.8 \%$ & $-87.0 \%$ \\
\hline & & HWFET & $-62.5 \%$ & $-62.5 \%$ & $-66.3 \%$ & $-69.5 \%$ & $-63.5 \%$ & $-68.2 \%$ & $-75.3 \%$ & $-66.1 \%$ & $-72.2 \%$ & $-80.1 \%$ & $-67.4 \%$ & $-73.5 \%$ & $-81.3 \%$ \\
\hline & & Combined & $-69.2 \%$ & $-69.2 \%$ & $-72.2 \%$ & $-74.8 \%$ & $-69.9 \%$ & $-73.9 \%$ & $-79.7 \%$ & $-72.2 \%$ & $-77.2 \%$ & $-84.2 \%$ & $-73.3 \%$ & $-78.2 \%$ & $-85.0 \%$ \\
\hline & \multirow{3}{*}{ Adjusted } & UDDS & $-68.9 \%$ & $-68.9 \%$ & $-71.4 \%$ & $-73.6 \%$ & $-69.4 \%$ & $-72.9 \%$ & $-77.7 \%$ & $-71.5 \%$ & $-75.7 \%$ & $-81.8 \%$ & $-72.4 \%$ & $-76.5 \%$ & $-82.4 \%$ \\
\hline & & HWFET & $-62.0 \%$ & $-62.0 \%$ & $-65.7 \%$ & $-68.8 \%$ & $-63.0 \%$ & $-67.6 \%$ & $-74.6 \%$ & $-65.5 \%$ & $-71.5 \%$ & $-79.4 \%$ & $-66.8 \%$ & $-72.9 \%$ & $-80.6 \%$ \\
\hline & & Combined & $-66.3 \%$ & $-66.3 \%$ & $-69.3 \%$ & $-71.8 \%$ & $-67.0 \%$ & $-70.9 \%$ & $-76.5 \%$ & $-69.2 \%$ & $-74.2 \%$ & $-80.9 \%$ & $-70.3 \%$ & $-75.1 \%$ & $-81.7 \%$ \\
\hline \multirow{6}{*}{ CI Split HEV } & \multirow{3}{*}{ Unadjusted } & UDDS & $-52.4 \%$ & $-52.4 \%$ & $-55.6 \%$ & $-59.7 \%$ & $-52.7 \%$ & $-59.1 \%$ & $-64.8 \%$ & $-56.6 \%$ & $-63.7 \%$ & $-71.3 \%$ & $-58.5 \%$ & $-65.5 \%$ & $-72.4 \%$ \\
\hline & & HWFET & $-19.9 \%$ & $-19.9 \%$ & $-26.7 \%$ & $-33.3 \%$ & $-20.9 \%$ & $-31.6 \%$ & $-40.6 \%$ & $-27.0 \%$ & $-38.8 \%$ & $-50.8 \%$ & $-30.3 \%$ & $-42.0 \%$ & $-53.5 \%$ \\
\hline & & Combined & $-40.8 \%$ & $-40.8 \%$ & $-45.3 \%$ & $-50.3 \%$ & $-41.4 \%$ & $-49.4 \%$ & $-56.2 \%$ & $-46.1 \%$ & $-54.9 \%$ & $-64.0 \%$ & $-48.5 \%$ & $-57.1 \%$ & $-65.7 \%$ \\
\hline & \multirow{3}{*}{ Adjusted } & \begin{tabular}{|l|} 
UDDS \\
\end{tabular} & $-49.5 \%$ & $-49.5 \%$ & $-52.6 \%$ & $-56.5 \%$ & $-49.9 \%$ & $-55.9 \%$ & $-61.3 \%$ & $-53.6 \%$ & $-60.3 \%$ & $-67.5 \%$ & $-55.4 \%$ & $-61.9 \%$ & $-68.5 \%$ \\
\hline & & HWFET & $-19.7 \%$ & $-19.7 \%$ & $-26.4 \%$ & $-33.0 \%$ & $-20.7 \%$ & $-31.4 \%$ & $-40.3 \%$ & $-26.8 \%$ & $-38.4 \%$ & $-50.3 \%$ & $-30.0 \%$ & $-41.6 \%$ & $-53.0 \%$ \\
\hline & & \begin{tabular}{|c|} 
Combined \\
\end{tabular} & $-38.3 \%$ & $-38.3 \%$ & $-42.8 \%$ & $-47.7 \%$ & $-38.9 \%$ & $-46.7 \%$ & $-53.4 \%$ & $-43.5 \%$ & $-52.1 \%$ & $-61.0 \%$ & $-45.8 \%$ & $-54.3 \%$ & $-62.7 \%$ \\
\hline \multirow{6}{*}{ CI Split HEV PHEV10 } & \multirow{3}{*}{ Unadjusted } & \begin{tabular}{|l|} 
UDDS \\
\end{tabular} & $-59.4 \%$ & $-59.4 \%$ & $-62.3 \%$ & $-65.7 \%$ & $-59.7 \%$ & $-64.8 \%$ & $-69.6 \%$ & $-62.6 \%$ & $-68.6 \%$ & $-75.1 \%$ & $-64.3 \%$ & $-70.1 \%$ & $-76.1 \%$ \\
\hline & & HWFET & $-38.6 \%$ & $-38.6 \%$ & $-43.7 \%$ & $-48.6 \%$ & $-39.2 \%$ & $-47.1 \%$ & $-55.0 \%$ & $-43.5 \%$ & $-53.5 \%$ & $-62.4 \%$ & $-46.0 \%$ & $-56.1 \%$ & $-64.7 \%$ \\
\hline & & Combined & $-52.0 \%$ & $-52.0 \%$ & $-55.7 \%$ & $-59.6 \%$ & $-52.4 \%$ & $-58.5 \%$ & $-64.4 \%$ & $-55.8 \%$ & $-63.2 \%$ & $-70.6 \%$ & $-57.8 \%$ & $-65.1 \%$ & $-72.1 \%$ \\
\hline & \multirow{3}{*}{ Adjusted } & \begin{tabular}{|l|} 
UDDS \\
\end{tabular} & $-56.3 \%$ & $-56.3 \%$ & $-59.0 \%$ & $-62.2 \%$ & $-56.5 \%$ & $-61.3 \%$ & $-65.9 \%$ & $-59.2 \%$ & $-64.9 \%$ & $-71.0 \%$ & $-60.8 \%$ & $-66.3 \%$ & $-72.0 \%$ \\
\hline & & HWFET & $-38.3 \%$ & $-38.3 \%$ & $-43.3 \%$ & $-48.1 \%$ & $-38.8 \%$ & $-46.7 \%$ & $-54.5 \%$ & $-43.1 \%$ & $-53.0 \%$ & $-61.8 \%$ & $-45.6 \%$ & $-55.6 \%$ & $-64.1 \%$ \\
\hline & & Combined & $-49.5 \%$ & $-49.5 \%$ & $-53.1 \%$ & $-56.9 \%$ & $-49.8 \%$ & $-55.8 \%$ & $-61.6 \%$ & $-53.2 \%$ & $-60.5 \%$ & $-67.6 \%$ & $-55.1 \%$ & $-62.3 \%$ & $-69.1 \%$ \\
\hline \multirow{6}{*}{ CI Split HEV PHEV2O } & \multirow{3}{*}{ Unadjusted } & \begin{tabular}{|l|} 
UDDS \\
\end{tabular} & $-71.6 \%$ & $-71.6 \%$ & $-73.5 \%$ & $-76.0 \%$ & $-71.6 \%$ & $-75.4 \%$ & $-79.0 \%$ & $-73.7 \%$ & $-78.3 \%$ & $-82.8 \%$ & $-74.9 \%$ & $-79.3 \%$ & $-83.6 \%$ \\
\hline & & HWFET & $-50.0 \%$ & $-50.0 \%$ & $-53.5 \%$ & $-57.7 \%$ & $-49.7 \%$ & $-56.8 \%$ & $-55.5 \%$ & $-53.4 \%$ & $-61.8 \%$ & $-63.1 \%$ & $-55.7 \%$ & $-56.5 \%$ & $-65.2 \%$ \\
\hline & & Combined & $-63.9 \%$ & $-63.9 \%$ & $-66.4 \%$ & $-69.5 \%$ & $-63.8 \%$ & $-68.8 \%$ & $-70.6 \%$ & $-66.5 \%$ & $-72.4 \%$ & $-75.8 \%$ & $-68.1 \%$ & $-71.2 \%$ & $-77.0 \%$ \\
\hline & \multirow{3}{*}{ Adjusted } & \begin{tabular}{|l|} 
UDDS \\
\end{tabular} & $-67.7 \%$ & $-67.7 \%$ & $-69.6 \%$ & $-71.9 \%$ & $-67.8 \%$ & $-71.4 \%$ & $-74.7 \%$ & $-69.7 \%$ & $-74.1 \%$ & $-78.4 \%$ & $-70.9 \%$ & $-75.0 \%$ & $-79.1 \%$ \\
\hline & & HWFET & $-49.5 \%$ & $-49.5 \%$ & $-53.0 \%$ & $-57.2 \%$ & $-49.3 \%$ & $-56.3 \%$ & $-55.0 \%$ & $-53.0 \%$ & $-61.3 \%$ & $-62.5 \%$ & $-55.2 \%$ & $-56.0 \%$ & $-64.6 \%$ \\
\hline & & Combined & $-60.9 \%$ & $-60.9 \%$ & $-63.4 \%$ & $-66.4 \%$ & $-60.8 \%$ & $-65.7 \%$ & $-67.3 \%$ & $-63.4 \%$ & $-69.3 \%$ & $-72.4 \%$ & $-65.0 \%$ & $-67.9 \%$ & $-73.6 \%$ \\
\hline
\end{tabular}




\begin{tabular}{|c|c|c|c|c|c|c|c|c|c|c|c|c|c|c|c|}
\hline & & & \multicolumn{13}{|c|}{ Midsize } \\
\hline & & & 2010 & & 2010 & & & 2015 & & & 2030 & & & 2045 & \\
\hline & & & Ref & low & avg & high & low & avg & high & low & avg & high & low & avg & high \\
\hline \multirow{6}{*}{ Cl Series HEV PHEV 30} & \multirow{3}{*}{ Unadjusted } & UDDS & $-69.2 \%$ & $-69.2 \%$ & $-71.9 \%$ & $-74.8 \%$ & $-69.8 \%$ & $-73.9 \%$ & $-78.3 \%$ & $-72.4 \%$ & $-77.6 \%$ & $-82.8 \%$ & $-73.7 \%$ & $-78.7 \%$ & $-83.6 \%$ \\
\hline & & HWFET & $-54.9 \%$ & $-54.9 \%$ & $-59.0 \%$ & $-63.5 \%$ & $-55.5 \%$ & $-62.4 \%$ & $-68.5 \%$ & $-59.4 \%$ & $-67.2 \%$ & $-73.8 \%$ & $-61.4 \%$ & $-68.9 \%$ & $-75.4 \%$ \\
\hline & & Combined & $-64.1 \%$ & $-64.1 \%$ & $-67.3 \%$ & $-70.7 \%$ & $-64.7 \%$ & $-69.8 \%$ & $-74.8 \%$ & $-67.8 \%$ & $-73.9 \%$ & $-79.6 \%$ & $-69.3 \%$ & $-75.2 \%$ & $-80.7 \%$ \\
\hline & \multirow{3}{*}{ Adjusted } & UDDS & $-65.5 \%$ & $-65.5 \%$ & $-68.0 \%$ & $-70.8 \%$ & $-66.0 \%$ & $-69.9 \%$ & $-74.1 \%$ & $-68.5 \%$ & $-73.5 \%$ & $-78.4 \%$ & $-69.7 \%$ & $-74.5 \%$ & $-79.1 \%$ \\
\hline & & HWFET & $-54.4 \%$ & $-54.4 \%$ & $-58.5 \%$ & $-62.9 \%$ & $-55.0 \%$ & $-61.8 \%$ & $-67.9 \%$ & $-58.8 \%$ & $-66.6 \%$ & $-73.2 \%$ & $-60.9 \%$ & $-68.2 \%$ & $-74.7 \%$ \\
\hline & & Combined & $-61.3 \%$ & $-61.3 \%$ & $-64.4 \%$ & $-67.8 \%$ & $-61.9 \%$ & $-66.9 \%$ & $-71.8 \%$ & $-64.9 \%$ & $-70.9 \%$ & $-76.4 \%$ & $-66.4 \%$ & $-72.2 \%$ & $-77.5 \%$ \\
\hline \multirow{6}{*}{$\mathrm{Cl}$ Series HEV PHEV40 } & \multirow{3}{*}{ Unadjusted } & UDDS & $-73.2 \%$ & $-73.2 \%$ & $-75.6 \%$ & $-78.0 \%$ & $-73.7 \%$ & $-77.4 \%$ & $-81.3 \%$ & $-76.1 \%$ & $-80.7 \%$ & $-85.4 \%$ & $-77.2 \%$ & $-81.6 \%$ & $-86.2 \%$ \\
\hline & & HWFET & $-63.1 \%$ & $-63.1 \%$ & $-66.8 \%$ & $-70.2 \%$ & $-63.7 \%$ & $-69.2 \%$ & $-74.4 \%$ & $-66.9 \%$ & $-73.3 \%$ & $-78.8 \%$ & $-68.6 \%$ & $-74.7 \%$ & $-80.3 \%$ \\
\hline & & Combined & $-69.6 \%$ & $-69.6 \%$ & $-72.5 \%$ & $-75.2 \%$ & $-70.1 \%$ & $-74.4 \%$ & $-78.8 \%$ & $-72.8 \%$ & $-78.1 \%$ & $-83.0 \%$ & $-74.1 \%$ & $-79.2 \%$ & $-84.1 \%$ \\
\hline & \multirow{3}{*}{ Adjusted } & UDDS & $-69.3 \%$ & $-69.3 \%$ & $-71.5 \%$ & $-73.8 \%$ & $-69.7 \%$ & $-73.2 \%$ & $-76.9 \%$ & $-72.0 \%$ & $-76.3 \%$ & $-80.8 \%$ & $-73.1 \%$ & $-77.3 \%$ & $-81.5 \%$ \\
\hline & & HWFET & $-62.6 \%$ & $-62.6 \%$ & $-66.2 \%$ & $-69.5 \%$ & $-63.1 \%$ & $-68.5 \%$ & $-73.7 \%$ & $-66.3 \%$ & $-72.7 \%$ & $-78.1 \%$ & $-68.0 \%$ & $-74.0 \%$ & $-79.6 \%$ \\
\hline & & \begin{tabular}{|c|} 
Combined \\
\end{tabular} & $-66.8 \%$ & $-66.8 \%$ & $-69.5 \%$ & $-72.2 \%$ & $-67.2 \%$ & $-71.4 \%$ & $-75.7 \%$ & $-69.8 \%$ & $-75.0 \%$ & $-79.8 \%$ & $-71.1 \%$ & $-76.0 \%$ & $-80.8 \%$ \\
\hline \multirow{6}{*}{ H2 Split HEV } & \multirow{3}{*}{ Unadjusted } & \begin{tabular}{|l|} 
UDDS \\
\end{tabular} & $-47.2 \%$ & $-47.2 \%$ & $-56.5 \%$ & $-62.3 \%$ & $-52.8 \%$ & $-61.8 \%$ & $-68.1 \%$ & $-58.6 \%$ & $-66.9 \%$ & $-74.3 \%$ & $-61.9 \%$ & $-69.3 \%$ & $-76.3 \%$ \\
\hline & & HWFET & $-11.6 \%$ & $-11.6 \%$ & $-27.5 \%$ & $-37.4 \%$ & $-21.2 \%$ & $-35.8 \%$ & $-45.8 \%$ & $-30.3 \%$ & $-44.0 \%$ & $-55.5 \%$ & $-35.8 \%$ & $-48.1 \%$ & $-59.6 \%$ \\
\hline & & Combined & $-34.5 \%$ & $-34.5 \%$ & $-46.2 \%$ & $-53.5 \%$ & $-41.6 \%$ & $-52.6 \%$ & $-60.2 \%$ & $-48.6 \%$ & $-58.8 \%$ & $-67.6 \%$ & $-52.6 \%$ & $-61.8 \%$ & $-70.4 \%$ \\
\hline & \multirow{3}{*}{ Adjusted } & UDDS & $-44.6 \%$ & $-44.6 \%$ & $-53.4 \%$ & $-59.0 \%$ & $-49.9 \%$ & $-58.5 \%$ & $-64.4 \%$ & $-55.5 \%$ & $-63.3 \%$ & $-70.3 \%$ & $-58.6 \%$ & $-65.6 \%$ & $-72.2 \%$ \\
\hline & & HWFET & $-11.5 \%$ & $-11.5 \%$ & $-27.2 \%$ & $-37.1 \%$ & $-21.1 \%$ & $-35.5 \%$ & $-45.4 \%$ & $-30.1 \%$ & $-43.6 \%$ & $-55.0 \%$ & $-35.5 \%$ & $-47.7 \%$ & $-59.1 \%$ \\
\hline & & \begin{tabular}{|l|} 
Combined \\
\end{tabular} & $-32.2 \%$ & $-32.2 \%$ & $-43.6 \%$ & $-50.8 \%$ & $-39.1 \%$ & $-49.9 \%$ & $-57.3 \%$ & $-45.9 \%$ & $-55.9 \%$ & $-64.5 \%$ & $-49.9 \%$ & $-58.9 \%$ & $-67.3 \%$ \\
\hline \multirow{6}{*}{ H2 Split HEV PHEV10 } & \multirow{3}{*}{ Unadjusted } & UDDS & $-57.3 \%$ & $-57.3 \%$ & $-64.7 \%$ & $-69.6 \%$ & $-61.8 \%$ & $-68.6 \%$ & $-73.5 \%$ & $-65.9 \%$ & $-72.5 \%$ & $-78.8 \%$ & $-68.6 \%$ & $-74.4 \%$ & $-80.4 \%$ \\
\hline & & HWFET & $-36.3 \%$ & $-36.3 \%$ & $-47.5 \%$ & $-54.7 \%$ & $-43.2 \%$ & $-53.5 \%$ & $-60.6 \%$ & $-49.0 \%$ & $-59.1 \%$ & $-67.1 \%$ & $-52.9 \%$ & $-62.1 \%$ & $-70.3 \%$ \\
\hline & & Combined & $-49.8 \%$ & $-49.8 \%$ & $-58.6 \%$ & $-64.3 \%$ & $-55.2 \%$ & $-63.2 \%$ & $-68.9 \%$ & $-59.9 \%$ & $-67.8 \%$ & $-74.6 \%$ & $-63.0 \%$ & $-70.0 \%$ & $-76.8 \%$ \\
\hline & \multirow{3}{*}{ Adjusted } & \begin{tabular}{|l|} 
UDDS \\
\end{tabular} & $-54.2 \%$ & $-54.2 \%$ & $-61.2 \%$ & $-65.8 \%$ & $-58.5 \%$ & $-64.9 \%$ & $-69.5 \%$ & $-62.4 \%$ & $-68.6 \%$ & $-74.5 \%$ & $-64.9 \%$ & $-70.4 \%$ & $-76.1 \%$ \\
\hline & & HWFET & $-36.0 \%$ & $-36.0 \%$ & $-47.1 \%$ & $-54.2 \%$ & $-42.8 \%$ & $-53.0 \%$ & $-60.1 \%$ & $-48.6 \%$ & $-58.6 \%$ & $-66.5 \%$ & $-52.4 \%$ & $-61.5 \%$ & $-69.7 \%$ \\
\hline & & \begin{tabular}{|c|} 
Combined \\
\end{tabular} & $-47.4 \%$ & $-47.4 \%$ & $-55.9 \%$ & $-61.5 \%$ & $-52.6 \%$ & $-60.5 \%$ & $-66.0 \%$ & $-57.2 \%$ & $-64.9 \%$ & $-71.5 \%$ & $-60.2 \%$ & $-67.0 \%$ & $-73.7 \%$ \\
\hline \multirow{6}{*}{ H2 Split HEV PHEV2O } & \multirow{3}{*}{ Unadjusted } & \begin{tabular}{|l|} 
UDDS \\
\end{tabular} & $-70.0 \%$ & $-70.0 \%$ & $-75.3 \%$ & $-78.9 \%$ & $-73.1 \%$ & $-78.2 \%$ & $-81.7 \%$ & $-76.2 \%$ & $-81.0 \%$ & $-82.3 \%$ & $-78.0 \%$ & $-82.3 \%$ & $-83.8 \%$ \\
\hline & & HWFET & $-47.3 \%$ & $-47.3 \%$ & $-56.7 \%$ & $-63.1 \%$ & $-52.9 \%$ & $-61.3 \%$ & $-61.6 \%$ & $-58.4 \%$ & $-59.8 \%$ & $-68.0 \%$ & $-61.1 \%$ & $-63.1 \%$ & $-71.1 \%$ \\
\hline & & Combined & $-61.9 \%$ & $-61.9 \%$ & $-68.7 \%$ & $-73.3 \%$ & $-65.9 \%$ & $-72.2 \%$ & $-74.6 \%$ & $-69.9 \%$ & $-73.5 \%$ & $-77.2 \%$ & $-72.0 \%$ & $-75.5 \%$ & $-79.3 \%$ \\
\hline & \multirow{3}{*}{ Adjusted } & \begin{tabular}{|l|} 
UDDS \\
\end{tabular} & $-66.2 \%$ & $-66.2 \%$ & $-71.2 \%$ & $-74.6 \%$ & $-69.2 \%$ & $-74.0 \%$ & $-77.3 \%$ & $-72.1 \%$ & $-76.7 \%$ & $-77.9 \%$ & $-73.8 \%$ & $-77.9 \%$ & $-79.3 \%$ \\
\hline & & HWFET & $-46.9 \%$ & $-46.9 \%$ & $-56.2 \%$ & $-62.5 \%$ & $-52.5 \%$ & $-60.8 \%$ & $-61.1 \%$ & $-57.9 \%$ & $-59.3 \%$ & $-67.4 \%$ & $-60.6 \%$ & $-62.5 \%$ & $-70.4 \%$ \\
\hline & & Combined & $-58.9 \%$ & $-58.9 \%$ & $-65.6 \%$ & $-70.1 \%$ & $-62.9 \%$ & $-69.1 \%$ & $-71.2 \%$ & $-66.8 \%$ & $-70.1 \%$ & $-73.9 \%$ & $-68.9 \%$ & $-72.1 \%$ & $-76.0 \%$ \\
\hline \multirow{6}{*}{ H2 Series HEV PHEV3O } & \multirow{3}{*}{ Unadjusted } & \begin{tabular}{|l|} 
UDDS \\
\end{tabular} & $-67.5 \%$ & $-67.5 \%$ & $-73.5 \%$ & $-77.4 \%$ & $-71.2 \%$ & $-76.7 \%$ & $-81.0 \%$ & $-74.7 \%$ & $-80.2 \%$ & $-84.5 \%$ & $-76.9 \%$ & $-81.4 \%$ & $-85.7 \%$ \\
\hline & & HWFET & $-53.1 \%$ & $-53.1 \%$ & $-61.8 \%$ & $-67.1 \%$ & $-58.3 \%$ & $-66.2 \%$ & $-72.3 \%$ & $-63.4 \%$ & $-71.0 \%$ & $-77.1 \%$ & $-66.4 \%$ & $-73.3 \%$ & $-79.2 \%$ \\
\hline & & \begin{tabular}{|l|} 
Combined \\
\end{tabular} & $-62.4 \%$ & $-62.4 \%$ & $-69.3 \%$ & $-73.8 \%$ & $-66.6 \%$ & $-72.9 \%$ & $-77.9 \%$ & $-70.7 \%$ & $-76.9 \%$ & $-81.9 \%$ & $-73.2 \%$ & $-78.5 \%$ & $-83.4 \%$ \\
\hline & \multirow{3}{*}{ Adjusted } & \begin{tabular}{|l|} 
UDDS \\
\end{tabular} & $-63.9 \%$ & $-63.9 \%$ & $-69.5 \%$ & $-73.2 \%$ & $-67.4 \%$ & $-72.5 \%$ & $-76.6 \%$ & $-70.7 \%$ & $-75.9 \%$ & $-80.0 \%$ & $-72.8 \%$ & $-77.0 \%$ & $-81.1 \%$ \\
\hline & & HWFET & $-52.6 \%$ & $-52.6 \%$ & $-61.2 \%$ & $-66.5 \%$ & $-57.8 \%$ & $-65.6 \%$ & $-71.7 \%$ & $-62.8 \%$ & $-70.4 \%$ & $-76.5 \%$ & $-65.8 \%$ & $-72.6 \%$ & $-78.5 \%$ \\
\hline & & Combined & $-59.6 \%$ & $-59.6 \%$ & $-66.4 \%$ & $-70.7 \%$ & $-63.8 \%$ & $-69.9 \%$ & $-74.8 \%$ & $-67.7 \%$ & $-73.8 \%$ & $-78.6 \%$ & $-70.2 \%$ & $-75.4 \%$ & $-80.1 \%$ \\
\hline
\end{tabular}




\begin{tabular}{|c|c|c|c|c|c|c|c|c|c|c|c|c|c|c|c|}
\hline & & & \multicolumn{13}{|c|}{ Midsize } \\
\hline & & & 2010 & & 2010 & & & 2015 & & & 2030 & & & 2045 & \\
\hline & & & Ref & low & avg & high & low & avg & high & low & avg & high & low & avg & high \\
\hline \multirow{6}{*}{ H2 Series HEV PHEV40 } & \multirow{3}{*}{ Unadjusted } & UDDS & $-71.4 \%$ & $-71.4 \%$ & $-77.0 \%$ & $-80.3 \%$ & $-75.0 \%$ & $-79.8 \%$ & $-83.5 \%$ & $-78.1 \%$ & $-82.8 \%$ & $-86.9 \%$ & $-79.9 \%$ & $-84.0 \%$ & $-87.9 \%$ \\
\hline & & HWFET & $-61.7 \%$ & $-61.7 \%$ & $-68.8 \%$ & $-73.4 \%$ & $-66.0 \%$ & $-72.4 \%$ & $-77.5 \%$ & $-70.1 \%$ & $-76.4 \%$ & $-81.5 \%$ & $-72.5 \%$ & $-78.3 \%$ & $-83.2 \%$ \\
\hline & & Combined & $-68.0 \%$ & $-68.0 \%$ & $-74.1 \%$ & $-77.8 \%$ & $-71.8 \%$ & $-77.1 \%$ & $-81.4 \%$ & $-75.3 \%$ & $-80.5 \%$ & $-85.0 \%$ & $-77.3 \%$ & $-82.0 \%$ & $-86.2 \%$ \\
\hline & \multirow{3}{*}{ Adjusted } & \begin{tabular}{|l|} 
UDDS \\
\end{tabular} & $-67.6 \%$ & $-67.6 \%$ & $-72.8 \%$ & $-76.0 \%$ & $-71.0 \%$ & $-75.5 \%$ & $-79.0 \%$ & $-73.9 \%$ & $-78.4 \%$ & $-82.3 \%$ & $-75.6 \%$ & $-79.5 \%$ & $-83.2 \%$ \\
\hline & & HWFET & $-61.2 \%$ & $-61.2 \%$ & $-68.2 \%$ & $-72.7 \%$ & $-65.4 \%$ & $-71.7 \%$ & $-76.8 \%$ & $-69.5 \%$ & $-75.7 \%$ & $-80.7 \%$ & $-71.9 \%$ & $-77.6 \%$ & $-82.5 \%$ \\
\hline & & \begin{tabular}{|l|} 
Combined \\
\end{tabular} & $-65.2 \%$ & $-65.2 \%$ & $-71.1 \%$ & $-74.8 \%$ & $-68.9 \%$ & $-74.1 \%$ & $-78.2 \%$ & $-72.3 \%$ & $-77.4 \%$ & $-81.7 \%$ & $-74.2 \%$ & $-78.8 \%$ & $-82.9 \%$ \\
\hline \multirow{6}{*}{ E85 Split HEV } & \multirow{3}{*}{ Unadjusted } & \begin{tabular}{|l|} 
UDDS \\
\end{tabular} & $-44.6 \%$ & $-44.6 \%$ & $-49.3 \%$ & $-53.5 \%$ & $-45.7 \%$ & $-53.0 \%$ & $-64.3 \%$ & $-50.1 \%$ & $-57.9 \%$ & $-70.3 \%$ & $-51.5 \%$ & $-59.6 \%$ & $-71.6 \%$ \\
\hline & & HWFET & $-8.7 \%$ & $-8.7 \%$ & $-17.4 \%$ & $-23.9 \%$ & $-10.5 \%$ & $-21.4 \%$ & $-39.9 \%$ & $-16.6 \%$ & $-29.3 \%$ & $-49.1 \%$ & $-19.1 \%$ & $-32.3 \%$ & $-52.1 \%$ \\
\hline & & Combined & $-31.8 \%$ & $-31.8 \%$ & $-38.0 \%$ & $-43.0 \%$ & $-33.2 \%$ & $-41.7 \%$ & $-55.6 \%$ & $-38.2 \%$ & $-47.8 \%$ & $-62.8 \%$ & $-40.0 \%$ & $-49.9 \%$ & $-64.7 \%$ \\
\hline & \multirow{3}{*}{ Adjusted } & \begin{tabular}{|l|} 
UDDS \\
\end{tabular} & $-42.2 \%$ & $-42.2 \%$ & $-46.7 \%$ & $-50.6 \%$ & $-43.2 \%$ & $-50.1 \%$ & $-60.9 \%$ & $-47.4 \%$ & $-54.8 \%$ & $-66.5 \%$ & $-48.8 \%$ & $-56.4 \%$ & $-67.7 \%$ \\
\hline & & HWFET & $-8.6 \%$ & $-8.6 \%$ & $-17.2 \%$ & $-23.7 \%$ & $-10.4 \%$ & $-21.2 \%$ & $-39.6 \%$ & $-16.5 \%$ & $-29.0 \%$ & $-48.7 \%$ & $-18.9 \%$ & $-32.0 \%$ & $-51.7 \%$ \\
\hline & & \begin{tabular}{|c|} 
Combined \\
\end{tabular} & $-29.6 \%$ & $-29.6 \%$ & $-35.6 \%$ & $-40.5 \%$ & $-30.9 \%$ & $-39.2 \%$ & $-52.9 \%$ & $-35.8 \%$ & $-45.1 \%$ & $-59.8 \%$ & $-37.5 \%$ & $-47.2 \%$ & $-61.7 \%$ \\
\hline \multirow{6}{*}{ E85 Split HEV PHEV10 } & \multirow{3}{*}{ Unadjusted } & UDDS & $-56.0 \%$ & $-56.0 \%$ & $-59.6 \%$ & $-62.9 \%$ & $-56.9 \%$ & $-61.8 \%$ & $-70.5 \%$ & $-59.2 \%$ & $-65.4 \%$ & $-75.5 \%$ & $-60.5 \%$ & $-66.6 \%$ & $-76.7 \%$ \\
\hline & & HWFET & $-33.9 \%$ & $-33.9 \%$ & $-39.7 \%$ & $-44.7 \%$ & $-35.3 \%$ & $-43.2 \%$ & $-56.2 \%$ & $-38.6 \%$ & $-48.6 \%$ & $-62.5 \%$ & $-40.7 \%$ & $-50.8 \%$ & $-64.9 \%$ \\
\hline & & Combined & $-48.1 \%$ & $-48.1 \%$ & $-52.5 \%$ & $-56.4 \%$ & $-49.2 \%$ & $-55.2 \%$ & $-65.4 \%$ & $-51.8 \%$ & $-59.4 \%$ & $-70.9 \%$ & $-53.5 \%$ & $-61.0 \%$ & $-72.5 \%$ \\
\hline & \multirow{3}{*}{ Adjusted } & UDDS & $-53.0 \%$ & $-53.0 \%$ & $-56.4 \%$ & $-59.5 \%$ & $-53.8 \%$ & $-58.5 \%$ & $-66.7 \%$ & $-56.0 \%$ & $-61.8 \%$ & $-71.4 \%$ & $-57.2 \%$ & $-63.1 \%$ & $-72.5 \%$ \\
\hline & & HWFET & $-33.6 \%$ & $-33.6 \%$ & $-39.3 \%$ & $-44.3 \%$ & $-35.0 \%$ & $-42.8 \%$ & $-55.7 \%$ & $-38.2 \%$ & $-48.2 \%$ & $-62.0 \%$ & $-40.3 \%$ & $-50.3 \%$ & $-64.4 \%$ \\
\hline & & Combined & $-45.7 \%$ & $-45.7 \%$ & $-50.0 \%$ & $-53.8 \%$ & $-46.8 \%$ & $-52.6 \%$ & $-62.5 \%$ & $-49.3 \%$ & $-56.7 \%$ & $-67.9 \%$ & $-50.9 \%$ & $-58.3 \%$ & $-69.5 \%$ \\
\hline \multirow{6}{*}{ E85 Split HEV PHEV2O } & \multirow{3}{*}{ Unadjusted } & UDDS & $-69.0 \%$ & $-69.0 \%$ & $-71.7 \%$ & $-74.2 \%$ & $-69.9 \%$ & $-73.5 \%$ & $-79.6 \%$ & $-71.4 \%$ & $-76.2 \%$ & $-79.7 \%$ & $-72.4 \%$ & $-77.0 \%$ & $-80.8 \%$ \\
\hline & & HWFET & $-45.2 \%$ & $-45.2 \%$ & $-50.3 \%$ & $-54.9 \%$ & $-47.1 \%$ & $-52.8 \%$ & $-57.2 \%$ & $-49.3 \%$ & $-49.3 \%$ & $-63.6 \%$ & $-51.0 \%$ & $-52.0 \%$ & $-65.8 \%$ \\
\hline & & Combined & $-60.5 \%$ & $-60.5 \%$ & $-64.1 \%$ & $-67.4 \%$ & $-61.8 \%$ & $-66.1 \%$ & $-71.6 \%$ & $-63.5 \%$ & $-66.6 \%$ & $-74.0 \%$ & $-64.8 \%$ & $-68.1 \%$ & $-75.5 \%$ \\
\hline & \multirow{3}{*}{ Adjusted } & UDDS & $-65.3 \%$ & $-65.3 \%$ & $-67.9 \%$ & $-70.3 \%$ & $-66.1 \%$ & $-69.5 \%$ & $-75.3 \%$ & $-67.5 \%$ & $-72.1 \%$ & $-75.4 \%$ & $-68.5 \%$ & $-72.9 \%$ & $-76.5 \%$ \\
\hline & & HWFET & $-44.8 \%$ & $-44.8 \%$ & $-49.9 \%$ & $-54.4 \%$ & $-46.7 \%$ & $-52.3 \%$ & $-56.7 \%$ & $-48.9 \%$ & $-48.9 \%$ & $-63.0 \%$ & $-50.6 \%$ & $-51.6 \%$ & $-65.2 \%$ \\
\hline & & \begin{tabular}{|c|} 
Combined \\
\end{tabular} & $-57.6 \%$ & $-57.6 \%$ & $-61.1 \%$ & $-64.3 \%$ & $-58.8 \%$ & $-63.1 \%$ & $-68.3 \%$ & $-60.5 \%$ & $-63.3 \%$ & $-70.8 \%$ & $-61.8 \%$ & $-64.9 \%$ & $-72.2 \%$ \\
\hline \multirow{6}{*}{ E85 Series HEV PHEV30 } & \multirow{3}{*}{ Unadjusted } & \begin{tabular}{|l|} 
UDDS \\
\end{tabular} & $-66.8 \%$ & $-66.8 \%$ & $-70.0 \%$ & $-72.8 \%$ & $-67.4 \%$ & $-71.9 \%$ & $-79.1 \%$ & $-70.1 \%$ & $-75.5 \%$ & $-83.1 \%$ & $-71.3 \%$ & $-76.4 \%$ & $-83.8 \%$ \\
\hline & & HWFET & $-51.1 \%$ & $-51.1 \%$ & $-56.0 \%$ & $-59.8 \%$ & $-52.5 \%$ & $-58.6 \%$ & $-69.4 \%$ & $-55.7 \%$ & $-63.5 \%$ & $-74.0 \%$ & $-57.5 \%$ & $-65.4 \%$ & $-75.6 \%$ \\
\hline & & Combined & $-61.2 \%$ & $-61.2 \%$ & $-65.0 \%$ & $-68.2 \%$ & $-62.1 \%$ & $-67.1 \%$ & $-75.7 \%$ & $-65.0 \%$ & $-71.2 \%$ & $-79.9 \%$ & $-66.4 \%$ & $-72.5 \%$ & $-80.9 \%$ \\
\hline & \multirow{3}{*}{ Adjusted } & UDDS & $-63.2 \%$ & $-63.2 \%$ & $-66.2 \%$ & $-68.9 \%$ & $-63.8 \%$ & $-68.0 \%$ & $-74.9 \%$ & $-66.3 \%$ & $-71.4 \%$ & $-78.6 \%$ & $-67.4 \%$ & $-72.3 \%$ & $-79.3 \%$ \\
\hline & & \begin{tabular}{|l|} 
HWFET \\
\end{tabular} & $-50.7 \%$ & $-50.7 \%$ & $-55.5 \%$ & $-59.3 \%$ & $-52.0 \%$ & $-58.1 \%$ & $-68.8 \%$ & $-55.2 \%$ & $-62.9 \%$ & $-73.4 \%$ & $-57.0 \%$ & $-64.8 \%$ & $-74.9 \%$ \\
\hline & & Combined & $-58.5 \%$ & $-58.5 \%$ & $-62.2 \%$ & $-65.3 \%$ & $-59.4 \%$ & $-64.3 \%$ & $-72.6 \%$ & $-62.1 \%$ & $-68.2 \%$ & $-76.7 \%$ & $-63.5 \%$ & $-69.5 \%$ & $-77.7 \%$ \\
\hline \multirow{6}{*}{ E85 Series HEV PHEV40 } & \multirow{3}{*}{ Unadjusted } & \begin{tabular}{|l|} 
UDDS \\
\end{tabular} & $-70.7 \%$ & $-70.7 \%$ & $-73.9 \%$ & $-76.2 \%$ & $-71.9 \%$ & $-75.6 \%$ & $-81.9 \%$ & $-74.0 \%$ & $-78.8 \%$ & $-85.7 \%$ & $-75.0 \%$ & $-79.6 \%$ & $-86.4 \%$ \\
\hline & & HWFET & $-60.1 \%$ & $-60.1 \%$ & $-64.1 \%$ & $-67.1 \%$ & $-61.2 \%$ & $-66.2 \%$ & $-75.1 \%$ & $-63.9 \%$ & $-70.3 \%$ & $-79.0 \%$ & $-65.4 \%$ & $-71.9 \%$ & $-80.2 \%$ \\
\hline & & Combined & $-66.9 \%$ & $-66.9 \%$ & $-70.4 \%$ & $-73.0 \%$ & $-68.1 \%$ & $-72.2 \%$ & $-79.5 \%$ & $-70.4 \%$ & $-75.7 \%$ & $-83.3 \%$ & $-71.6 \%$ & $-76.9 \%$ & $-84.2 \%$ \\
\hline & \multirow{3}{*}{ Adjusted } & \begin{tabular}{|l|} 
UDDS \\
\end{tabular} & $-66.9 \%$ & $-66.9 \%$ & $-69.9 \%$ & $-72.1 \%$ & $-68.1 \%$ & $-71.5 \%$ & $-77.5 \%$ & $-70.0 \%$ & $-74.5 \%$ & $-81.1 \%$ & $-71.0 \%$ & $-75.3 \%$ & $-81.8 \%$ \\
\hline & & HWFET & $-59.6 \%$ & $-59.6 \%$ & $-63.5 \%$ & $-66.5 \%$ & $-60.7 \%$ & $-65.6 \%$ & $-74.4 \%$ & $-63.3 \%$ & $-69.6 \%$ & $-78.3 \%$ & $-64.8 \%$ & $-71.2 \%$ & $-79.5 \%$ \\
\hline & & Combined & $-64.2 \%$ & $-64.2 \%$ & $-67.5 \%$ & $-70.0 \%$ & $-65.3 \%$ & $-69.3 \%$ & $-76.4 \%$ & $-67.5 \%$ & $-72.7 \%$ & $-80.0 \%$ & $-68.7 \%$ & $-73.8 \%$ & $-80.9 \%$ \\
\hline
\end{tabular}




\begin{tabular}{|c|c|c|c|c|c|c|c|c|c|c|c|c|c|c|c|}
\hline & & & \multicolumn{13}{|c|}{ Midsize } \\
\hline & & & 2010 & & 2010 & & & 2015 & & & 2030 & & & 2045 & \\
\hline & & & Ref & low & avg & high & low & avg & high & low & avg & high & low & avg & high \\
\hline \multirow{6}{*}{ FC HEV } & \multirow{3}{*}{ Unadjusted } & UDDS & $-51.8 \%$ & $-51.8 \%$ & $-60.3 \%$ & $-66.6 \%$ & $-59.0 \%$ & $-66.2 \%$ & $-71.3 \%$ & $-63.4 \%$ & $-70.2 \%$ & $-76.3 \%$ & $-65.4 \%$ & $-71.4 \%$ & $-77.4 \%$ \\
\hline & & HWFET & $-34.2 \%$ & $-34.2 \%$ & $-45.1 \%$ & $-52.9 \%$ & $-43.2 \%$ & $-51.7 \%$ & $-58.1 \%$ & $-47.7 \%$ & $-56.5 \%$ & $-64.2 \%$ & $-50.2 \%$ & $-58.4 \%$ & $-66.5 \%$ \\
\hline & & Combined & $-45.6 \%$ & $-45.6 \%$ & $-54.9 \%$ & $-61.8 \%$ & $-53.4 \%$ & $-61.0 \%$ & $-66.6 \%$ & $-57.8 \%$ & $-65.3 \%$ & $-72.0 \%$ & $-60.0 \%$ & $-66.8 \%$ & $-73.5 \%$ \\
\hline & \multirow{3}{*}{ Adjusted } & \begin{tabular}{|l|} 
UDDS \\
\end{tabular} & $-49.0 \%$ & $-49.0 \%$ & $-57.1 \%$ & $-63.0 \%$ & $-55.9 \%$ & $-62.6 \%$ & $-67.4 \%$ & $-60.0 \%$ & $-66.4 \%$ & $-72.2 \%$ & $-61.9 \%$ & $-67.6 \%$ & $-73.3 \%$ \\
\hline & & HWFET & $-33.9 \%$ & $-33.9 \%$ & $-44.7 \%$ & $-52.5 \%$ & $-42.8 \%$ & $-51.3 \%$ & $-57.6 \%$ & $-47.2 \%$ & $-56.0 \%$ & $-63.6 \%$ & $-49.7 \%$ & $-57.8 \%$ & $-65.9 \%$ \\
\hline & & Combined & $-43.3 \%$ & $-43.3 \%$ & $-52.4 \%$ & $-59.1 \%$ & $-50.9 \%$ & $-58.3 \%$ & $-63.7 \%$ & $-55.2 \%$ & $-62.5 \%$ & $-69.0 \%$ & $-57.3 \%$ & $-63.9 \%$ & $-70.5 \%$ \\
\hline \multirow{6}{*}{ FC PHEV10 } & \multirow{3}{*}{ Unadjusted } & \begin{tabular}{|l|} 
UDDS \\
\end{tabular} & $-61.9 \%$ & $-61.9 \%$ & $-68.1 \%$ & $-72.9 \%$ & $-67.2 \%$ & $-72.0 \%$ & $-76.0 \%$ & $-69.8 \%$ & $-75.2 \%$ & $-80.0 \%$ & $-71.3 \%$ & $-76.0 \%$ & $-80.9 \%$ \\
\hline & & HWFET & $-51.6 \%$ & $-51.6 \%$ & $-58.9 \%$ & $-64.4 \%$ & $-57.4 \%$ & $-63.3 \%$ & $-67.9 \%$ & $-60.1 \%$ & $-66.6 \%$ & $-72.3 \%$ & $-61.8 \%$ & $-68.0 \%$ & $-74.3 \%$ \\
\hline & & Combined & $-58.2 \%$ & $-58.2 \%$ & $-64.8 \%$ & $-69.8 \%$ & $-63.7 \%$ & $-68.9 \%$ & $-73.1 \%$ & $-66.3 \%$ & $-72.1 \%$ & $-77.3 \%$ & $-67.9 \%$ & $-73.1 \%$ & $-78.6 \%$ \\
\hline & \multirow{3}{*}{ Adjusted } & \begin{tabular}{|l|} 
UDDS \\
\end{tabular} & $-58.6 \%$ & $-58.6 \%$ & $-64.4 \%$ & $-69.0 \%$ & $-63.6 \%$ & $-68.2 \%$ & $-71.9 \%$ & $-66.0 \%$ & $-71.1 \%$ & $-75.7 \%$ & $-67.4 \%$ & $-71.9 \%$ & $-76.6 \%$ \\
\hline & & HWFET & $-51.1 \%$ & $-51.1 \%$ & $-58.3 \%$ & $-63.8 \%$ & $-56.9 \%$ & $-62.7 \%$ & $-67.3 \%$ & $-59.6 \%$ & $-66.1 \%$ & $-71.7 \%$ & $-61.3 \%$ & $-67.4 \%$ & $-73.6 \%$ \\
\hline & & Combined & $-55.8 \%$ & $-55.8 \%$ & $-62.1 \%$ & $-67.0 \%$ & $-61.1 \%$ & $-66.1 \%$ & $-70.2 \%$ & $-63.6 \%$ & $-69.2 \%$ & $-74.2 \%$ & $-65.1 \%$ & $-70.2 \%$ & $-75.5 \%$ \\
\hline \multirow{6}{*}{ FC PHEV20 } & \multirow{3}{*}{ Unadjusted } & \begin{tabular}{|l|} 
UDDS \\
\end{tabular} & $-68.2 \%$ & $-68.2 \%$ & $-73.4 \%$ & $-77.4 \%$ & $-72.7 \%$ & $-76.8 \%$ & $-80.1 \%$ & $-74.9 \%$ & $-79.4 \%$ & $-83.5 \%$ & $-76.1 \%$ & $-80.2 \%$ & $-84.3 \%$ \\
\hline & & HWFET & $-51.4 \%$ & $-51.4 \%$ & $-59.0 \%$ & $-64.7 \%$ & $-57.7 \%$ & $-63.6 \%$ & $-68.3 \%$ & $-60.5 \%$ & $-67.1 \%$ & $-72.9 \%$ & $-62.3 \%$ & $-68.5 \%$ & $-74.6 \%$ \\
\hline & & Combined & $-62.3 \%$ & $-62.3 \%$ & $-68.3 \%$ & $-72.9 \%$ & $-67.4 \%$ & $-72.1 \%$ & $-75.9 \%$ & $-69.8 \%$ & $-75.1 \%$ & $-79.7 \%$ & $-71.2 \%$ & $-76.0 \%$ & $-80.8 \%$ \\
\hline & \multirow{3}{*}{ Adjusted } & \begin{tabular}{|l|} 
UDDS \\
\end{tabular} & $-64.6 \%$ & $-64.6 \%$ & $-69.5 \%$ & $-73.3 \%$ & $-68.8 \%$ & $-72.7 \%$ & $-75.8 \%$ & $-70.9 \%$ & $-75.2 \%$ & $-79.0 \%$ & $-72.1 \%$ & $-75.9 \%$ & $-79.7 \%$ \\
\hline & & HWFET & $-51.0 \%$ & $-51.0 \%$ & $-58.5 \%$ & $-64.1 \%$ & $-57.2 \%$ & $-63.1 \%$ & $-67.7 \%$ & $-60.0 \%$ & $-66.5 \%$ & $-72.2 \%$ & $-61.8 \%$ & $-67.9 \%$ & $-73.9 \%$ \\
\hline & & Combined & $-59.5 \%$ & $-59.5 \%$ & $-65.3 \%$ & $-69.8 \%$ & $-64.4 \%$ & $-69.1 \%$ & $-72.8 \%$ & $-66.8 \%$ & $-71.9 \%$ & $-76.5 \%$ & $-68.2 \%$ & $-72.9 \%$ & $-77.6 \%$ \\
\hline \multirow{6}{*}{ FC PHEV30 } & \multirow{3}{*}{ Unadjusted } & \begin{tabular}{|l|} 
UDDS \\
\end{tabular} & $-77.8 \%$ & $-77.8 \%$ & $-81.3 \%$ & $-83.8 \%$ & $-80.7 \%$ & $-83.5 \%$ & $-85.9 \%$ & $-82.1 \%$ & $-85.4 \%$ & $-88.3 \%$ & $-83.1 \%$ & $-86.0 \%$ & $-88.8 \%$ \\
\hline & & HWFET & $-69.4 \%$ & $-69.4 \%$ & $-67.5 \%$ & $-71.6 \%$ & $-66.3 \%$ & $-70.9 \%$ & $-74.8 \%$ & $-68.4 \%$ & $-73.8 \%$ & $-78.3 \%$ & $-69.9 \%$ & $-75.0 \%$ & $-79.7 \%$ \\
\hline & & Combined & $-74.8 \%$ & $-74.8 \%$ & $-76.4 \%$ & $-79.5 \%$ & $-75.6 \%$ & $-79.0 \%$ & $-82.0 \%$ & $-77.3 \%$ & $-81.3 \%$ & $-84.7 \%$ & $-78.4 \%$ & $-82.1 \%$ & $-85.6 \%$ \\
\hline & \multirow{3}{*}{ Adjusted } & \begin{tabular}{|l|} 
UDDS \\
\end{tabular} & $-73.6 \%$ & $-73.6 \%$ & $-76.9 \%$ & $-79.3 \%$ & $-76.4 \%$ & $-79.0 \%$ & $-81.3 \%$ & $-77.7 \%$ & $-80.8 \%$ & $-83.5 \%$ & $-78.6 \%$ & $-81.4 \%$ & $-84.1 \%$ \\
\hline & & HWFET & $-68.8 \%$ & $-68.8 \%$ & $-66.9 \%$ & $-71.0 \%$ & $-65.7 \%$ & $-70.3 \%$ & $-74.1 \%$ & $-67.8 \%$ & $-73.1 \%$ & $-77.6 \%$ & $-69.3 \%$ & $-74.3 \%$ & $-79.0 \%$ \\
\hline & & Combined & $-71.8 \%$ & $-71.8 \%$ & $-73.1 \%$ & $-76.2 \%$ & $-72.4 \%$ & $-75.7 \%$ & $-78.6 \%$ & $-74.0 \%$ & $-77.9 \%$ & $-81.3 \%$ & $-75.1 \%$ & $-78.7 \%$ & $-82.2 \%$ \\
\hline \multirow{6}{*}{ FC PHEV40 } & \multirow{3}{*}{ Unadjusted } & \begin{tabular}{|l|} 
UDDS \\
\end{tabular} & $-80.7 \%$ & $-80.7 \%$ & $-83.8 \%$ & $-86.0 \%$ & $-83.3 \%$ & $-85.7 \%$ & $-87.9 \%$ & $-84.5 \%$ & $-87.4 \%$ & $-89.9 \%$ & $-85.4 \%$ & $-88.0 \%$ & $-90.4 \%$ \\
\hline & & HWFET & $-69.6 \%$ & $-69.6 \%$ & $-74.3 \%$ & $-77.6 \%$ & $-73.4 \%$ & $-77.1 \%$ & $-80.2 \%$ & $-75.1 \%$ & $-79.4 \%$ & $-83.0 \%$ & $-76.3 \%$ & $-80.3 \%$ & $-84.1 \%$ \\
\hline & & Combined & $-76.8 \%$ & $-76.8 \%$ & $-80.4 \%$ & $-83.0 \%$ & $-79.8 \%$ & $-82.6 \%$ & $-85.1 \%$ & $-81.2 \%$ & $-84.6 \%$ & $-87.5 \%$ & $-82.2 \%$ & $-85.3 \%$ & $-88.2 \%$ \\
\hline & \multirow{3}{*}{ Adjusted } & \begin{tabular}{|l|} 
UDDS \\
\end{tabular} & $-76.4 \%$ & $-76.4 \%$ & $-79.3 \%$ & $-81.4 \%$ & $-78.8 \%$ & $-81.1 \%$ & $-83.2 \%$ & $-80.0 \%$ & $-82.7 \%$ & $-85.1 \%$ & $-80.8 \%$ & $-83.3 \%$ & $-85.6 \%$ \\
\hline & & HWFET & $-69.0 \%$ & $-69.0 \%$ & $-73.7 \%$ & $-76.9 \%$ & $-72.7 \%$ & $-76.4 \%$ & $-79.5 \%$ & $-74.4 \%$ & $-78.7 \%$ & $-82.3 \%$ & $-75.6 \%$ & $-79.6 \%$ & $-83.3 \%$ \\
\hline & & Combined & $-73.6 \%$ & $-73.6 \%$ & $-77.2 \%$ & $-79.7 \%$ & $-76.5 \%$ & $-79.3 \%$ & $-81.8 \%$ & $-77.9 \%$ & $-81.2 \%$ & $-84.0 \%$ & $-78.9 \%$ & $-81.9 \%$ & $-84.7 \%$ \\
\hline
\end{tabular}


Small SUV Class 


\section{Fuel economy}

\begin{tabular}{|c|c|c|c|c|c|c|c|c|c|c|c|c|c|c|c|}
\hline & & & \multicolumn{13}{|c|}{ Small_SUV } \\
\hline & & & \multirow{2}{*}{$\begin{array}{c}2010 \\
\text { Ref }\end{array}$} & \multicolumn{3}{|c|}{2010} & \multicolumn{3}{|c|}{2015} & \multicolumn{3}{|c|}{2030} & \multicolumn{3}{|c|}{2045} \\
\hline & & & & low & avg & high & low & avg & high & low & avg & high & low & avg & high \\
\hline \multirow{6}{*}{ SI Conv } & \multirow{3}{*}{ Unadjusted } & UDDS & 25.2 & 25.2 & 27.8 & 29.4 & 26.6 & 28.9 & 36.7 & 27.1 & 30.8 & 44.0 & 27.3 & 31.3 & 45.7 \\
\hline & & HWFET & 33.7 & 33.7 & 36.7 & 39.0 & 35.5 & 38.1 & 49.6 & 35.1 & 41.6 & 58.7 & 36.6 & 42.4 & 61.5 \\
\hline & & Combined & 28.4 & 28.4 & 31.2 & 33.1 & 29.9 & 32.4 & 41.6 & 30.2 & 34.9 & 49.6 & 30.9 & 35.5 & 51.6 \\
\hline & \multirow{3}{*}{ Adjusted } & \begin{tabular}{|l|} 
UDDS \\
\end{tabular} & 19.5 & 19.5 & 21.5 & 22.6 & 20.5 & 22.3 & 27.8 & 20.9 & 23.6 & 32.9 & 21.1 & 23.9 & 34.0 \\
\hline & & HWFET & 23.8 & 23.8 & 25.9 & 27.6 & 25.1 & 26.9 & 35.0 & 24.8 & 29.3 & 41.3 & 25.9 & 29.9 & 43.2 \\
\hline & & Combined & 21.3 & 21.3 & 23.3 & 24.6 & 22.4 & 24.1 & 30.6 & 22.5 & 25.9 & 36.2 & 23.0 & 26.3 & 37.6 \\
\hline \multirow{6}{*}{$\mathrm{Cl}$ Conv } & \multirow{3}{*}{ Unadjusted } & \begin{tabular}{|l|} 
UDDS \\
\end{tabular} & 29.9 & 29.9 & 31.5 & 34.3 & 30.5 & 33.6 & 37.4 & 31.5 & 36.5 & 43.6 & 32.5 & 37.7 & 45.0 \\
\hline & & \begin{tabular}{|c|} 
HWFET \\
\end{tabular} & 38.2 & 38.2 & 40.8 & 44.8 & 39.0 & 43.6 & 49.7 & 40.0 & 48.4 & 57.3 & 42.5 & 50.4 & 59.9 \\
\hline & & Combined & 33.2 & 33.2 & 35.1 & 38.3 & 33.8 & 37.5 & 42.1 & 34.8 & 41.0 & 48.9 & 36.4 & 42.5 & 50.7 \\
\hline & \multirow{3}{*}{ Adjusted } & \begin{tabular}{|l|} 
UDDS \\
\end{tabular} & 23.0 & 23.0 & 24.1 & 26.1 & 23.4 & 25.6 & 28.3 & 24.1 & 27.7 & 32.6 & 24.9 & 28.5 & 33.6 \\
\hline & & \begin{tabular}{|c|} 
HWFET \\
\end{tabular} & 27.0 & 27.0 & 28.8 & 31.6 & 27.6 & 30.8 & 35.0 & 28.2 & 34.1 & 40.4 & 30.0 & 35.5 & 42.2 \\
\hline & & Combined & 24.6 & 24.6 & 26.0 & 28.3 & 25.1 & 27.7 & 31.0 & 25.8 & 30.2 & 35.7 & 27.0 & 31.3 & 37.0 \\
\hline \multirow{6}{*}{ H2 Conv } & \multirow{3}{*}{ Unadjusted } & \begin{tabular}{|l|} 
UDDS \\
\end{tabular} & 21.6 & 21.6 & 25.9 & 29.6 & 24.5 & 29.0 & 33.2 & 26.8 & 32.3 & 39.1 & 28.6 & 34.1 & 42.1 \\
\hline & & \begin{tabular}{|c|} 
HWFET \\
\end{tabular} & 31.3 & 31.3 & 38.1 & 43.7 & 35.5 & 42.6 & 48.7 & 38.9 & 47.3 & 56.6 & 41.7 & 50.3 & 61.6 \\
\hline & & Combined & 25.1 & 25.1 & 30.3 & 34.6 & 28.5 & 33.9 & 38.7 & 31.1 & 37.7 & 45.4 & 33.3 & 39.9 & 49.1 \\
\hline & \multirow{3}{*}{ Adjusted } & \begin{tabular}{|l|} 
UDDS \\
\end{tabular} & 16.9 & 16.9 & 20.1 & 22.8 & 19.0 & 22.3 & 25.3 & 20.7 & 24.7 & 29.5 & 22.0 & 26.0 & 31.6 \\
\hline & & \begin{tabular}{|c|} 
HWFET \\
\end{tabular} & 22.2 & 22.2 & 26.9 & 30.8 & 25.1 & 30.1 & 34.3 & 27.5 & 33.4 & 39.9 & 29.5 & 35.4 & 43.3 \\
\hline & & Combined & 18.9 & 18.9 & 22.7 & 25.8 & 21.3 & 25.2 & 28.7 & 23.3 & 28.0 & 33.4 & 24.9 & 29.5 & 36.0 \\
\hline \multirow{6}{*}{ E85 Conv } & \multirow{3}{*}{ Unadjusted } & \begin{tabular}{|l|} 
UDDS \\
\end{tabular} & 24.2 & 24.2 & 25.0 & 26.5 & 23.7 & 25.9 & 35.2 & 24.3 & 28.0 & 41.0 & 24.9 & 28.3 & 42.5 \\
\hline & & \begin{tabular}{|c|} 
HWFET \\
\end{tabular} & 30.9 & 30.9 & 32.0 & 34.1 & 30.3 & 33.2 & 44.0 & 30.6 & 34.9 & 50.9 & 30.8 & 35.6 & 53.2 \\
\hline & & Combined & 26.8 & 26.8 & 27.8 & 29.4 & 26.3 & 28.7 & 38.7 & 26.8 & 30.7 & 45.0 & 27.2 & 31.2 & 46.7 \\
\hline & \multirow{3}{*}{ Adjusted } & \begin{tabular}{|l|} 
UDDS \\
\end{tabular} & 18.8 & 18.8 & 19.4 & 20.5 & 18.4 & 20.1 & 26.8 & 18.8 & 21.6 & 30.8 & 19.3 & 21.8 & 31.8 \\
\hline & & \begin{tabular}{|c|} 
HWFET \\
\end{tabular} & 21.8 & 21.8 & 22.7 & 24.1 & 21.4 & 23.5 & 31.1 & 21.7 & 24.7 & 35.9 & 21.8 & 25.1 & 37.5 \\
\hline & & Combined & 20.1 & 20.1 & 20.7 & 22.0 & 19.7 & 21.5 & 28.5 & 20.0 & 22.9 & 32.9 & 20.3 & 23.2 & 34.1 \\
\hline \multirow{6}{*}{ SI Split HEV } & \multirow{3}{*}{ Unadjusted } & \begin{tabular}{|l|} 
UDDS \\
\end{tabular} & 45.4 & 45.4 & 50.0 & 54.5 & 46.8 & 53.6 & 66.6 & 49.2 & 59.9 & 83.2 & 50.6 & 62.5 & 86.9 \\
\hline & & \begin{tabular}{|c|} 
HWFET \\
\end{tabular} & 39.3 & 39.3 & 43.5 & 47.3 & 40.4 & 45.6 & 56.2 & 41.7 & 50.6 & 68.6 & 43.4 & 52.9 & 72.2 \\
\hline & & Combined & 42.4 & 42.4 & 46.9 & 51.0 & 43.7 & 49.7 & 61.5 & 45.5 & 55.3 & 75.9 & 47.1 & 57.8 & 79.6 \\
\hline & \multirow{3}{*}{ Adjusted } & \begin{tabular}{|l|} 
UDDS \\
\end{tabular} & 33.8 & 33.8 & 37.0 & 39.9 & 34.8 & 39.3 & 47.7 & 36.4 & 43.4 & 57.9 & 37.4 & 45.1 & 60.1 \\
\hline & & \begin{tabular}{|c|} 
HWFET \\
\end{tabular} & 27.8 & 27.8 & 30.7 & 33.4 & 28.5 & 32.2 & 39.5 & 29.5 & 35.6 & 48.2 & 30.7 & 37.3 & 50.7 \\
\hline & & Combined & 30.8 & 30.8 & 33.9 & 36.7 & 31.7 & 35.8 & 43.7 & 32.9 & 39.5 & 53.1 & 34.0 & 41.2 & 55.4 \\
\hline \multirow{6}{*}{ SI Split HEV PHEV10 } & & \begin{tabular}{|l|} 
UDDS \\
\end{tabular} & 57.1 & 57.1 & 63.2 & 68.5 & 59.1 & 66.4 & 81.6 & 60.7 & 73.4 & 101.4 & 62.7 & 76.2 & 106.0 \\
\hline & Unadjusted & \begin{tabular}{|l|} 
HWFET \\
\end{tabular} & 50.9 & 50.9 & 56.3 & 64.2 & 52.5 & 61.7 & 75.4 & 53.5 & 68.2 & 89.9 & 55.8 & 70.9 & 95.1 \\
\hline & & Combined & 54.2 & 54.2 & 59.9 & 66.5 & 55.9 & 64.2 & 78.7 & 57.2 & 70.9 & 95.9 & 59.4 & 73.7 & 100.8 \\
\hline & & \begin{tabular}{|l|} 
UDDS \\
\end{tabular} & 41.6 & 41.6 & 45.6 & 48.9 & 42.9 & 47.6 & 56.9 & 44.0 & 51.9 & 68.3 & 45.2 & 53.7 & 70.8 \\
\hline & Adjusted & HWFET & 35.9 & 35.9 & 39.7 & 45.1 & 37.0 & 43.4 & 52.9 & 37.7 & 47.9 & 62.8 & 39.3 & 49.7 & 66.4 \\
\hline & & Combined & 38.8 & 38.8 & 42.7 & 47.2 & 40.0 & 45.6 & 55.0 & 40.9 & 50.0 & 65.7 & 42.3 & 51.8 & 68.8 \\
\hline
\end{tabular}




\begin{tabular}{|c|c|c|c|c|c|c|c|c|c|c|c|c|c|c|c|}
\hline & & & \multicolumn{13}{|c|}{ Small_SUV } \\
\hline & & & \multirow{2}{*}{$\begin{array}{l}2010 \\
\text { Ref }\end{array}$} & \multicolumn{3}{|c|}{2010} & \multicolumn{3}{|c|}{2015} & \multicolumn{3}{|c|}{2030} & \multicolumn{3}{|c|}{2045} \\
\hline & & & & low & avg & high & low & avg & high & low & avg & high & low & avg & high \\
\hline \multirow{6}{*}{ SI Split HEV PHEV2O } & \multirow{3}{*}{ Unadjusted } & UDDS & 81.0 & 81.0 & 90.2 & 97.7 & 84.7 & 94.4 & 116.6 & 87.1 & 105.1 & 122.8 & 89.5 & 109.2 & 128.1 \\
\hline & & HWFET & 58.1 & 58.1 & 58.5 & 64.1 & 64.5 & 61.3 & 75.8 & 64.2 & 67.9 & 94.5 & 66.9 & 71.3 & 99.7 \\
\hline & & Combined & 68.8 & 68.8 & 72.5 & 79.0 & 74.3 & 76.0 & 93.8 & 75.0 & 84.3 & 108.2 & 77.7 & 88.1 & 113.5 \\
\hline & \multirow{3}{*}{ Adjusted } & \begin{tabular}{|l|} 
UDDS \\
\end{tabular} & 56.5 & 56.5 & 62.0 & 66.2 & 58.8 & 64.4 & 76.5 & 60.1 & 70.4 & 79.8 & 61.6 & 72.6 & 82.5 \\
\hline & & HWFET & 40.9 & 40.9 & 41.2 & 45.1 & 45.3 & 43.1 & 53.1 & 45.1 & 47.7 & 66.0 & 47.0 & 50.1 & 69.5 \\
\hline & & Combined & 48.2 & 48.2 & 50.5 & 54.7 & 51.9 & 52.7 & 63.9 & 52.3 & 58.0 & 72.9 & 54.0 & 60.4 & 76.1 \\
\hline \multirow{6}{*}{ SI Series HEV PHEV3O } & \multirow{3}{*}{ Unadjusted } & \begin{tabular}{|l|} 
UDDS \\
\end{tabular} & 73.7 & 73.7 & 83.4 & 92.9 & 77.1 & 89.8 & 115.4 & 81.0 & 104.0 & 147.4 & 84.4 & 108.6 & 154.7 \\
\hline & & HWFET & 54.0 & 54.0 & 61.0 & 67.2 & 56.1 & 64.9 & 81.3 & 58.3 & 73.3 & 100.3 & 61.3 & 76.9 & 106.2 \\
\hline & & Combined & 63.3 & 63.3 & 71.5 & 79.3 & 66.0 & 76.6 & 97.1 & 68.9 & 87.5 & 121.7 & 72.2 & 91.6 & 128.3 \\
\hline & \multirow{3}{*}{ Adjusted } & \begin{tabular}{|l|} 
UDDS \\
\end{tabular} & 52.1 & 52.1 & 58.0 & 63.5 & 54.2 & 61.7 & 75.9 & 56.5 & 69.8 & 92.0 & 58.6 & 72.3 & 95.5 \\
\hline & & HWFET & 38.0 & 38.0 & 42.9 & 47.2 & 39.5 & 45.6 & 56.9 & 41.0 & 51.4 & 69.9 & 43.1 & 53.9 & 73.9 \\
\hline & & Combined & 44.7 & 44.7 & 50.0 & 55.0 & 46.4 & 53.3 & 66.0 & 48.3 & 60.1 & 80.6 & 50.4 & 62.6 & 84.4 \\
\hline \multirow{6}{*}{ SI Series HEV PHEV40 } & \multirow{3}{*}{ Unadjusted } & \begin{tabular}{|l|} 
UDDS \\
\end{tabular} & 84.4 & 84.4 & 95.5 & 106.9 & 88.3 & 102.0 & 133.4 & 93.0 & 119.6 & 169.5 & 96.9 & 123.4 & 179.5 \\
\hline & & HWFET & 68.2 & 68.2 & 77.1 & 85.1 & 70.9 & 82.2 & 103.1 & 73.8 & 93.0 & 127.7 & 77.4 & 97.6 & 135.2 \\
\hline & & Combined & 76.3 & 76.3 & 86.2 & 95.8 & 79.5 & 92.0 & 117.8 & 83.2 & 105.9 & 147.7 & 87.0 & 110.3 & 156.4 \\
\hline & \multirow{3}{*}{ Adjusted } & \begin{tabular}{|l|} 
UDDS \\
\end{tabular} & 58.6 & 58.6 & 65.0 & 71.3 & 60.9 & 68.7 & 85.2 & 63.6 & 78.1 & 102.2 & 65.8 & 80.1 & 106.6 \\
\hline & & HWFET & 47.9 & 47.9 & 54.0 & 59.5 & 49.7 & 57.5 & 71.9 & 51.7 & 64.9 & 88.6 & 54.3 & 68.1 & 93.6 \\
\hline & & Combined & 53.2 & 53.2 & 59.6 & 65.5 & 55.3 & 63.2 & 78.6 & 57.6 & 71.6 & 95.6 & 60.0 & 74.2 & 100.3 \\
\hline \multirow{6}{*}{ Cl Split HEV } & \multirow{3}{*}{ Unadjusted } & \begin{tabular}{|l|} 
UDDS \\
\end{tabular} & 49.8 & 49.8 & 54.3 & 59.9 & 50.8 & 58.9 & 68.2 & 54.0 & 66.3 & 82.5 & 56.3 & 69.6 & 85.7 \\
\hline & & HWFET & 41.7 & 41.7 & 45.6 & 50.5 & 42.4 & 49.0 & 56.2 & 44.5 & 54.7 & 66.4 & 46.8 & 57.6 & 69.7 \\
\hline & & \begin{tabular}{|l|} 
Combined \\
\end{tabular} & 45.8 & 45.8 & 50.0 & 55.2 & 46.6 & 54.0 & 62.2 & 49.3 & 60.5 & 74.4 & 51.6 & 63.6 & 77.7 \\
\hline & \multirow{3}{*}{ Adjusted } & \begin{tabular}{|l|} 
UDDS \\
\end{tabular} & 36.8 & 36.8 & 39.8 & 43.4 & 37.5 & 42.8 & 48.7 & 39.6 & 47.5 & 57.5 & 41.1 & 49.6 & 59.3 \\
\hline & & HWFET & 29.4 & 29.4 & 32.2 & 35.6 & 29.9 & 34.6 & 39.6 & 31.4 & 38.6 & 46.6 & 33.0 & 40.5 & 48.9 \\
\hline & & \begin{tabular}{|l|} 
Combined \\
\end{tabular} & 33.1 & 33.1 & 36.0 & 39.5 & 33.7 & 38.7 & 44.1 & 35.4 & 43.0 & 52.0 & 37.0 & 45.1 & 54.2 \\
\hline \multirow{6}{*}{ CI Split HEV PHEV10 } & \multirow{3}{*}{ Unadjusted } & \begin{tabular}{|l|} 
UDDS \\
\end{tabular} & 58.2 & 58.2 & 64.1 & 70.6 & 59.8 & 68.5 & 78.9 & 62.7 & 76.9 & 95.5 & 65.5 & 80.4 & 99.6 \\
\hline & & HWFET & 51.6 & 51.6 & 59.1 & 65.7 & 54.7 & 63.2 & 72.5 & 54.8 & 70.8 & 84.7 & 57.5 & 74.3 & 89.6 \\
\hline & & \begin{tabular}{|l|} 
Combined \\
\end{tabular} & 55.0 & 55.0 & 61.7 & 68.3 & 57.4 & 66.0 & 75.9 & 58.9 & 74.0 & 90.3 & 61.6 & 77.6 & 94.8 \\
\hline & \multirow{3}{*}{ Adjusted } & \begin{tabular}{|l|} 
UDDS \\
\end{tabular} & 42.3 & 42.3 & 46.1 & 50.2 & 43.4 & 48.9 & 55.3 & 45.2 & 54.1 & 65.0 & 47.0 & 56.2 & 67.3 \\
\hline & & HWFET & 36.4 & 36.4 & 41.6 & 46.2 & 38.5 & 44.4 & 50.9 & 38.6 & 49.7 & 59.3 & 40.5 & 52.1 & 62.6 \\
\hline & & \begin{tabular}{|l|} 
Combined \\
\end{tabular} & 39.4 & 39.4 & 44.0 & 48.3 & 41.0 & 46.8 & 53.2 & 42.0 & 52.0 & 62.3 & 43.8 & 54.3 & 65.1 \\
\hline \multirow{6}{*}{ CI Split HEV PHEV2O } & & \begin{tabular}{|l|} 
UDDS \\
\end{tabular} & 83.1 & 83.1 & 91.7 & 101.0 & 85.7 & 98.0 & 112.7 & 89.9 & 109.5 & 139.1 & 93.9 & 115.1 & 145.0 \\
\hline & Unadjusted & \begin{tabular}{l|l|} 
HWFET \\
\end{tabular} & 61.2 & 61.2 & 59.2 & 79.4 & 54.7 & 63.4 & 87.7 & 56.6 & 85.4 & 88.7 & 73.1 & 74.1 & 93.6 \\
\hline & & \begin{tabular}{|c|} 
Combined \\
\end{tabular} & 71.6 & 71.6 & 73.6 & 90.0 & 68.3 & 78.7 & 99.9 & 71.1 & 97.2 & 110.8 & 83.2 & 92.2 & 116.2 \\
\hline & & \begin{tabular}{|l|} 
UDDS \\
\end{tabular} & 57.8 & 57.8 & 62.8 & 68.1 & 59.3 & 66.4 & 74.5 & 61.8 & 72.8 & 88.0 & 64.1 & 75.8 & 90.9 \\
\hline & Adjusted & HWFET & 43.1 & 43.1 & 41.7 & 55.6 & 38.5 & 44.6 & 61.3 & 39.8 & 59.7 & 62.0 & 51.2 & 52.0 & 65.4 \\
\hline & & Combined & 50.1 & 50.1 & 51.2 & 61.9 & 47.7 & 54.4 & 67.9 & 49.5 & 66.3 & 74.1 & 57.6 & 62.8 & 77.3 \\
\hline
\end{tabular}




\begin{tabular}{|c|c|c|c|c|c|c|c|c|c|c|c|c|c|c|c|}
\hline & & & \multicolumn{13}{|c|}{ Small_SUV } \\
\hline & & & \multirow{2}{*}{$\frac{2010}{\text { Ref }}$} & \multicolumn{3}{|c|}{2010} & \multicolumn{3}{|c|}{2015} & \multicolumn{3}{|c|}{2030} & \multicolumn{3}{|c|}{2045} \\
\hline & & & & low & avg & high & low & avg & high & low & avg & high & low & avg & high \\
\hline \multirow{6}{*}{ CI Series HEV PHEV3O } & \multirow{3}{*}{ Unadjusted } & UDDS & 75.3 & 75.3 & 84.9 & 94.9 & 77.7 & 92.0 & 110.9 & 83.3 & 107.3 & 138.4 & 87.4 & 112.1 & 144.4 \\
\hline & & HWFET & 55.1 & 55.1 & 61.6 & 68.9 & 56.5 & 66.6 & 78.5 & 60.0 & 76.4 & 94.7 & 63.6 & 80.8 & 100.3 \\
\hline & & Combined & 64.6 & 64.6 & 72.5 & 81.1 & 66.5 & 78.5 & 93.5 & 70.9 & 90.8 & 114.6 & 74.8 & 95.4 & 120.6 \\
\hline & \multirow{3}{*}{ Adjusted } & \begin{tabular}{|l|} 
UDDS \\
\end{tabular} & 53.1 & 53.1 & 58.9 & 64.6 & 54.6 & 63.0 & 73.5 & 57.9 & 71.6 & 87.7 & 60.3 & 74.2 & 90.6 \\
\hline & & HWFET & 38.8 & 38.8 & 43.3 & 48.4 & 39.8 & 46.8 & 55.0 & 42.2 & 53.5 & 66.1 & 44.7 & 56.6 & 70.0 \\
\hline & & Combined & 45.6 & 45.6 & 50.7 & 56.2 & 46.8 & 54.5 & 63.8 & 49.6 & 62.1 & 76.4 & 52.1 & 65.1 & 80.0 \\
\hline \multirow{6}{*}{ CI Series HEV PHEV40 } & \multirow{3}{*}{ Unadjusted } & \begin{tabular}{|l|} 
UDDS \\
\end{tabular} & 86.2 & 86.2 & 96.5 & 109.3 & 89.1 & 105.9 & 128.2 & 95.6 & 123.6 & 162.0 & 100.6 & 130.9 & 168.2 \\
\hline & & HWFET & 69.6 & 69.6 & 77.7 & 87.2 & 71.4 & 84.1 & 99.7 & 75.9 & 96.9 & 120.4 & 80.5 & 102.8 & 127.7 \\
\hline & & Combined & 77.9 & 77.9 & 87.0 & 98.1 & 80.2 & 94.8 & 113.6 & 85.6 & 110.0 & 140.2 & 90.4 & 116.5 & 147.2 \\
\hline & \multirow{3}{*}{ Adjusted } & \begin{tabular}{|l|} 
UDDS \\
\end{tabular} & 59.6 & 59.6 & 65.5 & 72.6 & 61.3 & 70.8 & 82.6 & 65.1 & 80.2 & 98.9 & 67.9 & 83.9 & 101.7 \\
\hline & & HWFET & 48.9 & 48.9 & 54.5 & 61.0 & 50.1 & 58.8 & 69.5 & 53.2 & 67.6 & 83.6 & 56.4 & 71.6 & 88.6 \\
\hline & & Combined & 54.3 & 54.3 & 60.0 & 66.9 & 55.7 & 64.9 & 76.1 & 59.1 & 74.0 & 91.3 & 62.2 & 77.9 & 95.3 \\
\hline \multirow{6}{*}{ H2 Split HEV } & \multirow{3}{*}{ Unadjusted } & \begin{tabular}{|l|} 
UDDS \\
\end{tabular} & 44.2 & 44.2 & 54.1 & 62.8 & 50.1 & 61.7 & 73.6 & 55.3 & 71.2 & 90.0 & 60.0 & 76.4 & 97.3 \\
\hline & & HWFET & 37.8 & 37.8 & 46.2 & 53.6 & 42.6 & 52.0 & 61.5 & 46.6 & 59.5 & 73.2 & 50.8 & 64.1 & 79.9 \\
\hline & & Combined & 41.1 & 41.1 & 50.2 & 58.3 & 46.4 & 56.9 & 67.6 & 51.0 & 65.4 & 81.6 & 55.5 & 70.3 & 88.6 \\
\hline & \multirow{3}{*}{ Adjusted } & \begin{tabular}{|l|} 
UDDS \\
\end{tabular} & 33.0 & 33.0 & 39.7 & 45.3 & 37.0 & 44.6 & 52.0 & 40.5 & 50.6 & 61.9 & 43.5 & 53.8 & 66.0 \\
\hline & & HWFET & 26.7 & 26.7 & 32.6 & 37.7 & 30.1 & 36.6 & 43.2 & 32.9 & 41.9 & 51.3 & 35.8 & 45.1 & 56.0 \\
\hline & & Combined & 29.9 & 29.9 & 36.1 & 41.6 & 33.5 & 40.6 & 47.7 & 36.7 & 46.2 & 56.6 & 39.7 & 49.5 & 61.1 \\
\hline \multirow{6}{*}{ H2 Split HEV PHEV10 } & \multirow{3}{*}{ Unadjusted } & \begin{tabular}{|l|} 
UDDS \\
\end{tabular} & 55.2 & 55.2 & 67.8 & 78.4 & 62.7 & 75.8 & 89.8 & 68.3 & 86.7 & 109.4 & 74.0 & 93.1 & 119.6 \\
\hline & & HWFET & 49.5 & 49.5 & 62.7 & 72.8 & 55.7 & 70.5 & 82.5 & 60.5 & 80.1 & 96.3 & 65.6 & 85.9 & 105.6 \\
\hline & & Combined & 52.5 & 52.5 & 65.4 & 75.8 & 59.3 & 73.3 & 86.3 & 64.6 & 83.6 & 103.1 & 70.0 & 89.7 & 112.9 \\
\hline & \multirow{3}{*}{ Adjusted } & \begin{tabular}{|l|} 
UDDS \\
\end{tabular} & 40.4 & 40.4 & 48.5 & 55.0 & 45.2 & 53.4 & 61.7 & 48.8 & 59.9 & 72.7 & 52.3 & 63.6 & 78.1 \\
\hline & & HWFET & 34.9 & 34.9 & 44.1 & 51.1 & 39.2 & 49.5 & 57.7 & 42.6 & 56.1 & 67.2 & 46.1 & 60.1 & 73.6 \\
\hline & & Combined & 37.7 & 37.7 & 46.4 & 53.2 & 42.3 & 51.5 & 59.9 & 45.8 & 58.1 & 70.1 & 49.3 & 62.0 & 76.0 \\
\hline \multirow{6}{*}{ H2 Split HEV PHEV2O } & \multirow{3}{*}{ Unadjusted } & \begin{tabular}{|l|} 
UDDS \\
\end{tabular} & 78.5 & 78.5 & 96.2 & 111.7 & 90.2 & 108.1 & 130.2 & 98.2 & 124.4 & 132.5 & 105.8 & 135.0 & 145.5 \\
\hline & & $\begin{array}{l}\text { HWFET } \\
\end{array}$ & 56.4 & 56.4 & 75.6 & 87.2 & 67.1 & 84.2 & 83.3 & 72.6 & 80.0 & 101.4 & 78.9 & 86.8 & 111.0 \\
\hline & & Combined & 66.7 & 66.7 & 85.7 & 99.2 & 78.1 & 95.9 & 103.8 & 84.7 & 99.5 & 116.5 & 91.8 & 108.0 & 127.7 \\
\hline & \multirow{3}{*}{ Adjusted } & \begin{tabular}{|l|} 
UDDS \\
\end{tabular} & 55.1 & 55.1 & 65.4 & 73.9 & 61.9 & 72.0 & 83.6 & 66.5 & 80.6 & 84.7 & 70.8 & 86.0 & 91.1 \\
\hline & & HWFET & 39.7 & 39.7 & 53.0 & 61.0 & 47.1 & 58.9 & 58.3 & 50.9 & 56.0 & 70.7 & 55.3 & 60.7 & 77.2 \\
\hline & & Combined & 46.9 & 46.9 & 59.2 & 67.5 & 54.3 & 65.5 & 69.9 & 58.4 & 67.3 & 77.8 & 62.9 & 72.4 & 84.3 \\
\hline \multirow{6}{*}{ H2 Series HEV PHEV3O } & & \begin{tabular}{|l|} 
UDDS \\
\end{tabular} & 71.6 & 71.6 & 90.1 & 106.1 & 81.9 & 102.2 & 125.7 & 91.2 & 121.4 & 156.5 & 100.4 & 129.9 & 169.5 \\
\hline & Unadjusted & HWFET & 52.9 & 52.9 & 66.0 & 77.1 & 60.2 & 74.4 & 89.8 & 66.3 & 86.7 & 107.7 & 72.8 & 93.9 & 117.9 \\
\hline & & Combined & 61.8 & 61.8 & 77.4 & 90.8 & 70.5 & 87.5 & 106.5 & 78.0 & 102.9 & 130.0 & 85.8 & 110.8 & 141.6 \\
\hline & & UDDS & 50.8 & 50.8 & 61.9 & 70.9 & 57.1 & 68.8 & 81.3 & 62.5 & 79.1 & 96.3 & 67.8 & 83.4 & 102.2 \\
\hline & Adjusted & HWFET & 37.3 & 37.3 & 46.3 & 54.1 & 42.4 & 52.2 & 62.7 & 46.6 & 60.6 & 75.0 & 51.0 & 65.6 & 81.9 \\
\hline & & Combined & 43.7 & 43.7 & 53.8 & 62.2 & 49.4 & 60.2 & 71.7 & 54.2 & 69.6 & 85.4 & 59.1 & 74.3 & 92.0 \\
\hline
\end{tabular}




\begin{tabular}{|c|c|c|c|c|c|c|c|c|c|c|c|c|c|c|c|}
\hline & & & \multicolumn{13}{|c|}{ Small_SUV } \\
\hline & & & \multirow{2}{*}{$\begin{array}{l}2010 \\
\operatorname{Ref} \\
\end{array}$} & \multicolumn{3}{|c|}{2010} & \multicolumn{3}{|c|}{2015} & \multicolumn{3}{|c|}{2030} & \multicolumn{3}{|c|}{2045} \\
\hline & & & & low & avg & high & low & avg & high & low & avg & high & low & avg & high \\
\hline \multirow{6}{*}{ H2 Series HEV PHEV40 } & \multirow{3}{*}{ Unadjusted } & UDDS & 82.1 & 82.1 & 103.6 & 121.6 & 94.2 & 117.7 & 145.1 & 104.8 & 140.6 & 182.1 & 114.3 & 150.6 & 198.6 \\
\hline & & HWFET & 66.9 & 66.9 & 83.2 & 97.6 & 76.1 & 94.3 & 113.7 & 83.9 & 110.3 & 137.1 & 91.9 & 119.3 & 150.4 \\
\hline & & Combined & 74.5 & 74.5 & 93.3 & 109.5 & 85.1 & 105.9 & 129.1 & 94.2 & 125.1 & 158.7 & 103.0 & 134.7 & 173.6 \\
\hline & \multirow{3}{*}{ Adjusted } & \begin{tabular}{|l|} 
UDDS \\
\end{tabular} & 57.2 & 57.2 & 69.5 & 79.2 & 64.3 & 77.1 & 90.9 & 70.2 & 88.8 & 107.8 & 75.3 & 93.5 & 114.7 \\
\hline & & \begin{tabular}{|l|} 
HWFET \\
\end{tabular} & 47.0 & 47.0 & 58.3 & 68.1 & 53.3 & 65.8 & 79.1 & 58.7 & 76.7 & $\begin{array}{ll}94.9 \\
\end{array}$ & 64.2 & 82.8 & 103.8 \\
\hline & & Combined & 52.1 & 52.1 & 64.0 & 73.8 & 58.8 & 71.6 & 85.2 & 64.5 & 82.9 & 101.5 & 69.9 & 88.4 & 109.5 \\
\hline \multirow{6}{*}{ E85 Split HEV } & \multirow{3}{*}{ Unadjusted } & \begin{tabular}{|l|} 
UDDS \\
\end{tabular} & 41.9 & 41.9 & 46.5 & 50.6 & 43.5 & 49.6 & 65.3 & 45.3 & 55.9 & 78.2 & 47.1 & 57.9 & 81.7 \\
\hline & & HWFET & 36.3 & 36.3 & 40.3 & 43.9 & 37.5 & 42.4 & 55.2 & 38.5 & 47.4 & 64.5 & 40.4 & 49.2 & 68.0 \\
\hline & & \begin{tabular}{|l|} 
Combined \\
\end{tabular} & 39.2 & 39.2 & 43.5 & 47.4 & 40.6 & 46.1 & 60.3 & 42.0 & 51.7 & 71.4 & 43.8 & 53.6 & 74.9 \\
\hline & \multirow{3}{*}{ Adjusted } & \begin{tabular}{|l|} 
UDDS \\
\end{tabular} & 31.5 & 31.5 & 34.6 & 37.4 & 32.5 & 36.7 & 46.9 & 33.8 & 40.9 & 54.9 & 35.0 & 42.2 & 57.0 \\
\hline & & HWFET & 25.6 & 25.6 & 28.5 & 31.0 & 26.5 & 29.9 & 38.9 & 27.2 & 33.4 & 45.3 & 28.5 & 34.7 & 47.7 \\
\hline & & \begin{tabular}{|l|} 
Combined \\
\end{tabular} & 28.5 & 28.5 & 31.5 & 34.2 & 29.5 & 33.3 & 42.9 & 30.5 & 37.1 & 50.1 & 31.7 & 38.4 & 52.4 \\
\hline \multirow{6}{*}{ E85 Split HEV PHEV10 } & \multirow{3}{*}{ Unadjusted } & \begin{tabular}{|l|} 
UDDS \\
\end{tabular} & 53.5 & 53.5 & 59.1 & 64.2 & 55.3 & 62.3 & 80.6 & 56.8 & 68.8 & 95.9 & 58.8 & 71.6 & 100.1 \\
\hline & & \begin{tabular}{|l|} 
HWFET \\
\end{tabular} & 47.6 & 47.6 & 55.0 & 60.1 & 48.7 & 57.7 & 74.4 & 49.7 & 63.7 & 84.9 & 52.0 & 66.4 & 89.7 \\
\hline & & \begin{tabular}{|l|} 
Combined \\
\end{tabular} & 50.7 & 50.7 & 57.2 & 62.3 & 52.2 & 60.2 & 77.7 & 53.4 & 66.4 & 90.6 & 55.6 & 69.2 & 95.2 \\
\hline & \multirow{3}{*}{ Adjusted } & UDDS & 39.3 & 39.3 & 42.9 & 46.2 & 40.5 & 45.0 & 56.3 & 41.4 & 49.1 & 65.2 & 42.8 & 50.9 & 67.6 \\
\hline & & HWFET & 33.6 & 33.6 & 38.7 & 42.2 & 34.4 & 40.6 & 52.2 & 35.0 & 44.8 & 59.4 & 36.7 & 46.6 & 62.7 \\
\hline & & \begin{tabular}{|c|} 
Combined \\
\end{tabular} & 36.5 & 36.5 & 40.9 & 44.3 & 37.5 & 42.9 & 54.4 & 38.3 & 47.1 & 62.5 & 39.8 & 48.9 & 65.3 \\
\hline \multirow{6}{*}{ E85 Split HEV PHEV2O } & \multirow{3}{*}{ Unadjusted } & \begin{tabular}{|l|} 
UDDS \\
\end{tabular} & 75.8 & 75.8 & 84.1 & 91.4 & 79.4 & 88.7 & 115.3 & 81.7 & 98.7 & 116.0 & 84.0 & 102.5 & 121.1 \\
\hline & & HWFET & 54.2 & 54.2 & 54.6 & 59.7 & 60.3 & 57.3 & 74.0 & 61.4 & 63.5 & 89.2 & 54.3 & 66.8 & 94.1 \\
\hline & & \begin{tabular}{|c|} 
Combined \\
\end{tabular} & 64.3 & 64.3 & 67.6 & 73.7 & 69.5 & 71.1 & 92.2 & 71.1 & 79.0 & 102.2 & 67.4 & 82.6 & 107.3 \\
\hline & \multirow{3}{*}{ Adjusted } & \begin{tabular}{|l|} 
UDDS \\
\end{tabular} & 53.4 & 53.4 & 58.4 & 62.6 & 55.6 & 61.1 & 75.9 & 56.9 & 66.8 & 76.2 & 58.4 & 68.9 & 78.9 \\
\hline & & HWFET & 38.2 & 38.2 & 38.4 & 42.0 & 42.4 & 40.3 & 51.9 & 43.2 & 44.6 & 62.3 & 38.2 & 46.9 & 65.7 \\
\hline & & Combined & 45.3 & 45.3 & 47.3 & 51.3 & 48.8 & 49.6 & 62.8 & 49.8 & 54.6 & 69.3 & 47.2 & 56.9 & 72.4 \\
\hline \multirow{6}{*}{ E85 Series HEV PHEV30 } & \multirow{3}{*}{ Unadjusted } & \begin{tabular}{|l|} 
UDDS \\
\end{tabular} & 68.8 & 68.8 & 78.2 & 87.3 & 72.3 & 83.5 & 114.6 & 76.1 & 97.6 & 137.7 & 79.3 & 100.5 & 145.9 \\
\hline & & HWFET & 50.7 & 50.7 & 57.3 & 63.2 & 52.7 & 61.0 & 80.6 & 54.8 & 69.0 & 95.1 & 57.7 & 72.4 & 100.7 \\
\hline & & \begin{tabular}{|l|} 
Combined \\
\end{tabular} & 59.3 & 59.3 & 67.1 & 74.5 & 61.9 & 71.6 & 96.3 & 64.8 & 82.2 & 114.6 & 67.8 & 85.5 & 121.3 \\
\hline & \multirow{3}{*}{ Adjusted } & \begin{tabular}{|l|} 
UDDS \\
\end{tabular} & 49.1 & 49.1 & 54.8 & 60.3 & 51.3 & 58.0 & 75.5 & 53.6 & 66.2 & 87.3 & 55.5 & 67.8 & 91.3 \\
\hline & & HWFET & 35.7 & 35.7 & 40.3 & 44.4 & 37.1 & 42.9 & 56.4 & 38.6 & 48.4 & 66.4 & 40.6 & 50.8 & 70.2 \\
\hline & & \begin{tabular}{|c|} 
Combined \\
\end{tabular} & 42.0 & 42.0 & 47.2 & 51.9 & 43.8 & 50.1 & 65.5 & 45.6 & 56.8 & 76.5 & 47.6 & 58.9 & 80.4 \\
\hline \multirow{6}{*}{ E85 Series HEV PHEV40 } & & UDDS & 78.7 & 78.7 & 89.6 & 100.6 & 83.0 & 96.0 & 132.5 & 87.1 & 112.8 & 160.9 & 91.1 & 116.1 & 170.9 \\
\hline & Unadjusted & HWFET & 64.1 & 64.1 & 72.2 & 80.0 & 66.6 & 77.3 & 102.3 & 69.3 & 87.5 & 121.0 & 72.8 & 91.9 & 128.2 \\
\hline & & \begin{tabular}{|l|} 
Combined \\
\end{tabular} & 71.3 & 71.3 & 80.8 & 90.1 & 74.7 & 86.6 & 116.9 & 78.1 & 99.8 & 140.1 & 81.9 & 103.8 & 148.6 \\
\hline & & UDDS & 55.1 & 55.1 & 61.6 & 67.8 & 57.8 & 65.3 & 84.8 & 60.2 & 74.5 & 98.3 & 62.5 & 76.3 & 102.9 \\
\hline & Adjusted & HWFET & 45.0 & 45.0 & 50.6 & 56.0 & 46.8 & 54.2 & 71.3 & 48.7 & 61.2 & 84.0 & 51.1 & 64.2 & 88.9 \\
\hline & & Combined & 50.1 & 50.1 & 56.1 & 62.0 & 52.2 & 59.8 & 78.1 & 54.4 & 67.9 & 91.3 & 56.8 & 70.3 & 96.1 \\
\hline
\end{tabular}




\begin{tabular}{|c|c|c|c|c|c|c|c|c|c|c|c|c|c|c|c|}
\hline & & & \multicolumn{13}{|c|}{ Small_SUV } \\
\hline & & & \multirow{2}{*}{$\begin{array}{c}2010 \\
\text { Ref }\end{array}$} & \multicolumn{3}{|c|}{2010} & \multicolumn{3}{|c|}{2015} & \multicolumn{3}{|c|}{2030} & \multicolumn{3}{|c|}{2045} \\
\hline & & & & low & avg & high & low & avg & high & low & avg & high & low & avg & high \\
\hline \multirow{6}{*}{ FC HEV } & \multirow{3}{*}{ Unadjusted } & UDDS & 48.7 & 48.7 & 59.9 & 71.6 & 58.6 & 70.4 & 82.3 & 63.2 & 79.8 & 98.8 & 66.8 & 83.0 & 103.6 \\
\hline & & HWFET & 48.8 & 48.8 & 58.8 & 69.0 & 57.1 & 66.8 & 76.2 & 59.6 & 74.1 & 87.5 & 62.9 & 76.9 & 92.6 \\
\hline & & Combined & 48.7 & 48.7 & 59.4 & 70.4 & 57.9 & 68.7 & 79.5 & 61.5 & 77.1 & 93.4 & 65.0 & 80.1 & 98.3 \\
\hline & \multirow{3}{*}{ Adjusted } & UDDS & 36.1 & 36.1 & 43.5 & 50.8 & 42.6 & 50.1 & 57.4 & 45.6 & 55.8 & 66.9 & 47.9 & 57.8 & 69.5 \\
\hline & & HWFET & 34.4 & 34.4 & 41.4 & 48.5 & 40.2 & 46.9 & 53.4 & 41.9 & 51.9 & 61.2 & 44.2 & 53.9 & 64.7 \\
\hline & & Combined & 35.3 & 35.3 & 42.5 & 49.7 & 41.5 & 48.6 & 55.5 & 43.9 & 54.0 & 64.2 & 46.2 & 55.9 & 67.3 \\
\hline \multirow{6}{*}{ FC PHEV10 } & \multirow{3}{*}{ Unadjusted } & UDDS & 61.6 & 61.6 & 74.7 & 88.2 & 72.6 & 85.5 & 99.2 & 76.9 & 96.3 & 118.2 & 80.9 & 99.6 & 123.8 \\
\hline & & HWFET & 63.9 & 63.9 & 75.7 & 88.1 & 73.2 & 84.8 & 96.2 & 75.4 & 93.6 & 108.9 & 79.4 & 97.0 & 115.6 \\
\hline & & Combined & 62.6 & 62.6 & 75.1 & 88.1 & 72.9 & 85.2 & 97.9 & 76.2 & 95.0 & 113.8 & 80.2 & 98.4 & 119.9 \\
\hline & \multirow{3}{*}{ Adjusted } & UDDS & 44.6 & 44.6 & 52.7 & 60.8 & 51.5 & 59.2 & 67.1 & 54.1 & 65.4 & 77.4 & 56.5 & 67.3 & 80.3 \\
\hline & & HWFET & 44.9 & 44.9 & 53.1 & 61.6 & 51.3 & 59.4 & 67.2 & 52.8 & 65.4 & 75.8 & 55.6 & 67.7 & 80.3 \\
\hline & & Combined & 44.7 & 44.7 & 52.9 & 61.1 & 51.4 & 59.3 & 67.1 & 53.5 & 65.4 & 76.7 & 56.1 & 67.5 & 80.3 \\
\hline \multirow{6}{*}{ FC PHEV 20} & \multirow{3}{*}{ Unadjusted } & \begin{tabular}{|l|} 
UDDS \\
\end{tabular} & 73.8 & 73.8 & 89.7 & 106.3 & 87.1 & 102.9 & 119.7 & 92.4 & 116.2 & 143.0 & 97.4 & 120.6 & 150.0 \\
\hline & & HWFET & 65.3 & 65.3 & 77.9 & 91.1 & 75.3 & 87.8 & 100.0 & 78.1 & 97.1 & 114.4 & 82.3 & 100.8 & 121.1 \\
\hline & & Combined & 69.7 & 69.7 & 84.0 & 98.8 & 81.4 & 95.5 & 110.0 & 85.4 & 106.8 & 128.6 & 89.9 & 110.8 & 135.4 \\
\hline & \multirow{3}{*}{ Adjusted } & UDDS & 52.2 & 52.2 & 61.7 & 71.0 & 60.2 & 69.1 & 78.2 & 63.2 & 76.3 & 89.9 & 66.0 & 78.6 & 93.2 \\
\hline & & HWFET & 45.9 & 45.9 & 54.6 & 63.6 & 52.8 & 61.4 & 69.8 & 54.7 & 67.8 & 79.6 & 57.6 & 70.3 & 84.1 \\
\hline & & Combined & 49.1 & 49.1 & 58.3 & 67.5 & 56.6 & 65.4 & 74.2 & 59.1 & 72.2 & 84.9 & 62.0 & 74.6 & 88.9 \\
\hline \multirow{6}{*}{ FC PHEV 30} & \multirow{3}{*}{ Unadjusted } & UDDS & 105.2 & 105.2 & 127.3 & 148.0 & 123.5 & 144.1 & 168.9 & 129.3 & 163.7 & 200.7 & 136.6 & 170.5 & 210.1 \\
\hline & & HWFET & 82.1 & 82.1 & 97.8 & 112.9 & 94.4 & 109.3 & 125.2 & 97.2 & 121.5 & 143.0 & 102.6 & 126.3 & 151.3 \\
\hline & & Combined & 93.4 & 93.4 & 112.1 & 129.8 & 108.5 & 126.1 & 146.0 & 112.5 & 141.6 & 169.8 & 118.8 & 147.3 & 178.8 \\
\hline & \multirow{3}{*}{ Adjusted } & UDDS & 70.4 & 70.4 & 82.1 & 92.3 & 80.2 & 90.5 & 102.0 & 83.1 & 99.6 & 115.5 & 86.8 & 102.7 & 119.3 \\
\hline & & HWFET & 57.5 & 57.5 & 68.3 & 78.5 & 65.9 & 76.1 & 86.9 & 67.8 & 84.4 & 98.8 & 71.5 & 87.6 & 104.4 \\
\hline & & Combined & 63.9 & 63.9 & 75.2 & 85.5 & 73.1 & 83.4 & 94.6 & 75.4 & 92.1 & 107.3 & 79.2 & 95.3 & 112.1 \\
\hline \multirow{6}{*}{ FC PHEV 40} & \multirow{3}{*}{ Unadjusted } & \begin{tabular}{|l|} 
UDDS \\
\end{tabular} & 121.0 & 121.0 & 147.0 & 171.1 & 142.5 & 166.7 & 196.0 & 149.4 & 189.9 & 233.2 & 157.9 & 198.2 & 244.5 \\
\hline & & \begin{tabular}{|c|} 
HWFET \\
\end{tabular} & 103.8 & 103.8 & 124.1 & 143.4 & 119.7 & 138.8 & 159.4 & 123.3 & 154.7 & 182.3 & 130.2 & 161.0 & 193.1 \\
\hline & & Combined & 112.6 & 112.6 & 135.7 & 157.4 & 131.2 & 152.8 & 177.7 & 136.4 & 172.3 & 207.2 & 144.1 & 179.6 & 218.3 \\
\hline & \multirow{3}{*}{ Adjusted } & UDDS & 78.9 & 78.9 & 91.8 & 103.0 & 89.6 & 101.0 & 113.6 & 93.0 & 111.1 & 128.1 & 96.9 & 114.5 & 132.2 \\
\hline & & HWFET & 72.4 & 72.4 & 86.1 & 99.1 & 83.1 & 96.0 & 109.8 & 85.6 & 106.6 & 125.0 & 90.2 & 110.9 & 132.0 \\
\hline & & Combined & 75.8 & 75.8 & 89.2 & 101.2 & 86.6 & 98.7 & 111.8 & 89.5 & 109.0 & 126.7 & 93.8 & 112.8 & 132.1 \\
\hline
\end{tabular}

\section{$\underline{\text { Pev details }}$}


Argonne National Laboratory Report - Light-Duty Vehicle Fuel Consumption Displacement Potential Up to 2045

\begin{tabular}{|c|c|c|c|c|c|c|c|c|c|c|c|c|c|c|c|c|}
\hline & & & & \multicolumn{13}{|c|}{ Small_SUV } \\
\hline & & & & \multirow{2}{*}{$\begin{array}{l}2010 \\
\text { Ref }\end{array}$} & \multicolumn{3}{|c|}{2010} & \multicolumn{3}{|c|}{2015} & \multicolumn{3}{|c|}{2030} & \multicolumn{3}{|c|}{2045} \\
\hline & & & & & low & avg & high & low & avg & high & low & avg & high & low & avg & high \\
\hline \multirow{25}{*}{ SI Split HEV PHEV10 } & \multirow{9}{*}{ UDDS } & \multirow{4}{*}{$C D$} & Wh/mile & 222.3 & 222.3 & 202.1 & 188.5 & 218.1 & 194.4 & 187.0 & 214.4 & 184.6 & 165.7 & 208.8 & 182.9 & 160.5 \\
\hline & & & Distance & 12.2 & 12.2 & 12.3 & 12.2 & 12.1 & 12.2 & 11.6 & 11.9 & 12.2 & 11.3 & 12.3 & 11.7 & 10.9 \\
\hline & & & MPGGE Unadjusted & 771.0 & 771.0 & 831.9 & 923.0 & 807.1 & 887.5 & 1660.3 & 826.5 & 1039.2 & 2918.4 & 824.9 & 1563.7 & 3642.9 \\
\hline & & & MPGGE Adjusted & 241.6 & 241.6 & 248.6 & 257.9 & 245.8 & 254.4 & 304.2 & 248.0 & 268.2 & 336.8 & 247.8 & 300.0 & 346.6 \\
\hline & & \multirow{2}{*}{ CS } & MPGGE Unadjusted & 48.0 & 48.0 & 53.1 & 57.6 & 49.7 & 55.9 & 68.3 & 51.0 & 61.6 & 84.7 & 52.7 & 63.8 & 88.4 \\
\hline & & & MPGGE Adjusted & 35.6 & 35.6 & 39.0 & 42.0 & 36.7 & 40.8 & 48.8 & 37.6 & 44.6 & 58.7 & 38.7 & 45.9 & 60.9 \\
\hline & & \multirow{3}{*}{$\mathrm{CD}+\mathrm{CS}$} & Wh/mile & 46.3 & 46.3 & 42.1 & 39.3 & 45.4 & 40.5 & 39.0 & 44.7 & 38.5 & 34.5 & 43.5 & 38.1 & 33.4 \\
\hline & & & MPGGE Unadjusted & 57.1 & 57.1 & 63.2 & 68.5 & 59.1 & 66.4 & 81.6 & 60.7 & 73.4 & 101.4 & 62.7 & 76.2 & 106.0 \\
\hline & & & MPGGE Adjusted & 41.6 & 41.6 & 45.6 & 48.9 & 42.9 & 47.6 & 56.9 & 44.0 & 51.9 & 68.3 & 45.2 & 53.7 & 70.8 \\
\hline & \multirow{9}{*}{ HWFET } & \multirow{4}{*}{$C D$} & Wh/mile & 209.5 & 209.5 & 189.4 & 223.5 & 206.0 & 226.8 & 209.6 & 203.8 & 215.2 & 182.4 & 192.8 & 207.8 & 173.9 \\
\hline & & & Distance & 12.4 & 12.4 & 12.4 & 10.0 & 12.4 & 10.6 & 9.8 & 12.3 & 10.0 & 8.5 & 12.6 & 9.7 & 8.6 \\
\hline & & & MPGGE Unadjusted & 130.4 & 130.4 & 143.1 & 320.8 & 134.9 & 280.3 & 389.7 & 136.9 & 353.5 & 417.9 & 137.5 & 359.6 & 456.3 \\
\hline & & & MPGGE Adjusted & 90.4 & 90.4 & 98.9 & 213.6 & 93.4 & 188.2 & 255.8 & 94.8 & 233.8 & 272.8 & 95.1 & 237.5 & 295.6 \\
\hline & & \multirow{2}{*}{ CS } & MPGGE Unadjusted & 42.7 & 42.7 & 47.3 & 51.3 & 44.0 & 49.5 & 60.1 & 44.9 & 54.3 & 72.1 & 47.0 & 56.5 & 76.1 \\
\hline & & & MPGGE Adjusted & 30.2 & 30.2 & 33.3 & 36.1 & 31.1 & 34.9 & 42.3 & 31.7 & 38.3 & 50.6 & 33.1 & 39.8 & 53.3 \\
\hline & & \multirow{3}{*}{$\mathrm{CD}+\mathrm{CS}$} & Wh/mile & 61.6 & 61.6 & 55.7 & 65.7 & 60.6 & 66.7 & 61.6 & 59.9 & 63.3 & 53.6 & 56.7 & 61.1 & 51.2 \\
\hline & & & MPGGE Unadjusted & 50.9 & 50.9 & 56.3 & 64.2 & 52.5 & 61.7 & 75.4 & 53.5 & 68.2 & 89.9 & 55.8 & 70.9 & 95.1 \\
\hline & & & MPGGE Adjusted & 35.9 & 35.9 & 39.7 & 45.1 & 37.0 & 43.4 & 52.9 & 37.7 & 47.9 & 62.8 & 39.3 & 49.7 & 66.4 \\
\hline & \multirow{7}{*}{ Combined } & \multirow{2}{*}{$C D$} & MPGGE Unadjusted & 240.1 & 240.1 & 262.8 & 500.4 & 248.9 & 449.4 & 672.9 & 253.1 & 554.8 & 790.3 & 253.8 & 623.7 & 879.4 \\
\hline & & & MPGGE Adjusted & 137.8 & 137.8 & 147.9 & 235.9 & 141.7 & 219.6 & 280.4 & 143.5 & 251.5 & 304.6 & 143.9 & 268.3 & 321.6 \\
\hline & & \multirow{2}{*}{ CS } & MPGGE Unadjusted & 45.5 & 45.5 & 50.3 & 54.6 & 47.0 & 52.8 & 64.3 & 48.1 & 58.1 & 78.5 & 49.9 & 60.3 & 82.4 \\
\hline & & & MPGGE Adjusted & 32.9 & 32.9 & 36.3 & 39.1 & 33.9 & 37.9 & 45.6 & 34.7 & 41.5 & 54.8 & 36.0 & 43.0 & 57.3 \\
\hline & & \multirow{3}{*}{$C D+C S$} & Wh/mile & 53.2 & 53.2 & 48.2 & 51.2 & 52.3 & 52.3 & 49.2 & 51.5 & 49.6 & 43.1 & 49.4 & 48.5 & 41.4 \\
\hline & & & MPGGE Unadjusted & 54.2 & 54.2 & 59.9 & 66.5 & 55.9 & 64.2 & 78.7 & 57.2 & 70.9 & 95.9 & 59.4 & 73.7 & 100.8 \\
\hline & & & MPGGE Adjusted & 38.8 & 38.8 & 42.7 & 47.2 & 40.0 & 45.6 & 55.0 & 40.9 & 50.0 & 65.7 & 42.3 & 51.8 & 68.8 \\
\hline
\end{tabular}


Argonne National Laboratory Report - Light-Duty Vehicle Fuel Consumption Displacement Potential Up to 2045

\begin{tabular}{|c|c|c|c|c|c|c|c|c|c|c|c|c|c|c|c|c|}
\hline & & & & \multicolumn{13}{|c|}{ Small_SUV } \\
\hline & & & & 2010 & & 2010 & & & 2015 & & & 2030 & & & 2045 & \\
\hline \multirow{26}{*}{ SI Split HEV PHEV2O } & & \multirow{5}{*}{$C D$} & & Ref & low & avg & high & low & avg & high & low & avg & high & low & avg & high \\
\hline & \multirow{9}{*}{ UDDS } & & Wh/mile & 224.5 & 224.5 & 210.2 & 192.3 & 228.1 & 198.1 & 177.5 & 223.6 & 182.6 & 163.5 & 215.2 & 177.8 & 156.8 \\
\hline & & & Distance & 24.1 & 24.1 & 23.9 & 24.0 & 23.8 & 24.0 & 23.7 & 23.9 & 23.9 & 22.3 & 24.0 & 23.5 & 22.1 \\
\hline & & & MPGGE Unadjusted & 733.8 & 733.8 & 973.1 & 943.5 & 992.8 & 902.5 & 1139.6 & 1008.3 & 1015.3 & 2860.3 & 912.8 & 1079.5 & 3147.1 \\
\hline & & & MPGGE Adjusted & 237.0 & 237.0 & 262.5 & 259.9 & 264.3 & 255.9 & 275.9 & 265.6 & 266.2 & 335.8 & 256.9 & 271.4 & 340.3 \\
\hline & & \multirow{2}{*}{ CS } & MPGGE Unadjusted & 47.7 & 47.7 & 52.7 & 57.3 & 49.3 & 55.4 & 68.3 & 50.7 & 61.6 & 84.6 & 52.4 & 63.9 & 88.2 \\
\hline & & & MPGGE Adjusted & 35.4 & 35.4 & 38.7 & 41.7 & 36.5 & 40.5 & 48.8 & 37.4 & 44.6 & 58.7 & 38.5 & 46.0 & 60.8 \\
\hline & & \multirow{3}{*}{$\mathrm{CD}+\mathrm{CS}$} & Wh/mile & 118.1 & 118.1 & 110.4 & 101.3 & 119.6 & 104.3 & 93.8 & 117.3 & 96.5 & 62.3 & 113.1 & 94.0 & 59.7 \\
\hline & & & MPGGE Unadjusted & 81.0 & 81.0 & 90.2 & 97.7 & 84.7 & 94.4 & 116.6 & 87.1 & 105.1 & 122.8 & 89.5 & 109.2 & 128.1 \\
\hline & & & MPGGE Adjusted & 56.5 & 56.5 & 62.0 & 66.2 & 58.8 & 64.4 & 76.5 & 60.1 & 70.4 & 79.8 & 61.6 & 72.6 & 82.5 \\
\hline & \multirow{9}{*}{ HWFET } & \multirow{4}{*}{$C D$} & Wh/mile & 209.8 & 209.8 & 237.7 & 228.6 & 255.2 & 228.5 & 220.5 & 236.5 & 217.7 & 224.9 & 228.3 & 219.6 & 212.6 \\
\hline & & & Distance & 24.8 & 24.8 & 20.2 & 20.1 & 21.2 & 20.2 & 19.4 & 22.0 & 19.7 & 16.0 & 22.4 & 19.3 & 16.1 \\
\hline & & & MPGGE Unadjusted & 127.0 & 127.0 & 268.2 & 347.5 & 232.9 & 279.9 & 525.1 & 189.6 & 370.1 & N/A & 197.5 & 496.6 & N/A \\
\hline & & & MPGGE Adjusted & 88.1 & 88.1 & 180.5 & 230.1 & 157.9 & 187.9 & 335.6 & 129.7 & 244.0 & $\mathrm{~N} / \mathrm{A}$ & 134.9 & 319.2 & N/A \\
\hline & & \multirow{2}{*}{ CS } & MPGGE Unadjusted & 42.4 & 42.4 & 46.9 & 51.0 & 43.7 & 49.2 & 59.6 & 44.5 & 54.0 & 71.8 & 46.5 & 56.2 & 75.7 \\
\hline & & & MPGGE Adjusted & 30.0 & 30.0 & 33.1 & 35.9 & 30.8 & 34.7 & 42.0 & 31.4 & 38.0 & 50.4 & 32.8 & 39.5 & 53.1 \\
\hline & & \multirow{3}{*}{$\mathrm{CD}+\mathrm{CS}$} & Wh/mile & 107.8 & 107.8 & 69.9 & 67.2 & 127.9 & 67.2 & 64.8 & 119.4 & 64.0 & 66.1 & 115.3 & 64.6 & 62.5 \\
\hline & & & MPGGE Unadjusted & 58.1 & 58.1 & 58.5 & 64.1 & 64.5 & 61.3 & 75.8 & 64.2 & 67.9 & 94.5 & 66.9 & 71.3 & 99.7 \\
\hline & & & MPGGE Adjusted & 40.9 & 40.9 & 41.2 & 45.1 & 45.3 & 43.1 & 53.1 & 45.1 & 47.7 & 66.0 & 47.0 & 50.1 & 69.5 \\
\hline & \multirow{7}{*}{ Combined } & \multirow{2}{*}{$C D$} & MPGGE Unadjusted & 232.9 & 232.9 & 445.8 & 532.5 & 402.3 & 451.0 & 746.5 & 342.5 & 569.0 & 5021.3 & 347.1 & 706.4 & 5505.6 \\
\hline & & & MPGGE Adjusted & 134.6 & 134.6 & 218.0 & 245.6 & 202.8 & 220.1 & 299.9 & 180.5 & 255.7 & 559.9 & 182.6 & 291.0 & 566.8 \\
\hline & & \multirow{2}{*}{ CS } & MPGGE Unadjusted & 45.1 & 45.1 & 49.9 & 54.2 & 46.6 & 52.4 & 64.1 & 47.7 & 57.9 & 78.3 & 49.5 & 60.2 & 82.1 \\
\hline & & & MPGGE Adjusted & 32.7 & 32.7 & 36.0 & 38.9 & 33.7 & 37.7 & 45.5 & 34.5 & 41.4 & 54.6 & 35.7 & 42.9 & 57.1 \\
\hline & & \multirow{3}{*}{$\mathrm{CD}+\mathrm{CS}$} & Wh/mile & 113.5 & 113.5 & 92.2 & 85.9 & 123.3 & 87.6 & 80.8 & 118.2 & 81.9 & 64.0 & 114.1 & 80.8 & 61.0 \\
\hline & & & MPGGE Unadjusted & 68.8 & 68.8 & 72.5 & 79.0 & 74.3 & 76.0 & 93.8 & 75.0 & 84.3 & 108.2 & 77.7 & 88.1 & 113.5 \\
\hline & & & MPGGE Adjusted & 48.2 & 48.2 & 50.5 & 54.7 & 51.9 & 52.7 & 63.9 & 52.3 & 58.0 & 72.9 & 54.0 & 60.4 & 76.1 \\
\hline
\end{tabular}


Argonne National Laboratory Report - Light-Duty Vehicle Fuel Consumption Displacement Potential Up to 2045

\begin{tabular}{|c|c|c|c|c|c|c|c|c|c|c|c|c|c|c|c|c|}
\hline & & & & \multicolumn{13}{|c|}{ Small_SUV } \\
\hline & & & & \multirow{2}{*}{$\begin{array}{l}2010 \\
\text { Ref }\end{array}$} & \multicolumn{3}{|c|}{2010} & \multicolumn{3}{|c|}{2015} & \multicolumn{3}{|c|}{2030} & \multicolumn{3}{|c|}{2045} \\
\hline & & & & & low & avg & high & low & avg & high & low & avg & high & low & avg & high \\
\hline \multirow{25}{*}{ SI Series HEV PHEV30 } & \multirow{9}{*}{ UDDS } & \multirow{4}{*}{$C D$} & Wh/mile & 320.7 & 320.7 & 292.2 & 271.6 & 311.9 & 280.2 & 246.3 & 305.1 & 253.6 & 213.2 & 298.0 & 245.4 & 204.1 \\
\hline & & & Distance & 30.9 & 30.9 & 30.9 & 30.9 & 30.9 & 30.9 & 30.9 & 30.9 & 30.9 & 31.4 & 30.9 & 30.9 & 30.7 \\
\hline & & & MPGGE Unadjusted & $\mathrm{N} / \mathrm{A}$ & $\mathrm{N} / \mathrm{A}$ & N/A & N/A & N/A & $\mathrm{N} / \mathrm{A}$ & N/A & $\mathrm{N} / \mathrm{A}$ & N/A & N/A & N/A & N/A & $\mathrm{N} / \mathrm{A}$ \\
\hline & & & MPGGE Adjusted & $\mathrm{N} / \mathrm{A}$ & $\mathrm{N} / \mathrm{A}$ & $\mathrm{N} / \mathrm{A}$ & N/A & N/A & N/A & N/A & $\mathrm{N} / \mathrm{A}$ & N/A & N/A & N/A & $\mathrm{N} / \mathrm{A}$ & $\mathrm{N} / \mathrm{A}$ \\
\hline & & \multirow{2}{*}{ CS } & MPGGE Unadjusted & 34.6 & 34.6 & 39.2 & 43.7 & 36.3 & 42.2 & 54.2 & 38.1 & 48.9 & 69.3 & 39.7 & 51.0 & 72.7 \\
\hline & & & MPGGE Adjusted & 26.4 & 26.4 & 29.6 & 32.7 & 27.5 & 31.7 & 39.7 & 28.8 & 36.2 & 49.4 & 29.9 & 37.6 & 51.5 \\
\hline & & \multirow{3}{*}{$\mathrm{CD}+\mathrm{CS}$} & Wh/mile & 206.4 & 206.4 & 188.1 & 174.9 & 200.7 & 180.4 & 159.1 & 196.3 & 163.8 & 138.2 & 191.8 & 158.4 & 132.3 \\
\hline & & & MPGGE Unadjusted & 73.7 & 73.7 & 83.4 & 92.9 & 77.1 & 89.8 & 115.4 & 81.0 & 104.0 & 147.4 & 84.4 & 108.6 & 154.7 \\
\hline & & & MPGGE Adjusted & 52.1 & 52.1 & 58.0 & 63.5 & 54.2 & 61.7 & 75.9 & 56.5 & 69.8 & 92.0 & 58.6 & 72.3 & 95.5 \\
\hline & \multirow{9}{*}{ HWFET } & \multirow{4}{*}{$C D$} & Wh/mile & 347.8 & 347.8 & 314.3 & 291.3 & 339.4 & 301.7 & 266.1 & 334.6 & 273.8 & 234.4 & 323.0 & 264.7 & 221.9 \\
\hline & & & Distance & 28.5 & 28.5 & 28.8 & 28.8 & 28.5 & 28.7 & 28.6 & 28.1 & 28.7 & 27.7 & 28.5 & 28.5 & 28.2 \\
\hline & & & MPGGE Unadjusted & $\mathrm{N} / \mathrm{A}$ & $\mathrm{N} / \mathrm{A}$ & $\mathrm{N} / \mathrm{A}$ & $\mathrm{N} / \mathrm{A}$ & $\mathrm{N} / \mathrm{A}$ & $\mathrm{N} / \mathrm{A}$ & $\mathrm{N} / \mathrm{A}$ & $\mathrm{N} / \mathrm{A}$ & $\mathrm{N} / \mathrm{A}$ & $\mathrm{N} / \mathrm{A}$ & $\mathrm{N} / \mathrm{A}$ & $\mathrm{N} / \mathrm{A}$ & $\mathrm{N} / \mathrm{A}$ \\
\hline & & & MPGGE Adjusted & N/A & N/A & N/A & N/A & N/A & N/A & N/A & N/A & N/A & N/A & N/A & N/A & $\mathrm{N} / \mathrm{A}$ \\
\hline & & \multirow{2}{*}{ CS } & MPGGE Unadjusted & 31.9 & 31.9 & 36.0 & 39.7 & 33.1 & 38.3 & 48.0 & 34.4 & 43.2 & 59.2 & 36.2 & 45.4 & 62.6 \\
\hline & & & MPGGE Adjusted & 22.6 & 22.6 & 25.4 & 28.0 & 23.4 & 27.1 & 33.8 & 24.3 & 30.5 & 41.6 & 25.6 & 32.0 & 44.0 \\
\hline & & \multirow{3}{*}{$\mathrm{CD}+\mathrm{CS}$} & Wh/mile & 179.2 & 179.2 & 162.0 & 150.1 & 174.9 & 155.5 & 137.2 & 172.5 & 141.1 & 120.8 & 166.5 & 136.5 & 114.4 \\
\hline & & & MPGGE Unadjusted & 54.0 & 54.0 & 61.0 & 67.2 & 56.1 & 64.9 & 81.3 & 58.3 & 73.3 & 100.3 & 61.3 & 76.9 & 106.2 \\
\hline & & & \begin{tabular}{|l|} 
MPGGE Adjusted \\
\end{tabular} & 38.0 & 38.0 & 42.9 & 47.2 & 39.5 & 45.6 & 56.9 & 41.0 & 51.4 & 69.9 & 43.1 & 53.9 & 73.9 \\
\hline & \multirow{7}{*}{ Combined } & \multirow{2}{*}{$C D$} & MPGGE Unadjusted & $\mathrm{N} / \mathrm{A}$ & $\mathrm{N} / \mathrm{A}$ & N/A & N/A & N/A & $\mathrm{N} / \mathrm{A}$ & N/A & $\mathrm{N} / \mathrm{A}$ & $\mathrm{N} / \mathrm{A}$ & N/A & $\mathrm{N} / \mathrm{A}$ & N/A & $\mathrm{N} / \mathrm{A}$ \\
\hline & & & MPGGE Adjusted & N/A & N/A & N/A & N/A & N/A & N/A & N/A & N/A & N/A & N/A & N/A & N/A & N/A \\
\hline & & \multirow{2}{*}{ CS } & MPGGE Unadjusted & 33.3 & 33.3 & 37.7 & 41.8 & 34.8 & 40.4 & 51.2 & 36.3 & 46.2 & 64.3 & 38.0 & 48.3 & 67.8 \\
\hline & & & MPGGE Adjusted & 24.5 & 24.5 & 27.5 & 30.4 & 25.5 & 29.4 & 36.9 & 26.6 & 33.4 & 45.6 & 27.8 & 34.9 & 47.9 \\
\hline & & \multirow{3}{*}{$C D+C S$} & Wh/mile & 194.1 & 194.1 & 176.4 & 163.7 & 189.1 & 169.2 & 149.2 & 185.6 & 153.6 & 130.4 & 180.4 & 148.5 & 124.2 \\
\hline & & & MPGGE Unadjusted & 63.3 & 63.3 & 71.5 & 79.3 & 66.0 & 76.6 & 97.1 & 68.9 & 87.5 & 121.7 & 72.2 & 91.6 & 128.3 \\
\hline & & & MPGGE Adjusted & 44.7 & 44.7 & 50.0 & 55.0 & 46.4 & 53.3 & 66.0 & 48.3 & 60.1 & 80.6 & 50.4 & 62.6 & 84.4 \\
\hline
\end{tabular}


Argonne National Laboratory Report - Light-Duty Vehicle Fuel Consumption Displacement Potential Up to 2045

\begin{tabular}{|c|c|c|c|c|c|c|c|c|c|c|c|c|c|c|c|c|}
\hline & & & & \multicolumn{13}{|c|}{ Small_SUV } \\
\hline & & & & 2010 & & 2010 & & & 2015 & & & 2030 & & & 2045 & \\
\hline \multirow{26}{*}{ SI Series HEV PHEV40 } & & \multirow{5}{*}{$C D$} & & Ref & low & avg & high & low & avg & high & low & avg & high & low & avg & high \\
\hline & \multirow{9}{*}{ UDDS } & & Wh/mile & 327.7 & 327.7 & 298.4 & 276.8 & 318.5 & 285.7 & 251.4 & 311.2 & 259.3 & 215.3 & 303.8 & 249.7 & 206.3 \\
\hline & & & Distance & 40.6 & 40.6 & 40.8 & 40.8 & 40.6 & 40.8 & 40.5 & 40.6 & 40.6 & 40.4 & 40.8 & 40.5 & 40.6 \\
\hline & & & MPGGE Unadjusted & N/A & N/A & $\mathrm{N} / \mathrm{A}$ & $\mathrm{N} / \mathrm{A}$ & $\mathrm{N} / \mathrm{A}$ & $\mathrm{N} / \mathrm{A}$ & $\mathrm{N} / \mathrm{A}$ & $\mathrm{N} / \mathrm{A}$ & $\mathrm{N} / \mathrm{A}$ & $\mathrm{N} / \mathrm{A}$ & $\mathrm{N} / \mathrm{A}$ & N/A & $\mathrm{N} / \mathrm{A}$ \\
\hline & & & MPGGE Adjusted & $\mathrm{N} / \mathrm{A}$ & $\mathrm{N} / \mathrm{A}$ & $\mathrm{N} / \mathrm{A}$ & $\mathrm{N} / \mathrm{A}$ & $\mathrm{N} / \mathrm{A}$ & $\mathrm{N} / \mathrm{A}$ & $\mathrm{N} / \mathrm{A}$ & $\mathrm{N} / \mathrm{A}$ & $\mathrm{N} / \mathrm{A}$ & $\mathrm{N} / \mathrm{A}$ & $\mathrm{N} / \mathrm{A}$ & N/A & N/A \\
\hline & & \multirow{2}{*}{ CS } & MPGGE Unadjusted & 33.8 & 33.8 & 38.2 & 42.8 & 35.3 & 40.8 & 53.4 & 37.2 & 47.8 & 67.8 & 38.8 & 49.4 & 71.8 \\
\hline & & & MPGGE Adjusted & 25.7 & 25.7 & 28.9 & 32.0 & 26.8 & 30.7 & 39.2 & 28.2 & 35.5 & 48.5 & 29.3 & 36.5 & 51.0 \\
\hline & & \multirow{3}{*}{$\mathrm{CD}+\mathrm{CS}$} & Wh/mile & 240.4 & 240.4 & 219.1 & 203.3 & 233.7 & 209.9 & 185.8 & 228.4 & 191.6 & 159.3 & 223.0 & 184.3 & 152.6 \\
\hline & & & MPGGE Unadjusted & 84.4 & 84.4 & 95.5 & 106.9 & 88.3 & 102.0 & 133.4 & 93.0 & 119.6 & 169.5 & 96.9 & 123.4 & 179.5 \\
\hline & & & MPGGE Adjusted & 58.6 & 58.6 & 65.0 & 71.3 & 60.9 & 68.7 & 85.2 & 63.6 & 78.1 & 102.2 & 65.8 & 80.1 & 106.6 \\
\hline & \multirow{9}{*}{ HWFET } & \multirow{4}{*}{$C D$} & Wh/mile & 351.0 & 351.0 & 317.0 & 293.3 & 342.3 & 303.9 & 267.4 & 337.3 & 275.4 & 235.8 & 325.5 & 265.9 & 223.3 \\
\hline & & & Distance & 37.7 & 37.7 & 38.0 & 38.1 & 37.6 & 38.0 & 37.8 & 37.2 & 37.9 & 36.8 & 37.7 & 37.9 & 37.5 \\
\hline & & & MPGGE Unadjusted & N/A & N/A & N/A & N/A & $\mathrm{N} / \mathrm{A}$ & $\mathrm{N} / \mathrm{A}$ & N/A & N/A & N/A & N/A & N/A & N/A & N/A \\
\hline & & & MPGGE Adjusted & N/A & N/A & $\mathrm{N} / \mathrm{A}$ & $\mathrm{N} / \mathrm{A}$ & $\mathrm{N} / \mathrm{A}$ & N/A & N/A & $\mathrm{N} / \mathrm{A}$ & N/A & N/A & N/A & N/A & $\mathrm{N} / \mathrm{A}$ \\
\hline & & \multirow{2}{*}{ CS } & MPGGE Unadjusted & 31.4 & 31.4 & 35.5 & 39.1 & 32.6 & 37.8 & 47.4 & 33.9 & 42.8 & 58.8 & 35.6 & 44.9 & 62.2 \\
\hline & & & MPGGE Adjusted & 22.2 & 22.2 & 25.1 & 27.7 & 23.1 & 26.7 & 33.5 & 24.0 & 30.2 & 41.3 & 25.2 & 31.7 & 43.7 \\
\hline & & \multirow{3}{*}{$\mathrm{CD}+\mathrm{CS}$} & Wh/mile & 237.0 & 237.0 & 214.1 & 198.1 & 231.1 & 205.3 & 180.6 & 227.7 & 186.0 & 159.4 & 219.8 & 179.6 & 150.9 \\
\hline & & & MPGGE Unadjusted & 68.2 & 68.2 & 77.1 & 85.1 & 70.9 & 82.2 & 103.1 & 73.8 & 93.0 & 127.7 & 77.4 & 97.6 & 135.2 \\
\hline & & & MPGGE Adjusted & 47.9 & 47.9 & 54.0 & 59.5 & 49.7 & 57.5 & 71.9 & 51.7 & 64.9 & 88.6 & 54.3 & 68.1 & 93.6 \\
\hline & \multirow{7}{*}{ Combined } & \multirow{2}{*}{$C D$} & MPGGE Unadjusted & $\mathrm{N} / \mathrm{A}$ & $\mathrm{N} / \mathrm{A}$ & $\mathrm{N} / \mathrm{A}$ & $\mathrm{N} / \mathrm{A}$ & N/A & $\mathrm{N} / \mathrm{A}$ & N/A & $\mathrm{N} / \mathrm{A}$ & $\mathrm{N} / \mathrm{A}$ & $\mathrm{N} / \mathrm{A}$ & $\mathrm{N} / \mathrm{A}$ & $\mathrm{N} / \mathrm{A}$ & $\mathrm{N} / \mathrm{A}$ \\
\hline & & & MPGGE Adjusted & $\mathrm{N} / \mathrm{A}$ & $\mathrm{N} / \mathrm{A}$ & $\mathrm{N} / \mathrm{A}$ & $\mathrm{N} / \mathrm{A}$ & $\mathrm{N} / \mathrm{A}$ & $\mathrm{N} / \mathrm{A}$ & $\mathrm{N} / \mathrm{A}$ & $\mathrm{N} / \mathrm{A}$ & $\mathrm{N} / \mathrm{A}$ & $\mathrm{N} / \mathrm{A}$ & $\mathrm{N} / \mathrm{A}$ & $\mathrm{N} / \mathrm{A}$ & $\mathrm{N} / \mathrm{A}$ \\
\hline & & \multirow{2}{*}{ CS } & MPGGE Unadjusted & 32.7 & 32.7 & 36.9 & 41.0 & 34.0 & 39.4 & 50.5 & 35.7 & 45.4 & 63.4 & 37.3 & 47.2 & 67.1 \\
\hline & & & MPGGE Adjusted & 24.0 & 24.0 & 27.0 & 29.9 & 25.0 & 28.8 & 36.4 & 26.1 & 32.9 & 45.0 & 27.3 & 34.2 & 47.4 \\
\hline & & \multirow{3}{*}{$\mathrm{CD}+\mathrm{CS}$} & Wh/mile & 238.9 & 238.9 & 216.8 & 201.0 & 232.5 & 207.8 & 183.5 & 228.1 & 189.1 & 159.3 & 221.6 & 182.1 & 151.8 \\
\hline & & & MPGGE Unadjusted & 76.3 & 76.3 & 86.2 & 95.8 & 79.5 & 92.0 & 117.8 & 83.2 & 105.9 & 147.7 & 87.0 & 110.3 & 156.4 \\
\hline & & & MPGGE Adjusted & 53.2 & 53.2 & 59.6 & 65.5 & 55.3 & 63.2 & 78.6 & 57.6 & 71.6 & 95.6 & 60.0 & 74.2 & 100.3 \\
\hline
\end{tabular}


Argonne National Laboratory Report - Light-Duty Vehicle Fuel Consumption Displacement Potential Up to 2045

\begin{tabular}{|c|c|c|c|c|c|c|c|c|c|c|c|c|c|c|c|c|}
\hline & & & & \multicolumn{13}{|c|}{ Small_SUV } \\
\hline & & & & \multirow{2}{*}{$\begin{array}{l}2010 \\
\text { Ref }\end{array}$} & \multicolumn{3}{|c|}{2010} & \multicolumn{3}{|c|}{2015} & \multicolumn{3}{|c|}{2030} & \multicolumn{3}{|c|}{2045} \\
\hline & & & & & low & avg & high & low & avg & high & low & avg & high & low & avg & high \\
\hline \multirow{25}{*}{ CI Split HEV PHEV10 } & \multirow{9}{*}{ UDDS } & \multirow{4}{*}{$C D$} & Wh/mile & 211.6 & 211.6 & 208.2 & 195.5 & 225.5 & 197.8 & 186.0 & 221.6 & 191.1 & 173.6 & 214.3 & 181.6 & 166.7 \\
\hline & & & Distance & 13.0 & 13.0 & 12.6 & 12.3 & 12.2 & 12.8 & 12.3 & 12.2 & 12.3 & 11.4 & 12.6 & 12.3 & 11.4 \\
\hline & & & MPGGE Unadjusted & 500.4 & 500.4 & 785.4 & 913.3 & 791.6 & 792.0 & 1064.6 & 828.5 & 1025.2 & 2644.4 & 803.9 & 1093.6 & 2822.4 \\
\hline & & & MPGGE Adjusted & 200.1 & 200.1 & 243.3 & 257.0 & 244.0 & 244.1 & 270.2 & 248.2 & 267.0 & 331.9 & 245.5 & 272.5 & 335.2 \\
\hline & & \multirow{2}{*}{ CS } & MPGGE Unadjusted & 49.2 & 49.2 & 53.9 & 59.4 & 50.2 & 57.7 & 66.4 & 52.7 & 64.6 & 79.7 & 55.1 & 67.6 & 83.2 \\
\hline & & & MPGGE Adjusted & 36.4 & 36.4 & 39.5 & 43.1 & 37.1 & 42.0 & 47.6 & 38.8 & 46.5 & 55.8 & 40.4 & 48.4 & 57.8 \\
\hline & & \multirow{3}{*}{$\mathrm{CD}+\mathrm{CS}$} & Wh/mile & 44.1 & 44.1 & 43.4 & 40.7 & 47.0 & 41.2 & 38.7 & 46.2 & 39.8 & 36.2 & 44.7 & 37.8 & 34.7 \\
\hline & & & MPGGE Unadjusted & 58.2 & 58.2 & 64.1 & 70.6 & 59.8 & 68.5 & 78.9 & 62.7 & 76.9 & 95.5 & 65.5 & 80.4 & 99.6 \\
\hline & & & MPGGE Adjusted & 42.3 & 42.3 & 46.1 & 50.2 & 43.4 & 48.9 & 55.3 & 45.2 & 54.1 & 65.0 & 47.0 & 56.2 & 67.3 \\
\hline & \multirow{9}{*}{ HWFET } & \multirow{4}{*}{$C D$} & Wh/mile & 212.5 & 212.5 & 238.4 & 230.1 & 251.1 & 229.6 & 213.9 & 205.8 & 219.8 & 190.7 & 194.7 & 212.9 & 182.4 \\
\hline & & & Distance & 12.6 & 12.6 & 11.0 & 10.7 & 11.1 & 11.0 & 10.6 & 12.6 & 10.7 & 8.6 & 13.0 & 10.5 & 8.9 \\
\hline & & & MPGGE Unadjusted & 130.9 & 130.9 & 263.2 & 347.7 & 221.1 & 282.1 & 391.1 & 138.6 & 381.4 & 438.7 & 140.0 & 401.9 & 487.6 \\
\hline & & & MPGGE Adjusted & 90.7 & 90.7 & 177.3 & 230.3 & 150.3 & 189.3 & 256.7 & 95.9 & 250.8 & 285.2 & 96.8 & 263.2 & 313.9 \\
\hline & & \multirow{2}{*}{ CS } & MPGGE Unadjusted & 43.3 & 43.3 & 47.5 & 52.3 & 44.2 & 50.7 & 57.7 & 46.0 & 56.3 & 67.5 & 48.5 & 59.1 & 71.2 \\
\hline & & & MPGGE Adjusted & 30.6 & 30.6 & 33.5 & 36.8 & 31.2 & 35.8 & 40.6 & 32.5 & 39.7 & 47.4 & 34.2 & 41.6 & 50.0 \\
\hline & & \multirow{3}{*}{$\mathrm{CD}+\mathrm{CS}$} & Wh/mile & 62.5 & 62.5 & 70.1 & 67.7 & 73.9 & 67.5 & 62.9 & 60.5 & 64.7 & 56.1 & 57.3 & 62.6 & 53.7 \\
\hline & & & MPGGE Unadjusted & 51.6 & 51.6 & 59.1 & 65.7 & 54.7 & 63.2 & 72.5 & 54.8 & 70.8 & 84.7 & 57.5 & 74.3 & 89.6 \\
\hline & & & MPGGE Adjusted & 36.4 & 36.4 & 41.6 & 46.2 & 38.5 & 44.4 & 50.9 & 38.6 & 49.7 & 59.3 & 40.5 & 52.1 & 62.6 \\
\hline & \multirow{7}{*}{ Combined } & \multirow{2}{*}{$C D$} & MPGGE Unadjusted & 220.5 & 220.5 & 414.9 & 527.3 & 366.2 & 436.8 & 599.8 & 255.7 & 582.6 & 810.5 & 256.6 & 616.3 & 894.7 \\
\hline & & & MPGGE Adjusted & 129.7 & 129.7 & 208.4 & 244.2 & 190.5 & 216.0 & 264.0 & 144.7 & 259.5 & 309.1 & 145.2 & 268.2 & 325.3 \\
\hline & & \multirow{2}{*}{ CS } & MPGGE Unadjusted & 46.4 & 46.4 & 50.8 & 56.0 & 47.3 & 54.4 & 62.2 & 49.5 & 60.6 & 73.7 & 51.9 & 63.5 & 77.3 \\
\hline & & & MPGGE Adjusted & 33.5 & 33.5 & 36.6 & 40.1 & 34.2 & 39.0 & 44.2 & 35.6 & 43.1 & 51.7 & 37.3 & 45.1 & 54.0 \\
\hline & & \multirow{3}{*}{$C D+C S$} & Wh/mile & 52.4 & 52.4 & 55.4 & 52.9 & 59.1 & 53.1 & 49.6 & 52.6 & 51.0 & 45.1 & 50.3 & 49.0 & 43.2 \\
\hline & & & MPGGE Unadjusted & 55.0 & 55.0 & 61.7 & 68.3 & 57.4 & 66.0 & 75.9 & 58.9 & 74.0 & 90.3 & 61.6 & 77.6 & 94.8 \\
\hline & & & MPGGE Adjusted & 39.4 & 39.4 & 44.0 & 48.3 & 41.0 & 46.8 & 53.2 & 42.0 & 52.0 & 62.3 & 43.8 & 54.3 & 65.1 \\
\hline
\end{tabular}


Argonne National Laboratory Report - Light-Duty Vehicle Fuel Consumption Displacement Potential Up to 2045

\begin{tabular}{|c|c|c|c|c|c|c|c|c|c|c|c|c|c|c|c|c|}
\hline & & & & \multicolumn{13}{|c|}{ Small_SUV } \\
\hline & & & & 2010 & & 2010 & & & 2015 & & & 2030 & & & 2045 & \\
\hline \multirow{26}{*}{ CI Split HEV PHEV2O } & & \multirow{5}{*}{$C D$} & & Ref & low & avg & high & low & avg & high & low & avg & high & low & avg & high \\
\hline & \multirow{9}{*}{ UDDS } & & Wh/mile & 234.7 & 234.7 & 220.6 & 203.5 & 236.7 & 209.8 & 183.9 & 232.4 & 188.8 & 169.8 & 227.0 & 184.0 & 162.9 \\
\hline & & & Distance & 24.0 & 24.0 & 23.9 & 23.9 & 23.9 & 24.0 & 23.9 & 23.9 & 24.0 & 22.3 & 23.9 & 23.8 & 22.3 \\
\hline & & & MPGGE Unadjusted & 778.3 & 778.3 & 1048.8 & 1088.1 & 1005.9 & 1044.2 & 1040.7 & 1049.4 & 1003.5 & 2613.2 & 1072.3 & 1073.2 & 2705.0 \\
\hline & & & MPGGE Adjusted & 242.5 & 242.5 & 269.0 & 272.1 & 265.4 & 268.6 & 268.3 & 269.0 & 265.2 & 331.3 & 270.8 & 270.9 & 333.1 \\
\hline & & \multirow{2}{*}{ CS } & MPGGE Unadjusted & 48.8 & 48.8 & 53.5 & 59.0 & 49.9 & 57.2 & 66.2 & 52.4 & 64.3 & 79.7 & 54.7 & 67.6 & 83.1 \\
\hline & & & MPGGE Adjusted & 36.1 & 36.1 & 39.2 & 42.9 & 36.9 & 41.7 & 47.4 & 38.5 & 46.3 & 55.8 & 40.1 & 48.3 & 57.8 \\
\hline & & \multirow{3}{*}{$\mathrm{CD}+\mathrm{CS}$} & Wh/mile & 123.5 & 123.5 & 115.7 & 106.9 & 124.2 & 110.1 & 97.2 & 121.9 & 99.8 & 89.7 & 119.0 & 97.3 & 86.1 \\
\hline & & & MPGGE Unadjusted & 83.1 & 83.1 & 91.7 & 101.0 & 85.7 & 98.0 & 112.7 & 89.9 & 109.5 & 139.1 & 93.9 & 115.1 & 145.0 \\
\hline & & & MPGGE Adjusted & 57.8 & 57.8 & 62.8 & 68.1 & 59.3 & 66.4 & 74.5 & 61.8 & 72.8 & 88.0 & 64.1 & 75.8 & 90.9 \\
\hline & \multirow{9}{*}{ HWFET } & \multirow{4}{*}{$C D$} & Wh/mile & 240.9 & 240.9 & 250.4 & 230.7 & 261.6 & 240.4 & 213.2 & 250.4 & 219.0 & 229.2 & 254.0 & 213.8 & 217.3 \\
\hline & & & Distance & 23.2 & 23.2 & 19.4 & 21.2 & 19.6 & 20.3 & 21.0 & 19.9 & 21.0 & 16.4 & 21.3 & 20.3 & 16.5 \\
\hline & & & MPGGE Unadjusted & 169.5 & 169.5 & 322.8 & 332.0 & 252.5 & 341.9 & 363.1 & 228.3 & 354.0 & 65207.3 & 291.2 & 398.2 & 65207.3 \\
\hline & & & MPGGE Adjusted & 116.5 & 116.5 & 214.9 & 220.6 & 170.5 & 226.7 & 239.7 & 155.0 & 234.1 & 3034.8 & 195.1 & 261.0 & 3034.8 \\
\hline & & \multirow{2}{*}{ CS } & MPGGE Unadjusted & 42.9 & 42.9 & 47.1 & 52.0 & 43.9 & 50.5 & 57.5 & 45.7 & 56.0 & 67.4 & 48.2 & 58.9 & 71.1 \\
\hline & & & MPGGE Adjusted & 30.3 & 30.3 & 33.2 & 36.7 & 31.0 & 35.6 & 40.5 & 32.3 & 39.4 & 47.4 & 34.0 & 41.5 & 49.9 \\
\hline & & \multirow{3}{*}{$\mathrm{CD}+\mathrm{CS}$} & Wh/mile & 122.0 & 122.0 & 73.6 & 118.8 & 76.9 & 70.7 & 109.8 & 73.6 & 112.9 & 67.4 & 130.4 & 62.9 & 63.9 \\
\hline & & & MPGGE Unadjusted & 61.2 & 61.2 & 59.2 & 79.4 & 54.7 & 63.4 & 87.7 & 56.6 & 85.4 & 88.7 & 73.1 & 74.1 & 93.6 \\
\hline & & & MPGGE Adjusted & 43.1 & 43.1 & 41.7 & 55.6 & 38.5 & 44.6 & 61.3 & 39.8 & 59.7 & 62.0 & 51.2 & 52.0 & 65.4 \\
\hline & \multirow{7}{*}{ Combined } & \multirow{2}{*}{$C D$} & MPGGE Unadjusted & 297.5 & 297.5 & 521.3 & 537.4 & 429.3 & 542.7 & 565.7 & 400.8 & 549.7 & 4600.5 & 485.9 & 608.9 & 4756.7 \\
\hline & & & MPGGE Adjusted & 163.1 & 163.1 & 241.6 & 246.2 & 212.2 & 248.0 & 254.6 & 202.1 & 250.3 & 553.0 & 230.6 & 266.4 & 555.7 \\
\hline & & \multirow{2}{*}{ CS } & MPGGE Unadjusted & 46.0 & 46.0 & 50.4 & 55.7 & 47.0 & 54.0 & 62.0 & 49.2 & 60.3 & 73.7 & 51.6 & 63.4 & 77.2 \\
\hline & & & MPGGE Adjusted & 33.3 & 33.3 & 36.3 & 39.8 & 34.0 & 38.7 & 44.0 & 35.4 & 42.9 & 51.7 & 37.1 & 45.0 & 54.0 \\
\hline & & \multirow{3}{*}{$\mathrm{CD}+\mathrm{CS}$} & Wh/mile & 122.8 & 122.8 & 96.8 & 112.3 & 102.9 & 92.4 & 102.9 & 100.2 & 105.7 & 79.7 & 124.2 & 81.8 & 76.1 \\
\hline & & & MPGGE Unadjusted & 71.6 & 71.6 & 73.6 & 90.0 & 68.3 & 78.7 & 99.9 & 71.1 & 97.2 & 110.8 & 83.2 & 92.2 & 116.2 \\
\hline & & & MPGGE Adjusted & 50.1 & 50.1 & 51.2 & 61.9 & 47.7 & 54.4 & 67.9 & 49.5 & 66.3 & 74.1 & 57.6 & 62.8 & 77.3 \\
\hline
\end{tabular}


Argonne National Laboratory Report - Light-Duty Vehicle Fuel Consumption Displacement Potential Up to 2045

\begin{tabular}{|c|c|c|c|c|c|c|c|c|c|c|c|c|c|c|c|c|}
\hline & & & & \multicolumn{13}{|c|}{ Small_SUV } \\
\hline & & & & \multirow{2}{*}{$\begin{array}{l}2010 \\
\text { Ref }\end{array}$} & \multicolumn{3}{|c|}{2010} & \multicolumn{3}{|c|}{2015} & \multicolumn{3}{|c|}{2030} & \multicolumn{3}{|c|}{2045} \\
\hline & & & & & low & avg & high & low & avg & high & low & avg & high & low & avg & high \\
\hline \multirow{25}{*}{ CI Series HEV PHEV 30} & \multirow{9}{*}{ UDDS } & \multirow{4}{*}{$C D$} & Wh/mile & 331.5 & 331.5 & 302.8 & 281.7 & 322.0 & 290.2 & 256.0 & 315.3 & 263.5 & 222.5 & 307.9 & 255.0 & 213.4 \\
\hline & & & Distance & 31.4 & 31.4 & 30.9 & 31.0 & 31.9 & 30.9 & 30.9 & 31.4 & 30.9 & 30.9 & 31.0 & 30.9 & 31.4 \\
\hline & & & MPGGE Unadjusted & $\mathrm{N} / \mathrm{A}$ & $\mathrm{N} / \mathrm{A}$ & N/A & N/A & N/A & $\mathrm{N} / \mathrm{A}$ & N/A & $\mathrm{N} / \mathrm{A}$ & N/A & N/A & N/A & N/A & $\mathrm{N} / \mathrm{A}$ \\
\hline & & & MPGGE Adjusted & $\mathrm{N} / \mathrm{A}$ & $\mathrm{N} / \mathrm{A}$ & $\mathrm{N} / \mathrm{A}$ & N/A & N/A & N/A & N/A & $\mathrm{N} / \mathrm{A}$ & N/A & N/A & N/A & $\mathrm{N} / \mathrm{A}$ & $\mathrm{N} / \mathrm{A}$ \\
\hline & & \multirow{2}{*}{ CS } & MPGGE Unadjusted & 35.4 & 35.4 & 39.9 & 44.6 & 36.5 & 43.2 & 52.1 & 39.1 & 50.4 & 65.1 & 41.1 & 52.7 & 67.8 \\
\hline & & & MPGGE Adjusted & 26.9 & 26.9 & 30.1 & 33.3 & 27.7 & 32.4 & 38.4 & 29.5 & 37.2 & 46.7 & 30.9 & 38.7 & 48.5 \\
\hline & & \multirow{3}{*}{$\mathrm{CD}+\mathrm{CS}$} & Wh/mile & 213.3 & 213.3 & 195.0 & 181.4 & 207.2 & 186.9 & 165.4 & 202.9 & 170.2 & 144.3 & 198.2 & 164.7 & 138.4 \\
\hline & & & MPGGE Unadjusted & 75.3 & 75.3 & 84.9 & 94.9 & 77.7 & 92.0 & 110.9 & 83.3 & 107.3 & 138.4 & 87.4 & 112.1 & 144.4 \\
\hline & & & MPGGE Adjusted & 53.1 & 53.1 & 58.9 & 64.6 & 54.6 & 63.0 & 73.5 & 57.9 & 71.6 & 87.7 & 60.3 & 74.2 & 90.6 \\
\hline & \multirow{9}{*}{ HWFET } & \multirow{4}{*}{$C D$} & Wh/mile & 355.0 & 355.0 & 321.3 & 297.9 & 346.1 & 308.3 & 272.3 & 341.5 & 280.2 & 240.1 & 329.6 & 270.8 & 227.5 \\
\hline & & & \begin{tabular}{|l} 
Distance \\
\end{tabular} & 29.4 & 29.4 & 29.3 & 29.2 & 29.2 & 29.3 & 28.9 & 28.6 & 28.9 & 28.5 & 29.2 & 28.9 & 28.8 \\
\hline & & & MPGGE Unadjusted & $\mathrm{N} / \mathrm{A}$ & $\mathrm{N} / \mathrm{A}$ & $\mathrm{N} / \mathrm{A}$ & $\mathrm{N} / \mathrm{A}$ & $\mathrm{N} / \mathrm{A}$ & $\mathrm{N} / \mathrm{A}$ & $\mathrm{N} / \mathrm{A}$ & $\mathrm{N} / \mathrm{A}$ & $\mathrm{N} / \mathrm{A}$ & $\mathrm{N} / \mathrm{A}$ & $\mathrm{N} / \mathrm{A}$ & $\mathrm{N} / \mathrm{A}$ & $\mathrm{N} / \mathrm{A}$ \\
\hline & & & MPGGE Adjusted & N/A & N/A & N/A & N/A & N/A & N/A & N/A & N/A & N/A & N/A & N/A & N/A & $\mathrm{N} / \mathrm{A}$ \\
\hline & & \multirow{2}{*}{ CS } & MPGGE Unadjusted & 32.5 & 32.5 & 36.3 & 40.7 & 33.3 & 39.3 & 46.3 & 35.4 & 45.1 & 55.8 & 37.5 & 47.7 & 59.2 \\
\hline & & & MPGGE Adjusted & 23.0 & 23.0 & 25.7 & 28.7 & 23.6 & 27.8 & 32.7 & 25.0 & 31.8 & 39.3 & 26.5 & 33.6 & 41.7 \\
\hline & & \multirow{3}{*}{$\mathrm{CD}+\mathrm{CS}$} & Wh/mile & 182.9 & 182.9 & 165.6 & 153.6 & 178.4 & 158.9 & 140.4 & 176.0 & 144.4 & 123.7 & 169.9 & 139.6 & 117.3 \\
\hline & & & MPGGE Unadjusted & 55.1 & 55.1 & 61.6 & 68.9 & 56.5 & 66.6 & 78.5 & 60.0 & 76.4 & 94.7 & 63.6 & 80.8 & 100.3 \\
\hline & & & MPGGE Adjusted & 38.8 & 38.8 & 43.3 & 48.4 & 39.8 & 46.8 & 55.0 & 42.2 & 53.5 & 66.1 & 44.7 & 56.6 & 70.0 \\
\hline & \multirow{7}{*}{ Combined } & \multirow{2}{*}{$C D$} & MPGGE Unadjusted & $\mathrm{N} / \mathrm{A}$ & $\mathrm{N} / \mathrm{A}$ & N/A & N/A & N/A & $\mathrm{N} / \mathrm{A}$ & N/A & $\mathrm{N} / \mathrm{A}$ & $\mathrm{N} / \mathrm{A}$ & N/A & $\mathrm{N} / \mathrm{A}$ & N/A & $\mathrm{N} / \mathrm{A}$ \\
\hline & & & MPGGE Adjusted & N/A & N/A & N/A & N/A & N/A & N/A & N/A & N/A & N/A & N/A & N/A & N/A & N/A \\
\hline & & \multirow{2}{*}{ CS } & MPGGE Unadjusted & 34.0 & 34.0 & 38.2 & 42.7 & 35.0 & 41.4 & 49.3 & 37.4 & 47.9 & 60.6 & 39.4 & 50.3 & 63.7 \\
\hline & & & MPGGE Adjusted & 25.0 & 25.0 & 27.9 & 31.1 & 25.7 & 30.1 & 35.6 & 27.3 & 34.6 & 43.1 & 28.8 & 36.3 & 45.2 \\
\hline & & \multirow{3}{*}{$C D+C S$} & Wh/mile & 199.6 & 199.6 & 181.8 & 168.9 & 194.3 & 174.3 & 154.1 & 190.8 & 158.6 & 135.0 & 185.5 & 153.4 & 128.9 \\
\hline & & & MPGGE Unadjusted & 64.6 & 64.6 & 72.5 & 81.1 & 66.5 & 78.5 & 93.5 & 70.9 & 90.8 & 114.6 & 74.8 & 95.4 & 120.6 \\
\hline & & & MPGGE Adjusted & 45.6 & 45.6 & 50.7 & 56.2 & 46.8 & 54.5 & 63.8 & 49.6 & 62.1 & 76.4 & 52.1 & 65.1 & 80.0 \\
\hline
\end{tabular}


Argonne National Laboratory Report - Light-Duty Vehicle Fuel Consumption Displacement Potential Up to 2045

\begin{tabular}{|c|c|c|c|c|c|c|c|c|c|c|c|c|c|c|c|c|}
\hline & & & & \multicolumn{13}{|c|}{ Small_SUV } \\
\hline & & & & 2010 & & 2010 & & & 2015 & & & 2030 & & & 2045 & \\
\hline \multirow{26}{*}{ CI Series HEV PHEV40 } & & \multirow{5}{*}{$C D$} & & Ref & low & avg & high & low & avg & high & low & avg & high & low & avg & high \\
\hline & \multirow{9}{*}{ UDDS } & & Wh/mile & 338.6 & 338.6 & 308.9 & 287.2 & 328.8 & 296.1 & 261.3 & 321.3 & 268.9 & 224.8 & 314.0 & 259.1 & 215.4 \\
\hline & & & Distance & 40.8 & 40.8 & 40.8 & 40.8 & 40.8 & 40.8 & 40.7 & 40.6 & 40.8 & 40.6 & 40.8 & 40.4 & 40.6 \\
\hline & & & MPGGE Unadjusted & N/A & N/A & N/A & N/A & N/A & N/A & N/A & N/A & N/A & N/A & N/A & N/A & N/A \\
\hline & & & MPGGE Adjusted & N/A & N/A & N/A & N/A & N/A & N/A & N/A & N/A & N/A & N/A & N/A & N/A & $\mathrm{N} / \mathrm{A}$ \\
\hline & & \multirow{2}{*}{ CS } & MPGGE Unadjusted & 34.5 & 34.5 & 38.6 & 43.7 & 35.6 & 42.4 & 51.3 & 38.2 & 49.5 & 64.8 & 40.3 & 52.3 & 67.3 \\
\hline & & & MPGGE Adjusted & 26.2 & 26.2 & 29.1 & 32.7 & 27.1 & 31.8 & 37.8 & 28.9 & 36.6 & 46.6 & 30.3 & 38.5 & 48.2 \\
\hline & & \multirow{3}{*}{$C D+C S$} & Wh/mile & 248.5 & 248.5 & 226.9 & 211.1 & 241.3 & 217.6 & 193.2 & 235.9 & 198.8 & 166.4 & 230.6 & 191.3 & 159.4 \\
\hline & & & MPGGE Unadjusted & 86.2 & 86.2 & 96.5 & 109.3 & 89.1 & 105.9 & 128.2 & 95.6 & 123.6 & 162.0 & 100.6 & 130.9 & 168.2 \\
\hline & & & MPGGE Adjusted & 59.6 & 59.6 & 65.5 & 72.6 & 61.3 & 70.8 & 82.6 & 65.1 & 80.2 & 98.9 & 67.9 & 83.9 & 101.7 \\
\hline & \multirow{9}{*}{ HWFET } & \multirow{4}{*}{$C D$} & Wh/mile & 358.2 & 358.2 & 323.9 & 300.0 & 349.1 & 310.7 & 273.8 & 344.0 & 281.7 & 241.6 & 332.1 & 271.7 & 228.8 \\
\hline & & & Distance & 38.1 & 38.1 & 38.7 & 38.8 & 38.0 & 38.7 & 38.7 & 37.7 & 38.7 & 37.7 & 38.3 & 38.7 & 38.1 \\
\hline & & & MPGGE Unadjusted & N/A & N/A & N/A & N/A & N/A & N/A & N/A & N/A & N/A & N/A & N/A & N/A & $\mathrm{N} / \mathrm{A}$ \\
\hline & & & MPGGE Adjusted & $\mathrm{N} / \mathrm{A}$ & N/A & N/A & N/A & N/A & N/A & N/A & N/A & N/A & $\mathrm{N} / \mathrm{A}$ & $\mathrm{N} / \mathrm{A}$ & N/A & N/A \\
\hline & & \multirow{2}{*}{ CS } & MPGGE Unadjusted & 32.0 & 32.0 & 35.7 & 40.1 & 32.9 & 38.7 & 45.8 & 34.9 & 44.6 & 55.4 & 37.0 & 47.3 & 58.8 \\
\hline & & & MPGGE Adjusted & 22.7 & 22.7 & 25.3 & 28.4 & 23.3 & 27.3 & 32.4 & 24.7 & 31.5 & 39.0 & 26.2 & 33.3 & 41.4 \\
\hline & & \multirow{3}{*}{$C D+C S$} & Wh/mile & 241.9 & 241.9 & 218.7 & 202.7 & 235.7 & 209.8 & 185.0 & 232.3 & 190.3 & 163.3 & 224.3 & 183.5 & 154.7 \\
\hline & & & MPGGE Unadjusted & 69.6 & 69.6 & 77.7 & 87.2 & 71.4 & 84.1 & 99.7 & 75.9 & 96.9 & 120.4 & 80.5 & 102.8 & 127.7 \\
\hline & & & MPGGE Adjusted & 48.9 & 48.9 & 54.5 & 61.0 & 50.1 & 58.8 & 69.5 & 53.2 & 67.6 & 83.6 & 56.4 & 71.6 & 88.6 \\
\hline & \multirow{7}{*}{ Combined } & \multirow{2}{*}{$C D$} & MPGGE Unadjusted & N/A & N/A & N/A & N/A & N/A & N/A & N/A & N/A & N/A & N/A & N/A & $\mathrm{N} / \mathrm{A}$ & N/A \\
\hline & & & MPGGE Adjusted & N/A & N/A & N/A & N/A & N/A & N/A & N/A & N/A & N/A & N/A & N/A & N/A & N/A \\
\hline & & \multirow{2}{*}{ CS } & MPGGE Unadjusted & 33.3 & 33.3 & 37.3 & 42.0 & 34.3 & 40.6 & 48.7 & 36.7 & 47.1 & 60.2 & 38.7 & 49.9 & 63.2 \\
\hline & & & MPGGE Adjusted & 24.5 & 24.5 & 27.3 & 30.6 & 25.2 & 29.6 & 35.1 & 26.8 & 34.1 & 42.8 & 28.3 & 36.0 & 44.8 \\
\hline & & \multirow{3}{*}{$C D+C S$} & Wh/mile & 245.5 & 245.5 & 223.2 & 207.3 & 238.8 & 214.1 & 189.5 & 234.3 & 195.0 & 165.0 & 227.7 & 187.8 & 157.3 \\
\hline & & & MPGGE Unadjusted & 77.9 & 77.9 & 87.0 & 98.1 & 80.2 & 94.8 & 113.6 & 85.6 & 110.0 & 140.2 & 90.4 & 116.5 & 147.2 \\
\hline & & & \begin{tabular}{|l} 
MPGGE Adjusted \\
\end{tabular} & 54.3 & 54.3 & 60.0 & 66.9 & 55.7 & 64.9 & 76.1 & 59.1 & 74.0 & 91.3 & 62.2 & 77.9 & 95.3 \\
\hline
\end{tabular}


Argonne National Laboratory Report - Light-Duty Vehicle Fuel Consumption Displacement Potential Up to 2045

\begin{tabular}{|c|c|c|c|c|c|c|c|c|c|c|c|c|c|c|c|c|}
\hline & & & & \multicolumn{13}{|c|}{ Small_SUV } \\
\hline & & & & \multirow{2}{*}{$\begin{array}{l}2010 \\
\text { Ref }\end{array}$} & \multicolumn{3}{|c|}{2010} & \multicolumn{3}{|c|}{2015} & \multicolumn{3}{|c|}{2030} & \multicolumn{3}{|c|}{2045} \\
\hline & & & & & low & avg & high & low & avg & high & low & avg & high & low & avg & high \\
\hline \multirow{25}{*}{ H2 Split HEV PHEV10 } & \multirow{9}{*}{ UDDS } & \multirow{4}{*}{$C D$} & Wh/mile & 225.3 & 225.3 & 202.3 & 187.5 & 217.2 & 193.3 & 184.5 & 215.6 & 183.3 & 162.9 & 207.2 & 181.4 & 166.5 \\
\hline & & & Distance & 12.2 & 12.2 & 12.3 & 12.2 & 12.5 & 12.3 & 11.6 & 12.1 & 12.1 & 11.3 & 12.3 & 11.6 & 10.2 \\
\hline & & & MPGGE Unadjusted & 748.1 & 748.1 & 910.1 & 1087.7 & 797.5 & 1042.9 & 1875.6 & 949.1 & 1271.6 & 3302.1 & 1002.3 & 2090.2 & $\mathrm{~N} / \mathrm{A}$ \\
\hline & & & MPGGE Adjusted & 238.8 & 238.8 & 256.7 & 272.0 & 244.7 & 268.5 & 312.2 & 260.4 & 284.7 & 342.4 & 265.1 & 318.9 & $\mathrm{~N} / \mathrm{A}$ \\
\hline & & \multirow{2}{*}{ CS } & MPGGE Unadjusted & 46.4 & 46.4 & 57.0 & 65.9 & 52.7 & 63.7 & 75.2 & 57.4 & 72.8 & 91.4 & 62.2 & 77.9 & 99.2 \\
\hline & & & MPGGE Adjusted & 34.5 & 34.5 & 41.6 & 47.3 & 38.8 & 45.9 & 53.0 & 41.8 & 51.6 & 62.6 & 44.9 & 54.7 & 67.1 \\
\hline & & \multirow{3}{*}{$\mathrm{CD}+\mathrm{CS}$} & Wh/mile & 46.9 & 46.9 & 42.2 & 39.1 & 45.3 & 40.3 & 38.4 & 44.9 & 38.2 & 33.9 & 43.2 & 37.8 & 34.7 \\
\hline & & & MPGGE Unadjusted & 55.2 & 55.2 & 67.8 & 78.4 & 62.7 & 75.8 & 89.8 & 68.3 & 86.7 & 109.4 & 74.0 & 93.1 & 119.6 \\
\hline & & & MPGGE Adjusted & 40.4 & 40.4 & 48.5 & 55.0 & 45.2 & 53.4 & 61.7 & 48.8 & 59.9 & 72.7 & 52.3 & 63.6 & 78.1 \\
\hline & \multirow{9}{*}{ HWFET } & \multirow{4}{*}{$C D$} & Wh/mile & 211.2 & 211.2 & 228.1 & 218.2 & 202.3 & 225.9 & 207.1 & 203.9 & 213.5 & 179.3 & 192.4 & 205.3 & 171.8 \\
\hline & & & Distance & 12.4 & 12.4 & 11.0 & 10.6 & 12.5 & 10.6 & 9.8 & 12.3 & 10.0 & 8.5 & 12.5 & 9.7 & 8.5 \\
\hline & & & MPGGE Unadjusted & 127.2 & 127.2 & 253.8 & 335.3 & 138.1 & 322.7 & 419.3 & 155.1 & 404.5 & 431.1 & 163.5 & 428.0 & 494.8 \\
\hline & & & MPGGE Adjusted & 88.2 & 88.2 & 171.3 & 222.6 & 95.5 & 214.8 & 273.6 & 106.9 & 264.8 & 280.7 & 112.5 & 278.8 & 318.1 \\
\hline & & \multirow{2}{*}{ CS } & MPGGE Unadjusted & 41.5 & 41.5 & 50.6 & 58.4 & 46.9 & 56.5 & 65.8 & 50.7 & 63.9 & 77.3 & 55.2 & 68.6 & 84.6 \\
\hline & & & MPGGE Adjusted & 29.3 & 29.3 & 35.7 & 41.1 & 33.1 & 39.8 & 46.2 & 35.8 & 44.9 & 54.2 & 38.9 & 48.2 & 59.2 \\
\hline & & \multirow{3}{*}{$\mathrm{CD}+\mathrm{CS}$} & Wh/mile & 62.1 & 62.1 & 67.1 & 64.2 & 59.5 & 66.5 & 60.9 & 60.0 & 62.8 & 52.7 & 56.6 & 60.4 & 50.5 \\
\hline & & & MPGGE Unadjusted & 49.5 & 49.5 & 62.7 & 72.8 & 55.7 & 70.5 & 82.5 & 60.5 & 80.1 & 96.3 & 65.6 & 85.9 & 105.6 \\
\hline & & & MPGGE Adjusted & 34.9 & 34.9 & 44.1 & 51.1 & 39.2 & 49.5 & 57.7 & 42.6 & 56.1 & 67.2 & 46.1 & 60.1 & 73.6 \\
\hline & \multirow{7}{*}{ Combined } & \multirow{2}{*}{$C D$} & MPGGE Unadjusted & 234.1 & 234.1 & 420.6 & 541.2 & 253.3 & 520.3 & 731.7 & 287.2 & 647.3 & 826.2 & 302.9 & 760.7 & 1089.4 \\
\hline & & & MPGGE Adjusted & 135.1 & 135.1 & 209.7 & 247.3 & 143.7 & 241.3 & 293.6 & 158.2 & 275.4 & 311.6 & 164.6 & 299.6 & 353.7 \\
\hline & & \multirow{2}{*}{ CS } & MPGGE Unadjusted & 44.1 & 44.1 & 54.0 & 62.3 & 49.9 & 60.2 & 70.6 & 54.2 & 68.5 & 84.4 & 58.8 & 73.4 & 92.1 \\
\hline & & & MPGGE Adjusted & 32.0 & 32.0 & 38.7 & 44.3 & 36.0 & 42.9 & 49.7 & 38.9 & 48.3 & 58.5 & 42.0 & 51.5 & 63.3 \\
\hline & & \multirow{3}{*}{$C D+C S$} & Wh/mile & 53.8 & 53.8 & 53.4 & 50.4 & 51.7 & 52.1 & 48.6 & 51.7 & 49.3 & 42.4 & 49.2 & 48.0 & 41.8 \\
\hline & & & MPGGE Unadjusted & 52.5 & 52.5 & 65.4 & 75.8 & 59.3 & 73.3 & 86.3 & 64.6 & 83.6 & 103.1 & 70.0 & 89.7 & 112.9 \\
\hline & & & MPGGE Adjusted & 37.7 & 37.7 & 46.4 & 53.2 & 42.3 & 51.5 & 59.9 & 45.8 & 58.1 & 70.1 & 49.3 & 62.0 & 76.0 \\
\hline
\end{tabular}


Argonne National Laboratory Report - Light-Duty Vehicle Fuel Consumption Displacement Potential Up to 2045

\begin{tabular}{|c|c|c|c|c|c|c|c|c|c|c|c|c|c|c|c|c|}
\hline & & & & \multicolumn{13}{|c|}{ Small_SUV } \\
\hline & & & & 2010 & & 2010 & & & 2015 & & & 2030 & & & 2045 & \\
\hline \multirow{26}{*}{ H2 Split HEV PHEV2O } & & \multirow{5}{*}{$C D$} & & Ref & low & avg & high & low & avg & high & low & avg & high & low & avg & high \\
\hline & \multirow{9}{*}{ UDDS } & & Wh/mile & 228.3 & 228.3 & 206.1 & 189.5 & 229.8 & 195.6 & 182.6 & 225.0 & 181.2 & 160.3 & 214.9 & 183.1 & 164.5 \\
\hline & & & Distance & 24.0 & 24.0 & 24.0 & 24.0 & 23.9 & 24.0 & 22.8 & 23.9 & 23.8 & 22.3 & 23.9 & 22.8 & 20.4 \\
\hline & & & MPGGE Unadjusted & 729.0 & 729.0 & 921.5 & 1060.7 & 1057.1 & 1015.2 & 1834.5 & 1145.0 & 1243.1 & 3238.0 & 1165.4 & 1920.3 & N/A \\
\hline & & & \begin{tabular}{|l|} 
MPGGE Adjusted \\
\end{tabular} & 236.4 & 236.4 & 257.8 & 269.9 & 269.6 & 266.2 & 310.8 & 276.3 & 282.9 & 341.6 & 277.7 & 313.7 & $\mathrm{~N} / \mathrm{A}$ \\
\hline & & \multirow{2}{*}{ CS } & MPGGE Unadjusted & 46.1 & 46.1 & 56.4 & 65.5 & 52.5 & 63.5 & 75.2 & 57.2 & 72.8 & 91.3 & 61.7 & 77.9 & 99.0 \\
\hline & & & MPGGE Adjusted & 34.3 & 34.3 & 41.2 & 47.0 & 38.6 & 45.7 & 53.1 & 41.7 & 51.6 & 62.6 & 44.6 & 54.7 & 67.0 \\
\hline & & \multirow{3}{*}{$C D+C S$} & Wh/mile & 120.2 & 120.2 & 108.5 & 99.8 & 120.5 & 103.1 & 96.5 & 118.0 & 95.8 & 61.0 & 112.9 & 96.7 & 62.6 \\
\hline & & & MPGGE Unadjusted & 78.5 & 78.5 & 96.2 & 111.7 & 90.2 & 108.1 & 130.2 & 98.2 & 124.4 & 132.5 & 105.8 & 135.0 & 145.5 \\
\hline & & & MPGGE Adjusted & 55.1 & 55.1 & 65.4 & 73.9 & 61.9 & 72.0 & 83.6 & 66.5 & 80.6 & 84.7 & 70.8 & 86.0 & 91.1 \\
\hline & \multirow{9}{*}{ HWFET } & \multirow{4}{*}{$C D$} & Wh/mile & 211.2 & 211.2 & 237.3 & 219.1 & 240.1 & 226.7 & 218.9 & 237.1 & 216.7 & 222.4 & 226.8 & 218.2 & 211.0 \\
\hline & & & Distance & 24.9 & 24.9 & 21.2 & 21.2 & 22.0 & 21.2 & 19.4 & 21.9 & 19.5 & 15.9 & 22.0 & 19.2 & 16.0 \\
\hline & & & MPGGE Unadjusted & 123.5 & 123.5 & 281.1 & 322.7 & 199.9 & 309.4 & 584.6 & 215.0 & 442.8 & N/A & 233.7 & 612.4 & $\mathrm{~N} / \mathrm{A}$ \\
\hline & & & MPGGE Adjusted & 85.7 & 85.7 & 188.7 & 214.8 & 136.5 & 206.5 & 369.3 & 146.4 & 287.7 & $\mathrm{~N} / \mathrm{A}$ & 158.5 & 384.7 & N/A \\
\hline & & \multirow{2}{*}{ CS } & MPGGE Unadjusted & 41.2 & 41.2 & 50.3 & 58.0 & 46.5 & 56.1 & 65.5 & 50.4 & 63.5 & 77.1 & 54.8 & 68.3 & 84.4 \\
\hline & & & MPGGE Adjusted & 29.1 & 29.1 & 35.4 & 40.8 & 32.8 & 39.5 & 46.0 & 35.5 & 44.7 & 54.0 & 38.6 & 48.0 & 59.0 \\
\hline & & \multirow{3}{*}{$C D+C S$} & Wh/mile & 108.5 & 108.5 & 122.1 & 112.8 & 121.3 & 116.7 & 64.4 & 119.7 & 63.7 & 65.4 & 114.5 & 64.2 & 62.1 \\
\hline & & & MPGGE Unadjusted & 56.4 & 56.4 & 75.6 & 87.2 & 67.1 & 84.2 & 83.3 & 72.6 & 80.0 & 101.4 & 78.9 & 86.8 & 111.0 \\
\hline & & & MPGGE Adjusted & 39.7 & 39.7 & 53.0 & 61.0 & 47.1 & 58.9 & 58.3 & 50.9 & 56.0 & 70.7 & 55.3 & 60.7 & 77.2 \\
\hline & \multirow{7}{*}{ Combined } & \multirow{2}{*}{$C D$} & MPGGE Unadjusted & 227.4 & 227.4 & 455.1 & 522.8 & 360.8 & 501.0 & 935.0 & 388.7 & 685.6 & 5658.4 & 417.2 & 979.2 & $\mathrm{~N} / \mathrm{A}$ \\
\hline & & & MPGGE Adjusted & 132.0 & 132.0 & 221.3 & 242.0 & 187.4 & 235.6 & 334.7 & 197.4 & 285.0 & 568.7 & 207.5 & 342.1 & 640.8 \\
\hline & & \multirow{2}{*}{ CS } & MPGGE Unadjusted & 43.8 & 43.8 & 53.5 & 61.9 & 49.6 & 59.9 & 70.5 & 53.9 & 68.3 & 84.3 & 58.4 & 73.3 & 91.8 \\
\hline & & & MPGGE Adjusted & 31.8 & 31.8 & 38.4 & 44.0 & 35.8 & 42.7 & 49.6 & 38.7 & 48.2 & 58.4 & 41.7 & 51.5 & 63.1 \\
\hline & & \multirow{3}{*}{$\mathrm{CD}+\mathrm{CS}$} & Wh/mile & 115.0 & 115.0 & 114.6 & 105.6 & 120.9 & 109.2 & 82.0 & 118.8 & 81.4 & 63.0 & 113.6 & 82.1 & 62.4 \\
\hline & & & MPGGE Unadjusted & 66.7 & 66.7 & 85.7 & 99.2 & 78.1 & 95.9 & 103.8 & 84.7 & 99.5 & 116.5 & 91.8 & 108.0 & 127.7 \\
\hline & & & MPGGE Adjusted & 46.9 & 46.9 & 59.2 & 67.5 & 54.3 & 65.5 & 69.9 & 58.4 & 67.3 & 77.8 & 62.9 & 72.4 & 84.3 \\
\hline
\end{tabular}


Argonne National Laboratory Report - Light-Duty Vehicle Fuel Consumption Displacement Potential Up to 2045

\begin{tabular}{|c|c|c|c|c|c|c|c|c|c|c|c|c|c|c|c|c|}
\hline & & & & \multicolumn{13}{|c|}{ Small_SUV } \\
\hline & & & & \multirow{2}{*}{$\begin{array}{l}2010 \\
\text { Ref }\end{array}$} & \multicolumn{3}{|c|}{2010} & \multicolumn{3}{|c|}{2015} & \multicolumn{3}{|c|}{2030} & \multicolumn{3}{|c|}{2045} \\
\hline & & & & & low & avg & high & low & avg & high & low & avg & high & low & avg & high \\
\hline \multirow{25}{*}{ H2 Series HEV PHEV 30} & \multirow{9}{*}{ UDDS } & \multirow{4}{*}{$C D$} & Wh/mile & 325.2 & 325.2 & 291.9 & 269.0 & 314.4 & 277.7 & 242.3 & 306.6 & 250.9 & 208.4 & 294.8 & 242.0 & 200.5 \\
\hline & & & Distance & 30.9 & 30.9 & 30.9 & 30.9 & 30.9 & 31.0 & 30.9 & 30.9 & 30.9 & 31.4 & 30.9 & 30.9 & 30.7 \\
\hline & & & MPGGE Unadjusted & $\mathrm{N} / \mathrm{A}$ & $\mathrm{N} / \mathrm{A}$ & N/A & N/A & N/A & $\mathrm{N} / \mathrm{A}$ & N/A & $\mathrm{N} / \mathrm{A}$ & N/A & N/A & N/A & N/A & $\mathrm{N} / \mathrm{A}$ \\
\hline & & & MPGGE Adjusted & $\mathrm{N} / \mathrm{A}$ & $\mathrm{N} / \mathrm{A}$ & $\mathrm{N} / \mathrm{A}$ & N/A & N/A & N/A & N/A & $\mathrm{N} / \mathrm{A}$ & N/A & N/A & N/A & $\mathrm{N} / \mathrm{A}$ & $\mathrm{N} / \mathrm{A}$ \\
\hline & & \multirow{2}{*}{ CS } & MPGGE Unadjusted & 33.6 & 33.6 & 42.3 & 49.9 & 38.5 & 48.0 & 59.1 & 42.9 & 57.1 & 73.6 & 47.2 & 61.0 & 79.7 \\
\hline & & & MPGGE Adjusted & 25.6 & 25.6 & 31.7 & 36.9 & 29.1 & 35.6 & 42.9 & 32.1 & 41.6 & 52.0 & 35.1 & 44.2 & 55.8 \\
\hline & & \multirow{3}{*}{$\mathrm{CD}+\mathrm{CS}$} & Wh/mile & 209.3 & 209.3 & 187.9 & 173.2 & 202.3 & 178.8 & 156.5 & 197.3 & 162.0 & 135.0 & 189.7 & 156.2 & 129.9 \\
\hline & & & MPGGE Unadjusted & 71.6 & 71.6 & 90.1 & 106.1 & 81.9 & 102.2 & 125.7 & 91.2 & 121.4 & 156.5 & 100.4 & 129.9 & 169.5 \\
\hline & & & MPGGE Adjusted & 50.8 & 50.8 & 61.9 & 70.9 & 57.1 & 68.8 & 81.3 & 62.5 & 79.1 & 96.3 & 67.8 & 83.4 & 102.2 \\
\hline & \multirow{9}{*}{ HWFET } & \multirow{4}{*}{$C D$} & Wh/mile & 350.8 & 350.8 & 314.1 & 289.6 & 341.0 & 300.1 & 263.6 & 335.7 & 272.0 & 231.5 & 320.9 & 262.6 & 219.7 \\
\hline & & & Distance & 28.7 & 28.7 & 28.7 & 28.7 & 28.5 & 28.7 & 28.3 & 28.1 & 28.5 & 27.5 & 28.4 & 28.3 & 27.8 \\
\hline & & & MPGGE Unadjusted & $\mathrm{N} / \mathrm{A}$ & $\mathrm{N} / \mathrm{A}$ & $\mathrm{N} / \mathrm{A}$ & $\mathrm{N} / \mathrm{A}$ & $\mathrm{N} / \mathrm{A}$ & $\mathrm{N} / \mathrm{A}$ & $\mathrm{N} / \mathrm{A}$ & $\mathrm{N} / \mathrm{A}$ & $\mathrm{N} / \mathrm{A}$ & $\mathrm{N} / \mathrm{A}$ & $\mathrm{N} / \mathrm{A}$ & $\mathrm{N} / \mathrm{A}$ & $\mathrm{N} / \mathrm{A}$ \\
\hline & & & MPGGE Adjusted & N/A & N/A & N/A & N/A & N/A & N/A & N/A & N/A & N/A & N/A & N/A & N/A & $\mathrm{N} / \mathrm{A}$ \\
\hline & & \multirow{2}{*}{ CS } & MPGGE Unadjusted & 31.2 & 31.2 & 38.9 & 45.5 & 35.5 & 43.9 & 53.0 & 39.1 & 51.2 & 63.5 & 42.9 & 55.4 & 69.6 \\
\hline & & & MPGGE Adjusted & 22.1 & 22.1 & 27.5 & 32.1 & 25.1 & 31.0 & 37.3 & 27.6 & 36.1 & 44.6 & 30.3 & 39.0 & 48.8 \\
\hline & & \multirow{3}{*}{$\mathrm{CD}+\mathrm{CS}$} & Wh/mile & 180.8 & 180.8 & 161.9 & 149.3 & 175.8 & 154.7 & 135.8 & 173.0 & 140.2 & 119.3 & 165.4 & 135.3 & 113.2 \\
\hline & & & MPGGE Unadjusted & 52.9 & 52.9 & 66.0 & 77.1 & 60.2 & 74.4 & 89.8 & 66.3 & 86.7 & 107.7 & 72.8 & 93.9 & 117.9 \\
\hline & & & MPGGE Adjusted & 37.3 & 37.3 & 46.3 & 54.1 & 42.4 & 52.2 & 62.7 & 46.6 & 60.6 & 75.0 & 51.0 & 65.6 & 81.9 \\
\hline & \multirow{7}{*}{ Combined } & \multirow{2}{*}{$C D$} & MPGGE Unadjusted & $\mathrm{N} / \mathrm{A}$ & $\mathrm{N} / \mathrm{A}$ & N/A & N/A & N/A & $\mathrm{N} / \mathrm{A}$ & N/A & $\mathrm{N} / \mathrm{A}$ & $\mathrm{N} / \mathrm{A}$ & N/A & $\mathrm{N} / \mathrm{A}$ & N/A & $\mathrm{N} / \mathrm{A}$ \\
\hline & & & MPGGE Adjusted & N/A & N/A & N/A & N/A & N/A & N/A & N/A & N/A & N/A & N/A & N/A & N/A & N/A \\
\hline & & \multirow{2}{*}{ CS } & MPGGE Unadjusted & 32.5 & 32.5 & 40.7 & 47.8 & 37.1 & 46.1 & 56.2 & 41.1 & 54.2 & 68.7 & 45.2 & 58.4 & 74.8 \\
\hline & & & MPGGE Adjusted & 23.9 & 23.9 & 29.7 & 34.6 & 27.2 & 33.4 & 40.2 & 29.9 & 38.9 & 48.4 & 32.8 & 41.7 & 52.4 \\
\hline & & \multirow{3}{*}{$C D+C S$} & Wh/mile & 196.4 & 196.4 & 176.2 & 162.4 & 190.3 & 167.9 & 147.2 & 186.3 & 152.2 & 127.9 & 178.8 & 146.8 & 122.4 \\
\hline & & & MPGGE Unadjusted & 61.8 & 61.8 & 77.4 & 90.8 & 70.5 & 87.5 & 106.5 & 78.0 & 102.9 & 130.0 & 85.8 & 110.8 & 141.6 \\
\hline & & & MPGGE Adjusted & 43.7 & 43.7 & 53.8 & 62.2 & 49.4 & 60.2 & 71.7 & 54.2 & 69.6 & 85.4 & 59.1 & 74.3 & 92.0 \\
\hline
\end{tabular}


Argonne National Laboratory Report - Light-Duty Vehicle Fuel Consumption Displacement Potential Up to 2045

\begin{tabular}{|c|c|c|c|c|c|c|c|c|c|c|c|c|c|c|c|c|}
\hline & & & & \multicolumn{13}{|c|}{ Small_SUV } \\
\hline & & & & 2010 & & 2010 & & & 2015 & & & 2030 & & & 2045 & \\
\hline \multirow{26}{*}{ H2 Series HEV PHEV 40} & & \multirow{5}{*}{$C D$} & & Ref & low & avg & high & low & avg & high & low & avg & high & low & avg & high \\
\hline & \multirow{9}{*}{ UDDS } & & Wh/mile & 332.3 & 332.3 & 298.0 & 274.3 & 320.9 & 283.3 & 247.8 & 312.8 & 256.3 & 210.7 & 300.6 & 246.0 & 202.5 \\
\hline & & & Distance & 40.6 & 40.6 & 40.6 & 40.8 & 40.6 & 40.8 & 40.6 & 40.6 & 40.6 & 40.4 & 40.8 & 40.9 & 40.5 \\
\hline & & & MPGGE Unadjusted & N/A & N/A & $\mathrm{N} / \mathrm{A}$ & $\mathrm{N} / \mathrm{A}$ & $\mathrm{N} / \mathrm{A}$ & $\mathrm{N} / \mathrm{A}$ & $\mathrm{N} / \mathrm{A}$ & $\mathrm{N} / \mathrm{A}$ & $\mathrm{N} / \mathrm{A}$ & $\mathrm{N} / \mathrm{A}$ & $\mathrm{N} / \mathrm{A}$ & N/A & $\mathrm{N} / \mathrm{A}$ \\
\hline & & & MPGGE Adjusted & $\mathrm{N} / \mathrm{A}$ & $\mathrm{N} / \mathrm{A}$ & $\mathrm{N} / \mathrm{A}$ & $\mathrm{N} / \mathrm{A}$ & $\mathrm{N} / \mathrm{A}$ & $\mathrm{N} / \mathrm{A}$ & $\mathrm{N} / \mathrm{A}$ & $\mathrm{N} / \mathrm{A}$ & $\mathrm{N} / \mathrm{A}$ & $\mathrm{N} / \mathrm{A}$ & $\mathrm{N} / \mathrm{A}$ & N/A & N/A \\
\hline & & \multirow{2}{*}{ CS } & MPGGE Unadjusted & 32.9 & 32.9 & 41.4 & 48.7 & 37.7 & 47.1 & 58.0 & 41.9 & 56.3 & 72.8 & 45.7 & 60.2 & 79.5 \\
\hline & & & MPGGE Adjusted & 25.1 & 25.1 & 31.1 & 36.0 & 28.5 & 35.0 & 42.2 & 31.4 & 41.1 & 51.6 & 34.1 & 43.7 & 55.6 \\
\hline & & \multirow{3}{*}{$\mathrm{CD}+\mathrm{CS}$} & Wh/mile & 243.9 & 243.9 & 218.8 & 201.5 & 235.5 & 208.0 & 183.1 & 229.6 & 189.4 & 155.8 & 220.7 & 181.5 & 149.8 \\
\hline & & & MPGGE Unadjusted & 82.1 & 82.1 & 103.6 & 121.6 & 94.2 & 117.7 & 145.1 & 104.8 & 140.6 & 182.1 & 114.3 & 150.6 & 198.6 \\
\hline & & & MPGGE Adjusted & 57.2 & 57.2 & 69.5 & 79.2 & 64.3 & 77.1 & 90.9 & 70.2 & 88.8 & 107.8 & 75.3 & 93.5 & 114.7 \\
\hline & \multirow{9}{*}{ HWFET } & \multirow{4}{*}{$C D$} & Wh/mile & 354.1 & 354.1 & 316.7 & 291.7 & 343.9 & 302.3 & 265.0 & 338.4 & 273.5 & 233.1 & 323.4 & 263.6 & 221.0 \\
\hline & & & Distance & 37.8 & 37.8 & 37.9 & 38.0 & 37.7 & 37.9 & 37.7 & 37.3 & 37.8 & 36.5 & 37.6 & 37.9 & 37.2 \\
\hline & & & MPGGE Unadjusted & N/A & N/A & N/A & N/A & $\mathrm{N} / \mathrm{A}$ & $\mathrm{N} / \mathrm{A}$ & N/A & N/A & N/A & N/A & N/A & N/A & N/A \\
\hline & & & MPGGE Adjusted & N/A & N/A & $\mathrm{N} / \mathrm{A}$ & $\mathrm{N} / \mathrm{A}$ & $\mathrm{N} / \mathrm{A}$ & N/A & N/A & $\mathrm{N} / \mathrm{A}$ & N/A & N/A & N/A & N/A & $\mathrm{N} / \mathrm{A}$ \\
\hline & & \multirow{2}{*}{ CS } & MPGGE Unadjusted & 30.8 & 30.8 & 38.3 & 44.9 & 35.0 & 43.4 & 52.3 & 38.6 & 50.7 & 63.1 & 42.3 & 54.9 & 69.2 \\
\hline & & & MPGGE Adjusted & 21.8 & 21.8 & 27.1 & 31.7 & 24.8 & 30.6 & 36.9 & 27.3 & 35.8 & 44.3 & 29.9 & 38.6 & 48.6 \\
\hline & & \multirow{3}{*}{$\mathrm{CD}+\mathrm{CS}$} & Wh/mile & 239.1 & 239.1 & 213.9 & 197.0 & 232.2 & 204.2 & 179.0 & 228.5 & 184.7 & 157.5 & 218.4 & 178.0 & 149.4 \\
\hline & & & MPGGE Unadjusted & 66.9 & 66.9 & 83.2 & 97.6 & 76.1 & 94.3 & 113.7 & 83.9 & 110.3 & 137.1 & 91.9 & 119.3 & 150.4 \\
\hline & & & MPGGE Adjusted & 47.0 & 47.0 & 58.3 & 68.1 & 53.3 & 65.8 & 79.1 & 58.7 & 76.7 & 94.9 & 64.2 & 82.8 & 103.8 \\
\hline & \multirow{7}{*}{ Combined } & \multirow{2}{*}{$C D$} & MPGGE Unadjusted & $\mathrm{N} / \mathrm{A}$ & $\mathrm{N} / \mathrm{A}$ & $\mathrm{N} / \mathrm{A}$ & $\mathrm{N} / \mathrm{A}$ & $\mathrm{N} / \mathrm{A}$ & $\mathrm{N} / \mathrm{A}$ & N/A & $\mathrm{N} / \mathrm{A}$ & $\mathrm{N} / \mathrm{A}$ & $\mathrm{N} / \mathrm{A}$ & $\mathrm{N} / \mathrm{A}$ & $\mathrm{N} / \mathrm{A}$ & N/A \\
\hline & & & MPGGE Adjusted & $\mathrm{N} / \mathrm{A}$ & $\mathrm{N} / \mathrm{A}$ & $\mathrm{N} / \mathrm{A}$ & $\mathrm{N} / \mathrm{A}$ & $\mathrm{N} / \mathrm{A}$ & $\mathrm{N} / \mathrm{A}$ & $\mathrm{N} / \mathrm{A}$ & $\mathrm{N} / \mathrm{A}$ & $\mathrm{N} / \mathrm{A}$ & $\mathrm{N} / \mathrm{A}$ & $\mathrm{N} / \mathrm{A}$ & $\mathrm{N} / \mathrm{A}$ & $\mathrm{N} / \mathrm{A}$ \\
\hline & & \multirow{2}{*}{ CS } & MPGGE Unadjusted & 31.9 & 31.9 & 40.0 & 46.9 & 36.4 & 45.3 & 55.3 & 40.4 & 53.6 & 68.1 & 44.1 & 57.7 & 74.5 \\
\hline & & & MPGGE Adjusted & 23.5 & 23.5 & 29.2 & 33.9 & 26.7 & 32.9 & 39.6 & 29.4 & 38.5 & 48.1 & 32.0 & 41.2 & 52.2 \\
\hline & & \multirow{3}{*}{$\mathrm{CD}+\mathrm{CS}$} & Wh/mile & 241.7 & 241.7 & 216.6 & 199.5 & 234.0 & 206.3 & 181.3 & 229.1 & 187.3 & 156.6 & 219.6 & 179.9 & 149.6 \\
\hline & & & MPGGE Unadjusted & 74.5 & 74.5 & 93.3 & 109.5 & 85.1 & 105.9 & 129.1 & 94.2 & 125.1 & 158.7 & 103.0 & 134.7 & 173.6 \\
\hline & & & MPGGE Adjusted & 52.1 & 52.1 & 64.0 & $\begin{array}{l}73.8 \\
\end{array}$ & 58.8 & 71.6 & 85.2 & 64.5 & 82.9 & 101.5 & 69.9 & 88.4 & 109.5 \\
\hline
\end{tabular}


Argonne National Laboratory Report - Light-Duty Vehicle Fuel Consumption Displacement Potential Up to 2045

\begin{tabular}{|c|c|c|c|c|c|c|c|c|c|c|c|c|c|c|c|c|}
\hline & & & & \multicolumn{13}{|c|}{ Small_SUV } \\
\hline & & & & \multirow{2}{*}{$\begin{array}{l}2010 \\
\text { Ref }\end{array}$} & \multicolumn{3}{|c|}{2010} & \multicolumn{3}{|c|}{2015} & \multicolumn{3}{|c|}{2030} & \multicolumn{3}{|c|}{2045} \\
\hline & & & & & low & avg & high & low & avg & high & low & avg & high & low & avg & high \\
\hline \multirow{25}{*}{ E85 Split HEV PHEV10 } & \multirow{9}{*}{ UDDS } & \multirow{4}{*}{$C D$} & Wh/mile & 222.3 & 222.3 & 202.5 & 188.7 & 215.7 & 194.5 & 183.8 & 209.6 & 184.9 & 165.9 & 208.9 & 182.7 & 159.4 \\
\hline & & & Distance & 12.2 & 12.2 & 12.3 & 12.2 & 12.6 & 12.2 & 11.9 & 12.6 & 12.2 & 11.4 & 12.3 & 11.7 & 11.2 \\
\hline & & & MPGGE Unadjusted & 725.3 & 725.3 & 783.5 & 870.7 & 704.0 & 837.4 & 1383.9 & 672.9 & 982.6 & 2775.3 & 779.7 & 1475.4 & 3069.4 \\
\hline & & & MPGGE Adjusted & 235.9 & 235.9 & 243.1 & 252.7 & 233.1 & 249.2 & 291.1 & 228.8 & 263.4 & 334.4 & 242.6 & 295.9 & 339.2 \\
\hline & & \multirow{2}{*}{ CS } & MPGGE Unadjusted & 45.0 & 45.0 & 49.7 & 54.0 & 46.6 & 52.4 & 67.5 & 47.8 & 57.8 & 80.1 & 49.5 & 60.0 & 83.6 \\
\hline & & & MPGGE Adjusted & 33.6 & 33.6 & 36.7 & 39.6 & 34.6 & 38.5 & 48.3 & 35.5 & 42.1 & 56.0 & 36.6 & 43.5 & 58.1 \\
\hline & & \multirow{3}{*}{$\mathrm{CD}+\mathrm{CS}$} & Wh/mile & 46.3 & 46.3 & 42.2 & 39.3 & 44.9 & 40.5 & 38.3 & 43.7 & 38.5 & 34.6 & 43.5 & 38.1 & 33.2 \\
\hline & & & MPGGE Unadjusted & 53.5 & 53.5 & 59.1 & 64.2 & 55.3 & 62.3 & 80.6 & 56.8 & 68.8 & 95.9 & 58.8 & 71.6 & 100.1 \\
\hline & & & MPGGE Adjusted & 39.3 & 39.3 & 42.9 & 46.2 & 40.5 & 45.0 & 56.3 & 41.4 & 49.1 & 65.2 & 42.8 & 50.9 & 67.6 \\
\hline & \multirow{9}{*}{ HWFET } & \multirow{4}{*}{$C D$} & Wh/mile & 210.2 & 210.2 & 236.0 & 223.7 & 200.4 & 227.1 & 209.8 & 198.0 & 215.5 & 182.7 & 194.0 & 207.8 & 174.3 \\
\hline & & & Distance & 12.3 & 12.3 & 10.7 & 10.0 & 12.5 & 10.6 & 9.8 & 12.5 & 10.0 & 8.5 & 12.5 & 9.7 & 8.6 \\
\hline & & & MPGGE Unadjusted & 122.6 & 122.6 & 251.3 & 302.6 & 119.0 & 264.2 & 387.5 & 120.8 & 333.8 & 396.8 & 129.4 & 338.1 & 433.4 \\
\hline & & & MPGGE Adjusted & 85.1 & 85.1 & 169.8 & 202.2 & 82.7 & 178.0 & 254.5 & 83.9 & 221.7 & 260.1 & 89.7 & 224.3 & 282.1 \\
\hline & & \multirow{2}{*}{ CS } & MPGGE Unadjusted & 39.9 & 39.9 & 44.1 & 47.9 & 41.1 & 46.3 & 59.3 & 41.9 & 50.8 & 68.0 & 43.8 & 52.9 & 71.8 \\
\hline & & & MPGGE Adjusted & 28.2 & 28.2 & 31.2 & 33.8 & 29.0 & 32.6 & 41.7 & 29.6 & 35.8 & 47.8 & 30.9 & 37.3 & 50.3 \\
\hline & & \multirow{3}{*}{$\mathrm{CD}+\mathrm{CS}$} & Wh/mile & 61.8 & 61.8 & 69.4 & 65.8 & 58.9 & 66.8 & 61.7 & 58.2 & 63.4 & 53.7 & 57.1 & 61.1 & 51.3 \\
\hline & & & MPGGE Unadjusted & 47.6 & 47.6 & 55.0 & 60.1 & 48.7 & 57.7 & 74.4 & 49.7 & 63.7 & 84.9 & 52.0 & 66.4 & 89.7 \\
\hline & & & MPGGE Adjusted & 33.6 & 33.6 & 38.7 & 42.2 & 34.4 & 40.6 & 52.2 & 35.0 & 44.8 & 59.4 & 36.7 & 46.6 & 62.7 \\
\hline & \multirow{7}{*}{ Combined } & \multirow{2}{*}{$C D$} & MPGGE Unadjusted & 225.8 & 225.8 & 401.2 & 472.0 & 219.2 & 423.7 & 641.5 & 220.2 & 524.1 & 750.6 & 239.1 & 586.9 & 821.3 \\
\hline & & & MPGGE Adjusted & 131.2 & 131.2 & 203.5 & 227.2 & 128.2 & 211.2 & 273.4 & 128.7 & 242.8 & 296.3 & 137.3 & 258.7 & 310.8 \\
\hline & & \multirow{2}{*}{ CS } & MPGGE Unadjusted & 42.5 & 42.5 & 47.0 & 51.1 & 43.9 & 49.5 & 63.5 & 45.0 & 54.4 & 74.2 & 46.7 & 56.6 & 77.8 \\
\hline & & & MPGGE Adjusted & 30.9 & 30.9 & 34.0 & 36.7 & 31.9 & 35.6 & 45.1 & 32.6 & 39.0 & 52.0 & 33.8 & 40.5 & 54.3 \\
\hline & & \multirow{3}{*}{$C D+C S$} & Wh/mile & 53.3 & 53.3 & 54.4 & 51.2 & 51.2 & 52.4 & 48.8 & 50.2 & 49.7 & 43.2 & 49.6 & 48.4 & 41.3 \\
\hline & & & MPGGE Unadjusted & 50.7 & 50.7 & 57.2 & 62.3 & 52.2 & 60.2 & 77.7 & 53.4 & 66.4 & 90.6 & 55.6 & 69.2 & 95.2 \\
\hline & & & MPGGE Adjusted & 36.5 & 36.5 & 40.9 & 44.3 & 37.5 & 42.9 & 54.4 & 38.3 & 47.1 & 62.5 & 39.8 & 48.9 & 65.3 \\
\hline
\end{tabular}


Argonne National Laboratory Report - Light-Duty Vehicle Fuel Consumption Displacement Potential Up to 2045

\begin{tabular}{|c|c|c|c|c|c|c|c|c|c|c|c|c|c|c|c|c|}
\hline & & & & \multicolumn{13}{|c|}{ Small_SUV } \\
\hline & & & & 2010 & & 2010 & & & 2015 & & & 2030 & & & 2045 & \\
\hline \multirow{26}{*}{ E85 Split HEV PHEV20 } & & \multirow{5}{*}{$C D$} & & Ref & low & avg & high & low & avg & high & low & avg & high & low & avg & high \\
\hline & \multirow{9}{*}{ UDDS } & & Wh/mile & 224.7 & 224.7 & 206.1 & 191.0 & 228.2 & 198.3 & 177.6 & 224.1 & 182.8 & 163.4 & 215.3 & 178.0 & 157.1 \\
\hline & & & Distance & 24.0 & 24.0 & 24.1 & 24.0 & 23.8 & 24.0 & 23.7 & 23.8 & 23.8 & 22.3 & 24.0 & 23.5 & 22.1 \\
\hline & & & MPGGE Unadjusted & 694.1 & 694.1 & 792.7 & 847.2 & 937.2 & 852.0 & 1132.9 & 963.5 & 958.8 & 2719.9 & 861.6 & 1018.7 & 2976.3 \\
\hline & & & MPGGE Adjusted & 231.7 & 231.7 & 244.2 & 250.3 & 259.3 & 250.8 & 275.4 & 261.7 & 261.3 & 333.4 & 251.8 & 266.5 & 337.7 \\
\hline & & \multirow{2}{*}{ CS } & MPGGE Unadjusted & 44.6 & 44.6 & 49.4 & 53.6 & 46.2 & 52.0 & 67.5 & 47.5 & 57.8 & 79.9 & 49.2 & 60.0 & 83.4 \\
\hline & & & MPGGE Adjusted & 33.3 & 33.3 & 36.5 & 39.4 & 34.4 & 38.3 & 48.3 & 35.3 & 42.1 & 55.9 & 36.4 & 43.5 & 58.0 \\
\hline & & \multirow{3}{*}{$C D+C S$} & Wh/mile & 118.2 & 118.2 & 108.5 & 100.6 & 119.7 & 104.4 & 93.9 & 117.6 & 96.6 & 62.2 & 113.2 & 94.1 & 59.8 \\
\hline & & & MPGGE Unadjusted & 75.8 & 75.8 & 84.1 & 91.4 & 79.4 & 88.7 & 115.3 & 81.7 & 98.7 & 116.0 & 84.0 & 102.5 & 121.1 \\
\hline & & & MPGGE Adjusted & 53.4 & 53.4 & 58.4 & 62.6 & 55.6 & 61.1 & 75.9 & 56.9 & 66.8 & 76.2 & 58.4 & 68.9 & 78.9 \\
\hline & \multirow{9}{*}{ HWFET } & \multirow{4}{*}{$C D$} & Wh/mile & 210.7 & 210.7 & 237.7 & 228.4 & 255.5 & 228.8 & 212.1 & 251.2 & 217.8 & 224.8 & 246.4 & 219.7 & 212.9 \\
\hline & & & Distance & 24.7 & 24.7 & 20.2 & 20.1 & 21.2 & 20.2 & 19.5 & 21.2 & 19.7 & 16.0 & 20.2 & 19.3 & 16.1 \\
\hline & & & MPGGE Unadjusted & 119.5 & 119.5 & 250.8 & 324.8 & 218.5 & 263.8 & 409.4 & 216.7 & 349.0 & N/A & 251.0 & 468.5 & $\mathrm{~N} / \mathrm{A}$ \\
\hline & & & MPGGE Adjusted & 83.0 & 83.0 & 169.4 & 216.1 & 148.6 & 177.7 & 267.7 & 147.4 & 231.1 & $\mathrm{~N} / \mathrm{A}$ & 169.5 & 302.8 & N/A \\
\hline & & \multirow{2}{*}{ CS } & MPGGE Unadjusted & 39.6 & 39.6 & 43.8 & 47.4 & 40.8 & 45.9 & 58.8 & 41.7 & 50.4 & 67.8 & 43.5 & 52.5 & 71.6 \\
\hline & & & MPGGE Adjusted & 28.0 & 28.0 & 30.9 & 33.5 & 28.8 & 32.4 & 41.4 & 29.4 & 35.6 & 47.6 & 30.7 & 37.0 & 50.2 \\
\hline & & \multirow{3}{*}{$C D+C S$} & Wh/mile & 108.3 & 108.3 & 69.9 & 67.2 & 128.0 & 67.3 & 62.4 & 125.9 & 64.1 & 66.1 & 72.5 & 64.6 & 62.6 \\
\hline & & & MPGGE Unadjusted & 54.2 & 54.2 & 54.6 & 59.7 & 60.3 & 57.3 & 74.0 & 61.4 & 63.5 & 89.2 & 54.3 & 66.8 & 94.1 \\
\hline & & & MPGGE Adjusted & 38.2 & 38.2 & 38.4 & 42.0 & 42.4 & 40.3 & 51.9 & 43.2 & 44.6 & 62.3 & 38.2 & 46.9 & 65.7 \\
\hline & \multirow{7}{*}{ Combined } & \multirow{2}{*}{$C D$} & MPGGE Unadjusted & 219.4 & 219.4 & 401.9 & 491.5 & 377.9 & 425.3 & 631.0 & 377.7 & 536.8 & 4782.9 & 411.3 & 666.5 & 5217.6 \\
\hline & & & \begin{tabular}{|l|} 
MPGGE Adjusted \\
\end{tabular} & 128.3 & 128.3 & 203.7 & 233.6 & 194.2 & 211.6 & 271.9 & 194.0 & 246.8 & 556.2 & 206.7 & 281.7 & 562.8 \\
\hline & & \multirow{2}{*}{ CS } & MPGGE Unadjusted & 42.2 & 42.2 & 46.7 & 50.7 & 43.6 & 49.1 & 63.3 & 44.7 & 54.2 & 74.0 & 46.4 & 56.4 & 77.6 \\
\hline & & & MPGGE Adjusted & 30.7 & 30.7 & 33.7 & 36.5 & 31.6 & 35.4 & 44.9 & 32.4 & 38.9 & 51.8 & 33.6 & 40.3 & 54.2 \\
\hline & & \multirow{3}{*}{$C D+C S$} & Wh/mile & 113.7 & 113.7 & 91.2 & 85.6 & 123.4 & 87.7 & 79.7 & 121.3 & 81.9 & 64.0 & 94.9 & 80.8 & 61.1 \\
\hline & & & MPGGE Unadjusted & 64.3 & 64.3 & 67.6 & 73.7 & 69.5 & 71.1 & 92.2 & 71.1 & 79.0 & 102.2 & 67.4 & 82.6 & 107.3 \\
\hline & & & \begin{tabular}{|l|} 
MPGEE Adjusted \\
\end{tabular} & 45.3 & 45.3 & 47.3 & 51.3 & 48.8 & 49.6 & 62.8 & 49.8 & 54.6 & 69.3 & 47.2 & 56.9 & 72.4 \\
\hline
\end{tabular}


Argonne National Laboratory Report - Light-Duty Vehicle Fuel Consumption Displacement Potential Up to 2045

\begin{tabular}{|c|c|c|c|c|c|c|c|c|c|c|c|c|c|c|c|c|}
\hline & & & & \multicolumn{13}{|c|}{ Small_SUV } \\
\hline & & & & \multirow{2}{*}{$\begin{array}{l}2010 \\
\text { Ref }\end{array}$} & \multicolumn{3}{|c|}{2010} & \multicolumn{3}{|c|}{2015} & \multicolumn{3}{|c|}{2030} & \multicolumn{3}{|c|}{2045} \\
\hline & & & & & low & avg & high & low & avg & high & low & avg & high & low & avg & high \\
\hline \multirow{25}{*}{ E85 Series HEV PHEV30 } & \multirow{9}{*}{ UDDS } & \multirow{4}{*}{$C D$} & Wh/mile & 320.9 & 320.9 & 292.5 & 271.8 & 312.1 & 280.4 & 246.5 & 305.3 & 253.8 & 213.3 & 298.2 & 245.6 & 204.4 \\
\hline & & & Distance & 30.9 & 30.9 & 30.9 & 30.9 & 30.9 & 30.9 & 30.9 & 30.9 & 30.9 & 31.4 & 30.9 & 30.9 & 30.7 \\
\hline & & & MPGGE Unadjusted & $\mathrm{N} / \mathrm{A}$ & $\mathrm{N} / \mathrm{A}$ & N/A & N/A & N/A & $\mathrm{N} / \mathrm{A}$ & N/A & $\mathrm{N} / \mathrm{A}$ & N/A & N/A & N/A & N/A & $\mathrm{N} / \mathrm{A}$ \\
\hline & & & MPGGE Adjusted & $\mathrm{N} / \mathrm{A}$ & $\mathrm{N} / \mathrm{A}$ & $\mathrm{N} / \mathrm{A}$ & N/A & N/A & N/A & N/A & $\mathrm{N} / \mathrm{A}$ & N/A & N/A & N/A & $\mathrm{N} / \mathrm{A}$ & $\mathrm{N} / \mathrm{A}$ \\
\hline & & \multirow{2}{*}{ CS } & MPGGE Unadjusted & 32.3 & 32.3 & 36.7 & 41.0 & 34.0 & 39.3 & 53.9 & 35.8 & 45.9 & 64.7 & 37.3 & 47.2 & 68.6 \\
\hline & & & MPGGE Adjusted & 24.7 & 24.7 & 27.8 & 30.8 & 25.9 & 29.6 & 39.5 & 27.2 & 34.2 & 46.5 & 28.2 & 35.1 & 48.9 \\
\hline & & \multirow{3}{*}{$\mathrm{CD}+\mathrm{CS}$} & Wh/mile & 206.5 & 206.5 & 188.3 & 175.0 & 200.9 & 180.5 & 159.2 & 196.4 & 163.9 & 138.3 & 191.9 & 158.5 & 132.5 \\
\hline & & & MPGGE Unadjusted & 68.8 & 68.8 & 78.2 & 87.3 & 72.3 & 83.5 & 114.6 & 76.1 & 97.6 & 137.7 & 79.3 & 100.5 & 145.9 \\
\hline & & & MPGGE Adjusted & 49.1 & 49.1 & 54.8 & 60.3 & 51.3 & 58.0 & 75.5 & 53.6 & 66.2 & 87.3 & 55.5 & 67.8 & 91.3 \\
\hline & \multirow{9}{*}{ HWFET } & \multirow{4}{*}{$C D$} & Wh/mile & 347.9 & 347.9 & 314.5 & 291.4 & 339.6 & 301.8 & 266.3 & 334.8 & 273.9 & 234.5 & 323.2 & 264.9 & 222.1 \\
\hline & & & Distance & 28.5 & 28.5 & 28.8 & 29.2 & 28.4 & 28.7 & 28.6 & 28.1 & 28.7 & 27.7 & 28.5 & 28.5 & 28.2 \\
\hline & & & MPGGE Unadjusted & $\mathrm{N} / \mathrm{A}$ & $\mathrm{N} / \mathrm{A}$ & $\mathrm{N} / \mathrm{A}$ & $\mathrm{N} / \mathrm{A}$ & $\mathrm{N} / \mathrm{A}$ & $\mathrm{N} / \mathrm{A}$ & $\mathrm{N} / \mathrm{A}$ & $\mathrm{N} / \mathrm{A}$ & $\mathrm{N} / \mathrm{A}$ & $\mathrm{N} / \mathrm{A}$ & $\mathrm{N} / \mathrm{A}$ & $\mathrm{N} / \mathrm{A}$ & $\mathrm{N} / \mathrm{A}$ \\
\hline & & & MPGGE Adjusted & N/A & N/A & N/A & N/A & N/A & N/A & N/A & N/A & N/A & N/A & N/A & N/A & $\mathrm{N} / \mathrm{A}$ \\
\hline & & \multirow{2}{*}{ CS } & MPGGE Unadjusted & 29.9 & 29.9 & 33.8 & 37.3 & 31.1 & 36.0 & 47.5 & 32.3 & 40.7 & 56.1 & 34.0 & 42.7 & 59.4 \\
\hline & & & MPGGE Adjusted & 21.2 & 21.2 & 23.9 & 26.4 & 22.0 & 25.5 & 33.5 & 22.9 & 28.8 & 39.5 & 24.1 & 30.2 & 41.8 \\
\hline & & \multirow{3}{*}{$\mathrm{CD}+\mathrm{CS}$} & Wh/mile & 179.3 & 179.3 & 162.1 & 150.2 & 175.0 & 155.6 & 137.2 & 172.5 & 141.2 & 120.9 & 166.5 & 136.5 & 114.5 \\
\hline & & & MPGGE Unadjusted & 50.7 & 50.7 & 57.3 & 63.2 & 52.7 & 61.0 & 80.6 & 54.8 & 69.0 & 95.1 & 57.7 & 72.4 & 100.7 \\
\hline & & & MPGGE Adjusted & 35.7 & 35.7 & 40.3 & 44.4 & 37.1 & 42.9 & 56.4 & 38.6 & 48.4 & 66.4 & 40.6 & 50.8 & 70.2 \\
\hline & \multirow{7}{*}{ Combined } & \multirow{2}{*}{$C D$} & MPGGE Unadjusted & $\mathrm{N} / \mathrm{A}$ & $\mathrm{N} / \mathrm{A}$ & N/A & N/A & N/A & $\mathrm{N} / \mathrm{A}$ & N/A & $\mathrm{N} / \mathrm{A}$ & $\mathrm{N} / \mathrm{A}$ & N/A & $\mathrm{N} / \mathrm{A}$ & N/A & $\mathrm{N} / \mathrm{A}$ \\
\hline & & & MPGGE Adjusted & N/A & N/A & N/A & N/A & N/A & N/A & N/A & N/A & N/A & N/A & N/A & N/A & N/A \\
\hline & & \multirow{2}{*}{ CS } & MPGGE Unadjusted & 31.2 & 31.2 & 35.3 & 39.3 & 32.6 & 37.7 & 50.8 & 34.1 & 43.4 & 60.6 & 35.7 & 45.1 & 64.1 \\
\hline & & & MPGGE Adjusted & 23.0 & 23.0 & 25.9 & 28.6 & 24.0 & 27.6 & 36.6 & 25.1 & 31.5 & 43.1 & 26.2 & 32.7 & 45.4 \\
\hline & & \multirow{3}{*}{$C D+C S$} & Wh/mile & 194.3 & 194.3 & 176.5 & 163.8 & 189.2 & 169.3 & 149.3 & 185.7 & 153.7 & 130.4 & 180.5 & 148.6 & 124.4 \\
\hline & & & MPGGE Unadjusted & 59.3 & 59.3 & 67.1 & 74.5 & 61.9 & 71.6 & 96.3 & 64.8 & 82.2 & 114.6 & 67.8 & 85.5 & 121.3 \\
\hline & & & MPGGE Adjusted & 42.0 & 42.0 & 47.2 & 51.9 & 43.8 & 50.1 & 65.5 & 45.6 & 56.8 & 76.5 & 47.6 & 58.9 & 80.4 \\
\hline
\end{tabular}


Argonne National Laboratory Report - Light-Duty Vehicle Fuel Consumption Displacement Potential Up to 2045

\begin{tabular}{|c|c|c|c|c|c|c|c|c|c|c|c|c|c|c|c|c|}
\hline & & & & \multicolumn{13}{|c|}{ Small_SUV } \\
\hline \multirow{27}{*}{ E85 Series HEV PHEV40 } & & & & 2010 & & 2010 & & & 2015 & & & 2030 & & & 2045 & \\
\hline & & \multirow{5}{*}{$C D$} & & Ref & low & avg & high & low & avg & high & low & avg & high & low & avg & high \\
\hline & \multirow{9}{*}{ UDDS } & & Wh/mile & 327.9 & 327.9 & 298.8 & 277.0 & 318.7 & 285.9 & 251.8 & 311.6 & 259.5 & 215.5 & 304.0 & 249.9 & 206.5 \\
\hline & & & Distance & 40.5 & 40.5 & 40.8 & 40.8 & 40.6 & 40.8 & 40.5 & 40.5 & 40.6 & 40.4 & 40.8 & 40.5 & 40.6 \\
\hline & & & MPGGE Unadjusted & N/A & N/A & N/A & N/A & N/A & N/A & N/A & N/A & N/A & N/A & N/A & N/A & $\mathrm{N} / \mathrm{A}$ \\
\hline & & & MPGGE Adjusted & N/A & N/A & N/A & N/A & N/A & $\mathrm{N} / \mathrm{A}$ & N/A & N/A & N/A & N/A & N/A & N/A & $\mathrm{N} / \mathrm{A}$ \\
\hline & & \multirow{2}{*}{ CS } & MPGGE Unadjusted & 31.5 & 31.5 & 35.8 & 40.2 & 33.2 & 38.4 & 53.0 & 34.9 & 45.1 & 64.4 & 36.4 & 46.5 & 68.4 \\
\hline & & & MPGGE Adjusted & 24.1 & 24.1 & 27.2 & 30.3 & 25.3 & 29.0 & 38.9 & 26.5 & 33.6 & 46.3 & 27.6 & 34.6 & 48.8 \\
\hline & & \multirow{3}{*}{$C D+C S$} & Wh/mile & 240.6 & 240.6 & 219.4 & 203.5 & 233.9 & 210.0 & 186.1 & 228.7 & 191.8 & 159.4 & 223.2 & 184.4 & 152.7 \\
\hline & & & MPGGE Unadjusted & 78.7 & 78.7 & 89.6 & 100.6 & 83.0 & 96.0 & 132.5 & 87.1 & 112.8 & 160.9 & 91.1 & 116.1 & 170.9 \\
\hline & & & MPGGE Adjusted & 55.1 & 55.1 & 61.6 & 67.8 & 57.8 & 65.3 & 84.8 & 60.2 & 74.5 & 98.3 & 62.5 & 76.3 & 102.9 \\
\hline & \multirow{9}{*}{ HWFET } & \multirow{4}{*}{$C D$} & Wh/mile & 351.2 & 351.2 & 317.2 & 293.5 & 342.5 & 304.1 & 267.6 & 337.6 & 275.5 & 235.9 & 325.6 & 266.0 & 223.4 \\
\hline & & & Distance & 37.7 & 37.7 & 38.0 & 38.2 & 37.6 & 38.0 & 37.9 & 37.2 & 37.9 & 36.8 & 37.8 & 38.0 & 37.5 \\
\hline & & & MPGGE Unadjusted & N/A & N/A & N/A & N/A & N/A & N/A & N/A & N/A & N/A & $\mathrm{N} / \mathrm{A}$ & N/A & N/A & N/A \\
\hline & & & MPGGE Adjusted & $\mathrm{N} / \mathrm{A}$ & $\mathrm{N} / \mathrm{A}$ & $\mathrm{N} / \mathrm{A}$ & $\mathrm{N} / \mathrm{A}$ & $\mathrm{N} / \mathrm{A}$ & $\mathrm{N} / \mathrm{A}$ & $\mathrm{N} / \mathrm{A}$ & N/A & N/A & $\mathrm{N} / \mathrm{A}$ & $\mathrm{N} / \mathrm{A}$ & $\mathrm{N} / \mathrm{A}$ & $\mathrm{N} / \mathrm{A}$ \\
\hline & & \multirow{2}{*}{ CS } & MPGGE Unadjusted & 29.5 & 29.5 & 33.2 & 36.8 & 30.6 & 35.5 & 47.0 & 31.9 & 40.3 & 55.7 & 33.5 & 42.3 & 59.0 \\
\hline & & & MPGGE Adjusted & 20.9 & 20.9 & 23.5 & 26.0 & 21.7 & 25.1 & 33.2 & 22.6 & 28.4 & 39.2 & 23.7 & 29.9 & 41.5 \\
\hline & & \multirow{3}{*}{$C D+C S$} & Wh/mile & 237.1 & 237.1 & 214.2 & 198.2 & 231.2 & 205.3 & 180.7 & 227.9 & 186.0 & 159.5 & 219.9 & 179.7 & 151.0 \\
\hline & & & MPGGE Unadjusted & 64.1 & 64.1 & 72.2 & 80.0 & 66.6 & 77.3 & 102.3 & 69.3 & 87.5 & 121.0 & 72.8 & 91.9 & 128.2 \\
\hline & & & MPGGE Adjusted & 45.0 & 45.0 & 50.6 & 56.0 & 46.8 & 54.2 & 71.3 & 48.7 & 61.2 & 84.0 & 51.1 & 64.2 & 88.9 \\
\hline & \multirow{7}{*}{ Combined } & \multirow{2}{*}{$C D$} & MPGGE Unadjusted & $\mathrm{N} / \mathrm{A}$ & $\mathrm{N} / \mathrm{A}$ & N/A & N/A & $\mathrm{N} / \mathrm{A}$ & $\mathrm{N} / \mathrm{A}$ & N/A & $\mathrm{N} / \mathrm{A}$ & N/A & $\mathrm{N} / \mathrm{A}$ & $\mathrm{N} / \mathrm{A}$ & $\mathrm{N} / \mathrm{A}$ & $\mathrm{N} / \mathrm{A}$ \\
\hline & & & MPGGE Adjusted & N/A & N/A & N/A & N/A & N/A & N/A & N/A & N/A & N/A & N/A & N/A & $\mathrm{N} / \mathrm{A}$ & N/A \\
\hline & & \multirow{2}{*}{ CS } & MPGGE Unadjusted & 30.5 & 30.5 & 34.6 & 38.6 & 32.0 & 37.1 & 50.1 & 33.4 & 42.8 & 60.1 & 35.1 & 44.5 & 63.8 \\
\hline & & & MPGGE Adjusted & 22.5 & 22.5 & 25.4 & 28.2 & 23.6 & 27.1 & 36.1 & 24.6 & 31.1 & 42.8 & 25.7 & 32.3 & 45.2 \\
\hline & & \multirow{3}{*}{$C D+C S$} & Wh/mile & 239.0 & 239.0 & 217.0 & 201.1 & 232.7 & 207.9 & 183.7 & 228.3 & 189.2 & 159.4 & 221.7 & 182.3 & 151.9 \\
\hline & & & MPGGE Unadjusted & 71.3 & 71.3 & 80.8 & 90.1 & 74.7 & 86.6 & 116.9 & 78.1 & 99.8 & 140.1 & 81.9 & 103.8 & 148.6 \\
\hline & & & MPGGE Adjusted & 50.1 & 50.1 & 56.1 & 62.0 & 52.2 & 59.8 & 78.1 & 54.4 & 67.9 & 91.3 & 56.8 & 70.3 & 96.1 \\
\hline
\end{tabular}


Argonne National Laboratory Report - Light-Duty Vehicle Fuel Consumption Displacement Potential Up to 2045

\begin{tabular}{|c|c|c|c|c|c|c|c|c|c|c|c|c|c|c|c|c|}
\hline & & & & \multicolumn{13}{|c|}{ Small_SUV } \\
\hline & & & & \multirow{2}{*}{$\begin{array}{l}2010 \\
\text { Ref }\end{array}$} & \multicolumn{3}{|c|}{2010} & \multicolumn{3}{|c|}{2015} & \multicolumn{3}{|c|}{2030} & \multicolumn{3}{|c|}{2045} \\
\hline & & & & & low & avg & high & low & avg & high & low & avg & high & low & avg & high \\
\hline \multirow{25}{*}{ FC HEV PHEV10 } & \multirow{9}{*}{ UDDS } & \multirow{4}{*}{$C D$} & Wh/mile & 321.4 & 321.4 & 274.2 & 244.0 & 297.6 & 252.1 & 222.6 & 281.3 & 229.5 & 188.2 & 267.1 & 219.4 & 179.9 \\
\hline & & & Distance & 10.2 & 10.2 & 10.2 & 10.2 & 10.2 & 10.2 & 10.2 & 10.2 & 10.2 & 10.2 & 10.2 & 10.2 & 10.2 \\
\hline & & & MPGGE Unadjusted & $\mathrm{N} / \mathrm{A}$ & $\mathrm{N} / \mathrm{A}$ & N/A & N/A & N/A & $\mathrm{N} / \mathrm{A}$ & N/A & $\mathrm{N} / \mathrm{A}$ & N/A & N/A & N/A & N/A & $\mathrm{N} / \mathrm{A}$ \\
\hline & & & MPGGE Adjusted & $\mathrm{N} / \mathrm{A}$ & $\mathrm{N} / \mathrm{A}$ & $\mathrm{N} / \mathrm{A}$ & N/A & N/A & N/A & N/A & $\mathrm{N} / \mathrm{A}$ & N/A & N/A & N/A & $\mathrm{N} / \mathrm{A}$ & $\mathrm{N} / \mathrm{A}$ \\
\hline & & \multirow{2}{*}{ CS } & MPGGE Unadjusted & 51.2 & 51.2 & 62.0 & 73.2 & 60.3 & 71.0 & 82.4 & 63.8 & 79.9 & 98.1 & 67.1 & 82.7 & 102.7 \\
\hline & & & MPGGE Adjusted & 37.7 & 37.7 & 44.8 & 51.8 & 43.7 & 50.4 & 57.4 & 46.0 & 55.9 & 66.5 & 48.1 & 57.6 & 69.1 \\
\hline & & \multirow{3}{*}{$\mathrm{CD}+\mathrm{CS}$} & Wh/mile & 67.0 & 67.0 & 57.1 & 50.8 & 62.0 & 52.5 & 46.4 & 58.6 & 47.8 & 39.2 & 55.6 & 45.7 & 37.5 \\
\hline & & & MPGGE Unadjusted & 61.6 & 61.6 & 74.7 & 88.2 & 72.6 & 85.5 & 99.2 & 76.9 & 96.3 & 118.2 & 80.9 & 99.6 & 123.8 \\
\hline & & & MPGGE Adjusted & 44.6 & 44.6 & 52.7 & 60.8 & 51.5 & 59.2 & 67.1 & 54.1 & 65.4 & 77.4 & 56.5 & 67.3 & 80.3 \\
\hline & \multirow{9}{*}{ HWFET } & \multirow{4}{*}{$C D$} & Wh/mile & 330.1 & 330.1 & 282.4 & 252.1 & 306.7 & 260.6 & 227.2 & 290.7 & 234.4 & 192.9 & 276.5 & 227.2 & 184.4 \\
\hline & & & \begin{tabular}{|l} 
Distance \\
\end{tabular} & 9.0 & 9.0 & 9.2 & 9.2 & 9.3 & 9.2 & 8.7 & 10.0 & 8.7 & 8.4 & 10.0 & 8.7 & 8.4 \\
\hline & & & MPGGE Unadjusted & 462.0 & 462.0 & 484.5 & 524.9 & 456.4 & 492.0 & 524.4 & 413.7 & 515.0 & 497.3 & 441.0 & 541.9 & 557.3 \\
\hline & & & MPGGE Adjusted & 299.0 & 299.0 & 312.2 & 335.5 & 295.6 & 316.5 & 335.2 & 270.3 & 329.8 & 319.6 & 286.6 & 345.2 & 353.9 \\
\hline & & \multirow{2}{*}{ CS } & MPGGE Unadjusted & 50.2 & 50.2 & 59.8 & 69.8 & 57.8 & 67.3 & 76.5 & 59.9 & 74.4 & 87.3 & 63.1 & 77.0 & 92.4 \\
\hline & & & MPGGE Adjusted & 35.4 & 35.4 & 42.1 & 49.0 & 40.7 & 47.2 & 53.6 & 42.1 & 52.1 & 61.1 & 44.3 & 54.0 & 64.6 \\
\hline & & \multirow{3}{*}{$\mathrm{CD}+\mathrm{CS}$} & Wh/mile & 97.1 & 97.1 & 83.0 & 74.1 & 90.2 & 76.7 & 66.8 & 85.5 & 68.9 & 56.7 & 81.3 & 66.8 & 54.2 \\
\hline & & & MPGGE Unadjusted & 63.9 & 63.9 & 75.7 & 88.1 & 73.2 & 84.8 & 96.2 & 75.4 & 93.6 & 108.9 & 79.4 & 97.0 & 115.6 \\
\hline & & & MPGGE Adjusted & 44.9 & 44.9 & 53.1 & 61.6 & 51.3 & 59.4 & 67.2 & 52.8 & 65.4 & 75.8 & 55.6 & 67.7 & 80.3 \\
\hline & \multirow{7}{*}{ Combined } & \multirow{2}{*}{$C D$} & MPGGE Unadjusted & $\mathrm{N} / \mathrm{A}$ & $\mathrm{N} / \mathrm{A}$ & N/A & N/A & $\mathrm{N} / \mathrm{A}$ & $\mathrm{N} / \mathrm{A}$ & $\mathrm{N} / \mathrm{A}$ & $\mathrm{N} / \mathrm{A}$ & $\mathrm{N} / \mathrm{A}$ & N/A & $\mathrm{N} / \mathrm{A}$ & $\mathrm{N} / \mathrm{A}$ & $\mathrm{N} / \mathrm{A}$ \\
\hline & & & MPGGE Adjusted & N/A & N/A & N/A & N/A & N/A & N/A & N/A & N/A & N/A & N/A & N/A & N/A & N/A \\
\hline & & \multirow{2}{*}{ CS } & MPGGE Unadjusted & 50.7 & 50.7 & 61.0 & 71.6 & 59.2 & 69.2 & 79.6 & 62.0 & 77.3 & 93.0 & 65.2 & 80.0 & 97.8 \\
\hline & & & MPGGE Adjusted & 36.6 & 36.6 & 43.5 & 50.5 & 42.3 & 48.9 & 55.6 & 44.2 & 54.1 & 63.9 & 46.3 & 55.9 & 67.0 \\
\hline & & \multirow{3}{*}{$\mathrm{CD}+\mathrm{CS}$} & Wh/mile & 80.5 & 80.5 & 68.8 & 61.3 & 74.7 & 63.4 & 55.6 & 70.7 & 57.3 & 47.1 & 67.2 & 55.2 & 45.0 \\
\hline & & & MPGGE Unadjusted & 62.6 & 62.6 & 75.1 & 88.1 & 72.9 & 85.2 & 97.9 & 76.2 & 95.0 & 113.8 & 80.2 & 98.4 & 119.9 \\
\hline & & & MPGGE Adjusted & 44.7 & 44.7 & 52.9 & 61.1 & 51.4 & 59.3 & 67.1 & 53.5 & 65.4 & 76.7 & 56.1 & 67.5 & 80.3 \\
\hline
\end{tabular}




\begin{tabular}{|c|c|c|c|c|c|c|c|c|c|c|c|c|c|c|c|c|}
\hline & & & & \multicolumn{13}{|c|}{ Small_SUV } \\
\hline & & & & 2010 & & 2010 & & & 2015 & & & 2030 & & & 2045 & \\
\hline \multirow{26}{*}{ FC HEV PHEV 20} & & \multirow{5}{*}{$C D$} & & Ref & low & avg & high & low & avg & high & low & avg & high & low & avg & high \\
\hline & \multirow{9}{*}{ UDDS } & & Wh/mile & 327.5 & 327.5 & 278.5 & 247.0 & 302.5 & 255.6 & 222.8 & 285.5 & 229.8 & 186.3 & 270.9 & 221.1 & 177.9 \\
\hline & & & Distance & 20.2 & 20.2 & 20.2 & 20.2 & 20.2 & 20.2 & 20.5 & 20.1 & 20.6 & 20.3 & 20.2 & 20.6 & 20.3 \\
\hline & & & MPGGE Unadjusted & N/A & N/A & N/A & N/A & N/A & N/A & N/A & N/A & N/A & N/A & N/A & $\mathrm{N} / \mathrm{A}$ & N/A \\
\hline & & & MPGGE Adjusted & N/A & $\mathrm{N} / \mathrm{A}$ & N/A & N/A & N/A & N/A & N/A & N/A & N/A & $\mathrm{N} / \mathrm{A}$ & $N / A$ & $\mathrm{~N} / \mathrm{A}$ & $\mathrm{N} / \mathrm{A}$ \\
\hline & & \multirow{2}{*}{ CS } & MPGGE Unadjusted & 50.2 & 50.2 & 61.0 & 72.3 & 59.2 & 70.0 & 81.4 & 62.8 & 79.0 & 97.3 & 66.2 & 82.0 & 102.0 \\
\hline & & & MPGGE Adjusted & 37.1 & 37.1 & 44.2 & 51.3 & 43.0 & 49.8 & 56.8 & 45.3 & 55.4 & 66.0 & 47.5 & 57.1 & 68.6 \\
\hline & & \multirow{3}{*}{$\mathrm{CD}+\mathrm{CS}$} & Wh/mile & 124.4 & 124.4 & 105.8 & 93.8 & 114.9 & 97.1 & 84.8 & 108.5 & 87.5 & 70.9 & 102.9 & 84.1 & 67.7 \\
\hline & & & MPGGE Unadjusted & 73.8 & 73.8 & 89.7 & 106.3 & 87.1 & 102.9 & 119.7 & 92.4 & 116.2 & 143.0 & 97.4 & 120.6 & 150.0 \\
\hline & & & MPGGE Adjusted & 52.2 & 52.2 & 61.7 & 71.0 & 60.2 & 69.1 & 78.2 & 63.2 & 76.3 & 89.9 & 66.0 & 78.6 & 93.2 \\
\hline & \multirow{9}{*}{ HWFET } & \multirow{4}{*}{$C D$} & Wh/mile & 373.6 & 373.6 & 324.3 & 292.1 & 354.0 & 303.6 & 266.5 & 342.0 & 274.5 & 235.1 & 324.5 & 264.5 & 221.9 \\
\hline & & & Distance & 17.4 & 17.4 & 18.2 & 16.9 & 17.1 & 16.9 & 16.8 & 16.7 & 16.9 & 16.0 & 16.7 & 16.8 & 16.1 \\
\hline & & & MPGGE Unadjusted & N/A & N/A & N/A & N/A & N/A & N/A & $\mathrm{N} / \mathrm{A}$ & N/A & N/A & N/A & N/A & N/A & N/A \\
\hline & & & MPGGE Adjusted & $\mathrm{N} / \mathrm{A}$ & $\mathrm{N} / \mathrm{A}$ & N/A & N/A & $\mathrm{N} / \mathrm{A}$ & N/A & $\mathrm{N} / \mathrm{A}$ & $\mathrm{N} / \mathrm{A}$ & $\mathrm{N} / \mathrm{A}$ & $\mathrm{N} / \mathrm{A}$ & $\mathrm{N} / \mathrm{A}$ & N/A & $\mathrm{N} / \mathrm{A}$ \\
\hline & & \multirow{2}{*}{ CS } & MPGGE Unadjusted & 49.6 & 49.6 & 59.2 & 69.2 & 57.3 & 66.7 & 76.0 & 59.3 & 73.8 & 87.0 & 62.5 & 76.6 & 92.1 \\
\hline & & & MPGGE Adjusted & 35.0 & 35.0 & 41.7 & 48.6 & 40.3 & 46.8 & 53.3 & 41.8 & 51.8 & 60.8 & 44.0 & 53.7 & 64.3 \\
\hline & & \multirow{3}{*}{$\mathrm{CD}+\mathrm{CS}$} & Wh/mile & 109.9 & 109.9 & 95.4 & 85.9 & 104.1 & 89.3 & 78.4 & 100.6 & 80.7 & 69.1 & 95.5 & 77.8 & 65.3 \\
\hline & & & MPGGE Unadjusted & 65.3 & 65.3 & 77.9 & 91.1 & 75.3 & 87.8 & 100.0 & 78.1 & 97.1 & 114.4 & 82.3 & 100.8 & 121.1 \\
\hline & & & MPGGE Adjusted & 45.9 & 45.9 & 54.6 & 63.6 & 52.8 & 61.4 & 69.8 & 54.7 & 67.8 & 79.6 & 57.6 & 70.3 & 84.1 \\
\hline & \multirow{7}{*}{ Combined } & \multirow{2}{*}{$C D$} & MPGGE Unadjusted & $\mathrm{N} / \mathrm{A}$ & $\mathrm{N} / \mathrm{A}$ & N/A & $\mathrm{N} / \mathrm{A}$ & $\mathrm{N} / \mathrm{A}$ & $\mathrm{N} / \mathrm{A}$ & $\mathrm{N} / \mathrm{A}$ & $\mathrm{N} / \mathrm{A}$ & $\mathrm{N} / \mathrm{A}$ & $\mathrm{N} / \mathrm{A}$ & N/A & $\mathrm{N} / \mathrm{A}$ & $\mathrm{N} / \mathrm{A}$ \\
\hline & & & MPGGE Adjusted & $\mathrm{N} / \mathrm{A}$ & $\mathrm{N} / \mathrm{A}$ & N/A & $\mathrm{N} / \mathrm{A}$ & $\mathrm{N} / \mathrm{A}$ & $\mathrm{N} / \mathrm{A}$ & $\mathrm{N} / \mathrm{A}$ & $\mathrm{N} / \mathrm{A}$ & $\mathrm{N} / \mathrm{A}$ & $\mathrm{N} / \mathrm{A}$ & $\mathrm{N} / \mathrm{A}$ & $\mathrm{N} / \mathrm{A}$ & $\mathrm{N} / \mathrm{A}$ \\
\hline & & \multirow{2}{*}{ CS } & MPGGE Unadjusted & 49.9 & 49.9 & 60.2 & 70.9 & 58.3 & 68.4 & 78.9 & 61.2 & 76.6 & 92.3 & 64.5 & 79.5 & 97.3 \\
\hline & & & MPGGE Adjusted & 36.1 & 36.1 & 43.0 & 50.0 & 41.8 & 48.4 & 55.2 & 43.6 & 53.7 & 63.6 & 45.8 & 55.5 & 66.6 \\
\hline & & \multirow{3}{*}{$\mathrm{CD}+\mathrm{CS}$} & Wh/mile & 117.9 & 117.9 & 101.1 & 90.3 & 110.1 & 93.6 & 81.9 & 104.9 & 84.4 & 70.1 & 99.6 & 81.3 & 66.6 \\
\hline & & & MPGGE Unadjusted & 69.7 & 69.7 & 84.0 & 98.8 & 81.4 & 95.5 & 110.0 & 85.4 & 106.8 & 128.6 & 89.9 & 110.8 & 135.4 \\
\hline & & & MPGGE Adjusted & 49.1 & 49.1 & 58.3 & 67.5 & 56.6 & 65.4 & 74.2 & 59.1 & 72.2 & 84.9 & 62.0 & 74.6 & 88.9 \\
\hline
\end{tabular}


Argonne National Laboratory Report - Light-Duty Vehicle Fuel Consumption Displacement Potential Up to 2045

\begin{tabular}{|c|c|c|c|c|c|c|c|c|c|c|c|c|c|c|c|c|}
\hline & & & & \multicolumn{13}{|c|}{ Small_SUV } \\
\hline & & & & \multirow{2}{*}{$\begin{array}{l}2010 \\
\text { Ref }\end{array}$} & \multicolumn{3}{|c|}{2010} & \multicolumn{3}{|c|}{2015} & \multicolumn{3}{|c|}{2030} & \multicolumn{3}{|c|}{2045} \\
\hline & & & & & low & avg & high & low & avg & high & low & avg & high & low & avg & high \\
\hline \multirow{25}{*}{ FC HEV PHEV 30} & \multirow{9}{*}{ UDDS } & \multirow{4}{*}{$C D$} & Wh/mile & 318.7 & 318.7 & 272.4 & 246.4 & 296.1 & 253.2 & 217.9 & 283.1 & 225.0 & 186.2 & 267.8 & 216.0 & 178.0 \\
\hline & & & Distance & 33.5 & 33.5 & 33.5 & 33.4 & 32.0 & 33.4 & 32.1 & 32.1 & 32.0 & 30.8 & 32.1 & 32.0 & 30.8 \\
\hline & & & MPGGE Unadjusted & $\mathrm{N} / \mathrm{A}$ & $\mathrm{N} / \mathrm{A}$ & N/A & N/A & N/A & $\mathrm{N} / \mathrm{A}$ & N/A & $\mathrm{N} / \mathrm{A}$ & N/A & N/A & N/A & N/A & $\mathrm{N} / \mathrm{A}$ \\
\hline & & & MPGGE Adjusted & $\mathrm{N} / \mathrm{A}$ & $\mathrm{N} / \mathrm{A}$ & $\mathrm{N} / \mathrm{A}$ & N/A & N/A & N/A & N/A & $\mathrm{N} / \mathrm{A}$ & N/A & N/A & N/A & $\mathrm{N} / \mathrm{A}$ & $\mathrm{N} / \mathrm{A}$ \\
\hline & & \multirow{2}{*}{ CS } & MPGGE Unadjusted & 49.4 & 49.4 & 59.8 & 69.6 & 58.1 & 67.8 & 79.4 & 60.8 & 76.9 & 94.3 & 64.2 & 80.1 & 98.7 \\
\hline & & & MPGGE Adjusted & 36.6 & 36.6 & 43.4 & 49.6 & 42.3 & 48.4 & 55.6 & 44.0 & 54.1 & 64.3 & 46.2 & 56.0 & 66.8 \\
\hline & & \multirow{3}{*}{$\mathrm{CD}+\mathrm{CS}$} & Wh/mile & 203.1 & 203.1 & 173.6 & 157.0 & 188.6 & 161.3 & 139.2 & 180.3 & 143.8 & 119.6 & 170.7 & 138.0 & 114.3 \\
\hline & & & MPGGE Unadjusted & 105.2 & 105.2 & 127.3 & 148.0 & 123.5 & 144.1 & 168.9 & 129.3 & 163.7 & 200.7 & 136.6 & 170.5 & 210.1 \\
\hline & & & MPGGE Adjusted & 70.4 & 70.4 & 82.1 & 92.3 & 80.2 & 90.5 & 102.0 & 83.1 & 99.6 & 115.5 & 86.8 & 102.7 & 119.3 \\
\hline & \multirow{9}{*}{ HWFET } & \multirow{4}{*}{$C D$} & Wh/mile & 359.1 & 359.1 & 312.2 & 284.8 & 340.5 & 294.2 & 258.1 & 331.2 & 265.9 & 227.6 & 313.6 & 255.8 & 215.2 \\
\hline & & & Distance & 27.2 & 27.2 & 26.7 & 26.5 & 26.5 & 26.4 & 26.0 & 26.2 & 26.0 & 25.4 & 26.2 & 25.9 & 25.6 \\
\hline & & & MPGGE Unadjusted & $\mathrm{N} / \mathrm{A}$ & $\mathrm{N} / \mathrm{A}$ & $\mathrm{N} / \mathrm{A}$ & $\mathrm{N} / \mathrm{A}$ & $\mathrm{N} / \mathrm{A}$ & $\mathrm{N} / \mathrm{A}$ & $\mathrm{N} / \mathrm{A}$ & $\mathrm{N} / \mathrm{A}$ & $\mathrm{N} / \mathrm{A}$ & $\mathrm{N} / \mathrm{A}$ & $\mathrm{N} / \mathrm{A}$ & $\mathrm{N} / \mathrm{A}$ & $\mathrm{N} / \mathrm{A}$ \\
\hline & & & MPGGE Adjusted & N/A & N/A & N/A & N/A & N/A & N/A & N/A & N/A & N/A & N/A & N/A & N/A & $\mathrm{N} / \mathrm{A}$ \\
\hline & & \multirow{2}{*}{ CS } & MPGGE Unadjusted & 48.4 & 48.4 & 57.7 & 66.6 & 55.7 & 64.5 & 73.9 & 57.3 & 71.7 & 84.4 & 60.5 & 74.5 & 89.3 \\
\hline & & & MPGGE Adjusted & 34.1 & 34.1 & 40.6 & 46.8 & 39.2 & 45.3 & 51.8 & 40.3 & 50.3 & 59.0 & 42.6 & 52.3 & 62.4 \\
\hline & & \multirow{3}{*}{$\mathrm{CD}+\mathrm{CS}$} & Wh/mile & 184.8 & 184.8 & 160.7 & 146.6 & 175.2 & 151.4 & 132.9 & 170.4 & 136.9 & 117.3 & 161.4 & 131.7 & 110.9 \\
\hline & & & MPGGE Unadjusted & 82.1 & 82.1 & 97.8 & 112.9 & 94.4 & 109.3 & 125.2 & 97.2 & 121.5 & 143.0 & 102.6 & 126.3 & 151.3 \\
\hline & & & \begin{tabular}{|l|} 
MPGGE Adjusted \\
\end{tabular} & 57.5 & 57.5 & 68.3 & 78.5 & 65.9 & 76.1 & 86.9 & 67.8 & 84.4 & 98.8 & 71.5 & 87.6 & 104.4 \\
\hline & \multirow{7}{*}{ Combined } & \multirow{2}{*}{$C D$} & MPGGE Unadjusted & $\mathrm{N} / \mathrm{A}$ & $\mathrm{N} / \mathrm{A}$ & $\mathrm{N} / \mathrm{A}$ & N/A & N/A & $\mathrm{N} / \mathrm{A}$ & N/A & $\mathrm{N} / \mathrm{A}$ & $\mathrm{N} / \mathrm{A}$ & N/A & $\mathrm{N} / \mathrm{A}$ & N/A & $\mathrm{N} / \mathrm{A}$ \\
\hline & & & MPGGE Adjusted & N/A & N/A & N/A & N/A & N/A & N/A & N/A & N/A & N/A & N/A & N/A & N/A & N/A \\
\hline & & \multirow{2}{*}{ CS } & MPGGE Unadjusted & 49.0 & 49.0 & 58.9 & 68.2 & 57.0 & 66.2 & 76.8 & 59.2 & 74.5 & 89.6 & 62.5 & 77.5 & 94.2 \\
\hline & & & MPGGE Adjusted & 35.4 & 35.4 & 42.1 & 48.3 & 40.8 & 47.0 & 53.8 & 42.3 & 52.3 & 61.8 & 44.5 & 54.3 & 64.8 \\
\hline & & \multirow{3}{*}{$C D+C S$} & Wh/mile & 194.8 & 194.8 & 167.7 & 152.3 & 182.6 & 156.8 & 136.4 & 175.9 & 140.7 & 118.6 & 166.5 & 135.2 & 112.8 \\
\hline & & & MPGGE Unadjusted & 93.4 & $\begin{array}{ll}93.4 \\
\end{array}$ & 112.1 & 129.8 & 108.5 & 126.1 & 146.0 & 112.5 & 141.6 & 169.8 & 118.8 & 147.3 & 178.8 \\
\hline & & & MPGGE Adjusted & 63.9 & 63.9 & 75.2 & 85.5 & 73.1 & $\begin{array}{l}83.4 \\
\end{array}$ & 94.6 & 75.4 & 92.1 & 107.3 & 79.2 & 95.3 & 112.1 \\
\hline
\end{tabular}




\begin{tabular}{|c|c|c|c|c|c|c|c|c|c|c|c|c|c|c|c|c|}
\hline & & & & \multicolumn{13}{|c|}{ Small_SUV } \\
\hline & & & & 2010 & & 2010 & & & 2015 & & & 2030 & & & 2045 & \\
\hline \multirow{26}{*}{ FC HEV PHEV 40} & & \multirow{5}{*}{$C D$} & & Ref & low & avg & high & low & avg & high & low & avg & high & low & avg & high \\
\hline & \multirow{9}{*}{ UDDS } & & Wh/mile & 325.3 & 325.3 & 277.1 & 250.3 & 301.5 & 257.1 & 220.1 & 287.8 & 227.3 & 189.1 & 272.1 & 217.7 & 180.6 \\
\hline & & & Distance & 41.9 & 41.9 & 41.1 & 41.1 & 41.1 & 41.1 & 40.9 & 41.1 & 41.0 & 40.6 & 41.1 & 41.0 & 40.6 \\
\hline & & & MPGGE Unadjusted & $\mathrm{N} / \mathrm{A}$ & N/A & N/A & N/A & $N / A$ & $N / A$ & N/A & N/A & N/A & N/A & N/A & N/A & N/A \\
\hline & & & MPGGE Adjusted & $\mathrm{N} / \mathrm{A}$ & $\mathrm{N} / \mathrm{A}$ & N/A & N/A & N/A & N/A & N/A & $\mathrm{N} / \mathrm{A}$ & N/A & N/A & N/A & N/A & N/A \\
\hline & & \multirow{2}{*}{ CS } & MPGGE Unadjusted & 48.4 & 48.4 & 58.8 & 68.5 & 57.0 & 66.7 & 78.4 & 59.8 & 76.0 & 93.3 & 63.1 & 79.3 & 97.8 \\
\hline & & & MPGGE Adjusted & 35.9 & 35.9 & 42.7 & 48.9 & 41.6 & 47.8 & 55.0 & 43.4 & 53.5 & 63.7 & 45.5 & 55.5 & 66.3 \\
\hline & & \multirow{3}{*}{$\mathrm{CD}+\mathrm{CS}$} & Wh/mile & 235.2 & 235.2 & 200.3 & 180.9 & 217.9 & 185.9 & 159.7 & 208.1 & 164.9 & 138.6 & 196.7 & 157.9 & 132.3 \\
\hline & & & MPGGE Unadjusted & 121.0 & 121.0 & 147.0 & 171.1 & 142.5 & 166.7 & 196.0 & 149.4 & 189.9 & 233.2 & 157.9 & 198.2 & 244.5 \\
\hline & & & MPGGE Adjusted & 78.9 & 78.9 & 91.8 & 103.0 & 89.6 & 101.0 & 113.6 & 93.0 & 111.1 & 128.1 & 96.9 & 114.5 & 132.2 \\
\hline & \multirow{9}{*}{ HWFET } & \multirow{4}{*}{$C D$} & Wh/mile & 364.1 & 364.1 & 315.8 & 287.7 & 344.6 & 297.2 & 260.2 & 334.8 & 268.1 & 228.4 & 316.8 & 257.5 & 215.7 \\
\hline & & & Distance & 36.7 & 36.7 & 38.7 & 35.5 & 38.7 & 35.3 & 35.5 & 39.0 & 37.6 & 34.4 & 35.2 & 36.0 & 34.6 \\
\hline & & & MPGGE Unadjusted & $\mathrm{N} / \mathrm{A}$ & N/A & $\mathrm{N} / \mathrm{A}$ & N/A & N/A & N/A & N/A & N/A & N/A & N/A & N/A & N/A & N/A \\
\hline & & & MPGGE Adjusted & $\mathrm{N} / \mathrm{A}$ & $\mathrm{N} / \mathrm{A}$ & $\mathrm{N} / \mathrm{A}$ & $\mathrm{N} / \mathrm{A}$ & $\mathrm{N} / \mathrm{A}$ & $\mathrm{N} / \mathrm{A}$ & N/A & N/A & N/A & $\mathrm{N} / \mathrm{A}$ & $\mathrm{N} / \mathrm{A}$ & $\mathrm{N} / \mathrm{A}$ & $\mathrm{N} / \mathrm{A}$ \\
\hline & & \multirow{2}{*}{ CS } & MPGGE Unadjusted & 47.8 & 47.8 & 57.1 & 65.9 & 55.0 & 63.9 & 73.3 & 56.7 & 71.2 & 83.9 & 59.9 & 74.1 & 88.8 \\
\hline & & & MPGGE Adjusted & 33.7 & 33.7 & 40.2 & 46.3 & 38.8 & 44.9 & 51.4 & 39.9 & 49.9 & 58.7 & 42.1 & 52.0 & 62.1 \\
\hline & & \multirow{3}{*}{$\mathrm{CD}+\mathrm{CS}$} & Wh/mile & 245.3 & 245.3 & 212.7 & 193.9 & 232.2 & 200.2 & 175.5 & 225.6 & 180.9 & 154.2 & 213.5 & 173.6 & 145.6 \\
\hline & & & MPGGE Unadjusted & 103.8 & 103.8 & 124.1 & 143.4 & 119.7 & 138.8 & 159.4 & 123.3 & 154.7 & 182.3 & 130.2 & 161.0 & 193.1 \\
\hline & & & MPGGE Adjusted & 72.4 & 72.4 & 86.1 & 99.1 & 83.1 & 96.0 & 109.8 & 85.6 & 106.6 & 125.0 & 90.2 & 110.9 & 132.0 \\
\hline & \multirow{7}{*}{ Combined } & \multirow{2}{*}{$C D$} & MPGGE Unadjusted & $\mathrm{N} / \mathrm{A}$ & $\mathrm{N} / \mathrm{A}$ & N/A & N/A & N/A & $\mathrm{N} / \mathrm{A}$ & $\mathrm{N} / \mathrm{A}$ & N/A & $\mathrm{N} / \mathrm{A}$ & $\mathrm{N} / \mathrm{A}$ & N/A & $\mathrm{N} / \mathrm{A}$ & N/A \\
\hline & & & MPGGE Adjusted & N/A & $\mathrm{N} / \mathrm{A}$ & N/A & N/A & N/A & N/A & N/A & N/A & N/A & N/A & N/A & N/A & N/A \\
\hline & & \multirow{2}{*}{ CS } & MPGGE Unadjusted & 48.1 & 48.1 & 58.0 & 67.3 & 56.1 & 65.4 & 76.0 & 58.4 & 73.7 & 88.8 & 61.6 & 76.9 & 93.5 \\
\hline & & & MPGGE Adjusted & 34.9 & 34.9 & 41.5 & 47.7 & 40.3 & 46.4 & 53.3 & 41.7 & 51.8 & 61.4 & 43.9 & 53.9 & 64.3 \\
\hline & & \multirow{3}{*}{$C D+C S$} & Wh/mile & 239.7 & 239.7 & 205.9 & 186.7 & 224.3 & 192.3 & 166.8 & 215.9 & 172.1 & 145.6 & 204.2 & 165.0 & 138.3 \\
\hline & & & MPGGE Unadjusted & 112.6 & 112.6 & 135.7 & 157.4 & 131.2 & 152.8 & 177.7 & 136.4 & 172.3 & 207.2 & 144.1 & 179.6 & 218.3 \\
\hline & & & MPGGE Adjusted & 75.8 & 75.8 & 89.2 & 101.2 & 86.6 & 98.7 & 111.8 & 89.5 & 109.0 & 126.7 & 93.8 & 112.8 & 132.1 \\
\hline \multirow{2}{*}{ EV } & UDDS & EV Only & Wh/mile & 321.9 & 321.9 & 288.7 & 264.9 & 312.3 & 274.9 & 228.3 & 303.8 & 235.4 & 189.1 & 293.7 & 224.4 & 179.9 \\
\hline & HWFET & EV Only & Wh/mile & 355.4 & 355.4 & 318.7 & 293.2 & 346.3 & 304.7 & 262.3 & 339.7 & 269.9 & 226.2 & 326.1 & 258.2 & 213.6 \\
\hline
\end{tabular}

\section{Fuel consumption}




\begin{tabular}{|c|c|c|c|c|c|c|c|c|c|c|c|c|c|c|c|}
\hline & & & \multicolumn{13}{|c|}{ Small_SUV } \\
\hline & & & 2010 & & 2010 & & & 2015 & & & 2030 & & & 2045 & \\
\hline & & & Ref & low & avg & high & low & avg & high & low & avg & high & low & avg & high \\
\hline \multirow{6}{*}{ SI Conv } & \multirow{3}{*}{ Unadjusted } & UDDS & 9.3 & 9.3 & 8.4 & 8.0 & 8.9 & 8.1 & 6.4 & 8.7 & 7.6 & 5.3 & 8.6 & 7.5 & 5.2 \\
\hline & & HWFET & 7.0 & 7.0 & 6.4 & 6.0 & 6.6 & 6.2 & 4.7 & 6.7 & 5.7 & 4.0 & 6.4 & 5.5 & 3.8 \\
\hline & & Combined & 8.3 & 8.3 & 7.5 & 7.1 & 7.9 & 7.3 & 5.7 & 7.8 & 6.7 & 4.7 & 7.6 & 6.6 & 4.6 \\
\hline & \multirow{3}{*}{ Adjusted } & \begin{tabular}{|l|} 
UDDS \\
\end{tabular} & 12.0 & 12.0 & 11.0 & 10.4 & 11.5 & 10.6 & 8.5 & 11.2 & 9.9 & 7.2 & 11.1 & 9.8 & 6.9 \\
\hline & & HWFET & 9.9 & 9.9 & 9.1 & 8.5 & 9.4 & 8.7 & 6.7 & 9.5 & 8.0 & 5.7 & 9.1 & 7.9 & 5.4 \\
\hline & & Combined & 11.1 & 11.1 & 10.1 & 9.6 & 10.5 & 9.7 & 7.7 & 10.4 & 9.1 & 6.5 & 10.2 & 8.9 & 6.3 \\
\hline \multirow{6}{*}{$\mathrm{Cl}$ Conv } & \multirow{3}{*}{ Unadjusted } & \begin{tabular}{|l|} 
UDDS \\
\end{tabular} & 7.9 & 7.9 & 7.5 & 6.9 & 7.7 & 7.0 & 6.3 & 7.5 & 6.4 & 5.4 & 7.2 & 6.2 & 5.2 \\
\hline & & HWFET & 6.2 & 6.2 & 5.8 & 5.3 & 6.0 & 5.4 & 4.7 & 5.9 & 4.9 & 4.1 & 5.5 & 4.7 & 3.9 \\
\hline & & Combined & 7.1 & 7.1 & 6.7 & 6.1 & 7.0 & 6.3 & 5.6 & 6.8 & 5.7 & 4.8 & 6.5 & 5.5 & 4.6 \\
\hline & \multirow{3}{*}{ Adjusted } & \begin{tabular}{|l|} 
UDDS \\
\end{tabular} & 10.2 & 10.2 & 9.7 & 9.0 & 10.1 & 9.2 & 8.3 & 9.8 & 8.5 & 7.2 & 9.5 & 8.2 & 7.0 \\
\hline & & HWFET & 8.7 & 8.7 & 8.2 & 7.4 & 8.5 & 7.6 & 6.7 & 8.3 & 6.9 & 5.8 & 7.8 & 6.6 & 5.6 \\
\hline & & Combined & 9.5 & 9.5 & 9.0 & 8.3 & 9.4 & 8.5 & 7.6 & 9.1 & 7.8 & 6.6 & 8.7 & 7.5 & 6.4 \\
\hline \multirow{6}{*}{$\mathrm{H} 2$ Conv } & \multirow{3}{*}{ Unadjusted } & \begin{tabular}{|l|} 
UDDS \\
\end{tabular} & 10.9 & 10.9 & 9.1 & 7.9 & 9.6 & 8.1 & 7.1 & 8.8 & 7.3 & 6.0 & 8.2 & 6.9 & 5.6 \\
\hline & & HWFET & 7.5 & 7.5 & 6.2 & 5.4 & 6.6 & 5.5 & 4.8 & 6.1 & 5.0 & 4.2 & 5.6 & 4.7 & 3.8 \\
\hline & & Combined & 9.4 & 9.4 & 7.8 & 6.8 & 8.3 & 6.9 & 6.1 & 7.6 & 6.2 & 5.2 & 7.1 & 5.9 & 4.8 \\
\hline & \multirow{3}{*}{ Adjusted } & \begin{tabular}{|l|} 
UDDS \\
\end{tabular} & 13.9 & 13.9 & 11.7 & 10.3 & 12.4 & 10.5 & 9.3 & 11.4 & 9.5 & 8.0 & 10.7 & 9.1 & 7.4 \\
\hline & & \begin{tabular}{l|l|} 
HWFET \\
\end{tabular} & 10.6 & 10.6 & 8.7 & 7.6 & 9.4 & 7.8 & 6.9 & 8.6 & 7.0 & 5.9 & 8.0 & 6.6 & 5.4 \\
\hline & & Combined & 12.4 & 12.4 & 10.4 & 9.1 & 11.0 & 9.3 & 8.2 & 10.1 & 8.4 & 7.0 & 9.5 & 8.0 & 6.5 \\
\hline \multirow{6}{*}{ E85 Conv } & \multirow{3}{*}{ Unadjusted } & \begin{tabular}{|l|} 
UDDS \\
\end{tabular} & 9.7 & 9.7 & 9.4 & 8.9 & 9.9 & 9.1 & 6.7 & 9.7 & 8.4 & 5.7 & 9.5 & 8.3 & 5.5 \\
\hline & & HWFET & 7.6 & 7.6 & 7.3 & 6.9 & 7.8 & 7.1 & 5.3 & 7.7 & 6.7 & 4.6 & 7.6 & 6.6 & 4.4 \\
\hline & & Combined & 8.8 & 8.8 & 8.5 & 8.0 & 9.0 & 8.2 & 6.1 & 8.8 & 7.7 & 5.2 & 8.6 & 7.5 & 5.0 \\
\hline & \multirow{3}{*}{ Adjusted } & \begin{tabular}{|l|} 
UDDS \\
\end{tabular} & 12.5 & 12.5 & 12.1 & 11.5 & 12.8 & 11.7 & 8.8 & 12.5 & 10.9 & 7.6 & 12.2 & 10.8 & 7.4 \\
\hline & & HWFET & 10.8 & 10.8 & 10.4 & 9.8 & 11.0 & 10.0 & 7.6 & 10.8 & 9.5 & 6.6 & 10.8 & 9.4 & 6.3 \\
\hline & & Combined & 11.7 & 11.7 & 11.3 & 10.7 & 12.0 & 11.0 & 8.2 & 11.7 & 10.3 & 7.1 & 11.6 & 10.1 & 6.9 \\
\hline \multirow{6}{*}{ SI Split HEV } & \multirow{3}{*}{ Unadjusted } & \begin{tabular}{|l|} 
UDDS \\
\end{tabular} & 5.2 & 5.2 & 4.7 & 4.3 & 5.0 & 4.4 & 3.5 & 4.8 & 3.9 & 2.8 & 4.6 & 3.8 & 2.7 \\
\hline & & HWFET & 6.0 & 6.0 & 5.4 & 5.0 & 5.8 & 5.2 & 4.2 & 5.6 & 4.7 & 3.4 & 5.4 & 4.4 & 3.3 \\
\hline & & Combined & 5.5 & 5.5 & 5.0 & 4.6 & 5.4 & 4.7 & 3.8 & 5.2 & 4.3 & 3.1 & 5.0 & 4.1 & 3.0 \\
\hline & \multirow{3}{*}{ Adjusted } & \begin{tabular}{|l|} 
UDDS \\
\end{tabular} & 7.0 & 7.0 & 6.4 & 5.9 & 6.8 & 6.0 & 4.9 & 6.5 & 5.4 & 4.1 & 6.3 & 5.2 & 3.9 \\
\hline & & HWFET & 8.5 & 8.5 & 7.7 & 7.0 & 8.2 & 7.3 & 5.9 & 8.0 & 6.6 & 4.9 & 7.7 & 6.3 & 4.6 \\
\hline & & Combined & 7.6 & 7.6 & 6.9 & 6.4 & 7.4 & 6.6 & 5.4 & 7.1 & 5.9 & 4.4 & 6.9 & 5.7 & 4.2 \\
\hline \multirow{6}{*}{ SI Split HEV PHEV10 } & \multirow{3}{*}{ Unadjusted } & \begin{tabular}{|l|} 
UDDS \\
\end{tabular} & 4.1 & 4.1 & 3.7 & 3.4 & 4.0 & 3.5 & 2.9 & 3.9 & 3.2 & 2.3 & 3.8 & 3.1 & 2.2 \\
\hline & & HWFET & 4.6 & 4.6 & 4.2 & 3.7 & 4.5 & 3.8 & 3.1 & 4.4 & 3.5 & 2.6 & 4.2 & 3.3 & 2.5 \\
\hline & & Combined & 4.3 & 4.3 & 3.9 & 3.5 & 4.2 & 3.7 & 3.0 & 4.1 & 3.3 & 2.5 & 4.0 & 3.2 & 2.3 \\
\hline & \multirow{3}{*}{ Adjusted } & UDDS & 5.6 & 5.6 & 5.2 & 4.8 & 5.5 & 4.9 & 4.1 & 5.4 & 4.5 & 3.4 & 5.2 & 4.4 & 3.3 \\
\hline & & HWFET & 6.6 & 6.6 & 5.9 & 5.2 & 6.4 & 5.4 & 4.4 & 6.2 & 4.9 & 3.7 & 6.0 & 4.7 & 3.5 \\
\hline & & Combined & 6.1 & 6.1 & 5.5 & 5.0 & 5.9 & 5.2 & 4.3 & 5.8 & 4.7 & 3.6 & 5.6 & 4.5 & 3.4 \\
\hline
\end{tabular}




\begin{tabular}{|c|c|c|c|c|c|c|c|c|c|c|c|c|c|c|c|}
\hline & & & \multicolumn{13}{|c|}{ Small_SUV } \\
\hline & & & \multirow{2}{*}{$\begin{array}{c}2010 \\
\text { Ref }\end{array}$} & \multicolumn{3}{|c|}{2010} & \multicolumn{3}{|c|}{2015} & \multicolumn{3}{|c|}{2030} & \multicolumn{3}{|c|}{2045} \\
\hline & & & & low & avg & high & low & avg & high & low & avg & high & low & avg & high \\
\hline \multirow{6}{*}{ SI Split HEV PHEV20 } & \multirow{3}{*}{ Unadjusted } & UDDS & 2.9 & 2.9 & 2.6 & 2.4 & 2.8 & 2.5 & 2.0 & 2.7 & 2.2 & 1.9 & 2.6 & 2.2 & 1.8 \\
\hline & & HWFET & 4.1 & 4.1 & 4.0 & 3.7 & 3.6 & 3.8 & 3.1 & 3.7 & 3.5 & 2.5 & 3.5 & 3.3 & 2.4 \\
\hline & & Combined & 3.4 & 3.4 & 3.2 & 3.0 & 3.2 & 3.1 & 2.5 & 3.1 & 2.8 & 2.2 & 3.0 & 2.7 & 2.1 \\
\hline & \multirow{3}{*}{ Adjusted } & UDDS & 4.2 & 4.2 & 3.8 & 3.6 & 4.0 & 3.7 & 3.1 & 3.9 & 3.3 & 2.9 & 3.8 & 3.2 & 2.9 \\
\hline & & HWFET & 5.8 & 5.8 & 5.7 & 5.2 & 5.2 & 5.5 & 4.4 & 5.2 & 4.9 & 3.6 & 5.0 & 4.7 & 3.4 \\
\hline & & Combined & 4.9 & 4.9 & 4.7 & 4.3 & 4.5 & 4.5 & 3.7 & 4.5 & 4.1 & 3.2 & 4.4 & 3.9 & 3.1 \\
\hline \multirow{6}{*}{ SI Series HEV PHEV30 } & \multirow{3}{*}{ Unadjusted } & \begin{tabular}{|l|} 
UDDS \\
\end{tabular} & 3.2 & 3.2 & 2.8 & 2.5 & 3.0 & 2.6 & 2.0 & 2.9 & 2.3 & 1.6 & 2.8 & 2.2 & 1.5 \\
\hline & & HWFET & 4.4 & 4.4 & 3.9 & 3.5 & 4.2 & 3.6 & 2.9 & 4.0 & 3.2 & 2.3 & 3.8 & 3.1 & 2.2 \\
\hline & & Combined & 3.7 & 3.7 & 3.3 & 3.0 & 3.6 & 3.1 & 2.4 & 3.4 & 2.7 & 1.9 & 3.3 & 2.6 & 1.8 \\
\hline & \multirow{3}{*}{ Adjusted } & UDDS & 4.5 & 4.5 & 4.1 & 3.7 & 4.3 & 3.8 & 3.1 & 4.2 & 3.4 & 2.6 & 4.0 & 3.3 & 2.5 \\
\hline & & HWFET & 6.2 & 6.2 & 5.5 & 5.0 & 6.0 & 5.2 & 4.1 & 5.7 & 4.6 & 3.4 & 5.5 & 4.4 & 3.2 \\
\hline & & Combined & 5.3 & 5.3 & 4.7 & 4.3 & 5.1 & 4.4 & 3.6 & 4.9 & 3.9 & 2.9 & 4.7 & 3.8 & 2.8 \\
\hline \multirow{6}{*}{ SI Series HEV PHEV40 } & \multirow{3}{*}{ Unadjusted } & \begin{tabular}{|l|} 
UDDS \\
\end{tabular} & 2.8 & 2.8 & 2.5 & 2.2 & 2.7 & 2.3 & 1.8 & 2.5 & 2.0 & 1.4 & 2.4 & 1.9 & 1.3 \\
\hline & & HWFET & 3.4 & 3.4 & 3.1 & 2.8 & 3.3 & 2.9 & 2.3 & 3.2 & 2.5 & 1.8 & 3.0 & 2.4 & 1.7 \\
\hline & & Combined & 3.1 & 3.1 & 2.7 & 2.5 & 3.0 & 2.6 & 2.0 & 2.8 & 2.2 & 1.6 & 2.7 & 2.1 & 1.5 \\
\hline & \multirow{3}{*}{ Adjusted } & \begin{tabular}{|l|} 
UDDS \\
\end{tabular} & 4.0 & 4.0 & 3.6 & 3.3 & 3.9 & 3.4 & 2.8 & 3.7 & 3.0 & 2.3 & 3.6 & 2.9 & 2.2 \\
\hline & & HWFET & 4.9 & 4.9 & 4.4 & 4.0 & 4.7 & 4.1 & 3.3 & 4.5 & 3.6 & 2.7 & 4.3 & 3.5 & 2.5 \\
\hline & & Combined & 4.4 & 4.4 & 3.9 & 3.6 & 4.3 & 3.7 & 3.0 & 4.1 & 3.3 & 2.5 & 3.9 & 3.2 & 2.3 \\
\hline \multirow{6}{*}{ CI Split HEV } & \multirow{3}{*}{ Unadjusted } & \begin{tabular}{|l|} 
UDDS \\
\end{tabular} & 4.7 & 4.7 & 4.3 & 3.9 & 4.6 & 4.0 & 3.5 & 4.4 & 3.5 & 2.8 & 4.2 & 3.4 & 2.7 \\
\hline & & HWFET & 5.6 & 5.6 & 5.2 & 4.7 & 5.5 & 4.8 & 4.2 & 5.3 & 4.3 & 3.5 & 5.0 & 4.1 & 3.4 \\
\hline & & Combined & 5.1 & 5.1 & 4.7 & 4.3 & 5.0 & 4.4 & 3.8 & 4.8 & 3.9 & 3.2 & 4.6 & 3.7 & 3.0 \\
\hline & \multirow{3}{*}{ Adjusted } & \begin{tabular}{|l|} 
UDDS \\
\end{tabular} & 6.4 & 6.4 & 5.9 & 5.4 & 6.3 & 5.5 & 4.8 & 5.9 & 5.0 & 4.1 & 5.7 & 4.7 & 4.0 \\
\hline & & \begin{tabular}{|l|} 
HWFET \\
\end{tabular} & 8.0 & 8.0 & 7.3 & 6.6 & 7.9 & 6.8 & 5.9 & 7.5 & 6.1 & 5.0 & 7.1 & 5.8 & 4.8 \\
\hline & & Combined & 7.1 & 7.1 & 6.5 & 6.0 & 7.0 & 6.1 & 5.3 & 6.6 & 5.5 & 4.5 & 6.4 & 5.2 & 4.3 \\
\hline \multirow{6}{*}{ CI Split HEV PHEV10 } & \multirow{3}{*}{ Unadjusted } & \begin{tabular}{|l|} 
UDDS \\
\end{tabular} & 4.0 & 4.0 & 3.7 & 3.3 & 3.9 & 3.4 & 3.0 & 3.8 & 3.1 & 2.5 & 3.6 & 2.9 & 2.4 \\
\hline & & \begin{tabular}{|l|} 
HWFET \\
\end{tabular} & 4.6 & 4.6 & 4.0 & 3.6 & 4.3 & 3.7 & 3.2 & 4.3 & 3.3 & 2.8 & 4.1 & 3.2 & 2.6 \\
\hline & & Combined & 4.3 & 4.3 & 3.8 & 3.4 & 4.1 & 3.6 & 3.1 & 4.0 & 3.2 & 2.6 & 3.8 & 3.0 & 2.5 \\
\hline & \multirow{3}{*}{ Adjusted } & \begin{tabular}{|l|} 
UDDS \\
\end{tabular} & 5.6 & 5.6 & 5.1 & 4.7 & 5.4 & 4.8 & 4.3 & 5.2 & 4.4 & 3.6 & 5.0 & 4.2 & 3.5 \\
\hline & & HWFET & 6.5 & 6.5 & 5.7 & 5.1 & 6.1 & 5.3 & 4.6 & 6.1 & 4.7 & 4.0 & 5.8 & 4.5 & 3.8 \\
\hline & & Combined & 6.0 & 6.0 & 5.3 & 4.9 & 5.7 & 5.0 & 4.4 & 5.6 & 4.5 & 3.8 & 5.4 & 4.3 & 3.6 \\
\hline \multirow{6}{*}{ CI Split HEV PHEV2O } & & \begin{tabular}{|l|} 
UDDS \\
\end{tabular} & 2.8 & 2.8 & 2.6 & 2.3 & 2.7 & 2.4 & 2.1 & 2.6 & 2.1 & 1.7 & 2.5 & 2.0 & 1.6 \\
\hline & Unadjusted & \begin{tabular}{|c|} 
HWFET \\
\end{tabular} & 3.8 & 3.8 & 4.0 & 3.0 & 4.3 & 3.7 & 2.7 & 4.2 & 2.8 & 2.7 & 3.2 & 3.2 & 2.5 \\
\hline & & Combined & 3.3 & 3.3 & 3.2 & 2.6 & 3.4 & 3.0 & 2.4 & 3.3 & 2.4 & 2.1 & 2.8 & 2.6 & 2.0 \\
\hline & & \begin{tabular}{|l|} 
UDDS \\
\end{tabular} & 4.1 & 4.1 & 3.7 & 3.5 & 4.0 & 3.5 & 3.2 & 3.8 & 3.2 & 2.7 & 3.7 & 3.1 & 2.6 \\
\hline & Adjusted & \begin{tabular}{|l|} 
HWFET \\
\end{tabular} & 5.5 & 5.5 & 5.6 & 4.2 & 6.1 & 5.3 & 3.8 & 5.9 & 3.9 & 3.8 & 4.6 & 4.5 & 3.6 \\
\hline & & Combined & 4.7 & 4.7 & 4.6 & 3.8 & 4.9 & 4.3 & 3.5 & 4.8 & 3.5 & 3.2 & 4.1 & 3.7 & 3.0 \\
\hline
\end{tabular}




\begin{tabular}{|c|c|c|c|c|c|c|c|c|c|c|c|c|c|c|c|}
\hline & & & \multicolumn{13}{|c|}{ Small_SUV } \\
\hline & & & \multirow{2}{*}{$\begin{array}{c}2010 \\
\text { Ref }\end{array}$} & \multicolumn{3}{|c|}{2010} & \multicolumn{3}{|c|}{2015} & \multicolumn{3}{|c|}{2030} & \multicolumn{3}{|c|}{2045} \\
\hline & & & & low & avg & high & low & avg & high & low & avg & high & low & avg & high \\
\hline \multirow{6}{*}{ CI Series HEV PHEV 30} & \multirow{3}{*}{ Unadjusted } & UDDS & 3.1 & 3.1 & 2.8 & 2.5 & 3.0 & 2.6 & 2.1 & 2.8 & 2.2 & 1.7 & 2.7 & 2.1 & 1.6 \\
\hline & & HWFET & 4.3 & 4.3 & 3.8 & 3.4 & 4.2 & 3.5 & 3.0 & 3.9 & 3.1 & 2.5 & 3.7 & 2.9 & 2.3 \\
\hline & & Combined & 3.6 & 3.6 & 3.2 & 2.9 & 3.5 & 3.0 & 2.5 & 3.3 & 2.6 & 2.1 & 3.1 & 2.5 & 2.0 \\
\hline & \multirow{3}{*}{ Adjusted } & \begin{tabular}{|l|} 
UDDS \\
\end{tabular} & 4.4 & 4.4 & 4.0 & 3.6 & 4.3 & 3.7 & 3.2 & 4.1 & 3.3 & 2.7 & 3.9 & 3.2 & 2.6 \\
\hline & & HWFET & 6.1 & 6.1 & 5.4 & 4.9 & 5.9 & 5.0 & 4.3 & 5.6 & 4.4 & 3.6 & 5.3 & 4.2 & 3.4 \\
\hline & & Combined & 5.2 & 5.2 & 4.6 & 4.2 & 5.0 & 4.3 & 3.7 & 4.7 & 3.8 & 3.1 & 4.5 & 3.6 & 2.9 \\
\hline \multirow{6}{*}{ CI Series HEV PHEV 40} & \multirow{3}{*}{ Unadjusted } & UDDS & 2.7 & 2.7 & 2.4 & 2.2 & 2.6 & 2.2 & 1.8 & 2.5 & 1.9 & 1.5 & 2.3 & 1.8 & 1.4 \\
\hline & & HWFET & 3.4 & 3.4 & 3.0 & 2.7 & 3.3 & 2.8 & 2.4 & 3.1 & 2.4 & 2.0 & 2.9 & 2.3 & 1.8 \\
\hline & & Combined & 3.0 & 3.0 & 2.7 & 2.4 & 2.9 & 2.5 & 2.1 & 2.7 & 2.1 & 1.7 & 2.6 & 2.0 & 1.6 \\
\hline & \multirow{3}{*}{ Adjusted } & UDDS & 3.9 & 3.9 & 3.6 & 3.2 & 3.8 & 3.3 & 2.8 & 3.6 & 2.9 & 2.4 & 3.5 & 2.8 & 2.3 \\
\hline & & HWFET & 4.8 & 4.8 & 4.3 & 3.9 & 4.7 & 4.0 & 3.4 & 4.4 & 3.5 & 2.8 & 4.2 & 3.3 & 2.7 \\
\hline & & Combined & 4.3 & 4.3 & 3.9 & 3.5 & 4.2 & 3.6 & 3.1 & 4.0 & 3.2 & 2.6 & 3.8 & 3.0 & 2.5 \\
\hline \multirow{6}{*}{ H2 Split HEV } & \multirow{3}{*}{ Unadjusted } & \begin{tabular}{|l|} 
UDDS \\
\end{tabular} & 5.3 & 5.3 & 4.3 & 3.7 & 4.7 & 3.8 & 3.2 & 4.3 & 3.3 & 2.6 & 3.9 & 3.1 & 2.4 \\
\hline & & HWFET & 6.2 & 6.2 & 5.1 & 4.4 & 5.5 & 4.5 & 3.8 & 5.0 & 4.0 & 3.2 & 4.6 & 3.7 & 2.9 \\
\hline & & Combined & 5.7 & 5.7 & 4.7 & 4.0 & 5.1 & 4.1 & 3.5 & 4.6 & 3.6 & 2.9 & 4.2 & 3.3 & 2.7 \\
\hline & \multirow{3}{*}{ Adjusted } & \begin{tabular}{|l|} 
UDDS \\
\end{tabular} & 7.1 & 7.1 & 5.9 & 5.2 & 6.4 & 5.3 & 4.5 & 5.8 & 4.7 & 3.8 & 5.4 & 4.4 & 3.6 \\
\hline & & HWFET & 8.8 & 8.8 & 7.2 & 6.2 & 7.8 & 6.4 & 5.4 & 7.2 & 5.6 & 4.6 & 6.6 & 5.2 & 4.2 \\
\hline & & Combined & 7.9 & 7.9 & 6.5 & 5.7 & 7.0 & 5.8 & 4.9 & 6.4 & 5.1 & 4.2 & 5.9 & 4.8 & 3.9 \\
\hline \multirow{6}{*}{ H2 Split HEV PHEV10 } & \multirow{3}{*}{ Unadjusted } & \begin{tabular}{|l|} 
UDDS \\
\end{tabular} & 4.3 & 4.3 & 3.5 & 3.0 & 3.8 & 3.1 & 2.6 & 3.4 & 2.7 & 2.1 & 3.2 & 2.5 & 2.0 \\
\hline & & HWFET & 4.8 & 4.8 & 3.8 & 3.2 & 4.2 & 3.3 & 2.9 & 3.9 & 2.9 & 2.4 & 3.6 & 2.7 & 2.2 \\
\hline & & Combined & 4.5 & 4.5 & 3.6 & 3.1 & 4.0 & 3.2 & 2.7 & 3.6 & 2.8 & 2.3 & 3.4 & 2.6 & 2.1 \\
\hline & \multirow{3}{*}{ Adjusted } & \begin{tabular}{|l|} 
UDDS \\
\end{tabular} & 5.8 & 5.8 & 4.9 & 4.3 & 5.2 & 4.4 & 3.8 & 4.8 & 3.9 & 3.2 & 4.5 & 3.7 & 3.0 \\
\hline & & HWFET & 6.7 & 6.7 & 5.3 & 4.6 & 6.0 & 4.8 & 4.1 & 5.5 & 4.2 & 3.5 & 5.1 & 3.9 & 3.2 \\
\hline & & Combined & 6.2 & 6.2 & 5.1 & 4.4 & 5.6 & 4.6 & 3.9 & 5.1 & 4.0 & 3.4 & 4.8 & 3.8 & 3.1 \\
\hline \multirow{6}{*}{ H2 Split HEV PHEV2O } & \multirow{3}{*}{ Unadjusted } & \begin{tabular}{|l|} 
UDDS \\
\end{tabular} & 3.0 & 3.0 & 2.4 & 2.1 & 2.6 & 2.2 & 1.8 & 2.4 & 1.9 & 1.8 & 2.2 & 1.7 & 1.6 \\
\hline & & HWFET & 4.2 & 4.2 & 3.1 & 2.7 & 3.5 & 2.8 & 2.8 & 3.2 & 2.9 & 2.3 & 3.0 & 2.7 & 2.1 \\
\hline & & Combined & 3.5 & 3.5 & 2.7 & 2.4 & 3.0 & 2.5 & 2.3 & 2.8 & 2.4 & 2.0 & 2.6 & 2.2 & 1.8 \\
\hline & \multirow{3}{*}{ Adjusted } & \begin{tabular}{|l|} 
UDDS \\
\end{tabular} & 4.3 & 4.3 & 3.6 & 3.2 & 3.8 & 3.3 & 2.8 & 3.5 & 2.9 & 2.8 & 3.3 & 2.7 & 2.6 \\
\hline & & HWFET & 5.9 & 5.9 & 4.4 & 3.9 & 5.0 & 4.0 & 4.0 & 4.6 & 4.2 & 3.3 & 4.3 & 3.9 & 3.0 \\
\hline & & Combined & 5.0 & 5.0 & 4.0 & 3.5 & 4.3 & 3.6 & 3.4 & 4.0 & 3.5 & 3.0 & 3.7 & 3.2 & 2.8 \\
\hline \multirow{6}{*}{ H2 Series HEV PHEV3O } & & \begin{tabular}{|l|} 
UDDS \\
\end{tabular} & 3.3 & 3.3 & 2.6 & 2.2 & 2.9 & 2.3 & 1.9 & 2.6 & 1.9 & 1.5 & 2.3 & 1.8 & 1.4 \\
\hline & Unadjusted & \begin{tabular}{|c|} 
HWFET \\
\end{tabular} & 4.4 & 4.4 & 3.6 & 3.0 & 3.9 & 3.2 & 2.6 & 3.5 & 2.7 & 2.2 & 3.2 & 2.5 & 2.0 \\
\hline & & Combined & 3.8 & 3.8 & 3.0 & 2.6 & 3.3 & 2.7 & 2.2 & 3.0 & 2.3 & 1.8 & 2.7 & 2.1 & 1.7 \\
\hline & & \begin{tabular}{|l|} 
UDDS \\
\end{tabular} & 4.6 & 4.6 & 3.8 & 3.3 & 4.1 & 3.4 & 2.9 & 3.8 & 3.0 & 2.4 & 3.5 & 2.8 & 2.3 \\
\hline & Adjusted & \begin{tabular}{|c|} 
HWFET \\
\end{tabular} & 6.3 & 6.3 & 5.1 & 4.4 & 5.6 & 4.5 & 3.7 & 5.0 & 3.9 & 3.1 & 4.6 & 3.6 & 2.9 \\
\hline & & Combined & 5.4 & 5.4 & 4.4 & 3.8 & 4.8 & 3.9 & 3.3 & 4.3 & 3.4 & 2.8 & 4.0 & 3.2 & 2.6 \\
\hline
\end{tabular}




\begin{tabular}{|c|c|c|c|c|c|c|c|c|c|c|c|c|c|c|c|}
\hline & & & \multicolumn{13}{|c|}{ Small_SUV } \\
\hline & & & \multirow{2}{*}{$\begin{array}{c}2010 \\
\text { Ref }\end{array}$} & \multicolumn{3}{|c|}{2010} & \multicolumn{3}{|c|}{2015} & \multicolumn{3}{|c|}{2030} & \multicolumn{3}{|c|}{2045} \\
\hline & & & & low & avg & high & low & avg & high & low & avg & high & low & avg & high \\
\hline \multirow{6}{*}{ H2 Series HEV PHEV40 } & \multirow{3}{*}{ Unadjusted } & UDDS & 2.9 & 2.9 & 2.3 & 1.9 & 2.5 & 2.0 & 1.6 & 2.2 & 1.7 & 1.3 & 2.1 & 1.6 & 1.2 \\
\hline & & HWFET & 3.5 & 3.5 & 2.8 & 2.4 & 3.1 & 2.5 & 2.1 & 2.8 & 2.1 & 1.7 & 2.6 & 2.0 & 1.6 \\
\hline & & Combined & 3.2 & 3.2 & 2.5 & 2.1 & 2.8 & 2.2 & 1.8 & 2.5 & 1.9 & 1.5 & 2.3 & 1.7 & 1.4 \\
\hline & \multirow{3}{*}{ Adjusted } & \begin{tabular}{|l|} 
UDDS \\
\end{tabular} & 4.1 & 4.1 & 3.4 & 3.0 & 3.7 & 3.0 & 2.6 & 3.4 & 2.6 & 2.2 & 3.1 & 2.5 & 2.1 \\
\hline & & HWFET & 5.0 & 5.0 & 4.0 & 3.5 & 4.4 & 3.6 & 3.0 & 4.0 & 3.1 & 2.5 & 3.7 & 2.8 & 2.3 \\
\hline & & Combined & 4.5 & 4.5 & 3.7 & 3.2 & 4.0 & 3.3 & 2.8 & 3.6 & 2.8 & 2.3 & 3.4 & 2.7 & 2.1 \\
\hline \multirow{6}{*}{ E85 Split HEV } & \multirow{3}{*}{ Unadjusted } & \begin{tabular}{|l|} 
UDDS \\
\end{tabular} & 5.6 & 5.6 & 5.1 & 4.6 & 5.4 & 4.7 & 3.6 & 5.2 & 4.2 & 3.0 & 5.0 & 4.1 & 2.9 \\
\hline & & HWFET & 6.5 & 6.5 & 5.8 & 5.4 & 6.3 & 5.6 & 4.3 & 6.1 & 5.0 & 3.6 & 5.8 & 4.8 & 3.5 \\
\hline & & Combined & 6.0 & 6.0 & 5.4 & 5.0 & 5.8 & 5.1 & 3.9 & 5.6 & 4.5 & 3.3 & 5.4 & 4.4 & 3.1 \\
\hline & \multirow{3}{*}{ Adjusted } & \begin{tabular}{|l|} 
UDDS \\
\end{tabular} & 7.5 & 7.5 & 6.8 & 6.3 & 7.2 & 6.4 & 5.0 & 7.0 & 5.8 & 4.3 & 6.7 & 5.6 & 4.1 \\
\hline & & HWFET & 9.2 & 9.2 & 8.3 & 7.6 & 8.9 & 7.9 & 6.1 & 8.6 & 7.0 & 5.2 & 8.2 & 6.8 & 4.9 \\
\hline & & Combined & 8.2 & 8.2 & 7.5 & 6.9 & 8.0 & 7.1 & 5.5 & 7.7 & 6.3 & 4.7 & 7.4 & 6.1 & 4.5 \\
\hline \multirow{6}{*}{ E85 Split HEV PHEV10 } & \multirow{3}{*}{ Unadjusted } & \begin{tabular}{|l|} 
UDDS \\
\end{tabular} & 4.4 & 4.4 & 4.0 & 3.7 & 4.3 & 3.8 & 2.9 & 4.1 & 3.4 & 2.5 & 4.0 & 3.3 & 2.3 \\
\hline & & HWFET & 4.9 & 4.9 & 4.3 & 3.9 & 4.8 & 4.1 & 3.2 & 4.7 & 3.7 & 2.8 & 4.5 & 3.5 & 2.6 \\
\hline & & Combined & 4.6 & 4.6 & 4.1 & 3.8 & 4.5 & 3.9 & 3.0 & 4.4 & 3.5 & 2.6 & 4.2 & 3.4 & 2.5 \\
\hline & \multirow{3}{*}{ Adjusted } & UDDS & 6.0 & 6.0 & 5.5 & 5.1 & 5.8 & 5.2 & 4.2 & 5.7 & 4.8 & 3.6 & 5.5 & 4.6 & 3.5 \\
\hline & & HWFET & 7.0 & 7.0 & 6.1 & 5.6 & 6.8 & 5.8 & 4.5 & 6.7 & 5.2 & 4.0 & 6.4 & 5.0 & 3.8 \\
\hline & & Combined & 6.4 & 6.4 & 5.7 & 5.3 & 6.3 & 5.5 & 4.3 & 6.1 & 5.0 & 3.8 & 5.9 & 4.8 & 3.6 \\
\hline \multirow{6}{*}{ E85 Split HEV PHEV20 } & \multirow{3}{*}{ Unadjusted } & \begin{tabular}{|l|} 
UDDS \\
\end{tabular} & 3.1 & 3.1 & 2.8 & 2.6 & 3.0 & 2.7 & 2.0 & 2.9 & 2.4 & 2.0 & 2.8 & 2.3 & 1.9 \\
\hline & & HWFET & 4.3 & 4.3 & 4.3 & 3.9 & 3.9 & 4.1 & 3.2 & 3.8 & 3.7 & 2.6 & 4.3 & 3.5 & 2.5 \\
\hline & & Combined & 3.7 & 3.7 & 3.5 & 3.2 & 3.4 & 3.3 & 2.6 & 3.3 & 3.0 & 2.3 & 3.5 & 2.8 & 2.2 \\
\hline & \multirow{3}{*}{ Adjusted } & \begin{tabular}{|l|} 
UDDS \\
\end{tabular} & 4.4 & 4.4 & 4.0 & 3.8 & 4.2 & 3.9 & 3.1 & 4.1 & 3.5 & 3.1 & 4.0 & 3.4 & 3.0 \\
\hline & & HWFET & 6.2 & 6.2 & 6.1 & 5.6 & 5.5 & 5.8 & 4.5 & 5.4 & 5.3 & 3.8 & 6.2 & 5.0 & 3.6 \\
\hline & & Combined & 5.2 & 5.2 & 5.0 & 4.6 & 4.8 & 4.7 & 3.7 & 4.7 & 4.3 & 3.4 & 5.0 & 4.1 & 3.2 \\
\hline \multirow{6}{*}{ E85 Series HEV PHEV30 } & \multirow{3}{*}{ Unadjusted } & \begin{tabular}{|l|} 
UDDS \\
\end{tabular} & 3.4 & 3.4 & 3.0 & 2.7 & 3.3 & 2.8 & 2.1 & 3.1 & 2.4 & 1.7 & 3.0 & 2.3 & 1.6 \\
\hline & & HWFET & 4.6 & 4.6 & 4.1 & 3.7 & 4.5 & 3.9 & 2.9 & 4.3 & 3.4 & 2.5 & 4.1 & 3.2 & 2.3 \\
\hline & & Combined & 4.0 & 4.0 & 3.5 & 3.2 & 3.8 & 3.3 & 2.4 & 3.6 & 2.9 & 2.1 & 3.5 & 2.7 & 1.9 \\
\hline & \multirow{3}{*}{ Adjusted } & \begin{tabular}{|l|} 
UDDS \\
\end{tabular} & 4.8 & 4.8 & 4.3 & 3.9 & 4.6 & 4.1 & 3.1 & 4.4 & 3.6 & 2.7 & 4.2 & 3.5 & 2.6 \\
\hline & & HWFET & 6.6 & 6.6 & 5.8 & 5.3 & 6.3 & 5.5 & 4.2 & 6.1 & 4.9 & 3.5 & 5.8 & 4.6 & 3.4 \\
\hline & & Combined & 5.6 & 5.6 & 5.0 & 4.5 & 5.4 & 4.7 & 3.6 & 5.2 & 4.1 & 3.1 & 4.9 & 4.0 & 2.9 \\
\hline \multirow{6}{*}{ E85 Series HEV PHEV40 } & & \begin{tabular}{|l|} 
UDDS \\
\end{tabular} & 3.0 & 3.0 & 2.6 & 2.3 & 2.8 & 2.4 & 1.8 & 2.7 & 2.1 & 1.5 & 2.6 & 2.0 & 1.4 \\
\hline & Unadjusted & \begin{tabular}{|c|} 
HWFET \\
\end{tabular} & 3.7 & 3.7 & 3.3 & 2.9 & 3.5 & 3.0 & 2.3 & 3.4 & 2.7 & 1.9 & 3.2 & 2.6 & 1.8 \\
\hline & & Combined & 3.3 & 3.3 & 2.9 & 2.6 & 3.1 & 2.7 & 2.0 & 3.0 & 2.4 & 1.7 & 2.9 & 2.3 & 1.6 \\
\hline & & \begin{tabular}{|l|} 
UDDS \\
\end{tabular} & 4.3 & 4.3 & 3.8 & 3.5 & 4.1 & 3.6 & 2.8 & 3.9 & 3.2 & 2.4 & 3.8 & 3.1 & 2.3 \\
\hline & Adjusted & \begin{tabular}{|l|} 
HWFET \\
\end{tabular} & 5.2 & 5.2 & 4.6 & 4.2 & 5.0 & 4.3 & 3.3 & 4.8 & 3.8 & 2.8 & 4.6 & 3.7 & 2.6 \\
\hline & & Combined & 4.7 & 4.7 & 4.2 & 3.8 & 4.5 & 3.9 & 3.0 & 4.3 & 3.5 & 2.6 & 4.1 & 3.3 & 2.4 \\
\hline
\end{tabular}




\begin{tabular}{|c|c|c|c|c|c|c|c|c|c|c|c|c|c|c|c|}
\hline & & & \multicolumn{13}{|c|}{ Small_SUV } \\
\hline & & & \multirow{2}{*}{$\begin{array}{c}2010 \\
\text { Ref }\end{array}$} & \multicolumn{3}{|c|}{2010} & \multicolumn{3}{|c|}{2015} & \multicolumn{3}{|c|}{2030} & \multicolumn{3}{|c|}{2045} \\
\hline & & & & low & avg & high & low & avg & high & low & avg & high & low & avg & high \\
\hline \multirow{6}{*}{ FC HEV } & \multirow{3}{*}{ Unadjusted } & UDDS & 4.8 & 4.8 & 3.9 & 3.3 & 4.0 & 3.3 & 2.9 & 3.7 & 2.9 & 2.4 & 3.5 & 2.8 & 2.3 \\
\hline & & HWFET & 4.8 & 4.8 & 4.0 & 3.4 & 4.1 & 3.5 & 3.1 & 3.9 & 3.2 & 2.7 & 3.7 & 3.1 & 2.5 \\
\hline & & Combined & 4.8 & 4.8 & 4.0 & 3.3 & 4.1 & 3.4 & 3.0 & 3.8 & 3.0 & 2.5 & 3.6 & 2.9 & 2.4 \\
\hline & \multirow{3}{*}{ Adjusted } & \begin{tabular}{|l|} 
UDDS \\
\end{tabular} & 6.5 & 6.5 & 5.4 & 4.6 & 5.5 & 4.7 & 4.1 & 5.2 & 4.2 & 3.5 & 4.9 & 4.1 & 3.4 \\
\hline & & HWFET & 6.8 & 6.8 & 5.7 & 4.9 & 5.9 & 5.0 & 4.4 & 5.6 & 4.5 & 3.8 & 5.3 & 4.4 & 3.6 \\
\hline & & Combined & 6.7 & 6.7 & 5.5 & 4.7 & 5.7 & 4.8 & 4.2 & 5.4 & 4.4 & 3.7 & 5.1 & 4.2 & 3.5 \\
\hline \multirow{6}{*}{ FC PHEV10 } & \multirow{3}{*}{ Unadjusted } & \begin{tabular}{|l|} 
UDDS \\
\end{tabular} & 3.8 & 3.8 & 3.1 & 2.7 & 3.2 & 2.8 & 2.4 & 3.1 & 2.4 & 2.0 & 2.9 & 2.4 & 1.9 \\
\hline & & HWFET & 3.7 & 3.7 & 3.1 & 2.7 & 3.2 & 2.8 & 2.4 & 3.1 & 2.5 & 2.2 & 3.0 & 2.4 & 2.0 \\
\hline & & Combined & 3.8 & 3.8 & 3.1 & 2.7 & 3.2 & 2.8 & 2.4 & 3.1 & 2.5 & 2.1 & 2.9 & 2.4 & 2.0 \\
\hline & \multirow{3}{*}{ Adjusted } & \begin{tabular}{|l|} 
UDDS \\
\end{tabular} & 5.3 & 5.3 & 4.5 & 3.9 & 4.6 & 4.0 & 3.5 & 4.4 & 3.6 & 3.0 & 4.2 & 3.5 & 2.9 \\
\hline & & \begin{tabular}{|c|} 
HWFET \\
\end{tabular} & 5.2 & 5.2 & 4.4 & 3.8 & 4.6 & 4.0 & 3.5 & 4.5 & 3.6 & 3.1 & 4.2 & 3.5 & 2.9 \\
\hline & & Combined & 5.3 & 5.3 & 4.4 & 3.8 & 4.6 & 4.0 & 3.5 & 4.4 & 3.6 & 3.1 & 4.2 & 3.5 & 2.9 \\
\hline \multirow{6}{*}{ FC PHEV20 } & \multirow{3}{*}{ Unadjusted } & UDDS & 3.2 & 3.2 & 2.6 & 2.2 & 2.7 & 2.3 & 2.0 & 2.5 & 2.0 & 1.6 & 2.4 & 2.0 & 1.6 \\
\hline & & \begin{tabular}{|c|} 
HWFET \\
\end{tabular} & 3.6 & 3.6 & 3.0 & 2.6 & 3.1 & 2.7 & 2.4 & 3.0 & 2.4 & 2.1 & 2.9 & 2.3 & 1.9 \\
\hline & & Combined & 3.4 & 3.4 & 2.8 & 2.4 & 2.9 & 2.5 & 2.1 & 2.8 & 2.2 & 1.8 & 2.6 & 2.1 & 1.7 \\
\hline & \multirow{3}{*}{ Adjusted } & \begin{tabular}{|l|} 
UDDS \\
\end{tabular} & 4.5 & 4.5 & 3.8 & 3.3 & 3.9 & 3.4 & 3.0 & 3.7 & 3.1 & 2.6 & 3.6 & 3.0 & 2.5 \\
\hline & & \begin{tabular}{|c|} 
HWFET \\
\end{tabular} & 5.1 & 5.1 & 4.3 & 3.7 & 4.5 & 3.8 & 3.4 & 4.3 & 3.5 & 3.0 & 4.1 & 3.3 & 2.8 \\
\hline & & Combined & 4.8 & 4.8 & 4.0 & 3.5 & 4.2 & 3.6 & 3.2 & 4.0 & 3.3 & 2.8 & 3.8 & 3.2 & 2.6 \\
\hline \multirow{6}{*}{ FC PHEV30 } & \multirow{3}{*}{ Unadjusted } & \begin{tabular}{|l|} 
UDDS \\
\end{tabular} & 2.2 & 2.2 & 1.8 & 1.6 & 1.9 & 1.6 & 1.4 & 1.8 & 1.4 & 1.2 & 1.7 & 1.4 & 1.1 \\
\hline & & \begin{tabular}{|c|} 
HWFET \\
\end{tabular} & 2.9 & 2.9 & 2.4 & 2.1 & 2.5 & 2.2 & 1.9 & 2.4 & 1.9 & 1.6 & 2.3 & 1.9 & 1.6 \\
\hline & & Combined & 2.5 & 2.5 & 2.1 & 1.8 & 2.2 & 1.9 & 1.6 & 2.1 & 1.7 & 1.4 & 2.0 & 1.6 & 1.3 \\
\hline & \multirow{3}{*}{ Adjusted } & \begin{tabular}{|l|} 
UDDS \\
\end{tabular} & 3.3 & 3.3 & 2.9 & 2.5 & 2.9 & 2.6 & 2.3 & 2.8 & 2.4 & 2.0 & 2.7 & 2.3 & 2.0 \\
\hline & & HWFET & 4.1 & 4.1 & 3.4 & 3.0 & 3.6 & 3.1 & 2.7 & 3.5 & 2.8 & 2.4 & 3.3 & 2.7 & 2.3 \\
\hline & & Combined & 3.7 & 3.7 & 3.1 & 2.7 & 3.2 & 2.8 & 2.5 & 3.1 & 2.6 & 2.2 & 3.0 & 2.5 & 2.1 \\
\hline \multirow{6}{*}{ FC PHEV 40} & \multirow{3}{*}{ Unadjusted } & \begin{tabular}{|l|} 
UDDS \\
\end{tabular} & 1.9 & 1.9 & 1.6 & 1.4 & 1.7 & 1.4 & 1.2 & 1.6 & 1.2 & 1.0 & 1.5 & 1.2 & 1.0 \\
\hline & & \begin{tabular}{|c|} 
HWFET \\
\end{tabular} & 2.3 & 2.3 & 1.9 & 1.6 & 2.0 & 1.7 & 1.5 & 1.9 & 1.5 & 1.3 & 1.8 & 1.5 & 1.2 \\
\hline & & Combined & 2.1 & 2.1 & 1.7 & 1.5 & 1.8 & 1.5 & 1.3 & 1.7 & 1.4 & 1.1 & 1.6 & 1.3 & 1.1 \\
\hline & \multirow{3}{*}{ Adjusted } & \begin{tabular}{|l|} 
UDDS \\
\end{tabular} & 3.0 & 3.0 & 2.6 & 2.3 & 2.6 & 2.3 & 2.1 & 2.5 & 2.1 & 1.8 & 2.4 & 2.1 & 1.8 \\
\hline & & HWFET & 3.3 & 3.3 & 2.7 & 2.4 & 2.8 & 2.5 & 2.1 & 2.7 & 2.2 & 1.9 & 2.6 & 2.1 & 1.8 \\
\hline & & Combined & 3.1 & 3.1 & 2.6 & 2.3 & 2.7 & 2.4 & 2.1 & 2.6 & 2.2 & 1.9 & 2.5 & 2.1 & 1.8 \\
\hline
\end{tabular}

Fuel Consumption Improvement versus the Conventional 2010 Reference 


\begin{tabular}{|c|c|c|c|c|c|c|c|c|c|c|c|c|c|c|c|}
\hline & & & \multicolumn{13}{|c|}{ Small_SUV } \\
\hline & & & \multirow{2}{*}{$\begin{array}{l}2010 \\
\text { Ref }\end{array}$} & \multicolumn{3}{|c|}{2010} & \multicolumn{3}{|c|}{2015} & \multicolumn{3}{|c|}{2030} & \multicolumn{3}{|c|}{2045} \\
\hline & & & & low & avg & high & low & avg & high & low & avg & high & low & avg & high \\
\hline \multirow{6}{*}{ SI Conv } & \multirow{3}{*}{ Unadjusted } & UDDS & $0.0 \%$ & $0.0 \%$ & $-9.5 \%$ & $-14.4 \%$ & $-5.1 \%$ & $-12.9 \%$ & $-31.4 \%$ & $-7.0 \%$ & $-18.3 \%$ & $-42.7 \%$ & $-7.8 \%$ & $-19.4 \%$ & $-44.8 \%$ \\
\hline & & HWFET & $0.0 \%$ & $0.0 \%$ & $-8.1 \%$ & $-13.6 \%$ & $-5.1 \%$ & $-11.6 \%$ & $-32.1 \%$ & $-4.1 \%$ & $-19.0 \%$ & $-42.7 \%$ & $-8.1 \%$ & $-20.6 \%$ & $-45.2 \%$ \\
\hline & & Combined & $0.0 \%$ & $0.0 \%$ & $-9.0 \%$ & $-14.1 \%$ & $-5.1 \%$ & $-12.4 \%$ & $-31.7 \%$ & $-5.9 \%$ & $-18.6 \%$ & $-42.7 \%$ & $-7.9 \%$ & $-19.8 \%$ & $-45.0 \%$ \\
\hline & \multirow{3}{*}{ Adjusted } & \begin{tabular}{|l|} 
UDDS \\
\end{tabular} & $0.0 \%$ & $0.0 \%$ & $-9.0 \%$ & $-13.7 \%$ & $-4.8 \%$ & $-12.2 \%$ & $-29.8 \%$ & $-6.6 \%$ & $-17.4 \%$ & $-40.6 \%$ & $-7.4 \%$ & $-18.4 \%$ & $-42.6 \%$ \\
\hline & & HWFET & $0.0 \%$ & $0.0 \%$ & $-8.1 \%$ & $-13.5 \%$ & $-5.0 \%$ & $-11.5 \%$ & $-31.9 \%$ & $-4.1 \%$ & $-18.8 \%$ & $-42.4 \%$ & $-8.0 \%$ & $-20.4 \%$ & $-44.9 \%$ \\
\hline & & \begin{tabular}{|l|} 
Combined \\
\end{tabular} & $0.0 \%$ & $0.0 \%$ & $-8.6 \%$ & $-13.6 \%$ & $-4.9 \%$ & $-11.9 \%$ & $-30.6 \%$ & $-5.6 \%$ & $-18.0 \%$ & $-41.3 \%$ & $-7.7 \%$ & $-19.2 \%$ & $-43.5 \%$ \\
\hline \multirow{6}{*}{ Cl Conv } & \multirow{3}{*}{ Unadjusted } & \begin{tabular}{|l|} 
UDDS \\
\end{tabular} & $-15.8 \%$ & $-15.8 \%$ & $-20.1 \%$ & $-26.5 \%$ & $-17.3 \%$ & $-25.1 \%$ & $-32.6 \%$ & $-19.9 \%$ & $-31.0 \%$ & $-42.2 \%$ & $-22.6 \%$ & $-33.2 \%$ & $-44.0 \%$ \\
\hline & & HWFET & $-11.9 \%$ & $-11.9 \%$ & $-17.4 \%$ & $-24.8 \%$ & $-13.7 \%$ & $-22.8 \%$ & $-32.2 \%$ & $-15.8 \%$ & $-30.4 \%$ & $-41.3 \%$ & $-20.9 \%$ & $-33.2 \%$ & $-43.8 \%$ \\
\hline & & Combined & $-14.3 \%$ & $-14.3 \%$ & $-19.1 \%$ & $-25.9 \%$ & $-15.9 \%$ & $-24.2 \%$ & $-32.5 \%$ & $-18.4 \%$ & $-30.7 \%$ & $-41.9 \%$ & $-21.9 \%$ & $-33.2 \%$ & $-43.9 \%$ \\
\hline & \multirow{3}{*}{ Adjusted } & UDDS & $-15.0 \%$ & $-15.0 \%$ & $-19.1 \%$ & $-25.2 \%$ & $-16.4 \%$ & $-23.8 \%$ & $-31.0 \%$ & $-18.9 \%$ & $-29.4 \%$ & $-40.1 \%$ & $-21.5 \%$ & $-31.5 \%$ & $-41.8 \%$ \\
\hline & & HWFET & $-11.8 \%$ & $-11.8 \%$ & $-17.3 \%$ & $-24.6 \%$ & $-13.6 \%$ & $-22.6 \%$ & $-32.0 \%$ & $-15.7 \%$ & $-30.1 \%$ & $-41.0 \%$ & $-20.7 \%$ & $-33.0 \%$ & $-43.5 \%$ \\
\hline & & \begin{tabular}{|l|} 
Combined \\
\end{tabular} & $-13.7 \%$ & $-13.7 \%$ & $-18.3 \%$ & $-25.0 \%$ & $-15.3 \%$ & $-23.3 \%$ & $-31.4 \%$ & $-17.6 \%$ & $-29.7 \%$ & $-40.5 \%$ & $-21.2 \%$ & $-32.1 \%$ & $-42.5 \%$ \\
\hline \multirow{6}{*}{ H2 Conv } & \multirow{3}{*}{ Unadjusted } & \begin{tabular}{|l|} 
UDDS \\
\end{tabular} & $16.6 \%$ & $16.6 \%$ & $-2.8 \%$ & $-14.9 \%$ & $2.9 \%$ & $-13.1 \%$ & $-24.1 \%$ & $-5.8 \%$ & $-21.9 \%$ & $-35.5 \%$ & $-12.0 \%$ & $-26.1 \%$ & $-40.2 \%$ \\
\hline & & HWFET & $7.5 \%$ & $7.5 \%$ & $-11.6 \%$ & $-22.9 \%$ & $-5.1 \%$ & $-20.9 \%$ & $-30.8 \%$ & $-13.4 \%$ & $-28.8 \%$ & $-40.5 \%$ & $-19.3 \%$ & $-33.0 \%$ & $-45.3 \%$ \\
\hline & & \begin{tabular}{|l|} 
Combined \\
\end{tabular} & $13.2 \%$ & $13.2 \%$ & $-6.1 \%$ & $-17.9 \%$ & $-0.1 \%$ & $-16.1 \%$ & $-26.6 \%$ & $-8.7 \%$ & $-24.5 \%$ & $-37.4 \%$ & $-14.7 \%$ & $-28.7 \%$ & $-42.1 \%$ \\
\hline & \multirow{3}{*}{ Adjusted } & UDDS & $15.8 \%$ & $15.8 \%$ & $-2.7 \%$ & $-14.2 \%$ & $2.8 \%$ & $-12.5 \%$ & $-22.9 \%$ & $-5.5 \%$ & $-20.8 \%$ & $-33.7 \%$ & $-11.4 \%$ & $-24.8 \%$ & $-38.2 \%$ \\
\hline & & HWFET & $7.5 \%$ & $7.5 \%$ & $-11.5 \%$ & $-22.7 \%$ & $-5.1 \%$ & $-20.8 \%$ & $-30.6 \%$ & $-13.3 \%$ & $-28.6 \%$ & $-40.2 \%$ & $-19.1 \%$ & $-32.8 \%$ & $-45.0 \%$ \\
\hline & & \begin{tabular}{|l|} 
Combined \\
\end{tabular} & $12.5 \%$ & $12.5 \%$ & $-6.2 \%$ & $-17.6 \%$ & $-0.4 \%$ & $-15.8 \%$ & $-26.0 \%$ & $-8.6 \%$ & $-24.0 \%$ & $-36.4 \%$ & $-14.5 \%$ & $-28.0 \%$ & $-40.9 \%$ \\
\hline \multirow{6}{*}{ E85 Conv } & \multirow{3}{*}{ Unadjusted } & UDDS & $4.0 \%$ & $4.0 \%$ & $0.7 \%$ & $-4.9 \%$ & $6.4 \%$ & $-2.7 \%$ & $-28.4 \%$ & $3.9 \%$ & $-9.9 \%$ & $-38.6 \%$ & $1.4 \%$ & $-11.1 \%$ & $-40.7 \%$ \\
\hline & & HWFET & $9.1 \%$ & $9.1 \%$ & $5.2 \%$ & $-1.2 \%$ & $11.3 \%$ & $1.4 \%$ & $-23.5 \%$ & $9.9 \%$ & $-3.5 \%$ & $-33.8 \%$ & $9.4 \%$ & $-5.3 \%$ & $-36.7 \%$ \\
\hline & & \begin{tabular}{|c|} 
Combined \\
\end{tabular} & $5.9 \%$ & $5.9 \%$ & $2.4 \%$ & $-3.5 \%$ & $8.2 \%$ & $-1.1 \%$ & $-26.5 \%$ & $6.2 \%$ & $-7.5 \%$ & $-36.8 \%$ & $4.4 \%$ & $-8.9 \%$ & $-39.2 \%$ \\
\hline & \multirow{3}{*}{ Adjusted } & \begin{tabular}{|l|} 
UDDS \\
\end{tabular} & $3.8 \%$ & $3.8 \%$ & $0.6 \%$ & $-4.6 \%$ & $6.0 \%$ & $-2.6 \%$ & $-27.0 \%$ & $3.7 \%$ & $-9.4 \%$ & $-36.7 \%$ & $1.3 \%$ & $-10.5 \%$ & $-38.7 \%$ \\
\hline & & HWFET & $9.0 \%$ & $9.0 \%$ & $5.2 \%$ & $-1.2 \%$ & $11.2 \%$ & $1.4 \%$ & $-23.3 \%$ & $9.9 \%$ & $-3.5 \%$ & $-33.6 \%$ & $9.3 \%$ & $-5.3 \%$ & $-36.4 \%$ \\
\hline & & \begin{tabular}{|c|} 
Combined \\
\end{tabular} & $5.9 \%$ & $5.9 \%$ & $2.5 \%$ & $-3.2 \%$ & $8.1 \%$ & $-1.0 \%$ & $-25.5 \%$ & $6.2 \%$ & $-7.0 \%$ & $-35.4 \%$ & $4.5 \%$ & $-8.4 \%$ & $-37.7 \%$ \\
\hline \multirow{6}{*}{ SI Split HEV } & \multirow{3}{*}{ Unadjusted } & UDDS & $-44.4 \%$ & $-44.4 \%$ & $-49.6 \%$ & $-53.8 \%$ & $-46.2 \%$ & $-53.0 \%$ & $-62.2 \%$ & $-48.8 \%$ & $-57.9 \%$ & $-69.7 \%$ & $-50.2 \%$ & $-59.7 \%$ & $-71.0 \%$ \\
\hline & & HWFET & $-14.3 \%$ & $-14.3 \%$ & $-22.5 \%$ & $-28.9 \%$ & $-16.7 \%$ & $-26.2 \%$ & $-40.1 \%$ & $-19.3 \%$ & $-33.4 \%$ & $-50.9 \%$ & $-22.5 \%$ & $-36.4 \%$ & $-53.4 \%$ \\
\hline & & \begin{tabular}{|l|} 
Combined \\
\end{tabular} & $-33.0 \%$ & $-33.0 \%$ & $-39.4 \%$ & $-44.3 \%$ & $-35.0 \%$ & $-42.8 \%$ & $-53.8 \%$ & $-37.6 \%$ & $-48.6 \%$ & $-62.6 \%$ & $-39.7 \%$ & $-50.8 \%$ & $-64.3 \%$ \\
\hline & \multirow{3}{*}{ Adjusted } & \begin{tabular}{|l|l|} 
UDDS \\
\end{tabular} & $-42.2 \%$ & $-42.2 \%$ & $-47.2 \%$ & $-51.1 \%$ & $-43.9 \%$ & $-50.3 \%$ & $-59.1 \%$ & $-46.3 \%$ & $-55.0 \%$ & $-66.3 \%$ & $-47.7 \%$ & $-56.7 \%$ & $-67.5 \%$ \\
\hline & & HWFET & $-14.2 \%$ & $-14.2 \%$ & $-22.4 \%$ & $-28.7 \%$ & $-16.6 \%$ & $-26.0 \%$ & $-39.8 \%$ & $-19.2 \%$ & $-33.2 \%$ & $-50.5 \%$ & $-22.3 \%$ & $-36.1 \%$ & $-53.0 \%$ \\
\hline & & Combined & $-31.0 \%$ & $-31.0 \%$ & $-37.2 \%$ & $-42.1 \%$ & $-32.9 \%$ & $-40.6 \%$ & $-51.3 \%$ & $-35.4 \%$ & $-46.2 \%$ & $-59.9 \%$ & $-37.5 \%$ & $-48.4 \%$ & $-61.7 \%$ \\
\hline \multirow{6}{*}{ SI Split HEV PHEV10 } & & \begin{tabular}{|l|} 
UDDS \\
\end{tabular} & $-55.9 \%$ & $-55.9 \%$ & $-60.1 \%$ & $-63.2 \%$ & $-57.4 \%$ & $-62.1 \%$ & $-69.1 \%$ & $-58.5 \%$ & $-65.7 \%$ & $-75.2 \%$ & $-59.8 \%$ & $-66.9 \%$ & $-76.2 \%$ \\
\hline & Unadjusted & HWFET & $-33.9 \%$ & $-33.9 \%$ & $-40.2 \%$ & $-47.6 \%$ & $-35.9 \%$ & $-45.5 \%$ & $-55.3 \%$ & $-37.1 \%$ & $-50.6 \%$ & $-62.6 \%$ & $-39.6 \%$ & $-52.5 \%$ & $-64.6 \%$ \\
\hline & & \begin{tabular}{|l|} 
Combined \\
\end{tabular} & $-47.5 \%$ & $-47.5 \%$ & $-52.6 \%$ & $-57.3 \%$ & $-49.2 \%$ & $-55.8 \%$ & $-63.9 \%$ & $-50.4 \%$ & $-59.9 \%$ & $-70.4 \%$ & $-52.1 \%$ & $-61.4 \%$ & $-71.8 \%$ \\
\hline & & UDDS & $-53.1 \%$ & $-53.1 \%$ & $-57.1 \%$ & $-60.1 \%$ & $-54.5 \%$ & $-59.0 \%$ & $-65.7 \%$ & $-55.6 \%$ & $-62.4 \%$ & $-71.4 \%$ & $-56.8 \%$ & $-63.6 \%$ & $-72.4 \%$ \\
\hline & Adjusted & HWFET & $-33.6 \%$ & $-33.6 \%$ & $-39.9 \%$ & $-47.2 \%$ & $-35.6 \%$ & $-45.1 \%$ & $-54.9 \%$ & $-36.8 \%$ & $-50.2 \%$ & $-62.1 \%$ & $-39.3 \%$ & $-52.1 \%$ & $-64.1 \%$ \\
\hline & & Combined & $-45.3 \%$ & $-45.3 \%$ & $-50.2 \%$ & $-54.9 \%$ & $-46.9 \%$ & $-53.4 \%$ & $-61.4 \%$ & $-48.0 \%$ & $-57.5 \%$ & $-67.7 \%$ & $-49.8 \%$ & $-59.0 \%$ & $-69.1 \%$ \\
\hline
\end{tabular}




\begin{tabular}{|c|c|c|c|c|c|c|c|c|c|c|c|c|c|c|c|}
\hline & & & \multicolumn{13}{|c|}{ Small_SUV } \\
\hline & & & 2010 & & 2010 & & & 2015 & & & 2030 & & & 2045 & \\
\hline & & & Ref & low & avg & high & low & avg & high & low & avg & high & low & avg & high \\
\hline \multirow{6}{*}{ SI Split HEV PHEV2O } & \multirow{3}{*}{ Unadjusted } & UDDS & $-68.9 \%$ & $-68.9 \%$ & $-72.1 \%$ & $-74.2 \%$ & $-70.3 \%$ & $-73.3 \%$ & $-78.4 \%$ & $-71.0 \%$ & $-76.0 \%$ & $-79.5 \%$ & $-71.8 \%$ & $-76.9 \%$ & $-80.3 \%$ \\
\hline & & HWFET & $-42.0 \%$ & $-42.0 \%$ & $-42.4 \%$ & $-47.5 \%$ & $-47.8 \%$ & $-45.1 \%$ & $-55.6 \%$ & $-47.5 \%$ & $-50.4 \%$ & $-64.4 \%$ & $-49.7 \%$ & $-52.8 \%$ & $-66.2 \%$ \\
\hline & & Combined & $-58.7 \%$ & $-58.7 \%$ & $-60.8 \%$ & $-64.0 \%$ & $-61.7 \%$ & $-62.6 \%$ & $-69.7 \%$ & $-62.1 \%$ & $-66.3 \%$ & $-73.7 \%$ & $-63.4 \%$ & $-67.8 \%$ & $-75.0 \%$ \\
\hline & \multirow{3}{*}{ Adjusted } & UDDS & $-65.5 \%$ & $-65.5 \%$ & $-68.5 \%$ & $-70.5 \%$ & $-66.8 \%$ & $-69.7 \%$ & $-74.5 \%$ & $-67.5 \%$ & $-72.2 \%$ & $-75.5 \%$ & $-68.3 \%$ & $-73.1 \%$ & $-76.3 \%$ \\
\hline & & HWFET & $-41.7 \%$ & $-41.7 \%$ & $-42.1 \%$ & $-47.1 \%$ & $-47.5 \%$ & $-44.8 \%$ & $-55.1 \%$ & $-47.2 \%$ & $-50.1 \%$ & $-63.9 \%$ & $-49.3 \%$ & $-52.4 \%$ & $-65.7 \%$ \\
\hline & & Combined & $-55.9 \%$ & $-55.9 \%$ & $-57.9 \%$ & $-61.1 \%$ & $-59.0 \%$ & $-59.7 \%$ & $-66.7 \%$ & $-59.3 \%$ & $-63.3 \%$ & $-70.8 \%$ & $-60.7 \%$ & $-64.8 \%$ & $-72.1 \%$ \\
\hline \multirow{6}{*}{ SI Series HEV PHEV 30} & \multirow{3}{*}{ Unadjusted } & UDDS & $-65.8 \%$ & $-65.8 \%$ & $-69.8 \%$ & $-72.9 \%$ & $-67.3 \%$ & $-71.9 \%$ & $-78.2 \%$ & $-68.9 \%$ & $-75.8 \%$ & $-82.9 \%$ & $-70.1 \%$ & $-76.8 \%$ & $-83.7 \%$ \\
\hline & & $\begin{array}{l}\text { HWFET } \\
\end{array}$ & $-37.6 \%$ & $-37.6 \%$ & $-44.8 \%$ & $-49.9 \%$ & $-39.9 \%$ & $-48.1 \%$ & $-58.6 \%$ & $-42.2 \%$ & $-54.1 \%$ & $-66.4 \%$ & $-45.1 \%$ & $-56.2 \%$ & $-68.3 \%$ \\
\hline & & Combined & $-55.1 \%$ & $-55.1 \%$ & $-60.3 \%$ & $-64.2 \%$ & $-56.9 \%$ & $-62.9 \%$ & $-70.7 \%$ & $-58.8 \%$ & $-67.5 \%$ & $-76.6 \%$ & $-60.6 \%$ & $-69.0 \%$ & $-77.9 \%$ \\
\hline & \multirow{3}{*}{ Adjusted } & UDDS & $-62.5 \%$ & $-62.5 \%$ & $-66.3 \%$ & $-69.3 \%$ & $-64.0 \%$ & $-68.4 \%$ & $-74.3 \%$ & $-65.5 \%$ & $-72.0 \%$ & $-78.8 \%$ & $-66.7 \%$ & $-73.0 \%$ & $-79.5 \%$ \\
\hline & & HWFET & $-37.4 \%$ & $-37.4 \%$ & $-44.4 \%$ & $-49.5 \%$ & $-39.6 \%$ & $-47.8 \%$ & $-58.2 \%$ & $-41.9 \%$ & $-53.7 \%$ & $-65.9 \%$ & $-44.7 \%$ & $-55.8 \%$ & $-67.8 \%$ \\
\hline & & Combined & $-52.4 \%$ & $-52.4 \%$ & $-57.5 \%$ & $-61.3 \%$ & $-54.2 \%$ & $-60.1 \%$ & $-67.8 \%$ & $-56.0 \%$ & $-64.6 \%$ & $-73.6 \%$ & $-57.9 \%$ & $-66.1 \%$ & $-74.8 \%$ \\
\hline \multirow{6}{*}{ SI Series HEV PHEV40 } & \multirow{3}{*}{ Unadjusted } & UDDS & $-70.1 \%$ & $-70.1 \%$ & $-73.6 \%$ & $-76.4 \%$ & $-71.5 \%$ & $-75.3 \%$ & $-81.1 \%$ & $-72.9 \%$ & $-78.9 \%$ & $-85.1 \%$ & $-74.0 \%$ & $-79.6 \%$ & $-86.0 \%$ \\
\hline & & HWFET & $-50.7 \%$ & $-50.7 \%$ & $-56.3 \%$ & $-60.4 \%$ & $-52.5 \%$ & $-59.0 \%$ & $-67.3 \%$ & $-54.4 \%$ & $-63.8 \%$ & $-73.6 \%$ & $-56.5 \%$ & $-65.5 \%$ & $-75.1 \%$ \\
\hline & & Combined & $-62.7 \%$ & $-62.7 \%$ & $-67.0 \%$ & $-70.3 \%$ & $-64.3 \%$ & $-69.1 \%$ & $-75.9 \%$ & $-65.9 \%$ & $-73.2 \%$ & $-80.8 \%$ & $-67.4 \%$ & $-74.2 \%$ & $-81.8 \%$ \\
\hline & \multirow{3}{*}{ Adjusted } & UDDS & $-66.6 \%$ & $-66.6 \%$ & $-69.9 \%$ & $-72.6 \%$ & $-67.9 \%$ & $-71.6 \%$ & $-77.1 \%$ & $-69.3 \%$ & $-75.0 \%$ & $-80.9 \%$ & $-70.3 \%$ & $-75.6 \%$ & $-81.7 \%$ \\
\hline & & HWFET & $-50.3 \%$ & $-50.3 \%$ & $-55.9 \%$ & $-60.0 \%$ & $-52.1 \%$ & $-58.6 \%$ & $-66.8 \%$ & $-54.0 \%$ & $-63.3 \%$ & $-73.1 \%$ & $-56.1 \%$ & $-65.0 \%$ & $-74.6 \%$ \\
\hline & & Combined & $-60.1 \%$ & $-60.1 \%$ & $-64.3 \%$ & $-67.5 \%$ & $-61.6 \%$ & $-66.3 \%$ & $-73.0 \%$ & $-63.1 \%$ & $-70.3 \%$ & $-77.8 \%$ & $-64.6 \%$ & $-71.4 \%$ & $-78.8 \%$ \\
\hline \multirow{6}{*}{ CI Split HEV } & \multirow{3}{*}{ Unadjusted } & UDDS & $-49.4 \%$ & $-49.4 \%$ & $-53.6 \%$ & $-57.9 \%$ & $-50.4 \%$ & $-57.2 \%$ & $-63.0 \%$ & $-53.4 \%$ & $-62.0 \%$ & $-69.5 \%$ & $-55.2 \%$ & $-63.8 \%$ & $-70.6 \%$ \\
\hline & & HWFET & $-19.2 \%$ & $-19.2 \%$ & $-26.2 \%$ & $-33.3 \%$ & $-20.6 \%$ & $-31.3 \%$ & $-40.1 \%$ & $-24.3 \%$ & $-38.5 \%$ & $-49.3 \%$ & $-28.0 \%$ & $-41.5 \%$ & $-51.7 \%$ \\
\hline & & Combined & $-37.9 \%$ & $-37.9 \%$ & $-43.2 \%$ & $-48.6 \%$ & $-39.1 \%$ & $-47.4 \%$ & $-54.3 \%$ & $-42.3 \%$ & $-53.1 \%$ & $-61.8 \%$ & $-44.9 \%$ & $-55.4 \%$ & $-63.4 \%$ \\
\hline & \multirow{3}{*}{ Adjusted } & UDDS & $-46.9 \%$ & $-46.9 \%$ & $-50.9 \%$ & $-55.0 \%$ & $-47.9 \%$ & $-54.4 \%$ & $-59.9 \%$ & $-50.7 \%$ & $-58.9 \%$ & $-66.0 \%$ & $-52.5 \%$ & $-60.6 \%$ & $-67.1 \%$ \\
\hline & & HWFET & $-19.1 \%$ & $-19.1 \%$ & $-26.0 \%$ & $-33.0 \%$ & $-20.4 \%$ & $-31.1 \%$ & $-39.8 \%$ & $-24.1 \%$ & $-38.2 \%$ & $-48.9 \%$ & $-27.8 \%$ & $-41.2 \%$ & $-51.3 \%$ \\
\hline & & Combined & $-35.7 \%$ & $-35.7 \%$ & $-40.9 \%$ & $-46.2 \%$ & $-36.9 \%$ & $-45.0 \%$ & $-51.8 \%$ & $-40.0 \%$ & $-50.6 \%$ & $-59.1 \%$ & $-42.6 \%$ & $-52.8 \%$ & $-60.8 \%$ \\
\hline \multirow{6}{*}{ CI Split HEV PHEV10 } & \multirow{3}{*}{ Unadjusted } & UDDS & $-56.7 \%$ & $-56.7 \%$ & $-60.7 \%$ & $-64.3 \%$ & $-57.8 \%$ & $-63.2 \%$ & $-68.1 \%$ & $-59.8 \%$ & $-67.2 \%$ & $-73.6 \%$ & $-61.5 \%$ & $-68.7 \%$ & $-74.7 \%$ \\
\hline & & HWFET & $-34.7 \%$ & $-34.7 \%$ & $-43.1 \%$ & $-48.7 \%$ & $-38.5 \%$ & $-46.7 \%$ & $-53.6 \%$ & $-38.6 \%$ & $-52.4 \%$ & $-60.3 \%$ & $-41.4 \%$ & $-54.7 \%$ & $-62.4 \%$ \\
\hline & & Combined & $-48.3 \%$ & $-48.3 \%$ & $-54.0 \%$ & $-58.4 \%$ & $-50.5 \%$ & $-56.9 \%$ & $-62.6 \%$ & $-51.7 \%$ & $-61.6 \%$ & $-68.5 \%$ & $-53.9 \%$ & $-63.4 \%$ & $-70.0 \%$ \\
\hline & \multirow{3}{*}{ Adjusted } & UDDS & $-53.8 \%$ & $-53.8 \%$ & $-57.6 \%$ & $-61.1 \%$ & $-55.0 \%$ & $-60.1 \%$ & $-64.7 \%$ & $-56.8 \%$ & $-63.9 \%$ & $-69.9 \%$ & $-58.5 \%$ & $-65.3 \%$ & $-71.0 \%$ \\
\hline & & HWFET & $-34.5 \%$ & $-34.5 \%$ & $-42.7 \%$ & $-48.4 \%$ & $-38.2 \%$ & $-46.4 \%$ & $-53.2 \%$ & $-38.3 \%$ & $-52.1 \%$ & $-59.8 \%$ & $-41.1 \%$ & $-54.3 \%$ & $-62.0 \%$ \\
\hline & & Combined & $-46.1 \%$ & $-46.1 \%$ & $-51.7 \%$ & $-56.0 \%$ & $-48.2 \%$ & $-54.6 \%$ & $-60.1 \%$ & $-49.4 \%$ & $-59.1 \%$ & $-65.9 \%$ & $-51.5 \%$ & $-60.9 \%$ & $-67.4 \%$ \\
\hline \multirow{6}{*}{ CI Split HEV PHEV2O } & \multirow{3}{*}{ Unadjusted } & UDDS & $-69.7 \%$ & $-69.7 \%$ & $-72.5 \%$ & $-75.1 \%$ & $-70.6 \%$ & $-74.3 \%$ & $-77.6 \%$ & $-72.0 \%$ & $-77.0 \%$ & $-81.9 \%$ & $-73.2 \%$ & $-78.1 \%$ & $-82.6 \%$ \\
\hline & & HWFET & $-45.0 \%$ & $-45.0 \%$ & $-43.2 \%$ & $-57.6 \%$ & $-38.4 \%$ & $-46.9 \%$ & $-61.6 \%$ & $-40.5 \%$ & $-60.6 \%$ & $-62.1 \%$ & $-53.9 \%$ & $-54.6 \%$ & $-64.0 \%$ \\
\hline & & Combined & $-60.3 \%$ & $-60.3 \%$ & $-61.4 \%$ & $-68.4 \%$ & $-58.4 \%$ & $-63.9 \%$ & $-71.5 \%$ & $-60.0 \%$ & $-70.8 \%$ & $-74.4 \%$ & $-65.9 \%$ & $-69.2 \%$ & $-75.6 \%$ \\
\hline & \multirow{3}{*}{ Adjusted } & UDDS & $-66.2 \%$ & $-66.2 \%$ & $-68.9 \%$ & $-71.3 \%$ & $-67.1 \%$ & $-70.6 \%$ & $-73.8 \%$ & $-68.4 \%$ & $-73.2 \%$ & $-77.8 \%$ & $-69.5 \%$ & $-74.2 \%$ & $-78.5 \%$ \\
\hline & & HWFET & $-44.7 \%$ & $-44.7 \%$ & $-42.8 \%$ & $-57.2 \%$ & $-38.2 \%$ & $-46.6 \%$ & $-61.2 \%$ & $-40.2 \%$ & $-60.1 \%$ & $-61.6 \%$ & $-53.5 \%$ & $-54.2 \%$ & $-63.5 \%$ \\
\hline & & Combined & $-57.6 \%$ & $-57.6 \%$ & $-58.4 \%$ & $-65.6 \%$ & $-55.5 \%$ & $-60.9 \%$ & $-68.7 \%$ & $-57.1 \%$ & $-67.9 \%$ & $-71.3 \%$ & $-63.1 \%$ & $-66.2 \%$ & $-72.5 \%$ \\
\hline
\end{tabular}




\begin{tabular}{|c|c|c|c|c|c|c|c|c|c|c|c|c|c|c|c|}
\hline & & & \multicolumn{13}{|c|}{ Small_SUV } \\
\hline & & & \multirow{2}{*}{$\begin{array}{c}2010 \\
\text { Ref }\end{array}$} & \multicolumn{3}{|c|}{2010} & \multicolumn{3}{|c|}{2015} & \multicolumn{3}{|c|}{2030} & \multicolumn{3}{|c|}{2045} \\
\hline & & & & low & avg & high & low & avg & high & low & avg & high & low & avg & high \\
\hline \multirow{6}{*}{ CI Series HEV PHEV3O } & \multirow{3}{*}{ Unadjusted } & UDDS & $-66.5 \%$ & $-66.5 \%$ & $-70.3 \%$ & $-73.4 \%$ & $-67.6 \%$ & $-72.6 \%$ & $-77.3 \%$ & $-69.7 \%$ & $-76.5 \%$ & $-81.8 \%$ & $-71.2 \%$ & $-77.5 \%$ & $-82.5 \%$ \\
\hline & & HWFET & $-38.9 \%$ & $-38.9 \%$ & $-45.3 \%$ & $-51.2 \%$ & $-40.4 \%$ & $-49.5 \%$ & $-57.1 \%$ & $-43.9 \%$ & $-55.9 \%$ & $-64.4 \%$ & $-47.0 \%$ & $-58.3 \%$ & $-66.4 \%$ \\
\hline & & Combined & $-56.0 \%$ & $-56.0 \%$ & $-60.8 \%$ & $-65.0 \%$ & $-57.3 \%$ & $-63.8 \%$ & $-69.6 \%$ & $-59.9 \%$ & $-68.7 \%$ & $-75.2 \%$ & $-62.0 \%$ & $-70.2 \%$ & $-76.4 \%$ \\
\hline & \multirow{3}{*}{ Adjusted } & UDDS & $-63.2 \%$ & $-63.2 \%$ & $-66.8 \%$ & $-69.8 \%$ & $-64.2 \%$ & $-69.0 \%$ & $-73.4 \%$ & $-66.3 \%$ & $-72.7 \%$ & $-77.7 \%$ & $-67.6 \%$ & $-73.7 \%$ & $-78.4 \%$ \\
\hline & & HWFET & $-38.6 \%$ & $-38.6 \%$ & $-45.0 \%$ & $-50.8 \%$ & $-40.1 \%$ & $-49.1 \%$ & $-56.7 \%$ & $-43.6 \%$ & $-55.5 \%$ & $-64.0 \%$ & $-46.7 \%$ & $-57.9 \%$ & $-66.0 \%$ \\
\hline & & Combined & $-53.3 \%$ & $-53.3 \%$ & $-58.1 \%$ & $-62.2 \%$ & $-54.5 \%$ & $-61.0 \%$ & $-66.7 \%$ & $-57.1 \%$ & $-65.8 \%$ & $-72.2 \%$ & $-59.2 \%$ & $-67.3 \%$ & $-73.4 \%$ \\
\hline \multirow{6}{*}{ CI Series HEV PHEV40 } & \multirow{3}{*}{ Unadjusted } & UDDS & $-70.8 \%$ & $-70.8 \%$ & $-73.9 \%$ & $-76.9 \%$ & $-71.7 \%$ & $-76.2 \%$ & $-80.3 \%$ & $-73.6 \%$ & $-79.6 \%$ & $-84.4 \%$ & $-75.0 \%$ & $-80.7 \%$ & $-85.0 \%$ \\
\hline & & $\begin{array}{l}\text { HWFET } \\
\end{array}$ & $-51.6 \%$ & $-51.6 \%$ & $-56.7 \%$ & $-61.4 \%$ & $-52.9 \%$ & $-59.9 \%$ & $-66.2 \%$ & $-55.6 \%$ & $-65.3 \%$ & $-72.0 \%$ & $-58.2 \%$ & $-67.2 \%$ & $-73.6 \%$ \\
\hline & & Combined & $-63.5 \%$ & $-63.5 \%$ & $-67.3 \%$ & $-71.0 \%$ & $-64.6 \%$ & $-70.0 \%$ & $-75.0 \%$ & $-66.8 \%$ & $-74.2 \%$ & $-79.7 \%$ & $-68.6 \%$ & $-75.6 \%$ & $-80.7 \%$ \\
\hline & \multirow{3}{*}{ Adjusted } & UDDS & $-67.2 \%$ & $-67.2 \%$ & $-70.2 \%$ & $-73.1 \%$ & $-68.1 \%$ & $-72.4 \%$ & $-76.3 \%$ & $-70.0 \%$ & $-75.7 \%$ & $-80.2 \%$ & $-71.2 \%$ & $-76.7 \%$ & $-80.8 \%$ \\
\hline & & HWFET & $-51.3 \%$ & $-51.3 \%$ & $-56.3 \%$ & $-61.0 \%$ & $-52.5 \%$ & $-59.5 \%$ & $-65.7 \%$ & $-55.2 \%$ & $-64.8 \%$ & $-71.5 \%$ & $-57.7 \%$ & $-66.7 \%$ & $-73.1 \%$ \\
\hline & & Combined & $-60.8 \%$ & $-60.8 \%$ & $-64.6 \%$ & $-68.2 \%$ & $-61.9 \%$ & $-67.2 \%$ & $-72.1 \%$ & $-64.1 \%$ & $-71.3 \%$ & $-76.7 \%$ & $-65.8 \%$ & $-72.7 \%$ & $-77.7 \%$ \\
\hline \multirow{6}{*}{ H2 Split HEV } & \multirow{3}{*}{ Unadjusted } & UDDS & $-43.0 \%$ & $-43.0 \%$ & $-53.4 \%$ & $-59.9 \%$ & $-49.7 \%$ & $-59.1 \%$ & $-65.7 \%$ & $-54.4 \%$ & $-64.6 \%$ & $-72.0 \%$ & $-58.0 \%$ & $-67.0 \%$ & $-74.1 \%$ \\
\hline & & HWFET & $-10.9 \%$ & $-10.9 \%$ & $-27.1 \%$ & $-37.2 \%$ & $-20.9 \%$ & $-35.2 \%$ & $-45.2 \%$ & $-27.7 \%$ & $-43.4 \%$ & $-54.0 \%$ & $-33.7 \%$ & $-47.5 \%$ & $-57.9 \%$ \\
\hline & & Combined & $-30.8 \%$ & $-30.8 \%$ & $-43.4 \%$ & $-51.2 \%$ & $-38.8 \%$ & $-50.1 \%$ & $-57.9 \%$ & $-44.3 \%$ & $-56.6 \%$ & $-65.2 \%$ & $-48.8 \%$ & $-59.6 \%$ & $-67.9 \%$ \\
\hline & \multirow{3}{*}{ Adjusted } & UDDS & $-40.9 \%$ & $-40.9 \%$ & $-50.8 \%$ & $-56.9 \%$ & $-47.2 \%$ & $-56.2 \%$ & $-62.5 \%$ & $-51.7 \%$ & $-61.4 \%$ & $-68.4 \%$ & $-55.1 \%$ & $-63.7 \%$ & $-70.4 \%$ \\
\hline & & HWFET & $-10.8 \%$ & $-10.8 \%$ & $-26.9 \%$ & $-36.9 \%$ & $-20.8 \%$ & $-35.0 \%$ & $-44.9 \%$ & $-27.5 \%$ & $-43.1 \%$ & $-53.6 \%$ & $-33.4 \%$ & $-47.1 \%$ & $-57.4 \%$ \\
\hline & & Combined & $-28.8 \%$ & $-28.8 \%$ & $-41.2 \%$ & $-48.9 \%$ & $-36.6 \%$ & $-47.7 \%$ & $-55.4 \%$ & $-42.0 \%$ & $-54.0 \%$ & $-62.5 \%$ & $-46.4 \%$ & $-57.0 \%$ & $-65.2 \%$ \\
\hline \multirow{6}{*}{ H2 Split HEV PHEV10 } & \multirow{3}{*}{ Unadjusted } & UDDS & $-54.4 \%$ & $-54.4 \%$ & $-62.8 \%$ & $-67.9 \%$ & $-59.8 \%$ & $-66.7 \%$ & $-71.9 \%$ & $-63.1 \%$ & $-70.9 \%$ & $-77.0 \%$ & $-66.0 \%$ & $-72.9 \%$ & $-78.9 \%$ \\
\hline & & HWFET & $-32.0 \%$ & $-32.0 \%$ & $-46.3 \%$ & $-53.8 \%$ & $-39.6 \%$ & $-52.2 \%$ & $-59.2 \%$ & $-44.3 \%$ & $-58.0 \%$ & $-65.0 \%$ & $-48.7 \%$ & $-60.8 \%$ & $-68.1 \%$ \\
\hline & & Combined & $-45.9 \%$ & $-45.9 \%$ & $-56.6 \%$ & $-62.5 \%$ & $-52.1 \%$ & $-61.2 \%$ & $-67.1 \%$ & $-56.0 \%$ & $-66.0 \%$ & $-72.4 \%$ & $-59.4 \%$ & $-68.3 \%$ & $-74.8 \%$ \\
\hline & \multirow{3}{*}{ Adjusted } & UDDS & $-51.7 \%$ & $-51.7 \%$ & $-59.7 \%$ & $-64.5 \%$ & $-56.8 \%$ & $-63.4 \%$ & $-68.4 \%$ & $-60.0 \%$ & $-67.4 \%$ & $-73.1 \%$ & $-62.7 \%$ & $-69.3 \%$ & $-75.0 \%$ \\
\hline & & HWFET & $-31.7 \%$ & $-31.7 \%$ & $-46.0 \%$ & $-53.4 \%$ & $-39.3 \%$ & $-51.8 \%$ & $-58.7 \%$ & $-44.0 \%$ & $-57.5 \%$ & $-64.5 \%$ & $-48.3 \%$ & $-60.4 \%$ & $-67.6 \%$ \\
\hline & & Combined & $-43.7 \%$ & $-43.7 \%$ & $-54.2 \%$ & $-60.0 \%$ & $-49.8 \%$ & $-58.8 \%$ & $-64.5 \%$ & $-53.6 \%$ & $-63.4 \%$ & $-69.7 \%$ & $-56.9 \%$ & $-65.7 \%$ & $-72.0 \%$ \\
\hline \multirow{6}{*}{ H2 Split HEV PHEV2O } & \multirow{3}{*}{ Unadjusted } & UDDS & $-67.9 \%$ & $-67.9 \%$ & $-73.8 \%$ & $-77.4 \%$ & $-72.0 \%$ & $-76.7 \%$ & $-80.6 \%$ & $-74.3 \%$ & $-79.7 \%$ & $-81.0 \%$ & $-76.2 \%$ & $-81.3 \%$ & $-82.7 \%$ \\
\hline & & HWFET & $-40.3 \%$ & $-40.3 \%$ & $-55.5 \%$ & $-61.4 \%$ & $-49.8 \%$ & $-60.0 \%$ & $-59.6 \%$ & $-53.6 \%$ & $-57.9 \%$ & $-66.8 \%$ & $-57.3 \%$ & $-61.2 \%$ & $-69.7 \%$ \\
\hline & & Combined & $-57.4 \%$ & $-57.4 \%$ & $-66.8 \%$ & $-71.3 \%$ & $-63.6 \%$ & $-70.4 \%$ & $-72.6 \%$ & $-66.5 \%$ & $-71.4 \%$ & $-75.6 \%$ & $-69.0 \%$ & $-73.7 \%$ & $-77.7 \%$ \\
\hline & \multirow{3}{*}{ Adjusted } & UDDS & $-64.5 \%$ & $-64.5 \%$ & $-70.1 \%$ & $-73.6 \%$ & $-68.5 \%$ & $-72.9 \%$ & $-76.6 \%$ & $-70.6 \%$ & $-75.8 \%$ & $-77.0 \%$ & $-72.4 \%$ & $-77.3 \%$ & $-78.6 \%$ \\
\hline & & HWFET & $-40.0 \%$ & $-40.0 \%$ & $-55.1 \%$ & $-61.0 \%$ & $-49.5 \%$ & $-59.6 \%$ & $-59.1 \%$ & $-53.2 \%$ & $-57.5 \%$ & $-66.3 \%$ & $-56.9 \%$ & $-60.8 \%$ & $-69.2 \%$ \\
\hline & & Combined & $-54.7 \%$ & $-54.7 \%$ & $-64.1 \%$ & $-68.5 \%$ & $-60.8 \%$ & $-67.5 \%$ & $-69.6 \%$ & $-63.6 \%$ & $-68.4 \%$ & $-72.7 \%$ & $-66.2 \%$ & $-70.7 \%$ & $-74.8 \%$ \\
\hline \multirow{6}{*}{ H2 Series HEV PHEV3O } & & UDDS & $-64.8 \%$ & $-64.8 \%$ & $-72.0 \%$ & $-76.2 \%$ & $-69.2 \%$ & $-75.3 \%$ & $-80.0 \%$ & $-72.4 \%$ & $-79.2 \%$ & $-83.9 \%$ & $-74.9 \%$ & $-80.6 \%$ & $-85.1 \%$ \\
\hline & Unadjusted & HWFET & $-36.4 \%$ & $-36.4 \%$ & $-49.0 \%$ & $-56.3 \%$ & $-44.1 \%$ & $-54.8 \%$ & $-62.5 \%$ & $-49.2 \%$ & $-61.2 \%$ & $-68.7 \%$ & $-53.7 \%$ & $-64.2 \%$ & $-71.4 \%$ \\
\hline & & Combined & $-54.0 \%$ & $-54.0 \%$ & $-63.3 \%$ & $-68.7 \%$ & $-59.7 \%$ & $-67.5 \%$ & $-73.3 \%$ & $-63.6 \%$ & $-72.4 \%$ & $-78.1 \%$ & $-66.9 \%$ & $-74.3 \%$ & $-79.9 \%$ \\
\hline & & UDDS & $-61.6 \%$ & $-61.6 \%$ & $-68.4 \%$ & $-72.4 \%$ & $-65.8 \%$ & $-71.6 \%$ & $-76.0 \%$ & $-68.8 \%$ & $-75.3 \%$ & $-79.7 \%$ & $-71.2 \%$ & $-76.6 \%$ & $-80.9 \%$ \\
\hline & Adjusted & HWFET & $-36.1 \%$ & $-36.1 \%$ & $-48.6 \%$ & $-55.9 \%$ & $-43.8 \%$ & $-54.4 \%$ & $-62.0 \%$ & $-48.9 \%$ & $-60.7 \%$ & $-68.2 \%$ & $-53.3 \%$ & $-63.7 \%$ & $-70.9 \%$ \\
\hline & & Combined & $-51.3 \%$ & $-51.3 \%$ & $-60.5 \%$ & $-65.8 \%$ & $-56.9 \%$ & $-64.7 \%$ & $-70.4 \%$ & $-60.8 \%$ & $-69.4 \%$ & $-75.1 \%$ & $-64.0 \%$ & $-71.4 \%$ & $-76.9 \%$ \\
\hline
\end{tabular}




\begin{tabular}{|c|c|c|c|c|c|c|c|c|c|c|c|c|c|c|c|}
\hline & & & \multicolumn{13}{|c|}{ Small_SUV } \\
\hline & & & \multirow{2}{*}{$\begin{array}{c}2010 \\
\text { Ref }\end{array}$} & \multicolumn{3}{|c|}{2010} & \multicolumn{3}{|c|}{2015} & \multicolumn{3}{|c|}{2030} & \multicolumn{3}{|c|}{2045} \\
\hline & & & & low & avg & high & low & avg & high & low & avg & high & low & avg & high \\
\hline \multirow{6}{*}{ H2 Series HEV PHEV40 } & \multirow{3}{*}{ Unadjusted } & UDDS & $-69.3 \%$ & $-69.3 \%$ & $-75.7 \%$ & $-79.3 \%$ & $-73.2 \%$ & $-78.6 \%$ & $-82.6 \%$ & $-75.9 \%$ & $-82.1 \%$ & $-86.2 \%$ & $-77.9 \%$ & $-83.3 \%$ & $-87.3 \%$ \\
\hline & & HWFET & $-49.6 \%$ & $-49.6 \%$ & $-59.5 \%$ & $-65.5 \%$ & $-55.8 \%$ & $-64.3 \%$ & $-70.4 \%$ & $-59.9 \%$ & $-69.5 \%$ & $-75.4 \%$ & $-63.4 \%$ & $-71.8 \%$ & $-77.6 \%$ \\
\hline & & Combined & $-61.8 \%$ & $-61.8 \%$ & $-69.5 \%$ & $-74.1 \%$ & $-66.6 \%$ & $-73.2 \%$ & $-78.0 \%$ & $-69.8 \%$ & $-77.3 \%$ & $-82.1 \%$ & $-72.4 \%$ & $-78.9 \%$ & $-83.6 \%$ \\
\hline & \multirow{3}{*}{ Adjusted } & UDDS & $-65.9 \%$ & $-65.9 \%$ & $-71.9 \%$ & $-75.3 \%$ & $-69.6 \%$ & $-74.7 \%$ & $-78.5 \%$ & $-72.2 \%$ & $-78.0 \%$ & $-81.9 \%$ & $-74.1 \%$ & $-79.1 \%$ & $-83.0 \%$ \\
\hline & & HWFET & $-49.3 \%$ & $-49.3 \%$ & $-59.1 \%$ & $-65.0 \%$ & $-55.3 \%$ & $-63.8 \%$ & $-69.9 \%$ & $-59.4 \%$ & $-69.0 \%$ & $-74.9 \%$ & $-62.9 \%$ & $-71.2 \%$ & $-77.0 \%$ \\
\hline & & Combined & $-59.2 \%$ & $-59.2 \%$ & $-66.8 \%$ & $-71.2 \%$ & $-63.9 \%$ & $-70.3 \%$ & $-75.0 \%$ & $-67.1 \%$ & $-74.4 \%$ & $-79.1 \%$ & $-69.6 \%$ & $-76.0 \%$ & $-80.6 \%$ \\
\hline \multirow{6}{*}{ E85 Split HEV } & \multirow{3}{*}{ Unadjusted } & UDDS & $-39.9 \%$ & $-39.9 \%$ & $-45.8 \%$ & $-50.2 \%$ & $-42.0 \%$ & $-49.2 \%$ & $-61.4 \%$ & $-44.4 \%$ & $-54.9 \%$ & $-67.8 \%$ & $-46.5 \%$ & $-56.5 \%$ & $-69.2 \%$ \\
\hline & & $\begin{array}{l}\text { HWFET } \\
\end{array}$ & $-7.2 \%$ & $-7.2 \%$ & $-16.5 \%$ & $-23.4 \%$ & $-10.2 \%$ & $-20.5 \%$ & $-39.0 \%$ & $-12.7 \%$ & $-29.0 \%$ & $-47.8 \%$ & $-16.6 \%$ & $-31.6 \%$ & $-50.5 \%$ \\
\hline & & Combined & $-27.5 \%$ & $-27.5 \%$ & $-34.7 \%$ & $-40.0 \%$ & $-30.0 \%$ & $-38.3 \%$ & $-52.9 \%$ & $-32.4 \%$ & $-45.1 \%$ & $-60.2 \%$ & $-35.1 \%$ & $-47.0 \%$ & $-62.1 \%$ \\
\hline & \multirow{3}{*}{ Adjusted } & UDDS & $-37.9 \%$ & $-37.9 \%$ & $-43.5 \%$ & $-47.7 \%$ & $-39.9 \%$ & $-46.7 \%$ & $-58.3 \%$ & $-42.2 \%$ & $-52.2 \%$ & $-64.4 \%$ & $-44.2 \%$ & $-53.7 \%$ & $-65.7 \%$ \\
\hline & & HWFET & $-7.1 \%$ & $-7.1 \%$ & $-16.4 \%$ & $-23.2 \%$ & $-10.2 \%$ & $-20.4 \%$ & $-38.7 \%$ & $-12.6 \%$ & $-28.8 \%$ & $-47.5 \%$ & $-16.5 \%$ & $-31.3 \%$ & $-50.1 \%$ \\
\hline & & Combined & $-25.5 \%$ & $-25.5 \%$ & $-32.6 \%$ & $-37.9 \%$ & $-28.0 \%$ & $-36.2 \%$ & $-50.4 \%$ & $-30.3 \%$ & $-42.8 \%$ & $-57.6 \%$ & $-33.1 \%$ & $-44.7 \%$ & $-59.5 \%$ \\
\hline \multirow{6}{*}{ E85 Split HEV PHEV10 } & \multirow{3}{*}{ Unadjusted } & UDDS & $-52.9 \%$ & $-52.9 \%$ & $-57.3 \%$ & $-60.7 \%$ & $-54.5 \%$ & $-59.6 \%$ & $-68.7 \%$ & $-55.6 \%$ & $-63.4 \%$ & $-73.7 \%$ & $-57.2 \%$ & $-64.8 \%$ & $-74.8 \%$ \\
\hline & & HWFET & $-29.2 \%$ & $-29.2 \%$ & $-38.8 \%$ & $-43.9 \%$ & $-30.9 \%$ & $-41.6 \%$ & $-54.7 \%$ & $-32.2 \%$ & $-47.2 \%$ & $-60.3 \%$ & $-35.3 \%$ & $-49.3 \%$ & $-62.5 \%$ \\
\hline & & Combined & $-43.9 \%$ & $-43.9 \%$ & $-50.3 \%$ & $-54.4 \%$ & $-45.5 \%$ & $-52.8 \%$ & $-63.4 \%$ & $-46.7 \%$ & $-57.2 \%$ & $-68.6 \%$ & $-48.9 \%$ & $-58.9 \%$ & $-70.1 \%$ \\
\hline & \multirow{3}{*}{ Adjusted } & UDDS & $-50.3 \%$ & $-50.3 \%$ & $-54.5 \%$ & $-57.7 \%$ & $-51.7 \%$ & $-56.6 \%$ & $-65.3 \%$ & $-52.9 \%$ & $-60.2 \%$ & $-70.1 \%$ & $-54.3 \%$ & $-61.6 \%$ & $-71.1 \%$ \\
\hline & & HWFET & $-29.0 \%$ & $-29.0 \%$ & $-38.5 \%$ & $-43.6 \%$ & $-30.7 \%$ & $-41.3 \%$ & $-54.3 \%$ & $-32.0 \%$ & $-46.8 \%$ & $-59.9 \%$ & $-35.0 \%$ & $-48.9 \%$ & $-62.0 \%$ \\
\hline & & Combined & $-41.7 \%$ & $-41.7 \%$ & $-48.1 \%$ & $-52.1 \%$ & $-43.3 \%$ & $-50.5 \%$ & $-60.9 \%$ & $-44.5 \%$ & $-54.8 \%$ & $-66.0 \%$ & $-46.6 \%$ & $-56.5 \%$ & $-67.5 \%$ \\
\hline \multirow{6}{*}{ E85 Split HEV PHEV20 } & \multirow{3}{*}{ Unadjusted } & UDDS & $-66.8 \%$ & $-66.8 \%$ & $-70.0 \%$ & $-72.4 \%$ & $-68.2 \%$ & $-71.6 \%$ & $-78.1 \%$ & $-69.1 \%$ & $-74.5 \%$ & $-78.3 \%$ & $-70.0 \%$ & $-75.4 \%$ & $-79.2 \%$ \\
\hline & & HWFET & $-37.9 \%$ & $-37.9 \%$ & $-38.3 \%$ & $-43.6 \%$ & $-44.2 \%$ & $-41.2 \%$ & $-54.5 \%$ & $-45.2 \%$ & $-47.0 \%$ & $-62.2 \%$ & $-37.9 \%$ & $-49.6 \%$ & $-64.2 \%$ \\
\hline & & Combined & $-55.8 \%$ & $-55.8 \%$ & $-58.0 \%$ & $-61.5 \%$ & $-59.1 \%$ & $-60.1 \%$ & $-69.2 \%$ & $-60.0 \%$ & $-64.0 \%$ & $-72.2 \%$ & $-57.8 \%$ & $-65.6 \%$ & $-73.5 \%$ \\
\hline & \multirow{3}{*}{ Adjusted } & UDDS & $-63.4 \%$ & $-63.4 \%$ & $-66.6 \%$ & $-68.8 \%$ & $-64.8 \%$ & $-68.0 \%$ & $-74.3 \%$ & $-65.7 \%$ & $-70.8 \%$ & $-74.4 \%$ & $-66.5 \%$ & $-71.6 \%$ & $-75.3 \%$ \\
\hline & & HWFET & $-37.6 \%$ & $-37.6 \%$ & $-38.0 \%$ & $-43.2 \%$ & $-43.8 \%$ & $-40.9 \%$ & $-54.1 \%$ & $-44.8 \%$ & $-46.6 \%$ & $-61.8 \%$ & $-37.7 \%$ & $-49.2 \%$ & $-63.8 \%$ \\
\hline & & Combined & $-53.1 \%$ & $-53.1 \%$ & $-55.1 \%$ & $-58.5 \%$ & $-56.4 \%$ & $-57.1 \%$ & $-66.2 \%$ & $-57.3 \%$ & $-61.1 \%$ & $-69.3 \%$ & $-54.9 \%$ & $-62.6 \%$ & $-70.6 \%$ \\
\hline \multirow{6}{*}{ E85 Series HEV PHEV30 } & \multirow{3}{*}{ Unadjusted } & UDDS & $-63.4 \%$ & $-63.4 \%$ & $-67.8 \%$ & $-71.1 \%$ & $-65.1 \%$ & $-69.8 \%$ & $-78.0 \%$ & $-66.9 \%$ & $-74.2 \%$ & $-81.7 \%$ & $-68.2 \%$ & $-74.9 \%$ & $-82.7 \%$ \\
\hline & & HWFET & $-33.6 \%$ & $-33.6 \%$ & $-41.2 \%$ & $-46.7 \%$ & $-36.1 \%$ & $-44.8 \%$ & $-58.2 \%$ & $-38.6 \%$ & $-51.2 \%$ & $-64.6 \%$ & $-41.6 \%$ & $-53.5 \%$ & $-66.6 \%$ \\
\hline & & Combined & $-52.1 \%$ & $-52.1 \%$ & $-57.7 \%$ & $-61.9 \%$ & $-54.1 \%$ & $-60.3 \%$ & $-70.5 \%$ & $-56.1 \%$ & $-65.4 \%$ & $-75.2 \%$ & $-58.1 \%$ & $-66.8 \%$ & $-76.6 \%$ \\
\hline & \multirow{3}{*}{ Adjusted } & UDDS & $-60.2 \%$ & $-60.2 \%$ & $-64.4 \%$ & $-67.6 \%$ & $-61.9 \%$ & $-66.3 \%$ & $-74.1 \%$ & $-63.5 \%$ & $-70.5 \%$ & $-77.6 \%$ & $-64.8 \%$ & $-71.2 \%$ & $-78.6 \%$ \\
\hline & & HWFET & $-33.4 \%$ & $-33.4 \%$ & $-40.9 \%$ & $-46.4 \%$ & $-35.8 \%$ & $-44.5 \%$ & $-57.8 \%$ & $-38.3 \%$ & $-50.8 \%$ & $-64.1 \%$ & $-41.3 \%$ & $-53.1 \%$ & $-66.1 \%$ \\
\hline & & Combined & $-49.4 \%$ & $-49.4 \%$ & $-55.0 \%$ & $-59.1 \%$ & $-51.4 \%$ & $-57.6 \%$ & $-67.6 \%$ & $-53.4 \%$ & $-62.6 \%$ & $-72.2 \%$ & $-55.4 \%$ & $-63.9 \%$ & $-73.6 \%$ \\
\hline \multirow{6}{*}{ E85 Series HEV PHEV40 } & & UDDS & $-68.0 \%$ & $-68.0 \%$ & $-71.9 \%$ & $-74.9 \%$ & $-69.6 \%$ & $-73.8 \%$ & $-81.0 \%$ & $-71.1 \%$ & $-77.7 \%$ & $-84.3 \%$ & $-72.3 \%$ & $-78.3 \%$ & $-85.3 \%$ \\
\hline & Unadjusted & HWFET & $-47.4 \%$ & $-47.4 \%$ & $-53.4 \%$ & $-57.9 \%$ & $-49.4 \%$ & $-56.4 \%$ & $-67.1 \%$ & $-51.4 \%$ & $-61.5 \%$ & $-72.2 \%$ & $-53.8 \%$ & $-63.4 \%$ & $-73.7 \%$ \\
\hline & & Combined & $-60.2 \%$ & $-60.2 \%$ & $-64.8 \%$ & $-68.5 \%$ & $-62.0 \%$ & $-67.2 \%$ & $-75.7 \%$ & $-63.6 \%$ & $-71.5 \%$ & $-79.7 \%$ & $-65.3 \%$ & $-72.6 \%$ & $-80.9 \%$ \\
\hline & & UDDS & $-64.6 \%$ & $-64.6 \%$ & $-68.3 \%$ & $-71.2 \%$ & $-66.2 \%$ & $-70.1 \%$ & $-77.0 \%$ & $-67.5 \%$ & $-73.8 \%$ & $-80.1 \%$ & $-68.7 \%$ & $-74.4 \%$ & $-81.0 \%$ \\
\hline & Adjusted & HWFET & $-47.1 \%$ & $-47.1 \%$ & $-53.0 \%$ & $-57.5 \%$ & $-49.1 \%$ & $-56.0 \%$ & $-66.6 \%$ & $-51.0 \%$ & $-61.1 \%$ & $-71.7 \%$ & $-53.4 \%$ & $-62.9 \%$ & $-73.2 \%$ \\
\hline & & Combined & $-57.6 \%$ & $-57.6 \%$ & $-62.1 \%$ & $-65.7 \%$ & $-59.3 \%$ & $-64.4 \%$ & $-72.8 \%$ & $-60.9 \%$ & $-68.7 \%$ & $-76.7 \%$ & $-62.6 \%$ & $-69.8 \%$ & $-77.9 \%$ \\
\hline
\end{tabular}


Argonne National Laboratory Report - Light-Duty Vehicle Fuel Consumption Displacement Potential Up to 2045

\begin{tabular}{|c|c|c|c|c|c|c|c|c|c|c|c|c|c|c|c|}
\hline & & & \multicolumn{13}{|c|}{ Small_SUV } \\
\hline & & & 2010 & & 2010 & & & 2015 & & & 2030 & & & 2045 & \\
\hline & & & Ref & low & avg & high & low & avg & high & low & avg & high & low & avg & high \\
\hline \multirow{6}{*}{ FC HEV } & \multirow{3}{*}{ Unadjusted } & UDDS & $-48.3 \%$ & $-48.3 \%$ & $-57.9 \%$ & $-64.8 \%$ & $-57.0 \%$ & $-64.2 \%$ & $-69.4 \%$ & $-60.1 \%$ & $-68.4 \%$ & $-74.5 \%$ & $-62.3 \%$ & $-69.7 \%$ & $-75.7 \%$ \\
\hline & & HWFET & $-31.0 \%$ & $-31.0 \%$ & $-42.7 \%$ & $-51.2 \%$ & $-41.0 \%$ & $-49.6 \%$ & $-55.8 \%$ & $-43.5 \%$ & $-54.5 \%$ & $-61.5 \%$ & $-46.5 \%$ & $-56.2 \%$ & $-63.6 \%$ \\
\hline & & Combined & $-41.7 \%$ & $-41.7 \%$ & $-52.2 \%$ & $-59.6 \%$ & $-50.9 \%$ & $-58.6 \%$ & $-64.2 \%$ & $-53.8 \%$ & $-63.2 \%$ & $-69.6 \%$ & $-56.3 \%$ & $-64.5 \%$ & $-71.1 \%$ \\
\hline & \multirow{3}{*}{ Adjusted } & UDDS & $-45.9 \%$ & $-45.9 \%$ & $-55.1 \%$ & $-61.6 \%$ & $-54.2 \%$ & $-61.0 \%$ & $-65.9 \%$ & $-57.2 \%$ & $-65.0 \%$ & $-70.8 \%$ & $-59.2 \%$ & $-66.2 \%$ & $-71.9 \%$ \\
\hline & & HWFET & $-30.7 \%$ & $-30.7 \%$ & $-42.4 \%$ & $-50.9 \%$ & $-40.7 \%$ & $-49.2 \%$ & $-55.4 \%$ & $-43.2 \%$ & $-54.1 \%$ & $-61.1 \%$ & $-46.1 \%$ & $-55.8 \%$ & $-63.2 \%$ \\
\hline & & Combined & $-39.8 \%$ & $-39.8 \%$ & $-50.0 \%$ & $-57.3 \%$ & $-48.8 \%$ & $-56.3 \%$ & $-61.7 \%$ & $-51.5 \%$ & $-60.7 \%$ & $-66.9 \%$ & $-54.0 \%$ & $-62.0 \%$ & $-68.4 \%$ \\
\hline \multirow{6}{*}{ FC PHEV10 } & \multirow{3}{*}{ Unadjusted } & UDDS & $-59.1 \%$ & $-59.1 \%$ & $-66.3 \%$ & $-71.4 \%$ & $-65.3 \%$ & $-70.5 \%$ & $-74.6 \%$ & $-67.2 \%$ & $-73.8 \%$ & $-78.7 \%$ & $-68.8 \%$ & $-74.7 \%$ & $-79.6 \%$ \\
\hline & & $\begin{array}{l}\text { HWFET } \\
\end{array}$ & $-47.3 \%$ & $-47.3 \%$ & $-55.5 \%$ & $-61.8 \%$ & $-54.0 \%$ & $-60.3 \%$ & $-65.0 \%$ & $-55.3 \%$ & $-64.0 \%$ & $-69.1 \%$ & $-57.6 \%$ & $-65.3 \%$ & $-70.9 \%$ \\
\hline & & Combined & $-54.6 \%$ & $-54.6 \%$ & $-62.2 \%$ & $-67.8 \%$ & $-61.0 \%$ & $-66.6 \%$ & $-71.0 \%$ & $-62.7 \%$ & $-70.1 \%$ & $-75.0 \%$ & $-64.6 \%$ & $-71.1 \%$ & $-76.3 \%$ \\
\hline & \multirow{3}{*}{ Adjusted } & UDDS & $-56.2 \%$ & $-56.2 \%$ & $-63.0 \%$ & $-67.9 \%$ & $-62.0 \%$ & $-67.0 \%$ & $-70.9 \%$ & $-63.9 \%$ & $-70.1 \%$ & $-74.8 \%$ & $-65.4 \%$ & $-71.0 \%$ & $-75.7 \%$ \\
\hline & & HWFET & $-46.9 \%$ & $-46.9 \%$ & $-55.1 \%$ & $-61.3 \%$ & $-53.6 \%$ & $-59.9 \%$ & $-64.5 \%$ & $-54.9 \%$ & $-63.5 \%$ & $-68.6 \%$ & $-57.2 \%$ & $-64.8 \%$ & $-70.3 \%$ \\
\hline & & Combined & $-52.5 \%$ & $-52.5 \%$ & $-59.8 \%$ & $-65.2 \%$ & $-58.7 \%$ & $-64.1 \%$ & $-68.3 \%$ & $-60.3 \%$ & $-67.5 \%$ & $-72.3 \%$ & $-62.1 \%$ & $-68.5 \%$ & $-73.5 \%$ \\
\hline \multirow{6}{*}{ FC PHEV2O } & \multirow{3}{*}{ Unadjusted } & UDDS & $-65.8 \%$ & $-65.8 \%$ & $-71.9 \%$ & $-76.3 \%$ & $-71.1 \%$ & $-75.5 \%$ & $-78.9 \%$ & $-72.7 \%$ & $-78.3 \%$ & $-82.4 \%$ & $-74.1 \%$ & $-79.1 \%$ & $-83.2 \%$ \\
\hline & & HWFET & $-48.4 \%$ & $-48.4 \%$ & $-56.8 \%$ & $-63.0 \%$ & $-55.3 \%$ & $-61.6 \%$ & $-66.3 \%$ & $-56.9 \%$ & $-65.3 \%$ & $-70.6 \%$ & $-59.1 \%$ & $-66.6 \%$ & $-72.2 \%$ \\
\hline & & Combined & $-59.2 \%$ & $-59.2 \%$ & $-66.2 \%$ & $-71.3 \%$ & $-65.1 \%$ & $-70.2 \%$ & $-74.2 \%$ & $-66.7 \%$ & $-73.4 \%$ & $-77.9 \%$ & $-68.4 \%$ & $-74.3 \%$ & $-79.0 \%$ \\
\hline & \multirow{3}{*}{ Adjusted } & UDDS & $-62.6 \%$ & $-62.6 \%$ & $-68.3 \%$ & $-72.5 \%$ & $-67.5 \%$ & $-71.7 \%$ & $-75.0 \%$ & $-69.1 \%$ & $-74.4 \%$ & $-78.3 \%$ & $-70.4 \%$ & $-75.2 \%$ & $-79.1 \%$ \\
\hline & & HWFET & $-48.0 \%$ & $-48.0 \%$ & $-56.4 \%$ & $-62.6 \%$ & $-54.9 \%$ & $-61.2 \%$ & $-65.9 \%$ & $-56.5 \%$ & $-64.9 \%$ & $-70.1 \%$ & $-58.7 \%$ & $-66.1 \%$ & $-71.7 \%$ \\
\hline & & Combined & $-56.7 \%$ & $-56.7 \%$ & $-63.5 \%$ & $-68.5 \%$ & $-62.5 \%$ & $-67.5 \%$ & $-71.3 \%$ & $-64.0 \%$ & $-70.6 \%$ & $-75.0 \%$ & $-65.7 \%$ & $-71.5 \%$ & $-76.1 \%$ \\
\hline \multirow{6}{*}{ FC PHEV30 } & \multirow{3}{*}{ Unadjusted } & UDDS & $-76.0 \%$ & $-76.0 \%$ & $-80.2 \%$ & $-83.0 \%$ & $-79.6 \%$ & $-82.5 \%$ & $-85.1 \%$ & $-80.5 \%$ & $-84.6 \%$ & $-87.4 \%$ & $-81.5 \%$ & $-85.2 \%$ & $-88.0 \%$ \\
\hline & & HWFET & $-59.0 \%$ & $-59.0 \%$ & $-65.6 \%$ & $-70.2 \%$ & $-64.3 \%$ & $-69.2 \%$ & $-73.1 \%$ & $-65.3 \%$ & $-72.3 \%$ & $-76.5 \%$ & $-67.2 \%$ & $-73.3 \%$ & $-77.7 \%$ \\
\hline & & Combined & $-69.6 \%$ & $-69.6 \%$ & $-74.6 \%$ & $-78.1 \%$ & $-73.8 \%$ & $-77.5 \%$ & $-80.5 \%$ & $-74.7 \%$ & $-79.9 \%$ & $-83.3 \%$ & $-76.1 \%$ & $-80.7 \%$ & $-84.1 \%$ \\
\hline & \multirow{3}{*}{ Adjusted } & UDDS & $-72.3 \%$ & $-72.3 \%$ & $-76.2 \%$ & $-78.8 \%$ & $-75.6 \%$ & $-78.4 \%$ & $-80.8 \%$ & $-76.5 \%$ & $-80.4 \%$ & $-83.1 \%$ & $-77.5 \%$ & $-81.0 \%$ & $-83.6 \%$ \\
\hline & & $\begin{array}{l}\text { HWFET } \\
\end{array}$ & $-58.5 \%$ & $-58.5 \%$ & $-65.1 \%$ & $-69.7 \%$ & $-63.9 \%$ & $-68.7 \%$ & $-72.6 \%$ & $-64.9 \%$ & $-71.8 \%$ & $-75.9 \%$ & $-66.7 \%$ & $-72.8 \%$ & $-77.2 \%$ \\
\hline & & Combined & $-66.8 \%$ & $-66.8 \%$ & $-71.7 \%$ & $-75.2 \%$ & $-70.9 \%$ & $-74.5 \%$ & $-77.5 \%$ & $-71.8 \%$ & $-76.9 \%$ & $-80.2 \%$ & $-73.1 \%$ & $-77.7 \%$ & $-81.0 \%$ \\
\hline \multirow{6}{*}{ FC PHEV40 } & \multirow{3}{*}{ Unadjusted } & UDDS & $-79.2 \%$ & $-79.2 \%$ & $-82.9 \%$ & $-85.3 \%$ & $-82.3 \%$ & $-84.9 \%$ & $-87.1 \%$ & $-83.1 \%$ & $-86.7 \%$ & $-89.2 \%$ & $-84.0 \%$ & $-87.3 \%$ & $-89.7 \%$ \\
\hline & & $\begin{array}{l}\text { HWFET } \\
\end{array}$ & $-67.6 \%$ & $-67.6 \%$ & $-72.9 \%$ & $-76.5 \%$ & $-71.9 \%$ & $-75.7 \%$ & $-78.9 \%$ & $-72.7 \%$ & $-78.2 \%$ & $-81.5 \%$ & $-74.1 \%$ & $-79.1 \%$ & $-82.6 \%$ \\
\hline & & Combined & $-74.8 \%$ & $-74.8 \%$ & $-79.1 \%$ & $-81.9 \%$ & $-78.3 \%$ & $-81.4 \%$ & $-84.0 \%$ & $-79.2 \%$ & $-83.5 \%$ & $-86.3 \%$ & $-80.3 \%$ & $-84.2 \%$ & $-87.0 \%$ \\
\hline & \multirow{3}{*}{ Adjusted } & UDDS & $-75.2 \%$ & $-75.2 \%$ & $-78.7 \%$ & $-81.0 \%$ & $-78.2 \%$ & $-80.7 \%$ & $-82.8 \%$ & $-79.0 \%$ & $-82.4 \%$ & $-84.8 \%$ & $-79.9 \%$ & $-82.9 \%$ & $-85.2 \%$ \\
\hline & & HWFET & $-67.1 \%$ & $-67.1 \%$ & $-72.3 \%$ & $-76.0 \%$ & $-71.3 \%$ & $-75.2 \%$ & $-78.3 \%$ & $-72.2 \%$ & $-77.7 \%$ & $-80.9 \%$ & $-73.6 \%$ & $-78.5 \%$ & $-82.0 \%$ \\
\hline & & Combined & $-72.0 \%$ & $-72.0 \%$ & $-76.2 \%$ & $-79.0 \%$ & $-75.4 \%$ & $-78.5 \%$ & $-81.0 \%$ & $-76.2 \%$ & $-80.5 \%$ & $-83.2 \%$ & $-77.3 \%$ & $-81.2 \%$ & $-83.9 \%$ \\
\hline
\end{tabular}




\section{$\underline{\text { Midsize SUV Class }}$}


Fuel economy

\begin{tabular}{|c|c|c|c|c|c|c|c|c|c|c|c|c|c|c|c|}
\hline & & & \multicolumn{13}{|c|}{ Midsize_SUV } \\
\hline & & & \multirow{2}{*}{$\frac{2010}{\text { Ref }}$} & \multicolumn{3}{|c|}{2010} & \multicolumn{3}{|c|}{2015} & \multicolumn{3}{|c|}{2030} & \multicolumn{3}{|c|}{2045} \\
\hline & & & & low & avg & high & low & avg & high & low & avg & high & low & avg & high \\
\hline \multirow{6}{*}{ SI Conv } & \multirow{3}{*}{ Unadjusted } & UDDS & 21.3 & 21.3 & 23.8 & 25.0 & 22.4 & 24.8 & 30.5 & 23.2 & 25.5 & 36.9 & 22.9 & 26.0 & 38.3 \\
\hline & & HWFET & 28.5 & 28.5 & 32.1 & 33.8 & 30.1 & 33.5 & 41.8 & 31.2 & 34.9 & 49.4 & 31.5 & 35.9 & 51.8 \\
\hline & & Combined & 24.0 & 24.0 & 26.9 & 28.3 & 25.3 & 28.1 & 34.8 & 26.3 & 29.0 & 41.6 & 26.1 & 29.7 & 43.4 \\
\hline & \multirow{3}{*}{ Adjusted } & UDDS & 16.6 & 16.6 & 18.5 & 19.4 & 17.5 & 19.2 & 23.4 & 18.1 & 19.8 & 27.9 & 17.8 & 20.1 & 28.9 \\
\hline & & HWFET & 20.2 & 20.2 & 22.7 & 23.9 & 21.3 & 23.7 & 29.6 & 22.1 & 24.7 & 34.9 & 22.3 & 25.4 & 36.5 \\
\hline & & Combined & 18.0 & 18.0 & 20.2 & 21.2 & 19.0 & 21.0 & 25.8 & 19.7 & 21.7 & 30.7 & 19.6 & 22.2 & 31.9 \\
\hline \multirow{6}{*}{ Cl Conv } & \multirow{3}{*}{ Unadjusted } & UDDS & 25.7 & 25.7 & 27.5 & 29.9 & 26.2 & 29.5 & 32.0 & 27.8 & 31.1 & 37.6 & 28.0 & 32.4 & 38.9 \\
\hline & & HWFET & 32.9 & 32.9 & 35.4 & 38.3 & 33.7 & 38.0 & 39.2 & 35.3 & 38.0 & 45.1 & 34.2 & 40.1 & 47.2 \\
\hline & & Combined & 28.5 & 28.5 & 30.6 & 33.2 & 29.1 & 32.8 & 34.9 & 30.7 & 33.9 & 40.6 & 30.5 & 35.5 & 42.2 \\
\hline & \multirow{3}{*}{ Adjusted } & \begin{tabular}{|l|} 
UDDS \\
\end{tabular} & 19.9 & 19.9 & 21.2 & 23.0 & 20.2 & 22.6 & 24.5 & 21.4 & 23.9 & 28.4 & 21.6 & 24.8 & 29.4 \\
\hline & & HWFET & 23.3 & 23.3 & 25.0 & 27.1 & 23.8 & 26.8 & 27.7 & 25.0 & 26.8 & 31.9 & 24.2 & 28.4 & 33.3 \\
\hline & & Combined & 21.3 & 21.3 & 22.8 & 24.7 & 21.7 & 24.4 & 25.8 & 22.9 & 25.1 & 29.9 & 22.7 & 26.3 & 31.0 \\
\hline \multirow{6}{*}{$\mathrm{H} 2$ Conv } & \multirow{3}{*}{ Unadjusted } & \begin{tabular}{|l|} 
UDDS \\
\end{tabular} & 18.1 & 18.1 & 22.2 & 25.3 & 20.5 & 24.9 & 28.0 & 23.2 & 27.2 & 33.3 & 24.1 & 28.9 & 35.9 \\
\hline & & HWFET & 27.3 & 27.3 & 31.9 & 36.2 & 30.9 & 35.8 & 41.0 & 33.1 & 39.7 & 47.7 & 35.6 & 42.7 & 51.8 \\
\hline & & Combined & 21.3 & 21.3 & 25.7 & 29.3 & 24.2 & 28.9 & 32.7 & 26.8 & 31.7 & 38.5 & 28.2 & 33.8 & 41.6 \\
\hline & \multirow{3}{*}{ Adjusted } & UDDS & 14.2 & 14.2 & 17.3 & 19.6 & 16.0 & 19.3 & 21.6 & 18.0 & 21.0 & 25.4 & 18.7 & 22.2 & 27.2 \\
\hline & & HWFET & 19.4 & 19.4 & 22.5 & 25.6 & 21.9 & 25.4 & 29.0 & 23.4 & 28.0 & 33.7 & 25.2 & 30.1 & 36.5 \\
\hline & & Combined & 16.2 & 16.2 & 19.3 & 21.9 & 18.2 & 21.6 & 24.4 & 20.1 & 23.7 & 28.5 & 21.2 & 25.2 & 30.8 \\
\hline \multirow{6}{*}{ E85 Conv } & \multirow{3}{*}{ Unadjusted } & UDDS & 20.5 & 20.5 & 21.6 & 22.7 & 20.0 & 22.4 & 29.5 & 21.1 & 23.3 & 34.8 & 21.0 & 23.8 & 36.2 \\
\hline & & \begin{tabular}{|c|} 
HWFET \\
\end{tabular} & 25.4 & 25.4 & 27.1 & 28.4 & 24.9 & 28.2 & 37.0 & 26.4 & 29.1 & 42.9 & 26.3 & 30.1 & 45.0 \\
\hline & & Combined & 22.5 & 22.5 & 23.8 & 24.9 & 22.0 & 24.7 & 32.5 & 23.2 & 25.6 & 38.0 & 23.1 & 26.3 & 39.7 \\
\hline & \multirow{3}{*}{ Adjusted } & \begin{tabular}{|l|} 
UDDS \\
\end{tabular} & 16.0 & 16.0 & 16.9 & 17.7 & 15.7 & 17.5 & 22.7 & 16.5 & 18.2 & 26.4 & 16.4 & 18.5 & 27.5 \\
\hline & & \begin{tabular}{|c|} 
HWFET \\
\end{tabular} & 18.0 & 18.0 & 19.2 & 20.1 & 17.7 & 20.0 & 26.2 & 18.7 & 20.6 & 30.3 & 18.6 & 21.3 & 31.7 \\
\hline & & Combined & 16.9 & 16.9 & 17.9 & 18.7 & 16.5 & 18.5 & 24.1 & 17.4 & 19.2 & 28.0 & 17.3 & 19.7 & 29.2 \\
\hline \multirow{6}{*}{ SI Split HEV } & \multirow{3}{*}{ Unadjusted } & \begin{tabular}{|l|} 
UDDS \\
\end{tabular} & 38.2 & 38.2 & 41.5 & 44.5 & 39.6 & 44.7 & 54.4 & 41.7 & 48.6 & 67.4 & 42.6 & 50.9 & 70.4 \\
\hline & & HWFET & 33.3 & 33.3 & 36.1 & 38.6 & 34.4 & 38.3 & 45.8 & 35.5 & 41.0 & 54.8 & 36.4 & 43.4 & 57.7 \\
\hline & & Combined & 35.8 & 35.8 & 38.9 & 41.6 & 37.1 & 41.6 & 50.2 & 38.7 & 44.9 & 61.1 & 39.6 & 47.2 & 64.0 \\
\hline & \multirow{3}{*}{ Adjusted } & \begin{tabular}{|l|} 
UDDS \\
\end{tabular} & 28.9 & 28.9 & 31.2 & 33.3 & 29.9 & 33.4 & 39.9 & 31.3 & 36.0 & 48.2 & 31.9 & 37.6 & 50.1 \\
\hline & & HWFET & 23.5 & 23.5 & 25.5 & 27.2 & 24.3 & 27.1 & 32.3 & 25.1 & 29.0 & 38.6 & 25.8 & 30.6 & 40.6 \\
\hline & & Combined & 26.2 & 26.2 & 28.3 & 30.3 & 27.1 & 30.2 & 36.1 & 28.2 & 32.5 & 43.4 & 28.8 & 34.1 & 45.3 \\
\hline \multirow{6}{*}{ SI Split HEV PHEV10 } & & \begin{tabular}{|l|} 
UDDS \\
\end{tabular} & 48.2 & 48.2 & 52.1 & 56.0 & 49.8 & 55.3 & 67.1 & 51.2 & 59.6 & 81.7 & 52.6 & 62.6 & 85.6 \\
\hline & Unadjusted & \begin{tabular}{|l|} 
HWFET \\
\end{tabular} & 43.5 & 43.5 & 46.8 & 49.9 & 44.9 & 49.5 & 58.4 & 45.8 & 52.5 & 69.1 & 47.1 & 54.9 & 73.5 \\
\hline & & Combined & 46.0 & 46.0 & 49.6 & 53.1 & 47.5 & 52.5 & 62.8 & 48.6 & 56.2 & 75.5 & 50.0 & 58.9 & 79.7 \\
\hline & & \begin{tabular}{|l|} 
UDDS \\
\end{tabular} & 35.7 & 35.7 & 38.3 & 40.9 & 36.8 & 40.4 & 48.0 & 37.7 & 43.3 & 57.0 & 38.7 & 45.2 & 59.2 \\
\hline & Adjusted & \begin{tabular}{|l|} 
HWFET \\
\end{tabular} & 30.7 & 30.7 & 33.0 & 35.2 & 31.7 & 34.9 & 41.1 & 32.3 & 37.0 & 48.5 & 33.2 & 38.7 & 51.6 \\
\hline & & Combined & 33.3 & 33.3 & 35.8 & 38.1 & 34.3 & 37.7 & 44.6 & 35.1 & 40.2 & 52.8 & 36.0 & 42.0 & 55.5 \\
\hline
\end{tabular}




\begin{tabular}{|c|c|c|c|c|c|c|c|c|c|c|c|c|c|c|c|}
\hline & & & \multicolumn{13}{|c|}{ Midsize_SUV } \\
\hline & & & \multirow{2}{*}{$\begin{array}{l}2010 \\
\text { Ref }\end{array}$} & \multicolumn{3}{|c|}{2010} & \multicolumn{3}{|c|}{2015} & \multicolumn{3}{|c|}{2030} & \multicolumn{3}{|c|}{2045} \\
\hline & & & & low & avg & high & low & avg & high & low & avg & high & low & avg & high \\
\hline \multirow{6}{*}{ SI Split HEV PHEV2O } & \multirow{3}{*}{ Unadjusted } & UDDS & 69.0 & 69.0 & 74.9 & 80.4 & 72.4 & 79.4 & 95.4 & 73.8 & 85.3 & 117.0 & 76.4 & 89.1 & 122.9 \\
\hline & & HWFET & 53.2 & 53.2 & 57.7 & 60.3 & 55.1 & 60.9 & 66.8 & 56.2 & 64.5 & 79.3 & 57.9 & 62.6 & 85.2 \\
\hline & & Combined & 60.9 & 60.9 & 66.0 & 69.9 & 63.4 & 69.9 & 80.0 & 64.7 & 74.5 & 96.4 & 66.8 & 74.8 & 102.5 \\
\hline & \multirow{3}{*}{ Adjusted } & UDDS & 49.2 & 49.2 & 52.9 & 56.2 & 51.3 & 55.6 & 64.9 & 52.2 & 59.1 & 76.8 & 53.8 & 61.3 & 79.9 \\
\hline & & HWFET & 37.5 & 37.5 & 40.6 & 42.4 & 38.8 & 42.8 & 46.9 & 39.5 & 45.3 & 55.5 & 40.7 & 44.0 & 59.6 \\
\hline & & \begin{tabular}{|l|} 
Combined \\
\end{tabular} & 43.2 & 43.2 & 46.5 & 49.0 & 44.8 & 49.0 & 55.4 & 45.6 & 52.0 & 65.5 & 47.0 & 52.1 & 69.3 \\
\hline \multirow{6}{*}{ SI Series HEV PHEV30 } & \multirow{3}{*}{ Unadjusted } & UDDS & 61.9 & 61.9 & 67.8 & 74.5 & 64.3 & 73.3 & 92.6 & 67.5 & 82.4 & 119.2 & 69.6 & 86.6 & 124.2 \\
\hline & & \begin{tabular}{|l|} 
HWFET \\
\end{tabular} & 45.9 & 45.9 & 50.4 & 54.3 & 47.8 & 53.8 & 65.9 & 49.7 & 58.7 & 79.8 & 51.3 & 62.7 & 84.2 \\
\hline & & \begin{tabular}{|l|} 
Combined \\
\end{tabular} & 53.5 & 53.5 & 58.6 & 63.8 & 55.6 & 63.0 & 78.3 & 58.1 & 69.7 & 97.5 & 60.0 & 73.9 & 102.3 \\
\hline & \multirow{3}{*}{ Adjusted } & \begin{tabular}{|l|} 
UDDS \\
\end{tabular} & 44.7 & 44.7 & 48.4 & 52.6 & 46.2 & 51.9 & 63.3 & 48.3 & 57.4 & 77.9 & 49.6 & 59.9 & 80.5 \\
\hline & & HWFET & 32.4 & 32.4 & 35.5 & 38.3 & 33.7 & 37.9 & 46.3 & 35.0 & 41.3 & 55.9 & 36.2 & 44.1 & 58.9 \\
\hline & & \begin{tabular}{|c|} 
Combined \\
\end{tabular} & 38.2 & 38.2 & 41.6 & 45.0 & 39.6 & 44.5 & 54.3 & 41.3 & 48.8 & 66.2 & 42.5 & 51.6 & 69.1 \\
\hline \multirow{6}{*}{ SI Series HEV PHEV40 } & \multirow{3}{*}{ Unadjusted } & \begin{tabular}{|l|} 
UDDS \\
\end{tabular} & 70.9 & 70.9 & 77.9 & 85.0 & 74.2 & 83.7 & 107.1 & 77.5 & 95.0 & 136.0 & 80.2 & 100.1 & 144.3 \\
\hline & & HWFET & 58.0 & 58.0 & 63.7 & 68.7 & 60.3 & 68.1 & 83.3 & 62.9 & 74.5 & 101.5 & 65.0 & 79.5 & 107.1 \\
\hline & & \begin{tabular}{|l|} 
Combined \\
\end{tabular} & 64.5 & 64.5 & 70.8 & 76.8 & 67.2 & 75.9 & 94.9 & 70.2 & 84.5 & 117.9 & 72.6 & 89.6 & 124.8 \\
\hline & \multirow{3}{*}{ Adjusted } & UDDS & 50.4 & 50.4 & 54.7 & 58.9 & 52.4 & 58.1 & 71.4 & 54.5 & 64.7 & 86.5 & 56.1 & 67.6 & 90.5 \\
\hline & & HWFET & 40.8 & 40.8 & 44.8 & 48.3 & 42.4 & 47.8 & 58.3 & 44.2 & 52.2 & 70.7 & 45.7 & 55.7 & 74.6 \\
\hline & & \begin{tabular}{|c|} 
Combined \\
\end{tabular} & 45.6 & 45.6 & 49.7 & 53.6 & 47.4 & 53.0 & 64.9 & 49.3 & 58.4 & 78.6 & 50.9 & 61.6 & 82.6 \\
\hline \multirow{6}{*}{ CI Split HEV } & \multirow{3}{*}{ Unadjusted } & \begin{tabular}{|l|} 
UDDS \\
\end{tabular} & 42.6 & 42.6 & 45.6 & 49.7 & 43.4 & 49.5 & 56.7 & 46.2 & 54.6 & 67.7 & 47.8 & 57.6 & 70.7 \\
\hline & & HWFET & 35.6 & 35.6 & 38.1 & 41.3 & 36.4 & 41.0 & 46.1 & 38.0 & 44.5 & 53.5 & 39.5 & 47.4 & 56.5 \\
\hline & & \begin{tabular}{|l|} 
Combined \\
\end{tabular} & 39.1 & 39.1 & 41.9 & 45.5 & 39.9 & 45.3 & 51.4 & 42.1 & 49.5 & 60.5 & 43.7 & 52.5 & 63.5 \\
\hline & \multirow{3}{*}{ Adjusted } & \begin{tabular}{|l|} 
UDDS \\
\end{tabular} & 31.9 & 31.9 & 33.9 & 36.7 & 32.5 & 36.6 & 41.4 & 34.4 & 40.0 & 48.4 & 35.5 & 41.9 & 50.3 \\
\hline & & HWFET & 25.2 & 25.2 & 26.9 & 29.2 & 25.7 & 29.0 & 32.5 & 26.8 & 31.4 & 37.7 & 27.9 & 33.4 & 39.7 \\
\hline & & \begin{tabular}{|c|} 
Combined \\
\end{tabular} & 28.5 & 28.5 & 30.4 & 32.9 & 29.1 & 32.7 & 36.9 & 30.5 & 35.6 & 42.9 & 31.6 & 37.6 & 44.9 \\
\hline \multirow{6}{*}{ CI Split HEV PHEV10 } & \multirow{3}{*}{ Unadjusted } & UDDS & 49.8 & 49.8 & 53.3 & 58.1 & 50.9 & 57.7 & 65.6 & 53.4 & 62.8 & 77.9 & 55.4 & 66.6 & 81.2 \\
\hline & & HWFET & 44.7 & 44.7 & 47.4 & 53.7 & 45.1 & 53.5 & 59.7 & 47.5 & 54.5 & 68.4 & 49.0 & 60.5 & 68.5 \\
\hline & & \begin{tabular}{|l|} 
Combined \\
\end{tabular} & 47.4 & 47.4 & 50.5 & 56.0 & 48.1 & 55.7 & 62.8 & 50.6 & 58.8 & 73.3 & 52.3 & 63.7 & 74.9 \\
\hline & \multirow{3}{*}{ Adjusted } & \begin{tabular}{|l|} 
UDDS \\
\end{tabular} & 36.8 & 36.8 & 39.2 & 42.3 & 37.5 & 42.0 & 47.1 & 39.2 & 45.3 & 54.7 & 40.5 & 47.7 & 56.7 \\
\hline & & HWFET & 31.6 & 31.6 & 33.4 & 37.8 & 31.9 & 37.7 & 42.0 & 33.5 & 38.4 & 48.0 & 34.5 & 42.5 & 48.1 \\
\hline & & Combined & 34.2 & 34.2 & 36.3 & 40.1 & 34.7 & 40.0 & 44.6 & 36.4 & 41.9 & 51.5 & 37.6 & 45.2 & 52.4 \\
\hline \multirow{6}{*}{ CI Split HEV PHEV2O } & & \begin{tabular}{|l|} 
UDDS \\
\end{tabular} & 71.2 & 71.2 & 77.1 & 83.7 & 73.1 & 82.5 & 93.9 & 76.9 & 89.8 & 112.2 & 79.5 & 95.0 & 116.3 \\
\hline & Unadjusted & HWFET & 54.7 & 54.7 & 49.7 & 52.9 & 55.8 & 52.7 & 68.3 & 58.0 & 65.4 & 78.3 & 51.0 & 68.9 & 78.5 \\
\hline & & Combined & 62.6 & 62.6 & 61.8 & 66.3 & 64.1 & 65.8 & 80.4 & 67.0 & 76.9 & 93.9 & 63.5 & 81.2 & 95.6 \\
\hline & & \begin{tabular}{|l|} 
UDDS \\
\end{tabular} & 50.6 & 50.6 & 54.2 & 58.1 & 51.7 & 57.5 & 64.1 & 54.1 & 61.7 & 74.2 & 55.7 & 64.7 & 76.4 \\
\hline & Adjusted & HWFET & 38.5 & 38.5 & 35.1 & 37.3 & 39.3 & 37.2 & 48.0 & 40.8 & 46.0 & 54.9 & 35.9 & 48.4 & 55.0 \\
\hline & & \begin{tabular}{|l|} 
Combined \\
\end{tabular} & 44.3 & 44.3 & 43.5 & 46.4 & 45.3 & 46.1 & 55.7 & 47.2 & 53.5 & 64.1 & 44.6 & 56.2 & 65.0 \\
\hline
\end{tabular}




\begin{tabular}{|c|c|c|c|c|c|c|c|c|c|c|c|c|c|c|c|}
\hline & & & \multicolumn{13}{|c|}{ Midsize_SUV } \\
\hline & & & \multirow{2}{*}{$\begin{array}{l}2010 \\
\text { Ref }\end{array}$} & \multicolumn{3}{|c|}{2010} & \multicolumn{3}{|c|}{2015} & \multicolumn{3}{|c|}{2030} & \multicolumn{3}{|c|}{2045} \\
\hline & & & & low & avg & high & low & avg & high & low & avg & high & low & avg & high \\
\hline \multirow{6}{*}{ CI Series HEV PHEV3O } & \multirow{3}{*}{ Unadjusted } & UDDS & 63.2 & 63.2 & 68.9 & 77.0 & 65.1 & 75.8 & 90.7 & 69.9 & 86.2 & 111.0 & 72.8 & 91.5 & 116.7 \\
\hline & & HWFET & 47.0 & 47.0 & 51.1 & 56.0 & 48.2 & 55.5 & 63.8 & 51.3 & 61.4 & 75.9 & 53.6 & 66.1 & 80.1 \\
\hline & & Combined & 54.8 & 54.8 & 59.6 & 65.9 & 56.2 & 65.0 & 76.3 & 60.1 & 72.9 & 91.9 & 62.7 & 78.0 & 96.8 \\
\hline & \multirow{3}{*}{ Adjusted } & UDDS & 45.6 & 45.6 & 49.1 & 54.1 & 46.8 & 53.4 & 62.3 & 49.8 & 59.6 & 73.6 & 51.6 & 62.7 & 76.6 \\
\hline & & HWFET & 33.2 & 33.2 & 36.0 & 39.4 & 34.0 & 39.1 & 44.9 & 36.2 & 43.2 & 53.2 & 37.7 & 46.5 & 56.1 \\
\hline & & \begin{tabular}{|l|} 
Combined \\
\end{tabular} & 39.0 & 39.0 & 42.2 & 46.4 & 40.0 & 45.8 & 53.0 & 42.6 & 50.9 & 62.8 & 44.3 & 54.2 & 65.8 \\
\hline \multirow{6}{*}{$\mathrm{Cl}$ Series HEV PHEV40 } & \multirow{3}{*}{ Unadjusted } & UDDS & 72.5 & 72.5 & 79.0 & 88.2 & 74.8 & 86.8 & 103.6 & 80.2 & 99.2 & 130.0 & 83.8 & 105.7 & 134.7 \\
\hline & & \begin{tabular}{|l|} 
HWFET \\
\end{tabular} & 59.4 & 59.4 & 64.6 & 70.9 & 60.9 & 70.2 & 80.9 & 64.8 & 77.9 & 96.5 & 67.8 & 83.8 & 101.6 \\
\hline & & \begin{tabular}{|l|} 
Combined \\
\end{tabular} & 66.0 & 66.0 & 71.8 & 79.5 & 67.8 & 78.5 & 92.0 & 72.4 & 88.4 & 112.5 & 75.8 & 94.6 & 117.5 \\
\hline & \multirow{3}{*}{ Adjusted } & \begin{tabular}{|l|} 
UDDS \\
\end{tabular} & 51.4 & 51.4 & 55.3 & 60.8 & 52.8 & 60.0 & 69.5 & 56.1 & 67.1 & 83.5 & 58.2 & 70.7 & 85.8 \\
\hline & & \begin{tabular}{|l|} 
HWFET \\
\end{tabular} & 41.8 & 41.8 & 45.4 & 49.7 & 42.8 & 49.3 & 56.7 & 45.5 & 54.6 & 67.4 & 47.6 & 58.7 & 70.8 \\
\hline & & \begin{tabular}{|c|} 
Combined \\
\end{tabular} & 46.6 & 46.6 & 50.4 & 55.3 & 47.8 & 54.6 & 63.1 & 50.8 & 60.8 & 75.4 & 52.9 & 64.7 & 78.4 \\
\hline \multirow{6}{*}{ H2 Split HEV } & \multirow{3}{*}{ Unadjusted } & \begin{tabular}{|l|} 
UDDS \\
\end{tabular} & 37.5 & 37.5 & 44.9 & 51.6 & 42.6 & 51.3 & 60.4 & 47.0 & 58.0 & 73.3 & 50.4 & 62.6 & 79.7 \\
\hline & & HWFET & 32.2 & 32.2 & 38.5 & 43.8 & 36.5 & 43.4 & 50.1 & 39.8 & 48.4 & 58.9 & 42.6 & 52.5 & 64.4 \\
\hline & & \begin{tabular}{|l|} 
Combined \\
\end{tabular} & 34.9 & 34.9 & 41.8 & 47.8 & 39.6 & 47.4 & 55.3 & 43.5 & 53.2 & 66.1 & 46.6 & 57.6 & 72.0 \\
\hline & \multirow{3}{*}{ Adjusted } & UDDS & 28.4 & 28.4 & 33.5 & 38.0 & 31.9 & 37.8 & 43.8 & 35.0 & 42.2 & 51.9 & 37.2 & 45.2 & 55.7 \\
\hline & & HWFET & 22.8 & 22.8 & 27.2 & 31.0 & 25.8 & 30.7 & 35.3 & 28.1 & 34.1 & 41.4 & 30.1 & 37.0 & 45.3 \\
\hline & & \begin{tabular}{|c|} 
Combined \\
\end{tabular} & 25.6 & 25.6 & 30.3 & 34.5 & 28.8 & 34.2 & 39.5 & 31.5 & 38.1 & 46.6 & 33.6 & 41.1 & 50.5 \\
\hline \multirow{6}{*}{ H2 Split HEV PHEV10 } & \multirow{3}{*}{ Unadjusted } & \begin{tabular}{|l|} 
UDDS \\
\end{tabular} & 46.8 & 46.8 & 56.0 & 64.1 & 53.2 & 63.2 & 73.8 & 58.0 & 70.5 & 88.8 & 62.0 & 76.2 & 96.5 \\
\hline & & HWFET & 42.5 & 42.5 & 50.4 & 57.1 & 48.0 & 56.5 & 64.2 & 51.9 & 61.9 & 77.4 & 55.5 & 67.0 & 84.8 \\
\hline & & \begin{tabular}{|l|} 
Combined \\
\end{tabular} & 44.7 & 44.7 & 53.3 & 60.7 & 50.7 & 60.0 & 69.1 & 55.1 & 66.3 & 83.3 & 58.9 & 71.8 & 90.9 \\
\hline & \multirow{3}{*}{ Adjusted } & UDDS & 34.8 & 34.8 & 40.9 & 46.1 & 39.1 & 45.6 & 52.2 & 42.2 & 50.1 & 61.1 & 44.8 & 53.7 & 65.6 \\
\hline & & \begin{tabular}{|l|} 
HWFET \\
\end{tabular} & 30.0 & 30.0 & 35.5 & 40.2 & 33.8 & 39.8 & 45.1 & 36.6 & 43.5 & 54.2 & 39.1 & 47.0 & 59.4 \\
\hline & & \begin{tabular}{|c|} 
Combined \\
\end{tabular} & 32.4 & 32.4 & 38.3 & 43.3 & 36.5 & 42.8 & 48.7 & 39.5 & 46.9 & 57.8 & 42.0 & 50.5 & 62.6 \\
\hline \multirow{6}{*}{ H2 Split HEV PHEV2O } & \multirow{3}{*}{ Unadjusted } & UDDS & 67.2 & 67.2 & 80.9 & 92.4 & 77.3 & 91.0 & 105.6 & 84.6 & 101.3 & 127.6 & 89.7 & 109.1 & 141.0 \\
\hline & & HWFET & 52.3 & 52.3 & 60.5 & 70.0 & 59.1 & 68.3 & 73.5 & 63.9 & 73.9 & 74.7 & 68.1 & 76.6 & 89.6 \\
\hline & & \begin{tabular}{|l|} 
Combined \\
\end{tabular} & 59.6 & 59.6 & 70.2 & 80.8 & 67.9 & 79.2 & 88.2 & 73.8 & 86.8 & 96.8 & 78.5 & 91.6 & 112.0 \\
\hline & \multirow{3}{*}{ Adjusted } & \begin{tabular}{|l|} 
UDDS \\
\end{tabular} & 48.1 & 48.1 & 56.5 & 63.2 & 54.3 & 62.4 & 70.6 & 58.7 & 68.3 & 82.3 & 61.7 & 72.5 & 88.9 \\
\hline & & HWFET & 36.9 & 36.9 & 42.5 & 49.2 & 41.6 & 48.0 & 51.6 & 44.9 & 51.8 & 52.4 & 47.8 & 53.7 & 62.6 \\
\hline & & \begin{tabular}{|l|} 
Combined \\
\end{tabular} & 42.3 & 42.3 & 49.2 & 56.0 & 47.7 & 55.0 & 60.5 & 51.5 & 59.7 & 65.5 & 54.6 & 62.6 & 74.8 \\
\hline \multirow{6}{*}{ H2 Series HEV PHEV30 } & & UDDS & 60.2 & 60.2 & 73.8 & 86.0 & 68.9 & 84.5 & 103.1 & 76.8 & 97.4 & 126.8 & 83.2 & 106.9 & 138.4 \\
\hline & Unadjusted & HWFET & 45.2 & 45.2 & 54.6 & 62.5 & 51.5 & 61.8 & 72.9 & 56.7 & 69.9 & 85.9 & 60.9 & 76.8 & 94.0 \\
\hline & & Combined & 52.4 & 52.4 & 63.7 & 73.5 & 59.8 & 72.5 & 86.9 & 66.2 & 82.7 & 104.4 & 71.4 & 90.9 & 114.2 \\
\hline & & \begin{tabular}{|l|} 
UDDS \\
\end{tabular} & 43.6 & 43.6 & 52.2 & 59.5 & 49.2 & 58.6 & 69.2 & 54.0 & 66.1 & 81.9 & 57.8 & 71.4 & 87.7 \\
\hline & Adjusted & HWFET & 31.9 & 31.9 & 38.4 & 43.9 & 36.3 & 43.5 & 51.1 & 39.9 & 49.0 & 60.1 & 42.8 & 53.8 & 65.7 \\
\hline & & \begin{tabular}{|l|} 
Combined \\
\end{tabular} & 37.4 & 37.4 & 44.9 & 51.3 & 42.4 & 50.7 & 59.7 & 46.6 & 57.1 & 70.4 & 50.0 & 62.2 & 76.2 \\
\hline
\end{tabular}




\begin{tabular}{|c|c|c|c|c|c|c|c|c|c|c|c|c|c|c|c|}
\hline & & & \multicolumn{13}{|c|}{ Midsize_SUV } \\
\hline & & & \multirow{2}{*}{$\begin{array}{l}2010 \\
\text { Ref }\end{array}$} & \multicolumn{3}{|c|}{2010} & \multicolumn{3}{|c|}{2015} & \multicolumn{3}{|c|}{2030} & \multicolumn{3}{|c|}{2045} \\
\hline & & & & low & avg & high & low & avg & high & low & avg & high & low & avg & high \\
\hline \multirow{6}{*}{ H2 Series HEV PHEV40 } & \multirow{3}{*}{ Unadjusted } & UDDS & 69.0 & 69.0 & 84.2 & 99.0 & 79.2 & 97.0 & 119.1 & 88.1 & 112.6 & 147.2 & 95.3 & 122.2 & 160.6 \\
\hline & & HWFET & 57.1 & 57.1 & 69.0 & 79.1 & 65.0 & 78.3 & 92.5 & 71.7 & $\begin{array}{l}88.4 \\
\end{array}$ & 109.1 & 77.2 & 97.5 & 119.8 \\
\hline & & Combined & 63.1 & 63.1 & 76.6 & 88.9 & 72.1 & 87.6 & 105.5 & 79.9 & 100.2 & 127.2 & 86.2 & 109.7 & 139.2 \\
\hline & \multirow{3}{*}{ Adjusted } & UDDS & 49.2 & 49.2 & 58.5 & 67.0 & 55.4 & 65.8 & 77.9 & 60.7 & 74.4 & 91.9 & 64.9 & 79.5 & 98.2 \\
\hline & & HWFET & 40.2 & 40.2 & 48.4 & 55.4 & 45.7 & 54.9 & 64.6 & 50.3 & 61.8 & 76.0 & 54.1 & 68.0 & 83.2 \\
\hline & & \begin{tabular}{|l|} 
Combined \\
\end{tabular} & 44.7 & 44.7 & 53.5 & 61.2 & 50.6 & 60.4 & 71.3 & 55.6 & 68.1 & 84.0 & 59.5 & 73.9 & 90.8 \\
\hline \multirow{6}{*}{ E85 Split HEV } & \multirow{3}{*}{ Unadjusted } & UDDS & 35.4 & 35.4 & 38.4 & 41.4 & 36.6 & 41.5 & 53.2 & 38.5 & 45.2 & 62.9 & 39.5 & 47.1 & 65.9 \\
\hline & & \begin{tabular}{|l|} 
HWFET \\
\end{tabular} & 30.9 & 30.9 & 33.5 & 35.9 & 31.8 & 35.6 & 44.9 & 33.0 & 38.3 & 51.4 & 33.9 & 40.2 & 54.4 \\
\hline & & \begin{tabular}{|l|} 
Combined \\
\end{tabular} & 33.2 & 33.2 & 36.0 & 38.7 & 34.3 & 38.6 & 49.1 & 35.8 & 41.8 & 57.2 & 36.8 & 43.8 & 60.2 \\
\hline & \multirow{3}{*}{ Adjusted } & \begin{tabular}{|l|} 
UDDS \\
\end{tabular} & 26.9 & 26.9 & 29.0 & 31.1 & 27.7 & 31.1 & 39.1 & 29.1 & 33.7 & 45.4 & 29.8 & 35.0 & 47.3 \\
\hline & & HWFET & 21.9 & 21.9 & 23.7 & 25.4 & 22.5 & 25.2 & 31.7 & 23.3 & 27.0 & 36.2 & 24.0 & 28.4 & 38.3 \\
\hline & & \begin{tabular}{|l|} 
Combined \\
\end{tabular} & 24.4 & 24.4 & 26.3 & 28.2 & 25.1 & 28.1 & 35.4 & 26.2 & 30.3 & 40.8 & 26.9 & 31.7 & 42.8 \\
\hline \multirow{6}{*}{ E85 Split HEV PHEV10 } & \multirow{3}{*}{ Unadjusted } & \begin{tabular}{|l|} 
UDDS \\
\end{tabular} & 45.1 & 45.1 & 48.8 & 52.8 & 46.7 & 52.1 & 66.3 & 48.0 & 55.9 & 77.3 & 49.2 & 58.7 & 80.9 \\
\hline & & HWFET & 40.6 & 40.6 & 43.8 & 46.7 & 41.9 & 46.3 & 57.3 & 42.8 & 49.0 & 65.3 & 44.1 & 51.6 & 69.4 \\
\hline & & Combined & 43.0 & 43.0 & 46.4 & 49.9 & 44.4 & 49.3 & 61.9 & 45.5 & 52.6 & 71.4 & 46.8 & 55.3 & 75.3 \\
\hline & \multirow{3}{*}{ Adjusted } & UDDS & 33.7 & 33.7 & 36.1 & 38.8 & 34.7 & 38.3 & 47.5 & 35.6 & 40.8 & 54.3 & 36.4 & 42.7 & 56.5 \\
\hline & & HWFET & 28.7 & 28.7 & 30.9 & 32.9 & 29.6 & 32.7 & 40.3 & 30.2 & 34.6 & 45.9 & 31.1 & 36.4 & 48.7 \\
\hline & & Combined & 31.2 & 31.2 & 33.6 & 35.9 & 32.2 & 35.6 & 44.0 & 33.0 & 37.8 & 50.2 & 33.8 & 39.6 & 52.7 \\
\hline \multirow{6}{*}{ E85 Split HEV PHEV20 } & \multirow{3}{*}{ Unadjusted } & \begin{tabular}{|l|} 
UDDS \\
\end{tabular} & 64.4 & 64.4 & 70.3 & 75.5 & 67.9 & 74.6 & 94.2 & 69.9 & 80.0 & 110.7 & 71.2 & 83.7 & 116.1 \\
\hline & & HWFET & 49.8 & 49.8 & 52.9 & 55.9 & 51.5 & 55.8 & 66.0 & 52.4 & 56.6 & 74.9 & 54.1 & 58.9 & 80.3 \\
\hline & & \begin{tabular}{|l|} 
Combined \\
\end{tabular} & 56.9 & 56.9 & 61.2 & 65.2 & 59.4 & 64.8 & 79.0 & 60.7 & 67.4 & 91.1 & 62.3 & 70.4 & 96.7 \\
\hline & \multirow{3}{*}{ Adjusted } & UDDS & 46.3 & 46.3 & 50.0 & 53.2 & 48.5 & 52.7 & 64.3 & 49.8 & 56.0 & 73.4 & 50.6 & 58.1 & 76.3 \\
\hline & & \begin{tabular}{|l|} 
HWFET \\
\end{tabular} & 35.1 & 35.1 & 37.3 & 39.4 & 36.3 & 39.3 & 46.4 & 36.9 & 39.8 & 52.5 & 38.1 & 41.5 & 56.3 \\
\hline & & \begin{tabular}{|l|} 
Combined \\
\end{tabular} & 40.5 & 40.5 & 43.4 & 45.9 & 42.1 & 45.7 & 54.8 & 43.0 & 47.3 & 62.3 & 44.1 & 49.2 & 65.8 \\
\hline \multirow{6}{*}{ E85 Series HEV PHEV30 } & \multirow{3}{*}{ Unadjusted } & UDDS & 58.0 & 58.0 & 63.7 & 70.1 & 60.4 & 68.4 & 91.8 & 63.5 & 77.4 & 111.4 & 65.5 & 81.4 & 117.9 \\
\hline & & HWFET & 43.1 & 43.1 & 47.3 & 51.1 & 44.9 & 50.6 & 65.3 & 46.7 & 55.2 & 75.6 & 48.3 & 58.9 & 79.8 \\
\hline & & \begin{tabular}{|l|} 
Combined \\
\end{tabular} & 50.2 & 50.2 & 55.1 & 60.0 & 52.3 & 59.1 & 77.6 & 54.7 & 65.6 & 91.8 & 56.4 & 69.5 & 97.0 \\
\hline & \multirow{3}{*}{ Adjusted } & UDDS & 42.2 & 42.2 & 45.9 & 49.9 & 43.8 & 48.9 & 62.9 & 45.8 & 54.4 & 73.8 & 47.0 & 56.8 & 77.2 \\
\hline & & HWFET & 30.5 & 30.5 & 33.4 & 36.0 & 31.7 & 35.7 & 45.9 & 33.0 & 38.9 & 53.0 & 34.1 & 41.5 & 55.9 \\
\hline & & \begin{tabular}{|l|} 
Combined \\
\end{tabular} & 36.0 & 36.0 & 39.2 & 42.5 & 37.4 & 41.9 & 53.9 & 39.0 & 46.1 & 62.7 & 40.1 & 48.7 & 65.9 \\
\hline \multirow{6}{*}{ E85 Series HEV PHEV40 } & & \begin{tabular}{|l|} 
UDDS \\
\end{tabular} & 66.0 & 66.0 & 73.0 & 79.9 & 69.5 & 78.5 & 106.0 & 72.9 & 88.8 & 129.0 & 75.3 & 94.2 & 137.7 \\
\hline & Unadjusted & HWFET & 54.5 & 54.5 & 59.8 & 64.6 & 56.7 & 64.0 & 82.7 & 59.1 & 70.1 & 96.1 & 61.1 & 74.8 & 101.6 \\
\hline & & Combined & 60.2 & 60.2 & 66.4 & 72.2 & 63.1 & 71.2 & 94.0 & 66.0 & 79.3 & 111.8 & 68.2 & 84.4 & 118.7 \\
\hline & & UDDS & 47.3 & 47.3 & 51.7 & 55.9 & 49.5 & 55.1 & 70.8 & 51.6 & 61.2 & 83.0 & 53.1 & 64.3 & 87.3 \\
\hline & Adjusted & HWFET & 38.4 & 38.4 & 42.1 & 45.4 & 39.9 & 45.0 & 57.9 & 41.6 & 49.2 & 67.1 & 43.0 & 52.5 & 70.8 \\
\hline & & \begin{tabular}{|l|} 
Combined \\
\end{tabular} & 42.8 & 42.8 & 46.9 & 50.7 & 44.7 & 50.0 & 64.3 & 46.6 & 55.1 & 75.0 & 48.0 & 58.4 & 79.0 \\
\hline
\end{tabular}


Argonne National Laboratory Report - Light-Duty Vehicle Fuel Consumption Displacement Potential Up to 2045

\begin{tabular}{|c|c|c|c|c|c|c|c|c|c|c|c|c|c|c|c|}
\hline & & & \multicolumn{13}{|c|}{ Midsize_SUV } \\
\hline & & & \multirow{2}{*}{$\begin{array}{c}2010 \\
\text { Ref }\end{array}$} & \multicolumn{3}{|c|}{2010} & \multicolumn{3}{|c|}{2015} & \multicolumn{3}{|c|}{2030} & \multicolumn{3}{|c|}{2045} \\
\hline & & & & low & avg & high & low & avg & high & low & avg & high & low & avg & high \\
\hline \multirow{6}{*}{ FC HEV } & \multirow{3}{*}{ Unadjusted } & UDDS & 40.7 & 40.7 & 49.4 & 58.6 & 49.1 & 58.7 & 67.5 & 53.5 & 64.9 & 80.3 & 56.1 & 68.0 & 84.2 \\
\hline & & HWFET & 41.0 & 41.0 & 48.4 & 55.8 & 48.2 & 55.5 & 61.7 & 50.5 & 59.4 & 69.7 & 52.5 & 62.6 & 73.7 \\
\hline & & Combined & 40.8 & 40.8 & 48.9 & 57.3 & 48.7 & 57.2 & 64.7 & 52.1 & 62.3 & 75.2 & 54.4 & 65.5 & 79.1 \\
\hline & \multirow{3}{*}{ Adjusted } & UDDS & 30.6 & 30.6 & 36.5 & 42.6 & 36.3 & 42.7 & 48.3 & 39.3 & 46.6 & 56.1 & 41.0 & 48.6 & 58.5 \\
\hline & & HWFET & 29.0 & 29.0 & 34.1 & 39.3 & 34.0 & 39.1 & 43.4 & 35.6 & 41.8 & 49.0 & 37.0 & 44.0 & 51.7 \\
\hline & & Combined & 29.9 & 29.9 & 35.4 & 41.1 & 35.2 & 41.0 & 45.9 & 37.5 & 44.3 & 52.6 & 39.1 & 46.4 & 55.2 \\
\hline \multirow{6}{*}{ FC PHEV10 } & \multirow{3}{*}{ Unadjusted } & UDDS & 52.1 & 52.1 & 62.0 & 72.4 & 61.5 & 71.5 & 81.6 & 65.2 & 78.4 & 96.2 & 68.2 & 82.0 & 100.9 \\
\hline & & \begin{tabular}{|l|} 
HWFET \\
\end{tabular} & 54.2 & 54.2 & 62.4 & 71.0 & 62.2 & 70.3 & 77.5 & 64.1 & 74.5 & 86.1 & 66.3 & 78.7 & 91.1 \\
\hline & & Combined & 53.0 & 53.0 & 62.2 & 71.8 & 61.8 & 71.0 & 79.7 & 64.7 & 76.6 & 91.4 & 67.3 & 80.5 & 96.2 \\
\hline & \multirow{3}{*}{ Adjusted } & \begin{tabular}{|l|} 
UDDS \\
\end{tabular} & 38.3 & 38.3 & 44.8 & 51.3 & 44.5 & 50.8 & 56.9 & 46.8 & 55.0 & 65.4 & 48.7 & 57.2 & 68.0 \\
\hline & & \begin{tabular}{|l|} 
HWFET \\
\end{tabular} & 38.2 & 38.2 & 43.9 & 49.8 & 43.7 & 49.4 & 54.3 & 45.1 & 52.3 & 60.3 & 46.6 & 55.2 & 63.7 \\
\hline & & Combined & 38.2 & 38.2 & 44.4 & 50.6 & 44.1 & 50.1 & 55.7 & 46.0 & 53.7 & 63.0 & 47.7 & 56.2 & 66.0 \\
\hline \multirow{6}{*}{ FC PHEV 20} & \multirow{3}{*}{ Unadjusted } & \begin{tabular}{|l|} 
UDDS \\
\end{tabular} & 62.4 & 62.4 & 74.3 & 87.1 & 73.8 & 86.1 & 98.7 & 78.4 & 94.6 & 116.4 & 82.2 & 99.1 & 122.2 \\
\hline & & \begin{tabular}{|l|} 
HWFET \\
\end{tabular} & 55.4 & 55.4 & 64.3 & 73.6 & 64.0 & 72.9 & 80.8 & 66.4 & 77.8 & 91.0 & 68.8 & 82.0 & 96.2 \\
\hline & & Combined & 59.0 & 59.0 & 69.4 & 80.4 & 69.0 & 79.6 & 89.7 & 72.5 & 86.2 & 103.4 & 75.6 & 90.6 & 108.9 \\
\hline & \multirow{3}{*}{ Adjusted } & \begin{tabular}{|l|} 
UDDS \\
\end{tabular} & 45.0 & 45.0 & 52.5 & 60.1 & 52.2 & 59.5 & 66.8 & 55.0 & 64.5 & 76.4 & 57.3 & 67.0 & 79.5 \\
\hline & & \begin{tabular}{|l|} 
HWFET \\
\end{tabular} & 39.0 & 39.0 & 45.2 & 51.6 & 45.0 & 51.1 & 56.6 & 46.6 & 54.5 & 63.6 & 48.3 & 57.4 & 67.1 \\
\hline & & Combined & 42.1 & 42.1 & 48.9 & 56.0 & 48.7 & 55.4 & 61.8 & 50.9 & 59.6 & 70.1 & 52.9 & 62.3 & 73.4 \\
\hline \multirow{6}{*}{ FC PHEV 30} & \multirow{3}{*}{ Unadjusted } & \begin{tabular}{|l|} 
UDDS \\
\end{tabular} & 89.5 & 89.5 & 105.8 & 121.7 & 105.1 & 120.8 & 139.2 & 110.1 & 133.4 & 163.2 & 115.4 & 140.5 & 171.3 \\
\hline & & \begin{tabular}{|l|} 
HWFET \\
\end{tabular} & 69.9 & 69.9 & 80.7 & 91.3 & 80.3 & 90.8 & 101.2 & 82.7 & 97.3 & 113.7 & 85.9 & 102.8 & 120.1 \\
\hline & & Combined & 79.5 & 79.5 & 92.8 & 105.8 & 92.3 & 105.2 & 119.1 & 95.8 & 114.3 & 136.5 & 99.9 & 120.6 & 143.7 \\
\hline & \multirow{3}{*}{ Adjusted } & \begin{tabular}{|l|} 
UDDS \\
\end{tabular} & 61.6 & 61.6 & 70.8 & 79.2 & 70.4 & 78.7 & 88.0 & 73.1 & 85.2 & 99.4 & 75.9 & 88.7 & 103.0 \\
\hline & & \begin{tabular}{|l|} 
HWFET \\
\end{tabular} & 49.1 & 49.1 & 56.5 & 63.8 & 56.3 & 63.5 & 70.6 & 57.9 & 67.9 & 79.1 & 60.1 & 71.7 & 83.4 \\
\hline & & Combined & 55.2 & 55.2 & 63.6 & 71.4 & 63.2 & 71.0 & 79.2 & 65.3 & 76.4 & 89.1 & 67.9 & 80.1 & 93.1 \\
\hline \multirow{6}{*}{ FC PHEV 40} & \multirow{3}{*}{ Unadjusted } & \begin{tabular}{|l|} 
UDDS \\
\end{tabular} & 103.0 & 103.0 & 122.1 & 140.6 & 121.2 & 139.6 & 161.4 & 127.1 & 154.8 & 189.5 & 133.5 & 163.1 & 199.2 \\
\hline & & \begin{tabular}{|l|} 
HWFET \\
\end{tabular} & 88.4 & 88.4 & 102.4 & 115.8 & 101.9 & 115.3 & 128.9 & 105.0 & 123.8 & 144.9 & 109.1 & 131.0 & 153.1 \\
\hline & & Combined & 95.9 & 95.9 & 112.4 & 128.3 & 111.7 & 127.5 & 144.9 & 116.1 & 139.1 & 166.4 & 121.2 & 146.9 & 175.5 \\
\hline & \multirow{3}{*}{ Adjusted } & UDDS & 69.2 & 69.2 & 79.4 & 88.7 & 79.0 & 88.3 & 98.6 & 82.0 & 95.5 & 110.9 & 85.2 & 99.3 & 114.9 \\
\hline & & \begin{tabular}{|l|} 
HWFET \\
\end{tabular} & 61.8 & 61.8 & 71.4 & 80.5 & 71.0 & 80.2 & 89.3 & 73.1 & 85.9 & 100.1 & 75.9 & 90.8 & 105.6 \\
\hline & & Combined & 65.7 & 65.7 & 75.6 & 84.8 & 75.2 & 84.4 & 94.2 & 77.8 & 90.9 & 105.8 & 80.8 & 95.3 & 110.5 \\
\hline
\end{tabular}


Argonne National Laboratory Report - Light-Duty Vehicle Fuel Consumption Displacement Potential Up to 2045

$\underline{\text { Pev details }}$ 
Argonne National Laboratory Report - Light-Duty Vehicle Fuel Consumption Displacement Potential Up to 2045

\begin{tabular}{|c|c|c|c|c|c|c|c|c|c|c|c|c|c|c|c|c|}
\hline & & & & \multicolumn{13}{|c|}{ Midsize_SUV } \\
\hline & & & & \multirow{2}{*}{$\begin{array}{c}2010 \\
\text { Ref }\end{array}$} & \multicolumn{3}{|c|}{2010} & \multicolumn{3}{|c|}{2015} & \multicolumn{3}{|c|}{2030} & \multicolumn{3}{|c|}{2045} \\
\hline & & & & & low & avg & high & low & avg & high & low & avg & high & low & avg & high \\
\hline \multirow{25}{*}{ SI Split HEV PHEV10 } & \multirow{9}{*}{ UDDS } & \multirow{4}{*}{$C D$} & Wh/mile & 241.9 & 241.9 & 224.5 & 211.5 & 236.1 & 214.2 & 215.8 & 231.8 & 209.1 & 190.6 & 228.8 & 208.9 & 183.3 \\
\hline & & & Distance & 12.2 & 12.2 & 13.0 & 13.0 & 12.8 & 13.0 & 12.2 & 12.5 & 12.9 & 12.3 & 12.5 & 12.6 & 12.2 \\
\hline & & & MPGGE Unadjusted & 431.8 & 431.8 & 456.9 & 491.7 & 443.4 & 484.1 & 870.0 & 454.3 & 543.9 & 1132.5 & 468.4 & 764.4 & 1257.7 \\
\hline & & & MPGGE Adjusted & 185.6 & 185.6 & 191.1 & 198.3 & 188.2 & 196.8 & 252.6 & 190.6 & 208.2 & 275.4 & 193.6 & 240.8 & 283.8 \\
\hline & & \multirow{2}{*}{ CS } & MPGGE Unadjusted & 40.8 & 40.8 & 44.1 & 47.4 & 42.1 & 46.8 & 56.4 & 43.3 & 50.4 & 68.6 & 44.5 & 52.7 & 71.8 \\
\hline & & & MPGGE Adjusted & 30.7 & 30.7 & 32.9 & 35.2 & 31.6 & 34.8 & 41.2 & 32.4 & 37.2 & 49.0 & 33.2 & 38.7 & 51.0 \\
\hline & & \multirow{3}{*}{$\mathrm{CD}+\mathrm{CS}$} & Wh/mile & 50.4 & 50.4 & 46.8 & 44.1 & 49.2 & 44.6 & 45.0 & 48.3 & 43.6 & 39.7 & 47.7 & 43.5 & 38.2 \\
\hline & & & MPGGE Unadjusted & 48.2 & 48.2 & 52.1 & 56.0 & 49.8 & 55.3 & 67.1 & 51.2 & 59.6 & 81.7 & 52.6 & 62.6 & 85.6 \\
\hline & & & MPGGE Adjusted & 35.7 & 35.7 & 38.3 & 40.9 & 36.8 & 40.4 & 48.0 & 37.7 & 43.3 & 57.0 & 38.7 & 45.2 & 59.2 \\
\hline & \multirow{9}{*}{ HWFET } & \multirow{4}{*}{$C D$} & Wh/mile & 245.3 & 245.3 & 227.2 & 215.5 & 239.8 & 217.1 & 200.4 & 237.4 & 207.7 & 180.1 & 233.5 & 191.8 & 180.6 \\
\hline & & & Distance & 12.5 & 12.5 & 12.5 & 12.5 & 12.6 & 12.5 & 12.4 & 12.5 & 12.4 & 12.2 & 12.5 & 12.8 & 11.8 \\
\hline & & & MPGGE Unadjusted & 111.0 & 111.0 & 117.5 & 125.0 & 113.8 & 123.8 & 146.9 & 115.8 & 132.0 & 173.6 & 119.4 & 131.1 & 203.7 \\
\hline & & & MPGGE Adjusted & 77.2 & 77.2 & 81.7 & 86.7 & 79.1 & 85.9 & 101.4 & 80.5 & 91.4 & 119.2 & 82.9 & 90.8 & 139.0 \\
\hline & & \multirow{2}{*}{ CS } & MPGGE Unadjusted & 36.5 & 36.5 & 39.4 & 42.0 & 37.7 & 41.6 & 49.0 & 38.5 & 44.1 & 58.1 & 39.6 & 46.4 & 61.2 \\
\hline & & & MPGGE Adjusted & 25.8 & 25.8 & 27.8 & 29.6 & 26.7 & 29.4 & 34.6 & 27.2 & 31.1 & 40.9 & 27.9 & 32.7 & 43.0 \\
\hline & & \multirow{3}{*}{$\mathrm{CD}+\mathrm{CS}$} & Wh/mile & 72.2 & 72.2 & 66.8 & 63.4 & 70.5 & 63.8 & 58.9 & 69.8 & 61.1 & 53.0 & 68.7 & 56.4 & 53.1 \\
\hline & & & MPGGE Unadjusted & 43.5 & 43.5 & 46.8 & 49.9 & 44.9 & 49.5 & 58.4 & 45.8 & 52.5 & 69.1 & 47.1 & 54.9 & 73.5 \\
\hline & & & MPGGE Adjusted & 30.7 & 30.7 & 33.0 & 35.2 & 31.7 & 34.9 & 41.1 & 32.3 & 37.0 & 48.5 & 33.2 & 38.7 & 51.6 \\
\hline & \multirow{7}{*}{ Combined } & \multirow{2}{*}{$C D$} & MPGGE Unadjusted & 187.7 & 187.7 & 198.7 & 211.9 & 192.5 & 209.6 & 270.5 & 196.3 & 226.2 & 324.8 & 202.3 & 240.9 & 377.9 \\
\hline & & & MPGGE Adjusted & 113.7 & 113.7 & 119.2 & 125.6 & 116.2 & 124.5 & 151.2 & 118.0 & 132.2 & 173.2 & 120.9 & 138.2 & 193.2 \\
\hline & & \multirow{2}{*}{ CS } & MPGGE Unadjusted & 38.7 & 38.7 & 41.8 & 44.8 & 40.0 & 44.3 & 52.8 & 41.0 & 47.4 & 63.5 & 42.1 & 49.7 & 66.6 \\
\hline & & & MPGGE Adjusted & 28.3 & 28.3 & 30.4 & 32.5 & 29.2 & 32.1 & 37.9 & 29.8 & 34.2 & 45.0 & 30.6 & 35.8 & 47.1 \\
\hline & & \multirow{3}{*}{$C D+C S$} & Wh/mile & 60.2 & 60.2 & 55.8 & 52.7 & 58.8 & 53.3 & 51.2 & 58.0 & 51.4 & 45.7 & 57.1 & 49.3 & 44.9 \\
\hline & & & MPGGE Unadjusted & 46.0 & 46.0 & 49.6 & 53.1 & 47.5 & 52.5 & 62.8 & 48.6 & 56.2 & 75.5 & 50.0 & 58.9 & 79.7 \\
\hline & & & MPGGE Adjusted & 33.3 & 33.3 & 35.8 & 38.1 & 34.3 & 37.7 & 44.6 & 35.1 & 40.2 & 52.8 & 36.0 & 42.0 & 55.5 \\
\hline
\end{tabular}


Argonne National Laboratory Report - Light-Duty Vehicle Fuel Consumption Displacement Potential Up to 2045

\begin{tabular}{|c|c|c|c|c|c|c|c|c|c|c|c|c|c|c|c|c|}
\hline & & & & \multicolumn{13}{|c|}{ Midsize_SUV } \\
\hline & & & & \multirow{2}{*}{$\begin{array}{l}2010 \\
\text { Ref }\end{array}$} & \multicolumn{3}{|c|}{2010} & \multicolumn{3}{|c|}{2015} & \multicolumn{3}{|c|}{2030} & \multicolumn{3}{|c|}{2045} \\
\hline \multirow{26}{*}{ SI Split HEV PHEV2O } & & \multirow{5}{*}{$C D$} & & & low & avg & high & low & avg & high & low & avg & high & low & avg & high \\
\hline & \multirow{9}{*}{ UDDS } & & Wh/mile & 269.9 & 269.9 & 254.2 & 238.6 & 274.6 & 241.6 & 213.2 & 263.8 & 224.8 & 188.2 & 265.6 & 211.9 & 180.8 \\
\hline & & & Distance & 23.9 & 23.9 & 24.0 & 24.0 & 23.4 & 24.0 & 24.1 & 23.9 & 24.0 & 23.6 & 23.4 & 24.0 & 23.4 \\
\hline & & & MPGGE Unadjusted & 706.0 & 706.0 & 812.7 & 859.7 & 1033.2 & 853.8 & 841.7 & 867.3 & 825.9 & 1118.2 & 1086.4 & 753.2 & 1232.4 \\
\hline & & & MPGGE Adjusted & 233.4 & 233.4 & 246.5 & 251.6 & 267.7 & 250.9 & 249.7 & 252.4 & 247.9 & 274.3 & 271.9 & 239.4 & 282.2 \\
\hline & & \multirow{2}{*}{ CS } & MPGGE Unadjusted & 40.5 & 40.5 & 43.8 & 47.0 & 41.9 & 46.4 & 56.2 & 43.0 & 50.1 & 68.6 & 44.2 & 52.6 & 71.9 \\
\hline & & & MPGGE Adjusted & 30.4 & 30.4 & 32.7 & 34.9 & 31.4 & 34.5 & 41.0 & 32.2 & 37.0 & 49.0 & 33.0 & 38.7 & 51.0 \\
\hline & & \multirow{3}{*}{$\mathrm{CD}+\mathrm{CS}$} & Wh/mile & 140.9 & 140.9 & 133.1 & 125.0 & 143.9 & 126.6 & 112.4 & 137.6 & 117.7 & 99.5 & 139.2 & 111.9 & 95.6 \\
\hline & & & MPGGE Unadjusted & 69.0 & 69.0 & 74.9 & 80.4 & 72.4 & 79.4 & 95.4 & 73.8 & 85.3 & 117.0 & 76.4 & 89.1 & 122.9 \\
\hline & & & MPGGE Adjusted & 49.2 & 49.2 & 52.9 & 56.2 & 51.3 & 55.6 & 64.9 & 52.2 & 59.1 & 76.8 & 53.8 & 61.3 & 79.9 \\
\hline & \multirow{9}{*}{ HWFET } & \multirow{4}{*}{$C D$} & Wh/mile & 302.4 & 302.4 & 285.4 & 257.3 & 299.3 & 272.0 & 201.5 & 294.3 & 258.9 & 182.8 & 289.9 & 193.5 & 183.4 \\
\hline & & & Distance & 21.4 & 21.4 & 21.4 & 22.0 & 21.4 & 21.3 & 24.8 & 21.4 & 21.1 & 23.5 & 21.4 & 25.0 & 22.5 \\
\hline & & & MPGGE Unadjusted & 186.4 & 186.4 & 208.7 & 186.9 & 199.2 & 218.6 & 145.4 & 197.7 & 228.9 & 172.6 & 206.0 & 130.2 & 202.6 \\
\hline & & & MPGGE Adjusted & 127.6 & 127.6 & 142.2 & 128.0 & 136.0 & 148.7 & 100.4 & 135.0 & 155.3 & 118.5 & 140.5 & 90.2 & 138.2 \\
\hline & & \multirow{2}{*}{ CS } & MPGGE Unadjusted & 36.2 & 36.2 & 39.1 & 41.6 & 37.3 & 41.3 & 48.9 & 38.2 & 43.8 & 57.9 & 39.3 & 46.3 & 61.0 \\
\hline & & & MPGGE Adjusted & 25.6 & 25.6 & 27.6 & 29.4 & 26.4 & 29.2 & 34.5 & 27.0 & 30.9 & 40.8 & 27.7 & 32.6 & 42.9 \\
\hline & & \multirow{3}{*}{$C D+C S$} & Wh/mile & 151.6 & 151.6 & 142.8 & 129.6 & 149.9 & 136.2 & 103.7 & 147.4 & 129.6 & 94.1 & 145.2 & 99.6 & 94.4 \\
\hline & & & MPGGE Unadjusted & 53.2 & 53.2 & 57.7 & 60.3 & 55.1 & 60.9 & 66.8 & 56.2 & 64.5 & 79.3 & 57.9 & 62.6 & 85.2 \\
\hline & & & MPGGE Adjusted & 37.5 & 37.5 & 40.6 & 42.4 & 38.8 & 42.8 & 46.9 & 39.5 & 45.3 & 55.5 & 40.7 & 44.0 & 59.6 \\
\hline & \multirow{7}{*}{ Combined } & \multirow{2}{*}{$C D$} & MPGGE Unadjusted & 313.1 & 313.1 & 353.0 & 328.1 & 358.2 & 370.0 & 266.8 & 343.6 & 380.0 & 322.7 & 371.6 & 238.8 & 374.8 \\
\hline & & & MPGGE Adjusted & 170.0 & 170.0 & 185.4 & 175.4 & 186.5 & 191.6 & 149.6 & 181.4 & 195.5 & 172.4 & 191.4 & 137.3 & 192.1 \\
\hline & & \multirow{2}{*}{ CS } & MPGGE Unadjusted & 38.4 & 38.4 & 41.5 & 44.4 & 39.7 & 44.0 & 52.6 & 40.7 & 47.1 & 63.4 & 41.8 & 49.5 & 66.6 \\
\hline & & & MPGGE Adjusted & 28.0 & 28.0 & 30.2 & 32.2 & 28.9 & 31.9 & 37.8 & 29.6 & 34.0 & 44.9 & 30.4 & 35.7 & 47.0 \\
\hline & & \multirow{3}{*}{$\mathrm{CD}+\mathrm{CS}$} & Wh/mile & 145.7 & 145.7 & 137.5 & 127.0 & 146.6 & 130.9 & 108.5 & 142.0 & 123.1 & 97.1 & 141.9 & 106.4 & 95.1 \\
\hline & & & MPGGE Unadjusted & 60.9 & 60.9 & 66.0 & 69.9 & 63.4 & 69.9 & 80.0 & 64.7 & 74.5 & 96.4 & 66.8 & 74.8 & 102.5 \\
\hline & & & MPGGE Adjusted & 43.2 & 43.2 & 46.5 & 49.0 & 44.8 & 49.0 & 55.4 & 45.6 & 52.0 & 65.5 & 47.0 & 52.1 & 69.3 \\
\hline
\end{tabular}


Argonne National Laboratory Report - Light-Duty Vehicle Fuel Consumption Displacement Potential Up to 2045

\begin{tabular}{|c|c|c|c|c|c|c|c|c|c|c|c|c|c|c|c|c|}
\hline & & & & \multicolumn{13}{|c|}{ Midsize_SUV } \\
\hline & & & & \multirow{2}{*}{$\begin{array}{c}2010 \\
\text { Ref }\end{array}$} & \multicolumn{3}{|c|}{2010} & \multicolumn{3}{|c|}{2015} & \multicolumn{3}{|c|}{2030} & \multicolumn{3}{|c|}{2045} \\
\hline & & & & & low & avg & high & low & avg & high & low & avg & high & low & avg & high \\
\hline \multirow{25}{*}{ SI Series HEV PHEV30 } & \multirow{9}{*}{ UDDS } & \multirow{4}{*}{$C D$} & Wh/mile & 375.2 & 375.2 & 349.3 & 327.6 & 364.8 & 332.4 & 296.8 & 356.5 & 308.8 & 258.7 & 350.9 & 295.7 & 247.3 \\
\hline & & & Distance & 31.4 & 31.4 & 30.9 & 30.9 & 31.9 & 30.9 & 30.9 & 30.9 & 30.9 & 31.8 & 30.9 & 30.9 & 31.8 \\
\hline & & & MPGGE Unadjusted & $\mathrm{N} / \mathrm{A}$ & $\mathrm{N} / \mathrm{A}$ & N/A & N/A & N/A & $\mathrm{N} / \mathrm{A}$ & N/A & $\mathrm{N} / \mathrm{A}$ & N/A & N/A & N/A & N/A & $\mathrm{N} / \mathrm{A}$ \\
\hline & & & MPGGE Adjusted & $\mathrm{N} / \mathrm{A}$ & $\mathrm{N} / \mathrm{A}$ & $\mathrm{N} / \mathrm{A}$ & N/A & N/A & N/A & N/A & $\mathrm{N} / \mathrm{A}$ & N/A & N/A & N/A & $\mathrm{N} / \mathrm{A}$ & $\mathrm{N} / \mathrm{A}$ \\
\hline & & \multirow{2}{*}{ CS } & MPGGE Unadjusted & 29.1 & 29.1 & 31.8 & 35.0 & 30.2 & 34.4 & 43.5 & 31.8 & 38.7 & 56.0 & 32.7 & 40.7 & 58.4 \\
\hline & & & MPGGE Adjusted & 22.4 & 22.4 & 24.4 & 26.6 & 23.2 & 26.2 & 32.5 & 24.3 & 29.2 & 40.9 & 25.0 & 30.6 & 42.5 \\
\hline & & \multirow{3}{*}{$\mathrm{CD}+\mathrm{CS}$} & Wh/mile & 241.4 & 241.4 & 224.8 & 210.9 & 234.8 & 214.0 & 191.7 & 229.4 & 199.4 & 167.5 & 225.9 & 190.8 & 160.1 \\
\hline & & & MPGGE Unadjusted & 61.9 & 61.9 & 67.8 & 74.5 & 64.3 & 73.3 & 92.6 & 67.5 & 82.4 & 119.2 & 69.6 & 86.6 & 124.2 \\
\hline & & & MPGGE Adjusted & 44.7 & 44.7 & 48.4 & 52.6 & 46.2 & 51.9 & 63.3 & 48.3 & 57.4 & 77.9 & 49.6 & 59.9 & 80.5 \\
\hline & \multirow{9}{*}{ HWFET } & \multirow{4}{*}{$C D$} & Wh/mile & 407.6 & 407.6 & 379.7 & 358.6 & 397.9 & 361.8 & 327.7 & 392.3 & 340.7 & 293.5 & 384.8 & 324.4 & 278.4 \\
\hline & & & \begin{tabular}{|l} 
Distance \\
\end{tabular} & 28.5 & 28.5 & 28.5 & 28.1 & 28.3 & 28.3 & 27.7 & 28.0 & 27.8 & 27.0 & 28.1 & 27.9 & 27.2 \\
\hline & & & MPGGE Unadjusted & $\mathrm{N} / \mathrm{A}$ & $\mathrm{N} / \mathrm{A}$ & $\mathrm{N} / \mathrm{A}$ & $\mathrm{N} / \mathrm{A}$ & N/A & $\mathrm{N} / \mathrm{A}$ & N/A & $\mathrm{N} / \mathrm{A}$ & $\mathrm{N} / \mathrm{A}$ & $\mathrm{N} / \mathrm{A}$ & $\mathrm{N} / \mathrm{A}$ & $\mathrm{N} / \mathrm{A}$ & $\mathrm{N} / \mathrm{A}$ \\
\hline & & & MPGGE Adjusted & N/A & N/A & N/A & N/A & N/A & N/A & N/A & N/A & N/A & N/A & N/A & N/A & $\mathrm{N} / \mathrm{A}$ \\
\hline & & \multirow{2}{*}{ CS } & MPGGE Unadjusted & 27.1 & 27.1 & 29.7 & 32.1 & 28.2 & 31.8 & 38.9 & 29.3 & 34.6 & 47.1 & 30.3 & 37.0 & 49.7 \\
\hline & & & MPGGE Adjusted & 19.2 & 19.2 & 21.0 & 22.7 & 20.0 & 22.5 & 27.5 & 20.8 & 24.5 & 33.2 & 21.4 & 26.2 & 35.0 \\
\hline & & \multirow{3}{*}{$\mathrm{CD}+\mathrm{CS}$} & Wh/mile & 210.1 & 210.1 & 195.7 & 184.8 & 205.1 & 186.5 & 168.9 & 202.1 & 175.6 & 151.2 & 198.3 & 167.2 & 143.4 \\
\hline & & & MPGGE Unadjusted & 45.9 & 45.9 & 50.4 & 54.3 & 47.8 & 53.8 & 65.9 & 49.7 & 58.7 & 79.8 & 51.3 & 62.7 & 84.2 \\
\hline & & & MPGGE Adjusted & 32.4 & 32.4 & 35.5 & 38.3 & 33.7 & 37.9 & 46.3 & 35.0 & 41.3 & 55.9 & 36.2 & 44.1 & 58.9 \\
\hline & \multirow{7}{*}{ Combined } & \multirow{2}{*}{$C D$} & MPGGE Unadjusted & $\mathrm{N} / \mathrm{A}$ & $\mathrm{N} / \mathrm{A}$ & N/A & N/A & N/A & $\mathrm{N} / \mathrm{A}$ & N/A & $\mathrm{N} / \mathrm{A}$ & $\mathrm{N} / \mathrm{A}$ & N/A & $\mathrm{N} / \mathrm{A}$ & N/A & $\mathrm{N} / \mathrm{A}$ \\
\hline & & & MPGGE Adjusted & N/A & N/A & N/A & N/A & N/A & N/A & N/A & N/A & N/A & N/A & N/A & N/A & N/A \\
\hline & & \multirow{2}{*}{ CS } & MPGGE Unadjusted & 28.2 & 28.2 & 30.8 & 33.6 & 29.3 & 33.2 & 41.3 & 30.6 & 36.8 & 51.6 & 31.6 & 38.9 & 54.1 \\
\hline & & & MPGGE Adjusted & 20.8 & 20.8 & 22.7 & 24.7 & 21.6 & 24.4 & 30.0 & 22.6 & 26.9 & 37.1 & 23.2 & 28.4 & 38.8 \\
\hline & & \multirow{3}{*}{$C D+C S$} & Wh/mile & 227.3 & 227.3 & 211.7 & 199.1 & 221.4 & 201.6 & 181.4 & 217.1 & 188.7 & 160.2 & 213.4 & 180.2 & 152.6 \\
\hline & & & MPGGE Unadjusted & 53.5 & 53.5 & 58.6 & 63.8 & 55.6 & 63.0 & 78.3 & 58.1 & 69.7 & 97.5 & 60.0 & 73.9 & 102.3 \\
\hline & & & MPGGE Adjusted & 38.2 & 38.2 & 41.6 & 45.0 & 39.6 & 44.5 & 54.3 & 41.3 & 48.8 & 66.2 & 42.5 & 51.6 & 69.1 \\
\hline
\end{tabular}




\begin{tabular}{|c|c|c|c|c|c|c|c|c|c|c|c|c|c|c|c|c|}
\hline & & & & \multicolumn{13}{|c|}{ Midsize_SUV } \\
\hline & & & & \multirow{2}{*}{$\begin{array}{c}2010 \\
\text { Ref }\end{array}$} & \multicolumn{3}{|c|}{2010} & \multicolumn{3}{|c|}{2015} & \multicolumn{3}{|c|}{2030} & \multicolumn{3}{|c|}{2045} \\
\hline \multirow{26}{*}{ SI Series HEV PHEV4O } & & \multirow{5}{*}{$C D$} & & & low & avg & high & low & avg & high & low & avg & high & low & avg & high \\
\hline & \multirow{9}{*}{ UDDS } & & Wh/mile & 383.3 & 383.3 & 356.5 & 334.2 & 372.4 & 339.3 & 302.5 & 363.8 & 314.8 & 261.6 & 357.4 & 300.6 & 249.8 \\
\hline & & & Distance & 40.7 & 40.7 & 40.6 & 40.6 & 40.6 & 40.7 & 40.8 & 40.7 & 40.8 & 40.4 & 40.6 & 40.5 & 40.4 \\
\hline & & & MPGGE Unadjusted & N/A & N/A & N/A & N/A & N/A & N/A & N/A & N/A & N/A & N/A & N/A & N/A & N/A \\
\hline & & & MPGGE Adjusted & N/A & N/A & N/A & N/A & N/A & N/A & N/A & N/A & N/A & N/A & N/A & N/A & $\mathrm{N} / \mathrm{A}$ \\
\hline & & \multirow{2}{*}{ CS } & MPGGE Unadjusted & 28.4 & 28.4 & 31.2 & 34.0 & 29.7 & 33.5 & 42.8 & 31.0 & 38.0 & 54.4 & 32.1 & 40.0 & 57.7 \\
\hline & & & MPGGE Adjusted & 21.8 & 21.8 & 23.9 & 25.9 & 22.8 & 25.5 & 32.1 & 23.8 & 28.7 & 39.9 & 24.5 & 30.1 & 42.0 \\
\hline & & \multirow{3}{*}{$C D+C S$} & Wh/mile & 281.2 & 281.2 & 261.7 & 245.4 & 273.3 & 249.1 & 223.4 & 267.0 & 232.4 & 193.3 & 262.4 & 221.7 & 184.5 \\
\hline & & & MPGGE Unadjusted & 70.9 & 70.9 & 77.9 & 85.0 & 74.2 & 83.7 & 107.1 & 77.5 & 95.0 & 136.0 & 80.2 & 100.1 & 144.3 \\
\hline & & & MPGGE Adjusted & 50.4 & 50.4 & 54.7 & 58.9 & 52.4 & 58.1 & 71.4 & 54.5 & 64.7 & 86.5 & 56.1 & 67.6 & 90.5 \\
\hline & \multirow{9}{*}{ HWFET } & \multirow{4}{*}{$C D$} & Wh/mile & 411.4 & 411.4 & 382.8 & 361.4 & 401.3 & 364.8 & 329.5 & 395.5 & 342.6 & 295.5 & 387.4 & 325.9 & 280.1 \\
\hline & & & Distance & 37.6 & 37.6 & 37.6 & 37.4 & 37.4 & 37.6 & 37.2 & 37.2 & 37.2 & 35.9 & 37.3 & 37.4 & 36.1 \\
\hline & & & MPGGE Unadjusted & N/A & N/A & N/A & N/A & N/A & N/A & N/A & N/A & N/A & N/A & N/A & N/A & $\mathrm{N} / \mathrm{A}$ \\
\hline & & & MPGGE Adjusted & $\mathrm{N} / \mathrm{A}$ & N/A & N/A & N/A & N/A & N/A & N/A & N/A & N/A & $\mathrm{N} / \mathrm{A}$ & $\mathrm{N} / \mathrm{A}$ & N/A & N/A \\
\hline & & \multirow{2}{*}{ CS } & MPGGE Unadjusted & 26.7 & 26.7 & 29.3 & 31.6 & 27.7 & 31.3 & 38.3 & 28.9 & 34.3 & 46.7 & 29.9 & 36.6 & 49.3 \\
\hline & & & MPGGE Adjusted & 18.9 & 18.9 & 20.7 & 22.4 & 19.7 & 22.2 & 27.1 & 20.5 & 24.2 & 32.9 & 21.2 & 25.9 & 34.8 \\
\hline & & \multirow{3}{*}{$C D+C S$} & Wh/mile & 277.7 & 277.7 & 258.5 & 244.0 & 270.9 & 246.3 & 222.5 & 267.0 & 231.3 & 199.6 & 261.6 & 220.0 & 189.2 \\
\hline & & & MPGGE Unadjusted & 58.0 & 58.0 & 63.7 & 68.7 & 60.3 & 68.1 & 83.3 & 62.9 & 74.5 & 101.5 & 65.0 & 79.5 & 107.1 \\
\hline & & & MPGGE Adjusted & 40.8 & 40.8 & 44.8 & 48.3 & 42.4 & 47.8 & 58.3 & 44.2 & 52.2 & 70.7 & 45.7 & 55.7 & 74.6 \\
\hline & \multirow{7}{*}{ Combined } & \multirow{2}{*}{$C D$} & MPGGE Unadjusted & N/A & N/A & N/A & N/A & N/A & N/A & N/A & N/A & N/A & N/A & N/A & $\mathrm{N} / \mathrm{A}$ & N/A \\
\hline & & & MPGGE Adjusted & N/A & N/A & N/A & N/A & N/A & N/A & N/A & N/A & N/A & N/A & N/A & N/A & N/A \\
\hline & & \multirow{2}{*}{ CS } & MPGGE Unadjusted & 27.6 & 27.6 & 30.3 & 32.9 & 28.8 & 32.5 & 40.7 & 30.0 & 36.2 & 50.6 & 31.1 & 38.4 & 53.6 \\
\hline & & & MPGGE Adjusted & 20.4 & 20.4 & 22.4 & 24.2 & 21.3 & 23.9 & 29.6 & 22.2 & 26.5 & 36.4 & 22.9 & 28.0 & 38.4 \\
\hline & & \multirow{3}{*}{$C D+C S$} & Wh/mile & 279.7 & 279.7 & 260.2 & 244.8 & 272.2 & 247.9 & 223.0 & 267.0 & 231.9 & 196.1 & 262.0 & 220.9 & 186.6 \\
\hline & & & MPGGE Unadjusted & 64.5 & 64.5 & 70.8 & 76.8 & 67.2 & 75.9 & 94.9 & 70.2 & 84.5 & 117.9 & 72.6 & 89.6 & 124.8 \\
\hline & & & MPGGE Adjusted & 45.6 & 45.6 & 49.7 & 53.6 & 47.4 & 53.0 & 64.9 & 49.3 & 58.4 & 78.6 & 50.9 & 61.6 & 82.6 \\
\hline
\end{tabular}


Argonne National Laboratory Report - Light-Duty Vehicle Fuel Consumption Displacement Potential Up to 2045

\begin{tabular}{|c|c|c|c|c|c|c|c|c|c|c|c|c|c|c|c|c|}
\hline & & & & \multicolumn{13}{|c|}{ Midsize_SUV } \\
\hline & & & & \multirow{2}{*}{$\begin{array}{c}2010 \\
\text { Ref }\end{array}$} & \multicolumn{3}{|c|}{2010} & \multicolumn{3}{|c|}{2015} & \multicolumn{3}{|c|}{2030} & \multicolumn{3}{|c|}{2045} \\
\hline & & & & & low & avg & high & low & avg & high & low & avg & high & low & avg & high \\
\hline \multirow{25}{*}{ CI Split HEV PHEV10 } & \multirow{9}{*}{ UDDS } & \multirow{4}{*}{$C D$} & Wh/mile & 249.7 & 249.7 & 233.2 & 217.1 & 244.0 & 235.4 & 223.7 & 239.3 & 217.1 & 198.1 & 236.1 & 216.5 & 190.2 \\
\hline & & & Distance & 12.3 & 12.3 & 12.6 & 12.4 & 12.3 & 12.2 & 12.1 & 12.3 & 12.3 & 11.9 & 12.3 & 12.4 & 12.3 \\
\hline & & & MPGGE Unadjusted & 440.6 & 440.6 & 468.4 & 486.4 & 448.9 & 661.8 & 851.1 & 469.0 & 567.3 & 1068.5 & 485.7 & 816.1 & 1132.6 \\
\hline & & & MPGGE Adjusted & 187.6 & 187.6 & 193.6 & 197.3 & 189.4 & 227.2 & 250.7 & 193.7 & 212.3 & 270.5 & 197.1 & 246.8 & 275.4 \\
\hline & & \multirow{2}{*}{ CS } & MPGGE Unadjusted & 42.1 & 42.1 & 45.1 & 49.2 & 43.1 & 48.6 & 55.1 & 45.2 & 53.1 & 65.5 & 46.9 & 56.1 & 68.2 \\
\hline & & & MPGGE Adjusted & 31.6 & 31.6 & 33.7 & 36.4 & 32.2 & 36.0 & 40.3 & 33.7 & 39.0 & 47.0 & 34.8 & 41.0 & 48.7 \\
\hline & & \multirow{3}{*}{$\mathrm{CD}+\mathrm{CS}$} & Wh/mile & 52.0 & 52.0 & 48.6 & 45.2 & 50.8 & 49.0 & 46.6 & 49.9 & 45.2 & 41.3 & 49.2 & 45.1 & 39.6 \\
\hline & & & MPGGE Unadjusted & 49.8 & 49.8 & 53.3 & 58.1 & 50.9 & 57.7 & 65.6 & 53.4 & 62.8 & 77.9 & 55.4 & 66.6 & 81.2 \\
\hline & & & MPGGE Adjusted & 36.8 & 36.8 & 39.2 & 42.3 & 37.5 & 42.0 & 47.1 & 39.2 & 45.3 & 54.7 & 40.5 & 47.7 & 56.7 \\
\hline & \multirow{9}{*}{ HWFET } & \multirow{4}{*}{$C D$} & Wh/mile & 261.1 & 261.1 & 231.4 & 276.6 & 243.6 & 284.5 & 264.2 & 252.8 & 211.1 & 232.0 & 236.0 & 242.0 & 173.6 \\
\hline & & & Distance & 12.4 & 12.4 & 13.0 & 10.8 & 12.9 & 10.7 & 10.6 & 12.4 & 12.9 & 10.5 & 13.0 & 11.1 & 12.5 \\
\hline & & & MPGGE Unadjusted & 123.8 & 123.8 & 119.2 & 250.2 & 114.5 & 275.3 & 320.4 & 130.7 & 137.1 & 322.4 & 123.3 & 238.7 & 171.5 \\
\hline & & & MPGGE Adjusted & 85.9 & 85.9 & 82.8 & 169.0 & 79.6 & 185.0 & 213.4 & 90.6 & 94.9 & 214.6 & 85.6 & 161.7 & 117.8 \\
\hline & & \multirow{2}{*}{ CS } & MPGGE Unadjusted & 37.2 & 37.2 & 39.8 & 43.0 & 37.9 & 42.7 & 47.5 & 39.5 & 45.8 & 54.8 & 41.1 & 48.9 & 57.6 \\
\hline & & & MPGGE Adjusted & 26.3 & 26.3 & 28.1 & 30.4 & 26.8 & 30.1 & 33.5 & 27.9 & 32.3 & 38.6 & 29.0 & 34.5 & 40.5 \\
\hline & & \multirow{3}{*}{$\mathrm{CD}+\mathrm{CS}$} & Wh/mile & 76.8 & 76.8 & 68.1 & 81.4 & 71.7 & 83.7 & 77.7 & 74.4 & 62.1 & 68.2 & 69.4 & 71.2 & 51.1 \\
\hline & & & MPGGE Unadjusted & 44.7 & 44.7 & 47.4 & 53.7 & 45.1 & 53.5 & 59.7 & 47.5 & 54.5 & 68.4 & 49.0 & 60.5 & 68.5 \\
\hline & & & \begin{tabular}{|l|} 
MPGGE Adjusted \\
\end{tabular} & 31.6 & 31.6 & 33.4 & 37.8 & 31.9 & 37.7 & 42.0 & 33.5 & 38.4 & 48.0 & 34.5 & 42.5 & 48.1 \\
\hline & \multirow{7}{*}{ Combined } & \multirow{2}{*}{$C D$} & MPGGE Unadjusted & 204.8 & 204.8 & 202.1 & 341.4 & 194.0 & 405.6 & 487.7 & 216.7 & 235.2 & 523.5 & 209.2 & 390.7 & 321.6 \\
\hline & & & MPGGE Adjusted & 122.4 & 122.4 & 120.8 & 183.5 & 116.9 & 206.1 & 232.4 & 128.1 & 136.4 & 242.2 & 124.3 & 199.5 & 171.9 \\
\hline & & \multirow{2}{*}{ CS } & MPGGE Unadjusted & 39.8 & 39.8 & 42.6 & 46.2 & 40.6 & 45.7 & 51.4 & 42.5 & 49.5 & 60.2 & 44.1 & 52.6 & 63.0 \\
\hline & & & MPGGE Adjusted & 29.0 & 29.0 & 30.9 & 33.4 & 29.5 & 33.1 & 37.0 & 30.8 & 35.7 & 42.8 & 32.0 & 37.8 & 44.7 \\
\hline & & \multirow{3}{*}{$\mathrm{CD}+\mathrm{CS}$} & Wh/mile & 63.2 & 63.2 & 57.4 & 61.5 & 60.2 & 64.6 & 60.6 & 60.9 & 52.8 & 53.4 & 58.3 & 56.8 & 44.8 \\
\hline & & & MPGGE Unadjusted & 47.4 & 47.4 & 50.5 & 56.0 & 48.1 & 55.7 & 62.8 & 50.6 & 58.8 & 73.3 & 52.3 & 63.7 & 74.9 \\
\hline & & & MPGGE Adjusted & 34.2 & 34.2 & 36.3 & 40.1 & 34.7 & 40.0 & 44.6 & 36.4 & 41.9 & 51.5 & 37.6 & 45.2 & 52.4 \\
\hline
\end{tabular}


Argonne National Laboratory Report - Light-Duty Vehicle Fuel Consumption Displacement Potential Up to 2045

\begin{tabular}{|c|c|c|c|c|c|c|c|c|c|c|c|c|c|c|c|c|}
\hline & & & & \multicolumn{13}{|c|}{ Midsize_SUV } \\
\hline & & & & \multirow{2}{*}{$\begin{array}{c}2010 \\
\text { Ref }\end{array}$} & \multicolumn{3}{|c|}{2010} & \multicolumn{3}{|c|}{2015} & \multicolumn{3}{|c|}{2030} & \multicolumn{3}{|c|}{2045} \\
\hline \multirow{26}{*}{ CI Split HEV PHEV2O } & & & & & low & avg & high & low & avg & high & low & avg & high & low & avg & high \\
\hline & \multirow{9}{*}{ UDDS } & \multirow{4}{*}{$C D$} & Wh/mile & 278.9 & 278.9 & 269.6 & 247.8 & 275.4 & 251.1 & 227.3 & 271.8 & 231.7 & 200.6 & 266.2 & 221.4 & 187.6 \\
\hline & & & \begin{tabular}{|l|} 
Distance \\
\end{tabular} & 24.0 & 24.0 & 23.8 & 24.0 & 24.0 & 24.0 & 23.9 & 23.9 & 24.0 & 23.4 & 24.0 & 24.0 & 23.7 \\
\hline & & & MPGGE Unadjusted & 724.9 & 724.9 & 1033.7 & 917.7 & 800.8 & 910.2 & 995.8 & 879.0 & 833.6 & 1272.4 & 863.1 & 845.8 & 1116.2 \\
\hline & & & \begin{tabular}{|l|} 
MPGGE Adjusted \\
\end{tabular} & 235.8 & 235.8 & 267.7 & 257.4 & 245.1 & 256.7 & 264.5 & 253.6 & 248.8 & 284.7 & 251.9 & 250.1 & 274.2 \\
\hline & & \multirow{2}{*}{ CS } & MPGGE Unadjusted & 41.7 & 41.7 & 44.7 & 48.8 & 42.7 & 48.2 & 54.9 & 44.9 & 52.9 & 65.4 & 46.5 & 55.9 & 68.2 \\
\hline & & & MPGGE Adjusted & 31.3 & 31.3 & 33.4 & 36.2 & 32.0 & 35.7 & 40.2 & 33.5 & 38.9 & 47.0 & 34.6 & 40.9 & 48.7 \\
\hline & & \multirow{3}{*}{$C D+C S$} & Wh/mile & 145.5 & 145.5 & 141.1 & 129.9 & 143.7 & 131.6 & 119.4 & 141.8 & 121.3 & 105.7 & 138.9 & 116.9 & 99.3 \\
\hline & & & MPGGE Unadjusted & 71.2 & 71.2 & 77.1 & 83.7 & 73.1 & 82.5 & 93.9 & 76.9 & 89.8 & 112.2 & 79.5 & 95.0 & 116.3 \\
\hline & & & MPGGE Adjusted & 50.6 & 50.6 & 54.2 & 58.1 & 51.7 & 57.5 & 64.1 & 54.1 & 61.7 & 74.2 & 55.7 & 64.7 & 76.4 \\
\hline & \multirow{9}{*}{ HWFET } & \multirow{4}{*}{$C D$} & Wh/mile & 310.8 & 310.8 & 303.3 & 265.8 & 307.5 & 277.1 & 241.0 & 302.3 & 244.6 & 214.5 & 299.4 & 225.9 & 176.3 \\
\hline & & & Distance & 21.4 & 21.4 & 19.5 & 20.1 & 21.4 & 20.0 & 21.8 & 21.4 & 22.5 & 21.4 & 19.7 & 23.3 & 23.8 \\
\hline & & & MPGGE Unadjusted & 201.3 & 201.3 & 272.4 & 208.2 & 212.8 & 236.3 & 209.6 & 214.8 & 188.9 & 226.9 & 238.9 & 184.8 & 170.7 \\
\hline & & & MPGGE Adjusted & 137.4 & 137.4 & 183.2 & 141.9 & 144.9 & 160.1 & 142.8 & 146.2 & 129.3 & 154.0 & 161.8 & 126.6 & 117.3 \\
\hline & & \multirow{2}{*}{ CS } & MPGGE Unadjusted & 37.0 & 37.0 & 39.5 & 42.8 & 37.6 & 42.4 & 47.2 & 39.2 & 45.6 & 54.6 & 40.8 & 48.7 & 57.4 \\
\hline & & & MPGGE Adjusted & 26.1 & 26.1 & 27.9 & 30.2 & 26.6 & 29.9 & 33.3 & 27.7 & 32.2 & 38.4 & 28.8 & 34.3 & 40.4 \\
\hline & & \multirow{3}{*}{$C D+C S$} & Wh/mile & 155.4 & 155.4 & 89.2 & 78.2 & 153.7 & 81.5 & 121.3 & 151.2 & 123.4 & 108.3 & 88.1 & 114.2 & 90.8 \\
\hline & & & MPGGE Unadjusted & 54.7 & 54.7 & 49.7 & 52.9 & 55.8 & 52.7 & 68.3 & 58.0 & 65.4 & 78.3 & 51.0 & 68.9 & 78.5 \\
\hline & & & MPGGE Adjusted & 38.5 & 38.5 & 35.1 & 37.3 & 39.3 & 37.2 & 48.0 & 40.8 & 46.0 & 54.9 & 35.9 & 48.4 & 55.0 \\
\hline & \multirow{7}{*}{ Combined } & \multirow{2}{*}{$C D$} & MPGGE Unadjusted & 334.0 & 334.0 & 457.9 & 362.2 & 356.9 & 398.7 & 370.5 & 367.6 & 328.7 & 413.9 & 396.7 & 324.1 & 319.6 \\
\hline & & & MPGGE Adjusted & 178.4 & 178.4 & 221.7 & 188.4 & 186.9 & 201.9 & 191.2 & 190.6 & 175.7 & 206.0 & 201.4 & 173.8 & 171.1 \\
\hline & & \multirow{2}{*}{ CS } & MPGGE Unadjusted & 39.4 & 39.4 & 42.2 & 45.9 & 40.2 & 45.4 & 51.2 & 42.1 & 49.4 & 60.0 & 43.8 & 52.4 & 62.9 \\
\hline & & & MPGGE Adjusted & 28.8 & 28.8 & 30.7 & 33.2 & 29.3 & 32.9 & 36.8 & 30.6 & 35.6 & 42.7 & 31.7 & 37.6 & 44.6 \\
\hline & & \multirow{3}{*}{$C D+C S$} & Wh/mile & 150.0 & 150.0 & 117.8 & 106.6 & 148.2 & 109.0 & 120.3 & 146.0 & 122.2 & 106.9 & 116.0 & 115.7 & 95.5 \\
\hline & & & MPGGE Unadjusted & 62.6 & 62.6 & 61.8 & 66.3 & 64.1 & 65.8 & 80.4 & 67.0 & 76.9 & 93.9 & 63.5 & 81.2 & 95.6 \\
\hline & & & MPGGE Adjusted & 44.3 & 44.3 & 43.5 & 46.4 & 45.3 & 46.1 & 55.7 & 47.2 & 53.5 & 64.1 & 44.6 & 56.2 & 65.0 \\
\hline
\end{tabular}


Argonne National Laboratory Report - Light-Duty Vehicle Fuel Consumption Displacement Potential Up to 2045

\begin{tabular}{|c|c|c|c|c|c|c|c|c|c|c|c|c|c|c|c|c|}
\hline & & & & \multicolumn{13}{|c|}{ Midsize_SUV } \\
\hline & & & & \multirow{2}{*}{$\begin{array}{c}2010 \\
\text { Ref }\end{array}$} & \multicolumn{3}{|c|}{2010} & \multicolumn{3}{|c|}{2015} & \multicolumn{3}{|c|}{2030} & \multicolumn{3}{|c|}{2045} \\
\hline & & & & & low & avg & high & low & avg & high & low & avg & high & low & avg & high \\
\hline \multirow{25}{*}{ CI Series HEV PHEV30 } & \multirow{9}{*}{ UDDS } & \multirow{4}{*}{$C D$} & Wh/mile & 385.8 & 385.8 & 359.6 & 337.9 & 375.0 & 342.9 & 306.8 & 366.5 & 318.8 & 268.5 & 360.7 & 305.5 & 257.2 \\
\hline & & & Distance & 31.4 & 31.4 & 31.4 & 31.4 & 31.4 & 31.4 & 30.9 & 31.4 & 30.9 & 30.9 & 31.4 & 30.9 & 30.9 \\
\hline & & & MPGGE Unadjusted & $\mathrm{N} / \mathrm{A}$ & $\mathrm{N} / \mathrm{A}$ & N/A & N/A & N/A & $\mathrm{N} / \mathrm{A}$ & N/A & $\mathrm{N} / \mathrm{A}$ & N/A & N/A & N/A & N/A & $\mathrm{N} / \mathrm{A}$ \\
\hline & & & MPGGE Adjusted & $\mathrm{N} / \mathrm{A}$ & $\mathrm{N} / \mathrm{A}$ & $\mathrm{N} / \mathrm{A}$ & N/A & N/A & N/A & N/A & $\mathrm{N} / \mathrm{A}$ & N/A & N/A & N/A & $\mathrm{N} / \mathrm{A}$ & $\mathrm{N} / \mathrm{A}$ \\
\hline & & \multirow{2}{*}{ CS } & MPGGE Unadjusted & 29.7 & 29.7 & 32.4 & 36.2 & 30.6 & 35.6 & 42.7 & 32.8 & 40.5 & 52.2 & 34.2 & 43.0 & 54.9 \\
\hline & & & MPGGE Adjusted & 22.8 & 22.8 & 24.7 & 27.5 & 23.5 & 27.0 & 32.0 & 25.1 & 30.5 & 38.4 & 26.0 & 32.2 & 40.2 \\
\hline & & \multirow{3}{*}{$\mathrm{CD}+\mathrm{CS}$} & Wh/mile & 248.3 & 248.3 & 231.5 & 217.5 & 241.3 & 220.8 & 198.2 & 235.9 & 205.9 & 173.9 & 232.2 & 197.1 & 166.6 \\
\hline & & & MPGGE Unadjusted & 63.2 & 63.2 & 68.9 & 77.0 & 65.1 & 75.8 & 90.7 & 69.9 & 86.2 & 111.0 & 72.8 & 91.5 & 116.7 \\
\hline & & & MPGGE Adjusted & 45.6 & 45.6 & 49.1 & 54.1 & 46.8 & 53.4 & 62.3 & 49.8 & 59.6 & 73.6 & 51.6 & 62.7 & 76.6 \\
\hline & \multirow{9}{*}{ HWFET } & \multirow{4}{*}{$C D$} & Wh/mile & 414.8 & 414.8 & 386.5 & 365.4 & 404.6 & 368.8 & 334.2 & 398.9 & 347.3 & 299.6 & 391.2 & 330.7 & 284.5 \\
\hline & & & \begin{tabular}{|l} 
Distance \\
\end{tabular} & 29.4 & 29.4 & 29.2 & 28.6 & 28.8 & 28.8 & 28.2 & 28.4 & 28.2 & 27.5 & 28.5 & 28.5 & 27.7 \\
\hline & & & MPGGE Unadjusted & $\mathrm{N} / \mathrm{A}$ & $\mathrm{N} / \mathrm{A}$ & $\mathrm{N} / \mathrm{A}$ & $\mathrm{N} / \mathrm{A}$ & N/A & $\mathrm{N} / \mathrm{A}$ & N/A & $\mathrm{N} / \mathrm{A}$ & N/A & $\mathrm{N} / \mathrm{A}$ & $\mathrm{N} / \mathrm{A}$ & $\mathrm{N} / \mathrm{A}$ & $\mathrm{N} / \mathrm{A}$ \\
\hline & & & MPGGE Adjusted & N/A & N/A & N/A & N/A & N/A & N/A & N/A & N/A & N/A & N/A & N/A & N/A & $\mathrm{N} / \mathrm{A}$ \\
\hline & & \multirow{2}{*}{ CS } & MPGGE Unadjusted & 27.8 & 27.8 & 30.2 & 33.0 & 28.4 & 32.7 & 37.7 & 30.3 & 36.2 & 44.8 & 31.6 & 39.0 & 47.3 \\
\hline & & & MPGGE Adjusted & 19.7 & 19.7 & 21.4 & 23.4 & 20.2 & 23.2 & 26.6 & 21.4 & 25.6 & 31.6 & 22.4 & 27.6 & 33.3 \\
\hline & & \multirow{3}{*}{$\mathrm{CD}+\mathrm{CS}$} & Wh/mile & 213.7 & 213.7 & 199.2 & 188.3 & 208.5 & 190.1 & 172.2 & 205.6 & 179.0 & 154.4 & 201.6 & 170.5 & 146.6 \\
\hline & & & MPGGE Unadjusted & 47.0 & 47.0 & 51.1 & 56.0 & 48.2 & 55.5 & 63.8 & 51.3 & 61.4 & 75.9 & 53.6 & 66.1 & 80.1 \\
\hline & & & \begin{tabular}{|l|} 
MPGGE Adjusted \\
\end{tabular} & 33.2 & 33.2 & 36.0 & 39.4 & 34.0 & 39.1 & 44.9 & 36.2 & 43.2 & 53.2 & 37.7 & 46.5 & 56.1 \\
\hline & \multirow{7}{*}{ Combined } & \multirow{2}{*}{$C D$} & MPGGE Unadjusted & N/A & $\mathrm{N} / \mathrm{A}$ & $\mathrm{N} / \mathrm{A}$ & N/A & $\mathrm{N} / \mathrm{A}$ & $\mathrm{N} / \mathrm{A}$ & $\mathrm{N} / \mathrm{A}$ & $\mathrm{N} / \mathrm{A}$ & $\mathrm{N} / \mathrm{A}$ & N/A & $\mathrm{N} / \mathrm{A}$ & $\mathrm{N} / \mathrm{A}$ & $\mathrm{N} / \mathrm{A}$ \\
\hline & & & MPGGE Adjusted & N/A & N/A & N/A & N/A & N/A & N/A & N/A & N/A & N/A & N/A & N/A & N/A & N/A \\
\hline & & \multirow{2}{*}{ CS } & MPGGE Unadjusted & 28.8 & 28.8 & 31.3 & 34.7 & 29.6 & 34.2 & 40.2 & 31.6 & 38.5 & 48.6 & 33.0 & 41.1 & 51.2 \\
\hline & & & MPGGE Adjusted & 21.3 & 21.3 & 23.1 & 25.5 & 21.8 & 25.1 & 29.3 & 23.3 & 28.1 & 35.0 & 24.3 & 29.9 & 36.8 \\
\hline & & \multirow{3}{*}{$C D+C S$} & Wh/mile & 232.7 & 232.7 & 216.9 & 204.4 & 226.6 & 207.0 & 186.5 & 222.2 & 193.8 & 165.1 & 218.4 & 185.1 & 157.6 \\
\hline & & & MPGGE Unadjusted & 54.8 & 54.8 & 59.6 & 65.9 & 56.2 & 65.0 & 76.3 & 60.1 & 72.9 & 91.9 & 62.7 & 78.0 & 96.8 \\
\hline & & & MPGGE Adjusted & 39.0 & 39.0 & 42.2 & 46.4 & 40.0 & 45.8 & 53.0 & 42.6 & 50.9 & 62.8 & 44.3 & 54.2 & 65.8 \\
\hline
\end{tabular}




\begin{tabular}{|c|c|c|c|c|c|c|c|c|c|c|c|c|c|c|c|c|}
\hline & & & & \multicolumn{13}{|c|}{ Midsize_SUV } \\
\hline & & & & \multirow{2}{*}{$\begin{array}{l}2010 \\
\text { Ref }\end{array}$} & \multicolumn{3}{|c|}{2010} & \multicolumn{3}{|c|}{2015} & \multicolumn{3}{|c|}{2030} & \multicolumn{3}{|c|}{2045} \\
\hline \multirow{26}{*}{$\mathrm{CI}$ Series HEV PHEV 40} & & \multirow{5}{*}{$C D$} & & & low & avg & high & low & avg & high & low & avg & high & low & avg & high \\
\hline & \multirow{9}{*}{ UDDS } & & Wh/mile & 394.2 & 394.2 & 367.2 & 344.4 & 383.1 & 349.4 & 312.7 & 373.9 & 325.0 & 271.4 & 367.7 & 310.5 & 259.9 \\
\hline & & & Distance & 40.7 & 40.7 & 40.8 & 40.8 & 40.7 & 40.8 & 40.6 & 40.8 & 40.8 & 40.6 & 40.8 & 40.5 & 40.6 \\
\hline & & & MPGGE Unadjusted & N/A & N/A & N/A & N/A & N/A & N/A & N/A & N/A & N/A & N/A & N/A & $\mathrm{N} / \mathrm{A}$ & N/A \\
\hline & & & MPGGE Adjusted & N/A & $\mathrm{N} / \mathrm{A}$ & N/A & N/A & N/A & N/A & N/A & $\mathrm{N} / \mathrm{A}$ & N/A & $\mathrm{N} / \mathrm{A}$ & $N / A$ & $\mathrm{~N} / \mathrm{A}$ & $\mathrm{N} / \mathrm{A}$ \\
\hline & & \multirow{2}{*}{ CS } & MPGGE Unadjusted & 29.0 & 29.0 & 31.6 & 35.3 & 29.9 & 34.7 & 41.4 & 32.1 & 39.7 & 52.0 & 33.5 & 42.3 & 53.9 \\
\hline & & & MPGGE Adjusted & 22.3 & 22.3 & 24.2 & 26.8 & 23.0 & 26.4 & 31.1 & 24.5 & 29.9 & 38.3 & 25.6 & 31.7 & 39.5 \\
\hline & & \multirow{3}{*}{$\mathrm{CD}+\mathrm{CS}$} & Wh/mile & 289.3 & 289.3 & 269.6 & 252.9 & 281.2 & 256.6 & 231.0 & 274.5 & 240.0 & 200.6 & 270.0 & 229.0 & 192.1 \\
\hline & & & MPGGE Unadjusted & 72.5 & 72.5 & 79.0 & 88.2 & 74.8 & 86.8 & 103.6 & 80.2 & 99.2 & 130.0 & 83.8 & 105.7 & 134.7 \\
\hline & & & MPGGE Adjusted & 51.4 & 51.4 & 55.3 & 60.8 & 52.8 & 60.0 & 69.5 & 56.1 & 67.1 & 83.5 & 58.2 & 70.7 & 85.8 \\
\hline & \multirow{9}{*}{ HWFET } & \multirow{4}{*}{$C D$} & Wh/mile & 418.6 & 418.6 & 389.9 & 368.1 & 408.3 & 371.4 & 336.0 & 402.1 & 349.2 & 301.6 & 394.2 & 332.2 & 286.4 \\
\hline & & & Distance & 37.9 & 37.9 & 38.0 & 37.8 & 37.8 & 37.9 & 37.5 & 37.5 & 37.7 & 36.4 & 37.7 & 37.8 & 36.6 \\
\hline & & & MPGGE Unadjusted & N/A & N/A & N/A & N/A & N/A & N/A & N/A & N/A & N/A & N/A & N/A & N/A & N/A \\
\hline & & & MPGGE Adjusted & $\mathrm{N} / \mathrm{A}$ & $\mathrm{N} / \mathrm{A}$ & N/A & N/A & $\mathrm{N} / \mathrm{A}$ & N/A & N/A & $\mathrm{N} / \mathrm{A}$ & $\mathrm{N} / \mathrm{A}$ & $\mathrm{N} / \mathrm{A}$ & $\mathrm{N} / \mathrm{A}$ & N/A & $\mathrm{N} / \mathrm{A}$ \\
\hline & & \multirow{2}{*}{ CS } & MPGGE Unadjusted & 27.3 & 27.3 & 29.7 & 32.6 & 28.0 & 32.3 & 37.2 & 29.8 & 35.8 & 44.4 & 31.2 & 38.5 & 46.7 \\
\hline & & & MPGGE Adjusted & 19.4 & 19.4 & 21.0 & 23.1 & 19.8 & 22.9 & 26.3 & 21.1 & 25.4 & 31.3 & 22.1 & 27.2 & 33.0 \\
\hline & & \multirow{3}{*}{$\mathrm{CD}+\mathrm{CS}$} & Wh/mile & 282.6 & 282.6 & 263.3 & 248.6 & 275.7 & 250.8 & 226.9 & 271.5 & 235.8 & 203.7 & 266.2 & 224.3 & 193.5 \\
\hline & & & MPGGE Unadjusted & 59.4 & 59.4 & 64.6 & 70.9 & 60.9 & 70.2 & 80.9 & 64.8 & 77.9 & 96.5 & 67.8 & 83.8 & 101.6 \\
\hline & & & MPGGE Adjusted & 41.8 & 41.8 & 45.4 & 49.7 & 42.8 & 49.3 & 56.7 & 45.5 & 54.6 & 67.4 & 47.6 & 58.7 & 70.8 \\
\hline & \multirow{7}{*}{ Combined } & \multirow{2}{*}{$C D$} & MPGGE Unadjusted & $\mathrm{N} / \mathrm{A}$ & $\mathrm{N} / \mathrm{A}$ & N/A & N/A & $\mathrm{N} / \mathrm{A}$ & $\mathrm{N} / \mathrm{A}$ & $\mathrm{N} / \mathrm{A}$ & $\mathrm{N} / \mathrm{A}$ & $\mathrm{N} / \mathrm{A}$ & $\mathrm{N} / \mathrm{A}$ & N/A & $\mathrm{N} / \mathrm{A}$ & $\mathrm{N} / \mathrm{A}$ \\
\hline & & & MPGGE Adjusted & $\mathrm{N} / \mathrm{A}$ & $\mathrm{N} / \mathrm{A}$ & N/A & $\mathrm{N} / \mathrm{A}$ & $\mathrm{N} / \mathrm{A}$ & $\mathrm{N} / \mathrm{A}$ & $\mathrm{N} / \mathrm{A}$ & $\mathrm{N} / \mathrm{A}$ & $\mathrm{N} / \mathrm{A}$ & $\mathrm{N} / \mathrm{A}$ & $\mathrm{N} / \mathrm{A}$ & $\mathrm{N} / \mathrm{A}$ & $\mathrm{N} / \mathrm{A}$ \\
\hline & & \multirow{2}{*}{ CS } & MPGGE Unadjusted & 28.2 & 28.2 & 30.7 & 34.0 & 29.0 & 33.6 & 39.4 & 31.0 & 37.9 & 48.3 & 32.4 & 40.5 & 50.4 \\
\hline & & & MPGGE Adjusted & 20.9 & 20.9 & 22.7 & 25.0 & 21.4 & 24.7 & 28.8 & 22.9 & 27.7 & 34.8 & 23.9 & 29.5 & 36.3 \\
\hline & & \multirow{3}{*}{$\mathrm{CD}+\mathrm{CS}$} & Wh/mile & 286.3 & 286.3 & 266.8 & 251.0 & 278.7 & 254.0 & 229.2 & 273.2 & 238.1 & 202.0 & 268.3 & 226.9 & 192.7 \\
\hline & & & MPGGE Unadjusted & 66.0 & 66.0 & 71.8 & 79.5 & 67.8 & 78.5 & 92.0 & 72.4 & 88.4 & 112.5 & 75.8 & 94.6 & 117.5 \\
\hline & & & MPGGE Adjusted & 46.6 & 46.6 & 50.4 & 55.3 & 47.8 & 54.6 & 63.1 & 50.8 & 60.8 & 75.4 & 52.9 & 64.7 & 78.4 \\
\hline
\end{tabular}


Argonne National Laboratory Report - Light-Duty Vehicle Fuel Consumption Displacement Potential Up to 2045

\begin{tabular}{|c|c|c|c|c|c|c|c|c|c|c|c|c|c|c|c|c|}
\hline & & & & \multicolumn{13}{|c|}{ Midsize_SUV } \\
\hline & & & & \multirow{2}{*}{$\begin{array}{c}2010 \\
\text { Ref }\end{array}$} & \multicolumn{3}{|c|}{2010} & \multicolumn{3}{|c|}{2015} & \multicolumn{3}{|c|}{2030} & \multicolumn{3}{|c|}{2045} \\
\hline & & & & & low & avg & high & low & avg & high & low & avg & high & low & avg & high \\
\hline \multirow{25}{*}{ H2 Split HEV PHEV10 } & \multirow{9}{*}{ UDDS } & \multirow{4}{*}{$C D$} & Wh/mile & 242.5 & 242.5 & 224.0 & 209.7 & 237.7 & 212.5 & 213.0 & 232.1 & 207.2 & 195.4 & 224.0 & 203.7 & 191.3 \\
\hline & & & Distance & 12.8 & 12.8 & 13.0 & 12.9 & 12.4 & 13.0 & 12.1 & 12.6 & 12.8 & 11.6 & 12.8 & 12.8 & 11.3 \\
\hline & & & MPGGE Unadjusted & 412.3 & 412.3 & 501.9 & 578.1 & 483.4 & 567.8 & 999.6 & 524.0 & 659.2 & 1953.0 & 537.9 & 908.2 & 2834.1 \\
\hline & & & MPGGE Adjusted & 181.1 & 181.1 & 200.3 & 214.2 & 196.7 & 212.4 & 264.9 & 204.6 & 226.8 & 314.8 & 207.1 & 256.5 & 335.4 \\
\hline & & \multirow{2}{*}{ CS } & MPGGE Unadjusted & 39.6 & 39.6 & 47.4 & 54.2 & 45.0 & 53.5 & 62.0 & 49.0 & 59.6 & 74.2 & 52.5 & 64.2 & 80.6 \\
\hline & & & MPGGE Adjusted & 29.8 & 29.8 & 35.2 & 39.8 & 33.6 & 39.2 & 44.8 & 36.3 & 43.2 & 52.5 & 38.6 & 46.2 & 56.3 \\
\hline & & \multirow{3}{*}{$\mathrm{CD}+\mathrm{CS}$} & Wh/mile & 50.5 & 50.5 & 46.7 & 43.7 & 49.5 & 44.3 & 44.4 & 48.4 & 43.2 & 40.7 & 46.7 & 42.4 & 39.9 \\
\hline & & & MPGGE Unadjusted & 46.8 & 46.8 & 56.0 & 64.1 & 53.2 & 63.2 & 73.8 & 58.0 & 70.5 & 88.8 & 62.0 & 76.2 & 96.5 \\
\hline & & & MPGGE Adjusted & 34.8 & 34.8 & 40.9 & 46.1 & 39.1 & 45.6 & 52.2 & 42.2 & 50.1 & 61.1 & 44.8 & 53.7 & 65.6 \\
\hline & \multirow{9}{*}{ HWFET } & \multirow{4}{*}{$C D$} & Wh/mile & 245.2 & 245.2 & 226.7 & 214.5 & 241.0 & 216.1 & 198.8 & 237.5 & 206.8 & 221.0 & 231.9 & 190.5 & 211.5 \\
\hline & & & Distance & 12.5 & 12.5 & 12.5 & 12.4 & 12.5 & 12.5 & 12.2 & 12.5 & 12.2 & 8.3 & 12.4 & 12.6 & 8.3 \\
\hline & & & MPGGE Unadjusted & 107.8 & 107.8 & 127.2 & 144.2 & 122.7 & 142.5 & 162.4 & 131.9 & 156.7 & 323.9 & 141.4 & 161.2 & 366.7 \\
\hline & & & MPGGE Adjusted & 75.0 & 75.0 & 88.2 & 99.6 & 85.2 & 98.5 & 111.8 & 91.4 & 108.0 & 215.6 & 97.7 & 111.0 & 241.9 \\
\hline & & \multirow{2}{*}{ CS } & MPGGE Unadjusted & 35.6 & 35.6 & 42.3 & 48.0 & 40.3 & 47.5 & 53.9 & 43.5 & 52.0 & 62.4 & 46.6 & 56.5 & 68.3 \\
\hline & & & MPGGE Adjusted & 25.2 & 25.2 & 29.9 & 33.8 & 28.4 & 33.5 & 38.0 & 30.7 & 36.6 & 43.9 & 32.9 & 39.8 & 47.9 \\
\hline & & \multirow{3}{*}{$\mathrm{CD}+\mathrm{CS}$} & Wh/mile & 72.1 & 72.1 & 66.7 & 63.1 & 70.9 & 63.6 & 58.5 & 69.9 & 60.8 & 65.0 & 68.2 & 56.0 & 62.2 \\
\hline & & & MPGGE Unadjusted & 42.5 & 42.5 & 50.4 & 57.1 & 48.0 & 56.5 & 64.2 & 51.9 & 61.9 & 77.4 & 55.5 & 67.0 & 84.8 \\
\hline & & & \begin{tabular}{|l|} 
MPGGE Adjusted \\
\end{tabular} & 30.0 & 30.0 & 35.5 & 40.2 & 33.8 & 39.8 & 45.1 & 36.6 & 43.5 & 54.2 & 39.1 & 47.0 & 59.4 \\
\hline & \multirow{7}{*}{ Combined } & \multirow{2}{*}{$C D$} & MPGGE Unadjusted & 181.5 & 181.5 & 215.9 & 245.5 & 208.1 & 242.4 & 301.1 & 224.2 & 269.9 & 598.5 & 237.8 & 294.4 & 703.6 \\
\hline & & & MPGGE Adjusted & 110.7 & 110.7 & 127.4 & 141.1 & 123.8 & 139.7 & 163.9 & 131.4 & 151.7 & 260.8 & 137.7 & 161.3 & 285.7 \\
\hline & & \multirow{2}{*}{ CS } & MPGGE Unadjusted & 37.7 & 37.7 & 45.0 & 51.2 & 42.7 & 50.6 & 58.1 & 46.4 & 55.9 & 68.4 & 49.6 & 60.5 & 74.5 \\
\hline & & & MPGGE Adjusted & 27.5 & 27.5 & 32.6 & 36.8 & 31.0 & 36.4 & 41.4 & 33.6 & 40.0 & 48.2 & 35.8 & 43.1 & 52.2 \\
\hline & & \multirow{3}{*}{$C D+C S$} & Wh/mile & 60.2 & 60.2 & 55.7 & 52.4 & 59.1 & 52.9 & 50.7 & 58.0 & 51.1 & 51.6 & 56.4 & 48.6 & 49.9 \\
\hline & & & MPGGE Unadjusted & 44.7 & 44.7 & 53.3 & 60.7 & 50.7 & 60.0 & 69.1 & 55.1 & 66.3 & 83.3 & 58.9 & 71.8 & 90.9 \\
\hline & & & MPGGE Adjusted & 32.4 & 32.4 & 38.3 & 43.3 & 36.5 & 42.8 & 48.7 & 39.5 & 46.9 & 57.8 & 42.0 & 50.5 & 62.6 \\
\hline
\end{tabular}


Argonne National Laboratory Report - Light-Duty Vehicle Fuel Consumption Displacement Potential Up to 2045

\begin{tabular}{|c|c|c|c|c|c|c|c|c|c|c|c|c|c|c|c|c|}
\hline & & & & \multicolumn{13}{|c|}{ Midsize_SUV } \\
\hline & & & & \multirow{2}{*}{$\begin{array}{c}2010 \\
\text { Ref }\end{array}$} & \multicolumn{3}{|c|}{2010} & \multicolumn{3}{|c|}{2015} & \multicolumn{3}{|c|}{2030} & \multicolumn{3}{|c|}{2045} \\
\hline \multirow{26}{*}{ H2 Split HEV PHEV2O } & & & & & low & avg & high & low & avg & high & low & avg & high & low & avg & high \\
\hline & \multirow{9}{*}{ UDDS } & \multirow{4}{*}{$C D$} & Wh/mile & 275.1 & 275.1 & 253.7 & 237.6 & 275.8 & 239.1 & 211.3 & 273.3 & 222.0 & 190.5 & 257.2 & 208.8 & 187.4 \\
\hline & & & Distance & 24.0 & 24.0 & 23.9 & 23.9 & 23.5 & 24.0 & 24.0 & 22.7 & 24.0 & 22.9 & 23.9 & 24.0 & 22.3 \\
\hline & & & MPGGE Unadjusted & 748.2 & 748.2 & 911.4 & 1063.8 & 1119.9 & 994.1 & 1006.6 & 1386.3 & 995.5 & 1544.4 & 1070.3 & 956.4 & 2623.2 \\
\hline & & & \begin{tabular}{|l|} 
MPGGE Adjusted \\
\end{tabular} & 238.8 & 238.8 & 256.8 & 270.2 & 274.5 & 264.4 & 265.5 & 291.3 & 264.5 & 299.2 & 270.7 & 261.0 & 331.5 \\
\hline & & \multirow{2}{*}{ CS } & MPGGE Unadjusted & 39.3 & 39.3 & 47.2 & 53.8 & 44.7 & 53.1 & 61.9 & 48.8 & 59.5 & 74.3 & 52.3 & 64.2 & 80.8 \\
\hline & & & MPGGE Adjusted & 29.6 & 29.6 & 35.0 & 39.5 & 33.4 & 39.0 & 44.7 & 36.1 & 43.2 & 52.5 & 38.5 & 46.2 & 56.4 \\
\hline & & \multirow{3}{*}{$C D+C S$} & Wh/mile & 143.6 & 143.6 & 133.0 & 124.6 & 144.5 & 125.3 & 111.6 & 143.0 & 116.2 & 100.0 & 134.2 & 110.3 & 99.0 \\
\hline & & & MPGGE Unadjusted & 67.2 & 67.2 & 80.9 & 92.4 & 77.3 & 91.0 & 105.6 & 84.6 & 101.3 & 127.6 & 89.7 & 109.1 & 141.0 \\
\hline & & & MPGGE Adjusted & 48.1 & 48.1 & 56.5 & 63.2 & 54.3 & 62.4 & 70.6 & 58.7 & 68.3 & 82.3 & 61.7 & 72.5 & 88.9 \\
\hline & \multirow{9}{*}{ HWFET } & \multirow{4}{*}{$C D$} & Wh/mile & 308.5 & 308.5 & 265.2 & 267.1 & 300.4 & 257.7 & 199.9 & 295.4 & 236.3 & 190.9 & 287.3 & 193.8 & 264.6 \\
\hline & & & Distance & 21.4 & 21.4 & 22.7 & 21.3 & 21.4 & 21.9 & 24.6 & 21.3 & 22.0 & 19.1 & 21.3 & 24.8 & 15.9 \\
\hline & & & MPGGE Unadjusted & 193.7 & 193.7 & 177.9 & 248.0 & 215.3 & 212.6 & 161.1 & 225.5 & 207.2 & 206.3 & 241.0 & 162.9 & $\mathrm{~N} / \mathrm{A}$ \\
\hline & & & MPGGE Adjusted & 132.5 & 132.5 & 122.0 & 167.6 & 146.5 & 144.8 & 110.9 & 153.1 & 141.2 & 140.7 & 163.2 & 112.1 & N/A \\
\hline & & \multirow{2}{*}{ CS } & MPGGE Unadjusted & 35.3 & 35.3 & 42.0 & 47.5 & 40.0 & 47.1 & 53.7 & 43.2 & 51.7 & 62.2 & 46.2 & 56.3 & 68.1 \\
\hline & & & MPGGE Adjusted & 25.0 & 25.0 & 29.7 & 33.5 & 28.2 & 33.2 & 37.8 & 30.5 & 36.4 & 43.7 & 32.6 & 39.7 & 47.8 \\
\hline & & \multirow{3}{*}{$C D+C S$} & Wh/mile & 154.4 & 154.4 & 133.9 & 133.8 & 150.4 & 129.8 & 102.8 & 148.9 & 119.5 & 56.2 & 144.0 & 99.7 & 77.8 \\
\hline & & & MPGGE Unadjusted & 52.3 & 52.3 & 60.5 & 70.0 & 59.1 & 68.3 & 73.5 & 63.9 & 73.9 & 74.7 & 68.1 & 76.6 & 89.6 \\
\hline & & & MPGGE Adjusted & 36.9 & 36.9 & 42.5 & 49.2 & 41.6 & 48.0 & 51.6 & 44.9 & 51.8 & 52.4 & 47.8 & 53.7 & 62.6 \\
\hline & \multirow{7}{*}{ Combined } & \multirow{2}{*}{$C D$} & MPGGE Unadjusted & 327.0 & 327.0 & 319.2 & 428.9 & 387.4 & 374.5 & 299.4 & 418.0 & 367.0 & 394.1 & 420.0 & 299.7 & 4618.2 \\
\hline & & & MPGGE Adjusted & 175.4 & 175.4 & 171.5 & 211.9 & 197.0 & 192.7 & 163.1 & 207.2 & 189.9 & 198.5 & 208.8 & 163.4 & 553.3 \\
\hline & & \multirow{2}{*}{ CS } & MPGGE Unadjusted & 37.4 & 37.4 & 44.7 & 50.8 & 42.4 & 50.2 & 57.9 & 46.1 & 55.7 & 68.3 & 49.4 & 60.4 & 74.5 \\
\hline & & & MPGGE Adjusted & 27.3 & 27.3 & 32.4 & 36.6 & 30.8 & 36.2 & 41.3 & 33.4 & 39.9 & 48.2 & 35.6 & 43.0 & 52.2 \\
\hline & & \multirow{3}{*}{$C D+C S$} & \begin{tabular}{|l} 
Wh/mile \\
\end{tabular} & 148.4 & 148.4 & 133.4 & 128.7 & 147.2 & 127.3 & 107.6 & 145.6 & 117.7 & 80.3 & 138.6 & 105.6 & 89.5 \\
\hline & & & MPGGE Unadjusted & 59.6 & 59.6 & 70.2 & 80.8 & 67.9 & 79.2 & 88.2 & 73.8 & 86.8 & 96.8 & 78.5 & 91.6 & 112.0 \\
\hline & & & MPGGE Adjusted & 42.3 & 42.3 & 49.2 & 56.0 & 47.7 & 55.0 & 60.5 & 51.5 & 59.7 & 65.5 & 54.6 & 62.6 & 74.8 \\
\hline
\end{tabular}


Argonne National Laboratory Report - Light-Duty Vehicle Fuel Consumption Displacement Potential Up to 2045

\begin{tabular}{|c|c|c|c|c|c|c|c|c|c|c|c|c|c|c|c|c|}
\hline & & & & \multicolumn{13}{|c|}{ Midsize_SUV } \\
\hline & & & & \multirow{2}{*}{$\begin{array}{c}2010 \\
\text { Ref }\end{array}$} & \multicolumn{3}{|c|}{2010} & \multicolumn{3}{|c|}{2015} & \multicolumn{3}{|c|}{2030} & \multicolumn{3}{|c|}{2045} \\
\hline & & & & & low & avg & high & low & avg & high & low & avg & high & low & avg & high \\
\hline \multirow{25}{*}{ H2 Series HEV PHEV30 } & \multirow{9}{*}{ UDDS } & \multirow{4}{*}{$C D$} & Wh/mile & 378.7 & 378.7 & 347.3 & 323.6 & 365.8 & 328.7 & 291.6 & 356.7 & 304.4 & 252.5 & 346.5 & 290.5 & 242.1 \\
\hline & & & Distance & 31.4 & 31.4 & 31.9 & 30.9 & 31.9 & 30.9 & 30.9 & 31.9 & 30.9 & 30.9 & 30.9 & 30.9 & 32.0 \\
\hline & & & MPGGE Unadjusted & $\mathrm{N} / \mathrm{A}$ & $\mathrm{N} / \mathrm{A}$ & N/A & N/A & N/A & $\mathrm{N} / \mathrm{A}$ & N/A & $\mathrm{N} / \mathrm{A}$ & N/A & N/A & N/A & N/A & $\mathrm{N} / \mathrm{A}$ \\
\hline & & & MPGGE Adjusted & $\mathrm{N} / \mathrm{A}$ & $\mathrm{N} / \mathrm{A}$ & $\mathrm{N} / \mathrm{A}$ & N/A & N/A & N/A & N/A & $\mathrm{N} / \mathrm{A}$ & N/A & N/A & N/A & $\mathrm{N} / \mathrm{A}$ & $\mathrm{N} / \mathrm{A}$ \\
\hline & & \multirow{2}{*}{ CS } & MPGGE Unadjusted & 28.3 & 28.3 & 34.7 & 40.4 & 32.4 & 39.7 & 48.5 & 36.1 & 45.8 & 59.6 & 39.1 & 50.3 & 65.0 \\
\hline & & & MPGGE Adjusted & 21.8 & 21.8 & 26.4 & 30.4 & 24.8 & 29.9 & 35.9 & 27.4 & 34.1 & 43.3 & 29.5 & 37.1 & 46.7 \\
\hline & & \multirow{3}{*}{$\mathrm{CD}+\mathrm{CS}$} & Wh/mile & 243.7 & 243.7 & 223.5 & 208.2 & 235.4 & 211.6 & 188.3 & 229.6 & 196.6 & 163.4 & 223.0 & 187.4 & 156.7 \\
\hline & & & MPGGE Unadjusted & 60.2 & 60.2 & 73.8 & 86.0 & 68.9 & 84.5 & 103.1 & 76.8 & 97.4 & 126.8 & 83.2 & 106.9 & 138.4 \\
\hline & & & MPGGE Adjusted & 43.6 & 43.6 & 52.2 & 59.5 & 49.2 & 58.6 & 69.2 & 54.0 & 66.1 & 81.9 & 57.8 & 71.4 & 87.7 \\
\hline & \multirow{9}{*}{ HWFET } & \multirow{4}{*}{$C D$} & Wh/mile & 410.0 & 410.0 & 378.3 & 355.9 & 398.6 & 359.4 & 324.4 & 392.4 & 337.9 & 289.6 & 381.8 & 321.1 & 275.1 \\
\hline & & & \begin{tabular}{|l} 
Distance \\
\end{tabular} & 28.6 & 28.6 & 28.4 & 27.9 & 28.4 & 28.2 & 27.6 & 28.0 & 27.6 & 26.7 & 27.9 & 27.7 & 26.9 \\
\hline & & & MPGGE Unadjusted & $\mathrm{N} / \mathrm{A}$ & $\mathrm{N} / \mathrm{A}$ & $\mathrm{N} / \mathrm{A}$ & $\mathrm{N} / \mathrm{A}$ & N/A & $\mathrm{N} / \mathrm{A}$ & N/A & $\mathrm{N} / \mathrm{A}$ & $\mathrm{N} / \mathrm{A}$ & $\mathrm{N} / \mathrm{A}$ & $\mathrm{N} / \mathrm{A}$ & $\mathrm{N} / \mathrm{A}$ & $\mathrm{N} / \mathrm{A}$ \\
\hline & & & MPGGE Adjusted & N/A & N/A & N/A & N/A & N/A & N/A & N/A & N/A & N/A & N/A & N/A & N/A & $\mathrm{N} / \mathrm{A}$ \\
\hline & & \multirow{2}{*}{ CS } & MPGGE Unadjusted & 26.7 & 26.7 & 32.2 & 36.9 & 30.4 & 36.5 & 43.0 & 33.5 & 41.2 & 50.7 & 35.9 & 45.3 & 55.5 \\
\hline & & & MPGGE Adjusted & 18.9 & 18.9 & 22.8 & 26.1 & 21.5 & 25.8 & 30.4 & 23.7 & 29.1 & 35.7 & 25.4 & 32.0 & 39.1 \\
\hline & & \multirow{3}{*}{$\mathrm{CD}+\mathrm{CS}$} & Wh/mile & 211.3 & 211.3 & 195.0 & 183.4 & 205.4 & 185.2 & 167.1 & 202.2 & 174.1 & 149.2 & 196.8 & 165.5 & 141.7 \\
\hline & & & MPGGE Unadjusted & 45.2 & 45.2 & 54.6 & 62.5 & 51.5 & 61.8 & 72.9 & 56.7 & 69.9 & 85.9 & 60.9 & 76.8 & 94.0 \\
\hline & & & \begin{tabular}{|l|} 
MPGGE Adjusted \\
\end{tabular} & 31.9 & 31.9 & 38.4 & 43.9 & 36.3 & 43.5 & 51.1 & 39.9 & 49.0 & 60.1 & 42.8 & 53.8 & 65.7 \\
\hline & \multirow{7}{*}{ Combined } & \multirow{2}{*}{$C D$} & MPGGE Unadjusted & N/A & $\mathrm{N} / \mathrm{A}$ & $\mathrm{N} / \mathrm{A}$ & N/A & $\mathrm{N} / \mathrm{A}$ & $\mathrm{N} / \mathrm{A}$ & $\mathrm{N} / \mathrm{A}$ & $\mathrm{N} / \mathrm{A}$ & $\mathrm{N} / \mathrm{A}$ & N/A & $\mathrm{N} / \mathrm{A}$ & $\mathrm{N} / \mathrm{A}$ & $\mathrm{N} / \mathrm{A}$ \\
\hline & & & MPGGE Adjusted & N/A & N/A & N/A & N/A & N/A & N/A & N/A & N/A & N/A & N/A & N/A & N/A & N/A \\
\hline & & \multirow{2}{*}{ CS } & MPGGE Unadjusted & 27.5 & 27.5 & 33.5 & 38.7 & 31.5 & 38.2 & 45.8 & 34.9 & 43.6 & 55.2 & 37.6 & 47.9 & 60.4 \\
\hline & & & MPGGE Adjusted & 20.4 & 20.4 & 24.6 & 28.3 & 23.2 & 27.9 & 33.2 & 25.6 & 31.7 & 39.5 & 27.5 & 34.6 & 42.9 \\
\hline & & \multirow{3}{*}{$\mathrm{CD}+\mathrm{CS}$} & Wh/mile & 229.1 & 229.1 & 210.7 & 197.1 & 221.9 & 199.7 & 178.8 & 217.3 & 186.4 & 157.0 & 211.2 & 177.5 & 150.0 \\
\hline & & & MPGGE Unadjusted & 52.4 & 52.4 & 63.7 & 73.5 & 59.8 & 72.5 & 86.9 & 66.2 & 82.7 & 104.4 & 71.4 & 90.9 & 114.2 \\
\hline & & & MPGGE Adjusted & 37.4 & 37.4 & 44.9 & 51.3 & 42.4 & 50.7 & 59.7 & 46.6 & 57.1 & 70.4 & 50.0 & 62.2 & 76.2 \\
\hline
\end{tabular}




\begin{tabular}{|c|c|c|c|c|c|c|c|c|c|c|c|c|c|c|c|c|}
\hline & & & & \multicolumn{13}{|c|}{ Midsize_SUV } \\
\hline & & & & \multirow{2}{*}{$\begin{array}{l}2010 \\
\text { Ref }\end{array}$} & \multicolumn{3}{|c|}{2010} & \multicolumn{3}{|c|}{2015} & \multicolumn{3}{|c|}{2030} & \multicolumn{3}{|c|}{2045} \\
\hline \multirow{26}{*}{ H2 Series HEV PHEV40 } & & \multirow{5}{*}{$C D$} & & & low & avg & high & low & avg & high & low & avg & high & low & avg & high \\
\hline & \multirow{9}{*}{ UDDS } & & Wh/mile & 386.5 & 386.5 & 354.5 & 330.0 & 373.6 & 335.2 & 297.3 & 364.0 & 310.5 & 255.3 & 353.0 & 295.5 & 244.7 \\
\hline & & & Distance & 40.7 & 40.7 & 40.7 & 40.6 & 40.7 & 40.6 & 40.6 & 40.7 & 40.6 & 40.4 & 40.6 & 40.5 & 40.4 \\
\hline & & & MPGGE Unadjusted & N/A & N/A & $N / A$ & N/A & N/A & N/A & N/A & N/A & N/A & N/A & N/A & N/A & N/A \\
\hline & & & MPGGE Adjusted & N/A & N/A & N/A & N/A & N/A & $\mathrm{N} / \mathrm{A}$ & N/A & N/A & N/A & N/A & N/A & N/A & $\mathrm{N} / \mathrm{A}$ \\
\hline & & \multirow{2}{*}{ CS } & MPGGE Unadjusted & 27.6 & 27.6 & 33.7 & 39.6 & 31.7 & 38.8 & 47.7 & 35.2 & 45.0 & 58.9 & 38.1 & 48.9 & 64.2 \\
\hline & & & MPGGE Adjusted & 21.3 & 21.3 & 25.7 & 29.8 & 24.2 & 29.3 & 35.4 & 26.8 & 33.6 & 42.8 & 28.8 & 36.2 & 46.2 \\
\hline & & \multirow{3}{*}{$\mathrm{CD}+\mathrm{CS}$} & Wh/mile & 283.7 & 283.7 & 260.2 & 242.3 & 274.2 & 246.1 & 219.5 & 267.2 & 229.1 & 188.5 & 259.1 & 217.8 & 180.6 \\
\hline & & & MPGGE Unadjusted & 69.0 & 69.0 & 84.2 & 99.0 & 79.2 & 97.0 & 119.1 & 88.1 & 112.6 & 147.2 & 95.3 & 122.2 & 160.6 \\
\hline & & & MPGGE Adjusted & 49.2 & 49.2 & 58.5 & 67.0 & 55.4 & 65.8 & 77.9 & 60.7 & 74.4 & 91.9 & 64.9 & 79.5 & 98.2 \\
\hline & \multirow{9}{*}{ HWFET } & \multirow{4}{*}{$C D$} & Wh/mile & 413.6 & 413.6 & 381.5 & 358.7 & 402.1 & 362.1 & 326.2 & 395.6 & 339.9 & 291.5 & 384.5 & 322.6 & 276.9 \\
\hline & & & Distance & 38.6 & 38.6 & 37.5 & 37.2 & 37.5 & 37.4 & 36.8 & 37.1 & 36.8 & 35.6 & 37.1 & 37.1 & 35.9 \\
\hline & & & MPGGE Unadjusted & N/A & N/A & N/A & N/A & N/A & N/A & N/A & N/A & N/A & N/A & N/A & N/A & $\mathrm{N} / \mathrm{A}$ \\
\hline & & & MPGGE Adjusted & N/A & N/A & N/A & N/A & N/A & N/A & N/A & N/A & N/A & $\mathrm{N} / \mathrm{A}$ & $\mathrm{N} / \mathrm{A}$ & N/A & N/A \\
\hline & & \multirow{2}{*}{ CS } & MPGGE Unadjusted & 26.3 & 26.3 & 31.7 & 36.4 & 29.9 & 36.0 & 42.5 & 33.0 & 40.6 & 50.2 & 35.5 & 44.9 & 55.1 \\
\hline & & & MPGGE Adjusted & 18.6 & 18.6 & 22.5 & 25.7 & 21.2 & 25.5 & 30.0 & 23.3 & 28.7 & 35.4 & 25.1 & 31.7 & 38.8 \\
\hline & & \multirow{3}{*}{$\mathrm{CD}+\mathrm{CS}$} & Wh/mile & 279.2 & 279.2 & 257.6 & 242.2 & 271.5 & 244.5 & 220.2 & 267.1 & 229.4 & 196.9 & 259.6 & 217.8 & 187.0 \\
\hline & & & MPGGE Unadjusted & 57.1 & 57.1 & 69.0 & 79.1 & 65.0 & 78.3 & 92.5 & 71.7 & 88.4 & 109.1 & 77.2 & 97.5 & 119.8 \\
\hline & & & MPGGE Adjusted & 40.2 & 40.2 & 48.4 & 55.4 & 45.7 & 54.9 & 64.6 & 50.3 & 61.8 & 76.0 & 54.1 & 68.0 & 83.2 \\
\hline & \multirow{7}{*}{ Combined } & \multirow{2}{*}{$C D$} & MPGGE Unadjusted & $\mathrm{N} / \mathrm{A}$ & $\mathrm{N} / \mathrm{A}$ & $\mathrm{N} / \mathrm{A}$ & $\mathrm{N} / \mathrm{A}$ & N/A & $\mathrm{N} / \mathrm{A}$ & N/A & $\mathrm{N} / \mathrm{A}$ & $\mathrm{N} / \mathrm{A}$ & $\mathrm{N} / \mathrm{A}$ & $\mathrm{N} / \mathrm{A}$ & $\mathrm{N} / \mathrm{A}$ & $\mathrm{N} / \mathrm{A}$ \\
\hline & & & MPGGE Adjusted & $\mathrm{N} / \mathrm{A}$ & $\mathrm{N} / \mathrm{A}$ & $\mathrm{N} / \mathrm{A}$ & $\mathrm{N} / \mathrm{A}$ & N/A & $\mathrm{N} / \mathrm{A}$ & $\mathrm{N} / \mathrm{A}$ & $\mathrm{N} / \mathrm{A}$ & $\mathrm{N} / \mathrm{A}$ & $\mathrm{N} / \mathrm{A}$ & $\mathrm{N} / \mathrm{A}$ & $\mathrm{N} / \mathrm{A}$ & $\mathrm{N} / \mathrm{A}$ \\
\hline & & \multirow{2}{*}{ CS } & MPGGE Unadjusted & 27.0 & 27.0 & 32.8 & 38.1 & 30.8 & 37.5 & 45.2 & 34.2 & 42.9 & 54.6 & 36.9 & 47.0 & 59.8 \\
\hline & & & MPGGE Adjusted & 20.0 & 20.0 & 24.1 & 27.8 & 22.8 & 27.4 & 32.8 & 25.1 & 31.2 & 39.1 & 27.0 & 34.0 & 42.6 \\
\hline & & \multirow{3}{*}{$\mathrm{CD}+\mathrm{CS}$} & Wh/mile & 281.7 & 281.7 & 259.0 & 242.2 & 272.9 & 245.4 & 219.8 & 267.2 & 229.3 & 192.3 & 259.3 & 217.8 & 183.5 \\
\hline & & & MPGGE Unadjusted & 63.1 & 63.1 & 76.6 & 88.9 & 72.1 & 87.6 & 105.5 & 79.9 & 100.2 & 127.2 & 86.2 & 109.7 & 139.2 \\
\hline & & & MPGGE Adjusted & 44.7 & 44.7 & 53.5 & 61.2 & 50.6 & 60.4 & 71.3 & 55.6 & 68.1 & 84.0 & 59.5 & 73.9 & 90.8 \\
\hline
\end{tabular}


Argonne National Laboratory Report - Light-Duty Vehicle Fuel Consumption Displacement Potential Up to 2045

\begin{tabular}{|c|c|c|c|c|c|c|c|c|c|c|c|c|c|c|c|c|}
\hline & & & & \multicolumn{13}{|c|}{ Midsize_SUV } \\
\hline & & & & \multirow{2}{*}{$\begin{array}{c}2010 \\
\text { Ref }\end{array}$} & \multicolumn{3}{|c|}{2010} & \multicolumn{3}{|c|}{2015} & \multicolumn{3}{|c|}{2030} & \multicolumn{3}{|c|}{2045} \\
\hline & & & & & low & avg & high & low & avg & high & low & avg & high & low & avg & high \\
\hline \multirow{25}{*}{ E85 Split HEV PHEV10 } & \multirow{9}{*}{ UDDS } & \multirow{4}{*}{$C D$} & Wh/mile & 241.8 & 241.8 & 225.1 & 226.9 & 236.6 & 229.8 & 213.8 & 232.0 & 209.8 & 190.7 & 229.3 & 208.9 & 183.5 \\
\hline & & & Distance & 12.8 & 12.8 & 13.0 & 12.3 & 12.9 & 12.4 & 12.6 & 12.5 & 12.9 & 12.3 & 12.5 & 12.6 & 12.2 \\
\hline & & & MPGGE Unadjusted & 402.8 & 402.8 & 431.0 & 646.4 & 418.1 & 633.5 & 806.7 & 428.6 & 514.1 & 1078.3 & 440.9 & 724.6 & 1191.9 \\
\hline & & & MPGGE Adjusted & 178.8 & 178.8 & 185.4 & 224.9 & 182.4 & 223.0 & 245.8 & 184.9 & 202.7 & 271.3 & 187.6 & 235.8 & 279.5 \\
\hline & & \multirow{2}{*}{ CS } & MPGGE Unadjusted & 38.2 & 38.2 & 41.3 & 44.4 & 39.5 & 43.8 & 55.8 & 40.6 & 47.2 & 64.9 & 41.7 & 49.4 & 67.9 \\
\hline & & & MPGGE Adjusted & 28.9 & 28.9 & 31.0 & 33.2 & 29.8 & 32.8 & 40.8 & 30.5 & 35.1 & 46.7 & 31.3 & 36.6 & 48.6 \\
\hline & & \multirow{3}{*}{$\mathrm{CD}+\mathrm{CS}$} & Wh/mile & 50.4 & 50.4 & 46.9 & 47.3 & 49.3 & 47.9 & 44.5 & 48.3 & 43.7 & 39.7 & 47.8 & 43.5 & 38.2 \\
\hline & & & MPGGE Unadjusted & 45.1 & 45.1 & 48.8 & 52.8 & 46.7 & 52.1 & 66.3 & 48.0 & 55.9 & 77.3 & 49.2 & 58.7 & 80.9 \\
\hline & & & MPGGE Adjusted & 33.7 & 33.7 & 36.1 & 38.8 & 34.7 & 38.3 & 47.5 & 35.6 & 40.8 & 54.3 & 36.4 & 42.7 & 56.5 \\
\hline & \multirow{9}{*}{ HWFET } & \multirow{4}{*}{$C D$} & Wh/mile & 245.1 & 245.1 & 228.2 & 216.2 & 240.7 & 217.8 & 194.5 & 238.4 & 208.7 & 180.6 & 234.3 & 194.0 & 181.2 \\
\hline & & & Distance & 12.5 & 12.5 & 12.5 & 12.5 & 12.5 & 12.5 & 12.9 & 12.5 & 12.4 & 12.2 & 12.5 & 12.6 & 11.8 \\
\hline & & & MPGGE Unadjusted & 103.3 & 103.3 & 110.6 & 117.8 & 106.8 & 116.6 & 137.0 & 109.0 & 124.2 & 164.7 & 112.4 & 125.9 & 193.2 \\
\hline & & & MPGGE Adjusted & 72.0 & 72.0 & 77.0 & 81.9 & 74.4 & 81.0 & 94.8 & 75.9 & 86.2 & 113.3 & 78.2 & 87.3 & 132.1 \\
\hline & & \multirow{2}{*}{ CS } & MPGGE Unadjusted & 34.1 & 34.1 & 36.8 & 39.2 & 35.1 & 38.9 & 48.4 & 35.9 & 41.2 & 54.9 & 37.0 & 43.5 & 57.7 \\
\hline & & & MPGGE Adjusted & 24.1 & 24.1 & 26.0 & 27.7 & 24.8 & 27.5 & 34.2 & 25.4 & 29.1 & 38.6 & 26.2 & 30.7 & 40.6 \\
\hline & & \multirow{3}{*}{$\mathrm{CD}+\mathrm{CS}$} & Wh/mile & 72.1 & 72.1 & 67.1 & 63.6 & 70.8 & 64.1 & 57.2 & 70.1 & 61.4 & 53.1 & 68.9 & 57.1 & 53.3 \\
\hline & & & MPGGE Unadjusted & 40.6 & 40.6 & 43.8 & 46.7 & 41.9 & 46.3 & 57.3 & 42.8 & 49.0 & 65.3 & 44.1 & 51.6 & 69.4 \\
\hline & & & MPGGE Adjusted & 28.7 & 28.7 & 30.9 & 32.9 & 29.6 & 32.7 & 40.3 & 30.2 & 34.6 & 45.9 & 31.1 & 36.4 & 48.7 \\
\hline & \multirow{7}{*}{ Combined } & \multirow{2}{*}{$C D$} & MPGGE Unadjusted & 174.8 & 174.8 & 187.1 & 214.1 & 180.9 & 211.6 & 252.1 & 184.8 & 213.1 & 308.3 & 190.5 & 230.7 & 358.4 \\
\hline & & & MPGGE Adjusted & 107.2 & 107.2 & 113.5 & 125.9 & 110.3 & 124.7 & 143.2 & 112.3 & 126.0 & 166.7 & 115.1 & 133.6 & 186.1 \\
\hline & & \multirow{2}{*}{ CS } & MPGGE Unadjusted & 36.2 & 36.2 & 39.1 & 41.9 & 37.4 & 41.5 & 52.2 & 38.3 & 44.3 & 60.0 & 39.4 & 46.6 & 62.9 \\
\hline & & & MPGGE Adjusted & 26.5 & 26.5 & 28.5 & 30.5 & 27.3 & 30.2 & 37.5 & 28.0 & 32.1 & 42.7 & 28.7 & 33.7 & 44.6 \\
\hline & & \multirow{3}{*}{$C D+C S$} & Wh/mile & 60.1 & 60.1 & 56.0 & 54.6 & 59.0 & 55.2 & 50.2 & 58.1 & 51.7 & 45.8 & 57.3 & 49.6 & 45.0 \\
\hline & & & MPGGE Unadjusted & 43.0 & 43.0 & 46.4 & 49.9 & 44.4 & 49.3 & 61.9 & 45.5 & 52.6 & 71.4 & 46.8 & 55.3 & 75.3 \\
\hline & & & MPGGE Adjusted & 31.2 & 31.2 & 33.6 & 35.9 & 32.2 & 35.6 & 44.0 & 33.0 & 37.8 & 50.2 & 33.8 & 39.6 & 52.7 \\
\hline
\end{tabular}


Argonne National Laboratory Report - Light-Duty Vehicle Fuel Consumption Displacement Potential Up to 2045

\begin{tabular}{|c|c|c|c|c|c|c|c|c|c|c|c|c|c|c|c|c|}
\hline & & & & \multicolumn{13}{|c|}{ Midsize_SUV } \\
\hline & & & & \multirow{2}{*}{$\begin{array}{c}2010 \\
\text { Ref }\end{array}$} & \multicolumn{3}{|c|}{2010} & \multicolumn{3}{|c|}{2015} & \multicolumn{3}{|c|}{2030} & \multicolumn{3}{|c|}{2045} \\
\hline \multirow{26}{*}{ E85 Split HEV PHEV20 } & & & & & low & avg & high & low & avg & high & low & avg & high & low & avg & high \\
\hline & \multirow{9}{*}{ UDDS } & \multirow{4}{*}{$C D$} & Wh/mile & 268.4 & 268.4 & 254.9 & 238.5 & 274.8 & 241.7 & 213.6 & 269.6 & 224.5 & 188.1 & 258.5 & 211.8 & 181.0 \\
\hline & & & Distance & 24.0 & 24.0 & 23.9 & 24.0 & 23.4 & 24.0 & 24.0 & 23.5 & 24.0 & 23.6 & 23.9 & 23.9 & 23.4 \\
\hline & & & MPGGE Unadjusted & 634.3 & 634.3 & 780.4 & 805.5 & 974.7 & 797.9 & 839.5 & 999.6 & 765.3 & 1063.6 & 793.7 & 713.1 & 1168.2 \\
\hline & & & \begin{tabular}{|l|} 
MPGGE Adjusted \\
\end{tabular} & 223.1 & 223.1 & 242.7 & 245.6 & 262.7 & 244.8 & 249.4 & 264.9 & 240.9 & 270.1 & 244.3 & 234.3 & 277.9 \\
\hline & & \multirow{2}{*}{ CS } & MPGGE Unadjusted & 37.8 & 37.8 & 41.0 & 44.1 & 39.3 & 43.6 & 55.5 & 40.4 & 47.0 & 64.9 & 41.5 & 49.3 & 67.9 \\
\hline & & & MPGGE Adjusted & 28.6 & 28.6 & 30.8 & 33.0 & 29.6 & 32.6 & 40.6 & 30.4 & 34.9 & 46.7 & 31.2 & 36.5 & 48.5 \\
\hline & & \multirow{3}{*}{$C D+C S$} & Wh/mile & 140.1 & 140.1 & 133.6 & 124.9 & 144.0 & 126.6 & 112.6 & 141.2 & 118.2 & 99.5 & 135.5 & 111.9 & 95.7 \\
\hline & & & MPGGE Unadjusted & 64.4 & $\begin{array}{ll}64.4 \\
\end{array}$ & 70.3 & 75.5 & 67.9 & 74.6 & 94.2 & 69.9 & 80.0 & 110.7 & 71.2 & 83.7 & 116.1 \\
\hline & & & MPGGE Adjusted & 46.3 & 46.3 & 50.0 & 53.2 & 48.5 & 52.7 & 64.3 & 49.8 & 56.0 & 73.4 & 50.6 & 58.1 & 76.3 \\
\hline & \multirow{9}{*}{ HWFET } & \multirow{4}{*}{$C D$} & Wh/mile & 302.9 & 302.9 & 271.7 & 252.7 & 299.6 & 259.9 & 202.3 & 294.4 & 216.2 & 183.3 & 290.1 & 195.6 & 184.0 \\
\hline & & & Distance & 21.3 & 21.3 & 22.0 & 22.5 & 21.4 & 22.0 & 24.7 & 21.3 & 24.4 & 23.5 & 21.3 & 24.8 & 22.5 \\
\hline & & & MPGGE Unadjusted & 175.2 & 175.2 & 164.7 & 164.3 & 186.7 & 173.7 & 144.5 & 184.6 & 129.5 & 163.8 & 192.7 & 124.9 & 192.2 \\
\hline & & & MPGGE Adjusted & 120.3 & 120.3 & 113.3 & 113.0 & 127.8 & 119.3 & 99.8 & 126.4 & 89.8 & 112.7 & 131.8 & 86.7 & 131.4 \\
\hline & & \multirow{2}{*}{ CS } & MPGGE Unadjusted & 33.8 & 33.8 & 36.5 & 38.9 & 34.8 & 38.5 & 48.2 & 35.6 & 41.0 & 54.7 & 36.7 & 43.4 & 57.5 \\
\hline & & & MPGGE Adjusted & 23.9 & 23.9 & 25.8 & 27.5 & 24.6 & 27.2 & 34.0 & 25.1 & 28.9 & 38.5 & 25.9 & 30.6 & 40.5 \\
\hline & & \multirow{3}{*}{$C D+C S$} & Wh/mile & 151.8 & 151.8 & 136.8 & 127.6 & 150.1 & 130.9 & 104.0 & 147.6 & 110.8 & 94.4 & 145.3 & 100.7 & 94.7 \\
\hline & & & MPGGE Unadjusted & 49.8 & 49.8 & 52.9 & 55.9 & 51.5 & 55.8 & 66.0 & 52.4 & 56.6 & 74.9 & 54.1 & 58.9 & 80.3 \\
\hline & & & MPGGE Adjusted & 35.1 & 35.1 & 37.3 & 39.4 & 36.3 & 39.3 & 46.4 & 36.9 & 39.8 & 52.5 & 38.1 & 41.5 & 56.3 \\
\hline & \multirow{7}{*}{ Combined } & \multirow{2}{*}{$C D$} & MPGGE Unadjusted & 291.1 & 291.1 & 291.0 & 292.2 & 336.1 & 304.9 & 265.3 & 334.6 & 238.5 & 306.3 & 330.3 & 228.6 & 355.6 \\
\hline & & & MPGGE Adjusted & 161.1 & 161.1 & 160.3 & 160.8 & 178.1 & 166.1 & 149.0 & 177.4 & 137.1 & 165.9 & 176.5 & 132.6 & 185.1 \\
\hline & & \multirow{2}{*}{ CS } & MPGGE Unadjusted & 35.9 & 35.9 & 38.9 & 41.6 & 37.1 & 41.1 & 52.0 & 38.1 & 44.1 & 59.9 & 39.2 & 46.5 & 62.8 \\
\hline & & & MPGGE Adjusted & 26.3 & 26.3 & 28.3 & 30.2 & 27.1 & 29.9 & 37.3 & 27.8 & 31.9 & 42.6 & 28.6 & 33.6 & 44.5 \\
\hline & & \multirow{3}{*}{$C D+C S$} & Wh/mile & 145.4 & 145.4 & 135.0 & 126.1 & 146.7 & 128.5 & 108.8 & 144.1 & 114.9 & 97.2 & 139.9 & 106.9 & 95.3 \\
\hline & & & MPGGE Unadjusted & 56.9 & 56.9 & 61.2 & 65.2 & 59.4 & 64.8 & 79.0 & 60.7 & 67.4 & 91.1 & 62.3 & 70.4 & 96.7 \\
\hline & & & \begin{tabular}{|l} 
MPGGE Adjusted \\
\end{tabular} & 40.5 & 40.5 & 43.4 & 45.9 & 42.1 & 45.7 & 54.8 & 43.0 & 47.3 & 62.3 & 44.1 & 49.2 & 65.8 \\
\hline
\end{tabular}


Argonne National Laboratory Report - Light-Duty Vehicle Fuel Consumption Displacement Potential Up to 2045

\begin{tabular}{|c|c|c|c|c|c|c|c|c|c|c|c|c|c|c|c|c|}
\hline & & & & \multicolumn{13}{|c|}{ Midsize_SUV } \\
\hline & & & & \multirow{2}{*}{$\begin{array}{c}2010 \\
\text { Ref }\end{array}$} & \multicolumn{3}{|c|}{2010} & \multicolumn{3}{|c|}{2015} & \multicolumn{3}{|c|}{2030} & \multicolumn{3}{|c|}{2045} \\
\hline & & & & & low & avg & high & low & avg & high & low & avg & high & low & avg & high \\
\hline \multirow{25}{*}{ E85 Series HEV PHEV30 } & \multirow{9}{*}{ UDDS } & \multirow{4}{*}{$C D$} & Wh/mile & 375.5 & 375.5 & 349.7 & 327.8 & 365.0 & 332.8 & 297.2 & 357.1 & 309.1 & 258.9 & 351.3 & 296.0 & 247.6 \\
\hline & & & Distance & 31.7 & 31.7 & 30.9 & 30.9 & 31.6 & 30.9 & 30.9 & 30.9 & 30.9 & 31.7 & 31.6 & 30.9 & 31.7 \\
\hline & & & MPGGE Unadjusted & $\mathrm{N} / \mathrm{A}$ & $\mathrm{N} / \mathrm{A}$ & N/A & N/A & N/A & $\mathrm{N} / \mathrm{A}$ & N/A & $\mathrm{N} / \mathrm{A}$ & N/A & N/A & N/A & N/A & $\mathrm{N} / \mathrm{A}$ \\
\hline & & & MPGGE Adjusted & $\mathrm{N} / \mathrm{A}$ & $\mathrm{N} / \mathrm{A}$ & $\mathrm{N} / \mathrm{A}$ & N/A & N/A & N/A & N/A & $\mathrm{N} / \mathrm{A}$ & N/A & N/A & N/A & $\mathrm{N} / \mathrm{A}$ & $\mathrm{N} / \mathrm{A}$ \\
\hline & & \multirow{2}{*}{ CS } & MPGGE Unadjusted & 27.3 & 27.3 & 29.9 & 33.0 & 28.4 & 32.2 & 43.2 & 29.8 & 36.4 & 52.3 & 30.8 & 38.3 & 55.4 \\
\hline & & & MPGGE Adjusted & 21.0 & 21.0 & 23.0 & 25.2 & 21.9 & 24.6 & 32.3 & 22.9 & 27.6 & 38.5 & 23.6 & 28.9 & 40.5 \\
\hline & & \multirow{3}{*}{$\mathrm{CD}+\mathrm{CS}$} & Wh/mile & 241.6 & 241.6 & 225.0 & 211.0 & 234.9 & 214.2 & 192.0 & 229.8 & 199.6 & 167.7 & 226.1 & 190.9 & 160.3 \\
\hline & & & MPGGE Unadjusted & 58.0 & 58.0 & 63.7 & 70.1 & 60.4 & 68.4 & 91.8 & 63.5 & 77.4 & 111.4 & 65.5 & 81.4 & 117.9 \\
\hline & & & MPGGE Adjusted & 42.2 & 42.2 & 45.9 & 49.9 & 43.8 & 48.9 & 62.9 & 45.8 & 54.4 & 73.8 & 47.0 & 56.8 & 77.2 \\
\hline & \multirow{9}{*}{ HWFET } & \multirow{4}{*}{$C D$} & Wh/mile & 407.9 & 407.9 & 379.9 & 358.7 & 398.1 & 362.1 & 328.0 & 392.6 & 340.9 & 293.7 & 385.0 & 324.6 & 278.6 \\
\hline & & & \begin{tabular}{|l} 
Distance \\
\end{tabular} & 28.5 & 28.5 & 28.5 & 28.1 & 28.3 & 28.4 & 27.8 & 28.0 & 27.8 & 27.0 & 28.1 & 27.9 & 27.2 \\
\hline & & & MPGGE Unadjusted & $\mathrm{N} / \mathrm{A}$ & $\mathrm{N} / \mathrm{A}$ & $\mathrm{N} / \mathrm{A}$ & $\mathrm{N} / \mathrm{A}$ & N/A & $\mathrm{N} / \mathrm{A}$ & N/A & N/A & $\mathrm{N} / \mathrm{A}$ & $\mathrm{N} / \mathrm{A}$ & $\mathrm{N} / \mathrm{A}$ & $\mathrm{N} / \mathrm{A}$ & $\mathrm{N} / \mathrm{A}$ \\
\hline & & & MPGGE Adjusted & N/A & N/A & N/A & N/A & N/A & N/A & N/A & N/A & N/A & N/A & N/A & N/A & $\mathrm{N} / \mathrm{A}$ \\
\hline & & \multirow{2}{*}{ CS } & MPGGE Unadjusted & 25.4 & 25.4 & 27.9 & 30.1 & 26.5 & 29.9 & 38.5 & 27.6 & 32.6 & 44.6 & 28.5 & 34.8 & 47.1 \\
\hline & & & MPGGE Adjusted & 18.0 & 18.0 & 19.8 & 21.3 & 18.8 & 21.1 & 27.2 & 19.5 & 23.1 & 31.5 & 20.2 & 24.6 & 33.2 \\
\hline & & \multirow{3}{*}{$\mathrm{CD}+\mathrm{CS}$} & Wh/mile & 210.2 & 210.2 & 195.8 & 184.9 & 205.1 & 186.6 & 169.0 & 202.3 & 175.7 & 151.3 & 198.4 & 167.3 & 143.5 \\
\hline & & & MPGGE Unadjusted & 43.1 & 43.1 & 47.3 & 51.1 & 44.9 & 50.6 & 65.3 & 46.7 & 55.2 & 75.6 & 48.3 & 58.9 & 79.8 \\
\hline & & & MPGGE Adjusted & 30.5 & 30.5 & 33.4 & 36.0 & 31.7 & 35.7 & 45.9 & 33.0 & 38.9 & 53.0 & 34.1 & 41.5 & 55.9 \\
\hline & \multirow{7}{*}{ Combined } & \multirow{2}{*}{$C D$} & MPGGE Unadjusted & N/A & $\mathrm{N} / \mathrm{A}$ & $\mathrm{N} / \mathrm{A}$ & N/A & $\mathrm{N} / \mathrm{A}$ & $\mathrm{N} / \mathrm{A}$ & $\mathrm{N} / \mathrm{A}$ & $\mathrm{N} / \mathrm{A}$ & $\mathrm{N} / \mathrm{A}$ & N/A & $\mathrm{N} / \mathrm{A}$ & $\mathrm{N} / \mathrm{A}$ & $\mathrm{N} / \mathrm{A}$ \\
\hline & & & MPGGE Adjusted & N/A & N/A & N/A & N/A & N/A & N/A & N/A & N/A & N/A & N/A & N/A & N/A & N/A \\
\hline & & \multirow{2}{*}{ CS } & MPGGE Unadjusted & 26.4 & 26.4 & 29.0 & 31.6 & 27.5 & 31.1 & 40.9 & 28.8 & 34.6 & 48.5 & 29.7 & 36.6 & 51.3 \\
\hline & & & MPGGE Adjusted & 19.6 & 19.6 & 21.4 & 23.3 & 20.4 & 22.9 & 29.8 & 21.3 & 25.4 & 35.0 & 21.9 & 26.8 & 36.9 \\
\hline & & \multirow{3}{*}{$\mathrm{CD}+\mathrm{CS}$} & Wh/mile & 227.5 & 227.5 & 211.9 & 199.2 & 221.5 & 201.8 & 181.6 & 217.4 & 188.8 & 160.3 & 213.6 & 180.3 & 152.8 \\
\hline & & & MPGGE Unadjusted & 50.2 & 50.2 & 55.1 & 60.0 & 52.3 & 59.1 & 77.6 & 54.7 & 65.6 & 91.8 & 56.4 & 69.5 & 97.0 \\
\hline & & & MPGGE Adjusted & 36.0 & 36.0 & 39.2 & 42.5 & 37.4 & 41.9 & 53.9 & 39.0 & 46.1 & 62.7 & 40.1 & 48.7 & 65.9 \\
\hline
\end{tabular}




\begin{tabular}{|c|c|c|c|c|c|c|c|c|c|c|c|c|c|c|c|c|}
\hline & & & & \multicolumn{13}{|c|}{ Midsize_SUV } \\
\hline & & & & \multirow{2}{*}{$\begin{array}{c}2010 \\
\text { Ref }\end{array}$} & \multicolumn{3}{|c|}{2010} & \multicolumn{3}{|c|}{2015} & \multicolumn{3}{|c|}{2030} & \multicolumn{3}{|c|}{2045} \\
\hline \multirow{26}{*}{ E85 Series HEV PHEV40 } & & & & & low & avg & high & low & avg & high & low & avg & high & low & avg & high \\
\hline & \multirow{9}{*}{ UDDS } & \multirow{4}{*}{$C D$} & Wh/mile & 383.7 & 383.7 & 356.9 & 334.4 & 372.6 & 339.6 & 302.8 & 364.3 & 315.1 & 261.8 & 357.9 & 300.9 & 250.1 \\
\hline & & & Distance & 40.7 & 40.7 & 40.6 & 40.5 & 40.6 & 40.6 & 40.8 & 40.6 & 40.6 & 40.4 & 40.6 & 40.5 & 40.4 \\
\hline & & & MPGGE Unadjusted & N/A & N/A & N/A & N/A & N/A & N/A & N/A & N/A & N/A & N/A & N/A & N/A & N/A \\
\hline & & & MPGGE Adjusted & N/A & N/A & N/A & N/A & N/A & $\mathrm{N} / \mathrm{A}$ & N/A & N/A & N/A & N/A & N/A & N/A & $\mathrm{N} / \mathrm{A}$ \\
\hline & & \multirow{2}{*}{ CS } & MPGGE Unadjusted & 26.4 & 26.4 & 29.2 & 32.0 & 27.8 & 31.4 & 42.4 & 29.1 & 35.5 & 51.6 & 30.1 & 37.7 & 55.1 \\
\hline & & & MPGGE Adjusted & 20.4 & 20.4 & 22.5 & 24.5 & 21.4 & 24.0 & 31.8 & 22.4 & 27.0 & 38.0 & 23.1 & 28.5 & 40.3 \\
\hline & & \multirow{3}{*}{$C D+C S$} & Wh/mile & 281.6 & 281.6 & 261.9 & 245.5 & 273.4 & 249.4 & 223.7 & 267.4 & 232.6 & 193.4 & 262.7 & 221.8 & 184.7 \\
\hline & & & MPGGE Unadjusted & 66.0 & 66.0 & 73.0 & 79.9 & 69.5 & 78.5 & 106.0 & 72.9 & 88.8 & 129.0 & 75.3 & 94.2 & 137.7 \\
\hline & & & MPGGE Adjusted & 47.3 & 47.3 & 51.7 & 55.9 & 49.5 & 55.1 & 70.8 & 51.6 & 61.2 & 83.0 & 53.1 & 64.3 & 87.3 \\
\hline & \multirow{9}{*}{ HWFET } & \multirow{4}{*}{$C D$} & Wh/mile & 411.7 & 411.7 & 383.1 & 361.6 & 401.4 & 365.0 & 329.7 & 395.8 & 342.8 & 295.6 & 387.7 & 326.0 & 280.3 \\
\hline & & & Distance & 37.6 & 37.6 & 37.6 & 37.3 & 37.4 & 37.6 & 37.2 & 37.2 & 37.1 & 35.9 & 37.3 & 37.4 & 36.1 \\
\hline & & & MPGGE Unadjusted & N/A & N/A & N/A & N/A & N/A & N/A & N/A & N/A & N/A & N/A & N/A & N/A & $\mathrm{N} / \mathrm{A}$ \\
\hline & & & MPGGE Adjusted & $\mathrm{N} / \mathrm{A}$ & N/A & N/A & N/A & N/A & N/A & N/A & $\mathrm{N} / \mathrm{A}$ & $\mathrm{N} / \mathrm{A}$ & $\mathrm{N} / \mathrm{A}$ & $\mathrm{N} / \mathrm{A}$ & N/A & N/A \\
\hline & & \multirow{2}{*}{ CS } & MPGGE Unadjusted & 25.1 & 25.1 & 27.5 & 29.7 & 26.1 & 29.4 & 38.0 & 27.2 & 32.2 & 44.2 & 28.1 & 34.4 & 46.7 \\
\hline & & & MPGGE Adjusted & 17.8 & 17.8 & 19.5 & 21.1 & 18.5 & 20.8 & 26.9 & 19.3 & 22.8 & 31.2 & 19.9 & 24.4 & 33.0 \\
\hline & & \multirow{3}{*}{$C D+C S$} & Wh/mile & 278.0 & 278.0 & 258.6 & 244.1 & 271.0 & 246.5 & 222.6 & 267.2 & 231.5 & 199.7 & 261.8 & 220.1 & 189.3 \\
\hline & & & MPGGE Unadjusted & 54.5 & 54.5 & 59.8 & 64.6 & 56.7 & 64.0 & 82.7 & 59.1 & 70.1 & 96.1 & 61.1 & 74.8 & 101.6 \\
\hline & & & MPGGE Adjusted & 38.4 & 38.4 & 42.1 & 45.4 & 39.9 & 45.0 & 57.9 & 41.6 & 49.2 & 67.1 & 43.0 & 52.5 & 70.8 \\
\hline & \multirow{7}{*}{ Combined } & \multirow{2}{*}{$C D$} & MPGGE Unadjusted & N/A & $\mathrm{N} / \mathrm{A}$ & N/A & N/A & $\mathrm{N} / \mathrm{A}$ & N/A & $\mathrm{N} / \mathrm{A}$ & $\mathrm{N} / \mathrm{A}$ & $\mathrm{N} / \mathrm{A}$ & N/A & N/A & $\mathrm{N} / \mathrm{A}$ & N/A \\
\hline & & & MPGGE Adjusted & N/A & $\mathrm{N} / \mathrm{A}$ & N/A & $\mathrm{N} / \mathrm{A}$ & $\mathrm{N} / \mathrm{A}$ & N/A & N/A & $\mathrm{N} / \mathrm{A}$ & N/A & N/A & N/A & $\mathrm{N} / \mathrm{A}$ & N/A \\
\hline & & \multirow{2}{*}{ CS } & MPGGE Unadjusted & 25.8 & 25.8 & 28.4 & 30.9 & 27.0 & 30.5 & 40.3 & 28.2 & 34.0 & 48.0 & 29.2 & 36.1 & 51.0 \\
\hline & & & MPGGE Adjusted & 19.1 & 19.1 & 21.0 & 22.8 & 20.0 & 22.5 & 29.4 & 20.9 & 24.9 & 34.6 & 21.6 & 26.5 & 36.6 \\
\hline & & \multirow{3}{*}{$C D+C S$} & Wh/mile & 279.9 & 279.9 & 260.4 & 244.9 & 272.3 & 248.1 & 223.2 & 267.3 & 232.1 & 196.2 & 262.3 & 221.0 & 186.8 \\
\hline & & & MPGGE Unadjusted & 60.2 & 60.2 & 66.4 & 72.2 & 63.1 & 71.2 & 94.0 & 66.0 & 79.3 & 111.8 & 68.2 & 84.4 & 118.7 \\
\hline & & & \begin{tabular}{|l} 
MPGGE Adjusted \\
\end{tabular} & 42.8 & 42.8 & 46.9 & 50.7 & 44.7 & 50.0 & 64.3 & 46.6 & 55.1 & 75.0 & 48.0 & 58.4 & 79.0 \\
\hline
\end{tabular}


Argonne National Laboratory Report - Light-Duty Vehicle Fuel Consumption Displacement Potential Up to 2045

\begin{tabular}{|c|c|c|c|c|c|c|c|c|c|c|c|c|c|c|c|c|}
\hline & & & & \multicolumn{13}{|c|}{ Midsize_SUV } \\
\hline & & & & \multirow{2}{*}{$\begin{array}{c}2010 \\
\text { Ref }\end{array}$} & \multicolumn{3}{|c|}{2010} & \multicolumn{3}{|c|}{2015} & \multicolumn{3}{|c|}{2030} & \multicolumn{3}{|c|}{2045} \\
\hline & & & & & low & avg & high & low & avg & high & low & avg & high & low & avg & high \\
\hline \multirow{25}{*}{ FC HEV PHEV10 } & \multirow{9}{*}{ UDDS } & \multirow{4}{*}{$C D$} & Wh/mile & 380.7 & 380.7 & 331.4 & 298.2 & 351.5 & 302.1 & 271.0 & 331.7 & 282.2 & 231.6 & 317.2 & 269.3 & 220.9 \\
\hline & & & Distance & 11.3 & 11.3 & 11.1 & 10.2 & 10.3 & 10.2 & 10.2 & 11.1 & 10.1 & 10.1 & 10.2 & 10.2 & 10.1 \\
\hline & & & MPGGE Unadjusted & $\mathrm{N} / \mathrm{A}$ & $\mathrm{N} / \mathrm{A}$ & N/A & N/A & N/A & $\mathrm{N} / \mathrm{A}$ & N/A & $\mathrm{N} / \mathrm{A}$ & N/A & N/A & N/A & $\mathrm{N} / \mathrm{A}$ & N/A \\
\hline & & & MPGGE Adjusted & $\mathrm{N} / \mathrm{A}$ & $\mathrm{N} / \mathrm{A}$ & $\mathrm{N} / \mathrm{A}$ & N/A & N/A & N/A & N/A & $\mathrm{N} / \mathrm{A}$ & N/A & N/A & N/A & $\mathrm{N} / \mathrm{A}$ & N/A \\
\hline & & \multirow{2}{*}{ CS } & MPGGE Unadjusted & 43.2 & 43.2 & 51.4 & 60.1 & 51.0 & 59.4 & 67.7 & 54.1 & 65.1 & 79.8 & 56.6 & 68.1 & 83.7 \\
\hline & & & MPGGE Adjusted & 32.3 & 32.3 & 37.9 & 43.6 & 37.6 & 43.1 & 48.4 & 39.7 & 46.8 & 55.9 & 41.3 & 48.6 & 58.2 \\
\hline & & \multirow{3}{*}{$\mathrm{CD}+\mathrm{CS}$} & Wh/mile & 79.3 & 79.3 & 69.0 & 62.1 & 73.2 & 62.9 & 56.5 & 69.1 & 58.8 & 48.3 & 66.1 & 56.1 & 46.0 \\
\hline & & & MPGGE Unadjusted & 52.1 & 52.1 & 62.0 & 72.4 & 61.5 & 71.5 & 81.6 & 65.2 & 78.4 & 96.2 & 68.2 & 82.0 & 100.9 \\
\hline & & & MPGGE Adjusted & 38.3 & 38.3 & 44.8 & 51.3 & 44.5 & 50.8 & 56.9 & 46.8 & 55.0 & 65.4 & 48.7 & 57.2 & 68.0 \\
\hline & \multirow{9}{*}{ HWFET } & \multirow{4}{*}{$C D$} & Wh/mile & 390.8 & 390.8 & 341.3 & 308.6 & 362.1 & 312.0 & 277.3 & 343.2 & 288.1 & 237.7 & 328.4 & 276.6 & 226.6 \\
\hline & & & Distance & 9.2 & 9.2 & 9.3 & 9.2 & 9.3 & 9.2 & 8.5 & 9.3 & 8.7 & 8.3 & 10.0 & 8.5 & 8.3 \\
\hline & & & MPGGE Unadjusted & 402.5 & 402.5 & 388.7 & 391.1 & 394.3 & 391.4 & 385.9 & 361.7 & 370.8 & 355.3 & 358.2 & 412.2 & 386.0 \\
\hline & & & MPGGE Adjusted & 263.6 & 263.6 & 255.2 & 256.7 & 258.7 & 256.9 & 253.6 & 238.8 & 244.4 & 234.9 & 236.7 & 269.4 & 253.6 \\
\hline & & \multirow{2}{*}{ CS } & MPGGE Unadjusted & 42.6 & 42.6 & 49.3 & 56.4 & 49.1 & 55.8 & 61.9 & 50.9 & 59.5 & 69.5 & 52.7 & 62.7 & 73.4 \\
\hline & & & MPGGE Adjusted & 30.1 & 30.1 & 34.8 & 39.7 & 34.6 & 39.3 & 43.5 & 35.9 & 41.9 & 48.8 & 37.2 & 44.1 & 51.5 \\
\hline & & \multirow{3}{*}{$\mathrm{CD}+\mathrm{CS}$} & Wh/mile & 114.9 & 114.9 & 100.4 & 90.8 & 106.5 & 91.8 & 81.6 & 101.0 & 84.7 & 69.9 & 96.6 & 81.4 & 66.6 \\
\hline & & & MPGGE Unadjusted & 54.2 & 54.2 & 62.4 & 71.0 & 62.2 & 70.3 & 77.5 & 64.1 & 74.5 & 86.1 & 66.3 & 78.7 & 91.1 \\
\hline & & & MPGGE Adjusted & 38.2 & 38.2 & 43.9 & 49.8 & 43.7 & 49.4 & 54.3 & 45.1 & 52.3 & 60.3 & 46.6 & 55.2 & 63.7 \\
\hline & \multirow{7}{*}{ Combined } & \multirow{2}{*}{$C D$} & MPGGE Unadjusted & N/A & N/A & $\mathrm{N} / \mathrm{A}$ & N/A & $\mathrm{N} / \mathrm{A}$ & N/A & $\mathrm{N} / \mathrm{A}$ & $\mathrm{N} / \mathrm{A}$ & $\mathrm{N} / \mathrm{A}$ & $\mathrm{N} / \mathrm{A}$ & $\mathrm{N} / \mathrm{A}$ & N/A & $\mathrm{N} / \mathrm{A}$ \\
\hline & & & MPGGE Adjusted & N/A & N/A & N/A & N/A & N/A & N/A & N/A & N/A & N/A & N/A & N/A & N/A & N/A \\
\hline & & \multirow{2}{*}{ CS } & MPGGE Unadjusted & 42.9 & 42.9 & 50.5 & 58.4 & 50.2 & 57.7 & 64.9 & 52.6 & 62.5 & 74.8 & 54.8 & 65.5 & 78.7 \\
\hline & & & MPGGE Adjusted & 31.3 & 31.3 & 36.4 & 41.8 & 36.2 & 41.3 & 46.1 & 37.9 & 44.4 & 52.4 & 39.3 & 46.5 & 55.0 \\
\hline & & \multirow{3}{*}{$\mathrm{CD}+\mathrm{CS}$} & Wh/mile & 95.3 & 95.3 & 83.1 & 75.0 & 88.2 & 75.9 & 67.7 & 83.4 & 70.5 & 58.0 & 79.8 & 67.5 & 55.3 \\
\hline & & & MPGGE Unadjusted & 53.0 & 53.0 & 62.2 & 71.8 & 61.8 & 71.0 & 79.7 & 64.7 & 76.6 & 91.4 & 67.3 & 80.5 & 96.2 \\
\hline & & & MPGGE Adjusted & 38.2 & 38.2 & 44.4 & 50.6 & 44.1 & 50.1 & 55.7 & 46.0 & 53.7 & 63.0 & 47.7 & 56.2 & 66.0 \\
\hline
\end{tabular}




\begin{tabular}{|c|c|c|c|c|c|c|c|c|c|c|c|c|c|c|c|c|}
\hline & & & & \multicolumn{13}{|c|}{ Midsize_SUV } \\
\hline & & & & \multirow{2}{*}{$\begin{array}{l}2010 \\
\text { Ref } \\
\end{array}$} & \multicolumn{3}{|c|}{2010} & \multicolumn{3}{|c|}{2015} & \multicolumn{3}{|c|}{2030} & \multicolumn{3}{|c|}{2045} \\
\hline \multirow{26}{*}{ FC HEV PHEV 20} & & \multirow{5}{*}{$C D$} & & & low & avg & high & low & avg & high & low & avg & high & low & avg & high \\
\hline & \multirow{9}{*}{ UDDS } & & Wh/mile & 387.4 & 387.4 & 336.8 & 302.5 & 357.2 & 306.4 & 271.1 & 336.7 & 283.1 & 229.6 & 321.4 & 269.5 & 218.8 \\
\hline & & & Distance & 20.2 & 20.2 & 20.2 & 20.2 & 20.2 & 20.2 & 20.5 & 20.6 & 20.5 & 20.3 & 20.2 & 20.6 & 20.4 \\
\hline & & & MPGGE Unadjusted & $\mathrm{N} / \mathrm{A}$ & $\mathrm{N} / \mathrm{A}$ & $\mathrm{N} / \mathrm{A}$ & $\mathrm{N} / \mathrm{A}$ & $\mathrm{N} / \mathrm{A}$ & $\mathrm{N} / \mathrm{A}$ & $\mathrm{N} / \mathrm{A}$ & $\mathrm{N} / \mathrm{A}$ & $\mathrm{N} / \mathrm{A}$ & N/A & $\mathrm{N} / \mathrm{A}$ & $\mathrm{N} / \mathrm{A}$ & $\mathrm{N} / \mathrm{A}$ \\
\hline & & & MPGGE Adjusted & $\mathrm{N} / \mathrm{A}$ & $\mathrm{N} / \mathrm{A}$ & $\mathrm{N} / \mathrm{A}$ & $\mathrm{N} / \mathrm{A}$ & $\mathrm{N} / \mathrm{A}$ & $\mathrm{N} / \mathrm{A}$ & N/A & $\mathrm{N} / \mathrm{A}$ & $\mathrm{N} / \mathrm{A}$ & $\mathrm{N} / \mathrm{A}$ & $\mathrm{N} / \mathrm{A}$ & $\mathrm{N} / \mathrm{A}$ & $\mathrm{N} / \mathrm{A}$ \\
\hline & & \multirow{2}{*}{ CS } & MPGGE Unadjusted & 42.4 & 42.4 & 50.6 & 59.2 & 50.2 & 58.5 & 67.1 & 53.3 & 64.3 & 79.1 & 55.9 & 67.4 & 83.1 \\
\hline & & & MPGGE Adjusted & 31.8 & 31.8 & 37.3 & 43.0 & 37.1 & 42.6 & 48.0 & 39.1 & 46.3 & 55.4 & 40.8 & 48.2 & 57.8 \\
\hline & & \multirow{3}{*}{$C D+C S$} & Wh/mile & 147.2 & 147.2 & 127.9 & 114.9 & 135.7 & 116.4 & 103.2 & 127.9 & 107.7 & 87.4 & 122.1 & 102.5 & 83.2 \\
\hline & & & MPGGE Unadjusted & 62.4 & 62.4 & 74.3 & 87.1 & 73.8 & 86.1 & 98.7 & 78.4 & 94.6 & 116.4 & 82.2 & 99.1 & 122.2 \\
\hline & & & MPGGE Adjusted & 45.0 & 45.0 & 52.5 & 60.1 & 52.2 & 59.5 & 66.8 & 55.0 & 64.5 & 76.4 & 57.3 & 67.0 & 79.5 \\
\hline & \multirow{9}{*}{ HWFET } & \multirow{4}{*}{$C D$} & Wh/mile & 440.6 & 440.6 & 394.1 & 362.8 & 416.9 & 366.1 & 330.6 & 402.6 & 344.2 & 296.5 & 388.2 & 325.9 & 280.6 \\
\hline & & & Distance & 19.5 & 19.5 & 17.1 & 16.7 & 18.5 & 16.8 & 16.6 & 16.7 & 16.6 & 15.7 & 16.6 & 16.7 & 15.8 \\
\hline & & & MPGGE Unadjusted & N/A & N/A & N/A & N/A & N/A & N/A & $\mathrm{N} / \mathrm{A}$ & N/A & N/A & N/A & N/A & N/A & $\mathrm{N} / \mathrm{A}$ \\
\hline & & & MPGGE Adjusted & N/A & $\mathrm{N} / \mathrm{A}$ & N/A & N/A & N/A & N/A & $\mathrm{N} / \mathrm{A}$ & N/A & $\mathrm{N} / \mathrm{A}$ & $\mathrm{N} / \mathrm{A}$ & $\mathrm{N} / \mathrm{A}$ & N/A & N/A \\
\hline & & \multirow{2}{*}{ CS } & MPGGE Unadjusted & 42.1 & 42.1 & 48.8 & 55.9 & 48.6 & 55.4 & 61.4 & 50.4 & 59.1 & 69.2 & 52.3 & 62.4 & 73.1 \\
\hline & & & MPGGE Adjusted & 29.7 & 29.7 & 34.4 & 39.4 & 34.3 & 39.0 & 43.2 & 35.6 & 41.6 & 48.6 & 36.9 & 43.8 & 51.3 \\
\hline & & \multirow{3}{*}{$C D+C S$} & Wh/mile & 129.6 & 129.6 & 115.9 & 106.7 & 122.6 & 107.7 & 97.3 & 118.4 & 101.2 & 87.2 & 114.2 & 95.9 & 82.5 \\
\hline & & & MPGGE Unadjusted & 55.4 & 55.4 & 64.3 & 73.6 & 64.0 & 72.9 & 80.8 & 66.4 & 77.8 & 91.0 & 68.8 & 82.0 & 96.2 \\
\hline & & & MPGGE Adjusted & 39.0 & 39.0 & 45.2 & 51.6 & 45.0 & 51.1 & 56.6 & 46.6 & 54.5 & 63.6 & 48.3 & 57.4 & 67.1 \\
\hline & \multirow{7}{*}{ Combined } & \multirow{2}{*}{$C D$} & MPGGE Unadjusted & $\mathrm{N} / \mathrm{A}$ & N/A & N/A & $\mathrm{N} / \mathrm{A}$ & N/A & $\mathrm{N} / \mathrm{A}$ & $\mathrm{N} / \mathrm{A}$ & $\mathrm{N} / \mathrm{A}$ & $\mathrm{N} / \mathrm{A}$ & $\mathrm{N} / \mathrm{A}$ & N/A & $\mathrm{N} / \mathrm{A}$ & N/A \\
\hline & & & MPGGE Adjusted & $\mathrm{N} / \mathrm{A}$ & $\mathrm{N} / \mathrm{A}$ & N/A & $\mathrm{N} / \mathrm{A}$ & $\mathrm{N} / \mathrm{A}$ & N/A & $\mathrm{N} / \mathrm{A}$ & $\mathrm{N} / \mathrm{A}$ & $\mathrm{N} / \mathrm{A}$ & $\mathrm{N} / \mathrm{A}$ & $\mathrm{N} / \mathrm{A}$ & $\mathrm{N} / \mathrm{A}$ & N/A \\
\hline & & \multirow{2}{*}{ CS } & MPGGE Unadjusted & 42.3 & 42.3 & 49.8 & 57.7 & 49.5 & 57.1 & 64.4 & 52.0 & 61.9 & 74.3 & 54.2 & 65.0 & 78.3 \\
\hline & & & MPGGE Adjusted & 30.8 & 30.8 & 36.0 & 41.3 & 35.8 & 40.9 & 45.7 & 37.4 & 44.0 & 52.1 & 38.9 & 46.1 & 54.7 \\
\hline & & \multirow{3}{*}{$C D+C S$} & Wh/mile & 139.3 & 139.3 & 122.5 & 111.2 & 129.8 & 112.5 & 100.5 & 123.6 & 104.8 & 87.3 & 118.5 & 99.5 & 82.9 \\
\hline & & & MPGGE Unadjusted & 59.0 & 59.0 & 69.4 & 80.4 & 69.0 & 79.6 & 89.7 & 72.5 & 86.2 & 103.4 & 75.6 & 90.6 & 108.9 \\
\hline & & & MPGGE Adjusted & 42.1 & 42.1 & 48.9 & 56.0 & 48.7 & 55.4 & $\begin{array}{l}61.8 \\
\end{array}$ & 50.9 & 59.6 & 70.1 & 52.9 & 62.3 & 73.4 \\
\hline
\end{tabular}


Argonne National Laboratory Report - Light-Duty Vehicle Fuel Consumption Displacement Potential Up to 2045

\begin{tabular}{|c|c|c|c|c|c|c|c|c|c|c|c|c|c|c|c|c|}
\hline & & & & \multicolumn{13}{|c|}{ Midsize_SUV } \\
\hline & & & & \multirow{2}{*}{$\begin{array}{c}2010 \\
\text { Ref }\end{array}$} & \multicolumn{3}{|c|}{2010} & \multicolumn{3}{|c|}{2015} & \multicolumn{3}{|c|}{2030} & \multicolumn{3}{|c|}{2045} \\
\hline & & & & & low & avg & high & low & avg & high & low & avg & high & low & avg & high \\
\hline \multirow{25}{*}{ FC HEV PHEV30 } & \multirow{9}{*}{ UDDS } & \multirow{4}{*}{$C D$} & Wh/mile & 374.6 & 374.6 & 328.2 & 300.5 & 348.1 & 302.8 & 265.1 & 332.5 & 276.8 & 229.2 & 317.2 & 262.7 & 218.6 \\
\hline & & & Distance & 33.6 & 33.6 & 32.0 & 32.1 & 33.6 & 33.5 & 32.0 & 32.1 & 32.0 & 32.0 & 32.0 & 32.0 & 32.0 \\
\hline & & & MPGGE Unadjusted & $\mathrm{N} / \mathrm{A}$ & $\mathrm{N} / \mathrm{A}$ & N/A & N/A & N/A & $\mathrm{N} / \mathrm{A}$ & N/A & $\mathrm{N} / \mathrm{A}$ & N/A & N/A & N/A & N/A & $\mathrm{N} / \mathrm{A}$ \\
\hline & & & MPGGE Adjusted & $\mathrm{N} / \mathrm{A}$ & $\mathrm{N} / \mathrm{A}$ & $\mathrm{N} / \mathrm{A}$ & N/A & N/A & N/A & N/A & $\mathrm{N} / \mathrm{A}$ & N/A & N/A & N/A & $\mathrm{N} / \mathrm{A}$ & $\mathrm{N} / \mathrm{A}$ \\
\hline & & \multirow{2}{*}{ CS } & MPGGE Unadjusted & 42.1 & 42.1 & 49.7 & 57.2 & 49.4 & 56.8 & 65.4 & 51.7 & 62.7 & 76.7 & 54.3 & 66.0 & 80.5 \\
\hline & & & MPGGE Adjusted & 31.6 & 31.6 & 36.8 & 41.7 & 36.5 & 41.4 & 47.0 & 38.1 & 45.3 & 54.0 & 39.8 & 47.3 & 56.2 \\
\hline & & \multirow{3}{*}{$\mathrm{CD}+\mathrm{CS}$} & Wh/mile & 238.7 & 238.7 & 209.1 & 191.5 & 221.8 & 192.9 & 169.4 & 211.8 & 176.9 & 147.1 & 202.1 & 167.9 & 140.3 \\
\hline & & & MPGGE Unadjusted & 89.5 & 89.5 & 105.8 & 121.7 & 105.1 & 120.8 & 139.2 & 110.1 & 133.4 & 163.2 & 115.4 & 140.5 & 171.3 \\
\hline & & & MPGGE Adjusted & 61.6 & 61.6 & 70.8 & 79.2 & 70.4 & 78.7 & 88.0 & 73.1 & 85.2 & 99.4 & 75.9 & 88.7 & 103.0 \\
\hline & \multirow{9}{*}{ HWFET } & \multirow{4}{*}{$C D$} & Wh/mile & 422.0 & 422.0 & 378.5 & 352.5 & 400.3 & 354.2 & 319.4 & 389.3 & 332.5 & 286.1 & 374.7 & 314.5 & 270.9 \\
\hline & & & \begin{tabular}{|l} 
Distance \\
\end{tabular} & 27.1 & 27.1 & 26.5 & 28.8 & 28.4 & 26.2 & 28.8 & 28.8 & 25.7 & 25.0 & 28.8 & 25.7 & 25.1 \\
\hline & & & MPGGE Unadjusted & $\mathrm{N} / \mathrm{A}$ & $\mathrm{N} / \mathrm{A}$ & $\mathrm{N} / \mathrm{A}$ & $\mathrm{N} / \mathrm{A}$ & N/A & $\mathrm{N} / \mathrm{A}$ & N/A & $\mathrm{N} / \mathrm{A}$ & N/A & $\mathrm{N} / \mathrm{A}$ & $\mathrm{N} / \mathrm{A}$ & $\mathrm{N} / \mathrm{A}$ & $\mathrm{N} / \mathrm{A}$ \\
\hline & & & MPGGE Adjusted & N/A & N/A & N/A & N/A & N/A & N/A & N/A & N/A & N/A & N/A & N/A & N/A & $\mathrm{N} / \mathrm{A}$ \\
\hline & & \multirow{2}{*}{ CS } & MPGGE Unadjusted & 41.2 & 41.2 & 47.6 & 53.9 & 47.4 & 53.6 & 59.7 & 48.8 & 57.4 & 67.1 & 50.7 & 60.7 & 70.9 \\
\hline & & & MPGGE Adjusted & 29.1 & 29.1 & 33.6 & 37.9 & 33.4 & 37.7 & 42.0 & 34.4 & 40.4 & 47.1 & 35.7 & 42.7 & 49.7 \\
\hline & & \multirow{3}{*}{$\mathrm{CD}+\mathrm{CS}$} & Wh/mile & 217.2 & 217.2 & 194.7 & 181.4 & 206.0 & 182.3 & 164.5 & 200.4 & 171.2 & 147.4 & 192.8 & 161.9 & 139.6 \\
\hline & & & MPGGE Unadjusted & 69.9 & 69.9 & 80.7 & 91.3 & 80.3 & 90.8 & 101.2 & 82.7 & 97.3 & 113.7 & 85.9 & 102.8 & 120.1 \\
\hline & & & MPGGE Adjusted & 49.1 & 49.1 & 56.5 & 63.8 & 56.3 & 63.5 & 70.6 & 57.9 & 67.9 & 79.1 & 60.1 & 71.7 & 83.4 \\
\hline & \multirow{7}{*}{ Combined } & \multirow{2}{*}{$C D$} & MPGGE Unadjusted & $\mathrm{N} / \mathrm{A}$ & $\mathrm{N} / \mathrm{A}$ & N/A & N/A & N/A & $\mathrm{N} / \mathrm{A}$ & N/A & $\mathrm{N} / \mathrm{A}$ & $\mathrm{N} / \mathrm{A}$ & N/A & $\mathrm{N} / \mathrm{A}$ & N/A & $\mathrm{N} / \mathrm{A}$ \\
\hline & & & MPGGE Adjusted & N/A & N/A & N/A & N/A & N/A & N/A & N/A & N/A & N/A & N/A & N/A & N/A & N/A \\
\hline & & \multirow{2}{*}{ CS } & MPGGE Unadjusted & 41.7 & 41.7 & 48.8 & 55.6 & 48.5 & 55.3 & 62.7 & 50.4 & 60.2 & 72.0 & 52.6 & 63.5 & 75.8 \\
\hline & & & MPGGE Adjusted & 30.4 & 30.4 & 35.3 & 39.9 & 35.1 & 39.7 & 44.6 & 36.3 & 42.9 & 50.6 & 37.8 & 45.1 & 53.1 \\
\hline & & \multirow{3}{*}{$\mathrm{CD}+\mathrm{CS}$} & Wh/mile & 229.0 & 229.0 & 202.6 & 186.9 & 214.6 & 188.1 & 167.2 & 206.7 & 174.3 & 147.2 & 197.9 & 165.2 & 140.0 \\
\hline & & & MPGGE Unadjusted & 79.5 & 79.5 & 92.8 & 105.8 & 92.3 & 105.2 & 119.1 & 95.8 & 114.3 & 136.5 & 99.9 & 120.6 & 143.7 \\
\hline & & & MPGGE Adjusted & 55.2 & 55.2 & 63.6 & 71.4 & 63.2 & 71.0 & 79.2 & 65.3 & 76.4 & 89.1 & 67.9 & 80.1 & 93.1 \\
\hline
\end{tabular}




\begin{tabular}{|c|c|c|c|c|c|c|c|c|c|c|c|c|c|c|c|c|}
\hline & & & & \multicolumn{13}{|c|}{ Midsize_SUV } \\
\hline & & & & 2010 & & 2010 & & & 2015 & & & 2030 & & & 2045 & \\
\hline \multirow{26}{*}{ FC HEV PHEV40 } & & \multirow{5}{*}{$C D$} & & Ref & low & avg & high & low & avg & high & low & avg & high & low & avg & high \\
\hline & \multirow{9}{*}{ UDDS } & & Wh/mile & 382.4 & 382.4 & 334.1 & 305.5 & 354.3 & 307.5 & 267.8 & 338.0 & 279.6 & 232.9 & 322.1 & 265.1 & 222.4 \\
\hline & & & Distance & 41.8 & 41.8 & 41.3 & 41.2 & 41.3 & 41.1 & 41.0 & 41.3 & 41.0 & 40.7 & 41.2 & 41.0 & 40.5 \\
\hline & & & MPGGE Unadjusted & $\mathrm{N} / \mathrm{A}$ & N/A & N/A & N/A & $N / A$ & $N / A$ & N/A & N/A & N/A & N/A & N/A & $\mathrm{N} / \mathrm{A}$ & N/A \\
\hline & & & MPGGE Adjusted & $\mathrm{N} / \mathrm{A}$ & $\mathrm{N} / \mathrm{A}$ & N/A & N/A & N/A & N/A & N/A & $\mathrm{N} / \mathrm{A}$ & N/A & $\mathrm{N} / \mathrm{A}$ & $\mathrm{N} / \mathrm{A}$ & N/A & $\mathrm{N} / \mathrm{A}$ \\
\hline & & \multirow{2}{*}{ CS } & MPGGE Unadjusted & 41.2 & 41.2 & 48.8 & 56.2 & 48.5 & 55.9 & 64.6 & 50.9 & 61.9 & 75.8 & 53.4 & 65.2 & 79.7 \\
\hline & & & MPGGE Adjusted & 31.0 & 31.0 & 36.2 & 41.1 & 35.9 & 40.8 & 46.4 & 37.5 & 44.7 & 53.4 & 39.2 & 46.9 & 55.8 \\
\hline & & \multirow{3}{*}{$\mathrm{CD}+\mathrm{CS}$} & Wh/mile & 276.4 & 276.4 & 241.5 & 220.8 & 256.1 & 222.3 & 194.2 & 244.3 & 202.8 & 170.5 & 232.9 & 192.3 & 163.0 \\
\hline & & & MPGGE Unadjusted & 103.0 & 103.0 & 122.1 & 140.6 & 121.2 & 139.6 & 161.4 & 127.1 & 154.8 & 189.5 & 133.5 & 163.1 & 199.2 \\
\hline & & & MPGGE Adjusted & 69.2 & 69.2 & 79.4 & 88.7 & 79.0 & 88.3 & 98.6 & 82.0 & 95.5 & 110.9 & 85.2 & 99.3 & 114.9 \\
\hline & \multirow{9}{*}{ HWFET } & \multirow{4}{*}{$C D$} & Wh/mile & 427.9 & 427.9 & 382.9 & 356.2 & 405.0 & 357.8 & 322.1 & 393.6 & 335.2 & 287.4 & 378.5 & 316.7 & 271.9 \\
\hline & & & Distance & 38.7 & 38.7 & 38.6 & 38.6 & 38.6 & 38.6 & 36.0 & 39.0 & 37.6 & 33.5 & 39.0 & 36.8 & 33.7 \\
\hline & & & MPGGE Unadjusted & $\mathrm{N} / \mathrm{A}$ & $\mathrm{N} / \mathrm{A}$ & $\mathrm{N} / \mathrm{A}$ & $\mathrm{N} / \mathrm{A}$ & $\mathrm{N} / \mathrm{A}$ & N/A & N/A & $\mathrm{N} / \mathrm{A}$ & $\mathrm{N} / \mathrm{A}$ & $\mathrm{N} / \mathrm{A}$ & N/A & N/A & $\mathrm{N} / \mathrm{A}$ \\
\hline & & & MPGGE Adjusted & $\mathrm{N} / \mathrm{A}$ & $\mathrm{N} / \mathrm{A}$ & $\mathrm{N} / \mathrm{A}$ & $\mathrm{N} / \mathrm{A}$ & $\mathrm{N} / \mathrm{A}$ & $\mathrm{N} / \mathrm{A}$ & $\mathrm{N} / \mathrm{A}$ & $\mathrm{N} / \mathrm{A}$ & $\mathrm{N} / \mathrm{A}$ & $\mathrm{N} / \mathrm{A}$ & $\mathrm{N} / \mathrm{A}$ & $\mathrm{N} / \mathrm{A}$ & $\mathrm{N} / \mathrm{A}$ \\
\hline & & \multirow{2}{*}{ CS } & MPGGE Unadjusted & 40.7 & 40.7 & 47.1 & 53.3 & 46.9 & 53.1 & 59.3 & 48.3 & 57.0 & 66.6 & 50.2 & 60.3 & 70.4 \\
\hline & & & MPGGE Adjusted & 28.7 & 28.7 & 33.2 & 37.5 & 33.1 & 37.4 & 41.7 & 34.0 & 40.1 & 46.8 & 35.4 & 42.4 & 49.4 \\
\hline & & \multirow{3}{*}{$\mathrm{CD}+\mathrm{CS}$} & Wh/mile & 288.3 & 288.3 & 258.0 & 240.0 & 272.9 & 241.1 & 217.2 & 265.1 & 226.0 & 193.9 & 255.0 & 213.5 & 183.5 \\
\hline & & & MPGGE Unadjusted & 88.4 & 88.4 & $\begin{array}{l}102.4 \\
\end{array}$ & 115.8 & 101.9 & 115.3 & 128.9 & 105.0 & 123.8 & 144.9 & 109.1 & 131.0 & 153.1 \\
\hline & & & MPGGE Adjusted & 61.8 & 61.8 & 71.4 & 80.5 & 71.0 & 80.2 & 89.3 & 73.1 & 85.9 & 100.1 & 75.9 & 90.8 & 105.6 \\
\hline & \multirow{7}{*}{ Combined } & \multirow{2}{*}{$C D$} & MPGGE Unadjusted & $\mathrm{N} / \mathrm{A}$ & $\mathrm{N} / \mathrm{A}$ & N/A & $\mathrm{N} / \mathrm{A}$ & $\mathrm{N} / \mathrm{A}$ & N/A & $\mathrm{N} / \mathrm{A}$ & $\mathrm{N} / \mathrm{A}$ & $\mathrm{N} / \mathrm{A}$ & $\mathrm{N} / \mathrm{A}$ & $\mathrm{N} / \mathrm{A}$ & $\mathrm{N} / \mathrm{A}$ & $\mathrm{N} / \mathrm{A}$ \\
\hline & & & MPGGE Adjusted & $\mathrm{N} / \mathrm{A}$ & N/A & $\mathrm{N} / \mathrm{A}$ & $\mathrm{N} / \mathrm{A}$ & $\mathrm{N} / \mathrm{A}$ & $\mathrm{N} / \mathrm{A}$ & N/A & $\mathrm{N} / \mathrm{A}$ & N/A & $\mathrm{N} / \mathrm{A}$ & $\mathrm{N} / \mathrm{A}$ & N/A & N/A \\
\hline & & \multirow{2}{*}{ CS } & MPGGE Unadjusted & 41.0 & 41.0 & 48.0 & 54.9 & 47.7 & 54.6 & 62.1 & 49.7 & 59.6 & 71.4 & 51.9 & 62.9 & 75.2 \\
\hline & & & MPGGE Adjusted & 29.9 & 29.9 & 34.8 & 39.4 & 34.6 & 39.2 & 44.2 & 35.9 & 42.5 & 50.2 & 37.4 & 44.7 & 52.7 \\
\hline & & \multirow{3}{*}{$C D+C S$} & Wh/mile & 281.8 & 281.8 & 248.9 & 229.5 & 263.6 & 230.7 & 204.6 & 253.7 & 213.3 & 181.0 & 242.8 & 201.8 & 172.2 \\
\hline & & & MPGGE Unadjusted & 95.9 & 95.9 & 112.4 & 128.3 & 111.7 & 127.5 & 144.9 & 116.1 & 139.1 & 166.4 & 121.2 & 146.9 & 175.5 \\
\hline & & & MPGGE Adjusted & 65.7 & 65.7 & 75.6 & 84.8 & 75.2 & 84.4 & 94.2 & 77.8 & 90.9 & 105.8 & 80.8 & 95.3 & 110.5 \\
\hline \multirow{2}{*}{ EV } & UDDS & EV Only & Wh/mile & 378.4 & 378.4 & 348.9 & 324.5 & 367.2 & 329.7 & 278.9 & 356.9 & 291.0 & 234.7 & 348.0 & 274.2 & 222.7 \\
\hline & HWFET & EV Only & Wh/mile & 417.7 & 417.7 & 386.9 & 363.0 & 407.1 & 366.6 & 324.3 & 399.2 & 337.7 & 285.4 & 389.3 & 318.0 & 269.1 \\
\hline
\end{tabular}


Fuel consumption

\begin{tabular}{|c|c|c|c|c|c|c|c|c|c|c|c|c|c|c|c|}
\hline & & & \multicolumn{13}{|c|}{ Midsize_SUV } \\
\hline & & & \multirow{2}{*}{$\frac{2010}{\text { Ref }}$} & \multicolumn{3}{|c|}{2010} & \multicolumn{3}{|c|}{2015} & \multicolumn{3}{|c|}{2030} & \multicolumn{3}{|c|}{2045} \\
\hline & & & & low & avg & high & low & avg & high & low & avg & high & low & avg & high \\
\hline \multirow{6}{*}{ SI Conv } & \multirow{3}{*}{ Unadjusted } & UDDS & 11.1 & 11.1 & 9.9 & 9.4 & 10.5 & 9.5 & 7.7 & 10.1 & 9.2 & 6.4 & 10.3 & 9.0 & 6.1 \\
\hline & & HWFET & 8.3 & 8.3 & 7.3 & 7.0 & 7.8 & 7.0 & 5.6 & 7.5 & 6.7 & 4.8 & 7.5 & 6.5 & 4.5 \\
\hline & & Combined & 9.8 & 9.8 & 8.7 & 8.3 & 9.3 & 8.4 & 6.8 & 9.0 & 8.1 & 5.6 & 9.0 & 7.9 & 5.4 \\
\hline & \multirow{3}{*}{ Adjusted } & UDDS & 14.2 & 14.2 & 12.7 & 12.1 & 13.5 & 12.2 & 10.0 & 13.0 & 11.9 & 8.4 & 13.2 & 11.7 & 8.1 \\
\hline & & HWFET & 11.7 & 11.7 & 10.4 & 9.8 & 11.1 & 9.9 & 8.0 & 10.7 & 9.5 & 6.7 & 10.6 & 9.3 & 6.4 \\
\hline & & Combined & 13.0 & 13.0 & 11.7 & 11.1 & 12.4 & 11.2 & 9.1 & 11.9 & 10.8 & 7.7 & 12.0 & 10.6 & 7.4 \\
\hline \multirow{6}{*}{$\mathrm{Cl}$ Conv } & \multirow{3}{*}{ Unadjusted } & UDDS & 9.2 & 9.2 & 8.5 & 7.9 & 9.0 & 8.0 & 7.3 & 8.5 & 7.6 & 6.3 & 8.4 & 7.3 & 6.0 \\
\hline & & HWFET & 7.1 & 7.1 & 6.7 & 6.1 & 7.0 & 6.2 & 6.0 & 6.7 & 6.2 & 5.2 & 6.9 & 5.9 & 5.0 \\
\hline & & Combined & 8.3 & 8.3 & 7.7 & 7.1 & 8.1 & 7.2 & 6.7 & 7.7 & 6.9 & 5.8 & 7.7 & 6.6 & 5.6 \\
\hline & \multirow{3}{*}{ Adjusted } & \begin{tabular}{|l|} 
UDDS \\
\end{tabular} & 11.8 & 11.8 & 11.1 & 10.2 & 11.6 & 10.4 & 9.6 & 11.0 & 9.9 & 8.3 & 10.9 & 9.5 & 8.0 \\
\hline & & \begin{tabular}{|c|} 
HWFET \\
\end{tabular} & 10.1 & 10.1 & 9.4 & 8.7 & 9.9 & 8.8 & 8.5 & 9.4 & 8.8 & 7.4 & 9.7 & 8.3 & 7.1 \\
\hline & & Combined & 11.0 & 11.0 & 10.3 & 9.5 & 10.8 & 9.7 & 9.1 & 10.3 & 9.4 & 7.9 & 10.4 & 9.0 & 7.6 \\
\hline \multirow{6}{*}{ H2 Conv } & \multirow{3}{*}{ Unadjusted } & \begin{tabular}{|l|} 
UDDS \\
\end{tabular} & 13.0 & 13.0 & 10.6 & 9.3 & 11.5 & 9.4 & 8.4 & 10.2 & 8.7 & 7.1 & 9.7 & 8.1 & 6.6 \\
\hline & & HWFET & 8.6 & 8.6 & 7.4 & 6.5 & 7.6 & 6.6 & 5.7 & 7.1 & 5.9 & 4.9 & 6.6 & 5.5 & 4.5 \\
\hline & & Combined & 11.0 & 11.0 & 9.1 & 8.0 & 9.7 & 8.1 & 7.2 & 8.8 & 7.4 & 6.1 & 8.3 & 7.0 & 5.6 \\
\hline & \multirow{3}{*}{ Adjusted } & \begin{tabular}{|l|} 
UDDS \\
\end{tabular} & 16.5 & 16.5 & 13.6 & 12.0 & 14.7 & 12.2 & 10.9 & 13.0 & 11.2 & 9.3 & 12.5 & 10.6 & 8.6 \\
\hline & & \begin{tabular}{|c|} 
HWFET \\
\end{tabular} & 12.2 & 12.2 & 10.4 & 9.2 & 10.7 & 9.3 & 8.1 & 10.1 & 8.4 & 7.0 & 9.4 & 7.8 & 6.4 \\
\hline & & Combined & 14.6 & 14.6 & 12.2 & 10.7 & 12.9 & 10.9 & 9.6 & 11.7 & 9.9 & 8.2 & 11.1 & 9.3 & 7.6 \\
\hline \multirow{6}{*}{ E85 Conv } & \multirow{3}{*}{ Unadjusted } & \begin{tabular}{|l|} 
UDDS \\
\end{tabular} & 11.5 & 11.5 & 10.9 & 10.4 & 11.7 & 10.5 & 8.0 & 11.1 & 10.1 & 6.8 & 11.2 & 9.9 & 6.5 \\
\hline & & \begin{tabular}{|c|} 
HWFET \\
\end{tabular} & 9.2 & 9.2 & 8.7 & 8.3 & 9.4 & 8.3 & 6.4 & 8.9 & 8.1 & 5.5 & 8.9 & 7.8 & 5.2 \\
\hline & & \begin{tabular}{|l|} 
Combined \\
\end{tabular} & 10.5 & 10.5 & 9.9 & 9.4 & 10.7 & 9.5 & 7.2 & 10.1 & 9.2 & 6.2 & 10.2 & 9.0 & 5.9 \\
\hline & \multirow{3}{*}{ Adjusted } & \begin{tabular}{|l|} 
UDDS \\
\end{tabular} & 14.7 & 14.7 & 13.9 & 13.3 & 15.0 & 13.5 & 10.4 & 14.2 & 13.0 & 8.9 & 14.4 & 12.7 & 8.6 \\
\hline & & \begin{tabular}{|l|} 
HWFET \\
\end{tabular} & 13.0 & 13.0 & 12.2 & 11.7 & 13.3 & 11.8 & 9.0 & 12.6 & 11.4 & 7.8 & 12.6 & 11.0 & 7.4 \\
\hline & & Combined & 13.9 & 13.9 & 13.2 & 12.6 & 14.2 & 12.7 & 9.7 & 13.5 & 12.3 & 8.4 & 13.6 & 12.0 & 8.0 \\
\hline \multirow{6}{*}{ SI Split HEV } & \multirow{3}{*}{ Unadjusted } & \begin{tabular}{|l|} 
UDDS \\
\end{tabular} & 6.2 & 6.2 & 5.7 & 5.3 & 5.9 & 5.3 & 4.3 & 5.6 & 4.8 & 3.5 & 5.5 & 4.6 & 3.3 \\
\hline & & HWFET & 7.1 & 7.1 & 6.5 & 6.1 & 6.8 & 6.1 & 5.1 & 6.6 & 5.7 & 4.3 & 6.5 & 5.4 & 4.1 \\
\hline & & Combined & 6.6 & 6.6 & 6.0 & 5.6 & 6.3 & 5.7 & 4.7 & 6.1 & 5.2 & 3.9 & 5.9 & 5.0 & 3.7 \\
\hline & \multirow{3}{*}{ Adjusted } & \begin{tabular}{|l|} 
UDDS \\
\end{tabular} & 8.1 & 8.1 & 7.5 & 7.1 & 7.9 & 7.0 & 5.9 & 7.5 & 6.5 & 4.9 & 7.4 & 6.3 & 4.7 \\
\hline & & \begin{tabular}{|c|} 
HWFET \\
\end{tabular} & 10.0 & 10.0 & 9.2 & 8.6 & 9.7 & 8.7 & 7.3 & 9.4 & 8.1 & 6.1 & 9.1 & 7.7 & 5.8 \\
\hline & & Combined & 9.0 & 9.0 & 8.3 & 7.8 & 8.7 & 7.8 & 6.5 & 8.3 & 7.2 & 5.4 & 8.2 & 6.9 & 5.2 \\
\hline \multirow{6}{*}{ SI Split HEV PHEV10 } & & \begin{tabular}{|l|} 
UDDS \\
\end{tabular} & 4.9 & 4.9 & 4.5 & 4.2 & 4.7 & 4.3 & 3.5 & 4.6 & 3.9 & 2.9 & 4.5 & 3.8 & 2.7 \\
\hline & Unadjusted & \begin{tabular}{|c|} 
HWFET \\
\end{tabular} & 5.4 & 5.4 & 5.0 & 4.7 & 5.2 & 4.8 & 4.0 & 5.1 & 4.5 & 3.4 & 5.0 & 4.3 & 3.2 \\
\hline & & Combined & 5.1 & 5.1 & 4.7 & 4.4 & 5.0 & 4.5 & 3.7 & 4.8 & 4.2 & 3.1 & 4.7 & 4.0 & 3.0 \\
\hline & & \begin{tabular}{|l|} 
UDDS \\
\end{tabular} & 6.6 & 6.6 & 6.1 & 5.7 & 6.4 & 5.8 & 4.9 & 6.2 & 5.4 & 4.1 & 6.1 & 5.2 & 4.0 \\
\hline & Adjusted & \begin{tabular}{|l|} 
HWFET \\
\end{tabular} & 7.7 & 7.7 & 7.1 & 6.7 & 7.4 & 6.7 & 5.7 & 7.3 & 6.4 & 4.8 & 7.1 & 6.1 & 4.6 \\
\hline & & Combined & 7.1 & 7.1 & 6.6 & 6.2 & 6.9 & 6.2 & 5.3 & 6.7 & 5.9 & 4.5 & 6.5 & 5.6 & 4.2 \\
\hline
\end{tabular}




\begin{tabular}{|c|c|c|c|c|c|c|c|c|c|c|c|c|c|c|c|}
\hline & & & \multicolumn{13}{|c|}{ Midsize_SUV } \\
\hline & & & \multirow{2}{*}{$\begin{array}{l}2010 \\
\operatorname{Ref}\end{array}$} & \multicolumn{3}{|c|}{2010} & \multicolumn{3}{|c|}{2015} & \multicolumn{3}{|c|}{2030} & \multicolumn{3}{|c|}{2045} \\
\hline & & & & low & avg & high & low & avg & high & low & avg & high & low & avg & high \\
\hline \multirow{6}{*}{ SI Split HEV PHEV2O } & \multirow{3}{*}{ Unadjusted } & UDDS & 3.4 & 3.4 & 3.1 & 2.9 & 3.2 & 3.0 & 2.5 & 3.2 & 2.8 & 2.0 & 3.1 & 2.6 & 1.9 \\
\hline & & \begin{tabular}{|c|} 
HWFET \\
\end{tabular} & 4.4 & 4.4 & 4.1 & 3.9 & 4.3 & 3.9 & 3.5 & 4.2 & 3.6 & 3.0 & 4.1 & 3.8 & 2.8 \\
\hline & & Combined & 3.9 & 3.9 & 3.6 & 3.4 & 3.7 & 3.4 & 2.9 & 3.6 & 3.2 & 2.4 & 3.5 & 3.1 & 2.3 \\
\hline & \multirow{3}{*}{ Adjusted } & UDDS & 4.8 & 4.8 & 4.4 & 4.2 & 4.6 & 4.2 & 3.6 & 4.5 & 4.0 & 3.1 & 4.4 & 3.8 & 2.9 \\
\hline & & HWFET & 6.3 & 6.3 & 5.8 & 5.5 & 6.1 & 5.5 & 5.0 & 5.9 & 5.2 & 4.2 & 5.8 & 5.3 & 3.9 \\
\hline & & Combined & 5.5 & 5.5 & 5.1 & 4.8 & 5.2 & 4.8 & 4.2 & 5.2 & 4.5 & 3.6 & 5.0 & 4.5 & 3.4 \\
\hline \multirow{6}{*}{ SI Series HEV PHEV30 } & \multirow{3}{*}{ Unadjusted } & UDDS & 3.8 & 3.8 & 3.5 & 3.2 & 3.7 & 3.2 & 2.5 & 3.5 & 2.9 & 2.0 & 3.4 & 2.7 & 1.9 \\
\hline & & HWFET & 5.1 & 5.1 & 4.7 & 4.3 & 4.9 & 4.4 & 3.6 & 4.7 & 4.0 & 2.9 & 4.6 & 3.8 & 2.8 \\
\hline & & Combined & 4.4 & 4.4 & 4.0 & 3.7 & 4.2 & 3.7 & 3.0 & 4.0 & 3.4 & 2.4 & 3.9 & 3.2 & 2.3 \\
\hline & \multirow{3}{*}{ Adjusted } & UDDS & 5.3 & 5.3 & 4.9 & 4.5 & 5.1 & 4.5 & 3.7 & 4.9 & 4.1 & 3.0 & 4.7 & 3.9 & 2.9 \\
\hline & & HWFET & 7.3 & 7.3 & 6.6 & 6.1 & 7.0 & 6.2 & 5.1 & 6.7 & 5.7 & 4.2 & 6.5 & 5.3 & 4.0 \\
\hline & & Combined & 6.2 & 6.2 & 5.7 & 5.2 & 5.9 & 5.3 & 4.3 & 5.7 & 4.8 & 3.6 & 5.5 & 4.6 & 3.4 \\
\hline \multirow{6}{*}{ SI Series HEV PHEV40 } & \multirow{3}{*}{ Unadjusted } & UDDS & 3.3 & 3.3 & 3.0 & 2.8 & 3.2 & 2.8 & 2.2 & 3.0 & 2.5 & 1.7 & 2.9 & 2.4 & 1.6 \\
\hline & & HWFET & 4.1 & 4.1 & 3.7 & 3.4 & 3.9 & 3.5 & 2.8 & 3.7 & 3.2 & 2.3 & 3.6 & 3.0 & 2.2 \\
\hline & & Combined & 3.6 & 3.6 & 3.3 & 3.1 & 3.5 & 3.1 & 2.5 & 3.4 & 2.8 & 2.0 & 3.2 & 2.6 & 1.9 \\
\hline & \multirow{3}{*}{ Adjusted } & \begin{tabular}{|l|} 
UDDS \\
\end{tabular} & 4.7 & 4.7 & 4.3 & 4.0 & 4.5 & 4.0 & 3.3 & 4.3 & 3.6 & 2.7 & 4.2 & 3.5 & 2.6 \\
\hline & & HWFET & 5.8 & 5.8 & 5.3 & 4.9 & 5.5 & 4.9 & 4.0 & 5.3 & 4.5 & 3.3 & 5.1 & 4.2 & 3.2 \\
\hline & & Combined & 5.2 & 5.2 & 4.7 & 4.4 & 5.0 & 4.4 & 3.6 & 4.8 & 4.0 & 3.0 & 4.6 & 3.8 & 2.8 \\
\hline \multirow{6}{*}{ Cl Split HEV } & \multirow{3}{*}{ Unadjusted } & UDDS & 5.5 & 5.5 & 5.2 & 4.7 & 5.4 & 4.7 & 4.2 & 5.1 & 4.3 & 3.5 & 4.9 & 4.1 & 3.3 \\
\hline & & HWFET & 6.6 & 6.6 & 6.2 & 5.7 & 6.5 & 5.7 & 5.1 & 6.2 & 5.3 & 4.4 & 6.0 & 5.0 & 4.2 \\
\hline & & Combined & 6.0 & 6.0 & 5.6 & 5.2 & 5.9 & 5.2 & 4.6 & 5.6 & 4.8 & 3.9 & 5.4 & 4.5 & 3.7 \\
\hline & \multirow{3}{*}{ Adjusted } & \begin{tabular}{|l|} 
UDDS \\
\end{tabular} & 7.4 & 7.4 & 6.9 & 6.4 & 7.2 & 6.4 & 5.7 & 6.8 & 5.9 & 4.9 & 6.6 & 5.6 & 4.7 \\
\hline & & HWFET & 9.3 & 9.3 & 8.7 & 8.1 & 9.1 & 8.1 & 7.2 & 8.8 & 7.5 & 6.2 & 8.4 & 7.0 & 5.9 \\
\hline & & Combined & 8.3 & 8.3 & 7.7 & 7.1 & 8.1 & 7.2 & 6.4 & 7.7 & 6.6 & 5.5 & 7.4 & 6.2 & 5.2 \\
\hline \multirow{6}{*}{ CI Split HEV PHEV10 } & \multirow{3}{*}{ Unadjusted } & UDDS & 4.7 & 4.7 & 4.4 & 4.1 & 4.6 & 4.1 & 3.6 & 4.4 & 3.7 & 3.0 & 4.2 & 3.5 & 2.9 \\
\hline & & \begin{tabular}{|l|} 
HWFET \\
\end{tabular} & 5.3 & 5.3 & 5.0 & 4.4 & 5.2 & 4.4 & 3.9 & 5.0 & 4.3 & 3.4 & 4.8 & 3.9 & 3.4 \\
\hline & & Combined & 5.0 & 5.0 & 4.7 & 4.2 & 4.9 & 4.2 & 3.7 & 4.7 & 4.0 & 3.2 & 4.5 & 3.7 & 3.1 \\
\hline & \multirow{3}{*}{ Adjusted } & \begin{tabular}{|l|} 
UDDS \\
\end{tabular} & 6.4 & 6.4 & 6.0 & 5.6 & 6.3 & 5.6 & 5.0 & 6.0 & 5.2 & 4.3 & 5.8 & 4.9 & 4.2 \\
\hline & & \begin{tabular}{|l|} 
HWFET \\
\end{tabular} & 7.5 & 7.5 & 7.0 & 6.2 & 7.4 & 6.2 & 5.6 & 7.0 & 6.1 & 4.9 & 6.8 & 5.5 & 4.9 \\
\hline & & Combined & 6.9 & 6.9 & 6.5 & 5.9 & 6.8 & 5.9 & 5.3 & 6.5 & 5.6 & 4.6 & 6.3 & 5.2 & 4.5 \\
\hline \multirow{6}{*}{ CI Split HEV PHEV20 } & & UDDS & 3.3 & 3.3 & 3.0 & 2.8 & 3.2 & 2.8 & 2.5 & 3.1 & 2.6 & 2.1 & 3.0 & 2.5 & 2.0 \\
\hline & Unadjusted & \begin{tabular}{|l|} 
HWFET \\
\end{tabular} & 4.3 & 4.3 & 4.7 & 4.4 & 4.2 & 4.5 & 3.4 & 4.1 & 3.6 & 3.0 & 4.6 & 3.4 & 3.0 \\
\hline & & Combined & 3.8 & 3.8 & 3.8 & 3.5 & 3.7 & 3.6 & 2.9 & 3.5 & 3.1 & 2.5 & 3.7 & 2.9 & 2.5 \\
\hline & & \begin{tabular}{|l|} 
UDDS \\
\end{tabular} & 4.7 & 4.7 & 4.3 & 4.0 & 4.5 & 4.1 & 3.7 & 4.3 & 3.8 & 3.2 & 4.2 & 3.6 & 3.1 \\
\hline & Adjusted & HWFET & 6.1 & 6.1 & 6.7 & 6.3 & 6.0 & 6.3 & 4.9 & 5.8 & 5.1 & 4.3 & 6.5 & 4.9 & 4.3 \\
\hline & & Combined & 5.3 & 5.3 & 5.4 & 5.1 & 5.2 & 5.1 & 4.2 & 5.0 & 4.4 & 3.7 & 5.3 & 4.2 & 3.6 \\
\hline
\end{tabular}




\begin{tabular}{|c|c|c|c|c|c|c|c|c|c|c|c|c|c|c|c|}
\hline & & & \multicolumn{13}{|c|}{ Midsize_SUV } \\
\hline & & & \multirow{2}{*}{$\begin{array}{l}2010 \\
\operatorname{Ref}\end{array}$} & \multicolumn{3}{|c|}{2010} & \multicolumn{3}{|c|}{2015} & \multicolumn{3}{|c|}{2030} & \multicolumn{3}{|c|}{2045} \\
\hline & & & & low & avg & high & low & avg & high & low & avg & high & low & avg & high \\
\hline \multirow{6}{*}{ CI Series HEV PHEV 30} & \multirow{3}{*}{ Unadjusted } & UDDS & 3.7 & 3.7 & 3.4 & 3.1 & 3.6 & 3.1 & 2.6 & 3.4 & 2.7 & 2.1 & 3.2 & 2.6 & 2.0 \\
\hline & & \begin{tabular}{|c|} 
HWFET \\
\end{tabular} & 5.0 & 5.0 & 4.6 & 4.2 & 4.9 & 4.2 & 3.7 & 4.6 & 3.8 & 3.1 & 4.4 & 3.6 & 2.9 \\
\hline & & Combined & 4.3 & 4.3 & 3.9 & 3.6 & 4.2 & 3.6 & 3.1 & 3.9 & 3.2 & 2.6 & 3.8 & 3.0 & 2.4 \\
\hline & \multirow{3}{*}{ Adjusted } & UDDS & 5.2 & 5.2 & 4.8 & 4.3 & 5.0 & 4.4 & 3.8 & 4.7 & 3.9 & 3.2 & 4.6 & 3.8 & 3.1 \\
\hline & & HWFET & 7.1 & 7.1 & 6.5 & 6.0 & 6.9 & 6.0 & 5.2 & 6.5 & 5.4 & 4.4 & 6.2 & 5.1 & 4.2 \\
\hline & & Combined & 6.0 & 6.0 & 5.6 & 5.1 & 5.9 & 5.1 & 4.4 & 5.5 & 4.6 & 3.7 & 5.3 & 4.3 & 3.6 \\
\hline \multirow{6}{*}{ CI Series HEV PHEV 40} & \multirow{3}{*}{ Unadjusted } & \begin{tabular}{|l|} 
UDDS \\
\end{tabular} & 3.2 & 3.2 & 3.0 & 2.7 & 3.1 & 2.7 & 2.3 & 2.9 & 2.4 & 1.8 & 2.8 & 2.2 & 1.7 \\
\hline & & HWFET & 4.0 & 4.0 & 3.6 & 3.3 & 3.9 & 3.3 & 2.9 & 3.6 & 3.0 & 2.4 & 3.5 & 2.8 & 2.3 \\
\hline & & Combined & 3.6 & 3.6 & 3.3 & 3.0 & 3.5 & 3.0 & 2.6 & 3.2 & 2.7 & 2.1 & 3.1 & 2.5 & 2.0 \\
\hline & \multirow{3}{*}{ Adjusted } & \begin{tabular}{|l|} 
UDDS \\
\end{tabular} & 4.6 & 4.6 & 4.3 & 3.9 & 4.5 & 3.9 & 3.4 & 4.2 & 3.5 & 2.8 & 4.0 & 3.3 & 2.7 \\
\hline & & HWFET & 5.6 & 5.6 & 5.2 & 4.7 & 5.5 & 4.8 & 4.2 & 5.2 & 4.3 & 3.5 & 4.9 & 4.0 & 3.3 \\
\hline & & Combined & 5.0 & 5.0 & 4.7 & 4.3 & 4.9 & 4.3 & 3.7 & 4.6 & 3.9 & 3.1 & 4.4 & 3.6 & 3.0 \\
\hline \multirow{6}{*}{ H2 Split HEV } & \multirow{3}{*}{ Unadjusted } & UDDS & 6.3 & 6.3 & 5.2 & 4.6 & 5.5 & 4.6 & 3.9 & 5.0 & 4.1 & 3.2 & 4.7 & 3.8 & 3.0 \\
\hline & & HWFET & 7.3 & 7.3 & 6.1 & 5.4 & 6.4 & 5.4 & 4.7 & 5.9 & 4.9 & 4.0 & 5.5 & 4.5 & 3.7 \\
\hline & & Combined & 6.7 & 6.7 & 5.6 & 4.9 & 5.9 & 5.0 & 4.3 & 5.4 & 4.4 & 3.6 & 5.1 & 4.1 & 3.3 \\
\hline & \multirow{3}{*}{ Adjusted } & UDDS & 8.3 & 8.3 & 7.0 & 6.2 & 7.4 & 6.2 & 5.4 & 6.7 & 5.6 & 4.5 & 6.3 & 5.2 & 4.2 \\
\hline & & HWFET & 10.3 & 10.3 & 8.6 & 7.6 & 9.1 & 7.7 & 6.7 & 8.4 & 6.9 & 5.7 & 7.8 & 6.4 & 5.2 \\
\hline & & Combined & 9.2 & 9.2 & 7.8 & 6.8 & 8.2 & 6.9 & 5.9 & 7.5 & 6.2 & 5.0 & 7.0 & 5.7 & 4.7 \\
\hline \multirow{6}{*}{ H2 Split HEV PHEV10 } & \multirow{3}{*}{ Unadjusted } & \begin{tabular}{|l|} 
UDDS \\
\end{tabular} & 5.0 & 5.0 & 4.2 & 3.7 & 4.4 & 3.7 & 3.2 & 4.1 & 3.3 & 2.7 & 3.8 & 3.1 & 2.4 \\
\hline & & HWFET & 5.5 & 5.5 & 4.7 & 4.1 & 4.9 & 4.2 & 3.7 & 4.5 & 3.8 & 3.0 & 4.2 & 3.5 & 2.8 \\
\hline & & Combined & 5.3 & 5.3 & 4.4 & 3.9 & 4.6 & 3.9 & 3.4 & 4.3 & 3.5 & 2.8 & 4.0 & 3.3 & 2.6 \\
\hline & \multirow{3}{*}{ Adjusted } & UDDS & 6.8 & 6.8 & 5.7 & 5.1 & 6.0 & 5.2 & 4.5 & 5.6 & 4.7 & 3.8 & 5.3 & 4.4 & 3.6 \\
\hline & & HWFET & 7.8 & 7.8 & 6.6 & 5.9 & 6.9 & 5.9 & 5.2 & 6.4 & 5.4 & 4.3 & 6.0 & 5.0 & 4.0 \\
\hline & & Combined & 7.3 & 7.3 & 6.1 & 5.4 & 6.4 & 5.5 & 4.8 & 6.0 & 5.0 & 4.1 & 5.6 & 4.7 & 3.8 \\
\hline \multirow{6}{*}{ H2 Split HEV PHEV2O } & \multirow{3}{*}{ Unadjusted } & UDDS & 3.5 & 3.5 & 2.9 & 2.5 & 3.0 & 2.6 & 2.2 & 2.8 & 2.3 & 1.8 & 2.6 & 2.2 & 1.7 \\
\hline & & \begin{tabular}{|l|} 
HWFET \\
\end{tabular} & 4.5 & 4.5 & 3.9 & 3.4 & 4.0 & 3.4 & 3.2 & 3.7 & 3.2 & 3.1 & 3.5 & 3.1 & 2.6 \\
\hline & & Combined & 3.9 & 3.9 & 3.3 & 2.9 & 3.5 & 3.0 & 2.7 & 3.2 & 2.7 & 2.4 & 3.0 & 2.6 & 2.1 \\
\hline & \multirow{3}{*}{ Adjusted } & \begin{tabular}{|l|} 
UDDS \\
\end{tabular} & 4.9 & 4.9 & 4.2 & 3.7 & 4.3 & 3.8 & 3.3 & 4.0 & 3.4 & 2.9 & 3.8 & 3.2 & 2.6 \\
\hline & & \begin{tabular}{|l|} 
HWFET \\
\end{tabular} & 6.4 & 6.4 & 5.5 & 4.8 & 5.7 & 4.9 & 4.6 & 5.2 & 4.5 & 4.5 & 4.9 & 4.4 & 3.8 \\
\hline & & Combined & 5.6 & 5.6 & 4.8 & 4.2 & 4.9 & 4.3 & 3.9 & 4.6 & 3.9 & 3.6 & 4.3 & 3.8 & 3.1 \\
\hline \multirow{6}{*}{ H2 Series HEV PHEV3O } & & \begin{tabular}{|l|} 
UDDS \\
\end{tabular} & 3.9 & 3.9 & 3.2 & 2.7 & 3.4 & 2.8 & 2.3 & 3.1 & 2.4 & 1.9 & 2.8 & 2.2 & 1.7 \\
\hline & Unadjusted & \begin{tabular}{|l|} 
HWFET \\
\end{tabular} & 5.2 & 5.2 & 4.3 & 3.8 & 4.6 & 3.8 & 3.2 & 4.1 & 3.4 & 2.7 & 3.9 & 3.1 & 2.5 \\
\hline & & Combined & 4.5 & 4.5 & 3.7 & 3.2 & 3.9 & 3.2 & 2.7 & 3.6 & 2.8 & 2.3 & 3.3 & 2.6 & 2.1 \\
\hline & & \begin{tabular}{|l|} 
UDDS \\
\end{tabular} & 5.4 & 5.4 & 4.5 & 4.0 & 4.8 & 4.0 & 3.4 & 4.4 & 3.6 & 2.9 & 4.1 & 3.3 & 2.7 \\
\hline & Adjusted & HWFET & 7.4 & 7.4 & 6.1 & 5.4 & 6.5 & 5.4 & 4.6 & 5.9 & 4.8 & 3.9 & 5.5 & 4.4 & 3.6 \\
\hline & & Combined & 6.3 & 6.3 & 5.2 & 4.6 & 5.5 & 4.6 & 3.9 & 5.0 & 4.1 & 3.3 & 4.7 & 3.8 & 3.1 \\
\hline
\end{tabular}




\begin{tabular}{|c|c|c|c|c|c|c|c|c|c|c|c|c|c|c|c|}
\hline & & & \multicolumn{13}{|c|}{ Midsize_SUV } \\
\hline & & & \multirow{2}{*}{$\frac{2010}{\text { Ref }}$} & \multicolumn{3}{|c|}{2010} & \multicolumn{3}{|c|}{2015} & \multicolumn{3}{|c|}{2030} & \multicolumn{3}{|c|}{2045} \\
\hline & & & & low & avg & high & low & avg & high & low & avg & high & low & avg & high \\
\hline \multirow{6}{*}{ H2 Series HEV PHEV 40} & \multirow{3}{*}{ Unadjusted } & UDDS & 3.4 & 3.4 & 2.8 & 2.4 & 3.0 & 2.4 & 2.0 & 2.7 & 2.1 & 1.6 & 2.5 & 1.9 & 1.5 \\
\hline & & \begin{tabular}{|c|} 
HWFET \\
\end{tabular} & 4.1 & 4.1 & 3.4 & 3.0 & 3.6 & 3.0 & 2.5 & 3.3 & 2.7 & 2.2 & 3.0 & 2.4 & 2.0 \\
\hline & & Combined & 3.7 & 3.7 & 3.1 & 2.6 & 3.3 & 2.7 & 2.2 & 2.9 & 2.3 & 1.8 & 2.7 & 2.1 & 1.7 \\
\hline & \multirow{3}{*}{ Adjusted } & UDDS & 4.8 & 4.8 & 4.0 & 3.5 & 4.2 & 3.6 & 3.0 & 3.9 & 3.2 & 2.6 & 3.6 & 3.0 & 2.4 \\
\hline & & HWFET & 5.8 & 5.8 & 4.9 & 4.2 & 5.1 & 4.3 & 3.6 & 4.7 & 3.8 & 3.1 & 4.3 & 3.5 & 2.8 \\
\hline & & Combined & 5.3 & 5.3 & 4.4 & 3.8 & 4.7 & 3.9 & 3.3 & 4.2 & 3.5 & 2.8 & 4.0 & 3.2 & 2.6 \\
\hline \multirow{6}{*}{ E85 Split HEV } & \multirow{3}{*}{ Unadjusted } & UDDS & 6.6 & 6.6 & 6.1 & 5.7 & 6.4 & 5.7 & 4.4 & 6.1 & 5.2 & 3.7 & 5.9 & 5.0 & 3.6 \\
\hline & & HWFET & 7.6 & 7.6 & 7.0 & 6.5 & 7.4 & 6.6 & 5.2 & 7.1 & 6.1 & 4.6 & 6.9 & 5.8 & 4.3 \\
\hline & & Combined & 7.1 & 7.1 & 6.5 & 6.1 & 6.9 & 6.1 & 4.8 & 6.6 & 5.6 & 4.1 & 6.4 & 5.4 & 3.9 \\
\hline & \multirow{3}{*}{ Adjusted } & \begin{tabular}{|l|} 
UDDS \\
\end{tabular} & 8.8 & 8.8 & 8.1 & 7.6 & 8.5 & 7.6 & 6.0 & 8.1 & 7.0 & 5.2 & 7.9 & 6.7 & 5.0 \\
\hline & & HWFET & 10.8 & 10.8 & 9.9 & 9.3 & 10.4 & 9.3 & 7.4 & 10.1 & 8.7 & 6.5 & 9.8 & 8.3 & 6.1 \\
\hline & & Combined & 9.7 & 9.7 & 8.9 & 8.3 & 9.4 & 8.4 & 6.6 & 9.0 & 7.8 & 5.8 & 8.7 & 7.4 & 5.5 \\
\hline \multirow{6}{*}{ E85 Split HEV PHEV10 } & \multirow{3}{*}{ Unadjusted } & \begin{tabular}{|l|} 
UDDS \\
\end{tabular} & 5.2 & 5.2 & 4.8 & 4.5 & 5.0 & 4.5 & 3.5 & 4.9 & 4.2 & 3.0 & 4.8 & 4.0 & 2.9 \\
\hline & & HWFET & 5.8 & 5.8 & 5.4 & 5.0 & 5.6 & 5.1 & 4.1 & 5.5 & 4.8 & 3.6 & 5.3 & 4.6 & 3.4 \\
\hline & & Combined & 5.5 & 5.5 & 5.1 & 4.7 & 5.3 & 4.8 & 3.8 & 5.2 & 4.5 & 3.3 & 5.0 & 4.3 & 3.1 \\
\hline & \multirow{3}{*}{ Adjusted } & UDDS & 7.0 & 7.0 & 6.5 & 6.1 & 6.8 & 6.1 & 5.0 & 6.6 & 5.8 & 4.3 & 6.5 & 5.5 & 4.2 \\
\hline & & HWFET & 8.2 & 8.2 & 7.6 & 7.1 & 8.0 & 7.2 & 5.8 & 7.8 & 6.8 & 5.1 & 7.6 & 6.5 & 4.8 \\
\hline & & Combined & 7.5 & 7.5 & 7.0 & 6.5 & 7.3 & 6.6 & 5.3 & 7.1 & 6.2 & 4.7 & 6.9 & 5.9 & 4.5 \\
\hline \multirow{6}{*}{ E85 Split HEV PHEV20 } & \multirow{3}{*}{ Unadjusted } & \begin{tabular}{|l|} 
UDDS \\
\end{tabular} & 3.7 & 3.7 & 3.3 & 3.1 & 3.5 & 3.2 & 2.5 & 3.4 & 2.9 & 2.1 & 3.3 & 2.8 & 2.0 \\
\hline & & HWFET & 4.7 & 4.7 & 4.4 & 4.2 & 4.6 & 4.2 & 3.6 & 4.5 & 4.2 & 3.1 & 4.4 & 4.0 & 2.9 \\
\hline & & Combined & 4.1 & 4.1 & 3.8 & 3.6 & 4.0 & 3.6 & 3.0 & 3.9 & 3.5 & 2.6 & 3.8 & 3.3 & 2.4 \\
\hline & \multirow{3}{*}{ Adjusted } & \begin{tabular}{|l|} 
UDDS \\
\end{tabular} & 5.1 & 5.1 & 4.7 & 4.4 & 4.8 & 4.5 & 3.7 & 4.7 & 4.2 & 3.2 & 4.7 & 4.0 & 3.1 \\
\hline & & HWFET & 6.7 & 6.7 & 6.3 & 6.0 & 6.5 & 6.0 & 5.1 & 6.4 & 5.9 & 4.5 & 6.2 & 5.7 & 4.2 \\
\hline & & Combined & 5.8 & 5.8 & 5.4 & 5.1 & 5.6 & 5.2 & 4.3 & 5.5 & 5.0 & 3.8 & 5.3 & 4.8 & 3.6 \\
\hline \multirow{6}{*}{ E85 Series HEV PHEV30 } & \multirow{3}{*}{ Unadjusted } & \begin{tabular}{|l|} 
UDDS \\
\end{tabular} & 4.1 & 4.1 & 3.7 & 3.4 & 3.9 & 3.4 & 2.6 & 3.7 & 3.0 & 2.1 & 3.6 & 2.9 & 2.0 \\
\hline & & \begin{tabular}{|l|} 
HWFET \\
\end{tabular} & 5.5 & 5.5 & 5.0 & 4.6 & 5.2 & 4.6 & 3.6 & 5.0 & 4.3 & 3.1 & 4.9 & 4.0 & 2.9 \\
\hline & & Combined & 4.7 & 4.7 & 4.3 & 3.9 & 4.5 & 4.0 & 3.0 & 4.3 & 3.6 & 2.6 & 4.2 & 3.4 & 2.4 \\
\hline & \multirow{3}{*}{ Adjusted } & \begin{tabular}{|l|} 
UDDS \\
\end{tabular} & 5.6 & 5.6 & 5.1 & 4.7 & 5.4 & 4.8 & 3.7 & 5.1 & 4.3 & 3.2 & 5.0 & 4.1 & 3.0 \\
\hline & & \begin{tabular}{|l|} 
HWFET \\
\end{tabular} & 7.7 & 7.7 & 7.0 & 6.5 & 7.4 & 6.6 & 5.1 & 7.1 & 6.0 & 4.4 & 6.9 & 5.7 & 4.2 \\
\hline & & Combined & 6.5 & 6.5 & 6.0 & 5.5 & 6.3 & 5.6 & 4.4 & 6.0 & 5.1 & 3.8 & 5.9 & 4.8 & 3.6 \\
\hline \multirow{6}{*}{ E85 Series HEV PHEV40 } & & \begin{tabular}{|l|} 
UDDS \\
\end{tabular} & 3.6 & 3.6 & 3.2 & 2.9 & 3.4 & 3.0 & 2.2 & 3.2 & 2.6 & 1.8 & 3.1 & 2.5 & 1.7 \\
\hline & Unadjusted & \begin{tabular}{|c|} 
HWFET \\
\end{tabular} & 4.3 & 4.3 & 3.9 & 3.6 & 4.2 & 3.7 & 2.8 & 4.0 & 3.4 & 2.4 & 3.8 & 3.1 & 2.3 \\
\hline & & Combined & 3.9 & 3.9 & 3.5 & 3.3 & 3.7 & 3.3 & 2.5 & 3.6 & 3.0 & 2.1 & 3.4 & 2.8 & 2.0 \\
\hline & & \begin{tabular}{|l|} 
UDDS \\
\end{tabular} & 5.0 & 5.0 & 4.5 & 4.2 & 4.7 & 4.3 & 3.3 & 4.6 & 3.8 & 2.8 & 4.4 & 3.7 & 2.7 \\
\hline & Adjusted & \begin{tabular}{|c|} 
HWFET \\
\end{tabular} & 6.1 & 6.1 & 5.6 & 5.2 & 5.9 & 5.2 & 4.1 & 5.7 & 4.8 & 3.5 & 5.5 & 4.5 & 3.3 \\
\hline & & Combined & 5.5 & 5.5 & 5.0 & 4.6 & 5.3 & 4.7 & 3.7 & 5.1 & 4.3 & 3.1 & 4.9 & 4.0 & 3.0 \\
\hline
\end{tabular}




\begin{tabular}{|c|c|c|c|c|c|c|c|c|c|c|c|c|c|c|c|}
\hline & & & \multicolumn{13}{|c|}{ Midsize_SUV } \\
\hline & & & \multirow{2}{*}{$\frac{2010}{\text { Ref }}$} & \multicolumn{3}{|c|}{2010} & \multicolumn{3}{|c|}{2015} & \multicolumn{3}{|c|}{2030} & \multicolumn{3}{|c|}{2045} \\
\hline & & & & low & avg & high & low & avg & high & low & avg & high & low & avg & high \\
\hline \multirow{6}{*}{ FC HEV } & \multirow{3}{*}{ Unadjusted } & UDDS & 5.8 & 5.8 & 4.8 & 4.0 & 4.8 & 4.0 & 3.5 & 4.4 & 3.6 & 2.9 & 4.2 & 3.5 & 2.8 \\
\hline & & HWFET & 5.7 & 5.7 & 4.9 & 4.2 & 4.9 & 4.2 & 3.8 & 4.7 & 4.0 & 3.4 & 4.5 & 3.8 & 3.2 \\
\hline & & Combined & 5.8 & 5.8 & 4.8 & 4.1 & 4.8 & 4.1 & 3.6 & 4.5 & 3.8 & 3.1 & 4.3 & 3.6 & 3.0 \\
\hline & \multirow{3}{*}{ Adjusted } & UDDS & 7.7 & 7.7 & 6.4 & 5.5 & 6.5 & 5.5 & 4.9 & 6.0 & 5.0 & 4.2 & 5.7 & 4.8 & 4.0 \\
\hline & & HWFET & 8.1 & 8.1 & 6.9 & 6.0 & 6.9 & 6.0 & 5.4 & 6.6 & 5.6 & 4.8 & 6.4 & 5.3 & 4.5 \\
\hline & & Combined & 7.9 & 7.9 & 6.6 & 5.7 & 6.7 & 5.7 & 5.1 & 6.3 & 5.3 & 4.5 & 6.0 & 5.1 & 4.3 \\
\hline \multirow{6}{*}{ FC PHEV10 } & \multirow{3}{*}{ Unadjusted } & UDDS & 4.5 & 4.5 & 3.8 & 3.2 & 3.8 & 3.3 & 2.9 & 3.6 & 3.0 & 2.4 & 3.4 & 2.9 & 2.3 \\
\hline & & HWFET & 4.3 & 4.3 & 3.8 & 3.3 & 3.8 & 3.3 & 3.0 & 3.7 & 3.2 & 2.7 & 3.5 & 3.0 & 2.6 \\
\hline & & Combined & 4.4 & 4.4 & 3.8 & 3.3 & 3.8 & 3.3 & 3.0 & 3.6 & 3.1 & 2.6 & 3.5 & 2.9 & 2.4 \\
\hline & \multirow{3}{*}{ Adjusted } & UDDS & 6.1 & 6.1 & 5.3 & 4.6 & 5.3 & 4.6 & 4.1 & 5.0 & 4.3 & 3.6 & 4.8 & 4.1 & 3.5 \\
\hline & & \begin{tabular}{|l|} 
HWFET \\
\end{tabular} & 6.2 & 6.2 & 5.4 & 4.7 & 5.4 & 4.8 & 4.3 & 5.2 & 4.5 & 3.9 & 5.0 & 4.3 & 3.7 \\
\hline & & Combined & 6.1 & 6.1 & 5.3 & 4.6 & 5.3 & 4.7 & 4.2 & 5.1 & 4.4 & 3.7 & 4.9 & 4.2 & 3.6 \\
\hline \multirow{6}{*}{ FC PHEV 20} & \multirow{3}{*}{ Unadjusted } & UDDS & 3.8 & 3.8 & 3.2 & 2.7 & 3.2 & 2.7 & 2.4 & 3.0 & 2.5 & 2.0 & 2.9 & 2.4 & 1.9 \\
\hline & & HWFET & 4.2 & 4.2 & 3.7 & 3.2 & 3.7 & 3.2 & 2.9 & 3.5 & 3.0 & 2.6 & 3.4 & 2.9 & 2.4 \\
\hline & & Combined & 4.0 & 4.0 & 3.4 & 2.9 & 3.4 & 3.0 & 2.6 & 3.2 & 2.7 & 2.3 & 3.1 & 2.6 & 2.2 \\
\hline & \multirow{3}{*}{ Adjusted } & UDDS & 5.2 & 5.2 & 4.5 & 3.9 & 4.5 & 4.0 & 3.5 & 4.3 & 3.6 & 3.1 & 4.1 & 3.5 & 3.0 \\
\hline & & HWFET & 6.0 & 6.0 & 5.2 & 4.6 & 5.2 & 4.6 & 4.2 & 5.0 & 4.3 & 3.7 & 4.9 & 4.1 & 3.5 \\
\hline & & Combined & 5.6 & 5.6 & 4.8 & 4.2 & 4.8 & 4.2 & 3.8 & 4.6 & 3.9 & 3.4 & 4.4 & 3.8 & 3.2 \\
\hline \multirow{6}{*}{ FC PHEV 30} & \multirow{3}{*}{ Unadjusted } & UDDS & 2.6 & 2.6 & 2.2 & 1.9 & 2.2 & 1.9 & 1.7 & 2.1 & 1.8 & 1.4 & 2.0 & 1.7 & 1.4 \\
\hline & & \begin{tabular}{|l|} 
HWFET \\
\end{tabular} & 3.4 & 3.4 & 2.9 & 2.6 & 2.9 & 2.6 & 2.3 & 2.8 & 2.4 & 2.1 & 2.7 & 2.3 & 2.0 \\
\hline & & Combined & 3.0 & 3.0 & 2.5 & 2.2 & 2.5 & 2.2 & 2.0 & 2.5 & 2.1 & 1.7 & 2.4 & 2.0 & 1.6 \\
\hline & \multirow{3}{*}{ Adjusted } & UDDS & 3.8 & 3.8 & 3.3 & 3.0 & 3.3 & 3.0 & 2.7 & 3.2 & 2.8 & 2.4 & 3.1 & 2.7 & 2.3 \\
\hline & & \begin{tabular}{|c|} 
HWFET \\
\end{tabular} & 4.8 & 4.8 & 4.2 & 3.7 & 4.2 & 3.7 & 3.3 & 4.1 & 3.5 & 3.0 & 3.9 & 3.3 & 2.8 \\
\hline & & Combined & 4.3 & 4.3 & 3.7 & 3.3 & 3.7 & 3.3 & 3.0 & 3.6 & 3.1 & 2.6 & 3.5 & 2.9 & 2.5 \\
\hline \multirow{6}{*}{ FC PHEV40 } & \multirow{3}{*}{ Unadjusted } & UDDS & 2.3 & 2.3 & 1.9 & 1.7 & 1.9 & 1.7 & 1.5 & 1.9 & 1.5 & 1.2 & 1.8 & 1.4 & 1.2 \\
\hline & & HWFET & 2.7 & 2.7 & 2.3 & 2.0 & 2.3 & 2.0 & 1.8 & 2.2 & 1.9 & 1.6 & 2.2 & 1.8 & 1.5 \\
\hline & & Combined & 2.5 & 2.5 & 2.1 & 1.8 & 2.1 & 1.8 & 1.6 & 2.0 & 1.7 & 1.4 & 1.9 & 1.6 & 1.3 \\
\hline & \multirow{3}{*}{ Adjusted } & \begin{tabular}{|l|} 
UDDS \\
\end{tabular} & 3.4 & 3.4 & 3.0 & 2.7 & 3.0 & 2.7 & 2.4 & 2.9 & 2.5 & 2.1 & 2.8 & 2.4 & 2.0 \\
\hline & & HWFET & 3.8 & 3.8 & 3.3 & 2.9 & 3.3 & 2.9 & 2.6 & 3.2 & 2.7 & 2.4 & 3.1 & 2.6 & 2.2 \\
\hline & & Combined & 3.6 & 3.6 & 3.1 & 2.8 & 3.1 & 2.8 & 2.5 & 3.0 & 2.6 & 2.2 & 2.9 & 2.5 & 2.1 \\
\hline
\end{tabular}


Fuel Consumption Improvement versus the Conventional 2010 Reference

\begin{tabular}{|c|c|c|c|c|c|c|c|c|c|c|c|c|c|c|c|}
\hline & & & \multicolumn{13}{|c|}{ Midsize_SUV } \\
\hline & & & 2010 & & 2010 & & & 2015 & & & 2030 & & & 2045 & \\
\hline & & & Ref & low & avg & high & low & avg & high & low & avg & high & low & avg & high \\
\hline \multirow{6}{*}{ SI Conv } & \multirow{3}{*}{ Unadjusted } & UDDS & $0.0 \%$ & $0.0 \%$ & $-10.5 \%$ & $-15.1 \%$ & $-5.1 \%$ & $-14.2 \%$ & $-30.4 \%$ & $-8.6 \%$ & $-16.8 \%$ & $-42.3 \%$ & $-7.0 \%$ & $-18.2 \%$ & $-44.5 \%$ \\
\hline & & HWFET & $0.0 \%$ & $0.0 \%$ & $-11.2 \%$ & $-15.7 \%$ & $-5.2 \%$ & $-14.9 \%$ & $-31.9 \%$ & $-8.6 \%$ & $-18.3 \%$ & $-42.4 \%$ & $-9.5 \%$ & $-20.7 \%$ & $-45.0 \%$ \\
\hline & & Combined & $0.0 \%$ & $0.0 \%$ & $-10.8 \%$ & $-15.3 \%$ & $-5.1 \%$ & $-14.5 \%$ & $-31.0 \%$ & $-8.6 \%$ & $-17.4 \%$ & $-42.4 \%$ & $-7.9 \%$ & $-19.2 \%$ & $-44.7 \%$ \\
\hline & \multirow{3}{*}{ Adjusted } & UDDS & $0.0 \%$ & $0.0 \%$ & $-10.0 \%$ & $-14.4 \%$ & $-4.9 \%$ & $-13.6 \%$ & $-29.1 \%$ & $-8.2 \%$ & $-16.1 \%$ & $-40.6 \%$ & $-6.7 \%$ & $-17.5 \%$ & $-42.6 \%$ \\
\hline & & HWFET & $0.0 \%$ & $0.0 \%$ & $-11.2 \%$ & $-15.6 \%$ & $-5.2 \%$ & $-14.9 \%$ & $-31.7 \%$ & $-8.6 \%$ & $-18.2 \%$ & $-42.1 \%$ & $-9.4 \%$ & $-20.6 \%$ & $-44.7 \%$ \\
\hline & & Combined & $0.0 \%$ & $0.0 \%$ & $-10.5 \%$ & $-14.9 \%$ & $-5.0 \%$ & $-14.1 \%$ & $-30.1 \%$ & $-8.3 \%$ & $-16.9 \%$ & $-41.2 \%$ & $-7.8 \%$ & $-18.7 \%$ & $-43.5 \%$ \\
\hline \multirow{6}{*}{$\mathrm{Cl}$ Conv } & \multirow{3}{*}{ Unadjusted } & UDDS & $-17.2 \%$ & $-17.2 \%$ & $-22.8 \%$ & $-28.9 \%$ & $-18.8 \%$ & $-27.8 \%$ & $-33.6 \%$ & $-23.4 \%$ & $-31.7 \%$ & $-43.4 \%$ & $-24.0 \%$ & $-34.5 \%$ & $-45.3 \%$ \\
\hline & & HWFET & $-13.5 \%$ & $-13.5 \%$ & $-19.4 \%$ & $-25.7 \%$ & $-15.3 \%$ & $-24.9 \%$ & $-27.4 \%$ & $-19.4 \%$ & $-24.9 \%$ & $-36.9 \%$ & $-16.7 \%$ & $-29.0 \%$ & $-39.6 \%$ \\
\hline & & Combined & $-15.8 \%$ & $-15.8 \%$ & $-21.5 \%$ & $-27.7 \%$ & $-17.5 \%$ & $-26.7 \%$ & $-31.2 \%$ & $-21.9 \%$ & $-29.2 \%$ & $-40.9 \%$ & $-21.2 \%$ & $-32.4 \%$ & $-43.2 \%$ \\
\hline & \multirow{3}{*}{ Adjusted } & \begin{tabular}{|l|} 
UDDS \\
\end{tabular} & $-16.5 \%$ & $-16.5 \%$ & $-21.8 \%$ & $-27.7 \%$ & $-18.0 \%$ & $-26.6 \%$ & $-32.2 \%$ & $-22.4 \%$ & $-30.4 \%$ & $-41.6 \%$ & $-22.9 \%$ & $-33.0 \%$ & $-43.4 \%$ \\
\hline & & HWFET & $-13.4 \%$ & $-13.4 \%$ & $-19.3 \%$ & $-25.5 \%$ & $-15.2 \%$ & $-24.8 \%$ & $-27.2 \%$ & $-19.3 \%$ & $-24.8 \%$ & $-36.7 \%$ & $-16.6 \%$ & $-28.9 \%$ & $-39.4 \%$ \\
\hline & & Combined & $-15.3 \%$ & $-15.3 \%$ & $-20.8 \%$ & $-26.8 \%$ & $-16.9 \%$ & $-25.9 \%$ & $-30.2 \%$ & $-21.1 \%$ & $-28.1 \%$ & $-39.6 \%$ & $-20.4 \%$ & $-31.3 \%$ & $-41.8 \%$ \\
\hline \multirow{6}{*}{ H2 Conv } & \multirow{3}{*}{ Unadjusted } & \begin{tabular}{|l|} 
UDDS \\
\end{tabular} & $17.4 \%$ & $17.4 \%$ & $-4.3 \%$ & $-16.0 \%$ & $3.7 \%$ & $-14.7 \%$ & $-24.0 \%$ & $-8.2 \%$ & $-21.8 \%$ & $-36.1 \%$ & $-11.9 \%$ & $-26.4 \%$ & $-40.7 \%$ \\
\hline & & HWFET & $4.3 \%$ & $4.3 \%$ & $-10.6 \%$ & $-21.4 \%$ & $-7.9 \%$ & $-20.5 \%$ & $-30.5 \%$ & $-13.9 \%$ & $-28.2 \%$ & $-40.3 \%$ & $-19.9 \%$ & $-33.3 \%$ & $-45.1 \%$ \\
\hline & & Combined & $12.5 \%$ & $12.5 \%$ & $-6.7 \%$ & $-18.0 \%$ & $-0.7 \%$ & $-16.9 \%$ & $-26.5 \%$ & $-10.4 \%$ & $-24.2 \%$ & $-37.7 \%$ & $-15.0 \%$ & $-29.0 \%$ & $-42.4 \%$ \\
\hline & \multirow{3}{*}{ Adjusted } & \begin{tabular}{|l|} 
UDDS \\
\end{tabular} & $16.7 \%$ & $16.7 \%$ & $-4.1 \%$ & $-15.3 \%$ & $3.6 \%$ & $-14.1 \%$ & $-23.0 \%$ & $-7.9 \%$ & $-20.9 \%$ & $-34.6 \%$ & $-11.4 \%$ & $-25.3 \%$ & $-39.0 \%$ \\
\hline & & HWFET & $4.3 \%$ & $4.3 \%$ & $-10.5 \%$ & $-21.2 \%$ & $-7.8 \%$ & $-20.4 \%$ & $-30.4 \%$ & $-13.8 \%$ & $-28.1 \%$ & $-40.0 \%$ & $-19.8 \%$ & $-33.0 \%$ & $-44.8 \%$ \\
\hline & & Combined & $11.7 \%$ & $11.7 \%$ & $-6.7 \%$ & $-17.7 \%$ & $-1.0 \%$ & $-16.6 \%$ & $-26.0 \%$ & $-10.3 \%$ & $-23.8 \%$ & $-36.8 \%$ & $-14.8 \%$ & $-28.4 \%$ & $-41.3 \%$ \\
\hline \multirow{6}{*}{ E85 Conv } & \multirow{3}{*}{ Unadjusted } & UDDS & $3.8 \%$ & $3.8 \%$ & $-1.7 \%$ & $-6.2 \%$ & $6.1 \%$ & $-5.2 \%$ & $-28.0 \%$ & $0.6 \%$ & $-8.9 \%$ & $-38.9 \%$ & $1.5 \%$ & $-10.7 \%$ & $-41.3 \%$ \\
\hline & & HWFET & $11.9 \%$ & $11.9 \%$ & $5.1 \%$ & $0.2 \%$ & $14.2 \%$ & $1.1 \%$ & $-23.0 \%$ & $8.1 \%$ & $-2.3 \%$ & $-33.6 \%$ & $8.3 \%$ & $-5.3 \%$ & $-36.6 \%$ \\
\hline & & Combined & $6.9 \%$ & $6.9 \%$ & $0.8 \%$ & $-3.7 \%$ & $9.2 \%$ & $-2.8 \%$ & $-26.1 \%$ & $3.4 \%$ & $-6.4 \%$ & $-36.8 \%$ & $4.1 \%$ & $-8.6 \%$ & $-39.5 \%$ \\
\hline & \multirow{3}{*}{ Adjusted } & \begin{tabular}{|l|} 
UDDS \\
\end{tabular} & $3.6 \%$ & $3.6 \%$ & $-1.7 \%$ & $-5.9 \%$ & $5.8 \%$ & $-4.9 \%$ & $-26.8 \%$ & $0.5 \%$ & $-8.5 \%$ & $-37.2 \%$ & $1.4 \%$ & $-10.2 \%$ & $-39.5 \%$ \\
\hline & & \begin{tabular}{|c|} 
HWFET \\
\end{tabular} & $11.9 \%$ & $11.9 \%$ & $5.0 \%$ & $0.2 \%$ & $14.1 \%$ & $1.1 \%$ & $-22.9 \%$ & $8.0 \%$ & $-2.3 \%$ & $-33.4 \%$ & $8.3 \%$ & $-5.3 \%$ & $-36.4 \%$ \\
\hline & & Combined & $6.9 \%$ & $6.9 \%$ & $1.0 \%$ & $-3.4 \%$ & $9.2 \%$ & $-2.5 \%$ & $-25.2 \%$ & $3.6 \%$ & $-6.0 \%$ & $-35.7 \%$ & $4.2 \%$ & $-8.2 \%$ & $-38.3 \%$ \\
\hline \multirow{6}{*}{ SI Split HEV } & \multirow{3}{*}{ Unadjusted } & UDDS & $-44.4 \%$ & $-44.4 \%$ & $-48.8 \%$ & $-52.3 \%$ & $-46.4 \%$ & $-52.5 \%$ & $-60.9 \%$ & $-49.0 \%$ & $-56.3 \%$ & $-68.4 \%$ & $-50.1 \%$ & $-58.3 \%$ & $-69.8 \%$ \\
\hline & & \begin{tabular}{|l|} 
HWFET \\
\end{tabular} & $-14.4 \%$ & $-14.4 \%$ & $-21.1 \%$ & $-26.1 \%$ & $-17.2 \%$ & $-25.6 \%$ & $-37.8 \%$ & $-19.8 \%$ & $-30.6 \%$ & $-48.0 \%$ & $-21.8 \%$ & $-34.4 \%$ & $-50.6 \%$ \\
\hline & & Combined & $-33.0 \%$ & $-33.0 \%$ & $-38.3 \%$ & $-42.4 \%$ & $-35.3 \%$ & $-42.3 \%$ & $-52.2 \%$ & $-37.9 \%$ & $-46.5 \%$ & $-60.7 \%$ & $-39.4 \%$ & $-49.2 \%$ & $-62.5 \%$ \\
\hline & \multirow{3}{*}{ Adjusted } & \begin{tabular}{|l|} 
UDDS \\
\end{tabular} & $-42.5 \%$ & $-42.5 \%$ & $-46.7 \%$ & $-50.1 \%$ & $-44.4 \%$ & $-50.3 \%$ & $-58.4 \%$ & $-46.9 \%$ & $-53.9 \%$ & $-65.5 \%$ & $-48.0 \%$ & $-55.8 \%$ & $-66.8 \%$ \\
\hline & & HWFET & $-14.3 \%$ & $-14.3 \%$ & $-21.0 \%$ & $-25.9 \%$ & $-17.1 \%$ & $-25.5 \%$ & $-37.6 \%$ & $-19.7 \%$ & $-30.4 \%$ & $-47.8 \%$ & $-21.7 \%$ & $-34.1 \%$ & $-50.3 \%$ \\
\hline & & Combined & $-31.2 \%$ & $-31.2 \%$ & $-36.3 \%$ & $-40.4 \%$ & $-33.4 \%$ & $-40.3 \%$ & $-50.0 \%$ & $-36.0 \%$ & $-44.4 \%$ & $-58.4 \%$ & $-37.4 \%$ & $-47.1 \%$ & $-60.2 \%$ \\
\hline \multirow{6}{*}{ SI Split HEV PHEV10 } & \multirow{3}{*}{ Unadjusted } & \begin{tabular}{|l|} 
UDDS \\
\end{tabular} & $-55.9 \%$ & $-55.9 \%$ & $-59.2 \%$ & $-62.1 \%$ & $-57.3 \%$ & $-61.5 \%$ & $-68.3 \%$ & $-58.4 \%$ & $-64.3 \%$ & $-74.0 \%$ & $-59.6 \%$ & $-66.0 \%$ & $-75.1 \%$ \\
\hline & & HWFET & $-34.5 \%$ & $-34.5 \%$ & $-39.2 \%$ & $-42.9 \%$ & $-36.6 \%$ & $-42.4 \%$ & $-51.2 \%$ & $-37.8 \%$ & $-45.7 \%$ & $-58.8 \%$ & $-39.5 \%$ & $-48.1 \%$ & $-61.2 \%$ \\
\hline & & Combined & $-47.8 \%$ & $-47.8 \%$ & $-51.6 \%$ & $-54.8 \%$ & $-49.4 \%$ & $-54.3 \%$ & $-61.8 \%$ & $-50.6 \%$ & $-57.3 \%$ & $-68.2 \%$ & $-52.0 \%$ & $-59.2 \%$ & $-69.9 \%$ \\
\hline & \multirow{3}{*}{ Adjusted } & \begin{tabular}{|l|} 
UDDS \\
\end{tabular} & $-53.5 \%$ & $-53.5 \%$ & $-56.7 \%$ & $-59.4 \%$ & $-54.9 \%$ & $-58.9 \%$ & $-65.4 \%$ & $-56.0 \%$ & $-61.6 \%$ & $-70.8 \%$ & $-57.0 \%$ & $-63.2 \%$ & $-72.0 \%$ \\
\hline & & HWFET & $-34.3 \%$ & $-34.3 \%$ & $-38.9 \%$ & $-42.7 \%$ & $-36.3 \%$ & $-42.1 \%$ & $-50.9 \%$ & $-37.6 \%$ & $-45.5 \%$ & $-58.4 \%$ & $-39.3 \%$ & $-47.8 \%$ & $-60.9 \%$ \\
\hline & & Combined & $-45.8 \%$ & $-45.8 \%$ & $-49.5 \%$ & $-52.7 \%$ & $-47.4 \%$ & $-52.2 \%$ & $-59.6 \%$ & $-48.6 \%$ & $-55.1 \%$ & $-65.8 \%$ & $-49.9 \%$ & $-57.0 \%$ & $-67.5 \%$ \\
\hline
\end{tabular}


Argonne National Laboratory Report - Light-Duty Vehicle Fuel Consumption Displacement Potential Up to 2045

\begin{tabular}{|c|c|c|c|c|c|c|c|c|c|c|c|c|c|c|c|}
\hline & & & \multicolumn{13}{|c|}{ Midsize_SUV } \\
\hline & & & 2010 & & 2010 & & & 2015 & & & 2030 & & & 2045 & \\
\hline & & & Ref & low & avg & high & low & avg & high & low & avg & high & low & avg & high \\
\hline \multirow{6}{*}{ SI Split HEV PHEV2O } & \multirow{3}{*}{ Unadjusted } & UDDS & $-69.2 \%$ & $-69.2 \%$ & $-71.6 \%$ & $-73.6 \%$ & $-70.6 \%$ & $-73.2 \%$ & $-77.7 \%$ & $-71.2 \%$ & $-75.1 \%$ & $-81.8 \%$ & $-72.2 \%$ & $-76.1 \%$ & $-82.7 \%$ \\
\hline & & HWFET & $-46.5 \%$ & $-46.5 \%$ & $-50.6 \%$ & $-52.8 \%$ & $-48.3 \%$ & $-53.2 \%$ & $-57.4 \%$ & $-49.3 \%$ & $-55.8 \%$ & $-64.1 \%$ & $-50.8 \%$ & $-54.5 \%$ & $-66.5 \%$ \\
\hline & & Combined & $-60.6 \%$ & $-60.6 \%$ & $-63.7 \%$ & $-65.7 \%$ & $-62.2 \%$ & $-65.7 \%$ & $-70.0 \%$ & $-62.9 \%$ & $-67.8 \%$ & $-75.1 \%$ & $-64.1 \%$ & $-67.9 \%$ & $-76.6 \%$ \\
\hline & \multirow{3}{*}{ Adjusted } & UDDS & $-66.3 \%$ & $-66.3 \%$ & $-68.6 \%$ & $-70.5 \%$ & $-67.6 \%$ & $-70.1 \%$ & $-74.4 \%$ & $-68.2 \%$ & $-71.9 \%$ & $-78.4 \%$ & $-69.1 \%$ & $-72.9 \%$ & $-79.2 \%$ \\
\hline & & HWFET & $-46.2 \%$ & $-46.2 \%$ & $-50.3 \%$ & $-52.4 \%$ & $-48.0 \%$ & $-52.9 \%$ & $-57.0 \%$ & $-49.0 \%$ & $-55.5 \%$ & $-63.7 \%$ & $-50.4 \%$ & $-54.1 \%$ & $-66.1 \%$ \\
\hline & & \begin{tabular}{|l|} 
Combined \\
\end{tabular} & $-58.2 \%$ & $-58.2 \%$ & $-61.2 \%$ & $-63.2 \%$ & $-59.7 \%$ & $-63.2 \%$ & $-67.4 \%$ & $-60.4 \%$ & $-65.3 \%$ & $-72.4 \%$ & $-61.6 \%$ & $-65.4 \%$ & $-73.9 \%$ \\
\hline \multirow{6}{*}{ SI Series HEV PHEV30 } & \multirow{3}{*}{ Unadjusted } & \begin{tabular}{|l|} 
UDDS \\
\end{tabular} & $-65.7 \%$ & $-65.7 \%$ & $-68.6 \%$ & $-71.5 \%$ & $-66.9 \%$ & $-71.0 \%$ & $-77.0 \%$ & $-68.5 \%$ & $-74.2 \%$ & $-82.2 \%$ & $-69.4 \%$ & $-75.5 \%$ & $-82.9 \%$ \\
\hline & & HWFET & $-38.0 \%$ & $-38.0 \%$ & $-43.4 \%$ & $-47.6 \%$ & $-40.4 \%$ & $-47.1 \%$ & $-56.7 \%$ & $-42.7 \%$ & $-51.5 \%$ & $-64.3 \%$ & $-44.5 \%$ & $-54.6 \%$ & $-66.2 \%$ \\
\hline & & Combined & $-55.2 \%$ & $-55.2 \%$ & $-59.1 \%$ & $-62.4 \%$ & $-56.9 \%$ & $-61.9 \%$ & $-69.3 \%$ & $-58.7 \%$ & $-65.6 \%$ & $-75.4 \%$ & $-60.0 \%$ & $-67.5 \%$ & $-76.5 \%$ \\
\hline & \multirow{3}{*}{ Adjusted } & UDDS & $-62.9 \%$ & $-62.9 \%$ & $-65.7 \%$ & $-68.4 \%$ & $-64.1 \%$ & $-68.0 \%$ & $-73.8 \%$ & $-65.6 \%$ & $-71.1 \%$ & $-78.7 \%$ & $-66.5 \%$ & $-72.3 \%$ & $-79.4 \%$ \\
\hline & & HWFET & $-37.7 \%$ & $-37.7 \%$ & $-43.1 \%$ & $-47.3 \%$ & $-40.1 \%$ & $-46.8 \%$ & $-56.4 \%$ & $-42.4 \%$ & $-51.1 \%$ & $-63.9 \%$ & $-44.2 \%$ & $-54.2 \%$ & $-65.7 \%$ \\
\hline & & Combined & $-52.8 \%$ & $-52.8 \%$ & $-56.6 \%$ & $-59.9 \%$ & $-54.4 \%$ & $-59.4 \%$ & $-66.8 \%$ & $-56.3 \%$ & $-63.0 \%$ & $-72.7 \%$ & $-57.5 \%$ & $-65.0 \%$ & $-73.9 \%$ \\
\hline \multirow{6}{*}{ SI Series HEV PHEV40 } & \multirow{3}{*}{ Unadjusted } & UDDS & $-70.0 \%$ & $-70.0 \%$ & $-72.7 \%$ & $-75.0 \%$ & $-71.3 \%$ & $-74.6 \%$ & $-80.1 \%$ & $-72.6 \%$ & $-77.6 \%$ & $-84.4 \%$ & $-73.5 \%$ & $-78.8 \%$ & $-85.3 \%$ \\
\hline & & HWFET & $-50.9 \%$ & $-50.9 \%$ & $-55.3 \%$ & $-58.5 \%$ & $-52.7 \%$ & $-58.1 \%$ & $-65.8 \%$ & $-54.7 \%$ & $-61.7 \%$ & $-71.9 \%$ & $-56.2 \%$ & $-64.2 \%$ & $-73.4 \%$ \\
\hline & & Combined & $-62.8 \%$ & $-62.8 \%$ & $-66.1 \%$ & $-68.8 \%$ & $-64.3 \%$ & $-68.4 \%$ & $-74.7 \%$ & $-65.8 \%$ & $-71.6 \%$ & $-79.6 \%$ & $-66.9 \%$ & $-73.2 \%$ & $-80.8 \%$ \\
\hline & \multirow{3}{*}{ Adjusted } & \begin{tabular}{|l|} 
UDDS \\
\end{tabular} & $-67.0 \%$ & $-67.0 \%$ & $-69.6 \%$ & $-71.8 \%$ & $-68.3 \%$ & $-71.4 \%$ & $-76.7 \%$ & $-69.5 \%$ & $-74.3 \%$ & $-80.8 \%$ & $-70.4 \%$ & $-75.4 \%$ & $-81.7 \%$ \\
\hline & & HWFET & $-50.6 \%$ & $-50.6 \%$ & $-54.9 \%$ & $-58.2 \%$ & $-52.4 \%$ & $-57.8 \%$ & $-65.4 \%$ & $-54.3 \%$ & $-61.4 \%$ & $-71.5 \%$ & $-55.8 \%$ & $-63.8 \%$ & $-73.0 \%$ \\
\hline & & Combined & $-60.4 \%$ & $-60.4 \%$ & $-63.7 \%$ & $-66.3 \%$ & $-61.9 \%$ & $-65.9 \%$ & $-72.2 \%$ & $-63.4 \%$ & $-69.1 \%$ & $-77.0 \%$ & $-64.5 \%$ & $-70.7 \%$ & $-78.2 \%$ \\
\hline \multirow{6}{*}{ CI Split HEV } & \multirow{3}{*}{ Unadjusted } & UDDS & $-50.1 \%$ & $-50.1 \%$ & $-53.3 \%$ & $-57.2 \%$ & $-51.1 \%$ & $-57.1 \%$ & $-62.5 \%$ & $-53.9 \%$ & $-61.0 \%$ & $-68.6 \%$ & $-55.6 \%$ & $-63.1 \%$ & $-69.9 \%$ \\
\hline & & HWFET & $-20.0 \%$ & $-20.0 \%$ & $-25.2 \%$ & $-31.1 \%$ & $-21.7 \%$ & $-30.5 \%$ & $-38.2 \%$ & $-25.0 \%$ & $-35.9 \%$ & $-46.8 \%$ & $-27.9 \%$ & $-39.9 \%$ & $-49.5 \%$ \\
\hline & & \begin{tabular}{|l|} 
Combined \\
\end{tabular} & $-38.7 \%$ & $-38.7 \%$ & $-42.7 \%$ & $-47.3 \%$ & $-39.9 \%$ & $-47.0 \%$ & $-53.3 \%$ & $-43.0 \%$ & $-51.5 \%$ & $-60.3 \%$ & $-45.1 \%$ & $-54.3 \%$ & $-62.2 \%$ \\
\hline & \multirow{3}{*}{ Adjusted } & UDDS & $-48.0 \%$ & $-48.0 \%$ & $-51.1 \%$ & $-54.8 \%$ & $-48.9 \%$ & $-54.7 \%$ & $-59.8 \%$ & $-51.7 \%$ & $-58.5 \%$ & $-65.7 \%$ & $-53.2 \%$ & $-60.4 \%$ & $-67.0 \%$ \\
\hline & & HWFET & $-19.9 \%$ & $-19.9 \%$ & $-25.1 \%$ & $-30.9 \%$ & $-21.5 \%$ & $-30.3 \%$ & $-38.0 \%$ & $-24.8 \%$ & $-35.7 \%$ & $-46.5 \%$ & $-27.7 \%$ & $-39.6 \%$ & $-49.2 \%$ \\
\hline & & \begin{tabular}{|l|} 
Combined \\
\end{tabular} & $-36.7 \%$ & $-36.7 \%$ & $-40.6 \%$ & $-45.1 \%$ & $-37.9 \%$ & $-44.9 \%$ & $-51.0 \%$ & $-40.9 \%$ & $-49.3 \%$ & $-58.0 \%$ & $-43.0 \%$ & $-52.1 \%$ & $-59.8 \%$ \\
\hline \multirow{6}{*}{ CI Split HEV PHEV10 } & \multirow{3}{*}{ Unadjusted } & UDDS & $-57.3 \%$ & $-57.3 \%$ & $-60.1 \%$ & $-63.4 \%$ & $-58.2 \%$ & $-63.1 \%$ & $-67.6 \%$ & $-60.2 \%$ & $-66.1 \%$ & $-72.7 \%$ & $-61.6 \%$ & $-68.1 \%$ & $-73.8 \%$ \\
\hline & & HWFET & $-36.3 \%$ & $-36.3 \%$ & $-39.9 \%$ & $-46.9 \%$ & $-36.9 \%$ & $-46.8 \%$ & $-52.3 \%$ & $-40.0 \%$ & $-47.7 \%$ & $-58.3 \%$ & $-41.8 \%$ & $-52.9 \%$ & $-58.4 \%$ \\
\hline & & Combined & $-49.3 \%$ & $-49.3 \%$ & $-52.4 \%$ & $-57.1 \%$ & $-50.1 \%$ & $-56.9 \%$ & $-61.8 \%$ & $-52.5 \%$ & $-59.2 \%$ & $-67.3 \%$ & $-54.1 \%$ & $-62.3 \%$ & $-68.0 \%$ \\
\hline & \multirow{3}{*}{ Adjusted } & \begin{tabular}{|l|} 
UDDS \\
\end{tabular} & $-54.9 \%$ & $-54.9 \%$ & $-57.6 \%$ & $-60.7 \%$ & $-55.7 \%$ & $-60.5 \%$ & $-64.7 \%$ & $-57.7 \%$ & $-63.3 \%$ & $-69.6 \%$ & $-59.0 \%$ & $-65.2 \%$ & $-70.7 \%$ \\
\hline & & HWFET & $-36.1 \%$ & $-36.1 \%$ & $-39.6 \%$ & $-46.6 \%$ & $-36.7 \%$ & $-46.5 \%$ & $-52.0 \%$ & $-39.7 \%$ & $-47.4 \%$ & $-58.0 \%$ & $-41.5 \%$ & $-52.6 \%$ & $-58.0 \%$ \\
\hline & & \begin{tabular}{|l|} 
Combined \\
\end{tabular} & $-47.3 \%$ & $-47.3 \%$ & $-50.3 \%$ & $-55.0 \%$ & $-48.1 \%$ & $-54.8 \%$ & $-59.6 \%$ & $-50.4 \%$ & $-56.9 \%$ & $-64.9 \%$ & $-52.0 \%$ & $-60.1 \%$ & $-65.6 \%$ \\
\hline \multirow{6}{*}{ CI Split HEV PHEV2O } & \multirow{3}{*}{ Unadjusted } & \begin{tabular}{|l|} 
UDDS \\
\end{tabular} & $-70.1 \%$ & $-70.1 \%$ & $-72.4 \%$ & $-74.6 \%$ & $-70.9 \%$ & $-74.2 \%$ & $-77.4 \%$ & $-72.4 \%$ & $-76.3 \%$ & $-81.1 \%$ & $-73.3 \%$ & $-77.6 \%$ & $-81.7 \%$ \\
\hline & & HWFET & $-47.9 \%$ & $-47.9 \%$ & $-42.7 \%$ & $-46.1 \%$ & $-49.0 \%$ & $-46.0 \%$ & $-58.3 \%$ & $-50.8 \%$ & $-56.5 \%$ & $-63.6 \%$ & $-44.1 \%$ & $-58.7 \%$ & $-63.7 \%$ \\
\hline & & Combined & $-61.7 \%$ & $-61.7 \%$ & $-61.2 \%$ & $-63.8 \%$ & $-62.6 \%$ & $-63.5 \%$ & $-70.1 \%$ & $-64.2 \%$ & $-68.8 \%$ & $-74.4 \%$ & $-62.2 \%$ & $-70.4 \%$ & $-74.9 \%$ \\
\hline & \multirow{3}{*}{ Adjusted } & \begin{tabular}{|l|} 
UDDS \\
\end{tabular} & $-67.2 \%$ & $-67.2 \%$ & $-69.4 \%$ & $-71.4 \%$ & $-67.9 \%$ & $-71.1 \%$ & $-74.1 \%$ & $-69.3 \%$ & $-73.1 \%$ & $-77.6 \%$ & $-70.2 \%$ & $-74.3 \%$ & $-78.3 \%$ \\
\hline & & HWFET & $-47.6 \%$ & $-47.6 \%$ & $-42.4 \%$ & $-45.8 \%$ & $-48.7 \%$ & $-45.7 \%$ & $-57.9 \%$ & $-50.5 \%$ & $-56.1 \%$ & $-63.2 \%$ & $-43.8 \%$ & $-58.3 \%$ & $-63.3 \%$ \\
\hline & & Combined & $-59.3 \%$ & $-59.3 \%$ & $-58.5 \%$ & $-61.1 \%$ & $-60.2 \%$ & $-60.9 \%$ & $-67.6 \%$ & $-61.7 \%$ & $-66.3 \%$ & $-71.8 \%$ & $-59.6 \%$ & $-67.9 \%$ & $-72.2 \%$ \\
\hline
\end{tabular}


Argonne National Laboratory Report - Light-Duty Vehicle Fuel Consumption Displacement Potential Up to 2045

\begin{tabular}{|c|c|c|c|c|c|c|c|c|c|c|c|c|c|c|c|}
\hline & & & \multicolumn{13}{|c|}{ Midsize_SUV } \\
\hline & & & 2010 & & 2010 & & & 2015 & & & 2030 & & & 2045 & \\
\hline & & & Ref & low & avg & high & low & avg & high & low & avg & high & low & avg & high \\
\hline \multirow{6}{*}{$\mathrm{Cl}$ Series HEV PHEV 30} & \multirow{3}{*}{ Unadjusted } & UDDS & $-66.4 \%$ & $-66.4 \%$ & $-69.1 \%$ & $-72.4 \%$ & $-67.3 \%$ & $-71.9 \%$ & $-76.6 \%$ & $-69.6 \%$ & $-75.3 \%$ & $-80.8 \%$ & $-70.8 \%$ & $-76.8 \%$ & $-81.8 \%$ \\
\hline & & HWFET & $-39.4 \%$ & $-39.4 \%$ & $-44.3 \%$ & $-49.1 \%$ & $-40.9 \%$ & $-48.6 \%$ & $-55.4 \%$ & $-44.5 \%$ & $-53.6 \%$ & $-62.5 \%$ & $-46.8 \%$ & $-56.9 \%$ & $-64.4 \%$ \\
\hline & & Combined & $-56.2 \%$ & $-56.2 \%$ & $-59.7 \%$ & $-63.6 \%$ & $-57.3 \%$ & $-63.1 \%$ & $-68.5 \%$ & $-60.1 \%$ & $-67.1 \%$ & $-73.9 \%$ & $-61.7 \%$ & $-69.2 \%$ & $-75.2 \%$ \\
\hline & \multirow{3}{*}{ Adjusted } & UDDS & $-63.6 \%$ & $-63.6 \%$ & $-66.2 \%$ & $-69.3 \%$ & $-64.5 \%$ & $-68.9 \%$ & $-73.3 \%$ & $-66.6 \%$ & $-72.1 \%$ & $-77.4 \%$ & $-67.8 \%$ & $-73.5 \%$ & $-78.3 \%$ \\
\hline & & HWFET & $-39.2 \%$ & $-39.2 \%$ & $-44.0 \%$ & $-48.8 \%$ & $-40.7 \%$ & $-48.3 \%$ & $-55.0 \%$ & $-44.2 \%$ & $-53.3 \%$ & $-62.1 \%$ & $-46.5 \%$ & $-56.6 \%$ & $-64.0 \%$ \\
\hline & & \begin{tabular}{|l|} 
Combined \\
\end{tabular} & $-53.8 \%$ & $-53.8 \%$ & $-57.3 \%$ & $-61.1 \%$ & $-54.9 \%$ & $-60.6 \%$ & $-66.0 \%$ & $-57.6 \%$ & $-64.5 \%$ & $-71.3 \%$ & $-59.2 \%$ & $-66.7 \%$ & $-72.6 \%$ \\
\hline \multirow{6}{*}{$\mathrm{Cl}$ Series HEV PHEV 40} & \multirow{3}{*}{ Unadjusted } & UDDS & $-70.7 \%$ & $-70.7 \%$ & $-73.1 \%$ & $-75.9 \%$ & $-71.6 \%$ & $-75.5 \%$ & $-79.5 \%$ & $-73.5 \%$ & $-78.6 \%$ & $-83.6 \%$ & $-74.6 \%$ & $-79.9 \%$ & $-84.2 \%$ \\
\hline & & HWFET & $-52.1 \%$ & $-52.1 \%$ & $-55.9 \%$ & $-59.8 \%$ & $-53.2 \%$ & $-59.4 \%$ & $-64.8 \%$ & $-56.0 \%$ & $-63.4 \%$ & $-70.5 \%$ & $-58.0 \%$ & $-66.0 \%$ & $-72.0 \%$ \\
\hline & & \begin{tabular}{|l|} 
Combined \\
\end{tabular} & $-63.6 \%$ & $-63.6 \%$ & $-66.6 \%$ & $-69.8 \%$ & $-64.6 \%$ & $-69.4 \%$ & $-73.9 \%$ & $-66.9 \%$ & $-72.8 \%$ & $-78.7 \%$ & $-68.3 \%$ & $-74.6 \%$ & $-79.6 \%$ \\
\hline & \multirow{3}{*}{ Adjusted } & \begin{tabular}{|l|} 
UDDS \\
\end{tabular} & $-67.7 \%$ & $-67.7 \%$ & $-70.0 \%$ & $-72.7 \%$ & $-68.5 \%$ & $-72.3 \%$ & $-76.1 \%$ & $-70.4 \%$ & $-75.2 \%$ & $-80.1 \%$ & $-71.5 \%$ & $-76.5 \%$ & $-80.6 \%$ \\
\hline & & HWFET & $-51.7 \%$ & $-51.7 \%$ & $-55.6 \%$ & $-59.4 \%$ & $-52.9 \%$ & $-59.1 \%$ & $-64.4 \%$ & $-55.7 \%$ & $-63.1 \%$ & $-70.1 \%$ & $-57.6 \%$ & $-65.6 \%$ & $-71.5 \%$ \\
\hline & & \begin{tabular}{|l|} 
Combined \\
\end{tabular} & $-61.3 \%$ & $-61.3 \%$ & $-64.2 \%$ & $-67.4 \%$ & $-62.2 \%$ & $-67.0 \%$ & $-71.4 \%$ & $-64.5 \%$ & $-70.3 \%$ & $-76.1 \%$ & $-65.9 \%$ & $-72.1 \%$ & $-77.0 \%$ \\
\hline \multirow{6}{*}{ H2 Split HEV } & \multirow{3}{*}{ Unadjusted } & \begin{tabular}{|l|} 
UDDS \\
\end{tabular} & $-43.3 \%$ & $-43.3 \%$ & $-52.6 \%$ & $-58.8 \%$ & $-50.0 \%$ & $-58.6 \%$ & $-64.8 \%$ & $-54.8 \%$ & $-63.3 \%$ & $-71.0 \%$ & $-57.8 \%$ & $-66.1 \%$ & $-73.3 \%$ \\
\hline & & HWFET & $-11.6 \%$ & $-11.6 \%$ & $-26.0 \%$ & $-35.0 \%$ & $-21.9 \%$ & $-34.4 \%$ & $-43.2 \%$ & $-28.5 \%$ & $-41.1 \%$ & $-51.6 \%$ & $-33.1 \%$ & $-45.8 \%$ & $-55.8 \%$ \\
\hline & & Combined & $-31.3 \%$ & $-31.3 \%$ & $-42.5 \%$ & $-49.8 \%$ & $-39.4 \%$ & $-49.4 \%$ & $-56.6 \%$ & $-44.8 \%$ & $-54.9 \%$ & $-63.7 \%$ & $-48.5 \%$ & $-58.4 \%$ & $-66.7 \%$ \\
\hline & \multirow{3}{*}{ Adjusted } & \begin{tabular}{|l|} 
UDDS \\
\end{tabular} & $-41.4 \%$ & $-41.4 \%$ & $-50.4 \%$ & $-56.3 \%$ & $-47.9 \%$ & $-56.1 \%$ & $-62.1 \%$ & $-52.5 \%$ & $-60.7 \%$ & $-68.0 \%$ & $-55.4 \%$ & $-63.3 \%$ & $-70.2 \%$ \\
\hline & & HWFET & $-11.6 \%$ & $-11.6 \%$ & $-25.8 \%$ & $-34.8 \%$ & $-21.8 \%$ & $-34.2 \%$ & $-42.9 \%$ & $-28.3 \%$ & $-40.8 \%$ & $-51.3 \%$ & $-32.9 \%$ & $-45.5 \%$ & $-55.4 \%$ \\
\hline & & Combined & $-29.4 \%$ & $-29.4 \%$ & $-40.5 \%$ & $-47.6 \%$ & $-37.4 \%$ & $-47.3 \%$ & $-54.4 \%$ & $-42.8 \%$ & $-52.7 \%$ & $-61.3 \%$ & $-46.3 \%$ & $-56.1 \%$ & $-64.3 \%$ \\
\hline \multirow{6}{*}{ H2 Split HEV PHEV10 } & \multirow{3}{*}{ Unadjusted } & UDDS & $-54.5 \%$ & $-54.5 \%$ & $-62.1 \%$ & $-66.8 \%$ & $-60.0 \%$ & $-66.4 \%$ & $-71.2 \%$ & $-63.3 \%$ & $-69.8 \%$ & $-76.0 \%$ & $-65.7 \%$ & $-72.1 \%$ & $-78.0 \%$ \\
\hline & & HWFET & $-32.9 \%$ & $-32.9 \%$ & $-43.5 \%$ & $-50.1 \%$ & $-40.6 \%$ & $-49.6 \%$ & $-55.6 \%$ & $-45.1 \%$ & $-54.0 \%$ & $-63.2 \%$ & $-48.7 \%$ & $-57.5 \%$ & $-66.4 \%$ \\
\hline & & \begin{tabular}{|l|} 
Combined \\
\end{tabular} & $-46.3 \%$ & $-46.3 \%$ & $-55.0 \%$ & $-60.5 \%$ & $-52.7 \%$ & $-60.0 \%$ & $-65.3 \%$ & $-56.4 \%$ & $-63.8 \%$ & $-71.2 \%$ & $-59.2 \%$ & $-66.6 \%$ & $-73.6 \%$ \\
\hline & \multirow{3}{*}{ Adjusted } & UDDS & $-52.2 \%$ & $-52.2 \%$ & $-59.4 \%$ & $-64.0 \%$ & $-57.5 \%$ & $-63.5 \%$ & $-68.2 \%$ & $-60.6 \%$ & $-66.9 \%$ & $-72.8 \%$ & $-62.9 \%$ & $-69.1 \%$ & $-74.7 \%$ \\
\hline & & HWFET & $-32.7 \%$ & $-32.7 \%$ & $-43.2 \%$ & $-49.8 \%$ & $-40.4 \%$ & $-49.3 \%$ & $-55.3 \%$ & $-44.8 \%$ & $-53.6 \%$ & $-62.8 \%$ & $-48.4 \%$ & $-57.1 \%$ & $-66.0 \%$ \\
\hline & & Combined & $-44.4 \%$ & $-44.4 \%$ & $-52.9 \%$ & $-58.3 \%$ & $-50.6 \%$ & $-57.8 \%$ & $-63.0 \%$ & $-54.3 \%$ & $-61.5 \%$ & $-68.8 \%$ & $-57.1 \%$ & $-64.2 \%$ & $-71.2 \%$ \\
\hline \multirow{6}{*}{ H2 Split HEV PHEV2O } & \multirow{3}{*}{ Unadjusted } & UDDS & $-68.4 \%$ & $-68.4 \%$ & $-73.7 \%$ & $-77.0 \%$ & $-72.5 \%$ & $-76.6 \%$ & $-79.9 \%$ & $-74.9 \%$ & $-79.0 \%$ & $-83.3 \%$ & $-76.3 \%$ & $-80.5 \%$ & $-84.9 \%$ \\
\hline & & HWFET & $-45.5 \%$ & $-45.5 \%$ & $-52.9 \%$ & $-59.3 \%$ & $-51.8 \%$ & $-58.3 \%$ & $-61.2 \%$ & $-55.4 \%$ & $-61.4 \%$ & $-61.9 \%$ & $-58.2 \%$ & $-62.8 \%$ & $-68.2 \%$ \\
\hline & & Combined & $-59.7 \%$ & $-59.7 \%$ & $-65.8 \%$ & $-70.3 \%$ & $-64.6 \%$ & $-69.7 \%$ & $-72.8 \%$ & $-67.5 \%$ & $-72.3 \%$ & $-75.2 \%$ & $-69.4 \%$ & $-73.8 \%$ & $-78.6 \%$ \\
\hline & \multirow{3}{*}{ Adjusted } & \begin{tabular}{|l|} 
UDDS \\
\end{tabular} & $-65.5 \%$ & $-65.5 \%$ & $-70.6 \%$ & $-73.7 \%$ & $-69.4 \%$ & $-73.4 \%$ & $-76.5 \%$ & $-71.7 \%$ & $-75.7 \%$ & $-79.8 \%$ & $-73.1 \%$ & $-77.1 \%$ & $-81.3 \%$ \\
\hline & & \begin{tabular}{|l|} 
HWFET \\
\end{tabular} & $-45.3 \%$ & $-45.3 \%$ & $-52.6 \%$ & $-59.0 \%$ & $-51.4 \%$ & $-57.9 \%$ & $-60.9 \%$ & $-55.0 \%$ & $-61.0 \%$ & $-61.5 \%$ & $-57.8 \%$ & $-62.4 \%$ & $-67.8 \%$ \\
\hline & & \begin{tabular}{|l|} 
Combined \\
\end{tabular} & $-57.3 \%$ & $-57.3 \%$ & $-63.3 \%$ & $-67.8 \%$ & $-62.2 \%$ & $-67.2 \%$ & $-70.2 \%$ & $-65.0 \%$ & $-69.8 \%$ & $-72.4 \%$ & $-66.9 \%$ & $-71.2 \%$ & $-75.9 \%$ \\
\hline \multirow{6}{*}{ H2 Series HEV PHEV30 } & \multirow{3}{*}{ Unadjusted } & \begin{tabular}{|l|} 
UDDS \\
\end{tabular} & $-64.7 \%$ & $-64.7 \%$ & $-71.2 \%$ & $-75.3 \%$ & $-69.2 \%$ & $-74.8 \%$ & $-79.4 \%$ & $-72.3 \%$ & $-78.2 \%$ & $-83.2 \%$ & $-74.4 \%$ & $-80.1 \%$ & $-84.6 \%$ \\
\hline & & HWFET & $-37.0 \%$ & $-37.0 \%$ & $-47.8 \%$ & $-54.4 \%$ & $-44.7 \%$ & $-53.9 \%$ & $-60.9 \%$ & $-49.8 \%$ & $-59.2 \%$ & $-66.8 \%$ & $-53.2 \%$ & $-62.9 \%$ & $-69.7 \%$ \\
\hline & & Combined & $-54.2 \%$ & $-54.2 \%$ & $-62.3 \%$ & $-67.4 \%$ & $-59.9 \%$ & $-66.9 \%$ & $-72.4 \%$ & $-63.8 \%$ & $-71.0 \%$ & $-77.0 \%$ & $-66.4 \%$ & $-73.6 \%$ & $-79.0 \%$ \\
\hline & \multirow{3}{*}{ Adjusted } & \begin{tabular}{|l|} 
UDDS \\
\end{tabular} & $-61.9 \%$ & $-61.9 \%$ & $-68.2 \%$ & $-72.1 \%$ & $-66.2 \%$ & $-71.7 \%$ & $-76.0 \%$ & $-69.3 \%$ & $-74.9 \%$ & $-79.7 \%$ & $-71.3 \%$ & $-76.7 \%$ & $-81.1 \%$ \\
\hline & & HWFET & $-36.7 \%$ & $-36.7 \%$ & $-47.5 \%$ & $-54.1 \%$ & $-44.4 \%$ & $-53.6 \%$ & $-60.5 \%$ & $-49.5 \%$ & $-58.8 \%$ & $-66.4 \%$ & $-52.9 \%$ & $-62.5 \%$ & $-69.3 \%$ \\
\hline & & Combined & $-51.8 \%$ & $-51.8 \%$ & $-59.9 \%$ & $-64.8 \%$ & $-57.5 \%$ & $-64.4 \%$ & $-69.8 \%$ & $-61.3 \%$ & $-68.4 \%$ & $-74.4 \%$ & $-63.9 \%$ & $-71.0 \%$ & $-76.3 \%$ \\
\hline
\end{tabular}


Argonne National Laboratory Report - Light-Duty Vehicle Fuel Consumption Displacement Potential Up to 2045

\begin{tabular}{|c|c|c|c|c|c|c|c|c|c|c|c|c|c|c|c|}
\hline & & & \multicolumn{13}{|c|}{ Midsize_SUV } \\
\hline & & & 2010 & & 2010 & & & 2015 & & & 2030 & & & 2045 & \\
\hline & & & Ref & low & avg & high & low & avg & high & low & avg & high & low & avg & high \\
\hline \multirow{6}{*}{ H2 Series HEV PHEV40 } & \multirow{3}{*}{ Unadjusted } & UDDS & $-69.2 \%$ & $-69.2 \%$ & $-74.8 \%$ & $-78.5 \%$ & $-73.1 \%$ & $-78.1 \%$ & $-82.2 \%$ & $-75.9 \%$ & $-81.1 \%$ & $-85.6 \%$ & $-77.7 \%$ & $-82.6 \%$ & $-86.8 \%$ \\
\hline & & HWFET & $-50.1 \%$ & $-50.1 \%$ & $-58.7 \%$ & $-64.0 \%$ & $-56.2 \%$ & $-63.6 \%$ & $-69.2 \%$ & $-60.3 \%$ & $-67.8 \%$ & $-73.9 \%$ & $-63.1 \%$ & $-70.8 \%$ & $-76.2 \%$ \\
\hline & & Combined & $-62.0 \%$ & $-62.0 \%$ & $-68.7 \%$ & $-73.0 \%$ & $-66.7 \%$ & $-72.6 \%$ & $-77.2 \%$ & $-70.0 \%$ & $-76.1 \%$ & $-81.1 \%$ & $-72.1 \%$ & $-78.1 \%$ & $-82.8 \%$ \\
\hline & \multirow{3}{*}{ Adjusted } & \begin{tabular}{|l|} 
UDDS \\
\end{tabular} & $-66.3 \%$ & $-66.3 \%$ & $-71.6 \%$ & $-75.2 \%$ & $-70.0 \%$ & $-74.8 \%$ & $-78.7 \%$ & $-72.7 \%$ & $-77.7 \%$ & $-81.9 \%$ & $-74.4 \%$ & $-79.1 \%$ & $-83.1 \%$ \\
\hline & & HWFET & $-49.8 \%$ & $-49.8 \%$ & $-58.3 \%$ & $-63.6 \%$ & $-55.8 \%$ & $-63.2 \%$ & $-68.8 \%$ & $-59.9 \%$ & $-67.3 \%$ & $-73.4 \%$ & $-62.7 \%$ & $-70.3 \%$ & $-75.7 \%$ \\
\hline & & \begin{tabular}{|l|} 
Combined \\
\end{tabular} & $-59.6 \%$ & $-59.6 \%$ & $-66.3 \%$ & $-70.5 \%$ & $-64.3 \%$ & $-70.1 \%$ & $-74.7 \%$ & $-67.5 \%$ & $-73.5 \%$ & $-78.5 \%$ & $-69.7 \%$ & $-75.6 \%$ & $-80.1 \%$ \\
\hline \multirow{6}{*}{ E85 Split HEV } & \multirow{3}{*}{ Unadjusted } & \begin{tabular}{|l|} 
UDDS \\
\end{tabular} & $-39.9 \%$ & $-39.9 \%$ & $-44.6 \%$ & $-48.6 \%$ & $-41.9 \%$ & $-48.7 \%$ & $-60.1 \%$ & $-44.8 \%$ & $-53.0 \%$ & $-66.2 \%$ & $-46.2 \%$ & $-54.9 \%$ & $-67.8 \%$ \\
\hline & & HWFET & $-7.7 \%$ & $-7.7 \%$ & $-14.9 \%$ & $-20.7 \%$ & $-10.5 \%$ & $-19.9 \%$ & $-36.6 \%$ & $-13.6 \%$ & $-25.5 \%$ & $-44.6 \%$ & $-16.1 \%$ & $-29.2 \%$ & $-47.6 \%$ \\
\hline & & \begin{tabular}{|l|} 
Combined \\
\end{tabular} & $-27.7 \%$ & $-27.7 \%$ & $-33.3 \%$ & $-38.0 \%$ & $-30.0 \%$ & $-37.8 \%$ & $-51.1 \%$ & $-33.0 \%$ & $-42.6 \%$ & $-58.0 \%$ & $-34.8 \%$ & $-45.1 \%$ & $-60.1 \%$ \\
\hline & \multirow{3}{*}{ Adjusted } & UDDS & $-38.2 \%$ & $-38.2 \%$ & $-42.7 \%$ & $-46.5 \%$ & $-40.1 \%$ & $-46.7 \%$ & $-57.5 \%$ & $-42.9 \%$ & $-50.8 \%$ & $-63.4 \%$ & $-44.3 \%$ & $-52.6 \%$ & $-64.9 \%$ \\
\hline & & HWFET & $-7.7 \%$ & $-7.7 \%$ & $-14.8 \%$ & $-20.5 \%$ & $-10.4 \%$ & $-19.8 \%$ & $-36.3 \%$ & $-13.5 \%$ & $-25.4 \%$ & $-44.3 \%$ & $-16.0 \%$ & $-29.0 \%$ & $-47.3 \%$ \\
\hline & & \begin{tabular}{|l|} 
Combined \\
\end{tabular} & $-25.9 \%$ & $-25.9 \%$ & $-31.5 \%$ & $-36.1 \%$ & $-28.2 \%$ & $-35.8 \%$ & $-49.0 \%$ & $-31.1 \%$ & $-40.5 \%$ & $-55.7 \%$ & $-32.9 \%$ & $-43.1 \%$ & $-57.8 \%$ \\
\hline \multirow{6}{*}{ E85 Split HEV PHEV10 } & \multirow{3}{*}{ Unadjusted } & UDDS & $-52.9 \%$ & $-52.9 \%$ & $-56.4 \%$ & $-59.7 \%$ & $-54.5 \%$ & $-59.2 \%$ & $-67.9 \%$ & $-55.7 \%$ & $-61.9 \%$ & $-72.5 \%$ & $-56.8 \%$ & $-63.8 \%$ & $-73.7 \%$ \\
\hline & & HWFET & $-29.8 \%$ & $-29.8 \%$ & $-35.0 \%$ & $-39.0 \%$ & $-32.0 \%$ & $-38.5 \%$ & $-50.3 \%$ & $-33.4 \%$ & $-41.9 \%$ & $-56.4 \%$ & $-35.4 \%$ & $-44.8 \%$ & $-58.9 \%$ \\
\hline & & Combined & $-44.1 \%$ & $-44.1 \%$ & $-48.3 \%$ & $-51.9 \%$ & $-45.9 \%$ & $-51.3 \%$ & $-61.2 \%$ & $-47.3 \%$ & $-54.3 \%$ & $-66.4 \%$ & $-48.7 \%$ & $-56.6 \%$ & $-68.1 \%$ \\
\hline & \multirow{3}{*}{ Adjusted } & \begin{tabular}{|l|} 
UDDS \\
\end{tabular} & $-50.7 \%$ & $-50.7 \%$ & $-54.0 \%$ & $-57.2 \%$ & $-52.2 \%$ & $-56.7 \%$ & $-65.0 \%$ & $-53.3 \%$ & $-59.3 \%$ & $-69.4 \%$ & $-54.4 \%$ & $-61.1 \%$ & $-70.6 \%$ \\
\hline & & HWFET & $-29.6 \%$ & $-29.6 \%$ & $-34.8 \%$ & $-38.7 \%$ & $-31.8 \%$ & $-38.2 \%$ & $-50.0 \%$ & $-33.2 \%$ & $-41.6 \%$ & $-56.0 \%$ & $-35.2 \%$ & $-44.6 \%$ & $-58.6 \%$ \\
\hline & & Combined & $-42.2 \%$ & $-42.2 \%$ & $-46.3 \%$ & $-49.8 \%$ & $-44.0 \%$ & $-49.3 \%$ & $-59.0 \%$ & $-45.2 \%$ & $-52.2 \%$ & $-64.0 \%$ & $-46.7 \%$ & $-54.4 \%$ & $-65.8 \%$ \\
\hline \multirow{6}{*}{ E85 Split HEV PHEV20 } & \multirow{3}{*}{ Unadjusted } & UDDS & $-67.0 \%$ & $-67.0 \%$ & $-69.7 \%$ & $-71.8 \%$ & $-68.7 \%$ & $-71.5 \%$ & $-77.4 \%$ & $-69.6 \%$ & $-73.4 \%$ & $-80.8 \%$ & $-70.1 \%$ & $-74.6 \%$ & $-81.7 \%$ \\
\hline & & HWFET & $-42.8 \%$ & $-42.8 \%$ & $-46.2 \%$ & $-49.0 \%$ & $-44.7 \%$ & $-48.9 \%$ & $-56.8 \%$ & $-45.6 \%$ & $-49.6 \%$ & $-62.0 \%$ & $-47.3 \%$ & $-51.7 \%$ & $-64.5 \%$ \\
\hline & & Combined & $-57.8 \%$ & $-57.8 \%$ & $-60.8 \%$ & $-63.2 \%$ & $-59.6 \%$ & $-62.9 \%$ & $-69.6 \%$ & $-60.5 \%$ & $-64.4 \%$ & $-73.7 \%$ & $-61.5 \%$ & $-65.9 \%$ & $-75.2 \%$ \\
\hline & \multirow{3}{*}{ Adjusted } & UDDS & $-64.1 \%$ & $-64.1 \%$ & $-66.8 \%$ & $-68.8 \%$ & $-65.8 \%$ & $-68.5 \%$ & $-74.2 \%$ & $-66.6 \%$ & $-70.3 \%$ & $-77.4 \%$ & $-67.2 \%$ & $-71.4 \%$ & $-78.2 \%$ \\
\hline & & HWFET & $-42.5 \%$ & $-42.5 \%$ & $-45.9 \%$ & $-48.7 \%$ & $-44.4 \%$ & $-48.6 \%$ & $-56.5 \%$ & $-45.3 \%$ & $-49.3 \%$ & $-61.6 \%$ & $-47.0 \%$ & $-51.3 \%$ & $-64.1 \%$ \\
\hline & & \begin{tabular}{|l|} 
Combined \\
\end{tabular} & $-55.4 \%$ & $-55.4 \%$ & $-58.4 \%$ & $-60.7 \%$ & $-57.2 \%$ & $-60.5 \%$ & $-67.1 \%$ & $-58.0 \%$ & $-61.9 \%$ & $-71.0 \%$ & $-59.0 \%$ & $-63.3 \%$ & $-72.6 \%$ \\
\hline \multirow{6}{*}{ E85 Series HEV PHEV30 } & \multirow{3}{*}{ Unadjusted } & UDDS & $-63.3 \%$ & $-63.3 \%$ & $-66.6 \%$ & $-69.7 \%$ & $-64.8 \%$ & $-68.9 \%$ & $-76.8 \%$ & $-66.5 \%$ & $-72.5 \%$ & $-80.9 \%$ & $-67.5 \%$ & $-73.9 \%$ & $-82.0 \%$ \\
\hline & & HWFET & $-33.9 \%$ & $-33.9 \%$ & $-39.8 \%$ & $-44.2 \%$ & $-36.6 \%$ & $-43.7 \%$ & $-56.4 \%$ & $-39.0 \%$ & $-48.4 \%$ & $-62.3 \%$ & $-41.0 \%$ & $-51.6 \%$ & $-64.3 \%$ \\
\hline & & Combined & $-52.2 \%$ & $-52.2 \%$ & $-56.4 \%$ & $-60.0 \%$ & $-54.1 \%$ & $-59.4 \%$ & $-69.1 \%$ & $-56.1 \%$ & $-63.4 \%$ & $-73.9 \%$ & $-57.5 \%$ & $-65.5 \%$ & $-75.3 \%$ \\
\hline & \multirow{3}{*}{ Adjusted } & \begin{tabular}{|l|} 
UDDS \\
\end{tabular} & $-60.6 \%$ & $-60.6 \%$ & $-63.8 \%$ & $-66.7 \%$ & $-62.1 \%$ & $-66.0 \%$ & $-73.6 \%$ & $-63.7 \%$ & $-69.5 \%$ & $-77.5 \%$ & $-64.7 \%$ & $-70.8 \%$ & $-78.5 \%$ \\
\hline & & HWFET & $-33.7 \%$ & $-33.7 \%$ & $-39.5 \%$ & $-44.0 \%$ & $-36.3 \%$ & $-43.4 \%$ & $-56.0 \%$ & $-38.8 \%$ & $-48.1 \%$ & $-61.9 \%$ & $-40.7 \%$ & $-51.3 \%$ & $-63.9 \%$ \\
\hline & & Combined & $-49.8 \%$ & $-49.8 \%$ & $-54.0 \%$ & $-57.6 \%$ & $-51.7 \%$ & $-56.9 \%$ & $-66.5 \%$ & $-53.7 \%$ & $-60.9 \%$ & $-71.2 \%$ & $-55.0 \%$ & $-62.9 \%$ & $-72.6 \%$ \\
\hline \multirow{6}{*}{ E85 Series HEV PHEV40 } & \multirow{3}{*}{ Unadjusted } & UDDS & $-67.8 \%$ & $-67.8 \%$ & $-70.9 \%$ & $-73.4 \%$ & $-69.4 \%$ & $-72.9 \%$ & $-79.9 \%$ & $-70.8 \%$ & $-76.1 \%$ & $-83.5 \%$ & $-71.8 \%$ & $-77.4 \%$ & $-84.6 \%$ \\
\hline & & HWFET & $-47.7 \%$ & $-47.7 \%$ & $-52.4 \%$ & $-55.9 \%$ & $-49.7 \%$ & $-55.5 \%$ & $-65.5 \%$ & $-51.8 \%$ & $-59.3 \%$ & $-70.4 \%$ & $-53.4 \%$ & $-61.9 \%$ & $-72.0 \%$ \\
\hline & & Combined & $-60.2 \%$ & $-60.2 \%$ & $-63.9 \%$ & $-66.8 \%$ & $-61.9 \%$ & $-66.3 \%$ & $-74.5 \%$ & $-63.6 \%$ & $-69.7 \%$ & $-78.5 \%$ & $-64.8 \%$ & $-71.6 \%$ & $-79.8 \%$ \\
\hline & \multirow{3}{*}{ Adjusted } & \begin{tabular}{|l|} 
UDDS \\
\end{tabular} & $-64.9 \%$ & $-64.9 \%$ & $-67.9 \%$ & $-70.3 \%$ & $-66.5 \%$ & $-69.8 \%$ & $-76.6 \%$ & $-67.8 \%$ & $-72.8 \%$ & $-80.0 \%$ & $-68.7 \%$ & $-74.2 \%$ & $-81.0 \%$ \\
\hline & & HWFET & $-47.4 \%$ & $-47.4 \%$ & $-52.0 \%$ & $-55.6 \%$ & $-49.4 \%$ & $-55.1 \%$ & $-65.1 \%$ & $-51.5 \%$ & $-59.0 \%$ & $-69.9 \%$ & $-53.1 \%$ & $-61.5 \%$ & $-71.5 \%$ \\
\hline & & Combined & $-57.9 \%$ & $-57.9 \%$ & $-61.5 \%$ & $-64.4 \%$ & $-59.6 \%$ & $-63.9 \%$ & $-72.0 \%$ & $-61.2 \%$ & $-67.3 \%$ & $-75.9 \%$ & $-62.4 \%$ & $-69.1 \%$ & $-77.2 \%$ \\
\hline
\end{tabular}


Argonne National Laboratory Report - Light-Duty Vehicle Fuel Consumption Displacement Potential Up to 2045

\begin{tabular}{|c|c|c|c|c|c|c|c|c|c|c|c|c|c|c|c|}
\hline & & & \multicolumn{13}{|c|}{ Midsize_SUV } \\
\hline & & & 2010 & & 2010 & & & 2015 & & & 2030 & & & 2045 & \\
\hline & & & Ref & low & avg & high & low & avg & high & low & avg & high & low & avg & high \\
\hline \multirow{6}{*}{ FC HEV } & \multirow{3}{*}{ Unadjusted } & UDDS & $-47.8 \%$ & $-47.8 \%$ & $-57.0 \%$ & $-63.7 \%$ & $-56.7 \%$ & $-63.8 \%$ & $-68.5 \%$ & $-60.2 \%$ & $-67.2 \%$ & $-73.5 \%$ & $-62.1 \%$ & $-68.7 \%$ & $-74.8 \%$ \\
\hline & & HWFET & $-30.6 \%$ & $-30.6 \%$ & $-41.1 \%$ & $-49.0 \%$ & $-40.9 \%$ & $-48.7 \%$ & $-53.8 \%$ & $-43.6 \%$ & $-52.0 \%$ & $-59.2 \%$ & $-45.8 \%$ & $-54.5 \%$ & $-61.3 \%$ \\
\hline & & Combined & $-41.2 \%$ & $-41.2 \%$ & $-51.0 \%$ & $-58.1 \%$ & $-50.7 \%$ & $-58.1 \%$ & $-62.9 \%$ & $-53.9 \%$ & $-61.5 \%$ & $-68.1 \%$ & $-55.9 \%$ & $-63.3 \%$ & $-69.7 \%$ \\
\hline & \multirow{3}{*}{ Adjusted } & UDDS & $-45.7 \%$ & $-45.7 \%$ & $-54.6 \%$ & $-61.0 \%$ & $-54.3 \%$ & $-61.1 \%$ & $-65.6 \%$ & $-57.7 \%$ & $-64.4 \%$ & $-70.4 \%$ & $-59.5 \%$ & $-65.8 \%$ & $-71.6 \%$ \\
\hline & & HWFET & $-30.4 \%$ & $-30.4 \%$ & $-40.9 \%$ & $-48.7 \%$ & $-40.6 \%$ & $-48.4 \%$ & $-53.5 \%$ & $-43.4 \%$ & $-51.7 \%$ & $-58.8 \%$ & $-45.5 \%$ & $-54.1 \%$ & $-61.0 \%$ \\
\hline & & \begin{tabular}{|l|} 
Combined \\
\end{tabular} & $-39.6 \%$ & $-39.6 \%$ & $-49.0 \%$ & $-56.0 \%$ & $-48.8 \%$ & $-56.0 \%$ & $-60.7 \%$ & $-51.9 \%$ & $-59.3 \%$ & $-65.7 \%$ & $-53.8 \%$ & $-61.1 \%$ & $-67.3 \%$ \\
\hline \multirow{6}{*}{ FC PHEV10 } & \multirow{3}{*}{ Unadjusted } & UDDS & $-59.2 \%$ & $-59.2 \%$ & $-65.7 \%$ & $-70.6 \%$ & $-65.4 \%$ & $-70.3 \%$ & $-73.9 \%$ & $-67.4 \%$ & $-72.9 \%$ & $-77.9 \%$ & $-68.8 \%$ & $-74.1 \%$ & $-78.9 \%$ \\
\hline & & HWFET & $-47.4 \%$ & $-47.4 \%$ & $-54.3 \%$ & $-59.9 \%$ & $-54.2 \%$ & $-59.5 \%$ & $-63.2 \%$ & $-55.6 \%$ & $-61.8 \%$ & $-66.9 \%$ & $-57.0 \%$ & $-63.8 \%$ & $-68.7 \%$ \\
\hline & & \begin{tabular}{|l|} 
Combined \\
\end{tabular} & $-54.7 \%$ & $-54.7 \%$ & $-61.4 \%$ & $-66.6 \%$ & $-61.2 \%$ & $-66.2 \%$ & $-69.9 \%$ & $-62.9 \%$ & $-68.7 \%$ & $-73.7 \%$ & $-64.4 \%$ & $-70.2 \%$ & $-75.1 \%$ \\
\hline & \multirow{3}{*}{ Adjusted } & UDDS & $-56.7 \%$ & $-56.7 \%$ & $-62.9 \%$ & $-67.7 \%$ & $-62.7 \%$ & $-67.3 \%$ & $-70.8 \%$ & $-64.6 \%$ & $-69.8 \%$ & $-74.6 \%$ & $-65.9 \%$ & $-70.9 \%$ & $-75.6 \%$ \\
\hline & & \begin{tabular}{|l|} 
HWFET \\
\end{tabular} & $-47.1 \%$ & $-47.1 \%$ & $-54.0 \%$ & $-59.5 \%$ & $-53.8 \%$ & $-59.1 \%$ & $-62.8 \%$ & $-55.2 \%$ & $-61.4 \%$ & $-66.5 \%$ & $-56.7 \%$ & $-63.4 \%$ & $-68.3 \%$ \\
\hline & & Combined & $-52.8 \%$ & $-52.8 \%$ & $-59.3 \%$ & $-64.4 \%$ & $-59.1 \%$ & $-64.0 \%$ & $-67.6 \%$ & $-60.8 \%$ & $-66.4 \%$ & $-71.3 \%$ & $-62.2 \%$ & $-67.9 \%$ & $-72.7 \%$ \\
\hline \multirow{6}{*}{ FC PHEV 20} & \multirow{3}{*}{ Unadjusted } & UDDS & $-65.9 \%$ & $-65.9 \%$ & $-71.4 \%$ & $-75.6 \%$ & $-71.2 \%$ & $-75.3 \%$ & $-78.5 \%$ & $-72.9 \%$ & $-77.5 \%$ & $-81.7 \%$ & $-74.1 \%$ & $-78.5 \%$ & $-82.6 \%$ \\
\hline & & HWFET & $-48.5 \%$ & $-48.5 \%$ & $-55.7 \%$ & $-61.3 \%$ & $-55.5 \%$ & $-60.9 \%$ & $-64.7 \%$ & $-57.1 \%$ & $-63.4 \%$ & $-68.7 \%$ & $-58.6 \%$ & $-65.3 \%$ & $-70.4 \%$ \\
\hline & & Combined & $-59.3 \%$ & $-59.3 \%$ & $-65.4 \%$ & $-70.2 \%$ & $-65.2 \%$ & $-69.8 \%$ & $-73.3 \%$ & $-66.9 \%$ & $-72.2 \%$ & $-76.8 \%$ & $-68.2 \%$ & $-73.5 \%$ & $-78.0 \%$ \\
\hline & \multirow{3}{*}{ Adjusted } & \begin{tabular}{|l|} 
UDDS \\
\end{tabular} & $-63.1 \%$ & $-63.1 \%$ & $-68.4 \%$ & $-72.4 \%$ & $-68.2 \%$ & $-72.1 \%$ & $-75.1 \%$ & $-69.8 \%$ & $-74.2 \%$ & $-78.3 \%$ & $-71.0 \%$ & $-75.2 \%$ & $-79.1 \%$ \\
\hline & & HWFET & $-48.2 \%$ & $-48.2 \%$ & $-55.3 \%$ & $-60.9 \%$ & $-55.1 \%$ & $-60.5 \%$ & $-64.3 \%$ & $-56.7 \%$ & $-63.0 \%$ & $-68.3 \%$ & $-58.2 \%$ & $-64.9 \%$ & $-69.9 \%$ \\
\hline & & Combined & $-57.1 \%$ & $-57.1 \%$ & $-63.1 \%$ & $-67.8 \%$ & $-62.9 \%$ & $-67.4 \%$ & $-70.8 \%$ & $-64.5 \%$ & $-69.7 \%$ & $-74.2 \%$ & $-65.9 \%$ & $-71.1 \%$ & $-75.4 \%$ \\
\hline \multirow{6}{*}{ FC PHEV30 } & \multirow{3}{*}{ Unadjusted } & UDDS & $-76.2 \%$ & $-76.2 \%$ & $-79.9 \%$ & $-82.5 \%$ & $-79.8 \%$ & $-82.4 \%$ & $-84.7 \%$ & $-80.7 \%$ & $-84.1 \%$ & $-87.0 \%$ & $-81.6 \%$ & $-84.9 \%$ & $-87.6 \%$ \\
\hline & & HWFET & $-59.2 \%$ & $-59.2 \%$ & $-64.7 \%$ & $-68.8 \%$ & $-64.5 \%$ & $-68.6 \%$ & $-71.9 \%$ & $-65.5 \%$ & $-70.7 \%$ & $-74.9 \%$ & $-66.8 \%$ & $-72.3 \%$ & $-76.3 \%$ \\
\hline & & \begin{tabular}{|l|} 
Combined \\
\end{tabular} & $-69.8 \%$ & $-69.8 \%$ & $-74.2 \%$ & $-77.3 \%$ & $-74.0 \%$ & $-77.2 \%$ & $-79.8 \%$ & $-74.9 \%$ & $-79.0 \%$ & $-82.4 \%$ & $-76.0 \%$ & $-80.1 \%$ & $-83.3 \%$ \\
\hline & \multirow{3}{*}{ Adjusted } & UDDS & $-73.0 \%$ & $-73.0 \%$ & $-76.5 \%$ & $-79.0 \%$ & $-76.4 \%$ & $-78.9 \%$ & $-81.1 \%$ & $-77.3 \%$ & $-80.5 \%$ & $-83.3 \%$ & $-78.1 \%$ & $-81.3 \%$ & $-83.9 \%$ \\
\hline & & HWFET & $-58.9 \%$ & $-58.9 \%$ & $-64.3 \%$ & $-68.4 \%$ & $-64.1 \%$ & $-68.2 \%$ & $-71.4 \%$ & $-65.1 \%$ & $-70.3 \%$ & $-74.5 \%$ & $-66.4 \%$ & $-71.8 \%$ & $-75.8 \%$ \\
\hline & & Combined & $-67.3 \%$ & $-67.3 \%$ & $-71.6 \%$ & $-74.7 \%$ & $-71.5 \%$ & $-74.6 \%$ & $-77.2 \%$ & $-72.4 \%$ & $-76.4 \%$ & $-79.7 \%$ & $-73.4 \%$ & $-77.5 \%$ & $-80.6 \%$ \\
\hline \multirow{6}{*}{ FC PHEV 40} & \multirow{3}{*}{ Unadjusted } & UDDS & $-79.4 \%$ & $-79.4 \%$ & $-82.6 \%$ & $-84.9 \%$ & $-82.5 \%$ & $-84.8 \%$ & $-86.8 \%$ & $-83.3 \%$ & $-86.3 \%$ & $-88.8 \%$ & $-84.1 \%$ & $-87.0 \%$ & $-89.3 \%$ \\
\hline & & HWFET & $-67.8 \%$ & $-67.8 \%$ & $-72.2 \%$ & $-75.4 \%$ & $-72.0 \%$ & $-75.3 \%$ & $-77.9 \%$ & $-72.9 \%$ & $-77.0 \%$ & $-80.3 \%$ & $-73.9 \%$ & $-78.3 \%$ & $-81.4 \%$ \\
\hline & & \begin{tabular}{|l|} 
Combined \\
\end{tabular} & $-75.0 \%$ & $-75.0 \%$ & $-78.6 \%$ & $-81.3 \%$ & $-78.5 \%$ & $-81.2 \%$ & $-83.4 \%$ & $-79.3 \%$ & $-82.7 \%$ & $-85.6 \%$ & $-80.2 \%$ & $-83.7 \%$ & $-86.3 \%$ \\
\hline & \multirow{3}{*}{ Adjusted } & UDDS & $-76.0 \%$ & $-76.0 \%$ & $-79.1 \%$ & $-81.3 \%$ & $-79.0 \%$ & $-81.2 \%$ & $-83.2 \%$ & $-79.8 \%$ & $-82.6 \%$ & $-85.0 \%$ & $-80.5 \%$ & $-83.3 \%$ & $-85.5 \%$ \\
\hline & & HWFET & $-67.4 \%$ & $-67.4 \%$ & $-71.7 \%$ & $-74.9 \%$ & $-71.6 \%$ & $-74.8 \%$ & $-77.4 \%$ & $-72.4 \%$ & $-76.5 \%$ & $-79.8 \%$ & $-73.4 \%$ & $-77.8 \%$ & $-80.9 \%$ \\
\hline & & Combined & $-72.5 \%$ & $-72.5 \%$ & $-76.1 \%$ & $-78.7 \%$ & $-76.0 \%$ & $-78.6 \%$ & $-80.8 \%$ & $-76.8 \%$ & $-80.2 \%$ & $-82.9 \%$ & $-77.7 \%$ & $-81.1 \%$ & $-83.7 \%$ \\
\hline
\end{tabular}




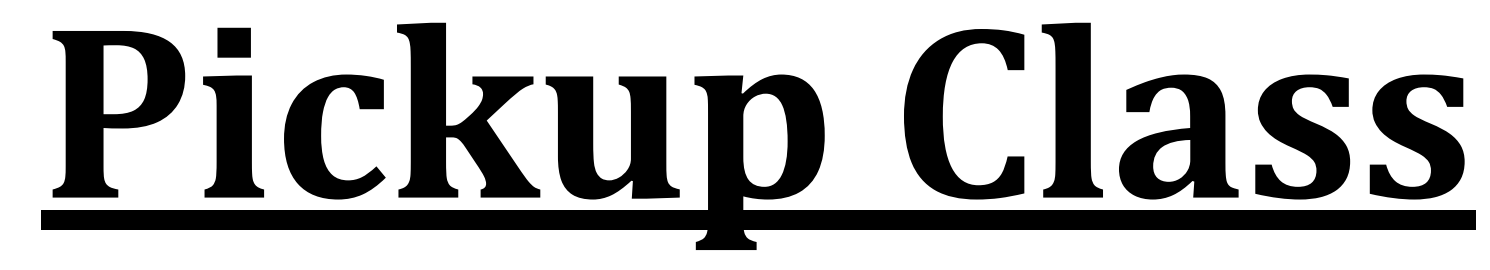




\section{Fuel economy}

\begin{tabular}{|c|c|c|c|c|c|c|c|c|c|c|c|c|c|c|c|}
\hline & & & \multicolumn{13}{|c|}{ Pickup } \\
\hline & & & \multirow{2}{*}{$\frac{2010}{\text { Ref }}$} & \multicolumn{3}{|c|}{2010} & \multicolumn{3}{|c|}{2015} & \multicolumn{3}{|c|}{2030} & \multicolumn{3}{|c|}{2045} \\
\hline & & & & low & avg & high & low & avg & high & low & avg & high & low & avg & high \\
\hline \multirow{6}{*}{ SI Conv } & \multirow{3}{*}{ Unadjusted } & UDDS & 18.5 & 18.5 & 20.0 & 21.3 & 19.5 & 21.1 & 26.8 & 19.9 & 22.5 & 32.1 & 20.0 & 22.8 & 33.7 \\
\hline & & HWFET & 23.9 & 23.9 & 25.9 & 27.9 & 25.4 & 27.3 & 34.8 & 25.9 & 29.0 & 40.3 & 26.4 & 29.4 & 41.9 \\
\hline & & Combined & 20.6 & 20.6 & 22.3 & 23.8 & 21.8 & 23.5 & 29.9 & 22.2 & 25.0 & 35.4 & 22.4 & 25.3 & 37.0 \\
\hline & \multirow{3}{*}{ Adjusted } & UDDS & 14.5 & 14.5 & 15.7 & 16.6 & 15.3 & 16.5 & 20.7 & 15.6 & 17.5 & 24.6 & 15.7 & 17.7 & 25.7 \\
\hline & & HWFET & 17.0 & 17.0 & 18.4 & 19.7 & 18.0 & 19.3 & 24.6 & 18.3 & 20.6 & 28.4 & 18.7 & 20.8 & 29.6 \\
\hline & & Combined & 15.5 & 15.5 & 16.8 & 17.9 & 16.4 & 17.6 & 22.3 & 16.7 & 18.8 & 26.2 & 16.9 & 19.0 & 27.3 \\
\hline \multirow{6}{*}{$\mathrm{Cl}$ Conv } & \multirow{3}{*}{ Unadjusted } & \begin{tabular}{|l|} 
UDDS \\
\end{tabular} & 22.5 & 22.5 & 23.8 & 25.6 & 23.0 & 25.5 & 28.3 & 24.2 & 27.6 & 32.8 & 24.8 & 28.6 & 34.2 \\
\hline & & HWFET & 27.8 & 27.8 & 29.4 & 31.0 & 28.7 & 31.7 & 34.0 & 30.2 & 33.1 & 38.1 & 30.2 & 34.4 & 39.6 \\
\hline & & Combined & 24.6 & 24.6 & 26.0 & 27.8 & 25.2 & 28.0 & 30.6 & 26.6 & 29.9 & 35.0 & 26.9 & 30.9 & 36.4 \\
\hline & \multirow{3}{*}{ Adjusted } & UDDS & 17.5 & 17.5 & 18.5 & 19.9 & 17.9 & 19.7 & 21.8 & 18.8 & 21.3 & 25.1 & 19.2 & 22.0 & 26.0 \\
\hline & & \begin{tabular}{|c|} 
HWFET \\
\end{tabular} & 19.7 & 19.7 & 20.8 & 22.0 & 20.3 & 22.5 & 24.0 & 21.4 & 23.4 & 26.9 & 21.4 & 24.3 & 27.9 \\
\hline & & Combined & 18.4 & 18.4 & 19.5 & 20.7 & 18.9 & 20.9 & 22.7 & 19.9 & 22.2 & 25.9 & 20.1 & 23.0 & 26.9 \\
\hline \multirow{6}{*}{ H2 Conv } & \multirow{3}{*}{ Unadjusted } & UDDS & 15.9 & 15.9 & 19.0 & 21.3 & 18.1 & 21.4 & 24.1 & 19.9 & 23.4 & 28.6 & 20.9 & 24.8 & 31.1 \\
\hline & & HWFET & 22.9 & 22.9 & 27.0 & 30.5 & 26.2 & 30.6 & 34.2 & 28.7 & 33.2 & 39.1 & 30.2 & 35.2 & 42.3 \\
\hline & & Combined & 18.5 & 18.5 & 21.9 & 24.6 & 21.0 & 24.8 & 27.8 & 23.1 & 27.0 & 32.5 & 24.2 & 28.6 & 35.3 \\
\hline & \multirow{3}{*}{ Adjusted } & UDDS & 12.6 & 12.6 & 14.9 & 16.6 & 14.3 & 16.7 & 18.7 & 15.6 & 18.2 & 22.0 & 16.3 & 19.2 & 23.8 \\
\hline & & HWFET & 16.3 & 16.3 & 19.2 & 21.6 & 18.5 & 21.7 & 24.2 & 20.3 & 23.5 & 27.6 & 21.4 & 24.9 & 29.9 \\
\hline & & Combined & 14.0 & 14.0 & 16.5 & 18.6 & 15.9 & 18.6 & 20.9 & 17.4 & 20.2 & 24.2 & 18.3 & 21.4 & 26.2 \\
\hline \multirow{6}{*}{ E85 Conv } & \multirow{3}{*}{ Unadjusted } & UDDS & 17.7 & 17.7 & 18.0 & 19.2 & 17.5 & 18.8 & 25.3 & 17.8 & 20.1 & 29.7 & 17.9 & 20.3 & 30.9 \\
\hline & & HWFET & 21.7 & 21.7 & 22.0 & 24.5 & 21.5 & 23.1 & 32.1 & 21.9 & 25.4 & 36.6 & 23.1 & 25.8 & 37.9 \\
\hline & & Combined & 19.3 & 19.3 & 19.6 & 21.2 & 19.1 & 20.6 & 27.9 & 19.4 & 22.2 & 32.4 & 20.0 & 22.4 & 33.7 \\
\hline & \multirow{3}{*}{ Adjusted } & \begin{tabular}{|l|} 
UDDS \\
\end{tabular} & 13.9 & 13.9 & 14.1 & 15.0 & 13.8 & 14.8 & 19.6 & 14.0 & 15.7 & 22.8 & 14.1 & 15.9 & 23.7 \\
\hline & & HWFET & 15.4 & 15.4 & 15.6 & 17.4 & 15.3 & 16.4 & 22.7 & 15.5 & 18.0 & 25.9 & 16.4 & 18.3 & 26.8 \\
\hline & & Combined & 14.6 & 14.6 & 14.8 & 16.0 & 14.4 & 15.5 & 20.9 & 14.7 & 16.7 & 24.1 & 15.1 & 16.9 & 25.0 \\
\hline \multirow{6}{*}{ SI Split HEV } & \multirow{3}{*}{ Unadjusted } & UDDS & 31.9 & 31.9 & 34.3 & 37.2 & 33.2 & 37.3 & 45.6 & 35.2 & 41.0 & 55.3 & 36.1 & 42.6 & 57.8 \\
\hline & & HWFET & 27.9 & 27.9 & 29.8 & 32.2 & 29.0 & 31.8 & 38.1 & 30.2 & 34.4 & 44.6 & 31.0 & 35.7 & 46.4 \\
\hline & & Combined & 30.0 & 30.0 & 32.1 & 34.7 & 31.2 & 34.6 & 41.9 & 32.8 & 37.7 & 49.9 & 33.6 & 39.2 & 52.1 \\
\hline & \multirow{3}{*}{ Adjusted } & UDDS & 24.4 & 24.4 & 26.1 & 28.1 & 25.3 & 28.2 & 34.0 & 26.8 & 30.8 & 40.5 & 27.4 & 31.9 & 42.1 \\
\hline & & HWFET & 19.8 & 19.8 & 21.1 & 22.8 & 20.6 & 22.5 & 26.9 & 21.4 & 24.3 & 31.5 & 22.0 & 25.2 & 32.8 \\
\hline & & Combined & 22.1 & 22.1 & 23.6 & 25.4 & 22.9 & 25.3 & 30.4 & 24.0 & 27.5 & 35.9 & 24.6 & 28.5 & 37.3 \\
\hline \multirow{6}{*}{ SI Split HEV PHEV10 } & & UDDS & 40.3 & 40.3 & 43.2 & 46.8 & 42.1 & 46.3 & 55.8 & 43.7 & 50.5 & 67.2 & 44.8 & 51.9 & 70.2 \\
\hline & Unadjusted & HWFET & 36.6 & 36.6 & 38.9 & 41.9 & 37.9 & 41.1 & 48.7 & 39.4 & 44.0 & 56.6 & 40.6 & 45.4 & 60.4 \\
\hline & & Combined & 38.5 & 38.5 & 41.2 & 44.5 & 40.1 & 43.8 & 52.4 & 41.6 & 47.3 & 62.0 & 42.8 & 48.8 & 65.4 \\
\hline & & UDDS & 30.3 & 30.3 & 32.3 & 34.8 & 31.6 & 34.4 & 40.8 & 32.7 & 37.3 & 48.1 & 33.5 & 38.2 & 49.9 \\
\hline & Adjusted & HWFET & 25.9 & 25.9 & 27.5 & 29.6 & 26.8 & 29.0 & 34.4 & 27.8 & 31.0 & 39.9 & 28.7 & 32.1 & 42.5 \\
\hline & & Combined & 28.1 & 28.1 & 30.0 & 32.2 & 29.2 & 31.8 & 37.6 & 30.3 & 34.2 & 44.0 & 31.1 & 35.2 & 46.3 \\
\hline
\end{tabular}




\begin{tabular}{|c|c|c|c|c|c|c|c|c|c|c|c|c|c|c|c|}
\hline & & & \multicolumn{13}{|c|}{ Pickup } \\
\hline & & & \multirow{2}{*}{$\begin{array}{l}2010 \\
\text { Ref }\end{array}$} & \multicolumn{3}{|c|}{2010} & \multicolumn{3}{|c|}{2015} & \multicolumn{3}{|c|}{2030} & \multicolumn{3}{|c|}{2045} \\
\hline & & & & low & avg & high & low & avg & high & low & avg & high & low & avg & high \\
\hline \multirow{6}{*}{ SI Split HEV PHEV2O } & \multirow{3}{*}{ Unadjusted } & UDDS & 58.1 & 58.1 & 62.2 & 67.7 & 60.8 & 67.1 & 81.0 & 63.2 & 72.9 & 95.8 & 64.8 & 74.9 & 100.3 \\
\hline & & HWFET & 44.4 & 44.4 & 47.0 & 50.9 & 46.4 & 50.5 & \begin{tabular}{ll|}
48.4 \\
\end{tabular} & 47.9 & 43.7 & 55.9 & 49.3 & 45.2 & 58.0 \\
\hline & & Combined & 51.0 & 51.0 & 54.3 & 58.9 & 53.3 & 58.4 & 62.1 & 55.2 & 56.1 & 72.5 & 56.8 & 57.8 & 75.5 \\
\hline & \multirow{3}{*}{ Adjusted } & \begin{tabular}{|l|} 
UDDS \\
\end{tabular} & 42.3 & 42.3 & 44.9 & 48.4 & 44.0 & 48.0 & 56.5 & 45.5 & 51.6 & 65.1 & 46.6 & 52.9 & 67.7 \\
\hline & & HWFET & 31.4 & 31.4 & 33.2 & 35.9 & 32.7 & 35.6 & 34.1 & 33.8 & 30.9 & 39.3 & 34.8 & 31.9 & 40.8 \\
\hline & & \begin{tabular}{|l|} 
Combined \\
\end{tabular} & 36.6 & 36.6 & 38.7 & 41.8 & 38.1 & 41.5 & 43.6 & 39.4 & 39.6 & 50.3 & 40.4 & 40.8 & 52.2 \\
\hline \multirow{6}{*}{ SI Series HEV PHEV3O } & \multirow{3}{*}{ Unadjusted } & UDDS & 51.1 & 51.1 & 55.8 & 61.8 & 53.9 & 60.6 & 76.6 & 57.3 & 68.9 & 95.8 & 59.0 & 71.4 & 101.7 \\
\hline & & HWFET & 37.8 & 37.8 & 40.8 & 44.6 & 39.8 & 44.3 & 54.1 & 41.9 & 48.6 & 64.0 & 43.4 & 50.6 & 66.5 \\
\hline & & Combined & 44.1 & 44.1 & 47.9 & 52.7 & 46.5 & 51.9 & 64.5 & 49.2 & 58.0 & 78.3 & 50.8 & 60.3 & 82.2 \\
\hline & \multirow{3}{*}{ Adjusted } & \begin{tabular}{|l|} 
UDDS \\
\end{tabular} & 37.7 & 37.7 & 40.8 & 44.7 & 39.5 & 43.9 & 53.9 & 41.7 & 49.2 & 65.2 & 42.9 & 50.7 & 68.5 \\
\hline & & \begin{tabular}{|l|} 
HWFET \\
\end{tabular} & 26.7 & 26.7 & 28.8 & 31.5 & 28.1 & 31.2 & 38.1 & 29.6 & 34.3 & 45.0 & 30.7 & 35.7 & 46.7 \\
\hline & & Combined & 31.8 & 31.8 & 34.4 & 37.6 & 33.4 & 37.1 & 45.4 & 35.2 & 41.1 & 54.2 & 36.4 & 42.6 & 56.6 \\
\hline \multirow{6}{*}{ SI Series HEV PHEV40 } & \multirow{3}{*}{ Unadjusted } & \begin{tabular}{|l|} 
UDDS \\
\end{tabular} & 58.5 & 58.5 & 64.1 & 70.5 & 61.9 & 69.6 & 88.5 & 65.9 & 79.4 & 110.2 & 67.9 & 82.5 & 116.3 \\
\hline & & \begin{tabular}{|l|} 
HWFET \\
\end{tabular} & 47.9 & 47.9 & 51.6 & 56.6 & 50.5 & 56.0 & 68.7 & 53.2 & 61.7 & 81.4 & 55.0 & 64.3 & 84.7 \\
\hline & & \begin{tabular}{|c|} 
Combined \\
\end{tabular} & 53.2 & 53.2 & 57.8 & 63.5 & 56.2 & 62.8 & 78.3 & 59.5 & 70.3 & 95.1 & 61.4 & 73.2 & 99.6 \\
\hline & \multirow{3}{*}{ Adjusted } & \begin{tabular}{|l|} 
UDDS \\
\end{tabular} & 42.5 & 42.5 & 46.1 & 50.1 & 44.7 & 49.6 & 61.0 & 47.3 & 55.6 & 73.1 & 48.5 & 57.4 & 76.4 \\
\hline & & HWFET & 33.8 & 33.8 & 36.4 & 39.9 & 35.6 & 39.4 & 48.2 & 37.5 & 43.4 & 57.0 & 38.7 & 45.2 & 59.3 \\
\hline & & Combined & 38.1 & 38.1 & 41.2 & 44.9 & 40.1 & 44.4 & 54.5 & 42.3 & 49.4 & 64.9 & 43.6 & 51.2 & 67.6 \\
\hline \multirow{6}{*}{ CI Split HEV } & \multirow{3}{*}{ Unadjusted } & \begin{tabular}{|l|} 
UDDS \\
\end{tabular} & 36.0 & 36.0 & 38.2 & 42.0 & 37.1 & 41.9 & 47.9 & 39.7 & 46.7 & 56.6 & 41.2 & 48.6 & 58.8 \\
\hline & & $\begin{array}{l}\text { HWFET } \\
\end{array}$ & 29.8 & 29.8 & 31.5 & 34.7 & 30.7 & 34.2 & 38.4 & 32.5 & 37.5 & 43.6 & 33.8 & 39.1 & 45.4 \\
\hline & & \begin{tabular}{|l|} 
Combined \\
\end{tabular} & 32.9 & 32.9 & 34.9 & 38.3 & 33.9 & 38.1 & 43.1 & 36.1 & 42.1 & 49.9 & 37.5 & 43.8 & 51.9 \\
\hline & \multirow{3}{*}{ Adjusted } & \begin{tabular}{|l|} 
UDDS \\
\end{tabular} & 27.3 & 27.3 & 28.9 & 31.5 & 28.1 & 31.4 & 35.5 & 29.9 & 34.7 & 41.3 & 30.9 & 36.0 & 42.8 \\
\hline & & HWFET & 21.1 & 21.1 & 22.3 & 24.5 & 21.7 & 24.2 & 27.1 & 23.0 & 26.5 & 30.8 & 23.9 & 27.7 & 32.1 \\
\hline & & \begin{tabular}{|l|} 
Combined \\
\end{tabular} & 24.1 & 24.1 & 25.5 & 27.9 & 24.8 & 27.7 & 31.2 & 26.4 & 30.5 & 35.8 & 27.3 & 31.7 & 37.2 \\
\hline \multirow{6}{*}{ CI Split HEV PHEV10 } & \multirow{3}{*}{ Unadjusted } & \begin{tabular}{|l|} 
UDDS \\
\end{tabular} & 42.0 & 42.0 & 44.6 & 49.0 & 43.3 & 48.5 & 55.2 & 46.0 & 53.7 & 64.6 & 47.7 & 55.9 & 67.4 \\
\hline & & \begin{tabular}{|l|} 
HWFET \\
\end{tabular} & 37.6 & 37.6 & 39.6 & 43.2 & 38.7 & 42.8 & 47.7 & 40.7 & 46.5 & 53.7 & 42.3 & 48.4 & 55.7 \\
\hline & & \begin{tabular}{|l|} 
Combined \\
\end{tabular} & 39.9 & 39.9 & 42.2 & 46.2 & 41.1 & 45.8 & 51.5 & 43.5 & 50.2 & 59.2 & 45.1 & 52.3 & 61.6 \\
\hline & \multirow{3}{*}{ Adjusted } & \begin{tabular}{|l|} 
UDDS \\
\end{tabular} & 31.5 & 31.5 & 33.3 & 36.3 & 32.4 & 35.9 & 40.4 & 34.3 & 39.4 & 46.5 & 35.4 & 40.9 & 48.2 \\
\hline & & HWFET & 26.5 & 26.5 & 28.0 & 30.5 & 27.3 & 30.2 & 33.6 & 28.7 & 32.8 & 37.8 & 29.9 & 34.1 & 39.2 \\
\hline & & \begin{tabular}{|l|} 
Combined \\
\end{tabular} & 29.1 & 29.1 & 30.7 & 33.4 & 29.9 & 33.1 & 37.0 & 31.5 & 36.1 & 42.1 & 32.7 & 37.5 & 43.7 \\
\hline \multirow{6}{*}{ CI Split HEV PHEV2O } & & \begin{tabular}{|l|} 
UDDS \\
\end{tabular} & 60.5 & 60.5 & 64.3 & 70.6 & 62.5 & 70.0 & 79.7 & 66.4 & 77.5 & 90.9 & 68.9 & 80.9 & 95.8 \\
\hline & Unadjusted & HWFET & 45.9 & 45.9 & 48.2 & 52.9 & 47.4 & 52.4 & 58.0 & 49.7 & 56.7 & 53.0 & 51.8 & 59.1 & 55.0 \\
\hline & & \begin{tabular}{|l|} 
Combined \\
\end{tabular} & 52.9 & 52.9 & 55.9 & 61.3 & 54.6 & 60.8 & 68.2 & 57.7 & 66.5 & 68.7 & 60.0 & 69.4 & 71.8 \\
\hline & & \begin{tabular}{|l|} 
UDDS \\
\end{tabular} & 43.8 & 43.8 & 46.3 & 50.2 & 45.1 & 49.8 & 55.8 & 47.6 & 54.5 & 62.4 & 49.2 & 56.5 & 65.2 \\
\hline & Adjusted & HWFET & 32.4 & 32.4 & 34.0 & 37.3 & 33.4 & 36.9 & 40.8 & 35.1 & 39.9 & 37.3 & 36.5 & 41.6 & 38.7 \\
\hline & & \begin{tabular}{|l|} 
Combined \\
\end{tabular} & 37.8 & 37.8 & 39.8 & 43.4 & 39.0 & 43.1 & 47.9 & 41.0 & 46.8 & 47.9 & 42.5 & 48.7 & 49.9 \\
\hline
\end{tabular}




\begin{tabular}{|c|c|c|c|c|c|c|c|c|c|c|c|c|c|c|c|}
\hline & & & \multicolumn{13}{|c|}{ Pickup } \\
\hline & & & \multirow{2}{*}{$\begin{array}{l}2010 \\
\text { Ref }\end{array}$} & \multicolumn{3}{|c|}{2010} & \multicolumn{3}{|c|}{2015} & \multicolumn{3}{|c|}{2030} & \multicolumn{3}{|c|}{2045} \\
\hline & & & & low & avg & high & low & avg & high & low & avg & high & low & avg & high \\
\hline \multirow{6}{*}{ CI Series HEV PHEV30 } & \multirow{3}{*}{ Unadjusted } & UDDS & 52.9 & 52.9 & 57.1 & 63.8 & 55.0 & 63.1 & 74.7 & 59.6 & 72.5 & 90.8 & 62.6 & 75.9 & 96.3 \\
\hline & & HWFET & 39.0 & 39.0 & 41.7 & 46.3 & 40.5 & 45.8 & 52.8 & 43.6 & 51.2 & 60.9 & 45.5 & 53.7 & 63.3 \\
\hline & & Combined & 45.6 & 45.6 & 49.0 & 54.5 & 47.4 & 54.0 & 62.9 & 51.2 & 61.1 & 74.4 & 53.5 & 64.0 & 78.0 \\
\hline & \multirow{3}{*}{ Adjusted } & \begin{tabular}{|l|} 
UDDS \\
\end{tabular} & 38.9 & 38.9 & 41.7 & 46.0 & 40.3 & 45.5 & 52.7 & 43.3 & 51.4 & 62.3 & 45.2 & 53.5 & 65.4 \\
\hline & & HWFET & 27.6 & 27.6 & 29.4 & 32.7 & 28.6 & 32.3 & 37.2 & 30.8 & 36.1 & 42.8 & 32.1 & 37.8 & 44.5 \\
\hline & & Combined & 32.8 & 32.8 & 35.1 & 38.8 & 34.0 & 38.5 & 44.4 & 36.6 & 43.2 & 51.7 & 38.2 & 45.1 & 54.0 \\
\hline \multirow{6}{*}{$\mathrm{Cl}$ Series HEV PHEV40 } & \multirow{3}{*}{ Unadjusted } & \begin{tabular}{|l|} 
UDDS \\
\end{tabular} & 60.7 & 60.7 & 65.6 & 73.8 & 63.2 & 73.0 & 86.2 & 68.5 & 83.7 & 105.2 & 71.4 & 87.8 & 111.5 \\
\hline & & HWFET & 49.4 & 49.4 & 52.8 & 58.5 & 51.2 & 58.0 & 67.1 & 55.1 & 65.1 & 77.1 & 57.5 & 68.4 & 80.4 \\
\hline & & Combined & 55.0 & 55.0 & 59.1 & 66.0 & 57.2 & 65.4 & 76.4 & 61.8 & 74.2 & 90.4 & 64.4 & 77.9 & 95.0 \\
\hline & \multirow{3}{*}{ Adjusted } & \begin{tabular}{|l|} 
UDDS \\
\end{tabular} & 44.0 & 44.0 & 47.1 & 52.2 & 45.6 & 51.7 & 59.6 & 48.9 & 58.2 & 70.4 & 50.7 & 60.6 & 73.9 \\
\hline & & \begin{tabular}{|l|} 
HWFET \\
\end{tabular} & 34.8 & 34.8 & 37.2 & 41.2 & 36.1 & 40.8 & 47.1 & 38.8 & 45.7 & 54.0 & 40.5 & 48.0 & 56.3 \\
\hline & & Combined & 39.3 & 39.3 & 42.0 & 46.6 & 40.8 & 46.1 & 53.3 & 43.8 & 51.8 & 62.0 & 45.5 & 54.2 & 64.8 \\
\hline \multirow{6}{*}{ H2 Split HEV } & \multirow{3}{*}{ Unadjusted } & \begin{tabular}{|l|} 
UDDS \\
\end{tabular} & 31.5 & 31.5 & 37.4 & 43.1 & 36.0 & 43.0 & 50.5 & 40.1 & 49.0 & 60.3 & 42.8 & 52.2 & 65.3 \\
\hline & & \begin{tabular}{|l|} 
HWFET \\
\end{tabular} & 27.1 & 27.1 & 31.9 & 36.6 & 30.9 & 36.2 & 41.7 & 34.0 & 40.5 & 47.9 & 36.3 & 43.1 & 51.7 \\
\hline & & Combined & 29.4 & 29.4 & 34.7 & 39.9 & 33.5 & 39.6 & 46.1 & 37.1 & 44.8 & 54.0 & 39.6 & 47.7 & 58.4 \\
\hline & \multirow{3}{*}{ Adjusted } & \begin{tabular}{|l|} 
UDDS \\
\end{tabular} & 24.1 & 24.1 & 28.3 & 32.2 & 27.3 & 32.2 & 37.3 & 30.2 & 36.2 & 43.7 & 32.1 & 38.4 & 46.9 \\
\hline & & HWFET & 19.2 & 19.2 & 22.6 & 25.9 & 21.9 & 25.6 & 29.5 & 24.1 & 28.6 & 33.8 & 25.7 & 30.5 & 36.5 \\
\hline & & Combined & 21.6 & 21.6 & 25.4 & 29.0 & 24.6 & 28.8 & 33.3 & 27.1 & 32.4 & 38.6 & 28.8 & 34.4 & 41.5 \\
\hline \multirow{6}{*}{ H2 Split HEV PHEV10 } & \multirow{3}{*}{ Unadjusted } & \begin{tabular}{|l|} 
UDDS \\
\end{tabular} & 39.3 & 39.3 & 46.6 & 53.6 & 45.0 & 53.0 & 61.8 & 49.5 & 59.9 & 72.8 & 53.1 & 63.6 & 78.8 \\
\hline & & HWFET & 36.0 & 36.0 & 42.0 & 48.0 & 41.0 & 47.4 & 54.0 & 44.8 & 52.6 & 62.5 & 47.8 & 55.9 & 67.3 \\
\hline & & \begin{tabular}{|l|} 
Combined \\
\end{tabular} & 37.7 & 37.7 & 44.4 & 50.9 & 43.1 & 50.3 & 58.0 & 47.2 & 56.3 & 67.8 & 50.6 & 59.9 & 73.2 \\
\hline & \multirow{3}{*}{ Adjusted } & \begin{tabular}{|l|} 
UDDS \\
\end{tabular} & 29.6 & 29.6 & 34.7 & 39.4 & 33.5 & 38.9 & 44.6 & 36.6 & 43.4 & 51.6 & 39.0 & 45.8 & 55.2 \\
\hline & & HWFET & 25.5 & 25.5 & 29.7 & 33.8 & 28.9 & 33.5 & 38.0 & 31.6 & 37.0 & 43.9 & 33.7 & 39.3 & 47.3 \\
\hline & & Combined & 27.6 & 27.6 & 32.2 & 36.7 & 31.3 & 36.3 & 41.4 & 34.2 & 40.3 & 47.8 & 36.4 & 42.6 & 51.3 \\
\hline \multirow{6}{*}{ H2 Split HEV PHEV2O } & \multirow{3}{*}{ Unadjusted } & \begin{tabular}{|l|} 
UDDS \\
\end{tabular} & 56.6 & 56.6 & 67.3 & 77.7 & 64.7 & 76.9 & 89.7 & 71.5 & 86.8 & 104.0 & 76.7 & 91.3 & 112.8 \\
\hline & & \begin{tabular}{|l|} 
HWFET \\
\end{tabular} & 43.7 & 43.7 & 50.9 & 47.7 & 49.7 & 47.1 & 53.6 & 54.4 & 51.7 & 60.0 & 58.1 & 55.1 & 64.9 \\
\hline & & Combined & 50.0 & 50.0 & 58.8 & 60.5 & 56.9 & 59.9 & 68.9 & 62.6 & 66.5 & 78.2 & 67.0 & 70.5 & 84.6 \\
\hline & \multirow{3}{*}{ Adjusted } & \begin{tabular}{|l|} 
UDDS \\
\end{tabular} & 41.3 & 41.3 & 48.2 & 54.5 & 46.5 & 54.1 & 61.7 & 50.8 & 60.0 & 69.7 & 53.9 & 62.6 & 74.5 \\
\hline & & HWFET & 30.9 & 30.9 & 35.9 & 33.6 & 35.0 & 33.3 & 37.8 & 38.3 & 36.4 & 42.2 & 40.9 & 38.8 & 45.6 \\
\hline & & Combined & 35.9 & 35.9 & 41.7 & 42.6 & 40.5 & 42.2 & 48.0 & 44.3 & 46.5 & 53.9 & 47.1 & 49.1 & 58.0 \\
\hline \multirow{6}{*}{ H2 Series HEV PHEV3O } & & \begin{tabular}{|l|} 
UDDS \\
\end{tabular} & 50.3 & 50.3 & 61.0 & 71.2 & 58.0 & 70.2 & 85.2 & 65.8 & 82.0 & 105.0 & 71.0 & 87.6 & 114.2 \\
\hline & Unadjusted & HWFET & 37.4 & 37.4 & 44.3 & 51.3 & 43.0 & 50.8 & 59.7 & 47.9 & 57.6 & 69.0 & 51.4 & 61.9 & 74.5 \\
\hline & & Combined & 43.6 & 43.6 & 52.1 & 60.6 & 50.1 & 59.9 & 71.4 & 56.4 & 68.9 & 85.0 & 60.6 & 73.8 & 92.1 \\
\hline & & \begin{tabular}{|l|} 
UDDS \\
\end{tabular} & 37.2 & 37.2 & 44.2 & 50.6 & 42.2 & 50.0 & 59.0 & 47.2 & 57.2 & 70.3 & 50.5 & 60.5 & 75.3 \\
\hline & Adjusted & HWFET & 26.5 & 26.5 & 31.2 & 36.1 & 30.4 & 35.8 & 42.0 & 33.8 & 40.6 & 48.4 & 36.2 & 43.5 & 52.3 \\
\hline & & \begin{tabular}{|l|} 
Combined \\
\end{tabular} & 31.4 & 31.4 & 37.2 & 42.9 & 35.9 & 42.4 & 49.9 & 40.1 & 48.3 & 58.4 & 42.9 & 51.5 & 62.8 \\
\hline
\end{tabular}




\begin{tabular}{|c|c|c|c|c|c|c|c|c|c|c|c|c|c|c|c|}
\hline & & & \multicolumn{13}{|c|}{ Pickup } \\
\hline & & & \multirow{2}{*}{$\begin{array}{l}2010 \\
\text { Ref }\end{array}$} & \multicolumn{3}{|c|}{2010} & \multicolumn{3}{|c|}{2015} & \multicolumn{3}{|c|}{2030} & \multicolumn{3}{|c|}{2045} \\
\hline & & & & low & avg & high & low & avg & high & low & avg & high & low & avg & high \\
\hline \multirow{6}{*}{ H2 Series HEV PHEV40 } & \multirow{3}{*}{ Unadjusted } & UDDS & 57.7 & 57.7 & 69.5 & 81.7 & 66.7 & 80.5 & 98.0 & 75.1 & 94.8 & 121.5 & 81.5 & 101.5 & 130.7 \\
\hline & & HWFET & 47.5 & 47.5 & 56.0 & 65.0 & 54.5 & 64.5 & 75.7 & 60.9 & 73.5 & 87.7 & 65.3 & 79.1 & 94.8 \\
\hline & & Combined & 52.6 & 52.6 & 62.7 & 73.2 & 60.6 & 72.4 & 86.6 & 67.9 & 83.8 & 103.5 & 73.3 & 90.0 & 111.6 \\
\hline & \multirow{3}{*}{ Adjusted } & \begin{tabular}{|l|} 
UDDS \\
\end{tabular} & 42.0 & 42.0 & 49.5 & 56.9 & 47.8 & 56.2 & 66.4 & 53.0 & 64.6 & 79.1 & 56.8 & 68.4 & 83.8 \\
\hline & & HWFET & 33.5 & 33.5 & 39.5 & 45.7 & 38.4 & 45.3 & 53.1 & 42.8 & 51.5 & 61.3 & 45.9 & 55.4 & 66.2 \\
\hline & & Combined & 37.7 & 37.7 & 44.4 & 51.3 & 43.1 & 50.8 & 59.7 & 47.9 & 58.0 & 70.0 & 51.3 & 61.9 & 74.8 \\
\hline \multirow{6}{*}{ E85 Split HEV } & \multirow{3}{*}{ Unadjusted } & \begin{tabular}{|l|} 
UDDS \\
\end{tabular} & 29.1 & 29.1 & 31.4 & 34.3 & 30.5 & 34.5 & 44.6 & 32.4 & 38.2 & 51.8 & 33.3 & 39.5 & 54.0 \\
\hline & & HWFET & 25.8 & 25.8 & 27.6 & 29.9 & 26.9 & 29.6 & 37.5 & 28.0 & 32.2 & 42.0 & 28.8 & 33.3 & 43.6 \\
\hline & & Combined & 27.5 & 27.5 & 29.6 & 32.2 & 28.8 & 32.1 & 41.1 & 30.3 & 35.2 & 46.9 & 31.1 & 36.4 & 48.8 \\
\hline & \multirow{3}{*}{ Adjusted } & \begin{tabular}{|l|} 
UDDS \\
\end{tabular} & 22.4 & 22.4 & 24.0 & 26.1 & 23.4 & 26.2 & 33.3 & 24.8 & 28.8 & 38.2 & 25.4 & 29.8 & 39.6 \\
\hline & & \begin{tabular}{|l|} 
HWFET \\
\end{tabular} & 18.3 & 18.3 & 19.5 & 21.1 & 19.1 & 21.0 & 26.5 & 19.9 & 22.8 & 29.7 & 20.4 & 23.5 & 30.8 \\
\hline & & Combined & 20.3 & 20.3 & 21.8 & 23.6 & 21.2 & 23.6 & 29.8 & 22.3 & 25.8 & 33.8 & 22.9 & 26.6 & 35.1 \\
\hline \multirow{6}{*}{ E85 Split HEV PHEV10 } & \multirow{3}{*}{ Unadjusted } & UDDS & 37.6 & 37.6 & 40.4 & 43.8 & 39.5 & 43.4 & 55.2 & 41.0 & 47.3 & 63.4 & 42.1 & 48.7 & 66.2 \\
\hline & & \begin{tabular}{|l|} 
HWFET \\
\end{tabular} & 34.2 & 34.2 & 36.1 & 38.9 & 35.7 & 38.5 & 48.1 & 36.5 & 41.3 & 53.5 & 37.6 & 42.6 & 57.1 \\
\hline & & Combined & 36.0 & 36.0 & 38.4 & 41.5 & 37.7 & 41.0 & 51.7 & 38.8 & 44.4 & 58.5 & 40.0 & 45.7 & 61.8 \\
\hline & \multirow{3}{*}{ Adjusted } & \begin{tabular}{|l|} 
UDDS \\
\end{tabular} & 28.5 & 28.5 & 30.4 & 32.8 & 29.7 & 32.5 & 40.4 & 30.8 & 35.1 & 45.7 & 31.6 & 36.1 & 47.5 \\
\hline & & HWFET & 24.2 & 24.2 & 25.5 & 27.5 & 25.2 & 27.2 & 33.9 & 25.8 & 29.2 & 37.7 & 26.6 & 30.1 & 40.2 \\
\hline & & Combined & 26.4 & 26.4 & 28.0 & 30.2 & 27.5 & 29.9 & 37.2 & 28.3 & 32.2 & 41.7 & 29.1 & 33.1 & 43.9 \\
\hline \multirow{6}{*}{ E85 Split HEV PHEV20 } & \multirow{3}{*}{ Unadjusted } & \begin{tabular}{|l|} 
UDDS \\
\end{tabular} & 54.2 & 54.2 & 58.4 & 63.5 & 57.0 & 62.8 & 80.0 & 59.1 & 68.3 & 90.7 & 60.8 & 70.4 & 94.8 \\
\hline & & HWFET & 41.4 & 41.4 & 44.1 & 47.7 & 43.3 & 47.2 & 47.8 & 44.8 & 41.1 & 52.8 & 46.1 & 42.4 & 54.9 \\
\hline & & \begin{tabular}{|l|} 
Combined \\
\end{tabular} & 47.6 & 47.6 & 51.0 & 55.3 & 49.9 & 54.7 & 61.4 & 51.7 & 52.6 & 68.5 & 53.2 & 54.3 & 71.4 \\
\hline & \multirow{3}{*}{ Adjusted } & UDDS & 39.8 & 39.8 & 42.5 & 45.7 & 41.6 & 45.3 & 56.0 & 43.0 & 48.8 & 62.3 & 44.0 & 50.1 & 64.6 \\
\hline & & HWFET & 29.2 & 29.2 & 31.2 & 33.7 & 30.6 & 33.3 & 33.7 & 31.6 & 29.0 & 37.2 & 32.5 & 29.9 & 38.6 \\
\hline & & Combined & 34.2 & 34.2 & 36.5 & 39.4 & 35.8 & 39.0 & 43.1 & 37.0 & 37.3 & 47.8 & 38.0 & 38.5 & 49.6 \\
\hline \multirow{6}{*}{ E85 Series HEV PHEV30 } & \multirow{3}{*}{ Unadjusted } & UDDS & 47.9 & 47.9 & 52.5 & 57.6 & 50.6 & 56.9 & 76.0 & 53.8 & 64.8 & 90.1 & 55.5 & 67.2 & 95.1 \\
\hline & & HWFET & 35.6 & 35.6 & 38.3 & 41.9 & 37.5 & 41.6 & 53.6 & 39.4 & 45.7 & 60.7 & 40.9 & 47.6 & 63.0 \\
\hline & & Combined & 41.4 & 41.4 & 45.0 & 49.3 & 43.7 & 48.8 & 64.0 & 46.2 & 54.6 & 74.0 & 47.8 & 56.7 & 77.4 \\
\hline & \multirow{3}{*}{ Adjusted } & UDDS & 35.6 & 35.6 & 38.6 & 42.0 & 37.3 & 41.5 & 53.5 & 39.5 & 46.6 & 61.9 & 40.6 & 48.1 & 64.8 \\
\hline & & HWFET & 25.1 & 25.1 & 27.1 & 29.6 & 26.5 & 29.4 & 37.8 & 27.8 & 32.3 & 42.7 & 28.9 & 33.6 & 44.3 \\
\hline & & Combined & 30.0 & 30.0 & 32.4 & 35.3 & 31.5 & 35.0 & 45.1 & 33.2 & 38.8 & 51.5 & 34.3 & 40.3 & 53.6 \\
\hline \multirow{6}{*}{ E85 Series HEV PHEV40 } & & \begin{tabular}{|l|} 
UDDS \\
\end{tabular} & 54.8 & 54.8 & 60.0 & 66.2 & 58.0 & 65.3 & 87.6 & 61.7 & 74.6 & 104.4 & 63.7 & 77.6 & 110.2 \\
\hline & Unadjusted & HWFET & 45.0 & 45.0 & 48.5 & 53.2 & 47.5 & 52.7 & 68.1 & 50.0 & 58.1 & 77.1 & 51.7 & 60.5 & 80.3 \\
\hline & & Combined & 49.9 & 49.9 & 54.2 & 59.7 & 52.7 & 59.0 & 77.6 & 55.8 & 66.1 & 90.0 & 57.7 & 68.9 & 94.4 \\
\hline & & \begin{tabular}{|l|} 
UDDS \\
\end{tabular} & 40.1 & 40.1 & 43.5 & 47.5 & 42.2 & 46.9 & 60.4 & 44.6 & 52.7 & 70.0 & 45.9 & 54.5 & 73.2 \\
\hline & Adjusted & HWFET & 31.8 & 31.8 & 34.2 & 37.5 & 33.5 & 37.1 & 47.8 & 35.3 & 40.9 & 54.0 & 36.5 & 42.6 & 56.2 \\
\hline & & \begin{tabular}{|l|} 
Combined \\
\end{tabular} & 35.9 & 35.9 & 38.8 & 42.4 & 37.8 & 41.9 & 54.0 & 39.9 & 46.6 & 61.8 & 41.1 & 48.4 & 64.4 \\
\hline
\end{tabular}




\begin{tabular}{|c|c|c|c|c|c|c|c|c|c|c|c|c|c|c|c|}
\hline & & & \multicolumn{13}{|c|}{ Pickup } \\
\hline & & & \multirow{2}{*}{$\begin{array}{l}2010 \\
\text { Ref }\end{array}$} & \multicolumn{3}{|c|}{2010} & \multicolumn{3}{|c|}{2015} & \multicolumn{3}{|c|}{2030} & \multicolumn{3}{|c|}{2045} \\
\hline & & & & low & avg & high & low & avg & high & low & avg & high & low & avg & high \\
\hline \multirow{6}{*}{ FC HEV } & \multirow{3}{*}{ Unadjusted } & UDDS & 33.7 & 33.7 & 40.9 & 48.9 & 41.2 & 49.0 & 56.6 & 45.4 & 55.0 & 66.2 & 47.7 & 56.7 & 69.3 \\
\hline & & HWFET & 33.7 & 33.7 & 39.3 & 45.9 & 40.1 & 45.4 & 50.8 & 42.6 & 49.3 & 55.9 & 44.3 & 50.7 & 58.1 \\
\hline & & Combined & 33.7 & 33.7 & 40.1 & 47.5 & 40.7 & 47.3 & 53.8 & 44.1 & 52.3 & 61.2 & 46.1 & 53.9 & 63.8 \\
\hline & \multirow{3}{*}{ Adjusted } & \begin{tabular}{|l|} 
UDDS \\
\end{tabular} & 25.7 & 25.7 & 30.7 & 36.2 & 31.0 & 36.2 & 41.3 & 33.8 & 40.2 & 47.5 & 35.4 & 41.4 & 49.4 \\
\hline & & HWFET & 23.9 & 23.9 & 27.7 & 32.4 & 28.3 & 32.1 & 35.8 & 30.1 & 34.8 & 39.4 & 31.2 & 35.8 & 40.9 \\
\hline & & \begin{tabular}{|l|} 
Combined \\
\end{tabular} & 24.9 & 24.9 & 29.3 & 34.4 & 29.7 & 34.2 & 38.6 & 32.0 & 37.6 & 43.5 & 33.4 & 38.7 & 45.2 \\
\hline \multirow{6}{*}{ FC PHEV10 } & \multirow{3}{*}{ Unadjusted } & \begin{tabular}{|l|} 
UDDS \\
\end{tabular} & 43.3 & 43.3 & 51.5 & 60.7 & 52.0 & 60.0 & 68.8 & 55.7 & 66.7 & 79.6 & 58.3 & 68.6 & 83.2 \\
\hline & & \begin{tabular}{|l|} 
HWFET \\
\end{tabular} & 44.5 & 44.5 & 50.4 & 58.1 & 51.7 & 57.5 & 63.3 & 54.0 & 61.5 & 68.4 & 55.8 & 63.2 & 70.9 \\
\hline & & Combined & 43.8 & 43.8 & 51.0 & 59.5 & 51.9 & 58.8 & 66.2 & 54.9 & 64.3 & 74.1 & 57.1 & 66.0 & 77.2 \\
\hline & \multirow{3}{*}{ Adjusted } & \begin{tabular}{|l|} 
UDDS \\
\end{tabular} & 32.4 & 32.4 & 37.9 & 44.0 & 38.3 & 43.5 & 49.1 & 40.7 & 47.8 & 55.7 & 42.4 & 49.0 & 57.8 \\
\hline & & \begin{tabular}{|l|} 
HWFET \\
\end{tabular} & 31.4 & $\begin{array}{ll}31.4 \\
\end{array}$ & 35.5 & 40.9 & 36.5 & 40.5 & 44.5 & 38.1 & 43.2 & 48.0 & 39.3 & 44.4 & 49.8 \\
\hline & & Combined & 31.9 & 31.9 & 36.8 & 42.5 & 37.4 & 42.1 & 46.9 & 39.5 & 45.6 & 52.0 & 40.9 & 46.8 & 53.9 \\
\hline \multirow{6}{*}{ FC PHEV20 } & \multirow{3}{*}{ Unadjusted } & \begin{tabular}{|l|} 
UDDS \\
\end{tabular} & 51.8 & 51.8 & 61.7 & 72.9 & 62.3 & 72.1 & 82.9 & 66.9 & 80.5 & 96.2 & 70.1 & 83.0 & 100.7 \\
\hline & & HWFET & 45.5 & 45.5 & 52.1 & 60.4 & 53.4 & 59.7 & 66.5 & 56.1 & 64.5 & 72.6 & 58.1 & 66.4 & 75.5 \\
\hline & & Combined & 48.8 & 48.8 & 57.0 & 66.7 & 57.9 & 65.9 & 74.6 & 61.6 & 72.4 & 83.9 & 64.1 & 74.6 & 87.5 \\
\hline & \multirow{3}{*}{ Adjusted } & \begin{tabular}{|l|} 
UDDS \\
\end{tabular} & 38.2 & 38.2 & 44.6 & 51.6 & 45.0 & 51.1 & 57.7 & 47.9 & 56.2 & 65.4 & 49.9 & 57.7 & 67.9 \\
\hline & & HWFET & 32.1 & 32.1 & 36.7 & 42.5 & 37.6 & 42.0 & 46.7 & 39.5 & 45.4 & 50.9 & 40.9 & 46.6 & 52.9 \\
\hline & & Combined & 35.2 & 35.2 & 40.7 & 47.1 & 41.3 & 46.6 & 52.2 & 43.7 & 50.8 & 58.0 & 45.4 & 52.1 & 60.2 \\
\hline \multirow{6}{*}{ FC PHEV30 } & \multirow{3}{*}{ Unadjusted } & \begin{tabular}{|l|} 
UDDS \\
\end{tabular} & 74.8 & 74.8 & 88.0 & 101.8 & 88.8 & 101.5 & 117.2 & 94.4 & 113.5 & 134.7 & 98.9 & 117.5 & 140.9 \\
\hline & & HWFET & 57.5 & 57.5 & 65.4 & 74.9 & 67.0 & 74.5 & 83.2 & 69.9 & 80.7 & 90.6 & 72.5 & 83.1 & 94.2 \\
\hline & & \begin{tabular}{|l|} 
Combined \\
\end{tabular} & 65.9 & 65.9 & 76.2 & 87.6 & 77.4 & 87.2 & 99.0 & 81.5 & 96.0 & 110.5 & 84.9 & 99.1 & 115.2 \\
\hline & \multirow{3}{*}{ Adjusted } & \begin{tabular}{|l|} 
UDDS \\
\end{tabular} & 52.8 & 52.8 & 60.7 & 68.5 & 61.1 & 68.4 & 76.8 & 64.4 & 74.9 & 85.8 & 66.9 & 77.0 & 88.9 \\
\hline & & HWFET & 40.5 & 40.5 & 46.0 & 52.5 & 47.0 & 52.2 & 58.2 & 49.1 & 56.5 & 63.3 & 50.8 & 58.2 & 65.8 \\
\hline & & Combined & 46.4 & 46.4 & 53.0 & 60.2 & 53.9 & 60.0 & 67.2 & 56.4 & 65.3 & 74.0 & 58.6 & 67.2 & 76.8 \\
\hline \multirow{6}{*}{ FC PHEV40 } & \multirow{3}{*}{ Unadjusted } & \begin{tabular}{|l|} 
UDDS \\
\end{tabular} & 85.9 & 85.9 & 101.4 & 117.6 & 102.4 & 117.3 & 135.8 & 109.0 & 131.6 & 156.4 & 114.3 & 136.5 & 163.8 \\
\hline & & HWFET & 72.7 & 72.7 & 82.9 & 95.0 & 84.9 & 94.6 & 105.8 & 88.7 & 102.7 & 115.5 & 92.1 & 105.9 & 120.2 \\
\hline & & Combined & 79.4 & 79.4 & 92.1 & 106.2 & 93.7 & 105.8 & 120.4 & 98.8 & 116.8 & 134.9 & 103.1 & 120.8 & 140.8 \\
\hline & \multirow{3}{*}{ Adjusted } & \begin{tabular}{|l|} 
UDDS \\
\end{tabular} & 59.5 & 59.5 & 68.3 & 77.1 & 68.9 & 76.9 & 86.4 & 72.5 & 84.3 & 96.3 & 75.3 & 86.7 & 99.7 \\
\hline & & HWFET & 51.0 & 51.0 & 58.0 & 66.3 & 59.4 & 66.0 & 73.7 & 62.0 & 71.6 & 80.3 & 64.3 & 73.8 & 83.5 \\
\hline & & Combined & 55.3 & 55.3 & 63.3 & 71.8 & 64.3 & 71.6 & 80.2 & 67.4 & 78.1 & $\begin{array}{l}88.4 \\
\end{array}$ & 69.9 & 80.4 & 91.7 \\
\hline
\end{tabular}




\section{$\underline{\text { Pev details }}$}

\begin{tabular}{|c|c|c|c|c|c|c|c|c|c|c|c|c|c|c|c|c|}
\hline & & & & \multicolumn{13}{|c|}{ Pickup } \\
\hline & & & & \multirow{2}{*}{$\begin{array}{c}2010 \\
\text { Ref }\end{array}$} & \multicolumn{3}{|c|}{2010} & \multicolumn{3}{|c|}{2015} & \multicolumn{3}{|c|}{2030} & \multicolumn{3}{|c|}{2045} \\
\hline & & & & & low & avg & high & low & avg & high & low & avg & high & low & avg & high \\
\hline \multirow{25}{*}{ SI Split HEV PHEV10 } & \multirow{9}{*}{ UDDS } & \multirow{4}{*}{$C D$} & Wh/mile & 281.1 & 281.1 & 264.7 & 251.7 & 271.8 & 252.7 & 234.3 & 263.3 & 241.9 & 213.2 & 260.3 & 237.7 & 205.1 \\
\hline & & & Distance & 12.4 & 12.4 & 12.4 & 12.3 & 12.4 & 12.8 & 12.3 & 12.6 & 12.8 & 12.3 & 12.8 & 12.6 & 12.2 \\
\hline & & & MPGGE Unadjusted & 326.4 & 326.4 & 349.6 & 402.4 & 339.7 & 391.3 & 466.8 & 350.1 & 427.4 & 619.1 & 361.0 & 453.3 & 660.0 \\
\hline & & & MPGGE Adjusted & 158.6 & 158.6 & 165.1 & 178.7 & 162.4 & 176.0 & 193.2 & 165.3 & 184.6 & 220.8 & 168.2 & 190.4 & 226.9 \\
\hline & & \multirow{2}{*}{ CS } & MPGGE Unadjusted & 34.1 & 34.1 & 36.6 & 39.6 & 35.7 & 39.2 & 47.3 & 37.1 & 42.7 & 56.9 & 38.0 & 43.9 & 59.3 \\
\hline & & & MPGGE Adjusted & 26.0 & 26.0 & 27.8 & 29.8 & 27.1 & 29.6 & 35.1 & 28.1 & 32.0 & 41.5 & 28.7 & 32.8 & 43.1 \\
\hline & & \multirow{3}{*}{$C D+C S$} & Wh/mile & 58.6 & 58.6 & 55.1 & 52.4 & 56.6 & 52.6 & 48.8 & 54.9 & 50.4 & 44.4 & 54.2 & 49.5 & 42.7 \\
\hline & & & MPGGE Unadjusted & 40.3 & 40.3 & 43.2 & 46.8 & 42.1 & 46.3 & 55.8 & 43.7 & 50.5 & 67.2 & 44.8 & 51.9 & 70.2 \\
\hline & & & MPGGE Adjusted & 30.3 & 30.3 & 32.3 & 34.8 & 31.6 & 34.4 & 40.8 & 32.7 & 37.3 & 48.1 & 33.5 & 38.2 & 49.9 \\
\hline & \multirow{9}{*}{ HWFET } & \multirow{4}{*}{$C D$} & Wh/mile & 309.7 & 309.7 & 293.9 & 275.5 & 285.6 & 263.9 & 242.5 & 292.4 & 249.4 & 235.8 & 289.0 & 242.7 & 263.2 \\
\hline & & & Distance & 12.4 & 12.4 & 12.2 & 12.2 & 12.6 & 12.5 & 12.4 & 12.2 & 12.5 & 11.6 & 12.2 & 12.4 & 8.1 \\
\hline & & & MPGGE Unadjusted & 100.6 & 100.6 & 106.6 & 114.8 & 95.2 & 102.7 & 121.7 & 107.5 & 109.8 & 154.0 & 111.7 & 113.5 & 230.1 \\
\hline & & & MPGGE Adjusted & 70.1 & 70.1 & 74.2 & 79.8 & 66.5 & 71.6 & 84.5 & 74.8 & 76.4 & 106.2 & 77.7 & 78.9 & 156.1 \\
\hline & & \multirow{2}{*}{ CS } & MPGGE Unadjusted & 30.5 & 30.5 & 32.4 & 34.9 & 31.8 & 34.5 & 41.0 & 32.8 & 37.0 & 47.2 & 33.8 & 38.2 & 49.0 \\
\hline & & & MPGGE Adjusted & 21.6 & 21.6 & 22.9 & 24.7 & 22.5 & 24.4 & 28.9 & 23.2 & 26.1 & 33.3 & 23.9 & 27.0 & 34.5 \\
\hline & & \multirow{3}{*}{$\mathrm{CD}+\mathrm{CS}$} & Wh/mile & 91.1 & 91.1 & 86.4 & 81.0 & 84.0 & 77.6 & 71.3 & 86.0 & 73.3 & 69.3 & 85.0 & 71.4 & 77.4 \\
\hline & & & MPGGE Unadjusted & 36.6 & 36.6 & 38.9 & 41.9 & 37.9 & 41.1 & 48.7 & 39.4 & 44.0 & 56.6 & 40.6 & 45.4 & 60.4 \\
\hline & & & MPGGE Adjusted & 25.9 & 25.9 & 27.5 & 29.6 & 26.8 & 29.0 & 34.4 & 27.8 & 31.0 & 39.9 & 28.7 & 32.1 & 42.5 \\
\hline & \multirow{7}{*}{ Combined } & \multirow{2}{*}{$C D$} & MPGGE Unadjusted & 162.4 & 162.4 & 172.5 & 189.1 & 157.6 & 172.8 & 205.1 & 173.6 & 185.7 & 262.4 & 180.1 & 193.1 & 358.5 \\
\hline & & & \begin{tabular}{|l|} 
MPGEE Adjusted \\
\end{tabular} & 101.2 & 101.2 & 106.5 & 114.7 & 98.5 & 106.3 & 122.4 & 107.0 & 112.8 & 148.6 & 110.3 & 116.4 & 188.5 \\
\hline & & \multirow{2}{*}{ CS } & MPGGE Unadjusted & 32.4 & 32.4 & 34.6 & 37.3 & 33.8 & 36.9 & 44.2 & 35.0 & 39.9 & 52.1 & 36.0 & 41.2 & 54.2 \\
\hline & & & MPGGE Adjusted & 23.8 & 23.8 & 25.4 & 27.3 & 24.8 & 27.0 & 32.0 & 25.7 & 29.1 & 37.3 & 26.3 & 29.9 & 38.8 \\
\hline & & \multirow{3}{*}{$\mathrm{CD}+\mathrm{CS}$} & Wh/mile & 73.2 & 73.2 & 69.2 & 65.3 & 68.9 & 63.9 & 58.9 & 68.9 & 60.7 & 55.6 & 68.1 & 59.4 & 58.3 \\
\hline & & & MPGGE Unadjusted & 38.5 & 38.5 & 41.2 & 44.5 & 40.1 & 43.8 & 52.4 & 41.6 & 47.3 & 62.0 & 42.8 & 48.8 & 65.4 \\
\hline & & & MPGGE Adjusted & 28.1 & 28.1 & 30.0 & 32.2 & 29.2 & 31.8 & 37.6 & 30.3 & 34.2 & 44.0 & 31.1 & 35.2 & 46.3 \\
\hline
\end{tabular}


Argonne National Laboratory Report - Light-Duty Vehicle Fuel Consumption Displacement Potential Up to 2045

\begin{tabular}{|c|c|c|c|c|c|c|c|c|c|c|c|c|c|c|c|c|}
\hline & & & & \multicolumn{13}{|c|}{ Pickup } \\
\hline & & & & 2010 & & 2010 & & & 2015 & & & 2030 & & & 2045 & \\
\hline \multirow{26}{*}{ SI Split HEV PHEV2O } & & \multirow{5}{*}{$C D$} & & Ref & low & avg & high & low & avg & high & low & avg & high & low & avg & high \\
\hline & \multirow{9}{*}{ UDDS } & & Wh/mile & 334.2 & 334.2 & 312.4 & 295.1 & 323.2 & 297.7 & 266.3 & 312.6 & 268.7 & 224.1 & 308.9 & 262.9 & 217.0 \\
\hline & & & Distance & 23.7 & 23.7 & 23.9 & 23.5 & 23.5 & 23.5 & 23.5 & 23.9 & 23.9 & 24.0 & 23.7 & 23.7 & 23.9 \\
\hline & & & MPGGE Unadjusted & 797.2 & 797.2 & 795.2 & 969.7 & 834.9 & 960.3 & 1003.6 & 841.9 & 787.9 & 799.6 & 861.8 & 802.6 & 890.1 \\
\hline & & & MPGGE Adjusted & 244.7 & 244.7 & 244.5 & 262.2 & 248.9 & 261.4 & 265.2 & 249.7 & 243.6 & 245.0 & 251.8 & 245.3 & 254.7 \\
\hline & & \multirow{2}{*}{ CS } & MPGGE Unadjusted & 33.7 & 33.7 & 36.2 & 39.2 & 35.3 & 38.9 & 47.2 & 36.7 & 42.7 & 56.7 & 37.7 & 43.8 & 59.2 \\
\hline & & & MPGGE Adjusted & 25.7 & 25.7 & 27.5 & 29.6 & 26.8 & 29.3 & 35.1 & 27.8 & 32.0 & 41.4 & 28.5 & 32.8 & 43.0 \\
\hline & & \multirow{3}{*}{$C D+C S$} & Wh/mile & 173.7 & 173.7 & 162.4 & 153.5 & 168.0 & 154.8 & 138.7 & 162.3 & 140.4 & 117.6 & 160.4 & 137.3 & 113.9 \\
\hline & & & MPGGE Unadjusted & 58.1 & 58.1 & 62.2 & 67.7 & 60.8 & 67.1 & 81.0 & 63.2 & 72.9 & 95.8 & 64.8 & 74.9 & 100.3 \\
\hline & & & MPGGE Adjusted & 42.3 & 42.3 & 44.9 & 48.4 & 44.0 & 48.0 & 56.5 & 45.5 & 51.6 & 65.1 & 46.6 & 52.9 & 67.7 \\
\hline & \multirow{9}{*}{ HWFET } & \multirow{4}{*}{$C D$} & Wh/mile & 364.7 & 364.7 & 344.7 & 323.8 & 353.4 & 326.6 & 243.1 & 345.2 & 249.6 & 226.9 & 340.1 & 244.3 & 219.1 \\
\hline & & & Distance & 21.7 & 21.7 & 21.6 & 21.4 & 21.6 & 21.4 & 20.2 & 21.5 & 20.2 & 19.3 & 21.4 & 20.0 & 19.2 \\
\hline & & & MPGGE Unadjusted & 152.8 & 152.8 & 158.5 & 172.7 & 159.5 & 171.1 & 120.4 & 163.8 & 108.9 & 138.3 & 169.1 & 112.6 & 144.1 \\
\hline & & & MPGGE Adjusted & 105.4 & 105.4 & 109.1 & 118.6 & 109.9 & 117.5 & 83.6 & 112.7 & 75.8 & 95.6 & 116.2 & 78.3 & 99.6 \\
\hline & & \multirow{2}{*}{ CS } & MPGGE Unadjusted & 30.3 & 30.3 & 32.1 & 34.6 & 31.6 & 34.3 & 40.7 & 32.5 & 36.8 & 47.0 & 33.5 & 38.0 & 48.8 \\
\hline & & & MPGGE Adjusted & 21.4 & 21.4 & 22.7 & 24.5 & 22.4 & 24.3 & 28.7 & 23.0 & 26.0 & 33.2 & 23.7 & 26.9 & 34.4 \\
\hline & & \multirow{3}{*}{$C D+C S$} & Wh/mile & 182.8 & 182.8 & 172.9 & 163.4 & 177.1 & 164.8 & 71.5 & 174.1 & 73.4 & 66.7 & 171.5 & 71.9 & 64.4 \\
\hline & & & MPGGE Unadjusted & 44.4 & 44.4 & 47.0 & 50.9 & 46.4 & 50.5 & 48.4 & 47.9 & 43.7 & 55.9 & 49.3 & 45.2 & 58.0 \\
\hline & & & MPGGE Adjusted & 31.4 & 31.4 & 33.2 & 35.9 & 32.7 & 35.6 & 34.1 & 33.8 & 30.9 & 39.3 & 34.8 & 31.9 & 40.8 \\
\hline & \multirow{7}{*}{ Combined } & \multirow{2}{*}{$C D$} & MPGGE Unadjusted & 275.1 & 275.1 & 283.2 & 315.2 & 287.4 & 312.2 & 233.4 & 294.1 & 207.0 & 253.6 & 303.1 & 213.6 & 267.3 \\
\hline & & & MPGGE Adjusted & 153.4 & 153.4 & 156.9 & 169.7 & 158.6 & 168.6 & 134.1 & 161.4 & 122.0 & 143.9 & 165.1 & 125.2 & 149.7 \\
\hline & & \multirow{2}{*}{ CS } & MPGGE Unadjusted & 32.1 & 32.1 & 34.2 & 37.0 & 33.5 & 36.7 & 44.0 & 34.7 & 39.8 & 51.9 & 35.7 & 41.0 & 54.0 \\
\hline & & & \begin{tabular}{|l|} 
MPGGE Adjusted \\
\end{tabular} & 23.6 & 23.6 & 25.1 & 27.1 & 24.6 & 26.8 & 31.9 & 25.4 & 29.0 & 37.2 & 26.1 & 29.8 & 38.7 \\
\hline & & \multirow{3}{*}{$C D+C S$} & Wh/mile & 177.8 & 177.8 & 167.1 & 157.9 & 172.1 & 159.3 & 108.5 & 167.6 & 110.2 & 94.7 & 165.4 & 107.8 & 91.6 \\
\hline & & & MPGGE Unadjusted & 51.0 & 51.0 & 54.3 & 58.9 & 53.3 & 58.4 & 62.1 & 55.2 & 56.1 & 72.5 & 56.8 & 57.8 & 75.5 \\
\hline & & & \begin{tabular}{|l|} 
MPGEE Adjusted \\
\end{tabular} & 36.6 & 36.6 & 38.7 & 41.8 & 38.1 & 41.5 & 43.6 & 39.4 & 39.6 & 50.3 & 40.4 & 40.8 & 52.2 \\
\hline
\end{tabular}


Argonne National Laboratory Report - Light-Duty Vehicle Fuel Consumption Displacement Potential Up to 2045

\begin{tabular}{|c|c|c|c|c|c|c|c|c|c|c|c|c|c|c|c|c|}
\hline & & & & \multicolumn{13}{|c|}{ Pickup } \\
\hline & & & & \multirow{2}{*}{$\begin{array}{c}2010 \\
\text { Ref }\end{array}$} & \multicolumn{3}{|c|}{2010} & \multicolumn{3}{|c|}{2015} & \multicolumn{3}{|c|}{2030} & \multicolumn{3}{|c|}{2045} \\
\hline & & & & & low & avg & high & low & avg & high & low & avg & high & low & avg & high \\
\hline \multirow{25}{*}{ SI Series HEV PHEV30 } & \multirow{9}{*}{ UDDS } & \multirow{4}{*}{$C D$} & Wh/mile & 444.2 & 444.2 & 416.3 & 387.8 & 427.4 & 392.2 & 349.4 & 413.3 & 359.8 & 309.8 & 406.9 & 349.7 & 296.3 \\
\hline & & & Distance & 31.9 & 31.9 & 31.4 & 31.9 & 31.4 & 32.0 & 30.9 & 31.4 & 30.9 & 31.8 & 31.4 & 30.9 & 31.8 \\
\hline & & & MPGGE Unadjusted & $\mathrm{N} / \mathrm{A}$ & $\mathrm{N} / \mathrm{A}$ & N/A & N/A & N/A & $\mathrm{N} / \mathrm{A}$ & N/A & $\mathrm{N} / \mathrm{A}$ & N/A & N/A & N/A & N/A & $\mathrm{N} / \mathrm{A}$ \\
\hline & & & MPGGE Adjusted & $\mathrm{N} / \mathrm{A}$ & $\mathrm{N} / \mathrm{A}$ & $\mathrm{N} / \mathrm{A}$ & N/A & N/A & N/A & N/A & $\mathrm{N} / \mathrm{A}$ & N/A & N/A & N/A & $\mathrm{N} / \mathrm{A}$ & $\mathrm{N} / \mathrm{A}$ \\
\hline & & \multirow{2}{*}{ CS } & MPGGE Unadjusted & 24.0 & 24.0 & 26.2 & 29.0 & 25.3 & 28.5 & 36.0 & 26.9 & 32.4 & 45.0 & 27.7 & 33.6 & 47.8 \\
\hline & & & MPGGE Adjusted & 18.7 & 18.7 & 20.3 & 22.3 & 19.6 & 21.9 & 27.3 & 20.8 & 24.8 & 33.6 & 21.4 & 25.6 & 35.5 \\
\hline & & \multirow{3}{*}{$\mathrm{CD}+\mathrm{CS}$} & Wh/mile & 285.7 & 285.7 & 267.8 & 249.5 & 274.9 & 252.3 & 225.5 & 265.9 & 232.2 & 200.4 & 261.8 & 225.4 & 191.6 \\
\hline & & & MPGGE Unadjusted & 51.1 & 51.1 & 55.8 & 61.8 & 53.9 & 60.6 & 76.6 & 57.3 & 68.9 & 95.8 & 59.0 & 71.4 & 101.7 \\
\hline & & & MPGGE Adjusted & 37.7 & 37.7 & 40.8 & 44.7 & 39.5 & 43.9 & 53.9 & 41.7 & 49.2 & 65.2 & 42.9 & 50.7 & 68.5 \\
\hline & \multirow{9}{*}{ HWFET } & \multirow{4}{*}{$C D$} & Wh/mile & 493.2 & 493.2 & 466.5 & 435.3 & 475.5 & 439.6 & 397.1 & 462.7 & 408.7 & 366.0 & 454.3 & 399.0 & 352.0 \\
\hline & & & \begin{tabular}{|l} 
Distance \\
\end{tabular} & 27.7 & 27.7 & 28.4 & 28.4 & 27.6 & 28.4 & 27.1 & 28.4 & 27.1 & 26.0 & 27.5 & 26.9 & 25.8 \\
\hline & & & MPGGE Unadjusted & $\mathrm{N} / \mathrm{A}$ & $\mathrm{N} / \mathrm{A}$ & $\mathrm{N} / \mathrm{A}$ & $\mathrm{N} / \mathrm{A}$ & N/A & $\mathrm{N} / \mathrm{A}$ & N/A & $\mathrm{N} / \mathrm{A}$ & N/A & $\mathrm{N} / \mathrm{A}$ & $\mathrm{N} / \mathrm{A}$ & $\mathrm{N} / \mathrm{A}$ & $\mathrm{N} / \mathrm{A}$ \\
\hline & & & MPGGE Adjusted & N/A & N/A & N/A & N/A & N/A & N/A & N/A & N/A & N/A & N/A & N/A & N/A & $\mathrm{N} / \mathrm{A}$ \\
\hline & & \multirow{2}{*}{ CS } & MPGGE Unadjusted & 22.3 & 22.3 & 24.1 & 26.3 & 23.5 & 26.1 & 31.9 & 24.7 & 28.7 & 37.8 & 25.6 & 29.9 & 39.3 \\
\hline & & & MPGGE Adjusted & 15.8 & 15.8 & 17.1 & 18.7 & 16.7 & 18.5 & 22.6 & 17.5 & 20.3 & 26.7 & 18.2 & 21.2 & 27.7 \\
\hline & & \multirow{3}{*}{$\mathrm{CD}+\mathrm{CS}$} & Wh/mile & 254.1 & 254.1 & 240.3 & 224.3 & 245.0 & 226.5 & 204.6 & 238.4 & 210.6 & 188.5 & 234.1 & 205.6 & 181.3 \\
\hline & & & MPGGE Unadjusted & 37.8 & 37.8 & 40.8 & 44.6 & 39.8 & 44.3 & 54.1 & 41.9 & 48.6 & 64.0 & 43.4 & 50.6 & 66.5 \\
\hline & & & MPGGE Adjusted & 26.7 & 26.7 & 28.8 & 31.5 & 28.1 & 31.2 & 38.1 & 29.6 & 34.3 & 45.0 & 30.7 & 35.7 & 46.7 \\
\hline & \multirow{7}{*}{ Combined } & \multirow{2}{*}{$C D$} & MPGGE Unadjusted & $\mathrm{N} / \mathrm{A}$ & $\mathrm{N} / \mathrm{A}$ & N/A & N/A & N/A & $\mathrm{N} / \mathrm{A}$ & N/A & $\mathrm{N} / \mathrm{A}$ & $\mathrm{N} / \mathrm{A}$ & N/A & $\mathrm{N} / \mathrm{A}$ & N/A & $\mathrm{N} / \mathrm{A}$ \\
\hline & & & MPGGE Adjusted & N/A & N/A & N/A & N/A & N/A & N/A & N/A & $\mathrm{N} / \mathrm{A}$ & N/A & N/A & N/A & N/A & N/A \\
\hline & & \multirow{2}{*}{ CS } & MPGGE Unadjusted & 23.2 & 23.2 & 25.2 & 27.7 & 24.5 & 27.4 & 34.0 & 25.9 & 30.6 & 41.4 & 26.7 & 31.8 & 43.5 \\
\hline & & & MPGGE Adjusted & 17.3 & 17.3 & 18.7 & 20.5 & 18.2 & 20.2 & 25.0 & 19.2 & 22.5 & 30.1 & 19.8 & 23.4 & 31.5 \\
\hline & & \multirow{3}{*}{$C D+C S$} & Wh/mile & 271.4 & 271.4 & 255.4 & 238.1 & 261.4 & 240.7 & 216.1 & 253.5 & 222.4 & 195.0 & 249.3 & 216.5 & 187.0 \\
\hline & & & MPGGE Unadjusted & 44.1 & 44.1 & 47.9 & 52.7 & 46.5 & 51.9 & 64.5 & 49.2 & 58.0 & 78.3 & 50.8 & 60.3 & 82.2 \\
\hline & & & MPGGE Adjusted & 31.8 & 31.8 & 34.4 & 37.6 & 33.4 & 37.1 & 45.4 & 35.2 & 41.1 & 54.2 & 36.4 & 42.6 & 56.6 \\
\hline
\end{tabular}




\begin{tabular}{|c|c|c|c|c|c|c|c|c|c|c|c|c|c|c|c|c|}
\hline & & & & \multicolumn{13}{|c|}{ Pickup } \\
\hline & & & & \multirow{2}{*}{$\begin{array}{l}\frac{2010}{\text { Ref }} \\
\end{array}$} & \multicolumn{3}{|c|}{2010} & \multicolumn{3}{|c|}{2015} & \multicolumn{3}{|c|}{2030} & \multicolumn{3}{|c|}{2045} \\
\hline \multirow{26}{*}{ SI Series HEV PHEV40 } & & & & & low & avg & high & low & avg & high & low & avg & high & low & avg & high \\
\hline & \multirow{9}{*}{ UDDS } & \multirow{4}{*}{$C D$} & Wh/mile & 454.2 & 454.2 & 425.1 & 395.6 & 436.5 & 400.4 & 356.5 & 421.8 & 367.1 & 312.8 & 414.7 & 356.2 & 299.3 \\
\hline & & & \begin{tabular}{|l|} 
Distance \\
\end{tabular} & 40.7 & 40.7 & 40.6 & 40.6 & 40.7 & 40.7 & 40.8 & 40.7 & 40.8 & 40.5 & 40.6 & 40.6 & 40.8 \\
\hline & & & MPGGE Unadjusted & N/A & N/A & N/A & N/A & N/A & N/A & $\mathrm{N} / \mathrm{A}$ & N/A & N/A & N/A & N/A & N/A & N/A \\
\hline & & & MPGGE Adjusted & N/A & N/A & N/A & N/A & N/A & N/A & $\mathrm{N} / \mathrm{A}$ & $\mathrm{N} / \mathrm{A}$ & N/A & N/A & N/A & N/A & $\mathrm{N} / \mathrm{A}$ \\
\hline & & \multirow{2}{*}{ CS } & MPGGE Unadjusted & 23.4 & 23.4 & 25.6 & 28.2 & 24.7 & 27.8 & 35.4 & 26.4 & 31.8 & 44.1 & 27.2 & 33.0 & 46.5 \\
\hline & & & MPGGE Adjusted & 18.2 & 18.2 & 19.9 & 21.7 & 19.2 & 21.5 & 26.9 & 20.4 & 24.3 & 32.9 & 21.0 & 25.2 & 34.6 \\
\hline & & \multirow{3}{*}{$C D+C S$} & Wh/mile & 333.0 & 333.0 & 311.7 & 290.2 & 320.1 & 293.8 & 263.0 & 309.4 & 270.7 & 230.6 & 304.3 & 262.5 & 220.6 \\
\hline & & & MPGGE Unadjusted & 58.5 & 58.5 & 64.1 & 70.5 & 61.9 & 69.6 & 88.5 & 65.9 & 79.4 & 110.2 & 67.9 & 82.5 & 116.3 \\
\hline & & & MPGGE Adjusted & 42.5 & 42.5 & 46.1 & 50.1 & 44.7 & 49.6 & 61.0 & 47.3 & 55.6 & 73.1 & 48.5 & 57.4 & 76.4 \\
\hline & \multirow{9}{*}{ HWFET } & \multirow{4}{*}{$C D$} & Wh/mile & 498.2 & 498.2 & 470.7 & 438.9 & 479.8 & 443.3 & 399.6 & 466.6 & 411.3 & 368.5 & 457.7 & 401.1 & 354.5 \\
\hline & & & Distance & 38.6 & 38.6 & 36.5 & 36.4 & 36.7 & 36.5 & 38.6 & 38.6 & 38.6 & 35.7 & 36.5 & 37.6 & 34.6 \\
\hline & & & MPGGE Unadjusted & N/A & N/A & N/A & N/A & N/A & $\mathrm{N} / \mathrm{A}$ & $\mathrm{N} / \mathrm{A}$ & N/A & N/A & N/A & N/A & N/A & N/A \\
\hline & & & MPGGE Adjusted & $\mathrm{N} / \mathrm{A}$ & N/A & $\mathrm{N} / \mathrm{A}$ & $\mathrm{N} / \mathrm{A}$ & N/A & N/A & N/A & $\mathrm{N} / \mathrm{A}$ & N/A & N/A & N/A & $\mathrm{N} / \mathrm{A}$ & $\mathrm{N} / \mathrm{A}$ \\
\hline & & \multirow{2}{*}{ CS } & MPGGE Unadjusted & 22.0 & 22.0 & 23.8 & 26.1 & 23.2 & 25.8 & 31.6 & 24.5 & 28.4 & 37.4 & 25.3 & 29.6 & 39.0 \\
\hline & & & MPGGE Adjusted & 15.6 & 15.6 & 16.8 & 18.5 & 16.5 & 18.3 & 22.4 & 17.4 & 20.1 & 26.5 & 17.9 & 21.0 & 27.5 \\
\hline & & \multirow{3}{*}{$C D+C S$} & Wh/mile & 336.3 & 336.3 & 317.7 & 296.3 & 323.9 & 299.3 & 269.7 & 314.9 & 277.6 & 248.8 & 309.0 & 270.7 & 239.3 \\
\hline & & & MPGGE Unadjusted & 47.9 & 47.9 & 51.6 & 56.6 & 50.5 & 56.0 & 68.7 & 53.2 & 61.7 & 81.4 & 55.0 & 64.3 & 84.7 \\
\hline & & & MPGGE Adjusted & 33.8 & 33.8 & 36.4 & 39.9 & 35.6 & 39.4 & 48.2 & 37.5 & 43.4 & 57.0 & 38.7 & 45.2 & 59.3 \\
\hline & \multirow{7}{*}{ Combined } & \multirow{2}{*}{$C D$} & MPGGE Unadjusted & $\mathrm{N} / \mathrm{A}$ & $\mathrm{N} / \mathrm{A}$ & $\mathrm{N} / \mathrm{A}$ & N/A & N/A & $\mathrm{N} / \mathrm{A}$ & $\mathrm{N} / \mathrm{A}$ & N/A & $\mathrm{N} / \mathrm{A}$ & N/A & $\mathrm{N} / \mathrm{A}$ & $\mathrm{N} / \mathrm{A}$ & $\mathrm{N} / \mathrm{A}$ \\
\hline & & & MPGGE Adjusted & $\mathrm{N} / \mathrm{A}$ & $\mathrm{N} / \mathrm{A}$ & N/A & N/A & N/A & $\mathrm{N} / \mathrm{A}$ & N/A & N/A & N/A & N/A & $\mathrm{N} / \mathrm{A}$ & $\mathrm{N} / \mathrm{A}$ & $\mathrm{N} / \mathrm{A}$ \\
\hline & & \multirow{2}{*}{ CS } & MPGGE Unadjusted & 22.8 & 22.8 & 24.8 & 27.2 & 24.0 & 26.9 & 33.6 & 25.5 & 30.2 & 40.8 & 26.3 & 31.4 & 42.8 \\
\hline & & & MPGGE Adjusted & 16.9 & 16.9 & 18.4 & 20.1 & 17.9 & 19.9 & 24.6 & 18.9 & 22.2 & 29.7 & 19.5 & 23.1 & 31.0 \\
\hline & & \multirow{3}{*}{$C D+C S$} & Wh/mile & 334.5 & 334.5 & 314.4 & 292.9 & 321.8 & 296.2 & 266.0 & 311.9 & 273.8 & 238.8 & 306.4 & 266.2 & 229.0 \\
\hline & & & MPGGE Unadjusted & 53.2 & 53.2 & 57.8 & 63.5 & 56.2 & 62.8 & 78.3 & 59.5 & 70.3 & 95.1 & 61.4 & 73.2 & 99.6 \\
\hline & & & \begin{tabular}{|l|} 
MPGGE Adjusted \\
\end{tabular} & 38.1 & 38.1 & 41.2 & 44.9 & 40.1 & 44.4 & 54.5 & 42.3 & 49.4 & 64.9 & 43.6 & 51.2 & 67.6 \\
\hline
\end{tabular}


Argonne National Laboratory Report - Light-Duty Vehicle Fuel Consumption Displacement Potential Up to 2045

\begin{tabular}{|c|c|c|c|c|c|c|c|c|c|c|c|c|c|c|c|c|}
\hline & & & & \multicolumn{13}{|c|}{ Pickup } \\
\hline & & & & \multirow{2}{*}{$\begin{array}{c}2010 \\
\text { Ref }\end{array}$} & \multicolumn{3}{|c|}{2010} & \multicolumn{3}{|c|}{2015} & \multicolumn{3}{|c|}{2030} & \multicolumn{3}{|c|}{2045} \\
\hline & & & & & low & avg & high & low & avg & high & low & avg & high & low & avg & high \\
\hline \multirow{25}{*}{ CI Split HEV PHEV10 } & \multirow{9}{*}{ UDDS } & \multirow{4}{*}{$C D$} & Wh/mile & 290.5 & 290.5 & 272.9 & 258.3 & 280.8 & 256.2 & 244.3 & 274.5 & 250.4 & 220.7 & 267.4 & 238.7 & 212.2 \\
\hline & & & Distance & 12.4 & 12.4 & 12.4 & 12.4 & 12.4 & 12.9 & 12.3 & 12.4 & 12.3 & 12.3 & 12.9 & 12.3 & 12.3 \\
\hline & & & MPGGE Unadjusted & 341.7 & 341.7 & 360.0 & 412.1 & 352.4 & 383.1 & 474.2 & 384.7 & 459.8 & 582.2 & 380.4 & 476.6 & 615.9 \\
\hline & & & MPGGE Adjusted & 163.0 & 163.0 & 168.0 & 181.0 & 165.9 & 173.9 & 194.8 & 174.4 & 191.7 & 214.8 & 173.3 & 195.3 & 220.3 \\
\hline & & \multirow{2}{*}{ CS } & MPGGE Unadjusted & 35.6 & 35.6 & 37.8 & 41.5 & 36.7 & 41.1 & 46.7 & 39.0 & 45.5 & 54.6 & 40.4 & 47.3 & 57.0 \\
\hline & & & MPGGE Adjusted & 27.0 & 27.0 & 28.6 & 31.2 & 27.8 & 30.9 & 34.8 & 29.4 & 33.9 & 40.0 & 30.4 & 35.2 & 41.5 \\
\hline & & \multirow{3}{*}{$\mathrm{CD}+\mathrm{CS}$} & Wh/mile & 60.5 & 60.5 & 56.9 & 53.8 & 58.5 & 53.4 & 50.9 & 57.2 & 52.2 & 46.0 & 55.7 & 49.7 & 44.2 \\
\hline & & & MPGGE Unadjusted & 42.0 & 42.0 & 44.6 & 49.0 & 43.3 & 48.5 & 55.2 & 46.0 & 53.7 & 64.6 & 47.7 & 55.9 & 67.4 \\
\hline & & & MPGGE Adjusted & 31.5 & 31.5 & 33.3 & 36.3 & 32.4 & 35.9 & 40.4 & 34.3 & 39.4 & 46.5 & 35.4 & 40.9 & 48.2 \\
\hline & \multirow{9}{*}{ HWFET } & \multirow{4}{*}{$C D$} & Wh/mile & 314.2 & 314.2 & 298.1 & 278.9 & 304.1 & 281.2 & 257.8 & 296.6 & 264.2 & 238.0 & 291.5 & 257.8 & 230.0 \\
\hline & & & Distance & 12.4 & 12.4 & 12.5 & 12.4 & 12.4 & 12.4 & 12.2 & 12.4 & 12.2 & 11.8 & 12.5 & 12.1 & 11.6 \\
\hline & & & MPGGE Unadjusted & 103.6 & 103.6 & 108.6 & 118.2 & 106.5 & 117.0 & 129.8 & 111.6 & 125.9 & 145.1 & 115.9 & 131.2 & 151.2 \\
\hline & & & MPGGE Adjusted & 72.2 & 72.2 & 75.6 & 82.1 & 74.2 & 81.3 & 89.9 & 77.7 & 87.3 & 100.2 & 80.6 & 90.9 & 104.3 \\
\hline & & \multirow{2}{*}{ CS } & MPGGE Unadjusted & 31.3 & 31.3 & 33.0 & 36.0 & 32.2 & 35.6 & 39.7 & 33.9 & 38.7 & 44.7 & 35.3 & 40.4 & 46.5 \\
\hline & & & MPGGE Adjusted & 22.1 & 22.1 & 23.3 & 25.5 & 22.8 & 25.2 & 28.1 & 24.0 & 27.4 & 31.6 & 24.9 & 28.5 & 32.8 \\
\hline & & \multirow{3}{*}{$\mathrm{CD}+\mathrm{CS}$} & Wh/mile & 92.4 & 92.4 & 87.7 & 82.0 & 89.4 & 82.7 & 75.8 & 87.2 & 77.7 & 70.0 & 85.7 & 75.8 & 67.6 \\
\hline & & & MPGGE Unadjusted & 37.6 & 37.6 & 39.6 & 43.2 & 38.7 & 42.8 & 47.7 & 40.7 & 46.5 & 53.7 & 42.3 & 48.4 & 55.7 \\
\hline & & & MPGGE Adjusted & 26.5 & 26.5 & 28.0 & 30.5 & 27.3 & 30.2 & 33.6 & 28.7 & 32.8 & 37.8 & 29.9 & 34.1 & 39.2 \\
\hline & \multirow{7}{*}{ Combined } & \multirow{2}{*}{$C D$} & MPGGE Unadjusted & 167.9 & 167.9 & 176.3 & 194.5 & 172.8 & 189.3 & 216.1 & 183.1 & 209.6 & 247.1 & 187.7 & 218.2 & 258.5 \\
\hline & & & MPGGE Adjusted & 104.1 & 104.1 & 108.4 & 117.4 & 106.6 & 115.0 & 127.7 & 111.7 & 124.6 & 141.8 & 114.1 & 128.8 & 146.8 \\
\hline & & \multirow{2}{*}{ CS } & MPGGE Unadjusted & 33.5 & 33.5 & 35.5 & 38.8 & 34.5 & 38.5 & 43.3 & 36.5 & 42.2 & 49.7 & 37.9 & 43.9 & 51.7 \\
\hline & & & MPGGE Adjusted & 24.6 & 24.6 & 26.0 & 28.3 & 25.3 & 28.1 & 31.4 & 26.7 & 30.6 & 35.7 & 27.7 & 31.8 & 37.1 \\
\hline & & \multirow{3}{*}{$C D+C S$} & Wh/mile & 74.9 & 74.9 & 70.7 & 66.5 & 72.4 & 66.6 & 62.1 & 70.7 & 63.7 & 56.8 & 69.2 & 61.5 & 54.7 \\
\hline & & & MPGGE Unadjusted & 39.9 & 39.9 & 42.2 & 46.2 & 41.1 & 45.8 & 51.5 & 43.5 & 50.2 & 59.2 & 45.1 & 52.3 & 61.6 \\
\hline & & & MPGGE Adjusted & 29.1 & 29.1 & 30.7 & 33.4 & 29.9 & 33.1 & 37.0 & 31.5 & 36.1 & 42.1 & 32.7 & 37.5 & 43.7 \\
\hline
\end{tabular}


Argonne National Laboratory Report - Light-Duty Vehicle Fuel Consumption Displacement Potential Up to 2045

\begin{tabular}{|c|c|c|c|c|c|c|c|c|c|c|c|c|c|c|c|c|}
\hline & & & & \multicolumn{13}{|c|}{ Pickup } \\
\hline & & & & \multirow{2}{*}{$\begin{array}{c}2010 \\
\text { Ref }\end{array}$} & \multicolumn{3}{|c|}{2010} & \multicolumn{3}{|c|}{2015} & \multicolumn{3}{|c|}{2030} & \multicolumn{3}{|c|}{2045} \\
\hline \multirow{26}{*}{ CI Split HEV PHEV2O } & & & & & low & avg & high & low & avg & high & low & avg & high & low & avg & high \\
\hline & \multirow{9}{*}{ UDDS } & \multirow{4}{*}{$C D$} & Wh/mile & 343.8 & 343.8 & 324.2 & 302.8 & 332.5 & 304.9 & 275.3 & 322.8 & 283.1 & 228.8 & 318.8 & 276.9 & 222.2 \\
\hline & & & Distance & 23.9 & 23.9 & 23.9 & 23.9 & 23.9 & 23.9 & 23.7 & 23.9 & 23.7 & 24.0 & 23.9 & 23.4 & 24.0 \\
\hline & & & MPGGE Unadjusted & 824.0 & 824.0 & 880.1 & 950.5 & 856.0 & 935.1 & 1007.1 & 914.1 & 983.3 & 651.9 & 956.9 & 1025.3 & 792.1 \\
\hline & & & MPGGE Adjusted & 247.7 & 247.7 & 253.7 & 260.5 & 251.2 & 259.1 & 265.5 & 257.1 & 263.5 & 225.8 & 261.1 & 267.0 & 244.1 \\
\hline & & \multirow{2}{*}{ CS } & MPGGE Unadjusted & 35.1 & 35.1 & 37.4 & 41.0 & 36.3 & 40.7 & 46.4 & 38.5 & 45.1 & 54.4 & 40.0 & 47.1 & 56.8 \\
\hline & & & MPGGE Adjusted & 26.7 & 26.7 & 28.3 & 30.8 & 27.5 & 30.6 & 34.5 & 29.1 & 33.7 & 39.9 & 30.1 & 35.0 & 41.4 \\
\hline & & \multirow{3}{*}{$C D+C S$} & Wh/mile & 178.7 & 178.7 & 168.5 & 157.3 & 172.8 & 158.4 & 143.5 & 167.8 & 147.5 & 119.8 & 165.7 & 144.3 & 116.6 \\
\hline & & & MPGGE Unadjusted & 60.5 & 60.5 & 64.3 & 70.6 & 62.5 & 70.0 & 79.7 & $\begin{array}{ll}66.4 \\
\end{array}$ & 77.5 & 90.9 & 68.9 & 80.9 & 95.8 \\
\hline & & & MPGGE Adjusted & 43.8 & 43.8 & 46.3 & 50.2 & 45.1 & 49.8 & 55.8 & 47.6 & 54.5 & 62.4 & 49.2 & 56.5 & 65.2 \\
\hline & \multirow{9}{*}{ HWFET } & \multirow{4}{*}{$C D$} & Wh/mile & 375.9 & 375.9 & 354.8 & 333.1 & 363.5 & 335.8 & 304.6 & 353.0 & 314.2 & 229.1 & 349.3 & 307.2 & 221.1 \\
\hline & & & Distance & 21.7 & 21.7 & 21.6 & 21.6 & 21.7 & 21.6 & 21.3 & 21.6 & 21.3 & 19.5 & 21.4 & 21.0 & 19.4 \\
\hline & & & MPGGE Unadjusted & 168.2 & 168.2 & 171.9 & 189.9 & 172.3 & 188.0 & 202.4 & 177.0 & 200.4 & 130.1 & 188.9 & 208.2 & 135.7 \\
\hline & & & MPGGE Adjusted & 115.6 & 115.6 & 118.1 & 130.0 & 118.3 & 128.7 & 138.1 & 121.5 & 136.8 & 90.2 & 129.3 & 141.9 & 93.9 \\
\hline & & \multirow{2}{*}{ CS } & MPGGE Unadjusted & 31.0 & 31.0 & 32.6 & 35.7 & 32.0 & 35.4 & 39.5 & 33.6 & 38.6 & 44.6 & 35.0 & 40.2 & 46.3 \\
\hline & & & MPGGE Adjusted & 21.9 & 21.9 & 23.1 & 25.3 & 22.6 & 25.0 & 27.9 & 23.8 & 27.3 & 31.5 & 24.7 & 28.4 & 32.7 \\
\hline & & \multirow{3}{*}{$C D+C S$} & Wh/mile & 189.1 & 189.1 & 178.6 & 167.7 & 182.9 & 169.0 & 152.4 & 177.7 & 157.1 & 67.4 & 175.7 & 153.6 & 65.0 \\
\hline & & & MPGGE Unadjusted & 45.9 & 45.9 & 48.2 & 52.9 & 47.4 & 52.4 & 58.0 & 49.7 & 56.7 & 53.0 & 51.8 & 59.1 & 55.0 \\
\hline & & & MPGGE Adjusted & 32.4 & 32.4 & 34.0 & 37.3 & 33.4 & 36.9 & 40.8 & 35.1 & 39.9 & 37.3 & 36.5 & 41.6 & 38.7 \\
\hline & \multirow{7}{*}{ Combined } & \multirow{2}{*}{$C D$} & MPGGE Unadjusted & 299.1 & 299.1 & 308.4 & 339.2 & 307.3 & 335.3 & 361.1 & 318.1 & 356.5 & 232.5 & 338.1 & 370.6 & 249.3 \\
\hline & & & MPGGE Adjusted & 163.6 & 163.6 & 167.3 & 179.4 & 166.9 & 177.9 & 187.6 & 171.1 & 186.0 & 134.7 & 179.0 & 191.2 & 142.0 \\
\hline & & \multirow{2}{*}{ CS } & MPGGE Unadjusted & 33.1 & 33.1 & 35.1 & 38.5 & 34.2 & 38.1 & 43.0 & 36.2 & 41.9 & 49.5 & 37.6 & 43.7 & 51.5 \\
\hline & & & MPGGE Adjusted & 24.3 & 24.3 & 25.7 & 28.1 & 25.1 & 27.8 & 31.2 & 26.5 & 30.4 & 35.6 & 27.4 & 31.7 & 37.0 \\
\hline & & \multirow{3}{*}{$C D+C S$} & Wh/mile & 183.4 & 183.4 & 173.0 & 162.0 & 177.3 & 163.1 & 147.5 & 172.2 & 151.8 & 96.2 & 170.2 & 148.5 & 93.4 \\
\hline & & & MPGGE Unadjusted & 52.9 & 52.9 & 55.9 & 61.3 & 54.6 & 60.8 & 68.2 & 57.7 & 66.5 & 68.7 & 60.0 & 69.4 & 71.8 \\
\hline & & & MPGGE Adjusted & 37.8 & 37.8 & 39.8 & 43.4 & 39.0 & 43.1 & 47.9 & 41.0 & 46.8 & 47.9 & 42.5 & 48.7 & 49.9 \\
\hline
\end{tabular}


Argonne National Laboratory Report - Light-Duty Vehicle Fuel Consumption Displacement Potential Up to 2045

\begin{tabular}{|c|c|c|c|c|c|c|c|c|c|c|c|c|c|c|c|c|}
\hline & & & & \multicolumn{13}{|c|}{ Pickup } \\
\hline & & & & \multirow{2}{*}{$\begin{array}{c}2010 \\
\text { Ref }\end{array}$} & \multicolumn{3}{|c|}{2010} & \multicolumn{3}{|c|}{2015} & \multicolumn{3}{|c|}{2030} & \multicolumn{3}{|c|}{2045} \\
\hline & & & & & low & avg & high & low & avg & high & low & avg & high & low & avg & high \\
\hline \multirow{25}{*}{ CI Series HEV PHEV30 } & \multirow{9}{*}{ UDDS } & \multirow{4}{*}{$C D$} & Wh/mile & 454.6 & 454.6 & 426.8 & 398.0 & 437.8 & 402.4 & 359.5 & 423.5 & 369.8 & 319.5 & 416.5 & 359.4 & 306.4 \\
\hline & & & Distance & 31.9 & 31.9 & 31.9 & 31.4 & 31.9 & 31.4 & 31.9 & 31.9 & 31.9 & 30.9 & 31.9 & 30.9 & 31.9 \\
\hline & & & MPGGE Unadjusted & $\mathrm{N} / \mathrm{A}$ & $\mathrm{N} / \mathrm{A}$ & N/A & N/A & N/A & $\mathrm{N} / \mathrm{A}$ & N/A & $\mathrm{N} / \mathrm{A}$ & N/A & N/A & N/A & N/A & $\mathrm{N} / \mathrm{A}$ \\
\hline & & & MPGGE Adjusted & $\mathrm{N} / \mathrm{A}$ & $\mathrm{N} / \mathrm{A}$ & $\mathrm{N} / \mathrm{A}$ & N/A & N/A & N/A & N/A & $\mathrm{N} / \mathrm{A}$ & N/A & N/A & N/A & $\mathrm{N} / \mathrm{A}$ & $\mathrm{N} / \mathrm{A}$ \\
\hline & & \multirow{2}{*}{ CS } & MPGGE Unadjusted & 24.9 & 24.9 & 26.9 & 30.0 & 25.9 & 29.7 & 35.1 & 28.0 & 34.1 & 42.7 & 29.4 & 35.7 & 45.2 \\
\hline & & & MPGGE Adjusted & 19.3 & 19.3 & 20.7 & 23.0 & 20.0 & 22.8 & 26.7 & 21.6 & 26.0 & 32.0 & 22.6 & 27.1 & 33.7 \\
\hline & & \multirow{3}{*}{$\mathrm{CD}+\mathrm{CS}$} & Wh/mile & 292.4 & 292.4 & 274.5 & 256.0 & 281.7 & 258.9 & 232.1 & 272.5 & 238.7 & 206.7 & 268.0 & 231.7 & 198.2 \\
\hline & & & MPGGE Unadjusted & 52.9 & 52.9 & 57.1 & 63.8 & 55.0 & 63.1 & 74.7 & 59.6 & 72.5 & 90.8 & 62.6 & 75.9 & 96.3 \\
\hline & & & MPGGE Adjusted & 38.9 & 38.9 & 41.7 & 46.0 & 40.3 & 45.5 & 52.7 & 43.3 & 51.4 & 62.3 & 45.2 & 53.5 & 65.4 \\
\hline & \multirow{9}{*}{ HWFET } & \multirow{4}{*}{$C D$} & Wh/mile & 500.2 & 500.2 & 473.5 & 442.1 & 482.4 & 446.3 & 403.7 & 469.5 & 415.2 & 372.2 & 460.7 & 405.4 & 358.4 \\
\hline & & & \begin{tabular}{|l} 
Distance \\
\end{tabular} & 28.0 & 28.0 & 27.7 & 27.7 & 27.9 & 27.7 & 27.4 & 27.7 & 27.4 & 26.3 & 27.8 & 27.2 & 26.2 \\
\hline & & & MPGGE Unadjusted & $\mathrm{N} / \mathrm{A}$ & $\mathrm{N} / \mathrm{A}$ & $\mathrm{N} / \mathrm{A}$ & $\mathrm{N} / \mathrm{A}$ & N/A & $\mathrm{N} / \mathrm{A}$ & N/A & $\mathrm{N} / \mathrm{A}$ & N/A & $\mathrm{N} / \mathrm{A}$ & $\mathrm{N} / \mathrm{A}$ & $\mathrm{N} / \mathrm{A}$ & $\mathrm{N} / \mathrm{A}$ \\
\hline & & & MPGGE Adjusted & N/A & N/A & N/A & N/A & N/A & N/A & N/A & N/A & N/A & N/A & N/A & N/A & $\mathrm{N} / \mathrm{A}$ \\
\hline & & \multirow{2}{*}{ CS } & MPGGE Unadjusted & 23.0 & 23.0 & 24.6 & 27.3 & 23.9 & 27.0 & 31.1 & 25.7 & 30.2 & 35.9 & 26.8 & 31.7 & 37.3 \\
\hline & & & MPGGE Adjusted & 16.3 & 16.3 & 17.4 & 19.4 & 17.0 & 19.2 & 22.0 & 18.2 & 21.4 & 25.4 & 19.0 & 22.4 & 26.4 \\
\hline & & \multirow{3}{*}{$\mathrm{CD}+\mathrm{CS}$} & Wh/mile & 257.7 & 257.7 & 244.0 & 227.8 & 248.6 & 230.0 & 208.0 & 241.9 & 214.0 & 191.7 & 237.4 & 208.9 & 184.6 \\
\hline & & & MPGGE Unadjusted & 39.0 & 39.0 & 41.7 & 46.3 & 40.5 & 45.8 & 52.8 & 43.6 & 51.2 & 60.9 & 45.5 & 53.7 & 63.3 \\
\hline & & & \begin{tabular}{|l|} 
MPGGE Adjusted \\
\end{tabular} & 27.6 & 27.6 & 29.4 & 32.7 & 28.6 & 32.3 & 37.2 & 30.8 & 36.1 & 42.8 & 32.1 & 37.8 & 44.5 \\
\hline & \multirow{7}{*}{ Combined } & \multirow{2}{*}{$C D$} & MPGGE Unadjusted & $\mathrm{N} / \mathrm{A}$ & $\mathrm{N} / \mathrm{A}$ & N/A & N/A & N/A & $\mathrm{N} / \mathrm{A}$ & N/A & $\mathrm{N} / \mathrm{A}$ & $\mathrm{N} / \mathrm{A}$ & N/A & $\mathrm{N} / \mathrm{A}$ & N/A & $\mathrm{N} / \mathrm{A}$ \\
\hline & & & MPGGE Adjusted & N/A & N/A & N/A & N/A & N/A & N/A & N/A & $\mathrm{N} / \mathrm{A}$ & N/A & N/A & N/A & N/A & N/A \\
\hline & & \multirow{2}{*}{ CS } & MPGGE Unadjusted & 24.0 & 24.0 & 25.8 & 28.7 & 24.9 & 28.4 & 33.2 & 26.9 & 32.2 & 39.4 & 28.2 & 33.8 & 41.3 \\
\hline & & & MPGGE Adjusted & 17.8 & 17.8 & 19.1 & 21.2 & 18.5 & 21.0 & 24.4 & 19.9 & 23.7 & 28.6 & 20.8 & 24.8 & 30.0 \\
\hline & & \multirow{3}{*}{$C D+C S$} & Wh/mile & 276.8 & 276.8 & 260.8 & 243.3 & 266.8 & 245.9 & 221.3 & 258.7 & 227.6 & 200.0 & 254.2 & 221.4 & 192.1 \\
\hline & & & MPGGE Unadjusted & 45.6 & 45.6 & 49.0 & 54.5 & 47.4 & 54.0 & 62.9 & 51.2 & 61.1 & 74.4 & 53.5 & 64.0 & 78.0 \\
\hline & & & MPGGE Adjusted & 32.8 & 32.8 & 35.1 & 38.8 & 34.0 & 38.5 & 44.4 & 36.6 & 43.2 & 51.7 & 38.2 & 45.1 & 54.0 \\
\hline
\end{tabular}




\begin{tabular}{|c|c|c|c|c|c|c|c|c|c|c|c|c|c|c|c|c|}
\hline & & & & \multicolumn{13}{|c|}{ Pickup } \\
\hline & & & & 2010 & & 2010 & & & 2015 & & & 2030 & & & 2045 & \\
\hline \multirow{26}{*}{ CI Series HEV PHEV40 } & & \multirow{5}{*}{$C D$} & & Ref & low & avg & high & low & avg & high & low & avg & high & low & avg & high \\
\hline & \multirow{9}{*}{ UDDS } & & Wh/mile & 464.8 & 464.8 & 435.7 & 406.0 & 446.8 & 410.3 & 366.4 & 432.0 & 377.1 & 322.6 & 424.7 & 366.1 & 309.3 \\
\hline & & & Distance & 40.7 & 40.7 & 40.7 & 40.7 & 40.7 & 40.7 & 40.6 & 40.7 & 40.6 & 40.5 & 40.7 & 40.8 & 40.5 \\
\hline & & & MPGGE Unadjusted & N/A & N/A & N/A & N/A & N/A & N/A & N/A & N/A & N/A & N/A & N/A & N/A & N/A \\
\hline & & & MPGGE Adjusted & N/A & N/A & N/A & N/A & N/A & $\mathrm{N} / \mathrm{A}$ & N/A & N/A & N/A & N/A & N/A & N/A & $\mathrm{N} / \mathrm{A}$ \\
\hline & & \multirow{2}{*}{ CS } & MPGGE Unadjusted & 24.3 & 24.3 & 26.2 & 29.5 & 25.3 & 29.2 & 34.5 & 27.4 & 33.5 & 42.1 & 28.6 & 35.1 & 44.6 \\
\hline & & & MPGGE Adjusted & 18.9 & 18.9 & 20.3 & 22.7 & 19.6 & 22.4 & 26.2 & 21.1 & 25.5 & 31.6 & 22.0 & 26.7 & 33.3 \\
\hline & & \multirow{3}{*}{$C D+C S$} & Wh/mile & 340.8 & 340.8 & 319.6 & 297.9 & 327.8 & 301.1 & 270.4 & 317.0 & 278.2 & 238.0 & 311.7 & 269.9 & 228.1 \\
\hline & & & MPGGE Unadjusted & 60.7 & 60.7 & 65.6 & 73.8 & 63.2 & 73.0 & 86.2 & 68.5 & $\begin{array}{l}83.7 \\
\end{array}$ & 105.2 & 71.4 & 87.8 & 111.5 \\
\hline & & & MPGGE Adjusted & 44.0 & 44.0 & 47.1 & 52.2 & 45.6 & 51.7 & 59.6 & 48.9 & 58.2 & 70.4 & 50.7 & 60.6 & 73.9 \\
\hline & \multirow{9}{*}{ HWFET } & \multirow{4}{*}{$C D$} & Wh/mile & 505.3 & 505.3 & 477.8 & 445.8 & 486.6 & 449.8 & 406.0 & 473.3 & 417.7 & 374.7 & 464.2 & 407.4 & 360.8 \\
\hline & & & Distance & 37.1 & 37.1 & 38.6 & 36.7 & 37.1 & 38.6 & 36.4 & 38.6 & 36.5 & 35.1 & 36.9 & 36.4 & 34.9 \\
\hline & & & MPGGE Unadjusted & N/A & N/A & N/A & N/A & N/A & N/A & N/A & N/A & N/A & N/A & N/A & N/A & $\mathrm{N} / \mathrm{A}$ \\
\hline & & & MPGGE Adjusted & $\mathrm{N} / \mathrm{A}$ & N/A & N/A & N/A & N/A & N/A & N/A & N/A & N/A & N/A & N/A & N/A & N/A \\
\hline & & \multirow{2}{*}{ CS } & MPGGE Unadjusted & 22.7 & 22.7 & 24.3 & 26.9 & 23.6 & 26.7 & 30.9 & 25.3 & 29.9 & 35.5 & 26.5 & 31.5 & 37.0 \\
\hline & & & MPGGE Adjusted & 16.1 & 16.1 & 17.2 & 19.1 & 16.7 & 18.9 & 21.8 & 18.0 & 21.2 & 25.1 & 18.8 & 22.3 & 26.2 \\
\hline & & \multirow{3}{*}{$C D+C S$} & Wh/mile & 341.1 & 341.1 & 322.5 & 301.0 & 328.5 & 303.7 & 274.1 & 319.5 & 282.0 & 253.0 & 313.4 & 275.0 & 243.6 \\
\hline & & & MPGGE Unadjusted & 49.4 & 49.4 & 52.8 & 58.5 & 51.2 & 58.0 & 67.1 & 55.1 & 65.1 & 77.1 & 57.5 & 68.4 & 80.4 \\
\hline & & & MPGGE Adjusted & 34.8 & 34.8 & 37.2 & 41.2 & 36.1 & 40.8 & 47.1 & 38.8 & 45.7 & 54.0 & 40.5 & 48.0 & 56.3 \\
\hline & \multirow{7}{*}{ Combined } & \multirow{2}{*}{$C D$} & MPGGE Unadjusted & N/A & N/A & N/A & N/A & $\mathrm{N} / \mathrm{A}$ & N/A & N/A & $\mathrm{N} / \mathrm{A}$ & $\mathrm{N} / \mathrm{A}$ & N/A & N/A & $\mathrm{N} / \mathrm{A}$ & N/A \\
\hline & & & MPGGE Adjusted & N/A & N/A & N/A & N/A & N/A & N/A & N/A & $\mathrm{N} / \mathrm{A}$ & N/A & N/A & N/A & $\mathrm{N} / \mathrm{A}$ & N/A \\
\hline & & \multirow{2}{*}{ CS } & MPGGE Unadjusted & 23.6 & 23.6 & 25.3 & 28.3 & 24.5 & 28.0 & 32.7 & 26.4 & 31.8 & 38.8 & 27.6 & 33.4 & 40.8 \\
\hline & & & MPGGE Adjusted & 17.5 & 17.5 & 18.8 & 20.9 & 18.2 & 20.7 & 24.1 & 19.6 & 23.4 & 28.3 & 20.4 & 24.5 & 29.7 \\
\hline & & \multirow{3}{*}{$\mathrm{CD}+\mathrm{CS}$} & Wh/mile & 340.9 & 340.9 & 320.9 & 299.3 & 328.1 & 302.2 & 272.0 & 318.1 & 279.9 & 244.7 & 312.5 & 272.2 & 235.1 \\
\hline & & & MPGGE Unadjusted & 55.0 & 55.0 & 59.1 & 66.0 & 57.2 & 65.4 & 76.4 & 61.8 & 74.2 & 90.4 & 64.4 & 77.9 & 95.0 \\
\hline & & & MPGGE Adjusted & 39.3 & 39.3 & 42.0 & 46.6 & 40.8 & 46.1 & 53.3 & 43.8 & 51.8 & 62.0 & 45.5 & 54.2 & 64.8 \\
\hline
\end{tabular}


Argonne National Laboratory Report - Light-Duty Vehicle Fuel Consumption Displacement Potential Up to 2045

\begin{tabular}{|c|c|c|c|c|c|c|c|c|c|c|c|c|c|c|c|c|}
\hline & & & & \multicolumn{13}{|c|}{ Pickup } \\
\hline & & & & \multirow{2}{*}{$\begin{array}{c}2010 \\
\text { Ref }\end{array}$} & \multicolumn{3}{|c|}{2010} & \multicolumn{3}{|c|}{2015} & \multicolumn{3}{|c|}{2030} & \multicolumn{3}{|c|}{2045} \\
\hline & & & & & low & avg & high & low & avg & high & low & avg & high & low & avg & high \\
\hline \multirow{25}{*}{ H2 Split HEV PHEV10 } & \multirow{9}{*}{ UDDS } & \multirow{4}{*}{$C D$} & Wh/mile & 284.3 & 284.3 & 267.2 & 246.3 & 272.2 & 251.2 & 234.9 & 263.0 & 243.6 & 209.2 & 261.3 & 236.2 & 206.2 \\
\hline & & & Distance & 12.3 & 12.3 & 12.3 & 12.4 & 12.5 & 12.3 & 12.3 & 12.8 & 12.2 & 12.2 & 12.4 & 12.3 & 12.1 \\
\hline & & & MPGGE Unadjusted & 328.8 & 328.8 & 407.0 & 454.1 & 370.1 & 465.2 & 569.6 & 404.1 & 562.3 & 693.5 & 461.6 & 590.2 & 853.1 \\
\hline & & & MPGGE Adjusted & 159.3 & 159.3 & 179.8 & 190.5 & 170.6 & 192.9 & 212.7 & 179.1 & 211.4 & 231.7 & 192.1 & 216.2 & 250.9 \\
\hline & & \multirow{2}{*}{ CS } & MPGGE Unadjusted & 33.3 & 33.3 & 39.5 & 45.4 & 38.1 & 44.9 & 52.2 & 41.9 & 50.6 & 61.5 & 45.0 & 53.8 & 66.5 \\
\hline & & & MPGGE Adjusted & 25.4 & 25.4 & 29.7 & 33.9 & 28.8 & 33.5 & 38.4 & 31.5 & 37.4 & 44.5 & 33.5 & 39.4 & 47.6 \\
\hline & & \multirow{3}{*}{$\mathrm{CD}+\mathrm{CS}$} & Wh/mile & 59.2 & 59.2 & 55.7 & 51.3 & 56.7 & 52.3 & 48.9 & 54.8 & 50.8 & 43.6 & 54.4 & 49.2 & 43.0 \\
\hline & & & MPGGE Unadjusted & 39.3 & 39.3 & 46.6 & 53.6 & 45.0 & 53.0 & 61.8 & 49.5 & 59.9 & 72.8 & 53.1 & 63.6 & 78.8 \\
\hline & & & MPGGE Adjusted & 29.6 & 29.6 & 34.7 & 39.4 & 33.5 & 38.9 & 44.6 & 36.6 & 43.4 & 51.6 & 39.0 & 45.8 & 55.2 \\
\hline & \multirow{9}{*}{ HWFET } & \multirow{4}{*}{$C D$} & Wh/mile & 312.0 & 312.0 & 292.9 & 273.3 & 300.1 & 276.0 & 252.4 & 292.1 & 260.8 & 269.1 & 286.1 & 253.0 & 258.6 \\
\hline & & & Distance & 12.2 & 12.2 & 12.2 & 12.2 & 12.2 & 12.2 & 12.2 & 12.2 & 11.7 & 8.0 & 12.2 & 12.1 & 7.8 \\
\hline & & & MPGGE Unadjusted & 99.9 & 99.9 & 115.5 & 131.9 & 112.8 & 130.4 & 147.4 & 122.5 & 144.1 & 232.6 & 131.5 & 152.7 & 247.1 \\
\hline & & & MPGGE Adjusted & 69.7 & 69.7 & 80.3 & 91.4 & 78.5 & 90.3 & 101.8 & 85.0 & 99.6 & 157.8 & 91.1 & 105.3 & 167.0 \\
\hline & & \multirow{2}{*}{ CS } & MPGGE Unadjusted & 29.9 & 29.9 & 35.0 & 40.0 & 34.1 & 39.5 & 45.0 & 37.3 & 43.8 & 50.8 & 39.8 & 46.5 & 54.7 \\
\hline & & & MPGGE Adjusted & 21.2 & 21.2 & 24.7 & 28.2 & 24.1 & 27.9 & 31.8 & 26.4 & 30.9 & 35.8 & 28.1 & 32.8 & 38.6 \\
\hline & & \multirow{3}{*}{$\mathrm{CD}+\mathrm{CS}$} & Wh/mile & 91.8 & 91.8 & 86.2 & 80.4 & 88.3 & 81.2 & 74.2 & 85.9 & 76.7 & 79.1 & 84.2 & 74.4 & 76.1 \\
\hline & & & MPGGE Unadjusted & 36.0 & 36.0 & 42.0 & 48.0 & 41.0 & 47.4 & 54.0 & 44.8 & 52.6 & 62.5 & 47.8 & 55.9 & 67.3 \\
\hline & & & MPGGE Adjusted & 25.5 & 25.5 & 29.7 & 33.8 & 28.9 & 33.5 & 38.0 & 31.6 & 37.0 & 43.9 & 33.7 & 39.3 & 47.3 \\
\hline & \multirow{7}{*}{ Combined } & \multirow{2}{*}{$C D$} & MPGGE Unadjusted & 161.9 & 161.9 & 190.6 & 216.3 & 182.7 & 215.8 & 248.8 & 198.6 & 243.9 & 366.6 & 216.7 & 257.8 & 405.5 \\
\hline & & & MPGGE Adjusted & 100.9 & 100.9 & 115.4 & 128.0 & 111.6 & 127.7 & 142.7 & 119.6 & 140.4 & 191.3 & 128.1 & 146.7 & 204.6 \\
\hline & & \multirow{2}{*}{ CS } & MPGGE Unadjusted & 31.7 & 31.7 & 37.3 & 42.8 & 36.2 & 42.3 & 48.7 & 39.7 & 47.3 & 56.2 & 42.5 & 50.2 & 60.6 \\
\hline & & & MPGGE Adjusted & 23.3 & 23.3 & 27.3 & 31.1 & 26.5 & 30.7 & 35.1 & 28.9 & 34.1 & 40.1 & 30.9 & 36.2 & 43.1 \\
\hline & & \multirow{3}{*}{$C D+C S$} & Wh/mile & 73.9 & 73.9 & 69.4 & 64.4 & 70.9 & 65.3 & 60.3 & 68.8 & 62.4 & 59.6 & 67.8 & 60.6 & 57.9 \\
\hline & & & MPGGE Unadjusted & 37.7 & 37.7 & 44.4 & 50.9 & 43.1 & 50.3 & 58.0 & 47.2 & 56.3 & 67.8 & 50.6 & 59.9 & 73.2 \\
\hline & & & MPGGE Adjusted & 27.6 & 27.6 & 32.2 & 36.7 & 31.3 & 36.3 & 41.4 & 34.2 & 40.3 & 47.8 & 36.4 & 42.6 & 51.3 \\
\hline
\end{tabular}


Argonne National Laboratory Report - Light-Duty Vehicle Fuel Consumption Displacement Potential Up to 2045

\begin{tabular}{|c|c|c|c|c|c|c|c|c|c|c|c|c|c|c|c|c|}
\hline & & & & \multicolumn{13}{|c|}{ Pickup } \\
\hline & & & & \multirow{2}{*}{$\begin{array}{c}2010 \\
\text { Ref }\end{array}$} & \multicolumn{3}{|c|}{2010} & \multicolumn{3}{|c|}{2015} & \multicolumn{3}{|c|}{2030} & \multicolumn{3}{|c|}{2045} \\
\hline \multirow{26}{*}{ H2 Split HEV PHEV2O } & & & & & low & avg & high & low & avg & high & low & avg & high & low & avg & high \\
\hline & \multirow{9}{*}{ UDDS } & \multirow{4}{*}{$C D$} & Wh/mile & 336.8 & 336.8 & 313.7 & 291.7 & 321.3 & 294.4 & 265.1 & 312.5 & 273.5 & 221.0 & 304.6 & 253.2 & 212.7 \\
\hline & & & Distance & 23.8 & 23.8 & 23.8 & 23.7 & 23.9 & 23.7 & 23.4 & 23.9 & 23.4 & 23.9 & 23.8 & 23.9 & 23.8 \\
\hline & & & MPGGE Unadjusted & 791.4 & 791.4 & 976.2 & 1134.0 & 843.5 & 1119.4 & 1291.5 & 970.7 & 1251.3 & 934.4 & 1040.5 & 874.9 & 1028.9 \\
\hline & & & MPGGE Adjusted & 244.0 & 244.0 & 262.8 & 275.5 & 249.9 & 274.4 & 285.9 & 262.3 & 283.4 & 259.0 & 268.3 & 253.2 & 267.3 \\
\hline & & \multirow{2}{*}{ CS } & MPGGE Unadjusted & 32.9 & 32.9 & 39.0 & 45.0 & 37.6 & 44.5 & 52.0 & 41.5 & 50.3 & 61.3 & 44.6 & 53.7 & 66.4 \\
\hline & & & MPGGE Adjusted & 25.1 & 25.1 & 29.4 & 33.6 & 28.5 & 33.2 & 38.3 & 31.2 & 37.2 & 44.4 & 33.3 & 39.4 & 47.6 \\
\hline & & \multirow{3}{*}{$C D+C S$} & Wh/mile & 175.1 & 175.1 & 163.1 & 151.7 & 167.0 & 153.1 & 138.2 & 162.3 & 142.6 & 116.0 & 158.2 & 132.5 & 111.6 \\
\hline & & & MPGGE Unadjusted & 56.6 & 56.6 & 67.3 & 77.7 & 64.7 & 76.9 & 89.7 & 71.5 & 86.8 & 104.0 & 76.7 & 91.3 & 112.8 \\
\hline & & & MPGGE Adjusted & 41.3 & 41.3 & 48.2 & 54.5 & 46.5 & 54.1 & 61.7 & 50.8 & 60.0 & $\begin{array}{ll}69.7 \\
\end{array}$ & 53.9 & 62.6 & 74.5 \\
\hline & \multirow{9}{*}{ HWFET } & \multirow{4}{*}{$C D$} & Wh/mile & 369.0 & 369.0 & 344.7 & 276.2 & 354.1 & 278.9 & 253.0 & 345.1 & 248.1 & 224.2 & 335.9 & 242.1 & 216.7 \\
\hline & & & Distance & 21.5 & 21.5 & 21.4 & 20.3 & 21.5 & 20.3 & 19.9 & 21.4 & 20.2 & 19.2 & 21.4 & 19.9 & 19.2 \\
\hline & & & MPGGE Unadjusted & 153.4 & 153.4 & 173.1 & 131.7 & 172.1 & 130.2 & 146.3 & 185.5 & 129.5 & 149.2 & 196.0 & 137.9 & 161.6 \\
\hline & & & MPGGE Adjusted & 105.8 & 105.8 & 118.9 & 91.2 & 118.2 & 90.2 & 101.0 & 127.1 & 89.7 & 103.0 & 134.0 & 95.4 & 111.3 \\
\hline & & \multirow{2}{*}{ CS } & MPGGE Unadjusted & 29.6 & 29.6 & 34.6 & 39.7 & 33.8 & 39.2 & 44.7 & 37.0 & 43.5 & 50.5 & 39.5 & 46.3 & 54.5 \\
\hline & & & MPGGE Adjusted & 21.0 & 21.0 & 24.5 & 28.0 & 23.9 & 27.7 & 31.6 & 26.1 & 30.7 & 35.6 & 27.9 & 32.7 & 38.4 \\
\hline & & \multirow{3}{*}{$C D+C S$} & Wh/mile & 186.0 & 186.0 & 173.9 & 81.2 & 177.4 & 82.0 & 74.4 & 174.0 & 73.0 & 65.9 & 169.4 & 71.2 & 63.7 \\
\hline & & & MPGGE Unadjusted & 43.7 & 43.7 & 50.9 & 47.7 & 49.7 & 47.1 & 53.6 & 54.4 & 51.7 & 60.0 & 58.1 & 55.1 & 64.9 \\
\hline & & & MPGGE Adjusted & 30.9 & 30.9 & 35.9 & 33.6 & 35.0 & 33.3 & 37.8 & 38.3 & 36.4 & 42.2 & 40.9 & 38.8 & 45.6 \\
\hline & \multirow{7}{*}{ Combined } & \multirow{2}{*}{$C D$} & MPGGE Unadjusted & 275.6 & 275.6 & 316.1 & 256.2 & 306.2 & 253.3 & 285.6 & 334.2 & 255.4 & 277.4 & 354.1 & 257.0 & 301.3 \\
\hline & & & MPGGE Adjusted & 153.7 & 153.7 & 170.1 & 144.3 & 166.5 & 143.0 & 156.8 & 177.4 & 143.8 & 154.0 & 184.9 & 145.2 & 163.9 \\
\hline & & \multirow{2}{*}{ CS } & MPGGE Unadjusted & 31.3 & 31.3 & 36.9 & 42.4 & 35.8 & 42.0 & 48.4 & 39.3 & 47.0 & 55.9 & 42.1 & 50.1 & 60.5 \\
\hline & & & MPGGE Adjusted & 23.0 & 23.0 & 27.0 & 30.8 & 26.2 & 30.5 & 34.9 & 28.7 & 33.9 & 39.9 & 30.6 & 36.1 & 43.0 \\
\hline & & \multirow{3}{*}{$C D+C S$} & Wh/mile & 180.0 & 180.0 & 168.0 & 120.0 & 171.7 & 121.1 & 109.5 & 167.5 & 111.3 & 93.4 & 163.3 & 104.9 & 90.1 \\
\hline & & & MPGGE Unadjusted & 50.0 & 50.0 & 58.8 & 60.5 & 56.9 & 59.9 & 68.9 & 62.6 & 66.5 & 78.2 & 67.0 & 70.5 & 84.6 \\
\hline & & & \begin{tabular}{|l|} 
MPGEE Adjusted \\
\end{tabular} & 35.9 & 35.9 & 41.7 & 42.6 & 40.5 & 42.2 & 48.0 & 44.3 & 46.5 & 53.9 & 47.1 & 49.1 & 58.0 \\
\hline
\end{tabular}


Argonne National Laboratory Report - Light-Duty Vehicle Fuel Consumption Displacement Potential Up to 2045

\begin{tabular}{|c|c|c|c|c|c|c|c|c|c|c|c|c|c|c|c|c|}
\hline & & & & \multicolumn{13}{|c|}{ Pickup } \\
\hline & & & & \multirow{2}{*}{$\begin{array}{c}2010 \\
\text { Ref }\end{array}$} & \multicolumn{3}{|c|}{2010} & \multicolumn{3}{|c|}{2015} & \multicolumn{3}{|c|}{2030} & \multicolumn{3}{|c|}{2045} \\
\hline & & & & & low & avg & high & low & avg & high & low & avg & high & low & avg & high \\
\hline \multirow{25}{*}{ H2 Series HEV PHEV30 } & \multirow{9}{*}{ UDDS } & \multirow{4}{*}{$C D$} & Wh/mile & 446.2 & 446.2 & 413.0 & 382.6 & 427.5 & 387.2 & 343.0 & 412.6 & 354.6 & 302.1 & 401.3 & 343.5 & 290.1 \\
\hline & & & Distance & 31.7 & 31.7 & 31.7 & 32.0 & 31.9 & 32.0 & 31.8 & 32.0 & 31.7 & 31.9 & 31.4 & 30.9 & 31.9 \\
\hline & & & MPGGE Unadjusted & $\mathrm{N} / \mathrm{A}$ & $\mathrm{N} / \mathrm{A}$ & N/A & N/A & N/A & $\mathrm{N} / \mathrm{A}$ & N/A & $\mathrm{N} / \mathrm{A}$ & N/A & N/A & N/A & N/A & $\mathrm{N} / \mathrm{A}$ \\
\hline & & & MPGGE Adjusted & $\mathrm{N} / \mathrm{A}$ & $\mathrm{N} / \mathrm{A}$ & $\mathrm{N} / \mathrm{A}$ & N/A & N/A & N/A & N/A & $\mathrm{N} / \mathrm{A}$ & N/A & N/A & N/A & $\mathrm{N} / \mathrm{A}$ & $\mathrm{N} / \mathrm{A}$ \\
\hline & & \multirow{2}{*}{ CS } & MPGGE Unadjusted & 23.7 & 23.7 & 28.7 & 33.5 & 27.3 & 33.0 & 40.0 & 31.0 & 38.6 & 49.3 & 33.4 & 41.2 & 53.7 \\
\hline & & & MPGGE Adjusted & 18.4 & 18.4 & 22.1 & 25.5 & 21.1 & 25.2 & 30.2 & 23.7 & 29.1 & 36.5 & 25.4 & 30.9 & 39.4 \\
\hline & & \multirow{3}{*}{$\mathrm{CD}+\mathrm{CS}$} & Wh/mile & 286.9 & 286.9 & 265.6 & 246.1 & 275.0 & 249.1 & 221.3 & 265.4 & 228.8 & 195.4 & 258.2 & 221.4 & 187.6 \\
\hline & & & MPGGE Unadjusted & 50.3 & 50.3 & 61.0 & 71.2 & 58.0 & 70.2 & 85.2 & 65.8 & 82.0 & 105.0 & 71.0 & 87.6 & 114.2 \\
\hline & & & MPGGE Adjusted & 37.2 & 37.2 & 44.2 & 50.6 & 42.2 & 50.0 & 59.0 & 47.2 & 57.2 & 70.3 & 50.5 & 60.5 & 75.3 \\
\hline & \multirow{9}{*}{ HWFET } & \multirow{4}{*}{$C D$} & Wh/mile & 494.5 & 494.5 & 464.2 & 431.8 & 475.6 & 436.2 & 392.9 & 462.2 & 405.3 & 361.1 & 450.6 & 395.0 & 348.1 \\
\hline & & & \begin{tabular}{|l} 
Distance \\
\end{tabular} & 27.7 & 27.7 & 28.4 & 27.3 & 27.6 & 27.4 & 26.9 & 28.4 & 26.9 & 25.8 & 28.4 & 26.7 & 25.7 \\
\hline & & & MPGGE Unadjusted & $\mathrm{N} / \mathrm{A}$ & $\mathrm{N} / \mathrm{A}$ & $\mathrm{N} / \mathrm{A}$ & $\mathrm{N} / \mathrm{A}$ & N/A & $\mathrm{N} / \mathrm{A}$ & N/A & $\mathrm{N} / \mathrm{A}$ & N/A & $\mathrm{N} / \mathrm{A}$ & $\mathrm{N} / \mathrm{A}$ & $\mathrm{N} / \mathrm{A}$ & $\mathrm{N} / \mathrm{A}$ \\
\hline & & & MPGGE Adjusted & N/A & N/A & N/A & N/A & N/A & N/A & N/A & N/A & N/A & N/A & N/A & N/A & $\mathrm{N} / \mathrm{A}$ \\
\hline & & \multirow{2}{*}{ CS } & MPGGE Unadjusted & 22.1 & 22.1 & 26.1 & 30.3 & 25.4 & 30.0 & 35.2 & 28.3 & 34.0 & 40.7 & 30.3 & 36.5 & 44.0 \\
\hline & & & MPGGE Adjusted & 15.7 & 15.7 & 18.5 & 21.4 & 18.0 & 21.2 & 24.9 & 20.0 & 24.1 & 28.8 & 21.5 & 25.8 & 31.0 \\
\hline & & \multirow{3}{*}{$\mathrm{CD}+\mathrm{CS}$} & Wh/mile & 254.8 & 254.8 & 239.2 & 222.5 & 245.0 & 224.8 & 202.4 & 238.1 & 208.8 & 186.0 & 232.2 & 203.5 & 179.3 \\
\hline & & & MPGGE Unadjusted & 37.4 & 37.4 & 44.3 & 51.3 & 43.0 & 50.8 & 59.7 & 47.9 & 57.6 & 69.0 & 51.4 & 61.9 & 74.5 \\
\hline & & & MPGGE Adjusted & 26.5 & 26.5 & 31.2 & 36.1 & 30.4 & 35.8 & 42.0 & 33.8 & 40.6 & 48.4 & 36.2 & 43.5 & 52.3 \\
\hline & \multirow{7}{*}{ Combined } & \multirow{2}{*}{$C D$} & MPGGE Unadjusted & $\mathrm{N} / \mathrm{A}$ & $\mathrm{N} / \mathrm{A}$ & N/A & N/A & N/A & $\mathrm{N} / \mathrm{A}$ & N/A & $\mathrm{N} / \mathrm{A}$ & $\mathrm{N} / \mathrm{A}$ & N/A & $\mathrm{N} / \mathrm{A}$ & N/A & $\mathrm{N} / \mathrm{A}$ \\
\hline & & & MPGGE Adjusted & N/A & N/A & N/A & N/A & N/A & N/A & N/A & $\mathrm{N} / \mathrm{A}$ & N/A & N/A & N/A & N/A & N/A \\
\hline & & \multirow{2}{*}{ CS } & MPGGE Unadjusted & 22.9 & 22.9 & 27.5 & 31.9 & 26.4 & 31.6 & 37.7 & 29.7 & 36.4 & 45.0 & 31.9 & 38.9 & 48.8 \\
\hline & & & MPGGE Adjusted & 17.1 & 17.1 & 20.3 & 23.5 & 19.5 & 23.2 & 27.5 & 21.9 & 26.6 & 32.5 & 23.5 & 28.4 & 35.1 \\
\hline & & \multirow{3}{*}{$C D+C S$} & Wh/mile & 272.5 & 272.5 & 253.7 & 235.5 & 261.5 & 238.2 & 212.8 & 253.1 & 219.8 & 191.1 & 246.5 & 213.4 & 183.9 \\
\hline & & & MPGGE Unadjusted & 43.6 & 43.6 & 52.1 & 60.6 & 50.1 & 59.9 & 71.4 & 56.4 & 68.9 & 85.0 & 60.6 & 73.8 & 92.1 \\
\hline & & & MPGGE Adjusted & 31.4 & 31.4 & 37.2 & 42.9 & 35.9 & 42.4 & 49.9 & 40.1 & 48.3 & 58.4 & 42.9 & 51.5 & 62.8 \\
\hline
\end{tabular}




\begin{tabular}{|c|c|c|c|c|c|c|c|c|c|c|c|c|c|c|c|c|}
\hline & & & & \multicolumn{13}{|c|}{ Pickup } \\
\hline & & & & \multirow{2}{*}{$\frac{2010}{\operatorname{Ref}}$} & \multicolumn{3}{|c|}{2010} & \multicolumn{3}{|c|}{2015} & \multicolumn{3}{|c|}{2030} & \multicolumn{3}{|c|}{2045} \\
\hline \multirow{26}{*}{ H2 Series HEV PHEV40 } & & & & & low & avg & high & low & avg & high & low & avg & high & low & avg & high \\
\hline & \multirow{9}{*}{ UDDS } & \multirow{4}{*}{$C D$} & Wh/mile & 456.3 & 456.3 & 421.8 & 390.4 & 436.6 & 395.2 & 349.9 & 420.9 & 361.5 & 305.2 & 409.0 & 350.1 & 292.9 \\
\hline & & & \begin{tabular}{|l|} 
Distance \\
\end{tabular} & 40.7 & 40.7 & 40.7 & 40.7 & 40.7 & 40.6 & 40.8 & 40.7 & 40.8 & 40.5 & 40.7 & 40.6 & 40.4 \\
\hline & & & MPGGE Unadjusted & N/A & N/A & N/A & N/A & N/A & N/A & $\mathrm{N} / \mathrm{A}$ & N/A & N/A & N/A & N/A & N/A & N/A \\
\hline & & & MPGGE Adjusted & N/A & N/A & N/A & N/A & N/A & N/A & $\mathrm{N} / \mathrm{A}$ & $\mathrm{N} / \mathrm{A}$ & N/A & N/A & N/A & N/A & $\mathrm{N} / \mathrm{A}$ \\
\hline & & \multirow{2}{*}{ CS } & MPGGE Unadjusted & 23.1 & 23.1 & 27.8 & 32.7 & 26.7 & 32.2 & 39.2 & 30.0 & 37.9 & 48.6 & 32.6 & 40.6 & 52.3 \\
\hline & & & MPGGE Adjusted & 18.0 & 18.0 & 21.4 & 24.9 & 20.6 & 24.6 & 29.6 & 23.0 & 28.7 & 36.0 & 24.9 & 30.5 & 38.5 \\
\hline & & \multirow{3}{*}{$C D+C S$} & Wh/mile & 334.5 & 334.5 & 309.3 & 286.4 & 320.2 & 289.9 & 258.0 & 308.8 & 266.6 & 225.0 & 300.0 & 258.0 & 215.9 \\
\hline & & & MPGGE Unadjusted & 57.7 & 57.7 & 69.5 & 81.7 & 66.7 & 80.5 & 98.0 & 75.1 & 94.8 & 121.5 & 81.5 & 101.5 & 130.7 \\
\hline & & & MPGGE Adjusted & 42.0 & 42.0 & 49.5 & 56.9 & 47.8 & 56.2 & 66.4 & 53.0 & 64.6 & 79.1 & 56.8 & 68.4 & 83.8 \\
\hline & \multirow{9}{*}{ HWFET } & \multirow{4}{*}{$C D$} & Wh/mile & 499.6 & 499.6 & 468.5 & 435.5 & 479.9 & 439.9 & 395.4 & 466.0 & 407.7 & 363.7 & 454.0 & 397.2 & 350.5 \\
\hline & & & Distance & 38.6 & 38.6 & 38.6 & 38.6 & 36.7 & 38.6 & 36.0 & 38.6 & 36.0 & 34.5 & 36.4 & 35.8 & 34.5 \\
\hline & & & MPGGE Unadjusted & N/A & N/A & N/A & N/A & N/A & $\mathrm{N} / \mathrm{A}$ & $\mathrm{N} / \mathrm{A}$ & N/A & N/A & N/A & N/A & N/A & N/A \\
\hline & & & MPGGE Adjusted & $\mathrm{N} / \mathrm{A}$ & N/A & $\mathrm{N} / \mathrm{A}$ & $\mathrm{N} / \mathrm{A}$ & N/A & N/A & N/A & $\mathrm{N} / \mathrm{A}$ & N/A & N/A & N/A & $\mathrm{N} / \mathrm{A}$ & $\mathrm{N} / \mathrm{A}$ \\
\hline & & \multirow{2}{*}{ CS } & MPGGE Unadjusted & 21.8 & 21.8 & 25.8 & 29.9 & 25.1 & 29.7 & 34.8 & 28.0 & 33.8 & 40.3 & 30.0 & 36.4 & 43.6 \\
\hline & & & MPGGE Adjusted & 15.5 & 15.5 & 18.3 & 21.2 & 17.8 & 21.0 & 24.6 & 19.8 & 23.9 & 28.5 & 21.3 & 25.7 & 30.8 \\
\hline & & \multirow{3}{*}{$C D+C S$} & Wh/mile & 337.2 & 337.2 & 316.2 & 294.0 & 323.9 & 296.9 & 266.8 & 314.6 & 275.2 & 245.5 & 306.5 & 268.0 & 236.5 \\
\hline & & & MPGGE Unadjusted & 47.5 & 47.5 & 56.0 & 65.0 & 54.5 & 64.5 & 75.7 & 60.9 & 73.5 & 87.7 & 65.3 & 79.1 & 94.8 \\
\hline & & & MPGGE Adjusted & 33.5 & 33.5 & 39.5 & 45.7 & 38.4 & 45.3 & 53.1 & 42.8 & 51.5 & 61.3 & 45.9 & 55.4 & 66.2 \\
\hline & \multirow{7}{*}{ Combined } & \multirow{2}{*}{$C D$} & MPGGE Unadjusted & $\mathrm{N} / \mathrm{A}$ & $\mathrm{N} / \mathrm{A}$ & $\mathrm{N} / \mathrm{A}$ & N/A & N/A & $\mathrm{N} / \mathrm{A}$ & $\mathrm{N} / \mathrm{A}$ & N/A & $\mathrm{N} / \mathrm{A}$ & N/A & $\mathrm{N} / \mathrm{A}$ & $\mathrm{N} / \mathrm{A}$ & $\mathrm{N} / \mathrm{A}$ \\
\hline & & & MPGGE Adjusted & $\mathrm{N} / \mathrm{A}$ & $\mathrm{N} / \mathrm{A}$ & N/A & N/A & N/A & $\mathrm{N} / \mathrm{A}$ & N/A & N/A & N/A & N/A & $\mathrm{N} / \mathrm{A}$ & $\mathrm{N} / \mathrm{A}$ & $\mathrm{N} / \mathrm{A}$ \\
\hline & & \multirow{2}{*}{ CS } & MPGGE Unadjusted & 22.5 & 22.5 & 26.9 & 31.4 & 25.9 & 31.0 & 37.1 & 29.1 & 35.9 & 44.5 & 31.4 & 38.6 & 48.0 \\
\hline & & & MPGGE Adjusted & 16.8 & 16.8 & 19.9 & 23.1 & 19.2 & 22.9 & 27.1 & 21.5 & 26.3 & 32.2 & 23.1 & 28.2 & 34.6 \\
\hline & & \multirow{3}{*}{$C D+C S$} & Wh/mile & 335.7 & 335.7 & 312.4 & 289.8 & 321.9 & 293.1 & 262.0 & 311.4 & 270.5 & 234.2 & 302.9 & 262.5 & 225.2 \\
\hline & & & MPGGE Unadjusted & 52.6 & 52.6 & 62.7 & 73.2 & 60.6 & 72.4 & 86.6 & 67.9 & 83.8 & 103.5 & 73.3 & 90.0 & 111.6 \\
\hline & & & \begin{tabular}{|l|} 
MPGGE Adjusted \\
\end{tabular} & 37.7 & 37.7 & 44.4 & 51.3 & 43.1 & 50.8 & 59.7 & 47.9 & 58.0 & 70.0 & 51.3 & 61.9 & 74.8 \\
\hline
\end{tabular}


Argonne National Laboratory Report - Light-Duty Vehicle Fuel Consumption Displacement Potential Up to 2045

\begin{tabular}{|c|c|c|c|c|c|c|c|c|c|c|c|c|c|c|c|c|}
\hline & & & & \multicolumn{13}{|c|}{ Pickup } \\
\hline & & & & \multirow{2}{*}{$\begin{array}{c}2010 \\
\text { Ref }\end{array}$} & \multicolumn{3}{|c|}{2010} & \multicolumn{3}{|c|}{2015} & \multicolumn{3}{|c|}{2030} & \multicolumn{3}{|c|}{2045} \\
\hline & & & & & low & avg & high & low & avg & high & low & avg & high & low & avg & high \\
\hline \multirow{25}{*}{ E85 Split HEV PHEV10 } & \multirow{9}{*}{ UDDS } & \multirow{4}{*}{$C D$} & Wh/mile & 281.5 & 281.5 & 265.2 & 251.4 & 272.0 & 252.7 & 234.7 & 263.7 & 243.7 & 213.5 & 260.2 & 238.1 & 205.6 \\
\hline & & & Distance & 12.4 & 12.4 & 12.4 & 12.3 & 12.4 & 12.8 & 12.3 & 12.6 & 12.6 & 12.3 & 12.9 & 12.6 & 12.2 \\
\hline & & & MPGGE Unadjusted & 307.1 & 307.1 & 329.6 & 376.0 & 320.5 & 368.5 & 464.6 & 329.6 & 413.9 & 588.7 & 338.8 & 428.0 & 627.8 \\
\hline & & & MPGGE Adjusted & 152.9 & 152.9 & 159.5 & 172.1 & 156.9 & 170.2 & 192.8 & 159.5 & 181.5 & 215.9 & 162.1 & 184.7 & 222.1 \\
\hline & & \multirow{2}{*}{ CS } & MPGGE Unadjusted & 31.9 & 31.9 & 34.3 & 37.1 & 33.5 & 36.8 & 46.7 & 34.8 & 40.0 & 53.6 & 35.7 & 41.2 & 56.0 \\
\hline & & & MPGGE Adjusted & 24.4 & 24.4 & 26.1 & 28.1 & 25.5 & 27.8 & 34.7 & 26.5 & 30.2 & 39.4 & 27.1 & 31.0 & 40.9 \\
\hline & & \multirow{3}{*}{$\mathrm{CD}+\mathrm{CS}$} & Wh/mile & 58.6 & 58.6 & 55.3 & 52.4 & 56.7 & 52.6 & 48.9 & 54.9 & 50.8 & 44.5 & 54.2 & 49.6 & 42.8 \\
\hline & & & MPGGE Unadjusted & 37.6 & 37.6 & 40.4 & 43.8 & 39.5 & 43.4 & 55.2 & 41.0 & 47.3 & 63.4 & 42.1 & 48.7 & 66.2 \\
\hline & & & MPGGE Adjusted & 28.5 & 28.5 & 30.4 & 32.8 & 29.7 & 32.5 & 40.4 & 30.8 & 35.1 & 45.7 & 31.6 & 36.1 & 47.5 \\
\hline & \multirow{9}{*}{ HWFET } & \multirow{4}{*}{$C D$} & Wh/mile & 310.3 & 310.3 & 280.7 & 262.8 & 300.9 & 264.4 & 243.2 & 279.4 & 250.1 & 236.3 & 275.0 & 243.5 & 263.5 \\
\hline & & & Distance & 12.4 & 12.4 & 12.5 & 12.5 & 12.2 & 12.5 & 12.4 & 12.5 & 12.5 & 11.6 & 12.6 & 12.4 & 8.1 \\
\hline & & & MPGGE Unadjusted & 94.4 & 94.4 & 91.1 & 98.1 & 98.6 & 96.6 & 120.7 & 91.9 & 103.7 & 146.3 & 95.0 & 106.9 & 218.6 \\
\hline & & & MPGGE Adjusted & 65.9 & 65.9 & 63.7 & 68.4 & 68.8 & 67.4 & 83.8 & 64.2 & 72.2 & 101.0 & 66.3 & 74.4 & 148.6 \\
\hline & & \multirow{2}{*}{ CS } & MPGGE Unadjusted & 28.5 & 28.5 & 30.3 & 32.7 & 29.7 & 32.4 & 40.4 & 30.7 & 34.7 & 44.6 & 31.6 & 35.8 & 46.3 \\
\hline & & & MPGGE Adjusted & 20.2 & 20.2 & 21.5 & 23.1 & 21.0 & 22.9 & 28.5 & 21.7 & 24.5 & 31.5 & 22.4 & 25.3 & 32.7 \\
\hline & & \multirow{3}{*}{$\mathrm{CD}+\mathrm{CS}$} & Wh/mile & 91.3 & 91.3 & 82.6 & 77.3 & 88.5 & 77.8 & 71.5 & 82.2 & 73.6 & 69.5 & 80.9 & 71.6 & 77.5 \\
\hline & & & MPGGE Unadjusted & 34.2 & 34.2 & 36.1 & 38.9 & 35.7 & 38.5 & 48.1 & 36.5 & 41.3 & 53.5 & 37.6 & 42.6 & 57.1 \\
\hline & & & MPGGE Adjusted & 24.2 & 24.2 & 25.5 & 27.5 & 25.2 & 27.2 & 33.9 & 25.8 & 29.2 & 37.7 & 26.6 & 30.1 & 40.2 \\
\hline & \multirow{7}{*}{ Combined } & \multirow{2}{*}{$C D$} & MPGGE Unadjusted & 152.4 & 152.4 & 151.3 & 165.3 & 159.2 & 162.6 & 203.5 & 152.3 & 176.4 & 249.3 & 157.2 & 181.9 & 340.7 \\
\hline & & & MPGGE Adjusted & 95.9 & 95.9 & 95.1 & 102.4 & 99.5 & 101.0 & 121.6 & 95.6 & 108.0 & 142.8 & 98.2 & 110.8 & 181.7 \\
\hline & & \multirow{2}{*}{ CS } & MPGGE Unadjusted & 30.3 & 30.3 & 32.4 & 35.0 & 31.6 & 34.6 & 43.7 & 32.8 & 37.4 & 49.1 & 33.7 & 38.6 & 51.2 \\
\hline & & & MPGGE Adjusted & 22.3 & 22.3 & 23.8 & 25.6 & 23.3 & 25.4 & 31.6 & 24.1 & 27.3 & 35.4 & 24.8 & 28.1 & 36.7 \\
\hline & & \multirow{3}{*}{$C D+C S$} & Wh/mile & 73.3 & 73.3 & 67.5 & 63.6 & 71.0 & 63.9 & 59.1 & 67.2 & 61.0 & 55.7 & 66.2 & 59.5 & 58.4 \\
\hline & & & MPGGE Unadjusted & 36.0 & 36.0 & 38.4 & 41.5 & 37.7 & 41.0 & 51.7 & 38.8 & 44.4 & 58.5 & 40.0 & 45.7 & 61.8 \\
\hline & & & MPGGE Adjusted & 26.4 & 26.4 & 28.0 & 30.2 & 27.5 & 29.9 & 37.2 & 28.3 & 32.2 & 41.7 & 29.1 & 33.1 & 43.9 \\
\hline
\end{tabular}


Argonne National Laboratory Report - Light-Duty Vehicle Fuel Consumption Displacement Potential Up to 2045

\begin{tabular}{|c|c|c|c|c|c|c|c|c|c|c|c|c|c|c|c|c|}
\hline & & & & \multicolumn{13}{|c|}{ Pickup } \\
\hline & & & & \multirow{2}{*}{$\begin{array}{c}2010 \\
\text { Ref }\end{array}$} & \multicolumn{3}{|c|}{2010} & \multicolumn{3}{|c|}{2015} & \multicolumn{3}{|c|}{2030} & \multicolumn{3}{|c|}{2045} \\
\hline \multirow{26}{*}{ E85 Split HEV PHEV20 } & & & & & low & avg & high & low & avg & high & low & avg & high & low & avg & high \\
\hline & \multirow{9}{*}{ UDDS } & \multirow{4}{*}{$C D$} & Wh/mile & 332.3 & 332.3 & 314.5 & 295.4 & 323.5 & 298.2 & 266.6 & 313.1 & 269.4 & 226.8 & 309.9 & 263.1 & 217.2 \\
\hline & & & Distance & 23.8 & 23.8 & 23.8 & 23.5 & 23.7 & 23.5 & 23.5 & 23.8 & 23.9 & 23.9 & 23.7 & 23.7 & 23.9 \\
\hline & & & MPGGE Unadjusted & 701.0 & 701.0 & 790.1 & 915.5 & 786.5 & 905.4 & 999.0 & 792.8 & 753.6 & 809.2 & 842.0 & 758.1 & 846.6 \\
\hline & & & MPGGE Adjusted & 232.7 & 232.7 & 243.9 & 257.2 & 243.5 & 256.2 & 264.8 & 244.2 & 239.5 & 246.1 & 249.7 & 240.0 & 250.2 \\
\hline & & \multirow{2}{*}{ CS } & MPGGE Unadjusted & 31.6 & 31.6 & 33.9 & 36.8 & 33.1 & 36.4 & 46.6 & 34.4 & 39.9 & 53.5 & 35.3 & 41.2 & 55.9 \\
\hline & & & MPGGE Adjusted & 24.2 & 24.2 & 25.8 & 27.9 & 25.2 & 27.6 & 34.7 & 26.2 & 30.1 & 39.3 & 26.8 & 31.0 & 40.9 \\
\hline & & \multirow{3}{*}{$C D+C S$} & Wh/mile & 172.7 & 172.7 & 163.4 & 153.7 & 168.1 & 155.1 & 138.8 & 162.6 & 140.8 & 119.0 & 161.1 & 137.4 & 114.0 \\
\hline & & & MPGGE Unadjusted & 54.2 & 54.2 & 58.4 & 63.5 & 57.0 & 62.8 & 80.0 & 59.1 & 68.3 & 90.7 & 60.8 & 70.4 & 94.8 \\
\hline & & & MPGGE Adjusted & 39.8 & 39.8 & 42.5 & 45.7 & 41.6 & 45.3 & 56.0 & 43.0 & 48.8 & 62.3 & 44.0 & 50.1 & 64.6 \\
\hline & \multirow{9}{*}{ HWFET } & \multirow{4}{*}{$C D$} & Wh/mile & 365.1 & 365.1 & 346.8 & 324.2 & 353.7 & 327.1 & 243.7 & 345.6 & 250.3 & 227.7 & 340.7 & 245.0 & 219.4 \\
\hline & & & Distance & 21.6 & 21.6 & 21.4 & 21.4 & 21.5 & 21.4 & 20.2 & 21.4 & 20.2 & 19.3 & 21.4 & 20.0 & 19.2 \\
\hline & & & MPGGE Unadjusted & 142.8 & 142.8 & 150.7 & 162.3 & 149.4 & 160.4 & 119.5 & 153.3 & 102.6 & 131.4 & 159.3 & 106.2 & 136.8 \\
\hline & & & MPGGE Adjusted & 98.7 & 98.7 & 104.0 & 111.7 & 103.1 & 110.4 & 83.0 & 105.7 & 71.5 & 91.0 & 109.7 & 74.0 & 94.6 \\
\hline & & \multirow{2}{*}{ CS } & MPGGE Unadjusted & 28.2 & 28.2 & 30.0 & 32.5 & 29.5 & 32.1 & 40.2 & 30.4 & 34.5 & 44.4 & 31.3 & 35.6 & 46.1 \\
\hline & & & MPGGE Adjusted & 20.0 & 20.0 & 21.2 & 23.0 & 20.9 & 22.7 & 28.4 & 21.5 & 24.4 & 31.3 & 22.2 & 25.2 & 32.5 \\
\hline & & \multirow{3}{*}{$C D+C S$} & Wh/mile & 183.0 & 183.0 & 175.0 & 163.6 & 177.3 & 165.1 & 71.7 & 174.3 & 73.6 & 67.0 & 171.8 & 72.1 & 64.5 \\
\hline & & & MPGGE Unadjusted & 41.4 & 41.4 & 44.1 & 47.7 & 43.3 & 47.2 & 47.8 & 44.8 & 41.1 & 52.8 & 46.1 & 42.4 & 54.9 \\
\hline & & & MPGGE Adjusted & 29.2 & 29.2 & 31.2 & 33.7 & 30.6 & 33.3 & 33.7 & 31.6 & 29.0 & 37.2 & 32.5 & 29.9 & 38.6 \\
\hline & \multirow{7}{*}{ Combined } & \multirow{2}{*}{$C D$} & MPGGE Unadjusted & 254.1 & 254.1 & 271.6 & 296.5 & 269.4 & 293.0 & 231.7 & 275.6 & 195.4 & 243.7 & 287.5 & 201.5 & 253.8 \\
\hline & & & MPGGE Adjusted & 144.4 & 144.4 & 151.9 & 162.2 & 151.0 & 160.7 & 133.4 & 153.6 & 116.4 & 139.3 & 158.6 & 119.4 & 143.8 \\
\hline & & \multirow{2}{*}{ CS } & MPGGE Unadjusted & 29.9 & 29.9 & 32.0 & 34.7 & 31.4 & 34.3 & 43.5 & 32.5 & 37.3 & 49.0 & 33.4 & 38.5 & 51.0 \\
\hline & & & MPGGE Adjusted & 22.1 & 22.1 & 23.5 & 25.4 & 23.1 & 25.2 & 31.5 & 23.9 & 27.2 & 35.3 & 24.5 & 28.1 & 36.7 \\
\hline & & \multirow{3}{*}{$C D+C S$} & Wh/mile & 177.3 & 177.3 & 168.6 & 158.1 & 172.3 & 159.6 & 108.6 & 167.9 & 110.6 & 95.6 & 165.9 & 108.0 & 91.7 \\
\hline & & & MPGGE Unadjusted & 47.6 & 47.6 & 51.0 & 55.3 & 49.9 & 54.7 & 61.4 & 51.7 & 52.6 & 68.5 & 53.2 & 54.3 & 71.4 \\
\hline & & & \begin{tabular}{|l|} 
MPGEE Adjusted \\
\end{tabular} & 34.2 & 34.2 & 36.5 & 39.4 & 35.8 & 39.0 & 43.1 & 37.0 & 37.3 & 47.8 & 38.0 & 38.5 & 49.6 \\
\hline
\end{tabular}


Argonne National Laboratory Report - Light-Duty Vehicle Fuel Consumption Displacement Potential Up to 2045

\begin{tabular}{|c|c|c|c|c|c|c|c|c|c|c|c|c|c|c|c|c|}
\hline & & & & \multicolumn{13}{|c|}{ Pickup } \\
\hline & & & & \multirow{2}{*}{$\begin{array}{c}2010 \\
\text { Ref }\end{array}$} & \multicolumn{3}{|c|}{2010} & \multicolumn{3}{|c|}{2015} & \multicolumn{3}{|c|}{2030} & \multicolumn{3}{|c|}{2045} \\
\hline & & & & & low & avg & high & low & avg & high & low & avg & high & low & avg & high \\
\hline \multirow{25}{*}{ E85 Series HEV PHEV30 } & \multirow{9}{*}{ UDDS } & \multirow{4}{*}{$C D$} & Wh/mile & 444.5 & 444.5 & 416.7 & 388.3 & 427.7 & 392.8 & 349.7 & 414.1 & 360.3 & 310.1 & 407.2 & 350.0 & 296.7 \\
\hline & & & Distance & 31.9 & 31.9 & 31.9 & 31.7 & 31.4 & 31.9 & 30.9 & 31.4 & 30.9 & 31.7 & 31.9 & 30.9 & 31.7 \\
\hline & & & MPGGE Unadjusted & $\mathrm{N} / \mathrm{A}$ & $\mathrm{N} / \mathrm{A}$ & N/A & N/A & N/A & $\mathrm{N} / \mathrm{A}$ & N/A & $\mathrm{N} / \mathrm{A}$ & N/A & N/A & N/A & N/A & $\mathrm{N} / \mathrm{A}$ \\
\hline & & & MPGGE Adjusted & $\mathrm{N} / \mathrm{A}$ & $\mathrm{N} / \mathrm{A}$ & $\mathrm{N} / \mathrm{A}$ & N/A & N/A & N/A & N/A & $\mathrm{N} / \mathrm{A}$ & N/A & N/A & N/A & N/A & $\mathrm{N} / \mathrm{A}$ \\
\hline & & \multirow{2}{*}{ CS } & MPGGE Unadjusted & 22.5 & 22.5 & 24.7 & 27.1 & 23.8 & 26.8 & 35.7 & 25.3 & 30.5 & 42.4 & 26.1 & 31.6 & 44.7 \\
\hline & & & MPGGE Adjusted & 17.6 & 17.6 & 19.1 & 20.9 & 18.5 & 20.7 & 27.1 & 19.6 & 23.4 & 31.8 & 20.2 & 24.2 & 33.4 \\
\hline & & \multirow{3}{*}{$\mathrm{CD}+\mathrm{CS}$} & Wh/mile & 285.9 & 285.9 & 268.0 & 249.8 & 275.1 & 252.7 & 225.7 & 266.4 & 232.5 & 200.6 & 262.0 & 225.7 & 191.9 \\
\hline & & & MPGGE Unadjusted & 47.9 & 47.9 & 52.5 & 57.6 & 50.6 & 56.9 & 76.0 & 53.8 & 64.8 & 90.1 & 55.5 & 67.2 & 95.1 \\
\hline & & & MPGGE Adjusted & 35.6 & 35.6 & 38.6 & 42.0 & 37.3 & 41.5 & 53.5 & 39.5 & 46.6 & 61.9 & 40.6 & 48.1 & 64.8 \\
\hline & \multirow{9}{*}{ HWFET } & \multirow{4}{*}{$C D$} & Wh/mile & 493.4 & 493.4 & 466.7 & 435.6 & 475.7 & 439.9 & 397.3 & 463.2 & 409.0 & 366.2 & 454.5 & 399.3 & 352.2 \\
\hline & & & \begin{tabular}{|l} 
Distance \\
\end{tabular} & 27.7 & 27.7 & 28.4 & 27.5 & 27.7 & 27.5 & 27.1 & 27.5 & 27.1 & 26.0 & 27.5 & 27.0 & 25.9 \\
\hline & & & MPGGE Unadjusted & $\mathrm{N} / \mathrm{A}$ & $\mathrm{N} / \mathrm{A}$ & $\mathrm{N} / \mathrm{A}$ & $\mathrm{N} / \mathrm{A}$ & N/A & $\mathrm{N} / \mathrm{A}$ & N/A & N/A & N/A & $\mathrm{N} / \mathrm{A}$ & $\mathrm{N} / \mathrm{A}$ & $\mathrm{N} / \mathrm{A}$ & $\mathrm{N} / \mathrm{A}$ \\
\hline & & & MPGGE Adjusted & N/A & N/A & N/A & N/A & N/A & N/A & N/A & N/A & N/A & N/A & N/A & N/A & $\mathrm{N} / \mathrm{A}$ \\
\hline & & \multirow{2}{*}{ CS } & MPGGE Unadjusted & 21.0 & 21.0 & 22.6 & 24.7 & 22.1 & 24.5 & 31.6 & 23.3 & 27.0 & 35.8 & 24.1 & 28.1 & 37.2 \\
\hline & & & MPGGE Adjusted & 14.9 & 14.9 & 16.0 & 17.5 & 15.7 & 17.4 & 22.4 & 16.5 & 19.1 & 25.3 & 17.1 & 19.9 & 26.3 \\
\hline & & \multirow{3}{*}{$\mathrm{CD}+\mathrm{CS}$} & Wh/mile & 254.2 & 254.2 & 240.5 & 224.4 & 245.1 & 226.7 & 204.7 & 238.7 & 210.7 & 188.6 & 234.2 & 205.7 & 181.4 \\
\hline & & & MPGGE Unadjusted & 35.6 & 35.6 & 38.3 & 41.9 & 37.5 & 41.6 & 53.6 & 39.4 & 45.7 & 60.7 & 40.9 & 47.6 & 63.0 \\
\hline & & & \begin{tabular}{|l|} 
MPGGE Adjusted \\
\end{tabular} & 25.1 & 25.1 & 27.1 & 29.6 & 26.5 & 29.4 & 37.8 & 27.8 & 32.3 & 42.7 & 28.9 & 33.6 & 44.3 \\
\hline & \multirow{7}{*}{ Combined } & \multirow{2}{*}{$C D$} & MPGGE Unadjusted & $\mathrm{N} / \mathrm{A}$ & $\mathrm{N} / \mathrm{A}$ & N/A & N/A & N/A & $\mathrm{N} / \mathrm{A}$ & N/A & $\mathrm{N} / \mathrm{A}$ & $\mathrm{N} / \mathrm{A}$ & N/A & $\mathrm{N} / \mathrm{A}$ & N/A & $\mathrm{N} / \mathrm{A}$ \\
\hline & & & MPGGE Adjusted & N/A & N/A & N/A & N/A & N/A & N/A & N/A & $\mathrm{N} / \mathrm{A}$ & N/A & N/A & N/A & N/A & N/A \\
\hline & & \multirow{2}{*}{ CS } & MPGGE Unadjusted & 21.8 & 21.8 & 23.7 & 26.0 & 23.0 & 25.7 & 33.8 & 24.3 & 28.8 & 39.1 & 25.2 & 29.9 & 41.0 \\
\hline & & & MPGGE Adjusted & 16.2 & 16.2 & 17.6 & 19.2 & 17.1 & 19.1 & 24.8 & 18.1 & 21.2 & 28.5 & 18.7 & 22.1 & 29.8 \\
\hline & & \multirow{3}{*}{$C D+C S$} & Wh/mile & 271.6 & 271.6 & 255.6 & 238.4 & 261.6 & 241.0 & 216.2 & 253.9 & 222.7 & 195.2 & 249.5 & 216.7 & 187.2 \\
\hline & & & MPGGE Unadjusted & 41.4 & 41.4 & 45.0 & 49.3 & 43.7 & 48.8 & 64.0 & 46.2 & 54.6 & 74.0 & 47.8 & 56.7 & 77.4 \\
\hline & & & MPGGE Adjusted & 30.0 & 30.0 & 32.4 & 35.3 & 31.5 & 35.0 & 45.1 & 33.2 & 38.8 & 51.5 & 34.3 & 40.3 & 53.6 \\
\hline
\end{tabular}




\begin{tabular}{|c|c|c|c|c|c|c|c|c|c|c|c|c|c|c|c|c|}
\hline & & & & \multicolumn{13}{|c|}{ Pickup } \\
\hline & & & & 2010 & & 2010 & & & 2015 & & & 2030 & & & 2045 & \\
\hline \multirow{26}{*}{ E85 Series HEV PHEV40 } & & \multirow{5}{*}{$C D$} & & Ref & low & avg & high & low & avg & high & low & avg & high & low & avg & high \\
\hline & \multirow{9}{*}{ UDDS } & & Wh/mile & 454.5 & 454.5 & 425.6 & 396.0 & 436.8 & 400.7 & 356.8 & 422.3 & 367.4 & 313.1 & 415.0 & 356.7 & 299.6 \\
\hline & & & Distance & 40.7 & 40.7 & 40.6 & 40.6 & 40.7 & 40.7 & 40.6 & 40.6 & 40.8 & 40.5 & 40.6 & 40.6 & 40.8 \\
\hline & & & MPGGE Unadjusted & N/A & N/A & N/A & N/A & N/A & N/A & N/A & N/A & N/A & N/A & N/A & N/A & N/A \\
\hline & & & MPGGE Adjusted & N/A & N/A & N/A & N/A & N/A & $\mathrm{N} / \mathrm{A}$ & N/A & N/A & N/A & N/A & N/A & N/A & $\mathrm{N} / \mathrm{A}$ \\
\hline & & \multirow{2}{*}{ CS } & MPGGE Unadjusted & 21.9 & 21.9 & 24.0 & 26.5 & 23.2 & 26.1 & 35.0 & 24.7 & 29.9 & 41.8 & 25.5 & 31.1 & 44.1 \\
\hline & & & MPGGE Adjusted & 17.1 & 17.1 & 18.7 & 20.5 & 18.1 & 20.2 & 26.6 & 19.1 & 22.9 & 31.3 & 19.7 & 23.8 & 33.0 \\
\hline & & \multirow{3}{*}{$C D+C S$} & Wh/mile & 333.2 & 333.2 & 312.1 & 290.5 & 320.4 & 294.0 & 263.2 & 309.9 & 271.0 & 230.9 & 304.5 & 262.8 & 220.9 \\
\hline & & & MPGGE Unadjusted & 54.8 & 54.8 & 60.0 & 66.2 & 58.0 & 65.3 & 87.6 & 61.7 & 74.6 & 104.4 & 63.7 & 77.6 & 110.2 \\
\hline & & & MPGGE Adjusted & 40.1 & 40.1 & 43.5 & 47.5 & 42.2 & 46.9 & 60.4 & 44.6 & 52.7 & 70.0 & 45.9 & 54.5 & 73.2 \\
\hline & \multirow{9}{*}{ HWFET } & \multirow{4}{*}{$C D$} & Wh/mile & 498.4 & 498.4 & 471.0 & 439.2 & 480.0 & 443.5 & 399.8 & 466.9 & 411.5 & 368.7 & 457.9 & 401.4 & 354.7 \\
\hline & & & Distance & 38.6 & 38.6 & 36.5 & 36.4 & 36.7 & 36.5 & 38.6 & 38.6 & 38.6 & 35.7 & 36.6 & 37.1 & 34.6 \\
\hline & & & MPGGE Unadjusted & N/A & N/A & N/A & N/A & N/A & N/A & N/A & N/A & N/A & N/A & N/A & N/A & $\mathrm{N} / \mathrm{A}$ \\
\hline & & & MPGGE Adjusted & $\mathrm{N} / \mathrm{A}$ & N/A & N/A & N/A & N/A & N/A & N/A & $\mathrm{N} / \mathrm{A}$ & N/A & $\mathrm{N} / \mathrm{A}$ & $\mathrm{N} / \mathrm{A}$ & N/A & $\mathrm{N} / \mathrm{A}$ \\
\hline & & \multirow{2}{*}{ CS } & MPGGE Unadjusted & 20.7 & 20.7 & 22.3 & 24.5 & 21.8 & 24.2 & 31.3 & 23.0 & 26.7 & 35.5 & 23.8 & 27.9 & 36.9 \\
\hline & & & MPGGE Adjusted & 14.7 & 14.7 & 15.8 & 17.4 & 15.5 & 17.2 & 22.2 & 16.3 & 18.9 & 25.1 & 16.9 & 19.7 & 26.1 \\
\hline & & \multirow{3}{*}{$C D+C S$} & Wh/mile & 336.4 & 336.4 & 317.9 & 296.4 & 324.0 & 299.4 & 269.9 & 315.2 & 277.7 & 248.9 & 309.1 & 270.9 & 239.4 \\
\hline & & & MPGGE Unadjusted & 45.0 & 45.0 & 48.5 & 53.2 & 47.5 & 52.7 & 68.1 & 50.0 & 58.1 & 77.1 & 51.7 & 60.5 & 80.3 \\
\hline & & & MPGGE Adjusted & 31.8 & 31.8 & 34.2 & 37.5 & 33.5 & 37.1 & 47.8 & 35.3 & 40.9 & 54.0 & 36.5 & 42.6 & 56.2 \\
\hline & \multirow{7}{*}{ Combined } & \multirow{2}{*}{$C D$} & MPGGE Unadjusted & N/A & N/A & N/A & N/A & $\mathrm{N} / \mathrm{A}$ & N/A & N/A & $\mathrm{N} / \mathrm{A}$ & $\mathrm{N} / \mathrm{A}$ & N/A & N/A & $\mathrm{N} / \mathrm{A}$ & N/A \\
\hline & & & MPGGE Adjusted & N/A & N/A & N/A & N/A & N/A & N/A & N/A & $\mathrm{N} / \mathrm{A}$ & N/A & N/A & N/A & $\mathrm{N} / \mathrm{A}$ & N/A \\
\hline & & \multirow{2}{*}{ CS } & MPGGE Unadjusted & 21.4 & 21.4 & 23.2 & 25.5 & 22.6 & 25.2 & 33.3 & 23.9 & 28.4 & 38.7 & 24.7 & 29.5 & 40.6 \\
\hline & & & MPGGE Adjusted & 15.9 & 15.9 & 17.3 & 18.9 & 16.8 & 18.7 & 24.4 & 17.8 & 20.9 & 28.2 & 18.3 & 21.8 & 29.5 \\
\hline & & \multirow{3}{*}{$\mathrm{CD}+\mathrm{CS}$} & Wh/mile & 334.7 & 334.7 & 314.7 & 293.2 & 322.0 & 296.4 & 266.2 & 312.3 & 274.0 & 239.0 & 306.6 & 266.4 & 229.2 \\
\hline & & & MPGGE Unadjusted & 49.9 & 49.9 & 54.2 & 59.7 & 52.7 & 59.0 & 77.6 & 55.8 & 66.1 & 90.0 & 57.7 & 68.9 & 94.4 \\
\hline & & & MPGGE Adjusted & 35.9 & 35.9 & 38.8 & 42.4 & 37.8 & 41.9 & 54.0 & 39.9 & 46.6 & 61.8 & 41.1 & 48.4 & 64.4 \\
\hline
\end{tabular}


Argonne National Laboratory Report - Light-Duty Vehicle Fuel Consumption Displacement Potential Up to 2045

\begin{tabular}{|c|c|c|c|c|c|c|c|c|c|c|c|c|c|c|c|c|}
\hline & & & & \multicolumn{13}{|c|}{ Pickup } \\
\hline & & & & \multirow{2}{*}{$\begin{array}{c}2010 \\
\text { Ref }\end{array}$} & \multicolumn{3}{|c|}{2010} & \multicolumn{3}{|c|}{2015} & \multicolumn{3}{|c|}{2030} & \multicolumn{3}{|c|}{2045} \\
\hline & & & & & low & avg & high & low & avg & high & low & avg & high & low & avg & high \\
\hline \multirow{25}{*}{ FC HEV PHEV10 } & \multirow{9}{*}{ UDDS } & \multirow{4}{*}{$C D$} & Wh/mile & 457.9 & 457.9 & 400.5 & 357.0 & 416.6 & 361.0 & 322.4 & 389.2 & 332.3 & 280.3 & 371.8 & 322.7 & 268.4 \\
\hline & & & Distance & 11.5 & 11.5 & 11.3 & 10.3 & 11.4 & 11.1 & 11.1 & 11.3 & 11.1 & 10.2 & 11.2 & 10.2 & 10.2 \\
\hline & & & MPGGE Unadjusted & $\mathrm{N} / \mathrm{A}$ & $\mathrm{N} / \mathrm{A}$ & N/A & N/A & N/A & $\mathrm{N} / \mathrm{A}$ & N/A & $\mathrm{N} / \mathrm{A}$ & N/A & N/A & N/A & N/A & $\mathrm{N} / \mathrm{A}$ \\
\hline & & & MPGGE Adjusted & $\mathrm{N} / \mathrm{A}$ & $\mathrm{N} / \mathrm{A}$ & $\mathrm{N} / \mathrm{A}$ & N/A & N/A & N/A & N/A & $\mathrm{N} / \mathrm{A}$ & N/A & N/A & N/A & $\mathrm{N} / \mathrm{A}$ & $\mathrm{N} / \mathrm{A}$ \\
\hline & & \multirow{2}{*}{ CS } & MPGGE Unadjusted & 36.0 & 36.0 & 42.7 & 50.4 & 43.2 & 49.8 & 57.1 & 46.2 & 55.4 & 66.1 & 48.4 & 56.9 & 69.0 \\
\hline & & & MPGGE Adjusted & 27.3 & 27.3 & 32.0 & 37.2 & 32.3 & 36.8 & 41.6 & 34.4 & 40.5 & 47.4 & 35.9 & 41.5 & 49.2 \\
\hline & & \multirow{3}{*}{$\mathrm{CD}+\mathrm{CS}$} & Wh/mile & 95.4 & 95.4 & 83.4 & 74.4 & 86.8 & 75.2 & 67.2 & 81.1 & 69.2 & 58.4 & 77.5 & 67.2 & 55.9 \\
\hline & & & MPGGE Unadjusted & 43.3 & 43.3 & 51.5 & 60.7 & 52.0 & 60.0 & 68.8 & 55.7 & 66.7 & 79.6 & 58.3 & 68.6 & 83.2 \\
\hline & & & MPGGE Adjusted & 32.4 & 32.4 & 37.9 & 44.0 & 38.3 & 43.5 & 49.1 & 40.7 & 47.8 & 55.7 & 42.4 & 49.0 & 57.8 \\
\hline & \multirow{9}{*}{ HWFET } & \multirow{4}{*}{$C D$} & Wh/mile & 470.1 & 470.1 & 414.5 & 370.6 & 430.0 & 376.3 & 330.0 & 403.7 & 340.2 & 293.4 & 385.8 & 332.5 & 281.1 \\
\hline & & & \begin{tabular}{|l} 
Distance \\
\end{tabular} & 10.6 & 10.6 & 10.5 & 10.0 & 10.6 & 10.0 & 10.0 & 10.0 & 10.0 & 8.0 & 10.0 & 9.7 & 8.0 \\
\hline & & & MPGGE Unadjusted & 302.3 & 302.3 & 282.0 & 296.0 & 307.8 & 299.1 & 282.4 & 292.1 & 275.4 & 261.4 & 283.9 & 283.9 & 265.5 \\
\hline & & & MPGGE Adjusted & 202.0 & 202.0 & 189.3 & 198.1 & 205.5 & 200.1 & 189.5 & 195.6 & 185.1 & 176.2 & 190.5 & 190.5 & 178.8 \\
\hline & & \multirow{2}{*}{ CS } & MPGGE Unadjusted & 35.0 & 35.0 & 40.0 & 46.3 & 41.0 & 45.8 & 50.8 & 43.0 & 49.4 & 55.5 & 44.5 & 50.7 & 57.6 \\
\hline & & & MPGGE Adjusted & 24.8 & 24.8 & 28.2 & 32.7 & 28.9 & 32.3 & 35.8 & 30.3 & 34.8 & 39.1 & 31.4 & 35.8 & 40.5 \\
\hline & & \multirow{3}{*}{$\mathrm{CD}+\mathrm{CS}$} & Wh/mile & 138.3 & 138.3 & 121.9 & 109.0 & 126.5 & 110.7 & 97.1 & 118.7 & 100.0 & 86.3 & 113.5 & 97.8 & 82.7 \\
\hline & & & MPGGE Unadjusted & 44.5 & 44.5 & 50.4 & 58.1 & 51.7 & 57.5 & 63.3 & 54.0 & 61.5 & 68.4 & 55.8 & 63.2 & 70.9 \\
\hline & & & \begin{tabular}{|l|} 
MPGGE Adjusted \\
\end{tabular} & 31.4 & 31.4 & 35.5 & 40.9 & 36.5 & 40.5 & 44.5 & 38.1 & 43.2 & 48.0 & 39.3 & 44.4 & 49.8 \\
\hline & \multirow{7}{*}{ Combined } & \multirow{2}{*}{$C D$} & MPGGE Unadjusted & N/A & $\mathrm{N} / \mathrm{A}$ & $\mathrm{N} / \mathrm{A}$ & N/A & $\mathrm{N} / \mathrm{A}$ & N/A & $\mathrm{N} / \mathrm{A}$ & $\mathrm{N} / \mathrm{A}$ & $\mathrm{N} / \mathrm{A}$ & $\mathrm{N} / \mathrm{A}$ & $\mathrm{N} / \mathrm{A}$ & N/A & $\mathrm{N} / \mathrm{A}$ \\
\hline & & & MPGGE Adjusted & N/A & N/A & N/A & N/A & N/A & N/A & N/A & N/A & N/A & N/A & N/A & N/A & N/A \\
\hline & & \multirow{2}{*}{ CS } & MPGGE Unadjusted & 35.5 & 35.5 & 41.4 & 48.5 & 42.1 & 47.9 & 54.1 & 44.7 & 52.5 & 60.8 & 46.6 & 54.0 & 63.4 \\
\hline & & & MPGGE Adjusted & 26.1 & 26.1 & 30.2 & 35.0 & 30.7 & 34.6 & 38.8 & 32.4 & 37.7 & 43.2 & 33.7 & 38.7 & 44.9 \\
\hline & & \multirow{3}{*}{$\mathrm{CD}+\mathrm{CS}$} & Wh/mile & 114.7 & 114.7 & 100.8 & 90.0 & 104.7 & 91.2 & 80.6 & 98.0 & 83.1 & 70.9 & 93.7 & 81.0 & 68.0 \\
\hline & & & MPGGE Unadjusted & 43.8 & 43.8 & 51.0 & 59.5 & 51.9 & 58.8 & 66.2 & 54.9 & 64.3 & 74.1 & 57.1 & 66.0 & 77.2 \\
\hline & & & MPGGE Adjusted & 31.9 & 31.9 & 36.8 & 42.5 & 37.4 & 42.1 & 46.9 & 39.5 & 45.6 & 52.0 & 40.9 & 46.8 & 53.9 \\
\hline
\end{tabular}




\begin{tabular}{|c|c|c|c|c|c|c|c|c|c|c|c|c|c|c|c|c|}
\hline & & & & \multicolumn{13}{|c|}{ Pickup } \\
\hline & & & & 2010 & & 2010 & & & 2015 & & & 2030 & & & 2045 & \\
\hline \multirow{26}{*}{ FC HEV PHEV 20} & & \multirow{5}{*}{$C D$} & & Ref & low & avg & high & low & avg & high & low & avg & high & low & avg & high \\
\hline & \multirow{9}{*}{ UDDS } & & Wh/mile & 466.5 & 466.5 & $\begin{array}{l}407.1 \\
\end{array}$ & 362.6 & 423.3 & 366.5 & 323.3 & 394.9 & 333.4 & 278.5 & 376.8 & 322.9 & 266.4 \\
\hline & & & Distance & 20.5 & 20.5 & 20.3 & 20.6 & 20.3 & 20.6 & 20.5 & 20.6 & 20.5 & 20.7 & 20.7 & 20.5 & 20.7 \\
\hline & & & MPGGE Unadjusted & N/A & N/A & N/A & N/A & N/A & N/A & N/A & N/A & N/A & N/A & N/A & N/A & N/A \\
\hline & & & MPGGE Adjusted & N/A & N/A & N/A & N/A & N/A & N/A & N/A & N/A & N/A & N/A & N/A & N/A & N/A \\
\hline & & \multirow{2}{*}{ CS } & MPGGE Unadjusted & 35.3 & 35.3 & 42.0 & 49.6 & 42.3 & 49.0 & 56.4 & 45.5 & 54.7 & 65.4 & 47.7 & 56.4 & 68.5 \\
\hline & & & MPGGE Adjusted & 26.8 & 26.8 & 31.5 & 36.6 & 31.7 & 36.3 & 41.2 & 33.9 & 40.1 & 47.0 & 35.4 & 41.2 & 48.9 \\
\hline & & \multirow{3}{*}{$C D+C S$} & Wh/mile & 177.2 & 177.2 & 154.7 & 137.7 & 160.8 & 139.2 & 123.0 & 150.0 & 126.9 & 105.9 & 143.2 & 122.9 & 101.3 \\
\hline & & & MPGGE Unadjusted & 51.8 & 51.8 & 61.7 & 72.9 & 62.3 & 72.1 & 82.9 & 66.9 & 80.5 & 96.2 & 70.1 & 83.0 & 100.7 \\
\hline & & & MPGGE Adjusted & 38.2 & 38.2 & 44.6 & 51.6 & 45.0 & 51.1 & 57.7 & 47.9 & 56.2 & 65.4 & 49.9 & 57.7 & 67.9 \\
\hline & \multirow{9}{*}{ HWFET } & \multirow{4}{*}{$C D$} & Wh/mile & 538.3 & 538.3 & 488.8 & 444.0 & 501.6 & 448.9 & 404.1 & 478.3 & 416.3 & 373.3 & 461.8 & 405.1 & 359.6 \\
\hline & & & Distance & 19.5 & 19.5 & 18.5 & 18.5 & 18.5 & 18.5 & 16.3 & 18.5 & 18.5 & 17.1 & 18.5 & 16.1 & 17.1 \\
\hline & & & MPGGE Unadjusted & N/A & N/A & N/A & N/A & N/A & $\mathrm{N} / \mathrm{A}$ & $\mathrm{N} / \mathrm{A}$ & N/A & N/A & N/A & N/A & N/A & N/A \\
\hline & & & MPGGE Adjusted & $\mathrm{N} / \mathrm{A}$ & N/A & N/A & $\mathrm{N} / \mathrm{A}$ & N/A & N/A & N/A & $\mathrm{N} / \mathrm{A}$ & N/A & N/A & $\mathrm{N} / \mathrm{A}$ & $\mathrm{N} / \mathrm{A}$ & N/A \\
\hline & & \multirow{2}{*}{ CS } & MPGGE Unadjusted & 34.6 & 34.6 & 39.6 & 45.9 & 40.6 & 45.4 & 50.5 & 42.6 & 49.1 & 55.2 & 44.2 & 50.5 & 57.3 \\
\hline & & & MPGGE Adjusted & 24.5 & 24.5 & 28.0 & 32.4 & 28.7 & 32.0 & 35.6 & 30.1 & 34.6 & 38.9 & 31.2 & 35.6 & 40.4 \\
\hline & & \multirow{3}{*}{$C D+C S$} & Wh/mile & 158.3 & 158.3 & 143.8 & 130.6 & 147.5 & 132.0 & 118.8 & 140.7 & 122.4 & 109.8 & 135.8 & 119.2 & 105.8 \\
\hline & & & MPGGE Unadjusted & 45.5 & 45.5 & 52.1 & 60.4 & 53.4 & 59.7 & 66.5 & 56.1 & 64.5 & 72.6 & 58.1 & 66.4 & 75.5 \\
\hline & & & MPGGE Adjusted & 32.1 & 32.1 & 36.7 & 42.5 & 37.6 & 42.0 & 46.7 & 39.5 & 45.4 & 50.9 & 40.9 & 46.6 & 52.9 \\
\hline & \multirow{7}{*}{ Combined } & \multirow{2}{*}{$C D$} & MPGGE Unadjusted & $\mathrm{N} / \mathrm{A}$ & $\mathrm{N} / \mathrm{A}$ & $\mathrm{N} / \mathrm{A}$ & N/A & N/A & $\mathrm{N} / \mathrm{A}$ & $\mathrm{N} / \mathrm{A}$ & N/A & $\mathrm{N} / \mathrm{A}$ & N/A & N/A & $\mathrm{N} / \mathrm{A}$ & N/A \\
\hline & & & MPGGE Adjusted & $\mathrm{N} / \mathrm{A}$ & N/A & N/A & N/A & N/A & N/A & N/A & N/A & N/A & N/A & N/A & N/A & N/A \\
\hline & & \multirow{2}{*}{ CS } & MPGGE Unadjusted & 35.0 & 35.0 & 40.9 & 47.8 & 41.5 & 47.3 & 53.6 & 44.2 & 52.0 & 60.4 & 46.0 & 53.6 & 63.0 \\
\hline & & & MPGGE Adjusted & 25.7 & 25.7 & 29.8 & 34.6 & 30.3 & 34.2 & 38.5 & 32.1 & 37.4 & 42.9 & 33.4 & 38.5 & 44.6 \\
\hline & & \multirow{3}{*}{$C D+C S$} & Wh/mile & 168.7 & 168.7 & 149.8 & 134.5 & 154.8 & 136.0 & 121.1 & 145.8 & 124.9 & 107.7 & 139.9 & 121.2 & 103.3 \\
\hline & & & MPGGE Unadjusted & 48.8 & 48.8 & 57.0 & 66.7 & 57.9 & 65.9 & 74.6 & 61.6 & 72.4 & 83.9 & 64.1 & 74.6 & 87.5 \\
\hline & & & \begin{tabular}{|l|} 
MPGGE Adjusted \\
\end{tabular} & 35.2 & 35.2 & 40.7 & 47.1 & 41.3 & 46.6 & 52.2 & 43.7 & 50.8 & 58.0 & 45.4 & 52.1 & 60.2 \\
\hline
\end{tabular}


Argonne National Laboratory Report - Light-Duty Vehicle Fuel Consumption Displacement Potential Up to 2045

\begin{tabular}{|c|c|c|c|c|c|c|c|c|c|c|c|c|c|c|c|c|}
\hline & & & & \multicolumn{13}{|c|}{ Pickup } \\
\hline & & & & \multirow{2}{*}{$\begin{array}{c}2010 \\
\text { Ref }\end{array}$} & \multicolumn{3}{|c|}{2010} & \multicolumn{3}{|c|}{2015} & \multicolumn{3}{|c|}{2030} & \multicolumn{3}{|c|}{2045} \\
\hline & & & & & low & avg & high & low & avg & high & low & avg & high & low & avg & high \\
\hline \multirow{25}{*}{ FC HEV PHEV30 } & \multirow{9}{*}{ UDDS } & \multirow{4}{*}{$C D$} & Wh/mile & 449.0 & 449.0 & 396.1 & 360.0 & 412.0 & 361.0 & 315.5 & 388.5 & 325.7 & 277.9 & 370.7 & 314.7 & 265.6 \\
\hline & & & Distance & 33.6 & 33.6 & 33.6 & 32.0 & 33.6 & 33.6 & 32.0 & 33.6 & 32.0 & 31.9 & 33.6 & 32.0 & 31.9 \\
\hline & & & MPGGE Unadjusted & $\mathrm{N} / \mathrm{A}$ & $\mathrm{N} / \mathrm{A}$ & N/A & N/A & N/A & $\mathrm{N} / \mathrm{A}$ & N/A & N/A & N/A & N/A & N/A & N/A & $\mathrm{N} / \mathrm{A}$ \\
\hline & & & MPGGE Adjusted & $\mathrm{N} / \mathrm{A}$ & $\mathrm{N} / \mathrm{A}$ & $\mathrm{N} / \mathrm{A}$ & N/A & N/A & N/A & N/A & $\mathrm{N} / \mathrm{A}$ & N/A & N/A & N/A & N/A & $\mathrm{N} / \mathrm{A}$ \\
\hline & & \multirow{2}{*}{ CS } & MPGGE Unadjusted & 35.1 & 35.1 & 41.3 & 47.8 & 41.7 & 47.7 & 55.1 & 44.4 & 53.4 & 63.3 & 46.5 & 55.2 & 66.2 \\
\hline & & & MPGGE Adjusted & 26.7 & 26.7 & 31.1 & 35.5 & 31.3 & 35.4 & 40.3 & 33.1 & 39.2 & 45.6 & 34.6 & 40.4 & 47.5 \\
\hline & & \multirow{3}{*}{$\mathrm{CD}+\mathrm{CS}$} & Wh/mile & 286.1 & 286.1 & 252.4 & 229.3 & 262.5 & 230.0 & 201.6 & 247.5 & 208.1 & 178.2 & 236.2 & 201.0 & 170.3 \\
\hline & & & MPGGE Unadjusted & 74.8 & 74.8 & 88.0 & 101.8 & 88.8 & 101.5 & 117.2 & 994.4 & 113.5 & 134.7 & 98.9 & 117.5 & 140.9 \\
\hline & & & MPGGE Adjusted & 52.8 & 52.8 & 60.7 & 68.5 & 61.1 & 68.4 & 76.8 & 64.4 & 74.9 & 85.8 & 66.9 & 77.0 & 88.9 \\
\hline & \multirow{9}{*}{ HWFET } & \multirow{4}{*}{$C D$} & Wh/mile & 513.3 & 513.3 & 467.6 & 430.1 & 480.5 & 432.3 & 388.7 & 460.9 & 400.6 & 358.3 & 444.2 & 389.1 & 344.5 \\
\hline & & & Distance & 28.8 & 28.8 & 28.8 & 28.8 & 28.8 & 28.8 & 25.2 & 28.8 & 25.2 & 25.8 & 28.8 & 25.0 & 25.3 \\
\hline & & & MPGGE Unadjusted & $\mathrm{N} / \mathrm{A}$ & $\mathrm{N} / \mathrm{A}$ & $\mathrm{N} / \mathrm{A}$ & $\mathrm{N} / \mathrm{A}$ & N/A & $\mathrm{N} / \mathrm{A}$ & N/A & N/A & N/A & $\mathrm{N} / \mathrm{A}$ & $\mathrm{N} / \mathrm{A}$ & $\mathrm{N} / \mathrm{A}$ & $\mathrm{N} / \mathrm{A}$ \\
\hline & & & MPGGE Adjusted & N/A & N/A & N/A & N/A & N/A & N/A & N/A & N/A & N/A & N/A & N/A & N/A & $\mathrm{N} / \mathrm{A}$ \\
\hline & & \multirow{2}{*}{ CS } & MPGGE Unadjusted & 33.9 & 33.9 & 38.6 & 44.2 & 39.5 & 43.9 & 49.1 & 41.2 & 47.6 & 53.5 & 42.8 & 49.0 & 55.6 \\
\hline & & & MPGGE Adjusted & 24.0 & 24.0 & 27.3 & 31.2 & 27.9 & 31.0 & 34.6 & 29.1 & 33.6 & 37.7 & 30.2 & 34.6 & 39.1 \\
\hline & & \multirow{3}{*}{$\mathrm{CD}+\mathrm{CS}$} & Wh/mile & 264.2 & 264.2 & 240.6 & 221.3 & 247.3 & 222.5 & 200.1 & 237.2 & 206.2 & 184.6 & 228.6 & 200.3 & 177.4 \\
\hline & & & MPGGE Unadjusted & 57.5 & 57.5 & 65.4 & 74.9 & 67.0 & 74.5 & 83.2 & 69.9 & 80.7 & 90.6 & 72.5 & 83.1 & 94.2 \\
\hline & & & MPGGE Adjusted & 40.5 & 40.5 & 46.0 & 52.5 & 47.0 & 52.2 & 58.2 & 49.1 & 56.5 & 63.3 & 50.8 & 58.2 & 65.8 \\
\hline & \multirow{7}{*}{ Combined } & \multirow{2}{*}{$C D$} & MPGGE Unadjusted & $\mathrm{N} / \mathrm{A}$ & $\mathrm{N} / \mathrm{A}$ & N/A & N/A & N/A & $\mathrm{N} / \mathrm{A}$ & N/A & $\mathrm{N} / \mathrm{A}$ & $\mathrm{N} / \mathrm{A}$ & N/A & $\mathrm{N} / \mathrm{A}$ & N/A & $\mathrm{N} / \mathrm{A}$ \\
\hline & & & MPGGE Adjusted & N/A & N/A & N/A & N/A & $\mathrm{N} / \mathrm{A}$ & N/A & N/A & $\mathrm{N} / \mathrm{A}$ & N/A & N/A & N/A & N/A & N/A \\
\hline & & \multirow{2}{*}{ CS } & MPGGE Unadjusted & 34.6 & 34.6 & 40.1 & 46.1 & 40.7 & 45.9 & 52.2 & 42.9 & 50.6 & 58.5 & 44.7 & 52.3 & 61.0 \\
\hline & & & MPGGE Adjusted & 25.4 & 25.4 & 29.2 & 33.4 & 29.7 & 33.3 & 37.5 & 31.2 & 36.4 & 41.7 & 32.4 & 37.6 & 43.3 \\
\hline & & \multirow{3}{*}{$C D+C S$} & Wh/mile & 276.2 & 276.2 & 247.1 & 225.7 & 255.6 & 226.6 & 200.9 & 242.8 & 207.3 & 181.1 & 232.8 & 200.7 & 173.5 \\
\hline & & & MPGGE Unadjusted & 65.9 & 65.9 & 76.2 & 87.6 & 77.4 & 87.2 & 99.0 & 81.5 & 96.0 & 110.5 & 84.9 & 99.1 & 115.2 \\
\hline & & & MPGGE Adjusted & 46.4 & 46.4 & 53.0 & 60.2 & 53.9 & 60.0 & 67.2 & 56.4 & 65.3 & 74.0 & 58.6 & 67.2 & 76.8 \\
\hline
\end{tabular}




\begin{tabular}{|c|c|c|c|c|c|c|c|c|c|c|c|c|c|c|c|c|}
\hline & & & & \multicolumn{13}{|c|}{ Pickup } \\
\hline & & & & 2010 & & 2010 & & & 2015 & & & 2030 & & & 2045 & \\
\hline \multirow{26}{*}{ FC HEV PHEV40 } & & \multirow{5}{*}{$C D$} & & Ref & low & avg & high & low & avg & high & low & avg & high & low & avg & high \\
\hline & \multirow{9}{*}{ UDDS } & & Wh/mile & 458.8 & 458.8 & 403.6 & 366.0 & 419.4 & 366.8 & 319.2 & 395.0 & 329.4 & 282.9 & 376.7 & 317.5 & 270.1 \\
\hline & & & Distance & 42.2 & 42.2 & 41.3 & 41.3 & 41.4 & 41.3 & 41.0 & 41.3 & 41.0 & 40.6 & 41.3 & 40.9 & 40.5 \\
\hline & & & MPGGE Unadjusted & $\mathrm{N} / \mathrm{A}$ & N/A & N/A & N/A & $N / A$ & N/A & N/A & N/A & N/A & N/A & N/A & N/A & N/A \\
\hline & & & MPGGE Adjusted & $\mathrm{N} / \mathrm{A}$ & $\mathrm{N} / \mathrm{A}$ & N/A & N/A & N/A & N/A & N/A & $\mathrm{N} / \mathrm{A}$ & N/A & N/A & $\mathrm{N} / \mathrm{A}$ & N/A & N/A \\
\hline & & \multirow{2}{*}{ CS } & MPGGE Unadjusted & 34.4 & 34.4 & 40.6 & 47.0 & 41.0 & 46.9 & 54.3 & 43.6 & 52.6 & 62.6 & 45.7 & 54.6 & 65.5 \\
\hline & & & MPGGE Adjusted & 26.2 & 26.2 & 30.5 & 34.9 & 30.8 & 34.9 & 39.8 & 32.6 & 38.7 & 45.2 & 34.0 & 40.0 & 47.0 \\
\hline & & \multirow{3}{*}{$\mathrm{CD}+\mathrm{CS}$} & Wh/mile & 331.6 & 331.6 & 291.8 & 264.6 & 303.2 & 265.1 & 231.4 & 285.5 & 238.9 & 207.0 & 272.3 & 230.2 & 197.6 \\
\hline & & & MPGGE Unadjusted & 85.9 & 85.9 & 101.4 & 117.6 & 102.4 & 117.3 & 135.8 & 109.0 & 131.6 & 156.4 & 114.3 & 136.5 & 163.8 \\
\hline & & & MPGGE Adjusted & 59.5 & 59.5 & 68.3 & 77.1 & 68.9 & 76.9 & 86.4 & 72.5 & 84.3 & 96.3 & 75.3 & 86.7 & 99.7 \\
\hline & \multirow{9}{*}{ HWFET } & \multirow{4}{*}{$C D$} & Wh/mile & 520.8 & 520.8 & 473.3 & 434.7 & 486.1 & 436.8 & 392.2 & 465.8 & 404.1 & 360.0 & 448.8 & 391.9 & 346.0 \\
\hline & & & Distance & 38.6 & 38.6 & 39.0 & 39.0 & 39.0 & 39.0 & 36.0 & 39.0 & 36.0 & 32.9 & 39.0 & 33.2 & 32.0 \\
\hline & & & MPGGE Unadjusted & $\mathrm{N} / \mathrm{A}$ & $\mathrm{N} / \mathrm{A}$ & $\mathrm{N} / \mathrm{A}$ & $\mathrm{N} / \mathrm{A}$ & $\mathrm{N} / \mathrm{A}$ & N/A & N/A & $\mathrm{N} / \mathrm{A}$ & $\mathrm{N} / \mathrm{A}$ & $\mathrm{N} / \mathrm{A}$ & N/A & N/A & $\mathrm{N} / \mathrm{A}$ \\
\hline & & & MPGGE Adjusted & $\mathrm{N} / \mathrm{A}$ & $\mathrm{N} / \mathrm{A}$ & $\mathrm{N} / \mathrm{A}$ & $\mathrm{N} / \mathrm{A}$ & $\mathrm{N} / \mathrm{A}$ & $\mathrm{N} / \mathrm{A}$ & $\mathrm{N} / \mathrm{A}$ & $\mathrm{N} / \mathrm{A}$ & $\mathrm{N} / \mathrm{A}$ & $\mathrm{N} / \mathrm{A}$ & $\mathrm{N} / \mathrm{A}$ & $\mathrm{N} / \mathrm{A}$ & $\mathrm{N} / \mathrm{A}$ \\
\hline & & \multirow{2}{*}{ CS } & MPGGE Unadjusted & 33.5 & 33.5 & 38.1 & 43.7 & 39.1 & 43.5 & 48.7 & 40.8 & 47.2 & 53.1 & 42.3 & 48.7 & 55.3 \\
\hline & & & MPGGE Adjusted & 23.7 & 23.7 & 27.0 & 30.9 & 27.6 & 30.7 & 34.3 & 28.8 & 33.3 & 37.4 & 29.9 & 34.4 & 38.9 \\
\hline & & \multirow{3}{*}{$\mathrm{CD}+\mathrm{CS}$} & Wh/mile & 350.8 & 350.8 & 318.9 & 292.9 & 327.5 & 294.3 & 264.4 & 313.8 & 272.5 & 242.8 & 302.3 & 264.1 & 233.4 \\
\hline & & & MPGGE Unadjusted & 72.7 & 72.7 & 82.9 & 95.0 & 84.9 & 94.6 & 105.8 & 88.7 & 102.7 & 115.5 & 92.1 & 105.9 & 120.2 \\
\hline & & & MPGGE Adjusted & 51.0 & 51.0 & 58.0 & 66.3 & 59.4 & 66.0 & 73.7 & 62.0 & 71.6 & 80.3 & 64.3 & 73.8 & 83.5 \\
\hline & \multirow{7}{*}{ Combined } & \multirow{2}{*}{$C D$} & MPGGE Unadjusted & $\mathrm{N} / \mathrm{A}$ & $\mathrm{N} / \mathrm{A}$ & N/A & $\mathrm{N} / \mathrm{A}$ & $\mathrm{N} / \mathrm{A}$ & N/A & $\mathrm{N} / \mathrm{A}$ & $\mathrm{N} / \mathrm{A}$ & N/A & N/A & $\mathrm{N} / \mathrm{A}$ & $\mathrm{N} / \mathrm{A}$ & N/A \\
\hline & & & MPGGE Adjusted & $\mathrm{N} / \mathrm{A}$ & N/A & $\mathrm{N} / \mathrm{A}$ & $\mathrm{N} / \mathrm{A}$ & $\mathrm{N} / \mathrm{A}$ & $\mathrm{N} / \mathrm{A}$ & N/A & $\mathrm{N} / \mathrm{A}$ & N/A & N/A & $\mathrm{N} / \mathrm{A}$ & N/A & N/A \\
\hline & & \multirow{2}{*}{ CS } & MPGGE Unadjusted & 33.9 & 33.9 & 39.4 & 45.5 & 40.1 & 45.3 & 51.6 & 42.3 & 50.1 & 57.9 & 44.1 & 51.8 & 60.5 \\
\hline & & & MPGGE Adjusted & 25.0 & 25.0 & 28.8 & 33.0 & 29.3 & 32.9 & 37.1 & 30.8 & 36.1 & 41.3 & 32.0 & 37.2 & 43.0 \\
\hline & & \multirow{3}{*}{$C D+C S$} & Wh/mile & 340.3 & 340.3 & 304.0 & 277.3 & 314.1 & 278.2 & 246.3 & 298.2 & 254.0 & 223.1 & 285.8 & 245.5 & 213.7 \\
\hline & & & MPGGE Unadjusted & 79.4 & 79.4 & 92.1 & 106.2 & 93.7 & 105.8 & 120.4 & 98.8 & 116.8 & 134.9 & 103.1 & 120.8 & 140.8 \\
\hline & & & MPGGE Adjusted & 55.3 & 55.3 & 63.3 & 71.8 & 64.3 & 71.6 & 80.2 & 67.4 & 78.1 & 88.4 & 69.9 & 80.4 & 91.7 \\
\hline \multirow{2}{*}{ EV } & UDDS & EV Only & Wh/mile & 456.0 & 456.0 & 424.2 & 391.2 & 436.6 & 395.6 & 333.7 & 418.4 & 343.3 & 286.2 & 407.9 & 329.4 & 272.4 \\
\hline & HWFET & EV Only & Wh/mile & 507.4 & 507.4 & 478.6 & 444.7 & 488.6 & 449.1 & 395.8 & 473.6 & 407.3 & 358.3 & 462.2 & 393.5 & 343.5 \\
\hline
\end{tabular}

\section{Fuel consumption}




\begin{tabular}{|c|c|c|c|c|c|c|c|c|c|c|c|c|c|c|c|}
\hline & & & \multicolumn{13}{|c|}{ Pickup } \\
\hline & & & 2010 & & 2010 & & & 2015 & & & 2030 & & & 2045 & \\
\hline & & & Ref & low & avg & high & low & avg & high & low & avg & high & low & avg & high \\
\hline \multirow{6}{*}{ SI Conv } & \multirow{3}{*}{ Unadjusted } & UDDS & 12.7 & 12.7 & 11.8 & 11.0 & 12.0 & 11.2 & 8.8 & 11.8 & 10.5 & 7.3 & 11.8 & 10.3 & 7.0 \\
\hline & & \begin{tabular}{ll|} 
HWFET \\
\end{tabular} & 9.8 & 9.8 & 9.1 & 8.4 & 9.3 & 8.6 & 6.8 & 9.1 & 8.1 & 5.8 & 8.9 & 8.0 & 5.6 \\
\hline & & Combined & 11.4 & 11.4 & 10.6 & 9.9 & 10.8 & 10.0 & 7.9 & 10.6 & 9.4 & 6.7 & 10.5 & 9.3 & 6.4 \\
\hline & \multirow{3}{*}{ Adjusted } & \begin{tabular}{|l|} 
UDDS \\
\end{tabular} & 16.2 & 16.2 & 15.0 & 14.1 & 15.4 & 14.3 & 11.3 & 15.1 & 13.4 & 9.6 & 15.0 & 13.3 & 9.2 \\
\hline & & HWFET & 13.9 & 13.9 & 12.8 & 11.9 & 13.1 & 12.2 & 9.6 & 12.8 & 11.4 & 8.3 & 12.6 & 11.3 & 7.9 \\
\hline & & Combined & 15.2 & 15.2 & 14.0 & 13.1 & 14.3 & 13.3 & 10.5 & 14.1 & 12.5 & 9.0 & 13.9 & 12.4 & 8.6 \\
\hline \multirow{6}{*}{$\mathrm{Cl}$ Conv } & \multirow{3}{*}{ Unadjusted } & \begin{tabular}{|l|} 
UDDS \\
\end{tabular} & 10.5 & 10.5 & 9.9 & 9.2 & 10.2 & 9.2 & 8.3 & 9.7 & 8.5 & 7.2 & 9.5 & 8.2 & 6.9 \\
\hline & & HWFET & 8.5 & 8.5 & 8.0 & 7.6 & 8.2 & 7.4 & 6.9 & 7.8 & 7.1 & 6.2 & 7.8 & 6.8 & 5.9 \\
\hline & & Combined & 9.6 & 9.6 & 9.0 & 8.5 & 9.3 & 8.4 & 7.7 & 8.8 & 7.9 & 6.7 & 8.7 & 7.6 & 6.5 \\
\hline & \multirow{3}{*}{ Adjusted } & UDDS & 13.4 & 13.4 & 12.7 & 11.8 & 13.1 & 11.9 & 10.8 & 12.5 & 11.0 & 9.4 & 12.2 & 10.7 & 9.0 \\
\hline & & \begin{tabular}{|l|} 
HWFET \\
\end{tabular} & 11.9 & 11.9 & 11.3 & 10.7 & 11.6 & 10.5 & 9.8 & 11.0 & 10.0 & 8.7 & 11.0 & 9.7 & 8.4 \\
\hline & & Combined & 12.8 & 12.8 & 12.1 & 11.3 & 12.4 & 11.3 & 10.3 & 11.8 & 10.6 & 9.1 & 11.7 & 10.2 & 8.8 \\
\hline \multirow{6}{*}{$\mathrm{H} 2$ Conv } & \multirow{3}{*}{ Unadjusted } & \begin{tabular}{|l|} 
UDDS \\
\end{tabular} & 14.7 & 14.7 & 12.4 & 11.0 & 13.0 & 11.0 & 9.8 & 11.8 & 10.1 & 8.2 & 11.3 & 9.5 & 7.6 \\
\hline & & \begin{tabular}{|l|} 
HWFET \\
\end{tabular} & 10.3 & 10.3 & 8.7 & 7.7 & 9.0 & 7.7 & 6.9 & 8.2 & 7.1 & 6.0 & 7.8 & 6.7 & 5.6 \\
\hline & & Combined & 12.7 & 12.7 & 10.7 & 9.5 & 11.2 & 9.5 & 8.5 & 10.2 & 8.7 & 7.2 & 9.7 & 8.2 & 6.7 \\
\hline & \multirow{3}{*}{ Adjusted } & \begin{tabular}{|l|} 
UDDS \\
\end{tabular} & 18.7 & 18.7 & 15.8 & 14.1 & 16.5 & 14.1 & 12.6 & 15.1 & 12.9 & 10.7 & 14.4 & 12.2 & 9.9 \\
\hline & & \begin{tabular}{|l|} 
HWFET \\
\end{tabular} & 14.5 & 14.5 & 12.3 & 10.9 & 12.7 & 10.9 & 9.7 & 11.6 & 10.0 & 8.5 & 11.0 & 9.4 & 7.9 \\
\hline & & Combined & 16.8 & 16.8 & 14.2 & 12.7 & 14.8 & 12.6 & 11.3 & 13.5 & 11.6 & 9.7 & 12.9 & 11.0 & 9.0 \\
\hline \multirow{6}{*}{ E85 Conv } & \multirow{3}{*}{ Unadjusted } & \begin{tabular}{|l|} 
UDDS \\
\end{tabular} & 13.3 & 13.3 & 13.1 & 12.3 & 13.5 & 12.5 & 9.3 & 13.2 & 11.7 & 7.9 & 13.1 & 11.6 & 7.6 \\
\hline & & HWFET & 10.8 & 10.8 & 10.7 & 9.6 & 10.9 & 10.2 & 7.3 & 10.7 & 9.2 & 6.4 & 10.2 & 9.1 & 6.2 \\
\hline & & \begin{tabular}{|l|} 
Combined \\
\end{tabular} & 12.2 & 12.2 & 12.0 & 11.1 & 12.3 & 11.4 & 8.4 & 12.1 & 10.6 & 7.2 & 11.8 & 10.5 & 7.0 \\
\hline & \multirow{3}{*}{ Adjusted } & \begin{tabular}{|l|} 
UDDS \\
\end{tabular} & 16.9 & 16.9 & 16.6 & 15.6 & 17.1 & 15.9 & 12.0 & 16.8 & 15.0 & 10.3 & 16.7 & 14.8 & 9.9 \\
\hline & & \begin{tabular}{|l|} 
HWFET \\
\end{tabular} & 15.3 & 15.3 & 15.1 & 13.5 & 15.4 & 14.3 & 10.3 & 15.1 & 13.0 & 9.1 & 14.3 & 12.9 & 8.8 \\
\hline & & Combined & 16.2 & 16.2 & 15.9 & 14.7 & 16.3 & 15.2 & 11.3 & 16.1 & 14.1 & 9.8 & 15.6 & 13.9 & 9.4 \\
\hline \multirow{6}{*}{ SI Split HEV } & \multirow{3}{*}{ Unadjusted } & \begin{tabular}{|l|} 
UDDS \\
\end{tabular} & 7.4 & 7.4 & 6.9 & 6.3 & 7.1 & 6.3 & 5.2 & 6.7 & 5.7 & 4.3 & 6.5 & 5.5 & 4.1 \\
\hline & & HWFET & 8.4 & 8.4 & 7.9 & 7.3 & 8.1 & 7.4 & 6.2 & 7.8 & 6.8 & 5.3 & 7.6 & 6.6 & 5.1 \\
\hline & & \begin{tabular}{|l|} 
Combined \\
\end{tabular} & 7.8 & 7.8 & 7.3 & 6.8 & 7.5 & 6.8 & 5.6 & 7.2 & 6.2 & 4.7 & 7.0 & 6.0 & 4.5 \\
\hline & \multirow{3}{*}{ Adjusted } & \begin{tabular}{|l|} 
UDDS \\
\end{tabular} & 9.6 & 9.6 & 9.0 & 8.4 & 9.3 & 8.3 & 6.9 & 8.8 & 7.6 & 5.8 & 8.6 & 7.4 & 5.6 \\
\hline & & HWFET & 11.9 & 11.9 & 11.2 & 10.3 & 11.4 & 10.4 & 8.7 & 11.0 & 9.7 & 7.5 & 10.7 & 9.3 & 7.2 \\
\hline & & \begin{tabular}{|l|} 
Combined \\
\end{tabular} & 10.7 & 10.7 & 10.0 & 9.2 & 10.3 & 9.3 & 7.7 & 9.8 & 8.6 & 6.6 & 9.5 & 8.3 & 6.3 \\
\hline \multirow{6}{*}{ SI Split HEV PHEV10 } & \multirow{3}{*}{ Unadjusted } & \begin{tabular}{|l|} 
UDDS \\
\end{tabular} & 5.8 & 5.8 & 5.4 & 5.0 & 5.6 & 5.1 & 4.2 & 5.4 & 4.7 & 3.5 & 5.2 & 4.5 & 3.4 \\
\hline & & HWFET & 6.4 & 6.4 & 6.0 & 5.6 & 6.2 & 5.7 & 4.8 & 6.0 & 5.3 & 4.2 & 5.8 & 5.2 & 3.9 \\
\hline & & Combined & 6.1 & 6.1 & 5.7 & 5.3 & 5.9 & 5.4 & 4.5 & 5.6 & 5.0 & 3.8 & 5.5 & 4.8 & 3.6 \\
\hline & \multirow{3}{*}{ Adjusted } & \begin{tabular}{|l|} 
UDDS \\
\end{tabular} & 7.8 & 7.8 & 7.3 & 6.8 & 7.5 & 6.8 & 5.8 & 7.2 & 6.3 & 4.9 & 7.0 & 6.2 & 4.7 \\
\hline & & HWFET & 9.1 & 9.1 & 8.6 & 7.9 & 8.8 & 8.1 & 6.8 & 8.5 & 7.6 & 5.9 & 8.2 & 7.3 & 5.5 \\
\hline & & \begin{tabular}{|l|} 
Combined \\
\end{tabular} & 8.4 & 8.4 & 7.9 & 7.3 & 8.1 & 7.4 & 6.3 & 7.8 & 6.9 & 5.3 & 7.6 & 6.7 & 5.1 \\
\hline
\end{tabular}




\begin{tabular}{|c|c|c|c|c|c|c|c|c|c|c|c|c|c|c|c|}
\hline & & & \multicolumn{13}{|c|}{ Pickup } \\
\hline & & & \multirow{2}{*}{$\begin{array}{l}2010 \\
\text { Ref }\end{array}$} & \multicolumn{3}{|c|}{2010} & \multicolumn{3}{|c|}{2015} & \multicolumn{3}{|c|}{2030} & \multicolumn{3}{|c|}{2045} \\
\hline & & & & low & avg & high & low & avg & high & low & avg & high & low & avg & high \\
\hline \multirow{6}{*}{ SI Split HEV PHEV2O } & \multirow{3}{*}{ Unadjusted } & UDDS & 4.0 & 4.0 & 3.8 & 3.5 & 3.9 & 3.5 & 2.9 & 3.7 & 3.2 & 2.5 & 3.6 & 3.1 & 2.3 \\
\hline & & \begin{tabular}{ll|} 
HWFET \\
\end{tabular} & 5.3 & 5.3 & 5.0 & 4.6 & 5.1 & 4.7 & 4.9 & 4.9 & 5.4 & 4.2 & 4.8 & 5.2 & 4.1 \\
\hline & & Combined & 4.6 & 4.6 & 4.3 & 4.0 & 4.4 & 4.0 & 3.8 & 4.3 & 4.2 & 3.2 & 4.1 & 4.1 & 3.1 \\
\hline & \multirow{3}{*}{ Adjusted } & \begin{tabular}{|l|} 
UDDS \\
\end{tabular} & 5.6 & 5.6 & 5.2 & 4.9 & 5.3 & 4.9 & 4.2 & 5.2 & 4.6 & 3.6 & 5.1 & 4.5 & 3.5 \\
\hline & & HWFET & 7.5 & 7.5 & 7.1 & 6.6 & 7.2 & 6.6 & 6.9 & 7.0 & 7.6 & 6.0 & 6.8 & 7.4 & 5.8 \\
\hline & & \begin{tabular}{|c|} 
Combined \\
\end{tabular} & 6.4 & 6.4 & 6.1 & 5.6 & 6.2 & 5.7 & 5.4 & 6.0 & 5.9 & 4.7 & 5.8 & 5.8 & 4.5 \\
\hline \multirow{6}{*}{ SI Series HEV PHEV3O } & \multirow{3}{*}{ Unadjusted } & \begin{tabular}{|l|} 
UDDS \\
\end{tabular} & 4.6 & 4.6 & 4.2 & 3.8 & 4.4 & 3.9 & 3.1 & 4.1 & 3.4 & 2.5 & 4.0 & 3.3 & 2.3 \\
\hline & & HWFET & 6.2 & 6.2 & 5.8 & 5.3 & 5.9 & 5.3 & 4.4 & 5.6 & 4.8 & 3.7 & 5.4 & 4.6 & 3.5 \\
\hline & & Combined & 5.3 & 5.3 & 4.9 & 4.5 & 5.1 & 4.5 & 3.6 & 4.8 & 4.1 & 3.0 & 4.6 & 3.9 & 2.9 \\
\hline & \multirow{3}{*}{ Adjusted } & UDDS & 6.2 & 6.2 & 5.8 & 5.3 & 6.0 & 5.4 & 4.4 & 5.6 & 4.8 & 3.6 & 5.5 & 4.6 & 3.4 \\
\hline & & \begin{tabular}{|l|} 
HWFET \\
\end{tabular} & 8.8 & 8.8 & 8.2 & 7.5 & 8.4 & 7.5 & 6.2 & 7.9 & 6.9 & 5.2 & 7.7 & 6.6 & 5.0 \\
\hline & & Combined & 7.4 & 7.4 & 6.8 & 6.3 & 7.0 & 6.3 & 5.2 & 6.7 & 5.7 & 4.3 & 6.5 & 5.5 & 4.2 \\
\hline \multirow{6}{*}{ SI Series HEV PHEV40 } & \multirow{3}{*}{ Unadjusted } & \begin{tabular}{|l|} 
UDDS \\
\end{tabular} & 4.0 & 4.0 & 3.7 & 3.3 & 3.8 & 3.4 & 2.7 & 3.6 & 3.0 & 2.1 & 3.5 & 2.9 & 2.0 \\
\hline & & \begin{tabular}{|l|} 
HWFET \\
\end{tabular} & 4.9 & 4.9 & 4.6 & 4.2 & 4.7 & 4.2 & 3.4 & 4.4 & 3.8 & 2.9 & 4.3 & 3.7 & 2.8 \\
\hline & & Combined & 4.4 & 4.4 & 4.1 & 3.7 & 4.2 & 3.7 & 3.0 & 4.0 & 3.3 & 2.5 & 3.8 & 3.2 & 2.4 \\
\hline & \multirow{3}{*}{ Adjusted } & \begin{tabular}{|l|} 
UDDS \\
\end{tabular} & 5.5 & 5.5 & 5.1 & 4.7 & 5.3 & 4.7 & 3.9 & 5.0 & 4.2 & 3.2 & 4.8 & 4.1 & 3.1 \\
\hline & & HWFET & 7.0 & 7.0 & 6.5 & 5.9 & 6.6 & 6.0 & 4.9 & 6.3 & 5.4 & 4.1 & 6.1 & 5.2 & 4.0 \\
\hline & & Combined & 6.2 & 6.2 & 5.7 & 5.2 & 5.9 & 5.3 & 4.3 & 5.6 & 4.8 & 3.6 & 5.4 & 4.6 & 3.5 \\
\hline \multirow{6}{*}{ CI Split HEV } & \multirow{3}{*}{ Unadjusted } & \begin{tabular}{|l|} 
UDDS \\
\end{tabular} & 6.5 & 6.5 & 6.2 & 5.6 & 6.3 & 5.6 & 4.9 & 5.9 & 5.0 & 4.2 & 5.7 & 4.8 & 4.0 \\
\hline & & HWFET & 7.9 & 7.9 & 7.5 & 6.8 & 7.7 & 6.9 & 6.1 & 7.2 & 6.3 & 5.4 & 7.0 & 6.0 & 5.2 \\
\hline & & Combined & 7.1 & 7.1 & 6.7 & 6.1 & 6.9 & 6.2 & 5.5 & 6.5 & 5.6 & 4.7 & 6.3 & 5.4 & 4.5 \\
\hline & \multirow{3}{*}{ Adjusted } & \begin{tabular}{|l|} 
UDDS \\
\end{tabular} & 8.6 & 8.6 & 8.1 & 7.5 & 8.4 & 7.5 & 6.6 & 7.9 & 6.8 & 5.7 & 7.6 & 6.5 & 5.5 \\
\hline & & HWFET & 11.1 & 11.1 & 10.5 & 9.6 & 10.8 & 9.7 & 8.7 & 10.2 & 8.9 & 7.6 & 9.8 & 8.5 & 7.3 \\
\hline & & \begin{tabular}{|l|} 
Combined \\
\end{tabular} & 9.8 & 9.8 & 9.2 & 8.4 & 9.5 & 8.5 & 7.5 & 8.9 & 7.7 & 6.6 & 8.6 & 7.4 & 6.3 \\
\hline \multirow{6}{*}{ CI Split HEV PHEV10 } & \multirow{3}{*}{ Unadjusted } & \begin{tabular}{|l|} 
UDDS \\
\end{tabular} & 5.6 & 5.6 & 5.3 & 4.8 & 5.4 & 4.8 & 4.3 & 5.1 & 4.4 & 3.6 & 4.9 & 4.2 & 3.5 \\
\hline & & HWFET & 6.3 & 6.3 & 5.9 & 5.4 & 6.1 & 5.5 & 4.9 & 5.8 & 5.1 & 4.4 & 5.6 & 4.9 & 4.2 \\
\hline & & \begin{tabular}{|l|} 
Combined \\
\end{tabular} & 5.9 & 5.9 & 5.6 & 5.1 & 5.7 & 5.1 & 4.6 & 5.4 & 4.7 & 4.0 & 5.2 & 4.5 & 3.8 \\
\hline & \multirow{3}{*}{ Adjusted } & \begin{tabular}{|l|} 
UDDS \\
\end{tabular} & 7.5 & 7.5 & 7.1 & 6.5 & 7.3 & 6.5 & 5.8 & 6.9 & 6.0 & 5.1 & 6.6 & 5.8 & 4.9 \\
\hline & & HWFET & 8.9 & 8.9 & 8.4 & 7.7 & 8.6 & 7.8 & 7.0 & 8.2 & 7.2 & 6.2 & 7.9 & 6.9 & 6.0 \\
\hline & & \begin{tabular}{|c|} 
Combined \\
\end{tabular} & 8.1 & 8.1 & 7.7 & 7.0 & 7.9 & 7.1 & 6.4 & 7.5 & 6.5 & 5.6 & 7.2 & 6.3 & 5.4 \\
\hline \multirow{6}{*}{ CI Split HEV PHEV2O } & & \begin{tabular}{|l|} 
UDDS \\
\end{tabular} & 3.9 & 3.9 & 3.7 & 3.3 & 3.8 & 3.4 & 3.0 & 3.5 & 3.0 & 2.6 & 3.4 & 2.9 & 2.5 \\
\hline & Unadjusted & HWFET & 5.1 & 5.1 & 4.9 & 4.4 & 5.0 & 4.5 & 4.1 & 4.7 & 4.1 & 4.4 & 4.5 & 4.0 & 4.3 \\
\hline & & Combined & 4.4 & 4.4 & 4.2 & 3.8 & 4.3 & 3.9 & 3.4 & 4.1 & 3.5 & 3.4 & 3.9 & 3.4 & 3.3 \\
\hline & & \begin{tabular}{|l|} 
UDDS \\
\end{tabular} & 5.4 & 5.4 & 5.1 & 4.7 & 5.2 & 4.7 & 4.2 & 4.9 & 4.3 & 3.8 & 4.8 & 4.2 & 3.6 \\
\hline & Adjusted & HWFET & 7.3 & 7.3 & 6.9 & 6.3 & 7.0 & 6.4 & 5.8 & 6.7 & 5.9 & 6.3 & 6.4 & 5.7 & 6.1 \\
\hline & & \begin{tabular}{|l|} 
Combined \\
\end{tabular} & 6.2 & 6.2 & 5.9 & 5.4 & 6.0 & 5.5 & 4.9 & 5.7 & 5.0 & 4.9 & 5.5 & 4.8 & 4.7 \\
\hline
\end{tabular}




\begin{tabular}{|c|c|c|c|c|c|c|c|c|c|c|c|c|c|c|c|}
\hline & & & \multicolumn{13}{|c|}{ Pickup } \\
\hline & & & \multirow{2}{*}{$\begin{array}{l}2010 \\
\text { Ref }\end{array}$} & \multicolumn{3}{|c|}{2010} & \multicolumn{3}{|c|}{2015} & \multicolumn{3}{|c|}{2030} & \multicolumn{3}{|c|}{2045} \\
\hline & & & & low & avg & high & low & avg & high & low & avg & high & low & avg & high \\
\hline \multirow{6}{*}{ CI Series HEV PHEV30 } & \multirow{3}{*}{ Unadjusted } & UDDS & 4.4 & 4.4 & 4.1 & 3.7 & 4.3 & 3.7 & 3.1 & 3.9 & 3.2 & 2.6 & 3.8 & 3.1 & 2.4 \\
\hline & & \begin{tabular}{ll|} 
HWFET \\
\end{tabular} & 6.0 & 6.0 & 5.6 & 5.1 & 5.8 & 5.1 & 4.5 & 5.4 & 4.6 & 3.9 & 5.2 & 4.4 & 3.7 \\
\hline & & Combined & 5.2 & 5.2 & 4.8 & 4.3 & 5.0 & 4.4 & 3.7 & 4.6 & 3.9 & 3.2 & 4.4 & 3.7 & 3.0 \\
\hline & \multirow{3}{*}{ Adjusted } & \begin{tabular}{|l|} 
UDDS \\
\end{tabular} & 6.0 & 6.0 & 5.6 & 5.1 & 5.8 & 5.2 & 4.5 & 5.4 & 4.6 & 3.8 & 5.2 & 4.4 & 3.6 \\
\hline & & HWFET & 8.5 & 8.5 & 8.0 & 7.2 & 8.2 & 7.3 & 6.3 & 7.6 & 6.5 & 5.5 & 7.3 & 6.2 & 5.3 \\
\hline & & Combined & 7.2 & 7.2 & 6.7 & 6.1 & 6.9 & 6.1 & 5.3 & 6.4 & 5.5 & 4.5 & 6.2 & 5.2 & 4.4 \\
\hline \multirow{6}{*}{$\mathrm{Cl}$ Series HEV PHEV40 } & \multirow{3}{*}{ Unadjusted } & \begin{tabular}{|l|} 
UDDS \\
\end{tabular} & 3.9 & 3.9 & 3.6 & 3.2 & 3.7 & 3.2 & 2.7 & 3.4 & 2.8 & 2.2 & 3.3 & 2.7 & 2.1 \\
\hline & & HWFET & 4.8 & 4.8 & 4.5 & 4.0 & 4.6 & 4.1 & 3.5 & 4.3 & 3.6 & 3.1 & 4.1 & 3.4 & 2.9 \\
\hline & & Combined & 4.3 & 4.3 & 4.0 & 3.6 & 4.1 & 3.6 & 3.1 & 3.8 & 3.2 & 2.6 & 3.7 & 3.0 & 2.5 \\
\hline & \multirow{3}{*}{ Adjusted } & \begin{tabular}{|l|} 
UDDS \\
\end{tabular} & 5.3 & 5.3 & 5.0 & 4.5 & 5.2 & 4.6 & 3.9 & 4.8 & 4.0 & 3.3 & 4.6 & 3.9 & 3.2 \\
\hline & & HWFET & 6.8 & 6.8 & 6.3 & 5.7 & 6.5 & 5.8 & 5.0 & 6.1 & 5.1 & 4.4 & 5.8 & 4.9 & 4.2 \\
\hline & & Combined & 6.0 & 6.0 & 5.6 & 5.0 & 5.8 & 5.1 & 4.4 & 5.4 & 4.5 & 3.8 & 5.2 & 4.3 & 3.6 \\
\hline \multirow{6}{*}{ H2 Split HEV } & \multirow{3}{*}{ Unadjusted } & \begin{tabular}{|l|} 
UDDS \\
\end{tabular} & 7.5 & 7.5 & 6.3 & 5.5 & 6.5 & 5.5 & 4.7 & 5.9 & 4.8 & 3.9 & 5.5 & 4.5 & 3.6 \\
\hline & & \begin{tabular}{|l|} 
HWFET \\
\end{tabular} & 8.7 & 8.7 & 7.4 & 6.4 & 7.6 & 6.5 & 5.6 & 6.9 & 5.8 & 4.9 & 6.5 & 5.5 & 4.5 \\
\hline & & Combined & 8.0 & 8.0 & 6.8 & 5.9 & 7.0 & 5.9 & 5.1 & 6.3 & 5.3 & 4.4 & 5.9 & 4.9 & 4.0 \\
\hline & \multirow{3}{*}{ Adjusted } & \begin{tabular}{|l|} 
UDDS \\
\end{tabular} & 9.7 & 9.7 & 8.3 & 7.3 & 8.6 & 7.3 & 6.3 & 7.8 & 6.5 & 5.4 & 7.3 & 6.1 & 5.0 \\
\hline & & HWFET & 12.2 & 12.2 & 10.4 & 9.1 & 10.8 & 9.2 & 8.0 & 9.8 & 8.2 & 7.0 & 9.2 & 7.7 & 6.5 \\
\hline & & Combined & 10.9 & 10.9 & 9.3 & 8.1 & 9.6 & 8.2 & 7.1 & 8.7 & 7.3 & 6.1 & 8.2 & 6.8 & 5.7 \\
\hline \multirow{6}{*}{ H2 Split HEV PHEV10 } & \multirow{3}{*}{ Unadjusted } & \begin{tabular}{|l|} 
UDDS \\
\end{tabular} & 6.0 & 6.0 & 5.0 & 4.4 & 5.2 & 4.4 & 3.8 & 4.8 & 3.9 & 3.2 & 4.4 & 3.7 & 3.0 \\
\hline & & HWFET & 6.5 & 6.5 & 5.6 & 4.9 & 5.7 & 5.0 & 4.4 & 5.3 & 4.5 & 3.8 & 4.9 & 4.2 & 3.5 \\
\hline & & \begin{tabular}{|l|} 
Combined \\
\end{tabular} & 6.2 & 6.2 & 5.3 & 4.6 & 5.5 & 4.7 & 4.1 & 5.0 & 4.2 & 3.5 & 4.7 & 3.9 & 3.2 \\
\hline & \multirow{3}{*}{ Adjusted } & \begin{tabular}{|l|} 
UDDS \\
\end{tabular} & 7.9 & 7.9 & 6.8 & 6.0 & 7.0 & 6.0 & 5.3 & 6.4 & 5.4 & 4.6 & 6.0 & 5.1 & 4.3 \\
\hline & & HWFET & 9.2 & 9.2 & 7.9 & 6.9 & 8.1 & 7.0 & 6.2 & 7.4 & 6.4 & 5.4 & 7.0 & 6.0 & 5.0 \\
\hline & & \begin{tabular}{|l|} 
Combined \\
\end{tabular} & 8.5 & 8.5 & 7.3 & 6.4 & 7.5 & 6.5 & 5.7 & 6.9 & 5.8 & 4.9 & 6.5 & 5.5 & 4.6 \\
\hline \multirow{6}{*}{ H2 Split HEV PHEV2O } & \multirow{3}{*}{ Unadjusted } & \begin{tabular}{|l|} 
UDDS \\
\end{tabular} & 4.2 & 4.2 & 3.5 & 3.0 & 3.6 & 3.1 & 2.6 & 3.3 & 2.7 & 2.3 & 3.1 & 2.6 & 2.1 \\
\hline & & HWFET & 5.4 & 5.4 & 4.6 & 4.9 & 4.7 & 5.0 & 4.4 & 4.3 & 4.5 & 3.9 & 4.1 & 4.3 & 3.6 \\
\hline & & \begin{tabular}{|l|} 
Combined \\
\end{tabular} & 4.7 & 4.7 & 4.0 & 3.9 & 4.1 & 3.9 & 3.4 & 3.8 & 3.5 & 3.0 & 3.5 & 3.3 & 2.8 \\
\hline & \multirow{3}{*}{ Adjusted } & \begin{tabular}{|l|} 
UDDS \\
\end{tabular} & 5.7 & 5.7 & 4.9 & 4.3 & 5.1 & 4.4 & 3.8 & 4.6 & 3.9 & 3.4 & 4.4 & 3.8 & 3.2 \\
\hline & & HWFET & 7.6 & 7.6 & 6.6 & 7.0 & 6.7 & 7.1 & 6.2 & 6.1 & 6.5 & 5.6 & 5.8 & 6.1 & 5.2 \\
\hline & & \begin{tabular}{|c|} 
Combined \\
\end{tabular} & 6.6 & 6.6 & 5.6 & 5.5 & 5.8 & 5.6 & 4.9 & 5.3 & 5.1 & 4.4 & 5.0 & 4.8 & 4.1 \\
\hline \multirow{6}{*}{ H2 Series HEV PHEV30 } & & \begin{tabular}{|l|} 
UDDS \\
\end{tabular} & 4.7 & 4.7 & 3.9 & 3.3 & 4.1 & 3.3 & 2.8 & 3.6 & 2.9 & 2.2 & 3.3 & 2.7 & 2.1 \\
\hline & Unadjusted & HWFET & 6.3 & 6.3 & 5.3 & 4.6 & 5.5 & 4.6 & 3.9 & 4.9 & 4.1 & 3.4 & 4.6 & 3.8 & 3.2 \\
\hline & & Combined & 5.4 & 5.4 & 4.5 & 3.9 & 4.7 & 3.9 & 3.3 & 4.2 & 3.4 & 2.8 & 3.9 & 3.2 & 2.6 \\
\hline & & \begin{tabular}{|l|} 
UDDS \\
\end{tabular} & 6.3 & 6.3 & 5.3 & 4.6 & 5.6 & 4.7 & 4.0 & 5.0 & 4.1 & 3.3 & 4.7 & 3.9 & 3.1 \\
\hline & Adjusted & HWFET & 8.9 & 8.9 & 7.5 & 6.5 & 7.7 & 6.6 & 5.6 & 7.0 & 5.8 & 4.9 & 6.5 & 5.4 & 4.5 \\
\hline & & \begin{tabular}{|l|} 
Combined \\
\end{tabular} & 7.5 & 7.5 & 6.3 & 5.5 & 6.5 & 5.5 & 4.7 & 5.9 & 4.9 & 4.0 & 5.5 & 4.6 & 3.7 \\
\hline
\end{tabular}




\begin{tabular}{|c|c|c|c|c|c|c|c|c|c|c|c|c|c|c|c|}
\hline & & & \multicolumn{13}{|c|}{ Pickup } \\
\hline & & & \multirow{2}{*}{$\begin{array}{l}2010 \\
\text { Ref }\end{array}$} & \multicolumn{3}{|c|}{2010} & \multicolumn{3}{|c|}{2015} & \multicolumn{3}{|c|}{2030} & \multicolumn{3}{|c|}{2045} \\
\hline & & & & low & avg & high & low & avg & high & low & avg & high & low & avg & high \\
\hline \multirow{6}{*}{ H2 Series HEV PHEV40 } & \multirow{3}{*}{ Unadjusted } & UDDS & 4.1 & 4.1 & 3.4 & 2.9 & 3.5 & 2.9 & 2.4 & 3.1 & 2.5 & 1.9 & 2.9 & 2.3 & 1.8 \\
\hline & & \begin{tabular}{ll|} 
HWFET \\
\end{tabular} & 5.0 & 5.0 & 4.2 & 3.6 & 4.3 & 3.6 & 3.1 & 3.9 & 3.2 & 2.7 & 3.6 & 3.0 & 2.5 \\
\hline & & Combined & 4.5 & 4.5 & 3.7 & 3.2 & 3.9 & 3.2 & 2.7 & 3.5 & 2.8 & 2.3 & 3.2 & 2.6 & 2.1 \\
\hline & \multirow{3}{*}{ Adjusted } & \begin{tabular}{|l|} 
UDDS \\
\end{tabular} & 5.6 & 5.6 & 4.7 & 4.1 & 4.9 & 4.2 & 3.5 & 4.4 & 3.6 & 3.0 & 4.1 & 3.4 & 2.8 \\
\hline & & HWFET & 7.0 & 7.0 & 6.0 & 5.1 & 6.1 & 5.2 & 4.4 & 5.5 & 4.6 & 3.8 & 5.1 & 4.2 & 3.6 \\
\hline & & Combined & 6.2 & 6.2 & 5.3 & 4.6 & 5.5 & 4.6 & 3.9 & 4.9 & 4.1 & 3.4 & 4.6 & 3.8 & 3.1 \\
\hline \multirow{6}{*}{ E85 Split HEV } & \multirow{3}{*}{ Unadjusted } & \begin{tabular}{|l|} 
UDDS \\
\end{tabular} & 8.1 & 8.1 & 7.5 & 6.9 & 7.7 & 6.8 & 5.3 & 7.3 & 6.2 & 4.5 & 7.1 & 6.0 & 4.4 \\
\hline & & HWFET & 9.1 & 9.1 & 8.5 & 7.9 & 8.7 & 7.9 & 6.3 & 8.4 & 7.3 & 5.6 & 8.2 & 7.1 & 5.4 \\
\hline & & Combined & 8.6 & 8.6 & 8.0 & 7.3 & 8.2 & 7.3 & 5.7 & 7.8 & 6.7 & 5.0 & 7.6 & 6.5 & 4.8 \\
\hline & \multirow{3}{*}{ Adjusted } & \begin{tabular}{|l|} 
UDDS \\
\end{tabular} & 10.5 & 10.5 & 9.8 & 9.0 & 10.1 & 9.0 & 7.1 & 9.5 & 8.2 & 6.2 & 9.3 & 7.9 & 5.9 \\
\hline & & HWFET & 12.9 & 12.9 & 12.0 & 11.1 & 12.3 & 11.2 & 8.9 & 11.8 & 10.3 & 7.9 & 11.5 & 10.0 & 7.6 \\
\hline & & Combined & 11.6 & 11.6 & 10.8 & 10.0 & 11.1 & 10.0 & 7.9 & 10.5 & 9.1 & 7.0 & 10.3 & 8.8 & 6.7 \\
\hline \multirow{6}{*}{ E85 Split HEV PHEV10 } & \multirow{3}{*}{ Unadjusted } & \begin{tabular}{|l|} 
UDDS \\
\end{tabular} & 6.3 & 6.3 & 5.8 & 5.4 & 6.0 & 5.4 & 4.3 & 5.7 & 5.0 & 3.7 & 5.6 & 4.8 & 3.6 \\
\hline & & \begin{tabular}{|l|} 
HWFET \\
\end{tabular} & 6.9 & 6.9 & 6.5 & 6.0 & 6.6 & 6.1 & 4.9 & 6.4 & 5.7 & 4.4 & 6.3 & 5.5 & 4.1 \\
\hline & & Combined & 6.5 & 6.5 & 6.1 & 5.7 & 6.2 & 5.7 & 4.5 & 6.1 & 5.3 & 4.0 & 5.9 & 5.1 & 3.8 \\
\hline & \multirow{3}{*}{ Adjusted } & \begin{tabular}{|l|} 
UDDS \\
\end{tabular} & 8.3 & 8.3 & 7.7 & 7.2 & 7.9 & 7.2 & 5.8 & 7.6 & 6.7 & 5.1 & 7.4 & 6.5 & 5.0 \\
\hline & & HWFET & 9.7 & 9.7 & 9.2 & 8.6 & 9.3 & 8.6 & 6.9 & 9.1 & 8.1 & 6.2 & 8.8 & 7.8 & 5.9 \\
\hline & & Combined & 8.9 & 8.9 & 8.4 & 7.8 & 8.5 & 7.9 & 6.3 & 8.3 & 7.3 & 5.6 & 8.1 & 7.1 & 5.4 \\
\hline \multirow{6}{*}{ E85 Split HEV PHEV20 } & \multirow{3}{*}{ Unadjusted } & \begin{tabular}{|l|} 
UDDS \\
\end{tabular} & 4.3 & 4.3 & 4.0 & 3.7 & 4.1 & 3.7 & 2.9 & 4.0 & 3.4 & 2.6 & 3.9 & 3.3 & 2.5 \\
\hline & & HWFET & 5.7 & 5.7 & 5.3 & 4.9 & 5.4 & 5.0 & 4.9 & 5.3 & 5.7 & 4.5 & 5.1 & 5.5 & 4.3 \\
\hline & & Combined & 4.9 & 4.9 & 4.6 & 4.3 & 4.7 & 4.3 & 3.8 & 4.6 & 4.5 & 3.4 & 4.4 & 4.3 & 3.3 \\
\hline & \multirow{3}{*}{ Adjusted } & \begin{tabular}{|l|} 
UDDS \\
\end{tabular} & 5.9 & 5.9 & 5.5 & 5.1 & 5.7 & 5.2 & 4.2 & 5.5 & 4.8 & 3.8 & 5.3 & 4.7 & 3.6 \\
\hline & & HWFET & 8.0 & 8.0 & 7.5 & 7.0 & 7.7 & 7.1 & 7.0 & 7.4 & 8.1 & 6.3 & 7.2 & 7.9 & 6.1 \\
\hline & & Combined & 6.9 & 6.9 & 6.4 & 6.0 & 6.6 & 6.0 & 5.5 & 6.4 & 6.3 & 4.9 & 6.2 & 6.1 & 4.7 \\
\hline \multirow{6}{*}{ E85 Series HEV PHEV30 } & \multirow{3}{*}{ Unadjusted } & \begin{tabular}{|l|} 
UDDS \\
\end{tabular} & 4.9 & 4.9 & 4.5 & 4.1 & 4.6 & 4.1 & 3.1 & 4.4 & 3.6 & 2.6 & 4.2 & 3.5 & 2.5 \\
\hline & & HWFET & 6.6 & 6.6 & 6.1 & 5.6 & 6.3 & 5.7 & 4.4 & 6.0 & 5.1 & 3.9 & 5.8 & 4.9 & 3.7 \\
\hline & & \begin{tabular}{|l|} 
Combined \\
\end{tabular} & 5.7 & 5.7 & 5.2 & 4.8 & 5.4 & 4.8 & 3.7 & 5.1 & 4.3 & 3.2 & 4.9 & 4.1 & 3.0 \\
\hline & \multirow{3}{*}{ Adjusted } & \begin{tabular}{|l|} 
UDDS \\
\end{tabular} & 6.6 & 6.6 & 6.1 & 5.6 & 6.3 & 5.7 & 4.4 & 6.0 & 5.0 & 3.8 & 5.8 & 4.9 & 3.6 \\
\hline & & HWFET & 9.4 & 9.4 & 8.7 & 7.9 & 8.9 & 8.0 & 6.2 & 8.4 & 7.3 & 5.5 & 8.1 & 7.0 & 5.3 \\
\hline & & \begin{tabular}{|c|} 
Combined \\
\end{tabular} & 7.8 & 7.8 & 7.3 & 6.7 & 7.5 & 6.7 & 5.2 & 7.1 & 6.1 & 4.6 & 6.9 & 5.8 & 4.4 \\
\hline \multirow{6}{*}{ E85 Series HEV PHEV40 } & & \begin{tabular}{|l|} 
UDDS \\
\end{tabular} & 4.3 & 4.3 & 3.9 & 3.6 & 4.1 & 3.6 & 2.7 & 3.8 & 3.2 & 2.3 & 3.7 & 3.0 & 2.1 \\
\hline & Unadjusted & HWFET & 5.2 & 5.2 & 4.8 & 4.4 & 5.0 & 4.5 & 3.5 & 4.7 & 4.1 & 3.1 & 4.5 & 3.9 & 2.9 \\
\hline & & Combined & 4.7 & 4.7 & 4.3 & 3.9 & 4.5 & 4.0 & 3.0 & 4.2 & 3.6 & 2.6 & 4.1 & 3.4 & 2.5 \\
\hline & & \begin{tabular}{|l|} 
UDDS \\
\end{tabular} & 5.9 & 5.9 & 5.4 & 5.0 & 5.6 & 5.0 & 3.9 & 5.3 & 4.5 & 3.4 & 5.1 & 4.3 & 3.2 \\
\hline & Adjusted & HWFET & 7.4 & 7.4 & 6.9 & 6.3 & 7.0 & 6.3 & 4.9 & 6.7 & 5.8 & 4.4 & 6.5 & 5.5 & 4.2 \\
\hline & & \begin{tabular}{|l|} 
Combined \\
\end{tabular} & 6.6 & 6.6 & 6.1 & 5.5 & 6.2 & 5.6 & 4.4 & 5.9 & 5.0 & 3.8 & 5.7 & 4.9 & 3.7 \\
\hline
\end{tabular}




\begin{tabular}{|c|c|c|c|c|c|c|c|c|c|c|c|c|c|c|c|}
\hline & & & \multicolumn{13}{|c|}{ Pickup } \\
\hline & & & \multirow{2}{*}{$\frac{2010}{\text { Ref }}$} & \multicolumn{3}{|c|}{2010} & \multicolumn{3}{|c|}{2015} & \multicolumn{3}{|c|}{2030} & \multicolumn{3}{|c|}{2045} \\
\hline & & & & low & avg & high & low & avg & high & low & avg & high & low & avg & high \\
\hline \multirow{6}{*}{ FC HEV } & \multirow{3}{*}{ Unadjusted } & UDDS & 7.0 & 7.0 & 5.8 & 4.8 & 5.7 & 4.8 & 4.2 & 5.2 & 4.3 & 3.6 & 4.9 & 4.1 & 3.4 \\
\hline & & HWFET & 7.0 & 7.0 & 6.0 & 5.1 & 5.9 & 5.2 & 4.6 & 5.5 & 4.8 & 4.2 & 5.3 & 4.6 & 4.0 \\
\hline & & Combined & 7.0 & 7.0 & 5.9 & 4.9 & 5.8 & 5.0 & 4.4 & 5.3 & 4.5 & 3.8 & 5.1 & 4.4 & 3.7 \\
\hline & \multirow{3}{*}{ Adjusted } & \begin{tabular}{|l|} 
UDDS \\
\end{tabular} & 9.1 & 9.1 & 7.7 & 6.5 & 7.6 & 6.5 & 5.7 & 7.0 & 5.8 & 5.0 & 6.6 & 5.7 & 4.8 \\
\hline & & HWFET & 9.9 & 9.9 & 8.5 & 7.3 & 8.3 & 7.3 & 6.6 & 7.8 & 6.8 & 6.0 & 7.5 & 6.6 & 5.7 \\
\hline & & Combined & 9.5 & 9.5 & 8.0 & 6.8 & 7.9 & 6.9 & 6.1 & 7.3 & 6.3 & 5.4 & 7.0 & 6.1 & 5.2 \\
\hline \multirow{6}{*}{ FC PHEV10 } & \multirow{3}{*}{ Unadjusted } & \begin{tabular}{|l|} 
UDDS \\
\end{tabular} & 5.4 & 5.4 & 4.6 & 3.9 & 4.5 & 3.9 & 3.4 & 4.2 & 3.5 & 3.0 & 4.0 & 3.4 & 2.8 \\
\hline & & HWFET & 5.3 & 5.3 & 4.7 & 4.1 & 4.5 & 4.1 & 3.7 & 4.4 & 3.8 & 3.4 & 4.2 & 3.7 & 3.3 \\
\hline & & Combined & 5.4 & 5.4 & 4.6 & 4.0 & 4.5 & 4.0 & 3.6 & 4.3 & 3.7 & 3.2 & 4.1 & 3.6 & 3.0 \\
\hline & \multirow{3}{*}{ Adjusted } & \begin{tabular}{|l|} 
UDDS \\
\end{tabular} & 7.3 & 7.3 & 6.2 & 5.4 & 6.1 & 5.4 & 4.8 & 5.8 & 4.9 & 4.2 & 5.5 & 4.8 & 4.1 \\
\hline & & \begin{tabular}{|l|} 
HWFET \\
\end{tabular} & 7.5 & 7.5 & 6.6 & 5.8 & 6.5 & 5.8 & 5.3 & 6.2 & 5.4 & 4.9 & 6.0 & 5.3 & 4.7 \\
\hline & & Combined & 7.4 & 7.4 & 6.4 & 5.5 & 6.3 & 5.6 & 5.0 & 6.0 & 5.2 & 4.5 & 5.7 & 5.0 & 4.4 \\
\hline \multirow{6}{*}{ FC PHEV 20} & \multirow{3}{*}{ Unadjusted } & UDDS & 4.5 & 4.5 & 3.8 & 3.2 & 3.8 & 3.3 & 2.8 & 3.5 & 2.9 & 2.4 & 3.4 & 2.8 & 2.3 \\
\hline & & HWFET & 5.2 & 5.2 & 4.5 & 3.9 & 4.4 & 3.9 & 3.5 & 4.2 & 3.6 & 3.2 & 4.0 & 3.5 & 3.1 \\
\hline & & Combined & 4.8 & 4.8 & 4.1 & 3.5 & 4.1 & 3.6 & 3.2 & 3.8 & 3.2 & 2.8 & 3.7 & 3.2 & 2.7 \\
\hline & \multirow{3}{*}{ Adjusted } & UDDS & 6.2 & 6.2 & 5.3 & 4.6 & 5.2 & 4.6 & 4.1 & 4.9 & 4.2 & 3.6 & 4.7 & 4.1 & 3.5 \\
\hline & & HWFET & 7.3 & 7.3 & 6.4 & 5.5 & 6.3 & 5.6 & 5.0 & 6.0 & 5.2 & 4.6 & 5.8 & 5.0 & 4.4 \\
\hline & & Combined & 6.7 & 6.7 & 5.8 & 5.0 & 5.7 & 5.0 & 4.5 & 5.4 & 4.6 & 4.1 & 5.2 & 4.5 & 3.9 \\
\hline \multirow{6}{*}{ FC PHEV 30} & \multirow{3}{*}{ Unadjusted } & UDDS & 3.1 & 3.1 & 2.7 & 2.3 & 2.7 & 2.3 & 2.0 & 2.5 & 2.1 & 1.7 & 2.4 & 2.0 & 1.7 \\
\hline & & \begin{tabular}{|c|} 
HWFET \\
\end{tabular} & 4.1 & 4.1 & 3.6 & 3.1 & 3.5 & 3.2 & 2.8 & 3.4 & 2.9 & 2.6 & 3.2 & 2.8 & 2.5 \\
\hline & & Combined & 3.6 & 3.6 & 3.1 & 2.7 & 3.0 & 2.7 & 2.4 & 2.9 & 2.5 & 2.1 & 2.8 & 2.4 & 2.0 \\
\hline & \multirow{3}{*}{ Adjusted } & UDDS & 4.5 & 4.5 & 3.9 & 3.4 & 3.8 & 3.4 & 3.1 & 3.7 & 3.1 & 2.7 & 3.5 & 3.1 & 2.6 \\
\hline & & \begin{tabular}{|c|} 
HWFET \\
\end{tabular} & 5.8 & 5.8 & 5.1 & 4.5 & 5.0 & 4.5 & 4.0 & 4.8 & 4.2 & 3.7 & 4.6 & 4.0 & 3.6 \\
\hline & & Combined & 5.1 & 5.1 & 4.4 & 3.9 & 4.4 & 3.9 & 3.5 & 4.2 & 3.6 & 3.2 & 4.0 & 3.5 & 3.1 \\
\hline \multirow{6}{*}{ FC PHEV 40} & \multirow{3}{*}{ Unadjusted } & UDDS & 2.7 & 2.7 & 2.3 & 2.0 & 2.3 & 2.0 & 1.7 & 2.2 & 1.8 & 1.5 & 2.1 & 1.7 & 1.4 \\
\hline & & \begin{tabular}{|c|} 
HWFET \\
\end{tabular} & 3.2 & 3.2 & 2.8 & 2.5 & 2.8 & 2.5 & 2.2 & 2.7 & 2.3 & 2.0 & 2.6 & 2.2 & 2.0 \\
\hline & & Combined & 3.0 & 3.0 & 2.6 & 2.2 & 2.5 & 2.2 & 2.0 & 2.4 & 2.0 & 1.7 & 2.3 & 1.9 & 1.7 \\
\hline & \multirow{3}{*}{ Adjusted } & UDDS & 4.0 & 4.0 & 3.4 & 3.1 & 3.4 & 3.1 & 2.7 & 3.2 & 2.8 & 2.4 & 3.1 & 2.7 & 2.4 \\
\hline & & \begin{tabular}{|l|} 
HWFET \\
\end{tabular} & 4.6 & 4.6 & 4.1 & 3.5 & 4.0 & 3.6 & 3.2 & 3.8 & 3.3 & 2.9 & 3.7 & 3.2 & 2.8 \\
\hline & & Combined & 4.3 & 4.3 & 3.7 & 3.3 & 3.7 & 3.3 & 2.9 & 3.5 & 3.0 & 2.7 & 3.4 & 2.9 & 2.6 \\
\hline
\end{tabular}


Fuel Consumption Improvement versus the Conventional 2010 Reference

\begin{tabular}{|c|c|c|c|c|c|c|c|c|c|c|c|c|c|c|c|}
\hline & & & \multicolumn{13}{|c|}{ Pickup } \\
\hline & & & \multirow{2}{*}{$\begin{array}{c}2010 \\
\text { Ref }\end{array}$} & \multicolumn{3}{|c|}{2010} & \multicolumn{3}{|c|}{2015} & \multicolumn{3}{|c|}{2030} & \multicolumn{3}{|c|}{2045} \\
\hline & & & & Iow & avg & high & low & avg & high & Iow & avg & high & low & avg & high \\
\hline \multirow{6}{*}{ SI Conv } & \multirow{3}{*}{ Unadjusted } & UDDS & $0.0 \%$ & $0.0 \%$ & $-7.7 \%$ & $-13.4 \%$ & $-5.5 \%$ & $-12.4 \%$ & $-31.2 \%$ & $-7.1 \%$ & $-17.9 \%$ & $-42.6 \%$ & $-7.7 \%$ & $-18.9 \%$ & $-45.2 \%$ \\
\hline & & \begin{tabular}{|l|} 
HWFET \\
\end{tabular} & $0.0 \%$ & $0.0 \%$ & $-7.6 \%$ & $-14.1 \%$ & $-5.8 \%$ & $-12.3 \%$ & $-31.2 \%$ & $-7.5 \%$ & $-17.5 \%$ & $-40.6 \%$ & $-9.2 \%$ & $-18.7 \%$ & $-42.9 \%$ \\
\hline & & Combined & $0.0 \%$ & $0.0 \%$ & $-7.7 \%$ & $-13.7 \%$ & $-5.6 \%$ & $-12.3 \%$ & $-31.2 \%$ & $-7.2 \%$ & $-17.7 \%$ & $-41.8 \%$ & $-8.3 \%$ & $-18.8 \%$ & $-44.3 \%$ \\
\hline & \multirow{3}{*}{ Adjusted } & \begin{tabular}{|l|} 
UDDS \\
\end{tabular} & $0.0 \%$ & $0.0 \%$ & $-7.4 \%$ & $-12.9 \%$ & $-5.3 \%$ & $-11.9 \%$ & $-30.0 \%$ & $-6.8 \%$ & $-17.2 \%$ & $-41.0 \%$ & $-7.4 \%$ & $-18.2 \%$ & $-43.6 \%$ \\
\hline & & \begin{tabular}{|c|} 
HWFET \\
\end{tabular} & $0.0 \%$ & $0.0 \%$ & $-7.6 \%$ & $-14.0 \%$ & $-5.8 \%$ & $-12.3 \%$ & $-31.0 \%$ & $-7.4 \%$ & $-17.4 \%$ & $-40.3 \%$ & $-9.2 \%$ & $-18.6 \%$ & $-42.7 \%$ \\
\hline & & Combined & $0.0 \%$ & $0.0 \%$ & $-7.5 \%$ & $-13.4 \%$ & $-5.5 \%$ & $-12.1 \%$ & $-30.4 \%$ & $-7.1 \%$ & $-17.3 \%$ & $-40.7 \%$ & $-8.2 \%$ & $-18.3 \%$ & $-43.2 \%$ \\
\hline \multirow{6}{*}{$\mathrm{Cl}$ Conv } & \multirow{3}{*}{ Unadjusted } & \begin{tabular}{|l|} 
UDDS \\
\end{tabular} & $-17.8 \%$ & $-17.8 \%$ & $-22.3 \%$ & $-28.0 \%$ & $-19.7 \%$ & $-27.5 \%$ & $-34.7 \%$ & $-23.9 \%$ & $-33.2 \%$ & $-43.8 \%$ & $-25.5 \%$ & $-35.5 \%$ & $-46.0 \%$ \\
\hline & & \begin{tabular}{|l|} 
HWFET \\
\end{tabular} & $-14.0 \%$ & $-14.0 \%$ & $-18.5 \%$ & $-22.8 \%$ & $-16.5 \%$ & $-24.6 \%$ & $-29.6 \%$ & $-20.8 \%$ & $-27.7 \%$ & $-37.2 \%$ & $-20.7 \%$ & $-30.4 \%$ & $-39.5 \%$ \\
\hline & & Combined & $-16.3 \%$ & $-16.3 \%$ & $-20.8 \%$ & $-26.0 \%$ & $-18.4 \%$ & $-26.4 \%$ & $-32.7 \%$ & $-22.7 \%$ & $-31.1 \%$ & $-41.2 \%$ & $-23.6 \%$ & $-33.5 \%$ & $-43.5 \%$ \\
\hline & \multirow{3}{*}{ Adjusted } & \begin{tabular}{|l|} 
UDDS \\
\end{tabular} & $-17.1 \%$ & $-17.1 \%$ & $-21.5 \%$ & $-27.0 \%$ & $-18.9 \%$ & $-26.5 \%$ & $-33.4 \%$ & $-23.0 \%$ & $-32.0 \%$ & $-42.2 \%$ & $-24.5 \%$ & $-34.1 \%$ & $-44.3 \%$ \\
\hline & & \begin{tabular}{|c|} 
HWFET \\
\end{tabular} & $-13.9 \%$ & $-13.9 \%$ & $-18.4 \%$ & $-22.7 \%$ & $-16.4 \%$ & $-24.5 \%$ & $-29.4 \%$ & $-20.7 \%$ & $-27.6 \%$ & $-37.0 \%$ & $-20.6 \%$ & $-30.2 \%$ & $-39.3 \%$ \\
\hline & & Combined & $-15.8 \%$ & $-15.8 \%$ & $-20.2 \%$ & $-25.2 \%$ & $-17.9 \%$ & $-25.7 \%$ & $-31.8 \%$ & $-22.0 \%$ & $-30.2 \%$ & $-40.0 \%$ & $-22.9 \%$ & $-32.5 \%$ & $-42.2 \%$ \\
\hline \multirow{6}{*}{ H2 Conv } & \multirow{3}{*}{ Unadjusted } & UDDS & $15.8 \%$ & $15.8 \%$ & $-2.6 \%$ & $-13.3 \%$ & $1.8 \%$ & $-13.8 \%$ & $-23.4 \%$ & $-7.3 \%$ & $-21.0 \%$ & $-35.3 \%$ & $-11.5 \%$ & $-25.5 \%$ & $-40.5 \%$ \\
\hline & & \begin{tabular}{|c|} 
HWFET \\
\end{tabular} & $4.4 \%$ & $4.4 \%$ & $-11.5 \%$ & $-21.5 \%$ & $-8.5 \%$ & $-21.8 \%$ & $-30.1 \%$ & $-16.5 \%$ & $-27.9 \%$ & $-38.8 \%$ & $-20.7 \%$ & $-32.0 \%$ & $-43.4 \%$ \\
\hline & & Combined & $11.4 \%$ & $11.4 \%$ & $-6.0 \%$ & $-16.5 \%$ & $-2.2 \%$ & $-16.9 \%$ & $-26.0 \%$ & $-10.9 \%$ & $-23.7 \%$ & $-36.7 \%$ & $-15.1 \%$ & $-28.0 \%$ & $-41.7 \%$ \\
\hline & \multirow{3}{*}{ Adjusted } & \begin{tabular}{|l|} 
UDDS \\
\end{tabular} & $15.2 \%$ & $15.2 \%$ & $-2.5 \%$ & $-12.8 \%$ & $1.7 \%$ & $-13.3 \%$ & $-22.6 \%$ & $-7.0 \%$ & $-20.2 \%$ & $-34.0 \%$ & $-11.1 \%$ & $-24.6 \%$ & $-39.0 \%$ \\
\hline & & \begin{tabular}{|l|} 
HWFET \\
\end{tabular} & $4.3 \%$ & $4.3 \%$ & $-11.4 \%$ & $-21.4 \%$ & $-8.5 \%$ & $-21.7 \%$ & $-29.9 \%$ & $-16.5 \%$ & $-27.7 \%$ & $-38.6 \%$ & $-20.6 \%$ & $-31.9 \%$ & $-43.2 \%$ \\
\hline & & Combined & $10.7 \%$ & $10.7 \%$ & $-6.2 \%$ & $-16.4 \%$ & $-2.5 \%$ & $-16.8 \%$ & $-25.6 \%$ & $-10.9 \%$ & $-23.3 \%$ & $-35.9 \%$ & $-15.0 \%$ & $-27.6 \%$ & $-40.8 \%$ \\
\hline \multirow{6}{*}{ E85 Conv } & \multirow{3}{*}{ Unadjusted } & \begin{tabular}{|l|} 
UDDS \\
\end{tabular} & $4.3 \%$ & $4.3 \%$ & $2.7 \%$ & $-3.7 \%$ & $5.7 \%$ & $-2.0 \%$ & $-26.9 \%$ & $3.8 \%$ & $-7.9 \%$ & $-37.9 \%$ & $3.0 \%$ & $-8.9 \%$ & $-40.2 \%$ \\
\hline & & HWFET & $10.2 \%$ & $10.2 \%$ & $8.8 \%$ & $-2.3 \%$ & $11.2 \%$ & $3.4 \%$ & $-25.5 \%$ & $9.2 \%$ & $-5.9 \%$ & $-34.6 \%$ & $3.4 \%$ & $-7.2 \%$ & $-36.8 \%$ \\
\hline & & Combined & $6.6 \%$ & $6.6 \%$ & $5.1 \%$ & $-3.1 \%$ & $7.8 \%$ & $0.1 \%$ & $-26.4 \%$ & $5.9 \%$ & $-7.2 \%$ & $-36.6 \%$ & $3.1 \%$ & $-8.3 \%$ & $-38.9 \%$ \\
\hline & \multirow{3}{*}{ Adjusted } & UDDS & $4.1 \%$ & $4.1 \%$ & $2.6 \%$ & $-3.5 \%$ & $5.5 \%$ & $-2.0 \%$ & $-25.9 \%$ & $3.6 \%$ & $-7.6 \%$ & $-36.5 \%$ & $2.8 \%$ & $-8.6 \%$ & $-38.7 \%$ \\
\hline & & HWFET & $10.2 \%$ & $10.2 \%$ & $8.8 \%$ & $-2.3 \%$ & $11.2 \%$ & $3.4 \%$ & $-25.4 \%$ & $9.1 \%$ & $-5.9 \%$ & $-34.4 \%$ & $3.4 \%$ & $-7.2 \%$ & $-36.6 \%$ \\
\hline & & Combined & $6.6 \%$ & $6.6 \%$ & $5.1 \%$ & $-3.0 \%$ & $7.8 \%$ & $0.3 \%$ & $-25.7 \%$ & $5.9 \%$ & $-6.9 \%$ & $-35.6 \%$ & $3.1 \%$ & $-8.0 \%$ & $-37.8 \%$ \\
\hline \multirow{6}{*}{ SI Split HEV } & \multirow{3}{*}{ Unadjusted } & \begin{tabular}{|l|} 
UDDS \\
\end{tabular} & $-42.1 \%$ & $-42.1 \%$ & $-46.2 \%$ & $-50.3 \%$ & $-44.4 \%$ & $-50.5 \%$ & $-59.5 \%$ & $-47.6 \%$ & $-54.9 \%$ & $-66.6 \%$ & $-48.8 \%$ & $-56.6 \%$ & $-68.1 \%$ \\
\hline & & HWFET & $-14.3 \%$ & $-14.3 \%$ & $-19.7 \%$ & $-25.6 \%$ & $-17.5 \%$ & $-24.8 \%$ & $-37.2 \%$ & $-20.7 \%$ & $-30.4 \%$ & $-46.4 \%$ & $-22.9 \%$ & $-32.9 \%$ & $-48.5 \%$ \\
\hline & & Combined & $-31.3 \%$ & $-31.3 \%$ & $-35.9 \%$ & $-40.8 \%$ & $-34.0 \%$ & $-40.6 \%$ & $-50.9 \%$ & $-37.2 \%$ & $-45.4 \%$ & $-58.8 \%$ & $-38.8 \%$ & $-47.4 \%$ & $-60.5 \%$ \\
\hline & \multirow{3}{*}{ Adjusted } & \begin{tabular}{|l|} 
UDDS \\
\end{tabular} & $-40.5 \%$ & $-40.5 \%$ & $-44.5 \%$ & $-48.5 \%$ & $-42.7 \%$ & $-48.6 \%$ & $-57.3 \%$ & $-45.8 \%$ & $-52.9 \%$ & $-64.2 \%$ & $-47.0 \%$ & $-54.5 \%$ & $-65.6 \%$ \\
\hline & & HWFET & $-14.2 \%$ & $-14.2 \%$ & $-19.6 \%$ & $-25.4 \%$ & $-17.4 \%$ & $-24.7 \%$ & $-37.0 \%$ & $-20.6 \%$ & $-30.2 \%$ & $-46.1 \%$ & $-22.7 \%$ & $-32.7 \%$ & $-48.2 \%$ \\
\hline & & Combined & $-29.7 \%$ & $-29.7 \%$ & $-34.2 \%$ & $-39.0 \%$ & $-32.3 \%$ & $-38.8 \%$ & $-49.0 \%$ & $-35.4 \%$ & $-43.6 \%$ & $-56.7 \%$ & $-37.0 \%$ & $-45.6 \%$ & $-58.4 \%$ \\
\hline \multirow{6}{*}{ SI Split HEV PHEV10 } & & \begin{tabular}{|l|} 
UDDS \\
\end{tabular} & $-54.1 \%$ & $-54.1 \%$ & $-57.3 \%$ & $-60.5 \%$ & $-56.1 \%$ & $-60.1 \%$ & $-66.9 \%$ & $-57.8 \%$ & $-63.4 \%$ & $-72.5 \%$ & $-58.8 \%$ & $-64.4 \%$ & $-73.7 \%$ \\
\hline & Unadjusted & HWFET & $-34.6 \%$ & $-34.6 \%$ & $-38.5 \%$ & $-42.9 \%$ & $-36.8 \%$ & $-41.7 \%$ & $-50.9 \%$ & $-39.2 \%$ & $-45.6 \%$ & $-57.7 \%$ & $-41.0 \%$ & $-47.3 \%$ & $-60.4 \%$ \\
\hline & & Combined & $-46.6 \%$ & $-46.6 \%$ & $-50.0 \%$ & $-53.7 \%$ & $-48.6 \%$ & $-53.0 \%$ & $-60.7 \%$ & $-50.6 \%$ & $-56.5 \%$ & $-66.8 \%$ & $-51.9 \%$ & $-57.8 \%$ & $-68.5 \%$ \\
\hline & & UDDS & $-52.1 \%$ & $-52.1 \%$ & $-55.1 \%$ & $-58.3 \%$ & $-54.0 \%$ & $-57.9 \%$ & $-64.4 \%$ & $-55.6 \%$ & $-61.1 \%$ & $-69.9 \%$ & $-56.6 \%$ & $-62.0 \%$ & $-71.0 \%$ \\
\hline & Adjusted & HWFET & $-34.4 \%$ & $-34.4 \%$ & $-38.3 \%$ & $-42.7 \%$ & $-36.6 \%$ & $-41.5 \%$ & $-50.6 \%$ & $-39.0 \%$ & $-45.3 \%$ & $-57.4 \%$ & $-40.8 \%$ & $-47.1 \%$ & $-60.1 \%$ \\
\hline & & Combined & $-44.9 \%$ & $-44.9 \%$ & $-48.2 \%$ & $-51.9 \%$ & $-46.8 \%$ & $-51.1 \%$ & $-58.7 \%$ & $-48.8 \%$ & $-54.6 \%$ & $-64.7 \%$ & $-50.1 \%$ & $-55.9 \%$ & $-66.5 \%$ \\
\hline
\end{tabular}




\begin{tabular}{|c|c|c|c|c|c|c|c|c|c|c|c|c|c|c|c|}
\hline & & & \multicolumn{13}{|c|}{ Pickup } \\
\hline & & & \multirow{2}{*}{$\begin{array}{c}2010 \\
\text { Ref }\end{array}$} & \multicolumn{3}{|c|}{2010} & \multicolumn{3}{|c|}{2015} & \multicolumn{3}{|c|}{2030} & \multicolumn{3}{|c|}{2045} \\
\hline & & & & low & avg & high & low & avg & high & low & avg & high & low & avg & high \\
\hline \multirow{6}{*}{ SI Split HEV PHEV2O } & \multirow{3}{*}{ Unadjusted } & UDDS & $-68.2 \%$ & $-68.2 \%$ & $-70.3 \%$ & $-72.7 \%$ & $-69.6 \%$ & $-72.5 \%$ & $-77.2 \%$ & $-70.8 \%$ & $-74.7 \%$ & $-80.7 \%$ & $-71.5 \%$ & $-75.3 \%$ & $-81.6 \%$ \\
\hline & & HWFET & $-46.1 \%$ & $-46.1 \%$ & $-49.1 \%$ & $-53.0 \%$ & $-48.4 \%$ & $-52.6 \%$ & $-50.5 \%$ & $-50.0 \%$ & $-45.3 \%$ & $-57.1 \%$ & $-51.5 \%$ & $-47.1 \%$ & $-58.7 \%$ \\
\hline & & Combined & $-59.7 \%$ & $-59.7 \%$ & $-62.1 \%$ & $-65.1 \%$ & $-61.4 \%$ & $-64.8 \%$ & $-66.9 \%$ & $-62.7 \%$ & $-63.3 \%$ & $-71.6 \%$ & $-63.8 \%$ & $-64.4 \%$ & $-72.7 \%$ \\
\hline & \multirow{3}{*}{ Adjusted } & \begin{tabular}{|l|} 
UDDS \\
\end{tabular} & $-65.7 \%$ & $-65.7 \%$ & $-67.7 \%$ & $-70.0 \%$ & $-67.0 \%$ & $-69.8 \%$ & $-74.3 \%$ & $-68.1 \%$ & $-71.9 \%$ & $-77.7 \%$ & $-68.9 \%$ & $-72.6 \%$ & $-78.6 \%$ \\
\hline & & HWFET & $-45.9 \%$ & $-45.9 \%$ & $-48.9 \%$ & $-52.7 \%$ & $-48.1 \%$ & $-52.3 \%$ & $-50.3 \%$ & $-49.7 \%$ & $-45.0 \%$ & $-56.8 \%$ & $-51.2 \%$ & $-46.8 \%$ & $-58.4 \%$ \\
\hline & & Combined & $-57.6 \%$ & $-57.6 \%$ & $-60.0 \%$ & $-62.9 \%$ & $-59.3 \%$ & $-62.6 \%$ & $-64.4 \%$ & $-60.6 \%$ & $-60.9 \%$ & $-69.1 \%$ & $-61.6 \%$ & $-62.0 \%$ & $-70.3 \%$ \\
\hline \multirow{6}{*}{ SI Series HEV PHEV3O } & \multirow{3}{*}{ Unadjusted } & \begin{tabular}{|l|} 
UDDS \\
\end{tabular} & $-63.9 \%$ & $-63.9 \%$ & $-66.9 \%$ & $-70.1 \%$ & $-65.7 \%$ & $-69.5 \%$ & $-75.9 \%$ & $-67.8 \%$ & $-73.2 \%$ & $-80.7 \%$ & $-68.7 \%$ & $-74.1 \%$ & $-81.8 \%$ \\
\hline & & HWFET & $-36.7 \%$ & $-36.7 \%$ & $-41.3 \%$ & $-46.4 \%$ & $-39.9 \%$ & $-45.9 \%$ & $-55.7 \%$ & $-42.9 \%$ & $-50.8 \%$ & $-62.6 \%$ & $-44.9 \%$ & $-52.7 \%$ & $-64.0 \%$ \\
\hline & & Combined & $-53.4 \%$ & $-53.4 \%$ & $-57.0 \%$ & $-60.9 \%$ & $-55.7 \%$ & $-60.4 \%$ & $-68.1 \%$ & $-58.1 \%$ & $-64.5 \%$ & $-73.7 \%$ & $-59.5 \%$ & $-65.8 \%$ & $-74.9 \%$ \\
\hline & \multirow{3}{*}{ Adjusted } & \begin{tabular}{|l|} 
UDDS \\
\end{tabular} & $-61.5 \%$ & $-61.5 \%$ & $-64.4 \%$ & $-67.5 \%$ & $-63.3 \%$ & $-66.9 \%$ & $-73.1 \%$ & $-65.3 \%$ & $-70.5 \%$ & $-77.8 \%$ & $-66.2 \%$ & $-71.4 \%$ & $-78.8 \%$ \\
\hline & & HWFET & $-36.5 \%$ & $-36.5 \%$ & $-41.1 \%$ & $-46.1 \%$ & $-39.7 \%$ & $-45.7 \%$ & $-55.4 \%$ & $-42.7 \%$ & $-50.5 \%$ & $-62.3 \%$ & $-44.6 \%$ & $-52.4 \%$ & $-63.7 \%$ \\
\hline & & Combined & $-51.2 \%$ & $-51.2 \%$ & $-54.8 \%$ & $-58.7 \%$ & $-53.6 \%$ & $-58.2 \%$ & $-65.8 \%$ & $-56.0 \%$ & $-62.3 \%$ & $-71.4 \%$ & $-57.3 \%$ & $-63.6 \%$ & $-72.6 \%$ \\
\hline \multirow{6}{*}{ SI Series HEV PHEV40 } & \multirow{3}{*}{ Unadjusted } & \begin{tabular}{|l|} 
UDDS \\
\end{tabular} & $-68.4 \%$ & $-68.4 \%$ & $-71.2 \%$ & $-73.8 \%$ & $-70.1 \%$ & $-73.5 \%$ & $-79.1 \%$ & $-72.0 \%$ & $-76.8 \%$ & $-83.2 \%$ & $-72.8 \%$ & $-77.6 \%$ & $-84.1 \%$ \\
\hline & & HWFET & $-50.0 \%$ & $-50.0 \%$ & $-53.7 \%$ & $-57.7 \%$ & $-52.6 \%$ & $-57.3 \%$ & $-65.2 \%$ & $-55.0 \%$ & $-61.2 \%$ & $-70.6 \%$ & $-56.5 \%$ & $-62.8 \%$ & $-71.7 \%$ \\
\hline & & \begin{tabular}{|l|} 
Combined \\
\end{tabular} & $-61.3 \%$ & $-61.3 \%$ & $-64.4 \%$ & $-67.6 \%$ & $-63.4 \%$ & $-67.2 \%$ & $-73.7 \%$ & $-65.4 \%$ & $-70.7 \%$ & $-78.3 \%$ & $-66.5 \%$ & $-71.9 \%$ & $-79.3 \%$ \\
\hline & \multirow{3}{*}{ Adjusted } & \begin{tabular}{|l|} 
UDDS \\
\end{tabular} & $-65.9 \%$ & $-65.9 \%$ & $-68.6 \%$ & $-71.1 \%$ & $-67.6 \%$ & $-70.8 \%$ & $-76.2 \%$ & $-69.3 \%$ & $-73.9 \%$ & $-80.2 \%$ & $-70.1 \%$ & $-74.7 \%$ & $-81.0 \%$ \\
\hline & & HWFET & $-49.8 \%$ & $-49.8 \%$ & $-53.4 \%$ & $-57.4 \%$ & $-52.3 \%$ & $-57.0 \%$ & $-64.8 \%$ & $-54.8 \%$ & $-60.9 \%$ & $-70.2 \%$ & $-56.2 \%$ & $-62.5 \%$ & $-71.4 \%$ \\
\hline & & Combined & $-59.3 \%$ & $-59.3 \%$ & $-62.3 \%$ & $-65.5 \%$ & $-61.3 \%$ & $-65.1 \%$ & $-71.5 \%$ & $-63.3 \%$ & $-68.6 \%$ & $-76.1 \%$ & $-64.4 \%$ & $-69.7 \%$ & $-77.0 \%$ \\
\hline \multirow{6}{*}{ CI Split HEV } & \multirow{3}{*}{ Unadjusted } & \begin{tabular}{|l|} 
UDDS \\
\end{tabular} & $-48.7 \%$ & $-48.7 \%$ & $-51.7 \%$ & $-56.0 \%$ & $-50.2 \%$ & $-55.9 \%$ & $-61.5 \%$ & $-53.5 \%$ & $-60.4 \%$ & $-67.4 \%$ & $-55.1 \%$ & $-62.0 \%$ & $-68.6 \%$ \\
\hline & & HWFET & $-19.7 \%$ & $-19.7 \%$ & $-24.1 \%$ & $-31.0 \%$ & $-22.0 \%$ & $-30.1 \%$ & $-37.7 \%$ & $-26.4 \%$ & $-36.2 \%$ & $-45.2 \%$ & $-29.2 \%$ & $-38.8 \%$ & $-47.3 \%$ \\
\hline & & \begin{tabular}{|l|} 
Combined \\
\end{tabular} & $-37.5 \%$ & $-37.5 \%$ & $-41.0 \%$ & $-46.3 \%$ & $-39.3 \%$ & $-45.9 \%$ & $-52.3 \%$ & $-43.0 \%$ & $-51.1 \%$ & $-58.8 \%$ & $-45.1 \%$ & $-53.0 \%$ & $-60.4 \%$ \\
\hline & \multirow{3}{*}{ Adjusted } & \begin{tabular}{|l|} 
UDDS \\
\end{tabular} & $-46.9 \%$ & $-46.9 \%$ & $-49.8 \%$ & $-54.0 \%$ & $-48.4 \%$ & $-53.9 \%$ & $-59.2 \%$ & $-51.5 \%$ & $-58.2 \%$ & $-64.9 \%$ & $-53.1 \%$ & $-59.7 \%$ & $-66.1 \%$ \\
\hline & & HWFET & $-19.6 \%$ & $-19.6 \%$ & $-24.0 \%$ & $-30.8 \%$ & $-21.9 \%$ & $-29.9 \%$ & $-37.5 \%$ & $-26.3 \%$ & $-36.0 \%$ & $-44.9 \%$ & $-29.0 \%$ & $-38.6 \%$ & $-47.1 \%$ \\
\hline & & \begin{tabular}{|l|} 
Combined \\
\end{tabular} & $-35.6 \%$ & $-35.6 \%$ & $-39.2 \%$ & $-44.4 \%$ & $-37.5 \%$ & $-44.0 \%$ & $-50.3 \%$ & $-41.1 \%$ & $-49.1 \%$ & $-56.7 \%$ & $-43.2 \%$ & $-51.0 \%$ & $-58.3 \%$ \\
\hline \multirow{6}{*}{ CI Split HEV PHEV10 } & \multirow{3}{*}{ Unadjusted } & \begin{tabular}{|l|} 
UDDS \\
\end{tabular} & $-56.0 \%$ & $-56.0 \%$ & $-58.6 \%$ & $-62.3 \%$ & $-57.4 \%$ & $-61.9 \%$ & $-66.6 \%$ & $-59.9 \%$ & $-65.6 \%$ & $-71.4 \%$ & $-61.3 \%$ & $-67.0 \%$ & $-72.6 \%$ \\
\hline & & HWFET & $-36.3 \%$ & $-36.3 \%$ & $-39.6 \%$ & $-44.7 \%$ & $-38.1 \%$ & $-44.1 \%$ & $-49.8 \%$ & $-41.2 \%$ & $-48.5 \%$ & $-55.4 \%$ & $-43.5 \%$ & $-50.6 \%$ & $-57.1 \%$ \\
\hline & & \begin{tabular}{|l|} 
Combined \\
\end{tabular} & $-48.4 \%$ & $-48.4 \%$ & $-51.2 \%$ & $-55.5 \%$ & $-49.9 \%$ & $-55.0 \%$ & $-60.1 \%$ & $-52.6 \%$ & $-59.0 \%$ & $-65.2 \%$ & $-54.4 \%$ & $-60.6 \%$ & $-66.6 \%$ \\
\hline & \multirow{3}{*}{ Adjusted } & \begin{tabular}{|l|} 
UDDS \\
\end{tabular} & $-53.9 \%$ & $-53.9 \%$ & $-56.4 \%$ & $-60.0 \%$ & $-55.2 \%$ & $-59.7 \%$ & $-64.1 \%$ & $-57.7 \%$ & $-63.2 \%$ & $-68.8 \%$ & $-59.0 \%$ & $-64.5 \%$ & $-69.9 \%$ \\
\hline & & HWFET & $-36.1 \%$ & $-36.1 \%$ & $-39.4 \%$ & $-44.4 \%$ & $-37.9 \%$ & $-43.8 \%$ & $-49.5 \%$ & $-41.0 \%$ & $-48.2 \%$ & $-55.1 \%$ & $-43.2 \%$ & $-50.3 \%$ & $-56.8 \%$ \\
\hline & & Combined & $-46.6 \%$ & $-46.6 \%$ & $-49.4 \%$ & $-53.6 \%$ & $-48.1 \%$ & $-53.1 \%$ & $-58.1 \%$ & $-50.8 \%$ & $-57.1 \%$ & $-63.1 \%$ & $-52.5 \%$ & $-58.7 \%$ & $-64.5 \%$ \\
\hline \multirow{6}{*}{ CI Split HEV PHEV2O } & & \begin{tabular}{|l|} 
UDDS \\
\end{tabular} & $-69.5 \%$ & $-69.5 \%$ & $-71.3 \%$ & $-73.8 \%$ & $-70.4 \%$ & $-73.6 \%$ & $-76.8 \%$ & $-72.2 \%$ & $-76.2 \%$ & $-79.7 \%$ & $-73.2 \%$ & $-77.2 \%$ & $-80.7 \%$ \\
\hline & Unadjusted & \begin{tabular}{|l|} 
HWFET \\
\end{tabular} & $-47.9 \%$ & $-47.9 \%$ & $-50.4 \%$ & $-54.7 \%$ & $-49.5 \%$ & $-54.3 \%$ & $-58.7 \%$ & $-51.9 \%$ & $-57.8 \%$ & $-54.8 \%$ & $-53.8 \%$ & $-59.5 \%$ & $-56.5 \%$ \\
\hline & & Combined & $-61.1 \%$ & $-61.1 \%$ & $-63.2 \%$ & $-66.5 \%$ & $-62.3 \%$ & $-66.1 \%$ & $-69.8 \%$ & $-64.3 \%$ & $-69.1 \%$ & $-70.1 \%$ & $-65.7 \%$ & $-70.3 \%$ & $-71.4 \%$ \\
\hline & & \begin{tabular}{|l|} 
UDDS \\
\end{tabular} & $-66.9 \%$ & $-66.9 \%$ & $-68.7 \%$ & $-71.1 \%$ & $-67.8 \%$ & $-70.9 \%$ & $-74.0 \%$ & $-69.5 \%$ & $-73.4 \%$ & $-76.7 \%$ & $-70.5 \%$ & $-74.3 \%$ & $-77.7 \%$ \\
\hline & Adjusted & HWFET & $-47.6 \%$ & $-47.6 \%$ & $-50.1 \%$ & $-54.5 \%$ & $-49.2 \%$ & $-54.0 \%$ & $-58.4 \%$ & $-51.6 \%$ & $-57.5 \%$ & $-54.5 \%$ & $-53.5 \%$ & $-59.2 \%$ & $-56.2 \%$ \\
\hline & & Combined & $-59.0 \%$ & $-59.0 \%$ & $-61.0 \%$ & $-64.3 \%$ & $-60.2 \%$ & $-64.0 \%$ & $-67.6 \%$ & $-62.1 \%$ & $-66.8 \%$ & $-67.6 \%$ & $-63.5 \%$ & $-68.1 \%$ & $-68.9 \%$ \\
\hline
\end{tabular}




\begin{tabular}{|c|c|c|c|c|c|c|c|c|c|c|c|c|c|c|c|}
\hline & & & \multicolumn{13}{|c|}{ Pickup } \\
\hline & & & \multirow{2}{*}{$\begin{array}{c}2010 \\
\operatorname{Ref} \\
\end{array}$} & \multicolumn{3}{|c|}{2010} & \multicolumn{3}{|c|}{2015} & \multicolumn{3}{|c|}{2030} & \multicolumn{3}{|c|}{2045} \\
\hline & & & & low & avg & high & low & avg & high & low & avg & high & low & avg & high \\
\hline \multirow{6}{*}{ CI Series HEV PHEV30 } & \multirow{3}{*}{ Unadjusted } & UDDS & $-65.1 \%$ & $-65.1 \%$ & $-67.7 \%$ & $-71.1 \%$ & $-66.4 \%$ & $-70.7 \%$ & $-75.3 \%$ & $-69.0 \%$ & $-74.5 \%$ & $-79.7 \%$ & $-70.5 \%$ & $-75.7 \%$ & $-80.8 \%$ \\
\hline & & HWFET & $-38.7 \%$ & $-38.7 \%$ & $-42.6 \%$ & $-48.3 \%$ & $-40.9 \%$ & $-47.8 \%$ & $-54.6 \%$ & $-45.1 \%$ & $-53.3 \%$ & $-60.7 \%$ & $-47.4 \%$ & $-55.4 \%$ & $-62.2 \%$ \\
\hline & & Combined & $-54.9 \%$ & $-54.9 \%$ & $-58.0 \%$ & $-62.3 \%$ & $-56.6 \%$ & $-61.9 \%$ & $-67.3 \%$ & $-59.8 \%$ & $-66.3 \%$ & $-72.3 \%$ & $-61.5 \%$ & $-67.8 \%$ & $-73.6 \%$ \\
\hline & \multirow{3}{*}{ Adjusted } & \begin{tabular}{|l|} 
UDDS \\
\end{tabular} & $-62.7 \%$ & $-62.7 \%$ & $-65.2 \%$ & $-68.4 \%$ & $-64.0 \%$ & $-68.1 \%$ & $-72.5 \%$ & $-66.5 \%$ & $-71.8 \%$ & $-76.7 \%$ & $-67.9 \%$ & $-72.9 \%$ & $-77.8 \%$ \\
\hline & & HWFET & $-38.5 \%$ & $-38.5 \%$ & $-42.4 \%$ & $-48.1 \%$ & $-40.7 \%$ & $-47.5 \%$ & $-54.4 \%$ & $-44.8 \%$ & $-53.0 \%$ & $-60.4 \%$ & $-47.1 \%$ & $-55.1 \%$ & $-61.8 \%$ \\
\hline & & Combined & $-52.7 \%$ & $-52.7 \%$ & $-55.8 \%$ & $-60.1 \%$ & $-54.4 \%$ & $-59.7 \%$ & $-65.0 \%$ & $-57.6 \%$ & $-64.0 \%$ & $-70.0 \%$ & $-59.3 \%$ & $-65.6 \%$ & $-71.3 \%$ \\
\hline \multirow{6}{*}{ CI Series HEV PHEV40 } & \multirow{3}{*}{ Unadjusted } & \begin{tabular}{|l|} 
UDDS \\
\end{tabular} & $-69.6 \%$ & $-69.6 \%$ & $-71.8 \%$ & $-75.0 \%$ & $-70.8 \%$ & $-74.7 \%$ & $-78.6 \%$ & $-73.1 \%$ & $-77.9 \%$ & $-82.4 \%$ & $-74.1 \%$ & $-79.0 \%$ & $-83.4 \%$ \\
\hline & & HWFET & $-51.5 \%$ & $-51.5 \%$ & $-54.7 \%$ & $-59.1 \%$ & $-53.3 \%$ & $-58.7 \%$ & $-64.3 \%$ & $-56.6 \%$ & $-63.2 \%$ & $-69.0 \%$ & $-58.4 \%$ & $-65.0 \%$ & $-70.2 \%$ \\
\hline & & Combined & $-62.6 \%$ & $-62.6 \%$ & $-65.2 \%$ & $-68.8 \%$ & $-64.0 \%$ & $-68.5 \%$ & $-73.1 \%$ & $-66.7 \%$ & $-72.3 \%$ & $-77.2 \%$ & $-68.1 \%$ & $-73.6 \%$ & $-78.3 \%$ \\
\hline & \multirow{3}{*}{ Adjusted } & UDDS & $-67.0 \%$ & $-67.0 \%$ & $-69.2 \%$ & $-72.2 \%$ & $-68.2 \%$ & $-71.9 \%$ & $-75.7 \%$ & $-70.4 \%$ & $-75.1 \%$ & $-79.4 \%$ & $-71.4 \%$ & $-76.1 \%$ & $-80.4 \%$ \\
\hline & & HWFET & $-51.2 \%$ & $-51.2 \%$ & $-54.4 \%$ & $-58.8 \%$ & $-53.0 \%$ & $-58.4 \%$ & $-64.0 \%$ & $-56.3 \%$ & $-62.9 \%$ & $-68.6 \%$ & $-58.1 \%$ & $-64.7 \%$ & $-69.9 \%$ \\
\hline & & Combined & $-60.5 \%$ & $-60.5 \%$ & $-63.1 \%$ & $-66.7 \%$ & $-61.9 \%$ & $-66.4 \%$ & $-70.9 \%$ & $-64.6 \%$ & $-70.1 \%$ & $-75.0 \%$ & $-65.9 \%$ & $-71.4 \%$ & $-76.0 \%$ \\
\hline \multirow{6}{*}{ H2 Split HEV } & \multirow{3}{*}{ Unadjusted } & \begin{tabular}{|l|} 
UDDS \\
\end{tabular} & $-41.5 \%$ & $-41.5 \%$ & $-50.6 \%$ & $-57.1 \%$ & $-48.7 \%$ & $-57.0 \%$ & $-63.4 \%$ & $-53.9 \%$ & $-62.3 \%$ & $-69.4 \%$ & $-56.9 \%$ & $-64.6 \%$ & $-71.7 \%$ \\
\hline & & \begin{tabular}{|l|} 
HWFET \\
\end{tabular} & $-11.7 \%$ & $-11.7 \%$ & $-24.9 \%$ & $-34.7 \%$ & $-22.5 \%$ & $-33.9 \%$ & $-42.6 \%$ & $-29.7 \%$ & $-40.9 \%$ & $-50.0 \%$ & $-34.1 \%$ & $-44.5 \%$ & $-53.7 \%$ \\
\hline & & Combined & $-30.0 \%$ & $-30.0 \%$ & $-40.7 \%$ & $-48.4 \%$ & $-38.6 \%$ & $-48.1 \%$ & $-55.4 \%$ & $-44.5 \%$ & $-54.0 \%$ & $-61.9 \%$ & $-48.1 \%$ & $-56.8 \%$ & $-64.8 \%$ \\
\hline & \multirow{3}{*}{ Adjusted } & \begin{tabular}{|l|} 
UDDS \\
\end{tabular} & $-39.9 \%$ & $-39.9 \%$ & $-48.7 \%$ & $-55.0 \%$ & $-46.9 \%$ & $-54.9 \%$ & $-61.1 \%$ & $-51.9 \%$ & $-60.0 \%$ & $-66.8 \%$ & $-54.8 \%$ & $-62.2 \%$ & $-69.1 \%$ \\
\hline & & HWFET & $-11.7 \%$ & $-11.7 \%$ & $-24.8 \%$ & $-34.5 \%$ & $-22.4 \%$ & $-33.7 \%$ & $-42.4 \%$ & $-29.5 \%$ & $-40.7 \%$ & $-49.8 \%$ & $-33.9 \%$ & $-44.3 \%$ & $-53.5 \%$ \\
\hline & & Combined & $-28.3 \%$ & $-28.3 \%$ & $-38.9 \%$ & $-46.6 \%$ & $-36.8 \%$ & $-46.2 \%$ & $-53.4 \%$ & $-42.7 \%$ & $-52.1 \%$ & $-59.8 \%$ & $-46.2 \%$ & $-54.8 \%$ & $-62.6 \%$ \\
\hline \multirow{6}{*}{ H2 Split HEV PHEV10 } & \multirow{3}{*}{ Unadjusted } & \begin{tabular}{|l|} 
UDDS \\
\end{tabular} & $-53.0 \%$ & $-53.0 \%$ & $-60.4 \%$ & $-65.6 \%$ & $-58.9 \%$ & $-65.2 \%$ & $-70.1 \%$ & $-62.7 \%$ & $-69.2 \%$ & $-74.6 \%$ & $-65.2 \%$ & $-71.0 \%$ & $-76.6 \%$ \\
\hline & & HWFET & $-33.5 \%$ & $-33.5 \%$ & $-43.0 \%$ & $-50.1 \%$ & $-41.6 \%$ & $-49.5 \%$ & $-55.7 \%$ & $-46.5 \%$ & $-54.5 \%$ & $-61.7 \%$ & $-49.9 \%$ & $-57.2 \%$ & $-64.4 \%$ \\
\hline & & Combined & $-45.4 \%$ & $-45.4 \%$ & $-53.7 \%$ & $-59.6 \%$ & $-52.2 \%$ & $-59.1 \%$ & $-64.5 \%$ & $-56.4 \%$ & $-63.5 \%$ & $-69.6 \%$ & $-59.3 \%$ & $-65.6 \%$ & $-71.9 \%$ \\
\hline & \multirow{3}{*}{ Adjusted } & UDDS & $-51.0 \%$ & $-51.0 \%$ & $-58.1 \%$ & $-63.1 \%$ & $-56.8 \%$ & $-62.8 \%$ & $-67.5 \%$ & $-60.4 \%$ & $-66.6 \%$ & $-71.9 \%$ & $-62.8 \%$ & $-68.3 \%$ & $-73.7 \%$ \\
\hline & & HWFET & $-33.3 \%$ & $-33.3 \%$ & $-42.8 \%$ & $-49.9 \%$ & $-41.3 \%$ & $-49.3 \%$ & $-55.4 \%$ & $-46.3 \%$ & $-54.2 \%$ & $-61.4 \%$ & $-49.7 \%$ & $-56.9 \%$ & $-64.1 \%$ \\
\hline & & Combined & $-43.7 \%$ & $-43.7 \%$ & $-51.8 \%$ & $-57.7 \%$ & $-50.4 \%$ & $-57.2 \%$ & $-62.5 \%$ & $-54.6 \%$ & $-61.5 \%$ & $-67.6 \%$ & $-57.4 \%$ & $-63.6 \%$ & $-69.8 \%$ \\
\hline \multirow{6}{*}{ H2 Split HEV PHEV2O } & \multirow{3}{*}{ Unadjusted } & UDDS & $-67.4 \%$ & $-67.4 \%$ & $-72.6 \%$ & $-76.2 \%$ & $-71.5 \%$ & $-76.0 \%$ & $-79.4 \%$ & $-74.2 \%$ & $-78.7 \%$ & $-82.2 \%$ & $-75.9 \%$ & $-79.8 \%$ & $-83.6 \%$ \\
\hline & & HWFET & $-45.3 \%$ & $-45.3 \%$ & $-53.0 \%$ & $-49.8 \%$ & $-51.8 \%$ & $-49.2 \%$ & $-55.4 \%$ & $-56.0 \%$ & $-53.7 \%$ & $-60.1 \%$ & $-58.8 \%$ & $-56.6 \%$ & $-63.1 \%$ \\
\hline & & Combined & $-58.8 \%$ & $-58.8 \%$ & $-65.0 \%$ & $-66.0 \%$ & $-63.9 \%$ & $-65.6 \%$ & $-70.1 \%$ & $-67.1 \%$ & $-69.1 \%$ & $-73.7 \%$ & $-69.3 \%$ & $-70.8 \%$ & $-75.7 \%$ \\
\hline & \multirow{3}{*}{ Adjusted } & \begin{tabular}{|l|} 
UDDS \\
\end{tabular} & $-64.9 \%$ & $-64.9 \%$ & $-69.9 \%$ & $-73.4 \%$ & $-68.8 \%$ & $-73.2 \%$ & $-76.5 \%$ & $-71.4 \%$ & $-75.8 \%$ & $-79.2 \%$ & $-73.1 \%$ & $-76.8 \%$ & $-80.5 \%$ \\
\hline & & HWFET & $-45.0 \%$ & $-45.0 \%$ & $-52.7 \%$ & $-49.5 \%$ & $-51.5 \%$ & $-49.0 \%$ & $-55.1 \%$ & $-55.7 \%$ & $-53.4 \%$ & $-59.8 \%$ & $-58.5 \%$ & $-56.3 \%$ & $-62.8 \%$ \\
\hline & & Combined & $-56.7 \%$ & $-56.7 \%$ & $-62.8 \%$ & $-63.6 \%$ & $-61.7 \%$ & $-63.2 \%$ & $-67.7 \%$ & $-65.0 \%$ & $-66.6 \%$ & $-71.2 \%$ & $-67.1 \%$ & $-68.4 \%$ & $-73.2 \%$ \\
\hline \multirow{6}{*}{ H2 Series HEV PHEV3O } & & UDDS & $-63.3 \%$ & $-63.3 \%$ & $-69.8 \%$ & $-74.1 \%$ & $-68.2 \%$ & $-73.7 \%$ & $-78.3 \%$ & $-72.0 \%$ & $-77.5 \%$ & $-82.4 \%$ & $-74.0 \%$ & $-78.9 \%$ & $-83.8 \%$ \\
\hline & Unadjusted & HWFET & $-36.0 \%$ & $-36.0 \%$ & $-45.9 \%$ & $-53.3 \%$ & $-44.3 \%$ & $-52.9 \%$ & $-59.9 \%$ & $-50.1 \%$ & $-58.5 \%$ & $-65.3 \%$ & $-53.4 \%$ & $-61.3 \%$ & $-67.9 \%$ \\
\hline & & Combined & $-52.8 \%$ & $-52.8 \%$ & $-60.5 \%$ & $-66.0 \%$ & $-59.0 \%$ & $-65.7 \%$ & $-71.2 \%$ & $-63.5 \%$ & $-70.1 \%$ & $-75.8 \%$ & $-66.0 \%$ & $-72.1 \%$ & $-77.7 \%$ \\
\hline & & UDDS & $-61.0 \%$ & $-61.0 \%$ & $-67.2 \%$ & $-71.3 \%$ & $-65.7 \%$ & $-71.0 \%$ & $-75.4 \%$ & $-69.3 \%$ & $-74.6 \%$ & $-79.4 \%$ & $-71.3 \%$ & $-76.0 \%$ & $-80.7 \%$ \\
\hline & Adjusted & HWFET & $-35.9 \%$ & $-35.9 \%$ & $-45.7 \%$ & $-53.0 \%$ & $-44.1 \%$ & $-52.6 \%$ & $-59.6 \%$ & $-49.8 \%$ & $-58.2 \%$ & $-65.0 \%$ & $-53.1 \%$ & $-61.0 \%$ & $-67.5 \%$ \\
\hline & & Combined & $-50.6 \%$ & $-50.6 \%$ & $-58.3 \%$ & $-63.8 \%$ & $-56.8 \%$ & $-63.4 \%$ & $-68.9 \%$ & $-61.3 \%$ & $-67.9 \%$ & $-73.4 \%$ & $-63.8 \%$ & $-69.8 \%$ & $-75.3 \%$ \\
\hline
\end{tabular}




\begin{tabular}{|c|c|c|c|c|c|c|c|c|c|c|c|c|c|c|c|}
\hline & & & \multicolumn{13}{|c|}{ Pickup } \\
\hline & & & \multirow{2}{*}{$\begin{array}{c}2010 \\
\text { Ref } \\
\end{array}$} & \multicolumn{3}{|c|}{2010} & \multicolumn{3}{|c|}{2015} & \multicolumn{3}{|c|}{2030} & \multicolumn{3}{|c|}{2045} \\
\hline & & & & low & avg & high & low & avg & high & low & avg & high & low & avg & high \\
\hline \multirow{6}{*}{ H2 Series HEV PHEV40 } & \multirow{3}{*}{ Unadjusted } & UDDS & $-68.0 \%$ & $-68.0 \%$ & $-73.4 \%$ & $-77.4 \%$ & $-72.3 \%$ & $-77.1 \%$ & $-81.2 \%$ & $-75.4 \%$ & $-80.5 \%$ & $-84.8 \%$ & $-77.3 \%$ & $-81.8 \%$ & $-85.9 \%$ \\
\hline & & HWFET & $-49.6 \%$ & $-49.6 \%$ & $-57.3 \%$ & $-63.2 \%$ & $-56.1 \%$ & $-62.9 \%$ & $-68.4 \%$ & $-60.7 \%$ & $-67.4 \%$ & $-72.7 \%$ & $-63.3 \%$ & $-69.8 \%$ & $-74.8 \%$ \\
\hline & & Combined & $-60.9 \%$ & $-60.9 \%$ & $-67.2 \%$ & $-71.9 \%$ & $-66.1 \%$ & $-71.6 \%$ & $-76.2 \%$ & $-69.7 \%$ & $-75.5 \%$ & $-80.1 \%$ & $-71.9 \%$ & $-77.1 \%$ & $-81.6 \%$ \\
\hline & \multirow{3}{*}{ Adjusted } & \begin{tabular}{|l|} 
UDDS \\
\end{tabular} & $-65.5 \%$ & $-65.5 \%$ & $-70.7 \%$ & $-74.5 \%$ & $-69.6 \%$ & $-74.2 \%$ & $-78.2 \%$ & $-72.6 \%$ & $-77.5 \%$ & $-81.7 \%$ & $-74.5 \%$ & $-78.8 \%$ & $-82.7 \%$ \\
\hline & & HWFET & $-49.3 \%$ & $-49.3 \%$ & $-57.0 \%$ & $-62.8 \%$ & $-55.8 \%$ & $-62.6 \%$ & $-68.0 \%$ & $-60.4 \%$ & $-67.1 \%$ & $-72.3 \%$ & $-63.0 \%$ & $-69.4 \%$ & $-74.4 \%$ \\
\hline & & Combined & $-58.8 \%$ & $-58.8 \%$ & $-65.1 \%$ & $-69.7 \%$ & $-64.0 \%$ & $-69.4 \%$ & $-74.0 \%$ & $-67.6 \%$ & $-73.2 \%$ & $-77.8 \%$ & $-69.8 \%$ & $-74.9 \%$ & $-79.3 \%$ \\
\hline \multirow{6}{*}{ E85 Split HEV } & \multirow{3}{*}{ Unadjusted } & UDDS & $-36.5 \%$ & $-36.5 \%$ & $-41.2 \%$ & $-46.2 \%$ & $-39.5 \%$ & $-46.4 \%$ & $-58.6 \%$ & $-43.1 \%$ & $-51.6 \%$ & $-64.4 \%$ & $-44.5 \%$ & $-53.2 \%$ & $-65.8 \%$ \\
\hline & & HWFET & $-7.2 \%$ & $-7.2 \%$ & $-13.3 \%$ & $-19.9 \%$ & $-11.2 \%$ & $-19.1 \%$ & $-36.2 \%$ & $-14.7 \%$ & $-25.6 \%$ & $-43.0 \%$ & $-16.9 \%$ & $-28.0 \%$ & $-45.1 \%$ \\
\hline & & Combined & $-25.1 \%$ & $-25.1 \%$ & $-30.4 \%$ & $-36.0 \%$ & $-28.5 \%$ & $-35.9 \%$ & $-49.9 \%$ & $-32.1 \%$ & $-41.6 \%$ & $-56.1 \%$ & $-33.9 \%$ & $-43.5 \%$ & $-57.8 \%$ \\
\hline & \multirow{3}{*}{ Adjusted } & UDDS & $-35.1 \%$ & $-35.1 \%$ & $-39.7 \%$ & $-44.5 \%$ & $-38.0 \%$ & $-44.7 \%$ & $-56.4 \%$ & $-41.5 \%$ & $-49.7 \%$ & $-62.0 \%$ & $-42.9 \%$ & $-51.2 \%$ & $-63.4 \%$ \\
\hline & & HWFET & $-7.2 \%$ & $-7.2 \%$ & $-13.2 \%$ & $-19.8 \%$ & $-11.1 \%$ & $-19.0 \%$ & $-36.0 \%$ & $-14.6 \%$ & $-25.5 \%$ & $-42.8 \%$ & $-16.8 \%$ & $-27.9 \%$ & $-44.9 \%$ \\
\hline & & Combined & $-23.6 \%$ & $-23.6 \%$ & $-28.8 \%$ & $-34.3 \%$ & $-26.9 \%$ & $-34.1 \%$ & $-48.0 \%$ & $-30.4 \%$ & $-39.7 \%$ & $-54.1 \%$ & $-32.2 \%$ & $-41.6 \%$ & $-55.8 \%$ \\
\hline \multirow{6}{*}{ E85 Split HEV PHEV10 } & \multirow{3}{*}{ Unadjusted } & UDDS & $-50.9 \%$ & $-50.9 \%$ & $-54.3 \%$ & $-57.9 \%$ & $-53.2 \%$ & $-57.5 \%$ & $-66.5 \%$ & $-55.0 \%$ & $-61.0 \%$ & $-70.9 \%$ & $-56.1 \%$ & $-62.1 \%$ & $-72.1 \%$ \\
\hline & & HWFET & $-30.1 \%$ & $-30.1 \%$ & $-33.7 \%$ & $-38.5 \%$ & $-32.9 \%$ & $-37.8 \%$ & $-50.2 \%$ & $-34.4 \%$ & $-42.0 \%$ & $-55.3 \%$ & $-36.4 \%$ & $-43.8 \%$ & $-58.1 \%$ \\
\hline & & Combined & $-42.9 \%$ & $-42.9 \%$ & $-46.3 \%$ & $-50.4 \%$ & $-45.3 \%$ & $-49.9 \%$ & $-60.2 \%$ & $-47.0 \%$ & $-53.6 \%$ & $-64.8 \%$ & $-48.5 \%$ & $-55.0 \%$ & $-66.7 \%$ \\
\hline & \multirow{3}{*}{ Adjusted } & UDDS & $-49.1 \%$ & $-49.1 \%$ & $-52.3 \%$ & $-55.7 \%$ & $-51.2 \%$ & $-55.3 \%$ & $-64.1 \%$ & $-52.9 \%$ & $-58.7 \%$ & $-68.3 \%$ & $-54.1 \%$ & $-59.8 \%$ & $-69.4 \%$ \\
\hline & & HWFET & $-29.9 \%$ & $-29.9 \%$ & $-33.5 \%$ & $-38.3 \%$ & $-32.7 \%$ & $-37.6 \%$ & $-50.0 \%$ & $-34.2 \%$ & $-41.8 \%$ & $-55.0 \%$ & $-36.2 \%$ & $-43.6 \%$ & $-57.8 \%$ \\
\hline & & Combined & $-41.2 \%$ & $-41.2 \%$ & $-44.6 \%$ & $-48.5 \%$ & $-43.6 \%$ & $-48.0 \%$ & $-58.3 \%$ & $-45.2 \%$ & $-51.7 \%$ & $-62.8 \%$ & $-46.7 \%$ & $-53.1 \%$ & $-64.6 \%$ \\
\hline \multirow{6}{*}{ E85 Split HEV PHEV20 } & \multirow{3}{*}{ Unadjusted } & \begin{tabular}{|l|} 
UDDS \\
\end{tabular} & $-66.0 \%$ & $-66.0 \%$ & $-68.4 \%$ & $-70.9 \%$ & $-67.6 \%$ & $-70.6 \%$ & $-76.9 \%$ & $-68.8 \%$ & $-73.0 \%$ & $-79.7 \%$ & $-69.6 \%$ & $-73.8 \%$ & $-80.5 \%$ \\
\hline & & HWFET & $-42.2 \%$ & $-42.2 \%$ & $-45.8 \%$ & $-49.9 \%$ & $-44.8 \%$ & $-49.3 \%$ & $-49.9 \%$ & $-46.6 \%$ & $-41.7 \%$ & $-54.7 \%$ & $-48.1 \%$ & $-43.6 \%$ & $-56.4 \%$ \\
\hline & & Combined & $-56.8 \%$ & $-56.8 \%$ & $-59.6 \%$ & $-62.8 \%$ & $-58.8 \%$ & $-62.3 \%$ & $-66.5 \%$ & $-60.2 \%$ & $-60.9 \%$ & $-70.0 \%$ & $-61.3 \%$ & $-62.1 \%$ & $-71.2 \%$ \\
\hline & \multirow{3}{*}{ Adjusted } & UDDS & $-63.5 \%$ & $-63.5 \%$ & $-65.8 \%$ & $-68.3 \%$ & $-65.1 \%$ & $-68.0 \%$ & $-74.1 \%$ & $-66.2 \%$ & $-70.3 \%$ & $-76.7 \%$ & $-67.1 \%$ & $-71.1 \%$ & $-77.5 \%$ \\
\hline & & HWFET & $-41.9 \%$ & $-41.9 \%$ & $-45.5 \%$ & $-49.6 \%$ & $-44.5 \%$ & $-49.0 \%$ & $-49.6 \%$ & $-46.3 \%$ & $-41.5 \%$ & $-54.4 \%$ & $-47.9 \%$ & $-43.3 \%$ & $-56.1 \%$ \\
\hline & & Combined & $-54.6 \%$ & $-54.6 \%$ & $-57.5 \%$ & $-60.6 \%$ & $-56.6 \%$ & $-60.2 \%$ & $-64.0 \%$ & $-58.0 \%$ & $-58.4 \%$ & $-67.5 \%$ & $-59.2 \%$ & $-59.6 \%$ & $-68.7 \%$ \\
\hline \multirow{6}{*}{ E85 Series HEV PHEV30 } & \multirow{3}{*}{ Unadjusted } & \begin{tabular}{|l|} 
UDDS \\
\end{tabular} & $-61.5 \%$ & $-61.5 \%$ & $-64.8 \%$ & $-67.9 \%$ & $-63.5 \%$ & $-67.6 \%$ & $-75.7 \%$ & $-65.7 \%$ & $-71.5 \%$ & $-79.5 \%$ & $-66.7 \%$ & $-72.5 \%$ & $-80.6 \%$ \\
\hline & & HWFET & $-32.7 \%$ & $-32.7 \%$ & $-37.5 \%$ & $-42.9 \%$ & $-36.1 \%$ & $-42.5 \%$ & $-55.4 \%$ & $-39.3 \%$ & $-47.7 \%$ & $-60.5 \%$ & $-41.4 \%$ & $-49.8 \%$ & $-62.0 \%$ \\
\hline & & Combined & $-50.3 \%$ & $-50.3 \%$ & $-54.2 \%$ & $-58.3 \%$ & $-52.9 \%$ & $-57.8 \%$ & $-67.8 \%$ & $-55.5 \%$ & $-62.3 \%$ & $-72.2 \%$ & $-56.9 \%$ & $-63.7 \%$ & $-73.4 \%$ \\
\hline & \multirow{3}{*}{ Adjusted } & UDDS & $-59.2 \%$ & $-59.2 \%$ & $-62.4 \%$ & $-65.4 \%$ & $-61.2 \%$ & $-65.1 \%$ & $-72.9 \%$ & $-63.3 \%$ & $-68.9 \%$ & $-76.6 \%$ & $-64.3 \%$ & $-69.8 \%$ & $-77.6 \%$ \\
\hline & & HWFET & $-32.5 \%$ & $-32.5 \%$ & $-37.3 \%$ & $-42.7 \%$ & $-35.9 \%$ & $-42.2 \%$ & $-55.1 \%$ & $-39.1 \%$ & $-47.4 \%$ & $-60.2 \%$ & $-41.2 \%$ & $-49.5 \%$ & $-61.7 \%$ \\
\hline & & Combined & $-48.2 \%$ & $-48.2 \%$ & $-52.1 \%$ & $-56.1 \%$ & $-50.8 \%$ & $-55.7 \%$ & $-65.6 \%$ & $-53.3 \%$ & $-60.0 \%$ & $-69.8 \%$ & $-54.8 \%$ & $-61.5 \%$ & $-71.1 \%$ \\
\hline \multirow{6}{*}{ E85 Series HEV PHEV40 } & & UDDS & $-66.3 \%$ & $-66.3 \%$ & $-69.2 \%$ & $-72.1 \%$ & $-68.2 \%$ & $-71.7 \%$ & $-78.9 \%$ & $-70.1 \%$ & $-75.3 \%$ & $-82.3 \%$ & $-71.0 \%$ & $-76.2 \%$ & $-83.3 \%$ \\
\hline & Unadjusted & HWFET & $-46.8 \%$ & $-46.8 \%$ & $-50.7 \%$ & $-55.0 \%$ & $-49.6 \%$ & $-54.6 \%$ & $-64.9 \%$ & $-52.2 \%$ & $-58.8 \%$ & $-69.0 \%$ & $-53.7 \%$ & $-60.5 \%$ & $-70.2 \%$ \\
\hline & & Combined & $-58.8 \%$ & $-58.8 \%$ & $-62.1 \%$ & $-65.5 \%$ & $-61.0 \%$ & $-65.1 \%$ & $-73.5 \%$ & $-63.1 \%$ & $-68.9 \%$ & $-77.1 \%$ & $-64.3 \%$ & $-70.1 \%$ & $-78.2 \%$ \\
\hline & & UDDS & $-63.9 \%$ & $-63.9 \%$ & $-66.7 \%$ & $-69.4 \%$ & $-65.7 \%$ & $-69.1 \%$ & $-76.0 \%$ & $-67.5 \%$ & $-72.5 \%$ & $-79.3 \%$ & $-68.4 \%$ & $-73.4 \%$ & $-80.2 \%$ \\
\hline & Adjusted & HWFET & $-46.6 \%$ & $-46.6 \%$ & $-50.4 \%$ & $-54.8 \%$ & $-49.3 \%$ & $-54.3 \%$ & $-64.5 \%$ & $-51.9 \%$ & $-58.5 \%$ & $-68.6 \%$ & $-53.5 \%$ & $-60.2 \%$ & $-69.8 \%$ \\
\hline & & Combined & $-56.7 \%$ & $-56.7 \%$ & $-60.0 \%$ & $-63.4 \%$ & $-58.9 \%$ & $-63.0 \%$ & $-71.3 \%$ & $-61.1 \%$ & $-66.7 \%$ & $-74.9 \%$ & $-62.2 \%$ & $-67.9 \%$ & $-75.9 \%$ \\
\hline
\end{tabular}




\begin{tabular}{|c|c|c|c|c|c|c|c|c|c|c|c|c|c|c|c|}
\hline & & & \multicolumn{13}{|c|}{ Pickup } \\
\hline & & & 2010 & & 2010 & & & 2015 & & & 2030 & & & 2045 & \\
\hline & & & Ref & low & avg & high & low & avg & high & low & avg & high & low & avg & high \\
\hline \multirow{6}{*}{ FC HEV } & \multirow{3}{*}{ Unadjusted } & UDDS & $-45.3 \%$ & $-45.3 \%$ & $-54.8 \%$ & $-62.3 \%$ & $-55.2 \%$ & $-62.3 \%$ & $-67.4 \%$ & $-59.3 \%$ & $-66.4 \%$ & $-72.1 \%$ & $-61.3 \%$ & $-67.5 \%$ & $-73.4 \%$ \\
\hline & & HWFET & $-29.1 \%$ & $-29.1 \%$ & $-39.0 \%$ & $-47.9 \%$ & $-40.3 \%$ & $-47.3 \%$ & $-52.9 \%$ & $-43.8 \%$ & $-51.5 \%$ & $-57.2 \%$ & $-45.9 \%$ & $-52.8 \%$ & $-58.8 \%$ \\
\hline & & \begin{tabular}{|l|} 
Combined \\
\end{tabular} & $-39.0 \%$ & $-39.0 \%$ & $-48.7 \%$ & $-56.7 \%$ & $-49.4 \%$ & $-56.5 \%$ & $-61.8 \%$ & $-53.3 \%$ & $-60.6 \%$ & $-66.4 \%$ & $-55.4 \%$ & $-61.8 \%$ & $-67.7 \%$ \\
\hline & \multirow{3}{*}{ Adjusted } & \begin{tabular}{|l|} 
UDDS \\
\end{tabular} & $-43.6 \%$ & $-43.6 \%$ & $-52.8 \%$ & $-60.0 \%$ & $-53.2 \%$ & $-60.0 \%$ & $-64.9 \%$ & $-57.1 \%$ & $-64.0 \%$ & $-69.5 \%$ & $-59.1 \%$ & $-65.0 \%$ & $-70.6 \%$ \\
\hline & & HWFET & $-28.9 \%$ & $-28.9 \%$ & $-38.8 \%$ & $-47.6 \%$ & $-40.1 \%$ & $-47.1 \%$ & $-52.6 \%$ & $-43.6 \%$ & $-51.2 \%$ & $-56.9 \%$ & $-45.7 \%$ & $-52.5 \%$ & $-58.5 \%$ \\
\hline & & \begin{tabular}{|l|} 
Combined \\
\end{tabular} & $-37.6 \%$ & $-37.6 \%$ & $-47.1 \%$ & $-54.9 \%$ & $-47.8 \%$ & $-54.7 \%$ & $-59.8 \%$ & $-51.5 \%$ & $-58.7 \%$ & $-64.3 \%$ & $-53.6 \%$ & $-59.9 \%$ & $-65.7 \%$ \\
\hline \multirow{6}{*}{ FC PHEV10 } & \multirow{3}{*}{ Unadjusted } & UDDS & $-57.4 \%$ & $-57.4 \%$ & $-64.1 \%$ & $-69.6 \%$ & $-64.5 \%$ & $-69.2 \%$ & $-73.2 \%$ & $-66.8 \%$ & $-72.3 \%$ & $-76.8 \%$ & $-68.3 \%$ & $-73.1 \%$ & $-77.8 \%$ \\
\hline & & HWFET & $-46.2 \%$ & $-46.2 \%$ & $-52.5 \%$ & $-58.8 \%$ & $-53.7 \%$ & $-58.4 \%$ & $-62.2 \%$ & $-55.7 \%$ & $-61.1 \%$ & $-65.0 \%$ & $-57.1 \%$ & $-62.1 \%$ & $-66.3 \%$ \\
\hline & & Combined & $-53.0 \%$ & $-53.0 \%$ & $-59.6 \%$ & $-65.4 \%$ & $-60.3 \%$ & $-65.0 \%$ & $-68.9 \%$ & $-62.5 \%$ & $-68.0 \%$ & $-72.2 \%$ & $-64.0 \%$ & $-68.8 \%$ & $-73.3 \%$ \\
\hline & \multirow{3}{*}{ Adjusted } & UDDS & $-55.2 \%$ & $-55.2 \%$ & $-61.8 \%$ & $-67.0 \%$ & $-62.1 \%$ & $-66.7 \%$ & $-70.4 \%$ & $-64.4 \%$ & $-69.7 \%$ & $-74.0 \%$ & $-65.8 \%$ & $-70.4 \%$ & $-74.9 \%$ \\
\hline & & HWFET & $-45.9 \%$ & $-45.9 \%$ & $-52.2 \%$ & $-58.5 \%$ & $-53.5 \%$ & $-58.1 \%$ & $-61.9 \%$ & $-55.4 \%$ & $-60.8 \%$ & $-64.7 \%$ & $-56.8 \%$ & $-61.8 \%$ & $-65.9 \%$ \\
\hline & & Combined & $-51.4 \%$ & $-51.4 \%$ & $-57.8 \%$ & $-63.5 \%$ & $-58.6 \%$ & $-63.1 \%$ & $-66.9 \%$ & $-60.7 \%$ & $-66.0 \%$ & $-70.1 \%$ & $-62.1 \%$ & $-66.8 \%$ & $-71.2 \%$ \\
\hline \multirow{6}{*}{ FC PHEV20 } & \multirow{3}{*}{ Unadjusted } & UDDS & $-64.4 \%$ & $-64.4 \%$ & $-70.1 \%$ & $-74.7 \%$ & $-70.3 \%$ & $-74.4 \%$ & $-77.7 \%$ & $-72.4 \%$ & $-77.1 \%$ & $-80.8 \%$ & $-73.7 \%$ & $-77.7 \%$ & $-81.7 \%$ \\
\hline & & HWFET & $-47.4 \%$ & $-47.4 \%$ & $-54.1 \%$ & $-60.4 \%$ & $-55.2 \%$ & $-59.9 \%$ & $-64.0 \%$ & $-57.3 \%$ & $-62.9 \%$ & $-67.0 \%$ & $-58.8 \%$ & $-63.9 \%$ & $-68.3 \%$ \\
\hline & & \begin{tabular}{|l|} 
Combined \\
\end{tabular} & $-57.8 \%$ & $-57.8 \%$ & $-63.9 \%$ & $-69.1 \%$ & $-64.5 \%$ & $-68.8 \%$ & $-72.4 \%$ & $-66.6 \%$ & $-71.6 \%$ & $-75.5 \%$ & $-67.9 \%$ & $-72.4 \%$ & $-76.5 \%$ \\
\hline & \multirow{3}{*}{ Adjusted } & \begin{tabular}{|l|} 
UDDS \\
\end{tabular} & $-62.0 \%$ & $-62.0 \%$ & $-67.5 \%$ & $-71.9 \%$ & $-67.7 \%$ & $-71.6 \%$ & $-74.9 \%$ & $-69.7 \%$ & $-74.2 \%$ & $-77.8 \%$ & $-70.9 \%$ & $-74.9 \%$ & $-78.6 \%$ \\
\hline & & HWFET & $-47.2 \%$ & $-47.2 \%$ & $-53.8 \%$ & $-60.0 \%$ & $-54.9 \%$ & $-59.6 \%$ & $-63.6 \%$ & $-57.0 \%$ & $-62.6 \%$ & $-66.7 \%$ & $-58.5 \%$ & $-63.6 \%$ & $-67.9 \%$ \\
\hline & & \begin{tabular}{|l|} 
Combined \\
\end{tabular} & $-55.9 \%$ & $-55.9 \%$ & $-61.8 \%$ & $-67.0 \%$ & $-62.4 \%$ & $-66.7 \%$ & $-70.2 \%$ & $-64.5 \%$ & $-69.4 \%$ & $-73.2 \%$ & $-65.8 \%$ & $-70.2 \%$ & $-74.2 \%$ \\
\hline \multirow{6}{*}{ FC PHEV30 } & \multirow{3}{*}{ Unadjusted } & UDDS & $-75.3 \%$ & $-75.3 \%$ & $-79.0 \%$ & $-81.9 \%$ & $-79.2 \%$ & $-81.8 \%$ & $-84.2 \%$ & $-80.4 \%$ & $-83.7 \%$ & $-86.3 \%$ & $-81.3 \%$ & $-84.3 \%$ & $-86.9 \%$ \\
\hline & & HWFET & $-58.4 \%$ & $-58.4 \%$ & $-63.4 \%$ & $-68.0 \%$ & $-64.3 \%$ & $-67.9 \%$ & $-71.2 \%$ & $-65.8 \%$ & $-70.3 \%$ & $-73.6 \%$ & $-67.0 \%$ & $-71.2 \%$ & $-74.6 \%$ \\
\hline & & Combined & $-68.8 \%$ & $-68.8 \%$ & $-73.0 \%$ & $-76.5 \%$ & $-73.4 \%$ & $-76.4 \%$ & $-79.2 \%$ & $-74.8 \%$ & $-78.6 \%$ & $-81.4 \%$ & $-75.8 \%$ & $-79.2 \%$ & $-82.1 \%$ \\
\hline & \multirow{3}{*}{ Adjusted } & UDDS & $-72.5 \%$ & $-72.5 \%$ & $-76.1 \%$ & $-78.8 \%$ & $-76.3 \%$ & $-78.8 \%$ & $-81.1 \%$ & $-77.5 \%$ & $-80.6 \%$ & $-83.1 \%$ & $-78.3 \%$ & $-81.2 \%$ & $-83.7 \%$ \\
\hline & & HWFET & $-58.1 \%$ & $-58.1 \%$ & $-63.1 \%$ & $-67.7 \%$ & $-63.9 \%$ & $-67.5 \%$ & $-70.9 \%$ & $-65.4 \%$ & $-70.0 \%$ & $-73.2 \%$ & $-66.6 \%$ & $-70.8 \%$ & $-74.2 \%$ \\
\hline & & \begin{tabular}{|l|} 
Combined \\
\end{tabular} & $-66.6 \%$ & $-66.6 \%$ & $-70.7 \%$ & $-74.2 \%$ & $-71.2 \%$ & $-74.1 \%$ & $-76.9 \%$ & $-72.5 \%$ & $-76.3 \%$ & $-79.0 \%$ & $-73.5 \%$ & $-76.9 \%$ & $-79.8 \%$ \\
\hline \multirow{6}{*}{ FC PHEV40 } & \multirow{3}{*}{ Unadjusted } & UDDS & $-78.5 \%$ & $-78.5 \%$ & $-81.8 \%$ & $-84.3 \%$ & $-82.0 \%$ & $-84.3 \%$ & $-86.4 \%$ & $-83.1 \%$ & $-86.0 \%$ & $-88.2 \%$ & $-83.8 \%$ & $-86.5 \%$ & $-88.7 \%$ \\
\hline & & HWFET & $-67.1 \%$ & $-67.1 \%$ & $-71.1 \%$ & $-74.8 \%$ & $-71.8 \%$ & $-74.7 \%$ & $-77.4 \%$ & $-73.0 \%$ & $-76.7 \%$ & $-79.3 \%$ & $-74.0 \%$ & $-77.4 \%$ & $-80.1 \%$ \\
\hline & & \begin{tabular}{|l|} 
Combined \\
\end{tabular} & $-74.1 \%$ & $-74.1 \%$ & $-77.7 \%$ & $-80.6 \%$ & $-78.0 \%$ & $-80.6 \%$ & $-82.9 \%$ & $-79.2 \%$ & $-82.4 \%$ & $-84.7 \%$ & $-80.0 \%$ & $-83.0 \%$ & $-85.4 \%$ \\
\hline & \multirow{3}{*}{ Adjusted } & UDDS & $-75.6 \%$ & $-75.6 \%$ & $-78.8 \%$ & $-81.2 \%$ & $-78.9 \%$ & $-81.1 \%$ & $-83.2 \%$ & $-80.0 \%$ & $-82.8 \%$ & $-84.9 \%$ & $-80.7 \%$ & $-83.3 \%$ & $-85.4 \%$ \\
\hline & & HWFET & $-66.7 \%$ & $-66.7 \%$ & $-70.8 \%$ & $-74.4 \%$ & $-71.4 \%$ & $-74.3 \%$ & $-77.0 \%$ & $-72.6 \%$ & $-76.3 \%$ & $-78.9 \%$ & $-73.6 \%$ & $-77.0 \%$ & $-79.7 \%$ \\
\hline & & Combined & $-72.0 \%$ & $-72.0 \%$ & $-75.5 \%$ & $-78.4 \%$ & $-75.9 \%$ & $-78.3 \%$ & $-80.6 \%$ & $-77.0 \%$ & $-80.1 \%$ & $-82.4 \%$ & $-77.8 \%$ & $-80.7 \%$ & $-83.1 \%$ \\
\hline
\end{tabular}




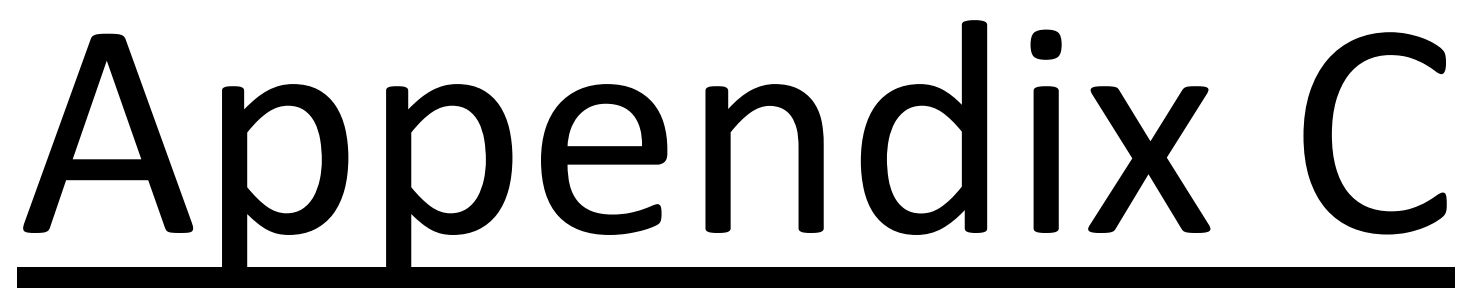

Vehicle Cost Tables 
Compact Class 


\section{Incremental Cost versus the Conventional 2010 Reference}

\begin{tabular}{|c|c|c|c|c|c|c|c|c|c|c|c|c|c|c|}
\hline & & \multicolumn{13}{|c|}{ Compact } \\
\hline & & \multirow{2}{*}{$\frac{2010}{\text { Ref }}$} & \multicolumn{3}{|c|}{2010} & \multicolumn{3}{|c|}{2015} & \multicolumn{3}{|c|}{2030} & \multicolumn{3}{|c|}{2045} \\
\hline & & & low & avg & high & low & avg & high & low & avg & high & low & avg & high \\
\hline \multirow{3}{*}{ SI Conv } & Vehicle Cost LOW & 0 & 0 & -38 & -36 & -17 & -44 & -63 & 802 & 765 & 780 & 1240 & 1177 & 1142 \\
\hline & Vehicle Cost AVG & 0 & 0 & -38 & -36 & 784 & 760 & 742 & 1118 & 1078 & 1091 & 1220 & 1160 & 1125 \\
\hline & Vehicle Cost HIGH & 0 & 0 & -38 & -36 & 148 & 122 & 102 & 605 & 567 & 581 & 680 & 619 & 584 \\
\hline \multirow{3}{*}{ Cl Conv } & Vehicle Cost LOW & 1928 & 1928 & 1865 & 1838 & 1701 & 1693 & 1595 & 2523 & 2428 & 2398 & 2609 & 2514 & 2412 \\
\hline & Vehicle Cost AVG & 1873 & 1873 & 1815 & 1794 & 2551 & 2549 & 2462 & 2634 & 2551 & 2531 & 2642 & 2560 & 2472 \\
\hline & Vehicle Cost $\mathrm{HIGH}$ & 1598 & 1598 & 1549 & 1540 & 1596 & 1603 & 1533 & 1980 & 1907 & 1895 & 1929 & 1858 & 1782 \\
\hline \multirow{3}{*}{ H2 Conv } & Vehicle Cost LOW & -435 & -435 & -464 & -430 & -522 & -496 & -519 & 266 & 233 & 243 & 527 & 491 & 452 \\
\hline & Vehicle Cost AVG & 2577 & 2577 & 2547 & 2580 & 2244 & 2270 & 2248 & 2243 & 2208 & 2215 & 951 & 914 & 874 \\
\hline & Vehicle Cost HIGH & 2022 & 2022 & 1992 & 2025 & 856 & 879 & 853 & 1091 & 1048 & 1047 & 623 & 576 & 526 \\
\hline \multirow{3}{*}{ E85 Conv } & Vehicle Cost LOW & -45 & -45 & -80 & -74 & -58 & -63 & -113 & 789 & 733 & 727 & 1209 & 1138 & 1070 \\
\hline & Vehicle Cost AVG & -44 & -44 & -79 & -73 & 746 & 743 & 695 & 1104 & 1045 & 1035 & 1191 & 1122 & 1056 \\
\hline & Vehicle Cost HIGH & -45 & -45 & -80 & -74 & 106 & 102 & 52 & 590 & 532 & 524 & 647 & 578 & 511 \\
\hline \multirow{3}{*}{ SI Split HEV } & Vehicle Cost LOW & 3403 & 3403 & 3187 & 2988 & 2744 & 2504 & 2198 & 2885 & 2533 & 2128 & 3091 & 2709 & 2238 \\
\hline & Vehicle Cost AVG & 3277 & 3277 & 3066 & 2872 & 2802 & 2611 & 2365 & 2588 & 2297 & 1959 & 2540 & 2226 & 1840 \\
\hline & Vehicle Cost HIGH & 2285 & 2285 & 2121 & 1968 & 1355 & 1200 & 1010 & 1243 & 1046 & 814 & 988 & 794 & 560 \\
\hline \multirow{3}{*}{ SI Split HEV PHEV10 } & Vehicle Cost LOW & 5931 & 5931 & 5592 & 5267 & 4239 & 3889 & 3354 & 3895 & 3380 & 2857 & 3673 & 3233 & 2757 \\
\hline & Vehicle Cost AVG & 5296 & 5296 & 4991 & 4699 & 3846 & 3583 & 3197 & 3377 & 3001 & 2605 & 3200 & 2830 & 2424 \\
\hline & Vehicle Cost $\mathrm{HIGH}$ & 4188 & 4188 & 3937 & 3700 & 1827 & 1654 & 1348 & 1628 & 1346 & 1077 & 1250 & 995 & 786 \\
\hline \multirow{3}{*}{ SI Split HEV PHEV2O } & Vehicle Cost LOW & 7956 & 7956 & 7497 & 7003 & 5510 & 5035 & 4230 & 4701 & 3984 & 3269 & 4086 & 3512 & 2954 \\
\hline & Vehicle Cost AVG & 6992 & 6992 & 6588 & 6153 & 4690 & 4343 & 3774 & 3795 & 3310 & 2808 & 3532 & 3053 & 2581 \\
\hline & Vehicle Cost HIGH & 5482 & 5482 & 5159 & 4809 & 2711 & 2454 & 1973 & 2256 & 1826 & 1419 & 1710 & 1327 & 1029 \\
\hline \multirow{3}{*}{ SI Series HEV PHEV30 } & Vehicle Cost LOW & 16751 & 16751 & 15785 & 14789 & 11804 & 10865 & 9049 & 9347 & 7815 & 6465 & 7732 & 6523 & 5462 \\
\hline & Vehicle Cost AVG & 14745 & 14745 & 13889 & 13011 & 9394 & 8703 & 7404 & 7305 & 6257 & 5293 & 6626 & 5612 & 4713 \\
\hline & Vehicle Cost $\mathrm{HIGH}$ & 11796 & 11796 & 11101 & 10394 & 5468 & 4988 & 3963 & 4159 & 3296 & 2588 & 3108 & 2359 & 1856 \\
\hline \multirow{3}{*}{ SI Series HEV PHEV40 } & Vehicle Cost LOW & 19599 & 19599 & 18450 & 17264 & 13695 & 12537 & 10325 & 10545 & 8719 & 7080 & 8402 & 6982 & 5804 \\
\hline & Vehicle Cost AVG & 17150 & 17150 & 16137 & 15098 & 10673 & 9832 & 8265 & 7976 & 6759 & 5634 & 7173 & 5986 & 4991 \\
\hline & Vehicle Cost HIGH & 13649 & 13649 & 12833 & 12000 & 6719 & 6097 & 4814 & 5024 & 3953 & 3037 & 3745 & 2800 & 2188 \\
\hline \multirow{3}{*}{ CI Split HEV } & Vehicle Cost LOW & 5791 & 5791 & 5542 & 5313 & 4758 & 4581 & 4247 & 4912 & 4495 & 4062 & 4831 & 4388 & 3906 \\
\hline & Vehicle Cost AVG & 5638 & 5638 & 5399 & 5178 & 4787 & 4648 & 4383 & 4395 & 4061 & 3714 & 4294 & 3936 & 3550 \\
\hline & Vehicle Cost HIGH & 4301 & 4301 & 4118 & 3949 & 3041 & 2936 & 2740 & 2831 & 2608 & 2376 & 2465 & 2257 & 2037 \\
\hline
\end{tabular}




\begin{tabular}{|c|c|c|c|c|c|c|c|c|c|c|c|c|c|c|}
\hline & & \multicolumn{13}{|c|}{ Compact } \\
\hline & & \multirow{2}{*}{$\begin{array}{l}2010 \\
\text { Ref }\end{array}$} & \multicolumn{3}{|c|}{2010} & \multicolumn{3}{|c|}{2015} & \multicolumn{3}{|c|}{2030} & \multicolumn{3}{|c|}{2045} \\
\hline & & & low & avg & high & low & avg & high & low & avg & high & low & avg & high \\
\hline \multirow{3}{*}{ CI Split HEV PHEV10 } & Vehicle Cost LOW & 8518 & 8518 & 8175 & 7829 & 6522 & 6135 & 5495 & 6074 & 5484 & 4913 & 5543 & 5050 & 4506 \\
\hline & Vehicle Cost AVG & 7818 & 7818 & 7508 & 7199 & 6059 & 5768 & 5303 & 5344 & 4916 & 4490 & 5093 & 4683 & 4226 \\
\hline & Vehicle Cost HIGH & 6392 & 6392 & 6142 & 5894 & 3613 & 3424 & 3072 & 3264 & 2957 & 2669 & 2779 & 2512 & 2290 \\
\hline \multirow{3}{*}{ CI Split HEV PHEV2O } & Vehicle Cost LOW & 10609 & 10609 & 10187 & 9720 & 7868 & 7399 & 6456 & 6971 & 6132 & 5363 & 5989 & 5369 & 4738 \\
\hline & Vehicle Cost AVG & 9569 & 9569 & 9197 & 8783 & 6953 & 6609 & 5938 & 5818 & 5249 & 4718 & 5452 & 4940 & 4411 \\
\hline & Vehicle Cost HIGH & 7728 & 7728 & 7432 & 7101 & 4548 & 4299 & 3749 & 3950 & 3466 & 3030 & 3277 & 2865 & 2551 \\
\hline \multirow{3}{*}{ CI Series HEV PHEV3O } & Vehicle Cost LOW & 19328 & 19328 & 18299 & 17277 & 14024 & 13043 & 11133 & 11440 & 9848 & 8418 & 9469 & 8223 & 7145 \\
\hline & \begin{tabular}{|l|} 
Vehicle Cost AVG \\
\end{tabular} & 17219 & 17219 & 16309 & 15410 & 11554 & 10831 & 9462 & 9161 & 8081 & 7073 & 8401 & 7364 & 6457 \\
\hline & Vehicle Cost HIGH & 13941 & 13941 & 13209 & 12489 & 7255 & 6756 & 5684 & 5792 & 4901 & 4155 & 4629 & 3861 & 3370 \\
\hline \multirow{3}{*}{$\mathrm{CI}$ Series HEV PHEV40 } & Vehicle Cost LOW & 22298 & 22298 & 21140 & 19911 & 16029 & 14799 & 12489 & 12697 & 10784 & 9085 & 10159 & 8698 & 7480 \\
\hline & Vehicle Cost AVG & 19725 & 19725 & 18706 & 17631 & 12911 & 12019 & 10378 & 9864 & 8598 & 7441 & 8962 & 7749 & 6729 \\
\hline & Vehicle Cost HIGH & 15870 & 15870 & 15053 & 14197 & 8579 & 7916 & 6586 & 6694 & 5583 & 4642 & 5285 & 4321 & 3700 \\
\hline \multirow{3}{*}{ H2 Split HEV } & Vehicle Cost LOW & 3490 & 3490 & 3175 & 2928 & 2647 & 2365 & 2012 & 2677 & 2286 & 1871 & 2706 & 2329 & 1934 \\
\hline & \begin{tabular}{|l|} 
Vehicle Cost AVG \\
\end{tabular} & 6370 & 6370 & 6064 & 5823 & 4625 & 4404 & 4126 & 4042 & 3726 & 3392 & 2578 & 2272 & 1953 \\
\hline & Vehicle Cost HIGH & 4760 & 4760 & 4526 & 4341 & 2397 & 2228 & 2021 & 1999 & 1785 & 1562 & 1193 & 1013 & 828 \\
\hline \multirow{3}{*}{ H2 Split HEV PHEV10 } & Vehicle Cost LOW & 6361 & 6361 & 5881 & 5478 & 4432 & 3939 & 3334 & 3909 & 3283 & 2718 & 3500 & 3049 & 2554 \\
\hline & Vehicle Cost AVG & 8694 & 8694 & 8261 & 7898 & 5917 & 5546 & 5108 & 5042 & 4581 & 4153 & 3426 & 3049 & 2629 \\
\hline & Vehicle Cost $\mathrm{HIGH}$ & 6944 & 6944 & 6591 & 6297 & 2986 & 2760 & 2441 & 2465 & 2158 & 1891 & 1534 & 1293 & 1099 \\
\hline \multirow{3}{*}{ H2 Split HEV PHEV2O } & Vehicle Cost LOW & 8379 & 8379 & 7759 & 7250 & 5790 & 5149 & 4211 & 4783 & 3886 & 3131 & 3924 & 3304 & 2729 \\
\hline & Vehicle Cost AVG & 10384 & 10384 & 9833 & 9381 & 6823 & 6354 & 5686 & 5507 & 4888 & 4360 & 3768 & 3251 & 2767 \\
\hline & Vehicle Cost HIGH & 8234 & 8234 & 7790 & 7428 & 3914 & 3585 & 3062 & 3123 & 2636 & 2224 & 2002 & 1616 & 1333 \\
\hline \multirow{3}{*}{ H2 Series HEV PHEV 30} & Vehicle Cost LOW & 16966 & 16966 & 15679 & 14576 & 11767 & 10606 & 8753 & 9146 & 7504 & 6102 & 7285 & 6099 & 5081 \\
\hline & \begin{tabular}{|l} 
Vehicle Cost AVG \\
\end{tabular} & 17927 & 17927 & 16789 & 15817 & 11269 & 10419 & 9100 & 8768 & 7649 & 6662 & 6616 & 5626 & 4767 \\
\hline & \begin{tabular}{|l|} 
Vehicle Cost HIGH \\
\end{tabular} & 14358 & 14358 & 13439 & 12659 & 6540 & 5960 & 4933 & 4918 & 4021 & 3299 & 3298 & 2576 & 2109 \\
\hline \multirow{3}{*}{ H2 Series HEV PHEV40 } & Vehicle Cost LOW & 19845 & 19845 & 18340 & 17019 & 13659 & 12239 & 10012 & 10349 & 8392 & 6725 & 7935 & 6543 & 5410 \\
\hline & Vehicle Cost AVG & 20358 & 20358 & 19033 & 17876 & 12546 & 11520 & 9947 & 9439 & 8140 & 7004 & 7145 & 5987 & 5034 \\
\hline & \begin{tabular}{|l|} 
Vehicle Cost HIGH \\
\end{tabular} & 16229 & 16229 & 15166 & 14242 & 7790 & 7041 & 5771 & 5782 & 4666 & 3754 & 3924 & 3007 & 2432 \\
\hline \multirow{3}{*}{ E85 Split HEV } & Vehicle Cost LOW & 3421 & 3421 & 3226 & 3057 & 2738 & 2568 & 2216 & 2922 & 2555 & 2161 & 3111 & 2730 & 2279 \\
\hline & Vehicle Cost AVG & 3295 & 3295 & 3106 & 2942 & 2802 & 2664 & 2384 & 2624 & 2319 & 1992 & 2560 & 2246 & 1875 \\
\hline & Vehicle Cost HIGH & 2303 & 2303 & 2155 & 2028 & 1355 & 1249 & 1027 & 1273 & 1065 & 840 & 1004 & 810 & 583 \\
\hline
\end{tabular}




\begin{tabular}{|c|c|c|c|c|c|c|c|c|c|c|c|c|c|c|}
\hline & & \multicolumn{13}{|c|}{ Compact } \\
\hline & & \multirow{2}{*}{$\begin{array}{c}2010 \\
\operatorname{Ref}\end{array}$} & \multicolumn{3}{|c|}{2010} & \multicolumn{3}{|c|}{2015} & \multicolumn{3}{|c|}{2030} & \multicolumn{3}{|c|}{2045} \\
\hline & & & low & avg & high & low & avg & high & low & avg & high & low & avg & high \\
\hline \multirow{3}{*}{ E85 Split HEV PHEV10 } & Vehicle Cost LOW & 5952 & 5952 & 5608 & 5287 & 4260 & 3908 & 3377 & 3917 & 3392 & 2877 & 3695 & 3257 & 2779 \\
\hline & Vehicle Cost AVG & 5317 & 5317 & 5007 & 4720 & 3866 & 3603 & 3220 & 3399 & 3018 & 2627 & 3221 & 2853 & 2445 \\
\hline & Vehicle Cost HIGH & 4208 & 4208 & 3953 & 3719 & 1844 & 1672 & 1366 & 1646 & 1356 & 1093 & 1266 & 1012 & 802 \\
\hline \multirow{3}{*}{ E85 Split HEV PHEV20 } & Vehicle Cost LOW & 7977 & 7977 & 7518 & 7024 & 5530 & 5058 & 4253 & 4719 & 4004 & 3288 & 4109 & 3536 & 2975 \\
\hline & Vehicle Cost AVG & 7013 & 7013 & 6610 & 6174 & 4710 & 4366 & 3796 & 3814 & 3331 & 2829 & 3555 & 3076 & 2601 \\
\hline & Vehicle Cost HIGH & 5502 & 5502 & 5179 & 4828 & 2729 & 2473 & 1992 & 2273 & 1843 & 1435 & 1727 & 1344 & 1045 \\
\hline \multirow{3}{*}{ E85 Series HEV PHEV30 } & Vehicle Cost LOW & 16774 & 16774 & 15808 & 14813 & 11827 & 10893 & 9075 & 9369 & 7840 & 6486 & 7755 & 6549 & 5486 \\
\hline & Vehicle Cost AVG & 14768 & 14768 & 13912 & 13035 & 9416 & 8728 & 7429 & 7327 & 6281 & 5314 & 6648 & 5637 & 4736 \\
\hline & \begin{tabular}{|l|} 
Vehicle cost HIGH \\
\end{tabular} & 11817 & 11817 & 11122 & 10416 & 5487 & 5009 & 3983 & 4177 & 3315 & 2605 & 3125 & 2377 & 1873 \\
\hline \multirow{3}{*}{ E85 Series HEV PHEV40 } & Vehicle Cost LOW & 19643 & 19643 & 18475 & 17288 & 13718 & 12551 & 10363 & 10567 & 8741 & 7101 & 8426 & 7008 & 5826 \\
\hline & Vehicle Cost AVG & 17190 & 17190 & 16162 & 15122 & 10695 & 9849 & 8298 & 7998 & 6781 & 5656 & 7195 & 6011 & 5013 \\
\hline & Vehicle cost HIGH & 13683 & 13683 & 12855 & 12022 & 6738 & 6107 & 4842 & 5042 & 3971 & 3054 & 3763 & 2818 & 2205 \\
\hline \multirow{3}{*}{ FC HEV } & Vehicle Cost LOW & 8302 & 8302 & 6716 & 5788 & 7505 & 5822 & 4618 & 3389 & 2472 & 1718 & 1812 & 1269 & 714 \\
\hline & Vehicle Cost AVG & 12228 & 12228 & 9953 & 8467 & 7477 & 5974 & 4849 & 4490 & 3393 & 2691 & 1739 & 1210 & 740 \\
\hline & Vehicle Cost HIGH & 9688 & 9688 & 7814 & 6588 & 3593 & 2681 & 1986 & 2191 & 1490 & 1013 & 353 & 17 & -264 \\
\hline \multirow{3}{*}{ FC HEV PHEV10 } & Vehicle Cost LOW & 9897 & 9897 & 8289 & 7251 & 7894 & 6607 & 5331 & 4439 & 3300 & 2421 & 2661 & 2042 & 1345 \\
\hline & Vehicle Cost AVG & 13051 & 13051 & 10978 & 9456 & 7896 & 6662 & 5486 & 5184 & 3984 & 3266 & 2477 & 1886 & 1304 \\
\hline & Vehicle Cost HIGH & 10376 & 10376 & 8670 & 7415 & 3538 & 2811 & 2064 & 2303 & 1537 & 1066 & 508 & 119 & -186 \\
\hline \multirow{3}{*}{ FC HEV PHEV2O } & Vehicle Cost LOW & 12380 & 12380 & 10541 & 9274 & 9552 & 7932 & 6396 & 5388 & 3985 & 2863 & 3127 & 2365 & 1543 \\
\hline & Vehicle Cost AVG & 15194 & 15194 & 12925 & 11204 & 9038 & 7571 & 6216 & 5714 & 4366 & 3492 & 2859 & 2152 & 1462 \\
\hline & \begin{tabular}{|l|} 
Vehicle Cost HIGH \\
\end{tabular} & 12019 & 12019 & 10163 & 8759 & 4672 & 3733 & 2788 & 3048 & 2075 & 1411 & 1026 & 483 & 56 \\
\hline \multirow{3}{*}{ FC HEV PHEV 30} & Vehicle Cost LOW & 17652 & 17652 & 15639 & 14259 & 13192 & 11555 & 9531 & 8365 & 6637 & 5188 & 5490 & 4360 & 3346 \\
\hline & Vehicle Cost AVG & 19905 & 19905 & 17472 & 15671 & 12096 & 10570 & 8829 & 8171 & 6587 & 5371 & 4946 & 3917 & 3036 \\
\hline & Vehicle Cost HIGH & 15992 & 15992 & 13997 & 12523 & 5841 & 4907 & 3705 & 3910 & 2786 & 1968 & 1648 & 905 & 431 \\
\hline \multirow{3}{*}{ FC HEV PHEV40 } & Vehicle Cost LOW & 20460 & 20460 & 18124 & 16495 & 15001 & 13058 & 10621 & 9445 & 7391 & 5759 & 6035 & 4738 & 3647 \\
\hline & Vehicle Cost AVG & 22396 & 22396 & 19669 & 17637 & 13389 & 11636 & 9607 & 8821 & 7053 & 5688 & 5399 & 4233 & 3281 \\
\hline & Vehicle Cost HIGH & 17919 & 17919 & 15695 & 14042 & 7061 & 5923 & 4438 & 4726 & 3347 & 2376 & 2200 & 1280 & 721 \\
\hline \multirow{3}{*}{ EV } & \begin{tabular}{|l|} 
Vehicle Cost LOW \\
\end{tabular} & 43649 & 43649 & 40907 & 37558 & 28156 & 25101 & 18121 & 18608 & 13238 & 9401 & 10649 & 7229 & 5267 \\
\hline & Vehicle Cost AVG & 36550 & 36550 & 34228 & 31399 & 19434 & 17337 & 12602 & 10667 & 7628 & 5415 & 8557 & 5762 & 4144 \\
\hline & Vehicle Cost $\mathrm{HIGH}$ & 28050 & 28050 & 26235 & 24036 & 16108 & 14218 & 9721 & 5159 & 3216 & 1851 & 4638 & 2516 & 1446 \\
\hline
\end{tabular}




\section{Cost saving per powertrain}

\begin{tabular}{|c|c|c|c|c|c|c|c|c|c|c|c|c|c|c|}
\hline & & \multicolumn{13}{|c|}{ Compact } \\
\hline & & \multirow{2}{*}{$\frac{2010}{\text { Ref }}$} & \multicolumn{3}{|c|}{2010} & \multicolumn{3}{|c|}{2015} & \multicolumn{3}{|c|}{2030} & \multicolumn{3}{|c|}{2045} \\
\hline & & & low & avg & high & low & avg & high & low & avg & high & low & avg & high \\
\hline \multirow{3}{*}{ SI Conv } & Vehicle Cost LOW & 0 & 0 & -38 & -36 & -17 & -44 & -63 & 802 & 765 & 780 & 1240 & 1177 & 1142 \\
\hline & Vehicle Cost AVG & 0 & 0 & -38 & -36 & 784 & 760 & 742 & 1118 & 1078 & 1091 & 1220 & 1160 & 1125 \\
\hline & Vehicle Cost HIGH & 0 & 0 & -38 & -36 & 148 & 122 & 102 & 605 & 567 & 581 & 680 & 619 & 584 \\
\hline \multirow{3}{*}{$\mathrm{Cl}$ Conv } & Vehicle Cost LOW & 0 & 0 & -64 & -91 & -227 & -236 & -333 & 595 & 500 & 470 & 681 & 585 & 484 \\
\hline & Vehicle Cost AVG & 0 & 0 & -59 & -80 & 678 & 676 & 588 & 761 & 678 & 657 & 769 & 687 & 599 \\
\hline & Vehicle Cost HIGH & 0 & 0 & -49 & -58 & -2 & 5 & -66 & 382 & 309 & 297 & 331 & 260 & 184 \\
\hline \multirow{3}{*}{ H2 Conv } & Vehicle Cost LOW & 0 & 0 & -29 & 5 & -87 & -61 & -84 & 701 & 668 & 678 & 963 & 927 & 887 \\
\hline & Vehicle Cost AVG & 0 & 0 & -30 & 3 & -333 & -306 & -329 & -334 & -369 & -362 & -1626 & -1663 & -1703 \\
\hline & Vehicle Cost HIGH & 0 & 0 & -30 & 3 & -1166 & -1143 & -1169 & -931 & -974 & -975 & -1399 & -1446 & -1496 \\
\hline \multirow{3}{*}{ E85 Conv } & Vehicle Cost LOW & 0 & 0 & -35 & -29 & -13 & -18 & -68 & 835 & 779 & 773 & 1254 & 1183 & 1115 \\
\hline & Vehicle Cost AVG & 0 & 0 & -35 & -29 & 791 & 788 & 740 & 1149 & 1089 & 1079 & 1235 & 1166 & 1101 \\
\hline & Vehicle Cost HIGH & 0 & 0 & -35 & -29 & 152 & 147 & 97 & 635 & 577 & 569 & 692 & 623 & 557 \\
\hline \multirow{3}{*}{ SI Split HEV } & Vehicle Cost LOW & 0 & 0 & -216 & -416 & -659 & -899 & -1206 & -518 & -871 & -1275 & -313 & -694 & -1165 \\
\hline & Vehicle Cost AVG & 0 & 0 & -210 & -405 & -475 & -666 & -911 & -689 & -980 & -1317 & -737 & -1051 & -1437 \\
\hline & Vehicle Cost HIGH & 0 & 0 & -164 & -317 & -930 & -1085 & -1276 & -1042 & -1239 & -1472 & -1298 & -1492 & -1725 \\
\hline \multirow{3}{*}{ SI Split HEV PHEV10 } & Vehicle Cost LOW & 0 & 0 & -339 & -665 & -1692 & -2042 & -2577 & -2036 & -2552 & -3074 & -2258 & -2698 & -3174 \\
\hline & Vehicle Cost AVG & 0 & 0 & -306 & -597 & -1450 & -1713 & -2099 & -1920 & -2295 & -2691 & -2097 & -2466 & -2873 \\
\hline & Vehicle Cost HIGH & 0 & 0 & -251 & -488 & -2362 & -2535 & -2840 & -2560 & -2843 & -3111 & -2939 & -3193 & -3402 \\
\hline \multirow{3}{*}{ SI Split HEV PHEV20 } & Vehicle Cost LOW & 0 & 0 & -460 & -954 & -2446 & -2922 & -3727 & -3255 & -3973 & -4687 & -3871 & -4444 & -5002 \\
\hline & Vehicle Cost AVG & 0 & 0 & -404 & -839 & -2302 & -2649 & -3218 & -3197 & -3682 & -4183 & -3459 & -3939 & -4411 \\
\hline & Vehicle Cost HIGH & 0 & 0 & -323 & -673 & -2771 & -3028 & -3509 & -3226 & -3656 & -4064 & -3772 & -4156 & -4453 \\
\hline \multirow{3}{*}{ SI Series HEV PHEV30 } & Vehicle Cost LOW & 0 & 0 & -966 & -1962 & -4947 & -5886 & -7702 & -7404 & -8936 & -10286 & -9019 & -10228 & -11289 \\
\hline & Vehicle Cost AVG & 0 & 0 & -856 & -1734 & -5351 & -6042 & -7341 & -7440 & -8488 & -9452 & -8119 & -9133 & -10032 \\
\hline & Vehicle Cost $\mathrm{HIGH}$ & 0 & 0 & -695 & -1402 & -6327 & -6808 & -7833 & -7636 & -8499 & -9207 & -8688 & -9437 & -9940 \\
\hline \multirow{3}{*}{ SI Series HEV PHEV40 } & Vehicle Cost LOW & 0 & 0 & -1149 & -2336 & -5904 & -7062 & -9274 & -9054 & -10880 & -12520 & -11197 & -12617 & -13795 \\
\hline & Vehicle Cost AVG & 0 & 0 & -1012 & -2052 & -6477 & -7317 & -8885 & -9174 & -10390 & -11516 & -9977 & -11163 & -12158 \\
\hline & Vehicle Cost HIGH & 0 & 0 & -817 & -1649 & -6931 & -7552 & -8835 & -8625 & -9696 & -10612 & -9904 & -10849 & -11461 \\
\hline \multirow{3}{*}{ CI Split HEV } & Vehicle Cost LOW & 0 & 0 & -249 & -478 & -1034 & -1210 & -1544 & -879 & -1296 & -1729 & -960 & -1403 & -1885 \\
\hline & Vehicle Cost AVG & 0 & 0 & -240 & -461 & -851 & -991 & -1255 & -1243 & -1577 & -1924 & -1345 & -1702 & -2089 \\
\hline & Vehicle Cost HIGH & 0 & 0 & -183 & -352 & -1259 & -1365 & -1560 & -1470 & -1693 & -1925 & -1836 & -2044 & -2264 \\
\hline
\end{tabular}




\begin{tabular}{|c|c|c|c|c|c|c|c|c|c|c|c|c|c|c|}
\hline & & \multicolumn{13}{|c|}{ Compact } \\
\hline & & \multirow{2}{*}{$\frac{2010}{\text { Ref }}$} & \multicolumn{3}{|c|}{2010} & \multicolumn{3}{|c|}{2015} & \multicolumn{3}{|c|}{2030} & \multicolumn{3}{|c|}{2045} \\
\hline & & & low & avg & high & low & avg & high & low & avg & high & low & avg & high \\
\hline \multirow{3}{*}{ CI Split HEV PHEV10 } & Vehicle Cost LOW & 0 & 0 & -344 & -690 & -1996 & -2383 & -3023 & -2445 & -3034 & -3606 & -2975 & -3468 & -4012 \\
\hline & Vehicle Cost AVG & 0 & 0 & -310 & -619 & -1759 & -2050 & -2515 & -2473 & -2902 & -3328 & -2724 & -3134 & -3592 \\
\hline & Vehicle Cost HIGH & 0 & 0 & -250 & -498 & -2779 & -2968 & -3320 & -3128 & -3435 & -3723 & -3612 & -3880 & -4102 \\
\hline \multirow{3}{*}{ CI Split HEV PHEV2O } & Vehicle Cost LOW & 0 & 0 & -421 & -889 & -2741 & -3210 & -4153 & -3637 & -4477 & -5246 & -4619 & -5239 & -5871 \\
\hline & Vehicle Cost AVG & 0 & 0 & -372 & -786 & -2616 & -2960 & -3630 & -3751 & -4320 & -4851 & -4116 & -4629 & -5157 \\
\hline & Vehicle Cost HIGH & 0 & 0 & -296 & -626 & -3180 & -3429 & -3979 & -3777 & -4261 & -4697 & -4451 & -4863 & -5177 \\
\hline \multirow{3}{*}{ CI Series HEV PHEV3O } & Vehicle Cost LOW & 0 & 0 & -1029 & -2052 & -5304 & -6285 & -8196 & -7888 & -9480 & -10910 & -9859 & -11105 & -12183 \\
\hline & Vehicle Cost AVG & 0 & 0 & -910 & -1809 & -5665 & -6388 & -7757 & -8058 & -9137 & -10146 & -8818 & -9855 & -10762 \\
\hline & Vehicle Cost HIGH & 0 & 0 & -732 & -1453 & -6686 & -7185 & -8257 & -8150 & -9040 & -9786 & -9313 & -10080 & -10572 \\
\hline \multirow{3}{*}{ CI Series HEV PHEV40 } & Vehicle Cost LOW & 0 & 0 & -1158 & -2387 & -6269 & -7499 & -9809 & -9601 & -11514 & -13213 & -12139 & -13600 & -14818 \\
\hline & Vehicle Cost AVG & 0 & 0 & -1019 & -2094 & -6814 & -7706 & -9347 & -9861 & -11127 & -12284 & -10763 & -11976 & -12996 \\
\hline & Vehicle cost HIGH & 0 & 0 & -818 & -1673 & -7291 & -7954 & -9284 & -9176 & -10287 & -11228 & -10585 & -11549 & -12170 \\
\hline \multirow{3}{*}{ H2 Split HEV } & Vehicle Cost LOW & 0 & 0 & -315 & -562 & -843 & -1125 & -1478 & -813 & -1204 & -1619 & $\begin{array}{ll}-784 \\
\end{array}$ & -1160 & -1556 \\
\hline & Vehicle Cost AVG & 0 & 0 & -306 & -547 & -1745 & -1967 & -2245 & -2329 & -2645 & -2979 & -3792 & -4098 & -4418 \\
\hline & Vehicle Cost HIGH & 0 & 0 & -234 & -419 & -2362 & -2532 & -2739 & -2761 & -2975 & -3197 & -3567 & -3747 & -3932 \\
\hline \multirow{3}{*}{ H2 Split HEV PHEV10 } & Vehicle Cost LOW & 0 & 0 & -480 & -883 & -1929 & -2422 & -3027 & -2452 & -3078 & -3643 & -2861 & -3312 & -3807 \\
\hline & Vehicle Cost AVG & 0 & 0 & -433 & -796 & -2777 & -3147 & -3586 & -3652 & -4113 & -4541 & -5267 & -5645 & -6065 \\
\hline & Vehicle Cost HIGH & 0 & 0 & -353 & $\begin{array}{l}-648 \\
\end{array}$ & -3958 & -4184 & $\begin{array}{l}-4503 \\
\end{array}$ & $\begin{array}{l}-4479 \\
\end{array}$ & -4786 & -5053 & -5410 & $\begin{array}{l}-5651 \\
\end{array}$ & -5845 \\
\hline \multirow{3}{*}{ H2 Split HEV PHEV2O } & Vehicle Cost LOW & 0 & 0 & -621 & -1129 & -2589 & -3231 & -4168 & -3597 & -4493 & -5248 & -4455 & -5076 & -5650 \\
\hline & Vehicle Cost AVG & 0 & 0 & -551 & -1003 & -3561 & -4030 & $\begin{array}{l}-4698 \\
\end{array}$ & -4877 & -5496 & -6023 & -6616 & -7133 & -7617 \\
\hline & Vehicle Cost $\mathrm{HIGH}$ & 0 & 0 & -444 & -806 & -4320 & -4649 & -5172 & -5111 & -5598 & -6010 & -6232 & -6618 & -6901 \\
\hline \multirow{3}{*}{ H2 Series HEV PHEV3O } & Vehicle Cost LOW & 0 & 0 & -1286 & -2390 & -5198 & -6360 & -8212 & -7819 & -9462 & -10864 & -9680 & -10866 & -11884 \\
\hline & Vehicle Cost AVG & 0 & 0 & -1139 & -2110 & -6658 & -7508 & -8828 & -9160 & -10278 & -11266 & -11311 & -12302 & -13161 \\
\hline & Vehicle Cost HIGH & 0 & 0 & -919 & -1699 & -7818 & -8399 & -9426 & -9440 & -10337 & -11059 & -11061 & -11782 & -12250 \\
\hline \multirow{3}{*}{ H2 Series HEV PHEV40 } & Vehicle Cost LOW & 0 & 0 & -1505 & -2826 & -6186 & -7607 & -9833 & -9496 & -11453 & -13120 & -11910 & -13302 & -14435 \\
\hline & Vehicle Cost AVG & 0 & 0 & -1325 & -2481 & -7811 & -8837 & -10411 & -10919 & -12218 & -13353 & -13213 & -14371 & -15324 \\
\hline & Vehicle Cost HIGH & 0 & 0 & -1063 & -1987 & -8439 & -9189 & -10459 & -10447 & -11563 & -12476 & -12306 & -13223 & -13798 \\
\hline \multirow{3}{*}{ E85 Split HEV } & Vehicle Cost LOW & 0 & 0 & -195 & -364 & -683 & -854 & -1206 & -500 & -867 & -1260 & -310 & $\begin{array}{l}-692 \\
\end{array}$ & -1142 \\
\hline & Vehicle Cost AVG & 0 & 0 & -190 & -353 & -493 & $\begin{array}{l}-631 \\
\end{array}$ & $\begin{array}{l}-911 \\
\end{array}$ & -672 & -976 & -1303 & -735 & -1049 & -1420 \\
\hline & Vehicle Cost HIGH & 0 & 0 & -148 & -275 & $\begin{array}{l}-948 \\
\end{array}$ & -1054 & -1276 & -1030 & -1238 & -1463 & -1299 & -1493 & -1720 \\
\hline
\end{tabular}




\begin{tabular}{|c|c|c|c|c|c|c|c|c|c|c|c|c|c|c|}
\hline & & \multicolumn{13}{|c|}{ Compact } \\
\hline & & \multirow{2}{*}{$\frac{2010}{\operatorname{Ref}}$} & \multicolumn{3}{|c|}{2010} & \multicolumn{3}{|c|}{2015} & \multicolumn{3}{|c|}{2030} & \multicolumn{3}{|c|}{2045} \\
\hline & & & low & avg & high & low & avg & high & low & avg & high & low & avg & high \\
\hline \multirow{3}{*}{ E85 Split HEV PHEV10 } & Vehicle Cost LOW & 0 & 0 & -344 & -665 & -1692 & -2043 & -2575 & -2035 & -2560 & -3074 & -2257 & -2695 & -3173 \\
\hline & \begin{tabular}{|l} 
Vehicle Cost AVG \\
.
\end{tabular} & 0 & 0 & -310 & -597 & -1451 & -1714 & $\begin{array}{l}-2098 \\
\end{array}$ & -1918 & -2299 & -2690 & -2096 & -2464 & -2872 \\
\hline & Vehicle Cost HIGH & 0 & 0 & -255 & -489 & -2363 & -2536 & -2841 & -2562 & -2851 & -3114 & -2941 & -3195 & -3405 \\
\hline \multirow{3}{*}{ E85 Split HEV PHEV20 } & Vehicle Cost LOW & 0 & 0 & -460 & -954 & -2447 & -2919 & -3724 & -3259 & -3973 & -4689 & -3868 & -4441 & -5002 \\
\hline & 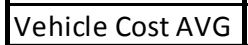 & 0 & 0 & -404 & -839 & -2303 & -2647 & -3217 & -3199 & -3682 & -4184 & $\begin{array}{l}-3458 \\
\end{array}$ & -3937 & -4412 \\
\hline & Vehicle Cost HIGH & 0 & 0 & -323 & -674 & -2773 & -3028 & -3510 & -3229 & -3658 & -4067 & -3775 & -4158 & -4457 \\
\hline \multirow{3}{*}{ E85 Series HEV PHEV30 } & Vehicle Cost LOW & 0 & 0 & -966 & -1962 & -4948 & -5882 & -7699 & -7406 & -8935 & -10289 & -9019 & -10226 & -11288 \\
\hline & \begin{tabular}{|l|} 
Vehicle Cost AVG \\
Cehicle Cost
\end{tabular} & 0 & 0 & -856 & -1733 & -5352 & -6040 & -7340 & -7441 & -8487 & $\begin{array}{l}-9454 \\
\end{array}$ & -8120 & -9132 & -10032 \\
\hline & \begin{tabular}{|l|} 
Vehicle Cost HIGH \\
Cehicle Cost
\end{tabular} & 0 & 0 & -695 & -1401 & -6330 & -6808 & -7834 & -7640 & -8502 & -9212 & -8692 & -9440 & -9944 \\
\hline \multirow{3}{*}{ E85 Series HEV PHEV40 } & Vehicle Cost LOW & 0 & 0 & -1168 & -2355 & -5925 & -7092 & $\begin{array}{l}-9279 \\
\end{array}$ & $\begin{array}{l}-9076 \\
\end{array}$ & -10902 & -12542 & -11217 & -12635 & -13817 \\
\hline & \begin{tabular}{|l|} 
Vehicle Cost AVG \\
\end{tabular} & 0 & 0 & -1028 & -2068 & -6495 & -7341 & -8892 & $\begin{array}{l}-9192 \\
\end{array}$ & -10408 & -11534 & $\begin{array}{ll}-9995 \\
\end{array}$ & -11179 & -12177 \\
\hline & 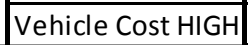 & 0 & 0 & -829 & -1662 & $\begin{array}{l}-6945 \\
\end{array}$ & $\begin{array}{l}-7576 \\
\end{array}$ & -8842 & -8642 & $\begin{array}{l}-9713 \\
\end{array}$ & -10629 & $\begin{array}{l}-9920 \\
\end{array}$ & -10865 & -11479 \\
\hline \multirow{3}{*}{ FC HEV } & Vehicle Cost LOW & $\overline{0}$ & $\overline{0}$ & -1586 & $\begin{array}{l}-2514 \\
\end{array}$ & -797 & -2480 & $\begin{array}{l}-3684 \\
\end{array}$ & -4913 & -5830 & -6584 & -6490 & -7033 & -7588 \\
\hline & Vehicle Cost AVG & 0 & 0 & -2275 & -3760 & -4750 & -6253 & -7378 & -7738 & -8835 & -9536 & -10489 & -11017 & -11487 \\
\hline & Vehicle Cost HIGH & 0 & 0 & -1874 & -3100 & -6095 & -7007 & -7702 & -7497 & -8199 & -8675 & -9335 & $\begin{array}{l}-9671 \\
\end{array}$ & -9952 \\
\hline \multirow{3}{*}{ FC HEV PHEV10 } & Vehicle Cost LOW & 0 & 0 & -1608 & -2647 & -2003 & -3291 & -4566 & -5459 & -6597 & -7476 & -7236 & -7856 & -8553 \\
\hline & \begin{tabular}{|l} 
Vehicle Cost AVG \\
\end{tabular} & 0 & 0 & -2073 & -3595 & -5155 & -6389 & -7565 & -7867 & -9067 & $\begin{array}{l}-9785 \\
\end{array}$ & -10574 & -11165 & -11747 \\
\hline & Vehicle Cost HIGH & 0 & 0 & $\begin{array}{l}-1706 \\
\end{array}$ & -2961 & $\begin{array}{ll}-6838 \\
\end{array}$ & -7565 & -8312 & $\begin{array}{l}-8073 \\
\end{array}$ & $\begin{array}{l}-8839 \\
\end{array}$ & $\begin{array}{l}-9311 \\
\end{array}$ & $\begin{array}{l}-9868 \\
\end{array}$ & -10257 & -10563 \\
\hline \multirow{3}{*}{ FC HEV PHEV 20} & Vehicle Cost LOW & 0 & 0 & -1839 & -3106 & -2828 & -4448 & -5984 & -6993 & -8395 & -9517 & -9254 & -10015 & -10838 \\
\hline & \begin{tabular}{|l|} 
Vehicle Cost AVG \\
\end{tabular} & 0 & 0 & -2269 & -3989 & -6156 & -7623 & $\begin{array}{l}-8978 \\
\end{array}$ & $\begin{array}{l}-9479 \\
\end{array}$ & -10827 & -11702 & -12335 & -13041 & -13731 \\
\hline & Vehicle Cost $\mathrm{HIGH}$ & 0 & 0 & -1856 & -3260 & -7347 & -8286 & -9231 & -8971 & $\begin{array}{l}-9944 \\
\end{array}$ & -10609 & -10993 & -11536 & -11963 \\
\hline \multirow{3}{*}{ FC HEV PHEV 30} & Vehicle Cost LOW & 0 & 0 & -2012 & -3393 & -4460 & -6097 & -8120 & $\begin{array}{l}-9287 \\
\end{array}$ & -11015 & -12464 & -12162 & -13292 & -14306 \\
\hline & Vehicle Cost AVG & 0 & 0 & -2433 & -4234 & -7810 & $\begin{array}{l}-9336 \\
\end{array}$ & -11076 & -11734 & -13318 & -14534 & -14960 & -15989 & -16870 \\
\hline & Vehicle Cost HIGH & 0 & 0 & -1995 & -3469 & -10151 & -11085 & -12287 & -12083 & -13206 & -14025 & -14344 & -15087 & -15561 \\
\hline \multirow{3}{*}{ FC HEV PHEV40 } & Vehicle Cost LOW & 0 & 0 & -2336 & -3964 & -5458 & -7402 & $\begin{array}{l}-9839 \\
\end{array}$ & -11014 & -13069 & -14701 & -14425 & -15722 & -16813 \\
\hline & Vehicle Cost AVG & 0 & 0 & -2727 & -4759 & $\begin{array}{l}-9007 \\
\end{array}$ & -10760 & -12789 & -13575 & -15343 & -16708 & -16997 & -18163 & -19116 \\
\hline & Vehicle Cost HIGH & 0 & 0 & -2224 & -3876 & -10857 & -11995 & -13480 & -13193 & -14572 & -15543 & -15719 & -16639 & -17198 \\
\hline \multirow{3}{*}{ EV } & Vehicle Cost LOW & 0 & 0 & -2741 & -6091 & -15493 & -18548 & -25528 & -25041 & -30410 & -34247 & -33000 & -36419 & -38382 \\
\hline & Vehicle Cost AVG & 0 & 0 & -2322 & -5151 & -17117 & -19213 & -23948 & -25884 & -28922 & -31135 & -27993 & -30788 & -32407 \\
\hline & Vehicle Cost HIGH & 0 & 0 & -1815 & -4014 & -11942 & -13832 & -18329 & -22891 & -24834 & -26199 & -23412 & -25534 & -26604 \\
\hline
\end{tabular}


Percentage decrease cost per powertrain

\begin{tabular}{|c|c|c|c|c|c|c|c|c|c|c|c|c|c|c|}
\hline & & \multicolumn{13}{|c|}{ Compact } \\
\hline & & \multirow{2}{*}{$\frac{2010}{\text { Ref }}$} & \multicolumn{3}{|c|}{2010} & \multicolumn{3}{|c|}{2015} & \multicolumn{3}{|c|}{2030} & \multicolumn{3}{|c|}{2045} \\
\hline & & & low & avg & high & low & avg & high & low & avg & high & low & avg & high \\
\hline \multirow{3}{*}{ SI Conv } & Vehicle Cost LOW & $0.0 \%$ & $0.0 \%$ & $0.3 \%$ & $0.3 \%$ & $0.1 \%$ & $0.3 \%$ & $0.5 \%$ & $-5.8 \%$ & $-5.5 \%$ & $-5.7 \%$ & $-9.0 \%$ & $-8.5 \%$ & $-8.3 \%$ \\
\hline & Vehicle Cost AVG & $0.0 \%$ & $0.0 \%$ & $-0.3 \%$ & $-0.3 \%$ & $5.7 \%$ & $5.5 \%$ & $5.4 \%$ & $8.1 \%$ & $7.8 \%$ & $7.9 \%$ & $8.8 \%$ & $8.4 \%$ & $8.2 \%$ \\
\hline & Vehicle Cost HIGH & $0.0 \%$ & $0.0 \%$ & $-0.3 \%$ & $-0.3 \%$ & $1.0 \%$ & $0.8 \%$ & $0.7 \%$ & $4.2 \%$ & $3.9 \%$ & $4.0 \%$ & $4.7 \%$ & $4.3 \%$ & $4.0 \%$ \\
\hline \multirow{3}{*}{$\mathrm{Cl}$ Conv } & Vehicle Cost LOW & $0.0 \%$ & $0.0 \%$ & $-0.4 \%$ & $-0.6 \%$ & $-1.4 \%$ & $-1.5 \%$ & $-2.1 \%$ & $3.8 \%$ & $3.2 \%$ & $3.0 \%$ & $4.3 \%$ & $3.7 \%$ & $3.1 \%$ \\
\hline & Vehicle Cost AVG & $0.0 \%$ & $0.0 \%$ & $-0.4 \%$ & $-0.5 \%$ & $4.3 \%$ & $4.3 \%$ & $3.8 \%$ & $4.9 \%$ & $4.3 \%$ & $4.2 \%$ & $4.9 \%$ & $4.4 \%$ & $3.8 \%$ \\
\hline & Vehicle Cost HIGH & $0.0 \%$ & $0.0 \%$ & $-0.3 \%$ & $-0.4 \%$ & $0.0 \%$ & $0.0 \%$ & $-0.4 \%$ & $2.4 \%$ & $1.9 \%$ & $1.9 \%$ & $2.1 \%$ & $1.6 \%$ & $1.1 \%$ \\
\hline \multirow{3}{*}{ H2 Conv } & Vehicle Cost LOW & $0.0 \%$ & $0.0 \%$ & $-0.2 \%$ & $0.0 \%$ & $-0.6 \%$ & $-0.5 \%$ & $-0.6 \%$ & $5.2 \%$ & $5.0 \%$ & $5.1 \%$ & $7.2 \%$ & $6.9 \%$ & $6.6 \%$ \\
\hline & Vehicle Cost AVG & $0.0 \%$ & $0.0 \%$ & $-0.2 \%$ & $0.0 \%$ & $-2.0 \%$ & $-1.9 \%$ & $-2.0 \%$ & $-2.0 \%$ & $-2.3 \%$ & $-2.2 \%$ & $-9.9 \%$ & $-10.2 \%$ & $-10.4 \%$ \\
\hline & Vehicle cost HIGH & $0.0 \%$ & $0.0 \%$ & $-0.2 \%$ & $0.0 \%$ & $-7.1 \%$ & $-6.9 \%$ & $-7.1 \%$ & $-5.7 \%$ & $-5.9 \%$ & $-5.9 \%$ & $-8.5 \%$ & $-8.8 \%$ & $-9.1 \%$ \\
\hline \multirow{3}{*}{ E85 Conv } & Vehicle Cost LOW & $0.0 \%$ & $0.0 \%$ & $-0.3 \%$ & $-0.2 \%$ & $-0.1 \%$ & $-0.1 \%$ & $-0.5 \%$ & $6.1 \%$ & $5.7 \%$ & $5.6 \%$ & $9.1 \%$ & $8.6 \%$ & $8.1 \%$ \\
\hline & Vehicle cost AVG & $0.0 \%$ & $0.0 \%$ & $-0.3 \%$ & $-0.2 \%$ & $5.7 \%$ & $5.7 \%$ & $5.4 \%$ & $8.3 \%$ & $7.9 \%$ & $7.8 \%$ & $9.0 \%$ & $8.5 \%$ & $8.0 \%$ \\
\hline & Vehicle Cost HIGH & $0.0 \%$ & $0.0 \%$ & $-0.2 \%$ & $-0.2 \%$ & $1.1 \%$ & $1.0 \%$ & $0.7 \%$ & $4.4 \%$ & $4.0 \%$ & $3.9 \%$ & $4.8 \%$ & $4.3 \%$ & $3.9 \%$ \\
\hline \multirow{3}{*}{ SI Split HEV } & Vehicle Cost LOW & $0.0 \%$ & $0.0 \%$ & $-1.3 \%$ & $-2.4 \%$ & $-3.8 \%$ & $-5.2 \%$ & $-7.0 \%$ & $-3.0 \%$ & $-5.1 \%$ & $-7.4 \%$ & $-1.8 \%$ & $-4.0 \%$ & $-6.8 \%$ \\
\hline & Vehicle Cost AVG & $0.0 \%$ & $0.0 \%$ & $-1.2 \%$ & $-2.4 \%$ & $-2.8 \%$ & $-3.9 \%$ & $-5.3 \%$ & $-4.0 \%$ & $-5.7 \%$ & $-7.7 \%$ & $-4.3 \%$ & $-6.2 \%$ & $-8.4 \%$ \\
\hline & Vehicle Cost HIGH & $0.0 \%$ & $0.0 \%$ & $-1.0 \%$ & $-1.9 \%$ & $-5.6 \%$ & $-6.5 \%$ & $-7.6 \%$ & $-6.2 \%$ & $-7.4 \%$ & $-8.8 \%$ & $-7.8 \%$ & $-8.9 \%$ & $-10.3 \%$ \\
\hline \multirow{3}{*}{ SI Split HEV PHEV10 } & Vehicle Cost LOW & $0.0 \%$ & $0.0 \%$ & $-1.7 \%$ & $-3.4 \%$ & $-8.6 \%$ & $-10.4 \%$ & $-13.1 \%$ & $-10.3 \%$ & $-12.9 \%$ & $-15.6 \%$ & $-11.4 \%$ & $-13.7 \%$ & $-16.1 \%$ \\
\hline & Vehicle Cost AVG & $0.0 \%$ & $0.0 \%$ & $-1.6 \%$ & $-3.1 \%$ & $-7.6 \%$ & $-9.0 \%$ & $-11.0 \%$ & $-10.1 \%$ & $-12.0 \%$ & $-14.1 \%$ & $-11.0 \%$ & $-12.9 \%$ & $-15.0 \%$ \\
\hline & Vehicle Cost HIGH & $0.0 \%$ & $0.0 \%$ & $-1.3 \%$ & $-2.6 \%$ & $-12.7 \%$ & $-13.6 \%$ & $-15.2 \%$ & $-13.7 \%$ & $-15.3 \%$ & $-16.7 \%$ & $-15.8 \%$ & $-17.1 \%$ & $-18.3 \%$ \\
\hline \multirow{3}{*}{ SI Split HEV PHEV2O } & Vehicle Cost LOW & $0.0 \%$ & $0.0 \%$ & $-2.1 \%$ & $-4.4 \%$ & $-11.2 \%$ & $-13.4 \%$ & $-17.1 \%$ & $-15.0 \%$ & $-18.3 \%$ & $-21.5 \%$ & $-17.8 \%$ & $-20.4 \%$ & $-23.0 \%$ \\
\hline & Vehicle cost AVG & $0.0 \%$ & $0.0 \%$ & $-1.9 \%$ & $-4.0 \%$ & $-11.1 \%$ & $-12.7 \%$ & $-15.5 \%$ & $-15.4 \%$ & $-17.7 \%$ & $-20.1 \%$ & $-16.6 \%$ & $-18.9 \%$ & $-21.2 \%$ \\
\hline & Vehicle Cost HIGH & $0.0 \%$ & $0.0 \%$ & $-1.6 \%$ & $-3.4 \%$ & $-13.9 \%$ & $-15.2 \%$ & $-17.6 \%$ & $-16.2 \%$ & $-18.3 \%$ & $-20.4 \%$ & $-18.9 \%$ & $-20.9 \%$ & $-22.3 \%$ \\
\hline \multirow{3}{*}{ SI Series HEV PHEV30 } & Vehicle Cost LOW & $0.0 \%$ & $0.0 \%$ & $-3.2 \%$ & $-6.4 \%$ & $-16.2 \%$ & $-19.3 \%$ & $-25.2 \%$ & $-24.2 \%$ & $-29.3 \%$ & $-33.7 \%$ & $-29.5 \%$ & $-33.5 \%$ & $-37.0 \%$ \\
\hline & Vehicle Cost AVG & $0.0 \%$ & $0.0 \%$ & $-3.0 \%$ & $-6.1 \%$ & $-18.7 \%$ & $-21.2 \%$ & $-25.7 \%$ & $-26.1 \%$ & $-29.7 \%$ & $-33.1 \%$ & $-28.4 \%$ & $-32.0 \%$ & $-35.1 \%$ \\
\hline & Vehicle Cost $\mathrm{HIGH}$ & $0.0 \%$ & $0.0 \%$ & $-2.6 \%$ & $-5.3 \%$ & $-24.1 \%$ & $-25.9 \%$ & $-29.8 \%$ & $-29.1 \%$ & $-32.4 \%$ & $-35.1 \%$ & $-33.1 \%$ & $-36.0 \%$ & $-37.9 \%$ \\
\hline \multirow{3}{*}{ SI Series HEV PHEV40 } & Vehicle Cost LOW & $0.0 \%$ & $0.0 \%$ & $-3.4 \%$ & $-7.0 \%$ & $-17.7 \%$ & $-21.1 \%$ & $-27.8 \%$ & $-27.1 \%$ & $-32.6 \%$ & $-37.5 \%$ & $-33.5 \%$ & $-37.8 \%$ & $-41.3 \%$ \\
\hline & Vehicle Cost AVG & $0.0 \%$ & $0.0 \%$ & $-3.3 \%$ & $-6.6 \%$ & $-20.9 \%$ & $-23.6 \%$ & $-28.7 \%$ & $-29.6 \%$ & $-33.6 \%$ & $-37.2 \%$ & $-32.2 \%$ & $-36.1 \%$ & $-39.3 \%$ \\
\hline & Vehicle Cost HIGH & $0.0 \%$ & $0.0 \%$ & $-2.9 \%$ & $-5.9 \%$ & $-24.7 \%$ & $-26.9 \%$ & $-31.4 \%$ & $-30.7 \%$ & $-34.5 \%$ & $-37.8 \%$ & $-35.3 \%$ & $-38.6 \%$ & $-40.8 \%$ \\
\hline \multirow{3}{*}{ CI Split HEV } & Vehicle Cost LOW & $0.0 \%$ & $0.0 \%$ & $-1.3 \%$ & $-2.4 \%$ & $-5.3 \%$ & $-6.2 \%$ & $-7.9 \%$ & $-4.5 \%$ & $-6.6 \%$ & $-8.8 \%$ & $-4.9 \%$ & $-7.2 \%$ & $-9.6 \%$ \\
\hline & Vehicle Cost AVG & $0.0 \%$ & $0.0 \%$ & $-1.2 \%$ & $-2.4 \%$ & $-4.4 \%$ & $-5.1 \%$ & $-6.5 \%$ & $-6.4 \%$ & $-8.1 \%$ & $-9.9 \%$ & $-6.9 \%$ & $-8.8 \%$ & $-10.7 \%$ \\
\hline & Vehicle Cost HIGH & $0.0 \%$ & $0.0 \%$ & $-1.0 \%$ & $-1.9 \%$ & $-6.7 \%$ & $-7.3 \%$ & $-8.3 \%$ & $-7.8 \%$ & $-9.0 \%$ & $-10.3 \%$ & $-9.8 \%$ & $-10.9 \%$ & $-12.1 \%$ \\
\hline
\end{tabular}




\begin{tabular}{|c|c|c|c|c|c|c|c|c|c|c|c|c|c|c|}
\hline & & \multicolumn{13}{|c|}{ Compact } \\
\hline & & \multirow{2}{*}{$\begin{array}{c}2010 \\
\text { Ref }\end{array}$} & \multicolumn{3}{|c|}{2010} & \multicolumn{3}{|c|}{2015} & \multicolumn{3}{|c|}{2030} & \multicolumn{3}{|c|}{2045} \\
\hline & & & low & avg & high & low & avg & high & low & avg & high & low & avg & high \\
\hline \multirow{3}{*}{ CI Split HEV PHEV10 } & Vehicle Cost LOW & $0.0 \%$ & $0.0 \%$ & $-1.5 \%$ & $-3.1 \%$ & $-8.9 \%$ & $-10.7 \%$ & $-13.5 \%$ & $-11.0 \%$ & $-13.6 \%$ & $-16.2 \%$ & $-13.3 \%$ & $-15.5 \%$ & $-18.0 \%$ \\
\hline & Vehicle Cost AVG & $0.0 \%$ & $0.0 \%$ & $-1.4 \%$ & $-2.9 \%$ & $-8.1 \%$ & $-9.5 \%$ & $-11.6 \%$ & $-11.4 \%$ & $-13.4 \%$ & $-15.4 \%$ & $-12.6 \%$ & $-14.5 \%$ & $-16.6 \%$ \\
\hline & Vehicle Cost HIGH & $0.0 \%$ & $0.0 \%$ & $-1.2 \%$ & $-2.4 \%$ & $-13.3 \%$ & $-14.2 \%$ & $-15.9 \%$ & $-15.0 \%$ & $-16.5 \%$ & $-17.9 \%$ & $-17.3 \%$ & $-18.6 \%$ & $-19.7 \%$ \\
\hline \multirow{3}{*}{ CI Split HEV PHEV2O } & Vehicle Cost LOW & $0.0 \%$ & $0.0 \%$ & $-1.7 \%$ & $-3.6 \%$ & $-11.2 \%$ & $-13.2 \%$ & $-17.0 \%$ & $-14.9 \%$ & $-18.3 \%$ & $-21.5 \%$ & $-18.9 \%$ & $-21.5 \%$ & $-24.1 \%$ \\
\hline & Vehicle Cost AVG & $0.0 \%$ & $0.0 \%$ & $-1.6 \%$ & $-3.4 \%$ & $-11.2 \%$ & $-12.7 \%$ & $-15.5 \%$ & $-16.0 \%$ & $-18.5 \%$ & $-20.8 \%$ & $-17.6 \%$ & $-19.8 \%$ & $-22.1 \%$ \\
\hline & Vehicle Cost HIGH & $0.0 \%$ & $0.0 \%$ & $-1.3 \%$ & $-2.8 \%$ & $-14.3 \%$ & $-15.5 \%$ & $-17.9 \%$ & $-17.0 \%$ & $-19.2 \%$ & $-21.2 \%$ & $-20.1 \%$ & $-21.9 \%$ & $-23.3 \%$ \\
\hline \multirow{3}{*}{ CI Series HEV PHEV3O } & Vehicle Cost LOW & $0.0 \%$ & $0.0 \%$ & $-3.1 \%$ & $-6.2 \%$ & $-16.0 \%$ & $-19.0 \%$ & $-24.7 \%$ & $-23.8 \%$ & $-28.6 \%$ & $-32.9 \%$ & $-29.8 \%$ & $-33.5 \%$ & $-36.8 \%$ \\
\hline & \begin{tabular}{|l} 
Vehicle Cost AVG \\
(ehic Cost
\end{tabular} & $0.0 \%$ & $0.0 \%$ & $-2.9 \%$ & $-5.8 \%$ & $-18.3 \%$ & $-20.6 \%$ & $-25.0 \%$ & $-26.0 \%$ & $-29.5 \%$ & $-32.7 \%$ & $-28.4 \%$ & $-31.8 \%$ & $-34.7 \%$ \\
\hline & Vehicle Cost HIGH & $0.0 \%$ & $0.0 \%$ & $-2.6 \%$ & $-5.1 \%$ & $-23.6 \%$ & $-25.3 \%$ & $-29.1 \%$ & $-28.7 \%$ & $-31.8 \%$ & $-34.5 \%$ & $-32.8 \%$ & $-35.5 \%$ & $-37.2 \%$ \\
\hline \multirow{3}{*}{ CI Series HEV PHEV40 } & Vehicle Cost LOW & $0.0 \%$ & $0.0 \%$ & $-3.2 \%$ & $-6.6 \%$ & $-17.4 \%$ & $-20.8 \%$ & $-27.2 \%$ & $-26.6 \%$ & $-31.9 \%$ & $-36.6 \%$ & $-33.6 \%$ & $-37.7 \%$ & $-41.1 \%$ \\
\hline & Vehicle Cost AVG & $0.0 \%$ & $0.0 \%$ & $-3.0 \%$ & $-6.2 \%$ & $-20.3 \%$ & $-23.0 \%$ & $-27.9 \%$ & $-29.4 \%$ & $-33.2 \%$ & $-36.6 \%$ & $-32.1 \%$ & $-35.7 \%$ & $-38.8 \%$ \\
\hline & Vehicle Cost HIGH & $0.0 \%$ & $0.0 \%$ & $-2.7 \%$ & $-5.5 \%$ & $-24.0 \%$ & $-26.2 \%$ & $-30.6 \%$ & $-30.3 \%$ & $-33.9 \%$ & $-37.0 \%$ & $-34.9 \%$ & $-38.1 \%$ & $-40.1 \%$ \\
\hline \multirow{3}{*}{ H2 Split HEV } & Vehicle Cost LOW & $0.0 \%$ & $0.0 \%$ & $-1.8 \%$ & $-3.3 \%$ & $-4.9 \%$ & $-6.5 \%$ & $-8.6 \%$ & $-4.7 \%$ & $-7.0 \%$ & $-9.4 \%$ & $-4.5 \%$ & $-6.7 \%$ & $-9.0 \%$ \\
\hline & \begin{tabular}{|l} 
Vehicle Cost AVG \\
\end{tabular} & $0.0 \%$ & $0.0 \%$ & $-1.5 \%$ & $-2.7 \%$ & $-8.6 \%$ & $-9.7 \%$ & $-11.1 \%$ & $-11.5 \%$ & $-13.1 \%$ & $-14.8 \%$ & $-18.8 \%$ & $-20.3 \%$ & $-21.9 \%$ \\
\hline & Vehicle Cost HIGH & $0.0 \%$ & $0.0 \%$ & $-1.2 \%$ & $-2.2 \%$ & $-12.3 \%$ & $-13.2 \%$ & $-14.3 \%$ & $-14.4 \%$ & $-15.5 \%$ & $-16.6 \%$ & $-18.6 \%$ & $-19.5 \%$ & $-20.5 \%$ \\
\hline \multirow{3}{*}{ H2 Split HEV PHEV10 } & Vehicle Cost LOW & $0.0 \%$ & $0.0 \%$ & $-2.4 \%$ & $-4.4 \%$ & $-9.6 \%$ & $-12.0 \%$ & $-15.0 \%$ & $-12.2 \%$ & $-15.3 \%$ & $-18.1 \%$ & $-14.2 \%$ & $-16.4 \%$ & $-18.9 \%$ \\
\hline & 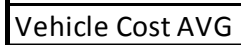 & $0.0 \%$ & $0.0 \%$ & $-1.9 \%$ & $-3.5 \%$ & $-12.3 \%$ & $-14.0 \%$ & $-15.9 \%$ & $-16.2 \%$ & $-18.3 \%$ & $-20.2 \%$ & $-23.4 \%$ & $-25.1 \%$ & $-27.0 \%$ \\
\hline & Vehicle Cost HIGH & $0.0 \%$ & $0.0 \%$ & $-1.7 \%$ & $-3.0 \%$ & $-18.5 \%$ & $-19.6 \%$ & $-21.1 \%$ & $-20.9 \%$ & $-22.4 \%$ & $-23.6 \%$ & $-25.3 \%$ & $-26.4 \%$ & $-27.3 \%$ \\
\hline \multirow{3}{*}{ H2 Split HEV PHEV2O } & Vehicle Cost LOW & $0.0 \%$ & $0.0 \%$ & $-2.8 \%$ & $-5.1 \%$ & $-11.7 \%$ & $-14.6 \%$ & $-18.8 \%$ & $-16.2 \%$ & $-20.3 \%$ & $-23.7 \%$ & $-20.1 \%$ & $-22.9 \%$ & $-25.5 \%$ \\
\hline & Vehicle Cost AVG & $0.0 \%$ & $0.0 \%$ & $-2.3 \%$ & $-4.1 \%$ & $-14.7 \%$ & $-16.7 \%$ & $-19.4 \%$ & $-20.2 \%$ & $-22.7 \%$ & $-24.9 \%$ & $-27.4 \%$ & $-29.5 \%$ & $-31.5 \%$ \\
\hline & Vehicle Cost HIGH & $0.0 \%$ & $0.0 \%$ & $-2.0 \%$ & $-3.6 \%$ & $-19.0 \%$ & $-20.5 \%$ & $-22.8 \%$ & $-22.5 \%$ & $-24.7 \%$ & $-26.5 \%$ & $-27.5 \%$ & $-29.2 \%$ & $-30.4 \%$ \\
\hline \multirow{3}{*}{ H2 Series HEV PHEV3O } & Vehicle Cost LOW & $0.0 \%$ & $0.0 \%$ & $-4.2 \%$ & $-7.8 \%$ & $-16.9 \%$ & $-20.7 \%$ & $-26.7 \%$ & $-25.4 \%$ & $-30.8 \%$ & $-35.3 \%$ & $-31.5 \%$ & $-35.3 \%$ & $-38.6 \%$ \\
\hline & Vehicle Cost AVG & $0.0 \%$ & $0.0 \%$ & $-3.6 \%$ & $-6.7 \%$ & $-21.0 \%$ & $-23.7 \%$ & $-27.8 \%$ & $-28.9 \%$ & $-32.4 \%$ & $-35.5 \%$ & $-35.6 \%$ & $-38.8 \%$ & $-41.5 \%$ \\
\hline & Vehicle Cost HIGH & $0.0 \%$ & $0.0 \%$ & $-3.2 \%$ & $-5.9 \%$ & $-27.1 \%$ & $-29.2 \%$ & $-32.7 \%$ & $-32.8 \%$ & $-35.9 \%$ & $-38.4 \%$ & $-38.4 \%$ & $-40.9 \%$ & $-42.5 \%$ \\
\hline \multirow{3}{*}{ H2 Series HEV PHEV40 } & Vehicle Cost LOW & $0.0 \%$ & $0.0 \%$ & $-4.5 \%$ & $-8.4 \%$ & $-18.4 \%$ & $-22.6 \%$ & $-29.2 \%$ & $-28.2 \%$ & $-34.0 \%$ & $-39.0 \%$ & $-35.4 \%$ & $-39.5 \%$ & $-42.9 \%$ \\
\hline & \begin{tabular}{|l} 
Vehicle Cost AVG \\
\end{tabular} & $0.0 \%$ & $0.0 \%$ & $-3.9 \%$ & $-7.3 \%$ & $-22.9 \%$ & $-25.9 \%$ & $-30.5 \%$ & $-32.0 \%$ & $-35.8 \%$ & $-39.1 \%$ & $-38.7 \%$ & $-42.1 \%$ & $-44.9 \%$ \\
\hline & \begin{tabular}{|l|} 
Vehicle Cost HIGH \\
\end{tabular} & $0.0 \%$ & $0.0 \%$ & $-3.5 \%$ & $-6.5 \%$ & $-27.5 \%$ & $-30.0 \%$ & $-34.1 \%$ & $-34.1 \%$ & $-37.7 \%$ & $-40.7 \%$ & $-40.1 \%$ & $-43.1 \%$ & $-45.0 \%$ \\
\hline \multirow{3}{*}{ E85 Split HEV } & Vehicle Cost LOW & $0.0 \%$ & $0.0 \%$ & $-1.1 \%$ & $-2.1 \%$ & $-4.0 \%$ & $-5.0 \%$ & $-7.0 \%$ & $-2.9 \%$ & $-5.0 \%$ & $-7.3 \%$ & $-1.8 \%$ & $-4.0 \%$ & $-6.6 \%$ \\
\hline & \begin{tabular}{|l} 
Vehicle Cost AVG \\
\end{tabular} & $0.0 \%$ & $0.0 \%$ & $-1.1 \%$ & $-2.1 \%$ & $-2.9 \%$ & $-3.7 \%$ & $-5.3 \%$ & $-3.9 \%$ & $-5.7 \%$ & $-7.6 \%$ & $-4.3 \%$ & $-6.1 \%$ & $-8.3 \%$ \\
\hline & Vehicle Cost HIGH & $0.0 \%$ & $0.0 \%$ & $-0.9 \%$ & $-1.6 \%$ & $-5.7 \%$ & $-6.3 \%$ & $-7.6 \%$ & $-6.2 \%$ & $-7.4 \%$ & $-8.7 \%$ & $-7.8 \%$ & $-8.9 \%$ & $-10.3 \%$ \\
\hline
\end{tabular}




\begin{tabular}{|c|c|c|c|c|c|c|c|c|c|c|c|c|c|c|}
\hline & & \multicolumn{13}{|c|}{ Compact } \\
\hline & & \multirow{2}{*}{$\begin{array}{l}2010 \\
\text { Ref } \\
\end{array}$} & \multicolumn{3}{|c|}{2010} & \multicolumn{3}{|c|}{2015} & \multicolumn{3}{|c|}{2030} & \multicolumn{3}{|c|}{2045} \\
\hline & & & low & avg & high & low & avg & high & low & avg & high & low & avg & high \\
\hline \multirow{3}{*}{ E85 Split HEV PHEV10 } & Vehicle Cost LOW & $0.0 \%$ & $0.0 \%$ & $-1.7 \%$ & $-3.4 \%$ & $-8.6 \%$ & $-10.3 \%$ & $-13.0 \%$ & $-10.3 \%$ & $-13.0 \%$ & $-15.6 \%$ & $-11.4 \%$ & $-13.6 \%$ & $-16.1 \%$ \\
\hline & Vehicle Cost AVG & $0.0 \%$ & $0.0 \%$ & $-1.6 \%$ & $-3.1 \%$ & $-7.6 \%$ & $-9.0 \%$ & $-11.0 \%$ & $-10.0 \%$ & $-12.0 \%$ & $-14.1 \%$ & $-11.0 \%$ & $-12.9 \%$ & $-15.0 \%$ \\
\hline & Vehicle Cost HIGH & $0.0 \%$ & $0.0 \%$ & $-1.4 \%$ & $-2.6 \%$ & $-12.7 \%$ & $-13.6 \%$ & $-15.2 \%$ & $-13.7 \%$ & $-15.3 \%$ & $-16.7 \%$ & $-15.8 \%$ & $-17.1 \%$ & $-18.3 \%$ \\
\hline \multirow{3}{*}{ E85 Split HEV PHEV20 } & Vehicle Cost LOW & $0.0 \%$ & $0.0 \%$ & $-2.1 \%$ & $-4.4 \%$ & $-11.2 \%$ & $-13.4 \%$ & $-17.1 \%$ & $-15.0 \%$ & $-18.2 \%$ & $-21.5 \%$ & $-17.8 \%$ & $-20.4 \%$ & $-23.0 \%$ \\
\hline & Vehicle Cost AVG & $0.0 \%$ & $0.0 \%$ & $-1.9 \%$ & $-4.0 \%$ & $-11.1 \%$ & $-12.7 \%$ & $-15.5 \%$ & $-15.4 \%$ & $-17.7 \%$ & $-20.1 \%$ & $-16.6 \%$ & $-18.9 \%$ & $-21.2 \%$ \\
\hline & Vehicle Cost HIGH & $0.0 \%$ & $0.0 \%$ & $-1.6 \%$ & $-3.4 \%$ & $-13.9 \%$ & $-15.2 \%$ & $-17.6 \%$ & $-16.2 \%$ & $-18.3 \%$ & $-20.4 \%$ & $-18.9 \%$ & $-20.8 \%$ & $-22.3 \%$ \\
\hline \multirow{3}{*}{ E85 Series HEV PHEV30 } & Vehicle Cost LOW & $0.0 \%$ & $0.0 \%$ & $-3.2 \%$ & $-6.4 \%$ & $-16.2 \%$ & $-19.2 \%$ & $-25.2 \%$ & $-24.2 \%$ & $-29.2 \%$ & $-33.7 \%$ & $-29.5 \%$ & $-33.5 \%$ & $-36.9 \%$ \\
\hline & Vehicle cost AVG & $0.0 \%$ & $0.0 \%$ & $-3.0 \%$ & $-6.1 \%$ & $-18.7 \%$ & $-21.1 \%$ & $-25.7 \%$ & $-26.0 \%$ & $-29.7 \%$ & $-33.1 \%$ & $-28.4 \%$ & $-32.0 \%$ & $-35.1 \%$ \\
\hline & \begin{tabular}{|l|} 
Vehicle Cost HIGH \\
\end{tabular} & $0.0 \%$ & $0.0 \%$ & $-2.6 \%$ & $-5.3 \%$ & $-24.1 \%$ & $-25.9 \%$ & $-29.8 \%$ & $-29.1 \%$ & $-32.4 \%$ & $-35.1 \%$ & $-33.1 \%$ & $-35.9 \%$ & $-37.9 \%$ \\
\hline \multirow{3}{*}{ E85 Series HEV PHEV40 } & Vehicle Cost LOW & $0.0 \%$ & $0.0 \%$ & $-3.5 \%$ & $-7.0 \%$ & $-17.7 \%$ & $-21.2 \%$ & $-27.8 \%$ & $-27.1 \%$ & $-32.6 \%$ & $-37.5 \%$ & $-33.5 \%$ & $-37.8 \%$ & $-41.3 \%$ \\
\hline & Vehicle Cost AVG & $0.0 \%$ & $0.0 \%$ & $-3.3 \%$ & $-6.7 \%$ & $-21.0 \%$ & $-23.7 \%$ & $-28.7 \%$ & $-29.7 \%$ & $-33.6 \%$ & $-37.2 \%$ & $-32.2 \%$ & $-36.1 \%$ & $-39.3 \%$ \\
\hline & Vehicle Cost HIGH & $0.0 \%$ & $0.0 \%$ & $-2.9 \%$ & $-5.9 \%$ & $-24.7 \%$ & $-26.9 \%$ & $-31.4 \%$ & $-30.7 \%$ & $-34.5 \%$ & $-37.8 \%$ & $-35.3 \%$ & $-38.6 \%$ & $-40.8 \%$ \\
\hline \multirow{3}{*}{ FC HEV } & Vehicle Cost LOW & $0.0 \%$ & $0.0 \%$ & $-7.2 \%$ & $-11.4 \%$ & $-3.6 \%$ & $-11.2 \%$ & $-16.7 \%$ & $-22.2 \%$ & $-26.4 \%$ & $-29.8 \%$ & $-29.4 \%$ & $-31.8 \%$ & $-34.3 \%$ \\
\hline & Vehicle Cost AVG & $0.0 \%$ & $0.0 \%$ & $-8.7 \%$ & $-14.4 \%$ & $-18.2 \%$ & $-24.0 \%$ & $-28.3 \%$ & $-29.7 \%$ & $-33.9 \%$ & $-36.6 \%$ & $-40.3 \%$ & $-42.3 \%$ & $-44.1 \%$ \\
\hline & Vehicle Cost HIGH & $0.0 \%$ & $0.0 \%$ & $-7.8 \%$ & $-12.8 \%$ & $-25.3 \%$ & $-29.0 \%$ & $-31.9 \%$ & $-31.1 \%$ & $-34.0 \%$ & $-35.9 \%$ & $-38.7 \%$ & $-40.1 \%$ & $-41.2 \%$ \\
\hline \multirow{3}{*}{ FC HEV PHEV10 } & Vehicle Cost LOW & $0.0 \%$ & $0.0 \%$ & $-6.8 \%$ & $-11.2 \%$ & $-8.5 \%$ & $-13.9 \%$ & $-19.3 \%$ & $-23.0 \%$ & $-27.8 \%$ & $-31.6 \%$ & $-30.5 \%$ & $-33.2 \%$ & $-36.1 \%$ \\
\hline & Vehicle Cost AVG & $0.0 \%$ & $0.0 \%$ & $-7.7 \%$ & $-13.4 \%$ & $-19.2 \%$ & $-23.8 \%$ & $-28.2 \%$ & $-29.3 \%$ & $-33.8 \%$ & $-36.4 \%$ & $-39.4 \%$ & $-41.6 \%$ & $-43.7 \%$ \\
\hline & Vehicle cost HIGH & $0.0 \%$ & $0.0 \%$ & $-6.9 \%$ & $-11.9 \%$ & $-27.5 \%$ & $-30.5 \%$ & $-33.5 \%$ & $-32.5 \%$ & $-35.6 \%$ & $-37.5 \%$ & $-39.8 \%$ & $-41.3 \%$ & $-42.6 \%$ \\
\hline \multirow{3}{*}{ FC HEV PHEV2O } & Vehicle Cost LOW & $0.0 \%$ & $0.0 \%$ & $-7.0 \%$ & $-11.9 \%$ & $-10.8 \%$ & $-17.0 \%$ & $-22.9 \%$ & $-26.7 \%$ & $-32.1 \%$ & $-36.4 \%$ & $-35.4 \%$ & $-38.3 \%$ & $-41.4 \%$ \\
\hline & \begin{tabular}{|l|} 
Vehicle Cost AVG \\
\end{tabular} & $0.0 \%$ & $0.0 \%$ & $-7.8 \%$ & $-13.8 \%$ & $-21.2 \%$ & $-26.3 \%$ & $-31.0 \%$ & $-32.7 \%$ & $-37.3 \%$ & $-40.4 \%$ & $-42.5 \%$ & $-45.0 \%$ & $-47.4 \%$ \\
\hline & Vehicle Cost HIGH & $0.0 \%$ & $0.0 \%$ & $-7.0 \%$ & $-12.3 \%$ & $-27.8 \%$ & $-31.3 \%$ & $-34.9 \%$ & $-33.9 \%$ & $-37.6 \%$ & $-40.1 \%$ & $-41.5 \%$ & $-43.6 \%$ & $-45.2 \%$ \\
\hline \multirow{3}{*}{ FC HEV PHEV 30} & Vehicle Cost LOW & $0.0 \%$ & $0.0 \%$ & $-6.4 \%$ & $-10.8 \%$ & $-14.2 \%$ & $-19.4 \%$ & $-25.8 \%$ & $-29.5 \%$ & $-35.0 \%$ & $-39.6 \%$ & $-38.7 \%$ & $-42.3 \%$ & $-45.5 \%$ \\
\hline & Vehicle Cost AVG & $0.0 \%$ & $0.0 \%$ & $-7.2 \%$ & $-12.6 \%$ & $-23.2 \%$ & $-27.7 \%$ & $-32.9 \%$ & $-34.8 \%$ & $-39.5 \%$ & $-43.1 \%$ & $-44.4 \%$ & $-47.4 \%$ & $-50.0 \%$ \\
\hline & Vehicle Cost HIGH & $0.0 \%$ & $0.0 \%$ & $-6.6 \%$ & $-11.4 \%$ & $-33.3 \%$ & $-36.4 \%$ & $-40.4 \%$ & $-39.7 \%$ & $-43.4 \%$ & $-46.1 \%$ & $-47.1 \%$ & $-49.6 \%$ & $-51.1 \%$ \\
\hline \multirow{3}{*}{ FC HEV PHEV40 } & Vehicle Cost LOW & $0.0 \%$ & $0.0 \%$ & $-6.8 \%$ & $-11.6 \%$ & $-15.9 \%$ & $-21.6 \%$ & $-28.7 \%$ & $-32.2 \%$ & $-38.2 \%$ & $-42.9 \%$ & $-42.1 \%$ & $-45.9 \%$ & $-49.1 \%$ \\
\hline & Vehicle Cost AVG & $0.0 \%$ & $0.0 \%$ & $-7.5 \%$ & $-13.1 \%$ & $-24.9 \%$ & $-29.7 \%$ & $-35.3 \%$ & $-37.5 \%$ & $-42.4 \%$ & $-46.2 \%$ & $-47.0 \%$ & $-50.2 \%$ & $-52.8 \%$ \\
\hline & Vehicle Cost HIGH & $0.0 \%$ & $0.0 \%$ & $-6.9 \%$ & $-12.0 \%$ & $-33.5 \%$ & $-37.1 \%$ & $-41.7 \%$ & $-40.8 \%$ & $-45.0 \%$ & $-48.0 \%$ & $-48.6 \%$ & $-51.4 \%$ & $-53.1 \%$ \\
\hline \multirow{3}{*}{ EV } & Vehicle Cost LOW & $0.0 \%$ & $0.0 \%$ & $-4.8 \%$ & $-10.6 \%$ & $-27.0 \%$ & $-32.3 \%$ & $-44.4 \%$ & $-43.6 \%$ & $-52.9 \%$ & $-59.6 \%$ & $-57.4 \%$ & $-63.4 \%$ & $-66.8 \%$ \\
\hline & Vehicle Cost AVG & $0.0 \%$ & $0.0 \%$ & $-4.6 \%$ & $-10.2 \%$ & $-34.0 \%$ & $-38.2 \%$ & $-47.6 \%$ & $-51.4 \%$ & $-57.4 \%$ & $-61.8 \%$ & $-55.6 \%$ & $-61.1 \%$ & $-64.4 \%$ \\
\hline & Vehicle Cost $\mathrm{HIGH}$ & $0.0 \%$ & $0.0 \%$ & $-4.3 \%$ & $-9.4 \%$ & $-28.1 \%$ & $-32.5 \%$ & $-43.1 \%$ & $-53.9 \%$ & $-58.4 \%$ & $-61.6 \%$ & $-55.1 \%$ & $-60.1 \%$ & $-62.6 \%$ \\
\hline
\end{tabular}




\section{$\underline{\text { Midsize Class }}$}

Incremental Cost versus the Conventional 2010 Reference 


\begin{tabular}{|c|c|c|c|c|c|c|c|c|c|c|c|c|c|c|}
\hline & & \multicolumn{13}{|c|}{ Midsize } \\
\hline & & \multirow{2}{*}{$\frac{2010}{\operatorname{Ref}}$} & \multicolumn{3}{|c|}{2010} & \multicolumn{3}{|c|}{2015} & \multicolumn{3}{|c|}{2030} & \multicolumn{3}{|c|}{2045} \\
\hline & & & low & avg & high & low & avg & high & low & avg & high & low & avg & high \\
\hline \multirow{3}{*}{ SI Conv } & Vehicle Cost LOW & 0 & 0 & -40 & -31 & 38 & 12 & -17 & 830 & 790 & 745 & 1223 & 1169 & 1090 \\
\hline & Vehicle Cost AVG & 0 & 0 & -40 & -31 & 837 & 813 & 786 & 1153 & 1107 & 1057 & 1201 & 1149 & 1073 \\
\hline & Vehicle Cost HIGH & 0 & 0 & -40 & -31 & 204 & 178 & 148 & 637 & 594 & 546 & 661 & 609 & 532 \\
\hline \multirow{3}{*}{ Cl Conv } & Vehicle Cost LOW & 1992 & 1992 & 1913 & 1901 & 1821 & 1755 & 1705 & 2556 & 2483 & 2392 & 2585 & 2535 & 2399 \\
\hline & Vehicle Cost AVG & 1928 & 1928 & 1854 & 1846 & 2656 & 2601 & 2560 & 2653 & 2594 & 2519 & 2609 & 2571 & 2454 \\
\hline & Vehicle Cost HIGH & 1633 & 1633 & 1572 & 1571 & 1676 & 1638 & 1612 & 1988 & 1940 & 1878 & 1888 & 1861 & 1760 \\
\hline \multirow{3}{*}{ H2 Conv } & Vehicle Cost LOW & -525 & -525 & -564 & -557 & -578 & -594 & -590 & 137 & 123 & 113 & 369 & 366 & 316 \\
\hline & \begin{tabular}{|l} 
Vehicle Cost AVG \\
\end{tabular} & 2488 & 2488 & 2447 & 2453 & 2182 & 2169 & 2174 & 2113 & 2097 & 2086 & 793 & 789 & 738 \\
\hline & Vehicle Cost HIGH & 1933 & 1933 & 1892 & 1898 & 803 & 783 & 784 & 971 & 944 & 922 & 474 & 458 & 394 \\
\hline \multirow{3}{*}{ E85 Conv } & Vehicle Cost LOW & -25 & -25 & -65 & -64 & 14 & -16 & -45 & 811 & 752 & 707 & 1196 & 1141 & 1043 \\
\hline & Vehicle Cost AVG & -24 & -24 & -65 & -63 & 815 & 787 & 761 & 1133 & 1068 & 1017 & 1175 & 1123 & 1029 \\
\hline & Vehicle Cost HIGH & -25 & -25 & -65 & -64 & 179 & 149 & 120 & 616 & 553 & 505 & 632 & 579 & 484 \\
\hline \multirow{3}{*}{ SI Split HEV } & Vehicle Cost LOW & 3876 & 3876 & 3624 & 3397 & 3086 & 2815 & 2524 & 3214 & 2741 & 2236 & 3390 & 2895 & 2343 \\
\hline & Vehicle Cost AVG & 3730 & 3730 & 3484 & 3263 & 3040 & 2822 & 2588 & 2825 & 2432 & 2014 & 2749 & 2341 & 1888 \\
\hline & Vehicle Cost HIGH & 2593 & 2593 & 2400 & 2227 & 1473 & 1305 & 1126 & 1340 & 1070 & 786 & 1035 & 781 & 506 \\
\hline \multirow{3}{*}{ SI Split HEV PHEV10 } & Vehicle Cost LOW & 6632 & 6632 & 6261 & 5885 & 4737 & 4323 & 3687 & 4211 & 3590 & 2991 & 3938 & 3430 & 2835 \\
\hline & Vehicle Cost AVG & 5911 & 5911 & 5579 & 5242 & 4178 & 3869 & 3408 & 3594 & 3139 & 2683 & 3396 & 2970 & 2462 \\
\hline & Vehicle Cost HIGH & 4656 & 4656 & 4388 & 4112 & 1941 & 1729 & 1370 & 1645 & 1308 & 1000 & 1225 & 925 & 683 \\
\hline \multirow{3}{*}{ SI Split HEV PHEV2O } & Vehicle Cost LOW & 8917 & 8917 & 8373 & 7803 & 6185 & 5580 & 4656 & 5128 & 4273 & 3438 & 4409 & 3729 & 3051 \\
\hline & Vehicle Cost AVG & 7828 & 7828 & 7347 & 6848 & 5138 & 4701 & 4047 & 4071 & 3495 & 2905 & 3776 & 3208 & 2633 \\
\hline & Vehicle Cost HIGH & 6123 & 6123 & 5736 & 5337 & 2951 & 2608 & 2061 & 2359 & 1841 & 1369 & 1751 & 1288 & 948 \\
\hline \multirow{3}{*}{ SI Series HEV PHEV3O } & Vehicle Cost LOW & 18510 & 18510 & 17207 & 16153 & 12980 & 11790 & 9756 & 10098 & 8311 & 6745 & 8316 & 6875 & 5692 \\
\hline & Vehicle Cost AVG & 16275 & 16275 & 15127 & 14197 & 10212 & 9337 & 7881 & 7818 & 6587 & 5469 & 7090 & 5881 & 4878 \\
\hline & Vehicle Cost HIGH & 12991 & 12991 & 12065 & 11315 & 5945 & 5333 & 4192 & 4406 & 3410 & 2593 & 3260 & 2385 & 1830 \\
\hline \multirow{3}{*}{ SI Series HEV PHEV4O } & Vehicle Cost LOW & 21767 & 21767 & 20226 & 18963 & 15067 & 13672 & 11162 & 11446 & 9304 & 7429 & 9059 & 7379 & 6059 \\
\hline & Vehicle Cost AVG & 19023 & 19023 & 17673 & 16567 & 11623 & 10608 & 8828 & 8572 & 7140 & 5849 & 7696 & 6292 & 5177 \\
\hline & Vehicle Cost HIGH & 15107 & 15107 & 14025 & 13139 & 7329 & 6584 & 5131 & 5380 & 4134 & 3091 & 3969 & 2873 & 2191 \\
\hline \multirow{3}{*}{ CI Split HEV } & Vehicle Cost LOW & 6359 & 6359 & 6049 & 5681 & 5277 & 4945 & 4532 & 5233 & 4727 & 4172 & 5107 & 4632 & 4034 \\
\hline & Vehicle Cost AVG & 6177 & 6177 & 5879 & 5524 & 5159 & 4896 & 4568 & 4610 & 4205 & 3761 & 4474 & 4092 & 3612 \\
\hline & Vehicle Cost HIGH & 4662 & 4662 & 4434 & 4163 & 3252 & 3058 & 2818 & 2901 & 2631 & 2338 & 2478 & 2258 & 1983 \\
\hline
\end{tabular}




\begin{tabular}{|c|c|c|c|c|c|c|c|c|c|c|c|c|c|c|}
\hline & & \multicolumn{13}{|c|}{ Midsize } \\
\hline & & \multirow{2}{*}{$\begin{array}{l}2010 \\
\text { Ref }\end{array}$} & \multicolumn{3}{|c|}{2010} & \multicolumn{3}{|c|}{2015} & \multicolumn{3}{|c|}{2030} & \multicolumn{3}{|c|}{2045} \\
\hline & & & low & avg & high & low & avg & high & low & avg & high & low & avg & high \\
\hline \multirow{3}{*}{ CI Split HEV PHEV10 } & Vehicle Cost LOW & 9366 & 9366 & 8929 & 8504 & 7085 & 6632 & 5904 & 6440 & 5764 & 5058 & 5855 & 5275 & 4610 \\
\hline & Vehicle Cost AVG & 8565 & 8565 & 8173 & 7793 & 6443 & 6102 & 5572 & 5598 & 5109 & 4571 & 5325 & 4843 & 4282 \\
\hline & Vehicle Cost HIGH & 6961 & 6961 & 6648 & 6340 & 3747 & 3523 & 3125 & 3295 & 2936 & 2598 & 2762 & 2447 & 2192 \\
\hline \multirow{3}{*}{ CI Split HEV PHEV2O } & Vehicle Cost LOW & 11767 & 11767 & 11154 & 10590 & 8625 & 6073 & 6940 & 7412 & 6474 & 5561 & 6352 & 5600 & 4874 \\
\hline & Vehicle Cost AVG & 10576 & 10576 & 10037 & 9539 & 7465 & 5795 & 6256 & 6105 & 5472 & 4832 & 5725 & 5103 & 4494 \\
\hline & Vehicle Cost HIGH & 8494 & 8494 & 8069 & 7672 & 4819 & 3387 & 3859 & 4046 & 3496 & 2993 & 3310 & 2829 & 2476 \\
\hline \multirow{3}{*}{ CI Series HEV PHEV 30} & Vehicle Cost LOW & 21135 & 21135 & 19799 & 18670 & 15220 & 14009 & 11873 & 12205 & 10370 & 8736 & 10062 & 8597 & 7378 \\
\hline & Vehicle Cost AVG & 18789 & 18789 & 17611 & 16620 & 12390 & 11497 & 9965 & 9681 & 8426 & 7270 & 8867 & 7647 & 6622 \\
\hline & Vehicle Cost HIGH & 15161 & 15161 & 14217 & 13423 & 7734 & 7117 & 5929 & 6036 & 5025 & 4177 & 4776 & 3906 & 3342 \\
\hline \multirow{3}{*}{ CI Series HEV PHEV40 } & Vehicle Cost LOW & 24555 & 24555 & 22927 & 21623 & 17390 & 15990 & 13354 & 13621 & 11407 & 9466 & 10843 & 9106 & 7758 \\
\hline & Vehicle Cost AVG & 21673 & 21673 & 20249 & 19109 & 13857 & 12836 & 10964 & 10469 & 9002 & 7675 & 9503 & 8060 & 6931 \\
\hline & Vehicle Cost HIGH & 17379 & 17379 & 16245 & 15337 & 9170 & 8431 & 6914 & 7059 & 5777 & 4707 & 5521 & 4395 & 3708 \\
\hline \multirow{3}{*}{ H2 Split HEV } & Vehicle Cost LOW & 3870 & 3870 & 3498 & 3211 & 2913 & 2583 & 2225 & 2854 & 2360 & 1890 & 2846 & 2422 & 1919 \\
\hline & Vehicle cost AVG & 6732 & 6732 & 6369 & 6089 & 4780 & 4521 & 4239 & 4127 & 3731 & 3353 & 2638 & 2295 & 1889 \\
\hline & Vehicle Cost HIGH & 4974 & 4974 & 4696 & 4482 & 2437 & 2241 & 2030 & 1963 & 1698 & 1448 & 1113 & 912 & 679 \\
\hline \multirow{3}{*}{ H2 Split HEV PHEV10 } & Vehicle Cost LOW & 7048 & 7048 & 6460 & 6007 & 4853 & 4313 & 3576 & 4200 & 3426 & 2783 & 3694 & 3151 & 2543 \\
\hline & Vehicle Cost AVG & 9289 & 9289 & 8759 & 8353 & 6171 & 5769 & 5230 & 5225 & 4647 & 4160 & 3552 & 3097 & 2580 \\
\hline & Vehicle Cost HIGH & 7384 & 7384 & 6954 & 6623 & 3013 & 2755 & 2380 & 2409 & 2043 & 1739 & 1428 & 1140 & 918 \\
\hline \multirow{3}{*}{ H2 Split HEV PHEV2O } & Vehicle Cost LOW & 9379 & 9379 & 8578 & 7954 & 6317 & 5587 & 4535 & 5123 & 4110 & 3222 & 4159 & 3434 & 2758 \\
\hline & \begin{tabular}{|l|} 
Vehicle Cost AVG \\
\end{tabular} & 11241 & 11241 & 10533 & 9982 & 7149 & 6614 & 5863 & 5704 & 5007 & 4379 & 3926 & 3322 & 2751 \\
\hline & Vehicle Cost HIGH & 8874 & 8874 & 8307 & 7865 & 4010 & 3643 & 3060 & 3125 & 2568 & 2096 & 1944 & 1495 & 1176 \\
\hline \multirow{3}{*}{ H2 Series HEV PHEV 30} & Vehicle Cost LOW & 18563 & 18563 & 16947 & 15782 & 12787 & 11384 & 9326 & 9784 & 7883 & 6302 & 7742 & 6344 & 5217 \\
\hline & Vehicle Cost AVG & 19305 & 19305 & 17880 & 16854 & 11950 & 10924 & 9457 & 9172 & 7871 & 6751 & 6961 & 5792 & 4841 \\
\hline & Vehicle Cost HIGH & 15414 & 15414 & 14269 & 13445 & 6895 & 6193 & 5056 & 5068 & 4040 & 3236 & 3350 & 2515 & 2012 \\
\hline \multirow{3}{*}{ H2 Series HEV PHEV40 } & Vehicle Cost LOW & 21896 & 21896 & 19908 & 18497 & 14915 & 13247 & 10704 & 11135 & 8859 & 6962 & 8464 & 6830 & 5561 \\
\hline & Vehicle Cost AVG & 22114 & 22114 & 20377 & 19142 & 13385 & 12180 & 10384 & 9921 & 8409 & 7114 & 7548 & 6187 & 5120 \\
\hline & Vehicle Cost HIGH & 17574 & 17574 & 16189 & 15204 & 8304 & 7428 & 5974 & 6045 & 4749 & 3718 & 4040 & 2989 & 2348 \\
\hline \multirow{3}{*}{ E85 Split HEV } & Vehicle Cost LOW & 3911 & 3911 & 3643 & 3416 & 3132 & 2886 & 2497 & 3192 & 2764 & 2294 & 3413 & 2954 & 2383 \\
\hline & Vehicle Cost AVG & 3765 & 3765 & 3504 & 3283 & 3080 & 2880 & 2573 & 2809 & 2455 & 2065 & 2772 & 2394 & 1924 \\
\hline & Vehicle Cost HIGH & 2623 & 2623 & 2419 & 2246 & 1510 & 1359 & 1111 & 1329 & 1089 & 821 & 1053 & 820 & 534 \\
\hline
\end{tabular}




\begin{tabular}{|c|c|c|c|c|c|c|c|c|c|c|c|c|c|c|}
\hline & & \multicolumn{13}{|c|}{ Midsize } \\
\hline & & \multirow{2}{*}{$\frac{2010}{\operatorname{Ref}}$} & \multicolumn{3}{|c|}{2010} & \multicolumn{3}{|c|}{2015} & \multicolumn{3}{|c|}{2030} & \multicolumn{3}{|c|}{2045} \\
\hline & & & low & avg & high & low & avg & high & low & avg & high & low & avg & high \\
\hline \multirow{3}{*}{ E85 Split HEV PHEV10 } & Vehicle Cost LOW & 6657 & 6657 & 6286 & 5910 & 4760 & 4347 & 3710 & 4238 & 3612 & 3013 & 3964 & 3455 & 2861 \\
\hline & Vehicle Cost AVG & 5936 & 5936 & 5605 & 5267 & 4200 & 3892 & 3431 & 3621 & 3163 & 2706 & 3421 & 2994 & 2486 \\
\hline & Vehicle Cost HIGH & 4679 & 4679 & 4411 & 4135 & 1960 & 1748 & 1388 & 1665 & 1326 & 1018 & 1244 & 942 & 700 \\
\hline \multirow{3}{*}{ E85 Split HEV PHEV20 } & \begin{tabular}{|l|} 
Vehicle Cost LOW \\
\end{tabular} & 8938 & 8938 & 8399 & 7828 & 6210 & 5642 & 4680 & 5156 & 4297 & 3462 & 4436 & 3749 & 3074 \\
\hline & Vehicle Cost AVG & 7849 & 7849 & 7373 & 6873 & 5162 & 4750 & 4070 & 4106 & 3518 & 2929 & 3801 & 3228 & 2655 \\
\hline & Vehicle Cost HIGH & 6143 & 6143 & 5759 & 5360 & 2971 & 2655 & 2080 & 2365 & 1860 & 1386 & 1770 & 1305 & 966 \\
\hline \multirow{3}{*}{ E85 Series HEV PHEV 30} & Vehicle Cost LOW & 18546 & 18546 & 17253 & 16185 & 13006 & 11819 & 9786 & 10134 & 8341 & 6770 & 8345 & 6900 & 5720 \\
\hline & \begin{tabular}{|l} 
Vehicle Cost AVG \\
\end{tabular} & 16310 & 16310 & 15170 & 14228 & 10236 & 9364 & 7909 & 7852 & 6616 & 5494 & 7117 & 5905 & 4904 \\
\hline & \begin{tabular}{|l|} 
Vehicle Cost HIGH \\
\end{tabular} & 13021 & 13021 & 12102 & 11343 & 5965 & 5355 & 4214 & 4431 & 3432 & 2612 & 3280 & 2404 & 1849 \\
\hline \multirow{3}{*}{ E85 Series HEV PHEV40 } & Vehicle Cost LOW & 21782 & 21782 & 20282 & 18996 & 15097 & 13702 & 11195 & 11483 & 9334 & 7457 & 9090 & 7406 & 6087 \\
\hline & \begin{tabular}{|l|} 
Vehicle Cost AVG \\
\end{tabular} & 19040 & 19040 & 17724 & 16598 & 11650 & 10636 & 8858 & 8606 & 7168 & 5875 & 7726 & 6317 & 5203 \\
\hline & Vehicle Cost HIGH & 15125 & 15125 & 14068 & 13167 & 7352 & 6607 & 5155 & 5406 & 4155 & 3111 & 3991 & 2892 & 2210 \\
\hline \multirow{3}{*}{ FC HEV } & Vehicle Cost LOW & 9593 & 9593 & 7779 & 6675 & 8955 & 6846 & 5404 & 3960 & 2889 & 1884 & 2080 & 1418 & 731 \\
\hline & Vehicle Cost AVG & 14063 & 14063 & 11368 & 9650 & 8699 & 6791 & 5473 & 5114 & 3835 & 2756 & 1972 & 1327 & 707 \\
\hline & Vehicle Cost HIGH & 11152 & 11152 & 8931 & 7515 & 4254 & 3110 & 2290 & 2513 & 1694 & 985 & 402 & -5 & -390 \\
\hline \multirow{3}{*}{ FC HEV PHEV10 } & Vehicle Cost LOW & 11355 & 11355 & 9465 & 8277 & 9146 & 7555 & 6059 & 4988 & 3673 & 2685 & 3027 & 2257 & 1420 \\
\hline & Vehicle Cost AVG & 14924 & 14924 & 12428 & 10713 & 8976 & 7463 & 6094 & 5785 & 4392 & 3416 & 2796 & 2065 & 1324 \\
\hline & Vehicle Cost HIGH & 11877 & 11877 & 9822 & 8410 & 4063 & 3160 & 2293 & 2532 & 1648 & 1044 & 547 & 78 & -312 \\
\hline \multirow{3}{*}{ FC HEV PHEV2O } & Vehicle Cost LOW & 14390 & 14390 & 12086 & 10573 & 11075 & 9035 & 7265 & 6097 & 4447 & 3143 & 3550 & 2620 & 1633 \\
\hline & Vehicle Cost AVG & 17564 & 17564 & 14691 & 12697 & 10293 & 8477 & 6919 & 6414 & 4822 & 3642 & 3225 & 2361 & 1494 \\
\hline & Vehicle Cost HIGH & 13909 & 13909 & 11567 & 9936 & 5346 & 4192 & 3112 & 3389 & 2255 & 1410 & 1129 & 483 & -54 \\
\hline \multirow{3}{*}{ FC HEV PHEV 30} & Vehicle Cost LOW & 19681 & 19681 & 17209 & 15680 & 14666 & 12645 & 10379 & 9144 & 7152 & 5476 & 5964 & 4632 & 3504 \\
\hline & Vehicle Cost AVG & 22244 & 22244 & 19222 & 17239 & 13363 & 11481 & 9539 & 8913 & 7068 & 5530 & 5360 & 4146 & 3129 \\
\hline & Vehicle Cost HIGH & 17858 & 17858 & 15384 & 13764 & 6491 & 5334 & 3989 & 4244 & 2949 & 1935 & 1739 & 883 & 328 \\
\hline \multirow{3}{*}{ FC HEV PHEV40 } & Vehicle Cost LOW & 22868 & 22868 & 19889 & 18119 & 16628 & 14298 & 11588 & 10341 & 7975 & 6077 & 6604 & 5046 & 3823 \\
\hline & 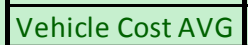 & 25061 & 25061 & 21590 & 19378 & 14759 & 12660 & 10399 & 9637 & 7579 & 5861 & 5891 & 4491 & 3389 \\
\hline & Vehicle Cost HIGH & 20034 & 20034 & 17215 & 15416 & 7817 & 6453 & 4805 & 5148 & 3566 & 2369 & 2392 & 1291 & 637 \\
\hline \multirow{3}{*}{ EV } & Vehicle Cost LOW & 48935 & 48935 & 44713 & 41331 & 31148 & 27404 & 19713 & 20415 & 14287 & 9953 & 11635 & 7727 & 5582 \\
\hline & Vehicle cost AVG & 40984 & 40984 & 37413 & 34552 & 21416 & 18845 & 13628 & 11637 & 8166 & 5666 & 9324 & 6130 & 4361 \\
\hline & Vehicle cost HIGH & 31432 & 31432 & 28656 & 26432 & 17821 & 15510 & 10555 & 5616 & 3397 & 1855 & 5044 & 2633 & 1460 \\
\hline
\end{tabular}




\section{Cost saving per powertrain}

\begin{tabular}{|c|c|c|c|c|c|c|c|c|c|c|c|c|c|c|}
\hline & & \multicolumn{13}{|c|}{ Midsize } \\
\hline & & \multirow{2}{*}{$\frac{2010}{\operatorname{Ref}}$} & \multicolumn{3}{|c|}{2010} & \multicolumn{3}{|c|}{2015} & \multicolumn{3}{|c|}{2030} & \multicolumn{3}{|c|}{2045} \\
\hline & & & low & avg & high & low & avg & high & low & avg & high & low & avg & high \\
\hline \multirow{3}{*}{ SI Conv } & Vehicle Cost LOW & 0 & 0 & -40 & -31 & 38 & 12 & -17 & 830 & 790 & 745 & 1223 & 1169 & 1090 \\
\hline & Vehicle Cost AVG & 0 & 0 & -40 & -31 & 837 & 813 & 786 & 1153 & 1107 & 1057 & 1201 & 1149 & 1073 \\
\hline & Vehicle Cost $\mathrm{HIGH}$ & 0 & 0 & -40 & -31 & 204 & 178 & 148 & 637 & 594 & 546 & 661 & 609 & 532 \\
\hline \multirow{3}{*}{$\mathrm{Cl}$ Conv } & Vehicle Cost LOW & 0 & 0 & -79 & -91 & -171 & -237 & -287 & 564 & 491 & 399 & 593 & 543 & 407 \\
\hline & Vehicle Cost AVG & 0 & 0 & -73 & -81 & 728 & 673 & 632 & 726 & 666 & 591 & 682 & 644 & 527 \\
\hline & Vehicle Cost HIGH & 0 & 0 & -61 & -62 & 43 & 5 & -21 & 355 & 307 & 245 & 255 & 228 & 127 \\
\hline \multirow{3}{*}{$\mathrm{H} 2$ Conv } & Vehicle Cost LOW & 0 & 0 & -39 & -32 & -54 & -70 & -65 & 661 & 647 & 638 & 894 & 890 & 841 \\
\hline & Vehicle Cost AVG & 0 & 0 & -40 & -35 & -305 & -319 & -313 & -374 & -390 & -402 & -1695 & -1699 & -1749 \\
\hline & Vehicle Cost HIGH & 0 & 0 & -40 & -35 & -1130 & -1150 & -1149 & -962 & -988 & -1011 & -1459 & -1475 & -1538 \\
\hline \multirow{3}{*}{ E85 Conv } & Vehicle Cost LOW & 0 & 0 & -41 & -39 & 39 & 9 & -20 & 836 & 777 & 732 & 1221 & 1165 & 1068 \\
\hline & Vehicle Cost AVG & 0 & 0 & -41 & -39 & 839 & 811 & 785 & 1157 & 1092 & 1041 & 1200 & 1147 & 1053 \\
\hline & Vehicle Cost $\mathrm{HIGH}$ & 0 & 0 & -41 & -39 & 204 & 174 & 145 & 640 & 578 & 530 & 657 & 604 & 509 \\
\hline \multirow{3}{*}{ SI Split HEV } & Vehicle Cost LOW & 0 & 0 & -252 & -479 & -790 & -1060 & -1351 & -662 & -1134 & -1640 & -485 & -980 & -1533 \\
\hline & \begin{tabular}{|l|} 
Vehicle Cost AVG \\
\end{tabular} & 0 & 0 & -246 & -467 & -690 & -908 & -1142 & -905 & -1298 & -1716 & -981 & -1389 & -1842 \\
\hline & Vehicle Cost HIGH & 0 & 0 & -193 & -366 & -1120 & -1288 & -1468 & -1253 & -1524 & -1807 & -1558 & -1813 & -2088 \\
\hline \multirow{3}{*}{ SI Split HEV PHEV10 } & Vehicle Cost LOW & 0 & 0 & -371 & -747 & -1895 & -2309 & -2945 & -2421 & -3042 & -3642 & -2694 & -3202 & -3797 \\
\hline & Vehicle Cost AVG & 0 & 0 & -332 & -669 & -1734 & -2043 & -2503 & -2317 & -2772 & -3228 & -2515 & -2941 & -3450 \\
\hline & Vehicle Cost HIGH & 0 & 0 & -269 & -544 & -2715 & -2928 & -3287 & -3011 & -3348 & -3656 & -3431 & -3732 & -3974 \\
\hline \multirow{3}{*}{ SI Split HEV PHEV2O } & Vehicle Cost LOW & 0 & 0 & -544 & -1113 & -2732 & -3337 & -4260 & -3788 & -4643 & -5479 & -4508 & -5188 & -5866 \\
\hline & Vehicle Cost AVG & 0 & 0 & -481 & -980 & -2690 & -3126 & -3781 & -3757 & -4333 & -4922 & -4052 & -4619 & -5194 \\
\hline & Vehicle Cost HIGH & 0 & 0 & -387 & -786 & -3172 & -3515 & -4062 & -3764 & -4282 & -4754 & -4372 & -4835 & -5175 \\
\hline \multirow{3}{*}{ SI Series HEV PHEV30 } & Vehicle Cost LOW & 0 & 0 & -1303 & -2358 & -5530 & -6720 & -8754 & -8413 & -10200 & -11765 & -10194 & -11635 & -12818 \\
\hline & Vehicle Cost AVG & 0 & 0 & -1149 & -2079 & -6064 & -6938 & -8394 & -8457 & -9688 & -10806 & -9185 & -10394 & -11397 \\
\hline & Vehicle Cost HIGH & 0 & 0 & -926 & -1676 & -7046 & -7658 & -8799 & -8586 & -9581 & -10398 & -9731 & -10606 & -11161 \\
\hline \multirow{3}{*}{ SI Series HEV PHEV40 } & Vehicle Cost LOW & 0 & 0 & -1541 & -2804 & -6700 & -8095 & -10606 & -10322 & -12463 & -14338 & -12709 & -14388 & -15708 \\
\hline & Vehicle Cost AVG & 0 & 0 & -1350 & -2456 & -7401 & -8415 & -10195 & -10451 & -11883 & -13174 & -11327 & -12731 & -13846 \\
\hline & Vehicle Cost HIGH & 0 & 0 & -1082 & -1968 & -7778 & -8523 & -9976 & -9727 & -10974 & -12016 & -11138 & -12234 & -12916 \\
\hline \multirow{3}{*}{ CI Split HEV } & Vehicle Cost LOW & 0 & 0 & -310 & -677 & -1082 & -1414 & -1827 & -1126 & -1632 & -2187 & -1252 & -1727 & -2325 \\
\hline & Vehicle Cost AVG & 0 & 0 & -298 & -653 & -1017 & -1281 & -1609 & -1567 & -1972 & -2416 & -1703 & -2085 & -2565 \\
\hline & Vehicle Cost $\mathrm{HIGH}$ & 0 & 0 & -228 & -499 & -1410 & -1604 & -1844 & -1761 & -2031 & -2325 & -2184 & -2404 & -2679 \\
\hline
\end{tabular}




\begin{tabular}{|c|c|c|c|c|c|c|c|c|c|c|c|c|c|c|}
\hline & & \multicolumn{13}{|c|}{ Midsize } \\
\hline & & \multirow{2}{*}{$\frac{2010}{\text { Ref }}$} & \multicolumn{3}{|c|}{2010} & \multicolumn{3}{|c|}{2015} & \multicolumn{3}{|c|}{2030} & \multicolumn{3}{|c|}{2045} \\
\hline & & & low & avg & high & low & avg & high & low & avg & high & low & avg & high \\
\hline \multirow{3}{*}{ CI Split HEV PHEV10 } & Vehicle Cost LOW & 0 & 0 & -437 & -862 & -2281 & -2734 & -3462 & -2925 & -3601 & -4308 & -3511 & -4091 & -4756 \\
\hline & Vehicle Cost AVG & 0 & 0 & -391 & -772 & -2122 & -2463 & -2993 & -2967 & -3456 & -3994 & -3239 & -3721 & -4282 \\
\hline & Vehicle Cost HIGH & 0 & 0 & -313 & -620 & -3214 & -3438 & -3836 & -3666 & -4025 & -4363 & -4198 & -4514 & -4769 \\
\hline \multirow{3}{*}{ CI Split HEV PHEV20 } & Vehicle Cost LOW & 0 & 0 & -613 & -1178 & -3143 & -5694 & -4827 & -4355 & -5294 & -6207 & -5415 & -6167 & -6894 \\
\hline & Vehicle Cost AVG & 0 & 0 & -539 & -1037 & -3111 & -4781 & -4320 & -4471 & -5104 & -5744 & -4850 & -5473 & -6081 \\
\hline & Vehicle Cost HIGH & 0 & 0 & -425 & -822 & -3675 & -5108 & -4635 & -4448 & -4998 & -5501 & -5184 & -5665 & -6018 \\
\hline \multirow{3}{*}{ CI Series HEV PHEV 30} & Vehicle Cost LOW & 0 & 0 & -1337 & -2466 & -5915 & -7127 & -9262 & -8930 & -10765 & -12400 & -11074 & -12538 & -13757 \\
\hline & Vehicle Cost AVG & 0 & 0 & -1178 & -2169 & -6399 & -7292 & -8825 & -9108 & -10363 & -11519 & -9922 & -11142 & -12167 \\
\hline & Vehicle Cost HIGH & 0 & 0 & -944 & -1738 & -7427 & -8044 & -9232 & -9124 & -10136 & -10984 & -10384 & -11254 & -11819 \\
\hline \multirow{3}{*}{ CI Series HEV PHEV40 } & Vehicle Cost LOW & 0 & 0 & -1628 & -2933 & -7166 & -8566 & -11201 & -10935 & -13148 & -15090 & -13712 & -15449 & -16797 \\
\hline & \begin{tabular}{|l|} 
Vehicle Cost AVG \\
\end{tabular} & 0 & 0 & -1424 & -2564 & -7816 & -8837 & -10709 & -11204 & -12672 & -13998 & -12171 & -13613 & -14742 \\
\hline & Vehicle Cost HIGH & 0 & 0 & -1134 & -2042 & -8208 & -8948 & -10465 & -10320 & -11601 & -12672 & -11858 & -12984 & -13671 \\
\hline \multirow{3}{*}{ H2 Split HEV } & Vehicle Cost LOW & 0 & 0 & -372 & -659 & -958 & -1287 & -1645 & -1017 & -1510 & -1981 & -1025 & -1449 & -1952 \\
\hline & Vehicle Cost AVG & 0 & 0 & -363 & -643 & -1952 & -2211 & -2493 & -2605 & -3001 & -3379 & -4094 & -4437 & -4843 \\
\hline & Vehicle Cost HIGH & 0 & 0 & -278 & -492 & -2537 & -2733 & -2944 & -3011 & -3276 & -3526 & -3861 & -4062 & -4296 \\
\hline \multirow{3}{*}{ H2 Split HEV PHEV10 } & Vehicle Cost LOW & 0 & 0 & -588 & -1041 & -2195 & -2735 & -3472 & -2848 & -3622 & -4265 & -3354 & -3897 & -4505 \\
\hline & Vehicle Cost AVG & 0 & 0 & -529 & -936 & -3117 & -3519 & -4058 & -4064 & -4641 & -5129 & -5737 & -6192 & -6708 \\
\hline & Vehicle Cost $\mathrm{HIGH}$ & 0 & 0 & -430 & -760 & -4371 & -4629 & -5004 & -4975 & -5340 & -5645 & -5956 & -6243 & -6465 \\
\hline \multirow{3}{*}{ H2 Split HEV PHEV 20} & Vehicle Cost LOW & 0 & 0 & -801 & -1425 & -3063 & -3793 & -4845 & -4256 & -5269 & -6158 & -5220 & -5945 & -6622 \\
\hline & Vehicle Cost AVG & 0 & 0 & -708 & -1259 & -4092 & -4628 & -5378 & -5537 & -6234 & -6862 & -7315 & -7919 & -8490 \\
\hline & Vehicle Cost HIGH & 0 & 0 & -567 & -1009 & -4864 & -5231 & -5814 & -5749 & -6306 & -6778 & -6931 & -7379 & -7698 \\
\hline \multirow{3}{*}{ H2 Series HEV PHEV 30} & Vehicle Cost LOW & 0 & 0 & -1616 & -2781 & -5776 & -7179 & -9238 & -8779 & -10681 & -12261 & -10822 & -12219 & -13346 \\
\hline & \begin{tabular}{|l|} 
Vehicle Cost AVG \\
\end{tabular} & 0 & 0 & -1425 & -2451 & -7355 & -8382 & -9849 & -10133 & -11435 & -12554 & -12345 & -13513 & -14464 \\
\hline & \begin{tabular}{|l|} 
Vehicle Cost HIGH \\
\end{tabular} & 0 & 0 & -1145 & -1969 & -8519 & -9221 & -10358 & -10346 & -11375 & -12178 & -12064 & -12899 & -13402 \\
\hline \multirow{3}{*}{ H2 Series HEV PHEV40 } & Vehicle Cost LOW & 0 & 0 & -1988 & -3399 & -6980 & -8648 & -11192 & -10760 & -13037 & -14933 & -13432 & -15066 & -16335 \\
\hline & \begin{tabular}{|l|} 
Vehicle Cost AVG \\
\end{tabular} & 0 & 0 & -1738 & -2972 & -8729 & -9935 & -11730 & -12193 & -13705 & -15000 & -14566 & -15927 & -16994 \\
\hline & Vehicle Cost HIGH & 0 & 0 & -1385 & -2371 & -9270 & -10147 & -11600 & -11529 & -12826 & -13857 & -13534 & -14585 & -15227 \\
\hline \multirow{3}{*}{ E85 Split HEV } & Vehicle Cost LOW & 0 & 0 & -268 & -495 & -779 & -1025 & -1414 & -719 & -1147 & -1617 & -498 & -957 & -1528 \\
\hline & Vehicle Cost AVG & 0 & 0 & -261 & -482 & -685 & -885 & -1193 & -956 & -1310 & -1700 & -994 & -1371 & -1841 \\
\hline & Vehicle Cost HIGH & 0 & 0 & -204 & -378 & -1114 & -1264 & -1512 & -1294 & -1534 & -1802 & -1570 & -1803 & -2090 \\
\hline
\end{tabular}




\begin{tabular}{|c|c|c|c|c|c|c|c|c|c|c|c|c|c|c|}
\hline & & \multicolumn{13}{|c|}{ Midsize } \\
\hline & & \multirow{2}{*}{$\frac{2010}{\operatorname{Ref}}$} & \multicolumn{3}{|c|}{2010} & \multicolumn{3}{|c|}{2015} & \multicolumn{3}{|c|}{2030} & \multicolumn{3}{|c|}{2045} \\
\hline & & & low & avg & high & low & avg & high & low & avg & high & low & avg & high \\
\hline \multirow{3}{*}{ E85 Split HEV PHEV10 } & Vehicle Cost LOW & 0 & 0 & -370 & -747 & -1896 & -2310 & -2947 & -2418 & -3044 & -3644 & -2693 & -3202 & -3796 \\
\hline & \begin{tabular}{|l} 
Vehicle Cost AVG \\
.
\end{tabular} & 0 & 0 & -331 & -669 & -1736 & -2044 & -2505 & -2315 & -2773 & -3230 & -2515 & -2942 & -3450 \\
\hline & \begin{tabular}{|l|} 
Vehicle Cost HIGH \\
\end{tabular} & 0 & 0 & -268 & -544 & -2718 & -2931 & -3291 & -3014 & -3353 & -3661 & -3435 & -3737 & -3978 \\
\hline \multirow{3}{*}{ E85 Split HEV PHEV20 } & Vehicle Cost LOW & 0 & 0 & -539 & -1109 & -2728 & -3296 & -4258 & -3782 & -4641 & -5476 & -4502 & -5189 & -5864 \\
\hline & 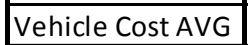 & 0 & 0 & -476 & $\begin{array}{l}-976 \\
\end{array}$ & -2687 & -3099 & -3780 & -3743 & -4331 & -4920 & -4048 & -4621 & -5194 \\
\hline & Vehicle Cost HIGH & 0 & 0 & -383 & -782 & -3171 & -3488 & -4063 & -3777 & -4282 & -4756 & -4373 & -4837 & -5177 \\
\hline \multirow{3}{*}{ E85 Series HEV PHEV30 } & Vehicle Cost LOW & 0 & 0 & -1293 & -2361 & -5540 & -6727 & -8760 & -8412 & -10205 & -11776 & -10201 & -11646 & -12826 \\
\hline & \begin{tabular}{|l|} 
Vehicle Cost AVG \\
Cehicle Cost
\end{tabular} & 0 & 0 & -1140 & -2082 & -6073 & -6946 & -8401 & -8458 & $\begin{array}{l}-9694 \\
\end{array}$ & -10816 & $\begin{array}{l}-9192 \\
\end{array}$ & -10405 & -11405 \\
\hline & \begin{tabular}{|l|} 
Vehicle Cost HIGH \\
Cehicle Cost
\end{tabular} & 0 & 0 & -919 & -1679 & -7056 & -7667 & -8808 & -8590 & -9589 & -10410 & -9741 & -10618 & -11172 \\
\hline \multirow{3}{*}{ E85 Series HEV PHEV40 } & Vehicle Cost LOW & 0 & 0 & -1500 & -2787 & -6685 & -8080 & -10588 & -10299 & -12448 & -14326 & -12692 & -14377 & -15696 \\
\hline & \begin{tabular}{|l|} 
Vehicle Cost AVG \\
\end{tabular} & 0 & 0 & -1317 & -2442 & -7390 & -8405 & -10182 & -10434 & -11872 & -13165 & -11315 & -12723 & -13837 \\
\hline & 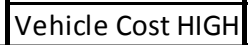 & 0 & 0 & -1057 & -1958 & $\begin{array}{l}-7773 \\
\end{array}$ & $\begin{array}{l}-8518 \\
\end{array}$ & $\begin{array}{l}-9970 \\
\end{array}$ & $\begin{array}{l}-9718 \\
\end{array}$ & -10969 & -12014 & -11134 & -12233 & -12914 \\
\hline \multirow{3}{*}{ FC HEV } & Vehicle Cost LOW & $\overline{0}$ & $\overline{0}$ & -1814 & -2918 & -638 & $\begin{array}{l}-2748 \\
\end{array}$ & -4189 & -5633 & -6704 & -7710 & -7513 & -8175 & -8862 \\
\hline & \begin{tabular}{|l} 
Vehicle Cost AVG \\
\end{tabular} & 0 & 0 & -2695 & -4413 & -5363 & -7271 & -8589 & -8949 & -10228 & -11307 & -12091 & -12736 & -13356 \\
\hline & Vehicle Cost HIGH & 0 & 0 & -2220 & -3637 & -6898 & -8042 & -8862 & -8639 & $\begin{array}{c}9458 \\
\end{array}$ & -10167 & -10750 & -11157 & -11542 \\
\hline \multirow{3}{*}{ FC HEV PHEV10 } & Vehicle Cost LOW & 0 & 0 & -1890 & -3078 & -2209 & -3800 & -5296 & -6367 & -7682 & -8670 & $\begin{array}{l}-8328 \\
\end{array}$ & $\begin{array}{ll}-9098 \\
\end{array}$ & $\begin{array}{l}-9934 \\
\end{array}$ \\
\hline & \begin{tabular}{|l} 
Vehicle Cost AVG \\
Vehich
\end{tabular} & 0 & 0 & -2496 & -4211 & -5947 & -7461 & -8830 & -9138 & -10532 & -11508 & -12127 & -12859 & -13600 \\
\hline & Vehicle Cost HIGH & 0 & 0 & -2055 & -3467 & $\begin{array}{l}-7814 \\
\end{array}$ & $\begin{array}{l}-8717 \\
\end{array}$ & $\begin{array}{l}-9584 \\
\end{array}$ & -9345 & -10229 & -10833 & -11330 & -11800 & -12190 \\
\hline \multirow{3}{*}{ FC HEV PHEV 20} & Vehicle Cost LOW & 0 & 0 & -2303 & -3817 & -3315 & -5354 & -7124 & -8293 & $\begin{array}{l}-9943 \\
\end{array}$ & -11247 & -10839 & -11770 & -12757 \\
\hline & \begin{tabular}{|l|} 
Vehicle Cost AVG \\
\end{tabular} & 0 & 0 & -2873 & -4866 & -7271 & -9087 & -10645 & -11150 & -12742 & -13922 & -14339 & -15203 & -16070 \\
\hline & Vehicle Cost $\mathrm{HIGH}$ & 0 & 0 & -2342 & -3974 & -8563 & -9717 & -10798 & -10520 & -11654 & -12499 & -12780 & -13426 & -13963 \\
\hline \multirow{3}{*}{ FC HEV PHEV 30} & Vehicle Cost LOW & 0 & 0 & -2472 & -4001 & -5015 & -7036 & $\begin{array}{l}-9302 \\
\end{array}$ & -10537 & -12529 & -14205 & -13717 & -15049 & -16177 \\
\hline & Vehicle Cost AVG & 0 & 0 & -3023 & -5006 & -8881 & -10763 & -12706 & -13331 & -15176 & -16714 & -16885 & -18099 & -19115 \\
\hline & Vehicle Cost HIGH & 0 & 0 & -2473 & -4094 & -11367 & -12524 & -13869 & -13613 & -14909 & -15923 & -16119 & -16975 & -17530 \\
\hline \multirow{3}{*}{ FC HEV PHEV40 } & Vehicle Cost LOW & 0 & 0 & -2979 & -4749 & -6241 & -8570 & -11280 & -12527 & -14893 & -16792 & -16264 & -17822 & -19045 \\
\hline & Vehicle Cost AVG & 0 & 0 & -3471 & -5683 & -10302 & -12400 & -14662 & -15424 & -17482 & -19200 & -19170 & -20570 & -21672 \\
\hline & Vehicle Cost HIGH & 0 & 0 & -2820 & -4618 & -12217 & -13581 & -15229 & -14887 & -16468 & -17665 & -17643 & -18744 & -19397 \\
\hline \multirow{3}{*}{ EV } & Vehicle Cost LOW & 0 & 0 & -4222 & -7605 & -17787 & -21531 & -29222 & -28521 & -34649 & -38982 & -37301 & -41208 & -43354 \\
\hline & Vehicle Cost AVG & 0 & 0 & -3570 & -6432 & $\begin{array}{l}-19568 \\
\end{array}$ & -22139 & -27356 & -29346 & -32818 & -35317 & -31659 & -34854 & -36623 \\
\hline & Vehicle Cost HIGH & 0 & 0 & -2776 & -5000 & -13611 & -15922 & -20877 & -25816 & -28035 & -29577 & -26388 & -28799 & -29972 \\
\hline
\end{tabular}




\section{Percentage decrease cost per powertrain}

\begin{tabular}{|c|c|c|c|c|c|c|c|c|c|c|c|c|c|c|}
\hline & & \multicolumn{13}{|c|}{ Midsize } \\
\hline & & \multirow{2}{*}{$\frac{2010}{\operatorname{Ref}}$} & \multicolumn{3}{|c|}{2010} & \multicolumn{3}{|c|}{2015} & \multicolumn{3}{|c|}{2030} & \multicolumn{3}{|c|}{2045} \\
\hline & & & low & avg & high & low & avg & high & low & avg & high & low & avg & high \\
\hline \multirow{3}{*}{ SI Conv } & Vehicle Cost LOW & $0.0 \%$ & $0.0 \%$ & $-0.3 \%$ & $-0.2 \%$ & $0.3 \%$ & $0.1 \%$ & $-0.1 \%$ & $5.7 \%$ & $5.5 \%$ & $5.1 \%$ & $8.4 \%$ & $8.1 \%$ & $7.5 \%$ \\
\hline & \begin{tabular}{|l|} 
Vehicle Cost AVG \\
\end{tabular} & $0.0 \%$ & $0.0 \%$ & $-0.3 \%$ & $-0.2 \%$ & $5.8 \%$ & $5.6 \%$ & $5.4 \%$ & $8.0 \%$ & $7.6 \%$ & $7.3 \%$ & $8.3 \%$ & $7.9 \%$ & $7.4 \%$ \\
\hline & Vehicle Cost HIGH & $0.0 \%$ & $0.0 \%$ & $-0.3 \%$ & $-0.2 \%$ & $1.3 \%$ & $1.2 \%$ & $1.0 \%$ & $4.2 \%$ & $3.9 \%$ & $3.6 \%$ & $4.4 \%$ & $4.0 \%$ & $3.5 \%$ \\
\hline \multirow{3}{*}{$\mathrm{Cl}$ Conv } & Vehicle Cost LOW & $0.0 \%$ & $0.0 \%$ & $-0.5 \%$ & $-0.6 \%$ & $-1.0 \%$ & $-1.4 \%$ & $-1.7 \%$ & $3.4 \%$ & $3.0 \%$ & $2.4 \%$ & $3.6 \%$ & $3.3 \%$ & $2.5 \%$ \\
\hline & Vehicle Cost AVG & $0.0 \%$ & $0.0 \%$ & $-0.4 \%$ & $-0.5 \%$ & $4.4 \%$ & $4.1 \%$ & $3.9 \%$ & $4.4 \%$ & $4.1 \%$ & $3.6 \%$ & $4.2 \%$ & $3.9 \%$ & $3.2 \%$ \\
\hline & Vehicle Cost HIGH & $0.0 \%$ & $0.0 \%$ & $-0.4 \%$ & $-0.4 \%$ & $0.3 \%$ & $0.0 \%$ & $-0.1 \%$ & $2.1 \%$ & $1.8 \%$ & $1.5 \%$ & $1.5 \%$ & $1.4 \%$ & $0.8 \%$ \\
\hline \multirow{3}{*}{ H2 Conv } & Vehicle Cost LOW & $0.0 \%$ & $0.0 \%$ & $-0.3 \%$ & $-0.2 \%$ & $-0.4 \%$ & $-0.5 \%$ & $-0.5 \%$ & $4.7 \%$ & $4.6 \%$ & $4.6 \%$ & $6.4 \%$ & $6.4 \%$ & $6.0 \%$ \\
\hline & Vehicle Cost AVG & $0.0 \%$ & $0.0 \%$ & $-0.2 \%$ & $-0.2 \%$ & $-1.8 \%$ & $-1.9 \%$ & $-1.8 \%$ & $-2.2 \%$ & $-2.3 \%$ & $-2.4 \%$ & $-10.0 \%$ & $-10.0 \%$ & $-10.3 \%$ \\
\hline & Vehicle Cost HIGH & $0.0 \%$ & $0.0 \%$ & $-0.2 \%$ & $-0.2 \%$ & $-6.6 \%$ & $-6.7 \%$ & $-6.7 \%$ & $-5.6 \%$ & $-5.8 \%$ & $-5.9 \%$ & $-8.5 \%$ & $-8.6 \%$ & $-9.0 \%$ \\
\hline \multirow{3}{*}{ E85 Conv } & Vehicle Cost LOW & $0.0 \%$ & $0.0 \%$ & $-0.3 \%$ & $-0.3 \%$ & $0.3 \%$ & $0.1 \%$ & $-0.1 \%$ & $5.8 \%$ & $5.4 \%$ & $5.1 \%$ & $8.4 \%$ & $8.1 \%$ & $7.4 \%$ \\
\hline & Vehicle Cost AVG & $0.0 \%$ & $0.0 \%$ & $-0.3 \%$ & $-0.3 \%$ & $5.8 \%$ & $5.6 \%$ & $5.4 \%$ & $8.0 \%$ & $7.5 \%$ & $7.2 \%$ & $8.3 \%$ & $7.9 \%$ & $7.3 \%$ \\
\hline & Vehicle Cost HIGH & $0.0 \%$ & $0.0 \%$ & $-0.3 \%$ & $-0.3 \%$ & $1.3 \%$ & $1.1 \%$ & $1.0 \%$ & $4.2 \%$ & $3.8 \%$ & $3.5 \%$ & $4.3 \%$ & $4.0 \%$ & $3.4 \%$ \\
\hline \multirow{3}{*}{ SI Split HEV } & Vehicle Cost LOW & $0.0 \%$ & $0.0 \%$ & $-1.4 \%$ & $-2.6 \%$ & $-4.3 \%$ & $-5.8 \%$ & $-7.4 \%$ & $-3.6 \%$ & $-6.2 \%$ & $-8.9 \%$ & $-2.6 \%$ & $-5.3 \%$ & $-8.4 \%$ \\
\hline & Vehicle Cost AVG & $0.0 \%$ & $0.0 \%$ & $-1.3 \%$ & $-2.6 \%$ & $-3.8 \%$ & $-5.0 \%$ & $-6.3 \%$ & $-5.0 \%$ & $-7.1 \%$ & $-9.4 \%$ & $-5.4 \%$ & $-7.6 \%$ & $-10.1 \%$ \\
\hline & Vehicle Cost $\mathrm{HIGH}$ & $0.0 \%$ & $0.0 \%$ & $-1.1 \%$ & $-2.1 \%$ & $-6.3 \%$ & $-7.3 \%$ & $-8.3 \%$ & $-7.1 \%$ & $-8.6 \%$ & $-10.2 \%$ & $-8.8 \%$ & $-10.2 \%$ & $-11.8 \%$ \\
\hline \multirow{3}{*}{ SI Split HEV PHEV10 } & Vehicle Cost LOW & $0.0 \%$ & $0.0 \%$ & $-1.8 \%$ & $-3.5 \%$ & $-9.0 \%$ & $-10.9 \%$ & $-14.0 \%$ & $-11.5 \%$ & $-14.4 \%$ & $-17.2 \%$ & $-12.8 \%$ & $-15.2 \%$ & $-18.0 \%$ \\
\hline & Vehicle Cost AVG & $0.0 \%$ & $0.0 \%$ & $-1.6 \%$ & $-3.3 \%$ & $-8.5 \%$ & $-10.0 \%$ & $-12.3 \%$ & $-11.4 \%$ & $-13.6 \%$ & $-15.8 \%$ & $-12.3 \%$ & $-14.4 \%$ & $-16.9 \%$ \\
\hline & Vehicle Cost HIGH & $0.0 \%$ & $0.0 \%$ & $-1.4 \%$ & $-2.7 \%$ & $-13.7 \%$ & $-14.8 \%$ & $-16.6 \%$ & $-15.2 \%$ & $-16.9 \%$ & $-18.5 \%$ & $-17.3 \%$ & $-18.9 \%$ & $-20.1 \%$ \\
\hline \multirow{3}{*}{ SI Split HEV PHEV20 } & Vehicle Cost LOW & $0.0 \%$ & $0.0 \%$ & $-2.3 \%$ & $-4.8 \%$ & $-11.7 \%$ & $-14.3 \%$ & $-18.2 \%$ & $-16.2 \%$ & $-19.8 \%$ & $-23.4 \%$ & $-19.3 \%$ & $-22.2 \%$ & $-25.1 \%$ \\
\hline & Vehicle Cost AVG & $0.0 \%$ & $0.0 \%$ & $-2.2 \%$ & $-4.4 \%$ & $-12.1 \%$ & $-14.0 \%$ & $-16.9 \%$ & $-16.8 \%$ & $-19.4 \%$ & $-22.1 \%$ & $-18.2 \%$ & $-20.7 \%$ & $-23.3 \%$ \\
\hline & Vehicle Cost HIGH & $0.0 \%$ & $0.0 \%$ & $-1.8 \%$ & $-3.7 \%$ & $-14.9 \%$ & $-16.5 \%$ & $-19.1 \%$ & $-17.7 \%$ & $-20.1 \%$ & $-22.4 \%$ & $-20.6 \%$ & $-22.7 \%$ & $-24.3 \%$ \\
\hline \multirow{3}{*}{ SI Series HEV PHEV30 } & Vehicle Cost LOW & $0.0 \%$ & $0.0 \%$ & $-4.0 \%$ & $-7.1 \%$ & $-16.8 \%$ & $-20.4 \%$ & $-26.5 \%$ & $-25.5 \%$ & $-30.9 \%$ & $-35.7 \%$ & $-30.9 \%$ & $-35.3 \%$ & $-38.9 \%$ \\
\hline & Vehicle Cost AVG & $0.0 \%$ & $0.0 \%$ & $-3.7 \%$ & $-6.8 \%$ & $-19.7 \%$ & $-22.6 \%$ & $-27.3 \%$ & $-27.5 \%$ & $-31.5 \%$ & $-35.1 \%$ & $-29.9 \%$ & $-33.8 \%$ & $-37.0 \%$ \\
\hline & Vehicle Cost HIGH & $0.0 \%$ & $0.0 \%$ & $-3.3 \%$ & $-6.0 \%$ & $-25.1 \%$ & $-27.2 \%$ & $-31.3 \%$ & $-30.5 \%$ & $-34.1 \%$ & $-37.0 \%$ & $-34.6 \%$ & $-37.7 \%$ & $-39.7 \%$ \\
\hline \multirow{3}{*}{ SI Series HEV PHEV40 } & Vehicle Cost LOW & $0.0 \%$ & $0.0 \%$ & $-4.3 \%$ & $-7.7 \%$ & $-18.5 \%$ & $-22.3 \%$ & $-29.3 \%$ & $-28.5 \%$ & $-34.4 \%$ & $-39.6 \%$ & $-35.1 \%$ & $-39.7 \%$ & $-43.3 \%$ \\
\hline & Vehicle Cost AVG & $0.0 \%$ & $0.0 \%$ & $-4.0 \%$ & $-7.3 \%$ & $-22.1 \%$ & $-25.1 \%$ & $-30.4 \%$ & $-31.2 \%$ & $-35.5 \%$ & $-39.3 \%$ & $-33.8 \%$ & $-38.0 \%$ & $-41.3 \%$ \\
\hline & Vehicle Cost $\mathrm{HIGH}$ & $0.0 \%$ & $0.0 \%$ & $-3.6 \%$ & $-6.5 \%$ & $-25.7 \%$ & $-28.2 \%$ & $-33.0 \%$ & $-32.2 \%$ & $-36.3 \%$ & $-39.7 \%$ & $-36.8 \%$ & $-40.5 \%$ & $-42.7 \%$ \\
\hline \multirow{3}{*}{ CI Split HEV } & Vehicle Cost LOW & $0.0 \%$ & $0.0 \%$ & $-1.5 \%$ & $-3.3 \%$ & $-5.2 \%$ & $-6.8 \%$ & $-8.8 \%$ & $-5.4 \%$ & $-7.8 \%$ & $-10.5 \%$ & $-6.0 \%$ & $-8.3 \%$ & $-11.2 \%$ \\
\hline & \begin{tabular}{|l|} 
Vehicle Cost AVG \\
\end{tabular} & $0.0 \%$ & $0.0 \%$ & $-1.4 \%$ & $-3.2 \%$ & $-4.9 \%$ & $-6.2 \%$ & $-7.8 \%$ & $-7.6 \%$ & $-9.5 \%$ & $-11.7 \%$ & $-8.2 \%$ & $-10.1 \%$ & $-12.4 \%$ \\
\hline & Vehicle Cost HIGH & $0.0 \%$ & $0.0 \%$ & $-1.2 \%$ & $-2.5 \%$ & $-7.1 \%$ & $-8.1 \%$ & $-9.3 \%$ & $-8.9 \%$ & $-10.3 \%$ & $-11.7 \%$ & $-11.0 \%$ & $-12.1 \%$ & $-13.5 \%$ \\
\hline
\end{tabular}




\begin{tabular}{|c|c|c|c|c|c|c|c|c|c|c|c|c|c|c|}
\hline & & \multicolumn{13}{|c|}{ Midsize } \\
\hline & & \multirow{2}{*}{$\begin{array}{l}2010 \\
\text { Ref }\end{array}$} & \multicolumn{3}{|c|}{2010} & \multicolumn{3}{|c|}{2015} & \multicolumn{3}{|c|}{2030} & \multicolumn{3}{|c|}{2045} \\
\hline & & & low & avg & high & low & avg & high & low & avg & high & low & avg & high \\
\hline \multirow{3}{*}{ CI Split HEV PHEV10 } & Vehicle Cost LOW & $0.0 \%$ & $0.0 \%$ & $-1.8 \%$ & $-3.6 \%$ & $-9.6 \%$ & $-11.5 \%$ & $-14.5 \%$ & $-12.3 \%$ & $-15.1 \%$ & $-18.1 \%$ & $-14.7 \%$ & $-17.2 \%$ & $-19.9 \%$ \\
\hline & Vehicle Cost AVG & $0.0 \%$ & $0.0 \%$ & $-1.7 \%$ & $-3.3 \%$ & $-9.2 \%$ & $-10.7 \%$ & $-13.0 \%$ & $-12.9 \%$ & $-15.0 \%$ & $-17.3 \%$ & $-14.1 \%$ & $-16.1 \%$ & $-18.6 \%$ \\
\hline & Vehicle Cost HIGH & $0.0 \%$ & $0.0 \%$ & $-1.4 \%$ & $-2.8 \%$ & $-14.5 \%$ & $-15.6 \%$ & $-17.4 \%$ & $-16.6 \%$ & $-18.2 \%$ & $-19.7 \%$ & $-19.0 \%$ & $-20.4 \%$ & $-21.6 \%$ \\
\hline \multirow{3}{*}{ CI Split HEV PHEV2O } & Vehicle Cost LOW & $0.0 \%$ & $0.0 \%$ & $-2.3 \%$ & $-4.5 \%$ & $-12.0 \%$ & $-21.7 \%$ & $-18.4 \%$ & $-16.6 \%$ & $-20.2 \%$ & $-23.6 \%$ & $-20.6 \%$ & $-23.5 \%$ & $-26.3 \%$ \\
\hline & Vehicle Cost AVG & $0.0 \%$ & $0.0 \%$ & $-2.2 \%$ & $-4.1 \%$ & $-12.4 \%$ & $-19.1 \%$ & $-17.2 \%$ & $-17.8 \%$ & $-20.4 \%$ & $-22.9 \%$ & $-19.4 \%$ & $-21.8 \%$ & $-24.3 \%$ \\
\hline & Vehicle Cost HIGH & $0.0 \%$ & $0.0 \%$ & $-1.8 \%$ & $-3.5 \%$ & $-15.6 \%$ & $-21.6 \%$ & $-19.6 \%$ & $-18.8 \%$ & $-21.2 \%$ & $-23.3 \%$ & $-21.9 \%$ & $-24.0 \%$ & $-25.5 \%$ \\
\hline \multirow{3}{*}{ CI Series HEV PHEV 30} & Vehicle Cost LOW & $0.0 \%$ & $0.0 \%$ & $-3.8 \%$ & $-6.9 \%$ & $-16.6 \%$ & $-20.0 \%$ & $-26.0 \%$ & $-25.1 \%$ & $-30.2 \%$ & $-34.8 \%$ & $-31.1 \%$ & $-35.2 \%$ & $-38.6 \%$ \\
\hline & Vehicle Cost AVG & $0.0 \%$ & $0.0 \%$ & $-3.5 \%$ & $-6.5 \%$ & $-19.2 \%$ & $-21.9 \%$ & $-26.5 \%$ & $-27.4 \%$ & $-31.1 \%$ & $-34.6 \%$ & $-29.8 \%$ & $-33.5 \%$ & $-36.6 \%$ \\
\hline & Vehicle Cost HIGH & $0.0 \%$ & $0.0 \%$ & $-3.1 \%$ & $-5.7 \%$ & $-24.5 \%$ & $-26.6 \%$ & $-30.5 \%$ & $-30.1 \%$ & $-33.5 \%$ & $-36.3 \%$ & $-34.3 \%$ & $-37.2 \%$ & $-39.0 \%$ \\
\hline \multirow{3}{*}{ CI Series HEV PHEV40 } & Vehicle Cost LOW & $0.0 \%$ & $0.0 \%$ & $-4.2 \%$ & $-7.5 \%$ & $-18.4 \%$ & $-21.9 \%$ & $-28.7 \%$ & $-28.0 \%$ & $-33.7 \%$ & $-38.7 \%$ & $-35.1 \%$ & $-39.6 \%$ & $-43.0 \%$ \\
\hline & \begin{tabular}{|l|} 
Vehicle Cost AVG \\
\end{tabular} & $0.0 \%$ & $0.0 \%$ & $-3.9 \%$ & $-7.1 \%$ & $-21.6 \%$ & $-24.4 \%$ & $-29.6 \%$ & $-31.0 \%$ & $-35.0 \%$ & $-38.7 \%$ & $-33.7 \%$ & $-37.6 \%$ & $-40.8 \%$ \\
\hline & Vehicle Cost HIGH & $0.0 \%$ & $0.0 \%$ & $-3.5 \%$ & $-6.3 \%$ & $-25.2 \%$ & $-27.5 \%$ & $-32.2 \%$ & $-31.7 \%$ & $-35.7 \%$ & $-39.0 \%$ & $-36.5 \%$ & $-39.9 \%$ & $-42.1 \%$ \\
\hline \multirow{3}{*}{ H2 Split HEV } & Vehicle Cost LOW & $0.0 \%$ & $0.0 \%$ & $-2.0 \%$ & $-3.6 \%$ & $-5.2 \%$ & $-7.0 \%$ & $-9.0 \%$ & $-5.5 \%$ & $-8.2 \%$ & $-10.8 \%$ & $-5.6 \%$ & $-7.9 \%$ & $-10.6 \%$ \\
\hline & Vehicle cost AVG & $0.0 \%$ & $0.0 \%$ & $-1.7 \%$ & $-3.0 \%$ & $-9.2 \%$ & $-10.4 \%$ & $-11.7 \%$ & $-12.3 \%$ & $-14.1 \%$ & $-15.9 \%$ & $-19.3 \%$ & $-20.9 \%$ & $-22.8 \%$ \\
\hline & Vehicle Cost HIGH & $0.0 \%$ & $0.0 \%$ & $-1.4 \%$ & $-2.4 \%$ & $-12.6 \%$ & $-13.6 \%$ & $-14.6 \%$ & $-15.0 \%$ & $-16.3 \%$ & $-17.5 \%$ & $-19.2 \%$ & $-20.2 \%$ & $-21.4 \%$ \\
\hline \multirow{3}{*}{ H2 Split HEV PHEV10 } & Vehicle Cost LOW & $0.0 \%$ & $0.0 \%$ & $-2.7 \%$ & $-4.8 \%$ & $-10.2 \%$ & $-12.7 \%$ & $-16.1 \%$ & $-13.2 \%$ & $-16.8 \%$ & $-19.8 \%$ & $-15.6 \%$ & $-18.1 \%$ & $-20.9 \%$ \\
\hline & Vehicle Cost AVG & $0.0 \%$ & $0.0 \%$ & $-2.2 \%$ & $-3.9 \%$ & $-13.1 \%$ & $-14.8 \%$ & $-17.1 \%$ & $-17.1 \%$ & $-19.5 \%$ & $-21.6 \%$ & $-24.1 \%$ & $-26.0 \%$ & $-28.2 \%$ \\
\hline & Vehicle Cost HIGH & $0.0 \%$ & $0.0 \%$ & $-1.9 \%$ & $-3.4 \%$ & $-19.4 \%$ & $-20.6 \%$ & $-22.2 \%$ & $-22.1 \%$ & $-23.7 \%$ & $-25.1 \%$ & $-26.5 \%$ & $-27.7 \%$ & $-28.7 \%$ \\
\hline \multirow{3}{*}{ H2 Split HEV PHEV2O } & Vehicle Cost LOW & $0.0 \%$ & $0.0 \%$ & $-3.4 \%$ & $-6.0 \%$ & $-12.8 \%$ & $-15.9 \%$ & $-20.3 \%$ & $-17.8 \%$ & $-22.1 \%$ & $-25.8 \%$ & $-21.9 \%$ & $-24.9 \%$ & $-27.8 \%$ \\
\hline & Vehicle Cost AVG & $0.0 \%$ & $0.0 \%$ & $-2.8 \%$ & $-4.9 \%$ & $-15.9 \%$ & $-18.0 \%$ & $-20.9 \%$ & $-21.5 \%$ & $-24.2 \%$ & $-26.7 \%$ & $-28.4 \%$ & $-30.8 \%$ & $-33.0 \%$ \\
\hline & \begin{tabular}{|l|} 
Vehicle Cost HIGH \\
\end{tabular} & $0.0 \%$ & $0.0 \%$ & $-2.4 \%$ & $-4.2 \%$ & $-20.3 \%$ & $-21.8 \%$ & $-24.2 \%$ & $-23.9 \%$ & $-26.3 \%$ & $-28.2 \%$ & $-28.9 \%$ & $-30.7 \%$ & $-32.1 \%$ \\
\hline \multirow{3}{*}{ H2 Series HEV PHEV3O } & Vehicle Cost LOW & $0.0 \%$ & $0.0 \%$ & $-4.9 \%$ & $-8.4 \%$ & $-17.5 \%$ & $-21.7 \%$ & $-28.0 \%$ & $-26.6 \%$ & $-32.3 \%$ & $-37.1 \%$ & $-32.7 \%$ & $-37.0 \%$ & $-40.4 \%$ \\
\hline & Vehicle Cost AVG & $0.0 \%$ & $0.0 \%$ & $-4.2 \%$ & $-7.3 \%$ & $-21.8 \%$ & $-24.8 \%$ & $-29.1 \%$ & $-30.0 \%$ & $-33.8 \%$ & $-37.1 \%$ & $-36.5 \%$ & $-40.0 \%$ & $-42.8 \%$ \\
\hline & Vehicle Cost $\mathrm{HIGH}$ & $0.0 \%$ & $0.0 \%$ & $-3.7 \%$ & $-6.4 \%$ & $-27.9 \%$ & $-30.2 \%$ & $-33.9 \%$ & $-33.9 \%$ & $-37.2 \%$ & $-39.9 \%$ & $-39.5 \%$ & $-42.2 \%$ & $-43.9 \%$ \\
\hline \multirow{3}{*}{ H2 Series HEV PHEV40 } & Vehicle Cost LOW & $0.0 \%$ & $0.0 \%$ & $-5.5 \%$ & $-9.3 \%$ & $-19.2 \%$ & $-23.8 \%$ & $-30.8 \%$ & $-29.6 \%$ & $-35.8 \%$ & $-41.1 \%$ & $-36.9 \%$ & $-41.4 \%$ & $-44.9 \%$ \\
\hline & Vehicle Cost AVG & $0.0 \%$ & $0.0 \%$ & $-4.7 \%$ & $-8.1 \%$ & $-23.8 \%$ & $-27.1 \%$ & $-32.0 \%$ & $-33.3 \%$ & $-37.4 \%$ & $-41.0 \%$ & $-39.8 \%$ & $-43.5 \%$ & $-46.4 \%$ \\
\hline & Vehicle Cost HIGH & $0.0 \%$ & $0.0 \%$ & $-4.2 \%$ & $-7.2 \%$ & $-28.3 \%$ & $-31.0 \%$ & $-35.5 \%$ & $-35.3 \%$ & $-39.2 \%$ & $-42.4 \%$ & $-41.4 \%$ & $-44.6 \%$ & $-46.6 \%$ \\
\hline \multirow{3}{*}{ E85 Split HEV } & Vehicle Cost LOW & $0.0 \%$ & $0.0 \%$ & $-1.5 \%$ & $-2.7 \%$ & $-4.2 \%$ & $-5.6 \%$ & $-7.7 \%$ & $-3.9 \%$ & $-6.2 \%$ & $-8.8 \%$ & $-2.7 \%$ & $-5.2 \%$ & $-8.3 \%$ \\
\hline & Vehicle Cost AVG & $0.0 \%$ & $0.0 \%$ & $-1.4 \%$ & $-2.6 \%$ & $-3.8 \%$ & $-4.8 \%$ & $-6.5 \%$ & $-5.2 \%$ & $-7.2 \%$ & $-9.3 \%$ & $-5.4 \%$ & $-7.5 \%$ & $-10.1 \%$ \\
\hline & Vehicle Cost HIGH & $0.0 \%$ & $0.0 \%$ & $-1.2 \%$ & $-2.1 \%$ & $-6.3 \%$ & $-7.1 \%$ & $-8.5 \%$ & $-7.3 \%$ & $-8.6 \%$ & $-10.2 \%$ & $-8.8 \%$ & $-10.2 \%$ & $-11.8 \%$ \\
\hline
\end{tabular}




\begin{tabular}{|c|c|c|c|c|c|c|c|c|c|c|c|c|c|c|}
\hline & & \multicolumn{13}{|c|}{ Midsize } \\
\hline & & \multirow{2}{*}{\begin{tabular}{|l|}
2010 \\
Ref
\end{tabular}} & \multicolumn{3}{|c|}{2010} & \multicolumn{3}{|c|}{2015} & \multicolumn{3}{|c|}{2030} & \multicolumn{3}{|c|}{2045} \\
\hline & & & low & avg & high & low & avg & high & low & avg & high & low & avg & high \\
\hline \multirow{3}{*}{ E85 Split HEV PHEV10 } & Vehicle Cost LOW & $0.0 \%$ & $0.0 \%$ & $-1.8 \%$ & $-3.5 \%$ & $-9.0 \%$ & $-10.9 \%$ & $-13.9 \%$ & $-11.4 \%$ & $-14.4 \%$ & $-17.2 \%$ & $-12.7 \%$ & $-15.2 \%$ & $-18.0 \%$ \\
\hline & Vehicle Cost AVG & $0.0 \%$ & $0.0 \%$ & $-1.6 \%$ & $-3.3 \%$ & $-8.5 \%$ & $-10.0 \%$ & $-12.3 \%$ & $-11.3 \%$ & $-13.6 \%$ & $-15.8 \%$ & $-12.3 \%$ & $-14.4 \%$ & $-16.9 \%$ \\
\hline & Vehicle Cost HIGH & $0.0 \%$ & $0.0 \%$ & $-1.4 \%$ & $-2.7 \%$ & $-13.7 \%$ & $-14.8 \%$ & $-16.6 \%$ & $-15.2 \%$ & $-16.9 \%$ & $-18.5 \%$ & $-17.3 \%$ & $-18.9 \%$ & $-20.1 \%$ \\
\hline \multirow{3}{*}{ E85 Split HEV PHEV20 } & Vehicle Cost LOW & $0.0 \%$ & $0.0 \%$ & $-2.3 \%$ & $-4.7 \%$ & $-11.6 \%$ & $-14.1 \%$ & $-18.2 \%$ & $-16.2 \%$ & $-19.8 \%$ & $-23.4 \%$ & $-19.2 \%$ & $-22.2 \%$ & $-25.0 \%$ \\
\hline & Vehicle Cost AVG & $0.0 \%$ & $0.0 \%$ & $-2.1 \%$ & $-4.4 \%$ & $-12.0 \%$ & $-13.9 \%$ & $-16.9 \%$ & $-16.8 \%$ & $-19.4 \%$ & $-22.0 \%$ & $-18.1 \%$ & $-20.7 \%$ & $-23.3 \%$ \\
\hline & Vehicle Cost $\mathrm{HIGH}$ & $0.0 \%$ & $0.0 \%$ & $-1.8 \%$ & $-3.7 \%$ & $-14.9 \%$ & $-16.4 \%$ & $-19.1 \%$ & $-17.8 \%$ & $-20.1 \%$ & $-22.4 \%$ & $-20.6 \%$ & $-22.7 \%$ & $-24.3 \%$ \\
\hline \multirow{3}{*}{ E85 Series HEV PHEV30 } & Vehicle Cost LOW & $0.0 \%$ & $0.0 \%$ & $-3.9 \%$ & $-7.1 \%$ & $-16.8 \%$ & $-20.4 \%$ & $-26.5 \%$ & $-25.5 \%$ & $-30.9 \%$ & $-35.7 \%$ & $-30.9 \%$ & $-35.3 \%$ & $-38.8 \%$ \\
\hline & Vehicle Cost AVG & $0.0 \%$ & $0.0 \%$ & $-3.7 \%$ & $-6.8 \%$ & $-19.7 \%$ & $-22.6 \%$ & $-27.3 \%$ & $-27.5 \%$ & $-31.5 \%$ & $-35.1 \%$ & $-29.8 \%$ & $-33.8 \%$ & $-37.0 \%$ \\
\hline & \begin{tabular}{|l|} 
Vehicle Cost HIGH \\
\end{tabular} & $0.0 \%$ & $0.0 \%$ & $-3.3 \%$ & $-6.0 \%$ & $-25.1 \%$ & $-27.2 \%$ & $-31.3 \%$ & $-30.5 \%$ & $-34.1 \%$ & $-37.0 \%$ & $-34.6 \%$ & $-37.7 \%$ & $-39.7 \%$ \\
\hline \multirow{3}{*}{ E85 Series HEV PHEV40 } & Vehicle Cost LOW & $0.0 \%$ & $0.0 \%$ & $-4.1 \%$ & $-7.7 \%$ & $-18.4 \%$ & $-22.3 \%$ & $-29.2 \%$ & $-28.4 \%$ & $-34.3 \%$ & $-39.5 \%$ & $-35.0 \%$ & $-39.6 \%$ & $-43.3 \%$ \\
\hline & Vehicle Cost AVG & $0.0 \%$ & $0.0 \%$ & $-3.9 \%$ & $-7.3 \%$ & $-22.0 \%$ & $-25.1 \%$ & $-30.4 \%$ & $-31.1 \%$ & $-35.4 \%$ & $-39.3 \%$ & $-33.7 \%$ & $-37.9 \%$ & $-41.3 \%$ \\
\hline & Vehicle Cost HIGH & $0.0 \%$ & $0.0 \%$ & $-3.5 \%$ & $-6.5 \%$ & $-25.7 \%$ & $-28.2 \%$ & $-33.0 \%$ & $-32.1 \%$ & $-36.3 \%$ & $-39.7 \%$ & $-36.8 \%$ & $-40.4 \%$ & $-42.7 \%$ \\
\hline \multirow{3}{*}{ FC HEV } & Vehicle Cost LOW & $0.0 \%$ & $0.0 \%$ & $-7.5 \%$ & $-12.1 \%$ & $-2.7 \%$ & $-11.4 \%$ & $-17.4 \%$ & $-23.4 \%$ & $-27.8 \%$ & $-32.0 \%$ & $-31.2 \%$ & $-34.0 \%$ & $-36.8 \%$ \\
\hline & Vehicle Cost AVG & $0.0 \%$ & $0.0 \%$ & $-9.4 \%$ & $-15.5 \%$ & $-18.8 \%$ & $-25.5 \%$ & $-30.1 \%$ & $-31.3 \%$ & $-35.8 \%$ & $-39.6 \%$ & $-42.3 \%$ & $-44.6 \%$ & $-46.8 \%$ \\
\hline & Vehicle Cost HIGH & $0.0 \%$ & $0.0 \%$ & $-8.4 \%$ & $-13.8 \%$ & $-26.2 \%$ & $-30.6 \%$ & $-33.7 \%$ & $-32.9 \%$ & $-36.0 \%$ & $-38.7 \%$ & $-40.9 \%$ & $-42.4 \%$ & $-43.9 \%$ \\
\hline \multirow{3}{*}{ FC HEV PHEV10 } & Vehicle Cost LOW & $0.0 \%$ & $0.0 \%$ & $-7.3 \%$ & $-11.9 \%$ & $-8.5 \%$ & $-14.7 \%$ & $-20.5 \%$ & $-24.6 \%$ & $-29.7 \%$ & $-33.6 \%$ & $-32.2 \%$ & $-35.2 \%$ & $-38.5 \%$ \\
\hline & Vehicle Cost AVG & $0.0 \%$ & $0.0 \%$ & $-8.5 \%$ & $-14.3 \%$ & $-20.2 \%$ & $-25.4 \%$ & $-30.0 \%$ & $-31.1 \%$ & $-35.8 \%$ & $-39.1 \%$ & $-41.2 \%$ & $-43.7 \%$ & $-46.2 \%$ \\
\hline & Vehicle Cost HIGH & $0.0 \%$ & $0.0 \%$ & $-7.6 \%$ & $-12.8 \%$ & $-28.9 \%$ & $-32.3 \%$ & $-35.5 \%$ & $-34.6 \%$ & $-37.9 \%$ & $-40.1 \%$ & $-41.9 \%$ & $-43.7 \%$ & $-45.1 \%$ \\
\hline \multirow{3}{*}{ FC HEV PHEV 20} & Vehicle Cost LOW & $0.0 \%$ & $0.0 \%$ & $-8.0 \%$ & $-13.2 \%$ & $-11.5 \%$ & $-18.5 \%$ & $-24.7 \%$ & $-28.7 \%$ & $-34.4 \%$ & $-39.0 \%$ & $-37.5 \%$ & $-40.8 \%$ & $-44.2 \%$ \\
\hline & \begin{tabular}{|l|} 
Vehicle Cost AVG \\
\end{tabular} & $0.0 \%$ & $0.0 \%$ & $-9.0 \%$ & $-15.2 \%$ & $-22.7 \%$ & $-28.3 \%$ & $-33.2 \%$ & $-34.8 \%$ & $-39.8 \%$ & $-43.4 \%$ & $-44.7 \%$ & $-47.4 \%$ & $-50.1 \%$ \\
\hline & Vehicle Cost $\mathrm{HIGH}$ & $0.0 \%$ & $0.0 \%$ & $-8.1 \%$ & $-13.7 \%$ & $-29.5 \%$ & $-33.5 \%$ & $-37.2 \%$ & $-36.2 \%$ & $-40.1 \%$ & $-43.0 \%$ & $-44.0 \%$ & $-46.2 \%$ & $-48.1 \%$ \\
\hline \multirow{3}{*}{ FC HEV PHEV 30} & Vehicle Cost LOW & $0.0 \%$ & $0.0 \%$ & $-7.2 \%$ & $-11.7 \%$ & $-14.7 \%$ & $-20.6 \%$ & $-27.2 \%$ & $-30.8 \%$ & $-36.7 \%$ & $-41.6 \%$ & $-40.2 \%$ & $-44.1 \%$ & $-47.4 \%$ \\
\hline & \begin{tabular}{|l|} 
Vehicle Cost AVG \\
\end{tabular} & $0.0 \%$ & $0.0 \%$ & $-8.2 \%$ & $-13.6 \%$ & $-24.2 \%$ & $-29.3 \%$ & $-34.6 \%$ & $-36.3 \%$ & $-41.3 \%$ & $-45.5 \%$ & $-46.0 \%$ & $-49.3 \%$ & $-52.0 \%$ \\
\hline & Vehicle Cost HIGH & $0.0 \%$ & $0.0 \%$ & $-7.5 \%$ & $-12.4 \%$ & $-34.5 \%$ & $-38.0 \%$ & $-42.0 \%$ & $-41.3 \%$ & $-45.2 \%$ & $-48.3 \%$ & $-48.9 \%$ & $-51.5 \%$ & $-53.1 \%$ \\
\hline \multirow{3}{*}{ FC HEV PHEV40 } & Vehicle Cost LOW & $0.0 \%$ & $0.0 \%$ & $-8.0 \%$ & $-12.7 \%$ & $-16.7 \%$ & $-22.9 \%$ & $-30.2 \%$ & $-33.5 \%$ & $-39.9 \%$ & $-45.0 \%$ & $-43.5 \%$ & $-47.7 \%$ & $-51.0 \%$ \\
\hline & \begin{tabular}{|l|} 
Vehicle Cost AVG \\
\end{tabular} & $0.0 \%$ & $0.0 \%$ & $-8.8 \%$ & $-14.4 \%$ & $-26.0 \%$ & $-31.4 \%$ & $-37.1 \%$ & $-39.0 \%$ & $-44.2 \%$ & $-48.5 \%$ & $-48.5 \%$ & $-52.0 \%$ & $-54.8 \%$ \\
\hline & Vehicle Cost HIGH & $0.0 \%$ & $0.0 \%$ & $-8.0 \%$ & $-13.1 \%$ & $-34.7 \%$ & $-38.6 \%$ & $-43.3 \%$ & $-42.3 \%$ & $-46.8 \%$ & $-50.2 \%$ & $-50.2 \%$ & $-53.3 \%$ & $-55.2 \%$ \\
\hline \multirow{3}{*}{ EV } & Vehicle Cost LOW & $0.0 \%$ & $0.0 \%$ & $-6.7 \%$ & $-12.0 \%$ & $-28.0 \%$ & $-34.0 \%$ & $-46.1 \%$ & $-45.0 \%$ & $-54.6 \%$ & $-61.5 \%$ & $-58.8 \%$ & $-65.0 \%$ & $-68.4 \%$ \\
\hline & Vehicle Cost AVG & $0.0 \%$ & $0.0 \%$ & $-6.4 \%$ & $-11.6 \%$ & $-35.3 \%$ & $-39.9 \%$ & $-49.3 \%$ & $-52.9 \%$ & $-59.2 \%$ & $-63.7 \%$ & $-57.1 \%$ & $-62.8 \%$ & $-66.0 \%$ \\
\hline & Vehicle Cost HIGH & $0.0 \%$ & $0.0 \%$ & $-6.0 \%$ & $-10.7 \%$ & $-29.2 \%$ & $-34.2 \%$ & $-44.8 \%$ & $-55.4 \%$ & $-60.2 \%$ & $-63.5 \%$ & $-56.7 \%$ & $-61.8 \%$ & $-64.4 \%$ \\
\hline
\end{tabular}


Small SUV Class 


\section{Incremental Cost versus the Conventional 2010 Reference}

\begin{tabular}{|c|c|c|c|c|c|c|c|c|c|c|c|c|c|c|}
\hline & & \multicolumn{13}{|c|}{ Small_SUV } \\
\hline & & \multirow{2}{*}{$\begin{array}{l}2010 \\
\operatorname{Ref}\end{array}$} & \multicolumn{3}{|c|}{2010} & \multicolumn{3}{|c|}{2015} & \multicolumn{3}{|c|}{2030} & \multicolumn{3}{|c|}{2045} \\
\hline & & & low & avg & high & low & avg & high & low & avg & high & low & avg & high \\
\hline \multirow{3}{*}{ SI Conv } & Vehicle Cost LOW & 0 & 0 & 99 & 49 & 11 & 91 & -641 & 999 & 191 & 87 & 1356 & 547 & 416 \\
\hline & \begin{tabular}{|l|} 
Vehicle Cost AVG \\
\end{tabular} & 0 & 0 & 99 & 49 & 826 & 908 & 129 & 1359 & 484 & 372 & 1299 & 495 & 368 \\
\hline & Vehicle Cost HIGH & 0 & 0 & 99 & 49 & 146 & 226 & -475 & 731 & -1 & -110 & 650 & -15 & -143 \\
\hline \multirow{3}{*}{$\mathrm{Cl}$ Conv } & Vehicle Cost LOW & 1348 & 1348 & 1464 & 1384 & 1123 & 1212 & 1050 & 2041 & 1857 & 1725 & 1965 & 1877 & 1736 \\
\hline & Vehicle Cost AVG & 1250 & 1250 & 1369 & 1296 & 1926 & 2022 & 1869 & 2101 & 1931 & 1816 & 1951 & 1875 & 1754 \\
\hline & Vehicle Cost HIGH & 981 & 981 & 1104 & 1043 & 974 & 1080 & 941 & 1461 & 1302 & 1201 & 1254 & 1188 & 1084 \\
\hline \multirow{3}{*}{ H2 Conv } & Vehicle Cost LOW & -529 & -529 & -1074 & -1100 & -1280 & -1169 & -1283 & -412 & -538 & -583 & -289 & -329 & -381 \\
\hline & Vehicle Cost AVG & 2489 & 2489 & 1907 & 1880 & 1450 & 1562 & 1449 & 1534 & 1406 & 1359 & 105 & 64 & 11 \\
\hline & Vehicle Cost $\mathrm{HIGH}$ & 1928 & 1928 & 1382 & 1355 & 102 & 209 & 91 & 425 & 286 & 229 & -180 & -232 & -299 \\
\hline \multirow{3}{*}{ E85 Conv } & Vehicle Cost LOW & -46 & -46 & 69 & -537 & -29 & -518 & -669 & 979 & 165 & 66 & 1329 & 521 & 398 \\
\hline & Vehicle Cost AVG & -45 & -45 & 70 & -566 & 790 & 252 & 103 & 1338 & 456 & 350 & 1274 & 470 & 351 \\
\hline & Vehicle Cost HIGH & -46 & -46 & 69 & -537 & 106 & -353 & -504 & 706 & -31 & -134 & 620 & -43 & -163 \\
\hline \multirow{3}{*}{ SI Split HEV } & Vehicle Cost LOW & 3678 & 3678 & 3395 & 3136 & 2807 & 2578 & 2116 & 2771 & 2332 & 1731 & 2965 & 2466 & 1855 \\
\hline & Vehicle Cost AVG & 3502 & 3502 & 3225 & 2972 & 2630 & 2447 & 2079 & 2325 & 1958 & 1456 & 2257 & 1842 & 1339 \\
\hline & Vehicle Cost $\mathrm{HIGH}$ & 2300 & 2300 & 2083 & 1883 & 1070 & 922 & 631 & 827 & 570 & 221 & 502 & 240 & -70 \\
\hline \multirow{3}{*}{ SI Split HEV PHEV10 } & Vehicle Cost LOW & 7186 & 7186 & 6672 & 6219 & 4925 & 4428 & 3659 & 4247 & 3440 & 2835 & 3834 & 3237 & 2601 \\
\hline & Vehicle Cost AVG & 6324 & 6324 & 5864 & 5457 & 4110 & 3739 & 3177 & 3440 & 2845 & 2396 & 3170 & 2671 & 2129 \\
\hline & Vehicle Cost HIGH & 4881 & 4881 & 4509 & 4178 & 1687 & 1431 & 1019 & 1303 & 871 & 526 & 796 & 421 & 156 \\
\hline \multirow{3}{*}{ SI Split HEV PHEV20 } & Vehicle Cost LOW & 9809 & 9809 & 9113 & 8475 & 6601 & 5921 & 4810 & 5336 & 4234 & 3283 & 4407 & 3566 & 2806 \\
\hline & Vehicle Cost AVG & 8522 & 8522 & 7908 & 7346 & 5220 & 4728 & 3944 & 4004 & 3264 & 2591 & 3631 & 2931 & 2288 \\
\hline & Vehicle Cost HIGH & 6557 & 6557 & 6067 & 5617 & 2863 & 2479 & 1811 & 2156 & 1479 & 950 & 1423 & 837 & 463 \\
\hline \multirow{3}{*}{ SI Series HEV PHEV 30} & Vehicle Cost LOW & 20304 & 20304 & 18589 & 17315 & 13930 & 12523 & 10220 & 10755 & 8504 & 6797 & 8609 & 6866 & 5587 \\
\hline & Vehicle Cost AVG & 17748 & 17748 & 16239 & 15116 & 10689 & 9656 & 8008 & 8074 & 6523 & 5309 & 7196 & 5736 & 4651 \\
\hline & Vehicle Cost HIGH & 14056 & 14056 & 12843 & 11939 & 6107 & 5380 & 4087 & 4411 & 3156 & 2256 & 3084 & 2012 & 1411 \\
\hline \multirow{3}{*}{ SI Series HEV PHEV40 } & Vehicle Cost LOW & 24013 & 24013 & 21985 & 20436 & 16270 & 14638 & 11783 & 12300 & 9612 & 7584 & 9478 & 7436 & 6017 \\
\hline & Vehicle Cost AVG & 20880 & 20880 & 19106 & 17749 & 12274 & 11087 & 9064 & 8943 & 7144 & 5748 & 7905 & 6199 & 5002 \\
\hline & Vehicle Cost HIGH & 16470 & 16470 & 15052 & 13966 & 7652 & 6781 & 5126 & 5523 & 3955 & 2827 & 3909 & 2558 & 1825 \\
\hline \multirow{3}{*}{ Cl Split HEV } & Vehicle Cost LOW & 6151 & 6151 & 5848 & 5529 & 4997 & 4642 & 4163 & 4830 & 4304 & 3707 & 4704 & 4179 & 3525 \\
\hline & Vehicle Cost AVG & 5929 & 5929 & 5637 & 5329 & 4744 & 4464 & 4086 & 4127 & 3704 & 3226 & 3989 & 3565 & 3040 \\
\hline & Vehicle Cost HIGH & 4339 & 4339 & 4115 & 3880 & 2824 & 2612 & 2330 & 2390 & 2101 & 1782 & 1933 & 1684 & 1384 \\
\hline
\end{tabular}




\begin{tabular}{|c|c|c|c|c|c|c|c|c|c|c|c|c|c|c|}
\hline & & \multicolumn{13}{|c|}{ Small SUV } \\
\hline & & \multirow{2}{*}{\begin{tabular}{|l|}
2010 \\
Ref
\end{tabular}} & \multicolumn{3}{|c|}{2010} & \multicolumn{3}{|c|}{2015} & \multicolumn{3}{|c|}{2030} & \multicolumn{3}{|c|}{2045} \\
\hline & & & low & avg & high & low & avg & high & low & avg & high & low & avg & high \\
\hline \multirow{3}{*}{ CI Split HEV PHEV10 } & Vehicle Cost LOW & 9783 & 9783 & 9222 & 8721 & 7175 & 6648 & 5866 & 6376 & 5582 & 4865 & 5660 & 5043 & 4331 \\
\hline & Vehicle Cost AVG & 8837 & 8837 & 8335 & 7887 & 6308 & 5914 & 5345 & 5352 & 4784 & 4250 & 5019 & 4512 & 3911 \\
\hline & Vehicle Cost HIGH & 7070 & 7070 & 6669 & 6308 & 3435 & 3166 & 2735 & 2903 & 2463 & 2093 & 2292 & 1911 & 1638 \\
\hline \multirow{3}{*}{ CI Split HEV PHEV2O } & Vehicle Cost LOW & 12525 & 12525 & 11780 & 11077 & 8927 & 8203 & 7015 & 7540 & 6371 & 5365 & 6246 & 5368 & 4559 \\
\hline & Vehicle Cost AVG & 11133 & 11133 & 10478 & 9859 & 7471 & 6944 & 6101 & 5966 & 5186 & 4480 & 5491 & 4767 & 4088 \\
\hline & Vehicle Cost HIGH & 8821 & 8821 & 8303 & 7810 & 4654 & 4257 & 3558 & 3792 & 3091 & 2540 & 2940 & 2345 & 1961 \\
\hline \multirow{3}{*}{ CI Series HEV PHEV 30} & Vehicle Cost LOW & 22987 & 22987 & 21217 & 19899 & 16199 & 14758 & 12361 & 12900 & 10580 & 8812 & 10367 & 8583 & 7275 \\
\hline & Vehicle Cost AVG & 20309 & 20309 & 18755 & 17594 & 12891 & 11830 & 10111 & 9951 & 8368 & 7120 & 8977 & 7492 & 6393 \\
\hline & Vehicle Cost HIGH & 16253 & 16253 & 15012 & 14082 & 7907 & 7170 & 5831 & 6058 & 4772 & 3851 & 4598 & 3510 & 2906 \\
\hline \multirow{3}{*}{ CI Series HEV PHEV40 } & Vehicle Cost LOW & 26833 & 26833 & 24745 & 23122 & 18641 & 16960 & 14009 & 14498 & 11724 & 9645 & 11270 & 9166 & 7719 \\
\hline & Vehicle Cost AVG & 23557 & 23557 & 21732 & 20313 & 14546 & 13320 & 11225 & 10848 & 9005 & 7584 & 9712 & 7966 & 6753 \\
\hline & Vehicle Cost HIGH & 18753 & 18753 & 17302 & 16173 & 9513 & 8624 & 6923 & 7203 & 5599 & 4453 & 5452 & 4072 & 3336 \\
\hline \multirow{3}{*}{ H2 Split HEV } & Vehicle Cost LOW & 3627 & 3627 & 3236 & 2913 & 2613 & 2196 & 1783 & 2451 & 1915 & 1360 & 2410 & 1933 & 1396 \\
\hline & Vehicle Cost AVG & 6459 & 6459 & 6076 & 5760 & 4341 & 4017 & 3695 & 3645 & 3211 & 2765 & 2128 & 1741 & 1307 \\
\hline & Vehicle Cost HIGH & 4635 & 4635 & 4341 & 4097 & 2000 & 1749 & 1501 & 1454 & 1154 & 858 & 554 & 325 & 74 \\
\hline \multirow{3}{*}{ H2 Split HEV PHEV10 } & Vehicle Cost LOW & 7507 & 7507 & 6736 & 6199 & 4947 & 4312 & 3482 & 4094 & 3203 & 2533 & 3476 & 2875 & 2220 \\
\hline & Vehicle Cost AVG & 9618 & 9618 & 8924 & 8441 & 6017 & 5545 & 4937 & 4937 & 4282 & 3783 & 3223 & 2723 & 2167 \\
\hline & Vehicle Cost HIGH & 7527 & 7527 & 6965 & 6573 & 2735 & 2430 & 2013 & 2030 & 1587 & 1244 & 968 & 616 & 372 \\
\hline \multirow{3}{*}{ H2 Split HEV PHEV2O } & Vehicle Cost LOW & 10170 & 10170 & 9174 & 8445 & 6667 & 5802 & 4626 & 5194 & 3978 & 3009 & 4055 & 3191 & 2432 \\
\hline & \begin{tabular}{|l|} 
Vehicle Cost AVG \\
\end{tabular} & 11848 & 11848 & 10966 & 10321 & 7162 & 6533 & 5701 & 5508 & 4687 & 4006 & 3690 & 2972 & 2332 \\
\hline & Vehicle Cost HIGH & 9228 & 9228 & 8522 & 8007 & 3920 & 3469 & 2791 & 2883 & 2182 & 1660 & 1587 & 1022 & 672 \\
\hline \multirow{3}{*}{ H2 Series HEV PHEV30 } & Vehicle Cost LOW & 20313 & 20313 & 18296 & 16899 & 13695 & 12091 & 9754 & 10389 & 8042 & 6318 & 7991 & 6301 & 5064 \\
\hline & \begin{tabular}{|l|} 
Vehicle Cost AVG \\
\end{tabular} & 20736 & 20736 & 18962 & 17731 & 12387 & 11215 & 9550 & 9374 & 7770 & 6555 & 7026 & 5615 & 4571 \\
\hline & Vehicle Cost $\mathrm{HIGH}$ & 16439 & 16439 & 15018 & 14029 & 7020 & 6216 & 4923 & 5034 & 3758 & 2873 & 3141 & 2116 & 1553 \\
\hline \multirow{3}{*}{ H2 Series HEV PHEV40 } & Vehicle Cost LOW & 24071 & 24071 & 21660 & 19997 & 16046 & 14174 & 11307 & 11942 & 9130 & 7089 & 8839 & 6862 & 5483 \\
\hline & \begin{tabular}{|l|} 
Vehicle Cost AVG \\
\end{tabular} & 23906 & 23906 & 21801 & 20343 & 13975 & 12621 & 10597 & 10242 & 8375 & 6983 & 7717 & 6071 & 4912 \\
\hline & \begin{tabular}{|l|} 
Vehicle Cost HIGH \\
\end{tabular} & 18879 & 18879 & 17203 & 16039 & 8570 & 7591 & 5952 & 6145 & 4541 & 3430 & 3950 & 2658 & 1958 \\
\hline \multirow{3}{*}{ E85 Split HEV } & Vehicle Cost LOW & 3737 & 3737 & 3434 & 3157 & 2829 & 2621 & 2157 & 2878 & 2327 & 1751 & 2989 & 2531 & 1876 \\
\hline & Vehicle Cost AVG & 3561 & 3561 & 3264 & 2994 & 2652 & 2485 & 2116 & 2419 & 1957 & 1477 & 2279 & 1900 & 1360 \\
\hline & Vehicle Cost HIGH & 2351 & 2351 & 2116 & 1904 & 1089 & 956 & 664 & 895 & 570 & 239 & 521 & 282 & -52 \\
\hline
\end{tabular}




\begin{tabular}{|c|c|c|c|c|c|c|c|c|c|c|c|c|c|c|}
\hline & & \multicolumn{13}{|c|}{ Small_SUV } \\
\hline & & \multirow{2}{*}{$\frac{2010}{\operatorname{Ref}}$} & \multicolumn{3}{|c|}{2010} & \multicolumn{3}{|c|}{2015} & \multicolumn{3}{|c|}{2030} & \multicolumn{3}{|c|}{2045} \\
\hline & & & low & avg & high & low & avg & high & low & avg & high & low & avg & high \\
\hline \multirow{3}{*}{ E85 Split HEV PHEV10 } & Vehicle Cost LOW & 7211 & 7211 & 6700 & 6243 & 4949 & 4452 & 3684 & 4273 & 3463 & 2859 & 3859 & 3294 & 2626 \\
\hline & Vehicle Cost AVG & 6349 & 6349 & 5892 & 5482 & 4133 & 3763 & 3201 & 3466 & 2868 & 2420 & 3193 & 2723 & 2153 \\
\hline & Vehicle Cost HIGH & 4904 & 4904 & 4534 & 4200 & 1706 & 1451 & 1038 & 1322 & 889 & 544 & 814 & 443 & 173 \\
\hline \multirow{3}{*}{ E85 Split HEV PHEV20 } & Vehicle Cost LOW & 9839 & 9839 & 9140 & 8502 & 6629 & 5948 & 4834 & 5361 & 4258 & 3334 & 4433 & 3591 & 2832 \\
\hline & Vehicle Cost AVG & 8551 & 8551 & 7935 & 7372 & 5246 & 4753 & 3967 & 4028 & 3288 & 2638 & 3657 & 2955 & 2312 \\
\hline & Vehicle Cost HIGH & 6583 & 6583 & 6091 & 5640 & 2883 & 2500 & 1830 & 2175 & 1497 & 973 & 1442 & 855 & 481 \\
\hline \multirow{3}{*}{ E85 Series HEV PHEV30 } & Vehicle Cost LOW & 20332 & 20332 & 18634 & 17352 & 13952 & 12549 & 10247 & 10781 & 8532 & 6822 & 8636 & 6892 & 5615 \\
\hline & Vehicle Cost AVG & 17776 & 17776 & 16281 & 15151 & 10711 & 9681 & 8033 & 8100 & 6550 & 5334 & 7222 & 5760 & 4678 \\
\hline & Vehicle Cost HIGH & 14082 & 14082 & 12880 & 11969 & 6125 & 5401 & 4108 & 4432 & 3176 & 2275 & 3104 & 2030 & 1430 \\
\hline \multirow{3}{*}{ E85 Series HEV PHEV40 } & Vehicle Cost LOW & 24037 & 24037 & 22038 & 20475 & 16300 & 14668 & 11814 & 12334 & 9638 & 7615 & 9510 & 7463 & 6043 \\
\hline & Vehicle Cost AVG & 20904 & 20904 & 19155 & 17785 & 12301 & 11114 & 9092 & 8974 & 7170 & 5778 & 7935 & 6226 & 5026 \\
\hline & Vehicle Cost HIGH & 16493 & 16493 & 15094 & 13998 & 7676 & 6804 & 5150 & 5548 & 3975 & 2849 & 3934 & 2578 & 1843 \\
\hline \multirow{3}{*}{ FC HEV } & Vehicle Cost LOW & 10269 & 10269 & 8253 & 6882 & 9239 & 7098 & 5595 & 3917 & 2657 & 1611 & 1821 & 1062 & 320 \\
\hline & Vehicle Cost AVG & 15511 & 15511 & 12425 & 10306 & 9038 & 7062 & 5662 & 5342 & 3774 & 2530 & 1707 & 953 & 236 \\
\hline & Vehicle Cost HIGH & 12256 & 12256 & 9718 & 7965 & 4205 & 3011 & 2140 & 2391 & 1400 & 601 & -5 & -484 & -936 \\
\hline \multirow{3}{*}{ FC HEV PHEV10 } & Vehicle Cost LOW & 12537 & 12537 & 10294 & 8905 & 10089 & 8073 & 6409 & 5182 & 3642 & 2344 & 2843 & 1880 & 955 \\
\hline & Vehicle Cost AVG & 16710 & 16710 & 13681 & 11682 & 9763 & 7892 & 6374 & 6109 & 4427 & 3043 & 2587 & 1665 & 792 \\
\hline & Vehicle Cost HIGH & 13240 & 13240 & 10743 & 9098 & 4343 & 3221 & 2247 & 2545 & 1460 & 600 & 224 & -384 & -878 \\
\hline \multirow{3}{*}{ FC HEV PHEV 20} & Vehicle Cost LOW & 16019 & 16019 & 13235 & 11418 & 12216 & 9841 & 7766 & 6476 & 4507 & 2994 & 3528 & 2283 & 1263 \\
\hline & Vehicle Cost AVG & 19755 & 19755 & 16229 & 13845 & 11226 & 9103 & 7301 & 6845 & 4908 & 3418 & 3150 & 1995 & 1045 \\
\hline & Vehicle Cost HIGH & 15584 & 15584 & 12703 & 10756 & 5792 & 4428 & 3168 & 3548 & 2140 & 1098 & 940 & 66 & -541 \\
\hline \multirow{3}{*}{ FC HEV PHEV30 } & Vehicle Cost LOW & 22105 & 22105 & 18998 & 17218 & 16159 & 13839 & 11239 & 10052 & 7482 & 5654 & 6301 & 4650 & 3418 \\
\hline & Vehicle Cost AVG & 25170 & 25170 & 21375 & 19037 & 14649 & 12503 & 10240 & 9805 & 7398 & 5677 & 5606 & 4089 & 2949 \\
\hline & Vehicle Cost $\mathrm{HIGH}$ & 20131 & 20131 & 17035 & 15124 & 7063 & 5737 & 4177 & 4606 & 2900 & 1773 & 1672 & 576 & -57 \\
\hline \multirow{3}{*}{ FC HEV PHEV40 } & Vehicle Cost LOW & 26046 & 26046 & 22168 & 20026 & 18466 & 15769 & 12611 & 11492 & 8433 & 6379 & 7056 & 5124 & 3794 \\
\hline & Vehicle Cost AVG & 28683 & 28683 & 24191 & 21559 & 16301 & 13880 & 11222 & 10687 & 7978 & 6121 & 6243 & 4487 & 3264 \\
\hline & Vehicle Cost $\mathrm{HIGH}$ & 22848 & 22848 & 19214 & 17074 & 8623 & 7042 & 5097 & 5689 & 3606 & 2309 & 2431 & 1043 & 313 \\
\hline \multirow{3}{*}{ EV } & Vehicle Cost LOW & 57651 & 57651 & 51645 & 47253 & 35919 & 31409 & 22479 & 23970 & 16010 & 11166 & 13443 & 8431 & 6044 \\
\hline & Vehicle Cost AVG & 48221 & 48221 & 43149 & 39438 & 24460 & 21359 & 15296 & 13435 & 8907 & 6114 & 10657 & 6559 & 4592 \\
\hline & Vehicle Cost $\mathrm{HIGH}$ & 36925 & 36925 & 32986 & 30102 & 20447 & 17674 & 11938 & 6407 & 3535 & 1809 & 5755 & 2665 & 1355 \\
\hline
\end{tabular}




\section{Cost saving per powertrain}

\begin{tabular}{|c|c|c|c|c|c|c|c|c|c|c|c|c|c|c|}
\hline & & \multicolumn{13}{|c|}{ Small_SUV } \\
\hline & & \multirow{2}{*}{$\frac{2010}{\text { Ref }}$} & \multicolumn{3}{|c|}{2010} & \multicolumn{3}{|c|}{2015} & \multicolumn{3}{|c|}{2030} & \multicolumn{3}{|c|}{2045} \\
\hline & & & low & avg & high & low & avg & high & low & avg & high & low & avg & high \\
\hline \multirow{3}{*}{ SI Conv } & Vehicle Cost LOW & 0 & 0 & 99 & 49 & 11 & 91 & -641 & 999 & 191 & 87 & 1356 & 547 & 416 \\
\hline & Vehicle Cost AVG & 0 & 0 & 99 & 49 & 826 & 908 & 129 & 1359 & 484 & 372 & 1299 & 495 & 368 \\
\hline & Vehicle Cost HIGH & 0 & 0 & 99 & 49 & 146 & 226 & -475 & 731 & -1 & -110 & 650 & -15 & -143 \\
\hline \multirow{3}{*}{$\mathrm{Cl}$ Conv } & Vehicle Cost LOW & 0 & 0 & 116 & 37 & -225 & -135 & -297 & 693 & 509 & 377 & 617 & 529 & 389 \\
\hline & Vehicle Cost AVG & 0 & 0 & 119 & 45 & 676 & 771 & 619 & 851 & 681 & 565 & 701 & 625 & 504 \\
\hline & Vehicle Cost HIGH & 0 & 0 & 124 & 62 & -7 & 99 & -40 & 480 & 321 & 220 & 273 & 207 & 103 \\
\hline \multirow{3}{*}{ H2 Conv } & Vehicle Cost LOW & 0 & 0 & -545 & -571 & -751 & -640 & -754 & 117 & -9 & -54 & 240 & 200 & 148 \\
\hline & Vehicle Cost AVG & 0 & 0 & -582 & -610 & -1039 & -927 & -1040 & -955 & -1083 & -1130 & -2384 & -2425 & -2478 \\
\hline & Vehicle Cost HIGH & 0 & 0 & -546 & -574 & -1826 & -1719 & -1837 & -1503 & -1642 & -1699 & -2108 & -2160 & -2227 \\
\hline \multirow{3}{*}{ E85 Conv } & Vehicle Cost LOW & 0 & 0 & 115 & -492 & 17 & -472 & -624 & 1025 & 211 & 111 & 1374 & 566 & 444 \\
\hline & \begin{tabular}{|l|} 
Vehicle Cost AVG \\
\end{tabular} & 0 & 0 & 115 & -522 & 834 & 296 & 148 & 1383 & 500 & 395 & 1319 & 514 & 396 \\
\hline & Vehicle Cost HIGH & 0 & 0 & 115 & -492 & 151 & -308 & -459 & 752 & 14 & -88 & 666 & 2 & -117 \\
\hline \multirow{3}{*}{ SI Split HEV } & Vehicle Cost LOW & 0 & 0 & -284 & -543 & -871 & -1100 & -1563 & -908 & -1347 & -1948 & -713 & -1212 & -1824 \\
\hline & Vehicle Cost AVG & 0 & 0 & -277 & -530 & -872 & -1055 & -1423 & -1176 & -1544 & -2046 & -1245 & -1660 & -2163 \\
\hline & Vehicle Cost HIGH & 0 & 0 & -217 & -417 & -1230 & -1378 & -1669 & -1473 & -1730 & -2079 & -1798 & -2061 & -2370 \\
\hline \multirow{3}{*}{ SI Split HEV PHEV10 } & Vehicle Cost LOW & 0 & 0 & -514 & -967 & -2261 & -2758 & -3527 & -2939 & -3746 & -4351 & -3352 & -3949 & -4585 \\
\hline & Vehicle Cost AVG & 0 & 0 & -460 & -867 & -2214 & -2585 & -3147 & -2884 & -3479 & -3928 & -3154 & -3653 & -4195 \\
\hline & Vehicle Cost HIGH & 0 & 0 & -372 & -703 & -3194 & -3449 & -3862 & -3578 & -4010 & -4354 & -4085 & -4460 & -4724 \\
\hline \multirow{3}{*}{ SI Split HEV PHEV20 } & Vehicle Cost LOW & 0 & 0 & -696 & -1334 & -3208 & -3888 & -4999 & -4473 & -5575 & -6526 & -5402 & -6243 & -7003 \\
\hline & Vehicle Cost AVG & 0 & 0 & -614 & -1175 & -3302 & -3794 & -4578 & -4518 & -5257 & -5931 & -4890 & -5590 & -6234 \\
\hline & Vehicle Cost HIGH & 0 & 0 & -490 & -940 & -3694 & -4078 & -4746 & -4401 & -5078 & -5607 & -5134 & -5720 & -6094 \\
\hline \multirow{3}{*}{ SI Series HEV PHEV 30} & Vehicle Cost LOW & 0 & 0 & -1716 & -2989 & -6374 & -7781 & -10084 & -9549 & -11800 & -13507 & -11695 & -13438 & -14717 \\
\hline & Vehicle Cost AVG & 0 & 0 & -1509 & -2632 & -7059 & -8092 & -9740 & -9674 & -11225 & -12439 & -10552 & -12013 & -13097 \\
\hline & Vehicle Cost HIGH & 0 & 0 & -1213 & -2118 & -7949 & -8676 & -9969 & -9645 & -10901 & -11800 & -10973 & -12045 & -12646 \\
\hline \multirow{3}{*}{ SI Series HEV PHEV40 } & Vehicle Cost LOW & 0 & 0 & -2029 & -3577 & -7743 & -9375 & -12230 & -11713 & -14401 & -16430 & -14536 & -16578 & -17996 \\
\hline & Vehicle Cost AVG & 0 & 0 & -1774 & -3131 & -8606 & -9793 & -11816 & -11937 & -13736 & -15132 & -12975 & -14680 & -15878 \\
\hline & Vehicle Cost HIGH & 0 & 0 & -1418 & -2504 & -8818 & -9689 & -11344 & -10947 & -12515 & -13643 & -12561 & -13912 & -14645 \\
\hline \multirow{3}{*}{ Cl Split HEV } & Vehicle Cost LOW & 0 & 0 & -303 & -622 & -1154 & -1509 & -1987 & -1321 & -1846 & -2443 & -1446 & -1971 & -2625 \\
\hline & Vehicle Cost AVG & 0 & 0 & -292 & -600 & -1186 & -1465 & -1843 & -1802 & -2226 & -2704 & -1940 & -2364 & -2889 \\
\hline & Vehicle Cost HIGH & 0 & 0 & -224 & -459 & -1515 & -1727 & -2009 & -1949 & -2238 & -2557 & -2406 & -2655 & -2955 \\
\hline
\end{tabular}




\begin{tabular}{|c|c|c|c|c|c|c|c|c|c|c|c|c|c|c|}
\hline & & \multicolumn{13}{|c|}{ Small SUV } \\
\hline & & \multirow{2}{*}{$\frac{2010}{\text { Ref }}$} & \multicolumn{3}{|c|}{2010} & \multicolumn{3}{|c|}{2015} & \multicolumn{3}{|c|}{2030} & \multicolumn{3}{|c|}{2045} \\
\hline & & & low & avg & high & low & avg & high & low & avg & high & low & avg & high \\
\hline \multirow{3}{*}{ CI Split HEV PHEV10 } & Vehicle Cost LOW & 0 & 0 & -561 & -1063 & -2608 & -3136 & -3918 & -3408 & -4201 & -4918 & -4124 & -4740 & -5452 \\
\hline & Vehicle Cost AVG & 0 & 0 & -501 & -950 & -2529 & -2923 & -3491 & -3485 & -4053 & -4586 & -3817 & -4324 & -4925 \\
\hline & Vehicle Cost HIGH & 0 & 0 & -400 & -762 & -3635 & -3904 & -4335 & -4167 & -4607 & -4977 & -4778 & -5159 & -5432 \\
\hline \multirow{3}{*}{ CI Split HEV PHEV2O } & Vehicle Cost LOW & 0 & 0 & -745 & -1447 & -3597 & -4322 & -5510 & -4985 & -6154 & -7160 & -6279 & -7157 & -7965 \\
\hline & Vehicle Cost AVG & 0 & 0 & -655 & -1274 & -3662 & -4190 & -5032 & -5167 & -5948 & $\begin{array}{l}-6653 \\
\end{array}$ & -5642 & -6366 & -7045 \\
\hline & Vehicle Cost HIGH & 0 & 0 & -519 & -1012 & -4167 & -4564 & -5263 & -5029 & -5730 & -6281 & -5881 & -6476 & -6861 \\
\hline \multirow{3}{*}{ CI Series HEV PHEV 30} & Vehicle Cost LOW & 0 & 0 & -1770 & -3089 & -6788 & -8229 & -10626 & -10088 & -12408 & -14176 & -12620 & -14405 & -15713 \\
\hline & Vehicle Cost AVG & 0 & 0 & -1554 & -2716 & -7418 & -8479 & -10198 & -10359 & -11942 & -13190 & -11332 & -12817 & -13917 \\
\hline & Vehicle Cost HIGH & 0 & 0 & -1241 & -2171 & -8346 & -9083 & -10421 & -10194 & -11481 & -12402 & -11655 & -12742 & -13347 \\
\hline \multirow{3}{*}{ CI Series HEV PHEV40 } & Vehicle Cost LOW & 0 & 0 & -2088 & -3712 & -8192 & $\begin{array}{l}-9873 \\
\end{array}$ & -12824 & -12336 & -15109 & -17188 & -15563 & -17668 & -19114 \\
\hline & Vehicle Cost AVG & 0 & 0 & -1825 & -3244 & -9011 & -10237 & -12332 & -12709 & -14552 & -15973 & -13845 & -15591 & -16804 \\
\hline & Vehicle Cost HIGH & 0 & 0 & -1451 & -2580 & -9240 & -10130 & -11830 & -11550 & -13154 & -14300 & -13301 & -14681 & -15417 \\
\hline \multirow{3}{*}{ H2 Split HEV } & Vehicle Cost LOW & 0 & 0 & -391 & -714 & -1014 & -1431 & -1844 & -1176 & -1712 & -2267 & -1218 & -1694 & -2231 \\
\hline & Vehicle Cost AVG & 0 & 0 & -383 & -699 & -2118 & -2442 & -2763 & -2814 & -3247 & -3693 & -4331 & -4718 & -5152 \\
\hline & Vehicle Cost HIGH & 0 & 0 & -294 & -538 & -2635 & -2886 & -3133 & -3181 & -3480 & -3777 & -4081 & -4310 & -4561 \\
\hline \multirow{3}{*}{ H2 Split HEV PHEV10 } & Vehicle Cost LOW & 0 & 0 & -771 & -1308 & -2560 & -3195 & -4025 & -3413 & -4304 & -4974 & -4031 & -4631 & -5287 \\
\hline & Vehicle Cost AVG & 0 & 0 & -693 & -1176 & -3601 & -4072 & -4680 & -4681 & -5336 & -5835 & -6395 & -6895 & -7451 \\
\hline & Vehicle Cost HIGH & 0 & 0 & -562 & -953 & -4792 & -5097 & -5514 & -5497 & -5940 & -6283 & $\begin{array}{l}-6559 \\
\end{array}$ & $\begin{array}{l}-6911 \\
\end{array}$ & -7155 \\
\hline \multirow{3}{*}{ H2 Split HEV PHEV2O } & Vehicle Cost LOW & 0 & 0 & -995 & -1725 & -3502 & -4368 & -5544 & -4975 & -6192 & -7161 & -6115 & -6979 & -7738 \\
\hline & \begin{tabular}{|l} 
Vehicle Cost AVG \\
\end{tabular} & 0 & 0 & -882 & -1527 & -4687 & -5315 & -6147 & -6340 & -7161 & -7842 & -8159 & -8876 & -9516 \\
\hline & Vehicle Cost HIGH & 0 & 0 & -707 & -1222 & -5308 & -5759 & -6437 & -6345 & $\begin{array}{l}-7046 \\
\end{array}$ & $\begin{array}{l}-7568 \\
\end{array}$ & -7642 & -8206 & -8557 \\
\hline \multirow{3}{*}{ H2 Series HEV PHEV3O } & Vehicle Cost LOW & 0 & 0 & -2017 & -3415 & -6618 & -8222 & -10559 & $\begin{array}{l}-9924 \\
\end{array}$ & -12271 & -13995 & -12322 & -14012 & -15249 \\
\hline & Vehicle Cost AVG & 0 & 0 & -1774 & -3005 & -8348 & -9520 & -11185 & -11362 & -12966 & -14181 & -13710 & -15121 & -16165 \\
\hline & Vehicle Cost HIGH & 0 & 0 & -1421 & -2410 & -9418 & -10222 & -11515 & -11404 & -12681 & -13565 & -13298 & -14322 & -14886 \\
\hline \multirow{3}{*}{ H2 Series HEV PHEV40 } & Vehicle Cost LOW & 0 & 0 & -2410 & -4074 & -8024 & $\begin{array}{l}-9896 \\
\end{array}$ & -12763 & -12129 & -14941 & -16981 & -15231 & -17209 & -18587 \\
\hline & Vehicle Cost AVG & 0 & 0 & -2106 & -3563 & $\begin{array}{l}-9931 \\
\end{array}$ & -11285 & -13309 & -13664 & -15531 & -16923 & -16189 & -17836 & -18994 \\
\hline & Vehicle Cost HIGH & 0 & 0 & -1677 & -2840 & -10309 & -11289 & -12927 & -12734 & -14339 & -15450 & -14929 & -16221 & -16922 \\
\hline \multirow{3}{*}{ E85 Split HEV } & Vehicle Cost LOW & 0 & 0 & -303 & -580 & -909 & -1117 & -1580 & -860 & -1411 & -1987 & -749 & -1206 & -1861 \\
\hline & Vehicle Cost AVG & 0 & 0 & -297 & -567 & -910 & -1077 & -1446 & -1142 & -1604 & -2084 & -1282 & -1661 & -2202 \\
\hline & Vehicle Cost HIGH & 0 & 0 & -235 & -447 & -1262 & -1395 & $\begin{array}{l}-1687 \\
\end{array}$ & -1456 & -1781 & -2112 & -1830 & -2069 & -2403 \\
\hline
\end{tabular}




\begin{tabular}{|c|c|c|c|c|c|c|c|c|c|c|c|c|c|c|}
\hline & & \multicolumn{13}{|c|}{ Small_SUV } \\
\hline & & \multirow{2}{*}{$\begin{array}{l}2010 \\
\text { Ref }\end{array}$} & \multicolumn{3}{|c|}{2010} & \multicolumn{3}{|c|}{2015} & \multicolumn{3}{|c|}{2030} & \multicolumn{3}{|c|}{2045} \\
\hline & & & low & avg & high & low & avg & high & low & avg & high & low & avg & high \\
\hline \multirow{3}{*}{ E85 Split HEV PHEV10 } & Vehicle Cost LOW & 0 & 0 & -511 & -968 & -2262 & -2759 & -3528 & -2939 & -3748 & -4353 & -3353 & -3917 & -4585 \\
\hline & Vehicle Cost AVG & 0 & 0 & -457 & -867 & -2216 & -2586 & -3148 & -2883 & -3481 & -3929 & -3156 & -3626 & -4196 \\
\hline & Vehicle Cost HIGH & 0 & 0 & -369 & -703 & -3198 & -3453 & -3865 & -3581 & -4015 & -4359 & -4089 & -4461 & -4730 \\
\hline \multirow{3}{*}{ E85 Split HEV PHEV20 } & Vehicle Cost LOW & 0 & 0 & -699 & -1337 & -3210 & -3891 & -5005 & -4478 & -5581 & -6505 & -5406 & -6248 & -7007 \\
\hline & Vehicle Cost AVG & 0 & 0 & -616 & -1179 & -3305 & -3798 & -4584 & -4523 & -5263 & -5913 & -4894 & -5596 & -6239 \\
\hline & Vehicle Cost HIGH & 0 & 0 & -492 & -943 & -3700 & -4083 & -4753 & -4408 & -5086 & -5610 & -5142 & -5729 & -6102 \\
\hline \multirow{3}{*}{ E85 Series HEV PHEV30 } & Vehicle Cost LOW & 0 & 0 & -1699 & -2980 & -6380 & -7783 & -10086 & -9551 & -11800 & -13510 & -11697 & -13440 & -14717 \\
\hline & Vehicle Cost AVG & 0 & 0 & -1495 & -2625 & -7065 & -8094 & -9743 & -9676 & -11226 & -12442 & -10554 & -12016 & -13098 \\
\hline & \begin{tabular}{|l} 
Vehicle Cost HIGH \\
\end{tabular} & 0 & 0 & -1202 & -2112 & -7956 & -8680 & $\begin{array}{l}-9973 \\
\end{array}$ & -9650 & -10906 & -11806 & -10978 & -12051 & -12652 \\
\hline \multirow{3}{*}{ E85 Series HEV PHEV40 } & Vehicle Cost LOW & 0 & 0 & $\begin{array}{ll}-1999 \\
\end{array}$ & -3562 & -7737 & -9369 & -12223 & -11703 & -14399 & -16422 & -14527 & -16574 & -17994 \\
\hline & \begin{tabular}{|l|} 
Vehicle Cost AVG \\
\end{tabular} & 0 & 0 & $\begin{array}{l}-1749 \\
\end{array}$ & -3119 & -8603 & -9790 & -11813 & -11930 & -13735 & -15127 & -12969 & -14679 & -15878 \\
\hline & Vehicle Cost HIGH & 0 & 0 & -1399 & -2495 & -8817 & $\begin{array}{l}-9689 \\
\end{array}$ & -11343 & -10945 & -12518 & -13644 & -12558 & -13915 & -14649 \\
\hline \multirow{3}{*}{ FC HEV } & Vehicle Cost LOW & 0 & 0 & -2016 & -3387 & -1030 & -3171 & -4675 & -6353 & -7612 & -8658 & -8449 & -9207 & -9949 \\
\hline & \begin{tabular}{|l|} 
Vehicle Cost AVG \\
\end{tabular} & 0 & 0 & -3086 & -5205 & -6474 & -8450 & $\begin{array}{l}-9849 \\
\end{array}$ & -10169 & -11738 & -12982 & -13804 & -14559 & -15275 \\
\hline & Vehicle Cost HIGH & 0 & 0 & -2539 & -4291 & -8051 & -9246 & -10116 & -9865 & -10857 & -11655 & -12261 & -12740 & -13193 \\
\hline \multirow{3}{*}{ FC HEV PHEV10 } & Vehicle Cost LOW & 0 & 0 & -2244 & -3632 & -2448 & -4464 & -6128 & -7355 & -8895 & -10193 & $\begin{array}{l}-9695 \\
\end{array}$ & -10658 & -11582 \\
\hline & Vehicle Cost AVG & 0 & 0 & -3029 & -5028 & -6947 & -8817 & -10336 & -10601 & -12283 & -13667 & -14123 & -15045 & -15918 \\
\hline & Vehicle Cost $\mathrm{HIGH}$ & 0 & 0 & -2496 & -4142 & -8897 & -10019 & -10992 & -10695 & -11780 & -12640 & -13015 & -13624 & -14117 \\
\hline \multirow{3}{*}{ FC HEV PHEV 20} & Vehicle Cost LOW & 0 & 0 & -2785 & -4602 & -3804 & -6178 & -8254 & -9544 & -11512 & -13025 & -12491 & -13736 & -14756 \\
\hline & Vehicle Cost AVG & 0 & 0 & -3526 & -5910 & -8529 & -10652 & -12455 & -12910 & -14847 & -16338 & -16605 & -17760 & -18710 \\
\hline & Vehicle Cost HIGH & 0 & 0 & -2881 & -4829 & -9792 & -11156 & -12416 & -12036 & -13444 & -14486 & -14644 & -15518 & -16125 \\
\hline \multirow{3}{*}{ FC HEV PHEV 30} & Vehicle Cost LOW & 0 & 0 & -3107 & -4887 & -5946 & -8266 & -10866 & -12053 & -14623 & -16451 & -15804 & -17455 & -18687 \\
\hline & Vehicle Cost AVG & 0 & 0 & -3795 & -6132 & -10521 & -12666 & -14930 & -15365 & -17772 & -19492 & -19564 & -21081 & -22220 \\
\hline & Vehicle Cost HIGH & 0 & 0 & -3096 & -5007 & -13068 & -14394 & -15954 & -15525 & -17231 & -18358 & -18458 & -19555 & -20188 \\
\hline \multirow{3}{*}{ FC HEV PHEV 40} & Vehicle Cost LOW & 0 & 0 & -3878 & -6020 & -7580 & -10277 & -13435 & -14555 & -17613 & -19667 & -18990 & -20922 & -22252 \\
\hline & Vehicle Cost AVG & 0 & 0 & -4492 & -7124 & -12382 & -14803 & -17461 & -17996 & -20706 & -22562 & -22440 & -24196 & -25419 \\
\hline & Vehicle Cost HIGH & 0 & 0 & -3635 & -5774 & -14226 & -15806 & -17751 & -17159 & -19243 & -20539 & -20418 & -21805 & -22536 \\
\hline \multirow{3}{*}{ EV } & Vehicle Cost LOW & 0 & 0 & -6007 & -10398 & -21732 & -26242 & -35172 & -33681 & -41642 & -46485 & -44209 & -49220 & -51607 \\
\hline & Vehicle Cost AVG & 0 & 0 & $\begin{array}{l}-5073 \\
\end{array}$ & -8783 & -23761 & -26862 & -32925 & -34786 & -39315 & -42107 & -37564 & -41662 & -43629 \\
\hline & Vehicle Cost HIGH & 0 & 0 & $\begin{array}{l}-3939 \\
\end{array}$ & -6823 & -16478 & -19251 & -24987 & -30518 & -33390 & -35116 & -31170 & -34261 & -35570 \\
\hline
\end{tabular}




\section{Percentage decrease cost per powertrain}

\begin{tabular}{|c|c|c|c|c|c|c|c|c|c|c|c|c|c|c|}
\hline & & \multicolumn{13}{|c|}{ Small_SUV } \\
\hline & & \multirow{2}{*}{$\begin{array}{c}2010 \\
\text { Ref }\end{array}$} & \multicolumn{3}{|c|}{2010} & \multicolumn{3}{|c|}{2015} & \multicolumn{3}{|c|}{2030} & \multicolumn{3}{|c|}{2045} \\
\hline & & & low & avg & high & low & avg & high & low & avg & high & low & avg & high \\
\hline \multirow{3}{*}{ SI Conv } & Vehicle Cost LOW & $0.0 \%$ & $0.0 \%$ & $0.6 \%$ & $0.3 \%$ & $0.1 \%$ & $0.6 \%$ & $-4.1 \%$ & $6.4 \%$ & $1.2 \%$ & $0.6 \%$ & $8.7 \%$ & $3.5 \%$ & $2.7 \%$ \\
\hline & Vehicle Cost AVG & $0.0 \%$ & $0.0 \%$ & $0.6 \%$ & $0.3 \%$ & $5.3 \%$ & $5.8 \%$ & $0.8 \%$ & $8.7 \%$ & $3.1 \%$ & $2.4 \%$ & $8.3 \%$ & $3.2 \%$ & $2.4 \%$ \\
\hline & Vehicle Cost HIGH & $0.0 \%$ & $0.0 \%$ & $0.6 \%$ & $0.3 \%$ & $0.9 \%$ & $1.4 \%$ & $-2.9 \%$ & $4.5 \%$ & $0.0 \%$ & $-0.7 \%$ & $4.0 \%$ & $-0.1 \%$ & $-0.9 \%$ \\
\hline \multirow{3}{*}{$\mathrm{Cl}$ Conv } & Vehicle Cost LOW & $0.0 \%$ & $0.0 \%$ & $0.7 \%$ & $0.2 \%$ & $-1.3 \%$ & $-0.8 \%$ & $-1.8 \%$ & $4.1 \%$ & $3.0 \%$ & $2.2 \%$ & $3.6 \%$ & $3.1 \%$ & $2.3 \%$ \\
\hline & Vehicle Cost AVG & $0.0 \%$ & $0.0 \%$ & $0.7 \%$ & $0.3 \%$ & $4.0 \%$ & $4.6 \%$ & $3.7 \%$ & $5.0 \%$ & $4.0 \%$ & $3.3 \%$ & $4.1 \%$ & $3.7 \%$ & $3.0 \%$ \\
\hline & Vehicle Cost HIGH & $0.0 \%$ & $0.0 \%$ & $0.7 \%$ & $0.4 \%$ & $0.0 \%$ & $0.6 \%$ & $-0.2 \%$ & $2.8 \%$ & $1.9 \%$ & $1.3 \%$ & $1.6 \%$ & $1.2 \%$ & $0.6 \%$ \\
\hline \multirow{3}{*}{ H2 Conv } & Vehicle Cost LOW & $0.0 \%$ & $0.0 \%$ & $-3.6 \%$ & $-3.8 \%$ & $-5.0 \%$ & $-4.2 \%$ & $-5.0 \%$ & $0.8 \%$ & $-0.1 \%$ & $-0.4 \%$ & $1.6 \%$ & $1.3 \%$ & $1.0 \%$ \\
\hline & Vehicle Cost AVG & $0.0 \%$ & $0.0 \%$ & $-3.2 \%$ & $-3.4 \%$ & $-5.7 \%$ & $-5.1 \%$ & $-5.7 \%$ & $-5.3 \%$ & $-6.0 \%$ & $-6.2 \%$ & $-13.2 \%$ & $-13.4 \%$ & $-13.7 \%$ \\
\hline & Vehicle Cost HIGH & $0.0 \%$ & $0.0 \%$ & $-3.0 \%$ & $-3.2 \%$ & $-10.0 \%$ & $-9.5 \%$ & $-10.1 \%$ & $-8.3 \%$ & $-9.0 \%$ & $-9.3 \%$ & $-11.6 \%$ & $-11.9 \%$ & $-12.2 \%$ \\
\hline \multirow{3}{*}{ E85 Conv } & Vehicle Cost LOW & $0.0 \%$ & $0.0 \%$ & $0.7 \%$ & $-3.2 \%$ & $0.1 \%$ & $-3.0 \%$ & $-4.0 \%$ & $6.6 \%$ & $1.4 \%$ & $0.7 \%$ & $8.8 \%$ & $3.6 \%$ & $2.9 \%$ \\
\hline & Vehicle Cost AVG & $0.0 \%$ & $0.0 \%$ & $0.7 \%$ & $-3.3 \%$ & $5.3 \%$ & $1.9 \%$ & $0.9 \%$ & $8.9 \%$ & $3.2 \%$ & $2.5 \%$ & $8.5 \%$ & $3.3 \%$ & $2.5 \%$ \\
\hline & Vehicle Cost HIGH & $0.0 \%$ & $0.0 \%$ & $0.7 \%$ & $-3.0 \%$ & $0.9 \%$ & $-1.9 \%$ & $-2.8 \%$ & $4.6 \%$ & $0.1 \%$ & $-0.5 \%$ & $4.1 \%$ & $0.0 \%$ & $-0.7 \%$ \\
\hline \multirow{3}{*}{ SI Split HEV } & Vehicle Cost LOW & $0.0 \%$ & $0.0 \%$ & $-1.5 \%$ & $-2.8 \%$ & $-4.5 \%$ & $-5.7 \%$ & $-8.1 \%$ & $-4.7 \%$ & $-7.0 \%$ & $-10.1 \%$ & $-3.7 \%$ & $-6.3 \%$ & $-9.5 \%$ \\
\hline & Vehicle Cost AVG & $0.0 \%$ & $0.0 \%$ & $-1.4 \%$ & $-2.8 \%$ & $-4.6 \%$ & $-5.5 \%$ & $-7.4 \%$ & $-6.1 \%$ & $-8.1 \%$ & $-10.7 \%$ & $-6.5 \%$ & $-8.7 \%$ & $-11.3 \%$ \\
\hline & Vehicle Cost HIGH & $0.0 \%$ & $0.0 \%$ & $-1.2 \%$ & $-2.2 \%$ & $-6.6 \%$ & $-7.4 \%$ & $-9.0 \%$ & $-7.9 \%$ & $-9.3 \%$ & $-11.2 \%$ & $-9.7 \%$ & $-11.1 \%$ & $-12.8 \%$ \\
\hline \multirow{3}{*}{ SI Split HEV PHEV10 } & Vehicle Cost LOW & $0.0 \%$ & $0.0 \%$ & $-2.3 \%$ & $-4.2 \%$ & $-9.9 \%$ & $-12.1 \%$ & $-15.5 \%$ & $-12.9 \%$ & $-16.4 \%$ & $-19.1 \%$ & $-14.7 \%$ & $-17.3 \%$ & $-20.1 \%$ \\
\hline & Vehicle Cost AVG & $0.0 \%$ & $0.0 \%$ & $-2.1 \%$ & $-3.9 \%$ & $-10.1 \%$ & $-11.8 \%$ & $-14.3 \%$ & $-13.1 \%$ & $-15.8 \%$ & $-17.9 \%$ & $-14.4 \%$ & $-16.6 \%$ & $-19.1 \%$ \\
\hline & Vehicle Cost HIGH & $0.0 \%$ & $0.0 \%$ & $-1.8 \%$ & $-3.3 \%$ & $-15.1 \%$ & $-16.3 \%$ & $-18.3 \%$ & $-16.9 \%$ & $-19.0 \%$ & $-20.6 \%$ & $-19.3 \%$ & $-21.1 \%$ & $-22.4 \%$ \\
\hline \multirow{3}{*}{ SI Split HEV PHEV20 } & Vehicle Cost LOW & $0.0 \%$ & $0.0 \%$ & $-2.7 \%$ & $-5.2 \%$ & $-12.6 \%$ & $-15.3 \%$ & $-19.7 \%$ & $-17.6 \%$ & $-21.9 \%$ & $-25.7 \%$ & $-21.3 \%$ & $-24.6 \%$ & $-27.6 \%$ \\
\hline & Vehicle Cost AVG & $0.0 \%$ & $0.0 \%$ & $-2.5 \%$ & $-4.9 \%$ & $-13.7 \%$ & $-15.7 \%$ & $-18.9 \%$ & $-18.7 \%$ & $-21.8 \%$ & $-24.5 \%$ & $-20.2 \%$ & $-23.1 \%$ & $-25.8 \%$ \\
\hline & Vehicle Cost HIGH & $0.0 \%$ & $0.0 \%$ & $-2.1 \%$ & $-4.1 \%$ & $-16.2 \%$ & $-17.9 \%$ & $-20.8 \%$ & $-19.3 \%$ & $-22.3 \%$ & $-24.6 \%$ & $-22.5 \%$ & $-25.1 \%$ & $-26.7 \%$ \\
\hline \multirow{3}{*}{ SI Series HEV PHEV 30} & Vehicle Cost LOW & $0.0 \%$ & $0.0 \%$ & $-4.8 \%$ & $-8.3 \%$ & $-17.8 \%$ & $-21.7 \%$ & $-28.1 \%$ & $-26.6 \%$ & $-32.9 \%$ & $-37.6 \%$ & $-32.6 \%$ & $-37.4 \%$ & $-41.0 \%$ \\
\hline & Vehicle Cost AVG & $0.0 \%$ & $0.0 \%$ & $-4.5 \%$ & $-7.9 \%$ & $-21.1 \%$ & $-24.2 \%$ & $-29.2 \%$ & $-29.0 \%$ & $-33.6 \%$ & $-37.3 \%$ & $-31.6 \%$ & $-36.0 \%$ & $-39.2 \%$ \\
\hline & Vehicle Cost HIGH & $0.0 \%$ & $0.0 \%$ & $-4.0 \%$ & $-7.0 \%$ & $-26.2 \%$ & $-28.6 \%$ & $-32.9 \%$ & $-31.8 \%$ & $-36.0 \%$ & $-38.9 \%$ & $-36.2 \%$ & $-39.7 \%$ & $-41.7 \%$ \\
\hline \multirow{3}{*}{ SI Series HEV PHEV40 } & Vehicle Cost LOW & $0.0 \%$ & $0.0 \%$ & $-5.1 \%$ & $-9.0 \%$ & $-19.5 \%$ & $-23.7 \%$ & $-30.9 \%$ & $-29.6 \%$ & $-36.4 \%$ & $-41.5 \%$ & $-36.7 \%$ & $-41.9 \%$ & $-45.4 \%$ \\
\hline & Vehicle Cost AVG & $0.0 \%$ & $0.0 \%$ & $-4.9 \%$ & $-8.6 \%$ & $-23.6 \%$ & $-26.8 \%$ & $-32.4 \%$ & $-32.7 \%$ & $-37.6 \%$ & $-41.4 \%$ & $-35.5 \%$ & $-40.2 \%$ & $-43.5 \%$ \\
\hline & Vehicle Cost HIGH & $0.0 \%$ & $0.0 \%$ & $-4.3 \%$ & $-7.7 \%$ & $-26.9 \%$ & $-29.6 \%$ & $-34.7 \%$ & $-33.5 \%$ & $-38.2 \%$ & $-41.7 \%$ & $-38.4 \%$ & $-42.5 \%$ & $-44.8 \%$ \\
\hline \multirow{3}{*}{ CI Split HEV } & Vehicle Cost LOW & $0.0 \%$ & $0.0 \%$ & $-1.4 \%$ & $-2.9 \%$ & $-5.3 \%$ & $-6.9 \%$ & $-9.1 \%$ & $-6.1 \%$ & $-8.5 \%$ & $-11.2 \%$ & $-6.6 \%$ & $-9.1 \%$ & $-12.1 \%$ \\
\hline & Vehicle Cost AVG & $0.0 \%$ & $0.0 \%$ & $-1.4 \%$ & $-2.8 \%$ & $-5.5 \%$ & $-6.8 \%$ & $-8.5 \%$ & $-8.4 \%$ & $-10.3 \%$ & $-12.5 \%$ & $-9.0 \%$ & $-11.0 \%$ & $-13.4 \%$ \\
\hline & Vehicle Cost HIGH & $0.0 \%$ & $0.0 \%$ & $-1.1 \%$ & $-2.2 \%$ & $-7.4 \%$ & $-8.4 \%$ & $-9.8 \%$ & $-9.5 \%$ & $-10.9 \%$ & $-12.4 \%$ & $-11.7 \%$ & $-12.9 \%$ & $-14.4 \%$ \\
\hline
\end{tabular}




\begin{tabular}{|c|c|c|c|c|c|c|c|c|c|c|c|c|c|c|}
\hline & & \multicolumn{13}{|c|}{ Small SUV } \\
\hline & & \multirow{2}{*}{$\frac{2010}{\operatorname{Ref}}$} & \multicolumn{3}{|c|}{2010} & \multicolumn{3}{|c|}{2015} & \multicolumn{3}{|c|}{2030} & \multicolumn{3}{|c|}{2045} \\
\hline & & & low & avg & high & low & avg & high & low & avg & high & low & avg & high \\
\hline \multirow{3}{*}{ CI Split HEV PHEV10 } & Vehicle Cost LOW & $0.0 \%$ & $0.0 \%$ & $-2.2 \%$ & $-4.2 \%$ & $-10.3 \%$ & $-12.4 \%$ & $-15.4 \%$ & $-13.4 \%$ & $-16.6 \%$ & $-19.4 \%$ & $-16.2 \%$ & $-18.7 \%$ & $-21.5 \%$ \\
\hline & Vehicle Cost AVG & $0.0 \%$ & $0.0 \%$ & $-2.0 \%$ & $-3.9 \%$ & $-10.3 \%$ & $-11.9 \%$ & $-14.3 \%$ & $-14.2 \%$ & $-16.6 \%$ & $-18.7 \%$ & $-15.6 \%$ & $-17.7 \%$ & $-20.1 \%$ \\
\hline & Vehicle Cost HIGH & $0.0 \%$ & $0.0 \%$ & $-1.7 \%$ & $-3.3 \%$ & $-15.6 \%$ & $-16.7 \%$ & $-18.6 \%$ & $-17.9 \%$ & $-19.8 \%$ & $-21.3 \%$ & $-20.5 \%$ & $-22.1 \%$ & $-23.3 \%$ \\
\hline \multirow{3}{*}{ CI Split HEV PHEV20 } & Vehicle Cost LOW & $0.0 \%$ & $0.0 \%$ & $-2.6 \%$ & $-5.1 \%$ & $-12.8 \%$ & $-15.4 \%$ & $-19.6 \%$ & $-17.7 \%$ & $-21.9 \%$ & $-25.5 \%$ & $-22.3 \%$ & $-25.4 \%$ & $-28.3 \%$ \\
\hline & Vehicle Cost AVG & $0.0 \%$ & $0.0 \%$ & $-2.4 \%$ & $-4.8 \%$ & $-13.7 \%$ & $-15.6 \%$ & $-18.8 \%$ & $-19.3 \%$ & $-22.2 \%$ & $-24.9 \%$ & $-21.1 \%$ & $-23.8 \%$ & $-26.3 \%$ \\
\hline & Vehicle Cost $\mathrm{HIGH}$ & $0.0 \%$ & $0.0 \%$ & $-2.1 \%$ & $-4.0 \%$ & $-16.6 \%$ & $-18.2 \%$ & $-21.0 \%$ & $-20.1 \%$ & $-22.9 \%$ & $-25.1 \%$ & $-23.5 \%$ & $-25.8 \%$ & $-27.4 \%$ \\
\hline \multirow{3}{*}{ CI Series HEV PHEV 30} & Vehicle Cost LOW & $0.0 \%$ & $0.0 \%$ & $-4.6 \%$ & $-8.0 \%$ & $-17.6 \%$ & $-21.3 \%$ & $-27.5 \%$ & $-26.1 \%$ & $-32.2 \%$ & $-36.7 \%$ & $-32.7 \%$ & $-37.3 \%$ & $-40.7 \%$ \\
\hline & Vehicle Cost AVG & $0.0 \%$ & $0.0 \%$ & $-4.3 \%$ & $-7.6 \%$ & $-20.6 \%$ & $-23.6 \%$ & $-28.4 \%$ & $-28.8 \%$ & $-33.2 \%$ & $-36.7 \%$ & $-31.5 \%$ & $-35.7 \%$ & $-38.7 \%$ \\
\hline & Vehicle Cost HIGH & $0.0 \%$ & $0.0 \%$ & $-3.8 \%$ & $-6.7 \%$ & $-25.7 \%$ & $-27.9 \%$ & $-32.1 \%$ & $-31.4 \%$ & $-35.3 \%$ & $-38.2 \%$ & $-35.9 \%$ & $-39.2 \%$ & $-41.1 \%$ \\
\hline \multirow{3}{*}{ Cl Series HEV PHEV40 } & Vehicle Cost LOW & $0.0 \%$ & $0.0 \%$ & $-4.9 \%$ & $-8.7 \%$ & $-19.3 \%$ & $-23.3 \%$ & $-30.2 \%$ & $-29.1 \%$ & $-35.6 \%$ & $-40.5 \%$ & $-36.7 \%$ & $-41.6 \%$ & $-45.0 \%$ \\
\hline & Vehicle Cost AVG & $0.0 \%$ & $0.0 \%$ & $-4.7 \%$ & $-8.3 \%$ & $-23.0 \%$ & $-26.1 \%$ & $-31.5 \%$ & $-32.4 \%$ & $-37.1 \%$ & $-40.8 \%$ & $-35.3 \%$ & $-39.8 \%$ & $-42.9 \%$ \\
\hline & Vehicle Cost HIGH & $0.0 \%$ & $0.0 \%$ & $-4.1 \%$ & $-7.4 \%$ & $-26.4 \%$ & $-28.9 \%$ & $-33.8 \%$ & $-33.0 \%$ & $-37.6 \%$ & $-40.9 \%$ & $-38.0 \%$ & $-41.9 \%$ & $-44.0 \%$ \\
\hline \multirow{3}{*}{ H2 Split HEV } & Vehicle Cost LOW & $0.0 \%$ & $0.0 \%$ & $-2.0 \%$ & $-3.7 \%$ & $-5.3 \%$ & $-7.4 \%$ & $-9.6 \%$ & $-6.1 \%$ & $-8.9 \%$ & $-11.8 \%$ & $-6.3 \%$ & $-8.8 \%$ & $-11.6 \%$ \\
\hline & Vehicle Cost AVG & $0.0 \%$ & $0.0 \%$ & $-1.7 \%$ & $-3.2 \%$ & $-9.6 \%$ & $-11.1 \%$ & $-12.5 \%$ & $-12.7 \%$ & $-14.7 \%$ & $-16.7 \%$ & $-19.6 \%$ & $-21.4 \%$ & $-23.3 \%$ \\
\hline & Vehicle Cost HIGH & $0.0 \%$ & $0.0 \%$ & $-1.4 \%$ & $-2.6 \%$ & $-12.6 \%$ & $-13.8 \%$ & $-15.0 \%$ & $-15.2 \%$ & $-16.7 \%$ & $-18.1 \%$ & $-19.5 \%$ & $-20.6 \%$ & $-21.8 \%$ \\
\hline \multirow{3}{*}{ H2 Split HEV PHEV10 } & Vehicle Cost LOW & $0.0 \%$ & $0.0 \%$ & $-3.3 \%$ & $-5.7 \%$ & $-11.1 \%$ & $-13.8 \%$ & $-17.4 \%$ & $-14.8 \%$ & $-18.6 \%$ & $-21.5 \%$ & $-17.4 \%$ & $-20.0 \%$ & $-22.9 \%$ \\
\hline & Vehicle Cost AVG & $0.0 \%$ & $0.0 \%$ & $-2.7 \%$ & $-4.7 \%$ & $-14.3 \%$ & $-16.1 \%$ & $-18.5 \%$ & $-18.5 \%$ & $-21.1 \%$ & $-23.1 \%$ & $-25.3 \%$ & $-27.3 \%$ & $-29.5 \%$ \\
\hline & Vehicle Cost HIGH & $0.0 \%$ & $0.0 \%$ & $-2.4 \%$ & $-4.0 \%$ & $-20.2 \%$ & $-21.4 \%$ & $-23.2 \%$ & $-23.1 \%$ & $-25.0 \%$ & $-26.4 \%$ & $-27.6 \%$ & $-29.1 \%$ & $-30.1 \%$ \\
\hline \multirow{3}{*}{ H2 Split HEV PHEV2O } & Vehicle Cost LOW & $0.0 \%$ & $0.0 \%$ & $-3.9 \%$ & $-6.7 \%$ & $-13.6 \%$ & $-16.9 \%$ & $-21.5 \%$ & $-19.3 \%$ & $-24.0 \%$ & $-27.8 \%$ & $-23.7 \%$ & $-27.1 \%$ & $-30.0 \%$ \\
\hline & \begin{tabular}{|l|} 
Vehicle Cost AVG \\
\end{tabular} & $0.0 \%$ & $0.0 \%$ & $-3.2 \%$ & $-5.6 \%$ & $-17.1 \%$ & $-19.3 \%$ & $-22.4 \%$ & $-23.1 \%$ & $-26.1 \%$ & $-28.5 \%$ & $-29.7 \%$ & $-32.3 \%$ & $-34.6 \%$ \\
\hline & Vehicle Cost HIGH & $0.0 \%$ & $0.0 \%$ & $-2.8 \%$ & $-4.8 \%$ & $-20.8 \%$ & $-22.6 \%$ & $-25.3 \%$ & $-24.9 \%$ & $-27.7 \%$ & $-29.7 \%$ & $-30.0 \%$ & $-32.2 \%$ & $-33.6 \%$ \\
\hline \multirow{3}{*}{ H2 Series HEV PHEV3O } & Vehicle Cost LOW & $0.0 \%$ & $0.0 \%$ & $-5.6 \%$ & $-9.5 \%$ & $-18.4 \%$ & $-22.9 \%$ & $-29.4 \%$ & $-27.6 \%$ & $-34.2 \%$ & $-39.0 \%$ & $-34.3 \%$ & $-39.0 \%$ & $-42.5 \%$ \\
\hline & Vehicle Cost AVG & $0.0 \%$ & $0.0 \%$ & $-4.9 \%$ & $-8.3 \%$ & $-23.0 \%$ & $-26.2 \%$ & $-30.8 \%$ & $-31.2 \%$ & $-35.6 \%$ & $-39.0 \%$ & $-37.7 \%$ & $-41.6 \%$ & $-44.4 \%$ \\
\hline & Vehicle Cost HIGH & $0.0 \%$ & $0.0 \%$ & $-4.3 \%$ & $-7.4 \%$ & $-28.8 \%$ & $-31.3 \%$ & $-35.2 \%$ & $-34.9 \%$ & $-38.8 \%$ & $-41.5 \%$ & $-40.7 \%$ & $-43.8 \%$ & $-45.5 \%$ \\
\hline \multirow{3}{*}{ H2 Series HEV PHEV40 } & Vehicle Cost LOW & $0.0 \%$ & $0.0 \%$ & $-6.1 \%$ & $-10.3 \%$ & $-20.2 \%$ & $-24.9 \%$ & $-32.2 \%$ & $-30.6 \%$ & $-37.7 \%$ & $-42.8 \%$ & $-38.4 \%$ & $-43.4 \%$ & $-46.9 \%$ \\
\hline & Vehicle Cost AVG & $0.0 \%$ & $0.0 \%$ & $-5.3 \%$ & $-9.0 \%$ & $-25.1 \%$ & $-28.5 \%$ & $-33.7 \%$ & $-34.6 \%$ & $-39.3 \%$ & $-42.8 \%$ & $-40.9 \%$ & $-45.1 \%$ & $-48.0 \%$ \\
\hline & Vehicle Cost HIGH & $0.0 \%$ & $0.0 \%$ & $-4.8 \%$ & $-8.1 \%$ & $-29.3 \%$ & $-32.1 \%$ & $-36.8 \%$ & $-36.2 \%$ & $-40.8 \%$ & $-44.0 \%$ & $-42.5 \%$ & $-46.2 \%$ & $-48.2 \%$ \\
\hline \multirow{3}{*}{ E85 Split HEV } & Vehicle Cost LOW & $0.0 \%$ & $0.0 \%$ & $-1.6 \%$ & $-3.0 \%$ & $-4.7 \%$ & $-5.8 \%$ & $-8.2 \%$ & $-4.4 \%$ & $-7.3 \%$ & $-10.3 \%$ & $-3.9 \%$ & $-6.2 \%$ & $-9.6 \%$ \\
\hline & Vehicle Cost AVG & $0.0 \%$ & $0.0 \%$ & $-1.5 \%$ & $-3.0 \%$ & $-4.7 \%$ & $-5.6 \%$ & $-7.5 \%$ & $-5.9 \%$ & $-8.4 \%$ & $-10.9 \%$ & $-6.7 \%$ & $-8.7 \%$ & $-11.5 \%$ \\
\hline & Vehicle Cost HIGH & $0.0 \%$ & $0.0 \%$ & $-1.3 \%$ & $-2.4 \%$ & $-6.8 \%$ & $-7.5 \%$ & $-9.1 \%$ & $-7.8 \%$ & $-9.6 \%$ & $-11.4 \%$ & $-9.8 \%$ & $-11.1 \%$ & $-12.9 \%$ \\
\hline
\end{tabular}




\begin{tabular}{|c|c|c|c|c|c|c|c|c|c|c|c|c|c|c|}
\hline & & \multicolumn{13}{|c|}{ Small_SUV } \\
\hline & & \multirow{2}{*}{$\begin{array}{l}2010 \\
\text { Ref } \\
\end{array}$} & \multicolumn{3}{|c|}{2010} & \multicolumn{3}{|c|}{2015} & \multicolumn{3}{|c|}{2030} & \multicolumn{3}{|c|}{2045} \\
\hline & & & low & avg & high & low & avg & high & low & avg & high & low & avg & high \\
\hline \multirow{3}{*}{ E85 Split HEV PHEV10 } & Vehicle Cost LOW & $0.0 \%$ & $0.0 \%$ & $-2.2 \%$ & $-4.2 \%$ & $-9.9 \%$ & $-12.1 \%$ & $-15.5 \%$ & $-12.9 \%$ & $-16.4 \%$ & $-19.1 \%$ & $-14.7 \%$ & $-17.2 \%$ & $-20.1 \%$ \\
\hline & Vehicle Cost AVG & $0.0 \%$ & $0.0 \%$ & $-2.1 \%$ & $-3.9 \%$ & $-10.1 \%$ & $-11.8 \%$ & $-14.3 \%$ & $-13.1 \%$ & $-15.8 \%$ & $-17.9 \%$ & $-14.4 \%$ & $-16.5 \%$ & $-19.1 \%$ \\
\hline & Vehicle Cost HIGH & $0.0 \%$ & $0.0 \%$ & $-1.7 \%$ & $-3.3 \%$ & $-15.1 \%$ & $-16.3 \%$ & $-18.3 \%$ & $-16.9 \%$ & $-19.0 \%$ & $-20.6 \%$ & $-19.3 \%$ & $-21.1 \%$ & $-22.4 \%$ \\
\hline \multirow{3}{*}{ E85 Split HEV PHEV20 } & Vehicle Cost LOW & $0.0 \%$ & $0.0 \%$ & $-2.7 \%$ & $-5.3 \%$ & $-12.6 \%$ & $-15.3 \%$ & $-19.7 \%$ & $-17.6 \%$ & $-21.9 \%$ & $-25.6 \%$ & $-21.3 \%$ & $-24.6 \%$ & $-27.5 \%$ \\
\hline & Vehicle Cost AVG & $0.0 \%$ & $0.0 \%$ & $-2.5 \%$ & $-4.9 \%$ & $-13.7 \%$ & $-15.7 \%$ & $-18.9 \%$ & $-18.7 \%$ & $-21.8 \%$ & $-24.4 \%$ & $-20.2 \%$ & $-23.1 \%$ & $-25.8 \%$ \\
\hline & Vehicle Cost $\mathrm{HIGH}$ & $0.0 \%$ & $0.0 \%$ & $-2.2 \%$ & $-4.1 \%$ & $-16.2 \%$ & $-17.9 \%$ & $-20.8 \%$ & $-19.3 \%$ & $-22.3 \%$ & $-24.6 \%$ & $-22.5 \%$ & $-25.1 \%$ & $-26.7 \%$ \\
\hline \multirow{3}{*}{ E85 Series HEV PHEV30 } & Vehicle Cost LOW & $0.0 \%$ & $0.0 \%$ & $-4.7 \%$ & $-8.3 \%$ & $-17.8 \%$ & $-21.7 \%$ & $-28.1 \%$ & $-26.6 \%$ & $-32.8 \%$ & $-37.6 \%$ & $-32.6 \%$ & $-37.4 \%$ & $-41.0 \%$ \\
\hline & Vehicle Cost AVG & $0.0 \%$ & $0.0 \%$ & $-4.5 \%$ & $-7.9 \%$ & $-21.1 \%$ & $-24.2 \%$ & $-29.2 \%$ & $-29.0 \%$ & $-33.6 \%$ & $-37.2 \%$ & $-31.6 \%$ & $-36.0 \%$ & $-39.2 \%$ \\
\hline & Vehicle Cost $\mathrm{HIGH}$ & $0.0 \%$ & $0.0 \%$ & $-4.0 \%$ & $-7.0 \%$ & $-26.2 \%$ & $-28.6 \%$ & $-32.9 \%$ & $-31.8 \%$ & $-36.0 \%$ & $-38.9 \%$ & $-36.2 \%$ & $-39.7 \%$ & $-41.7 \%$ \\
\hline \multirow{3}{*}{ E85 Series HEV PHEV40 } & Vehicle Cost LOW & $0.0 \%$ & $0.0 \%$ & $-5.0 \%$ & $-9.0 \%$ & $-19.5 \%$ & $-23.6 \%$ & $-30.8 \%$ & $-29.5 \%$ & $-36.3 \%$ & $-41.4 \%$ & $-36.7 \%$ & $-41.8 \%$ & $-45.4 \%$ \\
\hline & Vehicle Cost AVG & $0.0 \%$ & $0.0 \%$ & $-4.8 \%$ & $-8.5 \%$ & $-23.5 \%$ & $-26.8 \%$ & $-32.3 \%$ & $-32.6 \%$ & $-37.6 \%$ & $-41.4 \%$ & $-35.5 \%$ & $-40.2 \%$ & $-43.5 \%$ \\
\hline & Vehicle Cost $\mathrm{HIGH}$ & $0.0 \%$ & $0.0 \%$ & $-4.3 \%$ & $-7.6 \%$ & $-26.9 \%$ & $-29.6 \%$ & $-34.6 \%$ & $-33.4 \%$ & $-38.2 \%$ & $-41.7 \%$ & $-38.4 \%$ & $-42.5 \%$ & $-44.7 \%$ \\
\hline \multirow{3}{*}{ FC HEV } & Vehicle Cost LOW & $0.0 \%$ & $0.0 \%$ & $-7.8 \%$ & $-13.1 \%$ & $-4.0 \%$ & $-12.3 \%$ & $-18.1 \%$ & $-24.6 \%$ & $-29.4 \%$ & $-33.5 \%$ & $-32.7 \%$ & $-35.6 \%$ & $-38.5 \%$ \\
\hline & Vehicle Cost AVG & $0.0 \%$ & $0.0 \%$ & $-9.9 \%$ & $-16.7 \%$ & $-20.8 \%$ & $-27.1 \%$ & $-31.6 \%$ & $-32.6 \%$ & $-37.7 \%$ & $-41.7 \%$ & $-44.3 \%$ & $-46.7 \%$ & $-49.0 \%$ \\
\hline & Vehicle Cost HIGH & $0.0 \%$ & $0.0 \%$ & $-8.9 \%$ & $-15.1 \%$ & $-28.2 \%$ & $-32.4 \%$ & $-35.5 \%$ & $-34.6 \%$ & $-38.1 \%$ & $-40.9 \%$ & $-43.0 \%$ & $-44.7 \%$ & $-46.3 \%$ \\
\hline \multirow{3}{*}{ FC HEV PHEV10 } & Vehicle Cost LOW & $0.0 \%$ & $0.0 \%$ & $-8.0 \%$ & $-12.9 \%$ & $-8.7 \%$ & $-15.9 \%$ & $-21.8 \%$ & $-26.1 \%$ & $-31.6 \%$ & $-36.2 \%$ & $-34.5 \%$ & $-37.9 \%$ & $-41.2 \%$ \\
\hline & Vehicle Cost AVG & $0.0 \%$ & $0.0 \%$ & $-9.4 \%$ & $-15.5 \%$ & $-21.5 \%$ & $-27.3 \%$ & $-32.0 \%$ & $-32.8 \%$ & $-38.0 \%$ & $-42.3 \%$ & $-43.7 \%$ & $-46.5 \%$ & $-49.2 \%$ \\
\hline & Vehicle Cost HIGH & $0.0 \%$ & $0.0 \%$ & $-8.5 \%$ & $-14.0 \%$ & $-30.2 \%$ & $-34.0 \%$ & $-37.3 \%$ & $-36.3 \%$ & $-39.9 \%$ & $-42.9 \%$ & $-44.1 \%$ & $-46.2 \%$ & $-47.9 \%$ \\
\hline \multirow{3}{*}{ FC HEV PHEV2O } & Vehicle Cost LOW & $0.0 \%$ & $0.0 \%$ & $-8.8 \%$ & $-14.6 \%$ & $-12.0 \%$ & $-19.5 \%$ & $-26.1 \%$ & $-30.2 \%$ & $-36.4 \%$ & $-41.2 \%$ & $-39.5 \%$ & $-43.4 \%$ & $-46.7 \%$ \\
\hline & Vehicle Cost AVG & $0.0 \%$ & $0.0 \%$ & $-10.0 \%$ & $-16.7 \%$ & $-24.1 \%$ & $-30.1 \%$ & $-35.2 \%$ & $-36.5 \%$ & $-41.9 \%$ & $-46.2 \%$ & $-46.9 \%$ & $-50.2 \%$ & $-52.9 \%$ \\
\hline & Vehicle Cost HIGH & $0.0 \%$ & $0.0 \%$ & $-9.1 \%$ & $-15.2 \%$ & $-30.8 \%$ & $-35.0 \%$ & $-39.0 \%$ & $-37.8 \%$ & $-42.2 \%$ & $-45.5 \%$ & $-46.0 \%$ & $-48.7 \%$ & $-50.7 \%$ \\
\hline \multirow{3}{*}{ FC HEV PHEV 30} & Vehicle Cost LOW & $0.0 \%$ & $0.0 \%$ & $-8.2 \%$ & $-13.0 \%$ & $-15.8 \%$ & $-21.9 \%$ & $-28.8 \%$ & $-32.0 \%$ & $-38.8 \%$ & $-43.6 \%$ & $-41.9 \%$ & $-46.3 \%$ & $-49.6 \%$ \\
\hline & Vehicle Cost AVG & $0.0 \%$ & $0.0 \%$ & $-9.3 \%$ & $-15.0 \%$ & $-25.8 \%$ & $-31.0 \%$ & $-36.6 \%$ & $-37.7 \%$ & $-43.5 \%$ & $-47.8 \%$ & $-47.9 \%$ & $-51.7 \%$ & $-54.4 \%$ \\
\hline & Vehicle Cost HIGH & $0.0 \%$ & $0.0 \%$ & $-8.5 \%$ & $-13.8 \%$ & $-35.9 \%$ & $-39.6 \%$ & $-43.9 \%$ & $-42.7 \%$ & $-47.4 \%$ & $-50.5 \%$ & $-50.7 \%$ & $-53.7 \%$ & $-55.5 \%$ \\
\hline \multirow{3}{*}{ FC HEV PHEV40 } & Vehicle Cost LOW & $0.0 \%$ & $0.0 \%$ & $-9.3 \%$ & $-14.5 \%$ & $-18.2 \%$ & $-24.7 \%$ & $-32.3 \%$ & $-34.9 \%$ & $-42.3 \%$ & $-47.2 \%$ & $-45.6 \%$ & $-50.2 \%$ & $-53.4 \%$ \\
\hline & \begin{tabular}{|l} 
Vehicle Cost AVG \\
\end{tabular} & $0.0 \%$ & $0.0 \%$ & $-10.1 \%$ & $-16.1 \%$ & $-27.9 \%$ & $-33.4 \%$ & $-39.4 \%$ & $-40.6 \%$ & $-46.7 \%$ & $-50.9 \%$ & $-50.6 \%$ & $-54.6 \%$ & $-57.4 \%$ \\
\hline & Vehicle Cost HIGH & $0.0 \%$ & $0.0 \%$ & $-9.3 \%$ & $-14.8 \%$ & $-36.4 \%$ & $-40.4 \%$ & $-45.4 \%$ & $-43.9 \%$ & $-49.2 \%$ & $-52.5 \%$ & $-52.2 \%$ & $-55.8 \%$ & $-57.6 \%$ \\
\hline \multirow{3}{*}{ EV } & Vehicle Cost LOW & $0.0 \%$ & $0.0 \%$ & $-8.2 \%$ & $-14.2 \%$ & $-29.7 \%$ & $-35.8 \%$ & $-48.0 \%$ & $-46.0 \%$ & $-56.8 \%$ & $-63.5 \%$ & $-60.4 \%$ & $-67.2 \%$ & $-70.5 \%$ \\
\hline & Vehicle Cost AVG & $0.0 \%$ & $0.0 \%$ & $-7.9 \%$ & $-13.8 \%$ & $-37.2 \%$ & $-42.1 \%$ & $-51.6 \%$ & $-54.5 \%$ & $-61.6 \%$ & $-65.9 \%$ & $-58.8 \%$ & $-65.2 \%$ & $-68.3 \%$ \\
\hline & Vehicle Cost HIGH & $0.0 \%$ & $0.0 \%$ & $-7.4 \%$ & $-12.8 \%$ & $-31.0 \%$ & $-36.2 \%$ & $-47.0 \%$ & $-57.4 \%$ & $-62.8 \%$ & $-66.0 \%$ & $-58.6 \%$ & $-64.4 \%$ & $-66.9 \%$ \\
\hline
\end{tabular}




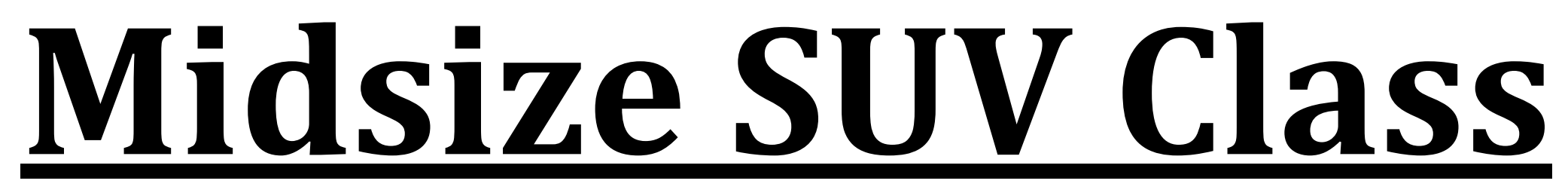


Incremental Cost versus the Conventional 2010 Reference

\begin{tabular}{|c|c|c|c|c|c|c|c|c|c|c|c|c|c|c|}
\hline & & \multicolumn{13}{|c|}{ Midsize_SUV } \\
\hline & & \multirow{2}{*}{$\begin{array}{c}2010 \\
\text { Ref }\end{array}$} & \multicolumn{3}{|c|}{2010} & \multicolumn{3}{|c|}{2015} & \multicolumn{3}{|c|}{2030} & \multicolumn{3}{|c|}{2045} \\
\hline & & & low & avg & high & low & avg & high & low & avg & high & low & avg & high \\
\hline \multirow{3}{*}{ SI Conv } & Vehicle Cost LOW & 0 & 0 & -108 & -165 & 2 & -123 & -93 & 797 & 783 & 13 & 1349 & 1210 & 348 \\
\hline & Vehicle Cost AVG & 0 & 0 & -108 & -165 & 810 & 690 & 721 & 1164 & 1147 & 307 & 1286 & 1152 & 295 \\
\hline & Vehicle Cost HIGH & 0 & 0 & -108 & -165 & 138 & 12 & 43 & 532 & 517 & -179 & 638 & 503 & -214 \\
\hline \multirow{3}{*}{$\mathrm{Cl}$ Conv } & Vehicle Cost LOW & 2494 & 2494 & 1340 & 1243 & 1139 & 1072 & 1006 & 1872 & 1785 & 1614 & 1906 & 1781 & 1616 \\
\hline & Vehicle Cost AVG & 2427 & 2427 & 1230 & 1140 & 1918 & 1862 & 1808 & 1914 & 1843 & 1693 & 1871 & 1764 & 1622 \\
\hline & Vehicle Cost HIGH & 2068 & 2068 & 936 & 861 & 928 & 890 & 853 & 1257 & 1200 & 1068 & 1157 & 1064 & 942 \\
\hline \multirow{3}{*}{ H2 Conv } & Vehicle Cost LOW & -735 & -735 & -758 & -788 & -825 & -853 & -1511 & -75 & -775 & -834 & 208 & -572 & -637 \\
\hline & Vehicle Cost AVG & 2283 & 2283 & 2259 & 2227 & 1954 & 1930 & 1215 & 1936 & 1169 & 1106 & 602 & -180 & -244 \\
\hline & Vehicle Cost HIGH & 1722 & 1722 & 1698 & 1666 & 555 & 521 & -133 & 741 & 63 & -13 & 274 & -458 & -540 \\
\hline \multirow{3}{*}{ E85 Conv } & Vehicle Cost LOW & -32 & -32 & -127 & -171 & -16 & -129 & -122 & 780 & 763 & -15 & 1321 & 1182 & 313 \\
\hline & Vehicle Cost AVG & -31 & -31 & -126 & -170 & 794 & 687 & 695 & 1147 & 1126 & 277 & 1259 & 1125 & 262 \\
\hline & Vehicle Cost HIGH & -32 & -32 & -127 & -171 & 118 & 6 & 13 & 512 & 492 & -211 & 607 & 472 & -251 \\
\hline \multirow{3}{*}{ SI Split HEV } & Vehicle Cost LOW & 4528 & 4528 & 4164 & 3859 & 3458 & 3077 & 2574 & 3240 & 2670 & 1996 & 3432 & 2776 & 2053 \\
\hline & Vehicle Cost AVG & 4325 & 4325 & 3969 & 3671 & 3081 & 2777 & 2374 & 2659 & 2184 & 1625 & 2583 & 2041 & 1447 \\
\hline & Vehicle Cost HIGH & 2874 & 2874 & 2594 & 2361 & 1345 & 1103 & 792 & 953 & 623 & 242 & 575 & 238 & -124 \\
\hline \multirow{3}{*}{ SI Split HEV PHEV10 } & Vehicle Cost LOW & 8640 & 8640 & 8080 & 7592 & 5939 & 5351 & 4438 & 4905 & 4016 & 3235 & 4417 & 3710 & 2875 \\
\hline & Vehicle Cost AVG & 7613 & 7613 & 7109 & 6671 & 4800 & 4357 & 3685 & 3901 & 3239 & 2653 & 3618 & 3028 & 2314 \\
\hline & Vehicle Cost HIGH & 5878 & 5878 & 5467 & 5109 & 1975 & 1693 & 1221 & 1412 & 956 & 528 & 829 & 397 & 77 \\
\hline \multirow{3}{*}{ SI Split HEV PHEV20 } & Vehicle Cost LOW & 11712 & 11712 & 11019 & 10349 & 7894 & 7120 & 5822 & 6217 & 5007 & 3812 & 5072 & 4063 & 3134 \\
\hline & Vehicle Cost AVG & 10186 & 10186 & 9571 & 8979 & 6092 & 5525 & 4604 & 4591 & 3766 & 2914 & 4143 & 3302 & 2515 \\
\hline & Vehicle Cost HIGH & 7837 & 7837 & 7342 & 6866 & 3356 & 2943 & 2185 & 2423 & 1707 & 1053 & 1571 & 905 & 454 \\
\hline \multirow{3}{*}{ SI Series HEV PHEV30 } & Vehicle Cost LOW & 23524 & 23524 & 21920 & 20534 & 16106 & 14622 & 12039 & 12280 & 9915 & 7842 & 9805 & 7815 & 6316 \\
\hline & Vehicle Cost AVG & 20556 & 20556 & 19141 & 17920 & 12209 & 11116 & 9269 & 9119 & 7491 & 6017 & 8158 & 6489 & 5219 \\
\hline & Vehicle Cost HIGH & 16257 & 16257 & 15117 & 14134 & 7032 & 6276 & 4819 & 4989 & 3667 & 2575 & 3478 & 2264 & 1556 \\
\hline \multirow{3}{*}{ SI Series HEV PHEV40 } & Vehicle Cost LOW & 27857 & 27857 & 25917 & 24302 & 18834 & 17140 & 13947 & 14090 & 11254 & 8801 & 10818 & 8494 & 6828 \\
\hline & Vehicle Cost AVG & 24215 & 24215 & 22515 & 21100 & 14055 & 12819 & 10557 & 10137 & 8238 & 6552 & 8984 & 7042 & 5637 \\
\hline & Vehicle Cost HIGH & 19077 & 19077 & 17716 & 16582 & 8834 & 7941 & 6088 & 6288 & 4639 & 3270 & 4439 & 2920 & 2050 \\
\hline \multirow{3}{*}{ Cl Split HEV } & Vehicle Cost LOW & 7120 & 7120 & 6732 & 6384 & 5716 & 5352 & 4730 & 5395 & 4719 & 3998 & 5178 & 4526 & 3720 \\
\hline & \begin{tabular}{|l|} 
Vehicle Cost AVG \\
\end{tabular} & 6853 & 6853 & 6479 & 6144 & 5242 & 4953 & 4463 & 4515 & 3971 & 3394 & 4306 & 3780 & 3132 \\
\hline & Vehicle Cost HIGH & 4967 & 4967 & 4681 & 4426 & 3106 & 2891 & 2523 & 2539 & 2171 & 1790 & 1976 & 1669 & 1296 \\
\hline
\end{tabular}




\begin{tabular}{|c|c|c|c|c|c|c|c|c|c|c|c|c|c|c|}
\hline & & \multicolumn{13}{|c|}{ Midsize_SUV } \\
\hline & & \multirow{2}{*}{$\frac{2010}{\operatorname{Ref}}$} & \multicolumn{3}{|c|}{2010} & \multicolumn{3}{|c|}{2015} & \multicolumn{3}{|c|}{2030} & \multicolumn{3}{|c|}{2045} \\
\hline & & & low & avg & high & low & avg & high & low & avg & high & low & avg & high \\
\hline \multirow{3}{*}{ CI Split HEV PHEV10 } & Vehicle Cost LOW & 11346 & 11346 & 10728 & 10186 & 8267 & 7623 & 6689 & 7122 & 6189 & 5340 & 6253 & 5568 & 4664 \\
\hline & Vehicle Cost AVG & 10216 & 10216 & 9662 & 9175 & 7063 & 6575 & 5891 & 5875 & 5190 & 4561 & 5468 & 4905 & 4141 \\
\hline & Vehicle Cost HIGH & 8130 & 8130 & 7683 & 7289 & 3740 & 3438 & 2947 & 3025 & 2550 & 2101 & 2317 & 1885 & 1557 \\
\hline \multirow{3}{*}{ CI Split HEV PHEV2O } & Vehicle Cost LOW & 14514 & 14514 & 13760 & 13029 & 10290 & 9478 & 8108 & 8447 & 7168 & 5946 & 6957 & 5933 & 4934 \\
\hline & Vehicle Cost AVG & 12869 & 12869 & 12200 & 11555 & 8402 & 7805 & 6829 & 6561 & 5693 & 4835 & 6034 & 5188 & 4349 \\
\hline & Vehicle Cost HIGH & 10150 & 10150 & 9615 & 9100 & 5164 & 4738 & 3947 & 4060 & 3322 & 2652 & 3085 & 2411 & 1954 \\
\hline \multirow{3}{*}{ CI Series HEV PHEV 30} & Vehicle Cost LOW & 26300 & 26300 & 24599 & 23219 & 18461 & 16943 & 14260 & 14449 & 12030 & 9897 & 11579 & 9552 & 8027 \\
\hline & Vehicle Cost AVG & 23194 & 23194 & 21697 & 20482 & 14477 & 13356 & 11435 & 11005 & 9354 & 7848 & 9942 & 8254 & 6972 \\
\hline & Vehicle Cost HIGH & 18500 & 18500 & 17302 & 16329 & 8865 & 8098 & 6595 & 6629 & 5290 & 4181 & 4983 & 3762 & 3055 \\
\hline \multirow{3}{*}{ CI Series HEV PHEV40 } & Vehicle Cost LOW & 30730 & 30730 & 28760 & 27088 & 21295 & 19516 & 16211 & 16325 & 13422 & 10886 & 12638 & 10258 & 8567 \\
\hline & Vehicle Cost AVG & 26934 & 26934 & 25210 & 23746 & 16399 & 15098 & 12753 & 12058 & 10127 & 8398 & 10804 & 8827 & 7411 \\
\hline & Vehicle Cost HIGH & 21379 & 21379 & 20005 & 18839 & 10730 & 9799 & 7888 & 7974 & 6299 & 4896 & 5981 & 4438 & 3569 \\
\hline \multirow{3}{*}{ H2 Split HEV } & Vehicle Cost LOW & 4294 & 4294 & 3826 & 3459 & 3065 & 2608 & 2084 & 2738 & 2094 & 1407 & 2693 & 2085 & 1398 \\
\hline & Vehicle Cost AVG & 7099 & 7099 & 6643 & 6284 & 4601 & 4242 & 3835 & 3792 & 3274 & 2723 & 2279 & 1785 & 1230 \\
\hline & Vehicle Cost HIGH & 5032 & 5032 & 4681 & 4406 & 2095 & 1825 & 1511 & 1419 & 1066 & 700 & 471 & 181 & -141 \\
\hline \multirow{3}{*}{ H2 Split HEV PHEV10 } & Vehicle Cost LOW & 8776 & 8776 & 8008 & 7421 & 5822 & 5094 & 4080 & 4662 & 3605 & 2817 & 3921 & 3215 & 2379 \\
\hline & Vehicle Cost AVG & 10730 & 10730 & 10037 & 9508 & 6571 & 6024 & 5273 & 5297 & 4502 & 3920 & 3535 & 2948 & 2239 \\
\hline & Vehicle Cost HIGH & 8354 & 8354 & 7791 & 7360 & 2898 & 2567 & 2090 & 2024 & 1546 & 1126 & 878 & 472 & 177 \\
\hline \multirow{3}{*}{ H2 Split HEV PHEV2O } & Vehicle Cost LOW & 12031 & 12031 & 10944 & 10158 & 7798 & 6859 & 5517 & 5943 & 4571 & 3396 & 4575 & 3572 & 2634 \\
\hline & Vehicle Cost AVG & 13460 & 13460 & 12496 & 11800 & 7879 & 7193 & 6242 & 5961 & 5012 & 4187 & 4061 & 3227 & 2436 \\
\hline & Vehicle Cost HIGH & 10440 & 10440 & 9665 & 9106 & 4279 & 3802 & 3040 & 3021 & 2277 & 1641 & 1608 & 967 & 546 \\
\hline \multirow{3}{*}{ H2 Series HEV PHEV3O } & Vehicle Cost LOW & 23329 & 23329 & 21394 & 19909 & 15676 & 14001 & 11397 & 11736 & 9275 & 7201 & 9004 & 7073 & 5631 \\
\hline & Vehicle Cost AVG & 23348 & 23348 & 21642 & 20334 & 13727 & 12501 & 10648 & 10246 & 8566 & 7105 & 7814 & 6201 & 4985 \\
\hline & Vehicle Cost HIGH & 18456 & 18456 & 17086 & 16036 & 7787 & 6954 & 5508 & 5460 & 4121 & 3055 & 3385 & 2230 & 1566 \\
\hline \multirow{3}{*}{ H2 Series HEV PHEV40 } & Vehicle Cost LOW & 27669 & 27669 & 25346 & 23602 & 18406 & 16437 & 13255 & 13534 & 10590 & 8131 & 9991 & 7736 & 6132 \\
\hline & Vehicle Cost AVG & 27011 & 27011 & 24976 & 23449 & 15571 & 14145 & 11900 & 11250 & 9297 & 7621 & 8619 & 6740 & 5393 \\
\hline & Vehicle Cost HIGH & 21276 & 21276 & 19651 & 18433 & 9587 & 8563 & 6740 & 6750 & 5068 & 3727 & 4324 & 2869 & 2050 \\
\hline \multirow{3}{*}{ E85 Split HEV } & Vehicle Cost LOW & 4555 & 4555 & 4219 & 3881 & 3507 & 3126 & 2597 & 3293 & 2687 & 2034 & 3457 & 2851 & 2072 \\
\hline & Vehicle Cost AVG & 4352 & 4352 & 4024 & 3695 & 3124 & 2820 & 2397 & 2709 & 2202 & 1663 & 2607 & 2108 & 1466 \\
\hline & Vehicle Cost $\mathrm{HIGH}$ & 2899 & 2899 & 2641 & 2382 & 1384 & 1142 & 812 & 993 & 639 & 273 & 595 & 287 & -108 \\
\hline
\end{tabular}




\begin{tabular}{|c|c|c|c|c|c|c|c|c|c|c|c|c|c|c|}
\hline & & \multicolumn{13}{|c|}{ Midsize_SUV } \\
\hline & & \multirow{2}{*}{$\begin{array}{c}2010 \\
\text { Ref }\end{array}$} & \multicolumn{3}{|c|}{2010} & \multicolumn{3}{|c|}{2015} & \multicolumn{3}{|c|}{2030} & \multicolumn{3}{|c|}{2045} \\
\hline & & & low & avg & high & low & avg & high & low & avg & high & low & avg & high \\
\hline \multirow{3}{*}{ E85 Split HEV PHEV10 } & Vehicle Cost LOW & 8675 & 8675 & 8112 & 7619 & 5964 & 5380 & 4464 & 4975 & 4043 & 3260 & 4446 & 3772 & 2901 \\
\hline & Vehicle Cost AVG & 7647 & 7647 & 7140 & 6697 & 4825 & 4384 & 3710 & 3965 & 3266 & 2678 & 3645 & 3084 & 2339 \\
\hline & Vehicle Cost HIGH & 5908 & 5908 & 5495 & 5133 & 1995 & 1714 & 1242 & 1441 & 976 & 546 & 849 & 420 & 95 \\
\hline \multirow{3}{*}{ E85 Split HEV PHEV20 } & Vehicle Cost LOW & 11750 & 11750 & 11051 & 10377 & 7920 & 7154 & 5854 & 6246 & 5035 & 3868 & 5100 & 4121 & 3162 \\
\hline & \begin{tabular}{|l|} 
Vehicle Cost AVG \\
\end{tabular} & 10223 & 10223 & 9603 & 9007 & 6117 & 5556 & 4633 & 4621 & 3794 & 2967 & 4170 & 3355 & 2541 \\
\hline & Vehicle Cost HIGH & 7870 & 7870 & 7371 & 6891 & 3377 & 2969 & 2209 & 2443 & 1728 & 1077 & 1591 & 924 & 474 \\
\hline \multirow{3}{*}{ E85 Series HEV PHEV30 } & Vehicle Cost LOW & 23564 & 23564 & 21959 & 20565 & 16135 & 14663 & 12076 & 12316 & 9945 & 7867 & 9835 & 7840 & 6344 \\
\hline & 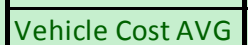 & 20594 & 20594 & 19178 & 17950 & 12236 & 11152 & 9302 & 9151 & 7520 & 6042 & 8186 & 6514 & 5245 \\
\hline & \begin{tabular}{|l} 
Vehicle Cost HIGH \\
\end{tabular} & 16291 & 16291 & 15149 & 14161 & 7054 & 6304 & 4845 & 5014 & 3688 & 2595 & 3499 & 2283 & 1575 \\
\hline \multirow{3}{*}{ E85 Series HEV PHEV40 } & Vehicle Cost LOW & 27913 & 27913 & 25959 & 24326 & 18863 & 17159 & 13980 & 14127 & 11284 & 8828 & 10853 & 8521 & 6857 \\
\hline & 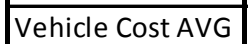 & 24267 & 24267 & 22555 & 21125 & 14083 & 12840 & 10587 & 10172 & 8269 & 6579 & 9018 & 7068 & 5664 \\
\hline & \begin{tabular}{|l} 
Vehicle Cost HIGH \\
Vehicle Cost
\end{tabular} & 19122 & 19122 & 17750 & 16606 & 8856 & 7955 & 6113 & 6314 & 4658 & 3291 & 4465 & 2941 & 2070 \\
\hline \multirow{3}{*}{ FC HEV } & Vehicle Cost LOW & 12966 & 12966 & 10332 & 8634 & 11694 & 8764 & 7148 & 4846 & 3387 & 2059 & 2342 & 1437 & 494 \\
\hline & \begin{tabular}{|l|} 
Vehicle Cost AVG \\
\end{tabular} & 19217 & 19217 & 15415 & 12853 & 11183 & 8597 & 7091 & 6487 & 4769 & 3204 & 2191 & 1307 & 404 \\
\hline & Vehicle Cost HIGH & 15240 & 15240 & 12111 & 9991 & 5374 & 3812 & 2879 & 2982 & 1882 & 876 & 147 & -411 & -981 \\
\hline \multirow{3}{*}{ FC HEV PHEV10 } & Vehicle Cost LOW & 15319 & 15319 & 12689 & 11091 & 12330 & 9887 & 8058 & 6321 & 4540 & 2965 & 3520 & 2371 & 1252 \\
\hline & \begin{tabular}{|l} 
Vehicle Cost AVG \\
Vehicle Cost
\end{tabular} & 20245 & 20245 & 16786 & 14500 & 11745 & 9547 & 7882 & 7367 & 5510 & 3829 & 3201 & 2118 & 1065 \\
\hline & Vehicle Cost HIGH & 16080 & 16080 & 13224 & 11340 & 5370 & 4058 & 2992 & 3145 & 1947 & 904 & 398 & -306 & -895 \\
\hline \multirow{3}{*}{ FC HEV PHEV 20} & Vehicle Cost LOW & 19428 & 19428 & 16256 & 14256 & 14844 & 12017 & 9522 & 7853 & 5661 & 3720 & 4253 & 2902 & 1630 \\
\hline & Vehicle Cost AVG & 23831 & 23831 & 19882 & 17237 & 13470 & 11005 & 8880 & 8242 & 6145 & 4252 & 3801 & 2553 & 1375 \\
\hline & Vehicle Cost $\mathrm{HIGH}$ & 18839 & 18839 & 15605 & 13444 & 7080 & 5511 & 4036 & 4331 & 2815 & 1495 & 1208 & 262 & -480 \\
\hline \multirow{3}{*}{ FC HEV PHEV 30} & Vehicle Cost LOW & 25839 & 25839 & 22649 & 20764 & 19001 & 16479 & 13580 & 11711 & 9028 & 6810 & 7391 & 5508 & 4074 \\
\hline & Vehicle Cost AVG & 29481 & 29481 & 25579 & 23103 & 17141 & 14826 & 12306 & 11432 & 8974 & 6870 & 6591 & 4864 & 3537 \\
\hline & Vehicle Cost HIGH & 23587 & 23587 & 20400 & 18375 & 8415 & 6985 & 5231 & 5441 & 3686 & 2299 & 2053 & 819 & 77 \\
\hline \multirow{3}{*}{ FC HEV PHEV 40} & Vehicle Cost LOW & 30486 & 30486 & 26516 & 24257 & 21687 & 18775 & 15249 & 13407 & 10199 & 7706 & 8280 & 6090 & 4543 \\
\hline & \begin{tabular}{|l} 
Vehicle Cost AVG \\
Vehicle Cost
\end{tabular} & 33623 & 33623 & 29021 & 26199 & 19063 & 16466 & 13506 & 12466 & 9697 & 7427 & 7336 & 5354 & 3931 \\
\hline & Vehicle Cost HIGH & 26791 & 26791 & 23063 & 20768 & 10231 & 8539 & 6358 & 6719 & 4562 & 2967 & 2943 & 1392 & 536 \\
\hline \multirow{3}{*}{ EV } & Vehicle Cost LOW & 67745 & 67745 & 62336 & 57876 & 42274 & 37718 & 27587 & 28132 & 19779 & 13785 & 15917 & 10226 & 7419 \\
\hline & Vehicle Cost AVG & 56699 & 56699 & 52128 & 48360 & 28713 & 25576 & 18701 & 15748 & 11000 & 7548 & 12625 & 7970 & 5658 \\
\hline & Vehicle Cost HIGH & 43431 & 43431 & 39876 & 36947 & 24152 & 21366 & 14847 & 7580 & 4565 & 2427 & 6913 & 3417 & 1865 \\
\hline
\end{tabular}




\section{Cost saving per powertrain}

\begin{tabular}{|c|c|c|c|c|c|c|c|c|c|c|c|c|c|c|}
\hline & & \multicolumn{13}{|c|}{ Midsize_SUV } \\
\hline & & \multirow{2}{*}{$\begin{array}{l}2010 \\
\text { Ref }\end{array}$} & \multicolumn{3}{|c|}{2010} & \multicolumn{3}{|c|}{2015} & \multicolumn{3}{|c|}{2030} & \multicolumn{3}{|c|}{2045} \\
\hline & & & low & avg & high & low & avg & high & low & avg & high & low & avg & high \\
\hline \multirow{3}{*}{ SI Conv } & Vehicle Cost LOW & 0 & 0 & -108 & -165 & 2 & -123 & -93 & 797 & 783 & 13 & 1349 & 1210 & 348 \\
\hline & Vehicle Cost AVG & 0 & 0 & -108 & -165 & 810 & 690 & 721 & 1164 & 1147 & 307 & 1286 & 1152 & 295 \\
\hline & Vehicle Cost HIGH & 0 & 0 & -108 & -165 & 138 & 12 & 43 & 532 & 517 & -179 & 638 & 503 & -214 \\
\hline \multirow{3}{*}{$\mathrm{Cl}$ Conv } & Vehicle Cost LOW & 0 & 0 & -1154 & -1252 & -1356 & -1423 & -1489 & -623 & -709 & -880 & -589 & -714 & -879 \\
\hline & Vehicle Cost AVG & 0 & 0 & -1197 & -1287 & -509 & -565 & -620 & -514 & -584 & -734 & -556 & -663 & -805 \\
\hline & Vehicle Cost HIGH & 0 & 0 & -1133 & -1207 & -1140 & -1178 & -1215 & -811 & -868 & -1000 & -911 & -1004 & -1127 \\
\hline \multirow{3}{*}{ H2 Conv } & Vehicle Cost LOW & 0 & 0 & -22 & -53 & -89 & -117 & -775 & 661 & -39 & -99 & 944 & 163 & 99 \\
\hline & Vehicle Cost AVG & 0 & 0 & -24 & -55 & -329 & -353 & -1067 & -347 & -1114 & -1176 & -1681 & -2462 & -2527 \\
\hline & Vehicle Cost HIGH & 0 & 0 & -24 & -55 & -1167 & -1200 & -1855 & -980 & -1659 & -1734 & -1448 & -2180 & -2262 \\
\hline \multirow{3}{*}{ E85 Conv } & Vehicle Cost LOW & 0 & 0 & -95 & -140 & 16 & -97 & -90 & 812 & 795 & 16 & 1353 & 1214 & 345 \\
\hline & Vehicle Cost AVG & 0 & 0 & -95 & -140 & 825 & 718 & 726 & 1178 & 1157 & 308 & 1290 & 1156 & 293 \\
\hline & Vehicle Cost HIGH & 0 & 0 & -95 & -140 & 150 & 37 & 45 & 544 & 524 & -179 & 639 & 504 & -219 \\
\hline \multirow{3}{*}{ SI Split HEV } & Vehicle Cost LOW & 0 & 0 & -364 & -670 & -1071 & -1451 & -1954 & -1288 & -1858 & -2533 & -1096 & -1752 & -2476 \\
\hline & Vehicle Cost AVG & 0 & 0 & -356 & -654 & -1244 & -1548 & -1950 & -1665 & -2141 & -2700 & -1742 & -2284 & -2878 \\
\hline & Vehicle Cost HIGH & 0 & 0 & -281 & -514 & -1530 & -1771 & -2083 & -1922 & -2251 & -2632 & -2299 & -2636 & -2999 \\
\hline \multirow{3}{*}{ SI Split HEV PHEV10 } & Vehicle Cost LOW & 0 & 0 & -560 & -1048 & -2701 & -3289 & -4202 & -3735 & -4624 & -5405 & -4223 & -4930 & -5765 \\
\hline & Vehicle Cost AVG & 0 & 0 & -504 & -942 & -2813 & -3256 & -3927 & -3712 & -4374 & -4960 & -3995 & -4585 & -5299 \\
\hline & Vehicle Cost $\mathrm{HIGH}$ & 0 & 0 & -411 & -769 & -3902 & -4185 & -4656 & -4465 & -4922 & -5349 & -5048 & -5480 & -5801 \\
\hline \multirow{3}{*}{ SI Split HEV PHEV20 } & Vehicle Cost LOW & 0 & 0 & -693 & -1363 & -3818 & -4592 & -5890 & -5495 & -6705 & -7900 & -6640 & -7648 & -8578 \\
\hline & Vehicle Cost AVG & 0 & 0 & -615 & -1206 & -4094 & -4661 & -5582 & -5595 & -6420 & -7272 & -6043 & -6884 & -7671 \\
\hline & Vehicle Cost HIGH & 0 & 0 & -495 & -971 & -4481 & -4895 & -5652 & -5414 & -6130 & -6784 & -6266 & -6932 & -7383 \\
\hline \multirow{3}{*}{ SI Series HEV PHEV 30} & Vehicle Cost LOW & 0 & 0 & -1603 & -2990 & -7418 & -8901 & -11485 & -11243 & -13609 & -15682 & -13719 & -15709 & -17208 \\
\hline & Vehicle Cost AVG & 0 & 0 & -1415 & -2636 & -8347 & -9440 & -11287 & -11437 & -13064 & -14539 & -12398 & -14067 & -15337 \\
\hline & Vehicle Cost $\mathrm{HIGH}$ & 0 & 0 & -1141 & -2124 & -9226 & -9981 & -11439 & -11269 & -12591 & -13682 & -12779 & -13993 & -14702 \\
\hline \multirow{3}{*}{ SI Series HEV PHEV40 } & Vehicle Cost LOW & 0 & 0 & -1940 & -3555 & -9023 & -10717 & -13910 & -13767 & -16602 & -19056 & -17039 & -19363 & -21028 \\
\hline & Vehicle Cost AVG & 0 & 0 & -1700 & -3115 & -10160 & -11395 & -13658 & -14077 & -15977 & -17663 & -15230 & -17173 & -18578 \\
\hline & Vehicle Cost HIGH & 0 & 0 & -1361 & -2495 & -10243 & -11136 & -12989 & -12789 & -14438 & -15807 & -14639 & -16157 & -17027 \\
\hline \multirow{3}{*}{ CI Split HEV } & Vehicle Cost LOW & 0 & 0 & -389 & -736 & -1404 & -1769 & -2390 & -1725 & -2402 & -3123 & -1942 & -2595 & -3400 \\
\hline & Vehicle Cost AVG & 0 & 0 & -374 & -709 & -1611 & -1900 & -2390 & -2339 & -2883 & -3459 & -2548 & -3073 & -3721 \\
\hline & Vehicle Cost HIGH & 0 & 0 & -286 & -542 & -1862 & -2077 & -2444 & -2428 & -2796 & -3178 & -2992 & -3298 & -3671 \\
\hline
\end{tabular}




\begin{tabular}{|c|c|c|c|c|c|c|c|c|c|c|c|c|c|c|}
\hline & & \multicolumn{13}{|c|}{ Midsize_SUV } \\
\hline & & \multirow{2}{*}{$\frac{2010}{\operatorname{Ref}}$} & \multicolumn{3}{|c|}{2010} & \multicolumn{3}{|c|}{2015} & \multicolumn{3}{|c|}{2030} & \multicolumn{3}{|c|}{2045} \\
\hline & & & low & avg & high & low & avg & high & low & avg & high & low & avg & high \\
\hline \multirow{3}{*}{ CI Split HEV PHEV10 } & Vehicle Cost LOW & 0 & 0 & -618 & -1160 & -3079 & -3723 & -4657 & -4224 & -5157 & -6006 & -5093 & -5778 & -6682 \\
\hline & Vehicle Cost AVG & 0 & 0 & -554 & -1040 & -3153 & -3641 & -4325 & -4341 & -5026 & -5655 & -4748 & -5311 & -6075 \\
\hline & Vehicle Cost HIGH & 0 & 0 & -448 & -841 & -4390 & -4692 & -5183 & -5105 & -5580 & -6029 & -5813 & -6245 & -6573 \\
\hline \multirow{3}{*}{ CI Split HEV PHEV2O } & Vehicle Cost LOW & 0 & 0 & -755 & -1486 & -4224 & -5037 & -6407 & -6068 & -7347 & -8568 & -7558 & -8582 & -9580 \\
\hline & Vehicle Cost AVG & 0 & 0 & -669 & -1313 & -4467 & -5064 & -6040 & -6308 & -7176 & -8033 & -6835 & -7680 & -8519 \\
\hline & Vehicle Cost HIGH & 0 & 0 & -535 & -1050 & -4986 & -5412 & -6203 & -6091 & $\begin{array}{l}-6828 \\
\end{array}$ & $\begin{array}{l}-7498 \\
\end{array}$ & -7065 & -7739 & -8196 \\
\hline \multirow{3}{*}{ CI Series HEV PHEV 30} & Vehicle Cost LOW & 0 & 0 & -1701 & -3081 & -7839 & -9357 & -12039 & -11851 & -14270 & -16402 & -14721 & -16748 & -18273 \\
\hline & \begin{tabular}{|l|} 
Vehicle Cost AVG \\
\end{tabular} & 0 & 0 & -1497 & -2712 & -8717 & $\begin{array}{l}-9838 \\
\end{array}$ & -11759 & -12189 & -13840 & -15346 & -13252 & -14941 & -16222 \\
\hline & Vehicle Cost HIGH & 0 & 0 & -1198 & -2172 & -9635 & -10403 & -11905 & -11871 & -13210 & -14320 & -13517 & -14738 & -15445 \\
\hline \multirow{3}{*}{ CI Series HEV PHEV40 } & Vehicle Cost LOW & 0 & 0 & -1970 & -3642 & -9435 & -11214 & -14519 & -14405 & -17308 & -19844 & -18092 & -20472 & -22163 \\
\hline & Vehicle Cost AVG & 0 & 0 & -1725 & -3188 & -10536 & -11837 & -14181 & -14877 & -16808 & -18536 & -16131 & -18107 & -19523 \\
\hline & Vehicle Cost $\mathrm{HIGH}$ & 0 & 0 & -1375 & -2540 & -10649 & -11580 & -13492 & -13405 & -15080 & -16484 & -15398 & -16941 & -17810 \\
\hline \multirow{3}{*}{ H2 Split HEV } & Vehicle Cost LOW & 0 & 0 & -467 & -835 & -1229 & $\begin{array}{c}-1686 \\
\end{array}$ & -2210 & -1556 & -2199 & -2886 & -1601 & -2209 & -2896 \\
\hline & Vehicle Cost AVG & 0 & 0 & -457 & -816 & -2498 & -2857 & -3264 & -3307 & -3826 & -4377 & -4821 & -5314 & -5869 \\
\hline & Vehicle Cost HIGH & 0 & 0 & -351 & -627 & -2938 & -3208 & -3521 & -3614 & -3967 & -4332 & -4561 & -4851 & -5174 \\
\hline \multirow{3}{*}{ H2 Split HEV PHEV10 } & Vehicle Cost LOW & 0 & 0 & -769 & -1356 & -2954 & -3682 & -4697 & -4115 & -5171 & -5959 & -4855 & -5562 & -6397 \\
\hline & Vehicle Cost AVG & 0 & 0 & -693 & -1222 & -4160 & -4706 & -5457 & -5433 & -6229 & -6810 & -7195 & -7782 & -8492 \\
\hline & Vehicle Cost HIGH & 0 & 0 & -563 & -994 & -5456 & -5787 & -6264 & -6330 & -6808 & -7228 & $\begin{array}{l}-7476 \\
\end{array}$ & -7882 & -8177 \\
\hline \multirow{3}{*}{ H2 Split HEV PHEV2O } & Vehicle Cost LOW & 0 & 0 & -1087 & -1874 & -4234 & -5172 & -6514 & -6088 & -7460 & -8635 & -7456 & -8459 & -9397 \\
\hline & \begin{tabular}{|l|} 
Vehicle Cost AVG \\
\end{tabular} & 0 & 0 & -964 & -1660 & -5582 & -6267 & -7219 & -7499 & -8448 & -9273 & -9399 & -10233 & -11025 \\
\hline & Vehicle Cost HIGH & 0 & 0 & -775 & -1334 & -6161 & -6639 & -7400 & $\begin{array}{l}-7419 \\
\end{array}$ & $\begin{array}{l}-8164 \\
\end{array}$ & $\begin{array}{l}-8799 \\
\end{array}$ & -8832 & -9473 & -9894 \\
\hline \multirow{3}{*}{ H2 Series HEV PHEV3O } & Vehicle Cost LOW & 0 & 0 & -1935 & -3420 & -7653 & -9328 & -11932 & -11592 & -14054 & -16128 & -14325 & -16256 & -17698 \\
\hline & Vehicle Cost AVG & 0 & 0 & -1706 & -3013 & $\begin{array}{l}-9620 \\
\end{array}$ & -10846 & -12700 & -13101 & -14782 & -16243 & -15533 & -17147 & -18362 \\
\hline & Vehicle Cost HIGH & 0 & 0 & -1370 & -2420 & -10669 & -11503 & -12949 & -12997 & -14335 & -15401 & -15071 & -16226 & -16890 \\
\hline \multirow{3}{*}{ H2 Series HEV PHEV40 } & Vehicle Cost LOW & 0 & 0 & -2323 & -4067 & -9263 & -11232 & -14414 & -14135 & -17080 & -19539 & -17678 & -19934 & -21537 \\
\hline & Vehicle Cost AVG & 0 & 0 & -2035 & -3562 & -11440 & -12866 & -15110 & -15761 & -17714 & -19389 & -18392 & -20270 & -21618 \\
\hline & Vehicle Cost HIGH & 0 & 0 & -1625 & -2843 & -11689 & -12713 & -14536 & -14526 & -16208 & -17549 & -16952 & -18408 & -19226 \\
\hline \multirow{3}{*}{ E85 Split HEV } & Vehicle Cost LOW & 0 & 0 & -336 & -673 & -1048 & -1428 & -1957 & -1262 & -1868 & -2520 & -1097 & -1703 & -2483 \\
\hline & Vehicle Cost AVG & 0 & 0 & -328 & $\begin{array}{l}-657 \\
\end{array}$ & -1228 & -1532 & $\begin{array}{l}-1955 \\
\end{array}$ & -1642 & -2150 & -2689 & -1745 & -2244 & -2886 \\
\hline & Vehicle Cost $\mathrm{HIGH}$ & 0 & 0 & -258 & -517 & -1515 & -1757 & -2087 & -1906 & -2260 & -2627 & -2304 & -2612 & -3007 \\
\hline
\end{tabular}




\begin{tabular}{|c|c|c|c|c|c|c|c|c|c|c|c|c|c|c|}
\hline & & \multicolumn{13}{|c|}{ Midsize_SUV } \\
\hline & & \multirow{2}{*}{$\begin{array}{l}2010 \\
\text { Ref }\end{array}$} & \multicolumn{3}{|c|}{2010} & \multicolumn{3}{|c|}{2015} & \multicolumn{3}{|c|}{2030} & \multicolumn{3}{|c|}{2045} \\
\hline & & & low & avg & high & low & avg & high & low & avg & high & low & avg & high \\
\hline \multirow{3}{*}{ E85 Split HEV PHEV10 } & Vehicle Cost LOW & 0 & 0 & -563 & -1056 & -2711 & -3295 & -4211 & -3700 & -4632 & -5415 & -4229 & -4903 & -5774 \\
\hline & Vehicle Cost AVG & 0 & 0 & -506 & -950 & -2822 & -3263 & -3937 & -3682 & -4381 & -4969 & -4002 & -4563 & -5308 \\
\hline & Vehicle Cost HIGH & 0 & 0 & -413 & -775 & -3913 & -4194 & -4666 & -4467 & -4932 & -5362 & -5059 & -5488 & -5813 \\
\hline \multirow{3}{*}{ E85 Split HEV PHEV20 } & Vehicle Cost LOW & 0 & 0 & -699 & -1374 & -3830 & -4596 & -5896 & -5504 & -6716 & -7882 & -6650 & -7629 & -8588 \\
\hline & Vehicle Cost AVG & 0 & 0 & -620 & -1216 & -4106 & -4667 & -5590 & -5602 & -6429 & -7256 & -6053 & -6868 & -7682 \\
\hline & Vehicle Cost HIGH & 0 & 0 & -499 & $\begin{array}{l}-979 \\
\end{array}$ & -4493 & -4901 & -5661 & -5427 & $\begin{array}{l}-6142 \\
\end{array}$ & -6793 & -6279 & -6946 & -7396 \\
\hline \multirow{3}{*}{ E85 Series HEV PHEV30 } & Vehicle Cost LOW & 0 & 0 & -1604 & -2999 & -7429 & -8900 & -11487 & -11247 & -13619 & -15697 & -13729 & -15723 & -17220 \\
\hline & Vehicle Cost AVG & 0 & 0 & -1416 & -2644 & -8358 & -9442 & -11291 & -11443 & -13074 & -14552 & -12408 & -14080 & -15349 \\
\hline & Vehicle Cost $\mathrm{HIGH}$ & 0 & 0 & -1141 & -2130 & -9237 & -9987 & -11446 & -11277 & -12602 & -13696 & -12791 & -14008 & -14716 \\
\hline \multirow{3}{*}{ E85 Series HEV PHEV40 } & Vehicle Cost LOW & 0 & 0 & -1955 & -3587 & -9050 & -10755 & -13933 & -13786 & -16629 & -19085 & -17060 & -19393 & -21056 \\
\hline & Vehicle Cost AVG & 0 & 0 & -1712 & -3142 & -10184 & -11427 & -13680 & -14095 & -15998 & -17688 & -15249 & -17199 & -18603 \\
\hline & Vehicle Cost HIGH & 0 & 0 & -1371 & -2516 & -10266 & -11167 & -13009 & -12808 & -14464 & -15831 & -14657 & -16181 & -17052 \\
\hline \multirow{3}{*}{ FC HEV } & Vehicle Cost LOW & 0 & 0 & -2634 & -4331 & -1271 & -4202 & -5818 & -8119 & -9578 & -10907 & -10624 & -11528 & -12472 \\
\hline & Vehicle Cost AVG & 0 & 0 & -3802 & -6364 & -8034 & -10620 & -12126 & -12730 & -14447 & -16013 & -17026 & -17910 & -18813 \\
\hline & Vehicle Cost HIGH & 0 & 0 & -3128 & -5249 & -9865 & -11427 & -12361 & -12257 & -13358 & -14364 & -15092 & -15651 & -16221 \\
\hline \multirow{3}{*}{ FC HEV PHEV10 } & Vehicle Cost LOW & 0 & 0 & -2629 & -4228 & -2989 & -5432 & -7261 & -8998 & -10779 & -12354 & -11799 & -12947 & -14067 \\
\hline & Vehicle Cost AVG & 0 & 0 & -3459 & -5746 & -8500 & -10698 & -12363 & -12878 & -14735 & -16417 & -17044 & -18127 & -19180 \\
\hline & Vehicle Cost $\mathrm{HIGH}$ & 0 & 0 & -2855 & -4739 & -10710 & -12022 & -13087 & -12935 & -14133 & -15175 & -15681 & -16385 & -16975 \\
\hline \multirow{3}{*}{ FC HEV PHEV2O } & Vehicle Cost LOW & 0 & 0 & -3172 & -5173 & -4584 & -7411 & -9906 & -11575 & -13767 & -15709 & -15176 & -16526 & -17798 \\
\hline & Vehicle Cost AVG & 0 & 0 & -3949 & $\begin{array}{l}-6594 \\
\end{array}$ & -10362 & -12826 & -14951 & -15589 & -17686 & -19579 & -20030 & -21279 & -22456 \\
\hline & Vehicle Cost $\mathrm{HIGH}$ & 0 & 0 & -3234 & -5395 & -11760 & -13328 & -14803 & -14508 & -16024 & -17344 & -17632 & -18577 & -19319 \\
\hline \multirow{3}{*}{ FC HEV PHEV30 } & Vehicle Cost LOW & 0 & 0 & -3190 & -5076 & $\begin{array}{l}-6838 \\
\end{array}$ & $\begin{array}{l}-9361 \\
\end{array}$ & -12259 & -14128 & -16811 & -19030 & -18449 & -20331 & -21766 \\
\hline & Vehicle Cost AVG & 0 & 0 & -3902 & -6378 & -12340 & -14655 & -17175 & -18049 & -20507 & -22611 & -22890 & -24617 & -25944 \\
\hline & Vehicle Cost HIGH & 0 & 0 & -3187 & -5212 & -15172 & -16602 & -18357 & -18146 & -19902 & -21288 & -21534 & -22768 & -23510 \\
\hline \multirow{3}{*}{ FC HEV PHEV40 } & Vehicle Cost LOW & 0 & 0 & -3970 & -6229 & -8799 & -11711 & -15237 & -17079 & -20287 & -22780 & -22206 & -24396 & -25943 \\
\hline & Vehicle Cost AVG & 0 & 0 & -4602 & -7424 & -14560 & -17157 & -20117 & -21157 & -23926 & -26196 & -26287 & -28269 & -29692 \\
\hline & \begin{tabular}{|l} 
Vehicle Cost HIGH \\
\end{tabular} & 0 & 0 & -3728 & -6023 & -16560 & -18252 & -20434 & -20072 & -22229 & -23824 & -23848 & -25399 & -26255 \\
\hline \multirow{3}{*}{ EV } & Vehicle Cost LOW & 0 & 0 & -5408 & -9869 & -25470 & -30027 & -40158 & -39612 & -47966 & -53959 & -51827 & -57519 & -60326 \\
\hline & Vehicle Cost AVG & 0 & 0 & -4571 & -8339 & -27986 & -31124 & -37998 & -40952 & -45699 & -49152 & -44075 & -48729 & -51042 \\
\hline & Vehicle Cost HIGH & 0 & 0 & -3555 & -6484 & -19280 & -22065 & -28584 & -35851 & -38866 & -41004 & -36519 & -40015 & -41566 \\
\hline
\end{tabular}




\section{Percentage decrease cost per powertrain}

\begin{tabular}{|c|c|c|c|c|c|c|c|c|c|c|c|c|c|c|}
\hline & & \multicolumn{13}{|c|}{ Midsize_SUV } \\
\hline & & \multirow{2}{*}{$\frac{2010}{\text { Ref }}$} & \multicolumn{3}{|c|}{2010} & \multicolumn{3}{|c|}{2015} & \multicolumn{3}{|c|}{2030} & \multicolumn{3}{|c|}{2045} \\
\hline & & & low & avg & high & low & avg & high & low & avg & high & low & avg & high \\
\hline \multirow{3}{*}{ SI Conv } & Vehicle Cost LOW & $0.0 \%$ & $0.0 \%$ & $0.7 \%$ & $1.0 \%$ & $0.0 \%$ & $0.7 \%$ & $0.6 \%$ & $-4.8 \%$ & $-4.8 \%$ & $-0.1 \%$ & $-8.2 \%$ & $-7.4 \%$ & $-2.1 \%$ \\
\hline & Vehicle Cost AVG & $-0.2 \%$ & $-0.2 \%$ & $0.4 \%$ & $0.8 \%$ & $-5.2 \%$ & $-4.4 \%$ & $-4.6 \%$ & $-7.3 \%$ & $-7.2 \%$ & $-2.1 \%$ & $-8.1 \%$ & $-7.3 \%$ & $-2.0 \%$ \\
\hline & Vehicle Cost HIGH & $-4.0 \%$ & $-4.0 \%$ & $-3.3 \%$ & $-3.0 \%$ & $-4.8 \%$ & $-4.0 \%$ & $-4.2 \%$ & $-7.2 \%$ & $-7.1 \%$ & $-2.9 \%$ & $-7.9 \%$ & $-7.0 \%$ & $-2.7 \%$ \\
\hline \multirow{3}{*}{$\mathrm{Cl}$ Conv } & Vehicle Cost LOW & $-15.2 \%$ & $-15.2 \%$ & $-8.2 \%$ & $-7.6 \%$ & $-6.9 \%$ & $-6.5 \%$ & $-6.1 \%$ & $-11.4 \%$ & $-10.9 \%$ & $-9.8 \%$ & $-11.6 \%$ & $-10.8 \%$ & $-9.8 \%$ \\
\hline & Vehicle Cost AVG & $-15.0 \%$ & $-15.0 \%$ & $-7.7 \%$ & $-7.2 \%$ & $-11.9 \%$ & $-11.6 \%$ & $-11.2 \%$ & $-11.9 \%$ & $-11.5 \%$ & $-10.5 \%$ & $-11.6 \%$ & $-11.0 \%$ & $-10.1 \%$ \\
\hline & Vehicle Cost HIGH & $-16.6 \%$ & $-16.6 \%$ & $-9.7 \%$ & $-9.2 \%$ & $-9.6 \%$ & $-9.4 \%$ & $-9.2 \%$ & $-11.6 \%$ & $-11.3 \%$ & $-10.5 \%$ & $-11.0 \%$ & $-10.4 \%$ & $-9.7 \%$ \\
\hline \multirow{3}{*}{ H2 Conv } & Vehicle Cost LOW & $4.5 \%$ & $4.5 \%$ & $4.6 \%$ & $4.8 \%$ & $5.0 \%$ & $5.2 \%$ & $9.2 \%$ & $0.5 \%$ & $4.7 \%$ & $5.1 \%$ & $-1.3 \%$ & $3.5 \%$ & $3.9 \%$ \\
\hline & Vehicle Cost AVG & $-14.1 \%$ & $-14.1 \%$ & $-14.0 \%$ & $-13.8 \%$ & $-12.1 \%$ & $-12.0 \%$ & $-7.6 \%$ & $-12.0 \%$ & $-7.4 \%$ & $-7.0 \%$ & $-3.9 \%$ & $0.8 \%$ & $1.2 \%$ \\
\hline & Vehicle Cost HIGH & $-14.4 \%$ & $-14.4 \%$ & $-14.3 \%$ & $-14.1 \%$ & $-7.3 \%$ & $-7.1 \%$ & $-3.2 \%$ & $-8.5 \%$ & $-4.4 \%$ & $-3.9 \%$ & $-5.6 \%$ & $-1.2 \%$ & $-0.7 \%$ \\
\hline \multirow{3}{*}{ E85 Conv } & Vehicle Cost LOW & $0.2 \%$ & $0.2 \%$ & $0.8 \%$ & $1.0 \%$ & $0.1 \%$ & $0.8 \%$ & $0.7 \%$ & $-4.7 \%$ & $-4.6 \%$ & $0.1 \%$ & $-8.0 \%$ & $-7.2 \%$ & $-1.9 \%$ \\
\hline & Vehicle Cost AVG & $-0.1 \%$ & $-0.1 \%$ & $0.5 \%$ & $0.8 \%$ & $-5.1 \%$ & $-4.4 \%$ & $-4.5 \%$ & $-7.2 \%$ & $-7.1 \%$ & $-1.9 \%$ & $-7.9 \%$ & $-7.1 \%$ & $-1.8 \%$ \\
\hline & Vehicle Cost HIGH & $-3.8 \%$ & $-3.8 \%$ & $-3.2 \%$ & $-2.9 \%$ & $-4.7 \%$ & $-4.0 \%$ & $-4.0 \%$ & $-7.1 \%$ & $-7.0 \%$ & $-2.7 \%$ & $-7.7 \%$ & $-6.8 \%$ & $-2.4 \%$ \\
\hline \multirow{3}{*}{ SI Split HEV } & Vehicle Cost LOW & $-27.6 \%$ & $-27.6 \%$ & $-25.3 \%$ & $-23.5 \%$ & $-21.0 \%$ & $-18.7 \%$ & $-15.7 \%$ & $-19.7 \%$ & $-16.2 \%$ & $-12.1 \%$ & $-20.9 \%$ & $-16.9 \%$ & $-12.5 \%$ \\
\hline & Vehicle Cost AVG & $-26.6 \%$ & $-26.6 \%$ & $-24.4 \%$ & $-22.6 \%$ & $-19.0 \%$ & $-17.1 \%$ & $-14.7 \%$ & $-16.4 \%$ & $-13.5 \%$ & $-10.1 \%$ & $-16.0 \%$ & $-12.7 \%$ & $-9.0 \%$ \\
\hline & Vehicle Cost HIGH & $-21.5 \%$ & $-21.5 \%$ & $-19.8 \%$ & $-18.3 \%$ & $-12.2 \%$ & $-10.7 \%$ & $-8.8 \%$ & $-9.8 \%$ & $-7.8 \%$ & $-5.4 \%$ & $-7.5 \%$ & $-5.4 \%$ & $-3.2 \%$ \\
\hline \multirow{3}{*}{ SI Split HEV PHEV10 } & Vehicle Cost LOW & $-52.6 \%$ & $-52.6 \%$ & $-49.2 \%$ & $-46.2 \%$ & $-36.1 \%$ & $-32.6 \%$ & $-27.0 \%$ & $-29.8 \%$ & $-24.4 \%$ & $-19.7 \%$ & $-26.9 \%$ & $-22.6 \%$ & $-17.5 \%$ \\
\hline & Vehicle Cost AVG & $-46.6 \%$ & $-46.6 \%$ & $-43.5 \%$ & $-40.8 \%$ & $-29.5 \%$ & $-26.8 \%$ & $-22.7 \%$ & $-24.0 \%$ & $-20.0 \%$ & $-16.4 \%$ & $-22.3 \%$ & $-18.7 \%$ & $-14.3 \%$ \\
\hline & Vehicle Cost HIGH & $-39.7 \%$ & $-39.7 \%$ & $-37.2 \%$ & $-35.1 \%$ & $-16.0 \%$ & $-14.3 \%$ & $-11.4 \%$ & $-12.6 \%$ & $-9.8 \%$ & $-7.2 \%$ & $-9.0 \%$ & $-6.4 \%$ & $-4.4 \%$ \\
\hline \multirow{3}{*}{ SI Split HEV PHEV2O } & Vehicle Cost LOW & $-71.3 \%$ & $-71.3 \%$ & $-67.1 \%$ & $-63.0 \%$ & $-48.0 \%$ & $-43.3 \%$ & $-35.4 \%$ & $-37.8 \%$ & $-30.5 \%$ & $-23.2 \%$ & $-30.9 \%$ & $-24.7 \%$ & $-19.1 \%$ \\
\hline & Vehicle Cost AVG & $-62.2 \%$ & $-62.2 \%$ & $-58.5 \%$ & $-54.9 \%$ & $-37.3 \%$ & $-33.9 \%$ & $-28.3 \%$ & $-28.2 \%$ & $-23.2 \%$ & $-18.0 \%$ & $-25.5 \%$ & $-20.3 \%$ & $-15.5 \%$ \\
\hline & Vehicle Cost HIGH & $-51.7 \%$ & $-51.7 \%$ & $-48.7 \%$ & $-45.8 \%$ & $-24.4 \%$ & $-21.9 \%$ & $-17.3 \%$ & $-18.7 \%$ & $-14.4 \%$ & $-10.4 \%$ & $-13.5 \%$ & $-9.5 \%$ & $-6.7 \%$ \\
\hline \multirow{3}{*}{ SI Series HEV PHEV 30} & Vehicle Cost LOW & $-143.1 \%$ & $-143.1 \%$ & $-133.4 \%$ & $-125.0 \%$ & $-98.0 \%$ & $-89.0 \%$ & $-73.3 \%$ & $-74.7 \%$ & $-60.3 \%$ & $-47.7 \%$ & $-59.7 \%$ & $-47.6 \%$ & $-38.4 \%$ \\
\hline & Vehicle Cost AVG & $-125.3 \%$ & $-125.3 \%$ & $-116.7 \%$ & $-109.3 \%$ & $-74.5 \%$ & $-67.9 \%$ & $-56.6 \%$ & $-55.7 \%$ & $-45.8 \%$ & $-36.9 \%$ & $-49.9 \%$ & $-39.7 \%$ & $-32.0 \%$ \\
\hline & Vehicle Cost HIGH & $-102.9 \%$ & $-102.9 \%$ & $-96.0 \%$ & $-90.0 \%$ & $-46.8 \%$ & $-42.2 \%$ & $-33.3 \%$ & $-34.3 \%$ & $-26.3 \%$ & $-19.6 \%$ & $-25.1 \%$ & $-17.7 \%$ & $-13.4 \%$ \\
\hline \multirow{3}{*}{ SI Series HEV PHEV40 } & Vehicle Cost LOW & $-169.5 \%$ & $-169.5 \%$ & $-157.7 \%$ & $-147.9 \%$ & $-114.6 \%$ & $-104.3 \%$ & $-84.9 \%$ & $-85.7 \%$ & $-68.5 \%$ & $-53.6 \%$ & $-65.8 \%$ & $-51.7 \%$ & $-41.6 \%$ \\
\hline & Vehicle Cost AVG & $-147.6 \%$ & $-147.6 \%$ & $-137.3 \%$ & $-128.6 \%$ & $-85.8 \%$ & $-78.3 \%$ & $-64.5 \%$ & $-61.9 \%$ & $-50.4 \%$ & $-40.1 \%$ & $-54.9 \%$ & $-43.1 \%$ & $-34.5 \%$ \\
\hline & Vehicle Cost HIGH & $-120.1 \%$ & $-120.1 \%$ & $-111.8 \%$ & $-104.9 \%$ & $-57.7 \%$ & $-52.3 \%$ & $-41.0 \%$ & $-42.2 \%$ & $-32.2 \%$ & $-23.9 \%$ & $-31.0 \%$ & $-21.7 \%$ & $-16.4 \%$ \\
\hline \multirow{3}{*}{ CI Split HEV } & Vehicle Cost LOW & $-43.3 \%$ & $-43.3 \%$ & $-41.0 \%$ & $-38.8 \%$ & $-34.8 \%$ & $-32.6 \%$ & $-28.8 \%$ & $-32.8 \%$ & $-28.7 \%$ & $-24.3 \%$ & $-31.5 \%$ & $-27.5 \%$ & $-22.6 \%$ \\
\hline & Vehicle Cost AVG & $-41.9 \%$ & $-41.9 \%$ & $-39.7 \%$ & $-37.6 \%$ & $-32.1 \%$ & $-30.4 \%$ & $-27.4 \%$ & $-27.7 \%$ & $-24.4 \%$ & $-20.9 \%$ & $-26.4 \%$ & $-23.2 \%$ & $-19.3 \%$ \\
\hline & Vehicle Cost HIGH & $-34.2 \%$ & $-34.2 \%$ & $-32.5 \%$ & $-30.9 \%$ & $-22.9 \%$ & $-21.6 \%$ & $-19.3 \%$ & $-19.4 \%$ & $-17.2 \%$ & $-14.9 \%$ & $-16.0 \%$ & $-14.1 \%$ & $-11.9 \%$ \\
\hline
\end{tabular}




\begin{tabular}{|c|c|c|c|c|c|c|c|c|c|c|c|c|c|c|}
\hline & & \multicolumn{13}{|c|}{ Midsize_SUV } \\
\hline & & \multirow{2}{*}{$\begin{array}{c}2010 \\
\text { Ref }\end{array}$} & \multicolumn{3}{|c|}{2010} & \multicolumn{3}{|c|}{2015} & \multicolumn{3}{|c|}{2030} & \multicolumn{3}{|c|}{2045} \\
\hline & & & low & avg & high & low & avg & high & low & avg & high & low & avg & high \\
\hline \multirow{3}{*}{ CI Split HEV PHEV10 } & Vehicle Cost LOW & $-69.0 \%$ & $-69.0 \%$ & $-65.3 \%$ & $-62.0 \%$ & $-50.3 \%$ & $-46.4 \%$ & $-40.7 \%$ & $-43.3 \%$ & $-37.7 \%$ & $-32.5 \%$ & $-38.1 \%$ & $-33.9 \%$ & $-28.4 \%$ \\
\hline & Vehicle Cost AVG & $-62.4 \%$ & $-62.4 \%$ & $-59.0 \%$ & $-56.1 \%$ & $-43.2 \%$ & $-40.3 \%$ & $-36.1 \%$ & $-36.0 \%$ & $-31.8 \%$ & $-28.0 \%$ & $-33.5 \%$ & $-30.1 \%$ & $-25.4 \%$ \\
\hline & Vehicle Cost HIGH & $-53.4 \%$ & $-53.4 \%$ & $-50.7 \%$ & $-48.3 \%$ & $-26.7 \%$ & $-24.9 \%$ & $-21.9 \%$ & $-22.4 \%$ & $-19.5 \%$ & $-16.8 \%$ & $-18.1 \%$ & $-15.4 \%$ & $-13.4 \%$ \\
\hline \multirow{3}{*}{ CI Split HEV PHEV2O } & Vehicle Cost LOW & $-88.3 \%$ & $-88.3 \%$ & $-83.7 \%$ & $-79.3 \%$ & $-62.6 \%$ & $-57.7 \%$ & $-49.3 \%$ & $-51.4 \%$ & $-43.6 \%$ & $-36.2 \%$ & $-42.3 \%$ & $-36.1 \%$ & $-30.0 \%$ \\
\hline & Vehicle Cost AVG & $-78.6 \%$ & $-78.6 \%$ & $-74.5 \%$ & $-70.6 \%$ & $-51.4 \%$ & $-47.7 \%$ & $-41.8 \%$ & $-40.2 \%$ & $-34.9 \%$ & $-29.7 \%$ & $-37.0 \%$ & $-31.8 \%$ & $-26.7 \%$ \\
\hline & Vehicle Cost HIGH & $-65.7 \%$ & $-65.7 \%$ & $-62.5 \%$ & $-59.3 \%$ & $-35.4 \%$ & $-32.8 \%$ & $-28.0 \%$ & $-28.7 \%$ & $-24.2 \%$ & $-20.1 \%$ & $-22.7 \%$ & $-18.6 \%$ & $-15.9 \%$ \\
\hline \multirow{3}{*}{ CI Series HEV PHEV3O } & Vehicle Cost LOW & $-160.0 \%$ & $-160.0 \%$ & $-149.7 \%$ & $-141.3 \%$ & $-112.3 \%$ & $-103.1 \%$ & $-86.8 \%$ & $-87.9 \%$ & $-73.2 \%$ & $-60.2 \%$ & $-70.5 \%$ & $-58.1 \%$ & $-48.8 \%$ \\
\hline & \begin{tabular}{|l|} 
Vehicle Cost AVG \\
Cehicle Cost
\end{tabular} & $-141.4 \%$ & $-141.4 \%$ & $-132.3 \%$ & $-124.9 \%$ & $-88.3 \%$ & $-81.5 \%$ & $-69.8 \%$ & $-67.2 \%$ & $-57.2 \%$ & $-48.0 \%$ & $-60.7 \%$ & $-50.5 \%$ & $-42.7 \%$ \\
\hline & Vehicle Cost $\mathrm{HIGH}$ & $-116.5 \%$ & $-116.5 \%$ & $-109.3 \%$ & $-103.3 \%$ & $-57.9 \%$ & $-53.2 \%$ & $-44.1 \%$ & $-44.3 \%$ & $-36.2 \%$ & $-29.4 \%$ & $-34.3 \%$ & $-26.9 \%$ & $-22.6 \%$ \\
\hline \multirow{3}{*}{ CI Series HEV PHEV40 } & Vehicle Cost LOW & $-187.0 \%$ & $-187.0 \%$ & $-175.0 \%$ & $-164.8 \%$ & $-129.6 \%$ & $-118.8 \%$ & $-98.6 \%$ & $-99.3 \%$ & $-81.7 \%$ & $-66.2 \%$ & $-76.9 \%$ & $-62.4 \%$ & $-52.1 \%$ \\
\hline & Vehicle Cost AVG & $-164.1 \%$ & $-164.1 \%$ & $-153.7 \%$ & $-144.7 \%$ & $-100.0 \%$ & $-92.1 \%$ & $-77.9 \%$ & $-73.6 \%$ & $-61.9 \%$ & $-51.3 \%$ & $-66.0 \%$ & $-54.0 \%$ & $-45.3 \%$ \\
\hline & Vehicle Cost HIGH & $-134.1 \%$ & $-134.1 \%$ & $-125.7 \%$ & $-118.6 \%$ & $-69.3 \%$ & $-63.6 \%$ & $-52.0 \%$ & $-52.5 \%$ & $-42.3 \%$ & $-33.8 \%$ & $-40.4 \%$ & $-31.0 \%$ & $-25.7 \%$ \\
\hline \multirow{3}{*}{ H2 Split HEV } & Vehicle Cost LOW & $-26.1 \%$ & $-26.1 \%$ & $-23.3 \%$ & $-21.0 \%$ & $-18.6 \%$ & $-15.9 \%$ & $-12.7 \%$ & $-16.7 \%$ & $-12.7 \%$ & $-8.6 \%$ & $-16.4 \%$ & $-12.7 \%$ & $-8.5 \%$ \\
\hline & \begin{tabular}{|l|} 
Vehicle Cost AVG \\
\end{tabular} & $-43.4 \%$ & $-43.4 \%$ & $-40.7 \%$ & $-38.5 \%$ & $-28.2 \%$ & $-26.1 \%$ & $-23.6 \%$ & $-23.3 \%$ & $-20.2 \%$ & $-16.8 \%$ & $-14.1 \%$ & $-11.1 \%$ & $-7.7 \%$ \\
\hline & Vehicle Cost HIGH & $-34.6 \%$ & $-34.6 \%$ & $-32.5 \%$ & $-30.8 \%$ & $-16.7 \%$ & $-15.1 \%$ & $-13.2 \%$ & $-12.6 \%$ & $-10.5 \%$ & $-8.2 \%$ & $-6.8 \%$ & $-5.1 \%$ & $-3.1 \%$ \\
\hline \multirow{3}{*}{ H2 Split HEV PHEV10 } & Vehicle Cost LOW & $-53.4 \%$ & $-53.4 \%$ & $-48.7 \%$ & $-45.2 \%$ & $-35.4 \%$ & $-31.0 \%$ & $-24.8 \%$ & $-28.4 \%$ & $-21.9 \%$ & $-17.1 \%$ & $-23.9 \%$ & $-19.6 \%$ & $-14.5 \%$ \\
\hline & Vehicle Cost AVG & $-65.5 \%$ & $-65.5 \%$ & $-61.3 \%$ & $-58.1 \%$ & $-40.2 \%$ & $-36.9 \%$ & $-32.3 \%$ & $-32.5 \%$ & $-27.6 \%$ & $-24.1 \%$ & $-21.8 \%$ & $-18.2 \%$ & $-13.9 \%$ \\
\hline & Vehicle Cost HIGH & $-54.8 \%$ & $-54.8 \%$ & $-51.4 \%$ & $-48.8 \%$ & $-21.6 \%$ & $-19.6 \%$ & $-16.7 \%$ & $-16.3 \%$ & $-13.4 \%$ & $-10.8 \%$ & $-9.3 \%$ & $-6.8 \%$ & $-5.0 \%$ \\
\hline \multirow{3}{*}{ H2 Split HEV PHEV2O } & Vehicle Cost LOW & $-73.2 \%$ & $-73.2 \%$ & $-66.6 \%$ & $-61.8 \%$ & $-47.5 \%$ & $-41.7 \%$ & $-33.6 \%$ & $-36.2 \%$ & $-27.8 \%$ & $-20.7 \%$ & $-27.8 \%$ & $-21.7 \%$ & $-16.0 \%$ \\
\hline & \begin{tabular}{|l} 
Vehicle Cost AVG \\
\end{tabular} & $-82.2 \%$ & $-82.2 \%$ & $-76.3 \%$ & $-72.1 \%$ & $-48.2 \%$ & $-44.0 \%$ & $-38.2 \%$ & $-36.5 \%$ & $-30.7 \%$ & $-25.7 \%$ & $-25.0 \%$ & $-19.9 \%$ & $-15.1 \%$ \\
\hline & Vehicle Cost HIGH & $-67.5 \%$ & $-67.5 \%$ & $-62.8 \%$ & $-59.4 \%$ & $-30.0 \%$ & $-27.1 \%$ & $-22.5 \%$ & $-22.4 \%$ & $-17.8 \%$ & $-14.0 \%$ & $-13.8 \%$ & $-9.9 \%$ & $-7.3 \%$ \\
\hline \multirow{3}{*}{ H2 Series HEV PHEV 30} & Vehicle Cost LOW & $-142.0 \%$ & $-142.0 \%$ & $-130.2 \%$ & $-121.2 \%$ & $-95.4 \%$ & $-85.2 \%$ & $-69.4 \%$ & $-71.4 \%$ & $-56.4 \%$ & $-43.8 \%$ & $-54.8 \%$ & $-43.0 \%$ & $-34.3 \%$ \\
\hline & \begin{tabular}{|l} 
Vehicle Cost AVG \\
\end{tabular} & $-142.3 \%$ & $-142.3 \%$ & $-131.9 \%$ & $-124.0 \%$ & $-83.8 \%$ & $-76.3 \%$ & $-65.0 \%$ & $-62.6 \%$ & $-52.4 \%$ & $-43.5 \%$ & $-47.8 \%$ & $-38.0 \%$ & $-30.6 \%$ \\
\hline & Vehicle Cost $\mathrm{HIGH}$ & $-116.3 \%$ & $-116.3 \%$ & $-107.9 \%$ & $-101.6 \%$ & $-51.4 \%$ & $-46.3 \%$ & $-37.5 \%$ & $-37.2 \%$ & $-29.0 \%$ & $-22.6 \%$ & $-24.6 \%$ & $-17.5 \%$ & $-13.5 \%$ \\
\hline \multirow{3}{*}{ H2 Series HEV PHEV 40} & Vehicle Cost LOW & $-168.4 \%$ & $-168.4 \%$ & $-154.2 \%$ & $-143.6 \%$ & $-112.0 \%$ & $-100.0 \%$ & $-80.7 \%$ & $-82.4 \%$ & $-64.4 \%$ & $-49.5 \%$ & $-60.8 \%$ & $-47.1 \%$ & $-37.3 \%$ \\
\hline & \begin{tabular}{|l} 
Vehicle Cost AVG \\
Vehicle Cost
\end{tabular} & $-164.6 \%$ & $-164.6 \%$ & $-152.2 \%$ & $-142.9 \%$ & $-95.0 \%$ & $-86.3 \%$ & $-72.7 \%$ & $-68.7 \%$ & $-56.8 \%$ & $-46.6 \%$ & $-52.7 \%$ & $-41.3 \%$ & $-33.1 \%$ \\
\hline & \begin{tabular}{|l|} 
Vehicle Cost HIGH \\
\end{tabular} & $-133.4 \%$ & $-133.4 \%$ & $-123.6 \%$ & $-116.1 \%$ & $-62.3 \%$ & $-56.1 \%$ & $-45.0 \%$ & $-45.0 \%$ & $-34.8 \%$ & $-26.7 \%$ & $-30.3 \%$ & $-21.4 \%$ & $-16.4 \%$ \\
\hline \multirow{3}{*}{ E85 Split HEV } & Vehicle Cost LOW & $-27.7 \%$ & $-27.7 \%$ & $-25.7 \%$ & $-23.6 \%$ & $-21.3 \%$ & $-19.0 \%$ & $-15.8 \%$ & $-20.0 \%$ & $-16.4 \%$ & $-12.4 \%$ & $-21.0 \%$ & $-17.3 \%$ & $-12.6 \%$ \\
\hline & Vehicle Cost AVG & $-26.7 \%$ & $-26.7 \%$ & $-24.7 \%$ & $-22.7 \%$ & $-19.3 \%$ & $-17.4 \%$ & $-14.8 \%$ & $-16.7 \%$ & $-13.6 \%$ & $-10.4 \%$ & $-16.1 \%$ & $-13.1 \%$ & $-9.2 \%$ \\
\hline & Vehicle Cost HIGH & $-21.6 \%$ & $-21.6 \%$ & $-20.0 \%$ & $-18.5 \%$ & $-12.4 \%$ & $-10.9 \%$ & $-8.9 \%$ & $-10.0 \%$ & $-7.9 \%$ & $-5.6 \%$ & $-7.6 \%$ & $-5.7 \%$ & $-3.3 \%$ \\
\hline
\end{tabular}




\begin{tabular}{|c|c|c|c|c|c|c|c|c|c|c|c|c|c|c|}
\hline & & \multicolumn{13}{|c|}{ Midsize_SUV } \\
\hline & & \multirow{2}{*}{$\begin{array}{l}2010 \\
\text { Ref } \\
\end{array}$} & \multicolumn{3}{|c|}{2010} & \multicolumn{3}{|c|}{2015} & \multicolumn{3}{|c|}{2030} & \multicolumn{3}{|c|}{2045} \\
\hline & & & low & avg & high & low & avg & high & low & avg & high & low & avg & high \\
\hline \multirow{3}{*}{ E85 Split HEV PHEV10 } & Vehicle Cost LOW & $-52.8 \%$ & $-52.8 \%$ & $-49.4 \%$ & $-46.4 \%$ & $-36.3 \%$ & $-32.7 \%$ & $-27.2 \%$ & $-30.3 \%$ & $-24.6 \%$ & $-19.8 \%$ & $-27.1 \%$ & $-23.0 \%$ & $-17.7 \%$ \\
\hline & Vehicle Cost AVG & $-46.8 \%$ & $-46.8 \%$ & $-43.7 \%$ & $-41.0 \%$ & $-29.6 \%$ & $-26.9 \%$ & $-22.8 \%$ & $-24.4 \%$ & $-20.1 \%$ & $-16.5 \%$ & $-22.4 \%$ & $-19.0 \%$ & $-14.5 \%$ \\
\hline & Vehicle Cost HIGH & $-39.9 \%$ & $-39.9 \%$ & $-37.4 \%$ & $-35.2 \%$ & $-16.1 \%$ & $-14.4 \%$ & $-11.5 \%$ & $-12.7 \%$ & $-9.9 \%$ & $-7.3 \%$ & $-9.1 \%$ & $-6.5 \%$ & $-4.5 \%$ \\
\hline \multirow{3}{*}{ E85 Split HEV PHEV20 } & Vehicle Cost LOW & $-71.5 \%$ & $-71.5 \%$ & $-67.3 \%$ & $-63.1 \%$ & $-48.2 \%$ & $-43.5 \%$ & $-35.6 \%$ & $-38.0 \%$ & $-30.6 \%$ & $-23.5 \%$ & $-31.0 \%$ & $-25.1 \%$ & $-19.2 \%$ \\
\hline & Vehicle Cost AVG & $-62.5 \%$ & $-62.5 \%$ & $-58.7 \%$ & $-55.1 \%$ & $-37.5 \%$ & $-34.1 \%$ & $-28.4 \%$ & $-28.4 \%$ & $-23.3 \%$ & $-18.3 \%$ & $-25.6 \%$ & $-20.7 \%$ & $-15.7 \%$ \\
\hline & Vehicle Cost $\mathrm{HIGH}$ & $-51.9 \%$ & $-51.9 \%$ & $-48.8 \%$ & $-45.9 \%$ & $-24.5 \%$ & $-22.0 \%$ & $-17.4 \%$ & $-18.8 \%$ & $-14.5 \%$ & $-10.5 \%$ & $-13.7 \%$ & $-9.6 \%$ & $-6.9 \%$ \\
\hline \multirow{3}{*}{ E85 Series HEV PHEV30 } & Vehicle Cost LOW & $-143.4 \%$ & $-143.4 \%$ & $-133.6 \%$ & $-125.1 \%$ & $-98.2 \%$ & $-89.2 \%$ & $-73.5 \%$ & $-74.9 \%$ & $-60.5 \%$ & $-47.9 \%$ & $-59.8 \%$ & $-47.7 \%$ & $-38.6 \%$ \\
\hline & Vehicle Cost AVG & $-125.6 \%$ & $-125.6 \%$ & $-116.9 \%$ & $-109.5 \%$ & $-74.7 \%$ & $-68.1 \%$ & $-56.9 \%$ & $-55.9 \%$ & $-46.0 \%$ & $-37.0 \%$ & $-50.1 \%$ & $-39.9 \%$ & $-32.2 \%$ \\
\hline & Vehicle Cost $\mathrm{HIGH}$ & $-103.1 \%$ & $-103.1 \%$ & $-96.2 \%$ & $-90.1 \%$ & $-46.9 \%$ & $-42.3 \%$ & $-33.5 \%$ & $-34.5 \%$ & $-26.4 \%$ & $-19.8 \%$ & $-25.3 \%$ & $-17.9 \%$ & $-13.6 \%$ \\
\hline \multirow{3}{*}{ E85 Series HEV PHEV40 } & Vehicle Cost LOW & $-169.9 \%$ & $-169.9 \%$ & $-158.0 \%$ & $-148.0 \%$ & $-114.8 \%$ & $-104.4 \%$ & $-85.1 \%$ & $-86.0 \%$ & $-68.7 \%$ & $-53.7 \%$ & $-66.0 \%$ & $-51.9 \%$ & $-41.7 \%$ \\
\hline & Vehicle Cost AVG & $-147.9 \%$ & $-147.9 \%$ & $-137.5 \%$ & $-128.8 \%$ & $-85.9 \%$ & $-78.4 \%$ & $-64.7 \%$ & $-62.1 \%$ & $-50.6 \%$ & $-40.3 \%$ & $-55.1 \%$ & $-43.3 \%$ & $-34.7 \%$ \\
\hline & Vehicle Cost HIGH & $-120.3 \%$ & $-120.3 \%$ & $-112.0 \%$ & $-105.0 \%$ & $-57.9 \%$ & $-52.4 \%$ & $-41.2 \%$ & $-42.4 \%$ & $-32.3 \%$ & $-24.0 \%$ & $-31.1 \%$ & $-21.9 \%$ & $-16.6 \%$ \\
\hline \multirow{3}{*}{ FC HEV } & Vehicle Cost LOW & $-78.9 \%$ & $-78.9 \%$ & $-62.9 \%$ & $-52.5 \%$ & $-71.2 \%$ & $-53.3 \%$ & $-43.5 \%$ & $-29.5 \%$ & $-20.6 \%$ & $-12.5 \%$ & $-14.3 \%$ & $-8.7 \%$ & $-3.0 \%$ \\
\hline & Vehicle Cost AVG & $-117.2 \%$ & $-117.2 \%$ & $-94.0 \%$ & $-78.5 \%$ & $-68.3 \%$ & $-52.6 \%$ & $-43.4 \%$ & $-39.7 \%$ & $-29.3 \%$ & $-19.7 \%$ & $-13.6 \%$ & $-8.2 \%$ & $-2.7 \%$ \\
\hline & Vehicle Cost HIGH & $-96.7 \%$ & $-96.7 \%$ & $-77.7 \%$ & $-64.8 \%$ & $-36.7 \%$ & $-27.2 \%$ & $-21.5 \%$ & $-22.1 \%$ & $-15.4 \%$ & $-9.3 \%$ & $-4.9 \%$ & $-1.5 \%$ & $2.0 \%$ \\
\hline \multirow{3}{*}{ FC HEV PHEV 10} & \begin{tabular}{|l} 
Vehicle Cost LOW \\
\end{tabular} & $-93.2 \%$ & $-93.2 \%$ & $-77.2 \%$ & $-67.5 \%$ & $-75.0 \%$ & $-60.2 \%$ & $-49.0 \%$ & $-38.5 \%$ & $-27.6 \%$ & $-18.0 \%$ & $-21.4 \%$ & $-14.4 \%$ & $-7.6 \%$ \\
\hline & Vehicle Cost AVG & $-123.4 \%$ & $-123.4 \%$ & $-102.4 \%$ & $-88.5 \%$ & $-71.7 \%$ & $-58.3 \%$ & $-48.2 \%$ & $-45.1 \%$ & $-33.8 \%$ & $-23.5 \%$ & $-19.7 \%$ & $-13.1 \%$ & $-6.7 \%$ \\
\hline & Vehicle Cost HIGH & $-101.8 \%$ & $-101.8 \%$ & $-84.4 \%$ & $-73.0 \%$ & $-36.6 \%$ & $-28.7 \%$ & $-22.2 \%$ & $-23.1 \%$ & $-15.8 \%$ & $-9.5 \%$ & $-6.4 \%$ & $-2.1 \%$ & $1.5 \%$ \\
\hline \multirow{3}{*}{ FC HEV PHEV2O } & Vehicle Cost LOW & $-118.2 \%$ & $-118.2 \%$ & $-98.9 \%$ & $-86.7 \%$ & $-90.3 \%$ & $-73.1 \%$ & $-57.9 \%$ & $-47.8 \%$ & $-34.5 \%$ & $-22.6 \%$ & $-25.9 \%$ & $-17.7 \%$ & $-9.9 \%$ \\
\hline & Vehicle Cost AVG & $-145.3 \%$ & $-145.3 \%$ & $-121.2 \%$ & $-105.1 \%$ & $-82.2 \%$ & $-67.2 \%$ & $-54.3 \%$ & $-50.4 \%$ & $-37.6 \%$ & $-26.1 \%$ & $-23.4 \%$ & $-15.8 \%$ & $-8.6 \%$ \\
\hline & Vehicle Cost $\mathrm{HIGH}$ & $-118.6 \%$ & $-118.6 \%$ & $-98.9 \%$ & $-85.8 \%$ & $-47.1 \%$ & $-37.5 \%$ & $-28.5 \%$ & $-30.3 \%$ & $-21.1 \%$ & $-13.1 \%$ & $-11.3 \%$ & $-5.6 \%$ & $-1.1 \%$ \\
\hline \multirow{3}{*}{ FC HEV PHEV 30} & Vehicle Cost LOW & $-157.2 \%$ & $-157.2 \%$ & $-137.8 \%$ & $-126.4 \%$ & $-115.6 \%$ & $-100.3 \%$ & $-82.6 \%$ & $-71.3 \%$ & $-54.9 \%$ & $-41.4 \%$ & $-45.0 \%$ & $-33.5 \%$ & $-24.8 \%$ \\
\hline & Vehicle Cost AVG & $-179.6 \%$ & $-179.6 \%$ & $-155.9 \%$ & $-140.8 \%$ & $-104.6 \%$ & $-90.5 \%$ & $-75.1 \%$ & $-69.8 \%$ & $-54.9 \%$ & $-42.1 \%$ & $-40.4 \%$ & $-29.8 \%$ & $-21.8 \%$ \\
\hline & Vehicle Cost HIGH & $-147.5 \%$ & $-147.5 \%$ & $-128.1 \%$ & $-115.8 \%$ & $-55.2 \%$ & $-46.5 \%$ & $-35.8 \%$ & $-37.1 \%$ & $-26.4 \%$ & $-18.0 \%$ & $-16.5 \%$ & $-9.0 \%$ & $-4.4 \%$ \\
\hline \multirow{3}{*}{ FC HEV PHEV40 } & Vehicle Cost LOW & $-185.5 \%$ & $-185.5 \%$ & $-161.4 \%$ & $-147.6 \%$ & $-132.0 \%$ & $-114.2 \%$ & $-92.8 \%$ & $-81.6 \%$ & $-62.1 \%$ & $-46.9 \%$ & $-50.4 \%$ & $-37.1 \%$ & $-27.6 \%$ \\
\hline & \begin{tabular}{|l} 
Vehicle Cost AVG \\
\end{tabular} & $-204.8 \%$ & $-204.8 \%$ & $-176.8 \%$ & $-159.7 \%$ & $-116.2 \%$ & $-100.4 \%$ & $-82.4 \%$ & $-76.1 \%$ & $-59.3 \%$ & $-45.4 \%$ & $-44.9 \%$ & $-32.8 \%$ & $-24.2 \%$ \\
\hline & \begin{tabular}{|l} 
Vehicle Cost HIGH \\
\end{tabular} & $-167.0 \%$ & $-167.0 \%$ & $-144.3 \%$ & $-130.4 \%$ & $-66.2 \%$ & $-55.9 \%$ & $-42.7 \%$ & $-44.9 \%$ & $-31.7 \%$ & $-22.0 \%$ & $-21.9 \%$ & $-12.4 \%$ & $-7.2 \%$ \\
\hline \multirow{3}{*}{ EV } & Vehicle Cost LOW & $-412.2 \%$ & $-412.2 \%$ & $-379.3 \%$ & $-352.2 \%$ & $-257.3 \%$ & $-229.5 \%$ & $-167.9 \%$ & $-171.2 \%$ & $-120.4 \%$ & $-83.9 \%$ & $-96.9 \%$ & $-62.2 \%$ & $-45.1 \%$ \\
\hline & Vehicle Cost AVG & $-345.3 \%$ & $-345.3 \%$ & $-317.5 \%$ & $-294.5 \%$ & $-175.0 \%$ & $-155.9 \%$ & $-114.0 \%$ & $-96.1 \%$ & $-67.2 \%$ & $-46.2 \%$ & $-77.1 \%$ & $-48.7 \%$ & $-34.7 \%$ \\
\hline & Vehicle Cost HIGH & $-268.3 \%$ & $-268.3 \%$ & $-246.6 \%$ & $-228.8 \%$ & $-150.9 \%$ & $-134.0 \%$ & $-94.3 \%$ & $-50.1 \%$ & $-31.8 \%$ & $-18.7 \%$ & $-46.0 \%$ & $-24.8 \%$ & $-15.3 \%$ \\
\hline
\end{tabular}




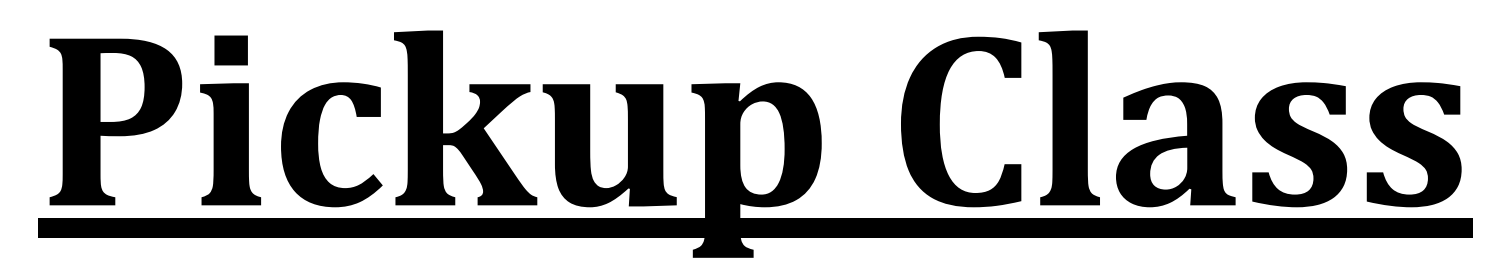


Incremental Cost versus the Conventional 2010 Reference

\begin{tabular}{|c|c|c|c|c|c|c|c|c|c|c|c|c|c|c|}
\hline & & \multicolumn{13}{|c|}{ Pickup } \\
\hline & & \multirow{2}{*}{$\begin{array}{c}2010 \\
\text { Ref }\end{array}$} & \multicolumn{3}{|c|}{2010} & \multicolumn{3}{|c|}{2015} & \multicolumn{3}{|c|}{2030} & \multicolumn{3}{|c|}{2045} \\
\hline & & & low & avg & high & low & avg & high & low & avg & high & low & avg & high \\
\hline \multirow{3}{*}{ SI Conv } & Vehicle Cost LOW & 0 & 0 & -63 & -155 & 2 & -99 & -229 & 825 & 653 & 492 & 1269 & 1088 & 870 \\
\hline & Vehicle Cost AVG & 0 & 0 & -63 & -155 & 803 & 706 & 580 & 1208 & 1027 & 855 & 1198 & 1024 & 813 \\
\hline & Vehicle Cost HIGH & 0 & 0 & -63 & -155 & 137 & 36 & -93 & 569 & 392 & 225 & 552 & 377 & 164 \\
\hline \multirow{3}{*}{$\mathrm{Cl}$ Conv } & Vehicle Cost LOW & 2534 & 2534 & 2422 & 2330 & 2261 & 2150 & 893 & 3021 & 1644 & 1478 & 2984 & 1626 & 1443 \\
\hline & Vehicle Cost AVG & 2452 & 2452 & 2348 & 2260 & 3131 & 3032 & 1677 & 3096 & 1684 & 1539 & 2979 & 1591 & 1434 \\
\hline & Vehicle Cost HIGH & 2061 & 2061 & 1975 & 1895 & 2001 & 1920 & 695 & 2264 & 1026 & 899 & 2087 & 877 & 741 \\
\hline \multirow{3}{*}{ H2 Conv } & Vehicle Cost LOW & -904 & -904 & -953 & -1019 & -1000 & -1059 & -1139 & -281 & -379 & -1145 & -92 & -152 & -957 \\
\hline & Vehicle Cost AVG & 2114 & 2114 & 2064 & 1997 & 1774 & 1717 & 1639 & 1728 & 1629 & 795 & 302 & 241 & -565 \\
\hline & Vehicle Cost HIGH & 1553 & 1553 & 1503 & 1436 & 382 & 318 & 235 & 549 & 437 & -313 & -9 & -87 & -850 \\
\hline \multirow{3}{*}{ E85 Conv } & Vehicle Cost LOW & -30 & -30 & -106 & -199 & -31 & -133 & -252 & 792 & 629 & 459 & 1231 & 1065 & 857 \\
\hline & Vehicle Cost AVG & -29 & -29 & -105 & -198 & 774 & 675 & 560 & 1173 & 1001 & 820 & 1163 & 1003 & 802 \\
\hline & Vehicle Cost $\mathrm{HIGH}$ & -30 & -30 & -106 & -199 & 104 & 1 & -117 & 531 & 364 & 187 & 512 & 350 & 148 \\
\hline \multirow{3}{*}{ SI Split HEV } & Vehicle Cost LOW & 5961 & 5961 & 5534 & 4615 & 4769 & 3726 & 3118 & 4421 & 3115 & 2249 & 4627 & 3162 & 2243 \\
\hline & Vehicle Cost AVG & 5763 & 5763 & 5347 & 4408 & 4234 & 3231 & 2749 & 3770 & 2515 & 1795 & 3645 & 2314 & 1559 \\
\hline & Vehicle Cost HIGH & 4043 & 4043 & 3717 & 2884 & 2254 & 1416 & 1031 & 1721 & 778 & 284 & 1257 & 307 & -152 \\
\hline \multirow{3}{*}{ SI Split HEV PHEV10 } & Vehicle Cost LOW & 10442 & 10442 & 9817 & 9122 & 7118 & 6455 & 5251 & 5758 & 4599 & 3748 & 5089 & 4289 & 3270 \\
\hline & Vehicle Cost AVG & 9218 & 9218 & 8655 & 8030 & 5625 & 5123 & 4222 & 4531 & 3638 & 3010 & 4150 & 3481 & 2609 \\
\hline & Vehicle Cost HIGH & 7140 & 7140 & 6681 & 6169 & 2390 & 2079 & 1512 & 1630 & 1115 & 623 & 939 & 472 & 93 \\
\hline \multirow{3}{*}{ SI Split HEV PHEV20 } & Vehicle Cost LOW & 14195 & 14195 & 13346 & 12457 & 9454 & 8609 & 7003 & 7269 & 5847 & 4493 & 5828 & 4722 & 3610 \\
\hline & Vehicle Cost AVG & 12364 & 12364 & 11614 & 10828 & 7170 & 6548 & 5408 & 5315 & 4342 & 3365 & 4741 & 3819 & 2876 \\
\hline & Vehicle Cost HIGH & 9534 & 9534 & 8931 & 8299 & 4034 & 3596 & 2659 & 2817 & 1984 & 1267 & 1807 & 1068 & 556 \\
\hline \multirow{3}{*}{ SI Series HEV PHEV30 } & Vehicle Cost LOW & 27463 & 27463 & 25756 & 23991 & 18645 & 17056 & 13966 & 13959 & 11302 & 9052 & 11062 & 8919 & 7225 \\
\hline & Vehicle Cost AVG & 23997 & 23997 & 22491 & 20934 & 14002 & 12832 & 10622 & 10285 & 8462 & 6860 & 9180 & 7384 & 5949 \\
\hline & Vehicle Cost HIGH & 18972 & 18972 & 17758 & 16504 & 8200 & 7388 & 5648 & 5684 & 4193 & 3012 & 3950 & 2625 & 1819 \\
\hline \multirow{3}{*}{ SI Series HEV PHEV40 } & Vehicle Cost LOW & 32649 & 32649 & 30538 & 28397 & 21863 & 20009 & 16225 & 16067 & 12880 & 10205 & 12240 & 9731 & 7855 \\
\hline & Vehicle Cost AVG & 28376 & 28376 & 26529 & 24654 & 16181 & 14830 & 12149 & 11472 & 9345 & 7506 & 10142 & 8046 & 6462 \\
\hline & Vehicle Cost HIGH & 22348 & 22348 & 20870 & 19369 & 10323 & 9340 & 7148 & 7199 & 5334 & 3846 & 5064 & 3405 & 2420 \\
\hline \multirow{3}{*}{ Cl Split HEV } & Vehicle Cost LOW & 8210 & 8210 & 7730 & 7212 & 6561 & 6079 & 5370 & 5987 & 5148 & 4295 & 5724 & 4910 & 3951 \\
\hline & \begin{tabular}{|l|} 
Vehicle Cost AVG \\
\end{tabular} & 7899 & 7899 & 7436 & 6936 & 5845 & 5461 & 4901 & 4939 & 4265 & 3582 & 4694 & 4038 & 3267 \\
\hline & Vehicle Cost HIGH & 5707 & 5707 & 5353 & 4969 & 3496 & 3214 & 2797 & 2743 & 2288 & 1832 & 2089 & 1707 & 1264 \\
\hline
\end{tabular}




\begin{tabular}{|c|c|c|c|c|c|c|c|c|c|c|c|c|c|c|}
\hline & & \multicolumn{13}{|c|}{ Pickup } \\
\hline & & \multirow{2}{*}{$\begin{array}{l}2010 \\
\text { Ref }\end{array}$} & \multicolumn{3}{|c|}{2010} & \multicolumn{3}{|c|}{2015} & \multicolumn{3}{|c|}{2030} & \multicolumn{3}{|c|}{2045} \\
\hline & & & low & avg & high & low & avg & high & low & avg & high & low & avg & high \\
\hline \multirow{3}{*}{ CI Split HEV PHEV10 } & Vehicle Cost LOW & 13151 & 13151 & 12520 & 11813 & 9494 & 8794 & 7586 & 7997 & 6825 & 5890 & 6965 & 6150 & 5081 \\
\hline & Vehicle Cost AVG & 11812 & 11812 & 11246 & 10613 & 7932 & 7401 & 6503 & 6511 & 5633 & 4936 & 6027 & 5355 & 4449 \\
\hline & Vehicle Cost HIGH & 9374 & 9374 & 8917 & 8404 & 4157 & 3833 & 3241 & 3235 & 2687 & 2198 & 2415 & 1932 & 1563 \\
\hline \multirow{3}{*}{ CI Split HEV PHEV2O } & Vehicle Cost LOW & 17024 & 17024 & 16108 & 15174 & 11866 & 10981 & 9331 & 9513 & 8030 & 6671 & 7740 & 6586 & 5441 \\
\hline & Vehicle Cost AVG & 15056 & 15056 & 14249 & 13425 & 9498 & 8844 & 7672 & 7287 & 6273 & 5314 & 6647 & 5693 & 4731 \\
\hline & Vehicle Cost HIGH & 11841 & 11841 & 11197 & 10538 & 5835 & 5384 & 4423 & 4438 & 3595 & 2863 & 3311 & 2558 & 2041 \\
\hline \multirow{3}{*}{ CI Series HEV PHEV3O } & Vehicle Cost LOW & 30326 & 30326 & 28552 & 26723 & 21082 & 19440 & 16246 & 16190 & 13452 & 11136 & 12858 & 10671 & 8955 \\
\hline & 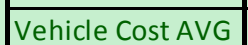 & 26706 & 26706 & 25145 & 23535 & 16336 & 15124 & 12836 & 12201 & 10338 & 8702 & 10972 & 9151 & 7710 \\
\hline & Vehicle Cost HIGH & 21254 & 21254 & 20005 & 18715 & 10063 & 9232 & 7443 & 7348 & 5824 & 4621 & 5450 & 4114 & 3314 \\
\hline \multirow{3}{*}{ CI Series HEV PHEV40 } & Vehicle Cost LOW & 35598 & 35598 & 33472 & 31286 & 24378 & 22442 & 18543 & 18357 & 15063 & 12320 & 14080 & 11508 & 9607 \\
\hline & \begin{tabular}{|l|} 
Vehicle Cost AVG \\
\end{tabular} & 31159 & 31159 & 29298 & 27386 & 18570 & 17156 & 14389 & 13420 & 11238 & 9363 & 11966 & 9832 & 8240 \\
\hline & \begin{tabular}{|l} 
Vehicle Cost HIGH \\
Vehicle Cost
\end{tabular} & 24682 & 24682 & 23201 & 21679 & 12233 & 11213 & 8965 & 8896 & 6985 & 5475 & 6595 & 4913 & 3933 \\
\hline \multirow{3}{*}{ H2 Split HEV } & Vehicle Cost LOW & 5695 & 5695 & 5183 & 4076 & 4313 & 3087 & 2492 & 3817 & 2359 & 1563 & 3737 & 2325 & 1484 \\
\hline & \begin{tabular}{|l|} 
Vehicle Cost AVG \\
\end{tabular} & 8513 & 8513 & 8014 & 6881 & 5697 & 4533 & 4070 & 4797 & 3423 & 2784 & 3202 & 1919 & 1239 \\
\hline & Vehicle Cost HIGH & 6170 & 6170 & 5787 & 4791 & 2971 & 1980 & 1626 & 2144 & 1069 & 641 & 1115 & 127 & -269 \\
\hline \multirow{3}{*}{ H2 Split HEV PHEV10 } & Vehicle Cost LOW & 10442 & 10442 & 9567 & 8820 & 6934 & 6052 & 4808 & 5391 & 4051 & 3154 & 4450 & 3631 & 2598 \\
\hline & Vehicle Cost AVG & 12207 & 12207 & 11417 & 10745 & 7317 & 6649 & 5719 & 5799 & 4769 & 4103 & 3930 & 3248 & 2368 \\
\hline & Vehicle Cost HIGH & 9488 & 9488 & 8843 & 8297 & 3226 & 2849 & 2295 & 2140 & 1590 & 1121 & 882 & 441 & 93 \\
\hline \multirow{3}{*}{ H2 Split HEV PHEV2O } & Vehicle Cost LOW & 14234 & 14234 & 13141 & 12059 & 9232 & 8216 & 6511 & 6869 & 5301 & 3924 & 5243 & 4092 & 2972 \\
\hline & Vehicle Cost AVG & 15383 & 15383 & 14414 & 13455 & 8833 & 8087 & 6872 & 6555 & 5474 & 4488 & 4568 & 3610 & 2666 \\
\hline & Vehicle Cost HIGH & 11907 & 11907 & 11129 & 10357 & 4851 & 4345 & 3402 & 3309 & 2454 & 1749 & 1739 & 1028 & 545 \\
\hline \multirow{3}{*}{ H2 Series HEV PHEV 30} & Vehicle Cost LOW & 27056 & 27056 & 25036 & 23168 & 18052 & 16268 & 13166 & 13273 & 10524 & 8265 & 10110 & 8025 & 6405 \\
\hline & \begin{tabular}{|l|} 
Vehicle Cost AVG \\
\end{tabular} & 26587 & 26587 & 24807 & 23161 & 15370 & 14063 & 11856 & 11267 & 9398 & 7808 & 8693 & 6953 & 5586 \\
\hline & \begin{tabular}{|l|} 
Vehicle Cost HIGH \\
Cehic Cost
\end{tabular} & 20986 & 20986 & 19556 & 18235 & 8816 & 7930 & 6207 & 6036 & 4530 & 3372 & 3737 & 2467 & 1721 \\
\hline \multirow{3}{*}{ H2 Series HEV PHEV 40} & Vehicle Cost LOW & 32256 & 32256 & 29757 & 27550 & 21255 & 19141 & 15374 & 15358 & 12066 & 9390 & 11258 & 8822 & 7022 \\
\hline & \begin{tabular}{|l} 
Vehicle Cost AVG \\
\end{tabular} & 30977 & 30977 & 28791 & 26858 & 17533 & 16004 & 13345 & 12433 & 10255 & 8433 & 9628 & 7601 & 6088 \\
\hline & \begin{tabular}{|l} 
Vehicle Cost HIGH \\
\end{tabular} & 24366 & 24366 & 22623 & 21080 & 10926 & 9821 & 7669 & 7531 & 5643 & 4182 & 4826 & 3230 & 2310 \\
\hline \multirow{3}{*}{ E85 Split HEV } & Vehicle Cost LOW & 6058 & 6058 & 5639 & 4679 & 4806 & 3794 & 3144 & 4478 & 3101 & 2271 & 4686 & 3211 & 2319 \\
\hline & Vehicle Cost AVG & 5861 & 5861 & 5453 & 4472 & 4269 & 3292 & 2773 & 3825 & 2506 & 1819 & 3699 & 2358 & 1625 \\
\hline & Vehicle Cost HIGH & 4125 & 4125 & 3804 & 2939 & 2285 & 1459 & 1053 & 1765 & 773 & 303 & 1298 & 340 & -109 \\
\hline
\end{tabular}




\begin{tabular}{|c|c|c|c|c|c|c|c|c|c|c|c|c|c|c|}
\hline & & \multicolumn{13}{|c|}{ Pickup } \\
\hline & & \multirow{2}{*}{$\frac{2010}{\operatorname{Ref}}$} & \multicolumn{3}{|c|}{2010} & \multicolumn{3}{|c|}{2015} & \multicolumn{3}{|c|}{2030} & \multicolumn{3}{|c|}{2045} \\
\hline & & & low & avg & high & low & avg & high & low & avg & high & low & avg & high \\
\hline \multirow{3}{*}{ E85 Split HEV PHEV10 } & Vehicle Cost LOW & 10520 & 10520 & 9813 & 9154 & 7211 & 6543 & 5275 & 5787 & 4626 & 3779 & 5120 & 4318 & 3298 \\
\hline & Vehicle Cost AVG & 9291 & 9291 & 8655 & 8062 & 5704 & 5199 & 4246 & 4560 & 3665 & 3040 & 4179 & 3508 & 2636 \\
\hline & Vehicle Cost HIGH & 7204 & 7204 & 6681 & 6198 & 2428 & 2115 & 1533 & 1651 & 1135 & 643 & 959 & 492 & 112 \\
\hline \multirow{3}{*}{ E85 Split HEV PHEV20 } & Vehicle Cost LOW & 14236 & 14236 & 13396 & 12492 & 9485 & 8642 & 7033 & 7300 & 5876 & 4521 & 5906 & 4751 & 3639 \\
\hline & Vehicle Cost AVG & 12404 & 12404 & 11661 & 10862 & 7199 & 6578 & 5436 & 5345 & 4370 & 3393 & 4810 & 3847 & 2903 \\
\hline & Vehicle Cost HIGH & 9569 & 9569 & 8971 & 8329 & 4057 & 3621 & 2681 & 2838 & 2005 & 1287 & 1838 & 1088 & 575 \\
\hline \multirow{3}{*}{ E85 Series HEV PHEV30 } & Vehicle Cost LOW & 27505 & 27505 & 25796 & 24039 & 18685 & 17111 & 14000 & 14007 & 11333 & 9081 & 11093 & 8949 & 7256 \\
\hline & \begin{tabular}{|l|} 
Vehicle Cost AVG \\
\end{tabular} & 24037 & 24037 & 22529 & 20980 & 14037 & 12877 & 10653 & 10325 & 8492 & 6889 & 9209 & 7412 & 5978 \\
\hline & Vehicle Cost HIGH & 19007 & 19007 & 17792 & 16543 & 8228 & 7427 & 5673 & 5719 & 4216 & 3033 & 3972 & 2646 & 1841 \\
\hline \multirow{3}{*}{ E85 Series HEV PHEV40 } & Vehicle Cost LOW & 32692 & 32692 & 30606 & 28452 & 21900 & 20050 & 16259 & 16107 & 12917 & 10235 & 12273 & 9765 & 7884 \\
\hline & Vehicle Cost AVG & 28417 & 28417 & 26592 & 24705 & 16215 & 14866 & 12179 & 11508 & 9380 & 7534 & 10172 & 8077 & 6490 \\
\hline & Vehicle Cost HIGH & 22384 & 22384 & 20923 & 19413 & 10351 & 9369 & 7173 & 7227 & 5360 & 3868 & 5087 & 3428 & 2441 \\
\hline \multirow{3}{*}{ FC HEV } & Vehicle Cost LOW & 15852 & 15852 & 12678 & 10508 & 14337 & 10989 & 8760 & 6013 & 4227 & 2707 & 2966 & 1922 & 801 \\
\hline & Vehicle Cost AVG & 23429 & 23429 & 18867 & 15613 & 13590 & 10633 & 8610 & 7924 & 5854 & 4112 & 2783 & 1776 & 712 \\
\hline & Vehicle Cost HIGH & 18659 & 18659 & 14904 & 12215 & 6736 & 4957 & 3695 & 3782 & 2449 & 1324 & 397 & -238 & -906 \\
\hline \multirow{3}{*}{ FC HEV PHEV10 } & Vehicle Cost LOW & 18595 & 18595 & 15408 & 13356 & 14810 & 12101 & 9669 & 7684 & 5481 & 3676 & 4315 & 3040 & 1638 \\
\hline & Vehicle Cost AVG & 24530 & 24530 & 20384 & 17461 & 14023 & 11577 & 9420 & 8873 & 6616 & 4744 & 3935 & 2742 & 1440 \\
\hline & Vehicle Cost HIGH & 19543 & 19543 & 16119 & 13711 & 6631 & 5179 & 3795 & 3947 & 2488 & 1334 & 692 & -80 & -798 \\
\hline \multirow{3}{*}{ FC HEV PHEV 20} & Vehicle Cost LOW & 23532 & 23532 & 19699 & 17155 & 17781 & 14639 & 11631 & 9477 & 6805 & 4598 & 5220 & 3631 & 2097 \\
\hline & Vehicle Cost AVG & 28841 & 28841 & 24113 & 20759 & 16070 & 13322 & 10763 & 9895 & 7364 & 5258 & 4679 & 3224 & 1817 \\
\hline & Vehicle Cost HIGH & 22862 & 22862 & 18987 & 16248 & 8657 & 6912 & 5133 & 5336 & 3512 & 2053 & 1664 & 578 & -292 \\
\hline \multirow{3}{*}{ FC HEV PHEV 30} & Vehicle Cost LOW & 30631 & 30631 & 27098 & 24613 & 22416 & 19606 & 16071 & 13611 & 10578 & 8183 & 8614 & 6566 & 4954 \\
\hline & Vehicle Cost AVG & 35074 & 35074 & 30711 & 27503 & 20175 & 17612 & 14544 & 13302 & 10540 & 8293 & 7704 & 5840 & 4344 \\
\hline & Vehicle Cost HIGH & 28090 & 28090 & 24529 & 21909 & 10194 & 8590 & 6442 & 6489 & 4489 & 3007 & 2588 & 1217 & 375 \\
\hline \multirow{3}{*}{ FC HEV PHEV40 } & Vehicle Cost LOW & 36214 & 36214 & 31734 & 28843 & 25545 & 22331 & 18065 & 15582 & 11951 & 9268 & 9636 & 7253 & 5525 \\
\hline & Vehicle Cost AVG & 40062 & 40062 & 34853 & 31249 & 22422 & 19564 & 16017 & 14510 & 11396 & 8968 & 8562 & 6411 & 4826 \\
\hline & Vehicle Cost $\mathrm{HIGH}$ & 31949 & 31949 & 27734 & 24805 & 12303 & 10429 & 7807 & 7974 & 5516 & 3821 & 3603 & 1887 & 937 \\
\hline \multirow{3}{*}{ EV } & Vehicle Cost LOW & 81567 & 81567 & 75765 & 69788 & 50378 & 45457 & 33299 & 33044 & 23567 & 16911 & 18671 & 12442 & 9165 \\
\hline & Vehicle Cost AVG & 68331 & 68331 & 63429 & 58378 & 34181 & 30795 & 22540 & 18519 & 13136 & 9311 & 14834 & 9742 & 7045 \\
\hline & Vehicle Cost HIGH & 52392 & 52392 & 48581 & 44653 & 29007 & 25991 & 18181 & 9075 & 5654 & 3275 & 8283 & 4437 & 2599 \\
\hline
\end{tabular}




\section{Cost saving per powertrain}

\begin{tabular}{|c|c|c|c|c|c|c|c|c|c|c|c|c|c|c|}
\hline & & \multicolumn{13}{|c|}{ Pickup } \\
\hline & & \multirow{2}{*}{$\frac{2010}{\operatorname{Ref}}$} & \multicolumn{3}{|c|}{2010} & \multicolumn{3}{|c|}{2015} & \multicolumn{3}{|c|}{2030} & \multicolumn{3}{|c|}{2045} \\
\hline & & & low & avg & high & low & avg & high & low & avg & high & low & avg & high \\
\hline \multirow{3}{*}{ SI Conv } & Vehicle Cost LOW & 0 & 0 & -63 & -155 & 2 & -99 & -229 & 825 & 653 & 492 & 1269 & 1088 & 870 \\
\hline & Vehicle Cost AVG & 0 & 0 & -63 & -155 & 803 & 706 & 580 & 1208 & 1027 & 855 & 1198 & 1024 & 813 \\
\hline & Vehicle Cost HIGH & 0 & 0 & -63 & -155 & 137 & 36 & -93 & 569 & 392 & 225 & 552 & 377 & 164 \\
\hline \multirow{3}{*}{$\mathrm{Cl}$ Conv } & Vehicle Cost LOW & 0 & 0 & -112 & -205 & -274 & -385 & -1641 & 487 & -890 & -1056 & 450 & -909 & -1091 \\
\hline & Vehicle Cost AVG & 0 & 0 & -103 & -192 & 679 & 580 & -774 & 644 & -768 & -913 & 528 & -861 & -1017 \\
\hline & Vehicle Cost HIGH & 0 & 0 & -86 & -167 & -61 & -142 & -1367 & 203 & -1035 & -1163 & 26 & -1185 & -1321 \\
\hline \multirow{3}{*}{ H2 Conv } & Vehicle Cost LOW & 0 & 0 & -49 & -115 & -95 & -155 & -234 & 623 & 525 & -240 & 812 & 752 & -53 \\
\hline & Vehicle Cost AVG & 0 & 0 & -50 & -117 & -340 & -397 & -475 & -386 & -485 & -1319 & -1812 & -1873 & -2679 \\
\hline & Vehicle Cost HIGH & 0 & 0 & -50 & -117 & -1171 & -1235 & -1318 & -1004 & -1116 & -1866 & -1562 & -1640 & -2403 \\
\hline \multirow{3}{*}{ E85 Conv } & Vehicle Cost LOW & 0 & 0 & -77 & -170 & -1 & -104 & -222 & 822 & 659 & 488 & 1261 & 1095 & 887 \\
\hline & Vehicle Cost AVG & 0 & 0 & -77 & -170 & 803 & 704 & 588 & 1201 & 1030 & 849 & 1192 & 1031 & 831 \\
\hline & Vehicle Cost HIGH & 0 & 0 & -77 & -170 & 134 & 31 & -88 & 561 & 393 & 217 & 542 & 380 & 178 \\
\hline \multirow{3}{*}{ SI Split HEV } & Vehicle Cost LOW & 0 & 0 & -427 & -1345 & -1192 & -2235 & -2842 & -1540 & -2846 & -3712 & -1334 & -2799 & -3718 \\
\hline & Vehicle Cost AVG & 0 & 0 & -416 & -1356 & -1529 & -2532 & -3014 & -1993 & -3248 & -3968 & -2118 & -3449 & -4204 \\
\hline & Vehicle Cost HIGH & 0 & 0 & -326 & -1159 & -1789 & -2627 & -3012 & -2322 & -3264 & -3759 & -2785 & -3736 & -4195 \\
\hline \multirow{3}{*}{ SI Split HEV PHEV10 } & Vehicle Cost LOW & 0 & 0 & -625 & -1321 & -3324 & -3988 & -5191 & -4684 & -5843 & -6694 & -5353 & -6153 & -7173 \\
\hline & Vehicle Cost AVG & 0 & 0 & -563 & -1188 & -3593 & -4094 & -4996 & -4687 & -5580 & -6208 & -5068 & -5737 & -6609 \\
\hline & Vehicle Cost HIGH & 0 & 0 & -460 & -972 & -4751 & -5061 & -5628 & -5511 & -6025 & -6517 & -6202 & -6669 & -7048 \\
\hline \multirow{3}{*}{ SI Split HEV PHEV20 } & Vehicle Cost LOW & 0 & 0 & -849 & -1738 & -4741 & -5586 & -7192 & -6926 & -8348 & -9702 & -8367 & -9473 & -10585 \\
\hline & Vehicle Cost AVG & 0 & 0 & -750 & -1536 & -5194 & -5816 & -6956 & -7049 & -8022 & -8999 & -7623 & -8545 & -9488 \\
\hline & Vehicle Cost HIGH & 0 & 0 & -603 & -1235 & -5499 & -5938 & -6874 & -6717 & -7550 & -8267 & -7726 & -8465 & -8978 \\
\hline \multirow{3}{*}{ SI Series HEV PHEV30 } & Vehicle Cost LOW & 0 & 0 & -1707 & -3472 & -8818 & -10407 & -13497 & -13505 & -16161 & -18411 & -16401 & -18544 & -20238 \\
\hline & Vehicle Cost AVG & 0 & 0 & -1506 & -3062 & -9995 & -11165 & -13375 & -13712 & -15535 & -17136 & -14816 & -16613 & -18048 \\
\hline & Vehicle Cost $\mathrm{HIGH}$ & 0 & 0 & -1214 & -2469 & -10772 & -11584 & -13324 & -13288 & -14779 & -15961 & -15022 & -16347 & -17153 \\
\hline \multirow{3}{*}{ SI Series HEV PHEV40 } & Vehicle Cost LOW & 0 & 0 & -2111 & -4251 & -10786 & -12639 & -16423 & -16582 & -19769 & -22444 & -20409 & -22917 & -24794 \\
\hline & Vehicle Cost AVG & 0 & 0 & -1848 & -3723 & -12195 & -13546 & -16228 & -16904 & -19031 & -20871 & -18235 & -20330 & -21914 \\
\hline & Vehicle Cost HIGH & 0 & 0 & -1478 & -2979 & -12025 & -13008 & -15200 & -15149 & -17014 & -18502 & -17284 & -18943 & -19927 \\
\hline \multirow{3}{*}{ Cl Split HEV } & Vehicle Cost LOW & 0 & 0 & -480 & -998 & -1649 & -2131 & -2840 & -2223 & -3062 & -3915 & -2486 & -3300 & -4259 \\
\hline & Vehicle Cost AVG & 0 & 0 & -463 & -963 & -2055 & -2438 & -2998 & -2960 & -3634 & -4318 & -3205 & -3862 & -4632 \\
\hline & Vehicle Cost HIGH & 0 & 0 & -355 & -738 & -2212 & -2493 & -2910 & -2965 & -3419 & -3875 & -3619 & -4000 & -4443 \\
\hline
\end{tabular}




\begin{tabular}{|c|c|c|c|c|c|c|c|c|c|c|c|c|c|c|}
\hline & & \multicolumn{13}{|c|}{ Pickup } \\
\hline & & \multirow{2}{*}{$\frac{2010}{\operatorname{Ref}}$} & \multicolumn{3}{|c|}{2010} & \multicolumn{3}{|c|}{2015} & \multicolumn{3}{|c|}{2030} & \multicolumn{3}{|c|}{2045} \\
\hline & & & low & avg & high & low & avg & high & low & avg & high & low & avg & high \\
\hline \multirow{3}{*}{ CI Split HEV PHEV10 } & Vehicle Cost LOW & 0 & 0 & -631 & -1338 & -3656 & -4357 & -5564 & -5154 & -6326 & -7261 & -6186 & -7001 & -8070 \\
\hline & Vehicle Cost AVG & 0 & 0 & -566 & -1200 & -3880 & -4411 & -5309 & -5301 & $\begin{array}{l}-6179 \\
\end{array}$ & -6876 & -5785 & $\begin{array}{l}-6457 \\
\end{array}$ & -7363 \\
\hline & Vehicle Cost HIGH & 0 & 0 & -457 & -969 & -5217 & -5541 & -6132 & -6139 & -6687 & -7176 & -6958 & -7442 & -7810 \\
\hline \multirow{3}{*}{ CI Split HEV PHEV2O } & Vehicle Cost LOW & 0 & 0 & -916 & -1850 & -5158 & -6043 & -7692 & -7510 & -8994 & -10353 & -9284 & -10438 & -11583 \\
\hline & Vehicle Cost AVG & 0 & 0 & -807 & -1632 & -5559 & -6212 & -7384 & -7769 & $\begin{array}{l}-8783 \\
\end{array}$ & $\begin{array}{l}-9743 \\
\end{array}$ & -8409 & $\begin{array}{l}-9363 \\
\end{array}$ & -10325 \\
\hline & Vehicle Cost HIGH & 0 & 0 & $\begin{array}{l}-644 \\
\end{array}$ & -1303 & -6006 & -6457 & -7418 & -7403 & -8246 & -8979 & -8530 & -9283 & -9800 \\
\hline \multirow{3}{*}{ CI Series HEV PHEV3O } & Vehicle Cost LOW & 0 & 0 & -1773 & -3603 & -9244 & -10886 & -14079 & -14136 & -16874 & -19190 & -17468 & -19655 & -21370 \\
\hline & \begin{tabular}{|l|} 
Vehicle Cost AVG \\
Cehicle Cost
\end{tabular} & 0 & 0 & -1561 & -3172 & -10370 & -11583 & -13871 & -14506 & -16369 & -18004 & -15735 & -17555 & -18997 \\
\hline & Vehicle Cost HIGH & 0 & 0 & -1250 & -2540 & -11192 & -12022 & -13811 & -13907 & -15430 & -16634 & -15805 & -17141 & -17940 \\
\hline \multirow{3}{*}{ CI Series HEV PHEV40 } & Vehicle Cost LOW & 0 & 0 & -2126 & -4312 & -11221 & -13156 & -17055 & -17241 & -20535 & -23278 & -21518 & -24090 & -25991 \\
\hline & Vehicle Cost AVG & 0 & 0 & -1860 & -3772 & -12589 & -14003 & -16770 & -17738 & -19921 & -21795 & -19193 & -21327 & -22919 \\
\hline & Vehicle Cost HIGH & 0 & 0 & -1481 & -3004 & -12449 & -13469 & -15717 & -15786 & -17697 & -19207 & -18087 & -19769 & -20749 \\
\hline \multirow{3}{*}{ H2 Split HEV } & Vehicle Cost LOW & 0 & 0 & -511 & -1619 & -1381 & -2607 & -3202 & -1878 & -3335 & -4132 & $\begin{array}{l}-1957 \\
\end{array}$ & -3370 & -4210 \\
\hline & \begin{tabular}{|l} 
Vehicle Cost AVG \\
\end{tabular} & 0 & 0 & -499 & -1632 & -2817 & -3980 & -4444 & -3716 & -5090 & -5730 & -5312 & -6594 & -7274 \\
\hline & Vehicle Cost HIGH & 0 & 0 & -383 & -1379 & -3200 & -4190 & -4544 & -4026 & -5101 & -5530 & -5055 & -6044 & -6439 \\
\hline \multirow{3}{*}{ H2 Split HEV PHEV10 } & Vehicle Cost LOW & 0 & 0 & -875 & -1622 & -3508 & -4390 & -5633 & -5051 & -6391 & -7288 & -5992 & -6810 & -7844 \\
\hline & Vehicle Cost AVG & 0 & 0 & -789 & -1462 & -4890 & -5557 & -6488 & -6408 & -7437 & -8104 & -8277 & -8959 & -9839 \\
\hline & Vehicle Cost HIGH & 0 & 0 & -645 & -1191 & -6262 & -6639 & $\begin{array}{l}-7194 \\
\end{array}$ & -7348 & -7898 & -8367 & -8606 & $\begin{array}{l}-9047 \\
\end{array}$ & -9395 \\
\hline \multirow{3}{*}{ H2 Split HEV PHEV2O } & Vehicle Cost LOW & 0 & 0 & -1093 & -2175 & -5002 & -6018 & -7723 & -7365 & -8933 & -10310 & -8991 & -10142 & -11262 \\
\hline & \begin{tabular}{|l} 
Vehicle Cost AVG \\
\end{tabular} & 0 & 0 & -969 & -1928 & -6551 & -7296 & -8511 & -8828 & -9909 & -10895 & -10815 & -11773 & -12718 \\
\hline & Vehicle cost HIGH & 0 & 0 & -778 & -1549 & -7055 & -7562 & -8504 & -8597 & -9452 & -10158 & -10167 & -10878 & -11362 \\
\hline \multirow{3}{*}{ H2 Series HEV PHEV 30} & Vehicle Cost LOW & 0 & 0 & -2021 & -3889 & -9004 & -10788 & -13890 & -13783 & -16533 & -18791 & -16946 & -19031 & -20652 \\
\hline & \begin{tabular}{|l} 
Vehicle Cost AVG \\
\end{tabular} & 0 & 0 & -1780 & -3426 & -11217 & -12524 & -14731 & -15320 & -17189 & -18779 & -17894 & -19635 & -21002 \\
\hline & Vehicle Cost HIGH & 0 & 0 & -1430 & -2751 & -12169 & -13056 & -14779 & -14950 & -16456 & -17614 & -17249 & -18519 & -19265 \\
\hline \multirow{3}{*}{ H2 Series HEV PHEV 40} & Vehicle Cost LOW & 0 & 0 & -2499 & -4706 & -11001 & -13115 & -16882 & -16898 & -20190 & -22866 & -20998 & -23434 & -25234 \\
\hline & \begin{tabular}{|l} 
Vehicle Cost AVG \\
\end{tabular} & 0 & 0 & -2186 & -4118 & -13444 & -14972 & -17631 & -18544 & -20722 & -22543 & -21349 & -23376 & -24889 \\
\hline & \begin{tabular}{|l|} 
Vehicle Cost HIGH \\
\end{tabular} & 0 & 0 & -1743 & -3286 & -13440 & -14545 & -16697 & -16835 & -18723 & -20184 & -19540 & -21136 & -22056 \\
\hline \multirow{3}{*}{ E85 Split HEV } & Vehicle Cost LOW & 0 & 0 & -418 & -1379 & -1252 & -2264 & -2914 & -1580 & -2956 & -3786 & -1372 & -2847 & -3738 \\
\hline & Vehicle Cost AVG & 0 & 0 & -408 & -1389 & -1592 & -2569 & $\begin{array}{ll}-3088 \\
\end{array}$ & -2037 & -3355 & -4042 & $\begin{array}{l}-2163 \\
\end{array}$ & -3503 & -4236 \\
\hline & Vehicle Cost HIGH & 0 & 0 & -321 & -1186 & -1840 & -2666 & -3072 & -2360 & -3352 & -3822 & -2827 & -3785 & -4234 \\
\hline
\end{tabular}




\begin{tabular}{|c|c|c|c|c|c|c|c|c|c|c|c|c|c|c|}
\hline & & \multicolumn{13}{|c|}{ Pickup } \\
\hline & & \multirow{2}{*}{$\begin{array}{l}2010 \\
\text { Ref } \\
\end{array}$} & \multicolumn{3}{|c|}{2010} & \multicolumn{3}{|c|}{2015} & \multicolumn{3}{|c|}{2030} & \multicolumn{3}{|c|}{2045} \\
\hline & & & low & avg & high & low & avg & high & low & avg & high & low & avg & high \\
\hline \multirow{3}{*}{ E85 Split HEV PHEV10 } & Vehicle Cost LOW & 0 & 0 & -706 & -1365 & -3309 & -3976 & -5244 & -4733 & -5894 & -6741 & -5400 & -6202 & -7221 \\
\hline & Vehicle Cost AVG & 0 & 0 & -636 & -1229 & -3587 & -4092 & -5045 & -4731 & -5626 & -6251 & -5112 & -5783 & -6654 \\
\hline & Vehicle Cost HIGH & 0 & 0 & -523 & -1006 & -4776 & -5089 & -5671 & -5554 & -6069 & -6561 & -6245 & -6712 & -7092 \\
\hline \multirow{3}{*}{ E85 Split HEV PHEV20 } & Vehicle Cost LOW & 0 & 0 & -840 & -1745 & -4751 & -5594 & -7203 & -6936 & -8361 & -9716 & -8330 & -9485 & -10597 \\
\hline & Vehicle Cost AVG & 0 & 0 & -743 & -1542 & -5205 & -5825 & -6967 & -7058 & -8034 & -9011 & -7594 & -8557 & -9500 \\
\hline & Vehicle Cost HIGH & 0 & 0 & -598 & -1240 & -5511 & -5948 & -6887 & -6730 & -7564 & -8282 & -7731 & -8481 & -8994 \\
\hline \multirow{3}{*}{ E85 Series HEV PHEV30 } & Vehicle Cost LOW & 0 & 0 & -1710 & -3466 & -8821 & -10395 & -13505 & -13498 & -16172 & -18424 & -16413 & -18557 & -20249 \\
\hline & Vehicle Cost AVG & 0 & 0 & -1508 & -3057 & -10000 & -11160 & -13384 & -13712 & -15545 & -17148 & -14828 & -16625 & -18059 \\
\hline & Vehicle Cost HIGH & 0 & 0 & -1215 & -2464 & -10779 & -11581 & -13334 & -13289 & -14791 & -15974 & -15035 & -16361 & -17167 \\
\hline \multirow{3}{*}{ E85 Series HEV PHEV40 } & Vehicle Cost LOW & 0 & 0 & -2086 & -4240 & -10792 & -12642 & -16433 & -16585 & -19775 & -22457 & -20419 & -22928 & -24808 \\
\hline & Vehicle Cost AVG & 0 & 0 & -1826 & -3712 & -12203 & -13551 & -16238 & -16909 & -19038 & -20883 & -18245 & -20340 & -21928 \\
\hline & Vehicle Cost HIGH & 0 & 0 & -1461 & -2971 & -12033 & -13014 & -15211 & -15157 & -17024 & -18516 & -17297 & -18956 & -19943 \\
\hline \multirow{3}{*}{ FC HEV } & Vehicle Cost LOW & 0 & 0 & -3173 & -5343 & -1515 & -4862 & -7091 & -9839 & -11625 & -13145 & -12886 & -13929 & -15050 \\
\hline & Vehicle Cost AVG & 0 & 0 & -4563 & -7816 & $\begin{array}{l}-9839 \\
\end{array}$ & -12796 & -14819 & -15506 & -17576 & -19318 & -20646 & -21653 & -22717 \\
\hline & Vehicle Cost HIGH & 0 & 0 & -3755 & -6443 & -11922 & -13701 & -14963 & -14877 & -16209 & -17335 & -18262 & -18897 & -19565 \\
\hline \multirow{3}{*}{ FC HEV PHEV10 } & Vehicle Cost LOW & 0 & 0 & -3187 & -5239 & -3785 & -6494 & -8926 & -10911 & -13114 & -14919 & -14280 & -15555 & -16957 \\
\hline & Vehicle Cost AVG & 0 & 0 & -4145 & -7069 & -10507 & -12952 & -15110 & -15657 & -17913 & -19785 & -20594 & -21788 & -23089 \\
\hline & Vehicle Cost $\mathrm{HIGH}$ & 0 & 0 & -3424 & -5832 & -12912 & -14364 & -15748 & -15596 & -17055 & -18209 & -18851 & -19623 & -20341 \\
\hline \multirow{3}{*}{ FC HEV PHEV2O } & Vehicle Cost LOW & 0 & 0 & -3833 & -6376 & -5751 & -8892 & -11900 & -14054 & -16727 & -18933 & -18311 & -19901 & -21434 \\
\hline & Vehicle Cost AVG & 0 & 0 & -4728 & -8082 & -12771 & -15520 & -18078 & -18946 & -21477 & -23583 & -24162 & -25617 & -27024 \\
\hline & Vehicle Cost HIGH & 0 & 0 & -3875 & -6614 & -14204 & -15950 & -17729 & -17526 & -19350 & -20809 & -21198 & -22283 & -23153 \\
\hline \multirow{3}{*}{ FC HEV PHEV30 } & Vehicle Cost LOW & 0 & 0 & -3534 & $\begin{array}{ll}-6018 \\
\end{array}$ & -8215 & -11025 & -14561 & -17021 & -20053 & -22448 & -22017 & -24066 & -25677 \\
\hline & Vehicle Cost AVG & 0 & 0 & -4363 & -7570 & -14899 & -17462 & -20530 & -21772 & -24533 & -26780 & -27369 & -29234 & -30730 \\
\hline & Vehicle Cost HIGH & 0 & 0 & $\begin{array}{l}-3561 \\
\end{array}$ & $\begin{array}{l}-6181 \\
\end{array}$ & -17895 & -19500 & -21648 & -21601 & -23601 & -25082 & -25502 & -26873 & -27714 \\
\hline \multirow{3}{*}{ FC HEV PHEV 40} & Vehicle Cost LOW & 0 & 0 & -4480 & -7372 & -10669 & -13883 & -18150 & -20632 & -24263 & -26946 & -26578 & -28961 & -30689 \\
\hline & Vehicle Cost AVG & 0 & 0 & -5209 & -8812 & -17640 & -20498 & -24045 & -25552 & -28666 & -31094 & -31500 & -33651 & -35236 \\
\hline & Vehicle Cost HIGH & 0 & 0 & -4215 & -7144 & -19646 & -21520 & -24142 & -23974 & -26432 & -28128 & -28345 & -30062 & -31011 \\
\hline \multirow{3}{*}{ EV } & Vehicle Cost LOW & 0 & 0 & -5802 & -11779 & -31189 & -36110 & -48269 & -48523 & -58001 & -64657 & -62897 & -69125 & -72403 \\
\hline & Vehicle Cost AVG & 0 & 0 & -4902 & -9953 & -34150 & -37536 & -45791 & -49811 & -55194 & -59019 & -53497 & -58588 & -61286 \\
\hline & Vehicle Cost HIGH & 0 & 0 & -3810 & -7738 & -23385 & -26400 & -34211 & -43316 & -46737 & -49116 & -44108 & -47955 & -49793 \\
\hline
\end{tabular}




\section{Percentage decrease cost per powertrain}

\begin{tabular}{|c|c|c|c|c|c|c|c|c|c|c|c|c|c|c|}
\hline & & \multicolumn{13}{|c|}{ Pickup } \\
\hline & & \multirow{2}{*}{$\begin{array}{l}2010 \\
\text { Ref } \\
\end{array}$} & \multicolumn{3}{|c|}{2010} & \multicolumn{3}{|c|}{2015} & \multicolumn{3}{|c|}{2030} & \multicolumn{3}{|c|}{2045} \\
\hline & & & low & avg & high & low & avg & high & low & avg & high & low & avg & high \\
\hline \multirow{3}{*}{ SI Conv } & Vehicle Cost LOW & $0.0 \%$ & $0.0 \%$ & $0.4 \%$ & $0.9 \%$ & $0.0 \%$ & $0.6 \%$ & $1.3 \%$ & $-4.8 \%$ & $-3.8 \%$ & $-2.9 \%$ & $-7.4 \%$ & $-6.3 \%$ & $-5.1 \%$ \\
\hline & Vehicle Cost AVG & $-0.2 \%$ & $-0.2 \%$ & $0.1 \%$ & $0.7 \%$ & $-4.9 \%$ & $-4.3 \%$ & $-3.6 \%$ & $-7.3 \%$ & $-6.2 \%$ & $-5.2 \%$ & $-7.2 \%$ & $-6.2 \%$ & $-5.0 \%$ \\
\hline & Vehicle Cost HIGH & $-3.8 \%$ & $-3.8 \%$ & $-3.4 \%$ & $-2.9 \%$ & $-4.6 \%$ & $-4.0 \%$ & $-3.3 \%$ & $-7.1 \%$ & $-6.1 \%$ & $-5.1 \%$ & $-7.0 \%$ & $-6.0 \%$ & $-4.8 \%$ \\
\hline \multirow{3}{*}{$\mathrm{Cl}$ Conv } & Vehicle Cost LOW & $-14.7 \%$ & $-14.7 \%$ & $-14.1 \%$ & $-13.6 \%$ & $-13.2 \%$ & $-12.5 \%$ & $-5.2 \%$ & $-17.6 \%$ & $-9.6 \%$ & $-8.6 \%$ & $-17.4 \%$ & $-9.5 \%$ & $-8.4 \%$ \\
\hline & Vehicle Cost AVG & $-14.5 \%$ & $-14.5 \%$ & $-13.9 \%$ & $-13.4 \%$ & $-18.5 \%$ & $-17.9 \%$ & $-10.0 \%$ & $-18.2 \%$ & $-10.0 \%$ & $-9.2 \%$ & $-17.6 \%$ & $-9.5 \%$ & $-8.6 \%$ \\
\hline & Vehicle Cost HIGH & $-15.8 \%$ & $-15.8 \%$ & $-15.3 \%$ & $-14.8 \%$ & $-15.4 \%$ & $-15.0 \%$ & $-7.8 \%$ & $-17.0 \%$ & $-9.8 \%$ & $-9.0 \%$ & $-15.9 \%$ & $-8.9 \%$ & $-8.1 \%$ \\
\hline \multirow{3}{*}{ H2 Conv } & Vehicle Cost LOW & $5.3 \%$ & $5.3 \%$ & $5.5 \%$ & $5.9 \%$ & $5.8 \%$ & $6.2 \%$ & $6.6 \%$ & $1.6 \%$ & $2.2 \%$ & $6.7 \%$ & $0.5 \%$ & $0.9 \%$ & $5.6 \%$ \\
\hline & Vehicle Cost AVG & $-12.5 \%$ & $-12.5 \%$ & $-12.2 \%$ & $-11.9 \%$ & $-10.6 \%$ & $-10.2 \%$ & $-9.8 \%$ & $-10.3 \%$ & $-9.7 \%$ & $-4.9 \%$ & $-2.0 \%$ & $-1.6 \%$ & $3.1 \%$ \\
\hline & Vehicle Cost HIGH & $-12.8 \%$ & $-12.8 \%$ & $-12.5 \%$ & $-12.2 \%$ & $-6.0 \%$ & $-5.6 \%$ & $-5.2 \%$ & $-7.0 \%$ & $-6.3 \%$ & $-2.0 \%$ & $-3.7 \%$ & $-3.3 \%$ & $1.1 \%$ \\
\hline \multirow{3}{*}{ E85 Conv } & Vehicle Cost LOW & $0.2 \%$ & $0.2 \%$ & $0.6 \%$ & $1.2 \%$ & $0.2 \%$ & $0.8 \%$ & $1.5 \%$ & $-4.6 \%$ & $-3.7 \%$ & $-2.7 \%$ & $-7.2 \%$ & $-6.2 \%$ & $-5.0 \%$ \\
\hline & Vehicle Cost AVG & $-0.1 \%$ & $-0.1 \%$ & $0.4 \%$ & $0.9 \%$ & $-4.7 \%$ & $-4.2 \%$ & $-3.5 \%$ & $-7.1 \%$ & $-6.1 \%$ & $-5.0 \%$ & $-7.0 \%$ & $-6.1 \%$ & $-4.9 \%$ \\
\hline & Vehicle Cost HIGH & $-3.6 \%$ & $-3.6 \%$ & $-3.2 \%$ & $-2.6 \%$ & $-4.4 \%$ & $-3.8 \%$ & $-3.1 \%$ & $-6.9 \%$ & $-5.9 \%$ & $-4.9 \%$ & $-6.8 \%$ & $-5.8 \%$ & $-4.7 \%$ \\
\hline \multirow{3}{*}{ SI Split HEV } & Vehicle Cost LOW & $-34.7 \%$ & $-34.7 \%$ & $-32.2 \%$ & $-26.9 \%$ & $-27.7 \%$ & $-21.7 \%$ & $-18.1 \%$ & $-25.7 \%$ & $-18.1 \%$ & $-13.1 \%$ & $-26.9 \%$ & $-18.4 \%$ & $-13.0 \%$ \\
\hline & Vehicle Cost AVG & $-33.8 \%$ & $-33.8 \%$ & $-31.3 \%$ & $-25.9 \%$ & $-24.9 \%$ & $-19.0 \%$ & $-16.2 \%$ & $-22.2 \%$ & $-14.9 \%$ & $-10.7 \%$ & $-21.4 \%$ & $-13.7 \%$ & $-9.3 \%$ \\
\hline & Vehicle Cost HIGH & $-27.3 \%$ & $-27.3 \%$ & $-25.4 \%$ & $-20.6 \%$ & $-16.9 \%$ & $-12.0 \%$ & $-9.8 \%$ & $-13.8 \%$ & $-8.3 \%$ & $-5.4 \%$ & $-11.1 \%$ & $-5.6 \%$ & $-2.9 \%$ \\
\hline \multirow{3}{*}{ SI Split HEV PHEV10 } & Vehicle Cost LOW & $-60.8 \%$ & $-60.8 \%$ & $-57.1 \%$ & $-53.1 \%$ & $-41.4 \%$ & $-37.6 \%$ & $-30.6 \%$ & $-33.5 \%$ & $-26.8 \%$ & $-21.8 \%$ & $-29.6 \%$ & $-25.0 \%$ & $-19.0 \%$ \\
\hline & Vehicle Cost AVG & $-53.9 \%$ & $-53.9 \%$ & $-50.6 \%$ & $-47.0 \%$ & $-33.0 \%$ & $-30.0 \%$ & $-24.8 \%$ & $-26.6 \%$ & $-21.4 \%$ & $-17.7 \%$ & $-24.4 \%$ & $-20.5 \%$ & $-15.4 \%$ \\
\hline & Vehicle Cost HIGH & $-45.3 \%$ & $-45.3 \%$ & $-42.7 \%$ & $-39.7 \%$ & $-17.7 \%$ & $-15.9 \%$ & $-12.6 \%$ & $-13.3 \%$ & $-10.3 \%$ & $-7.4 \%$ & $-9.3 \%$ & $-6.5 \%$ & $-4.3 \%$ \\
\hline \multirow{3}{*}{ SI Split HEV PHEV 20} & Vehicle Cost LOW & $-82.6 \%$ & $-82.6 \%$ & $-77.7 \%$ & $-72.5 \%$ & $-55.0 \%$ & $-50.1 \%$ & $-40.8 \%$ & $-42.3 \%$ & $-34.0 \%$ & $-26.1 \%$ & $-33.9 \%$ & $-27.5 \%$ & $-21.0 \%$ \\
\hline & Vehicle Cost AVG & $-72.2 \%$ & $-72.2 \%$ & $-67.8 \%$ & $-63.2 \%$ & $-42.0 \%$ & $-38.3 \%$ & $-31.7 \%$ & $-31.2 \%$ & $-25.5 \%$ & $-19.8 \%$ & $-27.8 \%$ & $-22.5 \%$ & $-17.0 \%$ \\
\hline & Vehicle Cost HIGH & $-59.3 \%$ & $-59.3 \%$ & $-55.8 \%$ & $-52.1 \%$ & $-27.3 \%$ & $-24.7 \%$ & $-19.3 \%$ & $-20.2 \%$ & $-15.3 \%$ & $-11.2 \%$ & $-14.3 \%$ & $-10.0 \%$ & $-7.0 \%$ \\
\hline \multirow{3}{*}{ SI Series HEV PHEV30 } & Vehicle Cost LOW & $-159.8 \%$ & $-159.8 \%$ & $-149.9 \%$ & $-139.6 \%$ & $-108.5 \%$ & $-99.2 \%$ & $-81.3 \%$ & $-81.2 \%$ & $-65.8 \%$ & $-52.7 \%$ & $-64.4 \%$ & $-51.9 \%$ & $-42.0 \%$ \\
\hline & Vehicle Cost AVG & $-139.9 \%$ & $-139.9 \%$ & $-131.1 \%$ & $-122.0 \%$ & $-81.7 \%$ & $-74.9 \%$ & $-62.0 \%$ & $-60.1 \%$ & $-49.5 \%$ & $-40.2 \%$ & $-53.7 \%$ & $-43.2 \%$ & $-34.8 \%$ \\
\hline & Vehicle Cost HIGH & $-114.2 \%$ & $-114.2 \%$ & $-107.1 \%$ & $-99.8 \%$ & $-51.5 \%$ & $-46.8 \%$ & $-36.7 \%$ & $-36.9 \%$ & $-28.2 \%$ & $-21.3 \%$ & $-26.8 \%$ & $-19.1 \%$ & $-14.4 \%$ \\
\hline \multirow{3}{*}{ SI Series HEV PHEV40 } & Vehicle Cost LOW & $-190.0 \%$ & $-190.0 \%$ & $-177.7 \%$ & $-165.2 \%$ & $-127.2 \%$ & $-116.4 \%$ & $-94.4 \%$ & $-93.5 \%$ & $-74.9 \%$ & $-59.4 \%$ & $-71.2 \%$ & $-56.6 \%$ & $-45.7 \%$ \\
\hline & Vehicle Cost AVG & $-165.3 \%$ & $-165.3 \%$ & $-154.6 \%$ & $-143.7 \%$ & $-94.4 \%$ & $-86.5 \%$ & $-70.9 \%$ & $-67.0 \%$ & $-54.6 \%$ & $-43.9 \%$ & $-59.2 \%$ & $-47.1 \%$ & $-37.8 \%$ \\
\hline & Vehicle Cost HIGH & $-133.8 \%$ & $-133.8 \%$ & $-125.2 \%$ & $-116.5 \%$ & $-63.9 \%$ & $-58.1 \%$ & $-45.4 \%$ & $-45.7 \%$ & $-34.8 \%$ & $-26.2 \%$ & $-33.3 \%$ & $-23.6 \%$ & $-17.9 \%$ \\
\hline \multirow{3}{*}{ CI Split HEV } & Vehicle Cost LOW & $-47.8 \%$ & $-47.8 \%$ & $-45.0 \%$ & $-42.0 \%$ & $-38.2 \%$ & $-35.4 \%$ & $-31.2 \%$ & $-34.8 \%$ & $-30.0 \%$ & $-25.0 \%$ & $-33.3 \%$ & $-28.6 \%$ & $-23.0 \%$ \\
\hline & Vehicle Cost AVG & $-46.2 \%$ & $-46.2 \%$ & $-43.5 \%$ & $-40.6 \%$ & $-34.2 \%$ & $-32.0 \%$ & $-28.8 \%$ & $-29.0 \%$ & $-25.1 \%$ & $-21.1 \%$ & $-27.5 \%$ & $-23.7 \%$ & $-19.2 \%$ \\
\hline & Vehicle Cost HIGH & $-37.0 \%$ & $-37.0 \%$ & $-34.9 \%$ & $-32.7 \%$ & $-24.1 \%$ & $-22.5 \%$ & $-20.1 \%$ & $-19.8 \%$ & $-17.1 \%$ & $-14.5 \%$ & $-16.0 \%$ & $-13.7 \%$ & $-11.2 \%$ \\
\hline
\end{tabular}


Argonne National Laboratory Report - Light-Duty Vehicle Fuel Consumption Displacement Potential Up to 2045

\begin{tabular}{|c|c|c|c|c|c|c|c|c|c|c|c|c|c|c|}
\hline & & \multicolumn{13}{|c|}{ Pickup } \\
\hline & & \multirow{2}{*}{$\begin{array}{c}2010 \\
\text { Ref } \\
\end{array}$} & \multicolumn{3}{|c|}{2010} & \multicolumn{3}{|c|}{2015} & \multicolumn{3}{|c|}{2030} & \multicolumn{3}{|c|}{2045} \\
\hline & & & low & avg & high & low & avg & high & low & avg & high & low & avg & high \\
\hline \multirow{3}{*}{ CI Split HEV PHEV10 } & Vehicle Cost LOW & $-76.5 \%$ & $-76.5 \%$ & $-72.8 \%$ & $-68.7 \%$ & $-55.2 \%$ & $-51.2 \%$ & $-44.1 \%$ & $-46.5 \%$ & $-39.7 \%$ & $-34.3 \%$ & $-40.5 \%$ & $-35.8 \%$ & $-29.6 \%$ \\
\hline & Vehicle Cost AVG & $-69.0 \%$ & $-69.0 \%$ & $-65.7 \%$ & $-62.0 \%$ & $-46.4 \%$ & $-43.3 \%$ & $-38.1 \%$ & $-38.1 \%$ & $-33.0 \%$ & $-29.0 \%$ & $-35.3 \%$ & $-31.4 \%$ & $-26.1 \%$ \\
\hline & Vehicle Cost HIGH & $-58.3 \%$ & $-58.3 \%$ & $-55.7 \%$ & $-52.7 \%$ & $-28.0 \%$ & $-26.1 \%$ & $-22.7 \%$ & $-22.6 \%$ & $-19.4 \%$ & $-16.6 \%$ & $-17.8 \%$ & $-15.0 \%$ & $-12.9 \%$ \\
\hline \multirow{3}{*}{ CI Split HEV PHEV20 } & Vehicle Cost LOW & $-99.1 \%$ & $-99.1 \%$ & $-93.7 \%$ & $-88.3 \%$ & $-69.0 \%$ & $-63.9 \%$ & $-54.3 \%$ & $-55.4 \%$ & $-46.7 \%$ & $-38.8 \%$ & $-45.0 \%$ & $-38.3 \%$ & $-31.7 \%$ \\
\hline & Vehicle Cost AVG & $-87.8 \%$ & $-87.8 \%$ & $-83.1 \%$ & $-78.3 \%$ & $-55.5 \%$ & $-51.7 \%$ & $-44.9 \%$ & $-42.6 \%$ & $-36.7 \%$ & $-31.2 \%$ & $-38.9 \%$ & $-33.4 \%$ & $-27.8 \%$ \\
\hline & Vehicle Cost HIGH & $-72.7 \%$ & $-72.7 \%$ & $-68.9 \%$ & $-65.1 \%$ & $-37.7 \%$ & $-35.1 \%$ & $-29.5 \%$ & $-29.6 \%$ & $-24.7 \%$ & $-20.5 \%$ & $-23.1 \%$ & $-18.7 \%$ & $-15.7 \%$ \\
\hline \multirow{3}{*}{ CI Series HEV PHEV 30} & Vehicle Cost LOW & $-176.5 \%$ & $-176.5 \%$ & $-166.1 \%$ & $-155.5 \%$ & $-122.7 \%$ & $-113.1 \%$ & $-94.5 \%$ & $-94.2 \%$ & $-78.3 \%$ & $-64.8 \%$ & $-74.8 \%$ & $-62.1 \%$ & $-52.1 \%$ \\
\hline & Vehicle Cost AVG & $-155.6 \%$ & $-155.6 \%$ & $-146.5 \%$ & $-137.2 \%$ & $-95.3 \%$ & $-88.2 \%$ & $-74.9 \%$ & $-71.2 \%$ & $-60.4 \%$ & $-50.9 \%$ & $-64.1 \%$ & $-53.5 \%$ & $-45.1 \%$ \\
\hline & Vehicle Cost HIGH & $-127.5 \%$ & $-127.5 \%$ & $-120.2 \%$ & $-112.7 \%$ & $-62.3 \%$ & $-57.5 \%$ & $-47.1 \%$ & $-46.6 \%$ & $-37.7 \%$ & $-30.7 \%$ & $-35.5 \%$ & $-27.7 \%$ & $-23.1 \%$ \\
\hline \multirow{3}{*}{ CI Series HEV PHEV40 } & Vehicle Cost LOW & $-207.1 \%$ & $-207.1 \%$ & $-194.8 \%$ & $-182.0 \%$ & $-141.8 \%$ & $-130.6 \%$ & $-107.9 \%$ & $-106.8 \%$ & $-87.6 \%$ & $-71.7 \%$ & $-81.9 \%$ & $-67.0 \%$ & $-55.9 \%$ \\
\hline & Vehicle Cost AVG & $-181.5 \%$ & $-181.5 \%$ & $-170.7 \%$ & $-159.6 \%$ & $-108.3 \%$ & $-100.1 \%$ & $-84.0 \%$ & $-78.3 \%$ & $-65.6 \%$ & $-54.7 \%$ & $-69.9 \%$ & $-57.4 \%$ & $-48.2 \%$ \\
\hline & Vehicle Cost HIGH & $-147.4 \%$ & $-147.4 \%$ & $-138.8 \%$ & $-129.9 \%$ & $-75.0 \%$ & $-69.0 \%$ & $-56.0 \%$ & $-55.6 \%$ & $-44.4 \%$ & $-35.7 \%$ & $-42.2 \%$ & $-32.4 \%$ & $-26.7 \%$ \\
\hline \multirow{3}{*}{ H2 Split HEV } & Vehicle Cost LOW & $-33.1 \%$ & $-33.1 \%$ & $-30.2 \%$ & $-23.7 \%$ & $-25.1 \%$ & $-18.0 \%$ & $-14.5 \%$ & $-22.2 \%$ & $-13.7 \%$ & $-9.1 \%$ & $-21.7 \%$ & $-13.5 \%$ & $-8.6 \%$ \\
\hline & \begin{tabular}{|l|} 
Vehicle Cost AVG \\
\end{tabular} & $-49.8 \%$ & $-49.8 \%$ & $-46.9 \%$ & $-40.3 \%$ & $-33.4 \%$ & $-26.6 \%$ & $-23.9 \%$ & $-28.1 \%$ & $-20.2 \%$ & $-16.4 \%$ & $-18.9 \%$ & $-11.4 \%$ & $-7.4 \%$ \\
\hline & Vehicle Cost HIGH & $-39.7 \%$ & $-39.7 \%$ & $-37.5 \%$ & $-31.7 \%$ & $-21.1 \%$ & $-15.3 \%$ & $-13.3 \%$ & $-16.3 \%$ & $-10.0 \%$ & $-7.5 \%$ & $-10.3 \%$ & $-4.5 \%$ & $-2.2 \%$ \\
\hline \multirow{3}{*}{ H2 Split HEV PHEV10 } & Vehicle Cost LOW & $-60.8 \%$ & $-60.8 \%$ & $-55.7 \%$ & $-51.3 \%$ & $-40.3 \%$ & $-35.2 \%$ & $-28.0 \%$ & $-31.4 \%$ & $-23.6 \%$ & $-18.4 \%$ & $-25.9 \%$ & $-21.1 \%$ & $-15.1 \%$ \\
\hline & Vehicle Cost AVG & $-71.3 \%$ & $-71.3 \%$ & $-66.7 \%$ & $-62.8 \%$ & $-42.8 \%$ & $-38.9 \%$ & $-33.5 \%$ & $-34.0 \%$ & $-28.0 \%$ & $-24.1 \%$ & $-23.1 \%$ & $-19.1 \%$ & $-14.0 \%$ \\
\hline & Vehicle Cost HIGH & $-59.0 \%$ & $-59.0 \%$ & $-55.3 \%$ & $-52.1 \%$ & $-22.6 \%$ & $-20.4 \%$ & $-17.1 \%$ & $-16.2 \%$ & $-13.0 \%$ & $-10.3 \%$ & $-8.9 \%$ & $-6.4 \%$ & $-4.3 \%$ \\
\hline \multirow{3}{*}{ H2 Split HEV PHEV2O } & Vehicle Cost LOW & $-82.8 \%$ & $-82.8 \%$ & $-76.5 \%$ & $-70.2 \%$ & $-53.7 \%$ & $-47.8 \%$ & $-37.9 \%$ & $-40.0 \%$ & $-30.8 \%$ & $-22.8 \%$ & $-30.5 \%$ & $-23.8 \%$ & $-17.3 \%$ \\
\hline & Vehicle Cost AVG & $-89.7 \%$ & $-89.7 \%$ & $-84.1 \%$ & $-78.5 \%$ & $-51.6 \%$ & $-47.3 \%$ & $-40.2 \%$ & $-38.4 \%$ & $-32.1 \%$ & $-26.3 \%$ & $-26.8 \%$ & $-21.2 \%$ & $-15.7 \%$ \\
\hline & Vehicle Cost HIGH & $-73.1 \%$ & $-73.1 \%$ & $-68.6 \%$ & $-64.1 \%$ & $-32.0 \%$ & $-29.1 \%$ & $-23.6 \%$ & $-23.1 \%$ & $-18.1 \%$ & $-14.0 \%$ & $-13.9 \%$ & $-9.8 \%$ & $-7.0 \%$ \\
\hline \multirow{3}{*}{ H2 Series HEV PHEV 30} & Vehicle Cost LOW & $-157.4 \%$ & $-157.4 \%$ & $-145.7 \%$ & $-134.8 \%$ & $-105.0 \%$ & $-94.7 \%$ & $-76.6 \%$ & $-77.2 \%$ & $-61.2 \%$ & $-48.1 \%$ & $-58.8 \%$ & $-46.7 \%$ & $-37.3 \%$ \\
\hline & Vehicle Cost AVG & $-154.9 \%$ & $-154.9 \%$ & $-144.6 \%$ & $-135.0 \%$ & $-89.7 \%$ & $-82.1 \%$ & $-69.2 \%$ & $-65.8 \%$ & $-54.9 \%$ & $-45.7 \%$ & $-50.8 \%$ & $-40.7 \%$ & $-32.7 \%$ \\
\hline & Vehicle Cost HIGH & $-125.9 \%$ & $-125.9 \%$ & $-117.6 \%$ & $-109.9 \%$ & $-55.1 \%$ & $-49.9 \%$ & $-39.9 \%$ & $-38.9 \%$ & $-30.2 \%$ & $-23.4 \%$ & $-25.5 \%$ & $-18.2 \%$ & $-13.8 \%$ \\
\hline \multirow{3}{*}{ H2 Series HEV PHEV40 } & Vehicle Cost LOW & $-187.7 \%$ & $-187.7 \%$ & $-173.1 \%$ & $-160.3 \%$ & $-123.7 \%$ & $-111.4 \%$ & $-89.5 \%$ & $-89.4 \%$ & $-70.2 \%$ & $-54.6 \%$ & $-65.5 \%$ & $-51.3 \%$ & $-40.9 \%$ \\
\hline & Vehicle Cost AVG & $-180.5 \%$ & $-180.5 \%$ & $-167.8 \%$ & $-156.5 \%$ & $-102.3 \%$ & $-93.4 \%$ & $-77.9 \%$ & $-72.6 \%$ & $-59.9 \%$ & $-49.3 \%$ & $-56.3 \%$ & $-44.5 \%$ & $-35.7 \%$ \\
\hline & Vehicle Cost HIGH & $-145.6 \%$ & $-145.6 \%$ & $-135.4 \%$ & $-126.5 \%$ & $-67.4 \%$ & $-60.9 \%$ & $-48.4 \%$ & $-47.6 \%$ & $-36.6 \%$ & $-28.1 \%$ & $-31.9 \%$ & $-22.6 \%$ & $-17.2 \%$ \\
\hline \multirow{3}{*}{ E85 Split HEV } & Vehicle Cost LOW & $-35.2 \%$ & $-35.2 \%$ & $-32.8 \%$ & $-27.2 \%$ & $-28.0 \%$ & $-22.1 \%$ & $-18.3 \%$ & $-26.1 \%$ & $-18.0 \%$ & $-13.2 \%$ & $-27.3 \%$ & $-18.7 \%$ & $-13.5 \%$ \\
\hline & Vehicle Cost AVG & $-34.3 \%$ & $-34.3 \%$ & $-32.0 \%$ & $-26.3 \%$ & $-25.1 \%$ & $-19.4 \%$ & $-16.4 \%$ & $-22.5 \%$ & $-14.8 \%$ & $-10.8 \%$ & $-21.8 \%$ & $-14.0 \%$ & $-9.7 \%$ \\
\hline & Vehicle Cost HIGH & $-27.8 \%$ & $-27.8 \%$ & $-25.9 \%$ & $-20.9 \%$ & $-17.1 \%$ & $-12.3 \%$ & $-9.9 \%$ & $-14.1 \%$ & $-8.3 \%$ & $-5.6 \%$ & $-11.3 \%$ & $-5.8 \%$ & $-3.2 \%$ \\
\hline
\end{tabular}


Argonne National Laboratory Report - Light-Duty Vehicle Fuel Consumption Displacement Potential Up to 2045

\begin{tabular}{|c|c|c|c|c|c|c|c|c|c|c|c|c|c|c|}
\hline & & \multicolumn{13}{|c|}{ Pickup } \\
\hline & & \multirow{2}{*}{$\frac{2010}{\text { Ref }}$} & \multicolumn{3}{|c|}{2010} & \multicolumn{3}{|c|}{2015} & \multicolumn{3}{|c|}{2030} & \multicolumn{3}{|c|}{2045} \\
\hline & & & low & avg & high & low & avg & high & low & avg & high & low & avg & high \\
\hline \multirow{3}{*}{ E85 Split HEV PHEV10 } & Vehicle Cost LOW & $-61.2 \%$ & $-61.2 \%$ & $-57.1 \%$ & $-53.3 \%$ & $-42.0 \%$ & $-38.1 \%$ & $-30.7 \%$ & $-33.7 \%$ & $-26.9 \%$ & $-22.0 \%$ & $-29.8 \%$ & $-25.1 \%$ & $-19.2 \%$ \\
\hline & \begin{tabular}{|l|} 
Vehicle Cost AVG \\
\end{tabular} & $-54.3 \%$ & $-54.3 \%$ & $-50.6 \%$ & $-47.1 \%$ & $-33.4 \%$ & $-30.5 \%$ & $-24.9 \%$ & $-26.8 \%$ & $-21.6 \%$ & $-17.9 \%$ & $-24.5 \%$ & $-20.6 \%$ & $-15.6 \%$ \\
\hline & \begin{tabular}{|l|} 
Vehicle Cost HIGH \\
\end{tabular} & $-45.7 \%$ & $-45.7 \%$ & $-42.7 \%$ & $-39.9 \%$ & $-17.9 \%$ & $-16.1 \%$ & $-12.7 \%$ & $-13.4 \%$ & $-10.4 \%$ & $-7.5 \%$ & $-9.4 \%$ & $-6.7 \%$ & $-4.4 \%$ \\
\hline \multirow{3}{*}{ E85 Split HEV PHEV20 } & Vehicle Cost LOW & $-82.8 \%$ & $-82.8 \%$ & $-77.9 \%$ & $-72.7 \%$ & $-55.2 \%$ & $-50.3 \%$ & $-40.9 \%$ & $-42.5 \%$ & $-34.2 \%$ & $-26.3 \%$ & $-34.4 \%$ & $-27.6 \%$ & $-21.2 \%$ \\
\hline & \begin{tabular}{|l|} 
Vehicle Cost AVG \\
\end{tabular} & $-72.4 \%$ & $-72.4 \%$ & $-68.1 \%$ & $-63.4 \%$ & $-42.1 \%$ & $-38.5 \%$ & $-31.9 \%$ & $-31.3 \%$ & $-25.7 \%$ & $-20.0 \%$ & $-28.2 \%$ & $-22.6 \%$ & $-17.1 \%$ \\
\hline & Vehicle Cost HIGH & $-59.5 \%$ & $-59.5 \%$ & $-56.0 \%$ & $-52.3 \%$ & $-27.4 \%$ & $-24.9 \%$ & $-19.4 \%$ & $-20.3 \%$ & $-15.5 \%$ & $-11.3 \%$ & $-14.5 \%$ & $-10.1 \%$ & $-7.1 \%$ \\
\hline \multirow{3}{*}{ E85 Series HEV PHEV30 } & Vehicle Cost LOW & $-160.0 \%$ & $-160.0 \%$ & $-150.1 \%$ & $-139.9 \%$ & $-108.7 \%$ & $-99.6 \%$ & $-81.5 \%$ & $-81.5 \%$ & $-65.9 \%$ & $-52.8 \%$ & $-64.5 \%$ & $-52.1 \%$ & $-42.2 \%$ \\
\hline & \begin{tabular}{|l|} 
Vehicle Cost AVG \\
\end{tabular} & $-140.1 \%$ & $-140.1 \%$ & $-131.3 \%$ & $-122.3 \%$ & $-81.9 \%$ & $-75.2 \%$ & $-62.2 \%$ & $-60.3 \%$ & $-49.6 \%$ & $-40.3 \%$ & $-53.8 \%$ & $-43.4 \%$ & $-35.0 \%$ \\
\hline & Vehicle Cost HIGH & $-114.4 \%$ & $-114.4 \%$ & $-107.3 \%$ & $-100.1 \%$ & $-51.7 \%$ & $-47.0 \%$ & $-36.8 \%$ & $-37.1 \%$ & $-28.3 \%$ & $-21.4 \%$ & $-26.9 \%$ & $-19.2 \%$ & $-14.5 \%$ \\
\hline \multirow{3}{*}{ E85 Series HEV PHEV40 } & Vehicle Cost LOW & $-190.2 \%$ & $-190.2 \%$ & $-178.1 \%$ & $-165.6 \%$ & $-127.4 \%$ & $-116.7 \%$ & $-94.6 \%$ & $-93.7 \%$ & $-75.2 \%$ & $-59.6 \%$ & $-71.4 \%$ & $-56.8 \%$ & $-45.9 \%$ \\
\hline & \begin{tabular}{|l|} 
Vehicle Cost AVG \\
\end{tabular} & $-165.6 \%$ & $-165.6 \%$ & $-155.0 \%$ & $-144.0 \%$ & $-94.6 \%$ & $-86.7 \%$ & $-71.1 \%$ & $-67.2 \%$ & $-54.8 \%$ & $-44.1 \%$ & $-59.4 \%$ & $-47.2 \%$ & $-38.0 \%$ \\
\hline & Vehicle Cost HIGH & $-134.0 \%$ & $-134.0 \%$ & $-125.5 \%$ & $-116.8 \%$ & $-64.0 \%$ & $-58.3 \%$ & $-45.5 \%$ & $-45.8 \%$ & $-35.0 \%$ & $-26.3 \%$ & $-33.4 \%$ & $-23.7 \%$ & $-18.0 \%$ \\
\hline \multirow{3}{*}{ FC HEV } & Vehicle Cost LOW & $-92.2 \%$ & $-92.2 \%$ & $-73.8 \%$ & $-61.1 \%$ & $-83.4 \%$ & $-63.9 \%$ & $-51.0 \%$ & $-35.0 \%$ & $-24.6 \%$ & $-15.8 \%$ & $-17.3 \%$ & $-11.2 \%$ & $-4.7 \%$ \\
\hline & \begin{tabular}{|l|} 
Vehicle Cost AVG \\
\end{tabular} & $-136.6 \%$ & $-136.6 \%$ & $-110.0 \%$ & $-91.1 \%$ & $-79.3 \%$ & $-62.1 \%$ & $-50.3 \%$ & $-46.3 \%$ & $-34.3 \%$ & $-24.2 \%$ & $-16.4 \%$ & $-10.6 \%$ & $-4.4 \%$ \\
\hline & Vehicle Cost HIGH & $-112.4 \%$ & $-112.4 \%$ & $-90.5 \%$ & $-74.9 \%$ & $-43.0 \%$ & $-32.6 \%$ & $-25.3 \%$ & $-25.8 \%$ & $-18.0 \%$ & $-11.5 \%$ & $-6.1 \%$ & $-2.4 \%$ & $1.5 \%$ \\
\hline \multirow{3}{*}{ FC HEV PHEV10 } & Vehicle Cost LOW & $-108.2 \%$ & $-108.2 \%$ & $-89.7 \%$ & $-77.7 \%$ & $-86.2 \%$ & $-70.4 \%$ & $-56.3 \%$ & $-44.7 \%$ & $-31.9 \%$ & $-21.4 \%$ & $-25.1 \%$ & $-17.7 \%$ & $-9.5 \%$ \\
\hline & \begin{tabular}{|l|} 
Vehicle Cost AVG \\
\end{tabular} & $-143.0 \%$ & $-143.0 \%$ & $-118.8 \%$ & $-101.8 \%$ & $-81.8 \%$ & $-67.6 \%$ & $-55.0 \%$ & $-51.9 \%$ & $-38.7 \%$ & $-27.8 \%$ & $-23.1 \%$ & $-16.2 \%$ & $-8.6 \%$ \\
\hline & Vehicle Cost HIGH & $-117.5 \%$ & $-117.5 \%$ & $-97.6 \%$ & $-83.6 \%$ & $-42.4 \%$ & $-33.9 \%$ & $-25.9 \%$ & $-26.8 \%$ & $-18.3 \%$ & $-11.6 \%$ & $-7.8 \%$ & $-3.3 \%$ & $0.8 \%$ \\
\hline \multirow{3}{*}{ FC HEV PHEV 20} & Vehicle Cost LOW & $-136.9 \%$ & $-136.9 \%$ & $-114.6 \%$ & $-99.8 \%$ & $-103.5 \%$ & $-85.2 \%$ & $-67.7 \%$ & $-55.1 \%$ & $-39.6 \%$ & $-26.8 \%$ & $-30.4 \%$ & $-21.1 \%$ & $-12.2 \%$ \\
\hline & Vehicle Cost AVG & $-168.0 \%$ & $-168.0 \%$ & $-140.5 \%$ & $-121.0 \%$ & $-93.7 \%$ & $-77.7 \%$ & $-62.9 \%$ & $-57.8 \%$ & $-43.1 \%$ & $-30.8 \%$ & $-27.5 \%$ & $-19.0 \%$ & $-10.8 \%$ \\
\hline & \begin{tabular}{|l|} 
Vehicle Cost HIGH \\
\end{tabular} & $-136.8 \%$ & $-136.8 \%$ & $-114.3 \%$ & $-98.3 \%$ & $-54.2 \%$ & $-44.0 \%$ & $-33.7 \%$ & $-34.8 \%$ & $-24.2 \%$ & $-15.7 \%$ & $-13.5 \%$ & $-7.2 \%$ & $-2.1 \%$ \\
\hline \multirow{3}{*}{ FC HEV PHEV 30} & Vehicle Cost LOW & $-178.2 \%$ & $-178.2 \%$ & $-157.7 \%$ & $-143.2 \%$ & $-130.4 \%$ & $-114.1 \%$ & $-93.5 \%$ & $-79.2 \%$ & $-61.5 \%$ & $-47.6 \%$ & $-50.1 \%$ & $-38.2 \%$ & $-28.8 \%$ \\
\hline & \begin{tabular}{|l|} 
Vehicle Cost AVG \\
\end{tabular} & $-204.3 \%$ & $-204.3 \%$ & $-178.9 \%$ & $-160.3 \%$ & $-117.6 \%$ & $-102.7 \%$ & $-84.9 \%$ & $-77.6 \%$ & $-61.6 \%$ & $-48.5 \%$ & $-45.1 \%$ & $-34.2 \%$ & $-25.5 \%$ \\
\hline & Vehicle Cost HIGH & $-167.2 \%$ & $-167.2 \%$ & $-146.5 \%$ & $-131.3 \%$ & $-63.1 \%$ & $-53.8 \%$ & $-41.3 \%$ & $-41.6 \%$ & $-29.9 \%$ & $-21.3 \%$ & $-18.9 \%$ & $-10.9 \%$ & $-6.0 \%$ \\
\hline \multirow{3}{*}{ FC HEV PHEV 40} & Vehicle Cost LOW & $-210.7 \%$ & $-210.7 \%$ & $-184.7 \%$ & $-167.8 \%$ & $-148.6 \%$ & $-129.9 \%$ & $-105.1 \%$ & $-90.7 \%$ & $-69.5 \%$ & $-53.9 \%$ & $-56.1 \%$ & $-42.2 \%$ & $-32.2 \%$ \\
\hline & \begin{tabular}{|l|} 
Vehicle Cost AVG \\
\end{tabular} & $-233.3 \%$ & $-233.3 \%$ & $-203.0 \%$ & $-182.1 \%$ & $-130.7 \%$ & $-114.1 \%$ & $-93.4 \%$ & $-84.7 \%$ & $-66.5 \%$ & $-52.4 \%$ & $-50.1 \%$ & $-37.5 \%$ & $-28.3 \%$ \\
\hline & Vehicle Cost HIGH & $-189.7 \%$ & $-189.7 \%$ & $-165.2 \%$ & $-148.1 \%$ & $-75.4 \%$ & $-64.5 \%$ & $-49.2 \%$ & $-50.2 \%$ & $-35.9 \%$ & $-26.0 \%$ & $-24.8 \%$ & $-14.8 \%$ & $-9.3 \%$ \\
\hline \multirow{3}{*}{ EV } & Vehicle Cost LOW & $-474.6 \%$ & $-474.6 \%$ & $-440.9 \%$ & $-406.1 \%$ & $-293.1 \%$ & $-264.5 \%$ & $-193.8 \%$ & $-192.3 \%$ & $-137.1 \%$ & $-98.4 \%$ & $-108.6 \%$ & $-72.4 \%$ & $-53.3 \%$ \\
\hline & \begin{tabular}{|l|} 
Vehicle Cost AVG \\
\end{tabular} & $-397.8 \%$ & $-397.8 \%$ & $-369.3 \%$ & $-339.9 \%$ & $-199.1 \%$ & $-179.4 \%$ & $-131.4 \%$ & $-108.0 \%$ & $-76.7 \%$ & $-54.4 \%$ & $-86.5 \%$ & $-56.9 \%$ & $-41.2 \%$ \\
\hline & Vehicle Cost HIGH & $-308.6 \%$ & $-308.6 \%$ & $-286.5 \%$ & $-263.6 \%$ & $-172.6 \%$ & $-155.0 \%$ & $-109.6 \%$ & $-56.6 \%$ & $-36.7 \%$ & $-22.9 \%$ & $-52.0 \%$ & $-29.6 \%$ & $-18.9 \%$ \\
\hline
\end{tabular}




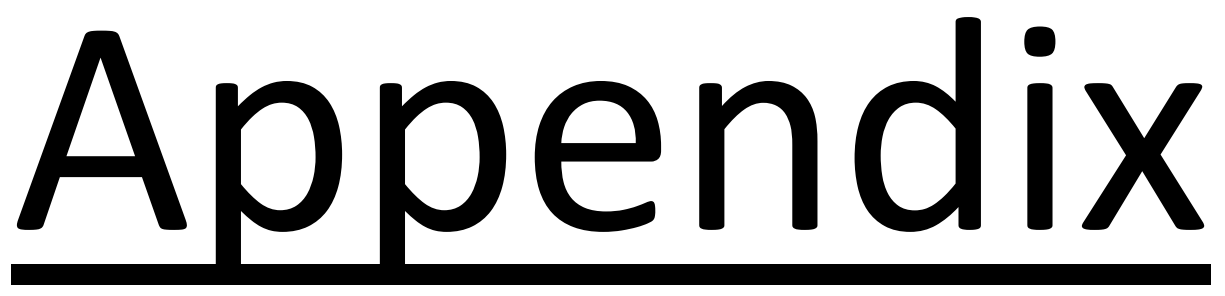

\section{Extras}


Conventional vehicle

A conventional vehicle uses a thermal engine to create power. The engine, by the mean of a transmission directly gives the power to the wheel. It is the most common configuration.

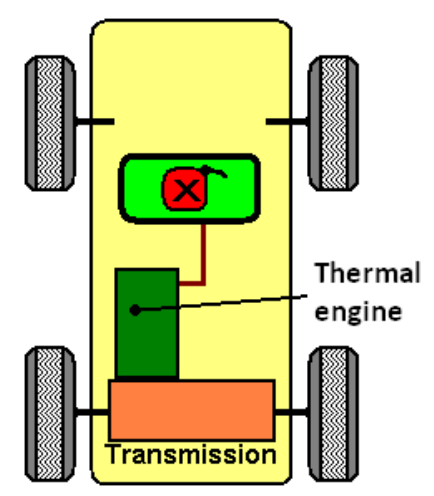

Figure 141 - Conventional vehicle
Electric vehicle

Electric vehicles only use batteries and electric motors to move.

This configuration has no direct emissions but has a limited range.

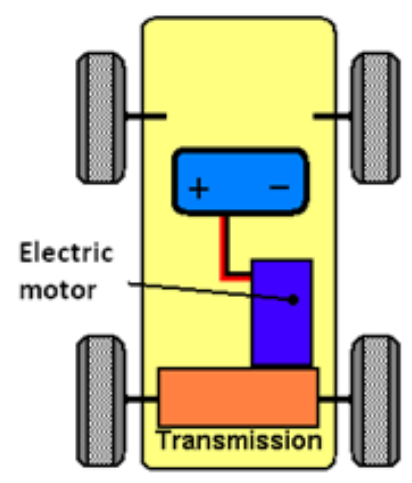

Figure 142 - Electric vehicle

Hybrid vehicle

Uses two or more distinct power sources to move the vehicle Most of the hybrid vehicle combines a thermal engine with electrical motors.

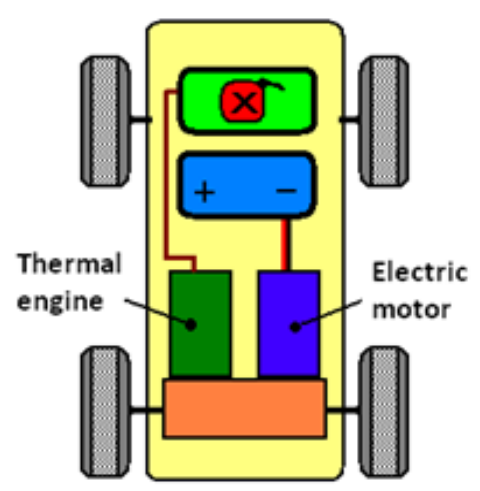

Figure 143 - Hybrid vehicle
$+$

Long vehicle range (up to 400 miles)

Quick fuel refill

Poor efficiency (peak efficiency is around 35\%)

Idling energy waste
Very good efficiency (electrical motor efficiency is above $\mathbf{8 0 \% )}$

Less pollution, less noise

Capture a part of the energy during braking

Low vehicle range

Long charging time

A short cycle life
It combines the advantages of conventional and electric vehicles (EVs), while minimizing their disadvantages.

Three main types of hybrid can be differentiated according to their configuration. 
Argonne National Laboratory Report - Light-Duty Vehicle Fuel Consumption Displacement Potential Up to 2045

\begin{tabular}{|c|c|c|c|c|}
\hline MPG & L/100km & - & L/100km & MPG \\
\hline 10 & 23.6 & - & 3 & 79 \\
\hline 15 & 15.7 & - & 3.5 & 67 \\
\hline 20 & 11.8 & - & 4 & 59 \\
\hline 25 & 9.4 & - & 4.5 & 52 \\
\hline 30 & 7.9 & - & 5 & 47 \\
\hline 35 & 6.7 & - & 5.5 & 43 \\
\hline 40 & 5.9 & - & 6 & 39 \\
\hline 45 & 5.2 & - & 7 & 34 \\
\hline 50 & 4.7 & - & 8 & 30 \\
\hline 55 & 4.3 & - & 9 & 26 \\
\hline 60 & 3.9 & - & 10 & 24 \\
\hline 65 & 3.6 & - & 11 & 21 \\
\hline 70 & 3.4 & - & 12 & 20 \\
\hline 75 & 3.1 & - & 13 & 18 \\
\hline 80 & 3.0 & - & 14 & 17 \\
\hline
\end{tabular}




\section{Argonne}

\section{Energy Systems Division}

Argonne National Laboratory

9700 South Cass Avenue, Bldg. 362

Argonne, IL 60439-4815

www.anl.gov

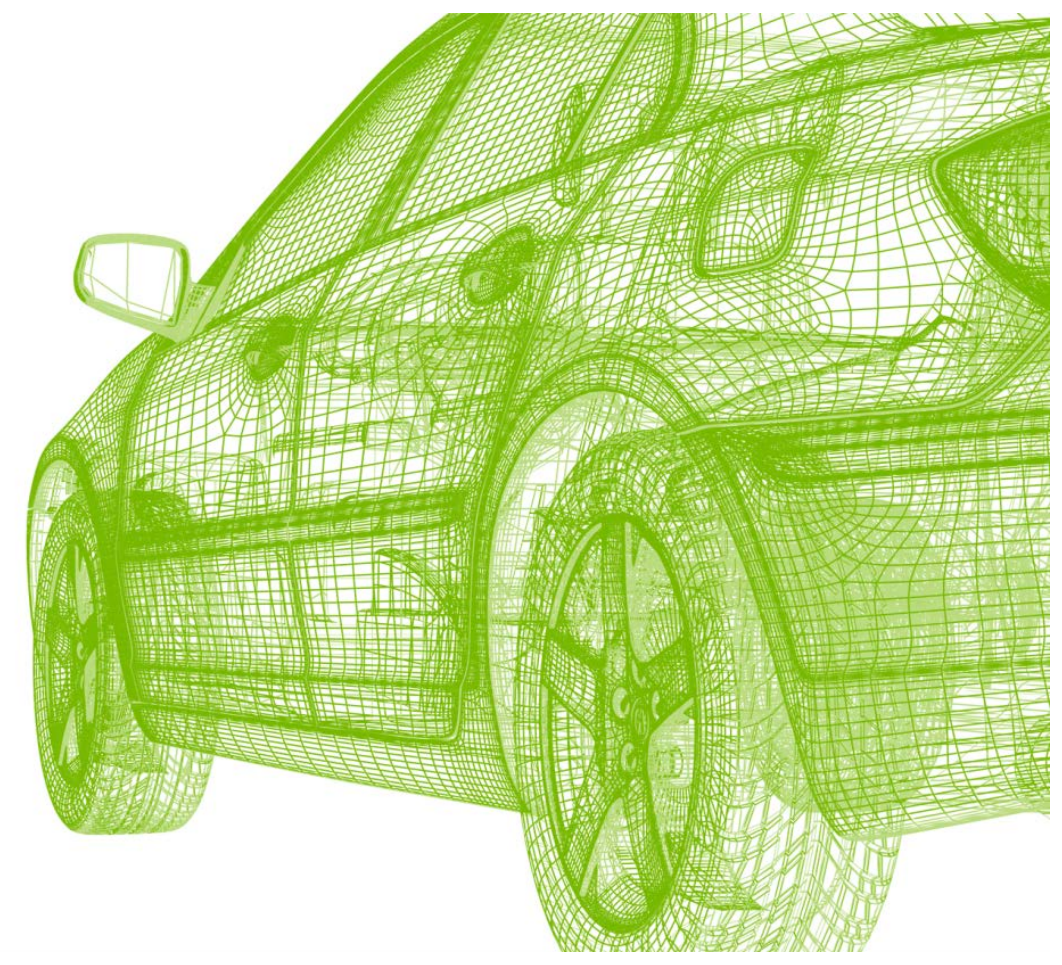

